DOE/RL-95-07

UC-630,721

\title{
Hanford Site Air Operating Permit Application
}

Date Published

May 1995.

United States

P.O. Box 550

Richland, Washington 99352 


\section{DISCLAIMER}

This report was prepared as an account of work sponsored by an agency of the United States Government. Neither the United States Government nor any agency thereof, nor any of their employees, make any warranty, express or implied, or assumes any legal liability or responsibility for the accuracy, completeness, or usefulness of any information, apparatus, product, or process disclosed, or represents that its use would not infringe privately owned rights. Reference herein to any specific commercial product, process, or service by trade name, trademark, manufacturer, or otherwise does not necessarily constitute or imply its endorsement, recommendation, or favoring by the United States Government or any agency thereof. The views and opinions of authors expressed herein do not necessarily state or reflect those of the United States Government or any agency thereof. 


\section{DISCLAIMER}

Portions of this document may be illegible in electronic image products. Images are produced from the best available original document. 
Hanford Site Air

Operating Permit Application
DOE/RL-95-07, Rev. 0 $05 / 95$

\section{DOCUMENT CONTENTS}

FOREWORD

GLOSSARY

PERMIT APPLICATION

I. GENERAL INFORMATION

II. REGULATORY REQUIREMENTS

III. COMPLIANCE PLAN SUMMARY

IV. INSIGNIFICANT EMISSIONS UNITS

V. PERMIT SHIELD SUMMARY

VI. REFERENCES

APPENDICES

A. CALCULATIONS

B. COMPLIANCE PLAN SCHEDULE

C. 100 AREAS

D. 200 EAST AREA

E. 200 WEST AREA

F. 300 AREA

G. 400 AREA

H. 600 AREA

I. MAINTENANCE

J. MISCELLANEOUS ITEMS

K. INSIGNIFICANT EMISSIONS UNITS 
This page intentionally left blank. 


\section{GLOSSARY}

AAQS

AEI

ALARA

BACT

BARCT

BART

BCCAA

BFDHD

BMP

BTU

CAA

CAS

CFC

CFR

DOE-HQ

DOH

DST

Ecology

EFSEC

EPA

ERC

FCAA

FR

gpd

HAP

HLAN

IEU

LAER

MEI

MOA

MOU

mrem ambient air quality standards

air emission inventory

as low as reasonably achievable

best available control technology

best available radionuclide control technology

best available radionuclide technology

Benton County Clean Air Authority

Benton-Franklin District Health Department

best management practice

British thermal unit

Clean Air Act of 1977

Chemical Abstract Service

chlorofluorocarbons

Code of Federal Regulations

U.S. Department of Energy-Headquarters

Washington State Department of Health

Double-Shell Tank System

Washington State Department of Ecology

Energy Facility Site Evaluation Council

U.S. Environmental Protection Agency

Environmental Remediation Contractor

Federal Clean Air Act

Federal Register

gallons per day

hazardous air pollutant

Hanford Local Area Network

insignificant emission units

lowest achievable emission rate

maximally exposed individual

memorandum-of-agreement

memorandum-of-understanding

millirem 
MVAC

NESHAPs

NOC

NSR

PL

PNL

POC

ppm

PSD

PTRAEU

PUREX

RACT

RCRA

RL

SEC

SEPA

SIC

SIP

SST

TAPs

T-BACT

TBD

TEDE

T-RACT

TRIGA

Tri-Party Agreement

TSD

USACE

UST

VOC

WAC

WHC

$\%$ motor vehicle air conditioning

Nation Emission Standards for Hazardous Air Pollutants

notice of construction

new source review

Public Law

Pacific Northwest Laboratory

point of contact

parts per million

prevention of significant deterioration

portable and temporary radionuclide airborne emission unit

plutonium-uranium extraction

reasonably available control technology

Resource Conservation and Recovery Act of 1976

U.S. Department of Energy, Richland Operations Office

section

State Environmental Policy Act of 1971

standard industrial classification

State Implementation Plan

single-shell tank

toxic air pollutants

best available control technology for toxics

to be determined

total effective dose equivalent

reasonably available control technology for toxics

Test Reactor and Isotope Production, General Atomics

Hanford Federal Facility Agreement and Consent Order

treatment, storage, and/or disposal

U.S. Army Corps of Engineers

underground storage tank

volatile organic compounds

Washington Administrative Code

Westinghouse Hanford Company

percent 


\section{DEFINITIONS}

Area Sources - Small [non-major (less than 10 tons per year of a hazardous air pollutant)] air emission sources usually of similar characteristics. They may be separated spatially. An example is underground storage tanks containing petroleum products on the Hanford Site.

Diffuse and Fugitive Emissions - See fugitive emissions. The Hanford Site often defines fugitive emissions of radionuclides as non-point source discharges from an enclosure or building that do not pass through a stack, vent or other point source. Diffuse emissions have been defined on the Hanford Site as emissions from surface contamination areas that may include cribs, ponds, or disposal sites.

Discharge Point - As defined in the Air Operating Permit Application Instructions, the point a regulated air pollutant enters the environment. By Ecology definition, a discharge point is understood to be a point source that emits pollutants from more than one emission point. See Emission Point.

Emission Units - Any part or activity of a stationary source that emits or has the potential to emit any regulated air pollutant or pollutant listed under Section 112(b) of the Federal Clean Air Act.

Emission Point - For this application, the point a regulated air pollutant enters the environment via an airborne pathway, including point sources, area sources, and fugitive emissions. The Air Operating Permit Application Instructions define emission point synonymously with source. See Discharge Point and Source.

Fugitive Emissions - These air emissions do not pass through a discrete emission point (vent, stack, chimney, or other functionally equivalent opening) and could not reasonably pass through such an opening [WAC 173-400-030(31)]. Examples include cribs; storage piles; ponds; and windows, doors, and other openings in buildings containing emission units. These air emissions are sometimes referred to as diffuse sources on the Hanford Site. This definition also applies to emissions of radioactive air pollutants.

Generation Point - As defined in Ecology Application instructions, it is the point at which a pollutant is generated. It includes individual pieces of equipment, units, and activities that generate pollutants that are not released directly to the outdoor ambient air. This term is synonymous with source in the Hanford Site Application.

Insignificant emission unit - For regulated air pollutants excluding radionuclides, it is an emission unit that meets the criteria of an insignificant emission unit as described in WAC 173-401-530. For radionuclides, it is those sources that do not have the potential to emit radionuclides in quantities that could cause any member of the public to receive a total effective dose equivalent in excess of 0.1 millirem per year. Insignificant radionuclide emission units are also referred to as minor stacks and vents or undesignated stacks or vents. 
Non-point Source - A location at which radioactive air emissions originate from an area, such as contaminated ground above a near-surface waste-disposal unit, whose extent may or may not be well defined [WAC 246-247-030(18)]. See fugitive emissions.

Point Sources - Discrete, well defined location from which radionuclide air emissions originate, such as a stack, vent or other functionally equivalent opening [WAC 246-247-030(20)]. The definition also applies to other sources of non-radioactive regulated pollutants.

Significant emission unit - For regulated air pollutants excluding radionuclides, it is an emission unit that does not meet the criteria of insignificant emission units as described in WAC 173-401-530. For radionuclide emission units, it is those sources that have the potential to emit radionuclides in quantities that could cause any member of the public to receive a total effective dose equivalent in excess of 0.1 millirem per year. Significant radionuclide emission units are also referred to as major stacks and vents or designated stacks or vents.

Source - For this application, the point at which regulated air pollutants are generated. Source includes emission units, area sources, and fugitive emissions. Source is synonymous with emission point as defined in the Air Operating Permit Application Instructions.

Stack - Means any point in a source designed to emit solids, liquids, or gases into the air, including a pipe or duct [WAC 173-400-030(71)]. 


\section{METRIC CONVERSION CHART}

The following conversion chart is provided to the reader as a tool to aid in conversion. Into metric units

Out of metric units

\begin{tabular}{|c|c|c|c|c|c|}
\hline If you know & $\begin{array}{l}\text { Multiply } \\
\text { by }\end{array}$ & To get & If you know & $\begin{array}{c}\text { Multiply } \\
\text { by }\end{array}$ & To get \\
\hline \multicolumn{3}{|c|}{ Length } & \multicolumn{3}{|c|}{ Length } \\
\hline inches & 25.40 & millimeters & millimeters & 0.0393 & inches \\
\hline inches & 2.54 & centimeters & centimeters & 0.393 & inches \\
\hline feet & 0.3048 & meters & meters & 3.2808 & feet \\
\hline yards & 0.914 & meters & meters & 1.09 & yards \\
\hline miles & 1.609 & kilometers & kilometers & 0.62 & miles \\
\hline \multicolumn{3}{|c|}{ Area } & \multicolumn{3}{|c|}{ Area } \\
\hline square inches & 6.4516 & $\begin{array}{l}\text { square } \\
\text { centimeters }\end{array}$ & $\begin{array}{l}\text { square } \\
\text { centimeters }\end{array}$ & 0.155 & $\begin{array}{l}\text { square } \\
\text { inches }\end{array}$ \\
\hline square feet & $\overline{0.092}$ & square meters & square meters & 10.7639 & square feet \\
\hline square yards & 0.836 & square meters & square meters & 1.20 & $\begin{array}{l}\text { square } \\
\text { yards }\end{array}$ \\
\hline square miles & 2.59 & $\begin{array}{l}\text { square } \\
\text { kilometers }\end{array}$ & $\begin{array}{l}\text { square } \\
\text { kilometers }\end{array}$ & 0.39 & $\begin{array}{l}\text { square } \\
\text { miles }\end{array}$ \\
\hline acres & 0.404 & hectares & hectares & 2.471 & acres \\
\hline \multicolumn{3}{|c|}{ Mass (weight) } & \multicolumn{3}{|c|}{ Mass (weight) } \\
\hline ounces & 28.35 & grams & grams & 0.0352 & ounces \\
\hline pounds & 0.453 & kilograms & kilograms & 2.2046 & pounds \\
\hline short ton & 0.907 & metric ton & metric ton & 1.10 & short ton \\
\hline \multicolumn{3}{|c|}{ Volume } & \multicolumn{3}{|c|}{ Volume } \\
\hline fluid ounces & 29.57 & milliliters & milliliters & 0.03 & fluid ounces \\
\hline quarts & 0.95 & liters & liters & 1.057 & quarts \\
\hline gallons & 3.79 & liters & liters & 0.26 & gallons \\
\hline cubic feet & 0.03 & cubic meters & cubic meters & 35.3147 & cubic feet \\
\hline cubic yards & 0.76 & cubic meters & cubic meters & 1.308 & cubic yards \\
\hline \multicolumn{3}{|c|}{ Temperature } & \multicolumn{3}{|c|}{ Temperature } \\
\hline Fahrenheit & $\begin{array}{l}\text { subtract } 32 \\
\text { then } \\
\text { multiply by } \\
\text { 5/9ths }\end{array}$ & Celsius & Celsius & $\begin{array}{l}\text { multiply by } \\
9 / 5 \text { ths, } \\
\text { then add } 32\end{array}$ & Fahrenheit \\
\hline
\end{tabular}

Source: Engineering Unit Conversions, M. R. Lindeburg, PE., Second Ed., 1990, Professional Publications, Inc., Belmont, California. 
Hanford Site Air

DOE/RL-95-07, Rev. 0

Operating Permit Application

05/95

This page intentionally left blank. 


\section{PERMIT APPLICATION ORGANIZATION}

Where possible, the Hanford Site Air Operating Permit Application (Application) follows the format defined by the Washington State Department of Ecology (Ecology) Air Quality Operating Permit Application and Instructions (Instructions). Several of the forms requesting plant and process specific information were not used in the Application for the reasons described below.

Much of the facility-specific information contained in Appendices $C$ through $\mathrm{J}$ was collected during a comprehensive air emissions inventory (AEI). This information was input to a database for data management and reporting purposes. The AEI Permit Application Reports (UP460) (AEI Reports) are considered equivalent to the Ecology form as allowed by the Instructions, and contain the same information which is to be input to the Ecology forms B-1 through C-4. The Ecology forms, referenced in the Instructions, are placed in the appropriate location in Chapters 1.0 through 4.0 with a reference to the Appendices.

To aid the permit writer, Table 1 lists the required data elements as found in Chapter 173-401 Washington Administrative Code and the Instructions, and the location of the information in the Application that fulfills the required data elements. Information in column one is not in numerical order. 
Table 1. Required Data Elements.

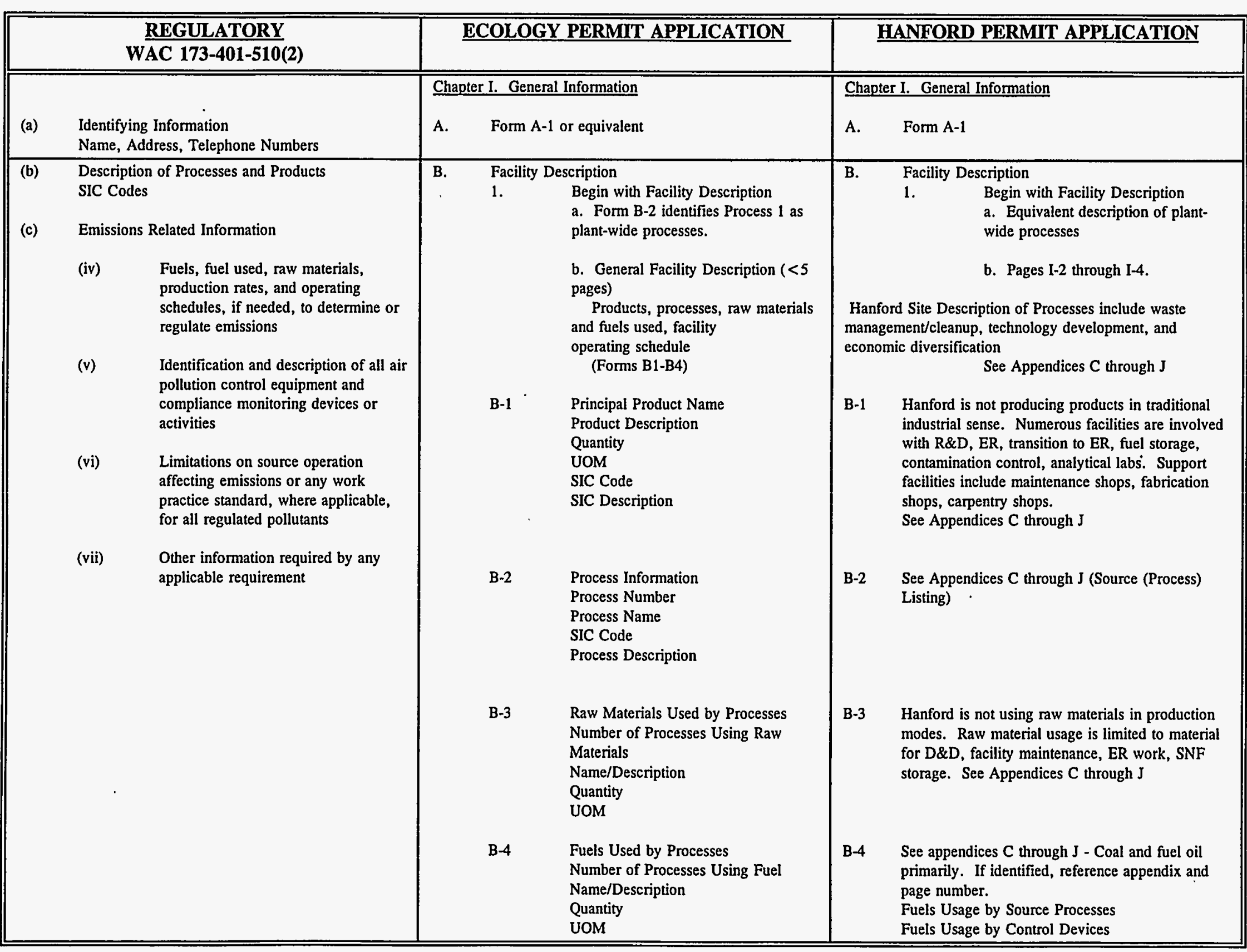


Table 1. Required Data Elements.

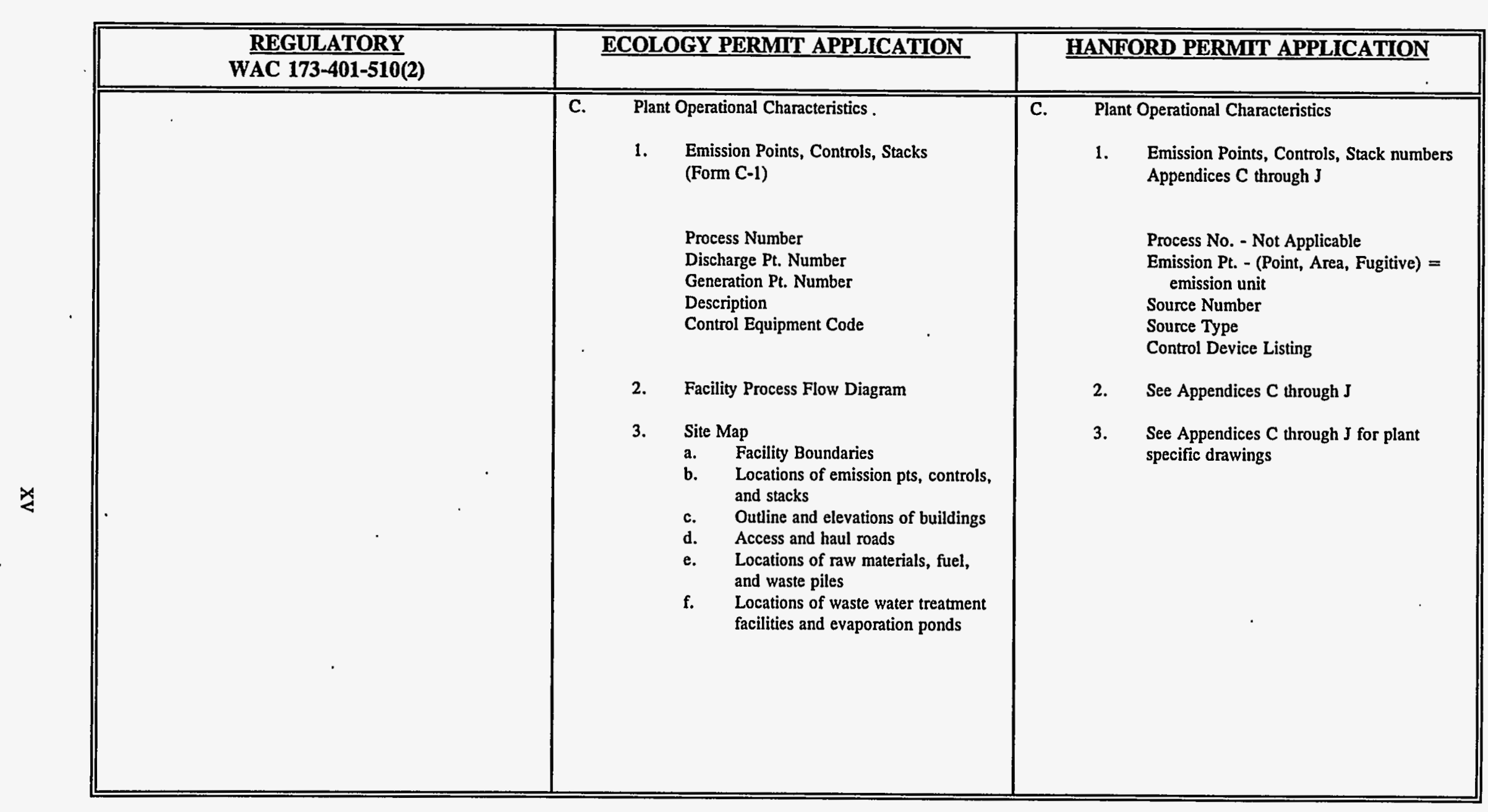


Table 1. Required Data Elements.

\begin{tabular}{|c|c|c|c|}
\hline & $\begin{array}{l}\text { REGULATORY } \\
\text { WAC 173-401-510(2) }\end{array}$ & ECOLOGY PERMIT APPLICATION & HANFORD PERMIT APPLICATION \\
\hline \multirow[t]{2}{*}{ (c) } & $\begin{array}{l}\text { Emissions Related Information } \\
\text { (i) all emissions for which source is } \\
\text { major \& all emissions of regulated } \\
\text { pollutants }\end{array}$ & $\begin{array}{l}\text { Chapter II. Regulatory Requirements } \\
\text { A. Description of Discharge Point and Emission Points }\end{array}$ & $\begin{array}{l}\text { Chapter II. Regulatory Requirements } \\
\text { A. Description of Discharge Point and Emission Points } \\
\text { See Appendices C through J }\end{array}$ \\
\hline & $\begin{array}{l}\text { (ii) Identification of all points of } \\
\text { emissions described in }(c(\text { i)) in } \\
\text { enough detail to establish fees and } \\
\text { applicable requirements }\end{array}$ (iii) $\begin{array}{l}\text { Emission rates in TPY and in terms } \\
\text { consistent with standard reference test } \\
\text { method }\end{array}$ & $\begin{array}{l}\text { B. Applicable Requirements } \\
\text { Complete Form C-2 or equivalent } \\
\text { Includes potential and actual } \\
\text { emissions, applicable requirements, } \\
\text { and compliance } \\
\text { 1. Requirement Citation } \\
\text { 2. Description of Compliance Methods } \\
\text { 3. Applicability Demonstration }\end{array}$ & $\begin{array}{l}\text { B. Applicable Requirements } \\
\text { See Tables II.B-1, II.B-2, and II.B-3 }\end{array}$ \\
\hline \multirow[t]{2}{*}{ (d) } & Air Pollution Control Requirements & & \\
\hline & $\begin{array}{l}\text { Citation and description of all } \\
\text { applicable requirements }\end{array}$ & & \\
\hline (e) & $\begin{array}{l}\text { Description of, or reference to any } \\
\text { applicable test method for determining } \\
\text { compliance with each applicable } \\
\text { requirement }\end{array}$ & & \\
\hline (f) & $\begin{array}{l}\text { An explanation of proposed exemptions from } \\
\text { otherwise applicable requirements }\end{array}$ & $\begin{array}{l}\text { C. Exemptions } \\
\text { Use this section for exemptions from otherwise } \\
\text { applicable requirements }\end{array}$ & C. Exemptions \\
\hline (k) & Inapplicable Requirements & $\begin{array}{l}\text { D. Inapplicable Requirements } \\
\text { 2. Brief Description } \\
\text { 3. Applicability Demonstration } \\
\text { 4. Permit Shield }\end{array}$ & $\begin{array}{l}\text { D. Inapplicable Requirements } \\
\text { See Table II.D-1 List of Inapplicable } \\
\text { Requirements, }\end{array}$ \\
\hline (h) & Compliance Plan & $\begin{array}{l}\text { E. Compliance Plan } \\
\text { 1. Status } \\
\text { 2. Compliance Method } \\
\text { 3. Compliance Certification } \\
\text { 4. Enhanced Monitoring } \\
\text { 5. Compliance Schedule }\end{array}$ & $\begin{array}{l}\text { E. Compliance Plan } \\
\text { See Tables II.B-1, II.B-2, } \\
\text { II.B-3, and } \\
\text { Section II.E.5 } \\
\text { See II.E.4 } \\
\text { See Appendix B }\end{array}$ \\
\hline (g) & $\begin{array}{l}\text { Additional information as determined to be } \\
\text { necessary by the permitting authority to define } \\
\text { alternative operation scenarios identified by the } \\
\text { source [WAC } 173-401-650 \text { (e) and } 173-401-722] \text {. }\end{array}$ & & F. Operational Flexibility \\
\hline
\end{tabular}


Table 1. Required Data Elements.

\begin{tabular}{|c|c|c|}
\hline $\begin{array}{l}\text { (c)(viii) Calculations on which the information in (c)(i) } \\
\text { through (c)(vii) are based }\end{array}$ & 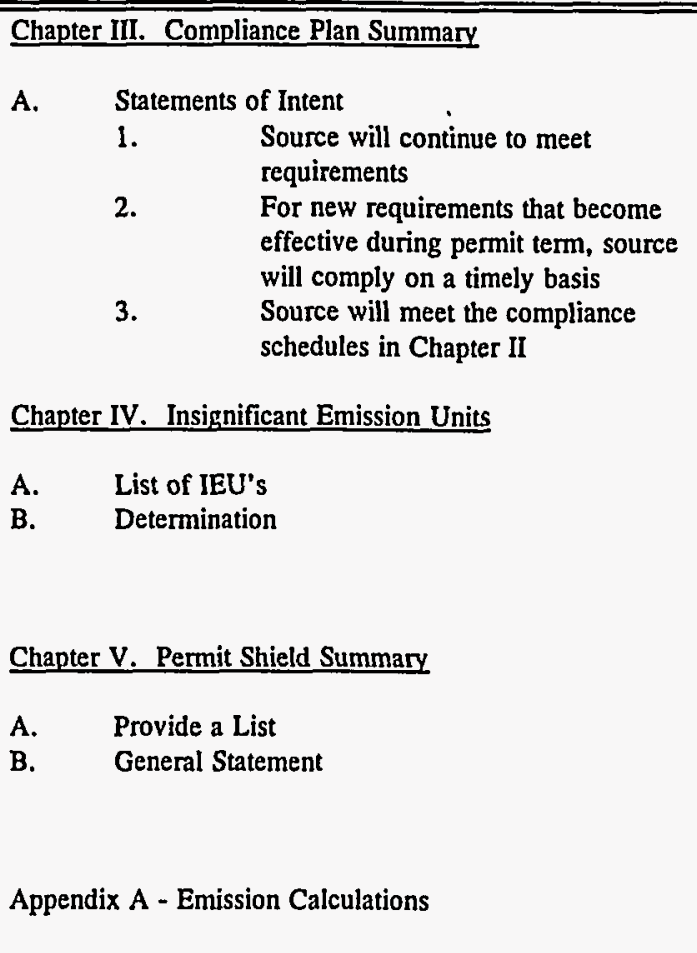 & 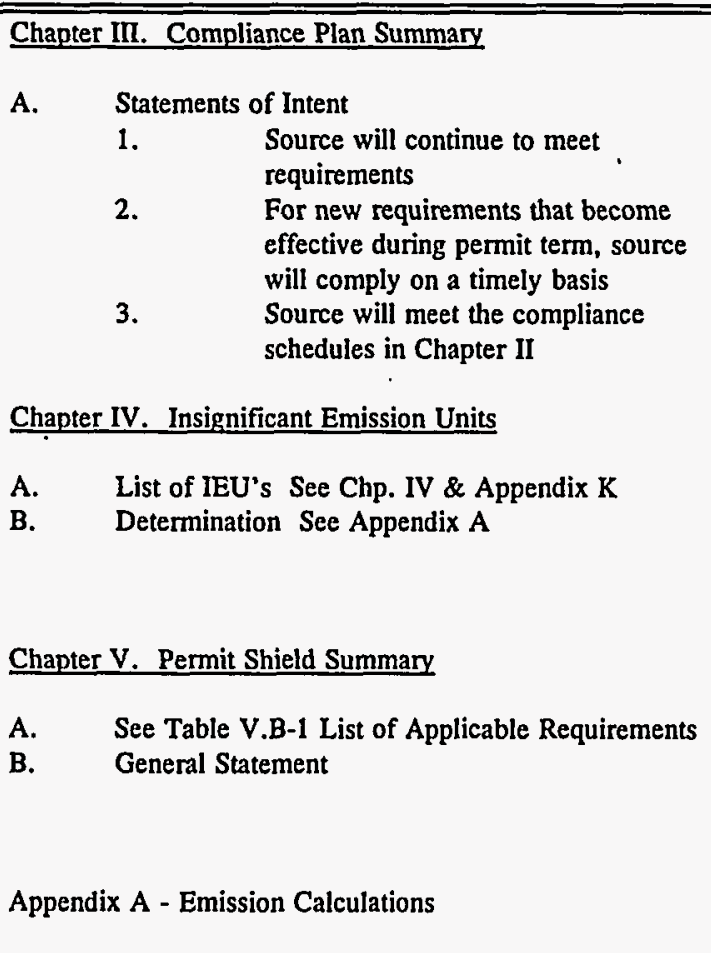 \\
\hline
\end{tabular}


Hanford Site Air

Operating Permit Application
DOE/RL-95-07, Rev. 0

05/95

This page intentionally left blank. 
Hanford Site Air

Operating Permit Application
DOE/RL-95-07, Rev. 0 05/95

\section{CHAPTER I}

\section{GENERAL INFORMATION.}




\section{CONTENTS}

I. GENERAL INFORMATION $\ldots \ldots \ldots \ldots \ldots \ldots \ldots \ldots \ldots$ I- . . . . . . .

I.A. IDENTIFYING INFORMATION AND CERTIFICATION $\ldots \ldots \ldots \ldots$ I-2

I.B. FACILITY DESCRIPTION . . . . . . . . . . . . . I-2

I.C. PLANT OPERATIONAL CHARACTERISTICS . . . . . . . . I-10

I.C.1. EMISSION POINTS, CONTROLS, STACKS: . . . . . . . I I-10

I.C.2. FACILITY PROCESS FLOW DIAGRAM $\ldots \ldots \ldots \ldots$ I-10

I.C.3. SITE MAP . . . . . . . . . . . . . . . I-10

I.C.4. POTENTIAL EMISSIONS SUMMARY . . . . . . . . I I-10

I.D. AIR EMISSIONS INVENTORY REPORT GUIDE . . . . . . . . . . . I-13

\section{FORMS}

Form A-1: General Information and Certification

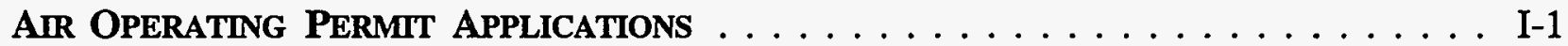

Form B-1: Facility SIC Codes and Associated Principal Products

AIr OPERATing Permit APPlications $\ldots \ldots \ldots \ldots \ldots \ldots \ldots$ I-5

Form B-2: Process Information

Air OPerating Permit ApPlications $\ldots \ldots \ldots \ldots \ldots \ldots \ldots \ldots$ I-6

Form B-3: Raw Materials Used by Processes

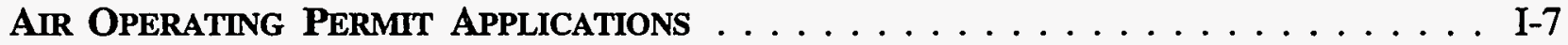

Form B-4: Fuels Used by Processes

Air OPERATING Permitt APPlications $\ldots \ldots \ldots \ldots \ldots \ldots \ldots$ I-8

Form C-1: Plant Operational Characteristics, Emission Point Descriptions

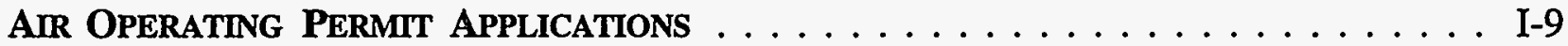

Form C-2: Regulations and Regulated Emissions

AIr OPERATing Permit APPlications $\ldots \ldots \ldots \ldots \ldots \ldots \ldots$ I-11

Form C-3: Insignificant Emissions

AIr OPERATING Permit APPlications $\ldots \ldots \ldots \ldots \ldots \ldots \ldots \ldots$ I-12

I-1. Hanford Site Map . . . . . . . . . . . . . . . . . . . I-37

\section{FIGURES}

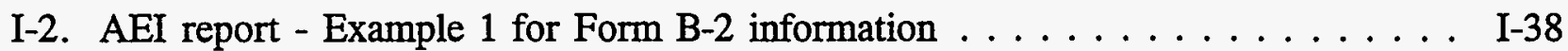

I-3. AEI report - Example 2 for Form B-4 information . . . . . . . . . . . . . I I-39

I-4. AEI report - Example 3 for Form C-1 information . . . . . . . . . . . . I-40 


\section{FIGURES (cont)}

I-5. Example of Discharge and Emission Point Definitions . . . . . . . . I-41

I-6. AEI report - Example 4, Emission Point Coordinates . . . . . . . . . I-42

I-7. AEI report - Example 5, Control Equipment Code $\ldots \ldots \ldots \ldots \ldots$. . . . . $\ldots$.

\section{TABLES}

I.D-1. Emission Points Identified in Application $\ldots \ldots \ldots \ldots \ldots$ I-16

I.D-2. Regulatory Identification Numbers $\ldots \ldots \ldots \ldots \ldots \ldots \ldots \ldots$ I-32 
Hanford Site Air

Operating Permit Application
DOE/RL-95-07, Rev. 0 05/95

This page intentionally left blank. 


\section{Form A-1: General Information and Certification Air OPERATINg PERMTt Applications}

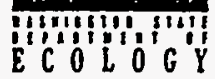

1. Company Name: U.S. Department of Energy Richland Operations Office

2. Plant or Facility Name: Hanford Site

3. Unified Business Identification Number (UBI\#): 91-0565159 (Tax Exempt Number)

4. Facility Address: 825 Jadwin Ave.

Richland. WA. 99352

5. Mailing Address: (if applicable) P.O. Box 550

County: Benton

Richland. WA. 99352

6. Owner: U.S. Department of Energy Richland Operations Office

Parent Company: Not Applicable

7. Facility contact who is familiar with the information contained in this application.

Name: James E. Rasmussen

Title: Director, Environmental Assurance, Permits. and Policy Division

Telephone: (509) 376-2247

8. Type of operating permit application: (check all that apply)

$\square$ Initial permit application

$\square$ Early reductions application

$\square$ Renewed permit application $\square$ General permit application

$\square$ Permit modification application

$\square$ Other (specify):

9. Reason for Application (tonnage of emission per year): $>100$ tons/year $\mathrm{CO}$. $\mathrm{NO}_{\mathrm{x}}$ $\underline{\mathrm{SO}}_{2}, \mathrm{PM}_{10}$

10. Claim of Confidentiality:

Some of the records and information contained in this application are $\square$ are not $\square$ (check one) unique to the applicant and/or are likely to adversely affect the competitive position of the applicant if released to the public or a competitor. If a claim of confidentiality is made for this application, provide a separate application for general distribution which is devoid of confidential information.

\section{Certification:}

I certify that I am the responsible official, as defined in WAC 173-401-200(27) for this facility. I further certify as required by WAC 173-401-520, that, based on information and belief formed after reasonable inquiry, the statements and information

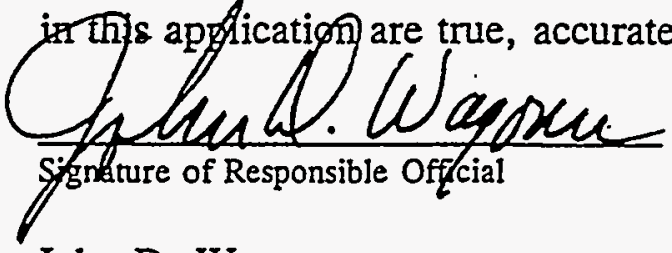

, and complete.

John D. Wagoner

Printed Name 


\section{CHAPTER I. GENERAL INFORMATION}

\section{I.A. IDENTIFYING INFORMATION AND CERTIFICATION \\ Refer to Page I-1.}

\section{I.B. FACILITY DESCRIPTION}

The Hanford Site, located in southcentral Washington State, is about 1,450 square kilometers (560 square miles) of semi-arid shrub and grasslands located just north of the confluence of the Snake and Yakima Rivers with the Columbia River. This land, with restricted public access, provides a buffer for the smaller areas historically used for the production of nuclear materials, waste storage, and waste disposal. About $6 \%$ of the land area has been disturbed and is actively used. This $6 \%$ is divided into the following areas:

- the 100-B/C, 100-D, 100-DR, 100-F, 100-H, 100-K, and 100-N Areas, which lie along the south shore of the Columbia River in the northern portion

- the 200-East and 200-West Areas, which lie in the center near the basalt outcrops of Gable Mountain and Gable Butte

- the 300 Area, near the southern border

- the 400 Area, between the 300 and 200 Areas

- the 1100 Area, a corridor northwest of the city of Richland used for vehicle maintenance and other support activities

- the 3000 Area, for sites used for research and technology development and administrative functions. These sites are not necessarily on the Hanford Site, and can be found adjacent to Richland, Kennewick, and Pasco, the Tri-Cities.

The 600 Area is the designation for land between the operational areas. Areas off the Hanford Site used for research and technology development and administrative functions can be found in Richland, Kennewick, and Pasco, the nearest cities.

The Hanford Site was acquired by the federal government in 1943 and for many years was dedicated primarily to the production of plutonium for national defense and the management of the resulting wastes. With the shutdown of the production facilities in the 1970s and 1980 s, missions were diversified to include research and development in the areas of energy, waste management, and environmental restoration. 
The current mission for the Hanford Site is:
- waste management/cleanup
- technology development
- economic diversification.

Current waste management activities include managing wastes with high and low levels of radioactivity (from the nuclear materials production activities) in the 200-East and 200-West Areas. Key waste management facilities include the waste storage tanks, Plutonium Uranium Extraction (PUREX) Plant, Plutonium Finishing Plant, Central Waste Complex, Low-Level Burial Grounds, B Plant, T Plant, and 242-A Evaporator. In addition, irradiated nuclear fuel is stored in the $100-\mathrm{K}$ Area in fuel storage basins.

Environmental restoration includes activities to decontaminate and decommission facilities and to cleanup or restore inactive waste sites. The surplus facilities program conducts surveillance and maintenance of such facilities, and has begun to clean up and dispose of more than 100 facilities.

Research and technology development activities are intended to improve the techniques and reduce the costs of waste management, environmental protection, and restoration.

Operations and activities on the Hanford Site are managed by the U.S. Department of Energy, Richland Operations Office (DOE-RL) through contractors and numerous subcontractors.

The principal contractors currently include:

- Westinghouse Hanford Company

- Battelle Memorial Institute

- Hanford Environmental Health Foundation

- Bechtel Hanford Incorporated.

Non-DOE-RL operations and activities include commercial power production by the Washington Public Power Supply System's WNP-2 Reactor (near the 400 Area) and commercial low-level radioactive waste burial at the site leased and licensed by Washington State and operated by US Ecology (near the 200 Areas). Siemens Power Corporation operates a commercial nuclear fuel fabrication facility, and Allied Technology Group Corporation operates a low-level radioactive waste decontamination, supercompaction, and packaging disposal facility near the southern boundary of the Hanford Site.

This air operating permit application for the Hanford Site has been prepared pursuant to Chapter 173-401, Washington Administrative Code, "Operating Permit Regulation". This permit application does not include non-DOE operations and activities mentioned in the previous paragraph. 
The air operating permit application for the Hanford Site is organized according to the six operational areas, (i.e., 100 Areas, 200 Areas, 300 Area, etc.) and the sources located within each area. The information on the sources located within each area is compiled as appendices to the permit application. For example, all information required for Sections B, $\mathrm{C}$, and $\mathrm{E}$ of the permit application pertaining to the PUREX Plant can be located in Appendix D. Therefore, the reader is referred to the appendices for required information. 
Form B-1: Facility SIC Codes and Associated Principal Products

air Operating Permit applications

Mingming

Facility Name:

Confidentiality Claim? Yes No (circle one)

\begin{tabular}{|c|c|c|c|c|c|}
\hline 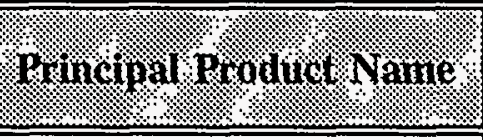 & 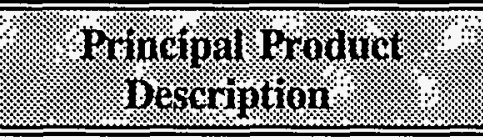 & Maxrinum & GOMU: & 8519809 & Ste besciption \\
\hline $\begin{array}{l}\text { No products are produced on the Hanford } \\
\text { Site that are markeced to the gencral public. } \\
\text { This section is considered not applicable to } \\
\text { the Hanford Site }\end{array}$ & $\begin{array}{l}\text { No products are produced on the Hanford } \\
\text { Site that are marketed to the gencral public. } \\
\text { This section is considered not applicable to } \\
\text { the Hanford Site }\end{array}$ & Not applicable & $\begin{array}{l}\text { Not } \\
\text { applicable }\end{array}$ & \multirow{3}{*}{$\begin{array}{l}\text { SIC Code } 9999 \text { is } \\
\text { accepted as the } \\
\text { sIC Code for all } \\
\text { Hanford Site } \\
\text { Operations. }\end{array}$} & \\
\hline & & & & & \\
\hline & & & & & \\
\hline & & & & & \\
\hline & & & & & \\
\hline & & & & & \\
\hline & & & & & \\
\hline & & & & & \\
\hline & & & & & \\
\hline & & & & & \\
\hline & & & & & \\
\hline & & & & & \\
\hline & & & & & \\
\hline & & & & & \\
\hline & & . & & & \\
\hline
\end{tabular}


Facility Name:

Process \#

\begin{tabular}{|c|c|c|c|}
\hline \%100ess. & \%lyoress & $\%$ & orocks \\
\hline
\end{tabular}

No process numbers were assigned to the multiple processes found on the Hanford Site. The Hanford Site Air Emissions Inventory was nearly complete when the Washington State Rule on the Air Operating Regulation, Chapter 173-401, WAC, became final. The rule focuses on process identification rather than emission point identification. The Hanford Site Air Emission Inventory was collected on an emission point basis as the Hanford Site Air Emission Inventory database did not allow input for process identification numbers. Following Form C-3 in this chapter, a guide is provided as to where the process name and process descriptions are contained

in the AEI reports located in the appendices for each emissions point (refer to Figure I-2). Additionally, SIC codes are not provided as the Hanford Site does not manufacture any products.

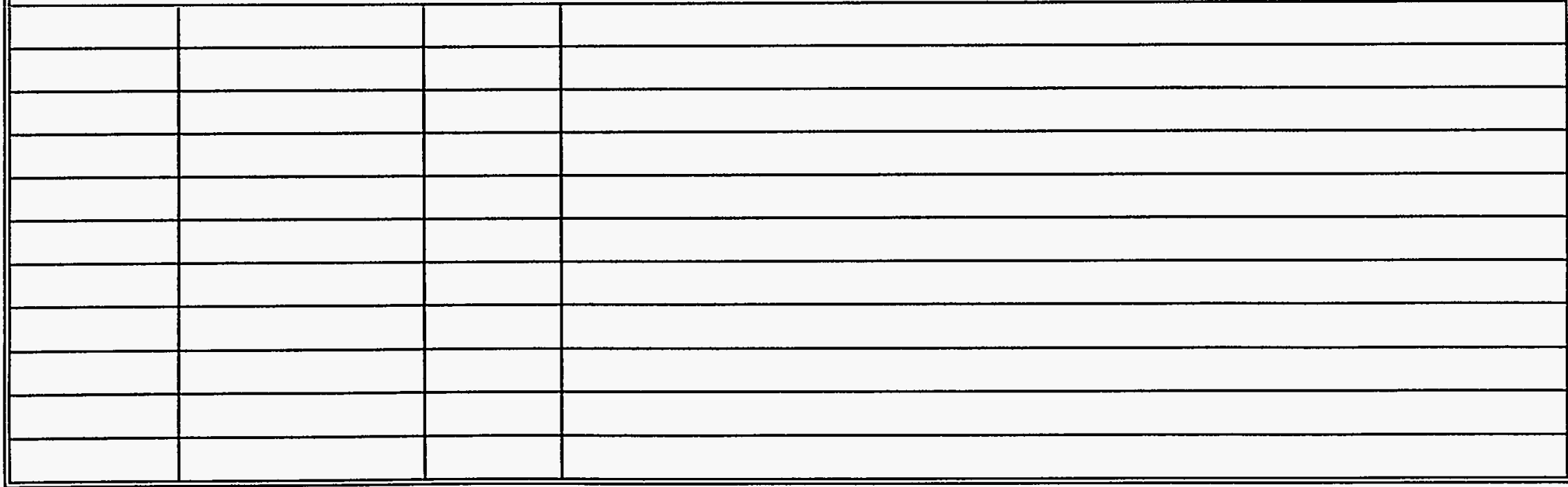


Hanford Site Air

Operating Permit Application

DOE/RL-95-07, Rev. 0

05/95

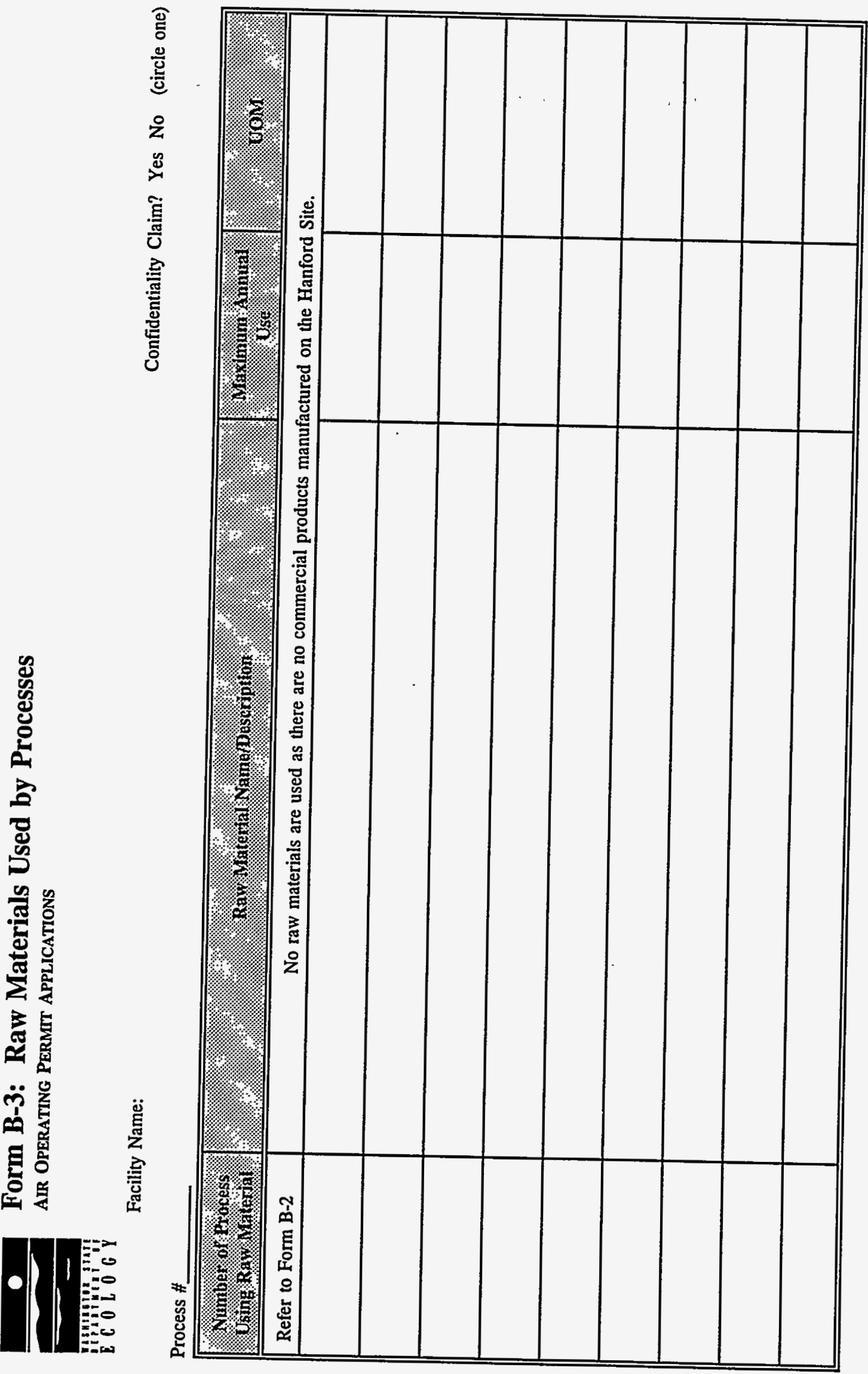




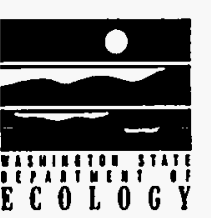

Form B-4: Fuels Used by Processes

air Operating Permit applications

Facility Name:

Process \#

\begin{tabular}{|l|l|l|l|l|}
\hline Refor to Form B-2 & Refer to the AEI report guide following Form C-3 of this chapter to identify where the Fuel Name/Description for each emission point is \\
\hline & located in the AEI reports in each subappendix (refer to Figure I-3). & & \\
\hline \hline & & & & \\
\hline & & & & \\
\hline & & & & \\
\hline & & & & \\
\hline
\end{tabular}

*Indicate if it is a primary, back-up, or emergency fuel. 


\section{Form C-1: Plant Operational Characteristics, Emission Point Descriptions}

Air Operating Permit Applications

Ectiog

Process \#

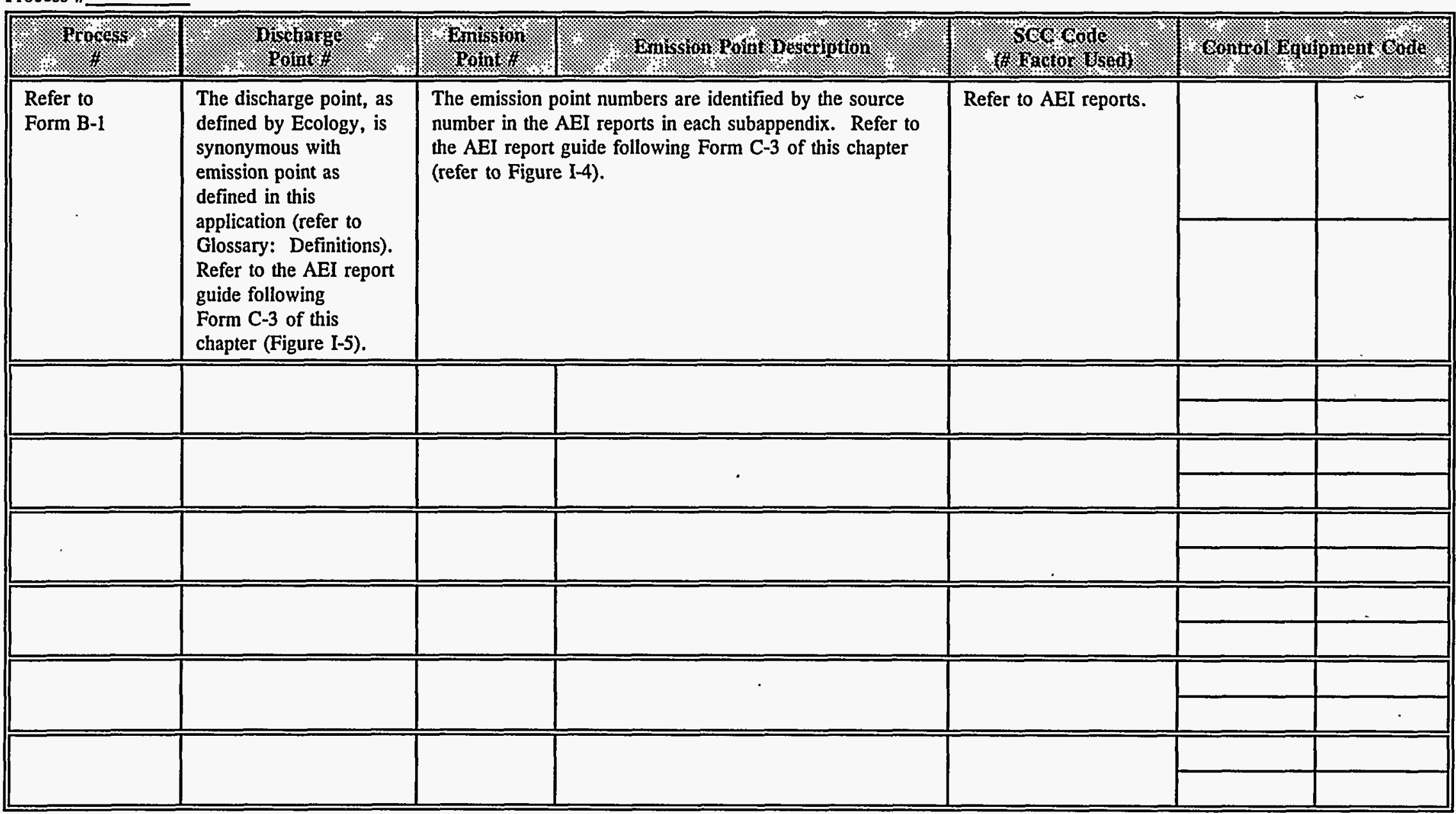




\section{I.C. PLANT OPERATIONAL CHARACTERISTICS}

\section{I.C.1. EMISSION POINTS, CONTROLS, STACKS:}

Refer to the AEI Permit Application Reports (UP460) (AIE Report) included in Appendices $\mathrm{C}$ through $\mathrm{K}$. The AEI Reports are considered equivalent to Form $\mathrm{C}-1$.

\section{I.C.2. FACILITY PROCESS FLOW DIAGRAM}

Refer to Appendices $\mathrm{C}$ through $\mathrm{K}$ for each emission point.

\section{I.C.3. SITE MAP}

Ecology requested the following to be shown on site maps:

- Facility boundaries

- Locations of emission points, controls and stacks

- Outline and elevations of buildings

- Access and haul roads

- Locations of raw material, fuel, and waste piles

- Locations of waste water treatment facilities and evaporation ponds.

Elevations of buildings are not provided on the site map. Input for building elevations was not supported by the AEI database. Consequently, this information was not collected. Also, the data collection process was complete before Ecology's identification of this requirement. The building elevation information can be provided on a case-by-case basis for specific buildings at Ecology's request.

Figure I-1 is a map of the Hanford Site. The map only shows the operational areas within the boundaries of the Hanford Site. Maps showing the above items are included in each subappendix located in Appendices $\mathrm{C}$ through $\mathrm{K}$.

\section{I.C.4. POTENTIAL EMISSIONS SUMMARY}

Refer to Form C-2 and Form C-3. 
Hanford Site Air

Operating Permit Application

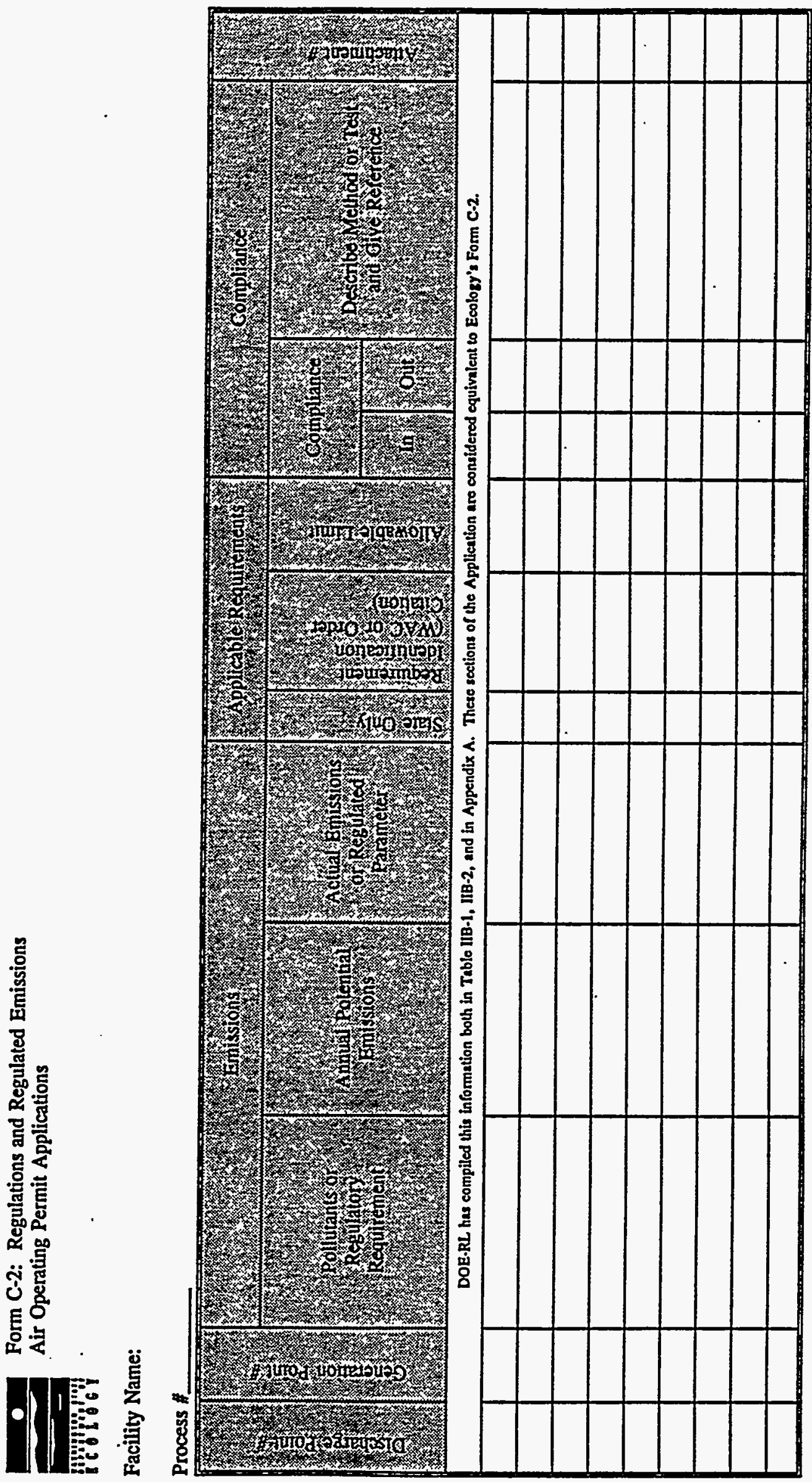




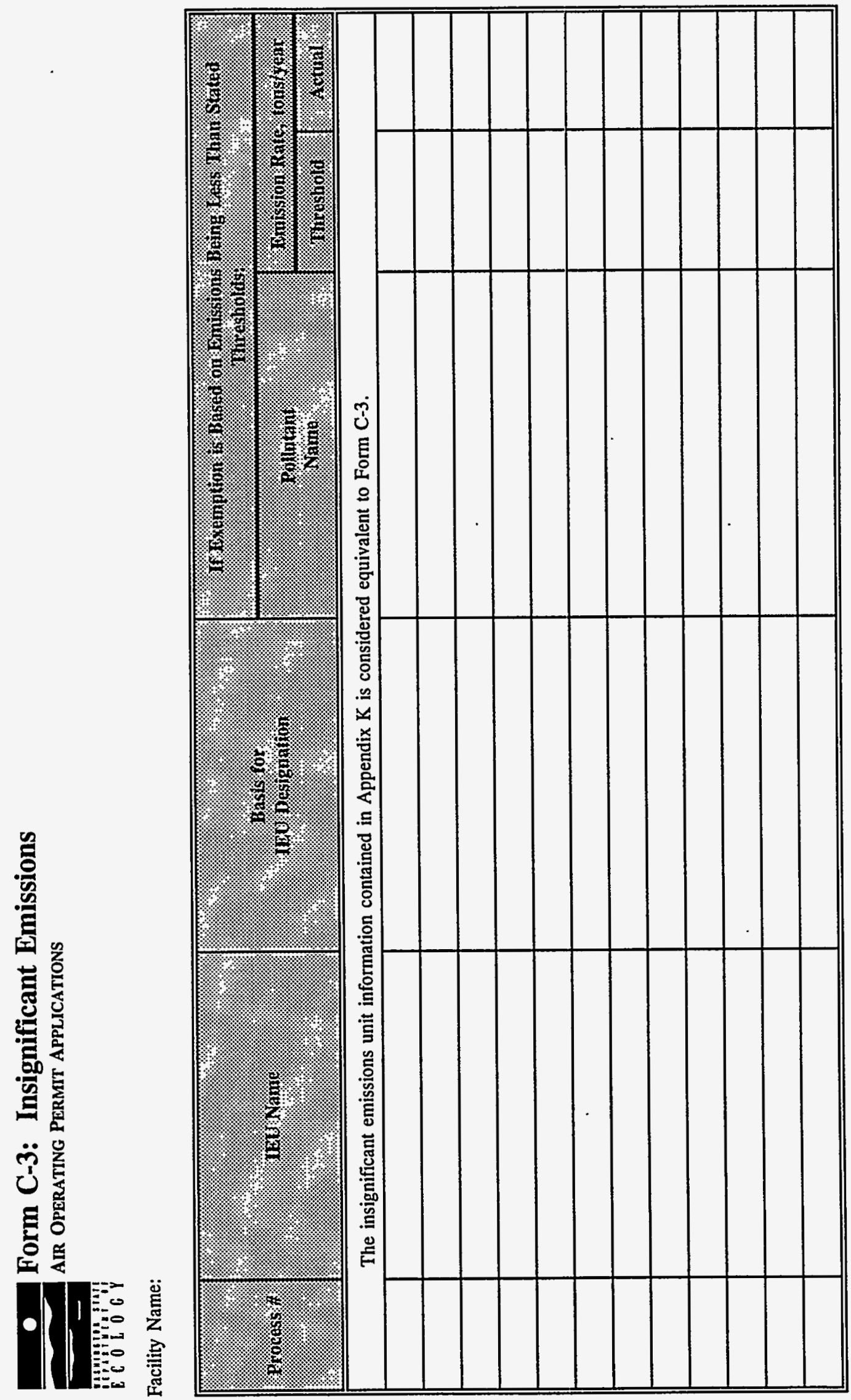




\section{I.D. AIR EMISSIONS INVENTORY REPORT GUIDE}

Form B-2: Process Information

Process names and descriptions are located under the subheading "Source (Process) Listing". Refer to AEI report Example 1, Figure I-2.

Form B-4: Fuels Used by Processes

Fuels Used by Processes are located under the subheading "Fuels Usage by Source Processes". Refer to AEI report Example 2, Figure I-3.

Form C-1: Plant Operational Characteristics, Emission Point Descriptions

Plant Operational Characteristics, Emission Point Descriptions are located under the subheading "Fuels Usage by Source Processes". Refer to AEI report Example 3, Figure I-4.

Coordinate Description

The coordinates of the emission points listed in the AEI reports are based on coordinate system Washington State North American Datum 83-91. Refer to AEI report Example 4, Figure I-6.

\section{Control Code Description}

Washington State Department of Ecology provided the following control equipment codes and equipment descriptions for control equipment on the Hanford Site. Refer to AEI report Example 5, Figure I-7.

\begin{tabular}{cl} 
Code & \multicolumn{1}{c}{ Description } \\
\hline 017 & Fabric Filter (Medium Temperature) \\
048 & Activated carbon adsorption \\
058 & Mat or panel filter \\
063 & Gravel bed filter \\
075 & Single cyclone \\
076 & Multiple cyclone without fly ash reinjection \\
099 & Miscellaneous control devices (high-efficiency \\
& particulate air filters are included in this category).
\end{tabular}




\section{Alpha-numeric Code Description}

The unique identification numbers assigned to all site AEI sources provides key information concerning location, type of emission point, and facilitates access to information within the AEI computer database. The identification code assigned to each emission point (point or fugitive) includes the operational area code, a category code, a building identifier, and a emission point identification code. For example, if the alpha-numeric code in the AEI reports for a given emission point is 200W H-271AW 001, the "200W" identifies the operational area, or 200 West Area, the $\mathrm{H}$ is the Category code " $\mathrm{H}$ " identifies the emission point as a Combustion Emission Point, 271AW is the building number, and "001" is the emission point identification code.
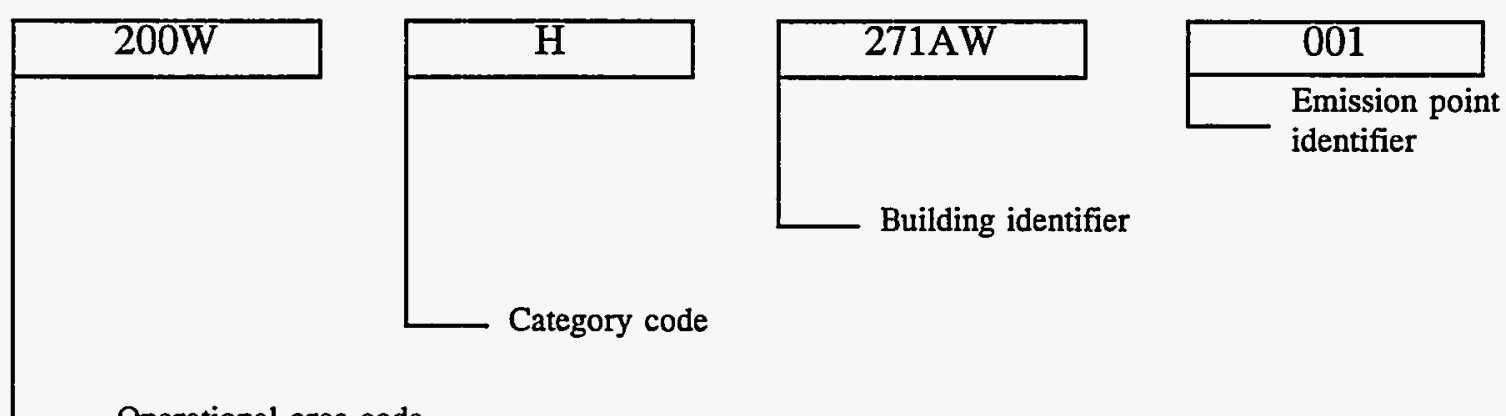

Building identifier

Operational area code

The category codes are as follows:

A - Administrative Emission Point - sanitary sewer vents, stove hoods

B - Administrative Emission Point - building exhausts (active or passive)

C - Administrative Emission Point - heating, ventilation, and air conditioning (HVAC)

D - Administrative Emission Point - steam releases

E - Combustion Emission Point - internal combustion (engines, turbines)

F - Combustion Emission Point - external (boilers only)

G - Gasoline Distribution Area

H - Combustion Emission Point - external (space heaters, hot water heaters)

J - Fugitive Emission Area - fugitive sources emitted directly to the ambient air (other than pump seals, valves flanges)

K - Paint Booth - Painting Operations

L - Smelter

M - Welding Booth

N - General Maintenance

P - Process Emission Point

R - Laboratory Emission Point - research

S - Laboratory Emission Point - process or QA/QC related

T - Tank or Storage Vessel Emission Point

U - Unassigned 
V - Fugitive Emission Area - fugitive sources (pump seals, valves, flanges)

W - Portable, temporary, radionuclide air emission unit

$X$ - Unassigned

$Y$ - Unassigned

$\mathrm{Z}$ - Other

Aside from the alpha-numeric code assigned during the AEI, each emission point is assigned a unique regulatory identification number. The following is a cross-reference for the AEI emission point codes and the regulatory identification number. The cross-reference also includes the location of the emission point information in the Application. The Table I.D-1 lists all of the emission points listed in the Application and provides a cross-reference with the regulatory identification numbers where appropriate. The Table I.D-2 provides a crossreference of the regulatory identification number and the AEI emission point identification numbers in the Application. The following two tables list the stationary source emission units that have been identified on the Hanford Site. These tables do not include temporary, insignificant emission units. For example, welding booths within certain facilities

(fabrication shops) have been identified with unique identification numbers. These emission units are included in the tables. Portable welders, however, that are used for a variety of construction, building modification, or maintenance activities have not been identified with unique emission point identification numbers and are not identified in the following tables. Many of these activities and emission units not specifically identified in the Application are associated with facility-wide construction, facility modification, and maintenance activities. These activities and emission units are considered insignificant emission units based on the criteria in WAC 173-401-530. The discussion of insignificant emission units is included in Chapter IV.

Not all of the emission categories reported under the "Facility Emissions" section of the Air Emission Inventory Permit Application Report (UP460), contain emission data. Where no emissions data is presented, no significant emissions of pollutants have been identified for that pollutant category. 
Hanford Site Air

DOE/RL-95-07, Rev. 0

Operating Permit Application

05/95

Table I.D-1. Emission Points Identified in Application

\begin{tabular}{|c|c|c|}
\hline AEI Identification Number & Regulatory ID. & Appendix \\
\hline $100 \mathrm{~K} N-1717 \mathrm{~K} 001$ & & C-1, K.3 \\
\hline 100K P-105KE 001 & $105-\mathrm{KE}$ & $\mathrm{C}-1$ \\
\hline $100 \mathrm{~K}$ P-105KE 002 & $105-\mathrm{KE}$ & $\mathrm{C}-1$ \\
\hline 100K P-105KE 003 & 105-KE & C-1 \\
\hline 100K P-105KE 004 & $105-\mathrm{KE}$ & $\mathrm{C}-1$ \\
\hline 100K P-105KW 001 & $105-\mathrm{KW}$ & $\mathrm{C}-1$ \\
\hline $100 \mathrm{~K} P-105 \mathrm{KW} 002$ & $105-\mathrm{KW}$ & $\mathrm{C}-1$ \\
\hline 100K P-105KW 003 & $105-\mathrm{KW}$ & C-1 \\
\hline 100K P-105KW 004 & $105-\mathrm{KW}$ & $\mathrm{C}-1$ \\
\hline $100 \mathrm{~K}$ R-1706 KE 001 & $1706 \mathrm{KE}$ & $\mathrm{C}-1$ \\
\hline 100K R-1706KEL 001 & 1706KEL & C-1, K.2 \\
\hline 100K R-1706KEL 002 & & $\mathrm{C}-1$ \\
\hline 100K R-1706KER 001 & $1706 \mathrm{KER}$ & $\mathrm{C}-1$ \\
\hline $100 \mathrm{~N} \mathrm{~K}-1143 \mathrm{~N} 015$ & & K.3 \\
\hline $100 \mathrm{~N} \mathrm{~K}-1517 \mathrm{~N} 021$ & & K.3 \\
\hline $100 \mathrm{~N} M-105 \mathrm{~N} 001$ & & K.2 \\
\hline $100 \mathrm{~N} M-1515 \mathrm{~N} 020$ & & K.3 \\
\hline $100 \mathrm{~N} M-1518 \mathrm{~N} 019$ & & K.3 \\
\hline $100 \mathrm{~N} M-1705 \mathrm{~N} 013$ & & K.3 \\
\hline $100 \mathrm{~N} \mathrm{~N}-1143 \mathrm{~N} 014$ & & $\mathrm{~K} .2$ \\
\hline $100 \mathrm{~N} \mathrm{~N}-1143 \mathrm{~N} 016$ & & K.3 \\
\hline $100 \mathrm{~N} \mathrm{~N}-1516 \mathrm{~N} 017$ & & $\mathrm{~K} .2$ \\
\hline $100 \mathrm{~N} N-1517 \mathrm{~N} 018$ & & K.3 \\
\hline $100 \mathrm{~N} P-105 \mathrm{~N} 002$ & $105-\mathrm{N}-14^{\prime} \mathrm{DR}$ & $\mathrm{C}-2$ \\
\hline $100 \mathrm{~N}$ P-107N 004 & $107-\mathrm{N}$ & $\mathrm{C}-2$ \\
\hline
\end{tabular}


Table I.D-1. Emission Points Identified in Application

\begin{tabular}{|c|c|c|}
\hline AEI Identification Number & Regulatory ID. & Appendix \\
\hline $100 \mathrm{~N} P-116 \mathrm{~N} 005$ & $116-\mathrm{N}$ & $\mathrm{C}-2$ \\
\hline $1100 \mathrm{G}-1172 \mathrm{~A} 001$ & & K.3 \\
\hline $1100 \mathrm{G}-1172 \mathrm{~A} 002$ & & K.2 \\
\hline $1100 \mathrm{G}-1172 \mathrm{~A} 003$ & & K.2 \\
\hline 1100 G-1172A 004 & & K.2 \\
\hline 1100 G-1172A 005 & & $\dot{\mathrm{K}} .2$ \\
\hline 1100 P-1167001 & & I-1 \\
\hline 200E F-284E 001 & 11 & D-1 \\
\hline 200E F-284E 002 & 11 & D-1 \\
\hline 200E F-284E 005 & & D-1 \\
\hline 200E G-2713E 001 & & K.3 \\
\hline 200E G-2713E 002 & & K.2 \\
\hline 200E G-2713E 003 & & K. 2 \\
\hline 200E H200E002 001 & & $\mathrm{I}-1$ \\
\hline 200E J-284E 003 & 16 & D-1 \\
\hline 200E J-284E 004 & 17 & D-1 \\
\hline 200E K-200E001 001 & & $\mathrm{I}-1$ \\
\hline 200E K-2715EC 001 & & K.2 \\
\hline 200E M-2244B 001 & & K.3 \\
\hline 200E M-2247B 001 & & K.3 \\
\hline 200E M-242AC 001 & & K.3 \\
\hline 200E M-277A 001 & & K.3 \\
\hline 200E N-2101M 001 & & K. 2 \\
\hline $.200 \mathrm{E} N-2101 \mathrm{M} 002$ & & K.2 \\
\hline
\end{tabular}


Table I.D-1. Emission Points Identified in Application

\begin{tabular}{|l|l|l|}
\hline AEI Identification Number & Regulatory ID. & Appendix \\
\hline 200E N-2242B 001 & & K.2 \\
\hline 200E N-2242B 002 & & K.3 \\
\hline 200E N-2715EC 003 & & K.2 \\
\hline 200E N-2721EA 001 & & K.3 \\
\hline 200E N-272AW 001 & & D-4, K.3 \\
\hline 200E N-272E 001 & & K.3 \\
\hline 200E N-275E 001 & & K.2 \\
\hline 200E N-275E 002 & 296-A-26 & K.3 \\
\hline 200E P-204AR 001 & & D-4 \\
\hline 200E P-241A101 001 & & D-4, K.2 \\
\hline 200E P-241A102 001 & & D-4, K.2 \\
\hline 200E P-241A103 001 & & D-4, K.2 \\
\hline 200E P-241A104 001 & & D-4, K.2 \\
\hline 200E P-241A106 001 & & D-4, K.2 \\
\hline 200E P-241A151 001 & & D-4 \\
\hline 200E P-241A302A 001 & & D-4 \\
\hline 200E P-241A350 001 & & D-4 \\
\hline 200E P-241A401 001 & & D-4 \\
\hline 200E P-241A417001 & & D-4 \\
\hline 200E P-241AA 001 & & D-4 \\
\hline 200E P-241AB 001 & & D-4 \\
\hline 200E P-241ANA 001 & & D-4 \\
\hline 200E P-241ANB 001 & & \\
\hline 200E P-241AP 001 & & \\
\hline
\end{tabular}


Hanford Site Air

Operating Permit Application

Table I.D-1. Emission Points Identified in Application

\begin{tabular}{|c|c|c|}
\hline AEI Identification Number & Regulatory ID. & Appendix \\
\hline 200E P-241AR151 001 & & $D-4$ \\
\hline 200E P-241AWA 001 & & $\mathrm{D}-4$ \\
\hline 200E P-241AWB 001 & & $\mathrm{D}-4$ \\
\hline 200E P-241AX101 001 & & D-4, K.2 \\
\hline 200E P-241AX102 001 & & D-4, K.2 \\
\hline 200E P-241AX103 001 & & D-4, K.2 \\
\hline 200E P-241AX104 001 & & D-4, K.2 \\
\hline 200E P-241AX152 001 & & D-4 \\
\hline 200E P-241AX155 001 & & D-4 \\
\hline 200E P-241AX501 001 & & D-4 \\
\hline 200E P-241AXA 001 & & $D-4$ \\
\hline 200E P-241AXB 001 & & $\mathrm{D}-4$ \\
\hline 200E P-241AY501 001 & & $\mathrm{D}-4$ \\
\hline 200E P-241AZ152 001 & & $D-4$ \\
\hline 200E P-241B101 001 & & D-4, K.2 \\
\hline 200E P-241B102 001 & & D-4, K.2 \\
\hline 200E P-241B103 001 & & D-4, K.2 \\
\hline 200E P-241B104 001 & & D-4, K.2 \\
\hline 200E P-241B105 001 & & D-4, K.2 \\
\hline 200E P-241B106 001 & & $\mathrm{D}-4, \mathrm{~K} .2$ \\
\hline 200E P-241B107 001 & & D-4, K.2 \\
\hline 200E P-241B108 001 & . & $D-4, K .2$ \\
\hline 200E P-241B109 001 & & $D-4, K .2$ \\
\hline 200E P-241B110 001 & & D-4, K.2 \\
\hline
\end{tabular}


Table I.D-1. Emission Points Identified in Application

\begin{tabular}{|c|c|c|}
\hline AEI Identification Number & Regulatory ID. & Appendix \\
\hline 200E P-241B111 001 & & D-4, K.2 \\
\hline 200E P-241B112 001 & & D-4, K.2 \\
\hline 200E P-241B201 001 & & D-4, K.2 \\
\hline 200E P-241B202 001 & & D-4, K.2 \\
\hline 200E P-241B203 001 & & D-4, K.2 \\
\hline 200E P-241B204 001 & & D-4, K.2 \\
\hline 200E P-241BX101 001 & & D-4, K.2 \\
\hline 200E P-241BX102001 & & D-4, K.2 \\
\hline 200E P-241BX103 001 & & D-4, K.2 \\
\hline 200E P-241BX104 001 & & D-4, K.2 \\
\hline 200E P-241BX105 001 & & D-4, K.2 \\
\hline 200E P-241BX106 001 & & D-4, K.2 \\
\hline 200E P-241BX107 001 & & $\mathrm{D}-4, \mathrm{~K} .2$ \\
\hline 200E P-241BX108 001 & & D-4, K.2 \\
\hline 200E P-241BX109 001 & & D-4, K.2 \\
\hline 200E P-241BX110 001 & & D-4, K.2 \\
\hline 200E P-241BX111 001 & & D-4, K.2 \\
\hline 200E P-241BX112 001 & & D-4, K.2 \\
\hline 200E P-241BY101 001 & & D-4, K.2 \\
\hline 200E P-241BY102 001 & & D-4, K.2 \\
\hline 200E P-241BY103 001 & & D-4, K.2 \\
\hline 200E P-241BY104 001 & & D-4, K.2 \\
\hline 200E P-241BY105 001 & & D-4, K.2 \\
\hline 200E P-241BY106 001 & & D-4, K.2 \\
\hline
\end{tabular}


Table I.D-1. Emission Points Identified in Application

\begin{tabular}{|c|c|c|}
\hline AEI Identification Number & Regulatory ID. & Appendix \\
\hline 200E P-241BY107 001 & & D-4, K.2 \\
\hline 200E P-241BY108 001 & & D-4, K.2 \\
\hline 200E P-241BY109 001 & & D-4, K.2 \\
\hline 200E P-241BY110 001 & & D-4, K.2 \\
\hline 200E P-241BY111 001 & & D-4, K.2 \\
\hline 200E P-241BY112001 & & D-4, K.2 \\
\hline 200E P-241C101 001 & & D-4, K.2 \\
\hline 200E P-241C102 001 & & D-4, K.2 \\
\hline 200E P-241C103 001 & & D-4, K.2 \\
\hline 200E P-241C104 001 & & D-4, K.2 \\
\hline 200E P-241C107 001 & & D-4, K.2 \\
\hline 200E P-241C108 001 & & $D-4, K .2$ \\
\hline 200E P-241C109 001 & & D-4, K.2 \\
\hline 200E P-241C110 001 & & D-4, K.2 \\
\hline 200E P-241C111 001 & & $D-4, K .2$ \\
\hline 200E P-241C112 001 & & D-4, K.2 \\
\hline 200E P-241C201 001 & & D-4, K.2 \\
\hline 200E P-241C202 001 & & $D-4, K .2$ \\
\hline 200E P-241C203 001 & & $D-4, K .2$ \\
\hline 200E P-241C204 001 & & D-4, K.2 \\
\hline 200E P-241ER151 001 & & $\mathrm{D}-4$ \\
\hline 200E P-241ER152 001 & & D-4 \\
\hline 200E P-241ER153 001 & & $D-4$ \\
\hline 200E P-241ER311 001 & & $D-4$ \\
\hline
\end{tabular}


Table I.D-1. Emission Points Identified in Application

\begin{tabular}{|c|c|c|}
\hline AEI Identification Number & Regulatory ID. & Appendix \\
\hline 200E P-242A 001 & $296-A-21$ & D-4, K.2 \\
\hline 200E P-242A 002 & $296-\mathrm{A}-22$ & $D-4, K .2$ \\
\hline 200E P-244A 001 & $296-\mathrm{A}-25$ & D-4 \\
\hline 200E P-244AR 001 & $296-A-12$ & $D-4$ \\
\hline 200E P-244AR 002 & $296-A-13$ & $D-4$ \\
\hline 200E P-244BX 001 & 296-B-28 & D-4 \\
\hline 200E P-244CR 001 & 296-C-05 & D-4 \\
\hline 200E P-291A001 001 & 291-A-01 & D-3 \\
\hline 200E P-291B001 001 & 291-B-01 & D-3 \\
\hline 200E P-296A 001 & & D-4, K.2 \\
\hline 200E P-296A001 001 & $296-\mathrm{A}-01$ & D-3 \\
\hline 200E P-296A002 001 & $296-\mathrm{A}-02$ & D-3 \\
\hline 200E P-296A003 001 & $296-\mathrm{A}-03$ & D-3 \\
\hline 200E P-296A006 001 & 296-A-06 & D-3 \\
\hline 200E P-296A007 001 & 296-A-07 & D-3 \\
\hline 200E P-296A008 001 & $296-\mathrm{A}-08$ & D-3 \\
\hline 200E P-296A010 001 & $296-\mathrm{A}-10$ & D-3 \\
\hline 200E P-296A014 001 & $296-\mathrm{A}-14$ & D-3 \\
\hline 200E P-296A018 001 & $296-A-18$ & D-4 \\
\hline 200E P-296A019 001 & 296-A-19 & D-4 \\
\hline 200E P-296A020 001 & 296-A-20 & $\mathrm{D}-4$ \\
\hline 200E P-296A028 001 & $296-\mathrm{A}-28$ & D-4 \\
\hline 200E P-296A030 001 & $296-\mathrm{A}-30$ & D-4 \\
\hline 200E P-296A041 001 & $296-\mathrm{A}-41$ & D-4 \\
\hline 200E P-296A05A 001 & 296-A-05A & D-3, K.2 \\
\hline 200E P-296A05B 001 & 296-A-05B & $\mathrm{D}-3, \mathrm{~K} .2$ \\
\hline
\end{tabular}


Table I.D-1. Emission Points Identified in Application

\begin{tabular}{|l|l|l|}
\hline AEI Identification Number & Regulatory ID. & Appendix \\
\hline 200E P-296AN 001 & 296-A-29 & D-4 \\
\hline 200E P-296AP 001 & 296-A-40 & D-4, K.2 \\
\hline 200E P-296AW 001 & 296-A-27 & D-4 \\
\hline 200E P-296AYAZ 001 & 296-A-17 & D-4 \\
\hline 200E P-296B005 001 & 296-B-05 & D-2 \\
\hline 200E P-296B010 001 & 296-B-10 & D-2 \\
\hline 200E P-296B013 001 & 296-B-13 & D-2 \\
\hline 200E P-296B022 001 & & D-2 \\
\hline 200E P-296B023 001 & & D-2 \\
\hline 200E P-296B024 001 & & D-2 \\
\hline 200E P-296B025 001 & & D-2 \\
\hline 200E P-296B026 001 & & D-2 \\
\hline 200E P-296B027 001 & & D-2 \\
\hline 200E P-296P016 001 & & D-4 \\
\hline 200E P-296P026 001 & 296-P-16 & D-4 \\
\hline 200E P-296P031 001 & 296-P-26 & D-4 \\
\hline 200E P-CORE 001 & 296-P-31 & J-1 \\
\hline 200E P-G001 001 & 296-P-32 & D-4 \\
\hline 200E P-LERF 001 & 296-G-01 & D-4 \\
\hline 200E T-AMUROOFVN 001 & D-3 \\
\hline 200E V-203A CHEM 001 & D-3 \\
\hline 200E V-211A CHEM 001 & D-3 \\
\hline 200W P-296S016 001 & & E-1 \\
\hline 200W P-296S023 001 & E-1, K.2 \\
\hline 200W F-284W 001 & E-2 \\
\hline
\end{tabular}


Table I.D-1. Emission Points Identified in Application

\begin{tabular}{|l|l|l|}
\hline AEI Identification Number & Regulatory ID. & Appendix \\
\hline 200W F-284W 002 & 12 & E-2 \\
\hline 200W F-284W 005 & 21 & E-2 \\
\hline 200W H-200W007 001 & & I-1 \\
\hline 200W J-284W 003 & 16 & E-2 \\
\hline 200W J-284W 004 & 17 & E-2 \\
\hline 200W K-200W003 001 & & I-1 \\
\hline 200W K-272S 001 & & E-7 \\
\hline 200W M 272WA & & E-7, K.3 \\
\hline 200W M-200W005 001 & & K.3 \\
\hline 200W M-200W006 001 & & K.3 \\
\hline 200W M-272W 001 & & K.3 \\
\hline 200W M-277W 001 & & K.3 \\
\hline 200W M-2722W 001 & & K.3 \\
\hline 200W N-200W001 001 & & K.2 \\
\hline 200W N-200W002 001 & & K.3 \\
\hline 200W N-200W004 001 & & K.3 \\
\hline 200W N-231Z 001 & & E-7 \\
\hline 200W N-275W 001 & E-7, K.3 \\
\hline 200W N-277W 002 & E-7 \\
\hline 200W N-277W 003 & E-7, K.3 \\
\hline 200W P 241S101 001 & E-7, K.2 \\
\hline 200W P 241S102 001 & E-7, K.2 \\
\hline 200W P 241S103 001 & & E-7, K.2 \\
\hline 200W P 241S104 001 & E-7, K.2 \\
\hline
\end{tabular}


Table I.D-1. Emission Points Identified in Application

\begin{tabular}{|l|l|l|}
\hline AEI Identification Number & Regulatory ID. & Appendix \\
\hline 200W P 241S105 001 & & E-7, K.2 \\
\hline 200W P 241S106 001 & & E-7, K.2 \\
\hline 200W P 241S107 001 & & E-7, K.2 \\
\hline 200W P 241S108 001 & & E-7, K.2 \\
\hline 200W P 241S109 001 & & E-7, K.2 \\
\hline 200W P 241S110 001 & & E-7, K.2 \\
\hline 200W P 241S111 001 & & E-7, K.2 \\
\hline 200W P 241S112 001 & & E-7, K.2 \\
\hline 200W P 241S151 001 & & E-7 \\
\hline 200W P 241S302A 001 & & E-7 \\
\hline 200W P 241SX113 001 & & E-7, K.2 \\
\hline 200W P 241SX115 001 & & E-7, K.2 \\
\hline 200W P 241SYA 001 & & E-7 \\
\hline 200W P 241SYB 001 & & E-7 \\
\hline 200W P 241T101 & & E-7, K.2 \\
\hline 200W P 241T102 & E-7, K.2 \\
\hline 200W P 241T103 & E-7, K.2 \\
\hline 200W P 241T104 & E-7, K.2 \\
\hline 200W P 241T105 & E-7, K.2 \\
\hline 200W P 241T106 & E-7, K.2 \\
\hline 200W P 241T107 & E-7, K.2 \\
\hline 200W P 241T108 & & E-7, K.2 \\
\hline 200W P 241T109 & E-7, K.2 \\
\hline 200W P 241T110 & & \\
\hline
\end{tabular}


Table I.D-1. Emission Points Identified in Application

\begin{tabular}{|l|l|l|}
\hline AEI Identification Number & Regulatory ID. & Appendix \\
\hline 200W P 241T111 & & E-7, K.2 \\
\hline 200W P 241T112 & & E-7, K.2 \\
\hline 200W P 241T201 & & E-7, K.2 \\
\hline 200W P 241T202 & & E-7, K.2 \\
\hline 200W P 241T203 & & E-7, K.2 \\
\hline 200W P 241T204 & & E-7, K.2 \\
\hline 200W P 241TX101 & & E-7, K.2 \\
\hline 200W P 241TX102 & & E-7, K.2 \\
\hline 200W P 241TX103 & & E-7, K.2 \\
\hline 200W P 241TX104 & & E-7, K.2 \\
\hline 200W P 241TX105 & & E-7, K.2 \\
\hline 200W P 241TX106 & & E-7, K.2 \\
\hline 200W P 241TX107 & & E-7, K.2 \\
\hline 200W P 241TX108 & & E-7, K.2 \\
\hline 200W P 241TX109 & & E-7, K.2 \\
\hline 200W P 241TX110 & & E-7, K.2 \\
\hline 200W P 241TX111 & E-7, K.2 \\
\hline 200W P 241TX112 & E-7, K.2 \\
\hline 200W P 241TX113 & E-7, K.2 \\
\hline 200W P 241TX114 & & E-7, K.2 \\
\hline 200W P 241TX115 & & E-7, K.2 \\
\hline 200W P 241TX116 & & E-7, K.2 K.2 K.2 \\
\hline 200W P 241TX117 & & \\
\hline 200W P 241TX118 & & \\
\hline
\end{tabular}


Table I.D-1. Emission Points Identified in Application

\begin{tabular}{|l|l|l|}
\hline AEI Identification Number & Regulatory ID. & Appendix \\
\hline 200W P 241TX152 001 & & E-7 \\
\hline 200W P 241TX154 001 & & E-7 \\
\hline 200W P 241TX302C 001 & & E-7 \\
\hline 200W P 241TY101 & & E-7, K.2 \\
\hline 200W P 241TY102 & & E-7, K.2 \\
\hline 200W P 241TY103 & & E-7, K.2 \\
\hline 200W P 241TY104 & & E-7, K.2 \\
\hline 200W P 241TY105 & & E-7, K.2 \\
\hline 200W P 241TY106 & & E-7, K.2 \\
\hline 200W P 241U101 & E-7, K.2 \\
\hline 200W P 241U102 & & E-7, K.2 \\
\hline 200W P 241U103 & & E-7, K.2 \\
\hline 200W P 241U104 & & E-7, K.2 \\
\hline 200W P 241U105 & & E-7, K.2 \\
\hline 200W P 241U106 & & E-7, K.2 \\
\hline 200W P 241U107 & & E-7, K.2 \\
\hline 200W P 241U108 & & E-7, K.2 \\
\hline 200W P 241U109 & & E-7, K.2 \\
\hline 200W P 241U110 & & E-7, K.2 K.2 \\
\hline 200W P 241U111 & E-7, K.2 \\
\hline 200W P 241U112 & & E-7 \\
\hline 200W P 241U151 001 & & E-7 \\
\hline 200W P 241U152 001 & & \\
\hline 200W P 241U201 & & \\
\hline
\end{tabular}


Hanford Site Air

Operating Permit Application

DOE/RL-95-07, Rev. 0

$05 / 95$

Table I.D-1. Emission Points Identified in Application

\begin{tabular}{|c|c|c|}
\hline AEI Identification Number & Regulatory ID. & Appendix \\
\hline $200 \mathrm{~W}$ P $241 \mathrm{U} 202$ & & $\mathrm{E}-7, \mathrm{~K} .2$ \\
\hline 200W P 241U203 & & E-7, K.2 \\
\hline 200W P $241 \mathrm{U} 204$ & & E-7, K.2 \\
\hline 200W P 241U301B 001 & & E-7 \\
\hline 200W P $241 \mathrm{UX154001}$ & & E-7 \\
\hline 200W P 241UX302A 001 & & E-7 \\
\hline $200 \mathrm{~W} P \quad 244 \mathrm{U} 001$ & 296-U-11 & E-7 \\
\hline 200W P 296SX & & E-7 \\
\hline 200W P 296SY & & E-7 \\
\hline 200W P-213W 001 & 296-W-03 & E-8 \\
\hline 200W P-241S101 & & K.2 \\
\hline 200W P-242S 001 & 296-S-18 & E-7 \\
\hline 200W P-242T 001 & 296-T-17 & E-7 \\
\hline 200W P-244S 001 & 296-S-22 & E-7 \\
\hline 200W P-244TX 001 & 296-T-18 & E-7 \\
\hline 200W P-291S001 001 & 291-S-01 & E-4 \\
\hline 200W P-291T001 001 & 291-T-01 & E-5 \\
\hline 200W P-291U001 001 & 291-U-01 & E-6 \\
\hline 200W P-291Z001 001 & 291-Z-01 & $\mathrm{E}-3, \mathrm{~K} .2$ \\
\hline 200W P-296P022 001 & 296-P-22 & E-7 \\
\hline 200W P-296P028 001 & 296-P-28 & E-7 \\
\hline 200W P-296S002 001 & 296-S-02 & E-4 \\
\hline 200W P-296S004 001 & 296-S-04 & E-4 \\
\hline 200W P-296\$006 001 & 296-S-06 & E-4 \\
\hline 200W P-296S016 001 & 296-S-16 & E-1 \\
\hline
\end{tabular}


Table I.D-1. Emission Points Identified in Application

\begin{tabular}{|c|c|c|}
\hline AEI Identification Number & Regulatory ID. & Appendix \\
\hline 200W P-296S021 001 & 296-S-21 & E-1, K.2 \\
\hline 200W P-296S07W 001 & $296-S-07 W$ & E-4 \\
\hline 200W P-296S025 001 & $296-\mathrm{S}-25$ & E-7 \\
\hline 200W P-296SX 001 & 296-S-15 & E-7 \\
\hline 200W P-296SY 001 & 296-P-23 & E-7 \\
\hline 200W P-296T007 001 & $296-\mathrm{T}-07$ & E-5 \\
\hline 200W P-296T011 001 & 296-T-11 & E-8 \\
\hline 200W P-296T012 001 & $296-\mathrm{T}-12$ & E-8 \\
\hline 200W P-296T013 001 & $296-\mathrm{T}-13$ & E-5 \\
\hline 200W P-296Z003 001 & 296-Z-03 & E-3 \\
\hline 200W P-296Z005 001 & 296-Z-05 & E-3 \\
\hline 200W P-296Z006 001 & 296-Z-06 & E-3 \\
\hline 200W P-296Z014 001 & 296-Z-14 & E-3 \\
\hline 200W P-296Z015 001 & & $E-3$ \\
\hline 200W S-222SA 001 & & E-1, K.2 \\
\hline 200W S-296S021 001 & $296-\mathrm{S}-21$ & E-1, K.2 \\
\hline 300 F-384 001 & $5,6,7$ & F-1 \\
\hline 300 F-384 002 & 4 & F-1 \\
\hline 300 F-384 005 & 19 & $F-1$ \\
\hline $300 \mathrm{~F}-384006$ & 8 & F-1 \\
\hline $300 \mathrm{~J}-384003$ & 20 & F-1 \\
\hline $300 \mathrm{~J}-384004$ & & $F-1$ \\
\hline $300 \mathrm{~K}-3709001$ & & $\mathrm{I}-1$ \\
\hline $300 \mathrm{M}-305 \mathrm{~A} 001$ & - & K.3 \\
\hline $300 \mathrm{M}-328003$ & & K.3 \\
\hline
\end{tabular}


Table I.D-1. Emission Points Identified in Application

\begin{tabular}{|c|c|c|}
\hline AEI Identification Number & Regulatory ID. & Appendix \\
\hline $300 \mathrm{M}-338001$ & & K.3 \\
\hline $300 \mathrm{~N}-305001$ & & K.3 \\
\hline $300 \mathrm{~N}-328001$ & & K.2 \\
\hline $300 \mathrm{~N}-328002$ & & K.2 \\
\hline $300 \mathrm{~N}-3713001$ & & K.2 \\
\hline $300 N-3713002$ & & K.3 \\
\hline $300 \mathrm{~N}-3717 \mathrm{~B} 001$ & & K.2 \\
\hline $300 \mathrm{~N}-3722001$ & & K.3 \\
\hline $300 N-3722002$ & & K.2 \\
\hline $300 \mathrm{~N}-3722003$ & & K.3 \\
\hline 300 P-306EULAB & 306-ULAB & F-1 \\
\hline 300 P-308TRIGA 001 & 308-TRIGA & F-1 \\
\hline 300 P-309PRTR 001 & 309-PR-TR & F-1 \\
\hline 300 P-340BLDG 001 & 340-B-BLDG & $\mathrm{F}-1$ \\
\hline 300 P-340DECON 001 & 340-DECON & F-1 \\
\hline 300 P-340NTEX 001 & 340-NT-EX & F-1 \\
\hline 300 P-377GEL 001 & 377-GEL & F-1 \\
\hline $400 \mathrm{~K}-4722 \mathrm{C} 001$ & & $\mathrm{I}-1$ \\
\hline $400 \mathrm{~N}-437001$ & & G-1 \\
\hline $400 \mathrm{~N}-4704 \mathrm{~N} 001$ & & K.3 \\
\hline $400 \mathrm{~N}-4713 \mathrm{~B} 001$ & & G-1 \\
\hline $400 \mathrm{~N}-4713 \mathrm{D} 001$ & & G-1 \\
\hline $400 \mathrm{~N}-4722 \mathrm{~B} 001$ & & K.3 \\
\hline $400 \mathrm{~N}-4722 \mathrm{~B} 002$ & & K.2 \\
\hline
\end{tabular}


Table I.D-1. Emission Points Identified in Application

\begin{tabular}{|l|l|l|}
\hline AEI Identification Number & Regulatory ID. & Appendix \\
\hline 400 N-4722C 002 & & K.3 \\
\hline 400 N-4722C 003 & & K.2 \\
\hline 400 P-408A 001 & & G-1 \\
\hline 400 P-408B 000 & & G-1 \\
\hline 400 P-408C 001 & & G-1 \\
\hline 400 P-437MN\&ST 001 & 437-MN\&ST & G-1 \\
\hline 400 P-FFTFCBEX 001 & FFTF-CB-EX & G-1 \\
\hline 400 P-FFTFHTTR 001 & FFTF-HT-TR & G-1 \\
\hline 400 P-FFTFRESB 001 & FFTF-RE-SB & G-1 \\
\hline 600 M-6290 001 & & K.3 \\
\hline 600 N-661 001 & & K.3 \\
\hline 600 N-MO222 001 & & K.2 \\
\hline 600 N-MO222 002 & & K.2 \\
\hline 600 N-MO302 001 & & K.3 \\
\hline 600 P-241EW151 001 & & H-1 \\
\hline 600 S-6266-001 & & H-2, K.2 \\
\hline 600 S-6266 002 & & H-2 \\
\hline 600 S-6269 001 & H-2 \\
\hline 600 Z-6266A 001 & H-2 \\
\hline
\end{tabular}


Table I.D-2. Regulatory Identification Numbers.

\begin{tabular}{|c|c|c|}
\hline Regulatory ID. & AEI ID. Number & Appendix \\
\hline $105-\mathrm{KE}$ & $100 \mathrm{~K}$ P-105KE 001 & $\mathrm{C}-1$ \\
\hline 105-KE & 100K P-105KE 002 & $C-1$ \\
\hline 105-KE & 100K P-105KE 003 & $\mathrm{C}-1$ \\
\hline $105-\mathrm{KE}$ & $100 \mathrm{~K}$ P-105KE 004 & $\mathrm{C}-1$ \\
\hline $105-\mathrm{KW}$ & 100K P-105KW 001 & $\mathrm{C}-1$ \\
\hline $105-\mathrm{KW}$ & 100K P-105KW 002 & $\mathrm{C}-1$ \\
\hline 105-KW & $100 \mathrm{~K}$ P-105KW 003 & $C-1$ \\
\hline $105-\mathrm{KW}$ & 100K P-105KW 004 & $\mathrm{C}-1$ \\
\hline $105-N-14^{\prime} \mathrm{DR}$ & $100 \mathrm{~N} P-105 \mathrm{~N} 002$ & $\mathrm{C}-2$ \\
\hline $107-\mathrm{N}$ & $100 N$ P-107N 004 & $\mathrm{C}-2$ \\
\hline $116-\mathrm{N}$ & $100 \mathrm{~N} P-116 \mathrm{~N} 005$ & $\mathrm{C}-2$ \\
\hline $1706 \mathrm{KE}$ & 100K R-1706 KE 001 & $C-1$ \\
\hline 1706 KER & 100K R-1706KER 001 & $\mathrm{C}-1$ \\
\hline $1706 \mathrm{KEL}$ & 100K R-1706KEL 001 & C-1, K.2 \\
\hline $291-A-01$ & 200E P-291A001 001 & D-3 \\
\hline 291-B-01 & 200E P-291B001 001 & $\mathrm{D}-3$ \\
\hline $291-S-01$ & 200W P-291S001 001 & E-4 \\
\hline 291-T-01 & 200W P-291T001 001 & E-5 \\
\hline 291-U-01 & 200W P-291U001 001 & E-6 \\
\hline 291-Z-01 & 200W P-291Z001 001 & $\mathrm{E}-3, \mathrm{~K} .2$ \\
\hline 296-A-01 & 200E P-296A001 001 & $\mathrm{D}-3$ \\
\hline 296-A-02 & 200E P-296A002 001 & $\mathrm{D}-3$ \\
\hline 296-A-03 & 200E P-296A003 001 & D-3 \\
\hline $296-A-05 A$ & 200E P-296A05A 001 & D-3, K.2 \\
\hline 296-A-05B & 200E P-296A05B 001 & D-3, K.2 \\
\hline 296-A-06 & 200E P-296A006 001 & $D-3$ \\
\hline
\end{tabular}


Table I.D-2. Regulatory Identification Numbers.

\begin{tabular}{|c|c|c|}
\hline Regulatory ID. & AEI ID. Number & Appendix \\
\hline 296-A-07 & 200E P-296A007 001 & D-3 \\
\hline 296-A-08 & 200E P-296A008 001 & $D-3$ \\
\hline $296-A-10$ & 200E P-296A010 001 & D-3 \\
\hline $296-A-12$ & 200E P-244AR 001 & $D-4$ \\
\hline $296-A-13$ & 200E P-244AR 002 & $D-4$ \\
\hline 296-A-14 & 200E P-296A014 001 & D-3 \\
\hline $296-A-17$ & 200E P-296AYAZ 001 & $D-4$ \\
\hline $296-A-18$ & 200E P-296A018 001 & D-4 \\
\hline 296-A-19 & 200E P-296A019 001 & $D-4$ \\
\hline $296-A-20$ & 200E P-296A020 001 & $D-4$ \\
\hline $296-A-21$ & $200 \mathrm{E}$ P-242A 001 & $\mathrm{D}-4, \mathrm{~K} .2$ \\
\hline $296-\mathrm{A}-22$ & 200E P-242A 002 & $\mathrm{D}-4, \mathrm{~K} .2$ \\
\hline $296-A-25$ & 200E P-244A 001 & $D-4$ \\
\hline $296-A-26$ & 200E P-204AR 001 & $D-4$ \\
\hline $296-A-27$ & 200E P-296AW 001 & $D-4$ \\
\hline 296-A-28 & 200E P-296A028 001 & D-4 \\
\hline $296-A-29$ & 200E P-296AN 001 & $D-4$ \\
\hline $296-A-30$ & 200E P-296A030 001 & $\mathrm{D}-4$ \\
\hline $296-A-40$ & 200E P-296AP 001 & D-4, K.2 \\
\hline $296-A-41$ & 200E P-296A041 001 & $\mathrm{D}-4$ \\
\hline 296-B-05 & 200E P-296B005 001 & D-2 \\
\hline 296-B-10 & 200E P-296B010 001 & $\mathrm{D}-2$ \\
\hline $296-B-13$ & 200E P-296B013 001 & $\mathrm{D}-2$ \\
\hline $296-B-28$ & 200E P-244BX 001 & $D-4$ \\
\hline 296-C-05 & 200E P-244CR 001 & $D-4$ \\
\hline $296-G-01$ & 200E P-G001 001 & $D-4$ \\
\hline
\end{tabular}


Table I.D-2. Regulatory Identification Numbers.

\begin{tabular}{|c|c|c|}
\hline Regulatory ID. & AEI ID. Number & Appendix \\
\hline $296-P-16$ & 200E P-296P016 001 & $D-4$ \\
\hline 296-P-22 & 200W P-296P022 001 & E-7 \\
\hline $296-\mathrm{P}-23$ & 200W P-296SY 001 & E-7 \\
\hline 296-P-26 & 200E P-296P026 001 & $D-4$ \\
\hline $296-P-28$ & 200W P-296P028 001 & E-7 \\
\hline 296-P-31 & 200Е Р-296Р031 001 & $\mathrm{D}-4$ \\
\hline 296-P-32 & 200E P-CORE 001 & $\mathrm{~J}-1$ \\
\hline 296-S-02 & 200W P-296S002001 & $\mathrm{E}-4$ \\
\hline 296-S-04 & 200W P-296S004 001 & $E-4$ \\
\hline 296-S-06 & 200W P-296S006 001 & E-4 \\
\hline 296-S-07W & 200W P-296S07W 001 & $E-4$ \\
\hline $296-S-15$ & 200W P-296SX 001 & E-7 \\
\hline 296-S-16 & 200W P-296S016 001 & E-1 \\
\hline 296-S-18 & 200W P-242S 001 & E-7 \\
\hline 296-S-21 & 200W P-296S021 001 & $\mathrm{E}-1, \mathrm{~K} .2$ \\
\hline $296-S-22$ & 200W P-244S 001 & E-7 \\
\hline $296-S-25$ & 200W P-296S025001 & E-7 \\
\hline $296-\mathrm{T}-07$ & 200W P-296T007 001 & E-5 \\
\hline $296-\mathrm{T}-11$ & 200W P-296T011001 & E-8 \\
\hline $296-\mathrm{T}-12$ & 200W P-296T012001 & E-8 \\
\hline $296-\mathrm{T}-13$ & 200W P-296T013 001 & E-5 \\
\hline $296-\mathrm{T}-17$ & 200W P-242T 001 & E-7 \\
\hline $296-\mathrm{T}-18$ & 200W P-244TX 001 & E-7 \\
\hline 296-U-11 & 200W P $244 U 001$ & $\mathrm{E}-7$ \\
\hline $296-W-03$ & $200 W$ P-213W 001 & E-8 \\
\hline $296-Z-03$ & 200W P-296Z003001 & E-3 \\
\hline
\end{tabular}


Table I.D-2. Regulatory Identification Numbers.

\begin{tabular}{|c|c|c|}
\hline Regulatory ID. & AEI ID. Number & Appendix \\
\hline $296-Z-05$ & 200W P-296Z005 001 & E-3 \\
\hline $296-Z-06$ & 200W P-296Z006 001 & E-3 \\
\hline 296-Z-14 & 200W P-296Z014 001 & E-3 \\
\hline 306-ULAB & 300 P-306EULAB & $\mathrm{F}-1$ \\
\hline 308-TRIGA & 300 P-308TRIGA 001 & $F-1$ \\
\hline 309-PR-TR & 300 P-309PRTR 001 & $\mathrm{~F}-1$ \\
\hline 340-B-BLDG & 300 P-340BLDG 001 & $F-1$ \\
\hline 340-DECON & 300 P-340DECON 001 & $F-1$ \\
\hline 340-NT-EX & 300 P-340NTEX 001 & $\mathrm{~F}-1$ \\
\hline 377-GEL & 300 P-377GEL 001 & $\mathrm{~F}-1 \cdot$ \\
\hline 437-MN\&ST & 400 P-437MN\&ST 001 & G-1 \\
\hline $5,6,7$ & 300 F-384 001 & $\mathrm{~F}-1$ \\
\hline FFTF-CB-EX & 400 P-FFTFCBEX 001 & G-1 \\
\hline FFTF-HT-TR & 400 P-FFTFHTTR 001 & G-1 \\
\hline FFTF-RE-SB & 400 P-FFTFRESB 001 & G-1 \\
\hline 4 & 300 F-384 002 & $\mathrm{~F}-1$ \\
\hline 8 & 300 F-384 006 & $\mathrm{~F}-1$ \\
\hline 11 & 200E F-284E 001 & D-1 \\
\hline 11 & 200E F-284E 002 & D-1 \\
\hline 12 & 200W F-284W 001 & $\mathrm{E}-2$ \\
\hline 12 & 200W F-284W 002 & $\mathrm{E}-2$ \\
\hline 16 & 200E J-284E 003 & $D-1$ \\
\hline 16 & $200 \mathrm{~W} \mathrm{~J}-284 \mathrm{~W} 003$ & $\mathrm{E}-2$ \\
\hline 17 & $200 \mathrm{E} J-284 \mathrm{E} 004$ & $D-1$ \\
\hline 17 & $200 \mathrm{~W} J-284 \mathrm{~W} 004$ & $\mathrm{E}-2$ \\
\hline 19 & 300 F-384 005 & $F-1$ \\
\hline
\end{tabular}


Table I.D-2. Regulatory Identification Numbers.

\begin{tabular}{|l|l|l|}
\hline Regulatory ID. & AEI ID. Number & Appendix \\
\hline 20 & 300 J-384 003 & F-1 \\
\hline 21 & 200 W F-284W 005 & E-2 \\
\hline
\end{tabular}




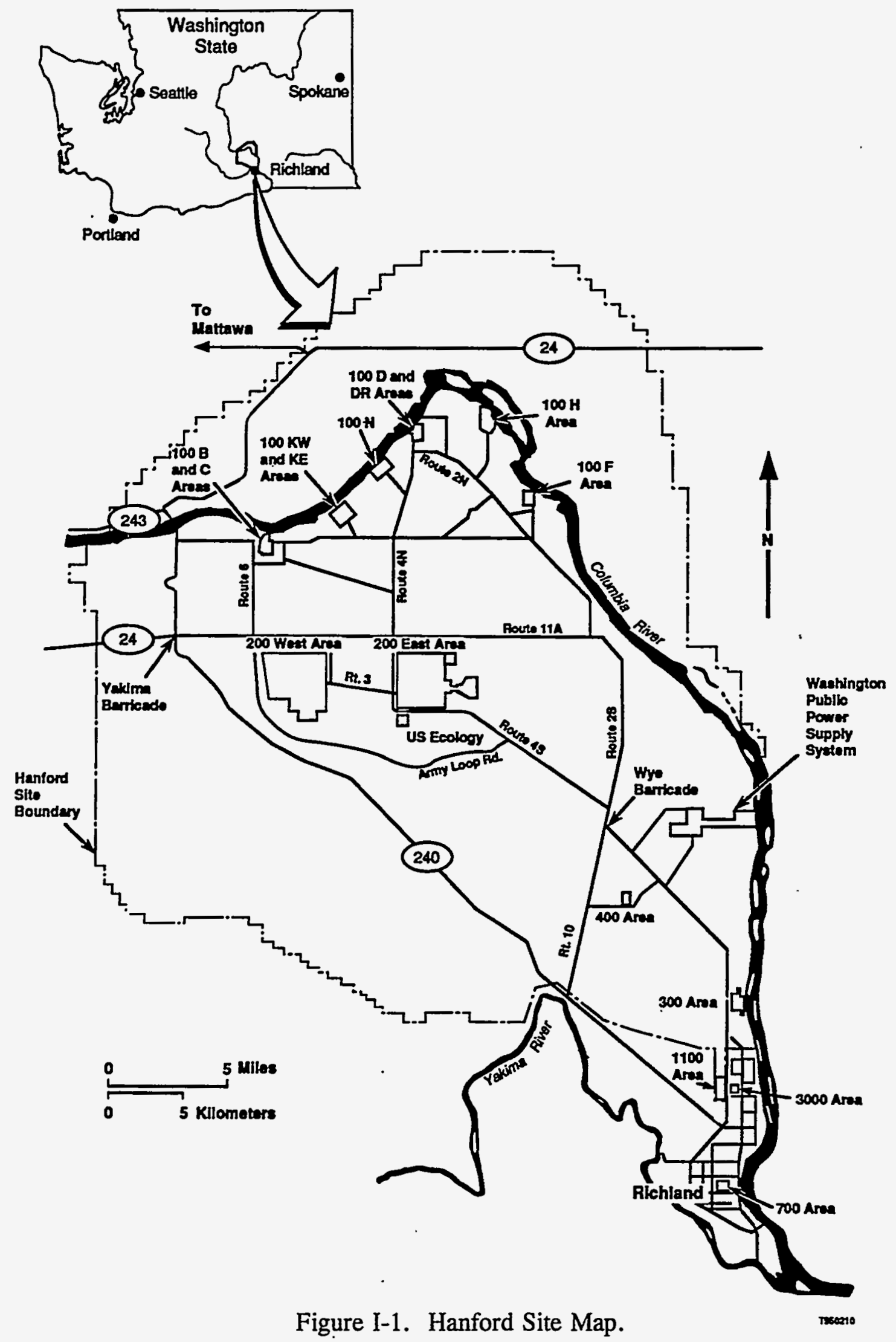


AIR EMISSIONS INVEMTORY PERHII APPLICATIOH REPORT (UP660)

Facilitr haMe: 384 Facllitiles and structures

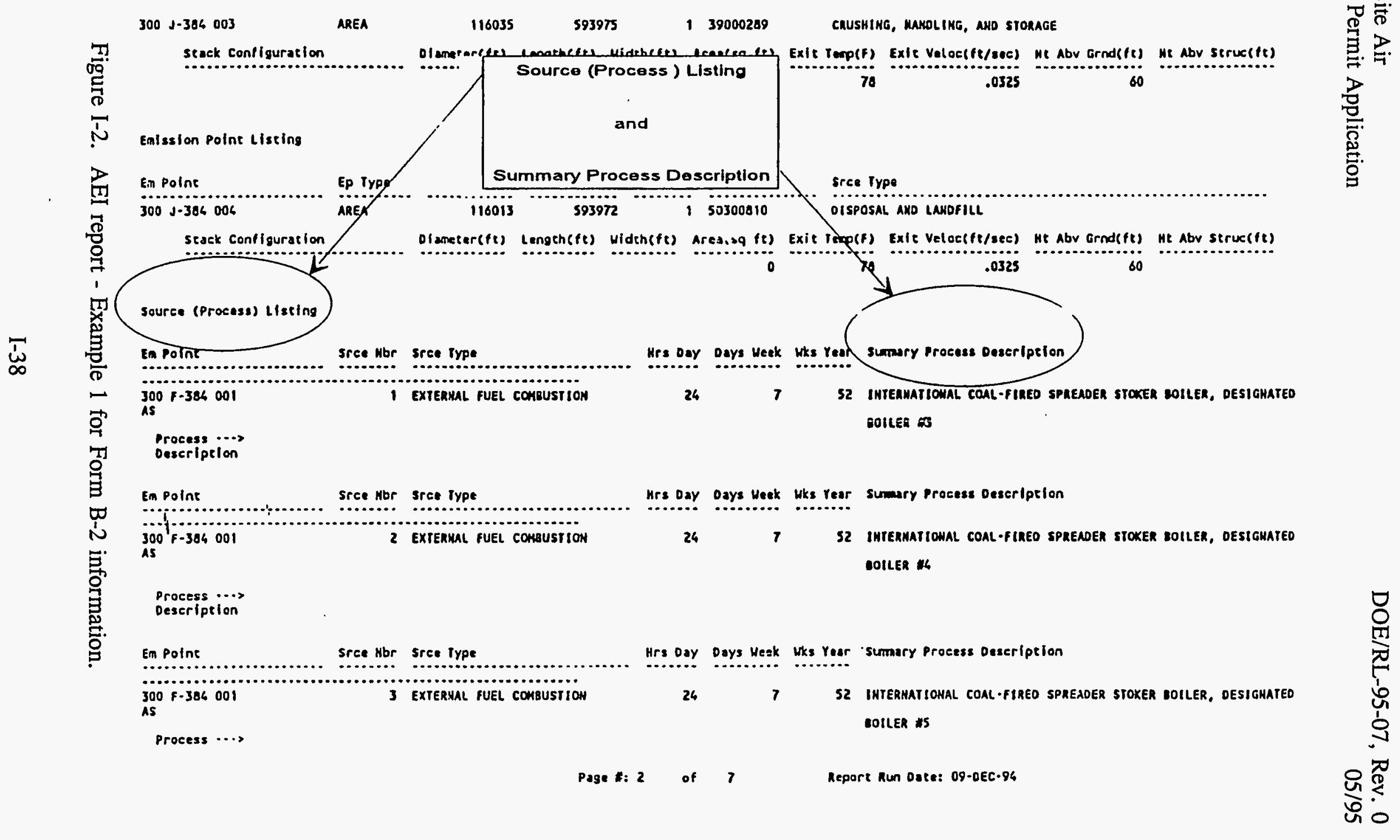


AIR EKISSIOWS IXVEHTORY PERHII APPLICATION REPORT (UP460) Facilitr haME: 384 facllitiles and structures

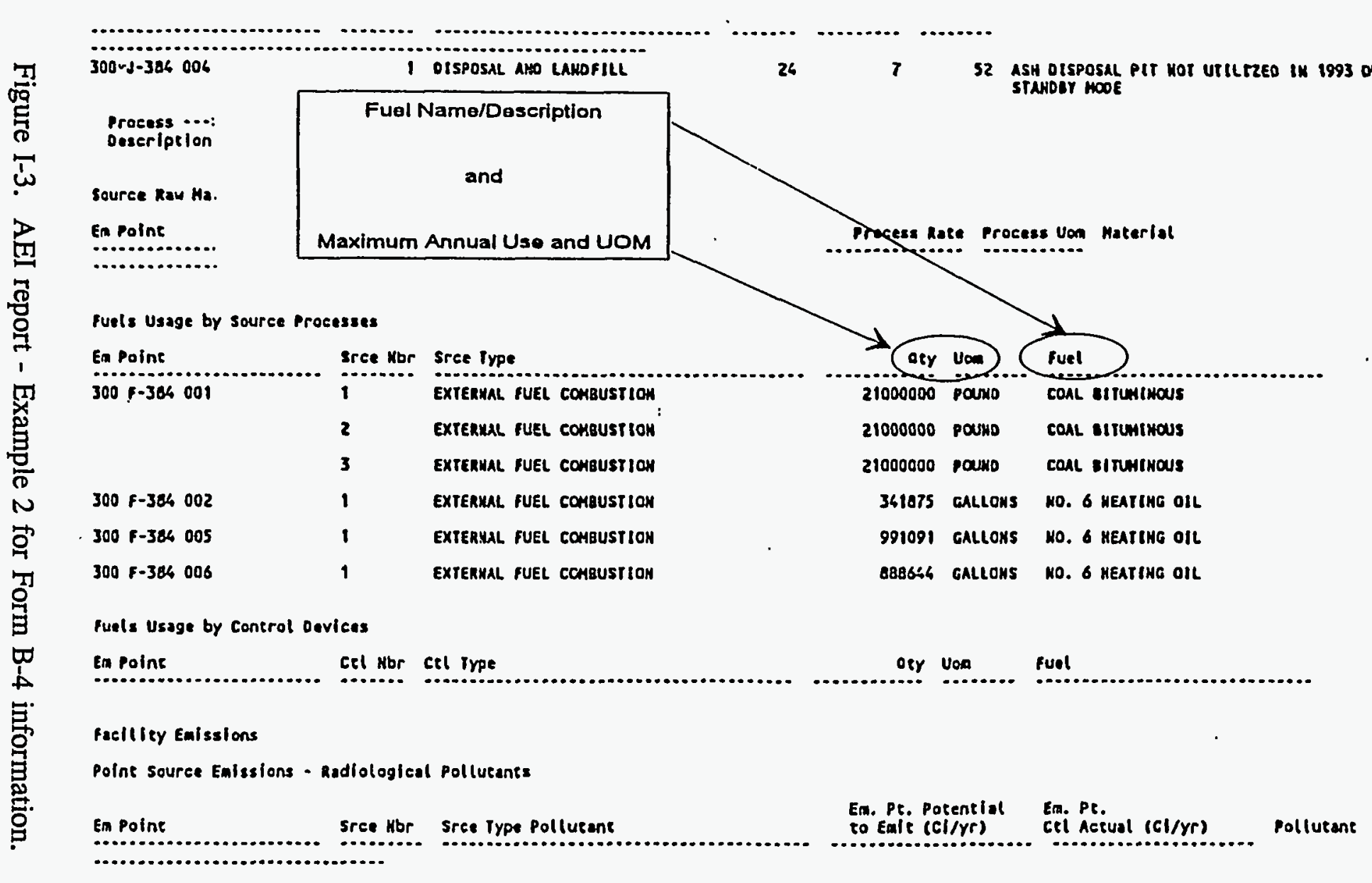

Point Source Emissions - HAP Pollutants

Em. Pt. Potential En. Pr.

Page : : 4 of 7 Repore Run Date: 09-DEC-94 
AIR EHISSIONS IHVEHTORY PERMIT APPLICATIOH REPORT (UP660)

FACILIIY HAME: 300 FACILITY 10 : 300 LACS

facillity guilding Llst

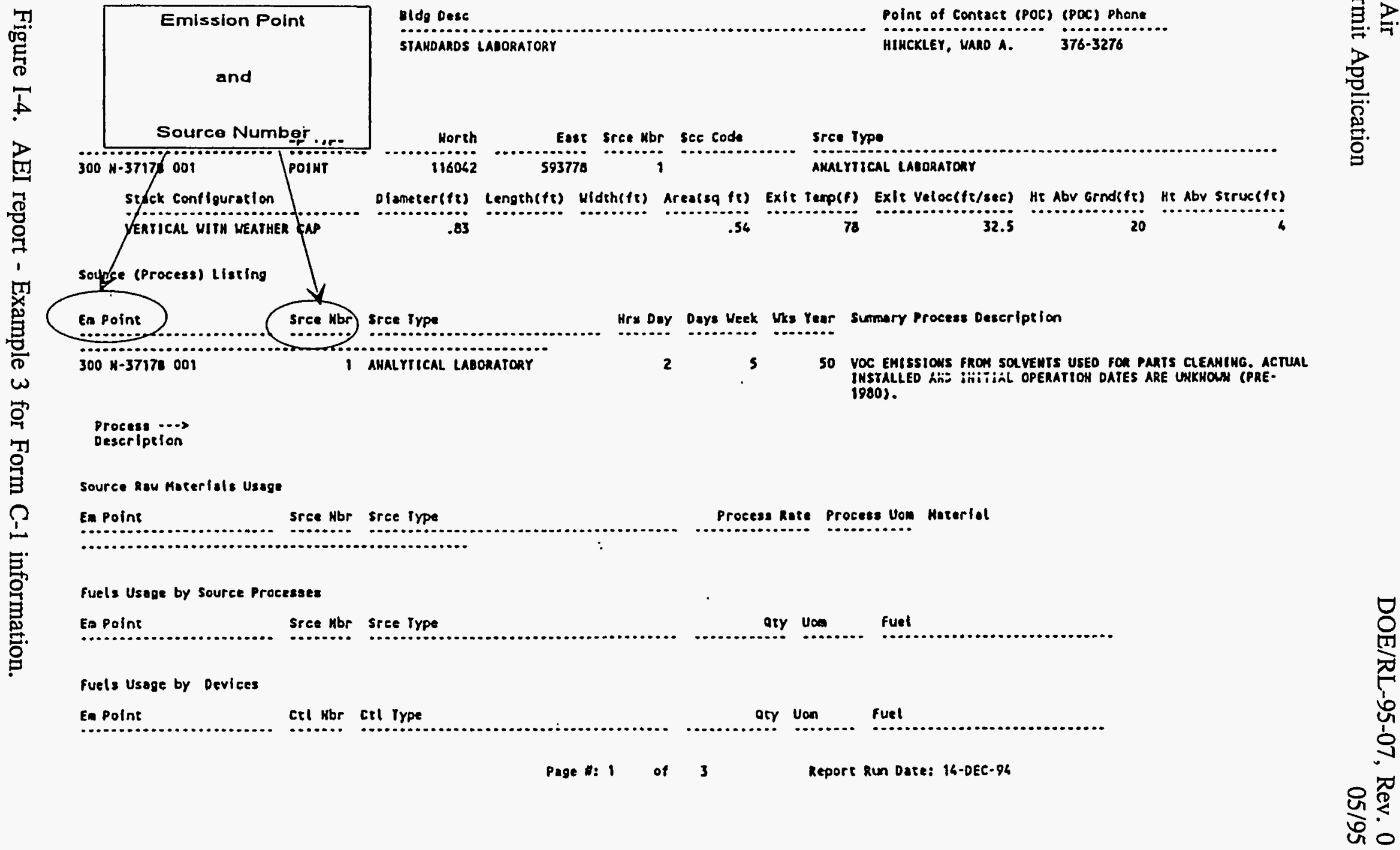




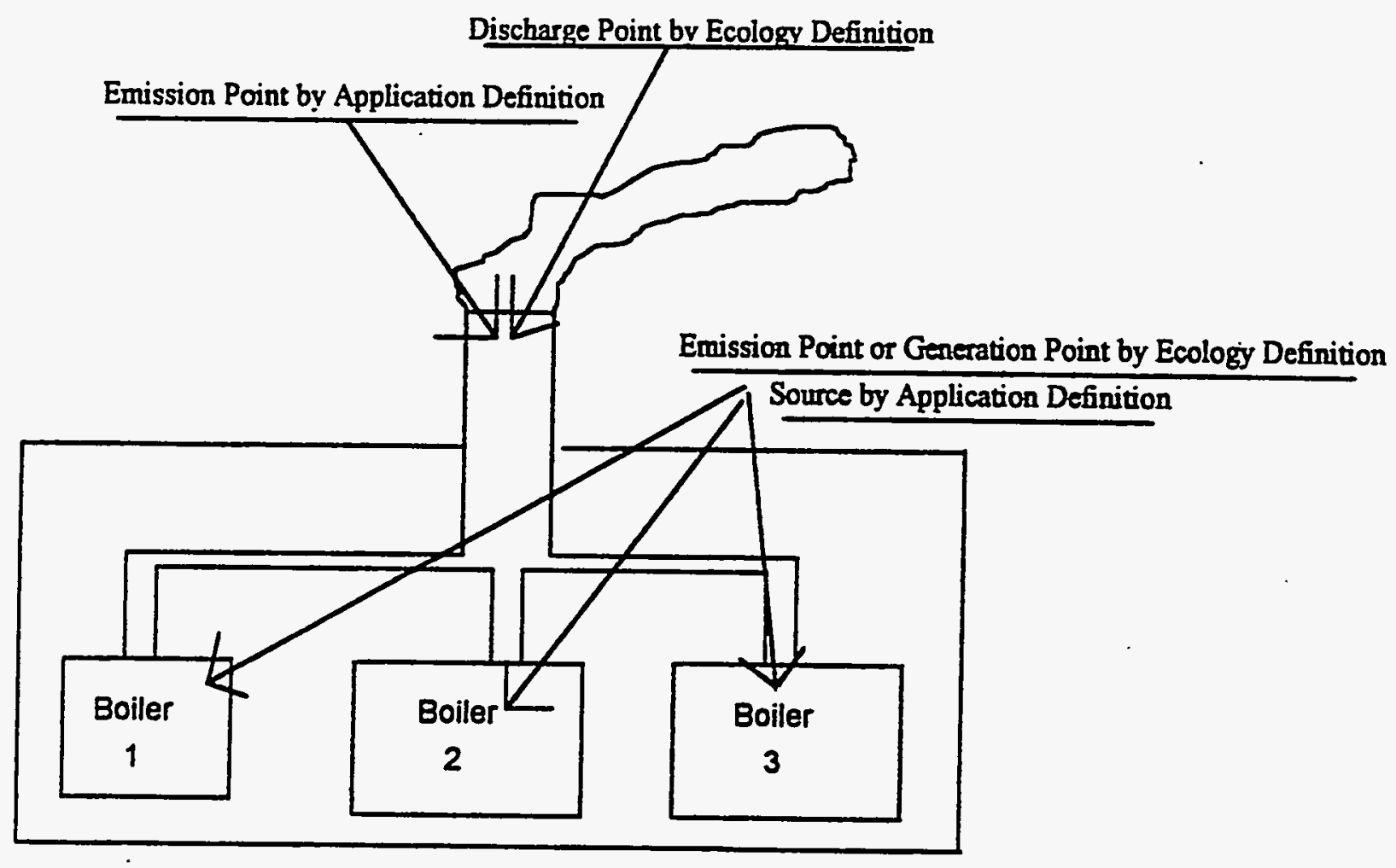

Figure I-5. Example of Discharge and Emission Point Definitions. 
AIR EHISSIONS INVENTORY PERRIT APPIICATION REPOAT (UP460)

FACILITY NAME: PACILITY ID : PUAREX

Emission Point Listing

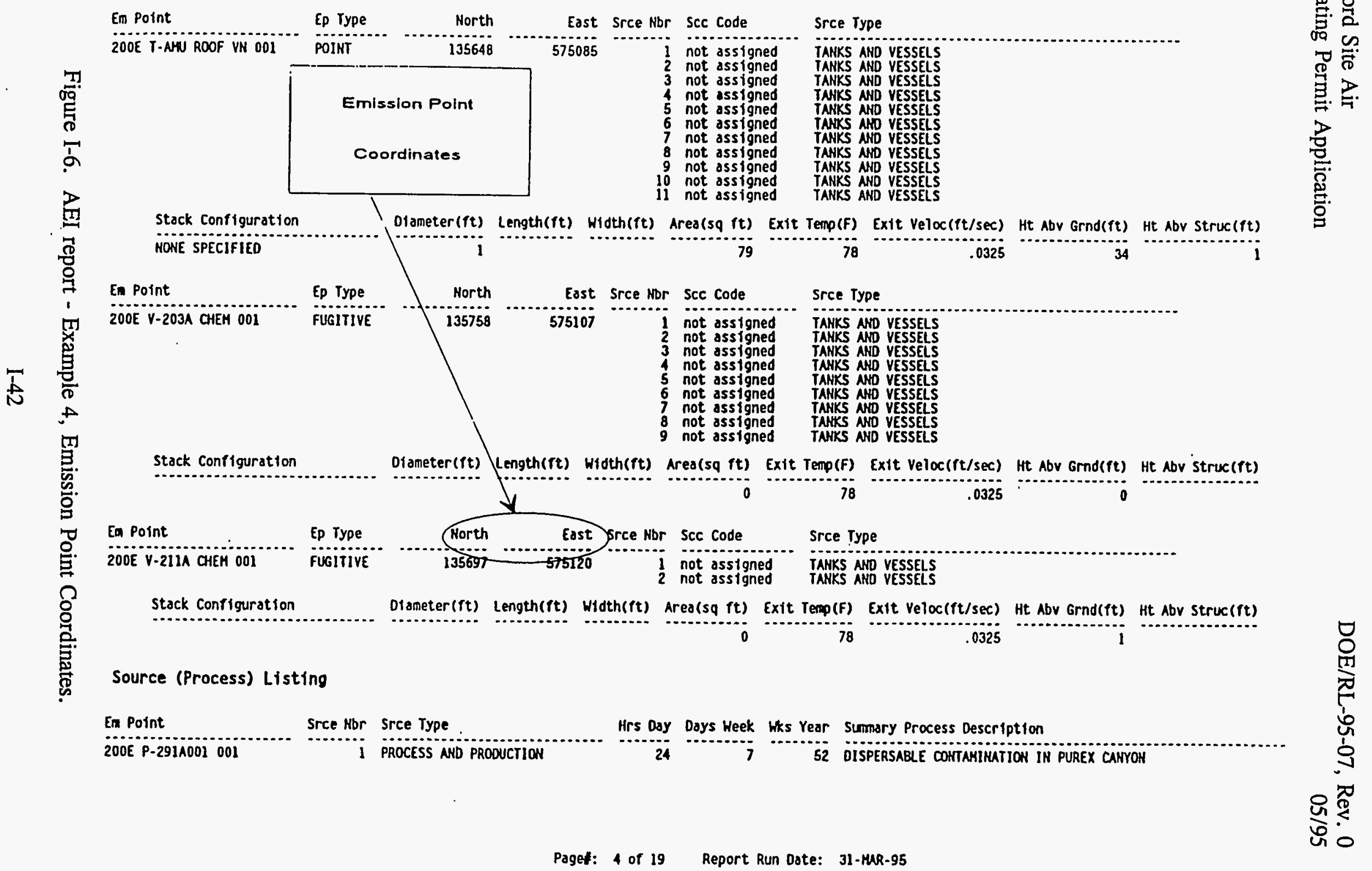


Insignificant Source Emissions - Radiological Pollutants

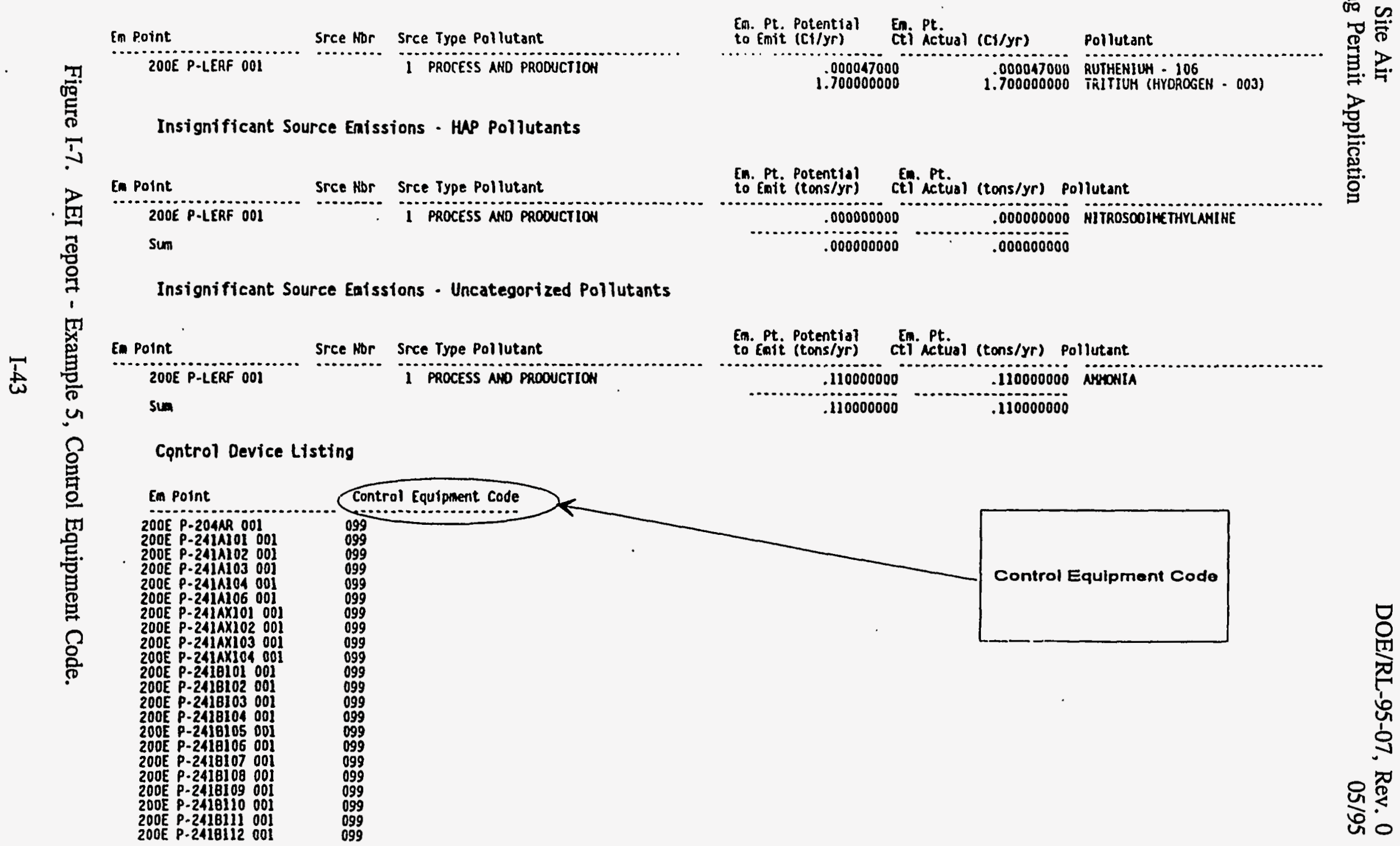

Page 1: 87 of $92^{\circ}$ Report Run Date: 31-Har-95 
Hanford Site Air

Operating Permit Application
DOE/RL-95-07, Rev. 0 05/95

CHAPTER II

REGULATORY REQUIREMENTS 


\section{CONTENTS}

II. REGULATORY REQUIREMENTS . . . . . . . . . . . . . . . II-1

II.A. DESCRIPTION OF EMISSION POINT $\ldots \ldots \ldots \ldots \ldots \ldots \ldots$. . . . .

II.B. APPLICABLE REQUIREMENTS . . . . . . . . . . . . . II-1

II.B.1. Radioactive Airborne Emissions and Notice of Construction Review . . . . . . . . . . II-1

II.B.2. Nonradioactive Airborne Emissions and Notice of Construction Review . . . . . . . . . . . II-2

II.B.3. Special Requirements $\ldots \ldots \ldots \ldots \ldots \ldots \ldots \ldots \ldots$. . . . . . .

II.C. EXEMPTIONS . . . . . . . . . . . . . . . . . II-3

II.D. INAPPLICABLE REQUIREMENTS $\ldots \ldots \ldots \ldots \ldots \ldots \ldots \ldots$ II-3

II.E. COMPLIANCE PLAN $\ldots \ldots \ldots \ldots \ldots \ldots \ldots \ldots \ldots \ldots$ II-3 $\ldots \ldots \ldots$

II.E.1. Status . . . . . . . . . . . . . . . . . . . II-3

II.E.2. Compliance Methods . . . . . . . . . . . . . . . . . . . II-3

II.E.2.1. Insignificant Emission Units and Activities . . . . . . II-3

II.E.2.2. Significant Emission Units and Activities . . . . . . . II-4

II.E.3. Compliance Certification and Reporting

Requirements . . . . . . . . . . . . . . . . II-6

II.E.3.1. Semiannual report $\ldots \ldots \ldots \ldots \ldots \ldots$. . . II-6

II.E.3.2. Annual Report . . . . . . . . . . . . . . . . . II-6

II.E.4. Enhanced Monitoring . . . . . . . . . . . . . . . . II-7

II.E.5. Compliance Plan and Schedule $\ldots \ldots \ldots \ldots \ldots \ldots \ldots \ldots$ II-8

II.F. OPERATIONAL FLEXIBILITY . . . . . . . . . . . . . . . . II-27

II.F.1 Reasonably Anticipated Operating Scenarios . . . . . . . . II-27

III.F.2 Emissions Trading . . . . . . . . . . . . . . . . II-31

II.F.3 Permit Modifications $\ldots \ldots \ldots \ldots \ldots \ldots \ldots \ldots$. . . . . . .

\section{TABLES}

II.B-1. Regulations and Regulated Emissions Air Operating Permit Applications . . II.B-1.1

II.B-2. Regulations and Regulated Emissions Air Operating Permit Applications . . II.B-2.1

II.B-3. Special Requirements . . . . . . . . . . . . . . . . . . . II.B-3.1

II.D-1. Air Operating Permit Inapplicable Requirements $\ldots \ldots \ldots \ldots \ldots$. . . . II.D-1.1 


\section{CHAPTER II. REGULATORY REQUIREMENTS}

This chapter identifies the applicable requirements for each emission point with emission limitations and other regulatory requirements that apply to airborne emissions of regulated pollutants on the Hanford Site. This chapter is intended to fulfill the requirements of Chapter II of Ecology's Instructions (Instructions). The first part of the chapter is organized as a series of tables that list emission point or process, the applicable requirements, the requirement citation, a brief description of the compliance methods, and the compliance status of the emission point or process. The tables have been designed to follow Form C-2 as much as possible.

For the benefit of the permitting agencies, the Chapter II.B tables are divided into separate tables of radionuclide airborne emission applicable requirements and hazardous air pollutant (HAP) applicable requirements. Tables II.B-1, II.B-2, and II.B-3 are intended to fulfill the requirements of Chapter II, Sections B of the Instructions. The Washington State Department of Health (DOH) regulates the radionuclide airborne emissions from the Hanford Site and the Washington State Department of Ecology (Ecology) regulates the criteria pollutants and HAP emissions. A memorandum of understanding between the two agencies provides the frame work for the dual nature of air emission regulation of the Hanford Site. In addition, Region 10 of the U.S. Environmental Protection Agency (EPA) also regulates airborne radionuclide emissions.

\section{II.A. DESCRIPTION OF EMISSION POINT}

The description for each emission point is contained in the AEI reports located in each subappendix for each operational area (Appendix $\mathrm{C}$ through $\mathrm{J}$ ). As explained previously, an equivalent method of reporting the same information was used.

\section{II.B. APPLICABLE REQUIREMENTS}

\section{II.B.1. Radioactive Airborne Emissions and Notice of Construction Review}

The DOH regulates airborne radionuclide emissions on the Hanford Site and has permitted the emissions through permit number FF-01. The FF-01 permit expires in 1997 or when replaced by the Air Operating Permit. Table II.B-1 provides a list of the radionuclide air emission units, demonstration of compliance status with Permit FF-01, and other applicable radionuclide air emission regulations and statutes. 
The first part of Table II.B-1 lists the applicable radionuclide emission limitations, the radionuclide emission units on the Hanford Site, and the compliance demonstration methods for the emission units. The second part of the table identifies specific projects and approval conditions specified through the Notice of Construction approval process. This section also provides descriptions of the compliance demonstration methods and the compliance status.

Where Table II.B-1 indicates an emission unit is not in compliance with the measurement requirements, please refer to Chapter II, Section II.E.5 for the Compliance Plan and Appendix B for the Compliance Schedule description.

\section{II.B.2. Nonradioactive Airborne Emissions and Notice of Construction Review}

Ecology regulates the nonradionuclide air emissions of regulated pollutants on the Hanford Site. A list of the nonradionuclide regulated pollutants is provided in Chapter V, Table V.A-2.

Table II.B-2 provides regulations and emission limitations of the regulated pollutants to specific emission units. The first part of the table provides the emission unit identification, the emission limits or regulations that apply to the emission unit, the compliance method, and the . compliance status. This table was designed to follow the format specified in Form C-2.

The second part of Table II.B-2 provides the list of emission units or projects with applicable requirements imposed through the Notice of Construction approval process. Included are the conditions of approval, allowable limits, the monitoring requirements, and a compliance demonstration.

Where indicated that an emission unit is not in compliance with the applicable requirement, refer to Chapter II, Section II.E.5 for the Compliance Plan and Appendix B for the Compliance Schedule description.

\section{II.B.3. Special Requirements}

Table II.B-3 identifies those requirements that fall under the jurisdiction of the local air pollution control authority. These requirements include such regulations as open burning and asbestos control. These items are included for completeness only because the local air pollution authority is not required to issue approval for the Application. 


\section{II.C. EXEMPTIONS}

The DOE-RL is not requesting any exemptions for the Hanford Site.

\section{II.D. INAPPLICABLE REQUTREMENTS}

The DOE-RL has compiled inapplicable requirements in Table II.D-1. The permit shield for inapplicable requirements is as follows:

Permit Shield Request:

The DOE-RL requests that it and its contractors be shielded from enforcement actions where identified rules are not applicable to a source.

\section{II.E. COMPLIANCE PLAN}

\section{II.E.1. Status}

The status of compliance with applicable requirements is provided in Tables II.B-1, II.B-2, and II.B-3 for source specific requirements.

\section{II.E.2. Compliance Methods}

\section{II.E.2.1 Insignificant Emission Units and Activities.}

The following limits or standards apply to insignificant emission units and activities on the Hanford Site. Compliance with the standards is required, however, no monitoring is required for insignificant emission units. The applicable requirements for radionuclide air emissin units is provided in the Emission Point Compliance Demonstration portion of Table II.B-1.

Applicable requirements Pollutant/ Limit/ Monitoring/ Compliance Method

Non-radiological emission units

WAC 173-400-040(1)

WAC 173-400-040(2)

WAC 173-400-040(3)

$\begin{array}{lll}\text { Opacity } & 20 \% & \text { None } \\ \text { Fallout } & \text { BMP } & \text { None } \\ \text { Fugitive } & \text { BMP } & \text { None } \\ \text { emissions } & & \end{array}$


WAC 173-400-040(4)

Odor

BMP

None

WAC $173-400-040$ (6)

$\mathrm{SO}_{2}$

WAC $173-400-040(8)$

Fugitive dust

$1,000 \mathrm{ppm}$

BMP

None

None

\section{II.E.2.2 Significant Emission Units and Activities}

The following limits or standards apply to emission sources not categorically exempt from inclusion in this Application or identified as insignificant emission units in Chapter IV. The limits also do not apply if an alternative limit or standard for the same pollutant is identified for an emission unit listed in the Notice of Construction Review portions of Tables II.B-1 or Table I.B-2. The applicable requirements for radionuclide air emissin units is provided in the Emission Point Compliance Demonstration portion of Table II.B-1.

Applicable requirements

$$
\begin{array}{cll}
\text { Pollutant/ } & \begin{array}{l}
\text { Limit/ } \\
\text { standameter }
\end{array} & \begin{array}{l}
\text { Monitoring/ } \\
\text { Compliance } \\
\text { Method }
\end{array} \\
\hline
\end{array}
$$

Frequency

Non-radiological emission units

WAC 173-400-040(1)

Opacity $\quad 20 \%$

Visual surveys

$1 / 6$ months

$\&$ excess emission reporting

WAC 173-400-040(2)

Fallout

BMP

Complaint investigation

\& recordkeeping

WAC $173-400-040(3)$

Fugitive

BMP

emissions

Visual survey, complaint investigation

$\&$ recordkeeping

WAC 173-400-040(4) Odor

BMP

Complaint investigation

\& recordkeeping 


\section{Opacity Monitoring}

Opacity monitoring for emission units will consist of a combination of visual surveys and excess emission reporting. Excess emission reporting will be according to the procedures previously established with Ecology.

\section{$\underline{B M P}$}

Best management practices (BMP) to control fugitive particulate emissions and fugitive dust include operational control programs such as wetting disturbed soil areas using water trucks, water wagons, or sprinkler systems to reduce fugitive dust and fugitive emissions.

$\mathrm{SO}_{2}$

This emission standard applies to all Hanford Site emission units. Not all Hanford Site emission units are tested for $\mathrm{SO}_{2}$ for the following reasons. It is assumed that the emission points on the Hanford Site with the greatest potential to emit $\mathrm{SO}_{2}$ are the combustion sources. Source tests are performed periodically at the Hanford Site steam generating facilities. The 200 Area coal-fired boilers are tested biannually and the results of the last test are presented in the Emission Point Compliance Review section of Table II.B-2 and demonstrate the these emission points are well below the $1,000 \mathrm{ppm}$ standard. The 200 Area package boiler, emission point $200 \mathrm{~W} \mathrm{F-284W} \mathrm{005,} \mathrm{test} \mathrm{requirements} \mathrm{are} \mathrm{also} \mathrm{identified} \mathrm{in} \mathrm{the} \mathrm{Notice} \mathrm{of}$ Construction Review section of Table II.B-2.

The oil-fired boilers in the 300 area are the largest combustion units in terms of No. 6 fuel oil usage. It is also assumed that No. 6 fuel oil represents the fuel with the highest sulfur content burned on the Hanford Site. The $\mathrm{SO}_{2}$ emissions from 300 F-384 005 were measured during a recent source test conducted on February $25-26,1995$ with written results of the tests received on about May 3,1995. This test of the 300 Area boiler demonstrated that the $\mathrm{SO}_{2}$ emissions from emission point 300 F-384 005 was 1,060 ppm, slightly higher than the 1,000 ppm limit. The results are reported in the Emission Point Compliance Review section of Table II.B-2. The compliance schedule milestone is presented in Appendix B. Based on the above assumptions and testing results described above, DOE/RL believes other Hanford Site combustion sources and emission units not burning No. 6 fuel oil, e.g. internal combustion engines, are in compliance with the $1,000 \mathrm{ppm} \mathrm{SO}_{2}$ limit.

\section{Frequency}

The frequency of Method 9 visual emission surveys shall be once every six months (1/6 months). The survey will be performed during normal operating conditions. This emission survey will document that the facility complies with the opacity standard during normal operations.

The limits or standards and the corresponding monitoring or compliance methods listed in this section apply to the following emission units unless an alternative limit is identified in Table II.B-1 or Table II.B-2. Historic reviews of the coal and oil-fired boilers have revealed that these units are grandfathered from the New Source Performance Standards. It has also been 
determined that there have been no past modifications to the boilers that would have required Prevention of Significant Deterioration review or permitting.

Emission Unit

200E F-284E 001

200E F-284E 002

200E H-200E002 001

200E K-200E001 001

200W F-284W 001

200W F-284W 002

200W H-200W007 001

200W K-200W003 001

100D F-525 001

200W E-947 001

200E E-670 001

200E E-500 001

200E E-600 001

200E E-565 001

200E E-565 002

300 E-900 001

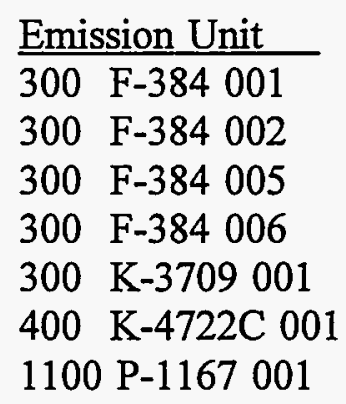

400 E-1500 002 (DG-2)

400 E-1500 001 (DG-1)

400 E-425 001 (G-3)

$300 \mathrm{E}-1000001$

300 E-1450 001

300 E-900 003

300 E-900 002

\section{II.E.3. Compliance Certification and Reporting Requirements}

\section{II.E.3.1. Semiannual report}

The semiannual report will contain the results of required and enhanced monitoring. The report will contain the monitoring results in curies from the radionuclide emission units designated as major stacks according to the most current quarterly report required by the compliance plan schedule (Appendix B).

The semiannual report also will include the semiannual requirement to report of portable and temporary radionuclide airborne emission unit (PTRAEU) usage. This report will also include monitoring results from enhanced monitoring programs when these programs become applicable to Hanford Site nonradionuclide major emission units. This report will also contain a compliance certification. For the period January 1 through June 30, the compliance certification will be provided by August 30 .

\section{II.E.3.2. Annual Report}

The annual report will consist of the annual Radionuclide Air Emission Report required by 40 CFR 61 Subpart H and WAC 246-247. The report also will consist of the Hanford Site 
annual report of emissions from the boilers, including the annual source test results for the 200 West Backup Boiler. The report also will consist of the second semi-annual PTRAEU report of usage. The annual report also will contain the second semi-annual compliance certification. For the period July 1 through December 31 , the compliance certification will be provided by June 30 to coincide with other required reporting requirements.

\section{II.E.4. Enhanced Monitoring}

The enhanced monitoring rule is not final at the time of Application preparation. When finalized, this rule will apply to major sources of hazardous air pollutant emissions. Those emission units that are affected by the enhanced monitoring rule will be reviewed at that time to determine compliance status with the requirements and the feasibility of applying the provisions to the units. The DOE-RL will comply with the requirements or terminate operation of the emission units within the timeframe allowed by the regulation, or develop and agree upon a compliance plan.

The emission units that might be impacted by the enhanced monitoring rule, when finalized, are as follows. (This list is not inclusive. Pending the final enhanced monitoring rules, this list might change.)

\begin{tabular}{|c|c|c|c|}
\hline Emission point & Pollutant & Emission point & Pollutant \\
\hline 200E F-284E 001 & $\begin{array}{l}\text { Carbon monoxide } \\
\mathrm{NOx} \\
\mathrm{SO}_{2}\end{array}$ & 300W F-384E 002 & $\begin{array}{l}\text { Carbon monoxide } \\
\mathrm{NOx} \\
\mathrm{SO}_{2}\end{array}$ \\
\hline 200E F-284E 002 & $\begin{array}{l}\text { Carbon monoxide } \\
\text { NOx } \\
\mathrm{SO}_{2}\end{array}$ & 300W F-384E 005 & $\begin{array}{l}\text { Carbon monoxide } \\
\mathrm{NOx} \\
\mathrm{SO}_{2}\end{array}$ \\
\hline 200W F-284E 001 & $\begin{array}{l}\text { Carbon monoxide } \\
\text { NOx } \\
\mathrm{SO}_{2}\end{array}$ & 300W F-384E 006 & $\begin{array}{l}\text { Carbon monoxide } \\
\mathrm{NOx} \\
\mathrm{SO}_{2}\end{array}$ \\
\hline 200W F-284E 002 & $\begin{array}{l}\text { Carbon monoxide } \\
\mathrm{NOx} \\
\mathrm{SO}_{2}\end{array}$ & & \\
\hline 200E H200E002 001 & Carbon monoxide & 200W H-200W007 001 & Carbon monoxide \\
\hline
\end{tabular}




\section{II.E.5. Compliance Plan and Schedule}

The following is the compliance plan for the Hanford Site. The compliance schedule is located in Appendix B.

\section{UNITED STATES ENVIRONMENTAL PROTECTION AGENCY, REGION 10 AND \\ WASHINGTON STATE DEPARTMENT OF ECOLOGY \\ AND \\ WASHINGTON DEPARTMENT OF HEALTH}

In the Matter of:

U.S. DEPARTMENT OF ENERGY

Richland Operations Office

Richland, Washington

$\begin{array}{ll} & \\ \text { FEDERAL FACILITY } \\ \text { COMPLIANCE PLAN } \\ \text { FOR WASHINGTON STATE } \\ \text { AIR OPERATING PERMIT }\end{array}$

\section{Introduction}

1. The United States Environmental Protection Agency (EPA), Washington State Department of Ecology (Ecology), Washington State Department of Health (DOH) and the U.S. Department of Energy, (DOE) Richland Operations Office (RL) are the parties to this Federal Facility Compliance Plan (Compliance Plan), entered into pursuant to Executive Order 12088, October 13, 1978, (43 Federal Register 47707) and the Clean Air Act (CAA), as amended, 42 United States Code (U.S.C.) 7401 et seq., and Revised Code of Washington (RCW) 70.94 et seq. The Office of Management and Budget and the Department of Justice will be notified of this Compliance Plan consistent with their respective duties under Executive Order 12088 to assure compliance at federal facilities with the environmental laws.

2. This Compliance Plan is entered into by the parties to bring the Hanford Site into compliance with the CAA, as amended, 42 U.S.C. 7401 et seq., and RCW 70.94 et seq., and their implementing regulations at 40 Code of Federal Regulations (CFR) Part 70, State Operating Permit Programs, and WAC 173-401, Operating Permit Regulations. The terms of this Compliance Plan shall apply to and be binding upon EPA, Ecology, $\mathrm{DOH}$ and DOE.

3. DOE shall notify its current agents, employees, and contractors, and any subsequent operating contractors, owners, operators, management, and lessees, who have responsibility for meeting operating permit requirements, of the existence of this compliance plan. DOE shall take all appropriate measures to ensure that its contractors 
performing work under this compliance plan act in a manner consistent with the terms of this Compliance Plan and the CAA and its implementing regulations. Except as stated, this Compliance Plan does not relieve DOE of any legal obligations under the CAA.

4. The duty of DOE to operate its facilities in compliance with the CAA is prescribed in Section 118 of the CAA, 42 U.S.C. 7418 and section 161 of RCW 70.94. Executive Order 12088 was issued to ensure federal compliance with applicable pollution control standards. This Compliance Plan is the "plan" as described in Section 1-601 of Executive Order 12088 to achieve and maintain compliance at the Hanford Site with applicable requirements under the CAA, as amended, its implementing regulations, and Executive Order 12088.

5. DOE agrees that EPA, Ecology and DOH have authority to enter its facilities on the Hanford Site, to bring enforcement actions, and to respond to emergency situations regarding matters covered by this Compliance Plan. DOE specifically reserves the right to contest any determinations, allegations, findings of fact, and conclusions of law in any proceeding other than actions brought by EPA, Ecology and DOH to enter or enforce this Compliance Plan or to respond to emergency situations regarding matters covered by this Compliance Plan. Nothing in this plan shall prevent any person from using for any purpose independent evidence that verifies the statement of facts contained herein.

\section{Definitions}

6. Except as provided below or otherwise explicitly stated herein, the definitions provided in the CAA and its implementing regulations shall control the meaning of the terms used in this Compliance Plan.

7. Compliance Plan shall mean this document and shall include all appendices to this document referred to herein. All such appendices shall be appended to and made an enforceable part of this Compliance Plan.

8. Days shall mean calendar days, unless business days are specified. Any submittal or written statement of dispute that under the terms of this Compliance Plan would be due on a Saturday, Sunday, or holiday shall be due the following business day.

9. DOE shall mean the U.S. Department of Energy, including its Richland Operations Office.

10. DOH shall mean the Washington Department of Health and its authorized representatives. 
11. Ecology shall mean the Washington State Department of Ecology and its authorized representatives.

12. EPA shall mean the United States Environmental Protection Agency, Region 10 and its authorized representatives.

13. Parties shall mean EPA, Ecology, DOH and DOE.

\section{Statement of Facts and Conclusions of Law}

14. DOE is a department, agency or instrumentality of the executive branch of the federal government and must comply with the requirements of the CAA, as amended, 42 U.S.C. 7401 et seq. and RCW 70.94 et seq., and their implementing regulations.

15. The Hanford Site is located in southeastern Washington State and is situated approximately 200 miles northeast of Portland, Oregon; 170 miles southeast of Seattle, Washington; and 120 miles southwest of Spokane, Washington. The site encompasses approximately 560 square miles.

16. The Hanford Site was acquired by the federal government in 1943 for construction and operation of facilities to produce plutonium for the atomic weapons program during and following World War II. Programs at the Hanford Site involve research and development, renewable energy technologies, waste disposal and cleanup of contamination from past practices.

17. The Hanford Site is owned and operated by the United States and was part of the DOE nuclear weapons complex.

18. DOE is an "owner or operator" of a "major source" covered by the air operating permit regulations as those terms are defined at 40 CFR Part 70 and WAC 173-401.

19. On July 21, 1992, EPA promulgated the State Operating Permit Program Regulations-40 CFR Part 70. On December 9, 1994, EPA delegated authority to implement the operating air permit program to Washington State. Under the Air Operating Permit rules and in addition to other requirements, major sources must certify compliance with all applicable CAA regulations. For items not in compliance major sources must schedule corrective actions.

20. WAC 173-401-615 refers to monitoring and related recordkeeping and reporting of major source emissions. These monitoring requirements become effective upon issuance of the air operating permit.

\section{Compliance Plan Schedule}


21. The Compliance Plan Schedule for the Hanford Site is intended to bring the Hanford Site into compliance as expeditiously as practical, pursuant to Section 1-601 of Executive Order 12088, and as set forth in the Compliance Schedule.

$h$ The DOE and EPA have entered into a separate agreement to bring the Hanford Site into compliance with the CAA, as amended, 42 U.S.C. 7401, et seq., and its implementing regulations at 40 CFR 61, National Emission Standards for Hazardous Air Pollutants (NESHAP). The terms of the NESHAP Agreement continue to appy to and to bind the DOE and EPA.

22. The Compliance Plan provides a schedule for DOE to review specified air emission sources to validate that those sources are compliant with all applicable regulations. For applicable requirements with which a source is in compliance, the sources will continue to meet such requirements barring force majeure, funding cuts, accident events, etcetera. The Compliance Plan establishes a schedule for DOE to come into compliance with all applicable regulations where non compliances have been identified and validated. DOE will come into compliance with newly promulgated regulations in a timely manner.

23. The Air Operating Permit Application provides the current list of those emission sources required to be registered under WAC 173-400-100 for the Hanford Site. Inclusion of an air emission source in the Air Operating Permit Application is equivalent to separate registration notification.

24. Prior failure to file miscellaneous reports or notifications for unknown minor modifications are not considered continuing violations of applicable requirements. The Compliance Plan addresses corrective actions designed to assure future compliance with all applicable notification and reporting requirements.

\section{Reporting}

25. DOE shall submit certified semi-annual progress reports to EPA, Ecology and DOH, as appropriate, beginning 180 days after the effective date of this compliance plan, that describe the progress made during the previous 6 months towards meeting the requirements of this compliance plan. The reports shall be submitted by the 10 th day of each month following the last day of the last month of the reporting period until compliance with the requirements contained in the Compliance Schedule has been achieved. The reports shall also indicate compliance or noncompliance with the schedule.

\section{Notification}

26. All communications between DOE, EPA, Ecology and DOH and all documents, progress reports, approvals, and other correspondence concerning the activities 
performed pursuant to the terms and conditions of this Compliance Plan shall be directed through the representatives listed below.

For EPA: (Name, title, address)

For Ecology: (Name, title, address) 


\author{
For DOH: $\quad$ Susan May, \\ Department of Health \\ Radiation Protection Division \\ Airdustrial Park, Bldg. 5 \\ P. O. Box 47827 \\ Olympia, WA $98504-7827$
}

For DOE: Paul Krupin, Acting Air Permitting Specialist

U.S. Department of Energy

Richland Operations Office

P.O. Box 550

Richland, Washington 99352

\title{
VII. Extensions
}

27. Any request for an extension of a deadline for 30 days or more shall be made in writing and received by EPA, Ecology or DOH at least 30 days prior to the deadline. EPA, Ecology or DOH will render its decision within 21 days of receipt of the extension request. Any request for an extension of a deadline for less than 30 days shall be made, either orally or in writing, at least seven days prior to the deadline. EPA, Ecology or $\mathrm{DOH}$ will respond to the request, either orally or in writing, prior to the due date. If EPA, Ecology or DOH respond orally they shall confirm their approval in writing. No more than one extension based on an oral request shall be given for each deadline. Any request for an extension shall be provided to the parties in accordance with Section VI (Notification). The request shall specify:

a. The deadline that is sought to be extended.

b. The length of the extension sought.

c. The justification for the extension, and

d. Any subsequent deadlines and/or related schedule(s) that would be affected if the extension was or was not granted.

28. Good cause may exist for an extension when sought in regard to:

a. An event of force majeure (an unexpected or unforeseeable event or circumstance which is beyond the control of DOE and which could not have been overcome by the due diligence of DOE). If DOE requests an extension of a deadline set forth in this Compliance Plan under this paragraph, DOE shall bear the burden of proof that the delay was caused by an event of force majeure. 
1. As soon as DOE becomes aware of an event of force majeure which may necessitate extending a deadline or deadlines, DOE shall notify within 3 business days the EPA, Ecology and DOH representative identified in paragraph 26 in writing. Such notification shall describe the cause and duration of the anticipated delay, the measures taken or to be taken to mitigate the anticipated delay, and the schedule for implementation of mitigation measures.

2. If EPA, Ecology or DOH determine that an event of force majeure necessitates extension of a deadline or deadlines, EPA, Ecology and DOH shall modify the associated deadline(s), as necessary, to conform with the delay.

3. If EPA, Ecology and DOH determine that the delay or anticipated delay has not been or will not be caused by a force majeure event, the existing deadline(s) shall remain in force. EPA, Ecology and DOH shall notify DOE of their determination in writing.

4. In the event that DOE disagrees with any determinations made by EPA, Ecology or DOH pursuant to this Section, DOE may utilize the Dispute Resolution section (Section IX) of this Compliance Plan to resolve such dispute.

b. A delay caused by the good faith invocation of dispute resolution or the initiation of administrative or judicial action.

c. A delay caused, or which is likely to be caused, by the grant of an extension in regard to another schedule.

d. A delay caused by additional work agreed to by the parties, and

e. Any other event or series of events mutually agreed to by the parties as constituting good cause.

29. Absent agreement of the parties with respect to the existence of good cause, the parties may seek and obtain a determination through the dispute resolution process whether or not good cause for an extension exists. DOE may invoke dispute resolution within 14 days of EPA's, Ecology's or DOH's decision on the extension request. If DOE fails to invoke dispute resolution within the 14 day period, DOE is deemed to accept EPA's, Ecology's or DOH's nonconcurrence and the existing schedule.

30. If EPA, Ecology or DOH determine that the requested extension is warranted, the parties shall extend the affected schedule accordingly. If EPA, Ecology, or DOH determines that all or part of the requested extension is not warranted, the schedule shall 
not be extended except as set forth in paragraph 26 of this section or in accordance with a determination resulting from the dispute resolution process.

31. When a timely request for an extension is made, EPA, Ecology and DOH shall refrain from requesting judicial enforcement against DOE or any of its contractors of the affected schedule until a decision is reached on whether the requested extension will be approved. Following the grant of an extension, judicial enforcement may be sought only to compel compliance with the schedule as most recently extended.

32. For extension requests by EPA, Ecology or DOH, if DOE does not deny or invoke dispute resolution within 14 days after written notice of the requested extension, the extension shall be deemed approved.

\section{Modification, Assignment and Termination of Compliance Plan}

33. This Compliance Plan may be modified only by agreement of the parties. All modifications shall be in writing and shall be effective when signed by all parties, or, in the case of an oral approval of an extension request, upon oral approval of the extension.

34. Except as otherwise set forth herein, this Compliance Plan shall terminate upon completion of the activities referenced in Section IV (Compliance Schedule).

35. This Compliance Plan may be terminated at any time upon mutual agreement of the parties.

36. Except as otherwise set forth in this Compliance Plan, the parties agree, subject to relevant considerations, including the facts, circumstances, and status of DOE's compliance with this Compliance Plan, to meet and negotiate in good faith the amendment of this Compliance Plan.

37. DOE warrants that it has exercised good faith and due diligence in identifying and providing information regarding major source air emissions at the Hanford Site. In the event that, despite the exercise of due diligence, previously unknown facts and conditions are discovered which demonstrate a material noncompliance with the air emission standards that has not previously been reported or known to EPA, Ecology or DOH, DOE shall notify EPA, Ecology or DOH, as appropriate, orally within 48 hours, and the parties agree to meet within 14 days to negotiate in good faith any modification of this Compliance Plan if needed to incorporate such facts and conditions. Any such modification agreed to under this section shall be governed by the provisions of this Compliance Plan.

38. Upon delegation of authority for 40 Code of Federal Regulation (CFR) 61 from EPA to the Washington State Department of Health (DOH), EPA's rights and responsibilities 
under this Compliance Plan are assigned to Ecology and DOH. No formal written record of the assignment to DOH shall be required from either EPA or DOE; the assignment shall be effected by the general delegation of program authority. Ecology and DOH shall provide to EPA and DOE written acceptance of the assignment within 30 days of delegation.

\section{Dispute Resolution}

39. Except as specifically set forth elsewhere in this Compliance Plan, if a dispute arises among the parties to this Compliance Plan, the procedures of this section (IX. Dispute Resolution) shall apply.

40. If a dispute arises, the disputing party/parties shall engage the other parties of interest in informal dispute resolution. Such interaction shall be between the appropriate EPA, Ecology, DOH and DOE representatives identified in Section VI (Notification) or their immediate supervisor for no more than 30 days. During this informal dispute resolution period, EPA, Ecology or DOH and DOE shall meet with each other and as many times as necessary to discuss and attempt resolution of the dispute.

41. Within 30 days (as identified in paragraph 40) after any action which leads to or generates a dispute, the disputing party shall submit to the other party/parties of interest a written statement of dispute setting forth the nature of the dispute, the work affected by the dispute, the disputing party's position with respect to the dispute and the information which the disputing party is relying upon to support its position.

42. If the dispute is not resolved informally between the interested parties, the disputing party shall forward the written statement of dispute to the Dispute Resolution Committee (DRC) for resolution. The written statement of dispute shall set forth the nature of the dispute, the disputing party's position, with respect to the dispute, the information the disputing party is relying on to support its position, and a description of the steps taken to try to resolve the dispute. Upon submission of a dispute to the DRC, the other party/parties of interest shall, within 14 days, submit a written statement formally establishing its/their position on the dispute.

43. The DRC shall serve as a forum for disputes that have not been resolved informally. The DRC shall be composed of one representative and one alternate representative from EPA, Ecology, DOH and DOE. The individuals designated to the DRC Committee shall be employed at the policy level or delegated authority to participate on the DRC for the purposes of dispute resolution. 
EPA's representative shall be the (title).

Ecology's representative shall be the (title).

DOH's representative shall be the (title).

DOE's representative shall be the (title).

If any delegation of this responsibility is made by a designated DRC representative, notification of such delegation shall be supplied to the other parties. The DRC Committee shall elect a Dispute Chairperson for each dispute.

44. Following elevation of a dispute to the DRC, the DRC shall have 21 days to unanimously resolve the dispute and issue a written position. If the DRC is unable to unanimously resolve the dispute within this 21-day period, the DRC Dispute Chairperson shall issue a written position on the dispute by the 28th day following submittal. Within 14 days after receipt of the DRC Dispute Chairperson's written position, The disputing party may submit a written notice of dispute to the Senior Executive Committee (SEC) for resolution. In the event that the dispute is not escalated to the SEC within the designated 14-day period, disputing party shall be deemed to have agreed with the DRC position with respect to the dispute.

45. The SEC shall serve as the forum for resolution of disputes for which no resolutions has been achieved in the DRC. The SEC shall be composed of a representative from EPA, Ecology, DOH and DOE.

EPA's SEC representative shall be the Administrator of EPA for Region 10.

Ecology's SEC representative shall be the Director of Ecology.

DOH's SEC representative shall be the Director of DOH.

DOE's SEC representative shall be the Richland Field Operations Manager.

The SEC representatives shall meet, confer, and make their best efforts to resolve the dispute and issue a written decision. If unanimous resolution of the dispute is not reached within 21 days, the Ecology Director shall issue a written position on the dispute within 14 days following the 21-day resolution process. Upon request and prior to resolution of the dispute the Ecology Director may meet with DOE and other interested parties to discuss the matter, but any such meeting will not automatically extend the deadline by which the director must make a decision. Time for issuing a final decision may be extended upon notice to the other parties. If the dispute involves a decision where EPA or DOH serve as the lead agency, the Ecology Director will consult with the Administrator of EPA Region 10 or the Director of the DOH before 
issuing a final decision. In the case of $\mathrm{DOE}$, all parties agree that this final decision or determination shall be deemed to have been decided as an adjudicative proceeding and that DOE may challenge Ecology's final decision or determination as provided by and subject to the standards contained in Chapter $34.05 \mathrm{RCW}$. If DOE objects to the decision or determination, DOE may file an appeal, at DOE's discretion in either the Pollution Control Hearings Board (PCHB) or in the courts. If DOE elects to file an appeal from the decision directly in the courts, Ecology agrees it will not raise an argument that initial jurisdiction of the matter should be with the PCHB.

46. DOE may, within 14 days of the receipt of the SEC written position, issue a written notice elevating the dispute to the Administrator of EPA, Ecology or DOH for resolution in accordance with all applicable laws and procedures. If DOE does not elevate the dispute to the Administrator of EPA, Ecology or DOH within the timeframe designated, DOE shall be deemed to have agreed with the SEC written position with respect to the dispute.

47. Upon escalation of a dispute to the Administrators pursuant to this section, the Administrators will review and resolve the dispute within 21 days. Upon request, and prior to resolving the dispute, the EPA, Ecology or DOH Administrators shall meet and confer with the Secretary of Energy to discuss the issue(s) under dispute. Upon resolution, the EPA, Ecology or DOH Administrator shall provide DOE with a written final decision setting forth resolution of the dispute.

48. The pendency of any dispute under this Section shall not affect the parties' timely performance of their respective responsibilities pursuant to this Compliance Plan, except that the time period for completion of work affected by such dispute(s) shall be extended for a period of time not to exceed the actual time taken to resolve any good faith dispute in accordance with the procedures specified herein. All elements of the work required by this Compliance Plan which are not affected by the dispute shall continue and be completed in accordance with the applicable schedule. The determination of elements of work, submittal, or actions affected by the dispute shall be determined by EPA, Ecology, DOH and DOE pending final resolution of the dispute.

49. When dispute resolution is in progress, work affected by the dispute will be immediately discontinued if the EPA, Ecology or DOH DRC representatives request, in writing, that work related to the dispute be stopped because, in EPA's, Ecology's or DOH's opinion, such work is inadequate or defective, and such inadequacy or defect is likely to yield an adverse effect on human health or the environment. To the extent possible, EPA, Ecology or DOH shall give the DOE DRC representative prior notification that a stop-work request is forthcoming. After stopping work, if DOE believes that the stop-work request is inappropriate or may have potential significant adverse impacts, DOE may meet with the EPA, Ecology and DOH DRC representatives to discuss the stop-work request. Following this meeting, and further consideration of the issues, the EPA, Ecology or DOH DRC representative will issue, in writing, a final 
decision with respect to the stop-work request. The final written decision to stop work may be immediately subjected to formal dispute resolution. Such dispute resolution may be brought directly to either the DRC or the SEC, at the discretion of DOE.

50. Within 30 days following the resolution of a dispute pursuant to the procedures specified in this section, DOE shall incorporate the resolution and final determination into the appropriate plan, schedule, or procedures and proceed to implement this Compliance Plan according to the amended plan, schedule, or procedures.

51. Resolution of a dispute pursuant to this section of the Compliance Plan shall be in accordance with all applicable laws, regulations, and requirements and constitutes a final resolution of any dispute arising under this Compliance Plan. The parties shall abide by all terms and conditions of any final resolution of dispute obtained pursuant to this section of the Compliance Plan. Such resolution shall contain a finding as to whether or not the invocation of this section was made in good faith.

\section{Funding}

52. DOE shall take all necessary steps to obtain timely funding in order to fully meet its obligations under this Compliance Plan. This shall be accomplished in the following manner:

A. In its annual budget request. DOE shall include estimated funding levels required to achieve full compliance with this agreement.

B. In the process of formulating its annual budget request, DOE may be subject to target funding guidance directed by the Office of Management and Budget (OMB). When DOE's target budget case differs from its full compliance funding case, the Parties agree to attempt to reach agreement regarding workscope, priorities, schedules/milestones, and Activity Data Sheet (ADS) funding levels required to accomplish the purpose of the Compliance Plan, provided satisfactory progress has been made in controlling costs in accordance with the cost efficiency initiatives. These discussions shall be conducted before DOE-RL submits its annual budget request and supporting ADSs to DOE Headquarters (DOE-HQ) under signature of the DOE-RL manager.

C. DOE-RL will submit its budget request with detailed ADSs, identifying both target and compliance funding levels, to DOE-HQ and identify any unresolved issues raised by Ecology and EPA. If these issues are not subsequently resolved prior to DOE's submission of its budget request to OMB, DOE-HQ will also identify these issues and the funding required for compliance to OMB.

D. In determining the workscope, priorities, and schedules, the Parties shall consider the values expressed by the Hanford stakeholders. 
E. The Parties recognize that successful implementation of this Compliance Plan is dependent upon the prudent use of resources, and that resource requirements and constraints should be considered during the work planning, budget formulation, and budget execution process. To ensure the development of responsible budget requests, consistent with the requirements of this Compliance Plan and applicable federal/state statutes, the Parties will work cooperatively and in good faith.

53. The purpose of this paragraph is to establish a mechanism that will help assure adequate progress toward meeting the requirements of this Compliance Plan. It provides for communication and consultation on work scope, priorities, schedules/milestones, and cost/funding matters. It further provides a means for performance measurement and for early identification of problems which could jeopardize compliance with the schedules and milestones of the Compliance Plan.

A. Within two weeks after DOE Headquarters (DOE-HQ) issuance of Environmental Management planning and/or budget guidance, including target level funding guidance, to the Richland Operations Office (DOE-RL), DOE-RL shall provide a copy of it to DOH, Ecology and EPA along with a preliminary assessment of its impacts. DOE-RL shall also provide a copy of its initial contractor budget guidance to $\mathrm{DOH}$, Ecology and EPA within two weeks after issuance.

B. DOH, EPA and Ecology agree not to release confidential budget information to any other entities prior to submission by the President of his budget request to Congress, unless authorized by DOE or required to do so by court order. DOE shall seek to intervene in any proceeding brought to compel or enjoin the release of this information. If allowed to intervene, DOE shall assert its interest in, and the legal basis for, maintaining the confidentiality of this information.

C. As soon as possible after DOE-HQ issuance of its initial planning guidance but no later than two weeks prior to DOE-RL's submission of its budget request and supporting Activity Data Sheets to DOE-HQ, DOH, Ecology and EPA shall be given: 1) a management level briefing at the ADS level on the budget request, including workscope, priorities, schedules/milestones, and five year target and compliance cost/funding projections. DOE-RL shall, to the extent it deems appropriate, revise its budget request and ADSs, including workscope, to address or resolve DOH, Ecology and EPA comments prior to transmittal to DOE-HQ. DOE-RL shall notify DOE-HQ in its budget request of any comments not fully resolved to the satisfaction of all Parties, and shall identify full compliance funding levels.

D. Within 30 days after the President's submission of the budget to Congress, DOE-RL shall brief DOH, Ecology and EPA on the President's budget request at the ADS level detail. At this briefing, DOE-RL shall notify DOH, Ecology and EPA of any differences between the target and compliance case workscope and cost/funding levels submitted in accordance with subparagraph C. above, and the actual workscope and funding levels included in the President's budget request to Congress. DOE-RL shall also provide DOH, Ecology and EPA its assessment of the impacts such differences may have on DOE's ability to meet milestones or satisfy other requirements of this Agreement. 
E. DOE shall notify and discuss with DOH, Ecology, and EPA, prior to transmittal to $\mathrm{OMB}$, any budget amendment, supplemental appropriation request or reprogramming request and any corresponding impacts upon the workscope and schedules, and DOE's ability to meet milestones or other requirements of this Agreement with and without the amendment, supplemental appropriation or reprogramming request.

F. Within 30 days after congressional budget appropriation, DOE-RL shall brief DOH, Ecology and EPA on the budget appropriation and subsequent funding allocations for the new fiscal year at ADS level detail. If there is a delay in congressional appropriation after the start of the fiscal year, DOE-RL shall inform DOH, Ecology, and EPA of any congressional continuing resolution action, and the potential impacts, if any, on progress to achieve milestones and other requirements of the Compliance Plan.

$\mathrm{DOH}$, Ecology and EPA will be given timely opportunity to review and comment on these budget appropriation and funding allocation actions, and to make recommendations for reallocation of available funds.

G. If the Congressional budget appropriation differs from the funding levels required to comply with any milestones or other requirements of the Compliance Plan, DOE-RL shall take whatever action is appropriate under the Compliance Plan. The Parties shall attempt to reach agreement on adjustments in workscope or milestones consistent with the Congressional appropriation which will minimize impacts on the requirements of this Compliance Plan. If agreement cannot be reached, DOH, Ecology, and EPA reserve the right to take appropriate action as provided for in this Compliance Plan.

H. DOH, Ecology and EPA project managers shall meet periodically throughout the budget execution year to discus the status of projects to be funded for the current fiscal year, and events that have affected, or may affect milestones or activity within such milestones.

I. In order to ensure continuing, effective and timely interface between DOE, DOH, Ecology and EPA regarding work scope planning/scheduling, budget/funding, current year performance status, milestone tracking, and notification of problem areas, DOE shall, unless otherwise agreed to, provide the following, or their equivalent, to DOH, Ecology and EPA:

1. Annual Multi-Year Program Plans, including ADS level funding projections, as soon as possible after their development;

2. Annual Fiscal Year Work Plans, including ADS level funding profiles, as soon as possible after start of each fiscal year;

3. The monthly Approved Funding Plan (AFP), at ADS level detail, within two weeks following the start of each month;

4. Monthly Site Management System reports shall be provided to DOH, Ecology and EPA to identify: any anticipated delays in meeting time schedules, the reason(s) for such delay and actions taken to prevent or mitigate the delay, and any potential problems that may result in a departure from the requirements and time schedules. In accomplishing this, the SMS reports shall, as a minimum, include for each program: monthly and cumulative budget, actual monthly and cumulative costs, performance 
measurement information including explanations of cost/schedule variances, progress in achievement of milestones, and notification of problems and program/project delays. The appropriate contractor program managers shall sign the monthly Site Management Systems report. The signature block shall contain the statement: "The information contained within this report is complete and accurate to the best of my knowledge." At the monthly milestone review meetings, the appropriate DOE program manager will provide DOE's assessment of milestone progress and the extent to which DOE agrees or disagrees with the preceding month's SMS report. The assessment will be documented in meeting minutes signed by the three parties. With regard to these assessments, signature of the minutes by DOH, Ecology and EPA shall indicate only that the assessment information was provided by DOE. The monthly Site Assessment System report shall also be placed in the Public Information Repositories as identified in Section 2.0 of the Action Plan.

5. Upon request, DOH, Ecology and EPA shall be provided access to available information below the ADS level of detail.

J. During the budget execution year, DOE-RL shall notify DOH, Ecology and EPA of any proposed action to internally reallocate funding at ADS levels, if such an action significantly affects workscope and schedules.

$\mathrm{K}$. Within 30 days following the completion of DOE's annual midyear management review (approximately April-May of each year), DOE-RL shall brief Ecology and EPA on any decisions that significantly affect milestones under this Compliance Plan.

L. As soon as possible following the end of each federal fiscal year, DOE-RL shall provide DOH, Ecology and EPA the fiscal year-end SMS report, and a summary briefing on the amount of funds that have been obligated and spent during the fiscal year ended and the work that has been performed. This summary shall include, at ADS level detail, actual versus planned expenditures for the fiscal year end; a summary of carryover amounts including those available for expenditures in the following budget execution year; and summaries/information explaining the extent of work planned versus work completed or performed during the year.

M. The parties agree to inform and involve the public and stakeholders at key stages of budget formulation and execution consistent with the Interim Report of the Federal Facilities Environmental Restoration Dialogue Committee.

N. The participation by DOH, Ecology and EPA in DOE's planning and budget formulation and execution process shall not affect DOE's authority over its budget and funding level submission.

\section{Other Applicable Laws}

54. All actions required to be taken pursuant to this Compliance Plan shall be undertaken in accordance with the requirements of all applicable local, state, and federal laws and regulations. DOE shall obtain or cause its representatives to obtain all permits and approvals necessary under such laws and regulations. 


\section{Other Claims}

55. Except as specifically provided for in this Compliance Plan, nothing in this Compliance Plan shall constitute or be construed as a release from any other claim, cause of action or demand in law or equity against any person, firm, partnership, or corporation for any liability it may have arising out of or relating in any way to the release of any pollutants or contaminants from the Facility.

\section{Access/Data/Document Availability}

56. EPA, Ecology or DOH will be permitted to enter all areas of Hanford which emit pollutants, or which contain information referred to in this section. EPA, Ecology or DOH will be permitted to inspect records, logs, and other documents relevant to implementation of this Compliance Plan; verify compliance by DOE with this Compliance Plan; review the progress of DOE, its contractors, and lessees in carrying out the activities under this Compliance Plan; conduct tests which EPA, Ecology or DOH deem necessary; and verify data submitted to EPA, Ecology or DOH by DOE. DOE shall honor all requests for access to the Hanford Site made by EPA, Ecology or $\mathrm{DOH}$, so long as the provisions of this section are fulfilled. When on site, EPA, Ecology or DOH shall comply with Occupational Safety and Health Administration (OSHA) rules, where applicable, and DOE site health and safety requirements. EPA, Ecology or DOH access shall be subject to the applicable requirements of the Atomic Energy Act (AEA), 42 U.S.C. 2011 et seq., and Executive Orders concerning the access to, and/or handling of unclassified controlled nuclear information, restricted data, and national security information.

57. DOE has provided to EPA, Ecology and DOH a protocol regarding health, safety, and security requirements at the Hanford Site and all appropriate application forms. The protocol includes a list of all necessary training courses, including agenda, to meet the requirements of the protocol. DOE shall provide notice to EPA, Ecology or DOH via certified mail, of any changes in that protocol at least 60 days before those changes become effective. Should DOE fail to provide Ecology or DOH with the required notice of change in protocol, DOE shall not deny EPA, Ecology or DOH access to the site so long as EPA, Ecology or DOH complies with the protocol in place immediately prior to those changes.

58. Information, records, or other documents produced under the terms of this Compliance Plan shall be available to the public except: (a) those identified to EPA, Ecology or DOH by DOE as classified, or unclassified but controlled, within the meaning of and in accordance with the AEA,

(b) those that could otherwise be withheld pursuant to the Freedom of Information Act or the Privacy Act, unless expressly authorized for release by the originating agency, (c) those still considered to be in draft or unfinished form, (d) those containing attorney 
work-product or attorney-client privileged material, or (e) those subject to business confidentiality claims. Documents or information so identified shall be handled in accordance with applicable regulations. No document marked draft may be made available to the public without prior written approval of the generating party. Unless otherwise restricted by Subsections (a), (b) and (d), if the document is draft final (pending public review) or final and no confidentiality claim under Subsection (e) accompanies information which is submitted to any party, then the information may be made available to the public without further notice to the originating party. EPA, Ecology or DOH reserve its right to seek or otherwise obtain access to such information or facilities in accordance with applicable law.

\section{Civil Enforcement Actions and Reservation of Rights}

59. Based on the facts and circumstances known to EPA, Ecology or DOH as of the effective date of this Compliance Plan, and as set forth in this Compliance Plan, EPA, Ecology and DOH hereby agree not to initiate any civil administrative enforcement action against DOE or any of its contractors, or to refer a civil judicial enforcement action against DOE or any of its contractors to the Department of Justice, for the air emission deficiencies at Hanford that are the subject of this Compliance Plan for so long as DOE is in compliance with the requirements of this Compliance Plan.

60. However, in the event that DOE is delayed in fulfilling its obligations as set forth in this Compliance Plan as a result of insufficient availability of funding, and the parties are unable to agree to an extension of schedules as provided for in Section VII (Extensions), the covenant set forth in paragraph 59 shall terminate in accordance with paragraph 67.

61. The Air Operating Permit Application provides the complete list of those emission sources required to be registered under WAC 173-400-100 for the Hanford Site. Inclusion of an air emission source in the Air Operating Permit Application is equivalent to separate registration notification.

62. Prior failure to file previous minor notifications or reports is not considered a violation of the Air Operating Permit. Included in the Compliance Plan Schedule is a corrective action which will assure that pertinent air management systems and procedures will be assessed and corrected so that failure to file such notices will not occur in the future without the collaboration of EPA, Ecology or DOH.

63. Further, nothing herein shall preclude any actions by EPA, Ecology or DOH to enforce the terms of this Compliance Plan, or to address or bring any legal or equitable claim for (1) any pre-existing, current or future violations or conditions at the facility not specifically covered by this Compliance Plan, or (2) any emergency condition or imminent hazard that may exist or arise at the facility. 
64. This covenant does not apply to any matters not covered by this Compliance Plan.

\section{Severability}

65. If any provision or authority of this Compliance Plan or the application of this Compliance Plan to any party or circumstance is held by a judicial or administrative authority to be invalid, the application of such provisions to other parties or circumstances and the remainder of the Compliance Plan shall remain in force and shall not be affected thereby.

\section{Sanctions}

66. In the event that DOE fails to bring the facility into full compliance with the regulatory requirements as specified in this Compliance Plan within the time periods indicated herein, subject to the Funding, Modification, Extensions, Dispute Resolution Sections or the terms and conditions of this Compliance Plan, EPA, Ecology and DOH reserve the right to pursue any remedies that it may have pursuant to Federal law.

67. In the event that DOE fails to comply with the schedules set forth herein, subject to the Funding, Modification, Extensions Dispute Resolution Sections or the terms and conditions of this Compliance Plan, the parties agree that EPA, Ecology and DOH shall have the right to terminate this Compliance Plan by written notice to the parties.

\section{Termination and Satisfaction}

68. Upon completion of the actions required under Section IV (Compliance Plan Schedule), DOE will submit a final progress report to EPA, Ecology and DOH that describes activities occurring subsequent to the last semi-annual progress report and will certify completion of DOE's obligations under this Compliance Plan. Compliance must be demonstrated by testing and reporting rather than the mere completion of construction of pollution abatement/monitoring facilities.

69. After review of the report and certification, EPA, Ecology and DOH shall issue a written certification of completion to DOE or indicate why DOE has not met the requirements. In the event that EPA, Ecology or DOH dispute that DOE's obligations under this Compliance Plan have been completed, then EPA, Ecology or DOH shall state with particularity the reasons therefore in writing to DOE. Such a statement disputing completion shall be subject to the provisions of Section IX (Dispute Resolution).

70. If EPA, Ecology or DOH fails to certify completion within 90 days of DOE's submittal, then DOE may seek such a certification through the dispute resolution process. 
71. Upon the demonstration of compliance by DOE, there will be a continuing obligation to comply with all applicable requirements of the CAA, as amended, and its implementing regulations.

\section{Effective Date}

72. The effective date of this Compliance Plan shall be the date on which this plan is submitted to Ecology. 


\section{II.F. Operational Flexibility}

\section{II.F.1 Reasonably Anticipated Operating Scenarios}

WAC 173-401-650(1) allows the facility to identify reasonably anticipated operating scenarios. Alternative operating scenarios that are included in the Permit can be implemented without a permit revision. These alternative operating scenarios must comply with all applicable requirements listed below and any terms or conditions of the Permit. In addition, the activities listed below in this section will be reviewed for new source review applicability. NOC's will be prepared for those activities requiring such. Any term or condition of the NOC approval that is a specific requirement of the NOC approval may require a Permit modification. Required Permit modifications will be obtained according to the procedures identified in II.F.3.

Applicable requirements.

WAC 173-400-040 General Standards for Maximum Emissions

WAC $173-401$

Air Operating Permit

WAC $173-460$

Toxic Air Pollutants

WAC 246-247

40 CFR 61 Subpart H NESHAPs for Radionuclides from DOE Facilities

Terms and conditions required by WAC 173-401-650 to be included in the permit include requiring each facility with permitted alternative operating scenarios to record in a $\log$ at the facility the operating scenario it is operating under. The permit shield shall be extended to all terms and conditions of the Permit applied to alternative operating scenarios identified in the Permit.

The following list is a compilation of reasonably anticipated operating scenarios.

\section{II.F.1.1 Environmental Restoration (ER)}

The cleanup of past-practice units will be conducted under the authority of the Comprehensive Environmental Response, Compensation, and Liability Act (CERCLA) or the Resource Conversation and Recovery Act (RCRA) process. CERCLA activities are not included in this Application (CERCLA). CERCLA has been identified as an inapplicable requirement in Table II.D-1, page II.D-1.5. The planned activities that are not conducted under CERCLA authority are listed in the following section and may be considered alternative operating scenarios when air operating permit applicable requirements are involved. The ER program is divided into the following activities:

- Closure of treatment, storage, and disposal (TSD) units (closure of inactive waste sites as defined in the Hanford Facility RCRA permit)

- Investigation and remediation of CERCLA Operable Units

- Decontamination \& Decommissioning (D\&D) activities for certain buildings 
- Surveillance and Maintenance activities for certain buildings Technology Demonstrations

- New cleanup disposal facilities (e.g. Environmental Restoration Disposal Facility) a CERCLA unit

\section{Alternative Operating Scenarios}

1) RCRA TSD unit closure.

Activities included as part of Environmental Restoration at the Hanford site, are closure of Treatment Storage and Disposal Facilities. As closure activities are pursued, emission estimates shall be documented with appropriate NOC applications as necessary. NOC approval conditions may require an Air Operating Permit Application Modification to incorporate the conditions into the Permit as well.

Interim stabilization of the B Ponds is also being conducted or planned. As closure activities are pursued, emission estimates shall be documented with appropriate NOC applications as necessary. NOC approval conditions may require an Air Operating Permit Application Modification to incorporate the conditions into the Permit as well. This operable unit contains five RCRA past practice units and five RCRA TSD waste management units. Interim stabilization (backfilling) activities are being performed for the 216-B-3 Main Pond and the 216-B-3-3 Ditch under a decommissioning work plan approved by Ecology. A closure plan has been submitted to EPA and Ecology.

There are no measurable airborne emissions at these inactive waste sites. As closure activities are pursued, emission estimates shall be documented with appropriate NOC applications as necessary. NOC approval conditions may require an Air Operating Permit Application Modification to incorporate the conditions into the Permit as well.

\section{2) Decontamination and Decommissioning Activities}

D\&D activities are planned or being conducted at certain facilities. Several decontamination methods have been proposed for the upcoming D\&D activities. Methods include but may not be limited to sandblasting, shot-peening, carbon dioxide pelletization, and chemical dissolution. As closure activities are pursued, emission estimates shall be documented with appropriate NOC applications as necessary. NOC approval conditions may require an Air Operating Permit Application Modification to incorporate the conditions into the Permit as well.

D\&D of the following facilities is currently underway as of January 1995: 189-D Mechanical Development Laboratory, 185-D Development Laboratory, 190-D Main Process Pump House, 190-DA Annex Facility, 195-D High Tower, 1250 Shipping and Receiving Warehouse, 1252 Shipping and Receiving Warehouse. D\&D of the following facilities is planned to be completed by the year 2000: 1240 Fabrication Facility, 1241 Plate Shop Facility, 1734N Gas Bottle Dock Storage Building, 105NC Hydrogen Mitigation Building, 104N Facilities 
Auxiliary Shop, 1112N Temporary Badgehouse, 183-C Filter Plant, 183-H Solar Evaporation Basins, and three square miles (area from Vernita Bridge to 100B/C).

\section{3) Surveillance and Maintenance Activities}

Periodic surveillance and routine maintenance are being performed at waste units and facilities that are inactive and awaiting $D \& D$. These inactive facilities and buildings have no measurable airborne emissions at this time. However, as these facilities are scheduled for $D \& D$ an emissions estimate will be conducted for each emission unit and activity. The results of the estimates will be used to determine whether a NOC application is required for the proposed activity. Again, previously unidentified NOC approval conditions will be incorporated into the Permit.

\section{II.F.1.2. PFP}

The normal operating scenarios at PFP are described in the process descriptions in Appendix E.3. The reasonably anticipated alternative operating scenarios are described below.

\section{Alternative Operating Scenarios}

1) Direct denitration of plutonium nitrate solutions using a vertical calciner producing about $20 \mathrm{Kg}$. of $\mathrm{NO}_{2}$ per day. A caustic scrubber will remove the majority of the $\mathrm{NO}_{2}$ prior to release to the atmosphere.

2) Increase the number of sludge stabilization furnaces used for solid sludge stabilization from 2 to up to 13 furnaces or install a semicontinuous furnace design with a similar capacity to the 13 sludge stabilization furnaces.

3) Install a pyrolysis furnace for stabilizing "polycubes" (polystyrene impregnated with plutonium), which would result in emissions of styrene and its combustion products.

4) Restart of glovebox HC-60 hydrolysis, which hydrolyzes organic-contaminated plutonium residues using boiling sodium hydroxide solution.

5) Nitric acid washing of contaminated surfaces in the 236-Z Canyon and possibly other areas. This process produces nitric acid fumes and NOx. This has historically been a routine PFP activity.

6) Operation of a solution concentrator in $236-\mathrm{Z}$ if large scale washing is conducted.

\section{II.F.1.3 Powerhouses}

The normal operating conditions at the powerhouses are described in Appendices D-1, E-2, and F-2. The reasonably alternative operating scenarios are described below. 


\section{Alternative Operating Scenario}

1) Portable package boilers to provide replacement steam or backup steam during outages or peak demand periods may be operated at numerous locations in the 200 and 300 areas. These package boilers may use diesel fuel, fuel oil, propane, or natural gases as the fuel. If the proposed package boilers operate on diesel fuel or fuel oil, they will be similar in design and operating characteristics to the package boiler identified as emission point 284W F-284W 005 in Appendix E-2. The applicable requirements will be the same as those described in Table II.B-2, for the 200 West Area package boiler.

\section{II.F.1.4 Rotary Mode Core Sampling Operations (RMCS)}

The normal operating conditions for the RMCS operations is described in Appendix J-1 and includes one rotary mode core sampling unit. The following describe alternative operating scenarios for Tank Farm RMCS purposes.

\section{Alternative Operating Scenarios}

1) The Rotary Mode Core Sampler described in Appendix J-1 is identified as emission point number 200 P-CORE 001 . There are currently plans to procure two additional RMCS sampling units. These units will be similar in design and function to the unit described in Appendix J-1. The emission unit numbers will be identified as $200 \mathrm{P}-\mathrm{CORE} 002$ and $200 \mathrm{P}-$ CORE 003. The applicable requirements will be identical to the requirements described in Table II.B-1, page II.B-1.24 and Table II.B-2, pages II.B-2.34 through II.B-2.37.

\section{II.F.1.5 Tank Farms}

The normal operating conditions at 200 East and 200 West Area Tank Farms are described in Appendices D-4 and E-7 respectively. The following are alternative operating scenarios for Tank Farms.

\section{Alternative Operating Scenarios}

1) This scenario involves the removal of the mixer pump in Tank 241-SY-101. The pollutants of concern that will be emitted during this activitiy are radionuclides. The potential offsite dose resulting from this activity is estimated to be $2.0 \mathrm{E}-06 \mathrm{mrem} / \mathrm{yr}$ to the maximally exposed individual.

2) This scenario involves removing equipment from waste tanks using a flexible receiver assembly. The pollutants of concern are radionuclides and the potential offsite dose from equipment removal using the receiver assembly is $0.083 \mathrm{mrem} / \mathrm{yr} / \mathrm{tank}$. 


\section{II.F.1.6 Solid Waste Management Facilities}

The normal operating scenarios at the Solid Waste Management Facilities are described in Appendix E.8. The reasonably anticipated alternative operating scenarios are described below.

\section{Alternative Operating Scenarios}

1) Perform waste verification activities at any waste management unit. These activities include the opening of waste containers, collecting samples for analysis, and performing in-situ field screening on the wastes.

This activity may involve temporary use of an enclosure, such as a green house or permacon. Depending on the radiological hazards present in the waste, HEPA filtration may be provided on the air venting system of the enclosure.

2) Perform waste sorting and repackaging at any waste management unit. This will involve opening waste containers, sorting the contents, and repackaging into new or original containers.

This activity may involve temporary use of an enclosure, such as a green house or permacon. Depending on the radiological hazards present in the waste, HEPA filtration may be provided on the air venting system of the enclosure.

3) Perform limited waste treatment, such as neutralization, solidification and adsorption, at any wate management unit.

This activity may involve temporary use of an enclosure, such as a green house or permacon. Depending on the radiological hazards present in the waste, HEPA filtration may be provided on the air venting system of the enclosure.

\section{II.F.2 Emissions Trading [WAC 173-401-650(2)]}

WAC 173-401-650(2) allows emissions trading within the facility if the Application includes a request that the Permit include terms and conditions for emissions trading. Any emission trading approved under the authority of this section will apply to emission trades on the Hanford Site only. Emissions trading implies that a facility such as the Hanford Site challenges a site-wide emissions limit or has an emission caps imposed. The $10 \mathrm{mrem} / \mathrm{yr}$ radionuclide standard is the only site-wide emissions limit idenitified in this application. The Hanford Site is several orders of magnitude below the limit, so emissions trading of radionuclide emissions does not seem to provide a significant benefit at this time.

The DOE-RL has not identified the necessity for emissions trades at the time of preparation of this Application and is not requesting permission for specific emission trades. However, as 
new regulations are developed to fully implement the Clean Air Act Amendments of 1990, the ability to trade emissions on the Hanford Site as allowed by the terms and conditions of the Permit may prove to be an important operational flexibility tool. Therefore, the DOE-RL requests that the Hanford Site Air Operating Permit include the terms and conditions to allow emissions trading as identified in WAC 173-401-650(2) without requiring a permit modification.

\section{II.F.3 Permit Modifications [WAC 173-401-725]}

At the time of preparation of the Application, the Environmental Protection Agency (EPA) was revising the 40 CFR 70 regulations pertaining to permit modifications. After 40 CFR 70 is revised, it is assumed that Ecology will revise WAC 173-401 to be consistent with 40 CFR 70. Because the rules governing permit modifications are in transition, it is difficult to predict the effect the rules changes will have on the permit modification process and operational flexibility. As much as possible, NOC applications or other air permitting activities will be conducted concurrently with the Air Operating Permit Modification in order to minimize the time and costs associated with air permitting. Emission units that begin operating or are modified after Permit issuance and determined to be insignificant emission units as described in WAC 173-401 will not be listed in the Permit, eliminating the need for permit modifications for insignificant emission units. As described in II.E.2.1, at least once during the permit term all insignificant emission units will be re-evaluated to confirm that the emission units continue to meet the criteria to be designated as an insignificant emission unit. 
Table IIB-1: Regulations and Regulated Emissions . Air Operating Permit Applications

Facility Name: Hanford Site, Radioactive Emissions

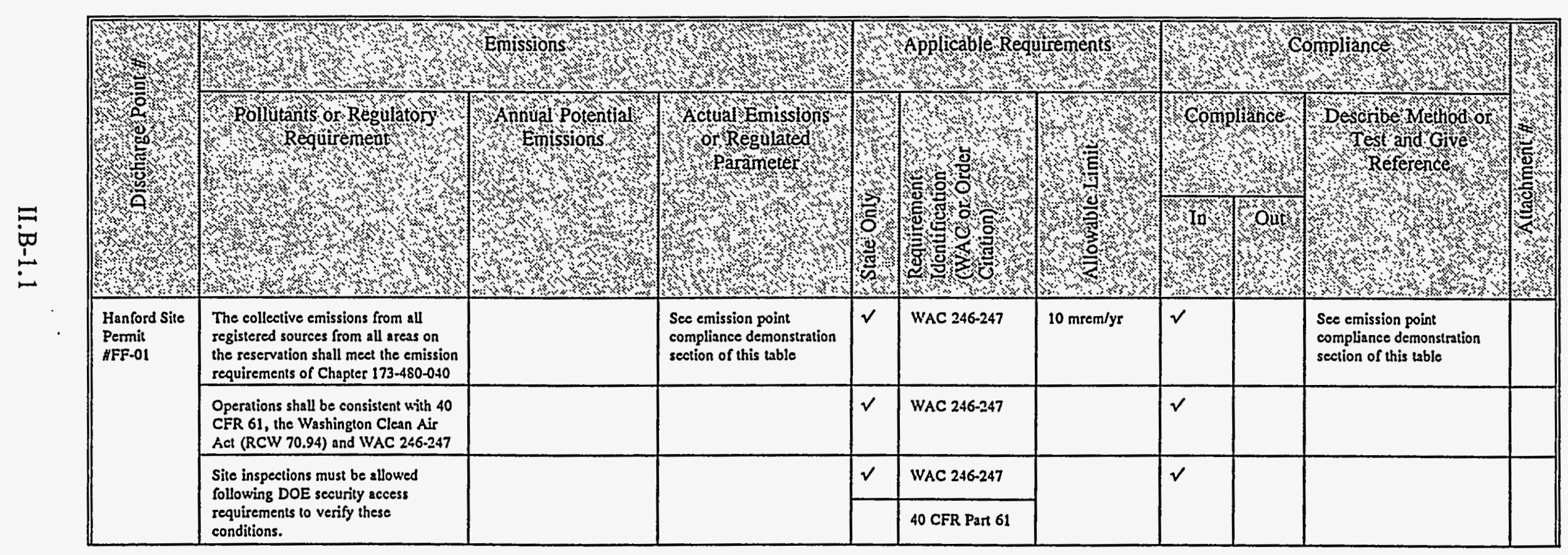



on
ये

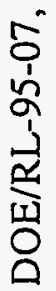

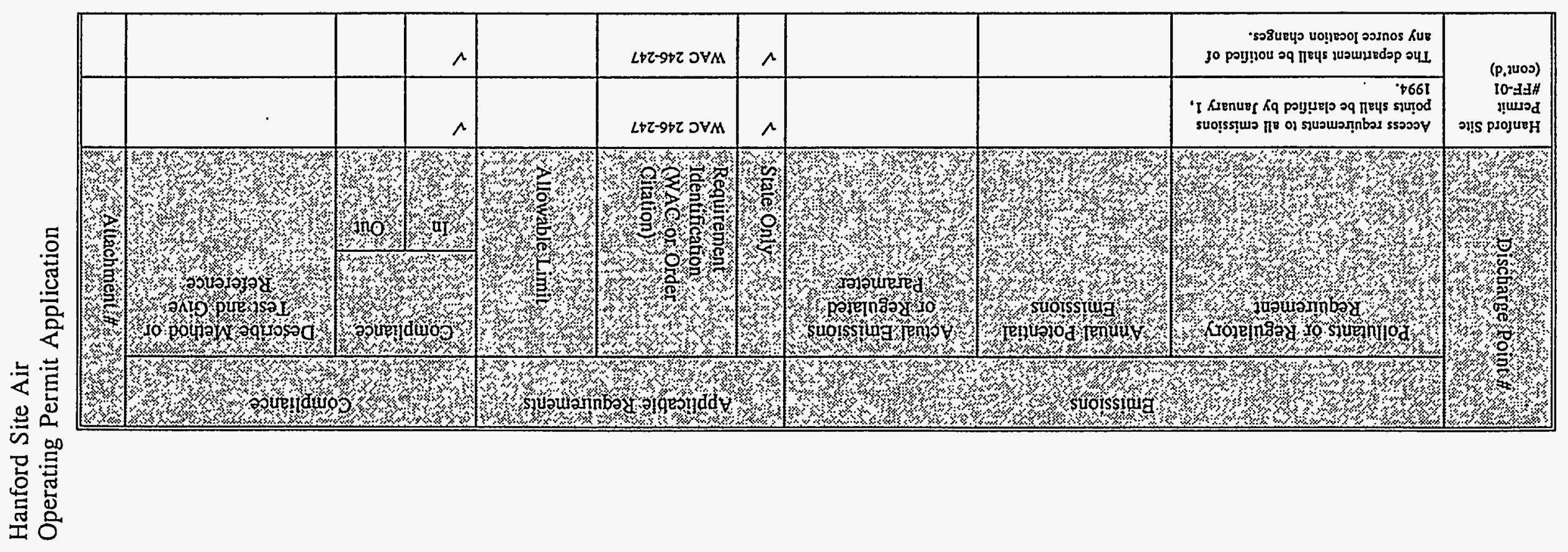

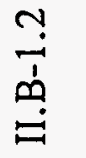


Hanford Site Air

Operating Permit Application

DOE/RL-95-07, Rev. 0
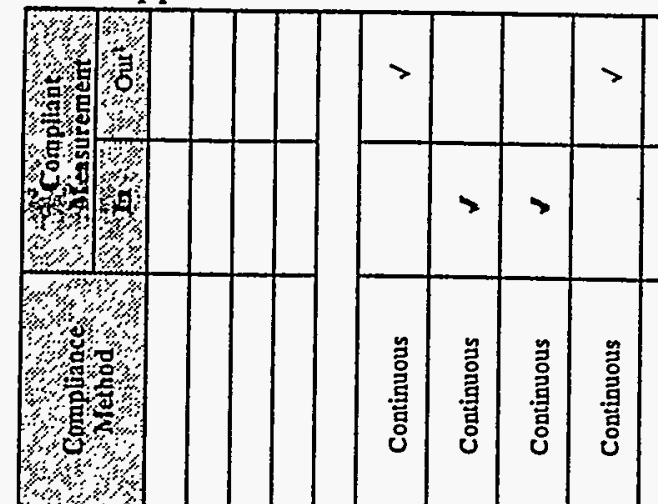

05/95

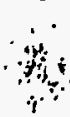

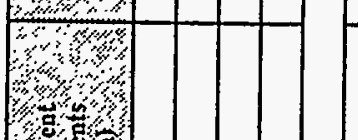

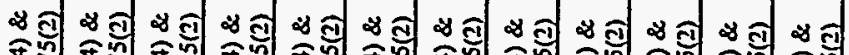

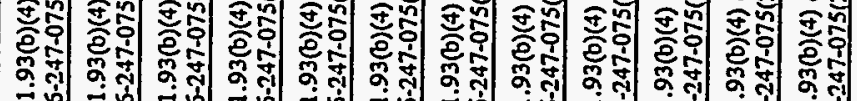

,

\%

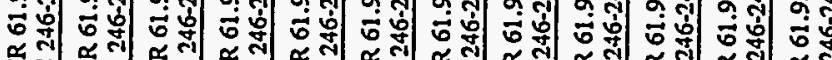

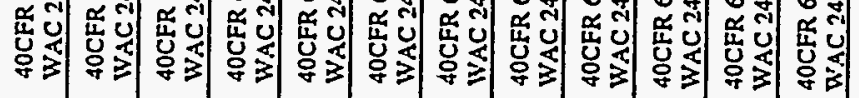

in

$\%$

\%

\%
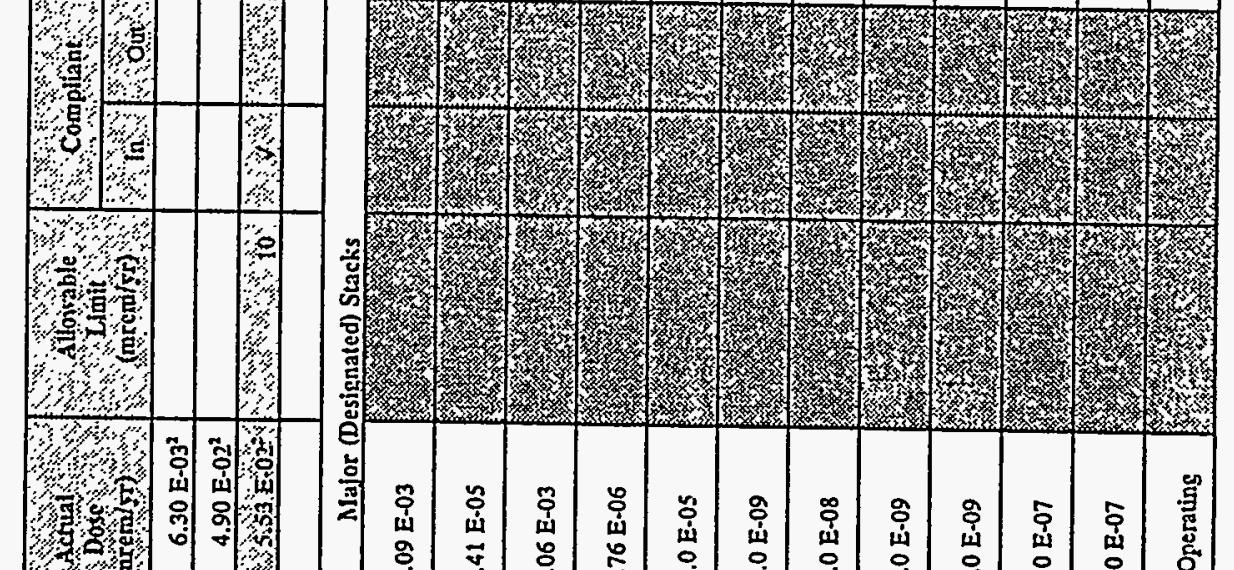

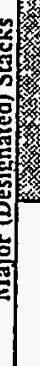

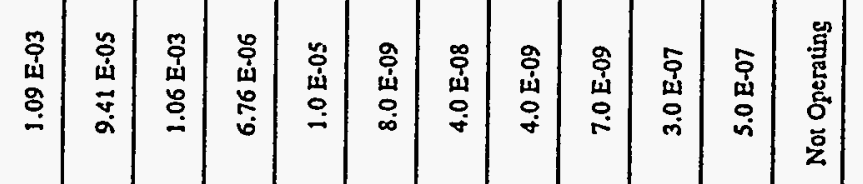

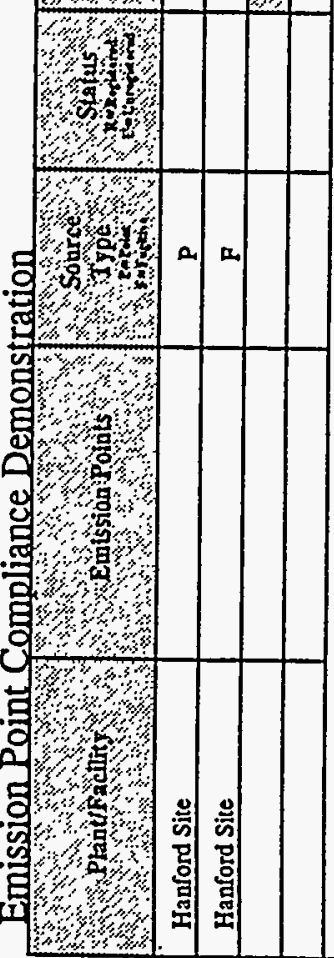

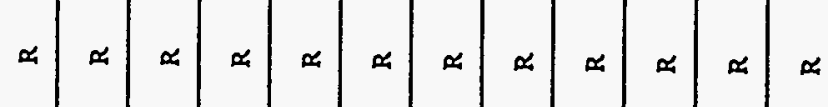

\begin{tabular}{|l|l|l|l|l|l|l|l|l|l|l|} 
& & & & & & & & & & \\
\hline & & & & & & & & & &
\end{tabular}

\begin{tabular}{|lllll|l|l|l|l|l|l|l|}
$\infty$ & a. & $\infty$ & $\infty$ & $\infty$ & $\infty$ & $\infty$ & $\infty$ & $\infty$ & $\infty$ & $\infty$ & $\infty$ \\
\hline
\end{tabular}

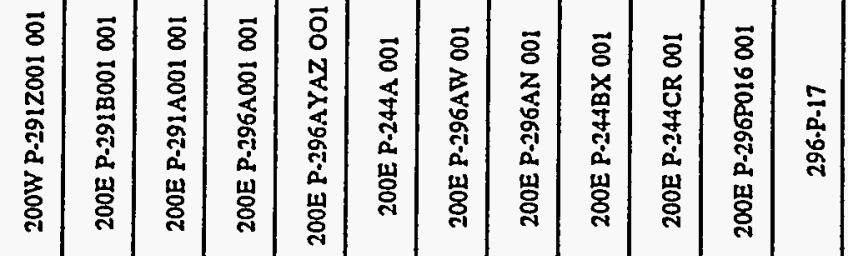




\begin{tabular}{|c|c|c|c|c|c|c|c|c|c|}
\hline 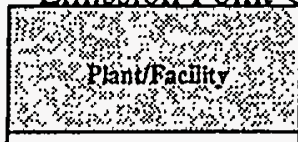 & 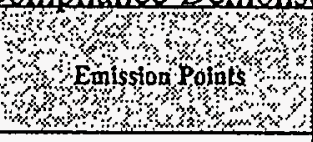 & 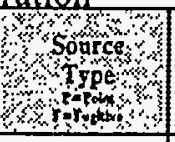 & 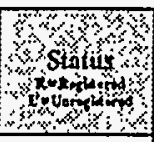 & 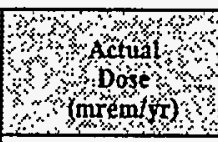 & 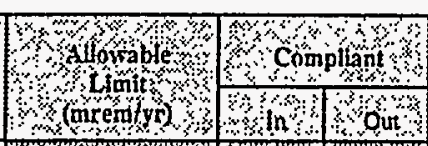 & 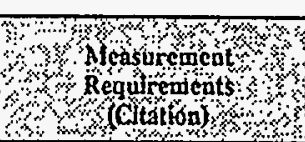 & 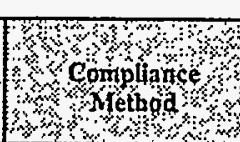 & 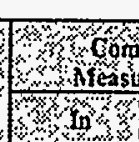 & 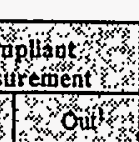 \\
\hline & 200E P-296SY 001 & $\mathrm{P}$ & $\mathbf{R}$ & $3.0 \mathrm{E}-08$ & 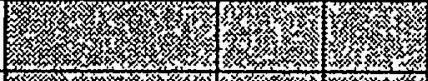 & $\begin{array}{l}\text { 40CFR } 61.93(6)(4) \& \\
\text { WAC 246-247-075(2) }\end{array}$ & Continuous & & $\checkmark$ \\
\hline & EP-296-P-26 & P & u & $1.0 \mathrm{E}-05$ & 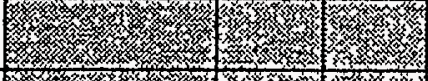 & $\begin{array}{l}\text { 40CFR 61.93(b)(4) \& } \\
\text { WAC 246-247.075(2) }\end{array}$ & Continuous & & $\checkmark$ \\
\hline & EP-296-P-28 & $\mathbf{P}$ & $\mathrm{u}$ & $3.0 \mathrm{E}-08$ & 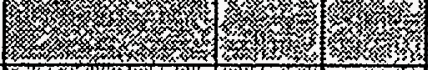 & $\begin{array}{l}\text { 40CFR 61.93(b)(4) \& } \\
\text { WAC 246-247-075(2) }\end{array}$ & Continuous & & $\checkmark$ \\
\hline & 200W P-2965X 001 & P & $\mathbf{R}$ & $2.0 \mathrm{E}-09$ & r.t. & $\begin{array}{l}\text { 40CFR 61.93(6)(4) \& } \\
\text { WAC 246-247-075(2) }\end{array}$ & Continuous & & $\checkmark$ \\
\hline & $200 \mathrm{~W} 244 \mathrm{~S} 001$ & $\mathbf{P}$ & $\mathbf{R}$ & $8.0 \mathrm{E}-08$ & $y_{x}$ & $\begin{array}{l}\text { 4OCFR 61.93(b)(4) \& } \\
\text { WAC 246-247-075(2) }\end{array}$ & Continuous & & $\checkmark$ \\
\hline & 200W 244TX 001 & $\mathrm{P}$ & $\mathbf{R}$ & $2.0 \mathrm{E}-08$ & $y_{3}$ & $\begin{array}{l}\text { 40CFR 61.93(b)(4) \& } \\
\text { WAC 246-247-075(2) }\end{array}$ & Continuous & & $\checkmark$ \\
\hline & EP-296-S-25 & $\mathbf{P}$ & $\mathbf{R}$ & $3.0 \mathrm{E}-08$ & 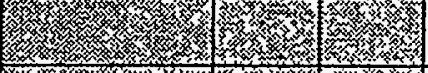 & $\begin{array}{l}\text { 40CFR 61.93(b)(4) \& } \\
\text { W.AC 246-247.075(2) } \\
\end{array}$ & Continuous & $\checkmark$ & \\
\hline & EP-996-P-32 & $\mathbf{P}$ & $\mathbf{R}$ & 3.3 E-12 & 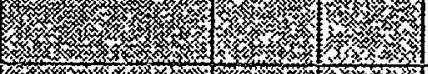 & $\begin{array}{l}\text { 40CFR 61.93(b)(4) \& } \\
\text { WAC 246.247.075(2) } \\
\end{array}$ & Continuous & $\checkmark$ & \\
\hline & EP-296-U-11 & $\mathbf{P}$ & $\mathbf{R}$ & Not Available & 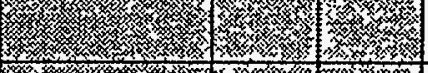 & $\begin{array}{l}\text { 40CFR 61.93(b)(4) \& } \\
\text { WAC 246.247.075(2) }\end{array}$ & Continuous & $\checkmark$ & \\
\hline & 200E P-242A 002 & $\mathbf{P}$ & $\mathbf{R}$ & Not Available & 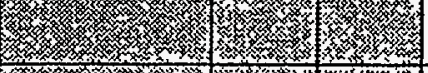 & $\begin{array}{l}\text { 40CFR 61.93(b)(4) \& } \\
\text { WWAC 246-247-075(2) } \\
\end{array}$ & Continuous & $\checkmark$ & \\
\hline & 200E P-:96.AP 001 & P & $\mathbf{R}$ & $2.0 \mathrm{E}-09$ & 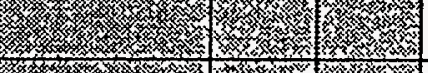 & $\begin{array}{l}\text { 40CFR 61.93(b)(4) \& } \\
\text { WAC 246-247-075(2) }\end{array}$ & Continuous & & $\checkmark$ \\
\hline 300 Area & 300 P-340NTEX 001 & $\mathrm{P}$ & $\mathbf{R}$ & $3.67 \mathrm{E}-06$ & 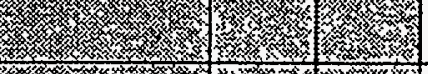 & $\begin{array}{l}\text { 40CFR 61.93(b)(4) \& } \\
\text { WAC 246-247-075(2) }\end{array}$ & Continuous & $\checkmark$ & \\
\hline & EP-324-01-S & P & $\mathbf{R}$ & $6.0 \mathrm{E}-04$ & 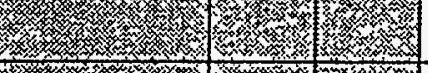 & $\begin{array}{l}\text { 40CFR 61.93(b)(4) \& } \\
\text { WAC 246-247-075(2) } \\
\end{array}$ & Continuous & $\checkmark$ & \\
\hline & EP-325-01-S & $\mathbf{P}$ & $\mathbf{R}$ & $4.0 \mathrm{E}-09$ & 1 & $\begin{array}{l}\text { 40CFR 61.93(b)(4) \& } \\
\text { WAC 246-247-075(2) }\end{array}$ & Continuous & $\checkmark$ & \\
\hline & EP-327-01-S & $\mathbf{P}$ & $\mathbf{R}$ & $3.0 \mathrm{E}-05$ & $1 \times 1 y_{2}$ & $\begin{array}{l}\text { 40CFR 61.93(0)(4) \& } \\
\text { W'AC 246-247.075(2) } \\
\end{array}$ & Continuous & $\checkmark$ & \\
\hline
\end{tabular}


ํ.

这骂

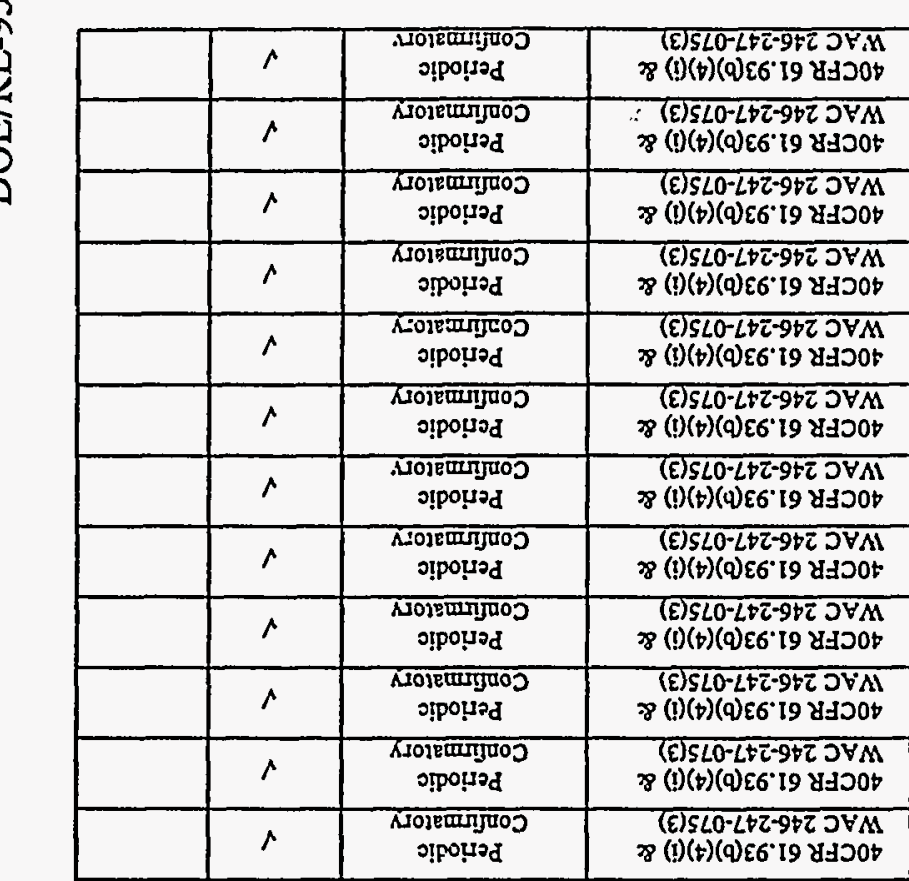

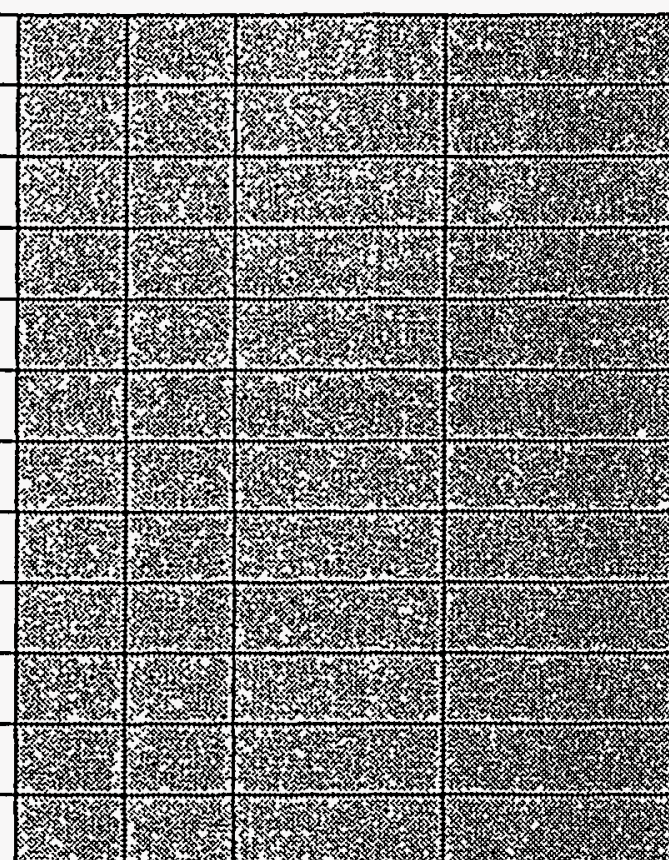

\begin{tabular}{|c|c|c|c|}
\hline $\mathrm{n}$ & d & $100210 \notin 96 i-d 300 \bar{\tau}$ & \\
\hline y & d & $100 \varepsilon 10896 \tau-d] 300 z$ & \\
\hline $\mathbf{y}$ & d & $100010896 \tau-d] 300 \tau$ & \\
\hline y & d & $100500896 \tau-d] 00 z$ & गणधा $\mathrm{Q}$ \\
\hline $\mathrm{n}$ & d & 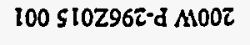 & \\
\hline \& & d & $100 \div 10 Z 96 i-d \mathrm{~d} A 00 \tau$ & \\
\hline y & d & $100900 Z 96 \overline{-d} \mathrm{~A} M 00 \bar{\tau}$ & \\
\hline y & d & $100500 z 96 \tau-d \equiv 00 z$ & \\
\hline y & d & $100 \varepsilon 00 Z 96 \sigma-d \mathrm{~d} M 00 \tau$ & (Jueld Z) dsd \\
\hline$\Omega$ & d & $100 \mathrm{LOOL} 96 \overline{\mathrm{C}} \mathrm{d} \mathrm{M} \mathrm{MOO \tau}$ & \\
\hline y & d & 100 ETOL $96 \tau-\mathrm{d} \triangle \mathrm{AOOZ}$ & \\
\hline y & d & $1001001.16 \tau-d \mathrm{~A} 1002$ & JEr|d I \\
\hline
\end{tabular}

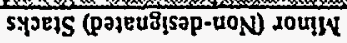

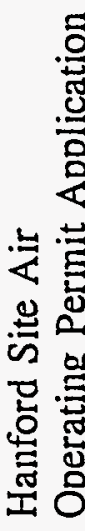

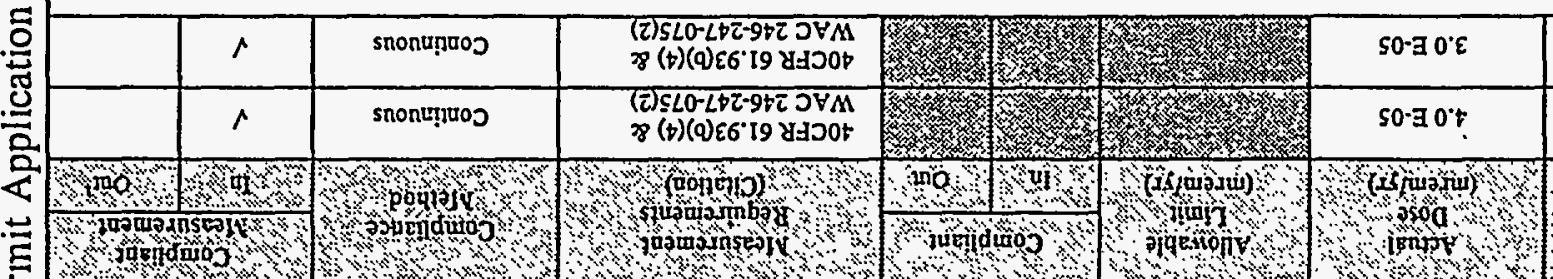

\&

云

는

온

공 
Emission Point Compliance Demonstration

\begin{tabular}{|c|c|c|c|c|c|c|c|c|c|}
\hline 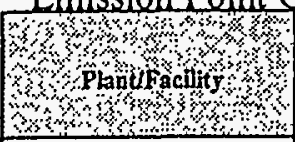 & 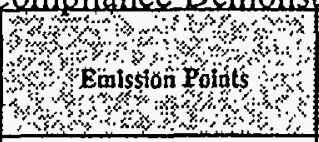 & 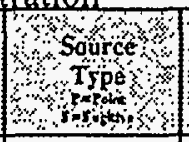 & 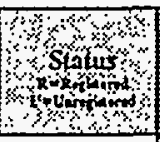 & 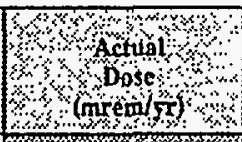 & 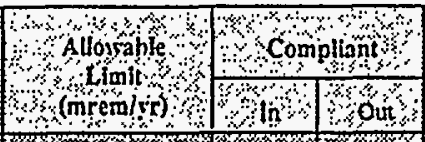 & 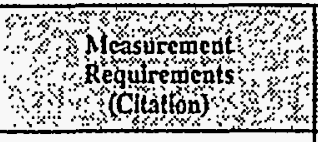 & 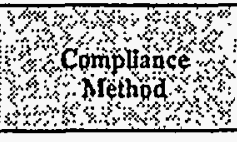 & 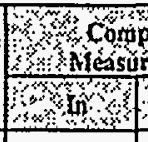 & 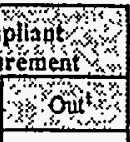 \\
\hline & 200E P-2968022 001 & $\mathbf{P}$ & $\mathbf{u}$ & & 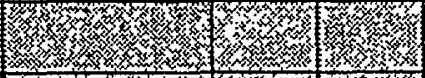 & $\begin{array}{l}\text { 40CFR 61.93(b)(4)(i) \& } \\
\text { WAC 246-247-075(3) }\end{array}$ & $\begin{array}{c}\text { Periodic } \\
\text { Confirmatory } \\
\end{array}$ & $\checkmark$ & \\
\hline & $200 \mathrm{E}$ P-296B023 001 & $\mathbf{P}$ & U & & 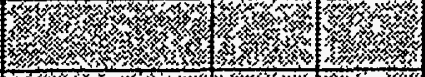 & $\begin{array}{l}\text { 40CFR 61.93(b)(4)(i) \& } \\
\text { WAC 246-247-075(3) }\end{array}$ & $\begin{array}{c}\text { Periodic } \\
\text { Confimatory }\end{array}$ & $\checkmark$ & \\
\hline & 200E P-296B024 001 & $\mathbf{P}$ & $\mathbf{U}$ & & 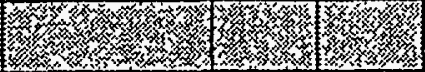 & $\begin{array}{l}\text { 40CFR 61.93(b)(4)(i) \& } \\
\text { WAC 246-247-075(3) }\end{array}$ & $\begin{array}{l}\text { Periodic } \\
\text { Confirmatory }\end{array}$ & $\checkmark$ & \\
\hline & 200E P-296B025 001 & $\mathbf{P}$ & $\mathbf{U}$ & & 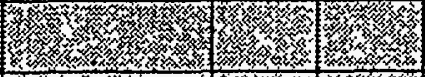 & $\begin{array}{l}\text { 40CFR } 61.93(b)(4)(\mathrm{i}) \text { \& } \\
\text { WAC 246-247.075(3) }\end{array}$ & $\begin{array}{c}\text { Periodic } \\
\text { Confirmatory }\end{array}$ & $\checkmark$ & \\
\hline & 200E P-296B026 001 & $\mathbf{P}$ & $\mathbf{U}$ & & 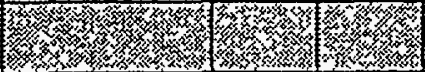 & $\begin{array}{l}\text { 40CFR 61.93(b)(4)(i) \& } \\
\text { WAC 246-247-075(3) }\end{array}$ & $\begin{array}{c}\text { Periodic } \\
\text { Confirmatory }\end{array}$ & $\checkmark$ & \\
\hline & 200E P-296B027 001 & P & $\mathbf{u}$ & & 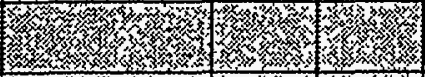 & $\begin{array}{l}\text { 40CFR 61.93(b)(4)(i) \& } \\
\text { WAC 246-247.075(3) }\end{array}$ & $\begin{array}{c}\text { Periodic } \\
\text { Confirmatory }\end{array}$ & $\checkmark$ & \\
\hline \multirow[t]{9}{*}{ PUREX } & 200E P-296A002 001 & $\mathbf{P}$ & $\mathbf{R}$ & & 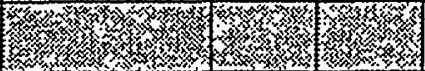 & $\begin{array}{l}\text { 40CFR } 61.93(0)(4)(i) \& \\
\text { WAC 246-247-075(3) }\end{array}$ & $\begin{array}{c}\text { Periodic } \\
\text { Confirmatory }\end{array}$ & $\checkmark$ & \\
\hline & 200E P-296A003 001 & $\mathbf{P}$ & $\mathbf{R}$ & & 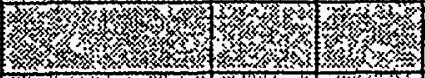 & $\begin{array}{l}\text { 40CFR 61.93(b)(4)(i) \& } \\
\text { WAC 246-247-075(3) }\end{array}$ & $\begin{array}{c}\text { Periodic } \\
\text { Confirmatory }\end{array}$ & $\checkmark$ & \\
\hline & 200E P-296A006 001 & $\mathbf{P}$ & $\mathbf{R}$ & & 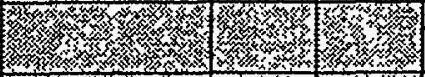 & $\begin{array}{l}\text { 40CFR 61.93(b)(4)(i) \& } \\
\text { IVAC 246-247-075(3) }\end{array}$ & $\begin{array}{c}\text { Periodic } \\
\text { Confinatory }\end{array}$ & $\checkmark$ & \\
\hline & 200E P-296A007 001 & $\mathbf{P}$ & $\mathbf{R}$ & & 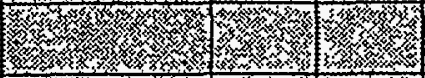 & $\begin{array}{l}\text { 40CFR 61.93(b)(4)(i) \& } \\
\text { WAC 246-247-075(3) }\end{array}$ & $\begin{array}{c}\text { Periodic } \\
\text { Confirmatory }\end{array}$ & $\checkmark$ & \\
\hline & 200E P-296.A008 001 & $\mathbf{P}$ & $\mathbf{R}$ & & 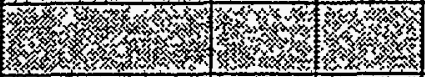 & $\begin{array}{l}\text { 40CFR } 61.93(0)(4)(i) \& \\
\text { WAC } 246-247.075(3)\end{array}$ & $\begin{array}{c}\text { Periodic } \\
\text { Confirmatory }\end{array}$ & $\checkmark$ & \\
\hline & 200E P-296,A010 001 & $\mathbf{P}$ & $\mathbf{R}$ & & 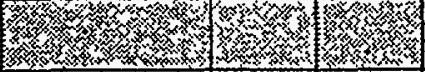 & $\begin{array}{l}\text { 40CFR } 61.93(b)(4)(i) \& \\
\text { WAC 246-247-075(3) }\end{array}$ & $\begin{array}{c}\text { Periodic } \\
\text { Confirmatory }\end{array}$ & $\checkmark$ & \\
\hline & 200E P-296A014 001 & $\mathbf{P}$ & $\mathbf{R}$ & & 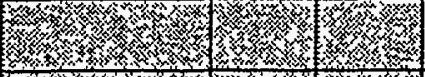 & $\begin{array}{l}\text { 40CFR } 61.93(b)(4)(\mathrm{i}) \& \\
\text { WAC 246-247-075(3) }\end{array}$ & $\begin{array}{l}\text { Periodic } \\
\text { Confirmatory }\end{array}$ & $\checkmark$ & \\
\hline & 200E P-296A05A 001 & $\mathbf{P}$ & $\mathbf{R}$ & & 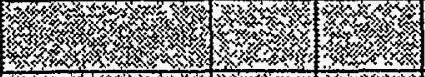 & $\begin{array}{l}\text { 40CFR 61.93(b)(4)(i) \& } \\
\text { WAC 246.247.075(3) }\end{array}$ & $\begin{array}{c}\text { Periodic } \\
\text { Confirmatory }\end{array}$ & $\checkmark$ & \\
\hline & 200E P-296A05B 001 & $\mathbf{P}$ & $\mathbf{R}$ & & xaty & $\begin{array}{l}\text { 40CFR 61.93(b)(4)(i) \& } \\
\text { WAC 246-247-075(3) }\end{array}$ & $\begin{array}{c}\text { Periodic } \\
\text { Confimmatory }\end{array}$ & $\checkmark$ & \\
\hline
\end{tabular}


Emission Point Compliance Demonstration

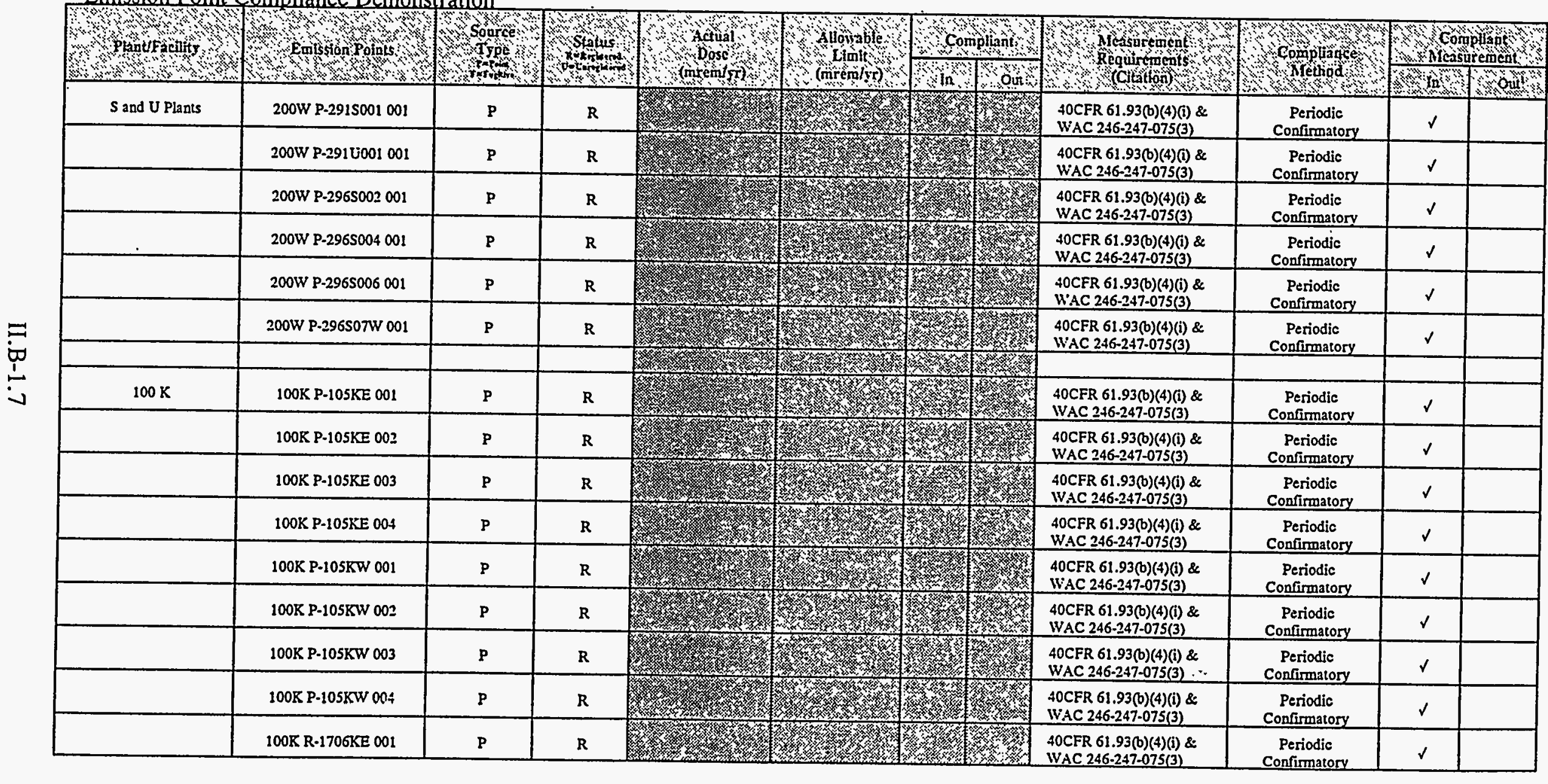


Emission Point Compliance Demonstration

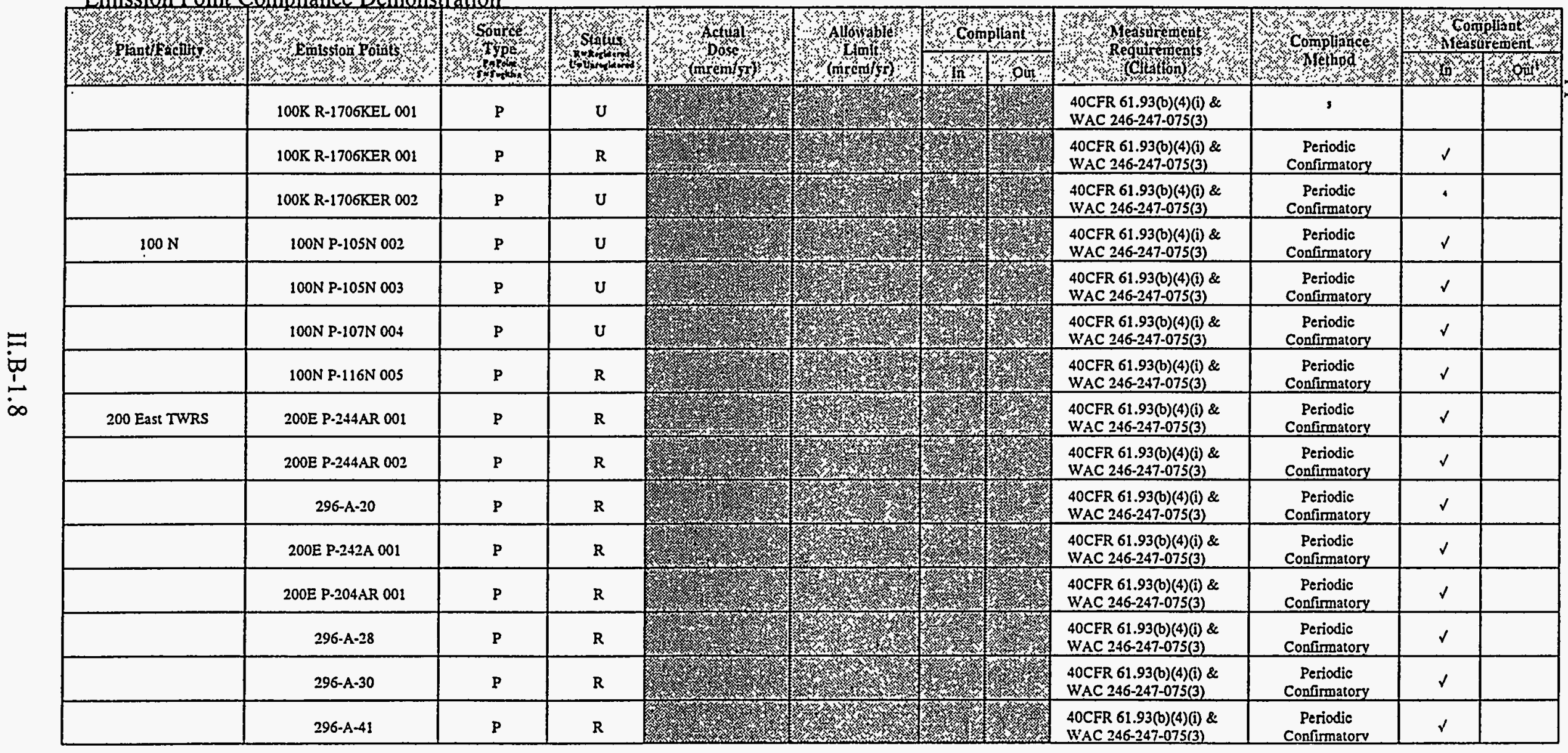




\begin{tabular}{|c|c|c|c|c|c|c|c|c|c|c|c|c|c|c|c|}
\hline ) & & & & & & & & & & & & & & & \\
\hline 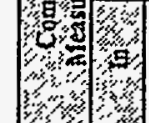 & $>$ & $>$ & $>$ & $>$ & $>$ & $>$ & $>$ & $>$ & $>$ & $>$ & $>$ & $>$ & $>$ & $>$ & $>$ \\
\hline \%y & 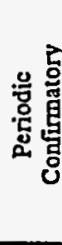 & 崽 & 每 & 宫 & 总 & 总 & 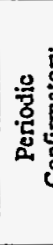 & 窇 & 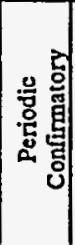 & 总总 & 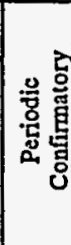 & 产 & & 总总 & 号 \\
\hline 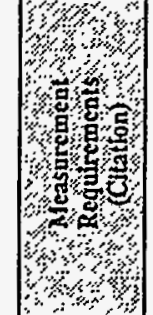 & 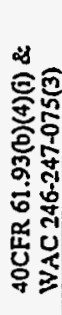 & 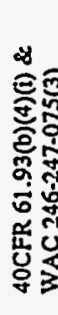 & $\begin{array}{l}0 \\
0 \\
0\end{array}$ & 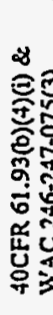 & 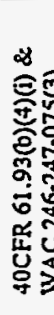 & 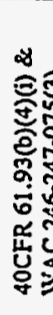 & 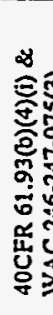 & 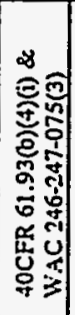 & 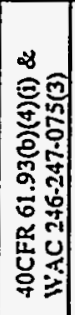 & 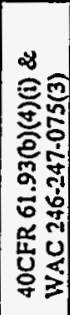 & 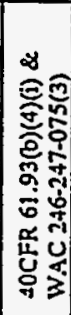 & 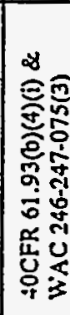 & & 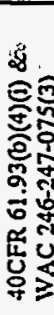 & 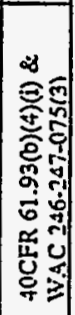 \\
\hline 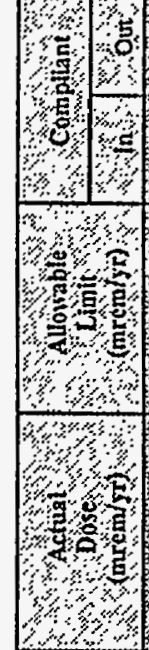 & 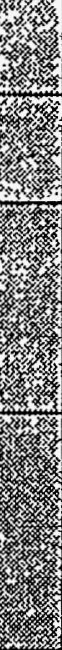 & xy & 极 & 聯 & $\frac{3}{3}$ & X & re & rex & $\frac{2}{3}$ & 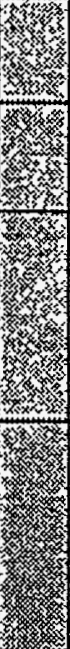 & 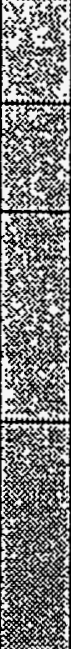 & 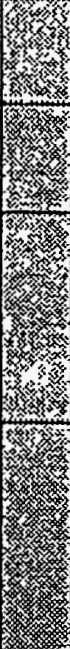 & 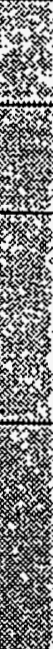 & x. & 3. \\
\hline W & $\infty$ & $\propto$ & $\propto$ & $\approx$ & $\approx$ & $\approx$ & $\propto$ & $\propto$ & & $\approx$ & $\propto$ & $\approx$ & $\alpha$ & $\propto$ & $D$ \\
\hline 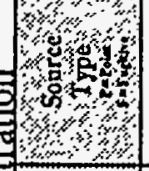 & A & $A$ & A & $a$ & a & a & a & A & a. & $a$ & $\infty$ & o & a & a & a \\
\hline 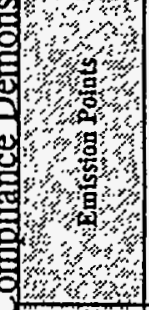 & 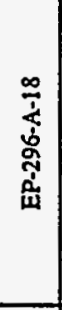 & 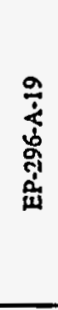 & 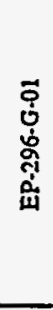 & 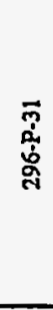 & 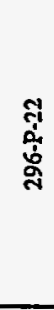 & 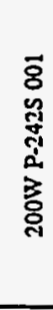 & 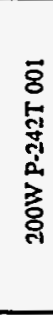 & 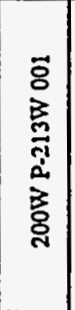 & 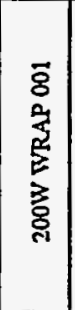 & 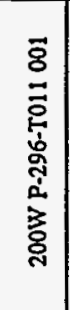 & 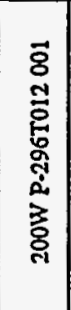 & 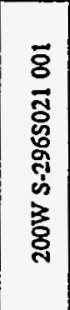 & 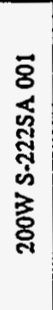 & 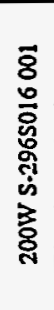 & 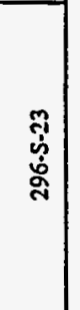 \\
\hline 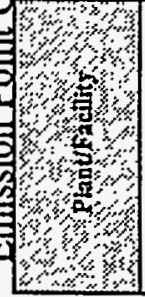 & & & & & 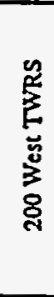 & & & 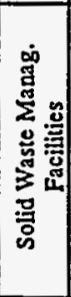 & & 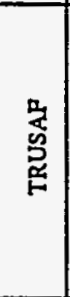 & & 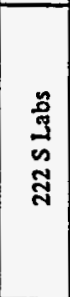 & & & \\
\hline
\end{tabular}


Emission Point Compliance Demonstration

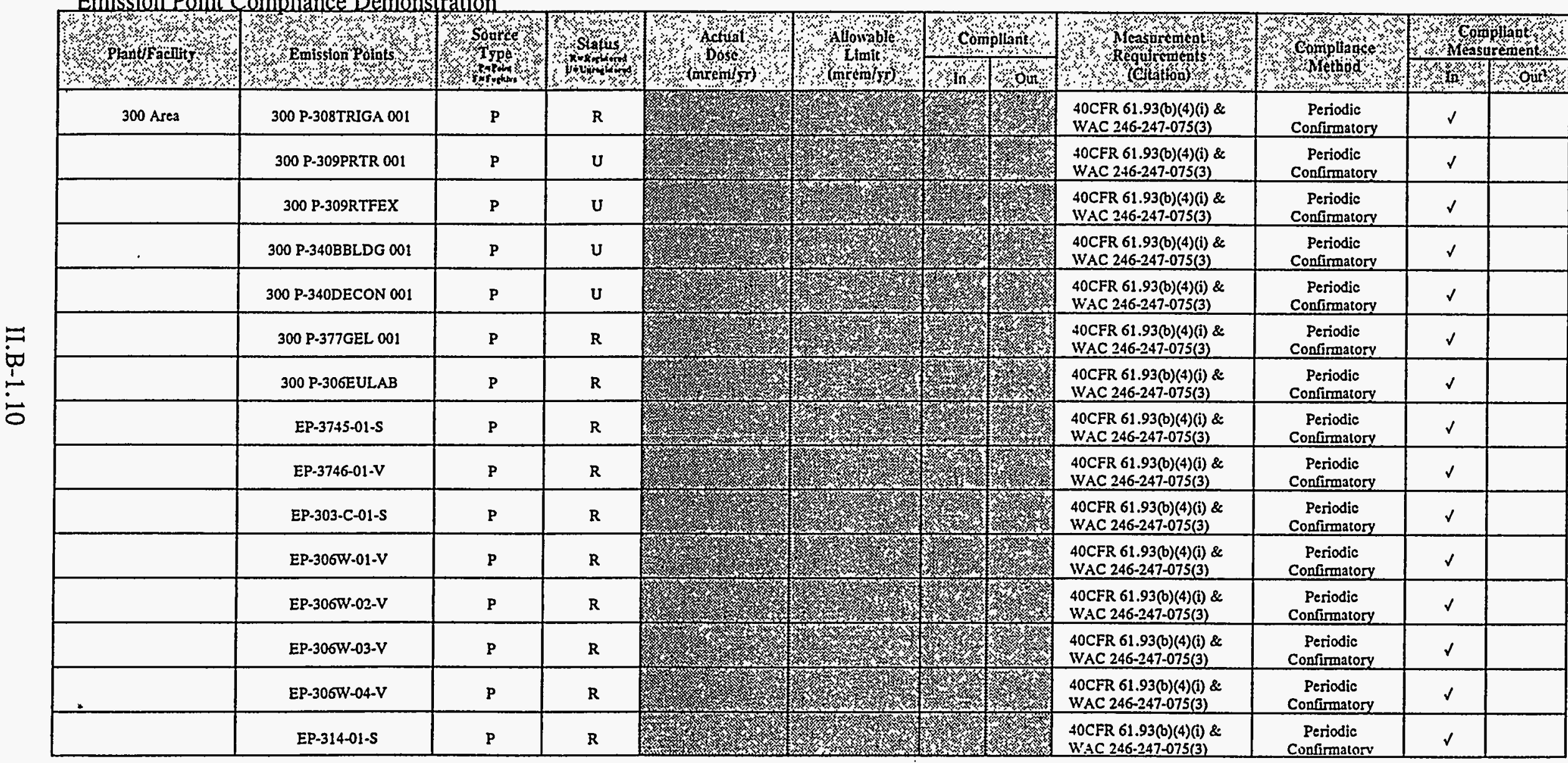




\begin{tabular}{|c|c|c|c|c|c|c|c|c|c|c|c|c|c|c|c|}
\hline \multicolumn{16}{|c|}{ 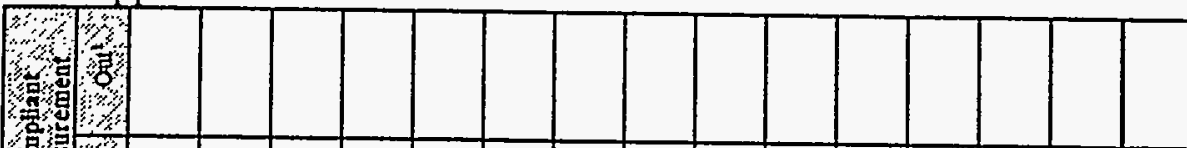 } \\
\hline \multicolumn{16}{|c|}{ 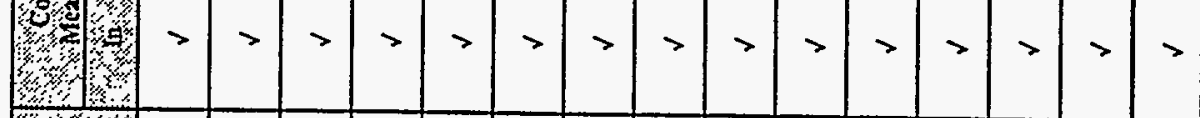 } \\
\hline & & & 5 & 5 & & & & & & & & & & & \\
\hline
\end{tabular}

\section{th:

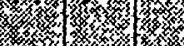

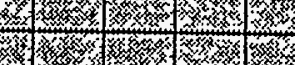

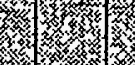

到

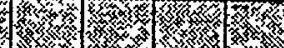

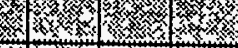

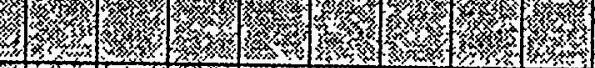

似

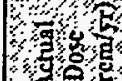

\%

\section{wis}

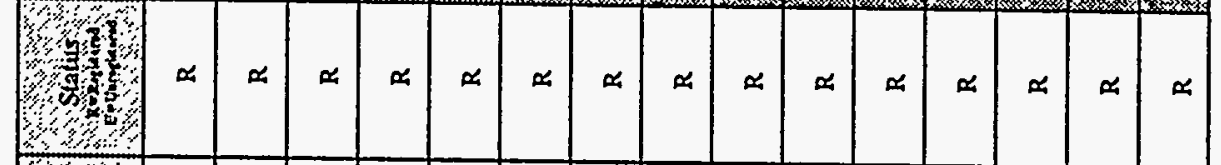

\begin{tabular}{|c|c|c|c|c|c|c|c|c|c|c|c|c|c|c|}
\hline 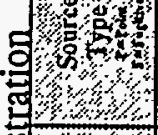 & a. & $\Delta$ & a & A & A. & a & a & a & a. & a & or & A & $a$ & $\infty$ \\
\hline 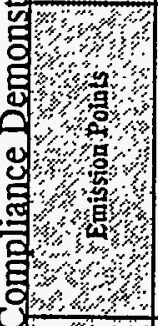 & 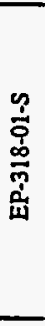 & 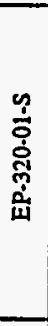 & 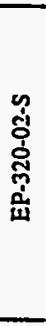 & 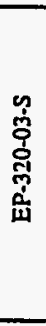 & 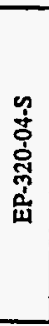 & 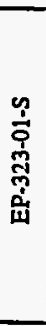 & 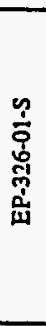 & 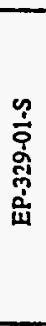 & 章 & 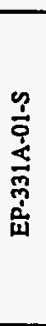 & 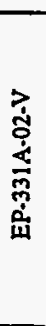 & 高 & 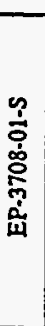 & 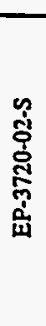 \\
\hline 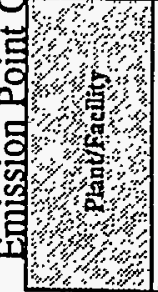 & & & & & & & & & & & & & & \\
\hline
\end{tabular}




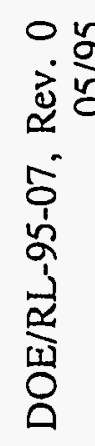

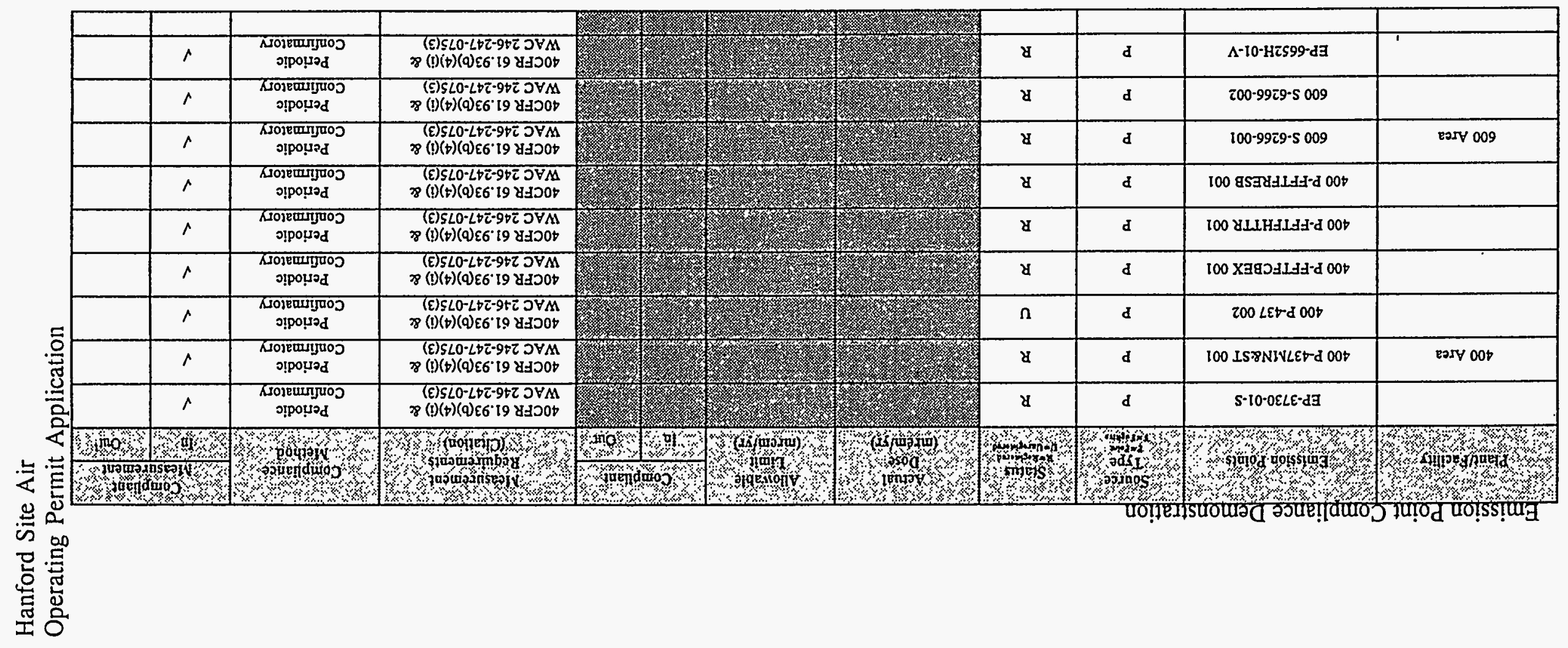



ำ
ऐं山े
ธ்
หั่
空
핑
口

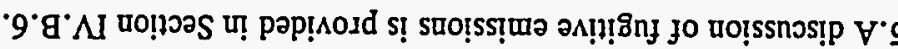

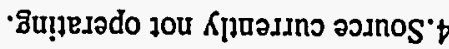

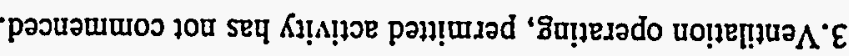

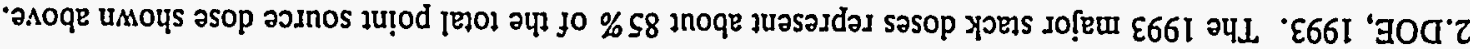
.

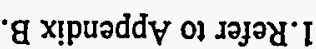




\begin{tabular}{|c|c|c|c|c|c|c|}
\hline 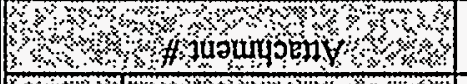 & & & & & & \\
\hline 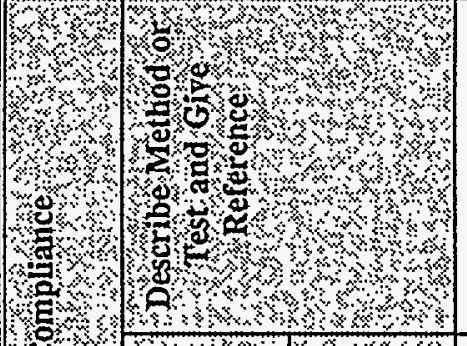 & & & 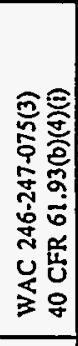 & 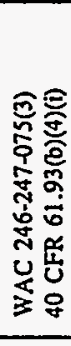 & 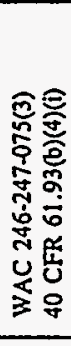 & 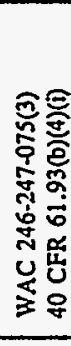 \\
\hline 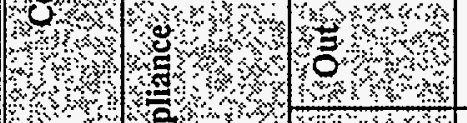 & & & & & & \\
\hline 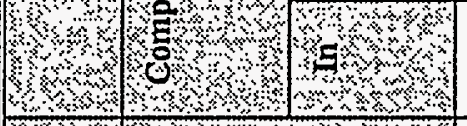 & & & $>$ & $>$ & $>$ & $>$ \\
\hline 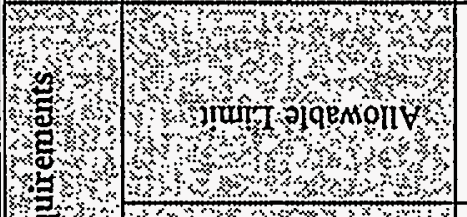 & & & 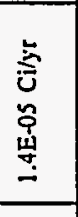 & 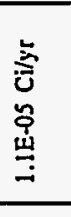 & 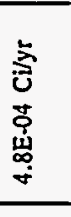 & 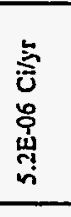 \\
\hline 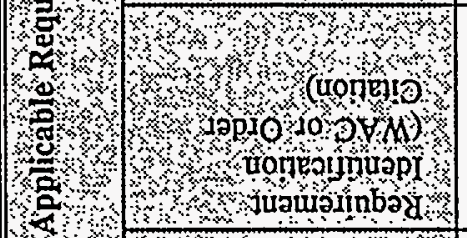 & & 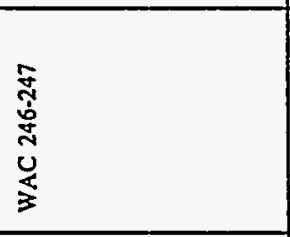 & 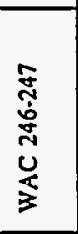 & 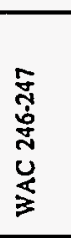 & 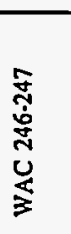 & 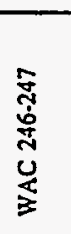 \\
\hline 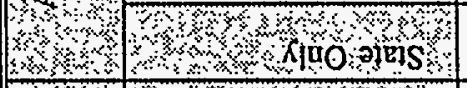 & & $>$ & $>$ & $>$ & $>$ & $>$ \\
\hline 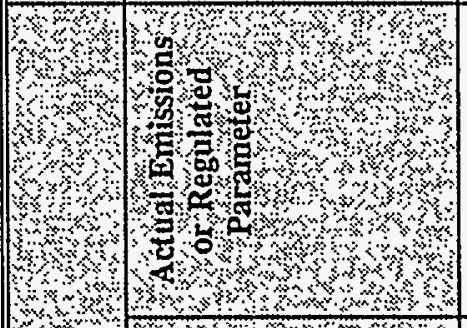 & & & & & & \\
\hline 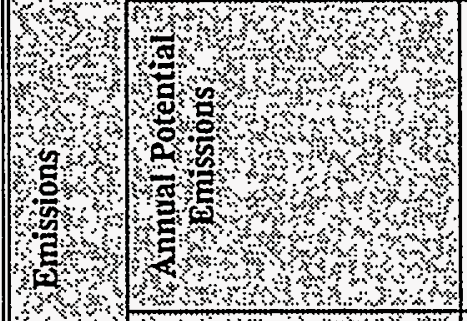 & & & 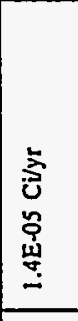 & 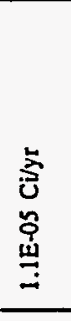 & 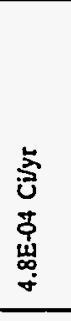 & 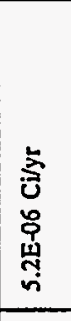 \\
\hline 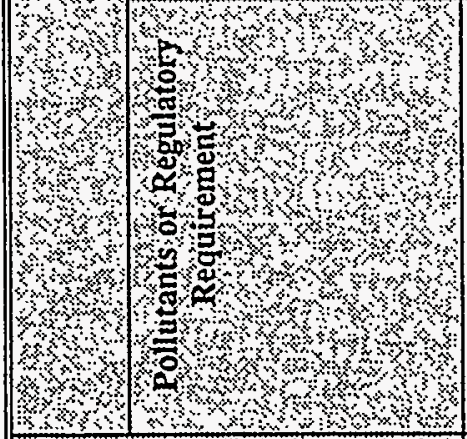 & & 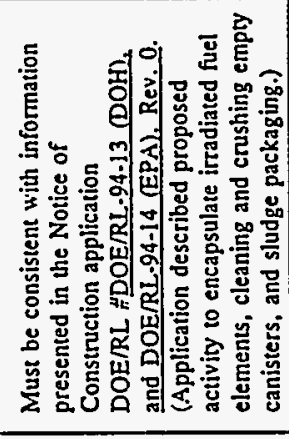 & 离 & نั่ & $\frac{\bar{m}}{\dot{\omega}}$ & 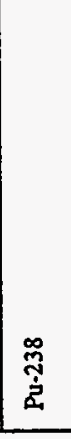 \\
\hline 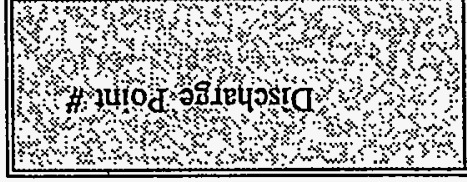 & 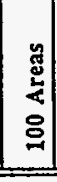 & 离 & & & & \\
\hline
\end{tabular}




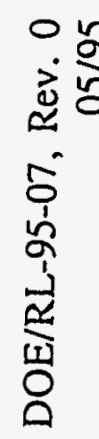

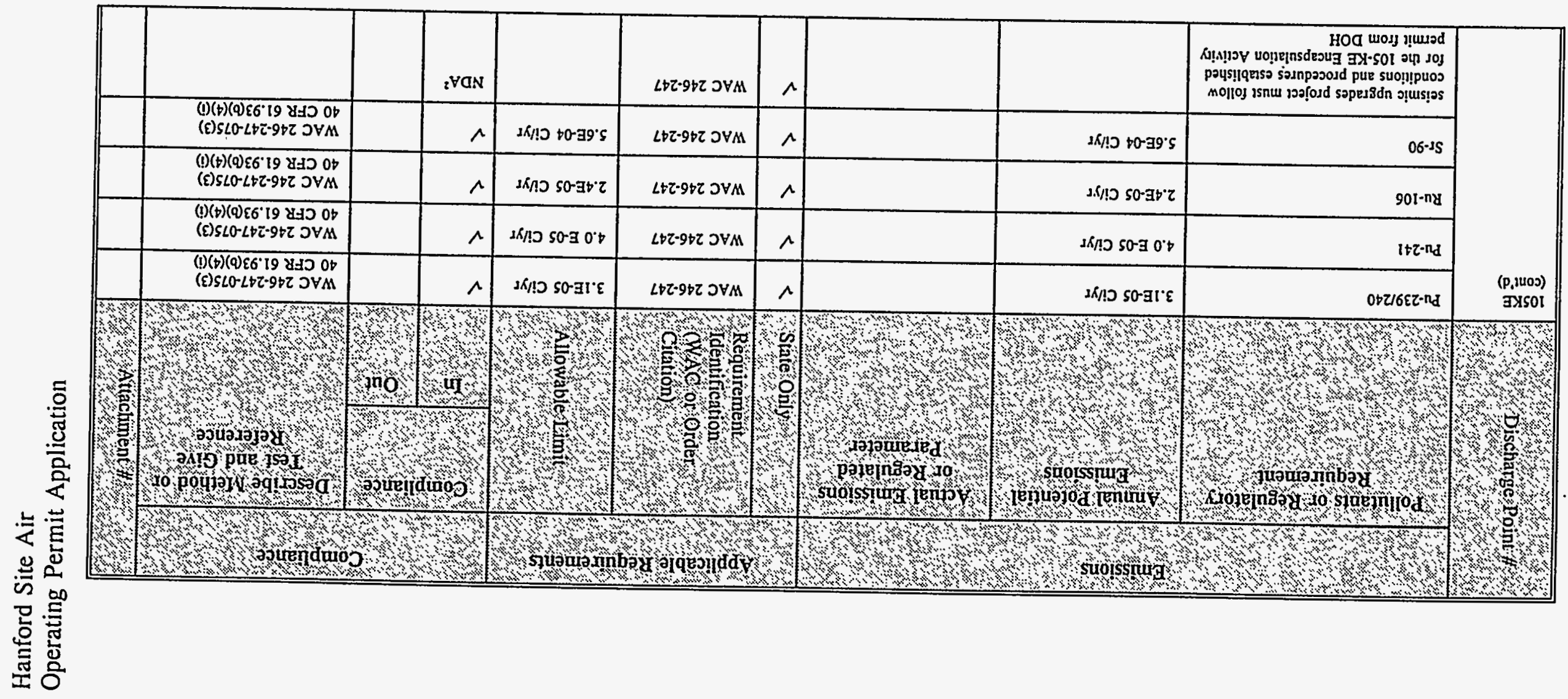




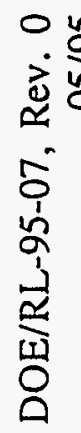

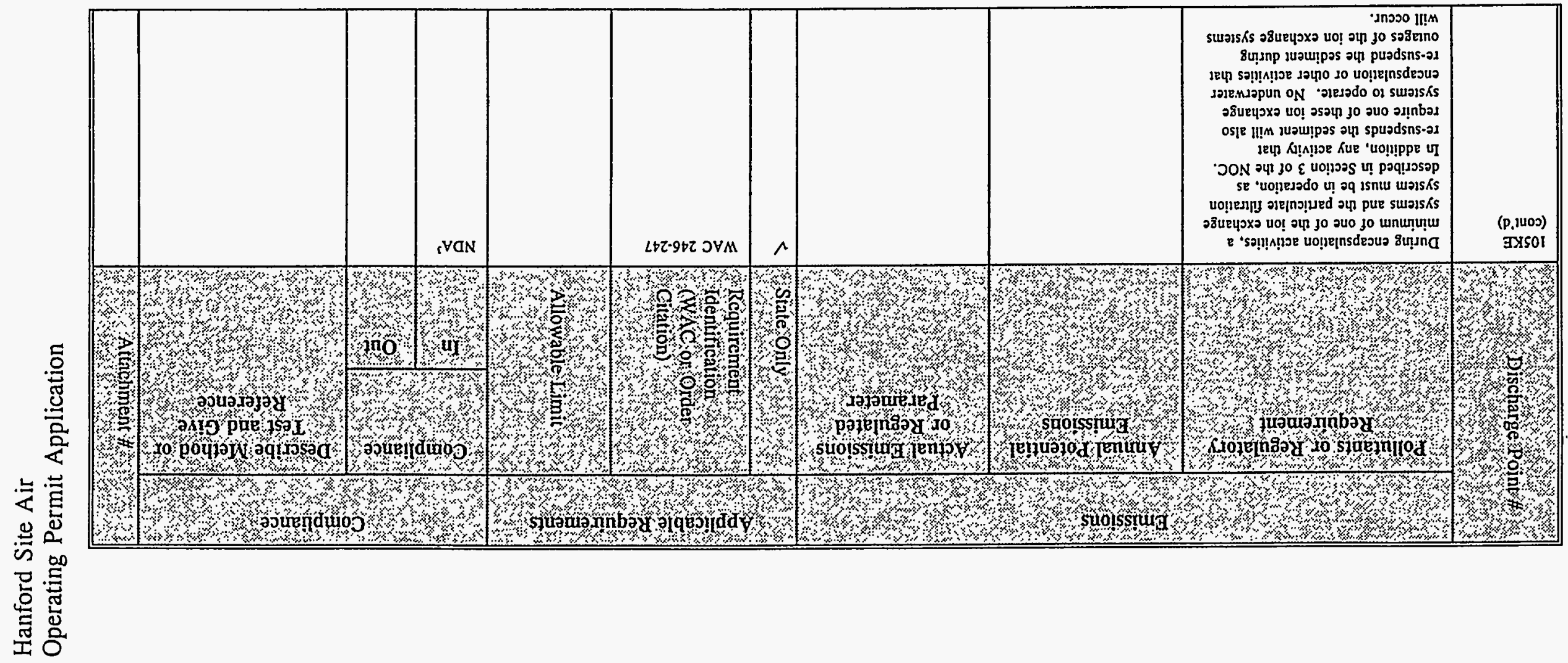

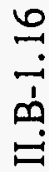




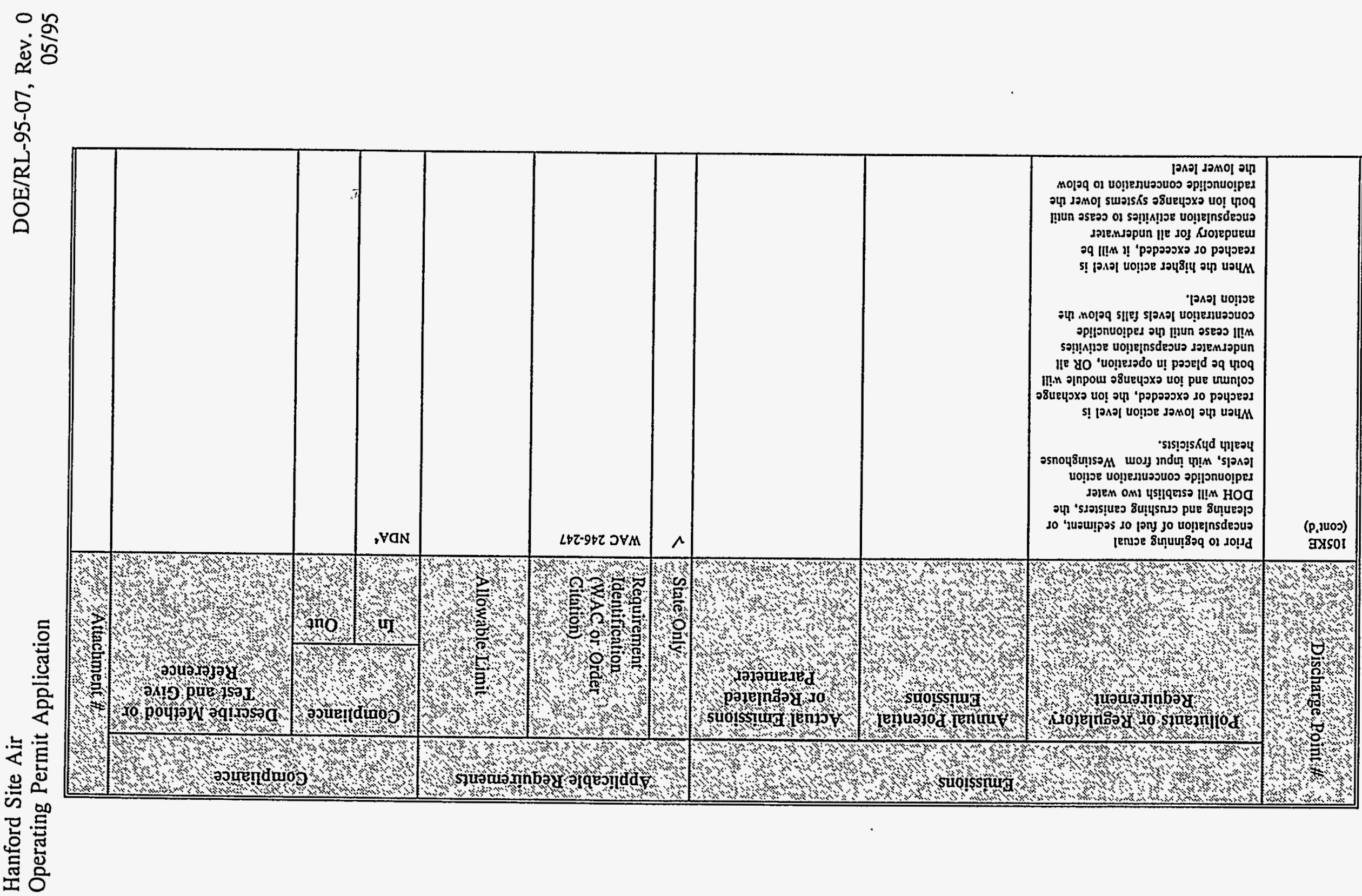

$\stackrel{\Xi}{\stackrel{\Xi}{\Xi}}$ 
वं

ㄹํㅇ

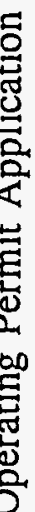

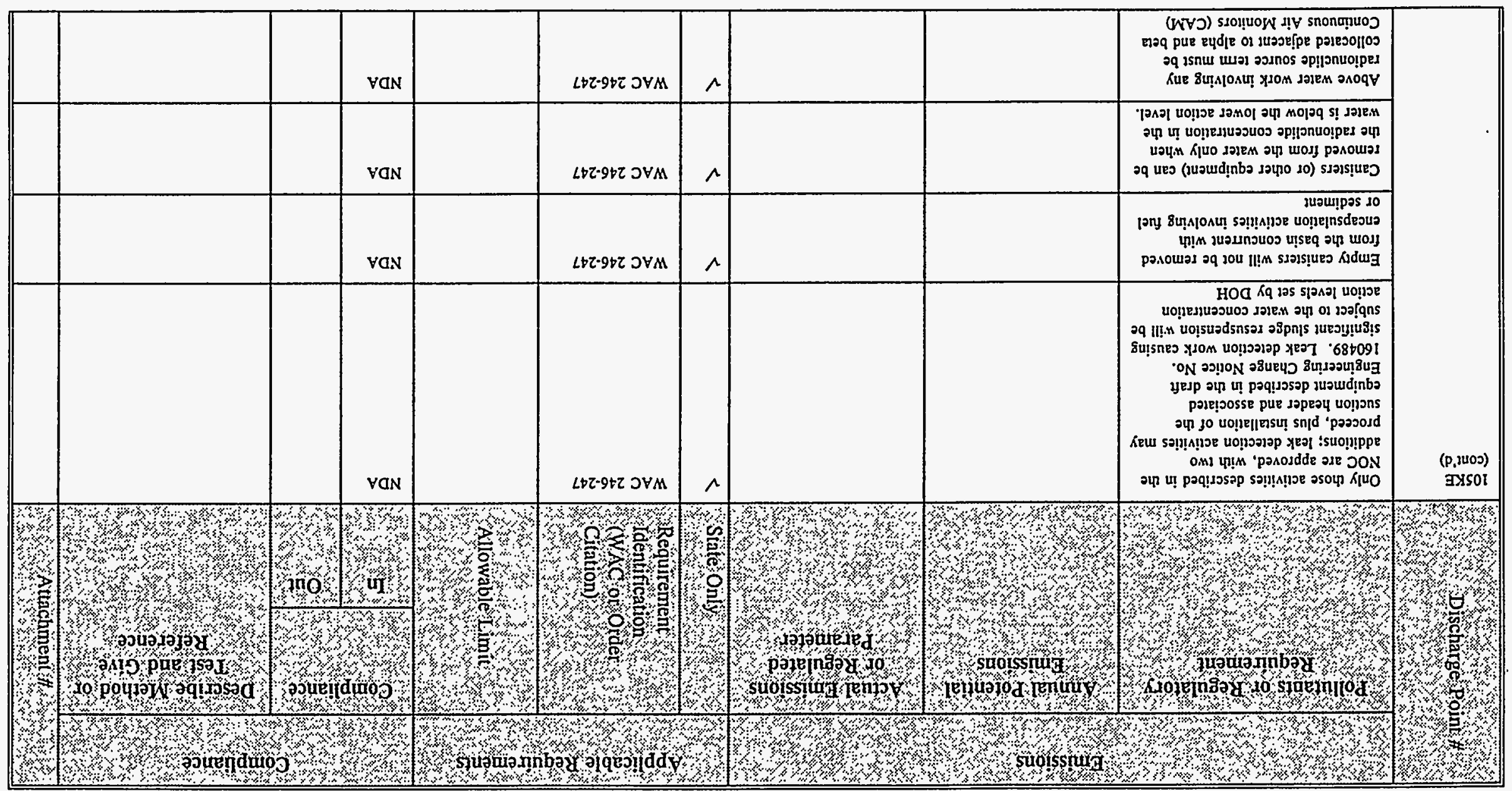




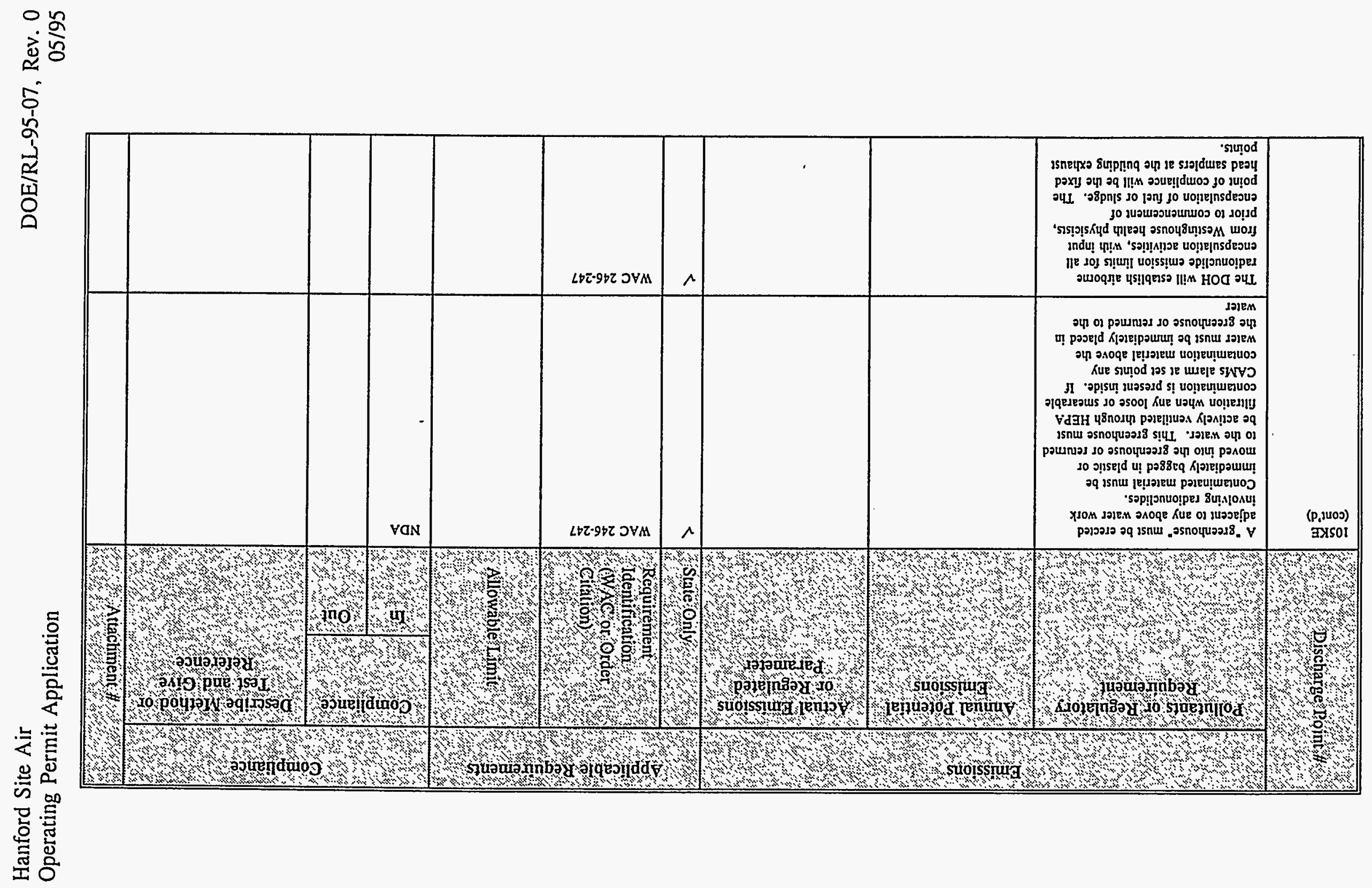




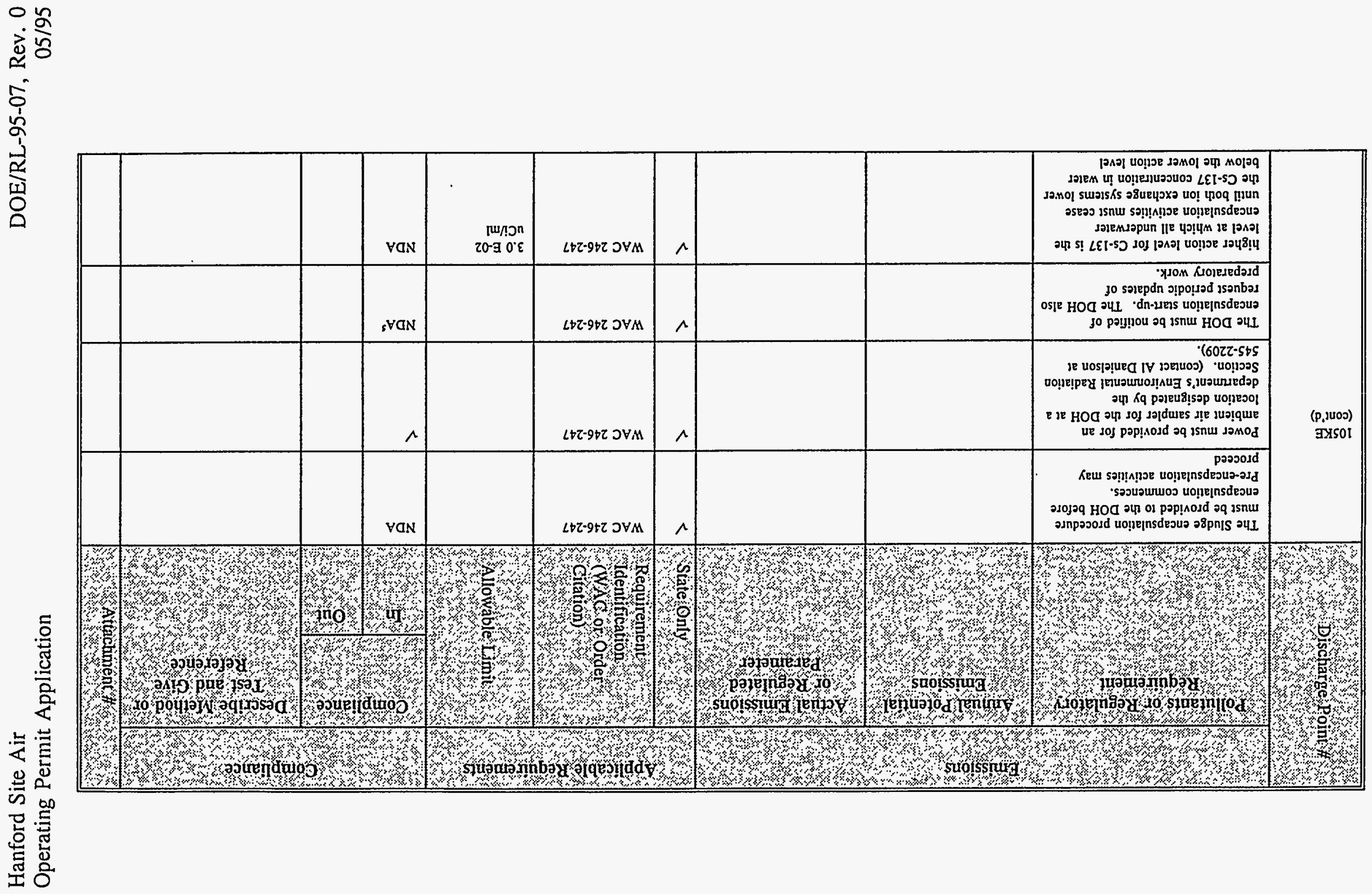

ঙำ 
뭉

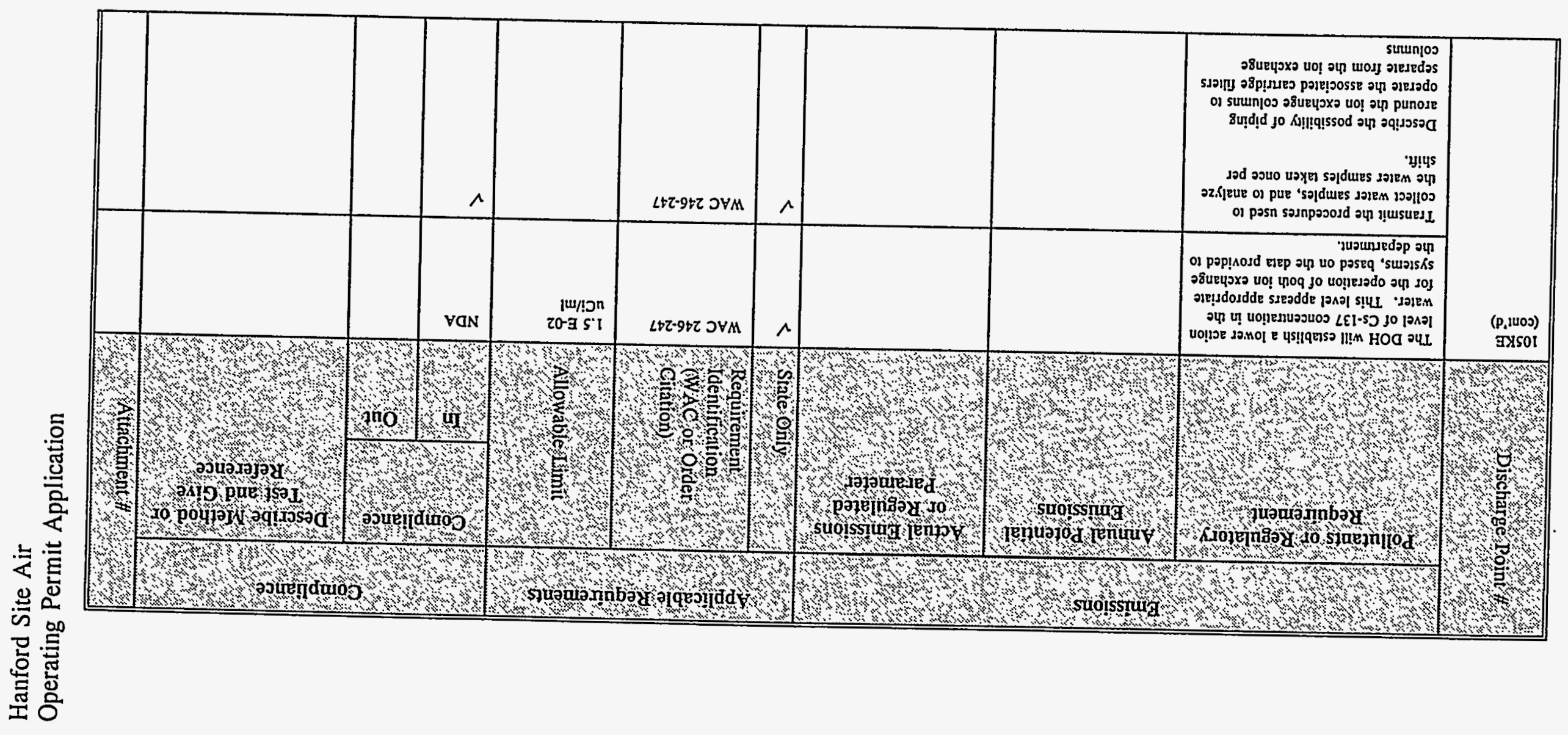




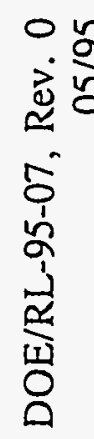

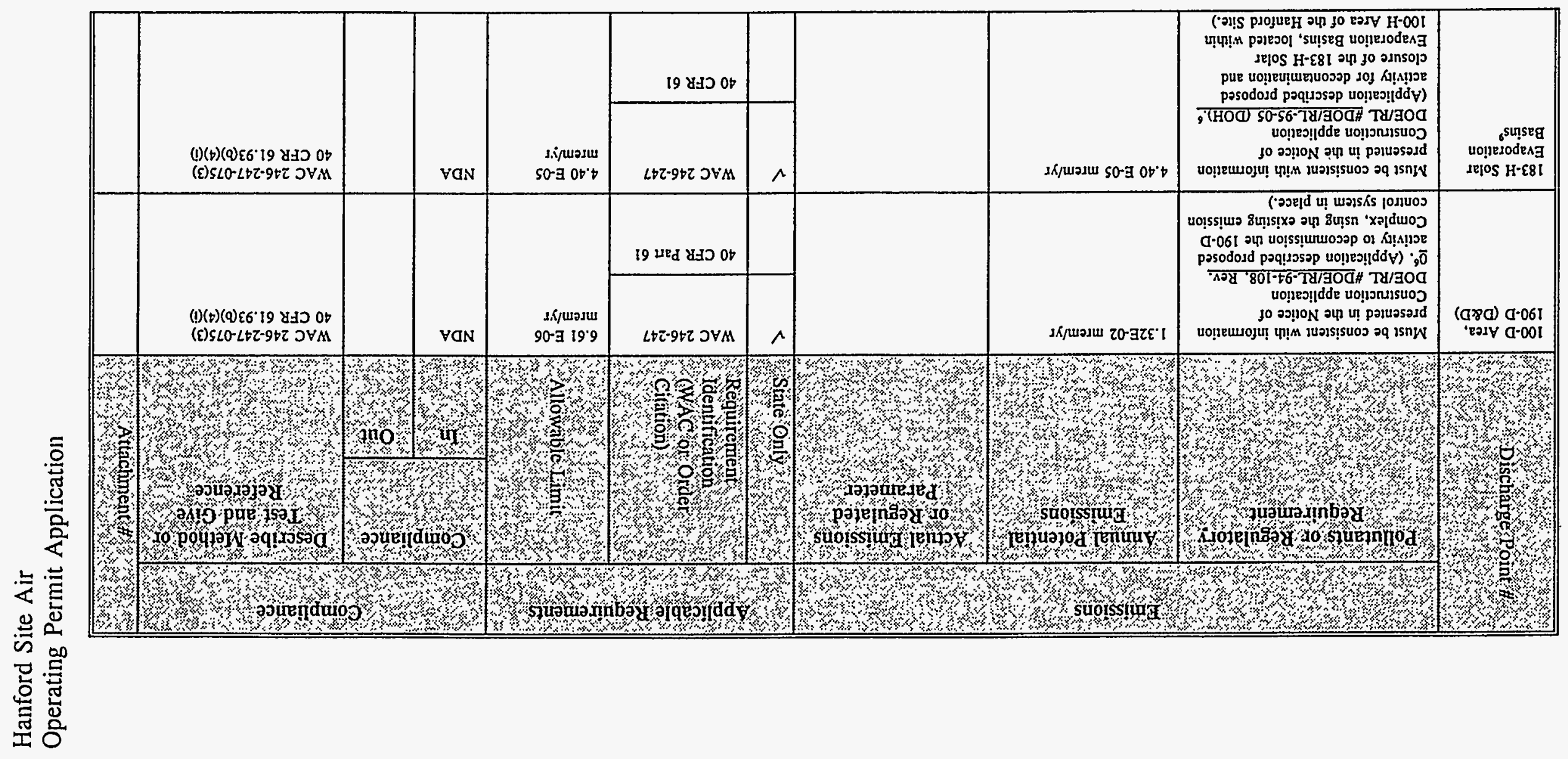


홈

ऐं

\begin{tabular}{|c|c|c|c|c|c|c|c|c|c|c|}
\hline & is & & $\mathrm{VQN}$ & & . & $\wedge$ & & & 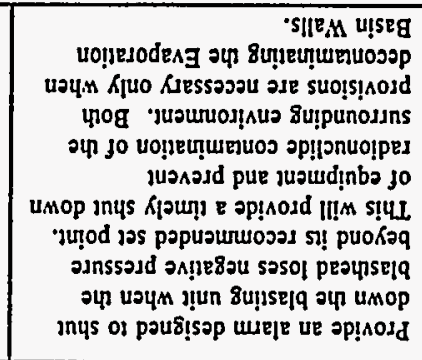 & \\
\hline & r & & $\mathrm{V} \triangle \mathrm{N}$ & & $\angle \nabla \tau-9 \Delta \tau \partial \nabla A \Delta$ & $\mu$ & & & 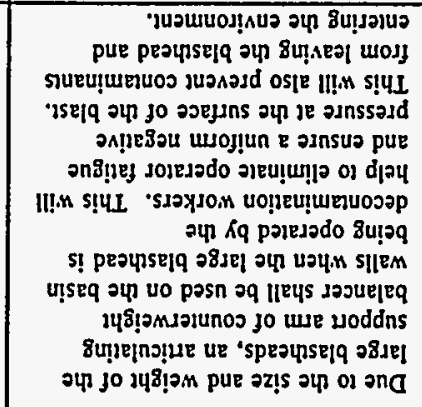 & 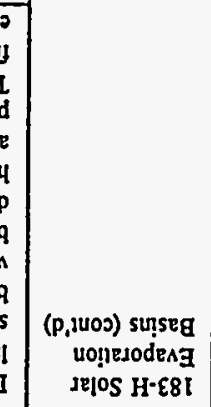 \\
\hline 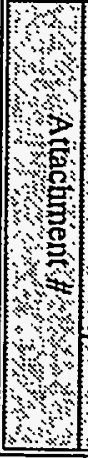 & 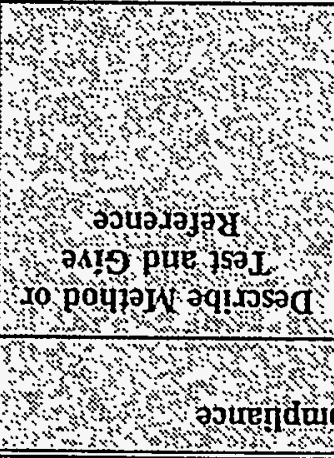 & 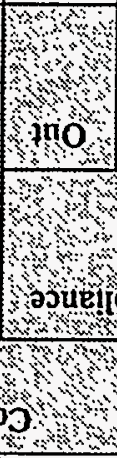 & 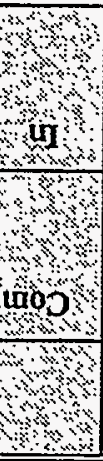 & 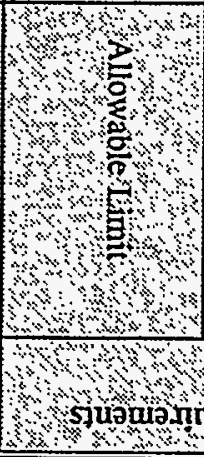 & 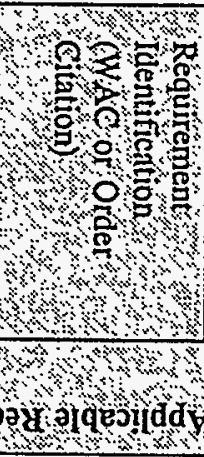 & (a) & 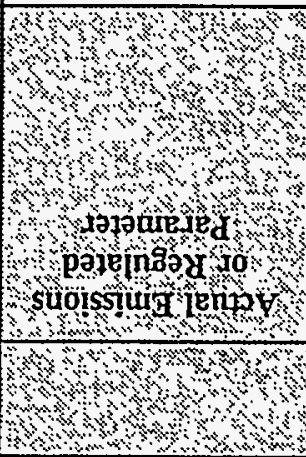 & 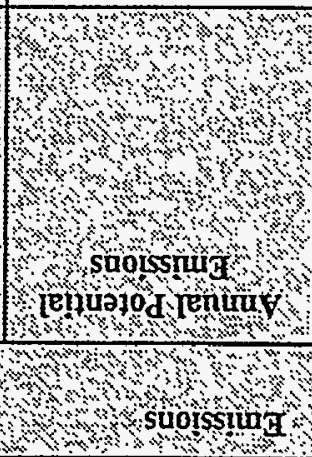 & 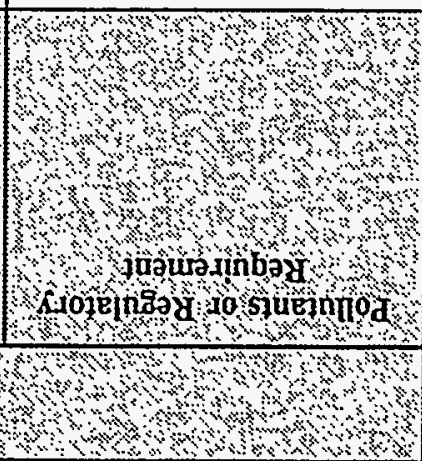 & 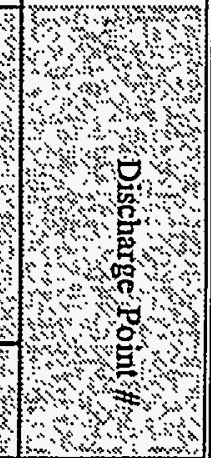 \\
\hline
\end{tabular}

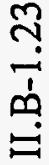

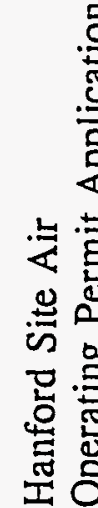


Hanford Site Air

DOE/RL-95-07, Rev. 0

Operating Permit Application

05/95

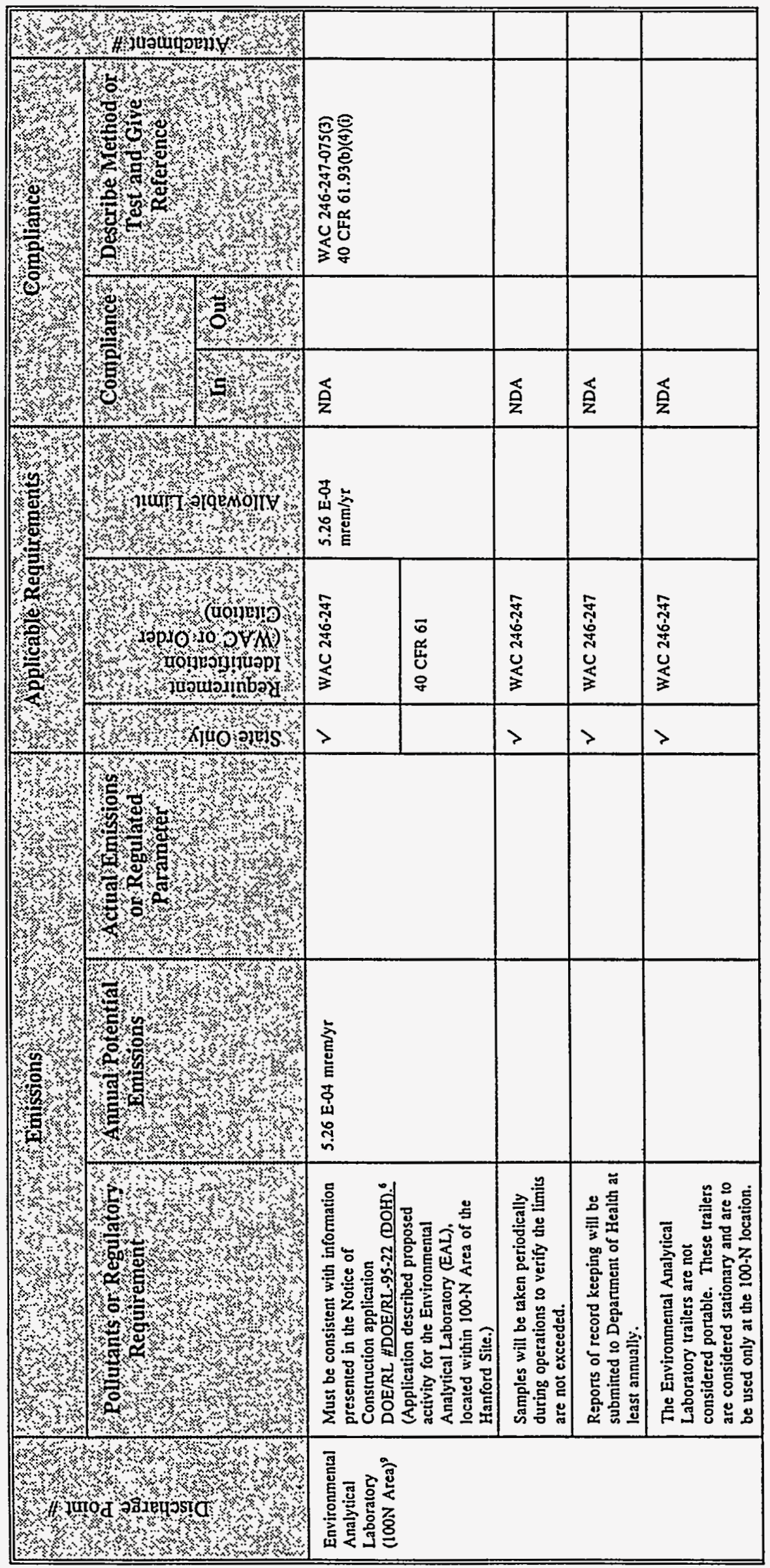

II. B-1.24 


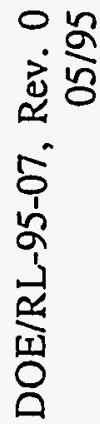
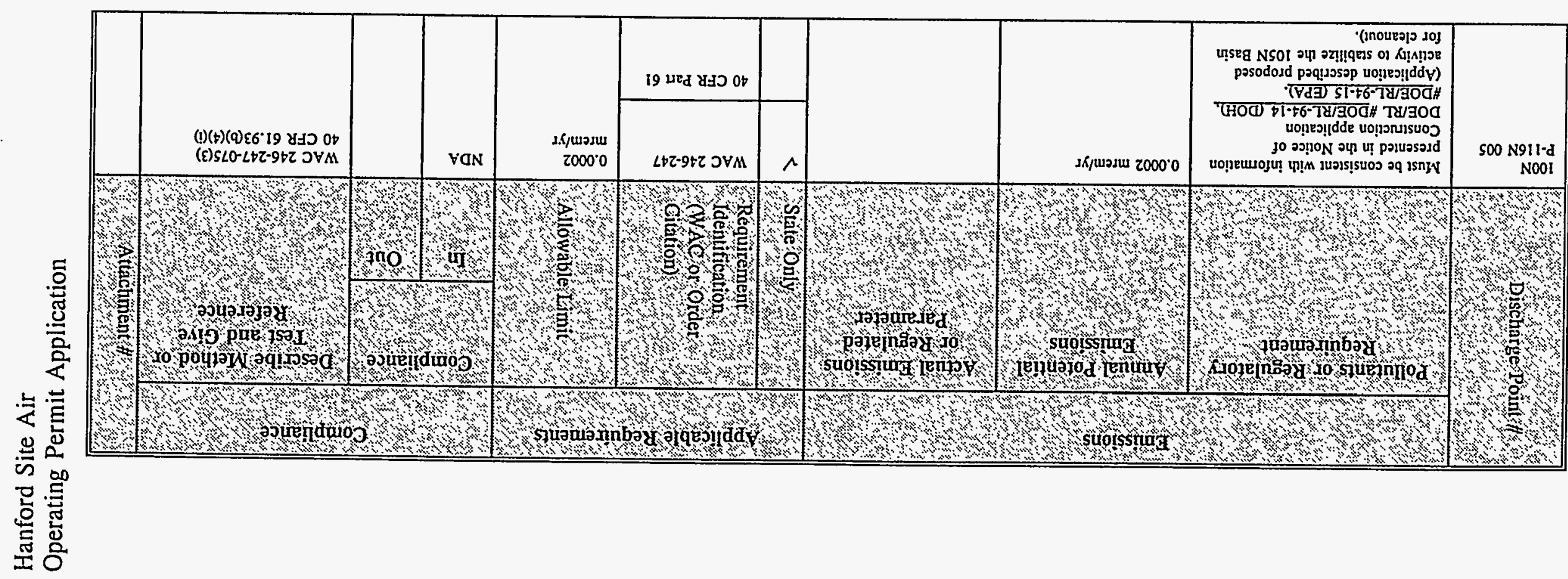
它

ये⿺

\begin{tabular}{|l|l|l|l|l|l|l|l|l|l|}
\hline & & & & & & & & & \\
L & & & & & & & & \\
oss
\end{tabular}

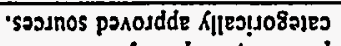

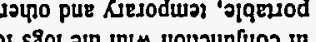

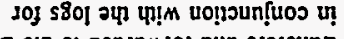
HOC

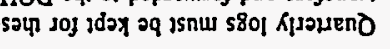

zusudinting posuos

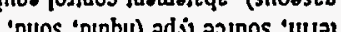

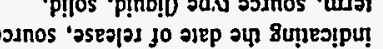
'pa880 pue pacoddde aq isnui

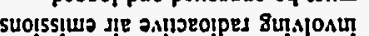

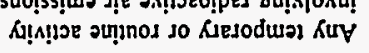

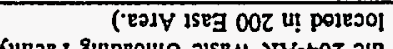
Ail!

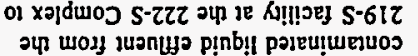

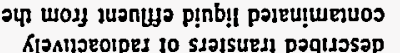

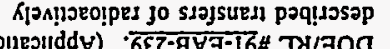

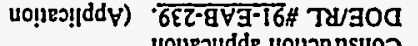
volyes!ldde uo!jonssuos

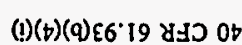

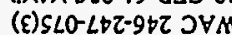

sh/wasur to.0

$\angle t 2-9 t 2$ วYM

sK/แองมี $50^{\circ} 0$

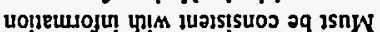

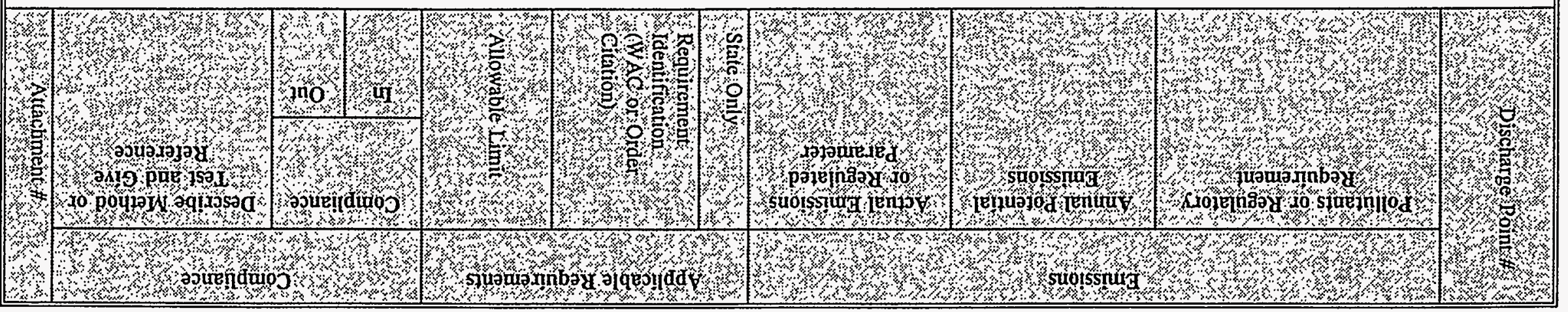

赵

용

氶 


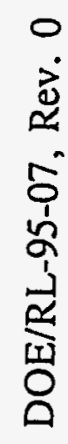

ये

\begin{tabular}{|c|c|c|c|c|c|c|c|c|c|}
\hline & 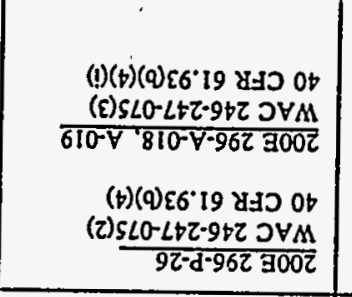 & ¿VAN & 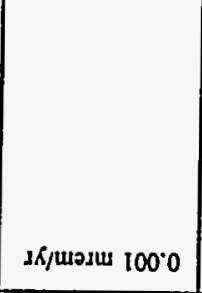 & $\begin{array}{c}\text { H Ledqns } \\
\text { '19 \&uכ ob }\end{array}$ & $\Lambda$ & & LS/ Rusasw t00 0 & 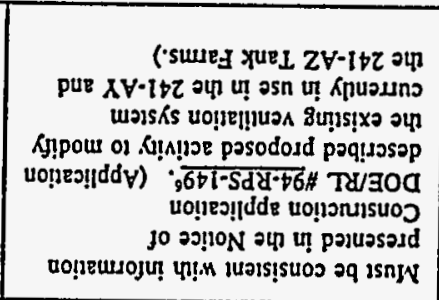 & 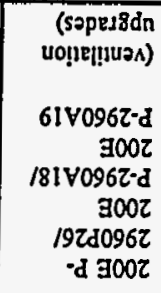 \\
\hline & א & $\mathrm{VaN}$ & 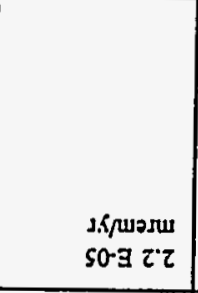 & $\angle \leftarrow \tau-9 \mathrm{Z} Z \mathrm{OVM}$ & $\Lambda$ & & 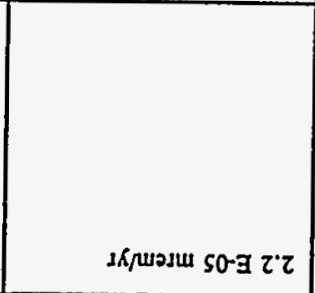 & 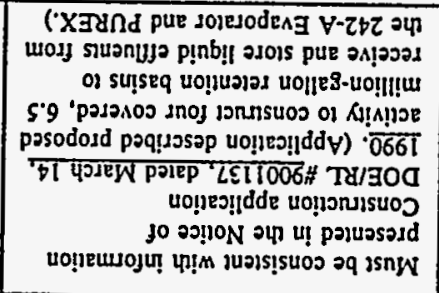 & 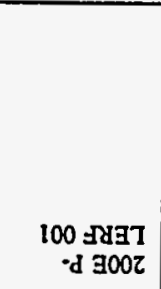 \\
\hline & & $\Lambda$ & & $\angle \forall Z-9 \nabla Z \supset \forall M$ & $\wedge$ & & & 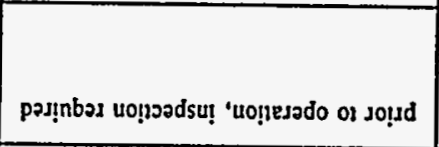 & 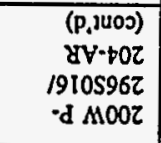 \\
\hline 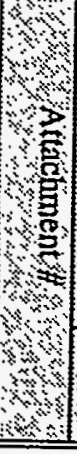 & 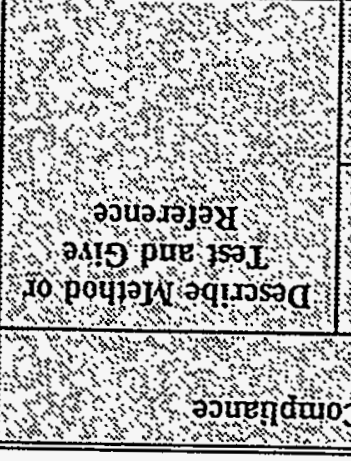 & 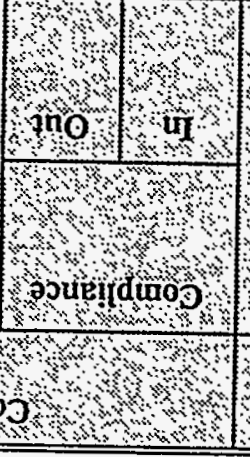 & (1) & 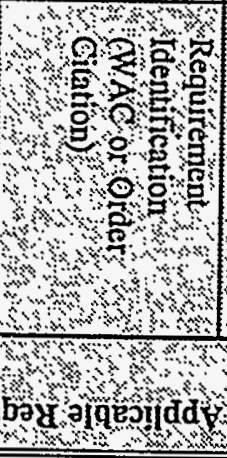 & (4) & 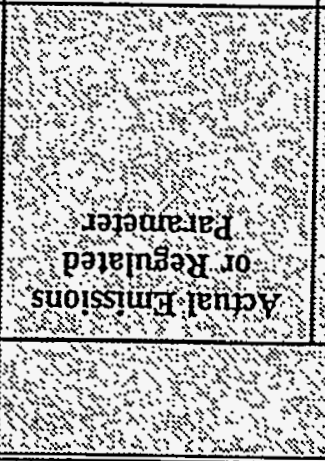 & 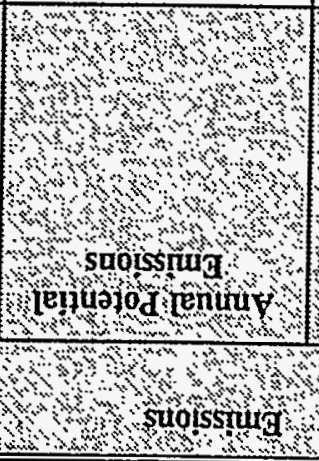 & 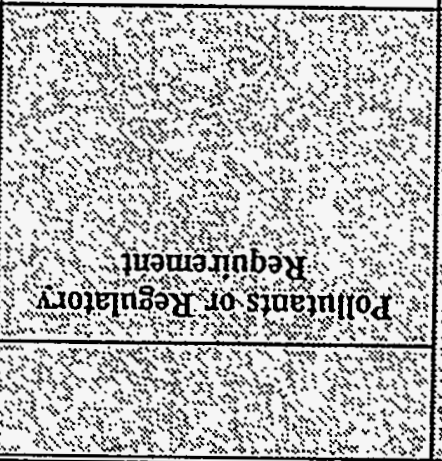 & 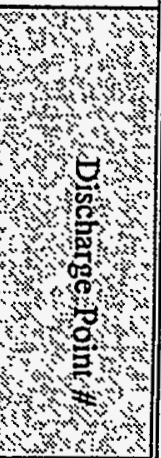 \\
\hline
\end{tabular}


文

¿่

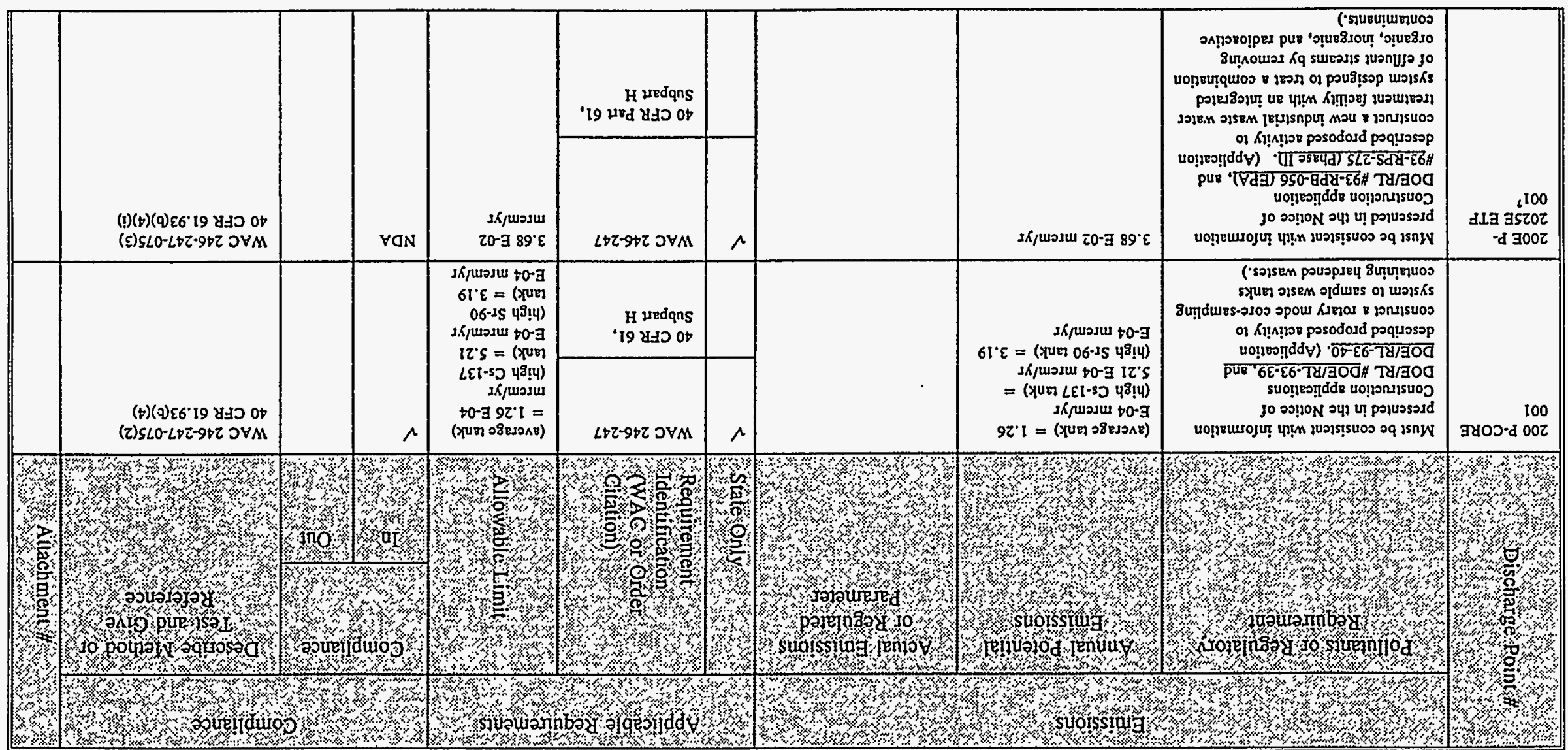

$\stackrel{Ð}{\circ}$

攽

댄.

坖

空 


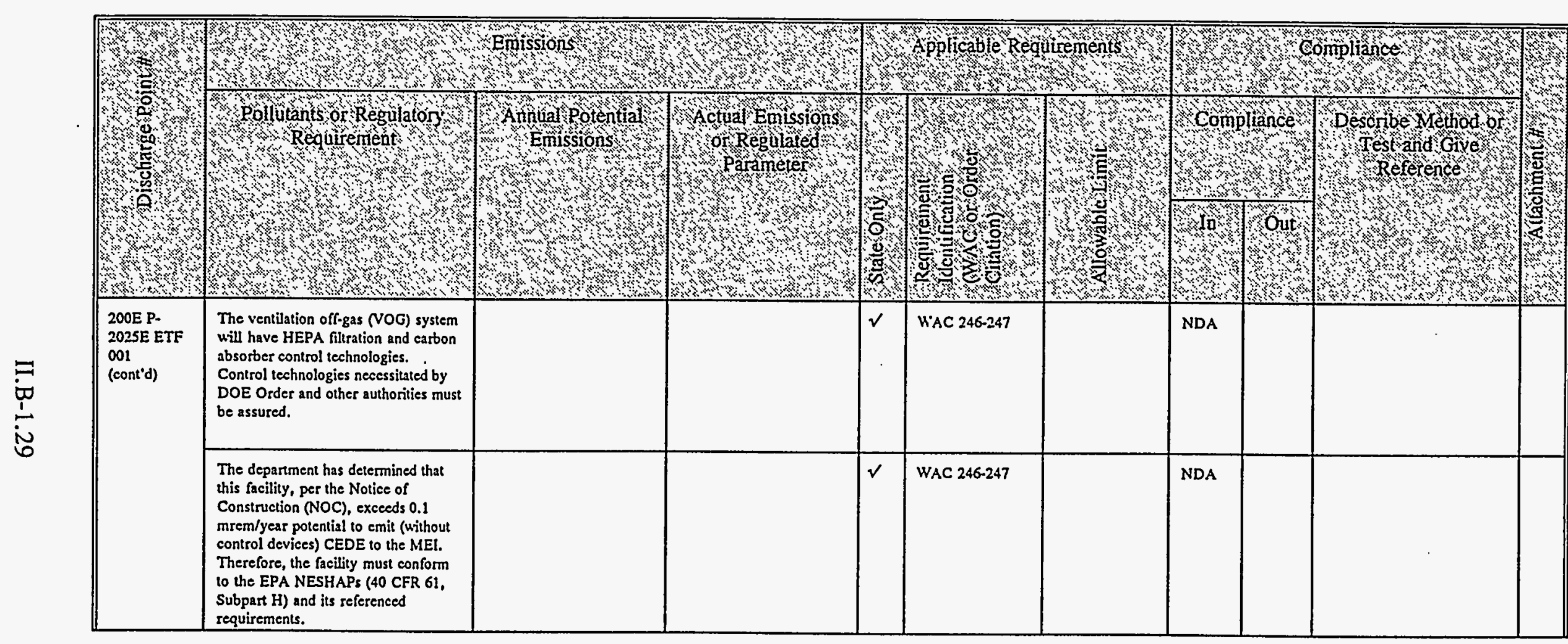




ऐั

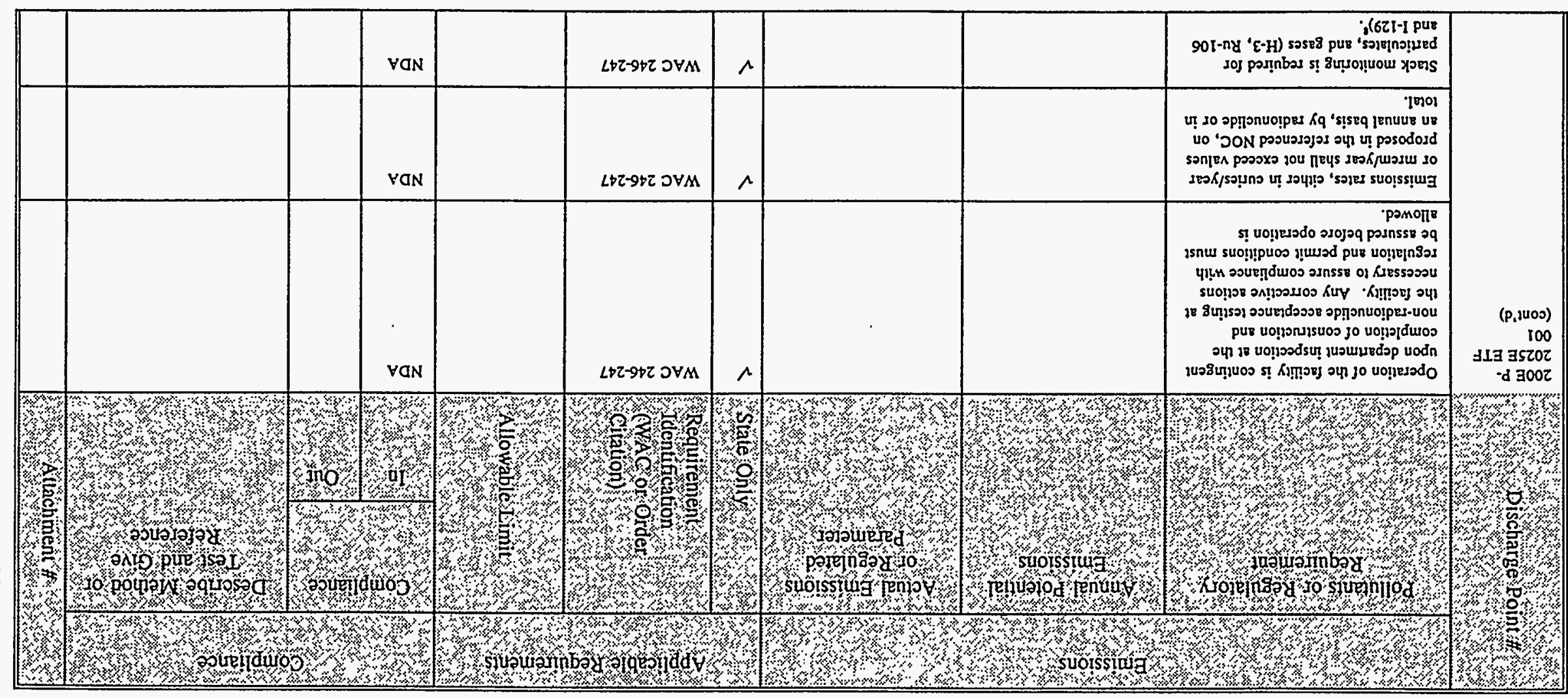

$\frac{\text { m. }}{\stackrel{\oplus}{\Xi}}$

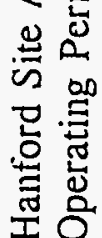


Hanford Site Air

Operating Permit Application

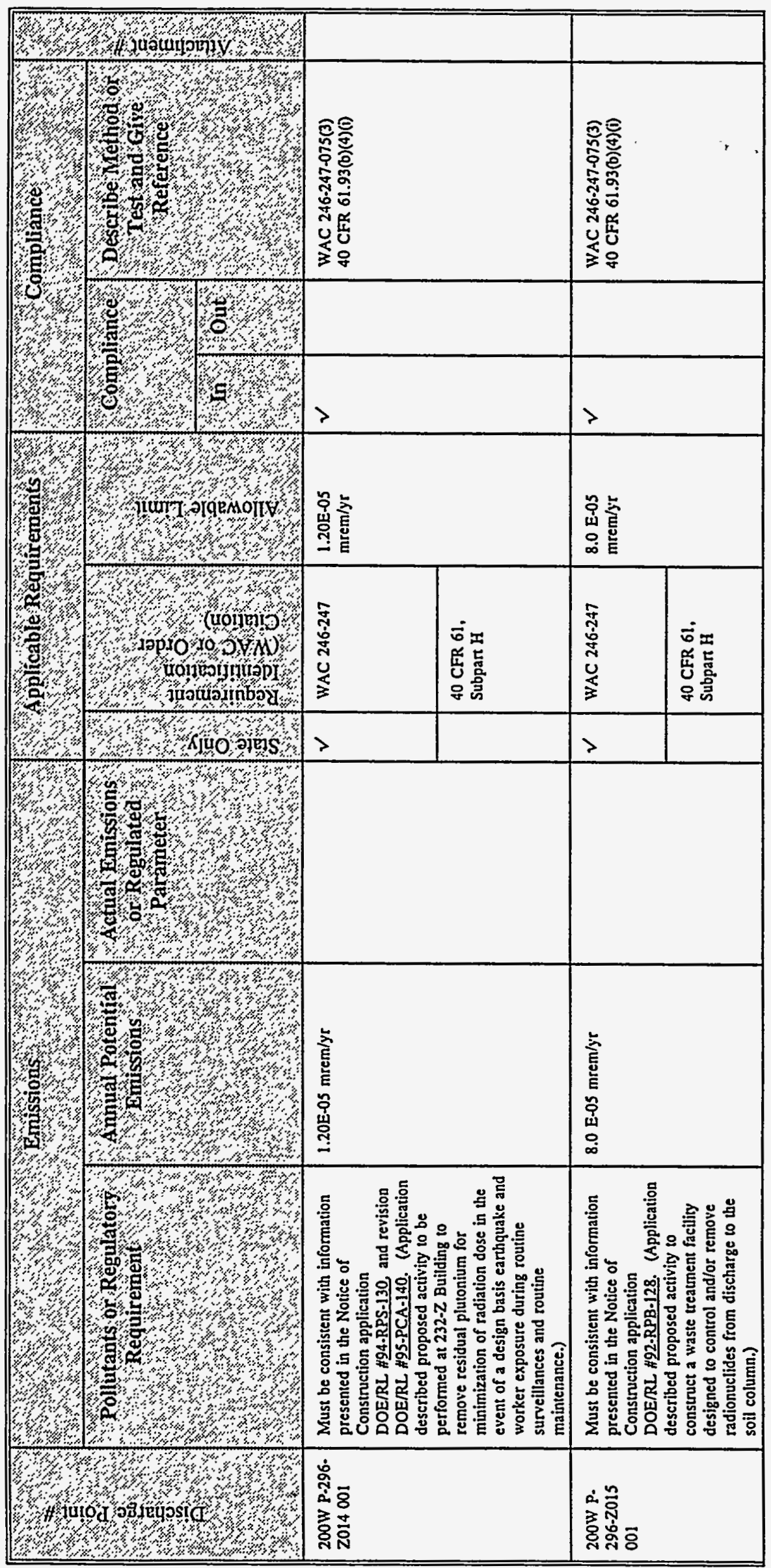


㐫

\begin{tabular}{|c|c|c|c|c|c|c|c|c|c|}
\hline & 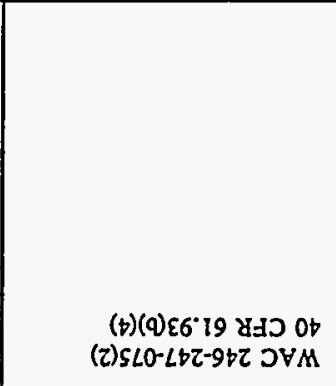 & GVON & 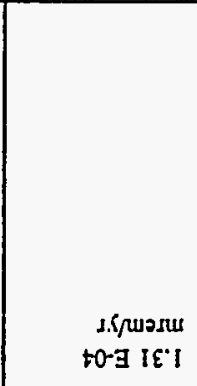 & 19 HEd प XIJ Ot & & & 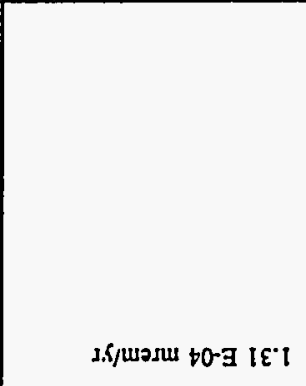 & 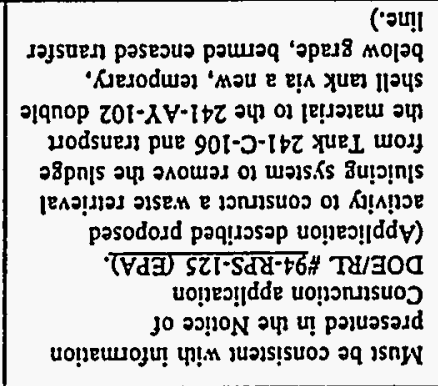 & $\begin{array}{r}100910 d 962 \\
-d 3002\end{array}$ \\
\hline & & $\Lambda$ & & $\angle b Z-9 b \tau$ JVM & $\wedge$ & & & 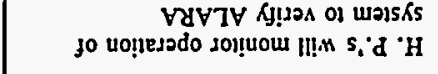 & \\
\hline & 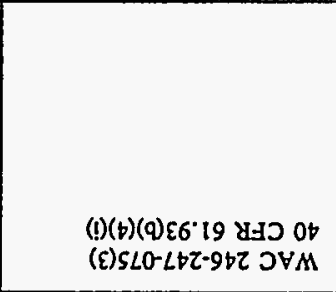 & $\mathrm{V} \square \mathrm{N}$ & $\begin{array}{l}\text { म.i, wasus } \\
80 \cdot \exists \tau \varepsilon^{\prime} 1\end{array}$ & $\angle D Z-9 b Z \supset Y M$ & $\wedge$ & & 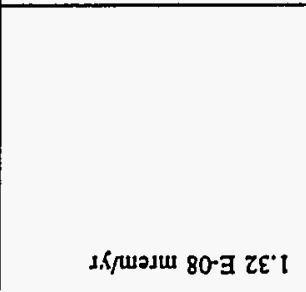 & 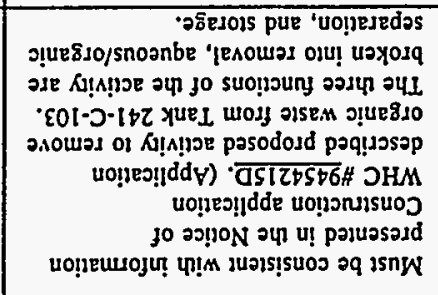 & $\begin{array}{r}100 \text { E0101 thz } \\
-d \mathrm{~d} 300 \tau\end{array}$ \\
\hline of & 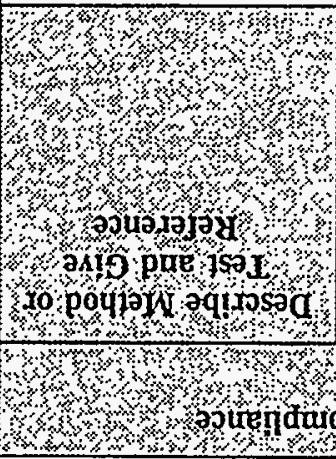 & 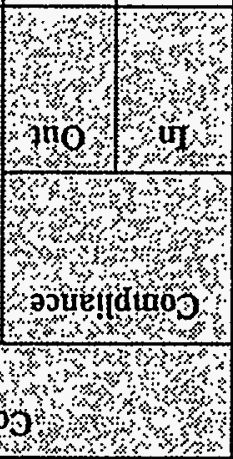 & 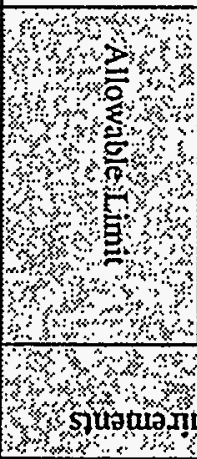 & 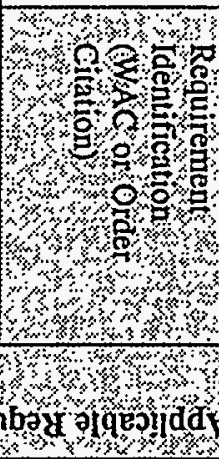 & 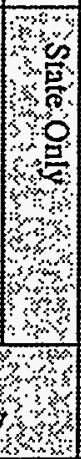 & 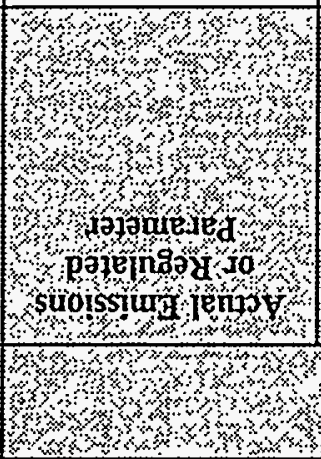 & 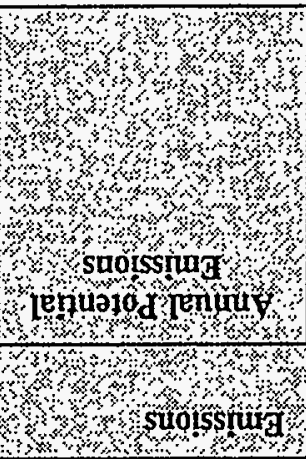 & 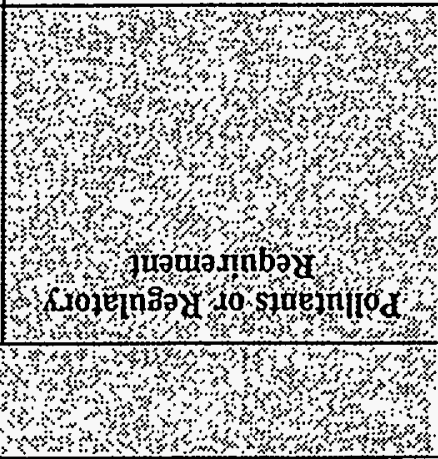 & (3) \\
\hline
\end{tabular}

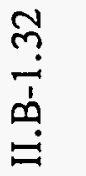

$<$

$\stackrel{0}{\circ}$

us

닝

高

조 ᄋ 


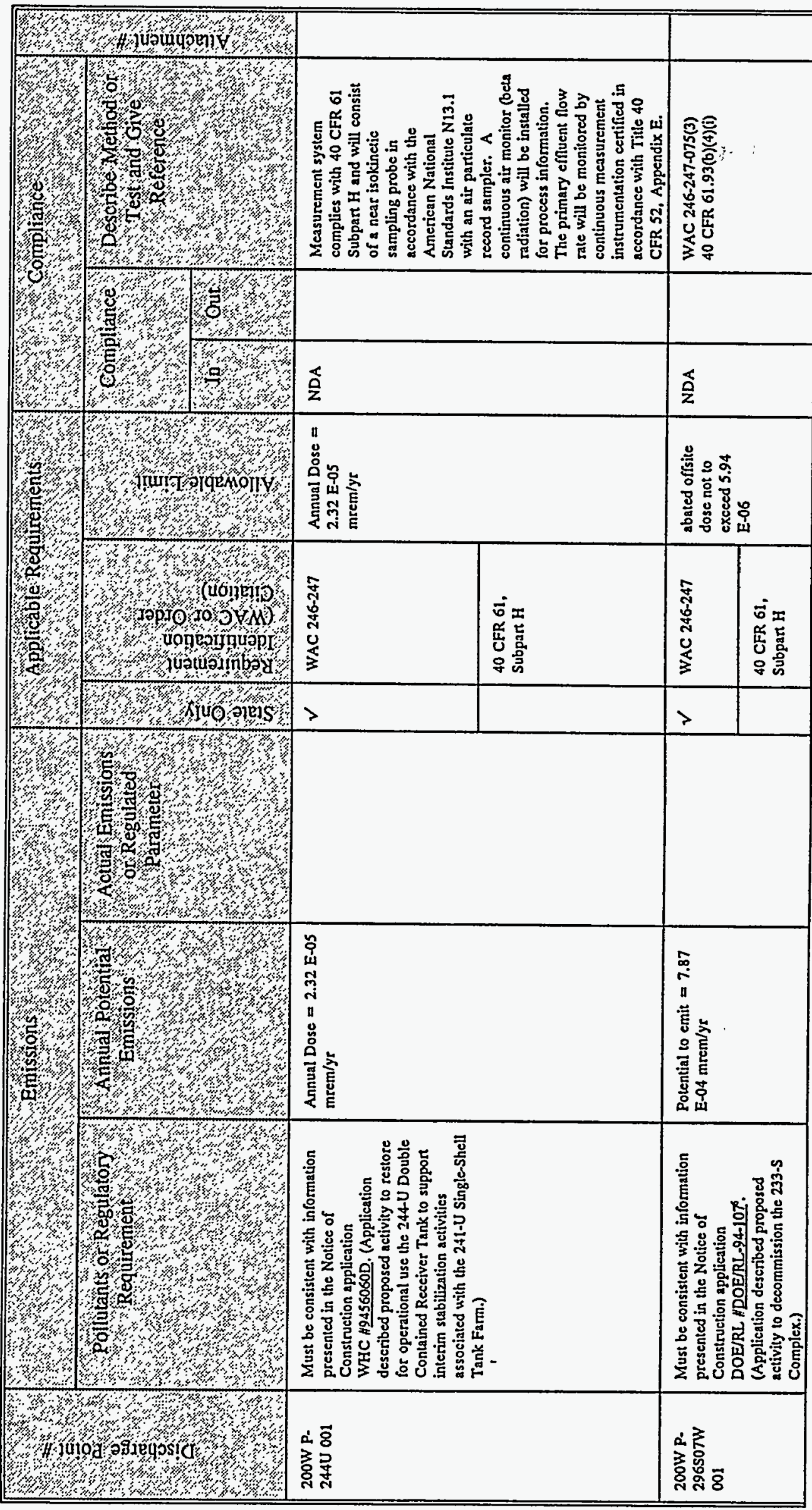




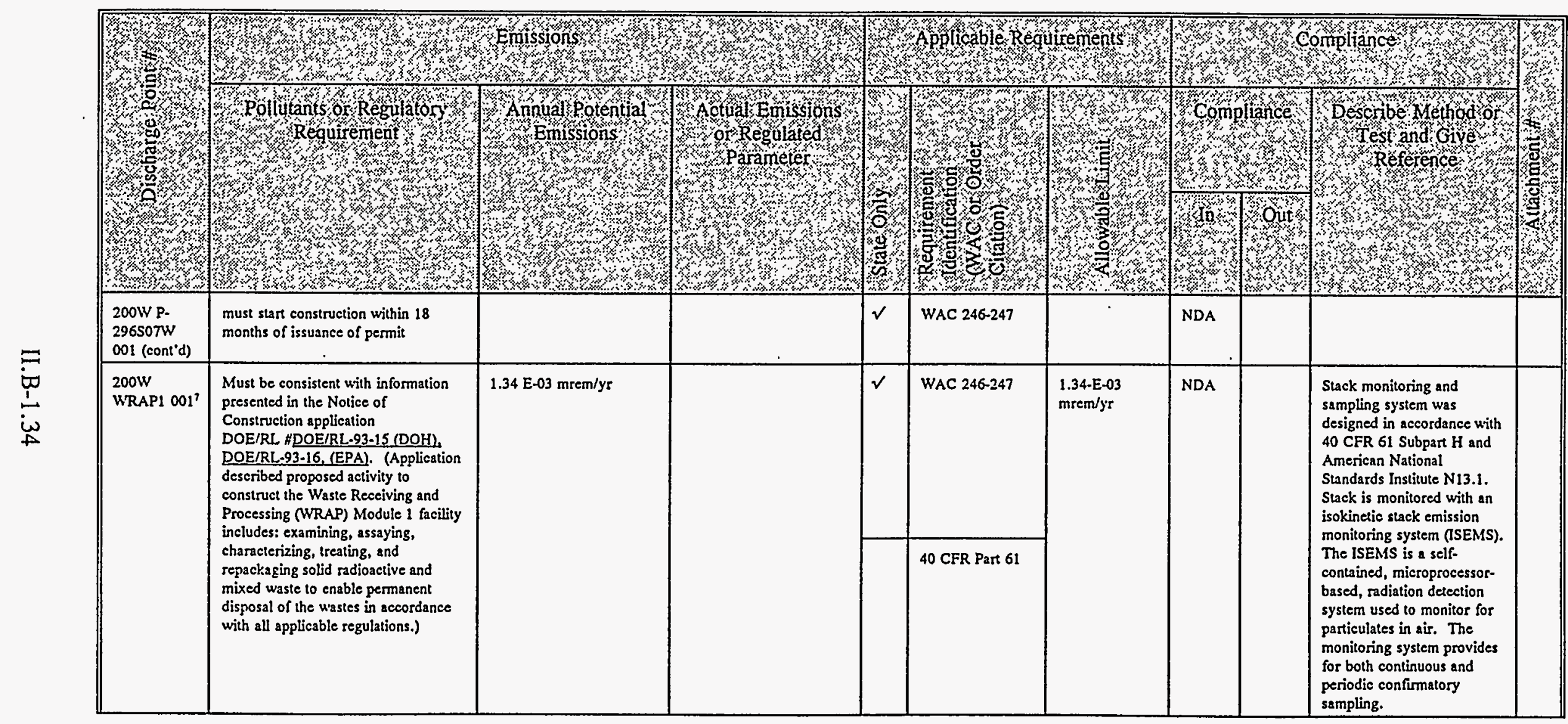


ํ.

ऐं

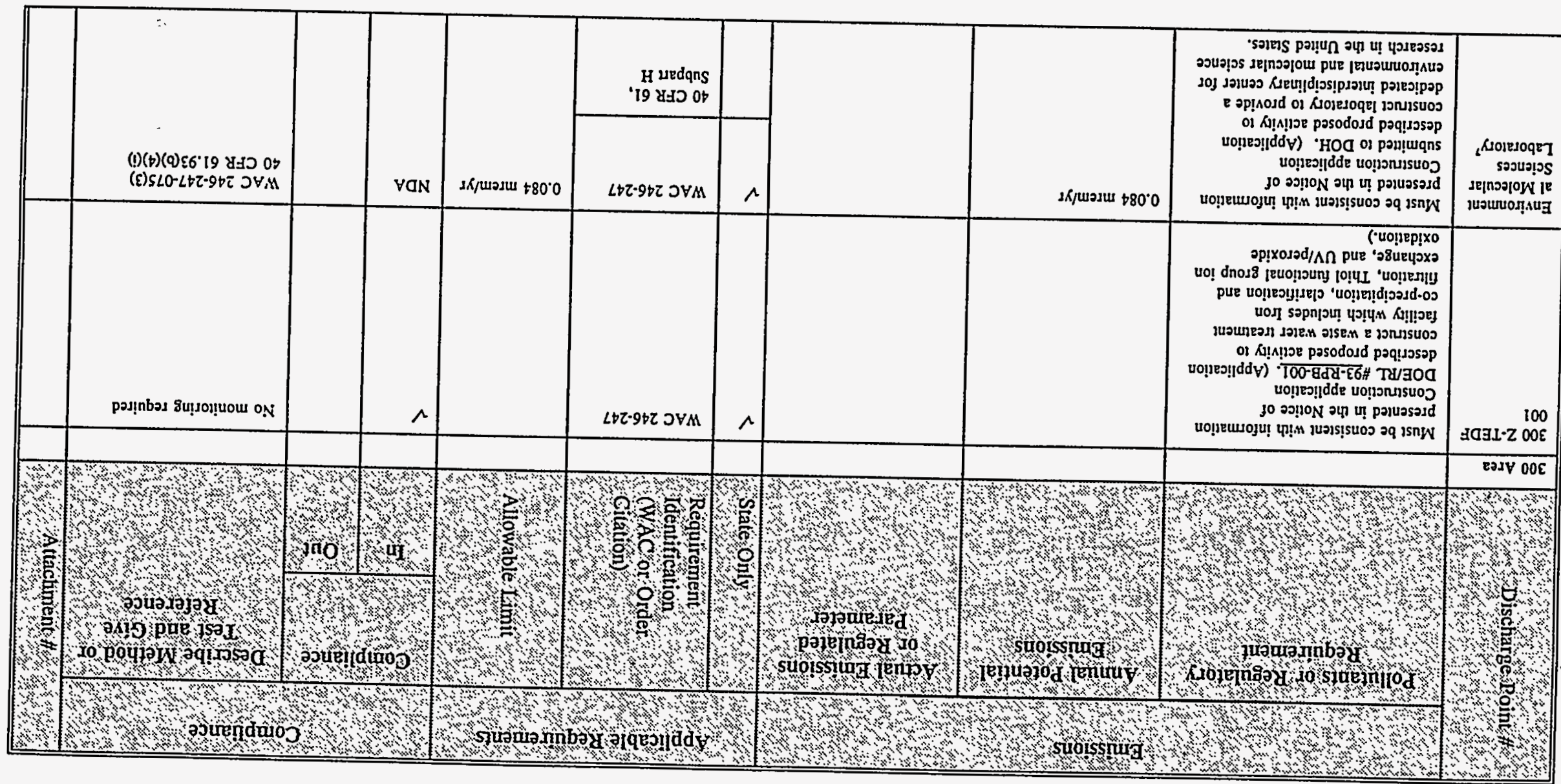




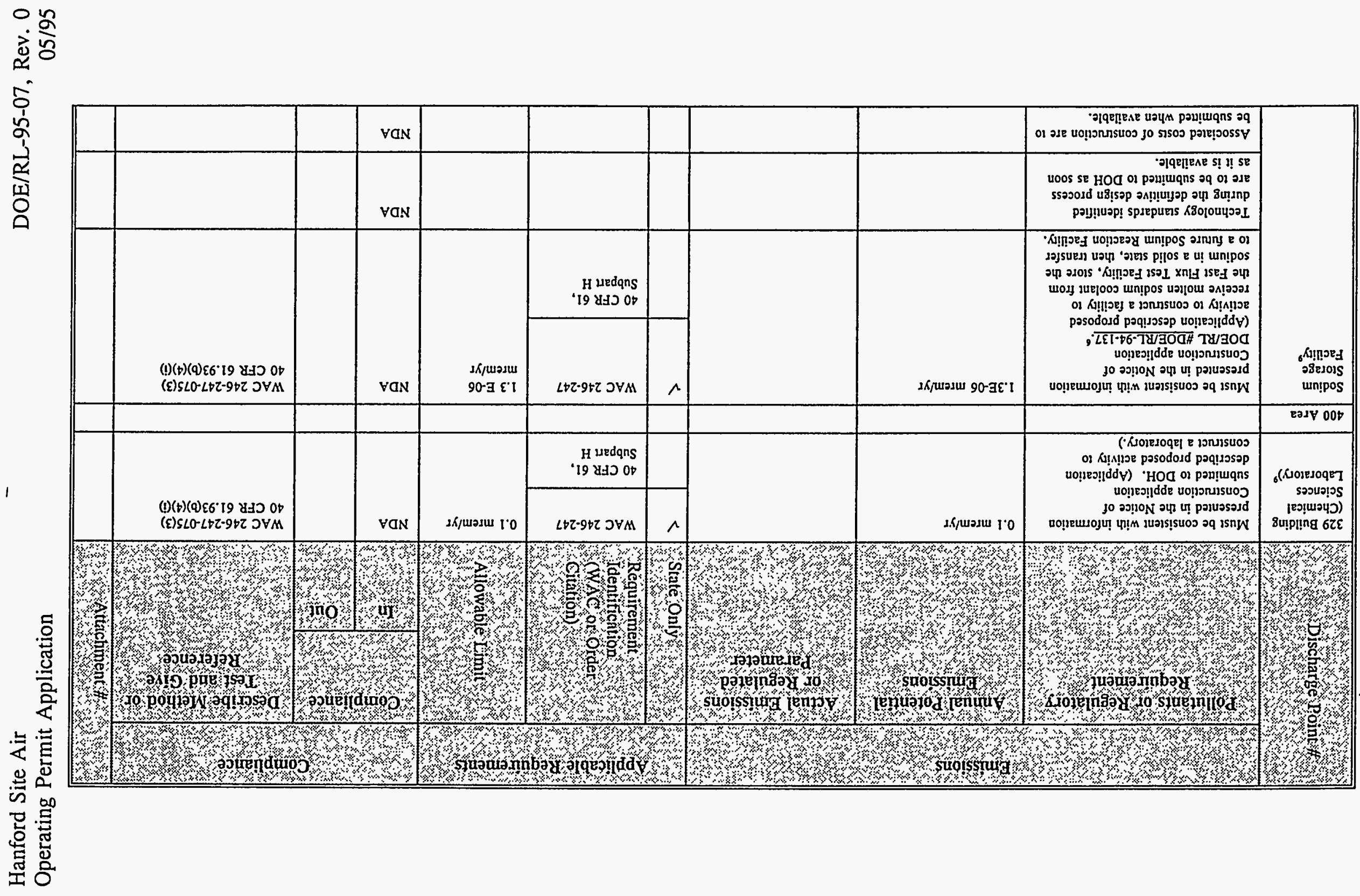


这

ㅊำ

\begin{tabular}{|c|c|c|c|c|c|}
\hline 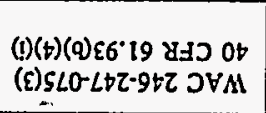 & VaN & $\angle b Z-9 b Z$ JVM & $\wedge$ & 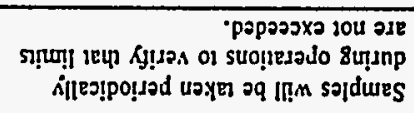 & \\
\hline \multirow[t]{2}{*}{. } & VaN & $\angle \nabla Z-9 b Z$ OYM & $\wedge$ & 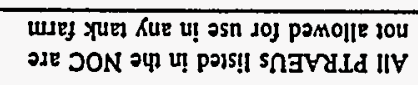 & \\
\hline & Van & 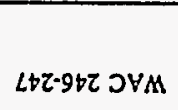 & $\Lambda$ & 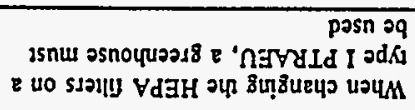 & \\
\hline \multirow[t]{3}{*}{ 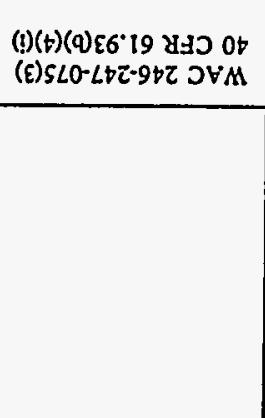 } & VON & $\angle \nabla Z \cdot 9 \forall Z \supset \forall M$ & $\mu$ & 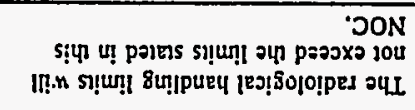 & \\
\hline & & 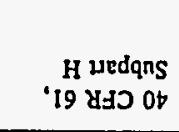 & & 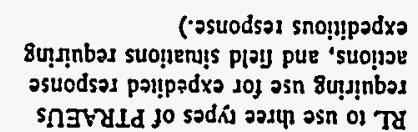 & 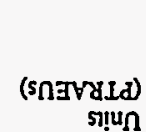 \\
\hline & ran & $\angle t \tau \cdot 9 t \tau$ כ $\forall M$ & $\Lambda$ & 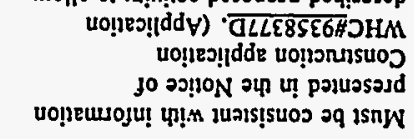 & 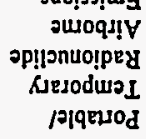 \\
\hline
\end{tabular}

ग5!5
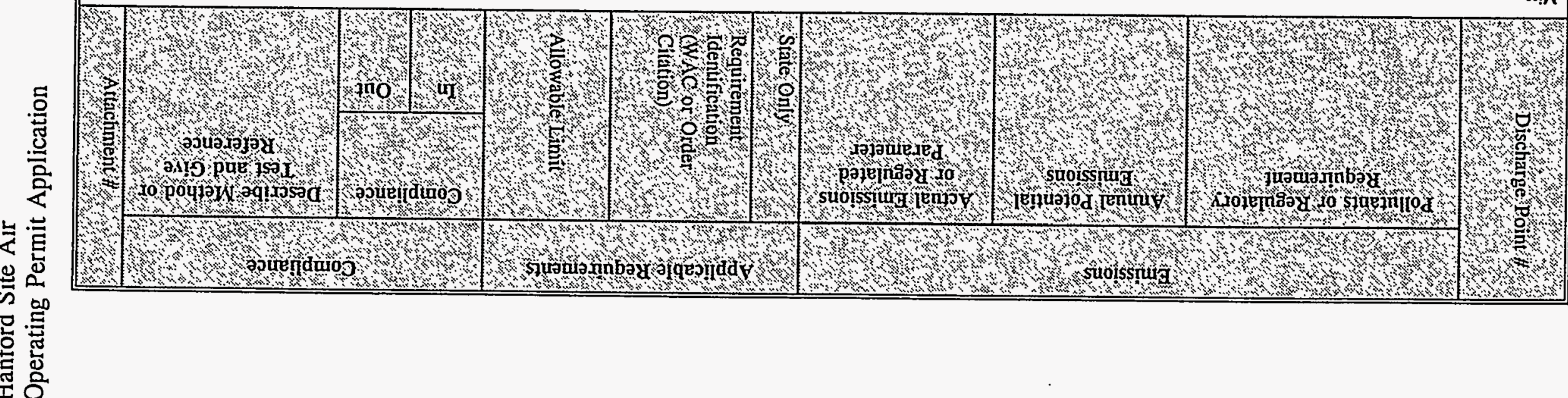
ํำ

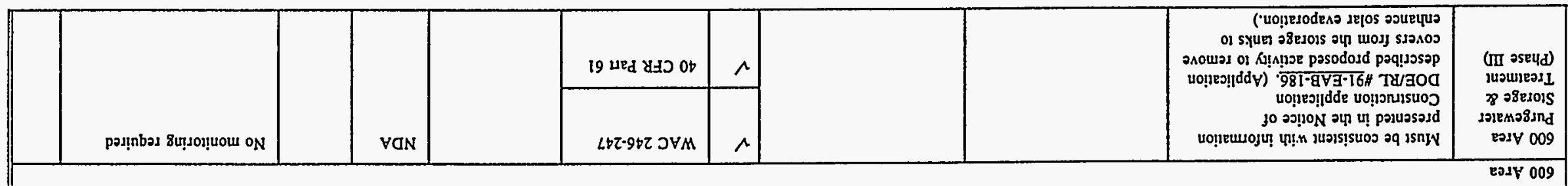

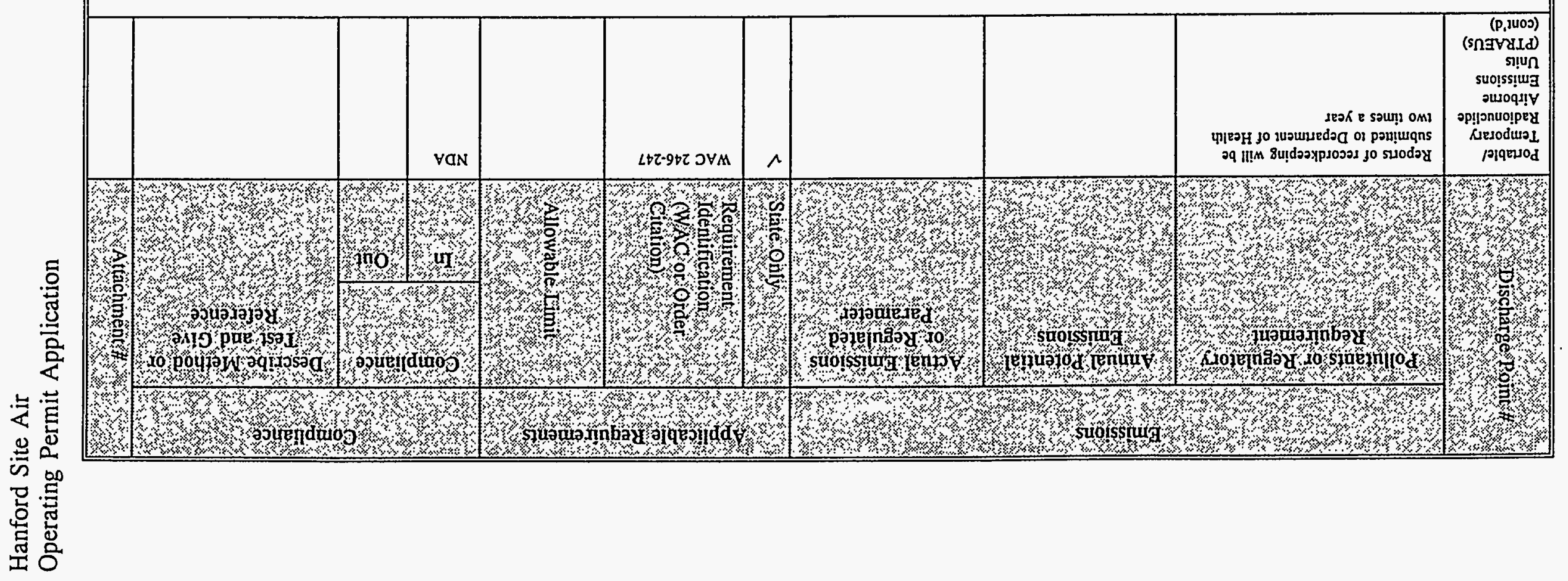




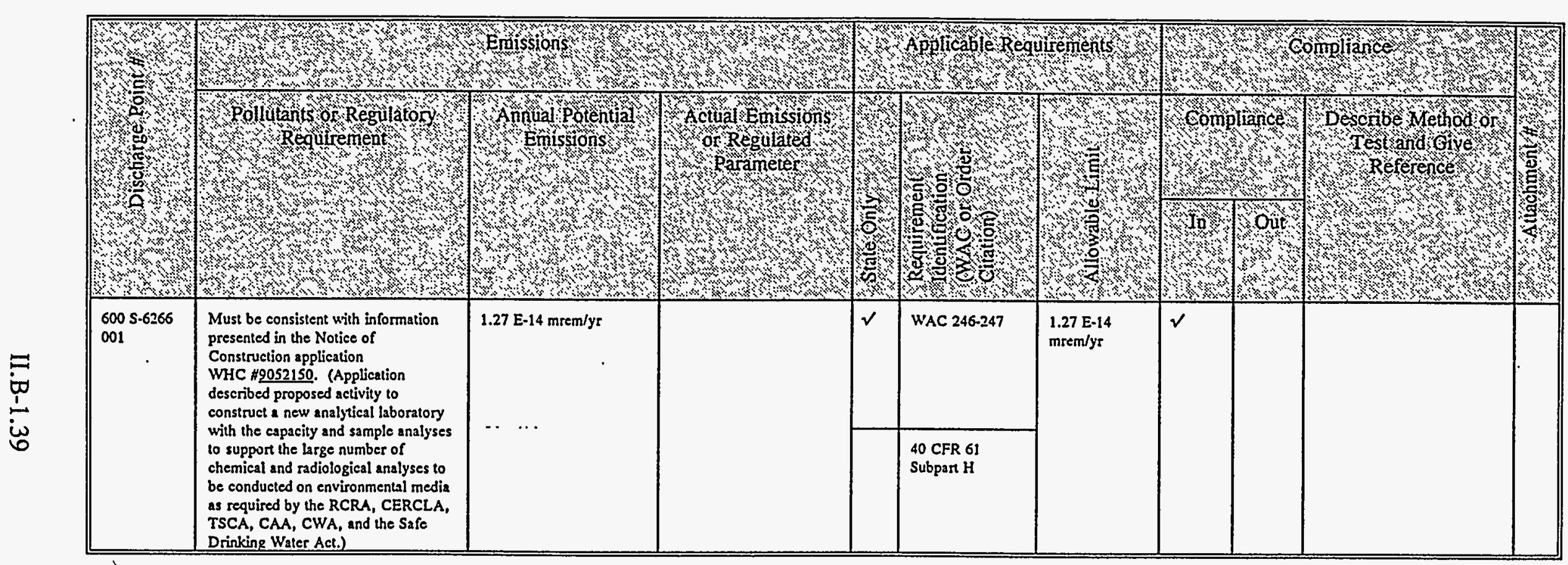

1.Approval for the 105KE Encapsulation Activity has been suspended per DOH letter \#AIR 94-605, Mr. A. W. Conklin, DOH, to Mr. S. H. Wisness, RL, dated June 7, 1994. However, the suspension does not affect other previously approved activities anticipated to occur in the 105KE Basin.

2.NDA = no data available. Project began in January 1995. Updated information will be provided to DOH.

3.Although the regulatory requirement states "During encapsulation activities...," this requirement applies to the other activities anticipated to occur in the 105KE Basin. This is aside from the fact that the $105 \mathrm{KE}$ Encapsulation Activity has been suspended. Refer to endnote 1.

4. Regulatory requirement applies to other anticipated activities in the 105KE Basin. 
5.105KE Encapsulation Activity suspended. Refer to endnote 1.

6.The notice of construction was sent to both the State of Washington, Department of Health and the U.S. Environmental Protection Agency, Region X.

7.Project construction is in progress. Upon startup, compliance with requirements will be assessed.

8. The State of Washington, Department of Health has been requested to change continuous stack monitoring to periodic confirmatory measurements.

9.Project construction has not begun. Upon startup, compliance with requirements will be assessed. 
Table IIB-2: Regulations and Regulated Emissions Air OpERATINg PERMtT Applications

Facility Name: Hanford Site, Non-Radioactive Emissions, Registered Emission Units

Emission Point Compliance Review

\begin{tabular}{|c|c|c|c|c|c|c|c|c|c|c|}
\hline 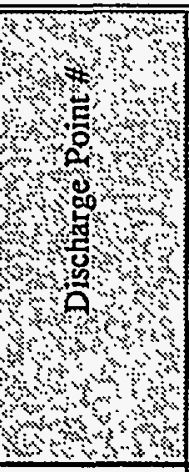 & 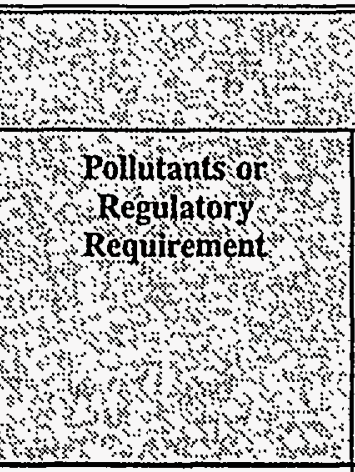 & 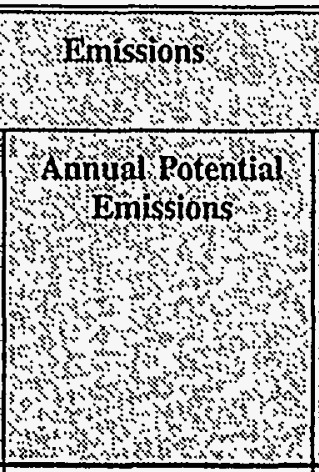 & 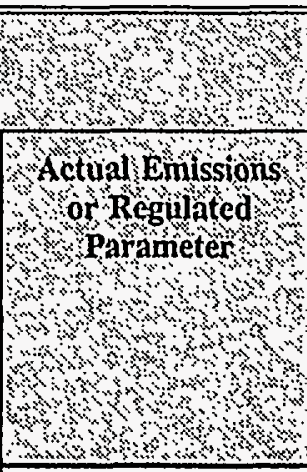 & 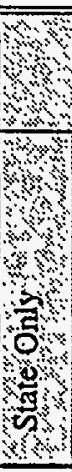 & 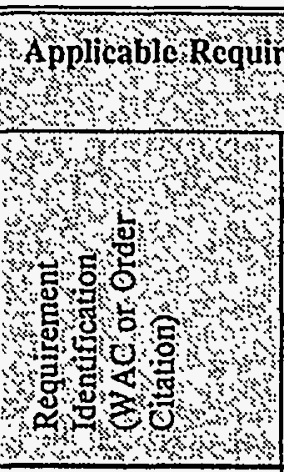 & $\frac{0}{\text { ements }}$ & 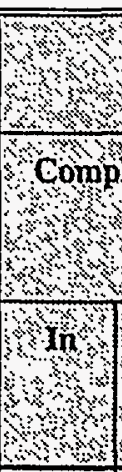 & 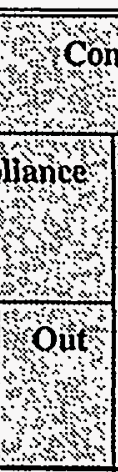 & 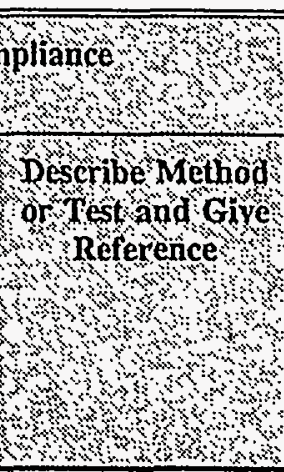 & s. \\
\hline \multirow{6}{*}{$\begin{array}{l}200 E \text { F-284E } \\
001\end{array}$} & Opacity & & & & WAC $173-400-040(1)$ & $20 \%$ & $\checkmark$ & & EPA Method 9! & \\
\hline & $\mathrm{SO}_{2}$ & & 88.1 & & WAC $173-400-040(6)$ & $1000 \mathrm{ppm}$ & $\checkmark$ & & EPA Method 8 & \\
\hline & Particulate Matter & & $0.03 \mathrm{gr} / \mathrm{dscf}$ & & WAC $173-400-050(1)$ & $0.2 \mathrm{gr} / \mathrm{dscf}$ & $\checkmark$ & & EPA Method 5 & \\
\hline & Registration & & & & WAC $173-400-100$ (dd) & & $\sqrt{ }$ & & & \\
\hline & Excess Emission Notification & & & & WAC $173-400 \cdot 107$ & & $\checkmark$ & & & \\
\hline & $\begin{array}{l}\text { Reporing, Monitoring, and } \\
\text { Recordkeeping }\end{array}$ & & & & WAC $173-400-105$ & & $\checkmark$ & & & \\
\hline \multirow{5}{*}{$\begin{array}{l}200 \mathrm{E} F-284 \mathrm{E} \\
002\end{array}$} & Opacity & & & & WAC $173-400-040(1)$ & $20 \%$ & $\checkmark$ & & EPA Method $9^{\prime}$ & \\
\hline & $\mathrm{SO}_{2}$ & & 88.1 & & WAC $173-400-040(6)$ & $1000 \mathrm{ppm}$ & $\sqrt{ }$ & & EPA Method 8 & \\
\hline & Particulate Matter & & $0.03 \mathrm{gr} / \mathrm{dscf}$ & & WAC $173-400-050(1)$ & $0.2 \mathrm{gr} / \mathrm{dscf}$ & $\checkmark$ & & EPA Method 5 & \\
\hline & Registration & & & & WAC $173-400 \cdot 100$ (dd) & & $\checkmark$ & & & \\
\hline & Excess Emission Notification & & & & WAC $173-400-107$ & & $\checkmark$ & & . & \\
\hline
\end{tabular}


官

这

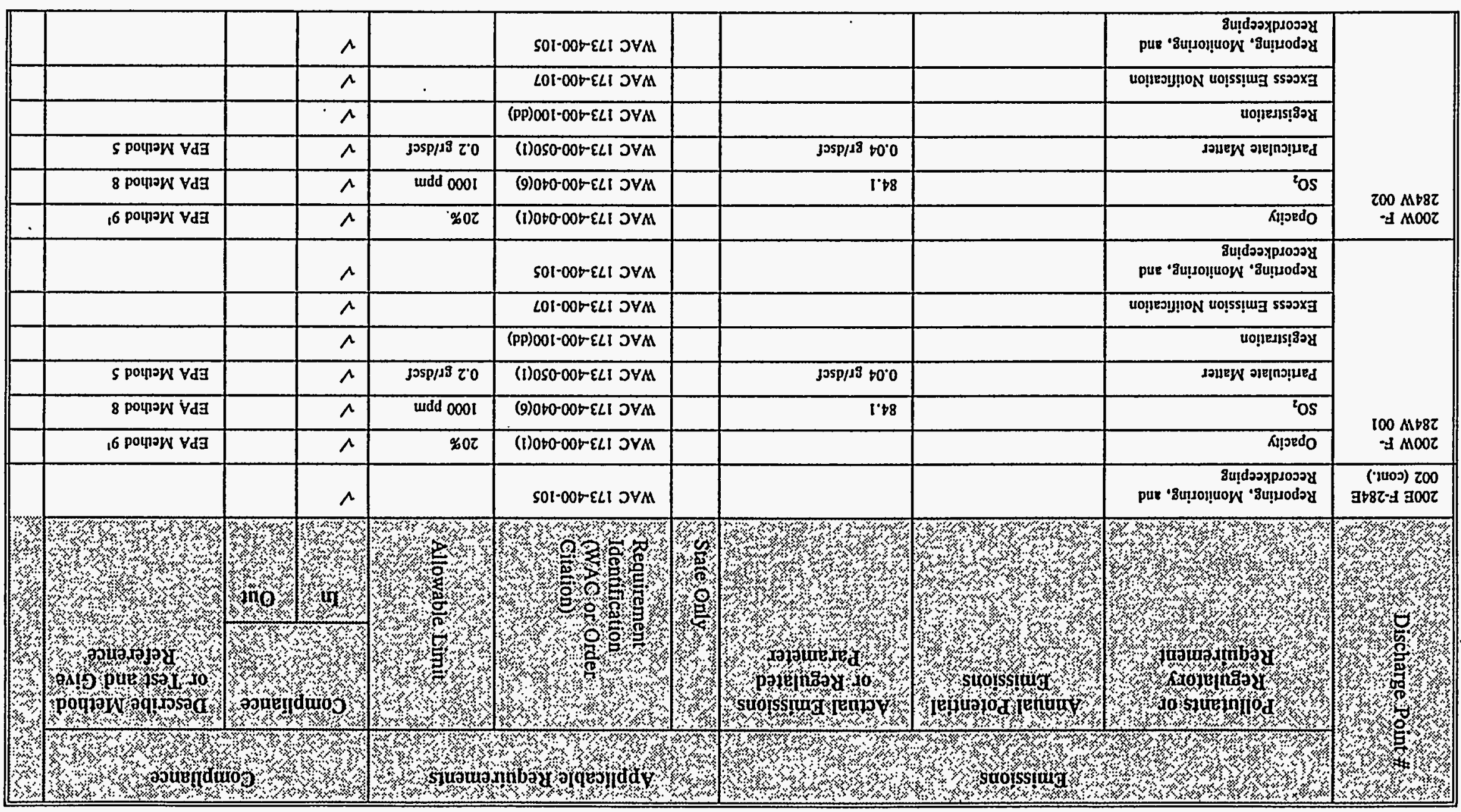

๓̃

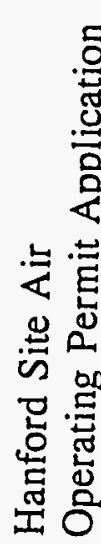




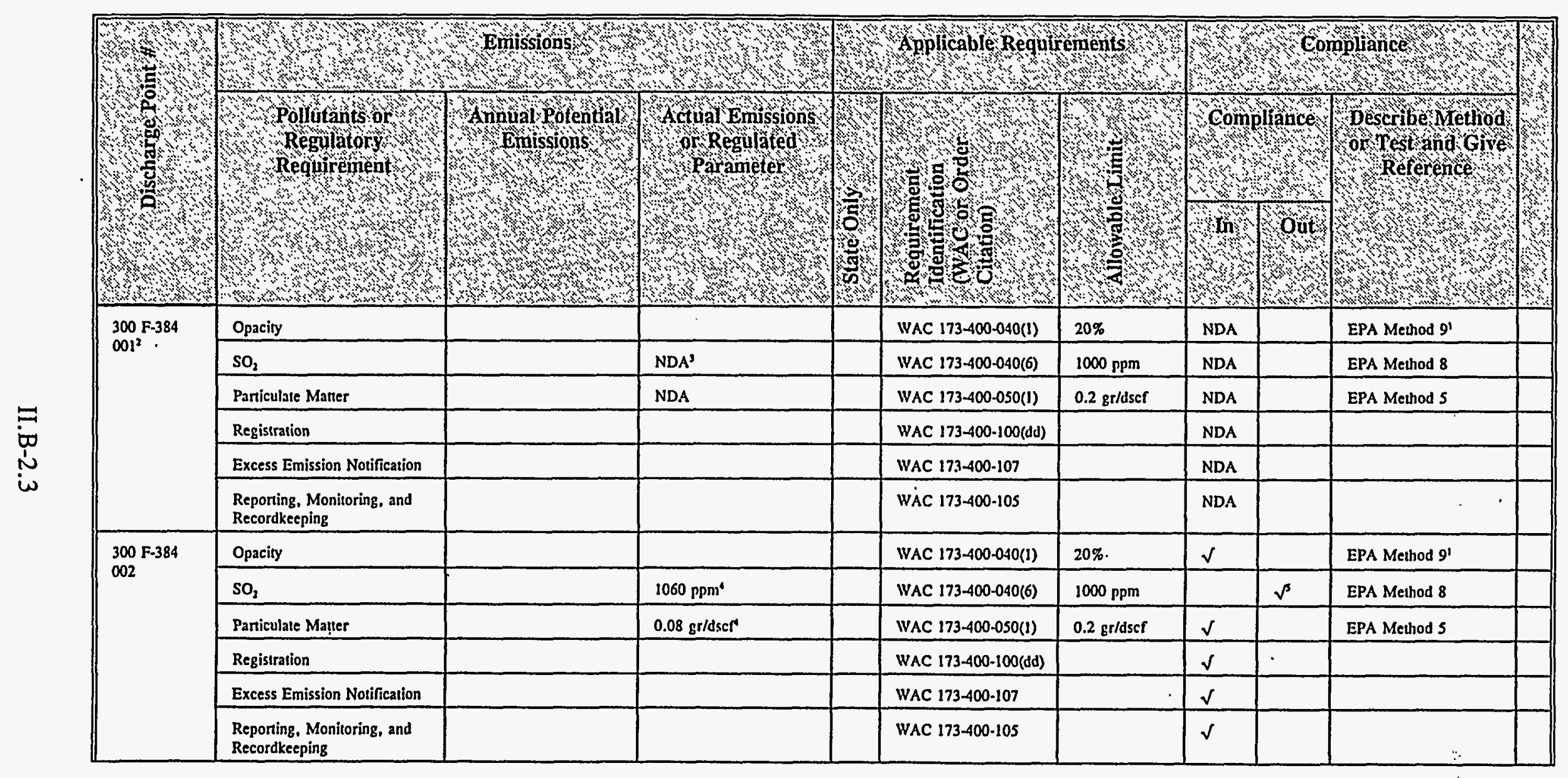




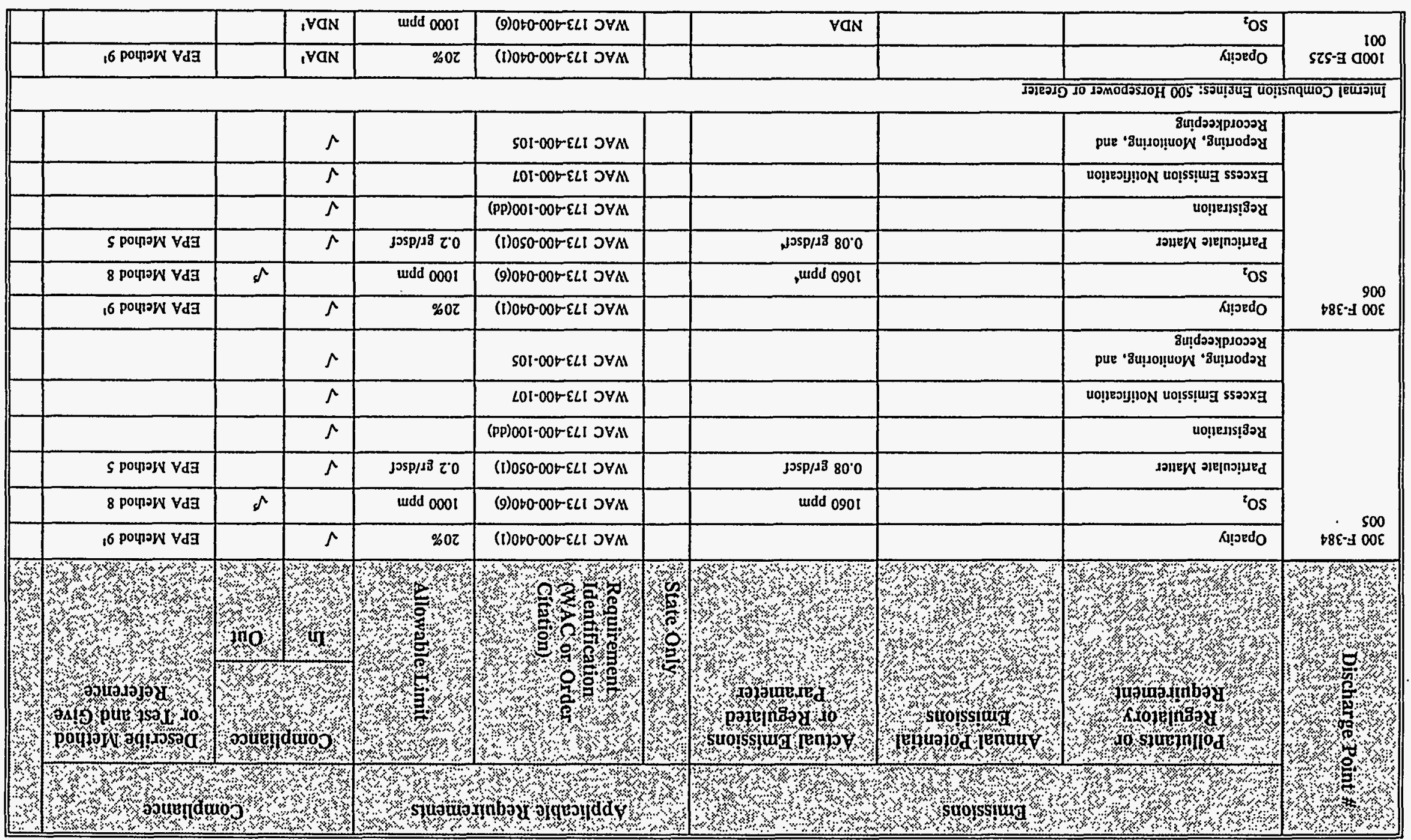




\begin{tabular}{|c|c|c|c|c|c|c|c|c|c|c|c|c|c|}
\hline \\
\hline \multicolumn{14}{|l|}{ 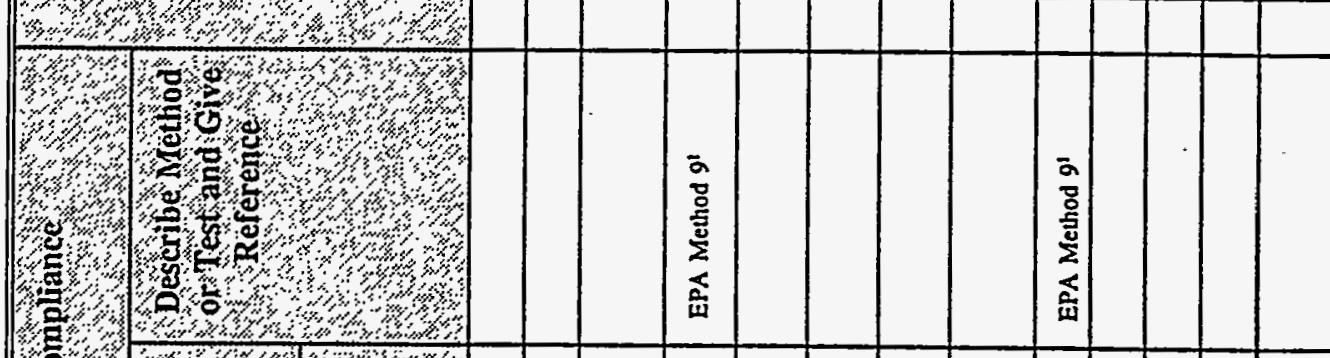 } \\
\hline (O) & & & & & & & & & & & & & \\
\hline mols & $s$ & $\widehat{\hat{a}}$ & $\widehat{\Sigma}$ & 高 & 产 & $s$ & $\widehat{\overbrace{}}$ & $\widehat{\S}$ & 髉 & 育 & $\xi$ & $\widehat{人}$ & $\overleftarrow{\Delta}$ \\
\hline 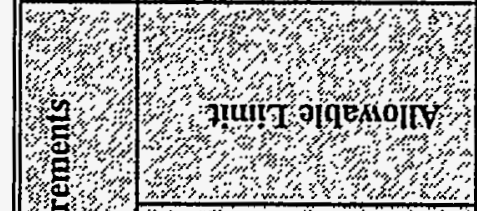 & & & & :̊ํ & 흘 & & & & 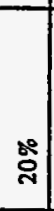 & 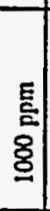 & & & \\
\hline 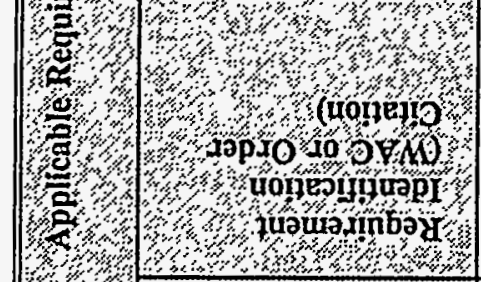 & 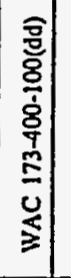 & 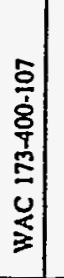 & 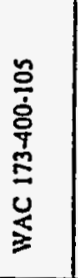 & 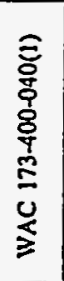 & 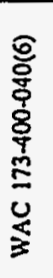 & 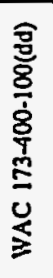 & 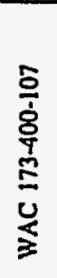 & 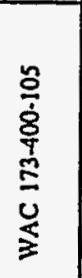 & 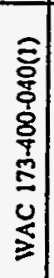 & 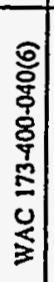 & 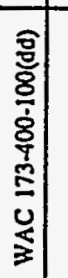 & 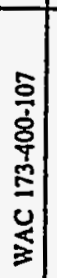 & 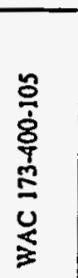 \\
\hline 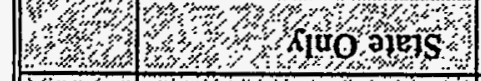 & & & & & & & & & & & & & \\
\hline 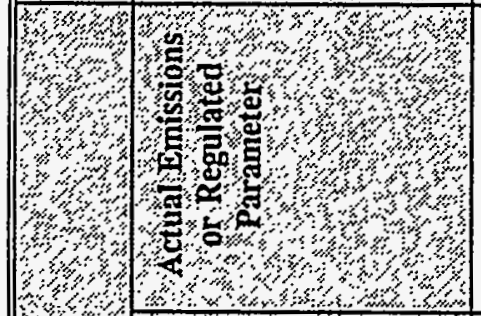 & & & & & $\widehat{\rho}$ & & & & & $\widehat{\widehat{c}}$ & & & \\
\hline 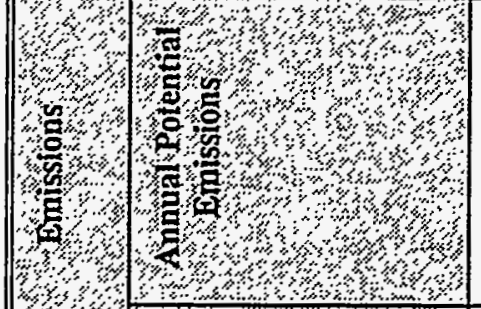 & & & & & & & & & & & & & \\
\hline \% & 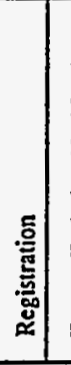 & 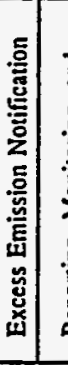 & 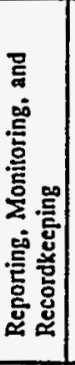 & $\begin{array}{l}\text { 产 } \\
\text { gू }\end{array}$ & in & 咅 & 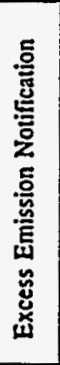 & 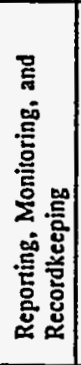 & 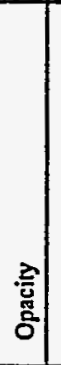 & & & 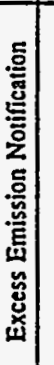 & 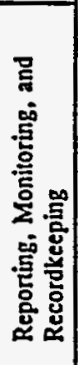 \\
\hline 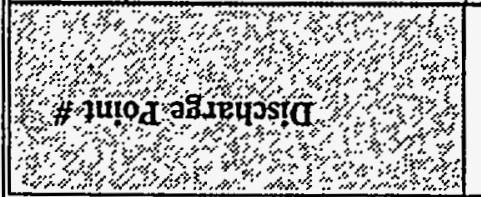 & \multicolumn{3}{|c|}{ 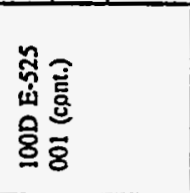 } & \multicolumn{5}{|l|}{ 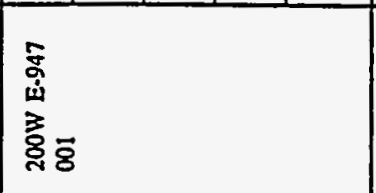 } & \multicolumn{5}{|c|}{ 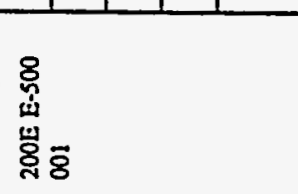 } \\
\hline
\end{tabular}




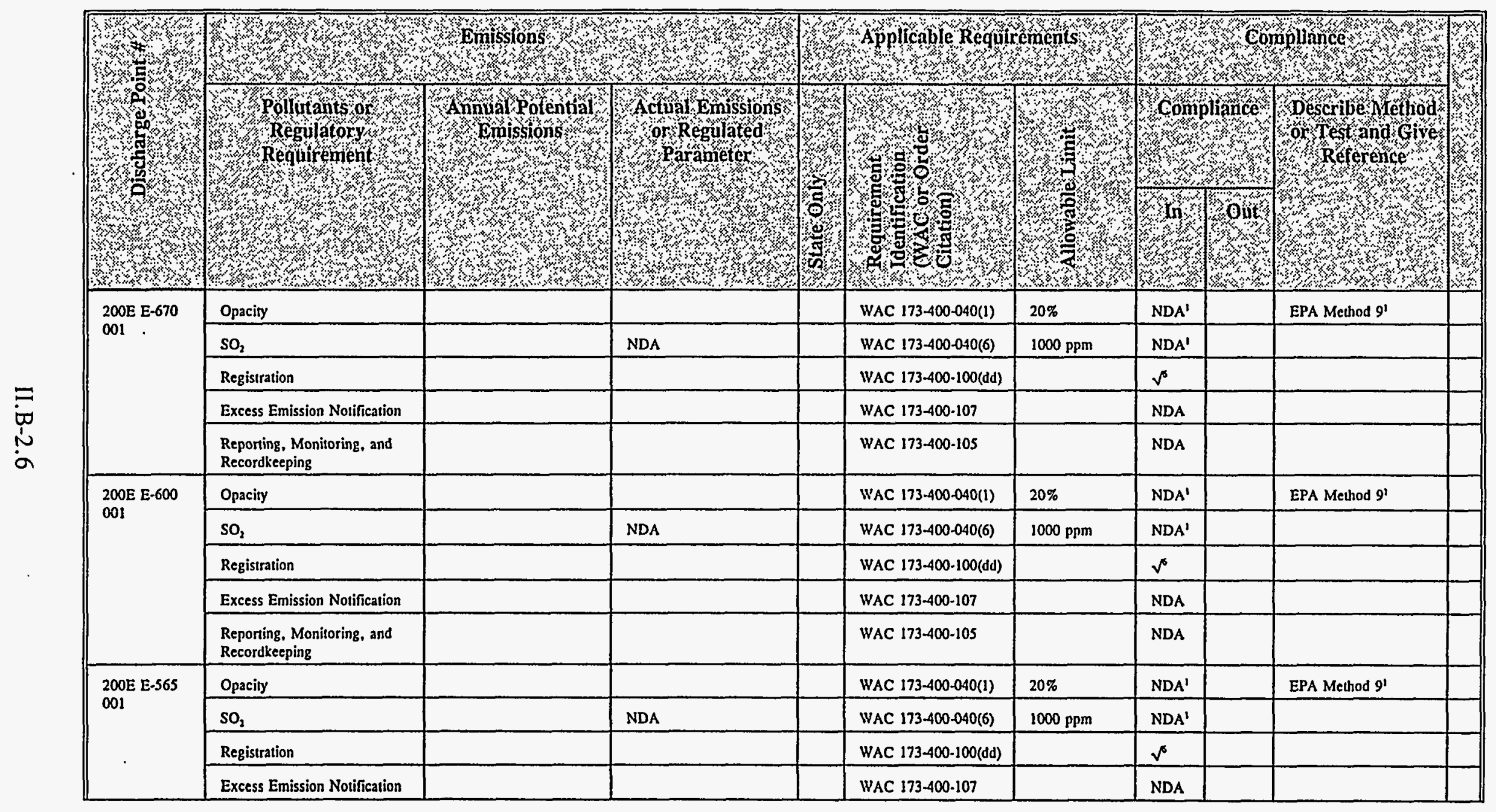




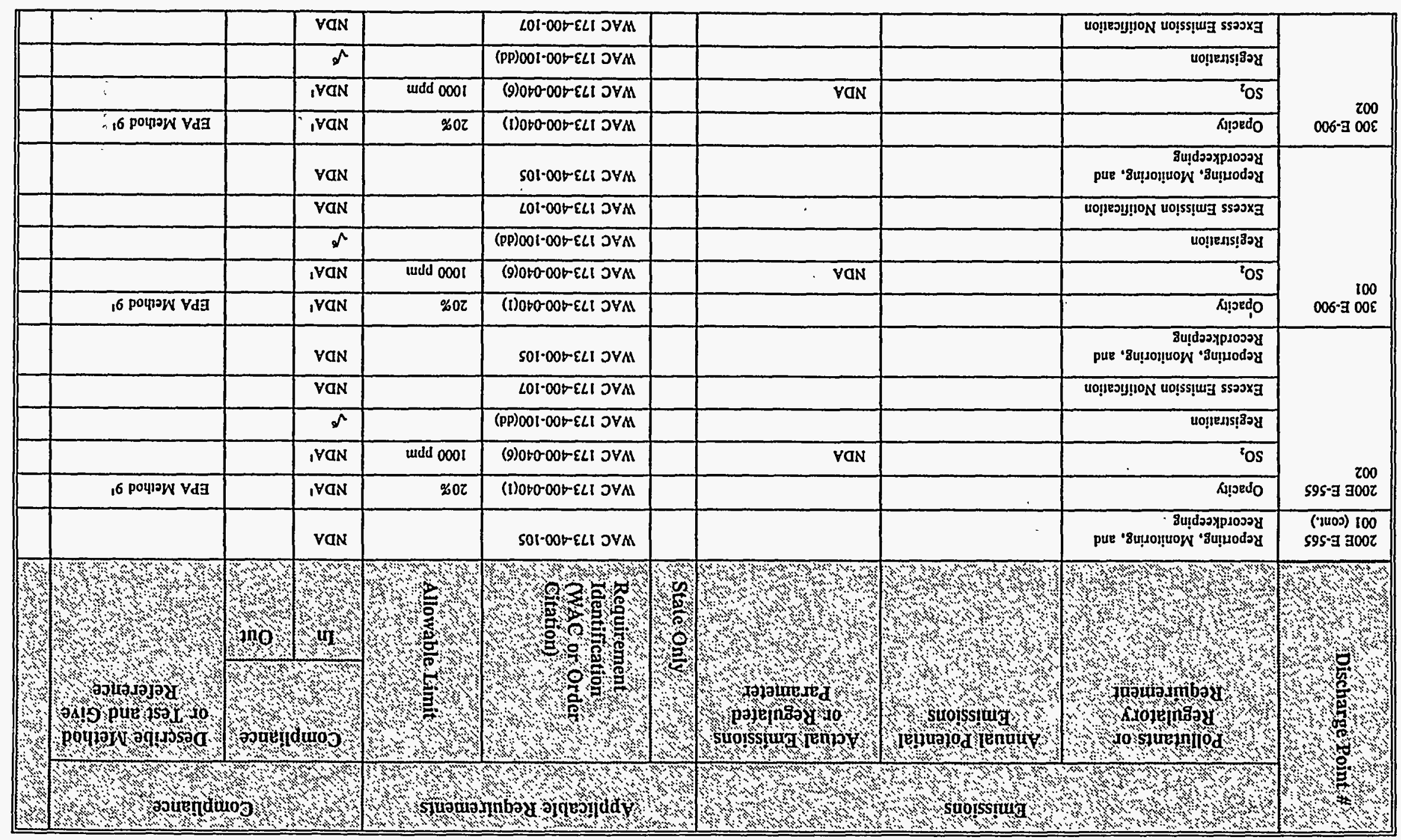




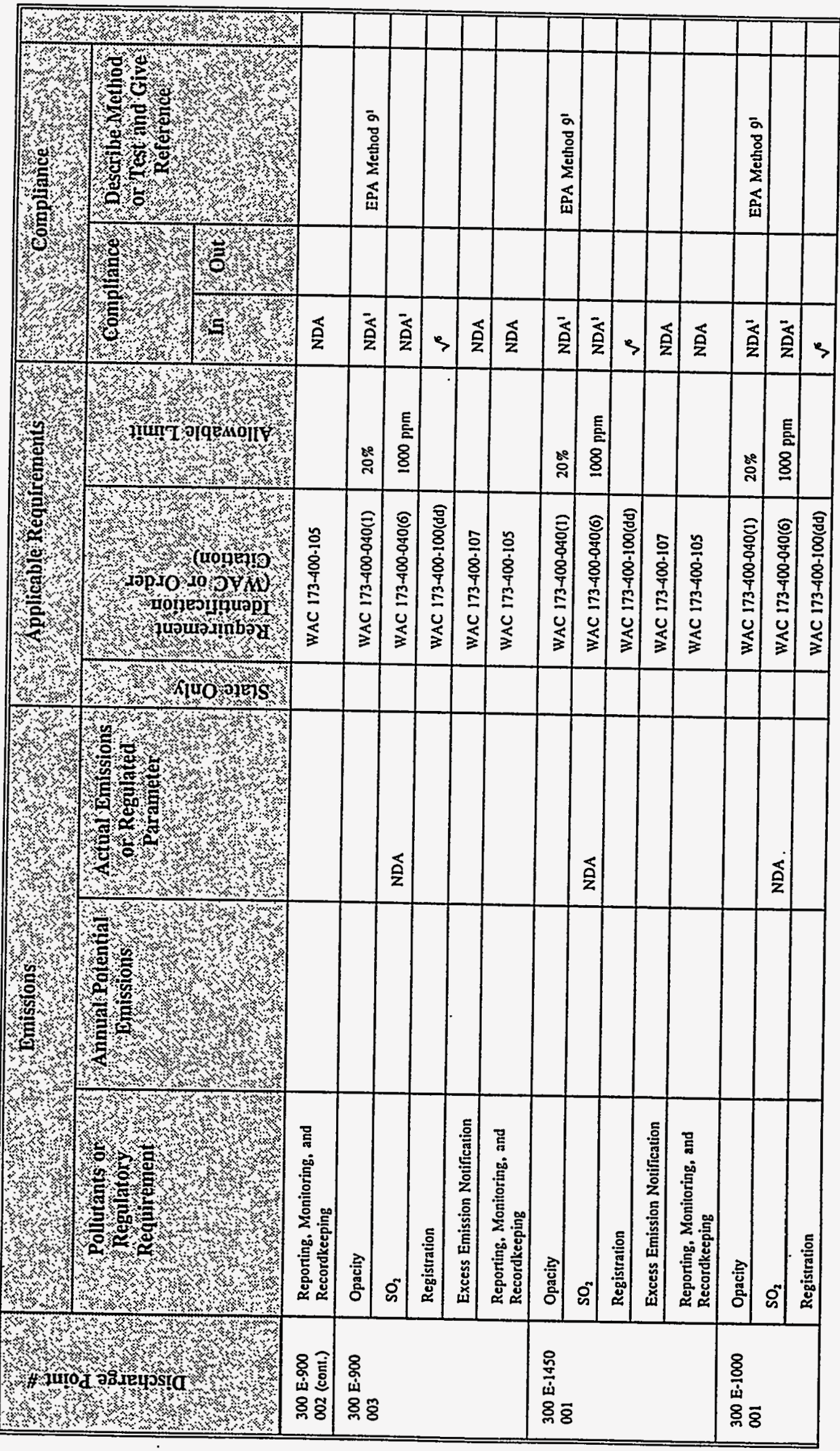


Hanford Site Air

DOE/RL-95-07, Rev. 0

Operating Permit Application

05/95

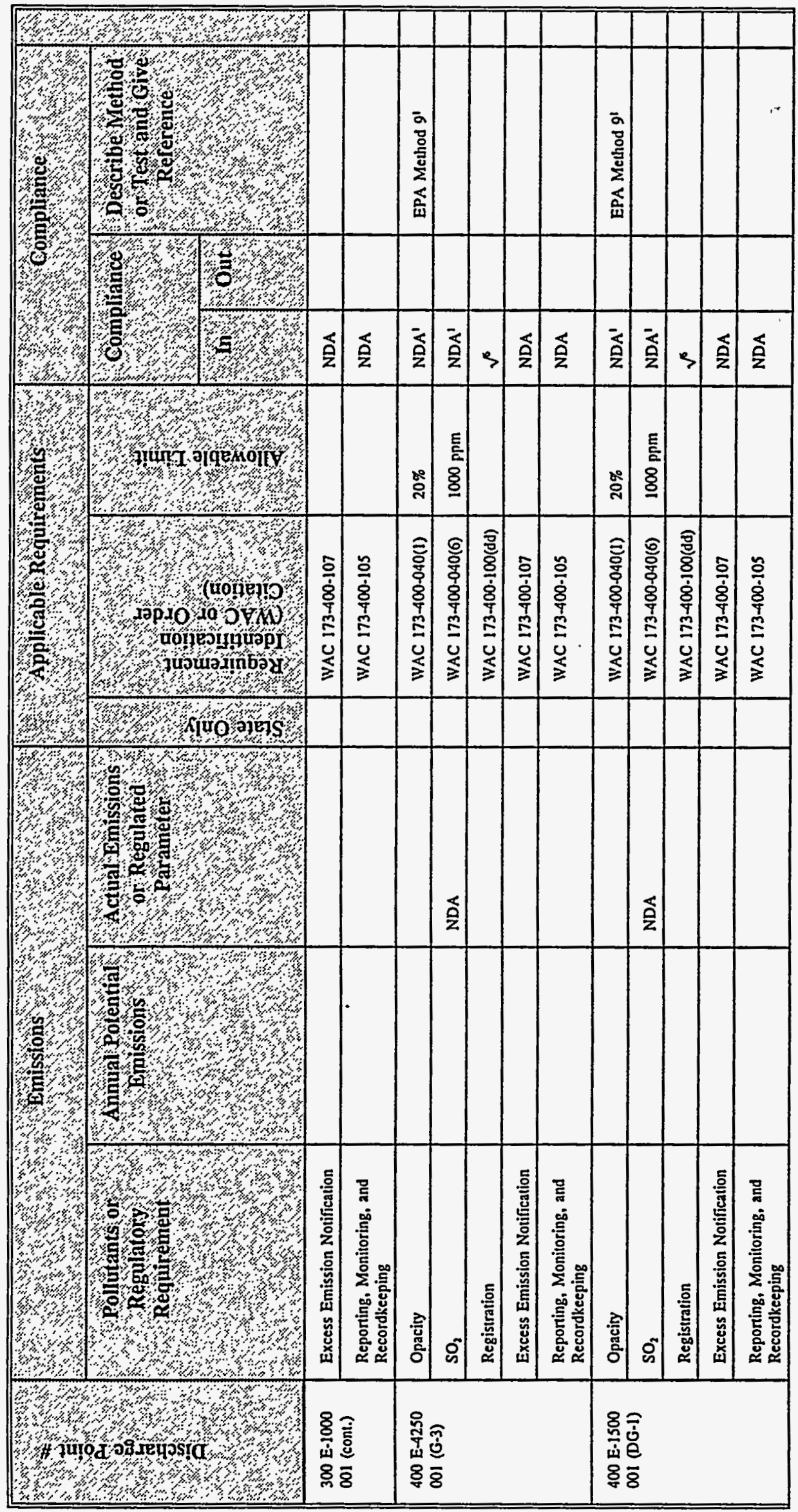

II.B-2.9 


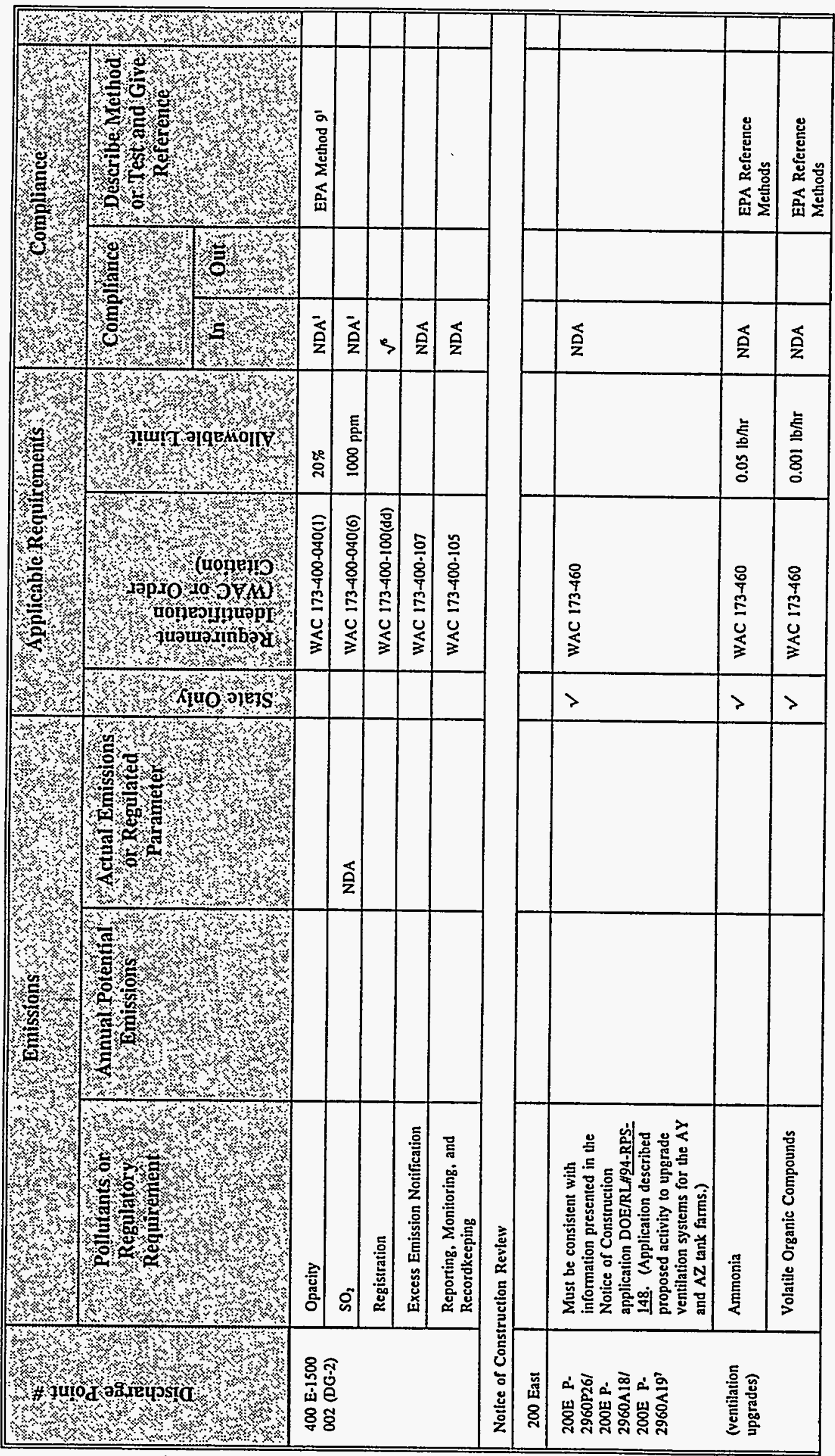




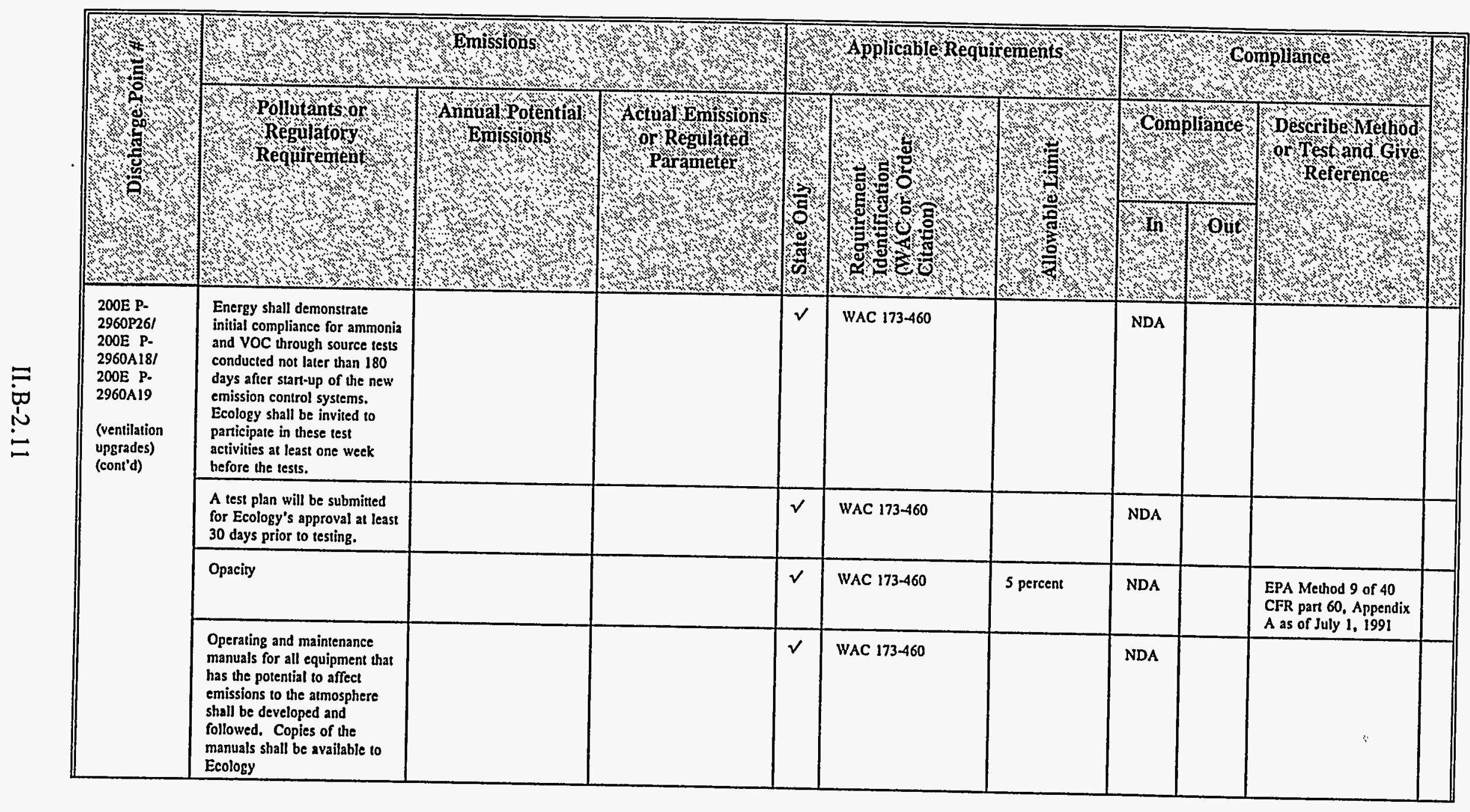




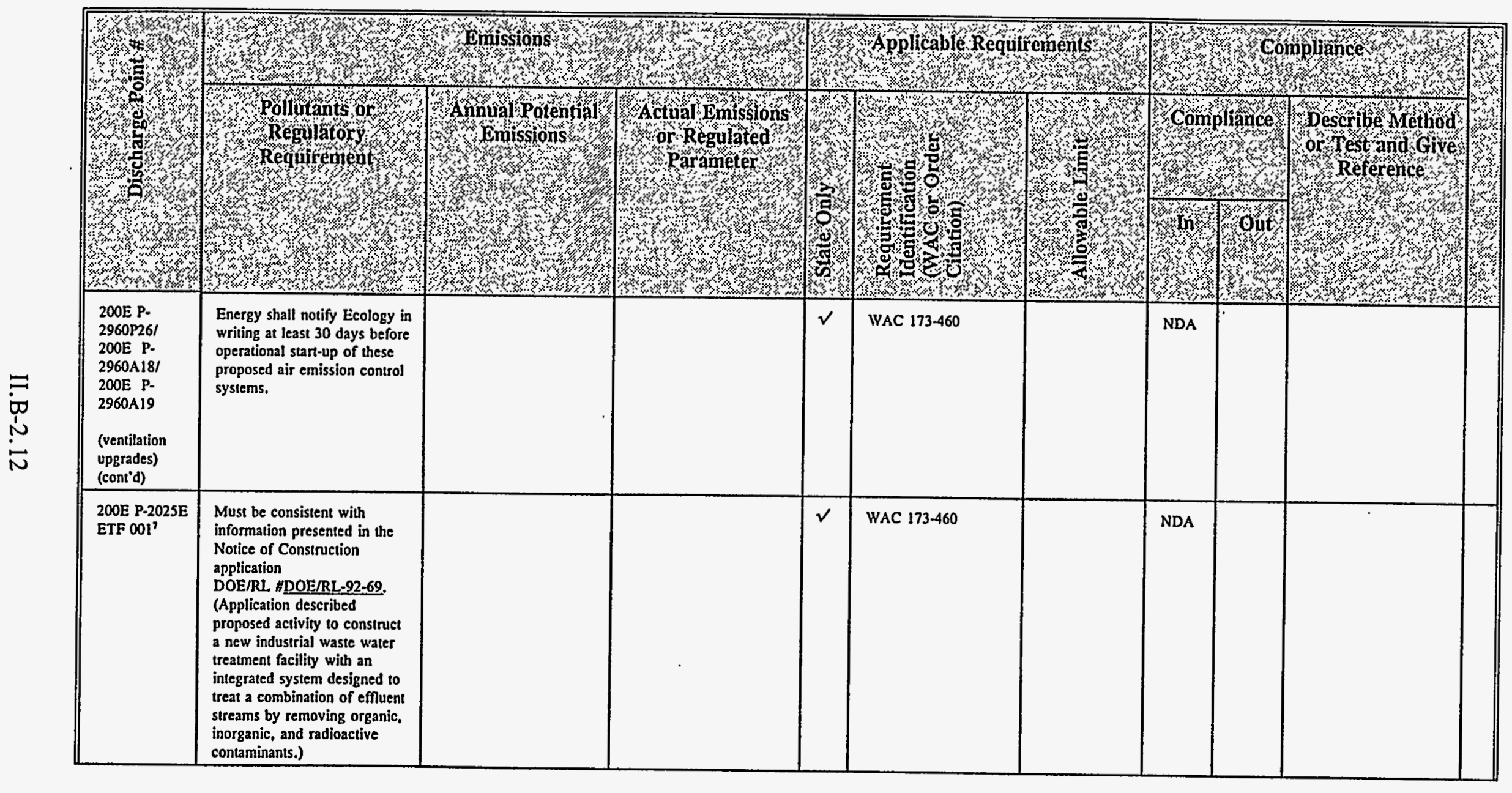




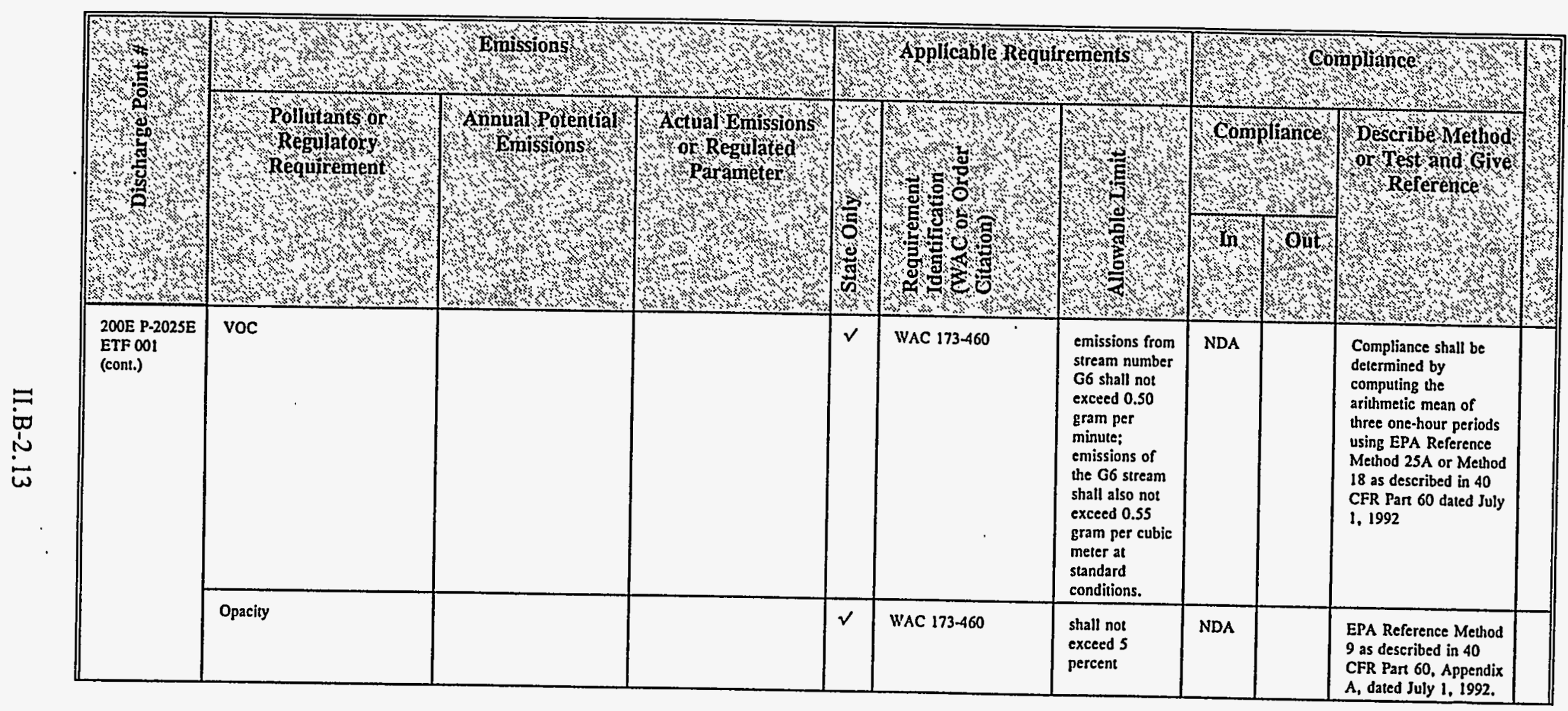




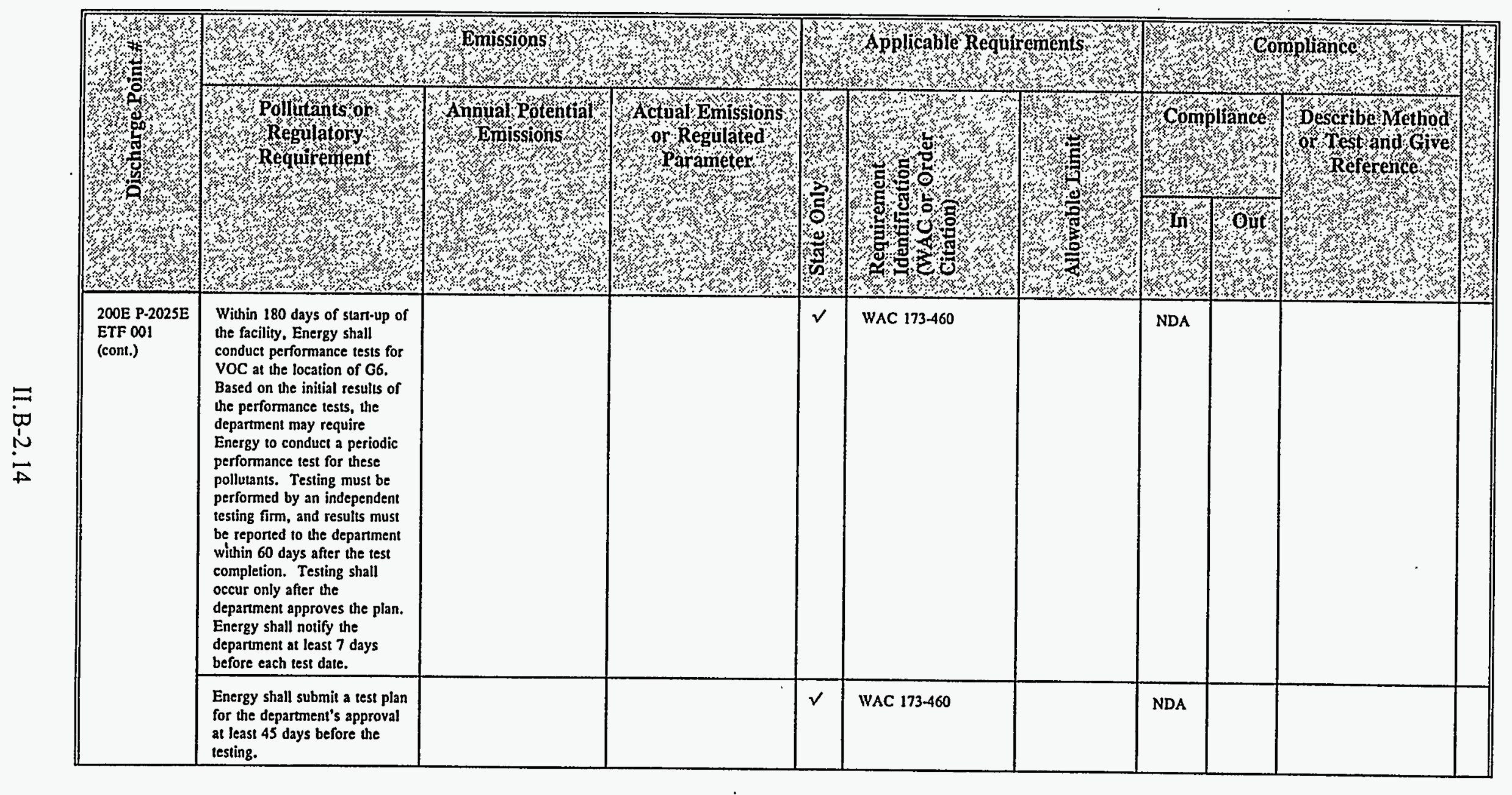

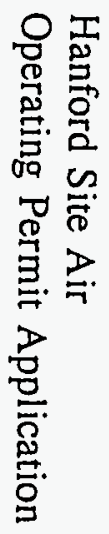




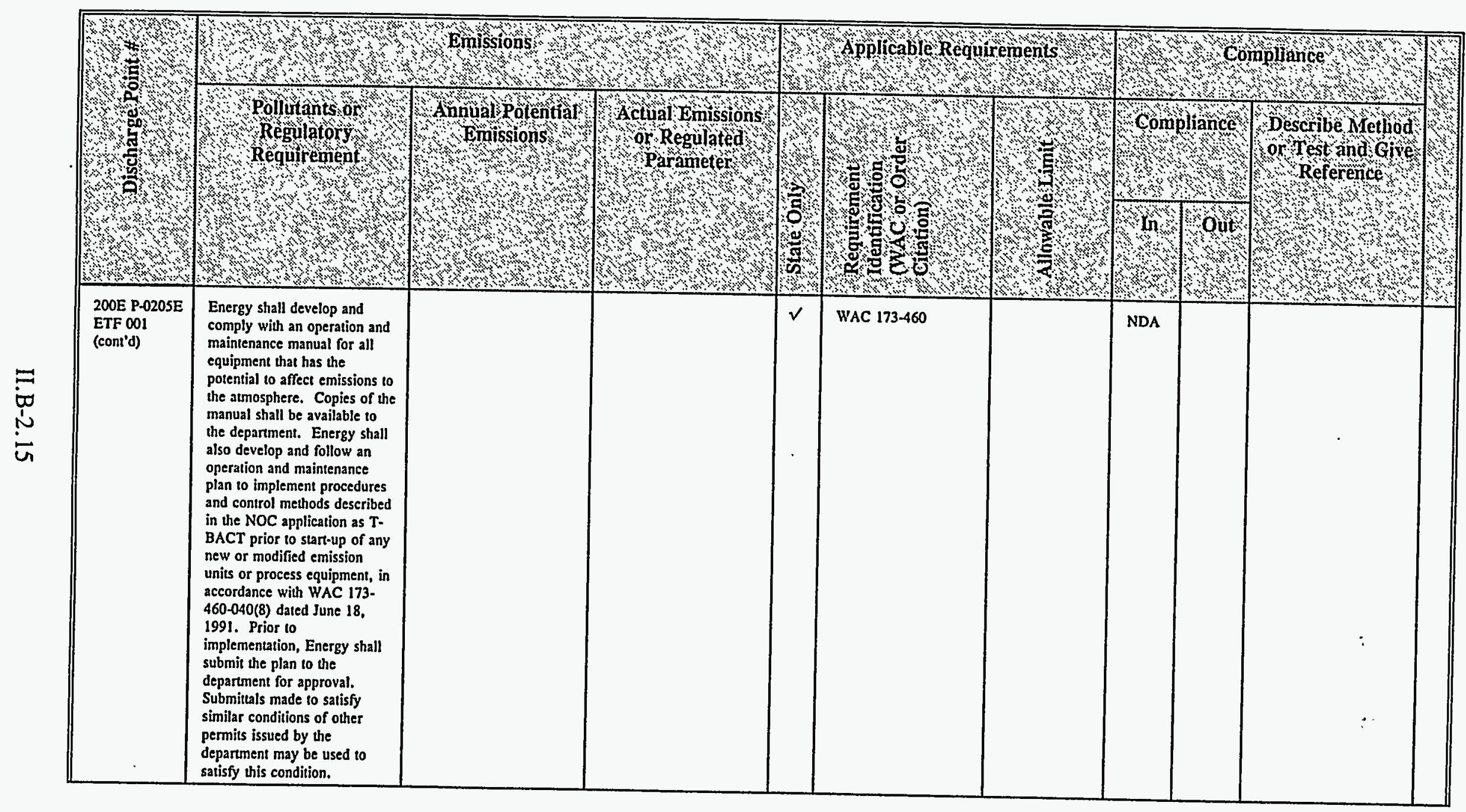


它

वे

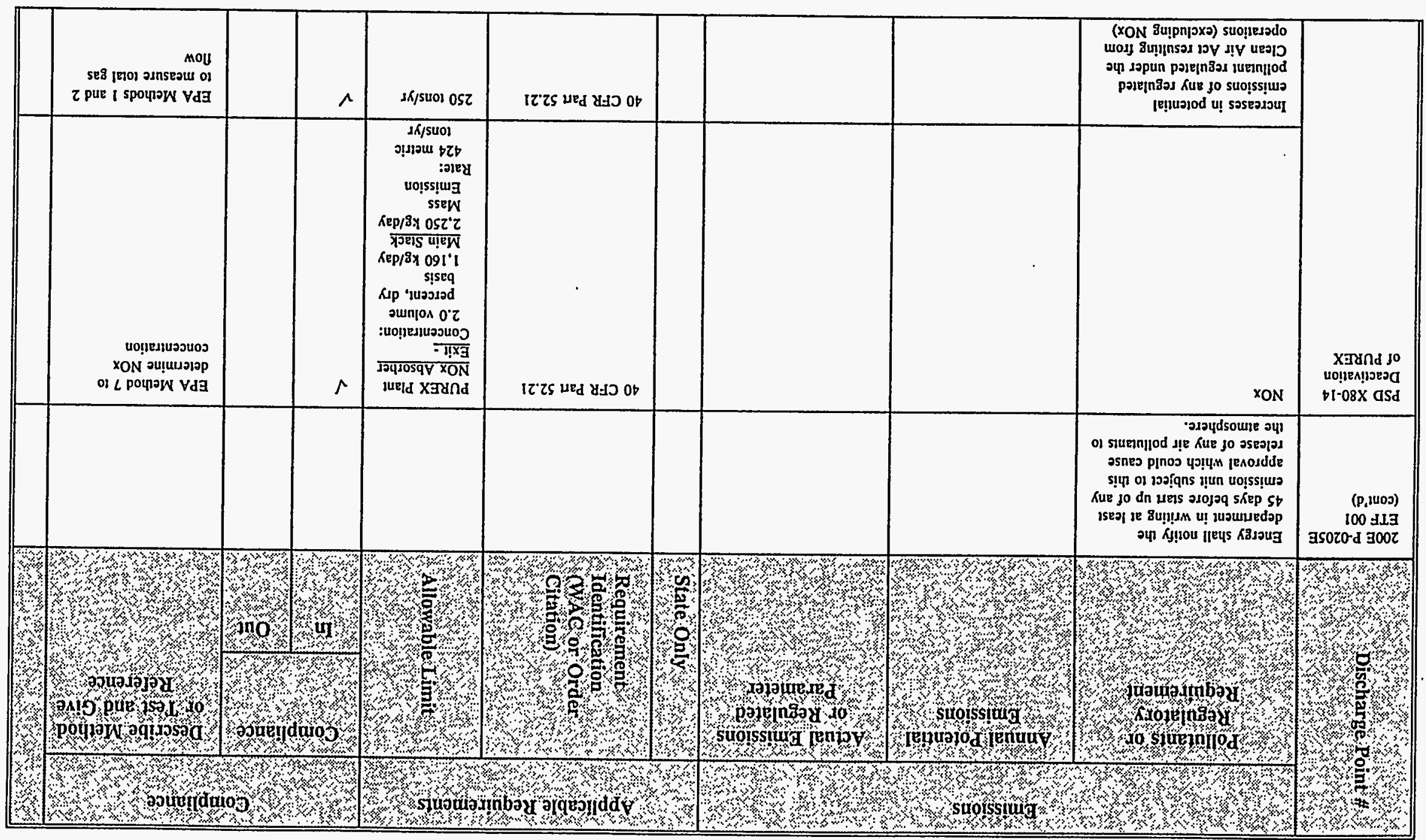


Hanford Site Air

Operating Permit Application

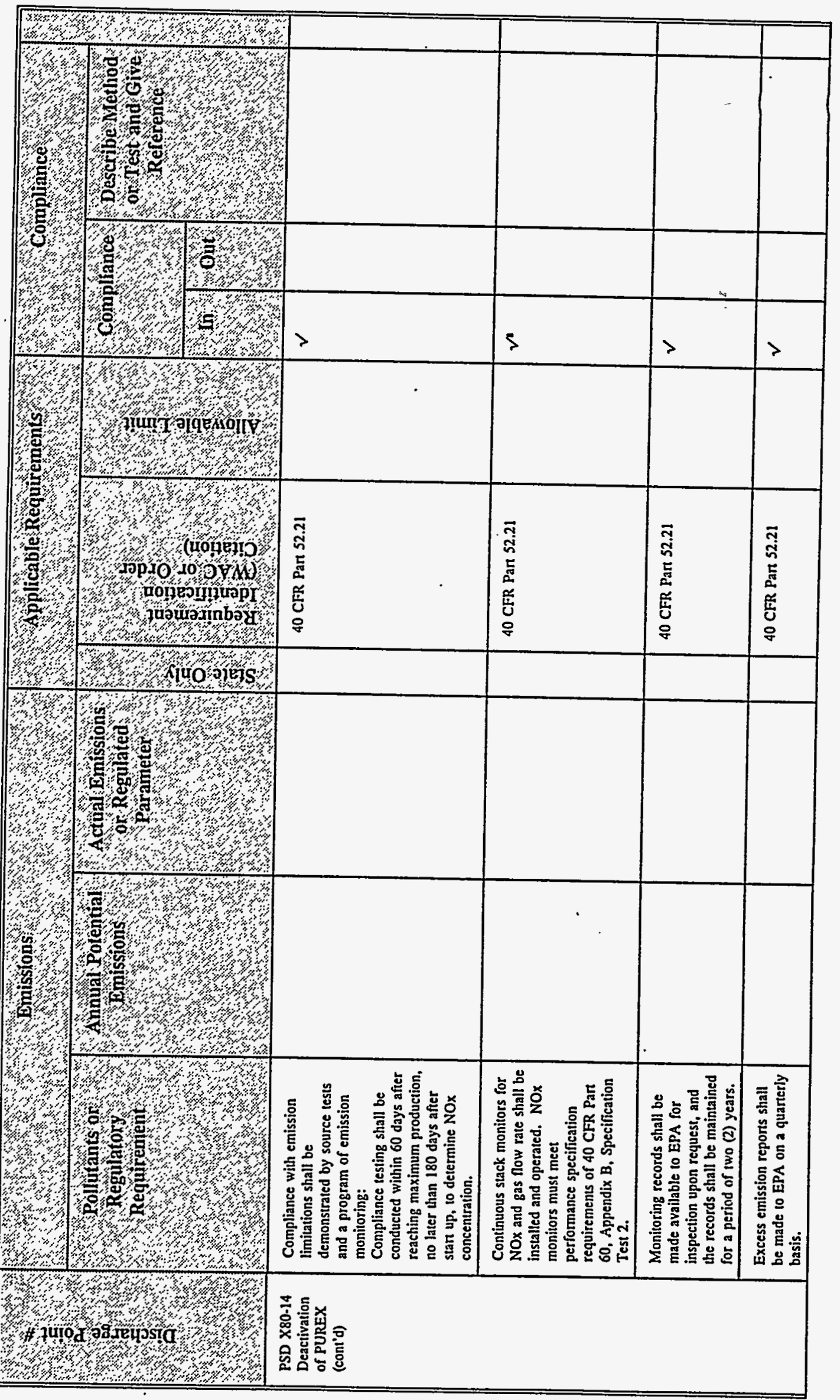


Hanford Site Air

Operating Permit Application
DOE/RL-95-07, Rev. 0

05/95

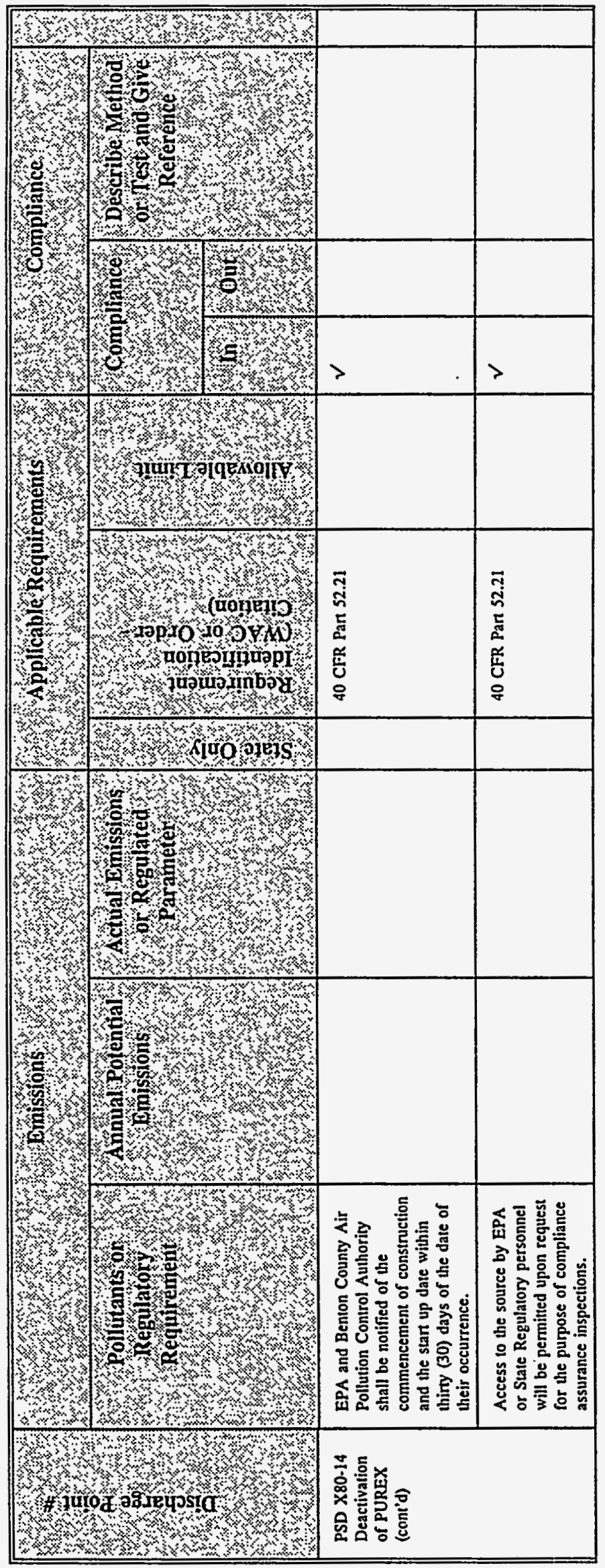

II.B-2.18 
Hanford Site Air

Operating Permit Application

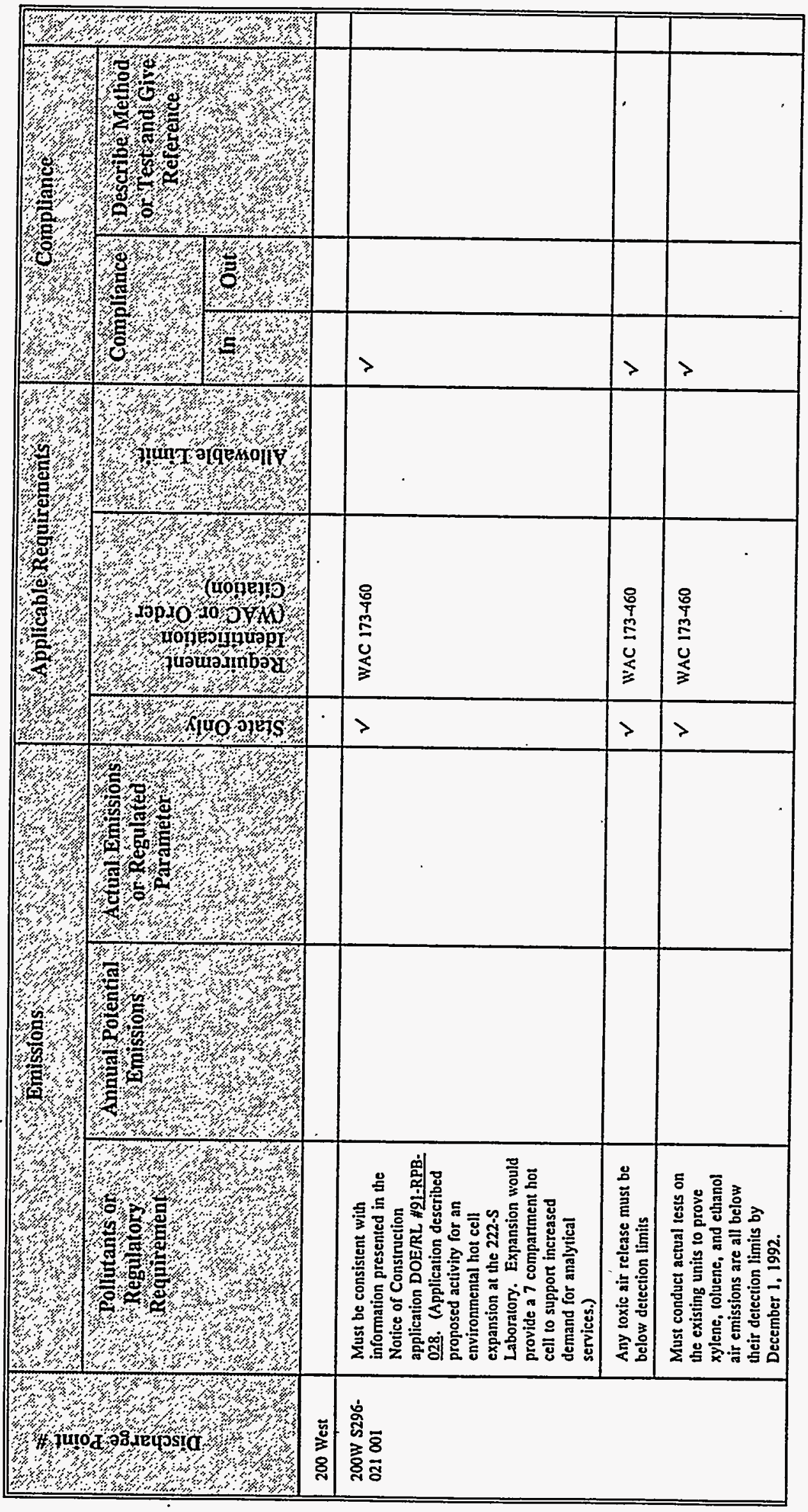

II.B-2.19 


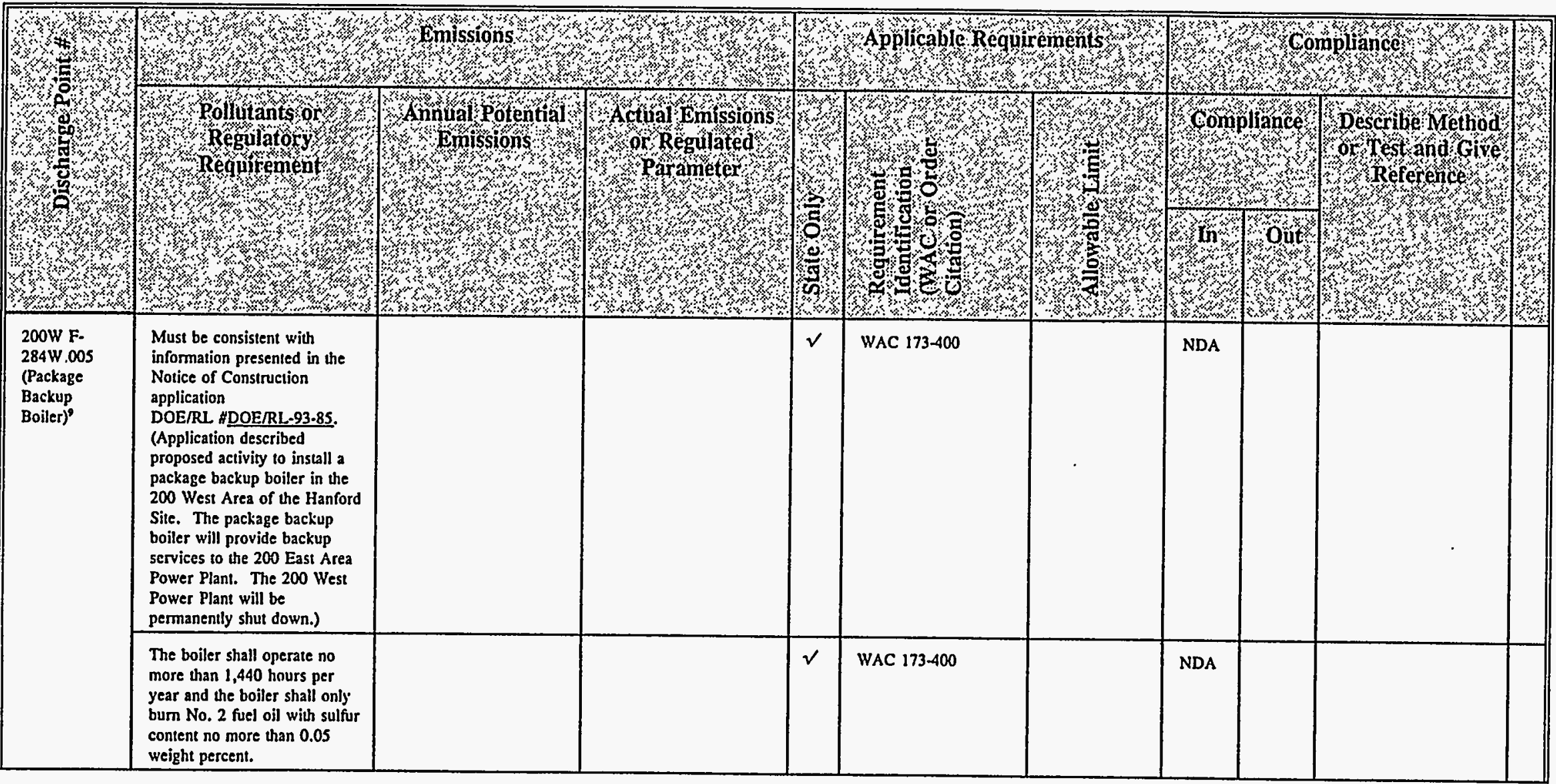


몽

这

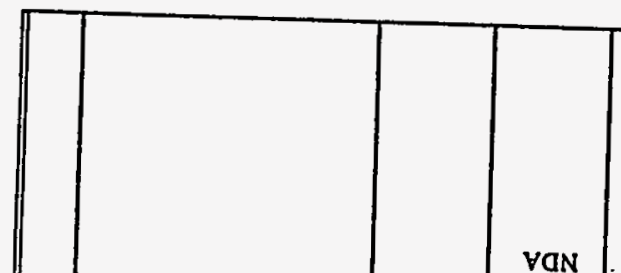

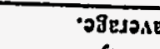

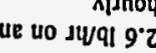

pur gosd 1028

2000 po5300

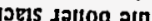

วอีอเวกร

4IE

pur uoge

\%L IE Apudda

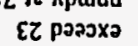

poppou jujeannbo

$101661^{\circ} \mathrm{s}$ sins jo se

lou lieys

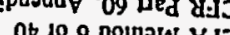

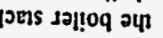

van

w013 suo!!sstur

(a)

ग8850.

Sisnoy
uo snou

dod spunod s.

pue nigww/qI

100 pazoso

poyroul juכjen!nbo ue

20 I66I' I Rinf jo se Y

xlpuoddy '09 Hed X

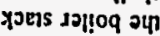

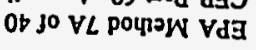

vaN

uod] suo!̣s!uo

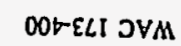

son

005-ELI כVM

OOt $\varepsilon L I$ DVM

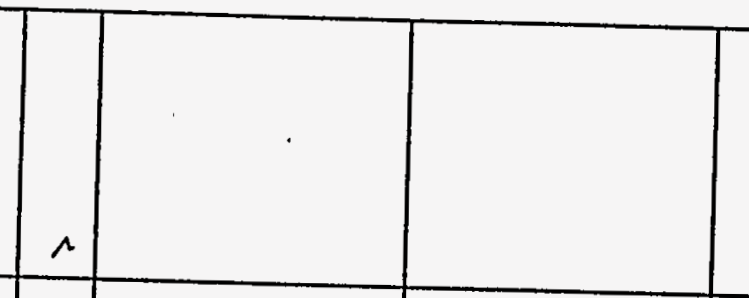

OI-WNd

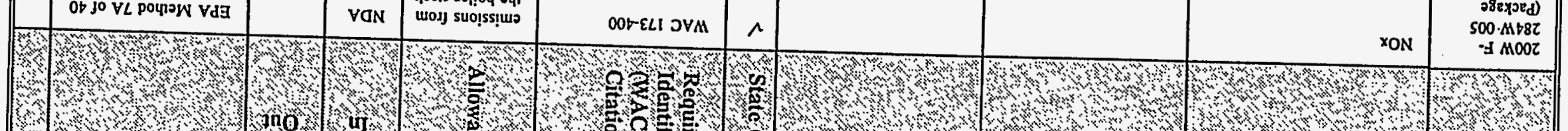

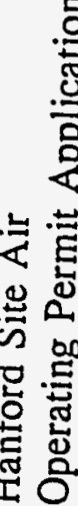

$$
20
$$

( 3 .

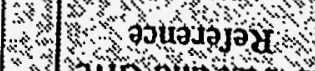

340 p pue 250 d

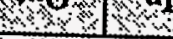

(3) 30 .

poypul aqussoa

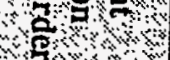

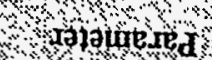

pajtitisay us

suolsş̧⿻

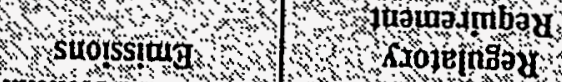

stopeingty

to siumanio.

snoissiug 


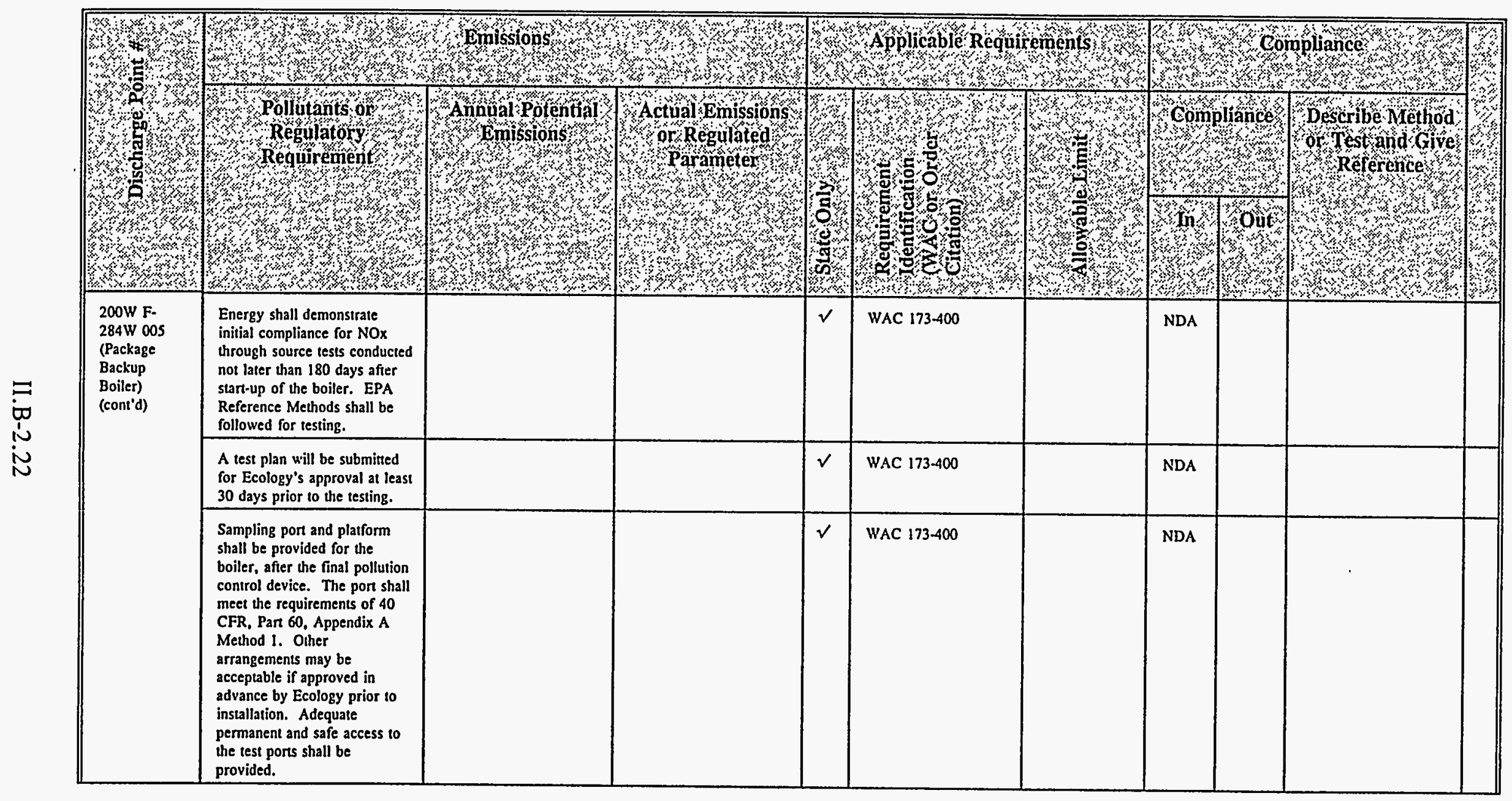


Hanford Site Air

\begin{tabular}{|c|c|c|}
\hline \multicolumn{3}{|l|}{ W०W } \\
\hline \multicolumn{3}{|l|}{ 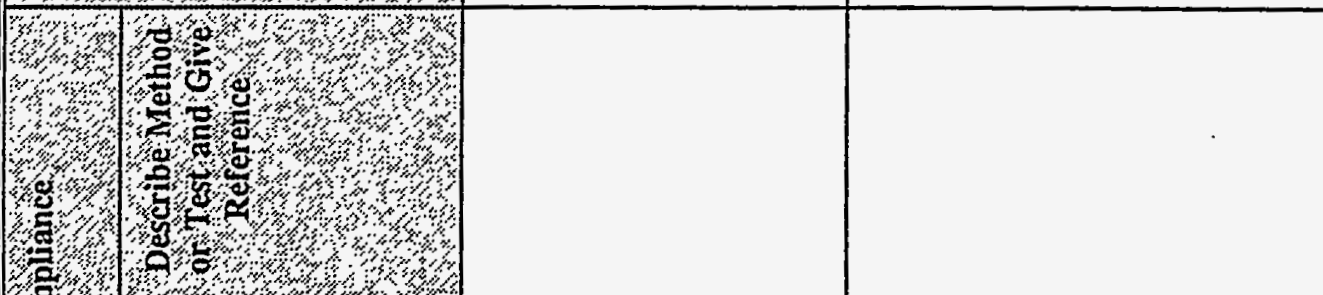 } \\
\hline \% & & \\
\hline 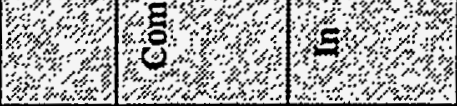 & 全 & $\widehat{\delta}$ \\
\hline 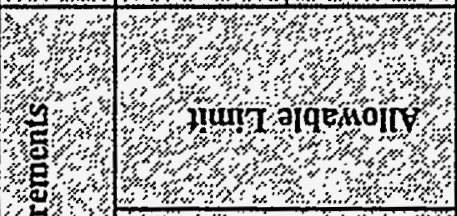 & & - \\
\hline 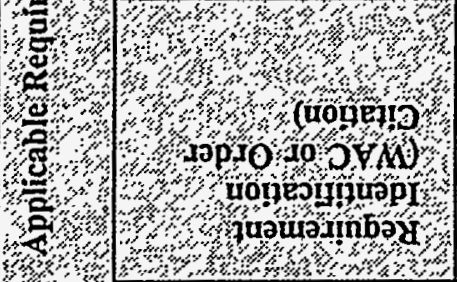 & 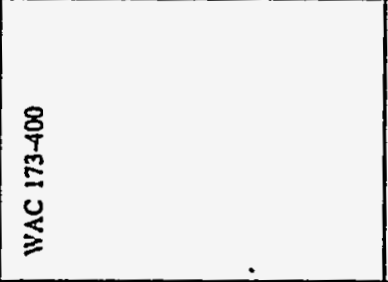 & 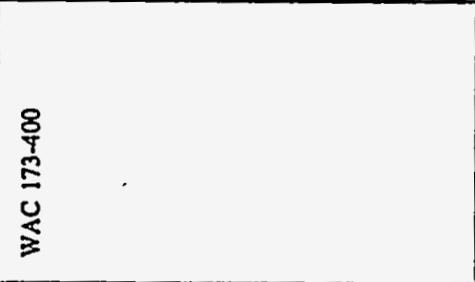 \\
\hline 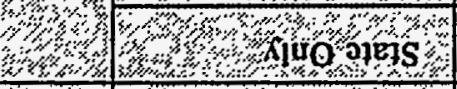 & $>$ & $>$ \\
\hline 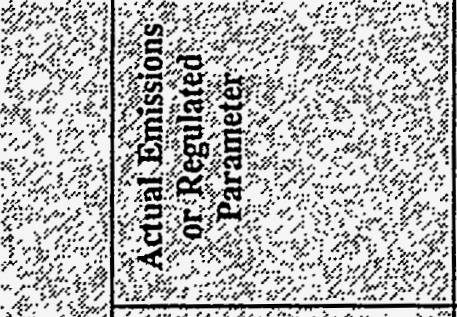 & & \\
\hline 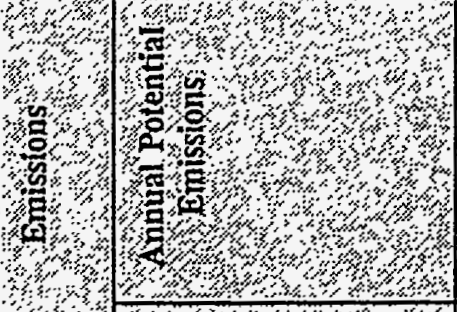 & & \\
\hline 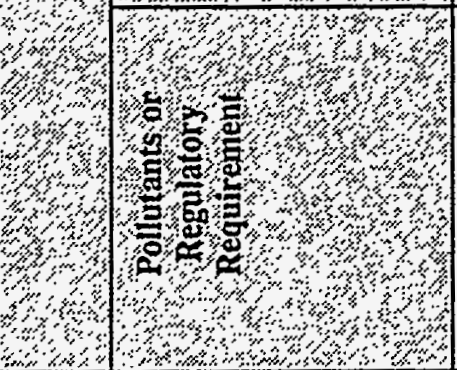 & 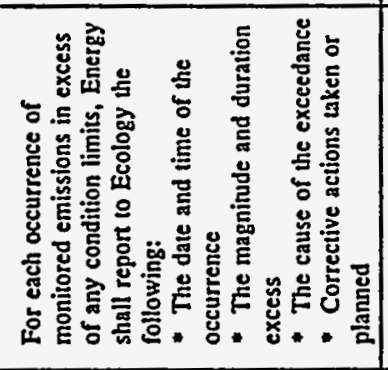 & 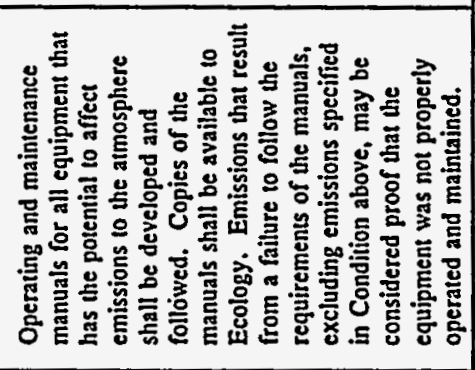 \\
\hline \%orom & 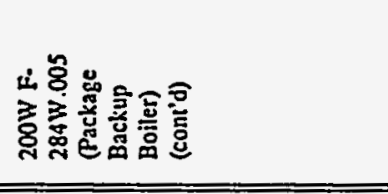 & \\
\hline
\end{tabular}


Hanford Site Air

Operating Permit Application

DOE/RL-95-07, Rev. 0

05/95

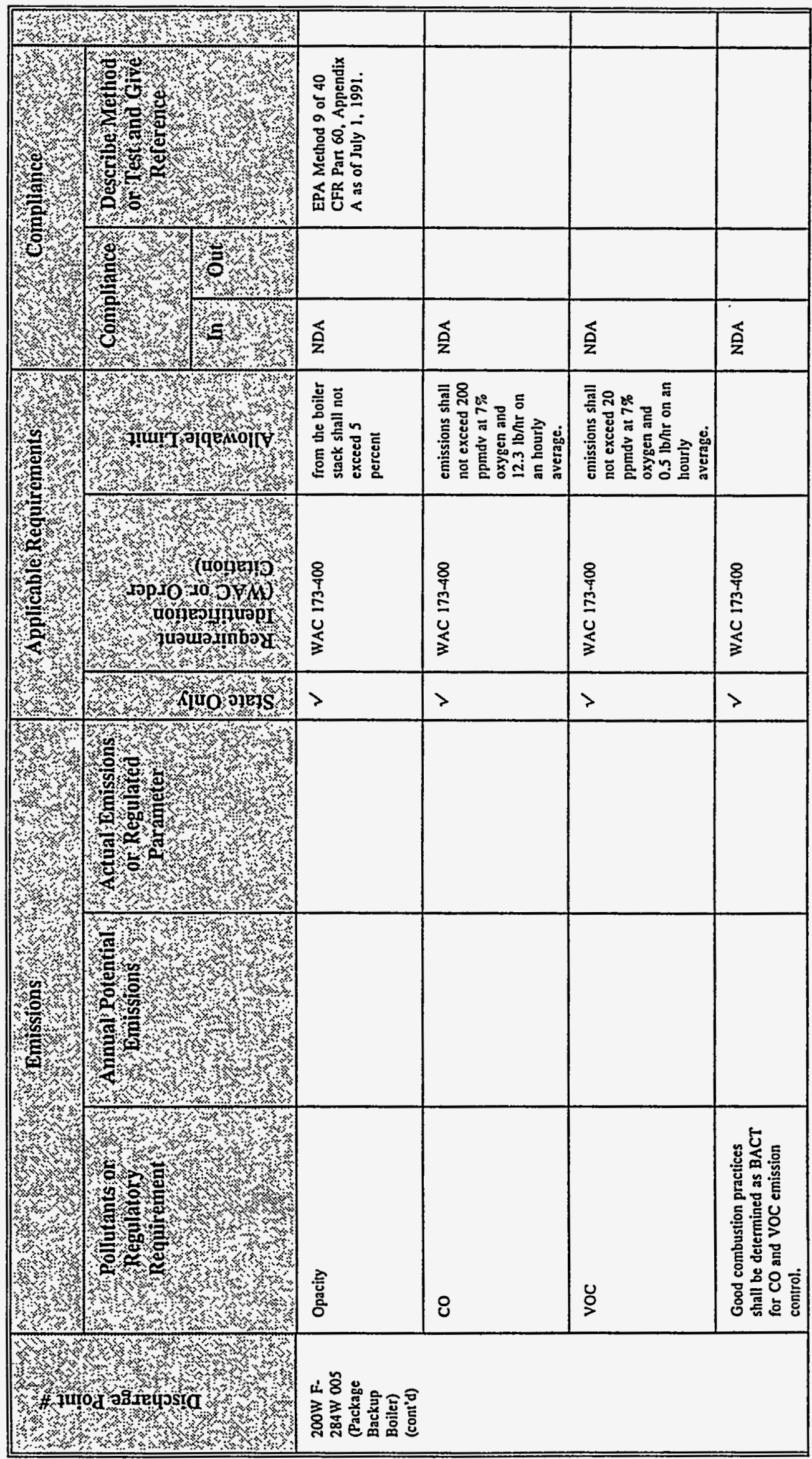




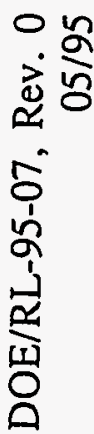

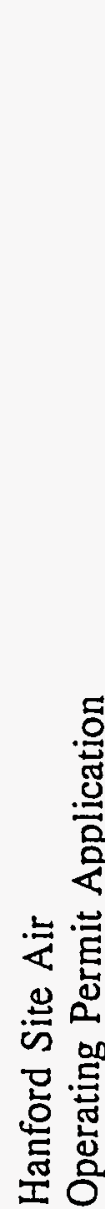

\begin{tabular}{|c|c|c|c|c|c|c|c|c|c|c|}
\hline & & & VaN & & OOFELI JVM & $\wedge$ & & & 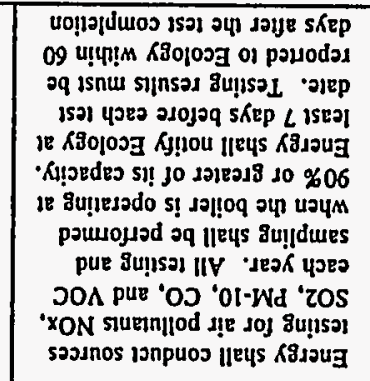 & \\
\hline 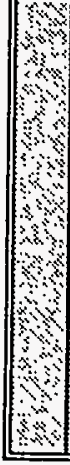 & 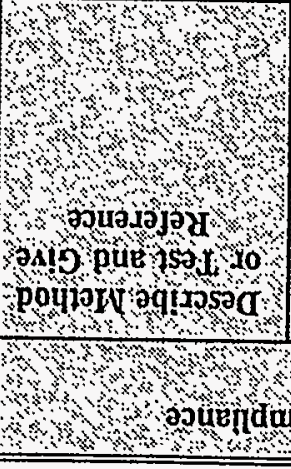 & 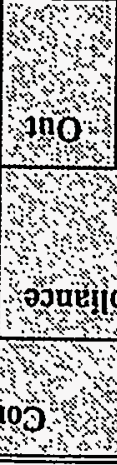 & $\frac{6}{6}$ & 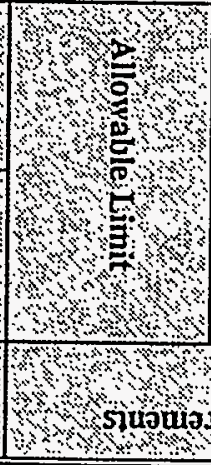 & 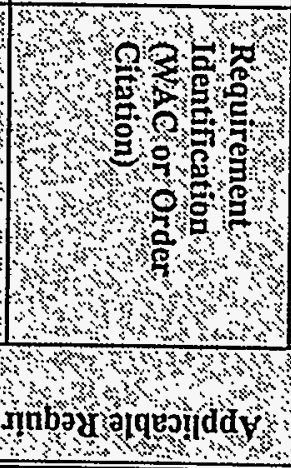 & $\frac{5}{5}$ & 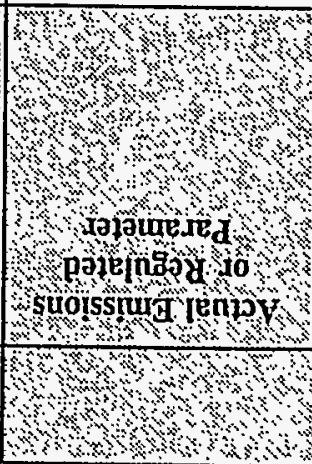 & 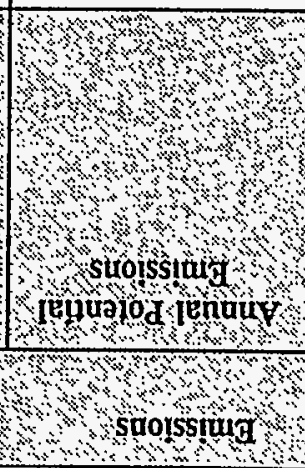 & 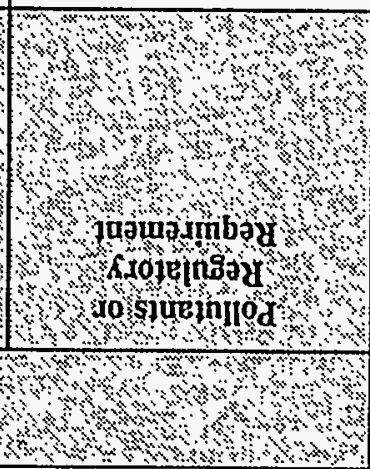 & 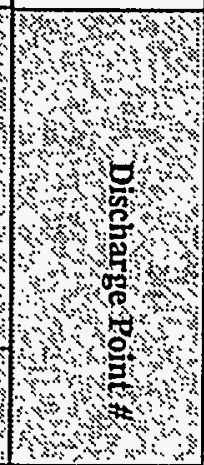 \\
\hline
\end{tabular}




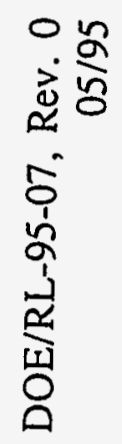

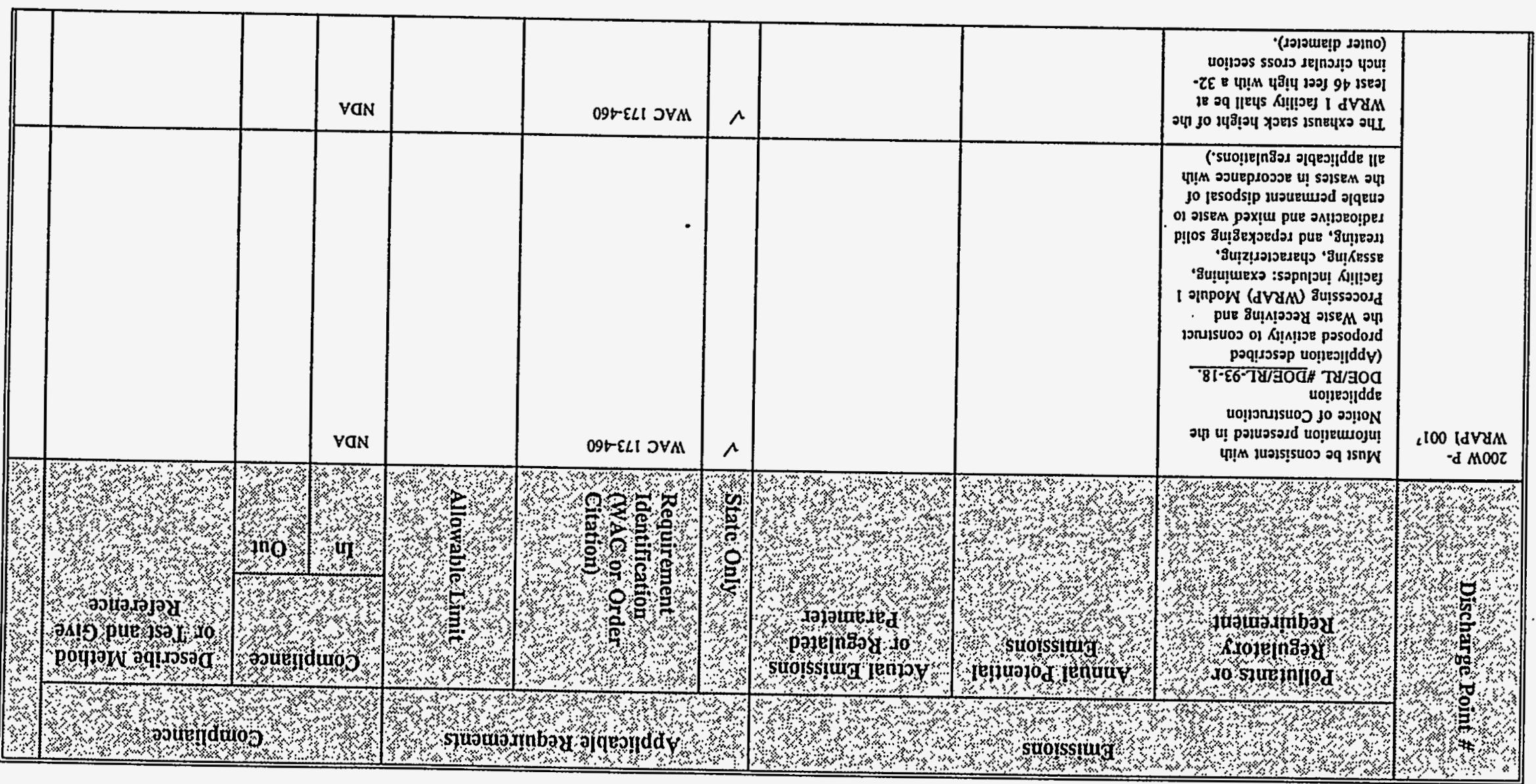


Hanford Site Air

Operating Permit Application

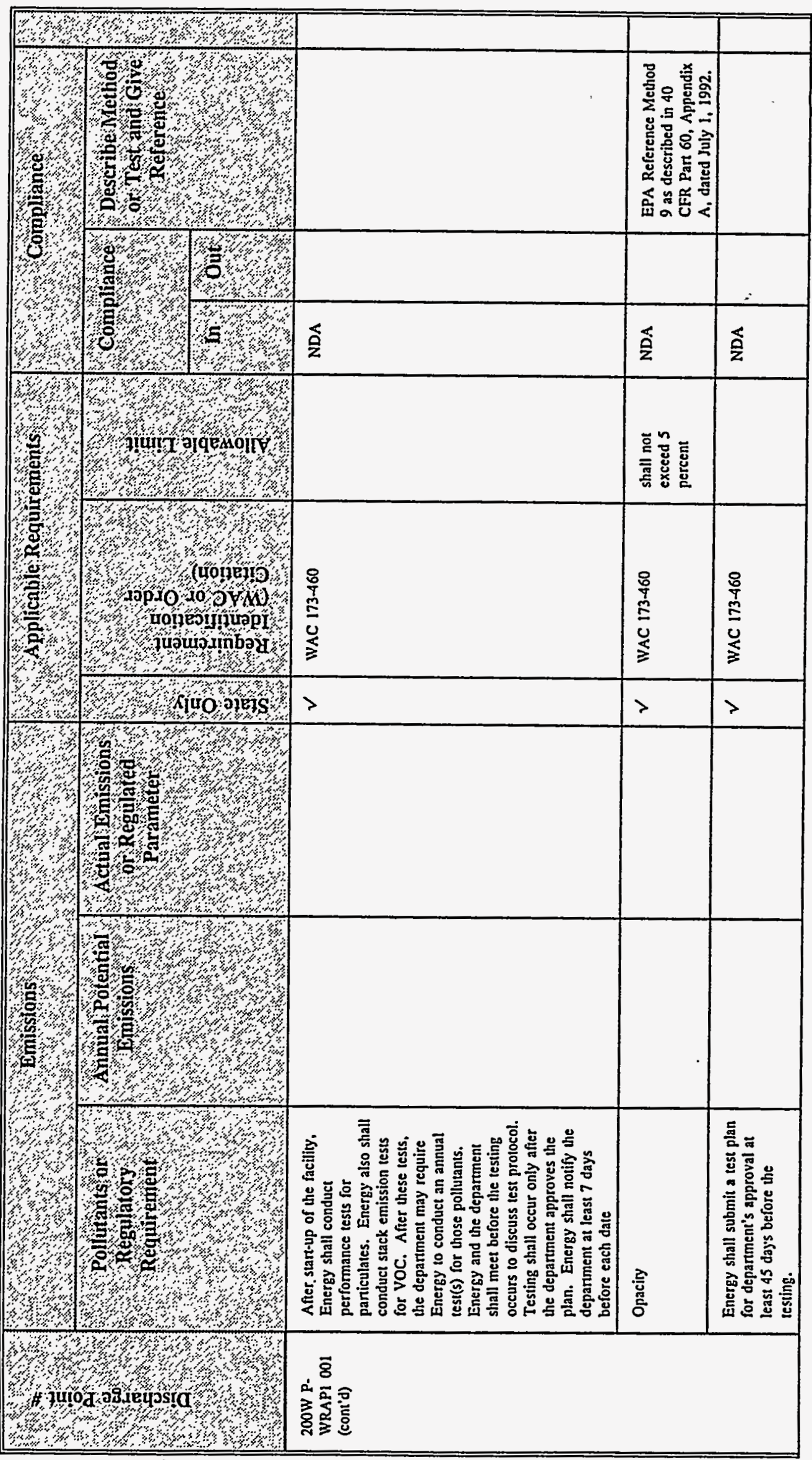


这

वे

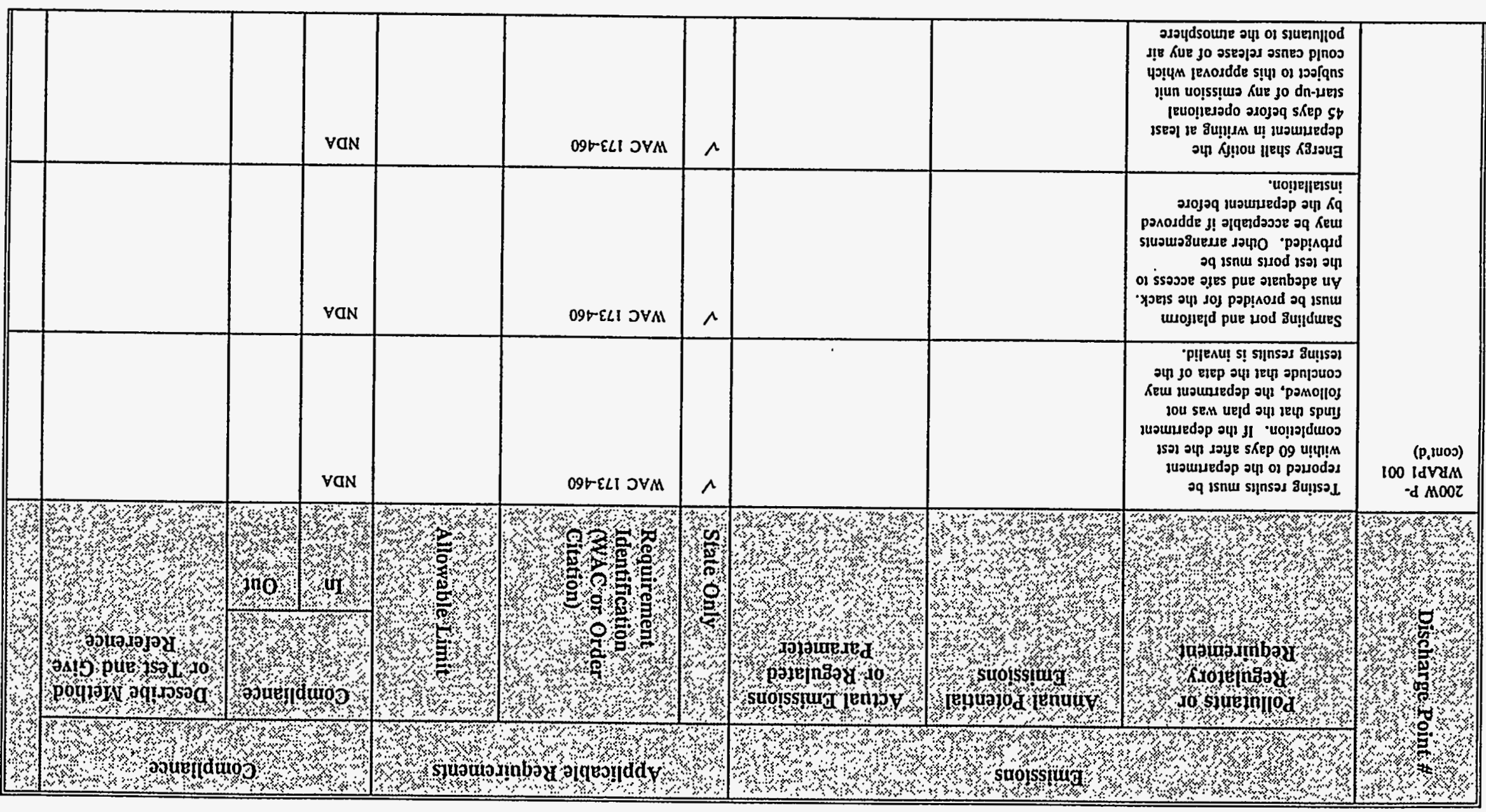


Hanford Site Air

Operating Permit Application

DOE/RL-95-07, Rev. 0

05/95

\begin{tabular}{|c|c|c|c|c|}
\hline 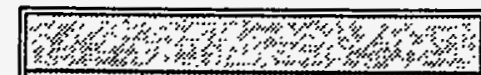 & & & & \\
\hline 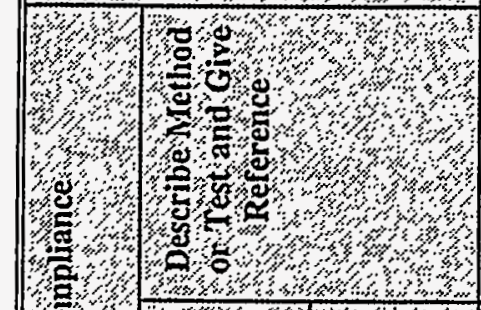 & & & & 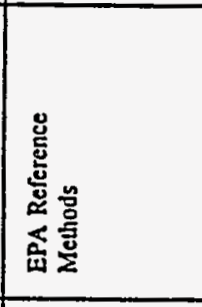 \\
\hline 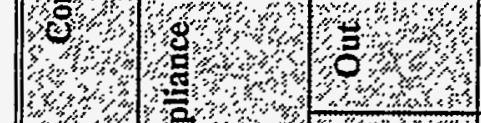 & & & & \\
\hline , & 会 & 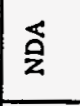 & 会 & 会 \\
\hline 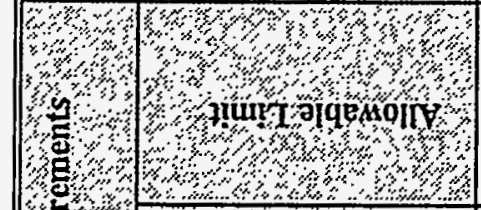 & & 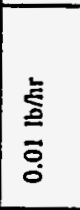 & 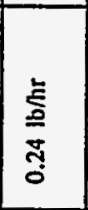 & \\
\hline 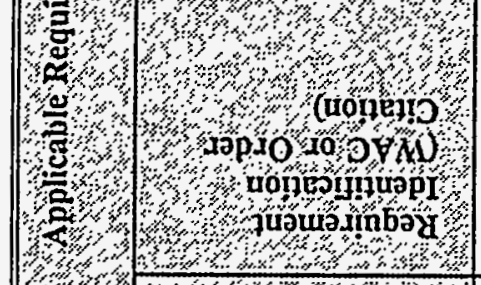 & 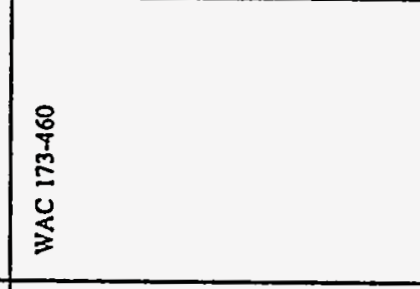 & 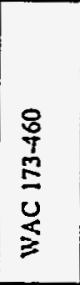 & 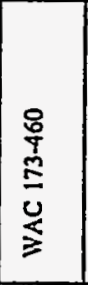 & 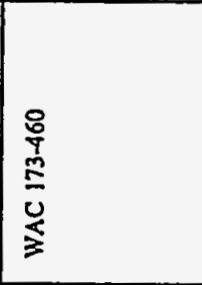 \\
\hline 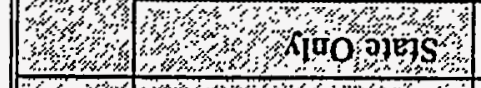 & $>$ & $>$ & $>$ & $>$ \\
\hline 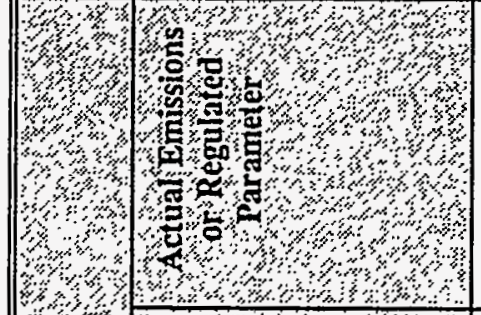 & & & & \\
\hline 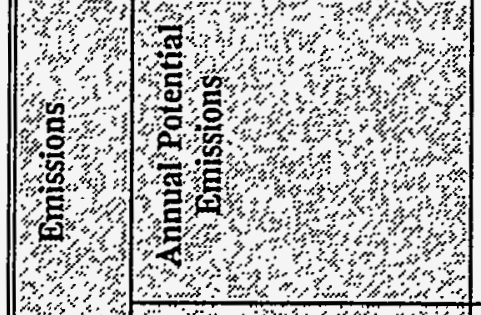 & & & & \\
\hline 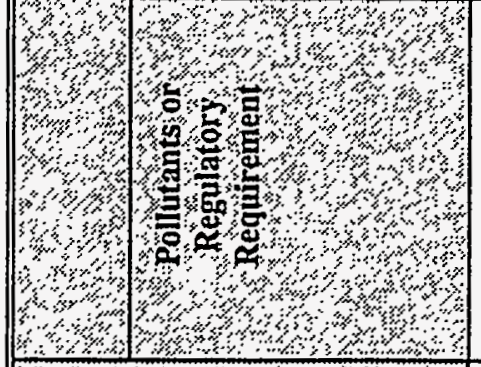 & 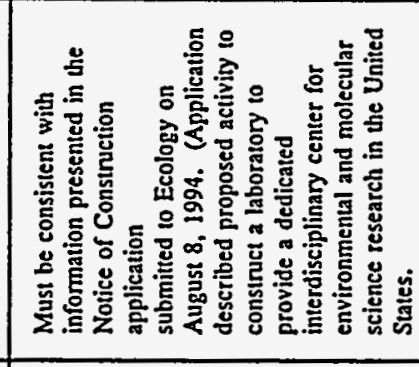 & 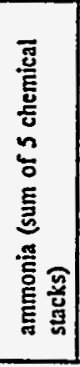 & 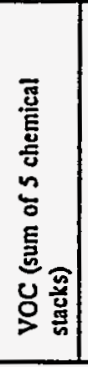 & 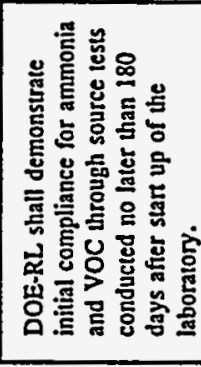 \\
\hline 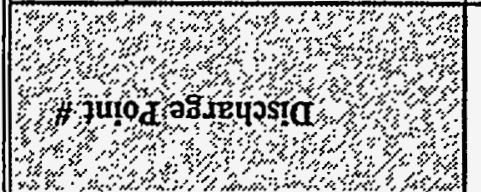 & 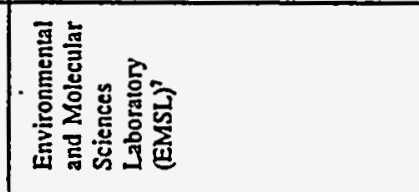 & & & \\
\hline
\end{tabular}




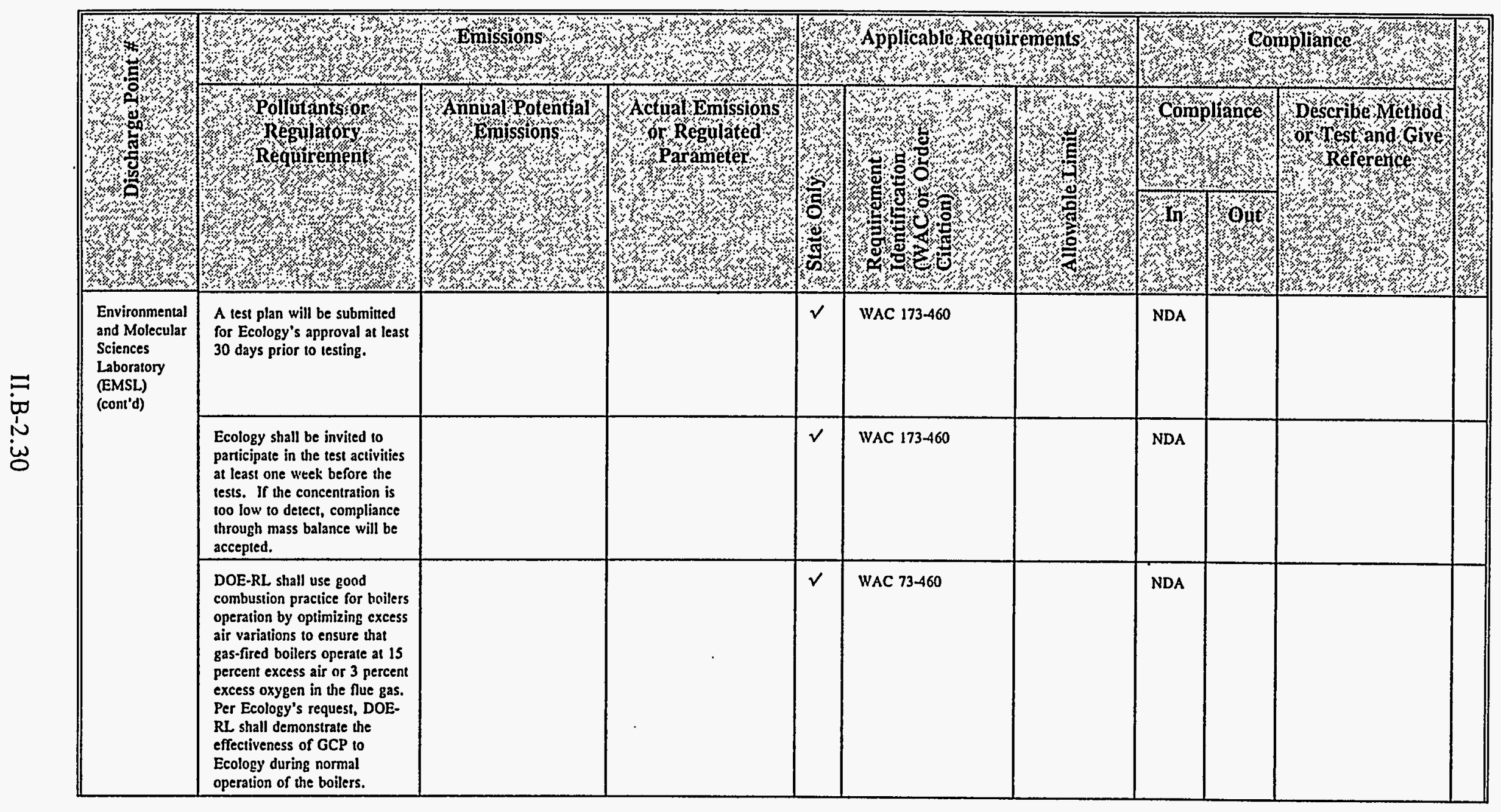




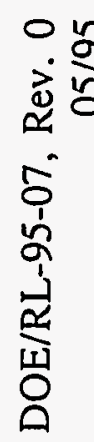

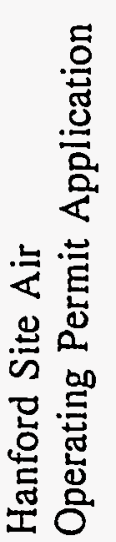

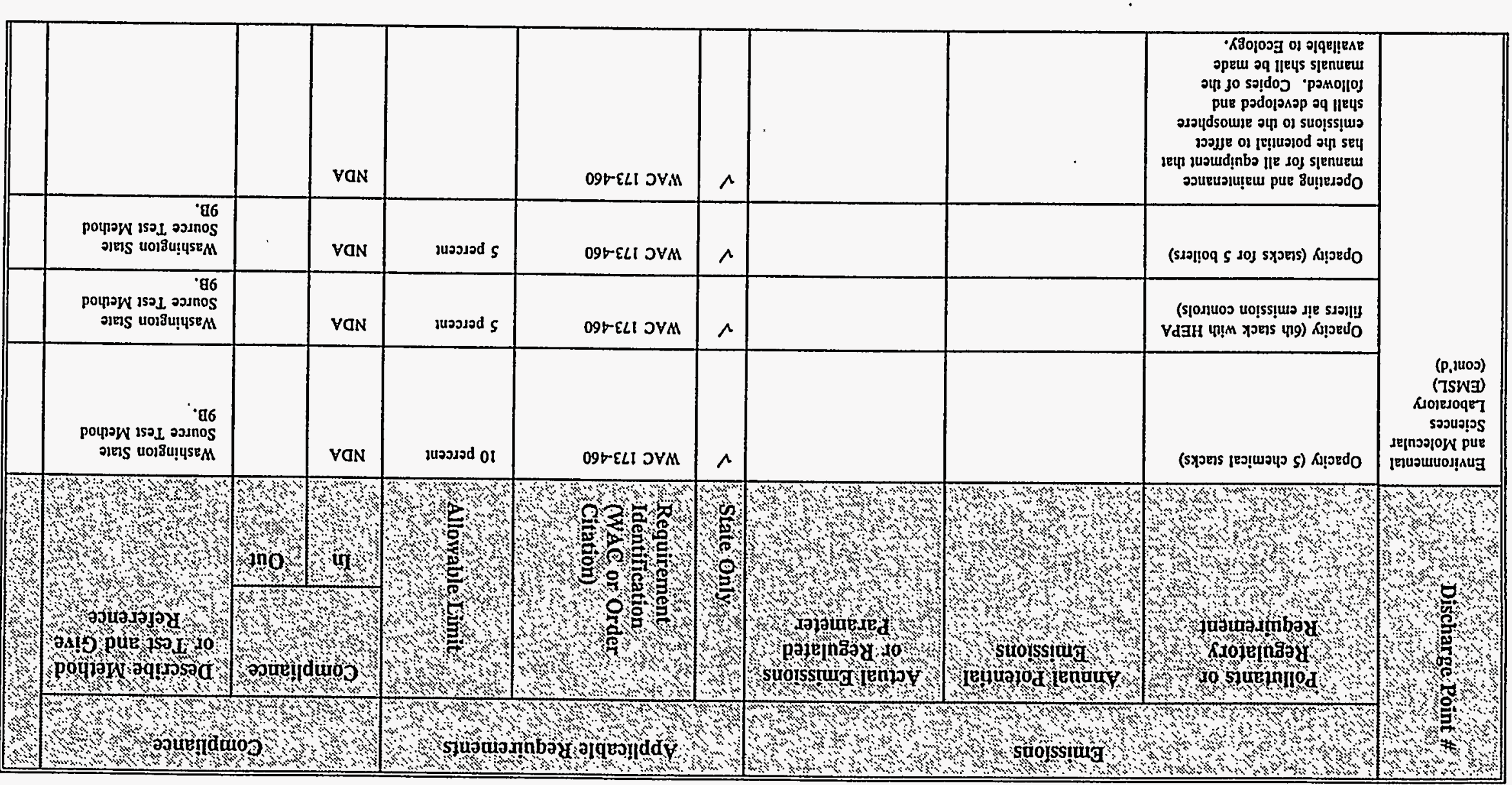


ํ.

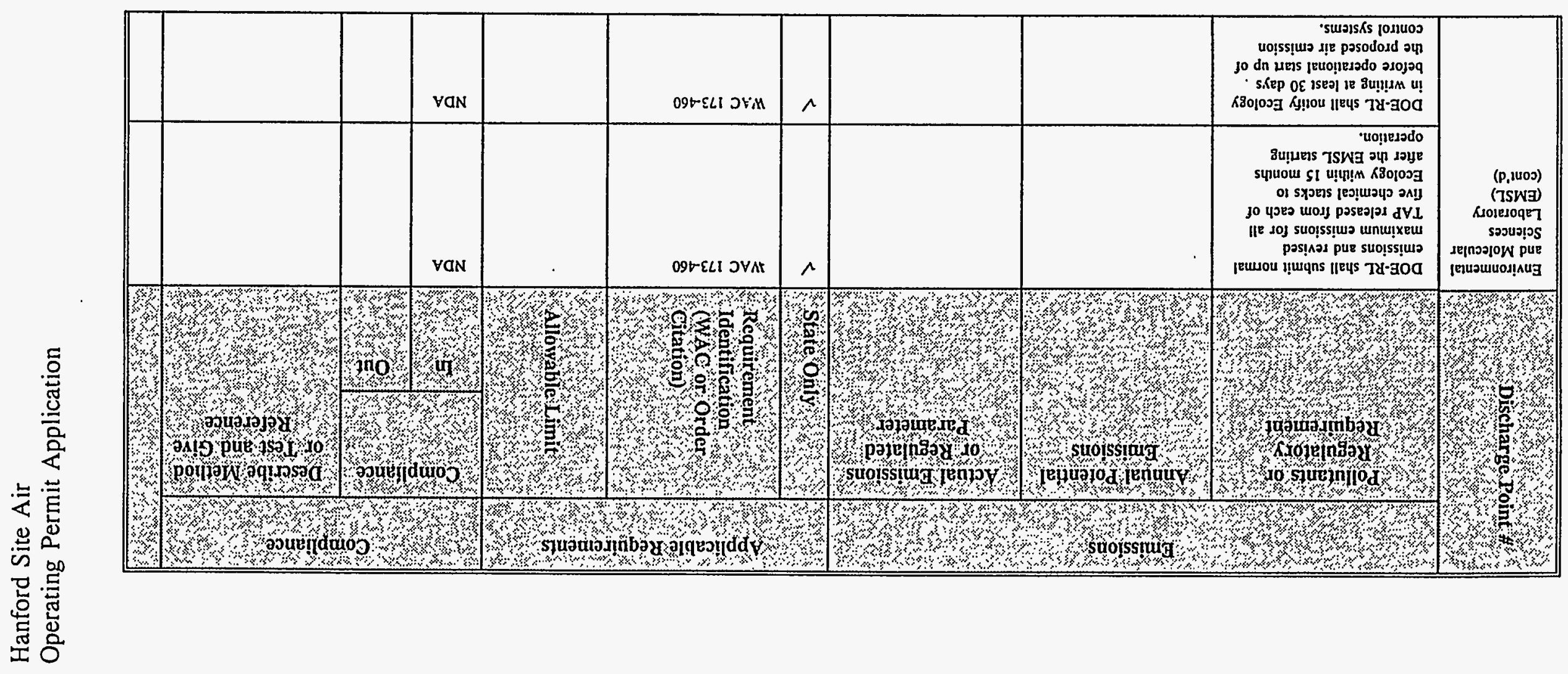

r.
ñ
๓่
$=$ 


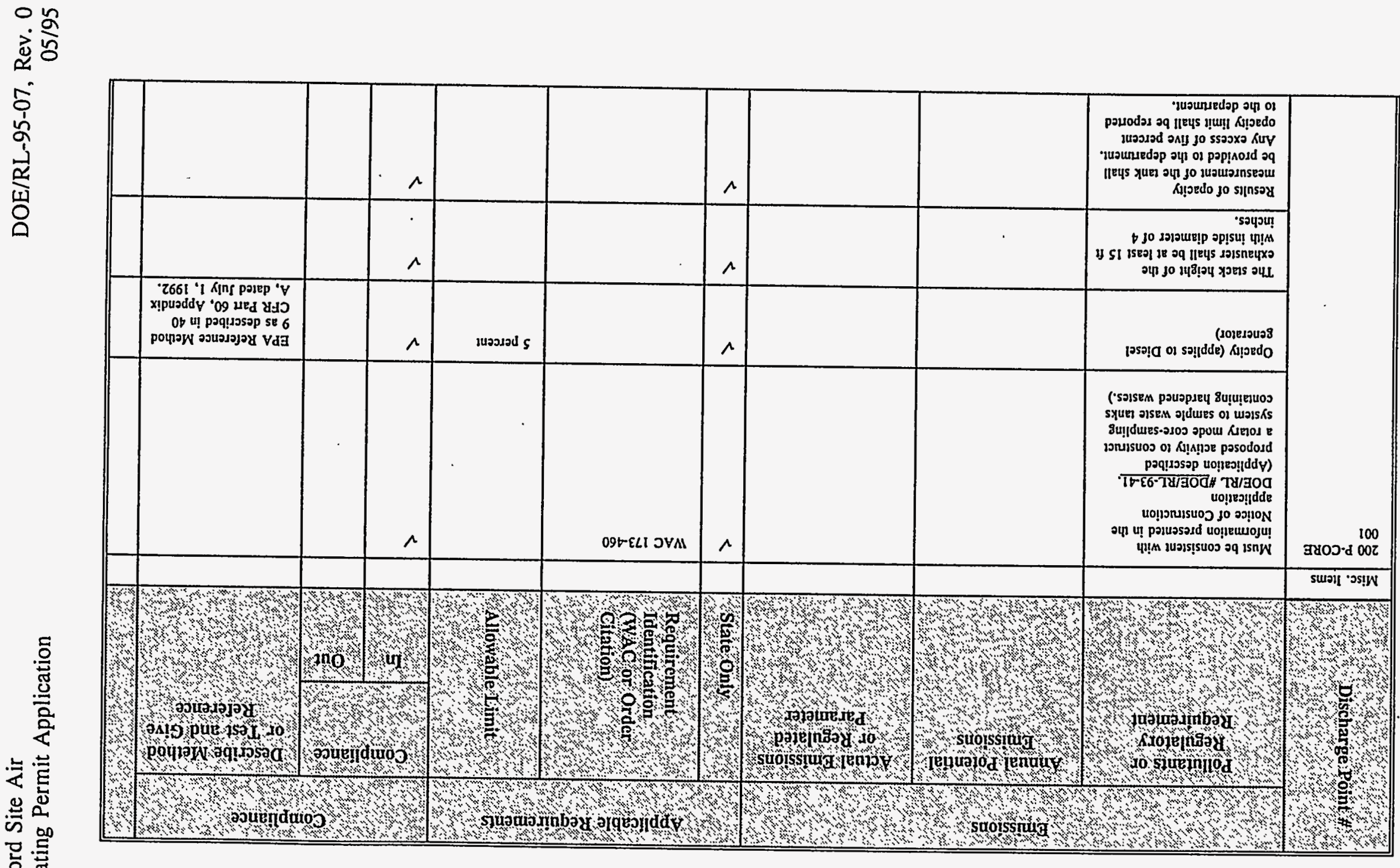


Hanford Site Air

Operating Permit Application

DOE/RL-95-07, Rev. 0

05/95

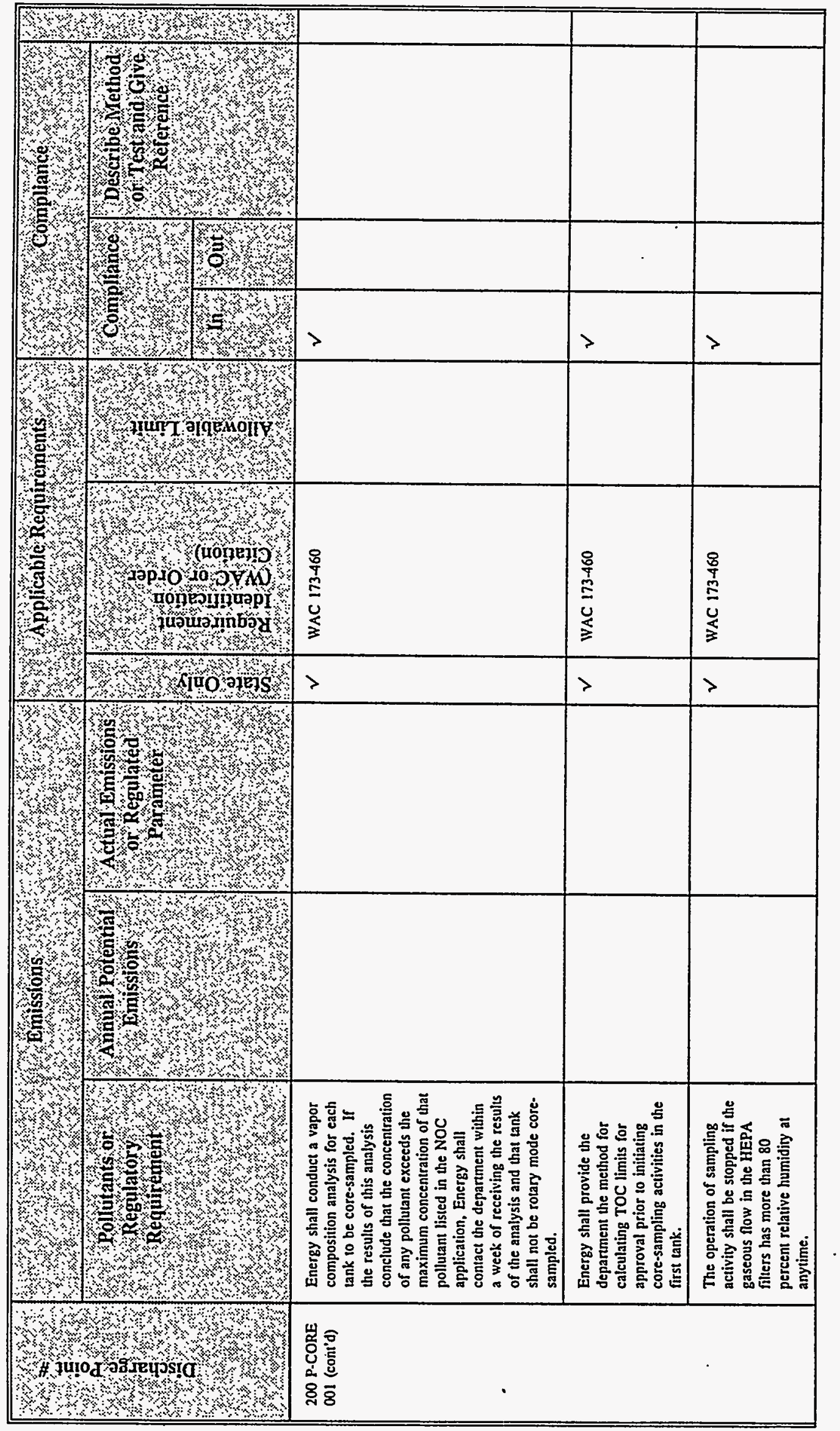


Hanford Site Air

Operating Permit Application

DOE/RL-95-07, Rev. 0

05/95

\begin{tabular}{|c|c|}
\hline \multicolumn{2}{|c|}{ 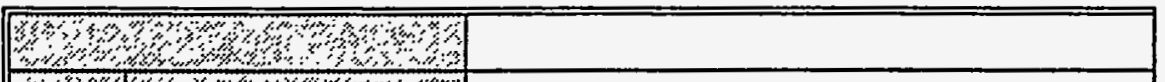 } \\
\hline \multicolumn{2}{|c|}{ 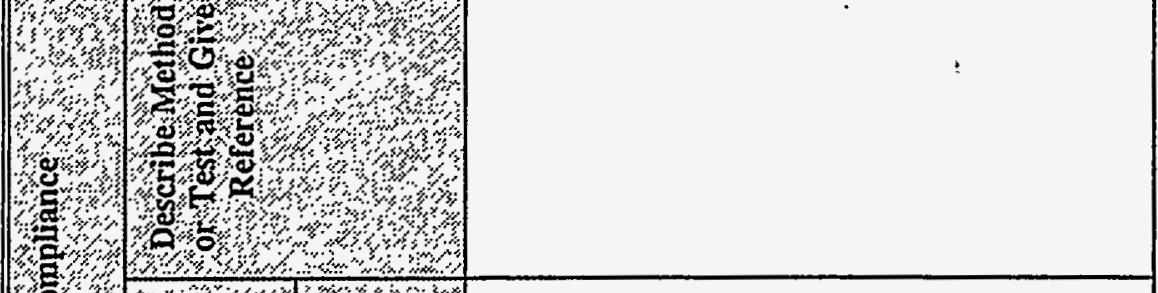 } \\
\hline \%, & 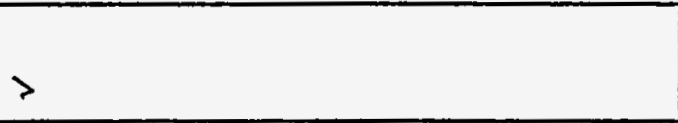 \\
\hline \multicolumn{2}{|l|}{ 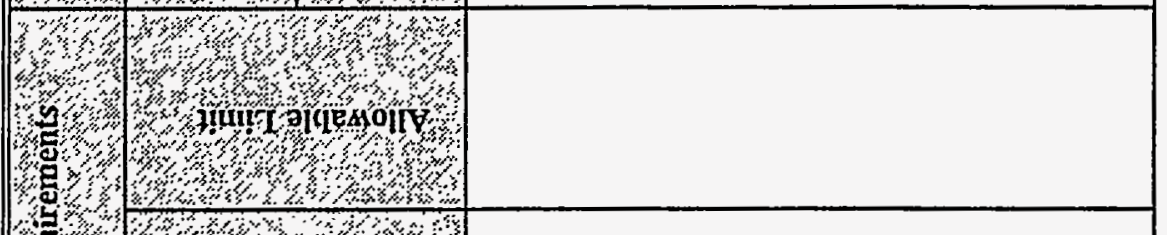 } \\
\hline 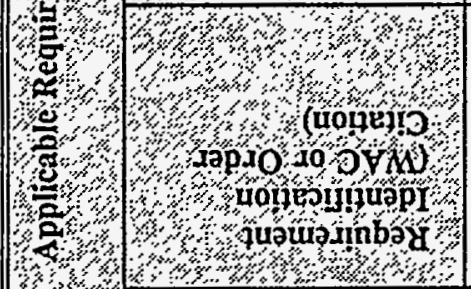 & \\
\hline & $>$ \\
\hline \multirow{2}{*}{\multicolumn{2}{|c|}{ 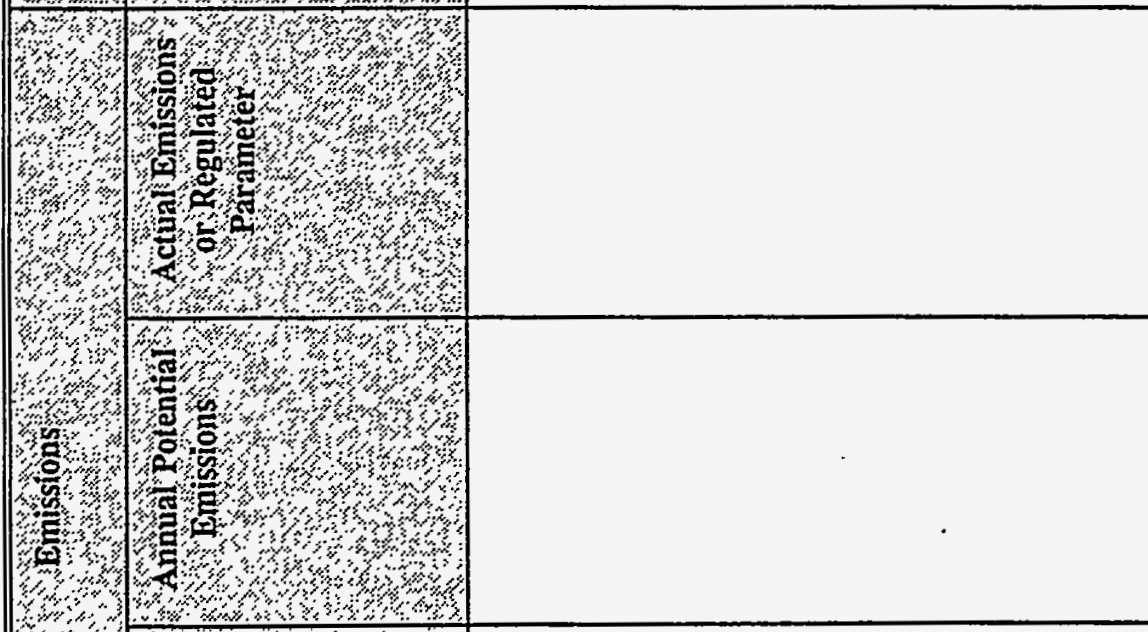 }} \\
\hline & \\
\hline 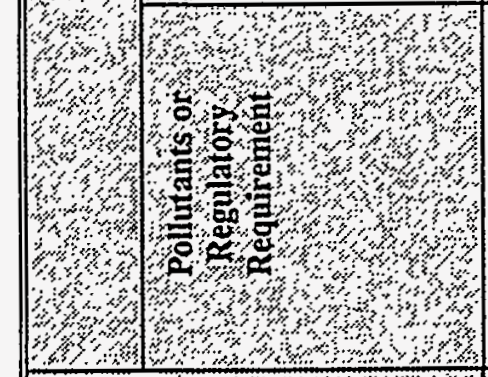 & 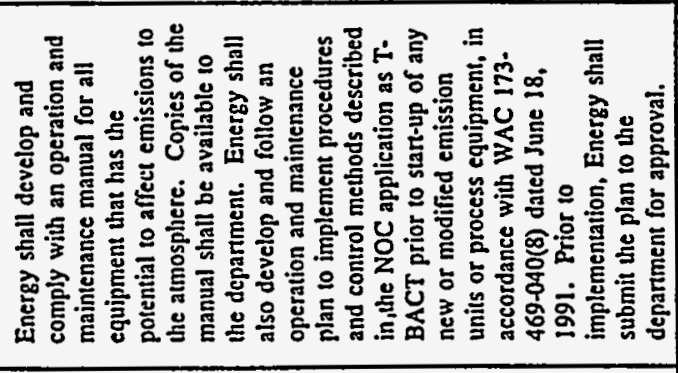 \\
\hline 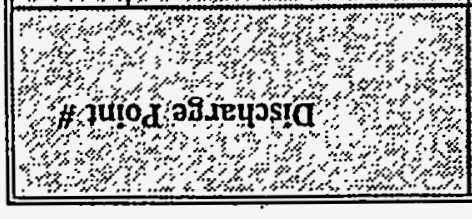 & 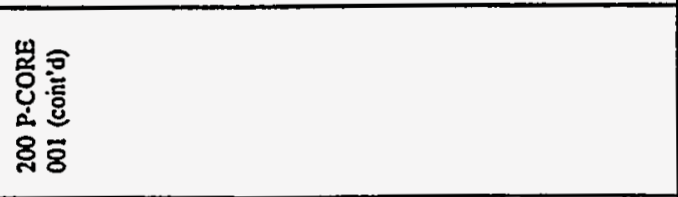 \\
\hline
\end{tabular}




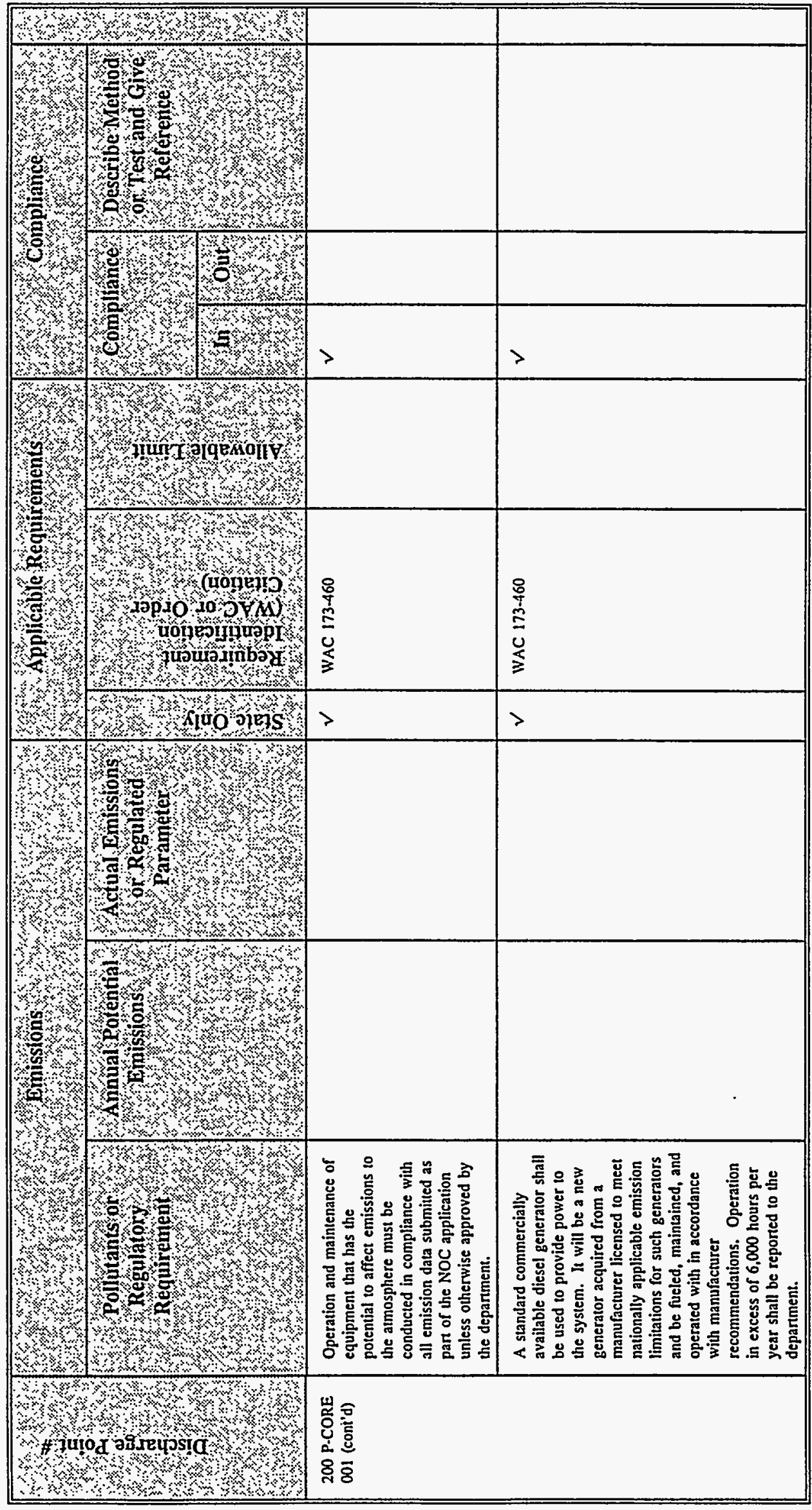

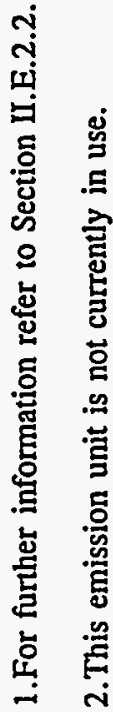




\section{NDA = No Data Available}

4.The reported result is from the 1995 source test of emission unit 300 F-384 005.

5.For information on the Compliance Plan and Schedule refer to Chapter II.E.5. and Appendix B, respectively.

6.Refer to Section II.E.5, subsection XIV., paragraph 61.

7.Project construction is in progress. Upon startup, compliance with requirements will be assessed.

8.Continuous stack monitoring for NOx operates only during NOx generating processes (e.g., denitration activities). Refer to Appendix B for milestone associated with clarification of continuous NOx monitoring requirements during deactivation activities.

9.Project has not begun startup activities. Upon startup, compliance with requirements will be assessed. 
Table II.B-3. Special Requirements.

\begin{tabular}{|c|c|c|c|c|c|}
\hline \multirow[t]{3}{*}{ Citation } & \multirow[t]{3}{*}{ Requirement } & \multicolumn{4}{|r|}{ Compliance } \\
\hline & & \multicolumn{2}{|c|}{ Compliance } & \multirow{2}{*}{\multicolumn{2}{|c|}{ Describe Method or Test and Give Reference }} \\
\hline & & In & Out & & \\
\hline \multirow[t]{4}{*}{$\begin{array}{l}40 \text { CFR } 61 \text {, Subpart } M \text {, } \\
\text { Asbesios }\end{array}$} & $\begin{array}{l}\text { National emission standard for demolition and } \\
\text { renovation of facilities involving regulated asbestos- } \\
\text { containing material (RACM) above or below (i.e., } \\
\text { large or small demolition) the following levels: 1) } 80 \\
\text { linear meters on pipes, 2) } 15 \text { square meters on facility } \\
\text { components, or 3) } 1 \text { cubic meter of facility components } \\
\text { where the length or area could not be measured } \\
\text { previously. } \\
\text { 1. Applicability of facilities being demolished, } \\
\quad \text { large or small notification requirements. }\end{array}$ & $\sqrt{ }$ & & \multirow[t]{4}{*}{$\begin{array}{l}1 . \\
2 . \\
3 .\end{array}$} & \multirow{4}{*}{$\begin{array}{l}\text { Facilities planned for demolition will be inspected to calculate the total estimated } \\
\text { RACM. } \\
\text { Notification is provided to the Benton County Clean Air Authority at least } 10 \text { working } \\
\text { days in advance of activity. An update, as required, is also provided when the amount } \\
\text { of asbestos affected changes by } 20 \text { percent. } \\
\text { The requirements and restrictions for removal, handling, encapsulation of asbestos } \\
\text { from facilities undergoing demolition will be followed in accordance with established } \\
\text { procedures for asbestos emission control. } \\
\text { At least one onsite demolition supervisor and/or foreman assigned to RACM activities } \\
\text { will be fully trained in accordance with the } 40 \text { CFR } 61.145 \text { requirements. Refresher } \\
\text { training will occur every two years. }\end{array}$} \\
\hline & 2. Notification requirements. & $\sqrt{ }$ & & & \\
\hline & $\begin{array}{l}\text { 3. Removal and handling of asbestos for } \\
\text { demolition activities. }\end{array}$ & $\sqrt{ }$ & & & \\
\hline & 4. Training of personnel. & $\sqrt{ }$ & & & \\
\hline \multirow[t]{3}{*}{$\begin{array}{l}\text { Benton County Clean Air } \\
\text { Authority, Regulation 1, } \\
\text { Article 8, Asbestos }\end{array}$} & $\begin{array}{l}\text { National emission standard for asbestos adopted by } \\
\text { reference as part of the Benton County Clean Air } \\
\text { Authority (BCCAA) regulations. } \\
\text { 1. Adoption of federal notification requirements } \\
\text { under } 40 \text { CFR } 61 \text {, Subpart M, by reference. }\end{array}$ & $\sqrt{ }$ & & & \multirow{3}{*}{$\begin{array}{l}\text { Notification is provided to the Benton County Clean Air Authority at least } 10 \text { working } \\
\text { days in advance of activity. An update, as required, is also provided when the amount } \\
\text { of asbestos affected changes by } 20 \text { percent. } \\
\text { Advance notification and fee payment, as applicable, will be made to the BCCAA in } \\
\text { accordance with the requirements of Regulation 1, Article } 8 \text { and Article } 10 \text {, and the } \\
\text { memorandum of agreement between DOE-RL and the BCCAA. } \\
\text { Facilities planned for demolition will be inspecied to calculate the total estimated } \\
\text { RACM. }\end{array}$} \\
\hline & $\begin{array}{l}\text { 2. Additional requirements of the BCCAA and fee } \\
\text { schedule described in Regulation } 1, \text { Article } 10 .\end{array}$ & $\sqrt{ }$ & & & \\
\hline & 3. Unexpected discovery of asbestos. & $\sqrt{ }$ & & & \\
\hline
\end{tabular}


Table II.B-3. Special Requirements.

\begin{tabular}{|c|c|c|c|c|}
\hline \multirow[t]{3}{*}{ Citation } & \multirow[t]{3}{*}{ Requirement } & \multicolumn{3}{|r|}{ Compliance } \\
\hline & & \multicolumn{2}{|c|}{ Compliance } & \multirow[t]{2}{*}{ Describe Method or Test and Give Reference } \\
\hline & & In & Out & \\
\hline \multirow[t]{4}{*}{$\begin{array}{l}\text { Benton County Clean Air } \\
\text { Authority, Regulation 1, } \\
\text { Aricle 8, Asbestos }\end{array}$} & $\begin{array}{l}\text { Memorandum of agreement between DOE-RL and } \\
\text { Benton County Clean Air Authority on removal and } \\
\text { encapsulation of asbestos materials. } \\
\text { 1. Disposal of regulated asbestos-containing } \\
\quad \text { material (RACM). }\end{array}$ & $\sqrt{ }$ & & \multirow{4}{*}{$\begin{array}{l}\text { 1. Asbestos containing waste will be disposed of at the Hanford Site Central Landfill or } \\
\text { other appropriate asbestos disposal facility in accordance with } 40 \text { CFR } 61.150 \\
\text { requirements, WAC 173-303, as applicable, and the memorandum of agreement signed } \\
\text { by DOE-RL and the BCCAA. } \\
\text { 2. Annual notifications will be made to the BCCAA in accordance with the requirements } \\
\text { set forth in the memorandum of agreement between DOE-RL and the BCCAA. Case- } \\
\text { by-case notifications for other asbestos projects will be provided to the BCCAA as } \\
\text { required. } \\
\text { 3. Payment of fees shall be billed to the DOE-RL contractor, as appropriate, for } \\
\text { notifications filled with the BCCAA. The appropriate DOE-RL contractor will provide } \\
\text { payment of billed fees for asbestos activities to the BCCAA within } 15 \text { working days } \\
\text { receipt of a valid invoice bill from the BCCAA. }\end{array}$} \\
\hline & 2. Notification requirements. & $\sqrt{ }$ & & \\
\hline & & & & \\
\hline & 3. Payment of fees. & $\sqrt{ }$ & & \\
\hline \multirow[t]{3}{*}{$\begin{array}{l}\text { WAC 173-425, } \\
\text { Open Burning }\end{array}$} & Prohibited materials. & $\sqrt{ }$ & & \multirow{3}{*}{$\begin{array}{l}\text { Prohibited materials listed under RCW } 70.94 .775 \text { and WAC } 173-425-040 \text { will not be } \\
\text { burned at the Hanford Site. } \\
\text { No outdoor fires will be ignited on days declared an air pollution episode by Ecology } \\
\text { or on days of impaired air quality as declared by Ecology/BCCAA. Existing fires will } \\
\text { not be given new fuel and allowed to burn down, with no visible smoke, within three } \\
\text { hours of a declaration of impaired air quality. } \\
\text { Burn permits for the Hanford Site are issued by the BCCAA. Burn permit conditions } \\
\text { are stated therein. }\end{array}$} \\
\hline & Currailment during episodes or impaired air quality. & $\sqrt{ }$ & & \\
\hline & Open buming permit requirements. & $\sqrt{ }$ & & \\
\hline \multirow{3}{*}{$\begin{array}{l}\text { Benton County Clean Air } \\
\text { Authority (BCCAA), open } \\
\text { burning requirements. } \\
\text { WAC } 173-425 \text { is adopied } \\
\text { by reference as part of } \\
\text { Regulation } 1 \text {, Article } 5 \text {. }\end{array}$} & $\begin{array}{l}\text { Observe "burn" or "no-burn" designations made by the } \\
\text { BCCAA." }\end{array}$ & $\sqrt{ }$ & & \multirow{3}{*}{$\begin{array}{l}\text { The Hanford Fire Department oversees all planned buming activities on site. Bum } \\
\text { day designations are observed and Special Burning Permits are applied for through the } \\
\text { BCCAA, as applicable, to support Hanford Site activities. } \\
\text { BCCAA burn requirements and restrictions are followed on the Hanford Site. The } \\
\text { Hanford Fire Department properly controls open burning to ensure compliance. } \\
\text { (Note: also WAC } 173-425 \text { ) }\end{array}$} \\
\hline & Follow BCCAA burn requirements and restrictions. & $\sqrt{ }$ & & \\
\hline & Requests for Special Burning Permit, as applicable. & $\sqrt{ }$ & & \\
\hline
\end{tabular}


Table II.B-3. Special Requirements.

\begin{tabular}{|c|c|c|c|c|c|}
\hline \multirow[t]{3}{*}{ Citation } & \multirow[t]{3}{*}{ Requirement } & \multicolumn{4}{|r|}{ Compliance } \\
\hline & & \multicolumn{2}{|c|}{ Compliance } & \multirow{2}{*}{\multicolumn{2}{|c|}{ Describe Method or Test and Give Reference }} \\
\hline & & In & Out & & \\
\hline \multirow[t]{3}{*}{$\begin{array}{l}\text { WAC } 173-491 \text {, } \\
\text { Emission Standards and } \\
\text { Controls for Sources } \\
\text { Emitting Gasoline Vapors }\end{array}$} & $\begin{array}{l}\text { Gasoline dispensing facilities shall register annually } \\
\text { with Ecology or the local air authority within } 60 \text { days } \\
\text { receipt of the registration form. A } \$ 100.00 \text { fee shall } \\
\text { accompany the registration for gasoline dispensing } \\
\text { facilities. }\end{array}$ & $\sqrt{ }$ & & \multirow[t]{3}{*}{$\begin{array}{l}1 . \\
2 . \\
3 .\end{array}$} & \multirow{3}{*}{$\begin{array}{l}\text { The Hanford Site fuel tanks are registered with Ecology and fees for the gasoline } \\
\text { dispensing facility category applicable will be paid to Ecology. } \\
\text { The DOE-RL maintains records of gasoline throughput on the Hanford Site. } \\
\text { Hanford Site fuel tanks qualify for and currently meet the Stage I requirements. New } \\
\text { gasoline storage tanks installed on the Hanford Site and over 10,000 gallons capacity } \\
\text { will comply with the Stage I requirements. }\end{array}$} \\
\hline & $\begin{array}{l}\text { Gasoline dispensing facilities shall maintain total annual } \\
\text { gasoline throughput records for the most recent two } \\
\text { calendar years. }\end{array}$ & $\sqrt{ }$ & & & \\
\hline & $\begin{array}{l}\text { Gasoline dispensing facilities with an annual gasoline } \\
\text { throughput greater than } 360,000 \text { gallons and all new } \\
\text { gasoline dispensing facilities with a total storage } \\
\text { capacity greater than } 10,000 \text { gallons will comply with } \\
\text { the Stage I requirements. }\end{array}$ & $\sqrt{ }$ & & & \\
\hline \multirow[t]{3}{*}{$\begin{array}{l}40 \text { CFR } 82 \text {, Subpart B, } \\
\text { Servicing Motor Vehicle } \\
\text { Air Conditioners }\end{array}$} & $\begin{array}{l}\text { Persons performing service on a motor vehicie } \\
\text { involving refrigerant in the motor vehicle air } \\
\text { conditioner. } \\
\text { Refrigerant recycling equipment must be certified by } \\
\text { the Administrator or an independent standards testing } \\
\text { organization approved by the Administrator. }\end{array}$ & $\sqrt{ }$ & & \multirow[t]{3}{*}{$\begin{array}{l}1 . \\
2 . \\
\text { 3. } \\
\text { 3. }\end{array}$} & \multirow{3}{*}{$\begin{array}{l}\text { The DOE-RL uses recovery/recycling equipment certified by the Underwriters' } \\
\text { Laboratory or determined by the EPA to be substantially identical to certified } \\
\text { equipment. MVAC recovery/recycling equipment certification forms submitted to } \\
\text { EPA Region } 10 \text { via DOE-RL in 1993. } \\
\text { Technicians are properly certified as part of the hiring practice. } \\
\text { Refrigerant currently is not sent offsite for reciamation. Persons performing MVAC } \\
\text { refrigerant service, maintenance, or repair must complete log sheets. A certified } \\
\text { technician fills out log sheet that includes: date of refrigerant recycling, vehicle } \\
\text { number, type of refrigerant, amount of refrigerant recycled, amount of new refrigerant } \\
\text { added, and the employee's name. (Note: Ecology agres the MVAC log system } \\
\text { satisfies the recordkeeping requirements under WAC 173-303-506. }\end{array}$} \\
\hline & $\begin{array}{l}\text { Technicians servicing MVAC units must be certified } \\
\text { through an EPA approved certification program. }\end{array}$ & $\sqrt{ }$ & & & \\
\hline & Recordkeeping requirements for MVAC refrigerants. & $\sqrt{ }$ & & & \\
\hline
\end{tabular}


Table II.B-3. Special Requirements.

\begin{tabular}{|c|c|c|c|c|}
\hline \multirow[t]{3}{*}{ Citation } & \multirow[t]{3}{*}{ Requirement } & \multicolumn{3}{|r|}{ Compliance } \\
\hline & & \multicolumn{2}{|c|}{ Compliance } & \multirow{2}{*}{ Describe Method or Test and Give Reference } \\
\hline & & In & Out & \\
\hline \multirow[t]{3}{*}{$\begin{array}{l}40 \text { CFR } 82, \text { Subpart D, } \\
\text { Federal Procurement }\end{array}$} & $\begin{array}{l}\text { Federal departments, agencies, and instrumentalities to } \\
\text { maximize procurement of safe alternatives pursuant to } \\
\text { the policies and requirements of the CAA Title VI. }\end{array}$ & $\sqrt{ }$ & & \multirow{3}{*}{$\begin{array}{l}\text { 1. Provisions of offsite contracts are specified in accordance with the federal acquisition } \\
\text { regulations and the DOE acquisition regulations. } \\
\text { 2. Ozone-depleting substances are purchased by certified technicians or a purchaser } \\
\text { representing and/or employing at least one certified technician. Class I or Class II } \\
\text { substances are substituted with safe alternatives to the maximum extent possible. No } \\
\text { purchases of a Class II substance are made for prohibited uses at the Hanford Site. } \\
\text { Reporting requirements have been met with a certification by the GSA that the Federal } \\
\text { Acquisition Regulation has been amended. }\end{array}$} \\
\hline & Procurement of contracted services. & & & \\
\hline & Procurement of refrigerants. & $\sqrt{ }$ & & \\
\hline \multirow[t]{4}{*}{$\begin{array}{l}40 \text { CFR 82, Subpart E, } \\
\text { Labeling of Products Using } \\
\text { Ozone-Depleting Substances }\end{array}$} & $\begin{array}{l}\text { Warning labels and markings on containers containing } \\
\text { certain ozone-depleting substances and refrigerant } \\
\text { recovery/recycling equipment. }\end{array}$ & $\sqrt{ }$ & & \multirow{4}{*}{$\begin{array}{l}\text { Ozone-depleting substance containers are not currently shipped offsite; however, a } \\
\text { labeling program currently is being evaluated. } \\
\text { Applicable equipment labeled in accordance with the requirements of Subpart E. }\end{array}$} \\
\hline & Container labeling & $\sqrt{ }$ & & \\
\hline & Recovery/Recycle equipment labeling & $\sqrt{ }$ & & \\
\hline & & & & \\
\hline
\end{tabular}


Table II.B-3. Special Requirements.

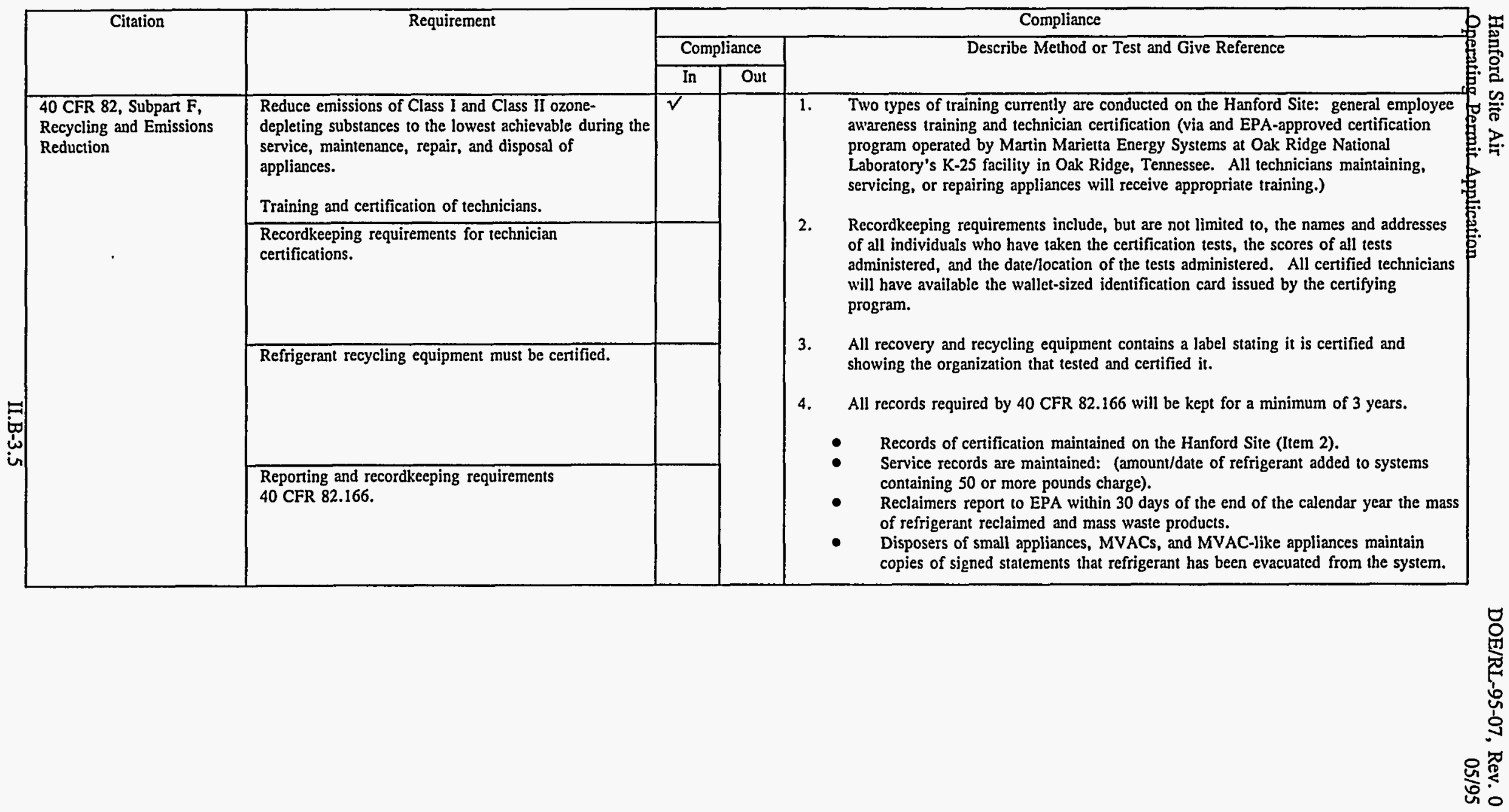


Table II.D-1. Air Operating Permit Inapplicable Requirements.

\begin{tabular}{|c|c|c|c|c|c|}
\hline \multirow[t]{2}{*}{ Requirement } & \multirow[t]{2}{*}{ Pollutant(s) } & \multicolumn{3}{|c|}{ Applicability } & \multirow[t]{2}{*}{ Comments } \\
\hline & & Federal & $\begin{array}{l}\text { Federal/ } \\
\text { State }\end{array}$ & State Only & \\
\hline WAC 173-405 Kraft Pulping Mills & & & Inapplicable & & This activity does not occur on the Hanford Site. \\
\hline WAC 173-410 Sulfite Pulping Mills & & & Inapplicable & & This activity does not occur on the Hanford Site. \\
\hline $\begin{array}{l}\text { WAC 173-415 Primary Aluminum } \\
\text { Plants }\end{array}$ & & & Inapplicable & & This activity does not occur on the Hanford Site. \\
\hline $\begin{array}{l}\text { WAC } 173-420 \text { Conformity of } \\
\text { Transportation Activities to Air Quality } \\
\text { Implementation Plans }\end{array}$ & & & & Inapplicable & \\
\hline $\begin{array}{l}\text { WAC } 173-422 \text { Motor Vehicle } \\
\text { Emission Inspection }\end{array}$ & & & Inapplicable & & $\begin{array}{l}\text { The Hanford Site is not located in a noncompliance area } \\
\text { or emission contributing area requiring a vehicle emission } \\
\text { inspection program. }\end{array}$ \\
\hline $\begin{array}{l}\text { WAC } 173-430 \text { Burning of Field and } \\
\text { Forage and Turf Grasses Grown for } \\
\text { Seed }\end{array}$ & & & Inapplicable & & This activity does not occur on the Hanford Site. \\
\hline $\begin{array}{l}\text { WAC 173-433 Solid Fuel Burning } \\
\text { Devices }\end{array}$ & & & Inapplicable & & $\begin{array}{l}\text { Applies to heating units less than } 1 \text { million BTU/hr and } \\
\text { space heating devices. The Hanford Site does not use } \\
\text { solid fuel burning devices for space heating. }\end{array}$ \\
\hline $\begin{array}{l}\text { WAC 173-435 Emergency Episode } \\
\text { Plan }\end{array}$ & & & Inapplicable & & $\begin{array}{l}\text { Director may request plan. } \\
\text { State-Only Requirement -070(1). }\end{array}$ \\
\hline
\end{tabular}


Table II.D-1. Air Operating Permit Inapplicable Requirements.

\begin{tabular}{|c|c|c|c|c|c|}
\hline \multirow[t]{2}{*}{ Requirement } & \multirow[t]{2}{*}{ Pollutant(s) } & \multicolumn{3}{|c|}{ Applicability } & \multirow[t]{2}{*}{ Comments } \\
\hline & & Federal & $\begin{array}{l}\text { Federal/ } \\
\text { State }\end{array}$ & State Only & \\
\hline WAC $173-440$ Sensitive Areas & & & Inapplicable & & $\begin{array}{l}\text { WAC } 173-440-040(3) \text { Designates } 1 \text { mile corridor along } \\
\text { Columbia River. Although the area along the Columbia } \\
\text { River is a sensitive area, no more restrictive standards for } \\
\text { sources in the listed categories apply to the Hanford Site. }\end{array}$ \\
\hline $\begin{array}{l}\text { WAC 173-470 Ambient Air Quality } \\
\text { Standards for Particulate Matter }\end{array}$ & & & Inapplicable & & $\begin{array}{l}\text { Applies to non-attainment areas; } \\
\text { Benton and Franklin Counties are in attainment of the } \\
\text { AAQS. }\end{array}$ \\
\hline $\begin{array}{l}\text { WAC } 173-490 \text { Emission Standards and } \\
\text { Controls for Sources Emitting Volatile } \\
\text { Organic Compounds }\end{array}$ & & & Inapplicable & & $\begin{array}{l}\text { Supplements WAC } 173-400 \text {. The Hanford Site is not } \\
\text { located in a designated ozone non-attainment area. }\end{array}$ \\
\hline $\begin{array}{l}\text { WAC } 463-39 \text { General Regulations } \\
\text { for Air Pollution Sources }\end{array}$ & & & Inapplicable & & $\begin{array}{l}\text { The Hanford Site is not subject to the EFSEC permitting } \\
\text { authority. }\end{array}$ \\
\hline 40 CFR 52.2479-52.2498 & & Inapplicable & & & $\begin{array}{l}\text { These sections apply to the SIP and not sources of air } \\
\text { pollution. }\end{array}$ \\
\hline $\begin{array}{l}\text { Preconstruction permits issued under } \\
\text { Title I Part D Plan Req's. for } \\
\text { Nonattainment Areas }\end{array}$ & & & Inapplicable & & $\begin{array}{l}\text { The Hanford site and surrounding areas are in attainment } \\
\text { of AAQS's. }\end{array}$ \\
\hline $\begin{array}{l}\text { Section } 111 \text { (NSPS) standards or } \\
\text { requirements, including Sec. 111(d) }\end{array}$ & $\begin{array}{l}\text { SO2 } \\
\text { NOx } \\
\text { PM10 }\end{array}$ & Inapplicable & & & $\begin{array}{l}\text { Applicable if triggered by construction of new sources or } \\
\text { modification of existing steam generating units meeting the } \\
\text { applicability criteria of } 40 \text { CFR } 61 \text {. }\end{array}$ \\
\hline
\end{tabular}


Table II.D-1. Air Operating Permit Inapplicable Requirements.

\begin{tabular}{|c|c|c|c|c|c|}
\hline \multirow[t]{2}{*}{ Requirement } & \multirow[t]{2}{*}{ Pollutant(s) } & \multicolumn{3}{|c|}{ Applicability } & \multirow[t]{2}{*}{ Comments } \\
\hline & & Federal & $\begin{array}{l}\text { Federal } / \\
\text { State }\end{array}$ & State Only & \\
\hline Title IV Acid Rain Control & $\begin{array}{l}\mathrm{SO} 2 \\
\mathrm{NOx}\end{array}$ & & Inapplicable & & $\begin{array}{l}\text { Steam generators on the Hanford Site are not included in } \\
\text { Phase I of Title IV program. }\end{array}$ \\
\hline $\begin{array}{l}\text { Standards or requirements governing } \\
\text { solid waste incineration under Sec. } 129 \\
\text { of the FCAA }\end{array}$ & & Inapplicable & & & This activity does not occur on the Hanford Site. \\
\hline $\begin{array}{l}\text { Consumer and commercial products, } \\
\text { under Sec. 183(e) of FCAA }\end{array}$ & & Inapplicable & & & $\begin{array}{l}\text { Federal Ozone Measures (Release of VOCs) - Regulated } \\
\text { entities defined as manufacturers, processors, wholesale } \\
\text { distributors, or importers of consumer or commercial } \\
\text { products for sale or distribution in interstate commerce. } \\
\text { The Hanford Site is not involved in these activities. }\end{array}$ \\
\hline $\begin{array}{l}\text { Standards or requirements governing } \\
\text { tank vessels, under Sec. } 183(\mathrm{f}) \text { of } \\
\text { FCAA }\end{array}$ & & Inapplicable & & & $\begin{array}{l}46 \text { USC } \$ 2101 \text { vessel that carries oil or hazardous } \\
\text { materials in bulk as cargo operating on the navigable } \\
\text { waters of the U.S. The Hanford Site is not involved in } \\
\text { these activities. }\end{array}$ \\
\hline $\begin{array}{l}\text { Outer continental shelfs, under Sec. } \\
328 \text { of FCAA }\end{array}$ & & Inapplicable & & & \\
\hline $\begin{array}{l}\text { Permits issued by local air pollution } \\
\text { control auth. and local orders or reg's. }\end{array}$ & & & Inapplicable & & $\begin{array}{l}\text { Refer to Table V-4 for special cases involving local air } \\
\text { pollution control agency jurisdiction. }\end{array}$ \\
\hline Chapter $80.50 \mathrm{RCW}$ & EFSEC & & Inapplicable & & $\begin{array}{l}\text { The Hanford Site is not subject to EFSEC permitting } \\
\text { authority. }\end{array}$ \\
\hline
\end{tabular}


Table II.D-1. Air Operating Permit Inapplicable Requirements.

\begin{tabular}{|c|c|c|c|c|c|}
\hline \multirow[t]{2}{*}{ Requirement } & \multirow[t]{2}{*}{ Pollutant(s) } & \multicolumn{3}{|c|}{ Applicability } & \multirow[t]{2}{*}{ Comments } \\
\hline & & Federal & $\begin{array}{l}\text { Federal } / \\
\text { State }\end{array}$ & State Only & \\
\hline $\begin{array}{l}\text { WAC 173-434 Solid Waste } \\
\text { Incineration }\end{array}$ & & & Inapplicable & & $\begin{array}{l}\text { No solid waste incineration facilities of the size threshold } \\
\text { identified in the regulation operate on the Hanford Site. } \\
\text { State-Only Requirements }-110,120,130(2) \text {. }\end{array}$ \\
\hline $\begin{array}{l}\text { WAC 173-450 Establishing } \\
\text { Requirements for Receipt of Financial } \\
\text { Aid }\end{array}$ & & & & Inapplicable & State procedural requirement. \\
\hline WAC 173-495 Weather Modification & & & & Inapplicable & This activity does not occur on the Hanford Site. \\
\hline $\begin{array}{l}\text { WAC 173-474 Ambient Air Quality } \\
\text { Standards for Sulfur Oxides }\end{array}$ & & & & Inapplicable & $\begin{array}{l}\text { No monitoring or compliance requirements apply to the } \\
\text { Hanford Site. }\end{array}$ \\
\hline $\begin{array}{l}\text { WAC 173-475 Ambient Air Quality } \\
\text { Standards for Carbon Monoxide, } \\
\text { Ozone, and Nitrogen Dioxide }\end{array}$ & & & & Inapplicable & $\begin{array}{l}\text { No monitoring or compliance requirements apply to the } \\
\text { Hanford Site. }\end{array}$ \\
\hline $\begin{array}{l}\text { WAC 173-481 Ambient Air Quality } \\
\text { and Environmental Standards for } \\
\text { Fluorides }\end{array}$ & & & & Inapplicable & $\begin{array}{l}\text { No monitoring or compliance requirements apply to the } \\
\text { Hanford Site. }\end{array}$ \\
\hline $\begin{array}{l}\text { Chapter } 43.37 \mathrm{RCW} \text { Weather } \\
\text { Modification }\end{array}$ & & & & Inapplicable & This activity does not occur on the Hanford Site. \\
\hline $\begin{array}{l}\text { WAC 173-492 Motor Fuel } \\
\text { Specifications for Oxygenated Gasoline }\end{array}$ & & & & Inapplicable & $\begin{array}{l}\text { The Hanford Site is not located in the control areas } \\
\text { requiring oxygenated gasoline use. }\end{array}$ \\
\hline
\end{tabular}


Table II.D-1. Air Operating Permit Inapplicable Requirements.

\begin{tabular}{|c|c|c|c|c|c|}
\hline \multirow[t]{2}{*}{ Requirement } & \multirow[t]{2}{*}{ Pollutant(s) } & \multicolumn{3}{|c|}{ Applicability } & \multirow[t]{2}{*}{ Comments } \\
\hline & & Federal & $\begin{array}{l}\text { Federal/ } \\
\text { State }\end{array}$ & State Only & \\
\hline $\begin{array}{l}\text { WAC } 173-400-060 \text { Emission Stds. for } \\
\text { general process units }\end{array}$ & Part. Matter & & Inapplicable & & $\begin{array}{l}\text { No facilities meeting the definition of general process } \\
\text { units [WAC 173-400-030(32)] operate on the Hanford } \\
\text { Site. }\end{array}$ \\
\hline $\begin{array}{l}\text { WAC 173-400-070 Emission Stds. for } \\
\text { certain source categories }\end{array}$ & Part. Matter & Inapplicable* & & & $\begin{array}{l}\text { * State Only Requirements (7)std. for acid mist from } \\
\text { sulfuric acid plants. No emission units in the source } \\
\text { categories are operated on the Hanford Site. }\end{array}$ \\
\hline $\begin{array}{l}\text { WAC 173-400-081 Startup and } \\
\text { Shutdown }\end{array}$ & & Inapplicable & & & $\begin{array}{l}\text { Applicable if RACT, BACT, LAER, or BART standards } \\
\text { provide special allowances during startup and shutdown. }\end{array}$ \\
\hline $\begin{array}{l}\text { WAC } 173-400-105(5) \text { Records, } \\
\text { Monitoring, and Reporting }\end{array}$ & Opacity & & Inapplicable & & $\begin{array}{l}\text { This section is not applicable to Hanford Site fossil } \\
\text { fuel-fired steam generators based on heat input ratings of } \\
\text { units. }\end{array}$ \\
\hline $\begin{array}{l}\text { WAC } 173-400-112 \text { Requirements for } \\
\text { new sources in nonattainment areas }\end{array}$ & & Inapplicable & & & $\begin{array}{l}\text { The Hanford Site is not located in any nonattainment } \\
\text { areas. }\end{array}$ \\
\hline $\begin{array}{l}\text { WAC 173-400-141 Prevention of } \\
\text { Significant Deterioration }\end{array}$ & & & & Inapplicable & $\begin{array}{l}\text { Applicable if triggered through source construction or } \\
\text { modification meeting definition. }\end{array}$ \\
\hline WAC 173-400-171 Public Involvement & & & & Inapplicable & Ecology procedural requirement. \\
\hline WAC $173-400-180$ Variance & & & & Inapplicable & Ecology procedural requirement. \\
\hline $\begin{array}{l}\text { WAC } 173-400-190 \text { Requirements for } \\
\text { Nonattainment Areas }\end{array}$ & & & & Inapplicable & The Hanford Site is not located in any nonattainment area. \\
\hline
\end{tabular}


Table II.D-1. Air Operating Permit Inapplicable Requirements.

\begin{tabular}{|c|c|c|c|c|c|}
\hline \multirow[t]{2}{*}{ Requirement } & \multirow[t]{2}{*}{ Pollutant(s) } & \multicolumn{3}{|c|}{ Applicability } & \multirow[t]{2}{*}{ Comments } \\
\hline & & Federal & $\begin{array}{l}\text { Federal } / \\
\text { State }\end{array}$ & State Only & \\
\hline $\begin{array}{l}\text { WAC 173-400-210 Emission } \\
\text { Requirements of Prior Decisions }\end{array}$ & & & & Inapplicable & $\begin{array}{l}\text { The Hanford Site air pollution jurisdiction has been } \\
\text { reserved by Ecology, except open-burning and asbestos } \\
\text { abatement. }\end{array}$ \\
\hline $\begin{array}{l}\text { WAĆ } 173-400-220 \text { Requirements for } \\
\text { Board Members }\end{array}$ & & & & Inapplicable & Ecology procedural requirement. \\
\hline WAC 173-400-260 Conflict of Interest & & & & Inapplicable & Ecology procedural requirement. \\
\hline $\begin{array}{l}\text { Comprehensive Environmental } \\
\text { Response, Compensation, and Liability } \\
\text { Act (CERCLA) of } 1980\end{array}$ & & Inapplicable & & & $\begin{array}{l}\text { Permitting is not a substantive requirement, Title V } \\
\text { permits are not applicable to CERCLA activities. }\end{array}$ \\
\hline $\begin{array}{l}\text { Resource Conservation and Recovery } \\
\text { Act (RCRA) of } 1976,40 \text { CFR } 264 \text { \& } \\
265 \text { Subparts AA, BB, and CC }\end{array}$ & & Inapplicable & & & These regulations are promulgated under RCRA authority. \\
\hline
\end{tabular}




\section{CHAPTER III}

\section{COMPLIANCE PLAN SUMMARY}




\section{CONTENTS}

III. COMPLIANCE PLAN SUMMARY $\ldots \ldots \ldots \ldots \ldots \ldots \ldots$ III-1

III.A. STATEMENTS OF INTENT $\ldots \ldots \ldots \ldots \ldots \ldots \ldots \ldots \ldots$ III-1

III.A. 1. Current Compliance . . . . . . . . . . . . . . . III-1

III.A.2. Future Compliance . . . . . . . . . . . . . . III-1

III.A.3. Noncompliance . . . . . . . . . . . . . . . . . III-1 


\section{CHAPTER III. COMPLIANCE PLAN SUMMARY}

\section{III.A. STATEMENTS OF INTENT}

\section{III.A.1. Current Compliance.}

For sources currently in compliance with all applicable requirements, DOE-RL shall ensure the sources continue such compliance.

\section{III.A.2. Future Compliance.}

For applicable requirements that become effective during the permit term, DOE-RL shall ensure the source will meet such requirements on a timely basis.

\section{III.A.3. Noncompliance.}

For sources not in compliance with all applicable requirements, DOE-RL shall ensure compliance with the Compliance Schedule (located in Appendix B) as developed under the Compliance Plan (Chapter II). 


\section{CHAPTER IV}

\section{INSIGNIFICANT EMISSIONS UNITS}




\section{CONTENTS}

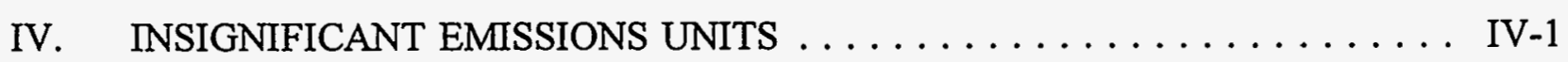

IV.A. LIST OF INSIGNIFICANT EMISSION UNITS $\ldots \ldots \ldots \ldots \ldots \ldots$ IV-1

IV.B. DETERMINATION $\ldots \ldots \ldots \ldots \ldots \ldots \ldots \ldots \ldots \ldots \ldots$ IV-1

IV.B.1. Introduction $\ldots \ldots \ldots \ldots \ldots \ldots \ldots \ldots \ldots$ IV-1

IV.B.2. Categorically exempt emission units (WAC 173-401-531) . . . IV-1

IV.B.3. Units or activities defined as insignificant based on

size or production rate (WAC 173-401-533) $\ldots \ldots \ldots \ldots$ IV-3

IV.B.3.1. Physical and analytical laboratories

[WAC-173-401-533(3)(c)] . . . . . . . . . . IV-3

IV.B.3.2. Industrial water chlorination facilities of not greater than 20 million gallons per day capacity [WAC 173-401-533(2)(p)] ......... IV-10

IV.B.3.3. Internal combustion engines greater than 100 horsepower and less than 500 horsepower . . . IV-10

IV.B.3.4. Operation, loading, and unloading of VOC storage tanks [WAC 173-401-533(2)(d)] . . . . . . . . . IV-11

IV.B.3.5. Operation, loading, and unloading storage of butane, propane, or liquified petroleum, storage tank, vessel capacity under 40,000 gallons [WAC 173-401-533(2)(e)] . . . . . . . . . . . . IV-11

IV.B.3.6. Space heaters and hot water heaters using natural gas, propane, kerosene and generating less than five million BTU/hr. [WAC 173-401-533(2)(r)] . . . . IV-11

IV.B.3.7. Cold Degreasers . . . . . . . . . . . . . . IV-11

IV.B.4. Emission units with actual emissions less than emission thesholds (WAC 173-401-530) . . . . . . . . . . . IV -12

IV.B.5. Fugitive emission sources [WAC 173-401-530(1)(d)] . . . . IV-12

IV.B.6. Fugitive emission sources [40 CFR 61 Subpart H] . . . . IV-15 


\section{CHAPTER IV}

\section{INSIGNIFICANT EMISSION UNITS}

\section{IV.A. LIST OF INSIGNIFICANT EMISSION UNITS}

The list of stationary insignificant emission units (IEUs) is located in Appendix K. This list is considered equivalent to Ecology's Form C-3.

\section{IV.B. DETERMINATION}

\section{IV.B.1. Introduction}

The Air Operating Permit regulations contain several criteria for identifying insignificant emission units or activities for purposes of the air operating permit program (WAC 173-401-530). The Hanford Site has evaluated its sources of regulated pollutants against the criteria of four of the methods allowed by the regulations. When a source or emission unit has been determined to be insignificant based on one of the criteria, the applicable requirements applied to those sources are reduced. For example, categorically exempt sources are not required to be listed in the Application. Other insignificant emission units must be listed in the Application, but the level of detail that must be provided is greatly reduced compared to the information required for significant sources.

Other requirements that do not apply to insignificant emission units include:

1) No testing, monitoring, reporting, or recordkeeping requirements will be required by the air operating permit [WAC-173-401-530-(2)(c)].

2) The annual compliance certification required by WAC 173-401-630(5) will not apply to insignificant emission units [WAC 173-401-530(2)(d)].

The following discussion fulfills the requirement to identify insignificant emission units and the methodologies used to determine their insignificance in adequate detail so that Ecology can evaluate the emission units to verify insignificance. The narrative descriptions of the methodologies are provided below. Specific emission units and other required information are listed in Appendix $\mathrm{K}$.

\section{IV.B.2. Categorically exempt emission units (WAC 173-401-532)}

Hanford Site property descriptions and, in many cases, facility inspections were used to determine the applicability of this category to particular facilities or activities. These emission units are not listed in the Application in accordance with Ecology instructions and regulations. 
Categorically exempt insignificant emission units may include, but are not limited to, the following types of emission units on the Hanford Site.

1) Mobile transport tanks on vehicles, except for those containing asphalt [WAC 173-401-532(2)].

2) Lubricating oil storage tanks [WAC 173-401-532(3)].

3) Storage tanks, reservoirs, and pumping and handling equipment of any size, limited to... hydraulic fluid... using appropriate lids and covers where there are no objectionable odor or airborne particulate [WAC 173-401-532(6)].

4) Pressurized storage of oxygen, nitrogen, carbon dioxide, air, or inert gases [WAC 173-401-532(5)].

5) Vehicle exhaust from auto maintenance and repair shops [WAC 173-401-532(7)].

6) Vents from continuous emissions monitors and other analyzers [WAC 173-401-532(8)].

7) Vents from rooms, buildings, and enclosures that contain permitted emissions units or activities from which local ventilation, controls and separate exhaust are provided [WAC 173-401-532(9)].

8) Internal combustion engines for propelling or powering a vehicle [WAC 173-401-532(10)].

9) Metal or glass heat-treating, in absence of molten materials, oils, or VOCs [WAC 173-401-532(17)].

10) Drop hammers or hydraulic presses for forging or metalworking [WAC 173-401-532(18)]

11) Plastic and resin curing equipment, excluding FRP [WAC 173-401-532(23)]

12) Plastic pipe welding [WAC 173-401-532(29)]

13) Extrusion equipment, metals, minerals, plastics, grain, or wood [WAC 173-401-532(121)].

14) Plant upkeep, including routine housekeeping, preparation for and paintingof structures and equipment, retarring roofs, applying insulation, to buildings in accordance with applicable environmental and health and safety requirements and paving or stripping parking lots [WAC 173-401-532(33).

15) Cleaning and sweeping of streets and paved surfaces [WAC 173-401-532(35)].

16) Steam cleaning operations[WAC 173-401-532(39)].

17) Lawn and landscaping activities [WAC 173-401-532(43)].

18) General vehicle maintenance, including vehicle exhaust from repair facilities [WAC 173-401-532(45)].

19) Comfort air conditioning or air cooling systems, not used to remove air contaminants from specific equipment [WAC 173-401-532(46)].

20) Natural and forced air vents and stacks for bathroom/toilet activities [WAC 173-401-532(48)].

21) Office activities [WAC 173-401-532(49)].

22) Fuel and exhaust emissions from vehicles in parking lots [WAC 173-401-532(54)].

23) Oxonation equipment [WAC 173-401-532(58)].

24) Photographic process equipment by which an image is reproduced upon material sensitized to radiant energy, e.g., blueprint activity, photocopiers, mimeograph, 
telefax, photographic developing, and microfiche [WAC 173-401-532(70)]

25) Sample gathering, preparation, and management [WAC 173-401-532(73)].

26) Repair and maintenance activities, not involving installation of an emission unit and not increasing potential emissions of a regulated pollutant [WAC 173-401-532(74)].

27) Steam vents and safety relief valves [WAC 173-401-532(87)].

28) Air compressors, pneumatically operated equipment, systems and hand tools [WAC 173-401-532(88)].

29) Steam leaks [WAC 173-401-532(89)].

30) Process water and white water storage tanks [WAC 173-401-532(94)].

31) Pond dredging [WAC 173-401-532(116)].

32) NonPCB oil filled circuit breakers, oil-filled transformers, and other equipment that is analgous to, but not considered to be a tank [WAC 173-401-532(118)].

33) Electric or steam-heated drying ovens and autoclaves [WAC 173-401-532(119)]

34) Water cooling towers processing exclusively noncontact cooling water [WAC 173-401-532(121)].

\section{IV.B.3. Units and activities defined as insignificant based on size or production rate (WAC 173-401-533)}

There are several types of emission units and activities that have been determined to be insignificant based on size or production rate. The individual emission units are listed in Appendix $\mathrm{K}$. The. types of emission units and the methodologies used to determine that the units are insignificant are discussed below.

\section{I .B.3.1. Physical and analytical laboratories [WAC-173-401-533(3)(c)]}

\section{IV.B.3.1.1. Introduction}

The regulation allows that physical and analytical laboratories may be insignificant based on size or production rate. However, the regulation does not define the criteria for determining that the laboratory is insignificant except to state that the determination will be made on a case-by-case basis. The methodology used to determine whether laboratories were insignificant based on size or production rate is described below.

WAC 173-401-530(1)(c), states that the emission unit or activity is insignificant if it is listed in WAC 173-401-533 and its size or production rate based on maximum rated capacity is below the level specified therein. WAC 173-401-533(3)(c) states that chemical or physical analytical laboratory operations may be determined to be insignificant on a case-by-case basis.

During discussions with Ecology staff, it had been indicated that the determination of insignificance on the basis of size or production rate for laboratories, including research and development laboratories, would be partly based on meeting the threshold values listed in WAC 173-401-530(4). The following discussion summarizes the methodology used to assess regulated pollutant emissions and provides a comparison of these emissions to the threshold 
values listed in WAC 173-401-530 (4).

\section{IV.B.3.1.2 Pacific Northwest Laboratory Operated Laboratories}

\section{IV.B.3.1.2.1 Methods and Assumptions}

The PNL facilities at the Hanford Site are predominantly chemical and physical analytical laboratories used to conduct research and development. The laboratories and their supporting offices, storage and utility areas, shops, etc., are contained in several buildings, primarily located in the 300 Area. Table K.1a in Appendix K lists PNL managed facilities on the contiguous Hanford site. The listing is derived from the PNL facilities database and identifies building number, name, usage code and description, and building size. The table lists the · total recent inventory of threshold chemicals in each building derived from the PNL Chemical Information System (CMS). The CMS is the laboratory central database used to track the current inventory of chemicals in the laboratory. This system tracks chemical containers from their introduction into the laboratory through Purchasing to the containers ultimate disposal as managed by PNL Waste Management. The CMS is maintained at an individual room/laboratory level inventory. Standard queries are available, and data files or hard copy printouts of inventories are readily accessible. The chemicals and chemical groups listed in WAC 173-401-530 were queried in the CMS, and the resulting inventories were the basis for the analysis provided herein.

The largest sources of chemical emissions are assumed to be from the use of chemicals in chemical analytical laboratories; consequently, comparison of PNL emissions to the chemical emission thresholds (other than carbon monoxide, nitrogen and sulfur oxides, $\mathrm{PM}_{10}$, and ozone depleting substance) is based on estimates of the releases at maximum rated capacity of the chemicals in the CMS inventory. The primary sources of CO, NOx and SOx are combustion sources using fossil fuels; therefore comparison to the thresholds for these emissions was based on identifying the largest combustion sources. These sources are comprised of installed facility heaters, boilers or engines. Compliance with the threshold limits for $\mathrm{CO}, \mathrm{NOx}$, and SOx was determined by estimating maximum rated capacity emissions for the sources identified in preventative maintenance records and during facility walkthroughs.

The VOC emissions estimate for Pacific Northwest Laboratories (PNL) was determined by reviewing the entire PNL inventory of liquids and gases in inventory. The review identified those liquids and gases that are volatile based on the scientifically accepted definition of VOC having a low solubility in water and a vapor pressure at 20 degrees centigrade of greater than one millimeter of mercury. Using this definition, the chemicals listed in Table K.1b were evaluated based on solubility and vapor pressure data. Compounds that met one or both of the criteria were defined as VOC.

Those chemicals defined as VOC were evaluated to determine which might participate in atmospheric photochemical reactions. Those compounds specifically identified as non-reactive in WAC 173-400, and those compounds that have similar chemical stability, were removed 
from the list. The remaining list identifies those chemicals defined as VOC and were included in the PNL estimate of VOC emissions.

This methodology is conservative in that if there was uncertainty as to volatility or photo reactivity the compound was considered to be both volatile and photo-reactive. Therefore, the current PNL VOC list will be subject to deletions as new data are obtained on the physicochemical properties of organic chemicals. The list may also expand as new compounds become part of the inventory with new research and development studies.

The primary sources of $\mathrm{PM}_{10}$ are expected to be shops used to construct research apparatus and maintain laboratory facilities. Compliance with the $\mathrm{PM}_{10}$ threshold was determined by identifying sources that would not be categorically exempt or insignificant based on size or production rate, estimating their emissions at maximum rated capacity and comparing them to the threshold.

Maximum-rated capacity chemical emissions (other than carbon monoxide, nitrogen and sulfur oxides, and $\mathrm{PM}_{10}$ ) were estimated for all PNL-managed buildings using chemical inventories in the CMS . Maximum-rated capacity of the laboratory operations and their emissions was estimated by assuming the chemical sources, primarily laboratories, could be operated at four times their current capacity, and for 24 hours per day rather than the actual nominal 8 hours per day. The annualized emission rates were determined by assuming that 100 percent of the chemical inventory was released to the air, as opposed to being distributed among air, liquid and solid waste streams.

The maximum-rated capacity for combustion sources would be based on the maximum number of hours the sources would operate for their designed use. As explained later, this analysis was not needed, as all sources are exempt. The maximum rated capacity for shops was based on the assumption that the shops could be operated at four times their current capacity and for 24 hours per day rather than the actual nominal 8 hours per day.

Walkthroughs of buildings and/or rooms listed as shops or physical laboratories were performed to identify categorically-exempt emission units (W AC 173-401-532), insignificant emission units based on size or production rates (WAC 173-401-533), and potentially significant emission units. These walk-through, inquiries, and inspections provided a sufficiently complete assessment to determine the sources were insignificant emission units. Maximum-rated capacity emission estimates were made for potentially significant emission units and compared with the threshold limits (WAC 173-401-530). All estimates were based on the most conservative usage estimates provided during the inquiries/inspections.

\section{IV.B.3.1.2.2. Results}

\section{Chemical Analytical Laboratories}

To perform the analysis for compliance with the threshold limits, an emission unit was 
defined as the individual room/laboratory in the facility. If it could initially be shown that for the entire building emission of any constituent was below the threshold value, no further room/laboratory analysis was performed. With this definition, the chemical emissions threshold determination was done in two steps. In the first step the total building emissions at maximum-rated capacity were compared to the threshold levels. This step assumed that 100 percent of the chemical inventory was used in one year and was released to air.

Table K.1b in Appendix K lists those buildings and those specific chemicals for which the total building emissions at maximum rated capacity exceeded the threshold levels under this conservative assumption. The buildings and chemicals that exceeded the threshold were further evaluated to determine if the total emissions from any individual room, operating at maximum rated capacity, exceeded the thresholds assuming that $1.0 \mathrm{E}-03$ of the liquids and 1.0 E-06 of the solids in inventory were released to air in a year. The 1.0 E-03 and 1.0 E-06 factors are taken from the analysis methodology used for radiological emission as outlined in 40 CFR 61. This method would give conservative values in lieu of evaluation done with individual vapor pressures and temperature analysis, and is far easier and reproducible in calculation. The results of this analysis are shown in Appendix K - Table K.1c. This analysis indicates that all building or rooms are insignificant emission units based on size or production rate with the possible exception of the 326 Building, Room 26-A. The analysis indicates a possible excess of fluorides. The indication of an excess was investigated to determine if a threshold was truly exceeded, or whether it was a result of the conservatism in the analysis approach. It was determined that the excess fluoride was sulfur hexafluoride (SF6) gas. The SF6 is used in the operation of an electron microscope. The actual use rate is less than 2 pounds per year rather than the inventory of 39 pounds as was conservatively assumed in the initial analysis. The maximum-rated capacity release rate would be less than 0.012 tons per year $(2 / 2000 \times 12)$, more than an order of magnitude less than the fluorides threshold of 0.15 tons per year.

\section{Physical Analytical Laboratories and Shops}

The PNL facility database identified space allocated to shops and laboratories. Primary shop activities are small-scale fabrication of assemblies used in laboratory research or for maintenance of the laboratories. Major physical laboratory activities identified include waste simulation studies in quarter-scale, double-shell tank mock ups (336 building); waste melter research (324 building); and depleted uranium metal working (306 W building).

Facility walkthroughs were performed to identify emission sources that would not be categorically exempt or insignificant based on size or production rate. Information obtained during the facility walkthroughs is presented below. The only significant $\mathrm{PM}_{10}$ source identified was the shop in the 350 building where wood cutting and sandblasting occur.

Using factors obtained from the U.S. Environmental Protection Agency Office of Air Quality Planning and Standards (OAQPS) database, the wood cutting emissions were estimated to be 43 pounds of $\mathrm{PM}_{10}$. This assumes all of the wood used is rendered to sawdust, and no emission controls are used. The emissions from sandblasting were estimated to be 1 pound 
of $\mathrm{PM}_{10}$. The total maximum-rated capacity for this facility is estimated at $528 \mathrm{lbs} /$ year $[(43+1) \times 12=528]$. The regulatory limit is 0.75 tons ( 1500 pounds) emitted annually. The emission rate of $\mathrm{PM}_{10}$ at maximum-rated capacity is well below the threshold limit.

The following buildings, listed in the Pacific Northwest Laboratory (PNL) property database, have shop space in the facility. Scoping inquiries and inspections of the facility to determine the disposition of the shop space were performed, and the facilities' potentially significant emission sources evaluated. Categorical exclusions under WAC 173-401-530, -532, and -533 are discussed for each facility.

350 Building (9556 square feet of shop space; 9556 total square feet in the facility.) The 350 building houses the general shop that supports all 300 Area PNL activities. It contains both a large metal-working area, including milling and fabrication equipment, and a sheet-metal shop. A small portion of the area is welding shop space. Some sandblasting of metal is done, using less than 2 tons of garnet sand annually. This building also contains a wood shop, where burial boxes that are used in the disposal of solid radioactive waste are assembled. Annual wood usage in all fabrications done in this shop are estimated at less than 120 tons. A small percentage of this amount would be released as sawdust $\left(\mathrm{PM}_{10}\right)$. Equipment in the wood shop is connected to a dust collection system which further reduces dust emissions. Estimates made using the factors available from the U.S. Environmental Protection Agency Office of Air Quality Planning and Standards (OAQPS) database gives approximately 43 pounds of $\mathrm{PM}_{10}$ emitted annually assuming the entire amount of wood is rendered to sawdust. Based on the criteria found in sections 22, 24, and 25 of WAC 173-401532 the facility is categorically exempt from the permit submission. Metal work is done primarily indoors. The facility has a dedicated ventilation system that exhausts the facility. Less than 300 pounds per year of welding rod is used, which is well below the limit established in WAC 173-401-533(i), excluding the welding done in the facility based on size or production rate. With less than 0.75 tons of $\mathrm{PM}_{10}$, the facility has insignificant emissions.

306W Building (5303 square feet of shop space; 22339 square feet of building space) This facility primarily machines depleted uranium billets. The facility contains most major pieces of metal shop equipment. There are several small electrically heated furnaces. The shop currently operates at a reduced level (no full scale machining operations), but is occupied Monday to Friday on a normal 8 hour day. The shop handles less than two tons of material per year. Based on WAC 173-401-532 (17)(18)(20)(22)(24) and (25) the facility is categorically exempt from the permit submission. All work is done indoors. The facility has a dedicated ventilation system that exhausts through HEPA filtration.

325 Building (4251 square feet of shop space; 41587 square feet of building space) This building contains a general metal shop and a small bench area for electronic test equipment and work. Small parts and assemblies in support of general facility maintenance and some research applications are produced. The shop contains several pieces of equipment for general metal work. The amount of welding done in this area uses less than 50 pounds of rod each year.

Based on WAC 173-401-532 (55) the facility is categorically exempt from the permit 
submission. All work is done indoors. The facility has a dedicated ventilation system that exhausts the area. Less than 100 pounds per year of welding rod is used, which is well below the limit established in WAC 173-401-533 (i), excluding the welding done in the facility based on size or production rate.

324 Building (4123 square feet of shop space; 32,136 square feet of building space) This building contains a general metal shop where small parts and assemblies in support of general facility maintenance and some research applications are produced. The shop contains several pieces of general metal working equipment. The amount of welding done in this area uses less than 100 pounds of rod each year. Based on the criteria found in WAC 173-401532 (55) the facility is categorically exempt from the permit submission. Work is done primarily indoors, with some welding activity done outside. The facility has a dedicated ventilation system that exhausts the area. Less than 100 pounds per year of welding rod are used, which is well below the WAC 173-401-533 (i) limit, excluding the welding done in the facility based on size or production rate.

622F Building (2660 square feet of shop space; 2660 square feet of building space) This building has three rooms that contain two small machine shop operations; and one room containing a small work bench used for electrical equipment testing. The machine shop equipment is used primarily for machining of small parts in support of research projects. This shop is used on a frequent, but not full- time basis. Based on WAC 173-401-532 (55), the facility is categorically exempt from the permit submission. All work is done indoors. No welding is done in the area.

3731A Building (2484 square feet of shop space; 2484 square feet of building space) This was formerly a graphite machining area, but is shut down. The facility is used for storage of the machining equipment.

327 Building (1424 square feet of shop space; 10,337 square feet of building space) The building contains a general metal shop where small parts and assemblies in support of general facility maintenance and some research applications are produced. The shop contains several pieces of general metal working equipment. No welding is done in this area. The air from this space is cycled through the facility HEPA filter system. Based on WAC 173-401532 (55) the facility is categorically exempt from the permit submission. All work is done indoors. No welding is done in the area.

3718A Trailer 4 (1310 square feet of shop space; 2025 square feet of building space) This space is an Instrument shop. The work done in this area is bench-type electronics tuning and repair, with no machining or production type work.

329 Building (1268 square feet of shop space; 13,817 square feet of building space) This space contains a general metal shop where small parts and assemblies in support of general facility maintenance and some research applications are produced. The shop contains several pieces of general metal working equipment. No welding is done in this area. Based on WAC 173-401-532 (55) the facility is categorically exempt from the permit submission. 
All work is done indoors.

331 Building (935 square feet of shop space; 44,478 square feet of building space)

The 331 building includes two spaces, one is a craft shop where a fitter, a millwright, and an electrician share an area for storage of equipment and general work; the other area is an Instrument shop, where small electrical equipment is calibrated and repaired. Activities performed in these areas are bench-type work, electronic tuning and repair, with no machining or production-type work.

350A Building (915 square feet of shop space; 915 square feet of building space) This building contains a paint shop with a walk-in-size paint booth. The ventilation system for the building operates at a slight negative pressure (approximately 0.5 inches water gauge) that draws outside air into the booth, and filters the discharge side. All air in the booth is filtered through a paper filter media which traps paint overspray. This building is used almost daily, for small jobs that can be easily moved in and out of the booth. On average, less than 2 gallons of paint a day is used, which is below the limit established in WAC 173-401-533 (q) and is excluded, based on size or production rate.

305B Building (896 square feet of shop space. 7012 square feet of building space) This facility is a permitted Treatment, Storage, and Disposal facility. The shop space in this facility has been dedicated to waste storage space. No shop activities occur in this facility.

6652C Building (770 square feet of shop space; 1430 square feet of building space) This facility is shut down. All equipment is being removed.

326 Building (727 square feet of shop space; 22,552 square feet of building space) This shop space has been converted to laboratory and office space. No shop activities occur in this building.

$622 \mathrm{R}$ Building (707 square feet of shop space; 3667 square feet of building space) This shop area is used as a general craft area; its primary function is as an electrical equipment test area used by an instrument technician. No milling, machining, or welding is done in this shop area.

320 Building (592 square feet of shop space; 11,963 square feet of building space) This shop area contains a metal lathe and a drill press, used primarily by research staff to machine small parts in support of research projects. This shop is used on a very infrequent basis. Based on WAC 173-401-532 (55) criteria, the activities in this shop area are categorically exempt from the permit submission. All work is done indoors.

306W Trailer 5 (364 square feet mislabeled as shop space; 364 square feet of building space)

This space is office space in an office trailer and was mislabeled as shop space.

3720 Building (352 square feet of shop space; 13,110 square feet of building space) 
This space is used as a material storage area. There are no shop activities at this facility.

3718A Building (352 square feet of shop space; 352 square feet of building space)

This space is used as a material storage area. There are no shop activities at this facility.

3745B Building (274 square feet of shop space; 3167 square feet of building space)

There is a small metal working area in this building that supports some small material/parts manufacturing needs particular to the work being done in the building. Based on the criteria found in WAC 173-401-532 (55) the facility is categorically exempt from the permit submission. All work is done indoors.

318 Building (209 square feet of shop space; 14,498 square of building space) The shop space in this facility is an electronics shop. The work in this area is bench-type work, with no machining or production-type work.

337 Building (178 square feet of shop space)

This space is occupied by the buildings computer support personnel and is an electronics repair shop. The work in this area is bench-type work, with no machining or production-type work.

\section{IV.B.3.2. Industrial water chlorination facilities of not greater than 20 million gallons per day capacity [WAC 173-401-533(2)(p)]}

Water filtration plants that chlorinate water are identified in the list below. These facilities are insignificant emissions units based on a comparison to the size or production rate threshold.

Facility

315 Filter Plant

283E Filter Plant

283W Filter Plant

183N Filter Plant

183KE Filter Plant
Capacity (gpc)

$5,040,000$

$2,160,000$

$2,160,000$

500,000

30,000

IV.B.3.3. Internal combustion engines greater than 100 horsepower and less than $\mathbf{5 0 0}$ horsepower

The issue of stationary internal combustion (IC) engines is not well defined in the regulations. Guidance from ecology on how to treat internal combustion engines less than 500 horsepower (HP) for this Application has indicated that providing numbers of units on-site would be appropriate. The number of units is listed below. 


$$
\frac{\text { Stationary IC Engines }}{>100 \mathrm{HP} \text { but }<500 \mathrm{HP}} \quad \frac{\text { No. of units (estimated) }}{200-250}
$$

These units are believed to be insignificant emission units based on size and hours of usage. Most of these units are emergency or backup generators and operate on a very limited basis. Except in emergencies or to provide backup or temporary power, the units are run intermittently for brief periods for maintenance or test purposes. These test periods generally do not exceed 4 hours per week. Some of these units may be used to provide temporary power and may operate for extended periods at temporary locations. For examle, the 185 horsepower diesel powered IC engine at $183 \mathrm{KE}$ in the $100 \mathrm{~K}$ Area runs continuously during warm weather to provide adequate water circulation for fish rearing activities

IV.B.3.4 Operation, loading, and unloading of VOC storage tanks (including gasoline storage tanks), ten thousand gallons capacity or less, with lids or other appropriate closure, vp not greater than $80 \mathrm{~mm} \mathrm{Hg}$ at $21^{\circ} \mathrm{C}$. [WAC 173-401-533(2)(d)]

There are numerous VOC storage tanks on the Hanford Site containing VOC's less than 10,000 gallons capacity and considered insignificant emission units. Many of these tanks store diesel fuel for the internal combustion engines identified in Table II.B-2 and in Section IV.B.3.3 above. These tanks have not been listed individually in this Application.

\section{V.B.3.5 Operation, loading, and unloading storage of butane, propane, or liquified petroleum (LPG), storage tank, vessel capacity under 40,000 gallons [WAC 173-401-533(2)(e)]}

There are propane storage tanks on the Hanford Site less than 40,000 gallons capacity. These tanks are used for space heating and as a supply for smaller, portable propane tanks used in construction and maintenance activities. These tanks have not been listed individually in this Application.

\section{IV.B.3.6 Space heaters and hot water heaters using natural gas, propane, or kerosene and generating less than five million BTU/hr. [WAC 173-401-533(2)(r)]}

There are numerous facilities on the Hanford Site that use space heaters and hot water heaters using propane and generating less than five million BTU/hr. These units have not been listed individually in this Application.

\section{IV.B.3.7. Cold Degreasers}

The insignificant emission unit regulations do not address this type of activity, however, guidance from Ecology has indicated that these units are considered insignificant emission units. There are about 50 cold degreasing units identified on the Hanford Site. These units are used primarily for parts washing in maintenance activities. 


\section{IV.B.4. Emission units with actual emissions less than emission thresholds (WAC 173-401-530)}

The actual emissions of numerous facilities were compared to the emission thresholds of the regulated pollutants identified in WAC 173-401-530 and WAC 173-401-531. The actual emissions for these emission units were estimated using calculational procedures. The emission units that have been determine to be insignificant based on the comparison to the threshold values are listed in Appendix K - Table K.2.

The table lists the emission unit, the pollutants, the CAS Number of the pollutant, the emission threshold, and the actual emissions. Table K.2 is intended to provide the information required by Form C-4 of the Ecology application instructions. Table C-4 was not used because of the large number of Hanford Site insignificant emission units, requiring a labor intensive effort to enter the information into the Ecology form. Table K-2 is equivalent to Form C-4, as allowed by the Ecology application instructions. The Ecology application instructions also require that the applicant provide a description of the methods used to determine actual emissions, including the calculations, assumptions, and input parameters used when emissions are estimated. The calculations used to estimate the actual emissions are described in Appendix A.

\section{IV.B.5. Fugitive emission sources [WAC 173-401-530(1)(d)]}

Emission units that have been identified on the Hanford Site that are insignificant because they are fugitive sources of regulated air pollutants are identified in Appendix K - Table K.3. The emission units listed in Table K.3 have also been assessed against the emission thresholds listed in WAC 173-401-530(4) and are insignificant based on estimated actual emissions as well.

\section{Facility-wide processes}

There are numerous activities on the Hanford Site that may generate fugitive air emissions. These activities are often associated with construction or facility modification and maintenance activities. Specific locations cannot be listed for the activities and emission units described below since these activities involve all areas and all facilities of the Hanford Site. At times the activities listed below may require operation of one or more point sources of regulated air pollutants in conjunction with the activities listed below. For example, a large construction project may require a concrete plant and an asphalt plant to supply materials for the construction and paving activities. These batch plants may not meet the criteria of an insignificant emission unit. These projects will be evaluated on a case-by-case basis to determine all applicable requirements associated with large construction projects.

Functions associated with new construction and facility modification and maintenance activities may include but are not limited to the following: 


\section{Site Preparation}

- Vegetation clearing.

- Land leveling, including preparing areas for foundations.

- Excavation

- $\quad$ Dredging

- Dust suppression

$\underline{\text { Roofing }}$
- $\quad$ Carpentry
- $\quad$ Concreting
- Coating
- Demolition
- $\quad$ Equipment and area cleaning
- Miscellaneous roofing activities

\section{Concreting and Paving}

- Construction of foundations, walls, floors, and other structural elements

- Construction of parking areas, roads, and other vehicular areas

\section{$\underline{\text { Structural construction }}$}

- Building framing - metal and/or wood

- Welding and cutting torch activities

- Interior construction and installation, e.g. walls, floors, ceilings, counters, and cabinets

- Installation of floor coverings

\section{Electrical Work}

- Interior lighting and power

- Exterior lighting and power

- Installation of temporary interior and exterior lighting

- Miscellaneous lighting and power

\section{Plumbing}

- $\quad$ Pipe threading

- Welding, brazing, soldering, or cutting torch activities

- $\quad$ Acid etching

- Application of protective coatings

- Equipment and area cleaning 


\section{Metal working activities}

- May include cutting, grinding, finishing, welding, drilling, machining, and other activities

\section{Heavy equipment usage}

$\begin{array}{ll}\text { - } & \text { Scrapers } \\ \text { - } & \text { Dulldozers } \\ \text { - } & \text { Front end loaders } \\ \text { - } & \text { Backhoes } \\ \text { - } & \text { Graders } \\ \text { - } & \text { Pranes } \\ \text { - Water trucks } \\ \text { - } \quad \text { Rater wagons } \\ \text { - } \quad \text { Rock crushers and pug mills } \\ \quad \text { Other heavy equipment as needed }\end{array}$

Agricultural and landscaping activities

- $\quad$ Site preparation

- Revegetation activities

- Application of agricultural and landscaping chemicals

- Application of surface coatings, e.g. rock, gravel, and plastics

\section{Miscellaneous construction activities}

- Installation of miscellaneous systems or equipment

- Installation and use of portable sanitation facilities

- Equipment and area cleaning

\section{Abatement activities}

- Lead abatement

- $\quad$ PCB equipment management and abatement

- $\quad$ Radiological contamination abatement

- Chemical contamination abatement

- Asbestos abatement (See Table II.B-3).

Demolition activities 
The activities listed above may be conducted in radiological and/or chemically contaminated areas and may be conducted in exhausted greenhouses. Activities conducted in contaminated areas are assessed to determine regulatory agency approvals that may be required prior to commencement of activities. Certain activities conducted in exhausted greenhouses may also be permitted under the portable and temporary radionuclide air emission unit notice of construction (NOC) approved by the Washington State Department of Health. The conditions associated with this NOC approval are described in Table II.B-1.

The activities or equipment listed above may include the use of fuels for propelling or powering equipment or the use of gasses, e.g. acetylene and oxygen for welding and cutting activities.

Certain of the above activities may generate fugitive emissions which are only subject to the General Standards for Maximum Emissions (WAC 173-400-040) and therefore may be considered insignificant emission units according to WAC 173-401-530(1)(d).

\section{IV.B.6 DIFFUSE AND FUGITIVE EMISSION SOURCES [40 CFR 61 Subpart H]}

In accordance with the conditions of the National Emission Standards for Hazardous Air Pollutants (NESHAP) Federal Facility Compliance Agreement (FFCA) signed on February 7, 1994, this Application contains the results of an assessment of diffuse and fugitive (fugitive) radionuclide emission dose from the Hanford Site (see Appendix B, Milestone Number A-10-1). The report, Diffuse and Fugitive Emission Dose Assessment on the Hanford Site (Davis et al. 1995) is included as a reference to this Application. A brief summary of the report follows.

Fugitive emission sources of radionuclide airborne emissions on the Hanford Site include cribs, ponds, ditches, trenches, retention basins, diversion boxes, valve pits, french drains and reverse or injection wells, tanks, burial grounds, decontamination and decommissioning activities, and radioactively contaminated surface areas (Davis et al. 1995). The following table is taken from the Davis et al. (1995) report. 
Types of Fugitive Emissions

Cribs*

Ponds

Ditches

Trenches*

Retention Basins

Diversion Boxes

Valve Pits

Reverse Wells

French Drains

Tanks

Burial Grounds
Total Number of Sites

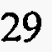

23

96

17

87

11

4

44

151

42

623

119
9

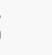

Total

*Cribs and trenches that are located in a contiguous complex, such as those within the BC Cribs and Trenches Area, were recorded as one site, rather than as individual sources.

Other potential sources of fugitive emissions not specifically identified in the table above are building penetrations such as access doors in facilities containing radioactively contaminated areas or surface contamination areas (DOE 1994). Another source of fugitive emissions includes maintenance activities performed at waste tanks in the 200 East and 200 West area. These activities are briefly described in Appendices D-4 and E-7, respectively.

The extent of the Hanford Site surface contamination is described below (Davis et al. 1995).

Hanford Site Area

100 Areas

200 Area

600 Area

300 Area (north)

Total
Surface Contamination, ${ }^{a}$ hectares

$$
\begin{array}{r}
65 \\
129 \\
1,063^{\mathrm{b}} \\
18 \\
1,275
\end{array}
$$

a Includes areas posted as Surface Contaminated Areas or Radiologically Controlled Areas.

b Includes BC Radiologically Controlled Area (RCA) south of the 200 East Area. Survey data of the BC RCA indicates this area may be significantly larger than currently characterized.

As reported in Table II.B-1, the dose to the maximally exposed individual from all fugitive sources was calculated to be $4.9 \mathrm{E}-02 \mathrm{mrem}$ for calendar year 1993. This is below the $0.1 \mathrm{mrem} / \mathrm{year}$ dose limit requiring continuous emissions monitoring. Consequently, the operational environmental monitoring program in conjunction with the radiological surveys of surface contamination areas constitute periodic confirmatory measurements of fugitive 
radionuclide emissions from the Hanford Site.

Additionally, the radionuclide emissions from the single-shell tanks and double-shell waste tanks in the 200 Area have been estimated and reported in Appendices D and E. These emission estimates contribute to the body of data that confirms the radionuclide emissions from all sources remain well below the $10 \mathrm{mrem} / \mathrm{yr}$. dose limit to the maximally exposed individual from all sources. 
Hanford Site Air

Operating Permit Application
DOE/RL-95-07, Rev. 0

05/95

This page intentionally left blank.

IV-18 
Hanford Site Air

Operating Permit Application
DOE/RL-95-07, Rev. 0 05/95

\section{CHAPTER V}

PERMIT SHIELD SUMMARY 


\section{CONTENTS}

V. PERMIT SHIELD SUMMARY $\ldots \ldots \ldots \ldots \ldots \ldots \ldots \ldots \ldots$ V-1

V.A. APPLICABLE REQUIREMENTS $\ldots \ldots \ldots \ldots \ldots \ldots \ldots \ldots$ V-1

V.B. PERMIT SHIELD REQUEST $\ldots \ldots \ldots \ldots \ldots \ldots \ldots \ldots \ldots \ldots$ V-1

\section{TABLES}

V.A-1. Applicable Requirements $\ldots \ldots \ldots \ldots \ldots \ldots \ldots \ldots \ldots$ V-2

V.A-2. Regulated Pollutants . . . . . . . . . . . . . . V-7 


\section{CHAPTER V. PERMIT SHIELD SUMMARY}

\section{V.A. APPLICABLE REQUIREMENTS}

The applicable requirements for which the DOE-RL requests a permit shield are located in Table V.A-1. Additionally, included as Table V.A-2, is a list of current and potential regulated pollutants. This list may change as a result of regulatory and Hanford Site process changes.

\section{V.B. PERMIT SHIELD REQUEST}

The DOE-RL requests that it and its contractors be shielded from enforcement actions alleging operation of an air emission source without a permit upon submittal of the Hanford Site Air Operating Permit Application. The DOE-RL also requests that a permit shield provision be incorporated within the air operating permit for all applicable and inapplicable requirements specified within the application and/or any appropriate modification of the application and/or permit. If granted, DOE-RL anticipates language similar to the following would be incorporated to the permit: Compliance with the permit conditions and compliance schedule contained within the permit is deemed compliance with any applicable air operating permit requirement, provided that such applicable requirements are included and are specifically identified in the permit upon its issuance.

Finally, DOE-RL requests that Ecology extend the permit shield to the permit application and/or permit for the inapplicable requirements specified within the air operating permit application. If granted, DOE-RL anticipates language similar to the following would be incorporated into the permit: Neither the permittee nor its contractors are required to comply with the inapplicable requirements specifically included and identified within the air operating permit application and/or any appropriate modification of the application or permit. 
Table V.A-1. Applicable Requirements.

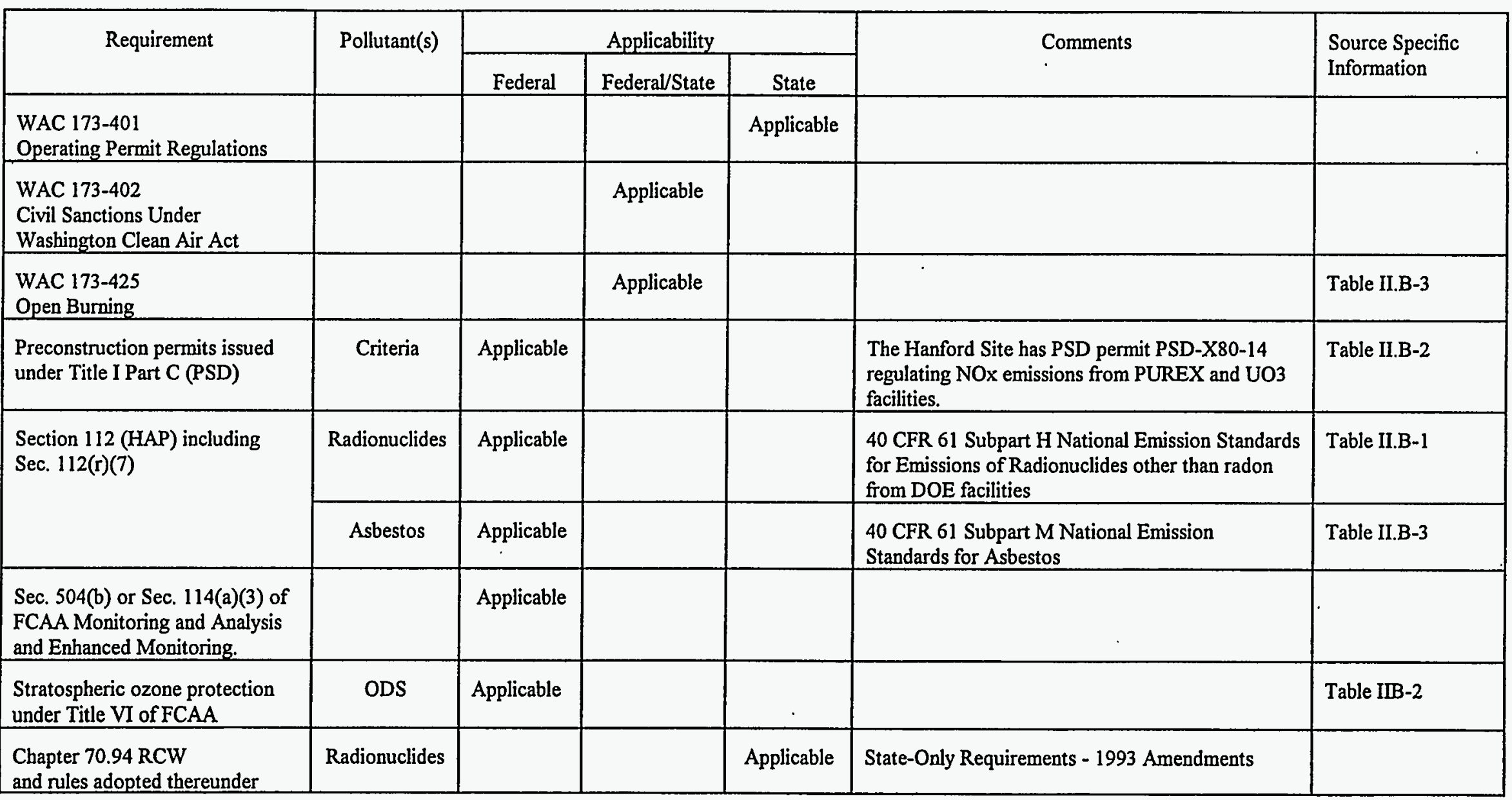


Table V.A-1. Applicable Requirements.

\begin{tabular}{|c|c|c|c|c|c|c|}
\hline \multirow[t]{2}{*}{ Requirement } & \multirow[t]{2}{*}{ Pollutant(s) } & \multicolumn{3}{|c|}{ Applicability } & \multirow[t]{2}{*}{ Comments } & \multirow{2}{*}{$\begin{array}{l}\text { Source Specific } \\
\text { Information }\end{array}$} \\
\hline & & Federal & Federal/State & State & & \\
\hline $\begin{array}{l}\text { Chapter } 70.98 \mathrm{RCW} \\
\text { and rules adopted thereunder }\end{array}$ & Radionuclides & & & Applicable & & \\
\hline $\begin{array}{l}\text { WAC } 173-421 \\
\text { Motor Vehicle Emission } \\
\text { Control Systems }\end{array}$ & & & & Applicable & & \\
\hline $\begin{array}{l}\text { WAC } 173-460 \\
\text { Controls for New Sources of } \\
\text { Toxic Air Pollutants }\end{array}$ & TAPs & & & Applicable & $\begin{array}{l}\text { Applicable if source is constructed or modified } \\
\text { causing increase in toxic air pollutants. }\end{array}$ & Table II.B-1 \\
\hline $\begin{array}{l}\text { WAC } 173-480 \\
\text { Ambient Air Quality Standards } \\
\text { and Emission Limits for } \\
\text { Radionuclides }\end{array}$ & Radionuclides & & & Applicable & $\begin{array}{l}\text { Gives Washington State Department of Health } \\
\text { authority to operate WAC 246-247 Radiation } \\
\text { Protection Program }\end{array}$ & \\
\hline $\begin{array}{l}\text { WAC } 246-247 \\
\text { Radiation Protection - } \\
\text { Air Emissions } 1\end{array}$ & Radionuclides & & & Applicable & & \\
\hline $\begin{array}{l}\text { WAC } 173-491 \\
\text { Emission Standards and } \\
\text { Controls for Sources Emitting } \\
\text { Gasoline Vapors }\end{array}$ & VOC & & & Applicable & $\begin{array}{l}\text { Stage } 1 \text { requirement for gasoline dispensing } \\
\text { facilities in eastern Washington counties. }\end{array}$ & Table II.B-3 \\
\hline
\end{tabular}


Table V.A-1. Applicable Requirements.

\begin{tabular}{|c|c|c|c|c|c|c|}
\hline \multirow[t]{2}{*}{ Requirement } & \multirow[t]{2}{*}{ Pollutant(s) } & \multicolumn{3}{|c|}{ Applicability } & \multirow[t]{2}{*}{ Comments } & \multirow{2}{*}{$\begin{array}{l}\text { Source Specific } \\
\text { Information }\end{array}$} \\
\hline & & Federal & Federal/State & State & & \\
\hline $\begin{array}{l}\text { WAC } 173-400-040 \\
\text { General Standards for } \\
\text { Maximum Emissions }\end{array}$ & $\begin{array}{l}\text { Opacity } \\
\text { SO2 } \\
\text { Fugitive } \\
\text { Emission } \\
\text { Fugitive Dust } \\
\text { Fallout } \\
\text { Odors }\end{array}$ & & Applicable* & & $\begin{array}{cl}\text { * State-Only } & \text { Requirements } \\
\text { (1)(c) } & \text { When two or more sources are } \\
& \text { connected to a common stack, } \\
& \text { Ecology may specify other soot } \\
& \text { blowing or grate cleaning } \\
& \text { allowable time limits } \\
\text { (1)(d) } & \text { alternate opacity limit per } \\
& \text { RCW 70.94.331(2)(c) } \\
(2) & \text { Fallout } \\
(4) & \text { Odors } \\
(6) & \text { second para. -exception to } \\
& 1,000 \text { ppm SO2 limit if } \\
& \text { compliance is not feasible and } \\
& \text { NAAQs will not be violated }\end{array}$ & Table II.B-2 \\
\hline $\begin{array}{l}\text { WAC } 173-400-050 \\
\text { Emission Standards for } \\
\text { combustion and incineration } \\
\text { unit }\end{array}$ & Part. Matter & & Applicable* & & $\begin{array}{l}\text { *State-Only Requirements alternative correction } \\
\text { factor }\end{array}$ & \\
\hline $\begin{array}{l}\text { WAC } 173-400-091 \\
\text { Voluntary Limits on emissions }\end{array}$ & $\begin{array}{l}\text { Criteria } \\
\text { HAPS }\end{array}$ & & Applicable & & & \\
\hline $\begin{array}{l}\text { WAC } 173-400-100 \\
\text { Registration }\end{array}$ & $\begin{array}{c}\text { Radionuclides } \\
\text { Criteria }\end{array}$ & & Applicable & & & $\begin{array}{l}\text { Table II.B-1 and } \\
\text { II.B-2 }\end{array}$ \\
\hline
\end{tabular}


Table V.A-1. Applicable Requirements.

\begin{tabular}{|c|c|c|c|c|c|c|}
\hline \multirow[t]{2}{*}{ Requirement } & \multirow[t]{2}{*}{ Pollutant(s) } & \multicolumn{3}{|c|}{ Applicability } & \multirow[t]{2}{*}{ Comments } & \multirow{2}{*}{$\begin{array}{l}\text { Source Specific } \\
\text { Information }\end{array}$} \\
\hline & & Federal & Federal/State & State & & \\
\hline $\begin{array}{l}\text { WAC } 173-400-105 \\
\text { Records, Monitoring, and } \\
\text { Recordkeeping }\end{array}$ & Criteria HAP & & Applicable & & Except WAC 173-400-105(5). & Table II.B-2 \\
\hline $\begin{array}{l}\text { WAC } 173-400-107 \\
\text { Excess emissions }\end{array}$ & & & Applicable & & & Table II.B-2 \\
\hline $\begin{array}{l}\text { WAC } 173-400-110 \\
\text { New Source Review (NSR) }\end{array}$ & $\begin{array}{l}\text { Criteria } \\
\text { TAPs } \\
\end{array}$ & & Applicable & & & $\begin{array}{l}\text { Table II.B-1 and } \\
\text { II.B-2 }\end{array}$ \\
\hline $\begin{array}{l}\text { WAC } 173-400-113 \\
\text { Requirements for new sources } \\
\text { in attainment or unclassifiable } \\
\text { area }\end{array}$ & Criteria & & Applicable & & . & \\
\hline $\begin{array}{l}\text { WAC } 173-400-114 \\
\text { Requirements for replacement } \\
\text { or substantial alteration of } \\
\text { emission control technology at } \\
\text { an existing stationary source }\end{array}$ & & & Applicable & & & \\
\hline $\begin{array}{l}\text { WAC } 173-400-115 \\
\text { Standards of performance for } \\
\text { new sources }\end{array}$ & & . & & Applicable & & \\
\hline $\begin{array}{l}\text { WAC 173-400-120 } \\
\text { Bubble Rules }\end{array}$ & & & & Applicable & & \\
\hline $\begin{array}{l}\text { WAC } 173-400-131 \\
\text { Issuance of emission reduction } \\
\text { credits }\end{array}$ & & & & Applicable & $\therefore$ & \\
\hline
\end{tabular}


Table V.A-1. Applicable Requirements.

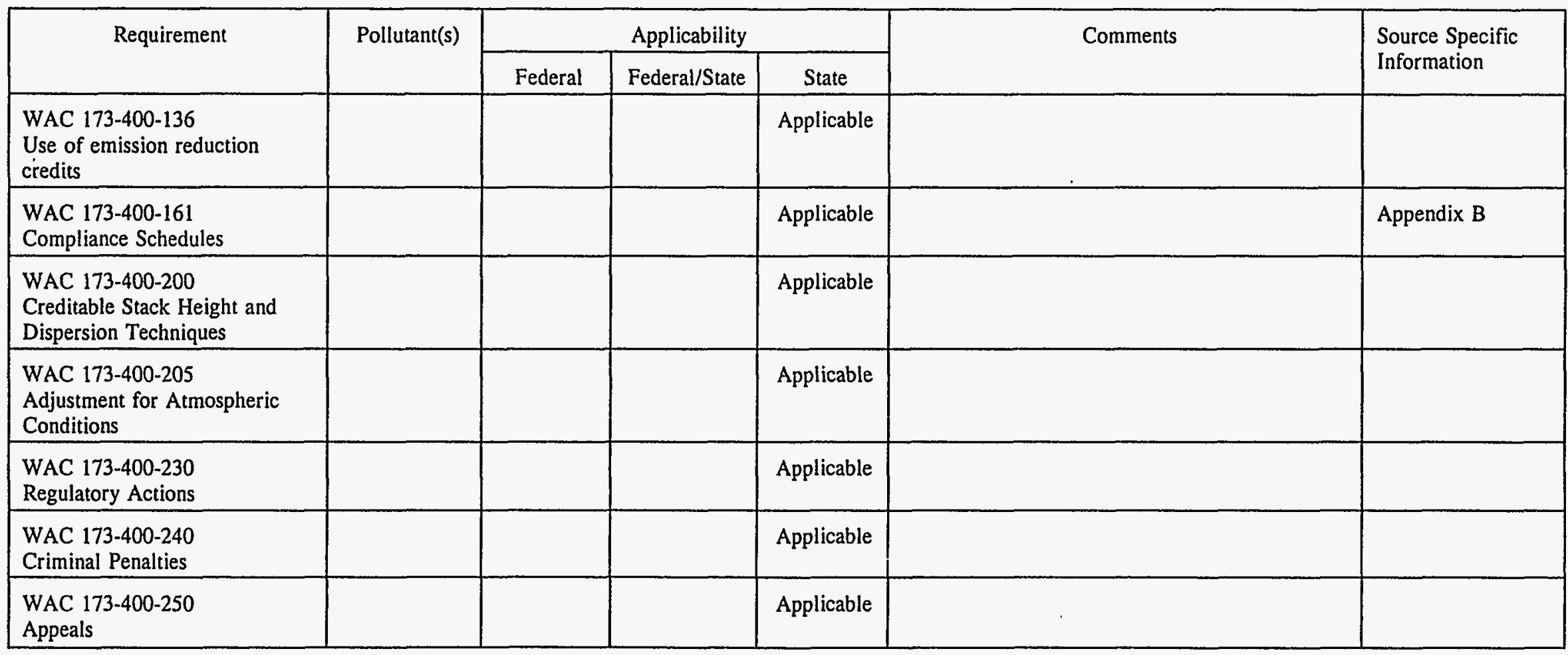


Table V.A-2. Regulated Pollutants.

\begin{tabular}{|l|c|c|c|c|c|c|}
\hline \multicolumn{1}{|c|}{ Pollutants } & $\begin{array}{c}\text { Pollutant } \\
\text { code }\end{array}$ & $\begin{array}{c}\text { CAS } \\
\text { number }\end{array}$ & Criteria & $\begin{array}{c}\text { Sec } \\
112(\mathrm{r})\end{array}$ & $\begin{array}{c}\text { WAC } \\
173-460\end{array}$ & $\begin{array}{c}\text { Sec } \\
112(\mathrm{~b})\end{array}$ \\
\hline 1,1,2-Trichloro-1,2,2-triflouroethane & 610 & $76-13-1$ & & & $\mathrm{X}$ & \\
\hline 1,3-Butadiene & 383 & $106-99-0$ & & $\mathrm{X}$ & $\mathrm{X}$ & $\mathrm{X}$ \\
\hline 2-Butoxy ethanol & 394 & $111-76-2$ & & & $\mathrm{X}$ & \\
\hline 2-Hexanone & 15 & $591-78-6$ & & & $\mathrm{X}$ & \\
\hline 2-Methylpropene & 910 & $115-11-7$ & & $\mathrm{X}$ & & \\
\hline 2,2,4-Trimethylpentane & 1340 & $540-84-1$ & & & $\mathrm{X}$ & $\mathrm{X}$ \\
\hline Acetic acid & 501 & $64-19-7$ & & & $\mathrm{X}$ & \\
\hline Acetone & 504 & $67-64-1$ & & & $\mathrm{X}$ & \\
\hline Acetonitrile & 506 & $75-5-8$ & & & $\mathrm{X}$ & $\mathrm{X}$ \\
\hline Ammonia & 475 & $7664-41-7$ & & $\mathrm{X}$ & $\mathrm{X}$ & \\
\hline Antimony & 487 & $7440-36-0$ & & & $\mathrm{X}$ & $\mathrm{X}$ \\
\hline Arsenic & 489 & $7440-38-2$ & & & $\mathrm{X}$ & $\mathrm{X}$ \\
\hline Benzene & 446 & $71-43-2$ & & & $\mathrm{X}$ & $\mathrm{X}$ \\
\hline Beryllium & 411 & $7440-41-7$ & & & $\mathrm{X}$ & $\mathrm{X}$ \\
\hline Bromine & 434 & $7726-95-6$ & & $\mathrm{X}$ & $\mathrm{X}$ & \\
\hline Butyl acetate & 398 & $123-86-4$ & & & $\mathrm{X}$ & $\mathrm{X}$ \\
\hline Butyl alcohol, tert- & 1670 & $75-65-0$ & & & $\mathrm{X}$ & $\mathrm{X}$ \\
\hline Cadmium & 1485 & $7440-43-9$ & & & $\mathrm{X}$ \\
\hline
\end{tabular}


Table V.A-2. Regulated Pollutants.

\begin{tabular}{|l|c|c|c|c|c|c|}
\hline \multicolumn{1}{|c|}{ Pollutants } & $\begin{array}{c}\text { Pollutant } \\
\text { code }\end{array}$ & $\begin{array}{c}\text { CAS } \\
\text { number }\end{array}$ & Criteria & $\begin{array}{c}\text { Sec } \\
112(\mathrm{r})\end{array}$ & $\begin{array}{c}\text { WAC } \\
173-460\end{array}$ & $\begin{array}{c}\text { Sec } \\
112(\mathrm{~b})\end{array}$ \\
\hline Carbon monoxide & 1665 & $630-8-0$ & $\mathrm{X}$ & & & \\
\hline Carbon tetrachloride & 368 & $56-23-5$ & & & $\mathrm{X}$ & $\mathrm{X}$ \\
\hline Chlorine & 318 & $7782-50-5$ & & $\mathrm{X}$ & $\mathrm{X}$ & $\mathrm{X}$ \\
\hline Chromium & 258 & $7440-47-3$ & & & $\mathrm{X}$ & $\mathrm{X}$ \\
\hline Cobalt & 1387 & $7440-48-4$ & & & $\mathrm{X}$ & $\mathrm{X}$ \\
\hline Copper & 269 & $7740-50-8$ & & & $\mathrm{X}$ & \\
\hline Cyclohexane & 230 & $110-82-7$ & & & $\mathrm{X}$ & \\
\hline Cyclohexanone & 1649 & $108-94-1$ & & & $\mathrm{X}$ & \\
\hline Dibutyl phthalate & 1498 & $87-74-2$ & & & $\mathrm{X}$ & $\mathrm{X}$ \\
\hline Ethyl alcohol & 82 & $64-17-5$ & & & $\mathrm{X}$ & \\
\hline Ethyl benzene & 1634 & $100-41-4$ & & & $\mathrm{X}$ & $\mathrm{X}$ \\
\hline Ethyl butyl ketone & -- & $106-35-4$ & & & $\mathrm{X}$ & \\
\hline Ethylene glycol & 95 & $107-21-1$ & & & $\mathrm{X}$ & $\mathrm{X}$ \\
\hline Formaldehyde & 108 & $50-0-0$ & & $\mathrm{X}$ & $\mathrm{X}$ & $\mathrm{X}$ \\
\hline Heptane & 1631 & $142-82-5$ & & & $\mathrm{X}$ & $\mathrm{X}$ \\
\hline Hexane, n-hexane & 12 & $110-54-3$ & & & $\mathrm{X}$ & $\mathrm{X}$ \\
\hline Hydrochloric acid & 1233 & $7647-1-0$ & & $\mathrm{X}$ & & \\
\hline Hydrogen cyanide & 25 & $74-90-8$ & & $\mathrm{X}$ & $\mathrm{X}$ & $\mathrm{X}$ \\
\hline
\end{tabular}


Table V.A-2. Regulated Pollutants.

\begin{tabular}{|l|c|c|c|c|c|c|}
\hline \multicolumn{1}{|c|}{ Pollutants } & $\begin{array}{c}\text { Pollutant } \\
\text { code }\end{array}$ & $\begin{array}{c}\text { CAS } \\
\text { number }\end{array}$ & Criteria & $\begin{array}{c}\text { Sec } \\
112(\mathrm{r})\end{array}$ & $\begin{array}{c}\text { WAC } \\
173-460\end{array}$ & $\begin{array}{c}\text { Sec } \\
112(\mathrm{~b})\end{array}$ \\
\hline Hydrogen sulfide & 1797 & $7783-6-4$ & & $\mathrm{X}$ & $\mathrm{X}$ & \\
\hline Isobutane & 908 & $75-28-5$ & & $\mathrm{X}$ & & \\
\hline Isopropyl alcohol & 1005 & $67-63-0$ & & & $\mathrm{X}$ & \\
\hline Lead & 1014 & $7439-92-1$ & $\mathrm{X}$ & & $\mathrm{X}$ & $\mathrm{X}$ \\
\hline Mercury & 970 & $7439-97-6$ & & & $\mathrm{X}$ & $\mathrm{X}$ \\
\hline Methane & 973 & $74-82-8$ & & $\mathrm{X}$ & & \\
\hline Methyl alcohol & 1738 & $67-56-1$ & & & $\mathrm{X}$ & \\
\hline Methyl chloride & 343 & $74-87-3$ & & $\mathrm{X}$ & $\mathrm{X}$ & $\mathrm{X}$ \\
\hline Methyl ether or dimethyl ether & 923 & $115-10-6$ & & $\mathrm{X}$ & & \\
\hline Methyl ethyl ketone (2-butone) & 924 & $78-93-3$ & & & $\mathrm{X}$ & $\mathrm{X}$ \\
\hline Methyl isobutyl ketone & 1396 & $108-10-1$ & & & $\mathrm{X}$ & $\mathrm{X}$ \\
\hline Methyl n-amyl ketone, 2-heptanone & 926 & $110-43-0$ & & & $\mathrm{X}$ & \\
\hline Methyl tert-butyl ether & 1739 & $1634-4-4$ & & & $\mathrm{X}$ & $\mathrm{X}$ \\
\hline Methylene chloride & 890 & $75-9-2$ & & & & $\mathrm{X}$ \\
\hline N-butyl aclohol & 388 & $71-36-3$ & & & $\mathrm{X}$ & \\
\hline N2O & 1288 & $10024-97-2$ & $\mathrm{X}$ & & & $\mathrm{X}$ \\
\hline Nickel & 870 & $7440-2-0$ & & & & $\mathrm{X}$ \\
\hline Nitrobenzene & 882 & $98-95-3$ & & $\mathrm{X}$ & $\mathrm{X}$ & $\mathrm{X}$ \\
\hline
\end{tabular}




\begin{tabular}{|c|c|c|c|c|c|c|}
\hline $\mathrm{X}$ & $\mathrm{X}$ & & & $\varepsilon-88-80 \mathrm{I}$ & It9 & әนәnן๐ \\
\hline & $\mathrm{X}$ & & & $6-66-60 I$ & 029 & uRInJoIрКчедәЈ \\
\hline & $\mathrm{X}$ & $\mathrm{X}$ & & $6-\varepsilon 6-t 99 L$ & E99 & p!oe ग!ny \\
\hline & & $\mathrm{X}$ & & 6-II-9DLL & 299 & ${ }^{\varepsilon} \mathrm{OS}$ \\
\hline & & $\mathrm{X}$ & $\mathrm{X}$ & $s-6-9 t L L$ & 099 & ${ }^{2} \mathrm{OS}$ \\
\hline $\mathrm{X}$ & $\mathrm{X}$ & & & $\tau-6 t-\tau 8 L L$ & 989 & 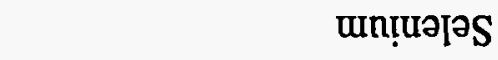 \\
\hline $\mathrm{X}$ & & & & & & səp!ןonuo!pey \\
\hline & $\mathrm{X}$ & & & I-98-0I I & $L E L$ & pu!p! I $\Lambda_{d}$ \\
\hline & & $\mathrm{X}$ & & $9-86-t L$ & $0 Z L$ & ourdo.Id \\
\hline $\mathrm{X}$ & & & & --- & SSL & WOd \\
\hline & & $\mathrm{X}$ & $\mathrm{X}$ & --- & LOZI & 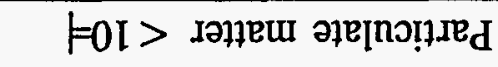 \\
\hline & $\mathrm{X}$ & & & $\tau-8 \varepsilon-t 99 L$ & $68 L$ & p!̣e ग!loqdsoपd \\
\hline & $X$ & $\mathrm{X}$ & & $0-99-60 I$ & $8 I 8$ & әนหุuว $\mathbf{d}$ \\
\hline & & & $\mathrm{X}$ & & & әuozo \\
\hline & $\mathrm{X}$ & & & 6-S9-III & $6+8$ & 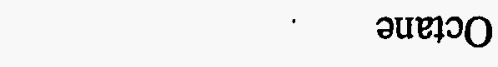 \\
\hline & & $X$ & $\mathrm{X}$ & $\cdots$ & $80 Z I$ & XON \\
\hline & $\mathrm{X}$ & & & Z-ヤ8-III & $t t 8$ & วuruoN \\
\hline $\mathrm{X}$ & $\mathrm{X}$ & & & 6-SL-Z9 & $\angle \varepsilon 8$ & 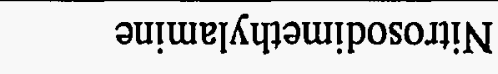 \\
\hline $\begin{array}{l}\text { (q) ZII } \\
\text { ग्S }\end{array}$ & $\begin{array}{c}09 \nabla-\varepsilon L I \\
\partial \forall M\end{array}$ & $\begin{array}{c}\text { (I)ZI I } \\
\text { OəS }\end{array}$ & 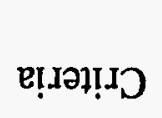 & $\begin{array}{l}\text { səquinu } \\
\text { SVD }\end{array}$ & $\begin{array}{c}\text { әроэ } \\
\text { furın!lod }\end{array}$ & słuęnIIOd \\
\hline
\end{tabular}

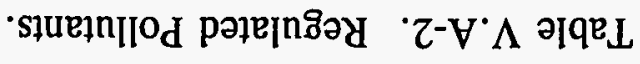


Table V.A-2. Regulated Pollutants.

\begin{tabular}{|l|c|c|c|c|c|c|}
\hline \multicolumn{1}{|c|}{ Pollutants } & $\begin{array}{c}\text { Pollutant } \\
\text { code }\end{array}$ & $\begin{array}{c}\text { CAS } \\
\text { number }\end{array}$ & Criteria & $\begin{array}{c}\text { Sec } \\
112(\mathrm{r})\end{array}$ & $\begin{array}{c}\text { WAC } \\
173-460\end{array}$ & $\begin{array}{c}\text { Sec } \\
112(\mathrm{~b})\end{array}$ \\
\hline Trichloroethylene & 601 & $79-1-6$ & & & $\mathrm{X}$ & $\mathrm{X}$ \\
\hline Volatile organic compound & 533 & --- & $\mathrm{X}$ & & & \\
\hline Xylene & 552 & $1330-20-7$ & & & $\mathrm{X}$ & $\mathrm{X}$ \\
\hline Yttrium & 556 & $7440-65-5$ & & & $\mathrm{X}$ & \\
\hline
\end{tabular}




\section{REFERENCES}

DOE-RL, 1993, Radionuclide Air Emission Report for the Hanford Site Calendar Year 1993, DOE/RL-94-51, U.S. Department of Energy, Richland Operations Office, Richland, Washington.

EPA, 1994, National Emissions Standards for Hazardous Air Pollutants Federal Facility Compliance Agreement, 94-RPS-128, U.S. Environmental Protection Agency, Region 10, Olympia, Washington.

PNL, 1993 Hanford Site Environmental Report for Calendar Year 1993, PNL-9823, Pacific Northwest Laboratory, Richland, Washington.

WAC 173-401, Operating Permit Regulation.

WHC, 1994a, Hanford Site Radionuclide National Emission Standards for Hazardous Air Pollutants Registered Stack Source Assessment, WHC-SD-EN-RPT-007, Rev. 0, Westinghouse Hanford Company, Richland, Washington.

WHC, 1994b, Hanford Site Radionuclide National Emission Standards for Hazardous Air Pollutants Unregistered Stack (Powered Exhaust) Source Assessment, WHC-SD-EN-RPT-25, Rev. 0, Westinghouse Hanford Company, Richland, Washington.

WHC, 1995, Diffuse and Fugitive Emission Dose Assessment on the Hanford Site, WHC-EP-0840, Westinghouse Hanford Company, Richland, Washington. 
Hanford Site Air

Operating Permit Application
DOE/RL-95-07, Rev. 0

05/95

This page intentionally left blank. 


\section{APPENDICES}
A. CALCULATIONS
B. COMPLIANCE PLAN SCHEDULE
C. 100 AREAS
D. 200 EAST AREA
E. 200 WEST AREA
F. 300 AREA
G. 400 AREA
H. 600 AREA
I. MAINTENANCE
J. MISCELLANEOUS ITEMS

K. INSIGNIFICANT EMISSIONS UNITS 
Hanford Site Air

Operating Permit Application
DOE/RL-95-07, Rev. 0 05/95

\section{APPENDIX A}

\section{CALCULATIONS}


Hanford Site Air

Operating Permit Application
DOE/RL-95-07, Rev. 0

05/95

This page intentionally left blank. 
This appendix either documents or references representative emission calculation methodologies for each classification of emission point as listed in the air emission inventory report. The classifications are briefly discussed below.

- All registered radionuclide stack emissions are referenced to the Hanford Site Radionuclide National Emission Standards for Hazardous Air Pollutants Registered Stack Source Assessment (WHC 1994a) and the Radionuclide Air Emission Report for the Hanford Site Calendar Year 1993 (DOE-RL 1993) and the Hanford Site Radionuclide National Emission Standards for Hazardous Air Pollutants Unregistered Stack Source Assessment (WHC 1994b).

- Diffuse and fugitive radionuclide emission calculations from tank farm singleshell tanks and tank farm transfer equipment are provided.

- Nonradiological emission calculations from both actively and passively ventilated single-shell and double-shell tanks are provided. (2)

- The remaining nonradiological emission categories are grouped by source type. Examples are underground petroleum storage tanks, carpentry shops, machine shops, laboratories, etc. A representative calculation is provided for each of these source types. (3) 
(1) Diffuse and fugitive radionuclide emission calculations from tank farm single-shell tanks and tank farm transfer equipment are provided. 
RAD EMISSIONS - ACTIVELY VENTILATED SSTs, DSTs, AND DCRTs

CALC. METHOD DATA:

Radionuclide inventory estimate of

component (i) from Davis 1994

40CFR61 release factor

number of HEPA filters

overall HEPA filter efficiency

emissions for each radionuclide component $(\mathrm{Ci} / \mathrm{yr})=$

eq\#1 $=$ uncontrolled emissions (potential to emit) for each radionuclide $(\mathrm{Ci} / \mathrm{yr})=[\mathrm{rad} \text { inventory }(\mathrm{Ci})]^{*}\left[\mathrm{release}\right.$ factor $\left.\left(\mathrm{yr}^{\wedge}{ }^{\wedge}-1\right)\right]$

eq $\# 2=$ decontamination factor (DF) for $n$ HEPA filters in series $=[1 /(1 \text {-efficiency }(\%) * .01)]^{\wedge} n$

eq\#3 = actual emissions (estimated) for each radionuclide (Ci/yr) $=$ [uncontrolled emissions (Ci/yr)]/[DF]

\begin{tabular}{l|l|l}
\hline Stack 296-A-12 & \#HEPA filters & Filler eff. (\%)
\end{tabular}

244-AR Vault

\begin{tabular}{|c|c|c|c|c|c|}
\hline \multirow{3}{*}{$\begin{array}{l}\text { Stack 296-A-12 } \\
\text { 244-AR Vault }\end{array}$} & $\#$ HEPA filters & \multicolumn{2}{|c|}{ Filter eff. (\%) } & & \\
\hline & 2 & \multicolumn{2}{|c|}{99.95} & & \\
\hline & \multicolumn{2}{|c|}{ decontamination factor $=$} & 4000000 & & \\
\hline Radionuclide & Symbol & Inventory $(\mathrm{Ci})$ & App. D Factor & $\begin{array}{l}\text { Potential to Emit } \\
\text { (Ci/yr) }\end{array}$ & $\begin{array}{c}\text { Actual Emissions } \\
(\mathrm{Ci} / \mathrm{yr})\end{array}$ \\
\hline Carbon 14 & 14-C & $5.5700 \mathrm{e}-04$ & $1 \mathrm{e}-03$ & $5.570 \mathrm{e}-07$ & $1.393 e-13$ \\
\hline Cobalt 60 & $60-\mathrm{Co}$ & $3.1500 \mathrm{e}-01$ & $1 e-03$ & $3.150 e-04$ & $7.875 \mathrm{e}-11$ \\
\hline Selenium 79 & $79-\mathrm{Se}$ & 0.0000 & $10-03$ & 0.000 & 0.000 \\
\hline Strontium $89 / 90$ & $89 / 90-\mathrm{Sr}$ & $1.1600 e+03$ & $10-03$ & $1.160 e+00$ & $2.900 e-07$ \\
\hline Yttrium 90 & $90-Y$ & $1.1600 \mathrm{e}+03$ & $1 \mathrm{e}-03$ & $1.160 e+00$ & $2.900 e-07$ \\
\hline Technetium 99 & 99-Tc & $1.2900 \mathrm{e}+01$ & $10-03$ & $1.290 \mathrm{e}-02$ & $3.225 e-09$ \\
\hline Ruthenium/Rhodium 106 & 106-Ru/Rh & 0.0000 & $1 e+00$ & 0.000 & 0.000 \\
\hline Antimony 125 & $125-\mathrm{Sb}$ & 0.0000 & $10-03$ & 0.000 & 0.000 \\
\hline Iodine 129 & $129-1$ & $2.7900 \mathrm{e}-04$ & $1 c+00$ & $2.790 e-04$ & $6.975 e-11$ \\
\hline Cesium 134 & 134-Cs & $3.0200 e+00$ & $10-03$ & $3.020 e-03$ & $7.5500-10$ \\
\hline Cesium 137 & $137-\mathrm{Cs}$ & $9.5400 e+02$ & $10-03$ & $9.540 \mathrm{e}-01$ & $2.385 e-07$ \\
\hline Cerium 143 & $143-\mathrm{Ce}$ & 0.0000 & $10-03$ & 0.000 & 0.000 \\
\hline Promethium 147 & $147-\mathrm{Pm}$ & 0.0000 & $1 e-03$ & 0.000 & 0.000 \\
\hline Europium 154 & $154-\mathrm{Eu}$ & $3.8100 e+00$ & $10-03$ & $3.810 e-03$ & $9.525 e-10$ \\
\hline Natural Uranium & Nat-U & $3.6800 \mathrm{e}-04$ & $10-03$ & $3.680 e-07$ & $9.200 e-14$ \\
\hline Neptunium 237 & $237-\mathrm{Np}$ & $4.5900 \mathrm{e}-04$ & $10-03$ & $4.590 \mathrm{e}-07$ & $1.148 \mathrm{e}-13$ \\
\hline Plutonium 238 & $238-\mathrm{Pu}$ & $3.1200 \mathrm{e}-02$ & $10-03$ & $3.120 \mathrm{e}-05$ & $7.800 e-12$ \\
\hline Plutonium 239/240 & 239/240-Pu & $1.4300 \mathrm{e}-01$ & $10-03$ & $1.430=-04$ & $3.575 e-11$ \\
\hline Plutonium 241 & $241-\mathrm{Pu}$ & $5.5500 \mathrm{e}-01$ & $10-03$ & $5.550 \mathrm{e}-04$ & $1.388 \mathrm{e}-10$ \\
\hline Americium 241 & 241-Am & $1.6000 e+02$ & 1003 & $1.600=-01$ & $4.000 \mathrm{e}-08$ \\
\hline Californium 242/244 & $242 / 244-\mathrm{Cm}$ & $1.0400 \mathrm{e}-02$ & 1003 & $1.040 e-05$ & $2.600 e-12$ \\
\hline
\end{tabular}


RAD EAISSIONSS - PASSIVEI.Y VE.NTIL ATED SINGLE SHELL TANKS

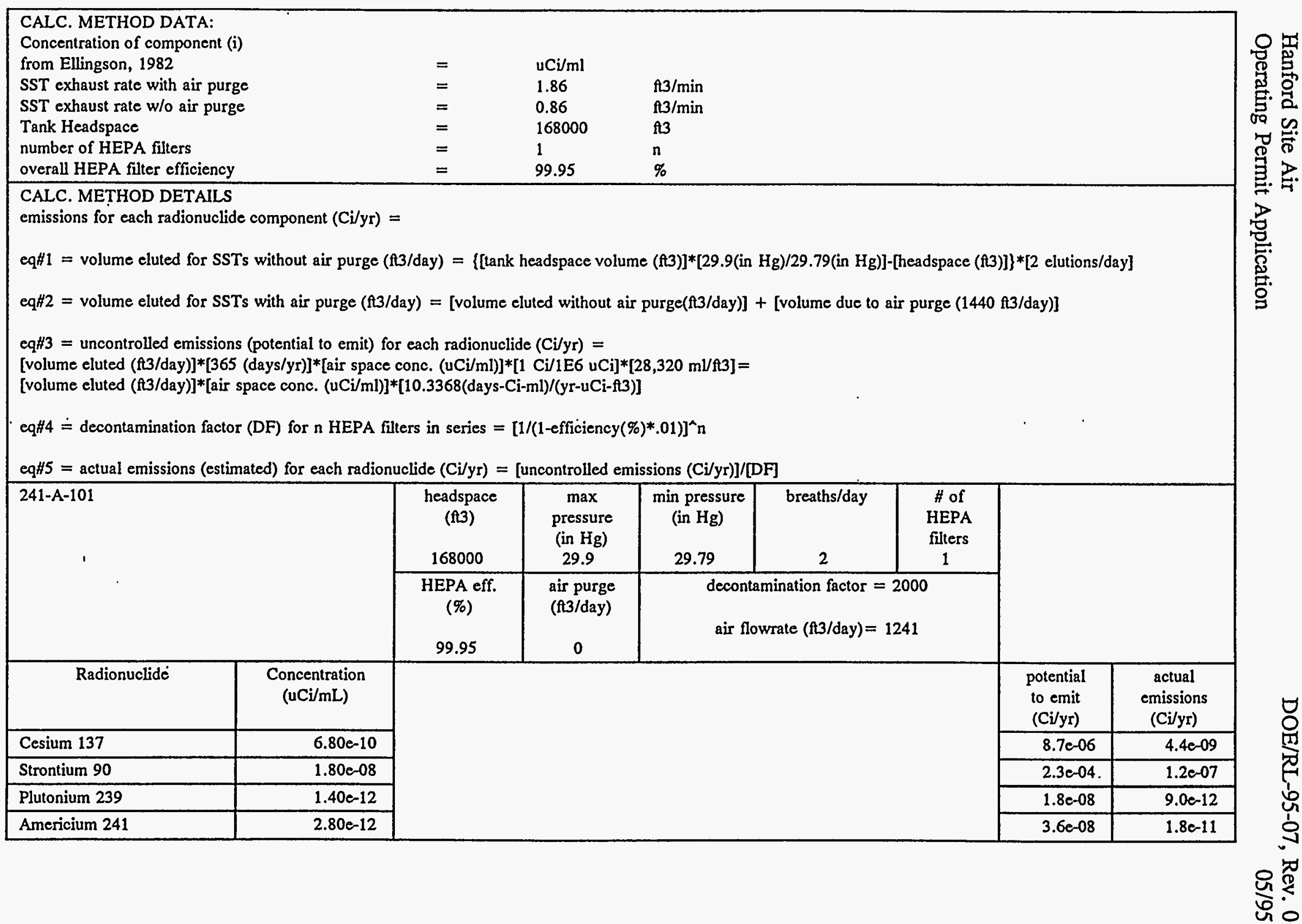


RAD EMISSIONS - CATCH TANKS, VALVE VAULTS, DIVERSION BOXES

\begin{tabular}{|c|c|c|c|c|c|c|c|c|c|c|c|}
\hline & & $\begin{array}{l}\text { CALC. METHOD \#1 DATA: } \\
\begin{array}{ll}\text { surface contamination (i) } & =\mathrm{dpm} / 100 \mathrm{~cm} 2 \\
\text { surface area } & =\mathrm{cm} 2\end{array}\end{array}$ & $\begin{array}{l}\text { yr^-1 } \\
4.51 \mathrm{e}-13\end{array}$ & $\mathrm{Ci} / \mathrm{dpm}$ & & & & & & & \\
\hline \multicolumn{12}{|c|}{$\begin{array}{ll}\text { CALC. METHOD } \# 2 \text { DATA: } & \\
\text { vault volume } & = \\
\text { initial pressure } & = \\
\text { final pressure } & = \\
\text { air concentration } & = \\
\end{array}$} \\
\hline \multicolumn{12}{|c|}{$\begin{array}{l}\text { CALC. METHOD \#1 DETAILS } \\
\text { emissions for cach radionuclide }(\mathrm{Ci} / \mathrm{yr})=[\text { surface contamination (i) }(\mathrm{dpm} / \mathrm{cm} 2)] *[\operatorname{area}(\mathrm{cm} 2)] *[\mathrm{release} \text { factor }(\mathrm{yr}-1)] *[4.5 \mathrm{E}-13(\mathrm{Ci} / \mathrm{dpm})]\end{array}$} \\
\hline \multicolumn{12}{|c|}{ 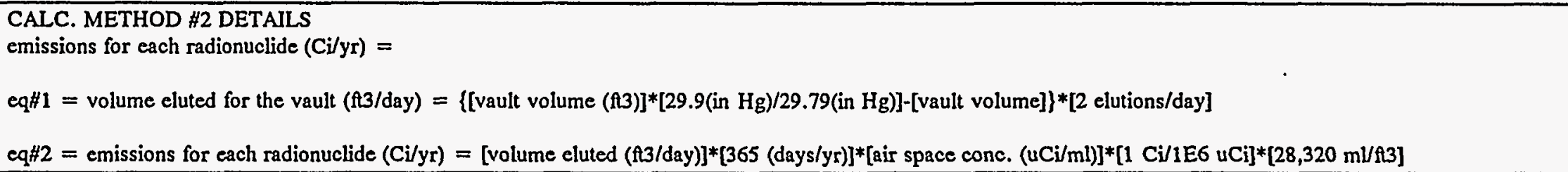 } \\
\hline \multicolumn{4}{|c|}{ Westinghouse Hanford Company } & \multicolumn{8}{|c|}{$\begin{array}{l}\text { P241AWA001 } \\
\text { 241-AW-A Valve Pit }\end{array}$} \\
\hline \multirow{2}{*}{$\begin{array}{c}\text { Source } \\
\text { ID } \\
\#\end{array}$} & \multirow[b]{2}{*}{ Radionuclide } & \multirow[b]{2}{*}{$\begin{array}{l}\text { CAS } \\
\text { Number }\end{array}$} & \multirow{2}{*}{$\begin{array}{l}\text { Gas, } \\
\text { Liquid, or } \\
\text { Solid }\end{array}$} & \multicolumn{2}{|c|}{ Uncontrolled Emissions } & & \multicolumn{2}{|c|}{ Controllẹd Emissions } & & & \\
\hline & & & & $\begin{array}{l}\text { Maximum } \\
(\mathrm{Ci} / \mathrm{yr})\end{array}$ & $\begin{array}{l}\text { Actual } \\
(\mathrm{Ci} / \mathrm{yr})\end{array}$ & & $\begin{array}{c}\text { Maximum } \\
(\mathrm{Ci} / \mathrm{yr})\end{array}$ & $\begin{array}{l}\text { Actual } \\
\text { (Ci/yr) }\end{array}$ & & & \\
\hline 001 & $\begin{array}{l}\text { Strontium } \\
89 / 90\end{array}$ & - & $\mathbf{S}$ & $2.84 e-10$ & $2.84 c-10$ & & $2.84 e-10$ & $2.84 \mathrm{e}-10$ & & & \\
\hline 001 & Yttrium 90 & $\begin{array}{c}10098-91- \\
6\end{array}$ & $\mathbf{S}$ & $2.84 e-10$ & $2.84 \mathrm{e}-10$ & & $2.84 e-10$ & $2.84 c-10$ & & & \\
\hline 001 & $\begin{array}{l}\text { Americium } \\
241\end{array}$ & $\begin{array}{c}14596-10- \\
2\end{array}$ & $S$ & $5.68 \mathrm{e}-12$ & $5.68 \mathrm{e}-12$ & & $5.68 e-12$ & $5.68 \mathrm{e}-12$ & & & \\
\hline \multicolumn{9}{|c|}{ Estimation of Surface Contamination Within Transfer Facilities } & & & \\
\hline & \multicolumn{4}{|c|}{ Facility Dimensions } & & \multirow{2}{*}{$\begin{array}{l}\text { Am-241 } \\
\text { Surface } \\
\text { Contaminat } \\
\text { ion } \\
\text { (Ci) }\end{array}$} & \multirow{2}{*}{$\begin{array}{c}\text { Sr-90 } \\
\text { Surface } \\
\text { Contaminati } \\
\text { on } \\
\text { (Ci) }\end{array}$} & \multirow{2}{*}{$\begin{array}{c}\text { Y-90 } \\
\text { Surface } \\
\text { Contaminat } \\
\text { ion } \\
\text { (Ci) }\end{array}$} & \multirow{2}{*}{$\begin{array}{c}\text { Am-241 } \\
\text { Emissio } \\
\text { ns } \\
(\mathrm{C} i / \mathrm{yr})\end{array}$} & \multirow{2}{*}{$\begin{array}{c}\text { Sr-90 } \\
\text { Emissio. } \\
\text { ns } \\
\text { (Ci/yr) }\end{array}$} & \multirow{2}{*}{$\begin{array}{c}\mathrm{Y}-90 \\
\text { Emission } \\
s \\
(\mathrm{Ci} / \mathrm{y} \mathrm{r})\end{array}$} \\
\hline Source & $\begin{array}{l}\text { Length } \\
\text { (ft) }\end{array}$ & $\begin{array}{l}\text { Width } \\
\text { (ft) }\end{array}$ & $\begin{array}{l}\text { Height } \\
\text { (ft) }\end{array}$ & $\begin{array}{l}\text { Diameter } \\
\text { (ft) }\end{array}$ & $\begin{array}{c}\text { Surface } \\
\text { Area } \\
\text { (ft2) }\end{array}$ & & & & & & \\
\hline $\begin{array}{l}241-A W-A \\
\text { Valve Pit }\end{array}$ & 14.0 & 12.0 & 6.6 & N/A & 678 & $5.68 \mathrm{e}-06$ & $2.84 e-04$ & $2.84 e-04$ & $\begin{array}{c}5.68 e-1 \\
2\end{array}$ & $\begin{array}{r}2.840-1 \\
0\end{array}$ & $2.84 c-10$ \\
\hline
\end{tabular}




\section{NON-RADIOLOGICAL AIR EMISSION ESTIMATES}

\subsection{Introduction}

Non-radiological (non-rad) emissions have been estimated for the major components in the 200 East and 200 West Tank Farms: single shell tanks, double shell tanks, and evaporators. Non-radiological emissions have not been estimated for the components in the waste retrieval operations which contain small inventories and]or are used infrequently. Non-radiological emissions from these minor components - valve pits, diversion boxes, DCRTs, transfer boxes, and diverter stations - are expected to be insignificant.

\subsection{Approach}

Ideally, non-rad emissions would be estimated using measured concentrations of air pollutants at the emission point and measured exhaust rates. Unfortunately, vapor characterization data which are available for the tank farm vessels in the 200 East and 200 West areas are extremely limited. Some liquid and solids sampling data are available, but due to the complex and heterogenous nature of the waste and the infrequent sampling there is a high degree of uncertainty associated with emissions estimates based on these concentration data. Therefore, emission estimates for all tanks are based on the small set of measured headspace concentrations, rather than extrapolation of liquid and]or solids data. A set of extremely conservative, worst ease assumptions has been used to estimate nomad emissions for all tanks.

The tank for which there is the most vapor characterization data is C-103. This tank is expected to represent the worst case for organic emissions, and was selected as the first tank for vapor characterization under the Tank Vapor Issue Resolution Program (Huckaby, 1994a). For the air emission inventory, emissions from this tank were estimated initially. It was assumed, as a worst-case condition, that organic vapor concentrations in other passively vented tanks would be equal to those in C-103, unless other vapor characterization data were available. This is an extremely conservative assumption, as only 4 or 5 other tanks on site may have organic concentrations of the same order of magnitude (Huckaby, 1994b).

The $\mathrm{C}-103$ data were adapted to provide emission estimates for actively vented tanks, again unless data specific to a tank were available. The methodology used for the various emissions' estimates is described below. 


\subsection{Emissions Estimates for Passively Vented Tanks}

\subsection{Identification of Pollutants}

A listing of all the chemicals which were included in the characterization for C-103 appears in Table 1. This table identifies which constituents are toxic air pollutants (TAPs) per Washington State code WAC 173-460, hazardous air-pollutants (HAPs) per the Clean Air Act, or criteria pollutants per 40 CFR Part 50. If a constituent fit into one or more of these categories, then it was included in the emissions estimates for C-103.

\subsection{Selection of Headspace Concentrations}

Table 2 presents the data for each of the air pollutants, as presented in the C-103 characterization report (Huckaby, 1994a). The last column identifies the value which was selected to be representative of $\mathrm{C}-103$ concentrations for the air emissions inventory. If the mean of all samples was reported, then it was applied. If the overall mean was not reported, the maximum of the mean values was selected. For cases in which either the pollutant was below the detection limit in all samples, or there was no significant difference between the samples and the blanks, the parameter was not included in emission estimates.

\subsection{Calculation of Gas Flowrate}

The majority of the single shell tanks have no active exhaust system. Emissions will occur from passively vented tanks in response to 'changes in the barometric pressure. When the barometric pressure drops, gas will flow out of the tank until the tank pressure has equilibrated with the ambient pressure. In order to estimate emissions from the passively vented tanks, the flowrate at which the gas will exit the tank was estimated. The calculations are based on an analysis from the report "Single Shell Tank Breather Filter Test Frequency" (Kriskovich, 1994).

Assumptions:

- The maximum' barometric pressure drop which is expected to occur over several hours at the Hanford Site is 0.11 inches of $\mathrm{Hg}$ (Kriskovich, 1994)

- The pressure drop occurs twice during a 24-hour period (Tranbarger, 1990).

- Tank headspace follows the Ideal Gas Law

- Temperature changes are neglected

- $\quad$ Base atmospheric pressure $=1 \mathrm{~atm}=29.9 \mathrm{in}$. $\mathrm{Hg}$

- Tank headspace $=168,000 \mathrm{ft}^{3}$ (Kriskovich, 1994). This is the maximum headspace volume for storage tanks in the 200 East and 200 West area (104-AX). 
Following the Ideal Gas Law ( $\mathrm{PV}=\mathrm{nRT}$ ), for constant temperature and moles of gas:

$$
\begin{aligned}
& \mathrm{P}_{1} * \mathrm{~V}_{1}=\mathrm{P}_{2} * \mathrm{~V}_{2} \quad \text { Therefore: } \mathrm{V}_{2}=\mathrm{V}_{1} * \mathrm{P}_{1} / \mathrm{P}_{2} \\
& \mathrm{P}_{2}=29.9 \text { in. } \mathrm{Hg}-\mathrm{O} .11 \text { in. } \mathrm{Hg}=29.79 \text { in. } \mathrm{Hg} \\
& \mathrm{V}_{2}=\mathrm{V}_{1} * 29.9 / 29.79=1.037 * \mathrm{~V}_{1} \\
& \mathrm{~V}_{1}=168,000 \mathrm{ft}^{3} \\
& \mathrm{~V}_{2}=1.0037 * 168,000 \mathrm{ft}^{3}=168,620 \mathrm{ft}^{3} \\
& \mathrm{~V}_{2}-\mathrm{V}_{1}=168,620-168,000=620 \mathrm{ft}^{3}
\end{aligned}
$$

Assuming a pressure drop twice a day:

$$
\text { Flowrate }=620 \mathrm{ft}^{3} / 12 \mathrm{hr}=51.7 \mathrm{ft} 3 / \mathrm{hr}(1 \mathrm{hr} / 60 \mathrm{~mm})=0.86 \mathrm{cfm}
$$

To be conservative in our estimate, one value of tank breathing rate is being applied to all tanks. In actuality, the breathing rate will be proportional to the tank vapor space, which is tank-specific. The tank with the greatest headspace has been used in the calculation, along with a high estimate of the diurnal barometric pressure drop, so the resulting flowrate is expected to overestimate actual breathing rates.

A second mechanism for exhaust from passively vented tanks is the air purge which is used on automated tank inventory level measurement. Single shell tanks have one of three types of level indication: manual tape, intrusion mode, or CASS (Crummel, 1990). The latter two methods have an air purge which is introduced into the tank headspace with a flowrate of approximately 50 cubic feet per hour (Huber, 1994). For tanks which have this air purge, a flowrate of $60 \mathrm{cfh}$ $=1.0 \mathrm{cfm}$ was added to the barometric pressure exhaust rate to obtain a total passively vented exhaust rate of $1.86 \mathrm{elm}$. Table 3 lists each passively vented tank, the type of level measurement, and whether or not it has the 1 elm air purge (sorted by tank and by air purge).

\subsection{Calculation of Emissions}

Calculations for all emissions were performed using Excel spreadsheet calculations. The formulas and sample calculations are provided on Calculation Sheet \#1, and summarized

The concentrations of components in the C-103 report were in units of ppmv. These concentrations were derived accounting for $42.2 \mathrm{mg} / \mathrm{l}$ of water in the headspace, which 
converts to 6 percent by volume, so water was included in the molecular weight of air. To convert percent by volume to percent by mass, the molecular weight of headspace and each component were used. Emissions were calculated using the equation:

$$
\begin{array}{ll}
\mathrm{E}=\mathrm{Q} * \mathrm{c} * \mathrm{~F} & \text { where } \mathrm{E}=\text { emission rate, } \mathrm{lb} / \mathrm{hr} \\
& \mathrm{Q}=\text { tank breathing rate, } \mathrm{cfm} \\
\mathrm{c}=\text { component concentration, weight } \% \\
\mathrm{~F}=\text { units conversion factor }
\end{array}
$$

Emission rates were then converted to units of $\mathrm{lb} /$ hour.

Assumptions made for the C-103 emissions estimates (and emissions from all other storage tanks) include:

- All components follow the Ideal Gas Law:-.

- All emissions are vapor phase constituents. Any particles which are present in the headspace are expected to have rad emissions associated with them. Therefore, particle emissions have been included in the inventory estimates for radiological components.

- There is no removal of vapor mass in the HEPA filters; controlled emissions are equal to uncontrolled emissions (See note below).

- Actual emissions are equal to maximum rate emissions. Data are not available for actual emissions versus a "design rate" for storage tanks. Instead, the estimates generated using extremely conservative assumptions have been applied to the design and actual emission categories.

- Emissions are constant for all three years, except when yearly data are available (ammonia in some actively vented tanks).

Note: Storage tank C-103 has a carbon adsorption filter installed on the exhaust. However, credit has not been taken for any removal of organics for several reasons. The exhaust is not sampled, so the removal efficiency and breakthrough point are not known. Not claiming removal leaves more flexibility for future operations.

Table 4 shows the completed emission estimates for C-103. 


\subsection{Emission Estimates for Other Passively Vented Tanks}

\section{Assumptions:}

- As noted above, data for headspace concentrations and emissions estimates is extremely limited. Therefore, emissions of organic constituents from all passively vented tanks were assumed to be equal to the $\mathrm{C}-103$ emissions, as a worst-case condition, unless data specific to a storage tank were available.

- For inorganic emission rates, C-103 may not represent the worst-case scenario. For inorganic constituents, the highest reported concentration or total mass emission rate for any passively vented tank was applied as the default for all tanks which do not have measured vapor phase concentrations.

- All passively vented tanks were assumed to have a breathing rate of $0.86 \mathrm{cfm}$ or $1.86 \mathrm{elm}$, depending on whether level indication instrumentation has an air purge.

Vapor phase concentration data which was available for passively vented tanks other than C-103 are described below by contaminant type:

\section{Organics}

Headspace concentration data were available for six other passively vented tanks: BY-104, BY-105, BY-106, TX-118, C-108, and C-111.

BY-104, BY-105, and BY-106: 13 organic compounds which are HAPs or TAPs were detected (Jenkins 1994a, 1994b, and 1994c). The concentrations were not compared with background concentrations.

For TX-118, C- 108, and C- 111, samples were analyzed for the 42 compounds specified by EPA Method TO-14 (Rasmussen, 1994). Results for these three tanks were:

TX-118: Concentrations were significantly above background for eight compounds which are TAPs or HAPs; Freon-11, benzene, toluene, carbon tetrachloride, methyl chloride, acetonitrile, $\mathrm{THF}$, and 3-heptanone. (Compounds present at concentrations greater than $5 \mathrm{ppbv}$.)

C-108: No compounds were present above ambient concentrations.

C-111: No compounds were present above ambient concentrations. 
Ammonia

Ammonia was measured in the headspace of BY-I04, BY-I05, and BY-I06 (Ligotke 1994a, $1994 \mathrm{~b}$, and 1994c). Concentrations were $248 \mathrm{ppmv}, 43 \mathrm{ppmv}$, and $74 \mathrm{ppmv}$, respectively. The only other ammonia concentration data which was available for the passively vented tanks was a spreadsheet reporting monitoring of emissions with Drager tubes during 1993 and 1994 (Hewlitt, 1994). The highest ammonia concentration in tank emissions was $260 \mathrm{ppm}$.

The ammonia concentration in C-103 of $304 \mathrm{ppmv}$, is the highest of the reported concentrations, so the $\mathrm{C}-103$ value was applied as the default value for tanks without measured concentrations.

\section{Carbon Monoxide}

The CO concentration for $\mathrm{C}-103$ was 26.7 ppmv. Concentrations of $\mathrm{CO}$ were available for seven other tanks: BY-I04, BY-I05, BY-106, C-104, TX-118, C-108, and C-111 [Rasmussen 1994a, 1994b, and 1994c). Reported CO concentrations in all of these tanks are less than 3 ppmv, so the $\mathrm{C}-103$ concentration was applied to all passively vented tanks for which $\mathrm{CO}$ was not measured.

NO and N02

In addition to C-103 data, the nitrogen components were measured for the three BY tanks 104, 105, and 106 (Jenkins 1994a, 1994b, and 1994c). Nitric oxide (NO) was 0.30 ppmv or below in the BY tanks, so the C-103 concentration of $26.7 \mathrm{ppmv}$ was used as the default value. Nitrogen dioxide (N02) was not detected in any of the passively vented tanks.

\section{$\underline{\mathrm{HCN}}$}

No $\mathrm{HCN}$ was detected in the $\mathrm{C}-103$ headspace, or in the Industrial Hygiene Drager tube monitoring. Per Jim Huckaby, HCN has not been detected in the headspace of any Hartford tanks, that he is aware of (Huckaby, 1994b). It was not included in emissions estimates.

\subsection{Emission Estimates for Actively Vented Tanks}

\subsection{Approach}

Emission data for actively vented (AV) tanks is even more limited than for the passively vented tanks. The only available vapor sampling data for organic emissions from actively vented ranks consist of total nonmethane organic carbon (TNMOC) concentrations in C- 
105 and C-106 (Huckaby, 1994b). No organic speciation data were located. Emission estimates for organic compounds were therefore derived from the C-103 headspace data.

The assumption that concentrations in the tank headspace are equal to those in C-103 is not appropriate for actively vented (AV) tanks. The air changeover rate is at least two orders of magnitude higher than that in the passively vented tanks, and concentrations of organics in the AV tanks are expected to be several orders of magnitude lower than in the C-103 headspace. Organics which are no longer being generated would have likely volatilized over time as the waste has aged. Additionally, any of the tanks contain waste with a low solids content, or a surface crust w[rich will slow volatilization rates for organics w[rich are still being generated.

Under these conditions, the rate limiting step in the transport of organic emissions from the waste to the tank vent stacks in the actively vented tanks is most likely the transport of the volatile species to the surface of the waste. Except for a few tanks in which boiling provides some mixing, there is no advective mechanism for contaminant transport. For these reasons, it has been assumed that the flux of organic contaminants from the actively vented tanks is equal to the flux of contaminants from C-103. The estimates of emissions from the AV tanks based on constant flux are expected to still be conservative, since C-103 has a much higher organic waste content than the AV tanks.

Using the assumption of equal flux, the flux of contaminants was used to calculate headspace concentrations; the methodology is explained below.

\subsection{Equal Flux Methodology}

The flux of a contaminant is equal to the mass emission rate per unit surface area. It was confirmed that all of the actively vented storage tanks have a diameter of 75 feet, so the assumption of equal flux simplifies to one of equal emission rates:

(3) $\mathrm{E}_{1}=\mathrm{E}_{2}=\mathrm{Q}_{2} * \mathrm{c}_{2}$

where $E_{1}=$ emission rate of component from C-103, mass/time

$\mathrm{E}_{2}=$ emission rate of component from AV tank, mass/time

$\mathrm{Q}_{2}=$ exhaust flowrate for a single AV tank, volume/time

$c_{2}=$ concentration of component in $\mathrm{AV}$ tank headspace, mass/volume

For each organic component, the emission rate for the AV tank was set equal to the C103 emission rate. Using the tank exhaust flowrate, Equation 3 was solved for concentration. The total emissions for a tank farm emission stack are equal to the emissions per tank multiplied by the number of tanks in the tank farm. Equations and sample calculations are shown on Calculation Sheet 
Assumptions for these calculations are:

- All tanks which vent to a common emission point have equal headspace concentrations

- All tanks which vent to a common emission point have equal exhaust rates

- Exhaust rates selected are the higher of a) the mean value of measured exhaust flowrates for each emission point (Crummel, 1994c) or b) the design exhaust flowrate (Crummel, 1994a). The flowrates for each actively exhausted emission point are shown in Table 5.

\section{Organics}

As discussed above, there were no available organic speciation data for actively vented tanks. The equal flux methodology was applied using $\mathrm{C}-103$ emission rates.

\section{Ammonia}

There were two sources of data for ammonia emissions from the AV tanks. The first is a set of annual ammonia emissions, in pounds, for four of the tank farm stacks for the years 1990 through 1993 (Crummel, 1994b). The second source was a collection of measured ammonia concentration and exhaust flowrates from tank SY-101 (Concentration and flowrate were measured for three months during the summer of 1993; the mean values were applied) (Wilkins, 1994). The ammonia concentrations, exhaust flowrates, and ammonia emission rates are shown in Table 6. Emissions from SY-101 are the highest, so this emission rate was applied to all AV stacks for which no ammonia data are available. Ammonia headspace concentrations were calculated using the same formulas and assumptions used for organic emission estimates. See Calculation Sheet

\section{Carbon Monoxide}

Carbon monoxide ( $\mathrm{CO}$ ) has been measured in two actively vented tanks; C-105 and C-106 (Rasmussen, 1994b). Exhaust rates were measured concurrently. The flux for each tank was calculated using equation 2. The tank with the higher flux rate is $\mathrm{C}-106$, so this flux rate was applied to all other AV tanks.

\section{$\underline{\mathrm{NO} \text { and } \mathrm{NO} 2}$}

The only concentrations available were for C-103; the flux from C-103 was applied to the actively vented tanks. 
$\underline{\mathrm{HCN}}$

No HCN was detected in the C-103 headspace, or in the Industrial Hygiene Drager tube monitoring. Per Jim Huckaby, HCN has not been detected in the headspace of tanks that he is aware of (Huckaby, 1994b). It was not included in emissions estimates.

\subsection{Emission Estimates for Evaporators}

\section{P-242-A-002 (Process ventilation)}

Emissions are based on data from the document "Facility Effluent Monitoring Plan for 242-A Evaporator Facility" (FEMP). In this document, values are provided for emissions from the vessel ventilation stack during normal operations. Five components are identified: acetone, 1-butanol, 2-hexanone, methylisobutyl ketone (MIBK), and ammonia. For the first four chemicals, the document contains emissions data for 24 hours of operation. These values were multiplied by 365 to give the maximum emission rate for 1994, when the evaporator was operating.

Ammonia data were are presented in the same document as an annual total mass. The highest annual mass was selected, and assumed to represent the actual emissions during 1994.

Actual emissions were calculated from the design rate emissions. The design rate emissions were multiplied by the ratio of the 1994 throughput (5.5 MM gallons) to the throughput at full-time, $100 \%$ of design, operation (73.6 MM gallons) to obtain actual emission rates.

Data are not available for emissions during 1992 and 1993, when the vessel was not operating. It has been assumed that the process emissions in 1992 and 1993 were equal to zero.

\section{$\underline{\mathrm{P}-242-\mathrm{A}-001}$ (Building ventilation)}

There are no nonradiological data available for emissions from the building stack. However, in the FEMP, data are presented for radiological (rad) emission components. Therefore, the nonrad emissions were based on the relative rad emissions from the process and the building. The total rad emissions for the building ventilation were divided by the total rad emissions for the process ventilation to give a building to process ratio. The nonrad emissions for the process stack were multiplied by this ratio to estimate nonrad emissions from the building during 1994.

During 1992 and 1993 the evaporator did not operate and it was assumed that there were no non-radiological emissions. 
Hanford Site Air

Operating Permit Application

$\underline{\mathrm{P}-242-\mathrm{S}-001}$

This evaporator is not in service emissions.

$\underline{\text { P-242-T-001 }}$

This evaporator is not in service emissions. It was assumed that there were no non-radiological It was assumed that there were no non-radiological
DOE/RL-95-07, Rev. 0

05/95 
Table 1. Chemicals Analyzed in C-103 Headspace.

\begin{tabular}{|c|c|c|c|}
\hline & & & Criteria \\
\hline Chemical (1) & HAP(2) & TAP (3) & Pollutant (4) \\
\hline Inorganics & & & \\
\hline \multicolumn{4}{|l|}{ Water } \\
\hline Ammonia & & $\bar{B}$ & \\
\hline \multicolumn{4}{|l|}{ Hydrogen } \\
\hline \multicolumn{4}{|l|}{ Nitrous Oxide (N2O) } \\
\hline Carbon Monoxide (CO) & & & $\bar{Y}$ \\
\hline Nitrogen Dioxide (NO2) & & & $\bar{Y}$ \\
\hline Nitric Oxide (NO) & & $\bar{B}$ & $\bar{Y}$ \\
\hline Sulfur Oxides & & & $\bar{Y}$ \\
\hline Hydrogen Cyanide & $\bar{Y}$ & $\bar{B}$ & \\
\hline \multicolumn{4}{|l|}{ Organics } \\
\hline Benzene & $\bar{Y}$ & $\overline{\mathrm{A}}$ & \\
\hline Toluene & $\bar{Y}$ & $\bar{B}$ & \\
\hline \multicolumn{4}{|l|}{ Butanal } \\
\hline \multicolumn{4}{|l|}{ n-Butanol } \\
\hline 1,3-Butadiene & $\bar{Y}$ & A & \\
\hline 1,1-DCE (vinylidene chloride) & $\bar{Y}$ & $\bar{B}$ & \\
\hline Methylene Chloride & $\bar{Y}$ & $\overline{\mathbf{A}}$ & \\
\hline Acetone (propanone) & & $\overline{\mathrm{B}}$ & \\
\hline 2-Pentanone & & $\bar{B}$ & \\
\hline 2-Hexanone & & $\overline{\mathrm{B}}$ & \\
\hline 2-Heptanone & & $\bar{B}$ & \\
\hline \multicolumn{4}{|l|}{ 2-Octanone } \\
\hline Acetonitrile & $\overline{\mathbf{Y}}$ & $\overline{\mathrm{B}}$ & \\
\hline \multicolumn{4}{|l|}{ Propanenitrile } \\
\hline \multicolumn{4}{|l|}{ Butanenitrile } \\
\hline \multicolumn{4}{|l|}{ Hexanenitrile } \\
\hline \multicolumn{4}{|l|}{ Heptanenitrile } \\
\hline \multicolumn{4}{|l|}{ Octanenitrile } \\
\hline \multicolumn{4}{|l|}{ Nonanenitrile } \\
\hline \multicolumn{4}{|l|}{ Methane } \\
\hline n-Hexane & $\bar{Y}$ & $\overline{\mathrm{B}}$ & \\
\hline n-Heptane & & $\overline{\mathrm{B}}$ & \\
\hline n-Octane & & B & \\
\hline n-Nonane & & $\bar{B}$ & \\
\hline \multicolumn{4}{|l|}{ n-Decane } \\
\hline \multicolumn{4}{|l|}{ n-Undecane } \\
\hline \multicolumn{4}{|l|}{ n-Dodecane } \\
\hline \multicolumn{4}{|l|}{ n-Tridecane } \\
\hline \multicolumn{4}{|l|}{ n-Tetradecane } \\
\hline \multicolumn{4}{|l|}{ Total Semivol NPH } \\
\hline \multicolumn{4}{|l|}{ DBBP (dibutylbutyl } \\
\hline Tributyphosphate & & $\bar{B}$ & \\
\hline \multicolumn{4}{|c|}{ 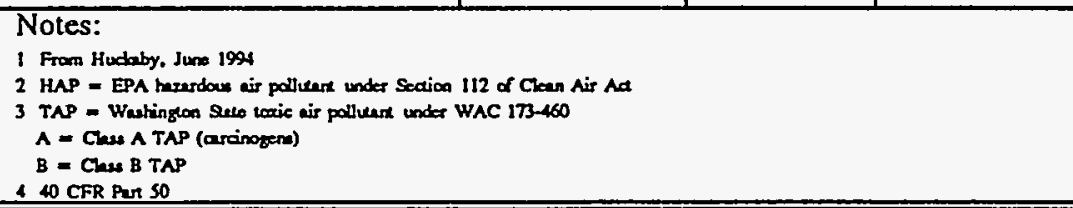 } \\
\hline
\end{tabular}


Table 2. C-103 Heads

\begin{tabular}{|c|c|c|c|c|c|c|}
\hline & & & & & & \\
\hline & Means & Means & all & Deviation & & Applied \\
\hline Chemical & ppmv & ppmv & ppmv & ppmv & Comments & ppmv \\
\hline 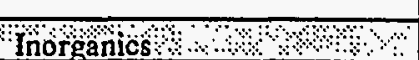 & मेक्ष & \%थ\% & १९७ & \%\$ & ঋ & ফি \\
\hline Ammonia & 296 & 310 & 304 & 11 & & 304 \\
\hline Carbon Monoxide (CO) & 22.3 & 28.6 & 26.7 & 2.3 & & 26.7 \\
\hline Sulfer Oxides & & & $<0.04$ & & Not detected & Not \\
\hline Nitric Oxide & & & $<0.02$ & & No signif. diff. between & Not \\
\hline $\mathrm{HCN}$ & 1.33 & 1.63 & 1.5 & 0.3 & & 1.5 \\
\hline & & & $<0.04$ & & No signif. diff. between & Not \\
\hline Organics & 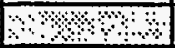 & ४४४४ & ४४४? & $8 \%,+3$ & 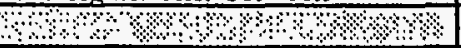 & कित \\
\hline Benzene & $<0.01$ & 0.33 & & & & \\
\hline Toluene & 0.02 & 0.057 & & & & 0.33 \\
\hline 1,3-Butadiene & $<0.02$ & 0.06 & & & & 0.057 \\
\hline 1,1-DCE (vinylidene & & & & & & 0.06 \\
\hline Methylene Chloride & $<0.02$ & 1.62 & & & Not detected & Not \\
\hline Acetone (propanone) & 8.8 & .19 .4 & & & Attribuled to use as a cleaning & Not \\
\hline 2-Pentanone & 1.09 & 1.09 & 1.09 & 0.64 & & 19.4 \\
\hline 2-Hexanone & 0.51 & 0.59 & & & & 1.09 \\
\hline 2-Heptanone & 0.56 & 0.56 & 0.56 & 0.1 & & 0.59 \\
\hline Acetonitrile & 9.1 & 13.2 & & & & 0.56 \\
\hline n-Hexane & 0.71 & 0.8 & & & & 13.2 \\
\hline $\mathrm{n}$-Heptane & 0.66 & 0.66 & 0.66 & 0.14 & & 0.8 \\
\hline n-Octane & 0.33 & 0.33 & 0.33 & 0.09 & & 0.66 \\
\hline n-Nonane & 0.28 & 0.28 & 0.28 & 0.08 & & 0.33 \\
\hline Tributylphosphate & 0.508 & 0.508 & 0.508 & 0.054 & & 0.28 \\
\hline & & & & & & 0.508 \\
\hline \multirow{2}{*}{\multicolumn{7}{|c|}{$\begin{array}{lll}\text { From Huckaby, June } 1994 & & \\
\text { HAP }=\text { EPA hazardous air pollutant under Section } 112 \text { of Clean Water Act }\end{array}$}} \\
\hline & & & & & & \\
\hline \multicolumn{7}{|c|}{\begin{tabular}{|l|l|} 
TAP $=$ Washington State toxic air pollutant under WAC $173-460$ & \\
\end{tabular}} \\
\hline \multicolumn{7}{|c|}{\begin{tabular}{|l|l|l}
$A=$ Class A TAP (carcinogens) & & \\
\end{tabular}} \\
\hline \multicolumn{7}{|l|}{\begin{tabular}{l|l}
$\mathrm{B}=$ Class B TAP & \\
\end{tabular}} \\
\hline \multirow{2}{*}{\multicolumn{7}{|c|}{ Chemicals which exhibited no significant difference between concentrations in the samples and those in the blanks were not }} \\
\hline When available, the overall me & an of samples & Swas applied & When this & was not availa & & eans was \\
\hline Methylene chloride concentrati & ons were attri & buted to con & amination by & the use of $\mathrm{M}$ & as a cleaning solvent in the sa & \\
\hline
\end{tabular}


Table 3. Level Indication of Single Shell Tanks

\begin{tabular}{|c|c|c|}
\hline Tank & Level & Air \\
\hline Name & Indication & Purge \\
\hline $241-A-101$ & Manual Tape & $\mathbf{N}$ \\
\hline $241-A-102$ & Intrusion Mode & $Y$ \\
\hline $241-A-103$ & Intrusion Mode & $Y$ \\
\hline $241-A-104$ & Manual Tape & $\mathbf{N}$ \\
\hline $241-A-105$ & Manual Tape & $N$ \\
\hline $241-A-106$ & Intrusion Mode & $Y$ \\
\hline $241-A X-101$ & Intrusion Mode & $\mathrm{Y}$ \\
\hline $241-\mathrm{AX}-102$ & Manual Tape & $\mathbf{N}$ \\
\hline $241-A X-103$ & CASS & $\mathbf{Y}$ \\
\hline $241-A X-104$ & Manual Tape & $\mathbf{N}$ \\
\hline $241-B-101$ & Intrusion Mode & $Y$ \\
\hline $241-B-102$ & CASS & $Y$ \\
\hline $241-\mathrm{B}-103$ & Intrusion Mode & $Y$ \\
\hline 241-B-104 & Manual Tape & $\mathbf{N}$ \\
\hline $241-B-105$ & Manual Tape & $\mathbf{N}$ \\
\hline 241-B-106 & CASS & $Y$ \\
\hline 241-B-107 & Manual Tape & $\mathbf{N}$ \\
\hline $241-\mathrm{B}-108$ & Intrusion Mode & $Y$ \\
\hline 241-B-109 & Manual Tape & $N$ \\
\hline $241-B-110$ & Manual Tape & $\mathbf{N}$ \\
\hline $241-B-111$ & Intrusion Mode & $\mathbf{Y}$ \\
\hline $241-B-112$ & CASS & $Y$ \\
\hline $241-\mathrm{B}-201$ & Manual Tape & $\mathbf{N}$ \\
\hline 241-B-202 & Manual Tape & $\mathbf{N}$ \\
\hline $241-B-203$ & Manual Tape & $\mathbf{N}$ \\
\hline 241-B-204 & Manual Tape & $\mathbf{N}$ \\
\hline $241-B X-101$ & Manual Tape & $N$ \\
\hline $241-\mathrm{BX}-102$ & Manunl Tape & $N$ \\
\hline $241-B X-103$ & CASS & $Y$ \\
\hline 241-BX-104 & CASS & $Y$ \\
\hline 241-BX-105 & Intrusion Mode & $Y$ \\
\hline 241-BX-106 & Manual Tape & $N$ \\
\hline $241-\mathrm{BX}-107$ & CASS & $Y$ \\
\hline $241-\mathrm{BX}-108$ & Manual Tape & $\mathrm{N}$ \\
\hline $241-\mathrm{BX}-109$ & CASS & $Y$ \\
\hline $241-B X-110$ & Manual Tape & $\mathrm{N}$ \\
\hline $241-B X-111$ & Manual Tape & $\mathbf{N}$ \\
\hline $241-\mathrm{BX}-112$ & CASS & $Y$ \\
\hline $241-\mathrm{BY}-101$ & Manual Tape & $\mathrm{N}$ \\
\hline 241-BY-102 & Manual Tape & $\mathbf{N}$ \\
\hline 241-BY-103 & Manual Tape & $\mathbf{N}$ \\
\hline 241-BY-104 & Manual Tape & $\mathbf{N}$ \\
\hline $241=\mathrm{BY}-105$ & Manual Tape & $N$ \\
\hline 241-BY-106 & Manual Tape & $\mathbf{N}$ \\
\hline
\end{tabular}


Table 3. Level Indication of Single Shell Tanks

\begin{tabular}{|c|c|c|}
\hline Tank & Level & Air \\
\hline Name & Indication & Purge \\
\hline & $:$ & \\
\hline 241-BY-107 & Manual Tape & $\mathbf{N}$ \\
\hline 241-BY-108 & Manual Tape & $\mathbf{N}$ \\
\hline 241-BY-109 & CASS & $Y$ \\
\hline $241-B Y-110$ & Manual Tape & $\mathbf{N}$ \\
\hline $241-B Y-111$ & Manual Tape & $\mathbf{N}$ \\
\hline 241-BY-112 & Manual Tape & $\mathbf{N}$ \\
\hline $241-C-101$ & Manual Tape & $\mathbf{N}$ \\
\hline $241-C-102$ & Intrusion Mode & $Y$ \\
\hline 241-C-103 & CASS & $\mathbf{N}$ \\
\hline $241-C-104$ & CASS & $Y$ \\
\hline 241-C-105 & CASS & $Y$ \\
\hline 241-C-106 & CASS & $Y$ \\
\hline 241-C-107 & CASS & $\mathbf{Y}$ \\
\hline 241-C-108 & Manual Tape & $\mathbf{N}$ \\
\hline 241-C-109 & Manual Tape & $\mathbf{N}$ \\
\hline $241-C-110$ & Manual Tape & $N$ \\
\hline 241-C-111 & Manual Tape & $\mathbf{N}$ \\
\hline $241-C-112$ & Manual Tape & $\mathbf{N}$ \\
\hline $241-C-201$ & Manual Tape & $N$ \\
\hline $241-C-202$ & Manual Tape & $\mathbf{N}$ \\
\hline $241-C=203$, मी। & Manual Tape & $\mathbf{N}$ \\
\hline 241-C-204 & Manual Tape & $\mathbf{N}$ \\
\hline $241-S-101$ & Intrusion Mode & $\mathbf{Y}$ \\
\hline $241-S-102$ & CASS & $Y$ \\
\hline $241-S-103$ & CASS & $\mathbf{N}$ \\
\hline $241-S-104$ & CASS & $N$ \\
\hline $241-S-105$ & Intrusion Mode & $\mathbf{Y}$ \\
\hline $241-S-106$ & CASS & $N$ \\
\hline $241-S-107$ & CASS & $N$ \\
\hline $241-S-108$ & Intrusion Mode & $Y$ \\
\hline $241-S-109$ & Intrusion Mode & $Y$ \\
\hline $241-S-110$ & Intrusion Mode & $Y$ \\
\hline $241-S-111$ & CASS & $\mathbf{N}$ \\
\hline $241-S-112$ & Intrusion Made & $\mathbf{N}$ \\
\hline $241-S X-101$ & Intrusion Mode & $Y$ \\
\hline 241-SX-102 & Intrusion Mode & $\mathbf{Y}$ \\
\hline $241-S X-103$ & CASS & $\bar{Y}$ \\
\hline 241-SX-104 & Intrusion Mode & $Y$ \\
\hline 241-SX-105 & Intrusion Mode & $\mathbf{Y}$ \\
\hline 241-SX-106 & CASS & $Y$ \\
\hline 241-SX-107 & Manual Tape & $\mathbf{N}$ \\
\hline
\end{tabular}


Table 3. Level Indication of Single Shell Tanks

\begin{tabular}{|c|c|c|}
\hline Tank & Level & Air \\
\hline Name $\quad \therefore$ & Indication & Purge \\
\hline $241-S X-108$ & Manual Tape & $\mathbf{N}$ \\
\hline $241-S X-109$ & Manual Tape & $\mathbf{N}$ \\
\hline $241-S X-110$ & Manual Tape & $\mathbf{N}$ \\
\hline $241-S X-111$ & Manual Tape & $\mathbf{N}$ \\
\hline $241-S X-112$ & Manual Tape & $\mathbf{N}$ \\
\hline $241-S X-113$ & Manual Tape & $\mathbf{N}$ \\
\hline $241-S X-114$ & Manual Tape & $\mathbf{N}$ \\
\hline $241-S X-115$ & Manual Tape & $\mathbf{N}$ \\
\hline $241-T-101$ & CASS & $\mathbf{Y}$ \\
\hline $241-T-102$ & CASS & $N$ \\
\hline $241-T-103$ & CASS & $\mathbf{Y}$ \\
\hline $241-T-104$ & Manual Tape & $\mathbf{N}$ \\
\hline $241-T-105$ & Intrusion Mode & $\mathbf{Y}$ \\
\hline $241-T-106$ & Intrusion Mode & $\mathrm{Y}$ \\
\hline $241-\mathrm{T}-107$ & CASS & $\mathbf{N}$ \\
\hline 241-T-108 & Manual Tape & $\mathbf{N}$ \\
\hline $241-T-109$ & Intrusion Mode & $\mathbf{N}$ \\
\hline $241-\mathrm{T}-110$ & Intrusion Mode & $\mathbf{Y}$ \\
\hline $241-\mathrm{T}-111$ & CASS & $\mathbf{Y}$ \\
\hline $241-T-112$ & CASS & $\mathbf{Y}$ \\
\hline $241-\mathrm{T}-201$ & Manual Tape & $\mathbf{N}$ \\
\hline $241-\mathrm{T}-202$ & Manual Tape & $\mathbf{N}$ \\
\hline 241-T-203 & Manual Tape & $N$ \\
\hline $241-T-204$ & Manual Tape & $\mathbf{N}$ \\
\hline 241-TX-101 & CASS & $\mathbf{Y}$ \\
\hline 241-TX-102 & Manual Tape & $\mathbf{N}$ \\
\hline 241-TX-103 & Intrusion Mode & $\mathrm{Y}$ \\
\hline 241-TX-104 & CASS & $\mathbf{Y}$ \\
\hline 241-TX-105 & Manual Tape & $N$ \\
\hline 241-TX-106 & Manual Tape & $N$ \\
\hline 241-TX-107 & CASS & $Y$ \\
\hline $241-T X-108$ & Intrusion Mode & $\mathbf{Y}$ \\
\hline 241-TX-109 & Intrusion Mode & $\mathbf{Y}$ \\
\hline 241-TX-110 & Manual Tape & $\mathbf{N}$ \\
\hline $241-T X-111, \cdots$ & Manual Tape & $\mathbf{N}$ \\
\hline $241-\mathrm{TX}-112$ & Manual Tape & $\mathbf{N}$ \\
\hline $241-\mathrm{TX}-113$ & Manual Tape & $\mathbf{N}$ \\
\hline $241-\mathrm{TX}-114$ & Manual Tape & $\mathbf{N}$ \\
\hline $241-T X-115$ & Manual Tape & $\mathbf{N}$ \\
\hline
\end{tabular}


Table 3. Level Indication of Single Shell Tanks

\begin{tabular}{|c|c|c|}
\hline Tank & Level & Air \\
\hline Name $/ \mathrm{l}$ & Indication & Purge \\
\hline 241-TX-116 & Manual Tape & $\mathbf{N}$ \\
\hline $241-T X-117$ & Manual Tape & $\mathbf{N}$ \\
\hline 241-TX-118 & Intrusion Mode & $\mathbf{Y}$ \\
\hline $241-T Y-101$ & Intrusion Mode & $Y$ \\
\hline 241-TY-102 & CASS & $\mathbf{Y}$ \\
\hline 241-TY-103 & - Intrusion Mode & $\mathbf{Y}$ \\
\hline $241-T Y-104$ & CASS & $Y$ \\
\hline 241-TY-105 & Manual Tape & $\mathbf{N}$ \\
\hline 241-TY-106 & Manual Tape & $N$ \\
\hline 241-U-101 & Manual Tape & $\mathbf{N}$ \\
\hline 241-U-102 & Intrusion Mode & $\mathbf{Y}$ \\
\hline $241-U-103$ & CASS & $\mathbf{N}$ \\
\hline $241-U-104$ & Manual Tape & $\mathbf{N}$ \\
\hline $241-\mathrm{U}-105$ & CASS & $\mathbf{N}$ \\
\hline 241-U-106 & CASS & $\mathbf{N}$ \\
\hline $241-U-107$ & CASS & $\mathbf{N}$ \\
\hline 241-U-108 & Intrusion Mode & $Y$ \\
\hline 241-U-109 & CASS & $\mathbf{N}$ \\
\hline $241-U-110$ & Intrusion Mode & $\mathbf{Y}$ \\
\hline 241-U-111 & Intrusion Mode & $\mathbf{Y}$ \\
\hline $241-U-112$ & Manual Tape & $\mathbf{N}$ \\
\hline $241-U-201$ & Manual Tape & $\mathbf{N}$ \\
\hline $241-U-202$ & Manual Tape & $\mathbf{N}$ \\
\hline $241-U-203$ & Manual Tape & $\mathbf{N}$ \\
\hline 241-U-204 & Manual Tape & $\mathbf{N}$ \\
\hline
\end{tabular}


Table 4. Emissions Spreadsheet for C-103

Weatiohouse Hanford Company

Emission point ID no. P241-C-103-001

Page 4 of 14

Emissions for CY 199

Chemical composition of emission stream for Non-Rediological Pollutrants

\begin{tabular}{|c|c|c|c|c|c|c|c|c|c|c|c|c|c|}
\hline \multirow{3}{*}{$\begin{array}{c}\text { Souroc } \\
\text { Ib } \\
\text { Number }\end{array}$} & \multirow{3}{*}{$\begin{array}{c}\text { CAS } \\
\text { Number }\end{array}$} & \multirow[b]{3}{*}{ Componere } & \multirow{3}{*}{$\begin{array}{c}\text { Consents. } \\
\text { (wright x) }\end{array}$} & \multirow{3}{*}{$\begin{array}{c}\text { Concentr. } \\
\text { (volume } x)\end{array}$} & \multirow{3}{*}{$\begin{array}{c}\text { Cas, } \\
\text { Liquid, } \\
\text { o Solid }\end{array}$} & \multicolumn{4}{|c|}{ Unoostrolled Emission Rate } & \multirow{2}{*}{\multicolumn{2}{|c|}{ Mex. Dexign }} & \multirow{2}{*}{\multicolumn{2}{|c|}{ Aaval Eminions }} \\
\hline & & & & & & \multicolumn{2}{|c|}{ Max. Datign } & \multicolumn{2}{|c|}{ Aatul Emisions } & & & & \\
\hline & & & & & & (Bba/tus) & $(\cos / y r)$ & $(\mathrm{b} / / \mathrm{m})$ & $(\operatorname{lon} / y x)$ & $\left(b_{n} / h_{x}\right)$ & $(\operatorname{lon} / y z)$ & $(\mathrm{be} / \mathrm{ta})$ & (lon/yz) \\
\hline \multicolumn{14}{|c|}{ ४। আ } \\
\hline \%l: & 7664011.7 & Ammon & W. $33 \mathrm{E} .02$ & 3.045 .02 & ô: & $\therefore 1500.03$ & $6.57 E .03$ & 1.50E-03 & $6.57 \mathrm{E}-03$ & $1.50 E-03$ & $6.5 \pi \mathrm{E}-03$ & $1.50 \mathrm{E} .03$ & $0.57 \mathrm{E} .03$ \\
\hline 1 & $630.08-0$ & Cabon Monoxide & 265E-03 & $20 \pi E .03$ & Cos & $2.17 E-04$ & 9.50E.0S & 2.17E-04 & 9.5OE.0A & 217E-OS & 9.50E-06 & 2.1TE-0. & 9.50E-04 \\
\hline 1 & 10102.43 & Nitric Oxike & $1.00 E-04$ & L.SOE.09 & ges & 1.31E.05 & $5.72 E .05$ & 1.31E.0S & S.DE.0s & 1.3JE-0S & S.ME.-OS & LWE.0S & $3.72=-05$ \\
\hline \multicolumn{14}{|c|}{$\beta_{1} / \ldots$} \\
\hline 1 & 1005.99 .0 & 1,3-Buendiene & 1.1SE.0S & $0.00 \varepsilon .06$ & Cas & $9.42 E-07$ & 4.13E.08 & 9.42E-07 & $4.13 \mathrm{E} .06$ & 9.42E-07 & 4.13E-06 & 9.42E-07 & 4.13E. 06 \\
\hline 1 & 110-43-0 & 2-Hepunane & $2.2 \pi 5-04$ & $3.60 \mathrm{E} .05$ & a. & $1.80 \mathrm{E}-05$ & 8.13E-05 & $1.86 E-05$ & $8.13 E-05$ & $1.86 E-05$ & 8.13E-0S & 1.86E-05 & 8.13E-0S \\
\hline 1 & $591-78-6$ & 2. Hexsnome & $2.10 E-04$ & $5.90 E-05$ & sess & $1.22 \mathrm{E}-05$ & $2.51 E=05$ & $1.72 E .05$ & 7.SIE-05 & 1 DE. 05 & 7.SIE.0S & $1.72 \mathrm{E} .05$ & ZSIE-0S \\
\hline $1 \%$ & 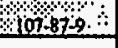 & 2 tentinone $\$$ & $33_{30} 04$ & WOSE.0SO & \% a & Y.725.05: & 1.19E.0A & $2.72 E .05$ & 1.19E.0s & $272 E-05$ & 1. I9E-OA & 2.72E.0S & 1.19E-0s \\
\hline 1 & 67.64 .1 & Aotone & $4.00 E=03$ & L.9NE-03 & One & 3.27 E. 04 & $1.43 E-03$ & 3.27E-04 & $1.43 E-03$ & 3.27E-04 & 1. $.43 E-03$ & $3.27 \mathrm{E}-0 \mathrm{k}$ & $1.43 \mathrm{E}-03$ \\
\hline 1 & 75.05 .8 & Aotomilitite & $1.92 \mathrm{E} .03$ & 1.32E.03 & Can & 1.5TE-06 & $6.89 E .04$ & $1.57 E .04$ & $6.80 E-01$ & 1.57E-OS & 6.86E-as & 1.STE. OS & $0.80 \mathrm{E} \cdot 04$ \\
\hline 1 & $71-3.2$ & Benzene & $2.248=02$ & $3.30 E=05$ & one & $2.48 \mathrm{E} \cdot 06$ & $3.28 E-05$ & 7.48E:06 & $3.28 E-0 S$ & $2,498.06$ & $3,28 E .05$ & $2.48 E \cdot 06$ & $3.28 E-05$ \\
\hline 1 & 142.82 .5 & n.Hexane & 2.3SE-04 & $6.60 \mathrm{E} .05$ & Cas & $1.92 \mathrm{E}-05$ & 8.40E.05 & $1.92 \mathrm{E} .05$ & 8.40E-0S & $1.92 E .05$ & 8.40 E. $0 \mathrm{~S}$ & 1.92E-05 & 8.40E-05. \\
\hline 1 & 100.54 .3 & n.Hexane & 2. $45 E-04$ & 8.00E-05 & ans & $200 \mathrm{E}=05$ & 8.76E.0S & $2.00 \mathrm{E} .05$ & 8.76E-0S & 200E.05 & $8.76 E .05$ & $200 E \cdot 05$ & $8.76 \mathrm{E}-05$ \\
\hline 1 & $111-84-2$ & n-Nomene & $1.27 \mathrm{E}-04$ & $2.80 \mathrm{E}-0 \mathrm{~s}$ & Cas & 1.04E.05 & 4.57E.0S & $1.04 \mathrm{E} .05$ & 4.STE-0S & 1.04E.05 & 4.STE-0S & 1.04E-05 & 4.5TE-0S \\
\hline 1 & $111-65.9$ & $n-0$ anse & L.34E.04 & $3,30 E-05$ & ane & $1,098.05$ & 4.79E-0s & $1.02 E-05$ & 1.79E-0s & 1.028 .05 & $4.79 E-05$ & L.02E-0S & 4.79E-0S \\
\hline 1 & 100.88 .3 & Tduene & $1.80 E-05$ & $5.70 \mathrm{E}-06$ & Cos & $1.52 E .06$ & $6.6 \mathrm{E}=.06$ & $1.52 \mathrm{E} .06$ & $6.67 E-06$ & $1.52 E=06$ & $6.6 \pi \mathrm{E}-\infty 6$ & 1.52E-0S & $6.67 E-06$ \\
\hline 1 & 126.73 .8 & Tributylphorphtale & $4.80 \mathrm{E}-04$ & $3.08 E-05$ & Cos & $3.93 E-05$ & 1.72E-04 & $3.93 \mathrm{E}-05$ & $1.72 E-04$ & $3.93 \mathrm{E}-05$ & 1.72E-O4 & 3.93E-05 & $1.72 E-04$ \\
\hline
\end{tabular}


Table 5. Flowrate Data for Actively Vented Storage Tanks

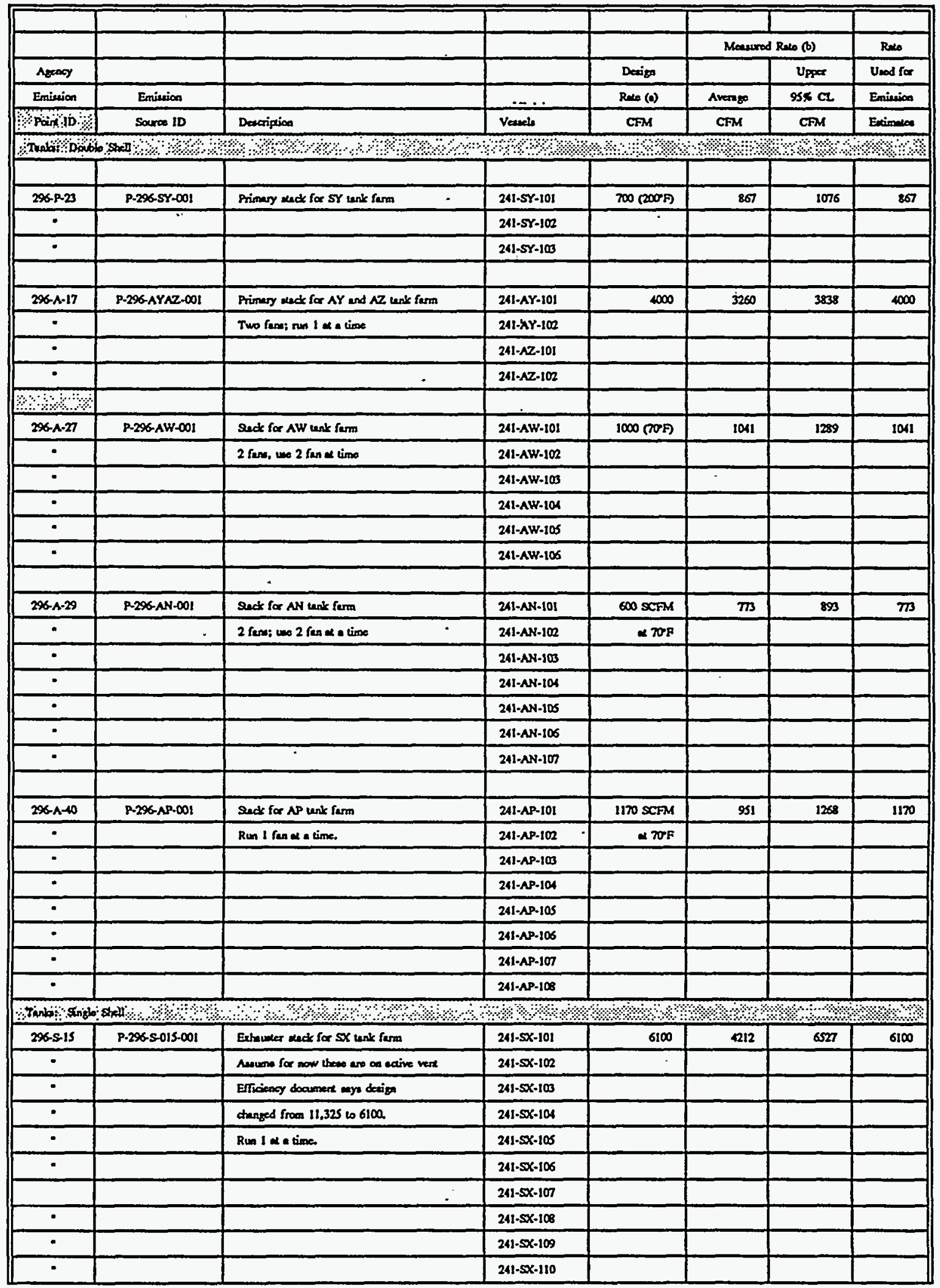


Table 5. Flowrate Data for Actively Vented Storage Tanks

\begin{tabular}{|c|c|c|c|c|c|c|c|}
\hline & & & & & & & \\
\hline & & & & & \multicolumn{2}{|c|}{ Measured Rere (b) } & Reve \\
\hline Astacy & & & & Deaigs & & $U_{p p e r}$ & Uned far \\
\hline Emiesion & Emincion & & & Rece (a) & Avernge & $95 \times \mathrm{CL}$ & Eminion \\
\hline Apin & Sarroe ID & Descripion & Vasele & CFM & CFM & CPM & Eximoles \\
\hline \multicolumn{8}{|c|}{ 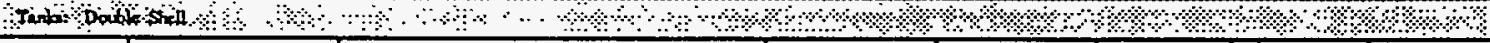 } \\
\hline$\cdot$ & & & 211-5x-111 & & & & \\
\hline$\cdot$ & & & $241 \cdot 5 x-112$ & & & & \\
\hline$\cdot$ & & & $211-5 x-114$ & & & & \\
\hline $206-\mathrm{P}-17$ & P-266-P-017-001 & 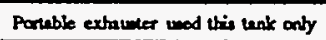 & $241-A-105$ & 7000 (70) & 1127 & 2234 & 7000 \\
\hline 296-P-16 & P-296-P-016-001 & Porreble exhumeres for C-10S/106 & $241 \cdot c-105$ & 7000 (60) & 3243 & 3993 & 7000 \\
\hline & & & & & & & \\
\hline
\end{tabular}

Table 6. Ammonia Emissions for Actively Vented Tanks

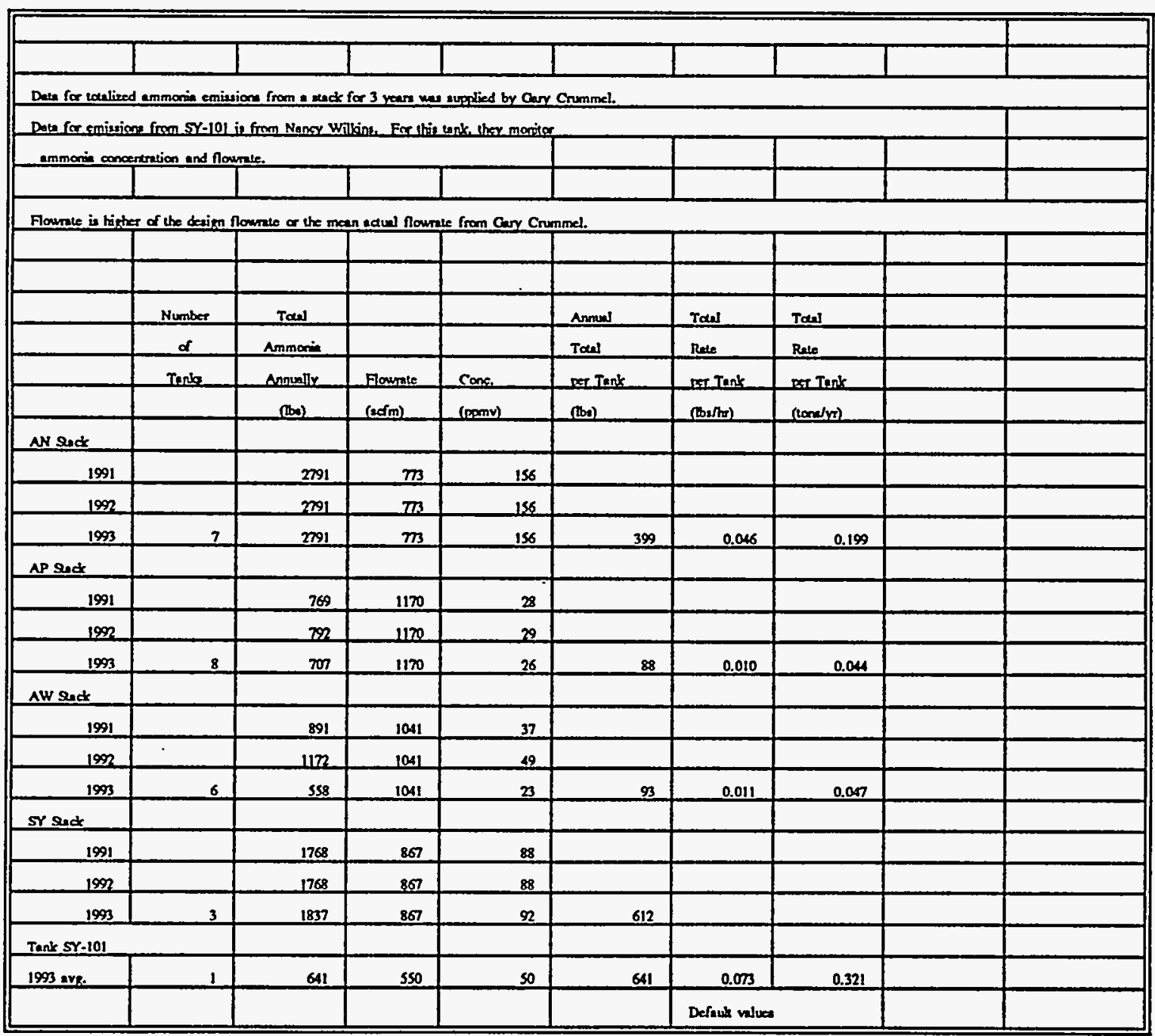


Calculation of Estimated Emissions from

$1 / 2$ Tank $\mathrm{C}-103$

* concentrations reported in ppm

* converting this to $\%$ by volume:

$$
\left(x_{p p m v}\right)\left(\frac{100 \%}{10^{6} \mathrm{ppm}}\right)=(x \text { pp mv })\left(10^{-4}\right) \% \text { volume of }
$$

* conversion of component $\%$ volume $\pi \%$ mass of Component (i) - Need to calculate the mole weight of Tank C-183

- neglect vapor constituent contribution To headspace mole-wt. assume $38^{\circ} \mathrm{C}\left(100^{\circ} \mathrm{F}\right.$ ) ten per aTure, then water
vapor content $=42.2 \mathrm{mg} / \mathrm{l}$
assume mole wt. of arrears

- assume mole wt. of dry air $=28.8 \mathrm{~g} / \mathrm{g}-\mathrm{mol}$

- Then the volume\% of water vapor in The

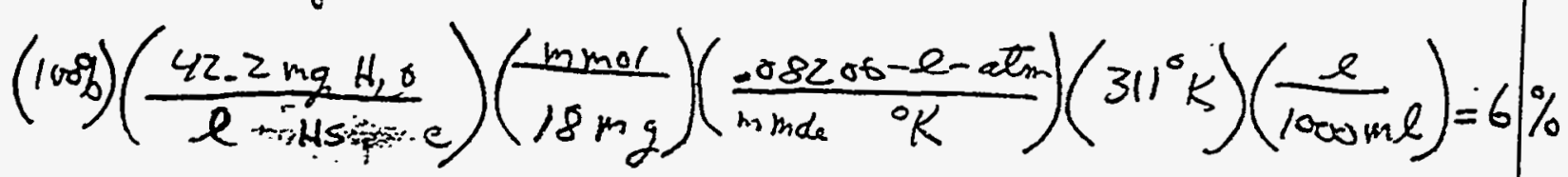

- Then the mole wt of headspace is:

$$
\begin{aligned}
& =-06(18 \mathrm{~g} / \mathrm{mal})+.97(28.8 \mathrm{~g} / \mathrm{mol}) \\
& =28.2 \mathrm{~g} / \mathrm{md}
\end{aligned}
$$

$\%$ assume volume $\%=$ mole $\%$

$\%$ mass of component( $i)=$

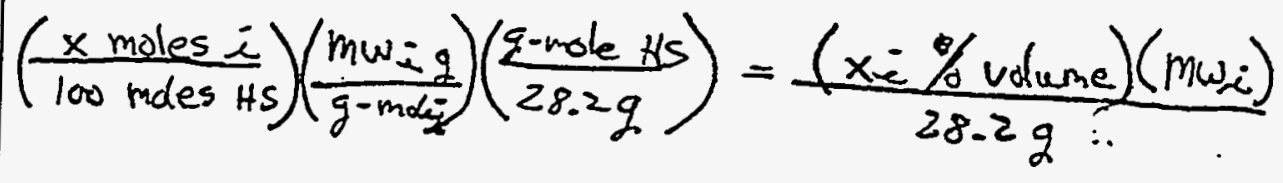

* Calculation of emissions

- Tank breathing rate $=Q$ F $7 / m$ in a $68^{\circ} \mathrm{F}$

- concentration of component i in tank in $\omega t^{\circ} \%=C_{i}$

- emission rate $=Q^{*} c_{i}=E_{i}$

where $E_{i}=$ emission rate of component is in $16 / h r$ 


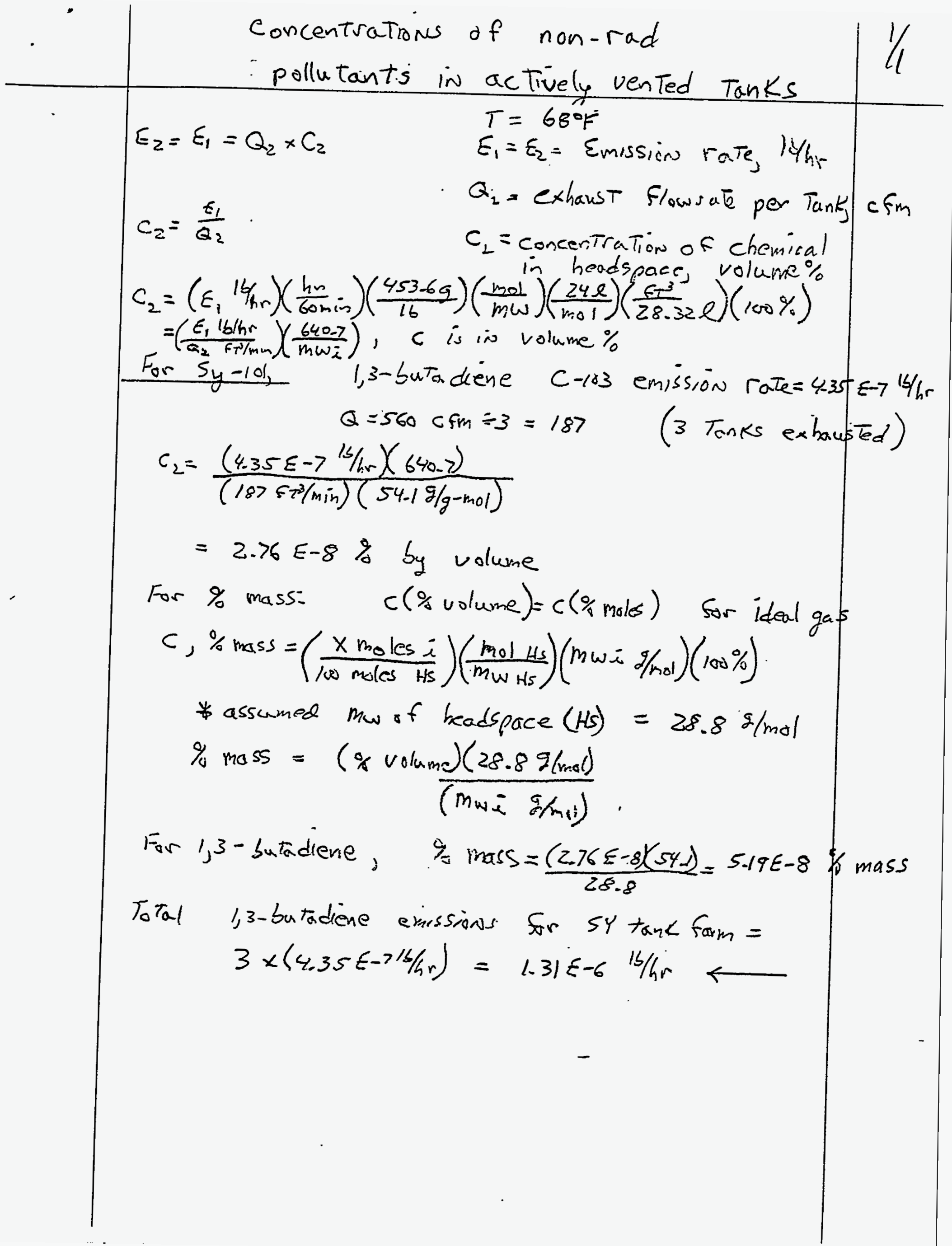




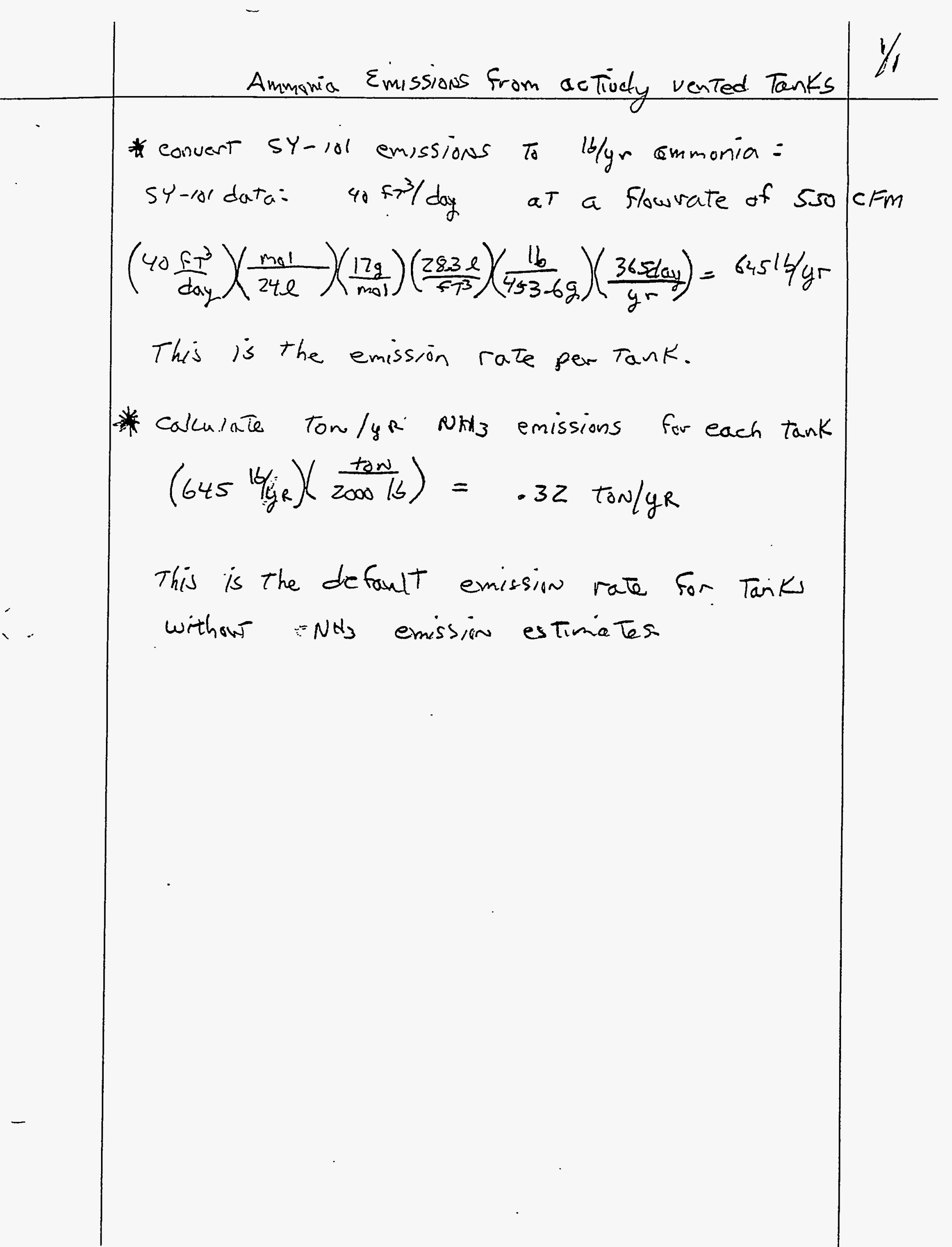




\subsection{REFERENCES Non-Radiological Emissions}

Crummel, G.M., "Single Shell Tank Farm Leak Detection Monitoring Frequencies", Westinghouse Hanford Company internal memorandum, November 26, 1990.

Crummel, G.M., and Gustavson, R.D., "Facility Effluent Monitoring Plan for 242-A Evaporator Facility", WHC-EP-0466-I, Revision 1, March 1993.

Crummel, G.M., 1994a. "Tank Farm Stack 'Sampling System Configuration and Efficiency Study", Westinghouse Hanford Company, WHC-SD-WM-ES-291, Rev 1, May 1994.

Crummel, G.M., 1994b. Table of annualized ammonia emissions taken from EPCRA 313 Release Data, June 23, 1994.

Crummel, G.M., 1994c. Table of measured exhaust rate statistics, September, 1994.

Hewlitt, E., spreadsheet of Industrial Monitoring Source Data for 1993 and 1994, June 21, '1994.

Huber, John., telephone conversation with S.L. Richards, October 13, 1994.

Huckaby, J.L., and Story, M.S., 1994a. "Vapor Characterization of Tank 241-C-103', WHC-EP-0780, June 1994.

Huckaby, J.L., 1994b. Telephone conversation with S.L. Richards, August 22, 1994.

Jenkins, Roger A., 1994a. 'Analysis of Tank 241-BY-105 Headspace Components", Report to Mr. Jerry Osborne, WHC, from Oak Ridge National Laboratory, September 2, 1994.

Jenkins, Roger A., 1994b. "Analysis of Tank 241-BY-104 Headspace Components", Report to Mi. Jerry Osborne, WHC, from Oak Ridge National Laboratory, September 7, 1994.

Jenkins, Roger A., 1994c. 'Analysis of Tank 241-BY-106 Headspace Components", Report to Mr . Jerry Osborne, WHC, from Oak Ridge National Laboratory, September 8, 1994.

Kriskovich, J.R., "Single Shell Tank Breather Filter Test Frequency", WHC-SD-WM-ES270, Rev O, January 1994. 
Ligotke, M.W., Pool, K.H., and Lemer, B.D., 1994a. "Vapor Space Characterization of Waste .Tank 241-BY-104: Inorganic Sample Results from 6/24/94", Preliminary Data Tables from Review Draft Prepared for U.S.D.O.E. by PNL, August 3, 1994.

Ligotke, M.W., Pool, K.H., and Lerner, B.D., 1994b. "Vapor Space Characterization of Waste Tank 241-BY-105: Inorganic Sample Results from 6/24/94", Preliminary Data Tables from Review' Draft Prepared for U.S.D.O.E. by PNL, August 3, 1994.

Ligotke, M.W., Pool, K.H., and Lerner, B.D., 1994c. "Vapor Space Characterization of Waste Tank 241-BY-106: Inorganic Sample Results from 6/24/94", Preliminary Data Tables from Review Draft Prepared for-U.S.D.O.E. by PNL, August 3, 1994.

Rasmussen, R.A., 1994a. "Hanford Storage Tank Vapor Headspace Canister Sample Analysis Report: Tank TX-118, Tank C-108, Tank C-Iii", Report No. SAND94-1568, May 1994.

Rasmussen, R.A., 1994b. "Air Samples Collected at Waste Tanks 241-C-104, -105, and 105 , on March 3 and February 15 and 16, 1994", portion of report sent by Jim Huckaby, August 22, 1994.

Rasmussen, R.A., 1994c. Tables 2, 3, and 4 labeled "Final Concentrations" for BY-I04, BY-I05, and BY-I06. Samples collected April 28. May 4, and May 9, 1994.

Tranbarger, R.K., "Past Vapor Releases from Single Shell Tanks 241-C-102 and -103", WHC Internal Memo, August 16, 1990.

Wilkins, N., telephone conversation with S.L. Richards, August 19 and 23, 1994. 
(3) The remaining nonradiological emission categories are grouped by source type. Examples are underground petroleum storage tanks, carpentry shops, machine shops, laboratories, etc. A representative calculation is provided for each of these source types. 


\section{EXAMPLE CALCULATIONS FOR CATEGORY F: COMBUSTION EMISSION POINT EXTERNAL (BOILERS ONLY)}

The example calculations presented in this section are for the 284E, 284W and 384 Powerhouses. A portion of the spreadsheet used to calculate estimated emissions is attached, showing the calculations for Boiler \#2 at the 384 Powerhouse, which is designated as emission unit 300 F-384-002. Example calculations for sulfur dioxide and lead are shown below.

\section{Assumptions}

Emission estimates are based on actual 1994 fuel usage, using emission factors from AP-42, or alternatively, from EPA-450/2-88-006a. Potential emissions are based on operating at the maximum hourly rate, for 24 hours per day, 365 days per year, with one exception. The potential emissions from the package boiler at the $284 \mathrm{~W}$ Powerhouse, emission unit 200W F-284W 005, are based on 1440 permitted operating hours.

At the 284E Powerhouse, the emission units 200E F-284E 001 and 002 represent two separate stacks, which operate independently, depending on which baghouse modules are in use, and which of the five coal-fired boilers are in use. The potential emissions are based on operating all five boilers at the maximum hourly rate. Actual emissions are based on total fuel usage, regardless of which boilers were used. The total emissions are assumed to be split evenly between the two stacks.

The same holds true for the $284 \mathrm{~W}$ Powerhouse, with its two stacks and four boilers, emission units 200W F-284W 001 and 002.

At the 384 Powerhouse, the three \#6 residual oil-fired boilers have separate stacks, and are separate emission units, 300 F-384 002, 005, and 006. The three inactive coal-fired boilers have a common stack.

Emissions are based on no controls in place, with the exception of particulates from coal combustion; both actual and potential particulate emissions are based on a removal efficiency of $99.8 \%$ for the baghouses.

Example calculations for emission unit 300 F-384-002:

1994 Fuel Usage $=679.24 \mathrm{kgal} ; \%$ Sulfur $=S=1.75 ;$ Heat content $=153,000 \mathrm{BTU} / \mathrm{gal}$

Emission Factor $_{\mathrm{SO} 2}=157 * \mathrm{~S} \mathrm{lb} / \mathrm{kgal}$

Emission Factor $\mathrm{P}_{\mathrm{P}}=194 \mathrm{lb} / 10^{\wedge} 12 \mathrm{BTU}$

Emissions $_{\mathrm{SO} 2}=($ Annual Fuel Usage $) *($ Emission Factor $)$

$=(679.24 \mathrm{kgal} / \mathrm{yr}) *(157 * 1.75 \mathrm{lb} / \mathrm{kgal}) / 2000 \mathrm{lb} / \mathrm{ton}$

$=93.31 \mathrm{ton} / \mathrm{yr}$ 
Hanford Site Air

Operating Permit Application
DOE/RL-95-07, Rev. 0

$05 / 95$

\section{Emissions $_{\mathrm{pb}}=($ Annual Fuel Usage $) *($ Emission Factor $)$}

$=(679.24 \mathrm{kgal} / \mathrm{yr}) *\left(194 \mathrm{lb} / 10^{\wedge} 12 \mathrm{BTU}\right) *(1000 \mathrm{gal} / \mathrm{kgal}) *(153,000 \mathrm{BTU} / \mathrm{gal})$

$(2000 \mathrm{lb} /$ ton $) *\left(1.0 \mathrm{E}+12 \mathrm{BTU} / 10^{\wedge} 12 \mathrm{BTU}\right)$

$$
=0.01 \mathrm{ton} / \mathrm{yr}
$$




\begin{tabular}{|c|c|c|c|c|c|c|c|c|c|c|c|c|}
\hline A & $A$ & 日 & $\begin{array}{c}\mathbf{C} \\
\end{array}$ & \begin{tabular}{|c|} 
D \\
\end{tabular} & E & $F$ & G & $\mathrm{H}$ & 1 & $J$ & $\mathrm{~K}$ & $L$ \\
\hline 2 & Facillity & Fuel Type & Hlcat Content & Sulfur Content & Ash Contenit & & & & & & & \\
\hline 3 & & & (BTU/gal) & $(w+\%)$ & $(w \%)$ & & & & & & & \\
\hline 4 & 300 Arca & & & & & & & & & & & \\
\hline 5 & Pawerlinulse & H6 Rasidfual Ficl Oil & 153000 & 1.75 & 0 & & & & & & & \\
\hline \multicolumn{13}{|l|}{$\frac{6}{7}$} \\
\hline 7 & & & & & & Actual Aimual & Dally Ave & Hourly Ave & Hourly Mlax & Alliuual Mlax & Max Ileat Impul & Max Sitenm Outpul \\
\hline 8 & Emulisslon l'olnt & Faclllity & AEI Descriplion & Soource Nunsher & SCC Code & Fucl Uxe (gal) & Finel Uxe (gal) & Fuel Use (gal) & Fuel lixe (gal) & Fuel Use (kgal) & (AIIITU) & (1'oundz Steram) \\
\hline \multicolumn{13}{|r|}{ 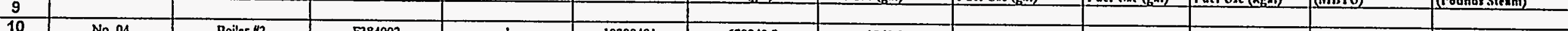 } \\
\hline 10 & No. 04 & Boiler $\mathrm{H2}$ & $F 384002$ & 1 & 10200401 & 679240.8 & 1860.9 & 77.5 & 915 & 8015 & 140 & 100000 \\
\hline 11 & No. 08 & Boiler 166 & F384006 & 1 & 10200401 & 418095.5 & 1145.5 & 47.7 & 730 & 6395 & 110 & 80000 \\
\hline 12 & No. 19 & Package Bniler & PB84005 & 1 & 10200101 & 889177.4 & $21,36.9$ & 101.5 & 550 & 1818 & 84 & 60000 \\
\hline \multicolumn{13}{|l|}{$\frac{13}{14}$} \\
\hline 14 & & Ho Fuel OII & Total Steam & 112 Boller & & H6 Boller & & 1'ackage Boflier & & H2 Boller & H6 Moller & mPackage Boller \\
\hline 15 & MONTII 1994 & Consumption (gal) & Genernited (B) & steam Generated & $\%$ or Steams & Sleam Generaled & $\%$ of Sieant & Sileam Generaled & $\%$ or Stean & Fuel Usage (gal) & Fuel Usage (gal) & Fuel Usage (gai) \\
\hline 16 & Jan & \begin{tabular}{|r|}
429347.33 \\
\end{tabular} & \begin{tabular}{|r|}
45203900 \\
\end{tabular} & 22071600 & $48.83 \%$ & \begin{tabular}{|r|}
8985986 \\
\end{tabular} & $19.88 \%$ & \begin{tabular}{|l|l|}
14146314 \\
\end{tabular} & $31.29 \%$ & \begin{tabular}{|r|}
209636 \\
\end{tabular} & $\begin{array}{r}85349 \\
\end{array}$ & 134362 \\
\hline 17 & Fob & 280207.48 & 39778398 & 18817200 & $47.31 \%$ & 3267000 & $8.21 \%$ & 17694198 & $44,48 \%$ & 132552 & 23013 & 124642 \\
\hline 18 & Mar & 219580.00 & 25243748 & 999600 & $3.96 \%$ & 6866000 & $27.20 \%$ & 17378148 & $68.81 \%$ & 8695 & 59723 & 151162 \\
\hline 19 & $\Delta p r$ & 157435.96 & 19966828 & 402000 & $2.01 \%$ & 4885000 & $24.47 \%$ & 14679828 & $73.52 \%$ & 3170 & 38518 & 115749 \\
\hline 20 & May & 111832.12 & 9171261 & 735600 & $7.76 \%$ & 1875000 & $51.16 \%$ & $.386,3604$ & $10.78 \%$ & $86 \times 3$ & 57541 & 45600 \\
\hline 21 & IIIII & 10207.36 & 2962578 & 118.56 & $40.02 \%$ & 0 & $0.00 \%$ & 1776978 & $.59 .98 \%$ & 16091 & 0 & 21117 \\
\hline 22 & S111 & 61910.73 & 3411816 & 762400 & $22.93 \%$ & 27000 & $0.79 \%$ & 2602116 & $76.28 \%$ & 14887 & 311 & 49516 \\
\hline 23 & Aug & 68192.80 & 945200 & 661200 & $69.95 \%$ & 284000 & $30.05 \%$ & 0 & $0.00 \%$ & 47703 & 20490 & 0 \\
\hline 24 & Sep & 58422.90 & 22924400 & 1952400 & $8.52 \%$ & 20972000 & $91.48 \%$ & 0 & $0.00 \%$ & 4976 & 53447 & 0 \\
\hline 25 & Oc! & 97193.20 & 8348714 & 4798800 & $57.48 \%$ & 266000 & $3.19 \%$ & 3283914 & $39.33 \%$ & 55866 & 3097 & 38230 \\
\hline 26 & Nov & 211120,66 & 23529780 & 17908800 & $76.11 \%$ & 9000 & $0.01 \%$ & 5611980 & $23.85 \%$ & 160686 & 81 & 30353 \\
\hline 27 & Dec & 248356.80 & 30194994 & 1981200 & $6.56 \%$ & 9279000 & $30.73 \%$ & 18934794 & $62.71 \%$ & 16296 & 76321 & 155741 \\
\hline \multicolumn{13}{|l|}{$\frac{28}{29}$} \\
\hline 29 & Tohal & 1986813.64 & 231984620 & 72296400 & & 59715986 & & 99972234 & & 679241 & 418095 & 889477 \\
\hline 30 & & & & & & & & & & & & \\
\hline 31 & & Tolal 300 Area & \begin{tabular}{|l} 
Total 300 Area \\
\end{tabular} & Hz Boller & 112 Boller \% & \#6 Boller & H6 Boller \% & Paskage Boller & I hog $1318 \%$ & H2 Hofler & H6 Boller & Package Boller \\
\hline 32 & Quarterly Totals & Fuel Consumed & Steam Generated & Steami Generaled & Seasonal use & Stcam Generated & Seasonal use & Steam Gencrated & Seaxonal uxe & Fuel Consumed & Fuel Consumed & Fuel Consumed \\
\hline \multicolumn{13}{|r|}{ Fuel Consumed } \\
\hline 34 & $1 \mathrm{QTR}$ & 929134.81 & 110226046 & 41888400 & $57.94 \%$ & 19118986 & $32.02 \%$ & 49218660 & $49.23 \%$ & 350884 & 168086 & 410166 \\
\hline 35 & $2 \mathrm{QTR}$ & 309475.74 & 32403670 & 2323200 & $3.21 \%$ & 9760000 & $16.34 \%$ & 20320470 & $20.33 \%$ & 27943 & 96061 & 185471 \\
\hline 36 & $3 \mathrm{QTR}$ & 191532.43 & 27281416 & 3396000 & $4.70 \%$ & 21283000 & $35.64 \%$ & 2602416 & $2.60 \%$ & 67566 & 74451 & 49516 \\
\hline 37 & $4 Q T R$ & 556670.66 & 62073488 & 24688800 & $34.15 \%$ & 9554000 & $16.00 \%$ & 27830688 & $27.84 \%$ & 232848 & 79498 & 244324 \\
\hline \multicolumn{13}{|l|}{$\begin{array}{l}38 \\
39\end{array}$} \\
\hline$\frac{39}{40}$ & & & & & & & & & & & & \\
\hline & & & & & & & & & & & & \\
\hline
\end{tabular}




\begin{tabular}{|c|c|c|c|c|c|c|c|c|c|c|c|c|}
\hline B & A & $\mathbf{B}$ & C & $\bar{D}$ & $\bar{E}$ & $\bar{F}$ & $\mathbf{G}$ & $H$ & $\mathrm{I}$ & $\mathbf{J}$ & $\bar{k}$ & $\mathbf{L}$ \\
\hline 2 & Facllity & & & & & & & & & & & \\
\hline \multicolumn{13}{|l|}{3} \\
\hline 4 & 300 Area & & & & & & & & & & & \\
\hline 5 & Powerhoilse & & & & & & & Actual & Potenlla! & & & \\
\hline 6 & & & Ar 42 Factor & Fuel Oll Use & Fuel Oll Use & Reference & Emisslons & Emlssions & Emlsslons & EQUATIONS USED & DESCRIPTION & FORMULA \\
\hline 7 & Emilssion Polnt & Pollutants (AED) & $(\mathrm{h} / \mathrm{kg}$ al) & $\left(g^{2} / y r\right)$ & $\left(\right.$ kga $\left.\mathrm{ll}_{y r r}\right)$ & Equatlon Used & $(\mathrm{h} / \mathrm{yr})$ & $(\operatorname{lon} / y r)$ & $(\operatorname{lon} / \mathrm{yr})$ & & & \\
\hline \multicolumn{13}{|l|}{8} \\
\hline 9 & No.04 & Carbon Monsoxide * & 5.000 & 679241 & 679.24 & 1 & 3396.20 & 1.70 & 20.0 & 1 & carbon monoxide: S Illokgal (Table 1.3-2) & 5 \\
\hline 10 & (F384002) & Conper ${ }^{\circ}$ & 0.043 & 679241 & 679.24 & 2 & 28.89 & 0.01 & 0.2 & 2 & conper: $278 \mathrm{lW} / 10^{\wedge} 12 \mathrm{BTU}$ (Page 4-161) & 0.042534 \\
\hline 11 & (source 1) & Melliane & 1.000 & 679241 & 679.24 & 3 & 679.24 & 0.34 & 4.0 & 3 & meliano:l lovkgal (Table 1.3-4) & 1 \\
\hline 12 & IBoiler 12 & Nox* & 55.000 & 679211 & 679.24 & 4 & 37358.21 & 18.68 & 220.4 & 4 & NOx: S5 Hikgal (Talle 1.3-2) & 35 \\
\hline 13 &. & Nitrons Oxide & 0.110 & 679241 & 679.21 & $s$ & 74.72 & 0.04 & 0.1 & $s$ & N2O:0.11 IllMkgal (Tallic 1.3.9) & 0.11 \\
\hline 14 & & Non-Melline Toc ${ }^{\circ}$ & 0.280 & 672211 & 679.21 & 6 & 190.19 & 0.10 & 1.1 & 6 & 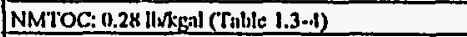 & 0.28 \\
\hline 15 & & Sulfur Dioxide* & 274.750 & 679241 & 679.21 & 1 & 186621.41 & 93.31 & 1101.1 & 7 & SOO2: 157 S HINkgal (Taulo 1.3-2) & 274.75 \\
\hline 16 & & Sulfur Trioxide & 3.500 & 679241 & 679.24 & 8 & 2377.34 & 1.19 & 14.0 & 8 & SO3: 2 S lb/kgal (Table 1.3-2) & 3.5 \\
\hline 17 & & TOC & 1.280 & 679241 & 679.24 & 9 & 869.43 & 0.43 & 5.1 & 9 & TOC: $1.28 \mathrm{H} / \mathrm{k}$ kgal (Talle $1.3-4)$ & 1.28 \\
\hline 18 & & Total PM & 19.303 & 679241 & 679.24 & 10 & 13111.05 & 6.56 & 77.4 & 10 & Total PM: $9.19(3)+3.2216 / 2 \mathrm{kgal}$ (Talle 1.3-2) & 19.3025 \\
\hline 19 & & PM10 & 16.778 & 679241 & 679.24 & 11 & 11396.17 & 5.70 & 67.2 & 11 & PM10: $7.17(1.12(\$)+0.38)$ llukgnil (Tnllie 1.3.6) & 16.7778 \\
\hline 20 & & Valtadium ** & 0.463 & 679241 & 679.21 & 12 & 314,16 & 0.16 & 1.9 & 12 & valladium: 1300 pe/J (1'agc 1-163) & 0.46252206 \\
\hline 21 & & Antinumuy & 0.0007 & 679211 & 679.21 & 13 & 4.78 & 0.00 & 0.0 & 13 & 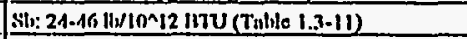 & 0.0070 .38 \\
\hline 22 & & Arenic & 0.017 & 679241 & 679.21 & 14 & 11.85 & 0.01 & 0.1 & 14 & $\Lambda s: 19.114 \mathrm{lW} / 10^{\wedge} 12$ BTU (Table $\left.1.3-11\right)$ & 0.017442 \\
\hline 23 & & Beryllium & 0.001 & 679241 & 679.24 & 15 & 0.44 & 0.00 & 0.0 & 15 & Be: $4.211 / 1012$ BTU (Table 1.3-11) & 0.0006426 \\
\hline 24 & & Cadmitum & 0.032 & 679241 & 679.24 & 16 & 21.93 & 0.01 & 0.1 & 16 & Cil: 16-211 I6/10012 BTU (Table 1.3-11) & 0.032283 \\
\hline 25 & & Cluroumim!n & 0.020 & 679211 & 679.24 & 17 & 13.30 & 0.01 & 0.1 & 17 & Cr: $21-128$ lW/10/12 13TU (Talle 1.3-11) & 0.019584 \\
\hline 26 & & Cobalt & 0.019 & 679241 & 679.21 & 18 & 12.57 & 0.01 & 0.1 & 18 & Co: $77-121$ ib/10112 BTU (Table 1.3-11) & 0.018513 \\
\hline 27 & & Fonmaldeliyde & 0.062 & 679241 & 679.24 & 19 & 42.09 & 0.02 & 0.2 & 19 & HCOII: $161-4051 \mathrm{~b} / 10 \cdot 12$ nTU (Taule $1.3-9)$ & 0.061965 \\
\hline 28 & & Lend * & 0.030 & 679241 & 679.24 & 20 & 20.16 & 0.01 & 0.1 & 20 & PU: $28-19416 / 10^{\wedge} 12$ B]U (Talle 1.3-9) & 0.029682 \\
\hline 29 & & Mrauganese & 0.011 & 679241 & 679.24 & 21 & 7.69 & 0.00 & 0.0 & 21 & Mu1: 23.74llb/10412 BTU (Table 1.3-11) & 0.011322 \\
\hline 30 & & Merctiry & 0.005 & 679241 & 679.24 & 22 & 3.33 & 0.00 & 0.0 & 22 & 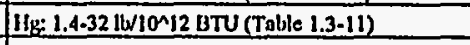 & 0.004896 \\
\hline 31 & & Nickel & 0.356 & 679241 & 679.24 & 23 & 242.14 & 0.12 & 1.4 & 23 & Ni: 837-2330 lb/10012 BTU (Table 1.3-11) & 0.35649 \\
\hline 32 & & POM & 8.400 & 679241 & 679.24 & 24 & 5705.62 & 2.85 & 33.7 & 24 & POM: 7.8-8.4 ll//kgan (Table 1.3-9) & $8.4^{\circ}$ \\
\hline 33 & & Selenium & 0.006 & 679241 & 679.24 & 25 & 3.95 & 0.00 & 0.0 & 25 & Se: 38 1U/10M/12 BTU (TaUle 1.3-11) & 0.005814 \\
\hline \multicolumn{13}{|l|}{34} \\
\hline 35 & & Total Crilerin Pollulants & & & & & & 119.83 & 1414.07 & & & \\
\hline \multicolumn{13}{|l|}{36} \\
\hline 37 & & - Criteria Pollutanls & & & & & & & & & & \\
\hline 38 & & $\because$ AP-42 Factor Not & & & & & & & & & & \\
\hline 39 & & Availabic; if roctor listed, & & & & & & & & & & \\
\hline 40 & & froin EPA-450/2-88-006a & & & & & & & & & & \\
\hline
\end{tabular}


The example calculations above and used in the spreadsheet are equivalent for the emission units listed below:

200E F-284E 001

200E F-284E 002

200E F-284E 005

200W F-284W 001

200W F-284W 002

200W F-284W 005

300 F-384 001

300 F-384 002

300 F-384 005

300 F-384 006 
EXAMPLE OF CALCULATIONS FOR CATEGORY G: GASOLINE DISTRIBUTION AREA (GASOLINE COMPONENT)

Example of generalized calculation using emission point \#G2713E001 for the gasoline component.

First, the total tank loss emission factor (pounds per 1,000 gallons of throughput) and the refueling loss emission factor were calculated.

$$
\begin{aligned}
& \operatorname{LoSS}_{f i 11}=11.5 \frac{1 b}{1000 \mathrm{gal}} \\
& \operatorname{LoSS}_{\text {breathing }}=1.0 \frac{1 \mathrm{~b}}{1000 \mathrm{gal}}
\end{aligned}
$$

Annual throughput $=152,187 \mathrm{gal}$

$\mathrm{EF}=$ Emission Factor

$$
\begin{gathered}
E F_{\text {tank }}=\text { LOSS }_{f i 11}+\text { LOSS }_{\text {breathing }} \\
E F_{\text {tank }}=11.5 \frac{I b}{1000 \mathrm{gaI}}+1.0 \frac{\mathrm{Ib}}{1000 \mathrm{gaI}}=12.5 \frac{1 \mathrm{~b}}{1000 \mathrm{gaI}} \\
. . \\
\text { LOSS }_{\text {displacement }}=11.0 \frac{\mathrm{Ib}}{1000 \mathrm{gaI}}
\end{gathered}
$$




$$
\begin{aligned}
& \text { LOSS }_{\text {spillage }}=0.7 \frac{1 b}{1000 \text { gaI }} \\
& E F_{\text {refueling }}=\text { LOSS }_{\text {displacement }}+\text { Loss } S_{\text {spillage }} \\
& E F_{\text {refueling }}=11.0 \frac{1 b}{1000 \mathrm{gaI}}+0.7 \frac{\mathrm{lb}}{1000 \mathrm{gaI}}=11.7 \frac{\mathrm{Ib}}{1000 \mathrm{gal}}
\end{aligned}
$$

Next, the emission factors were used to calculate the total VOC emissions by multiplying by the annual throughput in the tank.

$$
\text { Annual Emissions } \text { voc }=\frac{\text { Annual throughput } * E F_{\text {tank }}}{2000 \frac{1 b}{\text { ton }}}
$$

Annual Emissions voc $(\operatorname{tank})=\frac{152187 \mathrm{gal} * 12.5 \frac{1 \mathrm{~b}}{1000 \mathrm{gal}}}{2000 \frac{\mathrm{lb}}{\mathrm{ton}}}=0.951$ ton

$$
\text { Annual Emissions } \text { voc }(\text { refueling })=\frac{\text { Annual throughput } * E F_{\text {refueling }}}{2000 \frac{1 b}{\text { ton }}}
$$

$$
\text { Annual Emissions voc }(\text { refueling })=\frac{152187 \mathrm{gal} * 11.7 \frac{1 \mathrm{~b}}{1000 \mathrm{gal}}}{2000 \frac{1 \mathrm{~b}}{\mathrm{ton}}}=0.890 \mathrm{ton}
$$


Annual Emissions $_{v o c}\left(\right.$ total) $=$ Emissions $_{v o c}(\operatorname{tank})+$ Emissions $_{\text {voc }}($ refueling $)$

$$
\text { AnnualEmissions }_{\text {voc }}(\text { total })=0.951 \text { ton }+0.890 \text { ton }=1.841 \text { ton }
$$

The constituent weight emissions were determined by applying the weight fraction associated with each component in the vapor phase.

\begin{tabular}{||l|l||}
\hline Constituent & Weight Fraction \\
\hline Benzene & 0.1 \\
\hline ethylbenzene & 0.0028 \\
\hline hexane & 0.009 \\
\hline methyltertbutyl ether & 0.0288 \\
\hline toluene & 0.0103 \\
\hline 2,2,4-trimethylpentane & 0.0037 \\
\hline xylene & 0.0044 \\
\hline
\end{tabular}

WF $=$ Weight Fraction

$$
\begin{gathered}
W F_{\text {sumz }}=W F_{\text {benzene }}+W F_{\text {toluene }}+W F_{\text {ethylbenzene }}+W F_{\text {hexane }}+W F_{x y 1 e n \theta} \\
\qquad \\
W F_{\text {sum } 1}=0.01+0.0103+0.0028+0.009+0.0044=0.037 \\
W F_{\text {sumz }}=W F_{\text {methyltertbutylether }}+W F_{2,2,4-\text { trimethylpentane }} \\
W F_{\text {sumz }}=0.0288+0.0037=0.033
\end{gathered}
$$




$$
\begin{gathered}
W F_{\text {voc other }}=1-W F_{\text {sum1 }}-W F_{\text {sumz }} \\
W F_{\text {VOC other }}=1-0.037-0.033=0.931
\end{gathered}
$$

The weight fraction of each constituent was multiplied by the total VOC emissions. For example: Total annual emissions benzene $=W F_{\text {benzene }} *$ Annual Emission total voc Annual Total Emissions benzene $=0.01 * 1.841$ ton $=0.018$ ton

\begin{tabular}{|l|l|l|l||}
\hline Constituent & Weight Fraction & $\begin{array}{l}\text { total Annual } \\
\text { VOC emissions } \\
\text { (ton) }\end{array}$ & $\begin{array}{l}\text { Constituent } \\
\text { Annual } \\
\text { Emissions } \\
\text { (ton) }\end{array}$ \\
\hline Benzene & 0.1 & 1.841 & $1.8 \mathrm{E}-02$ \\
\hline ethylbenzene & 0.0028 & 1.841 & $5.516 \mathrm{E}-03$ \\
\hline hexane & 0.009 & 1.841 & $1.7 \mathrm{E}-02$ \\
\hline methyltertbutyl ether & 0.0288 & 1.841 & $5.3 \mathrm{E}-02$ \\
\hline toluene & 0.0103 & 1.841 & $1.9 \mathrm{E}-02$ \\
\hline $2,2,4$-trimethylpentane & 0.0037 & 1.841 & $6.813 \mathrm{E}-03$ \\
\hline xylene & 0.0044 & 1.841 & $8.102 \mathrm{E}-03$ \\
\hline Other VOC & 0.931 & 1.841 & 1.714 \\
\hline
\end{tabular}


Example of generalized calculation using emission point \#G2713E001 for the diesel component.

First, the total tank loss emission factor (pounds per 1,000 gallons of throughput) and the refueling loss emission factor were calculated.

$$
\begin{aligned}
& \operatorname{Losi}_{f i 11}=0.03 \frac{I b}{1000 g a I} \\
& \text { LOSS }_{\text {breathing }}=0.0 \frac{1 b}{1000 \mathrm{gal}}
\end{aligned}
$$

Annual throughput $=202,794 \mathrm{gal}$

$\mathrm{EF}=$ Emission Factor

$$
\begin{aligned}
& E F_{\text {tank }}=\text { LOSS }_{f i 11}+\text { LOSS }_{\text {breathing }} \\
& E F_{\text {tank }}=0.03 \frac{1 b}{1000 \mathrm{gaI}}+0.0 \frac{\mathrm{Ib}}{1000 \mathrm{gal}}=0.03 \frac{\mathrm{Ib}}{1000 \mathrm{gaI}} \\
& \text { LOSS }_{\text {displacement }}=0.3 \frac{1 \mathrm{~b}}{1000 \mathrm{gaI}}
\end{aligned}
$$




$$
\begin{gathered}
\text { LOSS }_{\text {spillage }}=0.7 \frac{1 \mathrm{~b}}{1000 \mathrm{gal}} \\
E F_{\text {refueling }}=\operatorname{LOSS}_{\text {displacement }}+\text { LOSS }_{\text {spillage }} \\
E F_{\text {refueling }}=0.03 \frac{\mathrm{lb}}{1000 \mathrm{gal}}+0.7 \frac{\mathrm{Ib}}{1000 \mathrm{gal}}=0.730 \frac{\mathrm{lb}}{1000 \mathrm{gaI}}
\end{gathered}
$$

Next, the emission factors were used to calculate the total VOC emissions by multiplying by the annual throughput in the tank.

$$
\text { Annual Emissions } \text { voc }=\frac{\text { Annual throughput } * E F_{\text {tank }}}{2000 \frac{1 b}{t o n}}
$$

Annual Emissions $v(\operatorname{tank})=\frac{202794 \mathrm{gal} * 0.03 \frac{\mathrm{lb}}{1000 \mathrm{gal}}}{2000 \frac{\mathrm{lb}}{\mathrm{ton}}}=0.003$ ton

Annual Emissions voc $($ refueling $)=\frac{\text { Annual throughput } * E F_{\text {refueling }}}{2000 \frac{1 b}{t o n}}$

Annual Emissions $v_{v o c}($ Iefueling $)=\frac{202794 \mathrm{gal} * 0.730 \frac{\mathrm{lb}}{1000 \mathrm{gal}}}{2000 \frac{\mathrm{lb}}{\mathrm{ton}}}=0.074 \mathrm{ton}$ 
Annual Emissions $_{\text {voc }}($ totaI $)=$ Emissions $_{\text {voc }}(\operatorname{tank})+$ Emissions $_{\text {voc }}($ refueling $)$

$$
\text { AnnualEmissions }_{\text {voc }}(\text { total })=0.003 \text { ton }+0.074 \text { ton }=0.077 \text { ton }
$$

The constituent weight emissions were determined by applying the weight fraction associated with each component in the vapor phase.

\begin{tabular}{||l|l|}
\hline Constituent & Weight Fraction \\
\hline Benzene & 0.1 \\
\hline hexane & 0.009 \\
\hline toluene & 0.0103 \\
\hline
\end{tabular}

$\mathrm{WF}=$ Weight Fraction

$$
\begin{gathered}
W F_{\text {VoC other }}=1-W F_{\text {benzene }}-W F_{\text {toluene }}-W F_{\text {hexane }} \\
. \\
W F_{\text {VOC other }}=1-0.01-0.0103-0.009=0.971
\end{gathered}
$$

The weight fraction of each constituent was multiplied by the total VOC emissions. For example:

Total annual emissions benzene $=W F_{\text {benzene }} *$ Annual Emission total voc

Annual Total Emissions benzene $=0.01 * 0.077$ ton $=7.70 E-04$ ton 


\begin{tabular}{||l|l|l|l||}
\hline \hline Constituent & Weight Fraction & $\begin{array}{l}\text { total Annual } \\
\text { VOC emissions } \\
\text { (ton) }\end{array}$ & $\begin{array}{l}\text { Constituent } \\
\text { Annual } \\
\text { Emissions } \\
\text { (ton) }\end{array}$ \\
\hline Benzene & 0.1 & 0.077 & $7.70 \mathrm{E}-04$ \\
\hline hexane & 0.009 & 0.077 & $6.94-04$ \\
\hline toluene & 0.0103 & 0.077 & $7.94 \mathrm{E}-04$ \\
\hline Other VOC & 0.971 & 0.077 & 0.075 \\
\hline
\end{tabular}

The example calculation is equivalent for the emission units listed below:

1100 G-1172A 001

$1100 \mathrm{G}-1172 \mathrm{~A} 002$

1100 G-1172A 003

1100 G-1172A 004

1100 G-1172A 005

200E G-2713E 001

200E G-2713E 002

200E G-2713E 003 


\section{EXAMPLE CALCULATION FOR CATEGORY H: COMBUSTION EMISSION POINT}

Example of generalized calculation using emission unit \#H200W007(200 West Burn Pit) for the burn of Russian Thistle (tumbleweed). The volume of tumbleweed burned per year is approximately $250 \mathrm{yd}^{3}$ per foot, and the maximum potential is $500 \mathrm{yd}^{3}$ per $\mathrm{yr}$.

$$
\begin{aligned}
& \text { Volume }_{\text {actual }}=250 \frac{y d^{3}}{y r} \\
& \text { Volume } \text { potential }_{1}=500 \frac{\mathrm{yd}^{3}}{\mathrm{yI}}
\end{aligned}
$$

The following emissions are produced from burning tumbleweed:

$$
\begin{aligned}
& \text { Particulate }=22 \frac{1 b}{\text { ton }} \\
& \text { Carbon Monoxide }=309 \frac{1 b}{t o n} \\
& \text { NonMethane } V O C=1.5 \frac{1 b}{t o n}
\end{aligned}
$$

The assumed specific gravity of tumbleweed is assumed to be that similar to hay.

$$
S p G_{\text {tumbleweed }}=0.32
$$

Based on the given specific gravity of tumbleweed, the density of tumbleweed is calculated as follow

$$
\rho_{\text {tumbleweed }}=S p G_{\text {tumblewieed }} \frac{g}{\mathrm{ml}} * \frac{1 \mathrm{lb}}{454 \mathrm{~g}} * 28317 \frac{\mathrm{ml}}{\mathrm{ft^{3 }}}=19.959 \frac{\mathrm{Ib}}{\mathrm{ft}^{3}}
$$


The time for the actual burning of tumbleweed is 1 hour per day, 1 day per week, 50 weeks per year.

$$
\text { Time }_{\text {actual }}=50 \mathrm{hr}
$$

The estimated amount of tumbleweed to burn each year is as follows:

$$
\begin{aligned}
& \text { Actual }=\text { Volume } e_{\text {actual }} * \frac{f t^{3}}{0.03704 y d^{3}} * \rho_{\text {tumbleweed }} \\
& \text { Actual }=250 \frac{y d^{3}}{y I} * \frac{f t^{3}}{0.03704 y d^{3}} * 19.959 \frac{l b}{f t^{3}}=134713 \frac{1 b}{y I} \\
& \text { Actual }=\left(\frac{134713 \mathrm{lb}}{y I}\right)\left(\frac{1 \mathrm{ton}}{2000 \mathrm{Ibs}}\right)=67.36 \frac{\mathrm{ton}}{\mathrm{yI}} \\
& \text { Potential }=\text { Volume } e_{\text {potential }} * \frac{f t^{3}}{0.03704 \mathrm{yd}^{3}} * \rho_{\text {tumbleweed }} \\
& \text { Potential }=500 \frac{y d^{3}}{y r} * \frac{f t^{3}}{0.03704 y d^{3}} * 19.959 \frac{1 b}{f t^{3}}=2.69 E+05 \frac{l b}{y r} \\
& \text { Potential }=2.69 E+05 \frac{1 b}{y r} * \frac{1 \text { ton }}{20001 b s}=134.7 \frac{\mathrm{ton}}{\mathrm{yr}}
\end{aligned}
$$

Using particulates as an example, the actual estimated emissions are as follows:

$$
\text { Emissions }_{\text {particulate }}=\text { Actual } * \text { Particulate }
$$




$$
\text { Emissions }_{\text {particulate }}=67.36 \frac{\text { ton }}{y x} * 22 \frac{1 b}{\operatorname{ton}} * \frac{1 \text { ton }}{20001 b}=0.741 \frac{\text { ton }}{y x}
$$

$$
\text { Emissions }_{\text {particulate rate }}=\frac{\text { Emissions }_{\text {particulate }}}{50 \frac{h r}{y x}}
$$

\begin{tabular}{|c|c|c|c|c|}
\hline Constituent & $\begin{array}{l}\text { Emissions } \\
\text { from Burning } \\
\text { Tumbleweeds } \\
\text { (lb/ton) }\end{array}$ & $\begin{array}{l}\text { Amount of } \\
\text { Tumbleweeds } \\
\text { burned } \\
\text { annually } \\
\text { (ton/yr) }\end{array}$ & $\begin{array}{l}\text { Estimated } \\
\text { Emission } \\
\underline{\text { Rate }} \\
\text { (ton/yr) }\end{array}$ & $\begin{array}{l}\text { Estimated } \\
\text { Emission } \\
\text { Rate } \\
\text { (lb/hr)* }\end{array}$ \\
\hline Particulate & 22 & 67.36 & 0.741 & 29.6 \\
\hline $\begin{array}{l}\text { Carbon } \\
\text { Monoxide }\end{array}$ & 309 & 67.36 & 10.4 & 416.3 \\
\hline $\begin{array}{l}\text { Non Methane } \\
\text { VOC }\end{array}$ & 1.5 & 67.36 & 0.051 & 2.02 \\
\hline
\end{tabular}

$$
\text { Emissions }_{\text {particulate rate }}=\frac{0.741 \frac{\mathrm{ton}}{\mathrm{yI}}}{50 \frac{\mathrm{hI}}{\mathrm{yI}}}\left(\frac{2000 \mathrm{Ibs}}{1 \mathrm{ton}}\right)=29.6 \frac{\mathrm{Ibs}}{\mathrm{hI}}
$$

* Based on 1 hour per day, 1 day per week, 50 weeks per year.

Using particulates as an example, the potential estimated emissions are as follows:

$$
\text { Emissions }_{\text {particulate }}=\text { Potential } * \text { Particulate }
$$

$$
\text { Emissions }_{\text {particulate }}=134.7 \frac{\text { ton }}{y r} * 22 \frac{1 b}{\operatorname{ton}} * \frac{1 \text { ton }}{20001 b}=1.48 \frac{\text { ton }}{y r}
$$




$$
\begin{aligned}
& \text { Emissions particulate rate }=\frac{\text { Emissions particulate }}{50 \frac{h r}{y I}} \\
& \text { Emissions particulate rate }=\frac{1.48 \frac{t o n}{y I}}{50 \frac{h r}{y r}}\left(\frac{2000 I b s}{1 t o n}\right)=59.2 \frac{I b s}{h r}
\end{aligned}
$$

\begin{tabular}{|l|l|l|l|l||}
\hline Constituent & $\begin{array}{l}\text { Emissions } \\
\text { from Burning } \\
\text { Tumbleweeds } \\
\text { (b/ton) }\end{array}$ & $\begin{array}{l}\text { Amount of } \\
\text { Tumbleweeds } \\
\text { burned } \\
\text { annually } \\
\text { (ton/yr) }\end{array}$ & $\begin{array}{l}\text { Estimated } \\
\text { Emission } \\
\frac{\text { Rate }}{\text { (ton/yr) }}\end{array}$ & $\begin{array}{l}\frac{\text { Estimated }}{\text { Emission }} \\
\underline{\text { Rate }} \\
(\mathrm{lb} / \mathrm{hr})^{*}\end{array}$ \\
\hline Particulate & 22 & 134.7 & 1.48 & 59.20 \\
\hline $\begin{array}{l}\text { Carbon } \\
\text { Monoxide }\end{array}$ & 309 & 134.7 & 20.81 & 832.5 \\
\hline $\begin{array}{l}\text { Non Methane } \\
\text { VOC }\end{array}$ & 1.5 & 134.7 & 0.101 & 4.041 \\
\hline
\end{tabular}

* Based on 1 hour per day, 1 day per week, 50 weeks per year.

The example calculation is equivalent for the emission units listed below:

200E H-200E002 001

200W H-200W007 001 


\section{EXAMPLE CALCULATIONS FOR CATEGORY J: FUGITTVE EMISSION AREA}

\section{Example Calculations for the Coal Pile Fugitive Emissions}

The example calculations presented in this section are for the coal piles at the $284 \mathrm{E}, 284 \mathrm{~W}$ and 384 Powerhouses. A portion of the spreadsheet used to calculate estimated emissions for particulates is attached, showing the inventory and the calculations for fugitive emissions at the 284E Powerhouse coal pile, which is designated as emission unit 200E J-284E-002.

\section{Assumptions}

Fugitive emission estimates for particulates are based on actual 1994 coal inventory and movement, using methodology found in supplement $\dot{C}$ of AP-42, sections 11.2.3 and 11.2.7. Actual emissions are based on the average inventory of the coal pile. Potential emissions are based on the maximum inventory during the year.

At the 284E Powerhouse, the coal pile was modeled as having a rectangular base, a flat top, and sides with a 45 degree slope. A linear relationship was developed between the volume and the surface area of the pile. Using a nominal density of $800 \mathrm{~kg} / \mathrm{m}^{3}$ for bituminous coal, the surface area of the pile was calculated from the storage inventory for tons of coal. The same approach was used for the coal pile at the 284W Powerhouse. At the 384 Powerhouse, the coal pile was modeled as having two conical piles with a slope of 45 degrees.

Metrology data was obtained from the 600 Area weather station, calculating the peak wind in meters/second at a reference height of 10 meters, and the equivalent friction velocity and erosion potential on a daily basis. Emissions produced when the threshold friction velocity of 1.12 meters/second was exceeded were totaled to estimate fugitive emissions from coal storage. Fugitive emissions from coal movement were also estimated based on loading/unloading operations from rail cars, and transfer operations from the storage pile to the coal bunkers. 
200E Area Coal Pile

\begin{tabular}{|c|c|c|c|c|c|c|c|c|c|c|}
\hline $\mathbf{E}$ & A & B & C & D & $\mathbf{E}$ & $\mathbf{F}$ & $\mathbf{G}$ & $\mathrm{H}$ & 1 & $\mathbf{J}$ \\
\hline 1 & 200E Area Coalpile & & Field & Field & Received & Received & Bunkers & Bunkers & Bunkers & \\
\hline 2 & & Date & Prev. ME In & ME InV & To Field & To Bunkers & Prev. ME In & ME Inv & From Field & Consumed \\
\hline 3 & & $01 / 26 / 94$ & 16425.59 & 16023.74 & 2465.55 & 890.90 & 482.00 & 384.00 & 2867.40 & 3856.30 \\
\hline 4 & & $02 / 23 / 94$ & 16023.74 & 20028.92 & 5528.78 & 1073.36 & 384.00 & 620.00 & 1523.60 & 2360.96 \\
\hline 5 & & $03 / 23 / 95$ & 20028.92 & 22336.90 & 4359.38 & 137.78 & 620.00 & 712.00 & 2051.40 & 2097.18 \\
\hline 6 & & $04 / 27 / 94$ & 22336.90 & 21030.35 & 164.65 & 93.90 & 712.00 & 490.00 & 1471.20 & 1787.10 \\
\hline 7 & & $05 / 24 / 94$ & 21030.35 & 19903.15 & 415.50 & 137.40 & 490.00 & 538.00 & 1542.70 & 1632.10 \\
\hline 8 & & $06 / 21 / 94$ & 19903.15 & 22652.30 & 3370.25 & 44.65 & 538.00 & 548.00 & 621.10 & 655.75 \\
\hline 9 & & $08 / 22 / 94$ & 22652.30 & 28110.55 & 5458.25 & 0.00 & 548.00 & 548.00 & 0.00 & 0.00 \\
\hline 10 & & $09 / 20 / 94$ & 28110.55 & 29960.65 & 1850.10 & 0.00 & 548.00 & 548.00 & 0.00 & 0.00 \\
\hline 11 & & $11 / 29 / 94$ & 29960.65 & 37306.70 & 7346.05 & 0.00 & 548.00 & 548.00 & 0.00 & 0.00 \\
\hline 12 & & $01 / 03 / 95$ & 37306.70 & 39314.55 & 2007.85 & 0.00 & 548.00 & 548.00 & 0.00 & 0.00 \\
\hline 13 & & $01 / 24 / 95$ & 39314.55 & 38268.40 & 178.45 & 641.90 & 548.00 & 368.00 & 1224.60 & 2046.50 \\
\hline 14 & & & & & & & & & & \\
\hline 15 & Totals & & 23377.89 & & 32966.36 & 2377.99 & & & 10077.40 & 12389.39 \\
\hline 16 & Transfer to West & $01 / 03 / 95$ & & -1305.00 & & & & & & \\
\hline 17 & Transfer from 300 & $11 / 29 / 94$ & & 2993.25 & & & & & & 12389.00 \\
\hline 18 & Transfer from 300 & $01 / 03 / 95$ & & 1592.80 & & & & & & \\
\hline 19 & & & & & & & & & & \\
\hline 20 & Average Inventory & & & 22504.24 & & & & & & \\
\hline 21 & Maximum Inventory & & & 40299.95 & & & & & & \\
\hline 22 & & & & & & & & & & \\
\hline 23 & Avg Volume $\left(m^{\wedge} 3\right)$ & & & 25573.00 & & & & & & \\
\hline 24 & Max Volume $\left(m^{\wedge} 3\right)$ & & & 45795.40 & & & & & & \\
\hline 25 & & & & & & & & & & \\
\hline 26 & Avg Surf Area $\left(m^{\wedge} 2\right)$ & & & 4394.26 & & & & & & \\
\hline 27 & Max Surf Area $\left(m^{\wedge} 2\right)$ & & & 6480.07 & & & & & & \\
\hline 28 & & & & & & & & & & \\
\hline 29 & Load/unload Coal & & & & 38857.41 & & & & & \\
\hline 30 & Transfer to Bunker & & & & & 12455.39 & & & & \\
\hline
\end{tabular}




\begin{tabular}{|c|c|c|c|c|c|c|c|c|c|c|c|c|c|c|c|c|}
\hline $\mathbf{c}$ & A & $\mathbf{B}$ & C & D & E & $\mathbf{F}$ & G & $\bar{H}$ & $T$ & $\mathrm{~J}$ & $\mathbf{K}$ & $\mathbf{L}$ & $M$ & $N$ & 0 & $\mathbf{P}$ \\
\hline 1 & Assumptlons & 200E Area & & & & & & & & & & & & & & \\
\hline 2 & \begin{tabular}{|l} 
Uncrusted \\
\end{tabular} & Coal Pile & & & & & & & & & & & & & & \\
\hline 3 & Particle Size & Mulliplier & 1 & & & & & $\dot{ }$ & & & & & & & & \\
\hline 4 & Threshold & Friclion Vel. & 1.12 & $\mathrm{~m} / \mathrm{s}$ & & & & & & & & & & & & \\
\hline 5 & & & & & & & & & & & & & & & & \\
\hline 6 & Height of & & & & & & & & & & & & & & & \\
\hline 7 & Anemometer & & & 50 & teet & & & Emissions & & & & & & & & \\
\hline 8 & us/ur & $\%$ & & 15.24 & melers & & & 1994 (tons) & $\mathbf{P M}$ & PM10 & & & & . & & \\
\hline 9 & 0.2 & 31 & & & & & & Storage & & & & & & & & \\
\hline 10 & 0.6 & 51 & & & & & & Aclual & 1.05 & 0.53 & & & & & & \\
\hline 11 & 0.9 & 15 & & & & & & Polenlial & 1.55 & 0.78 & & & & & & \\
\hline 12 & 1.1 & 3 & & & & & & Movement & & & & & & & & \\
\hline 13 & & & & & & & & Aclual & 0.15 & 0.07 & & & & & & \\
\hline 14 & Area of plle & & & & Maximum & & & & & & & & & & & \\
\hline 15 & Average Invenlory & & 22504.24 & Lons & Lenglh & 273 & II & Total & 1.20 & 0.60 & & & & & & \\
\hline 16 & Maximum Inventor & & 40299.95 & Ions & Width & 161 & II & & & & & & & & & \\
\hline 17 & Avg Surface Area & & 4,394 & sqmelers & HeighI & 40 & II & & & & & & & & & \\
\hline 18 & Max Surface Area & & 6,480 & sq molers & & & & & & & & & & & & \\
\hline 19 & & & & & & & & & & & & & & & & \\
\hline 20 & & Peak Wind & & Equivalont & & & & & & & & & & & & \\
\hline 21 & & & & Filclion & & & & Eroulon & & & & & & & & \\
\hline 22 & & Fautest milo & Fasteut millo, & Volocity. & & & & Polonllal & & & & & & & & \\
\hline 23 & & ut & $u \div(10 \mathrm{~m})$ & $u^{\circ}(\operatorname{m} / 0)$ & & & & $(\mathrm{g} \ln 2)$ & & & & & & & & \\
\hline 24 & Date & $(m / s)$ & $(\pi / 4)$ & unfur $=0.2$ & Helur $=0.6$ & uefur $=0.0$ & usfur $=1.1$ & uedur $=0.2$ & ue/ur $=0.6$ & ualur $=0.9$ & uelur $=1.1$ & uefur $=0.2$ & unfur $=0.6$ & ualur $=0.9$ & uelur $\mathrm{m} 1.1$ & Total \\
\hline 25 & $01-J a n-94$ & 4.917 & 4.66 & 0.09 & 0.28 & 0.42 & 0.51 & 0.00 & 000 & 0.00 & 0.00 & 0.00 & 0.00 & 0.00 & 0.00 & 0.00 \\
\hline 26 & 02-Jan-94 & 6.705 & 6.35 & 0.13 & 0.30 & 0.57 & 0.70 & 0.00 & 0.00 & 0.00 & 0.00 & 0.00 & 0.00 & 0.00 & 0.00 & 0.00 \\
\hline 27 & 03-Jan-94 & 6.258 & 5.93 & 0.12 & 0.36 & 0.53 & 065 & 0.00 & 000 & 0.00 & 0.00 & 0.00 & 0.00 & 0.00 & 0.00 & 0.00 \\
\hline 28 & 04-Jan-94 & 17.880 & 18.94 & 0.34 & 1.02 & 1.52 & 1.86 & 0.00 & 0.00 & 19.62 & 50.65 & 0.00 & 0.00 & 0.01 & .001 & 0.03 \\
\hline 29 & 05-Jan-94 & 12.069 & 11.44 & 0.23 & 0.69 & 1.03 & 1.26 & 0.00 & 0.00 & 0.00 & 4.55 & 0.00 & 0.00 & 000 & 0.00 & 0.00 \\
\hline 30 & 06-Jan-94 & 10.281 & 9.74 & 0.19 & 0.58 & 0.88 & 1.07 & 0.00 & 0.00 & 0.00 & 0.00 & 0.00 & 0.00 & 0.00 & 0.00 & 0.00 \\
\hline 31 & $07-\operatorname{Jan}-94$ & 5.364 & 5.08 & 0.10 & 0.30 & 0.46 & 0.56 & 000 & 000 & 0.00 & 0.00 & 0.00 & 0.00 & 0.00 & 0.00 & 0.00 \\
\hline 32 & 08-Jan-94 & 6.258 & 5.93 & 0.12 & 0.36 & 0.53 & 0.65 & 0.00 & 0.00 & 0.00 & 0.00 & 0.00 & 0.00 & 0.00 & 0.00 & 0.00 \\
\hline 33 & 09-Jan-94 & 8.493 & 8.05 & 0.16 & 0.48 & 0.72 & 0.89 & 0.00 & 0.00 & 0.00 & 0.00 & 0.00 & 0.00 & 0.00 & 0.00 & 0.00 \\
\hline 34 & 10-Jan-94 & 5.364 & 5.08 & 0.10 & 0.30 & 0.46 & 0.56 & 0.00 & 0.00 & 0.00 & 0.00 & 0.00 & 0.00 & 0.00 & 0.00 & 0.00 \\
\hline 35 & 11-Jan-94 & 8.046 & 7.62 & 0.15 & 0.46 & 0.69 & 0.84 & 0.00 & 0.00 & 0.00 & 0.00 & 0.00 & 0.00 & 0.00 & 0.00 & 0.00 \\
\hline 36 & 12-Jan-94 & 10.728 & 10.16 & 0.20 & 0.61 & 091 & 1.12 & 0.00 & 0.00 & 0.00 & 0.00 & 0.00 & 0.00 & 0.00 & 0.00 & 0.00 \\
\hline 37 & 13-Jan-94 & 4.470 & 4.21 & 0.08 & 0.25 & 0.38 & 0.47 & 0.00 & 0.00 & 0.00 & 0.00 & 0.00 & 0.00 & 0.00 & 0.00 & 0.00 \\
\hline 38 & 14-Jan-94 & 5.364 & 508 & 0.10 & 0.30 & 0.46 & 0.56 & 0.00 & 0.00 & 0.00 & 0.00 & 0.00 & 0.00 & 0.00 & 0.00 & 0.00 \\
\hline 39 & 15-Jan-94 & 10.728 & 10.16 & 0.20 & 0.61 & 0.91 & 1.12 & 0.00 & 0.00 & 0.00 & 0.00 & 0.00 & 0.00 & 0.00 & 0.00 & 0.00 \\
\hline 40 & 16-Jan-94 & 9.834 & 9.32 & 0.19 & 0.56 & 0.04 & 8.02 & 0.00 & 0.00 & 0.00 & 0.00 & 0.00 & 0.00 & 0.00 & 0.00 & 0.00 \\
\hline 41 & 17-Jan-94 & 4.917 & 4.66 & 0.09 & 0.28 & 0.42 & 0.51 & 0.00 & 0.00 & 0.00 & 000 & 0.00 & 0.00 & 0.00 & 0.00 & 0.00 \\
\hline 42 & 18-Jan-94 & 3.576 & 3.39 & 0.07 & 0.20 & 0.30 & 0.37 & 0.00 & 0.00 & 0.00 & 0.00 & 0.00 & 0.00 & 0.00 & 0.00 & 0.00 \\
\hline 43 & 19-Jan-94 & 6.258 & 5.93 & 0.12 & 0.36 & 0.53 & 0.65 & 0.00 & 0.00 & 0.00 & 0.00 & 0.00 & 0.00 & 0.00 & 0.00 & 0.00 \\
\hline 44 & 20-Jan-94 & 7.152 & 6.78 & 0.14 & 0.41 & 0.61 & 0.75 & 0.00 & 0.00 & 0.00 & 0.00 & 0.00 & 0.00 & 0,00 & 0.00 & 0.00 \\
\hline 45 & 21-Jan-94 & 4.470 & 4.24 & 0.08 & 0.25 & 0.38 & 0.47 & 0.00 & 0.00 & 0.00 & 0.00 & 0.00 & 0.00 & 0.00 & 0.00 & 0.00 \\
\hline 46 & 22-Jan-94 & 7.599 & 7.20 & 0.14 & 0.43 & 0.65 & 0.79 & 0.00 & 0.00 & 0.00 & 0.00 & 0.00 & 0.00 & 0.00 & 0.00 & 0.00 \\
\hline 47 & 23-Jan-94 & 7.152 & 6.78 & 0.14 & 0.41 & 0.61 & 0.75 & 0.00 & 0.00 & 0.00 & 0.00 & 0.00 & 0.00 & 0.00 & 0.00 & 0.00 \\
\hline 48 & 24-Jan-94 & 8.940 & 8.47 & 0.17 & 0.51 & 0.76 & 0.93 & 0.00 & 0.00 & 0.00 & 0.00 & 0.00 & 0.00 & 0.00 & 0.00 & 0.00 \\
\hline 49 & 25-Jan-94 & 4.023 & 3.01 & 0.08 & 0.23 & 0.34 & 0.42 & 0.00 & 0.00 & 0.00 & 0.00 & 0.00 & 0.00 & 0.00 & 0.00 & 0.00 \\
\hline 50 & 26-Jan-94 & 3.576 & 3.39 & 0.07 & 0.20 & 0.30 & 0.37 & 0.00 & 0.00 & 0.00 & 0.00 & 0.00 & 0.00 & 0.00 & 0.00 & 0.00 \\
\hline 51 & 27-Jan-94 & 4.023 & 3.81 & 0.08 & 0.23 & 0.34 & 0.12 & 0.00 & 0.00 & 0.00 & 0.00 & 0.00 & 0.00 & 0.00 & 0.00 & 0.00 \\
\hline 52 & 28-Jan-94 & 4.470 & 4.24 & 0.00 & 0.25 & 0.30 & 0.47 & 0.00 & 0.00 & 0.00 & 0.00 & 0.00 & 0.00 & 0.00 & 0.00 & 0.00 \\
\hline 53 & 29-Jan-94 & 5.364 & 5.08 & 0.10 & 0.30 & 0.46 & 0.56 & 0.00 & 0.00 & 0.00 & 0.00 & 0.00 & 0.00 & 0.00 & 0.00 & 0.00 \\
\hline 54 & 30-Jan-94 & 5.364 & 5.08 & 0.10 & 0.30 & 0.46 & 0.56 & 0.00 & 0.00 & 0.00 & 0.0 & 0.00 & 0.00 & 0.00 & 0.00 & 0.00 \\
\hline 55 & 31-Jan-94 & 4.023 & 3.89 & 0.08 & 0.23 & 0.34 & 0.42 & 0.00 & 0.00 & 0.00 & 0.00 & 0.00 & 0.00 & 0.00 & 0.00 & 0.00 \\
\hline
\end{tabular}




\section{EXAMPLE CALCULATIONS FOR CATEGORY K: PAINT BOOTHS}

The following example describes the methodology for calculating emissions related to solvent use in th. paint shops. Emissions from paint cleanup activities are from the solvents Mineral Spirits and Methyl Ethyl Ketone. The calculations assume that all the solvents are evaporated to the atmosphere. Annual usage of the solvents is 455 -gallon drums of Methyl Ethyl Ketone and 2 55-gallon drums of Mineral Spirits per year. The following example is for the 200 East Area Paint Shop.

$$
\begin{aligned}
& \text { Rate } e_{\text {methyl ethyl ketone }}=4 *\left(55 \frac{g a l}{y I}\right)=220 \frac{g a l}{y I} \\
& \text { Rate } e_{\text {mineral spirits }}=2 *\left(55 \frac{g a I}{y I}\right)=110 \frac{g a I}{y I}
\end{aligned}
$$

The density of Methyl Ethyl Ketone and Mineral Spirits is 6.72 and 6.62 pounds per gallon respectively. The annual usage in pounds per year is equal to the rate multiplied by the density for each material.

$$
\begin{aligned}
& \rho_{\text {methyl ethyl ketone }}=6.72 \frac{1 b}{g a l} \\
& \rho_{\text {mineral spirits }}=6.62 \frac{I b}{g a l} \\
& \text { Annual Usage }=\text { Rate } e_{\text {methyl ethyl ketone }} * \rho_{\text {methyl ethyl ketone }} \\
& \text { Annual Usage } e_{\text {methyl ethyl ketone }}=220 \frac{\mathrm{gal}}{\mathrm{yI}} * 6.72 \frac{\mathrm{Ib}}{\mathrm{gal}}=1478.4 \frac{\mathrm{Ib}}{\mathrm{yI}} \\
& \text { Annual Usage } \text { methyl ethyl ketone }=1478.4 \frac{1 b}{y r} * \frac{1 \text { ton }}{20001 \mathrm{bs}}=0.739 \frac{\text { ton }}{y r} \\
& \text { Annual Usage } e_{\text {mineral spirits }}=R a t e_{\text {mineral spirits }} * \rho_{\text {mineral spirits }}
\end{aligned}
$$




$$
\text { Annual Usage } e_{\text {mineral spirits }}=110 \frac{g a l}{y I} * 6.62 \frac{I b}{g a l}=728.2 \frac{I b}{y I}
$$

$$
\text { Annual Usage } e_{\text {mineral spirits }}=728.2 \frac{1 b}{y I} * \frac{1 \mathrm{ton}}{20001 \mathrm{bs}}=0.364 \frac{\mathrm{ton}}{\mathrm{yI}}
$$

The actual emissions on an annual basis are the same as the maximum emissions. The actual release per hour is calculated based on releases occurring 4 hours per day, 5 days per week, 50 weeks per year, or 1000 hours per year.

$$
\begin{aligned}
& \text { Emissions }_{\text {methyl ethyl ketone }}=\frac{\text { Aninual Usage }}{1000 \frac{h I}{y I}} \\
& \text { Emissions }_{\text {methyl ethyl ketone }}=\frac{1478.4 \frac{I b}{y I}}{1000 \frac{h I}{y I}}=1.478 \frac{l b}{h r} \\
& \text { Emissions }_{\text {mineral spirits }}=\frac{\text { Annual Usage }}{1000 \frac{h I}{y I}} \\
& \text { Emissions }_{\text {mineral spirits }}=\frac{728.2 \frac{1 b}{y r}}{1000 \frac{h r}{y r}}=0.728 \frac{1 b}{h r}
\end{aligned}
$$


The example calculation is equivalent for the emission units listed below:

$100 \mathrm{~N} \mathrm{~K}-1143015$

$100 \mathrm{~N} \mathrm{~K}-1517 \mathrm{~N} 021$

200E K-200E001 001

200W K-272S 001

200W K-200W003 001

200W K-2715EC 001

$300 \mathrm{~K}-3709001$

$400 \mathrm{~K}-4722 \mathrm{C} 001$ 


\section{EXAMPLE CALCULATIONS FOR CATEGORY M: WELDING BOOTH}

The following example describes the methodology for calculating emissions related to metal work. Emissions from metalworking activities is primarily in the form of particulates produced by abrasive cutting and grinding. The following example is for the 242AC Pipefitters Shop.

\section{Methodology}

1) Assume the concentration of particulate matter (PM) generated from the activity is equal to the Threshold Unit Valve (TLV) for particulates not otherwise classified (PNOC). The TLV for $\mathrm{PNOC}$ is 10 micrograms per cubic meter.

2) Estimate the total building volume for the applicable building.

3) - Estimate the building volume of the area where metalworking is performed.

4) Determine the percent of building exhaust volume containing metalworking emissions by ratio of metalworking building volume to total building volume.

5) Apply the metalworking volume percentage to the total building exhaust rate which is based on the total number of building exhaust units and the size of the exhaust units.

6) Multiply the total metalworking area exhaust rate by the PNOC TLV to determine mass emissions of particulate matter.

Example calculation:

$$
\text { Concentration of Particulate } \operatorname{Matter}\left(C_{P M}\right)=10 \frac{\mu g}{\mathrm{~m}^{3}}
$$

The total estimated building volume:

$$
\text { Volume (total building) }=24000 \mathrm{ft}^{3}
$$

The building volume of the area where metalworking is performed:

$$
\text { Volume (working area) }=24000 \mathrm{ft}^{3}
$$


The percent of building exhaust volume containing metalworking emissions:

$$
\operatorname{Ratio}(\text { Area })=\frac{\text { Volume }(\text { total building })}{\text { Volume }(\text { working area })}=\frac{24000 \mathrm{ft}^{3}}{24000 \mathrm{ft}^{3}}=1
$$

The building exhaust rate was determined by multiplying the total number of building exhausters by the average building exhauster flowrate. The following calculations give the potential to emit assum continuous operation.

$$
\begin{gathered}
\text { Number of Exhausters }=1 \\
\text { Flowrate of exhauster }=91900 \frac{f t^{3}}{h r}
\end{gathered}
$$

Total Flowrate=(number of exhausters) (Flowrate of exhausters)

$$
\begin{gathered}
\text { Total Flowrate }=(1)\left(91900 \frac{f t^{3}}{h I}\right) \\
\text { Flowrate (metalworking) }=\text { Ratio (Area)*Flowrate }(\text { total })=(1)\left(91900 \frac{f t^{3}}{h r}\right) \\
\text { Flowrate (metalworking activities })=91900 \frac{f t^{3}}{h r}
\end{gathered}
$$

Emissions (metalworking) $=C_{P M} *$ Flowrate (metalworkingactivities)

$$
\text { Emissions }=\left(10 \frac{\mu g}{m 3}\right)\left(91900 \frac{f t^{3}}{h r}\right)\left(\frac{0.028 m^{3}}{f t^{3}}\right)\left(\frac{g}{1.0 * 10^{06} \mu g}\right)\left(\frac{0.00220 \mathrm{lbs}}{g}\right)
$$




$$
\text { Emissions }=5.74 E-05 \frac{l b}{h I}
$$

$$
\text { Emissions }=\left(5.74 E-05 \frac{l b}{h r}\right)\left(\frac{1 \text { ton }}{20001 b s}\right)\left(\frac{24 h r s}{d a y}\right)\left(\frac{365 \text { days }}{y I}\right)=2.51 E-04 \frac{\text { ton }}{y r}
$$

Actual operations for the generation of particulates is 2 hours per day, 5 days per week, and 50 weeks per year, or 500 hours per year. There are 8760 hours in a year.

Time (actual operations) $=500 \mathrm{hrs}$

Time (annual) $=8760 \mathrm{hrs}$

$$
\text { Ratio }=\frac{\text { Time }(\text { actual })}{\text { Time }(\text { annual })}=\frac{500 h r}{8760 h r}=0.057
$$

Although the hourly emission rate estimate would not change during actual operation, the annual quantity emitted would change. The annual emissions were multiplied by the time ratio.

$$
\text { Emissions (actual) }=\text { Emissions (metalworking) (TimeRatio) }
$$

$$
\text { Emissions (actual) }=2.51 E-04 \frac{\text { ton }}{y r} * 0.57=1.44 E-05 \frac{\text { ton }}{y x}
$$


The emission calculations presented above are equivalent for the emission points listed below. The calculations for these emission points can be provided upon request.

\begin{tabular}{|c|c|c|}
\hline Emission Point & Emission Point & Emission Point \\
\hline $100 \mathrm{~N} M-105 \mathrm{~N} 001$ & 200E M-2244B 001 & $600 \mathrm{M}-6290001$ \\
\hline $100 \mathrm{~N} M-151 \mathrm{~N} 020$ & 200E M-2247B 001 & \\
\hline $100 \mathrm{~N}$ M-1518N 019 & 200E M-242AC 001 & \\
\hline 100N M-1705N 103 & 200E M-272E 001 & \\
\hline $200 \mathrm{~W} M-200 \mathrm{~W} 005001$ & $300 \mathrm{M}-305 \mathrm{~A} 001$ & \\
\hline $200 \mathrm{~W}$ M-200W006 001 & $300 \mathrm{M}-328003$ & \\
\hline 200W M-272W 001 & $300 \mathrm{M}-338001$ & \\
\hline 200W M-272WA 001 & & \\
\hline 200W M-277W 001 & $400 \mathrm{M}-4704 \mathrm{~N} 001$ & \\
\hline 200W M-275W 001 & & \\
\hline 200W M-2722W 001 & & \\
\hline
\end{tabular}


The following example calculation is for actual and potential emissions associated with sandblast operations. The primary emissions will consist of particulate from the breakdown of the blasting med Approximately $5-10 \%$ of the blast media (Idaho Garnet) will breakdown and become particul emissions during operations. The example calculation is for the 200W Carpenter Shop. Sandblast is done approximately 12 hours/week, 50 weeks/year, and the annual quantity of blast media used is approximately 45 ton/year.

$$
\begin{aligned}
& \text { Quantity }=45 \frac{\text { ton }}{y I} \\
& \text { BIeakdown } n_{\text {blast media }}=0.1 \\
& \text { Emissions }_{\text {actual }}=\text { Quantity } * \text { Breakdown } \text { blast media }_{\text {a }} \\
& \text { Emissions }_{\text {actual }}=45 \frac{\text { ton }}{y x} * 0.1=4.5 \frac{\text { ton }}{y r} \\
& \text { Time }_{\text {actual }}=12 \frac{h r S}{\text { week }} * 50 \frac{\text { weeks }}{y I}=600 \frac{h r S}{y I} \\
& \text { Time }_{\text {maximum }}=24 \frac{h r S}{d a y} * 365 \frac{\text { days }}{y r}=8760 \frac{h r S}{y r} \\
& \text { Emissions }_{\text {actual hourly }}=\frac{\text { Emissions }_{\text {actual }}}{\text { Time }_{\text {actual }}}
\end{aligned}
$$




$$
\begin{aligned}
& \text { Emissions }_{\text {actual hourly }}=\frac{4.5 \frac{\mathrm{ton}}{y r}}{600 \frac{h r}{y r}}=0.0075 \frac{\mathrm{ton}}{h r} \\
& \text { Emissions actual hourly }=0.0075 \frac{\mathrm{ton}}{y r} * 2000 \frac{\mathrm{Ibs}}{\mathrm{ton}}=15 \frac{\mathrm{lbs}}{\mathrm{hr}} \\
& \text { Emissions }_{\text {potential }}=\frac{\text { Time }_{\text {maximum }}}{\text { Time }_{\text {actual }}} * \text { Emissions }_{\text {actual }} \\
& \text { Emissions }_{\text {potential }}=\frac{8760 \frac{h r}{y I}}{600 \frac{h r}{y I}} * 4.5 \frac{\text { ton }}{y r}=65.7 \frac{\text { ton }}{y r}
\end{aligned}
$$

This example calculation is equivalent for the emission units listed below:

$100 \mathrm{~N} \mathrm{~N}-1516 \mathrm{~N} 017$

200E N-2242B 001

200E N-275E 001

200W N-2715EC 003

200W N-200W001 001

200W N-27TW 002

$300 \mathrm{~N}-328001$

$300 \mathrm{~N}-328002$

$300 \mathrm{~N}-3713001$

$300 \mathrm{~N}-3722002$

$400 \mathrm{~N}-4722 \mathrm{~B} 001$

$400 \mathrm{~N}-4722 \mathrm{C} 003$ 
EXAMPLE CALCULATION FOR CATEGORY P: PROCESS EMISSION POINT

The calculations presented in section 2 of this appendix demonstrate examples of Category $P$. 


\section{EXAMPLE CALCULATION FOR}

\section{CATEGORY R: LABORATORY EMISSION POINT}

Example calculation using emission unit $1706 \mathrm{KEL}$ for the hexane component based on calendar year 1994.

$$
\begin{gathered}
\text { Rate }=1.72 E+05 \frac{\mathrm{ml}}{\mathrm{yI}} \\
\rho=0.65937 \frac{\mathrm{g}}{\mathrm{mI}} \\
\text { Carbon } \text { effectiveness }=0.99 \\
D F=\frac{1}{1-\text { Carbon }_{\text {effectiveness }}} \\
D \quad \\
D F=\frac{1}{1-0.99}=100
\end{gathered}
$$

$\mathrm{DF}=$ Decontamination Factor

The actual emissions, controlled and uncontrolled, are based on 8 hours per day, 5 days per week, and 50 weeks per year operation.

$$
\begin{aligned}
& \text { Emissions } \text { uncontrolled }=\text { Rate } * \rho * \frac{11 b}{454 g} \\
& \text { Emissions }_{\text {uncontrolled }}=1.72 E+05 \frac{\mathrm{ml}}{\mathrm{yI}} * 0.65937 \frac{\mathrm{g}}{\mathrm{ml}} * \frac{1 \mathrm{lb}}{454 \mathrm{~g}}=250 \frac{\mathrm{Ib}}{\mathrm{yr}} \\
& \text { Emissions }_{\text {uncontrolled }}=250 \frac{1 b}{y I} * \frac{1 \text { ton }}{20001 b s}=0.125 \frac{\text { ton }}{y x} \\
& \text { APP A-54 }
\end{aligned}
$$




$$
\begin{aligned}
& \text { Emissions }_{\text {controlled }}=\frac{\text { Emissions }_{\text {uncontrolled }}}{D F}=\frac{0.125 \frac{\text { ton }}{y I}}{100}=1.25 E-03 \frac{\text { ton }}{y I} \\
& \text { Emissions }_{\text {uncontrolled }}=\frac{\text { Emissions }_{\text {uncontrolled }}}{250 \frac{d a y}{y r} * 8 \frac{h I}{d a y}} \\
& \text { Emissions }_{\text {uncontrolled }}=\frac{250 \frac{I b s}{y I}}{250 \frac{d a y}{y r} * 8 \frac{h r}{d a y}}=0.125 \frac{I b s}{h r}
\end{aligned}
$$

The potential emissions are based on 24 hours per day, 365 days per year of operation.

$$
\begin{aligned}
& \text { Emissions uncontrolled }\left(\text { potential) }=\text { Rate } * \rho * 1 \frac{1 b}{454 g} * \frac{24 \frac{h r}{d a y} * 365 \frac{d a y}{y r}}{8 \frac{h r}{d a y} * 250 \frac{d a y}{y I}}\right. \\
& \text { Emissions }_{\text {uncontrolled }}=1.72 E+05 \frac{\mathrm{ml}}{\mathrm{yI}} * 0.65937 \frac{\mathrm{g}}{\mathrm{ml}} * \frac{1 \mathrm{lb}}{454 \mathrm{~g}} * \frac{24 \frac{\mathrm{hr}}{\mathrm{day}} * 365 \frac{\mathrm{day}}{\mathrm{yr}}}{8 \frac{h I}{d a y} * 250 \frac{d a y}{y I}} \\
& \text { Emissions uncontrolled }\left(\text { potential) }=0.125 \frac{1 b}{h r}\right.
\end{aligned}
$$

Emissions $_{\text {uncontrolled }}($ potential $)=0.125 \frac{1 b}{h r} * \frac{1 \text { ton }}{20001 b s}=0.547 \frac{\text { ton }}{\text { year }}$ 
Hanford Site Air

Operating Permit Application

$$
\begin{gathered}
\text { Emissions } \text { controlled }(\text { potential })=\frac{\text { Emissions }_{\text {uncontrolled }} \text { (potential) }}{D F} \\
\text { Emissions } \text { controlled }_{\text {potential })}=\frac{0.125 \frac{\mathrm{lb}}{\mathrm{hI}}}{100}=1.25 \mathrm{E}-03 \frac{\mathrm{lb}}{\mathrm{hr}}
\end{gathered}
$$

Emissions $_{\text {controlled }}($ potential $)=1.25 E-03 \frac{l b}{h I} * \frac{\text { ton }}{20001 b s} * 8760 \frac{h r s}{y I}=5.47 E-03 \frac{\text { ton }}{y I}$

The example calculation is equivalent for the emission units listed below:

100K R-1706KEL 001 


\section{EXAMPLE CALCULATIONS FOR CATEGORY S: LABORATORIES}

(PROCESS OR QA/QC RELATED)

The example calculations presented in this section are for the WSCF Laboratory using the component hexane as an example.

\section{Assumptions}

Emission estimates are based upon actual chemical usage in the labs as provided by Westinghouse

Estimates assume $100 \%$ chemical loss to the atmosphere.

Emission estimates are equivalent for 1992, 1993, and 1994 with the exception of xylene and toluene

Compounds which have minimal vapor pressures such as nitric acid; hydrogen peroxide, phosphoric acid, potassium hydroxide, and other similar compounds were not included in the emission calculations. It is unlikely that emission of these chemicals would occur due to the amount and nature of their use in laboratories.

For ammonium compounds, it was assumed that 100 percent of the emissions are ammonia.

Pounds per hour $(\mathrm{lb} / \mathrm{hr})$ estimates are based on 8 hours per day, 5 days per week, 50 weeks per year.

It is assumed that actual $\mathrm{lb} / \mathrm{hr}$ emissions are potential $\mathrm{lb} / \mathrm{hr}$ emissions are equivalent.

sotential tons per year and pounds per year are based on 24 hours per day, 365 days per year.

No controls are present for non-radiological pollutants.

Actual Emissions

Pounds per hour $(\mathrm{lb} / \mathrm{hr})$ estimates are based on 8 hours per day, 5 days per week, 50 weeks per year.

$$
\text { Pounds Per Year }\left(\frac{I b}{y I}\right)=\text { Usage Rate }\left(\frac{m L}{y I}\right) * \operatorname{Density}\left(\frac{g}{m L}\right) *\left(\frac{1 I b}{454 g}\right)
$$

Using hexane as an example:

$$
\text { Pounds Per Year }=32,000 \frac{\mathrm{mL}}{\mathrm{year}} * 0.66 \frac{\mathrm{g}}{\mathrm{mL}} * \frac{1 \mathrm{lb}}{454 \mathrm{~g}}=46.52 \frac{1 \mathrm{~b}}{\mathrm{yI}} \text { (Hexane) }
$$


Tons Per Year $($ Hexane $)=$ Emissions $\left(\frac{I b}{y I}\right) *\left(\frac{1 \text { ton }}{2000 I b}\right)$

Tons Per Year $=\left(46.52 \frac{\mathrm{lbs}}{y r}\right) *\left(\frac{1 \text { ton }}{2000 \mathrm{lbs}}\right)=0.023\left(\frac{\text { tons }}{y r}\right)$ (hexane)

\section{Potential Emissions}

Potential tons per year and pounds per year are based on 24 hours per day, 365 days per year.

Pounds Per Hour $\left(\frac{l b s}{h r}\right)=$ Actual Pounds Per Hour $\left(\frac{l b}{h r}\right)$

Pounds Per $\operatorname{Year}\left(\frac{l b}{y I}\right)=$ Pounds Per Hour $\left(\frac{l b}{h I}\right) * 8760\left(\frac{h r S}{y I}\right)$

Pounds Pex $\operatorname{Year}\left(\frac{I b s}{y I}\right)=\left(0.23 \frac{I b s}{h I}\right) *\left(8760 \frac{h I}{y I}\right)=203.8\left(\frac{I b s}{y I}\right)$ (hexane)

Tons Per $\operatorname{Year}\left(\frac{\text { ton }}{y I}\right)=$ Potential Emissions $\left(\frac{l b s}{y I}\right) *\left(\frac{1 \text { ton }}{20001 b s}\right)$

Tons Per Year $=\left(203.8 \frac{l b s}{y I}\right) *\left(\frac{1 \text { ton }}{2000 I b s}\right)=\left(0.102 \frac{\text { ton }}{y I}\right)$ (hexane) 
The above calculations were performed for each of the components listed in the table below:

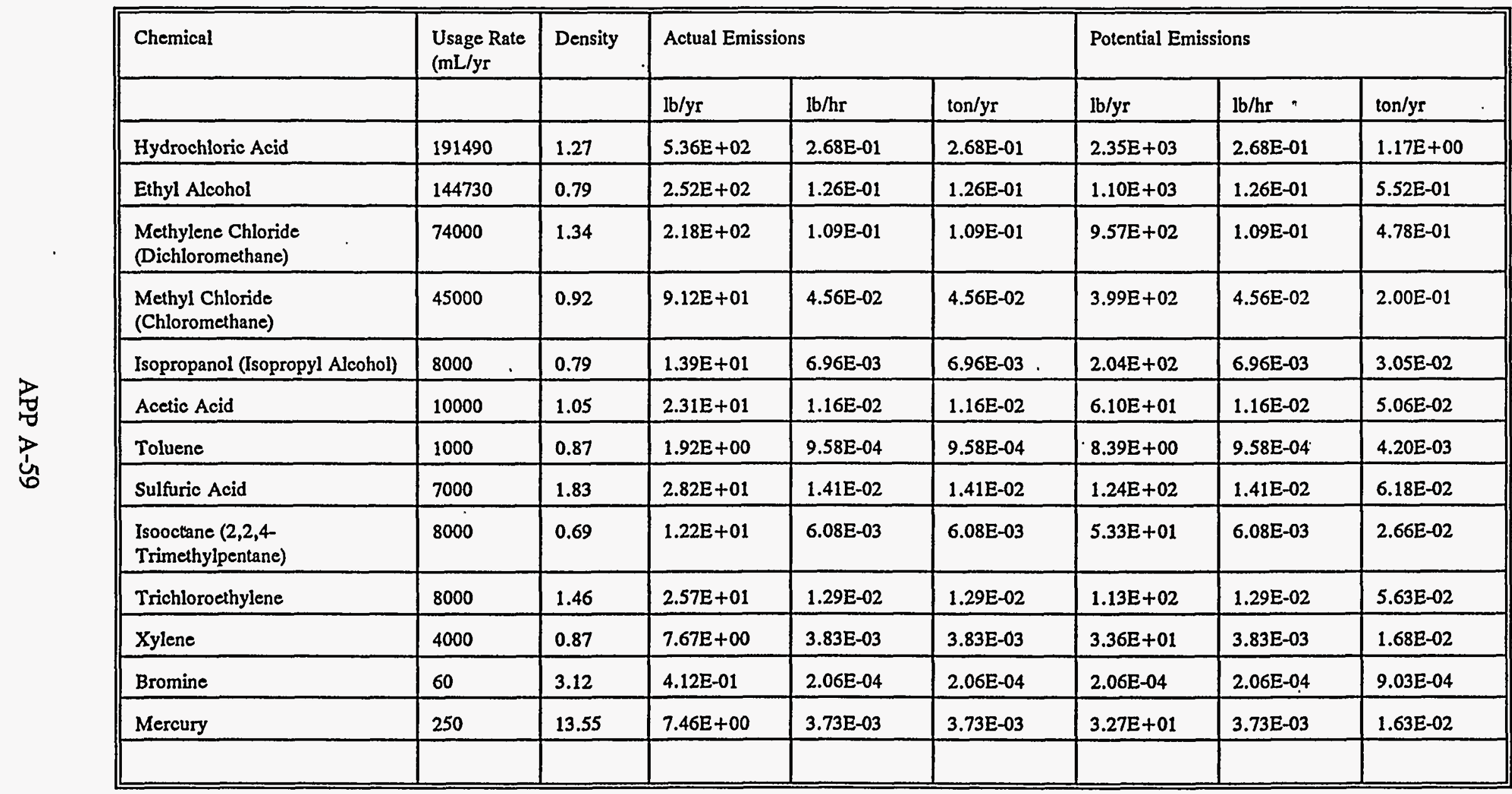


The following sources are also laboratories. The emissions calculations for these laboratories were calculated equivalent to those presented above. The calculations can be provided upon request.

200W S-222SA 001

200W S-296S021 001

$600 \mathrm{~S}-6266001$ 
APPENDIX B

COMPLIANCE PLAN SCHEDULE 
Hanford Site Air

Operating Permit Application
DOE/RL-95-07, Rev. 0 05/95

This page intentionally left blank. 


\section{COMPLIANCE PLAN SCHEDULE}

Milestone Number

A-10-01

A-20-01

A-20-02

A-20-03

A-30-xx

$A-40-x x$

A-50- $x x$
Description of Corrective Action

Assess and correct the various air management systems and procedures to ensure all notices and reports are submitted to the appropriate air agencies in a timely manner.

NESHAP Federal Facility Compliance Agreement (incorporated by reference, as signed February 7,1994 )

PUREX will assess and discuss with Ecology and EPA contimuous NOx monitoring requirements during deactivation

A schedule for re-assessing the $\mathrm{SO}_{2}$ emissions from the 300 Area boilers, emission points $300 \mathrm{~F}-384002$, 300 F-384 005, and 300 F-384 006 and to schedule the actions necessary to bring the emission units into compliance will be developed.
Target Date

$12 / 29 / 95$

As Agreed

08/01/95

08/01/95

TBD

TBD

TBD 
Hanford Site Air

Operating Permit Application
DOE/RL-95-07, Rev. 0

05/95

\section{APPENDIX C}

\section{AREAS}


Hanford Site Air

Operating Permit Application
DOE/RL-95-07, Rev. 0 $05 / 95$

\section{APPENDIX C}

\section{CONTENTS}

C-1 100K

C-2 100N 


\title{
APPENDIX C-1
}

\author{
$100 \mathrm{~K}$ \\ Chapter I. General Information
}

\section{I-B.1. Facility Description}

The 100K Basins were constructed in the early 1950's to store irradiated fuel from nuclear reactor operations. The $\mathrm{K}$ Basins consist of two irradiated fuel storage basins, $105 \mathrm{KE}$ and $105 \mathrm{KW}$, that adjoined the $105 \mathrm{KE}$ and $105 \mathrm{KW}$ reactor buildings. Other than the fuel storage basins, the reactor buildings are deactivated. Other significant $\mathrm{K}$ Basin structures consist of the $1717 \mathrm{~K}$ maintenance shop and the $1706 \mathrm{KE}$ development laboratory.

The 105KE and 105KW Basins presently contain 2,324 short tons of irradiated fuel. The fuel currently is stored under water in two reinforced concrete basins. Each basin is 125 feet long and 67 feet wide. Fuel canisters are stored in racks in the basins and water levels are maintained at a minimum of 10 feet above the canisters to cool the fuel and provide radiation shielding for personnel. The water in each basin is recirculated through a closed water cooling system. In addition, filters and ion exchange systems maintain water clarity and remove radionuclides.

The $105 \mathrm{KW}$ Basin presently contains zirconium clad irradiated fuel with enrichment up to and above 0.95 percent Uranium ${ }^{235}$. In the late 1970's, the $105 \mathrm{KW}$ Basin was emptied, decontaminated, and the basin walls coated with an epoxy. The 105KW Basin was placed back into operation in 1981 and all fuel stored within the basin has been encapsulated in canisters to prevent the spread of contamination from exposed degrading fuel. The $105 \mathrm{KE}$ Basin presently stores fuel in open containers with an operational fuel enrichment limit for the receipt and handling of irradiated fuel of less than or equal to 0.95 percent Uranium $^{235}$.

The fuel storage basins exhaust unfiltered air from four roof exhausters. Emissions are limited to radionuclides. The 1706KE laboratory exhausts HEPA-filtered air from contaminated areas. One hazardous air pollutant has been identified in one of the 1706KE exhaust streams. The $1717 \mathrm{~K}$ maintenance shop does not have an exhaust collection system, and small quantities of volatile organic compounds are used as a part of routine maintenance operations and are released as fugitive emissions.

\section{I-B.2. Products, Raw Materials, Fuels, Processes}

- Principal products - There are no products produced.

- Process information - There are two process activities at the $100 \mathrm{~K}$ Basins. 
- A cooling system for the water in the basins. The cooling system removes heat from the basin water and discharges the excess heat to the atmosphere by the use of an air cooled chiller.

- The ion exchange system provides ion removal that includes ionic radionuclides. There is no regeneration; therefore, spent ion exchange resin is disposed as radioactive waste.

- Raw materials used by processes - There are no raw materials used at the 100K Basins.

- Fuels used by processes - There are no fuels used at the 100K Basins.

\title{
I-C.1. Emission Points, Controls, Stacks
}

The emission point and stack information is found on pages 1 through 3 of the AEI report output. The control information is found on page 8 of the AEI report output.

\section{I-C.2. Facility Process Flow Diagrams}

Attached.

\author{
I-C.3. Site Map
}

Attached.

\section{Chapter II. Regulatory Requirements}

\section{II-A.1. Description of Discharge and Emission Points}

The emission point and stack information is found on pages 1 through 3 of the AEI report output. 
Facility Building List

\begin{tabular}{|c|c|c|c|c|c|c|c|}
\hline Bldg Id & North & East & Bldg Desc & Custodian (Contractor) & Cust Phone & DOE POC & DOE Phone \\
\hline $\begin{array}{l}105 \mathrm{KE} \\
105 \mathrm{KW} \\
1706 \mathrm{KE} \\
1706 \mathrm{KEL} \\
1706 \mathrm{KER} \\
1717 \mathrm{~K}\end{array}$ & $\begin{array}{l}146760 \\
146482 \\
146666 \\
146654 \\
146673 \\
146521\end{array}$ & $\begin{array}{l}569237 \\
568695 \\
569194 \\
569175 \\
569160 \\
569031\end{array}$ & $\begin{array}{l}\text { REACTOR FACILITY } \\
\text { REACTOR FACILITY } \\
\text { WATER STUDIES SEMIWORKS FACILITY } \\
\text { DEVELOPMENT LABORATORY } \\
\text { WATER STUDIES RECIRCULATION BUILOING } \\
\text { MAINTENANCE TRANSPORTATION SHOP BUILDING }\end{array}$ & $\begin{array}{l}\text { GANT. RICHARD } G . \\
\text { GANT. RICHARD G. } \\
\text { MACFARLAN, PAUL } 3 . \\
\text { MACFARLAN, PAUU } 3 . \\
\text { MACFARLAN; PAUL } 3 . \\
\text { DEBBAN, BRUCE L. }\end{array}$ & $\begin{array}{l}373-3781 \\
373-3781 \\
373-1589 \\
373-1589 \\
373-1589 \\
373-2168\end{array}$ & $\begin{array}{l}\text { JOE M. ESCAMILLO } \\
\text { JOE M. ESCAMILLO } \\
\text { JOE M. ESCAMILLO } \\
\text { JOE M. ESCAMILLO } \\
\text { JOE M. ESCAMLLO } \\
\text { JOE M. ESCAMILLO }\end{array}$ & $\begin{array}{l}376-4557 \\
376-4557 \\
376-4557 \\
376-4557 \\
376-4557 \\
376-4557\end{array}$ \\
\hline
\end{tabular}

\section{Emission Point Listing}

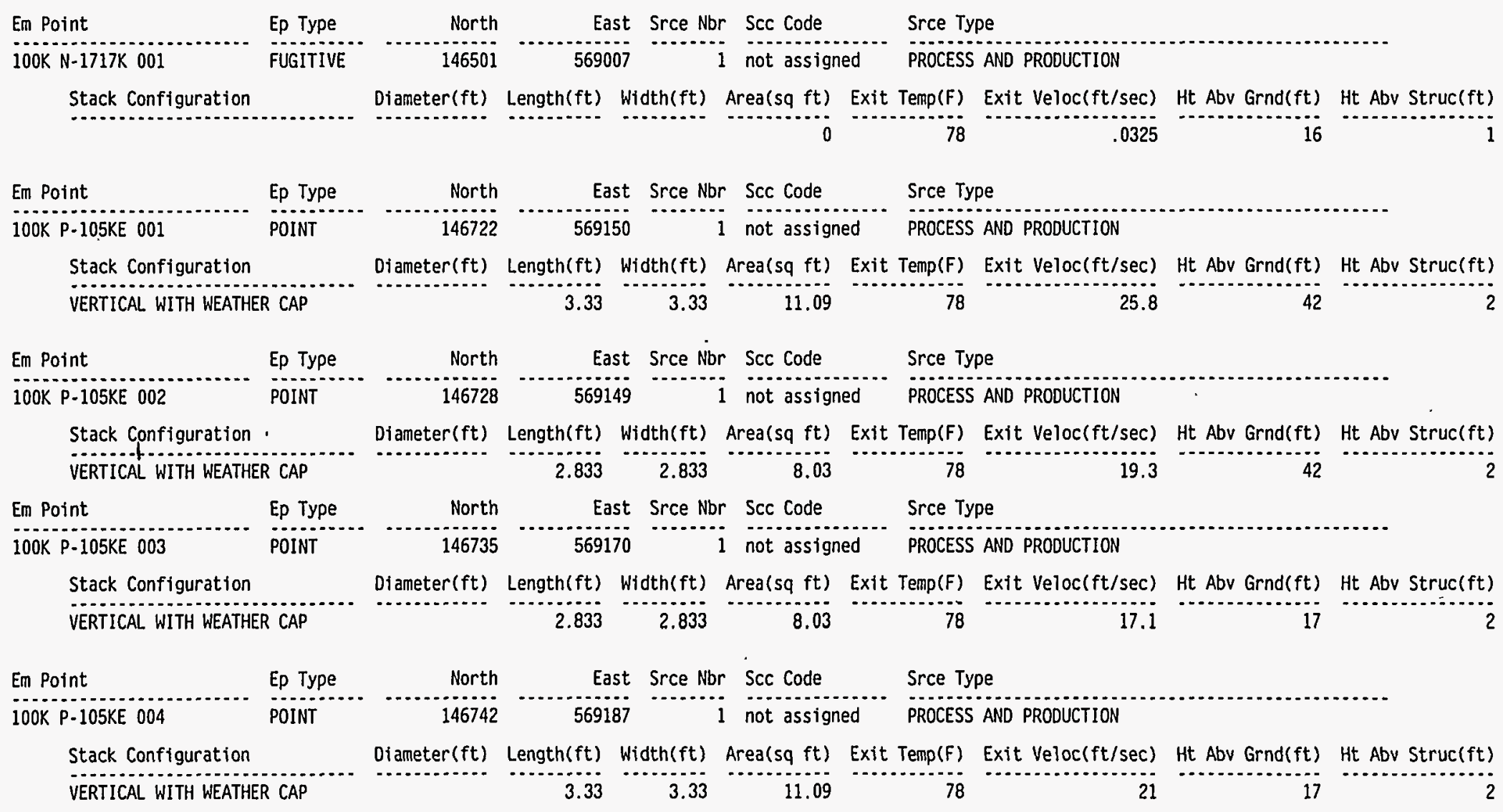

Page \#: 1 of 8 Report Run Date: 22-APR-95 
AIR EMISSIONS INVENTORY PERMIT APPLICATION REPORT (UP460) FACILITY NAME: 100K Facility and Related Structures

\section{Emission Point Listing}

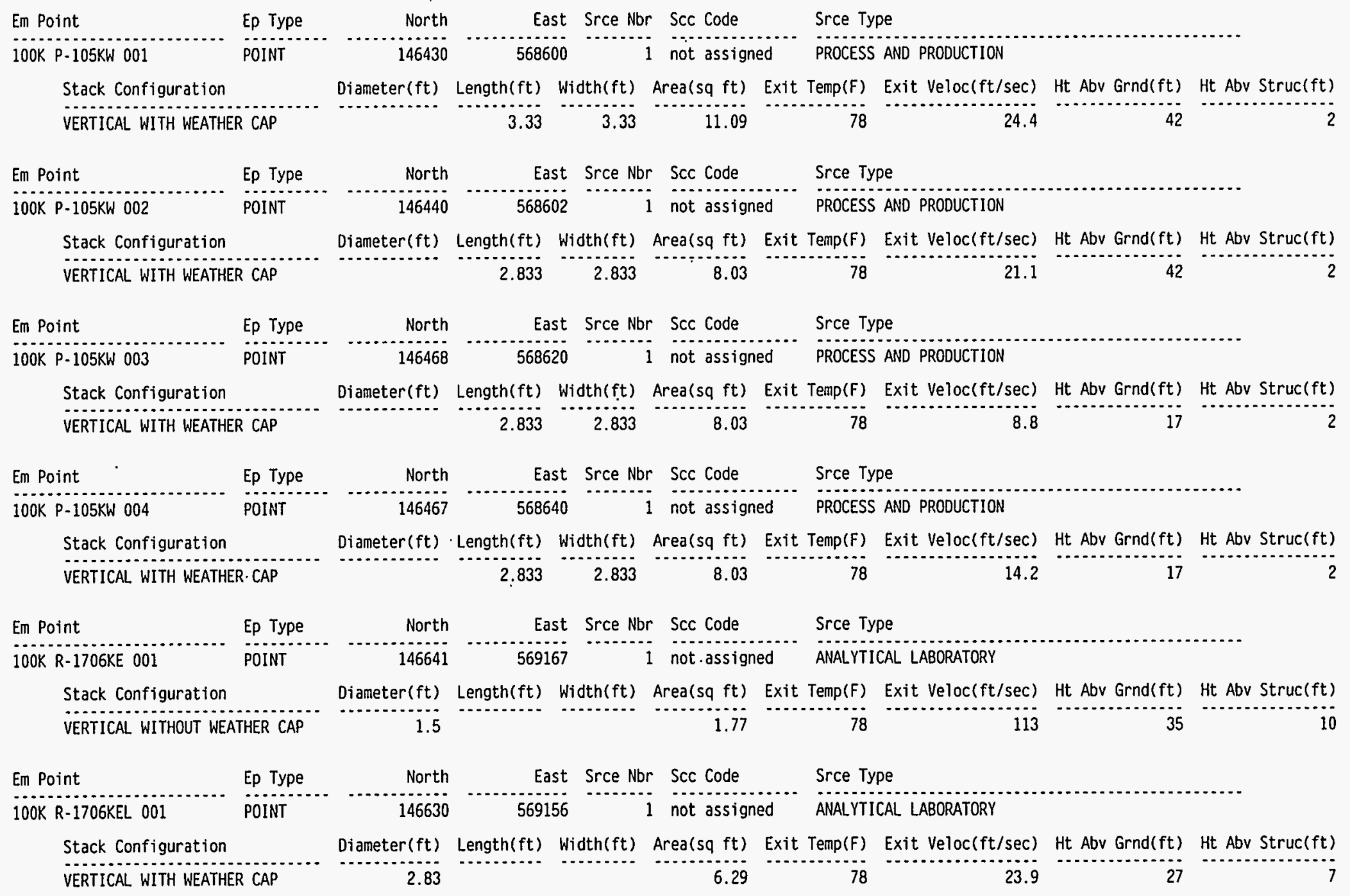


AIR EMISSIONS INVENTORY PERMIT APPLICATION REPORT (UP460)
FACILITY ID $100 \mathrm{~K}$ FACILITY NAME: 100K Facility and Related Structures

\section{Emission Point Listing}

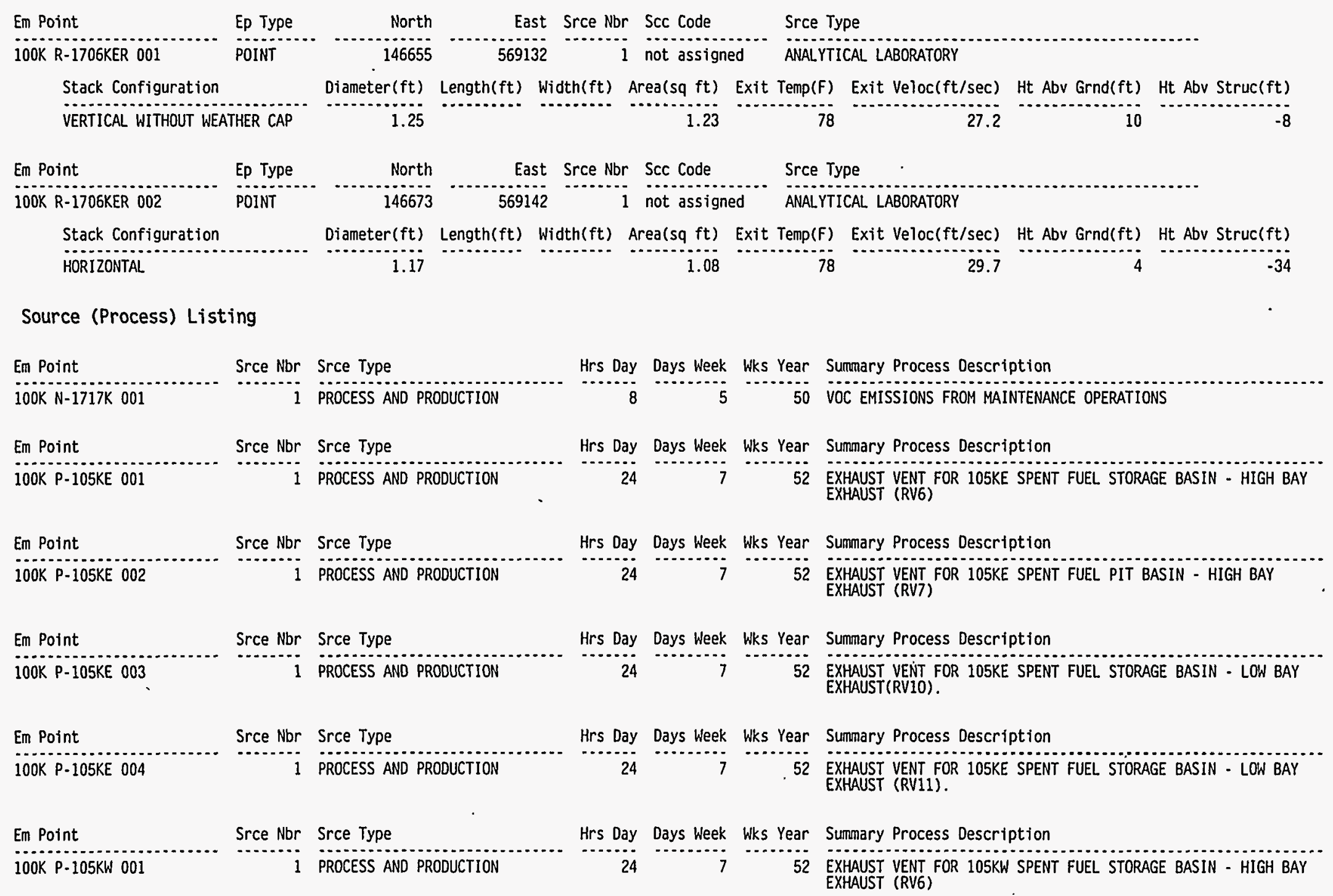


Source (Process) Listing

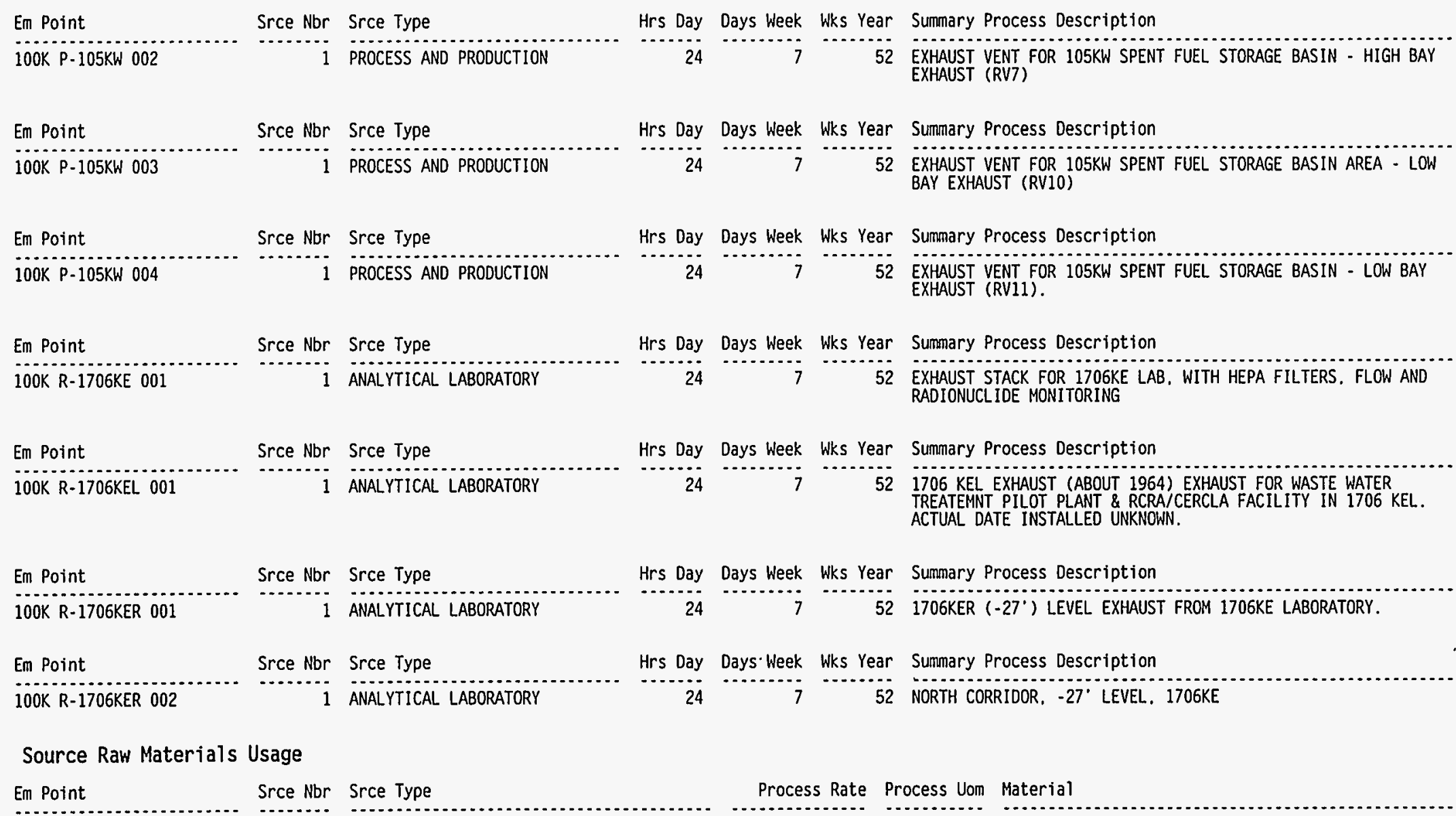

Fuels Usage by Source Processes

Em Point $\quad$ Srce Nbr Srce Type

Qty Uom

Fuel 
Fuels Usage by Control Devices

Em Point

$\mathrm{Ctl} \mathrm{Nbr}$ Ctl Type

Qty Uom

Fuel

Point Source Criteria Pollutants - Type: VOC

Em Point

100K R-1706KEL 001

- Sum

Point Source Emissions - Radiological Pollutants

Em Point

Srce Nbr Srce Type Pollutant

100K P-105KE 001

i PROCESS AND PRODUCTION

100K P-105KE 002

1 PROCESS AND PRODUCTION

100K P-105KE 003

1 PROCESS AND PRODUCTION

100K P-105KE 004

1 PROCESS AND PRODUCTION

100K P-105KW 001

100K P-105KW 002

100K P-105KW 003

100K P-105KW 004
1 PROCESS AND PRODUCTION

1 PROCESS AND PRODUCTION

1 PROCESS AND PRODUCTION

1 PROCESS AND PRODUCTION
Em. Pt. Potential Em. Pt.
to Emit (tons/yr) Ctl Actual (tons/yr) Pollutant

$\begin{array}{rr}.750000000 & .170000000 \\ .750000000 & .170000000\end{array}$

Em. Pt. Potential Em. Pt.

to Emit (Ci/yr) Pollutant

Refer to

WHC-SD-EN-RPT-007 $\quad$ Refer to 1 RL-94-51

Rev. 0 Page A-334 Table 2-1

Refer to

WHC-SD-EN-RPT-007 $\quad$ Refer to

Rev. O Page A-334

Table 2-1

Refer to

WHC-SD-EN-RPT-007

Refer to

DOE/RL-94-51

Refer to

WHC-SD-EN-RPT-007

Rev. 0 Page A-334

Refer to

Table 2-1

Refer to

Refer to

$\begin{array}{ll}\text { WHC-SD-EN-RPT-007 } & \text { DOE/RL-94-51 } \\ \text { Rev. O Page A-335 } & \text { Table 2-1 }\end{array}$

Refer to

WHC-SD-EN-RPT-007

Rev. 0 Page A-335

Refer to

Table 2-1

Refer to

WHC-SD-EN-RPT -007
Rev. 0 Page A-335

Refer to

DOE/RL. $-94-51$
Table $2-1$

Refer to

WHC-SD-EN-RPT-007

Refer to

DOE/RL $-94-51$

Table 2-1

Page \#: 5 of 8 Report Run Date: 22-APR-95 


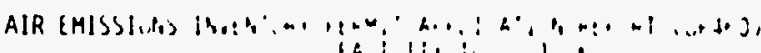

FACILITY NAME. LOUK focility and Reldated structures

Point Source Emissions - Radiological Pollutants

\begin{tabular}{|c|c|c|c|c|}
\hline Em Point & Srce Nbr & Srce Type Pollutant & $\begin{array}{l}\text { Em. Pt. Potential } \\
\text { to Emit (Ci/yr) }\end{array}$ & $\begin{array}{l}\mathrm{Em} \cdot{ }_{\mathrm{Ct}}{ }^{\mathrm{Pt}} \text { Actual }(\mathrm{Ci} / \mathrm{yr})\end{array}$ \\
\hline 100K R-1706KEL 001 & 1 & ANALYTIICAL LABORATORY & $\begin{array}{l}\text { Refer to } \\
\text { WHC-SD-EN-RPT-025 } \\
\text { Rev. O Page A-55 }\end{array}$ & $\begin{array}{l}\text { Refer to } \\
\text { WHC-SD-EN-RPT- } 025 \\
\text { Rev. O Page A-55 }\end{array}$ \\
\hline 100K R-1706KER 001 & 1 & ANALYTICAL LABORATORY & $\begin{array}{l}\text { Refer to } \\
\text { WHC-SD-EN-RPT-007 } \\
\text { Rev. 0 Page A-337 }\end{array}$ & $\begin{array}{l}\text { Refer to } \\
\text { DOE/RL-94-51 } \\
\text { Table 2-1 }\end{array}$ \\
\hline 100K R-1706KER 002 & 1 & ANALYTIICAL LABORATORY & $\begin{array}{l}\text { Refer to } \\
\text { WHC-SD-EN-RPT-007 } \\
\text { Rev. O Page A-337 }\end{array}$ & $\begin{array}{l}\text { Refer to } \\
\text { DOE/RL-94-51 } \\
\text { Table 2-1 }\end{array}$ \\
\hline
\end{tabular}

Point Source Emissions - HAP Pollutants

\begin{tabular}{|c|c|c|c|c|c|}
\hline Em Point & Srce Nbr & Srce Type Pollutant & $\begin{array}{l}\text { Em. Pt. Potential } \\
\text { to Emit (tons/yr) }\end{array}$ & $\begin{array}{l}\text { Em. Pt. } \\
\text { CtT Actual (tons/yr) }\end{array}$ & Pollutant \\
\hline $100 K \mathrm{R}-1706 \mathrm{KEL} 001$ & & 1 ANALYTICAL LABORATORY & .75000 & .1700000 & 00 N-HEXANE \\
\hline Sum & & & .75000 & .1700000 & \\
\hline
\end{tabular}

Point Source Emissions - Uncategorized Pollutants

Em Point

Srce Nbr Srce Type Pollutant

Em. Pt. Potential Em. Pt.

Sum

Fugitive Source Criteria Pollutants - Type: VOC

Fugitive Source Criteria Pollutants - Type: VOC

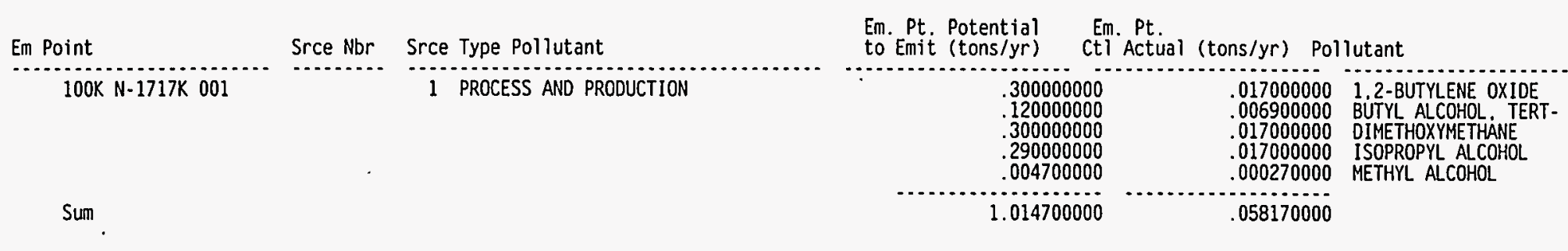

Page \#: 6 of 8 Report Run Date: 22-APR-95 


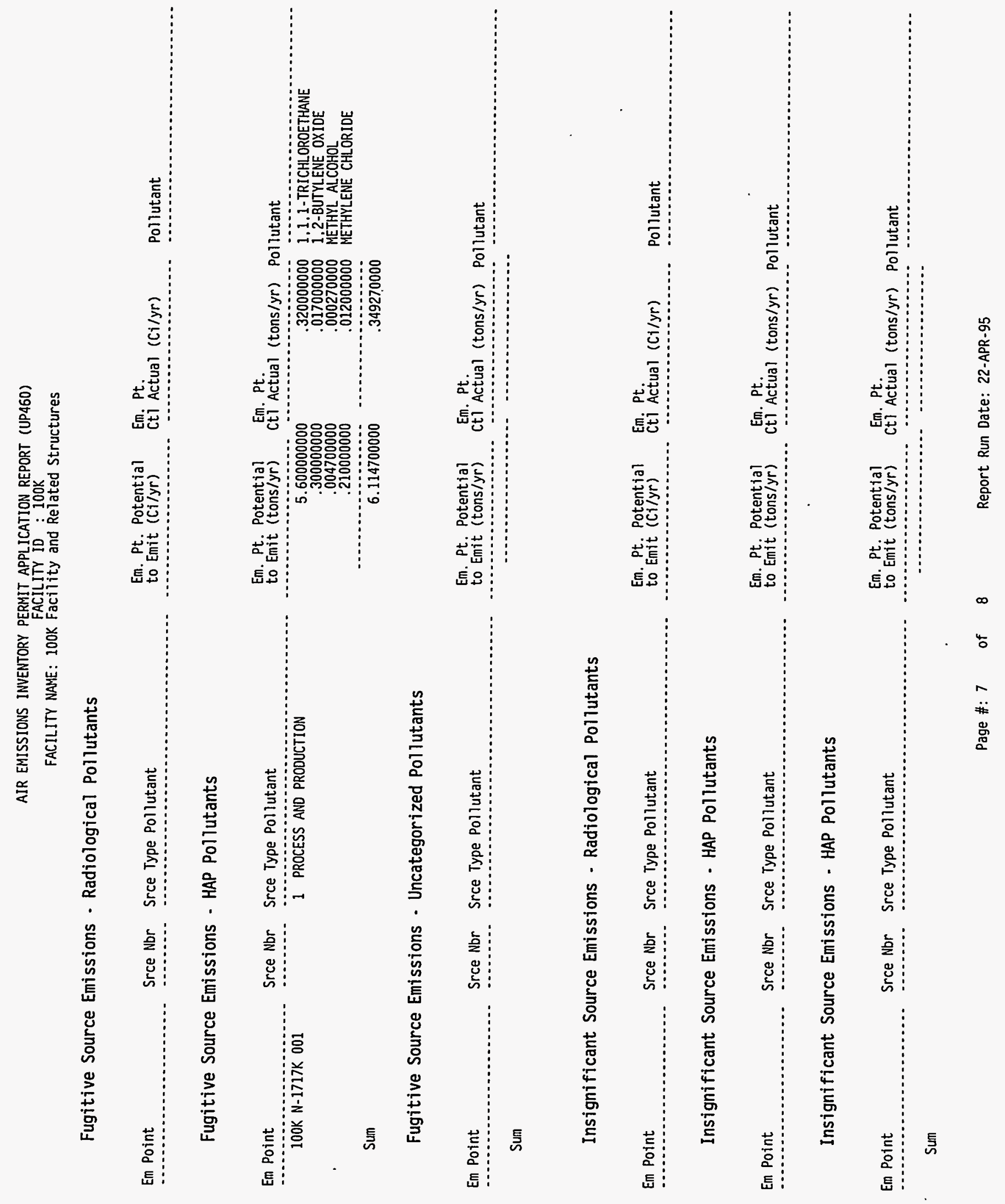


AIR EMISSIONS INVENTORY PERMIT APPLICATION REPORT (UP460)

FACILITY NAME: 100K Facility and Related Structures

Insignificant Source Emissions - Uncategorized Pollutants

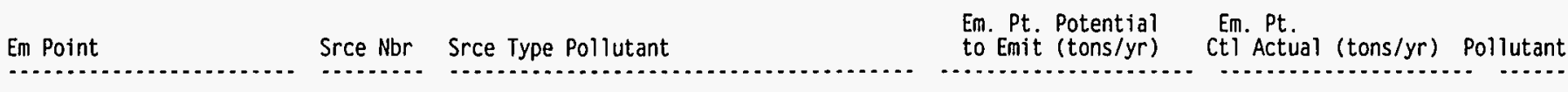

Sum

Control Device Listing

Em Point

Control Equipment Code

100K R-1706KE 00

$100 \mathrm{~K}$ R-1706KE 00

100K R-1706KEL 00

100K R-1706KER 001

100K R-1706KER 002

099

048
099

099

Monitors Listing 


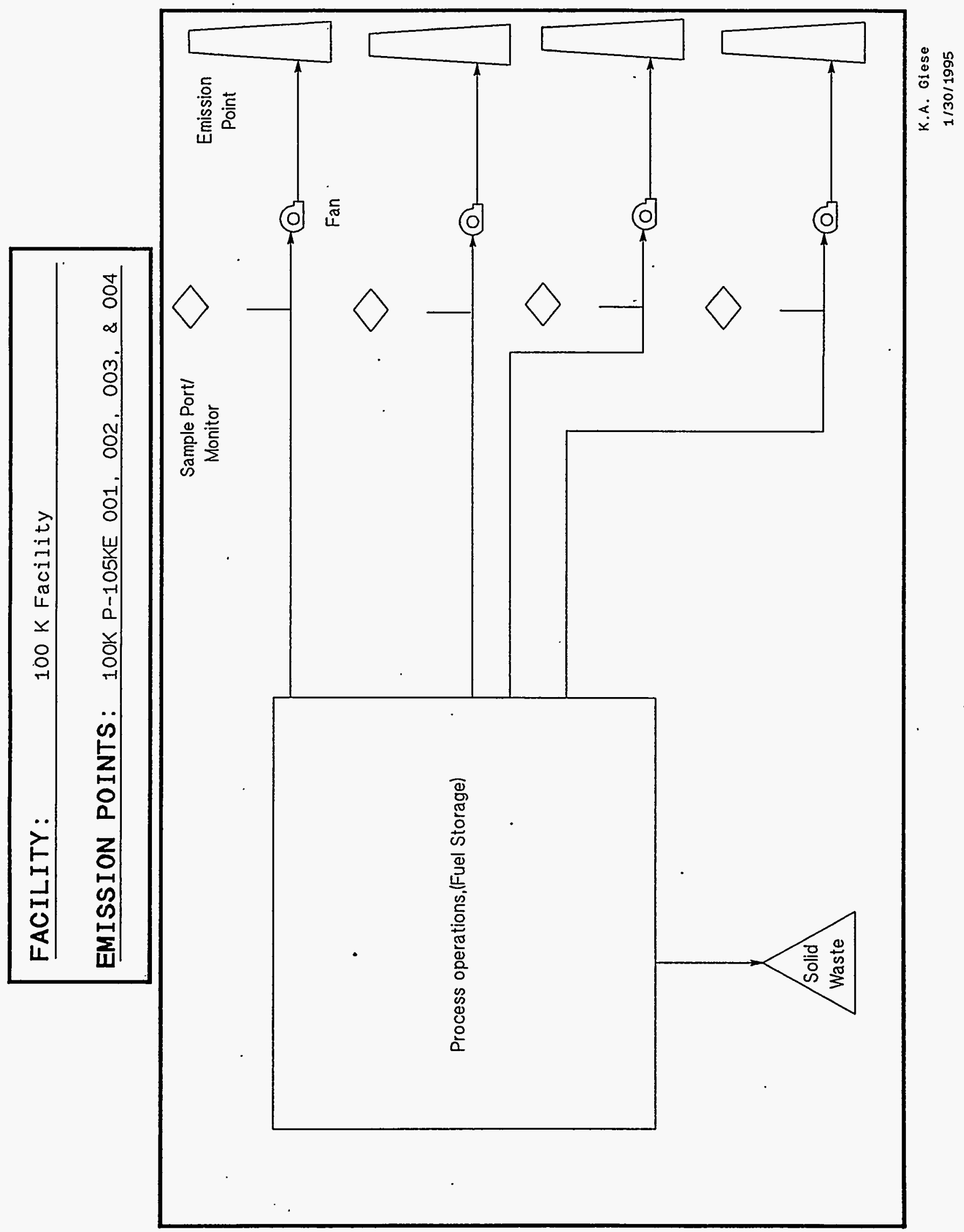




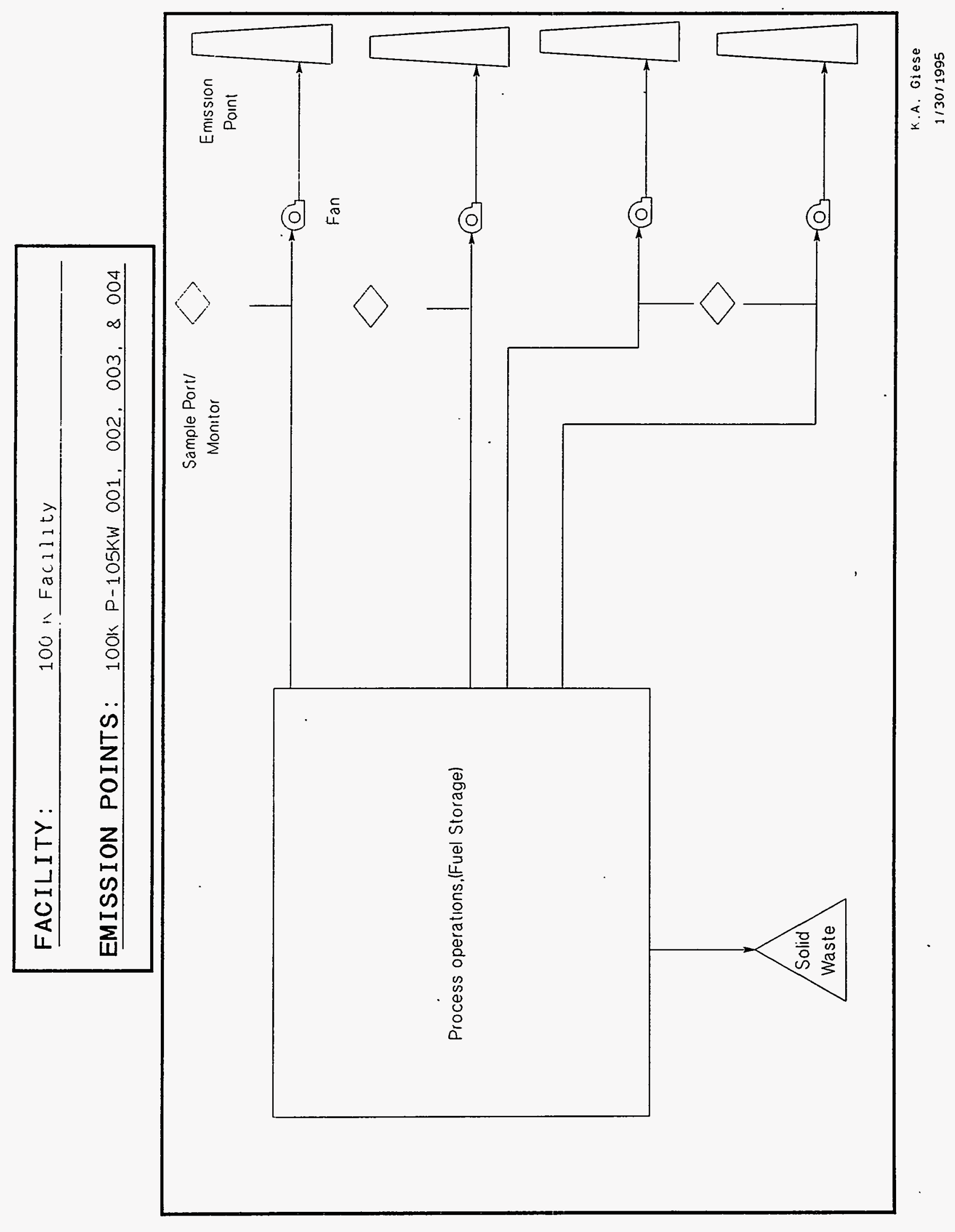




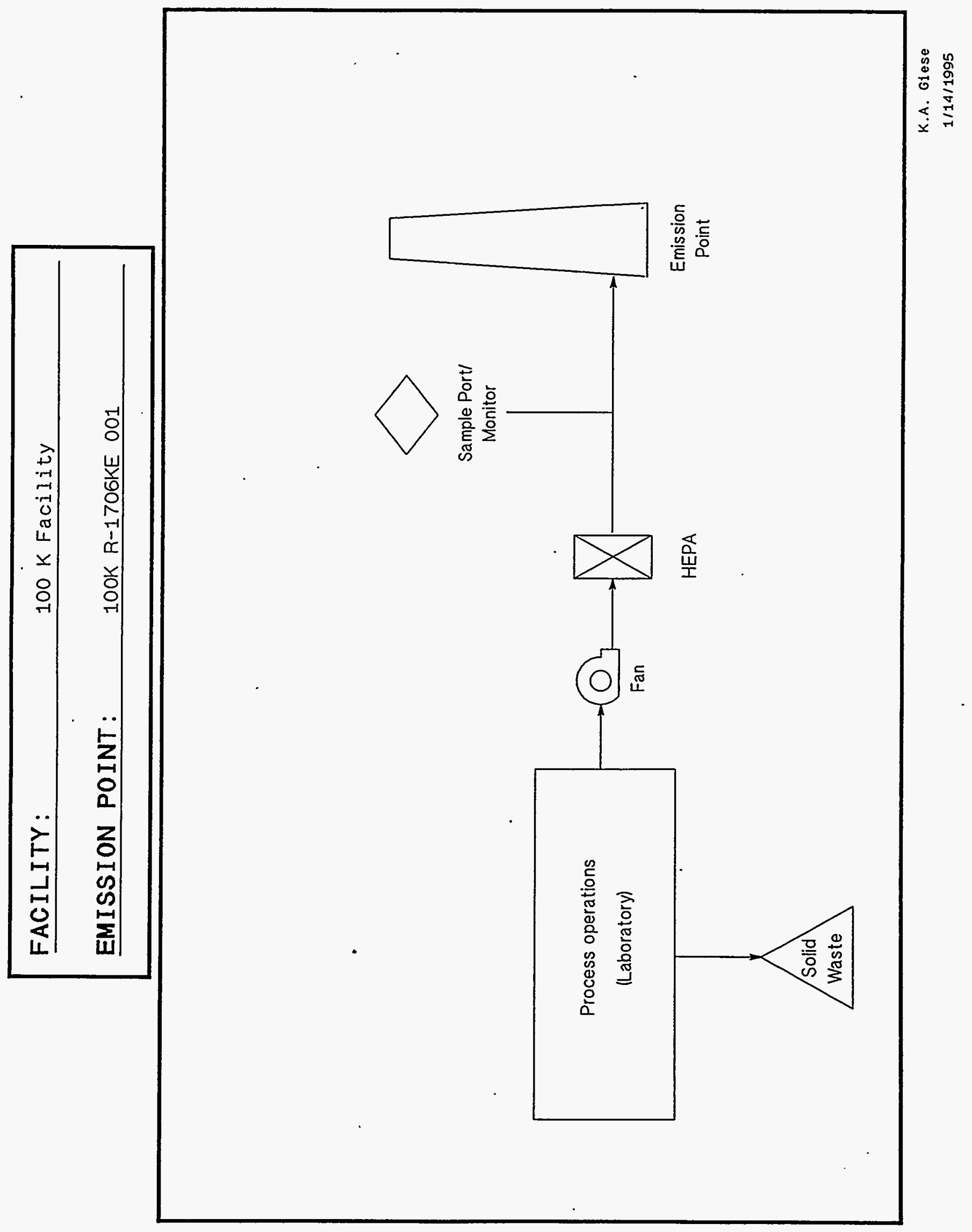




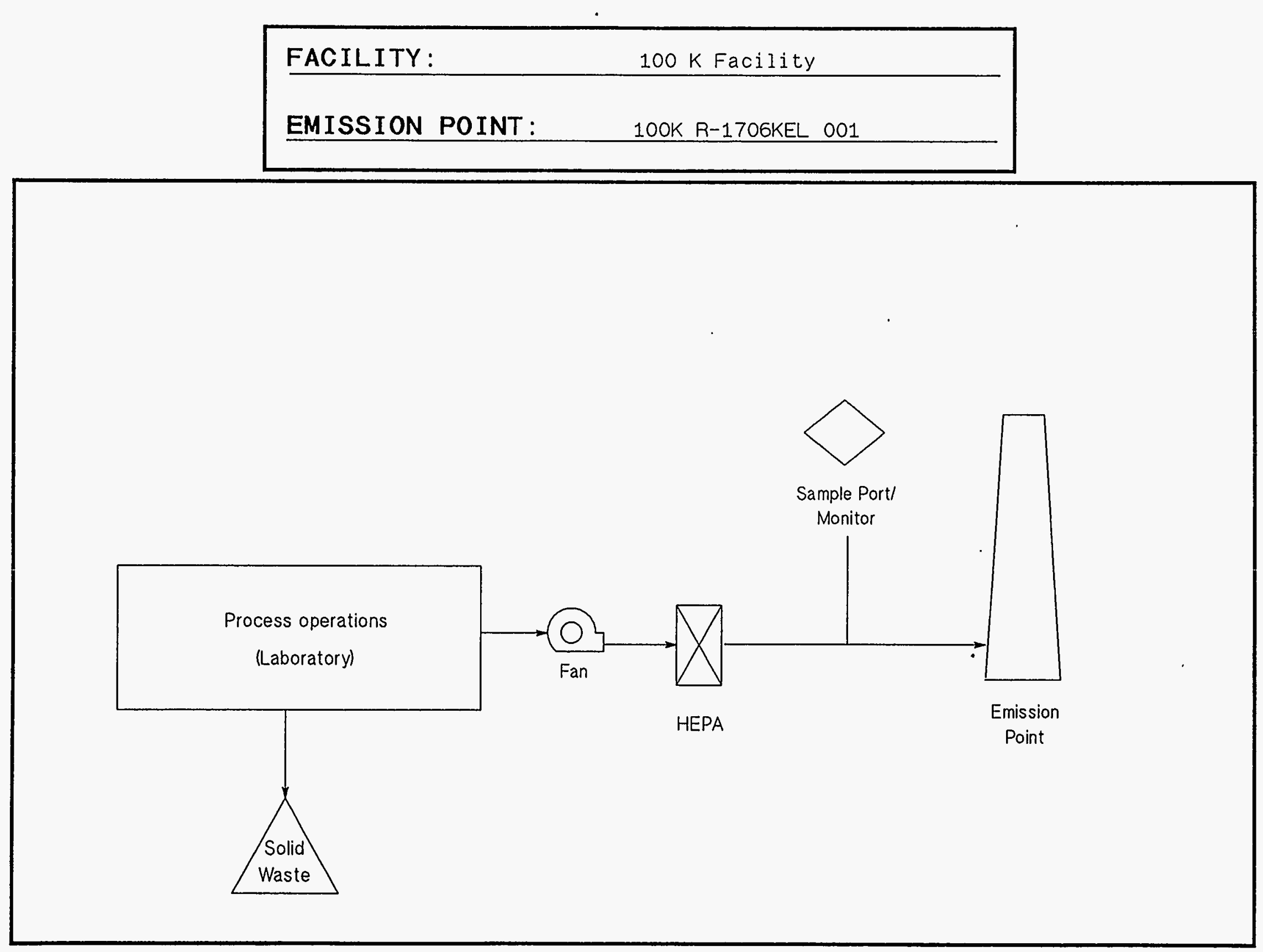

K.A. Giese 


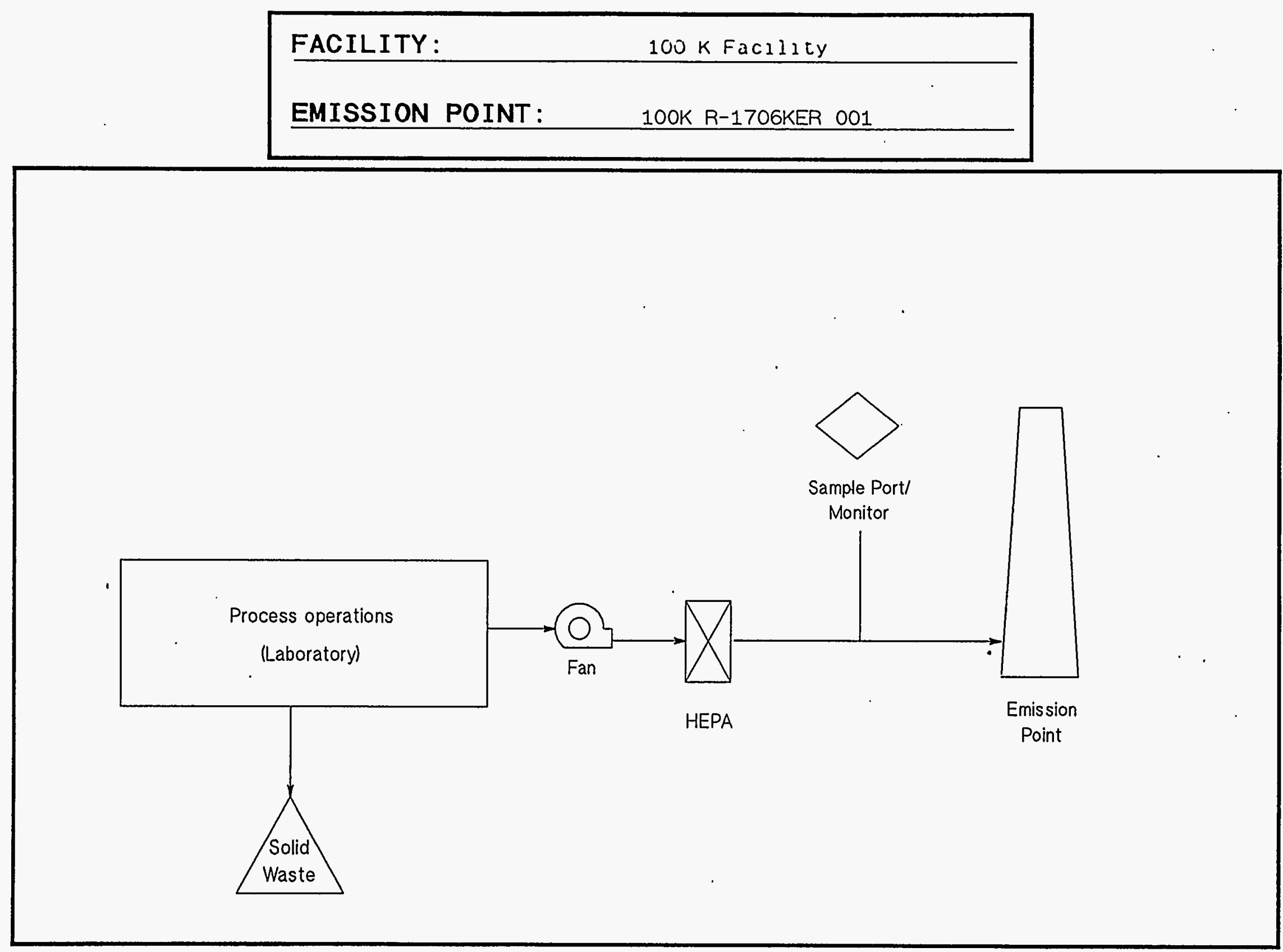

K.A. Giese

1/14/1995 


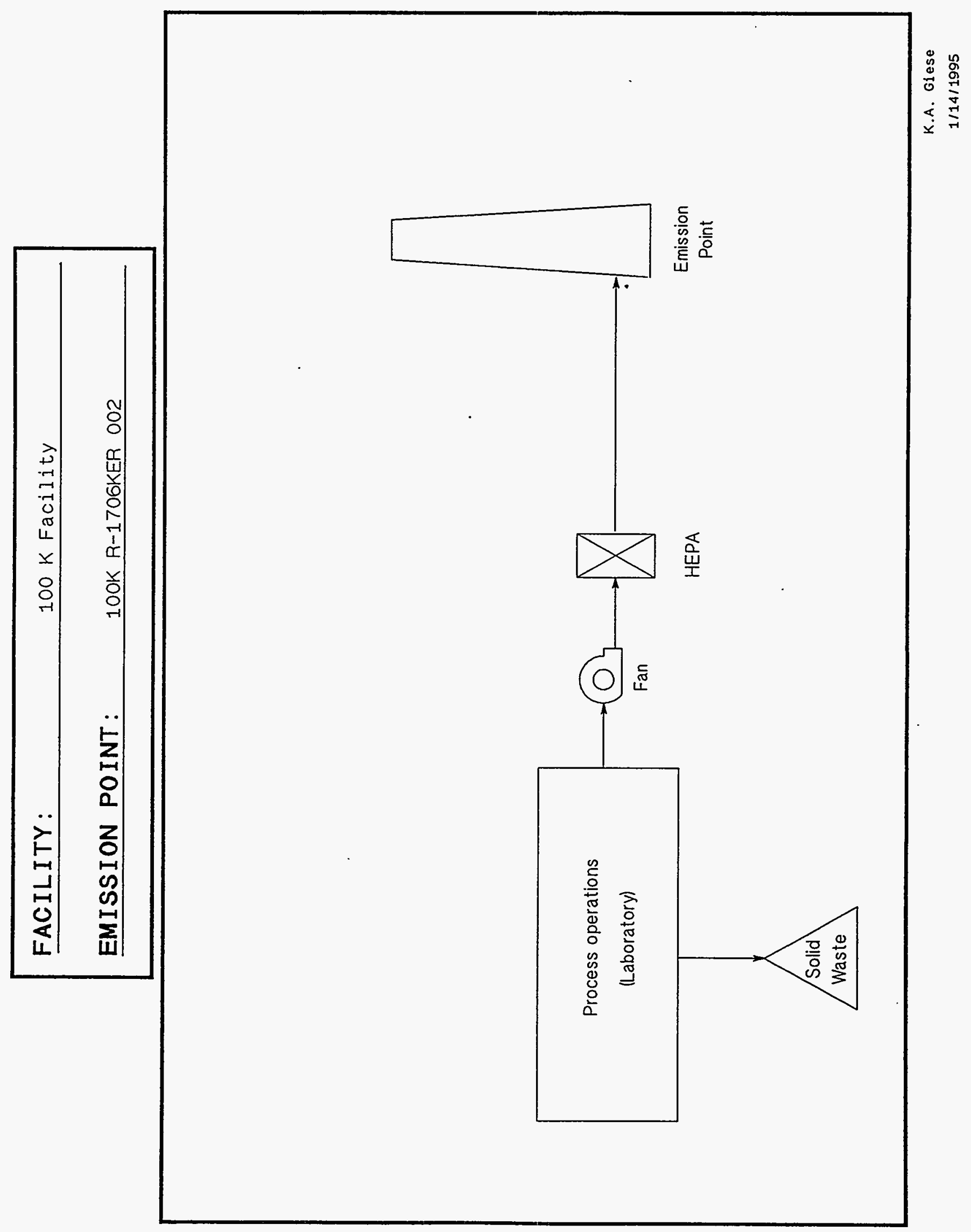




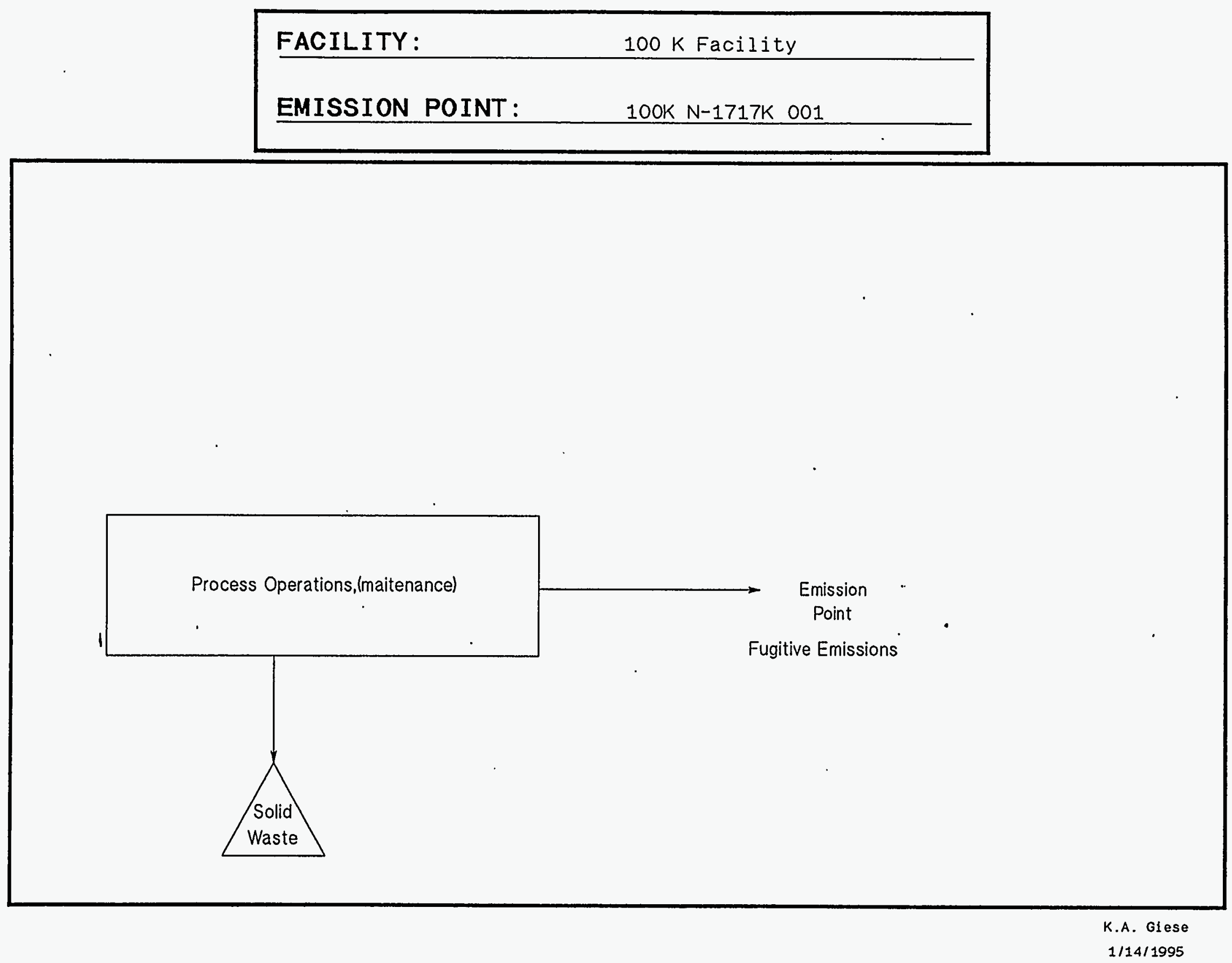




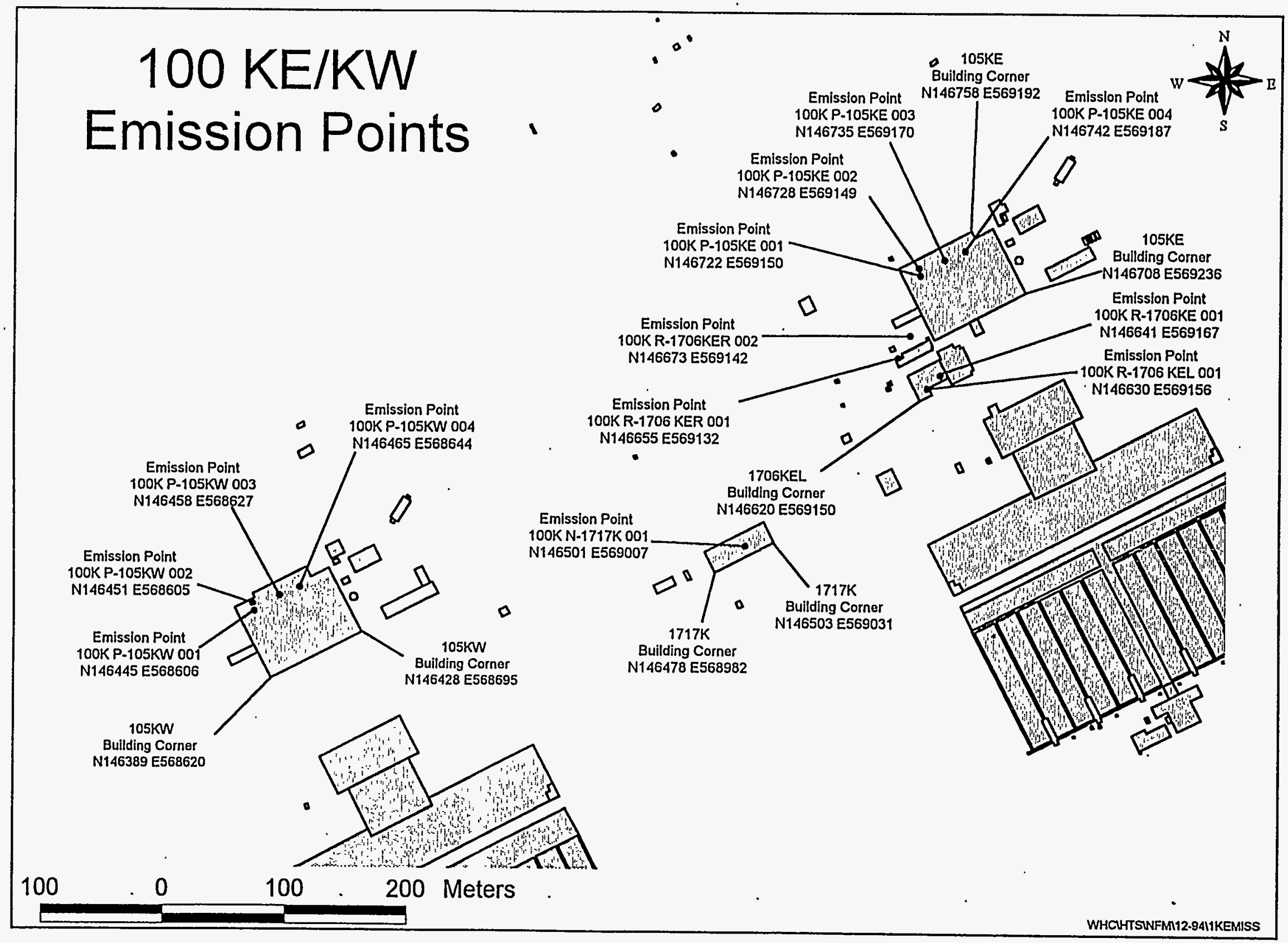




\section{APPENDIX C-2}

\section{$100 \mathrm{~N}$}

\section{Chapter I. General Information}

\section{I-B.1. Facility Description}

The N Reactor began operation in December 1963. The $\mathrm{N}$ Reactor was designed to produce special nuclear materials (SNM) and byproduct steam for electrical power generation. The 100-N Area reactor complex consists of 126 buildings for reactor operations, maintenance, support facilities, and an office complex. The 105-N Reactor Building contains the nuclear reactor itself, a pressurized water reactor (PWR), which was fueled with slightly enriched uranium and designed to operate at 1,825 pounds per square inch and $600^{\circ} \mathrm{F}$. The $\mathrm{N}$ Reactor produced approximately 4,000 megawatts of thermal power. The reactor process generated large quantities of heat that were removed by the primary coolant loop to the 109-N Heat Exchanger Building. This building transferred the heat from the primary loop to the secondary loop via steam generators. The secondary loop coolant, when it exited the steam generator, was in the form of steam and was carried to the Washington Public Power Supply System turbine generator building where the turbines were driven by the steam to produce electricity.

The N Reactor, by nature of its process, used large quantities of water for cooling purposes in the production of SNM and electricity. To support the large water usage, N Reactor used the 183-N Water Filtration Plant and the 163-N Demineralization Plant, which filtered and demineralized the water needed for operation.

During startup, the 184-N Plant Service Power House provided the initial plant steam necessary to start the plant before the steam generators provided plant steam. This power house was crucial to the $\mathrm{N}$ Reactor startup:

The 107-N Basin Recirculation Building filtered and demineralized the water from the $\mathrm{N}$ Basin Fuel Storage Facility, removing radionuclides from the water and reducing personnel radiation exposure. The $\mathrm{N}$ Basin stored the irradiated fuel from the reactor until the fuel was ready for transfer to the 200 Areas for chemical reprocessing.

The N Reactor and auxiliary facilities were operated until 1987 when DOE ordered $\mathrm{N}$ Reactor placed in a standby configuration. In 1991, DOE ordered the preservation activities to cease and identified steps to place $\mathrm{N}$ Reactor in a condition requiring only minimal surveillance and maintenance until final decontamination and decommissioning. 


\section{I-B.2. Products, Raw Materials, Fuels, Processes}

- Principle products - There are no products produced.

- Process information - There are no process activities.

- Raw materials used by processes - There are no raw materials used at $100 \mathrm{~N}$.

- Fuels used by processes - There are fuels used at $100 \mathrm{~N}$.

\section{I-C.1. Emission Points, Controls, Stacks}

The emission point and stack information is found on pages 1 and 2 of the AEI report output. The control information is found on page 8 of the AEI report output.

\section{I-C.2. Facility Process Flow Diagrams}

Attached.

\section{I-C.3. Site Map}

Attached.

\section{Chapter II. Regulatory Requirements}

\section{II-A.1. Description of Discharge and Emission Points}

The emission point and stack information is found on pages 1 and 2 of the AEI report output. 
AIR EMISSIONS INVENTORY PERMIT APPLICATION REPORT (UP460)

FACILITY NAME: 100N Facility and Related Structures

Facility Building List

\begin{tabular}{|c|c|c|c|c|c|c|c|}
\hline Bldg Id & North & East & Bldg Desc & Custodian(Contractor) & Cust Phone & DOE POC & DOE Phone \\
\hline $\begin{array}{l}105 \mathrm{~N} \\
107 N \\
116 \mathrm{~N}\end{array}$ & $\begin{array}{l}149572 \\
149587 \\
149698\end{array}$ & $\begin{array}{l}571309 \\
571117 \\
571224\end{array}$ & $\begin{array}{l}\text { REACTOR FACILITY } \\
\text { RECIRCULATION COOLING BUILDING } \\
\text { MAIN STACK AT } 100 \mathrm{~N}\end{array}$ & $\begin{array}{l}\text { TREVINO, REBIN A. } \\
\text { BLANKENSHIP. DORMAN } \\
\text { TREVINO, RUBIN A. }\end{array}$ & $\begin{array}{l}373-1663 \\
373-5456 \\
373-1663\end{array}$ & $\begin{array}{l}\text { ROGER D. FREEBERG } \\
\text { ROGER D. FREEBERG } \\
\text { ROGER D. FREEBERG }\end{array}$ & $\begin{array}{l}376-7167 \\
376-7167 \\
376-7167\end{array}$ \\
\hline
\end{tabular}

Emission Point Listing

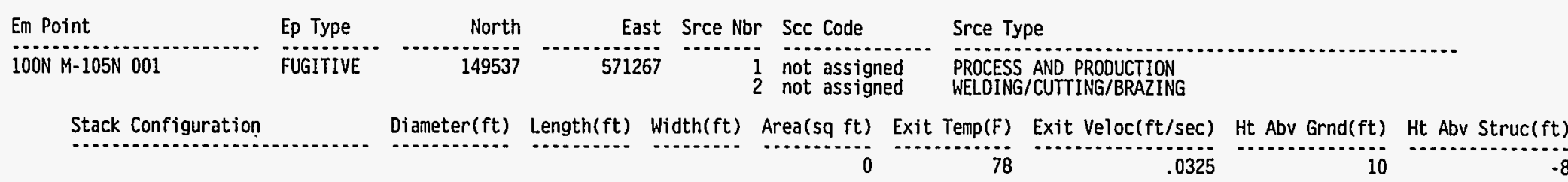

\begin{tabular}{|c|c|c|c|c|c|c|c|c|c|}
\hline Em Point & Ep Type & North & East & Srce $\mathrm{Nbr}$ & $r$ Scc Code & Srce Ts & & & \\
\hline $100 \mathrm{~N} P-105 \mathrm{~N} 002$ & POINT & 149508 & 571242 & & $i$ not assigne & PROCESS & AND PRODUCTION & & \\
\hline Stack Configuration & & Diameter ( $f t)$ & Length(ft) & Width(ft) & $\operatorname{Area}(s q \mathrm{ft})$ & Exit Temp(F) & Exit Veloc $(\mathrm{ft} / \mathrm{sec})$ & Ht Abv Grnd(ft) & $H t$ Abv Struc $(f t)$ \\
\hline VERTICAL WITHOUT WEA & THER CAP & & 1.5 & 1.5 & 2.25 & 70 & 47 & 35 & -36 \\
\hline
\end{tabular}

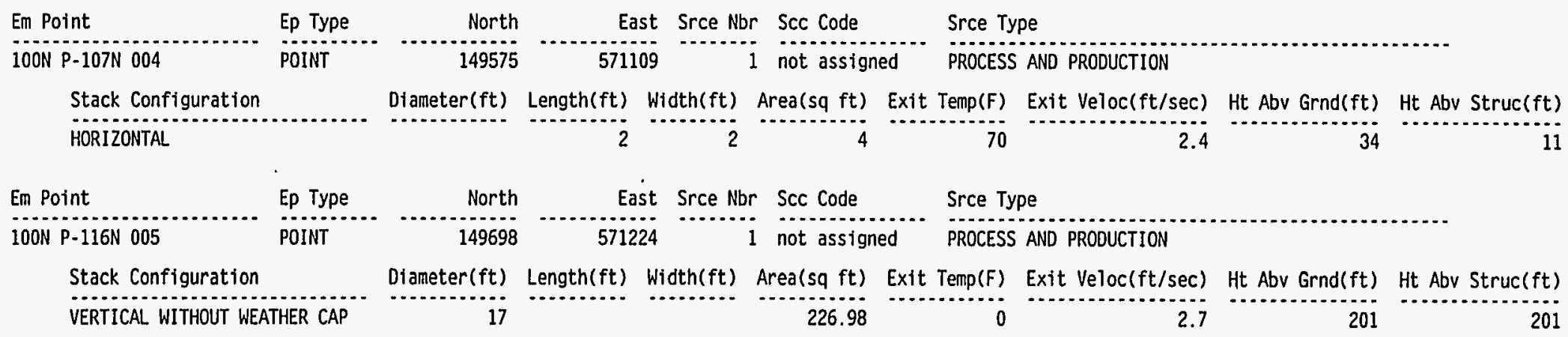




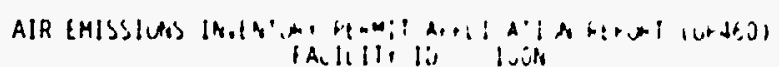

FACILITY NAME, IOON Facility and Related Structures

Source (Process) Listing

\begin{tabular}{|c|c|c|c|c|c|c|}
\hline Em Point & Srce Nbr & Srce Type & Hrs Day & Days Week & Wks Year & Summary Process Description \\
\hline $100 \mathrm{~N} \mathrm{M}-105 \mathrm{~N} 001$ & 1 & PROCESS AND PRODUCTION & 8 & 5 & 52 & PIPEFITTING AND METALWORKING OPERATIONS \\
\hline Em Point & Srce $\mathrm{Nbr}$ & Srce Type & Hrs Day & Days Week & Whs Year & Summary Process Description \\
\hline $100 \mathrm{~N} \mathrm{M}-105 \mathrm{~N} 001$ & 2 & WELDING/CUTTING/BRAZING & 8 & 5 & 52 & WELDING OPERATIONS \\
\hline \multicolumn{7}{|c|}{$\begin{array}{l}\text { ACTIVITY - WELDING, CUTTING \& BRAZING. PROCESS - BRAZING, OXY-ACETYLENE, OSY-ACETYLENE CUTTING, PLASMA ARC CUTTING, GAS TUNGSTEN ARC WELOING, \& } \\
\text { SHIELDEO METAL ARC WELDING. BASE METAL TYPE - ALUMINUM, CARBON STEEL. NICKEL BASED \& STAINLESS STEEL. TYPE OF FLUX REMOVAL - BRUSHING \& GRINDING. }\end{array}$} \\
\hline Em Point & Srce $\mathrm{Nbr}$ & Srce Type & Hrs Day & Days Week & Wks Year & Summary Process Description \\
\hline $100 \mathrm{~N} P-105 \mathrm{~N} 002$ & 1 & PROCESS AND PRODUCTION & 24 & 7 & 52 & $\begin{array}{l}\text { ROOMS } 305 / 306 \text { 14-FT STACK. DECONTAMINATION/SEGREGATION OF } \\
\text { RADIOACTIVE MATERIALS AND WASTES. NO VOLATILE EMISSIONS. }\end{array}$ \\
\hline Em Point & Srce $\mathrm{Nbr}$ & Srce Type & Hrs Day & Days Week & Wks Year & Summary Process Description \\
\hline $100 \mathrm{~N} P-107 \mathrm{~N} 004$ & 1 & PROCESS AND PRODUCTION & 24 & 7 & 52 & 107-N BASIN RECIRCULATION AREA STACK. STACK IS IN USE. \\
\hline Em Point & Srce $\mathrm{Nbr}$ & Srce Type & Hrs Day & Days Week & Whs Year & Summary Process Description \\
\hline $100 \mathrm{~N} \mathrm{P}-116 \mathrm{~N} 005$ & 1 & PROCESS AND PRODUCTION & 24 & 7 & 52 & 116-N AIR STACK. THE STACK IS IN USE. \\
\hline \multicolumn{7}{|c|}{ Source Raw Materials Usage } \\
\hline Em Point & Srce Nbr & Srce Type & & Process & $S$ Rate Pro & ocess Uom Material \\
\hline
\end{tabular}

Fuels Usage by Source Processes

Em Point

Srce Nbr Srce Type

Qty Uom

Fuel

Fuels Usage by Control Devices

Em Point

$\mathrm{Ctl} \mathrm{Nbr} \mathrm{Ctl}$ Type

Qty Uom Fuel 
AIR EMISSIONS INVENTORY PERMIT APPLICATION REPORT (UP460) FACILITY NAME: 100N Facility and Related Structures

Point Source Emissions - Radiological Pollutants

\begin{tabular}{|c|c|c|c|c|c|}
\hline Em Point & Srce Nbr & Srce Type Pollutant & $\begin{array}{l}\text { Em. Pt. Potential } \\
\text { to Emit (Ci/yr) }\end{array}$ & $\begin{array}{l}\text { Em. Pt. } \\
\text { Cti Actual (Ci/yr) }\end{array}$ & Pollutant \\
\hline $100 \mathrm{~N} P-116 \mathrm{~N} 005$ & i & PROCESS AND PRODUCTION & $\begin{array}{l}\text { Refer to } \\
\text { WHC-SD-EN-RPT-007 } \\
\text { Rev. } 0 \text { Page A-331 }\end{array}$ & $\begin{array}{l}\text { Refer to } \\
\text { DoE/RL-94-51 } \\
\text { Table 2-1 }\end{array}$ & \\
\hline
\end{tabular}

Point Source Emissions - HAP Pollutants

Em Point

Srce Nbr Srce Type Pollutant

Em. Pt. Potential Em. Pt.

(tons/yr) Pollutant

Sum

Point Source Emissions - Uncategorized Pollutants

Em Point

Srce Nbr Srce Type Pollutant

Em. Pt. Potential Em. Pt.

to Emit (tons/yr) ctl Actual (tons/yr) Pollutant

Sum

Fugitive Source Criteria Pollutants - Type: PM10

Em Point
Srce Nbr Srce Type Pollutant
$\begin{aligned} & \text { Sum } \\ & \text { M-100N } 001\end{aligned}$
1 PROCESS AND PRODUCTION

Em. Pt. Potential Em. Pt.
to Emit (tons/yr) Ctl Actual (tons/yr) Pollutant

Sum $.050000000 \quad 010000000$ PARTICULATE MATTER (10 MICRONS OR LESS) $.050000000 \quad .010000000$ 
AIR EMISSIONS INVENTORY PERMIT APPLICATION REPORT (UP460)

FACILITY NAME: $100 \mathrm{~N}$ Facility and Related Structures

Fugitive Source Emissions - Radiological Pollutants

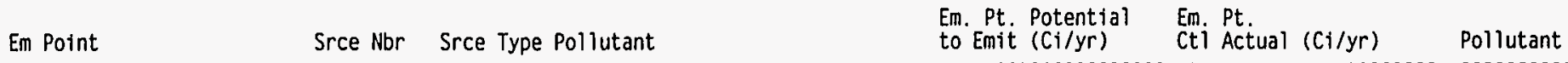

Fugitive Source Emissions - HAP Pollutants

\section{Em Point}

Srce Nbr Srce Type Pollutant

Sum

Fugitive Source Emissions - Uncategorized Pollutants

Em Point

Srce Nbr Srce Type Pollutant

Sum

Insignificant Source Emissions - Radiological Pollutants

\section{Em Point}

$100 \mathrm{~N}$ P. $105 \mathrm{~N}$ do2

Srce Nbr Srce Type Pollutant

.

Insignificant Source Emissions - Radiological Pollutants

$$
\text { Em Point }
$$

Srce Nbr Srce Type Pollutant

$100 \mathrm{~N}$ P-107N 004

1 PROCESS AND PRODUCTION

...

Em. Pt. Potential Em. Pt.

to Emit (tons/yr) Ctl Actual (tons/yr) Pollutant

100

\begin{tabular}{|c|c|c|}
\hline $\begin{array}{l}\text { Em. Pt. Potential } \\
\text { to Emit (Ci/yr) }\end{array}$ & $\begin{array}{l}\text { Em. Pt. } \\
\text { Cti Actual (Ci/yr) }\end{array}$ & Pollutant \\
\hline $\begin{array}{l}\text { Refer to } \\
\text { WHC-SD-EN-RPT-025 } \\
\text { Rev. 0 Page A-48 }\end{array}$ & $\begin{array}{l}\text { Refer to } \\
\text { DOE/RL-94-51 } \\
\text { Table 2-1 }\end{array}$ & \\
\hline $\begin{array}{l}\text { Em. Pt. Potential } \\
\text { to Emit } \begin{array}{l}\text { (Ci/yr) }\end{array}\end{array}$ & $\begin{array}{l}\text { Em. Pt. } \\
\text { Ctj Actual (Ci/yr) }\end{array}$ & Pollutant \\
\hline $\begin{array}{l}\text { Refer to } \\
\text { WHC-SD-EN-RPT-025 } \\
\text { Rev. O Page A-54 }\end{array}$ & $\begin{array}{l}\text { Refer to } \\
\text { DOE/RL-94-51 } \\
\text { Table 2-1 }\end{array}$ & \\
\hline
\end{tabular}

Page \#: 4 of 5 Report Run Date: 5-MAY-95 
AIR EMISSIONS INVENTORY PERMIT APPLICATION REPORT (UP460)

FACILITY NAME: 100N Facility and Related Structures

Insignificant Source Emissions - HAP Pollutants

Em Point

Srce Nbr Srce Type Pollutant

Sum

Insignificant Source Emissions - Uncategorized Pollutants

Em Point

Srce Nbr Srce Type Pollutant

Em. Pt. Potential Em. Pt.

(tons/yr) Pollutant

Control Device Listing

\begin{tabular}{lc} 
Em Point & Control Equipment Code \\
\hdashline $100 \mathrm{~N}$ P-105N 002 & 099 \\
$100 \mathrm{~N}$ P-107N 004 & 099 \\
$100 \mathrm{~N}$ P-116N 005 & 099
\end{tabular}

Em. Pt. Potential Em. Pt.

to Emit (tons/yr) Ctl Actual (tons/yr) Pollutant

\section{Sum}

099
099
099 


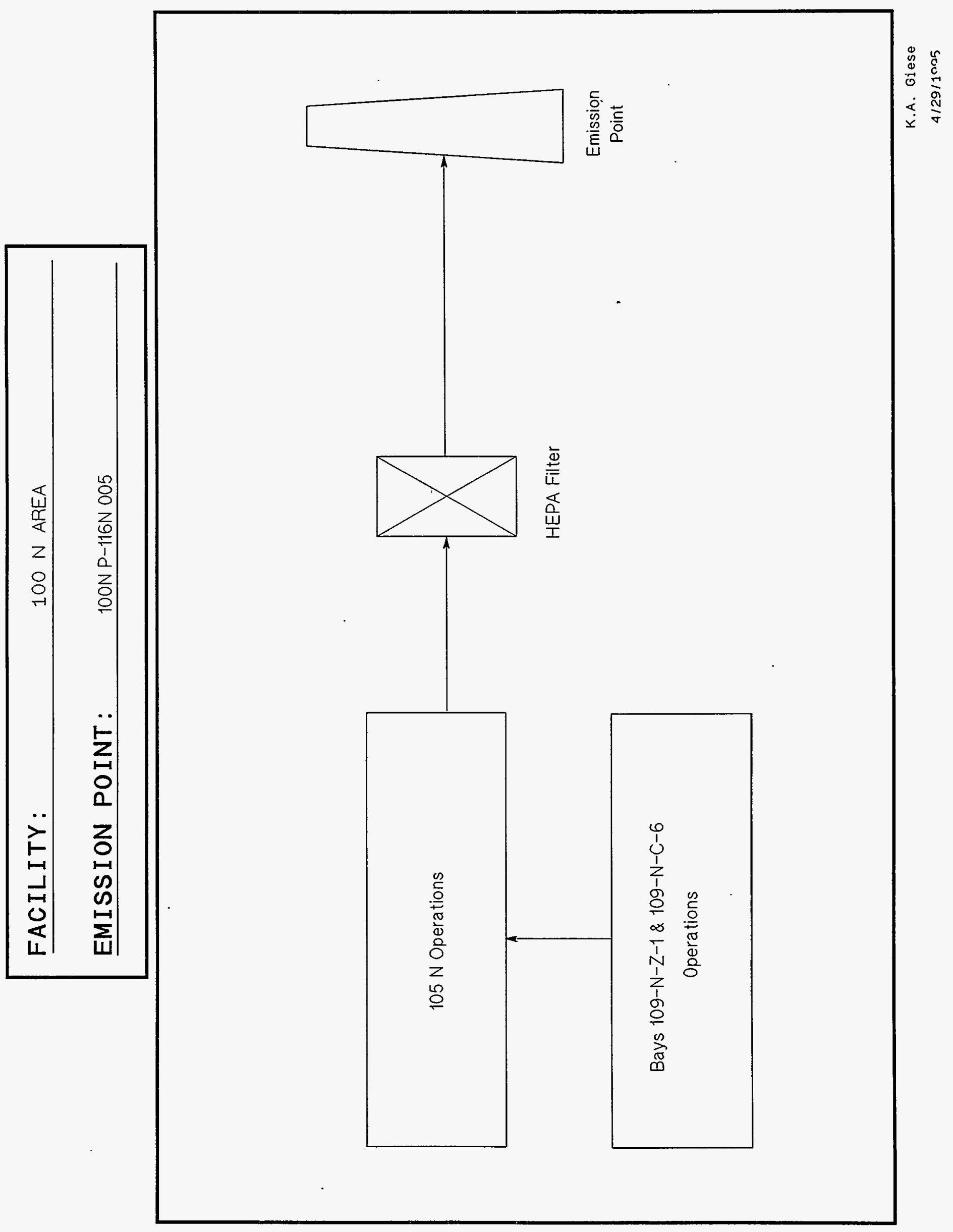




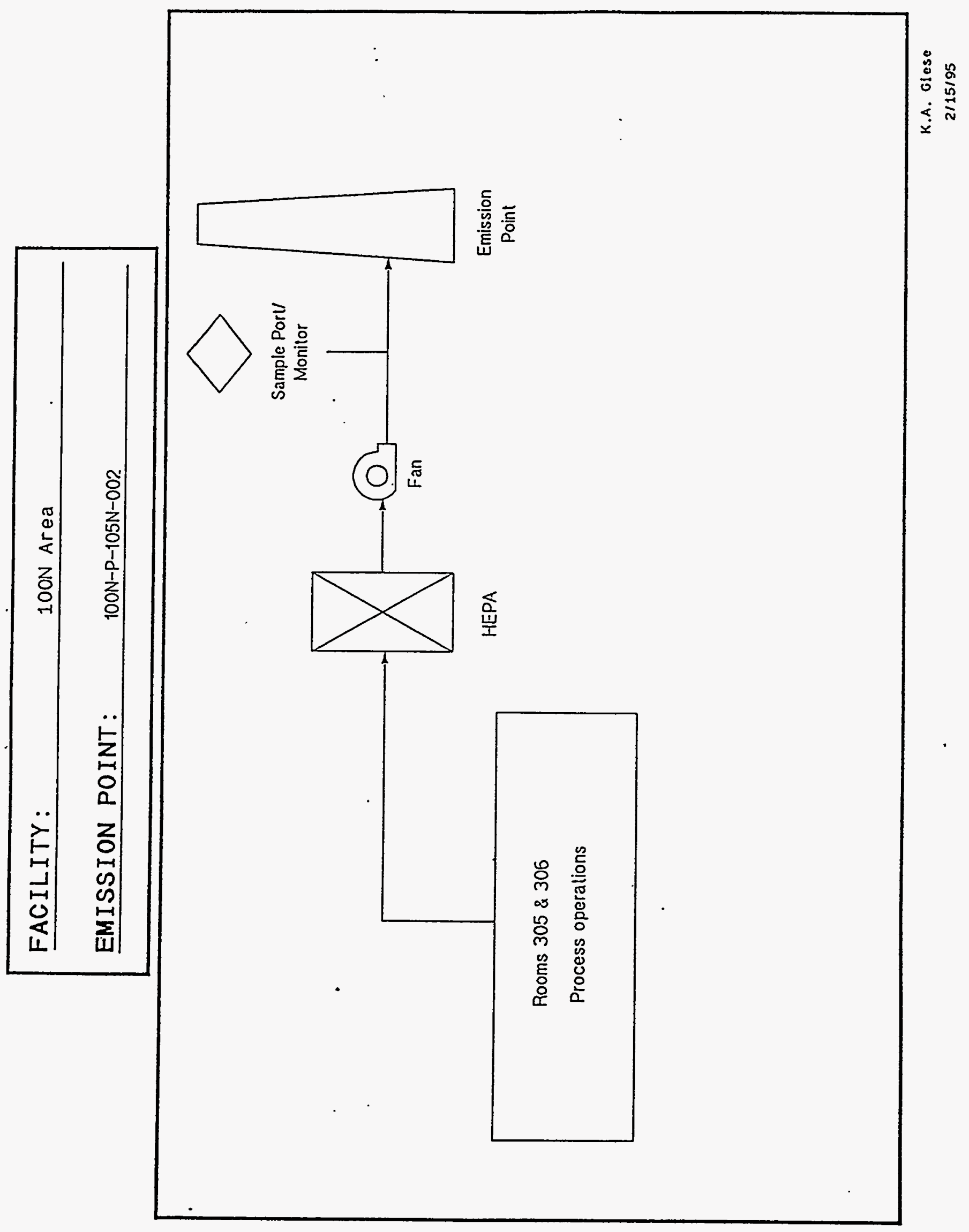




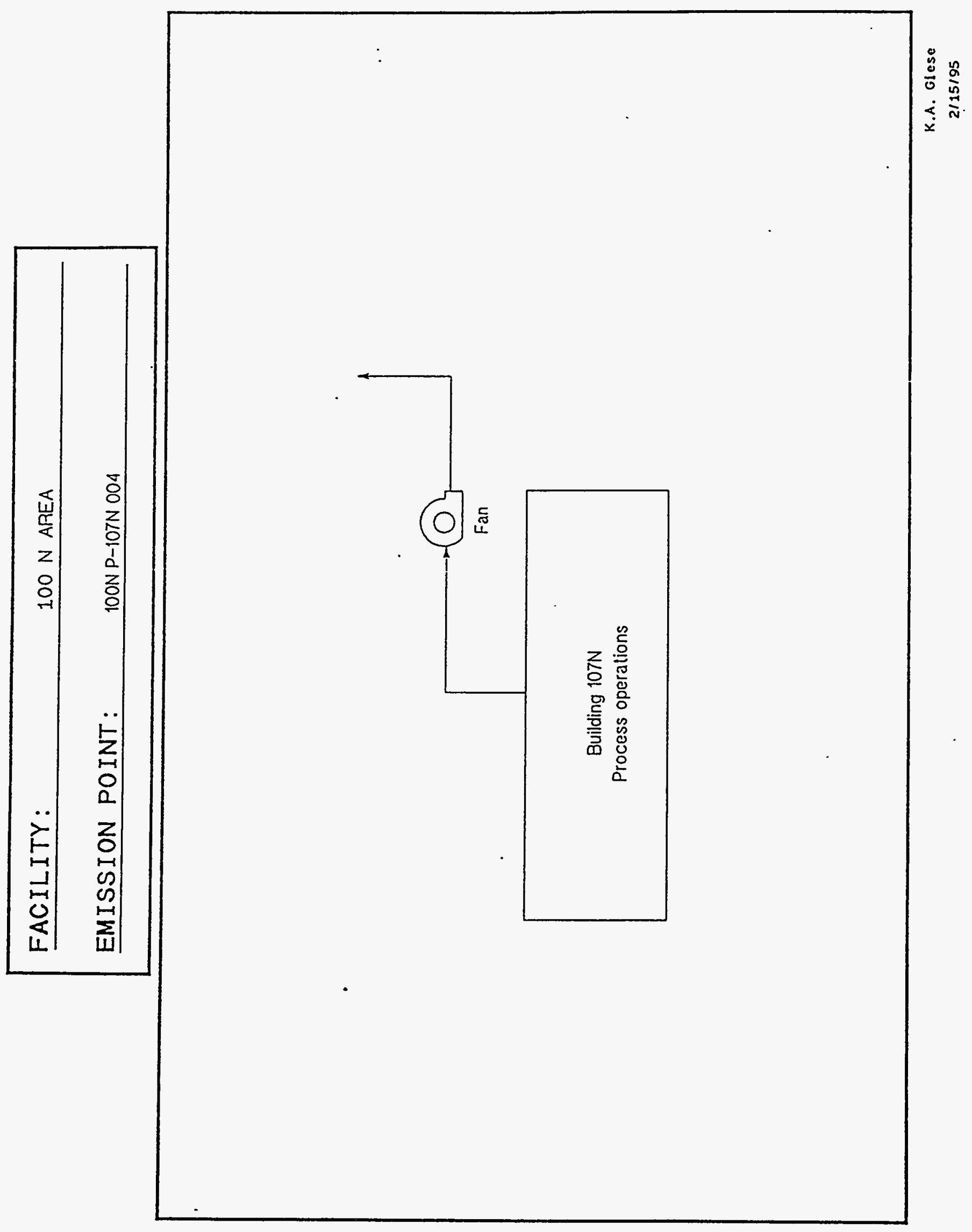




\section{N Area}

N149692

Emission Point

100N M-1705N 013

N149590 E571240

Building Corner N14958G E57110

Emission Point 100N P-107N 004

N149575 E571109

Emission Point

$100 \mathrm{~N}$ P-105N 003

N149526 571126

Emission Point
$100 \mathrm{~N}$ M-105N 001

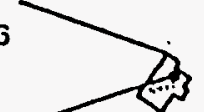

A 149537 E571267

Emission Point

100N T-166N 012

N149654 E571315
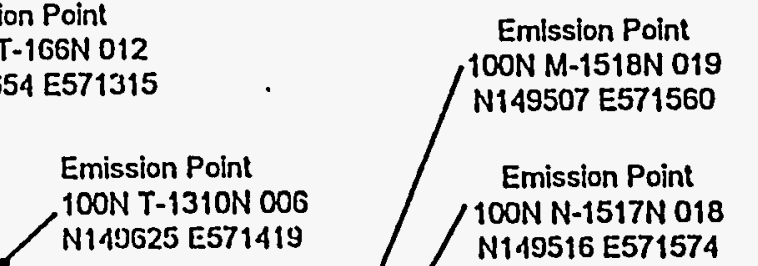

w

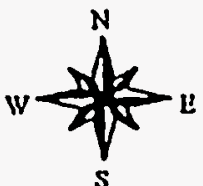

s

Emission Point

$100 \mathrm{~N} \mathrm{~N}-1517 \mathrm{~N} 018$

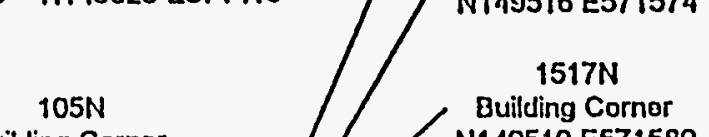

\section{$1517 \mathrm{~N}$}

Building Cornor N149537 E571308.

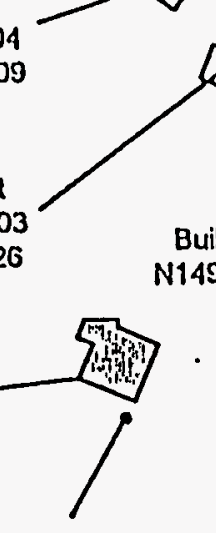

Emlssion Point

$182 \mathrm{~N}$

Building Corner

N149370 E571050

100N T-182N 007, 008

N149350 E571074

100N F-184N 011

N149400 E571270

ding Cornor

149459 E571263

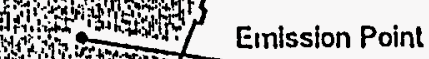

Einission Point

100N P-105N 002

308.

149510 Est

Emission Point

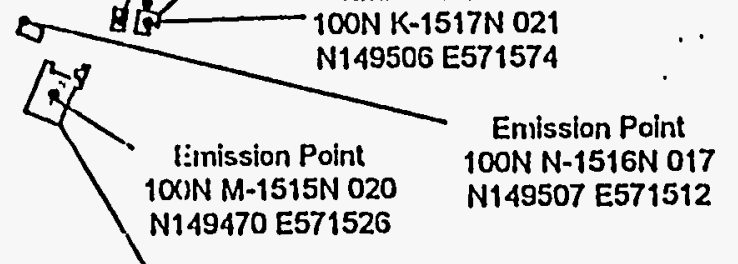

100N T-184N 009

N149426 E571292

$1515 \mathrm{~N}$

Uluilding Corner

Building Corne

N149453 E571520

Emission Point

$100 N$ F-184N.010

N149402 E571300

Emission Point

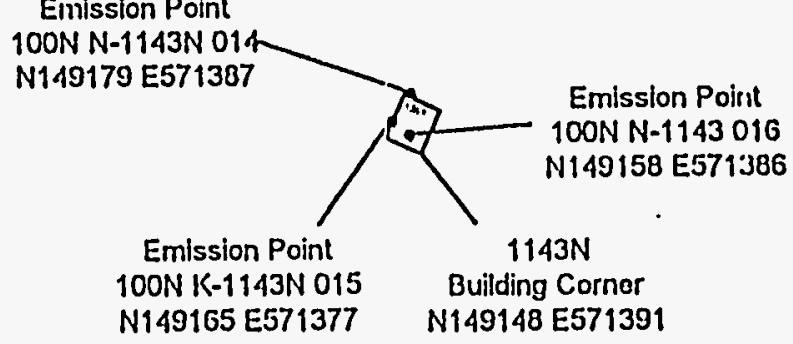


Hanford Site Air

Operating Permit Application
DOE/RL-95-07, Rev. 0

05/95

APPENDIX D

200 EAST AREA 


\section{APPENDIX D}

\section{CONTENTS}

D-1 284E POWERHOUSE

D-2 B PLANT

D-3 PUREX FACILITY

D-4 200 EAST AREA TANK FARMS

D-5 200 AREA EFFLUENT TREATMENT FACILITY 


\section{APPENDIX D-1}

\section{E POWERHOUSE}

\section{Chapter I. General Information}

\section{I-B.1. Facility Description}

The 284E Powerhouse is located in the 200 East Area. The 284E Powerhouse has five coalfired boilers in operation. All of the boilers are used exclusively to generate steam. Steam generated is used in nearby process facilities for heating and process operations. The total rated capacity of the five boilers is 310,000 pounds per hour of steam. An inoperable oilfired package boiler is also present at the 284E Powerhouse.

I-B.2. Products, Raw Materials, Fuels, Processes

- Principal products - The only product is $250-350$ pounds per square inch gauge steam for heating and process operations.

- Process information - Treated boiler feed water is prepared. Sanitary water is dechlorinate using sodium sulfite before entering sodium zeolite softener ion exchange units to remove presence of calcium and magnesium salts. Feed water chemistry control is performed using $<4 \%$ potassium hydroxide to prevent scaling and sedimentation. Sodium sulfite is used to scavenge oxygen. Amine is added to each boiler steam outlet line for condensate corrosion control.

- Raw materials used by processes - There are no raw materials used at the 284E Powerhouse.

- Fuels used by processes - All of the boilers use bituminous coal.

I-C.1. Emission Points, Controls, Stacks

The emission point and stack information is found on pages 1 and 2 of the AEI report output. The control information is found on page 7 of the AEI report output.

\section{I-C.2. Facility Process Flow Diagrams}

Attached.

I-C.3. Site Map

Attached. 
Hanford Site Air

Operating Permit Application
DOE/RL-95-07, Rev. 0

$05 / 95$

\section{Chapter II. Regulatory Requirements}

\section{II-A.1. Description of Discharge and Emission Points}

The emission point and stack information is found on pages 1 and 2 of the AEI report output. 
AIR EMISSIONS INVENTORY PERMIT APPLICATION REPORT (UP460)

FACILITY NAME: 284E Facilities and Structures

\section{Facility Building List}

\begin{tabular}{lrrlll} 
Bldg Id & North & East & Bldg Desc & Custodian(Contractor) Cust Phone DOE POC \\
\hline $284 E$ & 135674 & 573933 & POWER HOUSE AND STEAM PLANT & TRUE, GREGORY R. & DOE Phone
\end{tabular}

\section{Emission Point Listing}

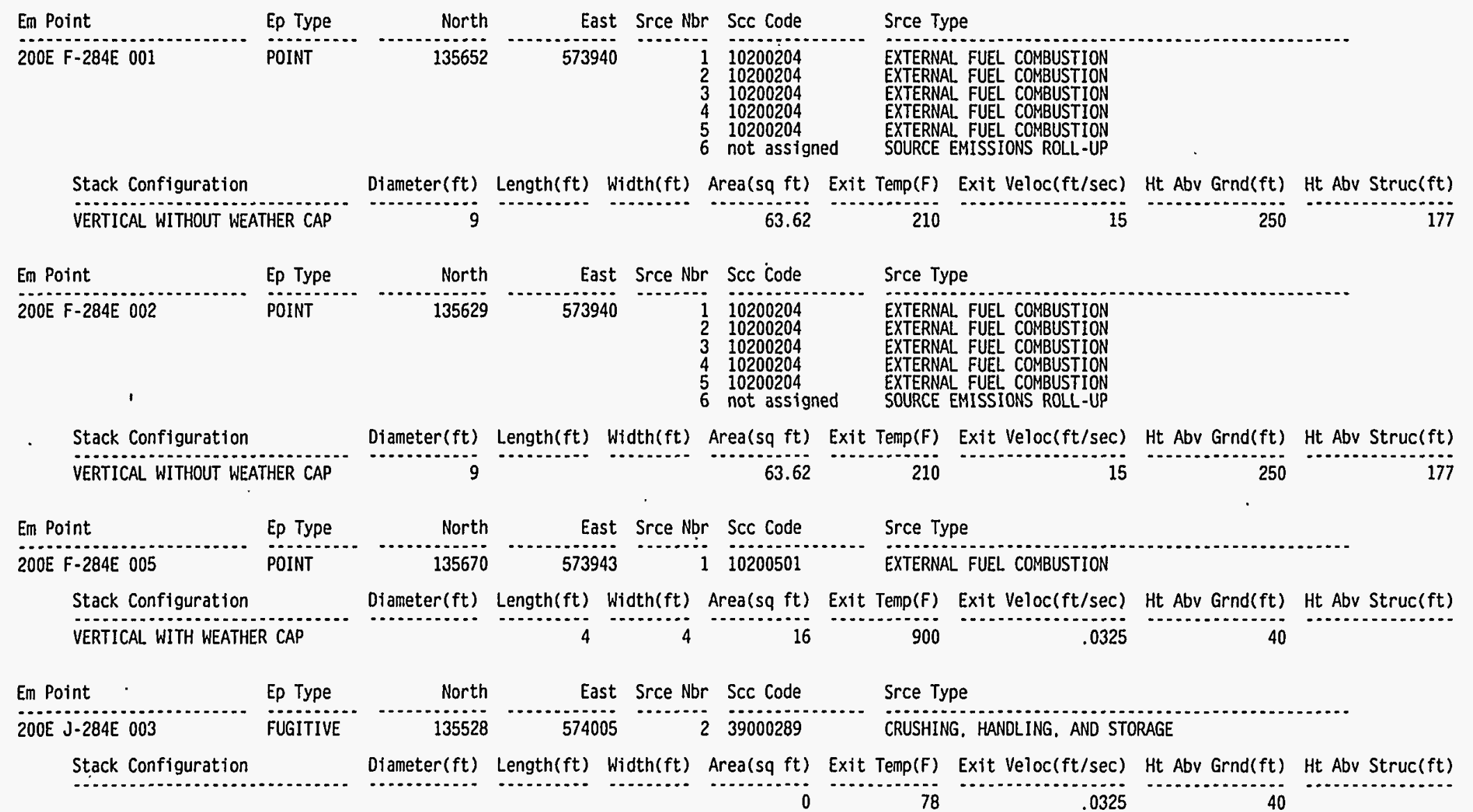


AIR EMISSIONS INVENTORY PERMIT APPLICATION REPORT (UP460) FACILITY NAME: 284E Facilities and Structures

\section{Emission Point Listing}

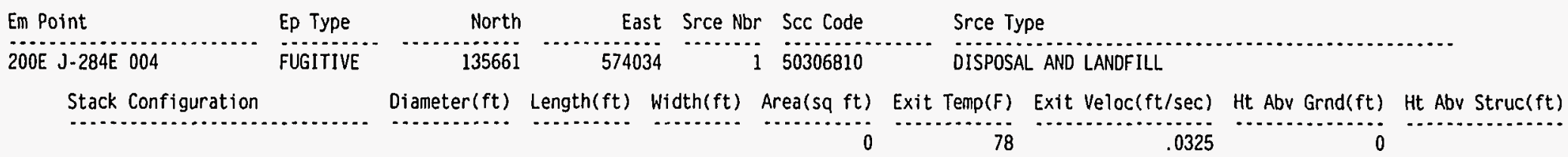

Source (Process) Listing

\begin{tabular}{|c|c|c|c|c|c|c|}
\hline Em Point & Srce Nbr & Srce Type & Hrs Day & Days Week & Wks Year & Summary Process Description \\
\hline $200 E F-284 E 001$ & 1 & EXTERNAL FUEL COMBUSTION & 24 & 7 & 52 & ERIE CITY, COAL-FIRED, SPREADER STOCKER BOILER. \\
\hline Em Point & Srce $\mathrm{Nbr}$ & Srce Type & Hrs Day & Days Week & Wks Year & Summary Process Description \\
\hline 200E F-284E 001 & 2 & EXTERNAL FUEL COMBUSTION & 24 & 7 & 52 & ERIE CITY COAL-FIRED SPREADER STOKER BOILER. \\
\hline En Point & Srce Nbr & Srce Type & Hrs Day & Days Week & Whs Year & Summary Process Description \\
\hline 200E F-284E 001 & 3 & EXTERNAL FUEL COMBUSTION & 24 & 7 & 52 & ERIE CITY COAL-FIRED SPREADER STOKER BOILER. \\
\hline Em Point & Srce Nbr & Srce Type & Hrs Day & Days Week & Wks Year & Summary Process Description \\
\hline $200 E \mathrm{~F}-284 \mathrm{E} 001$ & 4 & EXTERNAL FUEL COMBUSTION & 24 & 7 & 52 & RILEY COAL-FIRED SPREADER STOKER BOILER. \\
\hline Em Point & Srce Nbr & Srce Type & Hrs Day & Days Week & Wks Year & Summary Process Description \\
\hline $200 \mathrm{E} F-284 \mathrm{E} 001$ & 5 & EXTERNAL FUEL COMBUSTION & 24 & 7 & 52 & RILEY COAL-FIRED SPREADER STOKER BOILER. \\
\hline Em Point & Srce $\mathrm{Nbr}$ & Srce Type & Hrs Day & Days Week & Whs Year & Summary Process Description \\
\hline $200 \mathrm{E} F-284 \mathrm{E} 001$ & 6 & SOURCE EMISSIONS ROLL-UP & 24 & 7 & 52 & TOTAL EMISSIONS FOR EMISSION POINT F-284E-001 \\
\hline Em Point & Srce Nbr & Srce Type & Hrs Day & Days Week & Wks Year & Summary Process Description \\
\hline 200E F-284E 002 & 1 & EXTERNAL FUEL COMBUSTION & 24 & 7 & 52 & ERIE CITY COAL-FIRED SPREADER STOKER BOILER. \\
\hline Em Point & Srce Nbr & Srce Type & Hrs Day & Days Week & Whs Year & Summary Process Description \\
\hline 200E F-284E 002 & 2 & EXTERNAL FUEL COMBUSTION & 24 & 7 & 52 & ERIE CITY COAL-FIRED SPREADER STOKER BOILER. \\
\hline
\end{tabular}


AIR EMISSIONS INVENTORY PERMIT APPLICATION REPORT (UP460)

FACILITY NAME: $284 \mathrm{FACITY}$ Facilities and Structures

Source (Process) Listing

\begin{tabular}{|c|c|c|c|c|c|c|}
\hline Em Point & Srce $\mathrm{Nbr}$ & Srce Type & Hrs Day & Days Week & Wks Year & Summary Process Description \\
\hline 200E F-284E 002 & 3 & EXTERNAL FUEL COMBUSTION & 24 & 7 & 52 & ERIE CITY COAL-FIRED, SPREADER STOKER BOILER. \\
\hline Em Point & Srce $\mathrm{Nbr}$ & Srce Type & Hrs Day & Days Week & Wks Year & Summary Process Description \\
\hline 200E F-284E 002 & 4 & EXTERNAL FUEL COMBUSTION & 24 & 7 & 52 & ERIE CITY COAL-FIRED SPREADER STOKER BOILER. \\
\hline Em Point & Srce Nbr & Srce Type & Hrs Day & Days Week & Wks Year & Summary Process Description \\
\hline $200 E$ F-284E 002 & 5 & EXTERNAL FUEL COMBUSTION & 24 & 7 & 52 & ERIE CITY COAL-FIRED SPREADER STOKER BOILER. \\
\hline Em Point & Srce $\mathrm{Nbr}$ & Srce Type & Hrs Day & Days Week & Wks Year & Summary Process Description \\
\hline 200E F-284E 002 & 6 & SOURCE EMISSIONS ROLL-UP & 24 & 7 & 52 & TOTAL EMISSIONS FOR EMISSION POINT F-2B4E-002 \\
\hline Em Point & Srce Nbr & Srce Type & Hrs Day & Days Week & Wks Year & Summary Process Description \\
\hline $200 E F-284 E 005$ & $i$ & EXTERNAL FUEL COMBUSTION & & & & TRANE. NO. 2 OIL-FIRED PACKAGE BOILER, INOPERABLE \\
\hline Em Point & Srce $\mathrm{Nbr}$ & Srce Type & Hrs Day & Days Week & Wks Year & Summary Process Description \\
\hline $200 \mathrm{E} \mathrm{J}-284 \mathrm{E} 003$ & 2 & $\begin{array}{l}\text { CRUSHING, HANDLING, AND } \\
\text { STORAGE }\end{array}$ & 24 & 7 & 52 & COAL CRUSHER, FREQUENTLY INOPERABLE. ALSO COAL STORAGE. \\
\hline Em Point & Srce Nbr & Srce Type & Hrs Day & Days Week & Wks Year & Summary Process Description \\
\hline $200 \mathrm{E} \mathrm{J}-284 \mathrm{E} 004$ & 1 & DISPOSAL AND -LANDFILL & 24 & 7 & 52 & ASH OISPOSAL PIT \\
\hline \multicolumn{7}{|c|}{ Source Raw Materials Usage } \\
\hline Em Point & $\begin{array}{l}\text { Srce } \mathrm{Nbr} \\
\end{array}$ & Srce Type & & Proces: & ss Rate $\operatorname{Pr}$ & rocess Uom Material \\
\hline
\end{tabular}

Fuels Usage by Source Processes

Em Point

Srce Nbr Srce Type

200E F-284E 001

1 EXTERNAL FUEL COMBUSTION

Qty Uom Fuel

6000000 POUND COAL BITUMINOUS 


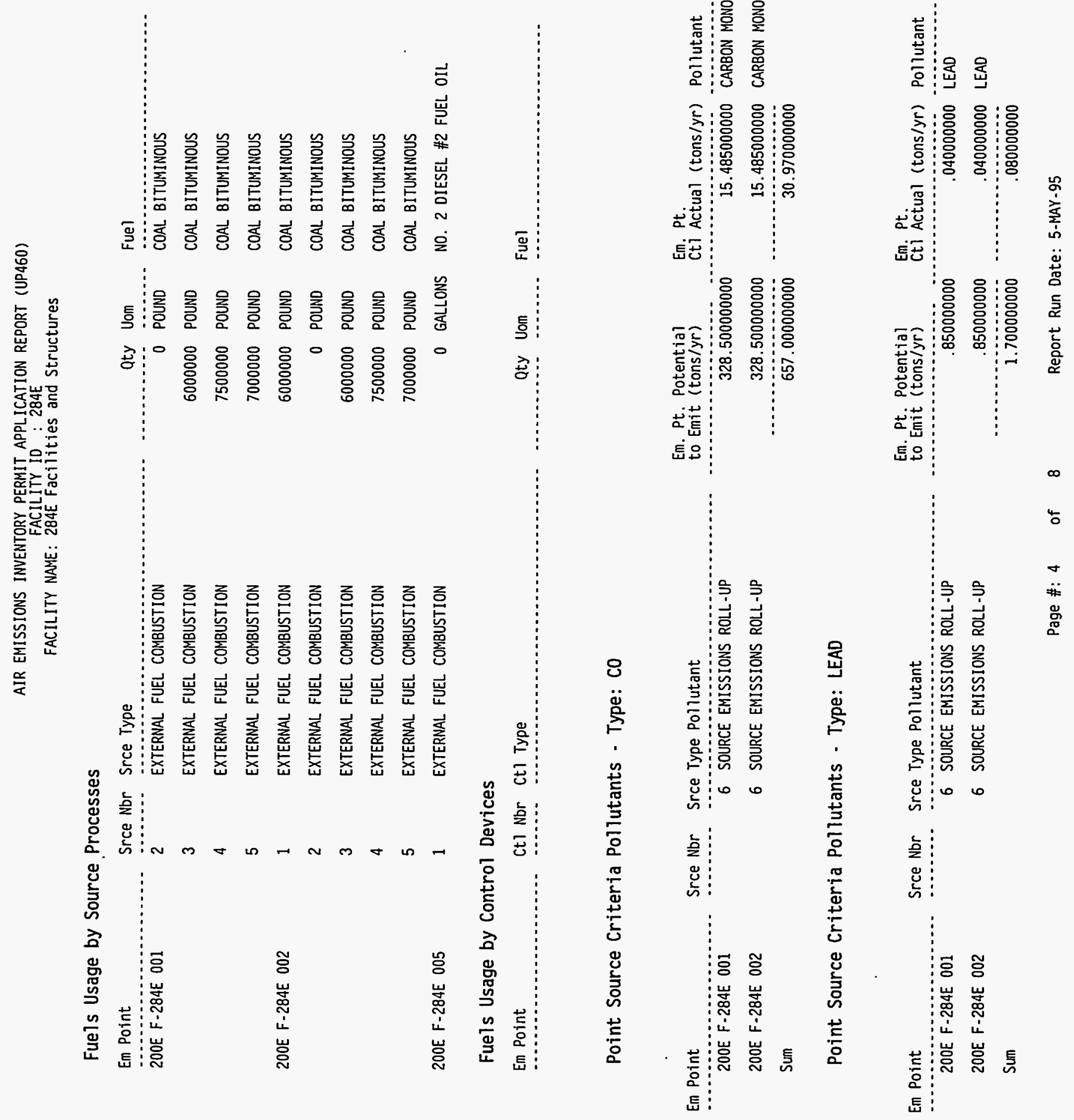


AIR EMISSIONS INVENTORY PERHIT APPLICAIION REPORT (UP460)

FACILITY NAME: 284E Facilities and Structures

Point Source Criteria Pollutants - Type: NOX

Em Point

200E F-284E 001

200E F-284E 002

Sum
Srce Nbr Srce Type Pollutant

6 SOURCE EMISSIONS ROLL-UP

6 SOURCE EMISSIONS ROLL-UP Em. Pt. Potential
to Emit (tons/yr) Em. Pt. Actual (tons/yr) Pollutant

$\begin{array}{rrr}900.000000000 & 42.430000000 & \text { NITROGEN OXIDES } \\ 900.000000000 & 42.430000000 & \text { NITROGEN OXIDES } \\ 1800.000000000 & 84.860000000 & \end{array}$

Point Source Criteria Pollutants - Type: PM

\begin{tabular}{|c|c|c|c|c|c|}
\hline Em Point & Srce Nbr & Srce Type Pollutant & $\begin{array}{l}\text { Em. Pt. Potential } \\
\text { to Emit (tons/yr) }\end{array}$ & $\begin{array}{l}\text { Em. Pt. } \\
\text { Cti Actual (tons/yr) }\end{array}$ & Pollutant \\
\hline $200 E$ F-284E 001 & & 6 SOURCE EMISSIONS ROLL-UP & 8.650000000 & .410000000 & TOTAL PARTICULATE MATTER \\
\hline 200E F-284E 002 & & 6 SOURCE EMISSIONS ROLL-UP & 8.650000000 & .410000000 & TOTAL PARTICULATE MATTER \\
\hline Sum & & & 17.300000000 & .820000000 & \\
\hline
\end{tabular}

Point Source Criteria Pollutants - Type: PM10

\begin{tabular}{|c|c|c|c|c|c|}
\hline Em Point & Srce Nbr & Srce Type Pollutant & $\begin{array}{l}\text { Em. Pt. Potential } \\
\text { to Emit (tons/yr) }\end{array}$ & $\begin{array}{l}\text { Em. Pt. } \\
\text { Cti Actual (tons/yr) }\end{array}$ & Pollutant \\
\hline 200E F-284E 001 & & 6 SOURCE EMISSIONS ROLL-UP & 1.750000000 & .080000000 & PARTICULATE MATTER ( 10 MICRONS OR LESS) \\
\hline 200E F-284E 002 & & 6 SOURCE EMISSIONS ROLL-UP & 1.750000000 & .080000000 & PARTICULATE MATTER (10 MICRONS OR L.ESS) \\
\hline Sum & & & 3.500000000 & .160000000 & \\
\hline
\end{tabular}

Point Source Criteria Pollutants - Type: SOX

\begin{tabular}{|c|c|c|c|c|c|}
\hline Em Point & Srce Nbr & Srce Type Pollutant & $\begin{array}{l}\text { Em. Pt. Potential } \\
\text { to Emit (tons/yr) }\end{array}$ & $\begin{array}{l}\text { Em. Pt. } \\
\text { Ct广 Actual (tons/yr) }\end{array}$ & Pollutant \\
\hline 200E F-284E .001 & & 6. SOURCE EMISSIONS ROLL-UP & 1310.500000000 & 62.000000000 & SULFUR DIOXIOE \\
\hline 200E F-284E 002 & & 6 SOURCE EMISSIONS ROLL-UP & 1310.500000000 & 62.000000000 & SULFUR DIOXIDE \\
\hline Sum & & & 2621.000000000 & 124.000000000 & \\
\hline
\end{tabular}


Point Source Criteria Pollutants - Type: VOC

\section{Em Point}

200E F-284E 001

200E F-284E 002

Sum

Point Source Emissions - Radiological Pollutants

Em Point

Srce Nbr Srce Type Pollutant

Em. Pt. Potential

to Emit (Ci/yr)

6 SOURCE EMISSIONS ROLL-UP

6 SOURCE EMISSIONS ROLL-UP

Em. Pt. Potential Em. Pt.

(tons/yr) Pollutant

\section{Point Source Emissions - HAP Pollutants}

Em Point

200E F-284E 001

Srce Nbr Srce Type Pollutant

6 SOURCE EMISSIONS ROLL-UP

200E F-284E 002

6 SOURCE EMISSIONS ROLL-UP

Sum

Em. Pt. Potential
to Emit (tons/yr) $\quad$ Cti Pt. Actual (tons/yr) Pollutant

$.900000000 \quad .040000000$ ARSENIC

$.100000000 \quad .005000000$ BERYLLIUM

$.050000000 \quad .005000000$ CADMIUM

$2.600000000 \quad .125000000$ CHROMIUM

$.350000000 \quad .015000000$ FORMALDEHYDE

3.6000000000 .170000000 MANGANESE

.05000000

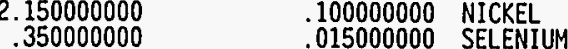


AIR EMISSIONS INVENTORY PERMIT APPLICATION REPORT (UP460)

FACILITY NAME: 284E Facilities and Structures

Point Source Emissions - Uncategorized Pollutants

\begin{tabular}{|c|c|c|c|c|c|}
\hline Em Point & Srce $\mathrm{Nbr}$ & Srce Type Pollutant & $\begin{array}{l}\text { Em. Pt. Potential } \\
\text { to Emit (tons/yr) }\end{array}$ & $\begin{array}{l}\text { Em. Pt. } \\
\text { Cti Actual (tons/yr) }\end{array}$ & Pollutant \\
\hline 200E F-284E 001 & & 6 SOURCE EMISSIONS ROLL-UP & $\begin{array}{r}1.650000000 \\
3.950000000 \\
5.900000000 \\
.200000000\end{array}$ & $\begin{array}{l}.075000000 \\
.185000000 \\
.280000000 \\
.010000000\end{array}$ & $\begin{array}{l}\text { COPPER } \\
\text { METHANE } \\
\text { NITROUS OXIDE } \\
\text { VANADIUM }\end{array}$ \\
\hline 200E F-284E 002 & & 6 SOURCE EMISSIONS ROLL-UP & $\begin{array}{r}1.650000000 \\
3.950000000 \\
5.90000000 \\
.200000000\end{array}$ & $\begin{array}{l}.075000000 \\
.185000000 \\
.280000000 \\
.010000000\end{array}$ & $\begin{array}{l}\text { COPPER } \\
\text { METHANE } \\
\text { NITROUS OXIDE } \\
\text { VANADIUM }\end{array}$ \\
\hline Sum & & & 23.400000000 & 1.100000000 & \\
\hline
\end{tabular}

Fugitive Source Criteria Pollutants - Type: PM

\begin{tabular}{|c|c|c|c|c|c|}
\hline Em Point & Srce $\mathrm{Nbr}$ & Srce Type Pollutant & $\begin{array}{l}\text { Em. Pt. Potential } \\
\text { to Emit (tons/yr) }\end{array}$ & $\begin{array}{l}\text { Em. Pt } \\
\text { Cti Actual (tons/yr) }\end{array}$ & Pollutant \\
\hline 200E J-284E 003 & & 2 CRUSHING, HANDLING, AND STORAGE & 2.000000000 & 2.000000000 & TOTAL PARTICULATE MATTER \\
\hline Sum & & & 2.000000000 & 2.000000000 & \\
\hline
\end{tabular}

Fugitive Source Emissions - Radiological Pollutants

Em Point

Srce $\mathrm{Nbr}$ Srce Type Pollutant

Em. Pt. Potential Em. Pt.
to Emit (Ci/yr)
Ctl Actual (Ci/yr) Pollutant

Fugitive Source Emissions - HAP Pollutants

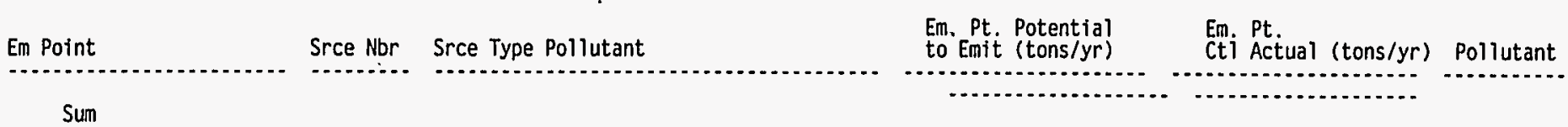

Fugitive Source Emissions - Uncategorized Pollutants

\begin{tabular}{|c|c|c|c|c|}
\hline Em Point & Srce $\mathrm{Nbr}$ & Srce Type Pollutant & $\begin{array}{l}\text { Em. Pt. Potential } \\
\text { to Emit (tons/yr) }\end{array}$ & $\begin{array}{l}\text { Em. Pt. } \\
\text { Cti Actual (tons/yr) }\end{array}$ \\
\hline
\end{tabular}


AIR EMISSIONS INVENTORY PERMIT APPLICATION REPORT (UP460)

FACILITY NAME: 284E Facilities and Structures

Insignificant Source Ėmissions - Radiological Pollutants

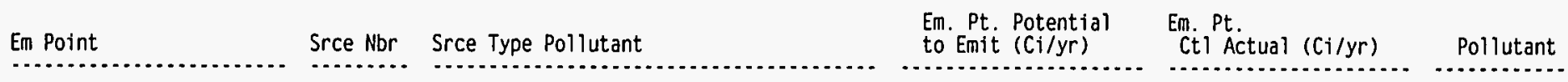

Insignificant Source Emissions - HAP Pollutants

Em Point

Srce $\mathrm{Nbr}$ Srce Type Pollutant

Sum

Insignificant Source Emissions - Uncategorized Pollutants

Em Point

Srce Nbr Srce Type Pollutant
Em. Pt. Potential

(tons/yr)

Em. Pt.

Em. Pt. Potential
to Emit $\begin{aligned} & \text { Em. Pt. } \\ & \text { (tons/yr) }\end{aligned}$ Cti Actual (tons/yr) Pollutant

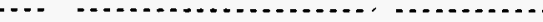

Sum

\section{Control Device Listing}

Em Point

Control Equipment Code

200E F-284E 001

016
016 


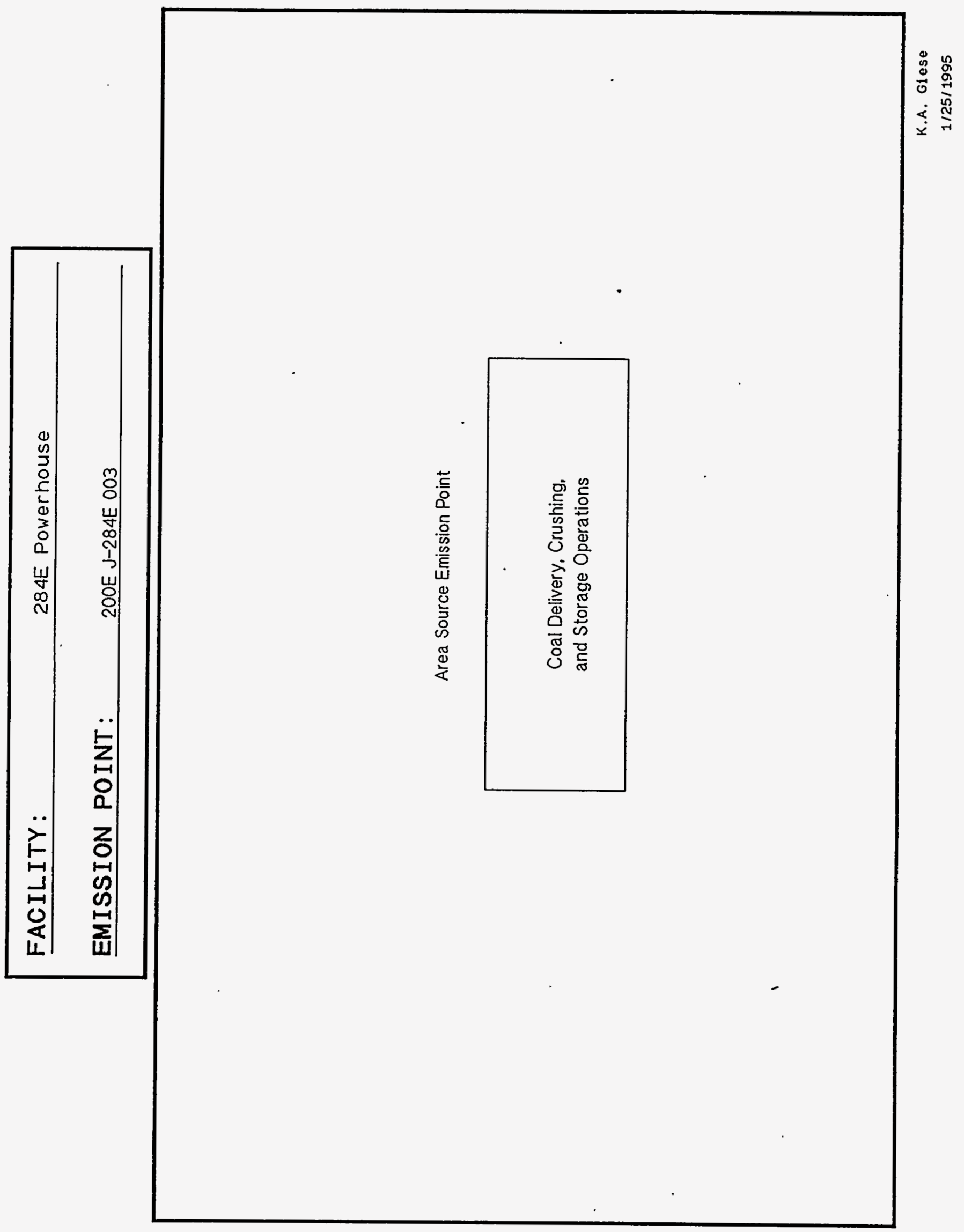




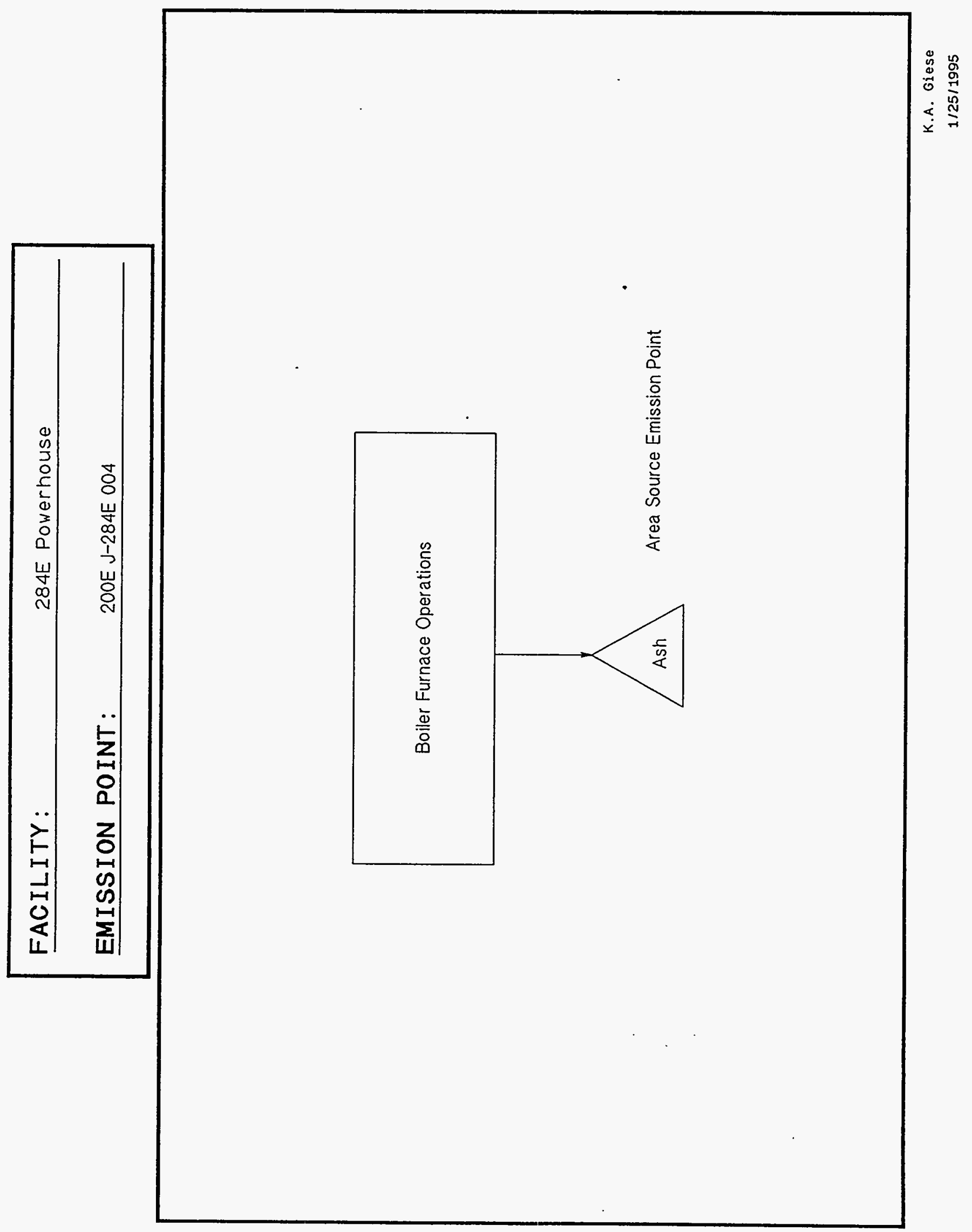


FACILITY:

EMISSION POINT:

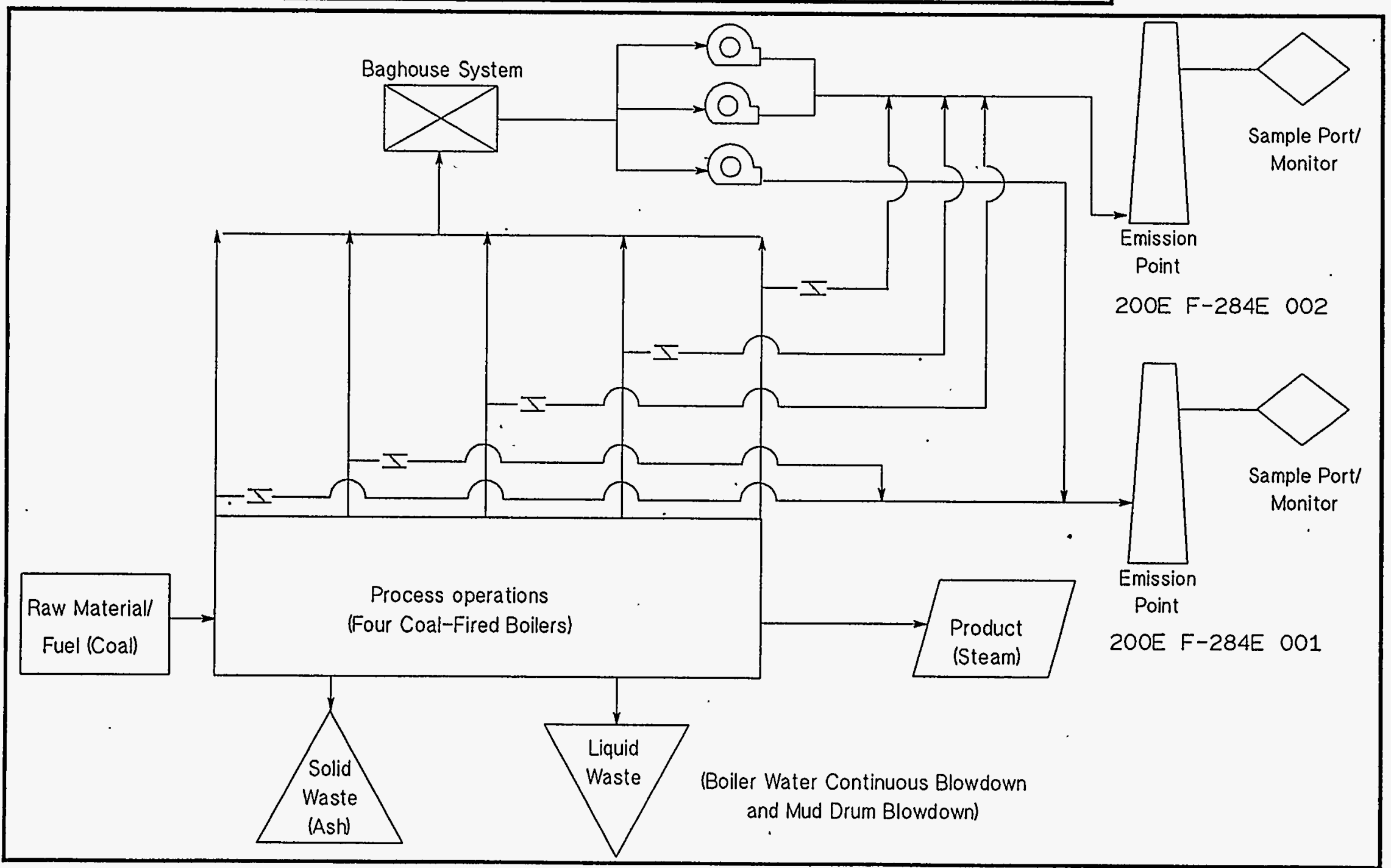

K.A. Giese 1/25/1995 


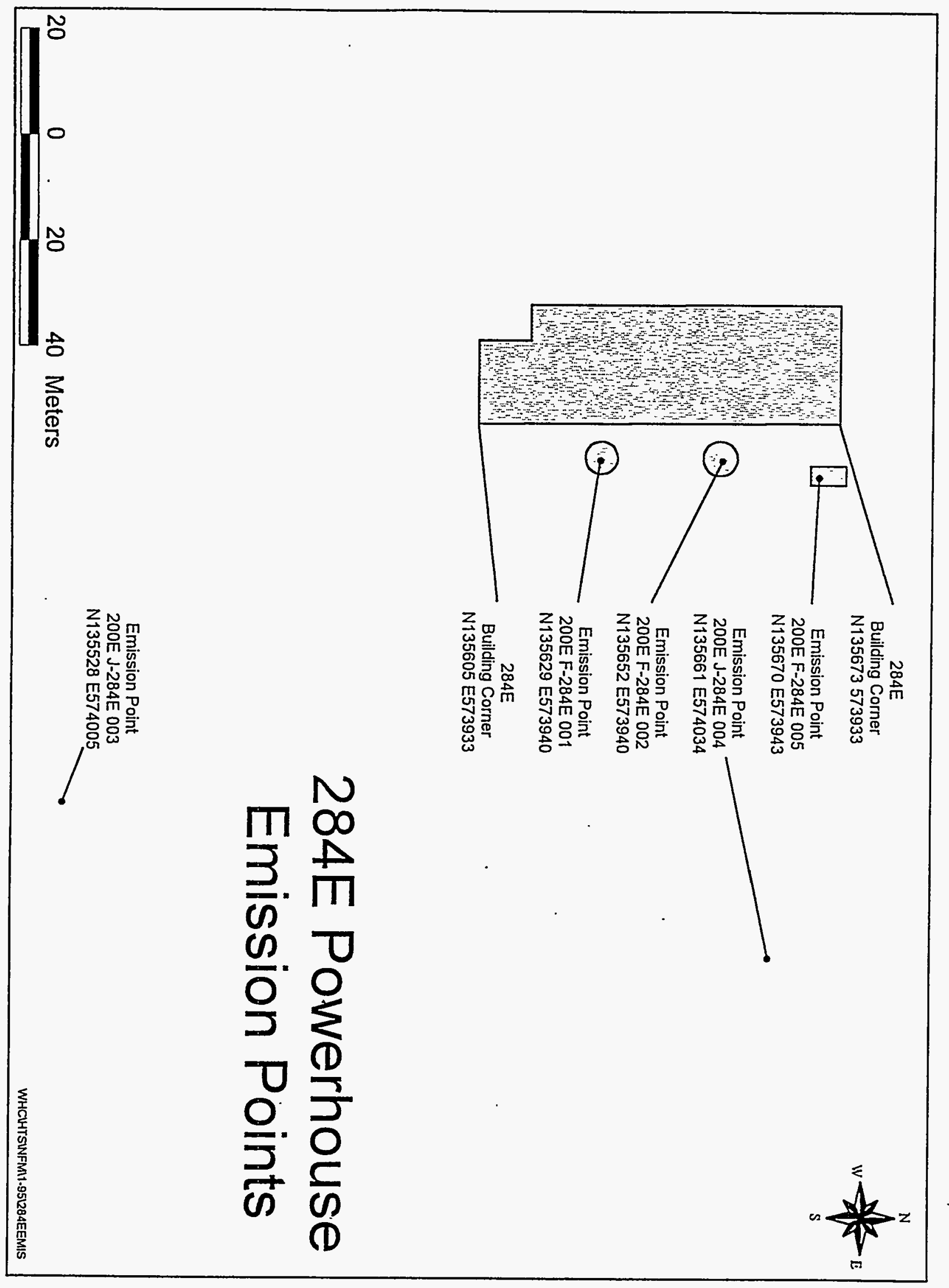




\title{
APPENDIX D-2
}

\section{B PLANT/WASTE ENCAPSULATION AND STORAGE FACILITY}

\author{
Chapter I. General Information
}

\section{I-B.1. Facility Description}

The B Plant was constructed between 1943-45 and originally was designed to process spent nuclear fuels in support of the Manhattan Project. The B Plant was modified from 19611967 for the separation of strontium and cesium from the fissile product waste stream following plutonium and uranium recovery from irradiated reactor fuels in a plutoniumuranium extraction process (PUREX). In 1974, the Waste Encapsulation and Storage Facility (WESF) was put into operation. The individual strontium and cesium solutions were transferred to WESF for conversion to solid compounds, the solid compounds were encapsulated, and the encapsulated compounds were stored in pool cells. This effort continued until 1985.

There are currently no production activities at B Plant/WESF. However, B Plant/WESF remains operational providing surveillance operations to ensure safe storage and management of the WESF cesium and strontium capsules, and of the other radiological inventory at B Plant/WESF. These operations include generation of demineralized water, treatment and storage of low-level radioactive waste, treatment and storage of organic solvent waste, waste transfers within and outside of B Plant/WESF, decontamination of equipment and facilities, various maintenance activities within the canyon and process cells in B Plant/WESF, and storage and transfer of dangerous chemicals inside and outside B Plant/WESF. The B Plant/WESF complex will continue these operations in a safe, cost-effective manner until final decontamination and decommissioning activities commence.

The B Plant/WESF is located in the 200 East Area of the Hanford Site. It is comprised of three main buildings: the 221-B Processing Building, the 271-B Service and Office Building, and 225-B the WESF Building. These are located on the site map in Section I.C.3. The B Plant/WESF complex is operated 24 hours a day.

I-B.2. Products, Raw Materials, Fuels, Processes

- Principal products - There are no products produced at B Plant.

- Process information.

- These are treatment and storage of hazardous and radioactive waste and demineralized water production. 
- Treatment and storage of low-level waste - The low-level waste is mixed with sodium hydroxide and sodium nitrate to meet storage criteria requirements at the Double-Shell Tank System.

- Demineralized water production - Deionized water is produced for use by a continuous deionization system. The process produces a concentrated ionic waste solution and deionized water.

- Treatment and storage of organic solvent waste. Treatment consists of radionuclide removal using caustic strikes.

- Raw materials used by processes - There are no raw materials consumed at B Plant.

- Fuels used by processes - There are no fuels used in the processes at B Plant.

I-C.1. Emission Points, Controls, Stacks

The emission point and stack information is found on pages 1 and 2 of the AEI report output. The control information is found on page 8 of the AEI report output.

I-C.2. Facility Process Flow Diagrams

Attached.

I-C.3. Site Map

Attached.

Chapter II. Regulatory Requirements

II-A.1. Description of Discharge and Emission Points

The emission point and stack information is found on pages 1 and 2 of the AEI report output. 


\section{Facility Building List}

\begin{tabular}{lr} 
Bldg Id & North \\
\hline$\ldots \ldots . . . . .$. & $\ldots . . . . . .$. \\
2918001 & 136382 \\
2968005 & 136444 \\
2968010 & 136433 \\
2968013 & 136381 \\
2968022 & 136469 \\
2968023 & 136469 \\
2968024 & 136484 \\
2968025 & 136469 \\
2968026 & 136469 \\
2968027 & 136469
\end{tabular}

East Bldg Desc

573571 B-PLANT MAIN STACK

573466 221-BB EXHAUST STACK

573361 WASTE ENCAPSULATION AND STORAGE FACILITY STACK

573358 221-BF EXHAUST STACK

573594 B-PLANT GALLERY EXHAUSTER

573559 B-PLANT GALLERY EXHAUSTER

573457 B-PLANT GALLERY EXHAUSTER

573424 B-PLANT GALLERY EXHAUSTER

573402 B-PLANT GALLERY EXHAUSTER
Custodian(Contractor) Cust Phone DOE POC

RIDGE. TINA M.

RIDGE. TINA M.

RIDGE. TINA M.

RIDGE. TINA M.

RIDGE; TINA $M$.

RIDGE. TINA $M$

RIDGE. TINA M.

RIDGE, TINA M.

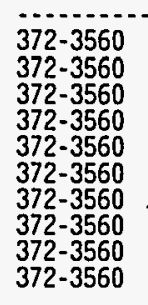

DOE Phone $372-0309$ 372-0309 $372-0309$ $372-0309$ $372-0309$ $372-0309$ $372-0309$ 372.0309 372.0309

\section{Emission Point Listing}

\begin{tabular}{|c|c|c|c|c|c|}
\hline Em Point & Ер Туре & North & East & Srce $\mathrm{Nbr}$ & Scc Code \\
\hline 200E P-291B001 001 & POINT & 136382 & 573571 & $\begin{array}{l}1 \\
1 \\
2 \\
3 \\
4 \\
5\end{array}$ & $\begin{array}{l}\text { not assigned } \\
\text { not assigned } \\
\text { not assigned } \\
\text { not assigned } \\
\text { not assigned }\end{array}$ \\
\hline
\end{tabular}

Srce Type

TANKS AND VESSELS

PROCESS AND PRODUCTION

PROCESS AND PRODUCTION

PROCESS AND PRODUCTION
SOURCE EMISSIONS ROLL-UP

Stack Configuration

Diameter (ft) Length(ft) Width(ft) Area(sq ft)

Exit Temp(F) Exit Veloc(ft/sec) Ht Abv Grnd(ft) Ht Abv Struc(ft)

VERTICAL WITHOUT WEATHER CAP

5

19.63

68

23.7

\begin{tabular}{|c|c|c|c|c|c|c|}
\hline Em Point & Ep Type & North & East & Srce $\mathrm{Nbr}$ & Scc Code & Srce Type \\
\hline 200E P-296B005'001 & POINT & 136444 & 573466 & $\begin{array}{l}1 \\
2 \\
3 \\
4 \\
5\end{array}$ & $\begin{array}{l}\text { not assigned } \\
\text { not assigned } \\
\text { not assigned } \\
\text { not assigned } \\
\text { not assigned }\end{array}$ & $\begin{array}{l}\text { TANNS AND VESSELS } \\
\text { TANKS AND VESSELS } \\
\text { PROCESS AND PRODUCTION } \\
\text { PROCESS AND PRODUCTION } \\
\text { SOURCE EMISSIONS ROLL-UP }\end{array}$ \\
\hline
\end{tabular}

Stack Configuration

Diameter(ft) Length(ft) Width(ft) Area(sq ft) Exit Temp(F) Exit Veloc(ft/sec) Ht Abv Grnd(ft) Ht Abv Struc(ft) VERTICAL WITHOUT WEATHER CAP 1.3 68 
AIR EMISSIONS INVENTORY PERMIT APPLICATION REPORT (UP460)
FACILITY ID : B-PIant

FACILITY NAME: B-Plant Facility and Associated Structures

\section{Emission Point Listing}

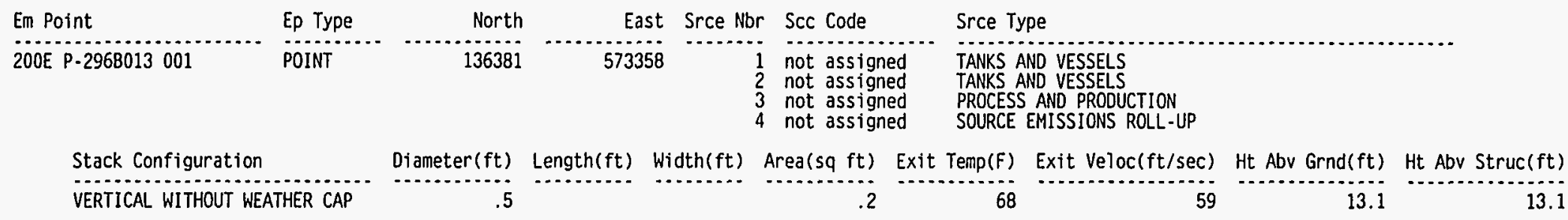

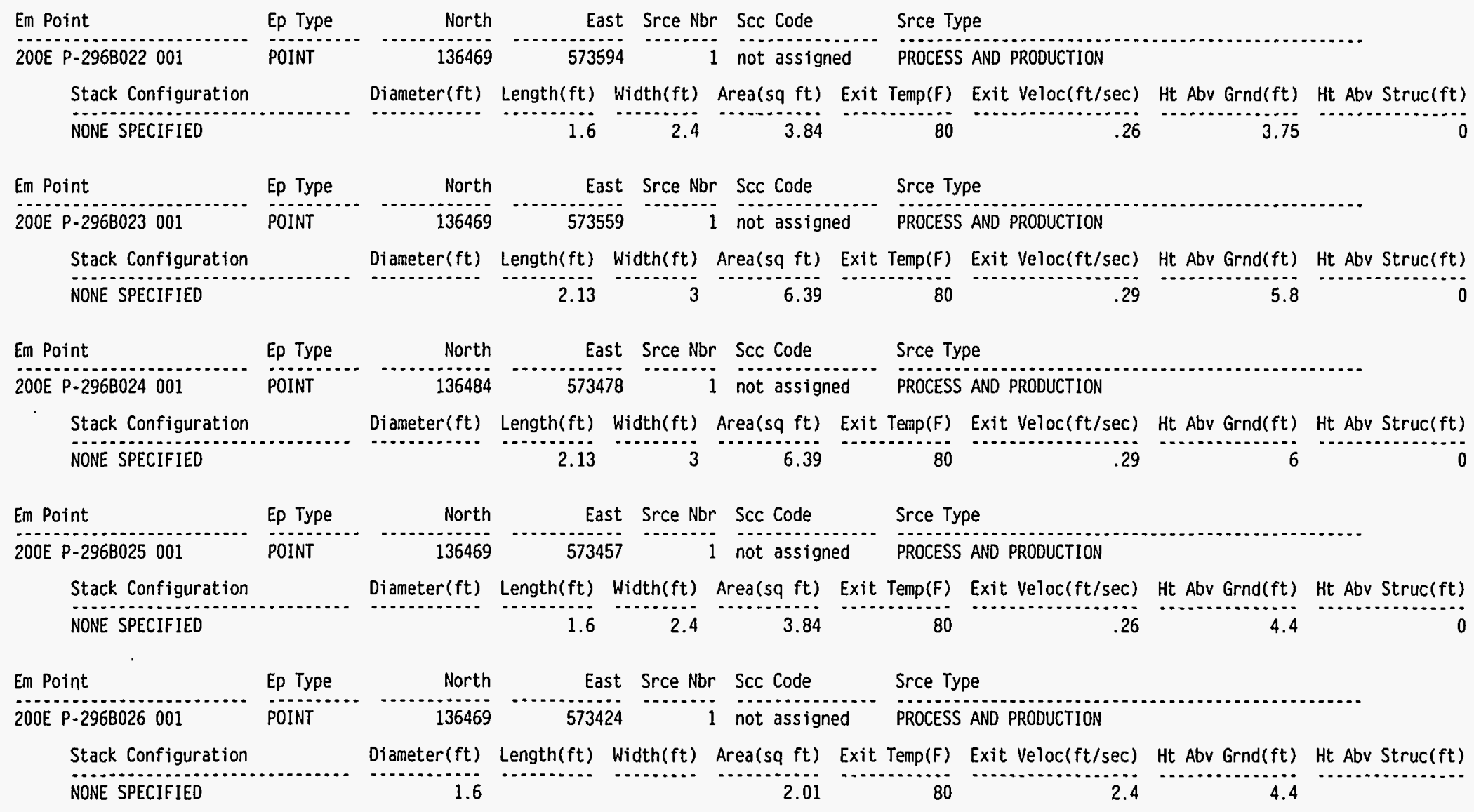

Page \#: 2 of $8 \quad$ Report Run Date: $29-A P R-95$ 


\section{Emission Point Listing}

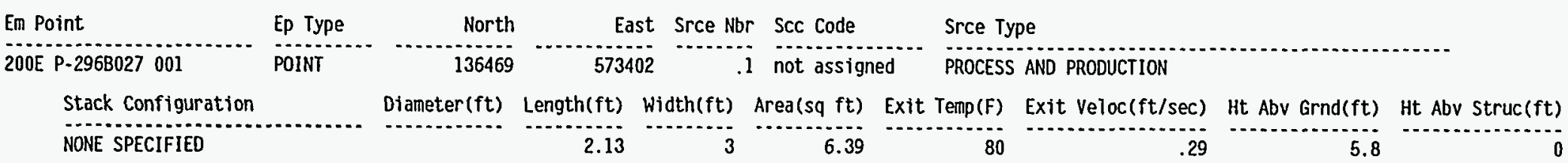

Source (Process) Listing

\begin{tabular}{|c|c|c|c|c|c|c|}
\hline Er Point & Srce Nbr & Srce Type & Hrs Day & Days Week & Wks Year & Sumnary Process Description \\
\hline $200 E$ P-291B001 001 & 1 & TANKS AND VESSELS & 24 & 7 & 52 & B-PLANT CANYON TANKS AND VESSELS \\
\hline
\end{tabular}

B-PLANT TANKS AND VESSELS CONSIST OF THE FOLLOWING MAJOR GROUPS:

1. LOW LEVEL WASTE (LLW) SYSTEM CONSISTS OF MULTIPLE TANKS. THE LLW IS CHEMICALLY ADJUSTED WITH NaNO2 AND NaOH TO MEET TANK FARM CRITERIA.

2. ORGANIC PHASE AND AQUEOUS PHASE SYSTEM IS MAOE UP OF MULTIPLE TANKS. THE ORGANIC PHASE. CONSISTS OF SALTS OF DH-2ethylheXY1 PhOSPhOrIC aCId

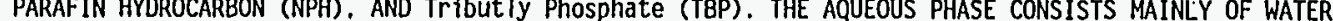

3. MULTIPLE TANKS WERE USED TO STORE AND TRANSFER THE NEUTRALIZED CURRENT ACID WASTE (NCAW) INVENTORY. THE NCAW WASTE WAS TRANSFERRED TO DOUBLE-SHELL TANK SYSTEM IN MAY 1993.

4. THERE ARE MULTIPLE OTHER TANKS LOCATED IN THE CANYON.

5. THE LLW CONCENTRATOR IN CELL 23 IS USED TO TREAT LLW WHEN REQUIRED.

$$
\begin{aligned}
& \text { Em Point Srce Nbr Srce Type Hrs Day Days Week Wks Year Sumnary Process Description } \\
& 52 \text { THE CANYON STORES VARIOUS RADIOACTIVE MATERIAL AND EQUIPMENT } \\
& \text { THAT CONTRIBUTE TO RADIOACTIVE EMISSIONS. PROCESS CELLS ALSO }
\end{aligned}
$$
PROCESSES THAT CONTRIBUTE TO RADIOACTIVE EMISSIONS INCLUDE THE TREATMENT AND STORAGE OF LOW LEVEL WASTE, ORGANIC PHASE WASTE. AND VARIOUS ACTIVITIES
CONDUCTED WITHIN THE CANYON AND PROCESS CELLS.
Em Point
Srce Nbr Srce Type
Hrs Day Days Week Wks Year Summary Process Description
200E P-291B001 001
3 PROCESS AND PRODUCTION
THE 224-B BUILDING CONTAINS EMPTY TANKS THAT CONTRIBUTE TO
THE 291BD01 RADIOACTIE EMISSIPNS THE TAT CONTRIBUTE TO PASSIVELY VENTILATED AND IS TIED INTO THE 221-8 WIND TUNNEL SYSTEM VIA A 24-IN. DIAMETER INTERCONNECTION.

ACTIVITIES THAT CONTRIBUTE TO THE $291 B 001$ RADIOACTIVE EMISSIONS INCLUDE WASTE TRANSFERS. DECONTAMINATION OF EQUIPMENT AND FACILITIES. AND VARIOUS OTHER MAINTENANCE ACTIVITIES WITHIN THE CANYON AND PROCESS CELLS. 
AIR EMISSIONS INVENTORY PERMIT APPLICATION REPORT (UP460)

FACILITY ID : B-Plant
FACILITY NAME: B-Plant Facility and Assocfated structures

Source (Process) Listing

\begin{tabular}{|c|c|c|c|c|c|c|}
\hline Em Point & Srce $\mathrm{Nbr}$ & Srce Type & Hrs Day & Days Week & Wks Year & Summary Process Description \\
\hline $200 E$ P-291B001 001 & 4 & PROCESS ANO PRODUCTION & 24 & 7 & 52 & $\begin{array}{l}\text { THE } 212 B \text { BUILDING CONTAINS A PROCESS CELL THAT CONTRIBUTES } \\
\text { TO THE } 291 B 001 \text { RADIOACTIVE STACK EMISSIONS. }\end{array}$ \\
\hline \multicolumn{7}{|c|}{$\begin{array}{l}\text { THE MAIN CANYON AND CELLS OF THE } 221 \text {-B BUILDING ARE EXHAUSTED VIA THE } 2918001 \text { STACK. THIS AIRFLOW SYSTEM REMOVES PROCESS-GENERATED HEAT AND CONTROLS } \\
\text { THE PATH OF THE RELEASE OF CONTAMINATED AIR FROM THE CANYON AND THE ADJACENT } 212-B \text { CASK STATION. }\end{array}$} \\
\hline Em Point & Srce Nbr & Srce Type & Hrs Day & Days Week & Wks Year & Summary Process Description \\
\hline 200E P-291B001 001 & 5 & SOURCE EMISSIONS ROLL-UP & 24 & 7 & 52 & TOTAL EMISSIONS FOR EMISSION POINT P-291B001-001. \\
\hline Em Point & Srce Nbr & Srce Type & Hrs Day & Days Week & Wks Year & Summary Process Description \\
\hline 200E P-296B005 001 & 1 & TANKS AND VESSELS & 24 & 7 & 52 & $\begin{array}{l}\text { BCP WEIR TANK PROCESS CONDENSATE EN ROUTE TO } 221-\mathrm{BF} \text {. THIS } \\
\text { TANK IS INACTIVE. }\end{array}$ \\
\hline Em Point & Srce $\mathrm{Nbr}$ & Srce Type & Hrs Day & Days Week & Whs Year & Summary Process Description \\
\hline 200E P-296B005 001 & 2 & TANKS AND VESSELS & 24 & 7 & 52 & $\begin{array}{l}\text { BCS WEIR TANK STEAM CONDENSATE EN ROUTE TO } 216-B-55 \text { CRIB OR } \\
216-B-64 \text { BASIN. THIS TANK IS ISOLATED FROM THE BCS TANK AT } \\
\text { THE DIVERTER PIT. THIS TANK IS INACTIVE. }\end{array}$ \\
\hline Em Point & Srce Nbr & Srce Type & Hrs Day & Days Week & Wks Year & Summary Process Description \\
\hline 200E P-296B005001 & 3 & PROCESS AND PRODUCTION & 24 & 7 & 52 & RESIDUAL RADIONUCLIOE CONTAMINATION IN THE WEIR TANK VAULT. \\
\hline En Point & Srce Nbr & Srce Type & Hrs Day & Days Week & Whs Year & Sumnary Process Description \\
\hline 200E P-296B005 001 & 4 & PROCESS AND PRODUCTION & 24 & 7 & 52 & NORMAL. FACILITY OPERATIONS \\
\hline Em Point & Srce Nbr & Srce Type & Hrs Day & Days Week & Wks Year & Summary Process Description \\
\hline $200 E$ P-296B005 001 & 5 & SOURCE EMISSIONS ROLL-UP & 24 & 7 & 52 & TOTAL EMISSIONS FOR EMISSION POINT P. \\
\hline
\end{tabular}




\section{Source (Process) Listing}

\begin{tabular}{|c|c|c|c|c|c|c|}
\hline Em Point & Srce Nbr & Srce Type & Hrs Day & Days Week & Wks Year & Summary Process Description \\
\hline 200E P-296B010 001 & 1 & PROCESS AND PRODUCTION & 24 & 7 & 52 & CESIUM CAPSULES ARE STORED UNDERWATER IN STORAGE POOLS. \\
\hline \multicolumn{7}{|c|}{$\begin{array}{l}\text { The 296-B-10 stack is an atmospheric discharge stack that handles exhaust of the HVAC systems (K-1. K-3 and K-4) of the 225-B (WESF) Building. The } \\
\text { ventilation system of WESF is designed to provide airflow patterns so that air movement throughout the building is from areas of lesser radioactivity } \\
\text { to areas of greater radioactivity All potentially contaminated areas are maintained at a negative pressure with respect to the atmosphere so that } \\
\text { air flows into the building at ali times. The air is not recirculated. }\end{array}$} \\
\hline \multicolumn{7}{|c|}{$\begin{array}{l}\text { The } K-1 \text { system provides ventilation for the high-risk operating and service areas with contaminated air being filtered through the K-1 filter } \\
\text { building. The } k-3 \text { HVAC system provides ventilation for the highly contaminated process cells and the canyon area. The K-4 HVAC system provides } \\
\text { ventilation for the capsule storage pool area. and the air is filtered through the K-1 filter building. }\end{array}$} \\
\hline \multicolumn{7}{|c|}{$\begin{array}{l}\text { Processes that contribute to radioactive emissions include generating low-level waste, waste transfers, decontamination of equipment and facilities. } \\
\text { and various other activities within the canyon. gallery. and process cells inside WESF. }\end{array}$} \\
\hline Em Point & Srce Nbr & Srce Type & Hrs Day & Days Week & Whs Year & Sumnary Process Description \\
\hline $200 E$ P-2968013 001 & $i$ & TANKS AND VESSELS & 24 & 7 & 52 & $\begin{array}{l}\text { RADIONUCLIDE EMISSION FROM B-PLANT PROCESS CONDENSATE STORED } \\
\text { IN TANK IN BUILDING } 221 B F\end{array}$ \\
\hline Em Point & Srce Nbr & Srce Type & Hrs Day & Days Week & Wks Year & Summary Process Description \\
\hline $200 \mathrm{P}-296 \mathrm{~B} 013001$ & 2 & TANKS AND VESSELS & 24 & 7 & 52 & $\begin{array}{l}\text { RADIONUCLIDE EMISSION FROM B-PLANT PROCESS CONDENSATE STORED } \\
\text { IN TANK IN BUILDING } 221 B F\end{array}$ \\
\hline Em Point & Srce $\mathrm{Nbr}$ & Sirce Type & Hrs Day & Days Week & Wks Year & Summary Process Description \\
\hline $200 E P-296 B 013001$ & 3 & PROCESS AND PROOUCTION & 24 & 7 & 52 & SMEAR CONTAMINATION INSIDE BUILDING \\
\hline Em Point & Srce $\mathrm{Nbr}$ & Srce Type & Hrs Day & Days Week & Wks Year & Summary Process Description \\
\hline 200E P-296B013 001 & 4 & SOURCE EMISSIONS ROLL-UP & 24 & $\dddot{7}$ & 52 & TOTAL EMISSIONS FOR EMISSION POINT P-296B013-001 \\
\hline Em Point & Srce Nbr & Srce Type & Hrs Day & $\begin{array}{l}\text { Days Week } \\
\text {........... }\end{array}$ & Wks Year & Summary Process Description \\
\hline $200 E$ P-296B022 001 & 1 & PROCESS AND PRODUCTION & 24 & 7 & 52 & B-PLANT GALLERY EXHAUSTER \\
\hline Em Point & Srce Nbr & Srce Type & Hrs Day & Days. Week & Wks Year & Summary Process Description \\
\hline 200E P-296B023 001 & 1 & PROCESS AND PRODUCTION & 24 & 7 & 52 & B-PLANT GALLERY EXHAUSTER \\
\hline Em Point & Srce Nbr & Srce Type & Hrs Day & Days Week & Wks Year & Summary Process Description \\
\hline $200 \mathrm{E}$ P-2968024 001 & & PROCESS AND PRODUCTION & 24 & 7 & & B-PLANT GALLERY EXHAUSTER \\
\hline
\end{tabular}


AIR EMISSIONS INVENTORY PERMIT APPLICATION REPORT (UP460)

FACILITY NAME: B.Plant Facility and Associated Structures

Source (Process) Listing

\begin{tabular}{|c|c|c|c|c|c|c|}
\hline Em Point & Srce Nbr & Srce Type & Hrs Day & Days Week & Whs Year & Summary Process Description \\
\hline 200E P-296B025 001 & 1 & PROCESS AND PRODUCTION & 24 & 7 & 52 & B-PLANT GALLERY EXHAUSTER \\
\hline Em Point & Srce Nbr & Srce Type & Hrs Day & Days Week & Wks Year & Summary Process Description \\
\hline $200 \mathrm{E}$ P-296B026 001 & 1 & PROCESS AND PRODUCTION & 24 & 7 & 52 & B-PLANT GALLERY EXHAUSTER \\
\hline Em Point & Srce $\mathrm{Nbr}$ & Srce Type & Hrs Day & Days Week & Whs Year & Summary Process Description \\
\hline 200E P-296B027 001 & 1 & PROCESS AND PRODUCTION & 24 & 7 & 52 & B-PLANT GALLERY EXHAUSTER \\
\hline
\end{tabular}

\section{Source Raw Materials Usage}

Em Point

Srce Nbr Srce Type

Process Rate Process Uom Materfal

Fuels Usage by Source Processes

Em Point

Srce $\mathrm{Nbr}$ Srce Type

Qty Uon

Fuel

Fuels Usage by Control Devices

Em Point

$\mathrm{Ctl} \mathrm{Nbr}$ Ctl Type

Qty Uom

Fuel

Point Source Emissions - Radiological Pollutants

\begin{tabular}{|c|c|c|c|c|c|}
\hline Em Point & Srce Nbr & Srce Type & $\begin{array}{l}\text { Em. Pt. Potential } \\
\text { to Emit (Ci/yr) }\end{array}$ & $\begin{array}{l}\text { Em. Pt. } \\
\text { Ctl Actual (Ci/yr) }\end{array}$ & Pollutant \\
\hline $200 E \mathrm{P}-291 \mathrm{~B} 001001$ & $\begin{array}{l}1 \\
2 \\
4 \\
7 \\
8\end{array}$ & $\begin{array}{l}\text { TANKS AND VESSELS } \\
\text { TANKS AND VESSELS } \\
\text { TANKS AND VESSELS } \\
\text { TANKS AND VESSELS } \\
\text { PROCESS AND PRODUCTION }\end{array}$ & $\begin{array}{l}\text { Refer to } \\
\text { WHC-SD-EN-RPT-007 } \\
\text { Rev. } 0 \text { Page A-33 }\end{array}$ & $\begin{array}{l}\text { Refer to } \\
\text { DOE/RL-94-51 } \\
\text { Table 2-1 }\end{array}$ & \\
\hline 200E P-296B005 001 & $\begin{array}{l}1 \\
2 \\
3\end{array}$ & $\begin{array}{l}\text { TANKS AND VESSELS } \\
\text { TANKS AND VESSELS } \\
\text { PROCESS AND PRODUCTION }\end{array}$ & $\begin{array}{l}\text { Refer to } \\
\text { WHC-SD-EN-RPT }-007 \\
\text { Rev. 0 Page A-34 }\end{array}$ & $\begin{array}{l}\text { Refer to } \\
\text { DoE/RL-94-51 } \\
\text { Table } 2-1\end{array}$ & \\
\hline 200E P-296B010 001 & 1 & PROCESS AND PRODUCTION & $\begin{array}{l}\text { Refer to } \\
\text { WHC-SD-EN-RPT-007 } \\
\text { Rev. 0 Page A-35 }\end{array}$ & $\begin{array}{c}\text { Refer to } \\
\text { DoE/RL-94-51 } \\
\text { Table 2-1 }\end{array}$ & \\
\hline 200E P-296B013 001 & $\begin{array}{l}1 \\
2 \\
3\end{array}$ & $\begin{array}{l}\text { TANKS AND VESSELS } \\
\text { TANKS AND VESSELS } \\
\text { PROCESS AND PRODUCTION }\end{array}$ & $\begin{array}{l}\text { Refer to } \\
\text { WHC-SD-EN-RPT-007 } \\
\text { Rev. 0 Page A-36 }\end{array}$ & $\begin{array}{c}\text { Refer to } \\
\text { DOE/RL-94-51 } \\
\text { Table 2-1 }\end{array}$ & \\
\hline
\end{tabular}




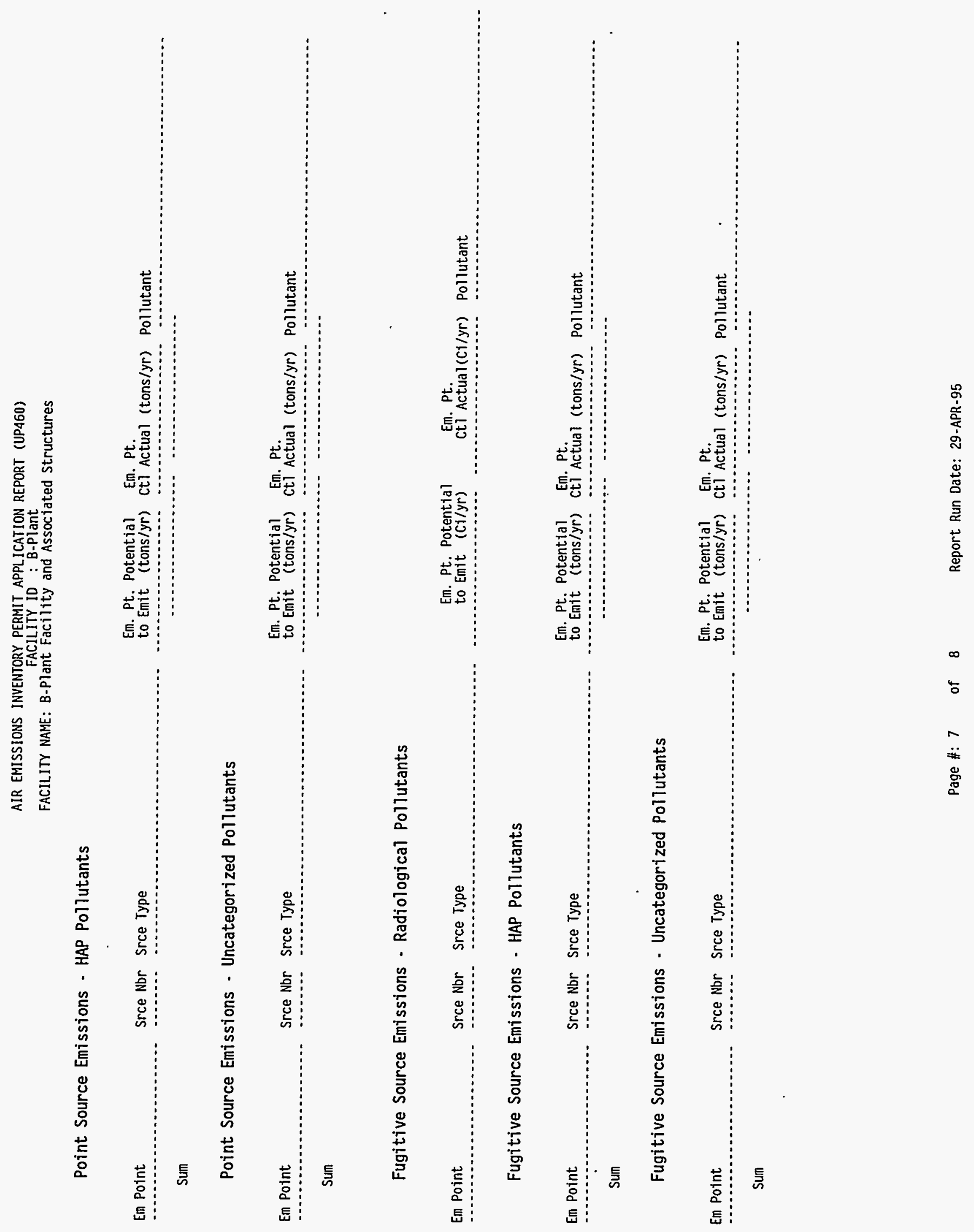


AIR EMISSIONS INVENTORY PERMIT APPLICATION REPORT (UP460)
FACILITY ID : B-Plant

FACILITY NAME: B-Plant Facility and Associated Structures

Insignificant Source Emissions - Radiological Pollutants

Em Point

Srce Nbr Srce Type

Em. Pt. Potential

to Emit (Ci/yr)

Em. Pt.
Ctl Actual(Ci/yr) Pollutant

Insignificant Source Emissions - HAP Pollutants

Em Point

Srce Nbr Srce Type

Sum

Insignificant Source Emissions - Uncategorized Pollutants

Em Point

Srce Nbr Srce Type

Sum

\section{Control Device Listing}

\section{Em Point}

Control Equipment Code

$200 E$ P-2918001 001

200E P-2918001 001

200E $P$ - 296B00 001

$200 \mathrm{E}$ P-296B013 001

$200 E$ P-2968022 001

200E P. 296023024

$200 E$ P. 2968025001

200E P-296B026 001
Em. Pt. Potential
to Emit
(tons/yr)

Em. Pt. Potential Em. Pt.

(tons/yr) Pollutant 


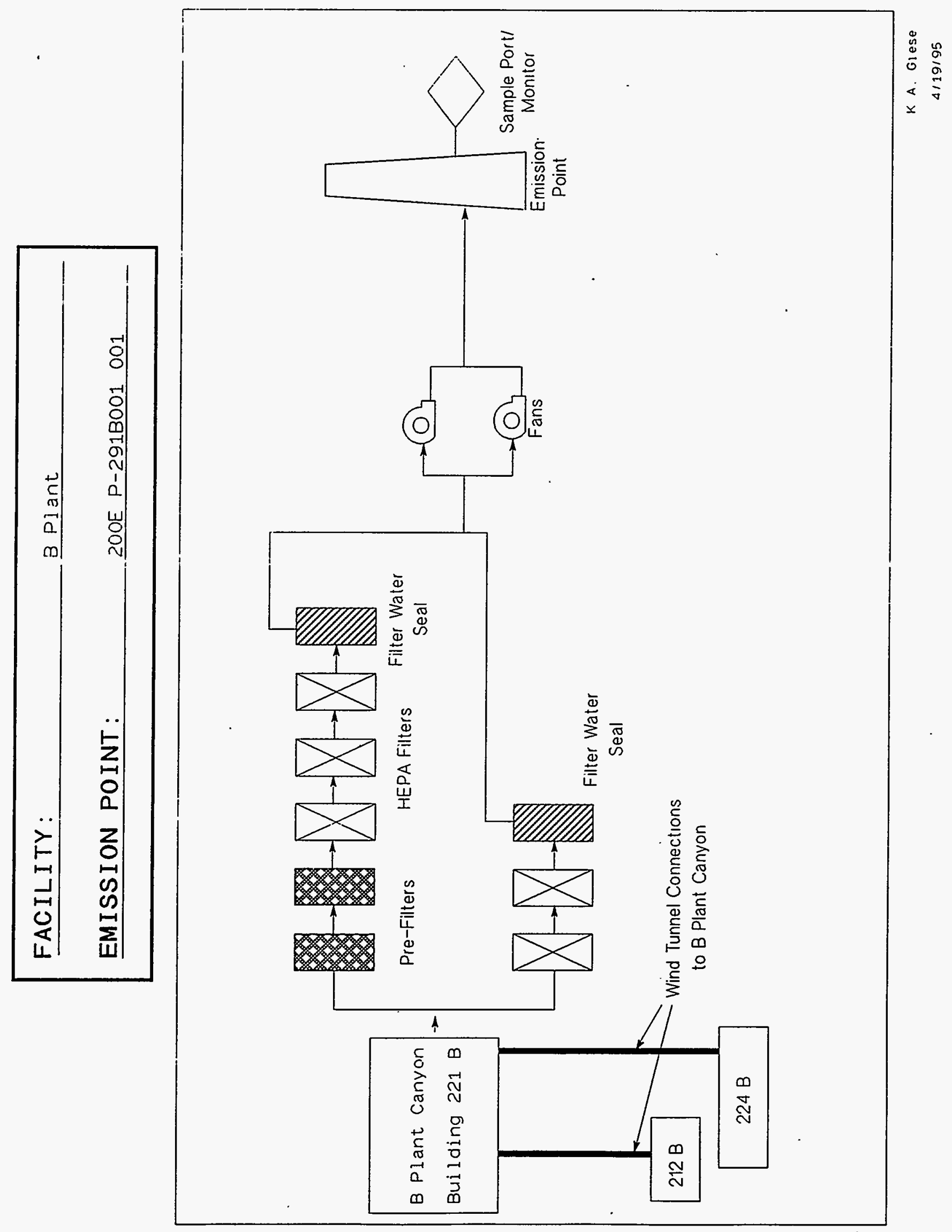




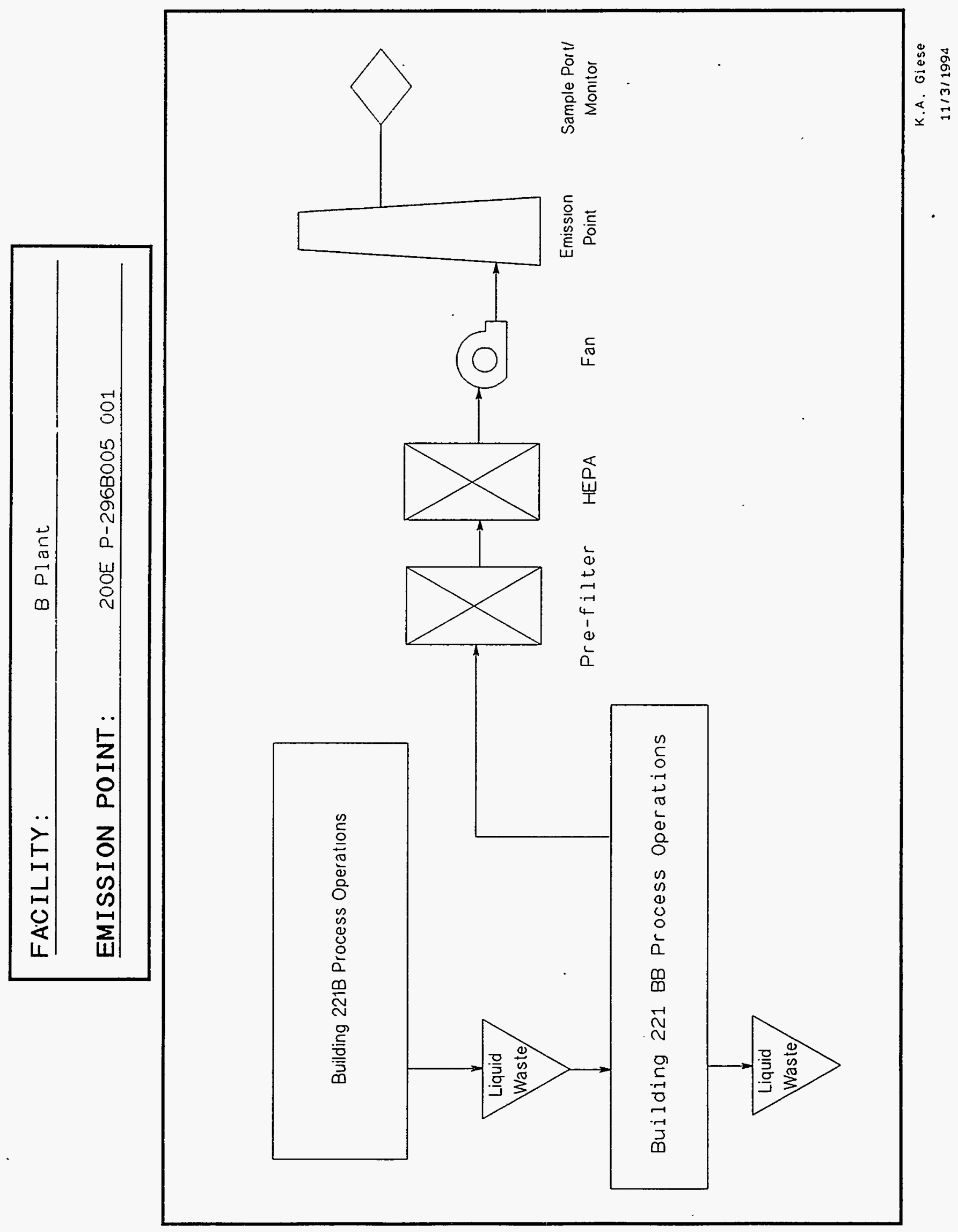




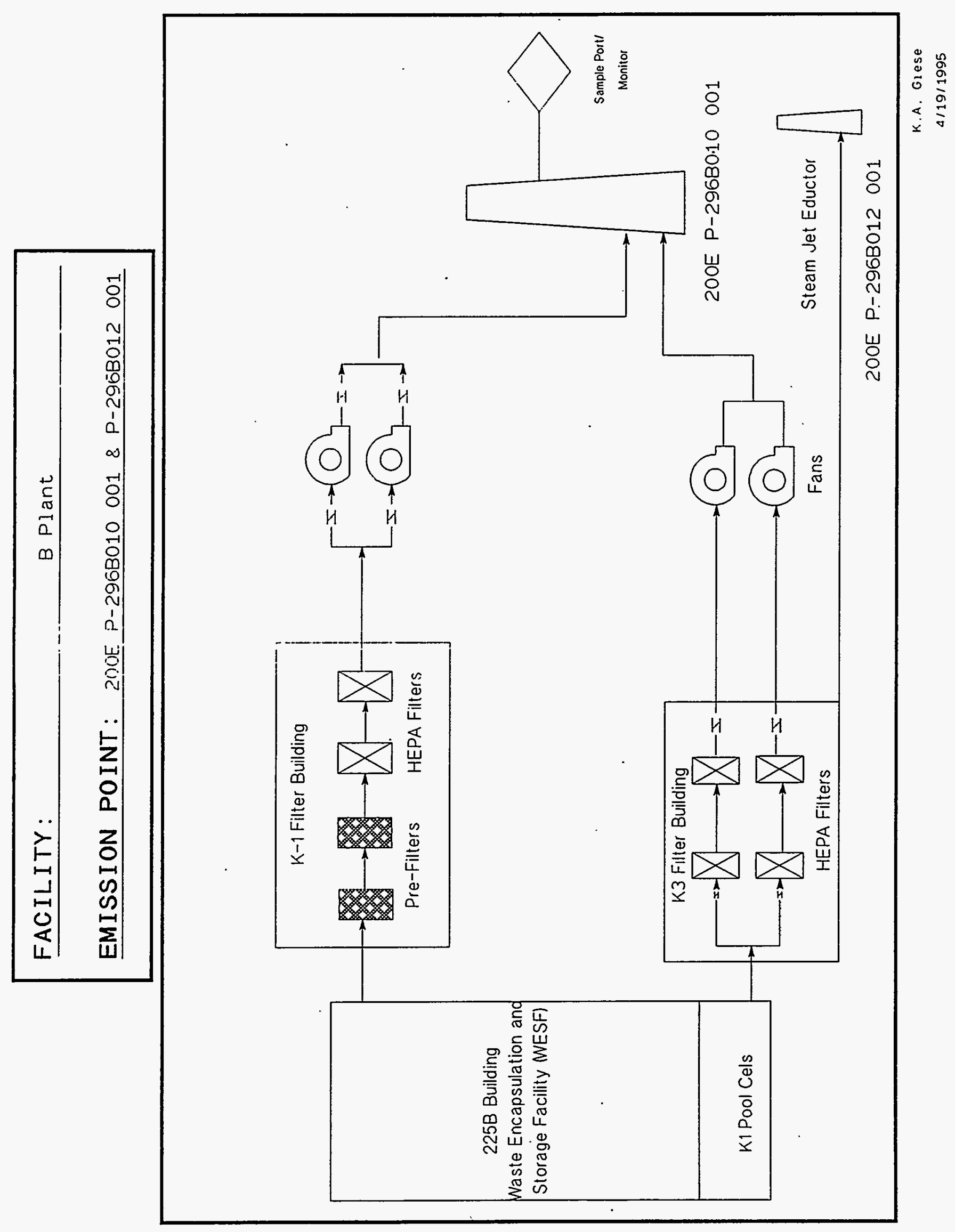



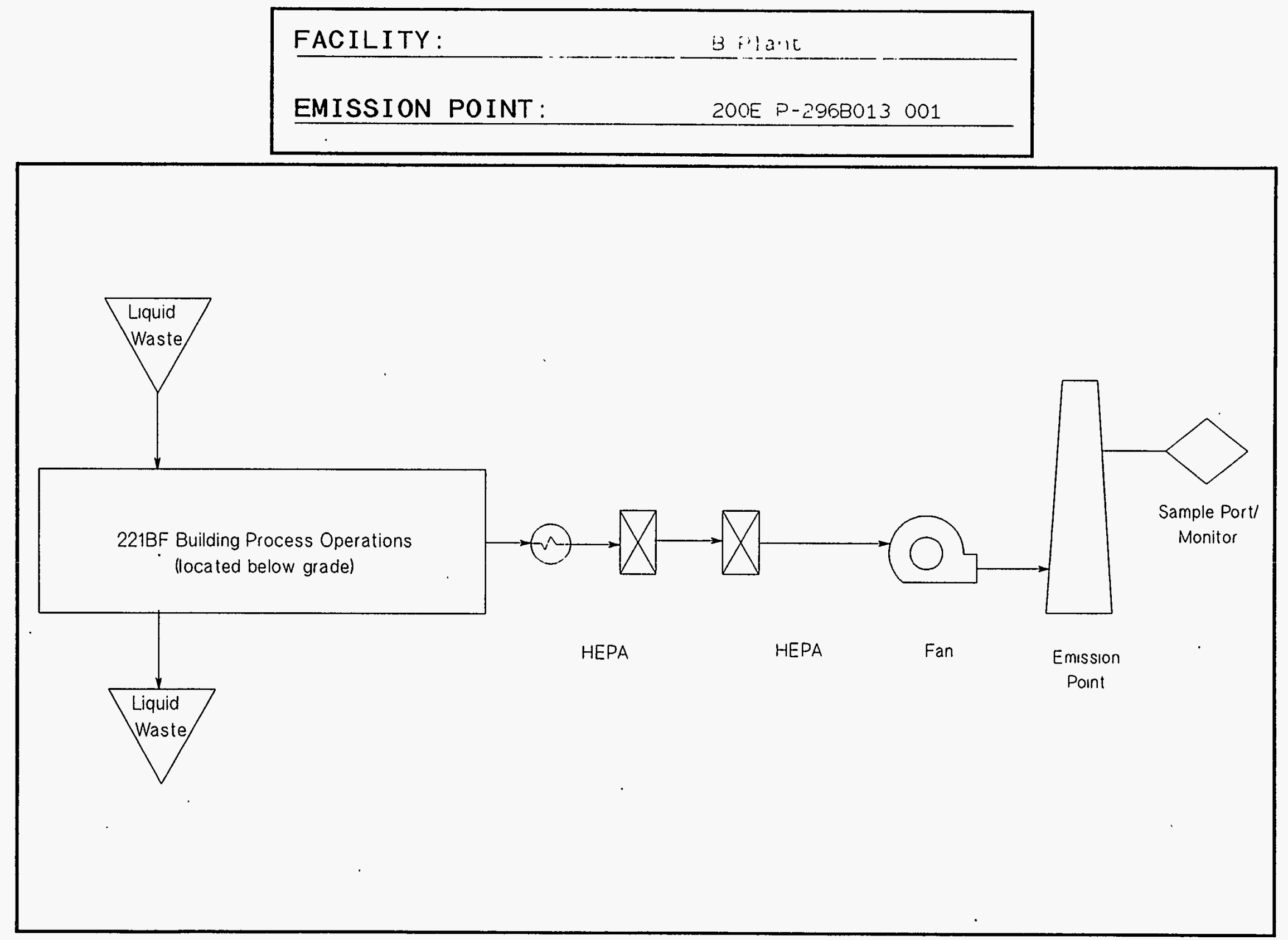


\section{FACILITY:}

B Plant

\section{EMISSION POINT:}

See Table

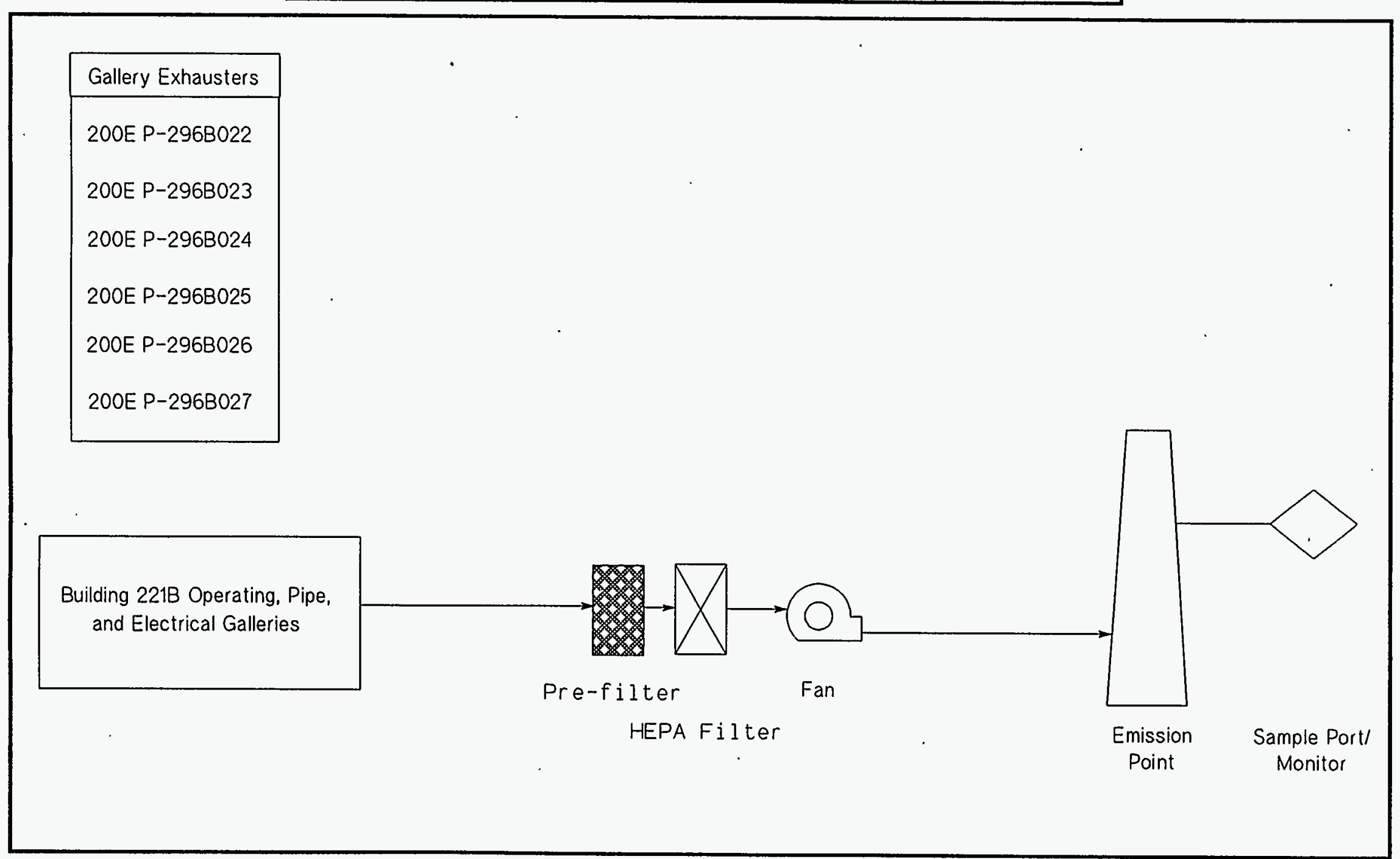




\section{B-Plant \& Waste Encapsulation Storage Facility (WESF) Emission Points}

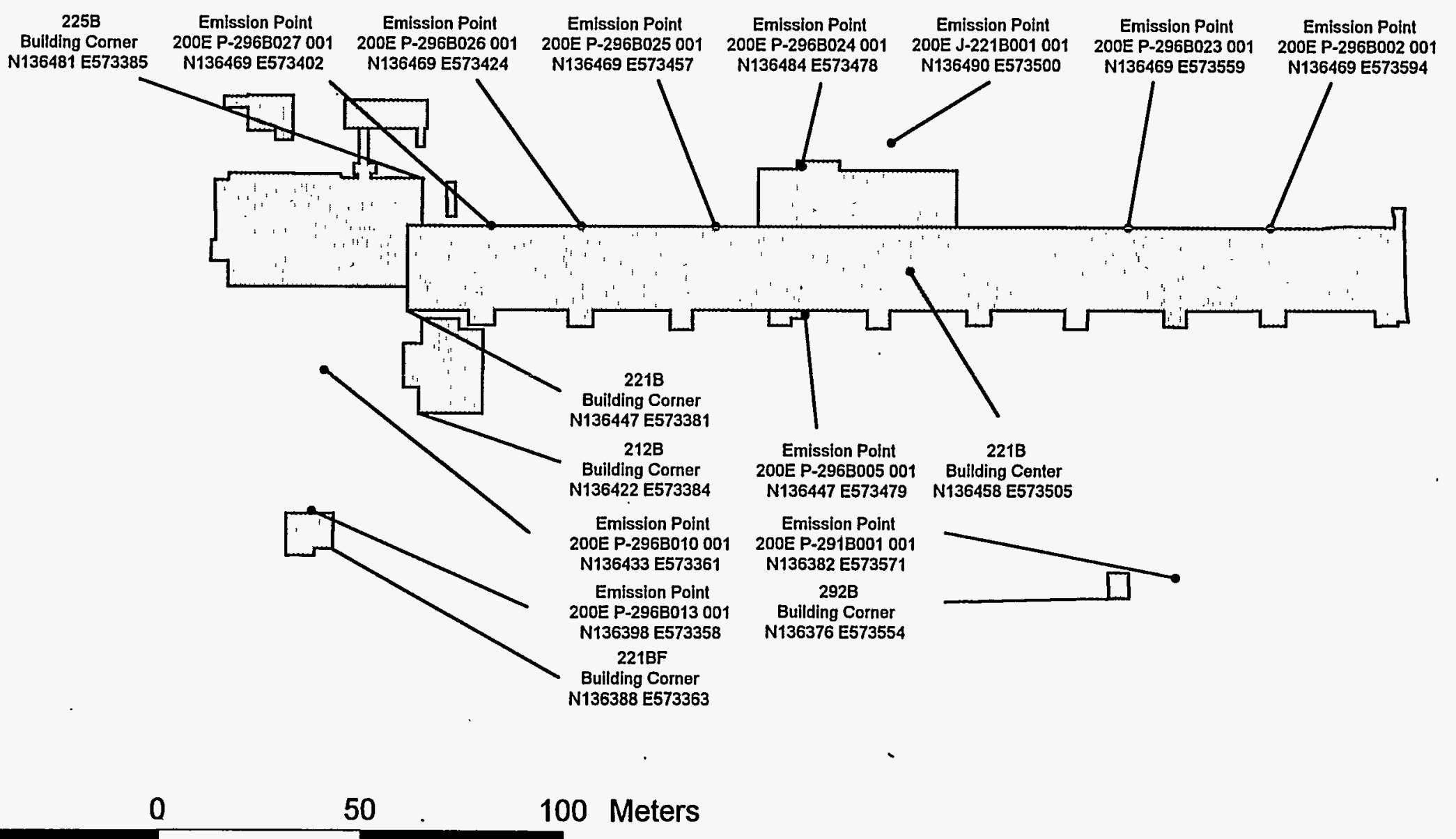




\section{APPENDIX D-3}

\section{PUREX FACILITY}

\section{Chapter I. General Information}

\section{I-B.1. Facility Description}

The Plutonium Uranium Extraction (PUREX) Facility began operations in 1955 to separate and recover usable actinides from fission products in spent nuclear fuel. The process entailed dissolving irradiated nuclear reactor fuel with acid solutions and then extracting the actinides from the resulting aqueous solutions through application of liquid-liquid solvent extraction technology.

The PUREX Facility used 11 stacks for the numerous process operations. Of the 11 PUREX stacks, only 10 stacks discharge at any one time as stacks $296-\mathrm{A}-5 \mathrm{~A}$ and $296-\mathrm{A}-5 \mathrm{~B}$ alternate weekly. There are four ventilation systems associated with the 11 stacks.

Currently, there are no production activities at the PUREX Facility; operations ceased in 1990. The PUREX Facility now is transitioning to a shutdown mode to place the PUREX Facility in a safe and environmentally secure configuration. In doing so, the PUREX Facility will concentrate on the removal, reduction, and stabilization of the radioactive and chemical materials remaining at the facility, and the shutdown of the utilities and effluents. The PUREX Facility will also consolidate the four ventilation systems into one by cascading air from one ventilation system to another. This will reduce the volume of air discharged and the number of stack monitoring stations needed during the long surveillance period following the completion of PUREX Facility deactivation. Final discharge of air will be through the main stack.

The PUREX Facility is located in the 200 East Area. The main building, 202-A, is a heavily shielded, reinforced-concrete structure known as a canyon building. This building contains the main equipment used in the PUREX process. Auxiliary PUREX equipment is located either outdoors or in one of several supporting buildings. PUREX is operated 24 hours a day.

\section{I-B.2 Products, Raw Materials, Fuels, Processes}

- Principal products - There are no products produced at PUREX.

- Process information - There are two operations at PUREX.

- There is an operation to concentrate liquid waste on an as-needed basis. The function of the concentrator is to reduce the liquid volume of waste that is generated by shutdown activities at PUREX. 
- There is an operation to flush all canyon and supporting tank farm process tanks and vessels. This flushing operation is designed to reduce chemical and radiological residues in the tanks and vessels. The removed chemical and radionuclide species mostly will be dissolved in the water solution.

- Raw materials used by processes - There are no raw materials used at the PUREX Facility.

- Fuels used by processes - There are no fuels used in the processes at the PUREX Facility.

\section{I-C.1. Emission Points, Controls, Stacks}

The emission point and stack information is found on pages 1 through 3 of the AEI report output. The control information is found on page 18 of the AEI report output.

\section{I-C.2. Facility Process Flow Diagrams}

Attached.

I-C.3. Site Map

Attached.

\section{Chapter II. Regulatory Requirements}

\section{II-A.1. Description of Discharge and Emission Points}

The emission point and stack information is found on pages 1 through 3 of the AEI report output. 
AIR EMISSIONS INVENTORY PERMIT APPLICATION REPORT (UP460)

FACILITY NAME: PUREX Facility and Related Structures

Facility Building List

\begin{tabular}{|c|c|c|c|c|c|c|c|c|}
\hline Bldg Id & North & East & Bldg Desc & Custodian(Contractor) & Cust Phone & DOE POC & & DOE Phone \\
\hline $\begin{array}{l}\text { 203A CHEM } \\
\text { 211A CHEM } \\
291 A 001 \\
296 A 001\end{array}$ & $\begin{array}{l}135758 \\
135697 \\
135564 \\
135667\end{array}$ & $\begin{array}{l}575107 \\
575120 \\
575224 \\
574973\end{array}$ & $\begin{array}{l}\text { PUREX, 203A TANK FARMS TANKS } \\
\text { PREREX, } 211 \text { A TANK FARM TANKS } \\
\text { PUREX MAIN BUILDING EXHAUST (BUILDING 202-A) } \\
\text { PRODUCT REMOVAL ROOM EXHAUST (LOCATED NEAR NW } \\
\text { CORNER 202-A PUREX) }\end{array}$ & $\begin{array}{l}\text { WIEGAND, DOTTIE L. } \\
\text { WIEGAND, DOTTIE L. } \\
\text { WIEGAND, DOTTIE L. } \\
\text { WIEGAND, DOTTIE L. }\end{array}$ & $\begin{array}{l}373-5279 \\
373-5279 \\
373-5279 \\
373-5279\end{array}$ & $\begin{array}{l}\text { R.X. (RICK) } \\
\text { R.X. (RICK) } \\
\text { R.X. (RICK) } \\
\text { R.X. (RICK) }\end{array}$ & $\begin{array}{l}\text { GONZALEZ } \\
\text { GONZALEZ } \\
\text { GONZALEZ } \\
\text { GONZALEZ }\end{array}$ & $\begin{array}{l}373-9922 \\
373-9922 \\
373-9922 \\
373-9922\end{array}$ \\
\hline $296 \mathrm{A002}$ & 135621 & 574971 & $\begin{array}{l}\text { STACK EXHAUSTS SAMPLER HOODS FROM WEST END OF } \\
\text { PUREX SAMPLE GALLERY (BLDG 202-A) }\end{array}$ & WIEGAND, DOTTIE L. & $373-5279$ & R.X. (RICK) & GONZALEZ & $373-9922$ \\
\hline $296 \mathrm{A003}$ & 135645 & 575243 & $\begin{array}{l}\text { EAST SAMPLE GALLERY HOOD EXHAUST FOR PUREX } \\
\text { (BUILDING 202-A. NE CORNER) }\end{array}$ & WIEGAND, DOTTIE L. & $373-5279$ & R.X. (RICK) & GONZALEZ & $373-9922$ \\
\hline $\begin{array}{l}296 A 006 \\
296 A 007 \\
296 A 008\end{array}$ & $\begin{array}{r}135641 \\
\cdot 135658 \\
135674\end{array}$ & $\begin{array}{l}575241 \\
575281 \\
574961\end{array}$ & $\begin{array}{l}\text { PUUREX EAST SAMPLE GALLERY AND U CELL EXHAUST } \\
\text { PUREX WEST SAMPLE GALERY AND R CELL EXHAUST } \\
\text { PUREX PIPE \& OPERATING GALLERY AND WHITE ROOM } \\
\text { EXHAUST }\end{array}$ & $\begin{array}{l}\text { WIEGAND. DOTTIE L. } \\
\text { WIEGAND, DOTTIE L. } \\
\text { WIEGAND, DOTTIE L. }\end{array}$ & $\begin{array}{l}373-5279 \\
376-5279 \\
373-5279\end{array}$ & $\begin{array}{l}\text { R.X. }(\text { RICK) } \\
\text { R.X. (RICK) } \\
\text { R.X. (RICK) }\end{array}$ & $\begin{array}{l}\text { GONZALEZ } \\
\text { GONZALEZ } \\
\text { GONZALEZ }\end{array}$ & $\begin{array}{l}373-9922 \\
373-9922 \\
373-9922\end{array}$ \\
\hline $\begin{array}{l}296 \mathrm{A010} \\
296 \mathrm{A014} \\
296 \mathrm{A05A}\end{array}$ & $\begin{array}{l}134986 \\
135593 \\
135641\end{array}$ & $\begin{array}{l}575288 \\
575146 \\
575170\end{array}$ & $\begin{array}{l}\text { PUREX TUNNEL NO. } 2 \text { EXHAUST } \\
\text { BACKUP FACILITY EXYAUST FOR BUILDING 293-A } \\
\text { PUREX WEST LABORATORY EXHAUST STACK (BUILDING 202- } \\
\text { A) }\end{array}$ & $\begin{array}{l}\text { WIEGAND, DOTTIE L. } \\
\text { WIEGAND, DOTTIE } L \text {. } \\
\text { WIEGAND, DOTTIE } L \text {. }\end{array}$ & $\begin{array}{l}373-5279 \\
373-5279 \\
373-5279\end{array}$ & $\begin{array}{l}\text { R.X. (RICK) } \\
\text { R.X. (RICK) } \\
\text { R.X. (RICK) }\end{array}$ & $\begin{array}{l}\text { GONZALEZ } \\
\text { GONZALEZ } \\
\text { GONZALEZ }\end{array}$ & $\begin{array}{l}373-9922 \\
373-9922 \\
373-9922\end{array}$ \\
\hline $5 A 05 B$ & 135641 & 575170 & PUREX EAST LABORATORY EXHAUST STACK (BUILDING 202- & WIEGAND, DOTTIE L. & $373-5279$ & R.X. (RICK) & GONZALEZ & $373-9922$ \\
\hline U ROOF VN & 135648 & 575085 & PUUREX EMISSION POINT, AMU ROOF VENT & WIEGAND. DOTTIE L. & $373-5279$ & R.X. (RICK) & ) GONZALEZ & $373-9922$ \\
\hline
\end{tabular}

\section{Emission Point Listing}

\begin{tabular}{|c|c|c|c|c|c|c|c|c|c|}
\hline Em Point & Eр Type & North & East & Srce Nbr & Scc Code & & Srce Type & & \\
\hline $200 E$ P-291A001 001 & POINT & 135564 . & 575224 & $\frac{1}{2}$ & $\begin{array}{l}\text { not assigned } \\
\text { not assigned } \\
\text { not assigned }\end{array}$ & & $\begin{array}{l}\text { PROCESS AND PRODUCTION } \\
\text { TANKS AND VESSELS } \\
\text { SOURCE EMISSIONS ROLL-UP }\end{array}$ & & \\
\hline Stack Configuration & & Diameter (ft) & Length $(f t)$ & Width(ft) & $\operatorname{Area}(s q \mathrm{ft})$ & Exit & Exit Veloc $(\mathrm{ft} / \mathrm{sec})$ & Ht Abv Grnd(ft) & Ht Abv Struc $(f t)$ \\
\hline VERTICAL WITHOUT WEAT & THER CAP & 7 & & & 38.48 & & 36.8 & 200 & 200 \\
\hline Em Point & Ep Type & North & East & Srce Nbr & Scc Code & & Srce Type & & \\
\hline 200E P-296A001 001 & POINT & 135667 & 574973 & & $\begin{array}{l}\text { not assigned } \\
\text { not assigned } \\
\text { not assigned } \\
\text { not assigned }\end{array}$ & & $\begin{array}{l}\text { PROCESS AND PRODUCTION } \\
\text { DRD/DEMOLITION } \\
\text { TANKS AND VESSELS } \\
\text { SOURCE EMISSIONS ROLL-UP }\end{array}$ & & \\
\hline Stack Configuration & & Diameter $(\mathrm{ft})$ & Length(ft) & Width $(f t)$ & $\operatorname{Area}(s q \mathrm{ft})$ & Exit & Exit Veloc $(\mathrm{ft} / \mathrm{sec})$ & Ht Abv Grnd(ft) & Ht Abv Struc(ft) \\
\hline VERTICAL WITHOUT WEAT\} & THER CAP & 1.8 & & & 2.54 & & 28.1 & 74 & 10 \\
\hline
\end{tabular}


AIR EMISSIONS INVENTORY PERMIT APPLICATION REPORT (UP460)

FACILITY NAME: FACILITY IO APPICATIO

\section{Emission Point Listing}

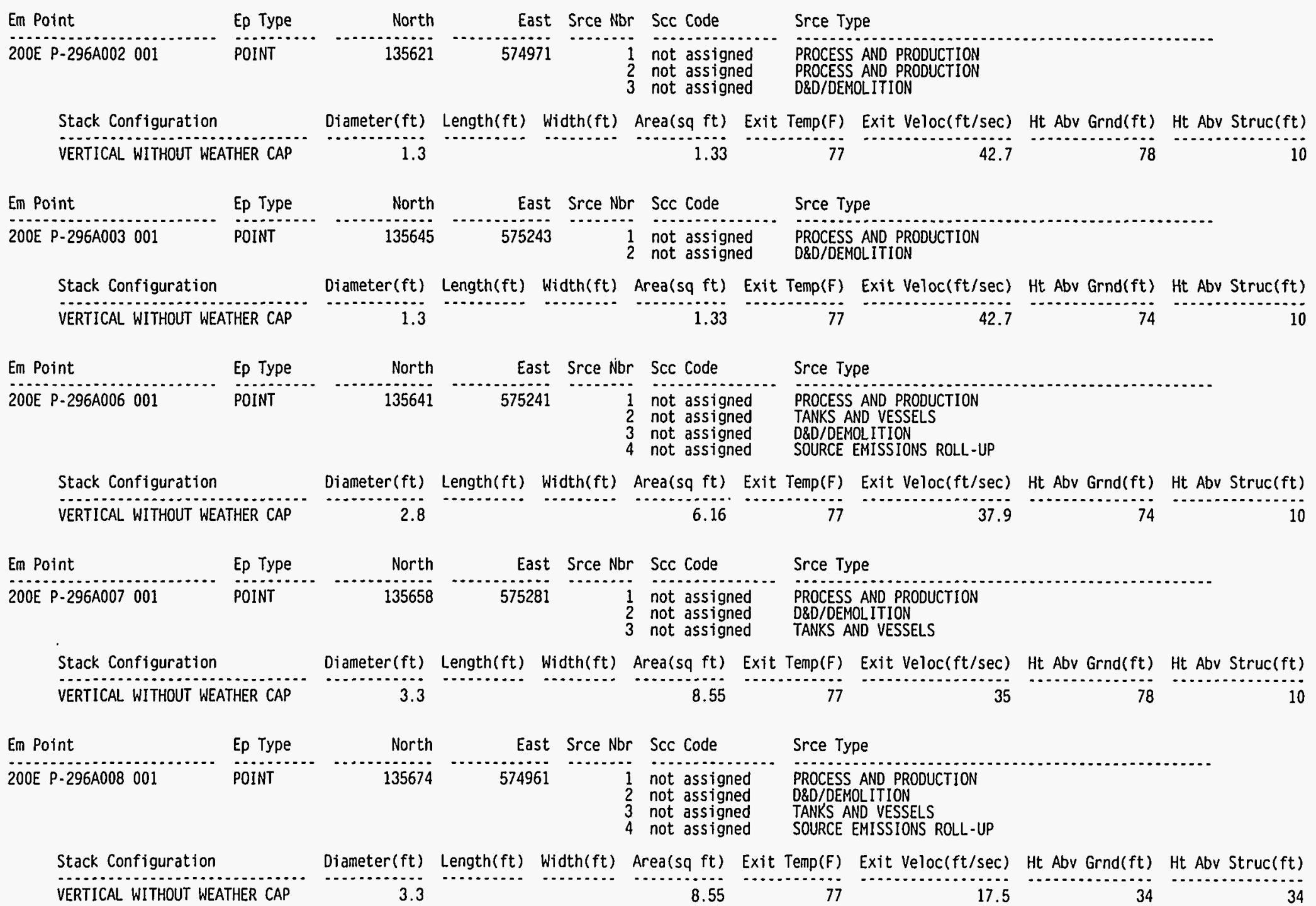


AIR EMISSIONS INVENTORY PERMIT APPLICATION REPORT (UP460)

FACILITY NAME: PUREX Factlity and Related Structures

\section{Emission Point Listing}

\begin{tabular}{|c|c|c|c|c|c|c|c|c|c|c|}
\hline Em Point & Ep Type & North & East & Srce Nbr & Scc Code & & Srce Ty & & & \\
\hline 200E P-296A010 001 & POINT & 134986 & 575288 & & not assigne & & PROCESS & AND PRODUCTION & & \\
\hline Stack Configuration & & Diameter ( $\mathrm{ft}$ ) & Length $(f t)$ & Width(ft) & Area $(s q \mathrm{ft})$ & Exit & $\operatorname{Temp}(F)$ & Exit Veloc $(\mathrm{ft} / \mathrm{sec})$ & Ht Abv Grnd(ft) & Ht Abv Struc(ft) \\
\hline VERTICAL WITHOUT WEA & THER CAP & 2 & & & 3.14 & & 68 & 18.8 & 20 & 20 \\
\hline Em Point & Ep Type & North & East & Srce Nbr & Scc Code & & \multicolumn{2}{|c|}{ Srce Type } & & \\
\hline 200E P-296A014 001 & POINT & 135593 & 575146 & 16 & not assigne & & PROCESS & AND PRODUCTION & & 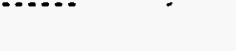 \\
\hline Stack Configuration & & Diameter ( $f t$ ) & Length(ft) & Width(ft) & Area $(s q f t)$ & Exit & Temp(F) & Exit Veloc(ft/sec) & Ht Abv Grnd(ft) & Ht Abv Struc(ft) \\
\hline VERTICAL WITHOUT WEAT & THER CAP & 2 & & & 3.14 & & 68 & 26.5 & 24.5 & 10 \\
\hline
\end{tabular}

Em Point

Ep Type

North

East Srce Nbr Scc Code

Srce Type

200E P-296A05A 001

POINT

135641

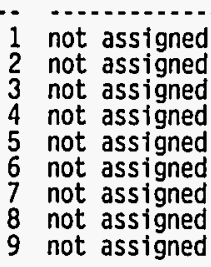

ANALYTICAL LABORATORY

ANALYTICAL LABORATORY

ANALYTCAL LABORATORY

ANAL YTICAL LABRARY

PROCESS AND PRODUCTION

PROCESS AND PRODUCTION

9 not assigned SOURCE EMISSIONS ROLL-UP

Stack Configuration

Diameter(ft) Length(ft) Width(ft) Area(sq ft) Exit Temp(F) Exit Veloc(ft/sec) Ht AbvGrnd(ft) Ht Abv Struc(ft) VERTICAL WITHOUT WEATHER CAP

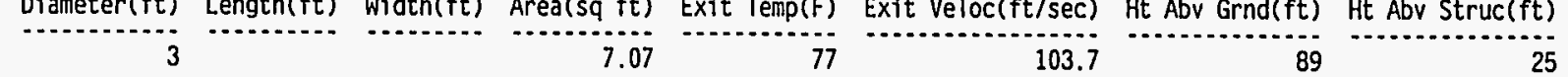

Em Point

Ep Type

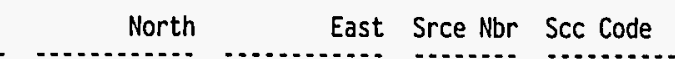

Srce Type

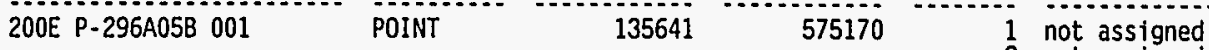

2 not assigned

4 not assigned

5 not assigned

7 not assigned

8 not assigned

ANALYTICAL LABORATORY

ANALYTCAL LABORATORY

ANAYTTCA LABORATORY

ANALYTICAL LABORATORY

ANALYTICAL LABORATORY

PROCESS AND PRODUCTION

PROCESS AND PRODUCTION

not assigned SOURCE EMISSIONS ROLL-UP

Stack Configuration

Diameter(ft) Length(ft) Width(ft) Area(sq ft) Exit Temp(F) Exit Veloc(ft/sec) Ht Abv Grnd(ft) Ht Abv Struc(ft) VERTICAL WITHOUT WEATHER CAP

3.5

77

76.2

89 
AIR EMISSIONS INVENTORY PERMIT APPLICATION REPORT (UP460)

FACILITY NAME: PUREX Facility and Related Structures

\section{Emission Point Listing}

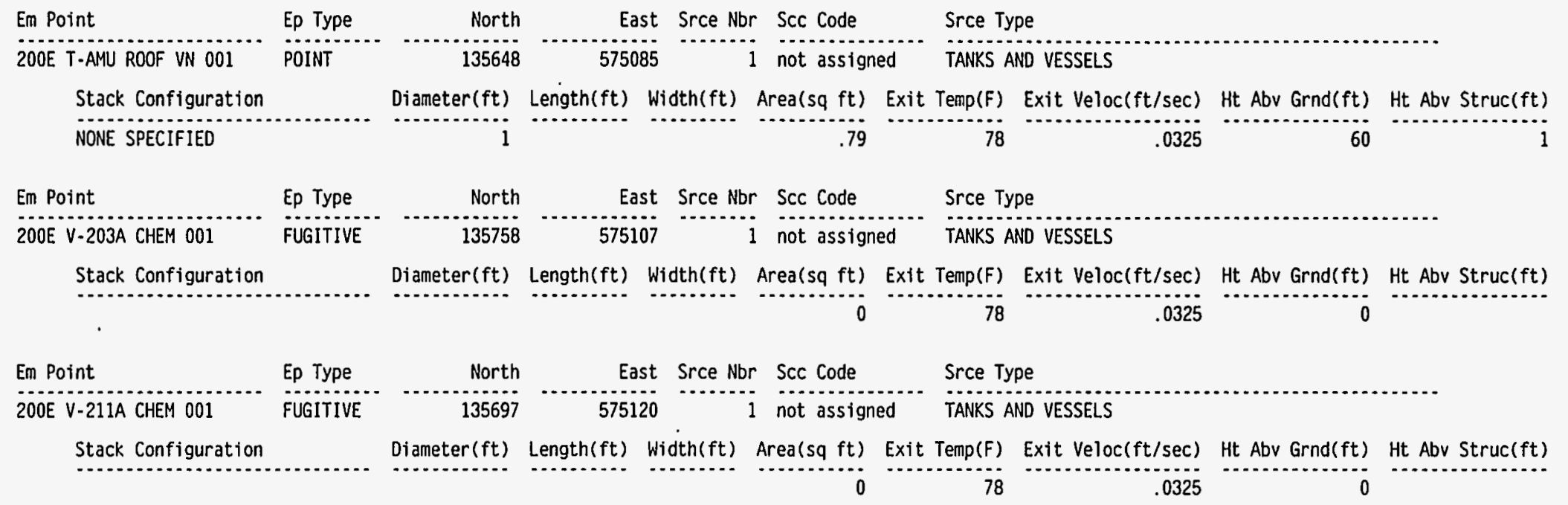

Source (Process) Listing

\begin{tabular}{|c|c|c|c|c|c|}
\hline Em Point & Srce $\mathrm{Nbr}$ & Srce Type & Hrs Day Days Week & Wks Year & Summary Process Description \\
\hline $200 E$ P-291A001 001 & 1 & PROCESS AND PRODUCTION & 24 & 52 & DISPERSABLE CONTAMINATION IN PUREX CANYON \\
\hline USING THE FSAR \& EPA FA & ACTORS, TH & E PREOICTED INVENTORY OF SOLID \& & LIQUID CONTAMINIATI & ON CAN BE & CALCULATED. THESE ARE: \\
\hline FSAR FACTORS & & EPA FACTORS & & & \\
\hline PREDICTED INVENTORY & (Ci) & PREDICTED INVENTORY (Ci) & & & \\
\hline $\begin{array}{l}\text { SOLID: } 8.25 \mathrm{E}+13 \\
\text { LIQUID: } 8.25 \mathrm{E}+10\end{array}$ & & $\begin{array}{l}4.12 E+10 \\
4.12 E+07\end{array}$ & & & \\
\hline Em Point & Srce Nbr & Srce Type & Hrs Day Days Week & Wks Year & Summary Process Description \\
\hline $200 E$ P-291A001 001 & $\ddot{2}$ & TANKS AND VESSELS & 24 & 52 & $\begin{array}{l}\text { MULTIPLE CANYON TANKS AND VESSELS USED TO SUPPORT PUREX } \\
\text { OPERATIONS. }\end{array}$ \\
\hline Em Point & Srce Nbr & Srce Type & Hrs Day Days Week & Wks Year & Summary Process Description \\
\hline $200 E$ P-291A001 001 & 3 & SOURCE EMISSIONS ROLL-UP & 24 & 52 & TOTAL EMISSIONS FOR EMISSION POINT P.291A001-001 \\
\hline
\end{tabular}


AIR EMISSIONS INVENTORY PERMIT APPLICATION REPORT (UP460)

FACILITY NAME: PUREX Facility and Related Structures

\section{Source (Process) Listing}

\begin{tabular}{|c|c|c|c|c|c|c|}
\hline Em Point & Srce Nbr & Srce Type & Hrs Day & Days Week & Wks Year & Summary Process Description \\
\hline 200E P-296A001 001 & 1 & PROCESS AND PRODUCTION & 24 & 7 & 52 & $\begin{array}{l}\text { N-CELL CONTAINS VARIOUS INACTIVE GLOVEBOXES THAT HAVE } 14000 \\
\text { GRAMS OF RESIDUAL PLUTONIUM OXIDE }\end{array}$ \\
\hline Em Point & Srce Nbr & Srce Type & Hrs Day & Days Week & Whs Year & Summary Process Description \\
\hline $200 \mathrm{E}$ P-296A001 001 & 2 & DQD/DEMOLITION & 24 & 7 & 52 & IN 1997 THE PUREX FACILITY WILL BE TURNED OVER TO D\&D \\
\hline Em Point & Srce Nbr & Srce Type & Hrs Day & Days Week & Wks Year & Summary Process Description \\
\hline 200E P-296A001 001 & 3 & TANKS AND VESSELS & 24 & 7 & 52 & $\begin{array}{l}\text { MULTIPLE TANKS AND VESSELS IN N-CELL USED TO SUPPORT PUREX } \\
\text { OPERATIONS }\end{array}$ \\
\hline Em Point. & Srce $\mathrm{Nbr}$ & Srce Type & Hrs Day & Days Week & Wks Year & Summary Process Description \\
\hline 200E P-296A001 001 & 4 & SOURCE EMISSIONS ROLL-UP & 24 & 7 & 52 & TOTAL EMISSIONS FOR EMISSION POINT P-296A001-001 \\
\hline Em Point & Srce $\mathrm{Nbr}$ & Srce Type & Hrs Day & Days Week & Wks Year & Summary Process Description \\
\hline $200 E$ P-296A002 001 & 1 & PROCESS AND PRODUCTION & 24 & 7 & 52 & $\begin{array}{l}\text { RESIDUAL CONTAMINATION OR INADVERTENT LEAKAGE ASSOCIATED } \\
\text { WITH REMOTE SAMPLERS. }\end{array}$ \\
\hline Em Point & Srce Nbr & Srce Type & Hrs Day & Days Week & Wks Year & Summary Process Description \\
\hline 200E P-296A002001 & 2 & PROCESS AND PRODUCTION & 8 & 4 & 52 & WASTE COMPACTOR \\
\hline Em Point & Srce Nbr & Srce Type & Hrs Day & Days Week & Wks Year & Summary Process Description \\
\hline 200E P-296A002001 & 3 & D\&D/OEMOLITION & & & & IN 1997 THE PUREX FACILITY WILL BE TURNED OVER TO D\&D. \\
\hline Em Point & Srce Nbr & Srce Type & Hrs Day & Days Week & Whs Year & Summary Process Description \\
\hline 200E P-296A003001 & 1 & PROCESS AND PRODUCTION & 24 & 7 & 52 & $\begin{array}{l}\text { RESIDUAL CONTAMINATION OR INADVERTENT LEAKAGE ASSOCIATED } \\
\text { WITH REMOTE SAMPLERS }\end{array}$ \\
\hline Em Point & Srce $\mathrm{Nbr}$ & Srce Type & Hrs Day & Days Week & Wks Year & Summary Process Description \\
\hline 200E P-296A003 001 & 2 & D\&D/DEMOLITION & & & & IN 1977 PUREX FACILITY WILL BE TURNEO OVER TO D\&D \\
\hline Em Point & Srce Nbr & Srce Type & Hrs Day & Days Week & Wks Year & Summary Process Description \\
\hline $200 E$ P.296A006 001 & 1 & PROCESS AND PROOUCTION & 24 & 7 & 52 & $\begin{array}{l}\text { THE SAMPLE GALLERY AND U-CELL CONTAIN TRACES OF } \\
\text { CONAMINATION THAT CONTRIBUTE TO THE RADIONUCLIDE EMISSIONS } \\
\text { FOR } 296-A-6 .\end{array}$ \\
\hline
\end{tabular}

Page\#: 5 of 15 Report Run Date: 22-APR-95 
AIR EMISSIONS INVENTORY PERMIT APPLICATION REPORT (UP460)

FACILITY NAME: PUREX Facility and Related Structures

\section{Source (Process) Listing}

\begin{tabular}{|c|c|c|c|c|c|c|}
\hline Em Point & Srce Nbr & Srce Type & Hrs Day & Days Week & Wks Year & Summary Process Description \\
\hline $200 E$ P-296A006 001 & 2 & TANKS AND VESSELS & 24 & 7 & 52 & MULTIPLE TANKS AND VESSELS USED TO SUPPORT PUREX OPERATIONS. \\
\hline Em Point & Srce $\mathrm{Nbr}$ & Srce Type & Hrs Day & Days Week & Wks Year & Summary Process Description \\
\hline 200E P-296A006 001 & 3 & D\&D/DEMOLITION & 24 & 7 & 52 & IN 1998 THE PUREX FACILITY WILL BE TURNED OVER TO D\&D. \\
\hline Em Point & Srce $\mathrm{Nbr}$ & Srce Type & Hrs Day & Days Week & Wks Year & Summary Process Description \\
\hline 200E P-296A006 001 & 4 & SOURCE EMISSIONS ROLL-UP & 24 & 7 & 52 & TOTAL EMISSIONS FOR EMISSION POINT P-296A006-001 \\
\hline Em Point & Srce $\mathrm{Nbr}$ & Srce Type & Hrs Day & Days Week & Wks Year & Summary Process Description \\
\hline 200E P.296A007001 & 1 & PROCESS AND PRODUCTION & 24 & 7 & 52 & TRACE CONTAMINANTS IN R CELL AND THE SAMPLE GALLERY \\
\hline Em Point & Srce $\mathrm{Nbr}$ & Srce Type & Hrs Day & Days Week & Wks Year & Summary Process Description \\
\hline 200E P-296A007 001 & 2 & D\&D/DEMOLITION & 24 & 7 & 52 & IN 1997 THE PUREX FACILITY WILL BE TURNED OVER TO D\&D. \\
\hline Em Point & Srce $\mathrm{Nbr}$ & Srce Type & Hrs Day & Days Week & Wks Year & Summary Process Description \\
\hline $200 E$ P-296A007 001 & 3 & TANKS AND VESSELS & & & & MULTIPLE TANKS AND VESSELS USED TO SUPPORT PUREX OPERATIONS \\
\hline Em Point & Srce $\mathrm{Nbr}$ & Srce Type & Hrs Day & Days Week & Whs Year & Summary Process Description \\
\hline $200 E$ P-296A008 001 & 1 & PROCESS AND PRODUCTION & 24 & 7 & 52 & PIPE AND OPERATING (P\&O) GALLERY AND WHITE ROOM \\
\hline Em Point & Srce $\mathrm{Nbr}$ & Srce Type & Hrs Day & Days Week & Wks Year & Summary Process Description \\
\hline $200 E$ P-296A008 001 & 2 & D\&D/DEMOLITION & 24 & 7 & 52 & IN 1997 THE PUREX FACILITY WILL BE TURNED OVER TO D\&D. \\
\hline Em Point & Srce Nbr & Srce Type & Hrs Day & Days Week & Wks Year & Summary Process Description \\
\hline 200E P.296A008001 & 3 & SOURCE EMISSIONS ROLL-UP & 24 & 7 & 52 & TOTAL EMISSIONS FOR EMISSION POINT P-296A08-001 \\
\hline Em Point $\ldots . . . \ldots . .$. & Srce $\mathrm{Nbr}$ & Srce Type & Hrs Day & Days Week & Wks Year & Summary Process Description \\
\hline 200E P-296A008 001 & 4 & TANKS AND VESSELS & & & & MULTIPLE TANKS AND VESSELS USEO TO SUPPORT PUREX \\
\hline
\end{tabular}


AIR EMISSIONS INVENTORY PERMIT APPLICATION REPORT (UP460)

FACILITY NAME: PUREX Facility and Related Structures

\section{Source (Process) Listing}

\begin{tabular}{|c|c|c|c|c|c|c|}
\hline Em Point & Srce Nbr & Srce Type & Hrs Day & Days Week & Wks Year & Summary Process Description \\
\hline 200E P-296A010001 & $\ddot{1}$ & PROCESS AND PRODUCTION & 24 & 7 & 52 & $\begin{array}{l}\text { RAILROAD CARS CARRYING FAILED, RADIOACTIVE, OR } \\
\text { NONSERVICEABLE EQUIPMENT }\end{array}$ \\
\hline \multicolumn{7}{|c|}{$\begin{array}{l}\text { STORAGE OF THE EQUIPMENT ALLOWS THE RADIOACTIVITY TO DECAY TO LESS DANGEROUS LEVELS. DURING } 1992.17 \text { OF THE } 40 \text { STORAGE SPACES WERE IN USE. NO } \\
\text { EQUIPMENT WAS PLACED IN THE TUNNEL DURING } 1992 \text {. DURING } 1993.17 \text { OF THE } 40 \text { STORAGE SPACES WERE IN USE. NO EQUIPMENT WAS PLACED IN THE TUNEL DURING } \\
1993 \text { ON APRIL } 8 \text {. } 1994 \text { POSITION } 18 \text { (OF } 40 \text { ) WAS FILLED WITH AN ACID ABSORBER. IT IS EXPECTED THAT FAILED EQUIPMENT FROM THE PUREX CANYON WILL BE } \\
\text { PLACED IN THE TUNNEL IN FUTURE YEARS. }\end{array}$} \\
\hline Em Point & Srce Nbr & Srce Type & Hrs Day & Days Week & Wks Year & Summary Process Description \\
\hline 200E P-296A014001 & 1 & PROCESS AND PRODUCTION & 24 & 7 & 52 & DISPERSIBLE CONTAMINATION IN 293-A \\
\hline Em Point & Srce Nbr & Srce Type & Hrs Day & Days Week & Wks Year & Summary Process Description \\
\hline $200 E$ P-296A05A 001 & 1 & ANALYTICAL LABORATORY & 24 & 7 & 52 & $\begin{array}{l}\text { LAB } 1: 8 \text { ARMPORT AND } 2 \text { OPENFACED HOODS ARE THE PRIMARY } \\
\text { SOURCE. THE ICP ANALYZER VENTS THROUGH A SEPARATE HEPA. } 2 \\
\text { PROCEDURES ARE CARRIED OUT IN THIS LAB. }\end{array}$ \\
\hline Em Point & Srce Nbr & Srce Type & Hrs Day & Days Week & Wks Year & Summary Process Description \\
\hline 200E P-296A05A 001 & 2 & ANALYTICAL LABORATORY & 24 & 7 & 52 & $\begin{array}{l}\text { LAB } 2: 7 \text { ARMPORT AND } 1 \text { OPENFACED HOODS EXHAUST FROM } 4 \\
\text { PROCEDURES THAT ARE CARRIEO OUT IN LAB } 2 \text {. }\end{array}$ \\
\hline Em Point & Srce Nbr & Srce Type & Hrs Day & Days Week & Wks Year & Summary Process Description \\
\hline 200E P-296A05A 001 & 3 & ANALYTICAL LABORATORY & $\dddot{24}$ & 7 & 52 & $\begin{array}{l}\text { LAB } 3: 7 \text { ARMPORT AND } 1 \text { OPENFACED HOOD EXHAUSTS THE } 17 \\
\text { PROCEDURES THAT ARE CARRIED OUT IN THIS LAB. }\end{array}$ \\
\hline Em Point & Srce $\mathrm{Nbr}$ & Srce Type & Hrs Day & Days Week & Wks Year & Summary Process Description \\
\hline $200 \mathrm{E}$ P-296A05A 001 & 4 & ANALYTICAL LABORATORY & 24 & 7 & 52 & $\begin{array}{l}\text { LAB } 4: 2 \text { ARMPORT AND } 4 \text { OPENFACED HOODS EXHAUSTS THE } 3 \\
\text { PROCEDURES THAT ARE CARRIED OUT IN THIS LAB. }\end{array}$ \\
\hline Em Point & Srce $\mathrm{Nbr}$ & Srce Type & Hrs Day & Days Week & Wks Year & Summary Process Description \\
\hline $200 E$ P-296A05A 001 & 5 & ANALYTICAL LABORATORY & 24 & 7 & $\overline{52}$ & $\begin{array}{l}\text { LAB } 6 \text { : FIVE SAMPLEHOODS EXHAUST } 3 \text { PROCEDURES THAT OCCUR IN } \\
\text { THIS LAB. }\end{array}$ \\
\hline Em Point & Srce $\mathrm{Nbr}$ & Srce Type & Hrs Day & Days Week & Wks Year & Summary Process Description \\
\hline 200E P-296A05A 001 & 6 & ANALYTICAL LABORATORY & 24 & 7 & 52 & $\begin{array}{l}\text { LAB } 5 \text { AND } 5 \text { HODS FROM LAB } 6 \text {. } 3 \text { PROCEDRES ARE CARRIED OUT } \\
\text { IN THIS LAB. FIVE ARMPORT AND ONE OPENFACED HOOD IN ADDITION } \\
\text { TO THE } 5 \text { HOODS FROM LAB } 6 \text { GO THROUGH THE SAME HEPA LAB } 5 \text { HAS } \\
6 \text { HOODS. }\end{array}$ \\
\hline
\end{tabular}


AIR EMISSIONS INVENTORY PERMIT APPLICATION REPORT (UP460)

FACILITY NAME: PUREX Facility and Related Structures

\section{Source (Process) Listing}

\begin{tabular}{|c|c|c|c|c|c|c|}
\hline Em Point & Srce Nbr & Srce Type & Hrs Day & Days Week & Wks Year & Summary Process Description \\
\hline $200 E$ P-296A05A 001 & 7 & PROCESS AND PRODUCTION & 24 & 7 & 52 & $\begin{array}{l}\text { DECON ROOM: } 4 \text { ARMPORT AND } 3 \text { OPENFACED HOODS EXHAUST FROM THE } \\
\text { DECONTAMINATION ROOM - SAMPLE RECEIVING IS VENTILLATED INTO }\end{array}$ \\
\hline Em Point & Srce $\mathrm{Nbr}$ & Srce Type & Hrs Day & Days Week & Wks Year & Summary Process Description \\
\hline 200E P-296A05A 001 & 8 & PROCESS AND PRODUCTION & 24 & 7 & 52 & $\begin{array}{l}\text { TWO SAMPLE STORAGE CABINETS AND THE HOT WASTE ROOM EXHAUST } \\
\text { NO HOT WASTES HAVE BEEN STORED. SAMPLE STORAGE INVENTORY IS } \\
\text { CHANGING. }\end{array}$ \\
\hline
\end{tabular}

APPROXIMATELY 5000 SAMPLES PER YEAR GO INTO THE SAMPLE STORAGE ROOM. THE HOT WASTE STORAGE ROOM CONTAINED A MUCH SMALLER VOLUME OF SAMPLES (<200) ANNUALLY. THE VOLUMES ARE:

HOT WASTE STORAGE
LABORATORY SAMPLES

\begin{tabular}{|c|c|c|c|c|c|c|}
\hline Em Point & Srce Nbr & Srce Type & Hrs Day & Days Week & Wks Year & Summary Process Description \\
\hline 200E P-296A05A 001 & 9 & SOURCE EMISSIONS ROLL-UP & 24 & 7 & 52 & TOTAL EMISSIONS FOR EMISSION POINT P-296A05A-001 \\
\hline Em Point & Srce $\mathrm{Nbr}$ & Srce Type & Hrs Day & Days Week & Wks Year & Summary Process Description \\
\hline $200 E$ P-296A05B 001 & 1 & ANALYTICAL LABORATORY & 24 & 7 & 52 & $\begin{array}{l}\text { LAB 1: } 8 \text { ARMPORT AND } 2 \text { OPENFACED HOODS ARE THE PRIMARY } \\
\text { SOURCE. THE ICP ANALYZER VENTS THROUGH A SEPARATE HEPA. } 2 \\
\text { PROCEDURES ARE CARRIED OUT IN THIS LAB. }\end{array}$ \\
\hline En Point & Srce $\mathrm{Nbr}$ & Srce Type & Hrs Day & Days Week & Whs Year & Summary Process Description \\
\hline $200 E$ P-296A05B 001 & 2 & ANALYTICAL LABORATORY & 24 & 7 & 52 & $\begin{array}{l}\text { LAB } 2: 7 \text { ARMPORT AND } 1 \text { OPENFACED HOODS EXHAUST FROM } 4 \\
\text { PROCEDURES THAT ARE CARRIED OUT IN LAB } 2 \text {. }\end{array}$ \\
\hline Em Point & Srce $\mathrm{Nbr}$ & Srce Type & Hrs Day & Days Week & Whs Year & Summary Process Description \\
\hline 200E P-296A05B O0I & 3 & ANALYTICAL LABORATORY & 24 & 7 & 52 & $\begin{array}{l}\text { LAB } 3: 7 \text { ARMPORT AND } 1 \text { OPENFACED HOOD EXHAUSTS THE } 17 \\
\text { PROCEDURES THAT ARE CARRIED OUT IN THIS LAB. }\end{array}$ \\
\hline Em Point & Srce Nbr & Srce Type & Hrs Day & Days Week & Wks Year & Summary Process Description \\
\hline $200 E$ P-296A05B 001 & 4 & ANALYTICAL LABORATORY & 24 & 7 & 52 & $\begin{array}{l}\text { LAB 4: } 2 \text { ARMPORT AND } 4 \text { OPENFACED HOODS EXHAUST THE } 3 \\
\text { PROCEDURES THAT ARE CARRIED OUT IN THIS LAB. }\end{array}$ \\
\hline Em Point & Srce Nbr & Srce Type & Hrs Day & Days Week & Wks Year & Sumary Process Description \\
\hline 200E P-296A05B 001 & 5 & ANALYTICAL LABORATORY & $\ddot{24}$ & 7 & 52 & $\begin{array}{l}\text { LAB } 6 \text { : FIVE SAMPLEHOODS EXHAUST } 3 \text { PROCEDURES THAT OCCUR IN } \\
\text { THIS LAB. }\end{array}$ \\
\hline
\end{tabular}


AIR EMISSIONS INVENTORY PERMIT APPLICATION REPORT (UP460)

FACILITY ID : PUREX
FACILITY NAME: PUREX Facility and Related Structures

Source (Process) Listing

\begin{tabular}{|c|c|c|c|c|c|c|}
\hline Em Point & Srce $\mathrm{Nbr}$ & Srce Type & Hrs Day & Days Week & Wks Year & Summary Process Description \\
\hline 200E P-296A05B 001 & 6 & ANALYTICAL LABORATORY & 24 & 7 & 52 & $\begin{array}{l}\text { LAB } 5 \text { AND } 5 \text { HOODS FROM LAB } 6 \text { : } 3 \text { PROCEDURES ARE CARRIED OUT } \\
\text { IN THIS LAB. FIVE ARMPORT AND ONE OPENFACED HOOD IN ADDITION } \\
\text { TO THE } 5 \text { HOODS FROM LAB } 6 \text { GO THROUGH THE SAME HEPA. LAB } 5 \\
\text { HAS } 6 \text { HOODS. }\end{array}$ \\
\hline Em Point & Srce Nbr & Srce Type & Hrs Day & Days Week & Wks Year & Summary Process Description \\
\hline 200E P-296A05B 001 & 7 & PROCESS AND PRODUCTION & 24 & 7 & 52 & $\begin{array}{l}\text { DECON ROOM : } 4 \text { ARMPORT AND } 3 \text { OPENFACED HOODS EXHAUST FROM THE } \\
\text { DECONTAMINATION ROOM. SAMPLE RECEIVING IS VENTILLATED INTO } \\
\text { THIS ROOM. DECONTAMINATION PROCESSES OCCUR IN THIS ROOM. }\end{array}$ \\
\hline Em Point & Srce $\mathrm{Nbr}$ & Srce Type & Hrs Day & Days Week & Wks Year & Summary Process Description \\
\hline 200 P-296А05B 001 & 8 & PROCESS ANO PRODUCTION & 24 & 7 & 52 & $\begin{array}{l}\text { TWO SAMPLE STORAGE CABINETS AND THE HOT WASTE ROOM EXHAUST } \\
\text { NO HOT WASTES HAVE BEEN STORED. SAMPLE STORAGE INVENTORY IS } \\
\text { CHANGING. }\end{array}$ \\
\hline \multicolumn{3}{|c|}{$\begin{array}{l}\text { APPROXIMATELY } 5000 \text { SAMPLES PER YEAR GO INTO THE SAMPLE STORAGE } \\
\text { ANNUALLY. THE VOLUMES ARE: }\end{array}$} & ROOM. THE I & HOT WASTE S & TORAGE ROON & M CONTAINED A. MUCH SMALLER VOLUME OF SAMPLES $(<200)$ \\
\hline $\begin{array}{l}\text { HOT WASTE STORAGE } \\
\text { LABORATORY SAMPLES }\end{array}$ & $\begin{array}{l}10 L \\
500 L\end{array}$ & & & & & · \\
\hline Em Point & Srce Nbr & Srce Type & Hrs Day & Days Week & Wks Year & Summary Process Description \\
\hline 200E P-296A05B 001 & 9 & SOURCE EMISSIONS ROLL-UP & 24 & $\dddot{7}$ & 52 & TOTAL EMISSIONS FOR EMISSION POINT P-296AO5B-001 \\
\hline Em Point & Srce Nbr & Srce Type & Hrs Day & Days Week & Whs Year & Summary Process Description \\
\hline 200E T-AMU ROOF VN 001 & 1 & TANKS AND VESSELS & 24 & 7 & 52 & $\begin{array}{l}\text { MULTIPLE AQUEOUS MAKE-UP TANKS USED TO SUPPORT PUREX } \\
\text { OPERATIONS. }\end{array}$ \\
\hline
\end{tabular}

Source (Process) Listing

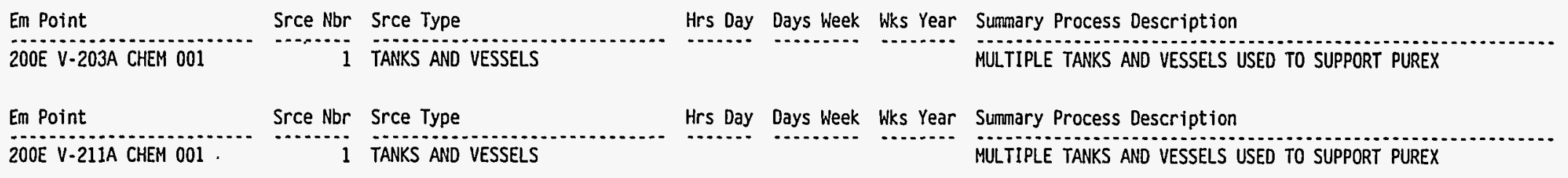


AIR EMISSIONS INVENTORY PERMIT APPLICATION REPORT (UP460)

FACILITY NAME: PAREX Facility and Related Structures

Source Raw Materials Usage

En Point

Srce Nbr Srce Type

Process Rate Process Uom Material

Fuels Usage by Source Processes

Em Point

Srce Nbr Srce Type

Qty Uom

Fuel

Fuels Usage by Control Devices

Em Point

Ct] $\mathrm{Nbr}$ Ct] Type

Qty Uom

Fuel

Point Source Criteria Pollutants - Type: H2S04

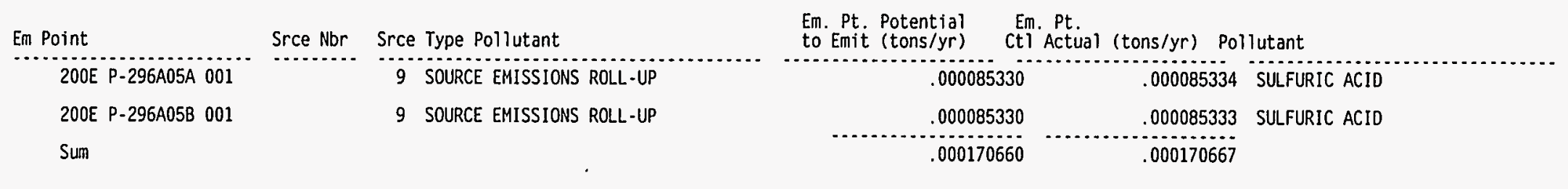

Point Source Criteria Pollutants - Type: VOC

\begin{tabular}{|c|c|c|}
\hline Em Point & Srce $\mathrm{Nbr}$ & Srce Type Pollutant \\
\hline 200E P-296A05A 001 & & 9 SOURCE EMISSIONS ROLL-UP \\
\hline 200E P-296A05B 001 & & 9 SOURCE EMISSIONS ROLL-UP \\
\hline
\end{tabular}

Sum

Em. Pt. Potential Em. Pt

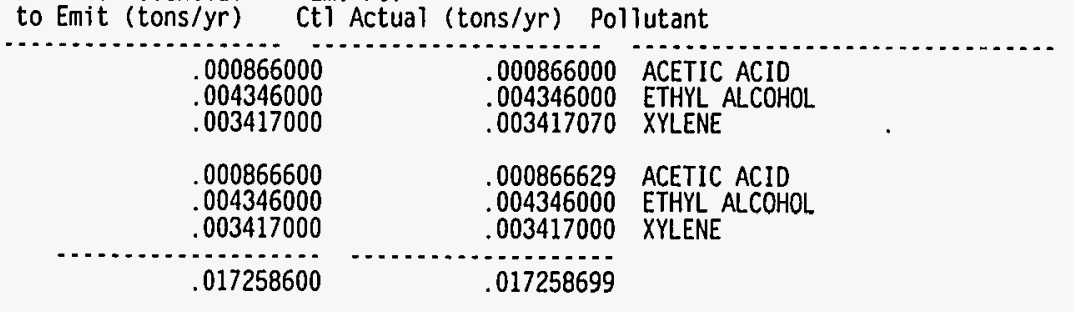

Page\# : 10 of 15 Report Run Date: 22-APR-95 
AIR EMISSIONS INVENTORY PERMIT APPLICATION REPORT (UP460)

FACILITY NAME: PUREX Facility and Related Structures

Point Source Emissions - Radiological Pollutants

\begin{tabular}{|c|c|c|c|c|}
\hline Em Point & Srce Nbr Srce Type Pollutant & $\begin{array}{l}\text { Em. Pt. Potential } \\
\text { to Emit }(\mathrm{Ci} / \mathrm{yr})\end{array}$ & $\begin{array}{l}\text { Em. Pt. } \\
\text { Cti Actual (Ci/yr) }\end{array}$ & Pollutant \\
\hline 200E P-291A001001 & $\begin{array}{ll}1 & \text { PROCESS. AND PRODUCTION } \\
2 & \text { TANKS AND VESSELS } \\
3 & \text { SOURCE EMISSION ROLLUUP }\end{array}$ & $\begin{array}{l}\text { Refer to } \\
\text { WHC-SD-EN-RPT-007 } \\
\text { Rev. O Page A-7 }\end{array}$ & $\begin{array}{l}\text { Refer to } \\
\text { DoE/RL-94-51 } \\
\text { Table } 2-1\end{array}$ & \\
\hline 200E P-296A001 001 & $\begin{array}{ll}1 & \text { PROCESS AND PRODUCTION } \\
2 & \text { D\&D/DEMOLITION } \\
3 & \text { TANKS \& VESSELS } \\
4 & \text { SOURCE EMISSION ROLLUP }\end{array}$ & $\begin{array}{l}\text { Refer to } \\
\text { WHC-SD-EN-RPT-007 } \\
\text { Rev. } 0 \text { Page A-20 }\end{array}$ & $\begin{array}{l}\text { Refer to } \\
\text { DoE/RL-94-51 } \\
\text { Table 2-1 }\end{array}$ & \\
\hline 200E P-296A002001 & $\begin{array}{ll}1 & \text { PROCESS AND PRODUCTION } \\
2 & \text { PROCESS AND PRODUCTION } \\
3 & \text { D\&D DEMOLITION }\end{array}$ & $\begin{array}{l}\text { Refer to } \\
\text { WHC-SD-EN-RPT-007 } \\
\text { Rev. } 0 \text { Page A-21 }\end{array}$ & $\begin{array}{l}\text { Refer to } \\
\text { DoE/RL-94-51 } \\
\text { Table 2-1 }\end{array}$ & \\
\hline 200E P-296A003 001 & $\begin{array}{l}1 \text { PROCESS AND PRODUCTION } \\
2 \text { D\&D DEMOLITION }\end{array}$ & $\begin{array}{l}\text { Refer to } \\
\text { WHC-SD-EN-RPT-007 } \\
\text { Rev. 0 Page A-22 }\end{array}$ & $\begin{array}{l}\text { Refer to } \\
\text { DoE/RL-94-51 } \\
\text { Table 2-1 }\end{array}$ & \\
\hline 200E P-296A006 001 & $\begin{array}{ll}1 & \text { PROCESS AND PRODUCTION } \\
2 & \text { TANKS AND VESSELS } \\
3 & \text { D\&D DEMOLITION } \\
4 & \text { SOURCE EMISSION ROLLUP }\end{array}$ & $\begin{array}{l}\text { Refer to } \\
\text { WHC-SD-EN-RPT- } 007 \\
\text { Rev. } 0 \text { Page A-26 }\end{array}$ & $\begin{array}{l}\text { Refer to } \\
\text { DOE/RL-94-51 } \\
\text { Table 2-1 }\end{array}$ & \\
\hline 200E P-296A008 001 & $\begin{array}{ll}1 & \text { PROCESS AND PRODUCTION } \\
2 & \text { D\&D/DEMOLITION } \\
3 & \text { TANKS AND VESSELS } \\
4 & \text { SOURCE EMISSION ROLLUP }\end{array}$ & $\begin{array}{l}\text { Refer to } \\
\text { WHC-SD-EN-RPT-007 } \\
\text { Rev. } 0 \text { Page A-28 }\end{array}$ & $\begin{array}{l}\text { Refer to } \\
\text { DOE/RL-94-51 } \\
\text { Table } 2-1\end{array}$ & \\
\hline $200 E$ P-296A010 001 & 1 PROCESS AND PRODUCTION & $\begin{array}{l}\text { Refer to } \\
\text { WHC-SD-EN-RPT-007 } \\
\text { Rev. 0 Page A-29 }\end{array}$ & $\begin{array}{l}\text { Refer to } \\
\text { DoE/RL-94-51 } \\
\text { Table } 2-1\end{array}$ & \\
\hline \multicolumn{5}{|c|}{ Point Source Emissions - Radiological Pollutants } \\
\hline Em Point & Srce Nbr Srce Type Pollutant & $\begin{array}{l}\text { Em. Pt. Potential } \\
\text { to Emit (Ci/yr) }\end{array}$ & $\begin{array}{l}\text { Em. Pt. } \\
\text { Cti Actual }(\mathrm{Ci} / \mathrm{yr})\end{array}$ & Pollutant \\
\hline 200E P-296A014 001 & 1 PROCESS AND PRODUCTION & $\begin{array}{l}\text { Refer to } \\
\text { WHC-SD-EN-RPT-007 } \\
\text { Rev. O Page A-30 }\end{array}$ & $\begin{array}{l}\text { Refer to } \\
\text { DOE/RL-94-51 } \\
\text { Table 2-1 }\end{array}$ & \\
\hline
\end{tabular}

Page\#: 11 of 15 Report Run Date: 22-APR-95 
AIR EMISSIONS INVENTORY PERMIT APPLICATION REPORT (UP460)

FACILITY NAME: PUREX Facility and Related Structures

Point Source Emissions - Radiological Pollutants

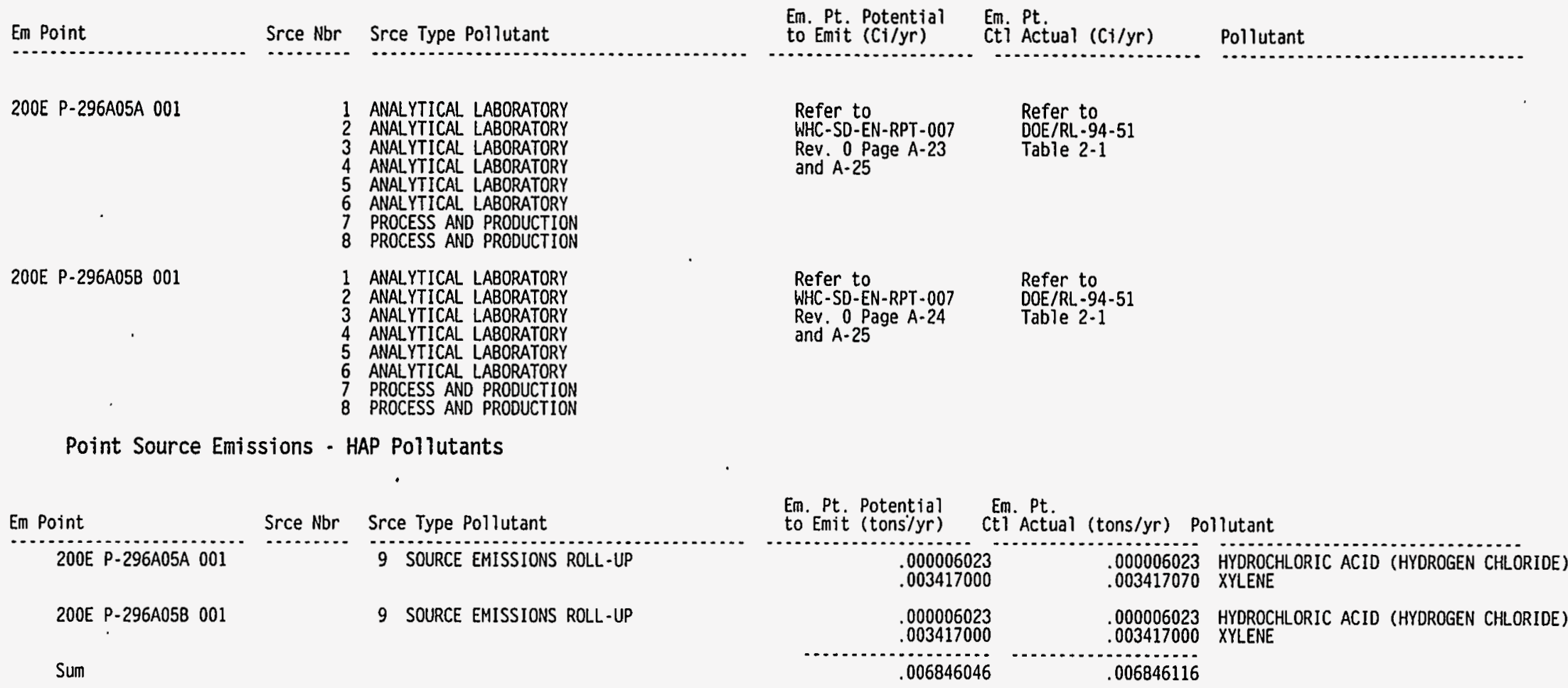


AIR EMISSIONS INVENTORY PERMIT APPLICATION REPORT (UP460)

FACILITY NAME: PUREX Facility and Related Structures

Point Source Emissions - Uncategorized Pollutants

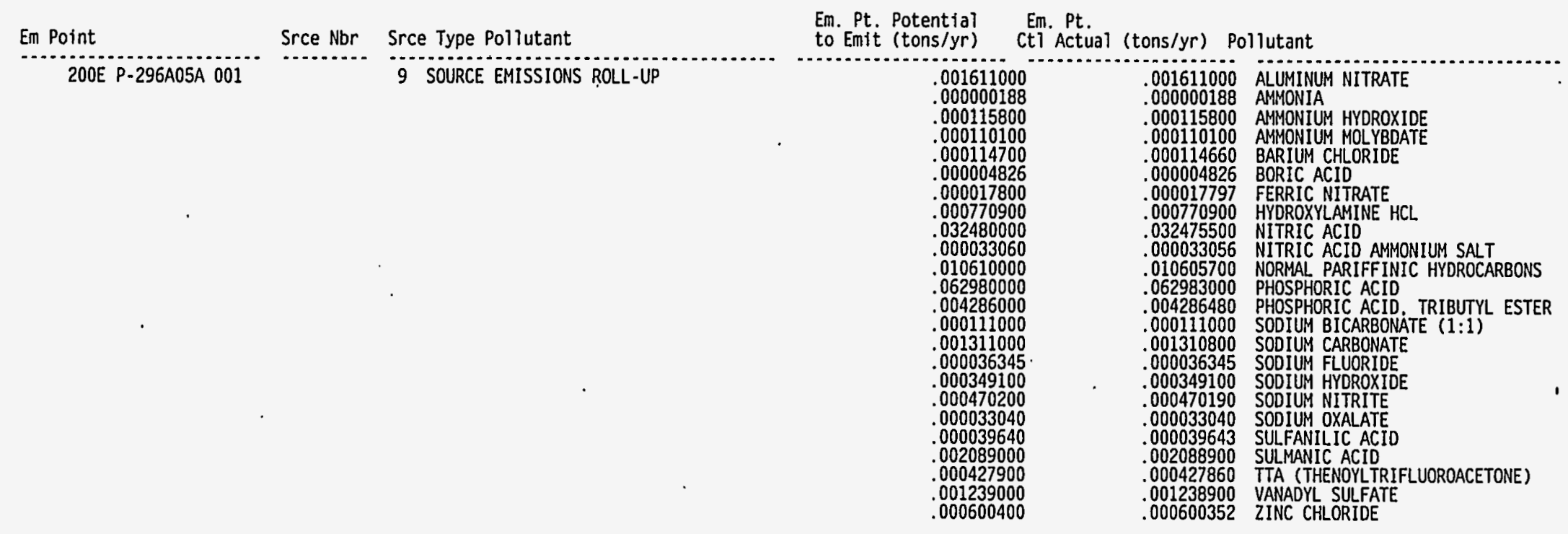

Page\#: 13 of 15 Report Run Date: 22-APR-95 
AIR EMISSIONS INVENTORY PERMIT APPLICATION REPORT (UP460)

FACILITY NAME: PUREX Facility and Related Structures

Point Source Emissions - Uncategorized Pollutants

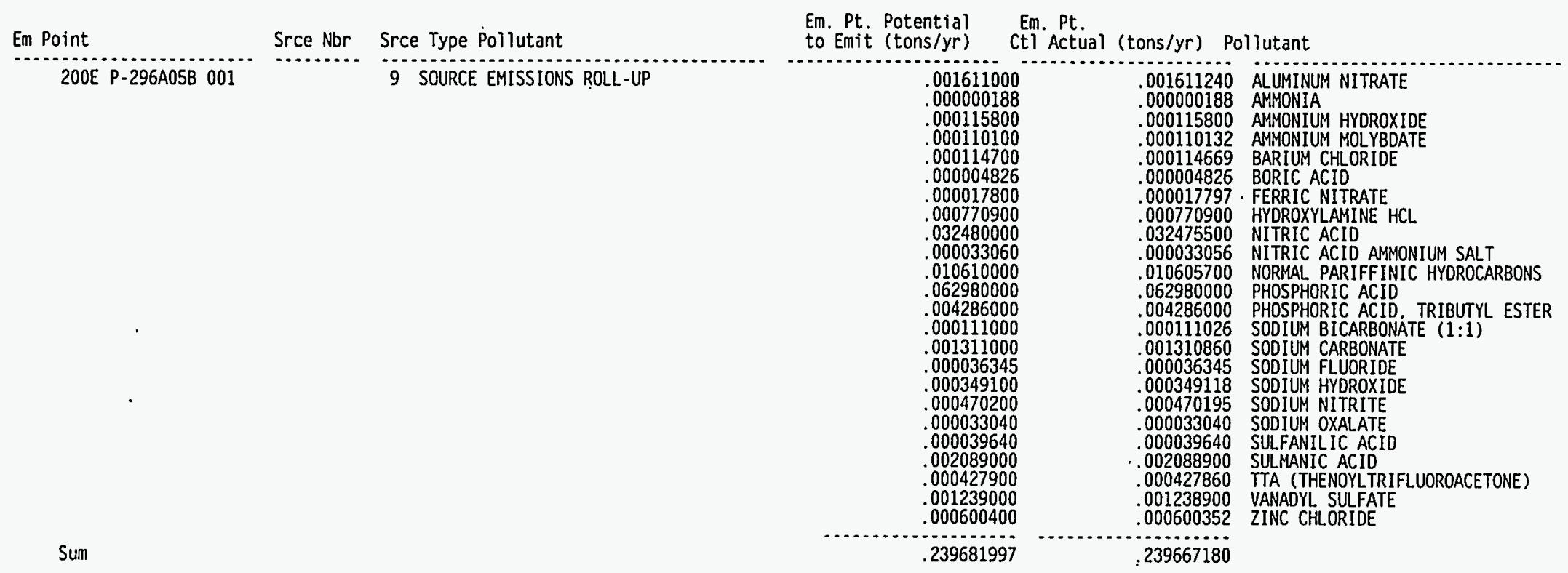

Fugitive Source Emissions - Radiological Pollutants

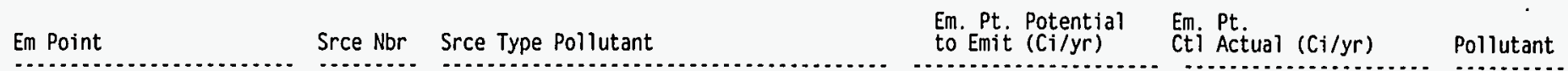

Fugitive Source Emissions - HAP Pollutants

Em Point

Srce Nbr Srce Type Pollutant

Em. Pt. Potential Em. Pt.
to Emit (tons/yr) Ct] Actual (tons/yr) Pollutant

Sum 
AIR EMISSIONS INVENTORY PERMIT APPLICATION REPORT (UP460)

FACILITY NAME: PUREX Facility and Related Structures

Fugitive Source Emissions - Uncategorized Pollutants

Em Point Srce Nbr Srce Type Pollutant

Sum

Insignificant Source Emissions - Radiological Pollutants

Em Point

Srce Nbr' Srce Type Pollutant

Em. Pt. Potential Em. Pt.
to Emit (Ci/yr)
Cti Actual (Ci/yr)

Poilutant

Insigṇificant Source Emissions - HAP Pollutants

Em Point

Srce Nbr Srce Type Pollutant

Sum

Insignificant Source Emissions - Uncategorized Pollutants

Em Point

Srce Nbr Srce Type Pollutant

Em. Pt. Potential Em. Pt.
to Emit (tons/yr) ctl Actual (tons/yr) Pollutant

to Emit (tons/yr) Ctl Actual (tons/yr) Pollutant

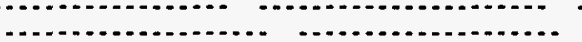
-.

Sum

Control Device Listing

Em Point

Control Equipment Code

200E P-291A001 001

200E P-296A001 001

200E $\mathrm{P}-296 \mathrm{0002} 001$

$200 E$ P. 2964006001

200E P-296A007 00

200E P-296A008 001

$200 E$ P.296A014 001

200E P-296A05A 001

Em. Pt. Potential Em. Pt. 


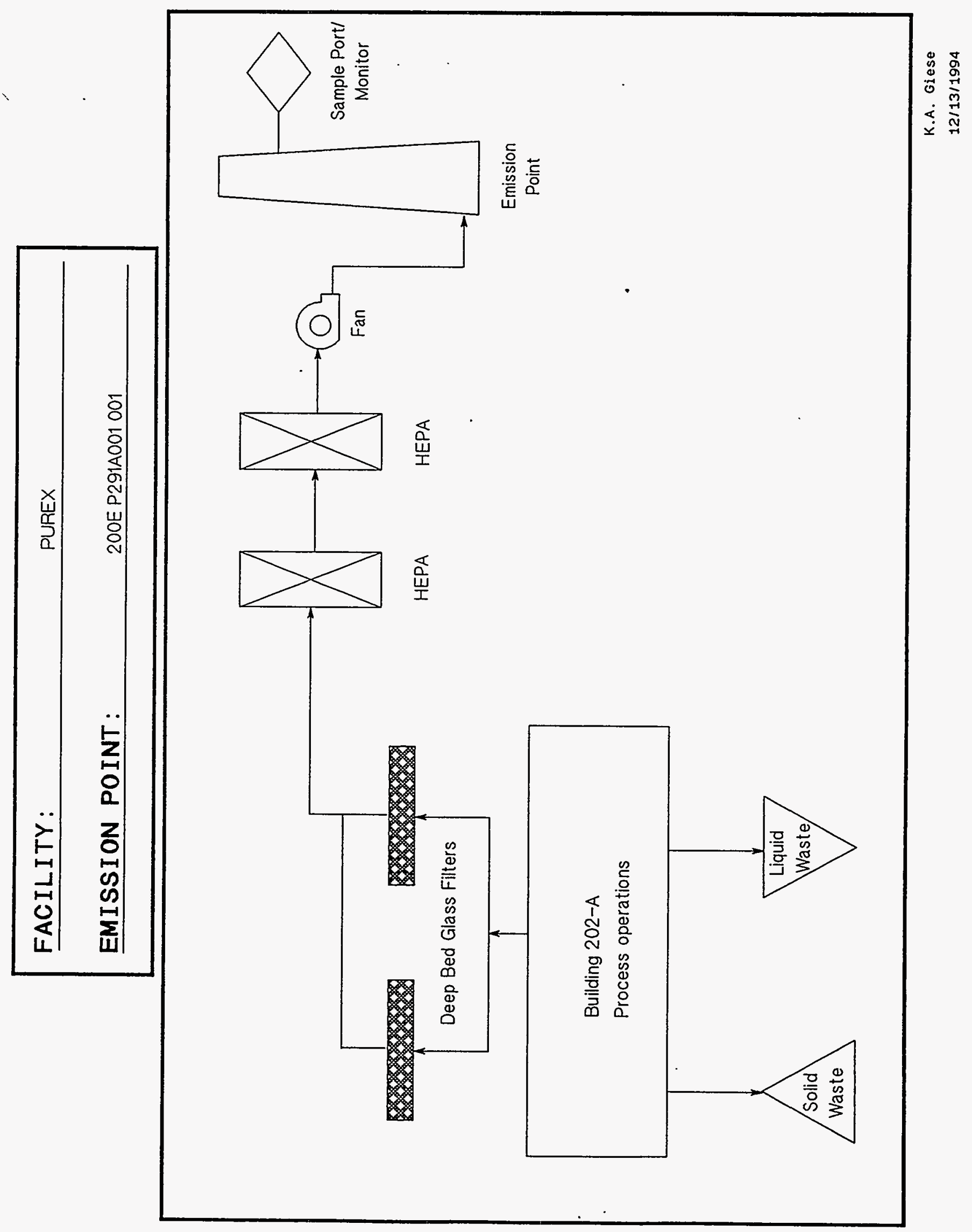




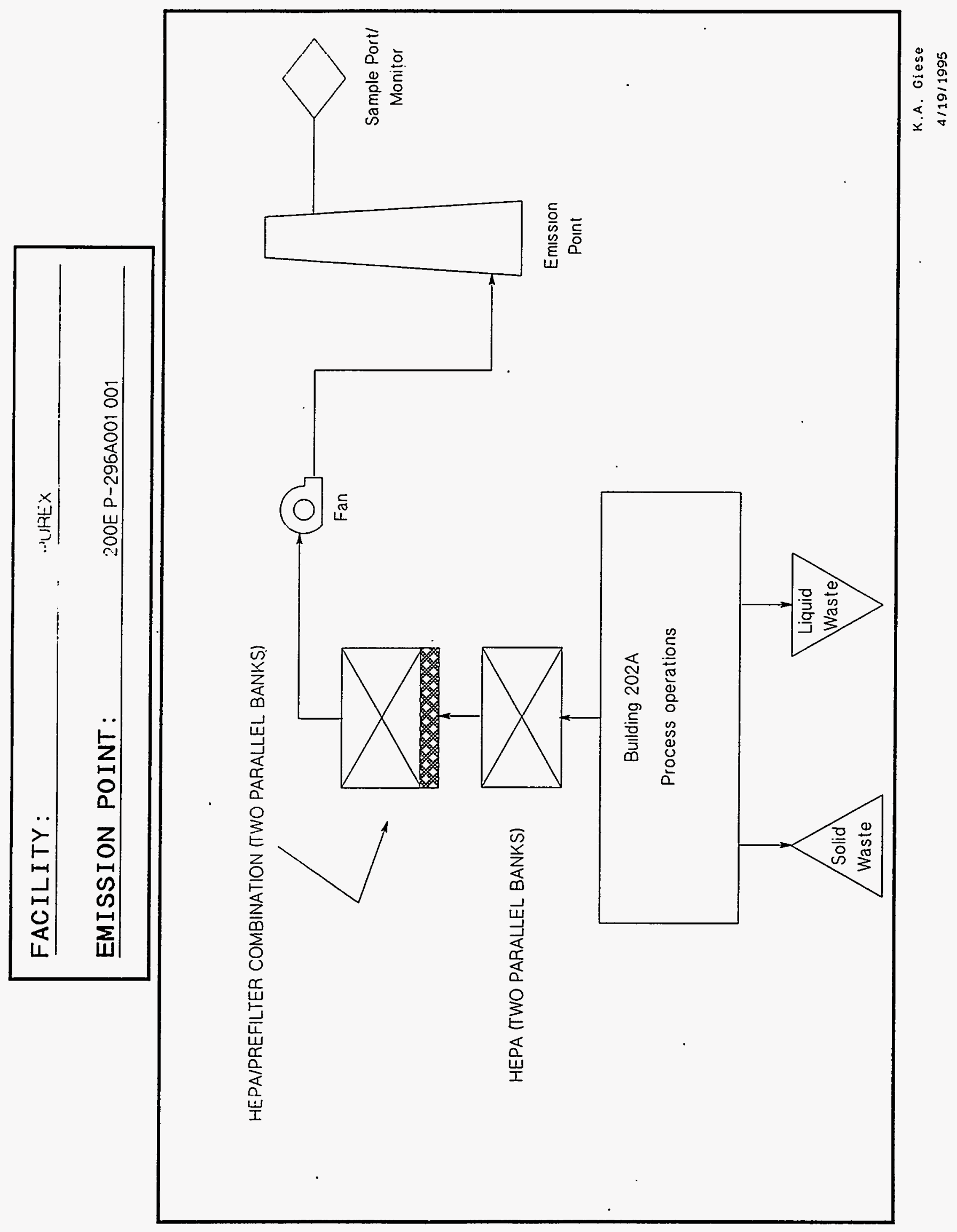




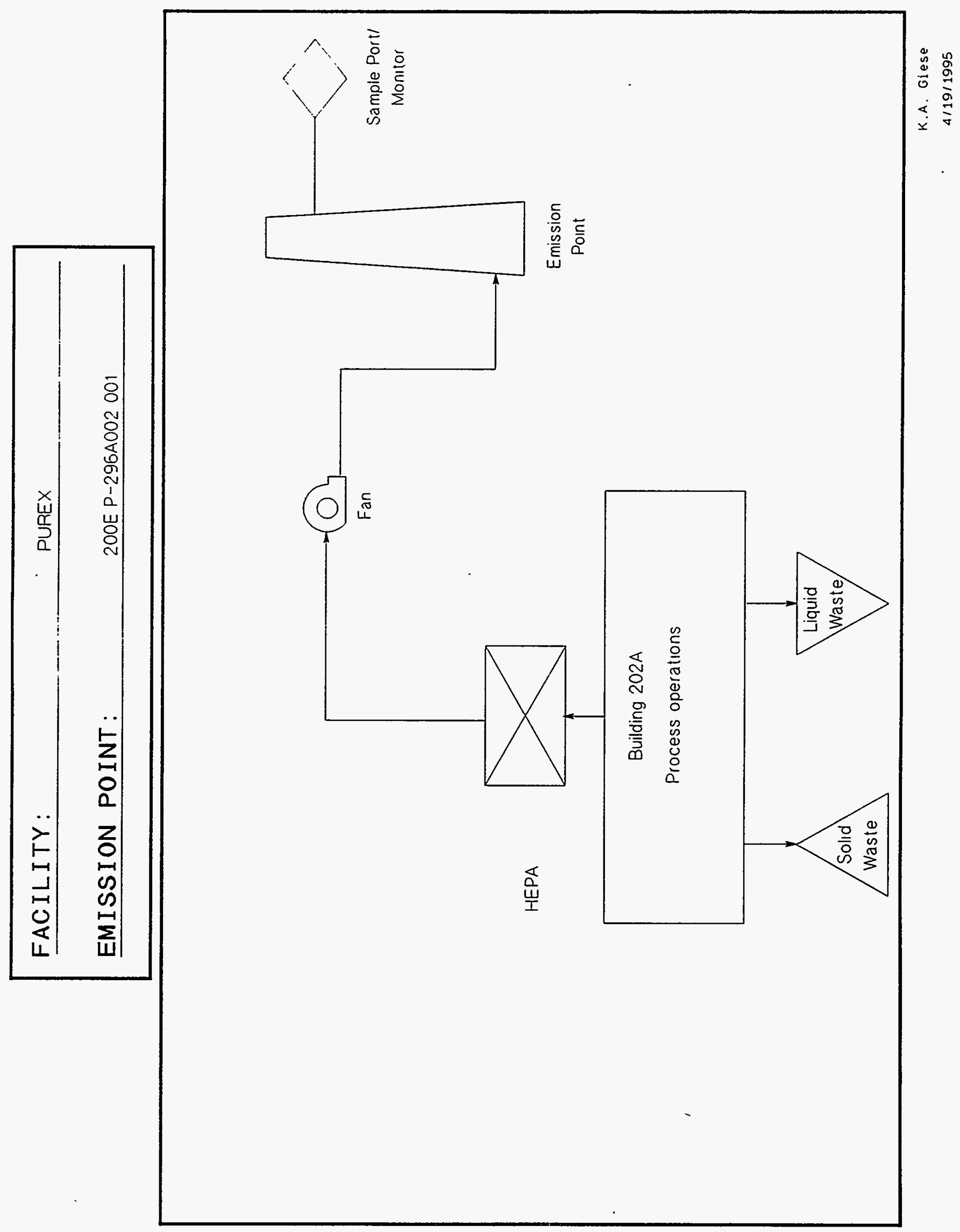




\begin{tabular}{|ll}
\hline FACILITY: & PUREX \\
\hline EMISSION POINT: & 200E P-296A003001 \\
\hline
\end{tabular}

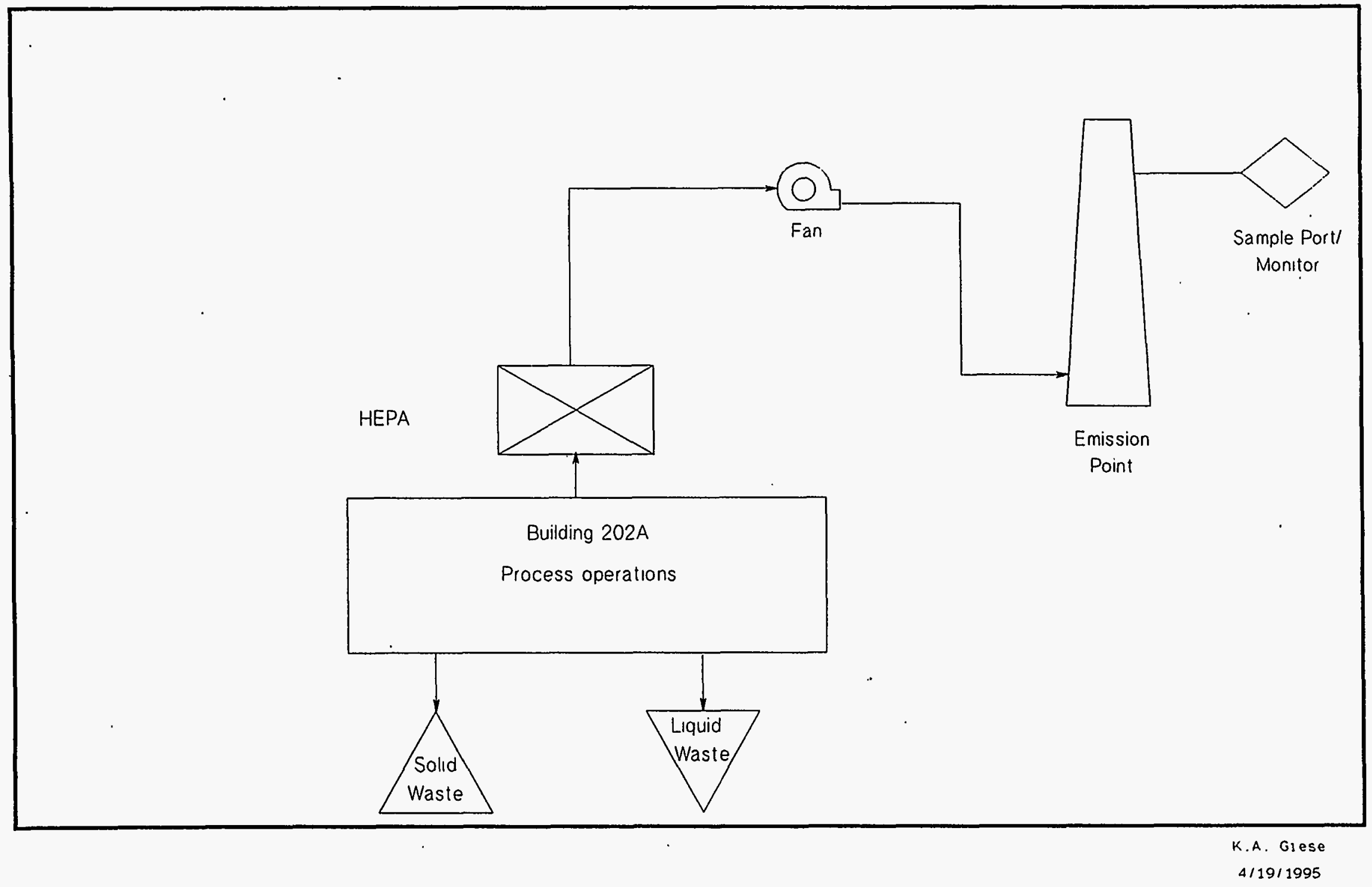




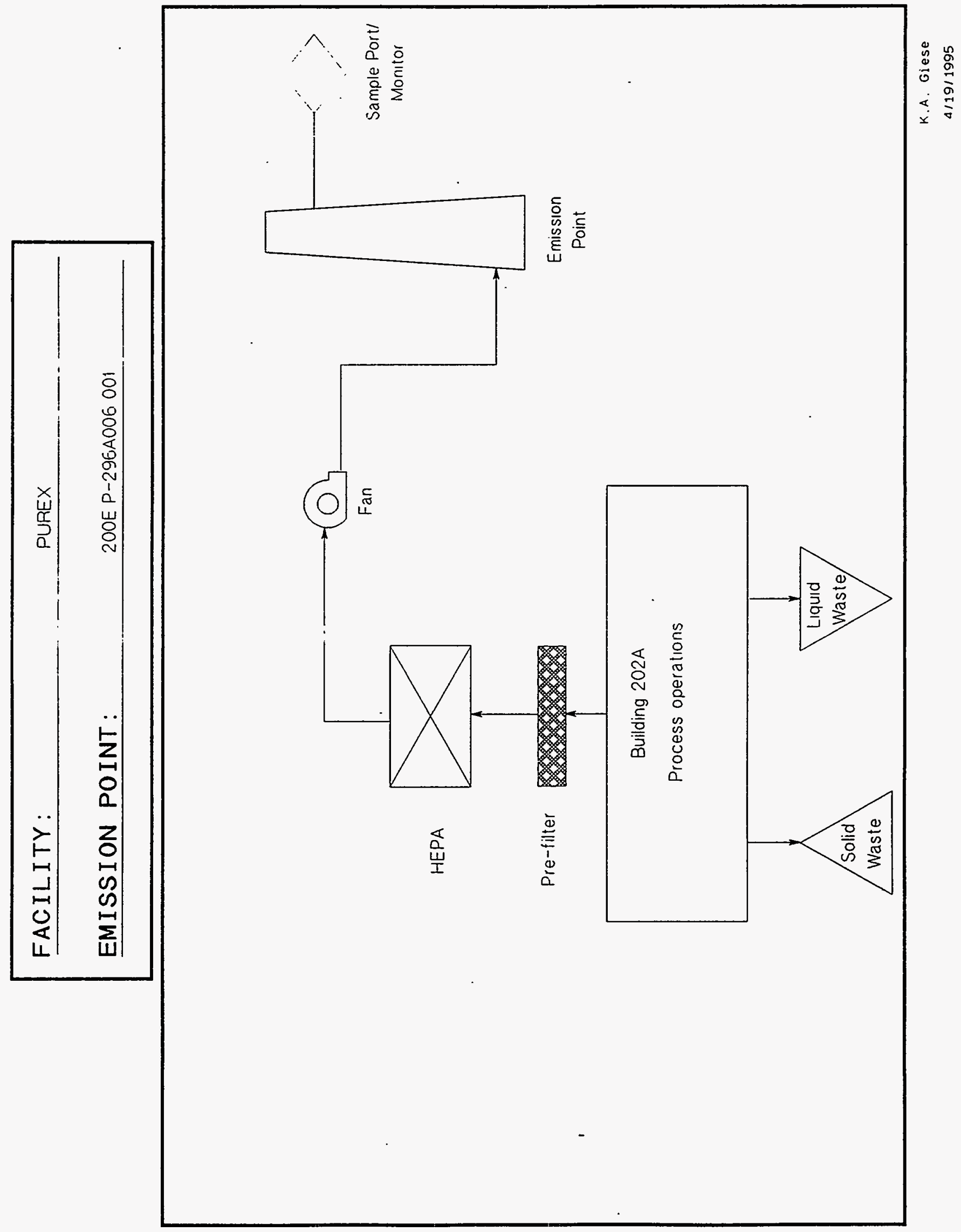




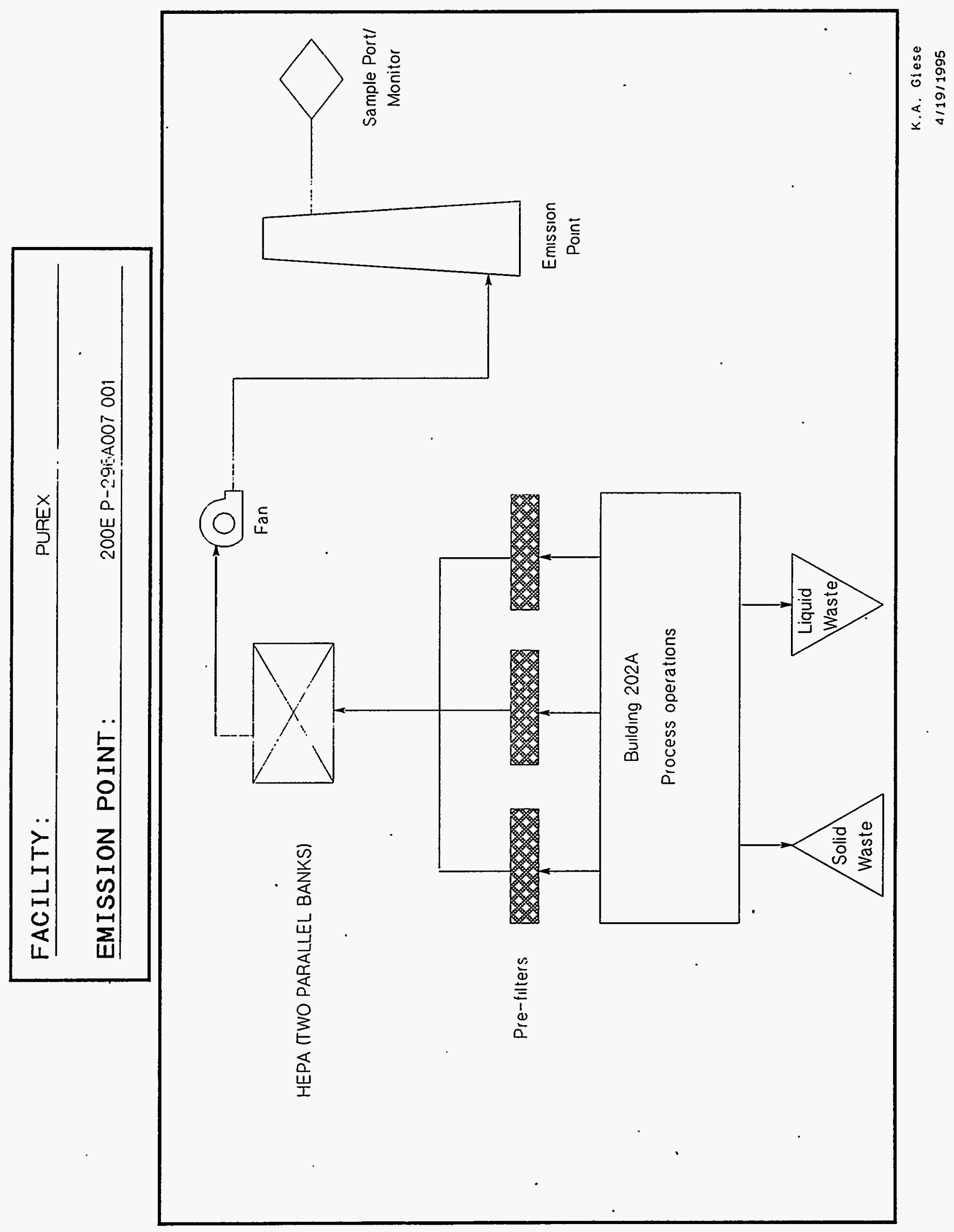




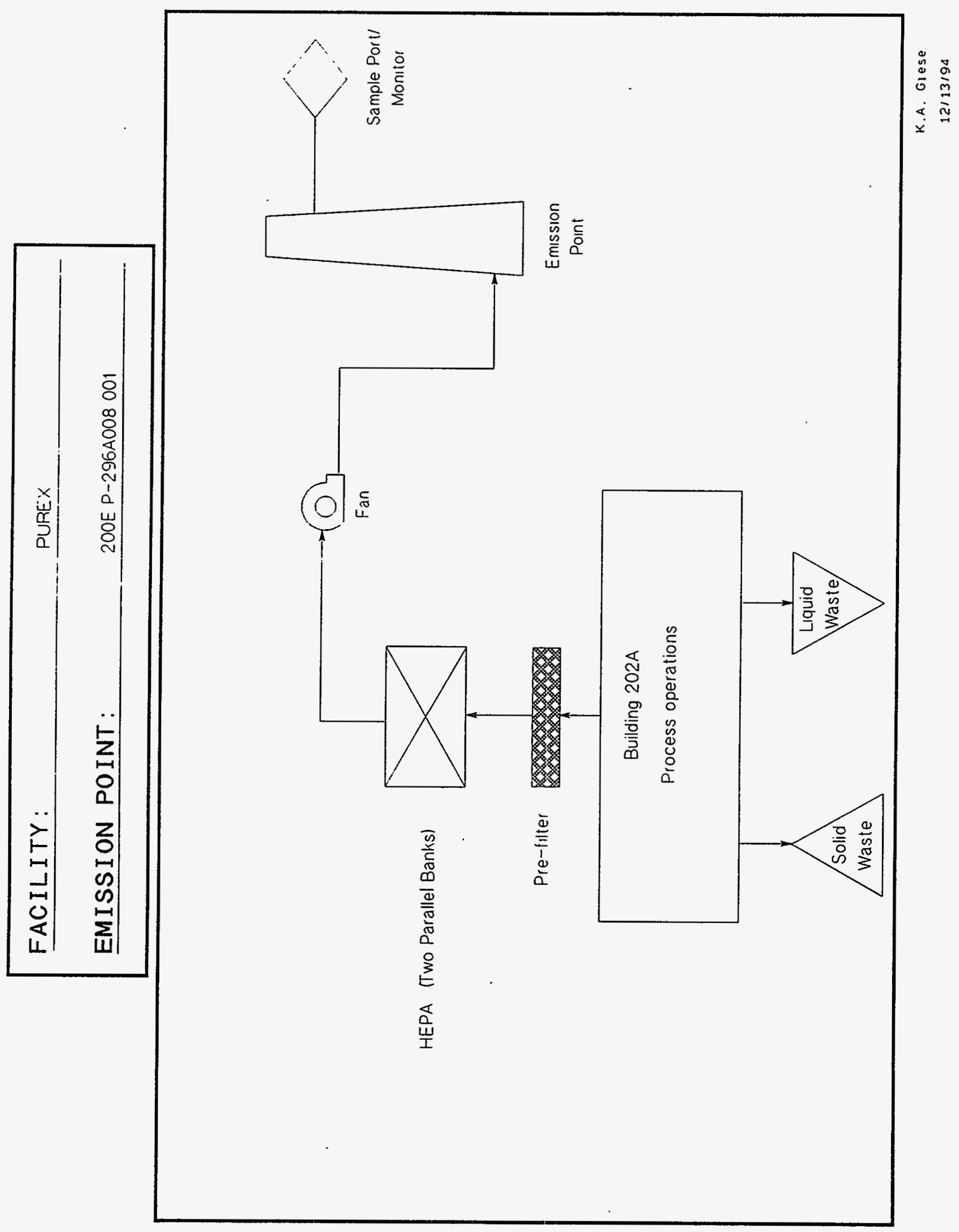




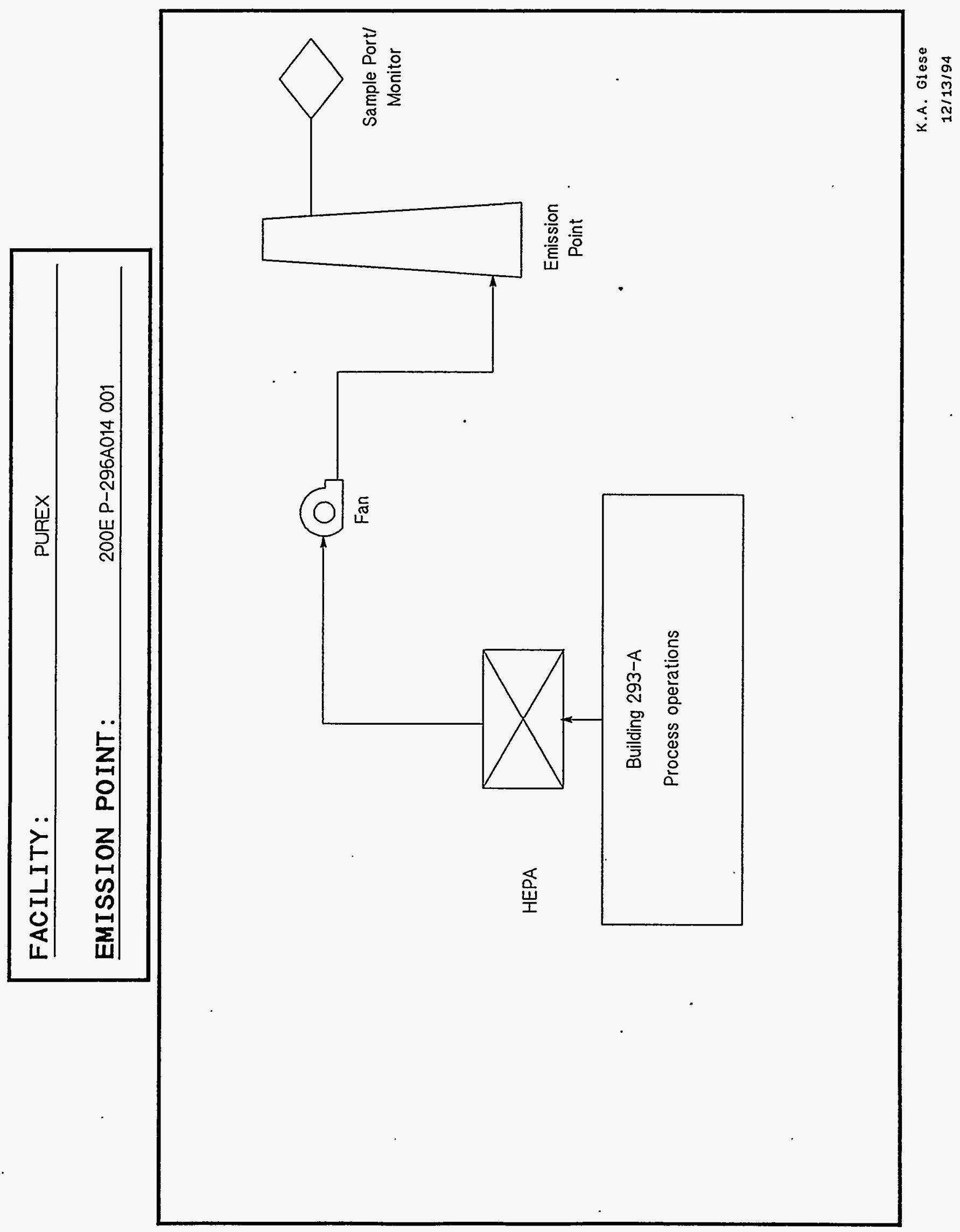




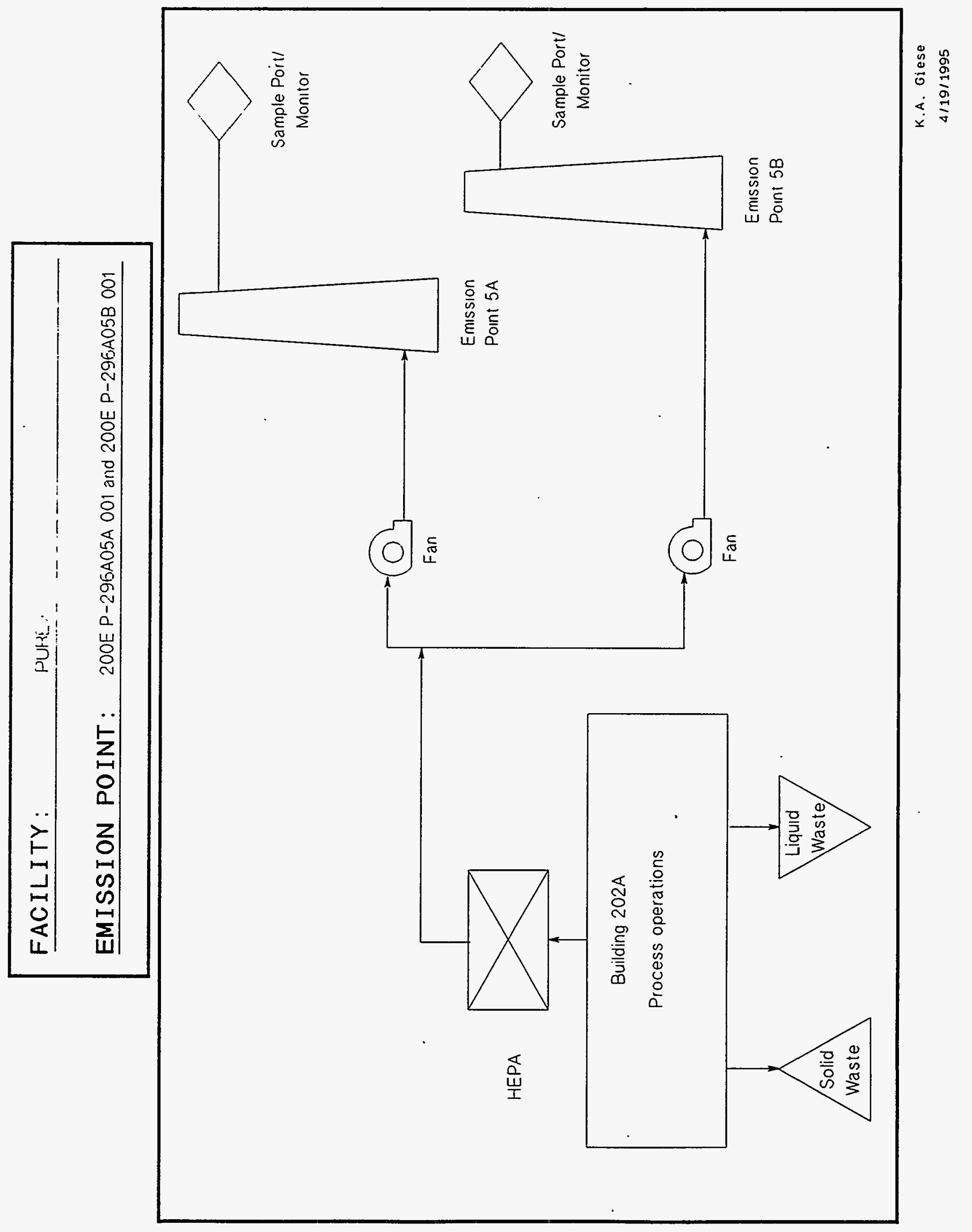




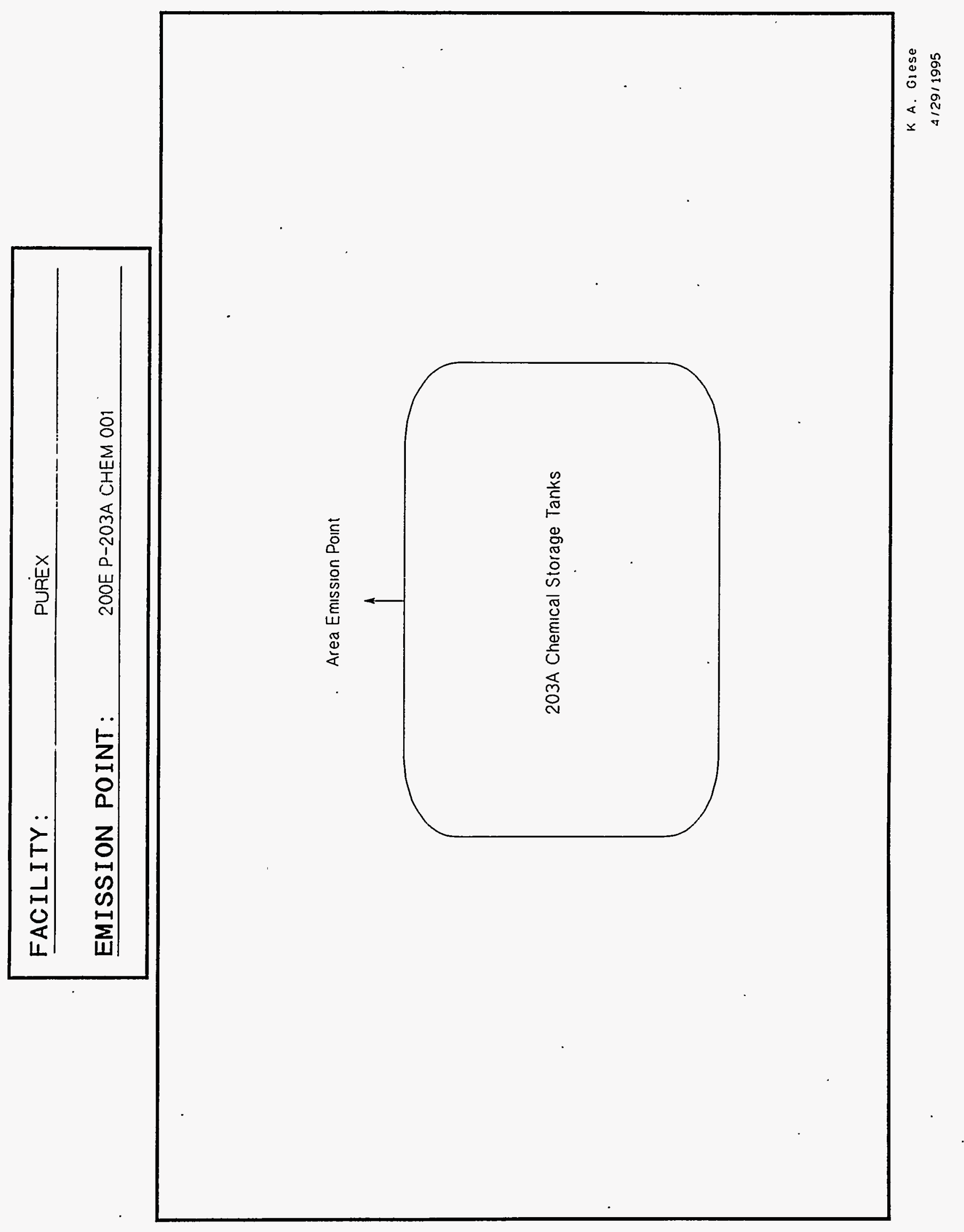




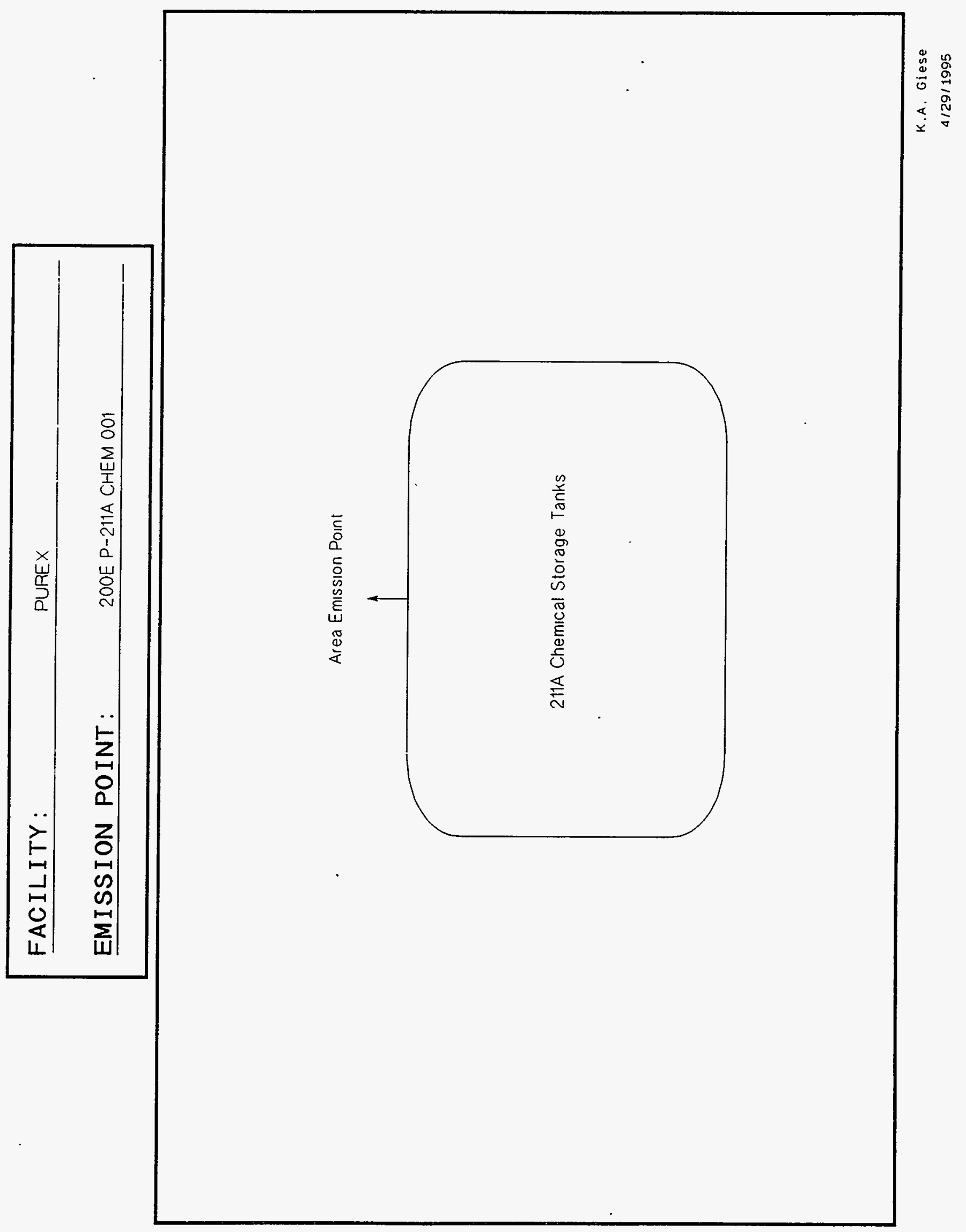


FACILITY: PUREX

EMISSION POINT: 200E P-AMU-ROOF-VENT

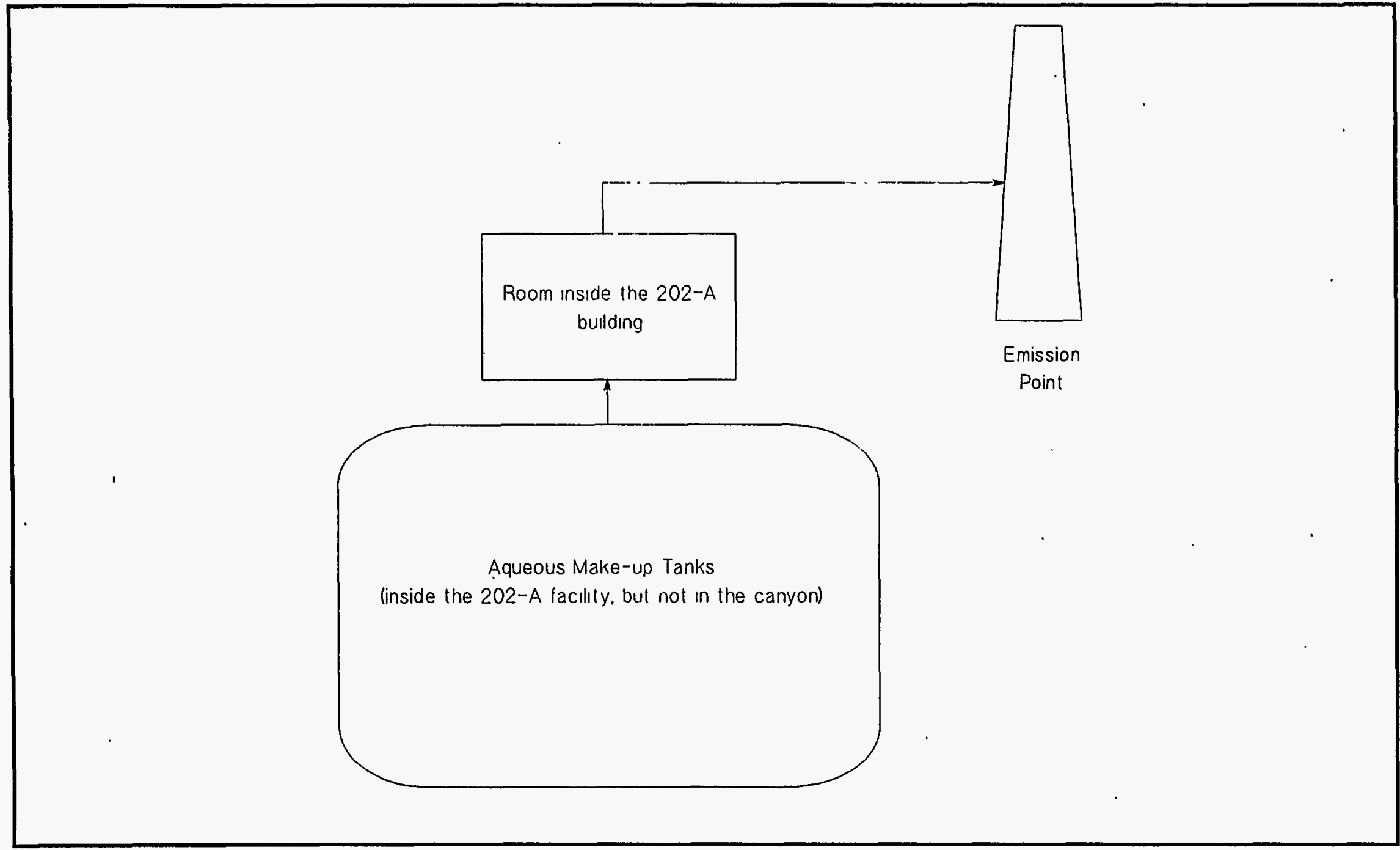




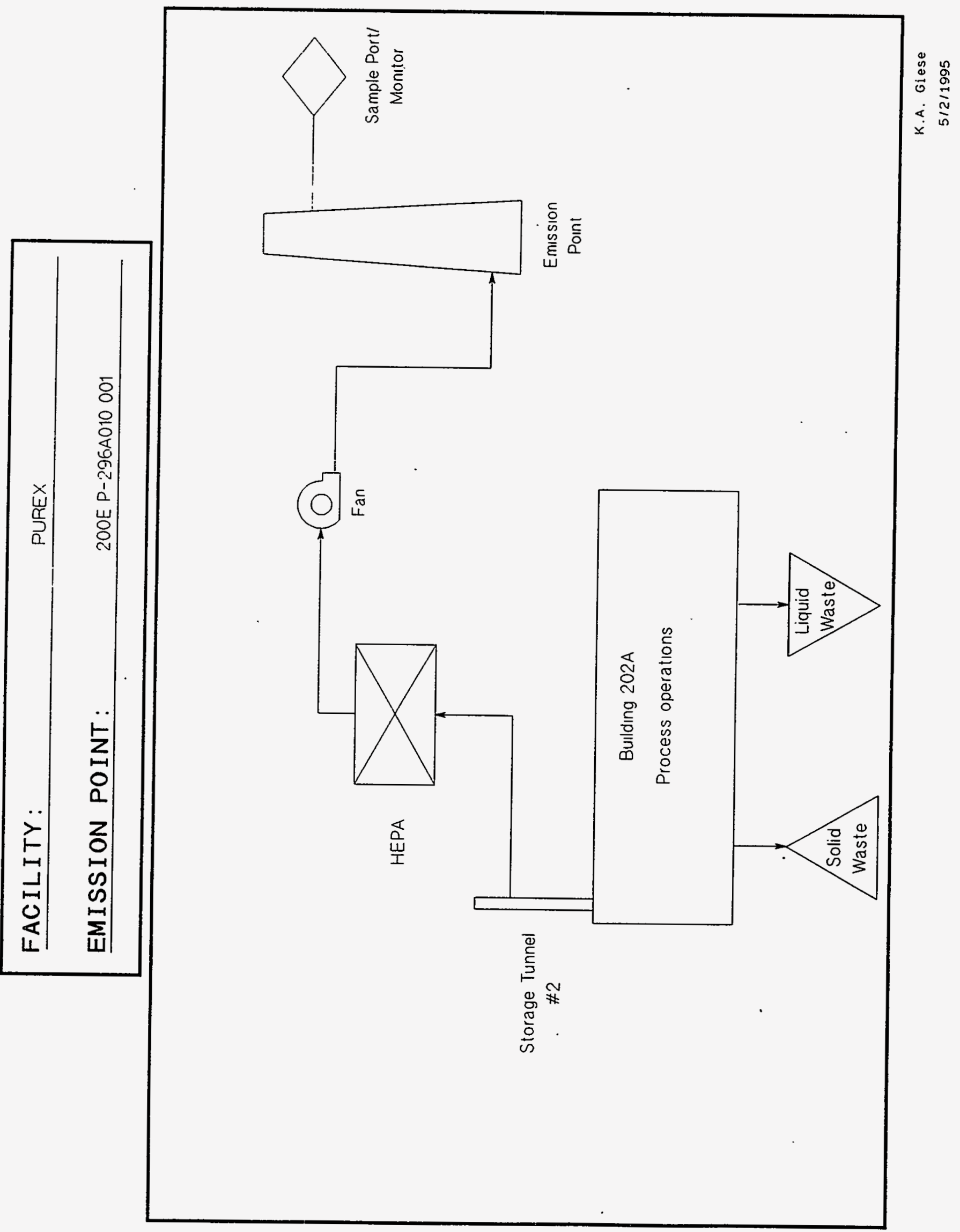




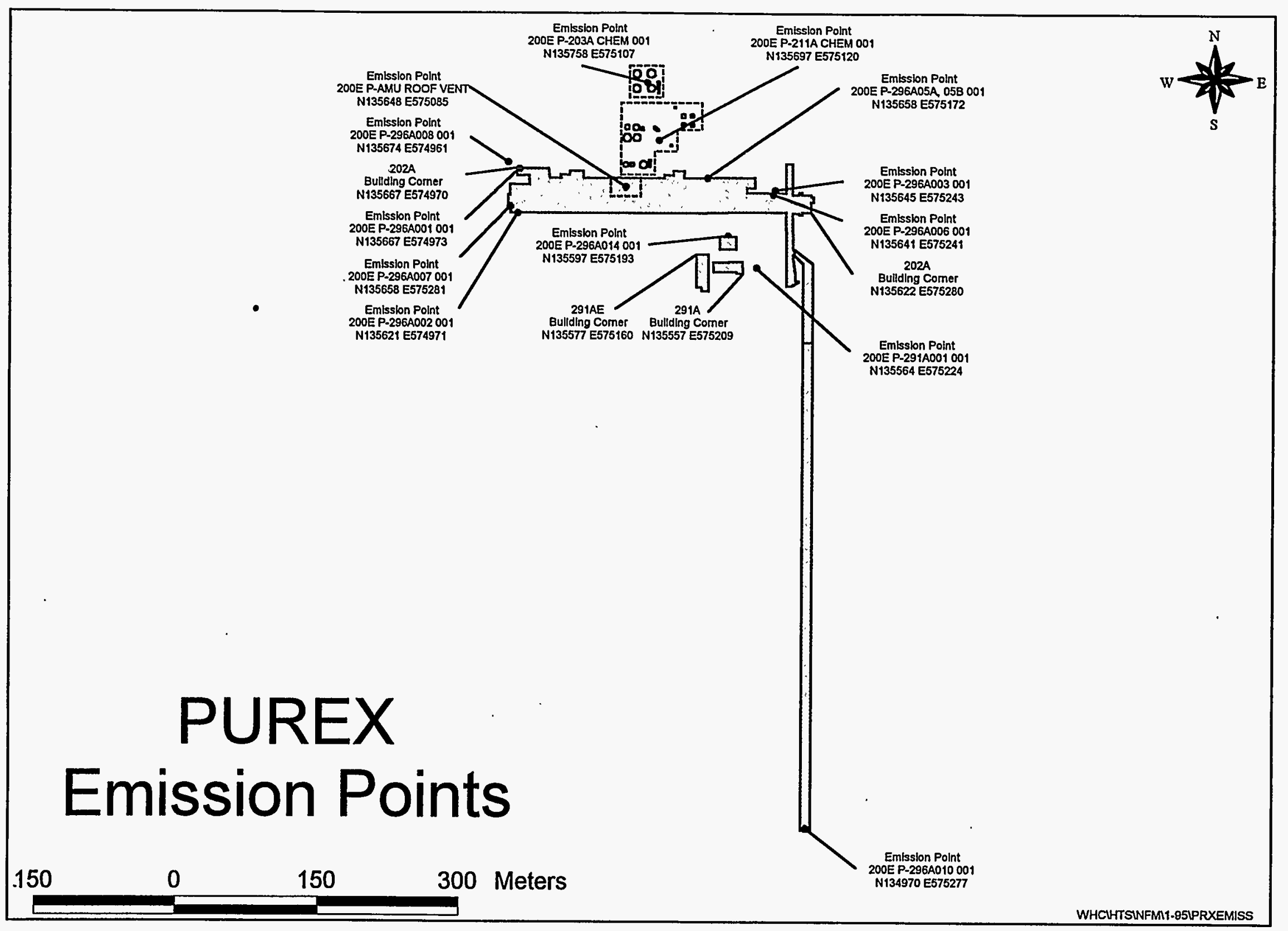




\title{
APPENDIX D-4
}

\section{EAST AREA TANK FARMS}

\author{
Chapter I. General Information
}

\section{I-B.1. Facility Description}

The single and double-shell tank farms initially were used for the storage and transfer of chemical and radiological wastes that were generated on the Hanford Site. These reprocessing plants performed chemical separations for the reprocessing of nuclear materials as well as radioactive waste storage and disposal. Operations at these plants, with the exception of shutdown and maintenance activities, has ceased. Shutdown and maintenance activities generate wastes that are transferred to the 200 East Area Tank Farms.

Currently, waste generated at the 100,300 , and 400 Areas is transported in railroad tank cars to the 204-AR Waste Unloading Station. There are two types of tanks in the 200 East Area tank farms, double-shell tanks (DSTs) and single-shell tanks (SSTs). There also are support operations associated with the 200 East Area Tank Farms such as receiving tanks and an evaporator.

The DST System treats and stores mixed waste before final treatment and disposal. The DST System includes double-contained receiver tanks (DCRTs) and ancillary equipment. The DSTs consist of 1-million gallon tanks designed for long-term storage (up to 50 years) of mixed waste. There are five DST Farms located in the 200 East Area with a total of 25 tanks. Liquid waste generated in the 200 Areas generally is transferred through underground pipelines and routed via DCRTs. The DCRTs serve various functions including receiver tanks for SST or DST waste, lift stations, and vent stations. The exhaust fans on the tank ventilation systems in the DCRTs keep a negative air pressure, which is monitored, inside the primary tank. The exhausted air passes through a heater, prefilter, HEPA filters, and is monitored for radiation. No offsite waste is accepted for storage in the DST System. The DSTs use separate ventilation systems for the primary tank and annulus ventilation. The primary tank ventilation system provides a negative pressure to prevent the escape of untreated dangerous and/or radioactive particulates to the atmosphere, removes heat generated by the tank contents, and removes water vapor to prevent tank corrosion. Additional tank space is used to support the environmental restoration and remediation mission (e.g., closure of single-shell tanks).

There are six SST Farms with a total of 66 tanks within the 200 East Area. Past-practice units are associated with the SSTs. The SST volumes vary from 55,000 to 1 million gallons. Addition of new waste to the SSTs was discontinued in November 1980. The SSTs currently are being interim stabilized and isolated. Interim stabilization involves the removal of supernate and interstitial liquid from the SSTs to minimize the spread of contamination if the tanks begin to leak. Interim isolation of a SST involves physical modifications to preclude 
the inadvertent addition of liquid to the tank. Forced ventilation provides cooling for some tanks containing materials which, through radioactive decay, generate heat that could exceed the established concrete temperature limits. Single-stage HEPA filters allow atmospheric breathing for tanks that do not require cooling. The SSTs contain mostly inorganic waste.

In addition to the SST and DST waste tank system emission points described in this appendix, there are numerous other sources from the SST and DST emission points that are potential sources of emissions. These points include access risers, pump pits, and diversion boxes on each waste tank. These points are normally covered or closed to control emissions from the waste tanks. Routine maintenance and operation activities performed periodically at each waste tank requires the cover blocks or other tank closures to be opened temporarily to allow access to the instrumentation and equipment located within each waste tank. These periods may allow unfiltered air exchanges between the waste tank vapor space or surface contamination areas with the environment. These emissions are considered fugitive emissions and constitute a portion of the radionuclide fugitive emissions from the Hanford Site described in Section IV.B.6.

There are other emission points associated with tank farm operations. These are the $209 \mathrm{E}$ Criticality Laboratory building; the Grout facility, which is currently inactive; the Liquid Effluent Retention Facilty (LERF), which is used to store liquid wastes prior to treatment, and the 242-A Evaporator treatment unit.

The 242-A Evaporator treatment unit concentrates the DST waste by removal of water and volatile and semivolatile organics through evaporation. The concentrated slurry is returned to the DST System and the process condensate is transferred for storage to LERF. Eventually the process condensate will be transferred to the 200 Area Effluent Treatment Facility.

The 200 East Area tank farms are operated for 24 hours per day, 365 days per year.

\section{I-B.2. Products, Raw Materials, Fuels, Processes}

- Principle products - There are no products produced at the 200 East Tank Farms.

- Process information - There is one process-related activity at the 200 East Tank Farms: an evaporation process.

The 242-A Evaporator unit concentrates DST waste by removal of water and volatile and semivolatile organics through evaporation. The concentrated slurry is returned to the DST System and the process condensate is transferred for storage to the Liquid Effluent Retention Facility. Eventually the process condensate will be transferred to the 200 Area Effluent Treatment Facility once this unit commences operation. 


\section{I-B.2. Products, Raw Materials, Fuels, Processes}

- Fuels used by processes - There are no fuels used at the 200 East Area Tank Farms.

- Raw materials used by processes - There are no raw materials used at the 200 East Area Tank Farms.

\section{I-C.1. Emission Points, Controls, Stacks}

The emission point and stack information is found on pages 4 through 21 of the AEI report output. The control information is found on page 92 of the AEI report output.

\section{I-C.2. Facility Process Flow Diagrams}

Attached.

I-C.3. Site Map

Attached.

\section{Chapter II. Regulatory Requirements}

\section{II-A.1. Description of Discharge and Emission Points}

The emission point and stack information is found on pages 4 through 21 of the AEI report output. 
Facility Building List

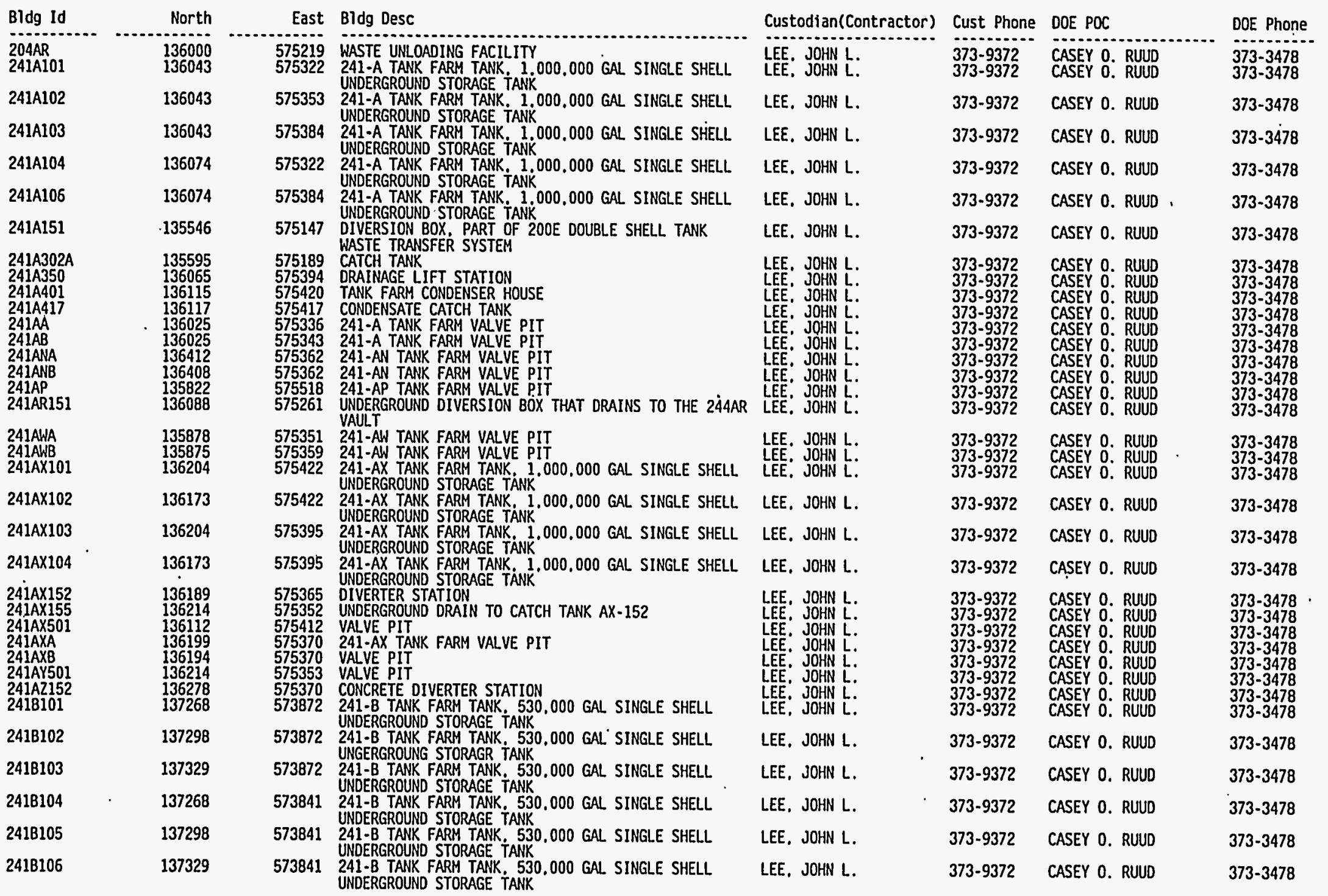




\begin{tabular}{|c|c|c|c|c|c|c|c|}
\hline $\begin{array}{l}\text { Facility } \\
\text { Bldg Id }\end{array}$ & $\begin{array}{l}\text { ng List } \\
\text { North }\end{array}$ & East & Bldg Desc & Custodian(Contractor) & Cust Phone & DOE POC & DOE Phone \\
\hline 2418107 & 137268 & 573811 & \multirow{7}{*}{ 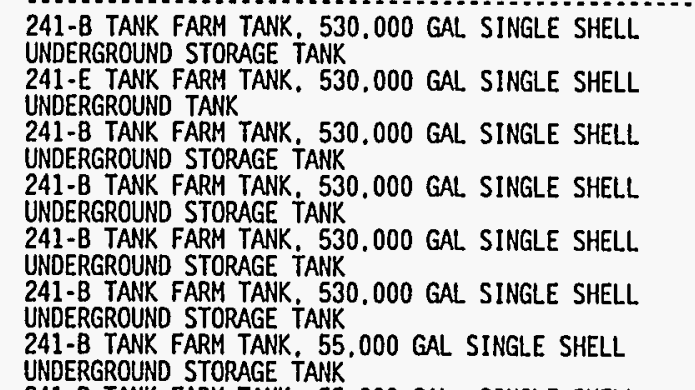 } & LEE, JOHN L. & $373-9372$ & CASEY 0 . RUUD & $373-3478$ \\
\hline $241 \mathrm{~B} 108$ & 137298 & 573811 & & LEE, JOHN L. & 373-9372 & CASEY O. RUUD & $373-3478$ \\
\hline 241B109 & 137329 & 573811 & & LEE, JOHN L. & 373-9372 & CASEY O. RUUD & 373-3478 \\
\hline 2418110 & 137268 & 573780 & & LEE, JOHN L. & 373-9372 & CASEY 0. RUUD & 373-3478 \\
\hline 2418111 & 137298 & 573780 & & LEE. JOHN L. & $373-9372$ & CASEY 0. RUUD & 373-3478 \\
\hline 241B112 & 137329 & 573780 & & LEE, JOHN L. & 373-9372 & CASEY 0. RUUD & 373-3478 \\
\hline 241B201 & 137359 & 573818 & & LEE. JOHN L. & 373-9372 & CASEY O. RUUD & $373-3478$ \\
\hline 241B202 & 137359 & 573803 & $\begin{array}{l}241 \text { - } 8 \text { TANK FARM TANK } 55.000 \text { GAL. SINGLE SHELL } \\
\text { UNDERGROUND STORAGE TANK }\end{array}$ & LEE, JOHN L. & $373-9372$ & CASEY 0. RUUD & 373-3478 \\
\hline $241 B 203$ & 137359 & -573788 & $\begin{array}{l}2 \text { 241-B TAN FARM TANK, } 55,000 \text { GAL. SINGLE SHELL. } \\
\text { UNDERGROUND STORAGE TANK }\end{array}$ & LEE. JOHN L. & 373-9372 & CASEY 0. RUUD & 373-3478 \\
\hline 241B204 & 137359 & 573773 & $\begin{array}{l}241-B \text { TANK FARM TANK } 55.000 \text { GAL. SINGLE SHELL } \\
\text { UNDERGROUND STORAGE TANK }\end{array}$ & LEE, JOHN L. & 373-9372 & CASEY 0. RUUD & 373-3478 \\
\hline $2418 \times 101$ & 137317 & 573659 & $\begin{array}{l}241 \text {-BX TANK FARM TANK. } 530.000 \text { GAL. SINGLE SHELL } \\
\text { UNDERGROUNO STORAGE TANK }\end{array}$ & LEE, JOHN L. & 373-9372 & CASEY 0. RUUD & 373-3478 \\
\hline $2418 \times 102$ & 137347 & 573659 & $\begin{array}{l}\text { 241-BX TANK FARM TANK } 530,000 \text { GAL. SINGLE SHELL } \\
\text { UNDERGOUND STORAGE TANK }\end{array}$ & LEE. JOHN L. & 373-9372 & CASEY O. RUUD & $373-3478$ \\
\hline $2418 \times 103$ & 137378 & 573659 & $\begin{array}{l}2 \text { 241-BX TAN FARM TANK, } 530.000 \text { GAL. SINGLE SHELL } \\
\text { UNDERGRONDD STORAGE TANK }\end{array}$ & LEE, JOHN L. & 373-9372 & CASEY 0. RUUD & 373-3478 \\
\hline 241BX104 & 137317 & 573629 & $\begin{array}{l}\text { 241-BX TANK FARM TANK. SINGLE SHELL UNDERGROUND } \\
\text { STORAGE TANK, } 530,000 \text { GALLON }\end{array}$ & LEE, JOHN L. & 373-9372 & CASEY 0. RUUD & $373-3478$ \\
\hline $2418 \times 105$ & 137347 & 573629 & $\begin{array}{l}\text { 241-XX TANK FARM TANK } 530,000 \text { GAL. SINGLE SHELL } \\
\text { UNDERGROUND STORAGE TANK }\end{array}$ & LEE. JOHN L. & 373-9372 & CASEY 0. RUUD & 373-3478 \\
\hline $2418 \times 106$ & 137378 & 573629 & $\begin{array}{l}241-B X \text { TAKK FARM TANK } 530.000 \text { GAL. SINGLE SHELL } \\
\text { UNDERGROUND STORAGE TANK }\end{array}$ & LEE, JOHN L. & 373-9372 & CASEY 0. RUUD & 373-3478 \\
\hline $2418 \times 107$ & 137317 & 573598 & $\begin{array}{l}241 \text { BX TANK FARM TANK } 530,000 \text { GAL. SINGLE SHELL } \\
\text { UNDERGROUND STORAGE TANK }\end{array}$ & LEE, JOHN L. & 373-9372 & CASEY 0. RUUD & $373-3478$ \\
\hline $2418 \times 108$ & 137347 & 573598 & $\begin{array}{l}241 \text {-BX TANK FARY TANK } 530,000 \text { GAL. SINGLE SHELL. } \\
\text { UNDERGROUND STORAGE TANK }\end{array}$ & LEE, JOHN L. & $373-9372$ & CASEY 0. RUUD & 373-3478 \\
\hline 2418X109 & 137378 & 573598 & $\begin{array}{l}241 \text {-BX TANK FARM TANK } 530,000 \text { GAL. SINGLE SHELL } \\
\text { UNDERGROUND STORAGE TANK }\end{array}$ & LEE. JOHN L: & 373-9372 & CASEY 0. RUUD & $373-3478$ \\
\hline $2418 \times 110$ & 137317 & 573568 & $\begin{array}{l}241 \text {-BX TANK FARM TANK } 530.000 \text { GAL. SINGLE SHELL } \\
\text { UNOERGROUND STORAGE TANK }\end{array}$ & LEE; JOHN L. & 373-9372 & CASEY 0. RUUD & $373-3478$ \\
\hline $2418 \times 111$ & 137347 & $573568^{\prime}$ & $\begin{array}{l}241 \text {-XX TAKK FARM TANK } 530.000 \text { GAL. SINGLE SHELL } \\
\text { UNDERGROUND STORAGE TANK }\end{array}$ & LEE, JOHN L. & $373-9372$ & CASEY 0. RUUD & $373-3478$ \\
\hline $2418 \times 112$ & 137378 & 573568 & $\begin{array}{l}241 \text {-BX TANK FARM TANK } 530.000 \text { GAL. SINGLE SHELL } \\
\text { UNDERGROUND STORAGE TANK }\end{array}$ & LEE. JOHN L. & 373-9372 & CASEY 0. RUUD & $373-3478$ \\
\hline 241BY101 & 137469 & 575660 & $\begin{array}{l}241 \text { - IY TANK FARM TANK } 750.000 \text { GAL. SINGLE SHELL } \\
\text { UNDERGROUND STORAGE TANK }\end{array}$ & LEE, JOHN L. & 373-9372 & CASEY 0. RUUD & 373-3478 \\
\hline 241BY102 & 137500 & 573660 & $\begin{array}{l}241 \text {-BY TANK FAR TANK } 750.000 \text { GAL. SINGLE SHELL } \\
\text { UNGERGROUND STORAGE TANK }\end{array}$ & LEE. JOHN L. & $373-9372$ & CASEY 0. RUUD & $373-3478$ \\
\hline $241 \mathrm{BY} 103$ & 137531 & 573660 & $\begin{array}{l}\text { 241-BY TANK FARM TANK } 750.000 \text { GAL. SINGLE SHELL } \\
\text { UNDERGROUND STORAGE TANK }\end{array}$ & LEE, JOHN L. & 373-9372 & CASEY 0. RUUD & $373-3478$ \\
\hline 241BY104 & 137469 & 573629 & $\begin{array}{l}\text { 241-BY TAMK FAPM TANK } 750,000 \text { GAL. SINGLE SHELL } \\
\text { UNDERGROUND STORAGE TANK }\end{array}$ & LEE, JOHN L. & $373-9372$ & CASEY 0. RUUD & $373-3478$ \\
\hline 241BY105 & 137500 & 573629 & $\begin{array}{l}\text { 241-BY TANK FARM TANK } 750.000 \text { GAL. SINGLE SHELL } \\
\text { UNDERGROUND STORAGE TANK }\end{array}$ & LEE, JOHN L. & $373-9372$ & CASEY O. RUUD & $373-3478$ \\
\hline 2418Y106 & 137531 & 573629 & $\begin{array}{l}\text { 241-BY TANK FARM TANK } 750.000 \text { GAL. SINGLE SHELL } \\
\text { UNDERGROUND STORAGE TANK }\end{array}$ & LEE, JOHN L. & 373-9372 & CASEY O. RUUD & $373-3478$ \\
\hline
\end{tabular}




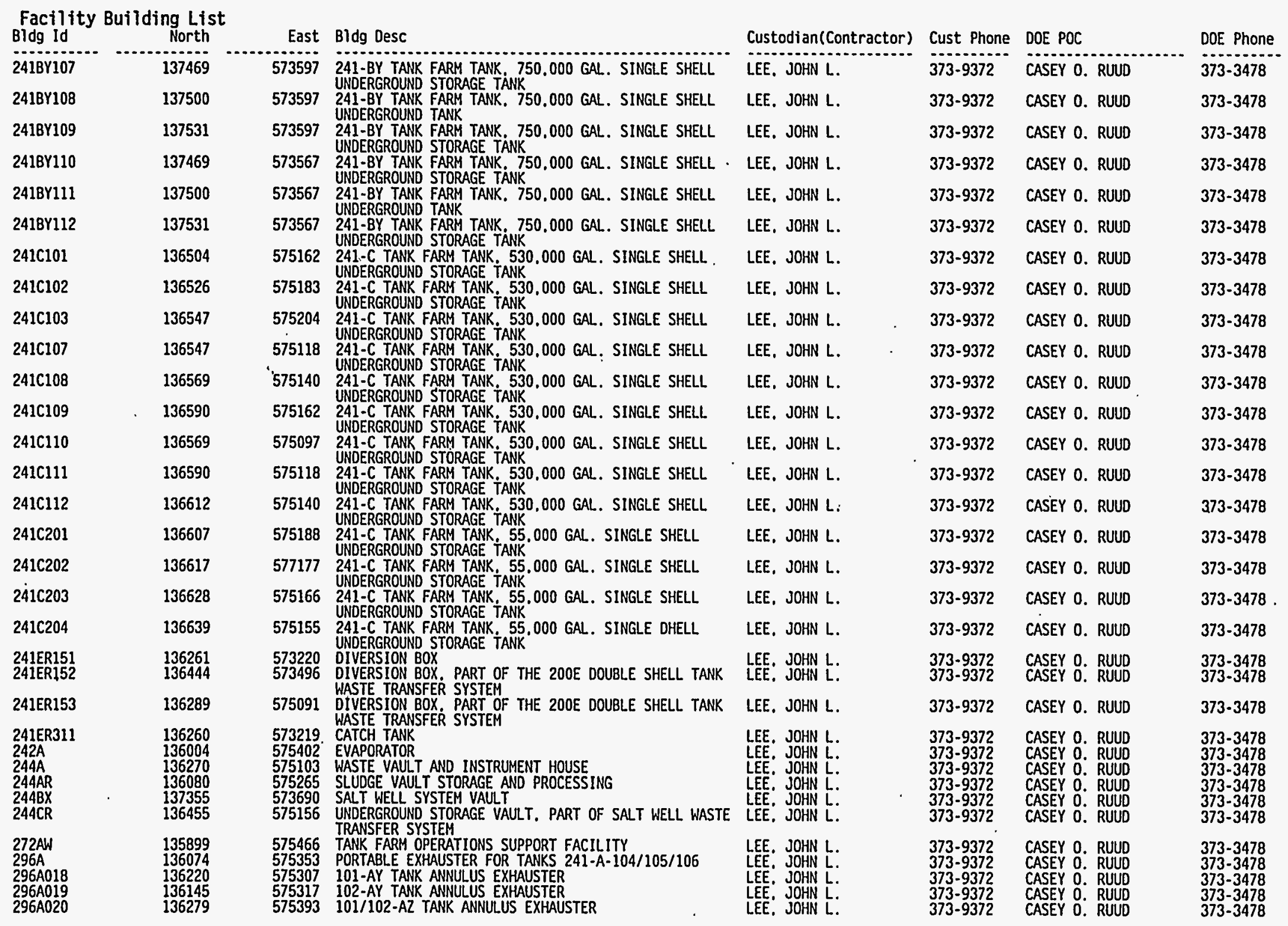




\section{Facility Building List}

\begin{tabular}{|c|c|c|c|c|c|c|c|}
\hline Bldg Id & North & East & Bldg Desc & Custodian(Contractor) & Cust Phone & DOE POC & DOE Phone \\
\hline 2964028 & 135841 & 575383 & 241-AN TANK ANNULUS EXHAUSTER FOR TANKS $101 / 1021$ & LEE. JOHN L. & 373.9372 & CASEY 0. RUUD & $373-3478$ \\
\hline $296 \mathrm{A030}$ & 136430 & 575395 & 241 -AN TANK ANNULUS EXHAUSTER FOR TANKS 101/102/ & LEE, JOHN L. & $373-9372$ & CASEY 0. RUUD & $373-3478$ \\
\hline $\begin{array}{l}296 A 041 \\
296 A N \\
296 A P \\
296 A N \\
296 A Y A Z \\
296 G 001 \\
296 P 016\end{array}$ & $\begin{array}{l}135815 \\
136432 \\
135817 \\
135842 \\
136150 \\
135979 \\
136561\end{array}$ & $\begin{array}{l}575597 \\
575396 \\
575602 \\
575383 \\
575389 \\
575900 \\
575162\end{array}$ & $\begin{array}{l}\text { 241-AP TANK ANNULUS EXHAUSTER } \\
\text { 241-AN TANK FARM PRIMARY VENTILATION STACK } \\
241 \text {-AP TANK FARM PRIMARY VENTILATION STACK } \\
241 \text {-AW TANK FARM PRIMARY VENTILATION STACK } \\
\text { 241-AYIAZ TANK FARM PRIMARY VENTILATION STACK } \\
\text { GROUT STACK } \\
\text { STACK. PORTABLE EXHAUSTER FOR TANKS } 241-C-104 / 105 /\end{array}$ & $\begin{array}{l}\text { LEE. JOHN L. } \\
\text { LEE, JOHN L. } \\
\text { LEE, JOHN L. } \\
\text { LEE. JOHN L. } \\
\text { LEE. JOHN L. } \\
\text { LEE. JOHN L. } \\
\text { LEE, JOHN L. }\end{array}$ & $\begin{array}{l}373-9372 \\
373-9372 \\
373-9372 \\
373-9372 \\
373-9372 \\
373-9372 \\
373-9372\end{array}$ & $\begin{array}{l}\text { CASEY } 0 . \text { RUUD } \\
\text { CASEY } 0 . \text { RUUD } \\
\text { CASEY } 0 . \text { RUUD } \\
\text { CASEY } 0 . \text { RUUD } \\
\text { CASEY } 0 . \text { RUUD } \\
\text { CASEY } 0 . \text { RUUD } \\
\text { CASEY } 0 . \text { RUUD }\end{array}$ & $\begin{array}{l}373-3478 \\
373-3478 \\
373-3478 \\
373-3478 \\
373-3478 \\
373-3478 \\
373-3478\end{array}$ \\
\hline $\begin{array}{l}66 P 026 \\
6 P 031 \\
R F\end{array}$ & $\begin{array}{l}136115 \\
136337 \\
137196\end{array}$ & $\begin{array}{l}575386 \\
574446 \\
575374\end{array}$ & $\begin{array}{l}\text { AY/AZ TANK BACKUP EXHAUSTER } \\
209-E \text { CRITICAL MASS LABORATORY BUILDING STACK } \\
\text { LIQUID EFFLUENT RETENTION FACILITY }\end{array}$ & $\begin{array}{l}\text { LEE. JOHN L. } \\
\text { LEE. JOHN L. } \\
\text { LEE. JOHN L. }\end{array}$ & $\begin{array}{l}373-9372 \\
373-9372 \\
373-9372\end{array}$ & $\begin{array}{l}\text { CASEY } 0 . \text { RUUD } \\
\text { CASEY O. RUUD } \\
\text { CASEY } 0 . \text { RUUD }\end{array}$ & $\begin{array}{l}373-3478 \\
373-3478 \\
373-3478\end{array}$ \\
\hline
\end{tabular}

\section{Emission Point Listing}

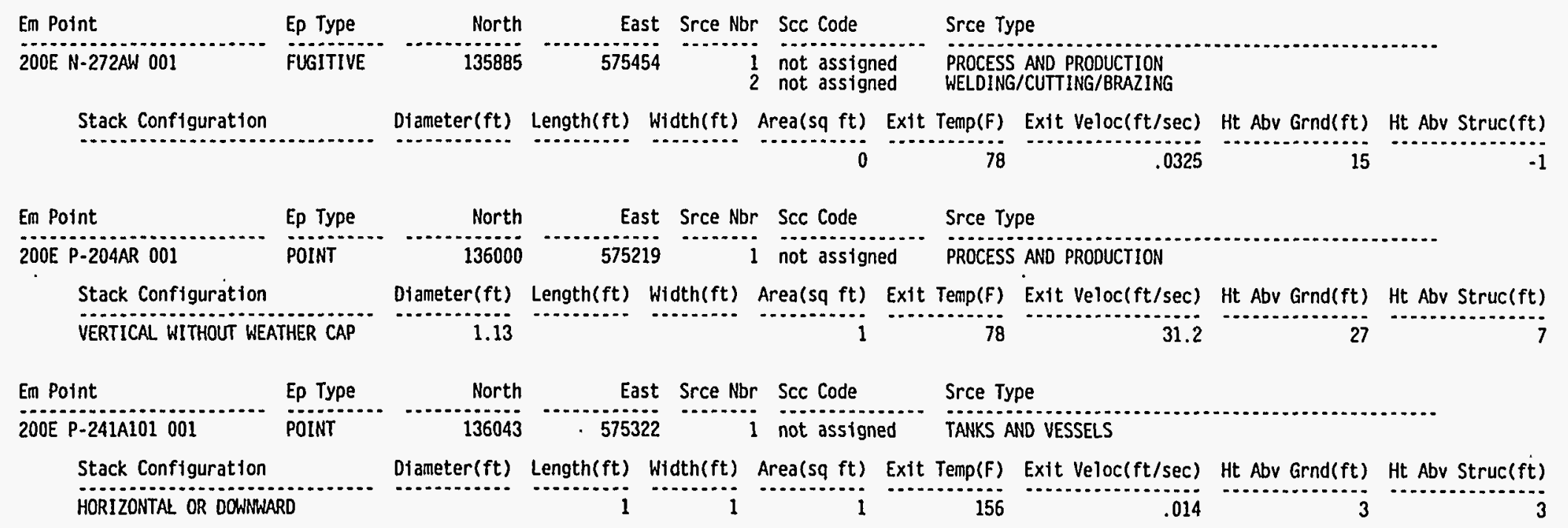


AIR EMISSIONS INVENTORY PERMIT APPLICATION REPORT (UP460)

FACILITY NAME: 200E Tank Farm and Associated Structures

\section{Emission Point Listing}

\begin{tabular}{|c|c|c|c|c|c|c|c|c|c|}
\hline Em Point & Ep Type & North & Srce Nbr & Scc Code & & Srce Ty & ype & & \\
\hline 200E P-241A102 001 & POINT & 136043 & 575353 & 1 not assigne & & TANKS AI & AND VESSELS & & \\
\hline Stack Configuration & & Diameter (ft) & Length $(\mathrm{ft})$ & Area $(s q \mathrm{ft})$ & Exit & $\operatorname{Temp}(F)$ & Exit Veloc $(\mathrm{ft} / \mathrm{sec})$ & Ht Abv Grnd(ft) & Ht Abv Struc (ft) \\
\hline HORIZONTAL OR DOWNWARD & & & 1 & 1 & & 90 & $.03 i$ & 3 & $\ddot{3}$ \\
\hline Em Point & Ep Type & North & Srce Nbr & $r$ Scc code & & Srce Ty & & & \\
\hline $200 E$ P-241A103 001 & POINT & 136043 & 575384 & 1 not assigne & & TANKS Al & AND VESSELS & & \\
\hline Stack Configuration & & Diameter (ft) & Length(ft) Width(ft) & Area $(s q f t)$ & Exit & Temp (F) & Exit Veloc $(\mathrm{ft} / \mathrm{sec})$ & Ht Abv Grnd(ft) & Ht Abv Struc(ft) \\
\hline HORIZONTAL OR DOWNWARD & & & 1 & 1 & & 116 & .031 & 3 & 3 \\
\hline Em Point & Ep Type & North & Srce Nbr & $r$ Scc code & & Srce Ty & & & \\
\hline 200E P-241A104 001 & POINT & 136074 & 575322 & 1 not assigne & & TANKS AI & AND VESSELS & & \\
\hline Stack Configuration & & Diameter $(f t)$ & Length(ft) Width(ft) & $\operatorname{Area}(s q \mathrm{ft})$ & Exit & Temp(F) & Exit Veloc $(\mathrm{ft} / \mathrm{sec})$ & Ht Abv Grnd(ft) & Ht Abv Struc(ft) \\
\hline HORIZONTAL OR DOWNWARD & & & $i$ & 1 & & 192 & .014 & 3 & 3 \\
\hline Em Point & Ep Type & North & East Srce Nbr & $r$ Scc Code & & Srce Ty & & & . \\
\hline 200E P-241A106 001 & POINT & 136074 & 575384 & not assigne & & TANKS AI & ND VESSELS & & \\
\hline Stack Configuration & & Diameter $(f t)$ & Length $(f t)$ Width $(f t)$ & Area $(s q f t)$ & Exit & Temp (F) & Exit Veloc $(\mathrm{ft} / \mathrm{sec})$ & Ht Abv Grnd(ft) & Ht Abv Struc(ft) \\
\hline HORIZONTAL OR DOWNWARD & & & 1 & & & 136 & .031 & 3 & 3 \\
\hline Em Point & Ep Type & North & East Srce $\mathrm{Nbr}$ & Scc Code & & Srce Typ & & & \\
\hline 200E P-241A151 001 & FUGITIVE & 135546 & 575147 & 1 not assigne & & PROCESS & AND PRODUCTION & & \\
\hline $\begin{array}{l}\text { Stack Configuration } \\
\end{array}$ & & Diameter (ft) & Length(ft) Width(ft) & $\begin{array}{r}\operatorname{Area}(s q \mathrm{ft}) \\
0\end{array}$ & Exit & $\begin{array}{r}\text { Temp (F) } \\
78\end{array}$ & $\begin{array}{r}\text { Exit } v e l o c(f t / s e c) \\
.0325\end{array}$ & Ht Abv Grnd(ft) & Ht Abv Struc(ft) \\
\hline Em Point & Ep Type & North & East srce Nbr & Scc Code & & Srce Typ & & & \\
\hline 200E P-24IA302A 001 & FUGITIVE & 135595 & 575189 & not assigne & & PROCESS & AND PRODUCTION & & \\
\hline Stack Configuration & & Diameter (ft) & Length(ft) Width(ft) & $\begin{array}{r}\operatorname{Area}(\mathrm{sq} f \mathrm{ft}) \\
0\end{array}$ & Exit & $\begin{array}{r}\text { Temp(F) } \\
78\end{array}$ & $\begin{array}{r}\text { Exit Veloc(ft/sec) } \\
.0325\end{array}$ & $\begin{array}{r}\text { Ht Abv Grnd(ft) } \\
1\end{array}$ & Ht Abv Struc (ft) \\
\hline
\end{tabular}


AIR EMISSIONS INVENTORY PERMIT APPLICATION REPORT (UP460)
FACILITY ID : 200E TANK

FACILITY NAME: 200E Tank Farm and Associated Structures

\section{Emission Point Listing}

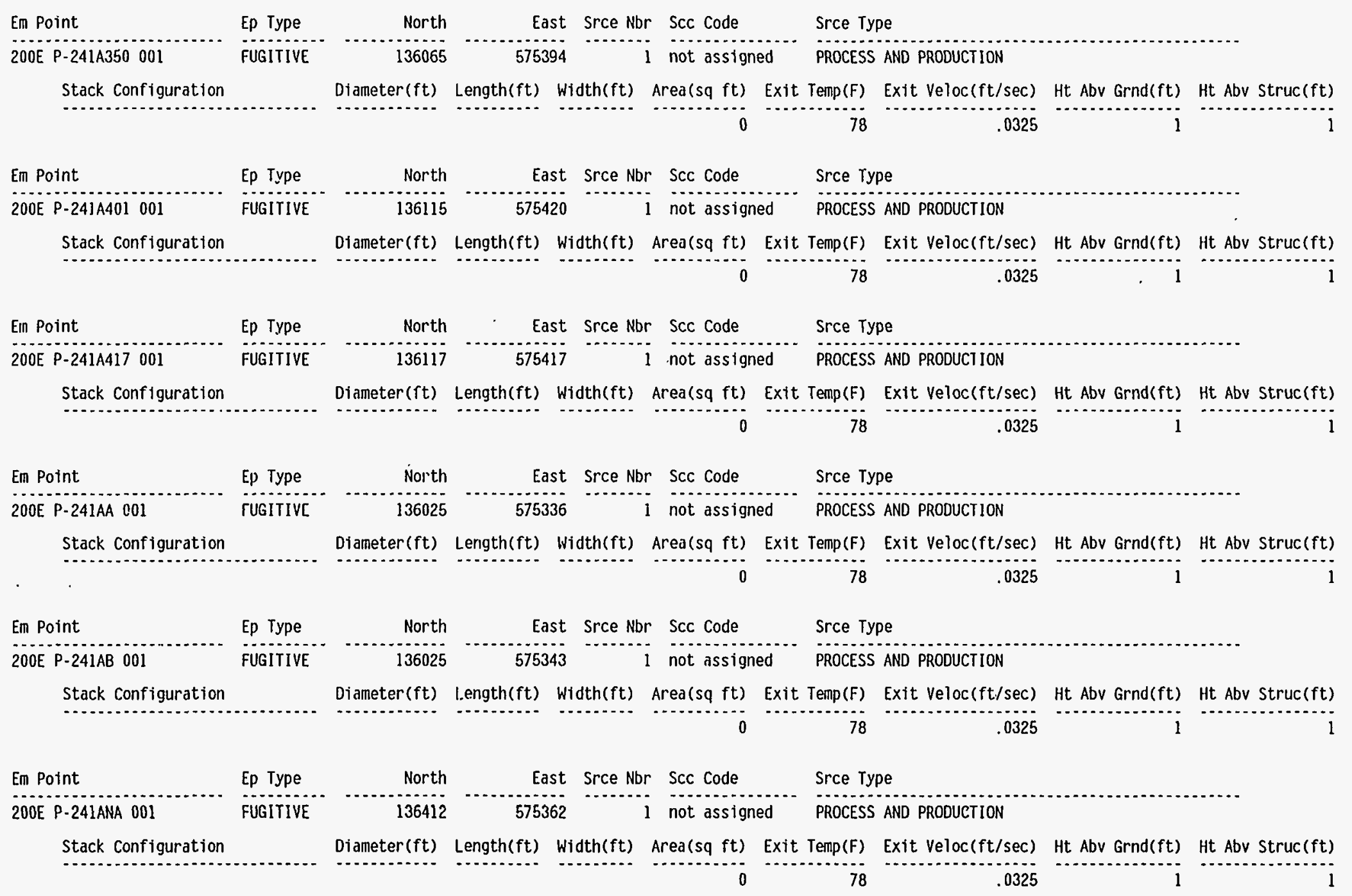

Page \#: 6 of 95 Report Run Date: 22-APR-95 
AIR EMISSIONS INVENTORY PERMIT APPLICATION REPORT (UP460) FACILITY NAME: 200 FACILITY Tank Farim and Associated structures

\section{Emission Point Listing}

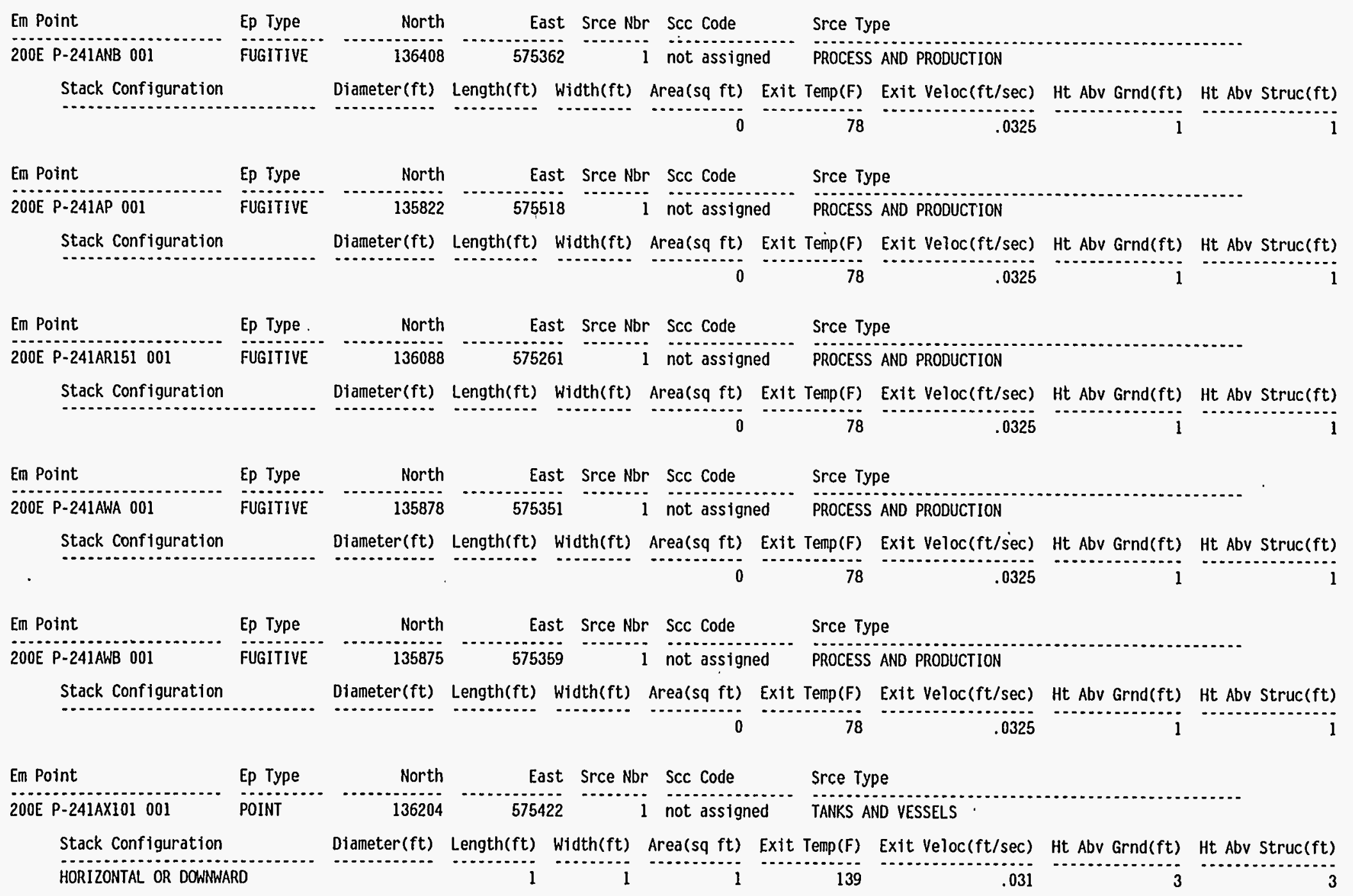


AIR EMISSIONS INVENTORY PERMIT APPLICATION REPORT (UP460) FACILITY NAME: 200 TACILITY ID Farm and Assoctated Structures

\section{Emission Point Listing}

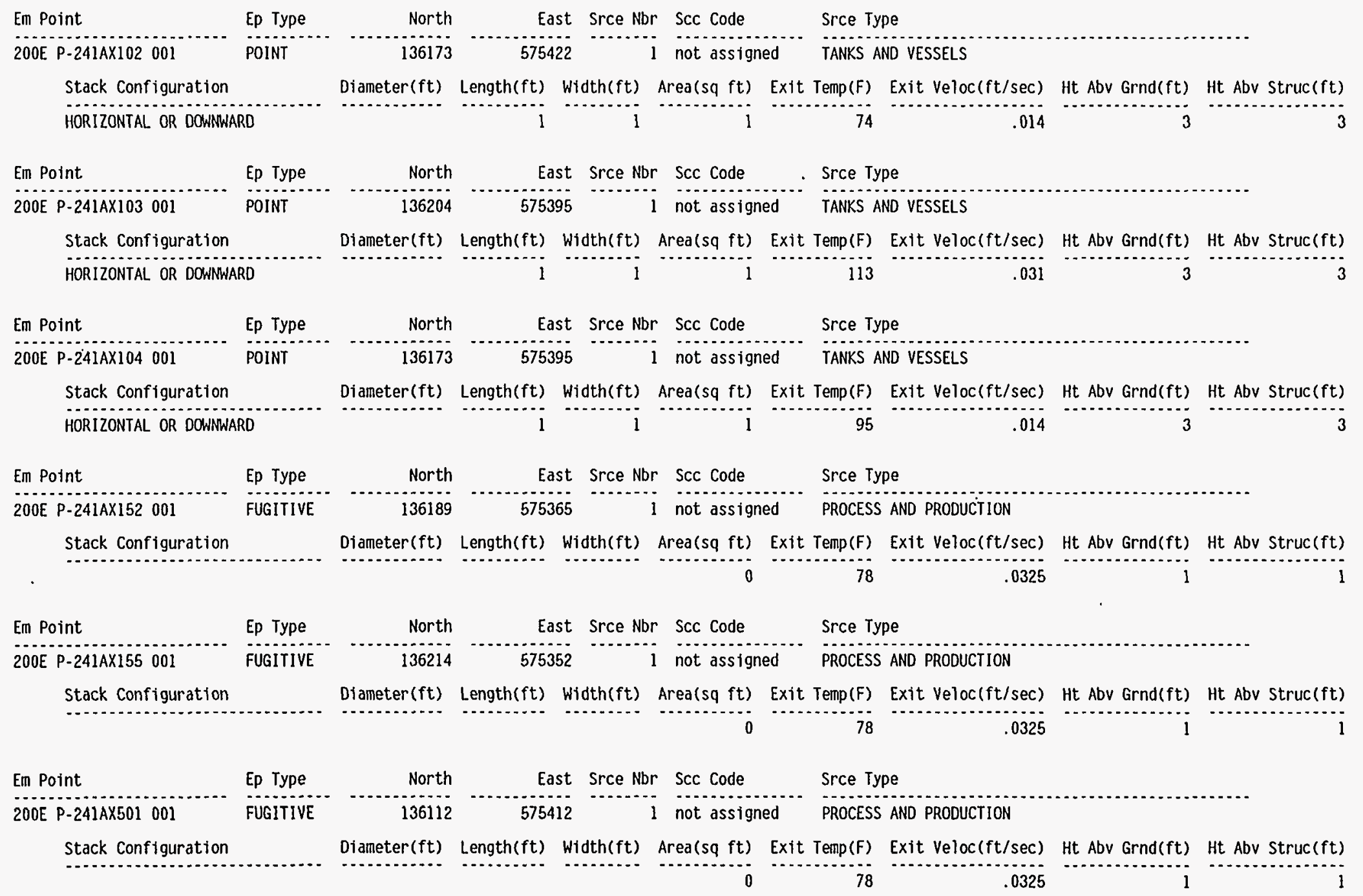

Page \#: 8 of 95 Report Run Date: 22-APR-95 
AIR EMISSIONS INVENTORY PERMIT APPLICATION REPORT (UP460) FACILITY NAME: 200 FACILITY ID : 200 Tank TANK

\section{Emission Point Listing}

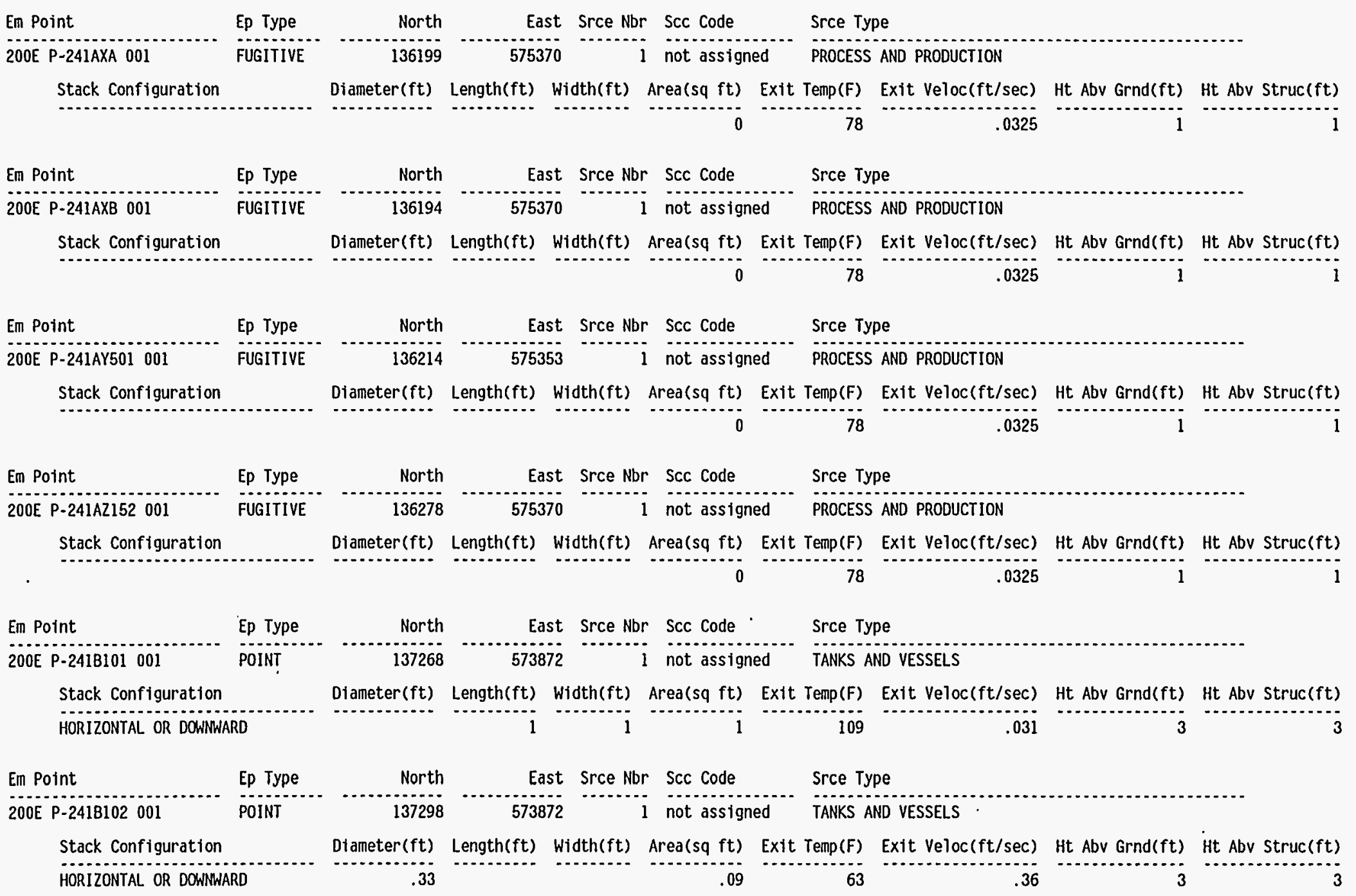


AIR EMISSIONS INVENTORY PERMIT APPLICATION REPORT (UP460)

FACILITY NAME: 200E Tank Farm and Associated structures

\section{Emission Point Listing}

\begin{tabular}{|c|c|c|c|c|c|c|c|c|}
\hline Em Point & Ep Type & North & East Srce Nbr & $r$ Scc Code & & Srce Type & & \\
\hline 200E P-241B103001 & POINT & 137329 & 573872 & not assigne & & TANKS AND VESSELS & & \\
\hline Stack Configuration & & Diameter ( $f t$ ) & Length(ft) & $\operatorname{Area}(s q \mathrm{ft})$ & Exit & Temp(F) Exit Veloc(ft/sec) & Ht Abv Grnd(ft) & Ht Abv Struc (ft) \\
\hline HORIZONTAL OR DOWNWARD & & .33 & & .09 & & .36 & 3 & 3 \\
\hline Em Point & Ep Type & North & Srce Nbr & Scc Code & & Srce Type & & \\
\hline 200E P-241B104001 & POINT & 137268 & 573841 & 1 not assigne & & TANKS AND VESSELS & & \\
\hline Stack Configuration & & Diameter (ft) & Length(ft) Width(ft) & $\operatorname{Area}(s q \mathrm{ft})$ & Exit & Temp(F) Exit Veloc (ft/sec) & Ht Abv Grnd(ft) & Ht Abv Struc $(f t)$ \\
\hline HORIZONTAL OR DOWNWARD & & 1 & & .79 & & .018 & 3 & 3 \\
\hline Em Point & Ep Type & North & East Srce $\mathrm{Nbr}$ & Scc Code & & Srce Type & & \\
\hline $200 E$ P-241B105 001 & POINT & 137298 & 573841 & i not assigne & & TANKS AND VESSELS & & \\
\hline Stack Configuration & & Diameter $(f t)$ & Length(ft) Width(ft) & Area $(s q f t)$ & Exit & Temp(F) Exit Veloc $(\mathrm{ft} / \mathrm{sec})$ & Ht Abv Grnd(ft) & Ht Abv Struc $(f t)$ \\
\hline HORIZONTAL OR DOWNWARD & & .33 & & .09 & & .17 & 3 & 3 \\
\hline Em Point & Ep Type & North & East Srce Nbr & Scc Code & & Srce Type & & \\
\hline $200 E P-241 B 106001$ & POINT & 137329 & 573841 & 1 not assigne & & TANKS AND VESSELS & & \\
\hline Stack Configuration & & Diameter $(\mathrm{ft})$ & Length(ft) Width(ft) & $\operatorname{Area}(s q \mathrm{ft})$ & Exit & Temp(F) Exit Veloc(ft/sec) & Ht Abv Grnd(ft) & Ht Abv Struc(ft) \\
\hline HORIZONTAL OR DOWNWARO & & .33 & & .09 & & .36 & 3 & 3 \\
\hline En Point & Ep Type & North & East Srce Nbr & Scc Code & & Srce Type & & \\
\hline $200 E \quad P-2418107001$ & POINT & 137268 & 573811 & 1 not assigne & & TANKS AND VESSELS & & \\
\hline Stack Configuration & & Diameter $(f t)$ & Length(ft) Width(ft) & $\operatorname{Area}(s q \mathrm{ft})$ & Exit & Temp(F) Exit Veloc (ft/sec) & Ht Abv Grnd(ft) & $H t$ Abv Struc $(f t)$ \\
\hline HORIZONTAL OR DOWNWARD & & & 1 & 1 & & .014 & 3 & 3 \\
\hline Em Point & Ep Type & North & Srce Nbr & $r$ Scc Code & & Srce Type & & \\
\hline 200E P-241B108 001 & POINT & 137298 & 573811 & 1 not assigne & & TANKS AND VESSELS & & \\
\hline Stack Configuration & & Diameter (ft) & Length(ft) Width(ft) & $\operatorname{Area}(s q \mathrm{ft})$ & Exit & Temp(F) Exit Veloc(ft/sec) & Ht Abv Grnd(ft) & Ht Abv Struc (ft) \\
\hline HORIZONTAL OR DOWNWARD & & .33 & & .09 & & .36 & 3 & 3 \\
\hline
\end{tabular}

Page \#: 10 of 95 Report Run Date: 22-APR-95 


\section{Emission Point Listing}

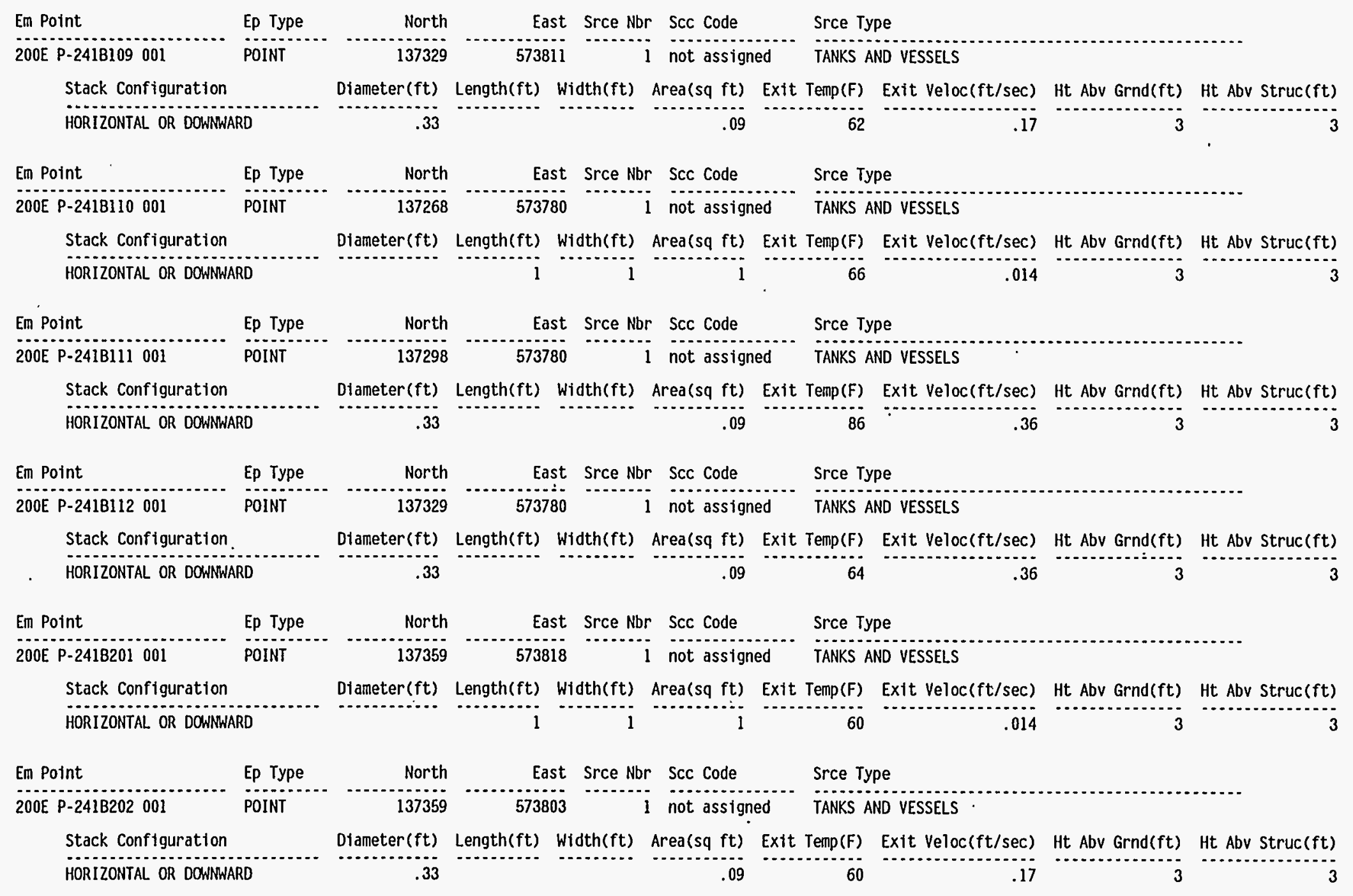

Page \#: 11 of 95 Report Run Date: 22-APR-95 
AIR EMISSIONS INVENTORY PERMIT APPLICATION REPORT (UP460)

FACILITY NAME: 200 Tank Farm and Associated Structures

\section{Emission Point Listing}

\begin{tabular}{|c|c|c|c|c|c|c|c|c|}
\hline Em Point & Ep Type & North & East & Srce Nbr & Scc Code & Srce Type & & \\
\hline 200E P-241B203001 & POINT & 137359 & 573788 & 8 & not assigned & TANKS AND VESSELS & & \\
\hline Stack Configuration & & Diameter $(\mathrm{ft})$ & Length(ft) & Width(ft) & $\operatorname{Area}(s q \mathrm{ft}$ ) & Exit $\operatorname{Veloc}(\mathrm{ft} / \mathrm{sec})$ & Ht Abv Grnd(ft) & $H t$ Abv Struc $(f t)$ \\
\hline HORIZONTAL OR DOWNWA & & .33 & & & .09 & .17 & 3 & \\
\hline
\end{tabular}

$\begin{array}{ll}\text { Em Point } & \text { Ep Type } \\ 200 E \text { P-2418204 } 001 & \text { POINT } \\ \text { Stack Configuration } & \end{array}$

......................

North East Srce Nbr Scc Code Srce Type

HORIZONTAL OR DOWNWARD

1 not assigned TANKS AND VESSELS

\begin{tabular}{|c|c|c|c|c|c|c|c|c|}
\hline Em Point & Ep Tyре & North & East Srce $\mathrm{Nbr}$ & $r$ Scc Code & & Srce Type & & \\
\hline 200E P-241BX101 001 & POINT & 137317 & 573659 & 1 not assigne & & TANKS AND VESSELS & & \\
\hline Stack Configuration & & Diameter $(\mathrm{ft})$ & Length(ft) Width(ft) & $\operatorname{Area}(s q \mathrm{ft})$ & Exit & Exit $\operatorname{Veloc}(\mathrm{ft} / \mathrm{sec})$ & Ht Abv Grnd(ft) & Ht Abv Struc $(f t)$ \\
\hline HORIZONTAL OR DOWNWAR & & & 1 & 1 & & .014 & 3 & 3 \\
\hline Em Point & Ep Type & North & East srce Nbr & r Scc Code & & Srce Type & & \\
\hline 200E P-241BX102001 & POINT & 137347 & 573659 & 1 not assigne & & TANKS AND VESSELS & & \\
\hline Stack Configuration & & Diameter (ft) & Length(ft) Width(ft) & Area $(s q f t)$ & Exit & $\operatorname{Temp}(F)$ Exit $\operatorname{Veloc}(\mathrm{ft} / \mathrm{sec})$ & Ht Abv Grnd(ft) & Ht Abv Struc ( $f t)$ \\
\hline HORIZONTAL OR DOWNWAR & & & $i$ & 1 & & .014 & 3 & 3 \\
\hline Em. Point & Ep Type & North & East Srce Nbr & $r$ Scc Code & & Srce Type & & \\
\hline 200E P-241BX103 001 & POINT & 137347 & 573659 & 1 not assigne & & TANKS AND VESSELS & & \\
\hline Stack Configuration & & Diameter (ft) & Length(ft) Width(ft) & Area(sq ft) & Exit & Temp (F) Exit Veloc (ft/sec) & Ht Abv Grnd(ft) & Ht Abv Struc (ft) \\
\hline HORIZONTAL OR DOWNWAR & & 1 & & .79 & & .039 & 3 & 3 \\
\hline Em Point & Ep Type & North & East Srce Nbr & $r$ Scc Code & & Srce Type & & \\
\hline 200E P-241BX104 001 & POINT & 137317 & 573629 & 1 not assigne & & TANKS AND VESSELS & & \\
\hline Stack Configuration & & Dlameter(ft) & Length(ft) Width(ft) & $\operatorname{Area}(s q \mathrm{ft})$ & Exit & Exit Veloc $(\mathrm{ft} / \mathrm{sec})$ & Ht Abv Grnd(ft) & Ht Abv Struc (ft) \\
\hline HORIZONTAL OR DOWNWAR & & .33 & & .09 & & .36 & 3 & 3 \\
\hline
\end{tabular}


AIR EMISSIONS INVENTORY PERMIT APPLICATION REPORT (UP460)

FACILITY NAME: 200 FACILITY Tank Farm and ASsOcjated Structures

\section{Emission Point Listing}

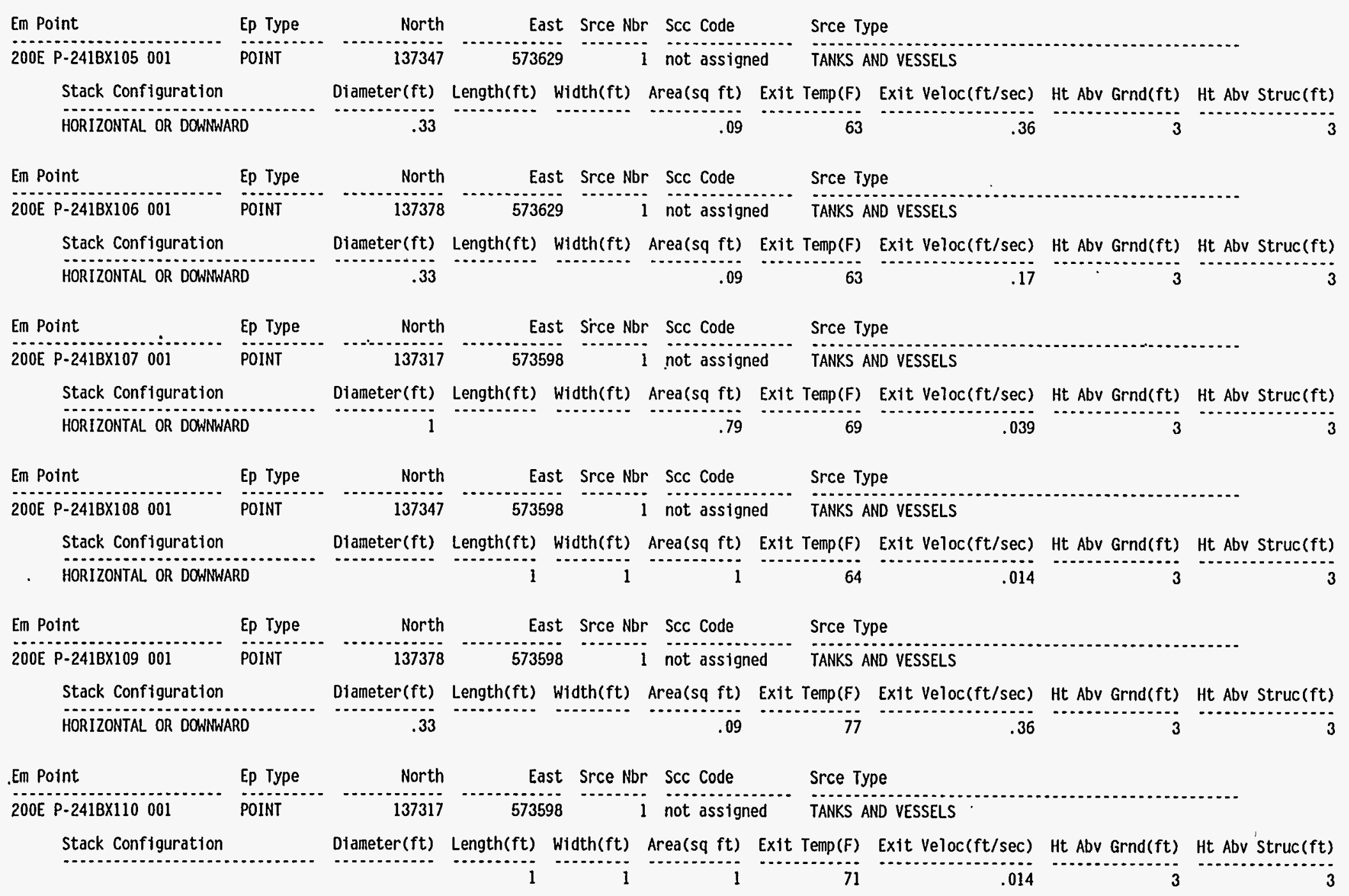


AIR EMISSIONS INVENTORY PERMIT APPLICATION REPORT (UP460) FACILITY NAME: FACILITY ID : 200E Tank Farm and Associated Structures

\section{Emission Point Listing}

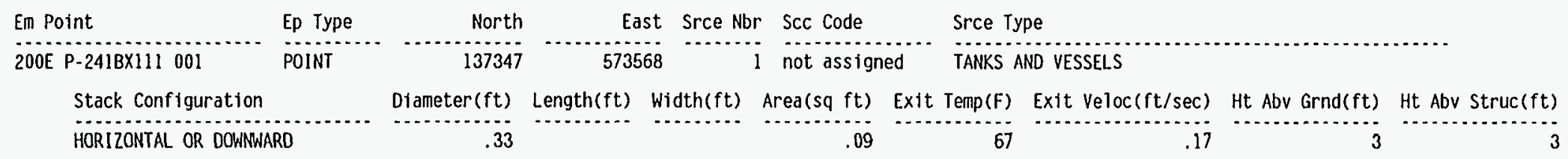




\section{Emission Point Listing}

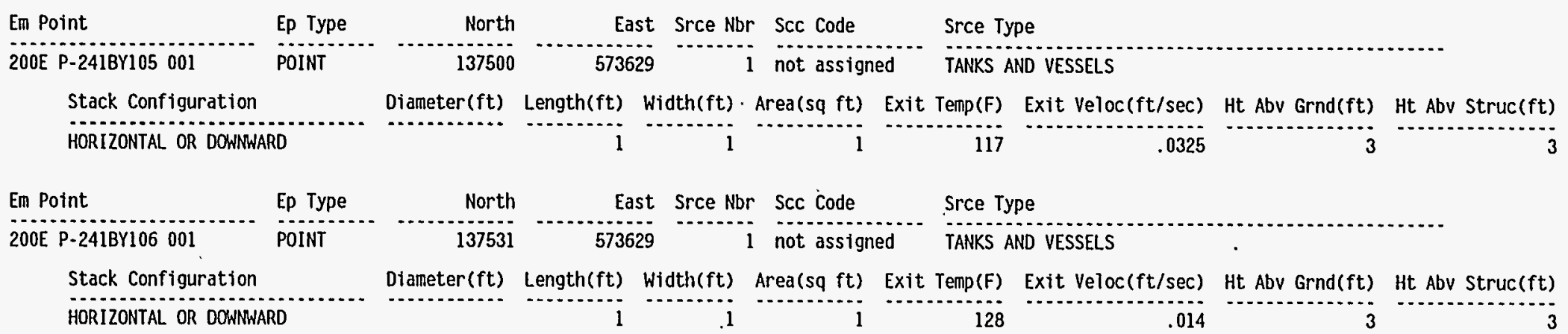

\begin{tabular}{ll} 
Em Point & Ep Type \\
\hline $200 E$ P-241BY107 001 & POINT \\
\multicolumn{2}{c}{ Stack Configuration }
\end{tabular}

North $\quad$ East Srce Nbr Scc Code Srce Type

Stack Configuration

HORIZONTAL OR DOWNARD

Dianeter(ft) Length(ft) Width(ft) Area(sq ft) Exit Temp(F) Exit Veloc(ft/sec) Ht Abv Grnd(ft) Ht Abv Struc(ft)

\begin{tabular}{|c|c|c|c|c|c|c|c|c|c|}
\hline Em Point & Ep Type & North & Eas & Srce Nbr & Scc Code & Srce Ty & & & \\
\hline 200E P-241BY108 001 & POINT & 137500 & 57359 & 97 & not assigne & TANKS A & ND VESSELS & & \\
\hline Stack Configuration & & Diameter (ft) & Length(ft) & Width(ft) & Area $(s q \mathrm{ft})$ & Exit Temp(F) & Exit Veloc (ft/sec) & Ht Abv Grnd(ft) & Ht Abv Struc(ft) \\
\hline HORIZONTAL OR DOWNW & & & 1 & 1 & 1 & 109 & .014 & & \\
\hline
\end{tabular}

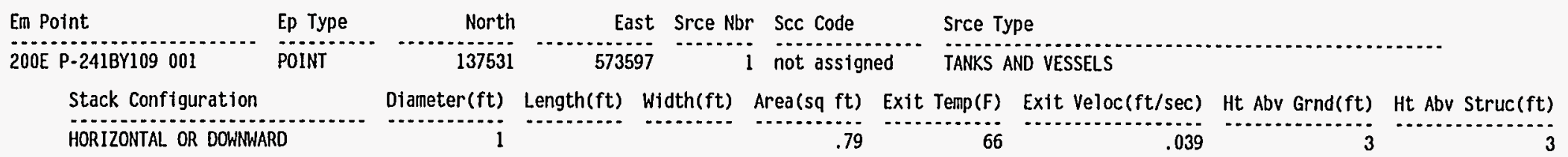

\begin{tabular}{|c|c|c|c|c|c|c|c|c|c|}
\hline Em Point & Ep Type & North & Eas & st Srce Nbr & Scc Code & Srce Ty & & & \\
\hline 200E P-241BY110 001 & POINT & 137469 & 57356 & 57 & not assigne & TANKS A & WND VESSELS & & \\
\hline Stack Configuration & & Diameter ( $f t)$ & Length $(f t)$ & Width (ft) & Area (sq ft) & Exit Temp (F) & Exit Veloc $(\mathrm{ft} / \mathrm{sec})$ & Ht Abv Grnd(ft) & Ht Abv Struc(ft) \\
\hline HORIZONTAL OR DOWNWA & & 1 & & & .79 & 118 & .018 & 3 & 3 \\
\hline
\end{tabular}




\section{Emission Point Listing}

\begin{tabular}{|c|c|c|c|c|c|c|c|c|c|}
\hline Em Point & Ep Type & North & East Srce $\mathrm{Nbr}$ & Ir Scc Code & & Srce Typ & & & \\
\hline $200 E$ P-241BY111 001 & POINT & 137500 & 573567 & not assigne & & TANKS AN & AND VESSELS & & \\
\hline Stack Configuration & & Diameter $(\mathrm{ft})$ & Length $(f t)$ Width(ft) & $\operatorname{Area}(s q \mathrm{ft})$ & Exit & Temp (F) & Exit Veloc(ft/sec) & Ht Abv Grnd(ft) & Ht Abv Struc $(f t)$ \\
\hline HORIZONTAL OR DOWNWARL & & .33 & & .09 & & 86 & .17 & 3 & 3 \\
\hline En Point & Ep Type & North & East Srce Nbr & or Scc Code & & Srce Typ & & & \\
\hline 200E P-241BY112 001 & POINT & 137531 & 573567 & 1 not assigne & & TANKS AI & AND VESSELS & & \\
\hline Stack Configuration & & Diameter (ft) & Length(ft) Width(ft) & $\operatorname{Area}(s q \mathrm{ft})$ & Exit & Temp (F) & Exit Veloc $(\mathrm{ft} / \mathrm{sec})$ & $H t$ Abv Grnd(ft) & $H t$ Abv $\operatorname{Struc}(f t)$ \\
\hline HORIZONTAL OR DOWNWARL & & .33 & & .09 & & 85 & .17 & 3 & 3 \\
\hline Em Point & Ep Type & North & East Srce Nbr & $r$ Scc Code & & Srce Typ & & & \\
\hline 200E P-241C101 001 & POINT & 136504 & 575162 & 1 not assigne & & TANKS AI & AND VESSELS & & \\
\hline Stack Configuration & & Diameter $(f t)$ & Length(ft) Width(ft) & $\operatorname{Area}(s q \mathrm{ft})$ & Exit & Temp (F) & Exit Veloc $(\mathrm{ft} / \mathrm{sec})$ & Ht. Abv Grnd(ft) & $H t$ Abv $\operatorname{Struc}(f t)$ \\
\hline HORIZONTAL OR OOWNWARL & & & $i$ & & & 87 & .014 & 3 & 3 \\
\hline Em Point & Ep Type & North & East Srce Nbr & r Scc code & & Srce Typ & & & \\
\hline 200E P-241C102001 & POINT & 136526 & 575183 & 1 not assigne & & TANKS AI & WD VESSELS & & \\
\hline Stack Configuration & & Diameter $(f t)$ & Length(ft) Width(ft) & $\operatorname{Area}(s q \mathrm{ft})$ & Exit & Temp(F) & Exit Veloc $(\mathrm{ft} / \mathrm{sec})$ & $H t$ Abv Grnd(ft) & Ht Abv Struc $(f t)$ \\
\hline HORIZONTAL OR DOWNWARI & & & 1 & & & 94 & .031 & 3 & 3 \\
\hline Em Point & Ep Type & North & East Srce Nbr & r Scc Code & & Srce Typ & & & \\
\hline 200E P-241C103001 & POINT & 136547 & 575204 & $i$ not assigne & & TANKS AI & ND VESSELS & & \\
\hline Stack Configuration & & Diameter $(f t)$ & Length(ft) Width(ft) & $\operatorname{Area}(s q \mathrm{ft})$ & Exit & Temp(F) & Exit Veloc $(\mathrm{ft} / \mathrm{sec})$ & Ht Abv Grnd(ft) & Ht Abv Struc(ft) \\
\hline HORIZONTAL OR DOWNWARI & & $i$ & & .79 & & 86 & .039 & 3 & 3 \\
\hline Em Point & Ep Type & North & Srce Nbr & Scc Code & & Srce Тур & & & \\
\hline 200E P-241C107 001 & POINT & 136547 & 575118 & 1 not assigne & & TANKS AI & WD VESSELS & & \\
\hline Stack Configuration & & Diameter $(f t)$ & Length(ft) Width(ft) & $\operatorname{Area}(s q \mathrm{ft})$ & Exit & $\operatorname{Temp}(\mathbf{F})$ & Exit Veloc $(\mathrm{ft} / \mathrm{sec})$ & Ht Abv Grnd(ft) & Ht Abv Struc (ft) \\
\hline HORIZONTAL OR DOWNWARL & & & 1 & $i$ & & 125 & .031 & 3 & \\
\hline
\end{tabular}


AIR EMISSIONS INVENTORY PERMIT APPLICATION REPORT (UP460)

FACILITY NAME: 200 Tank Farm and Associated structures

\section{Emission Point Listing}

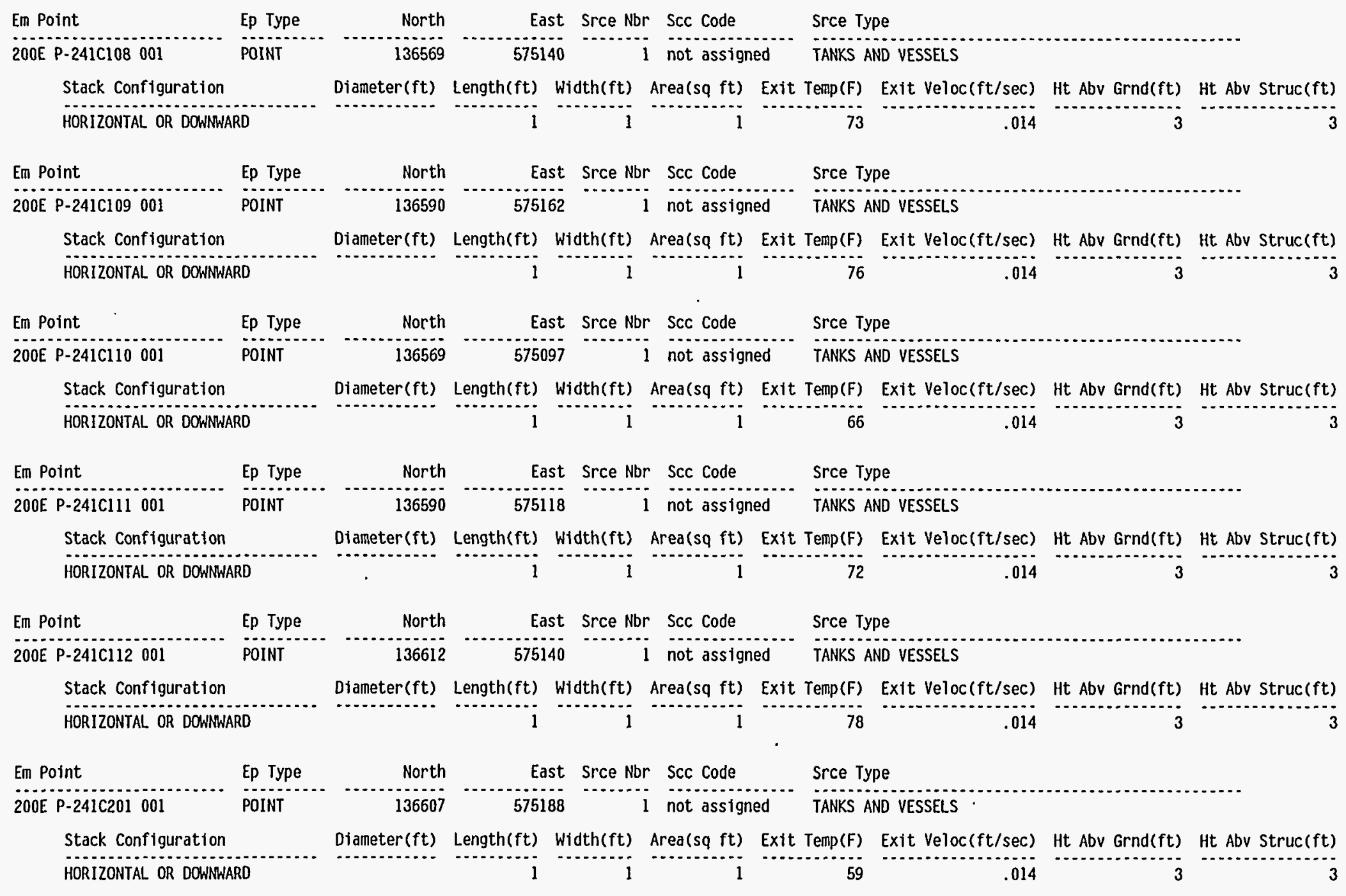




\section{Emission Point Listing}

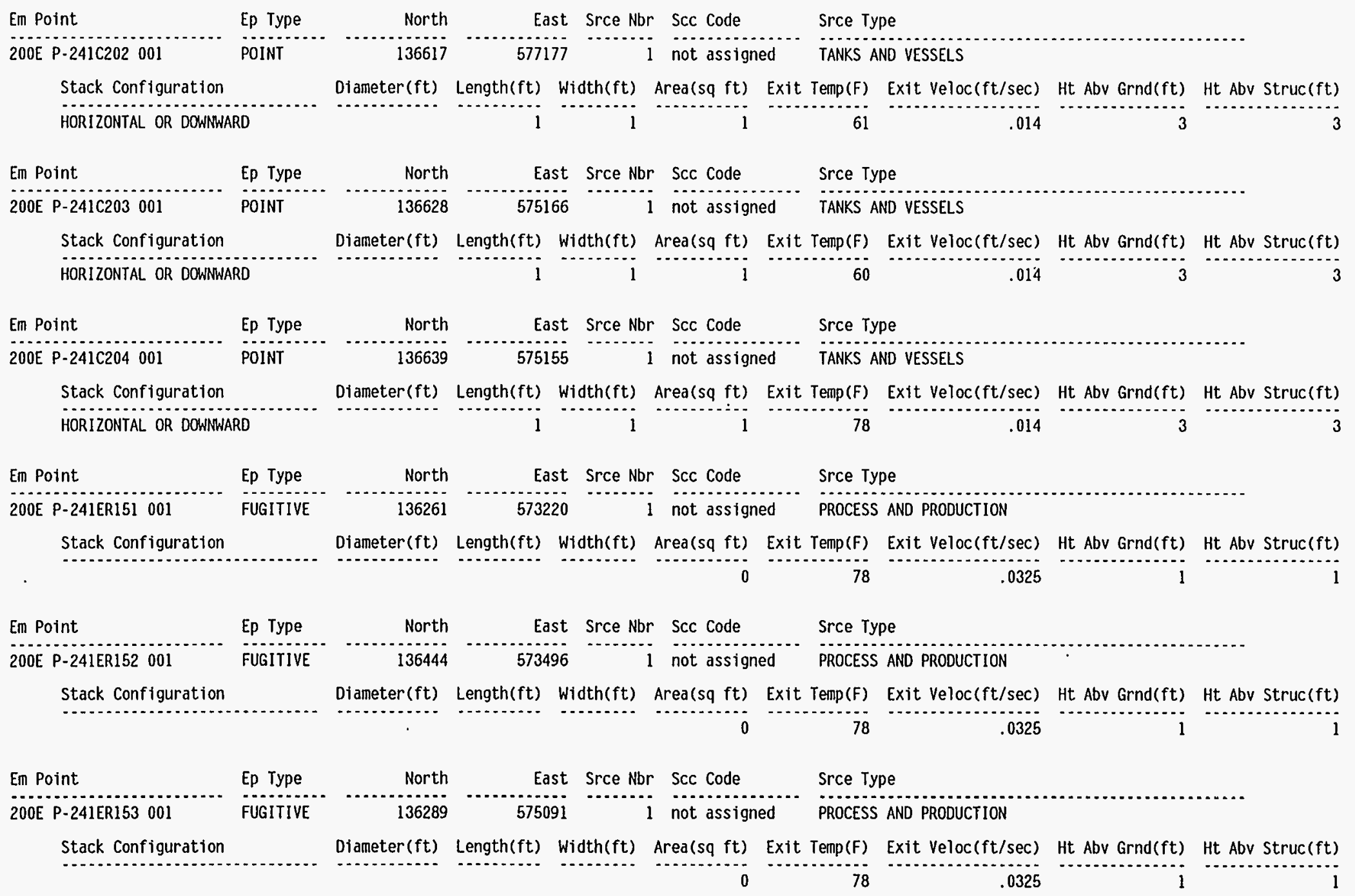


AIR EMISSIONS INVENTORY PERMIT APPLICATION REPORT (UP460) FACILITY. NAME: 200 Tank Farm and Associated structures

\section{Emission Point Listing}

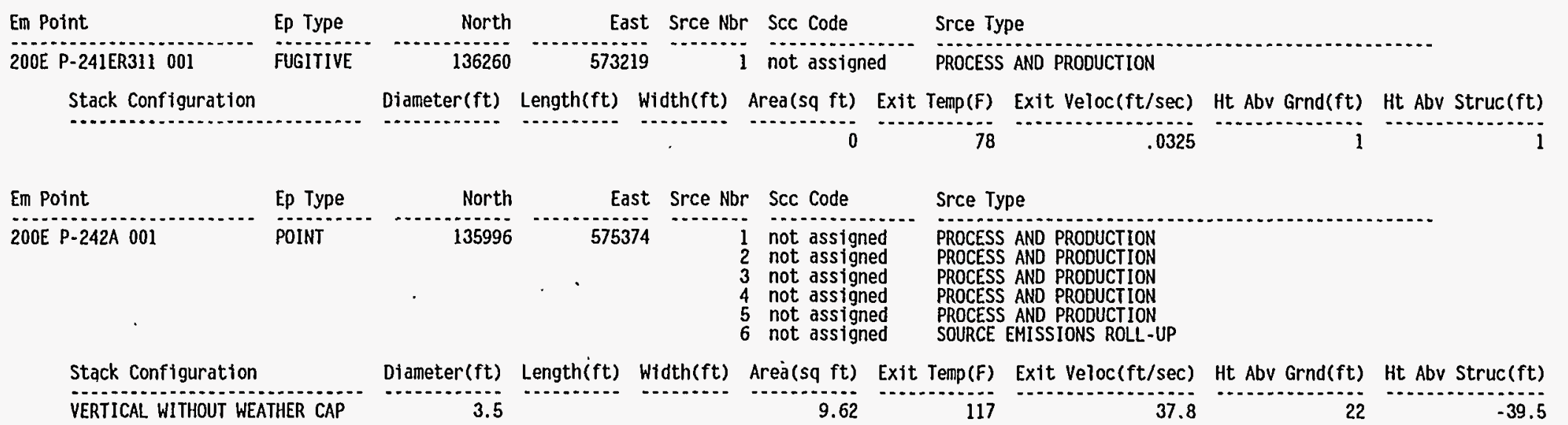

\section{Em Point} 200E P-242A 002 EP Type

\begin{tabular}{|rrr|r} 
North & East & Srce Nbr & Scc Code \\
\hline 135984 & 575385 & 1 & not assigned \\
& & 2 not assigned \\
3 & & not assigned \\
4 & not assigned \\
& & 5 & not assigned \\
& & 6 not assigned
\end{tabular}

Srce Type

PROCESS AND PRODUCTION

PROCESS AND PRODUCTION

PROCESS AND PRODUCTION

PROCESS AND PRODUCTION

6 not assigned SOURCE EMISSIONS ROLL-UP

Stack Configuration

Diameter(ft) Length(ft) Width(ft) Area(sq ft) Exit Temp(F) Exit Veloc(ft/sec) Ht Abv Grnd(ft) Ht Abv Struc(ft) VERTICAL WITHOUT WEATHER CAP .62 .3 120 120

\section{Em Point} Ep Type 200E P-244A 001 POINT 
AIR EMISSIONS INVENTORY PERMIT APPLICAIION REPORT (UP460)
FACILITY ID: 200E TANK

FACILITY NAME: 200E Tank Farin and Associated Structures

\section{Emission Point Listing}

\begin{tabular}{|c|c|c|c|c|c|c|c|c|}
\hline Em Point & Ep Type & North & Srce Nbr & $r$ Scc code & Srce Ty & & & \\
\hline 200E P-244AR 002 & POINT & 136090 & 575220 & 1 not assigne & PROCESS & AND PRODUCTION & & \\
\hline Stack Configuration & & Diameter $(\mathrm{ft})$ & Length(ft) Width(ft) & $\operatorname{Area}(s q \mathrm{ft})$ & Exit Temp(F) & Exit Veloc $(f t / s e c)$ & Ht Abv Grnd(ft) & Ht Abv Struc $(\mathrm{ft})$ \\
\hline VERTICAL WITHOUT WEA & THER CAP & 3 & & 7.07 & 78 & 16.4 & 61 & 21 \\
\hline
\end{tabular}

$\begin{array}{ll}\text { Em Point } & \text { Ep Type } \\ 200 \text { E P-244BX 001 } & \text { POINT }\end{array}$

North East Srce Nbr Scc Code Srce Type

200E P-244BX 001
Stack Configuration

$$
13735501573690 \quad 1 \text { not assigned PROCESS AND PRODUCTION }
$$

VERTICAL WITH WEATHER CAP

Diameter(ft) Length(ft) Width(ft) Area(sq ft) Exit Temp(F) Exit Veloc(ft/sec) Ht Abv Grnd(ft) Ht Abv Struc(ft)

En Point

Ep Type

.5

78

20.8

Em Point
200E P-244CR 001
Stack Configuration

North East Srce $\mathrm{Nbr}$ Scc Code Srce Type

$1364550-575156 \quad 1$ not assigned PROCESS AND PRODUCTION

VERTICAL WITH WEATHER CAP

Diameter $(f t)$ Length(ft) Width(ft) Area(sq ft) Exit Temp(F) Exit Veloc(ft/sec) Ht Abv Grnd(ft) Ht Abv Struc(ft)

Em Point

Ep Type

200E P-296A 001 POINT

North

North East Srce Nbr Scc Code

1.77 78

$39.5 \quad 50$

Stack Configuration

136074

Srce Type

Diameter(ft) Length(ft) Width(ft) Area(sq ft) Exit Temp(F) Exit Veloc (ft/sec) Ht Abv Grnd(ft) Ht Abv Struc(ft)

VERTICAL WITHOUT WEATHER CAP

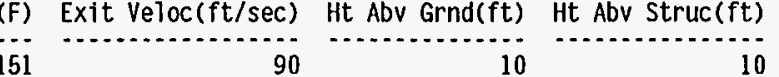

\section{Em Point}

Ep Type

1.33

1.39 15

200E P-296A018 001

POINT

\begin{tabular}{|c|c|c|c|c|}
\hline North & East & Srce Nbr & Scc Code & Srce Type \\
\hline 136220 & 575307 & 1 & not assigned & TANKS AND VESSELS \\
\hline
\end{tabular}

Stack Configuration

NONE SPECIFIED

Diameter(ft) Length(ft) Width(ft) Area(sq ft) Exit Temp(f) Exit Veloc(ft/sec) Ht Abv Grnd(ft) Ht Abv Struc(ft)

Em Point

Ep Type 1.25

1.23

68

12.5

Em Point
200E P-296A0ig 001
Stack Configuration

\begin{tabular}{|c|c|c|c|c|}
\hline North & East & Srce Nbr & Scc Code & Srce Type \\
\hline 136145 & 575317 & 1 & not assigned & TANKS AND VESSELS \\
\hline
\end{tabular}

Diameter(ft) Length(ft) Width(ft) Area(sq ft) Exit Temp(F) Exit Veloc(ft/sec) Ht Abv Grnd(ft) Ht Abv Struc(ft)

NONE SPECIFIED

1.25

1.23

68

16.4

12.9

Page \#: 20 of 95 Report Run Date: 22-APR-95 
AIR EMISSIONS INVENTORY PERMIT APPLICATION REPORT (UP460)

FACILITY NAME: $200 E$ Tank Farm and Associated Structures

\section{Emission Point Listing}

\begin{tabular}{|c|c|c|c|c|c|c|c|c|c|}
\hline Em Point & Ep Туре & North & East & Srce Nbr & - Scc code & & Srce Type & & \\
\hline 200E P-296A020 001 & POINT & 136279 & 575393 & & $\begin{array}{l}\text { not assigned } \\
\text { not assigned }\end{array}$ & & $\begin{array}{l}\text { TANKS AND VESSELS } \\
\text { TANKS AND VESSELS }\end{array}$ & & \\
\hline Stack Configuration & & Diameter $(f t)$ & Length(ft) & Width $(f t)$ & $\operatorname{Area}(s q \mathrm{ft})$ & Exit & Exit Veloc $(\mathrm{ft} / \mathrm{sec})$ & Ht Abv Grnd(ft) & Ht Abv Struc(ft) \\
\hline NONE SPECIFIED & & 2 & & & 3.14 & & 14.1 & 15.67 & \\
\hline Em Point & Ep Type & North & East & Srce Nbr & - Scc Code & & Srce Type & & \\
\hline 200E P-296A028 001 & POINT & 135841 & 575383 & ' & $\begin{array}{l}\text { not assigned } \\
\text { not assigned } \\
\text { not assigned } \\
\text { not assigned } \\
\text { not assigned } \\
\text { not assigned }\end{array}$ & & $\begin{array}{l}\text { TANKS AND VESSELS } \\
\text { TANKS AND VESSELS } \\
\text { TANKS AND VESSELS } \\
\text { TANKS AND VESSELS } \\
\text { TANKS AND VESSELS } \\
\text { TANKS AND VESSELS }\end{array}$ & & \\
\hline Stack Configuration & & Diameter (ft) & Length(ft) $h$ & Width(ft) & $\operatorname{Area}(s q \mathrm{ft})$ & Exit & Temp(F) Exit Veloc(ft/sec) & Ht Abv Grnd(ft) & Ht Abv Struc(ft) \\
\hline NONE SPECIFIED & & 2 & & & 3.14 & & 68 & 23 & \\
\hline En Point & Ep Type & North & East & t srce Nbr & Scc Code & & Srce Type & & \\
\hline 200E P-296A030 001 & POINT & 136430 & 575395 & & $\begin{array}{l}\text { not assigned } \\
\text { not assigned } \\
\text { not assigned } \\
\text { not assigned } \\
\text { not assigned } \\
\text { not assigned } \\
\text { not assigned }\end{array}$ & & $\begin{array}{l}\text { TANKS AND VESSELS } \\
\text { TANKS AND VESSELS } \\
\text { TANKS AND VESSELS } \\
\text { TANKS AND VESSELS } \\
\text { TANKS AND VESSELS } \\
\text { TANKS AND VESSELS } \\
\text { TANKS AND VESSELS }\end{array}$ & & \\
\hline Stack Configuration & & Diameter (ft) & Length(ft) & Width(ft) & $\operatorname{Area}(s q \mathrm{ft})$ & Exit & Temp(F) Exit Veloc(ft/sec) & $H t$ Abv Grnd(ft) & Ht Abv Struc (ft) \\
\hline NONE SPECIFIEO & & 2 & & & 3.14 & & 88.6 & 23 & \\
\hline Em Point & Ep Type & North & East & Srce Nbr & Scc Code & & Srce Type & & \\
\hline 200E P-296A041 001 & POINT & 135815 & 575597 & & $\begin{array}{l}\text { not assigned } \\
\text { not assigned } \\
\text { not assigned } \\
\text { not assigned } \\
\text { not assigned } \\
\text { not assigned } \\
\text { not assigned } \\
\text { not assigned }\end{array}$ & & $\begin{array}{l}\text { TANKS AND VESSELS } \\
\text { TANKS AND VESSELS } \\
\text { TANKS AND VESSELS } \\
\text { TANKS AND VESSELS } \\
\text { TANKS AND VESSELS } \\
\text { TANKS AND VESSELS } \\
\text { TANKS AND VESSELS } \\
\text { TANKS AND VESSELS }\end{array}$ & & \\
\hline Stack Configuration & & Diameter $(f t)$ & Length(ft) $h$ & Width(ft) & Area $(s q f t)$ & Exit & Temp(F) Exit Veloc(ft/sec) & Ht Abv Grnd(ft) & Ht Abv Struc (ft) \\
\hline NONE SPECIFIEO & & 2.3 & & & 4.15 & & 144.9 & 29.2 & \\
\hline
\end{tabular}

Page \#: 21 of 95 Report Run Date: 22-APR-95 
AIR EMISSIONS INVENTORY PERMIT APPLICATION REPORT (UP460)

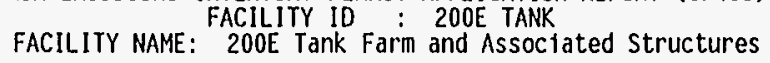

\section{Emission Point Listing}

\begin{tabular}{|c|c|c|c|c|c|c|c|c|c|}
\hline Em Point & Ep Type & North & East & Srce Nbr & Scc code & & Srce Type & & \\
\hline 200E P-296AN 001 & POINT & 136432 & 575396 & $\begin{array}{l}1 \\
2 \\
3 \\
4 \\
5 \\
6 \\
7 \\
8\end{array}$ & $\begin{array}{l}\text { not assigned } \\
\text { not assigned } \\
\text { not assigned } \\
\text { not assigned } \\
\text { not assigned } \\
\text { not assigned } \\
\text { not assigned } \\
\text { not assigned }\end{array}$ & & $\begin{array}{l}\text { TANKS AND VESSELS } \\
\text { TANKS AND VESSELS } \\
\text { TANKS AND VESSELS } \\
\text { TANKS AND VESSELS } \\
\text { TANKS AND VESSELS } \\
\text { TANKS AND VESELS } \\
\text { TANKS AND VESSELS } \\
\text { SOURCE EMISSIONS ROLL-UP }\end{array}$ & & \\
\hline Stack Configuration & & Diameter $(f t)$ & Length $(f t)$ & Width(ft) A & $\operatorname{Area}(s q \mathrm{ft})$ & Exit & Temp (F) Exit Veloc(ft/sec) & Ht Abv Grnd(ft) & Ht Abv Struc ( $f t)$ \\
\hline VERTICAL WITHOUT WEATI & THER CAP & .83 & & & .54 & & 18.2 & 14.92 & \\
\hline Em Point & Ep Type & North & East & Srce Nbr & Scc Code & & Srce Type & & \\
\hline 200E P-296AP 001 & POINT & 135817 & 575602 & $\begin{array}{l}1 \\
2 \\
3 \\
4 \\
5 \\
6 \\
7 \\
8 \\
9\end{array}$ & $\begin{array}{l}\text { not assigned } \\
\text { not assigned } \\
\text { not assigned } \\
\text { not assigned } \\
\text { not assigned } \\
\text { not assigned } \\
\text { not assigned } \\
\text { not assigned } \\
\text { not assigned }\end{array}$ & & $\begin{array}{l}\text { TANKS AND VESELS } \\
\text { TANKS AND VESSELS } \\
\text { TANKS AND VESSELS } \\
\text { TANKS AND VESSELS } \\
\text { TANKS AND VESSELS } \\
\text { TANKS AND VESELS } \\
\text { TANKS AND VESSELS } \\
\text { TANKS AND VESSELS } \\
\text { SOURCE EMISSIONS ROLL -UP }\end{array}$ & & \\
\hline Stack Configuration & & Diameter (ft) & Length(ft) & Width(ft) A & $\operatorname{Area}(s q \mathrm{ft})$ & Exit & Exit Veloc $(\mathrm{ft} / \mathrm{sec})$ & Ht Abv Grnd(ft) & Ht Abv Struc(ft) \\
\hline VERTICAL WITHOUT WEATI & THER CAP & .83 & & & .54 & & 35.8 & 13.33 & \\
\hline En Point & Ep Type & North & East & t Srce Nbr & Scc Code & & Srce Type & & \\
\hline 200E P-296AW 001 & POINT & 135842 & 575383 & $\begin{array}{l}1 \\
2 \\
3 \\
4 \\
5 \\
6 \\
7\end{array}$ & $\begin{array}{l}\text { not assigned } \\
\text { not assigned } \\
\text { not assigned } \\
\text { not assigned } \\
\text { not assigned } \\
\text { not assigned } \\
\text { not assigned }\end{array}$ & & $\begin{array}{l}\text { TANKS AND VESSELS } \\
\text { TANKS AND VESSELS } \\
\text { TANKS AND VESSELS } \\
\text { TANKS AND VESSELS } \\
\text { TANKS AND VESSES } \\
\text { TANKS AND VESSELS } \\
\text { SOURCE EMISSIONS ROLL-UP }\end{array}$ & & \\
\hline Stack Configuration & & Diameter $(\mathrm{ft})$ & Length(ft) & Width(ft) & $\operatorname{Area}(s q \mathrm{ft})$ & Exit & Exit Veloc $(\mathrm{ft} / \mathrm{sec})$ & Ht Abv Grnd(ft) & Ht Abv Struc(ft) \\
\hline VERTICAL WITHOUT WEAT & THER CAP & .83 & & & .54 & & 30.3 & 15.75 & \\
\hline
\end{tabular}


AIR EMISSIONS INVENTORY PERMIT APPLICATION REPORT (UP460) FACILITY NAME: 200E Tank Farm and Associated Structures

\section{Emission Point Listing}

\begin{tabular}{|c|c|c|c|c|c|c|c|c|}
\hline En Point & Ep Type & North & East Srce $\mathrm{Nbr}$ & Scc Code & & Srce Type & & \\
\hline 200E P-296AYAZ 001 & POINT & 136150 & 575389 & $\begin{array}{l}\text { not assigned } \\
\text { not assigned } \\
\text { not assigned } \\
4 \text { not assigned } \\
5 \text { not assigned }\end{array}$ & & $\begin{array}{l}\text { TANKS AND VESSELS - } \\
\text { TANKS AND VESSELS } \\
\text { TANKS AND VESSELS } \\
\text { TANKS AND VESSELS } \\
\text { SOURCE EMISSIONS ROLL-UP }\end{array}$ & & \\
\hline Stack Configuration & & Diameter $(f t)$ & Length(ft) Width(ft) & $\operatorname{Area}(s q \mathrm{ft}) \mathrm{E}$ & Exit & Temp(F) Exit Veloc(ft/sec) & Ht Abv Grnd(ft) & Ht Abv Struc(ft) \\
\hline VERTICAL WITHOUT WEATI & THER CAP & 1.5 & & 1.77 & & 31.91 & 50 & \\
\hline Em Point & Ep Type & North & East Srce Nbr & Scc Code & & Srce Type & & \\
\hline 200E P-296G001 001 & POINT & 135979 & 575900 & not assigned & & PROCESS AND PRODUCTION & & \\
\hline Stack Conftguration & & Diameter $(f t)$ & Length(ft) Width(ft) & $\operatorname{Area}(s q \mathrm{ft})$ & Exit & Temp(F) Exit Veloc (ft/sec) & Ht Abv Grnd(ft) & Ht Abv Struc(ft) \\
\hline NONE SPECIFIED & & .87 & & .59 & & 32.5 & 25 & \\
\hline Em Point & Ер Туре & North & East Srce Nbr & Scc Code & & Srce Type & & \\
\hline 200E P-296P016 001 & POINT & 136561 & 575162 & $\begin{array}{l}\frac{1}{2} \text { not assigned } \\
\text { not assigned } \\
\text { not assigned }\end{array}$ & & $\begin{array}{l}\text { TANKS AND VESSELS } \\
\text { TANKS AND VESSELS } \\
\text { SOURCE EMISSIONS ROLL-UP }\end{array}$ & & \\
\hline Stack Configuration & & Diameter (ft) & Width(ft) & $\operatorname{Area}(s q \mathrm{ft}) \quad E$ & Exit & Temp(F) Exit Veloc(ft/sec) & Ht Abv Grnd(ft) & Ht Abv Struc(ft) \\
\hline VERTICAL WITH WEATHER & R CAP & 1.3 & - & 1.33 & & 32.15 & 10 & 10 \\
\hline Em Point & Ep Type & North & East Srce $\mathrm{Nbr}$ & Scc Code & & Srce Type & & \\
\hline $200 E$ P-296P026 001 & POINT & 136115 & 575386 & $\begin{array}{l}\text { not assigned } \\
\text { not assigned } \\
\text { not assigned } \\
\text { not assigned }\end{array}$ & & $\begin{array}{l}\text { TANKS AND VESSELS } \\
\text { TANKS AND VESSELS } \\
\text { TANKS AND VESSELS } \\
\text { TANKS AND VESSELS }\end{array}$ & & \\
\hline Stack Configuration & & Diameter (ft) & Length(ft) & $\operatorname{Area}(s q \mathrm{ft})$ & Exit & Temp(F) Exit Veloc(ft/sec) & Ht Abv Grnd(ft) & Ht Abv Struc(ft) \\
\hline NONE SPECIFIED & & 1.3 & & 1.33 & & 88.6 & 10 & \\
\hline Em Point & Ep Type & North & East Srce $\mathrm{Nbr}$ & Scc Code & & Srce Type & & \\
\hline $200 E$ P-296P031 001 & POINT & 136337 & 574446 & not assigned & & PROCESS AND PRODUCTION & & \\
\hline Stack Configuration & & Diameter (ft) & Length $(f t)$ & $\operatorname{Area}(s q \mathrm{ft}) \mathrm{E}$ & Exit & Temp(F) Exit Veloc(ft/sec) & Ht Abv Grnd(ft) & Ht Abv Struc(ft) \\
\hline NONE SPECIFIED & & 2 & & 3.14 & & 32.5 & 32 & 10 \\
\hline
\end{tabular}




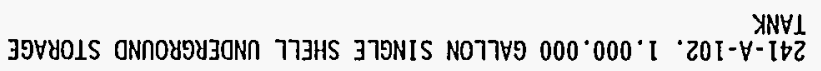
holfdinasag ssajodd Kaeuruns

29 $L$

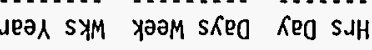

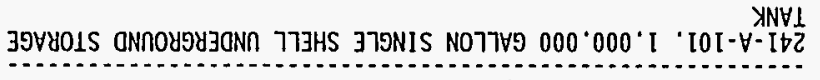
uolzd!Josao ssajodd Kapuruns $t z$ Jeas SXM XәOM SKeO KeO SJH
STISSIM ONY SXNYI I adk1 əכगS JQN STISSIM ONY SXNYI I

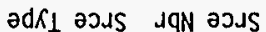

100 20IVItZ2-d 3002 quipod 43

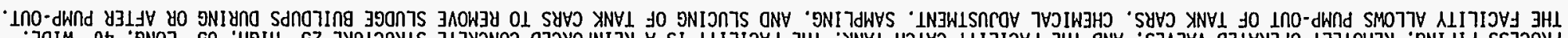

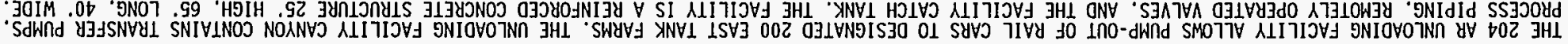

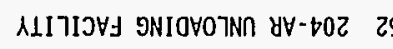
$\forall 2$ NOILJnOOYd ONY SSJJOYd I

100 \& $\forall 602-d 3002$

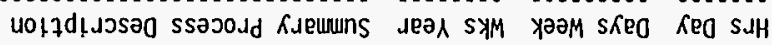

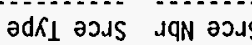
quilod $ய \exists$

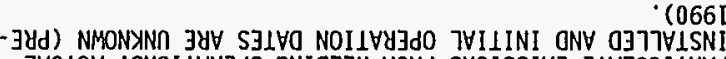

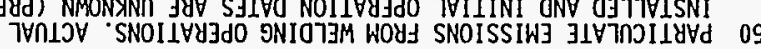

$g$

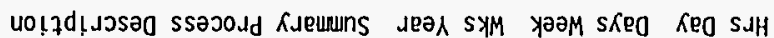

9NIZYYG/9NILIกJ/9NIOTJM 乙

100 MVZLZ-N $300 Z$

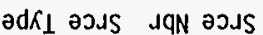
quiod wy

$\cdot(0661-3 \mathrm{yd})$

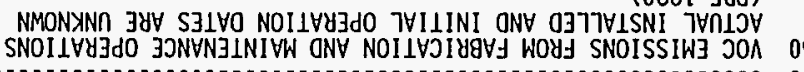

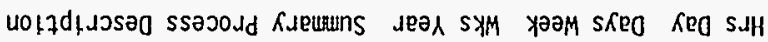

NOIJTOOOH ONY SSZJOYd I

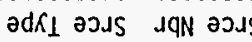

$100 M \forall Z \angle Z-N 300 Z$ quiod us

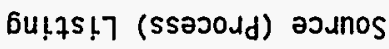

I $\angle L \quad 90^{\circ}$

92.

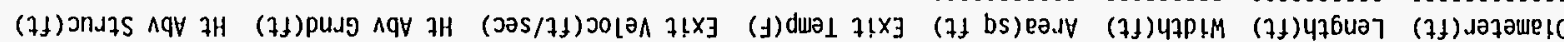

NOILONOOYd ONY SSJJOYd วd $\kappa_{\perp}$ วग」S (7t bs) ea.dy
I $\ldots \ldots . . \quad \forall \angle E S \angle G$ JqN OJJS 7SEJ
$96 \mathrm{I} \angle \varepsilon \mathrm{I}$

Y7.JON

....

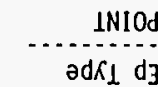

\section{7อ}

Iod $[00$ J437-d 3002

6u!̣s! 7 7u!̣od uo!ss!̣แ 
AIR EMISSIONS INVENTORY PLHMII APPLICAIION REPORT (UP460) FACILITY MAME. FACILITY lO : 200E TANK

Source (Process) Listing

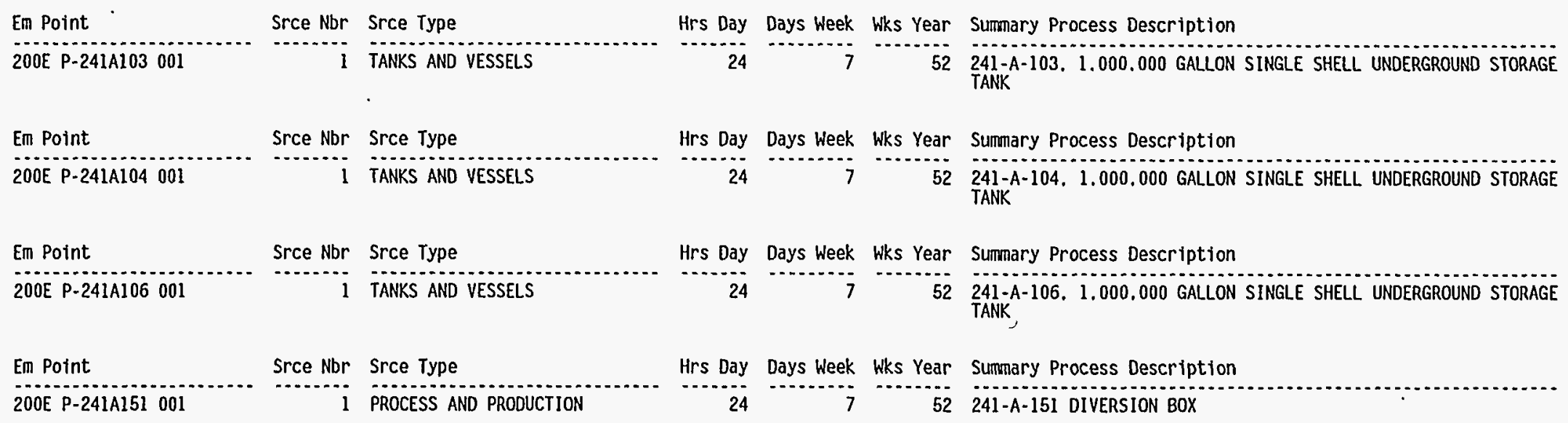

THE 241-A-151 DIVERSION BOX HAS INSIDE DIMENSIONS OF 54 FEET IN LENGTH. $71 / 2$ FEET IN WIDTH. AND 10 FEET DEEP. THE BOX DRAINS TO THE A-302A CATCH TYNKICAL WASTE TRANSFERRED VIA 241-A-151 CONSISTS OF PUREX COMPLEXED AND DECLADDING WASTES.

$\begin{array}{lrlrl}\text { Em Point } & \text { Srce Nbr Srce Type } & \text { Hrs Day Days Week Wks Year Summary Process Description } \\ 200 E \text { P-241A302A 001 } & \text { I PROCESS AND PRODUCTION } & 24 & 7 & 52 \text { 241-A-302A CATCH TANK }\end{array}$

THE 241-A-302A CATCH TANK RECEIVES FLUSH WATER AND LEAKS FROM THE 241-A-151 DIVERSION BOX. THE CATCH TANK CONSISTS OF A HORIZONTAL CVLINDRICAL CARBON STEEL VESSEL. A STEAM JET BOX RESTING ON THE TANK. AND A PUMP PIT ABOVE THE TANK. THE TANK IS 9.5 FEET IN DIAMETER AND 40 FEET LONG.

\footnotetext{
Em Point Srce Nbr Srce Type

Hrs Day Days Week Wks Year Summary Process Description

$200 E$ P-241A350 001 1 PROCESS AND PRODUCTION

THE REINFORCED CONCRETE PUMP PIT IS $9^{\circ} \times 9^{\circ} \times 11^{\circ}$ (LXWXD) INCLUDING THE COVER BLOCK (750 GAL. CAP.). THE PIT HAS $1^{\circ}$ THICK WALLS AND A 20" THICK FLOOR. EXCEPT FOR THE SUMP AREA WHERE THE FLOOR IS $28^{\prime \prime}$ THICK. THE COVER BLOCK IS 18" THICK. NINE FEET BELOW THE PIT FLOOR IS AN 8* O.D. 8. DEEP CAISSON LINER

THREE INCH DRAIN LINES 300. 307. 311. AND 317 CONNECT WITH NOZZLES IN THE PIT. EMPTYING INTO THE CATCH TANK. AN EXISTING 3" STAINLESS STEEL DRAIN SLOPES INTO THE CATCH TANK. H-2-70333 H-2-70538 H-2-70318.
} 


\begin{tabular}{|c|c|c|c|c|c|c|}
\hline Em Point & Srce Nbr & Srce Type & Hrs Day & Days Week & Whs Year & Summary Process Description \\
\hline 200E P-241A401 001 & 1 & PROCESS AND PRODUCTION & 24 & 7 & 52 & 241-A-401 DIVERTER CAISSON \\
\hline
\end{tabular}

A-401 DIVERTER CAISSON IS A $55^{\circ}$ DIAMETER. GALVANIZED, CORRUGATED, 14 GAGE CULVERT PIPE WITH A 14 GAGE, GALVANIZED STEEL. OCTAGON SHAPED COVER PLATE. THE PIT IS APPROXIMATELY 6 TO $8^{\prime \prime}$ ABOVE GRADE. THE UNDERSIDE OF THE COVER. AND 1 TO 2 . DOWN ON THE INSIDE SURFACE OF THE PIPE WALL. ARE INSULATED WITH WHERE THE LEAK DETECTOR IS LOCATED. THE CONCRETE FLOOR AND PIPING WERE PAINTED PER SPEC. B-180-Cl.

ALL PENETRATIONS TO THE CORRUGATED STEEL WERE SEALED WITH PERMATEX "FORM-A-GASKET" NO 2, H 2-73842.
Em Point
Srce $\mathrm{Nbr}$ Srce Type
Hrs Day Days Week Wks Year Summary Process Description
$200 E$ P-241A417 001
1 PROCESS AND PRODUCTION
24
52 241-A-417 CONDENSATE CATCH TANK

A-417 IS A CYLINDRICAL VESSEL ENCLOSED IN A CONCRETE VAULT. THE VAULT FLOOR IS 6" THICK CONCRETE, AND THE WALLS AND ROOF ARE 10" THICK. AN ALL WELD STEEL LINER. $24^{\circ} 6^{\prime \prime}$ IN DIAMETER, EXTENDS TO WITHIN $5^{\prime \prime}$ OF THE VAULT ROOF. THERE IS NO SPACE BETWEEN THE LINER AND THE CONCRETE WALLS AND FLOOR. LINER DETAILS COULD NOT BE FOUND. THERE ARE TWO OVERFLOW LINES TO THE LINER 9" FROM THE TOP TO PREVENT OVERFLOW OF THE CATCH TANK

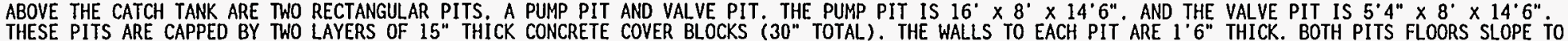
DRAINS THAT EMPTY INTO THE CATCH TANK BELOW. THESE DRAINS ARE $3^{\prime \prime}$. M5 PIPES, AND THEY ARE NOT LISTED ON THE PIPE SURUEY.

SIX RISERS PENETRATE THE CATCH TANK OUTSIDE THE PUMP PIT AND VALVE PIT: ONLY TWIO RISERS ARE USED ONE RISER IS FOR THE VENTILATION SYSTEM AND EXTENDS TO ABOVEGROUND HOUSING. AND THE OTHER IN ENCASED IS A CAISSON AND PROVIDES ACCESS TO THE TANK FOR SAMPLING.

LINER PENETRATIONS ARE WELOED TO THE LINER, H 2-(56800, 57302), SD-HS-SAR-010 REV 3.

Em Point Srce Nbr Srce Type

Hrs Day Days Week Wiks Year Summary Process Description $200 E$ P-241AA 001

1 PROCESS AND PRODUCTION

$24.52 \quad 241$ A-A VALVE PIT

A-A VALVE PIT IS A CONCRETE RECTANGULAR BOX WITH DIMENSIONS OF $12^{\circ} \times 10^{\circ} \times 4^{\circ} 9^{\prime \prime}($ L $X$ XWX $)$ LOCATED UNDERGROUND. WASTE TRANSPORTED THROUGH THE A-A VALVE
PIT INCLUDES RADIOACTIVE MIXED WASTE SOLUTIONS FROM PROCESSING AND DECONTAMINATION OPERATIONS AND B PLANT COMPLEXED AND NONCOMPLEXED WASTE.

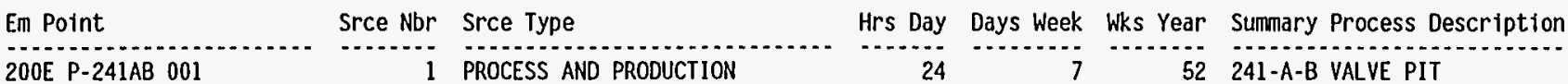

A-B VALVE PIT IS A CONCRETE RECTANGULAR BOX WITH INSIDE DIMENSIONS OF $12^{\prime} \times 10^{\prime} \times 4^{\circ} 9^{\prime \prime}($ LXWXD). AND IS LOCATED UNDERGROUND

A-8 VALVE PIT TRANSPORTS MIXED WASTE SOLUTIONS FROM PROCESSING AND DECONTAMINATION OPERATIONS IN ADDITION TO B PLANT COMPLEXED AND NON-COMPLEXED WASTE.
Em Point
Srce $\mathrm{Nbr}$ Srce Type
Hrs Day Days Week Wks Year Summary Process Description

200E P-241ANA 001

1 PROCESS AND PRODUCTION

$24 \quad 52 \quad 241-A N-A$ VALVE PIT

THE 241 AN-A VALVE PIT IS $14^{\circ} \times 14^{\circ} \times 6^{\circ} 8^{\prime \prime}(L X W \times D)$ AND IS LOCATEO UNDERGROUND. THE VALVE PIT DRAINS TO 102-AN.

GENERALLY. THE WASTES TRANSPORTED VIA THE 241 AN-A VALVE PIT ARE COMPLEX CONCENTRATE AND DOUBLE SHELL SLURRY. 
AIR EMISSIONS INVENTORY PERMIT APPLICATION REPORT (UP460)

FACILITY NAME: 200 Tank Farm and Associated Structures

\section{Source (Process) Listing}

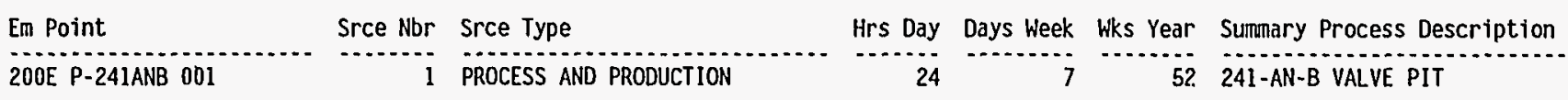

THE 241 -AN-B VALVE PIT IS $14^{\circ} \times 14^{\circ} \times 6^{\circ} 8^{\prime \prime}$ (LXWXD) AND IS LOCATED UNOERGROUND. THE PIT DRAINS TO TANK 102-AN. WASTES TRANSPORTED THROUGH THE PIT ARE COMPLEX CONCENTRATE AND DOUBLE SHELL SLURRY.

\begin{tabular}{|c|c|c|c|c|}
\hline En Point & Srce Nbr & Srce Type & Days Week Wks Year & Summary Process Description \\
\hline 200E P-241AP 001 & 1 & PROCESS AND PRODUCTION & 52 & 241-AP VALVE PIT \\
\hline \multicolumn{5}{|c|}{$\begin{array}{l}\text { THE CONCRETE VALVE PIT IS } 61^{\circ} \times 16^{\circ} \times 8^{\circ} \text { (LXWXD). WASTES TRANSPORTED VIA THE } 241 \text {-AP VALVE PIT ARE FROM TANKS 102-AP AND 104-AP PHOSPHATE SULPHATE } \\
\text { WASTES. AND TANKS } 105 \text {-AP AND 106-AP DOUBLE SHELL SLURRY FEED AND NON-COMPLEXED WASTE: }\end{array}$} \\
\hline En Point & Srce $\mathrm{Nbr}$ & Srce Type & Days Week Wks Year & Summary Process Description \\
\hline 200E P-241AR151001 & 1 & PROCESS AND PRODUCTION & 52 & 241-AR-151 DIVERSION BOX \\
\hline \multicolumn{5}{|c|}{$\begin{array}{l}\text { THE UNDERGROUND DIVERSION BOX IS } 7^{\circ} \times 5^{\circ} \times 1^{\circ} \text { (LXWXD) AND MADE OF CONCRETE. THE BOX DRAINS TO THE 244-AR VAULT. THE DIVERSION BOX TRANSPORTS MIXED } \\
\text { WASTE SOLUTIONS FROM PROCESSING AND DECONTAMINATION OPERATIONS. }\end{array}$} \\
\hline Em Point & Srce Nbr & Srce Type & Days Week Wks Year & Summary Process Description \\
\hline 200E P-241AWA 001 & 1 & PROCESS AND PRODUCTION & 52 & 241-AW-A VALVE PIT \\
\hline \multicolumn{5}{|c|}{$\begin{array}{l}\text { THE AW-A VALVE PIT IS } 14^{\circ} \times 12^{\circ} \times 6^{\circ} 7^{\prime \prime} \text { (LXWXD) LOCATED UNDERGROUND. THE PIT IS MADE OF CONCRETE. } \\
\text { THE WASTES TYPICALLY TRANSPORTED VIA THE AW-A VALVE PIT IS COMPLEX CONCENTRATE AND DOUBLE SHELL SLURRY FROM AW FARM. }\end{array}$} \\
\hline Em Point & Srce Nbr & Srce Type & Days Week Wks Year & Summary Process Description \\
\hline 200E P-241AWB 001 & 1 & PROCESS AND PRODUCTION & 52 & 241-AW-B VALVE PIT \\
\hline \multicolumn{5}{|c|}{$\begin{array}{l}\text { THE } 241 \text {-AW-B VALVE LOCATED UNDERGROUND IS A } 14^{\circ} \dot{\times} 12^{\circ} \times 6^{\circ} 7^{*}(\text { LXWXD) CONCRETE PIT. WASTES TRANSPORTED VIA THE } 241 \text {-AW-B VALVE PIT INCLUDES COMPLEX } \\
\text { CONCENTRATE AND DOUBLE SHELL. SLURRY TO/FROM AW FARM. }\end{array}$} \\
\hline Em Point & Srce Nbr & Srce Type & Days Week Wks Year & Sumnary Process Description \\
\hline 200E P-241AX101 001 & 1 & TANKS AND VESSELS & 52 & $\begin{array}{l}241-A X-101.1 .000 .000 \text { GALLON SINGLE SHELL UNDERGROUND } \\
\text { STORAGE TANK }\end{array}$ \\
\hline En Point & Srce Nbr & Srce Type & Days Week Wks Year & Summary Process Description \\
\hline 200E P-241AX102 001 & 1 & TANKS AND VESSELS & 52 & $\begin{array}{l}\text { 241-AX-102. } 1.000 .000 \text { GALLON SINGLE SHELL UNDERGROUND } \\
\text { STORAGE TANK }\end{array}$ \\
\hline Em Point & Srce Nbr & Srce Type & Days Week Wks Year & Summary Process Description \\
\hline 200E P-241AX103001 & 1 & TANKS AND VESSELS & 52 & $\begin{array}{l}241 \text {-AX-103, } 1.000 .000 \text { GALLON SINGLE SHELL UNDERGROUND } \\
\text { STORAGE TANK }\end{array}$ \\
\hline
\end{tabular}

Page \#: 27 of 95 Report Run Date: 22-APR-95 


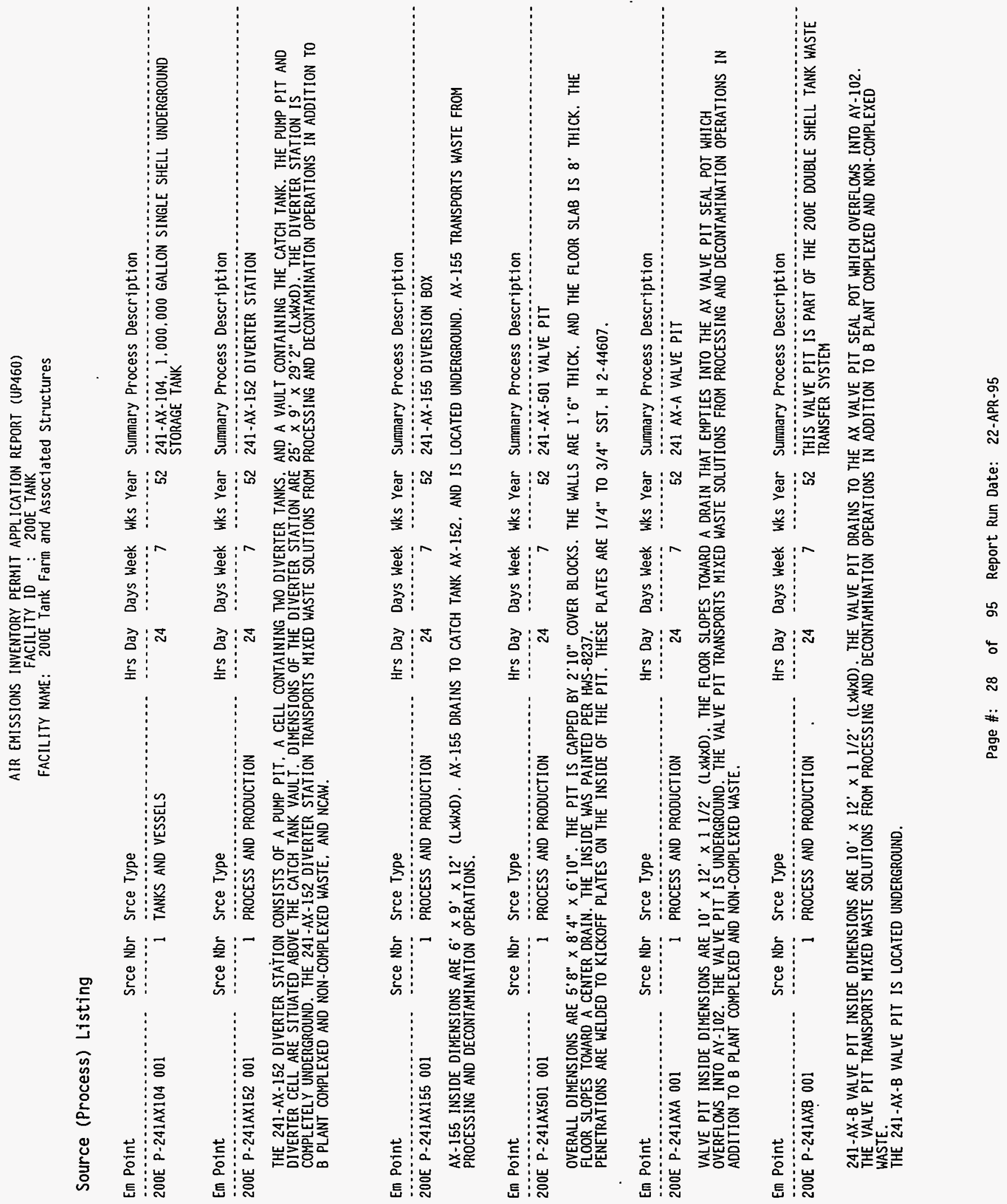


AIR EMISSIONS INVENTORY PERMIT APPLICATION REPORT (UP460)

FACILITY ID : 200E' TANK

Source (Process) Listing

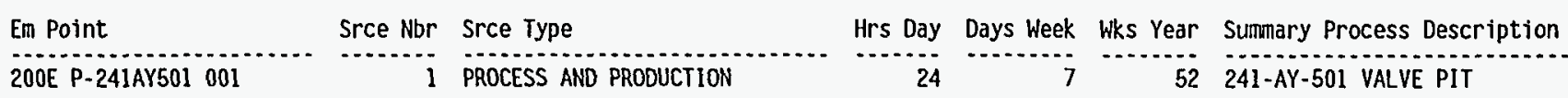

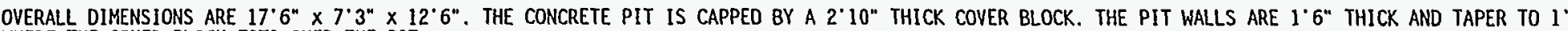

WHERE THE COVER BLOCK FITS OVER THE PIT.

PIT PENETRATIONS ARE WELDED TO FLANGES ON THE INSIDE OF THE PIT.

THE INSIDE SURFACES. INCLUDING THE COVER BLOCK. WERE ORIGINALLY COATED WITH 3 COATS OF AMERCOAT \#33, H 2-(64316, 64322), DOC. SD-HS-SAR-010 REV 3

\begin{tabular}{|c|c|c|c|c|c|c|}
\hline Em Point & Srce $\mathrm{Nbr}$ & Srce Type & Hrs Day & Days Week & Whs Year & Summary Process Description \\
\hline 200E P-241AZ152 001 & 1 & PROCESS AND PRODUCTION & 24 & 7 & 52 & 241-AZ-152 DIVERTER BOX \\
\hline \multicolumn{7}{|l|}{$\begin{array}{l}\text { THE } 241-A Z-152 \text { DIVERTER } \\
\text { IS LOCATED UNDERGROUND. }\end{array}$} \\
\hline Em Point & Srce Nbr & Srce Type & Mrs Day & Days Week & Wks Year & Summary Process Description \\
\hline $200 E$ P-241B101 001 & 1 & TANKS AND VESSELS & 24 & 7 & 52 & $\begin{array}{l}241-B-101,530.000 \text { GALLON SINGLE SHELL UNDERGROUND STORAGE } \\
\text { TANK }\end{array}$ \\
\hline Em Point & Srce $\mathrm{Nbr}$ & Srce Type & Hrs Day & Days Week & Wks Year & Sumnary Process Description \\
\hline 200E P-241B102001 & 1 & TANKS AND VESSELS & 24 & 7 & 52 & $\begin{array}{l}241-B-102,530.000 \text { GALLON SINGLE SHELL UNDERGROUND STORAGE } \\
\text { TANK }\end{array}$ \\
\hline Em Point & Srce Nbr & Srce Type & Hrs Day & Days Week & Wks Year & Summary Process Description \\
\hline 200E P-24IB103001 & 1 & TANKS AND VESSELS & 24 & 7 & 52 & $\begin{array}{l}241-8-103,530,000 \text { GALLON SINGLE SHELL UNDERGROUND STORAGE } \\
\text { TANK }\end{array}$ \\
\hline Em Point & Srce $\mathrm{Nbr}$ & Srce Type & Hrs Day & Days Week & Wks Year & Summary Process Description \\
\hline $200 E P-2418104001$ & 1 & TANKS AND VESSELS & 24 & 7 & 52 & $\begin{array}{l}\text { 241-B-104. 530.000 GALLON SINGLE SHELL UNDERGROUND STORAGE } \\
\text { TANK }\end{array}$ \\
\hline En Point & Srce $\mathrm{Nbr}$ & Srce Type & Hrs Day & Days Week & Wks Year & Surmary Process Description \\
\hline $200 E P-241 B 105001$ & 1 & TANKS AND VESSELS & 24 & 7 & 52 & $\begin{array}{l}241-B-105,530.000 \text { GALLON SINGLE SHELL UNDERGROUND STORAGE } \\
\text { TANK }\end{array}$ \\
\hline Em Point & Srce Nbr & Srce Type & Hrs Day & Days Week & Wks Year & Summary Process Description \\
\hline $200 \mathrm{E}$ P-241B106 001 & 1 & TANKS AND VESSELS & 24 & 7 & 52 & $\begin{array}{l}241-B-106.530 .000 \text { GALLON SINGLE SHELL UNDERGROUND STORAGE } \\
\text { TANK }\end{array}$ \\
\hline
\end{tabular}


AIR EMISSIONS INVENTORY PERMIT APPLICATION REPORT (UP460)
FACILITY ID: 200E TANK FACILITY NAME: $\begin{aligned} & \text { FACILITY ID } 200 \text { Tank Farm and Associated Structures } \\ & \text { (n) }\end{aligned}$

\section{Source (Process) Listing}

\begin{tabular}{|c|c|c|c|c|c|c|}
\hline Em Point & Srce Nbr & Srce Type & Hrs Day & Days Week & Wks Year & Sumnary Process Description \\
\hline 200E P-241B107001 & 1 & TANKS AND VESSELS & 24 & 7 & 52 & $\begin{array}{l}241-B-107.530 .000 \text { GALLON SINGLE SHELL UNDERGROUND STORAGE } \\
\text { TANK }\end{array}$ \\
\hline Ein Point & Srce Nbr & Srce Type & Hrs Day & Days Week & Wks Year & Summary Process Description \\
\hline $200 E$ P-241B108 001 & 1 & TANKS AND VESSELS & 24 & 7 & 52 & $\begin{array}{l}241-B-108,530.000 \text { GALLON SINGLE SHELL UNDERGROUND STORAGE } \\
\text { TANK }\end{array}$ \\
\hline Em Point & Srce Nbr & Srce Type & Hrs Day & Days Week & Whs Year & Suminary Process Description \\
\hline 200E P-241B109001 & 1 & TANKS AND VESSELS & 24 & 7 & 52 & $\begin{array}{l}241-B-109.530 .000 \text { GALLON SINGLE SHELL UNDERGROUND STORAGE } \\
\text { TANK }\end{array}$ \\
\hline Em Point & Srce Nbr & Srce Type & Hrs Day & Days Week & Wks Year & Sumnary Process Description \\
\hline 200E P-241B110 001 & 1 & TANKS AND VESSELS & 24 & 7 & 52 & $\begin{array}{l}241-B-110.530 .000 \text { GALLON SINGLE SHELL UNDERGROUND STORAGE } \\
\text { TANK }\end{array}$ \\
\hline Ein Point & Srce $\mathrm{Nbr}$ & Srce Type & Hrs Day & Days Week & Wks Year & Summary Process Description \\
\hline $200 E \mathrm{P}-241 \mathrm{~B} 111001$ & 1 & TANKS AND VESSELS & 24 & 7 & 52 & $\begin{array}{l}241-8-111,530.000 \text { GALLON SINGLE SHELL UNDERGROUND STORAGE } \\
\text { TANK }\end{array}$ \\
\hline Em Point & Srce Nbr & Srce Type & Hrs Day & Days Week & Wks Year & Summary Process Description \\
\hline 200E P-241B112001 & 1 & TANKS AND VESSELS & 24 & 7 & 52 & $\begin{array}{l}241-B-112,530.000 \text { GALLON SINGLE SHELL UNDERGROUND STORAGE } \\
\text { TANK }\end{array}$ \\
\hline Em Point & Srce Nbr & Srce Type & Hrs Day & Days Week & Wks Year & Summary Process Description \\
\hline $200 E \mathrm{P}-241 \mathrm{~B} 201001$ & 1 & TANKS AND VESSELS & 24 & 7 & 52 & $\begin{array}{l}241-B \cdot 201,55.000 \text { GALLON SINGLE SHELL UNDERGROUND STORAGE } \\
\text { TANK }\end{array}$ \\
\hline Em Point & Srce $\mathrm{Nbr}$ & Srce Type & Hrs Day & Days Week & Wks Year & Sumnary Process Description \\
\hline 200E P-241B202001 & 1 & TANKS AND VESSELS & 24 & 7 & 52 & $\begin{array}{l}241-B-202.55 .000 \text { GALLON SINGLE SHELL UNDERGROUND STORAGE } \\
\text { TANK }\end{array}$ \\
\hline Em Point & Srce Nbr & Srce Type & Hrs Day & Days Week & Wks Year & Summary Process Description \\
\hline $200 E \mathrm{P}-241 \mathrm{~B} 203001$ & 1 & TANKS AND VESSELS & 24 & 7 & 52 & $\begin{array}{l}241-B-203.55 .000 \text { GALLON SINGLE SHELL UNDERGROUND STORAGE } \\
\text { TANK }\end{array}$ \\
\hline
\end{tabular}


AIR EMISSIONS INVENTORY PERMIT APPLICATION REPORT (UP460)

FACILITY NAME: 200E Tank Farm and Associated Structures

\section{Source (Process) Listing}

\begin{tabular}{|c|c|c|c|c|c|c|}
\hline En Point & Srce Nbr & Srce Type & Hrs Day & Days Week & Whs Year & Summary Process Description \\
\hline $200 E$ P-241B204 001 & 1 & TANKS AND VESSELS & 24 & 7 & 52 & $\begin{array}{l}241-\mathrm{B}-204,55,000 \text { GALLON SINGLE SHELL UNDERGROUND STORAGE } \\
\text { TANK }\end{array}$ \\
\hline Em Point & Srce Nbr & Srce Type & Hrs Day & Days Week & Whs Year & Sumary Process Description \\
\hline 200E P-241BX101001 & 1 & TANKS AND VESSELS & 24 & 7 & 52 & $\begin{array}{l}241-B X-101.530 .000 \text { GALLON SINGLE SHELL UNDERGROUND STORAGE } \\
\text { TANK }\end{array}$ \\
\hline Em Point & Srce Nbr & Srce Type & Hrs Day & Days Week & Wks Year & Sumnary Process Description \\
\hline 200E P-241BX102 001 & 1 & TANKS AND VESSELS & 24 & 7 & 52 & $\begin{array}{l}241-B X-102,530.000 \text { GALLON SINGLE SHELL UNDERGROUNO STORAGE } \\
\text { TANK }\end{array}$ \\
\hline Em Point & Srce Nbr & Srce Type & Hrs Day & Days Week & Wks Year & Summary Process Description \\
\hline 200E P-241BX103001 & 1 & TANKS AND VESSELS & 24 & 7 & 52 & $\begin{array}{l}241-B X-103,530,000 \text { GALLON SINGLE SHELL UNDERGROUND STORAGE } \\
\text { TANK }\end{array}$ \\
\hline Em Point & Srce Nbr & Srce Type & Hrs Day & Days Week & Wks Year & Summary Process Description \\
\hline 200E P-24IBX104 001 & 1 & TANKS AND VESSELS & 24 & 7 & 52 & $\begin{array}{l}241-B X-104.530 .000 \text { GALLON SINGLE SHELL UNDERGROUND STORAGE } \\
\text { TANK }\end{array}$ \\
\hline En Point & Srce Nbr & Srce Type & Hrs Day & Days Week & Wks Year & Summary Process Description \\
\hline $200 E$ P-241BX105 001 & 1 & TANKS AND VESSELS & 24 & 7 & 52 & $\begin{array}{l}241-B X-105.530 .000 \text { GALLON SINGLE SHELL UNDERGROUND STORAGE } \\
\text { TANK }\end{array}$ \\
\hline Em Point & Srce Nbr & Srce Type & Hrs Day & Days Week & Wks Year & Summary Process Description \\
\hline 200E P-241BX106 001 & 1 & TANKS AND VESSELS & 24 & 7 & 52 & $\begin{array}{l}241-B X-106.530 .000 \text { GALLON SINGLE SHELL UNDERGROUND STORAGE } \\
\text { TANK }\end{array}$ \\
\hline Em Point & Srce Nbr & Srce Type & Hrs Day & Days Week & Whs Year & Sumnary Process Description \\
\hline 200E P-2418X107 001 & 1 & TANKS AND VESSELS & 24 & 7 & 52 & $\begin{array}{l}241-B X-107,530.000 \text { GALLON SINGLE SHELL UNDERGROUND STORAGE } \\
\text { TANK }\end{array}$ \\
\hline Em Point & Srce Nbr & Srce Type & Hrs Day & Days Week & Wks Year & Summary Process Description \\
\hline 200E P-241BX108 001 & 1 & TANKS AND VESSELSS & 24 & 7 & 52 & $\begin{array}{l}241-B X-108.530 .000 \text { GALLON SINGLE SHELL UNDERGROUND STORAGE } \\
\text { TANK }\end{array}$ \\
\hline
\end{tabular}


Source (Process) Listing

\begin{tabular}{|c|c|c|c|c|c|c|}
\hline Em Point & Srce Nbr & Srce Type & Hrs Day & Days Week & Whs Year & Sumnary Process Description \\
\hline 200E P-241BX109001 & 1 & TANKS AND VESSELSS & 24 & 7 & 52 & $\begin{array}{l}241-B X-109,530.000 \text { GALLON SINGLE SHELL UNDERGROUND STORAGE } \\
\text { TANK }\end{array}$ \\
\hline Em Point & Srce Nbr & Srce Type & Hrs Day & Days Week & Wks Year & Summary Process Description \\
\hline 200E P-241BX110 001 & 1 & TANKS AND VESSELSS & 24 & 7 & 52 & $\begin{array}{l}241-B X-110.530 .000 \text { GALLON SINGLE SHELL UNDERGROUND STORAGE } \\
\text { TANK }\end{array}$ \\
\hline En Point & Srce Nbr & Srce Type & Hrs Day & Days Week & Wks Year & Summary Process Description \\
\hline 200E P-241BX111 001 & 1 & TANKS AND VESSELS & 24 & 7 & 52 & $\begin{array}{l}241 \text {-BX-111, 530.000 GALLON SINGLE SHELL UNDERGROUND STORAGE } \\
\text { TANK }\end{array}$ \\
\hline Em Point & Srce Nbr & Srce Type & Hrs Day & Days Week & Wks Year & Sumnary Process Description \\
\hline $200 \mathrm{E}$ P-241BX112 001 & 1 & TANKS AND VESSELS & 24 & 7 & 52 & $\begin{array}{l}\text { 241-BX-112, 530.000 GALLON SINGLE SHELL. UNDERGROUND STORAGE } \\
\text { TANK }\end{array}$ \\
\hline En Point & Srce Nbr & Srce Type & Hrs Day & Days Week & Whs Year & Summary Process Description \\
\hline 200E P-241BY101 001 & 1 & TANKS AND VESSELS & 24 & 7 & 52 & $\begin{array}{l}241-B Y-101.750 .000 \text { GALLON SINGLE SHELL UNDERGROUND STORAGE } \\
\text { TANK }\end{array}$ \\
\hline Em Point & Srce Nbr & Srce Type & Hrs Day & Days Week & Wks Year & Summary Process Description \\
\hline 200E P-241BY102 001 & 1 & TANKS AND VESSELS & 24 & 7 & 52 & $\begin{array}{l}241 \text {-BY-102. } 750.000 \text { GALLON SINGLE SHELL UNDERGROUND STORAGE } \\
\text { TANK }\end{array}$ \\
\hline Em Point & Srce Nbr & Srce Type & Hrs Day & Days Week & Wks Year & Summary Process Description \\
\hline $200 E$ P-241BY103 001 & $\ddot{i}$ & TANKS AND VESSELS & 24 & 7 & 52 & $\begin{array}{l}241-B Y-103,750.000 \text { GALLON SINGLE SHELL UNDERGROUND STORAGE } \\
\text { TANK }\end{array}$ \\
\hline Em Point & Srce Nbr & Srce Type & Hrs Day & Days Week & Wks Year & Summary Process Description \\
\hline 200E P-241BY104 001 & 1 & TANKS AND VESSELS & 24 & 7 & 52 & $\begin{array}{l}241-B Y \cdot 104,750,000 \text { GALLON SINGLE SHELL UNOERGROUND STORAGE } \\
\text { TANK }\end{array}$ \\
\hline En Point & Srce Nbr & Srce Type & Hrs Day & Days Week & Wks Year & Summary Process Description \\
\hline $200 \mathrm{E}$ P-241BY105 001 & 1 & TANKS AND VESSELS & 24 & 7 & 52 & $\begin{array}{l}241-B Y=105,750,000 \text { GALLON SINGLE SHELL UNDERGROUND STORAGE } \\
\text { TANK }\end{array}$ \\
\hline
\end{tabular}


AIR EMISSIONS INVENTORY PERMIT APPLICATION REPORT (UP460)

FACILITY NAME: 200 Tank Farm and Associated Structures

Source (Process) Listing

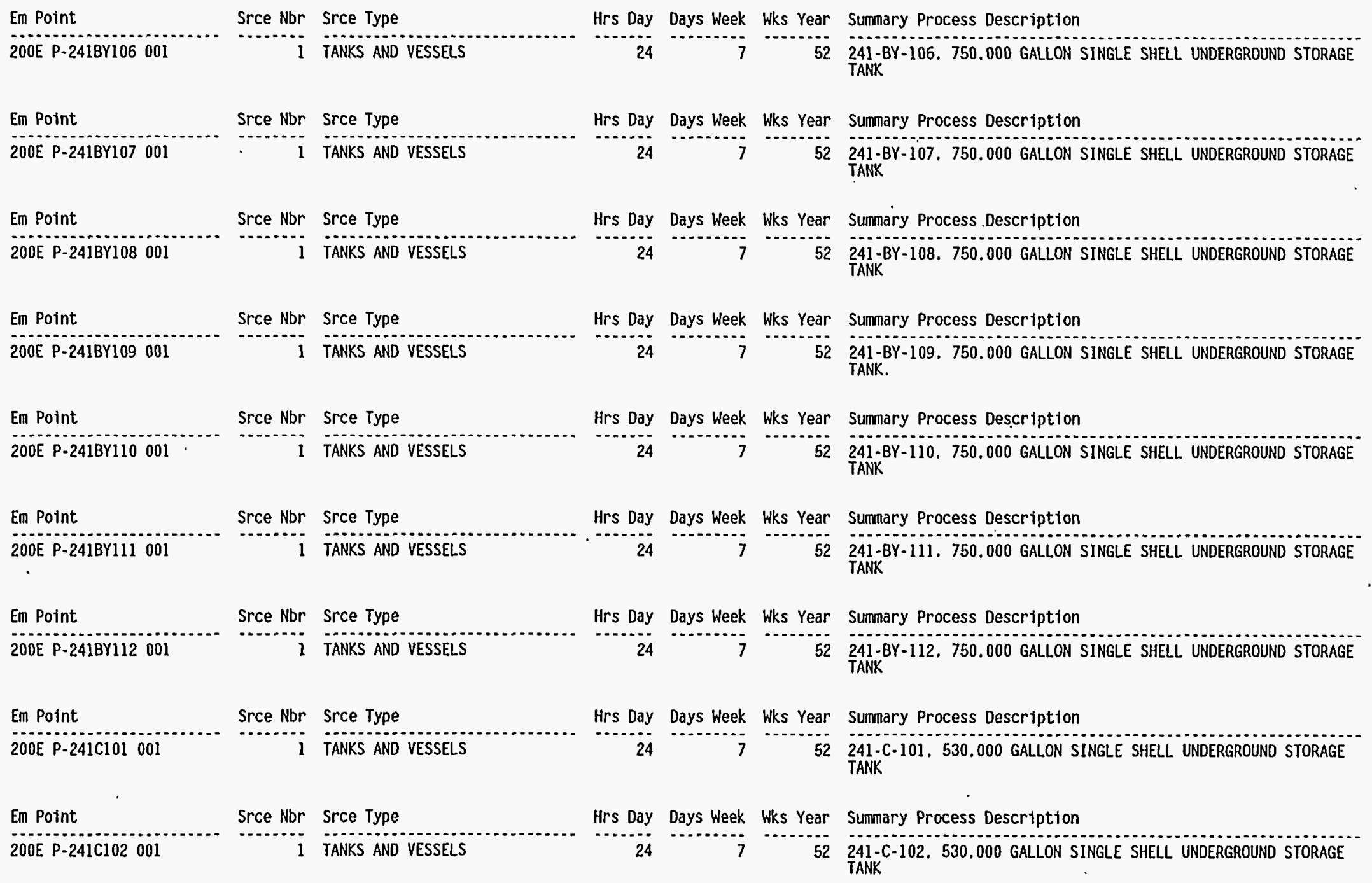


AIR EMISSIONS INVENTORY PERMIT APPLICATION REPORT (UP460)

FACILITY NAME: 200 Tank Farm and ASsociated Structures

\section{Source (Process) Listing}

\begin{tabular}{|c|c|c|c|c|c|c|}
\hline Em Point & Srce Nbr & Srce Type & Hrs Day & Days Week & Wks Year & Sumnary Process Description \\
\hline 200E P-241C103001 & 1 & TANKS AND VESSELS & 24 & 7 & 52 & $\begin{array}{l}241-C-103.530,000 \text { GALLON SINGLE-SHELL UNDERGROUND STORAGE } \\
\text { TANK }\end{array}$ \\
\hline En Point & Srce Nbr & Srce Type & Hrs Day & Days Week & Whs Year & Summary Process Description \\
\hline 200E P-241C107 001 & $i$ & TANKS AND VESSELS & 24 & 7 & 52 & $\begin{array}{l}241-C-107.530 .000 \text { GALLON SINGLE SHELL UNDERGROUND STORAGE } \\
\text { TANK }\end{array}$ \\
\hline Em Point & Srce Nbr & Srce Type & Hrs Day & Days Week & Wks Year & Summary Process Description \\
\hline $200 \mathrm{E}$ P-241C108 001 & $i$ & TANKS AND VESSELS & 24 & 7 & 52 & $\begin{array}{l}241-C-108,530,000 \text { GALLON SINGLE SHELL UNDERGROUND STORAGE } \\
\text { TANK }\end{array}$ \\
\hline Em Point & Srce $\mathrm{Nbr}$ & Srce Type & Hrs Day & Days Week & Whs Year & Summary Process Description \\
\hline $200 \mathrm{E}$ P-241C109 001 & $i$ & TANKS AND VESSELS & 24 & 7 & 52 & $\begin{array}{l}241-C-109.530 .000 \text { GALLON SINGLE SHELL UNDERGROUND STORAGE } \\
\text { TANK }\end{array}$ \\
\hline Em Point & Srce Nbr & Srce Type & Hrs Day & Days Week & Wks Year & Summary Process Description \\
\hline $200 \mathrm{P}-241 \mathrm{Cl10} 001$ & 1 & TANKS AND VESSELS & 24 & 7 & 52 & $\begin{array}{l}241-C-110.530 .000 \text { GALLON SINGLE SHELL UNDERGROUND STORAGE } \\
\text { TANK }\end{array}$ \\
\hline Em Point & Srce Nbr & Srce Type & Hrs Day & Days Week & Wks Year & Summary Process Description \\
\hline 200E P-241C111 001 & 1 & TANKS AND VESSELS & 24 & 7 & 52 & $\begin{array}{l}241-C-111.530 .000 \text { GALLON SINGLE SHELL UNDERGROUND STORAGE } \\
\text { TANK }\end{array}$ \\
\hline Em Point & Srce $\mathrm{Nbr}$ & Srce Type & Hrs Day & Days Week & Wks Year & Summary Process Description \\
\hline $200 E$ P-241C112001 & 1 & TANKS AND VESSELS & 24 & 7 & 52 & $\begin{array}{l}241-C-112.530 .000 \text { GALLON SINGLE SHELL UNDERGROUND STORAGE } \\
\text { TANK }\end{array}$ \\
\hline Em Point & Srce Nbr & Srce Type & Hrs Day & Days Week & Wks Year & Summary Process Description \\
\hline $200 E$ P-241C201 001 & 1 & TANKS AND VESSELS & 24 & 7 & 52 & $\begin{array}{l}241-C-201,55,000 \text { GALLON SINGLE SHELL UNDERGROUND STORAGE } \\
\text { TANK }\end{array}$ \\
\hline Em Point & Srce Nbr & Srce Type & Hrs Day & Days Week & Wks Year & Summary Process Description \\
\hline $200 E$ P-241C202 001 & 1 & TANKS AND VESSELS & 24 & 7 & 52 & $\begin{array}{l}241-\mathrm{C}-202.55 .000 \text { GALLON SINGLE SHELL UNDERGROUND STORAGE } \\
\text { TANK }\end{array}$ \\
\hline
\end{tabular}

Page \#: 34 of 95 Report Run Date: 22-APR-95 
AIR EMISSIONS INVENTORY PERMIT APPLICATION REPORT (UP460) FACILITY NAME: 200E Tank Farm and Associated Structures

Source (Process) Listing

\begin{tabular}{|c|c|c|c|c|c|c|}
\hline Em Point & Srce Nbr & Srce Type & Hrs Day & Days Week & Wks Year & Summary Process Description \\
\hline 200E P-241C203 001 & 1 & TANKS AND VESSELS & 24 & 7 & 52 & $\begin{array}{l}241-C-203,55.000 \text { GALLON SINGLE SHELL UNDERGROUND STORAGE } \\
\text { TANK }\end{array}$ \\
\hline Em Point & Srce Nbr & Srce Type & Hrs Day & Days Week & Wks Year & Summary Process Description \\
\hline 200E P-241C204 001 & 1 & TANKS AND VESSELS & 24 & 7 & 52 & $\begin{array}{l}241-C-204,55,000 \text { GALLON SINGLE SHELL UNDERGROUND STORAGE } \\
\text { TANK }\end{array}$ \\
\hline Em Point & Srce Nbr & Srce Type & Hrs Day & Days Week & Wks Year & Summary Process Description \\
\hline 200E P-241ER151 001 & $i$ & PROCESS AND PRODUC & 24 & 7 & 52 & 241-ER-151 DIVERSION BOX \\
\hline
\end{tabular}

THE DIVERSION BOX IS AN UNDERGROUND REINFORCED CONCRETE STRUCTURE WITH OVERALL DIMENSIONS OF $43^{\circ} \times 10^{\circ} \times 17^{\circ}$ (LXWXD). THE BOX ORAINS TO CATCH TANK 241 -ER-311. THE BOX HAS A LEAK DETECTOR THAT ALARMS IN 242 -A.
POSSIBLE WASTES INCLUDE FLUSH WATER MIXED WITH WASTE FROM SY FARM. B PLANT, AND B FARM.
Em Point
Srce Nbr Srce Type
Hrs Day Days Week Wks Year Summary Process Description
$200 \mathrm{E}$ P-241ER152 001
1 PROCESS AND PRODUCTION
$24 \quad 752$ 241-ER-152 DIVERSION BOX
THE DIVERSION BOX IS A REINFORCED CONCRETE STRUCTURE WITH DIMENSIONS OF $12^{\circ} \times 12^{\circ} \times 15^{\circ}$ (LXWXD). THE BOX DRAINS TO 241-ER-311. A LEAK DETECTOR ON THE WASTE TRANSFERRED THROUGH THE BOX INCLUDES FLUSH WATER MIXED WITH WASTES FROM SY FARM. B PLANT. AND B FARM.
Em Point
Srce Nbr Srce Type
200E P-241ER153 001
PROCESS AND PRODUCTION
Hrs Day Days Week Wks Year Summary Process Description

THE DIVERSION BOX IS REINFORCED CONCRETE WITH DIMENSIONS OF $15^{\circ} \times 15^{\circ} \times 12^{\circ}$ (LXWXD). THE BOX DRAINS TO LIFT STATION A-350. A LEAK DETECTOR IS MOUNTED WASTE SOURCES INCLUDE FLUSH WATER AND LEAKS FROM SY FARM. B PLANT, B FARM. AND C FARM.

\begin{tabular}{|c|c|c|c|c|c|c|}
\hline Em Point & Srce $\mathrm{Nbr}$ & Srce Type & Hrs Day & Days Week & Wks Year & Summary Process Description \\
\hline 200E P-24IER31I 001 & 1 & PROCESS AND PRODUCTION & 24 & 7 & 52 & 241-ER-311 CATCH TANK \\
\hline
\end{tabular}

THE 241 -ER-311 CATCH TANK CONSISTS OF A HORIZONTAL CYLINDRICAL CARBON STEEL VESSEL. A STEAM JET BOX RESTING ON THE TANK AND A PUMP PIT ABOVE THE

TANK. THE CATCH TANK SERVES 241-ER-151 AND 241-ER-152 DIVERSION BOXES. THE TANK HAS A 9.5 FOOT DIAMETER AND IS 40 FEET LONG. 
AIR EMISSIONS INVENTORY PERMIT APPLICATION REPORT (UP460)
FACILITY ID : 200E TANK

FACILITY NAME: 200E Tank Farm and ASSOCiated Structures

\section{Source (Process) Listing}

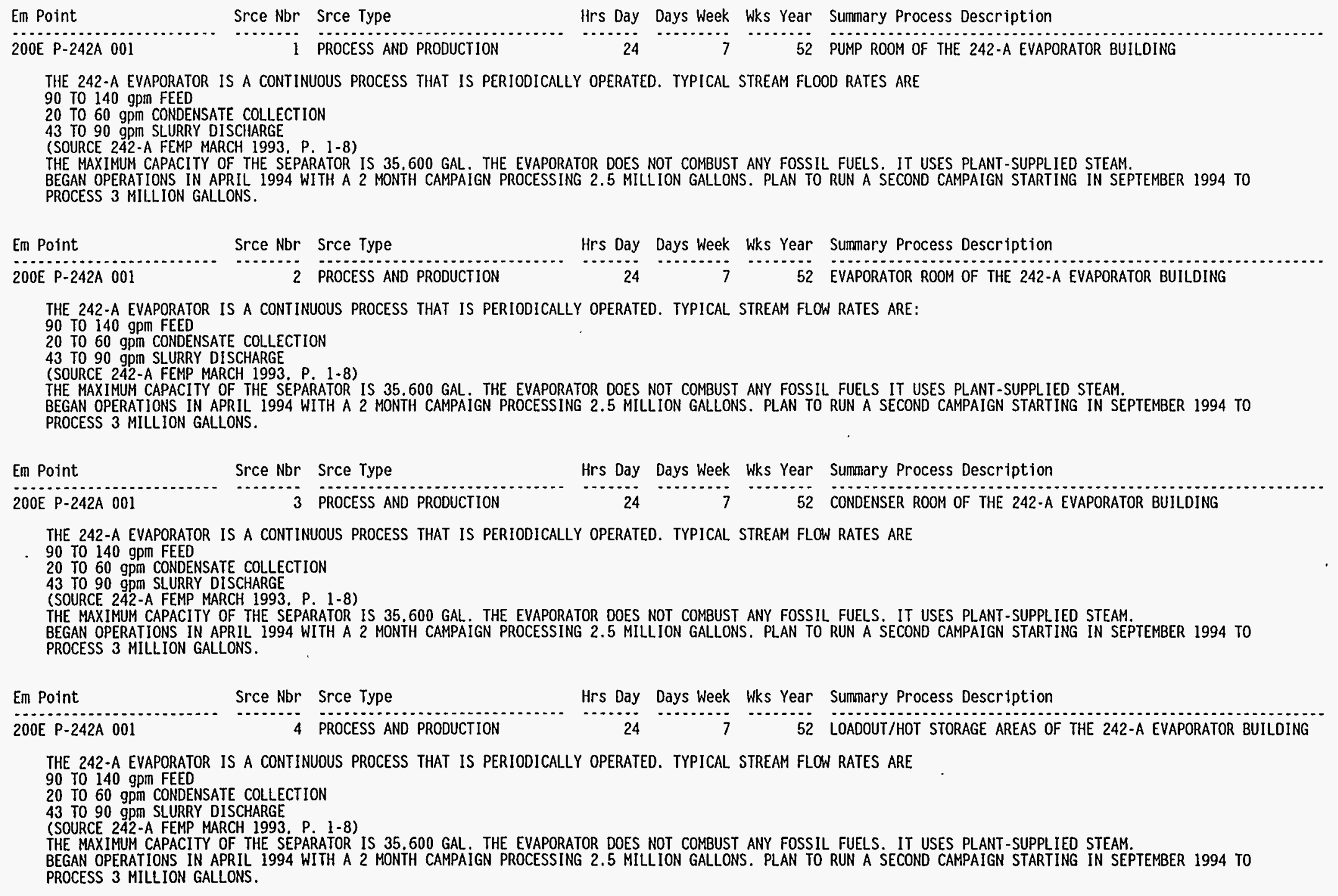




\section{Source (Process) Listing}

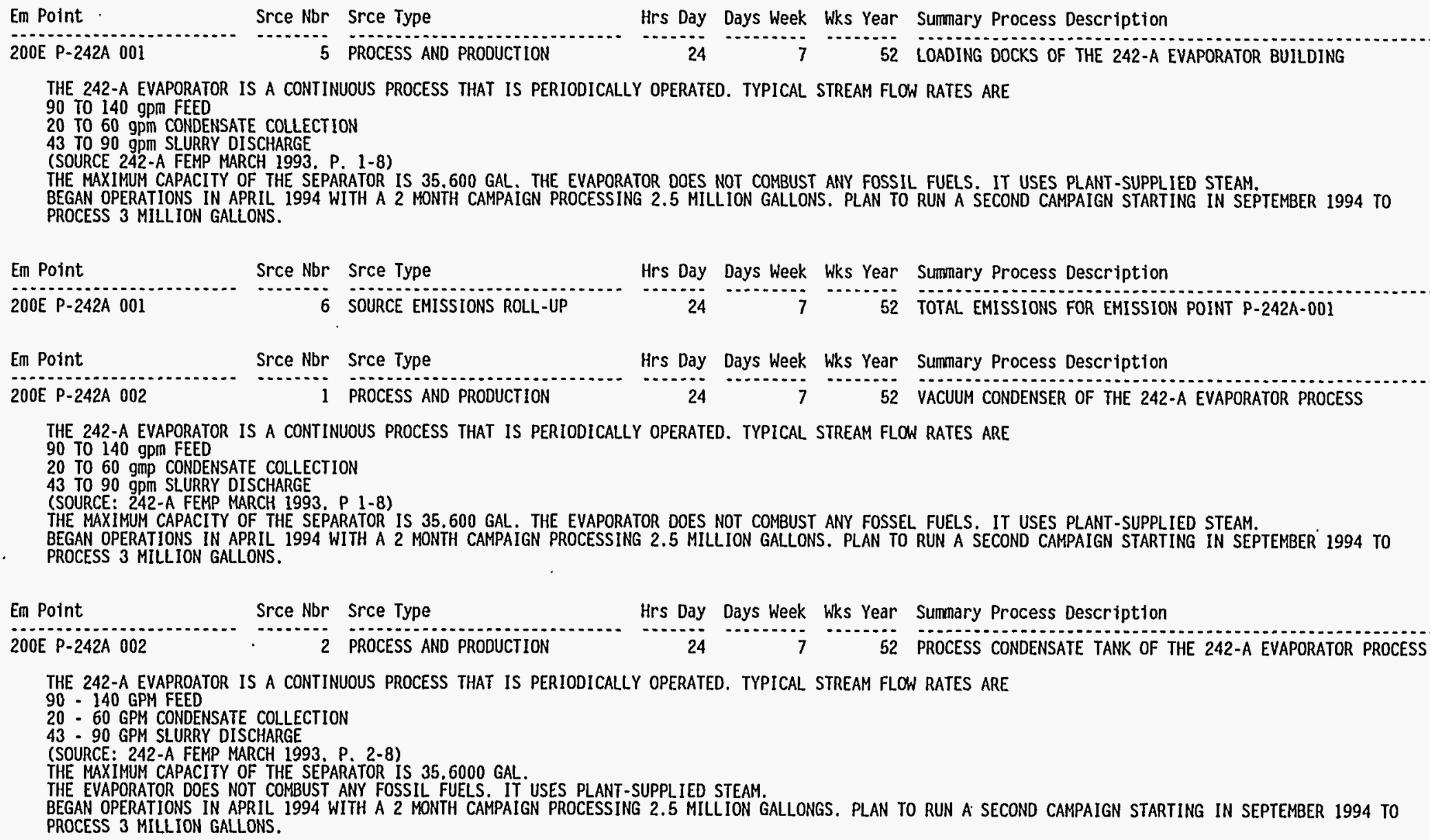


AIR EMISSIONS INVENTORY PERMIT APPLICATION REPORT (UP460)

FACILITY NAME: 200 Tank Farm and Associated structures

Source (Process) Listing

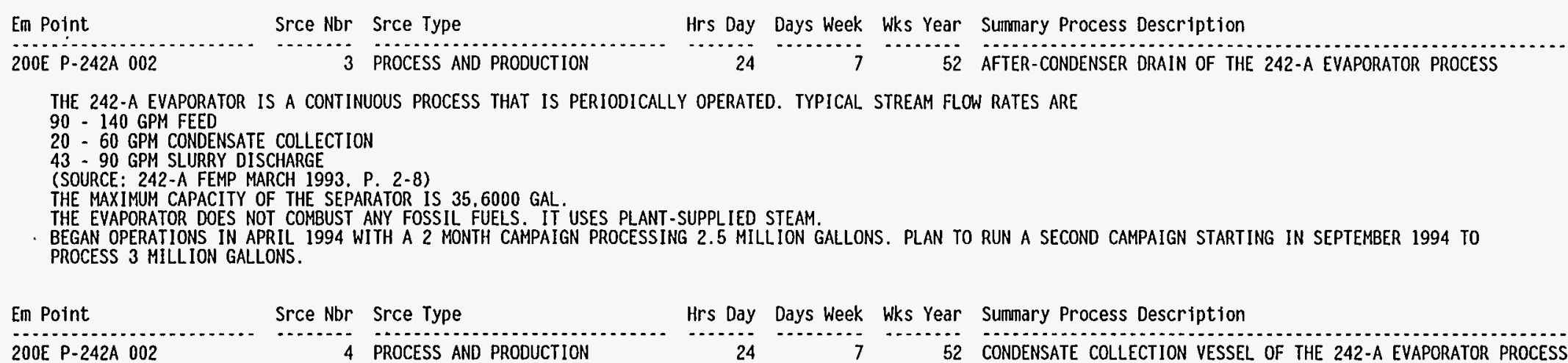

$200 \mathrm{P}-242 \mathrm{~A} 002$

4 PROCESS AND PRODUCTION

52 CONDENSATE COLLECTION VESSEL OF THE 242-A EVAPORATOR PROCESS

THE 242-A EVAPORATOR IS A CONTINUOUS PROCESS THAT IS PERIODICALLY OPERATED. TYPICAL STREAM FLOW RATES ARE

$90 \cdot 140 \mathrm{GPM}$ FEED

20 - 60 GPM CONDENSATE COLLECTION

43. 90 GPM SLURRY DISCHARGE

(SOURCE: 242-A FEMP MARCH 1993, P. 2-8)

THE MAXIMUM CAPACITY OF THE SEPARATOR IS $35,6000 \mathrm{GAL}$

A A 2 MONTH CAMPAign PROCESSING 2.5 MILLION GAllons. PLAN TO RUN A SECOND CAMPAign STARTING IN SEPTEMBER 1994 TO

PROCESS 3 MILLION GALLONS,

\begin{tabular}{|c|c|c|c|c|c|c|}
\hline Em Point & Srce Nbr & Srce Type & Hrs Day & Days Week & Wks Year & Summary Process Description \\
\hline $200 E$ P-242A 002 & 5 & PROCESS AND PRODUCTION & 24 & 7 & 52 & $\begin{array}{l}\text { SAMPLING PUMP EXHAUST FROM ROOM AIR SAMPLING FOR THE AMU } \\
\text { ROOM. CHANGE ROOM. CONTROL ROOM. CONDENSER ROOM. EVAPORATOR } \\
\text { ROOM. PUMP ROOM LOADOUT/NOT STORAGE, LOADING ROOM. AND ION } \\
\text { EXCHANGE COLUMN ROOM }\end{array}$ \\
\hline
\end{tabular}

THE 242-A EVAPROATOR IS A CONTINUOUS PROCESS THAT IS PERIODICALLY OPERATED. TYPICAL STREAM FLOW RATES ARE

90 - $140 \mathrm{GPM}$ CONDENSATE COLLECTION

43 - 90 GPM SLURRY DISCHARGE

(SOURCE: 242-A FEMP MARCH 1993, P. 2-8)

THE MAXIMUM CAPACITY OF THE SEPARATOR IS 35,6000 GAL

THE EVAPORATOR DOES NOT COMBUST ANY FOSSIL FUELS. IT USES PLANT-SUPPLIED STEAM

BEGAN OPERATIONS IN APRIL 1994 WITH A 2 MONTH CAMPAIGN PROCESSING 2.5 MILLION GALLONS. PLAN TO RUN A SECOND CAMPAIGN STARTING IN SEPTEMBER 1994 TO PROCESS 3 MILLION GALLONS.

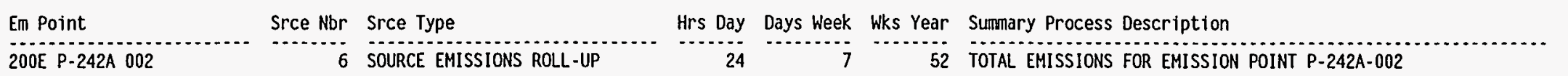




\begin{tabular}{|c|c|c|c|c|c|c|}
\hline Em Point & Srce Nbr & Srce Type & Hrs Day & Days Week & Whs Year & Summary Process Description \\
\hline $200 E$ P-244A 001 & 1 & PROCESS AND PRODUCTION & 24 & 7 & 52 & 244-A LIFT STATION (DCRT) \\
\hline
\end{tabular}

THE 244-A DCRT IS A SHORT TERM WASTE STORAGE FACILITY USED FOR INTERIM STORAGE OF LIQUID WASTES AND AS A VALVE PIT FOR WASTE TRANSFER OPERATIONS. IT IS AN UNDERGROUND CONCRETE STRUCTURE WITH A TANK VAULT. PUMP PIT. AND FILTER PIT. SALT WELL AND MISCELLANEOUS WEST AREA PLANT WASTES ARE ROUTED TO 200 EAST THROUGH THE 244-A DCRT.

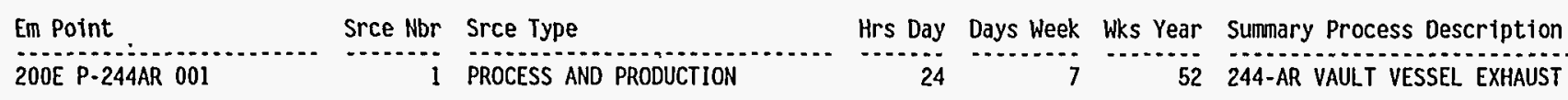

244 AR VAULT IS CURRENTLY USED FOR INTERIM STORAGE IN TRANSFER OF PUREX WASTE TO AND FROM B PLANT. THE VAULT CONTAINS (2) 50.000 GALLON STAINLESS STEEL TANKS WHICH ARE 20 FEET IN DIAMETER AND (2) 5.000 GALLON STAINLESS STEEL TANKS WHICH ARE 9.5 FEET IN DIAMETER. CHEMICAL MAKE-UP FACILITIES ARE

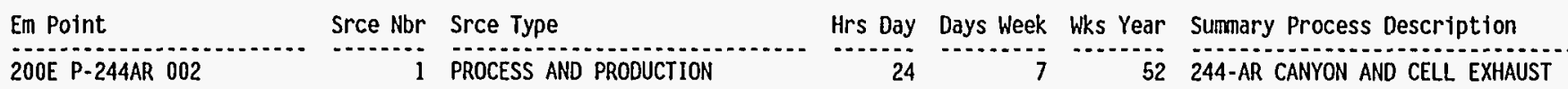

THE 244-AR CANYON AND CELLS ARE UTILIZED IN SUPPORT OF THE 244-AR VAULT. THE CANYON BUILDING IS A REINFORCED CONCRETE STRUCTURE WHICH MEASURES $94^{\circ} X$ $10^{\circ} \times 36^{\circ}$ (LXWXD). THREE PROCESS CELLS AND A FAILED EQUIPMENT STORAGE CELL ARE LOCATED BELOW THE CANYON DECK LEVEL.

Em Point Srce $\mathrm{Nbr}$ Srce Type Hrs Day Days Week Wks Year sumary Process Description $200 E$ P-244BX 001

1 PROCESS AND PRODUCTION $24 \quad 52$ 244-BX DOUBLE-CONTAINED RECEIVER TANK

THE 244-BX SALT WELL SYSTEM IS A RECTANGULAR VAULT FABRICATED OF REINFORCED CONCRETE CONSISTING OF THREE SECTIONS: A TANK VAULT. A PUMP PIT. AND WASTES FROM B. BX. AND BY TANK FARMS.

Em Point Srce Nbr Srce Type Hrs Day Days Week Wks Year Summary Process Description 200E $P$-244CR 001 1 PROCESS AND PRODUCTION

$$
24 \quad 7 \quad 52 \quad 244-C R \text { VAULT }
$$

244 CR VAULT IS AN UNDERGROUND STORAGE VAULT WHICH HOUSES TWO 40.000 GALLON TANKS. SALT WELL WASTE IS COLLECTED FROM C FARM AND TRANSFERRED TO 244 CR VAULT AND THEN TO A DESIGNATED DOUBLE SHELL TANK.

Em Point 200 P. $296 \mathrm{~A} 001$

Srce $\mathrm{Nbr}$ Srce Type 1 TANKS AND VESSELS
Hrs Day Days Week Whs Year Summary Process Description

$24 \quad 52$ TA1-A-105, $1,000,000$ GALLON SINGLE SHELL UNDERGROUND STORAGE

NO PROCESS DESCRIPTION 
AIR EMISSIONS INVENTORY PERMIT APPLICATION REPORT (UP460)

FACILITY NAME: 200E Tank Farm and Associated Structures

Source (Process) Listing

\begin{tabular}{|c|c|c|c|c|c|c|}
\hline Em Point & Srce Nbr & Srce Type & Mrs Day & Days Week & Wks Year & Summary Process Description \\
\hline 200E P-296A019 001 & 1 & TANKS AND VESSELS & 24 & 7 & 52 & 102-AY UNDERGROUND STORAGE TANK. \\
\hline Em Point & Srce $\mathrm{Nbr}$ & Srce Type & Hrs Day & Days Week & Wks Year & Summary Process Description \\
\hline 200E P-296A020 001 & 1 & TANKS AND VESSELS & 24 & 7 & 52 & 101-AZ UNDERGROUND STORAGE TANK \\
\hline Em Point & Srce Nbr & Srce Type & Hrs Day & Days Week & Wks Year & Summary Process Description \\
\hline 200E P-296A020 001 & 2 & TANKS AND VESSELS & 24 & 7 & 52 & 102-AZ UNDERGROUND STORAGE TANK \\
\hline Em Point & Srce $\mathrm{Nbr}$ & Srce Type & Hrs Day & Days Week & Wks Year & Summary Process Description \\
\hline 200E P-296A028 001 & 1 & TANKS AND VESSELS & 24 & 7 & 52 & 101 -AW UNDERGROUND STORAGE TANK. \\
\hline Em Point & Srce Nbr & Srce Type & Hrs Day & Days Week & Wks Year & Summary Process Description \\
\hline $200 E$ P-296A028 001 & 2 & TANKS AND VESSELS & 24 & $\overline{7}$ & 52 & 102-AW UNDERGROUND STORAGE TANK. \\
\hline Em Point & Srce Nbr & Srce Type & Hrs Day & Days Week & Wks Year & Summary Process Description \\
\hline Em Point & Srce Nbr & Srce Type & Hrs Day & Days Week & Wks Year & Summary Process Description \\
\hline $200 \mathrm{P}$ P-296A028 001 & 4 & TANKS AND VESSELS & 24 & 7 & 52 & 104-AW UNDERGROUND STORAGE TANK. \\
\hline Em Point & Srce Nbr & Srce Type & Hrs Day & Days Week & Whs Year & Summary Process Description \\
\hline $200 \mathrm{E}$ P-296A028001 & 5 & TANKS AND VESSELS & 24 & $\ddot{7}$ & 52 & 105-AW UNDERGROUND STORAGE TANK. \\
\hline Em Point & Srce Nbr & Srce Type & Hrs Day & Days Week & Wks Year & Summary Process Description \\
\hline 200E P-296A028001 & 6 & TANKS AND VESSELS & 24 & 7 & 52 & 106-AW UNDERGROUND STORAGE TANK. \\
\hline Em Point & Srce Nbr & Srce Type & Hrs Day & Days Week & Wks Year & Summary Process Description \\
\hline 200E P-296A030001 & 1 & TANKS AND VESSELS & 24 & 7 & 52 & 101-AN UNDERGROUND STORAGE TANK \\
\hline Em Point & Srce Nbr & Srce Type & Hrs Day & Days Week & Wks Year & Summary Process Description \\
\hline 200E P-296A030 001 & 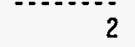 & TANKS AND VESSELS & 24 & 7 & 52 & 102-AN UNDERGROUND STORAGE TANK \\
\hline
\end{tabular}




\section{Source (Process) Listing}

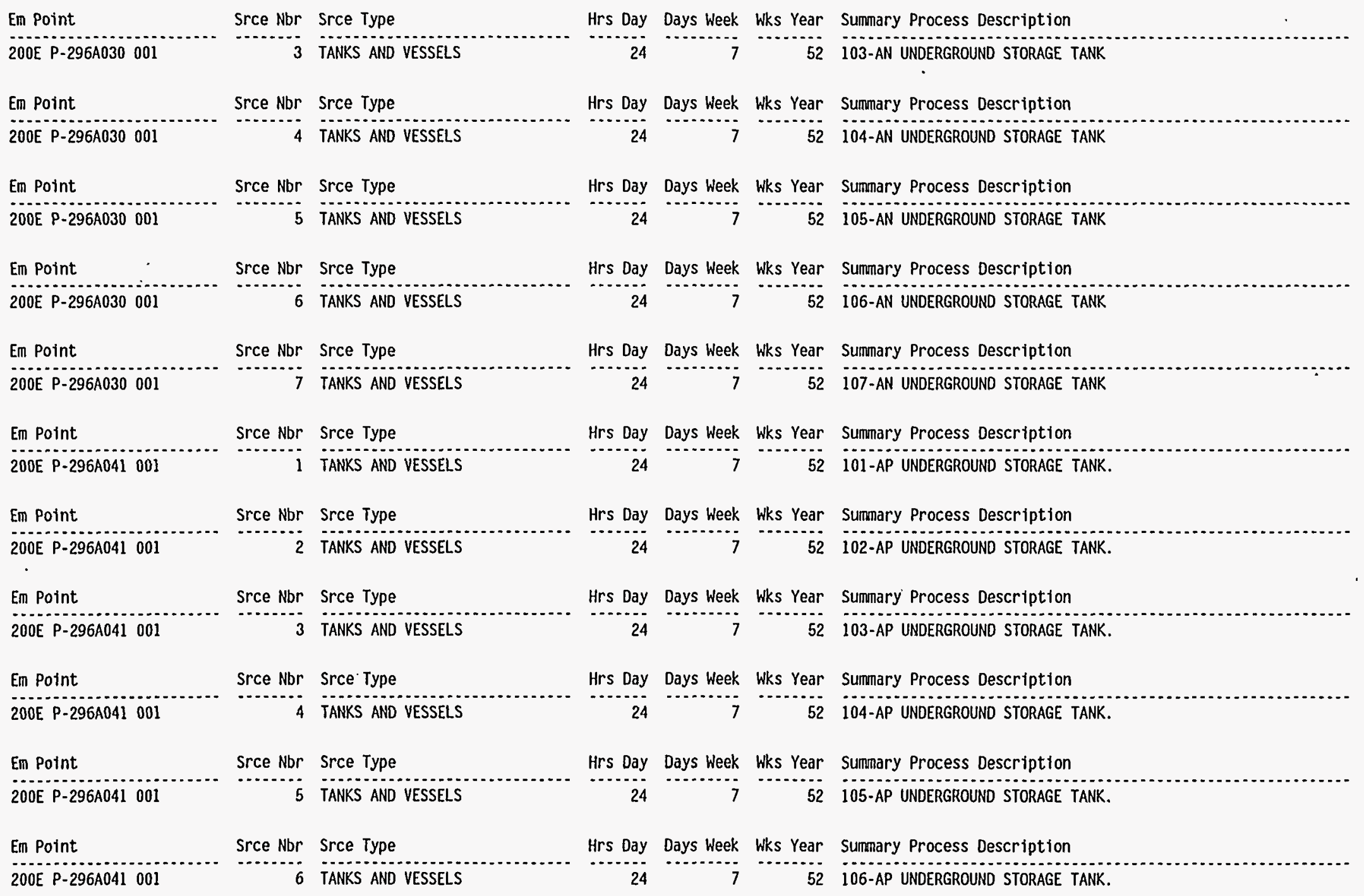




\section{Source (Process) Listing}

\begin{tabular}{|c|c|c|c|c|c|c|}
\hline Em Point & Srce Nbr & Srce Type & Hrs Day & Days Week & Wks Year & Summary Process Description \\
\hline 200E P-296A041 001 & 7 & TANKS AND VESSELS & 24 & 7 & 52. & 107-AP UNDERGROUND STORAGE TANK. \\
\hline Em Point & Srce $\mathrm{Nbr}$ & Srce Type & Hrs Day & Days Week & Wks Year & Sumnary Process Description \\
\hline $200 E$ P-296A041 001 & 8 & TANKS AND VESSELS & 24 & 7 & 52 & 108-AP UNDERGROUND STORAGE TANK. \\
\hline Em Point & Srce Nbr & Srce Type & Hrs Day & Days Week & Wks Year & Summary Process Description \\
\hline 200E P-296AN 001 & 1 & TANKS AND VESSELS & 24 & 7 & 52 & TANK 241 -AN-101 CONTAINS DILUTE NON-COMPLEXED (DN) WASTE. \\
\hline Em Point & Srce $\mathrm{Nbr}$ & Srce Type & Hrs Day & Days Week & Wks Year & Summary Process Description \\
\hline 200E P-296AN 001 & 2 & TANKS AND VESSELS & 24 & 7 & 52 & $\begin{array}{l}\text { TANK } 241 \text {-AN-102 CONTAINS SLUDGE AND COMPLEXANT CONCENTRATE } \\
\text { (CC) WASTE. }\end{array}$ \\
\hline Em Point & Srce Nbr & Srce Type & Hrs Day & Days Week & Wks Year & Summary Process Description \\
\hline $200 E$ P-296AN 001 & 3 & TANKS AND VESSELS & 24 & 7 & 52 & TANK 241-AN-103 CONTAINS DOUBLE-SHELL SLURRY (SL) WASTE. \\
\hline En Point & Srce $\mathrm{Nbr}$ & Srce Type & Hrs Day & Days Week & Wks Vear & Summary Process Description \\
\hline 200E P-296AN 001 & 4 & TANKS AND VESSELS & 24 & 7 & 52 & $\begin{array}{l}\text { TANK } 241 \text {-AN-104 CONTAINS DOUBLE-SHELL SLURRY FEED (SF) } \\
\text { WASTE. }\end{array}$ \\
\hline Em Point & Srce Nbr & Srce Type & Hrs Day & Days Week & Wks Year & Summary Process Description \\
\hline (1).................. & 3rce not & 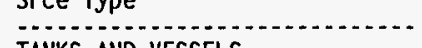 & nirs day & Days week & ......... & TANK 241 -AN-105 CONTAINS ROUBIF-SHEUI SUURPY FEFD (SF) \\
\hline 200E P-296AN 001 & 5 & TANKS AND VESSELS & 24 & 7 & 52 & $\begin{array}{l}\text { TANK } 241 \text {-AN-105 CONTAINS DOUBLE-SHELL SLURRY FEED (SF) } \\
\text { WASTE. }\end{array}$ \\
\hline Em Point & Srce Nbr & Srce Type & Hrs Day & Days Week & Wks Year & Summary Process Description \\
\hline $200 E$ P.296AN 001 & 6 & TANKS AND VESSELS & 24 & 7 & 52 & TANK 241-AN-106 CONTAINS CONCENTRATED PHOSPHATE WASTE (CP). \\
\hline Em Point & Srce $\mathrm{Nbr}$ & Srce Type & Hrs Day & Days Week & Wks Year & Summary Process Description \\
\hline 200E P-296AN 001 & 7 & TANKS AND VESSELS & 24 & 7 & 52 & $\begin{array}{l}\text { TANK } 241 \text {-AN-107 CONTAINS SLUDGE AND COMPLEXANT CONCENTRATE } \\
\text { (CC) WASTE. }\end{array}$ \\
\hline Em Point & Srce $\mathrm{Nbr}$ & Srce Type & Hrs Day & Days Week & Wks Year & Summary Process Description \\
\hline $200 E$ P-296AN 001 & 8 & SOURCE EMISSIONS ROLL-UP & 24 & 7 & 52 & TOTAL EMISSIONS FOR EMISSION POINT P-296AN-001 \\
\hline
\end{tabular}


AIR EMISSIONS INVENTORY PERMIT APPLICATION REPORT (UP460)

FACILITY NAME: 200E Tank Farm and Associated Structures

Source (Process) Listing

\begin{tabular}{|c|c|c|c|c|c|c|}
\hline En Point & Srce Nbr & Srce Type & Hrs Day & Days Week & Wks Year & Summary Process Description \\
\hline 200E P-296AP 001 & 1 & TANKS AND VESSELS & 24 & 7 & 52 & TANK 241-AN-101 CONTAINS DILUTE NON-COMPLEXED (DN) WASTE. \\
\hline Em Point & Srce Nbr & Srce Type & Hrs Day & Days Week & Whs Year & Sumnary Process Description \\
\hline 200E P-296AP 001 & 2 & TANKS AND VESSELS & 24 & 7 & 52 & TANK 241-AN-102 CONTAINS DILUTE NON-COMPLEXED (DN) WASTE. \\
\hline Em Point & Srce Nbr & Srce Type & Mrs Day & Days Week & Wks Year & Summary Process Description \\
\hline $200 E$ P-296AP 001 & 3 & TANKS AND VESSELS & 24 & 7 & 52 & TANK 241 -AN-103 CONTAINS DILUTE NON-COMPLEXED (DN) WASTE. \\
\hline Em Point & Srce Nbr & Srce Type & Hrs Day & Days Week & Whs Year & Summary Process Description \\
\hline 200E P-296AP 001 & 4 & TANKS AND VESSELS & 24 & 7 & 52 & TANK 241-AP-104 CONTAINS OILUTE NON-COMPLEXED (DN) WASTE. \\
\hline Em Point & Srce Nbr & Srce Type & Hrs Day & Days Week & Wks Year & Sumnary Process Description \\
\hline 200E P-296AP 001 & 5 & TANKS AND VESSELS & 24 & 7 & 52 & TANK 241-AP-105 CONTAINS DILUTE NON-COMPLEXEO (ON) WASTE. \\
\hline Em Point & Srce Nbr & Srce Type & Hrs Day & Days Week & Wks Year & Summary Process Description \\
\hline 200E P-296AP 001 & 6 & TANKS AND VESSELS & 24 & 7 & 52 & TANK 241-AP-106 CONTAINS DILUTE NON-COMPLEXED (DN) WASTE. \\
\hline Em Point & Srce Nbr & Srce Type & Hrs Day & Days Week & Wks Year & Summary Process Description \\
\hline 200E P-296AP 001 & 7 & TANKS AND VESSELS & 24 & 7 & 52 & TANK 241-AN-107 CONTAINS DILUTE NON-COMPLEXED (DN) WASTE. \\
\hline Em Point & Srce Nbr & Srce Type & Hrs Day & Days Week & Wks Year & Sunmary Process Description \\
\hline $200 E$ P-296AP 001 & 8 & TANKS ANO VESSELS & 24 & 7 & 52 & TANK 241-AN-108 CONTAINS DILUTE NON-COMPLEXED (DN) WASTE. \\
\hline Em Point & Srce Nbr & Srce Type & Hrs Day & Days Week & Wks Year & Sumuary Process Description \\
\hline $200 E \mathrm{P}-296 \mathrm{AP} 001$ & 9 & SOURCE EMISSIONS ROLL-UP & 24 & 7 & 52 & TOTAL EMISSIONS FOR EMISSION POINT P-296AP-001 \\
\hline Em Point & Srce Nbr & Srce Type & Hrs Day & Days Week & Wks Year & Summary Process Description \\
\hline $200 E$ P-296AW 001 & 1 & TANKS AND VESSELS & 24 & 7 & 52 & $\begin{array}{l}\text { TANK } 241 \text {-AW-101 CONTAINS SLUDGE AND DOUBLE-SHELL SLURRY FEEO } \\
\text { (SF) WASTE. }\end{array}$ \\
\hline Em Point & Srce Nbr & Srce Type & Hrs Day & Days Week & Wks Year & Surmary Process Description \\
\hline $200 E$ P-296AW 001 & 2 & TANKS AND VESSELS & 24 & 7 & 52 & TANK 241-AW- 102 CONTAINS DILUTE NON-COMPLEXED (DN) WASTE. \\
\hline
\end{tabular}


AIR EMISSIONS INVENTORY PERMIT APPLICATION REPORT (UP460)

FACILITY NAME: $200 \mathrm{E}$ Tank Farm and ASsociated structures

Source (Process) Listing

\begin{tabular}{|c|c|c|c|c|c|c|}
\hline En Point & Srce $\mathrm{Nbr}$ & Srce Type & Hrs Day & Days Week & Wks Year & Summary Process Description \\
\hline 200E P-296AW 001 & 3 & TANKS AND VESSELS & 24 & 7 & 52 & $\begin{array}{l}\text { TANK } 241 \text {-AW- } 103 \text { CONTAINS PUREX NEUTRALIZED CLADDING REMOVAL } \\
\text { WASTE SLUDGE AND DILUTE NON-COMPLEXED WASTE. }\end{array}$ \\
\hline Em Point & Srce Nbr & Srce Type & Hrs Day & Days Week & Wks Year & Sumnary Process Description \\
\hline $200 \mathrm{P}$ P-296AW 001 & 4 & TANKS AND VESSELS & 24 & 7 & 52 & $\begin{array}{l}\text { TANK } 241 \text {-AW-104 CONTAINS SLUDGE AND DILUTE NON-COMPLEXED } \\
\text { (DN) WASTE. }\end{array}$ \\
\hline Em Point & Srce $\mathrm{Nbr}$ & Srce Type & Hrs Day & Days Week & Wks Year & Summary Process Description \\
\hline 200E P-296AW 001 & 5 & TANKS AND VESSELS & 24 & 7 & 52 & $\begin{array}{l}\text { TANK } 241 \text {-AW-105 CONTAINS PUREX NCRW (NEUTRALIIED CLADDING } \\
\text { REMOVAL WASTE) SLUDGE (PD) AND DILUTE NON-COMPLEXED (DN) } \\
\text { WASTE. }\end{array}$ \\
\hline Em Point & Srce Nbr & Srce Type & Hrs Day & Days Week & Whs Year & Summary Process Description \\
\hline 200E P-296AW 001 & 6 & TANKS AND VESSELS & 24 & 7 & 52 & $\begin{array}{l}\text { TANK } 241 \text {-AW-106 CONTAINS SLUDGE, DOUBLE-SHELL SLURRY (SL), } \\
\text { AND DILUTE NON-COMPLEXED (DN) WASTE. }\end{array}$ \\
\hline Em Pornt & Srce Nbr & Srce Type & Hrs Day & Days Week & Wks Year & Summary Process Description \\
\hline Em Point & Srce $\mathrm{Nbr}$ & Srce Type & Hrs Day & Days Week & Wks Year & Summary Process Description \\
\hline 200E P-296AYAZ 001 & 1 & TANKS AND VESSELS & 24 & 7 & 52 & $\begin{array}{l}\text { TANK 241-AY-101 CONTAINS SLUDGE AND DILUTE COMPLEXED (DC) } \\
\text { WASTE }\end{array}$ \\
\hline Em Point & Srce $\mathrm{Nbr}$ & Srce Type & Hrs Day. & Days Week & Wks Year & Summary Process Description \\
\hline 200E P-296AYAZ 001 & 2 & TANKS AND VESSELS & 24 & 7 & 52 & $\begin{array}{l}\text { TANK 241-AY-102 CONTAINS SLUDGE AND DILUTE COMPLEXED (DC) } \\
\text { WASTE }\end{array}$ \\
\hline Em Point & Srce $\mathrm{Nbr}$ & Srce Type & Hrs Day & Days Week & Wks Year & Summary Process Description \\
\hline 200E P-296AYAZ 001 & 3 & TANKS AND VESSELS & 24 & 7 & 52 & $\begin{array}{l}\text { TANK 241-AZ-101 CONTAINS SLUDGE. NEUTRALIZED CURRENT ACIO } \\
\text { WASTE (AW). AND DILUTE NON-COMPLEXED WASTE }\end{array}$ \\
\hline Em Point & Srce Nbr & Srce Type & Hrs Day & Days Week & Wks Year & Sunnary Process Description \\
\hline $200 E$ P-296AYAZ 001 & 4 & TANKS AND VESSELS & 24 & 7 & 52 & $\begin{array}{l}\text { TANK } 241 \text {-AZ-102 CONTAINS SLUDGE NEUTRALIZEO CURRENT ACID } \\
\text { WASTE (AW). AND DILUTE NON-COMPLEXED (DN) WASTE }\end{array}$ \\
\hline
\end{tabular}

Page \#: 44 of 95 Report Run Date: 22-APR-95 
AIR EMISSIONS INVENTORY PERMIT APPLICATION REPORT (UP460)

FACILITY NAME: $200 E$ Tank Farm and Associated Structures

\section{Source (Process) Listing}

\begin{tabular}{|c|c|c|c|c|c|c|}
\hline Em Point & Srce $\mathrm{Nbr}$ & Srce Type & Hrs Day & Days Week & Wks Year & Summary Process Description \\
\hline 200E P-296AYAZ 001 & 5 & SOURCE EMISSIONS ROLL-UP & 24 & 7 & 52 & TOTAL EMISSIONS FOR EMISSION POINT P-296AYAZ-001 \\
\hline Em Point & Srce Nbr & Srce Type & Hrs Day & Days Week & Wks Year & Sumary Process Description \\
\hline $200 E$ P-2966001 001 & $i$ & PROCESS AND PRODUCTION & & & & NO LONGER OPERATING \\
\hline Em Point & Srce Nbr & Srce Type & Hrs Day & Days Week & Wks Year & Summary Process Description \\
\hline $200 E$ P-296P016 001 & 1 & TANKS AND VESSELS & 24 & 7 & 52 & $\begin{array}{l}241-\mathrm{C}-105,530.000 \text { GALLON SINGLE SHELL UNDERGROUND STORAGE } \\
\text { TANK }\end{array}$ \\
\hline THE ENTIRE BANK (OF 2 & FILTERS) Is & $S$ TESTED AS A SINGLE UNIT. WHICH & 1 MUST PASS & S THE 99.95 & S\% CRITERIA & \\
\hline Em Point & Srce $\mathrm{Nbr}$ & Srce Type & Hrs Day & Days Week & Wks Year & Summary Process Description \\
\hline $200 E$ P-296P016 001 & 2 & TANKS AND VESSELS & 24 & 7 & 52 & $\begin{array}{l}241-C-106.530 .000 \text { GALLON SINGLE SHELL UNDERGROUND STORAGE } \\
\text { TANK }\end{array}$ \\
\hline En Point & Srce Nbr & Srce Type & Hrs Day & Days Week & Wks Year & Sumnary Process Description \\
\hline $200 E$ P-296P016 001 & $\ddot{3}$ & SOURCE EMISSIONS ROLL-UP & 24 & 7 & 52 & TOTAL EMISSIONS FOR EMISSION POINT P-296P016-001 \\
\hline Em Point & Srce $\mathrm{Nbr}$ & Srce Type & Hrs Day & Days Week & Wks Year & Summary Process Description \\
\hline 200E P-296P026 001 & 1 & TANKS AND VESSELS & 24 & 7 & 52 & 101-AY UNDERGROUND STORAGE TANK. \\
\hline Em Point & Srce $\mathrm{Nbr}$ & Srce Type & Hrs Day & Days Week & Wks Year & Summary Process Description \\
\hline $200 \mathrm{P}-296 \mathrm{P} 026001$ & 2 & TANKS AND VESSELS & 24 & 7 & 52 & 102-AY UNDERGROUND STORAGE TANK. \\
\hline Em Point & Srce $\mathrm{Nbr}$ & Srce Type & Hrs Day & Days Week & Whs Year & Summary Process Description \\
\hline $200 E$ P-296P026 001 & 3 & TANKS AND VESSELS & 24 & 7 & 52 & 101-AZ UNDERGROUND STORAGE TANK. \\
\hline Em Point & Srce $\mathrm{Nbr}$ & Srce Type & Hirs Day & Days Week & Whs Year & Summary Process Description \\
\hline $200 E$ P-296P026 001 & 4 & TANKS AND VESSELS & 24 & 7 & 52 & 102-AZ UNDERGROUND STORAGE TANK. \\
\hline Em Point & Srce Nbr & Srce Type & Hrs Day & Days Week & Whs Year & Surmary Process Description \\
\hline $200 E$ P-296P031001 & 1 & PROCESS AND PRODUCTION & 24 & 7 & 52 & $\begin{array}{l}\text { PLANS ARE TO USE THE CRITICAL ASSEMBLY ROOM AND THE MIX ROOM } \\
\text { TO ASSAY. SAMPLE. AND REPACKAGE LOW-LEVEL RADIOACTIVE SOLIO } \\
\text { WASTE CONTAINERS. }\end{array}$ \\
\hline
\end{tabular}




\title{
Source (Process) Listing
}

\begin{abstract}
Em Point
Srce Nbr Srce Type

Hrs Day Days Week Wks Year Surmary Process Description

200E P-LERF OOI

1 PROCESS AND PRODUCTION

$24 \quad 52$ LIQUID EFFLUENT RETENTION FACILITY

THE LIQUID EFFLUENT RETENTION FACILITY (LERF) CONSISTS OF THREE IDENTICAL 24.6 ML (6.5 Mgal) SURFACE IMPOUNDMENTS (RETENTION BASINS). THESE BASINS ARE LOCATED ON A 157,956-H SQUARED (39-ACRE) SITE IN THE 200 AREA ON THE HANFORD SITE. THIS LOCATION IS APPROXIMATELY $1.2 \mathrm{~km}$ (0.75 mi) NORTH OF THE $242-A$ EVAPORATOR.

THE BASINS ARE CONSTRUCTED WITH PRIMARY AND SECONDARY COMPOSITE LINERS, A LEACHATE COLLECTION AND REMOVAL SYSTEM BETWEEN THE LINERS. AND A FLOATING COVER. IN ADDITION. THE LERF INCLUDES PIPING AND PUMPING SYSTEMS. UTILITIES. AND A BASIN OPERATIONS STRUCTURE.

EACH BASIN IS CONSTRUCTED WITH A PRIMARY AND SECONDARY LINER. THE UPPER (OR PRIMARY) LINER HAS A 1.52 -mII (60-mi]) HIGH-DENSITY POLYETHYLENE (HDPE) GEOMEMBRANE LAID DIRECTLY OVER AN IMPERMEABLE MANUFACTURED GEOTEXTILE/BENTONITE SANDWICH LAYER THE LOWER (OR SECONDARY) LINER IS ESSENTIALLY ANOTHER
HDPE LINER LAID ON $91.4 \mathrm{~cm}(36 \mathrm{IN}$.) OF SOIL/BENTONITE $(106.7 \mathrm{~cm}[42 \mathrm{in}$.$] ON THE SIDES OF THE BASIN). THE SOIL/BENTONITE LAYER ACTS AS A BARRIER TO$

ANY LIQUID THAT MIGHT FIND ITS WAY THROUGH OR UNDER THE PRIMARY LINER IS REMOVED BY THE LEACHATE COLLECTION SYSTEM. WHICH IS LOCATED BETWEEN THE SIDES OF THE SECONDARY IE IS SOPED TO A SUMP AT ONE CORNER OF THE BASIN WHERE A LEACHATE PEMOVAL RISER CONTAINIMG A DEDICATED PUMP IS LOCATED THE PUMP OPERATES WHENEVER THE LIQUID LEVEL IN THE SUMP REACHES $25.4 \mathrm{~cm}$ (10 in.). AND CONTINUES TO OPERATE UNTIL THE SUMP LIQUID LEVEL REACHES 5.1 CII (2 In.). THE LEACHATE IS PUMPED BACK INTO THE BASIN

A FLOATING GEOMEMBRANE COVER OF 1.52 - IMT (40-mi1) LOW-DENSITY POLYETHYLENE (LDPE) IS STRETCHED OVER EACH BASIN ABOVE THE PRIMARY LINER. THE COVER MATERIAL IS RESISTANT TO ULTRAVIOLET RADIATION. ACTIVATED CARBON BREATHER FILTERS ARE PROVIDED TO VENTILATE THE BASIN. THESE FILTERS ARE LOCATED IN ONE OF THE CORNERS OF EACH BASIN. THE COVERS AND LINERS ARE SECURED BY BATTEN STRIPS WITH ANCHOR BOLTS IMBEDDED IN CONCRETE FOOTINGS AT THE PERIMETER OF EACH BASIN. A MECHANICAL TENSIONING SYSTEM SUSPENDS THE COVER OVER THE BASIN AND ALLOWS THE COVER TO FLOAT ON THE EFFLUENT SURFACE.
\end{abstract}

\section{Source Raw Materia1s Usage}

Em Point

Srce Nbr Srce Type

Process Rate Process Vom Material

Fuels Usage by Source Processes

Em Point

Srce Nbr srce Type

Qty Uom

Fuel

Fuels Usage by Control Devices

Em Point

Ctl $\mathrm{Nbr}$ Ctl Type

Qty Uom

Fuel

Point Source Criteria Pollutants - Type: CO

\begin{tabular}{|c|c|c|c|c|c|}
\hline Em Point & Srce $\mathrm{Nbr}$ & Srce Type Pollutant & $\begin{array}{l}\text { Em. Pt. Potential } \\
\text { to Emit (tons/yr) }\end{array}$ & $\begin{array}{l}\text { Pt. } \\
\text { Actual (tons/yr) }\end{array}$ & lutant \\
\hline 200E P-241A101 001 & & 1 TANKS AND VESSELS & .000439000 & .000439000 & CARBON MONOXIDE \\
\hline $\begin{array}{l}\text { 200E P-241A102 } 001 \\
\text { 200E P-241A103 } \\
001\end{array}$ & & $\begin{array}{ll}1 & \text { TANKS AND VESSELS } \\
1 & \text { TANKS AND VESSELS }\end{array}$ & $\begin{array}{l}.000950000 \\
.000950000\end{array}$ & $\begin{array}{l}.000950000 \\
.000950000\end{array}$ & $\begin{array}{l}\text { CARBON MONOXIDE } \\
\text { CARBON MONOXIDE }\end{array}$ \\
\hline
\end{tabular}

Page \#: 46 of 95 Report Run Date: 22-APR-95 
AIR EMISSIONS INVENTORY PERMIT APPLICATION REPORT (UP460)

FACILITY NAME: 200E Tank Farm and Associated Structures

Point Source Criteria Pollutants - Type: CO

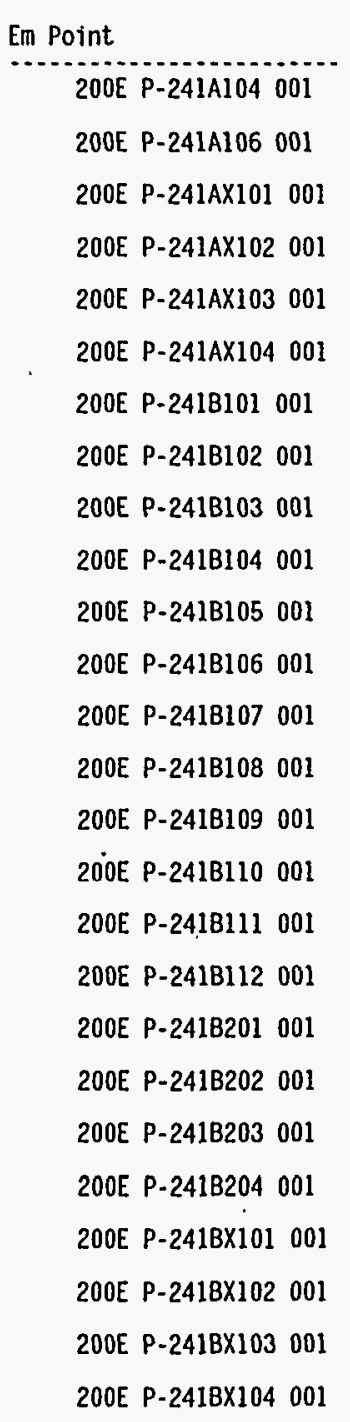

Srce Nbr Srce Type Pollutant

Em. Pt. Potential Em. Pt.

to Emit (tons/yr) ct] Actual (tons/yr) Pollutant

TANKS AND VESSELS

1 TANKS AND VESSELS

1 TANKS AND VESSELS

1 TANKS AND VESSELS

1 TANKS AND VESSELS

1 TANKS AND VESSELS

1 TANKS AND VESSELS

1 TANKS AND VESSELS

1 TANKS AND VESSELS

1 TANKS AND VESSELS

1 TANKS AND VESSELS

1 TANKS AND VESSELS

1 TANKS AND VESSELS

1 TANKS AND VESSELS

1 TANKS AND VESSELS

1 TANKS AND VESSELS

1 TANKS AND VESSELS

1 TANKS AND VESSELS

1 TANKS AND VESSELS

1 TANKS AND VESSELS

1 TANKS AND VESSELS

1 TANKS AND VESSELS

1 TANKS AND VESSELS

1 TANKS AND VESSELS

1 TANKS AND VESSELS

1 TANKS AND VESSELS

$\begin{array}{lll}.000439000 & .000439000 & \text { CARBON MONOXIDE } \\ .000950000 & .000950000 & \text { CARBON MONOXIDE } \\ .000950000 & .000950000 & \text { CARBON MONOXIDE } \\ .000439000 & .000439000 & \text { CARBON MONOXIDE } \\ .000950000 & .000905000 & \text { CARBON MONOXIDE } \\ .000439000 & .000439000 & \text { CARBON MONOXIDE } \\ .000950000 & .000950000 & \text { CARBON MONOXIDE } \\ .000950000 & .000950000 & \text { CARBON MONOXIDE } \\ .000950000 & .000950000 & \text { CARBON MONOXIDE } \\ .000439000 & .000439000 & \text { CARBON MONOXIDE } \\ .000439000 & .000439000 & \text { CARBON MONOXIDE } \\ .000950000 & .000950000 & \text { CARBON MONOXIDE } \\ .000439000 & .000439000 & \text { CARBON MONOXIDE } \\ .000950000 & .000950000 & \text { CARBON MONOXIDE } \\ .000439000 & .000439000 & \text { CARBON MONOXIDE } \\ .000439000 & .000439000 & \text { CARBON MONOXIDE } \\ .000950000 & .000950000 & \text { CARBON MONOXIDE } \\ .000950000 & .000950000 & \text { CARBON MONOXIDE } \\ .000439000 & .000439000 & \text { CARBON MONOXIDE } \\ .000439000 & .000439000 & \text { CARBON MONOXIDE } \\ .000439000 & .000439000 & \text { CARBON MONOXIDE } \\ .000439000 & .000439000 & \text { CARBON MONOXIDE } \\ .000439000 & .000439000 & \text { CARBON MONOXIDE } \\ .000439000 & .000439000 & \text { CARBON MONOXIDE } \\ .000950000 & .000950000 & \text { CARBON MONOXIDE } \\ .000950000 & .000950000 & \text { CARBON MONOXIDE }\end{array}$


Point Source Criteria Pollutants - Type: CO

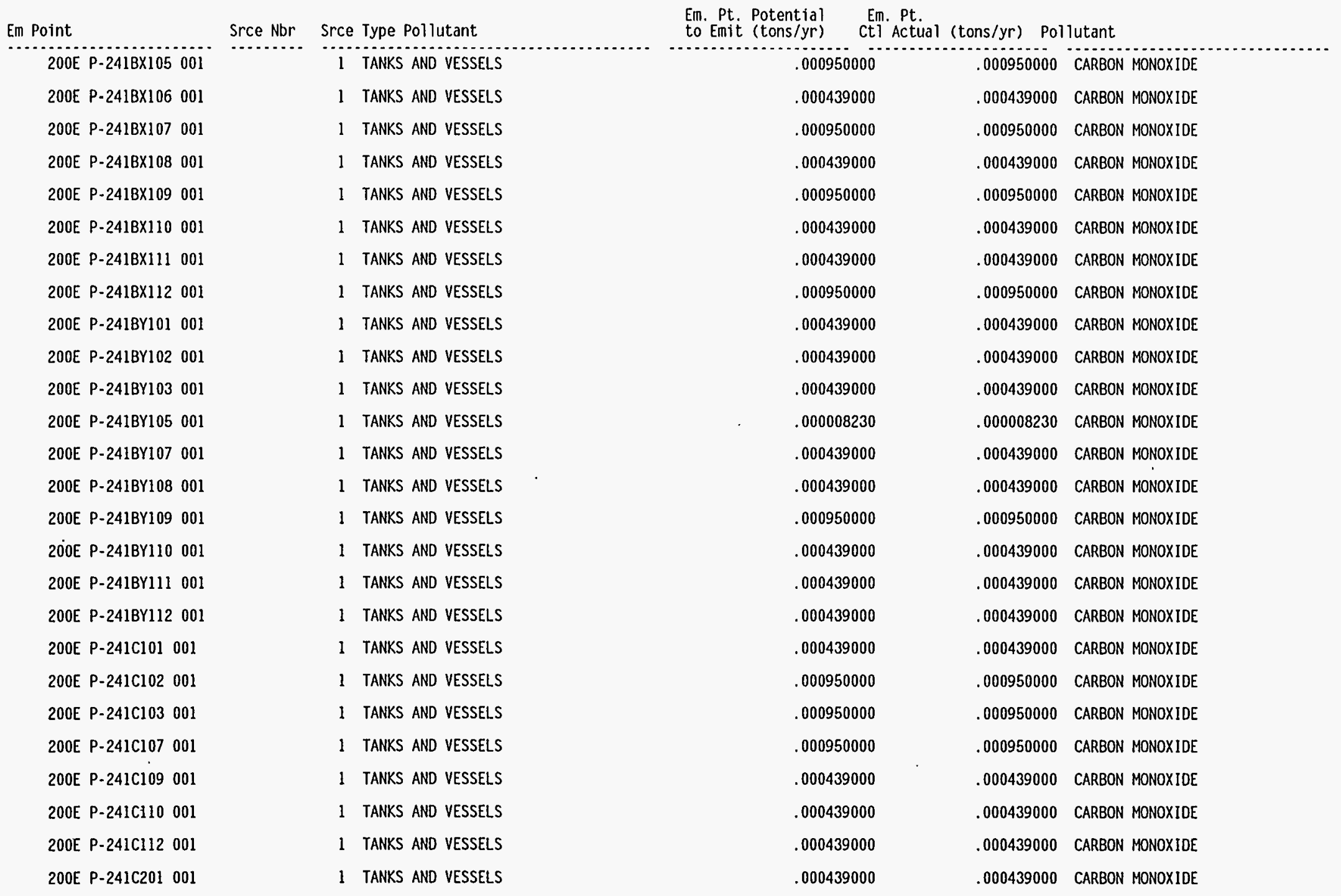


AIR EMISSIONS INVENTORY PERMIT APPLICATION REPORT (UP460)

FACILITY NAME: 200 Tank Farm and Associated structures

Point Source Criteria Pollutants - Type: co

\begin{tabular}{|c|c|c|c|c|c|c|}
\hline Em Point & Srce Nbr & Srce & Type Pollutant & $\begin{array}{l}\text { Em. Pt. Potential } \\
\text { to Emit (tons/yr) }\end{array}$ & $\begin{array}{l}\text { Em. Pt. } \\
\text { t1 Actual (tons/yr) }\end{array}$ & lutant \\
\hline 200E P-241C202 001 & & 1 & TANKS AND VESSELS & .000439000 & .000439000 & CARBON MONOXIDE \\
\hline 200E P-241C203 001 & & & TANKS AND VESSELS & .000439000 & .000439000 & CARBON MONOXIDE \\
\hline 200E P-241C204 001 & & 1 & TANKS AND VESSELS & .000439000 & .000439000 & CARBON MONOXIDE \\
\hline 200E P-296A 001 & & 1 & TANKS AND VESSELS & .017200000 & .017200000 & CARBON MONOXIDE \\
\hline 200E P-296AN 001 & & 8 & SOURCE EMISSIONS ROLL-UP & .120000000 & .120000000 & CARBON MONOXIDE \\
\hline 200E P-296AP 001 & & 9 & SOURCE EMISSIONS ROLL-UP & .138000000 & .138000000 & CARBON MONOXIDE \\
\hline 200E P-296AW 001 & & 7 & SOURCE EMISSIONS ROLL-UP & .103000000 & .103000000 & CARBON MONOXIDE \\
\hline 200E P-296AYAZ 001 & & 5 & SOURCE EMISSIONS ROLL-UP & .068800000 & .068800000 & CARBON MONOXIDE \\
\hline 200E P-296P016 001 & & 3 & SOURCE EMISSIONS ROLL-UP & .025520000 & .025520000 & CARBON MONOXIDE \\
\hline Sum & & & & .508793230 & .508748230 & \\
\hline
\end{tabular}


Point Source Criteria Pollutants - Type: NOX

\begin{tabular}{|c|c|c|c|c|c|c|}
\hline Em Point & Srce Nbr & Type Pollutant & $\begin{array}{l}\text { Em. Pt. Potential } \\
\text { to Emit (tons/yr) }\end{array}$ & (tons/yr) & lutant & \\
\hline 200E P-241A101 001 & 1 & TANKS AND VESSELS & .000026400 & .000026400 & NITROGEN OXIDE & (NO) \\
\hline 200E P-241A102001 & 1 & TANKS AND VESSELSS & .000057200 & .000057200 & NITROGEN OXIDE & $\{\mathrm{NO}\}$ \\
\hline 200E P-241A103001 & 1 & TANKS AND VESSELS & .000057200 & .000057200 & NITROGEN OXIDE & $\{\mathrm{NO}\}$ \\
\hline 200E P-241A104001 & 1 & TANKS AND VESSELS & .000026400 & .000026400 & NITROGEN OXIDE & $\{\mathrm{NO}\}$ \\
\hline $200 E \mathrm{P}-241 \mathrm{Al} 06001$ & 1 & TANKS AND VESSELS & .000057200 & .000057200 & NITROGEN OXIDE & $\{\mathrm{NO}\}$ \\
\hline 200E P-241AX101 001 & 1 & TANKS AND VESSELS & .000057200 & .000057200 & NITROGEN OXIDE & $\{\mathrm{NO}\}$ \\
\hline 200E P-241AX102001 & 1 & TANKS AND VESSELS & .000026400 & .000026400 & NITROGEN OXIDE & $\{N 0\}$ \\
\hline 200E P-241AX103001 & 1 & TANKS AND VESSELS & .000057200 & .000057200 & NITROGEN OXIDE & $\{\mathrm{NO}\}$ \\
\hline 200E P-241AX104001 & 1 & TANKS AND VESSELS & .000026400 & .000026400 & NITROGEN OXIDE & $\{\mathrm{NO}\}$ \\
\hline 200E P-241B101 001 & 1 & TANKS AND VESSELS & .000057200 & .000057200 & NITROGEN OXIDE & $\{\mathrm{NO}\}$ \\
\hline 200E P-241B102001 & 1 & TANKS AND VESSELS & .000057200 & .000057200 & NITROGEN OXIDE & $\{\mathrm{NO}\}$ \\
\hline 200E P-241B103001 & 1 & TANKS AND VESSELS & .000057200 & .000057200 & NITROGEN OXIDE & $\{\mathrm{NO}\}$ \\
\hline 200E P-241B104001 & 1 & TANKS AND VESSELS & .000026400 & .000026400 & NITROGEN OXIDE & $\{\mathrm{NO}\}$ \\
\hline 200E P-241B105001 & 1 & TANKS AND VESSELS & .000026400 & .000026400 & NITROGEN OXIDE & $\{\mathrm{NO}\}$ \\
\hline 200E P-241B106001 & 1 & TANKS AND VESSELS & .000057200 & .000057200 & NITROGEN OXIDE & $\{\mathrm{NO}\}$ \\
\hline 200E P-241B107001 & 1 & TANKS AND VESSELS & .000026400 & .000026400 & NITROGEN OXIDE & $\{\mathrm{NO}\}$ \\
\hline 200E P-241B108 001 & 1 & TANKS AND VESSELS & .000057200 & .000057200 & NITROGEN OXIDE & $\{\mathrm{NO}\}$ \\
\hline 200E P-241B109001 & 1 & TANKS AND VESSELS & .000026400 & .000026400 & NITROGEN OXIDE & $\{\mathrm{NO}\}$ \\
\hline $200 E \mathrm{P}-241 \mathrm{~B} 110001$ & 1 & TANKS AND VESSELS & .000026400 & .000026400 & NITROGEN OXIDE & $\{\mathrm{NO}\}$ \\
\hline 200E P-241B111 001 & 1 & TANKS AND VESSELS & .000057200 & .000057200 & NITROGEN OXIDE & $\{\mathrm{NO}\}$ \\
\hline 200E P-241B112001 & 1 & TANKS AND VESSELS & .000057200 & .000057200 & NITROGEN OXIDE & $\{\mathrm{NO}\}$ \\
\hline 200E P-241B201 001 & 1 & TANKS AND VESSELS & .000026400 & .000026400 & NITROGEN OXIDE & $\{\mathrm{NO}\}$ \\
\hline 200E P-241B202001 & 1 & TANKS AND VESSELS & .000026400 & .000026400 & NITROGEN OXIDE & $\{\mathrm{NO}\}$ \\
\hline 200E P-241B203 001 & 1 & TANKS AND VESSELS & .000026400 & .000026400 & NITROGEN OXIDE & $\{\mathrm{NO}\}$ \\
\hline 200E P-241B204 001 & 1 & TANKS AND VESSELS & .000026400 & .000026400 & NITROGEN OXIDE & $\{\mathrm{NO}\}$ \\
\hline 200E P-2418X101 001 & 1 & TANKS AND VESSELS & .000026400 & .000026400 & NITROGEN OXIDE & $\{\mathrm{NO}\}$ \\
\hline
\end{tabular}


Point Source Criteria Pollutants - Type: NOX

\begin{tabular}{|c|c|c|c|c|c|c|c|c|}
\hline En Point & Srce Nbr & Srce & Type Pollutant & $\begin{array}{l}\text { Em. Pt. Potential } \\
\text { to Emit (tons/yr) }\end{array}$ & $\begin{array}{l}\text { Em. Pt. } \\
\text { Ctl Actual }\end{array}$ & (tons/yr) $\mathrm{Po}_{0}$ & lutant & \\
\hline 200E P-241BX102 001 & & 1 & TANKS AND VESSELS & .00002640 & & .000026400 & NITROGEN OXIDE & \{NO\} \\
\hline 200E $P$-2418X103001 & & 1 & TANKS AND VESSELS & .00005720 & & .000057200 & NITROGEN OXIDE & $\{\mathrm{NO}\}$ \\
\hline 200E P-241BX104 001 & & 1 & TANKS AND VESSELS & .00005720 & & .000057200 & NITROGEN OXIDE & \{NO\} \\
\hline 200E P-241BX105001 & & 1 & TANKS AND VESSELS & .00005720 & & .000057200 & NITROGEN OXIDE & $\{\mathrm{NO}\}$ \\
\hline 200E P-241BX106 001 & & 1 & TANKS AND VESSELS & .00002640 & & .000026400 & NITROGEN OXIDE & (NO) \\
\hline 200E P-241BX107001 & & 1 & TANKS AND VESSELS & .00005720 & & .000057200 & NITROGEN OXIDE & \{NO\} \\
\hline 200E P-241BX108 001 & & 1 & TANKS AND VESSELS & .00002640 & & .000026400 & NITROGEN OXIDE & \{NO\} \\
\hline 200E P-241BX109001 & & 1 & TANKS AND VESSELS & $.00005720^{\circ}$ & & .000057200 & NITROGEN OXIOE & (NO) \\
\hline 200E P-241BX110 001 & & 1 & TANKS AND VESSELS & .00002640 & & .000026400 & NITROGEN OXIDE & $\{\mathrm{NO}\}$ \\
\hline 200E P-241BX111 001 & & 1 & TANKS AND VESSELS & .00002640 & & .000026400 & NITROGEN OXIDE & (NO) \\
\hline 200E P-241BX112001 & & 1 & TANKS AND VESSELS & .00005720 & & .000057200 & NITROGEN OXIDE & $\{\mathrm{NO}\}$ \\
\hline 200E P-241BY101 001 & & 1 & TANKS AND VESSELS & .00002640 & & .000026400 & NITROGEN OXIDE & $\{\mathrm{NO}\}$ \\
\hline 200E P-241BY102001 & & 1 & TANKS AND VESSELS & .00002640 & & .000026400 & NITROGEN OXIDE & $\{\mathrm{NO}\}$ \\
\hline 200E P-241BY103001 & & 1 & TANKS AND VESSELS & .00002640 & & .000026400 & NITROGEN OXIDE & $\{\mathrm{NO}\}$ \\
\hline 200E P-241BY104001 & & 1 & TANKS AND VESSELS & .00000529 & & .000005290 & NITROGEN OXIDE & $\{\mathrm{NO}\}$ \\
\hline 200E P-241BY105 001 & & 1 & TANKS AND VESSELS & .00000141 & & .000001410 & NITROGEN OXIDE & \{NO\} \\
\hline 200E P-241BY106 001 & & 1 & TANKS AND VESSELS & .00000176 & & .000001760 & NITROGEN OXIDE & $\{\mathrm{NO}\}$ \\
\hline 200E P-2418Y107001 & & 1 & TANKS AND VESSELS & .00002640 & & .000026400 & NITROGEN OXIDE & $\{\mathrm{NO}\}$ \\
\hline 200E P-241BY108.001 & & 1 & TANKS AND VESSELS & .00002640 & & .000026400 & NITROGEN OXIDE & $\{N O\}$ \\
\hline 200E P-241BY109001 & & 1 & TANKS AND VESSELS & .00005720 & & .000057200 & NITROGEN OXIDE & $\{\mathrm{NO}\}$ \\
\hline 200E P-241BY110 001 & & 1 & TANKS AND VESSELS & .00002640 & & .000026400 & NITROGEN OXIDE & $\{\mathrm{NO}\}$ \\
\hline 200E P-2418Y111 001 & & 1 & TANKS AND VESSELS & .00002640 & & .000026400 & NITROGEN OXIDE & $\{\mathrm{NO}\}$ \\
\hline 200E P-241BY112001 & & 1 & TANKS AND VESSELS & .00002640 & & .000026400 & NITROGEN OXIDE & $\{\mathrm{NO}\}$ \\
\hline 200E P-241C101 001 & & 1 & TANKS AND VESSELS & .00002640 & & .000026400 & NITROGEN OXIDE & $\{\mathrm{NO}\}$ \\
\hline 200E P-241C102 001 & & 1 & TANKS AND VESSELS & .00005720 & & .000057200 & NITROGEN OXIDE & $\{\mathrm{NO}\}$ \\
\hline 200E P-241C103 001 & & 1 & TANKS AND VESSELS & .00005720 & & .000057200 & NITROGEN OXIDE & $\{\mathrm{NO}\}$ \\
\hline
\end{tabular}


Point Source Criteria Pollutants - Type: NOX

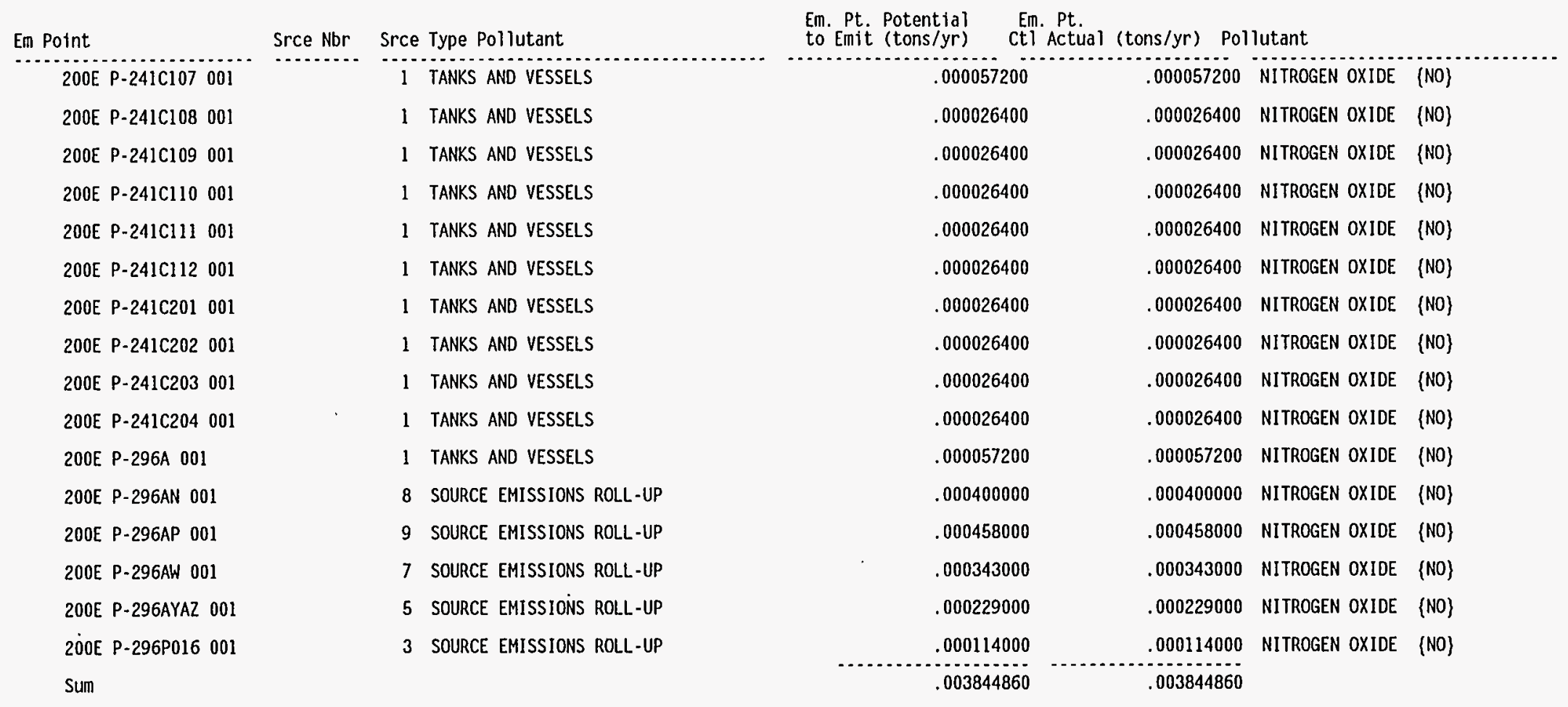


AIR EMISSIONS INVENIORY PERMII APPLICAIION REPORT (UP460)

FACILITY NAME: $200 \mathrm{E}$ Tank farm and Associated Structures

Point Source Criteria Pollutants - Type: voC

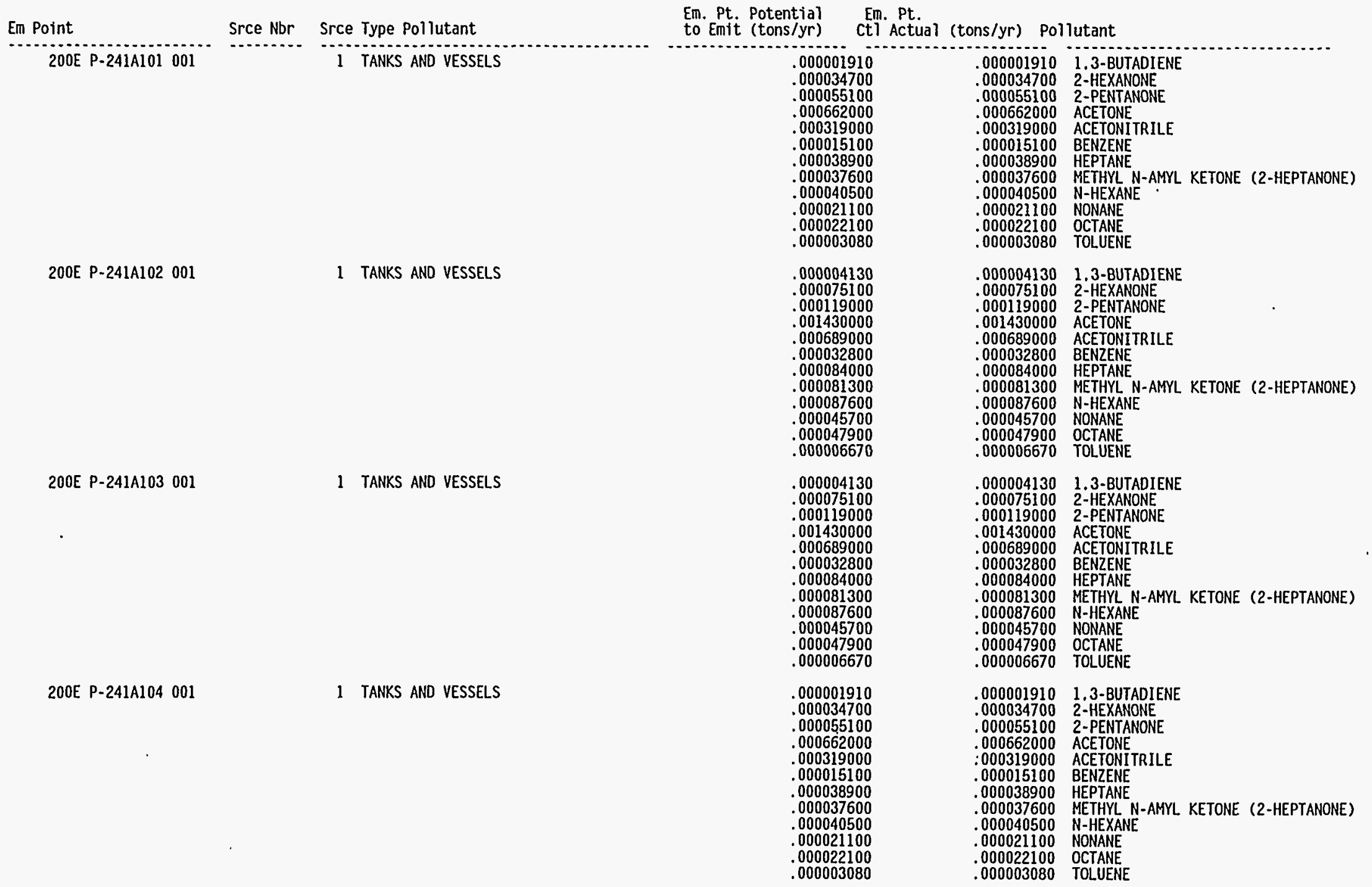


Point Source Criteria Pollutants - Type: VoC

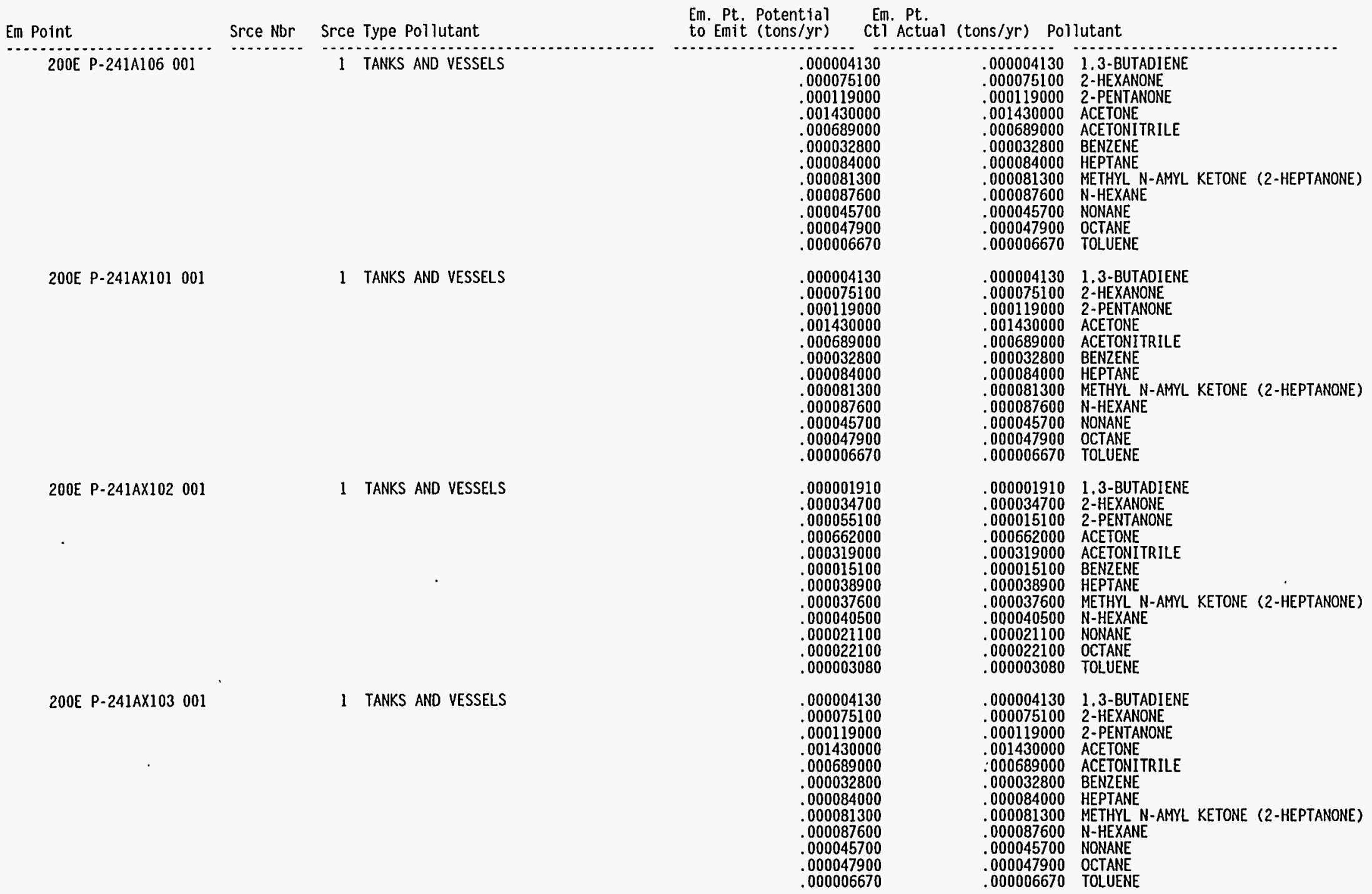


AIR EMISSIONS INVENTORY PERMIT APPLICATION REPORT (UP460)

FACILITY NAME: 200E Tank Farm and Associated Structures

Point Source Criteria Pollutants - Type: VOC

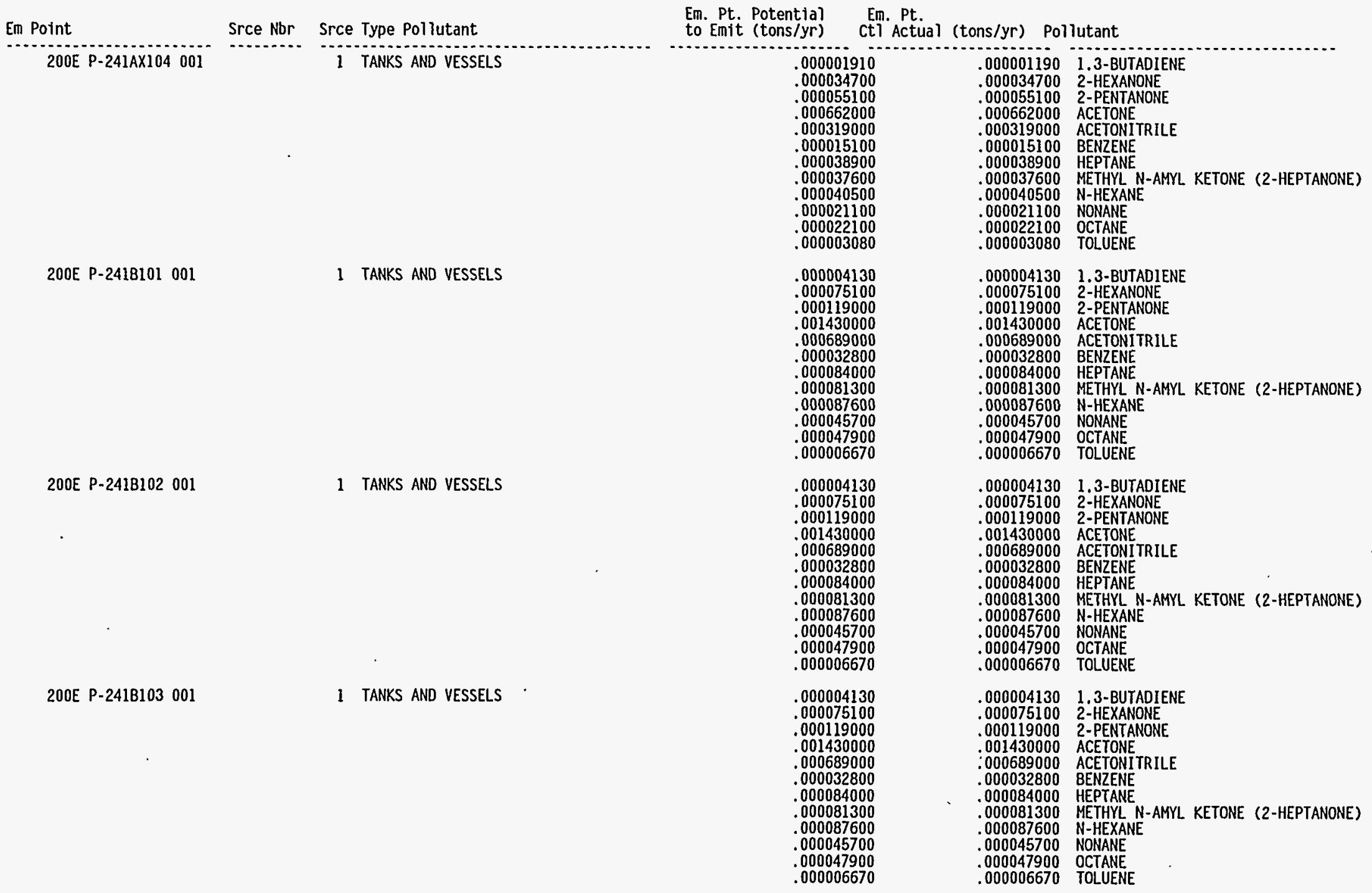

Page \#: 55 of 95 Report Run Date: 22-APR-95 
AIR EMISSIONS INVENTORY PERMIT APPLICATION REPORT (UP460)

FACILITY NAME: 200 FACILITY Tank Farm and ASsociated structures

Point Source Criteria Pollutants - Type: Voc

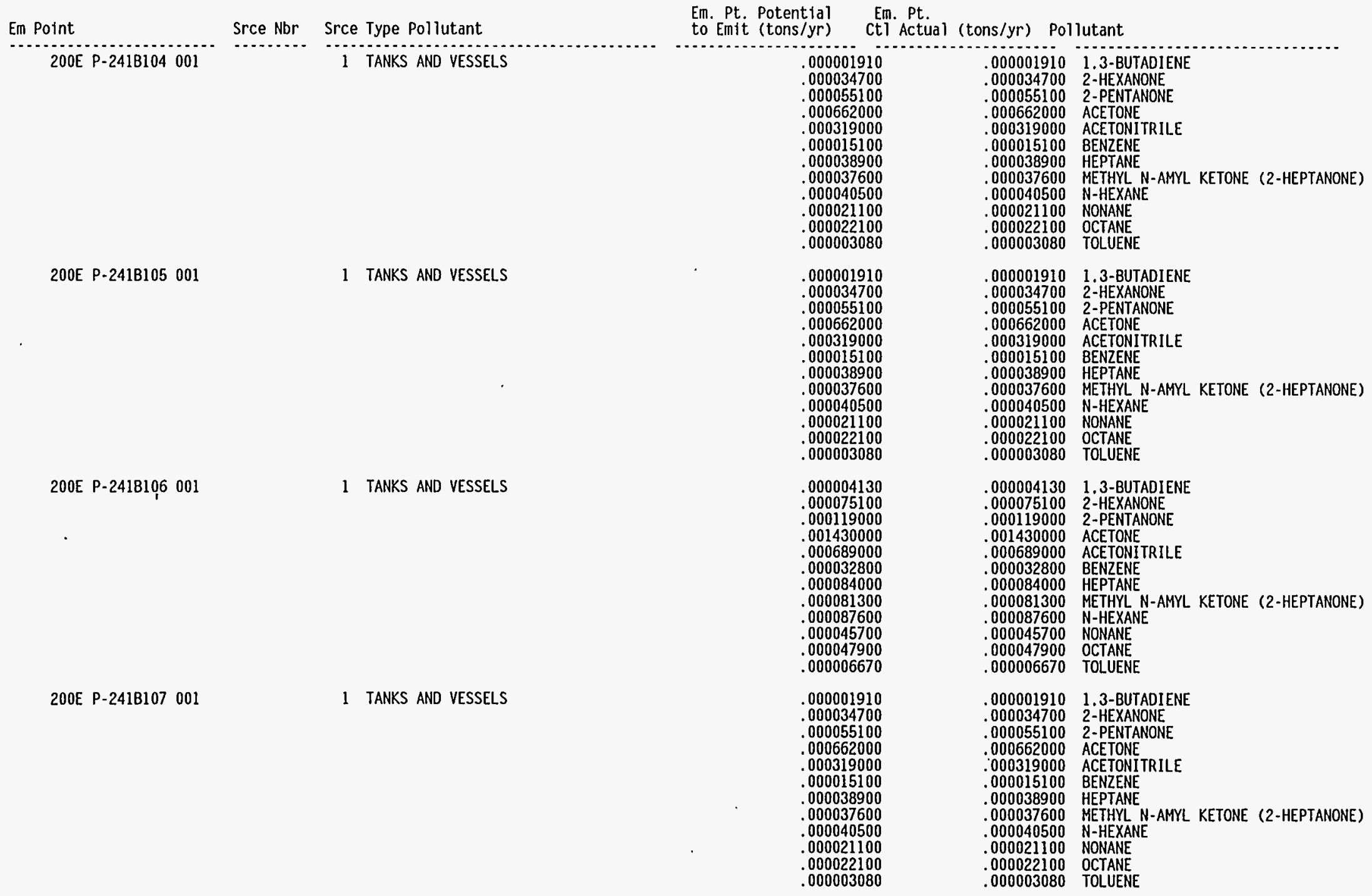

Page \#: 56 of 95 Report Run Date: 22-APR-95 
AIR EMISSIONS INVENTORY PERMIT APPLICATION REPORT (UP460)

FACILITY NAME: $200 E$ Tank Farm and Associated Structures

Point Source Criteria Pollutants - Type: VOC

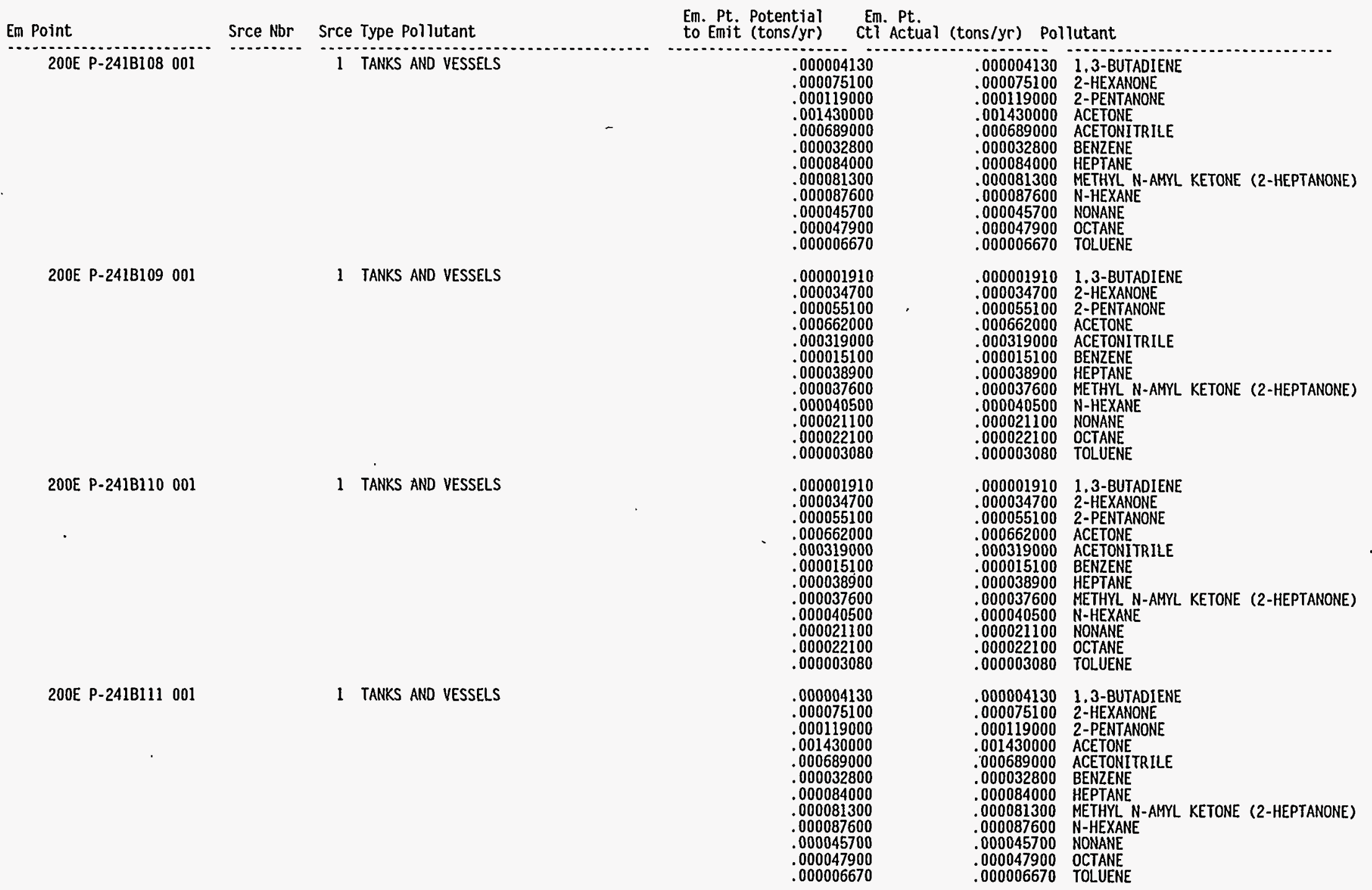

Page \#: 57 of 95 Report Run Date: 22-APR-95 
Point Source Criteria Pollutants - Type: VOC

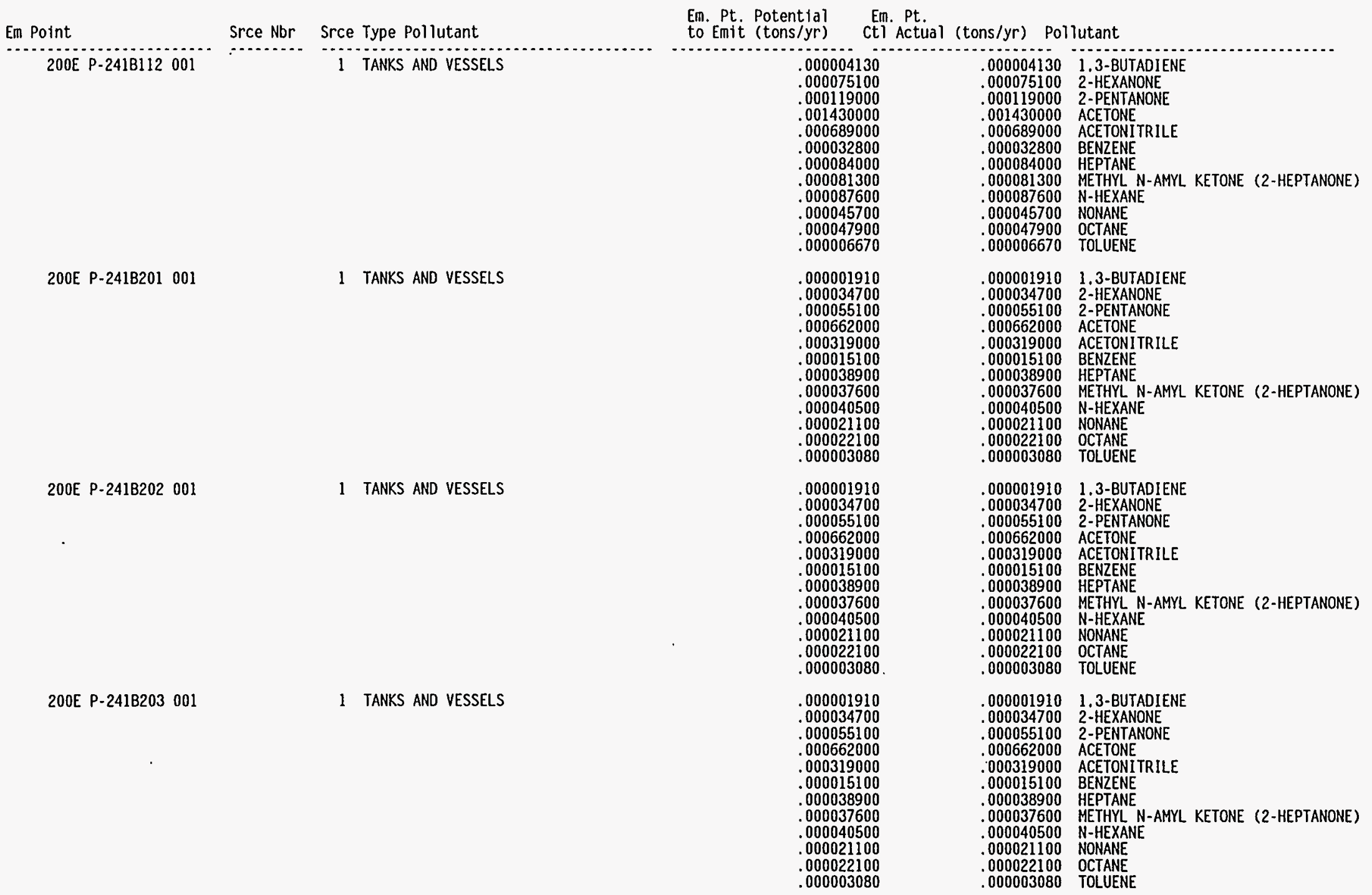


AIR EMISSIONS INVENTORY PERMIT APPLICATION REPORT (UP460)

FACILITY NAME: 200E Tank Farm and Associated Structures

Point Source Criteria Pollutants - Type: VOC

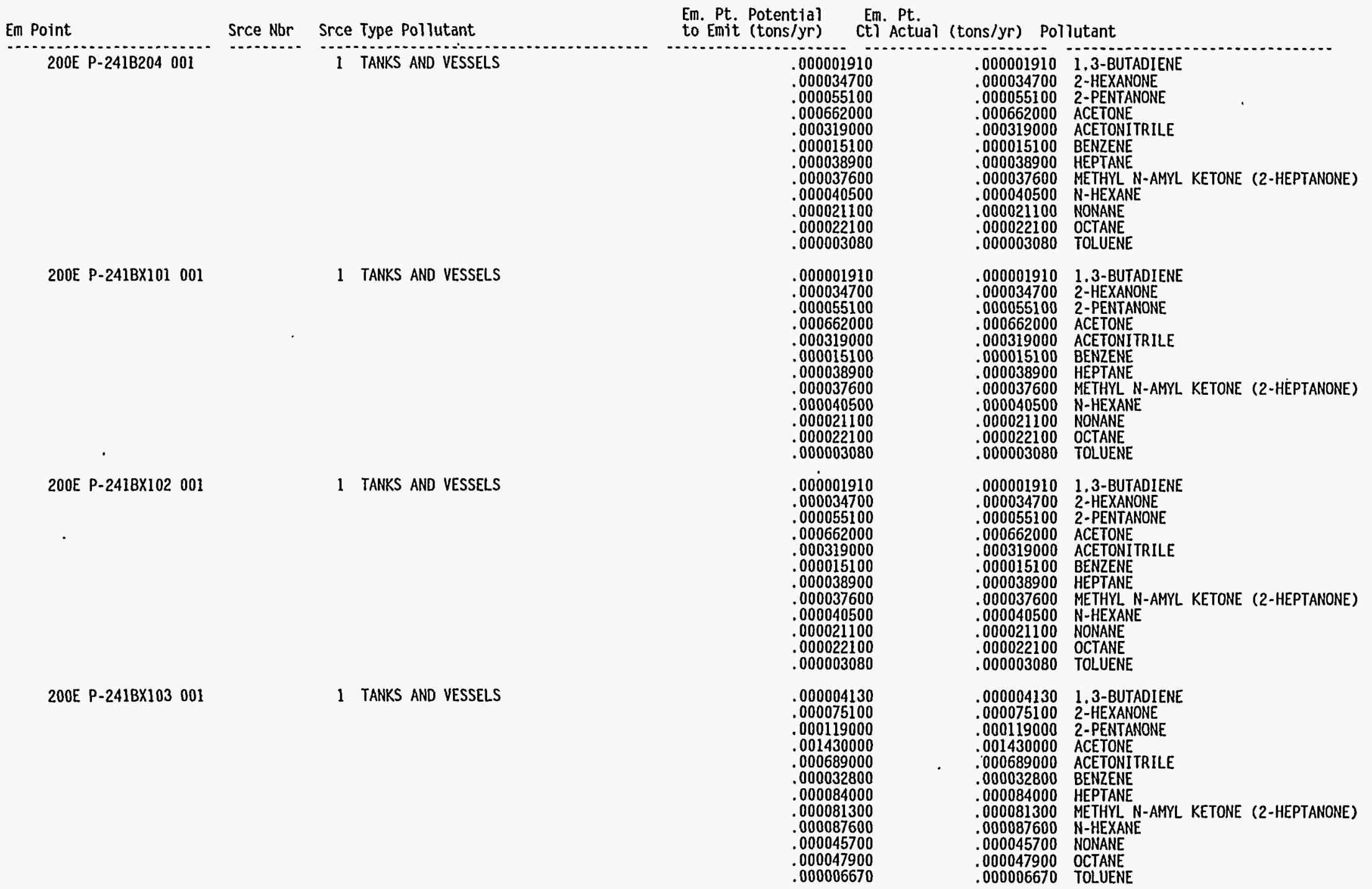

Page \#: 59 of 95 Report Run Date: 22-APR-95 
AIR EMISSIONS INVENIOKY PLKMII AP'LLICAIION REPORI (UP460)

FACILITY NAME: $200 E$ Tank Farm and ASsOCiated Structures

Point Source Criteria Pollutants - Type: VoC

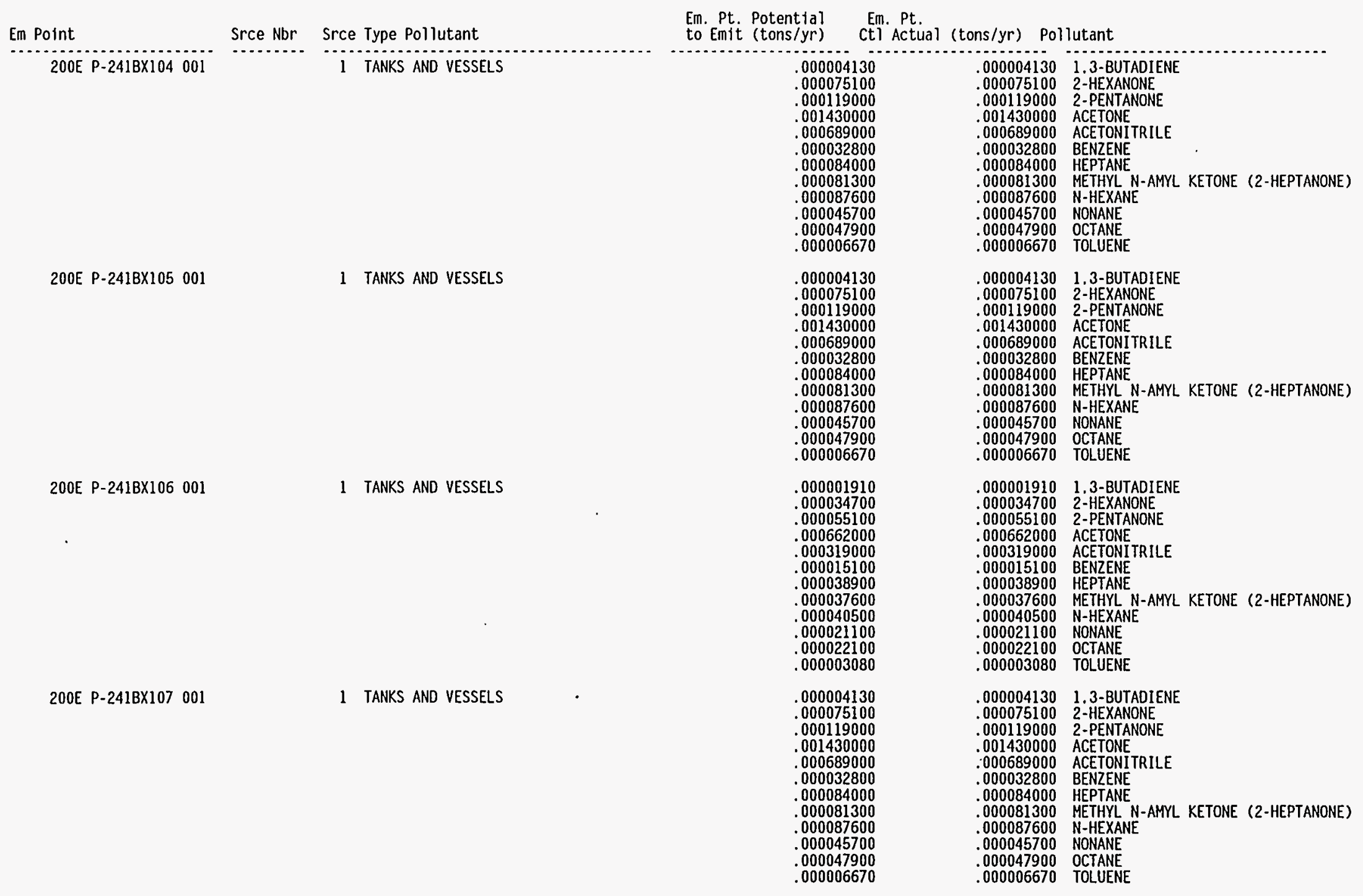

Page \#: 60 of 95 Report Run Date: 22 -APR-95 
AIR EMISSIONS INVENTORY PERMIT APPLICATION REPORT (UP460)

FACILITY NAME: $200 E$ Tank Farm and Associated Structures

Point Source Criteria Pollutants - Type: VOC

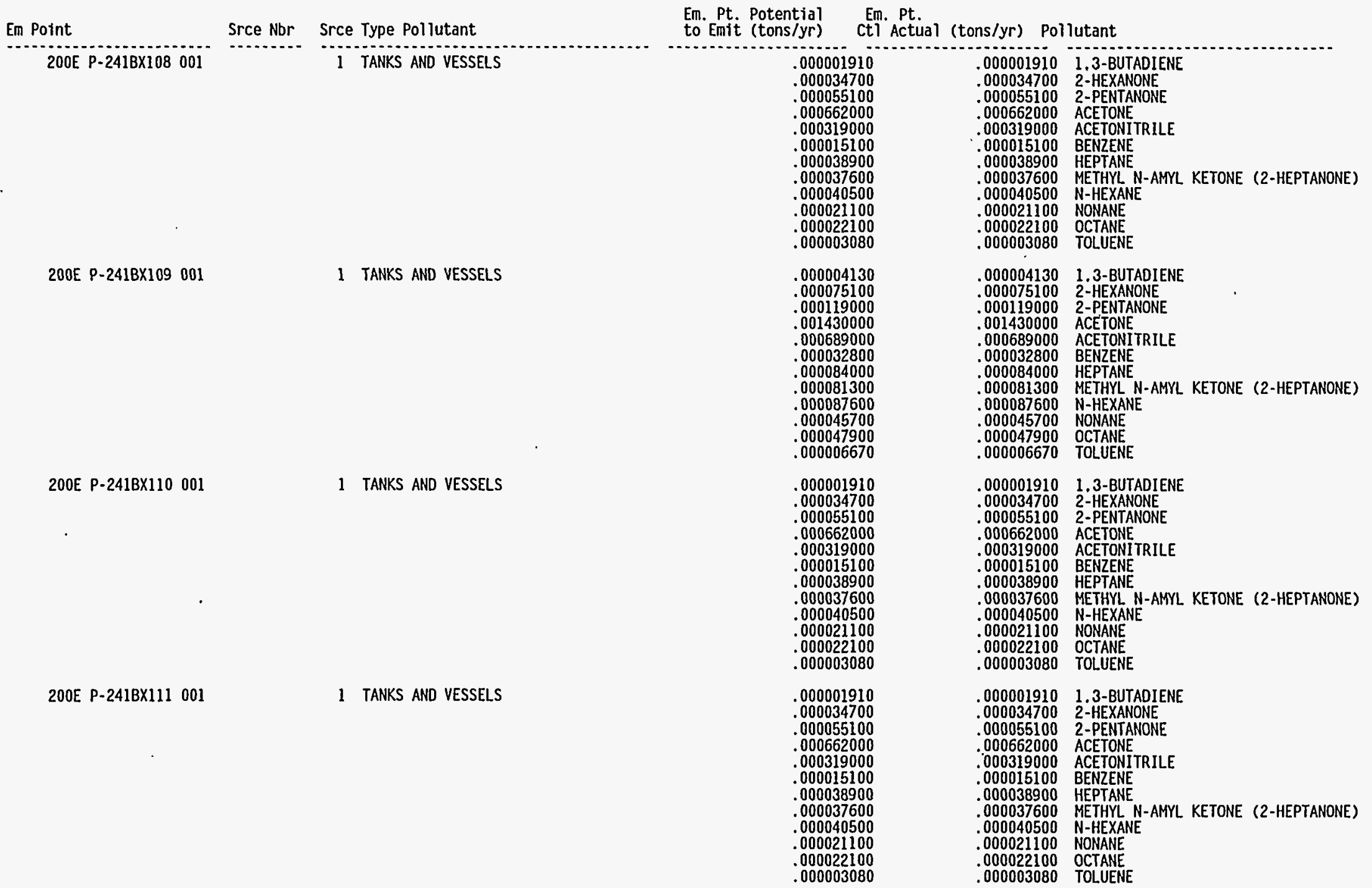

Page \#: 61 of 95 Report Run Date: 22-APR-95 
Point Source Criteria Pollutants - Type: VOC

\begin{tabular}{|c|c|c|c|c|c|c|c|}
\hline Em Point & Srce Nbr & Srce & Type Pollutant & & $\begin{array}{l}\text { Em. Pt. Potential } \\
\text { to Emit (tons/yr) }\end{array}$ & $\begin{array}{l}\text { Em. Pt. } \\
\text { Ctl Actual (tons/yr) }\end{array}$ & lutant \\
\hline $200 E$ P-241BX112001 & & 1 & TANKS AND VESSELS & & $\begin{array}{l}.000004130 \\
.000075100 \\
.000119000 \\
.001430000 \\
.000689000 \\
.000032800 \\
.000084000 \\
.000081300 \\
.000087600 \\
.000045700 \\
.000047900 \\
.000006670\end{array}$ & $\begin{array}{l}.000004130 \\
.000075100 \\
.000119000 \\
.001430000 \\
.000689000 \\
.000032800 \\
.000084000 \\
.000081300 \\
.000087600 \\
.000045700 \\
.000047900 \\
.000006670\end{array}$ & $\begin{array}{l}\text { 1.3-BUTADIENE } \\
\text { 2-HEXANONE } \\
\text { 2-PENTANONE } \\
\text { ACETONE } \\
\text { ACETONITRILE } \\
\text { BENZENE } \\
\text { HEPTANE } \\
\text { METHYL N-AMYL KETONE (2-HEPTANONE) } \\
\text { N-HEXANE } \\
\text { NONANE } \\
\text { OCTANE } \\
\text { TOLUENE }\end{array}$ \\
\hline 200E P-241BY101 001 & & 1 & TANKS AND VESSELLS & & $\begin{array}{l}.000001910 \\
.000034700 \\
.000055100 \\
.000662000 \\
.000319000 \\
.000015100 \\
.000038900 \\
.000037600 \\
.000040500 \\
.000021100 \\
.000022100 \\
.000003080\end{array}$ & $\begin{array}{l}.000001910 \\
.000034700 \\
.000055100 \\
.000662000 \\
.000319000 \\
.000015100 \\
.000038900 \\
.000037600 \\
.000040500 \\
.000021100 \\
.000022100 \\
.000003080\end{array}$ & $\begin{array}{l}\text { 1.3-BUTADIENE } \\
\text { 2-HEXANONE } \\
\text { 2-PENTANONE } \\
\text { ACETONE } \\
\text { ACETONITRILE } \\
\text { BENZENE } \\
\text { HEPTANE } \\
\text { METHY N-AMYL KETONE (2-HEPTANONE) } \\
\text { N-HEXANE } \\
\text { NONANE } \\
\text { OCTANE } \\
\text { TOLUENE }\end{array}$ \\
\hline 200E P-241BY102 001 & & 1 & TANKS AND VESSELS & & $\begin{array}{l}.000001910 \\
.000034700 \\
.000055100 \\
.000662000 \\
.000319000 \\
.000015100 \\
.000038900 \\
.000037600 \\
.000040500 \\
.000021100 \\
.000022100 \\
.000003080\end{array}$ & $\begin{array}{l}.000001910 \\
.000034700 \\
.000055100 \\
.000662000 \\
.000319000 \\
.000015100 \\
.000038900 \\
.000037600 \\
.000040500 \\
.000021100 \\
.000022100 \\
.000003080\end{array}$ & $\begin{array}{l}\text { 1.3-BUTADIENE } \\
\text { 2-HEXANONE } \\
\text { 2-PENTANONE } \\
\text { ACETONE } \\
\text { ACETONITRILE } \\
\text { BENZENE } \\
\text { HEPTANE } \\
\text { METHYL N-AMYL KETONE (2-HEPTANONE) } \\
\text { N-HEXANE } \\
\text { NONANE } \\
\text { OCTANE } \\
\text { TOLUENE }\end{array}$ \\
\hline 200E P-2418Y103 001 & & 1 & TANKS AND VESSELS & . & $\begin{array}{l}.000001910 \\
.000034700 \\
.000055100 \\
.000662000 \\
.000319000 \\
.000015100 \\
.000038900 \\
.000037600 \\
.000040500 \\
.000021100 \\
.000022100 \\
.000003080\end{array}$ & $\begin{array}{l}.000001910 \\
.000034700 \\
.000055100 \\
.000662000 \\
.000319000 \\
.000015100 \\
.000038900 \\
.000037600 \\
.000040500 \\
.000021100 \\
.000022100 \\
.000003080\end{array}$ & $\begin{array}{l}\text { 1.3-BUTADIENE } \\
\text { 2-HEXANONE } \\
\text { 2-PENTANONE } \\
\text { ACETONE } \\
\text { ACETONITRILE } \\
\text { BENZENE } \\
\text { HEPTANE } \\
\text { METHYL N-AMYL KETONE (2-HEPTANONE) } \\
\text { N-HEXANE } \\
\text { NONANE } \\
\text { OCTANE } \\
\text { TOLUENE }\end{array}$ \\
\hline
\end{tabular}


AIR EMISSIONS INVENTORY PERMIT APPLICATION REPORT (UP460)

FACILITY NAME: 200E Tank Farm and Associated Structures

Point Source Criteria Pollutants - Type: VOC

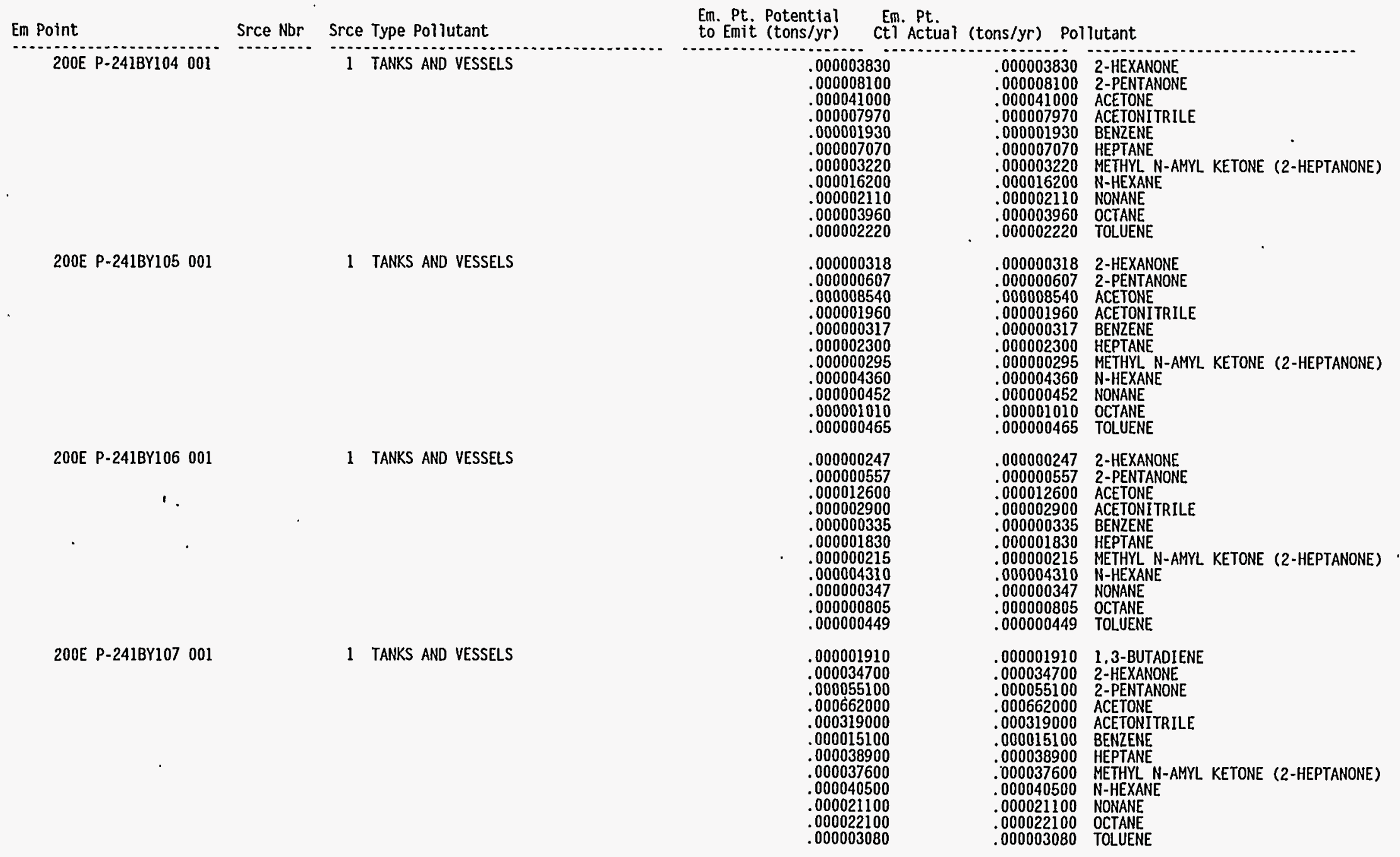


Point Source Criteria Pollutants - Type: VOC

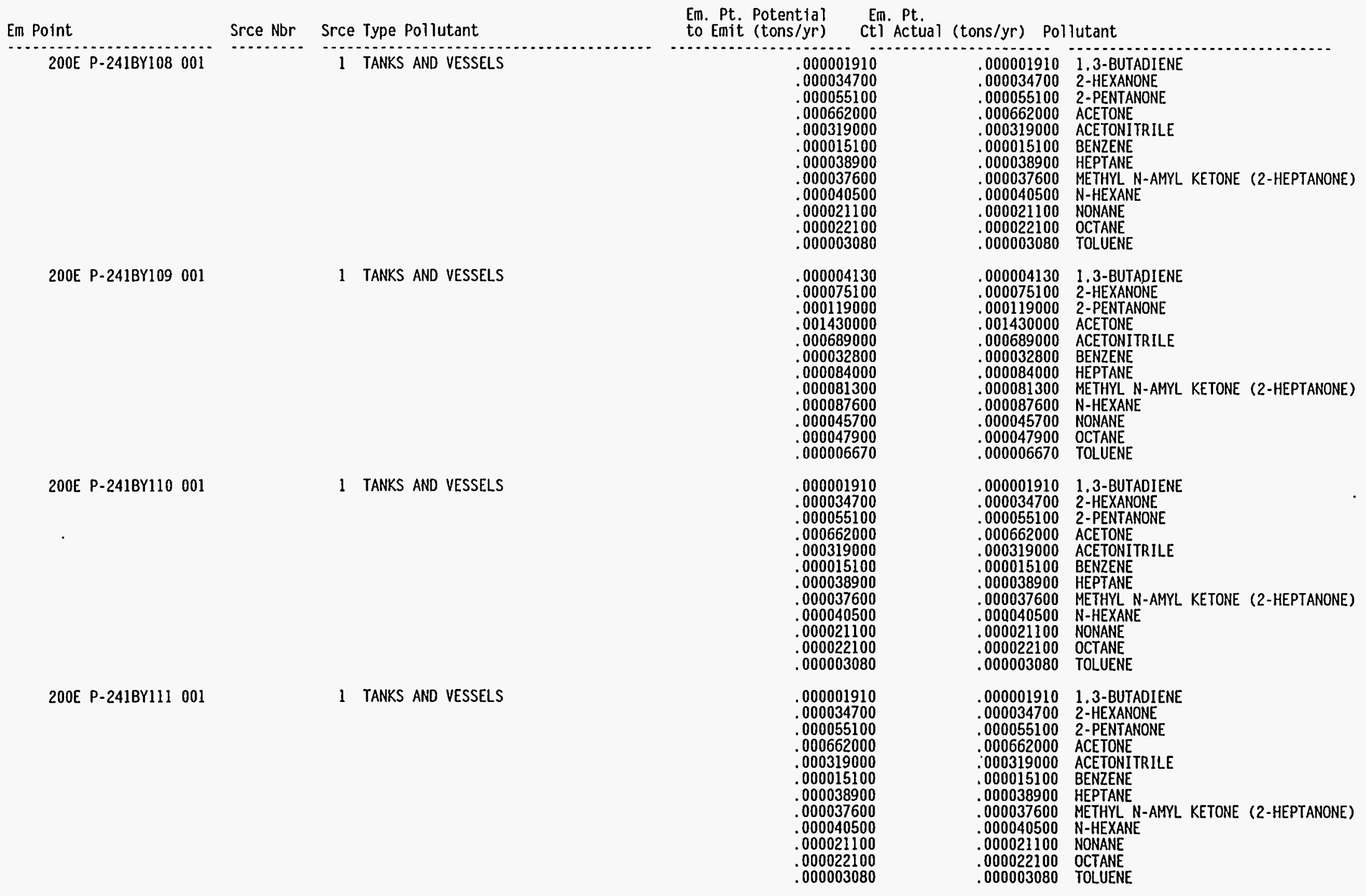


AIR EMISSIONS INVENTORY PERMIT APPLICATION REPORT (UP460)

FACILITY NAME: 200E Tank Farm and Associated Structures

Point Source Criteria Pollutants - Type: VOC

\begin{tabular}{|c|c|c|c|c|c|}
\hline \multirow{2}{*}{$\begin{array}{l}\text { Em Point } \\
\text { 200E P-241BY112 } 001\end{array}$} & \multirow{2}{*}{ Srce Nbr } & \multirow{2}{*}{$\begin{array}{l}\text { Srce Type Pollutant } \\
1 \text { TANKS AND VESSELS }\end{array}$} & \multicolumn{3}{|c|}{$\begin{array}{l}\text { Em. Pt. } \\
\text { ctl Actual (tons/yr) Pollutant. }\end{array}$} \\
\hline & & & $\begin{array}{l}.000001910 \\
.000034700 \\
.000055100 \\
.000662000 \\
.000319000 \\
.000015100 \\
.000038900 \\
.000037600 \\
.000040500 \\
.000021100 \\
.000022100 \\
.000003080\end{array}$ & $\begin{array}{l}.000001910 \\
.000034700 \\
.000055100 \\
.000662000 \\
.000319000 \\
.000015100 \\
.000038900 \\
.000037600 \\
.000040500 \\
.00002100 \\
.00022100 \\
.000003080\end{array}$ & $\begin{array}{l}\text { 1.3-BUTADIENE } \\
\text { 2-HEXANONE } \\
\text { 2-PENTANONE } \\
\text { ACEONE } \\
\text { ACETONITRILE } \\
\text { BENZENE } \\
\text { HEPTANE } \\
\text { METHY N-AMYL KETONE (2-HEPTANONE) } \\
\text { N-HEXANE } \\
\text { NONANE } \\
\text { OCTANE } \\
\text { TOLUENE }\end{array}$ \\
\hline 200E P-241C101 001 & & 1 TANKS AND VESSELS & $\begin{array}{l}.000001910 \\
.000034700 \\
.000055100 \\
.000662000 \\
.000319000 \\
.000015100 \\
.000038900 \\
.000037600 \\
.000040500 \\
.000021100 \\
.000022100 \\
.000003080\end{array}$ & $\begin{array}{l}.000001910 \\
.000034700 \\
.000055100 \\
.000662000 \\
.000319000 \\
.000015100 \\
.000038900 \\
.000037600 \\
.000040500 \\
.000021100 \\
.00002100 \\
.000003080\end{array}$ & $\begin{array}{l}\text { 1.3-BUTADIENE } \\
\text { 2-HEXANONE } \\
\text { 2-PENTANONE } \\
\text { ACETONE } \\
\text { ACETONITRILE } \\
\text { BENZENE } \\
\text { HEPTANE } \\
\text { METHY N-AMYL KETONE (2-HEPTANONE) } \\
\text { N-HEXANE } \\
\text { NONANE } \\
\text { OCTANE } \\
\text { TOLUENE }\end{array}$ \\
\hline $200 E$ P-241C102 001 & & I TANKS AND VESSELS & $\begin{array}{l}.000004130 \\
.000075100 \\
.00019900 \\
.001430000 \\
.000689000 \\
.000032800 \\
.000084000 \\
.000081300 \\
.000087600 \\
.000045700 \\
.000047900 \\
.000006670\end{array}$ & $\begin{array}{l}.000004130 \\
.000075100 \\
.000119000 \\
.001430000 \\
.000689000 \\
.000032800 \\
.000084000 \\
.000081300 \\
.000087600 \\
.000045700 \\
.000047900 \\
.000006670\end{array}$ & $\begin{array}{l}\text { 1.3-BUTADIENE } \\
\text { 2-HEXANONE } \\
\text { 2-PENTANONE } \\
\text { ACETONE } \\
\text { ACETONITRILE } \\
\text { BENZENE } \\
\text { HEPTANE } \\
\text { METHYL N-AMYL KETONE (2-HEPTANONE) } \\
\text { N-HEXANE } \\
\text { NONANE } \\
\text { OCTANE } \\
\text { TOLUENE }\end{array}$ \\
\hline 200E P-241C103 001 & & 1 TANKS AND VESSELS & $\begin{array}{l}.000004130 \\
.000075100 \\
.000119000 \\
.001430000 \\
.000689000 \\
.000032800 \\
.000084000 \\
.000081300 \\
.000087600 \\
.000045700 \\
.00004790 \\
.000006670\end{array}$ & $\begin{array}{l}.000004130 \\
.000075100 \\
.000119000 \\
.001430000 \\
.000689000 \\
.000032800 \\
.000084000 \\
.000081300 \\
.000087600 \\
.000045700 \\
.000047900 \\
.000006670\end{array}$ & $\begin{array}{l}1.3 \text {-BUTADIENE } \\
\text { 2-HEXANONE } \\
\text { 2-PENTANONE } \\
\text { ACEONE } \\
\text { ACEOONITRILE } \\
\text { BENZENE } \\
\text { HEPTANE } \\
\text { METHY N-AMYL KETONE (2-HEPTANONE) } \\
\text { N-HEXANE } \\
\text { NONANE } \\
\text { OCTANE } \\
\text { TOLUENE }\end{array}$ \\
\hline
\end{tabular}

Page \#: 65 of 95 Report Run Date: 22-APR-95 
AIR EMISSIONS INVENTORY PERMIT APPLICATION REPORT (UP460)

FACILITY NAME: $200 \mathrm{E}$ Tank Farm and ASsociated Structures

Point Source Criteria Pollutants - Type: VoC

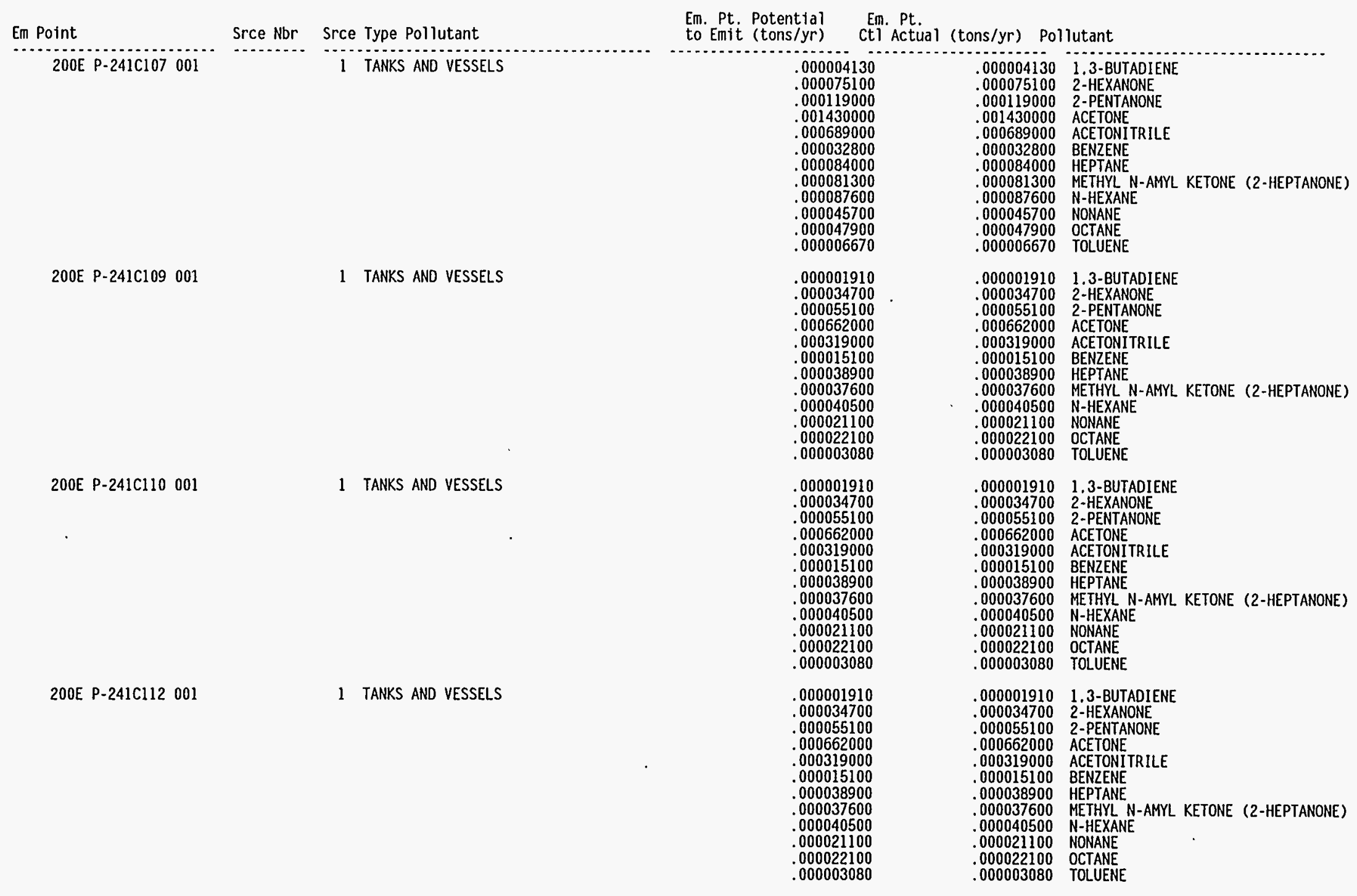

Page \#: 66 of 95 Report Run Date: 22-APR-95 
AIR EMISSIONS INVENTORY PERMIT APPLICAIION REPORT (UP460)

FACILITY 1D : 200E TANK

FACILITY NAME: 200E Tank Farm and Associated Structures

Point Source Criteria Pollutants - Type: VOC

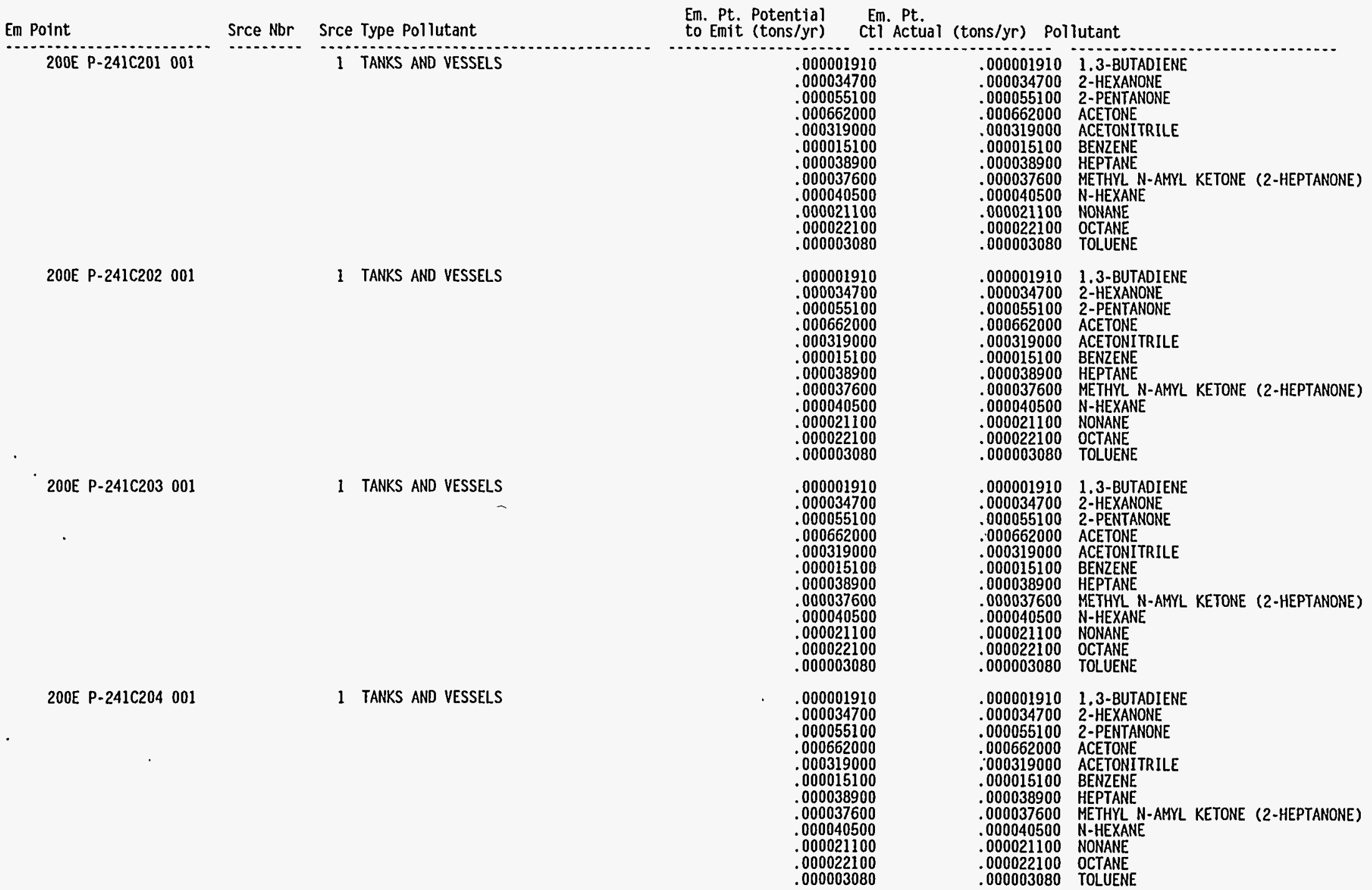

Page \#: 67 of 95 Report Run Date: 22-APR-95 
Point Source Criteria Pollutants - Type: VoC

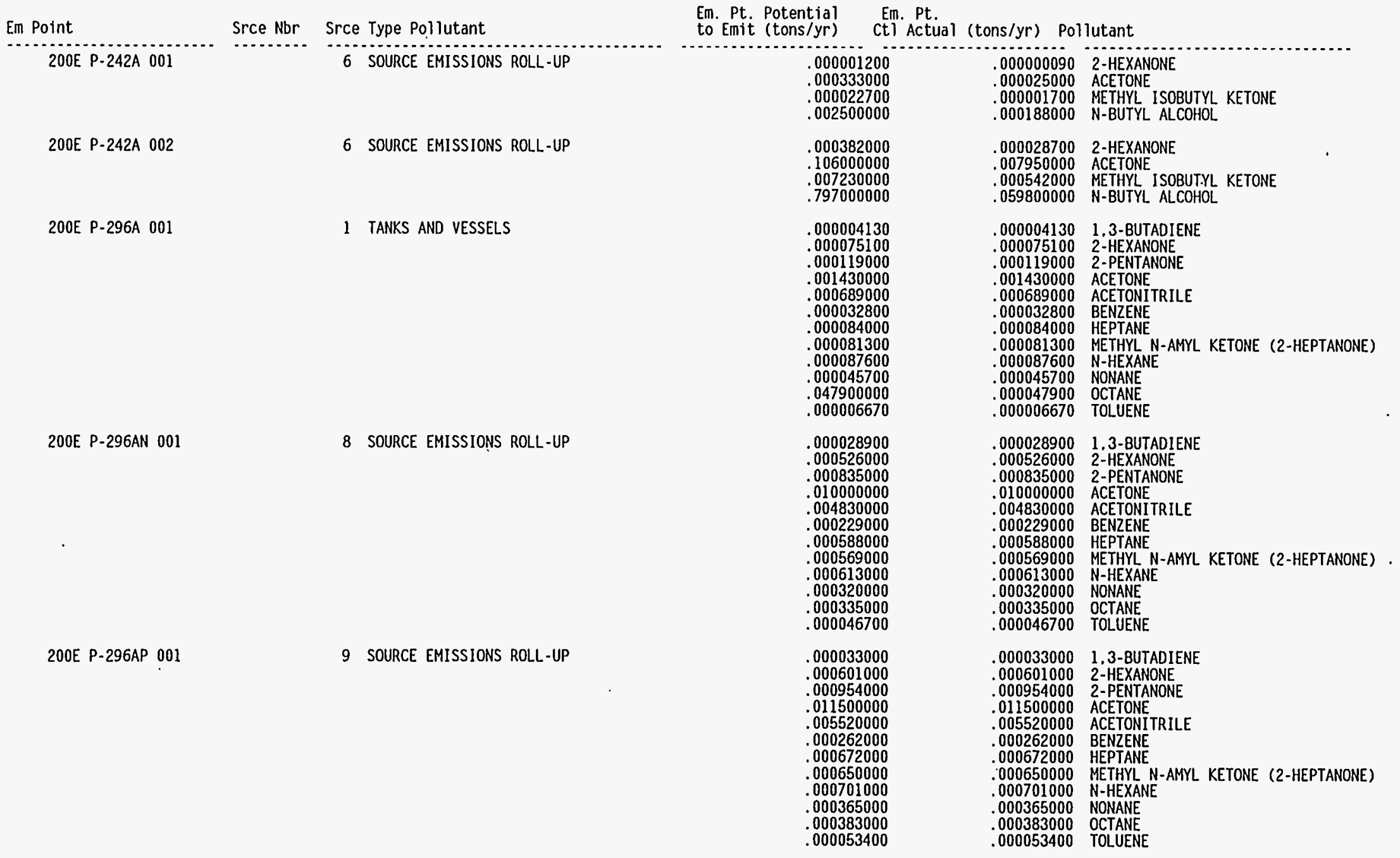


Point Source Criteria Pollutants - Type: VoC

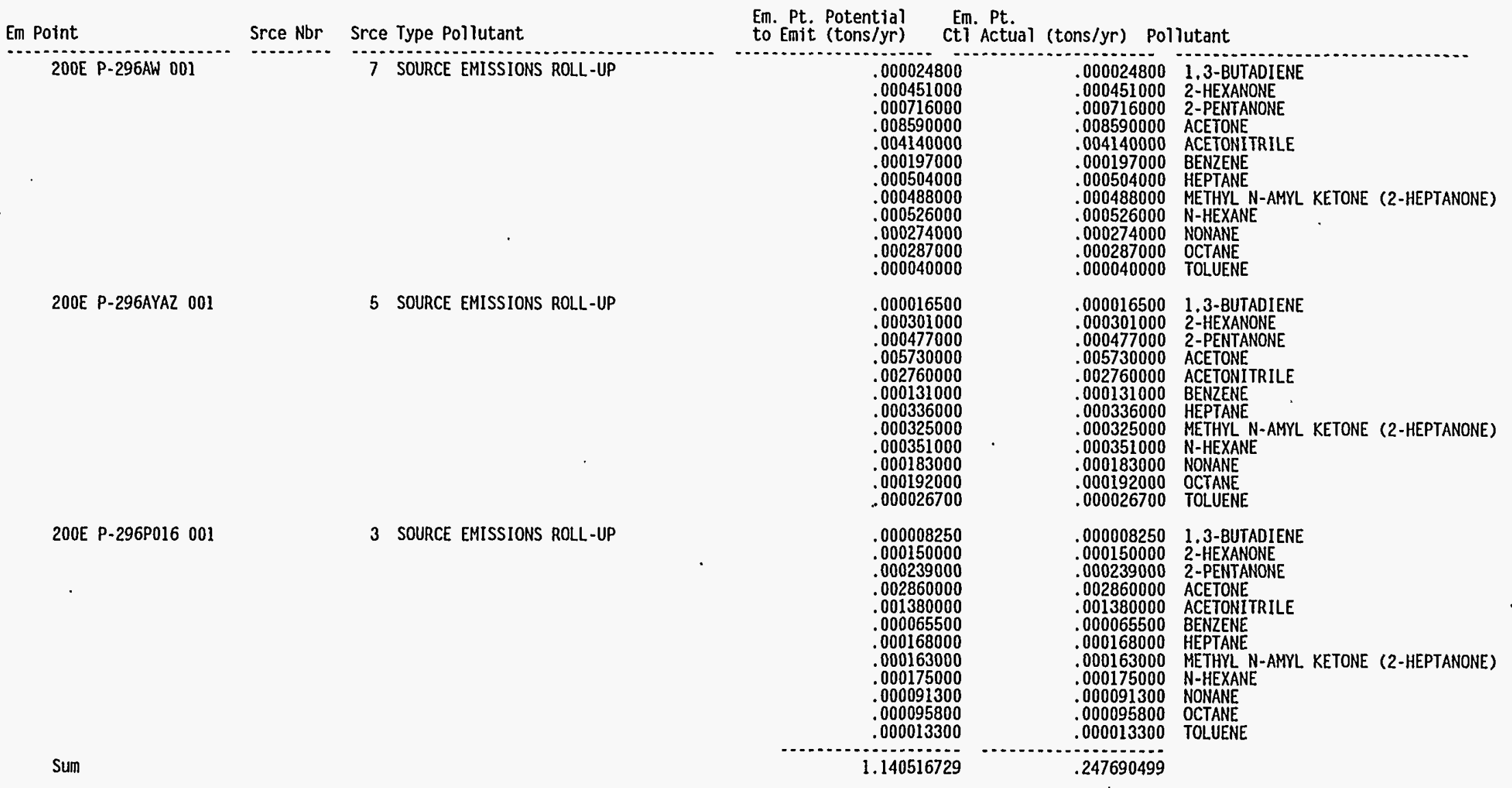

Point Source Emissions - Radiological Pollutants

\begin{tabular}{|c|c|c|c|c|c|}
\hline Em Point & Srce Nbr & Srce Type Pollutant & $\begin{array}{l}\text { Em. Pt. Potential } \\
\text { to Emit }(\mathrm{Ci} / \mathrm{yr})\end{array}$ & $\begin{array}{l}\text { Em. Pt. } \\
\text { Cti Actual (Ci/yr) }\end{array}$ & Pollutant \\
\hline 200E P-204AR 001 & 1 & PROCESS AND PRODUCTION & $\begin{array}{l}\text { Refer to } \\
\text { WHC-SO-EN-RPT-007 } \\
\text { Rev. 0 Page A-82 }\end{array}$ & $\begin{array}{l}\text { Refer to } \\
\text { D0E/RL-94-51 } \\
\text { Table 2-1 }\end{array}$ & \\
\hline
\end{tabular}

Page \#: 69 of 95 Report Run Date: 22-APR-95 
AIR EMISSIONS INVENTORY PERMIT APPLICATION REPORT (UP460)

FACILITY ID : 200 TANK
FACILITY NAME: 200E Tank Farm and Assoclated Structures

Point Source Emissions - Radiological Pollutants

\begin{tabular}{|c|c|c|c|c|c|}
\hline Em Point & Srce Nbr & Type Pollutant & $\begin{array}{l}\text { Em. Pt. Potential } \\
\text { to Emit }(\mathrm{Ci} / \mathrm{yr})\end{array}$ & $(\mathrm{Ci} / \mathrm{yr})$ & Pollutant \\
\hline 200E P-241A101 001 & 1 & TANKS AND VESSELS & $\begin{array}{l}.000000036 \\
.000008700 \\
.000000018 \\
.000230000\end{array}$ & $\begin{array}{l}.000000000 \\
.000000004 \\
.000000000 \\
.000000120\end{array}$ & $\begin{array}{l}\text { AMERICIUM - } 241 \\
\text { CESIUM - } 137 \\
\text { PLUTONIUM - } 239 \\
\text { STRONTIUM - } 090\end{array}$ \\
\hline $200 E$ P-241A102001 & 1 & TANKS AND VESSELS & $\begin{array}{l}.000000078 \\
.000019000 \\
.000000039 \\
.000500000\end{array}$ & $\begin{array}{l}.000000000 \\
.000000009 \\
.000000000 \\
.000000250\end{array}$ & $\begin{array}{l}\text { AMERICIUM - } 241 \\
\text { CESIUM }-137 \\
\text { PLUTONIUM - } 239 \\
\text { STRONTIUM }-090\end{array}$ \\
\hline 200E P-241A103001 & 1 & TANKS AND VESSELS & $\begin{array}{l}.000000078 \\
.000019000 \\
.000000039 \\
.000500000\end{array}$ & $\begin{array}{l}.000000000 \\
.000000009 \\
.000000000 \\
.000000250\end{array}$ & $\begin{array}{l}\text { AMERICIUM }-241 \\
\text { CESIUM }-137 \\
\text { PLUTONIUM }-239 \\
\text { STRONTIUM }-090\end{array}$ \\
\hline 200E P-241A104 001 & 1 & TANKS AND VESSELS & $\begin{array}{l}.000000036 \\
.000008700 \\
.000000018 \\
.000230000\end{array}$ & $\begin{array}{l}.000000000 \\
.000000004 \\
.000000000 \\
.000000120\end{array}$ & $\begin{array}{l}\text { AMERICIUM - } 241 \\
\text { CESIUM - } 137 \\
\text { PLUTONIUM - } 239 \\
\text { STRONTIUM - } 090\end{array}$ \\
\hline 200E P-241A106 001 & 1 & TANKS AND VESSELS & $\begin{array}{l}.000000078 \\
.000019000 \\
.000000039 \\
.000500000\end{array}$ & $\begin{array}{l}.000000000 \\
.000000006 \\
.000000000 \\
.000000170\end{array}$ & $\begin{array}{l}\text { AMERICIUM }-241 \\
\text { CESIUM - } 137 \\
\text { PLUTONIUM - } 239 \\
\text { STRONTIUM - } 090\end{array}$ \\
\hline 200E P-241AX102 001 & 1 & TANKS AND VESSELS & $\begin{array}{l}.000000036 \\
.000008700 \\
.000000018 \\
.000230000\end{array}$ & $\begin{array}{l}.000000000 \\
.000000004 \\
.000000000 \\
.000000120\end{array}$ & $\begin{array}{l}\text { AMERICIUM - } 241 \\
\text { CESIUM - } 137 \\
\text { PLUTONIUM - } 239 \\
\text { STRONTIUM - } 090\end{array}$ \\
\hline 200E P-241AX103001 & 1 & TANKS AND VESSELS & $\begin{array}{l}.000000078 \\
.000019000 \\
.000000039 \\
.000500000\end{array}$ & $\begin{array}{l}.000000000 \\
.000000009 \\
.000000000 \\
.000000250\end{array}$ & $\begin{array}{l}\text { AMERICIUM - } 241 \\
\text { CESIUM }-137 \\
\text { PLUTONIUM }-239 \\
\text { STRONTIUM }-090\end{array}$ \\
\hline 200E P-241AX104 001 & 1 & TANKS AND VESSELLS & $\begin{array}{l}.000000036 \\
.000008700 \\
.000000018 \\
.000230000\end{array}$ & $\begin{array}{l}.000000000 \\
.000000004 \\
.000000000 \\
.000000120\end{array}$ & $\begin{array}{l}\text { AMERICIUM - } 241 \\
\text { CESIUM - } 137 \\
\text { PLUTONIUM - } 239 \\
\text { STRONTIUM }-090\end{array}$ \\
\hline 200E P-241B102 001 & 1 & TANKS AND VESSELS & $\begin{array}{l}.000000078 \\
.000019000 \\
.000000039 \\
.000500000 \\
.000000078 \\
.000019000 \\
.000000039 \\
.000500000\end{array}$ & $\begin{array}{l}.000000000 \\
.000000009 \\
.000000000 \\
.000000250 \\
.000000000 \\
.000000009 \\
.000000000 \\
.000000250\end{array}$ & $\begin{array}{l}\text { AMERICIUM - } 241 \\
\text { CESIUM - } 137 \\
\text { PLUTONIUM }-239 \\
\text { STRONTIUM }-090 \\
\text { AMERICIUM }-241 \\
\text { CESIUM }-137 \\
\text { PLUTONIUM - } 239 \\
\text { STRONTIUM }-090\end{array}$ \\
\hline
\end{tabular}

Page \#: 70 of 95 Report Run Date: 22-APR-95 
AIR EMISSIONS INVENTORY PERMIT APPLICATION REPORT (UP460)

FACILITY NAME: $200 E$ Tank Farm and ASsociated Structures

Point Source Emissions - Radiological Pollutants

\begin{tabular}{|c|c|c|c|c|c|c|c|}
\hline Em Point & Srce Nbr & Srce & Type Pollutant & & $\begin{array}{l}\text { itial } \\
\text { (r) }\end{array}$ & $\begin{array}{l}\text { Pt. } \\
\text { Actual (Ci/yr) }\end{array}$ & Pollutant \\
\hline $200 E P-241 B 103001$ & & 1 & TANKS AND VESSELS & & $\begin{array}{l}.000000078 \\
.000019000 \\
.000000039 \\
.000500000\end{array}$ & $\begin{array}{l}.000000000 \\
.000000009 \\
.000000000 \\
.000000250\end{array}$ & $\begin{array}{l}\text { AMERICIUM - } 241 \\
\text { CESIUM - } 137 \\
\text { PLUTONIUM - } 239 \\
\text { STRONTIUM - } 090\end{array}$ \\
\hline 200E P-241B104 001 & & 1 & TANKS AND VESSELS & & $\begin{array}{l}.000000036 \\
.000008700 \\
.000000018 \\
.000230000\end{array}$ & $\begin{array}{l}.000000000 \\
.000000004 \\
.000000000 \\
.000000120\end{array}$ & $\begin{array}{l}\text { AMERICIUM - } 241 \\
\text { CESIUM - } 137 \\
\text { PLUTONIUM - } 239 \\
\text { STRONTIUM - } 090\end{array}$ \\
\hline 200E P-241B105 001 & & 1 & TANKS AND VESSELS & - & $\begin{array}{l}.000000000 \\
.000000330 \\
.000000000 \\
.000000110\end{array}$ & $\begin{array}{l}.000000000 \\
.000000000 \\
.000000000 \\
.000000000\end{array}$ & $\begin{array}{l}\text { AMERICIUM - } 241 \\
\text { CESIUM - } 137 \\
\text { PLUTONIUM - } 239 \\
\text { STRONTIUM - } 090\end{array}$ \\
\hline 200E P-2418106001 & & 1 & TANKS AND VESSELSS & & $\begin{array}{l}.000000078 \\
.000019000 \\
.000000039 \\
.000500000\end{array}$ & $\begin{array}{l}.000000000 \\
.000000009 \\
.000000000 \\
.000000250\end{array}$ & $\begin{array}{l}\text { AMERICIUM - } 241 \\
\text { CESIUM - } 137 \\
\text { PLUTONIUM - } 239 \\
\text { STRONTIUM - } 090\end{array}$ \\
\hline 200E P-241B107001 & & 1 & TANKS AND VESSELS & & $\begin{array}{l}.000000036 \\
.000008700 \\
.000000018 \\
.000230000\end{array}$ & $\begin{array}{l}.000000000 \\
.000000004 \\
.000000000 \\
.000000120\end{array}$ & $\begin{array}{l}\text { AMERICIUM - } 241 \\
\text { CESIUM - } 137 \\
\text { PLUTONIUM }-239 \\
\text { STRONTIUM }-090\end{array}$ \\
\hline 200E P-241B108 001 & & 1 & TANKS AND VESSELS & & $\begin{array}{l}.000000078 \\
.000019000 \\
.000000039 \\
.000500000\end{array}$ & $\begin{array}{l}.000000000 \\
.000000009 \\
.000000000 \\
.000000250\end{array}$ & $\begin{array}{l}\text { AMERICIUM - } 241 \\
\text { CESIUM - } 137 \\
\text { PLUTONIUM - } 239 \\
\text { STRONTIUM - } 090\end{array}$ \\
\hline 200E P-241B109001 & & 1 & TANKS AND VESSELS & & $\begin{array}{l}.000000036 \\
.000008700 \\
.000000018 \\
.000230000\end{array}$ & $\begin{array}{l}.000000000 \\
.000000004 \\
.000000000 \\
.000000120\end{array}$ & $\begin{array}{l}\text { AMERICIUM - } 241 \\
\text { CESIUM - } 137 \\
\text { PLUTONIUM - } 239 \\
\text { STRONTIUM - } 090\end{array}$ \\
\hline 200E P-241B110 001 & & 1 & TANKS AND VESSELS & & $\begin{array}{l}.000000036 \\
.000008700 \\
.000000018 \\
.000230000\end{array}$ & $\begin{array}{l}.000000000 \\
.000000004 \\
.000000000 \\
.000000120\end{array}$ & $\begin{array}{l}\text { AMERICIUM - } 241 \\
\text { CESIUM - } 137 \\
\text { PLUTONIUM }-239 \\
\text { STRONTIUM }-090\end{array}$ \\
\hline 200E P-241B111 001 & & 1 & TANKS AND VESSELS & & $\begin{array}{l}.000000078 \\
.000019000 \\
.000000039 \\
.000500000\end{array}$ & $\begin{array}{l}.000000000 \\
.000000009 \\
.000000000 \\
.000000250\end{array}$ & $\begin{array}{l}\text { AMERICIUM - } 241 \\
\text { CESIUM - } 137 \\
\text { PLUTONIUM }-239 \\
\text { STRONTIUM }-090\end{array}$ \\
\hline 200E P-241B201 001 & & 1 & TANKS AND VESSELS & & $\begin{array}{l}.000000078 \\
.000019000 \\
.00000039 \\
.000500000 \\
.000000036 \\
.000008700 \\
.000000018 \\
.000230000\end{array}$ & $\begin{array}{l}.000000000 \\
.000000009 \\
.000000000 \\
.000000250 \\
.000000000 \\
.000000004 \\
.000000000 \\
.000000120\end{array}$ & $\begin{array}{l}\text { AMERICIUM - } 241 \\
\text { CESIUM - } 137 \\
\text { PLUTONIUM - } 239 \\
\text { STRONTIUM }-090 \\
\text { AMERICIUM }-241 \\
\text { CESIUM - } 137 \\
\text { PLUTONIUM - } 239 \\
\text { STRONTIUM }-090\end{array}$ \\
\hline
\end{tabular}

Page \#: 71 of 95 Report Run Date: 22-APR-95 
AIR EMISSIONS INVENTORY PERMIT APPLICATION REPORT (UP460)

FACILITY NAME: 200E Tank Farm and Associated Structures

Point Source Emissions - Radiological Pollutants

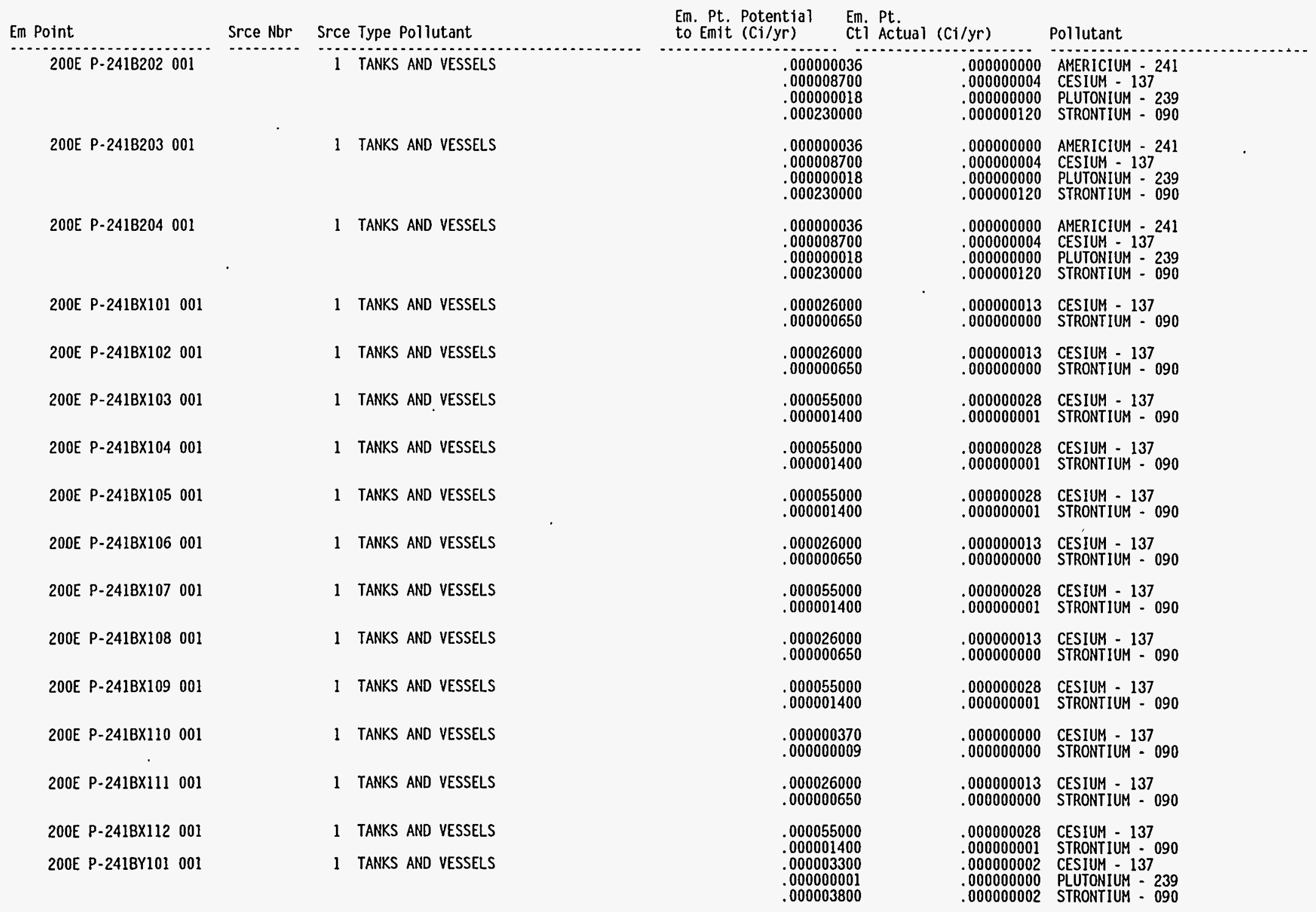

Page \#: 72 of 95 Report Run Date: 22-APR-95 
AIR EMISSIONS INVENTORY PERMIT APPLICATION REPORT (UP460)

FACILITY NAME: 200 Tank Farm and Associated Structures

Point Source Emissions - Radiological Pollutants

\begin{tabular}{|c|c|c|c|c|c|c|}
\hline Em Point & Srce Nbr & Srce & Type Pollutant & $\begin{array}{l}\text { Em. Pt. Potential } \\
\text { to Emit }(\mathrm{Ci} / \mathrm{yr})\end{array}$ & 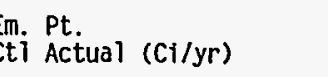 & Pollutant \\
\hline $200 E$ P-241BY102001 & & 1 & TANKS AND VESSELS & $\begin{array}{l}.000003300 \\
.000000001 \\
.000003800\end{array}$ & $\begin{array}{l}.000000002 \\
.000000000 \\
.000000002\end{array}$ & $\begin{array}{l}\text { CESIUM - } 137 \\
\text { PLUTONIUM - } 239 \\
\text { STRONTIUM - } 090\end{array}$ \\
\hline $200 E$ P-241BY104 001 & & 1 & TANKS AND VESSELS & $\begin{array}{l}.000003300 \\
.000000001 \\
.000003800\end{array}$ & $\begin{array}{l}.000000002 \\
.000000000 \\
.000000002\end{array}$ & $\begin{array}{l}\text { CESIUM - } 137 \\
\text { PLUTONIUM - } 239 \\
\text { STRONTIUM - } 090\end{array}$ \\
\hline 200E P-2418Y105001 & & 1 & TANKS AND VESSELS & $\begin{array}{l}.000003300 \\
.000000001 \\
.000003800\end{array}$ & $\begin{array}{l}.000000002 \\
.000000000 \\
.000000002\end{array}$ & $\begin{array}{l}\text { CESIUM - } 137 \\
\text { PLUTONIUM }-239 \\
\text { STRONTIUM }-090\end{array}$ \\
\hline 200E P-241BY106 001 & & 1 & TANKS AND VESSELS & $\begin{array}{l}.000003300 \\
.000000001 \\
.000003800\end{array}$ & $\begin{array}{l}.000000002 \\
.000000000 \\
.000000002\end{array}$ & $\begin{array}{l}\text { CESIUM - } 137 \\
\text { PLUTONIUM - } 239 \\
\text { STRONTIUM - } 090\end{array}$ \\
\hline $\begin{array}{c}\text { 200E P-241BY107 } 001 \\
\text {. }\end{array}$ & & 1 & TANKS AND VESSELS & $\begin{array}{l}.000003300 \\
.000000001 \\
.000003800\end{array}$ & $\begin{array}{l}.000000002 \\
.000000000 \\
.000000002\end{array}$ & $\begin{array}{l}\text { CESIUM - } 137 \\
\text { PLUTONIUM - } 239 \\
\text { STRONTIUM - } 090\end{array}$ \\
\hline 200E P-241BY109001 & & 1 & TANKS AND VESSELS & $\begin{array}{l}.000007200 \\
.000000002 \\
.000008300\end{array}$ & $\begin{array}{l}.000000004 \\
.000000000 \\
.000000004\end{array}$ & $\begin{array}{l}\text { CESIUM - } 137 \\
\text { PLUTONIUM - } 239 \\
\text { STRONTIUM }-090\end{array}$ \\
\hline $200 E$ P-241BY110 001 & & 1 & TANKS AND VESSELS & $\begin{array}{l}.000003300 \\
.000000001 \\
.000003800\end{array}$ & $\begin{array}{l}.000000002 \\
.000000000 \\
.000000002\end{array}$ & $\begin{array}{l}\text { CESIUM - } 137 \\
\text { PLUTONIUM - } 239 \\
\text { STRONTIUM }-090\end{array}$ \\
\hline 200E P-241BY111 001 & & 1 & TANKS AND VESSELS & $\begin{array}{l}.000003300 \\
.000000001 \\
.000003800\end{array}$ & $\begin{array}{l}.000000002 \\
.000000000 \\
.000000002\end{array}$ & $\begin{array}{l}\text { CESIUM - } 137 \\
\text { PLUTONIUM }-239 \\
\text { STRONTIUM }-090\end{array}$ \\
\hline 200E P-241BY112001 & & 1 & TANKS AND VESSELS & $\begin{array}{l}.000003300 \\
.000000001 \\
.000003800\end{array}$ & $\begin{array}{l}.000000002 \\
.000000000 \\
.000000002\end{array}$ & $\begin{array}{l}\text { CESIUM - } 137 \\
\text { PLUTONIUM - } 239 \\
\text { STRONTIUM - } 090\end{array}$ \\
\hline 200E P-241C101 001 & & 1 & TANKS AND VESSELS & $\begin{array}{l}.000000008 \\
.000000290 \\
.000000096 \\
.000002900\end{array}$ & $\begin{array}{l}.000000000 \\
.00000000 \\
.000000000 \\
.000000002\end{array}$ & $\begin{array}{l}\text { AMERICIUM - } 241 \\
\text { CESIUM }-137 \\
\text { PLUTONIUM - } 239 \\
\text { STRONTIUM } 090\end{array}$ \\
\hline 200E P-241C102 001 & & 1 & TANKS AND VESSELS & $\begin{array}{l}.000000018 \\
.000000640 \\
.000000210 \\
.000006400\end{array}$ & $\begin{array}{l}.000000000 \\
.000000000 \\
.000000000 \\
.000000003\end{array}$ & $\begin{array}{l}\text { AMERICIUM - } 241 \\
\text { CESIUM - } 137 \\
\text { PLUTONIUM }-239 \\
\text { STRONTIUM }-090\end{array}$ \\
\hline
\end{tabular}

Page \#: 73 of 95 Report Run Date: 22-APR-95 


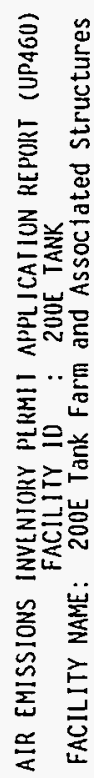

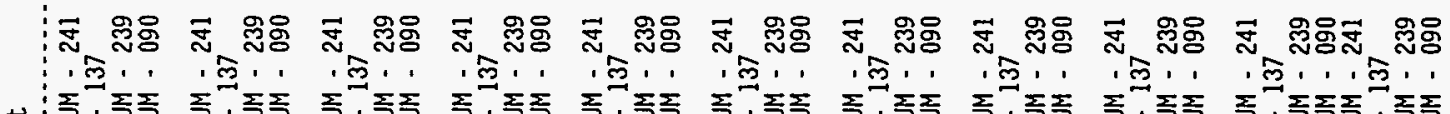

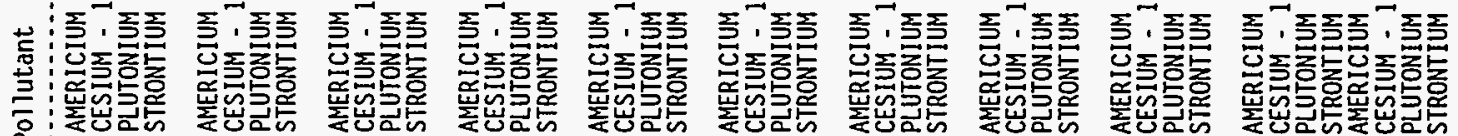

。

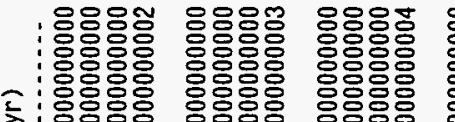

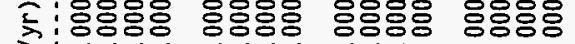
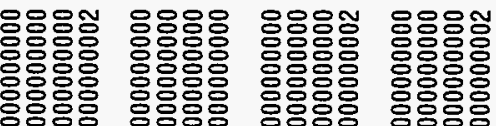

ㅇํㅇำ 용ํํ요요ำ

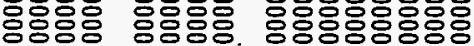

可

\section{턴 융융유요}

テ
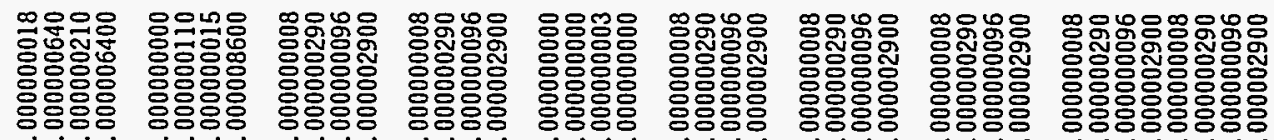

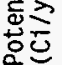

要要

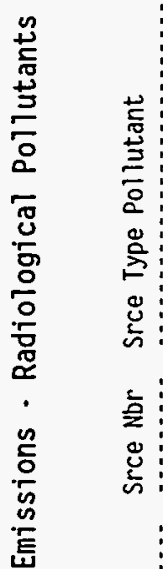

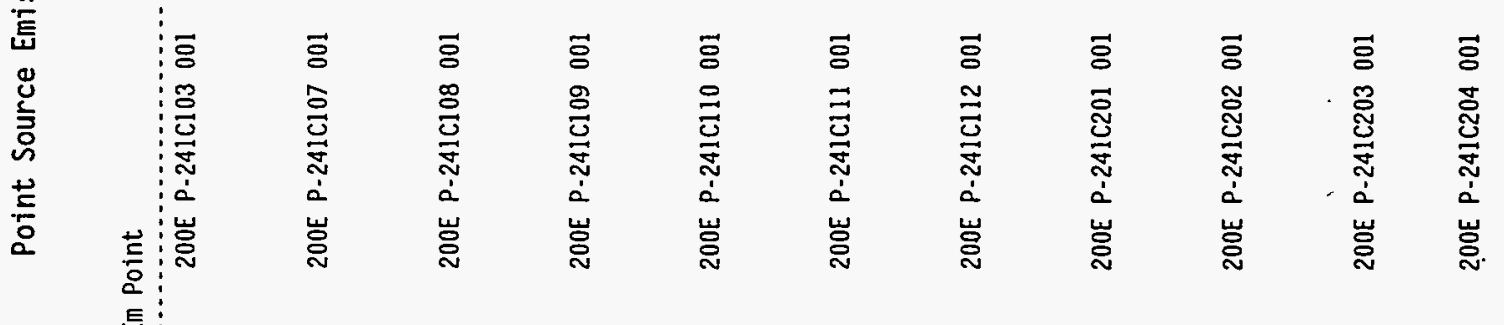


AIR EMISSIONS INVENTORY PERMIT APPLICATION REPORT (UP460)

FACILITY NAME: 200 Tank Farm and Associated Structures

Point Source Emissions - Radiological Pollutants

\begin{tabular}{|c|c|c|c|c|c|}
\hline Em Point & Srce Nbr & Srce Type Pollutant & $\begin{array}{l}\text { Em. Pt. Potential } \\
\text { to Emit (Ci/yr) }\end{array}$ & $\begin{array}{l}\text { Em. Pt. } \\
\text { Cti Actual (Ci/yr) }\end{array}$ & Pollutant \\
\hline 200E P-242A 001 & $\begin{array}{l}1 \\
2 \\
3 \\
4 \\
5\end{array}$ & $\begin{array}{l}\text { PROCESS AND PRODUCTION } \\
\text { PROCESS AND PRODUCTION } \\
\text { PROCESS AND PRODUCTION } \\
\text { PROCESS AND PRODUCTION } \\
\text { PROCESS AND PRODUCTION }\end{array}$ & $\begin{array}{l}\text { Refer to } \\
\text { WHC-SD-EN-RPT-007 } \\
\text { Rev. O Page A-73 }\end{array}$ & $\begin{array}{l}\text { Refer to } \\
\text { DoE/RL-94-51 } \\
\text { Table } 2-1\end{array}$ & \\
\hline 200E P-242A 002 & $\begin{array}{l}1 \\
2 \\
3 \\
4 \\
5\end{array}$ & $\begin{array}{l}\text { PROCESS AND PROOUCTION } \\
\text { PROCESS AND PRODUCION } \\
\text { PROCESS AND PRRDUCIION } \\
\text { PROCESS AND PRODUCIION } \\
\text { PROCESS AND PRODUCTION }\end{array}$ & $\begin{array}{l}\text { Refer to } \\
\text { WHC-SD-EN-RPT- } 007 \\
\text { Rev. O Page A-75 }\end{array}$ & $\begin{array}{l}\text { Refer to } \\
\text { DOE/RL-94-51 } \\
\text { Table 2-1 }\end{array}$ & \\
\hline 200E P-244A 001 & 1 & PROCESS AND PRODUCTION & $\begin{array}{l}\text { Refer to } \\
\text { WHC-SD-EN-RPT-007 } \\
\text { Rev. 0 Page A-78 }\end{array}$ & $\begin{array}{l}\text { Refer to } \\
\text { DOE/RL-94-51 } \\
\text { TabTe 2-1 }\end{array}$ & \\
\hline 200E P-244AR 001 & 1 & PROCESS AND PRODUCTION & $\begin{array}{l}\text { Refer to } \\
\text { WHC-SD-EN-RPT-007 } \\
\text { Rev. 0 Page A-58 }\end{array}$ & $\begin{array}{l}\text { Refer to } \\
\text { DOE/RL-94-51 } \\
\text { Table } 2-1\end{array}$ & \\
\hline 200E P-244AR 002 & 1 & PROCESS AND PRODUCTION & $\begin{array}{l}\text { Refer to } \\
\text { WHC-SD-EN-RPT-007 } \\
\text { Rev. 0 Page A-62 }\end{array}$ & $\begin{array}{l}\text { Refer to } \\
\text { DOE/RL-94-51 } \\
\text { Table } 2-1\end{array}$ & \\
\hline 200E P-244BX 001 & 1 & PROCESS AND PRODUCTION & $\begin{array}{l}\text { Refer to } \\
\text { WHC-SD-EN-RPT-007 } \\
\text { Rev. O Page A-97 }\end{array}$ & $\begin{array}{l}\text { Refer to } \\
\text { DOE/RL-94-51 } \\
\text { Table 2-1 }\end{array}$ & \\
\hline 200E P-244CR 001 & 1 & PROCESS AND PRODUCTION & $\begin{array}{l}\text { Refer to } \\
\text { WHC-SD-EN-RPT-007 } \\
\text { Rev. 0 Page A-104 }\end{array}$ & $\begin{array}{l}\text { Refer to } \\
\text { DOE/RL-94-51 } \\
\text { Table } 2-1\end{array}$ & \\
\hline 200E P-296A 001 & 1 & TANKS AND VESSELS & $\begin{array}{l}\text { Refer to } \\
\text { WHC-SD-EN-RPT-007 } \\
\text { Rev. 0 Page A-111 }\end{array}$ & $\begin{array}{l}\text { Refer to } \\
\text { DoE/RL-94-51 } \\
\text { Table 2-1 }\end{array}$ & \\
\hline 200E P-296AN 001 & $\begin{array}{l}1 \\
2 \\
3 \\
4 \\
5 \\
6 \\
7\end{array}$ & $\begin{array}{l}\text { TANKS AND VESSELS } \\
\text { TANKS AND VESSELS } \\
\text { TANKS AND VESSELS } \\
\text { TANKS AND VESSELS } \\
\text { TANKS AND VESSELS } \\
\text { TANKS AND VESSELS } \\
\text { TANKS AND VESSELS }\end{array}$ & $\begin{array}{l}\text { Refer to } \\
\text { WHC-SD-EN-RPT-007 } \\
\text { Rev. O Page A-88 }\end{array}$ & $\begin{array}{l}\text { Refer to } \\
\text { DoE/RL-94-51 } \\
\text { Table } 2-1\end{array}$ & \\
\hline 200E P-296A41 & $\begin{array}{l}1 \\
2 \\
3 \\
4 \\
5 \\
6 \\
7\end{array}$ & $\begin{array}{l}\text { TANKS AND VESSELS } \\
\text { TANKS AND VESSELS } \\
\text { TANKS AND VESSELS } \\
\text { TANKS AND VESSELS } \\
\text { TANKS AND VESSELS } \\
\text { TANKS AND VESSELS } \\
\text { TANKS AND VESSELS }\end{array}$ & $\begin{array}{l}\text { Refer to } \\
\text { WHC-SD-EN-RPT-007 } \\
\text { Rev. O Page A-95 }\end{array}$ & $\begin{array}{l}\text { Refer to } \\
\text { DoE/RL-94-51 } \\
\text { Table } 2-1\end{array}$ & \\
\hline
\end{tabular}

Page \#: 75 of 95 Report Run Date: 22-APR-95 
AIR EMISSIONS INVENTORY PERMIT APPLICATION REPORT (UP460)

FACILITY NAME: 200 EACILITY IO : 200E TANK Farim and Associated Structures

Point Source Emissions - Radiological Pollutants

\begin{tabular}{|c|c|c|c|c|c|}
\hline Em Point & Srce Nbr & Srce Type Pollutant & $\begin{array}{l}\text { Em. Pt. Potential } \\
\text { to Emit }(\mathrm{C} 1 / \mathrm{yr})\end{array}$ & $\begin{array}{l}\text { Em. Pt. } \\
\text { Cti Actual (Ci/yr) }\end{array}$ & Pollutant \\
\hline 200E P-296AP 001 & $\begin{array}{l}1 \\
2 \\
3 \\
4 \\
5 \\
6 \\
7 \\
8\end{array}$ & $\begin{array}{l}\text { TANKS AND VESSELS } \\
\text { TANKS AND VESSELS } \\
\text { TANKS AND VESSELS } \\
\text { TANKS AND VESSELS } \\
\text { TANKS AND VESSELS } \\
\text { TANKS AND VESSELS } \\
\text { TANKS AND VESSELS } \\
\text { TANKS AND VESSELS }\end{array}$ & $\begin{array}{l}\text { Refer to } \\
\text { WHC-SD-EN-RPT-007 } \\
\text { Rev. O Page A-92 }\end{array}$ & $\begin{array}{l}\text { Refer to } \\
\text { DOE/RL-94-51 } \\
\text { Table 2-1 }\end{array}$ & \\
\hline 200E P-296A030 & $\begin{array}{l}1 \\
2 \\
3 \\
4 \\
5 \\
6 \\
7 \\
8\end{array}$ & $\begin{array}{l}\text { TANKS AND VESSELS } \\
\text { TANKS AND VESSELS } \\
\text { TANKS AND VESSELS } \\
\text { TANKS AND VESSELS } \\
\text { TANKS AND VESSELS } \\
\text { TANSS AND VESSES } \\
\text { TANKS AND VESEELS } \\
\text { TANKS AND VESSELS }\end{array}$ & $\begin{array}{l}\text { Refer to } \\
\text { WHC-SD-EN-RPT-007 } \\
\text { Rev. O Page A-90 }\end{array}$ & $\begin{array}{l}\text { Refer to } \\
\text { DOE/RL-94-51 } \\
\text { Table } 2-1\end{array}$ & . \\
\hline 200E P-296A020 & $\frac{1}{2}$ & $\begin{array}{l}\text { TANKS AND VESSELS } \\
\text { TANKS AND VESSELS }\end{array}$ & $\begin{array}{l}\text { Refer to } \\
\text { WHC-SD-EN-RPT-007 } \\
\text { Rev. O Page A-71 }\end{array}$ & $\begin{array}{l}\text { Refer to } \\
\text { DOE/RL-94-51 } \\
\text { Table } 2-1\end{array}$ & \\
\hline 200E P-296A018 & 1 & TANKS AND VESSELS & $\begin{array}{l}\text { Refer to } \\
\text { WHC-SD-EN-RPT-007 } \\
\text { Rev. O Page A- } 67\end{array}$ & $\begin{array}{l}\text { Refer to } \\
\text { DOE/RL-94-51 } \\
\text { Table } 2-1\end{array}$ & \\
\hline 200E Р-296А019 & 1 & TANKS AND VESSELS & $\begin{array}{l}\text { Refer to } \\
\text { WHC-SD-EN-RPT- } 007 \\
\text { Rev. O Page A- } 69\end{array}$ & $\begin{array}{l}\text { Refer to } \\
\text { DoE/RL-94-51 } \\
\text { Table 2-1 }\end{array}$ & . \\
\hline 200E P-296AW 001 & $\begin{array}{l}1 \\
2 \\
3 \\
4 \\
5 \\
6\end{array}$ & $\begin{array}{l}\text { TANKS AND VESSELS } \\
\text { TANKS AND VESSELS } \\
\text { TANKS AND VESSELS } \\
\text { TANKS AND VESSELS } \\
\text { TANKS AND VESSELS } \\
\text { TANKS AND VESSELS }\end{array}$ & $\begin{array}{l}\text { Refer to } \\
\text { WHC-SD-EN-RPT-007 } \\
\text { Rev. O Page A-84 }\end{array}$ & $\begin{array}{l}\text { Refer to } \\
\text { DOE/RL-94-51 } \\
\text { Table 2-1 }\end{array}$ & \\
\hline 200E P-296A028 & $\begin{array}{l}1 \\
2 \\
3 \\
4 \\
5 \\
6\end{array}$ & $\begin{array}{l}\text { TANKS AND VESSELS } \\
\text { TANKS AND VESSELS } \\
\text { TANKS AND VESSELS } \\
\text { TANKS AND VESSELS } \\
\text { TANKS AND VESSELS } \\
\text { TANKS AND VESSELS }\end{array}$ & $\begin{array}{l}\text { Refer to } \\
\text { WHC-SO-EN-RPT-007 } \\
\text { Rev. } 0 \text { Page A-86 }\end{array}$ & $\begin{array}{l}\text { Refer to } \\
\text { DOE/RL-94-51 } \\
\text { Table 2-1 }\end{array}$ & \\
\hline 200E P-296AYAZ 001 & $\begin{array}{l}1 \\
1 \\
2 \\
3 \\
4\end{array}$ & $\begin{array}{l}\text { TANKS AND VESSELS } \\
\text { TANKS AND VESSELS } \\
\text { TANKS AND VESSELS } \\
\text { TANKS AND VESSELS }\end{array}$ & $\begin{array}{l}\text { Refer to } \\
\text { WHC-SD-EN-RPT-007 } \\
\text { Rev. 0 Page A-64 }\end{array}$ & $\begin{array}{l}\text { Refer to } \\
\text { DOE/RL-94-51 } \\
\text { Table 2-1 }\end{array}$ & \\
\hline 200E P-296P026001 & $\begin{array}{l}1 \\
2 \\
3 \\
4\end{array}$ & $\begin{array}{l}\text { TANKS AND VESSELS } \\
\text { TANKS AND VESSELS } \\
\text { TANKS AND VESSELS } \\
\text { TANKS AND VESSELS }\end{array}$ & $\begin{array}{l}\text { Refer to } \\
\text { WHC-SD-EN-RPT-007 } \\
\text { Rev. 0 Page A-117 }\end{array}$ & $\begin{array}{l}\text { Refer to } \\
\text { DOE/RL-94-51 } \\
\text { Table 2-1. Note: } \\
\text { not additive to }\end{array}$ & $\begin{array}{l}\text { these emissions are equivalent. but } \\
\text { the } 296-\mathrm{A}-17 \text { stack. }\end{array}$ \\
\hline
\end{tabular}

Page \#: 76 of 95 Report Run Date: 22-APR-95 
AIR EMISSIONS INVENTORY PERMIT APPLICATION REPORT (UP460)

FACILITY NAME: 200E Tank Farm and Associated Structures

Point Source Emissions - Radiological Pollutants

200E P-296P031

200E P-296P016 001 $\begin{array}{ll}1 & \text { TANKS AND VESSELS } \\ 2 & \text { TANKS AND VESSELS } \\ 3 & \text { TANKS AND VESSELS }\end{array}$

$\begin{array}{ll}1 & \text { TANKS AND VESSELS } \\ 2 & \text { TANKS AND VESSELS }\end{array}$
Refer to

WHC-SD-EN-RPT-007

Refer to

WHC-SD-EN-RPT-007
Rev. 0 Page A-108

Em. Pt. Potential Em. Pt.
to Emit (tons/yr) Ctl Actual (tons/yr) Pollutant
Refer to

Table 2-1

Refer to

DOE/RL-94-51

Table 2-1
Em Point

Srce Nbr Srce Type Pollutant

200E P-241A101 001

$$
1 \text { TANKS AND VESSELS }
$$

\section{Point Source Emissions - HAP Pollutants}

.0000019
.00031900
.0000151
.000040500

$\begin{array}{ll}.000001910 & 1.3-B U T A D I E N E \\ .000319000 & \text { ACETONITRILE } \\ .000015100 & \text { BENZENE } \\ .000040500 & \text { N-HEXANE } \\ .000003080 & \text { TOLEENE }\end{array}$

Point Source Emissions - HAP Pollutants

Em Point

200E P-241A102 001

200E P-241A103 001

200E P-241A104 001

200E P-241A106 001
Srce Nbr Srce Type Pollutant

1 TANKS AND VESSELS

1 TANKS AND VESSELS

1 TANKS AND VESSELS

1 TANKS AND VESSELS
Em. Pt. Potential Em. Pt.

to Emit (tons/yr) Ct). Actual (tons/yr) Pollutant

$\begin{array}{lll}.000004130 & .000004130 & 1.3-B U T A D I E N E \\ .000689000 & .000689000 & \text { ACETONITRILE } \\ .000032800 & .000032800 & \text { BENZENE } \\ .000087600 & .000087600 & \text { N-HEXANE } \\ .000006670 & .000006670 & \text { TOLUENE }\end{array}$

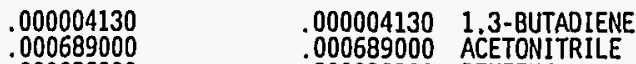

.00068900

.

.000087600

.000032800 BENZENE

.000087600 N-HEXANE

.000001910

.000319000

.00015100

.000003080

.000001910 1.3-BUTADIENE

.000319000 ACETONITRILE

O00015100 BENZERE

.

000004130

000004130 1.3-BUTADIENE

.000004130

.000032800

.000087600

O00689000 ACETONITR

DO0087600 M-HEXANE

.000006670 TOLUENE 
AIR EMISSIONS INVENTORY PERMIT APPLICATION REPORT (UP460)
FACILITY ID : 200E TANK

FACILITY NAME: 200 Tank Farm and Associated Structures

Point Source Emissions - HAP Pollutants

200E P-241AX101 001

TANKS AND VESSELS

200E P-241AX102 001

1 TANKS AND VESSELS

200E P-241AX103 001

1 TANKS AND VESSELS

200E P-241AX104 001

1 TANKS AND VESSELS

200E P-241B101 001

1 TANKS AND VESSELS
.000004130

000689000

.000032800

.000006670

.000001910

000319000

000015100

000003080

.000004130

000689000

00032800

00006670

000001910

000319000

000015100

000040500

000003080

.000004130

000689000

000032800

.000087600 $\begin{array}{ll}.000004130 & 1.3 \text {-BUTADIENE } \\ .000689000 & \text { ACETONITRILE } \\ .000032800 & \text { BENZENE } \\ .000087600 & \text { N-HEXANE } \\ .000006670 & \text { TOLUENE }\end{array}$

$.000001910 \quad 1.3$-BUTADIENE

000319000 ACETONITRILE

000015100 BENZENE

.000040500
000003080 TOLUEANE

000004130 1.3-BUTADIENE

000689000 ACETONITRILE

BENZENE

000006670 N.HEXANE

$.000001190 \quad 1.3$-BUTADIENE

.000319000 ACETONITRILE

.000015100 BENZENE

$.000040500 \quad N$-HEXANE

.000003080 TOLUENE

$.000004130 \quad 1.3$-BUTADIENE

.000689000 ACETONITRILE

.000032800 BENZENE

.000087600 N-HEXANE

Point Source Emissions - HAP Pollutants

\begin{tabular}{|c|c|c|c|c|c|}
\hline Em Point & Srce $\mathrm{Nbr}$ & Srce Type Pollutant & $\begin{array}{l}\text { Em. Pt. Potential } \\
\text { to Emit (tons/yr) }\end{array}$ & $\begin{array}{l}\text { Em. Pt. } \\
\text { Ctl Actual (tons/yr) }\end{array}$ & Pollutant \\
\hline 200E P-2418102001 & & 1 TANKS AND VESSELS & $\begin{array}{l}.000004130 \\
.000689000 \\
.000032800 \\
.000087600 \\
.000006670\end{array}$ & $\begin{array}{l}.000004130 \\
.000689000 \\
.000032800 \\
.000087600 \\
.000006670\end{array}$ & $\begin{array}{ll}0 & 1.3 \text {-BUTADIENE } \\
0 & \text { ACETONITRILE } \\
0 & \text { BENEENE } \\
0 & \text { N-HEXANE } \\
0 & \text { TOLUENE }\end{array}$ \\
\hline $200 E$ P-241B103 001 & & 1 TANKS AND VESSELS & $\begin{array}{l}.000004130 \\
.000689000 \\
.000032800 \\
.000087600 \\
.000006670\end{array}$ & $\begin{array}{l}.000004130 \\
.000689000 \\
.000032800 \\
.000087600 \\
.000006670\end{array}$ & $\begin{array}{ll}0 & 1.3 \text {-BUTADIENE } \\
0 & \text { ACETONITRILE } \\
0 & \text { BENZENE } \\
\text { N-HEXANE } \\
\text { TOLUENE } \\
\text { TOLEN }\end{array}$ \\
\hline
\end{tabular}

Page \#: 78 of 95 Report Run Date: 22-APR-95 
AIR EMISSIONS INVENTORY PERMIT APPLICATION REPORT (UP460)

FACILITY NAME: $200 E$ Tank Farm and Associated Structures

Point Source Emissions - HAP Pollutants

\begin{tabular}{|c|c|c|}
\hline 200E P-2418104 001 & 1 & TANKS AND VESSELS \\
\hline 200E P-241B105001 & 1 & TANKS AND VESSELS \\
\hline 200E P-241B106 001 & 1 & TANKS AND VESSELS \\
\hline 200E P-241B107 001 & 1 & TANKS AND VESSELS \\
\hline 200E P-241B108 001 & 1 & TANKS AND VESSELS \\
\hline $200 E$ P-241B109 001 & 1 & TANKS AND VESSELS \\
\hline & & \\
\hline $200 E$ P-241B110 001 & 1 & TANKS AND VESSELS \\
\hline
\end{tabular}

\section{.000001910 \\ 000319000 \\ . \\ .000003080}

000001910

00031900

000015100

.000003080

.000004130

000689000

0003800

.000006670

.000001910

.000319000

000015100

.000003080

.000004130

000689000

00032800

.0000806670

000001910

000319000

000015100

.000040500

.000001910

000319000

00015100

.000040500 $\begin{array}{ll}.000001910 & 1.3 \text {-BUTADIENE } \\ .000319000 & \text { ACETONITRILE }\end{array}$

.

D00040500 H HEXANE

.000003080 TOLUENE

.000001910 1.3-BUTADIENE

000319000 ACETONITRILE

0000015100 BENZENE

.000040500 N-HEXANE

.000003080 TOLUENE

.000004130 1.3-BUTADIENE

000689000 ACETONITRILE

000087600 N-HEXANE

000006670 TOLUENE

000001910 1.3-BUTADIENE

000319000 ACETONITRILE

.000015100 8ENZENE

000040500 N-HEXANE

.000003080 TOLUENE

$.000004130 \quad 1.3$-BUTADIENE

000689000 ACETONITRILE

D00087600 N-HEXANE

000006670 TOLUEA

$.000001910 \quad 1.3$-BUTADIENE

000319000 ACETONITRILE

000015100 BENZENE

000040500 N-HEXANE

.000003080 TOLUENE

$.000001910 \quad 1.3-B U T A D I E N E$

.000319000 ACETONITRILE

DON1510 BENZENE

000003080 TOLUENE 
AIR EMISSIONS INVENTORY PERMIT APPLICATION REPORT (UP460)

FACILITY NAME: 200 FACILITY ID : 200E TANK Farm and Associated structures

Point Source Emissions - HAP Pollutants

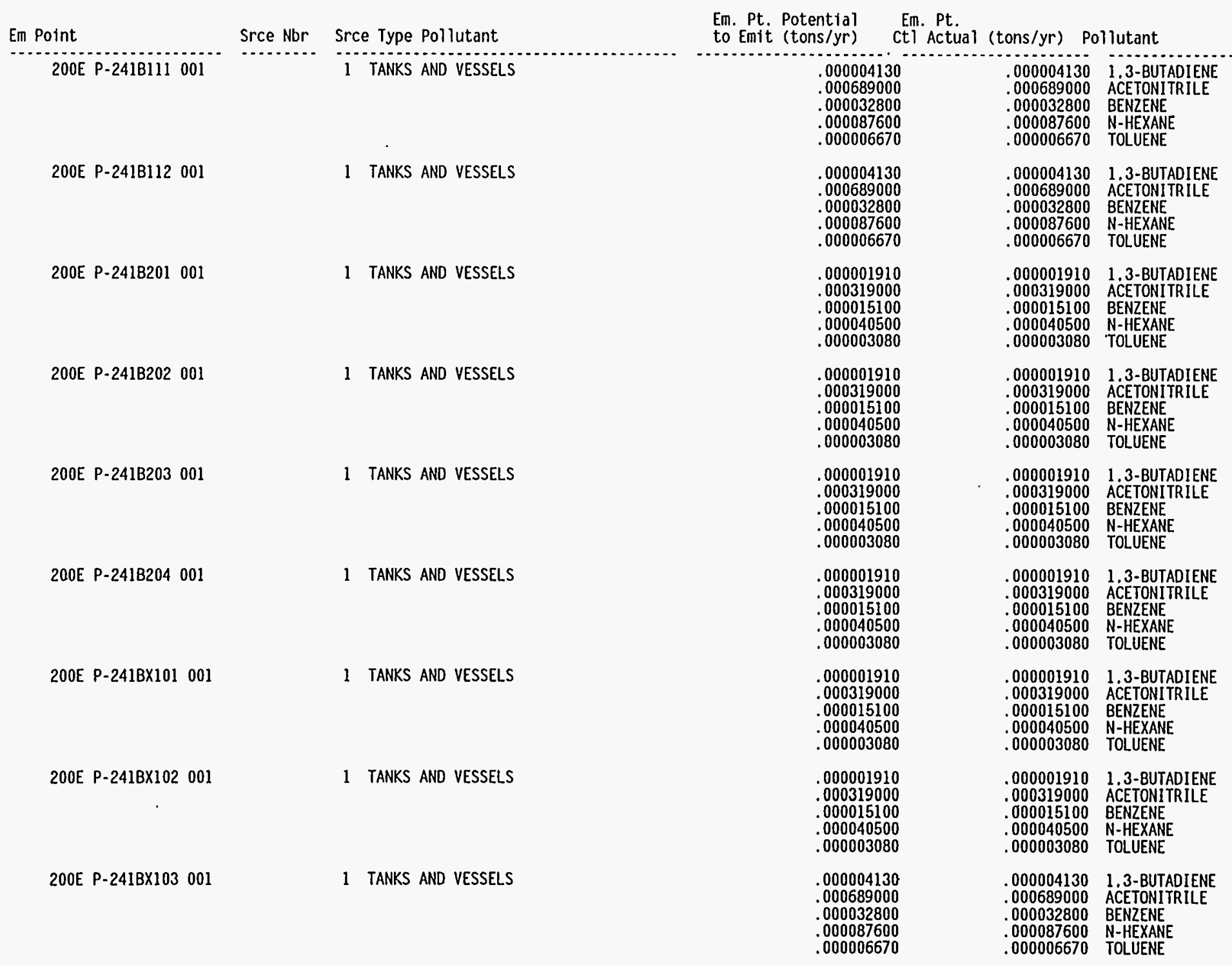

Page \#: 80 of 95 Report Run Date: 22-APR-95 
AIR EMISSIONS INVENIORY PERMII APPLICATION REPORT (UP460)

FACILITY NAME: 200E Tank Farm and Associated Structures

Point Source Emissions - HAP Pollutants

\begin{tabular}{|c|c|c|c|c|c|c|c|c|}
\hline Em Point & Srce Nbr & Src & Type Pollutant & $\begin{array}{l}\text { Em. Pt. Potential } \\
\text { to Emit (tons/yr) }\end{array}$ & $\begin{array}{l}\text { Em. Pt. } \\
\text { ctl Actual }\end{array}$ & (tons/yr) & ollutant & \\
\hline 200E P-241BX104001 & & 1 & TANKS AND VESSELS & $\begin{array}{l}.00000413 \\
.00068900 \\
.00003280 \\
.00008760 \\
.00000667\end{array}$ & & $\begin{array}{l}.000004130 \\
.000689000 \\
.000032800 \\
.000087600 \\
.000006670\end{array}$ & $\begin{array}{l}\text { 1.3-BUTADIENE } \\
\text { ACETONITRILE } \\
\text { BENZENE } \\
\text { N-HEXANE } \\
\text { TOLUENE }\end{array}$ & \\
\hline 200E P-241BX105 001 & & 1 & TANKS AND VESSELS & $\begin{array}{l}.00000413 \\
.00068900 \\
.00003280 \\
.00008760 \\
.00000667\end{array}$ & & $\begin{array}{l}.000004130 \\
.000689000 \\
.000032800 \\
.000087600 \\
.000006670\end{array}$ & $\begin{array}{l}\text { 1.3-BUTADIENE } \\
\text { ACETONITRILE } \\
\text { BENZENE } \\
\text { N-HEXANE } \\
\text { TOLUENE }\end{array}$ & \\
\hline 200E $P-241 B \times 106001$ & & 1 & TANKS AND VESSELS & $\begin{array}{l}.00000191 \\
.00031900 \\
.00001510 \\
.00004050 \\
.00000308\end{array}$ & & $\begin{array}{l}.000001910 \\
.000319000 \\
.000015100 \\
.000040500 \\
.000003080\end{array}$ & $\begin{array}{l}\text { 1.3-BUTADIENE } \\
\text { ACETONITRILE } \\
\text { BENZENE } \\
\text { N-HEXANE } \\
\text { TOLUENE }\end{array}$ & \\
\hline 200E P-241BX107 001 & & 1 & TANKS AND VESSELS & $\begin{array}{l}.00000413 \\
.00068900 \\
.00003280 \\
.00008760 \\
.00000667\end{array}$ & & $\begin{array}{l}.000004130 \\
.000689000 \\
.000032800 \\
.000087600 \\
.000006670\end{array}$ & $\begin{array}{l}\text { 1.3-BUTADIENE } \\
\text { ACETONITRILE } \\
\text { BENZENE } \\
\text { N-HEXANE } \\
\text { TOLUENE }\end{array}$ & \\
\hline 200E P-241BX108 001 & & 1 & TANKS AND VESSELS & $\begin{array}{l}.000001910 \\
.00031900 \\
.000015100 \\
.00004050 \\
.00000308\end{array}$ & & $\begin{array}{l}.000001910 \\
.000319000 \\
.000015100 \\
.000040500 \\
.000003080\end{array}$ & $\begin{array}{l}\text { 1.3-BUTADIENE } \\
\text { ACETONITRILE } \\
\text { BENZENE } \\
\text { N-HEXANE } \\
\text { TOLUENE }\end{array}$ & \\
\hline 200E P-241BX109 001 & & 1 & TANKS AND VESSELS & $\begin{array}{l}.00000413 \mathrm{C} \\
.00068900 \mathrm{c} \\
.00003280 \mathrm{C} \\
.00008760 \mathrm{c} \\
.00000667 \mathrm{c}\end{array}$ & & $\begin{array}{l}.000004130 \\
.000689000 \\
.000032800 \\
.000087600 \\
.000006670\end{array}$ & $\begin{array}{l}\text { 1.3-BUTADIENE } \\
\text { ACETONITRILE } \\
\text { BENZENE } \\
\text { N-HEXANE } \\
\text { TOLUENE }\end{array}$ & $\cdot$ \\
\hline 200E P-241BX110 001 & & 1 & TANKS AND VESSELS & $\begin{array}{l}.000001910 \\
.000319000 \\
.000015100 \\
.000040500 \\
.000003080\end{array}$ & & $\begin{array}{l}.000001910 \\
.000319000 \\
.000015100 \\
.000040500 \\
.000003080\end{array}$ & $\begin{array}{l}\text { 1.3-BUTADIENE } \\
\text { ACETONITRILE } \\
\text { BENZENE } \\
\text { N-HEXANE } \\
\text { TOLUENE }\end{array}$ & \\
\hline 200E P-241BX111 001 & & 1 & TANKS AND VESSELS & $\begin{array}{l}.000001910 \\
.000319000 \\
.000015100 \\
.000040500 \\
.000003080\end{array}$ & & $\begin{array}{l}.000001910 \\
.000319000 \\
.000015100 \\
.000040500 \\
.000003080\end{array}$ & $\begin{array}{l}\text { 1.3-BUTADIENE } \\
\text { ACETONITRILE } \\
\text { BENZENE } \\
\text { N-HEXANE } \\
\text { TOLUENE }\end{array}$ & \\
\hline 200E P-241BX112 001 & & 1 & TANKS AND VESSELS & $\begin{array}{l}.000004130 \\
.000689000 \\
.000032800 \\
.000087600 \\
.000006670\end{array}$ & & $\begin{array}{l}.000004130 \\
.000689000 \\
.000032800 \\
.000087600 \\
.000006670\end{array}$ & $\begin{array}{l}\text { 1.3-BUTADIENE } \\
\text { ACETONITR ILE } \\
\text { BENZENE } \\
\text { N-HEXANE } \\
\text { TOLUENE }\end{array}$ & \\
\hline
\end{tabular}

Page \#: 81 of 95 Report Run Date: 22-APR-95 
AIR EMISSIONS INVENTORY PERMIT APPLICATION REPORT (UP460)

FACILITY NAME: 200E Tank Farm and Associated Structures

Point Source Emissions - HAP Pollutants

\begin{tabular}{|c|c|c|c|c|c|}
\hline Em Point & Srce Nbr & Srce Type Pollutant & $\begin{array}{l}\text { Em. Pt. Potential } \\
\text { to Emit (tons/yr) }\end{array}$ & $\begin{array}{l}\text { En. Pt. } \\
\text { ctl Actual (tons/yr) }\end{array}$ & ollutant \\
\hline $200 E$ P-2418Y101 001 & & 1 TANKS AND VESSELS & $\begin{array}{l}.000001910 \\
.000319000 \\
.000015100 \\
.000040500 \\
.000003080\end{array}$ & $\begin{array}{l}.000001910 \\
.000319000 \\
.000015100 \\
.000040500 \\
.000003080\end{array}$ & $\begin{array}{l}\text { 1.3-BUTADIENE } \\
\text { ACETONITRILE } \\
\text { BENZENE } \\
\text { N-HEXANE } \\
\text { TOLUENE }\end{array}$ \\
\hline 200E P-241BY102 001 & & 1 TANKS AND VESSELS & $\begin{array}{l}.000001910 \\
.000319000 \\
.000015100 \\
.000040500 \\
.000003080\end{array}$ & $\begin{array}{l}.000001910 \\
.000319000 \\
.000015100 \\
.000040500 \\
.000003080\end{array}$ & $\begin{array}{l}\text { 1.3-BUTADIENE } \\
\text { ACETONITRILE } \\
\text { BENZENE } \\
\text { N-HEXANE } \\
\text { TOLUENE }\end{array}$ \\
\hline $200 E$ P-241BY103 001 & & 1 TANKS AND VESSELS & $\begin{array}{l}.000001910 \\
.000319000 \\
.000015100 \\
.000040500 \\
.000003080\end{array}$ & $\begin{array}{l}.000001910 \\
.000319000 \\
.000015100 \\
.000040500 \\
.000003080\end{array}$ & $\begin{array}{l}\text { 1.3-BUTADIENE } \\
\text { ACETONITRILE } \\
\text { BENZENE } \\
\text { N-HEXANE } \\
\text { TOLUENE }\end{array}$ \\
\hline 200E P-241BY104 001 & & 1 TANKS AND VESSELS & $\begin{array}{l}.000007970 \\
.000001930 \\
.000000090 \\
.000016200 \\
.000002220\end{array}$ & $\begin{array}{l}.000007970 \\
.000001930 \\
.000000090 \\
.000016200 \\
.000002220\end{array}$ & $\begin{array}{l}\text { ACETONITRILE } \\
\text { BENZENE } \\
\text { METHYLENE CHLORIDE } \\
\text { N-HEXANE } \\
\text { TOLUENE }\end{array}$ \\
\hline 200E P-2418Y105 001 & & 1 TANKS AND VESSELS & $\begin{array}{l}.000001960 \\
.000000317 \\
.000000259 \\
.000004360 \\
.000000465\end{array}$ & $\begin{array}{l}.000001960 \\
.000000317 \\
.000000259 \\
.000004360 \\
.000000465\end{array}$ & $\begin{array}{l}\text { ACETONITRILE } \\
\text { BENZENE } \\
\text { METHYLENE CHLORIDE } \\
\text { N-HEXANE } \\
\text { TOLUENE }\end{array}$ \\
\hline 200E P-241BY106 001 & & 1 TANKS AND VESSELS & $\begin{array}{l}.000002900 \\
.000000335 \\
.000000023 \\
.000004310 \\
.000000449\end{array}$ & $\begin{array}{l}.000002900 \\
.000000335 \\
.000000023 \\
.000004310 \\
.000000449\end{array}$ & $\begin{array}{l}\text { ACETONITRILE } \\
\text { BENZENE } \\
\text { METHYLENE CHLORIDE } \\
\text { N-HEXANE } \\
\text { TOLUENE }\end{array}$ \\
\hline 200E P-2418Y107 001 & & 1 TANKS AND VESSELS & $\begin{array}{l}.000001910 \\
.000319000 \\
.000015100 \\
.000040500 \\
.000003080\end{array}$ & $\begin{array}{l}.000001910 \\
.000319000 \\
.000015100 \\
.000040500 \\
.000003080\end{array}$ & $\begin{array}{l}\text { 1.3-BUTADIENE } \\
\text { ACETONITRILE } \\
\text { BENZENE } \\
\text { N-HEXANE } \\
\text { TOLUENE }\end{array}$ \\
\hline 200E P-241BY108 001 & & 1 TANKS AND VESSELS & $\begin{array}{l}.000001910 \\
.000319000 \\
.000015100 \\
.000040500 \\
.000003080\end{array}$ & $\begin{array}{l}.000001910 \\
.000319000 \\
.000015100 \\
.000040500 \\
.000003080\end{array}$ & $\begin{array}{l}\text { 1.3-BUTADIENE } \\
\text { ACETONITRILE } \\
\text { BENZENE } \\
\text { N-HEXANE } \\
\text { TOLUENE: }\end{array}$ \\
\hline 200E P-241BY109 001 & & 1 TANKS AND VESSELS & $\begin{array}{l}.000004130 \\
.000689000 \\
.000032800 \\
.000087600 \\
.000006670\end{array}$ & $\begin{array}{l}.000004130 \\
.000689000 \\
.000032800 \\
.000087600 \\
.000006670\end{array}$ & $\begin{array}{l}\text { 1.3-BUTADIENE } \\
\text { ACETONITRILE } \\
\text { BENZENE } \\
\text { N-HEXANE } \\
\text { TOLUENE }\end{array}$ \\
\hline
\end{tabular}

Page \#: 82 of 95 Report Run Date: 22-APR-95 
AIR EMISSIONS INVENTORY PERMIT APPLICATION REPORT (UP460)

FACILITY NAME: $200 E$ Tank Farm and Associated Structures

Point Source Emissions - HAP Pollutants

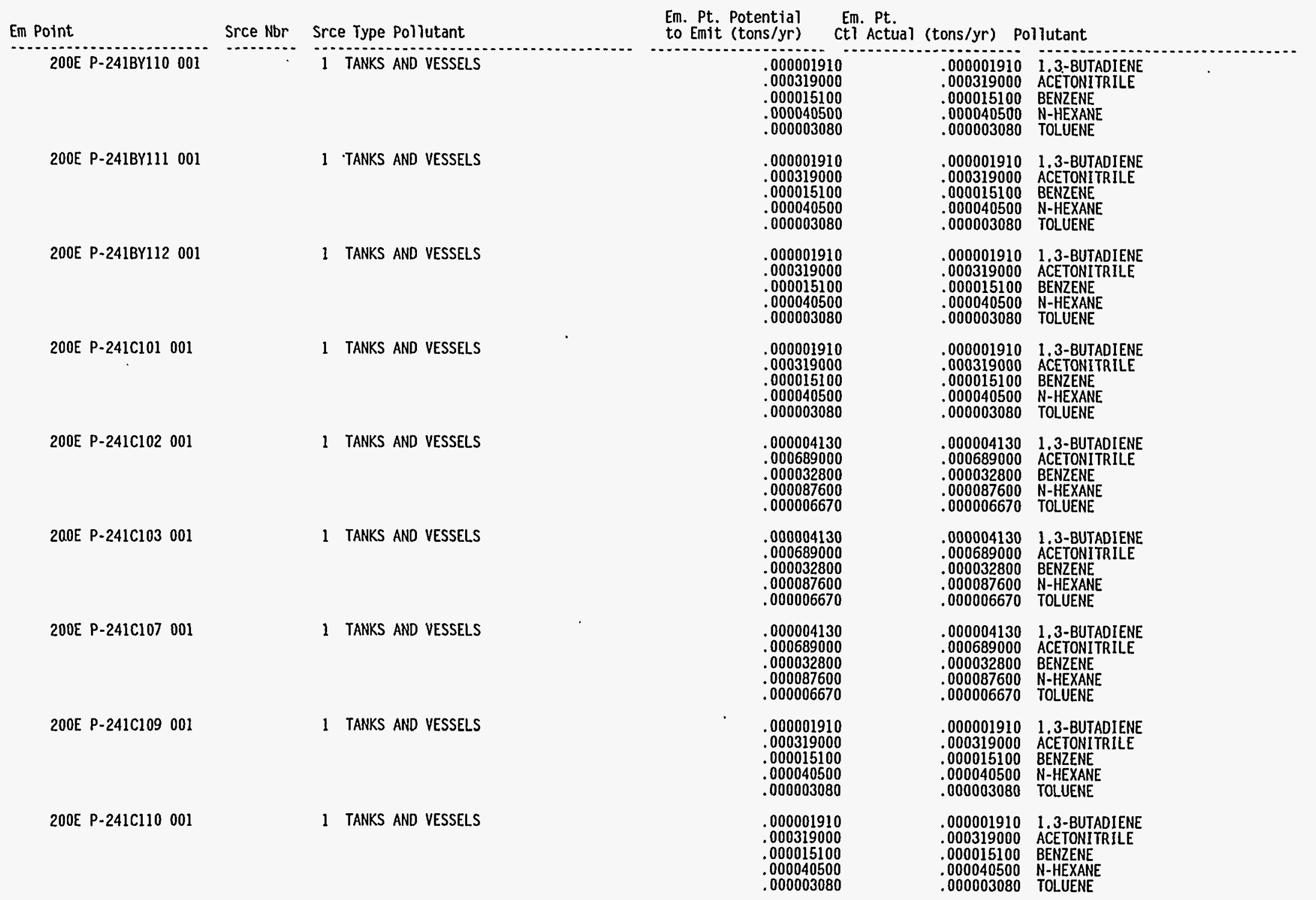

Page \#: 83 of 95 Report Run Date: 22-APR-95 
AIR EMISSIONS INVENTORY PERMIT APPLICATION REPORT (UP460)

FACILITY NAME: 200E Tank Farm and Assoclated Structures.

Point Source Emissions - HAP Pollutants

\begin{tabular}{|c|c|c|c|c|c|c|}
\hline Em Point & Srce Nbr & Src & Type Pollutant & $\begin{array}{l}\text { Em. Pt. Potential } \\
\text { to Emit (tons/yr) }\end{array}$ & $\begin{array}{l}\text { Em. Pt. } \\
\text { ctl Actual (tons/yr) Po }\end{array}$ & Pollutant \\
\hline 200E P-241C112001 & & 1 & TANKS AND VESSELS & $\begin{array}{l}.000001910 \\
.000319000 \\
.000015100 \\
.000040500 \\
.000003080\end{array}$ & $\begin{array}{l}.000001910 \\
.000319000 \\
.000015100 \\
.000040500 \\
.000003080\end{array}$ & $\begin{array}{l}\text { 1.3-BUTADI ENE } \\
\text { ACETONITRILE } \\
\text { BENZENE } \\
0 \text { N-HEXANE } \\
\text { TOLUENE }\end{array}$ \\
\hline 200E P-241C201 001 & & 1 & TANKS AND VESSELS & $\begin{array}{l}.000001910 \\
.000319000 \\
.000015100 \\
.000040500 \\
.000003080\end{array}$ & $\begin{array}{l}.000001910 \\
.000319000 \\
.000015100 \\
.000040500 \\
.000003080\end{array}$ & $\begin{array}{l}\text { 1,3-BUTADIENE } \\
\text { ACETONITRILE } \\
\text { BENZENE } \\
\text { BENEXANE } \\
\text { NOLUENE } \\
\text { TOLUENE }\end{array}$ \\
\hline 200E P-241C202 001 & & 1 & TANKS AND VESSELS & $\begin{array}{l}.000001910 \\
.000319000 \\
.000015100 \\
.000040500 \\
.000003080\end{array}$ & $\begin{array}{l}.000001910 \\
.000319000 \\
.000015100 \\
.000040500 \\
.000003080\end{array}$ & $\begin{array}{ll}\text { 1.3-BUTADIENE } \\
\text { ACETONITRILE } \\
\text { BENZENE } \\
\text { BENEXANE } \\
\text { TOLUENE } \\
\text { TOLUEN }\end{array}$ \\
\hline $200 E$ P-241C203 001 & & 1 & TANKS AND VESSELS & $\begin{array}{l}.000001910 \\
.000319000 \\
.000015100 \\
.000040500 \\
.000003080\end{array}$ & $\begin{array}{l}.000001910 \\
.000319000 \\
.000015100 \\
.000040500 \\
.000003080\end{array}$ & $\begin{array}{l}\text { 1.3-BUTADIENE } \\
\text { ACETONITRILE } \\
\text { BENZENE } \\
\text { N-HEXANE } \\
\text { TOLUENE }\end{array}$ \\
\hline 200E P-242A 001 & & 6 & SOURCE EMISSIONS ROLL-UP & .000022700 & .000001700 & METHYL ISOBUTYL KETONE \\
\hline 200E P-242A 002 & & 6 & SOURCE EMISSIONS ROLL-UP & .007230000 & .000542000 & METHYL ISOBUTYL KETONE \\
\hline 200E P-296A 001 & & 1 & TANKS AND VESSELS & $\begin{array}{l}.000004130 \\
.000689000 \\
.000032800 \\
.000087600 \\
.000006670\end{array}$ & $\begin{array}{l}.000004130 \\
.000689000 \\
.000032800 \\
.000087600 \\
.000006670\end{array}$ & $\begin{array}{l}\text { 1.3-BUTADIENE } \\
\text { ACETONITRILE } \\
\text { BENZENE } \\
\text { N-HEXANE } \\
\text { TOLUENE }\end{array}$ \\
\hline 200E P-296AN 001 & & 8 & SOURCE EMISSIONS ROLL-UP & $\begin{array}{l}.000028900 \\
.004830000 \\
.000229000 \\
.000613000 \\
.000046700\end{array}$ & $\begin{array}{l}.000028900 \\
.004830000 \\
.000229000 \\
.000613000 \\
.000046700\end{array}$ & $\begin{array}{l}\text { 1.3-BUTADIENE } \\
\text { ACETONITRILE } \\
\text { BENZENE } \\
\text { N-HEXANE } \\
\text { TOLUENE }\end{array}$ \\
\hline 200E P-296AP 001 & & 9 & SOURCE EMISSIONS ROLL-UP & $\begin{array}{l}.000033000 \\
.005520000 \\
.000262000 \\
.000701000 \\
.000053400\end{array}$ & $\begin{array}{l}.000033000 \\
.005520000 \\
.000262000 \\
.000701000 \\
.000053400\end{array}$ & $\begin{array}{l}\text { 1.3-BUTADIENE } \\
\text { ACETONITRILE } \\
\text { BENZENE } \\
\text { N-HEXANE } \\
\text { TOLUENE }\end{array}$ \\
\hline
\end{tabular}

Page \#: 84 of 95 Report Run Date: 22-APR-95 
AIR EMISSIONS INVENTORY PERMIT APPLICATION REPORT (UP460)

FACILITY NAME: $200 E$ Tank Farm and Associated Structures

Point Source Emissions - HAP Pollutants

\begin{tabular}{|c|c|c|c|c|c|}
\hline Em Point & Srce Nbr & Srce Type Pollutant & $\begin{array}{l}\text { Em. Pt. Potential } \\
\text { to Emit (tons/yr) }\end{array}$ & $\begin{array}{l}\text { Em. Pt. } \\
\text { ctl Actual (tons/yr) Po }\end{array}$ & ollutant \\
\hline 200E P-296AW 001 & & 7 SOURCE EMISSIONS ROLL-UP & $\begin{array}{l}.000024800 \\
.004140000 \\
.000197000 \\
.000526000 \\
.000040000\end{array}$ & $\begin{array}{l}.000024800 \\
.004140000 \\
.000197000 \\
.000526000 \\
.000040000\end{array}$ & $\begin{array}{l}\text { ACETONITRIENE } \\
\text { BENZENE } \\
\text { N-HEXANE } \\
\text { TOLUENE }\end{array}$ \\
\hline 200E P-296AYAZ 001 & & 5 SOURCE EMISSIONS ROLL-UP & $\begin{array}{l}.000016500 \\
.002760000 \\
.000131000 \\
.000351000 \\
.000026700\end{array}$ & $\begin{array}{l}.000016500 \\
.002760000 \\
.000131000 \\
.000351000 \\
.000026700\end{array}$ & $\begin{array}{l}\text { 1.3-BUTADIENE } \\
\text { ACETONITRILE } \\
\text { BENZENE } \\
\text { N-HEXANE } \\
\text { TOLUENE }\end{array}$ \\
\hline 200E P-296P016001 & & 3 SOURCE EMISSIONS ROLL-UP & $\begin{array}{l}.000008250 \\
.001380000 \\
.000065500 \\
.000175000 \\
.000013300\end{array}$ & $\begin{array}{l}.000008250 \\
.001380000 \\
.000065500 \\
.000175000 \\
.000013300\end{array}$ & $\begin{array}{l}\text { 1.3-8UTADIENE } \\
\text { ACETONITRILE } \\
\text { BENZENE } \\
\text { N-HEXANE } \\
\text { TOLUENE }\end{array}$ \\
\hline Sum & & & .061618788 & .054909068 & \\
\hline
\end{tabular}

Point Source Emissions - Uncategorized Pollutants

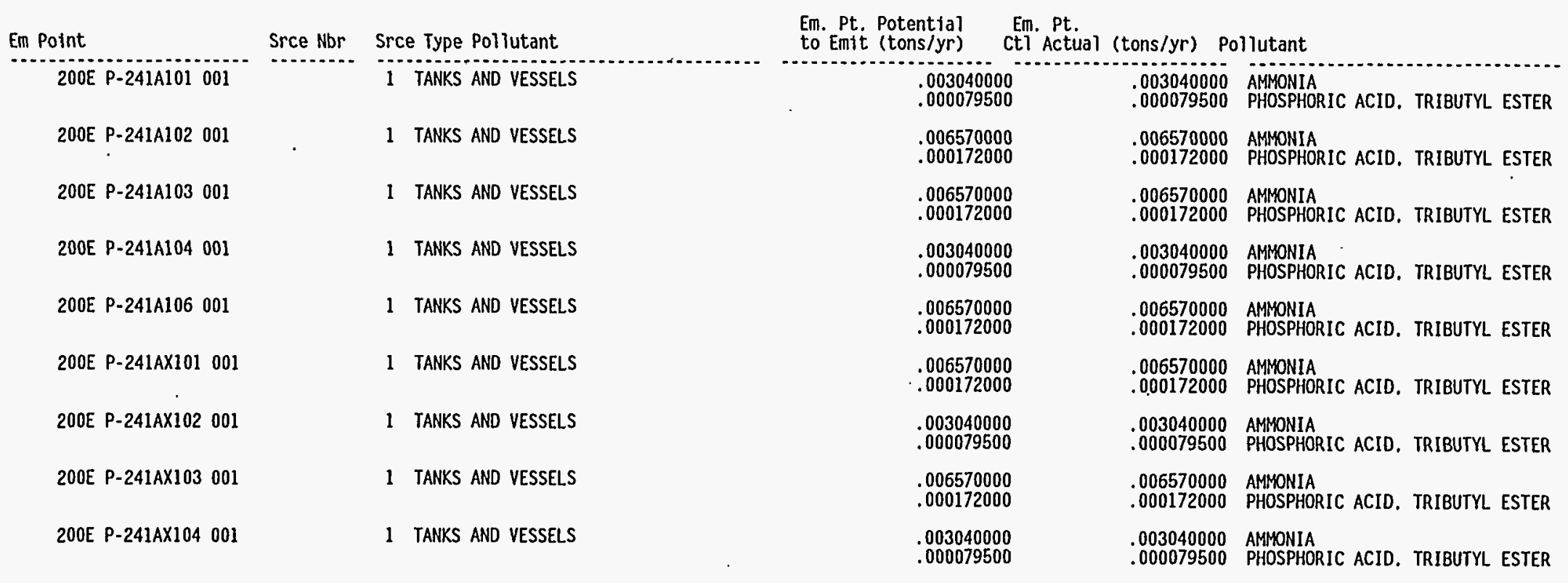

Page \#: 85 of 95 Report Run Date: 22-APR-95 
Point Source Emissions - Uncategorized Pollutants

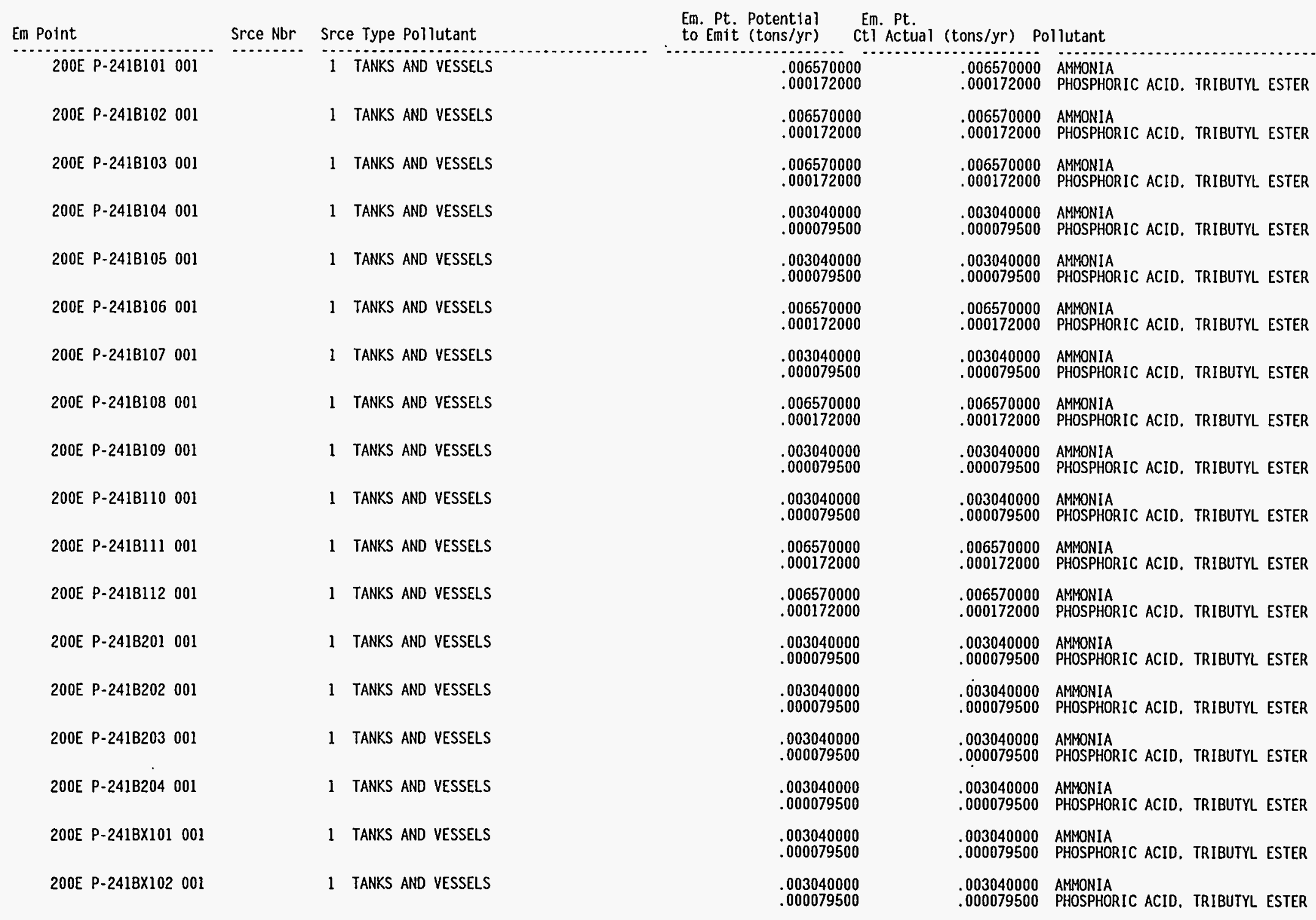


AIR EMISSIONS INVENTORY PERMIT APPLICATION REPORT (UP460)

FACILITY NAME: 200E Tank Farm and Associated Structures

Point Source Emissions - Uncategorized Pollutants

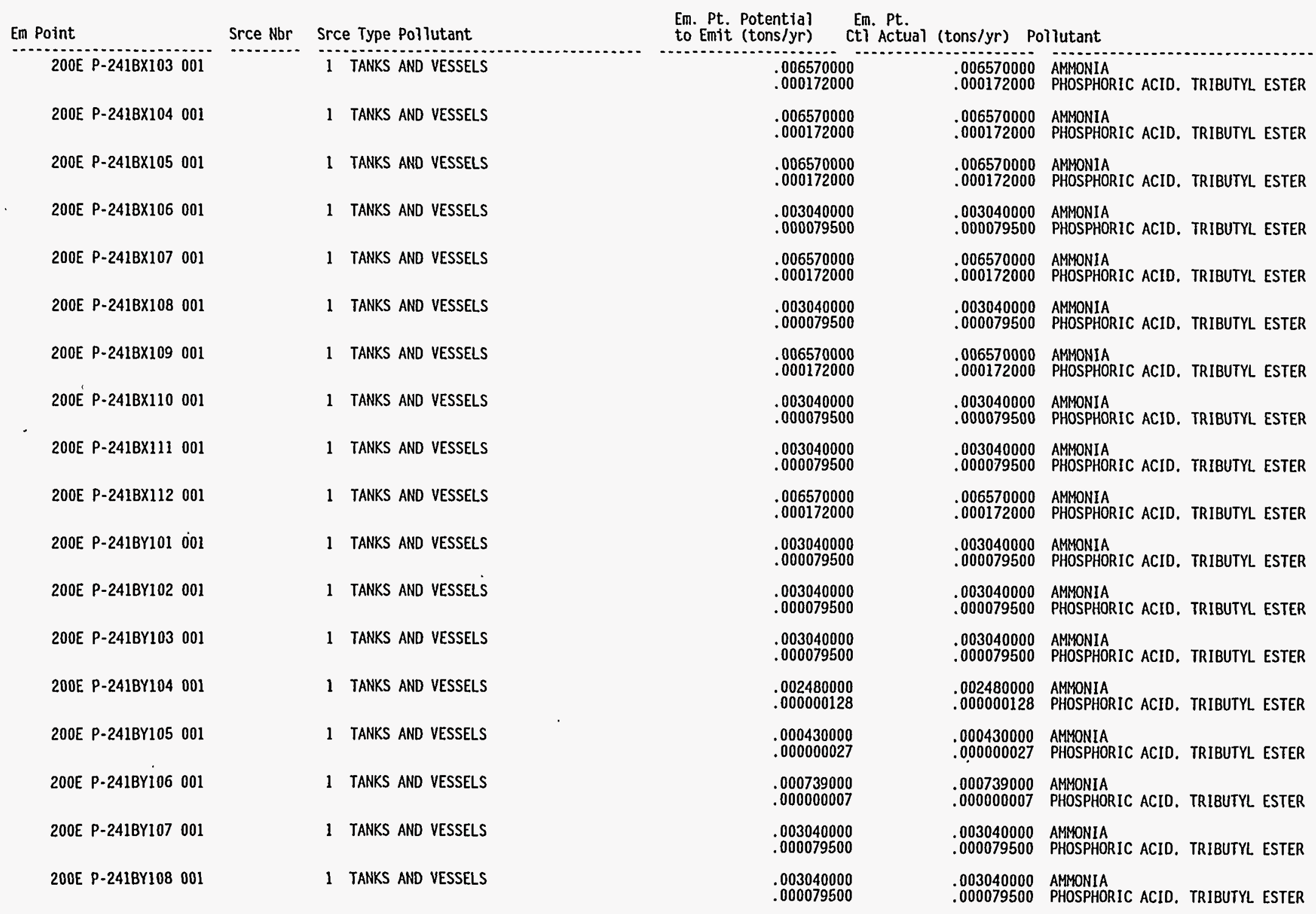

Page \#: 87 of 95 Report Run Date: 22-APR-95 
Point Source Emissions - Uncategorized Pollutants

\begin{tabular}{|c|c|c|c|c|c|c|}
\hline Em Point & Srce Nbr & Srce Type Pollutant & $\begin{array}{l}\text { Em. Pt. Potential } \\
\text { to Emit (tons/yr) }\end{array}$ & (tons/yr) & ollutant & \\
\hline 200E P-241BY109001 & & 1 TANKS AND VESSELS & $\begin{array}{l}.006570000 \\
.000172000\end{array}$ & $\begin{array}{l}.006570000 \\
.000172000\end{array}$ & $\begin{array}{l}\text { AMMONIA } \\
\text { PHOSPHORIC ACID. }\end{array}$ & TRIBUTYL ESTER \\
\hline 200E P-241BY110 001 & & 1 TANKS AND VESSELS & $\begin{array}{l}.003040000 \\
.000079500\end{array}$ & $\begin{array}{l}.003040000 \\
.000079500\end{array}$ & $\begin{array}{l}\text { AMMONIA } \\
\text { PHOSPHORIC ACID. }\end{array}$ & TRIBUTYL ESTER \\
\hline 200E P-241BY111 001 & & 1 TANKS AND VESSELS & $\begin{array}{l}.003040000 \\
.000079500\end{array}$ & $\begin{array}{l}.003040000 \\
.000079500\end{array}$ & $\begin{array}{l}\text { AMMONIA } \\
\text { PHOSPHORIC ACID. }\end{array}$ & TRIBUTYL ESTER \\
\hline 200E P-241BY112001 & & 1 TANKS AND VESSELS & $\begin{array}{l}.003040000 \\
.000079500\end{array}$ & $\begin{array}{l}.003040000 \\
.000079500\end{array}$ & $\begin{array}{l}\text { AMMONIA } \\
\text { PHOSPHORIC ACID. }\end{array}$ & TRIBUTYL ESTER \\
\hline 200E P-241C101 001 & & 1 TANKS AND VESSELS & $\begin{array}{l}.003040000 \\
.000079500\end{array}$ & $\begin{array}{l}.003040000 \\
.000079500\end{array}$ & $\begin{array}{l}\text { AMMONIA } \\
\text { PHOSPHORIC ACID. }\end{array}$ & TRIBUTYL ESTER \\
\hline 200E P-241C102 001 & & 1 TANKS AND VESSELS & $\begin{array}{l}.006570000 \\
.000172000\end{array}$ & $\begin{array}{l}.006570000 \\
.000172000\end{array}$ & $\begin{array}{l}\text { AMMONIA } \\
\text { PHOSPHORIC ACID. }\end{array}$ & TRIBUTYL ESTER \\
\hline $200 \mathrm{E} P-241 \mathrm{C} 103001$ & & 1 TANKS AND VESSELS & $\begin{array}{l}.006570000 \\
.000172000\end{array}$ & $\begin{array}{l}.006570000 \\
.000172000\end{array}$ & $\begin{array}{l}\text { AMMONIA } \\
\text { PHOSPHORIC ACID. }\end{array}$ & TRIBUTYL ESTER \\
\hline 200E P-241C107 001 & & 1 TANKS AND VESSELS & $\begin{array}{l}.006570000 \\
.000172000\end{array}$ & $\begin{array}{l}.006570000 \\
.000172000\end{array}$ & $\begin{array}{l}\text { AMMONIA } \\
\text { PHOSPHORIC ACIO. }\end{array}$ & TRIBUTYL ESTER \\
\hline $200 E \mathrm{P}-241 \mathrm{Cl} 08001$ & & 1 TANKS AND VESSELS & .003040000 & .003040000 & AMMONIA & \\
\hline 200E P-24IC109001 & & 1 TANKS AND VESSELLS & $\begin{array}{l}.003040000 \\
.000079500\end{array}$ & $\begin{array}{l}.003040000 \\
.000079500\end{array}$ & $\begin{array}{l}\text { AMMONIA } \\
\text { PHOSPHORIC ACID. }\end{array}$ & TRIBUTYL ESTER \\
\hline 200E P-241C110 001 & & 1 TANKS AND VESSELS & $\begin{array}{l}.003040000 \\
.000079500\end{array}$ & $\begin{array}{l}.003040000 \\
.000079500\end{array}$ & $\begin{array}{l}\text { AMMONIA } \\
\text { PHOSPHORIC ACID. }\end{array}$ & TRIBUTYL ESTER \\
\hline 200E P-241C111 001 & & 1 TANKS AND VESSELS & .003040000 & .003040000 & AMMONIA & \\
\hline 200E P-241C112001 & & 1 TANKS AND VESSELS & $\begin{array}{l}.003040000 \\
.000079500\end{array}$ & $\begin{array}{l}.003040000 \\
.000079500\end{array}$ & $\begin{array}{l}\text { AMMONIA } \\
\text { PHOSPHORIC ACID. }\end{array}$ & TRIBUTYL ESTER \\
\hline 200E P-241C201 001 & & 1 TANKS AND VESSELS & $\begin{array}{l}.003040000 \\
.000079500\end{array}$ & $\begin{array}{l}.003040000 \\
.000079500\end{array}$ & $\begin{array}{l}\text { AMHONIA } \\
\text { PHOSPHORIC ACID. }\end{array}$ & TRIBUTYL ESTER \\
\hline 200E P-241C202 001 & & 1 TANKS AND VESSELS & $\begin{array}{l}.003040000 \\
.000079500\end{array}$ & $\begin{array}{l}.003040000 \\
.000079500\end{array}$ & $\begin{array}{l}\text { AMMONIA } \\
\text { PHOSPHORIC ACID. }\end{array}$ & TRIBUTYL ESTER \\
\hline 200E P-241C203 001 & & 1 TANKS AND VESSELS & $\begin{array}{l}.003040000 \\
.000079500\end{array}$ & $\begin{array}{l}.003040000 \\
.000079500\end{array}$ & $\begin{array}{l}\text { AMMONIA } \\
\text { PHOSPHORIC ACID. }\end{array}$ & TRIBUTYL ESTER \\
\hline 200E P-241C204 001 & & 1 TANKS AND VESSELS & $\begin{array}{l}.003040000 \\
.000079500\end{array}$ & $\begin{array}{l}.003040000 \\
.000079500\end{array}$ & $\begin{array}{l}\text { AMMONIA } \\
\text { PHOSPHORIC ACID. }\end{array}$ & TRIBUTYL ESTER \\
\hline 200E P-242A 001 & & 6 SOURCE EMISSIONS ROLL-UP & .000314000 & .000023500 & AMMONIA & \\
\hline 200E P-242A 002 & & 6 SOURCE EMISSIONS ROLL-UP & .099900000 & .007490000 & AMMONIA & \\
\hline
\end{tabular}


AIR EMISSIONS INVENTORY PERMIT APPLICATION REPORT (UP460)

FACILITY NAME: FACILITY ID : 200E TANK

Point Source Emissions - Uncategorized Pollutants

\begin{tabular}{|c|c|c|c|c|c|c|c|}
\hline En Point & Srce Nbr & Srce & e Type Pollutant & $\begin{array}{l}\text { Em. Pt. Potential } \\
\text { to Emit (tons/yr) }\end{array}$ & $\begin{array}{l}\text { Em. Pt. } \\
\text { t] Actual (tons/yr) }\end{array}$ & ollutant & \\
\hline 200E P-296A 001 & & & TANKS AND VESSELS & $\begin{array}{l}.321000000 \\
.000172000\end{array}$ & $\begin{array}{l}.321000000 \\
.000172000\end{array}$ & $\begin{array}{l}\text { AMMONIA } \\
\text { PHOSPHORIC ACID. }\end{array}$ & TRIBUTYL ESTER \\
\hline 200E P-296AN 001 & & 8 & SOURCE EMISSIONS ROLL-UP & $\begin{array}{r}1.390000000 \\
.001200000\end{array}$ & $\begin{array}{r}1.390000000 \\
.001200000\end{array}$ & $\begin{array}{l}\text { AMMONIA } \\
\text { PHOSPHORIC ACID. }\end{array}$ & TRIBUTYL ESTER \\
\hline 200E P-296AP 001 & & 9 & SOURCE EMISSIONS ROLL-UP & $\begin{array}{l}.352000000 \\
.001380000\end{array}$ & $\begin{array}{l}.352000000 \\
.001380000\end{array}$ & $\begin{array}{l}\text { AMMONIA } \\
\text { PHOSPHORIC ACID. }\end{array}$ & TRIBUTYL ESTER \\
\hline 200E P-296AW 001 & & 7 & SOURCE EMISSIONS ROLL-UP & $\begin{array}{l}.282000000 \\
.001030000\end{array}$ & $\begin{array}{l}.282000000 \\
.001030000\end{array}$ & $\begin{array}{l}\text { AMMONIA } \\
\text { PHOSPHORIC ACID. }\end{array}$ & TRIBUTYL ESTER \\
\hline 200E P-296AYAZ 001 & & 5 & SOURCE EMISSIONS ROLL-UP & $\begin{array}{r}1.280000000 \\
.000688000\end{array}$ & $\begin{array}{r}1.280000000 \\
.000688000\end{array}$ & $\begin{array}{l}\text { AMMONIA } \\
\text { PHOSPHORIC ACIO. }\end{array}$ & TRIBUTYL ESTER \\
\hline 200E P-296P016 001 & & & SOURCE EMISSIONS ROLL-UP & $\begin{array}{l}.642000000 \\
.000344000\end{array}$ & $\begin{array}{l}.642000000 \\
.000344000\end{array}$ & $\begin{array}{l}\text { AMMONIA } \\
\text { PHOSPHORIC ACID. }\end{array}$ & TRIBUTYL ESTER \\
\hline sum & & & & 4.639263661 & 4.546563161 & & \\
\hline
\end{tabular}

Fugitive Source Criteria Pollutants - Type: VOC

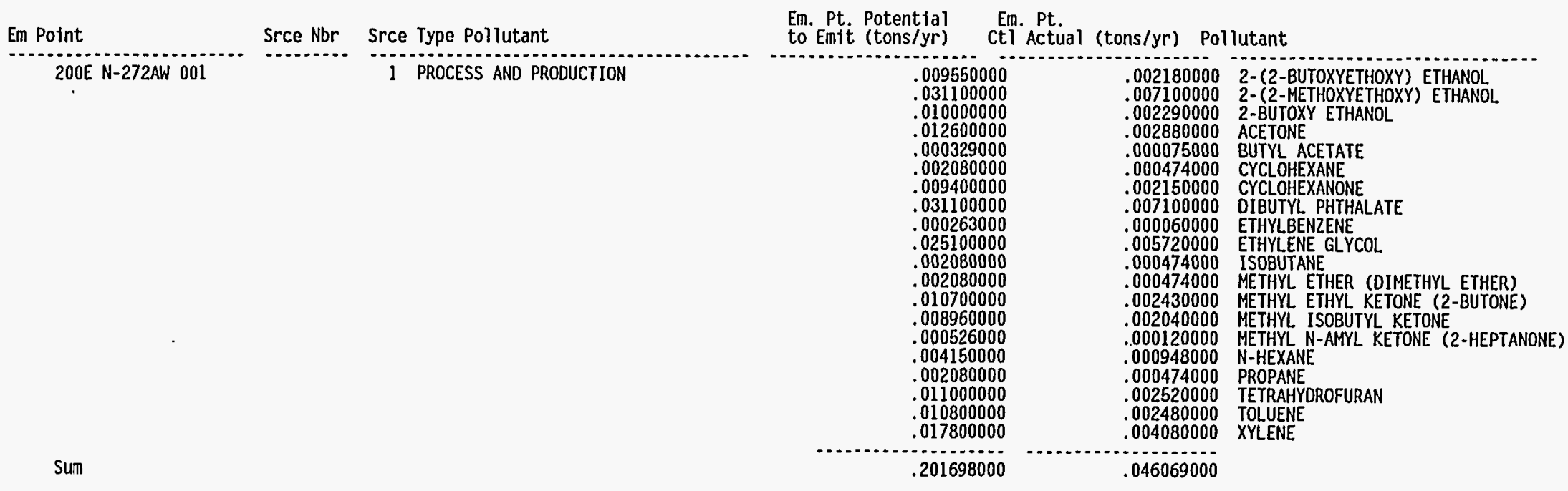


AIR EMISSIONS INVENTORY PERMIT APPLICATION REPORT (UP460)

FACILITY NAME: $200 E$ Tank Farm and Associated Structures

Fugitive Source Emissions - Radiological Pollutants

\begin{tabular}{|c|c|c|c|c|c|c|}
\hline Em Point & Srce Nbr & Srce & Type Pollutant & $\begin{array}{l}\text { Em. Pt. Potential } \\
\text { to Emit }(\mathrm{Ci} / \mathrm{yr})\end{array}$ & $(\mathrm{Cl} / \mathrm{yr})$ & Pollutant \\
\hline 200E P-241A151 001 & & 1 & PROCESS AND PRODUCTION & $\begin{array}{l}.000000000 \\
.000000002 \\
.000000002\end{array}$ & $\begin{array}{l}.000000000 \\
.000000001 \\
.000000001\end{array}$ & $\begin{array}{l}\text { AMERICIUM }-241 \\
\text { STRONTIUM } 89.90 \\
\text { YTTRIUM - } 090\end{array}$ \\
\hline 200E P-241A302A 001 & & 1 & PROCESS AND PRODUCTION & $\begin{array}{l}.000000000 \\
.000000001 \\
.000000001\end{array}$ & $\begin{array}{l}.000000000 \\
.000000001 \\
.000000001\end{array}$ & $\begin{array}{l}\text { AMERICIUM - } 241 \\
\text { STRONTIUM } 89.90 \\
\text { YTTRIUM - } 090\end{array}$ \\
\hline 200E P-241A350 001 & & 1 & PROCESS AND PRODUCTION & $\begin{array}{l}.000000000 \\
.000000000 \\
.000000000\end{array}$ & $\begin{array}{l}.000000000 \\
.000000000 \\
.000000000\end{array}$ & $\begin{array}{l}\text { AMERICIUM }-241 \\
\text { STRONTIUM } 89.90 \\
\text { YTTRIUM - } 090\end{array}$ \\
\hline 200E P-241A401 001 & & 1 & PROCESS AND PRODUCTION & $\begin{array}{l}.000000000 \\
.000000000 \\
.000000000\end{array}$ & $\begin{array}{l}.000000000 \\
.000000000 \\
.000000000\end{array}$ & $\begin{array}{l}\text { AMERICIUM }-241 \\
\text { STRONTIUM } 89.90 \\
\text { YTTRIUM - } 090\end{array}$ \\
\hline 200E P-241A417 001 & & 1 & PROCESS AND PRODUCTION & $\begin{array}{l}.000000000 \\
.000000002 \\
.000000002\end{array}$ & $\begin{array}{l}.000000000 \\
.000000002 \\
.000000002\end{array}$ & $\begin{array}{l}\text { AMERICIUM }-241 \\
\text { STRONTIUM } 89.90 \\
\text { YTTRIUM - } 090\end{array}$ \\
\hline 200E P-241AA 001 & & 1 & PROCESS AND PRODUCTION & $\begin{array}{l}.000000000 \\
.00000000 \\
.000000000\end{array}$ & $\begin{array}{l}.000000000 \\
.000000000 \\
.000000000\end{array}$ & $\begin{array}{l}\text { AMERICIUM }-241 \\
\text { STRONTIUM } 89.90 \\
\text { VTTRIUM - } 090\end{array}$ \\
\hline 200E P-241ANA 001 & & 1 & PROCESS AND PRODUCTION & $\begin{array}{l}.000000000 \\
.000000001 \\
.000000001\end{array}$ & $\begin{array}{l}.000000000 \\
.000000000 \\
.000000000\end{array}$ & $\begin{array}{l}\text { AMERICIUM }-241 \\
\text { STRONTIUM } 89.90 \\
\text { YTTRIUM }-090\end{array}$ \\
\hline 200E P-241ANB 001 & & 1 & PROCESS AND PRODUCTION & $\begin{array}{l}.000000000 \\
.000000000 \\
.000000000\end{array}$ & $\begin{array}{l}.000000000 \\
.000000000 \\
.000000000\end{array}$ & $\begin{array}{l}\text { AMERICIUM }-241 \\
\text { STRONTIUM } 89,90 \\
\text { YTTRIUM - } 090\end{array}$ \\
\hline 200E P-241AP 001 & & 1 & PROCESS AND PRODUCTION & $\begin{array}{l}.000000000 \\
.000000003 \\
.000000003\end{array}$ & $\begin{array}{l}.000000000 \\
.000000001 \\
.000000001\end{array}$ & $\begin{array}{l}\text { AMERICIUM }-241 \\
\text { STRONTIUM } 89.90 \\
\text { YTTRIUM - } 090\end{array}$ \\
\hline 200E P-241AR151 001 & & 1 & PROCESS AND PRODUCTION & $\begin{array}{l}.000000000 \\
.000000000 \\
.000000000\end{array}$ & $\begin{array}{l}.000000000 \\
.000000000 \\
.000000000\end{array}$ & $\begin{array}{l}\text { AMERICIUM }-241 \\
\text { STRONTIUM } 89.90 \\
\text { YTTRIUM }-090\end{array}$ \\
\hline 200E P-241AWA 001 & & 1 & PROCESS AND PROOUCTION & $\begin{array}{l}.000000000 \\
.000000000 \\
.000000000\end{array}$ & $\begin{array}{l}.000000000 \\
.000000000 \\
.000000000\end{array}$ & $\begin{array}{l}\text { AMERICIUM }-241 \\
\text { STRONTUUM } 89.90 \\
\text { YTTRIUM - } 090\end{array}$ \\
\hline 200E P-241AWB 001 & & 1 & PROCESS AND PRODUCTION & $\begin{array}{l}.001330000 \\
.177000000\end{array}$ & $\begin{array}{l}.000000000 \\
.000000000 \\
.000000000\end{array}$ & $\begin{array}{l}\text { AMERICIUM - } 241 \\
\text { STRONTIUM } 89.90 \\
\text { YTTRIUM - } 090\end{array}$ \\
\hline
\end{tabular}


AIR EMISSIONS INVENTORY PERMIT APPLICATION REPORT (UP460)

FACILITY ID : 200E TANK

FACILITV NAME: 200E Tank Farm and Associated Structures

Fugitive Source Emissions - Radiological Pollutants

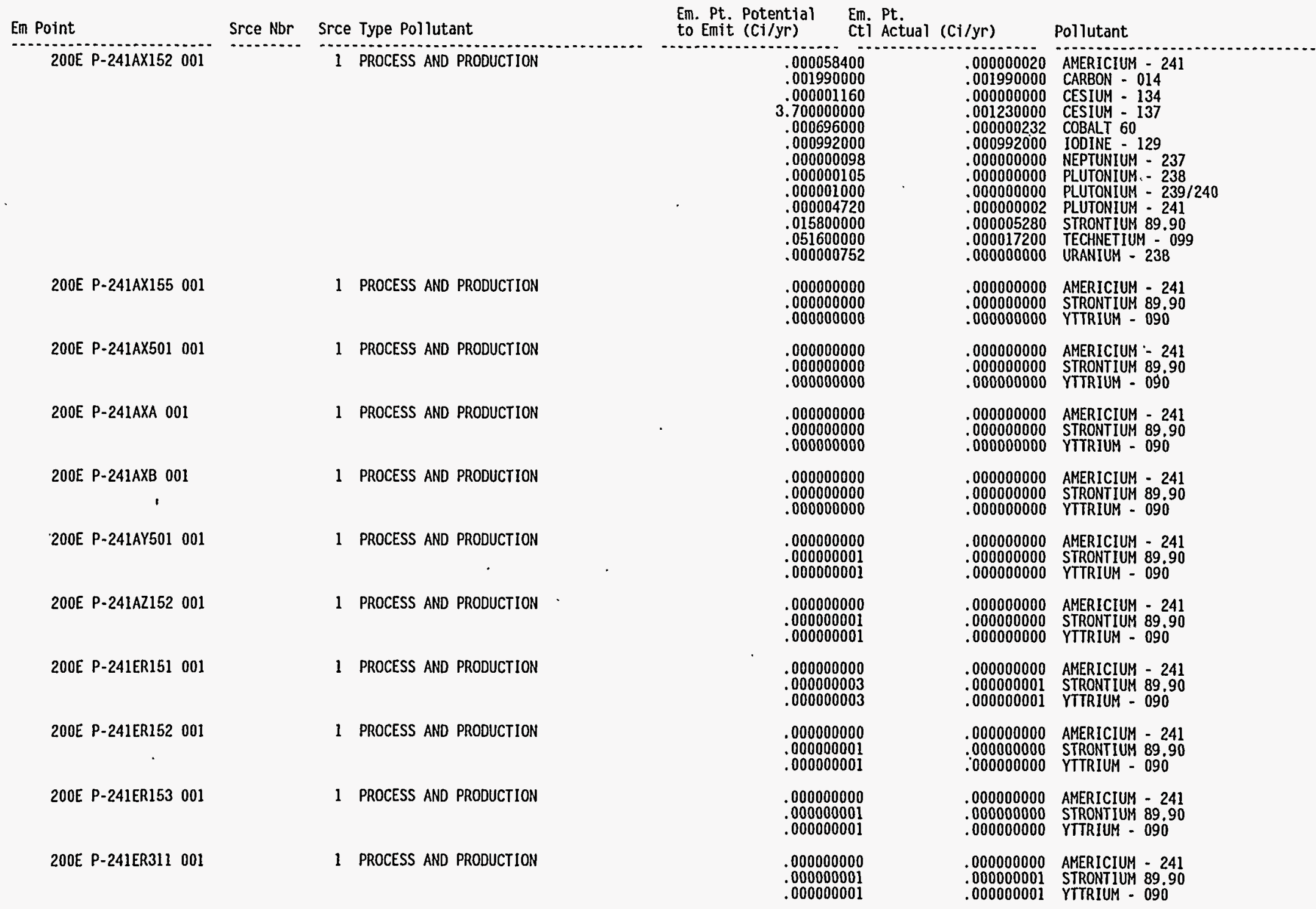

Page \#: 91 of 95 Report Run Date: 22-APR-95 
AIR EMISSIONS INVENTORY PERMIT APPLICATION REPORT (UP460)

FACILITY ID : 200E TANK
FACILITY NAME: 200E Tank Farm and Associated structures

Fugitive Source Emissions - HAP Pollutants

Em Point

Srce Nbr Srce Type Pollutant

$200 \mathrm{E} N-272 \mathrm{AW} 00$

1 PROCESS AND PRODUCTION

sum

Fugitive Source Emissions - Uncategorized Pollutants

Em Point

200E N-272AW 001

Srce Nbr Srce Type Pollutant

1 PROCESS AND PRODUCTION

200E P-241AX152 001

1 PROCESS AND PRODUCTION

Sum

Insignificant Source Criteria Pollutants - Type: VOC
Em. Pt. Potential Em. Pt.
to Emit (tons/yr) ctl Actual (tons/yr) Pollutant

\begin{tabular}{|c|c|c|}
\hline $\begin{array}{l}.009550000 \\
.031100000 \\
.010000000 \\
.031100000 \\
.000263000 \\
.025100000 \\
.010700000 \\
.008960000 \\
.004150000 \\
.010800000 \\
.017800000\end{array}$ & $\begin{array}{l}.002180000 \\
.007100000 \\
.002290000 \\
.007100000 \\
.000060000 \\
.005720000 \\
.002430000 \\
.002040000 \\
.000948000 \\
.002480000 \\
.004080000\end{array}$ & $\begin{array}{l}\text { 2-(2-BUTOXYETHOXY) ETHANOL } \\
\text { 2-(2-METHOXYETHOXY) ETHANOL } \\
\text { 2-BUTOXY ETHANOL } \\
\text { DIBUTYL PHTHALATE } \\
\text { ETHYLBENZENE } \\
\text { ETHYLENE GLYCOL } \\
\text { METHYL ETHYL KETONE (2-BUTONE) } \\
\text { METHYL ISOBUTYL KETONE } \\
\text { N-HEXANE } \\
\text { TOLUENE } \\
\text { XYLENE }\end{array}$ \\
\hline .159523000 & .036428000 & \\
\hline
\end{tabular}

Em. Pt. Potential Em. Pt.
to Emit (tons/yr) Ctl Actual (tons/yr) Pollutant

\begin{tabular}{|c|c|c|}
\hline .010000000 & .002290000 & \multirow{4}{*}{$\begin{array}{l}2,2,4 \text {-TRIMETHYL-1.3-PENTANEDIOL } \\
\text { MONOISOBUTYRATE } \\
\text { ESTER ALCOHOL (REPORTED AS CAS 25264-77- } \\
\text { 4) } \\
\text { LIGROINE OR VMEP NAPHTHA, OR PETROLEUM } \\
\text { NAPHTHA } \\
\text { PHOSPHORIC ACID } \\
\text { POLYYINYL CHLORIDE LATEX } \\
\text { VM\&P NAPHTHA }\end{array}$} \\
\hline .031100000 & .007100000 & \\
\hline .018800000 & .004280000 & \\
\hline $\begin{array}{l}.011100000 \\
.001520000 \\
.000788000\end{array}$ & $\begin{array}{l}.002540000 \\
.000347000 \\
.000180000\end{array}$ & \\
\hline $\begin{array}{l}286.000000000 \\
600.000000000\end{array}$ & $\begin{array}{l}286.000000000 \\
600.000000000\end{array}$ & \multirow[t]{2}{*}{$\begin{array}{l}\text { EUROPIUM } \\
\text { YTTRIUM }\end{array}$} \\
\hline 886.073308000 & 886.016737000 & \\
\hline
\end{tabular}

Em. Pt. Potential Em. Pt.
to Emit (tons/yr) ctl Actual

to Emit (tons/yr) ctl Actual (tons/yr) Pollutant

\begin{tabular}{|c|c|c|}
\hline $\begin{array}{l}.000012000 \\
.000030000 \\
.000000000\end{array}$ & $\begin{array}{l}.000012000 \\
.000030000 \\
.000000000\end{array}$ & $\begin{array}{l}\text { ISOPROPYL ALCOHOL } \\
\text { N-BUTY AL COHOL } \\
\text { NITROSODIMETHYLAMINE }\end{array}$ \\
\hline narasona & 000042000 & \\
\hline
\end{tabular}

Page \#: 92 of 95 Report Run Date: 22-APR-95 
AIR EMISSIONS INVENTORY PERMIT APPLICATION REPORT (UP460)

FACILITY NAME: 200 Tank Farm and Associated Structures

Insignificant Source Emissions - Radiological Pollutants

\begin{tabular}{|c|c|c|c|c|c|}
\hline Em Point & Srce $\mathrm{Nbr}$ & Srce Type Pollutant & $\begin{array}{l}\text { Em. Pt. Potential } \\
\text { to Emit }(\mathrm{Ci} / \mathrm{yr})\end{array}$ & $\begin{array}{l}\text { Em. Pt. } \\
\text { Cti Actual (Ci/yr) }\end{array}$ & Pollutant \\
\hline 200E P-LERF 001 & & 1 PROCESS AND PRODUCTION & $\begin{array}{l}.00004 \\
1.70000\end{array}$ & $\begin{array}{l}.000047000 \\
1.700000000\end{array}$ & $\begin{array}{l}\text { RUTHENIUM - } 106 \\
\text { TRITIUM (HYOROGEN - 003) }\end{array}$ \\
\hline
\end{tabular}

Insignificant Source Emissions - HAP Pollutants

\begin{tabular}{l} 
Em Point \\
\hline 200 Srce Nbr \\
SUm
\end{tabular}

\begin{tabular}{|c|c|c|}
\hline 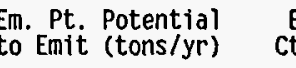 & $\begin{array}{l}\text { Em. Pt. } \\
\text { atf Actual (tons/yr) }\end{array}$ & Pollutant \\
\hline .000000000 & .00000000 & NITROSODIMETHYLAMINE \\
\hline .000000000 & .000000000 & \\
\hline
\end{tabular}

Insignificant Source Emissions - Uncategorized Pollutants

\begin{tabular}{|c|c|c|c|c|c|}
\hline Em Point & Srce Nbr & Srce Type Pollutant & $\begin{array}{l}\text { Em. Pt. Potential } \\
\text { to Emit (tons/yr) }\end{array}$ & $\begin{array}{l}\text { Em. Pt. } \\
\text { ctl Actual (tons/yr) }\end{array}$ & Pollutant \\
\hline 200E P-LERF 001 & & 1 PROCESS AND PRODUCTION & .11000 & .1100000 & AMMONIA \\
\hline Sum & & & .11000 & .1100000 & \\
\hline
\end{tabular}

Control Device Listing

Em Point

Control Equipment Code

200E P-204AR 001

200E P-204AR 001

200E P-241A103 001

$200 E$ P-241A104 001

$200 E$ P-241A106 001

200E P-241AX101 001

$200 E$ P-241AX102 00

$200 E$ P-241AX103 00

$200 E$ P-241AX104 O01

200E P-241B101 001

200E P-241BI02 001

200E $P-2418103$ 001

$200 E$ P.241B105 001

$200 E$ P-241B106 001

$200 E$ P.241B107 001

$200 E$ P. $241 B 108$ OOI

$200 E$ P.241BI09 001

200E P-241B110 001

200E P-241B111 001

099
099
099
099
099
099
099
099
099
099
099
099
099
099
099
099
099
099
099
099
099 


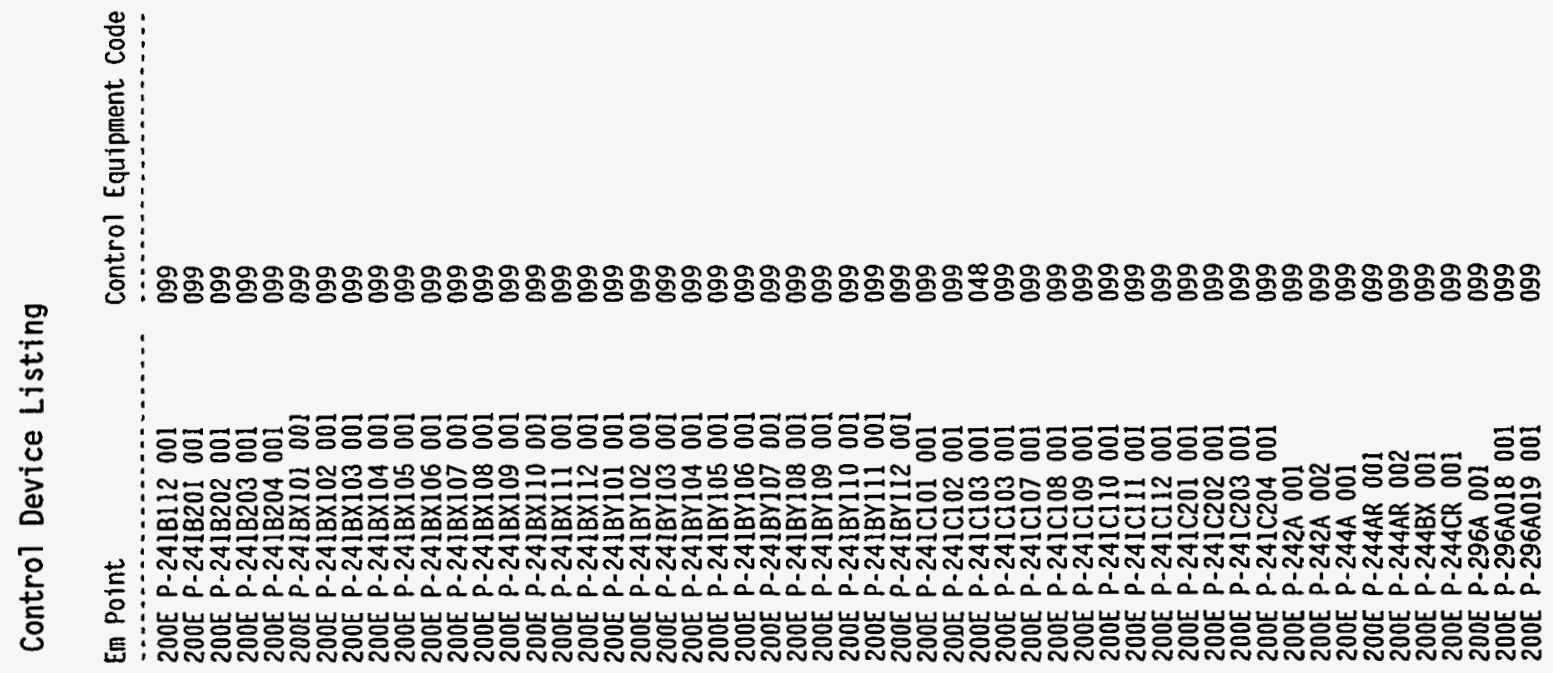



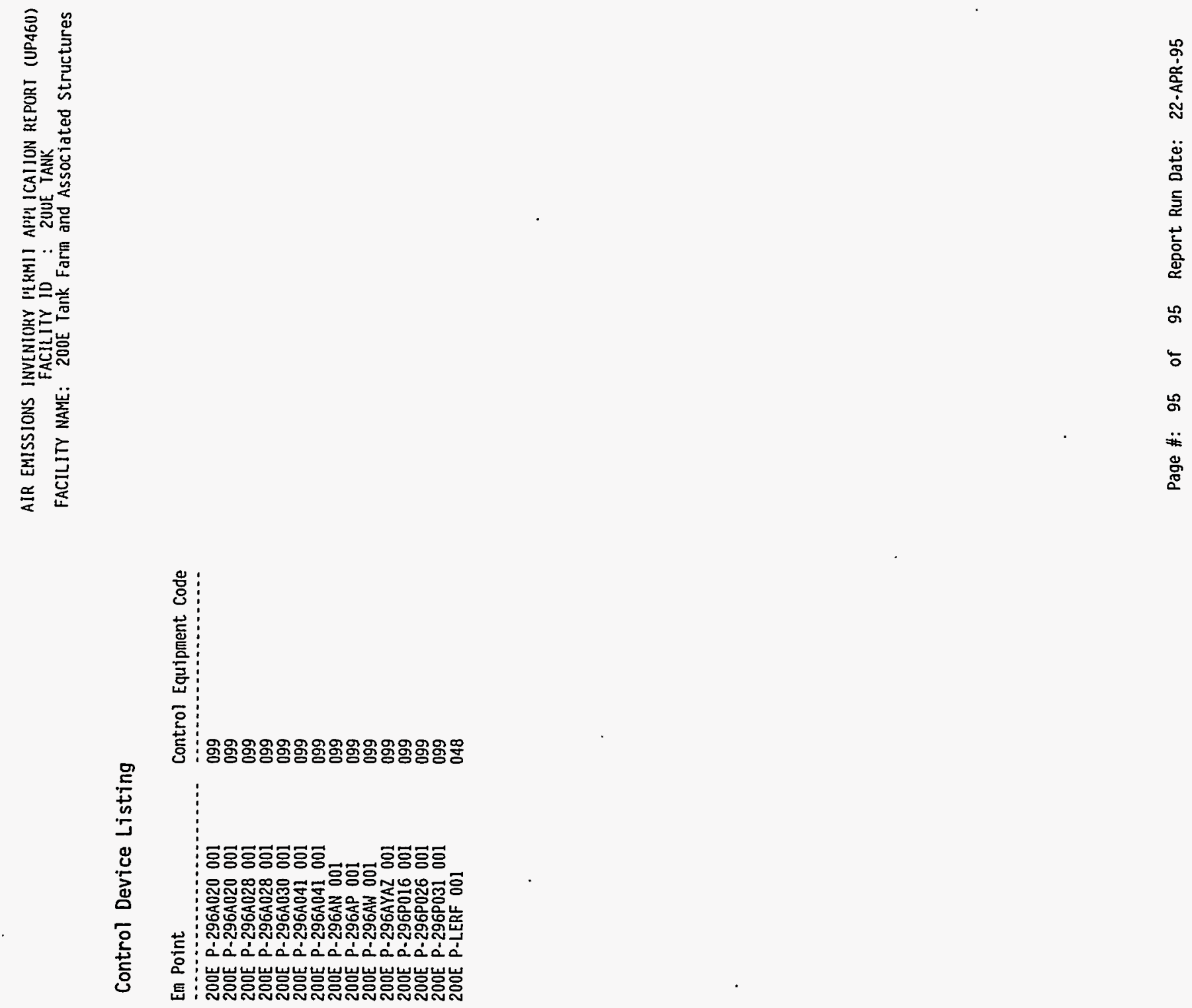


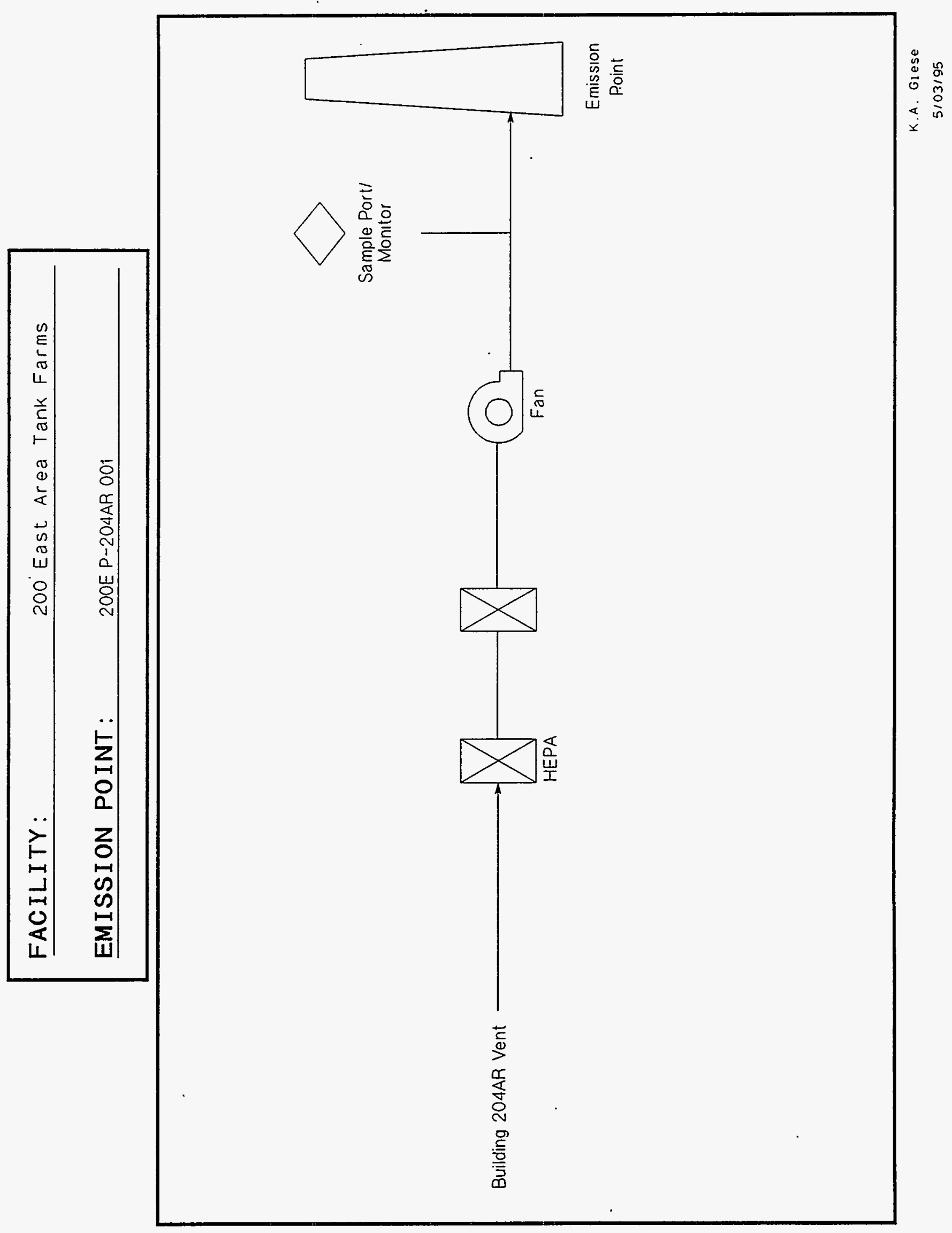




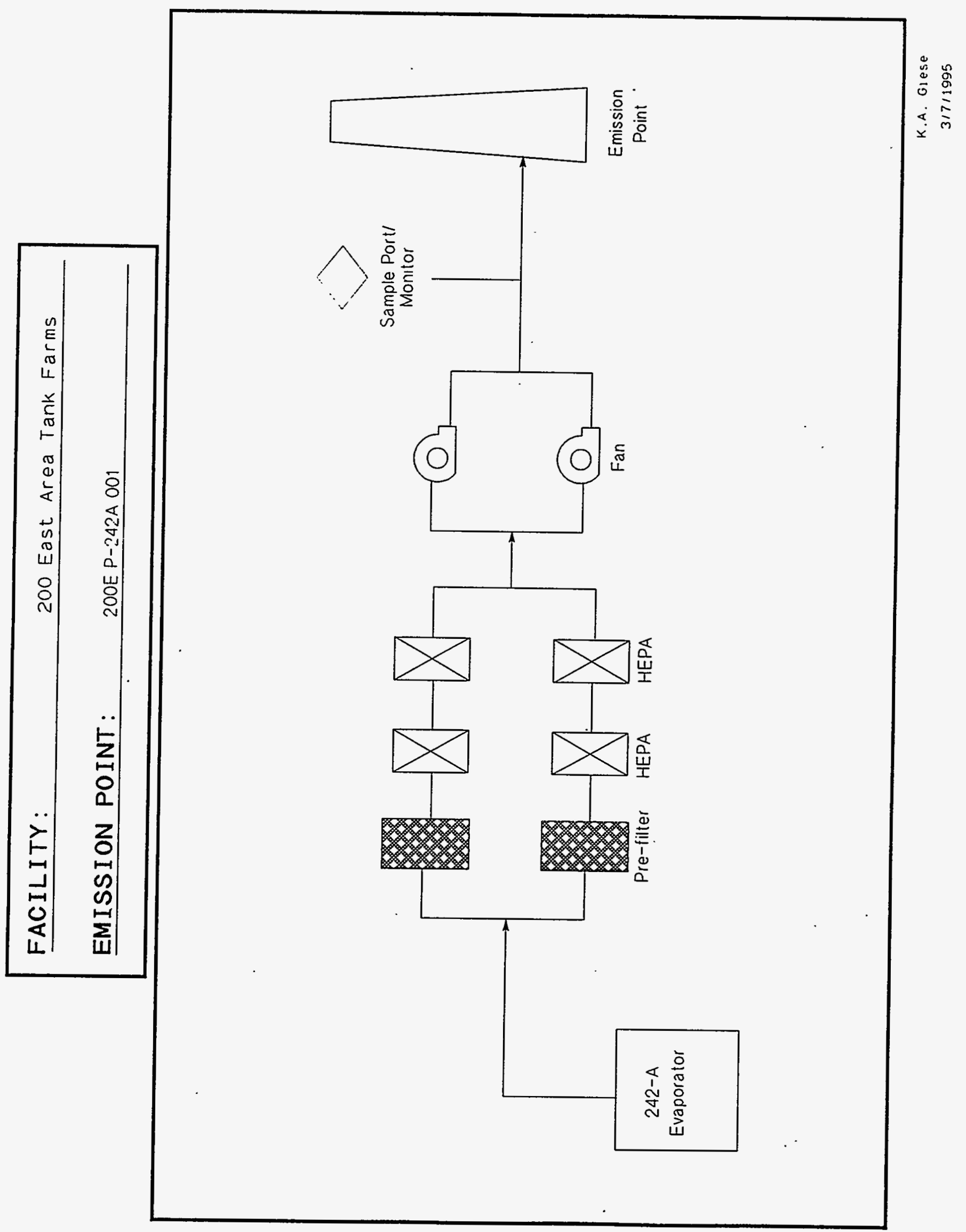




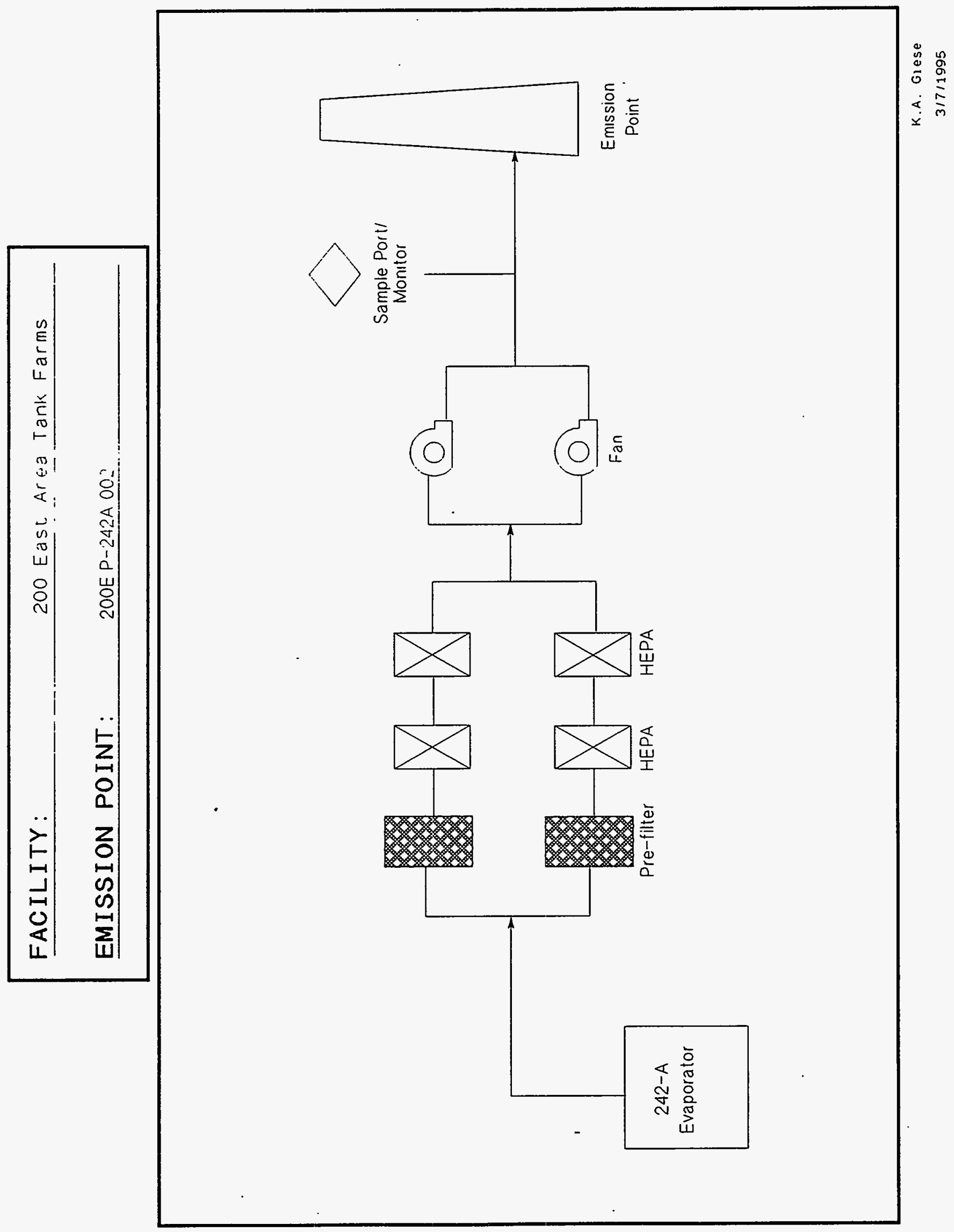




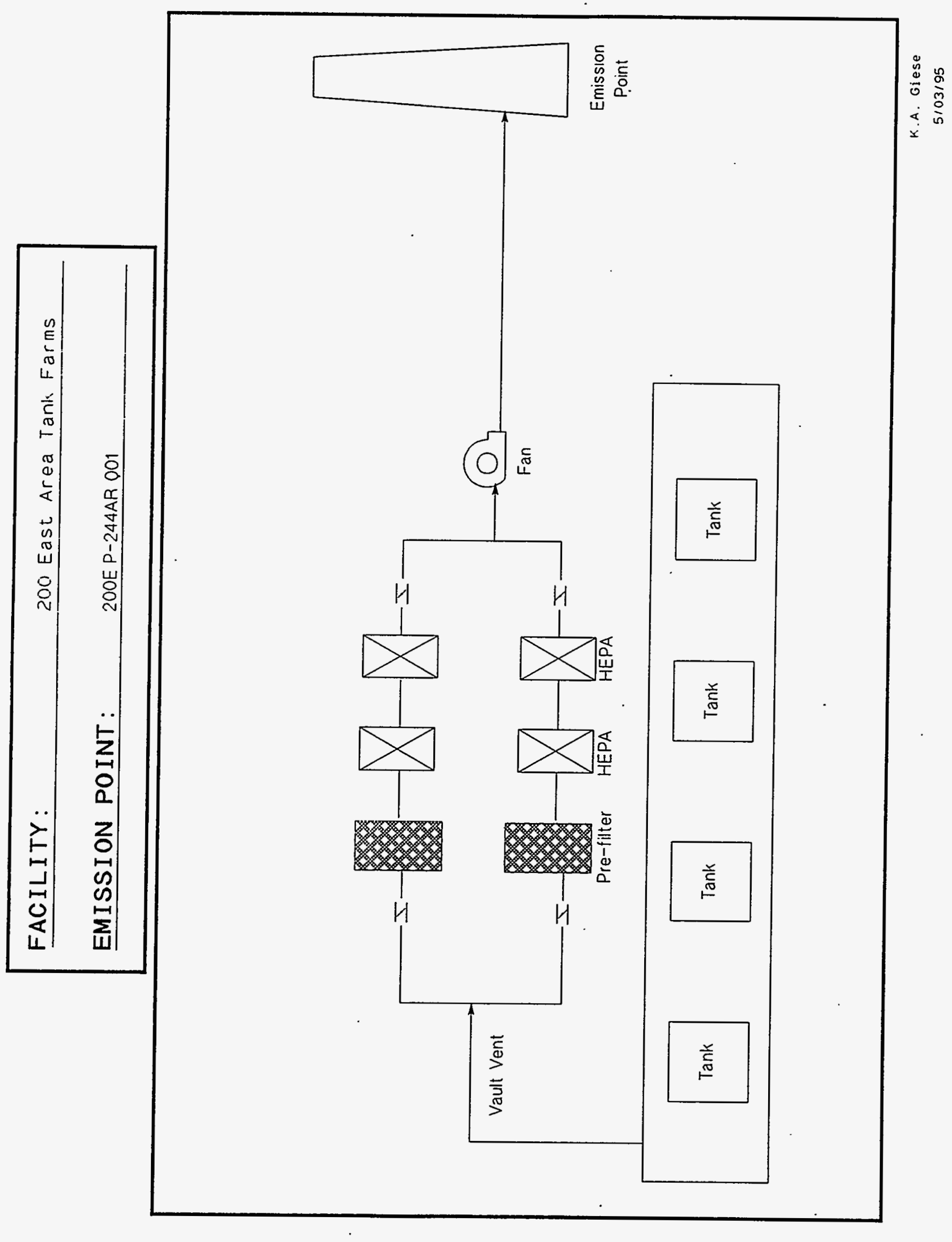




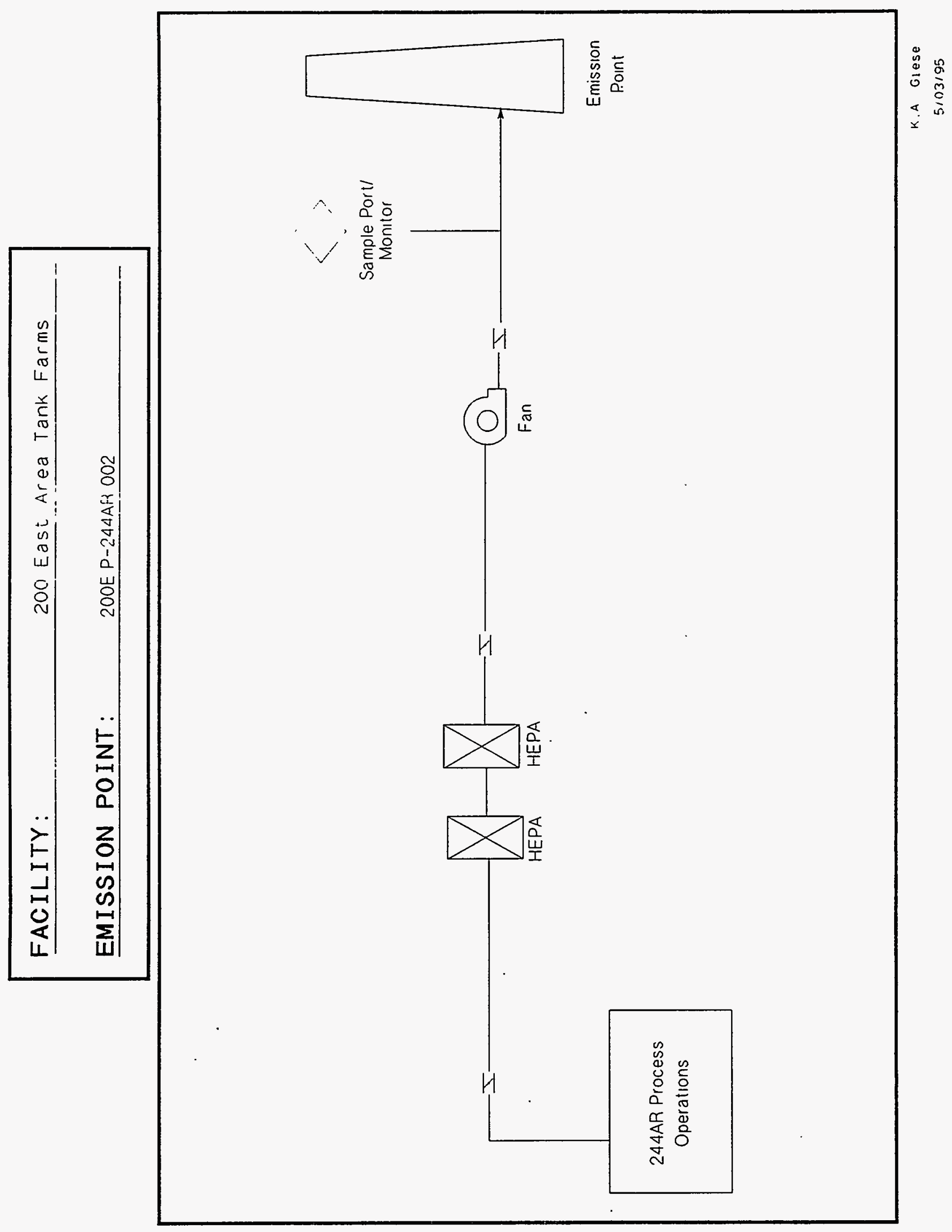




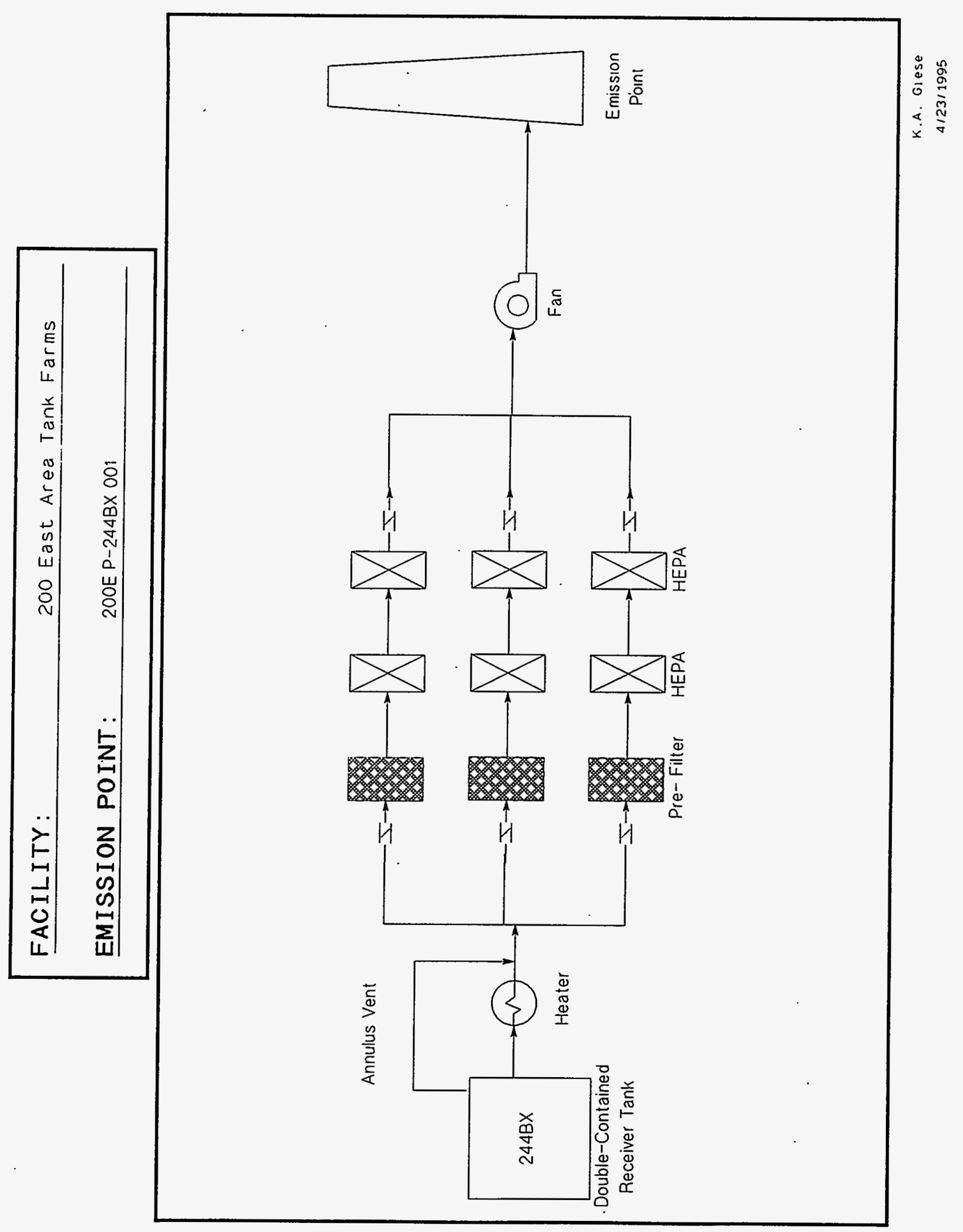




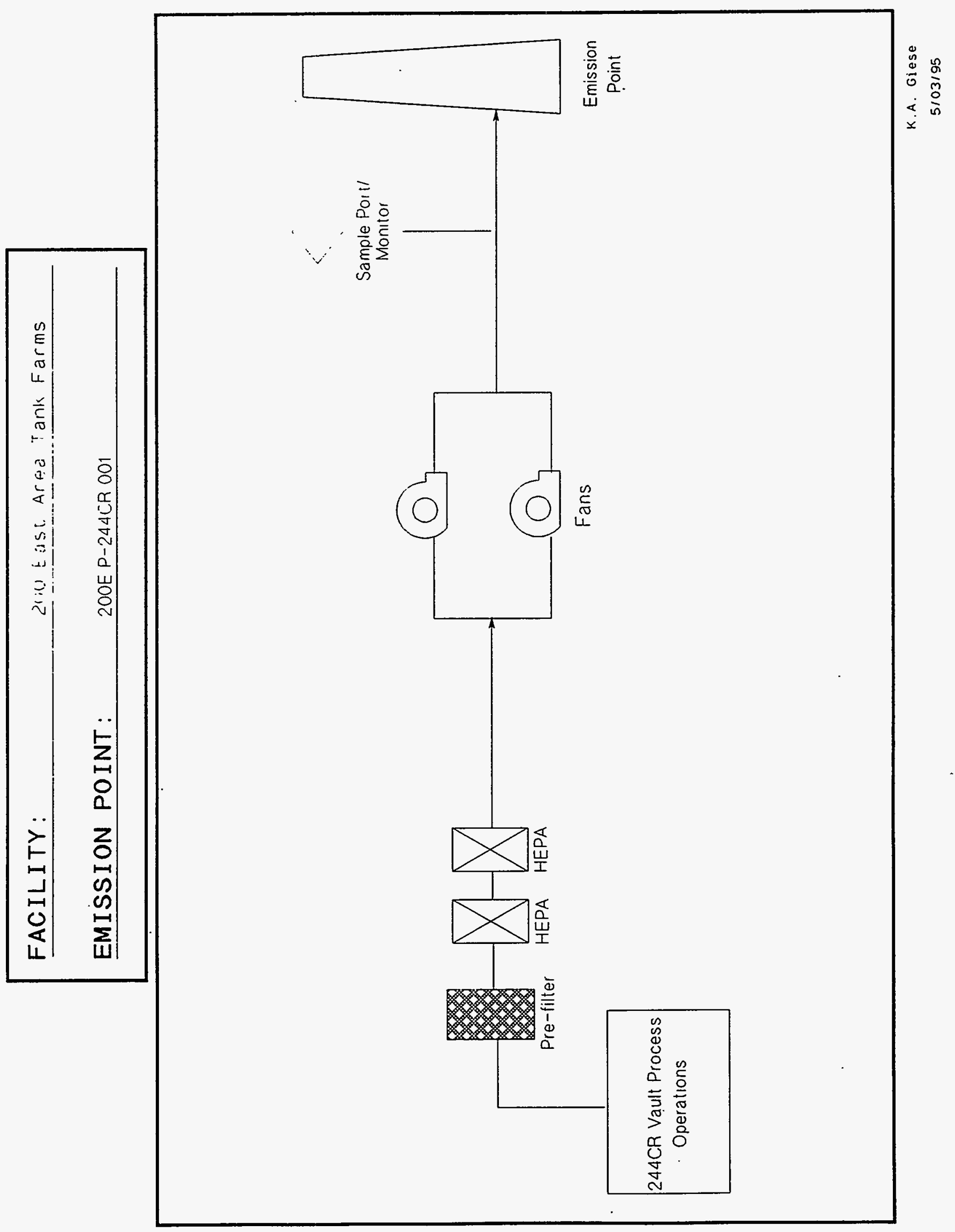




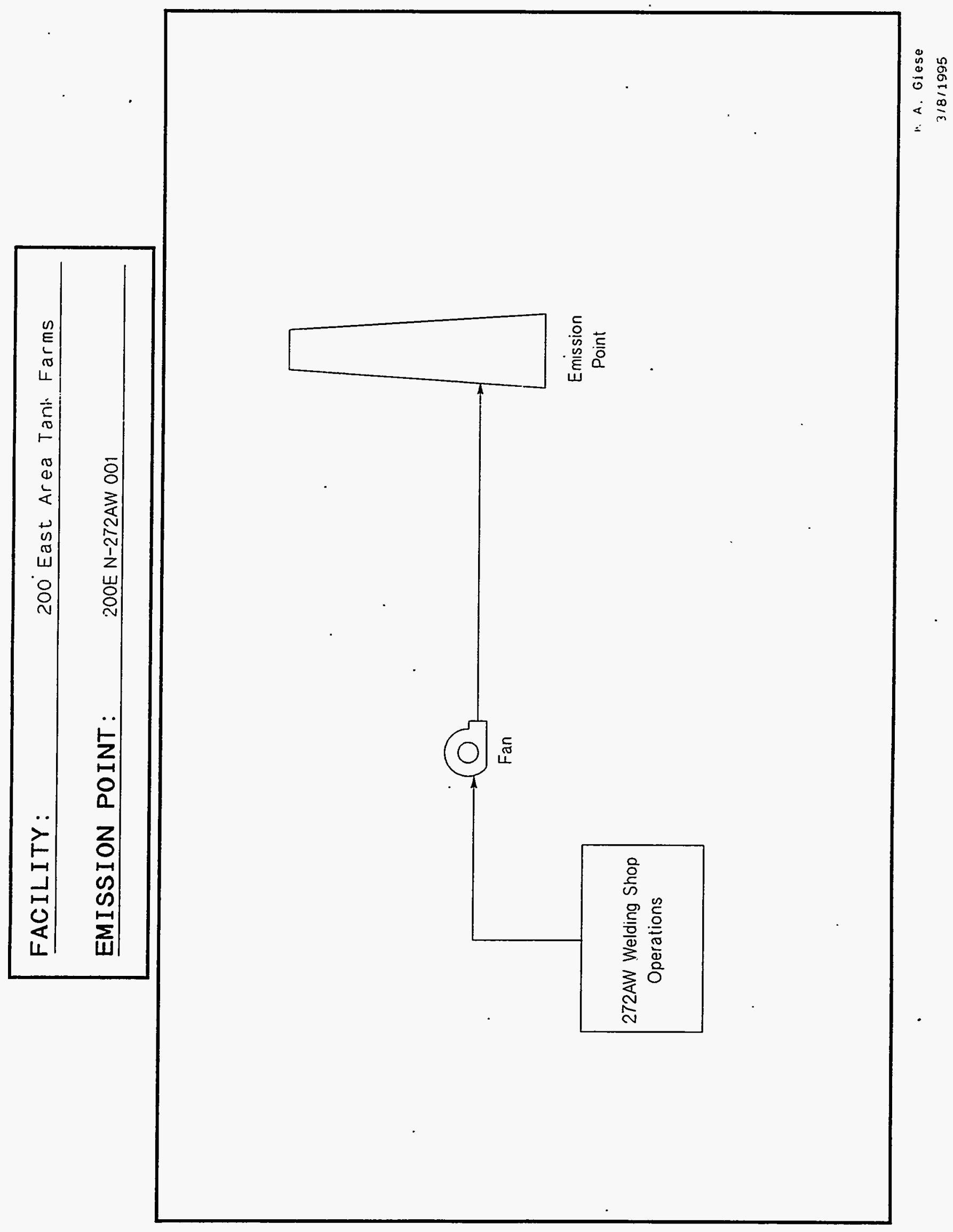




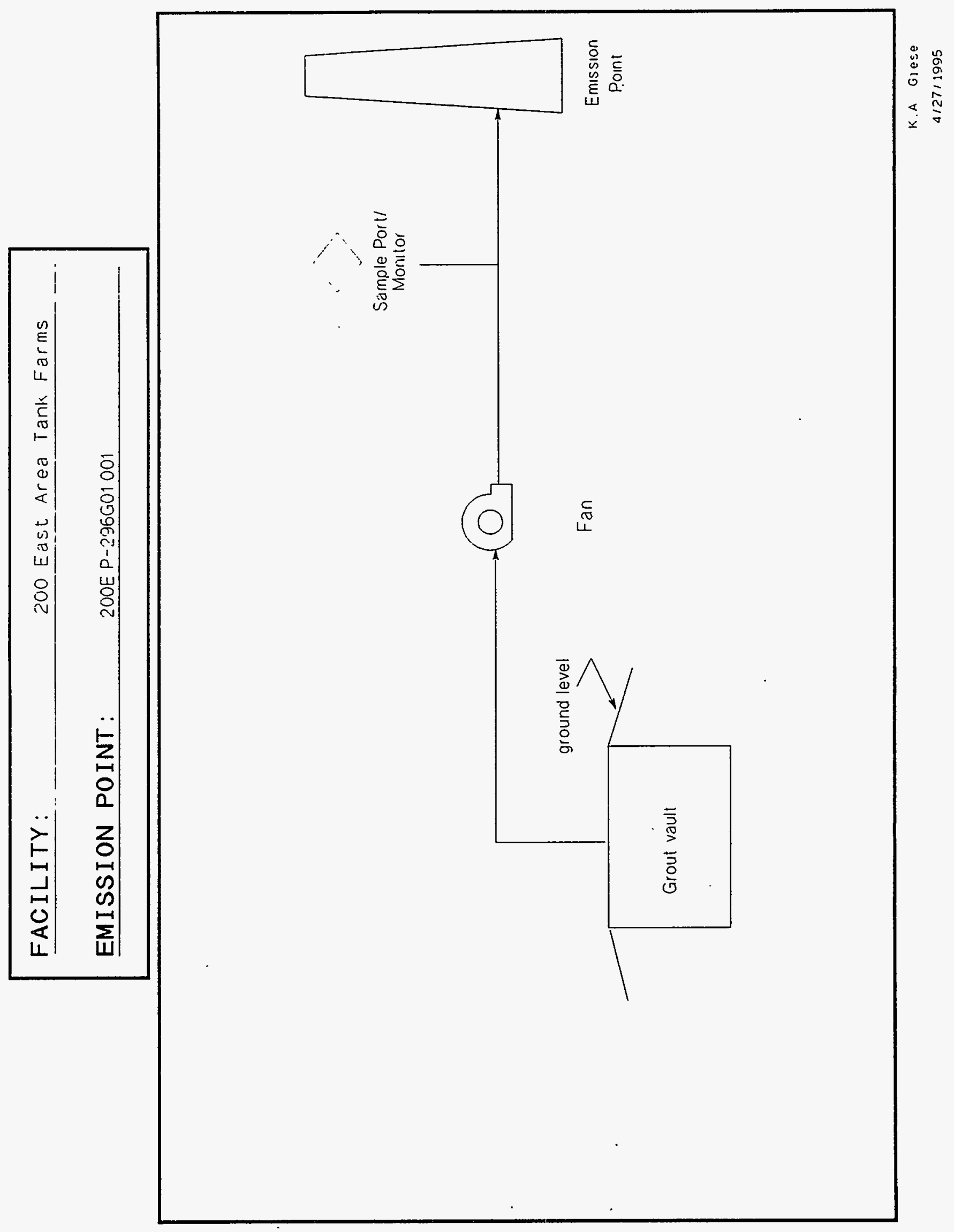




$\begin{array}{ll}\text { FACILITY: } & \text { 200E Area Tank }=\text { arms } \\ \text { EMISSION POINT: } & \text { 200E P-296P016 00! }\end{array}$

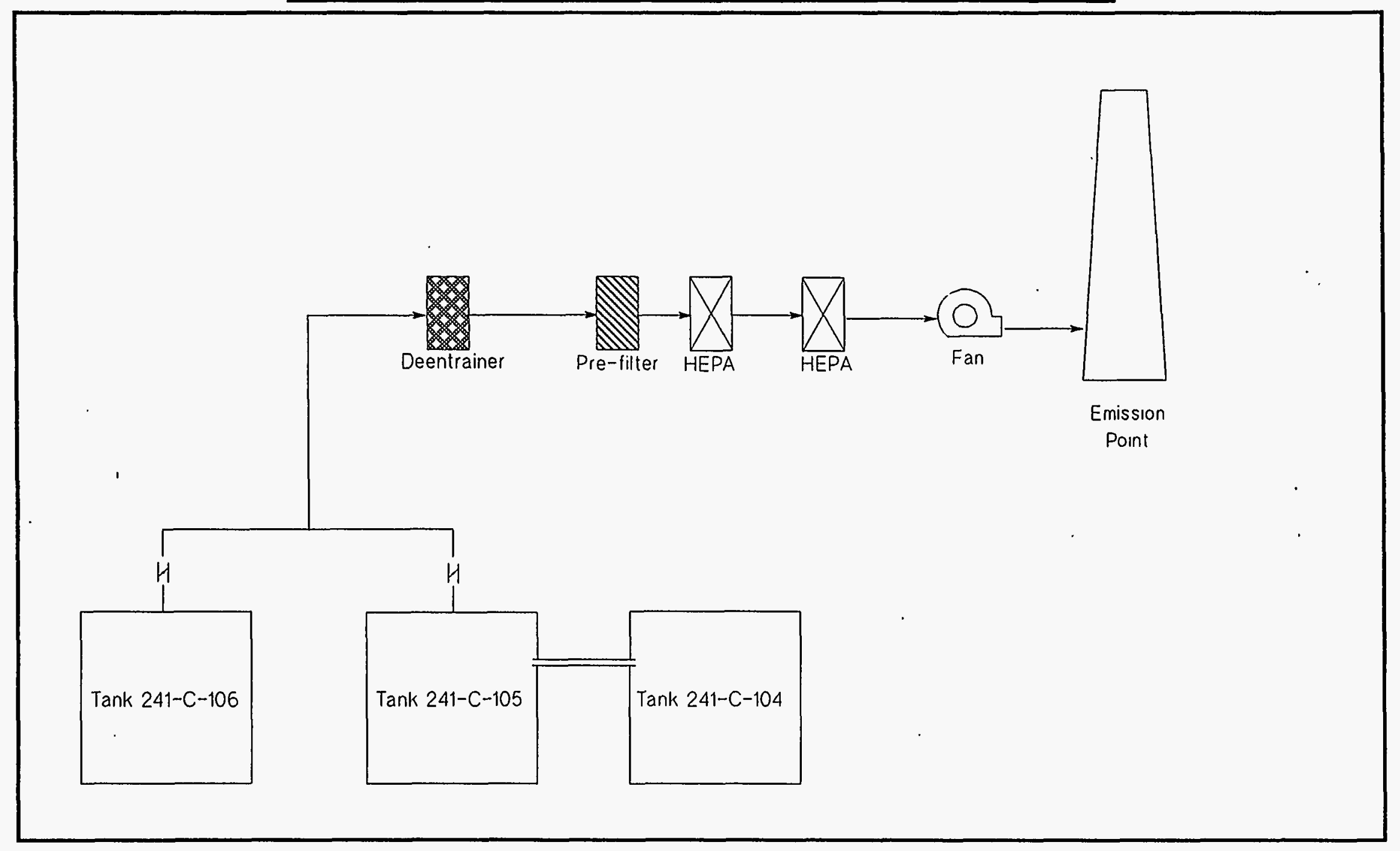



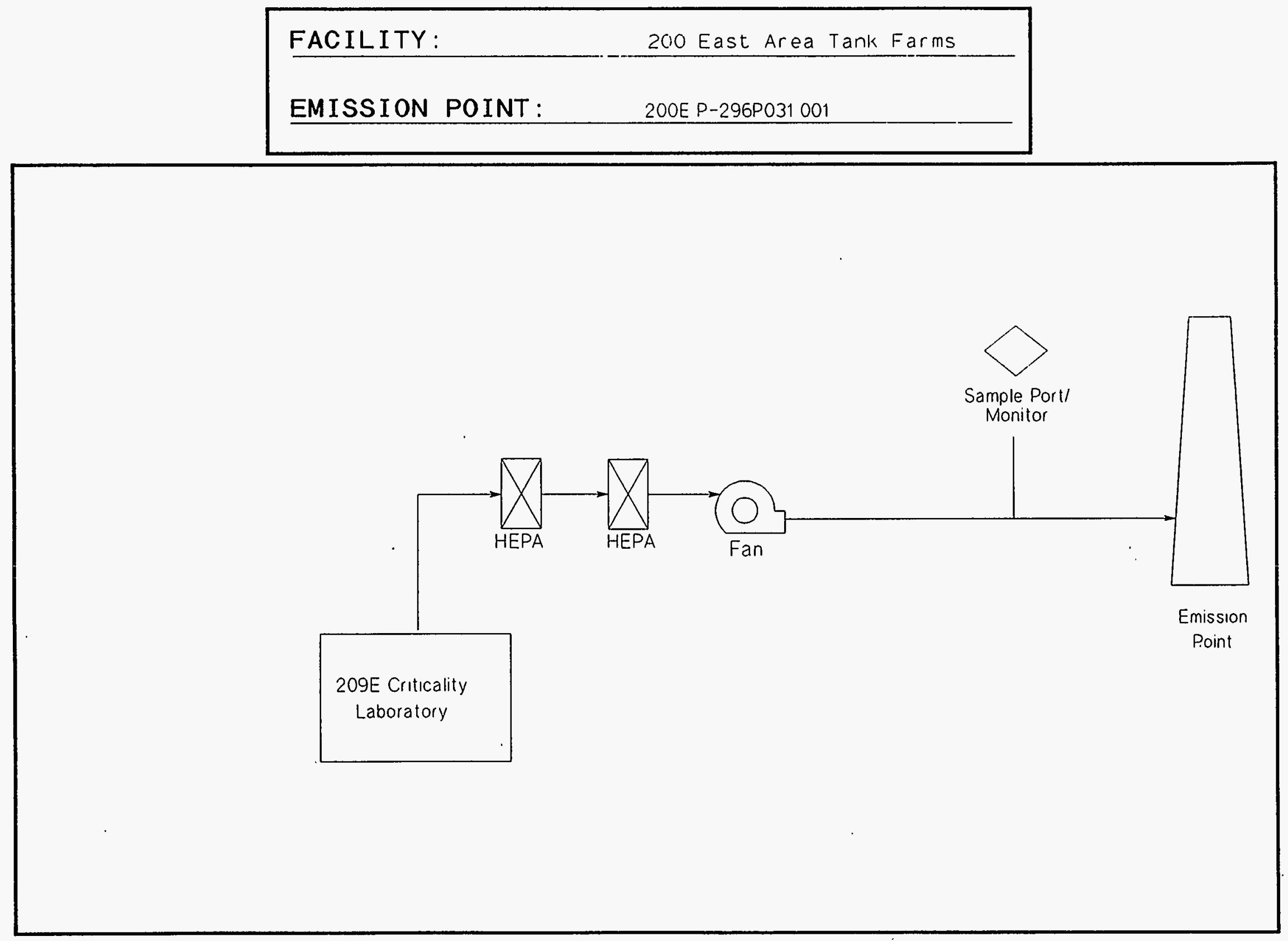

K.A. Glese 


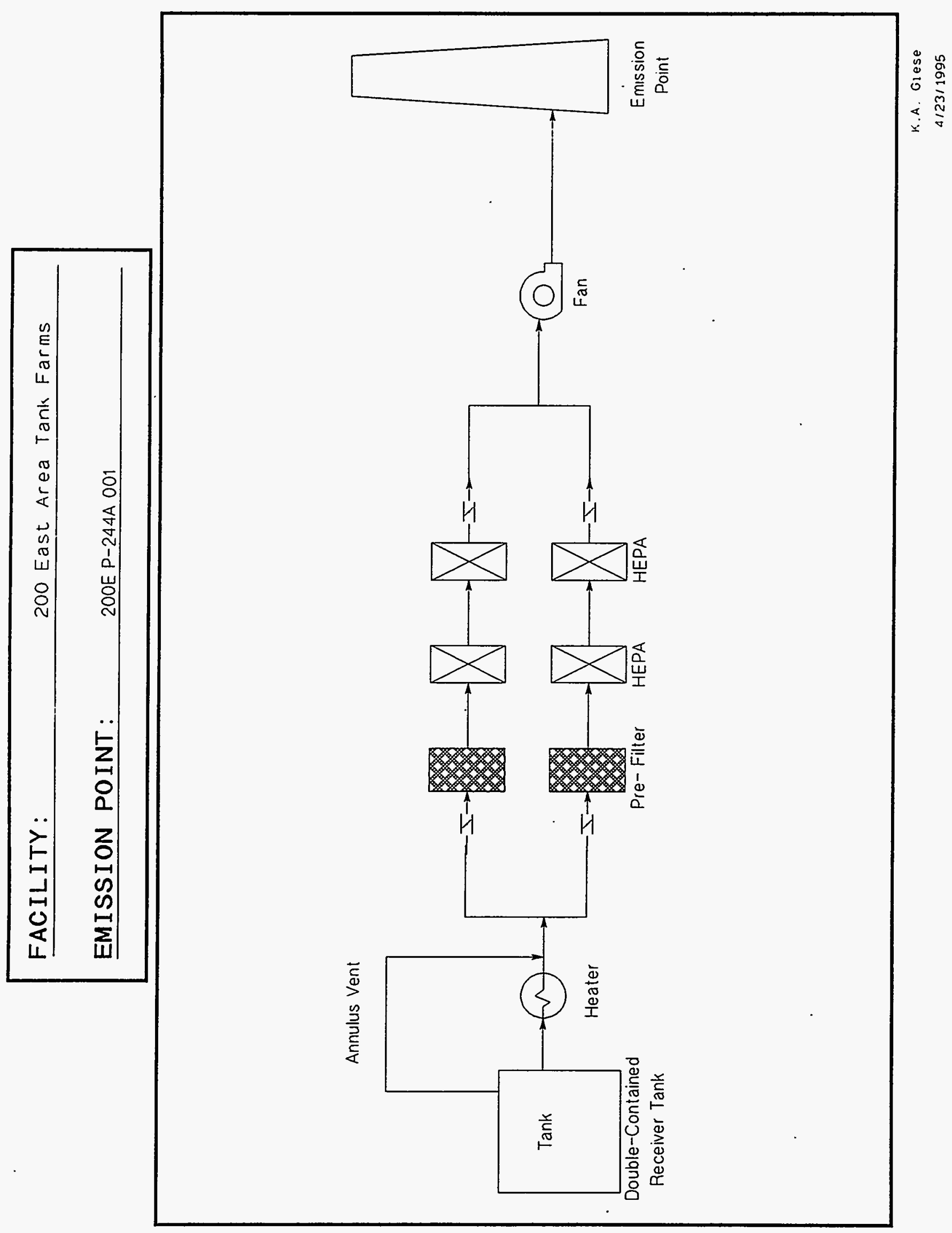




\begin{tabular}{ll|}
\hline FACILITY: & 200 East Area Tank Farms \\
\hline EMISSION POINT: & See Table \\
\hline
\end{tabular}

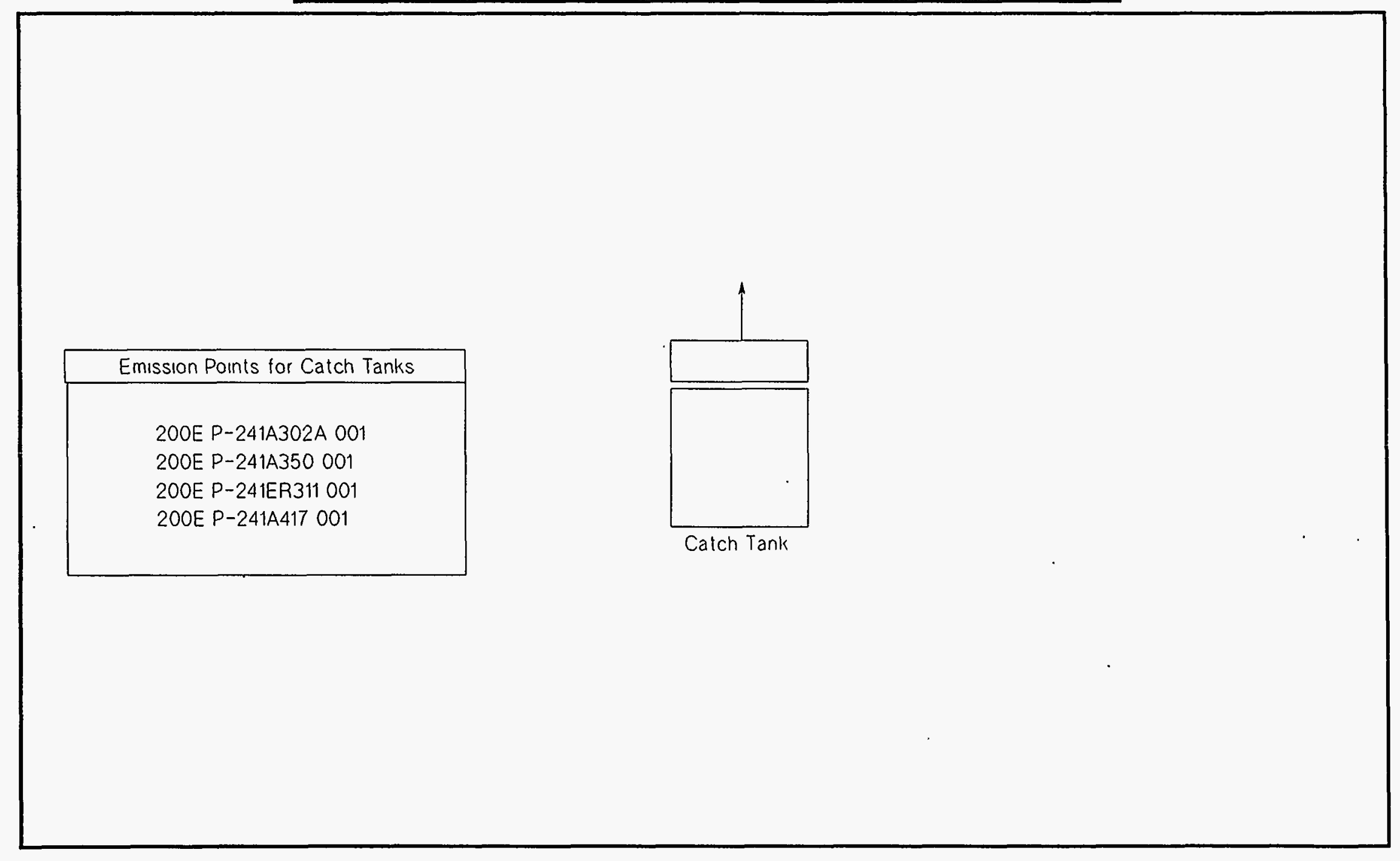


FACILITY:

:N: East Area Tank: arms

EMISSION POINT:

See Table

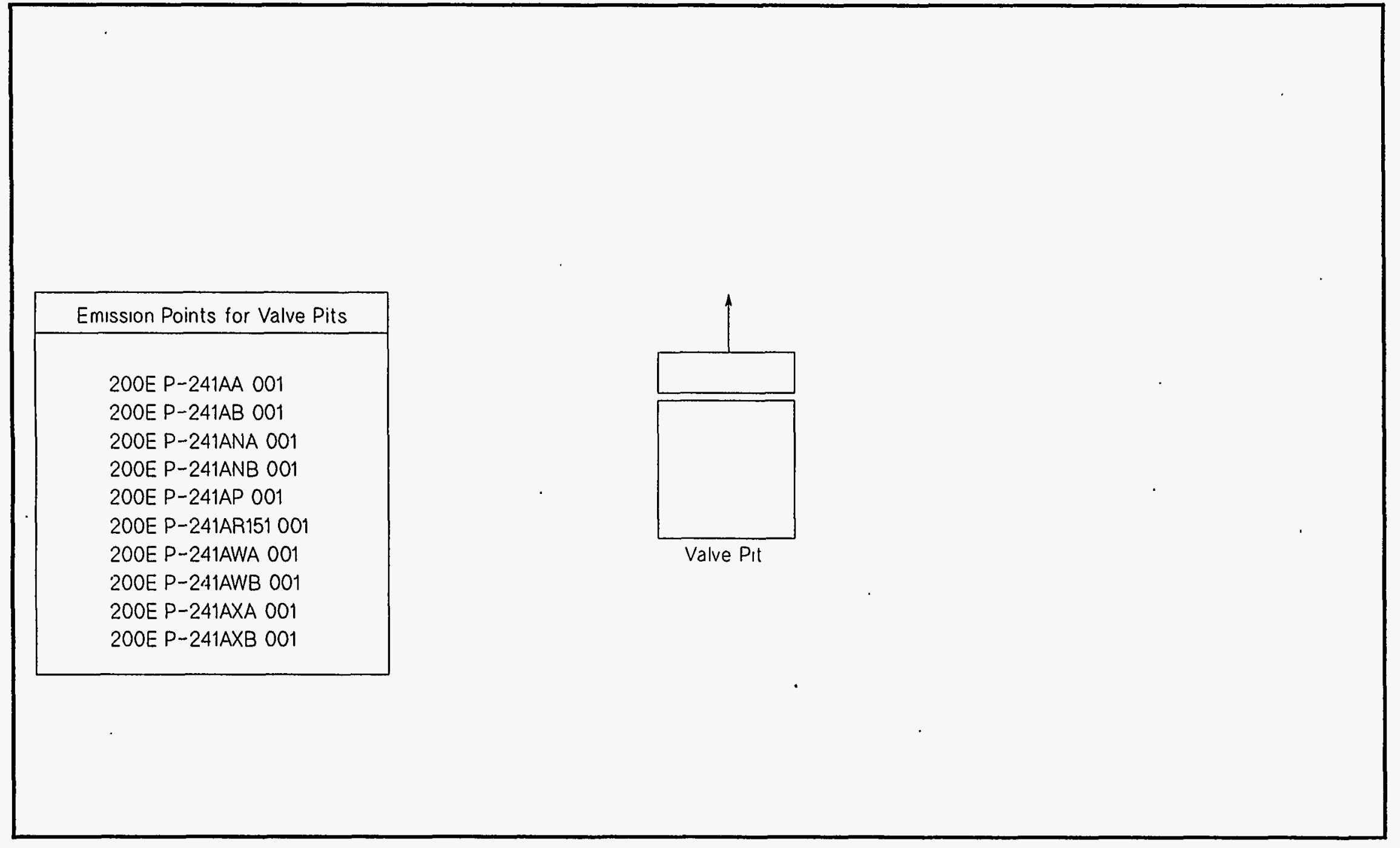




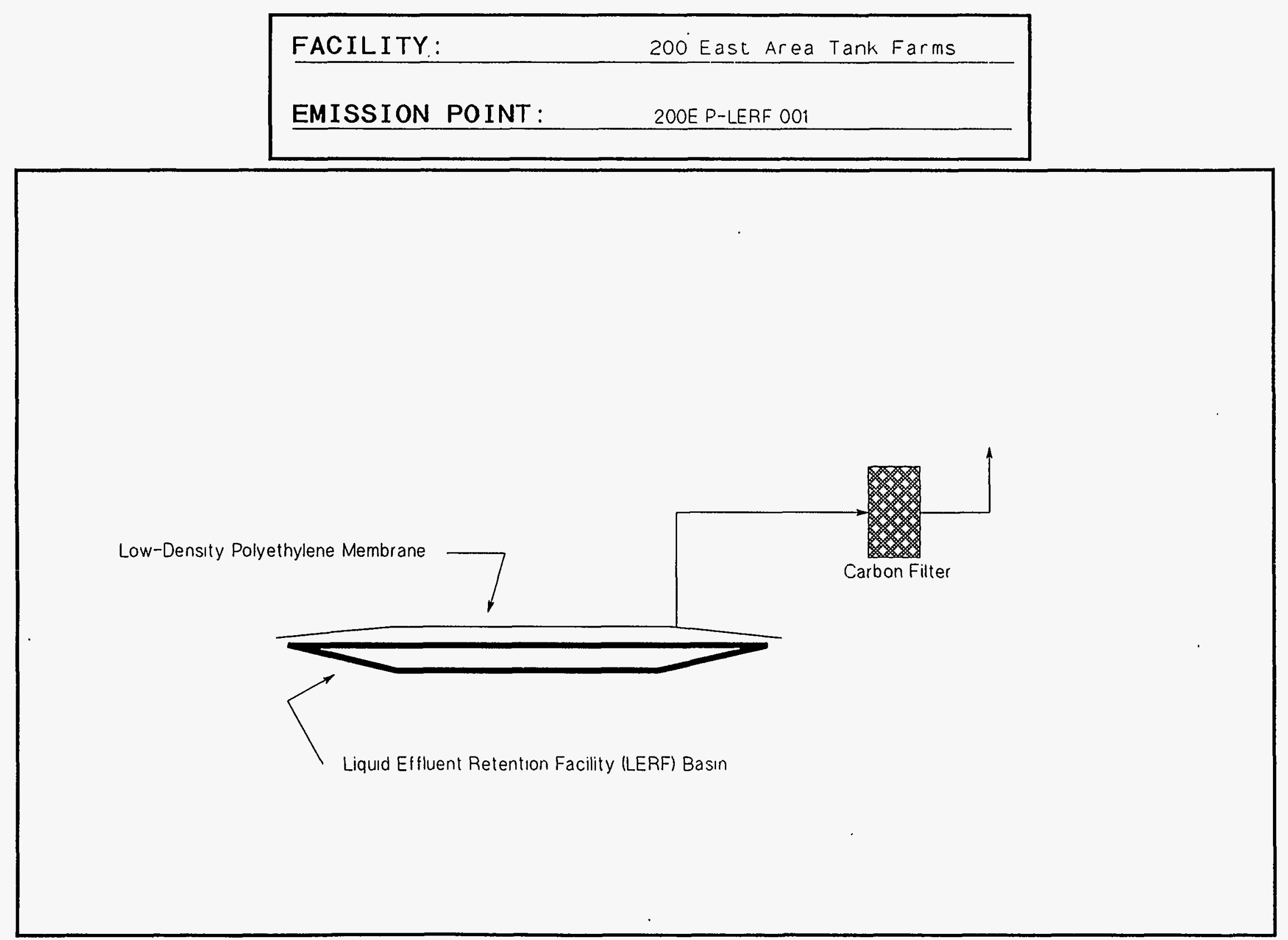

K.A. Glese 


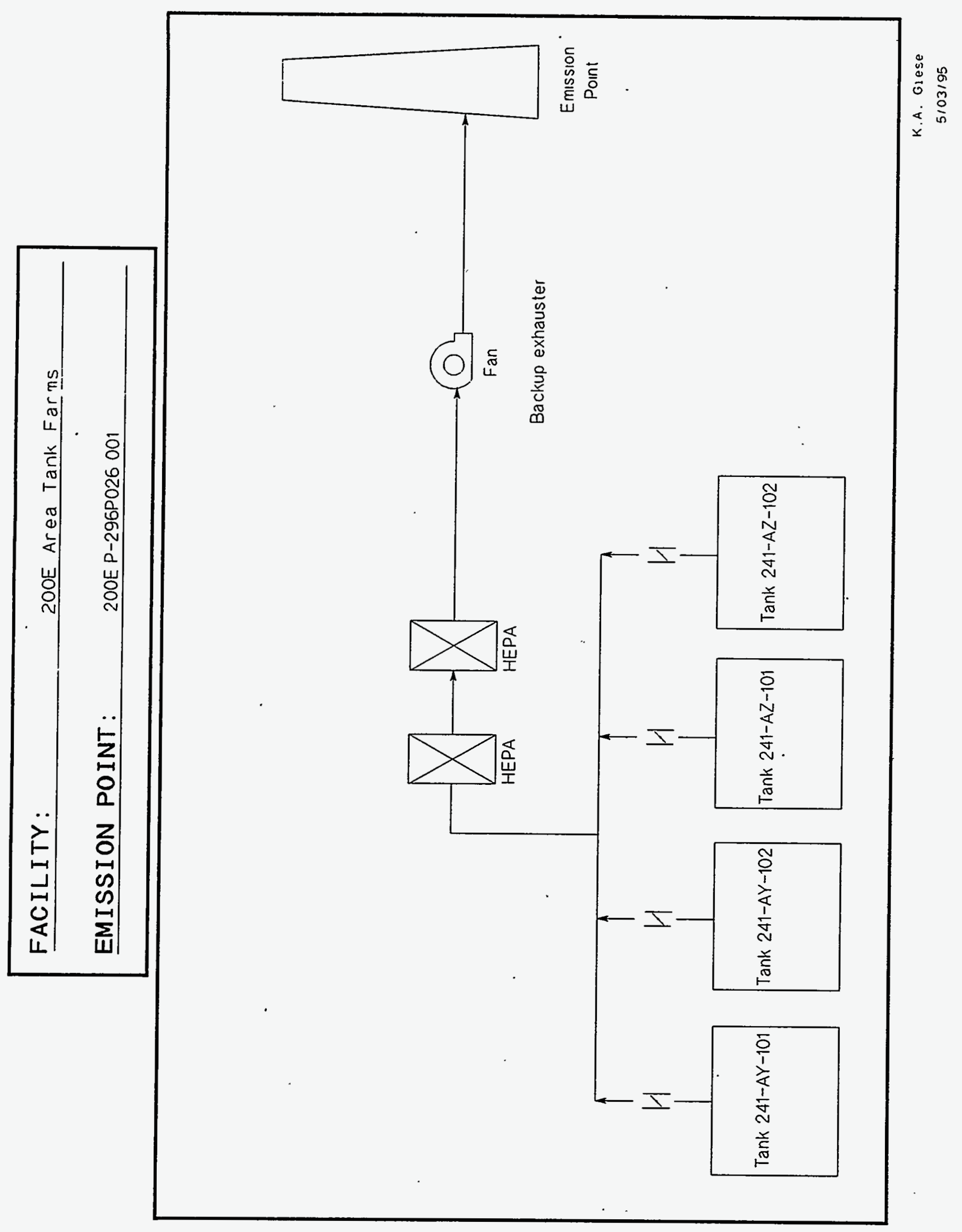



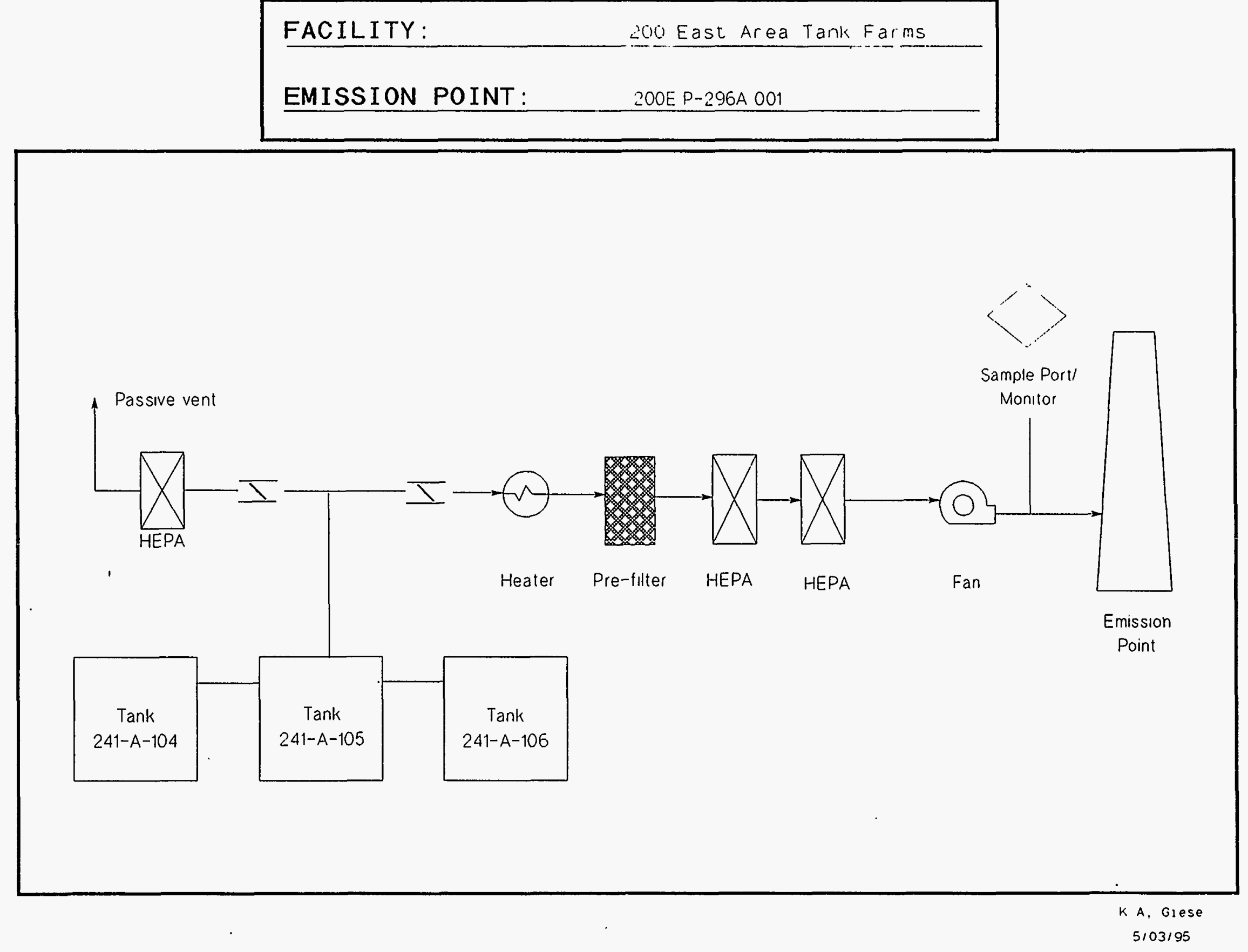


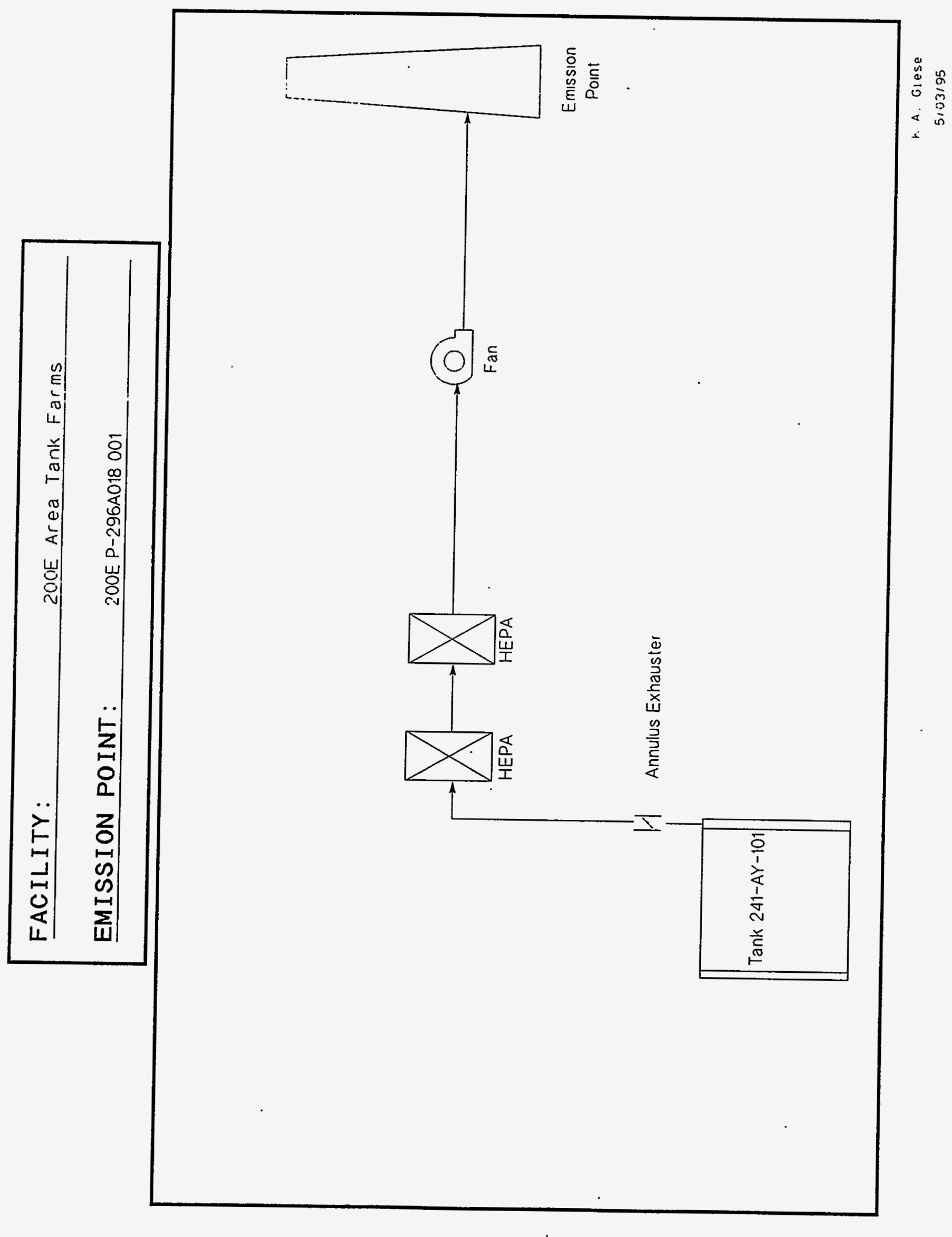




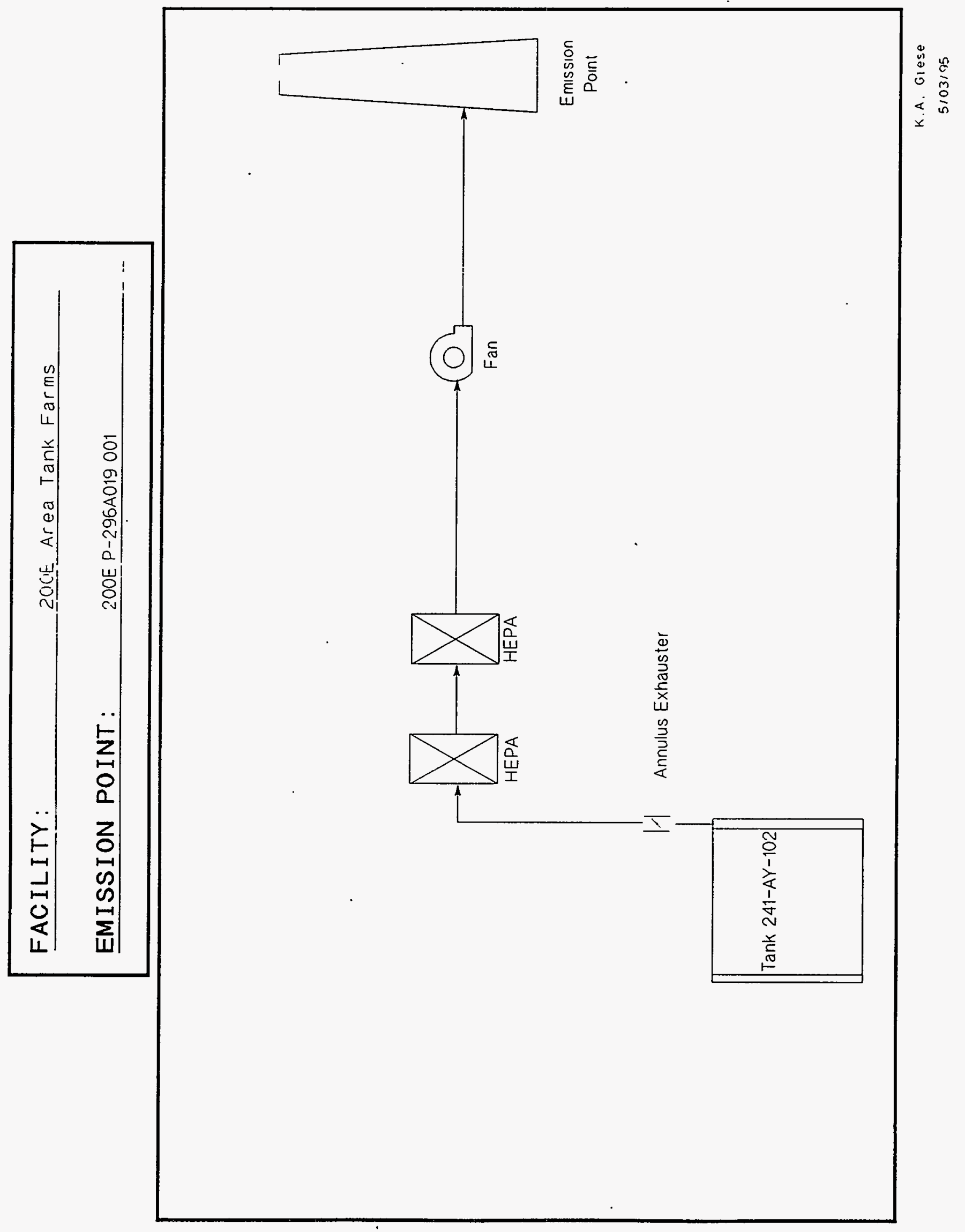




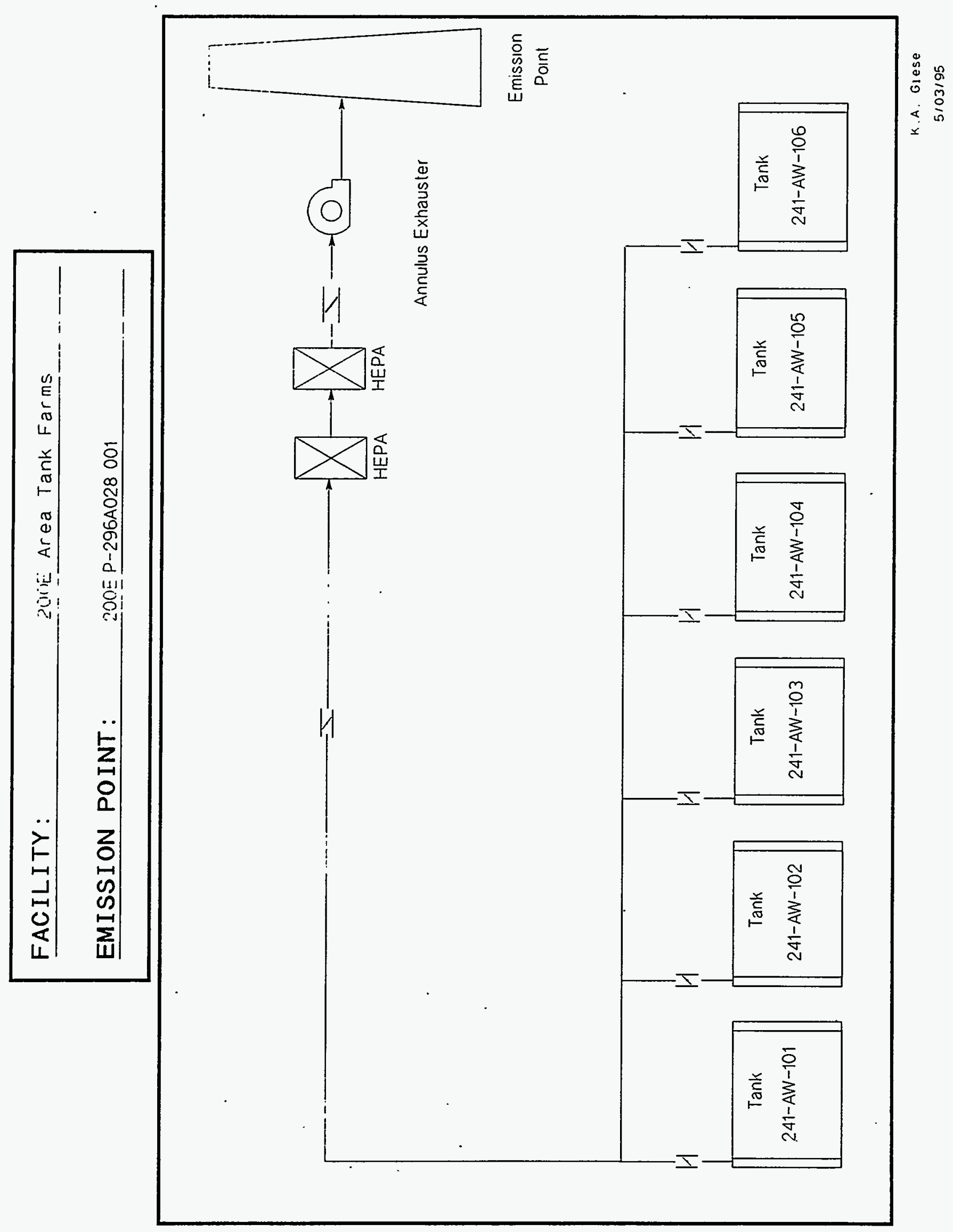



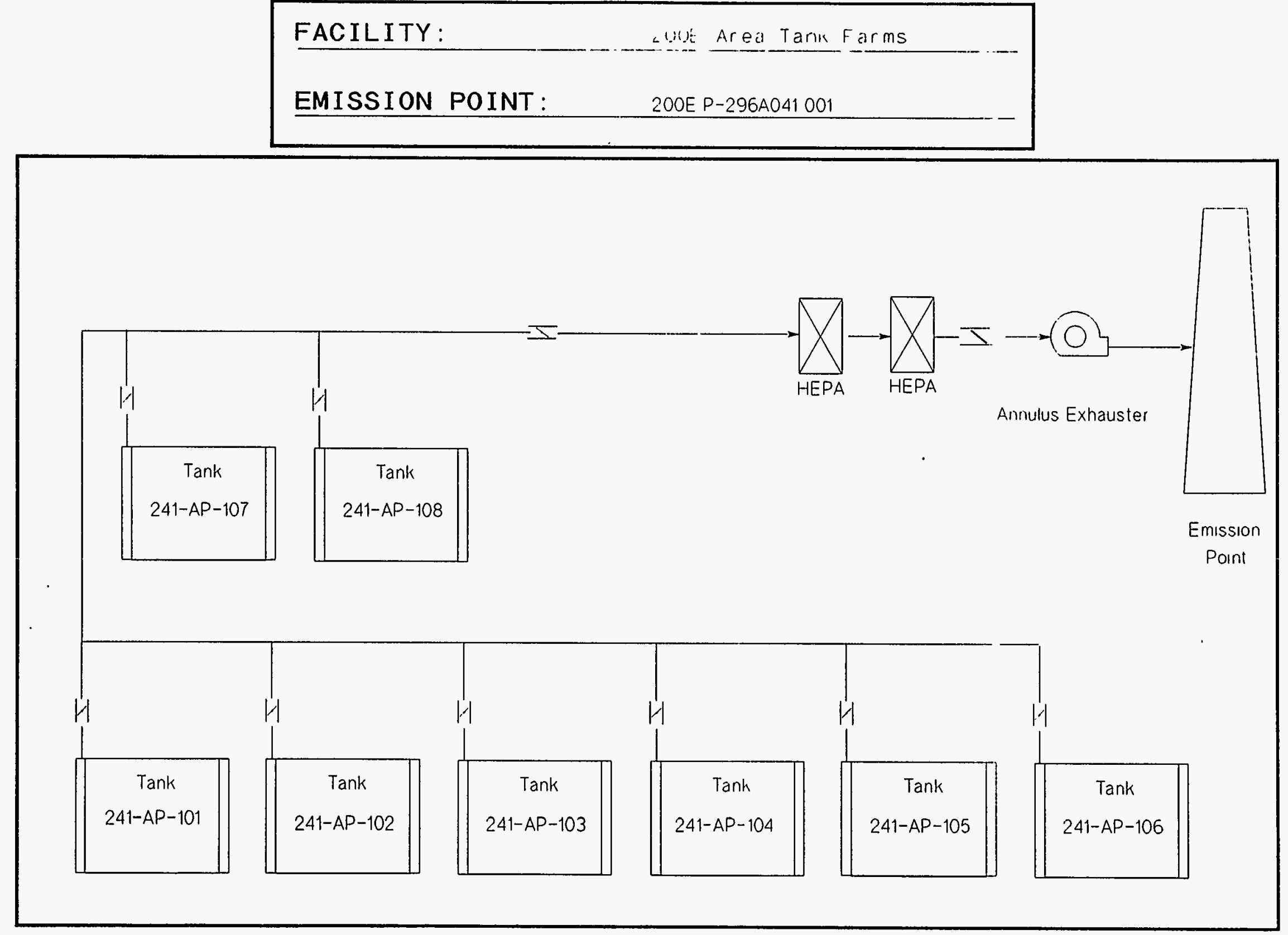


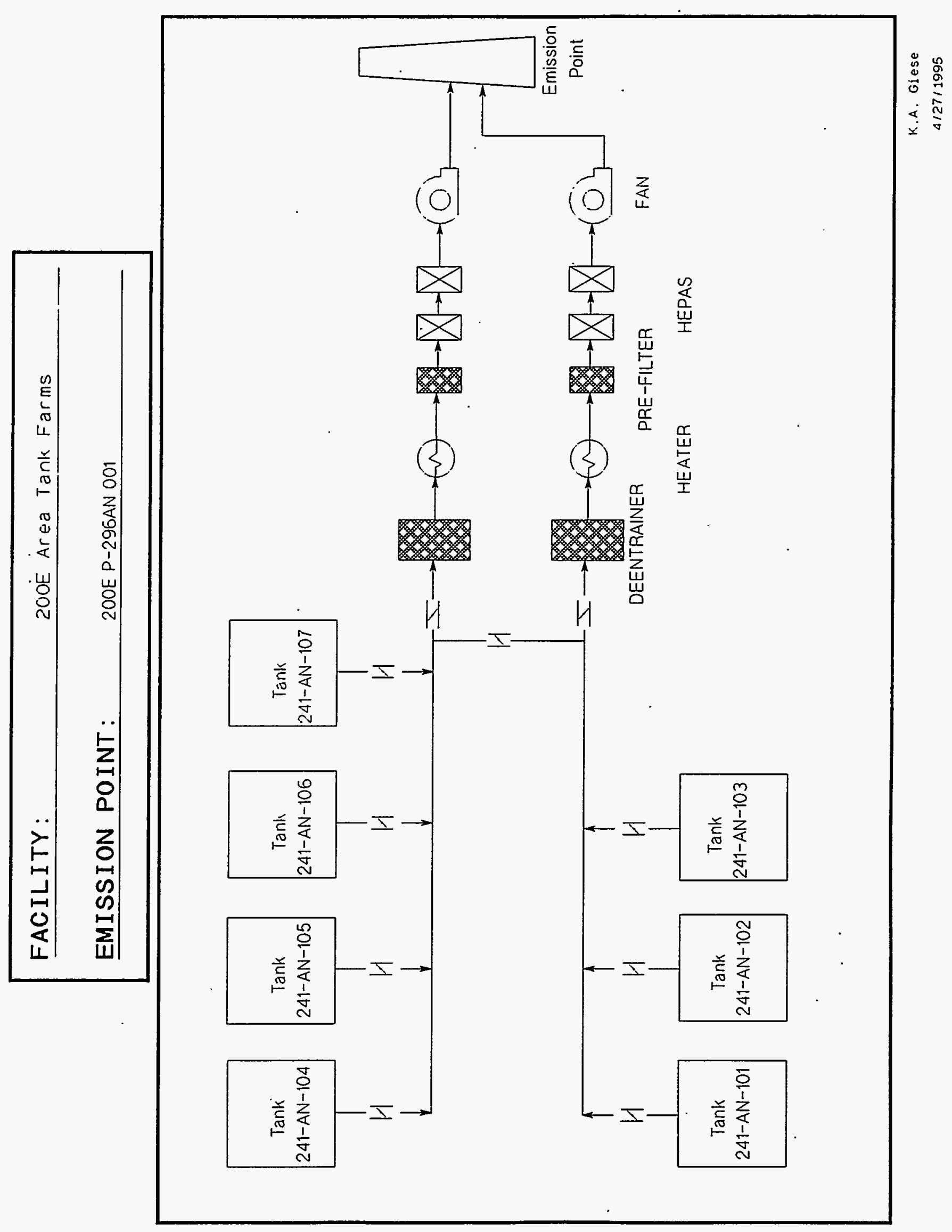




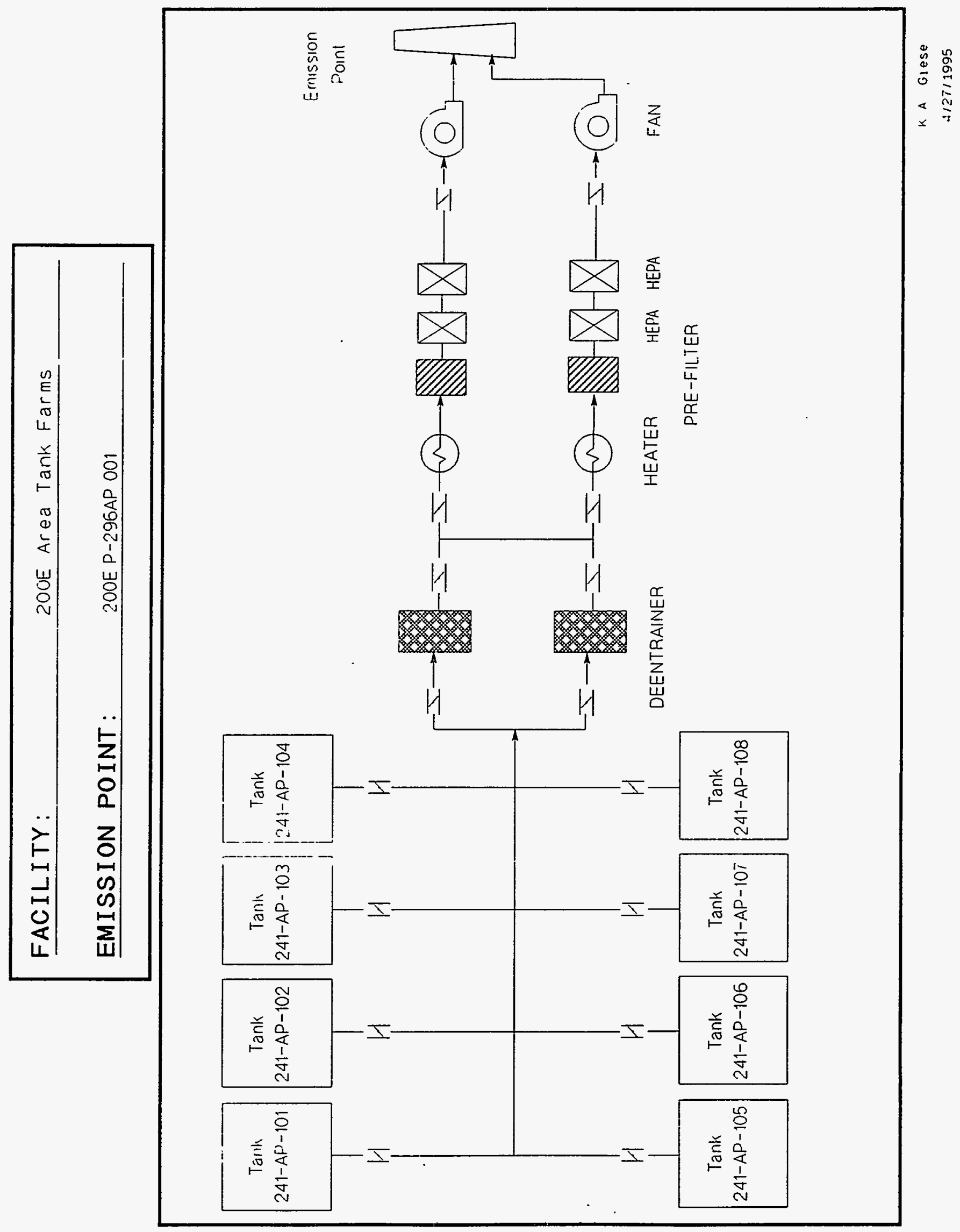




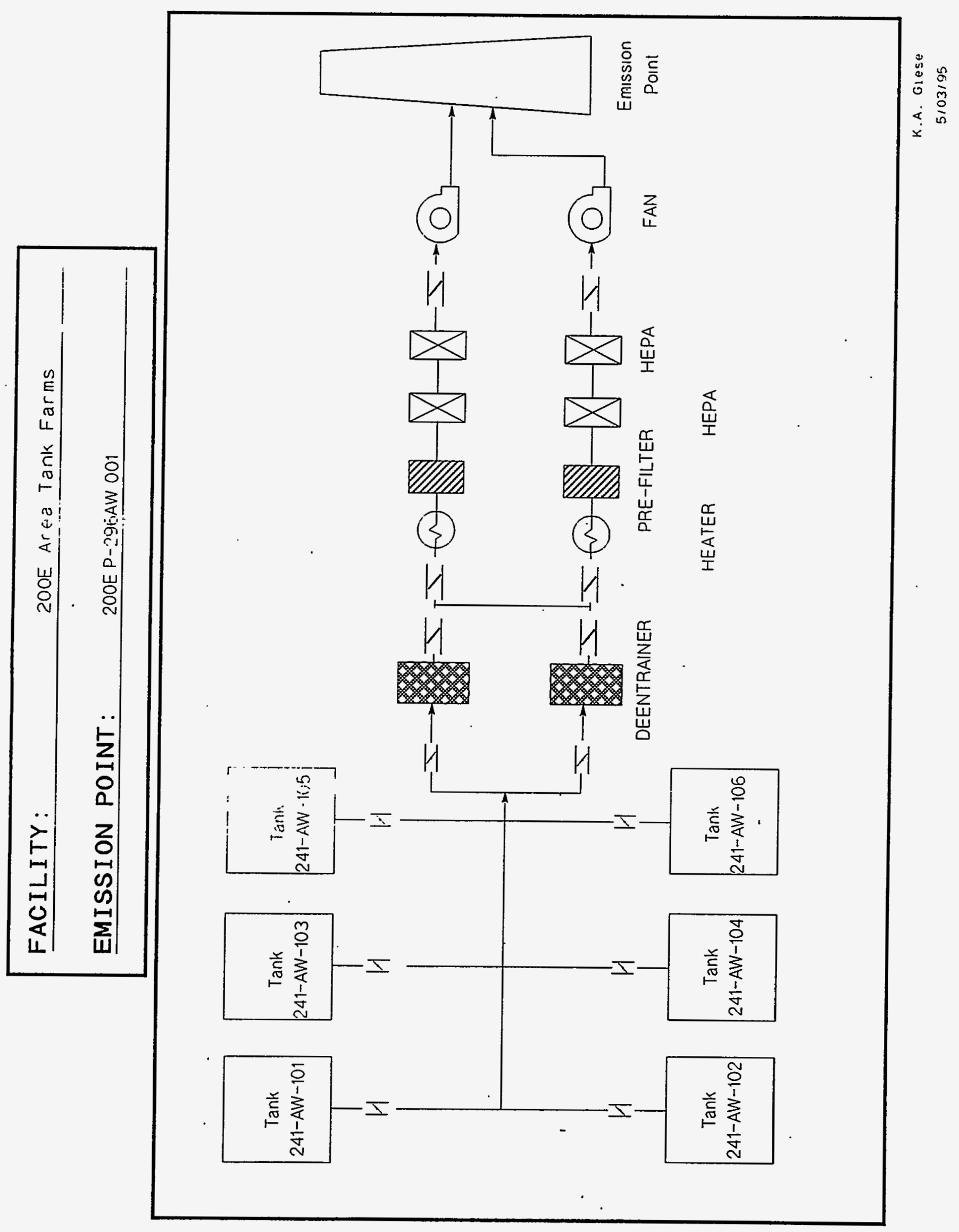




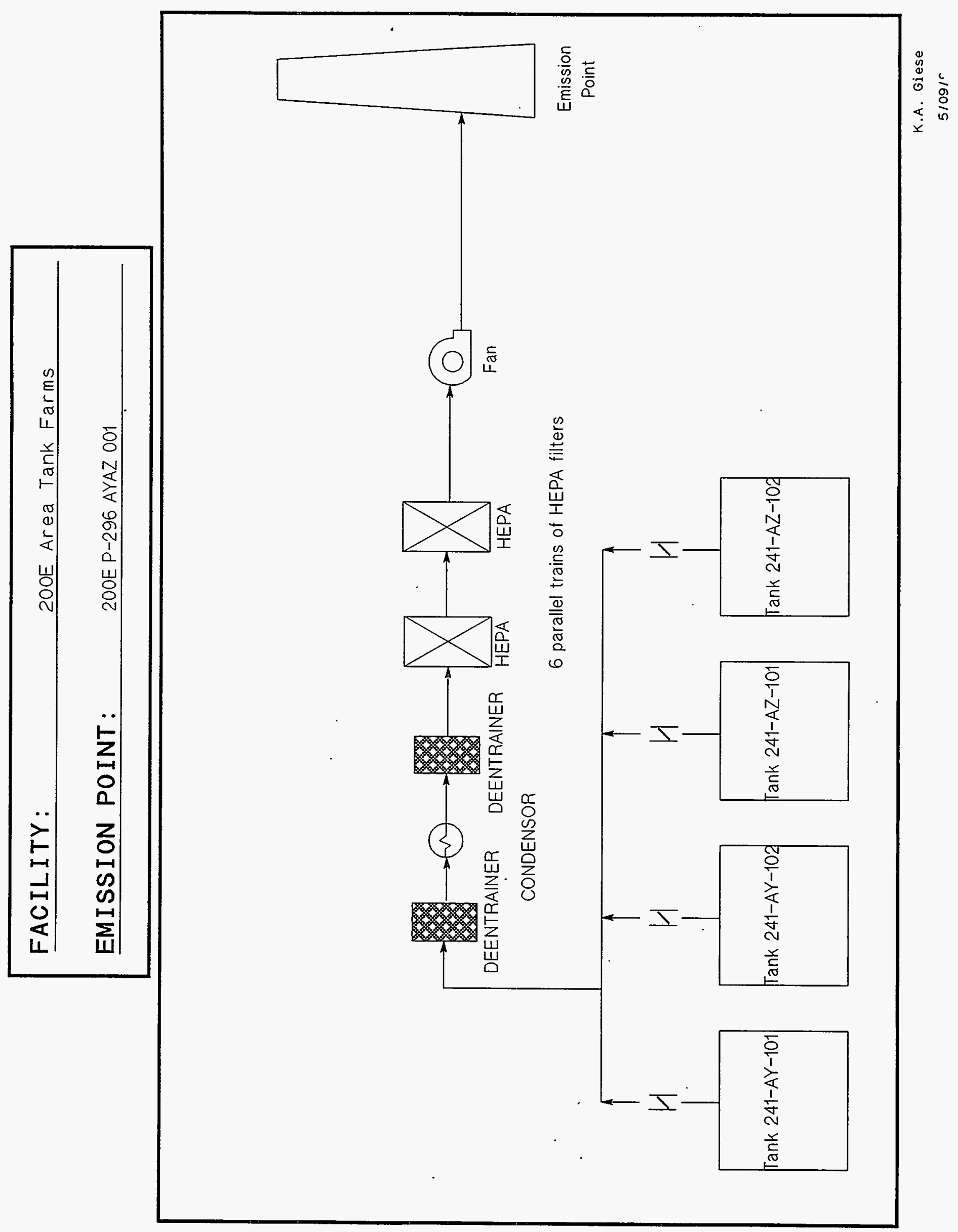




\begin{tabular}{|c|c|}
\hline FACILITY: & 200 East Area Tank Farms \\
\hline EMISSION POINT: & SEE INSET TABLE \\
\hline
\end{tabular}

200 East Area Single-Shell Tanks That Are Passively Vented

\begin{tabular}{|c|c|c|}
\hline 200E P-24IA101001 & $200 E P-2418201001$ & 200E P-24,1BY10E 001 \\
\hline 200E P-241A102 001 & $200 E P-241 B 202001$ & $200 E \mathrm{P}-241 \mathrm{BY} 107001$ \\
\hline $200 E$ P-241A103 001 & $200 E P-241 B 203001$ & $200 E$ P-241BY108 001 \\
\hline 200E P-241A104001 & $200 E P-2418: 204001$ & $200 E P-241 B Y 109001$ \\
\hline 200E P-241A106 001 & $200 E \mathrm{P}-241 \mathrm{~B} \times 101001$ & $200 E \mathrm{P}-241 \mathrm{BY} 110001$ \\
\hline 200E P-241AX101001 & $200 E P-241 B \times 102001$ & $200 E$ P-241BY111 001 \\
\hline $200 E P-241 A X 102001$ & 200E P-241BX103001 & 200E P-241BY112001 \\
\hline 200E P-241AX103001 & 200E P-241BX104 001 & $200 E$ P-241C101 001 \\
\hline 200E P-241AX104001 & 200E P-241BX105001 & $200 E$ P-241C102 001 \\
\hline 200E P-241B101 001 & $200 E P-2418 \times 106001$ & 200E P-241C103001 \\
\hline 200E P-241B102 001 & $200 E P-241 B \times 107001$ & 200E P-241C107 001 \\
\hline $200 E$ P-241B103 001 & 200E P-241BX108 001 & $200 E$ P-241C108 001 \\
\hline 200E P-241B104 001 & 200E P-241BX109001 & $200 E P-241 C 109001$ \\
\hline 200E P-241B105001 & 200E P-241BX110 001 & $200 E P-241 C 110001$ \\
\hline 200E P-241B106 001 & $\begin{array}{l}200 E P-241 B \times 111001 \\
200 E P-241 B X 112001\end{array}$ & $200 E P-241 C 111001$ \\
\hline 200E P-241B107 001 & 200E P-241BY101 001 & $200 E \mathrm{P}-241 \mathrm{C112} 001$ \\
\hline $\begin{array}{l}200 E P-241 B 108001 \\
200 E P-241 B 109001\end{array}$ & $200 E P-241 B Y 102001$ & $200 E P-241 C 201001$ \\
\hline $200 E \mathrm{P}-241 \mathrm{~B} 110001$ & 200E P-241BY103 001 & $200 E \mathrm{P}-241 \mathrm{C} 202001$ \\
\hline $200 E P-241 B 111001$ & $200 E$ P-241BY104 001 & $200 E P-241 C 203001$ \\
\hline 200E P-241B112 001 & $200 E P-241 B Y 105001$ & 200E P-241C204 001 \\
\hline
\end{tabular}




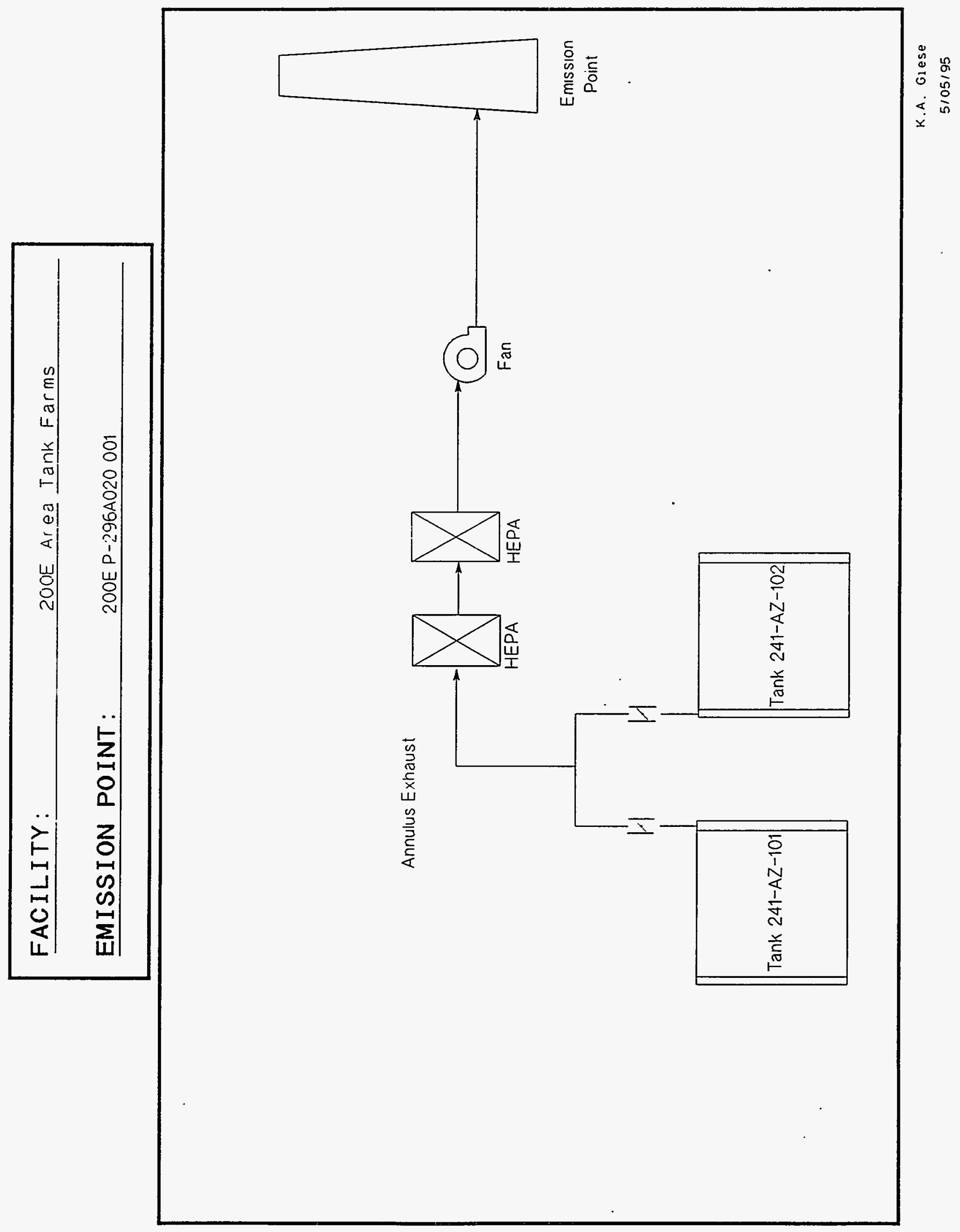




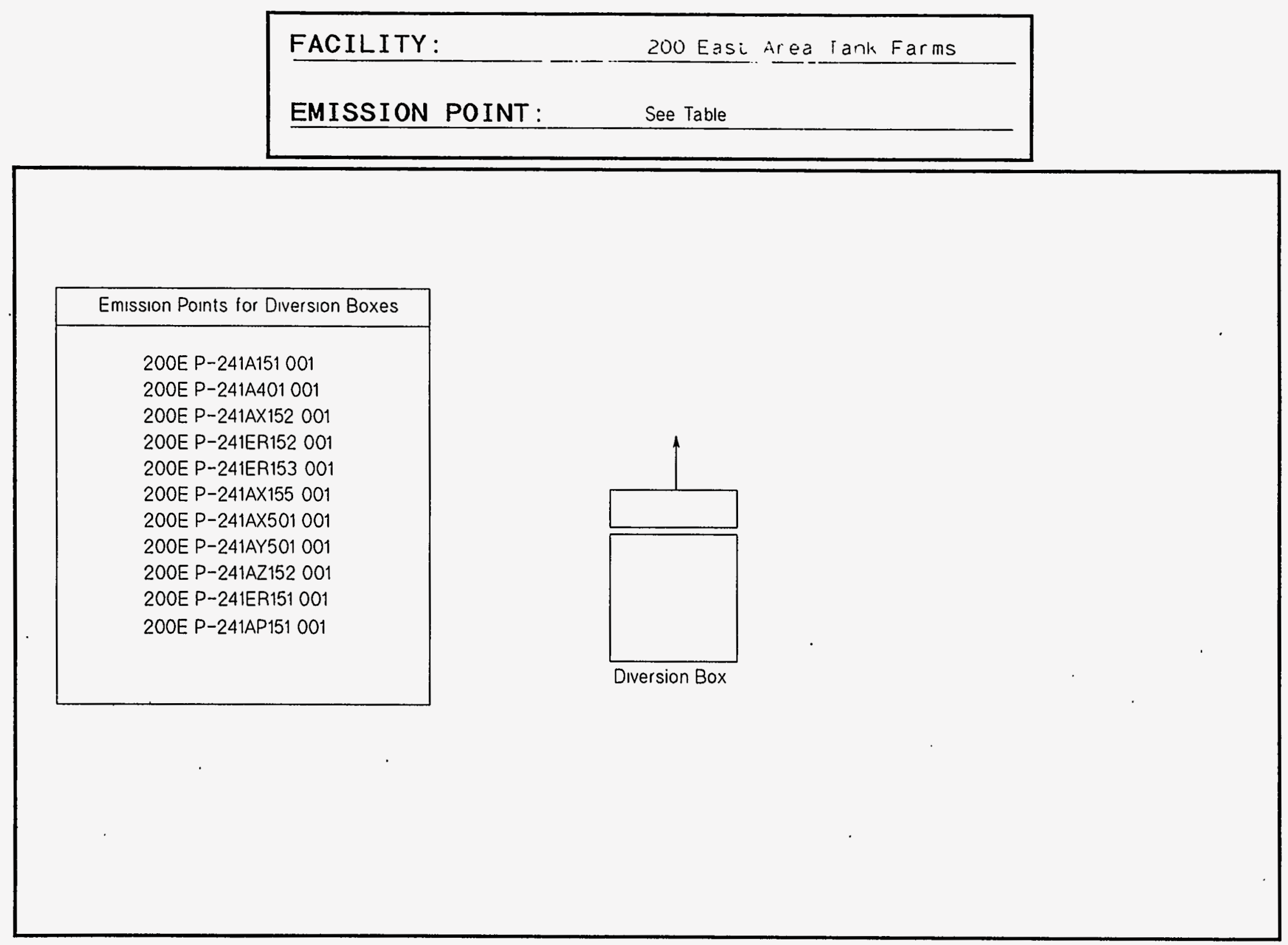




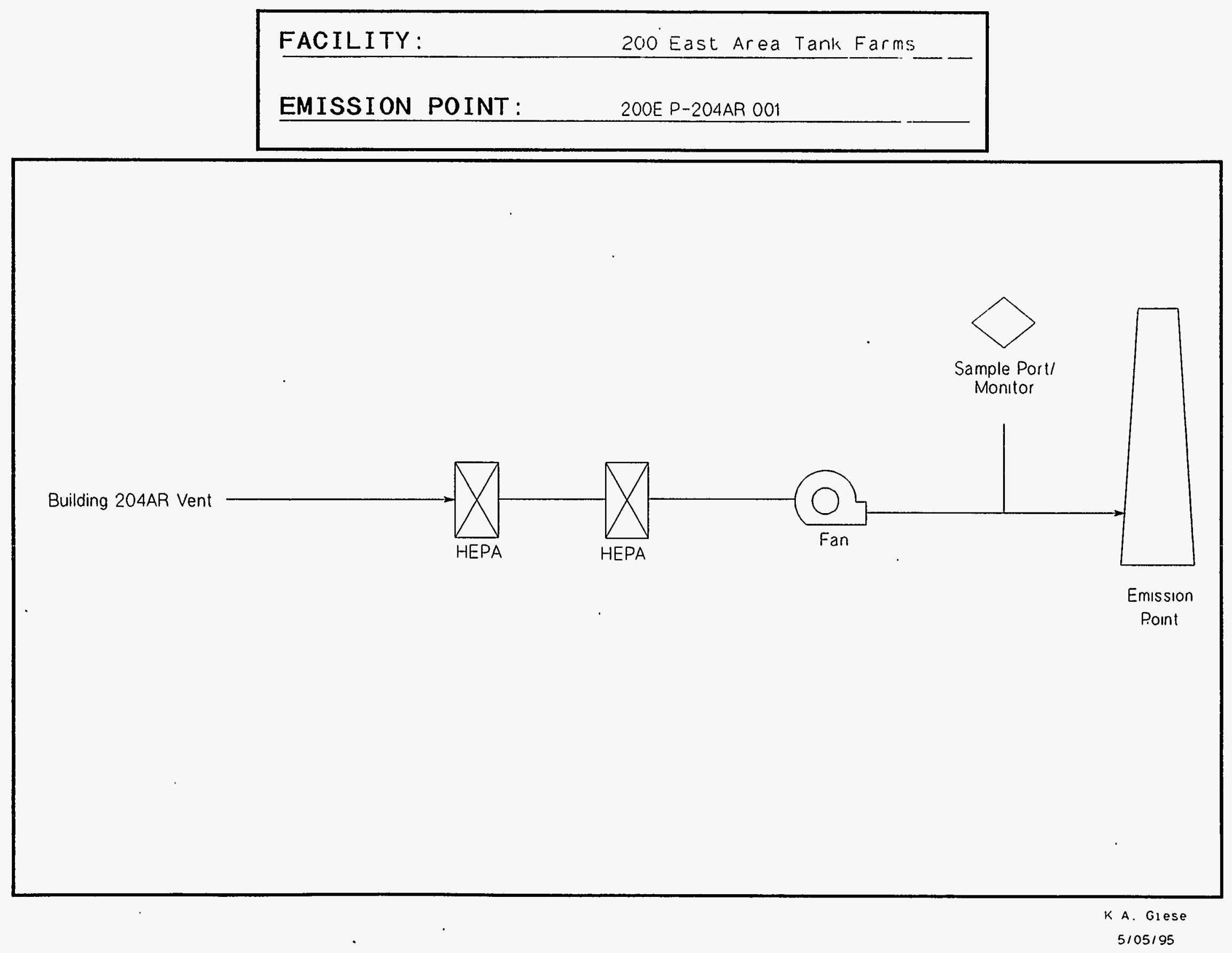




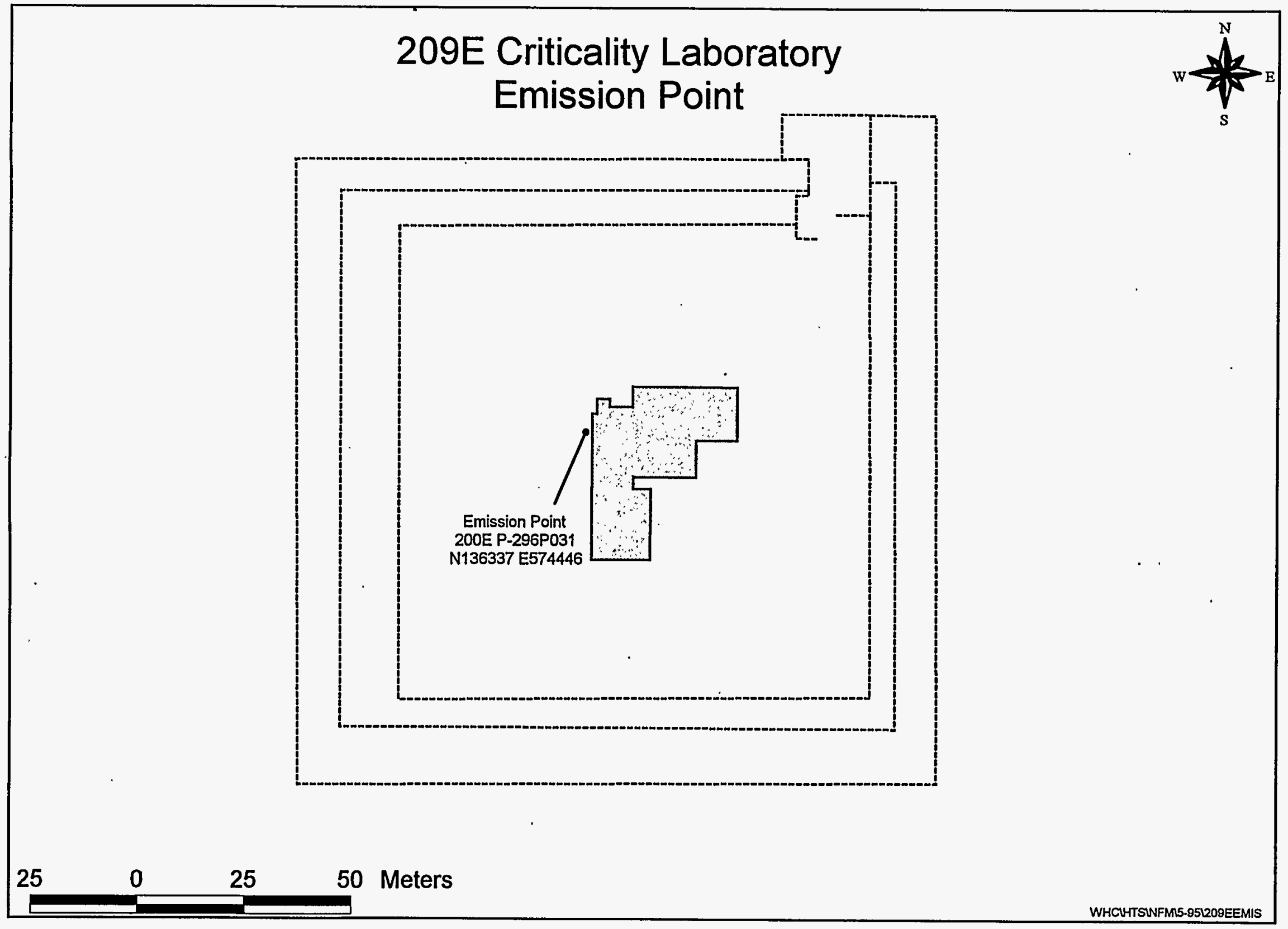


11 is

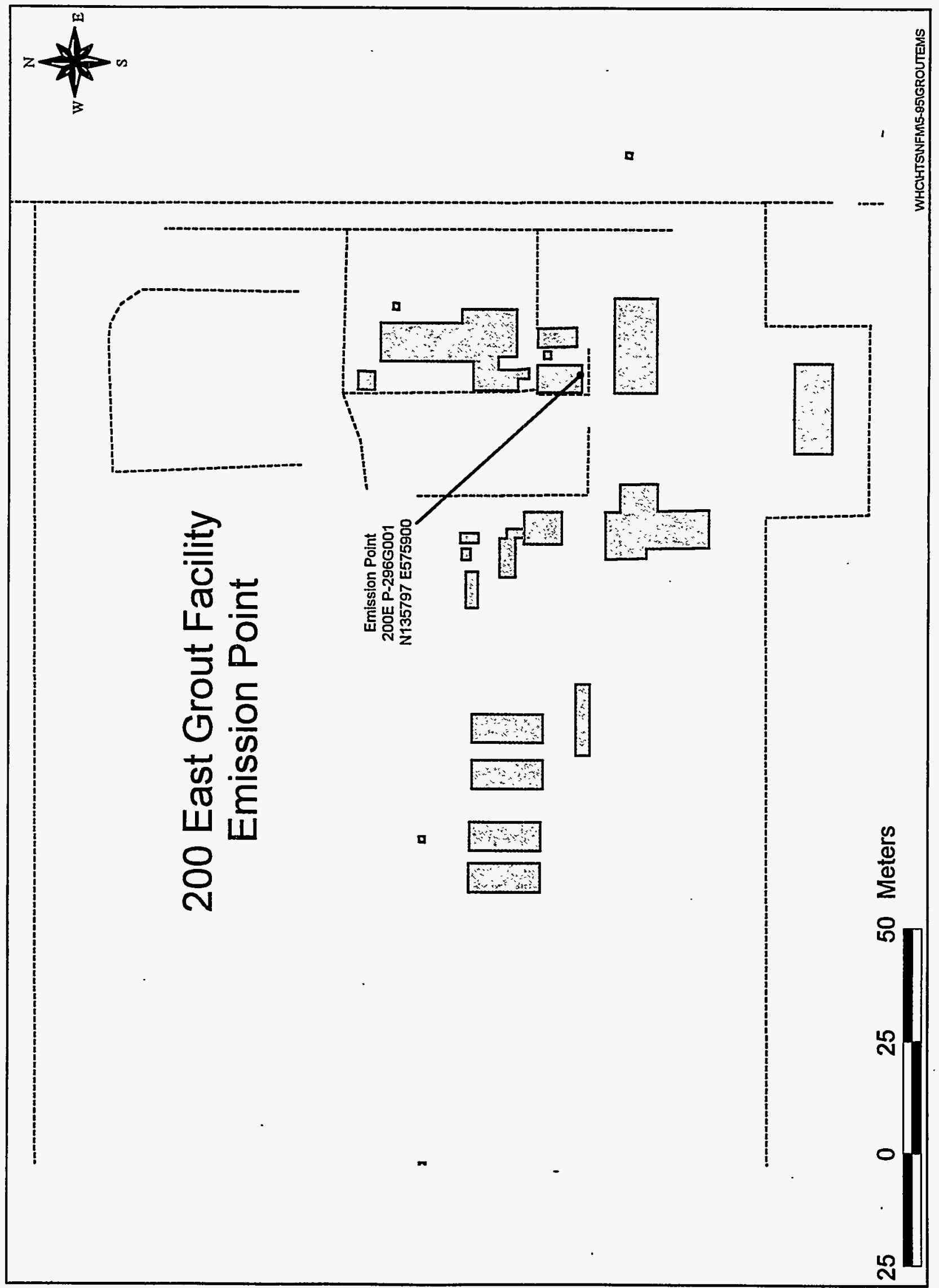




\section{A Tank Farm Emission Points}

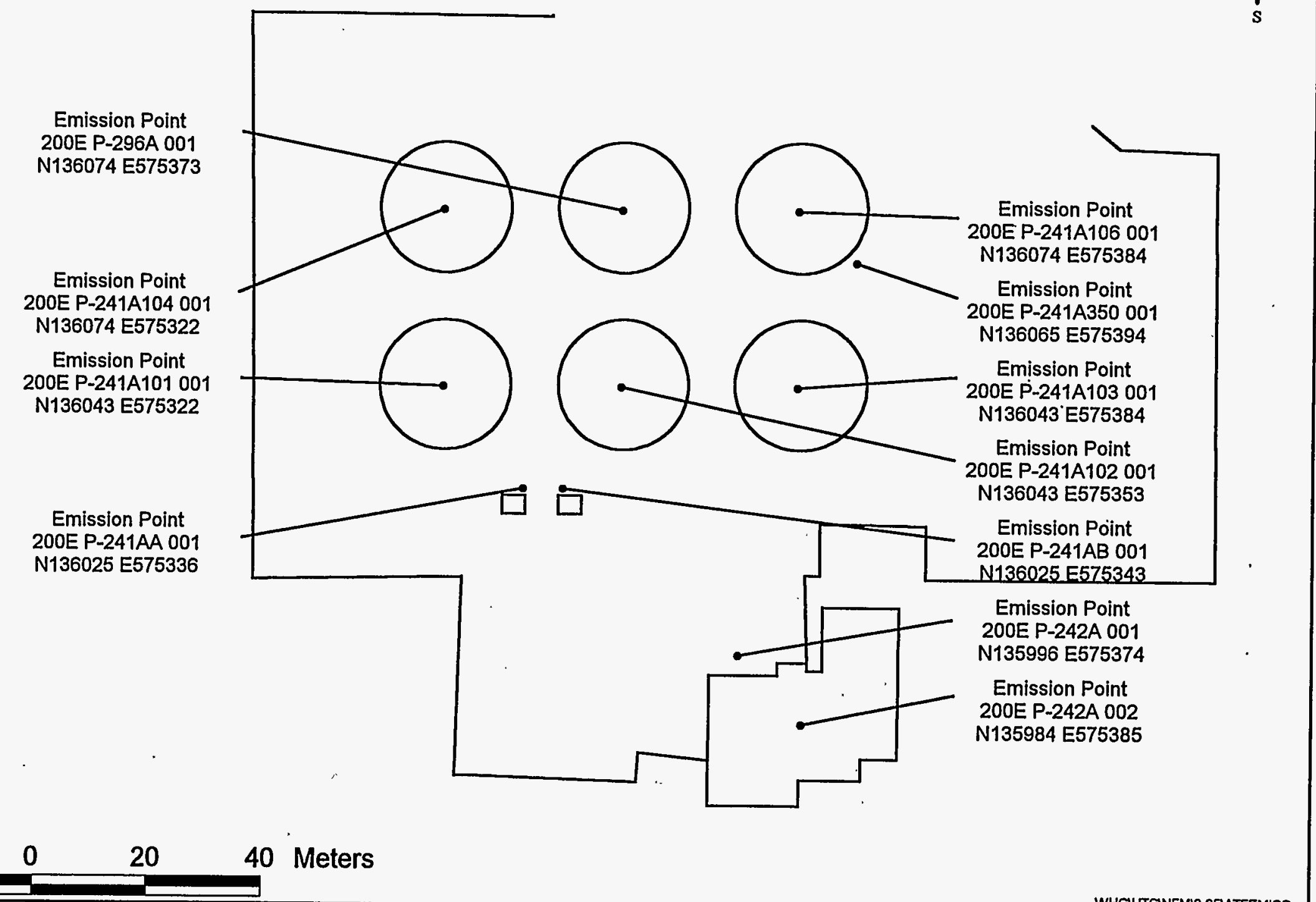




\section{AN/AZ Tank Farm Emission Points}
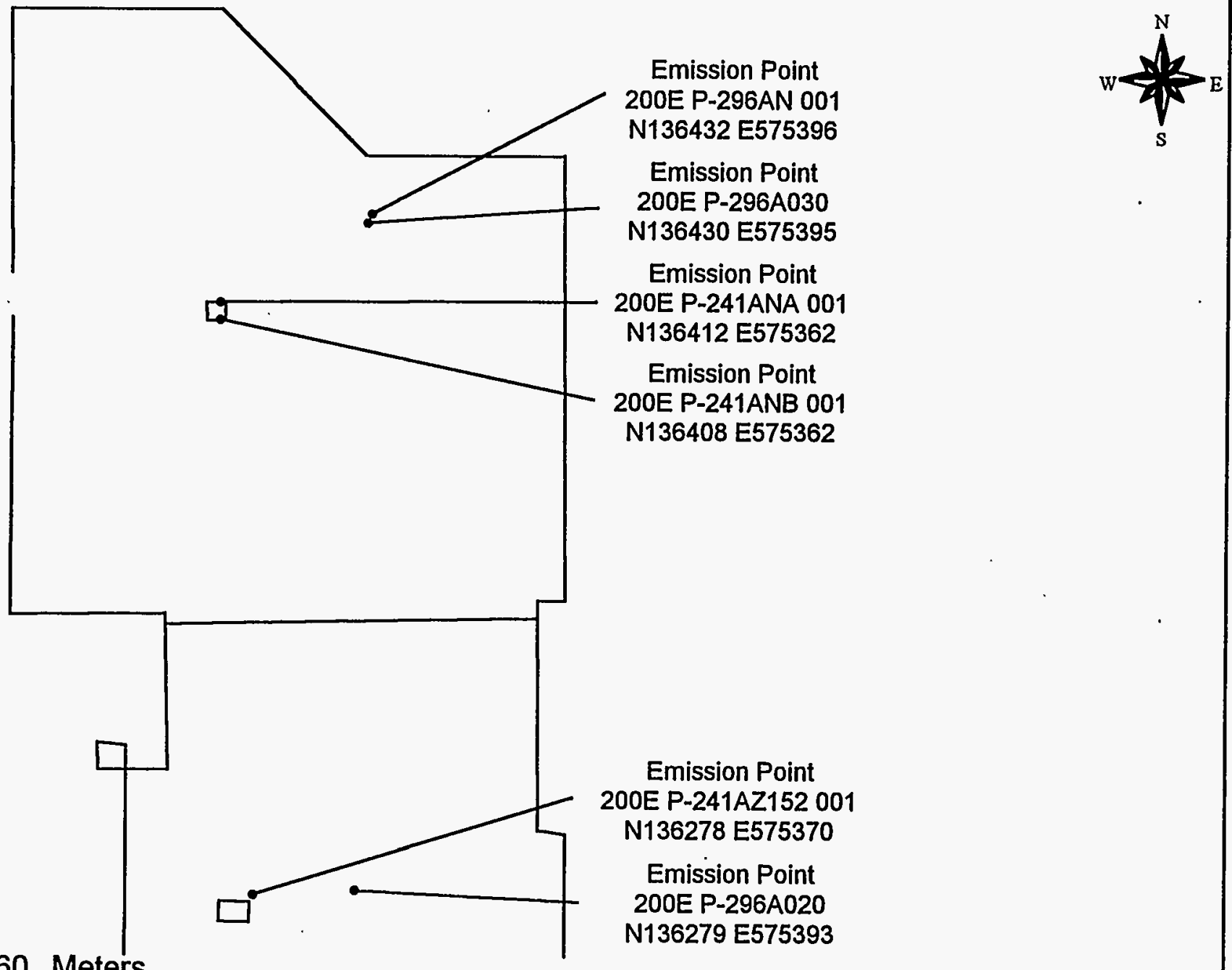


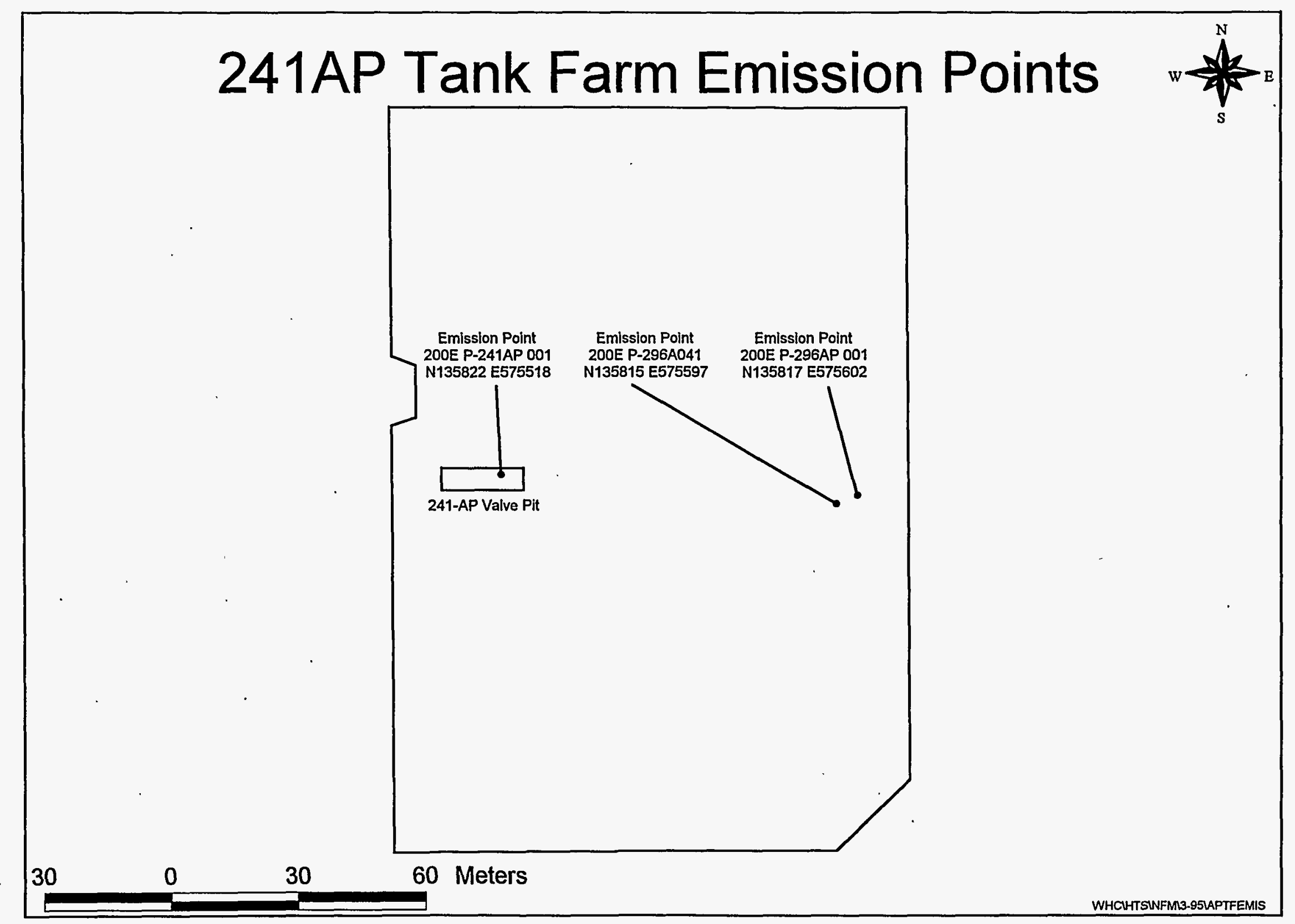




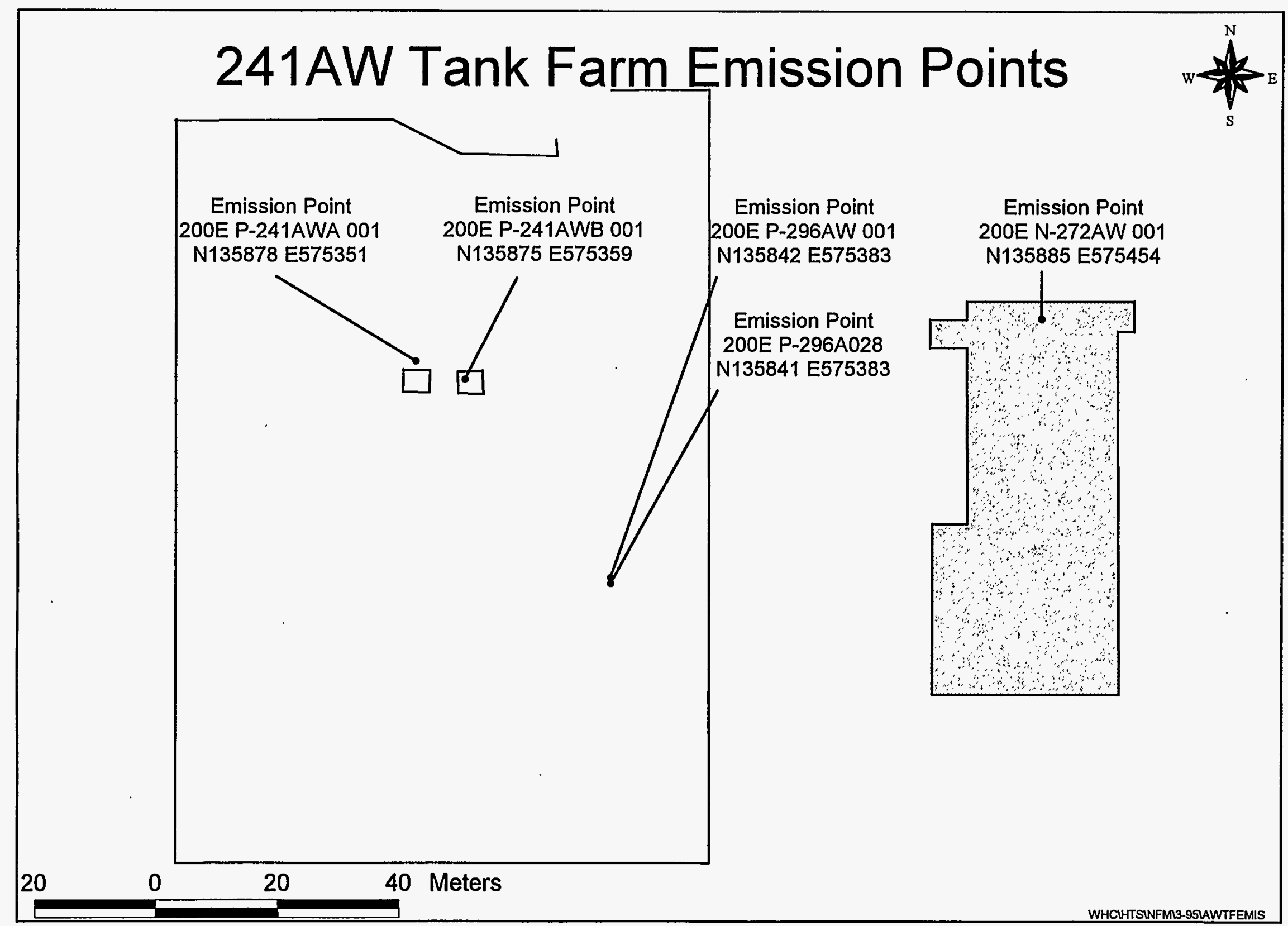




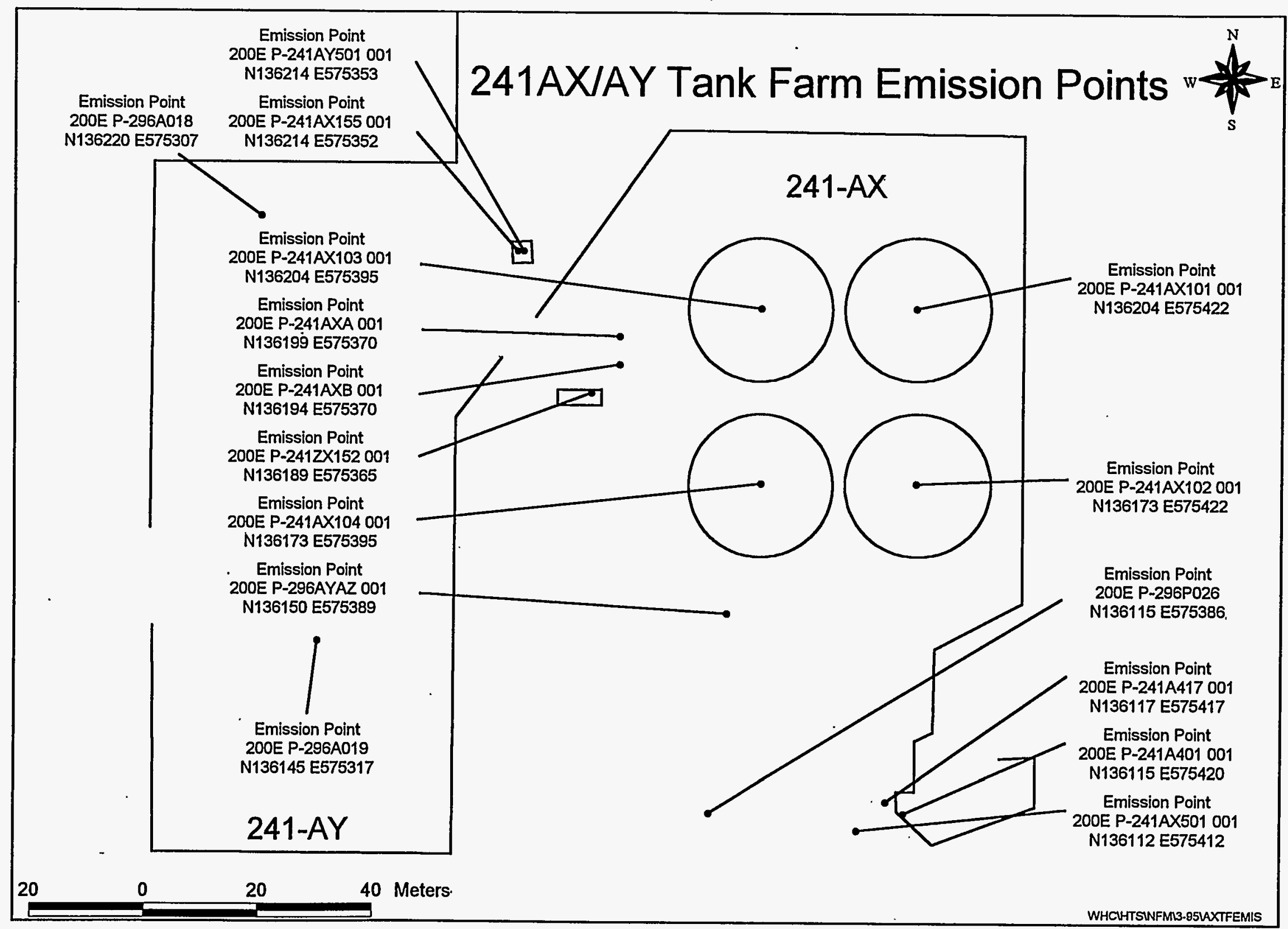




\section{B Tank Farm Emission Points}

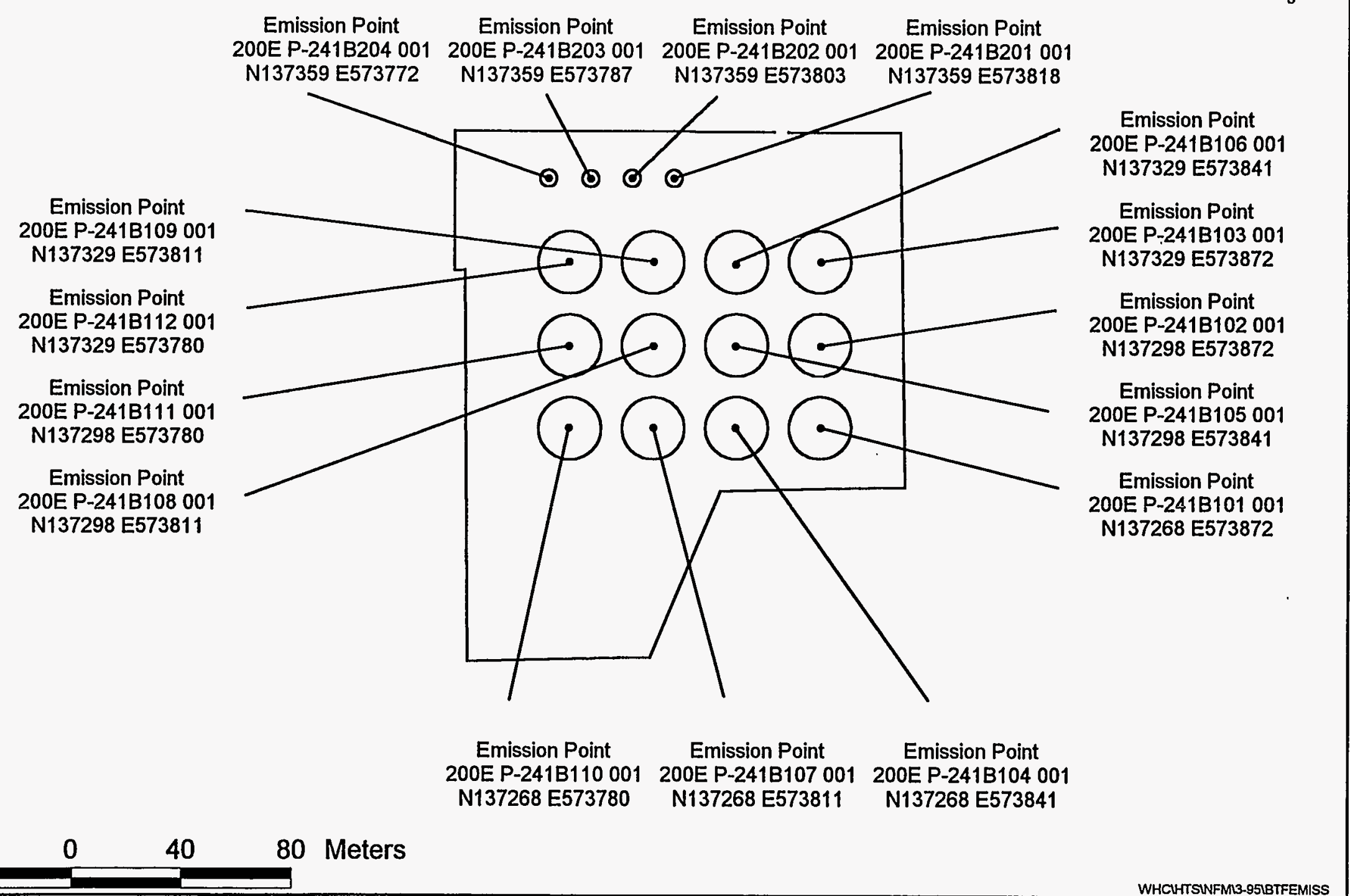




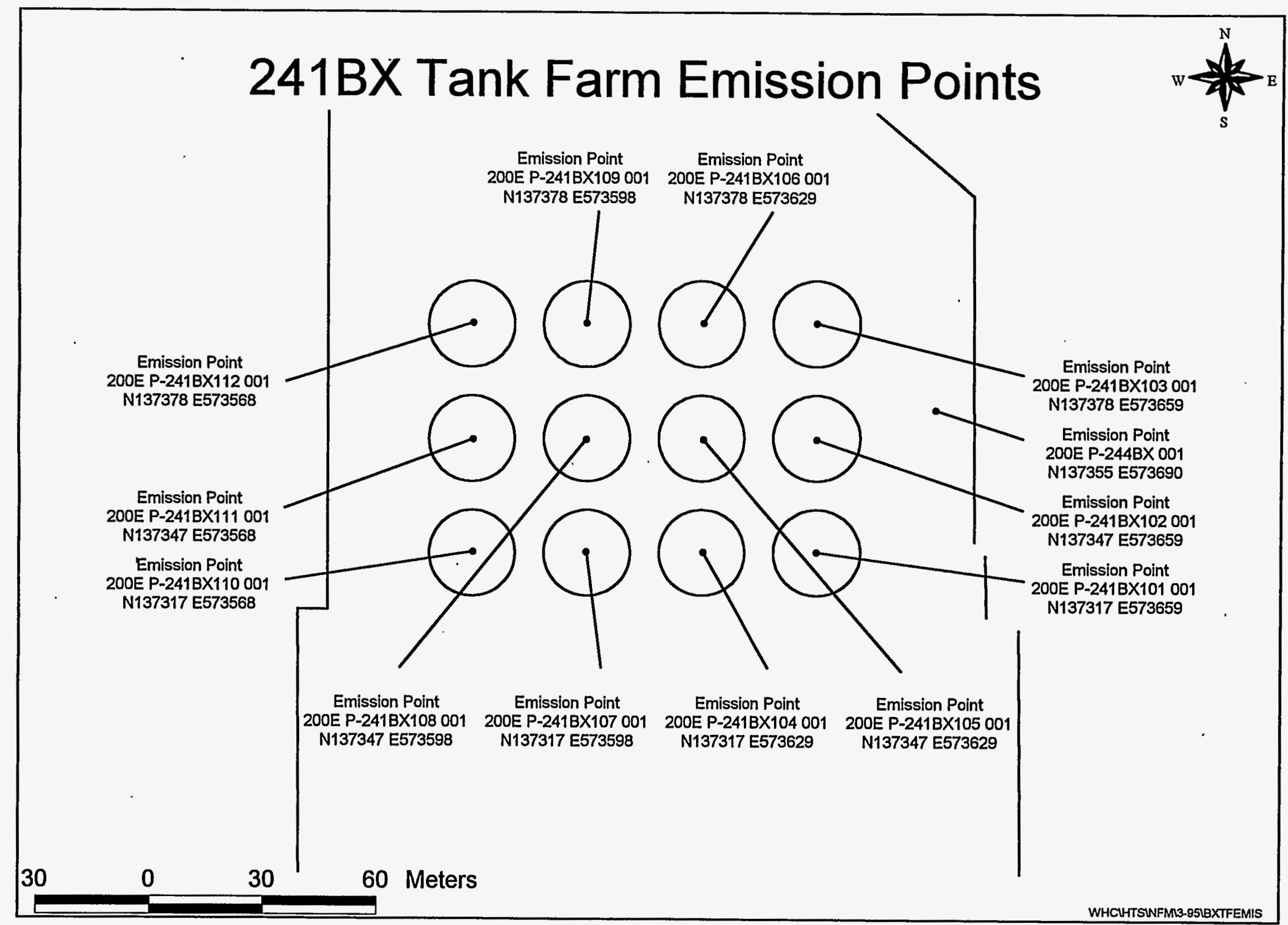




\section{BY Tank Farm Emission Points}

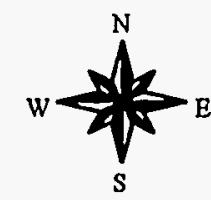

Emission Point

200E P-241BY109 001

N137531 E573597

Emission Point 200E P-241BY112 001 N137531 E573567

Emission Point 200E P-241BY108 001 N137500 E573597

Emission Point 200E P-241BY111 001 N137500 E573567

Emission Point 200E P-241BY110 001 N137469 E537567

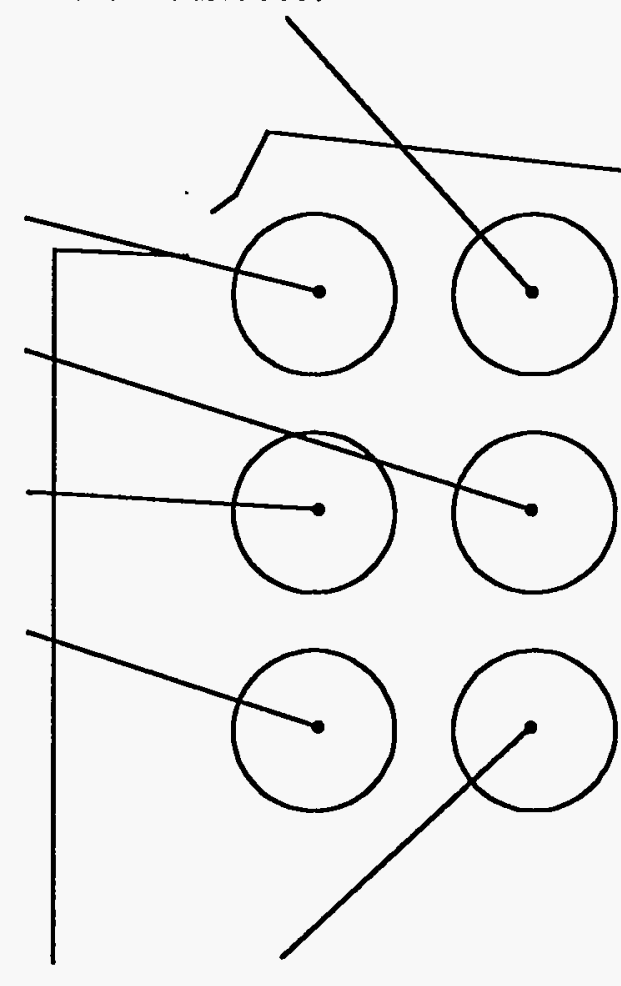

Emission Point 200E P-241BY107 001 N137469 E573597<smiles>CN</smiles>

Emission Point

200E P-241BY106 001

N137531 E573629

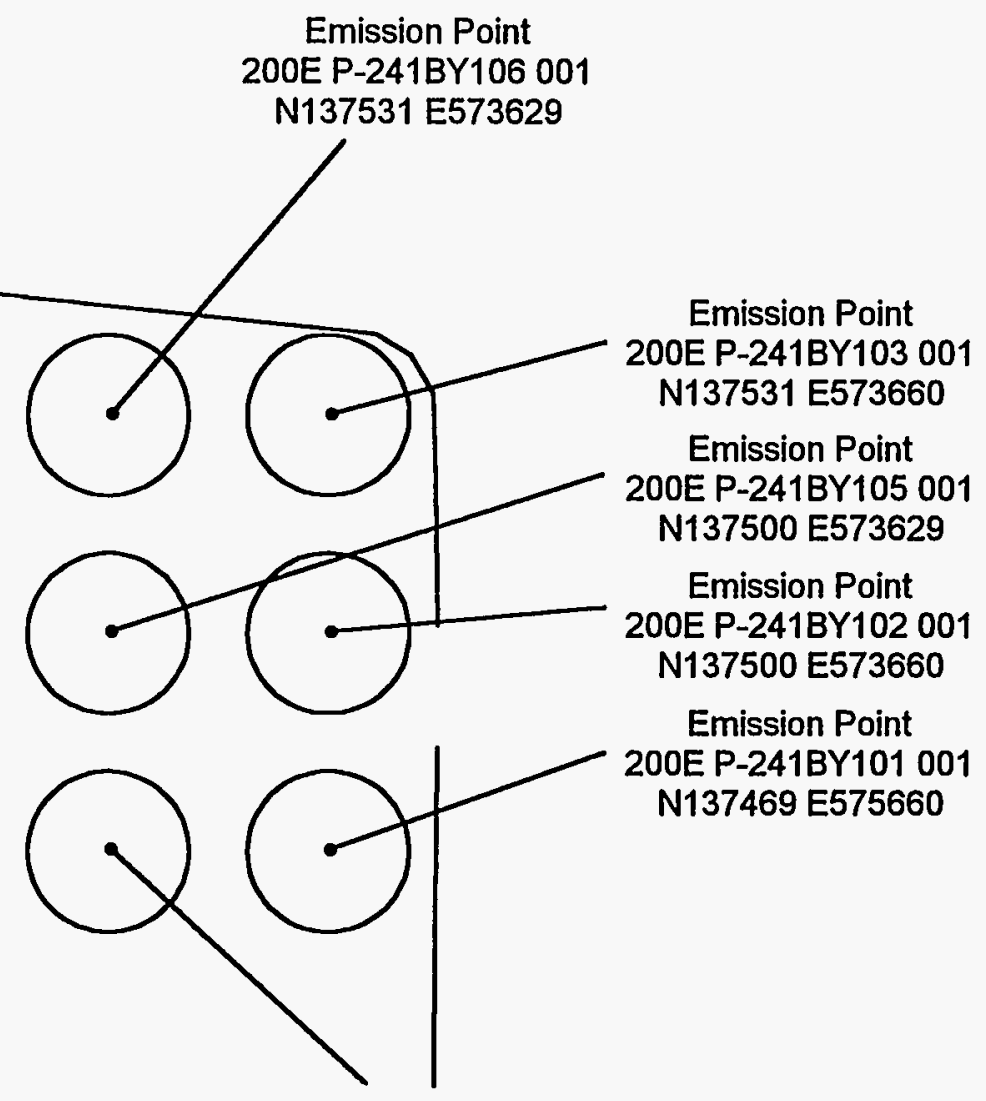

Emission Point 200E P-241BY104 001 N137469 E573629 


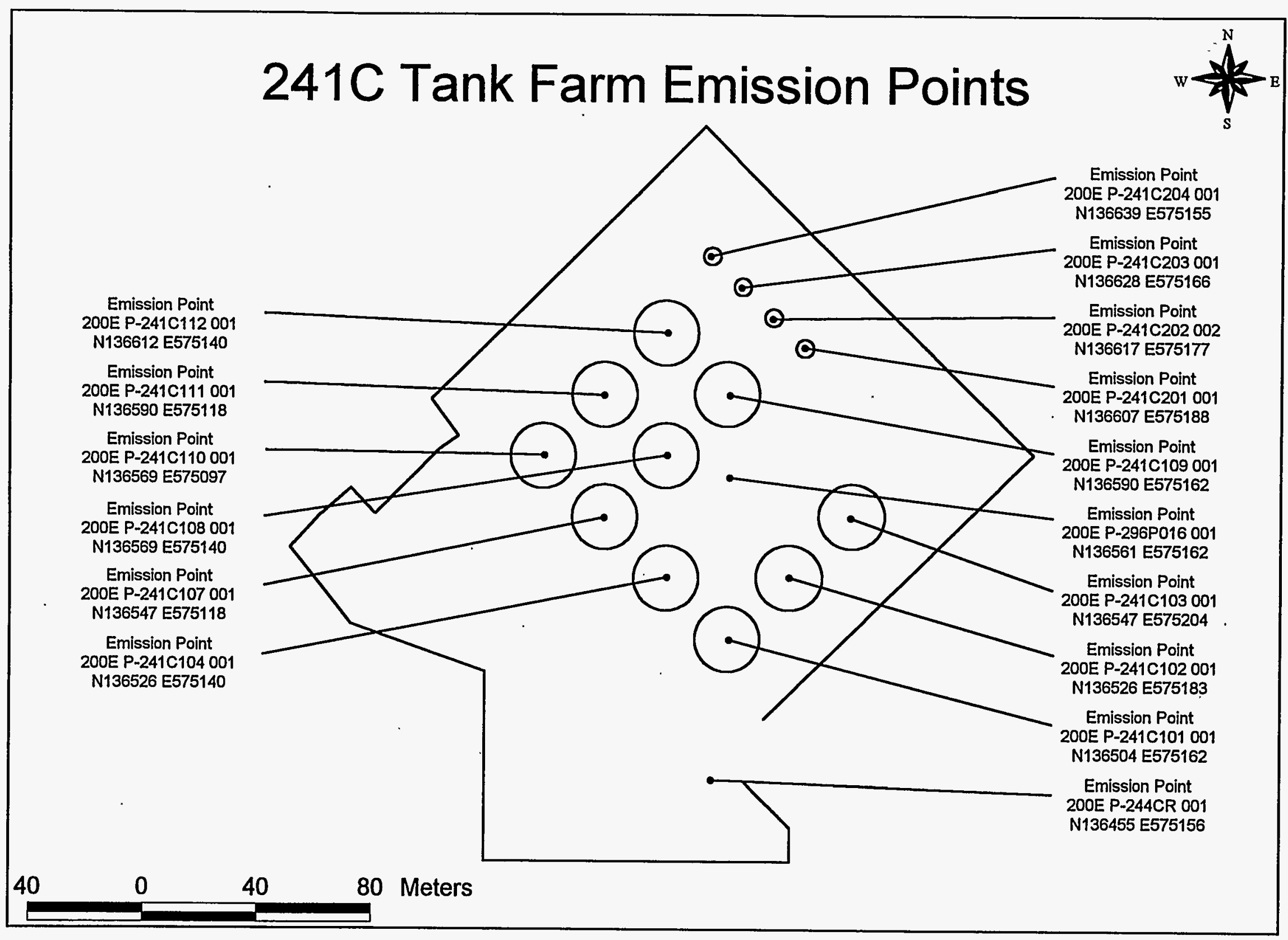




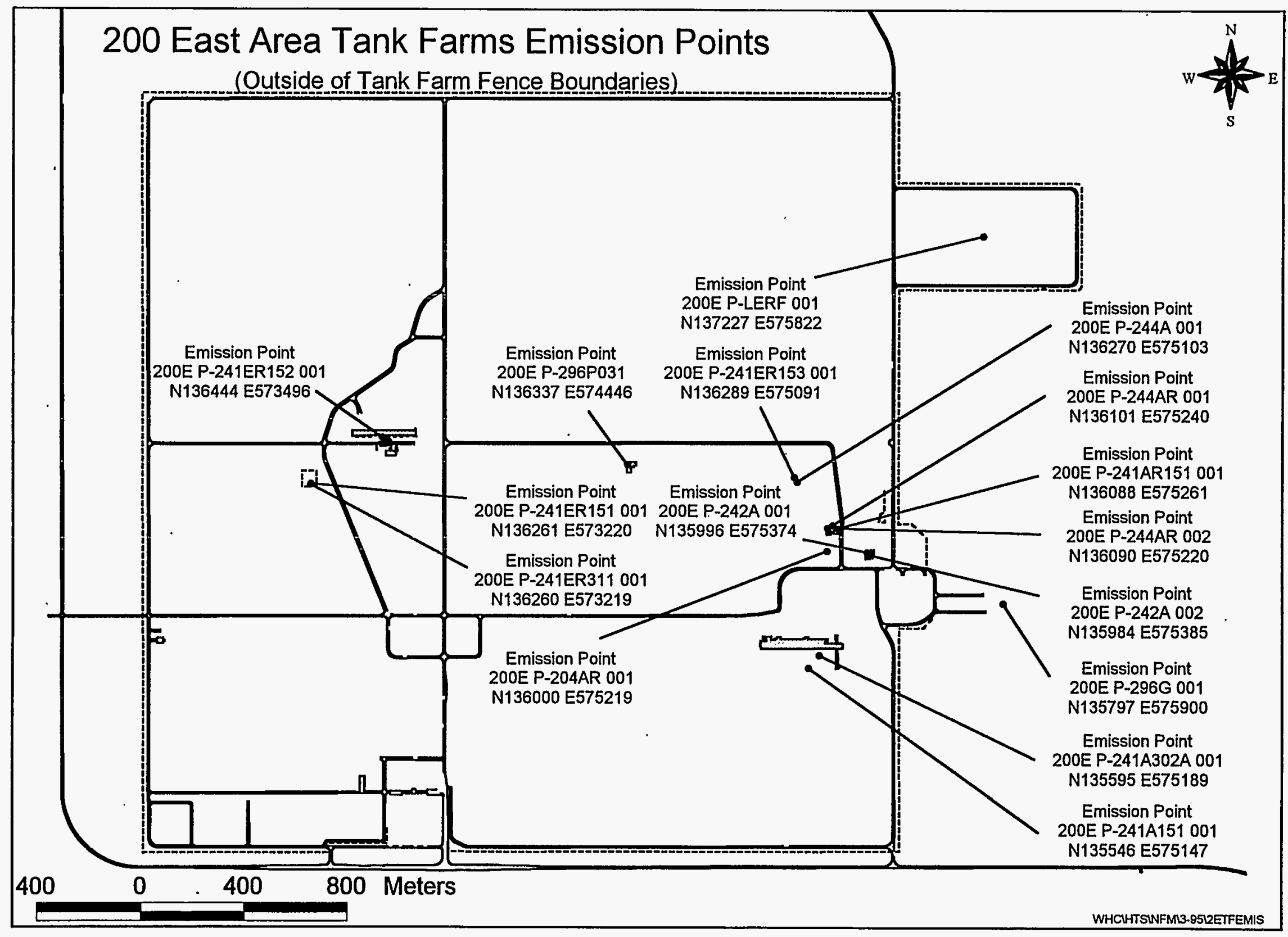




\section{APPENDIX D-5}

\section{AREA EFFLUENT TREATMENT FACILITY}

\section{Chapter I. General Information}

\section{I-B.1. Facility Description}

The 200 Area Effluent Treatment Facility (ETF) provides treatment for Hanford Site waste water generated in the 200 Areas by removing organic, inorganic, and radioactive contaminants. Once in operation, the 200 Area ETF will treat the effluent stream discharged from the 242-A Evaporator, a waste concentrator for a large volume of Hanford Site liquid waste forms stored in underground storage tanks. It is anticipated that the 200 Area ETF will treat other waste waters generated on the Hanford Site.

The 200 Area ETF is 13,400 square meters (44,000 square feet). The facility includes an engineered steel building that will house waste water treatment equipment. Some of the support systems include a vessel vent system, process piping and tanks, an integrated monitoring and control system, utility system, chemical makeup and distribution system, and a secondary waste packaging system.

The main unit operation in conjunction with on-line monitoring and sampling, ensure that treated waste waters are acceptable for discharge to the soil column in the 200 West Area. The main unit operation combines processes remove or destroy the heavy metals, organics and radionuclides that are identified as requiring treatment.

The 200 Area ETF is located in the 200 East Area. The main building, 2025E, houses the main unit operation. The 200 Area ETF is operated 24 hours per day.

\section{I-B.2. Products, Raw Materials, Fuels, Processes}

- Principal Products - There are no products produced at the 200 Area ETF

- Process Information - There is a main unit operation

- The main unit operation is a single treatment train with feed storage, suspended solids removal (rough, to $2 \mathrm{um}$ ), ultraviolet/oxidation with hydrogen peroxide, $\mathrm{pH}$ adjustment, suspended solids removal (fine, to $0.5 \mathrm{um}$ ), degasification (carbon dioxide removal), two stages of reverse osmosis (RO), mixed-bed ionexchange polishing, final $\mathrm{pH}$ adjustment. 
- Raw materials used by processes - There are no raw materials used at the 200 Area ETF.

- Fuels used by processes - There are no fuels used in the processes at the 200 Area ETF.

\section{I-C.1. Emission Points, Controls, Stacks}

The emission point and stack information are found on page 1 of the AEI report output. The control information is found on page 1 of the AEI report output.

\section{I-C.2. Facility Process Flow Diagrams}

Attached.

\section{I-C.3. Site Map}

Attached.

\section{Chapter II. Regulatory Requirements}

\section{II-A.1. Description of Discharge and Emission Points}

The emission point and stack information are found on page 1 of the AEI report output. 


\section{Facility Building List}

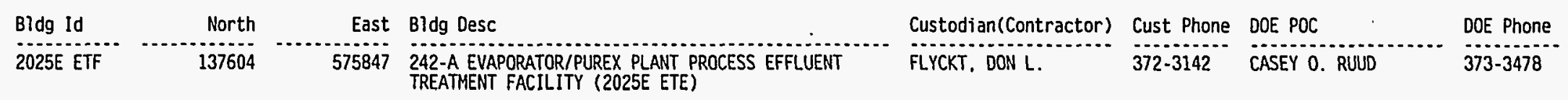

\section{Emission Point Listing}

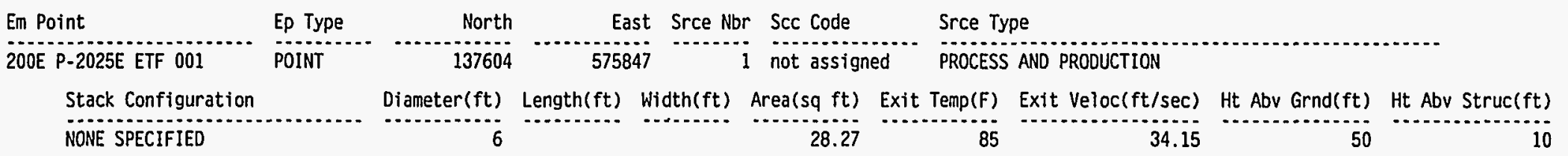

Source (Process) Listing

\begin{tabular}{|c|c|c|c|c|c|c|}
\hline Em Point & Srce $\mathrm{Nbr}$ & Srce Type & Hrs Day & Days Week & Wks Year & Summary Process Description \\
\hline 200E P-2025E ETF 001 & 1 & PROCESS AND PRODUCTION & 24 & 7 & 52 & $\begin{array}{l}\text { THE } 2025 \text { ETF WILL TREAT } 1 \text { INDUSTRAL WASTEWATER STREAM BY } \\
\text { REMOVING ORGANIC INORGANIC AND RADIOACTIVE CONTAMINANTS. } \\
\text { THE I WASTE STREAM IS THE } 242-A \text { EVAPORATOR PROCESS } \\
\text { CONDENSATE. }\end{array}$ \\
\hline
\end{tabular}

\section{Source Raw Materials Usage}

Em Point

Srce Nbr Srce Type

Process Rate Process Uom Material

Fuels Usage by Source Processes

Em Point Srce Nbr Srce Type

Qty Uom

Fuel

Fuels Usage by Control Devices

Em Point 
Point Source Emissions - Radiological Pollutants

\begin{tabular}{|c|c|c|c|c|}
\hline Em Point & Srce Nbr & Srce Type Pollutant & $\begin{array}{l}\text { Em. Pt. Potential } \\
\text { to Emit }(\mathrm{C} i / \mathrm{yr})\end{array}$ & $\begin{array}{l}\text { Em. Pt. } \\
\text { Cti Actual (Ci/yr) }\end{array}$ \\
\hline
\end{tabular}

Point Source Emissions - HAP Pollutants

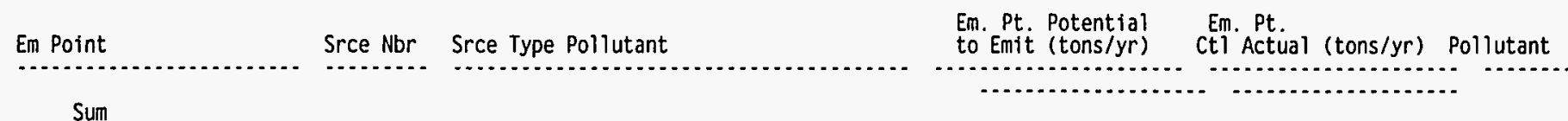

Point Source Emissions : Uncategorized Pollutants

Em Point

Srce Nbr Srce Type Pollutant

Em. Pt. Potential Em. Pt.

Em Point

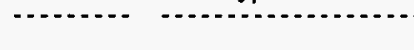

to Emit (tons/yr) Ctl Actual (tons/yr) Pollutant

Sum

Fugitive Source Emissions - Radiological Pollutants

\begin{tabular}{|c|c|c|c|c|}
\hline int & Srce Nbr & Srce Type Pollutant & $\begin{array}{l}\text { Em. Pt. Potential } \\
\text { to Emit }(\mathrm{Ci} / \mathrm{yr})\end{array}$ & $\begin{array}{l}\text { Em. Pt. } \\
\text { Cti Actual }(\mathrm{Ci} / \mathrm{yr})\end{array}$ \\
\hline
\end{tabular}

Fugitive Source Emissions - HAP Pollutants

\begin{tabular}{|c|c|c|c|c|}
\hline Em Point & Srce $\mathrm{Nbr}$ & Srce Type Pollutant & $\begin{array}{l}\text { Em. Pt. Potential } \\
\text { to Emit (tons/yr) }\end{array}$ & $\begin{array}{l}\text { Em. Pt. } \\
\text { Cti Actual (tons/yr) }\end{array}$ \\
\hline
\end{tabular}

Sum

Fugitive Source Emissions - Uncategorized Pollutants

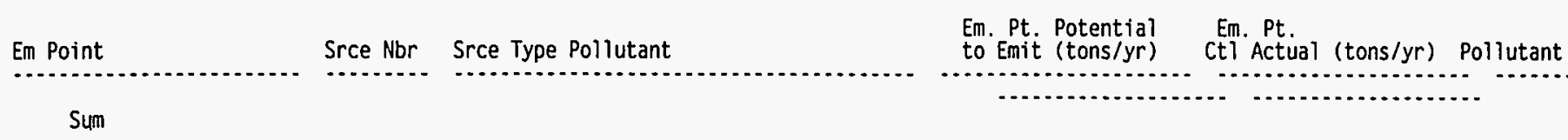


AIR EMISSIONS INVENTORY PERMIT APPLICATION REPORT (UP460)

FACILITY NAME: 200 EAST AREA EFFLUENT TREATMENT FACILITY

Insignificant Source Emissions - Radiological Pollutants

Em Point Srce Nbr Srce Type Pollutant

Insignificant Source Emissions - HAP Pollutants

Em Point

Srce Nbr Srce Type Pollutant

Insignificant Source Emissions - HAP Pollutants

Em Point

Srce Nbr Srce Type Pollutant

Em. Pt. Potential Em. Pt.

Pollutant

Sum

Insignificant Source Emissions - Uncategorized Pollutants

$$
\text { Em Point }
$$

Srce Nbr Srce Type Pollutant

Em. Pt. Potential Em. Pt.

to Emit (tons/yr) Cti Actual (tons/yr) Pollutant

Em. Pt. Potential Em. Pt.

Em. Pt. Potential Em. Pt.

to Emit (tons/yr) Ctl Actual (tons/yr) Pollutant

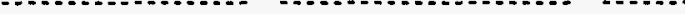

Sum

Control Device Listing
Em Point
Control Equipment Code
200E P-2025E ETF 001
099 


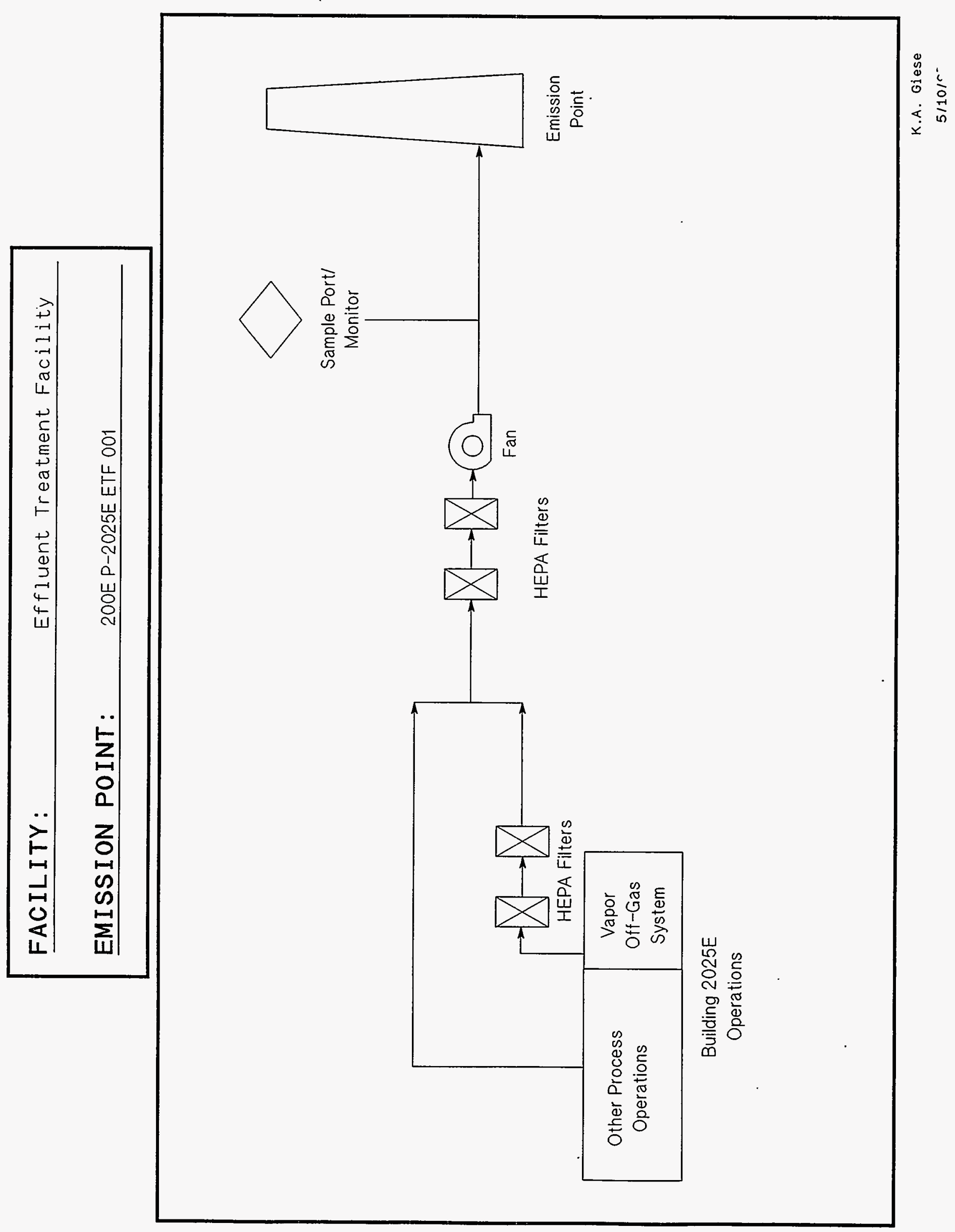




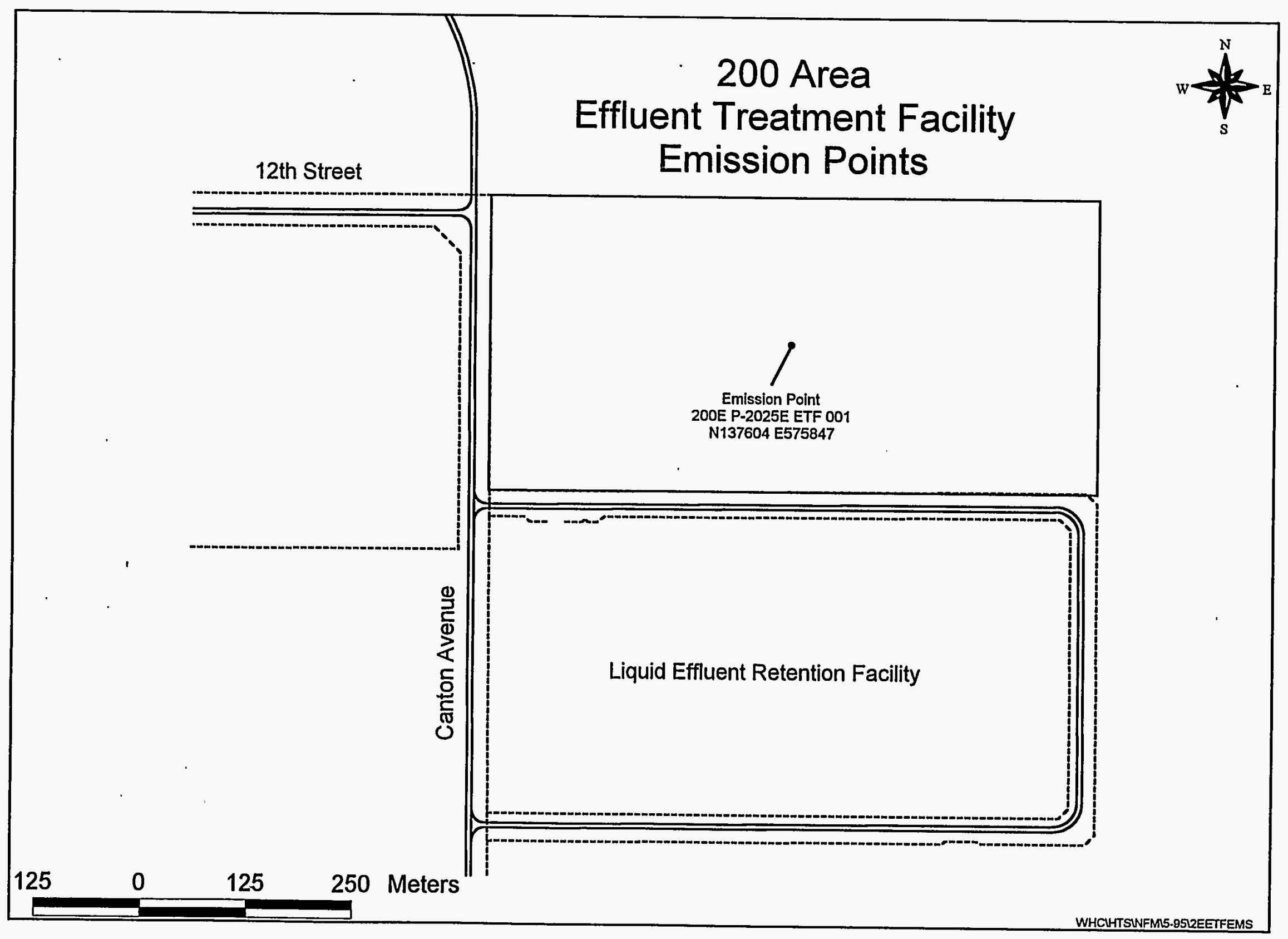


Hanford Site Air

Operating Permit Application
DOE/RL-95-07, Rev. 0

05/95

\section{APPENDIX E}

200 WEST AREA 


\section{APPENDIX E}

\section{CONTENTS}

E-1 222-S LABORATORY COMPLEX

E-2 284W POWERHOUSE

E-3 PLUTONIUM FINISHING PLANT

E-4 S PLANT

E-5 T PLANT

E-6 U PLANT

E-7 200 WEST AREA TANK FARMS

E-8 SOLID WASTE MANAGEMENT FACILITIES

E-9 WASTE RECEIVING AND PROCESSING FACILITY 


\title{
APPENDIX E-1
}

\section{2-S LABORATORY COMPLEX}

\author{
Chapter I. General Information
}

\section{I-B.1. Facility Description}

The 222-S Laboratory Complex, in the southeast corner of the 200 West Area, consists of the 222-S Laboratory, the 222-SA Standards Laboratory, and several ancillary facilities. The main facility of the complex is the 222-S Laboratory, which provides analytical chemistry and radiological services in support of clean up activities on the Hanford site.

Currently, 222-S Laboratory activities are to characterize the waste stored in single shell and double shell tanks that are located on the Hanford site. A 4,000 square foot addition was added to the east end of the 222-S Laboratory building in 1994. This addition houses a seven compartment hot cell and increases the area of the facility to approximately 39,000 square feet. These seven hot cells will be used for receiving, extruding and preparing high level radioactive core samples, mainly from single-shell and double-shell waste characterization activities.

The 222-SA Laboratory is a modular building located southeast of the 222-S Laboratory. Part of this laboratory prepares nonradioactive standards for other laboratories on the Hanford site. The other section of the laboratory is used for cold (nonradioactive) process development work and standards preparation.

The 219-S Waste Handling Facility has three storage tanks (Tanks 101, $102 \& 103$ ) in which liquid waste from $222-S$ can be received, stored temporarily, and neutralized. The radioactive waste from this facility is transferred to the Hanford site Tank Farms. A 700gallon sodium-hydroxide supply tank is also located in this facility to assist in the neutralization of the waste. The 219-S Facility is equipped with a sampling gallery which allows samples to be drawn from Tank 102 and 103 for analysis.

222-S Laboratory Complex is operated for 24 hours per day, and 365 days per year. 


\section{I-B.2. Products, Raw Materials, Fuels, Processes}

- Principle products - There are no products at the 222-S Laboratory Complex

- Process information - Outside of typical laboratory activities which are listed on page 2 of the AEI report, there is one process activity at the 222-S Laboratory Complex, water purification:

Water is purified for laboratory use by demineralization.

- Raw materials used by processes - There are no raw materials used at the 222-S Laboratory Complex.

- Fuels used by processes - There are no fuels used at the 222-S Laboratory Complex

\section{I-C.1. Emission Points, Controls, Stacks}

The emission point and stack information is found on page 1 of the AEI report output. The control information is found on page 5 of the AEI report output.

\section{I-C.2. Facility Process Flow Diagrams}

Attached.

I-C.3. Site Map

Attached.

\section{Chapter II. Regulatory Requirements}

\section{II-A.1. Description of Discharge and Emission Points}

The emission point and stack information is found on page 1 of the AEI report output. 


\section{Facility Building List}

\begin{tabular}{|c|c|c|c|c|c|c|c|}
\hline Bidg Id & North & East & Bldg Desc & Custodian(Contractor) & Cust Phone & DOE POC & DOE Phone \\
\hline $\begin{array}{l}222 S A \\
296 S 016 \\
296 S 021 \\
296 S 023\end{array}$ & $\begin{array}{l}133857 \\
133923 \\
133907 \\
133928\end{array}$ & $\begin{array}{l}567527 \\
567469 \\
567475 \\
567477\end{array}$ & $\begin{array}{l}\text { STANDARDS/PROCESS DEV LABORATORY } \\
\text { STACK VENTING } 219-5 \text { BUILDING } \\
\text { MAIN EXHAUST STACK FOR BUILDING 222-S } \\
219-S \text { WASTE HANDLING FACILITY. SAMPLE GALLERY } \\
\text { WASTE SAMPLING HOOD }\end{array}$ & $\begin{array}{l}\text { HALL. MICHAEL J. } \\
\text { HALL: MICHAEL j. } \\
\text { HALL: MICHAEL j. } \\
\text { HALL. MICHAEL j. }\end{array}$ & $\begin{array}{l}373-5719 \\
373-5719 \\
373-5719 \\
373-5719\end{array}$ & $\begin{array}{l}\text { STEVE VEITENHEIMER } \\
\text { STEVE VEITENHEIMER } \\
\text { STEVE VEITENHEIMER } \\
\text { STEVE VEITENHEIMER }\end{array}$ & $\begin{array}{l}373-9725 \\
373-9725 \\
373-9725 \\
373-9725\end{array}$ \\
\hline
\end{tabular}

\section{Emission Point Listing}

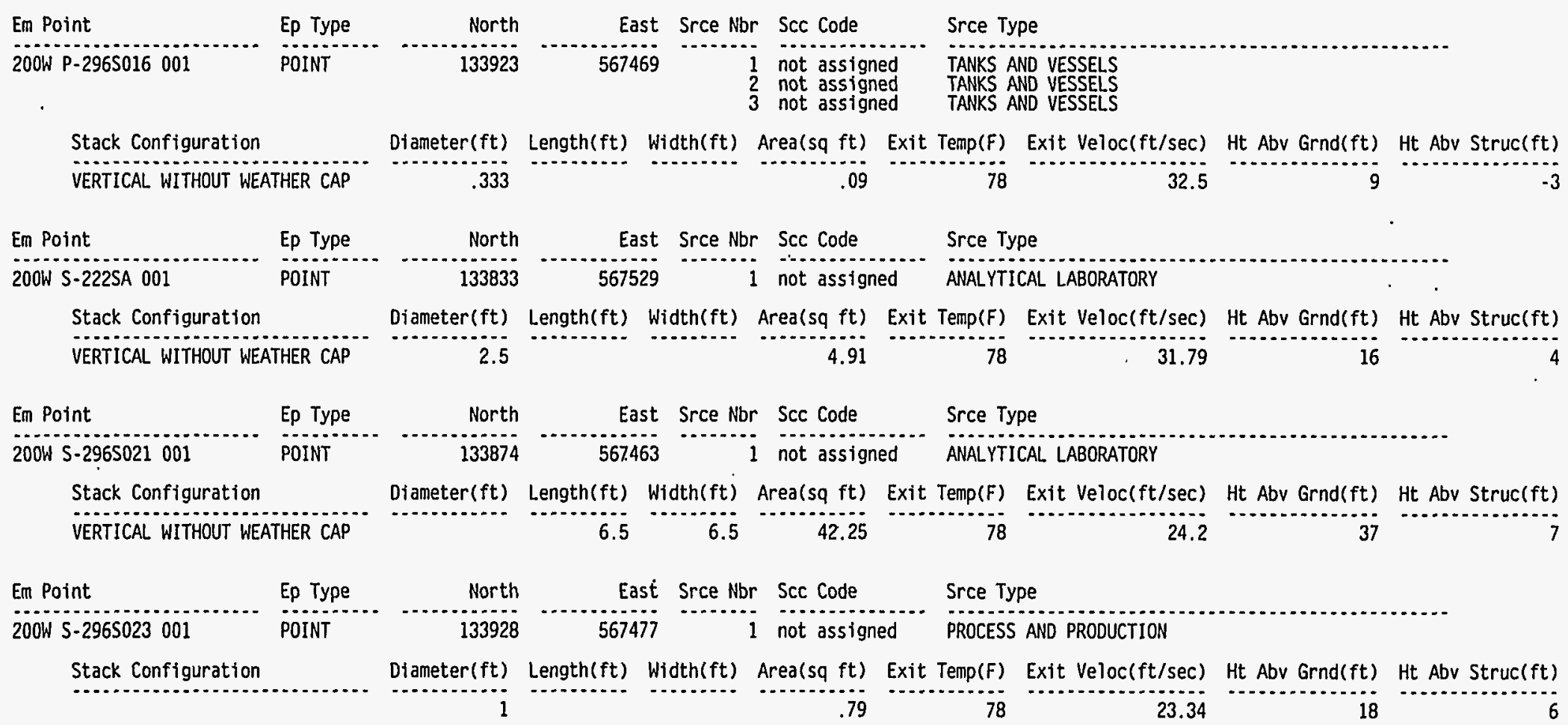




\section{Source (Process) Listing}

\begin{tabular}{|c|c|c|c|c|c|c|}
\hline Em Point & Srce Nbr & Srce Type & Hrs Day & Days Week & Whs Year & Summary Process Description \\
\hline 200W P-296S016 001 & 1 & TANKS AND VESSELS & 24 & 7 & 52 & 219-5 WASTE HANDLING FACILITY TANK 101 \\
\hline Em Point & Srce Nbr & Srce Type & Hrs Day & Days Week & Wks Year & Summary Process Description \\
\hline $200 \mathrm{~W} P-2965016001$ & 2 & TANKS AND VESSELS & 24 & 7 & 52 & 219-S WASTE HANDLING FACILITY TANK 102 \\
\hline Em Point & Srce Nbr & Srce Type & Hrs Day & Days Week & Whs Year & Summary Process Description \\
\hline 200W P-296S016 001 & 3 & TANKS AND VESSELS & 24 & 7 & 52 & 219-S WASTE HANDLING FACILITY TANK 103 \\
\hline Em Point & Srce Nbr & Srce Type & Hrs Day & Days Week & Whs Year & Summary Process Description \\
\hline 200W S-222SA 001 & 1 & ANALYTICAL LABORATORY & 8 & 5 & 50 & NON-RADIONUCLIDE CHEMICAL ANALYSIS LABORATORY \\
\hline Em Point & Srce $\mathrm{Nbr}$ & Srce Type & Hrs Day & Days Week & Whs Year & Summary Process Description \\
\hline $200 \mathrm{~W}$ S-296S021 001 & .1 & ANALYTICAL LABORATORY & 24 & 7 & 52 & PROCESS AND ANALYTICAL SUPPORT LABORATORY \\
\hline \multicolumn{7}{|c|}{$\begin{array}{l}\text { PROCEDURES AND ANALYSES PERFORMED INCLUDE: GAS CHROMATOGRAPHY, MASS SPECTROSCOPY. INDUCTIVELY COUPLED PLASMA SPECTROSCOPY, ION CHROMATOGRAPHY. THERMU } \\
\text { GRAVIMETRIC ANALYSIS, ATOMIC ABSORPTION SPECTROSCOPY. ION SPECIFIC ELECTRODE, FLLUORIMETRY TOTAL ORGANIC CARBON, PH, TOTAL INORGANIC CARBON ANALYSIS, } \\
\text { MULTIPLE RADIONUCLIDE ANALYSIS, GAMMA ENERGY. ALPHA ENERGY ANALYSIS. TOTAL BETA COUTING AND LIQUID SCINTILLATION COUNTING. }\end{array}$} \\
\hline Em Point & Srce Nbr & Srce Type & Hrs Day & Days Week & Wks Year & Summary Process Description \\
\hline $200 W$ S-296S023 001 & 1 & PROCESS AND PRODUCTION & 1 & $i$ & 12 & 219-S WASTE HANDLING FACILITY. SAMPLE GALLERY HOOO \\
\hline \multicolumn{7}{|c|}{ Source Raw Materials Usage } \\
\hline Em Point & $\begin{array}{l}\text { Srce } \mathrm{Nbr} \\
-. . . . . .\end{array}$ & Srce Type & & Process & s Rate Pro & ocess Vom Material \\
\hline
\end{tabular}

\section{Fuels Usage by Source Processes}

Em Point 
AIR EMISSIONS INVENTORY PERMIT APPLICATION REPORT (UP460)

FACILITY NAME: 200 W Laboratories and ASSOCIat

Fuels Usage by Control Devices

Em Point

Ctl Nbr Ctl Type

Qty Uom

Fuel

Point Source Criteria Pollutants - Type: H2S04

Em Point

$200 \mathrm{~W}$ S-2965021 001
Srce Nbr Srce Type Pollutant

I ANALYTICAL LABORATORY
Em. Pt. Potential Em. Pt.

(tons/yr) Pollutant $062000000 \quad 014000000$ SULFURIC ACID

Point Source Criteria Pollutants - Type: H2S04

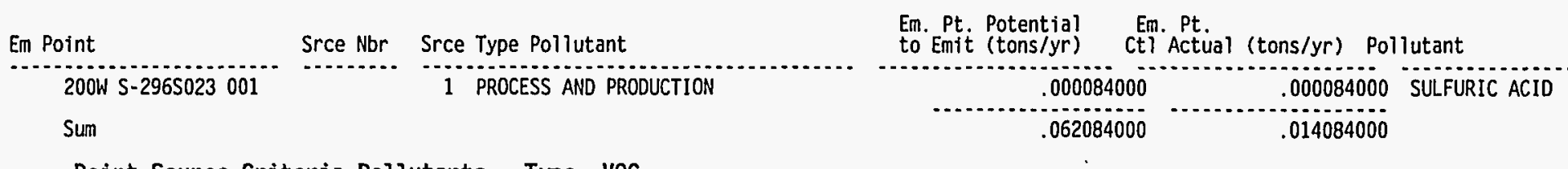

Point Source Criteria Pollutants - Type: VOC

Em Point

200W S-2965021 001

Srce Nbr Srce Type Pollutant

1 ANALYTICAL LABORATORY

200W S-2965023001

1 PROCESS AND PRODUCTION

Sum
Em. Pt. Potential Em. Pt.
to Emit (tons/yr) Ctl Actual (tons/yr) Pollutant

\section{$.027000000 \quad .006100000 \quad 2,2,4$-TRIMETHYLPENTANE}

$.051000000^{\circ} \quad .012000000$ ACETIC ACID

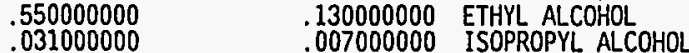

031000000

10000000

1004200000

.056000000

.007000000
.046000000
METTHYPY CHLORIDE

.023000000 N-HEXANE

013000000 TRICHLOROETHYLENE

$017000000 \quad 003800000$ XYLENE

$.000072000 \quad .000072000$ ACETIC ACID

000780000

.000042000

000042000 ISOPROPYL ALCOHOL

1.037094000

.242754000 


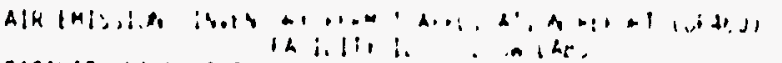

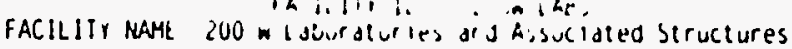

Point Source Emissions - Radiological Pollutants

\begin{tabular}{|c|c|c|c|c|c|}
\hline Em Point & Srce Nbr & Srce Type Pollutant & $\begin{array}{l}\text { Em. Pt. Potential } \\
\text { to Emit }(\mathrm{Ci} / \mathrm{yr})\end{array}$ & $\begin{array}{l}\text { Em. Pt. } \\
\mathrm{Cti} \text { Actual }(\mathrm{Ci} / \mathrm{yr})\end{array}$ & Pollutant \\
\hline 200W P-2965016001 & 1 & TANKS AND VESSELS & $\begin{array}{l}\text { Refer to } \\
\text { WHC-SD-EN-RPT-007 } \\
\text { Rev. } 0 \text { Page A-245 }\end{array}$ & $\begin{array}{l}\text { Refer to } \\
\text { DOE/RL-94-51 } \\
\text { Table 2-1 }\end{array}$ & \\
\hline 200W S-296Ṣ021 001 & 1 & ANALYTICAL LABORATORY & $\begin{array}{l}\text { Refer to } \\
\text { WHC-SD-EN-RPT-007 } \\
\text { Rev. } 0 \text { Page A-202 }\end{array}$ & $\begin{array}{l}\text { Refer to } \\
\text { DOE/RL-94-51 } \\
\text { Table } 2-1\end{array}$ & \\
\hline 200W S-296S023001 & 1 & TANKS AND VESSELS & $\begin{array}{l}\text { Refer to } \\
\text { WHC-SD-EN-RPT-025 } \\
\text { Rev. 0 Page A- } 19\end{array}$ & $\begin{array}{l}\text { Refer to } \\
\text { WHC-SD-EN-RPT-025 } \\
\text { Rev. O Page A-19 }\end{array}$ & \\
\hline
\end{tabular}

Point Source Emissions - HAP Pollutants

Em Point Srce Nbr Srce Type Pollutant

Em. Pt. Potential Em. Pt.

200W S-296S021 001 1 ANALYTICAL LABORATORY

to Ectual (tons/yr) Pollutant

.027000000
1.200000000
.016000000
.200000000
.480000000
.100000000
.004200000

O6) 006100000
033300000
HYDROCHLLRIC ACID (HYDROGEN CHLORIDE) .000000014 MERCURY METHY CHLORIDE TETHLENE CHLORIDE 000960000 TOXANE 013000000 TRICHLOROETHYLENE

.003800000 XYLENE

200W S-2965023001 1 PROCESS AND PRODUCTION

Sum

\begin{tabular}{|c|c|c|}
\hline $\begin{array}{l}.000200000 \\
.000000000\end{array}$ & $\begin{array}{l}.000200000 \\
.000000000\end{array}$ & $\begin{array}{l}\text { HYOROCHLORIC ACIO (HYOROGEN CHLORIDE) } \\
\text { MERCURY }\end{array}$ \\
\hline 2.100400000 & 236360014 & \\
\hline
\end{tabular}

Point Source Emissions - Uncategorized Pollutants

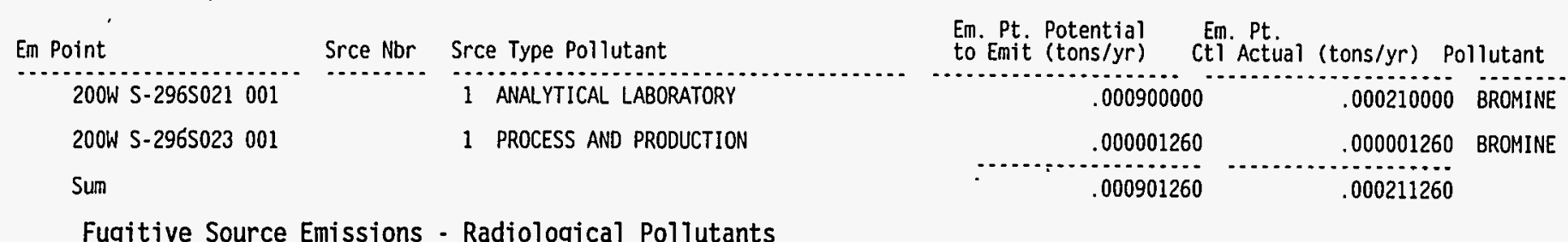

Fugitive Source Emissions - Radiological Pollutants

Em. Pt. Potential Em. Pt. 
AIR EMISSIONS INVENTORY PERMIT APPLICATION REPORT (UP460)

FACILITY NAME: 200 W Laboratories and Associated Structures

Fugitive Source Emissions - HAP Pollutants

Em Point

Srce Nbr Srce Type Pollutant

Sum

Fugitive Source Emissions - Uncategorized Pollutants

Em Point

Srce Nbr Srce Type Pollutant

Sum

Insignificant Source Criteria Pollutants - Type: H2SO4

\begin{tabular}{|c|c|c|}
\hline Em Point & Srce Nbr & Srce Type Pollutant \\
\hline 200W S-222SA 001 & & 1 ANALYTICAL LABORATORY \\
\hline Sum & & \\
\hline
\end{tabular}

Insignificant Source Criteria Pollutants - Type: VOC
En. Pt. Potential Em. Pt.

to Emit (tons/yr) Cti Actual (tons/yr) Pollutant

Em. Pt. Potential Em. Pt.
to Emit (tons/yr) Ct Actual (tons/yr) Pollutant

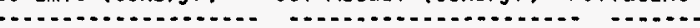


AIR EMISSIONS INVENTORY PERMIT APPLICATION REPORT (UP460)

FACILITY ID : 200W LABS

FACILITY ID : 200W LABS

Insignificant Source Emissions - HAP Pollutants

\begin{tabular}{|c|c|c|c|c|c|}
\hline Em Point & Srce $\mathrm{Nbr}$ & Srce Type Pollutant & $\begin{array}{l}\text { Em. Pt. Potential } \\
\text { to Emit (tons/yr) }\end{array}$ & $\begin{array}{l}\text { Em. Pt. } \\
\text { Ct1 Actual (tons/yr) }\end{array}$ & Pollutant \\
\hline 200W S-222SA 001 & & 1 ANALYTICAL LABORATORY & $\begin{array}{l}.092000000 \\
.270000000 \\
.007700000 \\
.091000000 \\
.023000000 \\
.004200000 \\
.017000000\end{array}$ & $\begin{array}{l}.021000000 \\
.063000000 \\
.001800000 \\
.021000000 \\
.005300000 \\
.000960000 \\
.003800000\end{array}$ & $\begin{array}{ll}0 & \text { HYROCHLORIC ACIO (HYDROGEN CHLORIOE) } \\
0 & \text { METHYL ALCOHOL } \\
0 & \text { METHYL ISOBUY KETONE } \\
0 & \text { METHYLENE CHLORIDE } \\
0 & \text { NITROBENZENE } \\
0 & \text { TOLUENE } \\
0 & \text { XYLENE }\end{array}$ \\
\hline Sum & & & .504900000 & .116860000 & \\
\hline
\end{tabular}

Insignificant Source Emissions - Uncategorized Pollutants

Em Point Srce Nbr Srce Type Pollutant

200W S-222SA 001

Sum

Control Device Listing

Em Point

Control Equipment Code

200W P.2965016 001

200W S-296S021 001

099

099
099

1

Em. Pt. Potential Em. Pt.
to Emit (tons/yr) CtI Actual (tons/yr) Pollutant

$\begin{array}{rrr}.300000000 & .069000000 & \text { AMMONIUM HYOROXIDE } \\ .015000000 & .003500000 & \text { PETROLEUM BENZIN } \\ .100000000 & .023000000 & \text { PHOSPHORIC ACID, TRIBUTYL ESTER } \\ .415000000 & .095500000 & \end{array}$




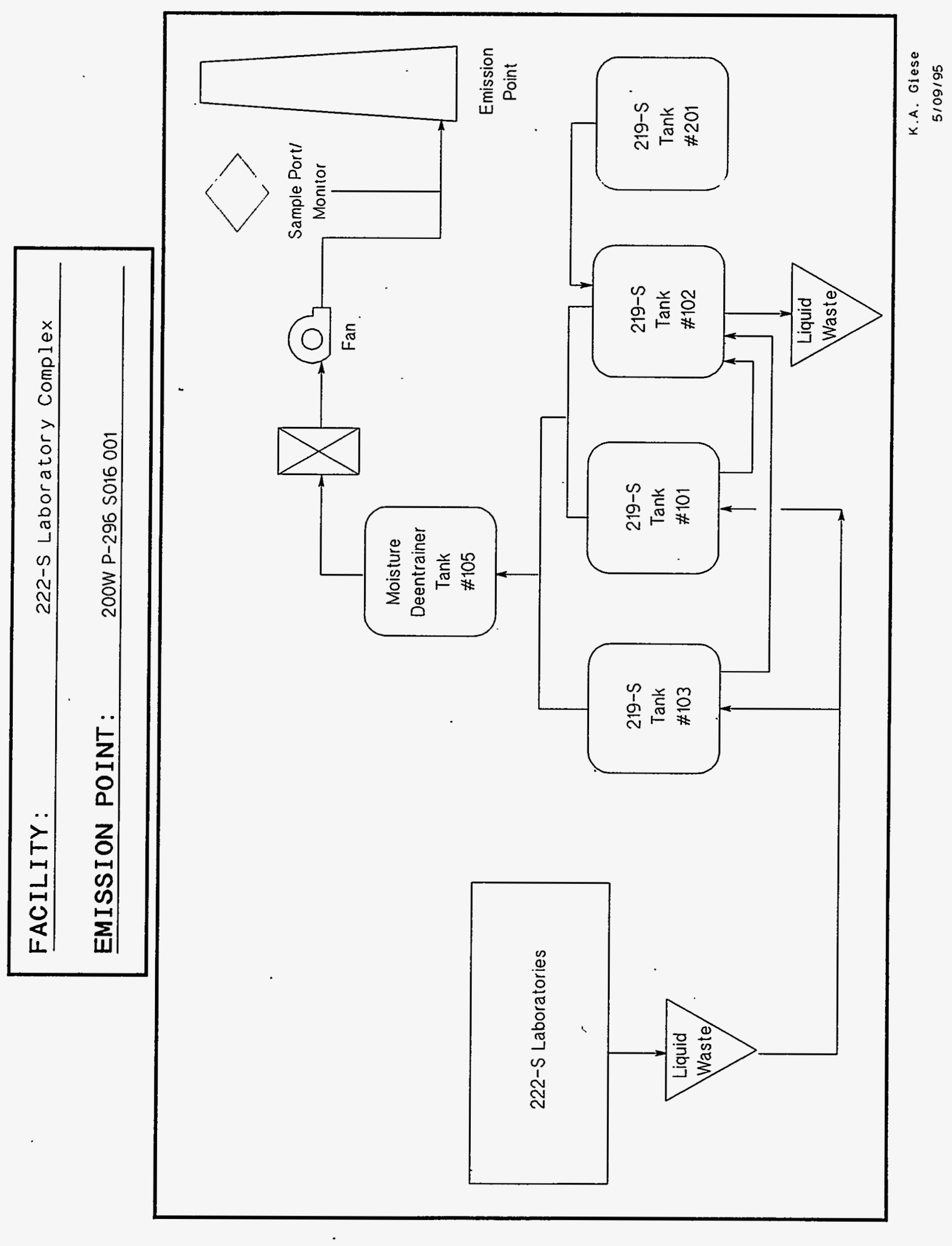




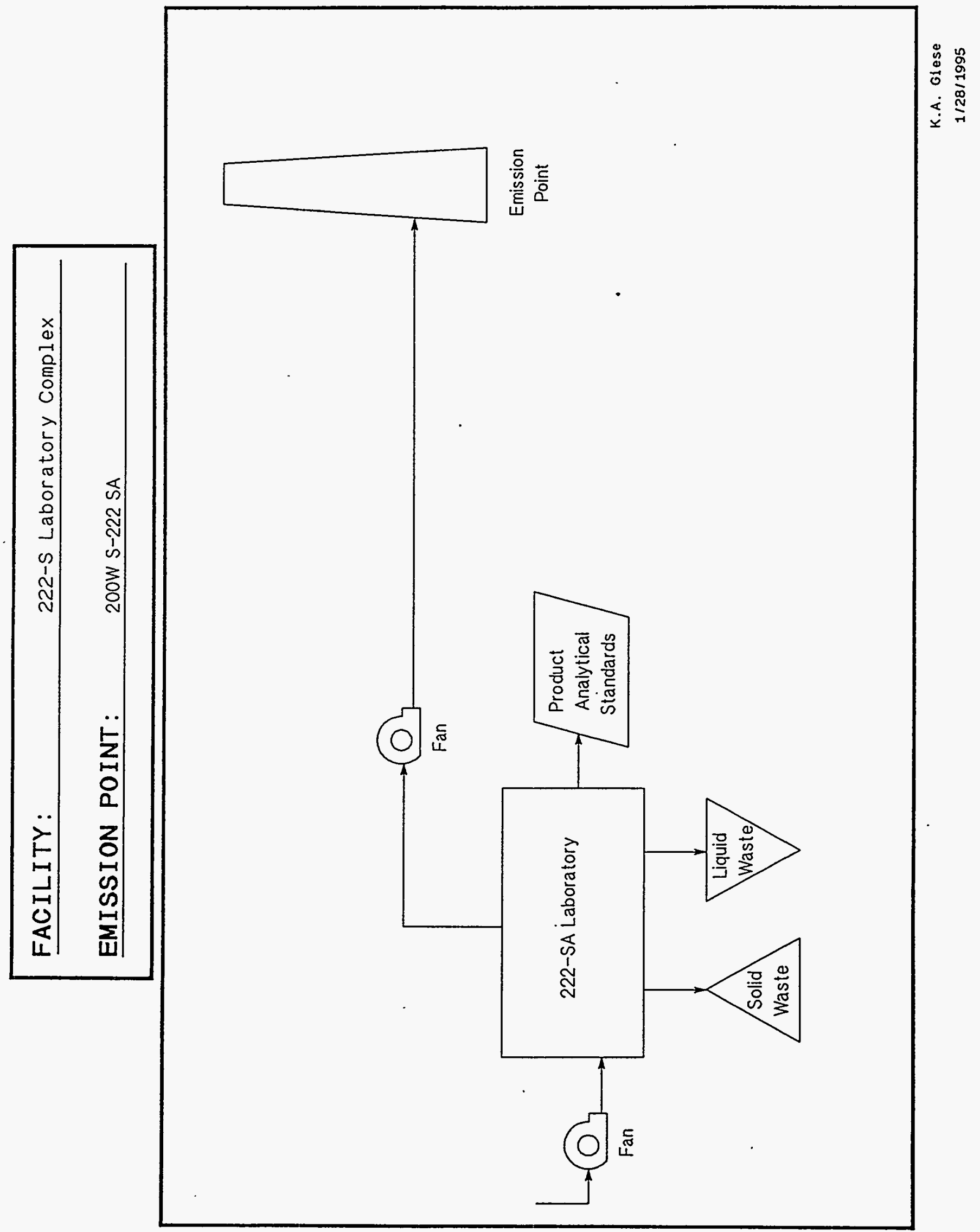




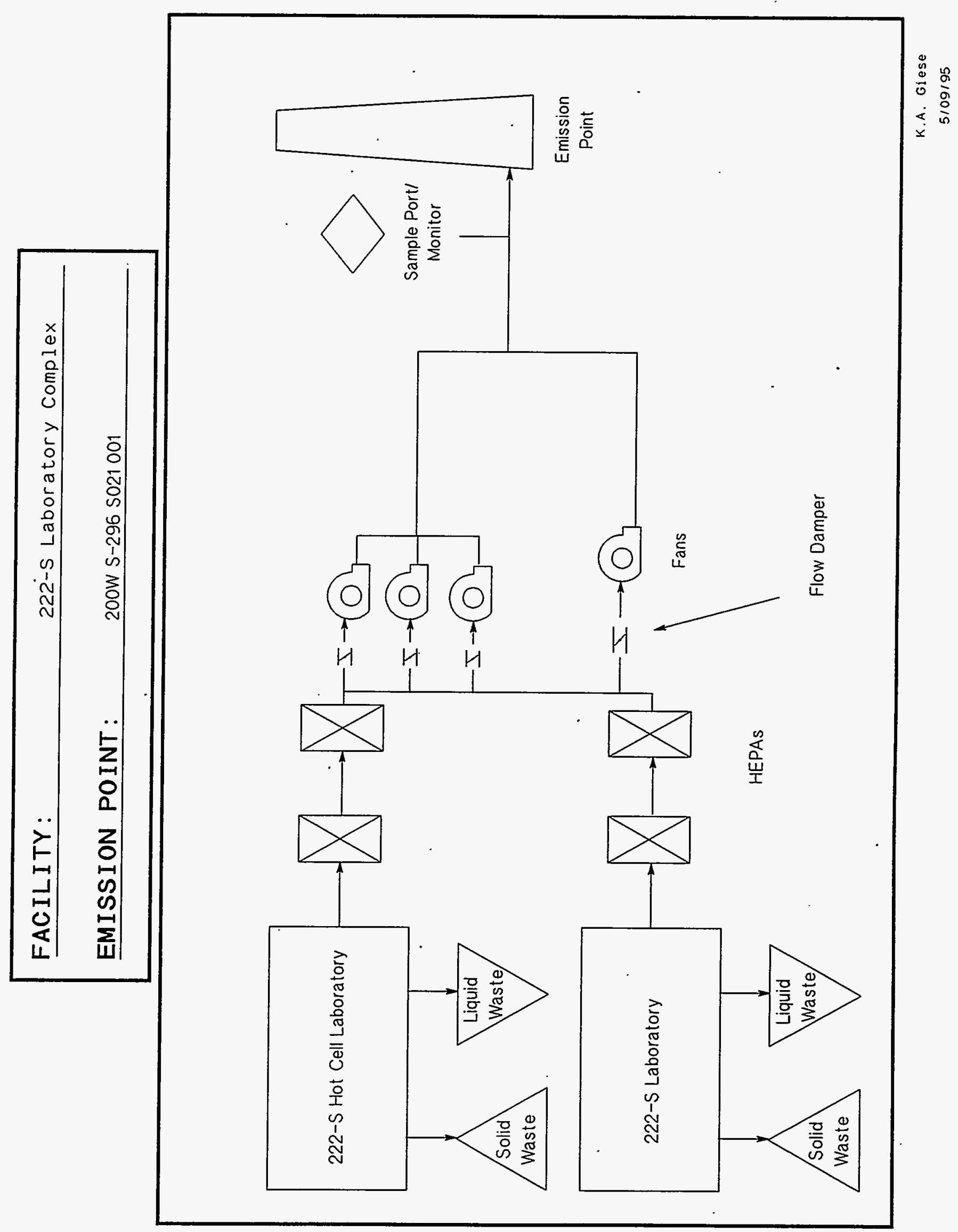




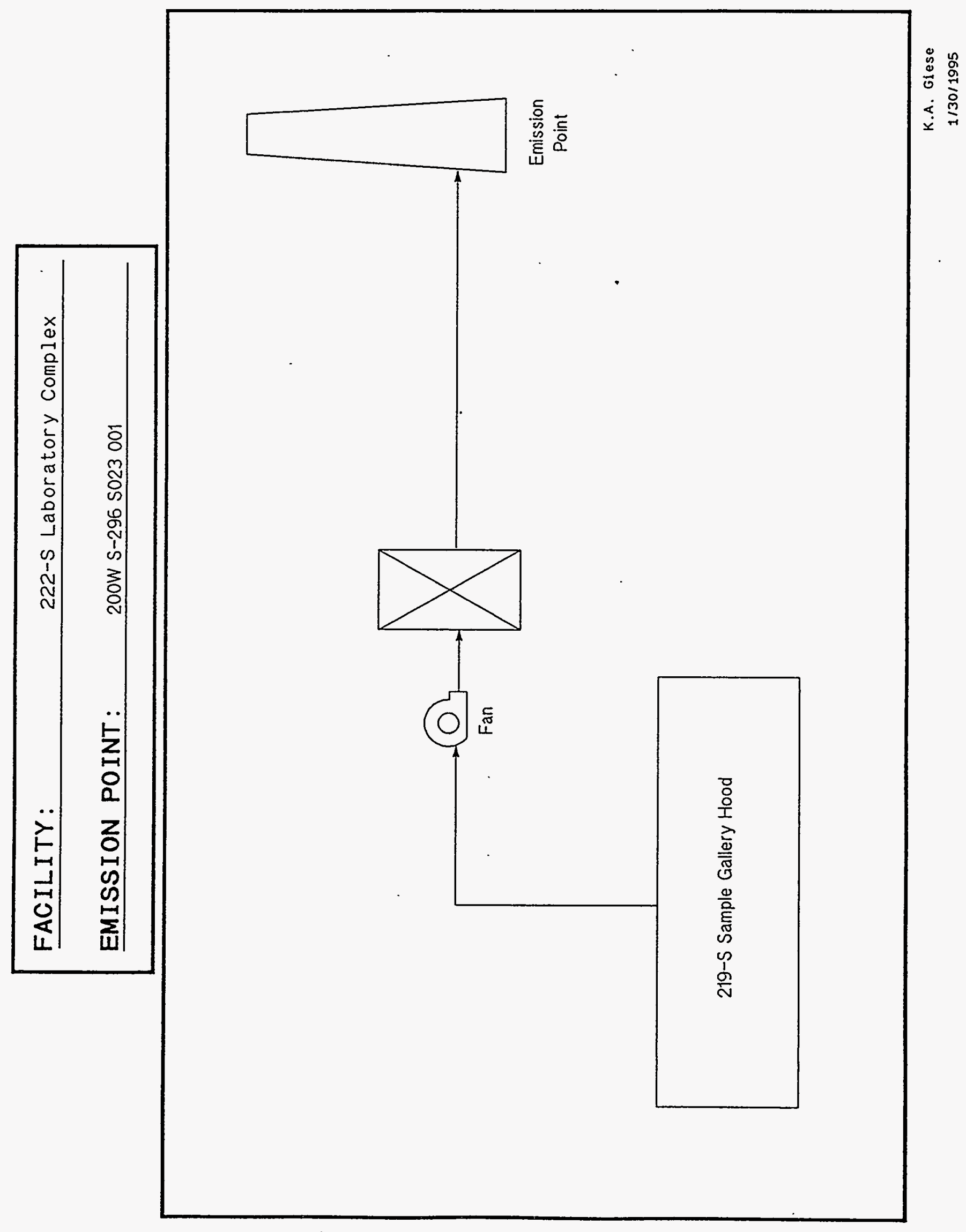




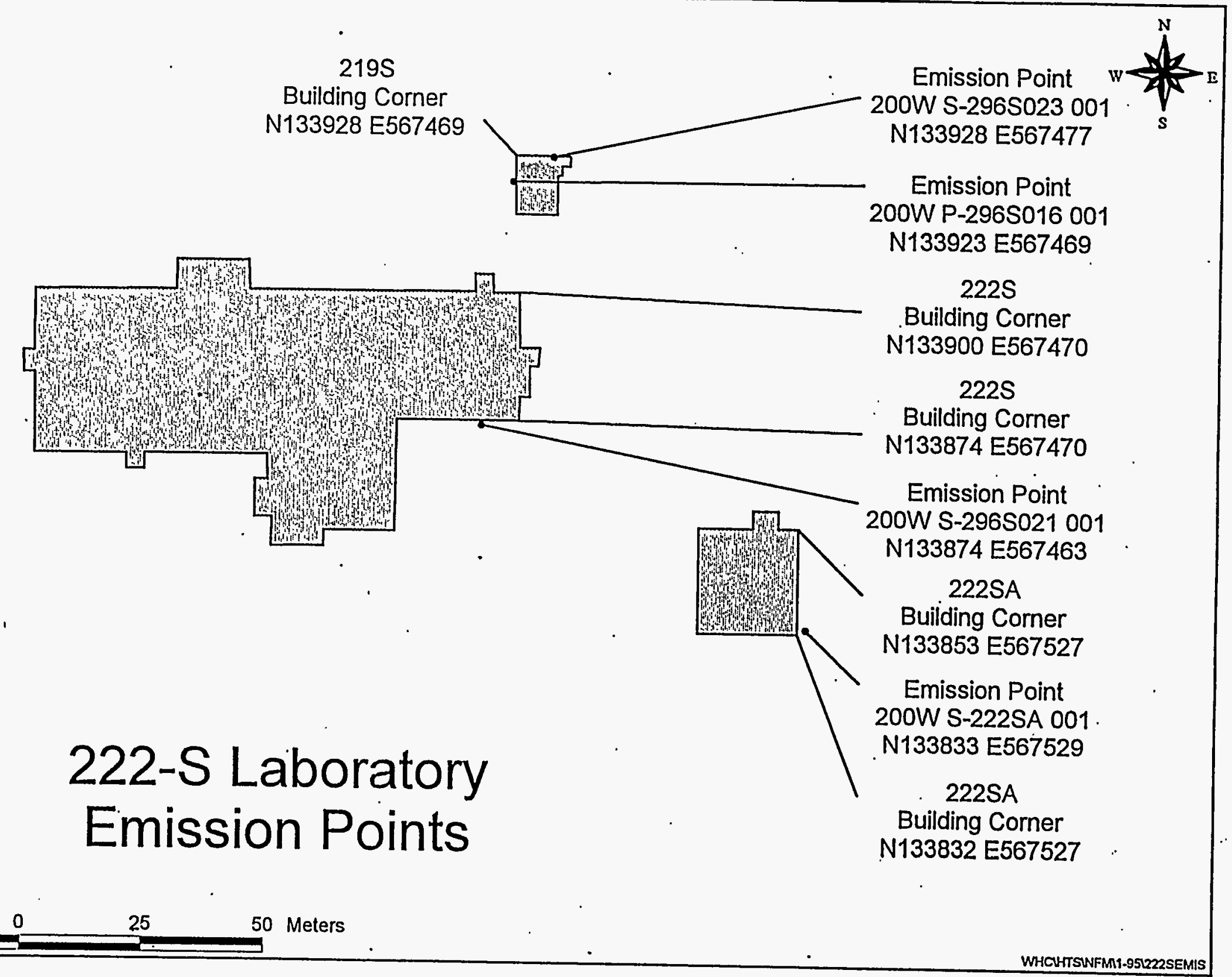




\section{APPENDIX E-2}

\section{W POWERHOUSE}

Chapter I. General Information

\section{I-B.1. Facility Description}

The $284 \mathrm{~W}$ Powerhouse is located in the 200 West Area. The 284W Powerhouse has five boilers in operation. Four of the boilers use bituminous coal, and the fifth boiler is a back-up package boiler that operates on diesel fuel. The package boiler did not operate in 1994 , although startup testing commenced in the fourth quarter of 1994. All of the boilers are used to generate steam exclusively. Steam generated is used in nearby process facilities for heating and process operations. The total rated capacity of the four coal-fired boilers is 280,000 pounds per hour and the diesel-fired boiler is 50,000 pounds per hour of steam, respectively.

\section{I-B.2. Products, Raw Materials, Fuels, Processes}

- Principal products - The only product is 250 to 350 pounds per square inch gauge steam for heating and process operations.

- Process information - Treated boiler feed water is prepared. Sanitary water is dechlorinate using sodium sulfite before entering sodium zeolite softeners ion exchange units to remove the presence of calcium and magnesium salts. Feed water chemistry control is performed using $<4 \%$ potassium hydroxide to prevent scaling and sedimentation. Sodium sulfite is used to scavenge oxygen. Amine is added to each boiler steam outlet line for condensate corrosion control.

- Raw materials used by processes - There are no raw materials used at the 284W Powerhouse.

\section{I-C.1. Emission Points, Controls, Stacks}

The emission point and stack information is found on pages 1 and 2 of the AEI report output. The control information is found on page 7 of the AEI report output.

\section{I-C.2. Facility Process Flow Diagrams}

Attached.

I-C.3. Site Map

Attached. 


\section{Chapter II. Regulatory Requirements}

\section{II-A.1. Description of Discharge and Emission Points}

The emission point and stack information is found on pages 1 and 2 of the AEI report output. 
AIR EMISSIONS INVENTORY PERMIT APPLICATION REPORT (UP460)

FACILITY NAME: 284W Facilities and Structures

\section{Facility Building List}

\begin{tabular}{|c|c|c|c|c|c|c|c|}
\hline Bidg Id & North & East & Bldg Desc & Custodian(Contractor) & Cust Phone & DOE POC & DOE Phone \\
\hline $284 \mathrm{~W}$ & 135991 & 567688 & POWER HOUSE STEAM PLANT & TRUE, GREGORY R. & $373-9038$ & DAVID G. MURILLO & $373-9179$ \\
\hline
\end{tabular}

\section{Emission Point Listing}

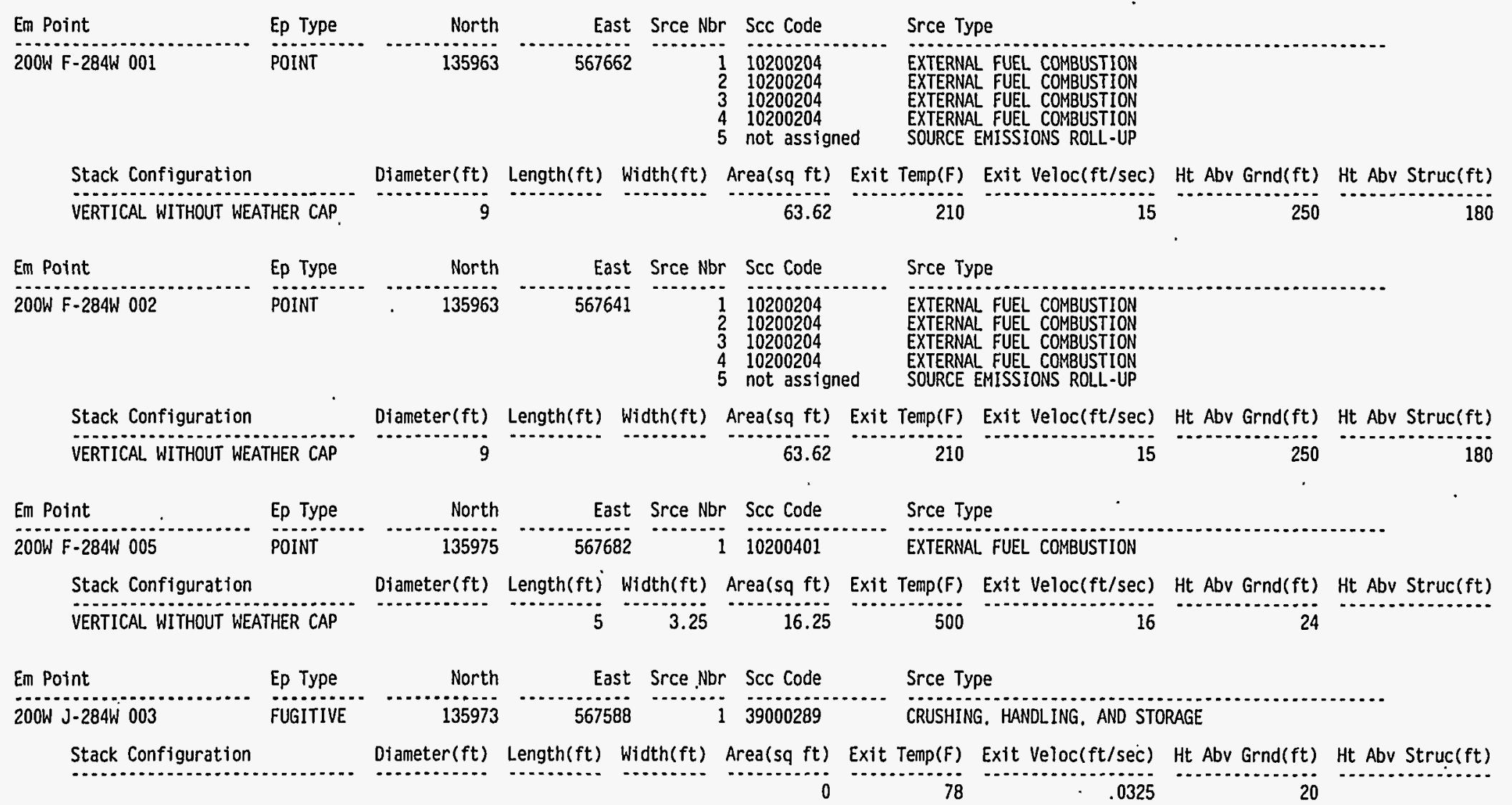

Page \#: 1 of 9 Report Run Date: 5-MAY-95 
AIR EMISSIONS INVENTORY PERMIT APPLICATION REPORT (UP460)

FACILITY NAME: 284W Facilities and Structures

Emission Point Listing

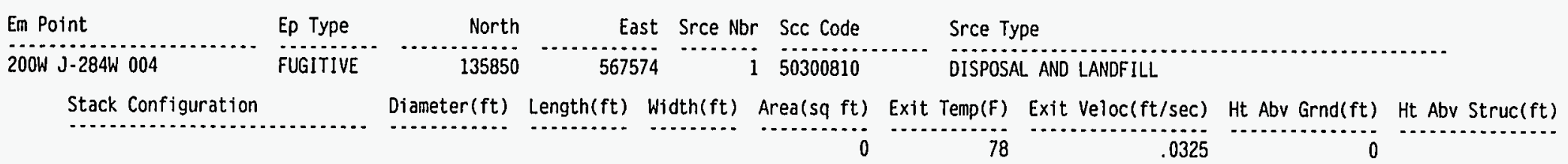

\section{Source (Process) Listing}

\begin{tabular}{|c|c|c|c|c|c|c|}
\hline Em Point & Srce Nbr & Srce Type & Hrs Day & Days Week & Wks Year & Summary Process Description \\
\hline 200W F-284W 001 & 1 & EXTERNAL FUEL COMBUSTION & 24 & 7 & 52 & $\begin{array}{l}\text { ERIE CITY, WATER-TUBE, STOKER-FIRED, STERLING-TYPE, DUMPING } \\
\text { GRATE COAL BOILER. }\end{array}$ \\
\hline Em Point & Srce Nbr & Srce Type & Hrs Day & Days Week & Wks Year & Summary Process Description \\
\hline 200W F-284W 001 & 2 & EXTERNAL FUEL COMBUSTION & 24 & 7 & 52 & $\begin{array}{l}\text { ERIE CITY, WATER-TUBE, STOKER-FIRED, STERLING-TYPE, DUMPING } \\
\text { GRATE COAL BOILER. }\end{array}$ \\
\hline Em Point & Srce $\mathrm{Nbr}$ & Srce Type & Hrs Day & Days Week & Wks Year & Summary Process Description \\
\hline 200W F-284W 001 & 3 & EXTERNAL FUEL COMBUSTION & 24 & 7 & 52 & $\begin{array}{l}\text { ERIE CITY, WATER-TUBE, STOKER-FIRED, STERLING-TYPE, DUMPING } \\
\text { GRATE COAL BOILER. }\end{array}$ \\
\hline Em Point & Srce Nbr & Srce Type & Hrs Day & Days Week & Wks Year & Summary Process Description \\
\hline 200W F-284W 001 & 4 & EXTERNAL FUEL COMBUSTION & 24 & 7 & 52 & $\begin{array}{l}\text { ERIE CITY, WATER-TUBE, STOKER-FIRED, STERLING TYPE, DUMP } \\
\text { GRATE COAL BOILER. }\end{array}$ \\
\hline Em Point & Srce $\mathrm{Nbr}$ & Srce Type & Hrs Day & Days Week & Wks Year & Summary Process Description \\
\hline 200W F-284W 001 & 5 & SOURCE EMISSIONS ROLL-UP & 24 & 7 & 52 & TOTAL EMISSIONS FOR EMISSION POINT F-284W-001 \\
\hline Em Point & Srce $\mathrm{Nbr}$ & Srce Type & Hrs Day & Days Week & Wks Year & Summary Process Description \\
\hline 200W F-284W 002 & 1 & EXTERNAL FUEL COMBUSTION & 24 & 7 & 52 & $\begin{array}{l}\text { ERIE CITY WATER-TUBE, STOKER-FIRED, STERLING TYPE, DUMPING } \\
\text { GRATE COAL BOILER. }\end{array}$ \\
\hline Em Point & Srce $\mathrm{Nbr}$ & Srce Type & Hrs Day & Days Week & Wks Year & Summary Process Description \\
\hline $200 \mathrm{~W} F-284 \mathrm{~W} 002$ & 2 & EXTERNAL FUEL COMBUSTION & 24 & 7 & 52 & $\begin{array}{l}\text { ERIE CITY, WATER-TUBE, STOKER-FIRED, STERLING TYPE, DUMPING } \\
\text { GRATE COAL BOILER. }\end{array}$ \\
\hline
\end{tabular}


AIR EMISSIONS INVENTORY PERMIT APPLICATION REPORT (UP460)

FACILITY NAME: 28ACW Facilities and Structures

Source (Process) Listing

\begin{tabular}{|c|c|c|c|c|c|c|}
\hline Em Point & Srce $\mathrm{Nbr}$ & Srce Type & Hrs Day & Days Week & Wks Year & Summary Process Description \\
\hline 200W F-284W 002 & 3 & EXTERNAL FUEL COMBUSTION & 24 & 7 & 52 & $\begin{array}{l}\text { ERIE CITY, WATER-TUBE, STOKER-FIRED, STERLING-TYPE, DUMPING } \\
\text { GRATE COAL BOILER. }\end{array}$ \\
\hline Em Point & Srce Nbr & Srce Type & Hrs Day & Days Week & Wks Year & Summary Process Description \\
\hline $200 \mathrm{~W} F-284 \mathrm{~W} 002$ & 4 & EXTERNAL FUEL COMBUSTION & 24 & 7 & 52 & $\begin{array}{l}\text { ERIE CITY WATER-TUBE, STOKER-FIRED, STERLING-TYPE, OUMP } \\
\text { GRATE COAL BOILER. }\end{array}$ \\
\hline Em Point & Srce Nbr & Srce Type & Hrs Day & Days Week & Wks Year & Summary Process Description \\
\hline $200 W F-284 W 002$ & 5 & SOURCE EMISSIONS ROLL-UP & 24 & 7 & 52 & TOTAL EMISSIONS FOR EMISSION POINT F-284W-002 \\
\hline Em Point & Srce Nbr & Srce Type & Hrs Day & Days Week & Wks Year & Sumnary Process Description \\
\hline $200 \mathrm{~W} F-284 \mathrm{~W} 005$ & $i$ & EXTERNAL FUEL COMBUSTION & 3.96 & 7 & 52 & PACKAGE BOILER. NO. 2 FUEL OIL \\
\hline Em Point & Srce $\mathrm{Nbr}$ & Srce Type & Hrs Day & Days Week & Whs Year & Summary Process Description \\
\hline $200 \mathrm{~W} \mathrm{~J}-284 \mathrm{~W} 003$ & 1 & $\begin{array}{l}\text { CRUSHING. HANDLING. AND } \\
\text { STORAGE }\end{array}$ & 24 & 7 & 52 & BITUMINOUS COAL STORAGE AREA \\
\hline Em Point & Srce Nbr & Srce Type & Hrs Day & Days Week & Whs Year & Sumnary Process Description \\
\hline $200 \mathrm{~W} \mathrm{~J}-284 \mathrm{~W} 004$ & 1 & DISPOSAL AND LANDFILL & 24 & 7 & 52 & ASH DISPOSAL PIT \\
\hline \multicolumn{7}{|c|}{ Source Raw Materials Usage · } \\
\hline Em Point & $\begin{array}{l}\text { Srce } \mathrm{Nbr} \\
\text { C........ }\end{array}$ & Srce Type & & Proces & is Rate $\mathrm{Pr}$ & ocess Uom Material \\
\hline
\end{tabular}

Fuels Usage by Source Processes

Em Point

Srce Nbr Srce Type

200W F-284W 001

1 EXTERNAL FUEL COMBUSTION

Qty Vom

Fuel

6500000 POUND

COAL BITUMINOUS

1650000 POUND COAL BITUMINOUS

EXTERNAL FUEL COMBUSTION

12500000 POUND COAL BITUMINOUS

EXTERNAL FUEL COMBUSTION

EXTERNAL FUEL COMBUSTION

4950000 POUND

COAL. BITUMINOUS 


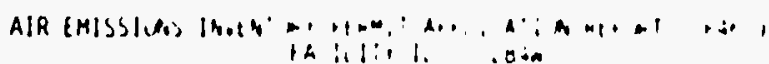

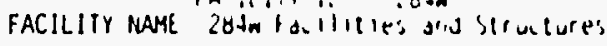

Fuels Usage by Source Processes

\begin{tabular}{|c|c|c|c|c|c|}
\hline Em Point & Srce Nbr & Srce Type & Qty & Uom & Fuel \\
\hline \multirow[t]{4}{*}{$200 W F-284 W 002$} & 1 & EXTERNAL FUEL COMBUSTION & 6500000 & POUND & COAL BITUMINOUS \\
\hline & 2 & EXTERNAL FUEL COMBUSTION & 1650000 & POUND & COAL BITUMINOUS \\
\hline & 3 & EXTERNAL FUEL COMBUSTION & 12500000 & POUND & COAL BITUMINOUS \\
\hline & 4 & EXTERNAL FUEL COMBUSTION & 4950000 & POUND & COAL BITUMINOUS \\
\hline 200W F-284W 005 & 1 & EXTERNAL FUEL COMBUSTION & 0 & GALLONS & NO. 2 DIESEL \#2 FUEL OIL \\
\hline
\end{tabular}

Fuels Usage by Control Devices

Em Point

Ctl Nbr Ctl Type

Qty Uom

Fuel

Point Source Criteria Pollutants - Type: Co

\begin{tabular}{|c|c|c|c|c|c|}
\hline Em Point & Srce Nbr & Srce Type Pollutant & $\begin{array}{l}\text { Em. Pt. Potential } \\
\text { to Emit (tons/yr) }\end{array}$ & $\begin{array}{l}\text { Em. Pt. } \\
\text { Cti Actual (tons/yr) }\end{array}$ & Pollutant \\
\hline 200W F-284W 001 & & 5 SOURCE EMISSIONS ROLL-UP & 262.800000000 & 43.350000000 & CARBON MONOXIDE \\
\hline 200W F-284W 002 & & 5 SOURCE EMISSIONS ROLL-UP & 262.800000000 & 43.350000000 & CARBON MONOXIDE \\
\hline 200W F-284W b05 & & 1 EXTERNAL FUEL COMBUSTION & 1.500000000 & .000000000 & CARBON MONOXIDE \\
\hline Sum & & & 527.100000000 & 86.700000000 & \\
\hline
\end{tabular}

Point Source Criteria Pollutants - Type: LEAD

\begin{tabular}{|c|c|c|c|c|c|}
\hline Em Point & Srce $\mathrm{Nbr}$ & Srce Type Pollutant & $\begin{array}{l}\text { Em. Pt. Potential } \\
\text { to Emit (tons/yr) }\end{array}$ & $\begin{array}{l}\text { Em. Pt. } \\
\text { Cti Actual (tons/yr) }\end{array}$ & Pollutant \\
\hline 200W F-284W 001 & & 5 SOURCE EMISSIONS ROLL-UP & .650000000 & .110000000 & LEAD \\
\hline $200 W F-284 W 002$ & & 5 SOURCE EMISSIONS ROLL-UP & .650000000 & .110000000 & LEAD \\
\hline 200W F-284W 005 & & 1 EXTERNAL FUEL COMBUSTION & .000400000 & .000000000 & LEAD \\
\hline Sum & & & 1.300400000 & .220000000 & \\
\hline
\end{tabular}

Page \#: 4 of 9 Report Run Date: 5-MAY-95 
AIR EMISSIONS INVENTORY PERMIT APPLICATION REPORT (UP460)

FACILITY NAME: $284 \mathrm{~W}$ Facilities and Structures

Point Source Criteria Pollutants - Type: NOX

\begin{tabular}{|c|c|c|c|c|c|}
\hline Em Point & Srce $\mathrm{Nbr}$ & Srce Type Pollutant & $\begin{array}{l}\text { Em. Pt. Potential } \\
\text { to Emit (tons/yr) }\end{array}$ & $\begin{array}{l}\text { Em. Pt. } \\
\text { Cti Actual (tons/yr) }\end{array}$ & Pollutant \\
\hline $200 \mathrm{~W} F-284 \mathrm{~W} 002$ & & 5 SOURCE EMISSIONS ROLL-UP & 720.000000000 & 118.850000000 & NITROGEN OXIDE \\
\hline 200W F-284W 005 & & 1 EXTERNAL FUEL COMBUSTION & 5.900000000 & $.000000000^{\circ}$ & NITROGEN OXIL \\
\hline Sum & & & 1445.900000000 & 237.700000000 & \\
\hline
\end{tabular}

Point Source Criteria Pollutants - Type: PM

\begin{tabular}{|c|c|c|}
\hline Em Point & Srce Nbr & Srce Type Pollutant \\
\hline 200W F-284W 001 & & 5 SOURCE EMISSIONS ROLL-UP \\
\hline 200W F-284W 002 & & 5 SOURCE EMISSIONS ROLL-UP \\
\hline 200W F-284W 005 & & 1 EXTERNAL FUEL COMBUSTION \\
\hline
\end{tabular}

Sum

Point Source Criteria Pollutants - Type: PM10
Em. Pt. Potential Em. Pt.

to Emit (tons/yr) Cti Actual (tons/yr) Pollutant

$\begin{array}{rrr}6.950000000 & 1.145000000 & \text { TOTAL PARTICULATE MATTER } \\ 6.950000000 & 1.145000000 & \text { TOTAL PARTICULATE MATTER } \\ .600000000 & .000000000 & \text { TOTAL PARTICULATE MATTER } \\ 14.500000000 & 2.290000000 & \\ & & \end{array}$

\begin{tabular}{lrl} 
Em Point & Srce Nbr & Srce Type Pollutant \\
\hline 200W F-284W 001 & & 5 SOURCE EMISSIONS ROLL-UP \\
200W F-284W 002 & 5 SOURCE EMISSIONS ROLL-UP \\
200W F-284W 005 & 1 EXTERNAL FUEL COMBUSTION
\end{tabular}

Sum
Em. Pt. Potential
to Emit (tons/yr)

Em. Pt.

Cti Actual (tons/yr) Pollutant

$.400000000 \quad 230000000$ PARTICULATE MATTER (10 MICRONS OR LESS)

$1.400000000 \cdot .230000000$ PARTICULATE MATTER (10 MICRONS OR LESS)

$.300000000 \quad .000000000$ PARTICULATE MATTER (10 MICRONS OR LESS)

3.100000000 
AIR EMISSIONS INVENTORY PERMIT APPLICATION REPORT (UP460)

FACILITY NAME: 284W Facilities and Structures

Point Source Criteria Pollutants - Type: SOX

\begin{tabular}{|c|c|c|c|c|c|}
\hline Em Point & Srce Nbr & Srce Type Pollutant & $\begin{array}{l}\text { Em. Pt. Potential } \\
\text { to Emit (tons/yr) }\end{array}$ & $\begin{array}{l}\text { Em. Pt. } \\
\text { Cti Actual (tons/yr) }\end{array}$ & Pollutant \\
\hline $200 \mathrm{~W}-284 \mathrm{~W} 001$ & & 5 SOURCE EMISSIONS ROLL-UP & 1048.500000000 & 173.000000000 & SULFUR DIOXIDE \\
\hline $200 W$ F-284W 002 & & 5 SOURCE EMISSIONS ROLL-UP & 1048.500000000 & 173.000000000 & SULFUR DIOXIDE \\
\hline 200W F-284W 005 & & 1 EXTERNAL FUEL COMBUSTION & 2.100000000 & .000000000 & SULFUR DIOXIDE \\
\hline Sum & & & 2099.100000000 & 346.000000000 & \\
\hline
\end{tabular}

Point Source Criteria Pollutants - Type: VOC

Em Point
Srce Nbr

Sun

Point Source Emissions - Radiological Pollutants
Em. Pt. Potential
to Emit (tons/yr)
Cti Pt Actual

\begin{tabular}{|c|c|c|}
\hline $\begin{array}{l}.300000000 \\
2.650000000\end{array}$ & $\begin{array}{l}.050000000 \\
.435000000\end{array}$ & $\begin{array}{l}\text { FORMALDEHYDE } \\
\text { NON-METHANE VOLATILE ORGANICS }\end{array}$ \\
\hline $\begin{array}{r}.300000000 \\
2.650000000\end{array}$ & $\begin{array}{l}.050000000 \\
.435000000\end{array}$ & $\begin{array}{l}\text { FORMALOEHYDE } \\
\text { NON-METHANE VOLATILE ORGANICS }\end{array}$ \\
\hline $\begin{array}{r}.020000000 \\
.060000000 \\
6.500000000\end{array}$ & $\begin{array}{l}.000000000 \\
.000000000 \\
.000000000\end{array}$ & $\begin{array}{l}\text { FORMALDEHYDE } \\
\text { NON-METHANE VOLATILE ORGANICS } \\
\text { POLYCYCLIC ORGANIC MATTER }\end{array}$ \\
\hline 12.480000000 & .970000000 & \\
\hline
\end{tabular}

Em Point $\quad 1 \quad$ Srce Nbr Srce Type Pollutant

Em. Pt. Potential Em. Pt.
to Emit (Ci/yr)
Cti Actual (Ci/yr) Pollutant

Point Source Emissions - HAP Pollutants

Em Point

Srce Nbr Srce Type Pollutant

200W F-284W 001
5 SOURCE EMISSIONS ROLL-UP
Em. Pt. Potential

to Emit (tons/yr)

Em. Pt.

$\begin{array}{rll}.700000000 & .120000000 & \text { ARSENIC } \\ .100000000 & .015000000 & \text { BERYLLIUM } \\ .050000000 & .010000000 & \text { CADMIUM } \\ 2.100000000 & .345000000 & \text { CHROMIUM } \\ .300000000 & .050000000 & \text { FORMALDEHYDE } \\ .650000000 & .110000000 & \text { LEAD } \\ 2.900000000 & .475000000 & \text { MANGANESE } \\ .020000000 & .005000000 & \text { MERCURY } \\ 1.700000000 & .285000000 & \text { NICKEL } \\ .250000000 & .045000000 & \text { SELENIUM }\end{array}$


AIR EMISSIONS INVENTORY PERMIT APPLICATION REPORT (UP460)

FACILITY NAME: 284W Facilities and Structures

\section{Point Source Emissions - HAP Pollutants}

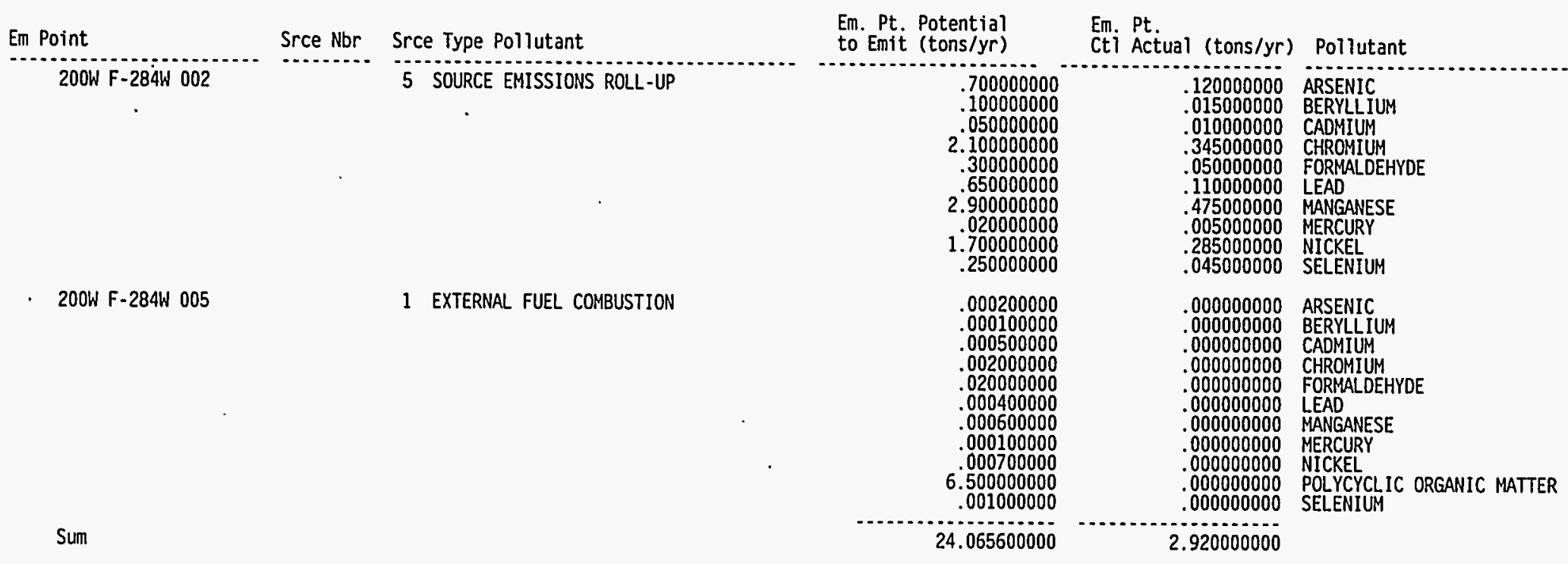

Point Source Emissions - Uncategorized Pollutants

\begin{tabular}{|c|c|c|c|c|c|}
\hline Em Point & Srce Nbr & Srce Type Pollutant & $\begin{array}{l}\text { Em. Pt. Potentia) } \\
\text { to Emit (tons/yr) }\end{array}$ & $\begin{array}{l}\text { Em. Pt. } \\
\text { Cti Actual (tons/yr) }\end{array}$ & Pollutant \\
\hline 200W F-284W 001 & & 5 SOURCE EMISSIONS ROLL-UP & $\begin{array}{r}1.300000000 \\
3.150000000 \\
4.750000000 \\
.200000000\end{array}$ & $\begin{array}{l}.215000000 \\
.520000000 \\
.780000000 \\
.030000000\end{array}$ & $\begin{array}{l}\text { COPPER } \\
\text { METHANE } \\
\text { NITROUS OXIDE } \\
\text { VANADIUM }\end{array}$ \\
\hline 200W F-284W 002 & & 5 SOURCE EMISSIONS ROLL-UP & $\begin{array}{r}1.300000000 \\
3.150000000 \\
4.750000000 \\
.200000000\end{array}$ & $\begin{array}{l}.215000000 \\
.520000000 \\
.780000000 \\
.030000000\end{array}$ & $\begin{array}{l}\text { COPPER } \\
\text { METHANE } \\
\text { NITROUS OXIDE } \\
\text { VANADIUM }\end{array}$ \\
\hline 200W F-284W 005 & & 1 EXTERNAL FUEL COMBUSTION & $\begin{array}{l}.010000000 \\
.020000000 \\
.030000000 \\
.003000000\end{array}$ & $\begin{array}{l}.000000000 \\
.000000000 \\
.000000000 \\
.000000000\end{array}$ & $\begin{array}{l}\text { COPPER } \\
\text { METHANE } \\
\text { NITROUS OXIDE } \\
\text { VANADIUM }\end{array}$ \\
\hline Sum & & & 18.863000000 & 3.090000000 & \\
\hline
\end{tabular}

Fugitive Source Criteria Pollutants - Type: PM 


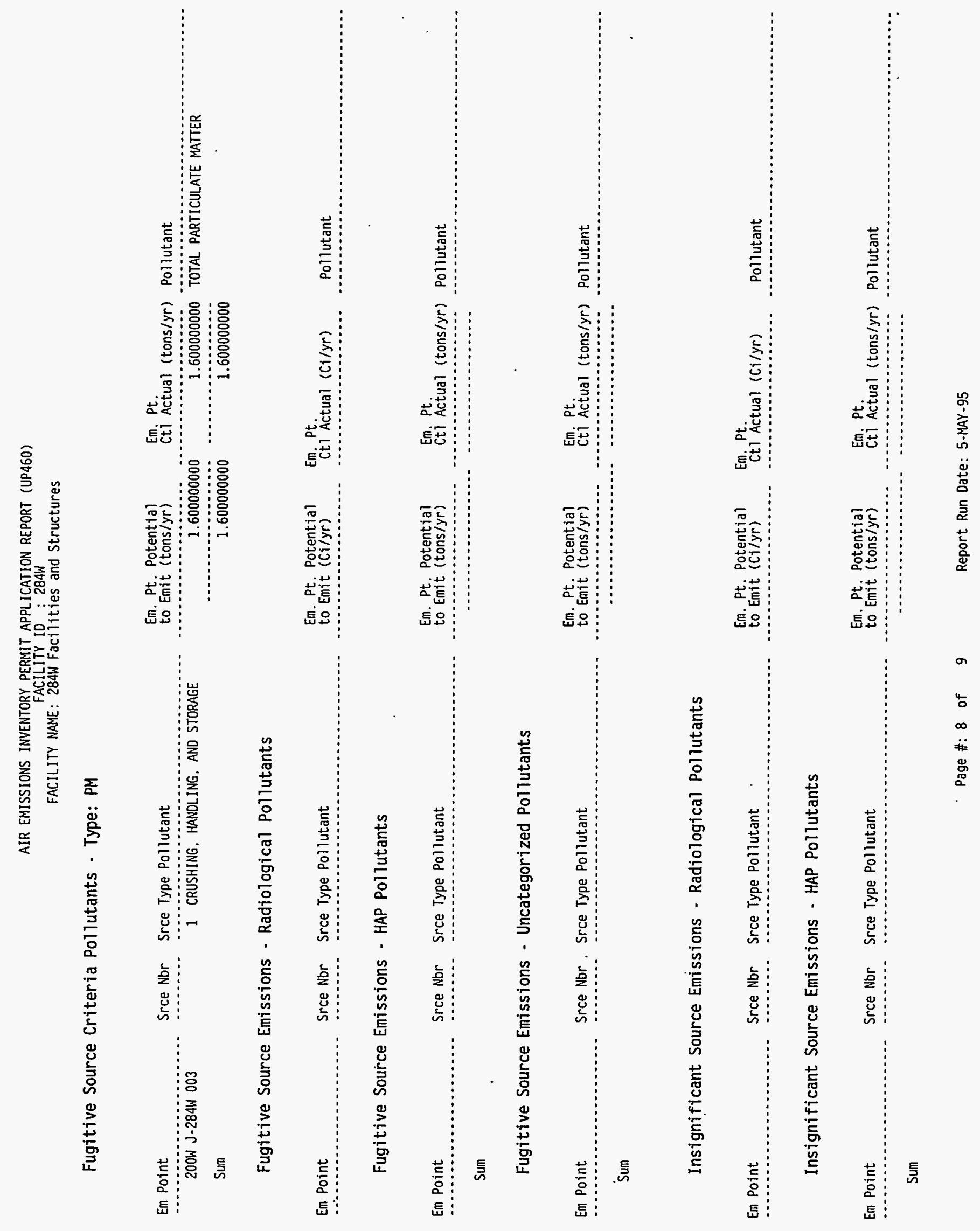


AIR EMISSIONS INVENTORY PERMIT APPLICATION REPORT (UP460)

FACILITY NAME: 284W Facilities and Structures

Insignificant Source Emissions - Uncategorized Pollutants

Em Point

Srce Nbr Srce Type Pollutant

Em. Pt. Potential

Em. Pt.

Ctl Actual (tons/yr) Pollutant

Sum

Control Device Listing

Em Point

Control Equipment Code

200W F-284W 001

200W F-284W 002

016 


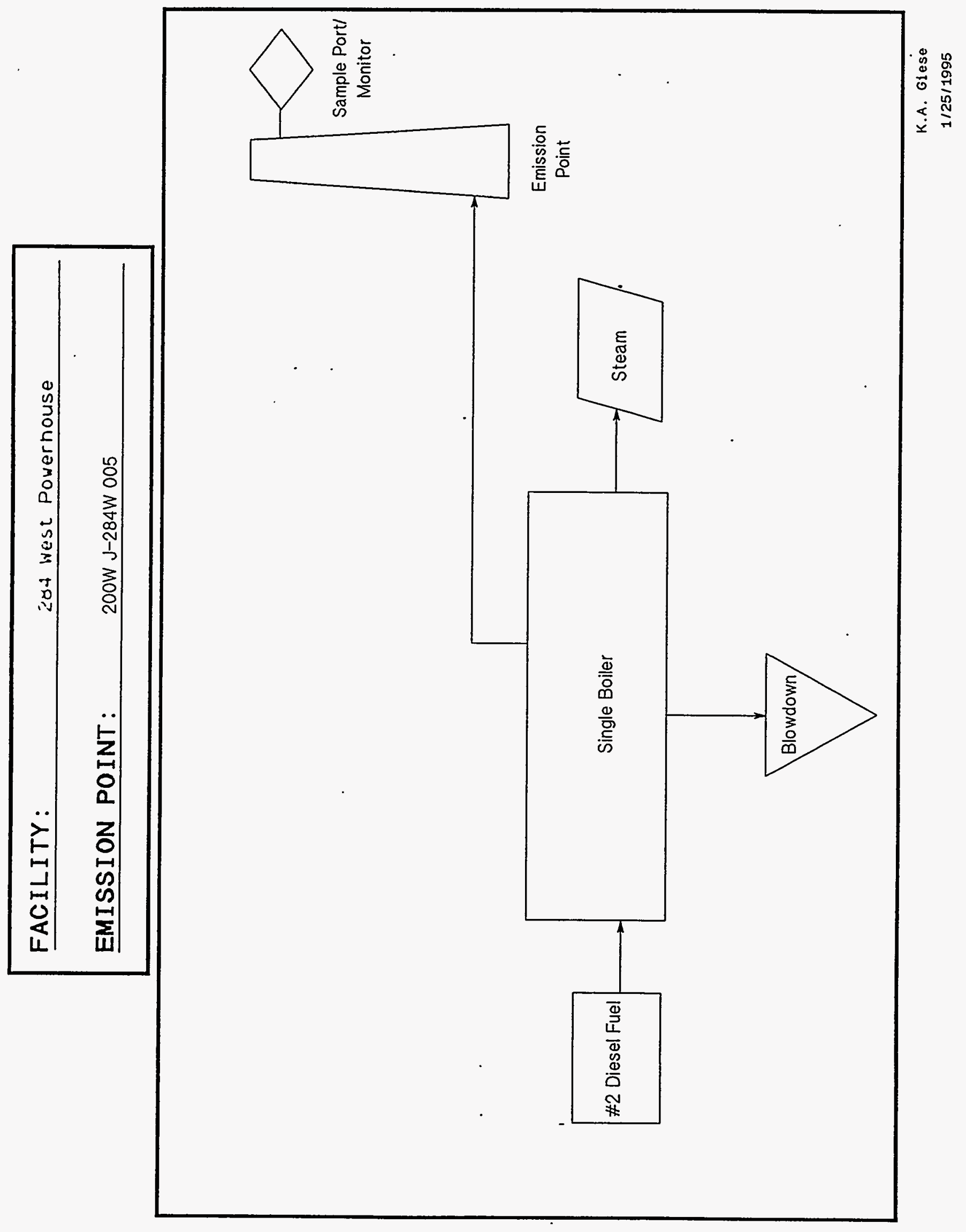




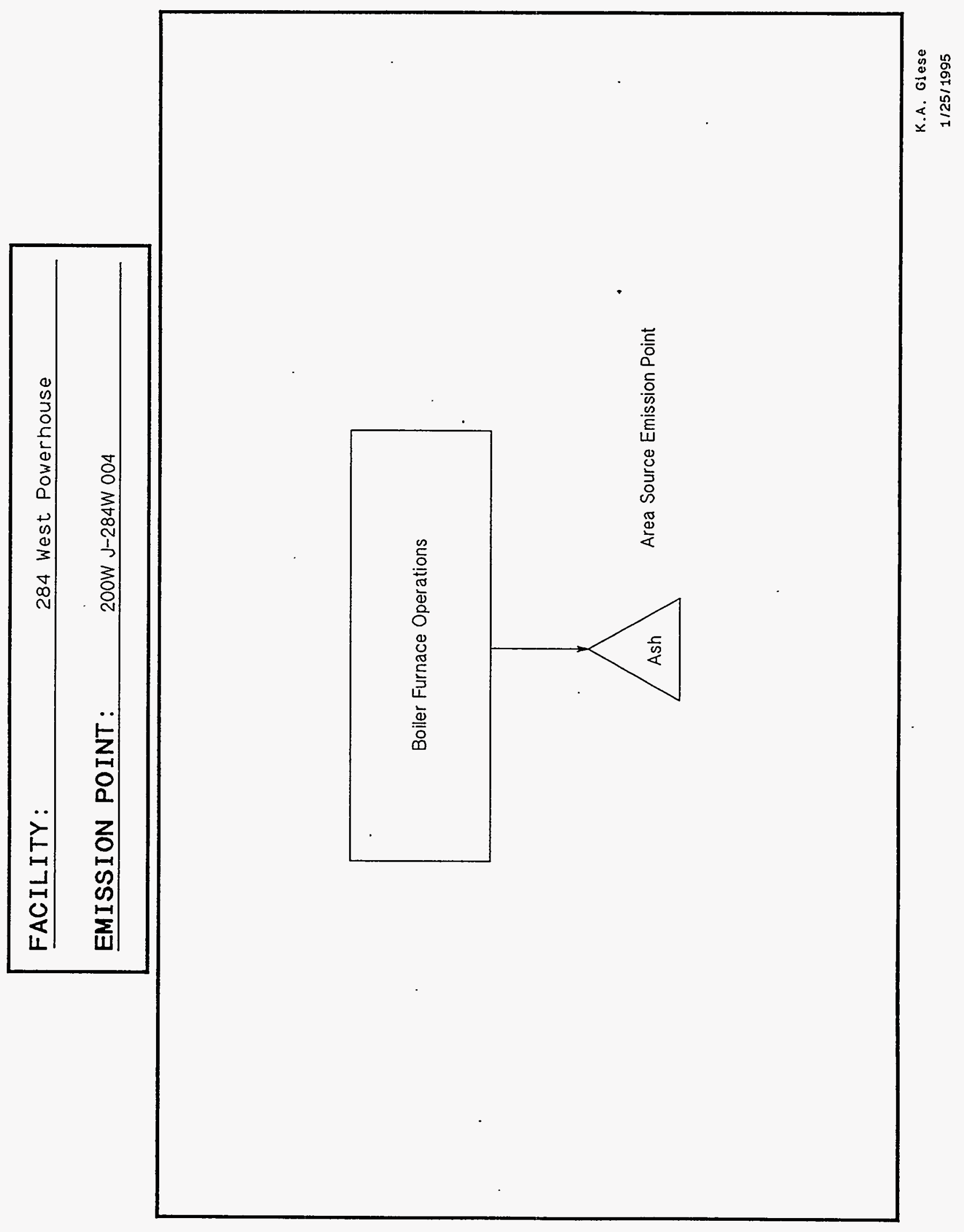


FACILITY:

EMISSION POINT:
284 West Powerhouse

200W J-284W 003

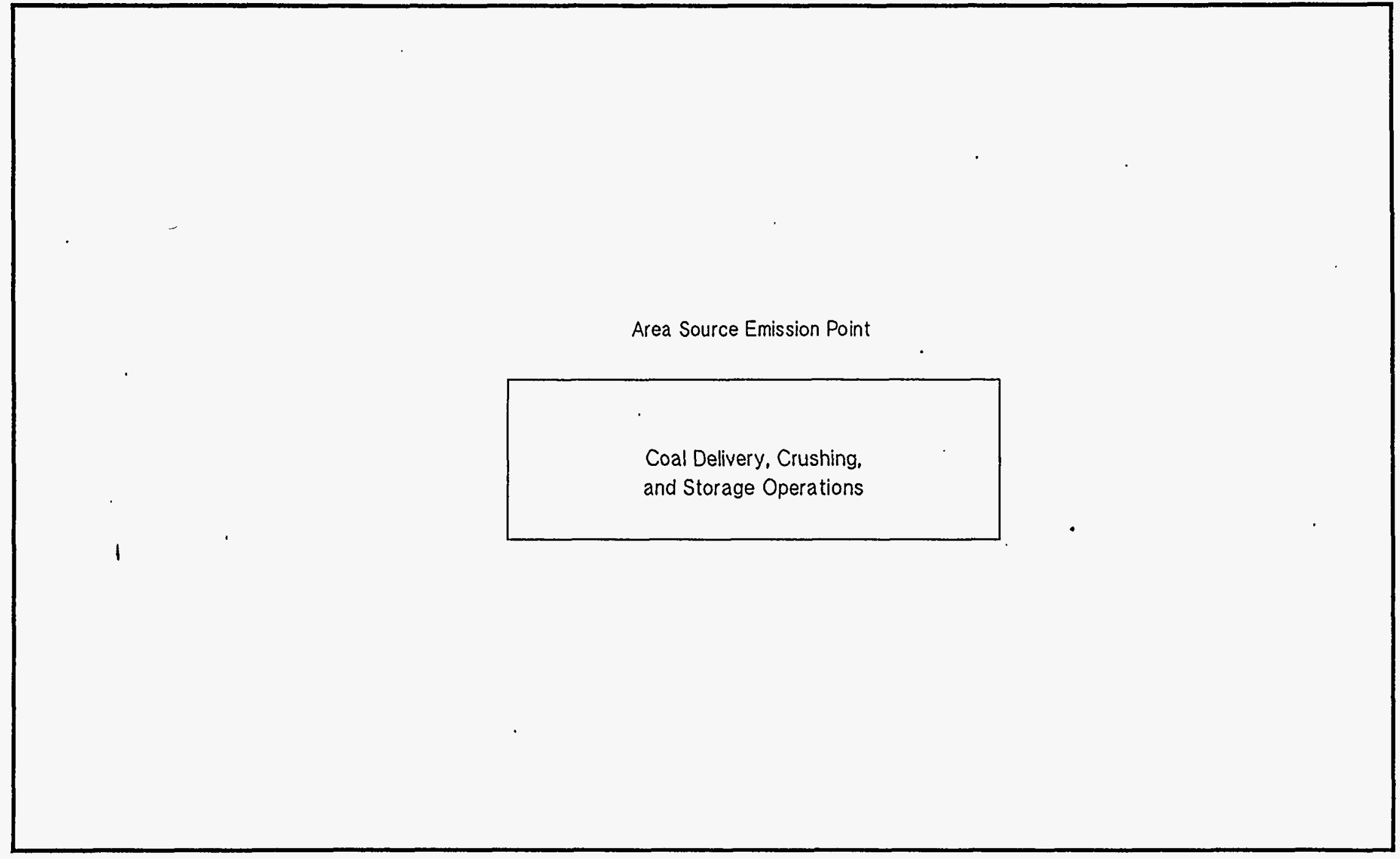




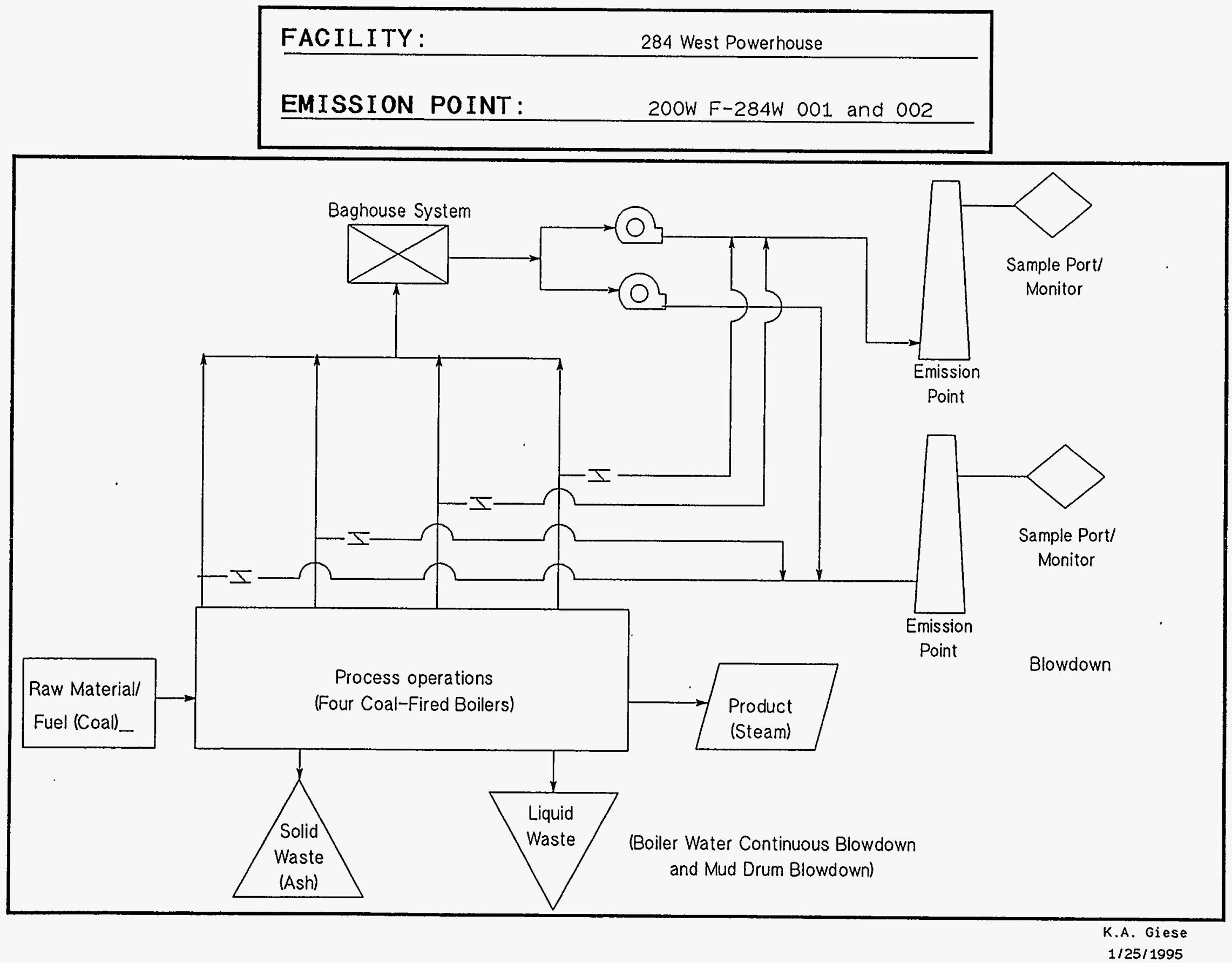




\section{W Powerhouse Area}
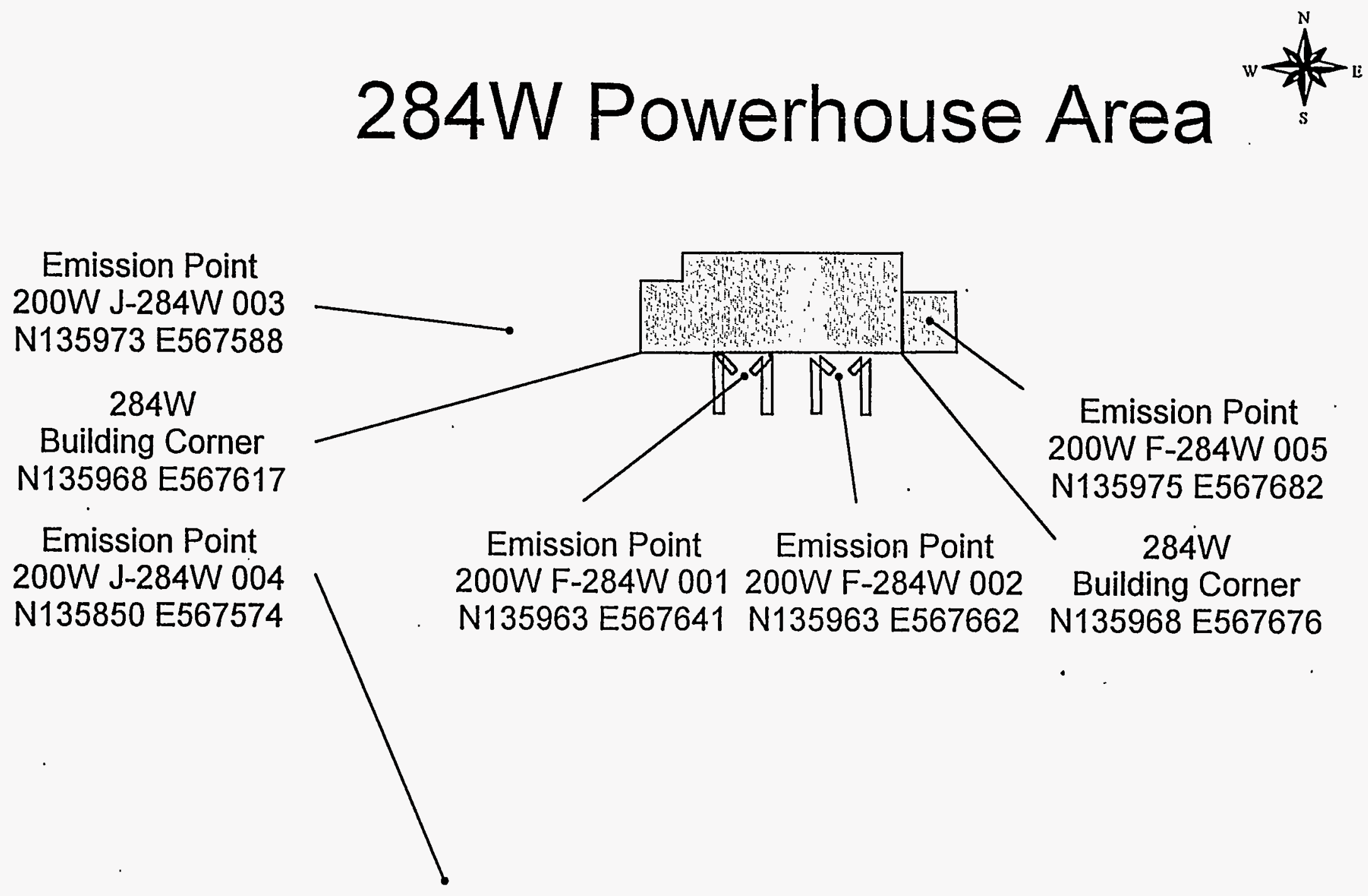


\title{
APPENDIX E-3
}

\section{PLUTONIUM FINISHING PLANT}

\author{
Chapter I. General Information
}

\section{I-B.1. Facility Description}

The Plutonium Finishing Plant (PFP) Complex consists of facilities as described in section I-B.2, plus several small support structures, constructed from 1949 to 1994. The PFP operations were designed for the recovery and chemical conversion of plutonium to a form suitable for weapons production.

Operations to purify plutonium nitrate and to convert the plutonium nitrate to metallic plutonium ceased by 1990. Current operations are low-level wastewater treatment; low-level waste, transuranic waste, hazardous waste, and radioactive mixed waste handling, packaging, and shipping; waste transfer to tank farms; SNM storage, stabilization, and management; and shipping and transfer of plutonium products and miscellaneous solid scrap materials. Future operations will include waste treatment, and decontamination and decommissioning operations.

The PFP is located in the 200 West Area. The PFP is located on the site map in Section I.C.3. The PFP is operated 24 hours a day, 365 days a year.

I-B.2. Products, Raw Materials, Fuels, Processes

- Principal products - There are no products produced at PFP.

- Process information - The current process operations at PFP are as follows:

- Building 234-5Z operations - Past operations converted plutonium nitrate solution to plutonium metal. Current operations include sludge stabilization activities in Glovebox HC-21C, downloading and testing of corrosive Pu bearing liquid in the PPSL, laboratory operations at the Analytical Laboratory, surveillance activities of unstable Pu bearing material stored in process gloveboxes in the Remote Mechanical C Line, RCRA and non-RCRA solid waste handling operations, the LLWTF operation, and the HVAC system operation. Additional plutonium stabilization activities and facility cleanout activities are planned or are ongoing.

- Building 243-Z - the Low-Level Waste Treatment Facility (LLWTF) receives and treats low-activity waste water from various parts of the PFP. The waste water stream is treated for organics, metal ions, and radionuclides passing through a series of filter trains consisting of granular activated carbon, bone char, and ion exchange resins. The purpose of the LLWTF is to produce water suitable for 
Effluent Disposal Facility.

- Building 236-Z operations - Past operations in 236-Z building recovered and purify plutonium bearing scrap materials and sludges into plutonium nitrate. The current operations in $236-\mathrm{Z}$ building include surveillance activities of stored unstable Pu scrap materials in process gloveboxes and cleanout activities of some process gloveboxes.

- Building 232-Z operations - The past operation in this building was incineration of $\mathrm{Pu}$ bearing waste. Currently, $\mathrm{Pu}$ removal in the ventilation duct and process gloveboxes and deactivation activities are ongoing in this building.

- Building 242-Z operations - The past operation in this building was the recovery of plutonium and americium in the low level liquid waste stream. Currently, this building is locked out and awaiting final decontamination.

- Building 2736-Z operations - SNM storage and surveillance activities are ongoing in this building.

- Building 2736-ZB operations - All SNM product handling operations pertaining to shipments, receipts, and storage of SNM are done in this building.

- Building 241-Z - a Waste Treatment Facility that receives liquid wastes generated at PFP. The liquid wastes are then transferred to tank farms.

- Raw materials used by processes - There are no raw materials used at PFP.

- Fuels used by processes - There are no fuels used in the processes at PFP.

I-C.1. Emission Points, Controls, Stacks

The emission point listing and stack information is found on pages 1 and 2, and the control device listing is found on page 7 of the AEI report output.

\section{I-C.2. Facility Process Flow Diagram}

Attached.

I-C.3. Site Map

Attached. 


\section{Chapter II. Regulatory Requirements}

\section{II-A.1. Description of Discharge and Emission Points}

The emission point listing and stack information is found on pages 1 and 2, and the control device listing is found on page 7 of the AEI report output. 
AIR EMISSIONS INVENTORY PERMIT APPLICATION REPORT (UP460)

FACILITY NAME: Plutonium Finishing Plant(PFP) and Structures

\section{Facility Building List}

\begin{tabular}{|c|c|c|c|}
\hline Bldg Id & North & East & Bldg Desc \\
\hline $\begin{array}{l}2912001 \\
2962003 \\
2962005 \\
2962006 \\
2962014 \\
2962015\end{array}$ & $\begin{array}{l}135564 \\
135526 \\
135607 \\
135633 \\
135561 \\
135586\end{array}$ & $\begin{array}{l}566488 \\
566501 \\
566434 \\
566431 \\
566455 \\
566510\end{array}$ & $\begin{array}{l}\text { STACK FOR BLDGS. 234-5Z, 236-Z, \& 242-Z } \\
\text { STACK FOR BLDGS. 241-Z., 241-2A. \& 241-2B } \\
2736-Z B \text { STACK } \\
\text { STACK FOR BLDGS. 2736-ZA \& 2736-Z } \\
232-Z \text { STACK } \\
243-Z \text { STACK }\end{array}$ \\
\hline
\end{tabular}

Custodian(Contractor) MCBRIDE. DON J. MCBRIDE. DON $\mathrm{j}$. MCBRIDE, DON $\mathrm{J}$. MCBRIDE. DON $j$. MCBRIDE, DON $\mathrm{J}$.

DOE Phone

Emission Point Listing

Em Point Ep Type 200W P-2912001 001 Ep Type North East Srce Nbr Scc Code Srce Type

135564

-

........

$\begin{array}{ll}1 & \text { not assigned } \\ 2 & \text { not assigned } \\ 3 & \text { not assigned } \\ 4 & \text { not assigned } \\ 5 & \text { not assigned } \\ 6 & \text { not assigned } \\ 7 & \text { not assigned } \\ 8 & \text { not assigned }\end{array}$

ANALYTICAL LABORATORY

PROCESS AND PRODUCTION

D\&D/DEMOLITION

O\&D/DEMOLITION

D\&O/DEMOLITION

SOURCE EMISSIONS ROLL-UP

8 not assigned ANALYTICAL LABORATORY

Stack Configuration

Diameter(ft) Length(ft) Width(ft) Area(sq ft) $\quad$ Exit Temp(F) Exit Veloc(ft/sec) Ht Abv Grnd(ft) Ht Abv Struc(ft)

Em Point Ep Type
POINT

North
135526

East Srce Nbr Scc Code

Srce Type

200W P-2962003 001

135526

$\begin{array}{rrr}566501 & 1 & \begin{array}{l}\text { not assigned } \\ \text { not assigned }\end{array} \\ & 3 & \text { not assigned }\end{array}$

TANKS AND

TANKS AND VESSELS

PROCESS AND PRODUCTION
SOURCE EMISSIONS ROLL-UP

Stack Configuration

Diameter (ft) Length(ft) Width(ft) Area(sq ft) Exit Temp(F) Exit Veloc(ft/sec)

VERTICAL WITHOUT WEATHER CAP

$1.13 \quad 120$

$373-5698$
$373-5698$
$373-5698$
$373-5698$
$373-5698$
$373-5698$

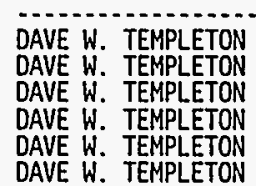

$373-2966$ $373-2966$ $373-2966$ $373-2966$

\begin{tabular}{|c|c|c|c|c|c|c|c|c|c|c|}
\hline Em Po & oint & Ep Type & North & East & Srce $\mathrm{Nbr}$ & Scc Code & Srce $T$. & & & \\
\hline $200 \mathrm{~W}$ & P-296Z005 001 & POINT & 135607 & 566434 & $\begin{array}{l}1 \\
2 \\
3 \\
4\end{array}$ & $\begin{array}{l}\text { not assigned } \\
\text { not assigned } \\
\text { not assignec } \\
\text { not assigned }\end{array}$ & $\begin{array}{l}\text { PROCES } \\
\text { PROCES } \\
\text { PROCES } \\
\text { SOURCE }\end{array}$ & $\begin{array}{l}\text { AND PRODUCTION } \\
\text { AND PRODUCTION } \\
\text { AND PRODUCTION } \\
\text { EMISSIONS ROLL-UP }\end{array}$ & & \\
\hline & Stack Configuration & & Di ameter $(\mathrm{ft})$ & Length(ft) & Width $(f t)$ & $\operatorname{Area}(s q \mathrm{ft})$ & Exit Temp(F) & Exit Veloc $(\mathrm{ft} / \mathrm{sec})$ & Ht Abv Grnd(ft) & Ht Abv Struc $(f t)$ \\
\hline & VERTICAL WITHOUT WEA & THER CAP & 2.8 & & & 6.16 & 68 & 36.5 & 27.9 & 15 \\
\hline
\end{tabular}




\section{Emission Point Listing}

\begin{tabular}{|c|c|c|c|c|c|c|c|c|c|c|}
\hline Em Point & Ep Type & North & East & Srce Nbr & Scc Code & & Srce Ty & & & \\
\hline 200W P-2962006 001 & POINT & 135633 & 566431 & 1 & not assigne & & PROCESS & AND PRODUCTION & & \\
\hline Stack Configuration & & Di ameter $(\mathrm{ft})$ & Length $(f t)$. W & Width(ft) & $\operatorname{Area}(s q f t)$ & Exit & $\operatorname{Temp}(F)$ & Exit Veloc $(\mathrm{ft} / \mathrm{sec})$ & Ht Abv Grnd(ft) & Ht Abv Struc(ft) \\
\hline VERTICAL WITHOUT WEAT & THER CAP & & 3 & 1.83 & 5.49 & & 68 & 36.4 & 14.67 & 2.5 \\
\hline Em Point & Ep Type & North & East & Srce Nbr & Scc Code & & Srce Ty & & & \\
\hline $200 \mathrm{~W}$ P-2962014 001 & POINT & 135561 & 566455 & & not assigne & & D\&D/DEM & MOLITION & & \\
\hline Stack Configuration & & Di ameter ( $\mathrm{ft}$ ) & Length(ft) & Width(ft) & $\operatorname{Area}(s q \mathrm{ft}$ ) & Exit & Temp(F) & Exit Veloc $(\mathrm{ft} / \mathrm{sec})$ & Ht Abv Grnd(ft) & Ht Abv Struc(ft) \\
\hline VERTICAL WITHOUT WEAT & THER CAP & $\ddot{i}$ & & & .79 & & 68 & 27.3 & 21 & 21 \\
\hline Em Point & Ep Type & North & East & Srce Nbr & - Scc Code & & Srce Typ & & & \\
\hline $200 W$ P-296z015001 & POINT & 135586 & 566510 & & not assigne & & PROCESS & AND PRODUCTION & & \\
\hline Stack Configuration & & Diameter $(\mathrm{ft})$ & Length $(f t)$ & Width(ft) & $\operatorname{Area}(s q \mathrm{ft})$ & Exit & $\operatorname{Temp}(F)$ & Exit Veloc $(\mathrm{ft} / \mathrm{sec})$ & Ht Abv Grnd(ft) & Ht Abv Struc(ft) \\
\hline VERTICAL WITHOUT WEA & & & & & .79 & & 68 & 34 & 42 & 26 \\
\hline
\end{tabular}

\section{Source (Process) Listing}

Em Point

Srce Nbr Srce Type

$200 \mathrm{~W}$ P-2912001 001

Em Point

200W P-2912001 001

Em Point

$200 \mathrm{~W}$ P-2912001 001

Em Point 200W P-2912001 001 i ANALYTICAL LABOPATORY Srce Nbr Srce Type 2 ANALYTICAL LABORATORY Srce Nbr Srce Type 3 PROCESS AND PRODUCTION Srce Nbr Srce Type 4 D\&D/DEMOLITION
Hrs Day Days Week Wks Year Summary Process Description $24 \quad 52$ Z-PLANT ANALYTICAL LABORATORY Hrs Day Days Week Wks Year Summary Process Description $24 \quad 52$ BACKSIDE DEVELOPMENT LAB Hrs Day Days Week Wks Year Summary Process Description $24 \quad 52$ ZONE 4 GLOVEBOXES AND VENT HOODS

Hrs Day Days Week Wks Year Summary Process Description $24 \quad 52$ D\&D OF THE 242-2 BUILDING (ASSUMED RUN ALL YEAR/WORST CASE) DURING THE LIFESPAN OF THIS PERMIT. 242-2 WILL UNDERGO D\&D - CONTRACTOR UNKNOWN NOW. METHODS HAVE NOT YET BEEN DETERMINED. 
AIR EMISSIONS INVENTORY PERMIT AP.PLICATION REPORT (UP460)

FACILITY NAME: Plutonium Finishing Plant(PFP) and Structures

\section{Source (Process) Listing}

\begin{tabular}{|c|c|c|c|c|c|c|}
\hline Em Point & Srce Nbr & Srce Type & Hrs Day & Days Week & Wks Year & Summary Process \\
\hline $200 \mathrm{~W}$ P-2912001 001 & 5 & D\&D/DEMOLITION & 24 & 7 & 52 & D\&D OF THE $234-5$ \\
\hline
\end{tabular}

OURING THE LIFESPAN OF THIS PERMIT, 234-5Z WILL UNDERGO D\&D - CONTRACTOR UNKNOWN NOW. METHODS HAVE NOT YET BEEN OETERMINEO.

\begin{tabular}{|c|c|c|c|c|c|c|}
\hline En Point & Srce Nbr & Srce Type & Hrs Day & Days Week & Wks Year & Summary Process \\
\hline 200W P-2912001 001 & 6 & D\&D/DEMOLITION & 24 & 7 & 52 & D\&D OF THE $236 \mathrm{~B}$ \\
\hline
\end{tabular}

DURING THE LIFESPAN OF THIS PERMIT, 236-Z WILL UNDERGO D\&D - CONTRACTOR UNKNOWN NOW. METHODS HAVE NOT YET BEEN DETERMINED.

\begin{tabular}{|c|c|c|c|c|c|c|}
\hline Em Point & Srce Nbr & Srce Type & Hrs Day & Days Week & Wks Year & Summary Process Description \\
\hline 200W P-29̣12001 001 & 7 & SOURCE EMISSIONS ROLL-UP & 24 & 7 & 52 & TOTAL EMISSIONS FOR EMISSION POINT P-2912001-001 \\
\hline Em Point & Srce Nbr & Srce Type & Hrs Day & Days Week & Wks Year & Summary Process Description \\
\hline 200W P-2912001 001 & 8 & ANALYTICAL LABORATORY & 24 & 7 & 52 & THIS IS A STANDARDS LABORATORY \\
\hline Em Point & Srce Nbr & Srce Type & Hrs Day & Days Week & Wks Year & Summary Process Description \\
\hline $200 W \mathrm{~W}-2962003001$ & 1 & TANKS AND VESSELS & 24 & 7 & 52 & $\begin{array}{l}\text { THE } 241-2 \text { WASTE TREATMENT FACILITY WHICH INCLUDES TANKS } 0-4 \text {. } \\
5,6,7,8 \text { AND THE RESPECTIVE TANK SUMPS. TANK D-9. GLOVEBOX } \\
\text { IN } 241-2 A \text {. AND ALL ASSOCIATED PIPING AND VENTILATION } \\
\text { DUCTWORK. }\end{array}$ \\
\hline \multicolumn{7}{|c|}{$\begin{array}{l}\text { THIS FACILITY IS USED TO HANDLE, TREAT, TEMPORILY STORE, AND TRANSFER AQUEOUS RADIOACTIVE WASTES FROM } 236-2 \text { AND } 234-52 . \text { WASTES ARE TREATED WITH } \\
\text { SODIUM HYDROXIDE OR POTASSIUM HYOROXIDE. SODIUM NITRITE AND FERRIC NITRATE ARE ADDED PRIOR TO WASTE TRANSFER TO UST. }\end{array}$} \\
\hline Em Point & Srce Nbr & Srce Type & Hrs Day & Days Week & Whs Year & Summary Process Description \\
\hline 200W P-2962003 001 & 2 & PROCESS AND PRODUCTION & 24 & 7 & 52 & $\begin{array}{l}\text { RADIONUCLIDE SMEARABLE CONTAMINATION WITHIN TANK VESSELS, } \\
\text { SUMPS. BUILDING. }\end{array}$ \\
\hline $200 \mathrm{~W}$ P-2962003 001 & 3 & SOURCE EMISSIONS ROLL-UP & 24 & 7 & 52 & TOTAL EMISSIONS FOR EMISSION POINT P-2962003-001 \\
\hline Em Point & $\begin{array}{l}\text { Srce Nbr } \\
. . . . . . . .\end{array}$ & $\begin{array}{l}\text { Srce Type } \\
\end{array}$ & Hrs Day & Days Week & Wks Year & Summary Process Description \\
\hline 200W P-2962005001 & 1 & PROCESS AND PRODUCTION & 24 & 7 & 52 & $\begin{array}{l}\text { STORAGE AND STAGING AREA, NON-DESTRUCTIVE ASSESSMENT FACILITY. } \\
\text { REPACKAGING FACILITY. BLDG. } 2736-Z B\end{array}$ \\
\hline
\end{tabular}




\section{Source (Process) Listing}

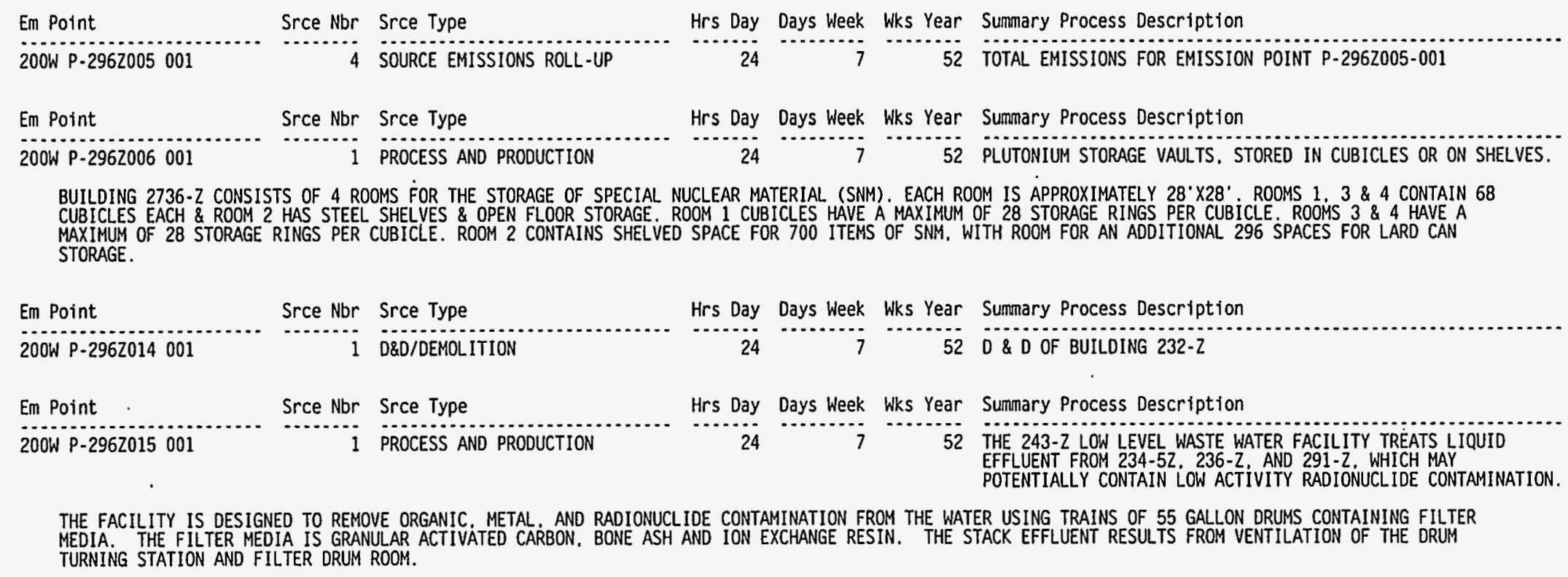

Fuels Usage by Source Processes

Em Point Srce Nbr Srce Type Qty Uom Fuel

Fuels Usage by Control Devices

Em Point Ctl Nbr Ctl Type Fuel

Point Source Criteria Pollutants - Type: H2SO4 


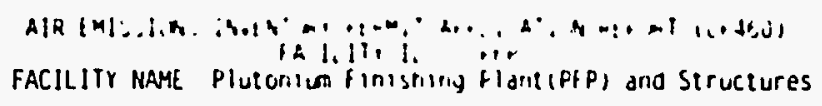

Point Source Criteria Pollutants - Type: voC

\begin{tabular}{|c|c|c|c|c|}
\hline int & Srce $\mathrm{Nbr}$ & Srce Type Pollutant & $\begin{array}{l}\text { Em. Pt. Potential } \\
\text { to Emit (tons/yr) }\end{array}$ & $\begin{array}{l}\text { Em. Pt. } \\
\text { Cti Actual (tons/yr) pollutant }\end{array}$ \\
\hline
\end{tabular}

Point Source Emissions - Radiological Pollutants

\begin{tabular}{|c|c|c|c|c|c|}
\hline Em Point & Srce Nbr & Srce Type Pollutant & $\begin{array}{l}\text { Em. Pt. Potentia) } \\
\text { to Emit (Ci/yr) }\end{array}$ & $\begin{array}{l}\text { Em. Pt. } \\
\text { Cti Actual (Ci/yr) }\end{array}$ & Pollutant \\
\hline 200W P-2912001 001 & $\begin{array}{l}1 \\
2 \\
3 \\
4 \\
5 \\
6\end{array}$ & $\begin{array}{l}\text { ANALYTICAL LABORATORY } \\
\text { ANALYTICAL LABORATORY } \\
\text { PROCESS AND PRODUCTION } \\
\text { D\&D/DEMOLITION } \\
\text { D\&D/DEMOLITION } \\
\text { D\&D/DEMOLITION }\end{array}$ & $\begin{array}{l}\text { Refer to } \\
\text { WHC-SD-EN-RPT-007 } \\
\text { Rev. } 0 \text { Page A-284 }\end{array}$ & $\begin{array}{l}\text { Refer to } \\
\text { DOE/RL-94-51 } \\
\text { Table } 2 \cdot 1\end{array}$ & \\
\hline $200 W$ P-2962003 001 & $\frac{1}{2}$ & $\begin{array}{l}\text { PROCESS AND PRODUCTION } \\
\text { TANKS AND VESSELS }\end{array}$ & $\begin{array}{l}\text { Refer to } \\
\text { WHC-SD-EN-RPT-007 } \\
\text { Rev. O Page A-285 }\end{array}$ & $\begin{array}{l}\text { Refer to } \\
\text { DOE/RL-94-51 } \\
\text { Table 2-1 }\end{array}$ & \\
\hline 200W P-296Z005 001 & $\begin{array}{l}1 \\
2 \\
3\end{array}$ & $\begin{array}{l}\text { PROCESS AND PRODUCTION } \\
\text { PROCESS AND PRODUCTION } \\
\text { PROCESS AND PRODUCTION }\end{array}$ & $\begin{array}{l}\text { Refer to } \\
\text { WHC-SD-EN-RPT-007 } \\
\text { Rev. 0 Page A-286 }\end{array}$ & $\begin{array}{l}\text { Refer to } \\
\text { DOE/RL-94-51 } \\
\text { Table 2-1 }\end{array}$ & \\
\hline
\end{tabular}

Point Source Emissions - Radiological Pollutants

\begin{tabular}{|c|c|c|c|c|c|}
\hline Em Point & Srce Nbr & Srce Type & $\begin{array}{l}\text { Em. Pt. Potential } \\
\text { to Emit (Ci/yr) }\end{array}$ & Ct1 Actual (Ci/yr) & Pollutant \\
\hline 200W P-296Z006 001 & 1 & PROCESS AND PRODUCTION & $\begin{array}{l}\text { Refer to } \\
\text { WHC-SD-EN-RPT-007 } \\
\text { Rev. O Page A-287 }\end{array}$ & $\begin{array}{l}\text { Refer to } \\
\text { DOE/RL. }-94-51 \\
\text { Table 2-1 }\end{array}$ & \\
\hline 200W P-2962014001 & 1 & D\&D/DEMOLITION & $\begin{array}{l}\text { Refer to } \\
\text { WHC-SD-EN-RPT-007 } \\
\text { Rev. O Page A-288 }\end{array}$ & $\begin{array}{l}\text { Refer to } \\
\text { DOE/RL-94-51 } \\
\text { Table } 2-1\end{array}$ & \\
\hline
\end{tabular}


AIR EMISSIONS INVENTORY PERMIT APPLICATION REPORT (UP460)

FACILITY NAME: Plutonium Finishing Plant(PFP) and Structures

Point Source Emissions - HAP Pollutants

Point Source Emissions - Uncategorized Pollutants

Fugitive Source Emissions - Radiological Pollutants

Fugitive Source Emissions - HAP Pollutants

\section{Em Point} Srce Nbr Srce Type Pollutant

Sum

Fugitive Source Emissions - Uncategorized Pollutants

\section{Em Point}

Srce Nbr Srce Type Pollutant

Em. Pt. Potential Em. Pt.

Em. Pt. Potential Em. Pt.

to Emit (tons/yr) ctl Actual (tons/yr) Pollutant

Sum

Insignificant Source Emissions - Radiological Pollutants

to Emit (Ci/yr) Cti Actual (Ci/yr) Pollutant


AIR EMISSIONS INVENTORY PERMIT APPLICATION REPORT (UP460)

FACILITY NAME: Plutonium Finishing Plant(PFP) and Structures

Insignificant Source Emissions - HAP Pollutants

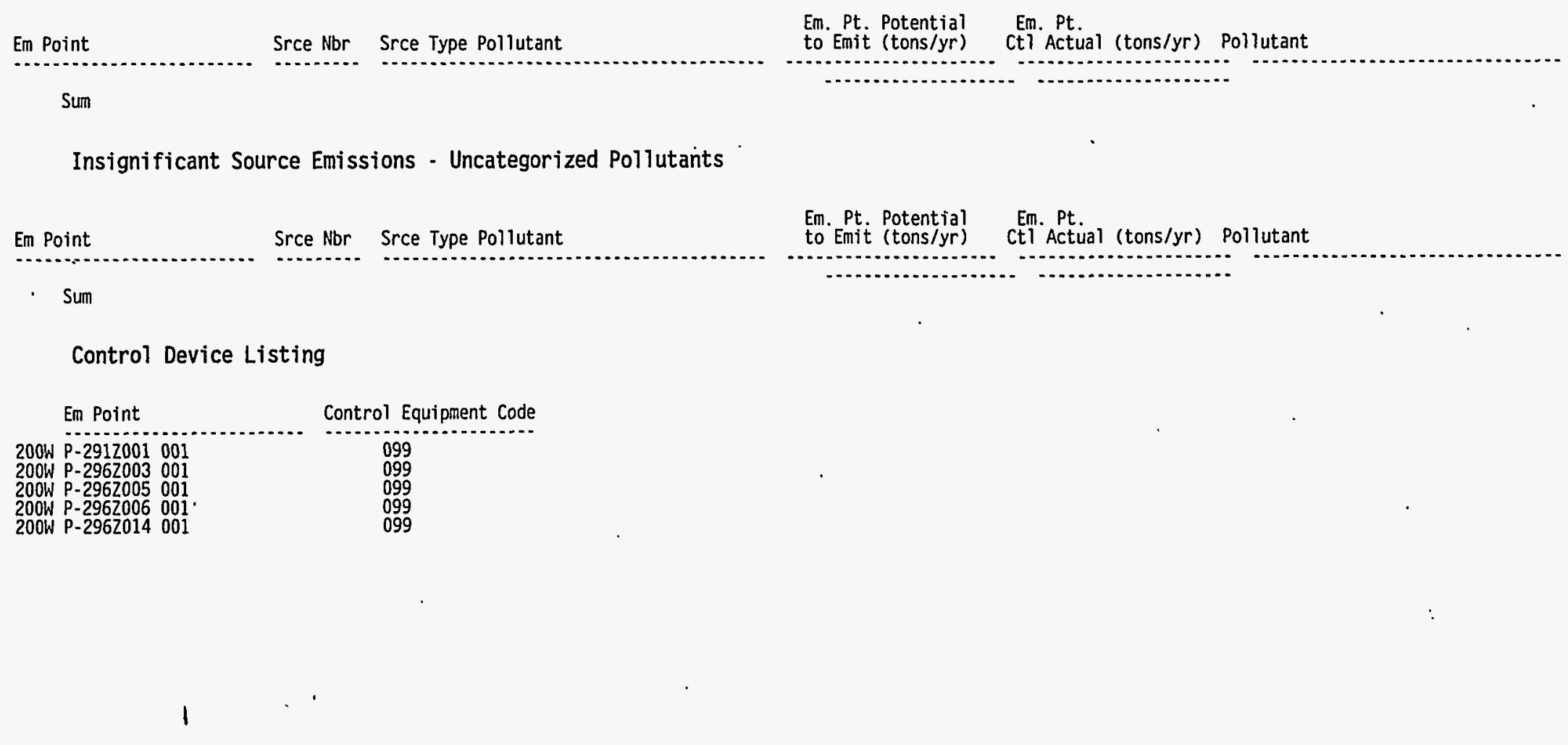

Page \#: 7 of 7 Report Run Date: 22-APR-95 


\section{FACILITY:}

Plutonium Finishing Plant

\section{EMISSION POINT:} 200W P-291Z001 001

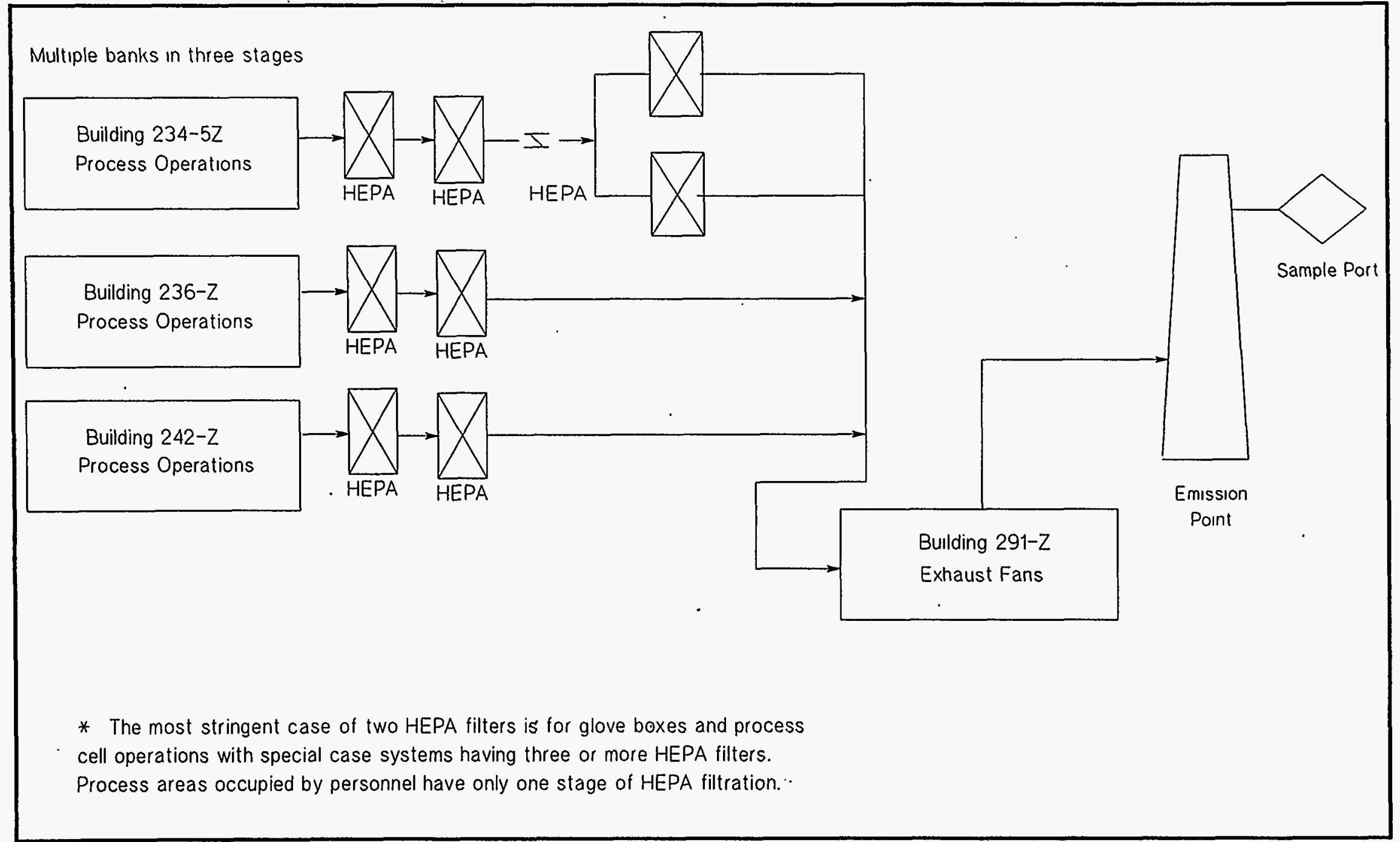




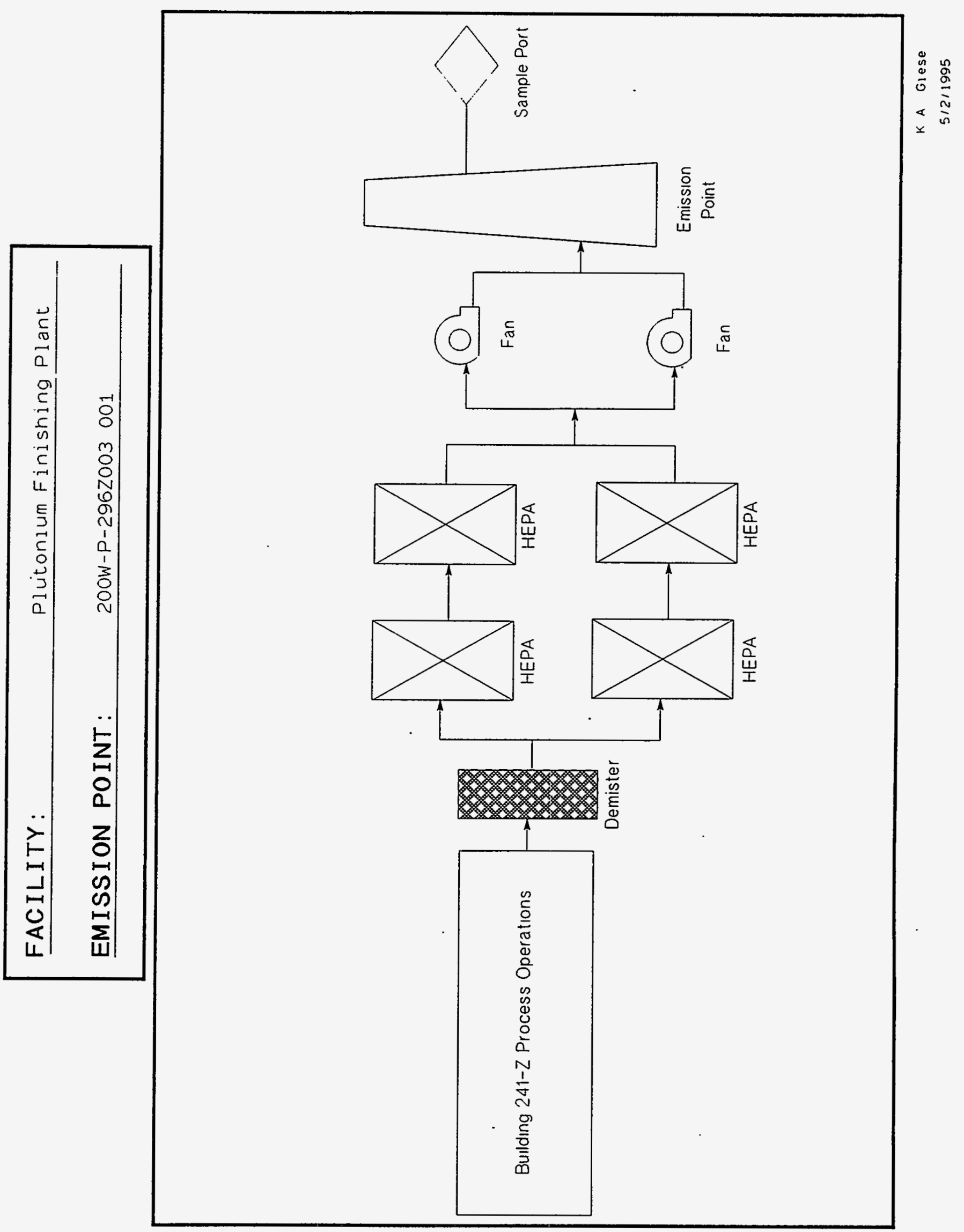




\begin{tabular}{|c|c|}
\hline FACILITY: & Plutonlum Finishing $\mathrm{Plant}$ \\
\hline EMISSION POINT: & $200 W$ P-2962005 001 \\
\hline
\end{tabular}

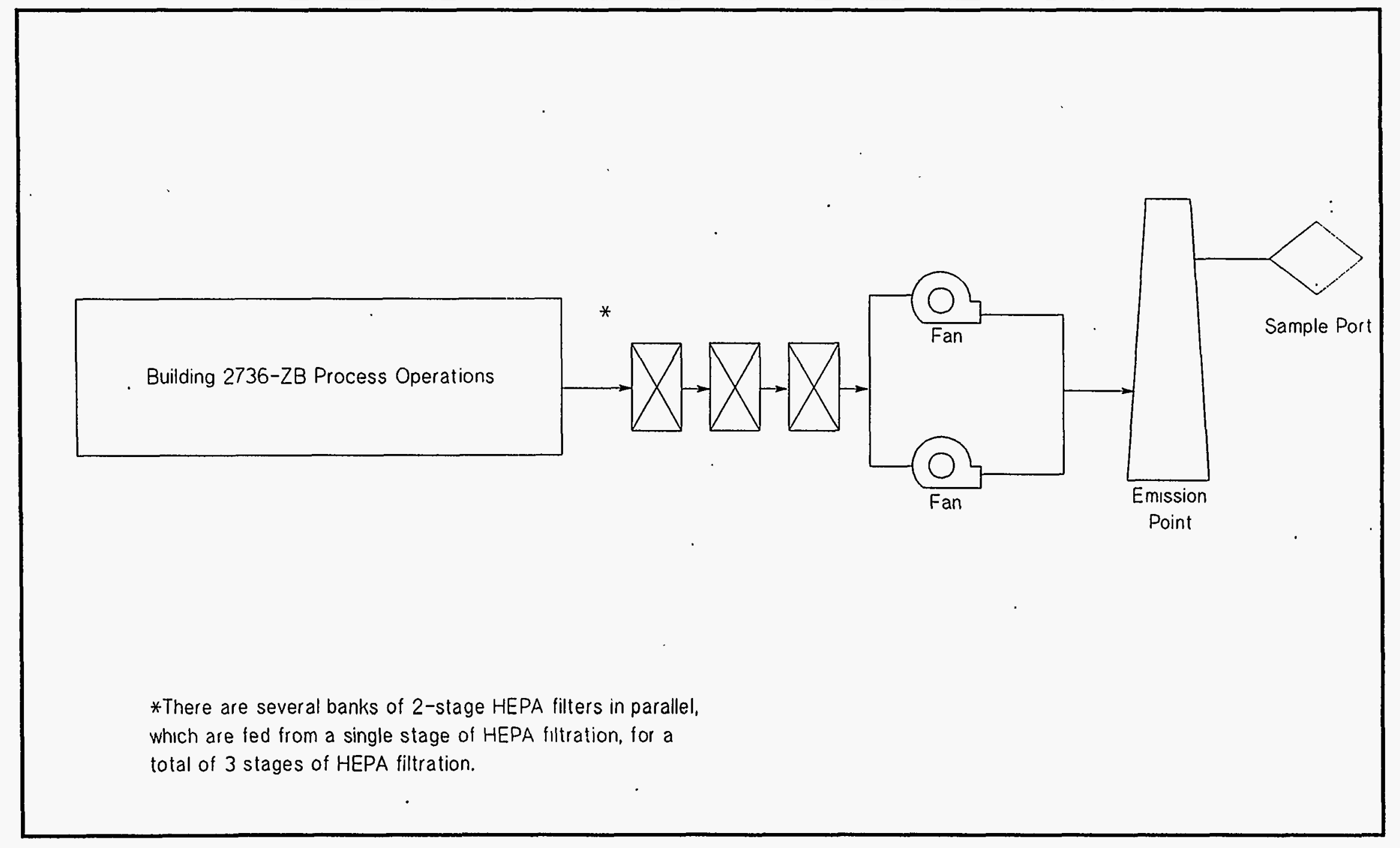




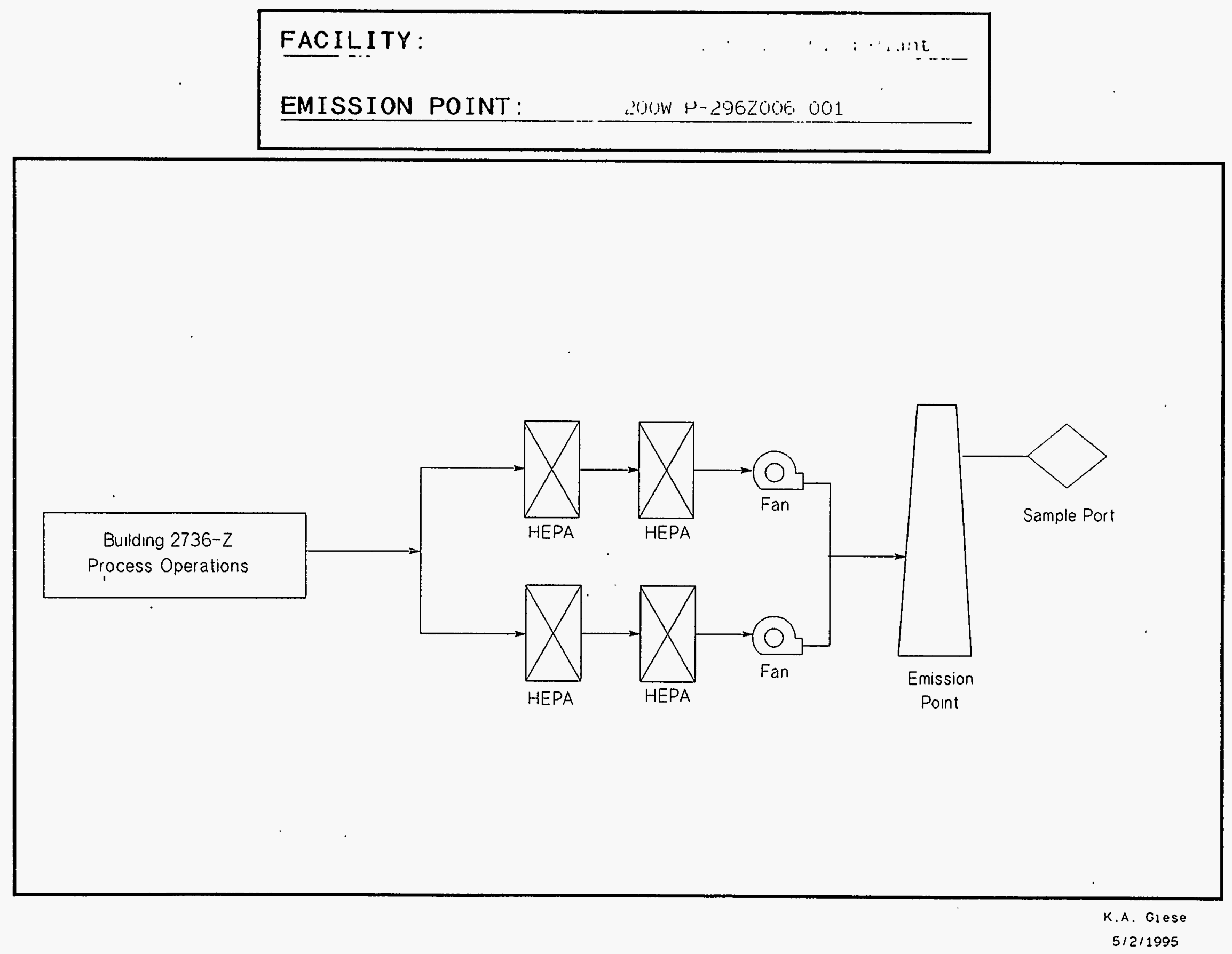




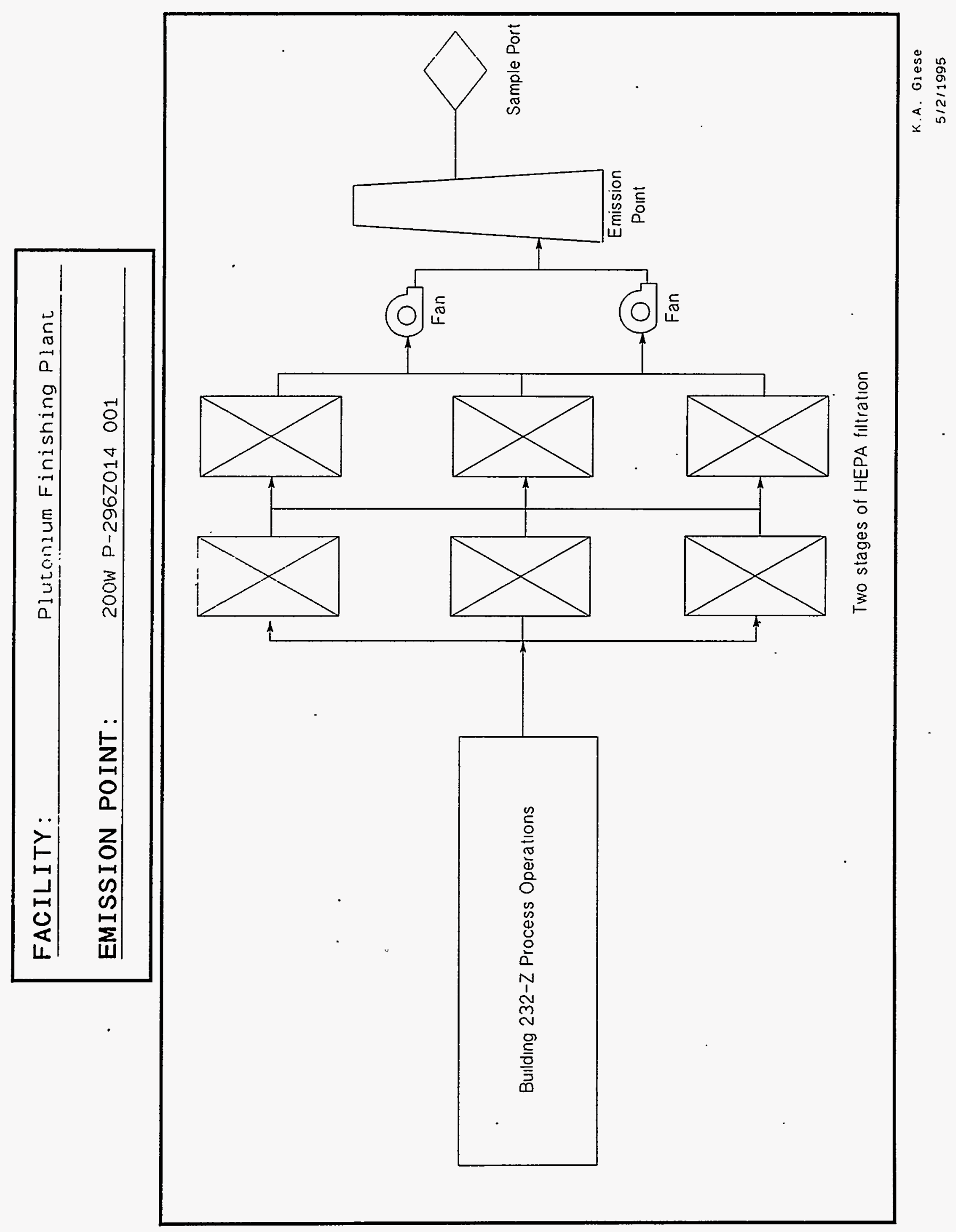




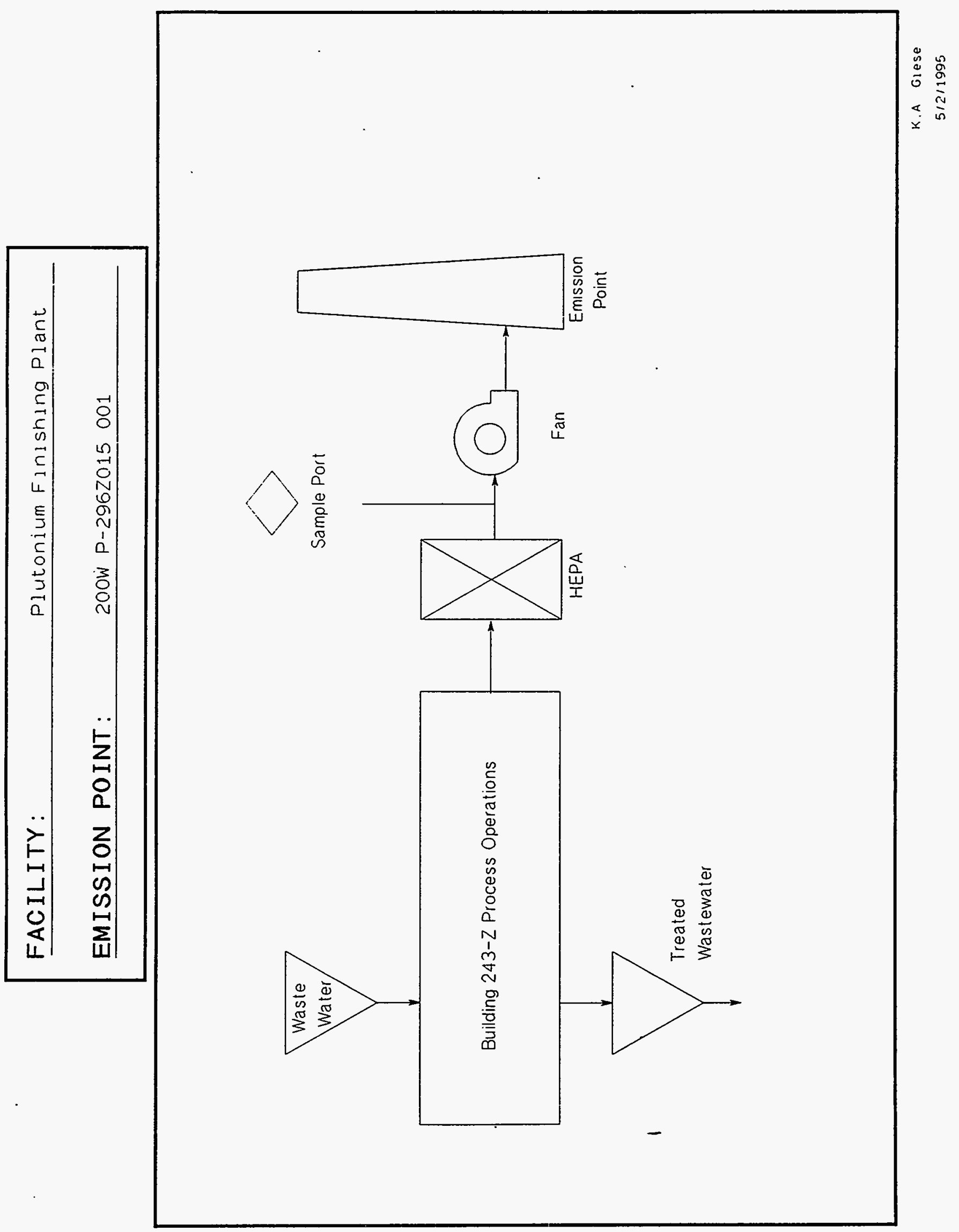




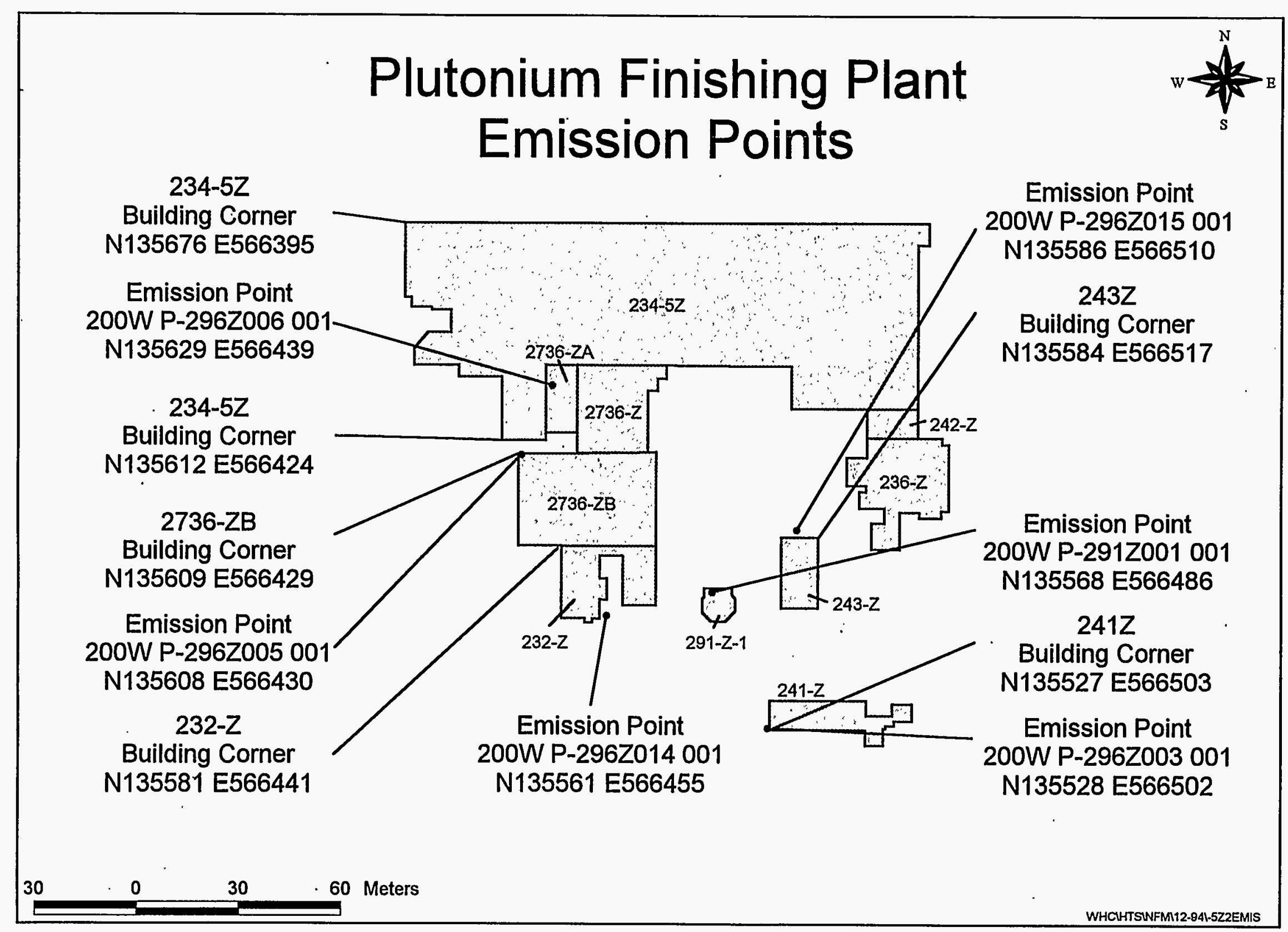




\title{
APPENDLX E-4
}

\author{
S PLANT
}

\section{Chapter I. General Information}

\section{I-B.1. Facility Description}

The S Plant [REDOX (reduction-oxidation)] is located in the 200 West Area. The S Plant was constructed from 1950 to 1952 and became the first large-scale, continuous flow, solvent extraction process plant built in the United States for the recovery of plutonium from irradiated uranium fuel. The process, which replaced the batch precipitation methods, was designed to separate uranium, plutonium, and neptunium as individual product streams from the fission products associated with irradiated fuel. This separation was accomplished by controlling the relative distribution of the activated components between aluminum nitratesalted aqueous solutions and an immiscible organic extractant, hexone (methyl isobutyl ketone).

The canyon portion of S Plant contains equipment for handling radioactive materials such as fuel dissolution, feed preparation, solvent distillation, and waste concentration and neutralization. The silo area, located at the west end of the canyon, houses the solvent extraction columns and aqueous makeup vessels.

All process activities at REDOX ceased in 1967. Presently, the canyon is used as a radioactive confinement area for the original equipment used in the REDOX process. The S Plant is scheduled for decommissioning in 2016.

\section{3-S Facility}

The 233-S Plutonium Concentration Facility, adjacent to and directly north of the REDOX canyon building, was built in 1954-1955 and placed into operation March 1955. During the initial process, a dilute plutonium nitrate solution was transferred from the 202-S Building to the 233-S Facility. Within the 233-S Facility, the plutonium solution was concentrated and loaded into product removal cans for transportation to other facilities for further reprocessing.

All process activities at 233-S Facility ceased in 1967. Presently, the 233-S Facility serves no purpose and is currently in the initial stages of decommissioning.

\section{I-B.2. Products, Raw Materials, Fuels, Processes}

There are no products, raw materials, fuels, or processes at S Plant or the 233-S Facility. 


\section{I-C.1. Emission Points, Controls, Stacks}

The emission point and stack information is found on pages 1 and 2 of the AEI report output.

\section{I-C.2. Facility Process Flow Diagram}

Attached.

I-C.3. Site Map

Attached.

\section{Chapter II. Regulatory Requirements}

\section{II-A.1. Description of Discharge and Emission Points}

The emission point and stack information is found on pages 1 and 2 of the AEI report output. 


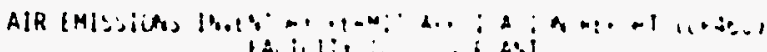

faCILIir hivit "̈siblus idalities

Facility Building List

\begin{tabular}{|c|c|c|c|c|c|}
\hline Bldg Id & North & East & Bidg Desc & Point of Contact ( $P O C)$ & (POC) Phone \\
\hline $\begin{array}{l}2915001 \\
2965002 \\
2965004 \\
2965006 \\
296 S 07 W\end{array}$ & $\begin{array}{l}134039 \\
134992 \\
134966 \\
134985 \\
135020\end{array}$ & $\begin{array}{l}567546 \\
567413 \\
567460 \\
567343 \\
567356\end{array}$ & $\begin{array}{l}\text { 202-S MAIN STACK } \\
202-5 \text { (REDOX) N\&S SAMPLE GALLERY EXHAUST STACK } \\
202-5 \text { (REDOX) OECONTAMINATION ROOM EXHAUST STACK } \\
202-5 \text { REDOX SILO STACK } \\
233-5 \text { PLUTONIUM CONCENTRATION FACILITY STACK }\end{array}$ & $\begin{array}{l}\text { Egge. Robert } G \text {. } \\
\text { Egge. Robert } G \text {. } \\
\text { Egge. Robert } G \text {. } \\
\text { Egge, Robert } G \text {. } \\
\text { Egge, Robert } G \text {. }\end{array}$ & $\begin{array}{l}373-1914 \\
373-1914 \\
373-1914 \\
373-1914 \\
373-1914\end{array}$ \\
\hline
\end{tabular}

\section{Emission Point Listing}

\begin{tabular}{|c|c|c|c|c|c|c|c|c|c|}
\hline Em Point & Ep Type & North & Eas & st Srce $\mathrm{Nbr}$ & Scc Code & Srce Ty & & & \\
\hline 200W P-2915001 001 & POINT & 135029 & 56753 & & & PROCESS & AND PRODUCTION & & \\
\hline Stack Configuration & & Diameter ( $f t)$ & Length(ft) & Width(ft) & Area $(s q f t)$ & Exit Temp(F) & Exit Veloc $(\mathrm{ft} / \mathrm{sec})$ & Ht Abv Grnd(ft) & Ht Abv Struc(ft) \\
\hline VERTICAL WITHOUT WE & THER CAP & 3.75 & & & 11.04 & 68 & 30.3 & 200 & \\
\hline
\end{tabular}

\begin{tabular}{|c|c|c|c|c|c|c|c|}
\hline Em Point & Ep Type & 'North & Srce $\mathrm{Nbr}$ & Scc Code & Srce Type & & \\
\hline $200 \mathrm{~W}$ P-2965002 001 & POINT & 134992 & 567403 & $\frac{1}{2}$ & $\begin{array}{l}\text { PROCESS AND PRODUCTION } \\
\text { PROCESS AND PRODUCTION }\end{array}$ & & \\
\hline Stack Configuration & & Diameter $(\mathrm{ft})$ & Length $(\mathrm{ft})$ & Area(sq ft) & Exit Veloc $(\mathrm{ft} / \mathrm{sec})$ & Ht Abv Grnd(ft) & Ht Abv Struc(ft) \\
\hline VERTICAL WITHOUT WE & THER CAP & 1.2 & & 1.13 & 9.3 & & 5 \\
\hline
\end{tabular}

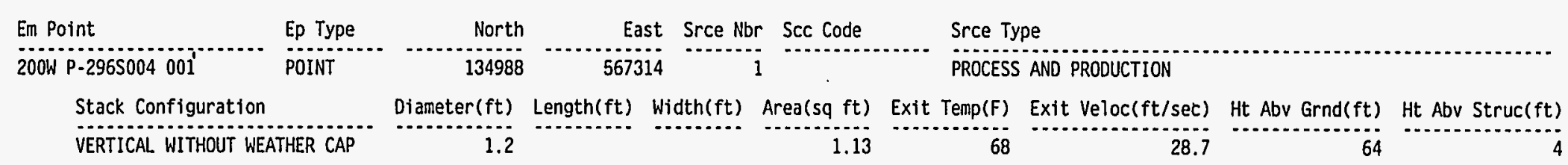

\begin{tabular}{|c|c|c|c|c|c|c|c|c|c|}
\hline Em Point & Ep Type & North & Eas & st Srce $\mathrm{Nbr}$ & - Scc Code & Srce Ty & & & \\
\hline $200 \mathrm{~W}$ P-2965006 001 & POINT & 135888 & 56789 & 90 & & PROCESS & AND PRODUCTION & & \\
\hline Stack Configuration & & Diameter $(f t)$ & Length(ft) & Width(ft) & Areạ(sq ft) & Exit Temp(F) & Exit Veloc $(\mathrm{ft} / \mathrm{sec})$ & Ht Abv Grnd(ft) & Ht Abv Struc(ft) \\
\hline VERTICAL, WITHOUT WEA & THER CAP & 2.25 & & & 3.98 & 68 & 8.6 & 128.5 & \\
\hline
\end{tabular}


AIR EMISSIONS INVENTORY PERMIT APPLICATION REPORT (UP460)

FACILITY ID : S PLANT
FACILITY NAME: Surplus Facilities

\section{Emission Point Listing}

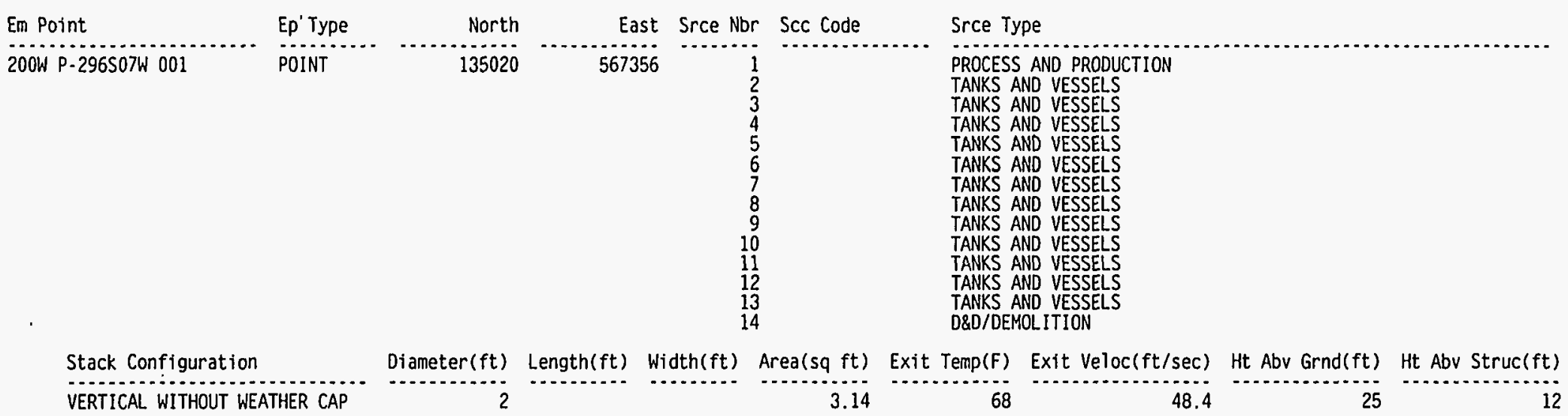

\section{Source (Process) Listing}

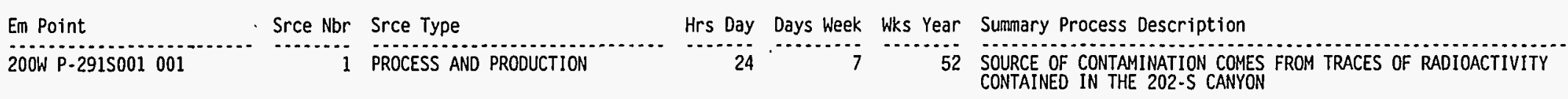

Process.$-->>$
Description

Em Point
$200 \mathrm{~W}$ P-2965002 001
Process $\ldots . .$.

Srce Nbr Srce Type

Hrs Day Days Week Wks Year Summary Process Description

Process -..>
Description

Em Point Srce Nbr Srce Type

Hrs Day Days Week Wks Year Summary Process Description

200W P-296S002 001

2 PROCESS AND PRODUCTION 52 RESIDUAL CONTAMINATION IN SAMPLE HOODS IN NORTH AND SOUTH
SAMPLE GALLRIES

Process $\ldots$-..>

Description 
AIR EMISSIONS INVENTORY PERMIT APPLICATION REPORT (UP460) FACILITY

\section{Source (Process) Listing}

\begin{tabular}{|c|c|c|c|c|c|c|}
\hline Em Point & Srce Nbr & Srce Type & Hrs Day & Days Week & Wks Year & Summary Process Description \\
\hline 200W P-296S004 001 & 1 & PROCESS AND PRODUCTION & 24 & 7 & 52 & $\begin{array}{l}\text { CONTAMINATION IN THE REGULATED SHOP AND THE DECON ROOM IN } \\
202-5\end{array}$ \\
\hline \multicolumn{7}{|l|}{$\begin{array}{l}\text { Process } \cdots . . .> \\
\text { Description }\end{array}$} \\
\hline Em Point & Srce Nbr & Srce Type & Hrs Day & Days Week & Wks Year & Summary Process Description \\
\hline $200 W$ P-296S006 001 & 1 & PROCESS AND PRODUCTION & 24 & 7 & 52 & VENTILATES THE CLEAN (UNCONTAMINATED) SILO AREA \\
\hline \multicolumn{7}{|l|}{$\begin{array}{l}\text { Process }-\rightarrow>> \\
\text { Description }\end{array}$} \\
\hline Em Point & Srce Nbr & Srce Type & Hrs Day & Days Week & Wks Year & Summary Process Description \\
\hline 200W P-296S07W 001 & 1 & PROCESS AND PRODUCTION & 24 & 7 & 52 & SCHEDULED FOR D\&D \\
\hline \multicolumn{7}{|l|}{$\begin{array}{l}\text { Process } \cdots . .> \\
\text { Description }\end{array}$} \\
\hline Em Point & Srce Nbr & Srce Type & Hrs Day & Days Week & Wks Year & Summary Process Description \\
\hline 200W P-296S07W 001 & 2 & TANKS AND VESSELS & 24 & 7 & 52 & SCHEDULED FOR D\&D \\
\hline \multicolumn{7}{|l|}{$\begin{array}{l}\text { Process ....> } \\
\text { Déscription }\end{array}$} \\
\hline Em Point & Srce Nbr & Srce Type & Hrs Day & Days Week & Wks Year & Sumary Process Description \\
\hline 200W P-296S07W 001 & 3 & TANKS AND VESSELS & 24 & 7 & 52 & SCHEDULED FOR D\&D \\
\hline \multicolumn{7}{|l|}{$\begin{array}{l}\text { Process } \ldots . .>\quad 1 \\
\text { Description }\end{array}$} \\
\hline Em Point & Srce $\mathrm{Nbr}$ & Srce Type & Hrs Day & Days Week & Wks Year & Summary Process Description \\
\hline 200W P-296S07W 001 & 4 & TANKS AND VESSELS & 24 & 7 & 52 & SCHEDULED FOR D\&D \\
\hline \multicolumn{7}{|l|}{$\begin{array}{l}\text { Process } \ldots . .> \\
\text { Description }\end{array}$} \\
\hline $\begin{array}{l}\text { Em Point } \\
\text {....................... }\end{array}$ & Srce Nbr & Srce Type & $\begin{array}{l}\text { Hrs Day } \\
\text {-........ }\end{array}$ & Days Week & Wks Year & Summary Process Description \\
\hline $200 W$ P-296507W 001 & 5 & TANKS AND VESSELS & 24 & 7 & 52 & SCHEDULED FOR D\&D \\
\hline
\end{tabular}

Page \#: 3 of 7 Report Run Date: 11-NOV-94 
AIR EMISSIONS INVENTORY PERMIT APPLICATION REPORT (UP460) FACI FACIT NAME: SURPlUS FaC

\section{Source (Process) Listing}

\begin{tabular}{|c|c|c|c|c|c|c|}
\hline Em Point & Srce Nbr & Srce Type & Hrs Day & Days Week & Wks Year & Summary Process Description \\
\hline 200W P-296507W 001 & 6 & TANKS AND VESSELS & 24 & 7 & 52 & SCHEDULED FOR D\&D \\
\hline \multicolumn{7}{|l|}{$\begin{array}{l}\text { Process ...> } \\
\text { Description }\end{array}$} \\
\hline Em Point & Srce Nbr & Srce Type & Hrs Day & Days Week & Wks Year & Summary Process Description \\
\hline 200W P-296S07W 001 & 7 & TANKS AND VESSELS & 24 & 7 & 52 & SCHEDULED FOR D\&D \\
\hline $\begin{array}{l}\text { Process -...> } \\
\text { Description }\end{array}$ & & - & & & & \\
\hline Em Point & Srce Nbr & Srce Type & Hrs Day & Days Week & Wks Year & Summary Process Description \\
\hline $200 W$ P-296507W 001 & 8 & TANKS AND VESSELS & 24 & 7 & 52 & SCHEDULED FOR D\&D \\
\hline \multicolumn{7}{|l|}{$\begin{array}{l}\text { Process --.> } \\
\text { Description }\end{array}$} \\
\hline Em Point & Srce Nbr & Srce Type & Hrs Day' & Days Week & Wks Year & Summary Process Description \\
\hline 200W P-296S07W 001 . & 9 & TANKS AND VESSELS & 24 & 7 & 52 & SCHEDULED FOR D\&D \\
\hline \multicolumn{7}{|l|}{$\begin{array}{l}\text { Process ...-> } \\
\text { Description }\end{array}$} \\
\hline Em Point & Srce Nbr & Srce Type & Hrs Day & Days Week & Wks Year & Summary Process Description \\
\hline 200W P-296S07W 001 & 10 & TANKS AND VESSELS & 24 & 7 & 52 & SCHEDULED FOR D\&D \\
\hline \multicolumn{7}{|l|}{$\begin{array}{l}\text { Process -...> } \\
\text { Description }\end{array}$} \\
\hline Em Point & Srce Nbr & Srce Type & Hrs Day & Days Week & Wks Year & Summary Process Description \\
\hline 200W P-296S07W 001 & 11 & TANKS AND VESSELS & 24 & 7 & - 52 & SCHEOULED FOR D\&D \\
\hline \multicolumn{7}{|l|}{$\begin{array}{l}\text { Process ....> } \\
\text { Description }\end{array}$} \\
\hline Em Point & Srce Nbr & Srce Type & Hrs Day & Days Week & Wks Year & Summary Process Description \\
\hline 200W P-296S07W 001 & 12 & TANKS AND VESSELS & 24 & 7 & 52 & SCHEDULED FOR D\&D \\
\hline
\end{tabular}

Page \#: 4 of 7 Report Run Date: 11-NOV-94 
AIR EMISSIONS INVENTORY PERMIT APPLICATION REPORT (UP460)

FACILITY NAME: SurpluS Facilities

\section{Source (Process) Listing}

Em Point Srce $\mathrm{Nbr}$ Srce Type

Hrs Day Days Week Wks Year Summary Process Description

200W P-296S07W 001

13 TANKS AND VESSELS

24

52 SCHEDULED FOR D\&D

Process $\ldots . .>$

Source Raw Materials Usage

Em Point

Srce Nbr Srce Type

Process Rate Process Uom Material

Fuels Usage by Source Processes

Em Point

Srce Nbr Srce Type

Qty Uom

Fuel

Fuels Usage by Control Devices

Em Point

Ctl $\mathrm{Nbr}$ Ctl Type

Qty Uom

Fuel

\section{Facility Emissions}

Point Source Emissions - Radiological Pollutants

\begin{tabular}{|c|c|c|}
\hline Em Point & Srce Nbr & Srce Type \\
\hline 200W P-2915001 001 & 1 & PROCESS AND PRODUCTION \\
\hline $200 \mathrm{~W} P-2965002001$ & $\frac{1}{2}$ & $\begin{array}{l}\text { PROCESS AND PRODUCTION } \\
\text { PROCESS AND PRODUCTION }\end{array}$ \\
\hline 200W P-296S004001 & 1 & PROCESS AND PRODUCTION \\
\hline 200W P-2965006 001 & 1 & PROCESS AND PRODUCTION \\
\hline
\end{tabular}

to Emitential to Emit ( $\mathrm{Ci} / \mathrm{yr})$

Refer to
WHC-SD-EN-RPT-007

Rev, 0 Page A-292

Em. Pt.

Refer to

WHC-SD-EN-RPT-007

Rev. 0 Page A-293

Refer to
DOE/RL $-94-5$

Table 2-1

Refer to

WHC-SD-EN-RPT-007

Rev. 0 Page A-294

Refer to

DOE/RL - $94-51$

Refer to

WHC-SO-EN-RPT-007

Refer to

Table 2-1

Rev. 0 Page A-295

Refer to
DOE/RL-94-51

Table 2-1

Page \#: 5 of 7 Report Run Date: 11-NOV-94 
Point Source Emissions - Radiological Pollutants

\begin{tabular}{|c|c|c|c|}
\hline Em Point & Srce Type & $\begin{array}{ll}\text { Em. Pt. Potential } \\
\text { to Emit } & (\mathrm{C} i / \mathrm{yr})\end{array}$ & $\begin{array}{l}\text { Em. Pt. } \\
\mathrm{CtT} \text { Actual (Ci/yr) }\end{array}$ \\
\hline $200 \mathrm{~W}$ P-296S07W 001 & 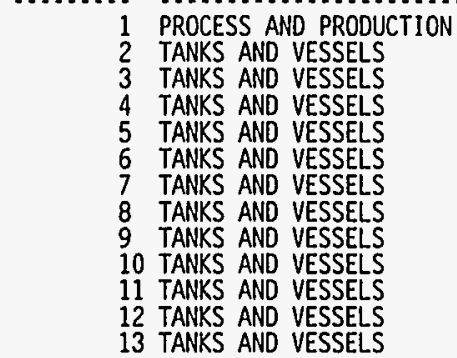 & $\begin{array}{l}\text { Refer to } \\
\text { WHC-SD-EN-RPT-007 } \\
\text { Rev. O Page A- } 296\end{array}$ & $\begin{array}{l}\text { Refer to } \\
\text { DOE/RL-94-51 } \\
\text { Table 2-1 }\end{array}$ \\
\hline
\end{tabular}

Point Source Emissions - HAP Pollutants

Em Point Srce Nbr Srce Type

Sum

Point Source Emissions - Uncategorized Pollutants

Em Point

Srce Nbr Srce Type

Sum

Fugitive Source Emissions - Radiological Pollutants

Em Point Srce Nbr Srce Type

Em. Pt. Potential Em. Pt

Em. Pt. Potential Em. Pt.

to Emit (tons/yr) . Ctl Actual Yr Pollutant

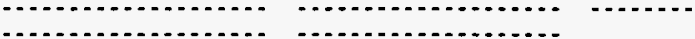

Em. Pt. Potential Em. Pt.

to Emit (tons/yr) Cti Actual (tons/yr) Pollutant

Fugitive Source Emissions : HAP Pollutants

Em Point $\quad$ Srce Nbr Srce Type

Em. Pt. Potential Em. Pt.

to Emit (tons/yr) ctî. Áctual (tons/yr) Pollutant

Sum

Fugitive Source Emissions - Uncategorized Pollutants

Em Point

Srce Nbr Srce Type

Em. Pt. Potential
to Emit (tons/yr) ctil Actual (tons/yr) Pollutant

Sum 
AIR EMISSIONS INVENTORY PERMIT APPLICATION REPORT (UP460) FACILITY ID : S PLANT
FACILITY NAME: Surplus Facilities

Insignificant Source Emissions - Radiological Pollutants

\begin{tabular}{|c|c|c|c|}
\hline Em Point & Srce Nbr Srce Type & $\begin{array}{l}\text { Em. Pt. Potential } \\
\text { to Emit (ci/yr) }\end{array}$ & $\begin{array}{l}\text { Em. Pt. } \\
\text { ctl Actual (ci/yr) }\end{array}$ \\
\hline
\end{tabular}

Insignificant Source Emissions - HAP Pollutants

Em Point

Srce Nbr Srce Type

Em. Pt. Potential Em. Pt.

to Emit (tons/yr) Cti Actual (tons/yr) Pollutant

Sum

Insignificant Source Emissions - Uncategorized Pollutants

Em Point

Srce Nbr Srce Type

Em. Pt. Potential Em. Pt.

to Emit (tons/yr) Ctl Actual (tons/yr) Pollutant

Sum

Control Device Listing

\begin{tabular}{|c|c|}
\hline Em Point & Control Equipment Code \\
\hline $\begin{array}{lll}200 \mathrm{~W} & \text { P-2915001 } & 001 \\
200 \mathrm{~W} & \mathrm{P}-2965002 & 001 \\
200 \mathrm{~W} & \mathrm{P}-2965002 & 001 \\
200 \mathrm{~W} & \mathrm{P}-2965002 & 001 \\
200 \mathrm{~W} & \mathrm{P}-2965004 & 001 \\
200 \mathrm{~W} & \mathrm{P}-296507 \mathrm{~W} & 001 \\
200 \mathrm{~W} & \mathrm{P}-296507 \mathrm{~W} & 001\end{array}$ & $\begin{array}{l}063 \\
099 \\
099 \\
099 \\
099 \\
099 \\
099\end{array}$ \\
\hline
\end{tabular}




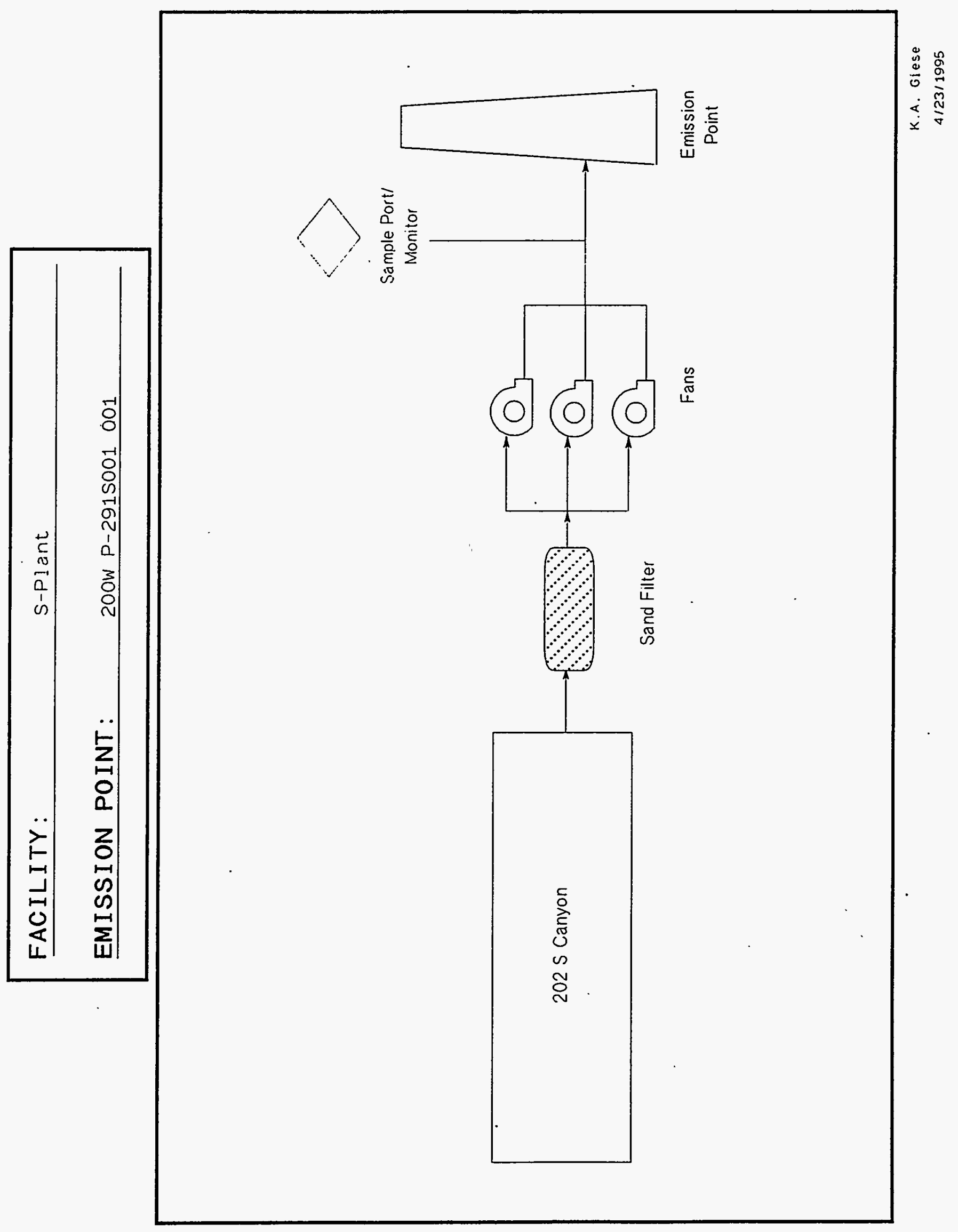




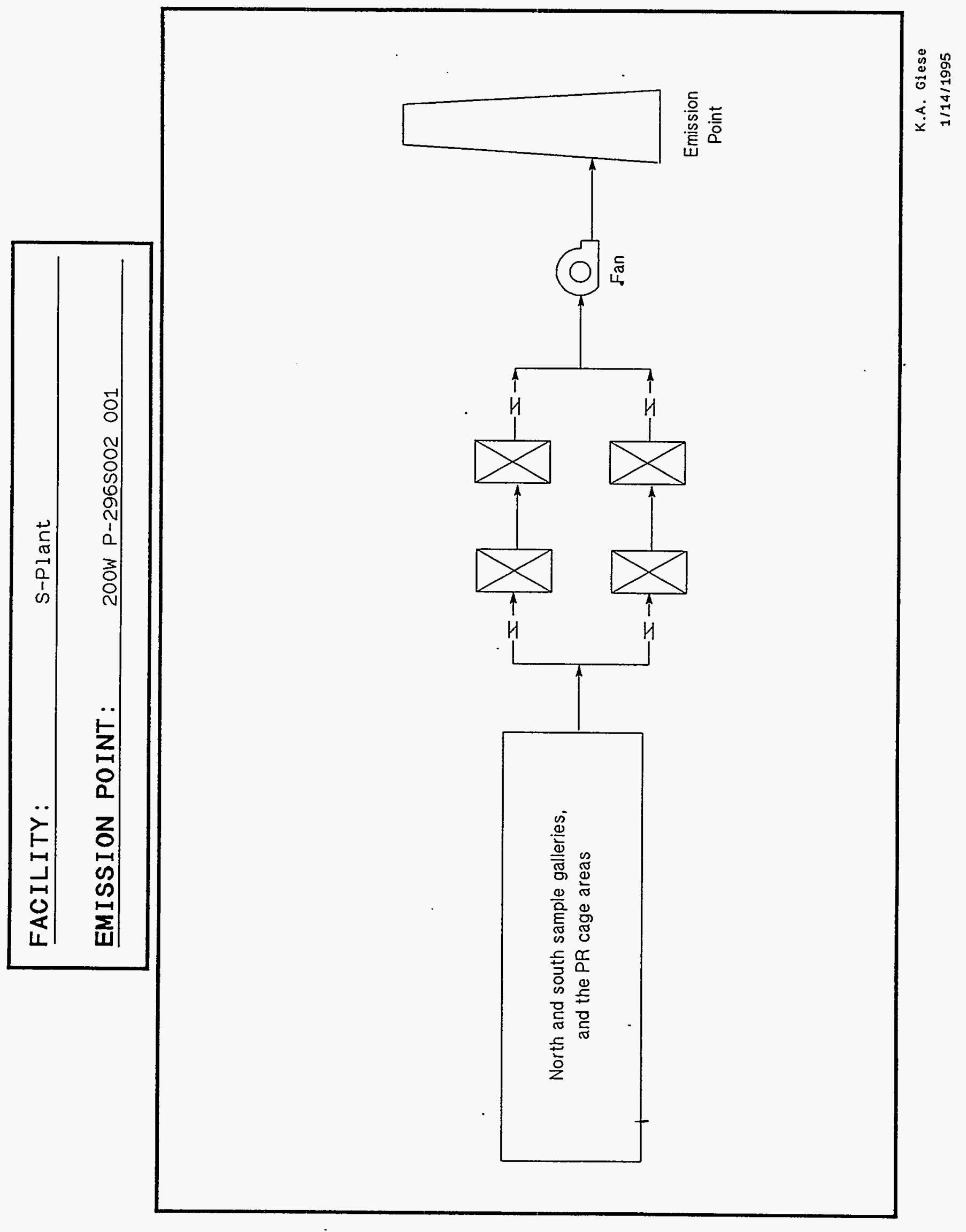




\begin{tabular}{|c|c|}
\hline FACILITY: & S-Plant \\
\hline EMISSION POINT: & $200 \mathrm{~W}$ P-2965004 001 \\
\hline
\end{tabular}

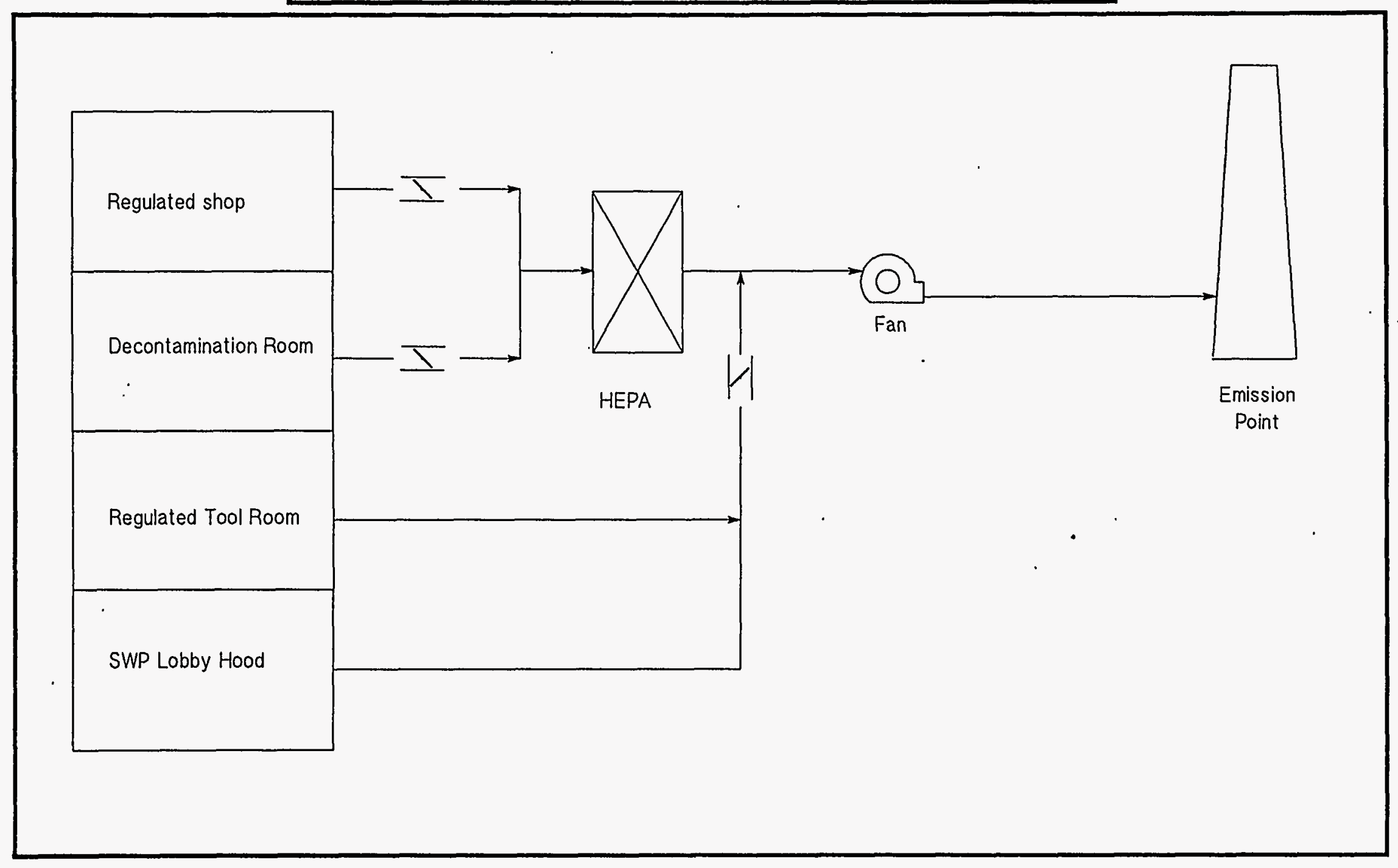




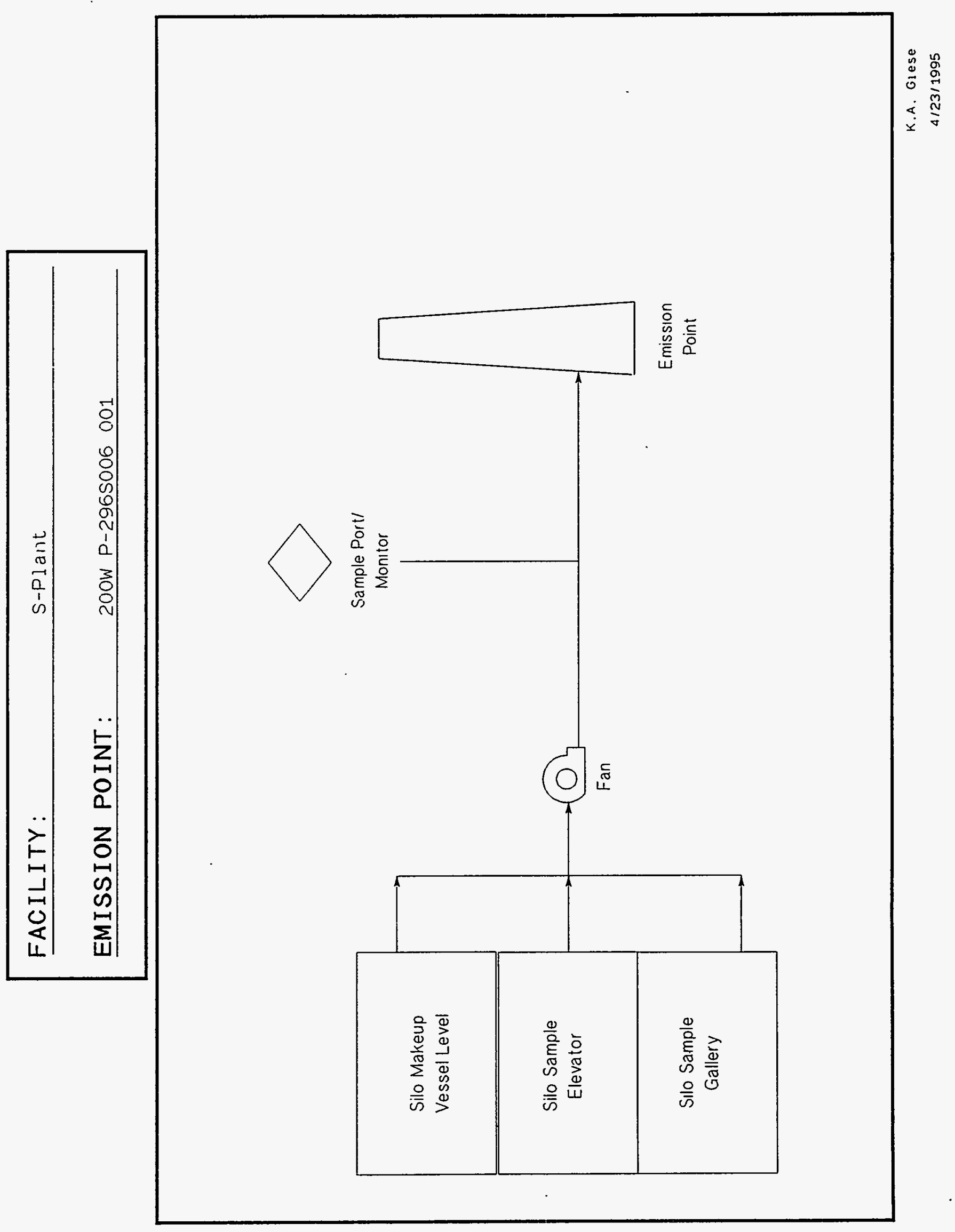




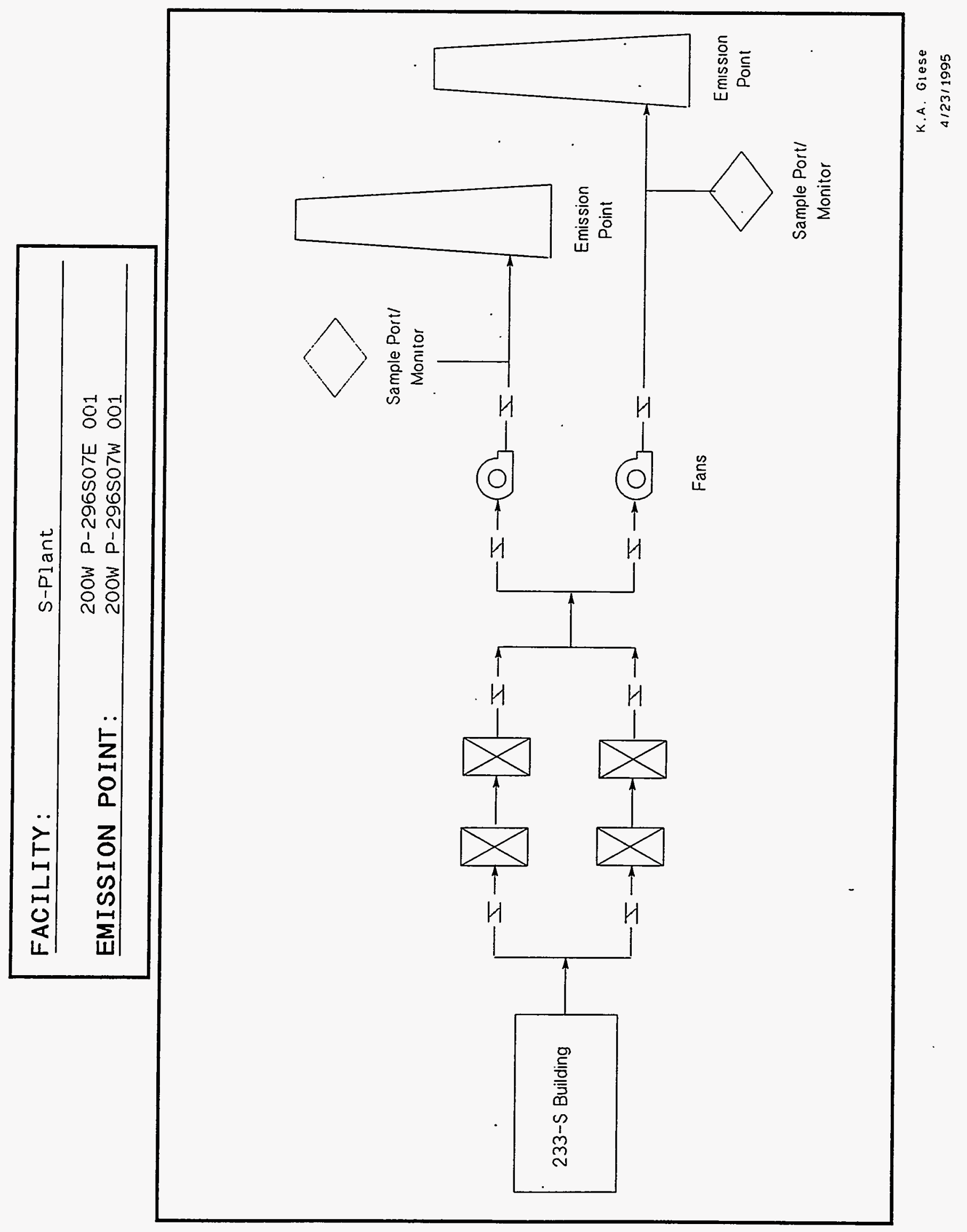




\section{S-Plant Emission Points}

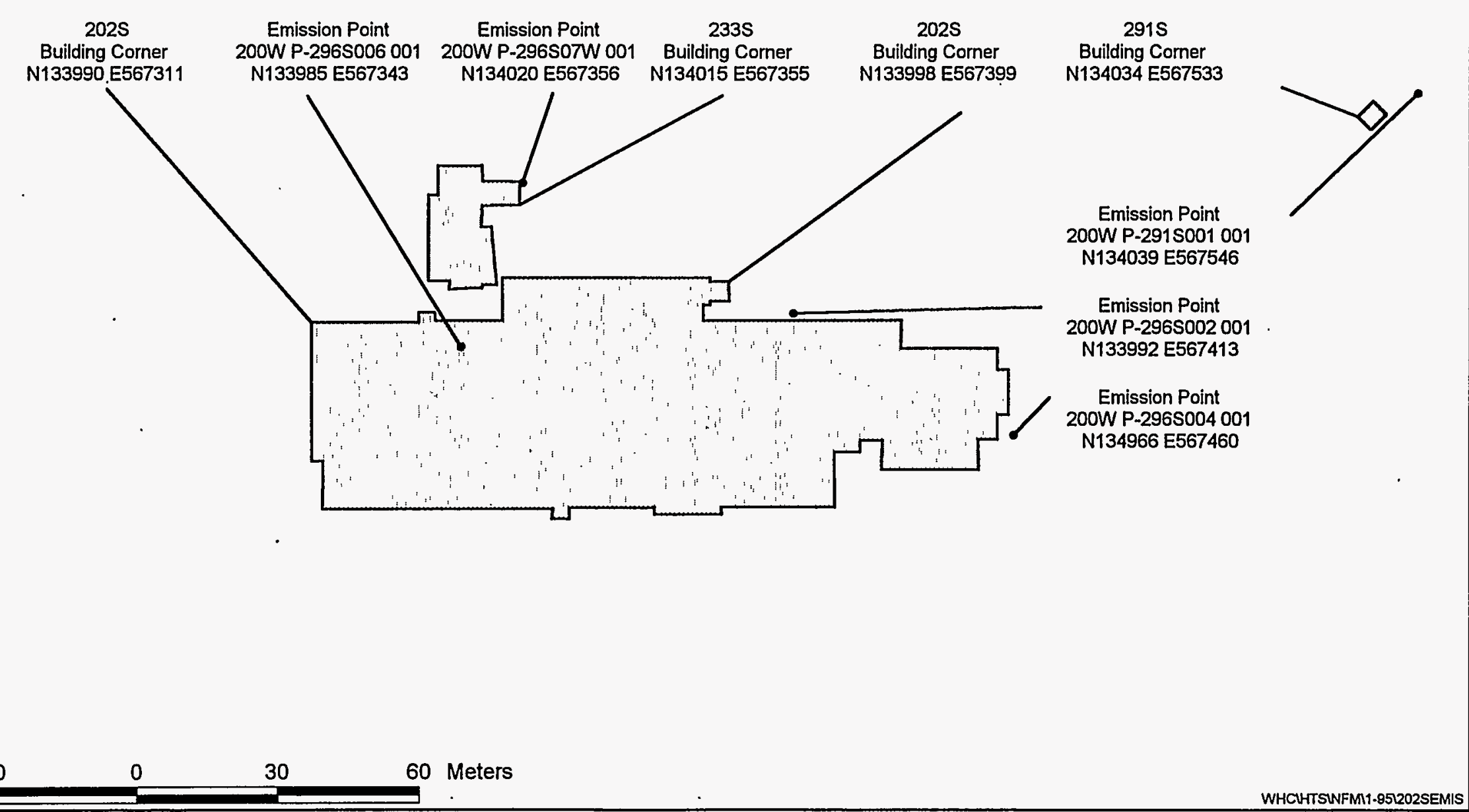




\section{APPENDIX E-5}

\section{T PLANT}

\section{Chapter I. General Information .}

\section{I-B.1. Facility Description}

The T Plant Complex was constructed in the mid 1940's to extract plutonium and uranium from spent reactor fuel using the bismuth phosphate process. The T Plant Complex performed this function until deactivation in 1956. In 1957, T Plant Complex became a betagamma decontamination and support complex for containment experiments. Currently, operations at $\mathrm{T}$ Plant Complex include equipment storage and decontamination, liquid waste tank car certification, and waste processing. The operations primarily occur in the 221-T and 2706-T Buildings.

The T Plant Complex consists of a number of buildings in the 200 West Area. The T Plant Complex is located on the site maps in Section I.C.3. The T Plant Complex is operated for $2+$ hours a day, 365 days a year.

\section{1-B.2. Products, Raw Materials, Fuels, Processes}

- Principal products - There are no products produced at $\mathrm{T}$ Plant.

- Process information - The current processes are discussed in the following:

- 221-T building operations - Provides storage and decontamination services for highly contaminated equipment. The decontamination processes include chemical spray, abrasive, immersion, and hand cleaning.

- 2706-T building operations - Provides decontamination of equipment with low levels of contamination and waste repackaging of containerized low level and mixed waste. The decontamination processes include abrasive, steam, and hand cleaning. The waste repackaging service entails verifying, sampling, repackaging, and shipping.

- Raw materials used by processes - There are no raw materials used at the T Plant Complex.

- Fuels used by processes - There are no fuels used in the processes at the T Plant Complex. 


\section{I-C.1. Emission Points, Controls, Stacks}

The emission point and stack information is found on pages 1 and 2 of the AEI report output. The control information is found on pages 5 and 6 of the AEI report output.

\section{I-C.2. Facility Process Flow Diagrams}

Attached.

\section{I-C.3. Site Map}

Attached.

\section{Chapter II. Regulatory Requirements}

\section{II-A.1. Description of Discharge and Emission Points}

The emission point and stack information is found on pages 1 and 2 of the AEI report output. 
AIR EMISSIONS INVENTORY PERMIT APPLICATION REPORT (UP460) FACILITY ID : T-Plant
FACILITY NAME: T-Plant Facility and Associated Structures

\section{Facility Building List}

\begin{tabular}{|c|c|c|c|c|c|c|c|}
\hline Bidg Id & North & East & Bldg Desc & Custodian(Contractor) & Cust Phone & DOE POC & DOE Phone \\
\hline $\begin{array}{l}291 T 001 \\
296 T 007 \\
296 T 013\end{array}$ & $\begin{array}{l}136867 \\
136911 \\
136901\end{array}$ & $\begin{array}{l}567676 \\
567513 \\
567605\end{array}$ & $\begin{array}{l}\text { T-PLANT MAIN STACK } \\
\text { STACK FOR } 2706 T \\
\text { T-PLANT AUXILLARY STACK }\end{array}$ & $\begin{array}{l}\text { SMITH, COLLIN L. } \\
\text { SMITH, COLLIN L. } \\
\text { SMITH, COLLIN L. }\end{array}$ & $\begin{array}{l}372-2933 \\
372-2933 \\
372-2933\end{array}$ & $\begin{array}{l}\text { KEVIN D. BASSELL } \\
\text { KEVIN D. BASSELL } \\
\text { KEVIN D. BASSELL }\end{array}$ & $\begin{array}{l}373-0463 \\
373-0463 \\
373-0463\end{array}$ \\
\hline
\end{tabular}

\section{Emission Point Listing}

\begin{tabular}{|c|c|c|c|c|c|c|c|c|c|}
\hline En Point & Ер Туре & North & Eas & Srce Nbr & Scc Code & Srce Ty & & & \\
\hline 200W P-291T001 001 & POINT & 136867 & 56767 & & $\begin{array}{l}\text { not assigne } \\
\text { not assigne } \\
\text { not assigne }\end{array}$ & $\begin{array}{l}\text { PROCESS } \\
\text { PROCESS } \\
\text { SOURCE }\end{array}$ & $\begin{array}{l}\text { AND PRODUCTION } \\
\text { AND PRODUCTION } \\
\text { EMISSIONS ROLL-UP }\end{array}$ & & \\
\hline Stack Configuration & & Diameter (ft) & Length(ft) & Width(ft) & $\operatorname{Area}(s q f t)$ & Exit Temp(F) & Exit Veloc $(\mathrm{ft} / \mathrm{sec})$ & Ht $A b v \operatorname{Grnd}(\mathrm{ft})$ & Ht. Abv Struc (ft) \\
\hline VERTICAL WITHOUT WE & R CAP & 5 & & & 19.63 & 78 & 30.6 & 200 & \\
\hline
\end{tabular}

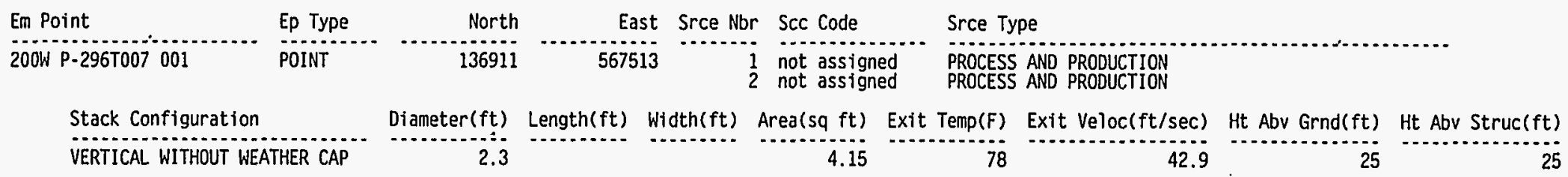

\begin{tabular}{|c|c|c|c|c|c|c|c|c|c|}
\hline Em Point & Ep Type & North & East & Srce $\mathrm{Nbr}$ & Scc Code & & Srce Type & & \\
\hline 200W P-296TO1B 001 & POINT & 136901 & 567605 & $\begin{array}{l}1 \\
2 \\
3\end{array}$ & $\begin{array}{l}\text { not assigne } \\
\text { not assigne } \\
\text { not assigne }\end{array}$ & & $\begin{array}{l}\text { PROCESS AND PRODUCTION } \\
\text { PROCESS AND PRODUCTION } \\
\text { SOURCE EMISSIONS ROLL-UP }\end{array}$ & & \\
\hline Stack Configuration & & Diameter $(f t)$ & Length $(f t)$ & Width(ft) & $\operatorname{Area}(s q \mathrm{ft}$ ) & Exit & Exit Veloc $(\mathrm{ft} / \mathrm{sec})$ & Ht Abv Grnd(ft) & Ht Abv Struc(ft) \\
\hline VERTICAL WITHOUT WE & ITHER CAP & & 2 & 2 & 4 & & 150 & 90 & \\
\hline
\end{tabular}


Source (Process) Listing

\begin{tabular}{|c|c|c|c|c|c|c|}
\hline Em Point & Srce Nbr & Srce Type & Hrs Day & Days Week & Whs Year & Summary Process Description \\
\hline 200W P-291T001 001 & 1 & PROCESS AND PRODUCTION & 24 & 7 & 52 & CANYON CELLS CONTAIN CONTAMINATED EQUIPMENT AND MATERIALS \\
\hline Em Point & Srce Nbr & Srce Type & Hrs Day & Days Week & Wks Year & Summary Process Description \\
\hline 200W P-291T001 001 & 2 & PROCESS AND PRODUCTION & 24 & 7 & 52 & DECONTAMINATION OF EQUIPMENT \\
\hline Em Point & Srce Nbr & Srce Type & Hrs Day & Days Week & Wks Year & Summary Process Description \\
\hline 200W P-291T001 001 & 3 & SOURCE EMISSIONS ROLL-UP & 24 & 7 & 52 & TOTAL EMISSIONS FOR EMISSION POINT P-291TO01-001 \\
\hline Em Point & Srce $\mathrm{Nbr}$ & Srce Type & Hrs Day & Days Week & Wks Year & Summary Process Description \\
\hline $200 \mathrm{~W}$ P-296T007 001 & 1 & PROCESS AND PRODUCTION & 8 & 5 & 52 & THIS FACILITY REPACKAGES LOW-LEVEL WASTE DRUMS. \\
\hline Em Point & Srce Nbr & Srce Type & Hrs Day & Days Week & Wks Year & Summary Process Description \\
\hline 200W P-296T007 001 & 2 & PROCESS AND PRODUCTION & 8 & 5 & 52 & DECONTAMINATION OF EQUIPMENT \\
\hline Em Point & Srce $\mathrm{Nbr}$ & 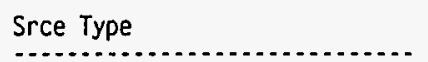 & Hrs Day & Days Week & Wks Year & Summary Process Description \\
\hline $200 \mathrm{~W}$ P-296T013001 & $i$ & PROCESS AND PROOUCTION & 24 & 7 & 52 & CANYON CELLS STORE CONTAMINATED EQUIPMENT AND MATERIALS. \\
\hline Em Point & Srce $\mathrm{Nbr}$ & Srce Type & Hrs Day & Days Week & Wks Year & Summary Process Description \\
\hline $200 \mathrm{~W}$ P.296T013 001 & 2 & PROCESS AND PRODUCTION & 24 & 7 & 52 & DECONTAMINATION OF EQUIPMENT \\
\hline Em Point & Srce Nbr & Srce Type & Hrs Day & Days Week & Wks Year & Summary Process Description \\
\hline $200 \mathrm{~W}$ P-296T013 001 & 3 & SOURCE EMISSIONS ROLL-UP & 24 & 7 & 52 & TOTAL EMISSIONS FOR EMISSION POINT P.296T013-001 \\
\hline \multicolumn{7}{|c|}{ Source Raw Materials Usage } \\
\hline Em Point & Srce Nbr & Srce Type & & Proces & s Rate Pro & ocess Uom Material \\
\hline
\end{tabular}

Fuels Usage by Source Processes
Em Point
Srce Nbr Srce Type
Qty Uom
Fuel

Fuels Usage by Control Devices

Em Point

Ctl $\mathrm{Nbr}$ Ctl Type

Qty Uom Fuel 
AIR EMISSIONS INVENTORY PERMIT APPLICATION REPORT (UP460)

FACILITY NAME: T-Plant Facility and Associated Structures

Point Source Emissions - Radiological Pollutants

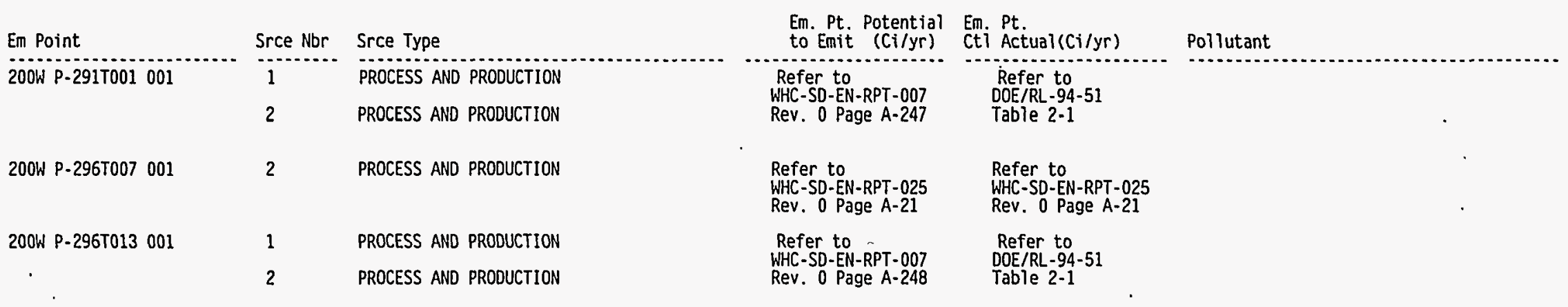

Point Source Emissions - HAP Pollutants

Em Point

Srce Nbr Srce Type Pollutant

Sum

Point Source Emissions - Uncategorized Pollutants

Em Point

Srce Nbr Srce Type Pollutant

Em. Pt. Potential Em. Pt.

to Emit (tons/yr) Ctl Actual (tons/yr) Pollutant

Sum

Fugitive Source Emissions - Radiological Pollutants

Em Point

Srce Nbr Srce Type

Em. Pt. Potential Em. Pt.

to Emit (Ci/yr) Cti Actual (Ci/yr)

Pollutant

Fugitive Source Emissions - HAP Pollutants

Em Point

Srce Nbr Srce Type Pollutant

Em. Pt. Potential Em. Pt.

to Emit (tons/yr) Cti Actual (tons/yr) Polrutant

Sum 
AIR EMISSIONS INVENTORY PERMIT APPLICATION REPORT (UP460)

FACILITY NAME: T-Plant Facility and Associated Structures

Fugitive Source Emissions - Uncategorized Pollutants

\begin{tabular}{|c|c|c|c|c|}
\hline Em Point & Srce Nbr & Srce Type Pollutant & $\begin{array}{l}\text { Em. Pt. Potential } \\
\text { to Emit (tons/yr) }\end{array}$ & $\begin{array}{l}\text { Em. Pt. } \\
\text { Ctl Actual (tons/yr) Pollutant }\end{array}$ \\
\hline
\end{tabular}

Sum

Insignificant Source Emissions - Radiological Pollutants

Em Point

Srce Nbr Srce Type

Em. Pt. Potential Em. Pt

to Emit (Ci/yr) Cti Actual(Ci/yr) Pollutant

Insignificant Source Emissions - HAP Pollutants

Em Point $\quad$. $\quad$ Srce Nbr Srce Type Pollutant

Em. Pt. Potential Em. Pt

to Emit (tons/yr) Ctl Actual (tons/yr) Pollutant

Sum

Insignificant Source Emissions - Uncategorized Pollutants

Em Point

Srce Nbr Srce Type Pollutant

Em. Pt. Potential Em. Pt.
to Emit (tons/yr) Ctl Actual (tons/yr) Pollutant

Sum

Control Device Listing

Em Point

Control Equipment Code

200W P-291T001 001

W P-296T007 00

017. 099

017.099

200W P-296T013 001

017.099 


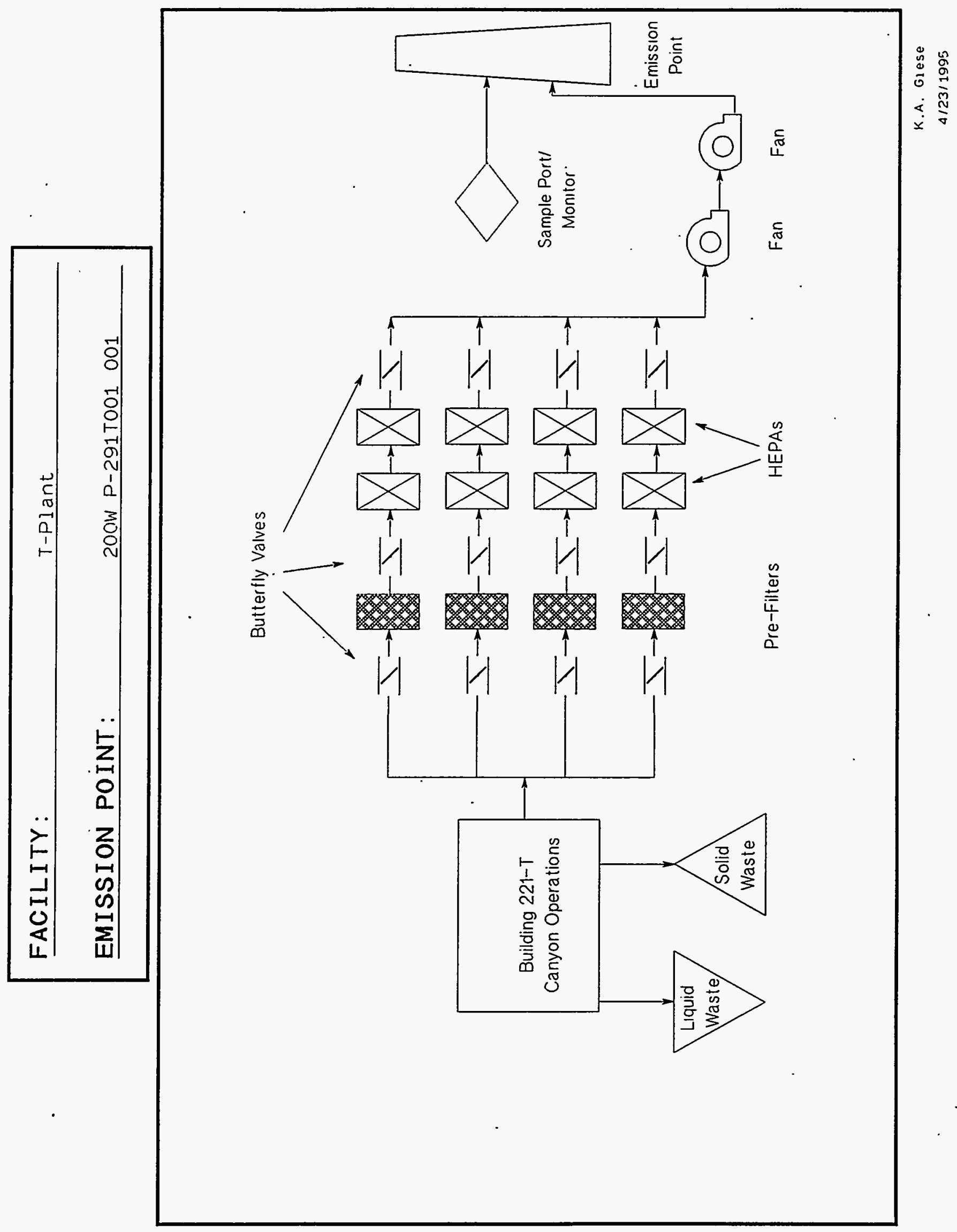




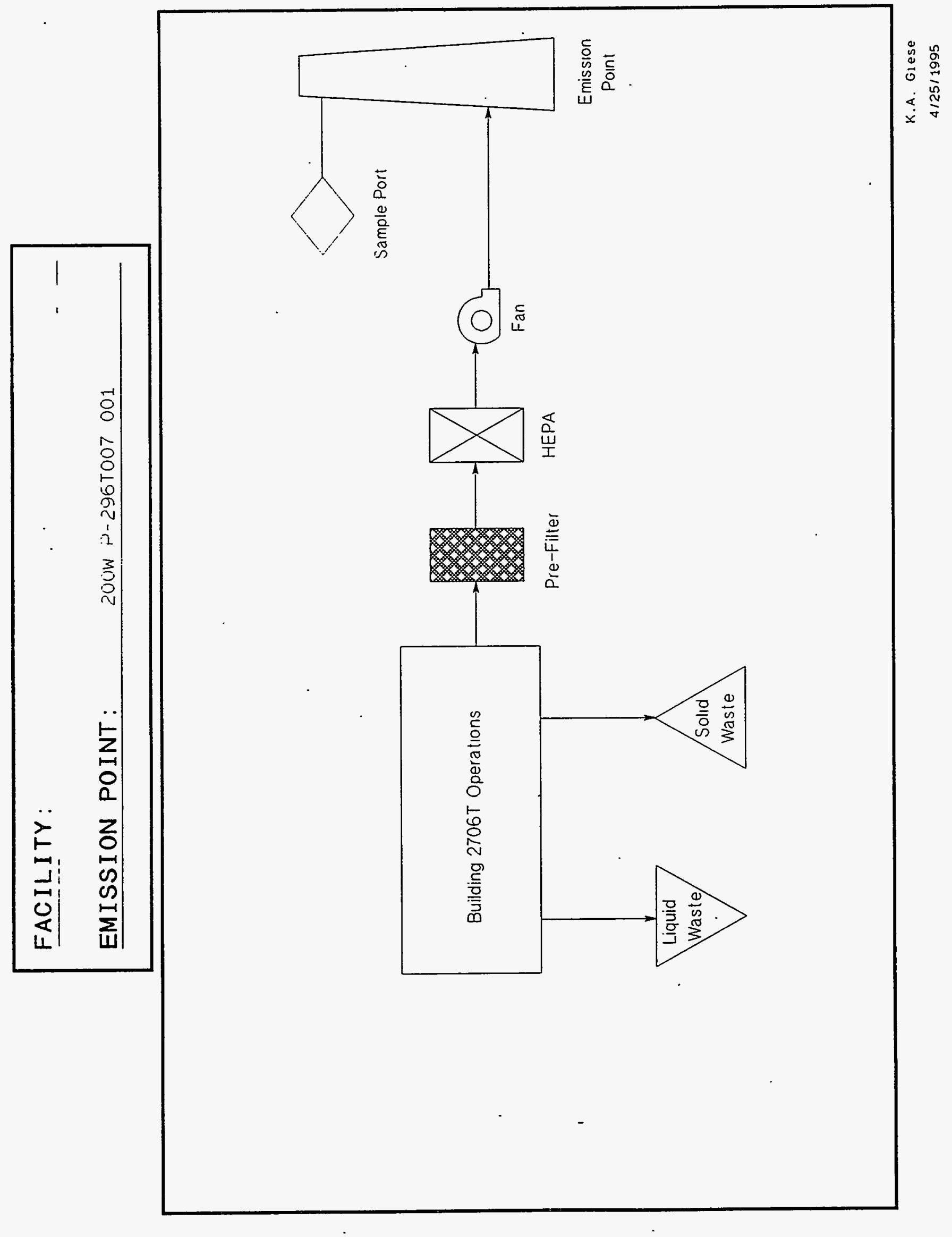




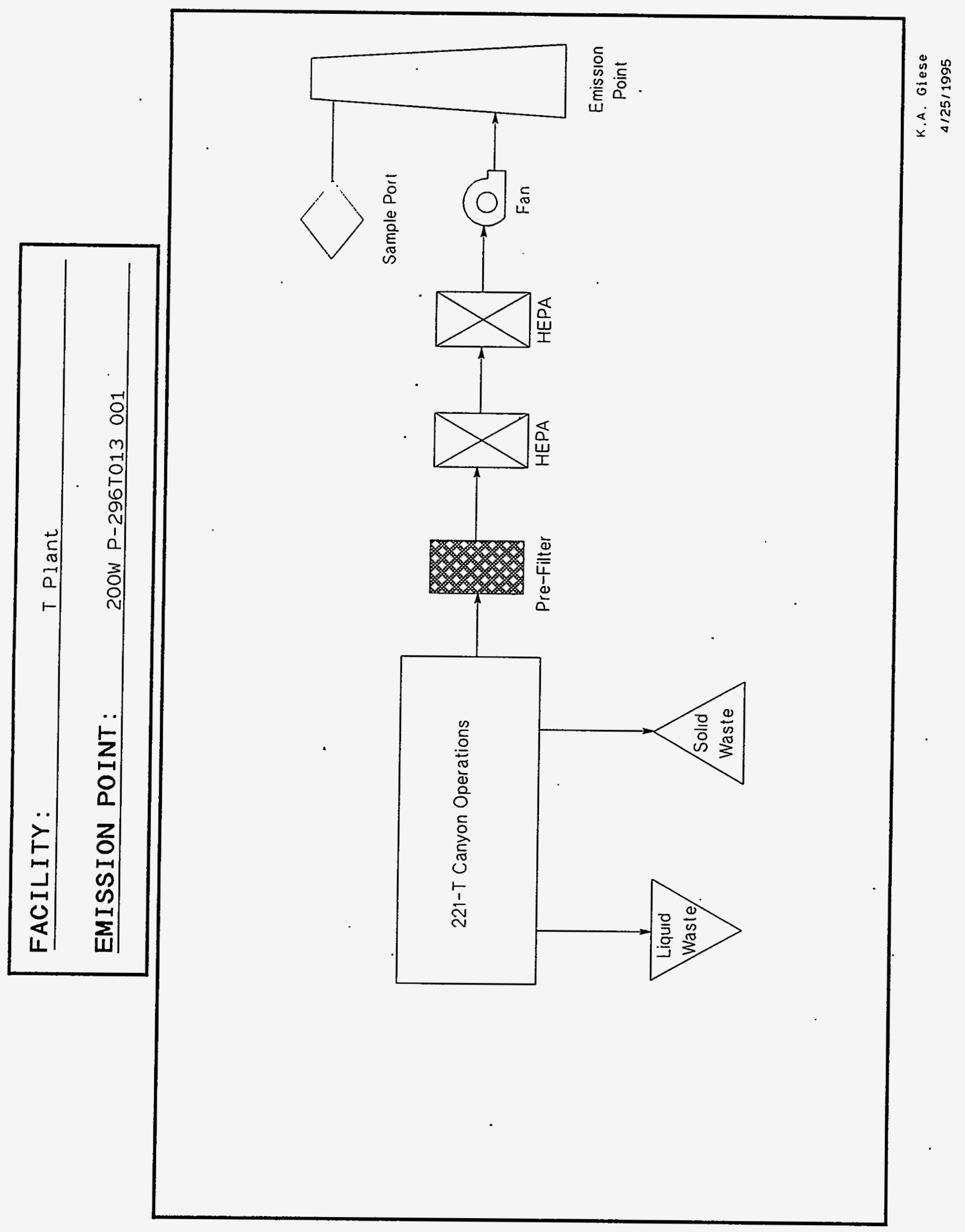




\section{T-Plant Emission Points}

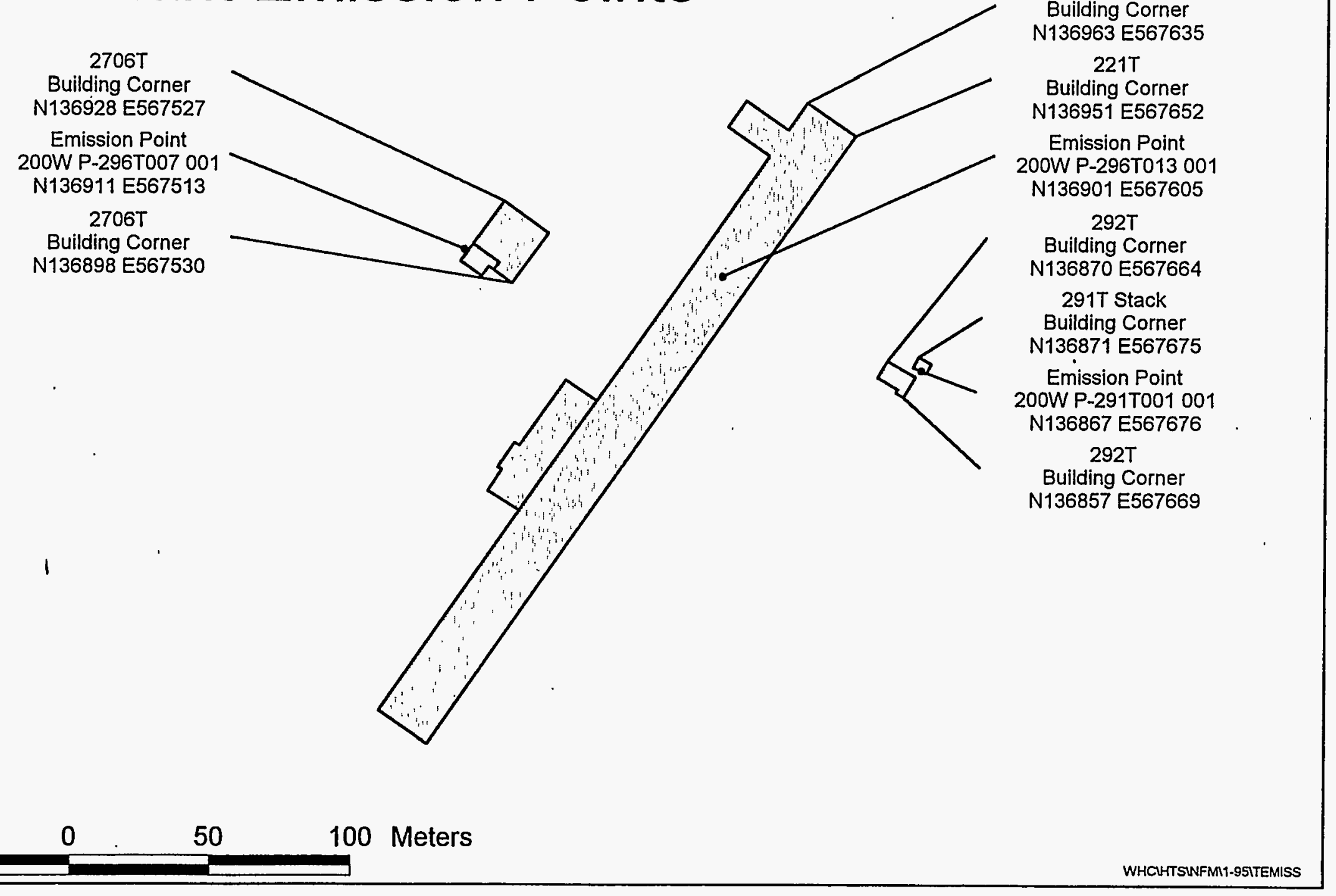




\section{APPENDIX E-6}

\section{U PLANT}

\section{Chapter I. General Information}

\section{I-B.1. Facility Description}

The U Plant is located in the 200 West Area. The reinforced concrete canyon building (221-U) originally was constructed from 1944-1945 as an integral part of the U Plant fuel separation facility, but was never used for that purpose. From 1950 through 1952, U Plant was extensively modified for a uranium metal recovery program. Modification included all new cell process equipment in the canyon building.

- From 1952 through 1958, the 221-U canyon building recovered uranium from high-level underground storage tank waste that contained liquid from the bismuth phosphate process (B Plant). The process involved solvent extraction using tributyl phosphate for the separation and decontamination of the uranium.

All process activities at U Plant ceased in 1964. Presently, the canyon is used as a radioactive confinement area for the storage of used process equipment from several processing plants onsite. The U Plant is scheduled for decommissioning in 2024.

\section{I-B.2. Products, Raw Materials, Fuels, Processes}

There are no products, raw materials, fuels, or processes at U Plant.

\section{I-C.1. Emission Points, Controls, Stacks}

The emission point and stack information is found on page 1 of the AEI report output.

\section{I-C.2. Facility Process Flow Diagram}

Attached.

I-C.3. Site Map

Attached.

\section{Chapter II. Regulatory Requirements}

\section{II-A.1. Description of Discharge and Emission Points}

The emission point and stack information is found on page 1 of the AEI report output. 
This page intentionally left blank. 
AIR EMISSIONS INVENTORY PERMIT APPLICATION REPORT (UP460) FACILITY ID : U PLANT
FACILITY NAME: Surplus Facilities

\section{Facility Building List}

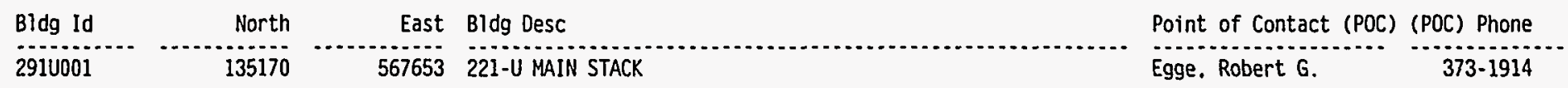

\section{Emission Point Listing}

\begin{tabular}{|c|c|c|c|c|c|c|c|}
\hline Em Point & Ep Tyре & North & East Srce Nbr & r Scc Code & Srce Type & & \\
\hline $200 W$ P-291U001 001 & POINT & 135170 & 567653 & 1 & PROCESS AND PRODUCTION & & \\
\hline Stack Configuration & & Diameter $(f t)$ & Length( $f t)$ & Area $(s q \mathrm{ft})$ & Exit Temp(F) Exit Veloc(ft/sec) & Ht Abv Grnd(ft) & Ht Abv Struc(ft) \\
\hline VERTICAL WITHOUT WEA & THER CAP & 3.75 & & 11.04 & 68 & 200 & \\
\hline
\end{tabular}

\section{Source (Process) Listing}

Em Point Srce Nbr Srce Type 200W P-2910001 001
Hrs Day Days Week Wks Year Summary Process Description 1 PROCESS AND PRODUCTION $\begin{aligned} & \text { Process } \ldots . .> \\ & \text { Description }\end{aligned}$
U-PLANT SERVES AS A STORAGE FACILITY FOR CONTAMINATED EQUIPMENT FROM AROUND THE HANFORD SITE. FINAL $0 \&$ D IS SCHEDULEO TO BEGIN IN 2023.

\section{Source Raw Materials Usage}

Em Point Srce Nbr Srce Type Process Rate Process Uom Material

\section{Fuels Usage by Source Processes}

Em Point Srce Nbr Srce Type Qty Uom Fuel

\section{Fuels Usage by Control Devices}

Em Point Ctl Nbr Ctl Type Qty Uom Fuel 


\section{Facility Emissions}

Point Source Emissions - Radiological Pollutants

Em Point

Srce Nbr Srce Type

1 PROCESS AND PRODUCTION
Em. Pt. Potential

to Emit ( $\mathrm{C} i / \mathrm{yr}$ )

Em. Pt

Ct) Actual(Ci/yr) Pollutant

$$
\begin{array}{ll}
\text { Refer to } & \text { Refer to } \\
\text { WHC-SD-EN-RPT-007 } & \text { DOE/RL-94-51 } \\
\text { Rev. 0 Page A- 297 } & \text { Table 2-1 }
\end{array}
$$

Point Source Emissions - HAP Pollutants

Em Point

Srce Nbr Srce Type

Em. Pt. Potential

to Emit (tons/yr)

Em. Pt.

Ctl Actual Yr Pollutant

Sum

Point Source Emissions - Uncategorized Pollutants

Em Point

Srce Nbr Srce Type

Em. Pt. Potential Em. Pt.

to Emit (tons/yr) cti Actual (tons/yr) Pollutant

Sum

Fugitive Source Emissions - Radiological Pollutants

Em Point

Srce Nbr Srce Type

Em. Pt. Potential

to Emit (ci/yr)

Em. Pt

Ctl Actuai (ci/yr) Pollutant

Fugitive Source Emissions - HAP Pollutants

Em Point

Srce Nbr Srce Type

Sum

Fugitive Source Emissions - Uncategorized Pollutants

Em Point

Srce Nbr Srce Type

Sum
Em. Pt. Potential Em. Pt.
to Emit (tons/yr) ctl Actual (tons/yr) Pollutant t

\section{Em. Pt. Potential Em. Pt.}

to Emit (tons/yr) ctl Actual (tons/yr) Pollutant 


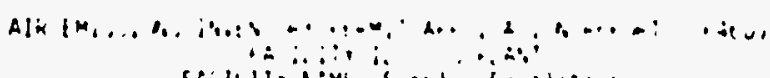

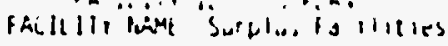

Insignificant Source Emissions - Radiological Pollutants

Em Point

Srce Nbr Srce Type

Em. Pt. Potential . Em. Pt.
to Emit (ci/yr)
Ctl Actual (ci/yr) Pollutant

Insignificant Source Emissions - HAP Pollutants

Em Point $\quad$ Srce Nbr Srce Type

$$
\begin{aligned}
& \text { Em. Pt. Potential Em. Pt. } \\
& \text { to Emit (tons/yr) cti Actua) (tons/yr) Pollutant }
\end{aligned}
$$

Sum

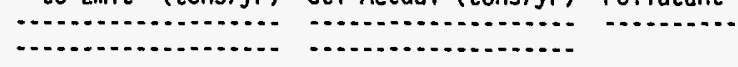

Insignificant Source Emissions - Uncategorized Pollutants

Em Point

Srce Nbr Srce Type

Em. Pt. Potential Em. Pt.

to Emit (tons/yr) Ctl Actual (tons/yr) Pollutant

Sum

\section{Control Device Listing}

Em Point Control Equipment Code

200W P-2910001 001 063

\section{Monitors Listing}

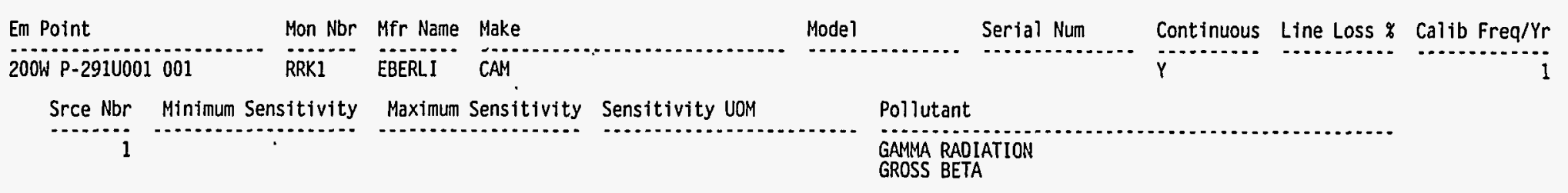




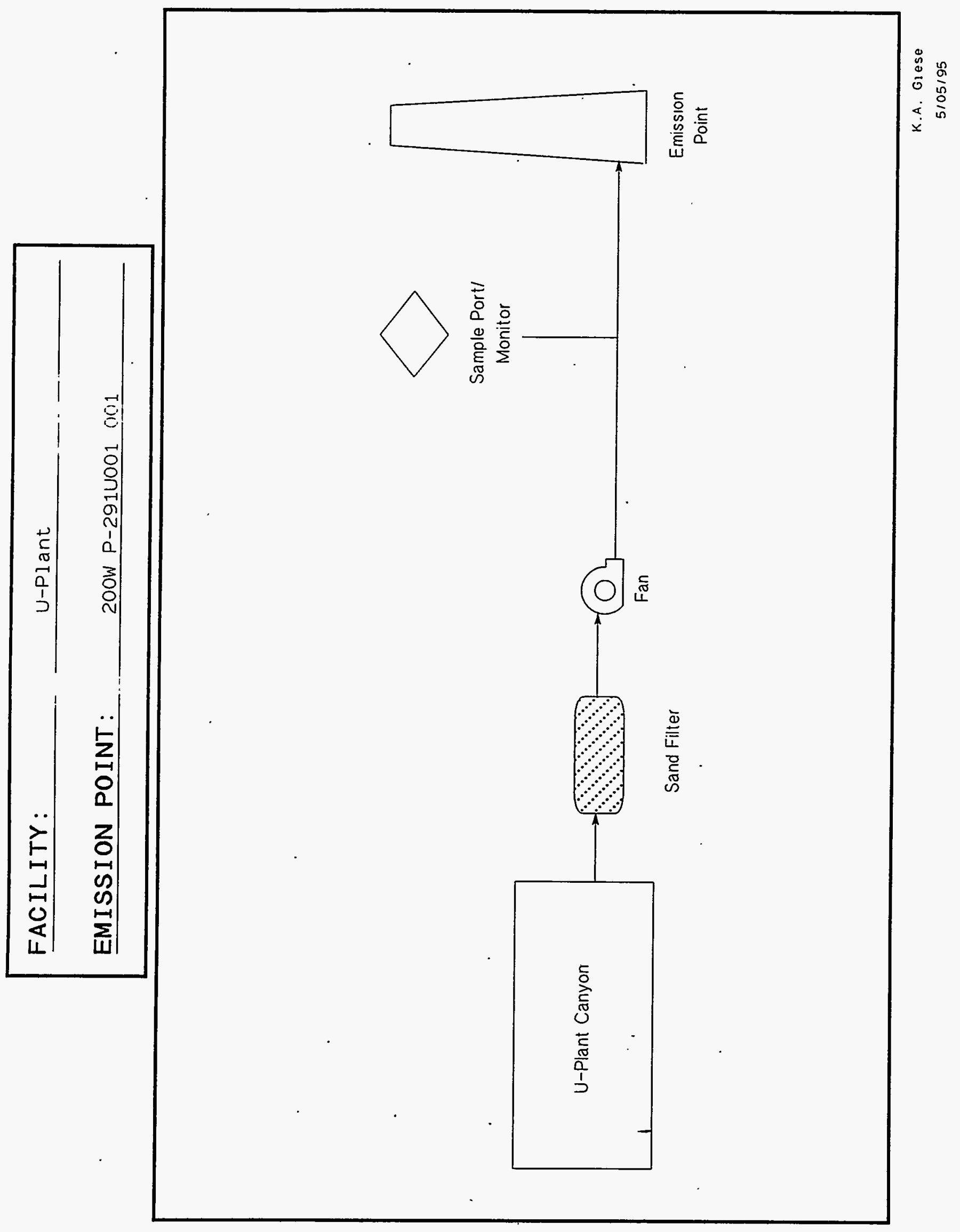




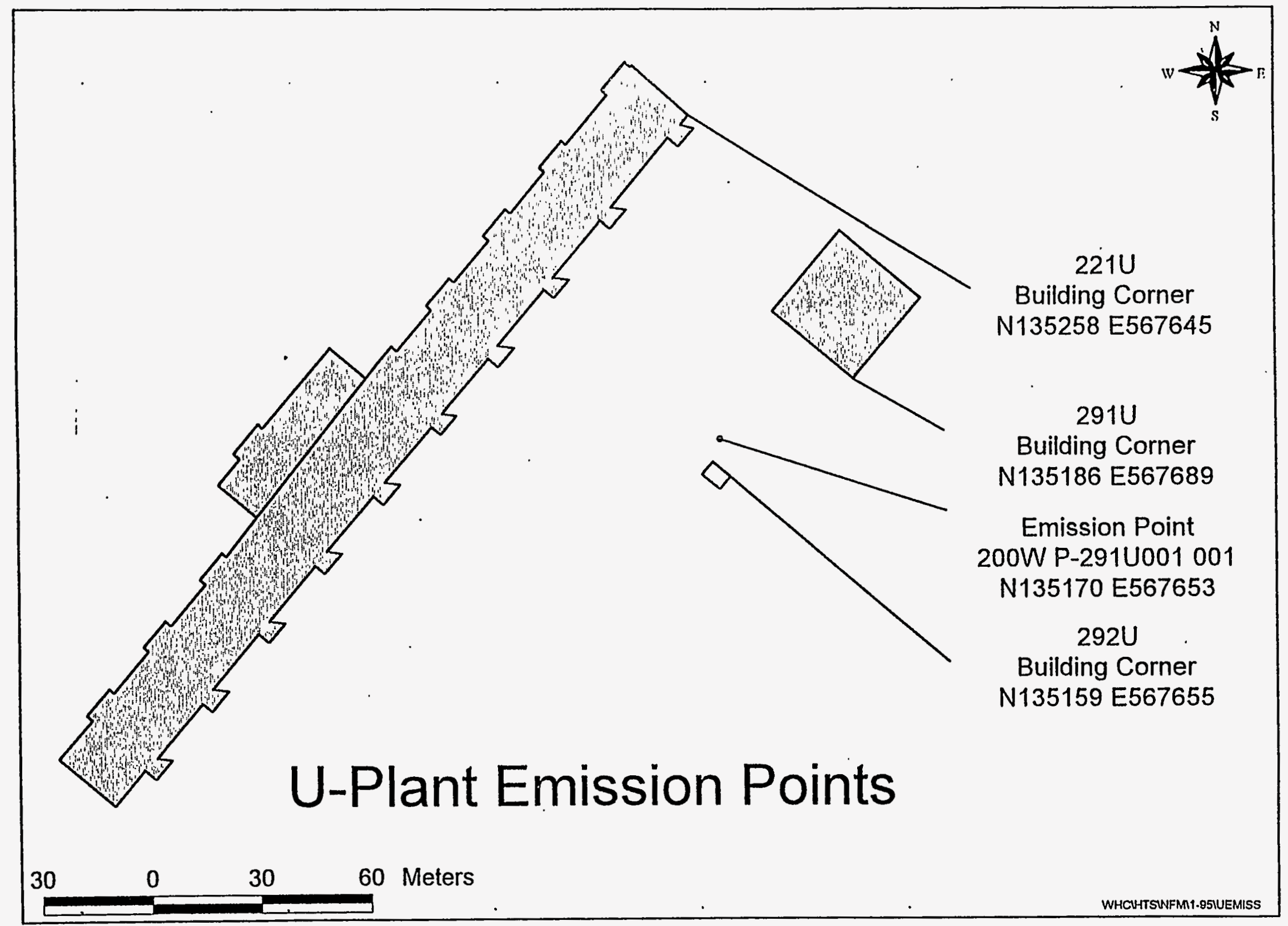




\section{APPENDIX E-7}

\section{WEST AREA TANK FARMS}

\section{Chapter I. General Information}

\section{I-B.1. Facility Description}

The tank farms initially were used for the transfer and storage of chemical and radiological wastes that were generated on the Hanford Site. Reprocessing plants performed chemical separations for the reprocessing of nuclear materials as well as radioactive waste storage and disposal. Operations at these plants, with the exception of shutdown and maintenance activities, have ceased. These shutdown and maintenance activities generate wastes that are transferred to the 200 West Area Tank Farms.

There are two types of tanks in the 200 West Area tank farms, DSTs and SSTs. There also are support operations associated with the 200 West Area Tank Farms such as receiving tanks.

The DST System treats and stores mixed waste before final treatment and disposal. The DST System consists of DCRTs and ancillary equipment. The DSTs consist of 1-million gallon tanks designed for long-term storage (up to 50 years) of mixed waste. There is one DST Farm located in the 200 West Area with a total of three tanks. Liquid waste generated in the 200 Areas generally is transferred through underground pipelines and routed via DCRTs. The DCRTs serve various functions including receiver tanks for SST or DST waste, lift stations, and vent stations. The exhaust fans on the tank ventilation systems in the DCRTs keep a negative air pressure, which is monitored, inside the primary tank. The exhausted air passes through a heater, prefilter, HEPA filters, and is monitored for radiation. No offsite waste is accepted for storage in the DST System. The DSTs use separate ventilation systems for the primary tank and annulus ventilation. The primary tank ventilation system provides a negative pressure in the tanks to prevent the escape of untreated dangerous and/or radioactive particulates to the atmosphere, removes heat generated by the tank contents, and removes water vapor to prevent tank corrosion. Additional tank space is used to support the environmental restoration and remediation mission (e.g., closure of SSTs).

There are six SST Farms with a total of 83 tanks within the 200 West Area. Past-practice units are associated with the SSTs. The SST volumes vary from 55,000 to 1 million gallons. Addition of new waste to the SSTs was discontinued in November 1980. The SSTs currently are being interim stabilized and isolated. Interim stabilization involves the removal of supernate and interstitial liquid from the SSTs to minimize the spread of contamination if the tanks begin to leak. Interim isolation of a SST involves physical modifications to preclude the inadvertent addition of liquid to the tank. Forced ventilation provides cooling for some tanks containing materials which, through radioactive decay, generate heat that could exceed 
the established concrete temperature limits. Single-stage HEPA filters allow atmospheric breathing for tanks that do not require cooling. The SSTs contain mostly inorganic waste.

In addition to the SST and DST waste tank system emission points described in this appendix, there are numerous other sources from the SST and DST emission points that are potential sources of emissions. These points include access risers, pump pits, and diversion boxes on each waste tank. These points are normally covered or closed to control emissions from the waste tanks. Routine maintenance and operation activities performed periodically at each waste tank requires the cover blocks or other tank closures to be opened temporarily to allow access to the instrumentation and equipment located within each waste tank. These periods may allow unfiltered air exchanges between the waste tank vapor space or surface contamination areas with the environment. These emissions are considered fugitive emissions and constitute a portion of the radionuclide fugitive emissions from the Hanford Site described in Section IV.B.6.

The 200 West Area tank farms are operated for 24 hours a day, 365 days a year.

\section{I-B.2. Products, Raw Materials, Fuels, Processes}

- Principle products - There are no products produced at the 200 West Tank Farms.

- Process information - There are no process-related activities at the 200 West Tank Farms.

- Raw materials used by processes - There are no raw materials used at the 200 West Area Tank Farms.

- Fuels used by processes - There are no fuels used at the 200 West Area Tank Farms.

\section{I-C.1. Emission Points, Controls, Stacks}

The emission point and stack information is found on pages 4 through 19 of the AEI report output. The control information is found on page 78 of the AEI report output.

\section{I-C.2. Facility Process Flow Diagrams}

Attached.

\section{I-C.3. Site Map}

Attached. 


\section{Chapter II. Regulatory Requirements}

\section{II-A.1. Description of Discharge and Emission Points}

The emission point and stack information is found on pages 4 through 19 of the AEI report output. 
Facility Building List

\begin{tabular}{|c|c|c|c|c|c|c|c|}
\hline Bldg Id & North & East & B]dg Desc & Custodian(Contractor) & Cust Phone & DOE POC & DOE Phone \\
\hline 241S101 & 134503 & 566835 & \multirow{24}{*}{ 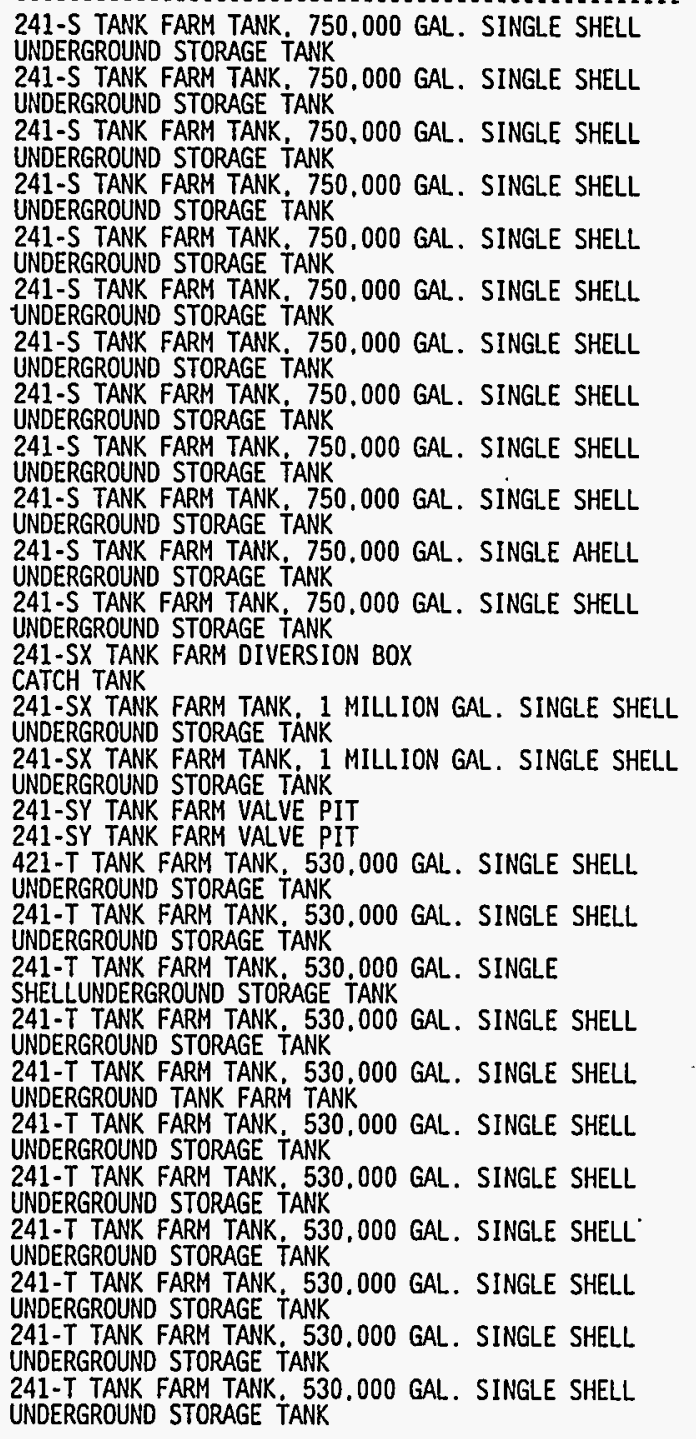 } & LEE. JOHN L. & $373-9372$ & CASEY O. RUUD & $373-3478$ \\
\hline $241 S 102$ & 134503 & 566804 & & LEE, JOHN L. & $373-9372$ & CASEY 0. RUUD & $373-3478$ \\
\hline 2415103 & 134503 & 566773 & & LEE, JOHN L. & $373-9372$ & CASEY O. RUUD & $373-3478$ \\
\hline 2415104 & 134472 & 566835 & & LEE, JOHN L. & $373-9372$ & CASEY O. RUUD & $373-3478$ \\
\hline 2415105 & 134472 & 566804 & & LEE, JOHN L. & $373-9372$ & CASEY 0. RUUD & $373-3478$ \\
\hline $241 S 106$ & 134472 & 566773 & & LEE, JOHN L. & $373-9372$ & CASEY 0. RUUD & $373-3478$ \\
\hline $241 \$ 107$ & 134441 & 566835 & & LEE. JOHN L. & $373-9372$ & CASEY 0. RUUD & $373-3478$ \\
\hline $241 S 108$ & 134441 & 566804 & & LEE, JOHN L. & $373-9372$ & CASEY 0. RUUD & $373-3478$ \\
\hline 2415109 & 134441 & 566773 & & LEE. JOHN L. & $373-9372$ & CASEY 0. RUUD & $373-3478$ \\
\hline 2415110 & 134410 & 566835 & & LEE, JOHN L. & $373-9372$ & CASEY 0. RUUD & $373-3478$ \\
\hline 2415111 & 134410 & 566804 & & LEE, JOHN L. & $373-9372$ & CASEY O. RUUD & $373-3478$ \\
\hline 2415112 & 134410 & 566773 & & LEE, JOHN L. & $373-9372$ & CASEY 0. RUUD & $373-3478$ \\
\hline $\begin{array}{l}241 S 151 \\
241 S 304 \\
2415 \times 113\end{array}$ & $\begin{array}{l}134320 \\
134336 \\
134173\end{array}$ & $\begin{array}{l}566901 \\
566923 \\
566836\end{array}$ & & $\begin{array}{l}\text { LEE, JOHN L. } \\
\text { LEE, JOHN L. } \\
\text { LEE, JOHN L. }\end{array}$ & $\begin{array}{l}373-9372 \\
373-9372 \\
373-9372\end{array}$ & 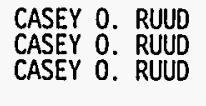 & $\begin{array}{l}373-3478 \\
373-3478 \\
373-3478\end{array}$ \\
\hline $241 S \times 115$ & 134173 & 566774 & & LEE, JOHN L. & $373-9372$ & CASEY 0. RUUD & $373-3478$ \\
\hline $\begin{array}{l}241 S Y A \\
241 S Y B \\
241 T 101\end{array}$ & $\begin{array}{l}134524 \\
134524 \\
136765\end{array}$ & $\begin{array}{l}566864 \\
566864 \\
566838\end{array}$ & & $\begin{array}{l}\text { LEE. JOHN L. } \\
\text { LEE. JOHN L. } \\
\text { LEE. JOHN L. }\end{array}$ & $\begin{array}{l}373-9372 \\
373-9372 \\
373-9372\end{array}$ & $\begin{array}{l}\text { CASEY } 0 . \text { RUUD } \\
\text { CASEY } 0 \text {. RUUD } \\
\text { CASEY } 0 \text {. RUUD }\end{array}$ & $\begin{array}{l}373-3478 \\
373-3478 \\
373-3478\end{array}$ \\
\hline $241 T 102$ & 136765 & 566807 & & LEE, JOHN L. & $373-9372$ & CASEY 0. RUUD & $373-3478$ \\
\hline 241T103 & 135765 & 566777 & & LEE, JỌN L. & $373-9372$ & CASEY 0. RUUD & $373-3478$ \\
\hline $241 T 104$ & 136734 & 566838 & & LEE. JOHN L. & $373-9372$ & CASEY 0. RUUD & $373-3478$ \\
\hline 241T105 & 136734 & 566807 & & LEE, JOHN L. & $373-9372$ & CASEY 0. RUUD & $373-3478$ \\
\hline 241T106 & 136734 & 566777 & & LEE, JOHN L. & $373-9372$ & CASEY 0. RUUD & $373-3478$ \\
\hline 241T107 & 136704 & 566838 & & LEE. JOHN L. & $373-9372$ & CASEY O. RUUD & $373-3478$ \\
\hline $241 T 108$ & 136704 & 566807 & & LEE, JOHN L. & $373-9372$ & CASEY O. RUUD & $373-3478$ \\
\hline 241T109 & 136704 & 566777 & & LEE. JOHN L. & $373-9372$ & CASEY O. RUUD & $373-3478$ \\
\hline $241 T 110$ & 136673 & 566838 & & LEE, JOHN L. & $373-9372$ & CASEY O. RUUD & $373-3478$ \\
\hline
\end{tabular}




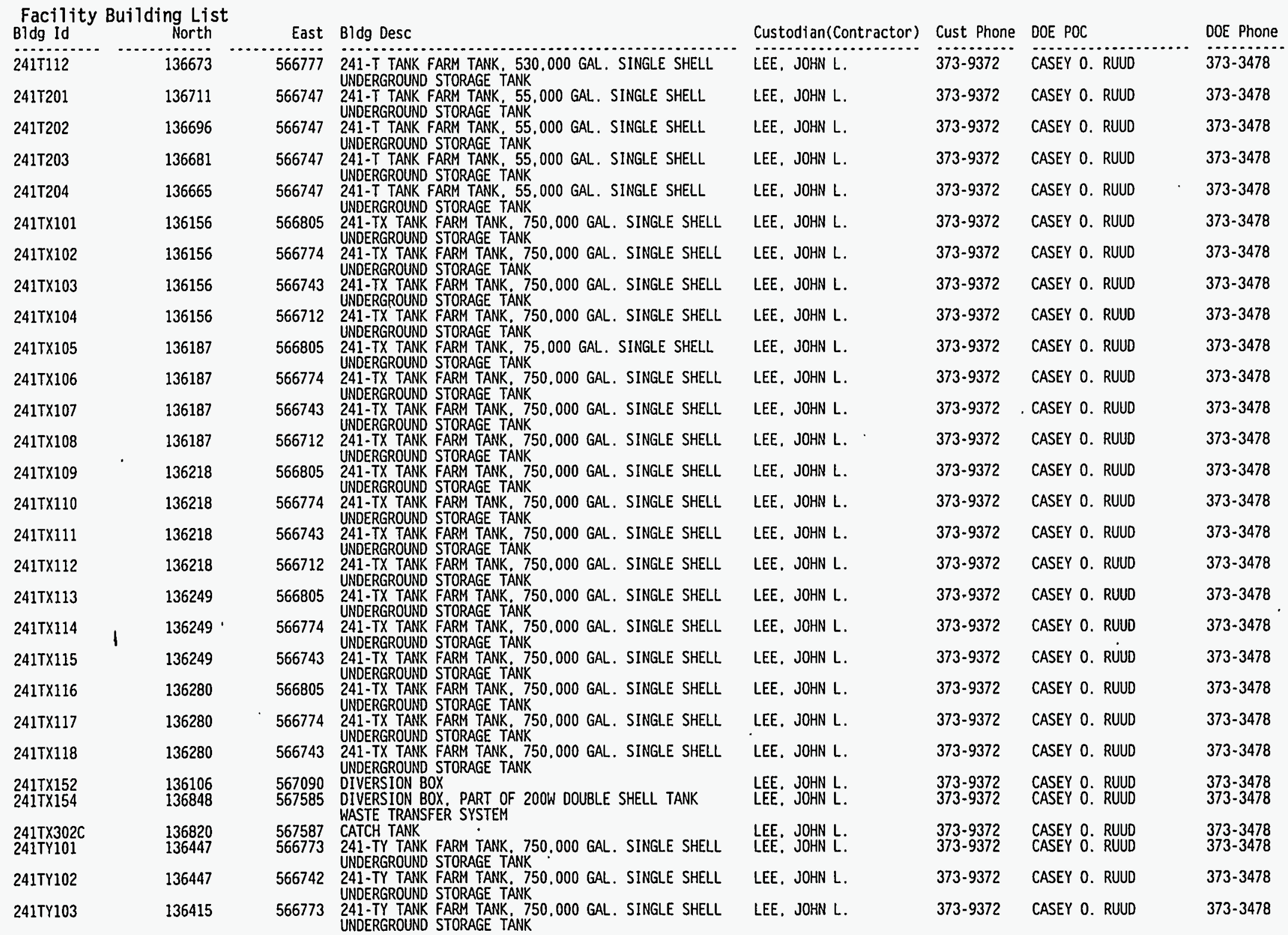




\begin{tabular}{|c|c|c|c|c|c|c|c|}
\hline $\begin{array}{l}\text { Facility } \\
\text { Bldg Id }\end{array}$ & Building List & East & Bldg Desc & Custodian(Contractor) & Cust Phone & DOE POC & DOE Phone \\
\hline $241 T Y 104$ & 136415 & 566742 & 241-TY TANK FARM TANK 750.000 GAL. SINGLE SHELL & LEE, JOHN L. & 373-9372 & CASEY 0 . RUUD & 373-3478 \\
\hline 241TY105 & 136384 & 566773 & 241-TY TANK FARM TANK 750,000 GAL. SINGLE SHELL & LEE, JOHN L. & 373-9372 & CASEY 0. RUUD & 373-3478 \\
\hline 241TY106 & 136384 & 566742 & $\begin{array}{l}241-\text { TY TANK FARM TANK } 750.000 \text { GAL. SINGLE SHELL } \\
\text { INDERGROUND STOBGE TONK }\end{array}$ & LEE, JOHN L. & 373-9372 & CASEY O. RUUD & $373-3478$ \\
\hline 241U101 & 135104 & 566842 & 241-U TANK FARM TANK, 530,000 GAL. SINGLE SHELL & LEE, JOHN L. & 373-9372 & CASEY 0. RUUD & 373-3478 \\
\hline 2410102 & 135104 & 566812 & $\begin{array}{l}241-U \text { TANK FARM TANE. } 530.000 \text { GAL. SINGLE SHELL } \\
\text { UNDERGROUND STORAGE TANK }\end{array}$ & LEE, JOHN L. & $373-9372$ & CASEY 0. RUUD & $373-3478$ \\
\hline 2410103 & 135104 & 566781 & 241-U TANK FARM TANK, 530.000 GAL. SINGLE SHELL & LEE. JOHN L. & 373-9372 & CASEY 0 . RUUD & $373-3478$ \\
\hline 2410104 & 135073 & 566842 & 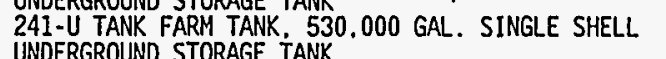 & LEE, JOHN L. & $373-9372$ & CASEY 0. RUUD & 373-3478 \\
\hline 2410105 & 135073 & 566812 & 241-U TAN FARM TANK 550.000 GAL. SINGLE SHELL & LEE, JOHN L. & 373-9372 & CASEY 0. RUUD & $373-3478$ \\
\hline 2410106 & 135073 & 566781 & 241-U TAN FARM TANK 530,000 GAL. SINGLE SHELL & LEE, JOHN L. & 373-9372 & CASEY 0. RUUD & 373-3478 \\
\hline 2410107 & 135043 & 566842 & $\begin{array}{l}241-U \text { TAK FARM TANK } 530,000 \text { GAL. SINGLE SHELL } \\
\text { UNDERGROUND STORAGE TANK }\end{array}$ & LEE, JOHN L. & 373-9372 & CASEY 0. RUUD & $373-3478$ \\
\hline 2410108 & 135043 & 566812 & 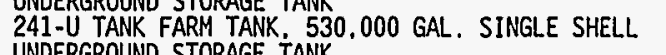 & LEE, JOHN L. & 373-9372 & CASEY 0. RUUD & $373-3478$ \\
\hline 2410109 & 135043 & 566781 & $\begin{array}{l}241-1 \text { TAAK FARM TANK } 530,000 \text { GAL. SINGLE SHELL } \\
\text { UNDERGROUND STORAGE TANK }\end{array}$ & LEE, JOHN L. & 373-9372 & CASEY 0. RUUD & $373-3478$ \\
\hline 2410110 & 135012 & 566842 & $\begin{array}{l}241-U \text { TANK FARM TANK } 530.000 \text { GAL. SINGLE SHELL } \\
\text { UNDERGPOUND STORAGE TANK }\end{array}$ & LEE, JOHN L. & 373-9372 & CASEY 0. RUUD & 373-3478 \\
\hline 241U111 & 135012 & 566812 & 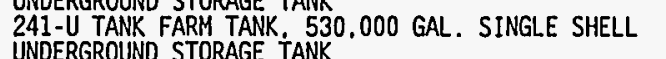 & LEE, JQHN L. & 373-9372 & CASEY 0. RUUD & 373-3478 \\
\hline 2410112 & 135012 & 566781 & 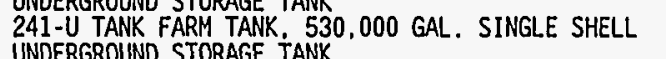 & LEE; JOHN L. & 373-9372 & CASEY 0. RUUD & $373-3478$ \\
\hline $\begin{array}{l}2410151 \\
2410152 \\
2410201\end{array}$ & $\begin{array}{l}134991 \\
134965 \\
135050\end{array}$ & $\begin{array}{l}566922 \\
566918 \\
566752\end{array}$ & 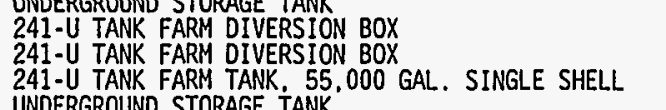 & $\begin{array}{l}\text { LEE, JOHN L. } \\
\text { LEE, JOHN L: } \\
\text { LEE, JOHN L. }\end{array}$ & $\begin{array}{l}373-9372 \\
373-9372 \\
373-9372\end{array}$ & $\begin{array}{l}\text { CASEY } 0 . \text { RUUD } \\
\text { CASEY } \\
\text { CASEY } \\
\text { C. RUUDD }\end{array}$ & $\begin{array}{l}373-3478 \\
373-3478 \\
373-3478\end{array}$ \\
\hline 241U202 & 135035 & 566752 & 241-U TANK FARM TANK 55.000 GAL. SINGLE SHELL & LEE. JOHN L. & $373-9372$ & CASEY 0. RUUD & 373-3478 \\
\hline 2410203 & 135020 & 566752 & $\begin{array}{l}241-1 \text { TANK FARM TANK, 55:000 GAL. SINGLE SHELL } \\
\text { UNDERGROUND STORAGE TANK }\end{array}$ & LEE, JOHN L. & 373-9372 & CASEY 0. RUUD & 373-3478 \\
\hline 2410204 & 135005 & 566752 & $\begin{array}{l}241-U \text { TAN FARM TANK, } 55,000 \text { GAL: SINGLE SHELL } \\
\text { UNDERGROND STORAGE TANK }\end{array}$ & LEE, JOHN L. & 373-9372 & CASEY 0. RUUD & 373-3478 \\
\hline $\begin{array}{l}24103018 \\
2410 \times 154\end{array}$ & $\begin{array}{r}85000 \\
135225\end{array}$ & $\begin{array}{l}600000 \\
567629\end{array}$ & $\begin{array}{l}\text { CACC TANK } \\
\text { DIRERSION BOX 2OOW DOUBLE SHELL TANK WASTE } \\
\text { TRANSEER SYSTEM }\end{array}$ & LEE, JOHN L. & $\begin{array}{l}373-9372 \\
373-9372\end{array}$ & $\begin{array}{l}\text { CASEY 0. RUUD } \\
\text { CASEY 0. RUUD }\end{array}$ & $\begin{array}{l}373-3478 \\
373-3478\end{array}$ \\
\hline $\begin{array}{l}2410 \times 302 A \\
2425 \\
242 T \\
2445 \\
244 \times 1 \times \\
2440 \\
2725 \\
2724 \mathrm{~A} \\
296022 \\
296028 \\
2965025 \\
2965 X\end{array}$ & $\begin{array}{l}134977 \\
134603 \\
136317 \\
134415 \\
13652 \\
134991 \\
13451 \\
135816 \\
134525 \\
134543 \\
134543 \\
134754\end{array}$ & $\begin{array}{l}566780 \\
566792 \\
566842 \\
566915 \\
566823 \\
566865 \\
566710 \\
565918 \\
566870 \\
566878 \\
566887 \\
566182\end{array}$ & 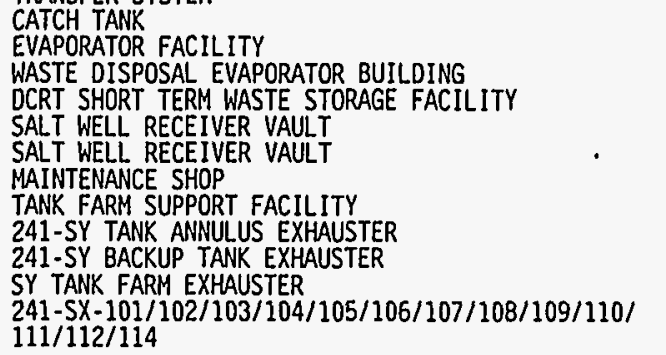 & 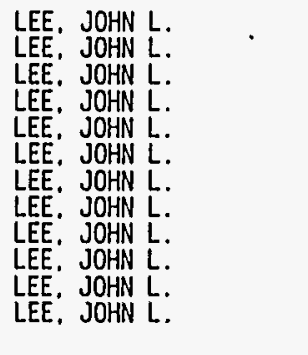 & $\begin{array}{l}373-9372 \\
373-9372 \\
373-9372 \\
373-9372 \\
373-9372 \\
373-9372 \\
373-9372 \\
373-9372 \\
373-99372 \\
373-9372 \\
373-9372 \\
373-9372\end{array}$ & $\begin{array}{l}\text { CASEY } 0 . \text { RUUD } \\
\text { CASEY O. RUUD } \\
\text { CASEY O. RUUD } \\
\text { CASEY O. RUUD } \\
\text { CASEY O. RUUD } \\
\text { CASEY O. RUUD } \\
\text { CASEY O. RUUD } \\
\text { CASEY O. RUUD } \\
\text { CASEY O. RUUD } \\
\text { CASEY O. RUUD } \\
\text { CASEY O. RUUD } \\
\text { CASEY }\end{array}$ & $\begin{array}{l}373-3478 \\
373-3478 \\
373-3478 \\
373-3478 \\
373-3478 \\
373-3478 \\
373-3478 \\
373-3478 \\
373-3478 \\
373-3478 \\
373-3478 \\
373-3478\end{array}$ \\
\hline
\end{tabular}




\section{Facility Building List}

\begin{tabular}{|c|c|c|c|c|c|c|c|}
\hline Bldg Id & North & East & Bldg Desc & Custodian(Contractor) & Cust Phone & DOE POC & DOE Phone \\
\hline $\begin{array}{l}2965 \mathrm{Y} \\
2960011\end{array}$ & $\begin{array}{l}134527 \\
134995\end{array}$ & $\begin{array}{l}566864 \\
566865\end{array}$ & $\begin{array}{l}241 \text {-SY TANK FARM PRIMARY VENTILATION STACK } \\
244-U \text { DOULE CONTAINMENT RECEIVER TANK (DCRT) } \\
\text { EXHAUSTER }\end{array}$ & $\begin{array}{l}\text { LEE, JOHN L. } \\
\text { LEE, JOHN L. }\end{array}$ & $\begin{array}{l}373-9732 \\
373-9372\end{array}$ & $\begin{array}{l}\text { CASEY } 0 . \text { RUUD } \\
\text { CASEY } 0 \text {. RUUD }\end{array}$ & $\begin{array}{l}373-3478 \\
373-3478\end{array}$ \\
\hline
\end{tabular}

\section{Emission Point Listing}

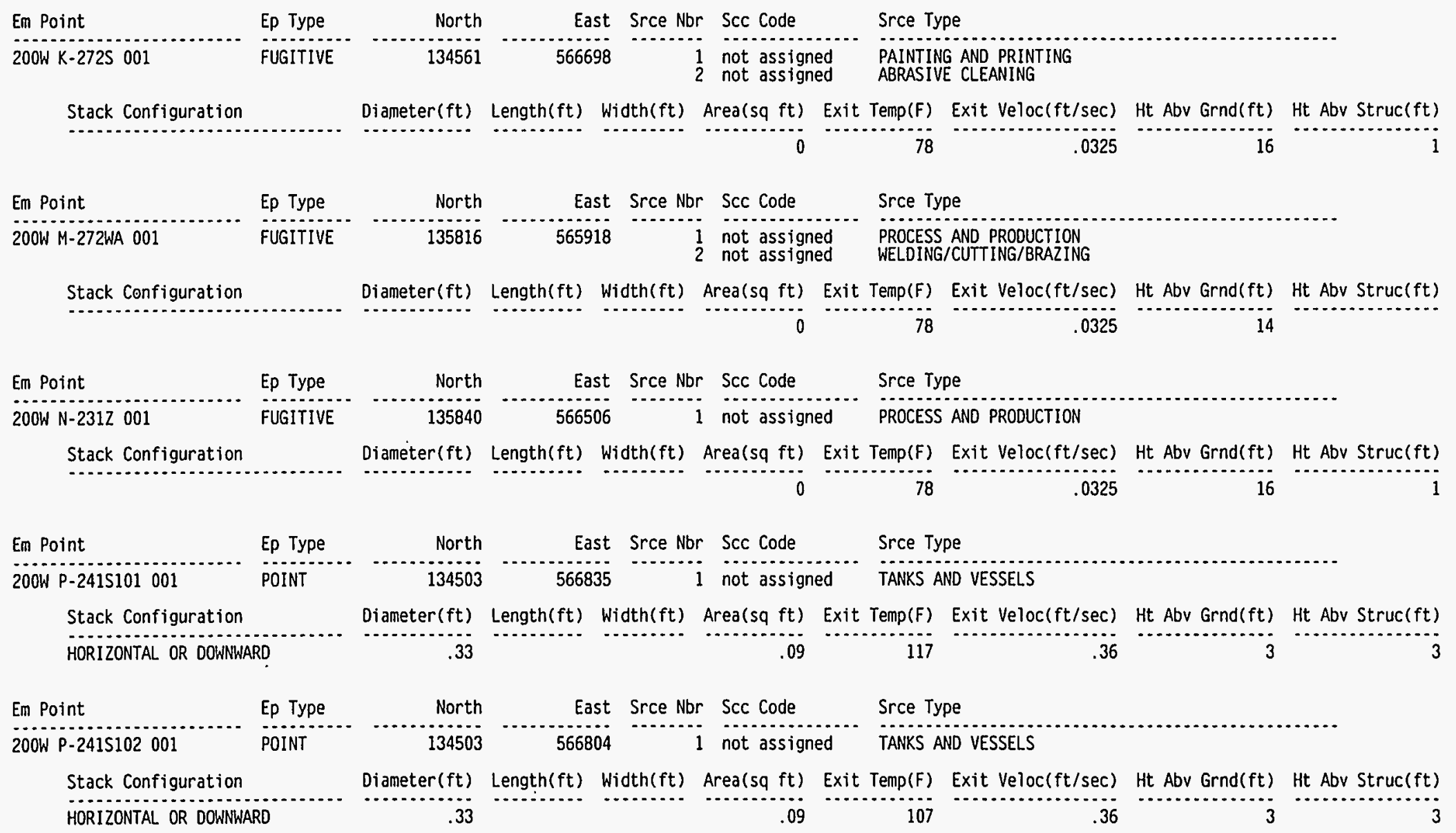


AIR EMISSIONS INVENTORY PERMIT APPLICATION REPORT (UP460)

\section{Emission Point Listing}

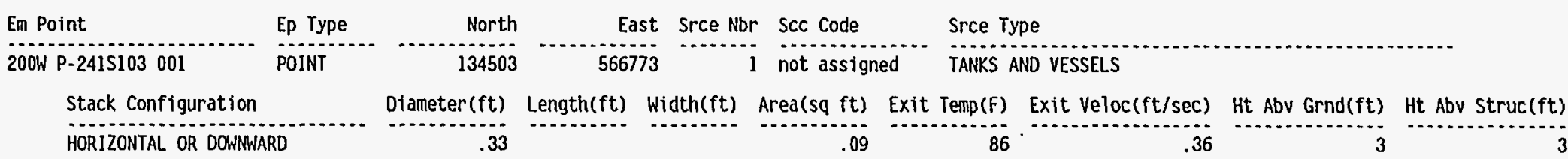

\begin{tabular}{ll} 
Em Point & Ep Type \\
\hline $200 \mathrm{~W}$ P-241S104 001 & POINT \\
\multicolumn{2}{c}{ Stack Configuration }
\end{tabular}

North East Srce Nbr Scc code Srce Type

Stack Configuration HORIZONTAL OR DOWWWARD

Diameter(ft) Length(ft) Width(ft) Area(sq ft) Exit Temp(F) Exit Veloc(ft/sec) Ht Abv Grnd(ft) Ht Abv Struc(ft)

Em Point
$200 \mathrm{~W}$ P-241S105 001 Type
Stack Configuration
HORIZONTAL OR DOWNWARD

North

33

106

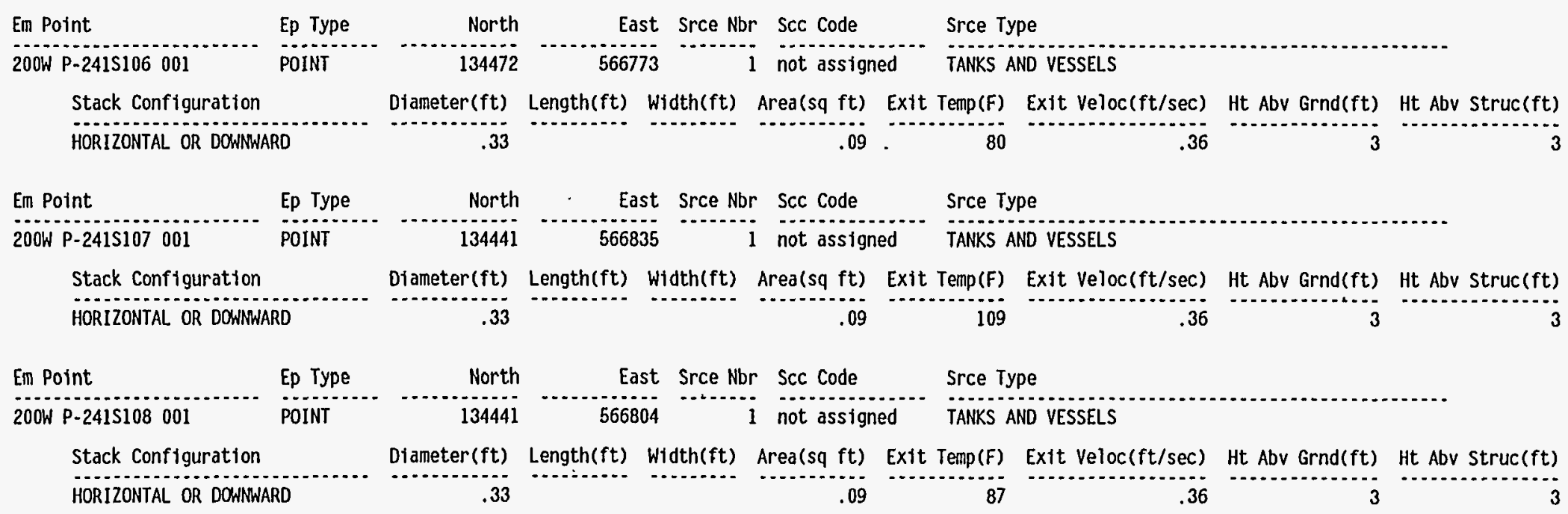




\section{Emission Point Listing}

\begin{tabular}{|c|c|c|c|c|c|c|c|c|}
\hline Em Point & Ep Type & North & Srce Nbr & r Scc Code & Srce Ty & & & \\
\hline $200 W$ P-241S109 001 & POINT & 134441 & 566773 & 1 not assigne & TANKS A & WND VESSELS & & \\
\hline Stack Configuration & & Diameter $(\mathrm{ft})$ & Length(ft) Width(ft) & $\operatorname{Area}(s q \mathrm{ft})$ & Exit $\operatorname{Temp}(F)$ & Exit $\operatorname{Veloc}(\mathrm{ft} / \mathrm{sec})$ & Ht Abv Grnd(ft) & Ht Abv Struc $(f t)$ \\
\hline HORIZONTAL OR DOWNWAR & & .33 & & .09 & 68 & .36 & 3 & 3 \\
\hline Em Point & Ep Type & North & Srce Nbr & $r$ Scc Code & Srce Ty & & & \\
\hline $200 \mathrm{~W}$ P-2415110 001 & POINT & 134410 & 566835 & 1 not assigne & TANKS A & AND VESSELS & & \\
\hline Stack Configuration & & Diameter $(\mathrm{ft})$ & Length(ft) Width(ft) & $\operatorname{Area}(s q \mathrm{ft})$ & Exit Temp(F) & Exit Veloc $(\mathrm{ft} / \mathrm{sec})$ & Ht Abv Grnd(ft) & Ht Abv Struc (ft) \\
\hline HORIZONTAL OR DOWNWA & & .33 & & .09 & 117 & .36 & 3 & \\
\hline
\end{tabular}

\begin{tabular}{|c|c|c|c|c|c|c|c|c|}
\hline Em Point & Ер Туре & North & Srce Nbr & r Scc Code & Srce $\mathrm{Ty}$ & & & \\
\hline $200 \mathrm{~W}$ P-2415111 001 & POINT & 134410 & 566804 & not assigne & TANKS A & AND VESSELS & & \\
\hline Stack Configuration & & Diameter (ft) & Length(ft) Width(ft) & $\operatorname{Area}(s q \mathrm{ft})$ & Exit Temp(F) & Exit Veloc $(\mathrm{ft} / \mathrm{sec})$ & Ht Abv Grnd(ft) & Ht Abv Struc(ft) \\
\hline HORIZONTAL OR DONNW & & .33 & & .09 & 92 & .36 & 3 & \\
\hline
\end{tabular}

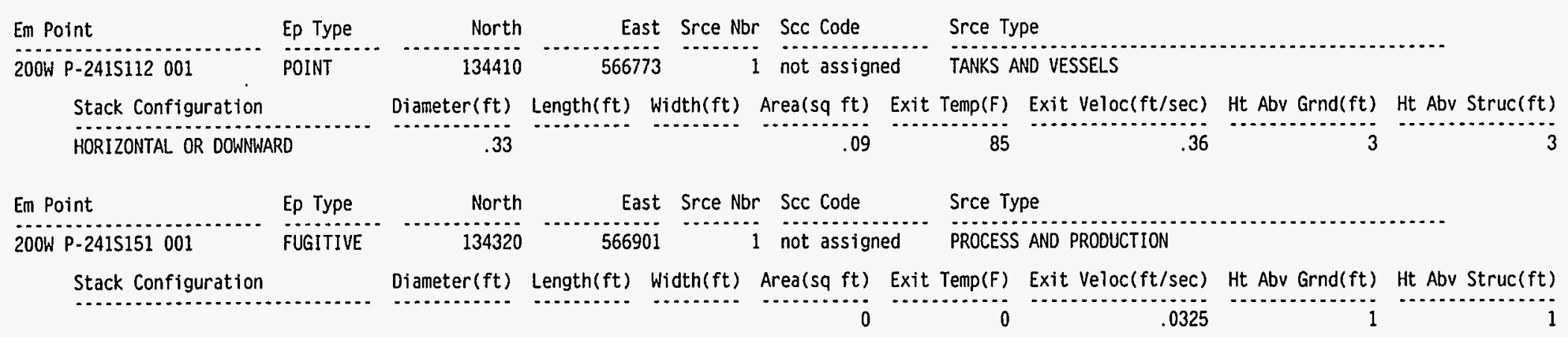

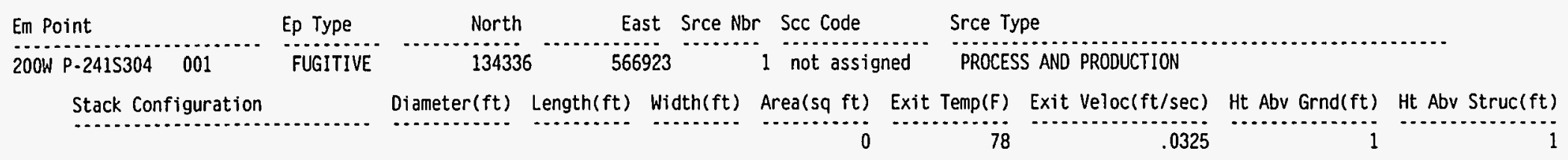




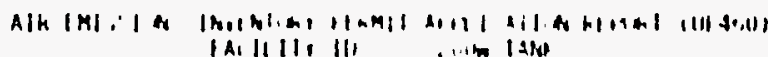

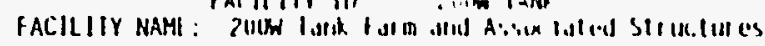

\section{Emission Point Listing}

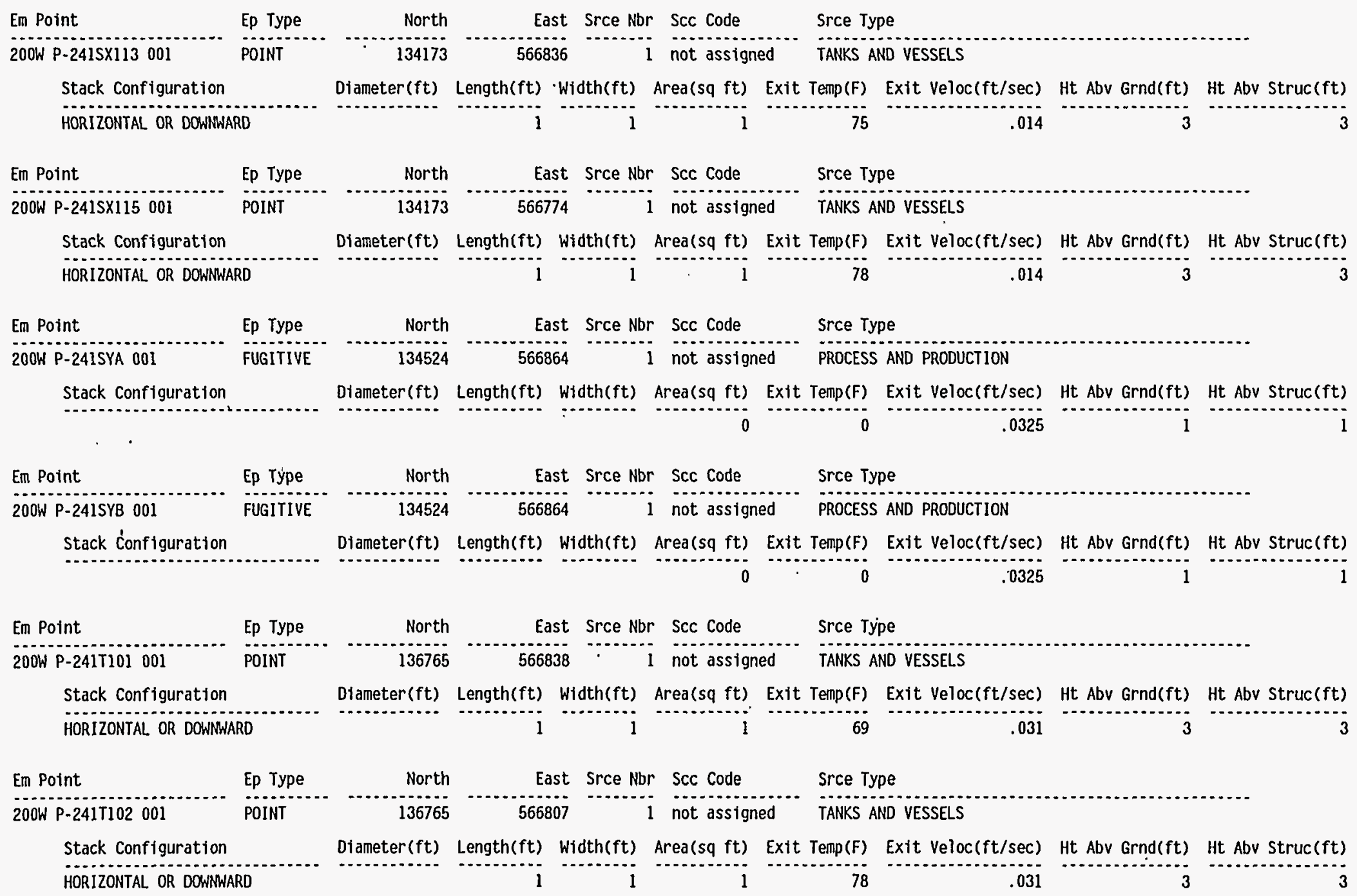


Emission Point Listing

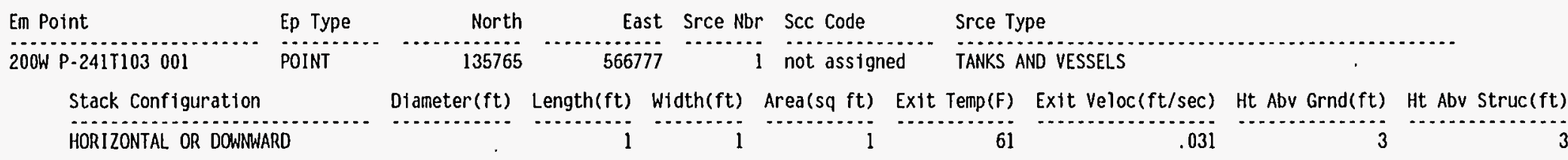

\begin{tabular}{|c|c|}
\hline Em Point & Ep Type \\
\hline $200 \mathrm{~W}$ P-241T104 001 & POINT \\
\hline
\end{tabular}

\begin{tabular}{|c|c|c|c|c|c|}
\hline North & Srce $\mathrm{Nbr}$ & Scc Code & Srce Type & & \\
\hline 136734 & 566838 & not assigned & TANKS AND VESSELS & & \\
\hline Diameter $(f t)$ & Length $(f t)$ & $\operatorname{Area}(s q \mathrm{ft})$ & Exit Veloc $(\mathrm{ft} / \mathrm{sec})$ & Ht Abv Grnd(ft) & Ht Abv Struc $(f t)$ \\
\hline & i & i & 014 & 3 & \\
\hline
\end{tabular}

Em Point 200W P-241T105 001

Ep Type
POINT

Stack Configuration HORIZONTAL OR DOWNARD D

North
136734

East Srce $\mathrm{Nbr}$ Scc Code

Diameter(ft) Length(ft) Width(ft) Area(sq ft) Exit Temp(F) Exit Veloc(ft/sec) Ht Abv Grnd(ft) Ht Abv Struc(ft)

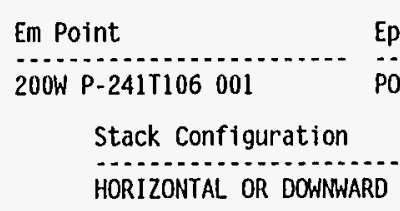

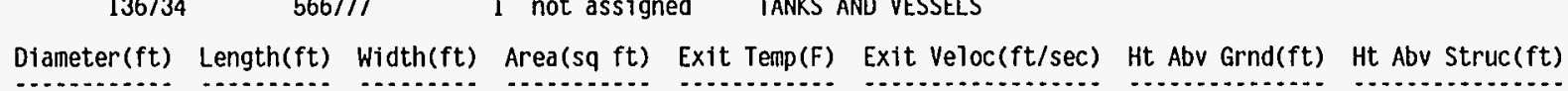
RD

\section{Em Point}

$200 \mathrm{~W}$ P-241T107 001

Ep Type POINT

Stack Configuration HORIZONTAL OR DOWNWARD

North
136704

Diameter(ft) Length(ft) Width(ft) Area(sq ft) Exit Temp(F) Exit Veloc(ft/sec) Ht Abv Grnd(ft) Ht Abv Struc(ft)

\section{Em Point}

Ep Type

200W P-241T108 001

POINT

North

East Srce Nbr Scc Code

....- -...... Srce Type

Stack Configuration

HORIZONTAL OR DOWNWARD
Diameter(ft) Length(ft) Width(ft) Area(sq ft) Exit Temp(F) Exit Veloc(ft/sec) Ht Abv Grnd(ft) Ht Abv $S t r u c(f t)$ 
AIR EMISSIONS INVENTORY PERMIT APPLICATION REPORT (UP460) FACILITY NAME: $200 \mathrm{~W}$ Tank Farm and Associated Structures

\section{Emission Point Listing}

\begin{tabular}{|c|c|c|c|c|c|c|c|c|c|}
\hline \multirow{2}{*}{$\begin{array}{l}\text { Em Point } \\
200 \mathrm{~W} \text { P-241T109 001 }\end{array}$} & \multirow{2}{*}{$\begin{array}{l}\text { Ep Type } \\
\text { POINT }\end{array}$} & \multirow{2}{*}{$\begin{array}{l}\text { North } \\
136704\end{array}$} & \multirow{2}{*}{\multicolumn{3}{|c|}{$\begin{array}{rrl}\text { East } & \text { Srce Nbr } & \text { Scc Code } \\
566777 & \ldots & \text { l not }\end{array}$}} & \multicolumn{4}{|c|}{ Srce Type } \\
\hline & & & & & & TANKS AI & ND VESSELS & & \\
\hline Stack Configuration & & Diameter $(f t)$ & Length(ft) Width(ft) & $\operatorname{Area}(s q \mathrm{ft})$ & Exit & Temp (F) & Exit veloc(ft/sec) & Ht Abv Grnd(ft) & Ht Abv Struc(ft) \\
\hline HORIZONTAL OR DOWNWAR & & & 1 & 1 & & 78 & 031 & 3 & 3 \\
\hline En Point & Ep Type & North & East Srce $\mathrm{Nbr}$ & Scc Code & & Srce Ty & & & \\
\hline $200 \mathrm{~W}$ P-241T110 001 & POINT & 136673 & 566838 & not assigne & & TANKS AI & ND VESSELS & & \\
\hline Stack Configuration & & Diameter $(f t)$ & Length(ft) Width(ft) & $\operatorname{Area}(s q f t)$ & Exit & Temp (F) & Exit Veloc $(\mathrm{ft} / \mathrm{sec})$ & Ht ADV Grnd(ft) & Ht Abv Struc(ft) \\
\hline HORIZONTAL OR DOWNWAR & & & $1 \quad 1$ & 1 & & 61 & 031 & 3 & 3 \\
\hline Em Point & Ep Type & North & East Srce Nbr & Scc code & & Srce Tyl & & & \\
\hline $200 \mathrm{~W}$ P-241T111 001 & POINT & 136673 & 566807 & not assigne & & TANKS Al & ND VESSELS & & \\
\hline Stack Configuration & & Diameter $(\mathrm{ft})$ & Length(ft) Width(ft) & $\operatorname{Area}(s q \mathrm{ft})$ & Exit & Temp $(F)$ & Exit Veloc $(\mathrm{ft} / \mathrm{sec})$ & Ht AbV Grnd(ft) & Ht Abv Struc $(f t)$ \\
\hline HORIZONTAL OR DOWNWAR & & & $1 \quad 1$ & 1 & & 61 & .031 & 3 & 3 \\
\hline Em Point & Ep Type & North & East srce $\mathrm{Nbr}$ & Scc Code & & Srce Tyl & pe & & \\
\hline $200 \mathrm{~W}$ P-241T112 001 & POINT & 136673 & 566777 & not assigne & & TANKS AI & ND VESSELS & & \\
\hline Stack Configuration & & Diameter $(\mathrm{ft})$ & Length(ft) Width(ft) & $\operatorname{Area}(s q \mathrm{ft})$ & Exit & Temp (F) & Exit Veloc(ft/sec) & Ht Abv Grnd(ft) & Ht Abv Struc $(f t)$ \\
\hline HORIZONTAL & & & $1 \quad 1$ & 1 & & 59 & .031 & 3 & 3 \\
\hline Em Point 1 & 'Ep Type & North & East Srce Nbr & Scc Code & & Srce Typ & & & \\
\hline $200 \mathrm{~W} P-241 T 201001$ & POINT & 136711 & 566747 & not assigne & & TANKS AI & ND VESSELS & & \\
\hline Stack Configuration & & Diameter $(\mathrm{ft})$ & Length(ft) Width(ft) & $\operatorname{Area}(s q \mathrm{ft})$ & Exit & Temp(F) & Exit $\operatorname{Veloc}(\mathrm{ft} / \mathrm{sec})$ & Ht Abv Grnd(ft) & Ht Abv Struc (ft) \\
\hline HORIZONTAL OR DOWNWAR & & & 1 & & & 60 & .014 & 3 & 3 \\
\hline Em Point & Ep Type & North & East Srce $\mathrm{Nbr}$ & Scc Code & & Srce Typ & & & \\
\hline 200W P-241T202 001 & POINT & 136696 & 566747 & not assigne & & TANKS AI & ND VESSELS & & \\
\hline Stack Configuration & & Diameter $(f t)$ & Length(ft) Wtdth(ft) & $\operatorname{Area}(s q \mathrm{ft})$ & Exit & Temp (F) & Exit veloc $(\mathrm{ft} / \mathrm{sec})$ & Ht Abv Grnd(ft) & Ht Abv Struc $(f t)$ \\
\hline HORIZONTAL OR DOWNWARI & & & $1 \quad 1$ & 1 & & 60 & .014 & 3 & \\
\hline
\end{tabular}


Emission Point Listing

\begin{tabular}{|c|c|c|c|c|c|c|c|c|}
\hline Em Point & Ep Type & North & East Srce Nbr & - Scc Code & & Srce Type & & \\
\hline $200 \mathrm{~W}$ P-241T203001 & POINT & 136681 & 566747 & not assigne & & TANKS AND VESSELS & & \\
\hline Stack Configuration & & Diameter $(f t)$ & Length(ft) Width(ft) & Area $(s q \mathrm{ft})$ & Exit & Temp(F) Exit Veloc(ft/sec) & Ht Abv Grnd(ft) & Ht Abv Struc $(f t)$ \\
\hline HORIZONTAL OR DOWNWAR & & & $\overline{1}$ & 1 & & .014 & 3 & 3 \\
\hline Em Point & Ep Type & North & Srce $\mathrm{Nbr}$ & Scc Code & & Srce Type & & \\
\hline 200W P-241T204001 & POINT & 136665 & 566747 & not assigne & & TANKS AND VESSELS & & \\
\hline Stack Configuration & & Diameter (ft) & Length(ft) Width(ft) & Area $(s q f t)$ & Exit & Temp(F) Exit Veloc (ft/sec) & Ht Abv Grnd(ft) & Ht Abv Struc(ft) \\
\hline HORIZONTAL OR DOWNWAR & & & 1 & 1 & & 62 & 3 & 3 \\
\hline Em Point & Ep Type & North & East Srce Nbr & Scc Code & & Srce Type & & \\
\hline $200 \mathrm{~W} P-241 \mathrm{~T} \times 101001$ & POINT & 136156 & 566805 & not assigne & & TANKS AND VESSELS & & \\
\hline Stack Configuration & & Diameter $(f t)$ & Length(ft) Width(ft) & $\operatorname{Area}(s q \mathrm{ft})$ & Exit & Temp(F) Exit Veloc $(\mathrm{ft} / \mathrm{sec})$ & Ht Abv Grnd(ft) & Ht Abv Struc $(f t)$ \\
\hline HORIZONTAL OR DOWNWAR & & .33 & & .09 & & .017 & 3 & 3 \\
\hline Em Point & Ep Type & North & East Srce Nbr & Scc Code & & Srce Type & & \\
\hline 200W P-241TX102 001 & POINT & 136156 & 566774 & not assigne & & TANKS AND VESSELS & & \\
\hline Stack Configuration & & Diameter $(f t)$ & Length(ft) Width(ft) & $\operatorname{Area}(s q \mathrm{ft})$ & Exit & Temp(F) Exit Veloc(ft/sec) & Ht Abv Grnd(ft) & Ht Abv Struc(ft) \\
\hline HORIZONTAL OR DOWNWAR & & 1.5 & & 1.77 & & .0079 & 3 & 3 \\
\hline Em Point & Ep Type & North & East Srce Nbr & Scc Code & & Srce Type & & \\
\hline $200 \mathrm{~W} P-241 \mathrm{~T} \times 103001$ & POINT & 136156 & 566743 & not assigne & & TANKS AND VESSELS & & \\
\hline Stack Configuration & & Diameter $(f t)$ & Length(ft) Width(ft) & Area(sq ft) & Exit & Temp(F) Exit Veloc(ft/sec) & Ht Abv Grnd(ft) & Ht Abv Struc(ft) \\
\hline HORIZONTAL OR DOWNWAR & & .33 & & .09 & & .36 & 3 & 3 \\
\hline Em Potnt & Ep Type & North & East Srce Nbr & Scc Code & & Srce Type & & \\
\hline $200 \mathrm{~W} P-241 \mathrm{TX} 104001$ & POINT & 136156 & 566712 & not assigne & & TANKS AND VESSELS & & \\
\hline Stack Configuration & & Diameter (ft) & Length $(f t)$ & Area $(s q f t)$ & Exit & Temp(F) Exit Veloc(ft/sec) & Ht Abv Grnd(ft) & Ht Abv Struc(ft) \\
\hline HORIZONTAL OR DOWNWAR & & .33 & & .09 & & .36 & 3 & 3 \\
\hline
\end{tabular}


AIR EMISSIONS INVENTORY PERMIT APPLICATION REPORT (UP460)

FACILITY NAME: 200W Tank Farm and Associated Structures

\section{Emission Point Listing}

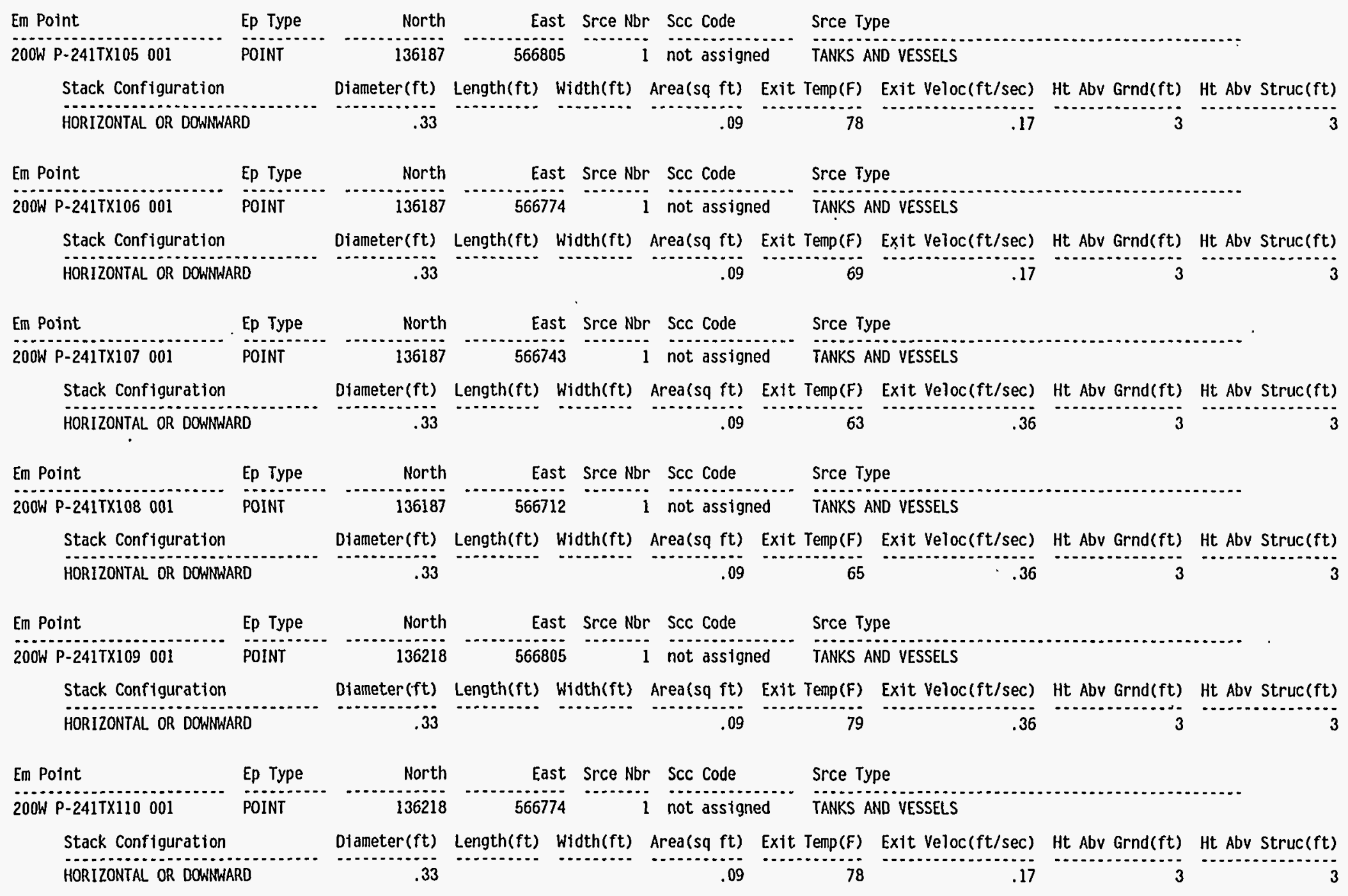




\section{Emission Point Listing}

\begin{tabular}{|c|c|c|c|c|c|c|c|c|c|}
\hline \multirow{2}{*}{$\begin{array}{l}\text { Em Point } \\
\text { 200W P-241TX111 001 }\end{array}$} & \multirow{2}{*}{$\begin{array}{l}\text { Ep Type } \\
\text { POINT }\end{array}$} & \multirow{2}{*}{$\begin{array}{l}\text { North } \\
136218\end{array}$} & \multirow{2}{*}{$\begin{array}{rr}\text { East } & \text { Srce Nbr } \\
566743 & 1\end{array}$} & \multirow{2}{*}{\multicolumn{2}{|c|}{$\begin{array}{l}\text { Scc Code } \\
\text { not assigned }\end{array}$}} & \multicolumn{4}{|c|}{ Srce Type } \\
\hline & & & & & & TANKS AI & IND VESSELS & & \\
\hline Stack Configuration & & Diameter $(f t)$ & Length(ft) Width(ft) & $\operatorname{Area}(s q \mathrm{ft})$ & Exit & Temp (F) & Exit Veloc(ft/sec) & Ht Abv Grnd(ft) & Ht Abv Struc $(f t)$ \\
\hline \multicolumn{2}{|l|}{ HORIZONTAL OR DOWNWARD } & .33 & & .09 & & 70 & .17 & 3 & 3 \\
\hline Em Point & Ep Type & North & East Srce Nbr & $r$ Scc code & & Srce Typ & & & \\
\hline $200 \mathrm{~W}$ P-241TX112001 & POINT & 136218 & 566712 & i not assigne & & TANKS AI & ND VESSELS & & \\
\hline \multicolumn{2}{|l|}{ Stack Configuration } & Diameter $(f t)$ & Length(ft) Width(ft) & $\operatorname{Area}(s q \mathrm{ft})$ & Exit & Temp (F) & Exit $\operatorname{Veloc}(\mathrm{ft} / \mathrm{sec})$ & Ht Abv Grnd(ft) & Ht Abv Struc(ft) \\
\hline \multicolumn{2}{|l|}{ HORIZONTAL OR DOWNWARD } & .33 & & .09 & & 70 & .17 & 3 & 3 \\
\hline Em Point & Ep Type & North & East Srce Nbr & Scc code & & Srce Typ & & & \\
\hline 200W P-241TX113 001 & POINT & 136249 & 566805 & not assigne & & TANKS AI & AND VESSELS & & \\
\hline \multicolumn{2}{|l|}{ Stack Configuration } & Diameter $(\mathrm{ft})$ & Length $(f t)$ Width $(f t)$ & $\operatorname{Area}(s q \mathrm{ft})$ & Exit & Temp (F) & Exit Veloc $(\mathrm{ft} / \mathrm{sec})$ & Ht. Abv Grnd(ft) & Ht Abv Struc $(\mathrm{ft})$ \\
\hline \multicolumn{2}{|l|}{ HORIZONTAL OR DOWNWARD } & $i$ & & .79 & & 69 & .018 & 3 & 3 \\
\hline Em Point & Ep Type & North & East Srce $\mathrm{Nbr}$ & Scc Code & & Srce Typ & pe & & \\
\hline $200 \mathrm{~W}$ P-241TX114 001 & POINT & 136249 & 566774 & not assigne & & TANKS AI & NO VESSELS & & \\
\hline \multicolumn{2}{|l|}{ Stack Configuration } & Diameter $(\mathrm{ft})$ & Length(ft) Width(ft) & Area $(s q \mathrm{ft})$ & Exit & Temp (F) & Exit Veloc $(\mathrm{ft} / \mathrm{sec})$ & Ht Abv Grnd(ft) & Ht Abv Struc(ft) \\
\hline \multicolumn{2}{|l|}{ HORIZONTAL OR DOWNWARD } & & 1 & 1 & & 78 & .014 & 3 & 3 \\
\hline Em Point & Ep Type & North & East Srce $\mathrm{Nbr}$ & Scc Code & & Srce Typ & & & \\
\hline 200W P-241TX115 001 & POINT & 136249 & 566743 & not assigne & & TANKS Al & ND VESSELS & & \\
\hline \multicolumn{2}{|l|}{ Stack Configuration } & Diameter $(f t)$ & Length(ft) Width(ft) & $\operatorname{Area}(s q \mathrm{ft})$ & Exit & $\operatorname{Temp}(F)$ & Exit Veloc $(\mathrm{ft} / \mathrm{sec})$ & Ht Abv Grnd(ft) & Ht Abv Struc (ft) \\
\hline \multicolumn{2}{|l|}{ HORIZONTAL OR DOWNWARD } & & 1 & 1 & & 69 & .014 & 3 & 3 \\
\hline Em Point & Ep Type & North & East Srce Nbr & Scc Code & & Srce Ty & & & \\
\hline $200 \mathrm{~W}$ P-241TX116 001 & POINT & 136280 & 566805 & not assigne & & TANKS Al & ND VESSELS & & \\
\hline Stack Configuration & & Diameter $(f t)$ & Length(ft) Width(ft) & $\operatorname{Area}(s q \mathrm{ft})$ & Exit & Temp (F) & Exit Veloc $(\mathrm{ft} / \mathrm{sec})$ & Ht Abv Grnd(ft) & Ht Abv Struc (ft) \\
\hline HORIZONTAL OR DOWNWARL & & .33 & & .09 & & 78 & .017 & 3 & 3 \\
\hline
\end{tabular}


AIR EMISSIONS INVENTORY PERMIT APPLICATION REPORT (UP460)

FACILITY NAME: $200 \mathrm{~W}$ Tank Farm and Associated Structures

Emission Point Listing

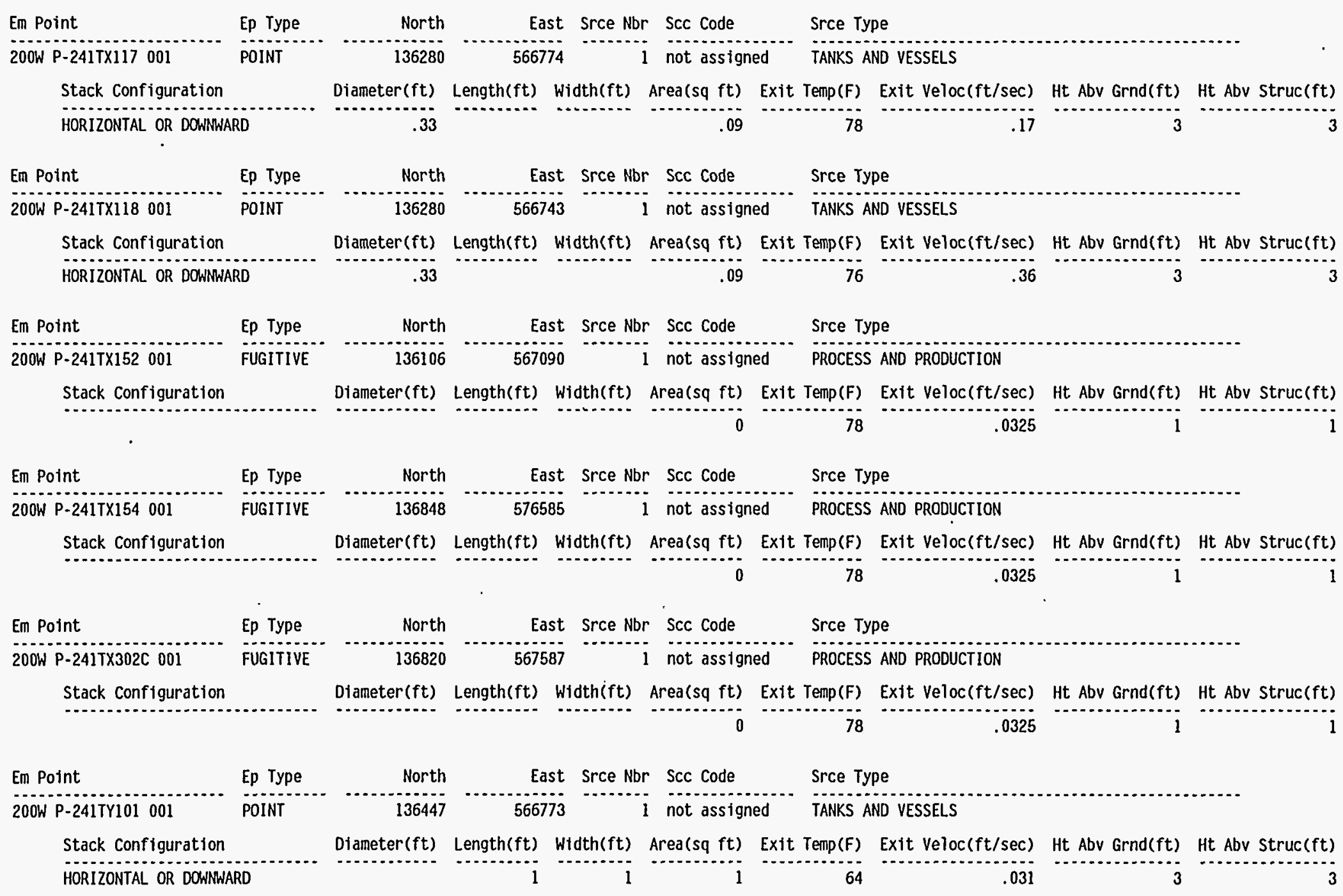

Page \#: 13 of 89 Report Run Date: 22-APR-95 


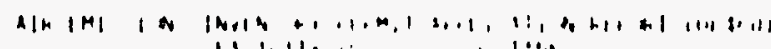

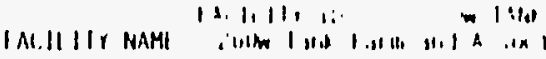

Emission Point Listing

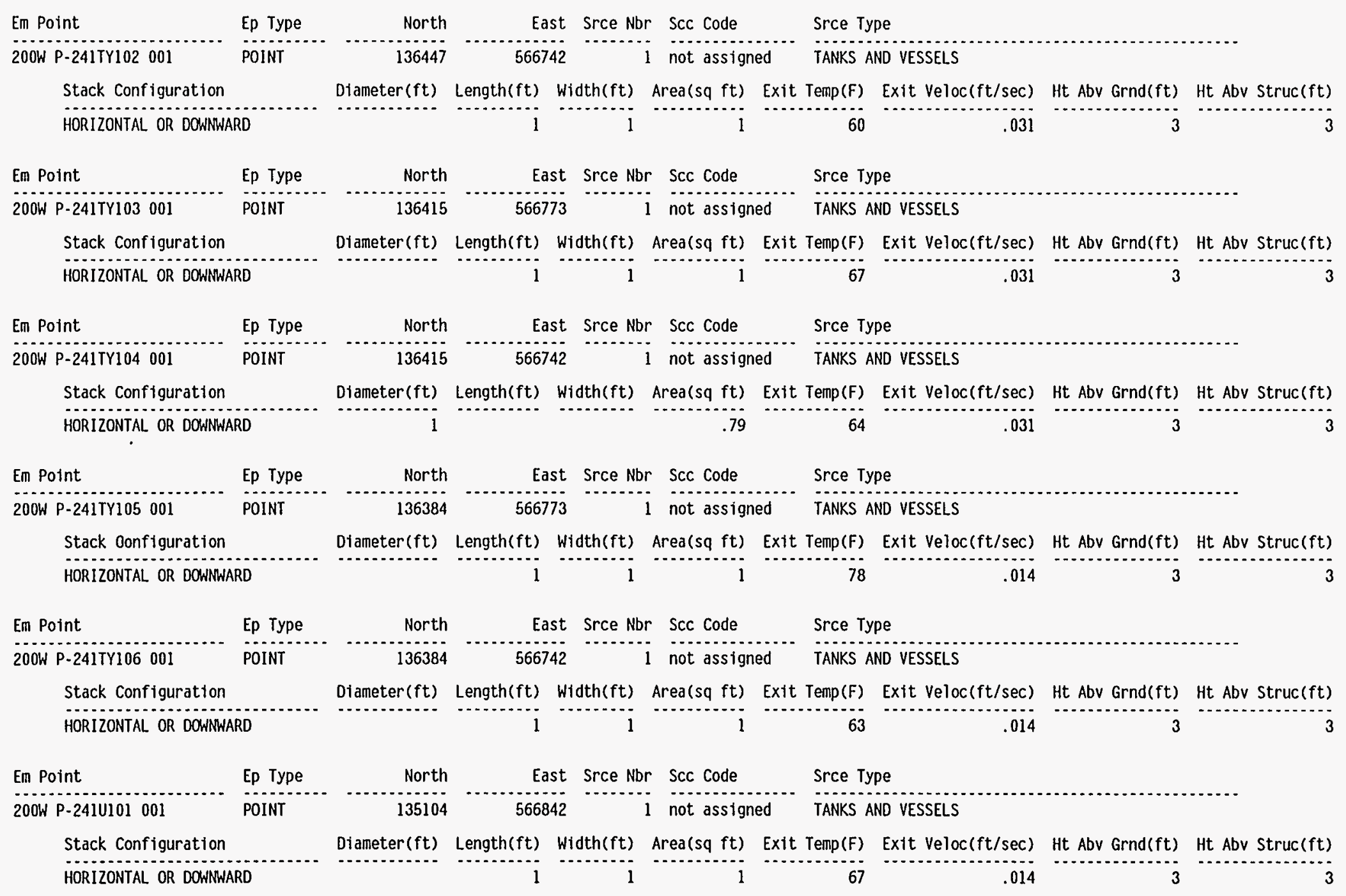


AIR EMISSIONS INVENTORY PERMIT APPLICATION REPORT (UP460)

FACILITY NAME: 200W Tank Farm and Associated Structures

\section{Emission Point Listing}

\begin{tabular}{|c|c|c|c|c|c|c|c|c|c|}
\hline Em Point & Eр Type & North & Eas & Srce Nbr & Scc Code & Srce Ty & & & \\
\hline 200W P-241U102001 & POINT & 135104 & 56681 & 12 & not assigne & TANKS A & WND VESSELS & & \\
\hline Stack Configuration & & Diameter $(f t)$ & Length $(f t)$ & Width(ft) & Area $(s q \mathrm{ft})$ & Exit Temp(F) & Exit Veloc (ft/sec) & HE Abv Grnd(ft) & Ht Abv Struc(ft) \\
\hline HORIZONTAL OR DOWNWA & & & 1 & 1 & 1 & 83 & .031 & 3 & \\
\hline
\end{tabular}

$\begin{array}{ll}\text { Em Point } & \text { Ep Type } \\ 200 \mathrm{~W} \text { P-2410103 } 001 & \text { POINT }\end{array}$

Stack Configuration

HORIZONTAL OR DOWWARD

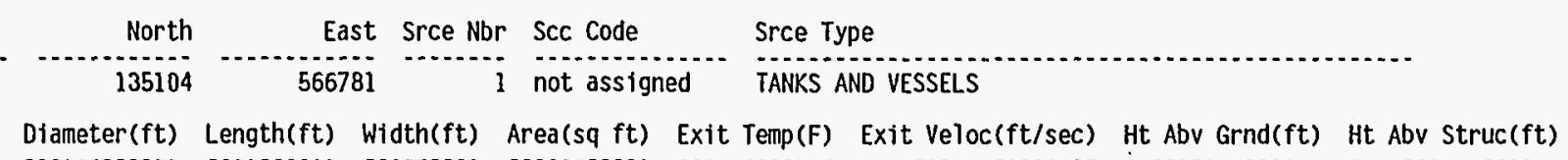

Em Point
$\begin{aligned} & \text { 200W P-2410104 } 001 \\ & \text { Stack Configuration }\end{aligned}$ HORIZONTAL OR DOWNWARD

Em Point
200W P-24lUI05 001 Ep Type
Stack Configuration
HORIZONTAL OR DOWNWARO

\begin{tabular}{|c|c|c|c|c|}
\hline North & East & Srce $\mathrm{Nbr}$ & Scc Code & Srce Type \\
\hline 135073 & 566842 & $i$ & not assigned & TANKS AND VESSELS \\
\hline
\end{tabular}

Diameter(ft) Length(ft) Width(ft) Area(sq ft) Exit Temp(F) Exit Veloc(ft/sec) Ht Abv Grnd(ft) Ht Abv Struc(ft) 1
1

\begin{tabular}{|c|c|c|c|c|c|c|c|c|}
\hline Em Point & Ep Type & North & Srce Nbr & Scc Code & Srce Ty & & & . \\
\hline $200 \mathrm{~W}$ P-2410106 001 & POINT & 135073 & 566781 & 1 not assigne & TANKS A & ND VESSELS & & \\
\hline Stack Configuration & & Diameter (ft) & Length(ft) Width(ft) & $\operatorname{Area}(s q \mathrm{ft})$ & Exit Temp(F) & Exit Veloc $(\mathrm{ft} / \mathrm{sec})$ & $H t$ Abv Grnd(ft) & Ht Abv Struc(ft) \\
\hline HORIZONTAL OR DOWNWAR & & & 1 & 1 & 76 & .031 & 3 & 3 \\
\hline Em Point & Ep Type & North & Srce Nbr & Scc code & Srce Ty & & & \\
\hline $200 \mathrm{~W}$ p-241u107 001 & POINT & 135043 & 566842 & 1 not assigne & TANKS AI & ND VESSELS & & \\
\hline Stack Configuration & & Diameter(ft) & Length(ft) Width(ft) & $\operatorname{Area}(s q \mathrm{ft})$ & Exit Temp(F) & Exit Veloc $(\mathrm{ft} / \mathrm{sec})$ & Ht $A b v G r n d(f t)$ & Ht Abv Struc $(f t)$ \\
\hline HORIZONTAL OR DOWNWAR & & & $\ddot{i}$ & 1 & 77 & .031 & 3 & 3 \\
\hline
\end{tabular}




\section{Emission Point Listing}

\begin{tabular}{|c|c|c|c|c|c|c|c|c|c|}
\hline Em Point & Ep Type & North & East Srce $\mathrm{Nbr}$ & r Scc Code & & Srce Ty & & & \\
\hline 200W P-241U108001 & POINT & 135043 & 566812 & 1 not assigne & & TANKS Al & NND VESSELS & & \\
\hline Stack Configuration & & Diameter $(\mathrm{ft})$ & Length $(f t)$ & $\operatorname{Area}(s q \mathrm{ft})$ & Exit & $\operatorname{Temp}(F)$ & Exit Veloc(ft/sec) & Ht $A b v \operatorname{Grnd}(f t)$ & Ht Abv Struc(ft) \\
\hline HORIZONTAL OR DOWNWAR & & & 1 & 1 & & 90 & .031 & 3 & 3 \\
\hline Em Point & Ep Type & North & Srce Nbr & $r$ Scc code & & Srce Ty & & & \\
\hline $200 \mathrm{~W}$ P-241U109 001 & POINT & 135043 & 566781 & i not assigne & & TANKS A & ND VESSELS & & \\
\hline Stack Configuration & & Diameter (ft) & Length(ft) Width(ft) & $\operatorname{Area}(s q \mathrm{ft})$ & Exit & Temp (F) & Exit Veloc(ft/sec) & $H t$ Abv Grnd(ft) & Ht Abv Struc(ft) \\
\hline HORIZONTAL OR DOWNWAR & & & 1 & i & & 92 & .031 & 3 & 3 \\
\hline Em Point & Ep Type & North & East Srce Nbr & $r$ Scc code & & Srce Ty & & & \\
\hline 200W P-241U110 001 & POINT & 135012 & 566842 & i not assigne & & TANKS A & ND VESSELS & & \\
\hline Stack Configuration & & Diameter $(\mathrm{ft})$ & Length(ft) Width(ft) & $\operatorname{Area}(s q \mathrm{ft})$ & Exit & $\operatorname{Temp}(F)$ & Exit $\operatorname{Veloc}(\mathrm{ft} / \mathrm{sec})$ & Ht Abv Grnd(ft) & Ht Abv Struc(ft) \\
\hline HORIZONTAL OR DOWNWAR & & & 1 & 1 & & 74 & .031 & 3 & 3 \\
\hline Em Point & Ep Type & North & East Srce $\mathrm{Nbr}$ & $r$ Scc code & & srce Ty & & & \\
\hline $200 \mathrm{~W}$ P-2410111 001 & POINT & 135012 & 566812 & 1 not assigned & & TANKS A & ND VESSELS & & \\
\hline Stack Configuration & & Diameter $(\mathrm{ft})$ & Length $(f t)$ Width $(f t)$ & $\operatorname{Area}(s q \mathrm{ft})$ & Exit & Temp(F) & Exit Veloc $(\mathrm{ft} / \mathrm{sec})$ & Ht Abv Grnd(ft) & Ht Abv Struc (ft) \\
\hline HORIZONTAL OR DOWNWAR & & & 1 & 1 & & 97 & .031 & 3 & \\
\hline Em Point & 'Ep Type & North & East Srce Nbr & $r$ Scc code & & Srce Ty & & & \\
\hline $200 \mathrm{~W}$ P-241U112 001 & POINT & 135012 & 566781 & 1 not assigned & & TANKS A & WND VESSELS & & \\
\hline Stack Configuration & & Diameter $(\mathrm{ft})$ & Length(ft) Width(ft) & $\operatorname{Area}(s q \mathrm{ft})$ & Exit & Temp(F) & Exit Veloc $(\mathrm{ft} / \mathrm{sec})$ & Ht Abv Grnd(ft) & Ht. Abv Struc(ft) \\
\hline HORIZONTAL OR DOWNWAR & & & 1 & 1 & & 62 & .014 & 3 & 3 \\
\hline Em Point & Ep Type & North & East Srce Nbr & Scc code & & Srce Ty & & & \\
\hline $200 \mathrm{~W}$ P-241U151 001 & FUGITIVE & 134991 & 566922 & not assigned & & PROCESS & AND PRODUCTION & & \\
\hline Stack Configuration & & Diameter $(\mathrm{ft})$ & Length(ft) Width(ft) & $\begin{array}{r}\text { Area }(s q \mathrm{ft}) \\
0 \\
0\end{array}$ & Exit & $\begin{array}{r}\text { Temp(F) } \\
0\end{array}$ & $\begin{array}{r}\text { Exit Veloc }(\mathrm{ft} / \mathrm{sec}) \\
.0325\end{array}$ & $\begin{array}{r}\text { Ht Abv Grnd(ft) } \\
1\end{array}$ & Ht Abv Struc (ft) \\
\hline
\end{tabular}


AIR EMISSIONS INVENTORY PERMIT APPLICATION REPORT (UP460)
FACILITY ID : 200W TANK FACILITY NAME: $200 \mathrm{~W}$ Tank Farm and ASsociated Structures

Emission Point Listing

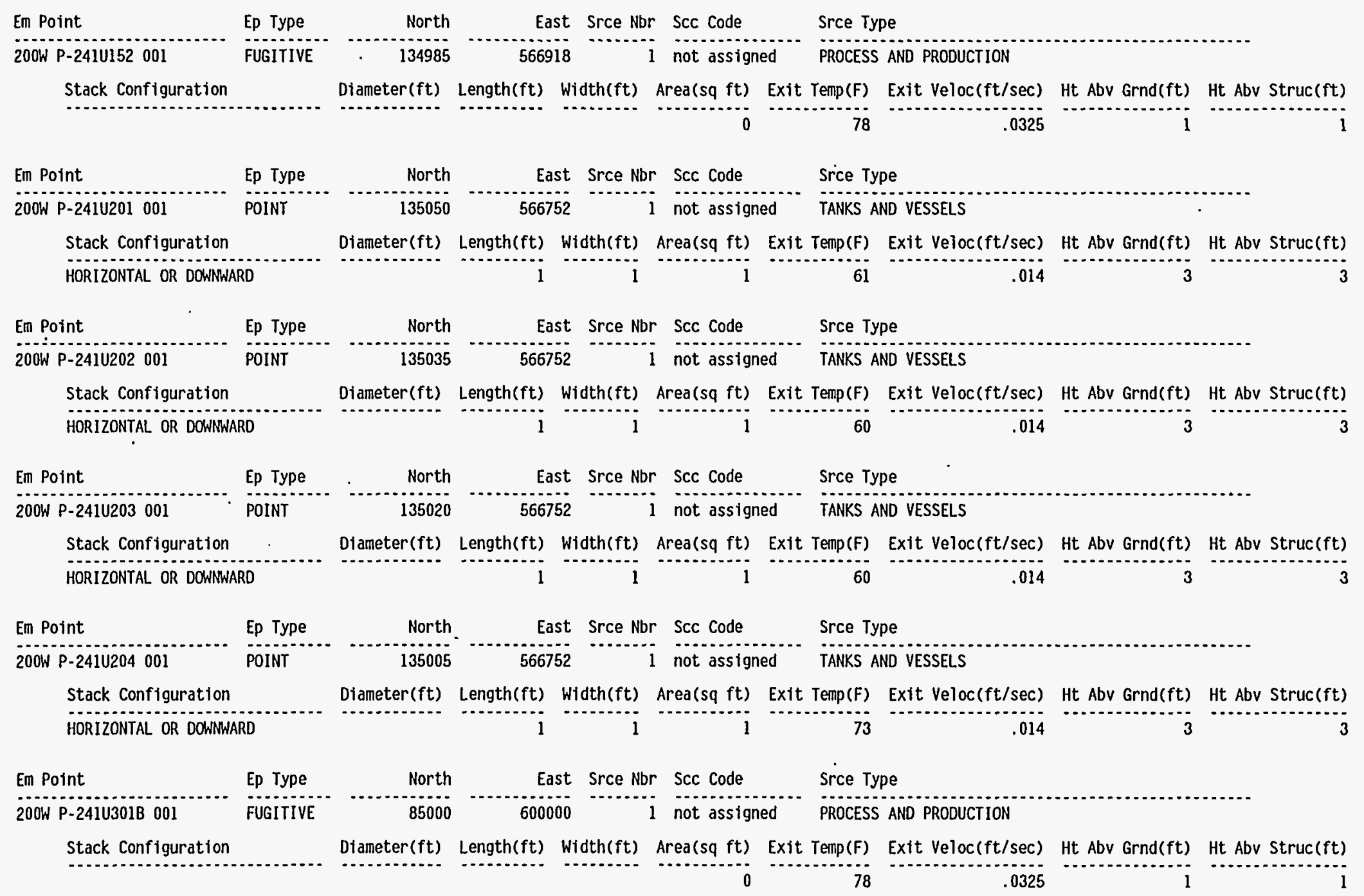


Emission Point Listing

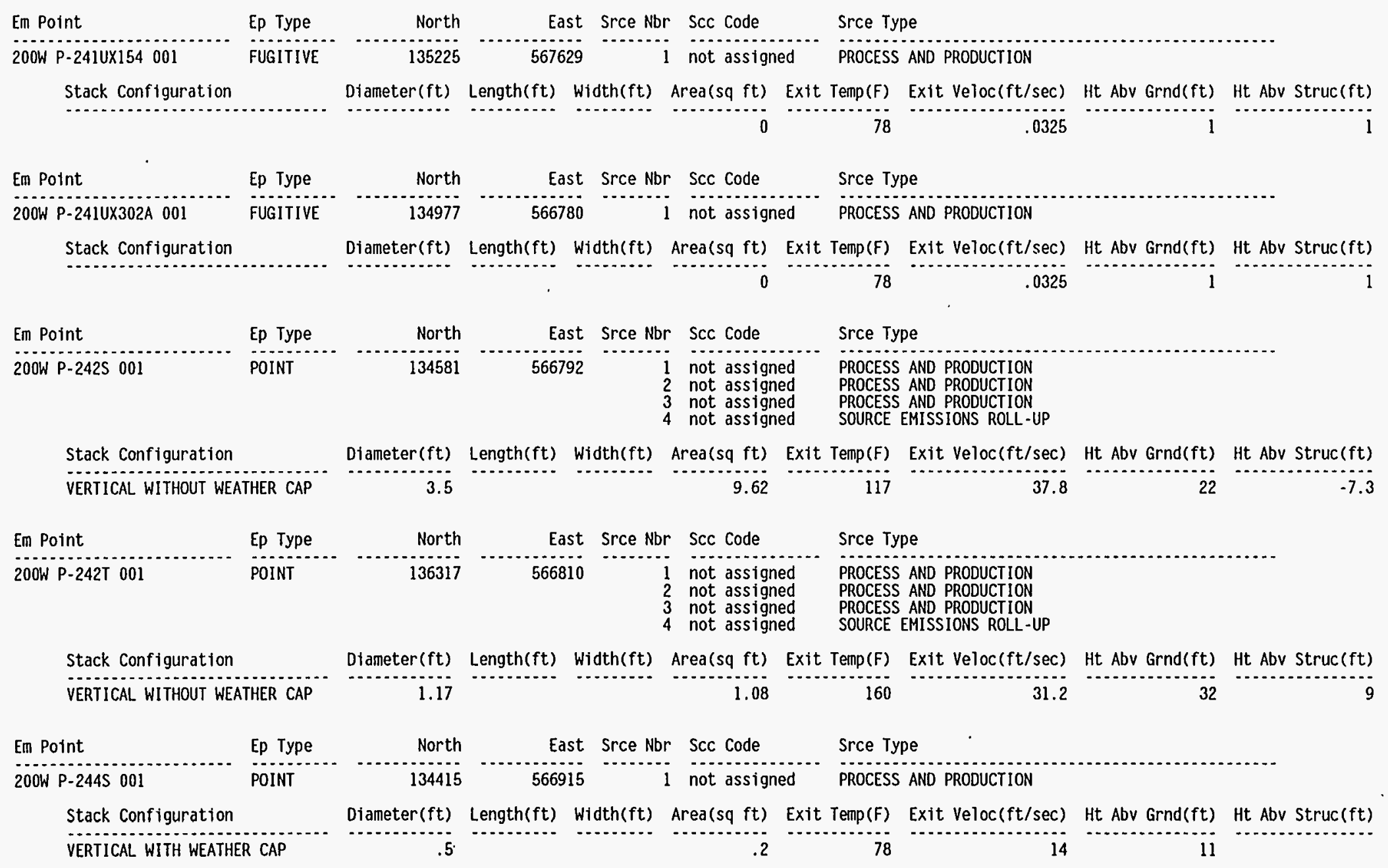


AIR EMISSIONS INVENTORY PERMIT APPLICATION REPORT (UP460)

FACILITY NAME: 200W Tank Farm and Associated Structures

\section{Emission Point Listing}

\begin{tabular}{|c|c|c|c|c|c|c|c|c|c|c|}
\hline Em Point & Ep Type & North & East & t Srce $\mathrm{Nbr}$ & - Scc Code & & Srce Ty & & & \\
\hline 200W P-244TX 001 & POINT & 136352 & 566823 & 3 & not assigne & & PROCESS & $S$ AND PRODUCTION & & \\
\hline Stack Configuration & & Diameter (ft) & Length(ft) $h$ & Width $(f t)$ & $\operatorname{Area}(s q \mathrm{ft})$ & Exit & $\operatorname{Temp}(F)$ & Exit Veloc $(\mathrm{ft} / \mathrm{sec})$ & Ht Abv Grnd(ft) & Ht Abv Struc(ft) \\
\hline VERTICAL WITH WEATHER & $R$ CAP & .5 & & & .2 & & 78 & 20.8 & 11 & \\
\hline Em Point & Ep Type & North & East & Srce Nbr & Scc Code & & Srce Ty & & & \\
\hline $200 \mathrm{~W} P-244 \mathrm{U} 001$ & POINT & 134087 & 56689 & $i$ & nat assigne & & PROCESS & $S$ AND PRODUCTION & & \\
\hline Stack Configuration & & Diameter (ft) & Length(ft) & Width(ft) & Area $(s q f t)$ & Exit & Temp (F) & Exit veloc $(\mathrm{ft} / \mathrm{sec})$ & Ht Abv Grnd(ft) & Ht Abv Struc(ft) \\
\hline VERTICAL WITH WEATHER & R CAP & .5 & & & .2 & & 78 & 14 & ii & \\
\hline Em Point & Ep Type & North & East & Srce Nbr & Scc code & & Srce Ty & & & \\
\hline $200 \mathrm{~W}$ P-296P022 001 & POINT & 134525 & 56687 & $\frac{1}{2}$ & $\begin{array}{l}\text { not assigne } \\
2 \text { not assigne } \\
\text { not assigne }\end{array}$ & & $\begin{array}{l}\text { TANKS A } \\
\text { TANKS A } \\
\text { TANKS A }\end{array}$ & $\begin{array}{l}\text { AND VESSELS } \\
\text { AND VESSELS } \\
\text { AND VESSELS }\end{array}$ & & - \\
\hline Stack Configuration & & Diameter $(f t)$ & Length(ft) & Width(ft) & Area (sq ft) & Exit & $\operatorname{Temp}(F)$ & Exit Veloc $(\mathrm{ft} / \mathrm{sec})$ & Ht Abv Grnd(ft) & Ht Abv Struc(ft) \\
\hline NONE SPECIFIED & & .67 & & & .35 & & 68 & 13.1 & 10.2 & \\
\hline Em Point & Ep Type & North & Eas & t srce $\mathrm{Nbr}$ & $r$ Scc code & & Srce Ty & ype & & \\
\hline $200 \mathrm{~W}$ P-296P028 001 & POINT & 134543 & 566878 & & $\begin{array}{l}\text { not assigne } \\
\text { not assigne } \\
3 \text { not assigne }\end{array}$ & & $\begin{array}{l}\text { TANKS A } \\
\text { TANKS A } \\
\text { TANKS A }\end{array}$ & $\begin{array}{l}\text { AND VESSELS } \\
\text { AND VESSELS } \\
\text { AND VESSELS }\end{array}$ & & \\
\hline Stack Configuration & & Diameter ( $f t)$ & Length(ft) & Width $(f t)$ & Area $(s q \mathrm{ft})$ & Exit & $\operatorname{Temp}(F)$ & Exit Veloc $(\mathrm{ft} / \mathrm{sec})$ & Ht Abv Grnd(ft) & Ht Abv Struc ( $f t$ ) \\
\hline NONE SPECIFIED & & .67 & & & .35 & & 68 & 12.5 & 11 & \\
\hline Em Point & Ep Type & North & Eas & t Srce Nbr & $r$ Scc code & & Srce Ty & & & \\
\hline $200 \mathrm{~W}$ P-296S025 001 & POINT & 134543 & 56688 & & $\begin{array}{l}\text { not assigne } \\
2 \text { not assigne } \\
3 \text { not assigne }\end{array}$ & & $\begin{array}{l}\text { TANKS A } \\
\text { TANKS A } \\
\text { TANKS A }\end{array}$ & $\begin{array}{l}\text { AND VESSELS } \\
\text { AND VESSELS } \\
\text { AND VESSELS }\end{array}$ & & \\
\hline Stack Configuration & & Diameter (ft) & Length(ft) & Width(ft) & $\operatorname{Area}(s q \mathrm{ft})$ & Exit & $\operatorname{Temp}(F)$ & Exit Veloc(ft/sec) & Ht Abv Grnd(ft) & Ht Abv Struc(ft) \\
\hline NONE SPECIFIED & & .67 & & & .35 & & 68 & 50.4 & 18 & \\
\hline
\end{tabular}


AIR EMISSIONS INVENTORY PERMIT APPLICATION REPORT (UP460)

FACILITY NAME: $200 \mathrm{~W}$ Tank Farm and ASsOCI

Emission Point Listing

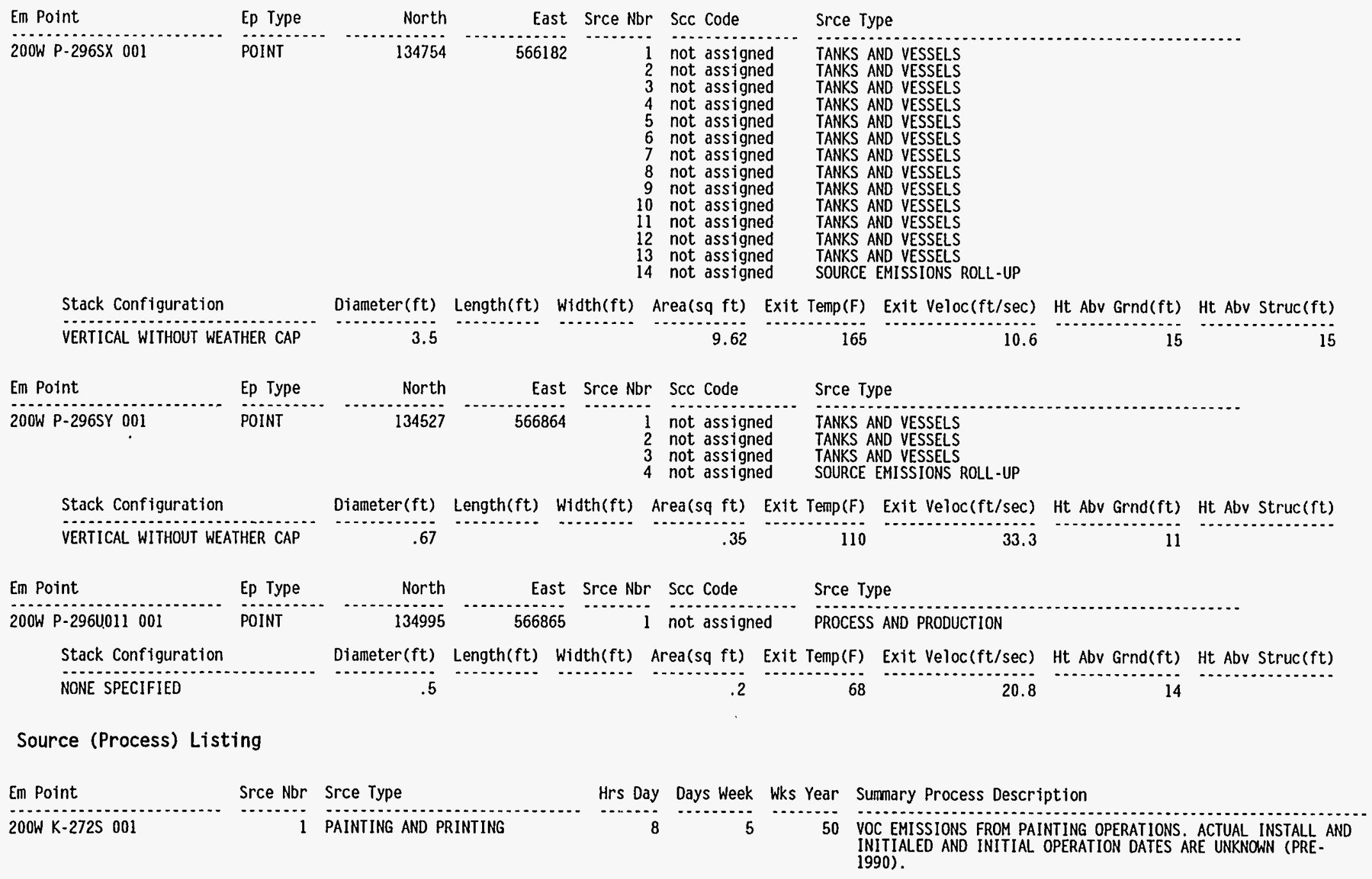

Page \#: 20 of 89 Report Run Date: 22-APR-95 


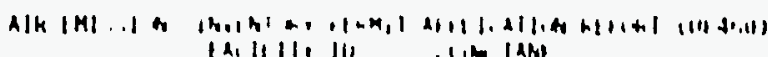

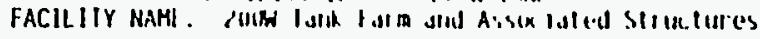

\section{Source (Process) Listing}

\begin{tabular}{|c|c|c|c|c|c|c|}
\hline Em Point & Srce Nbr & Srce Type & Hrs Day & Days Week & Wks Year & Sunmary Process Description \\
\hline $200 \mathrm{~W} \mathrm{~K}-2725001$ & 2 & ABRASIVE CLEANING & 8 & 1 & 50 & $\begin{array}{l}\text { OPEN AREA SAND BLASTING TO REMOVE RUST AND SCALE } \\
\text { ACCUMUATION. ACTUAL INSTALLED AND INITIAL OPERATION OATES } \\
\text { ARE UNKNOWN (PRE-1990). }\end{array}$ \\
\hline Em Point & Srce Nbr & Srce Type & Hrs Day & Days Week & Whs Year & Summary Process Description \\
\hline 200W M-272WA 001 & 1 & PROCESS AND PRODUCTION & 8 & 5 & 50 & $\begin{array}{l}\text { VOC EMISSIONS FROM FABRICATION AND MAINTENANCE OPERATIONS. } \\
\text { ACTUAL INSTALL AND INITIAL OPERATION DATES ARE UNKNOWN (PRE- } \\
1990 \text { ). }\end{array}$ \\
\hline
\end{tabular}

MISCELLANEOUS SOLVENT USAGE FOR MAINTENANCE PURPOSES.

\begin{tabular}{|c|c|c|c|c|c|c|}
\hline Em Point & Srce Nbr & Srce Type & Hrs Day & Days Week & Wks Year & Summary Process Description \\
\hline $200 \mathrm{~W} \mathrm{M}-272 \mathrm{WA} 00 \mathrm{I}$ & 2 & WELDING/CUTTING/BRAZING & 2 & $\overline{5}$ & 50 & $\begin{array}{l}\text { WELOING OPERATIONS ACTUAL INSTALL AND INITIAL OPERATION } \\
\text { DATES ARE UNKNOWN (PRE-1990). }\end{array}$ \\
\hline Em Point & Srce $\mathrm{Nbr}$ & Srce Type & Mrs Day & Days Week & Wks Year & Summary Process Description \\
\hline $200 \mathrm{~W} N-2312001$ & $i$ & PROCESS AND PRODUCTION & 4 & 5 & 52 & $\begin{array}{l}\text { SATELLITE CARPENTER SHOP, WOODWORKING (SAWING DRILLING. } \\
\text { SANDING), NO CONTROLS. ACTUAL INSTALLED AND INITIAL } \\
\text { OPERATION DATES ARE UNKNOWN (PRE-1980). }\end{array}$ \\
\hline En Point & Srce Nbr & Srce Type & Hrs Day & Days Week & Wks Year & Summary Process Description \\
\hline 200W P-241S101 001 & 1 & TANKS AND VESSELS & 24 & 7 & 52 & $\begin{array}{l}241-S-101.750 .000 \text { GALLON SINGLE SHELL UNDERGROUND STORAGE } \\
\text { TANK }\end{array}$ \\
\hline En Point & Srce $\mathrm{Nbr}$ & Srce Type & Hrs Day & Days Week & Wks Year & Surnary Process Description \\
\hline $200 \mathrm{~W}$ P-241S102 001 & 1 & TANKS AND VESSELS & 24 & 7 & 52 & $\begin{array}{l}241-S=102.750 .000 \text { GALLON SINGLE SHELL UNDERGROUND STORAGE } \\
\text { TANK }\end{array}$ \\
\hline Em Point & Srce $\mathrm{Nbr}$ & Srce Type & Hrs Day & Days Week & Wks Year & Summary Process Description \\
\hline $200 \mathrm{~W}$ P-241S103 001 & 1 & TANKS AND VESSELS & 24 & 7 & 52 & $\begin{array}{l}241-S-103,750.000 \text { GALLON SINGLE SHELL UNDERGROUND STORAGE } \\
\text { TANK }\end{array}$ \\
\hline Em Point & Srce $\mathrm{Nbr}$ & Srce Type & Hrs Day & Days Week & Wks Year & Sunmary Process Description \\
\hline $200 \mathrm{~W}$ P-2415104 001 & 1 & TANKS AND VESSELS & 24 & 7 & 52 & $\begin{array}{l}241-5-104.750 .000 \text { GALLON SINGLE SHELL UNDERGROUND STORAGE } \\
\text { TANK }\end{array}$ \\
\hline
\end{tabular}


Source (Process) Listing

\begin{tabular}{|c|c|c|c|c|c|c|}
\hline Em Point & Srce Nbr & Srce Type & Hrs Day & Days Week & Wks Year & Summary Process Description \\
\hline 200W P-241S105001 & 1 & TANKS AND VESSELS & 24 & 7 & 52 & $\begin{array}{l}241-S-105.750,000 \text { GALLON SINGLE SHELL UNDERGROUND STORAGE } \\
\text { TANK }\end{array}$ \\
\hline Em Point & Srce $\mathrm{Nbr}$ & Srce Type & Hrs Day & Days Week & Wks Year & Surmary Process Description \\
\hline $200 \mathrm{~W} \mathrm{P-241S106001}$ & 1 & TANKS AND VESSELS & 24 & 7 & 52 & $\begin{array}{l}241-S-106.750 .000 \text { GALLON SINGLE SHELL UNDERGROUND STORAGE } \\
\text { TANK }\end{array}$ \\
\hline Em Point & Srce Nbr & Srce Type & Hrs Day & Days Week & Wks Year & Sumnary Process Description \\
\hline $200 \mathrm{~W}$ P-241S107 001 & 1 & TANKS AND VESSELS & 24 & 7 & 52 & $\begin{array}{l}241-S-107.750 .000 \text { GALLON SINGLE SHELL UNDERGROUND STORAGE } \\
\text { TANK }\end{array}$ \\
\hline Em Point & Srce Nbr & Srce Type & Hrs Day & Days Week & Wks Year & Summary Process Description \\
\hline $200 \mathrm{~W}$ P-241S108 001 & $i$ & TANKS AND VESSELS & 24 & 7 & 52 & $\begin{array}{l}241-S-108.750 .000 \text { GALLON SINGLE-SHELL UNDERGROUND STORAGE } \\
\text { TANK }\end{array}$ \\
\hline Em Point & Srce Nbr & Srce Type & Hrs Day & Days Week & Wks Year & Sumnary Process Description \\
\hline $200 \mathrm{~W}$ P-241S109001 & 1 & TANKS AND VESSELS & 24 & 7 & 52 & $\begin{array}{l}241-S-109,750,000 \text { GALLON SINGLE SHELL UNDERGROUND STORAGE } \\
\text { TANK }\end{array}$ \\
\hline Em Point & Srce Nbr & Srce Type & Mrs Day & Days Week & Wks Year & Summary Process Description \\
\hline 200W P-241S110 001 & 1 & TANKS AND VESSELS & 24 & 7 & 52 & $\begin{array}{l}241-5-110,750,000 \text { GALLON SINGLE SHELL UNDERGROUND STORAGE } \\
\text { TANK }\end{array}$ \\
\hline Em Point & Srce Nbr & Srce Type & Hrs Day & Days Week & Wks Year & Sumary Process Description \\
\hline $200 \mathrm{~W}$ P-241S111 001 & 1 & TANKS AND VESSELS & 24 & 7 & 52 & $\begin{array}{l}241-S-111.750 .000 \text { GALLON SINGLE SHELL UNDERGROUND STORAGE } \\
\text { TANK }\end{array}$ \\
\hline Em Point & Srce $\mathrm{Nbr}$ & Srce Type & Hrs Day & Days Week & Wks Year & Summary Process Description \\
\hline $200 \mathrm{~W}$ P-241S112 001 & 1 & TANKS AND VESSELS & 24 & 7 & 52 & $\begin{array}{l}241-S-112,750.000 \text { GALLON SINGLE SHELL UNDERGROUND STORAGE } \\
\text { TANK }\end{array}$ \\
\hline Em Point & Srce Nbr & Srce Type & Hrs Day & Days Week & Wks Year & Summary Process Description \\
\hline $200 \mathrm{~W}$ P-241S151 001 & $i$ & PROCESS AND PRODUCTION & 24 & 7 & 52 & 241-S-151 DIVERSION BOX \\
\hline
\end{tabular}
200W P-241S151 001 1 PROCESS AND PRODUCTION$$
52 \text { 241-5-151 DIVERSION BOX }
$$

THE 241 -S-151 DIVERSION BOX. LOCATED UNDERGROUND, IS A REINFORCED CONCRETE STRUCTURE $56^{\circ} \times 24^{\circ} \times 17^{\circ}$ (LXWXD). THE BOX DRAINS TO CATCH TANK 241 -S-302A. THE DIVERSION BOX TRANSPORTS MIXED WASTE SOLUTIONS FROM PROCESSING AND DECONTAMINATION OPERATIONS. 


\begin{tabular}{|c|c|c|c|c|c|c|}
\hline Em Point & Srce Nbr & Srce Type & Hrs Day & Days Week & Wks Year & Summary Process Description \\
\hline 200W P-241S304 001 & 1 & PROCESS AND PRODUCTION & 24 & 7 & 52 & 241-S-302A CATCH TANK \\
\hline \multicolumn{7}{|c|}{$\begin{array}{l}\text { THE CATCH TANK SERVES THE } 241 \text {-S } 151 \text { TRANSFER BOX WHICH PROCESSES PROCESS CONDENSATE AND FLUSH WATER. THE CATCH TANK CONSISTS OF A HORIZONTAL } \\
\text { CYLINDICAL CARBN STEEL VESSEL. A STEAM JET BOX RESTING ON THE TANK. AND A PUMP PIT ABOVE THE TANK. THE DIAMETER OF THE TANK IS } 8 \text { FEET., } 10 \text { 7/8 INCHES } \\
\text { AND } 40 \text { FEET LONG. }\end{array}$} \\
\hline Em Point & Srce $\mathrm{Nbr}$ & Srce Type & Hrs Day & Days Week & Wks Year & Summary Process Description \\
\hline $200 \mathrm{~W} P-2415 \times 113001$ & $i$ & TANKS AND VESSELS & 24 & 7 & 52 & $\begin{array}{l}241-S X-113,1,000.000 \text { GALLON SINGLE SHELL UNDERGROUND } \\
\text { STORAGE TANK }\end{array}$ \\
\hline Em Point & Srce Nbr & Srce Type & Hrs Day & Days Week & Wks Year & Summary Process Description \\
\hline 200W P-241SX115001 & 1 & TANKS AND VESSELS & 24 & 7 & 52 & $\begin{array}{l}\text { 241-SX-115, } 1,000,000 \text { GALLON SINGLE SHELL UNDERGROUND } \\
\text { STORAGE TANK }\end{array}$ \\
\hline Em Point & Srce Nbr & Srce Type & Hrs Day & Days Week & Wks Year & Summary Process Description \\
\hline $200 \mathrm{~W} P$-241SYA 001 & $\ddot{i}$ & PROCESS AND PRODUCTION & 24 & 7 & 52 & 241-SY-A VALVE PIT \\
\hline \multicolumn{7}{|c|}{$\begin{array}{l}\text { THE CONCRETE VALVE PIT IS } 14^{\circ} \times 12^{\circ} \times 7^{\circ} 10^{\prime \prime}: \text { IS MADE OF CONCRETE, AND IS LOCATED UNDERGROUND. } \\
\text { WASTES TRANSFERRED VIA } 241^{-S Y}-A \text { INCLUDES LAB WASTE. COMPLEX CONCENTRATE. AND DOUBLE SHELL SLURRY. }\end{array}$} \\
\hline Em Point & Srce Nbr & Srce Type & Hrs Day & Days Week & Wks Year & Summary Process Description \\
\hline $200 \mathrm{~W}$-241SYB 001 & 1 & PROCESS AND PRODUCTION & 24 & 7 & 52 & 241-SY-B VALVE PIT \\
\hline \multicolumn{7}{|c|}{ THE $241-S Y-B$ VALVE PIT IS A $14^{\circ} \times 12^{\prime} \times 7^{\prime} 10^{\prime \prime}$ CONCRETE PIT UTILIZED TO TRANSFER SY TANK FARM LAB WASTE. COMPLEX CONCENTRATE. AND DOUBLE SHELL SLURRY. } \\
\hline Em Point 1 & 'Srce Nbr & Srce Type & Hrs Day & Days Week & Whs Year & Summary Process Description \\
\hline $200 \mathrm{~W}$ P-241T101 001 & $i$ & TANKS AND VESSELS & 24 & 7 & 52 & $\begin{array}{l}241-T-101.530 .000 \text { GALLON SINGLE SHELL. UNDERGROUND STORAGE } \\
\text { TANK }\end{array}$ \\
\hline Em Point & Srce $\mathrm{Nbr}$ & Srce Type & Hrs Day & Days Week & Wks Year & Summary Process Description \\
\hline $200 \mathrm{~W}$ P-241T102 001 & $i$ & TANKS AND VESSELS & 24 & 7 & 52 & $\begin{array}{l}241-T-102.530 .000 \text { GALLON SINGLE SHELL UNDERGROUND STORAGE } \\
\text { TANK }\end{array}$ \\
\hline Em Point & Srce Nbr & Srce Type & Hrs Day & Days Week & Wks Year & Summary Process Description \\
\hline $200 \mathrm{~W}$ P-241T103 001 & 1 & TANKS AND VESSELS & 24 & 7 & 52 & $\begin{array}{l}241-T-103.530 .000 \text { GALLON SINGLE SHELL UNDERGROUND STORAGE } \\
\text { TANK }\end{array}$ \\
\hline
\end{tabular}


AIR EMISSIONS INVENTORY PERMIT APPLICATION REPORT (UP460)

FACILITY ID : 200W TANK
FACILITY NAME: 200W Tank Farm and Associated Structures

Source (Process) Listing

\begin{tabular}{|c|c|c|c|c|c|c|}
\hline Em Point & Srce Nbr & Srce Type & Hrs Day & Days Week & Wks Year & Summary Process Description \\
\hline 200W P-241T104 001 & 1 & TANKS AND VESSELSS & 24 & 7 & 52 & $\begin{array}{l}241-T-104.530 .000 \text { GALLON SINGLE SHELL UNDERGROUND STORAGE } \\
\text { TANK }\end{array}$ \\
\hline Em Point & Srce Nbr & Srce Type & Hrs Day & Days Week & Wks Year & Summary Process Description \\
\hline $200 \mathrm{~W}$ P-241T105 001 & 1 & TANKS AND VESSELS & 24 & 7 & 52 & $\begin{array}{l}241-T-105,530.000 \text { GALLON SINGLE SHELL UNDERGROUND STORAGE } \\
\text { TANK }\end{array}$ \\
\hline Em Point & Srce $\mathrm{Nbr}$ & Srce Type & Hrs Day & Days Week & Wks Year & Summary Process Description \\
\hline 200W P-241T106 001 & 1 & TANKS AND VESSELS & 24 & 7 & 52 & $\begin{array}{l}241-T-106.530 .000 \text { GALLON SINGLE SHELL UNDERGROUND STORAGE } \\
\text { TANK }\end{array}$ \\
\hline Em Point & Srce $\mathrm{Nbr}$ & Srce Type & Hrs Day & Days Week & Wks Year & Summary Process Description \\
\hline $200 \mathrm{~W} P-241 \mathrm{T107} 001$ & 1 & TANKS AND VESSELS & 24 & $\dddot{7}$ & 52 & $\begin{array}{l}241-T-107.530 .000 \text { GALLON SINGLE SHELL UNDERGROUND STORAGE } \\
\text { TANK }\end{array}$ \\
\hline Em Point & Srce Nbr & Srce Type & Hrs Day & Days Week & Wks Year & Summary Process Description \\
\hline $200 \mathrm{~W} P-241 \mathrm{~T} 108001$ & 1 & TANKS AND VESSELS & 24 & 7 & 52 & $\begin{array}{l}241-T-108,530.000 \text { GALLON SINGLE SHELL UNDERGROUND STORAGE } \\
\text { TANK }\end{array}$ \\
\hline Em Point & Srce $\mathrm{Nbr}$ & Srce Type & Hrs Day & Days Week & Wks Year & Summary Process Description \\
\hline $200 \mathrm{~W}$ P-241T109 001 & $i$ & TANKS AND VESSELS & 24 & 7 & 52 & $\begin{array}{l}241-T-109.530 .000 \text { GALLON SINGLE SHELL UNDERGROUND STORAGE } \\
\text { TANK }\end{array}$ \\
\hline Em Point & Srce Nbr & Srce Type & Hrs Day & Days Week & Wks Year & Summary Process Description \\
\hline $200 \mathrm{~W} \mathrm{P}-241 \mathrm{T110} 001$ & $i$ & TANKS AND VESSELS & 24 & 7 & 52 & $\begin{array}{l}241-T-110.530 .000 \text { GALLON SINGLE SHELL UNDERGROUND STORAGE } \\
\text { TANK }\end{array}$ \\
\hline Em Point & Srce $\mathrm{Nbr}$ & Srce Type & Hrs Day & Days Week & Wks Year & Summary Process Description \\
\hline 200W P-241T111 001 & 1 & TANKS AND VESSELS & 24 & 7 & 52 & $\begin{array}{l}241-T-111.530 .000 \text { GALLON SINGLE SHELL UNDERGROUND STORAGE } \\
\text { TANK }\end{array}$ \\
\hline Em Point & Srce $\mathrm{Nbr}$ & Srce Type & Hrs Day & Days Week & Wks Year & Summary Process Description \\
\hline $200 \mathrm{~W}$ P-241T112 001 & 1 & TANKS AND VESSELS & 24 & 7 & 52 & $\begin{array}{l}241-T-112,530.000 \text { GALLON SINGLE SHELL UNDERGROUND STORAGE } \\
\text { TANK }\end{array}$ \\
\hline
\end{tabular}


AIR EMISSIONS INVENTORY PERMIT APPLICATION REPORT (UP460)

FACILITY NAME: 200W Tank Farm and Associated Structures

Source (Process) Listing

\begin{tabular}{|c|c|c|c|c|c|c|}
\hline Em Point & Srce Nbr & Srce Type & Hrs Day & Days Week & Wks Year & Summary Process Description \\
\hline 200W P-241T201 001 & 1 & TANKS AND VESSELS & 24 & 7 & 52 & $\begin{array}{l}241-T-201,55,000 \text { GALLON SINGLE SHELL UNDERGROUND STORAGE } \\
\text { TANK }\end{array}$ \\
\hline Em Point & Srce Nbr & Srce Type & Hrs Day & Days Week & Wks Year & Summary Process Description \\
\hline $200 \mathrm{~W}$ P-241T202 001 & i & TANKS AND VESSELS & 24 & 7 & 52 & $\begin{array}{l}241-T-201.55 .000 \text { GALLON SINGLE SHELL UNDERGROUND STORAGE } \\
\text { TANK }\end{array}$ \\
\hline Em Point & Srce $\mathrm{Nbr}$ & Srce Type & Hrs Day & Days Week & Wks Year & Surmary Process Description \\
\hline $200 \mathrm{~W}$ P-241T203 001 & 1 & TANKS AND VESSELS & 24 & 7 & 52 & $\begin{array}{l}241-T-203,55,000 \text { GALLON SINGLE SHELL UNDERGROUND STORAGE } \\
\text { TANK }\end{array}$ \\
\hline Em Point & Srce $\mathrm{Nbr}$ & Srce Type & Hrs Day & Days Week & Wks Year & Sumnary Process Description \\
\hline $200 \mathrm{~W}$ P-241T204 001 & $\ddot{i}$ & TANKS AND VESSELS & 24 & 7 & 52 & $\begin{array}{l}241-T-204,55,000 \text { GALLON SINGLE SHELL UNDERGROUND STORAGE } \\
\text { TANK }\end{array}$ \\
\hline Em Point & Srce Nbr & Srce Type & Hrs Day & Days Week & Wks Year & Sumnary Process Description \\
\hline $200 \mathrm{~W} P-241 \mathrm{~T} \times 101001$ & $i$ & TANKS AND VESSELS & 24 & 7 & 52 & $\begin{array}{l}241-T X-101,750.000 \text { GALLON SINGLE SHELL UNDERGROUND STORAGE } \\
\text { TANK }\end{array}$ \\
\hline Em Point & Srce Nbr & Srce Type & Hrs Day & Days Week & Wks Year & Surmary Process Description \\
\hline $200 \mathrm{~W}$ P-241TX102 001 & $\ddot{i}$ & TANKS AND VESSELS & 24 & 7 & 52 & $\begin{array}{l}241-T X-102,750,000 \text { GALLON SINGLE SHELLL UNDERGROUND STORAGE } \\
\text { TANK }\end{array}$ \\
\hline Em Point & Srce Nbr & Srce Type & Hrs Day & Days Week. & Wks Year & Summary Process Description \\
\hline $200 \mathrm{~W}$ P-241TX103 001 & $i$ & TANKS AND VESSELS & 24 & 7 & 52 & $\begin{array}{l}241-T X-103.750 .000 \text { GALLON SINGLE SHELL UNDERGROUND STORAGE } \\
\text { TANK }\end{array}$ \\
\hline Em Point & Srce Nbr & Srce Type & Hrs Day & Days Week & Wks Year & Sumnary Process Description \\
\hline $200 \mathrm{~W}$ P-241TX104001 & 1 & TANKS AND VESSELS & 24 & 7 & 52 & $\begin{array}{l}241-T X-104.750 .000 \text { GALLON SINGLE SHELL UNDERGROUND STORAGE } \\
\text { TANK }\end{array}$ \\
\hline Em Point & Srce Nbr & Srce Type & Hrs Day & Days Week & Wks Year & Sumnary Process Description \\
\hline $200 W$ P-241TX105001 & 1 & TANKS AND VESSELS & 24 & 7 & 52 & $\begin{array}{l}241-T X-105.750 .000 \text { GALLON SINGLE SHELL UNDERGROUND STORAGE } \\
\text { TANK }\end{array}$ \\
\hline
\end{tabular}


AIR EMISSIONS INVENTORY PERMIT APPLICATION REPORT (UP460)

FACILITY NAME: $200 \mathrm{~W}$ Tank Farm and Associated Structures

\section{Source (Process) Listing}

\begin{tabular}{|c|c|c|c|c|c|c|}
\hline Em Point & Srce Nbr & Srce Type & Hrs Day & Days Week & Wks Year & Summary Process Description \\
\hline 200W P-241TX106 001 & 1 & TANKS AND VESSELS & 24 & 7 & 52 & $\begin{array}{l}241-T X-106.750 .000 \text { GALLON SINGLE SHELL UNDERGROUND STORAGE } \\
\text { TANK }\end{array}$ \\
\hline Em Point & Srce Nbr & Srce Type & Hrs Day & Days Week & Wks Year & Sumnary Process Description \\
\hline 200W P-241TX107001 & 1 & TANKS AND VESSELS & 24 & 7 & 52 & $\begin{array}{l}241-T X-107,750.000 \text { GALLON SINGLE SHELL UNDERGROUND STORAGE } \\
\text { TANK }\end{array}$ \\
\hline Em Point & Srce Nbr & Srce Type & Hrs Day & Days Week & Whs Year & Summary Process Description \\
\hline Em Point & Srce Nbr & Srce Type & Hrs Day & Days Week & Wks Year & Sumnary Process Description \\
\hline $200 \mathrm{~W}$ P-241TX109 001 & 1 & TANKS AND VESSELS & 24 & 7 & 52 & $\begin{array}{l}241-T X-109.750 .000 \text { GALLON SINGLE SHELL UNDERGROUND STORAGE } \\
\text { TANK }\end{array}$ \\
\hline Em Point & Srce $\mathrm{Nbr}$ & Srce Type & Hrs Day & Days Week & Wks Year & Sumnary Process Description \\
\hline 200W P-241TX110 001 & 1 & TANKS AND VESSELS & 24 & 7 & 52 & $\begin{array}{l}241-T X-110.750 .000 \text { GALLON SINGLE SHELL UNDERGROUND STORAGE } \\
\text { TANK }\end{array}$ \\
\hline Em Point & Srce Nbr & Srce Type & Hrs Day & Days Week & Wks Year & Summary Process Description \\
\hline $200 \mathrm{~W} P-2417 \times 111001$ & 1 & TANKS AND VESSELS & 24 & 7 & 52 & $\begin{array}{l}241-T X-111.750 .000 \text { GALLON SINGLE SHELL UNDERGROUND STORAGE } \\
\text { TANK }\end{array}$ \\
\hline Em Point & Srce $\mathrm{Nbr}$ & Srce Type & Hrs Day & Days Week & Wks Year & Summary Process Description \\
\hline $200 \mathrm{~W}$ P-241TX112 001 & 1 & TANKS AND VESSELS & 24 & 7 & 52 & $\begin{array}{l}241-T X-112,750.000 \text { GALLON SINGLE SHELL UNDERGROUND STORAGE } \\
\text { TANK }\end{array}$ \\
\hline Em Point & Srce Nbr & Srce Type & Hrs Day & Days Week & Wks Year & Summary Process Description \\
\hline $200 \mathrm{~W}$ P-241TX113 001 & 1 & TANKS AND VESSELS & 24 & 7 & 52 & $\begin{array}{l}241-T X-113.750 .000 \text { GALLON SINGLE SHELL UNDERGROUND STORAGE } \\
\text { TANK }\end{array}$ \\
\hline Em Point & Srce Nbr & Srce Type & Hrs Day & Days Week & Wks Year & Summary Process Description \\
\hline $200 \mathrm{~W}$ P-241TX114001 & 1 & TANKS AND VESSELS & 24 & 7 & 52 & $\begin{array}{l}241-T X-114,750.000 \text { GALLON SINGLE SHELL UNOERGROUND STORAGE } \\
\text { TANK }\end{array}$ \\
\hline
\end{tabular}


AIR EMISSIONS INVENTORY PERMIT APPLICATION REPORT (UP460) FACILITY NAME: $200 \mathrm{~W}$ Tank Farm and Associated Structures

Source (Process) Listing

\begin{tabular}{|c|c|c|c|c|c|c|}
\hline En Point & Srce Nbr & Srce Type & Hrs Day & Days Week & Wks Year & Summary Process Description \\
\hline 200W P-241TX115001 & 1 & TANKS AND VESSELS & 24 & 7 & 52 & $\begin{array}{l}241-T X-115.750 .000 \text { GALLON SINGLE SHELL UNDERGROUND STORAGE } \\
\text { TANK }\end{array}$ \\
\hline Em Point & Srce Nbr & Srce Type & Hrs Day & Days Week & Wks Year & Summary Process Description \\
\hline $200 \mathrm{~W}$ P-241TX116 001 & i & TANKS AND VESSELS & 24 & $\overline{7}$ & 52 & $\begin{array}{l}241-T X-116,750.000 \text { GALLON SINGLE SHELL UNDERGROUND STORAGE } \\
\text { TANK }\end{array}$ \\
\hline Em Point & Srce Nbr & Srce Type & Hrs Day & Days Week & Wks Year & Summary Process Description \\
\hline 200W P-241TX117 001 & 1 & TANKS AND VESSELS & 24 & 7 & 52 & $\begin{array}{l}241-T X-117.750 .000 \text { GALLON SINGLE SHELL UNDERGROUND STORAGE } \\
\text { TANK }\end{array}$ \\
\hline Em Point & Srce Nbr & Srce Type & Hrs Day & Oays Week & Wks Year & Sumary Process Description \\
\hline $200 \mathrm{~W}$-241TX118 001 & 1 & TANKS AND VESSELS & 24 & 7 & 52 & $\begin{array}{l}241-T X-118.750 .000 \text { GALLON SINGLE SHELL UNDERGROUND STORAGE } \\
\text { TANK }\end{array}$ \\
\hline En Point & Srce Nbr & Srce Type & Hrs Day & Days Week & Wks Year & Summary Process Description \\
\hline $200 \mathrm{~W}$ P-241TX152001 & 1 & PROCESS AND PRODUCTION & 24 & & 52 & 241-TX-152 DIVERSION BOX \\
\hline
\end{tabular}

THE 241 -TX-152 DIVERSION BOX IS A REINFORCED CONCRETE STRUCTURE WITH OVERALL DIMENSIONS OF $~ 10.5$ FEET LONG. 8 FEET WIDE. AND 12 FEET DEEP. THE BOX DRAINS TO CATCH STATION $244-T X$ AND IS EQUIPPEO WITH A LEAK DETECTOR THAT ALARMS AT 244-S.
THE UNIT TRANSPORTS RADIOACTIVE WASTE SOLUTIONS FROM PROCESSING AND DECONTAMINATION OPERATIONS.

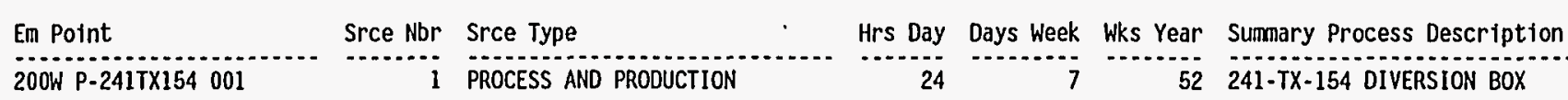

THE 241 -TX-154 DIVERSION BOX IS A REINFORCED CONCRETE STRUCTURE WITH OVERALL DIMENSIONS OF $\sim 36^{\circ} \times 10^{\circ} \times 18^{\circ}$ (LXWXD). THE BOX DRAINS TO CATCH TANK THE DIVERSION BOX TRANSPORTS RADIOACTIVE WASTE SOLUTIONS FROM PROCESSING AND DECONTAMINATION OPERATIONS.
En Point Srce Nbr Srce Type Hrs Day Days Week Wks Year Summary Process Description $200 \mathrm{~W}$ P-241TX302C 00 1 PROCESS AND PRODUCTION
$24-52$ 241-TX-302C CATCH TANK

THE CATCH TANK CONSISTS OF A HORIZONTAL CYLINDRICAL VESSEL MADE OF CARBON STEEL. A STEAM JET BOX RESTING ON THE TANK. AND A PUMP PIT ABOVE THE TANK. THE TANK SERVES DIVERSION BOX $241-T X-154$.

THE PUMP PIT IS 5'0 I.0. GALVANIZED CORRUGATED METAL PIPE WHICH IS 4.6" DEEP. A 12" SCH. 40 RISER ASTM A53 OESCENDS APPROXIMATELY 23' (TX) FROM THE

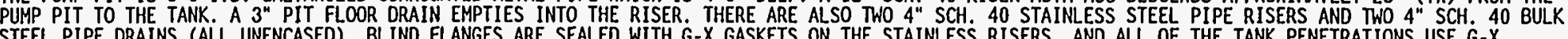
STEEL PIPE DRAINS (ALL UNENCASED). BLIND FLANGES ARE SEALED WITH G-X GASKETS ON THE STAINLESS RISERS. AND ALL OF THE TANK PENETRATIONS USE G- $X$

THE TANK ITSELF RESTS ON A SAND BED. IT IS MADE OF 9/16" THICK CARBON STEEL, 8.10 7/8" I.D. AND APPROXIMATELY 40 FT. LONG. A $1 "$ UNENCASED RAW WATER LINE SUPPLIES FLUSH WATER TO THE STEAM JET BOX RESTING ON THE TANK. THE INTERIOR OF THE TANK IS LEFT UNPAINTED. WHILE THE EXTERIOR HAS A COAL TAR PER H-2-833 NOTE 4. H-2-832, 833 H-2-71643 


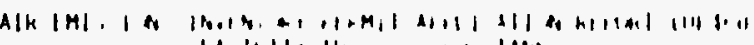

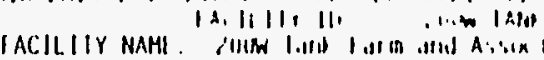

Source (Process) Listing

\begin{tabular}{|c|c|c|c|c|c|c|}
\hline Em Point & Srce Nbr & Srce Type & Hrs Day & Days Week & Wks Year & Surmary Process Description \\
\hline 200W P-241TY101 001 & 1 & TANKS AND VESSELS & 24 & 7 & 52 & $\begin{array}{l}241-\text { TY-101. } 750.000 \text { GALLON SINGLE SHELL UNDERGROUND STORAGE } \\
\text { TANK }\end{array}$ \\
\hline Em Point & Srce Nbr & Srce Type & Hrs Day & Days week & Wks Year & Sumunary Process Description \\
\hline 200W P-241TY102001 & 1 & TANKS AND VESSELS & 24 & 7 & 52 & $\begin{array}{l}241-\text { TY-102, } 750.000 \text { GALLON SINGLE SHELL UNDERGROUND STORAGE } \\
\text { TANK }\end{array}$ \\
\hline Em Point & Srce Nbr & Srce Type & Hrs Day & Days Week & Wks Year & Surnary Process Description \\
\hline 200W P-241TY103001 & 1 & TANKS AND VESSELS & 24 & 7 & 52 & $\begin{array}{l}241-T Y-103,750,000 \text { GALLON SINGLE SHELL UNDERGROUND STORAGE } \\
\text { TANK }\end{array}$ \\
\hline Em Point & Srce Nbr & Srce Type & Hrs Day & Days Week & Wks Year & Summary Process Description \\
\hline 200W P-241TY104001 & 1 & TANKS AND VESSELS & 24 & 7 & 52 & $\begin{array}{l}241-T Y-104.750 .000 \text { GALLON SINGLE SHELL UNDERGROUND STORAGE } \\
\text { TANK }\end{array}$ \\
\hline Em Point & Srce Nbr & Srce Type & Hrs Day & Days Week & Whs Year & Summary Process Description \\
\hline 200W P-241TY105001 & 1 & TANKS AND VESSELS & 24 & 7 & 52 & $\begin{array}{l}241-\text { TY-105. } 750.000 \text { GALLON SINGLE SHELL UNDERGROUND STORAGE } \\
\text { TANK }\end{array}$ \\
\hline Em Point , & Srce Nbr & Srce Type & Hrs Day & Days Week & Wks Year & Summary Process Description \\
\hline $200 \mathrm{~W}$ P-241TY106 001 & 1 & TANKS AND VESSELS & 24 & 7 & 52 & $\begin{array}{l}241-\text { TY- } 106,750.000 \text { GALLON SINGLE SHELL UNDERGROUND STORAGE } \\
\text { TANK }\end{array}$ \\
\hline Em Point & Srce Nbr & Srce Type & Hrs Day & Days Week & Wks Year & Summary Process Description \\
\hline 200W P-241U101 001 & 1 & TANKS AND VESSELS & 24 & 7 & 52 & $\begin{array}{l}241-U-101,530,000 \text { GALLON SINGLE SHELL UNDERGROUND STORAGE } \\
\text { TANK }\end{array}$ \\
\hline En Point & Srce Nbr & Srce Type & Hrs Day & Days Week & Wks Year & Sumnary Process Description \\
\hline $200 W$ P-241U102 001 & 1 & TANKS AND VESSELS & 24 & 7 & 52 & $\begin{array}{l}241-U-102.530 .000 \text { GALLON SINGLE SHELL UNDERGROUND STORAGE } \\
\text { TANK }\end{array}$ \\
\hline Em Point & Srce Nbr & Srce Type & Hrs Day & Days Week & Wks Year & Summary Process Description \\
\hline 200W P-241U103001 & 1 & TANKS AND VESSELS & 24 & 7 & 52 & $\begin{array}{l}241-U-103.530 .000 \text { GALLON SINGLE SHELL UNDERGROUND STORAGE } \\
\text { TANK }\end{array}$ \\
\hline
\end{tabular}

Page \#: 28 of 89 Report Run Date: 22-APR-95 
AIR EMISSIONS INVENTORY PERMIT APPLICATION REPORT (UP460)

FACILITY NAME: 200W Tank Farm and Associated Structures

\section{Source (Process) Listing}

\begin{tabular}{|c|c|c|c|c|c|c|}
\hline Em Point & Srce Nbr & Srce Type & Hrs Day & Days Week & Whs Year & Summary Process Description \\
\hline 200W P-241U104 001 & 1 & TANKS AND VESSELS & 24 & 7 & 52 & $\begin{array}{l}241-U-104.530 .000 \text { GALLON SINGLE SHELL UNDERGROUND STORAGE } \\
\text { TANK }\end{array}$ \\
\hline Em Point & Srce Nbr & Srce Type & Hrs Day & Days Week & Wks Year & Summary Process Description \\
\hline 200W P-241U105 001 & 1 & TANKS AND VESSELS & 24 & 7 & 52 & $\begin{array}{l}241-U-105,530.000 \text { GALLON SINGLE SHELL UNDERGROUND STORAGE } \\
\text { TANK }\end{array}$ \\
\hline Em Point & Srce Nbr & Srce Type & Hrs Day & Days Week & Whs Year & Summary Process Description \\
\hline 200W P-241U106 001 & 1 & TANKS AND VESSELS & 24 & 7 & 52 & $\begin{array}{l}241-U-106.530 .000 \text { GALLON SINGLE SHELL UNDERGROUND STORAGE } \\
\text { TANK }\end{array}$ \\
\hline Em Point & Srce Nbr & Srce Type & Hrs Day & Days Week & Wks Year & Surmary Process Description \\
\hline 200W P-241U107001 & 1 & TANKS AND VESSELS & 24 & 7 & 52 & $\begin{array}{l}241-U-107,530.000 \text { GALLON SINGLE SHELL UNDERGROUND STORAGE } \\
\text { TANK }\end{array}$ \\
\hline Em Point & Srce Nbr & Srce Type & Hrs Day & Days Week & Wks Year & Summary Process Description \\
\hline 200W P-241U108001 & 1 & TANKS AND VESSELS & 24 & 7 & 52 & $\begin{array}{l}241-U-108.530 .000 \text { GALLON SINGLE SHELL UNDERGROUND STORAGE } \\
\text { TANK }\end{array}$ \\
\hline Em Point & Srce Nbr & Srce Type & Hrs Day & Days Week & Wks Year & Summary Process Description \\
\hline 200W P-241U109001 & 1 & TANKS AND VESSELS & 24 & 7 & 52 & $\begin{array}{l}241-U-109,530,000 \text { GALLON SINGLE SHELL UNDERGROUND STORAGE } \\
\text { TANK }\end{array}$ \\
\hline Em Point & Srce Nbr & Srce Type & Hrs Day & Days Week & Whs Year & Surmary Process Description \\
\hline 200W P-241U110 001 & 1 & TANKS AND VESSELS & 24 & 7 & 52 & $\begin{array}{l}241-U-110.530,000 \text { GALLON SINGLE SHELL UNDERGROUND STORAGE } \\
\text { TANK }\end{array}$ \\
\hline Em Point & Srce Nbr & Srce Type & Hrs Day & Days Week & Wks Year & Summary Process Description \\
\hline 200W P-241U111 001 & 1 & TANKS AND VESSELS & 24 & 7 & 52 & $\begin{array}{l}241-U-111,530.000 \text { GALLON SINGLE SHELL UNDERGROUND STORAGE } \\
\text { TANK }\end{array}$ \\
\hline Em Point & Srce Nbr & Srce Type & Hrs Day & Days Week & Wks Year & Summary Process Description \\
\hline $200 \mathrm{~W}$ P-241U112001 & 1 & TANKS AND VESSELS & 24 & 7 & 52 & $\begin{array}{l}241-U-112.530 .000 \text { GALLON SINGLE-SHELL UNDERGROUND STORAGE } \\
\text { TANK }\end{array}$ \\
\hline
\end{tabular}


AIR EMISSIONS INVENTORY PERMIT APPLICATION REPORT (UP460)

FACILITY NAME: $200 \mathrm{~W}$ Tank Farm and Associated Structures

\section{Source (Process) Listing}

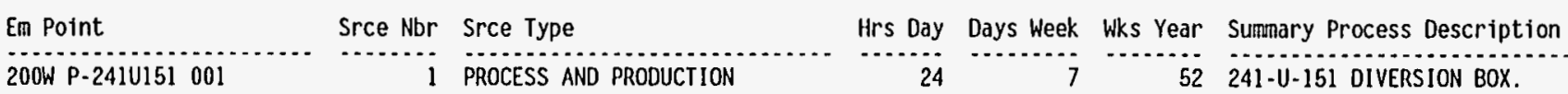

THE 241-U-151 DIVERSION BOX IS A REINFORCED CONCRETE STRUCTURE WITH DIMENSIONS OF $20^{\circ} \times 9^{\circ} \times 16^{\circ}$. THE BOX DRAINS TO CATCH TANK 241 -U-301. WASTE

TRANSPORTED VIA THE 241-U-151 DIVERSION BOX IS MIXED WASTE FROM PROCESSING AND DECONTAMINATION OPERATIONS.
Em Point
Srce $\mathrm{Nbr}$ Srce Type
Hrs Day Days Week Wks Year Sumnary Process Description
-.............
1 PROCESS AND PRODUCTION
$24 \quad 52 \quad 241-U-152$ DIVERSION BOX

THE $241-U-152$ DIVERSION BOX IS A REINFORCED CONCRETE STRUCTURE $28^{\circ} \times 9^{\circ} \times 17^{\circ}$ (LXWXD). THE BOX ORAINS TO CATCH TANK $241-U$ - 301 . THE BOX IS USED TO TRANSPORT MIXED WASTE SOLUTIONS FROM PROCESSING AND DECONTAMINATION OPERATIONS.

\begin{tabular}{|c|c|c|c|c|c|c|}
\hline Em Point & Srce $\mathrm{Nbr}$ & Srce Type & Hrs Day & Days Week & Wks Year & Summary Process Description \\
\hline 200W P-241U201 001 & 1 & TANKS AND VESSELS & 24 & 7 & 52 & $\begin{array}{l}241-U-201,55,000 \text { GALLON SINGLE SHELL UNDERGROUND STORAGE } \\
\text { TANK }\end{array}$ \\
\hline Em Point & Srce Nbr & Srce Type & Hrs Day & Days Week & Wks Year & Summary Process Description \\
\hline $200 W$ P-241U202 001 & 1 & TANKS AND VESSELS & 24 & 7 & 52 & $\begin{array}{l}241-U-202,55.000 \text { GALLON SINGLE SHELL UNDERGROUND STORAGE } \\
\text { TANK }\end{array}$ \\
\hline Em Point & Srce Nbr & Srce Type & Hrs Day & Days Week & Wks Year & Surmary Process Description \\
\hline 200W P-241U203001 & 1 & TANKS AND VESSELS & 24 & 7 & 52 & $\begin{array}{l}241-U-203.55,000 \text { GALLON SINGLE SHELL UNDERGROUND STORAGE } \\
\text { TANK }\end{array}$ \\
\hline En Point & Srce Nbr & Srce Type & Hrs Day & Days Week & Wks Year & Summary Process Description \\
\hline $200 \mathrm{~W}$ P-241U2Q4 001 & 1 & TANKS AND VESSELS & 24 & 7 & 52 & $\begin{array}{l}241-U-204.55 .000 \text { GALLON SINGLE SHELL UNDERGROUND STORAGE } \\
\text { TANK }\end{array}$ \\
\hline Em Point & Srce $\mathrm{Nbr}$. & Srce Type & Hrs Day & Days Week & Wks Year & Summary Process Description \\
\hline $200 \mathrm{~W} P-241 \mathrm{U} 301 \mathrm{~B} 001$ & 1 & PROCESS AND PRODUCTION & 24 & 7 & 52 & 241-U-301B CATCH TANK \\
\hline
\end{tabular}

241-U-301B CONSISTS OF A CATCH TANK AND A PUMP PIT DIRECTLY ABOVE THE CATCH TANK.

THE CATCH TANK IS A 36.000 GALLON UNLINED REINFORCED CONCRETE TANK APPROXIMATELY $66^{\circ}$ BELOW GRADE. THE WALL THICKNESS WAS NOT IDENTIFIED. BUT IT APPEARS TO BE APPROXIMATELY $6^{*}$ ON THE DRAWINGS LISTED BELOW. THE TANK DIAMETER IS $20^{\circ}$ AND THE BOTTOM IS FLAT. THE TANK OVERFLOWS THROUGH AN INLET LINE AT $15^{\prime} 6^{\prime \prime}$. THIS OVERFLOW LINE IS THE ORAIN FROM U-151 AND U-152.

THE PUMP PIT IS 5. IN DIAMETER, 5 DEEP. AND MADE OF 14 GAGE GALVANIZED CORRUGATED CULVERT PIPE. THE BASE IS 6' THICK CONCRETE. THE BASE IS NOT SHOWN TO BE SLOPING. THERE IS A 3"-M24 FLOOR DRAIN THAT EMPTIES INTO THE 12" RISER. THIS RISER IS LISTED IN THE PIPE SURVEY. H-2-(71643, 71653, 72903$)$ SDRE-TI $-0570-0$. 
AIR EMISSIONS INVENTORY PERMIT APPLICATION REPORT (UP460)

FACILITY NAME: $200 \mathrm{~W}$ Tank Farm and Associated structures

Source (Process) Listing

\begin{tabular}{|c|c|c|c|c|c|}
\hline Em Point & Srce $\mathrm{Nbr}$ & Srce Type & Hrs Day & Days Week Wks Year & Summary Process Description \\
\hline 200W P-241UX154001 & 1 & PROCESS AND PRODUCTION & 24 & 52 & 241-UX-154 DIVERSION BOX \\
\hline \multicolumn{6}{|c|}{$\begin{array}{l}\text { THE } 241 \text {-UX-154 DIVERSION BOX IS REINFORCED CONCRETE WITH OIMENSIONS OF } 48^{\circ} \times 10^{\circ} \times 17^{\circ} \text { (LXWXD). THE BOX DRAINS TO } 241 \text {-UX-302-A AND IS EQUIPPED WITH A } \\
\text { LEAK DETECTOR THAT ALARMS AT } 242-S \text {. THE DIVERSION BOX IS UNDERGROUND. THE BOX TRANSPORTS MIXED WASTE SOLUTIONS FROM PROCESSING AND DECONTAMINATION } \\
\text { OPERATIONS. }\end{array}$} \\
\hline Em Point & Srce Nbr & Srce Type & Hrs Day & Days Week Wks Year & Sumary Process Description \\
\hline 200W P-241UX302A 001 & 1 & PROCESS AND PRODUCTION & 24 & 52 & 241-UX-302A CATCH TANK \\
\hline \multicolumn{6}{|c|}{ 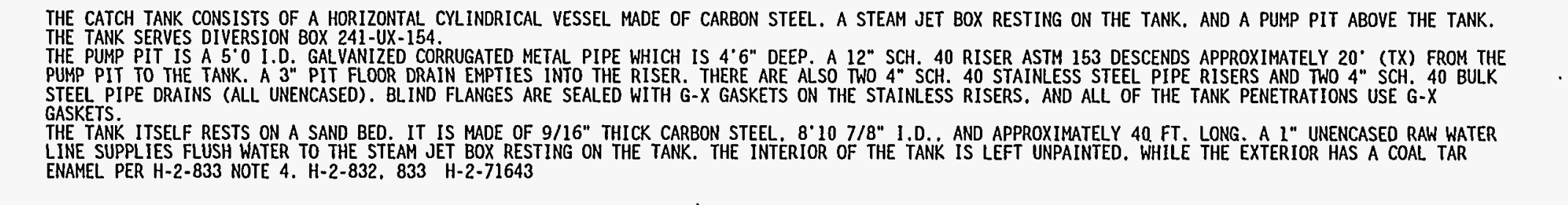 } \\
\hline Em Point & Srce Nbr & Srce Type & Hrs Day & Days Week Wks Year & Summary Process Description \\
\hline $200 \mathrm{~W}$ P-242S 001 & 1 & PROCESS AND PRODUCTION & & & $\begin{array}{l}\text { PUMP ROOM OF THE } 242-S \text { EVAPORATOR BUILDING. THIS IS IN A } \\
\text { STANOBY/SHUTDOWN MODE. }\end{array}$ \\
\hline Em Point & Srce Nbr & Srce Type & Hrs Day & Days Week Wks Year & Summary Process Description \\
\hline $200 \mathrm{~W}$ P-242S 001 & 2 & PROCESS AND PRODUCTION & & & $\begin{array}{l}\text { EVAPORATOR ROOM OF THE } 242-S \text { EVAPORATOR BUILDING. THIS IS IN } \\
\text { A STANDBY/SHUTDOWN MODE. }\end{array}$ \\
\hline Em Point & Srce $\mathrm{Nbr}$ & Srce Type & Hrs Day & Days Week Wks Year & Summary Process Description \\
\hline $200 \mathrm{~W} P-2425001$ & 3 & PROCESS AND PRODUCTION & & & $\begin{array}{l}\text { CONDENSER ROOM OF THE } 242-S \text { EVAPORATOR BUILDING. THIS IS IN } \\
\text { A STANDBY/SHUTDOWN MODE. }\end{array}$ \\
\hline Em Point & Srce $\mathrm{Nbr}$ & Srce Type & Hrs Day & Days Week Wks Year & Summary Process Description \\
\hline $200 W$ P-242S 001 & 4 & SOURCE EMISSIONS ROLL-UP & & & TOTAL EMISSIONS FOR EMISSION POINT P-242S-001 \\
\hline Em Point & Srce $\mathrm{Nbr}$ & Srce Type & Hrs Day & Days Week Wks Year & Surmary Process Description \\
\hline $200 \mathrm{~W} P-242 \mathrm{~T} 001$ & 1 & PROCESS AND PRODUCTION & & & $\begin{array}{l}\text { PUMP ROOM OF THE } 242-T \text { EVAPORATOR BUILDING PUT ON STANDBY- } \\
\text { SHUTDOWN ON APRIL } 15.1976 .\end{array}$ \\
\hline
\end{tabular}

Page \#: 31 of 89 Report Run Date: 22-APR-95 


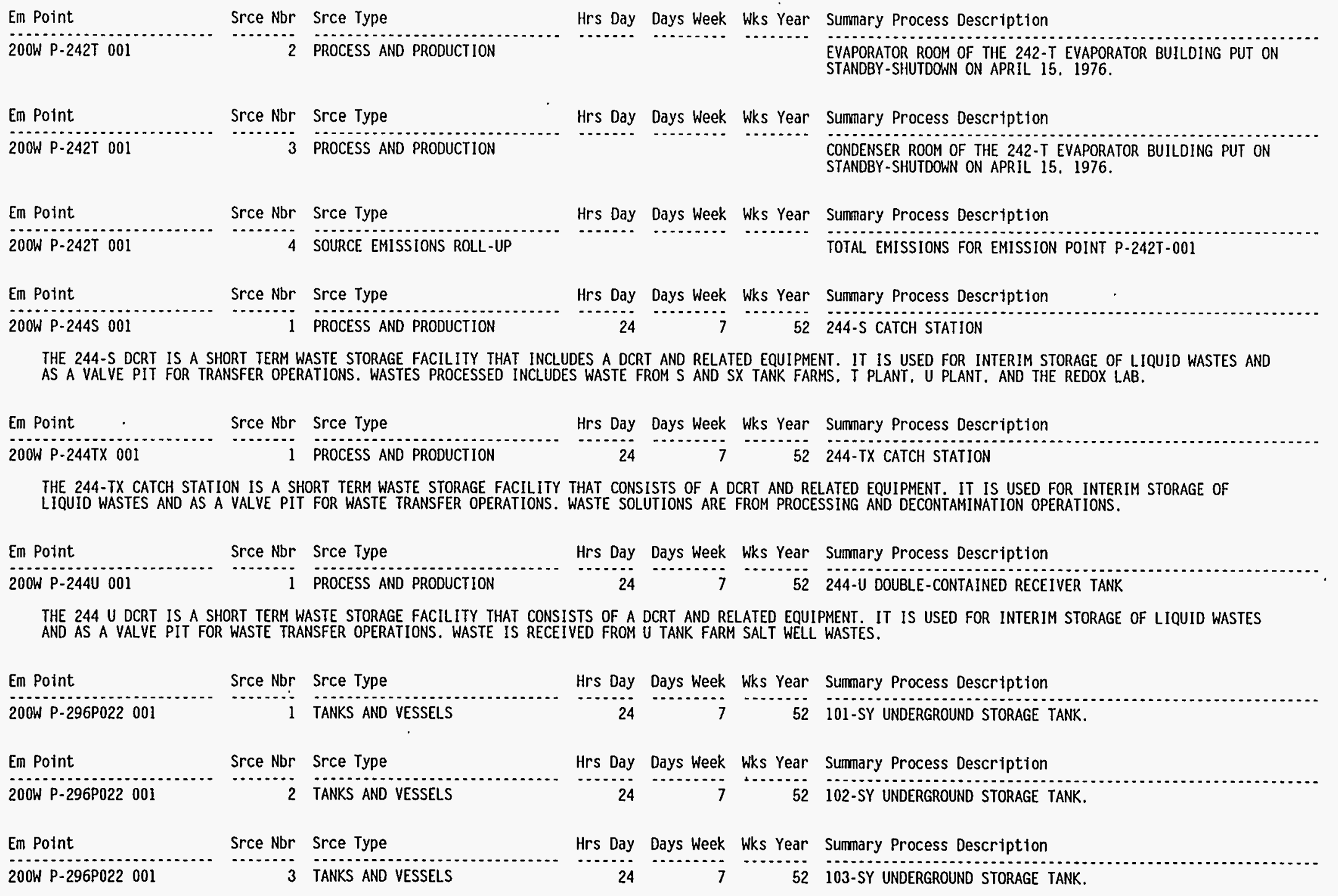


AIR EMISSIONS INVENTORY PERMIT APPLICATION REPORT (UP460) FACILITY NAME: 200W Tank Farm and Associated Structures

\section{Source (Process) Listing}

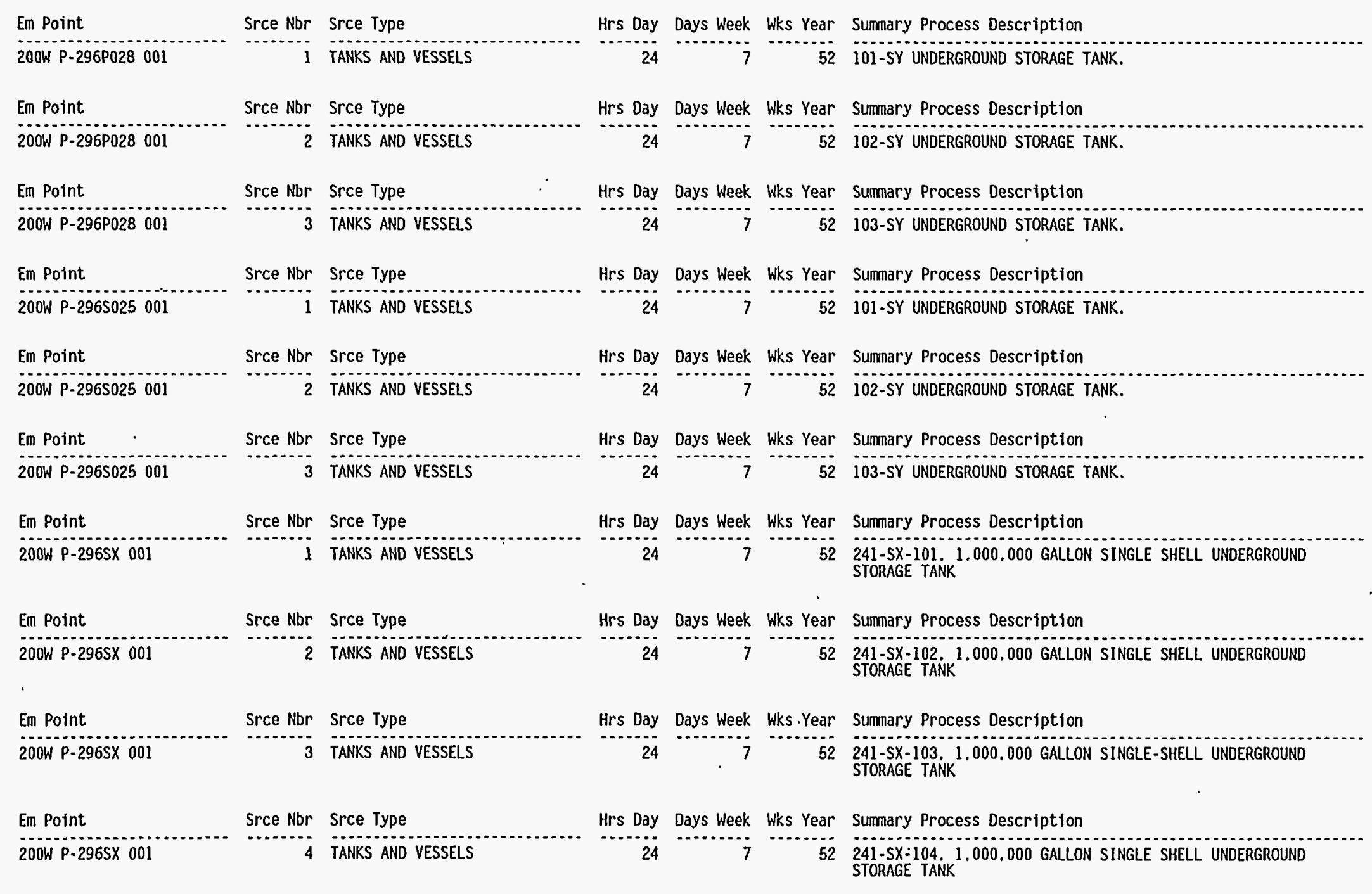


AIR EMISSIONS INVENTORY PERMIT APPLICATION REPORT (UP460)

FACILIIT NAME. FACILITY ID: $200 \mathrm{~W}$ TANK

Source (Process) Listing

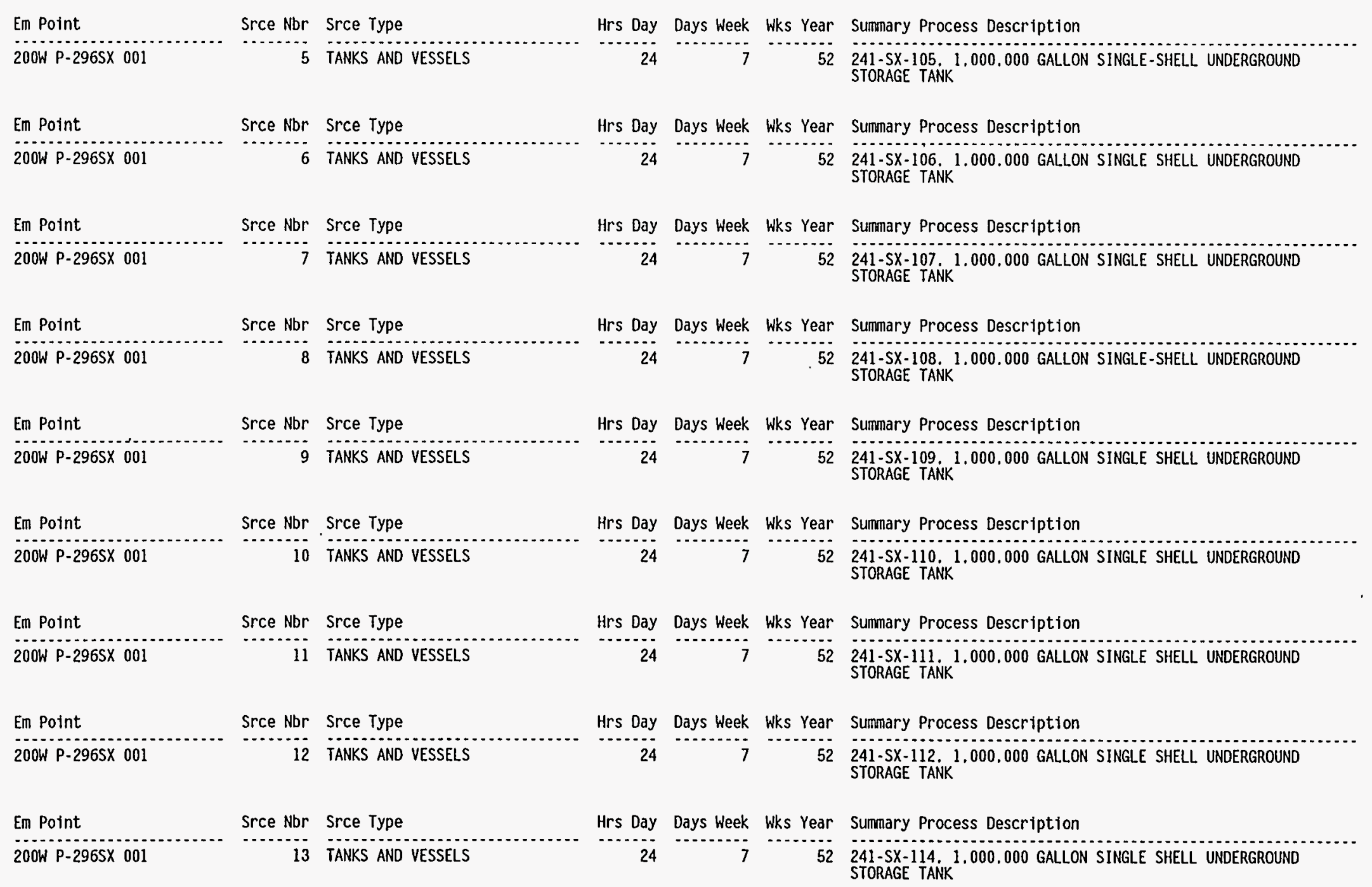

Page \#: 34 of 89 Report Run Date: 22 -APR-95 


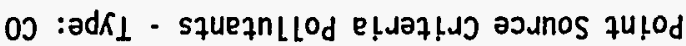

lang

won $\times 70$

.

lanj won

วdKl 175 JqN 175

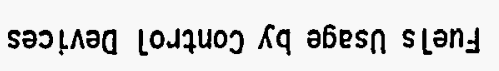

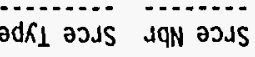

zulod wi

sassajodd asunos Kq abes

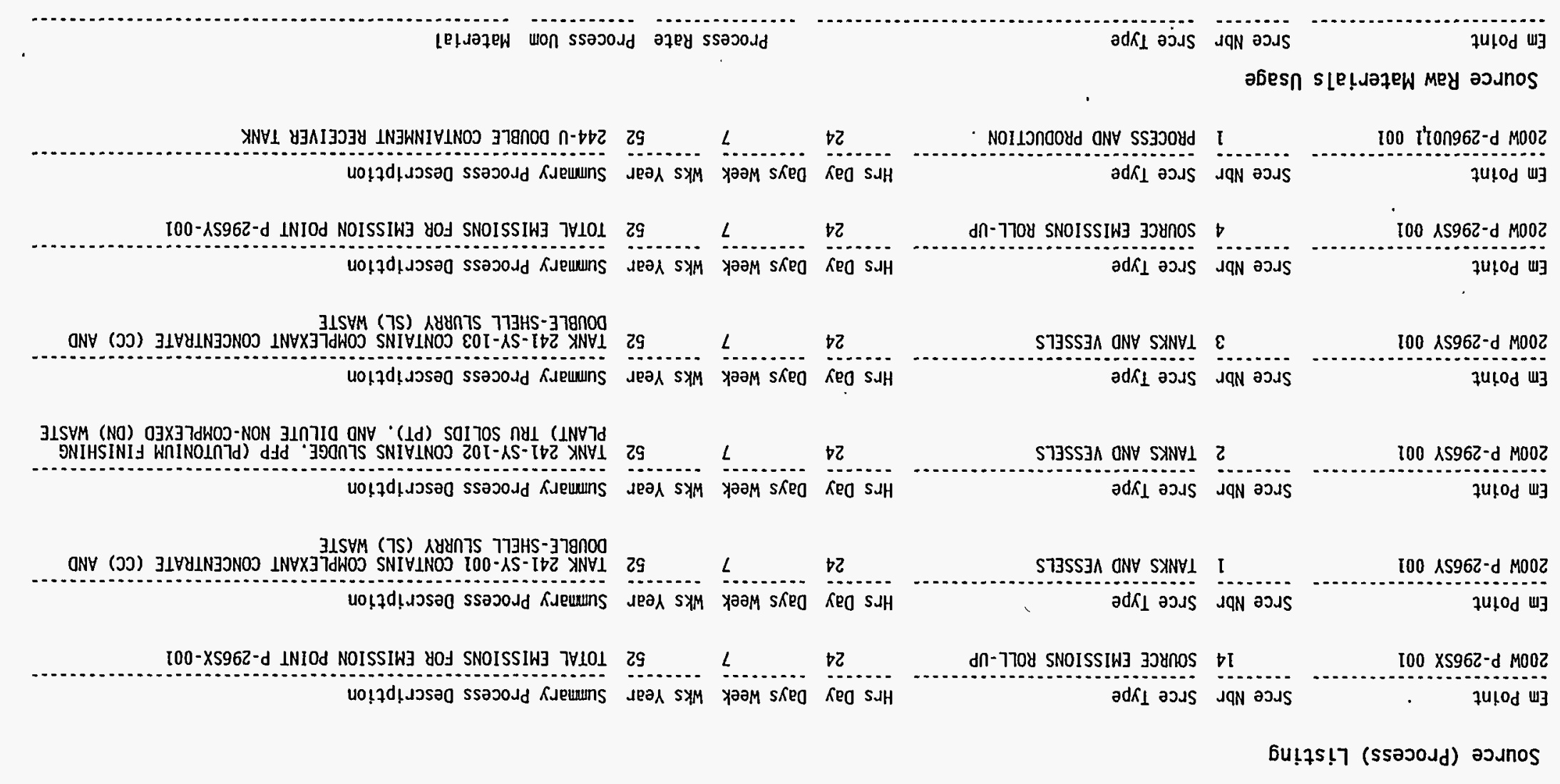

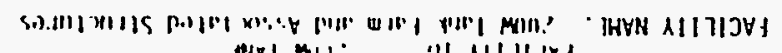

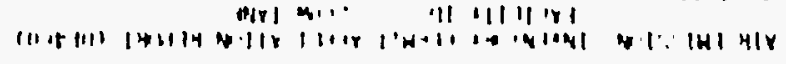


Point Source Criteria Pollutants - Type: Co

\begin{tabular}{|c|c|c|c|c|c|}
\hline Em Point & Srce Nbr & Type Pollutant & $\begin{array}{l}\text { Em. Pt. Potential } \\
\text { to Emit (tons/yr) }\end{array}$ & (tons/yr) & lutant \\
\hline $200 \mathrm{~W}$ P-241S101 001 & 1 & TANKS AND VESSELS & .000950000 & .000950000 & CARBON MONOXIDE \\
\hline 200W P-241S102001 & 1 & TANKS AND VESSELS & .000950000 & .000950000 & CARBON MONOXIDE \\
\hline 200W P-241S103001 & 1 & TANKS AND VESSELS & .000950000 & .000950000 & CARBON MONOXIDE \\
\hline 200W P-241S104001 & 1 & TANKS AND VESSELS & .000950000 & .000950000 & CARBON MONOXIDE \\
\hline 200W P-241S105 001 & 1 & IANKS AND VESSELS & .000950000 & .000950000 & CARBON MONOXIDE \\
\hline 200W P-241S106 001 & 1 & TANKS AND VESSELS & .000950000 & .000950000 & CARBON MONOXIDE \\
\hline 200W P-241S107001 & 1 & TANKS AND VESSELS & .000950000 & .000950000 & CARBON MONOXIDE \\
\hline 200W P-241S108 001 & 1 & TANKS AND VESSELS & .000950000 & .000950000 & CARBON MONOXIDE \\
\hline 200W P-241S109001 & 1 & TANKS AND VESSELS & .000950000 & .000950000 & CARBON MONOXIDE \\
\hline 200W P-241S110 001 & 1 & TANKS AND VESSELS & .000950000 & .000950000 & CARBON MONOXIDE \\
\hline 200W P-241S111 001 & 1 & TANKS AND VESSELS & .000950000 & .000950000 & CARBON MONOXIDE \\
\hline 200W P-241S112001 & 1 & TANKS AND VESSELS & .000950000 & .000950000 & CARBON MONOXIDE \\
\hline 200W P-241SX115001 & 1 & TANKS AND VESSELS & .000439000 & .000439000 & CARBON MONOXIDE \\
\hline 200W P-241T101 001 & 1 & TANKS AND VESSELS & .000950000 & .000950000 & CARBON MONOXIDE \\
\hline 200W P-241T102001 & 1 & TANKS AND VESSELS & .000950000 & .000950000 & CARBON MONOXIDE \\
\hline 200W P-241T103001 & 1 & TANKS AND VESSELS & .000950000 & .000950000 & CARBON MONOXIDE \\
\hline 200W P-241T104 001 & 1 & TANKS AND VESSELS & .000439000 & .000439000 & CARBON MONOXIDE \\
\hline 200W P-241T105 001 & 1 & TANKS AND VESSELS & .000950000 & .000950000 & CARBON MONOXIDE \\
\hline 200W P-241T106 001 & 1 & TANKS AND VESSELS & .000950000 & .000950000 & CARBON MONOXIDE \\
\hline 200W P-241T107001 & 1 & TANKS AND VESSELS & .000950000 & .000950000 & CARBON MONOXIDE \\
\hline 200W P-241T108 001 & 1 & TANKS AND VESSELS & . . .000439000 & .000439000 & CARBON MONOXIDE \\
\hline 200W P-241T109001 & 1 & TANKS AND VESSELS & .000950000 & .000950000 & CARBON MONOXIDE \\
\hline 200W P-241T110 001 & 1 & TANKS AND VESSELS & .000950000 & .000950000 & CARBON MONOXIDE \\
\hline 200W P-241T111 001 & 1 & TANKS AND VESSELS & .000950000 & .000950000 & CARBON MONOXIDE \\
\hline $200 \mathrm{~W} P-241 T 112001$ & 1 & TANKS AND VESSELS & .000950000 & .000950000 & CARBON MONOXIDE \\
\hline
\end{tabular}


Point Source Criteria Pollutants - Type: co

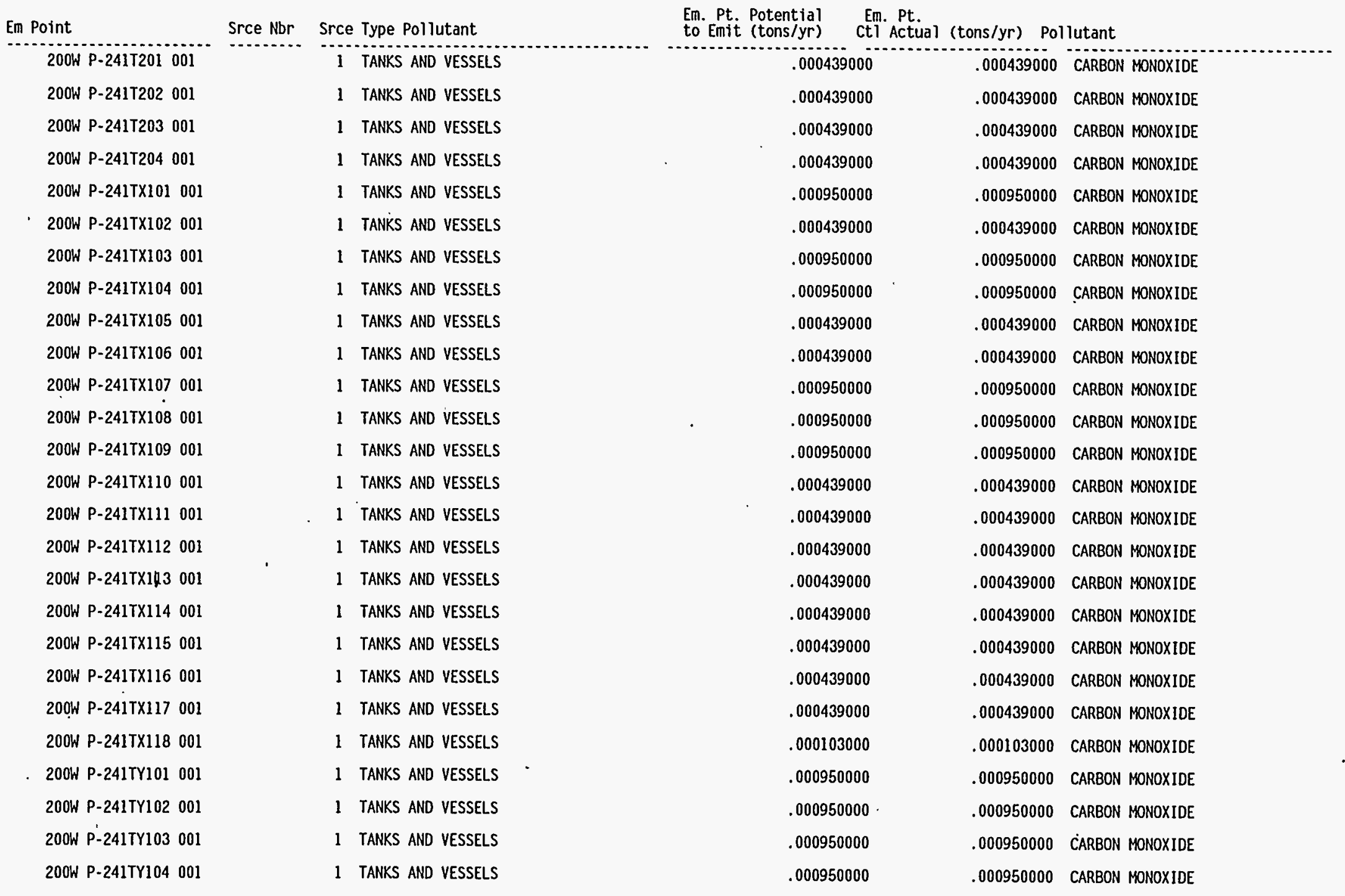


Point Source Criteria Pollutants - Type: CO

\begin{tabular}{|c|c|c|c|c|c|c|}
\hline Em Point & Srce Nbr & Srce & Type Pollutant & $\begin{array}{l}\text { Em. Pt. Potential } \\
\text { to Emit (tons/yr) }\end{array}$ & (tons/yr) & lutant \\
\hline 200W P-241TY105001 & & 1 & TANKS AND VESSELS & .000439000 & .000439000 & CARBON MONOXIDE \\
\hline 200W P-241TY106 001 & & 1 & TANKS AND VESSELS & .000439000 & .000439000 & CARBON MONOXIDE \\
\hline 200W P-241U101 001 & & 1 & TANKS AND VESSELS & .000439000 & .000439000 & CARBON MONOXIDE \\
\hline 200W P-241U102 001 & & 1 & TANKS ANO VESSELS & .000950000 & .000950000 & CARBON MONOXIDE \\
\hline 200W P-241U103001 & & 1 & TANKS AND VESSELS & .000950000 & .000950000 & CARBON MONOXIDE \\
\hline 200W P-241U104 001 & & 1 & TANKS AND VESSELS & .000439000 & .000439000 & CARBON MONOXIDE \\
\hline 200W P-241U105001 & & 1 & TANKS AND VESSELS & .000950000 & .000950000 & CARBON MONOXIDE \\
\hline 200W P-241U106 001 & & 1 & TANKS AND VESSELS & .000950000 & .000950000 & CARBON MONOXIDE \\
\hline 200W P-241U107 001 & & 1 & TANKS AND VESSELS & .000950000 & .000950000 & CARBON MONOXIDE \\
\hline 200W P-241U108001 & & 1 & TANKS AND VESSELS & .000950000 & .000950000 & CARBON MONOXIDE \\
\hline 200W P-241U109 001 & & 1 & TANKS AND VESSELS & .000950000 & .000950000 & CARBON MONOXIOE \\
\hline 200W P-241U110 001 & & 1 & TANKS AND VESSELS & .000950000 & .000950000 & CARBON MONOXIDE \\
\hline $200 \mathrm{~W} P-2410111001$ & & 1 & TANKS AND VESSELS & .000950000 & .000950000 & CARBON MONOXIDE \\
\hline 200W P-24lU112001 & & 1 & TANKS AND VESSELS & .000439000 & .000439000 & CARBON MONOXIDE \\
\hline 200W P-241U201 001 & & 1 & TANKS AND VESSELS & .000439000 & .000439000 & CARBON MONOXIDE \\
\hline 200W-P-241U202001 & & 1 & TANKS AND VESSELS & .000439000 & .000439000 & CARBON MONOXIDE \\
\hline 200W P-241U203 001 & & 1 & TANKS AND VESSELS & .000439000 & .000439000 & CARBON MONOXIDE \\
\hline 200W P-241U204 001 & & 1 & TANKS AND VESSELS & .000439000 & .000439000 & CARBON MONOXIDE \\
\hline $200 \mathrm{~W} P-2965 \times 001$ & & 14 & SOURCE EMISSIONS ROLL-UP & .224000000 & .224000000 & CARBON MONOXIDE \\
\hline 200W P-296SY 001 & & 4 & SOURCE EMISSIONS ROLL-UP & .051600000 & .051600000 & CARBON MONOXIDE \\
\hline Sum & & & & .326945000 & .326945000 & \\
\hline
\end{tabular}


Point Source Criteria Pollutants - Type: NOX

\begin{tabular}{|c|c|c|c|c|c|c|c|c|}
\hline Em Point & Srce Nbr & Srce & Type Pollutant & & $\begin{array}{l}\text { Em. Pt. Potential } \\
\text { to Emit (tons/yr) }\end{array}$ & (tons/yr) & liutant & \\
\hline 200W P-241S101 001 & & 1 & TANKS AND VESSELS & & .000057200 & .000057200 & NITROGEN OXIDE & $\{\mathrm{NO}\}$ \\
\hline 200W P-241S102001 & & 1 & TANKS AND VESSELS & & .000057200 & .000057200 & NITROGEN OXIDE & (NO) \\
\hline 200W P-241S103001 & & 1 & TANKS AND VESSELS & & .000057200 & .000057200 & NITROGEN OXIDE & $\{\mathrm{NO}\}$ \\
\hline 200W P-241S104001 & & 1 & TANKS AND VESSELS & & .000057200 & .000057200 & NITROGEN OXIDE & $\{\mathrm{NO}\}$ \\
\hline 200W P-241S105001 & & 1 & TANKS AND VESSELS & & .000057200 & .000057200 & NITROGEN OXIDE & $\{\mathrm{NO}\}$ \\
\hline $200 \mathrm{~W}$ P-241S107001 & & 1 & TANKS AND VESSELS & & .000057200 & .000057200 & NITROGEN OXIDE & $\{\mathrm{NO}\}$ \\
\hline $200 \mathrm{~W} P-241 S 108001$ & & 1 & TANKS AND VESSELS & & .000057200 & .000057200 & NITROGEN OXIDE & $\{\mathrm{NO}\}$ \\
\hline 200W P-241S109001 & & 1 & TANKS AND VESSELS & & .000057200 & .000057200 & NITROGEN OXIDE & $\{\mathrm{NO}\}$ \\
\hline $200 \mathrm{~W} P-241 S 110001$ & & 1 & TANKS AND VESSELS & & .000057200 & .000057200 & NITROGEN OXIDE & $\{\mathrm{NO}\}$ \\
\hline 200W P-241S111 001 & & 1 & TANKS AND VESSELS & & .000057200 & .000057200 & NITROGEN OXIDE & $\{\mathrm{NO}\}$ \\
\hline $200 \mathrm{~W}$ P-241S112001 & & 1 & TANKS AND VESSELS & & .000057200 & .000057200 & NITROGEN OXIDE & $\{\mathrm{NO}\}$ \\
\hline 200W P-241SX115001 & & 1 & TANKS AND VESSELS & & .000026400 & .000026400 & NITROGEN OXIDE & (NO) \\
\hline 200W P-241T101 001 & & 1 & TANKS AND VESSELS & & .000057200 & .000057200 & NITROGEN OXIDE & $\{\mathrm{NO}\}$ \\
\hline 200W P-241T102001 & & 1 & TANKS AND VESSELS & & .000057200 & .000057200 & NITROGEN OXIDE & $\{\mathrm{NO}\}$ \\
\hline 200W P-241T103 001 & & 1 & TANKS AND VESSELS & & .000057200 & .000057200 & NITROGEN OXIDE & $\{\mathrm{NO}\}$ \\
\hline 200W P-241T104 001 & & 1 & TANKS AND VESSELS & & .000026400 & .000026400 & NITROGEN OXIDE & $\{\mathrm{NO}\}$ \\
\hline 200W P-241T105 001 & & 1 & TANKS AND VESSELS & & .000057200 & .000057200 & NITROGEN OXIDE & $\{\mathrm{NO}\}$ \\
\hline 200W P-241T106001 & & 1 & TANKS AND VESSELS & · & .000057200 & .000057200 & NITROGEN OXIDE & (NO) \\
\hline 200W P-241T107001 & & 1 & TANKS AND VESSELS & & .000057200 & .000057200 & NITROGEN OXIDE & $\{\mathrm{NO}\}$ \\
\hline $200 \mathrm{~W}$ P-241T108 001 & & 1 & TANKS AND VESSELS & & .000026400 & .000026400 & NITROGEN OXIDE & (NO) \\
\hline 200W P-241T109001 & & 1 & TANKS AND VESSELS & & .000057200 & .000057200 & NITROGEN OXIOE & $\{\mathrm{NO}\}$ \\
\hline 200W P-241T110 001 & & 1 & TANKS AND VESSELS & & .000057200 & .000057200 & NITROGEN OXIOE & $\{\mathrm{N} 0\}$ \\
\hline 200W P-241T111 001 & & 1 & TANKS AND VESSELS & & .000057200 & .000057200 & NITROGEN OXIDE & $\{\mathrm{NO}\}$ \\
\hline 200W P-241T112001 & & 1 & TANKS AND VESSELS & & .000057200 & .000057200 & NITROGEN OXIDE & $\{\mathrm{NO}\}$ \\
\hline
\end{tabular}


Point Source Criteria Pollutants - Type: NOX

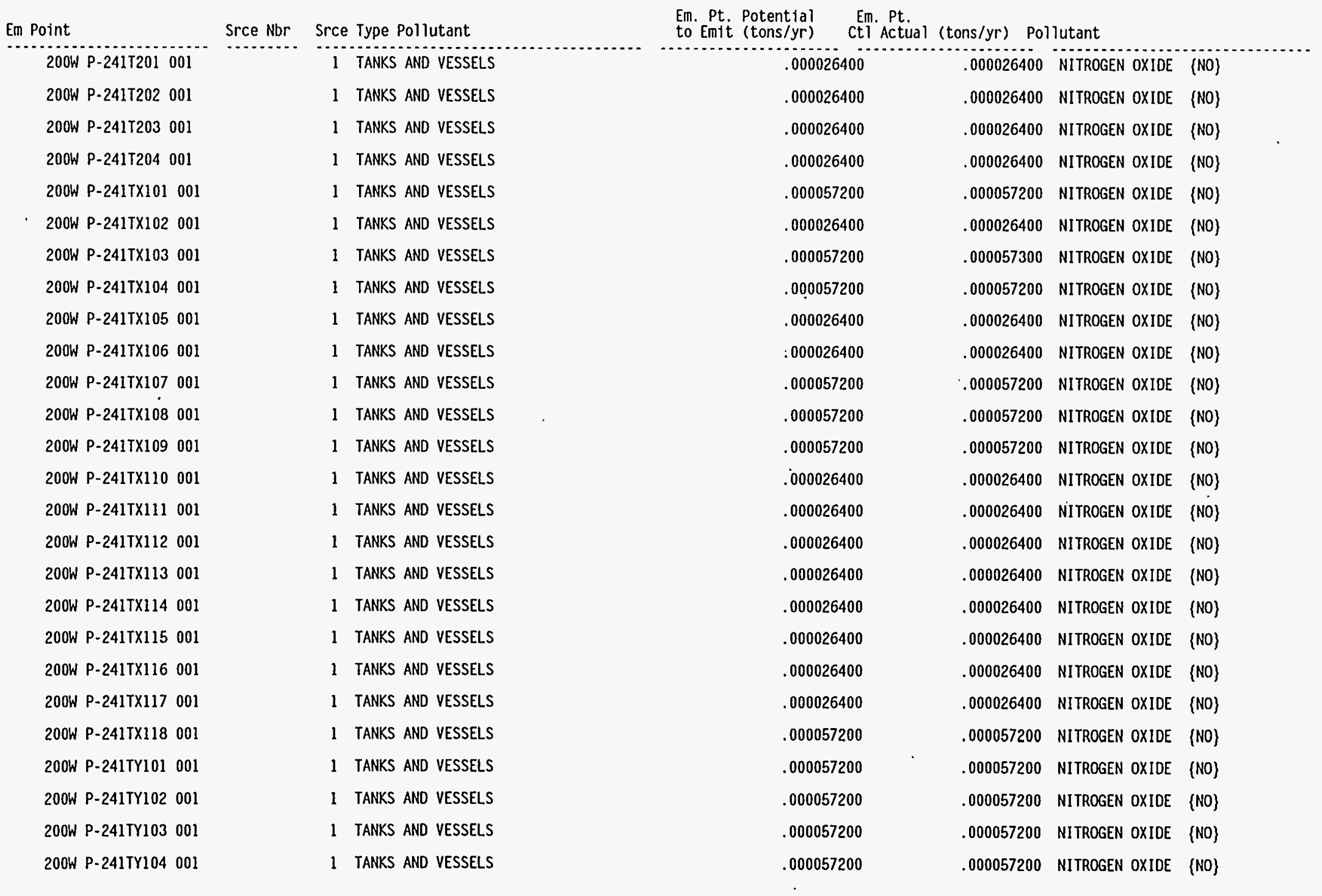


Point Source Criteria Pollutants - Type: NoX

\begin{tabular}{|c|c|c|c|c|c|c|}
\hline Em Point & Srce $\mathrm{Nbr}$ & Type Pollutant & $\begin{array}{l}\text { Em. Pt. Potential } \\
\text { to Emit (tons/yr) }\end{array}$ & (tons/yr) & lutant & \\
\hline 200W P-241TY105001 & 1 & TANKS AND VESSELS & .000026400 & .000026400 & NITROGEN OXIDE & (NO) \\
\hline 200W P-241TY106 001 & 1 & TANKS AND VESSELS & .000026400 & .000026400 & NITROGEN OXIDE & (NO) \\
\hline 200W P-241U101 001 & 1 & TANKS AND VESSELS & .000026400 & .000026400 & NITROGEN OXIDE & $\{N O\}$ \\
\hline 200W P-241U102001 & 1 & TANKS AND VESSELS & .000057200 & .000057200 & NITROGEN OXIDE & $\{N O\}$ \\
\hline 200W P-241U103001 & 1 & TANKS AND VESSELS & .000057200 & .000057200 & NITROGEN OXIOE & $\{N O\}$ \\
\hline $200 \mathrm{~W}$ P-241U104 001 & 1 & TANKS AND VESSELS & .000026400 & .000026400 & NITROGEN OXIDE & $\{N O\}$ \\
\hline 200W P-241U105001 & 1 & TANKS AND VESSELS & .000057200 & .000057200 & NITROGEN OXIDE & $\{N O\}$ \\
\hline 200W P-241U106 001 & 1 & TANKS AND VESSELS & .000057200 & .000057200 & NITROGEN OXIDE & $\{\mathrm{NO}\}$ \\
\hline 200W P-241U107001 & 1 & TANKS AND VESSELS & .000057200 & .000057200 & NITROGEN OXIDE & $\{\mathrm{NO}\}$ \\
\hline 200W P-241U108 001 & 1 & TANKS AND VESSELS & .000057200 & .000057200 & NITROGEN OXIDE & $\{\mathrm{NO}\}$ \\
\hline $200 \mathrm{~W}$ P-241U109001 & 1 & TANKS AND VESSELS & .000057200 & .000057200 & NITROGEN OXIDE & $\{\mathrm{NO}\}$ \\
\hline 200W P-241U110 001 & 1 & TANKS AND VESSELS & .000057200 & .000057200 & NITROGEN OXIDE & $\{\mathrm{NO}\}$ \\
\hline 200W P-241U111 001 & 1 & TANKS AND VESSELS & .000057200 & .000057200 & NITROGEN OXIDE & $\{N O\}$ \\
\hline 200W P-241U112001 & 1 & TANKS AND VESSELS & .000026400 & .000026400 & NITROGEN OXIDE & $\{N O\}$ \\
\hline 200W P-241U201 001 & 1 & TANKS AND VESSELLS & .000026400 & .000026400 & NITROGEN OXIDE & (NO) \\
\hline 200W P-241U202 001 & 1 & TANKS AND VESSELS & .000026400 & .000026400 & NITROGEN OXIDE & $\{N O\}$ \\
\hline 200W P-241U203 001 & 1 & TANKS AND VESSELS & .000026400 & .000026400 & NITROGEN OXIDE & $\langle N O\rangle$ \\
\hline 200W P-241U204 001 & 1 & TANKS AND VESSELS & .000026400 & .000026400 & NITROGEN OXIDE & (NO) \\
\hline 200W P-296SX 001 & 14 & -SOURCE EMISSIONS ROLL-UP & .000743000 & .000743000 & NITROGEN OXIDE & $\{\mathrm{NO}\}$ \\
\hline 200W P-296SY 00I & 4 & SOURCE EMISSIONS ROLL-UP & .000172000 & .000172000 & NITROGEN OXIDE & $\{\mathrm{NO}\}$ \\
\hline Sum & & & .004056600 & .004056700 & & \\
\hline
\end{tabular}




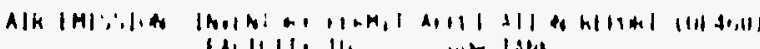

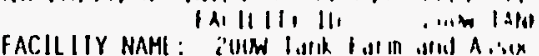

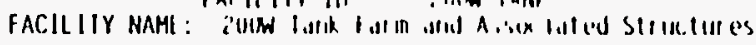

Point Source Criteria Pollutants - Type: VOC

\begin{tabular}{|c|c|c|c|c|c|}
\hline \multirow{2}{*}{$\begin{array}{l}\text { Em Polnt } \\
\text { 200W P-241S101 } 001\end{array}$} & \multirow{2}{*}{ Srce $\mathrm{Nbr}$} & \multirow{2}{*}{$\begin{array}{l}\text { Srce Type Pollutant } \\
1 \text { TANKS AND VESSELS }\end{array}$} & \multicolumn{3}{|c|}{$\begin{array}{l}\text { Em. Pt. } \\
\text { Ctl Actual (tons/yr) Pollutant }\end{array}$} \\
\hline & & & $\begin{array}{l}.000004130 \\
.000075100 \\
.000119000 \\
.001430000 \\
.000689000 \\
.000032800 \\
.000084000 \\
.000081300 \\
.000087600 \\
.000045700 \\
.000047900 \\
.000006670\end{array}$ & $\begin{array}{l}.000004130 \\
.000075100 \\
.000119000 \\
.001430000 \\
.000689000 \\
.000032800 \\
.000084000 \\
.000081300 \\
.000087600 \\
.000045700 \\
.000047900 \\
.000006670\end{array}$ & $\begin{array}{l}\text { 1.3-BUTADI ENE } \\
\text { 2-HEXANONE } \\
\text { 2-PENTANONE } \\
\text { ACETONE } \\
\text { ACETONITRILE } \\
\text { BENZENE } \\
\text { HEPTANE } \\
\text { METHYL N-AMYL KETONE (2-HEPTANONE) } \\
\text { N-HEXANE } \\
\text { NONANE } \\
\text { OCTANE } \\
\text { TOLUENE }\end{array}$ \\
\hline $200 \mathrm{~W} P-241 S 102001$ & & 1 TANKS AND VESSELS & $\begin{array}{l}.000004130 \\
.000075100 \\
.000119000 \\
.001430000 \\
.000689000 \\
.000032800 \\
.000084000 \\
.000081300 \\
.000087600 \\
.000045700 \\
.000047900 \\
.000006670\end{array}$ & $\begin{array}{l}.000004130 \\
.000075100 \\
.000119000 \\
.001430000 \\
.000689000 \\
.000032800 \\
.000084000 \\
.000081300 \\
.000087600 \\
.000045700 \\
.000047900 \\
.000006670\end{array}$ & $\begin{array}{l}\text { 1.3-BUTADIENE } \\
\text { 2-HEXANONE } \\
\text { 2-PENTANONE } \\
\text { ACETONE } \\
\text { ACETONITRILE } \\
\text { BENZENE } \\
\text { MEPTANE } \\
\text { METHYL N-AMTL KETONE (2-HEPTANONE) } \\
\text { N-HEXANE } \\
\text { NONANE } \\
\text { OCTANE } \\
\text { TOLUENE }\end{array}$ \\
\hline $200 \mathrm{~W} P-241 S 103001$ & & 1 TANKS AND VESSELS & $\begin{array}{l}.000004130 \\
.000075100 \\
.000119000 \\
.001430000 \\
.000689000 \\
.000032800 \\
.000084000 \\
.000081300 \\
.000087600 \\
.000045700 \\
.000047900 \\
.000006670\end{array}$ & $\begin{array}{l}.000004130 \\
.000075100 \\
.000119000 \\
.001430000 \\
.000689000 \\
.000032800 \\
.000084000 \\
.000081300 \\
.000087600 \\
.000045700 \\
.000047900 \\
.000006670\end{array}$ & $\begin{array}{l}\text { 1.3-BUTADIENE } \\
\text { 2-HEXANONE } \\
\text { 2-PENTANONE } \\
\text { ACETONE } \\
\text { ACETONITRILE } \\
\text { BENZENE } \\
\text { HEPTANE } \\
\text { METHYL N-AMYL KETONE (2-HEPTANONE) } \\
\text { N-HEXANE } \\
\text { NONANE } \\
\text { OCTANE } \\
\text { TOLUENE }\end{array}$ \\
\hline $200 \mathrm{~W} P-241 S 104001$ & & 1 TANKS AND VESSELS & $\begin{array}{l}.000004130 \\
.000075100 \\
.000119000 \\
.001430000 \\
.000689000 \\
.000032800 \\
.000084000 \\
.000081300 \\
.000087600 \\
.000045700 \\
.000047900 \\
.000006670\end{array}$ & $\begin{array}{l}.000004130 \\
.000075100 \\
.000119000 \\
.001430000 \\
.000689000 \\
.000032800 \\
.000084000 \\
.000081300 \\
.000087600 \\
.000045700 \\
.000047900 \\
.000006670\end{array}$ & $\begin{array}{l}\text { 1.3-BUTADIENE } \\
\text { 2-MEXANONE } \\
\text { 2-PENTANONE } \\
\text { ACETONE } \\
\text { ACETONITRILE } \\
\text { BENZENE } \\
\text { HEPTANE } \\
\text { METHYL N-AMYL KETONE (2-HEPTANONE) } \\
\text { N-HEXANE } \\
\text { NONANE } \\
\text { OCTANE } \\
\text { TOLUENE }\end{array}$ \\
\hline
\end{tabular}

Page \#: 42 of 89 Report Run Date: 22-APR-95 
AIR EMISSIONS INVENTORY PERMIT APPLICATION REPORT (UP460)

FACILITY NAME: 200W Tank Farm and Associated Structures

Point Source Criteria Pollutants - Type: VOC

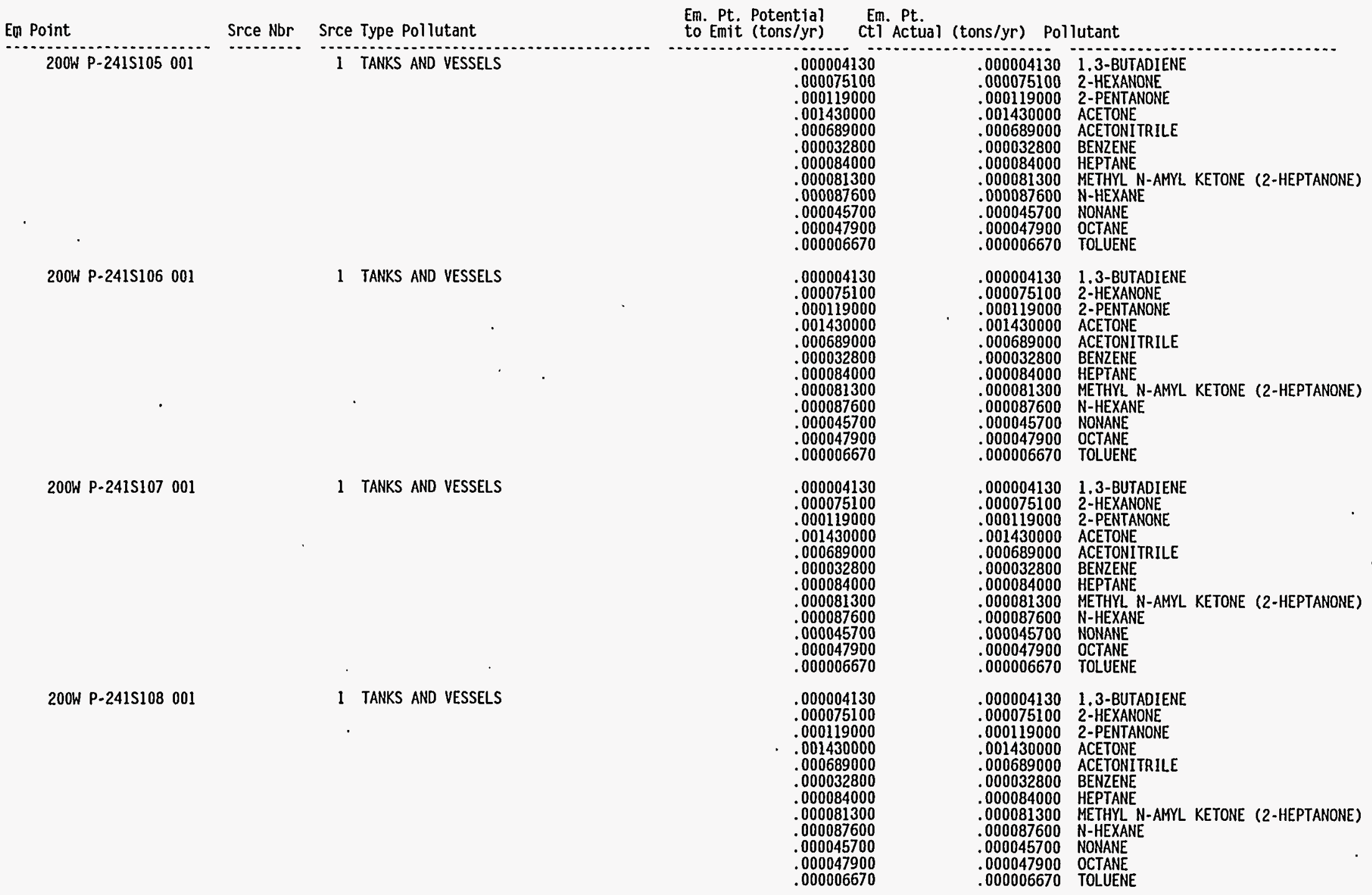

Page \#: 43 of 89 Report Run Date: 22-APR-95 
Point Source Criteria Pollutants - Type: VOC

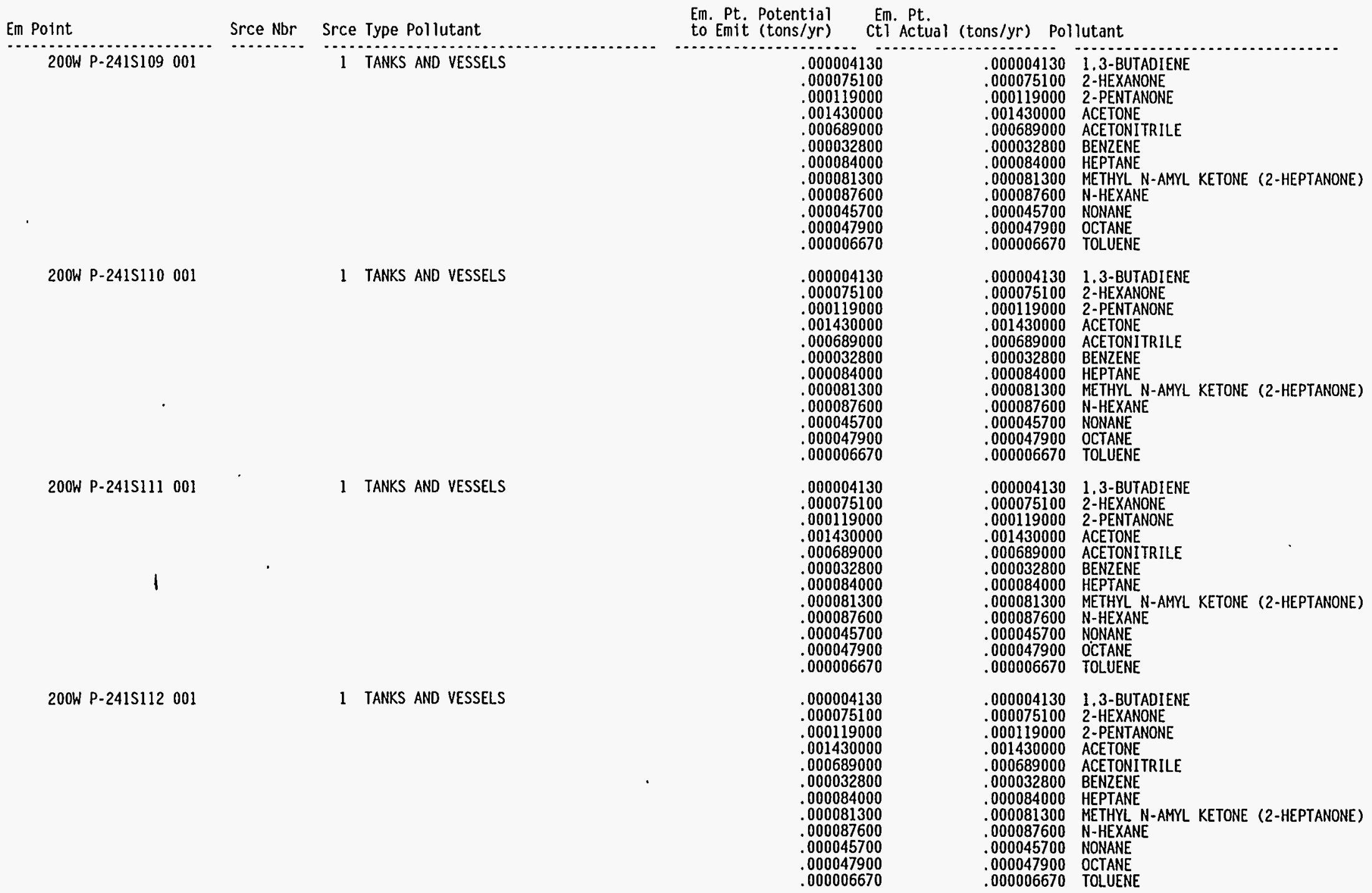


AIR EMISSIONS INVENTORY PERMIT APPLICATION REPORT (UP460)

FACILITY NAME: 20OW Tank Farm and Associated Structures

Point Source Criteria Pollutants - Type: voc

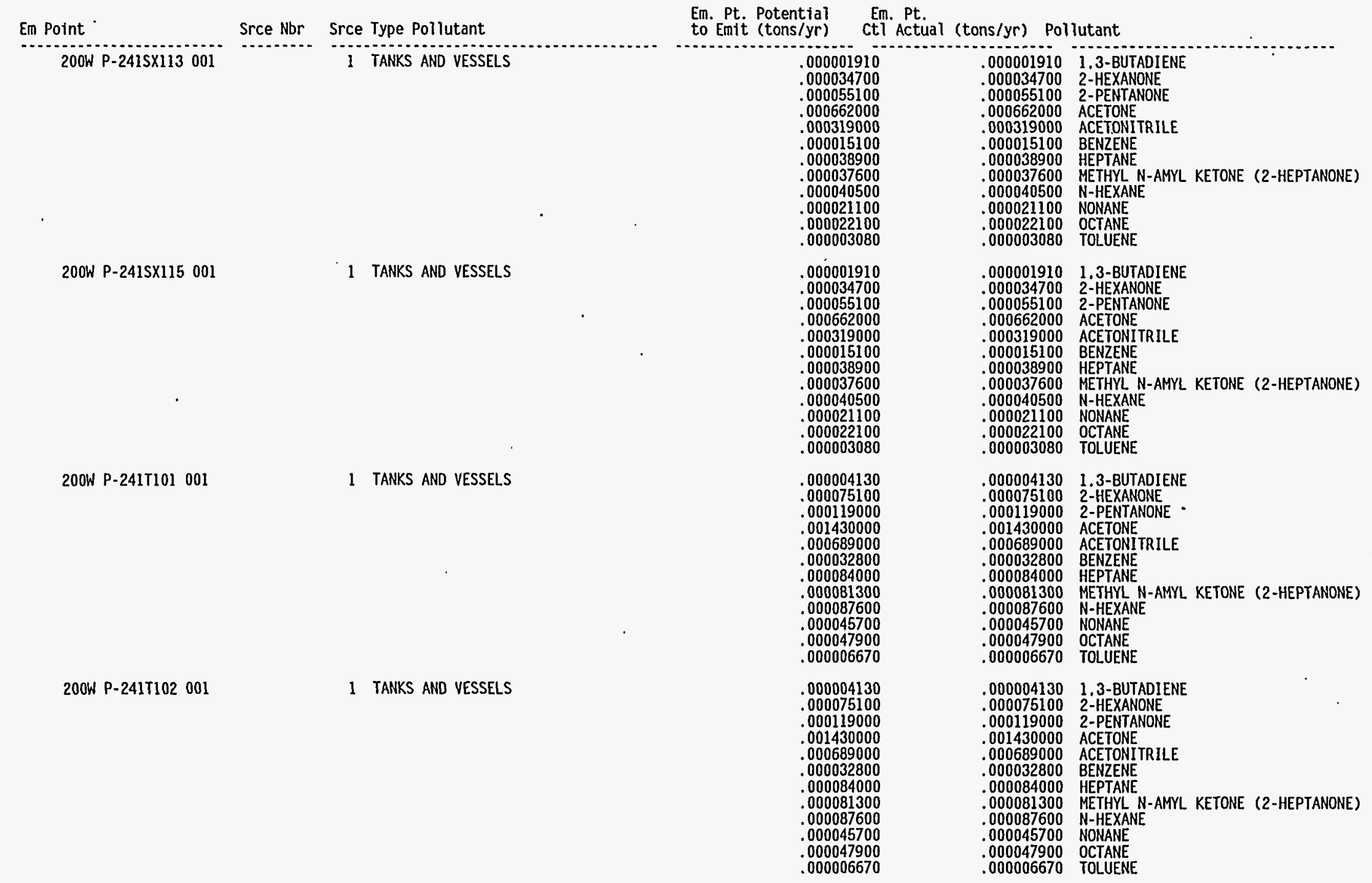


Point Source Criteria Pollutants - Type: Voc

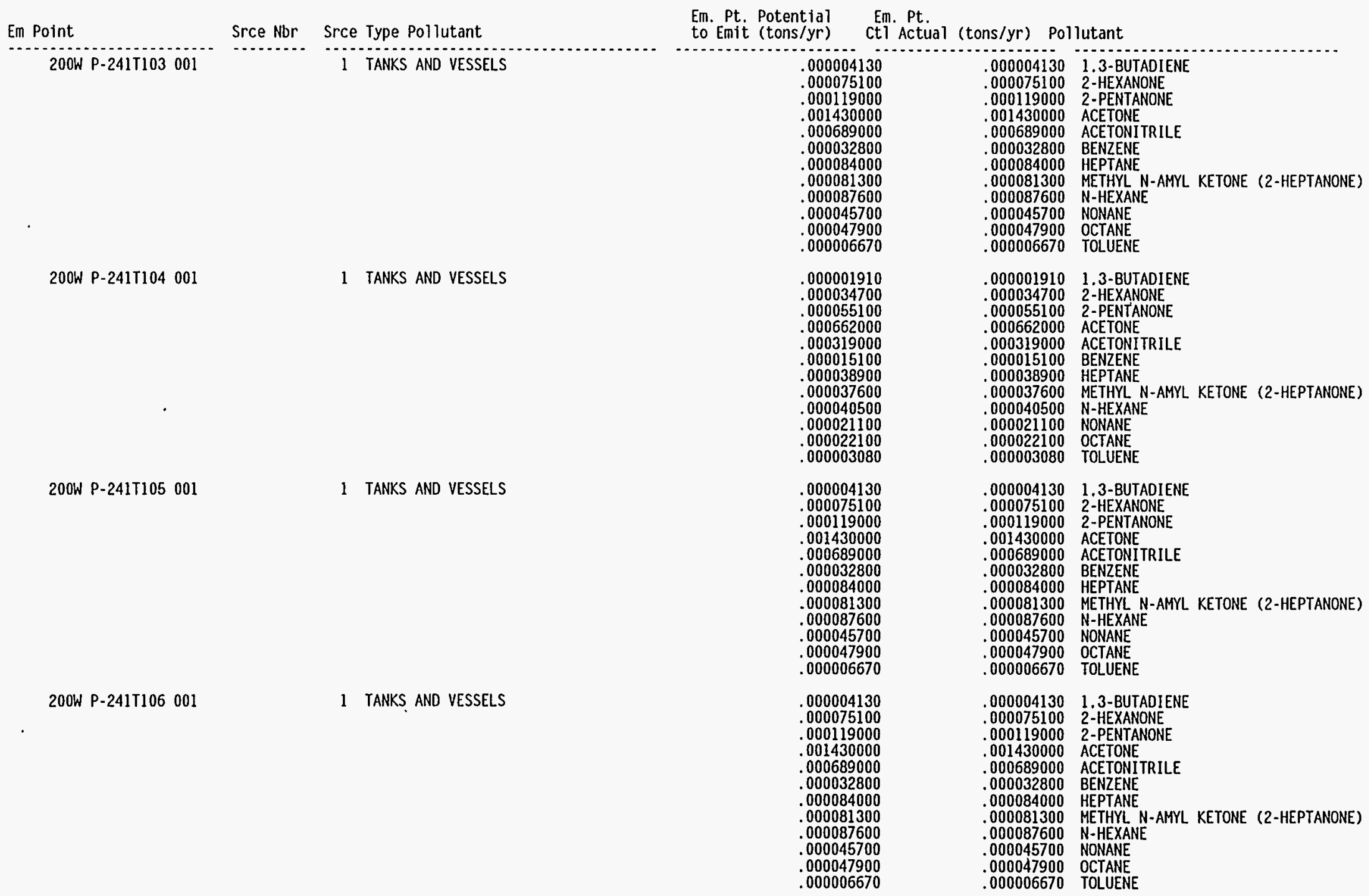


AIR EMISSIONS INVENTORY PERMIT APPLICATION REPORT (UP460)

FACILITY ID $: 200 \mathrm{~W}$ TANK
FACILITY NAME: $200 \mathrm{~W}$ Tank Farm and Associated Structures

Point Source Criteria Pollutants - Type: VOC

\begin{tabular}{|c|c|c|c|c|c|}
\hline Em Point & Srce Nbr & Srce Type Pollutant & $\begin{array}{l}\text { Em. Pt. Potential } \\
\text { to Emit (tons/yr) }\end{array}$ & $\begin{array}{l}\text { Pt. } \\
\text { ctual (tons/yr) Pol }\end{array}$ & lutant \\
\hline 200W P-241T107001 & & 1 TANKS AND VESSELS & $\begin{array}{l}.000004130 \\
.000075100 \\
.000119000 \\
.001430000 \\
.000689000 \\
.000032800 \\
.000084000 \\
.000081300 \\
.000087600 \\
.000045700 \\
.000047900 \\
.000006670\end{array}$ & $\begin{array}{l}.000004130 \\
.000075100 \\
.00011900 \\
.001430000 \\
.000689000 \\
.000032800 \\
.000084000 \\
.000081300 \\
.000087600 \\
.000045700 \\
.000047900 \\
.000006670\end{array}$ & $\begin{array}{l}\text { 1.3-BUTADIENE } \\
\text { 2-HEXANONE } \\
\text { 2-PENTANONE } \\
\text { ACETONE } \\
\text { ACETONITRILE } \\
\text { BENZENE } \\
\text { HEPTANE } \\
\text { METHYL N-AMYL KETONE (2-HEPTANONE) } \\
\text { N-HEXANE } \\
\text { NONANE } \\
\text { OCTANE } \\
\text { TOLUENE }\end{array}$ \\
\hline$\cdot$ & & 1 TANKS AND VESSELS & $\begin{array}{l}.000001910 \\
.000034700 \\
.000055100 \\
.000662000 \\
.000319000 \\
.000015100 \\
.000038900 \\
.000037600 \\
.000040500 \\
.000021100 \\
.000022100 \\
.000003080\end{array}$ & $\begin{array}{l}.000001910 \\
.000034700 \\
.000055100 \\
.000662000 \\
.000319000 \\
.000015100 \\
.000038900 \\
.000037600 \\
.000040500 \\
.000021100 \\
.000022100 \\
.000003080\end{array}$ & $\begin{array}{l}\text { 1.3-BUTADIENE } \\
\text { 2-HEXANONE } \\
\text { 2-PENTANONE } \\
\text { ACETONE } \\
\text { ACETONITRILE } \\
\text { BENZENE } \\
\text { HEPTANE } \\
\text { METHYL N-AMYL KETONE (2-HEPTANONE) } \\
\text { N-HEXANE } \\
\text { NONANE } \\
\text { OCTANE } \\
\text { TOLUENE }\end{array}$ \\
\hline 200W P-241T109 001 & & 1 TANKS AND VESSELS & $\begin{array}{l}.000004130 \\
.000075100 \\
.000119000 \\
.001430000 \\
.000689000 \\
.000032800 \\
.000084000 \\
.000081300 \\
.000087600 \\
.000045700 \\
.000047900 \\
.000006670\end{array}$ & $\begin{array}{l}.000004130 \\
.000075100 \\
.000119000 \\
.001430000 \\
.000689000 \\
.000032800 \\
.000084000 \\
.000081300 \\
.000087600 \\
.000045700 \\
.000047900 \\
.000006670\end{array}$ & $\begin{array}{l}\text { 1.3-BUTADIENE } \\
\text { 2-HEXANONE } \\
\text { 2-PENTANONE } \\
\text { ACETONE } \\
\text { ACETONITRILE } \\
\text { BENZENE } \\
\text { HEPTANE } \\
\text { METHYL N-AMYL KETONE (2-HEPTANONE) } \\
\text { N-HEXANE } \\
\text { NONANE } \\
\text { OCTANE } \\
\text { TOLUENE. }\end{array}$ \\
\hline 200W P-241T110 001 & & 1 TANKS AND VESSELS & $\begin{array}{l}.000004130 \\
.000075100 \\
.000119000 \\
.001430000 \\
.000689000 \\
.000032800 \\
.000084000 \\
.000081300 \\
.000087600 \\
.000045700 \\
.00004790 \\
.000006670\end{array}$ & $\begin{array}{l}.000004130 \\
.000075100 \\
.000119000 \\
.001430000 \\
.000689000 \\
.000032800 \\
.000084000 \\
.000081300 \\
.000087600 \\
.000045700 \\
.000047900 \\
.000006670\end{array}$ & $\begin{array}{l}\text { 1.3-BUTADIENE } \\
\text { 2-HEXANONE } \\
\text { 2-PENTANONE } \\
\text { ACETONE } \\
\text { ACETONITRILE } \\
\text { BENZENE } \\
\text { HEPTANE } \\
\text { METHYL N-AMYL KETONE (2-HEPTANONE) } \\
\text { N-HEXANE } \\
\text { NONANE } \\
\text { OCTANE } \\
\text { TOLUENE }\end{array}$ \\
\hline
\end{tabular}


AIR EMISSIONS INVENTORY PERMIT APPLICATION REPORT (UP460)

FACILITY ID $:$ 200W TANK
FACILITY NAME: 200W Tank Farm and Associated Structures

Point Source Criteria Pollutants - Type: VOC

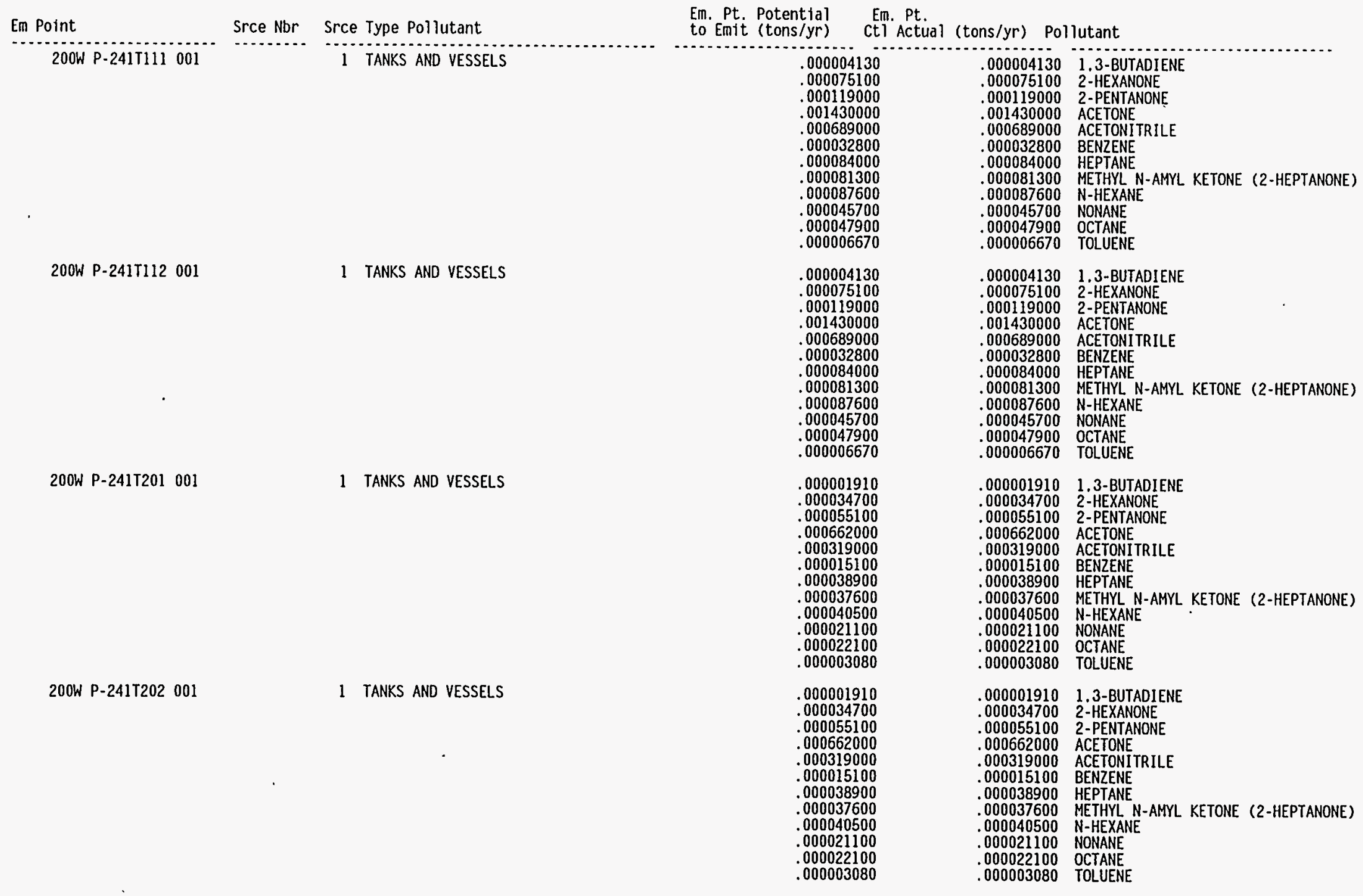

Page \#: 48 of 89 Report Run Date: 22-APR-95 


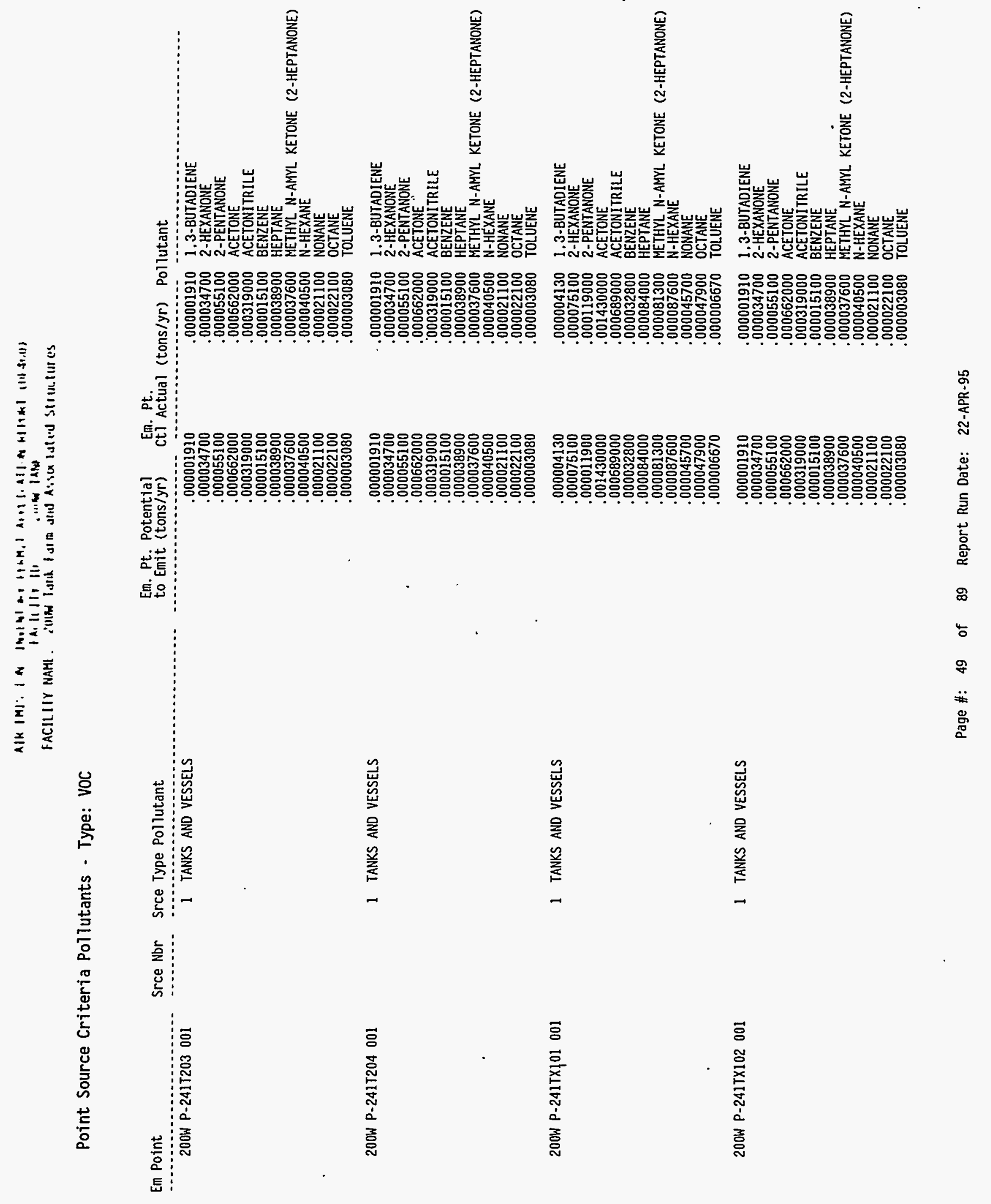


AIR EMISSIONS INVENTORY PERMIT APPLICATION REPORT (UP460)

FACILITY NAME: FACILITY ID : 20OW TANK

Point Source Criteria Pollutants - Type: Voc

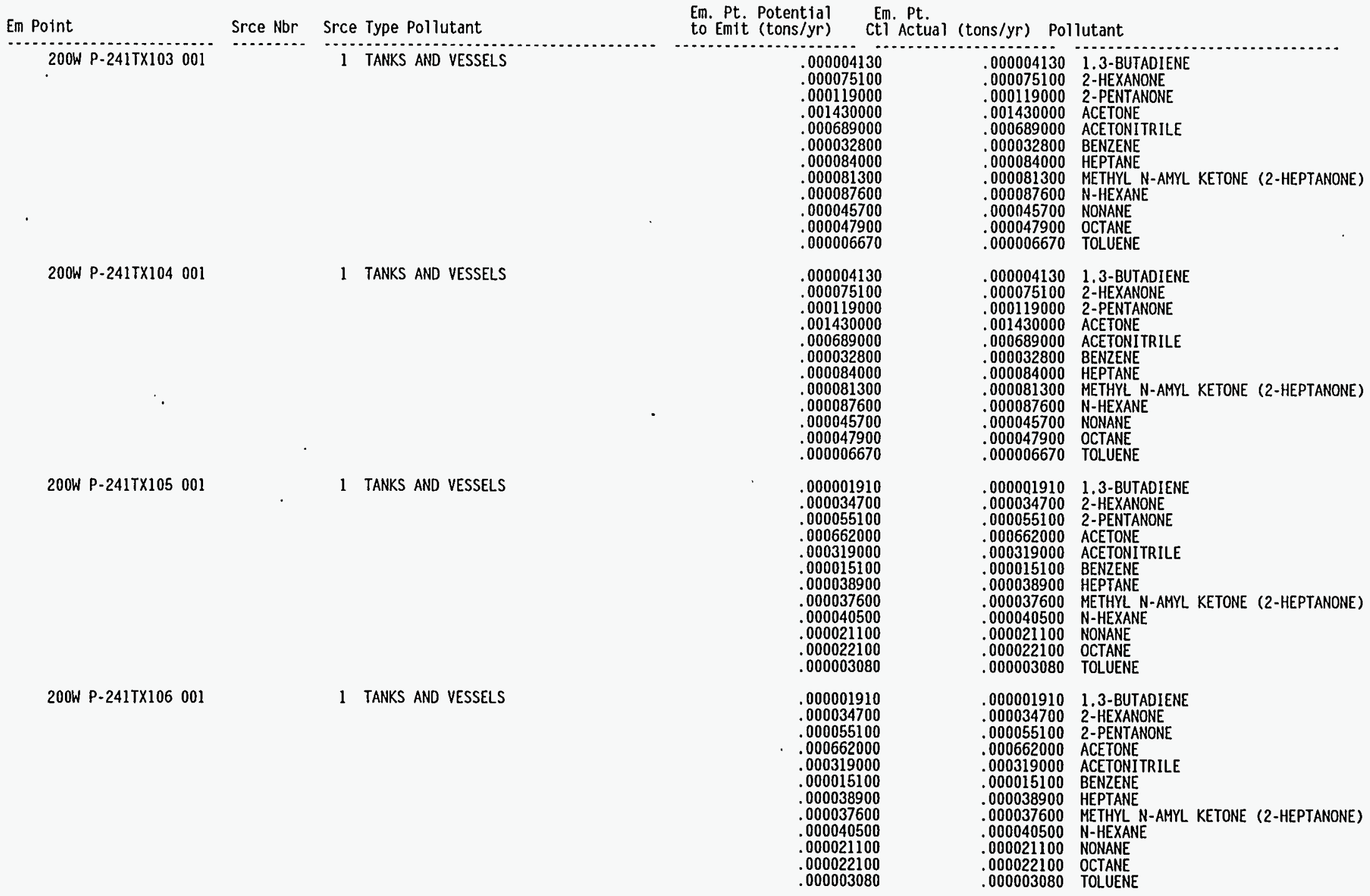

Page \#: 50 of 89 Report Run Date: 22-APR-95 
AIR EMISSIONS INVENTORY PERMIT APPLICATION REPORT (UP460)

FACILITY NAME: $200 \mathrm{~W}$ Tank Farm and Associated Structures

Point Source Criteria Pollutants - Type: Voc

\begin{tabular}{|c|c|c|c|c|c|c|}
\hline Em Point & Srce Nbr & Srce & Type Pollutant & $\begin{array}{l}\text { Em. Pt. Potential } \\
\text { to Emit (tons/yr) }\end{array}$ & $\begin{array}{l}\text { Em. Pt. } \\
\text { ctl Actual (tons/yr) }\end{array}$ & lutant \\
\hline 200W P-241TX107 001 & & 1 & TANKS AND VESSELS & $\begin{array}{l}.00000413 \\
.00007510 \\
.00011900 \\
.00143000 \\
.00068900 \\
.00003280 \\
.00008400 \\
.00008130 \\
.0008760 \\
.00004570 \\
.00004790 \\
.00000667\end{array}$ & $\begin{array}{l}.000004130 \\
.000075100 \\
.000119000 \\
.001430000 \\
.000689000 \\
.000032800 \\
.000084000 \\
.000081300 \\
.000087600 \\
.000045700 \\
.000047900 \\
.000006670\end{array}$ & $\begin{array}{l}\text { 1.3-BUTADIENE } \\
\text { 2-HEXANONE } \\
\text { 2-PENTANONE } \\
\text { ACETONE } \\
\text { ACETONITRILE } \\
\text { BENZENE } \\
\text { HEPTANE } \\
\text { METHYL N-AMYL KETONE (2-HEPTANONE) } \\
\text { N-HEXANE } \\
\text { NONANE } \\
\text { OCTANE } \\
\text { TOLUENE }\end{array}$ \\
\hline 200W P-241TX108 001 & & 1 & TANKS AND VESSELS & $\begin{array}{l}.00000413 \\
.00007510 \\
.00011900 \\
.00143000 \\
.00068900 \\
.00003280 \\
.00008400 \\
.00008130 \\
.00008760 \\
.00004570 \\
.00004790 \\
.00000667\end{array}$ & $\begin{array}{l}.000004130 \\
.000075100 \\
.000119000 \\
.001430000 \\
.000689000 \\
.000032800 \\
.000084000 \\
.000081300 \\
.000087600 \\
.000045700 \\
.000047900 \\
.000006670\end{array}$ & $\begin{array}{l}\text { 1.3-BUTADIENE } \\
\text { 2-HEXANONE } \\
\text { 2-PENTANONE } \\
\text { ACETONE } \\
\text { ACETONITRILE } \\
\text { BENZENE } \\
\text { HEPTANE } \\
\text { METHYL N-AMYL KETONE (2-HEPTANONE) } \\
\text { N-HEXANE } \\
\text { NONANE } \\
\text { OCTANE } \\
\text { TOLUENE }\end{array}$ \\
\hline 1 & ' & 1 & TANKS AND VESSELS & $\begin{array}{l}.00000413 \\
.00007510 \\
.00011900 \\
.00143000 \\
.00068900 \\
.00003280 \\
.000084000 \\
.000081300 \\
.000087600 \\
.000045700 \\
.00004790 \\
.00000667\end{array}$ & $\begin{array}{l}.000004130 \\
.000075100 \\
.000119000 \\
.001430000 \\
.000689000 \\
.000032800 \\
.000084000 \\
.000081300 \\
.000087600 \\
.000045700 \\
.000047900 \\
.000006670\end{array}$ & $\begin{array}{l}\text { 1.3-BUTADIENE } \\
\text { 2-HEXANONE } \\
\text { 2-PENTANONE } \\
\text { ACETONE } \\
\text { ACETONITRILE } \\
\text { BENZENE } \\
\text { HEPTANE } \\
\text { METHYL N-AMYL KETONE (2-HEPTANONE) } \\
\text { N-HEXANE } \\
\text { NONANE } \\
\text { OCTANE } \\
\text { TOLUENE }\end{array}$ \\
\hline 200W P-241TX110 001 & & 1 & TANKS AND VESSELS & $\begin{array}{l}.000001910 \\
.000034700 \\
.000055100 \\
.000662000 \\
.000319000 \\
.000015100 \\
.000038900 \\
.000037600 \\
.000040500 \\
.000021100 \\
.000022100 \\
.000003080\end{array}$ & $\begin{array}{l}.000001910 \\
.000034700 \\
.000055100 \\
.000662000 \\
.000319000 \\
.000015100 \\
.000038900 \\
.000037600 \\
.000040500 \\
.000021100 \\
.000022100 \\
.000003080\end{array}$ & $\begin{array}{l}\text { 1.3-BUTADIENE } \\
\text { 2-HEXANONE } \\
\text { 2-PENTANONE } \\
\text { ACETONE } \\
\text { ACETONITRILE } \\
\text { BENZENE } \\
\text { HEPTANE } \\
\text { METHYL N-AMYL KETONE (2-HEPTANONE) } \\
\text { N-HEXANE } \\
\text { NONANE } \\
\text { OCTANE } \\
\text { TOLUENE }\end{array}$ \\
\hline
\end{tabular}

Page \#: 51 of 89 Report Run Date: 22-APR-95 
AIR EMISSIONS INVENTORY PERMIT APPLICATION REPORT (UP460)

FACILITY NAME: 200W Tank Farm and Associated Structures

Point Source Criteria Pollutants - Type: VOC

\begin{tabular}{|c|c|c|c|c|c|c|}
\hline Em Point & Srce Nbr & Srce & Type Pollutant & $\begin{array}{l}\text { Em. Pt. Potential } \\
\text { to Emit (tons/yr) }\end{array}$ & \multicolumn{2}{|c|}{ Pollutant } \\
\hline 200W P-241TX111 001 & & 1 & TANKS AND VESSELS & $\begin{array}{l}.000001910 \\
.000034700 \\
.000055100 \\
.000662000 \\
.000319000 \\
.000015100 \\
.000038900 \\
.000037600 \\
.000040500 \\
.000021100 \\
.000022100 \\
.000003080\end{array}$ & $\begin{array}{l}.000001910 \\
.000034700 \\
.000055100 \\
.000662000 \\
.000319000 \\
.000015100 \\
.000038900 \\
.000037600 \\
.000040500 \\
.000021100 \\
.000022100 \\
.000003080\end{array}$ & $\begin{array}{l}\text { 1.3-BUTADIENE } \\
\text { 2-HEXANONE } \\
\text { 2-PENTANONE } \\
\text { ACETONE } \\
\text { ACETONITRILE } \\
\text { BENZENE } \\
\text { HEPTANE } \\
\text { METHYL N-AMYL KETONE (2-HEPTANONE) } \\
\text { N-HEXANE } \\
\text { NONANE } \\
\text { OCTANE } \\
\text { TOLUENE }\end{array}$ \\
\hline 200W P-241TX112 001 & & 1 & TANKS AND VESSELS & $\begin{array}{l}.000001910 \\
.0000347 .00 \\
.000055100 \\
.000662000 \\
.000319000 \\
.000015100 \\
.000038900 \\
.000037600 \\
.000040500 \\
.000021100 \\
.000022100 \\
.000003080\end{array}$ & $\begin{array}{l}.000001910 \\
.000034700 \\
.000055100 \\
.000662000 \\
.000319000 \\
.000015100 \\
.000038900 \\
.000037600 \\
.000040500 \\
.000021100 \\
.000022100 \\
.000003080\end{array}$ & $\begin{array}{l}\text { 1.3-BUTADIENE } \\
\text { 2-HEXANONE } \\
\text { 2-PENTANONE } \\
\text { ACETONE } \\
\text { ACETONITRILE } \\
\text { BENZENE } \\
\text { HEPTANE } \\
\text { METHYL N-AMYL KETONE (2-HEPTANONE) } \\
\text { N-HEXANE } \\
\text { NONANE } \\
\text { OCTANE } \\
\text { TOLUENE }\end{array}$ \\
\hline 200W P-241TX113 001 & & 1 & TANKS AND VESSELS & $\begin{array}{l}.000001910 \\
.000034700 \\
.000055100 \\
.000662000 \\
.000319000 \\
.000015100 \\
.000038900 \\
.000037600 \\
.000040500 \\
.000021100 \\
.000022100 \\
.000003080\end{array}$ & $\begin{array}{l}.000001910 \\
.000034700 \\
.000055100 \\
.000662000 \\
.000319000 \\
.000015100 \\
.000038900 \\
.000037600 \\
.000040500 \\
.000021100 \\
.000022100 \\
.000003080\end{array}$ & $\begin{array}{l}\text { 1.3-BUTADIENE } \\
\text { 2-HEXANONE } \\
\text { 2-PENTANONE } \\
\text { ACETONE } \\
\text { ACETONITRILE } \\
\text { BENZENE } \\
\text { HEPTANE } \\
\text { METHY N-AMYL KETONE (2-HEPTANONE) } \\
\text { N-HEXANE } \\
\text { NONANE } \\
\text { OCTANE } \\
\text { TOLUENE }\end{array}$ \\
\hline 200W P-241TX114 001 & & 1 & TANKS AND VESSELS & $\begin{array}{l}.000001910 \\
.000034700 \\
.000055100 \\
.000662000 \\
.000319000 \\
.000015100 \\
.000038900 \\
.000037600 \\
.000040500 \\
.000021100 \\
.000022100 \\
.000003080\end{array}$ & $\begin{array}{l}.000001910 \\
.000034700 \\
.000055100 \\
.000662000 \\
.000319000 \\
.000015100 \\
.000038900 \\
.000037600 \\
.000040500 \\
.000021100 \\
.000022100 \\
.000003080\end{array}$ & $\begin{array}{l}\text { 1.3-BUTADIENE } \\
\text { 2-HEXANONE } \\
\text { 2-PENTANONE } \\
\text { ACETONE } \\
\text { ACETONITRILE } \\
\text { BENZENE } \\
\text { HEPTANE } \\
\text { METHY N-AMYL KETONE (2-HEPTANONE) } \\
\text { N-HEXANE } \\
\text { NONANE } \\
\text { OCTANE } \\
\text { TOLUENE }\end{array}$ \\
\hline
\end{tabular}

Page \#: 52 of 89 Report Run Date: 22-APR-95 
AIR EMISSIONS INVENTORY PERMIT APPLICATION REPORT (UP460)

FACILITY NAME: 200W Tank Farm and Assocjated Structures

Point Source Criteria Pollutants - Type: VOC

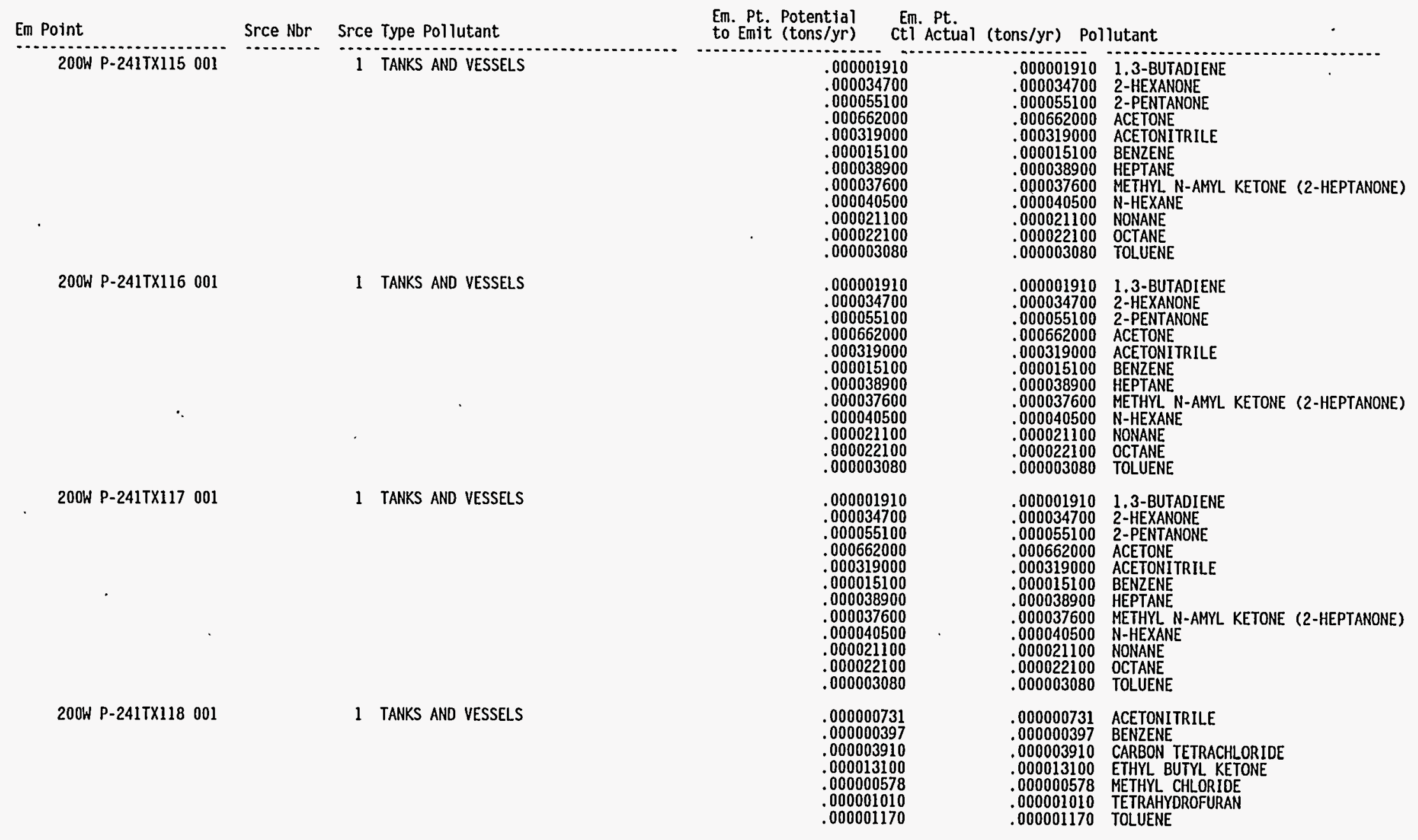

Page \#: 53 of 89 Report Run Date: 22-APR-95 
AIR EMISSIONS INVENTORY PERMIT APPLICATION REPORT (UP460)

FACILITY NAME: 200W Tank Farm and Associated Structures

Point Source Criteria Pollutants - Type: VoC

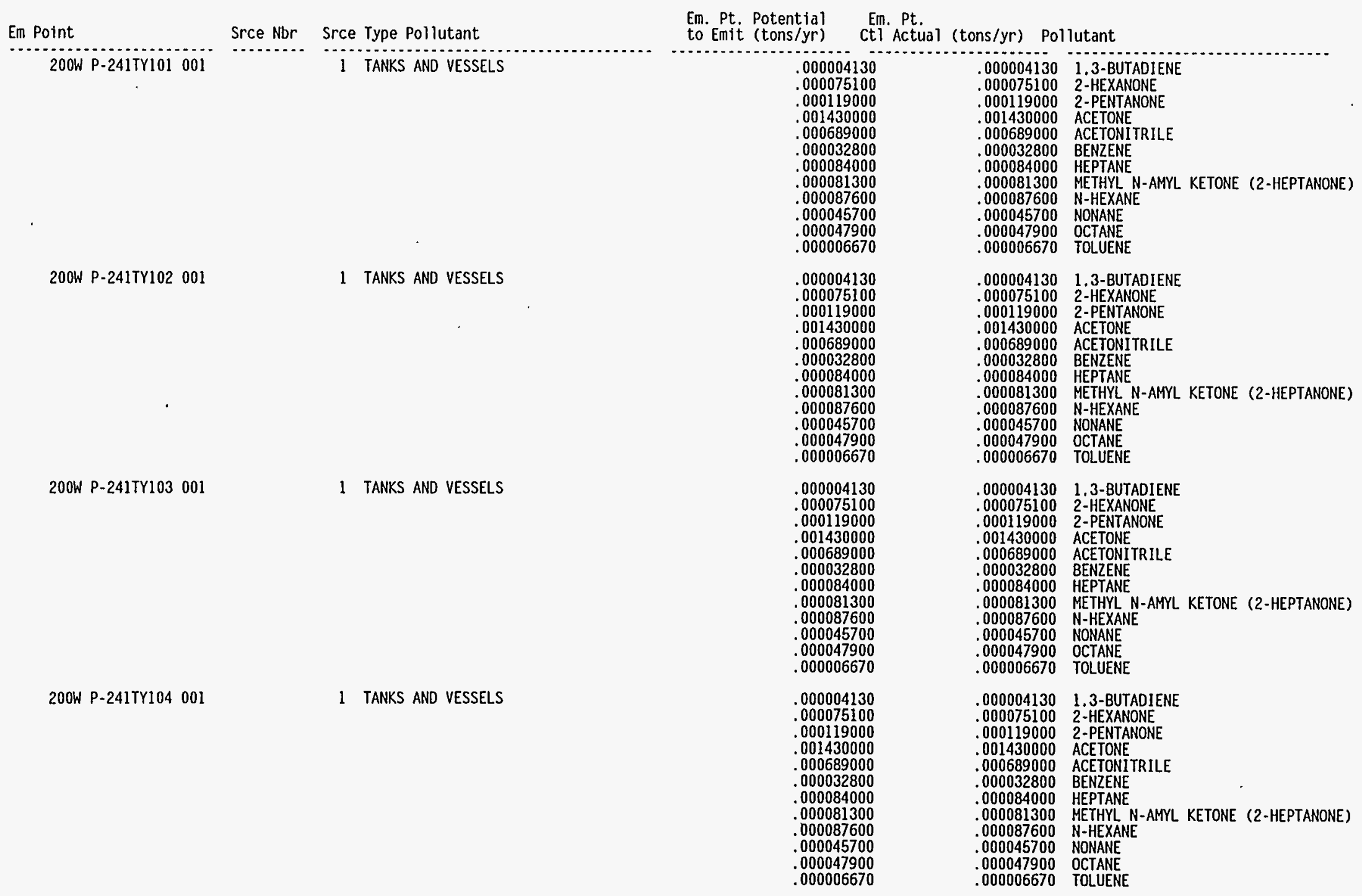

Page \#: 54 of 89 Report Run Date: 22-APR-95 
AIR EMISSIONS INVENTORY PERMIT APPLICATION REPORT (UP460)

FACILITY NAME: $200 \mathrm{~W}$ Tank Farm and Associated Structures

Point Source Criteria Pollutants - Type: VoC

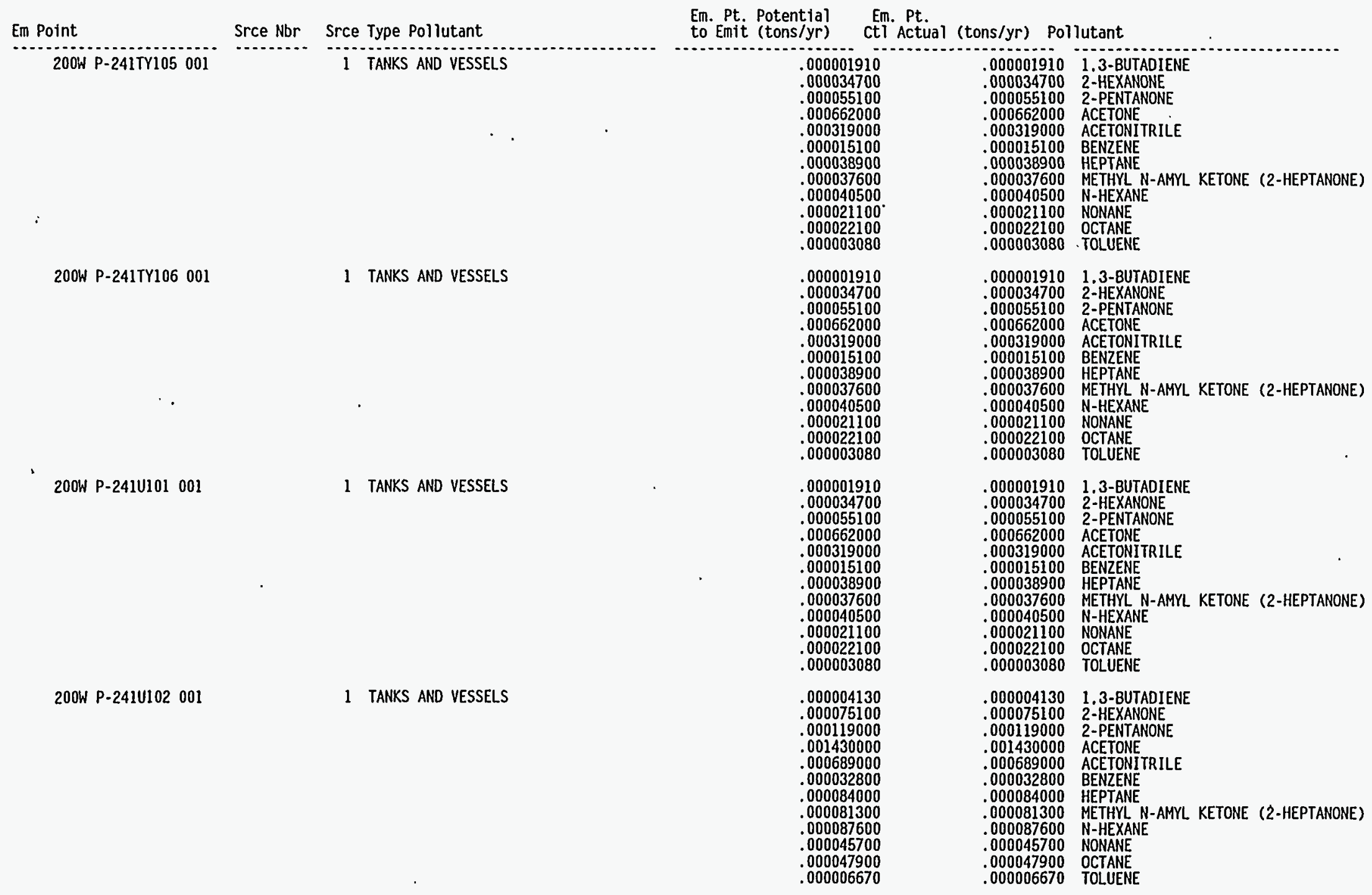

Page \#: 55 of 89 Report Run Date: 22-APR-95 


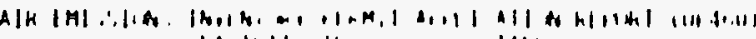

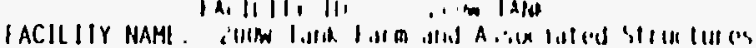

Point Source Criteria Pollutants - Type: VOC

Em Point 200W P-241U103 001

200W P.241U104001

200W P-241U105001

200W P-241U106 001
Srce Nbr Srce Type Pollutant

1 TANKS AND VESSELS

1 TANKS AND VESSELS

1 TANKS AND VESSELS

1 TANKS AND VESSELS
Em. Pt. Potential Em. Pt.

Ctl. Actual (tons/yr) Pollutant

to Emit (tons/yr) Ct i Actua) (tons/y

\begin{tabular}{|c|c|c|}
\hline $\begin{array}{l}.000004130 \\
000075100 \\
000119000 \\
001430000 \\
000689000 \\
000032800 \\
000084000 \\
000081300 \\
000087600 \\
000045700 \\
000047900 \\
000006670\end{array}$ & $\begin{array}{l}.000004130 \\
.000075100 \\
.000119000 \\
.001430000 \\
.000689000 \\
.000032800 \\
.000084000 \\
.000081300 \\
.000087600 \\
.000045700 \\
.000047900 \\
.000006670\end{array}$ & $\begin{array}{l}\text { 1.3-BUTADIENE } \\
\text { 2-HEXANONE } \\
\text { 2-PENTANONE } \\
\text { ACETONE } \\
\text { ACETONITRILE } \\
\text { BENZENE } \\
\text { HEPTANE } \\
\text { METHYL N-AMYL KETONE (2-HEPTANONE) } \\
\text { N-HEXANE } \\
\text { NONANE } \\
\text { OCTANE } \\
\text { TOLUENE }\end{array}$ \\
\hline $\begin{array}{l}.000001910 \\
.000034700 \\
.000055100 \\
.000662000 \\
.000319000 \\
.000015100 \\
.000038900 \\
.000037600 \\
.000040500 \\
.000021100 \\
.000022100 \\
.000003080\end{array}$ & $\begin{array}{l}.000001910 \\
.000034700 \\
.000055100 \\
.000662000 \\
.000319000 \\
.000015100 \\
.000038900 \\
.000037000 \\
.000040500 \\
.000021100 \\
.000022100 \\
.000003080\end{array}$ & $\begin{array}{l}\text { 1.3-BUTADIENE } \\
\text { 2-HEXANONE } \\
\text { 2-PENTANONE } \\
\text { ACETONE } \\
\text { ACETONITRILE } \\
\text { BENZENE } \\
\text { HEPAANE } \\
\text { METHYL N-AMYL KETONE (2-HEPTANONE) } \\
\text { N-HEXANE } \\
\text { NONANE } \\
\text { OCTANE } \\
\text { TOLUENE }\end{array}$ \\
\hline $\begin{array}{l}.000004130 \\
.000075100 \\
.000119000 \\
.001430000 \\
.000689000 \\
.000032800 \\
.000084000 \\
.000081300 \\
.000087600 \\
.000045700 \\
.000047900 \\
.000006670\end{array}$ & $\begin{array}{l}.000004130 \\
.000075100 \\
.000119000 \\
.001430000 \\
.000689000 \\
.000032800 \\
.000084000 \\
.000081300 \\
.000087600 \\
.000045700 \\
.000047900 \\
.000006670\end{array}$ & $\begin{array}{l}\text { 1.3-BUTADIENE } \\
\text { 2-HEXANONE } \\
\text { 2-PENTANONE } \\
\text { ACETONE } \\
\text { ACETONITRILE } \\
\text { BENZENE } \\
\text { HEPANE } \\
\text { METHYL N-AMYL KETONE (2-HEPTANONE) } \\
\text { N-HEXANE } \\
\text { NONANE } \\
\text { OCTANE } \\
\text { TOLUENE }\end{array}$ \\
\hline $\begin{array}{l}000004130 \\
000075100 \\
.000119000 \\
001430000 \\
.000689000 \\
000032800 \\
000084000 \\
000081300 \\
000087600 \\
000045700 \\
000047900 \\
.000006670\end{array}$ & $\begin{array}{l}.000004130 \\
.000075100 \\
.000119000 \\
.001430000 \\
.000689000 \\
.000032800 \\
.000084000 \\
.000081300 \\
.000087600 \\
.000045700 \\
.000047900 \\
.000006670\end{array}$ & $\begin{array}{l}\text { 1.3-BUTADIENE } \\
\text { 2-HEXANONE } \\
\text { 2-PENTANONE } \\
\text { ACEONE } \\
\text { ACETONITRILE } \\
\text { BENZENE } \\
\text { HEPTANE } \\
\text { METYY N-AMYL KETONE (2-HEPTANONE) } \\
\text { N-HEXANE } \\
\text { NONANE } \\
\text { OCTANE } \\
\text { TOLUENE }\end{array}$ \\
\hline
\end{tabular}

000004130 1.3-BUTADIENE

OC68000 ACETONITRILE

.

.

0087600 N-HEXA 
AIR EMISSIONS INVENTORY PERMIT APPLICATION REPORT (UP460)

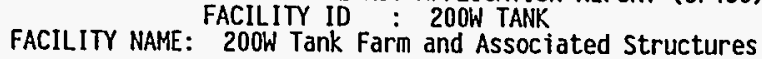

Point Source Criteria Pollutants - Type: VoC

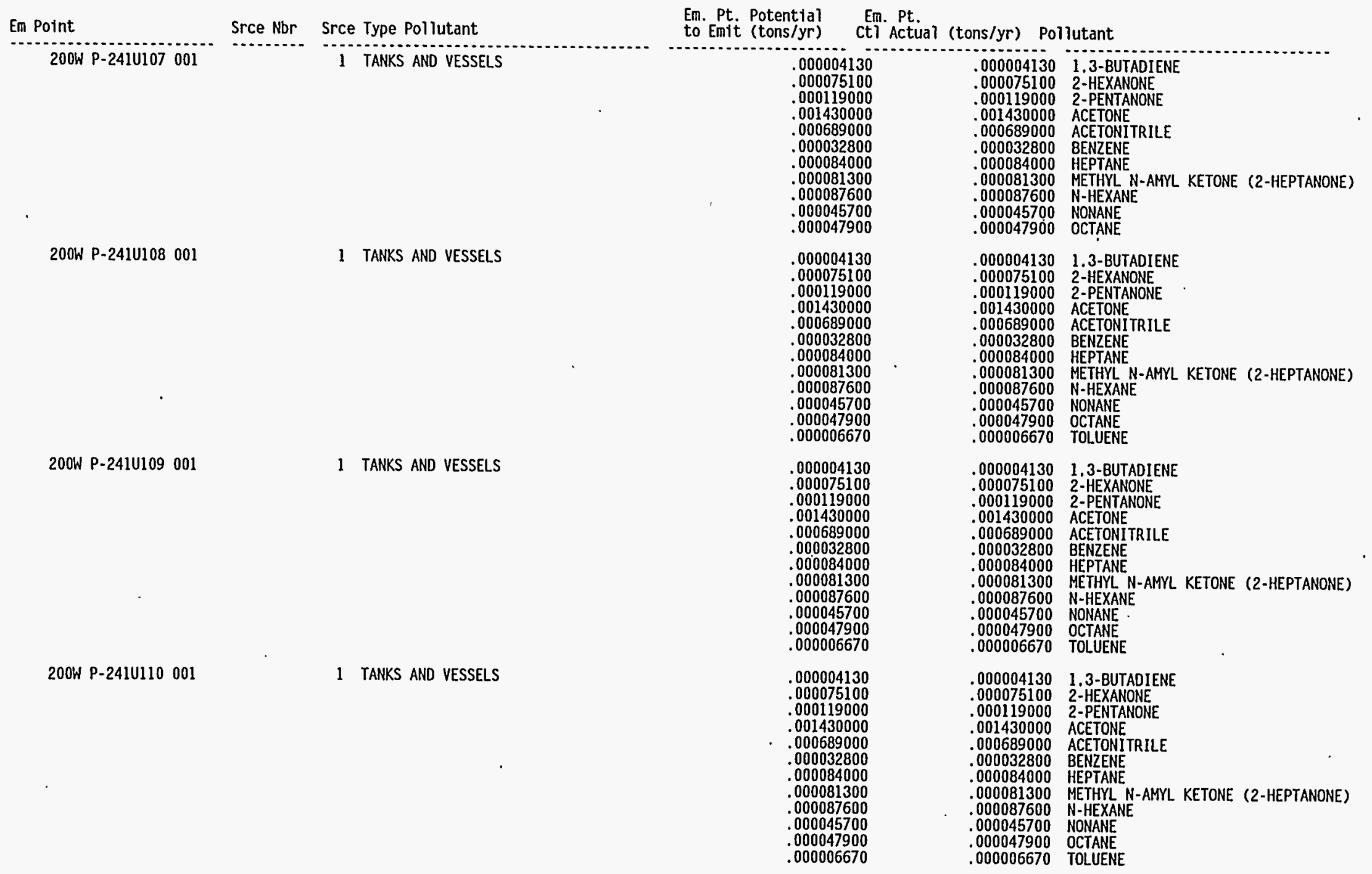

Page \#: 57 of 89 Report Run Date: 22-APR-95 
AIR EMISSIONS INVENTORY PERMIT APPLICATION REPORT (UP460)

FACILITY NAME: $200 \mathrm{~W}$ Tank Farm and Associated Structures

Point Source Criteria Pollutants - Type: Voc

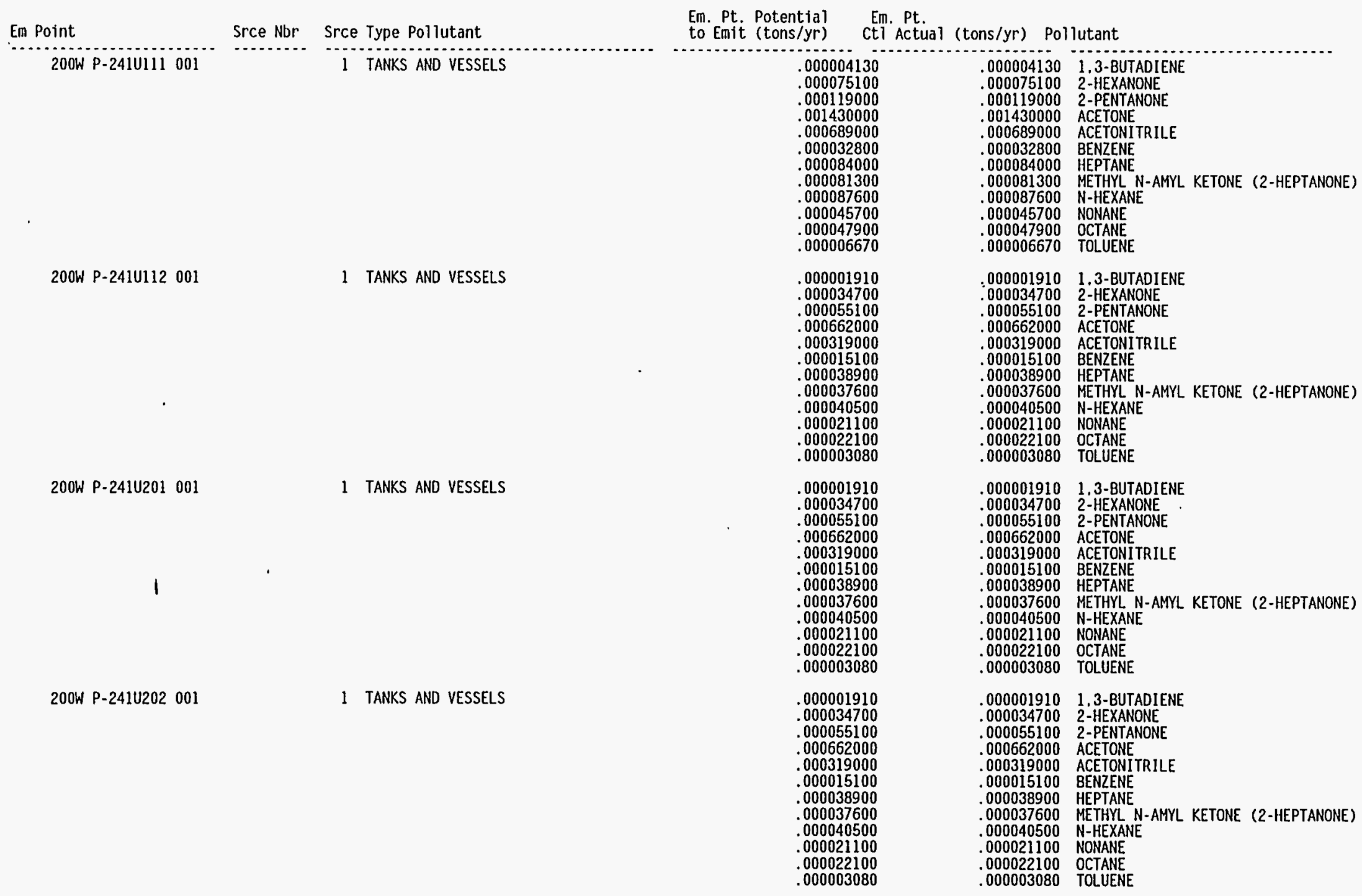

Page \#: 58 of 89 Report Run Date: 22-APR-95 
AIR EMISSIONS INVENTORY PERMIT APPLICATION REPORT (UP460)

FACILITY NAME: 200W Tank Farm and Associated structures

Point Source Criteria Pollutants - Type: VOC

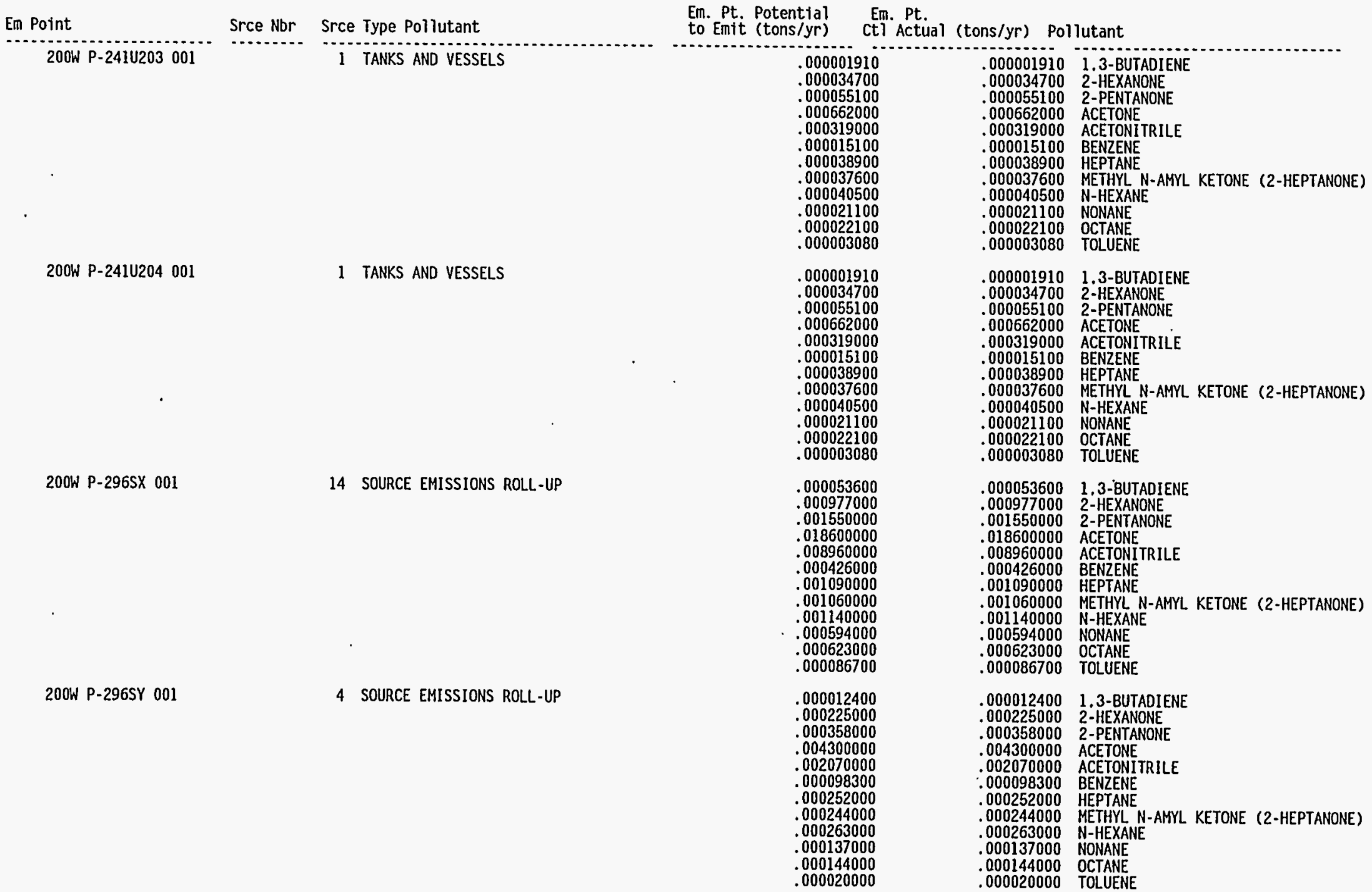


AIR EMISSIONS INVENTORY PERMIT APPLICATION REPORT (UP460)

FACILITY NAME: 200W Tank Farm and Associated Structures

Point Source Criteria Pollutants - Type: VoC

Em Point

Srce Nbr Srce Type Pollutant

Sum

Point Source Emissions - Radiological Pollutants

Em Point
200W P-241S102 001

200W P-241S103 001

Srce Nbr Srce Type Pollutant

1 TANKS AND VESSELS

1 TANKS AND VESSELS

200W P-241S105 001

200W P-241S106 001

200W P-2415107 001

200W P-241S108 001
1 .TANKS AND VESSELS

1 TANKS AND VESSELS

1 TANKS AND VESSELS

1 TANKS AND VESSELS

1 TANKS AND VESSELS

1 TANKS AND VESSELS
Em. Pt. Potential Em. Pt.

ctl Actual (tons/yr) Pollutant
.189159946
.189052846 Em. Pt. Potential Em. Pt.
to Emit (Ci/yr)
Cti Actual (Ci/yr) Pollutant

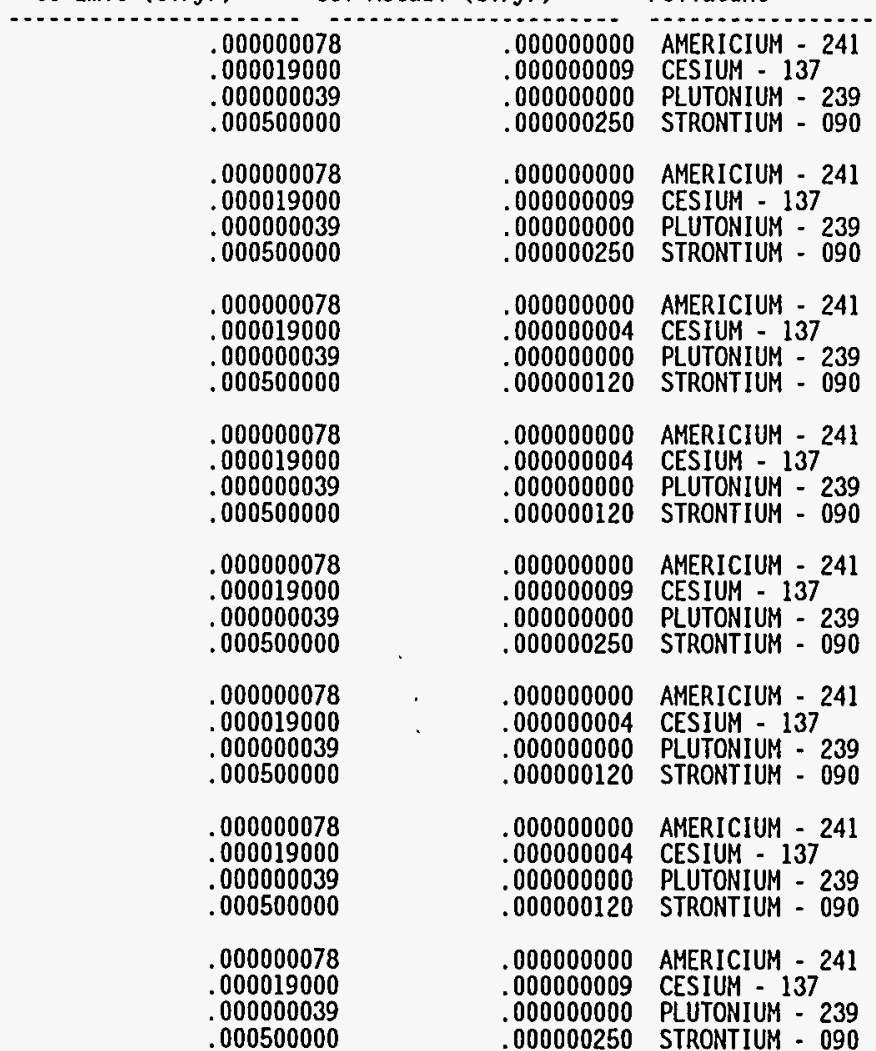


AIR EMISSIONS INVENTORY PERMIT APPLICATION REPORT (UP460)

FACILITY ID : $200 \mathrm{~W}$ TANK
FACILITY NAME: $200 \mathrm{~W}$ Tank Farm and Associated Structures

Point Source Emissions - Radiological Pollutants

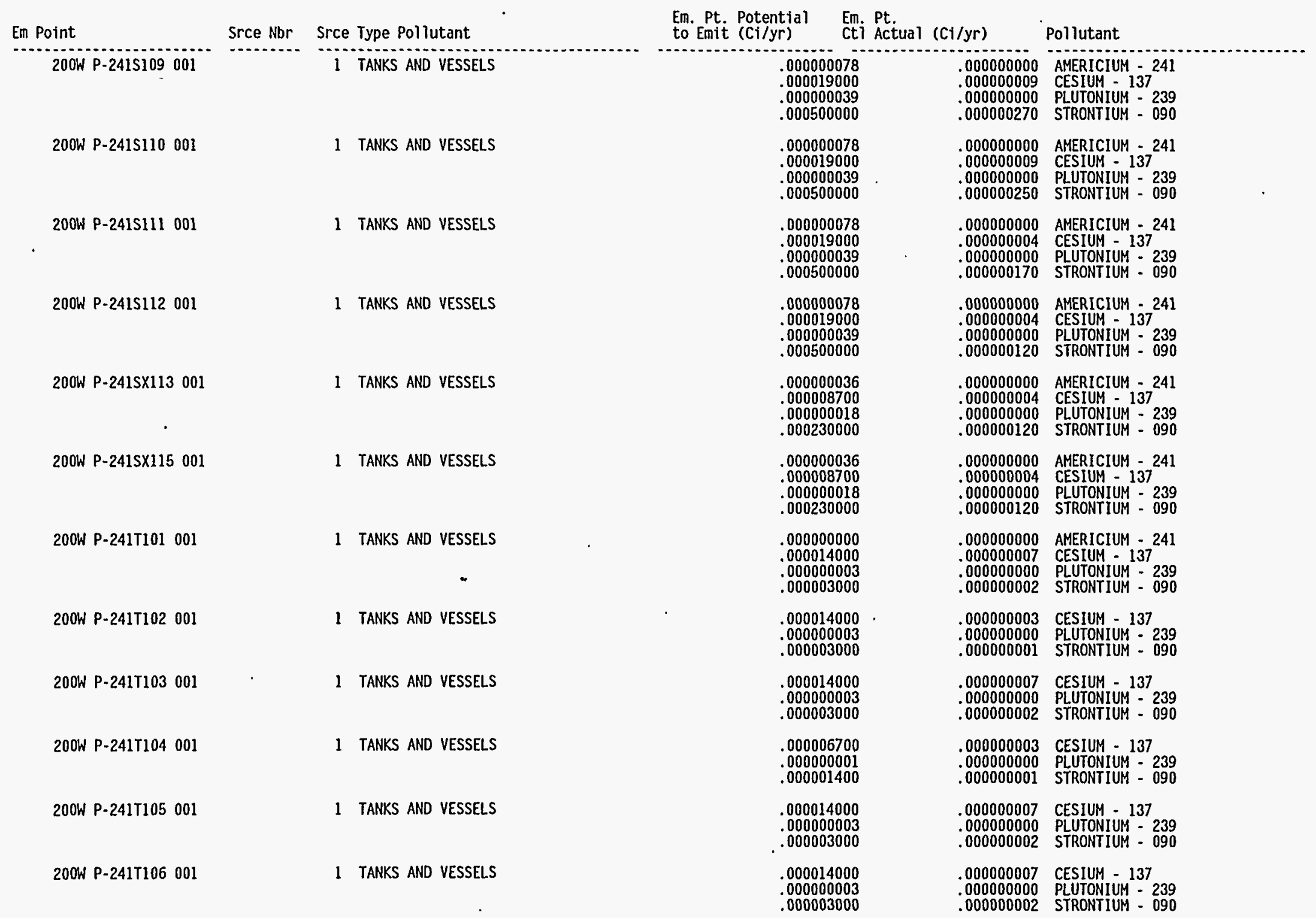

Page \#: 61 of 89 Report Run Date: 22-APR-95 
AIR EMISSIONS INVENTORY PERMIT APPLICATION REPORT (UP460) AIR EMISSIONS INVENTORY PERMIT APPLICATION REPORT (UP460)
FACILITY ID : 200W TANK
FACILITY NAME: 200W Tank Farm and Associated Structures

Point Source Emissions - Radiological Pollutants

\begin{tabular}{|c|c|c|c|c|c|}
\hline Em Point & Srce Nbr & Type Pollutant & $\begin{array}{l}\text { Em. Pt. Potential } \\
\text { to Emit }(\mathrm{C} i / \mathrm{yr})\end{array}$ & $\begin{array}{l}\text { m. Pt. } \\
\text { ti Actual (C1/yr) }\end{array}$ & Pollutant \\
\hline 200W P-241T107001 & 1 & TANKS AND VESSELS & $\begin{array}{l}.000014000 \\
.000000003 \\
.000003000\end{array}$ & $\begin{array}{l}.000000003 \\
.000000000 \\
.000000001\end{array}$ & $\begin{array}{l}\text { CESIUM 137 } \\
\text { PLUTONIUM - } 239 \\
\text { STRONTIUM }=090\end{array}$ \\
\hline 200W P-241T108 001 & 1 & TANKS AND VESSELS & $\begin{array}{l}.000006700 \\
.000000001 \\
.000001400\end{array}$ & $\begin{array}{l}.000000003 \\
.000000000 \\
.000000001\end{array}$ & $\begin{array}{l}\text { CESIUM - } 137 \\
\text { PLUTONIUM }-239 \\
\text { STRONTIUM }-090\end{array}$ \\
\hline 200W P-241T109 001 & 1 & TANKS AND VESSELS & $\begin{array}{l}.000014000 \\
.000000003 \\
.000003000\end{array}$ & $\begin{array}{l}.000000003 \\
.000000000 \\
.000000001\end{array}$ & $\begin{array}{l}\text { CESIUM - } 137 \\
\text { PLUTONIUM - } 239 \\
\text { STRONTIUM - 090 }\end{array}$ \\
\hline 200W P-241T110 001 & 1 & TANKS AND VESSELS & $\begin{array}{l}.000014000 \\
.000000003 \\
.000003000\end{array}$ & $\begin{array}{l}.000000007 \\
.000000000 \\
.000000002\end{array}$ & $\begin{array}{l}\text { CESIUM - } 137 \\
\text { PLUTONIUM - } 239 \\
\text { STRONTIUM - } 090\end{array}$ \\
\hline 200W P-241T111 001 & 1 & TANKS AND VESSELS & $\begin{array}{l}.000014000 \\
.000000003 \\
.000003000\end{array}$ & $\begin{array}{l}.000000007 \\
.000000000 \\
.000000002\end{array}$ & $\begin{array}{l}\text { CESIUM - } 137 \\
\text { PLUTONIUM }-239 \\
\text { STRONTIUM }-090\end{array}$ \\
\hline 200W P-241T112 001 & 1 & TANKS AND VESSELS & $\begin{array}{l}.000014000 \\
.000000003 \\
.000003000\end{array}$ & $\begin{array}{l}.000000007 \\
.00000000 \\
.000000002\end{array}$ & $\begin{array}{l}\text { CESIUM - } 137 \\
\text { PLUTONIUM }-239 \\
\text { STRONTIUM }-090\end{array}$ \\
\hline 200W P-241T201 001 & 1 & TANKS AND VESSELS & $\begin{array}{l}.000006700 \\
.000000001 \\
.000001400\end{array}$ & $\begin{array}{l}.000000003 \\
.000000000 \\
.000000001\end{array}$ & $\begin{array}{l}\text { CESIUM - } 137 \\
\text { PLUTONIUM - } 239 \\
\text { STRONTIUM - } 090\end{array}$ \\
\hline 200W P-241T202001 & 1 & TANKS AND VESSELS & $\begin{array}{l}.000006700 \\
.000000001 \\
.000001400\end{array}$ & $\begin{array}{l}.000000003 \\
.000000000 \\
.000000001\end{array}$ & $\begin{array}{l}\text { CESIUM - } 137 \\
\text { PLUTONIUM }-239 \\
\text { STRONTIUM }-090\end{array}$ \\
\hline 200W P-241T203001 & 1 & TANKS AND VESSELS & $\begin{array}{l}.000006700 \\
.000000001 \\
.000001400\end{array}$ & $\begin{array}{l}.000000003 \\
.000000000 \\
.000000001\end{array}$ & $\begin{array}{l}\text { CESIUM - } 137 \\
\text { PLUTONIUM - } 239 \\
\text { STRONTIUM }-090\end{array}$ \\
\hline 200W P-241T204001 & 1 & TANKS AND VESSELS & $\begin{array}{l}.000006700 \\
.000000001 \\
.000001400\end{array}$ & $\begin{array}{l}.000000003 \\
.00000000 \\
.000000001\end{array}$ & $\begin{array}{l}\text { CESIUM - } 137 \\
\text { PLUTONIUM - } 239 \\
\text { STRONTIUM - } 090\end{array}$ \\
\hline 200W P-241TX101 001 & 1 & TANKS AND VESSELS & $\begin{array}{l}.000016000 \\
.000000001 \\
.000000024\end{array}$ & $\begin{array}{l}.000000008 \\
.000000000 \\
.000000000\end{array}$ & $\begin{array}{l}\text { CESIUM - } 137 \\
\text { PLUTONIUM }-239 \\
\text { STRONTIUM }-090\end{array}$ \\
\hline 200W P-241TX102 001 & 1 & TANKS AND VESSELS & $\begin{array}{l}.000007300 \\
.000000001 \\
.000000011\end{array}$ & $\begin{array}{l}.000000004 \\
.000000000 \\
.000000000\end{array}$ & $\begin{array}{l}\text { CESIUM - } 137 \\
\text { PLUTONIUM - } 239 \\
\text { STRONTIUM - } 090\end{array}$ \\
\hline 200W P-241TX103001 & 1 & TANKS AND VESSELS & $\begin{array}{l}.000016000 \\
.000000001 \\
.000000024\end{array}$ & $\begin{array}{l}.000000008 \\
.000000000 \\
.000000000\end{array}$ & $\begin{array}{l}\text { CESIUM - } 137 \\
\text { PLUTONIUM - } 239 \\
\text { STRONTIUM - } 090\end{array}$ \\
\hline
\end{tabular}

Page \#: 62 of 89 Report Run Date: 22-APR-95 


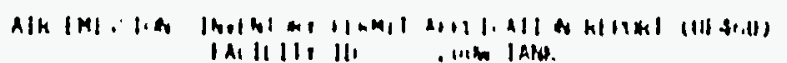

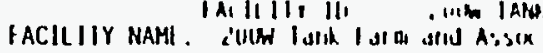

Point Source Emissions - Radiological Pollutants

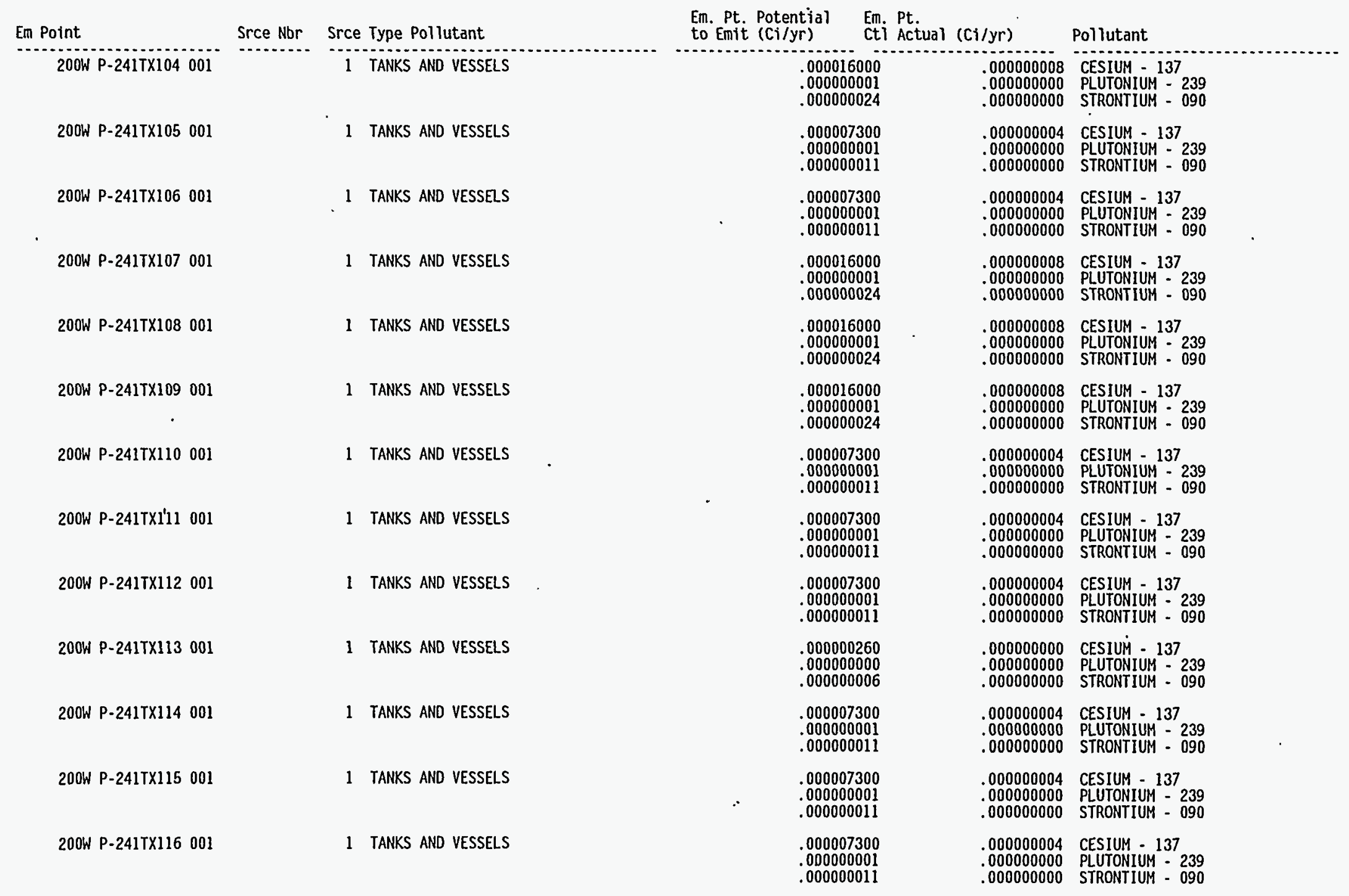


Point Source Emissions - Radiological Pollutants

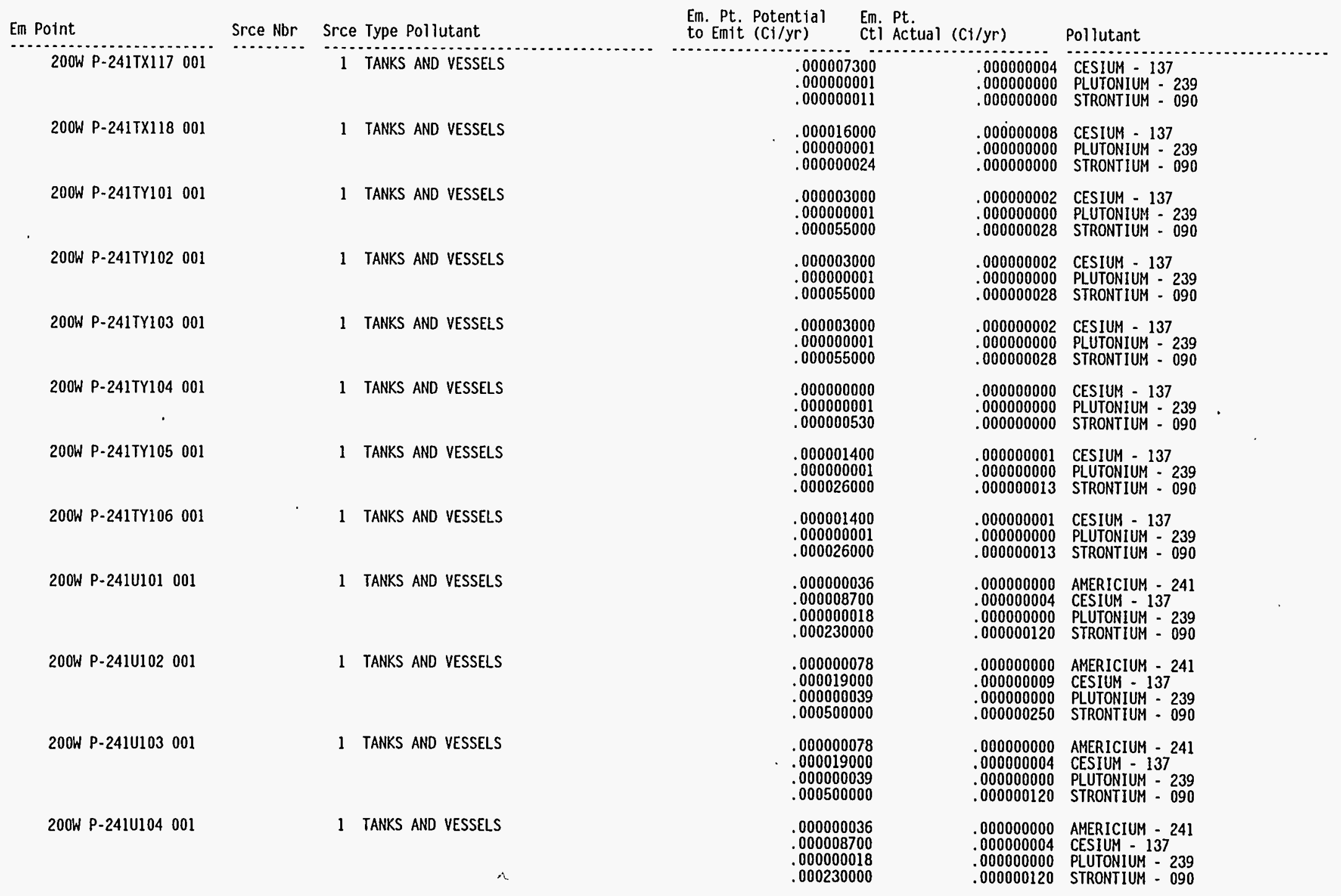


AIR EMISSIONS INVENTORY PERMIT APPLICATION REPORT (UP460)

FACILITY NAME: $200 \mathrm{~W}$ Tank Farm and Associated Structures

Point Source Emissions - Radiological Pollutants

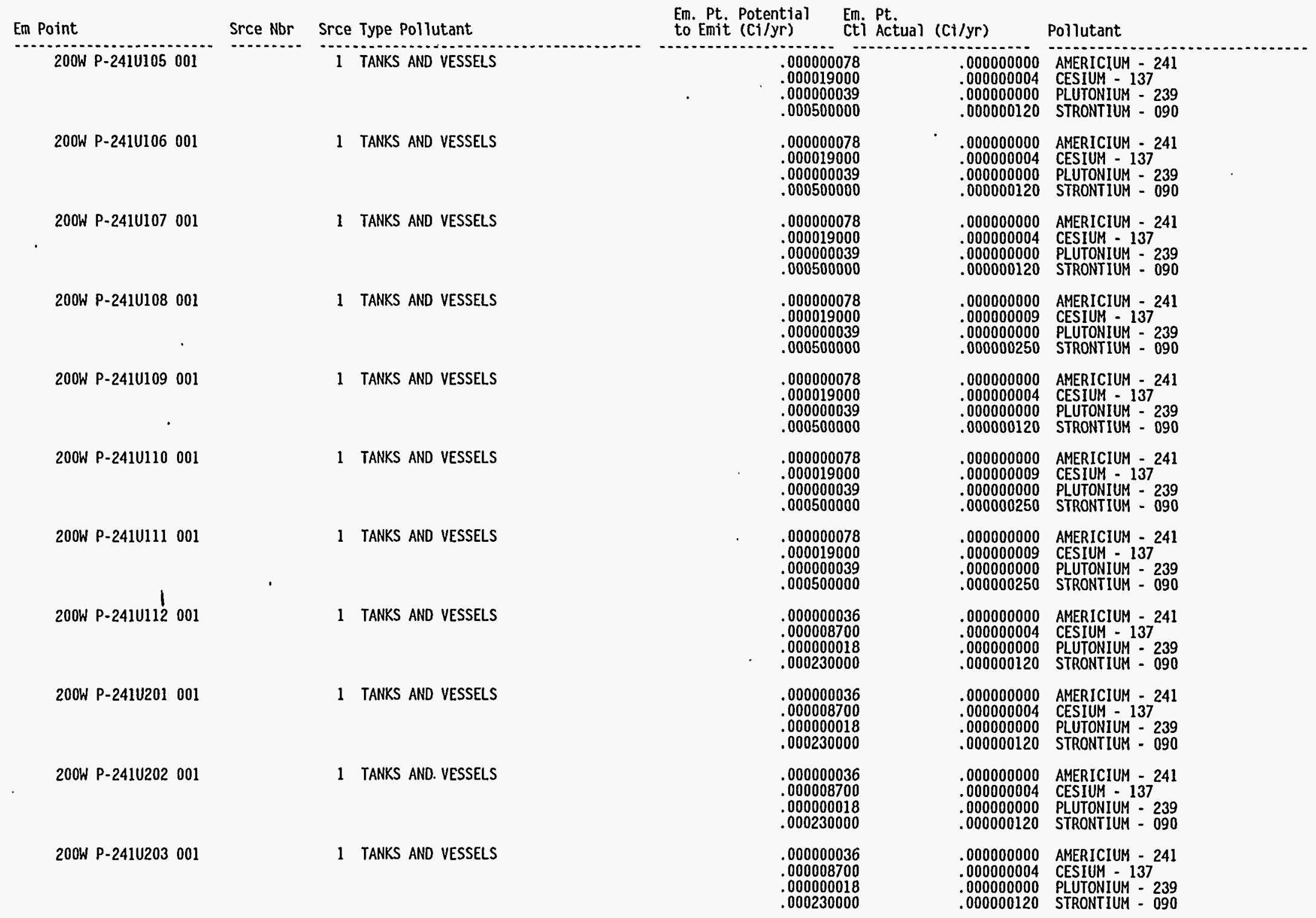

Page \#: 65 of 89 Report Run Date: 22-APR-95 
AIR EMISSIONS INVENTORY PERMIT APPLICATION REPORT (UP460)

FACILITY NAME: $200 \mathrm{~W}$ Tank Farm and Associated Structures

Point Source Emissions - Radiological Pollutants

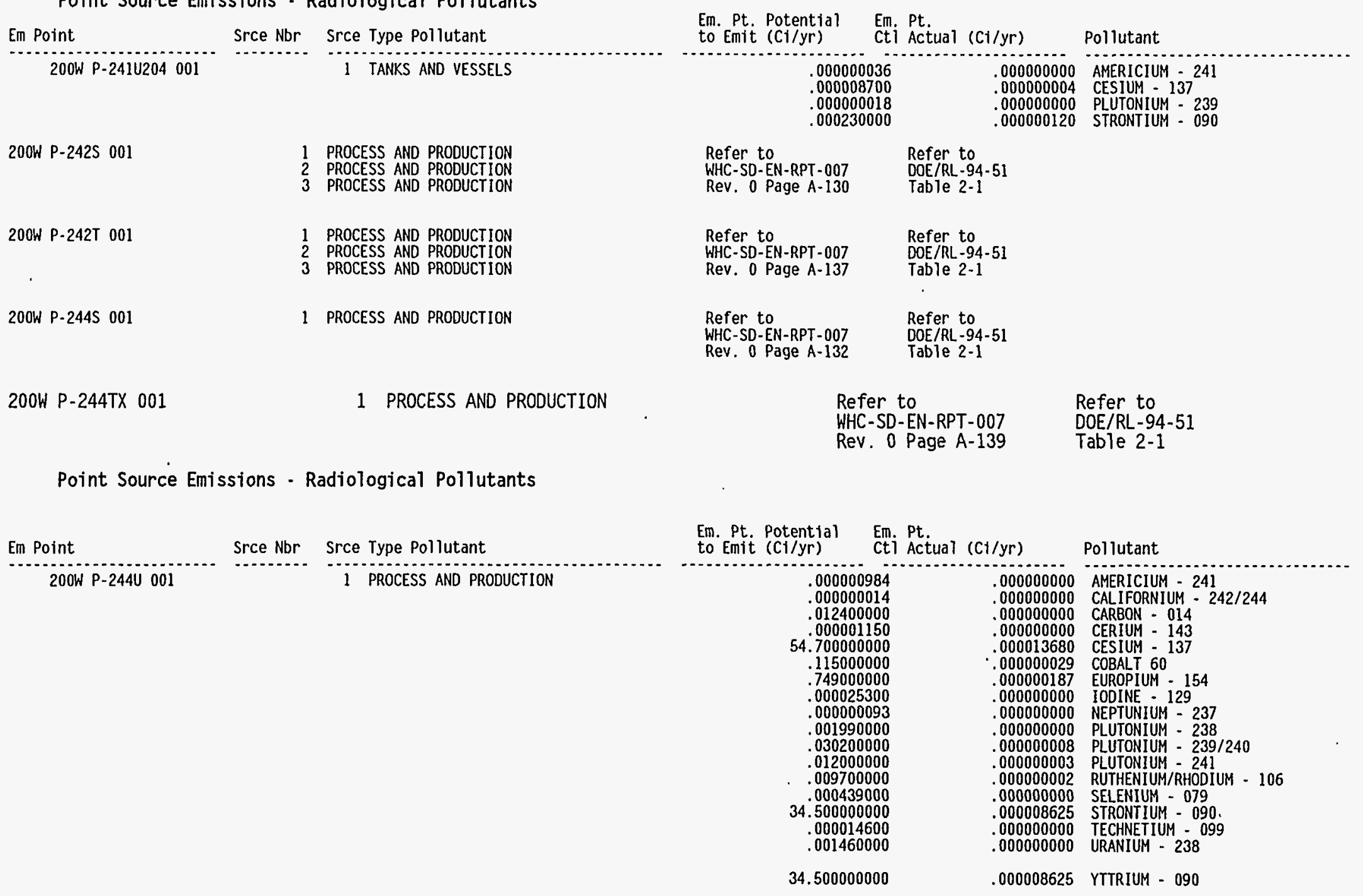


AIR EMISSIONS INVENTORY PERMIT APPLICATION REPORT (UP460)

FACILITY ID : 200W TANK

FACILITY NAME: 200W Tank Farm and Associated Structures

Point Source Enissions - Radiological Pollutants

\begin{tabular}{|c|c|c|c|}
\hline Em Point & Srce Nbr Srce Type Pollutant & $\begin{array}{l}\text { Em. Pt. Potential } \\
\text { to Emit (Ci/yr) }\end{array}$ & $\begin{array}{l}\text { Em. Pt. } \\
\text { Cti Actual (Ci/yr) }\end{array}$ \\
\hline 200W P-296SX 001 & $\begin{array}{ll}1 & \text { TANKS AND VESSELS } \\
2 & \text { TANKS AND VESSELS } \\
3 & \text { TANKS AND VESSELS } \\
4 & \text { TANKS AND VESSELS } \\
5 & \text { TANKS AND VESSELS } \\
6 & \text { TANKS AND VESSELS } \\
7 & \text { TANKS AND VESSELS } \\
8 & \text { TANKS AND VESSELS } \\
9 & \text { TANKS AND VESSELS } \\
10 & \text { TANKS AND VESSELS } \\
11 & \text { TANKS AND VESSELS } \\
12 & \text { TANKS AND VESSELS } \\
13 & \text { TANKS AND VESSELS }\end{array}$ & $\begin{array}{l}\text { Refer to } \\
\text { WHC-SD-EN-RPT-007 } \\
\text { Rev. O Page A-127 }\end{array}$ & $\begin{array}{l}\text { Refer to } \\
\text { DoE/RL-94-51 } \\
\text { Table 2-1 }\end{array}$ \\
\hline 200W P-296SY 001 & $\begin{array}{ll}1 & \text { TANKS AND VESSELS } \\
2 & \text { TANKS AND VESSELS } \\
3 & \text { TANKS AND VESSELS }\end{array}$ & $\begin{array}{l}\text { Refer to } \\
\text { WHC-SO-EN-RPT-007 } \\
\text { Rev. O Page A-115 }\end{array}$ & $\begin{array}{l}\text { Refer to } \\
\text { DOE/RL-94-51 } \\
\text { Table 2-1 }\end{array}$ \\
\hline 200W P-296P028 & $\begin{array}{ll}1 & \text { TANKS AND VESSELS } \\
2 & \text { TANKS AND VESELS } \\
3 & \text { TANKS AND VESSELS }\end{array}$ & $\begin{array}{l}\text { Refer to } \\
\text { WHC-SD-EN-RPT-007 } \\
\text { Rev. O Page A-115 }\end{array}$ & $\begin{array}{l}\text { Refer to } \\
\text { DOE/RL-94-51 } \\
\text { Table 2-1. Note: Emissions are equivalent. } \\
\text { but not addit tive to the } 296-\mathrm{P}-23 \text { primary } \\
\text { stack. Which is emission point } \\
200 \mathrm{p} \text {-296SY 001. }\end{array}$ \\
\hline $200 \mathrm{~W} 296 \mathrm{~S} 025$ & $\begin{array}{ll}1 & \text { TANKS AND VESSELS } \\
2 & \text { TANKS AND VESSELS } \\
3 & \text { TANKS AND VESSELS }\end{array}$ & $\begin{array}{l}\text { NOT YET } \\
\text { OPERATIONAL }\end{array}$ & $\begin{array}{l}\text { NOT YET } \\
\text { OPERATIONAL }\end{array}$ \\
\hline 200W 296U011 & 1 TANKS AND VESSELS & $\begin{array}{l}\text { Refer to } \\
\text { WHC-SD-EN-RPT-007 } \\
\text { Rev. O Page A-145 }\end{array}$ & $\begin{array}{l}\text { NOT YET } \\
\text { OPERATIONAL }\end{array}$ \\
\hline $200 \mathrm{~W} 296 \mathrm{P} 022$ & $\begin{array}{ll}1 & \text { TANKS AND VESSELS } \\
2 & \text { TANKS AND VESSELS } \\
3 & \text { TANKS AND VESSELS }\end{array}$ & $\begin{array}{l}\text { Refer to } \\
\text { WHC-SD-EN-RPT-007 } \\
\text { Rev. O Page A-113 }\end{array}$ & $\begin{array}{l}\text { Refer to } \\
\text { DOE/RL-94-51 } \\
\text { Table } 2-1\end{array}$ \\
\hline
\end{tabular}

Page \#: 67 of 89 Report Run Date: 22-APR-95 
Point Source Emissions - HAP Pollutants

\begin{tabular}{|c|c|c|c|c|c|c|c|}
\hline Em Point & Srce Nbr & Srce Type Pollutant & $\begin{array}{l}\text { Em. Pt. Potential } \\
\text { to Emit (tons/yr) }\end{array}$ & $\begin{array}{l}\text { Em. Pt. } \\
\text { ctl Actual }\end{array}$ & (tons/yr) & Pollutant & \\
\hline 200W P-241S101 001 & $\cdots$ & 1 TANKS AND VESSELS & $\begin{array}{l}.000004130 \\
.000689000\end{array}$ & & $\begin{array}{l}.000004130 \\
.000689000\end{array}$ & $\begin{array}{l}30 \\
0 \\
0\end{array}$ & \\
\hline \multicolumn{7}{|c|}{ Point Source Emissions - HAP Pollutants } & \\
\hline \multirow[t]{2}{*}{ En Point } & \multirow[t]{2}{*}{ Srce Nbr } & Srce Type Pollutant & $\begin{array}{l}\text { Em. Pt. Potential } \\
\text { to Emit (tons/yr) }\end{array}$ & \multirow[t]{2}{*}{$\begin{array}{l}\text { Em. Pt. } \\
\text { ctl Actual }\end{array}$} & (tons/yr) $\mathrm{P}$ & Pollutant & \\
\hline & & 1 TANKS AND VESSELS & $\begin{array}{l}.000032800 \\
.000087600 \\
.000006670\end{array}$ & & $\begin{array}{l}.000032800 \\
.000087600 \\
.000006670\end{array}$ & $\begin{array}{ll}0 & \text { BENZENE } \\
0 & \text { N-HEXANE } \\
0 & \text { TOLUENE }\end{array}$ & \\
\hline $200 W$ P-241S102001 & & 1 TANKS AND VESSELS & \multicolumn{2}{|c|}{$\begin{array}{l}.000004130 \\
.000689000 \\
.000032800 \\
.000087600 \\
.000006670\end{array}$} & $\begin{array}{l}.000004130 \\
.000689000 \\
.000032800 \\
.000087600 \\
.000006670\end{array}$ & $\begin{array}{ll}30 & 1.3 \text {-BUTADIENE } \\
0 & \text { ACETONITRILE } \\
0 & \text { BENZENE } \\
0 & \text { N-HEXANE } \\
0 & \text { TOLUENE }\end{array}$ & . \\
\hline 200W P-241S103001 & & 1 TANKS AND VESSELS & \multicolumn{2}{|c|}{$\begin{array}{l}.000004130 \\
.000689000 \\
.000032800 \\
.000087600 \\
.000006670\end{array}$} & $\begin{array}{l}.000004130 \\
.000689000 \\
.000032800 \\
.000087600 \\
.000006670\end{array}$ & $\begin{array}{ll}30 & 1.3 \text {-BUTAOIENE } \\
0 & \text { ACETONITRILE } \\
0 & \text { BENZENE } \\
0 & \text { N-HEXANE } \\
0 & \text { TOLUENE }\end{array}$ & \\
\hline 200W P-241S104001 & & 1 TANKS AND VESSELS & \multicolumn{2}{|c|}{$\begin{array}{l}.000004130 \\
.000689000 \\
.000032800 \\
.000087600 \\
.000006670\end{array}$} & $\begin{array}{l}.000004130 \\
.000689000 \\
.000032800 \\
.000087600 \\
.000006670\end{array}$ & $\begin{array}{ll}30 & 1.3 \text {-BUTADIENE } \\
0 & \text { ACETONITRILE } \\
0 & \text { BENZENE } \\
0 & \text { N-HEXANE } \\
0 & \text { TOLUENE }\end{array}$ & \\
\hline 200W P-241S105 001 & & 1 TANKS AND VESSELS & \multicolumn{2}{|c|}{$\begin{array}{l}.000004130 \\
.000689000 \\
.000032800 \\
.000087600 \\
.000006670\end{array}$} & $\begin{array}{l}.000004130 \\
.000689000 \\
.000032800 \\
.000087600 \\
.000006670\end{array}$ & $\begin{array}{ll}30 & 1.3 \text {-BUTADIENE } \\
0 & \text { ACETONITRILE } \\
0 & \text { BENZENE } \\
0 & \text { N-HEXANE } \\
0 & \text { TOLUENE }\end{array}$ & \\
\hline
\end{tabular}


AIR EMISSIONS INVENTORY PERMIT APPLICATION REPORT (UP460)

FACILITY NAME: FACILITY IO : 200W TANK

Point Source Emissions - HAP Pollutants

En Point

200W P-241S106 001

Srce Nbr Srce Type Pollutant

1 TANKS AND VESSELS

200W P-2415107 001

200W P-241S108001

200W P-2415109001

1 TANKS AND VESSELS

1 TANKS AND VESSELS
Em. Pt. Potential Em. Pt.
to Emit (tons/yr) Ctl Actual (tons/yr) Pollutant

\begin{tabular}{|c|c|}
\hline .000004130 & .000004130 \\
\hline
\end{tabular}

.000032800 BENZENE

.

00000670 TOLUENE

$.000004130 \quad: 000004130 \quad 1.3$-BUTADIENE

.000689000 ACETONITRIL

.000087600 N-HEXANE

$.000006670 \quad .000006670$ TOLUENE

$.000004130 \quad .000004130 \quad 1.3$-BUTADIENE

$.000689000 \quad .000689000$ ACETONITRIL

.00032800 BENZENE

.000087600 .00008\% N-HEXANE

.000004130

00006670 TOLUENE

.000032800

.0000087600

$000004130 \quad 1.3$-BUTADIENE

000689000 ACETONITRILE

000087600 BENLENE

000006670 TOLUENE

Point Source Emissions - HAP Pollutants

\begin{tabular}{|c|c|c|c|c|c|c|}
\hline Em Point & Srce Nbr & Srce Type Pollutant & $\begin{array}{l}\text { Em. Pt. Potential } \\
\text { to Emit (tons/yr) }\end{array}$ & $\begin{array}{l}\text { Em. Pt. } \\
\text { Ctl Actual }\end{array}$ & (tons/yr) & Pollutant \\
\hline $200 \mathrm{~W} P-2415110001$ & . & 1 TANKS AND VESSELS & $\begin{array}{l}.00000 \\
.00068 \\
.00003 \\
.00008 \\
.00000\end{array}$ & & $\begin{array}{l}.000004130 \\
.000689000 \\
.000032800 \\
.000087600 \\
.000006670\end{array}$ & $\begin{array}{ll}0 & 1.3 \text {-BUTADIEN } \\
0 & \text { ACETONITRILE } \\
0 & \text { BENZENE } \\
0 & \text { N-HEXANE } \\
0 & \text { TOLUENE }\end{array}$ \\
\hline
\end{tabular}




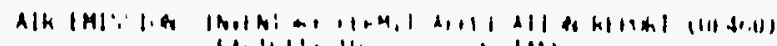

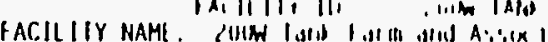

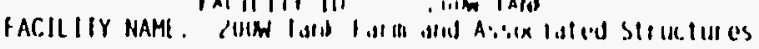

Point Source Emissions - HAP Pollutants

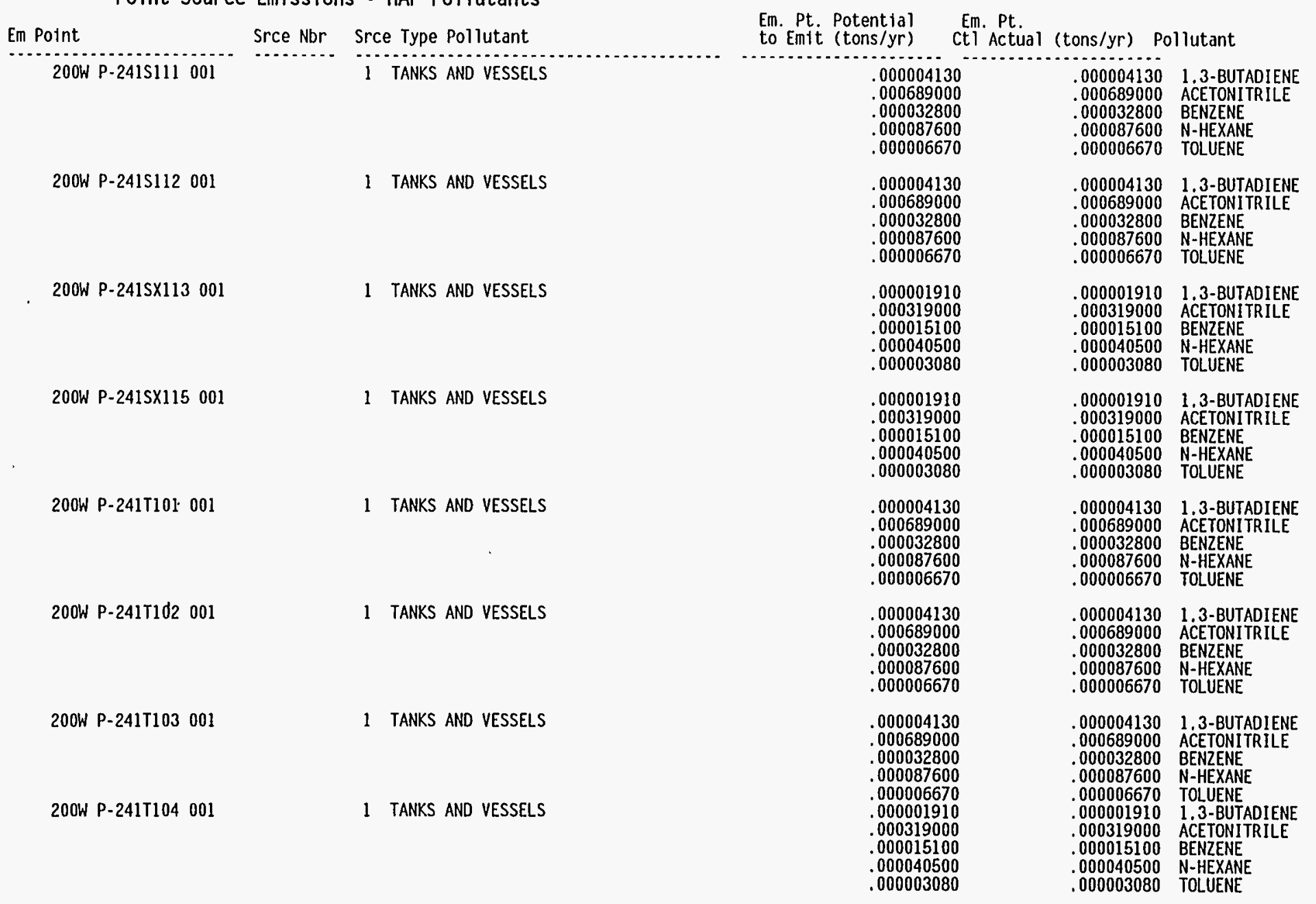

Page \#: 70 of 89 Report Run Date: 22-APR-95 


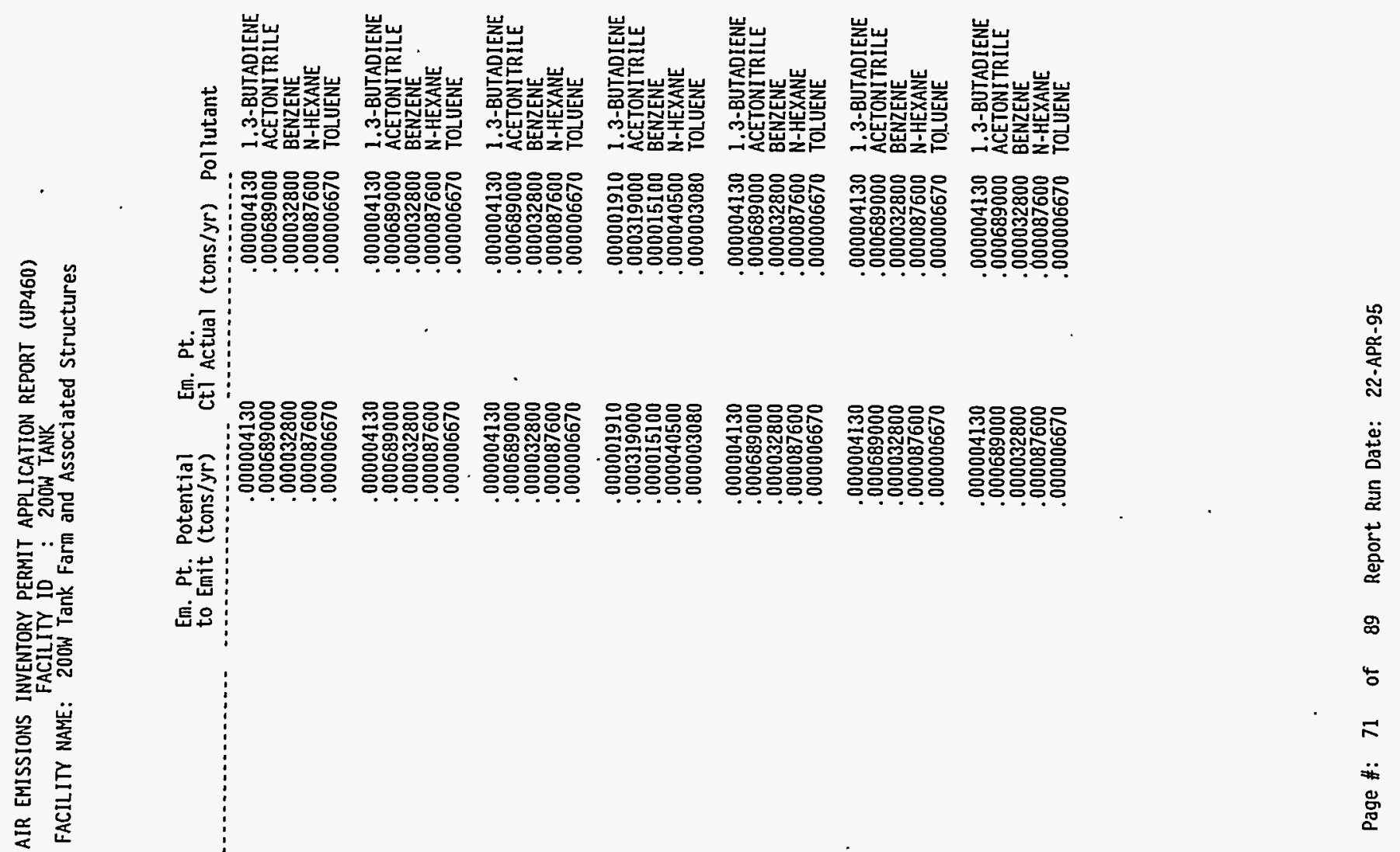

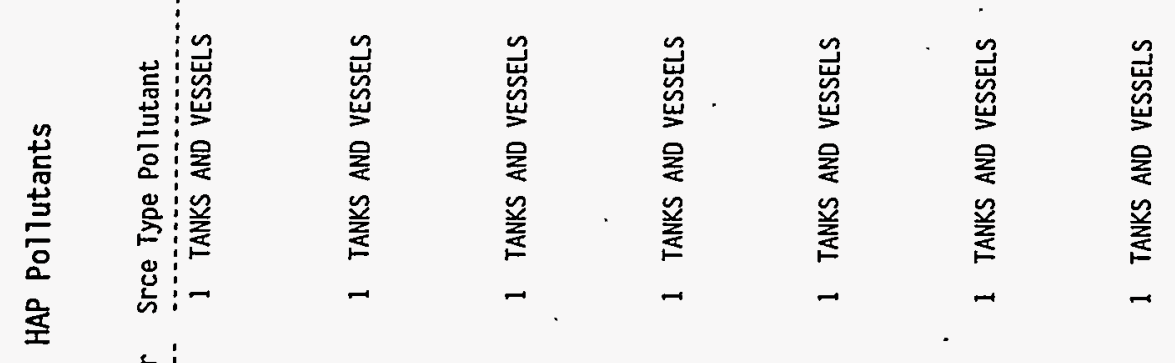

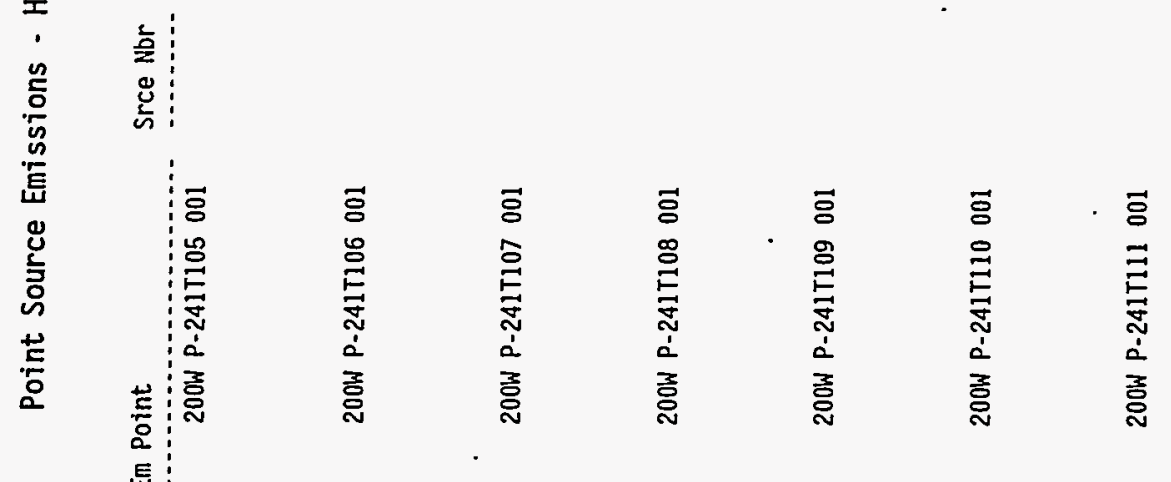


AIR EMISSIONS INVENTORY PERMIT APPLICATION REPORT (UP460)

FACILITY NAME: 200W Tank Farm and Associated Structures

Point Source Emissions - HAP Pollutants

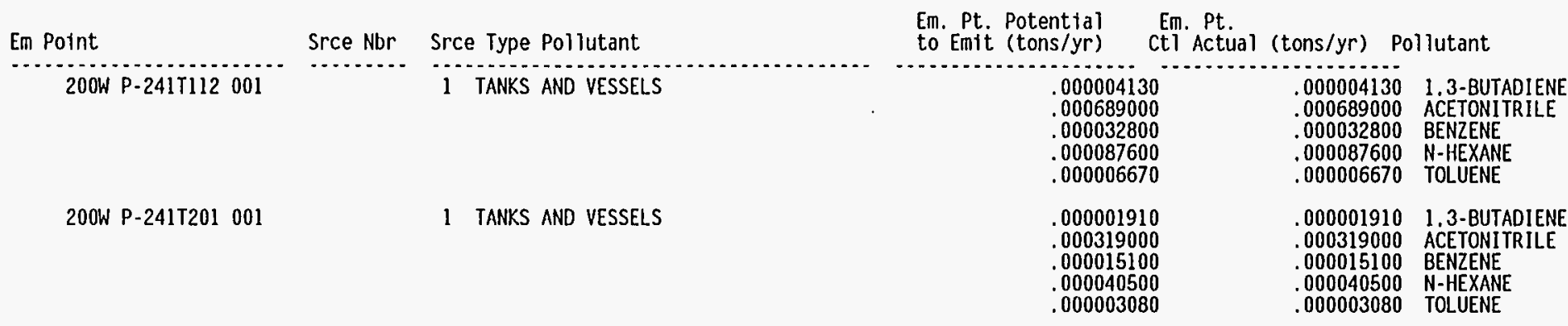

Point Source Emissions - HAP Pollutants

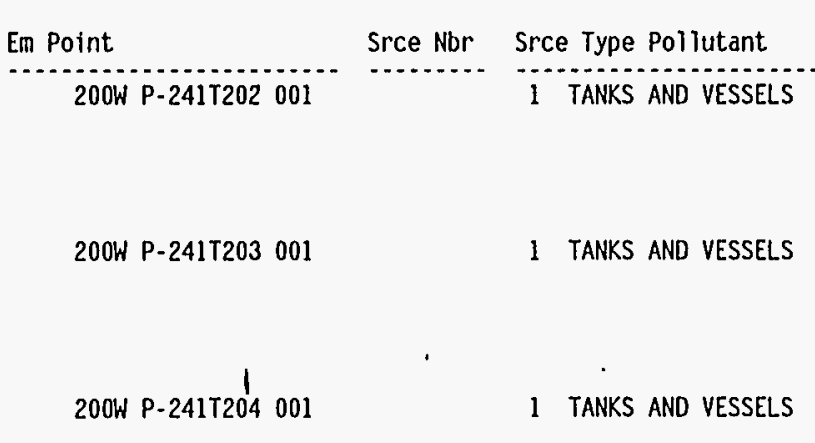

Em. Pt. Potential Em. Pt.
to Emit (tons/yr) Ctl Actual (tons/yr) Pollutant

$\begin{array}{lll}.000001910 & .000001910 & 1.3-B \text {-BUADIENE } \\ .00031900 & .000319000 & \text { ACETONITRILE } \\ .000015100 & .00015100 & \text { BENZENE } \\ .000040500 & .000040500 & \text { N-HEXANE } \\ .000003080 & .000003080 & \text { TOLUENE } \\ 0000001910 & .00001910 & 1.3 \text {-BUTADIENE } \\ .000031900 & .000319000 & \text { ACETONITRILE } \\ .000001500 & .00015100 & \text { BENZENE } \\ .000040500 & .000040500 & \text { N-HEXANE } \\ .000003080 & .000003080 & \text { TOLUENE } \\ .000001910 & .000001910 & 1.3 \text {-BUTADIENE } \\ .00031900 & .000319000 & \text { ACETONITRILE } \\ .00001500 & .00015100 & \text { 8ENZENE } \\ .000040500 & .00040500 & \text { N-HEXANE } \\ .000003080 & .000003080 & \text { TOLUENE }\end{array}$

Page \#: 72 of 89 Report Run Date: 22-APR-95 
AIR EMISSIONS INVENTORY PERMIT APPLICATION REPORT (UP460)

FACILITY NAME: $200 \mathrm{~W}$ Tank Farm and Associated Structures

Point Source Emissions - HAP Pollutants

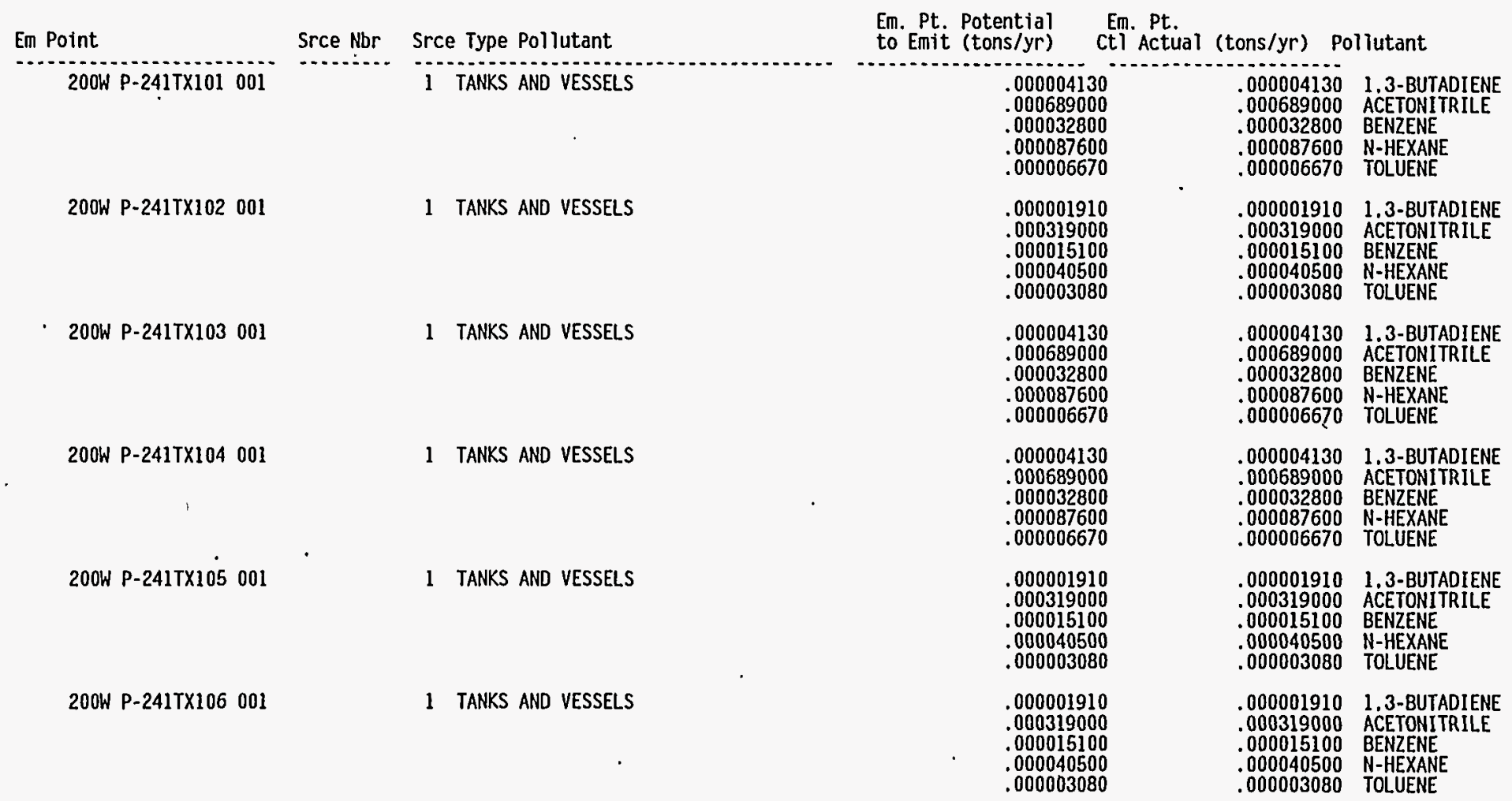

Page \#: 73 of 89 Report Run Date: 22-APR-95 
AIR EMISSIONS INVENTORY PERMIT APPLICATION REPORT (UP460)

FACILITY NAME: 200W Tank Farm and Associated Structures

Point Source Emissions - HAP Pollutants

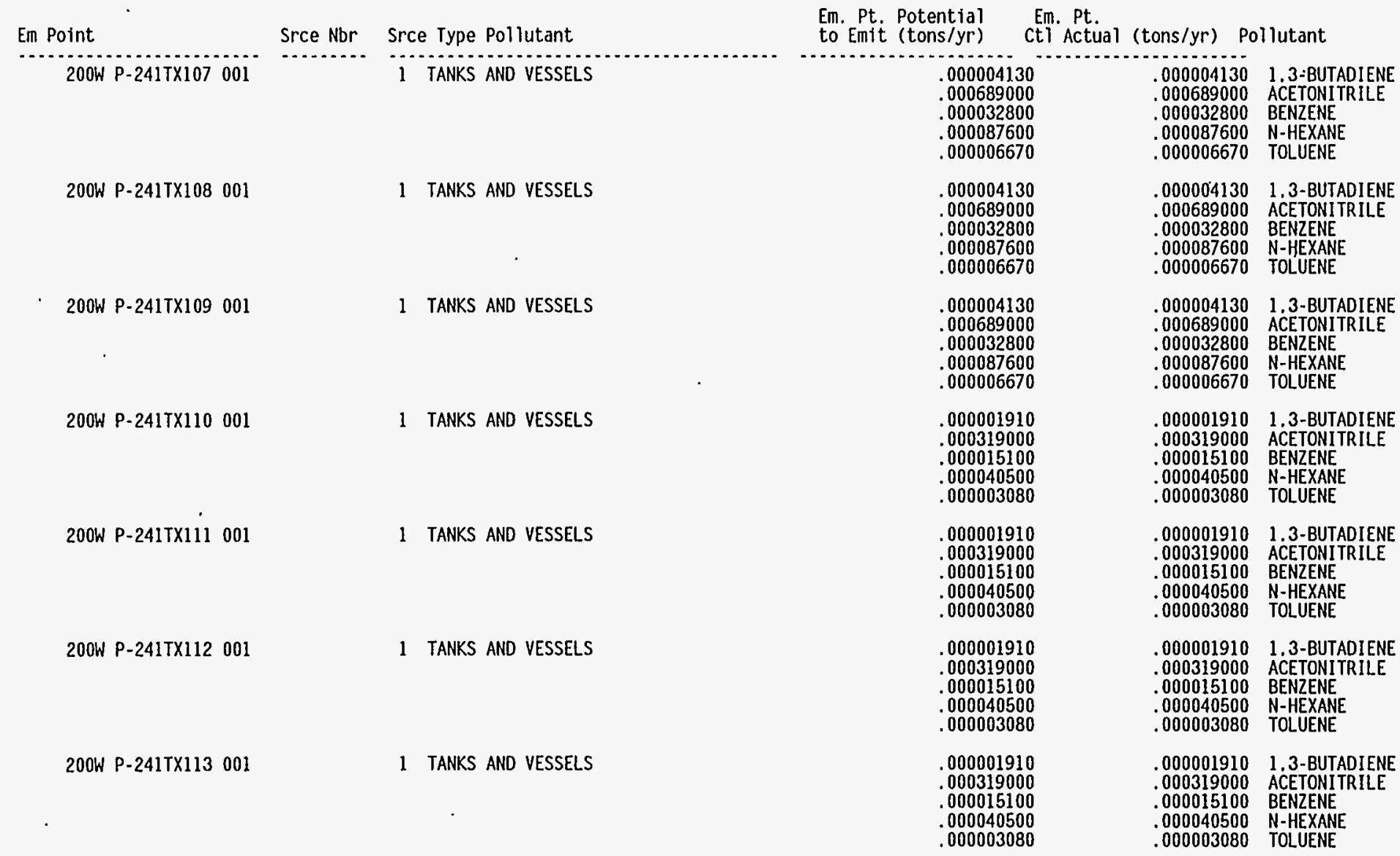

Page \#: 74 of 89 Report Run Date: 22-APR-95 
AIR EMISSIONS INVENTORY PERMIT APPLICATION REPORT (UP460)

FACILITY NAME: 200W Tank Farm and Associated Structures

Point Source Emissions - HAP Pollutants

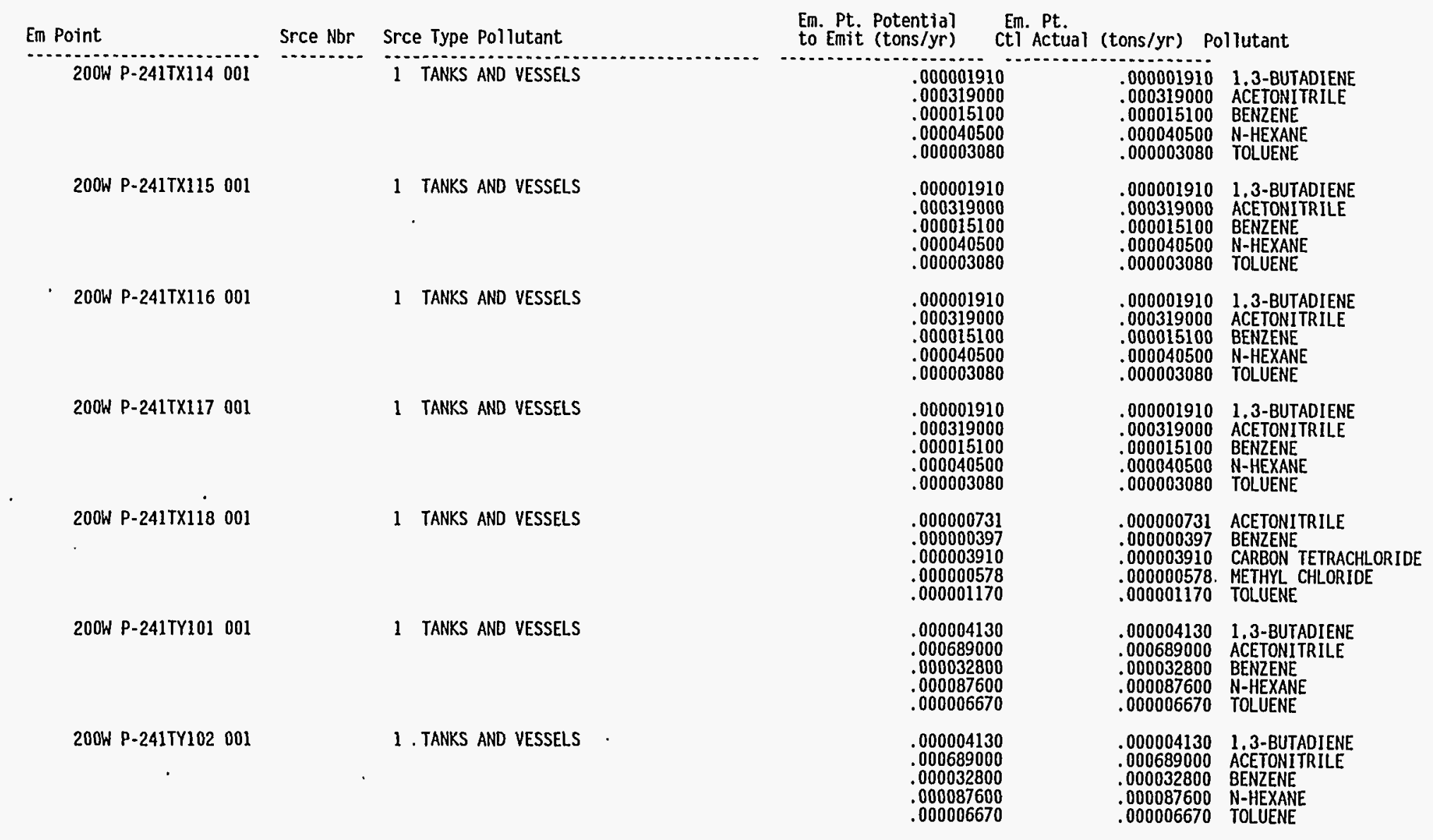

Page \#: 75 of 89 Report Run Date: 22-APR-95 
AIR EMISSIONS INVENTORY PERMIT APPLICATION REPORT (UP460)

FACILITY NAME: $200 \mathrm{~W}$ Tank Farm and Associated Structures

Point Source Emissions - HAP Pollutants

Em Point

Srce Nbr Srce Type Pollutant

1 TANKS AND VESSELS

200W P-241TY103001

200W P-241TY104 001

1 TANKS AND VESSELS

200W P-241TY105 001

1 TANKS AND VESSELS

200W P-241TY106 001

1 TANKS AND VESSELS
Em. Pt. Potential Em. Pt.
to Emit (tons/yr) ctl Actual (tons/yr) Pollutant

.

.000015100

.000040500

000004130
000689000

000032800

.000087600

.000006670

.000004130

000689000

00032800

000006670

.000001910

.000015100

000040500

000003080
$.000004130 \quad 1,3$-BUTADIENE

.000689000 ACETONITRILE

.000032800 BENZENE

.000087600
.000006670
NOLEXANE

$.000004130 \quad 1.3$-BUTADIENE

.000689000 ACETONITRILE

.00032800 BENZENE

.000006670 TOLUAE

000001910 13-8UTADIENE

000319000 ACETONITRILE

.000015100 BENZENE

.000040500 N-HEXANE

.000003080 TOLUENE

$.000001910 \quad$ 1.3-BUTADIENE

.000319000 ACETONITRILE

.000015100 BENZENE

.000040500 N-HEXANE

Point Source Emissions - HAP Pollutants

\begin{tabular}{|c|c|c|c|c|c|}
\hline Em Point & Srce Nbr & Srce Type Pollutant & $\begin{array}{l}\text { Em. Pt. Potential } \\
\text { to Emit (tons/yr) }\end{array}$ & $\begin{array}{l}\text { Em. Pt. } \\
\text { Ct1. Actual (tons/yr) }\end{array}$ & Pollutant \\
\hline $200 \mathrm{~W}$ P-241U101 001 & & 1 TANKS AND VESSELS & $\begin{array}{l}.00000 \\
.00031 \\
.00001 \\
.00004 \\
.00000\end{array}$ & $\begin{array}{l}.000001910 \\
.000319000 \\
.000015100 \\
.000040500 \\
.000003080\end{array}$ & $\begin{array}{ll}0 & 1.3 \text {-BUTADIENE } \\
0 & \text { ACETONITRILE } \\
0 & \text { BENZENE } \\
0 & \text { N-HEXANE } \\
0 & \text { TOLUENE }\end{array}$ \\
\hline
\end{tabular}

Page \#: 76 of 89 Report Run Date: 22-APR-95 


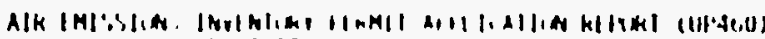

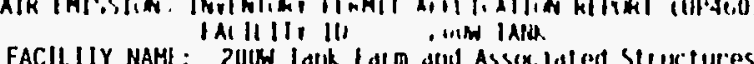

Point Source Emissions - HAP Pollutants

Em Point

200W P-241U102 001

Srce Nbr Srce Type Pollutant

1 TANKS AND VESSELS

200W P-241U103 001

1 TANKS AND VESSELS

200W P-241U104 001

1 TANKS AND VESSELS

200W P-241U105001

1 TANKS AND VESSELS

200W P-241U106 001

1 TANKS AND VESSELS

200W P-241010'7 001

1 TANKS AND VESSELS

200W P-241U108 001

1 TANKS AND VESSELS

200W P-241U109 001

Em. Pt. Potential Em. Pt.
to Emit (tons/yr) ctl Actual (tons/yr) Pollutant

.000004130 1.3-BUTADIENE

.000689000 ACETONITRILE

000087600 N-HEXANE

000006670 TOLUENE

00032800

.000087600

.000006670

$.000004130 \quad 1.3-B U T A D I E N E$

.000689000

.000032800

.000087600

.000689000 ACETONITRILE

.000032800 BENZENE

.000087600
.000006670
TOLUEXNE

.000001910

.000319000

.000015100

$.000001910 \quad 1.3$-BUTADIENE

.000003080

.000319000 ACETONITRILE

000015100 BENZENE

.000003080 TOLUENE

.000003080

.000004130

.000032800

.000087600

$.000004130 \quad 1.3$-BUTADIENE

.000689000 ACETONITRILE

000032800 BENZENE

.000006670 TOLUENE

.000004130

$.000004130 \quad 1.3$-BUTADIENE

.000689000

.000032800

.000087600

.000689000 ACETONITRILE

DO03280 BENZENE

.000006670 TOLUENE

.000004130

.000689000

.000032800
.000087600

.000004130 1.3-BUTADIENE

000032800 BENZENE

$000087600 \mathrm{~N}$-HEXANE

.000004130

.000689000

.000032800

.000087600

$.000004130 \quad 1,3$-BUTADIENE

.000689000 ACETONITRILE

.000032800 BENZENE

.000087600 N-HEXANE

.000004130

.000006670 TOLUENE

.000689000

.000087600

$.000004130 \quad 1.3$-BUTAQIENE

000689000 ACETONITRILE

BENZENE

.000006670 TOLUENE

Page \#: 77 of 89 Report Run Date: 22-APR-95 


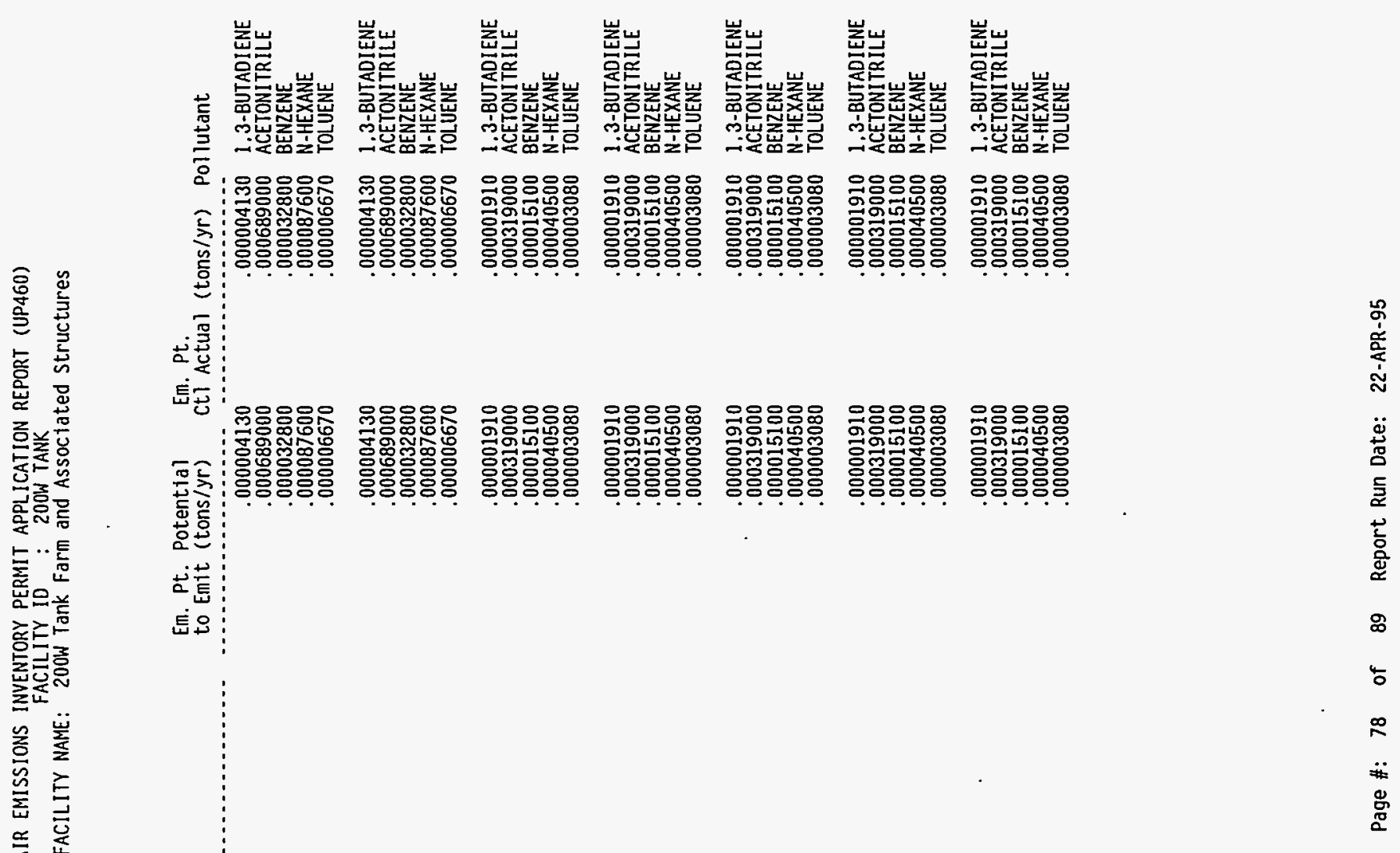

\begin{tabular}{|c|c|c|c|c|c|c|}
\hline & 岂岂 & 嵒 & 岀 & 荵 & 岂 & 岂 \\
\hline & 吾: 岕 & $\stackrel{\breve{M}}{\geq}$ & $\stackrel{心}{\longrightarrow}$ & $\stackrel{\mathscr{3}}{\longrightarrow}$ & $\stackrel{心}{\rightrightarrows}$ & $\stackrel{\mathscr{H}}{\Sigma}$ \\
\hline 旁 & 훙: & 운 & 웇 & 害 & 产 & 울 \\
\hline$\stackrel{\Xi}{\Xi}$ & 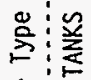 & 慈 & 美 & 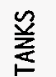 & 旁 & 茎 \\
\hline & 岕: & $\vec{r}$ & - & - & - & - \\
\hline
\end{tabular}

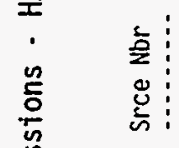

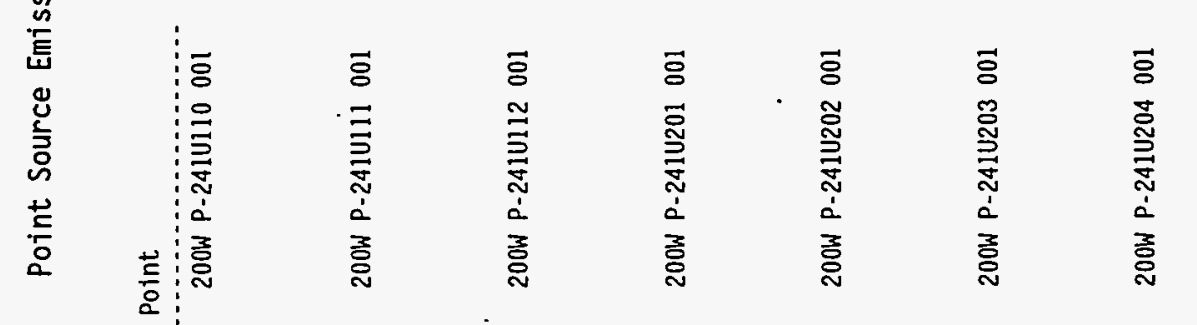


AIR EMISSIONS INVENTORY PERMIT APPLICATION REPORT (UP460)
FACILITY ID $: 200 \mathrm{~W}$ TANK

FACILITY NAME: $200 \mathrm{~W}$ Tank Farm and Associated Structures

Point Source Emissions - HAP Pollutants

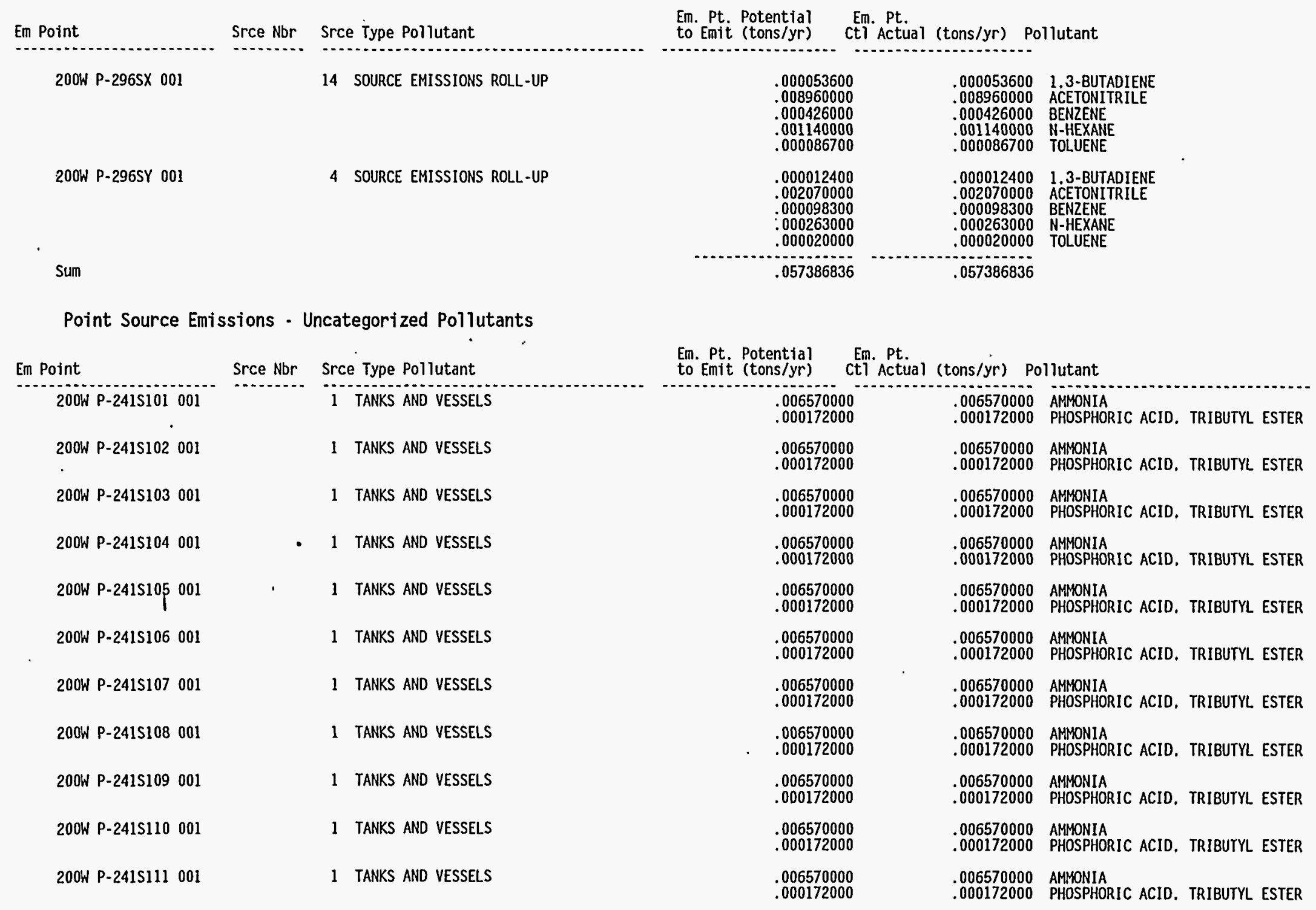


Point Source Emisstons - Uncategorized Pollutants

\begin{tabular}{|c|c|c|c|c|c|c|}
\hline Em Point & Srce $\mathrm{Nbr}$ & Srce Type Pollutant & $\begin{array}{l}\text { Em. Pt, Potential } \\
\text { to Emit (tons/yr) }\end{array}$ & (tons/yr) & ollutant & \\
\hline 200W P-241S112001 & & 1 TANKS AND VESSELS & $\begin{array}{l}.006570000 \\
.000172000\end{array}$ & $\begin{array}{l}.006570000 \\
.000172000\end{array}$ & $\begin{array}{l}\text { AMMONIA } \\
\text { PHOSPHORIC ACID. }\end{array}$ & TRIBUTYL ESTER \\
\hline 200W P-241SX113001 & & 1 TANKS AND VESSELS & $\begin{array}{l}.003040000 \\
.000079500\end{array}$ & $\begin{array}{l}.003040000 \\
.000079500\end{array}$ & $\begin{array}{l}\text { AMMONIA } \\
\text { PHOSPHORIC ACID. }\end{array}$ & TRIBUTYL ESTER \\
\hline 200W P-241SX115 001 & & 1 TANKS AND VESSELS & $\begin{array}{l}.003040000 \\
.000079500\end{array}$ & $\begin{array}{l}.003040000 \\
.000079500\end{array}$ & $\begin{array}{l}\text { AMMONIA } \\
\text { PHOSPHORIC ACID. }\end{array}$ & TRIBUTYL ESTER \\
\hline $200 H$ P-241T101 001 & & 1 TANKS AND VESSELS & $\begin{array}{l}.006570000 \\
.000172000\end{array}$ & $\begin{array}{l}.006570000 \\
.000172000\end{array}$ & $\begin{array}{l}\text { AMMONIA } \\
\text { PHOSPHORIC ACID. }\end{array}$ & TRIBUTYL ESTER \\
\hline
\end{tabular}

Point Source Emissions - Uncategorized Pollutants

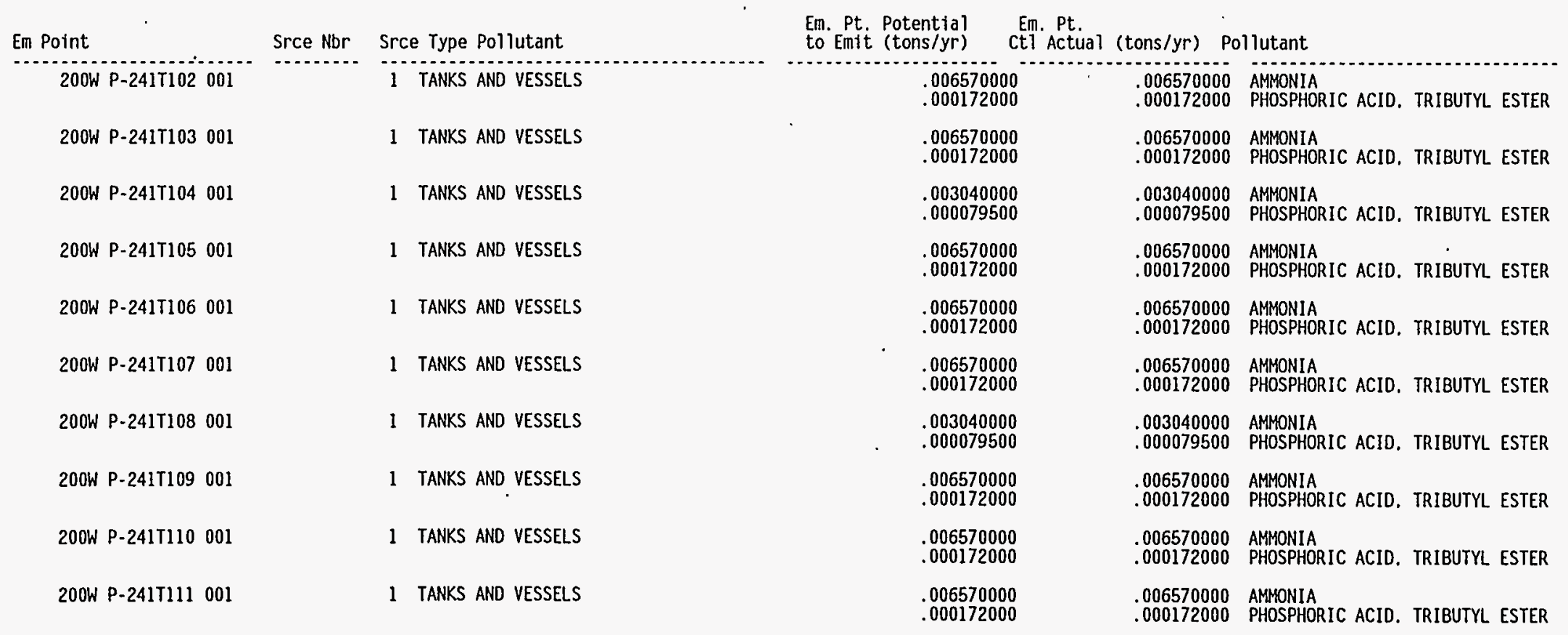


AIR EMISSIONS INVENTORY PERMIT APPLICATION REPORT (UP460)

FACILITY NAME: $200 \mathrm{~W}$ Tank Farm and Associated Structures

Point Source Emissions - Uncategorized Pollutants

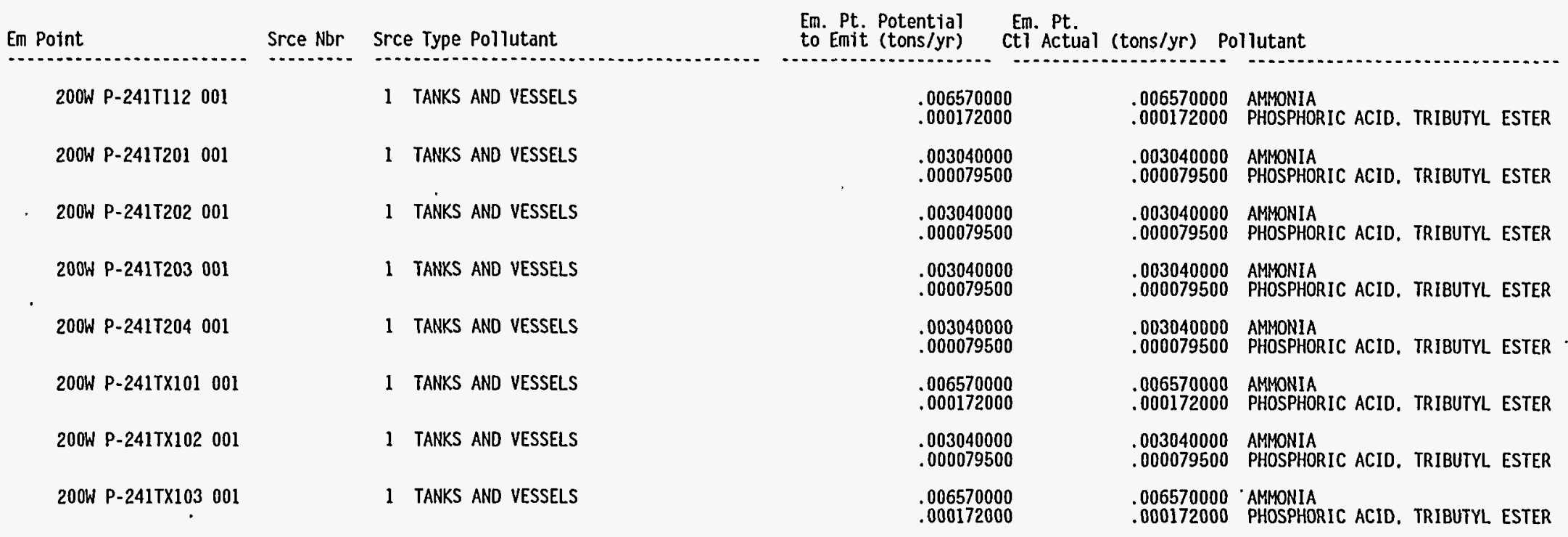

Point Source Emissions - Uncategorized Pollutants

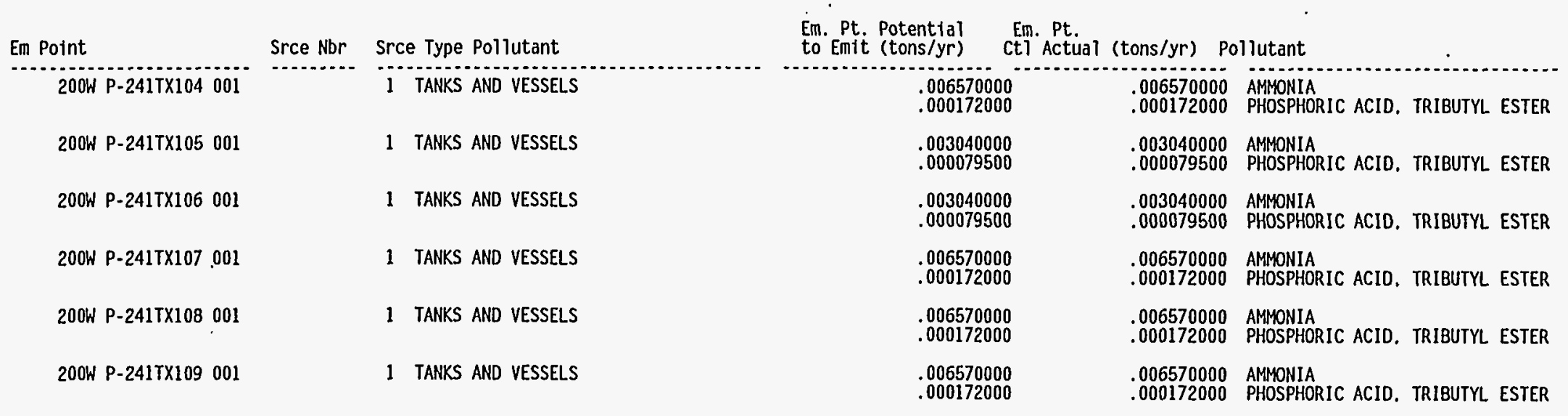

Page \#: 81 of 89 Report Run Date: 22-APR-95 
AIR EMISSIONS INVENTORY PERMIT APPLICATION REPORT (UP460)

FACILITY NAME: $200 \mathrm{~W}$ Tank Farm and Associated Structures

Point Source Emissions - Uncategorized Pollutants

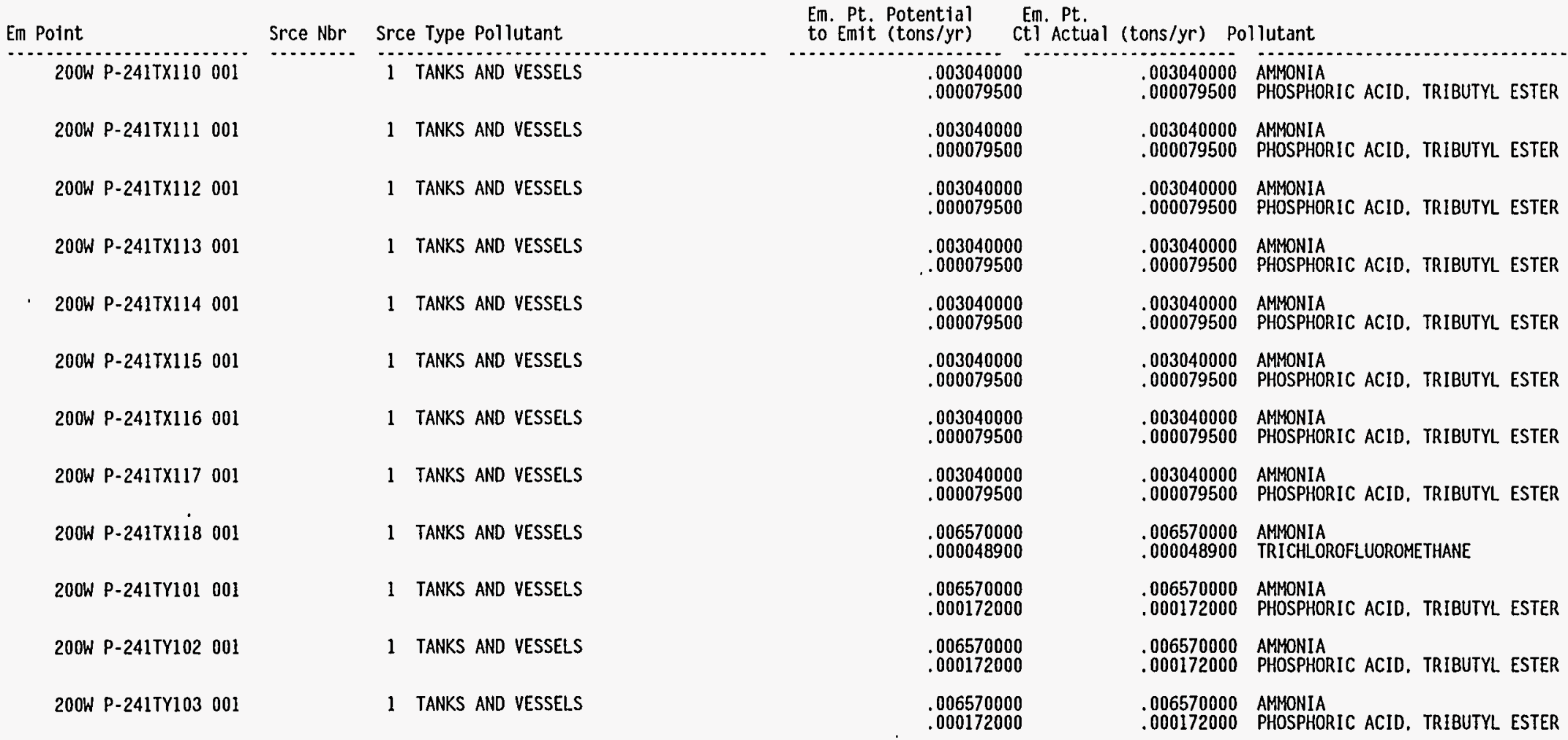

Point Source Emissions - Uncategorized Pollutants

\begin{tabular}{|c|c|c|c|c|c|c|}
\hline Em Point & Srce Nbr & Srce Type Pollutant & $\begin{array}{l}\text { Em. Pt. Potential } \\
\text { to Emit (tons/yr) }\end{array}$ & $\begin{array}{l}\text { Em. Pt. } \\
\text { Ctl Actual (tons/yr) }\end{array}$ & Pollutant & \\
\hline 200W P-241TY104 001 & & 1 TANKS AND VESSELS & $\begin{array}{l}.006570000 \\
.000172000\end{array}$ & $\begin{array}{l}.00657000 \\
.00017200\end{array}$ & $\begin{array}{l}\text { AMMONIA } \\
\text { PHOSPHORIC ACID. }\end{array}$ & TRIBUTYL ESTER \\
\hline 200W P-241TY105 001 & & 1 TANKS AND VESSELS & $\begin{array}{l}.003040000 \\
.000079500\end{array}$ & $\begin{array}{l}.00304000 \\
.00007950\end{array}$ & $\begin{array}{l}\text { AMMONIA } \\
\text { PHOSPHORIC ACID. }\end{array}$ & TRIBUTYL ESTER \\
\hline
\end{tabular}


AIR EMISSIONS INVENTORY PERMIT APPLICATION REPORT (UP460)
FACILITY IO : 200W TANK

FACILITY NAME: 200W Tank Farm and Associated Structures

Point Source Emissions - Uncategorized Pollutants

\begin{tabular}{|c|c|c|c|c|c|c|c|c|}
\hline Em Point & Srce Nbr & Src & Type Pollutant & & $\begin{array}{l}\text { Em. Pt. Potential } \\
\text { to Emit (tons/yr) }\end{array}$ & (tons/yr) & ollutant & \\
\hline 200W P-241TY106 001 & & 1 & TANKS AND VESSELS & & $\begin{array}{l}.003040000 \\
.000079500\end{array}$ & $\begin{array}{l}.003040000 \\
.000079500\end{array}$ & $\begin{array}{l}\text { AMMONIA } \\
\text { PHOSPHORIC ACID. }\end{array}$ & TRIBUTYL ESTER \\
\hline 200W P-241U101001 & & 1 & TANKS AND VESSELS & & $\begin{array}{l}.003040000 \\
.000079500\end{array}$ & $\begin{array}{l}.003040000 \\
.000079500\end{array}$ & $\begin{array}{l}\text { AMMONIA } \\
\text { PHOSPHORIC ACID. }\end{array}$ & TRIBUTYL ESTER \\
\hline 200W P-241U102 001 & & 1 & TANKS AND VESSELS & & $\begin{array}{l}.006570000 \\
.000172000\end{array}$ & $\begin{array}{l}.006570000 \\
.000172000\end{array}$ & $\begin{array}{l}\text { AMMONIA } \\
\text { PHOSPHORIC ACID. }\end{array}$ & TRIBUTYL ESTER \\
\hline 200W P-241U103001 & & 1 & TANKS AND VESSELS & & $\begin{array}{l}.006570000 \\
.000172000\end{array}$ & $\begin{array}{l}.006570000 \\
.000172000\end{array}$ & $\begin{array}{l}\text { AMMONIA } \\
\text { PHOSPHORIC ACID. }\end{array}$ & TRIBUTYL ESTER \\
\hline $200 W$ P-241U104 001 & & 1 & TANKS AND VESSELS & & $\begin{array}{l}.003040000 \\
.000079500\end{array}$ & $\begin{array}{l}.003040000 \\
.000079500\end{array}$ & $\begin{array}{l}\text { AMMONIA } \\
\text { PHOSPHORIC ACID. }\end{array}$ & TRIBUTYL ESTER \\
\hline 200W P-241U105 001 & & 1 & TANKS AND VESSELS & & $\begin{array}{l}.006570000 \\
.000172000\end{array}$ & $\begin{array}{l}.006570000 \\
.000172000\end{array}$ & $\begin{array}{l}\text { AMMONIA } \\
\text { PHOSPHORIC ACID. }\end{array}$ & TRIBUTYL ESTER \\
\hline 200W P-241U106 001 & & 1 & TANKS AND VESSELS & & $\begin{array}{l}.006570000 \\
.000172000\end{array}$ & $\begin{array}{l}.006570000 \\
.000172000\end{array}$ & $\begin{array}{l}\text { AMMONIA } \\
\text { PHOSPHORIC ACID. }\end{array}$ & -TRIBUTYL ESTER \\
\hline 200W P-241U107001 & & 1 & TANKS AND VESSELS & & $\begin{array}{l}.006570000 \\
.000172000\end{array}$ & $\begin{array}{l}.006570000 \\
.000172000\end{array}$ & $\begin{array}{l}\text { AMMONIA } \\
\text { PHOSPHORIC ACID. }\end{array}$ & TRIBUTYL ESTER \\
\hline 200W P-241U108 001 & & 1 & TANKS AND VESSELS & & $\begin{array}{l}.006570000 \\
.000172000\end{array}$ & $\begin{array}{l}.006570000 \\
.000172000\end{array}$ & $\begin{array}{l}\text { AMMONIA } \\
\text { PHOSPHORIC ACID. }\end{array}$ & TRIBUTYL ESTER \\
\hline 200W P-241U109001 & & 1 & TANKS AND VESSEL.S & & $\begin{array}{l}.006570000 \\
.000172000\end{array}$ & $\begin{array}{l}.006570000 \\
.000172000\end{array}$ & $\begin{array}{l}\text { AMMONIA } \\
\text { PHOSPHORIC ACID. }\end{array}$ & TRIBUTYL ESTER \\
\hline 200W P-241U110 001 & & 1 & TANKS AND VESSELS & & $\begin{array}{l}.006570000 \\
.000172000\end{array}$ & $\begin{array}{l}.006570000 \\
.000172000\end{array}$ & $\begin{array}{l}\text { AMMONIA } \\
\text { PHOSPHORIC ACID. }\end{array}$ & TRIBUTYL ESTER \\
\hline 200W P-241U111 001 & & 1 & TANKS AND VESSELS & & $\begin{array}{l}.006570000 \\
.000172000\end{array}$ & $\begin{array}{l}.006570000 \\
.000172000\end{array}$ & $\begin{array}{l}\text { AMMONIA } \\
\text { PHOSPHORIC ACID. }\end{array}$ & TRIBUTYL ESTER \\
\hline 200W P-241U112 001 & & 1 & TANKS AND VESSELS & & $\begin{array}{l}.003040000 \\
.000079500\end{array}$ & $\begin{array}{l}.003040000 \\
.000079500\end{array}$ & $\begin{array}{l}\text { AMMONIA } \\
\text { PHOSPHORIC ACID. }\end{array}$ & TRIBUTYL ESTER \\
\hline 200W P-241U201 001 & & 1 & TANKS AND VESSELS & & $\begin{array}{l}.003040000 \\
.000079500\end{array}$ & $\begin{array}{l}.003040000 \\
.000079500\end{array}$ & $\begin{array}{l}\text { AMMONIA } \\
\text { PHOSPHORIC ACID. }\end{array}$ & TRIBUTYL ESTER \\
\hline 200W P-241U202 001 & & 1 & TANKS AND VESSELS & & $\begin{array}{l}.003040000 \\
.000079500\end{array}$ & $\begin{array}{l}.003040000 \\
.000079500\end{array}$ & $\begin{array}{l}\text { AMMONIA } \\
\text { PHOSPHORIC ACIO. }\end{array}$ & TRIBUTYL ESTER \\
\hline 200W P-241U203 001 & & 1 & TANKS AND VESSELS & & $\begin{array}{l}.003040000 \\
.000079500\end{array}$ & $\begin{array}{l}.003040000 \\
.000079500\end{array}$ & $\begin{array}{l}\text { AMMONIA } \\
\text { PHOSPHORIC ACID. }\end{array}$ & TRIBUTYL ESTER \\
\hline
\end{tabular}




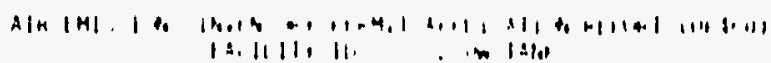

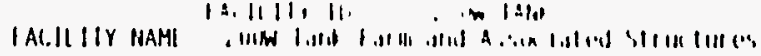

Point Source Emissions - Uncategorized Pollutants

\begin{tabular}{|c|c|c|c|c|c|}
\hline En Point & Srce Nbr & Srce Type Pollutant & $\begin{array}{l}\text { Em. Pt. Potentia) } \\
\text { to Enit (tons/yr) }\end{array}$ & $\begin{array}{l}\text { Em. Pt. } \\
\text { Ctl Actual (tons/yr) }\end{array}$ & ollutant \\
\hline 200W P-241U204001 & & 1 TANKS AND VESSELS & $\begin{array}{l}.003040000 \\
.000079500\end{array}$ & .003040000 & $\begin{array}{l}\text { AMMONIA } \\
\text { PHOSPHORIC ACID. TRIBUTYL ESTER }\end{array}$ \\
\hline 200W P-296SX 001 & & SOURCE EMISSIONS ROLL-UP & $\begin{array}{r}4.170000000 \\
.002240000\end{array}$ & $\begin{array}{r}4.170000000 \\
.002240000\end{array}$ & $\begin{array}{l}\text { AMMONIA } \\
\text { PHOSPHORIC ACIO. TRIBUTYL ESTER }\end{array}$ \\
\hline 200W P-296SY 001 & & 4 SOURCE EMISSIONS ROLL-UP & $\begin{array}{l}.963000000 \\
.000516000\end{array}$ & $\begin{array}{l}.963000000 \\
.000516000\end{array}$ & $\begin{array}{l}\text { AMMONIA } \\
\text { PHOSPHORIC ACID. TRIBUTYL ESTER }\end{array}$ \\
\hline Sum & & & 5.506142900 & 5.506142900 & \\
\hline
\end{tabular}

Fugitive Source Criteria Pollutants - Type: PM

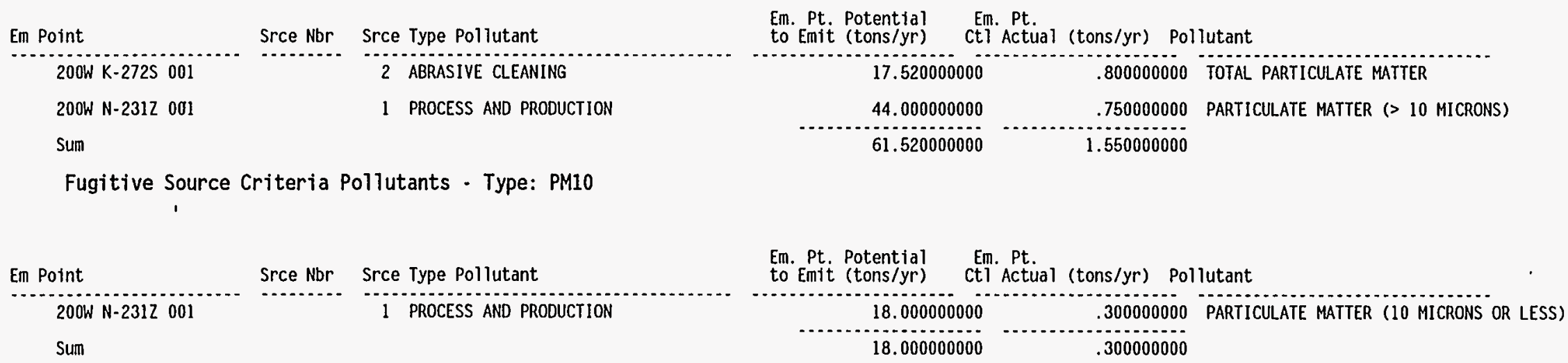


AIR EMISSIONS INVENTORY PERMIT APPLICATION REPORT (UP460)

FACILITY NAME: $200 \mathrm{~W}$ Tank Farm and Associated Structures

Fugitive Source Criteria Pollutants - Type: VoC

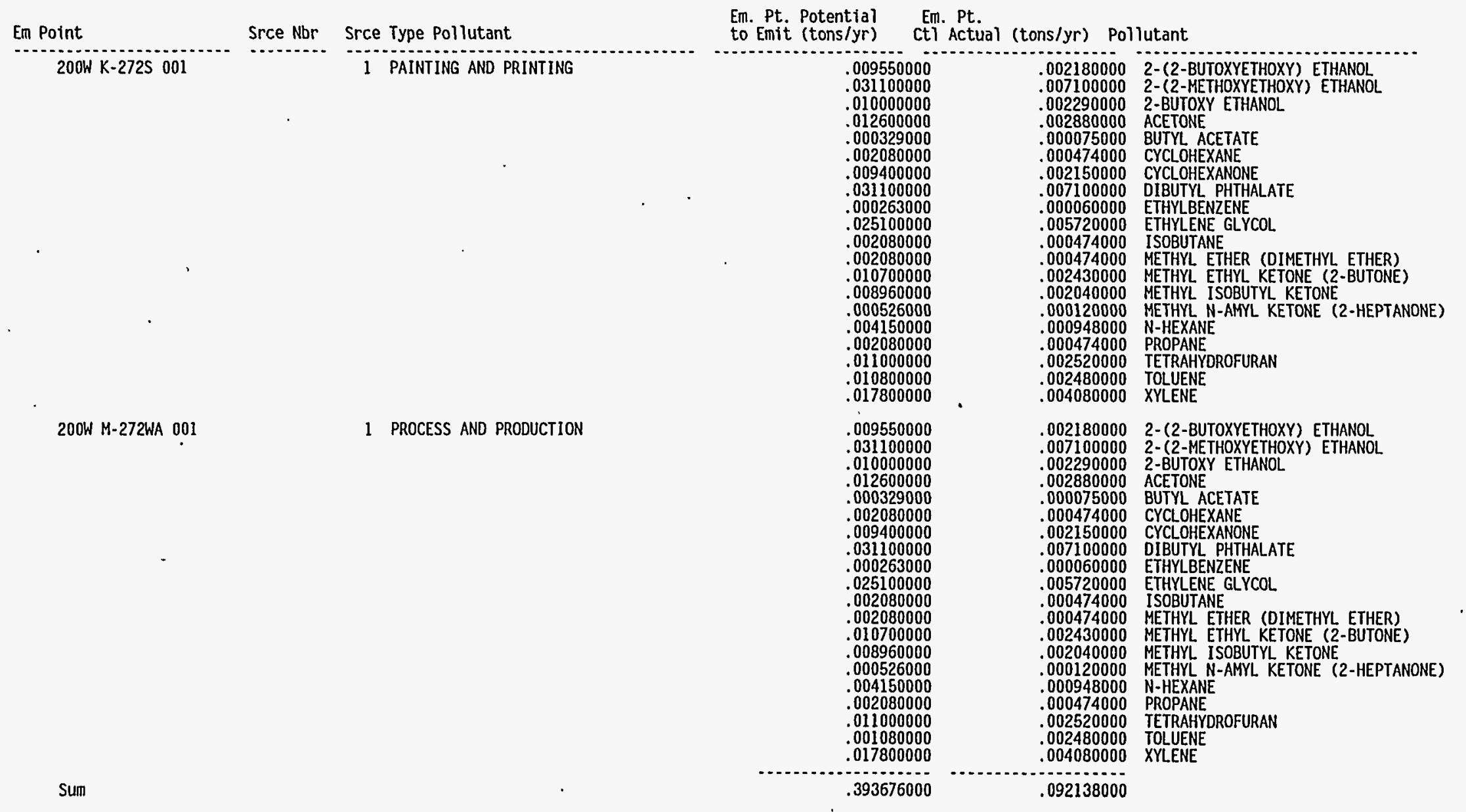

Fugitive Source Emissions - Radiological Pollutants

\begin{tabular}{|c|c|c|c|c|c|}
\hline Em Point & Srce $\mathrm{Nbr}$ & Srce Type Pollutant & $\begin{array}{l}\text { Em. Pt. Potential } \\
\text { to Emit }(\mathrm{C} 1 / \mathrm{yr})\end{array}$ & $\begin{array}{l}\text { Em. Pt. } \\
\text { Cti Actual (Ci/yr) }\end{array}$ & Pollutant \\
\hline 200W P-241S151 001 & & 1 PROCESS AND PRODUCTION & $\begin{array}{l}.0000 \\
.0000\end{array}$ & $\begin{array}{l}.000000000 \\
.000000002\end{array}$ & $\begin{array}{l}\text { AMERICIUM }-241 \\
\text { STRONTIUM } 89.90\end{array}$ \\
\hline
\end{tabular}

Page \#: 85 of 89 Report Run Date: 22-APR-95 
Fugitive Source Emissions - Radiological Pollutants

\begin{tabular}{|c|c|c|c|}
\hline Em Point & Srce Nbr & Srce & Type Pollutant \\
\hline 200W P-241S151 001 & & 1 & PROCESS AND PRODUCTION \\
\hline 200W P.241S304 001 & & 1 & PROCESS AND PRODUCTION \\
\hline 200W P-241SYA 001 & & 1 & PROCESS AND PRODUCTION \\
\hline 200W P-241SYB 001 & & 1 & PROCESS AND PRODUCTION \\
\hline 200W P-241TX152001 & & 1 & PROCESS AND PRODUCTION \\
\hline 200W P-241TX154001 & & 1 & PROCESS AND PRODUCTION \\
\hline 200W P-241TX302C 001 & & 1 & PROCESS AND PRODUCTION \\
\hline 200W P-241U151 001 & & 1 & PROCESS AND PRODUCTION \\
\hline 200W P-241U152 001 & & 1 & PROCESS AND PRODUCTION \\
\hline 200W P-241U301B 001 & . & 1 & PROCESS AND PRODUCTION \\
\hline 200W P-241UX154001 & & 1 & PROCESS AND PRODUCTION \\
\hline 200W P-241UX302A 001 & & 1 & PROCESS AND PRODUCTION \\
\hline
\end{tabular}

Fugitive Source Emissions - HAP Pollutants
Em. Pt. Potential Em. Pt.
to Emit (Ci/yr) $\quad$ Cti Actual (Ci/yr) Pollutant

.000000002 YTTRIUM - 090

.000000000 AMERICIUM - 241

000000001 STRONTIUM 89,90

000000001 YTTRIUM - 090

\begin{tabular}{|c|c|c|}
\hline $\begin{array}{l}.000000000 \\
.000000001 \\
.000000001\end{array}$ & $\begin{array}{l}.000000000 \\
.000000001 \\
.000000001\end{array}$ & $\begin{array}{l}\text { AMERICIUM - } 24 \\
\text { STRONTIUM } 89,9 \\
\text { YTRIUM - } 090^{\circ}\end{array}$ \\
\hline
\end{tabular}

$.000000000 \quad .000000000$ AMERICIUM -241 $.000000001 \quad .000000000$ YTTRIUM -090

$.000000000 \quad .000000000$ AMERICIUM -241

$.000000001 \quad .000000000$ STRONTIUM 89,90

$.000000001 \quad .000000000$ YTTRIUM - 090

$.000000000 \quad .000000000$ AMERICIUM -241 $.000000000 \quad .000000000$ STRONTIUM 89.90 $.000000000 \quad .000000000$ YTTRIUM - 090

$.000000000 \quad .000000000$ AMERICIUM -241 $.000000003 \quad .000000001$ STRONTIUM 89.90

.00000003 .0000001 VTRRIUH - 090

$.000000001 \quad .000000000$ AMERICIUM -241 $.000000001 \quad .000000001$ YTTRIUM $-090^{\circ}$

$.000000000 \quad .000000000$ AMERICIUM - 241 $.000000001 \quad .000000001$ STRONTIUM 89.90 $.000000001 \quad .000000001$ YTTRIUH -090

$.000000000 \quad .000000000$ AMERICIUM -241 $.00000002 \quad .000000001$ STRONTIUM 89.90 .00000002 .090

$.000000000 \quad .000000000$ AMERICIUM -241 .000000001 STRONTIUM 89,90

$.000000000 \quad .00000001$ YTRRIUT - 090

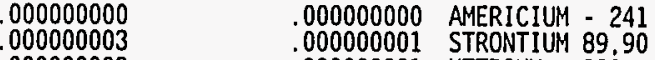
$.000000003 \quad .000000001$ YTTRIUM $-090^{\circ}$

$.000000000 \quad .000000000$ AMERICIUM $=241$

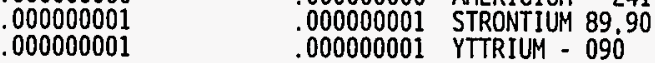

\begin{tabular}{|c|c|c|c|c|c|}
\hline Em Point & Srce Nbr & Srce Type Pollutant & $\begin{array}{l}\text { Em. Pt. Potential } \\
\text { to Emit (tons/yr) }\end{array}$ & $\begin{array}{l}\text { Em. Pt. } \\
\text { Ct? Actual (tons/yr) }\end{array}$ & Pollutant \\
\hline $200 \mathrm{~W} \mathrm{~K}-2725001$ & & 1 PAINTING AND PRINTING & $\begin{array}{l}.00955 \\
.03110\end{array}$ & $\begin{array}{l}.00218000 \\
.00710000\end{array}$ & $\begin{array}{ll}00 & 2-(2-B U T O X Y E T H O X Y) \\
00 & 2-(2-\text { EETHOXYETHOXY) ETHANOL }\end{array}$ \\
\hline
\end{tabular}

Page \#: 86 of 89 Report Run Date: 22-APR-95 
Fugitive Source Emissions - HAP Pollutants

\begin{tabular}{|c|c|c|c|c|c|}
\hline Em Point & Srce Nbr & Srce Type Pollutant & $\begin{array}{l}\text { Em. Pt. Potential } \\
\text { to Emit (tons/yr) }\end{array}$ & $\begin{array}{l}\text { Em. Pt. } \\
\text { ctl Actual (tons/yr) }\end{array}$ & ollutant \\
\hline $200 \mathrm{~W} \mathrm{~K}-2725001$ & & 1 PAINTING AND PRINTING & $\begin{array}{l}.010000000 \\
.031100000 \\
.000263000 \\
.025100000 \\
.010700000 \\
.008960000 \\
.004150000 \\
.010800000 \\
.017800000\end{array}$ & $\begin{array}{l}.002290000 \\
.007100000 \\
.000060000 \\
.005720000 \\
.002430000 \\
.002040000 \\
.000948000 \\
.002480000 \\
.004080000\end{array}$ & $\begin{array}{l}\text { 2-BUTOXY ETHANOL } \\
\text { OIBUTYL PHTHALATE } \\
\text { ETHYLBENZENE } \\
\text { ETHYLENE GLYCOL } \\
\text { METHYL ETHYL KETONE (2-BUTONE) } \\
\text { METHYL ISOBUTYL KETONE } \\
\text { N-HEXANE } \\
\text { TOLUENE } \\
\text { XYLENE }\end{array}$ \\
\hline 200W M-272WA 001 & & 1 PROCESS AND PRODUCTION & $\begin{array}{l}.009550000 \\
.031100000 \\
.010000000 \\
.031100000 \\
.000263000 \\
.025100000 \\
.010700000 \\
.008960000 \\
.004150000 \\
.001080000 \\
.017800000\end{array}$ & $\begin{array}{l}.002180000 \\
.007100000 \\
.002290000 \\
.007100000 \\
.000060000 \\
.005720000 \\
.002430000 \\
.002040000 \\
.000948000 \\
.002480000 \\
.004080000\end{array}$ & $\begin{array}{l}\text { 2-(2-BUTOXYETHOXY) ETHANOL } \\
\text { 2-(2-METHOXYETHOXY) ETHANOL } \\
\text { 2-BUTOXY ETHANOL } \\
\text { DIBUTYL PHTHALATE } \\
\text { ETHYLBENZENE } \\
\text { ETHYLENE GLYCOL } \\
\text { METHYL ETHYL KETONE (2-BUTONE) } \\
\text { METHYL ISOBUTYL KETONE } \\
\text { N-HEXANE } \\
\text { TOLUENE } \\
\text { XYLENE }\end{array}$ \\
\hline Sum & & & .309326000 & .072856000 & \\
\hline
\end{tabular}

Fugitive Source Emissions - Uncategorized Pollutants

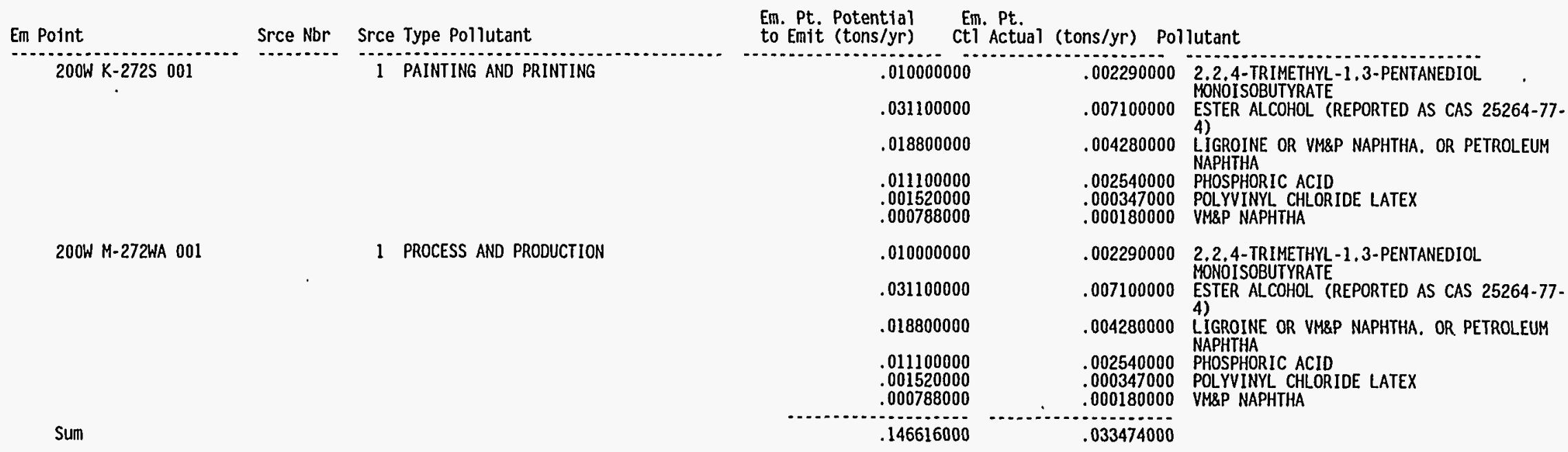




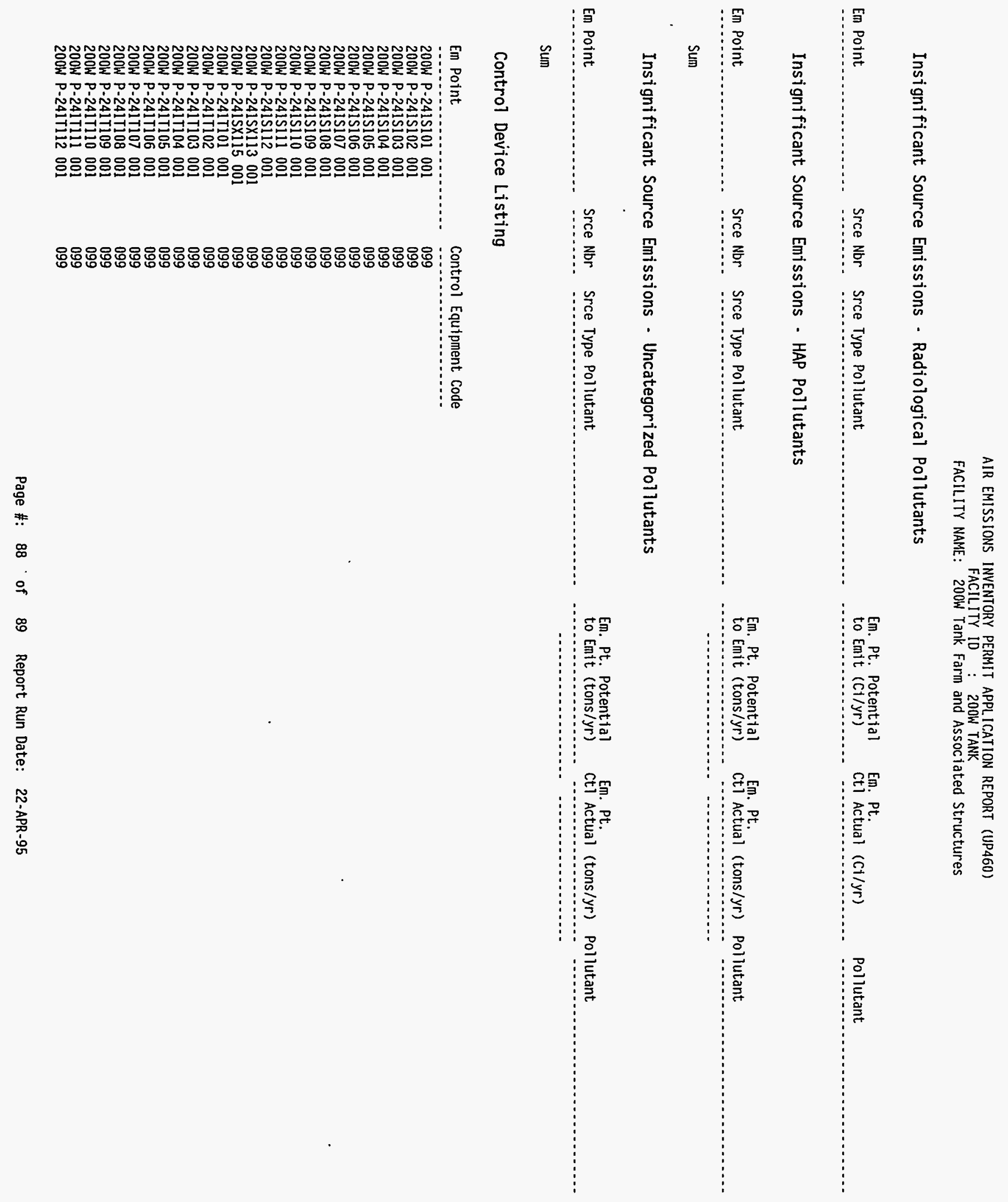


AIR EMISSIONS INVENTORY PERMIT APPLICATION REPORT (UP460)

FACILITY ID : 200W TANK
FACILITY NAME: 200W Tank Farm and Associated Structures

\section{Control Device Listing}

Em Point

200W P-241T201 001

200W P-241T202 001

200W P-241T203 001

200W P-241T204 001

200W P-241TX101 001

200W P-24IIX102 00

200W P-241TX104 001

200W P-241TX105 001

200W P-241TX106 00

$200 \mathrm{~W}$ P-241TX107 00

200W P-241TX108 00

200W P-241TX109 00

200W P-241TX110 00

200W P-241TX111 00

2004 - $417 \times 113001$

$200 \mathrm{P}-241 \mathrm{X} 11400$

$200 \mathrm{~W}$-241TX116 00

$200 \mathrm{~W}$ P.241TX117 00

$200 \mathrm{~W}$ P-241TX118 001

200W P-241TY101 001

200W P-241TY102 001

$200 \mathrm{~W}$ P-241TY103 001

200W P-241TY104 001

$200 \mathrm{~W}$ P-24ITY105 001

200W P-2417Y106 001

200W P-241U101 001

2004 P-2410102 001

200 P-2410103 001

$200 \mathrm{~W}$-241U105 001

$200 \mathrm{P}-2410105$ o01

200W P-241U107 001

$200 W$ P-241U108 001

200W P-24lu109 001

200W P-241U110 001

200W P-2410111 001

200W P-241U112 001

200W P-241U201 001

$200 \mathrm{~W}$ P-241U202 001

$200 \mathrm{~W}$ P-241U203 001

$200 W \mathrm{~W}-242 \mathrm{~S} 001$

200W P-242T 001

200W P-244TX 001

$200 \mathrm{~W}$ P.244U 001

200W

$200 W$ P-296P028 001

$200 W$ P.296S025 001

200W P-2965X 001

200W P-296SY DOI

\section{Control Equipment Code}

099

099

099

099

099

099

099

099

099

099

099
099

099

099

099

099

099

099
099

099
099

099

099

099.

099

099

099

099

099

099

099

099
099

099

099

099
099

099

099

099

099

099

099
099
099 
FAC IL I TY :

... ... . ism Farms

EMISSION POINT: - See Table

\begin{tabular}{|c|}
\hline Emission Points for Catch Tanks \\
\hline $200 W$ P-241UX302A 001 \\
\hline 200W P-241S304 001 \\
\hline 200W P-241TX302C 001 \\
\hline 200W P-241U301B 001 \\
\hline 200W P-241UX302A 001 \\
\hline 200W P-241S304 001 \\
\hline
\end{tabular}

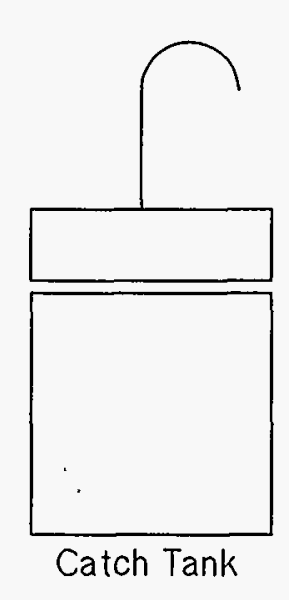




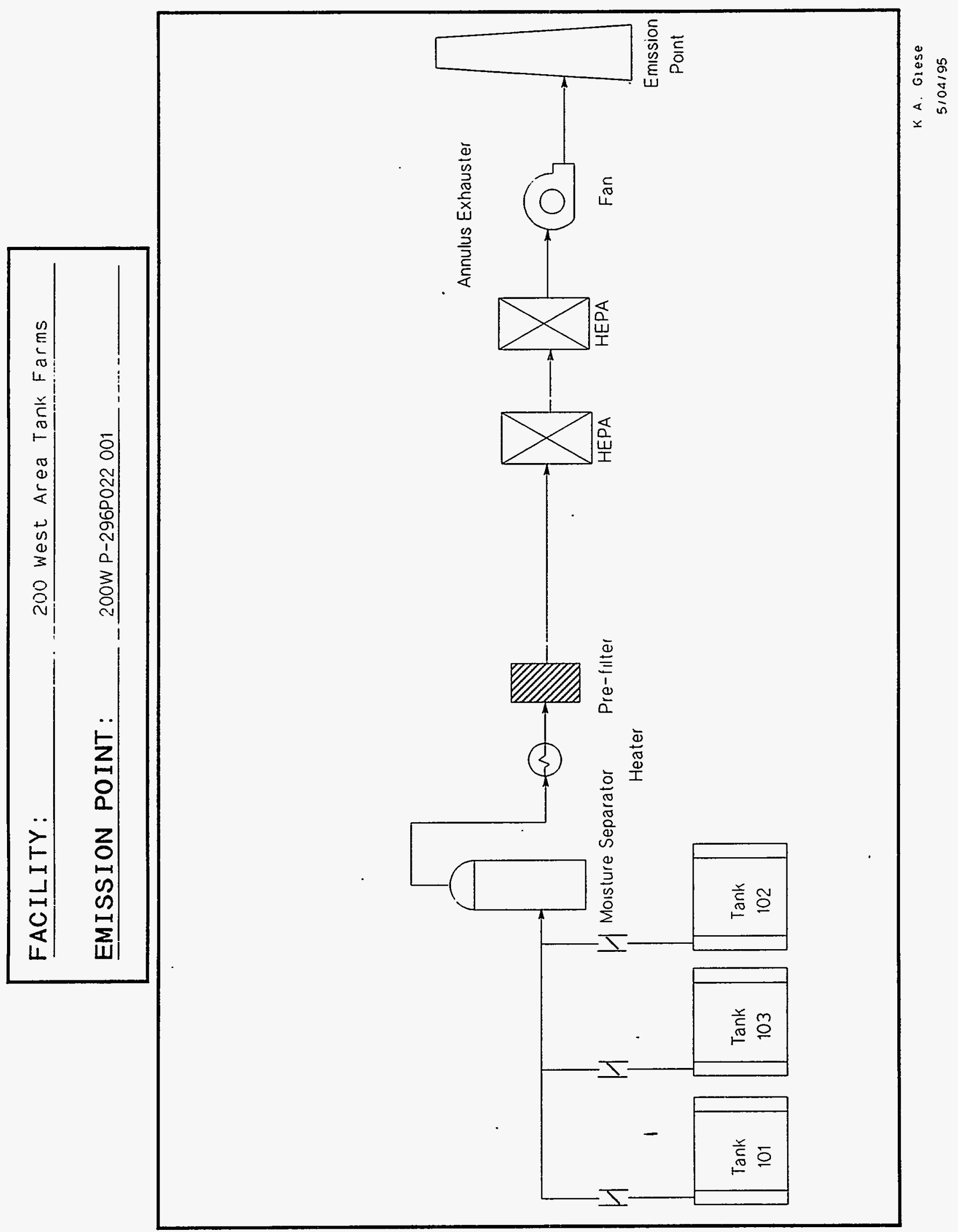




\begin{tabular}{|c|c|}
\hline FACILITY: & 200 West Area Tank Farms \\
\hline EMISSION POINT: & SEE INSET TABLE \\
\hline
\end{tabular}

200 West Area Single-Shell Tanks That Are Passively Vented

200W P-241S101001 200W P-241S102001

200W P-241S103 001

200W P-241S104 001

200W P-241S105 001

200W P-2415106 001

200W P-2415107 001

200W P-241S108 001

200W P-241S109 001

200W P-241S110 001

200W.P-241S111 001

200W P-241S112001

200W P-241SX113 001

200W P-241SX115 001

200W P-241T101001

200W P-241T102 001

200W P-241T103 001

200W P-241T104 001

200W P-241T105 001

200W P-241T106 001

200W P-241T107 001

200W P-241T108 001

200W P-241T109 001

200W P-241T110 001
200W P-241T111 001 200W P-241T112 001 200W P-241T:201001 .200W P-241T20? 001 200W P-241T203001 200W P-241T204 001 200W P-241TX101 001 200W P-241TX102 001 200W P-241TX103 001 200W P-241TX104 001 200W P-241TX105 001 200W P-241TX106 001 200W P-241TX107 001 200W P-241TX108 001 200W P-241TX109001 200W P-241TX110 001 200W P-241TX111 001 200W P-241TX112001 200W P-241TX113001 200W P-241TX114 001 200W P-241TX115 001 200W P-241TX116 001 200W P-241TX117001 200W P-241TX118 001
200W P-241TY101001 200W P-241TY102 001 200W P-241TY103 001 200W P-241TY104 001 200W P-24ITY 105001 200W P-241TY106 001 200W P-241U101001 200W P-241U102 001 200W P-241U103 001 .200W P-241U104 001 200W P-241U105 001 200W P-241U106 001 200W P-241U107 001 200W P-241U108 001 200W P-241U109001 200W P-241U110 001 200W P-241U111 001 200W P-241U112 001 200W P-241U201001 200W P-241U202 001 200W P-241U203 001 200W P-241U204001

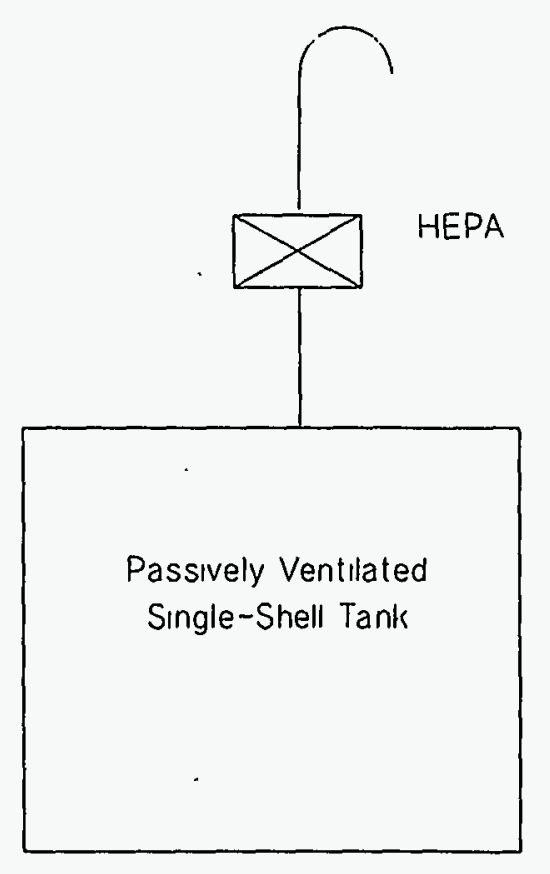




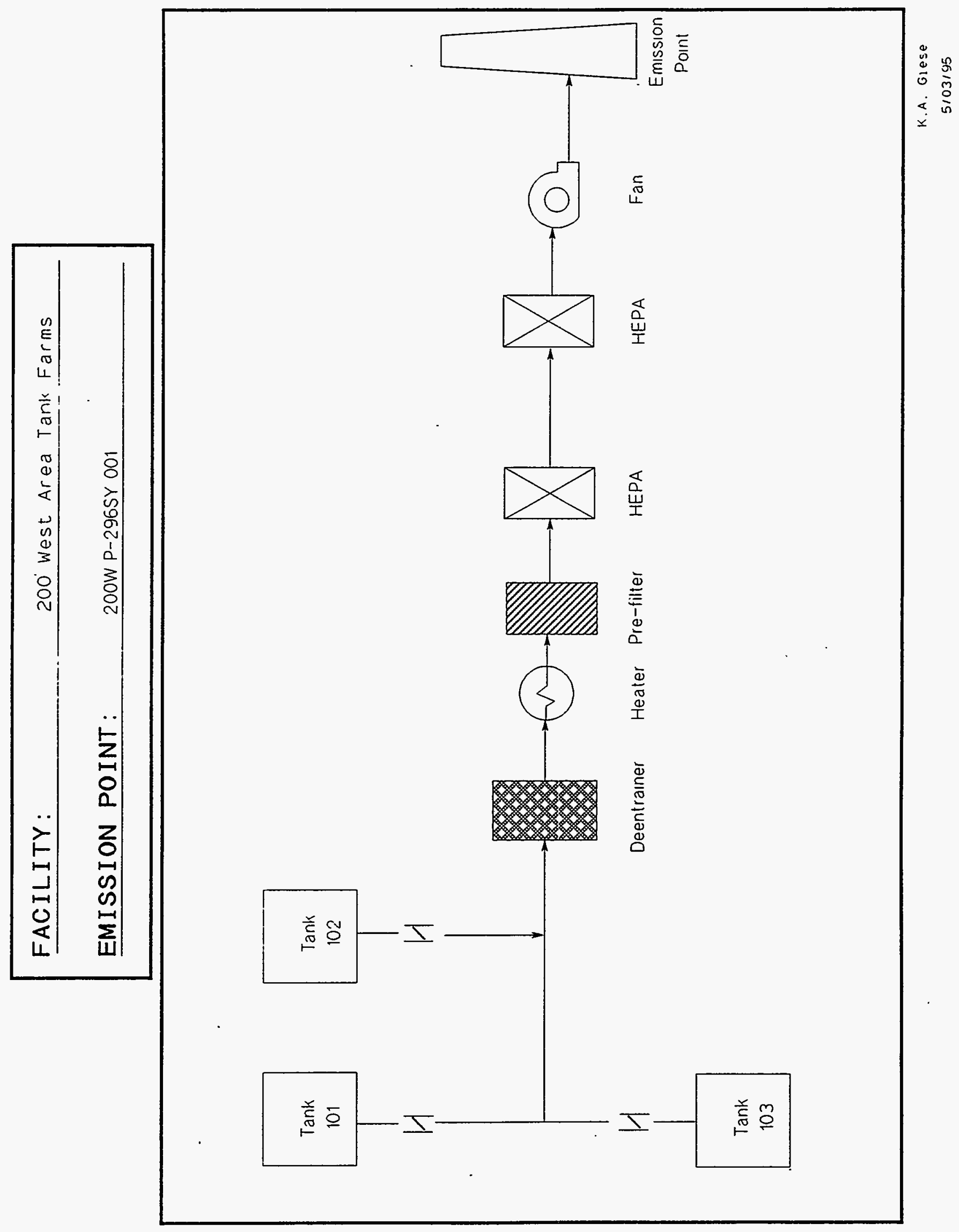




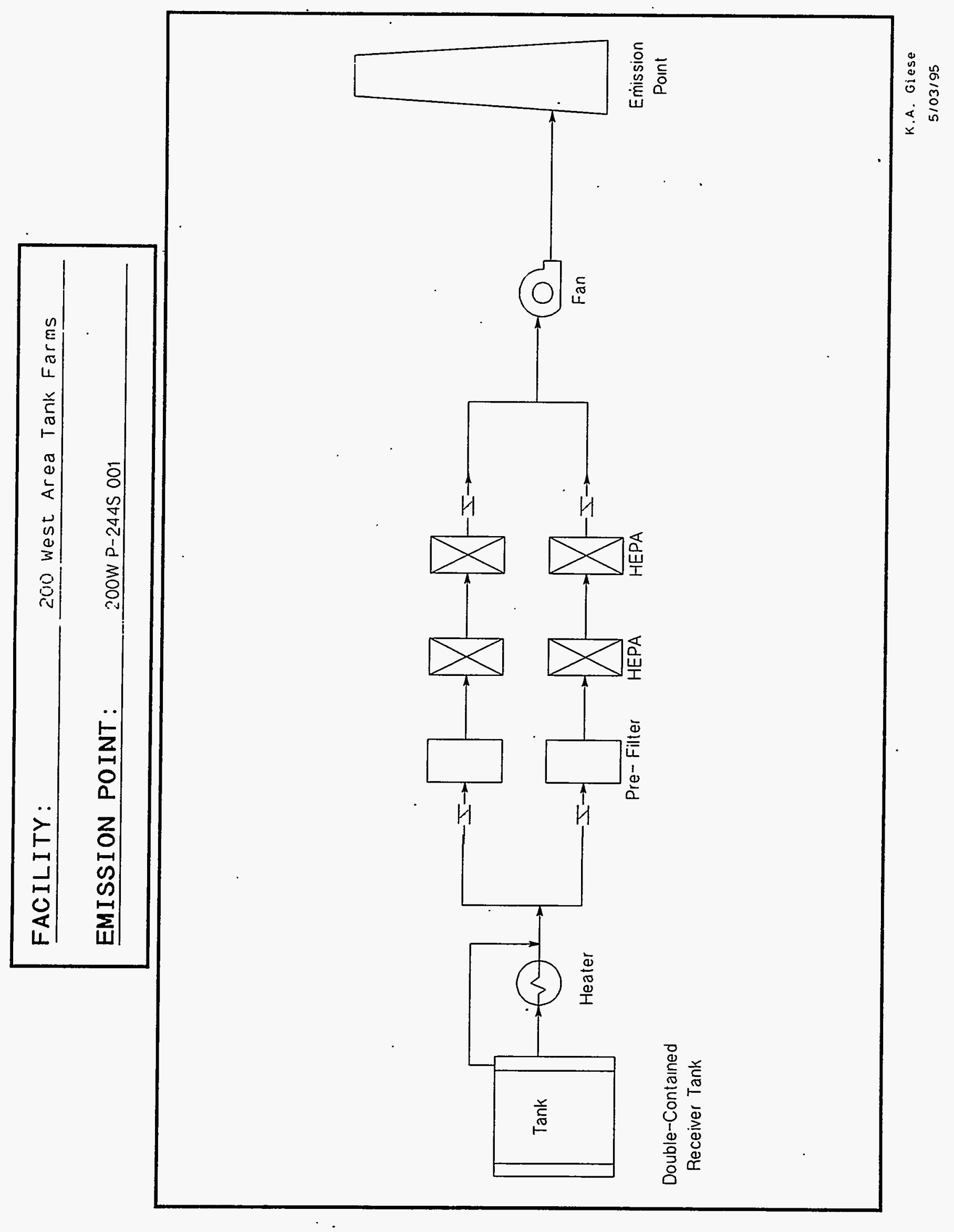




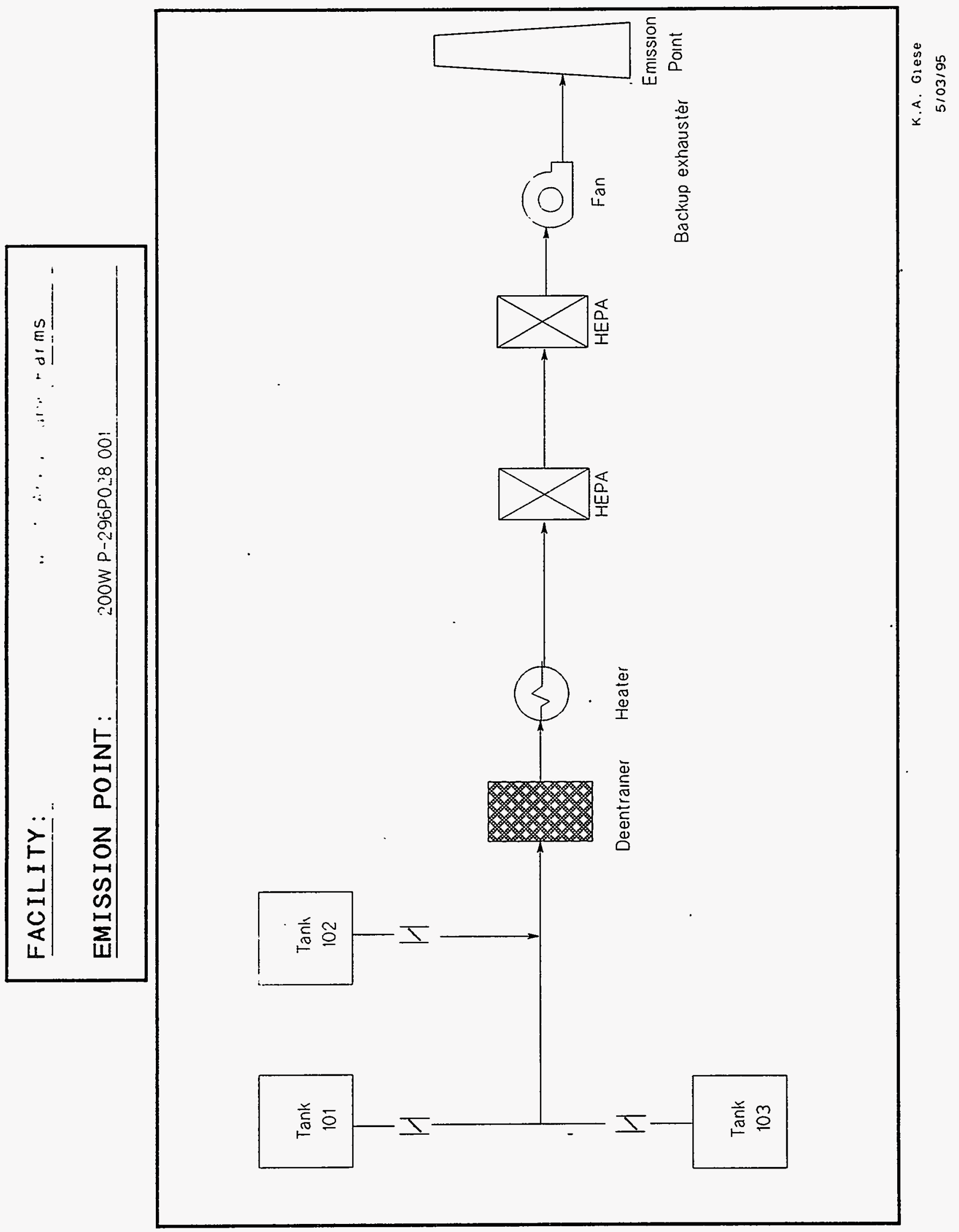




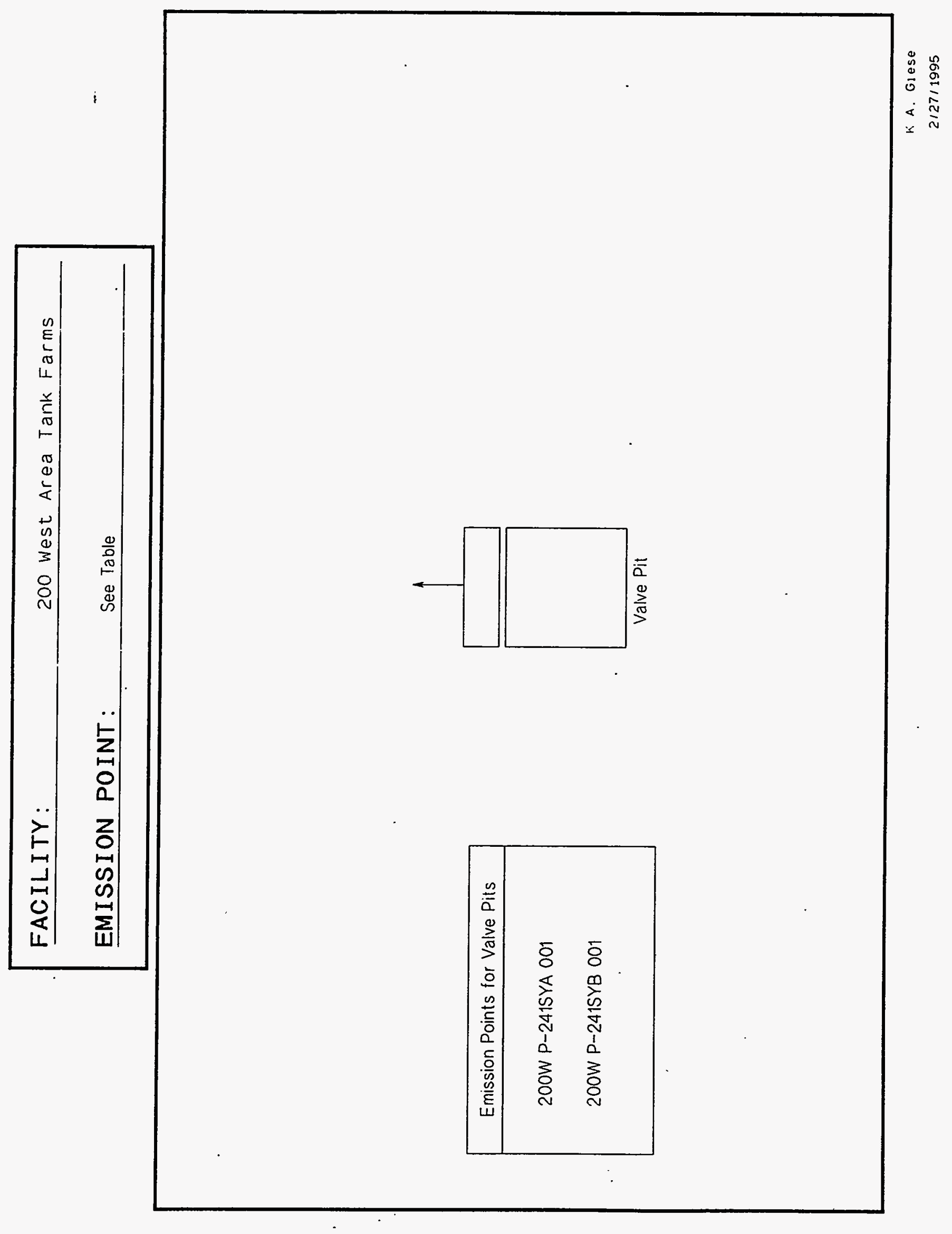




$\begin{array}{ll}\text { FACILITY: } & 200 \text { west Area Tank Farms } \\ \text { EMISSION POINT: } & \text { See Tabie }\end{array}$

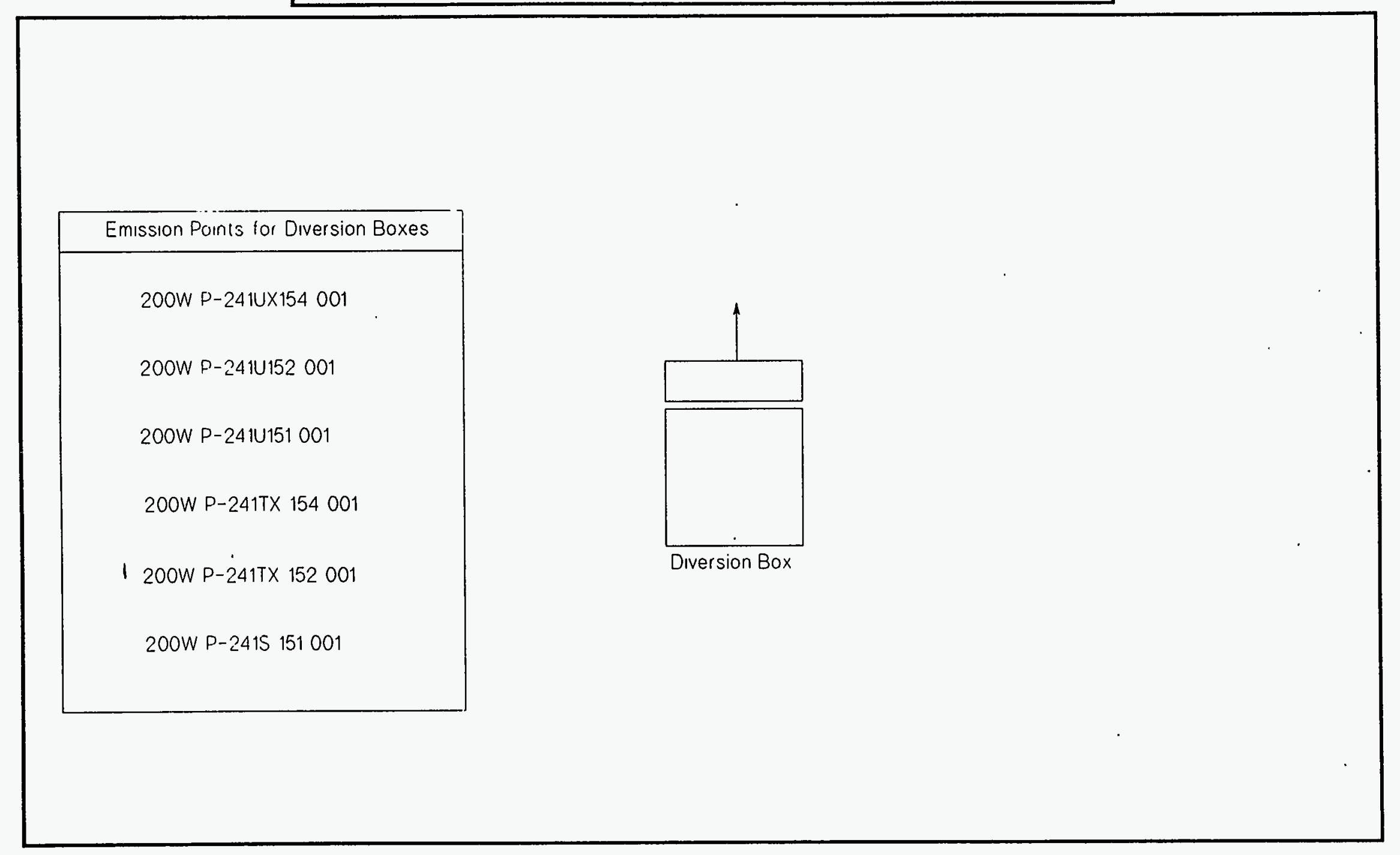




FACILITY:
EMISSION POINT:

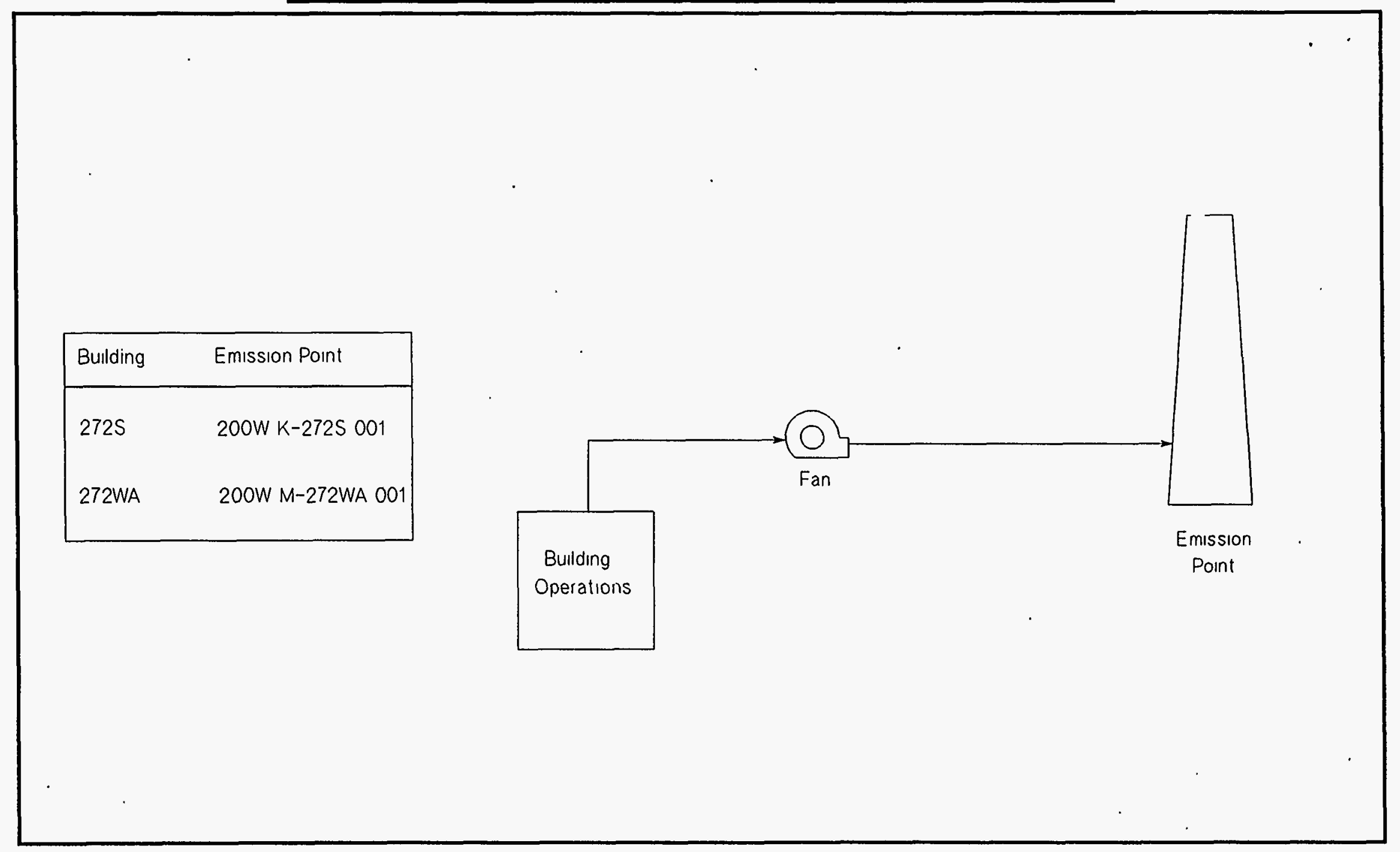




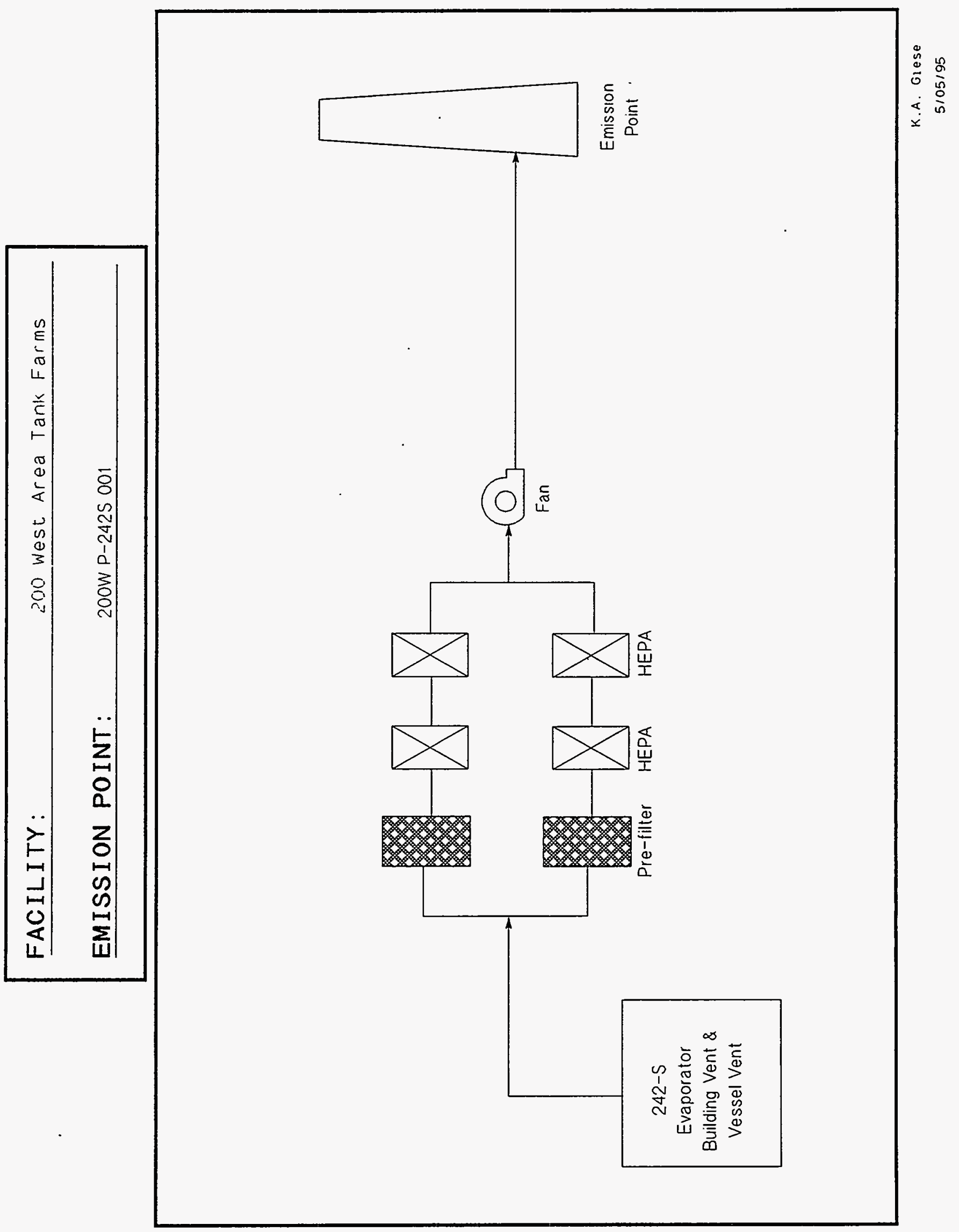




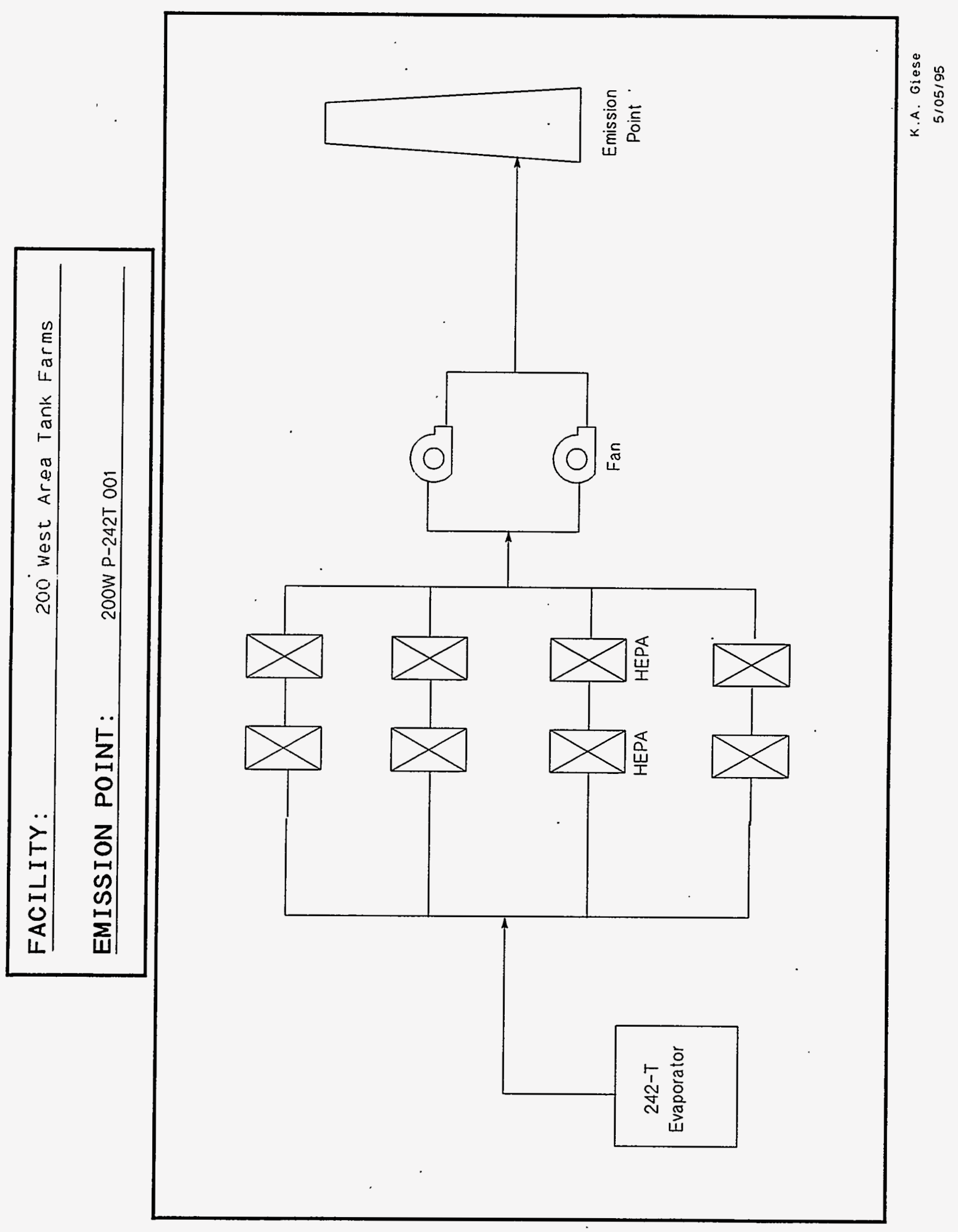




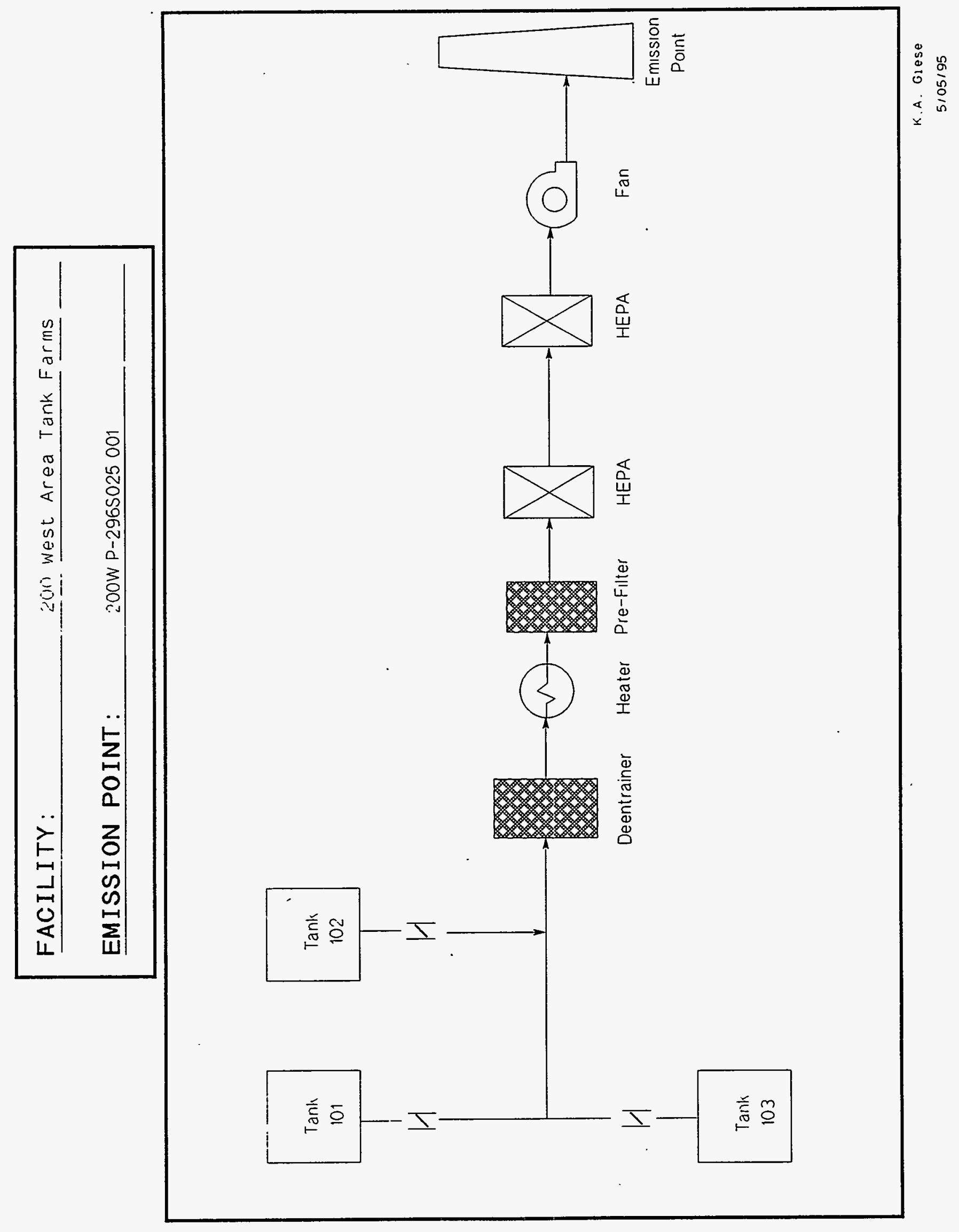




\section{FACILITY:}

$\therefore$

- $1 \cdots+41 \operatorname{tn}:$

EMISSION POINT: See Table

Emission Points for

double-contained receiver tanks

200W P-244U 001

200W P-244TX 001

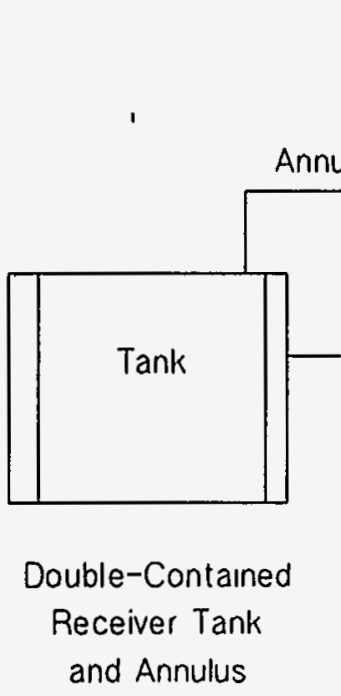

and Annulus

Heater
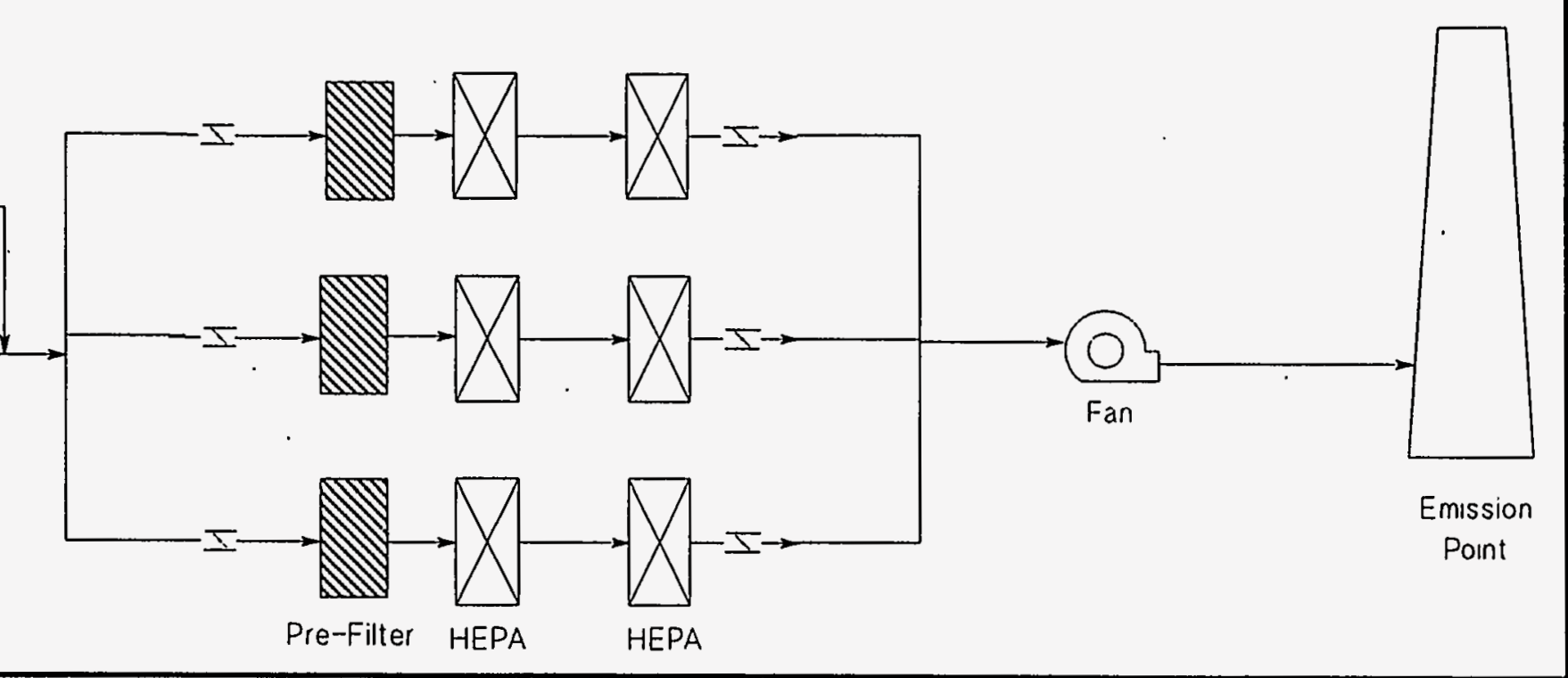


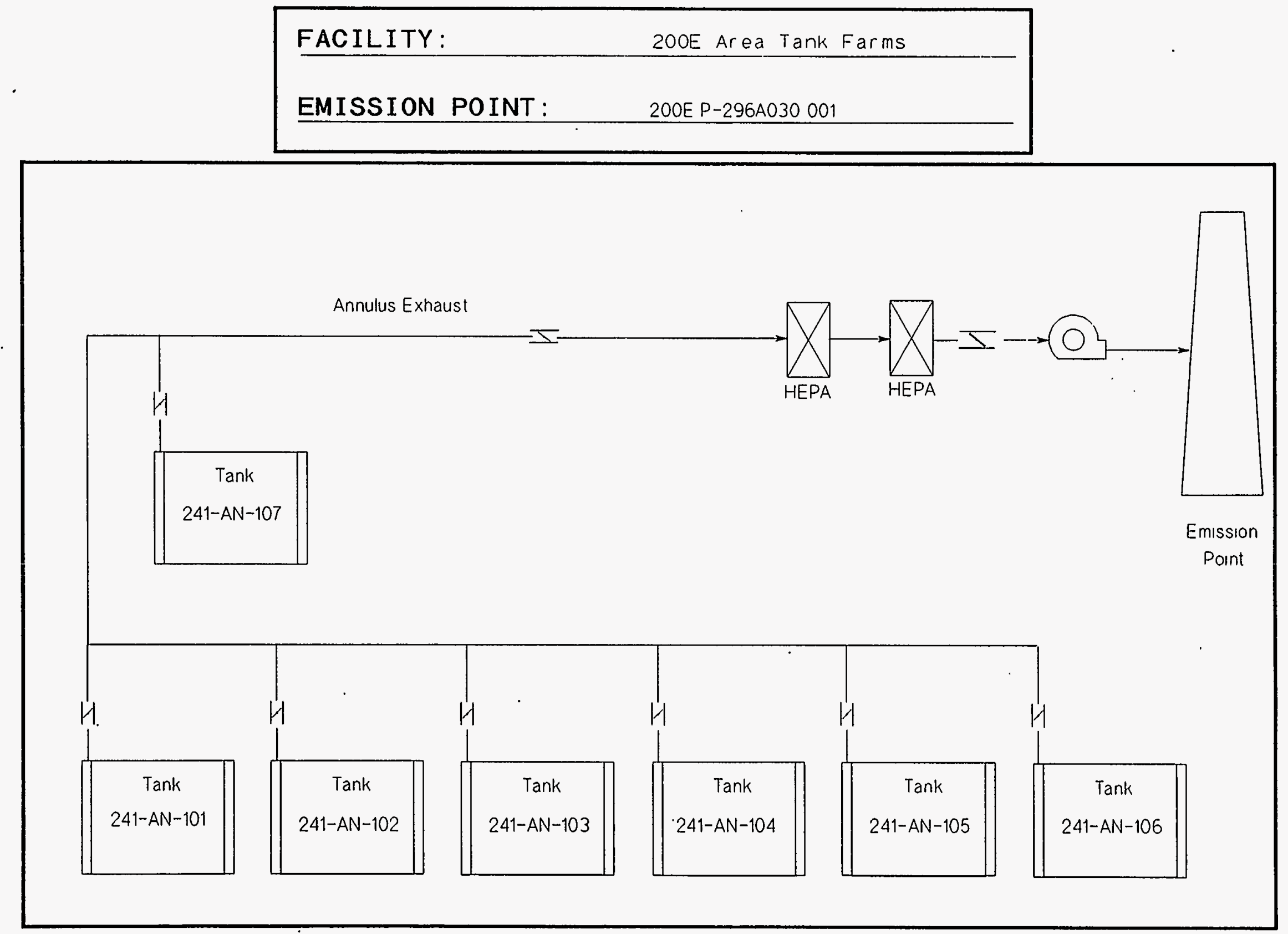



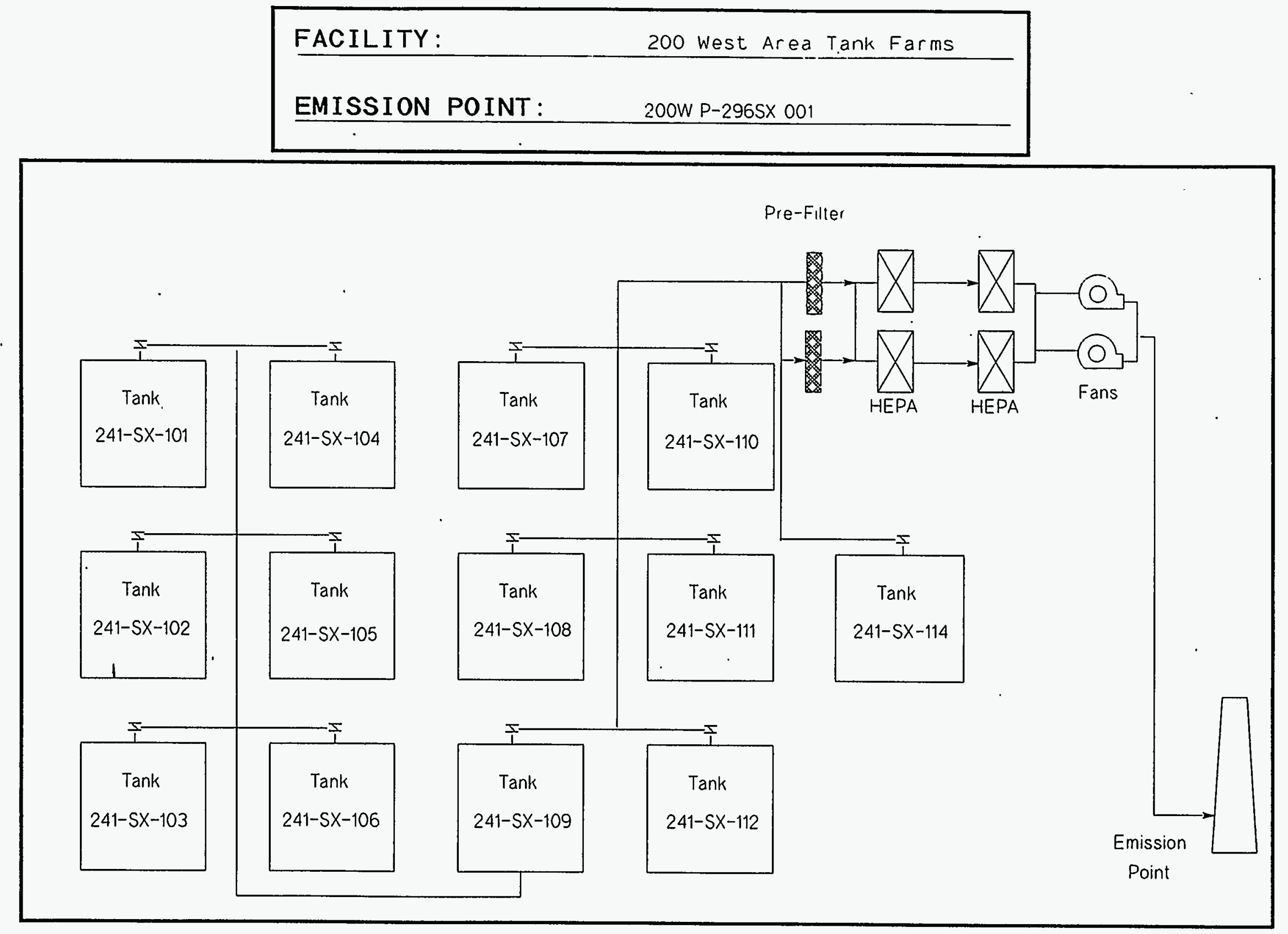


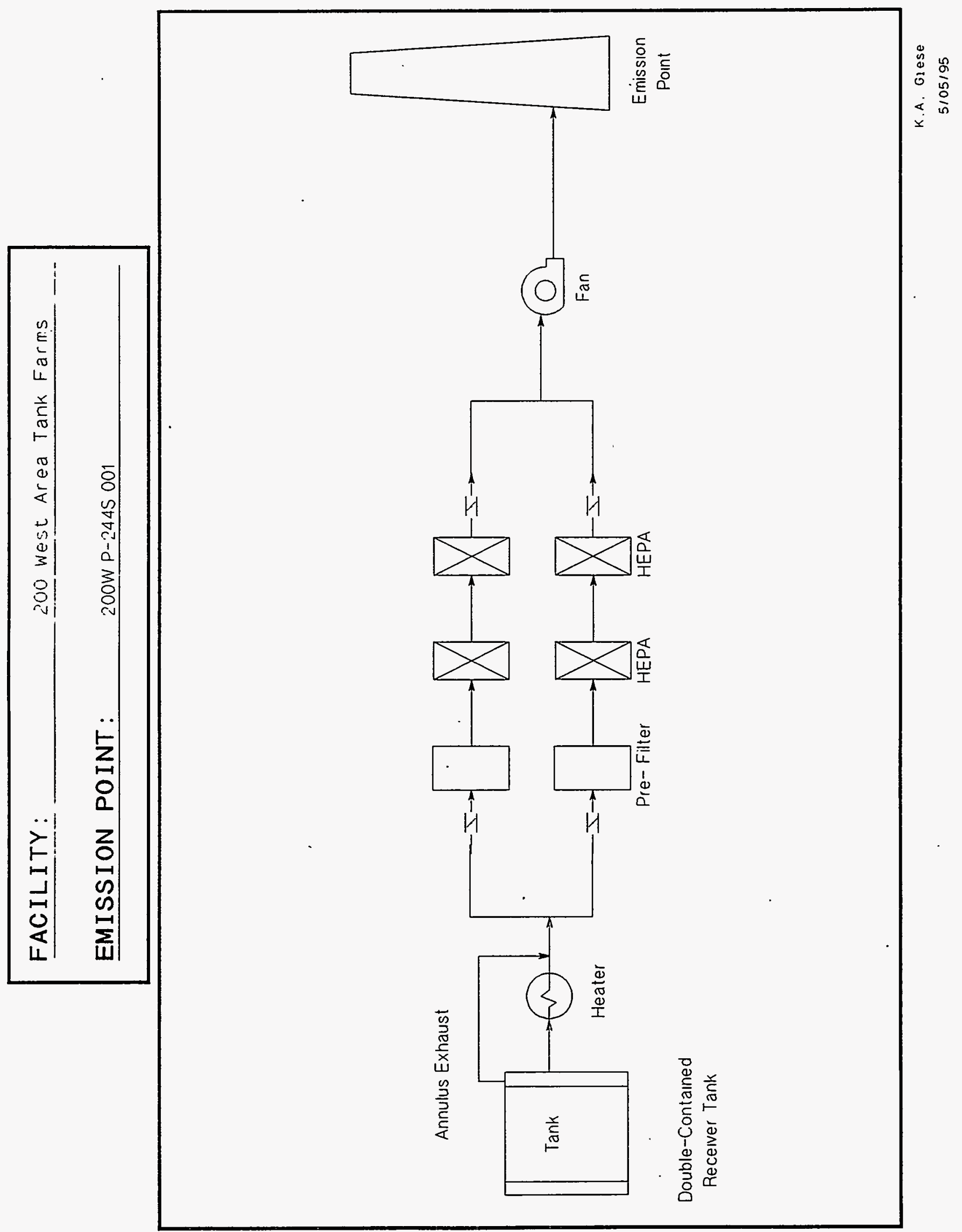




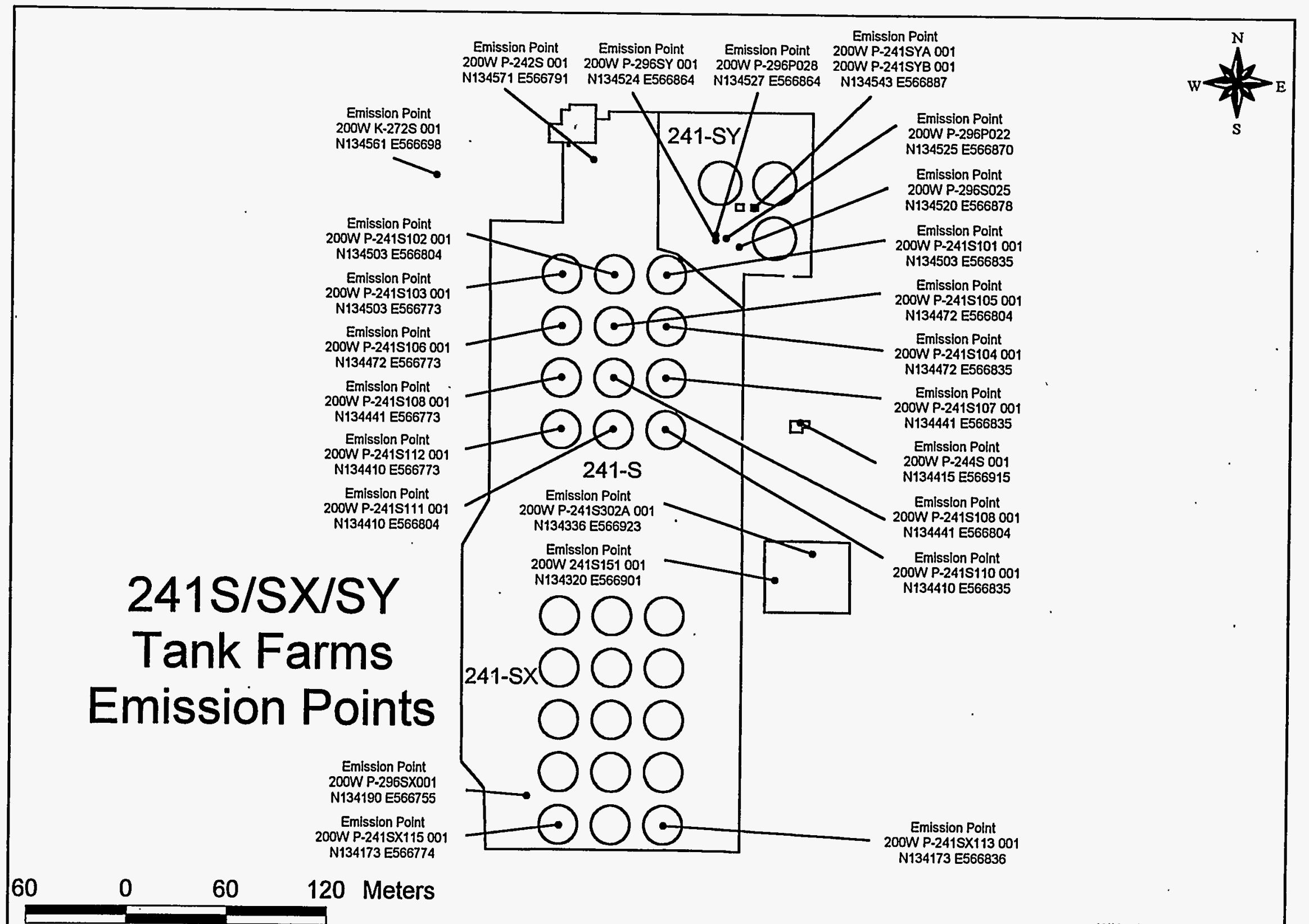




\section{T Tank Farm Emission Points}

Emission Point 200W P-241T103 001

N136765 E566777

Emission Point 200W P-241T106 001 N136734 E566777

Emission Point 200W P-241T109 001 N136704 E566777

Emission Point 200W P-241T201 001 N136711 E566747

Emission Point 200W P-241T202 001 N136696 E566747

Emission Point 200W P-241T203 001 N136681 E566747

Emission Point 200W P-241T112 001 N136673 E566777

Emission Point 200W P-241T204 001 N136665 E566747

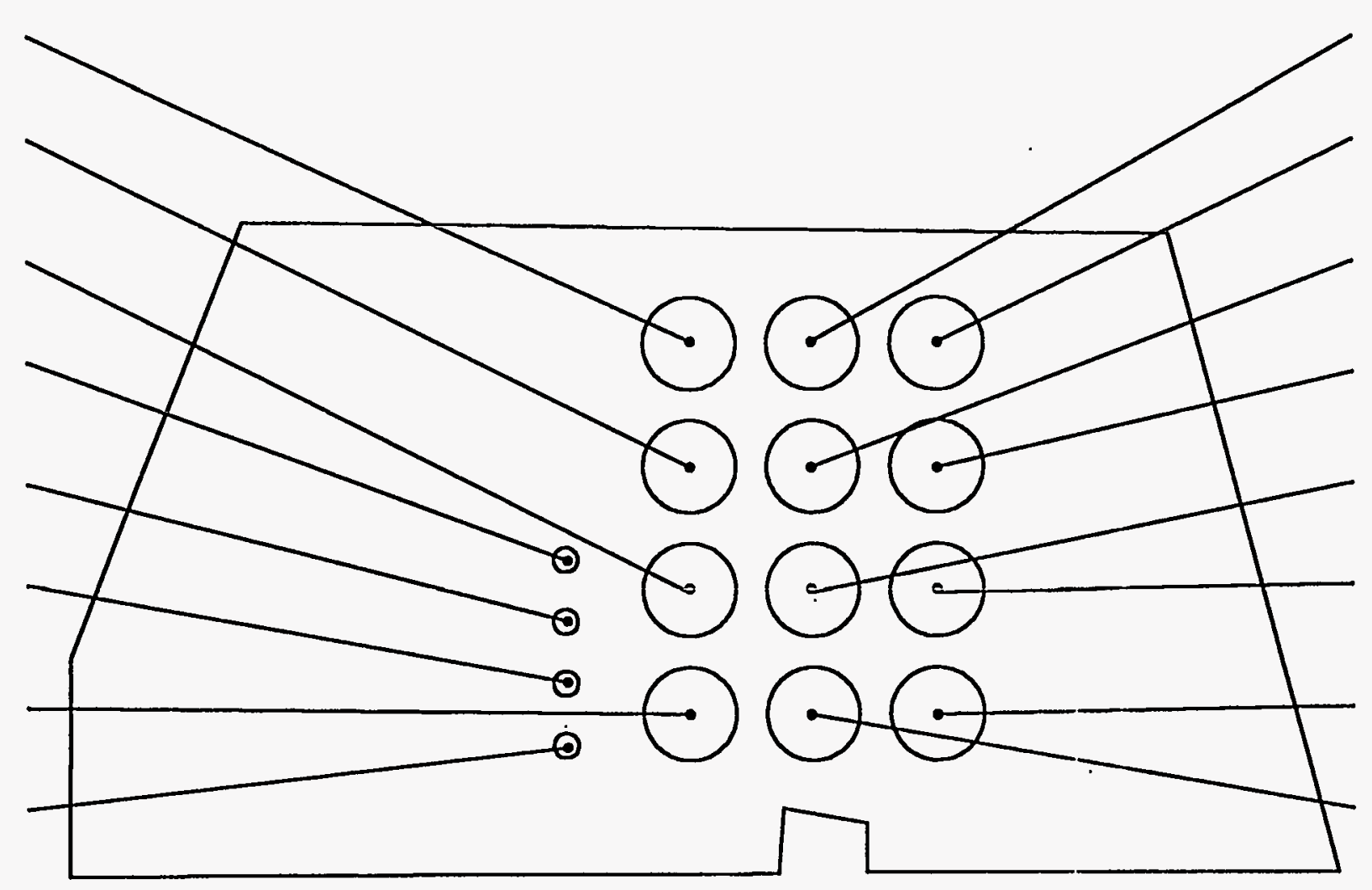

Emission Point 200W P-241T102 od 1 N136765 E566807

Emission Point 200W P-241T101 od 1 N136765 E566838

Emission Point 200W P-241T105 od1 N136734 E566807

Emission Point 200W P-241T104 od 1 N136734 E566838

Emission Point 200W P-241T108 od 1 N136704566807

Emission Point 200W P-241T107 od1 N136704 E566838

Emission Point 200W P-241T110 001 N136673 E566838

Emission Point 200W P-241T111 od 1 N136673 E566807 


\section{TXITY Tank Farms Emission Points}

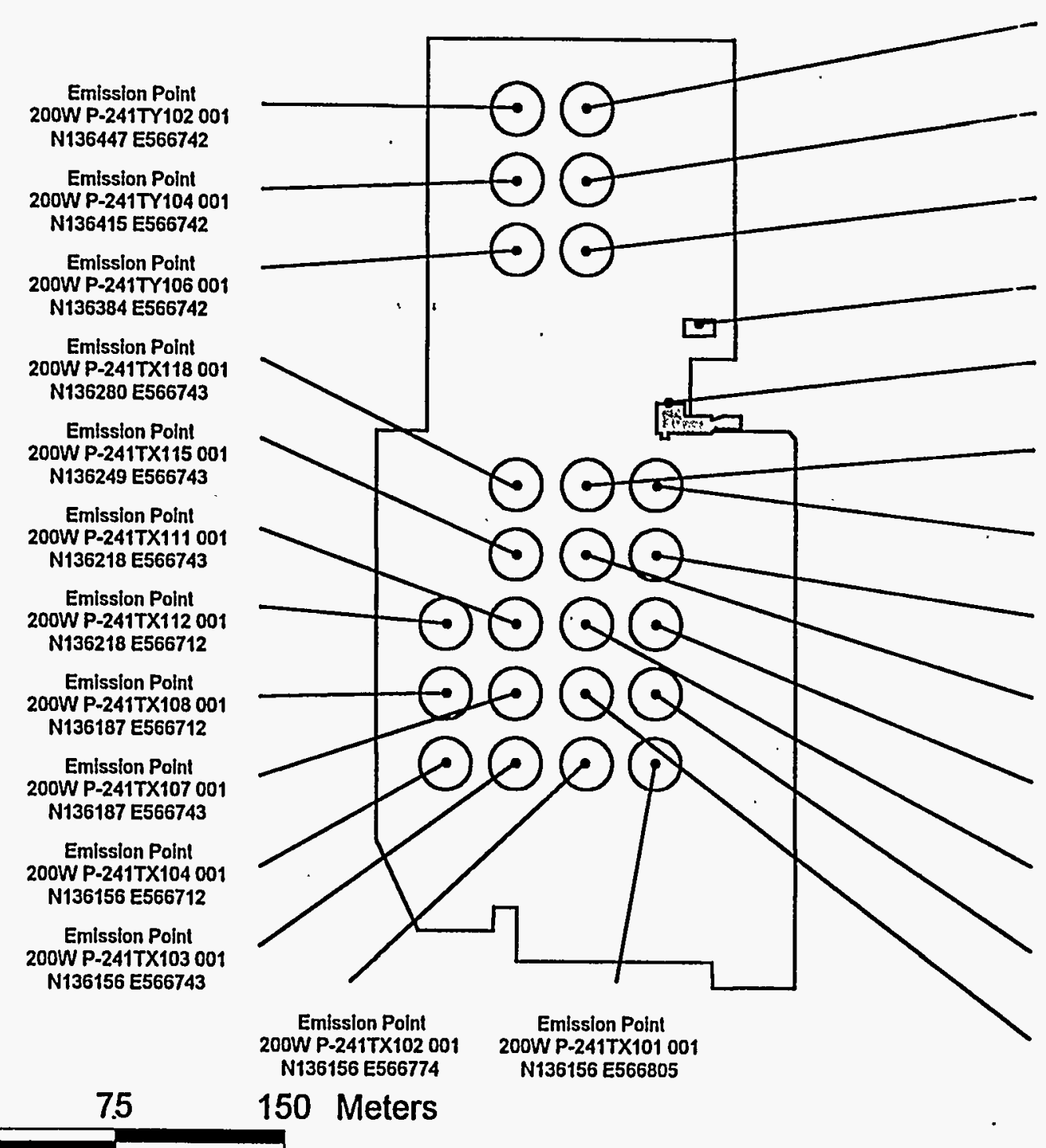

Emission Point

200W P-241TY101 001 N136447 E566773

- Emission Point

200W P-241TY103 001 N136415 E566773

Emission Point 200W P-241TY105 001 N136384 E566773

Emission Point 200W P-244TX 001 N136352 E566823

Emission Point 200W P-242T 001 N136317 E566810

Emission Point 200W P-241TX117 001 N136280 E566774

Emission Point 200W P-241TX116 001 N136280 E566805

Emission Point 200W P-241TX113 001 N136249 E566805

Emission Point 200W P-241TX114 001 N136249 E566774

Emission Point 200W P-241TX109001 N136218 E566805

Emission Point 200W P-241TX110 001 N136218 E566774

Emission Point 200W P-241TX105 00 N136187 E566805

Emission Point 200W P-241TX106 00 $\sum_{j}^{N}$ N136187 E566774 


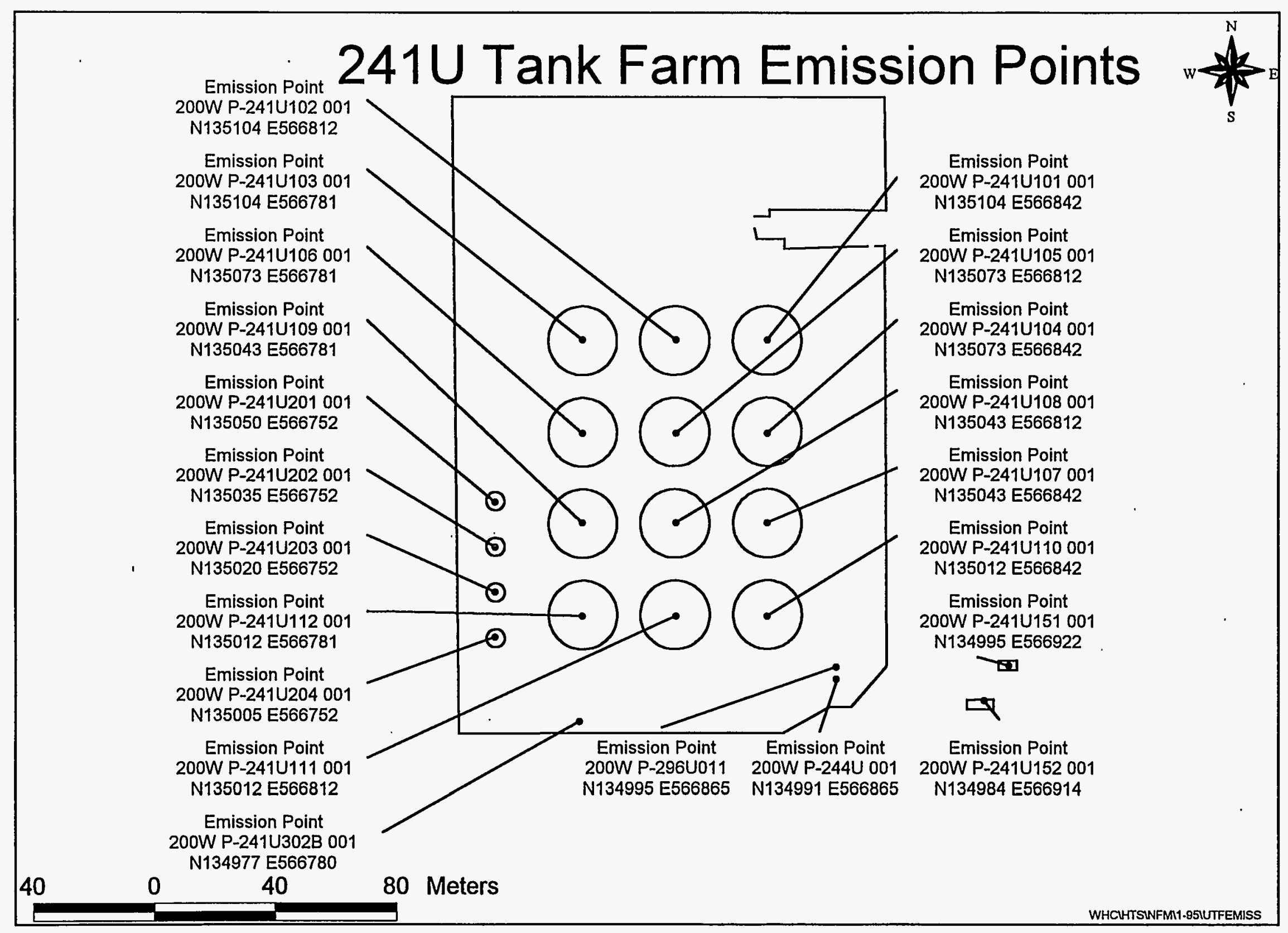




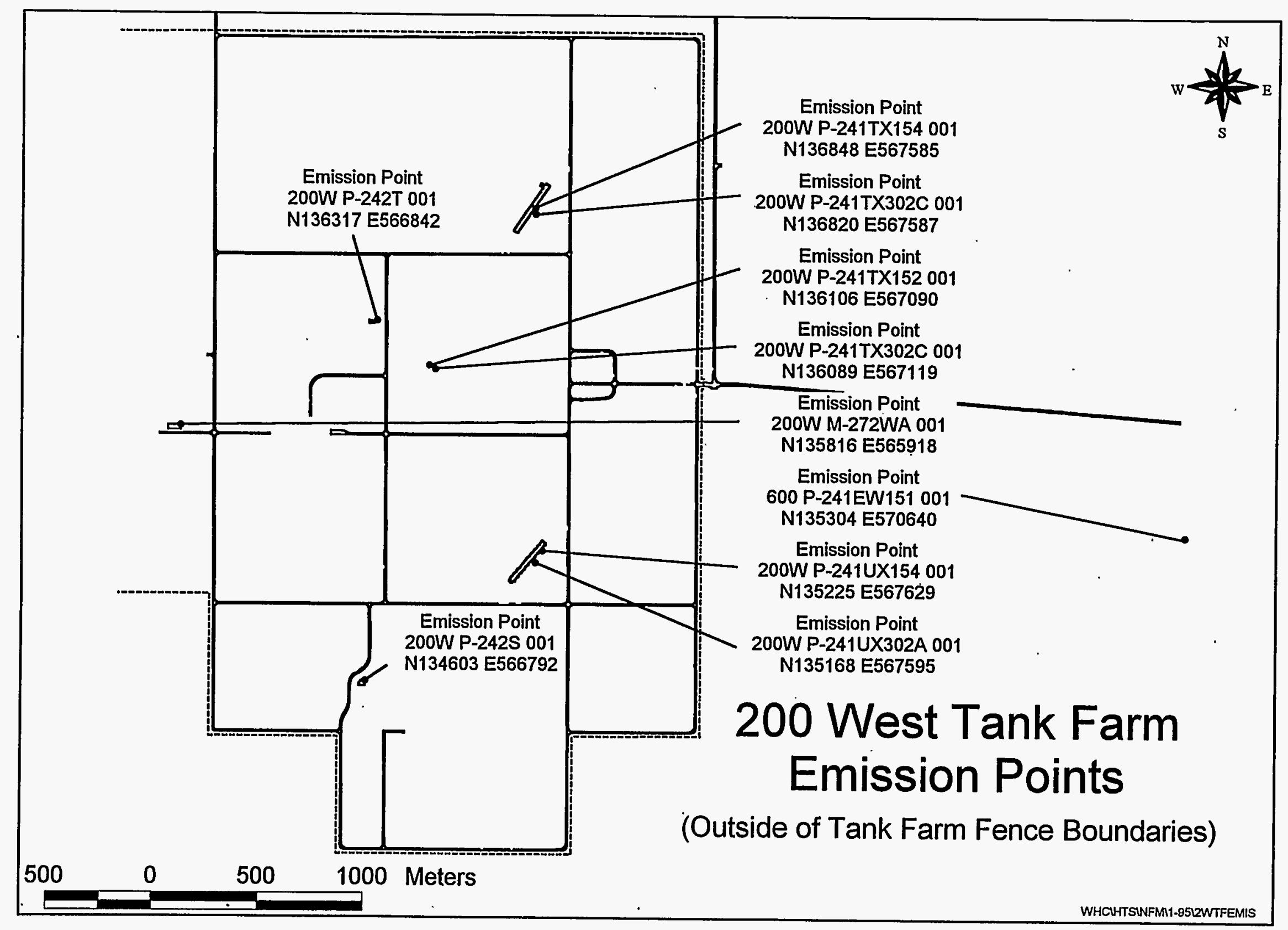




\title{
APPENDIX E-8
}

\section{SOLID WASTE MANAGEMENT FACILITIES}

\author{
Chapter I. General Information
}

\section{I-B.1. Facility Description}

The Solid Waste Management (SWM) facilities are located in the 200 Areas of the Hanford Site, and include the Central Waste Complex (CWC), 224-T Transuranic Storage and Assay Facility (TRUSAF), Low Level Burial Grounds, and Building 616. The two facilities that have the potential for airborne emissions are the 213-W Dry Waste Compactor Facility in the CWC and TRUSAF. The Low Level Burial Grounds have been identified as a fugitive source of emissions (refer to Section II.F of Chapter II).

\section{3-W Dry Waste Compactor Facility}

213-W Dry Waste Compactor Facility Waste compaction activities have ceased due to inefficiencies of the process. Until recently, the facility was used for waste confirmation and verification. The waste that was handled during the verification activities is low level waste and alpha-bearing non-transuranic waste. Currently, there are no SWM activities occurring in this facility.

\section{TRUSAF}

The 224-T TRUSAF provides a centralized storage unit for storage of transuranic waste and transuranic mixed waste and low level mixed waste from various Hanford Facility operations and from other U.S. Department of Energy and U.S. Department of Defense facilities. The transuranic waste will be prepared for transfer to, and disposal at the Waste Isolation Pilot Plant (WIPP) in Carlsbad, New Mexico or at another approved disposal site. The 224-T TRUSAF also will store retrieved containers of transuranic mixed waste from the Low-Level Burial Grounds (LLBG). The waste containers are $\mathrm{x}$-rayed to ensure that what can be identified is in general agreement with the shipping documentation.

The SWM facilities are operated for 8 hours a day, 5 days a week, 52 weeks per year.

\section{I-B.2. Products, Raw Materials, Fuels, Processes}

- Principle products - There are no products produced

- Process information - There are no process activities

- Raw materials used by processes - There are no raw materials used at the SWM facilities.

- Fuels used by processes - There are no fuels used at the SWM facilities. 
Hanford Site Air

Operating Permit Application
DOE/RL-95-07, Rev. 0

$05 / 95$

\section{I-C.1. Emission Points, Controls, Stacks}

The emission point and stack information is found on page 1 of the AEI report output. The control information is found on page 5 of the AEI report output.

\section{I-C.2. Facility Process Flow Diagrams}

Attached.

\section{I-C.3. Site Map}

Attached.

\section{Chapter II. Regulatory Requirements}

\section{II-A.1. Description of Discharge and Emission Points}

The emission point and stack information is found on page 1 of the AEI report output. 
Facility Building List

\begin{tabular}{|c|c|}
\hline Bldg Id & North \\
\hline $\begin{array}{l}213 \mathrm{~W} \\
296 \mathrm{~T} 011\end{array}$ & $\begin{array}{l}13583 \\
136699\end{array}$ \\
\hline 296T012 & $13670^{\circ}$ \\
\hline
\end{tabular}

East B]dg Desc

565900 WASTE COMPACTOR BUILDING

567547

567542

\section{WASTE COMPACTOR BUILDING}

224-T (TRUSAF) 224-T (TRUSAF)
PART OF PLENUM)
Custodian(Contractor)

CAMBEU SARAH CAMPBELL: SARAH

CAMPBELL, SARAH
Cust Phone DOE POC $372-0667$ 372-0667 372-0667
RUDOLPH GUERCIA
RUDOLPH GUERCIA

RUDOLPH GUERCIA
DOE Phone

$376-5494$ $376-5494$ 376-5494

\section{Emission Point Listing}

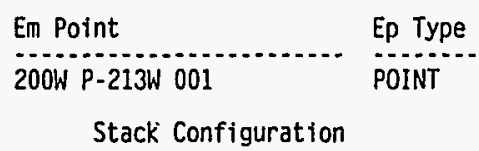

Stack Configuration VERTICAL WITHOUT WEATHER CAP

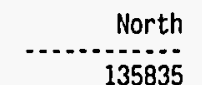

East Srce Nbr Scc Code

Srce Type

\begin{abstract}
Diameter(ft) Length(ft) Width(ft) Area(sq ft) Exit Temp(F) Exit Veloc(ft/sec) Ht Abv Grnd(ft) Ht Abv Struc(ft)
\end{abstract}

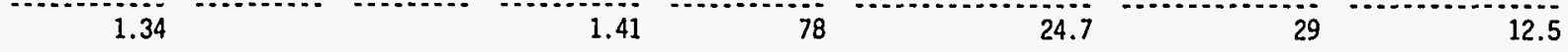

Em Point

Ep Type North East Srce $\mathrm{Nbr}$ Scc Code

Srce Type

200W P-296T011 001 POINT 567547

1 not assigned

PROCESS AND PRODUCTION

Stack Configuration

Diameter(ft) Length(ft) Width(ft) Area(sq ft) Exit Temp(F) Exit Veloc(ft/sec) Ht Abv Grnd(ft) Ht Abv Struc(ft)

HORIZONTAL

Em Point

Ep Type

200W P-296T012001 POINT

\section{North}

East Srce $\mathrm{Nbr}$ Scc Code

136703 .

567542 i not assigned

Srce Type

Stack Configuration Diameter(ft) Length(ft) Width(ft) Area(sq ft) Exit Temp(F) Exit Veloc(ft/sec) Ht Abv Grnd(ft) Ht Abv Struc(ft) HORIZONTAL OR DOWNWARD 2.67

Source (Process) Listing

Em Point

Srce Nbr Srce Type

Hrs Day Days Week Wks Year Summary Process Description

200W P-213W 001

1 PROCESS AND PRODUCTION 
Em Point 200W P-296T011 001
Srce Nbr Srce Type

1 PROCESS AND PRODUCTION
Hrs Day Days Week Wks Year Summary Process Description

$24752224-T$ IS CURRENTLY OPERATED AS A TRU WASTE STORAGE FACILITY.

The 244-T Transuranic Waste Storage ans Assay Facility (224-T TRUSAF) is located southeast of T-Plant in the 200W Area. The 224-T TRUSAF provides a centralized storage unit for storage of transuranic mixed waste and low level mixed waste from various Hanford Facility operations and from other U.S. Department of Energy and U.S. Department of Defense facilities. The transuranic mixed waste will be prepared for transfer to and disposal at the Waste Isolation Pilot Plant (WIPP) in Carlsbad. New Mexico or at another approved disposal site. The 224-T TRUSAF also will store retrieved containers of transuranic mixed waste

The 224-T TRUSAF storage unit capacity is approximately 110.000 gallons (416.400 liters) [2.000 55.gallon (208 liter) containers].

The potential transuranic mixed waste and transuranic waste managed could include waste from nonspecific sources, characteristic waste, listed waste, and state-only waste (extremely hazardous, dangerous and polychlorinated biphenyl contaminated waste). The estimated annual quantity of waste is based on gross estimates of mixed waste that could be stored at the 244-T TRUSAF.

There are waste storage modules on all three floors. These open-array storage modules are delineated by markings taped on the floor to prevent inadvertent commingling of waste forms. Incompatible dangerous waste is separated by being placed on different floors or in different rooms on the second floor. Transuranic mixed waste is stored based on both transuranic element content and dangerous waste constituents. All floors are serviced by normal ventilation. Ali floor drains have been sealed with nonshrinking concrete.

En Point Srce $\mathrm{Nbr}$ Srce Type Hrs Day Days Week Wks Year Summary Process Description

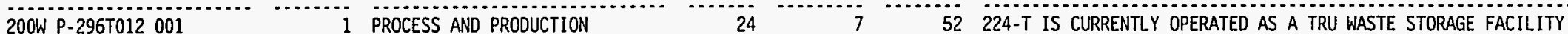

The 224-T Transuranic Waste Storage Facility (224-T TRUSAF) is located southeast of T-Plant in the 200W Area. The 224-T TRUSAF provides a centralized storage unit for storage of transuranic mixed waste and low level mixed waste from various Hanford Facility operations and from other $U . S$. Department of Energy and U.S. Department of Defense facilities. The transuranic mixed waste will be prepared transfer to and disposal at the Waste isolation Pilot Plant (WIPP) in Carlsbad. New Mexico or at another approved disposal site. The 224-T TRUSAF also will store retrieved containers of transuranic with the shipping documentation.

The 224-T TRUSAF storage unit capacity is approximately 110.000 gallons (416.400 liters) [2,000 55-gallon (208 1iter) containers]

The potential transuranic mixed and transuranic waste managed could include waste from nonspecific sources, characteristic waste. 1 isted waste, and state-only waste (extremely hazardous, danderous and polychlorinated biphenyl contaminated waste). The estimated annual quantity of waste is based on gross estimates of mixed waste that could be stored at the 224-T TRUSAF.

There are waste storage modules on all three floors. These open-array storage modules are delineated by markings taped on the floor to prevent inadvertent commingling of waste forms. Incompatible dangerous waste is separated by being placed on different floors or in different rooms on the second floor. Transuranic mixed waste is stored based on both transuranic element conte

\section{Source Raw Materials Usage}

Em Point

Srce Nbr Srce Type

Process Rate Process Uom Material 


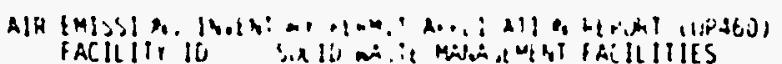

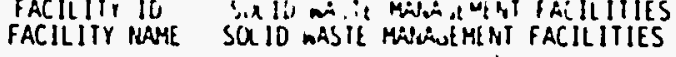

Fuels Usage by Source Processes

\section{Em Point}

Srce Nbr Srce Type

Fuels Usage by Control Devices

Em Point

Ctl $\mathrm{Nbr} C \mathrm{Ct}$ Type

Point Source Emissions - Radiological Pollutants

\begin{tabular}{ll} 
Em Point & Srce Nbr Srce Type Pollutant \\
\hline $200 W$ P-213W 001 & 1 PROCESS AND PRODUCTION
\end{tabular}

200W P-296T011 001

1 PROCESS AND PRODUCTION

1 PROCESS AND PRODUCTION

Point Source Emissions - HAP Pollutants

Em Point $\quad$ S $\quad$ Srce Nbr Srce Type Pollutant

Sum

Point Source Emissions - Uncategorized Pollutants

Em Point

Srce Nbr Srce Type Pollutant

Em. Pt. Potential Em. Pt.
to Emit (tons/yr) Ct Actua) (tons/yr) Pollutant

Sum

Fugitive Source Emissions - Radiological Pollutants

Em Point

Srce Nbr Srce Type Pollutant

Em. Pt. Potential Em. Pt.

to Emit (Ci/yr) $\quad$ Cti Actual (Ci/yr)

Pollutant

Page \#: 3 of $5 \quad$ Report Run Date: 22-APR-95 


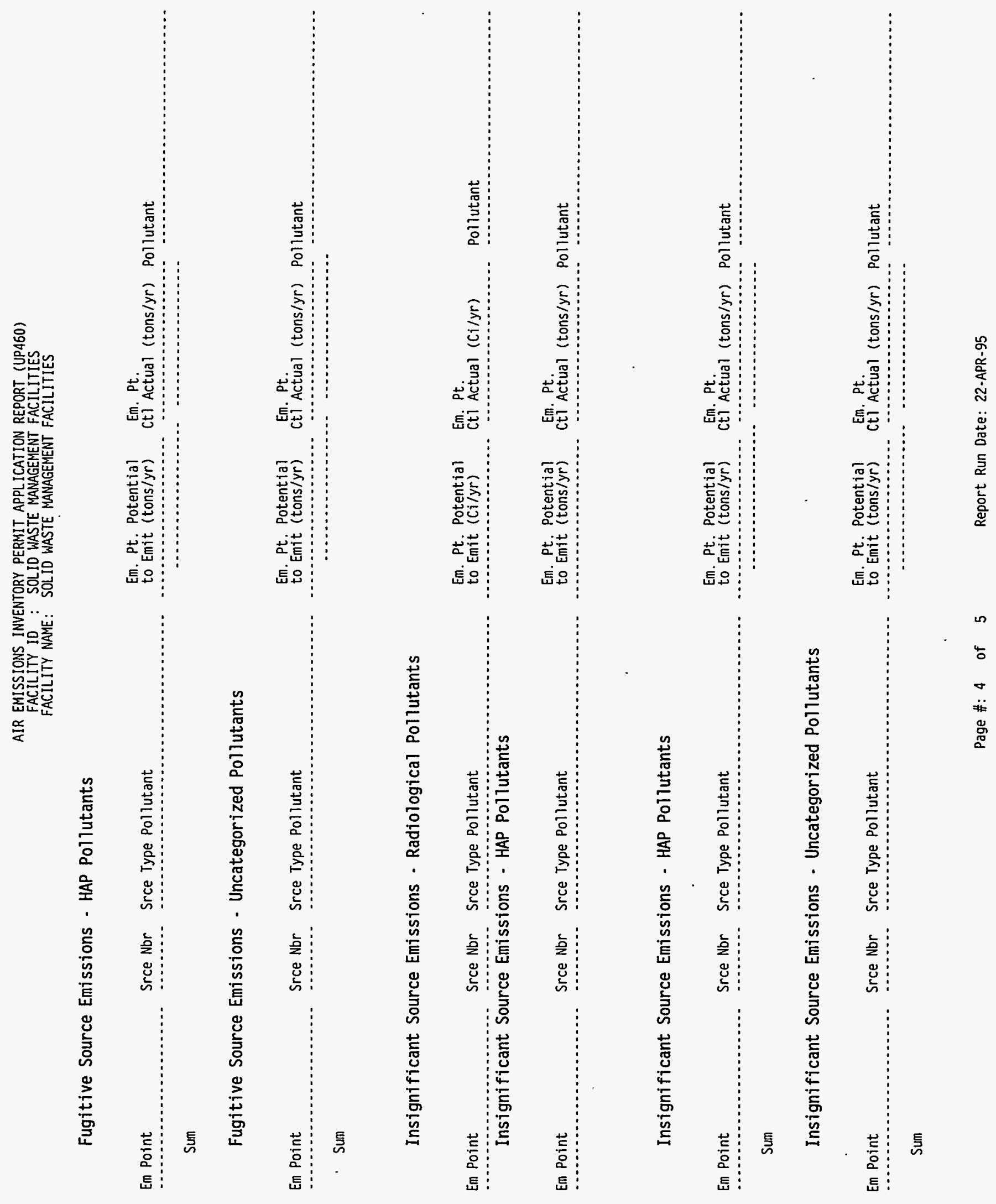


AIR EMISSIONS INVENTORY PERMIT APPLICATION REPORT (UP460) FACILITY ID : SOLID WASTE MANAGEMENT FACILITIES

Control Device Listing

Em Point

Control Equipment Code

200W P-213W 001 099

$\begin{array}{ll}\text { 200W P-296T011 } 001 & 099 \\ \text { 200W P-296T012 } 001 & 099\end{array}$

Page \#: 5 of 5 


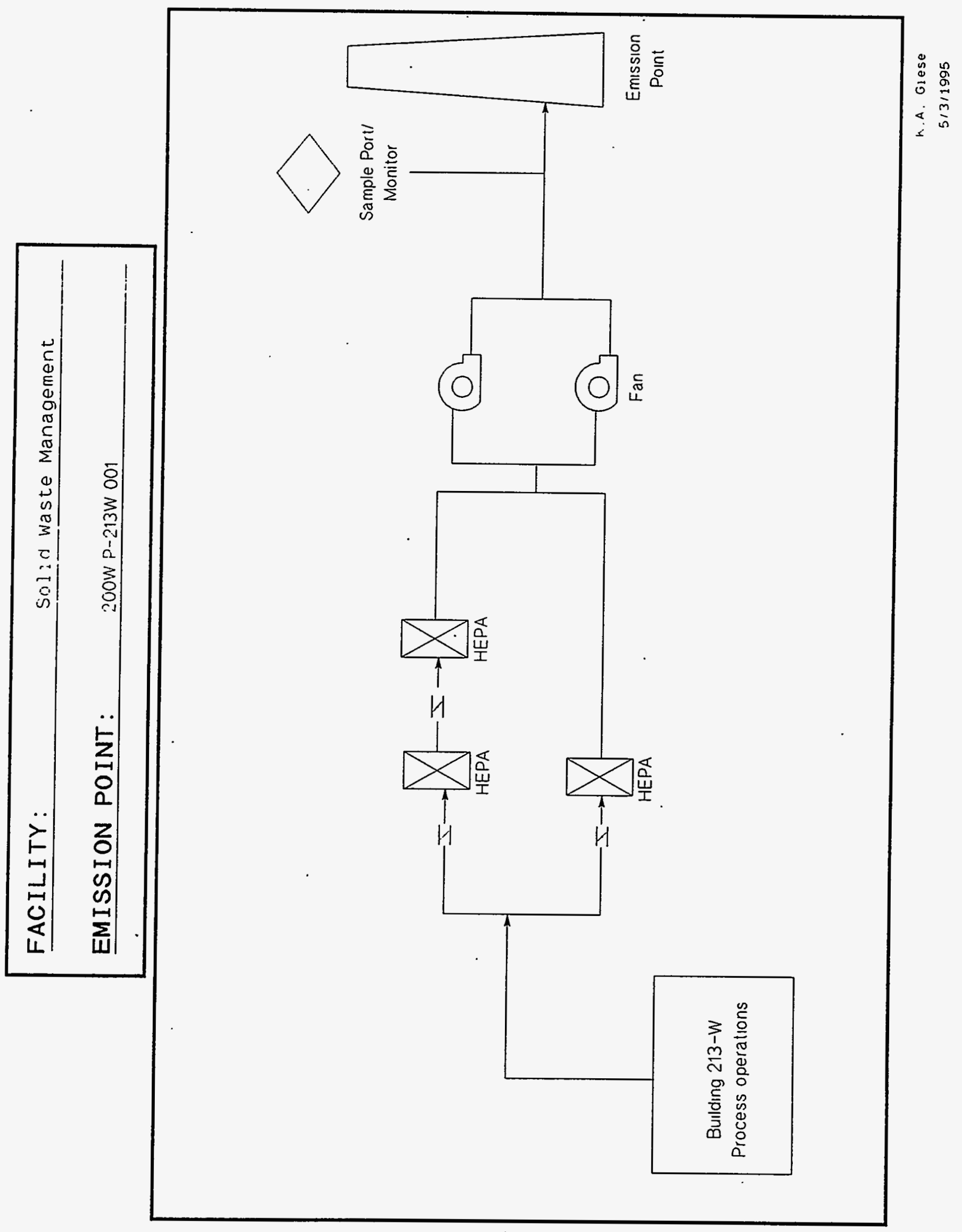




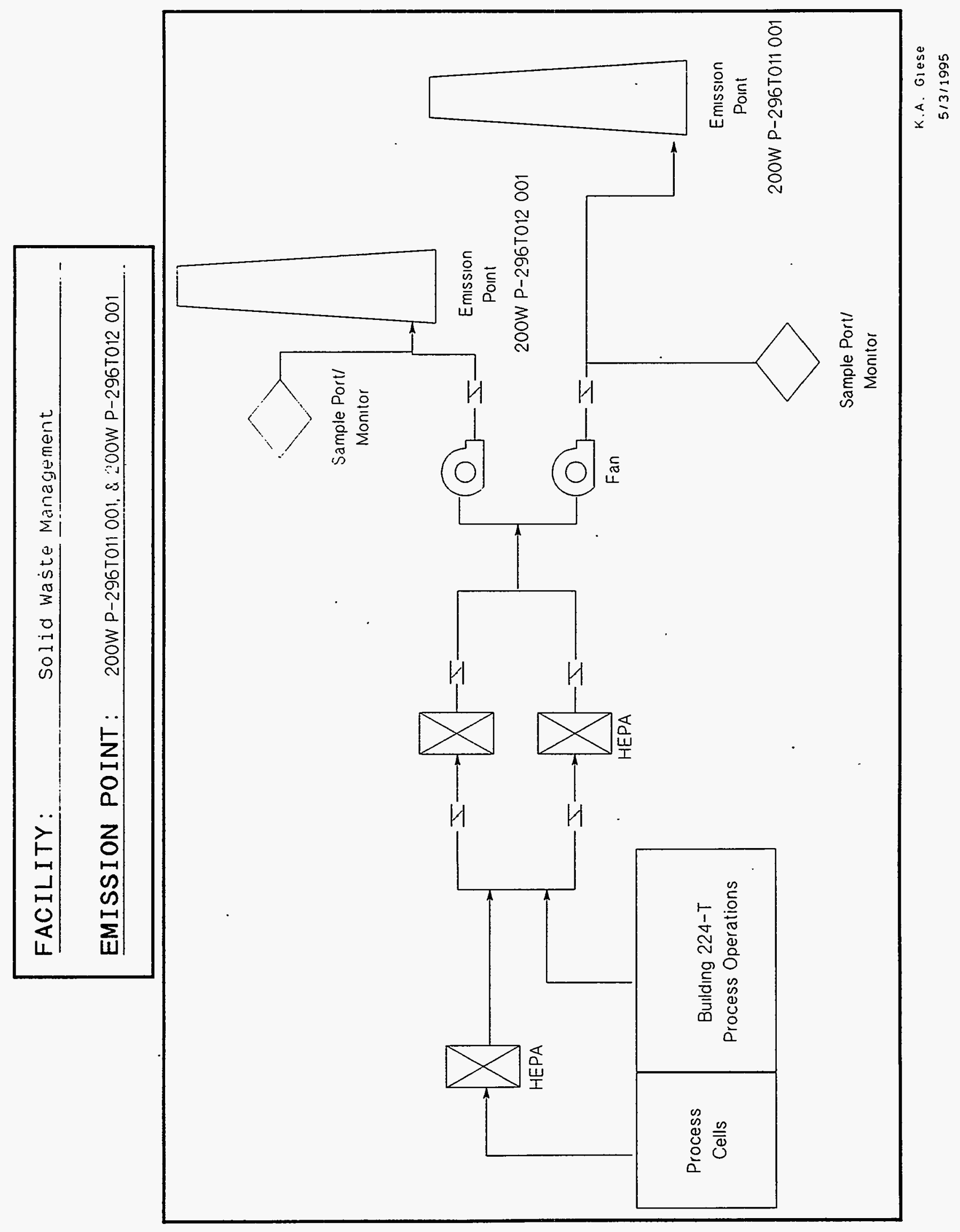




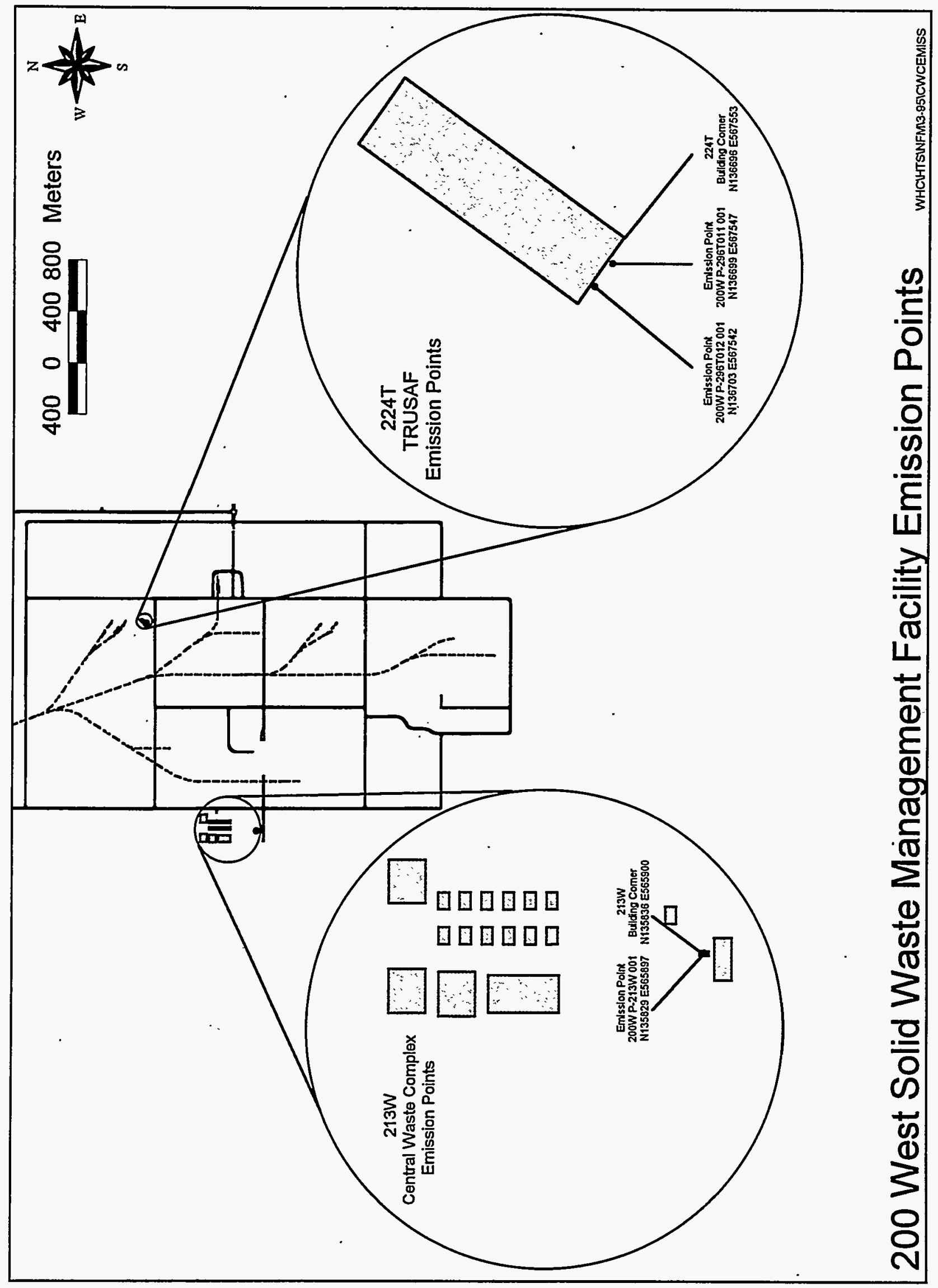




\section{APPENDIX E-9 \\ WASTE RECEIVING AND PROCESSING FACILITY}

\section{Chapter I. General Information}

\section{I-B.1. Facility Description}

The Waste Receiving and Processing Facility, upon completion of construction, will provide capabilities to examine, assay, characterize, treat, and repackage solid radioactive and mixed waste to enable permanent disposal of the waste in accordance with all applicable regulations. The solid wastes to be handled in the WRAP I facility include a low-level waste (LLW), Transuranic (TRU) waste, TRU mixed waste, and low-level mixed waste (LLMW).

The approximate size of the WRAP I building will be 4,766 square meters $\left(51,300 \mathrm{ft}^{2}\right)$. The WRAP I facility will be a metal building consisting of pre-insulated, prefinished metal, interlocking roof and wall sandwich panels. Ventilation exhaust points at WRAP I can be divided into two general categories; the exhaust stack, and miscellaneous vents.

The WRAP I facility is located in the 200 West Area. The 200 Area WRAP 1 Facility is operated 9 hours per day, 5 days per week.

\section{I-B.2. Products, Raw Materials, Fuels, Processes}

- Principal Products - There are no products produced at the WRAP I facility

- Process Information - There is a single operation at the WRAP I facility

- The operation of the WRAP I facility is to provide capabilities to examine, assay, characterize, treat, and repackage solid radioactive and mixed waste to enable permanent disposal of the waste in accordance with all applicable regulations.

- Raw materials used by processes - There are no raw materials used at the WRAP I facility

- Fuels used by processes - There are no fuels used in the processes at the WRAP I facility.

\section{I-C.1. Emission Points, Controls, Stacks}

The emission point and stack information are found on page 1 of the AEI report output. The control information is found on page 1 of the AEI report output. 


\section{I-C.2. Facility Process Flow Diagrams}

Attached.

\section{I-C.3. Site Map}

Attached.

\section{Chapter II. Regulatory Requirements}

\section{UI-A.1. Description of Discharge and Emission Points}

The emission point and stack information are found on page 1 of the AEI report output. 


\section{Facility Building List}

\begin{tabular}{|c|c|c|c|c|c|c|c|}
\hline Bldg Id & North & East & Bldg Desc & Custodian(Contractor) & Cust Phone & DOE POC & DOE Phone \\
\hline WRAP1 & 136576 & 565993 & WASTE RECEIVING AND PROCESSING FACILITY, MODULE 1 & MAYANCSIK, B. A. & $372-1342$ & RUDOLPH GUERCIA & $376-5494$ \\
\hline
\end{tabular}

\section{Emission Point Listing}

Em Point Ep Type

200W P-WRAPI 001 POINT

North East Srce Nbr Scc Code

Srce Type

565993 i not assigned

PROCESS AND PRODUCTION

Stack Configuration

Diameter(ft) Length(ft) Width(ft) Area(sq ft) Exit Temp(F) Exit Veloc(ft/sec) Ht Abv Grnd(ft) Ht Abv Struc(ft) NONE SPECIFIED

Source (Process) Listing

Em Point Srce $\mathrm{Nbr}$ Srce Type Hr....

200W P-WRAP1 001

I PROCESS AND PRODUCTION

Days Week Wks Year Summary Process Description

The mission of the Waste Receiving and pocessing Facility module i (WRAP1) includes: examining. assaying. characterizing. limited treating, and

repackaging of solid radioactive and mixed waste to enable permanent disposal of the waste. WRApl will be located in the $200 \mathrm{~W}$ area of the Hanford

site. south of $23 r d$ Street and west to Dayton Ave. It will be a $4.766 \mathrm{~m} 2$ metal building consisting of pre-insulated. pre-finished metal. interlocking

roof and wall sandwich pannels.

The solid waste to be handled in the WRAP1 facility include Low-level waste (LLW). Transuranic waste (TRU), TRU mixed wastes, and Low-level mixed waste (LLMW). The facility will only accept Contact Handled (CH) waste which is waste whose external dose rate does not exceed 200mrem/hr. The feed stream will consist of approximately 38,000 retrieved drums containing suspect TRU waste that were placed in storage beginning in 1970 (retrieved assay newly generated waste boxes as iarge as $2.5 \mathrm{~m}$ long by $1.5 \mathrm{~m}$ wide by $1.5 \mathrm{~m}$ high. However. the boxed waste will not be opened in the WRAP1 facility.

The incoming drums are only opened in the LLW and TRU gloveboxes that are located in the process area of the facility. The retrieved waste is dumped and sorted in these gloveboxes according to the results of the Non-destructive assay (NDA), and the Non-destructive Examination (NDE). The hazardous waste that is these areas, referred to as Zone I and Zone II, respectively, are exhausted through HEPA filter banks, and discharged through the one and only stack.

\section{Source Raw Materials Usage}

Em Point

Srce $\mathrm{Nbr} \cdot$ Srce Type

Process Rate Process Uom Material

Fuels Usage by Source Processes

Em Point Srce Nbr Srce Type

Qty Uom

Fuel 
Fuels Usage by Control Devices

Em Point

Ctl Nbr Ctl Type

Qty Vom

Fuel

Point Source Emissions - Radiological Pollutants

Em Point

Srce Nbr Srce Type Pollutant

to Emit (Ci/yr) Cti Actual (Ci/yr) Pollutant

Point Source Emissions - HAP Pollutants

Em Point

Srce Nbr Srce Type Pollutant

Em. Pt. Potential Em. Pt.

to Emit (tons/yr) Ctl Actual (tons/yr) Pollutant

Sum

Point Source Emissions - Uncategorized Pollutants

Em Point

Srce Nbr Srce Type Pollutant

Em. Pt. Potential Em. Pt.

to Emit (tons/yr) Ctl Actual (tons/yr) Pollutant

Sun

Fugitive Source Emissions - Radiological Pollutants

Em Point

Srce Nbr Srce Type Pollutant

Em. Pt. Potential Em. Pt.

Ctl Actual (Ci/yr)

Pollutant

Fugitive Source Emissions - HAP Pollutants

Em Point

Srce Nbr Srce Type Pollutant

Em. Pt. Potential Em. Pt.
to Emit (tons/yr) Ctl Actual (tons/yr) Pollutant

Sum 


\section{AIR EMISSIONS INVENTORY PERMIT APPLICATION REPORT (UP460) \\ FACILITY ID : WASTE RECEIVEING AND PACKAGING FACILITY}

FACILITY NAME: WRAPI

Fugitive Source Emissions - Uncategorized Pollutants

Em Point

Srce Nbr Srce Type Pollutant

Sum

Insignificant Source Emissions - Radiological Pollutants

Em Point $\quad$ Srce Nbr Srce Type Pollutant

Insignificant Source Emissions - HAP Pollutants

Em Point

Srce Nbr Srce Type Pollutant

Em. Pt. Potential Em. Pt

Cti Actual (Ci/yr)

Pollutant

Em. Pt. Potentiar Em. Pt.

Eomit (tons/yr) Ctl Actual (tons/yr) Pollutant

Insignificant Source Emissions - HAP Pollutants

Em Point

Srce Nbr Srce Type Pollutant

Sum

Insignificant Source Emissions - Uncategorized Pollutants

Em Point Srce $\mathrm{Nbr}$

Srce Type Pollutant
Em. Pt. Potential Em. Pt.

Eollutant

Sum

\section{Control Device Listing}

Em Point Control Equipment Code

200W P-WRAP1 001

099

Em. Pt. Potential Em. Pt.
to Emit (tons/yr) Ctl Actual (tons/yr) Pollutant

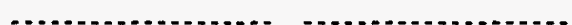

Em. Pt. Potential Em. Pt.
to Emit (tons/yr) Ctl Actual (tons/yr) Pollutant 


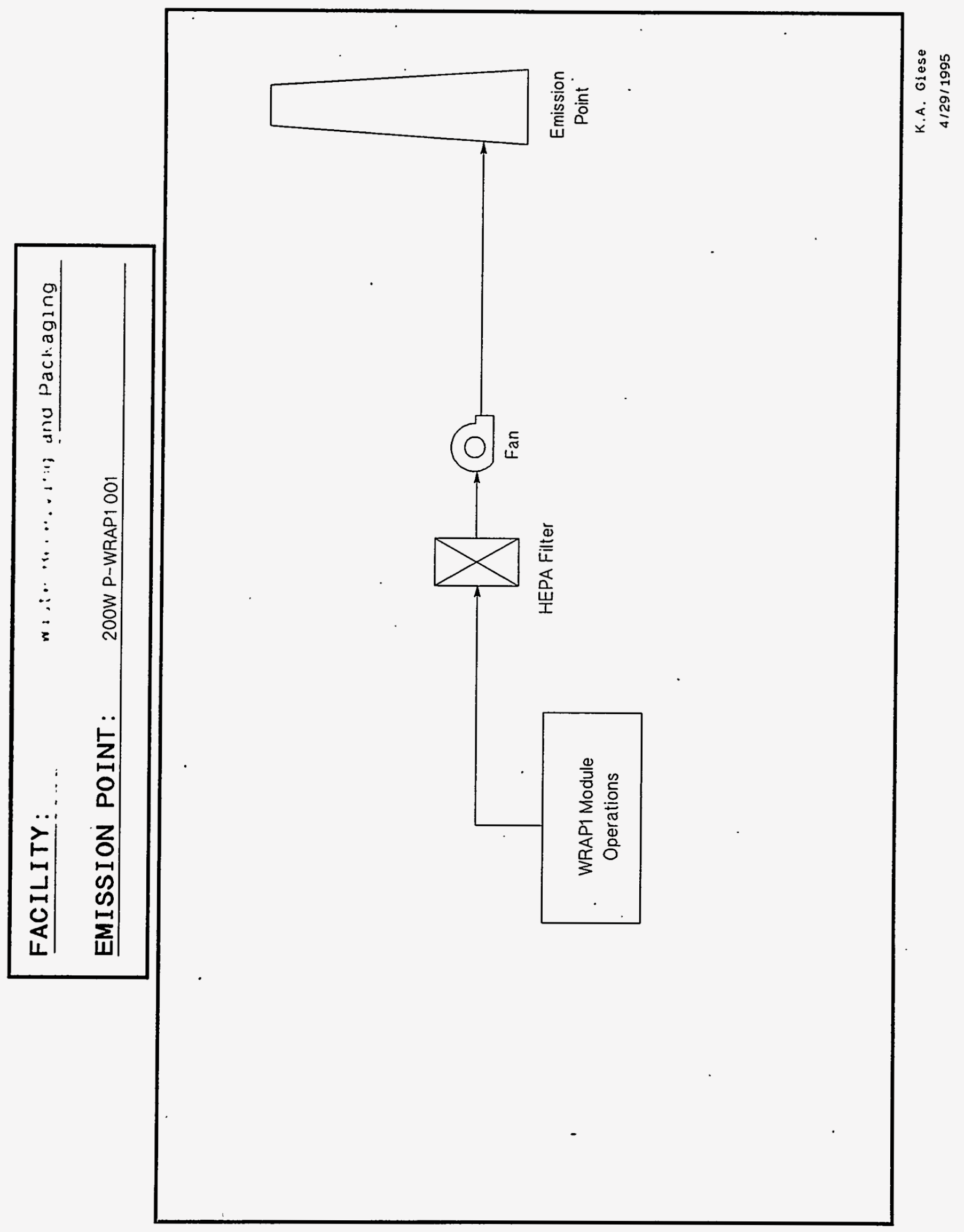




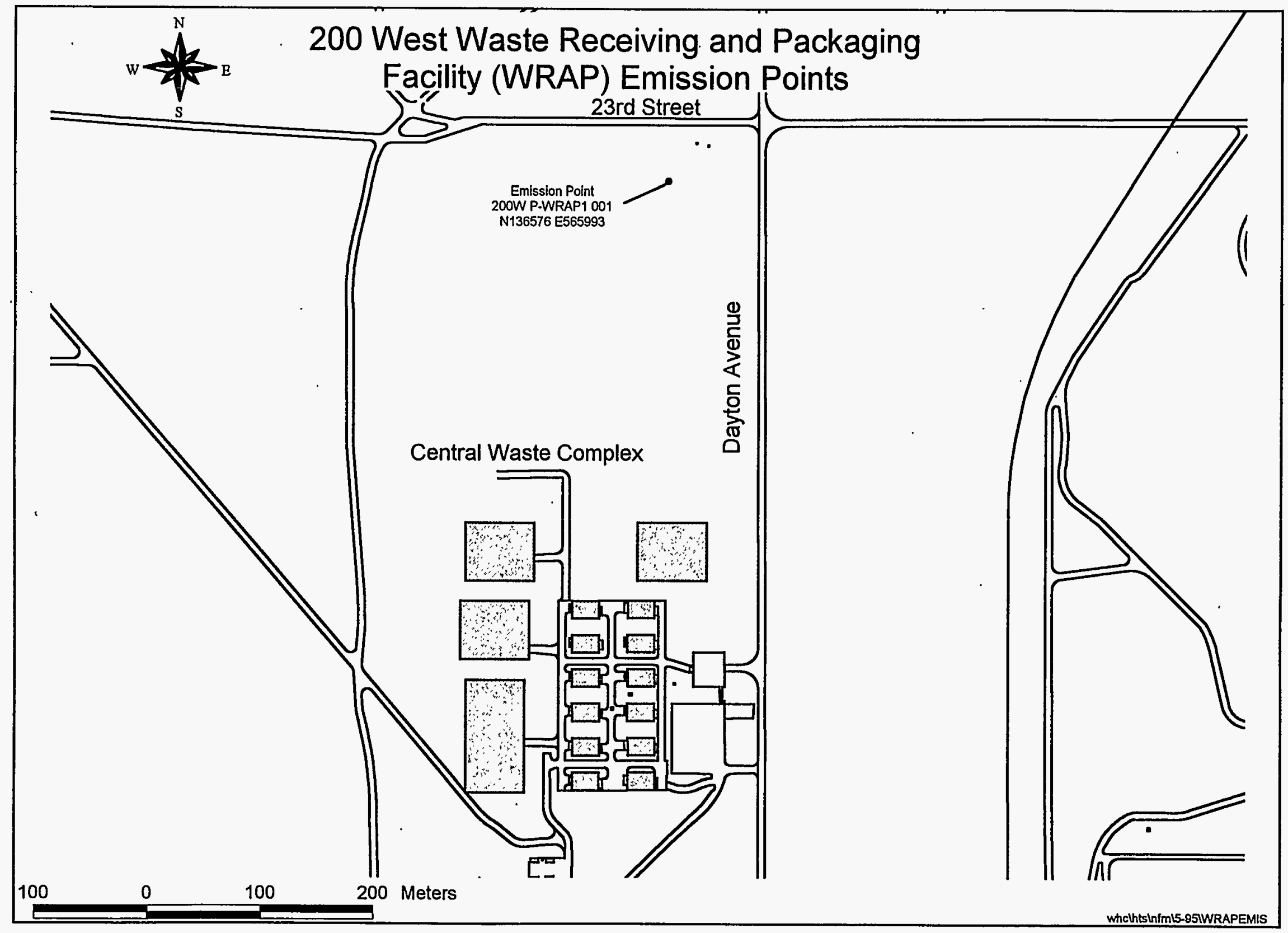


Hanford Site Air

Operating Permit Application
DOE/RL-95-07, Rev. 0

05/95

APPENDIX F

300 AREA

APP F-i 
APPENDIX F

CONTENTS

F-1 300 AREA

F-2 384 POWERHOUSE

F-3 300 AREA RESEARCH AND DEVELOPMENT FACIIITIES 


\title{
APPENDIX F-1
}

\author{
300 AREA \\ Chapter I. General Information
}

\section{I-B.1. Facility Description}

\section{5/306E/337B Buildings}

The 305/306E/337B Buildings house operations which design, fabricate and test prototype equipment for waste cleanup, waste sampling, and other projects associated with the Hanford Site.

The 306E Building at one time supported operation of the Fast Flux Test Facility (FFTF). The 306E-ULAB Stack Exhausts filtered air from past uranium oxide laboratory activities in rooms 158 and 159 . The laboratories are currently shut down. One of the ongoing activities in the $306 \mathrm{E}$ Building is nondestructive evaluation of materials.

\section{Building}

The 308 Building is a two-story structure referred to as the 308 Fuels Development Laboratory Building containing approximately 71,000 square feet of floor space within which a variety of technical activities have been performed since construction in 1960. Activities included plutonium fuels research, design, development, fabrication, assembly, inspection, engineering, and quality assurance. The 308 Building was expanded between 1975 and 1979, creating the 308 Annex. The 308 Annex contains test article assembly areas, shipping areas, and the Test Reactor and Isotope Production, General Atomics (TRIGA) reactor Fuel Storage Basin (FSB).

The 308 Building was deactivated and shutdown in March 1994. The 56 gloveboxes and open-faced hoods were cleaned out and stabilized with a nonhazardous acrylic coating to act as a fixative for residual holdup material. The glovebox ventilation/exhaust system was shutdown and physically blanked off, and the exhaust stack was deregistered. The laboratory . welding open-faced hood ventilation system also was shutdown and the exhaust stack deregistered. The 308 Building is unoccupied except for periodic surveillances to monitor shutdown conditions.

The 308 Annex ventilation system (308-TRIGA exhaust stack) remains operational as the annex has not been deactivated. The TRIGA reactor located in the 308 Annex has been shut down and defueled. The fuel is being stored in storage racks in the former TRIGA reactor water pool (FSB). The 308 Annex is unoccupied except for periodic surveillances to monitor shutdown conditions. Removal, handling, packaging, and shipping of the TRIGA fuel is expected to occur approximately in the 1996 time frame or beyond after the necessary . 
environmental assessments are completed and fuel storage casks are available. Deactivation of the 308 Annex will occur after fuel removal, with decommissioning activities to follow. The 308 Annex will be occupied during the fuel removal activity.

\section{Building}

The mission of the original 309 Building was to provide an operational test reactor to research and develop nuclear fuel technology. This test reactor was known as the Plutonium Recycle Test Reactor (PRTR). In 1962, the Plutonium Recycle Critical Facility (PRCF) was added to support the PRTR operations.

By 1975, the PRTR was deactivated, all of the fuel removed from the building, and the fuel storage basin decontaminated. In the late 1970s, the ground floor of the containment dome was emptied and converted into a fabrication shop. In the mid 1980s, an extensive cleanout effort removed most of the process equipment and vessels from the PRTR areas in the building.

The ground level of the containment dome currently is being used as an assembly shop. There is no further work scheduled for the containment vessel. The remainder of the building is used as general office space.

\section{Facility}

The 300 Area Treated Effluent Disposal Facility (TEDF) provides treatment for industrial waste waters generated in the 300 Area of the Hanford Site. The 300 Area TEDF recently began operation in December 1994. The 300 Area TEDF treats up to 300 gallons per minute (gpm) of process sewer waste waters from Hanford Site laboratories, along with support and inactive facilities. The laboratories services are particularly critical to the Hanford Site clean-up activities, including tank waste remediation efforts.

The main unit operation in conjunction with on-line monitoring and sampling, is to ensure that treated waste waters are acceptable for discharge to the Columbia River. The main unit operation combines processes to remove or destroy the heavy metals and organics that were identified as requiring treatment.

The 300 Area TEDF is located in the north part of the 300 Area. The main building, 310, houses the main unit operation. Diversion tanks, located adjacent to the 310 Building, provide capacity for extended facility shutdown and/or maintenance. The influent wastewater flowrate changes, but the average is about $110 \mathrm{gpm}$, which is far below the design flowrate. The 300 Area TEDF can be operated 24 hours per day. 


\section{Facility}

The 340 Facility is a complex of several building which include 340B, 340A, 340 Vault Tanks and 307 Water Basins. A radioactive (mixed) liquid waste system (RLWS) is managed in the 340 Facility. Waste is received via underground piping connecting Laboratory Buildings to the 340 Vault Tanks, or transported to the 340 Facility in containers or tank trucks for addition into the 340 Vault Tanks. There are two underground tanks; one tank is always on-line to receive waste, allowing the other tank to be isolated for transfer. Auxiliary storage is provided by six above ground storage (AGS) tanks located in the 340-A Building.

Railroad tanker car transfers of liquid mixed waste are made approximately every 90 days to the 204-AR Waste Unloading Station in the 200 East Area.

Solid mixed wastes are generated in the 340 Facility during the performance of routine operations, maintenance, and decontamination activities. A majority of the waste is packaged in containers, stored in the 340-B West Building, and transported by truck to the 200 Areas for burial or storage. Also, other 300 Area Facilities send their solid waste to the 340 Facility to be repackaged.

Decontamination activities take place in the 340 Building decontamination area that contains a hood, sink, and sump. The rest of the 340 Building consists of a control room, mechanical equipment room, and truck lock.

\section{Building}

The 377 Building, a 5,300 square foot structure located in the Northwest portion of the 300 Area, was constructed to allow examination of a failed nuclear steam.generator. The 377 Building was used in that capacity from 1983 to 1987 . The 377 Building was decontaminated in 1990, and currently serves as a laboratory for physical properties testing of contaminated soil and other samples.

Physical properties testing includes determination of particle size distribution, calcium carbonate content, hydraulic conductivity, specific gravity, moisture content, moisture retention, and retardation factors. Tests, other than those described, may be conducted in the future and will be evaluated on an as-needed basis for effluent monitoring requirements.

\section{I-B.2. Products, Raw Materials, Fuels, Processes}

- Principle products - There are no products produced at these 300 Area buildings. 
- Process information - There are process activities at the 310,340 and the 377 buildings:

- 310 Building - The main unit operation uses iron coprecipitation, gravity filtration, ion exchange, and UV oxidation to treat waste waters.

- 340 Building - Perform decontamination activities, waste water transfer operations, and low-level waste packaging operations.

- 377 Building - physical property testing of potentially radioactively contaminated soil samples.

- Raw materials used by processes - There are no raw materials consumed at these 300 Area buildings.

- Fuels used by processes - There are no fuels used in the processes at these 300 Area buildings.

\section{I-C.1. Emission Points, Controls, Stacks}

The emission point and stack information is found in the AEI report output. The control information is found on page 7 of the AEI report output.

\section{I-C.2. Facility Process Flow Diagrams}

Attached.

\section{I-C.3. Site Map}

Attached.

\section{Chapter II. Regulatory Requirements}

\section{II-A.1. Description of Discharge and Emission Points}

The emisșion point and stack information is found in the AEI report output. 
AIR EMISSIONS INVENTORY PERMIT APPLICATION REPORT (UP460)
FACILITY ID:300 AREA

FACILITY ID FACILITY NAME: 300 AREA Facility and Associated Structures
a

Rev.1 May 24. 1995

\section{Facility Building List}

\begin{tabular}{|c|c|c|c|}
\hline Bldg Id & North & East & Bldg Desc \\
\hline $\begin{array}{l}\text { 306E-ULAB } \\
\text { 308TRIGA }\end{array}$ & $\begin{array}{l}116099 \\
115813\end{array}$ & $\begin{array}{l}594009 \\
594174\end{array}$ & \multirow{3}{*}{$\begin{array}{l}306 E \text { BUILDING ROOMS } 158 \text { \& } 159 \\
\text { STACK FOR THE REACTOR HALL HOUSING THE TRIGA } \\
\text { REACTOR IN BUILDING } 308 \\
\text { STACK FOR THE PLUTONIUN RECYCLE TEST REACTOR } \\
\text { STACK. } 340 B \text { EAST BUILDING TANK CAR LOADOUT } \\
\text { FACILITY } \\
\text { STACK FOR THE } 340 \text { DECONTAMINATION FACILITY } \\
\text { BUILDING } 340 \text { TANK AND VAULT EXHAUST STACK } \\
\text { GEOTECHNICAL ENGINEERING LABORATORY } \\
300 \text { AREA TREATED EFFLUENT DISPOSAL FACILITY }\end{array}$} \\
\hline $\begin{array}{l}\text { 309PRTR } \\
\text { 340BBLDG }\end{array}$ & $\begin{array}{l}115672 \\
115958\end{array}$ & $\begin{array}{l}594161 \\
594160\end{array}$ & \\
\hline $\begin{array}{l}\text { 340DECON } \\
340 N T E X \\
377 \\
\text { TEOF }\end{array}$ & $\begin{array}{l}115939 \\
115926 \\
116162 \\
117343\end{array}$ & $\begin{array}{l}594165 \\
594172 \\
593655 \\
593642\end{array}$ & \\
\hline
\end{tabular}

Custodian(Contractor) Cust Phone DOE POC

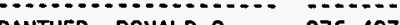
PANTHER, DONALD G. 376-4975 RASMUSSEN. DAVID E. $376-3288$ $\begin{array}{ll}\text { UTZ. EDWIN E. } & 376-0701 \\ \text { HAGGERTY. KEVIN J. } & 372-3133\end{array}$ $\begin{array}{ll}\text { HAGGERTY. KEVIN J. } & 372-3133 \\ \text { HAGGERTY. KEVIN J. } & 372-3133\end{array}$ HANTER JAMES A. $\quad 376-2356$ HALGREN, DALE L. $\quad$. 376-9988
DOE Phone …....... $\begin{array}{ll}\text { SHANNON HERRES } & 373-0908 \\ \text { SHANNON HERRES } & 373-0908\end{array}$ $\begin{array}{ll}\text { SHANNON HERRES } & 373-0908 \\ \text { ANNA BEARD } & 373-0908\end{array}$ ANNA BEARD $373-0908$ ANAA 373-0908 $373-0908$

\section{Emission Point Listing}

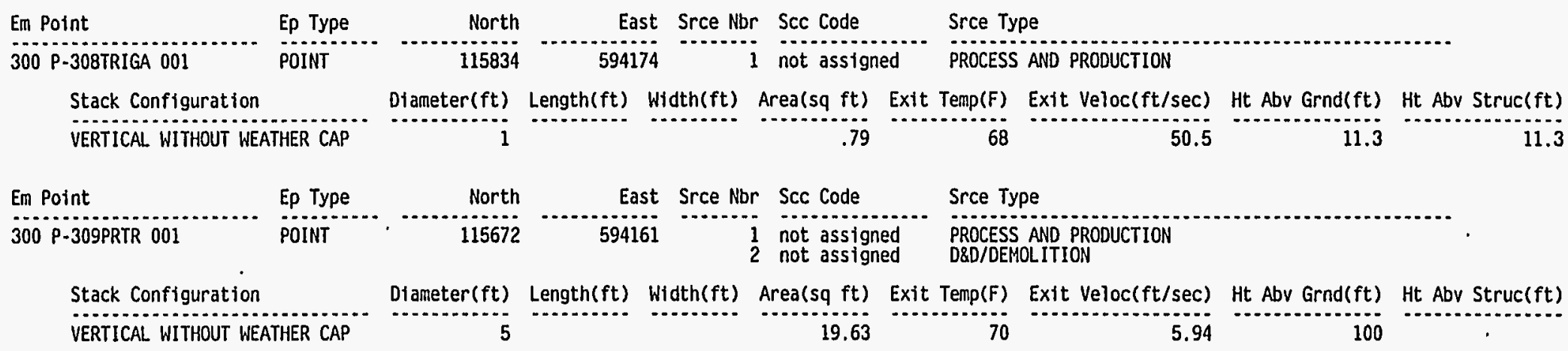

\begin{tabular}{|c|c|c|c|c|c|c|}
\hline Em Point & Ep Type & North & East & Srce Nbr & Scc Code & Srce Type \\
\hline 300 P-340BBLLG 001 & POINT & 115958 & 594160 & 1 & not assigned & FUELS/LIQUIDS LOADING RACK \\
\hline
\end{tabular}

Stack Configuration $\ldots$ Diameter(ft) Length(ft) Width(ft) Area(sq ft) Exit Temp(F) Exit Veloc(ft/sec) Ht Abv Grnd(ft) Ht Abv Struc(ft)

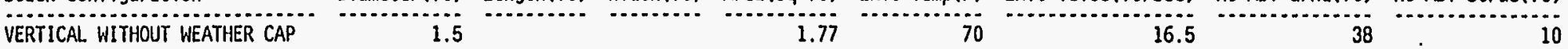

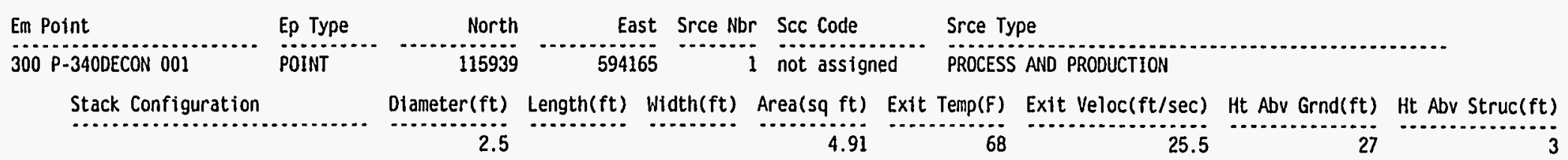




\section{Emission Point Listing}

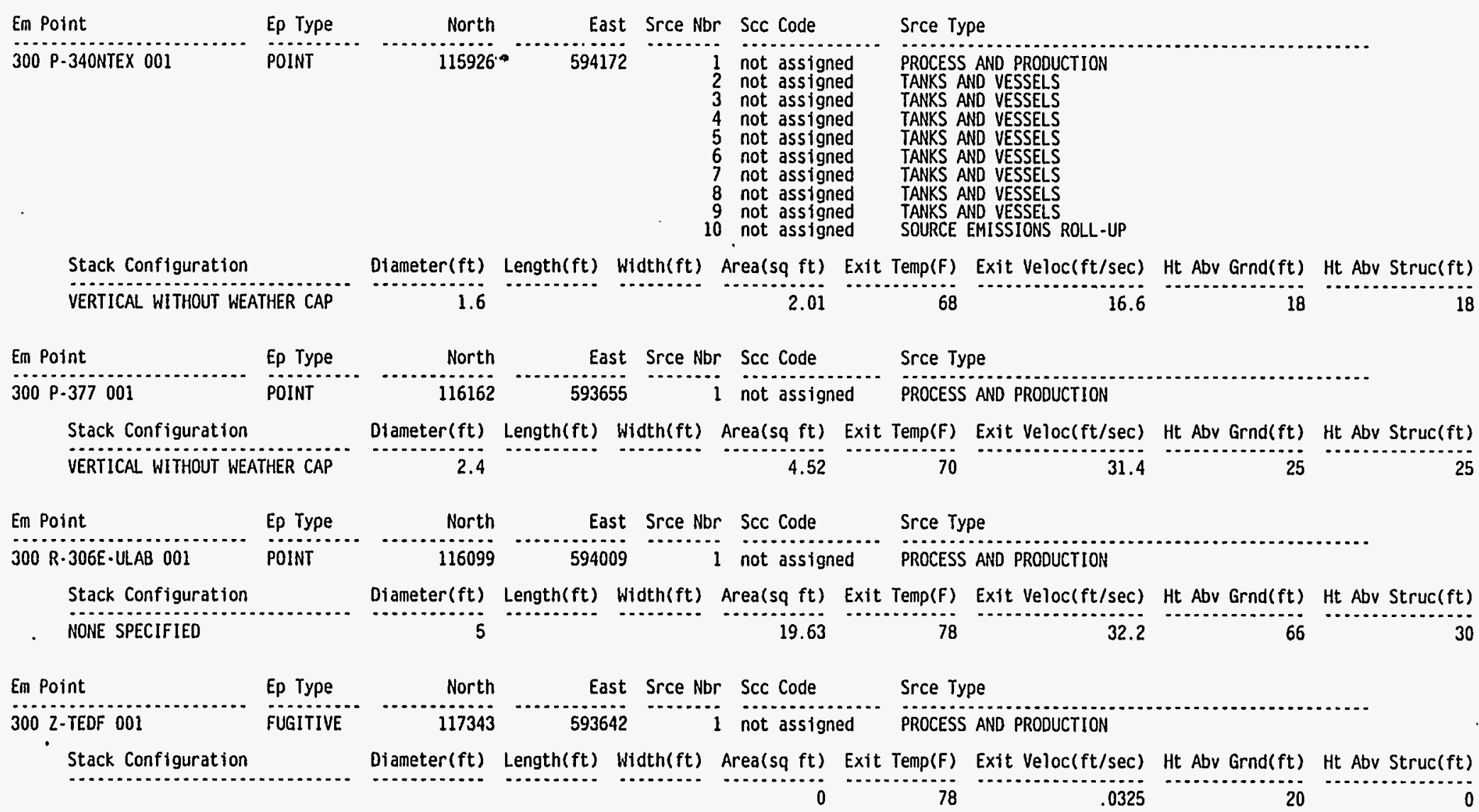

Source (Process) Listing

Em Point $\quad$ Srce Nbr Srce Type $\ldots \ldots \ldots \ldots \ldots \ldots$ Hrs Day Days Week Wks Year Summary Process Description

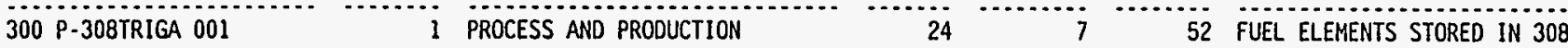


AIR EMISSIONS INVENTORY PERMIT APPLICATION REPORT (UP460)

FACILITY NAME: 300 AREA Facility and Associated Structures

\section{Source (Process) Listing}

$$
\text { Em Point }
$$

300 P-309PRTR 00

Em Point

300 P-309PRTR 001

Srce Nbr Srce Type

Em Point

300 P-340BBLDG 001

Em Point

30O P-34ODECON 001

Em Point 300 P-34ONTEX 001

Em Point

300 P-34ONTEX 001

En Point

300 P-34ONTEX 001

Em Point

300 P-340NTEX 001

Em Point

300 P-34ONTEX 001

Em Point

300 P-34ONTEX 001

Srce $\mathrm{Nbr}$ Srce Type

6 TANKS AND VESSELS

Em Point

300 P-34ONTEX 001

Srce Nbr Srce Type

Srce $\mathrm{Nbr}$ Srce Type

Srce Nbr Srce Type

Srce Nbr Srce Type

Srce Nbr Srce Type

Srce Nbr Srce Type

Srce Nor Srce Type
Srce Nbr Srce Type

Rev.1 May 24, 1995

Hrs Day Days Week Wks Year Summary Process Description

24 72 CONTAMINANT SOURCE IS A RESULT OF PLUTONIUM OXIDE CONTAINED IN VARIOUS PROCESS EQUIPMENT.

Hrs Day Days Week Wks Year Summary Process Description

50 D\&D OF THE 309 BUILDING WILL BEGIN IN CY 1995 AND IS EXPECTED TO END BY CY 1998.

Hrs Day Days Week Wks Year Summary Process Description

8 RADIOACTIVE LIQUID WASTE TRANSFER TO 20,000 GALLON RAILROAD TANK CAR.

Hrs Day Days Week Wks Year Summary Process Description 52 TRACE CONTAMINATION

Hrs Day Days Week Wks Year Summary Process Description

52 TRACE CONTAMINATION IN 340 VAULT

Hrs Day Days Week Wks Year Summary Process Description

$24 \quad 7 \quad 52$ TANK TK-1 IN 340 VAULT

Hrs Day Days Week Wks Year Summary Process Description

24 TANK TK-2 IN 340 VAULT

Hrs Day Days Week Wks Year Summary Process Description

$24 \quad 7 \quad 52$ TANK AGS-1

Hrs Day Days Week Wks Year Summary Process Description

Hrs Day Days Week Wks Year Summary Process Description

$24 \quad 7 \quad 52$ TANK AGS-3

Hrs Day Days Week Wks Year Summary Process Description

$24 \quad 7 \quad 52$ TANK AGS -4 
AIR EMISSIONS INVENTORY PERMIT APPLICATION REPORT (UP460)

FACILITY IO : 300 AREA
FACILITY NAME: 300 AREA Facility and Associated Structures

\section{Source (Process) Listing}

\begin{tabular}{|c|c|c|c|c|c|c|}
\hline Em Point & Srce Nbr & Srce Type & Hrs Day & Days Week & Wks Year & Sumary Process Description \\
\hline 300 P-340NTEX 001 & 8 & TANKS AND VESSELS & 24 & 7 & 52 & TANK AGS-5 \\
\hline En Point & Srce Nbr & Srce Type & Hrs Day & Days Week & Wks Year & Summary Process Description \\
\hline 300 P-340NTEX 001 & 9 & TANKS AND VESSELLS & 24 & 7 & 52 & TANK AGS-6 \\
\hline Em Point & Srce Nbr & Srce Type & Hrs Day & Days Week & Wks Year & Summary Process Description \\
\hline 300 P-340NTEX 001 & 10 & SOURCE EMISSIONS ROLL-UP & 24 & 7 & 52 & TOTAL EMISSIONS FOR EMISSION POINT P-340NTEX-001 \\
\hline Em Point & Srce Nbr & Srce Type & Hrs Day & Days Week & Wks Year & Summary Process Description \\
\hline $300 \quad P-377001$ & 1 & PROCESS AND PRODUCTION & 24 & 7 & 52 & GEOTECHNICAL TESTS ON RADIOLOGICALLY IMPACTED SOILS \\
\hline En Point & Srce Nbr & Srce Type & Hrs Day & Days Week & Wks Year & Summary Process Description \\
\hline 300 R-306E-ULAB 001 & 1 & PROCESS AND PRODUCTION & 24 & 7 & 52 & $\begin{array}{l}\text { THE 306E-ULAB STACK EXHAUSTS FILTERED AIR FROM PAST URANIUM } \\
\text { OXIDE LABORATORY ACTIVITIES IN ROOMS } 158 \text { AND } 159 . \text { THE } \\
\text { LABORATORIES ARE CURRENTLY SHUT DOWN. EMISSIONS MONITORING } \\
\text { CONSISTS OF A RECORD SAMPLER. }\end{array}$ \\
\hline
\end{tabular}


AIR EMISSIONS INVENTORY PERMIT APPLICATION REPORT (UP460)

FACILITY NAME: 300 AREA Facility and Associated Structures

\section{Source (Process) Listing}

\begin{tabular}{|c|c|c|c|c|c|}
\hline Em Point & Srce $\mathrm{Nbr}$ & Srce Type & Hrs Day & Days Week Wks Year & Summary Process Description \\
\hline 300 2-TEDF 001 & 1 & PROCESS AND PRODUCTION & & & $\begin{array}{l}\text { THE } 300 \text { AREA TEDF PROVIDES TREATMENT FOR INDUSTRIAL } \\
\text { WASTEWATERS GENERATED IN THE } 300 \text { AREA. THE TEDF TREATS UP TO } \\
300 \text { GAL/MIN OF PROCESS SEWER WASTEWATERS FROH SITE } \\
\text { LABORATORIES, ALONG WITH SUPPORT AND INACTIVE FACILITIES. }\end{array}$ \\
\hline
\end{tabular}

The TEDF consists of Best Available Technology for the constituents of concern in the wastewater. including trace quantities of aluminum. copper. cyanide, lead, mercury, and select organics. The main components of the system are:

WASTE COLLECTION SUMP collects flow from the 300 Area and pumps the liquid to the TEOF site. The sump is just north of the 300 Area and is connected to the TEDF by burried pipe.

EQUALIZATION TANK minimizes variations in flow and loading to the plant.

IRON COPRECIPITATION removes insoluable heavy metal precipitates through settiling. This process includes chemical additions (Chemical Mix Tank),

GRAVITY FILTERS use dual media (sand/coal) to remove suspended solids.

ION EXCHANGE removes mercury that forms insoluble metal sulfides with a thiol functional group resin.

UV OXIDATION destroys organics, bacteria, sulfides, nitrate, and cyanide by oxidizing them with ultraviolet light and hydroxal molecules (from hydrogen peroxide). Carbon dioxide and water are all that remain from organic reactions taken to completion.

DIVERSION TANKS provide capacity for extended plant shutdown and/or maintenance.

SLUDGE DEWATERING is performed using a sludge thickening tank. polymer addition. and plate and frame filter press. Dewatered sludge is placed in drums or bulk containers for disposal.

CENTRAL CONTROL SYSTEM uses industrial computers and programmable logic controllers to control a majority of operations. The capability for manual operations is built in throughout the facility.

Source Raw Materials Usage

En Point

Srce Nbr Srce Type

Process Rate Process Uom Material

Fuels Usage by Source Processes

En Point

Srce Nbr Srce Type

Qty Uom

Fuel

Fuels Usage by Control Devices

Em Point

$\mathrm{Ct}) \mathrm{Nbr}$ Ctl Type

Qty Uom

Fue

Page \#: 5 of $8 \quad$ Report Run Date: 29 -APR-95 
Point Source Emissions - Radiological Pollutants

\begin{tabular}{|c|c|c|c|c|c|}
\hline En Point & Srce $\mathrm{Nbr}$ & Srce Type Pollutant & $\begin{array}{l}\text { Em. Pt. Potential } \\
\text { to Emit (Ci/yr) }\end{array}$ & $\begin{array}{l}\text { Em. Pt. } \\
\text { Cti Actual }(C 1 / y r)\end{array}$ & Pollutant \\
\hline 300 P-30BTRIGA 001 & 1 & PROCESS AND PROOUCTION & $\begin{array}{l}\text { Refer to } \\
\text { WHC-SD-EN-RPT-007 } \\
\text { Rev. O Page A-321 }\end{array}$ & $\begin{array}{l}\text { Refer to } \\
\text { DOE/RL-94-51 } \\
\text { Table 2-1 }\end{array}$ & \\
\hline 300 P-309PRTR 001 & 1 & PROCESS AND PRODUCTION & $\begin{array}{l}\text { Refer to } \\
\text { WHC-SD-EN-RPT-025 } \\
\text { Rev. } 0 \text { Page A-41 }\end{array}$ & $\begin{array}{l}\text { Refer to } \\
\text { WHC-SD-EN-RPT-025 } \\
\text { Rev. } 0 \text { Page A-41 }\end{array}$ & \\
\hline 300 P-340BBLDG 001 & 1 & FUELS/LIQUIDS LOADING RACK & $\begin{array}{l}\text { Refer to } \\
\text { WHC-SD-EN-RPT- } 025 \\
\text { Rev. O Page A-43 }\end{array}$ & $\begin{array}{l}\text { Refer to } \\
\text { WHC-SD-EN-RPT-025 } \\
\text { Rev. O Page A- } 43\end{array}$ & \\
\hline 300 P-340DECON 001 & 1 & PROCESS AND PRODUCTION & $\begin{array}{l}\text { Refer to } \\
\text { WHC-SD-EN-RPT-025 } \\
\text { Rev. O Page A-44 }\end{array}$ & $\begin{array}{l}\text { Refer to } \\
\text { WHC-SD-EN-RPT-025 } \\
\text { Rev. } 0 \text { Page A-44 }\end{array}$ & \\
\hline 300 P-340NTEX 001 & $\begin{array}{l}1 \\
2 \\
3 \\
4 \\
5 \\
6 \\
7 \\
8 \\
9\end{array}$ & $\begin{array}{l}\text { PROCESS AND PRODUCTION } \\
\text { TANKS AND VESSELS } \\
\text { TANKS AND VESSELS } \\
\text { TANKS AND VESSELS } \\
\text { TANSS AND VESSELS } \\
\text { TANNSS AND VESSELS } \\
\text { TANNSS AND VESSELS } \\
\text { TANKS AND VESSELSS } \\
\text { TANKS AND VESSELS }\end{array}$ & $\begin{array}{l}\text { Refer to } \\
\text { WHC-SD-EN-RPT-007 } \\
\text { Rev. } 0 \text { Page A-324 }\end{array}$ & $\begin{array}{l}\text { Refer to } \\
\text { DOE/RL-94-51 } \\
\text { Table 2-1 }\end{array}$ & \\
\hline $\begin{array}{l}300 \text { P-377 } 001 \\
300 \text { R-306E-ULAB } 001 \\
\text {. }\end{array}$ & 1 & $\begin{array}{l}\text { PROCESS AND PRODUCTION } \\
\text { PROCESS AND PRODUCTION }\end{array}$ & $\begin{array}{l}\text { Refer to } \\
\text { WHC-SD-EN-RPT-007 } \\
\text { Rev.0 Page A-299 } \\
\text { Refer to } \\
\text { WHC-SD-EN-RPT-007 } \\
\text { Rev. 0 Page A-300 }\end{array}$ & $\begin{array}{l}\text { Refer to } \\
\text { DOE/RL-94-51 } \\
\text { Table 2-1 } \\
\text { Refer to } \\
\text { DOE/RL-94-51 } \\
\text { Table 2-1 }\end{array}$ & \\
\hline
\end{tabular}

Point Source Emissions - HAP Pollutants

\begin{tabular}{|c|c|c|c|c|c|}
\hline Em Point & Srce Nbr & Srce Type Pollutant & $\begin{array}{l}\text { Em. Pt. Potential } \\
\text { to Emit (tons/yr) }\end{array}$ & Em $\mathrm{Et}$ Pt Actual (tons/yr) & Pollutant \\
\hline \multicolumn{6}{|l|}{ Sum } \\
\hline \multicolumn{6}{|c|}{ Point Source Emissions - Uncategorized Pollutants } \\
\hline Em Point & Srce Nbr & Srce Type Pollutant & $\begin{array}{l}\text { Em. Pt. Potential } \\
\text { to Emit (tons/yr) }\end{array}$ & $\begin{array}{l}\text { Em. Pt. } \\
\text { Ctl Actual (tons/yr) }\end{array}$ & Pollutant \\
\hline
\end{tabular}

Sum 


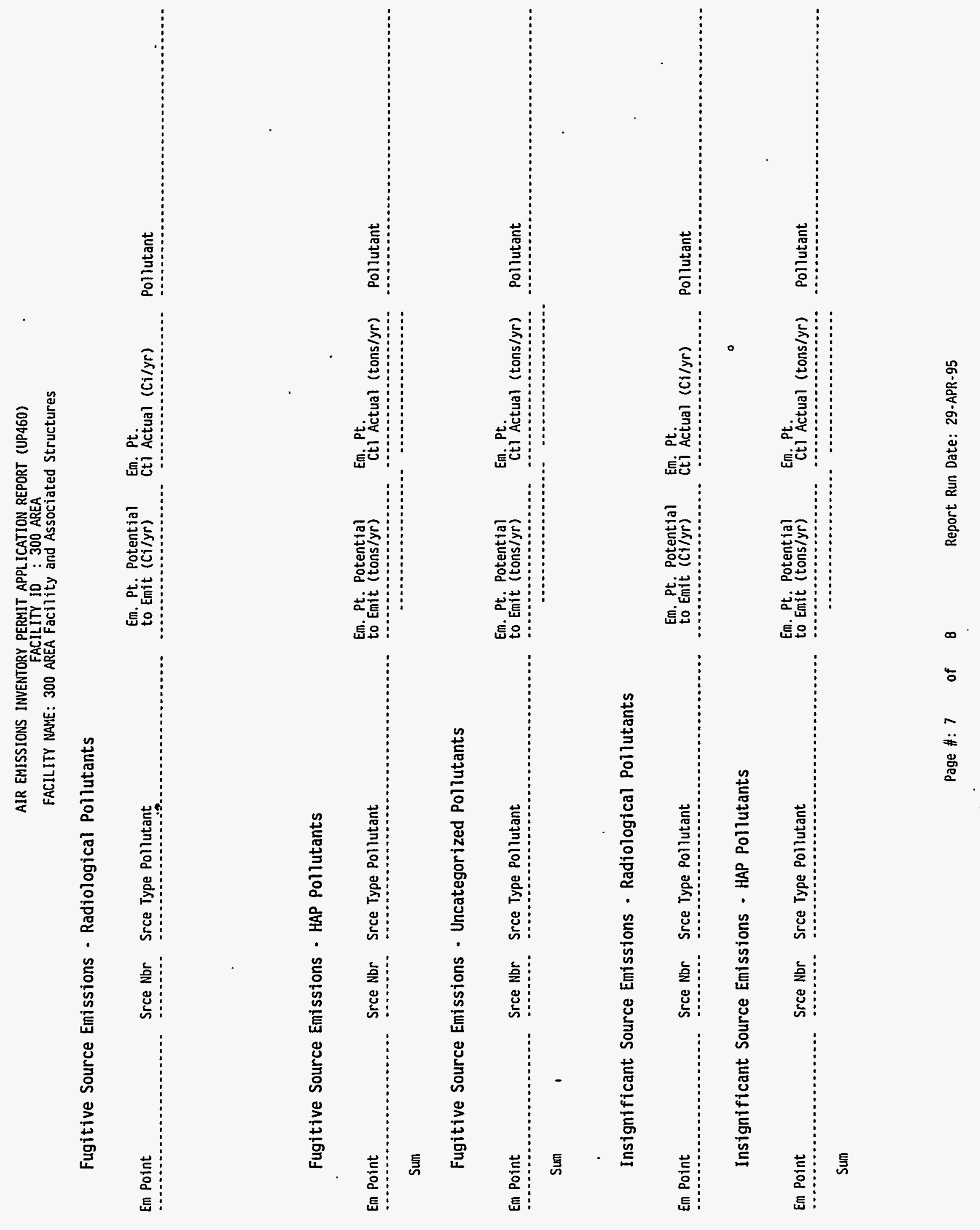


AIR EMISSIONS INVENTORY PERMIT APPLICATION REPORT (UP460)

FACILITY ID :300 AREA
FACILITY NAME: 300 AREA Facility and Associated Structures

Insignificant Source Emissions - Uncategorized Pollutants

\begin{tabular}{|c|c|c|c|c|}
\hline Point & Srce Nbr & Srce Type Pollutant & $\begin{array}{l}\text { Em. Pt. Potential } \\
\text { to Emit (tons/yr) }\end{array}$ & $\begin{array}{l}\text { Em. Pt. } \\
\mathrm{Ct} \text { l Actual (tons/yr) }\end{array}$ \\
\hline
\end{tabular}

Sum

\section{Control Device Listing}

Em Point

Control Equipment Code

$\begin{array}{ll}300 \text { P.308TRIGA 001 } & 099 \\ 300 \text { P.309PRTR 001 } & 099 \\ 300 \text { P-340BBLDG 001 } & 099 \\ 300 \text { P-340DECON 001 } & 099 \\ 300 \text { P-340NTEX 001 } & 099 \\ 300 \text { P-377GEL 001 } & 099 \\ 300 \text { P-377 001 } & 099 \\ 300 \text { R-306E-ULAB 001 } & 099\end{array}$

Page \#: 8 of 8 Report Run Date: $29-A$ PR-95 


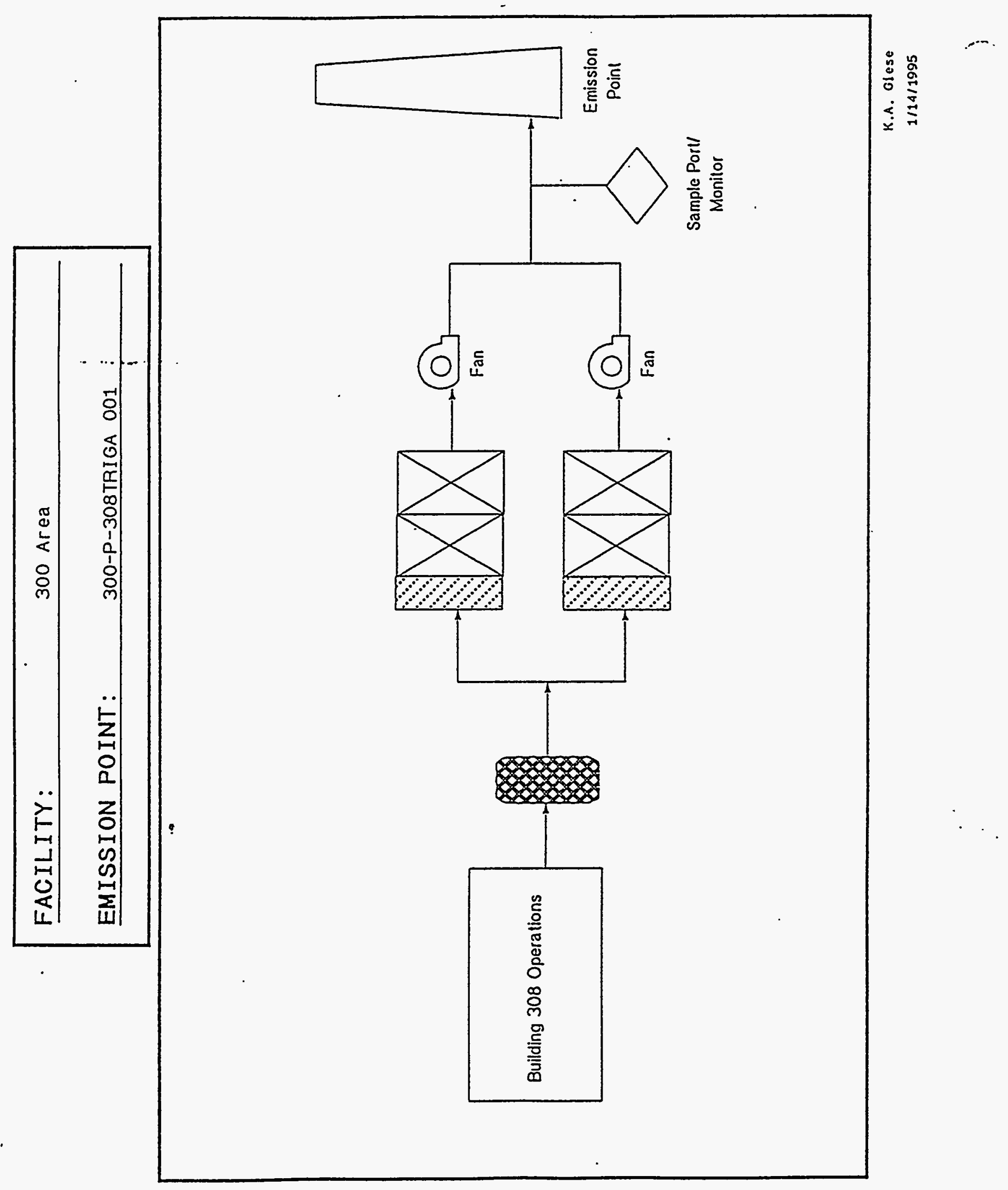




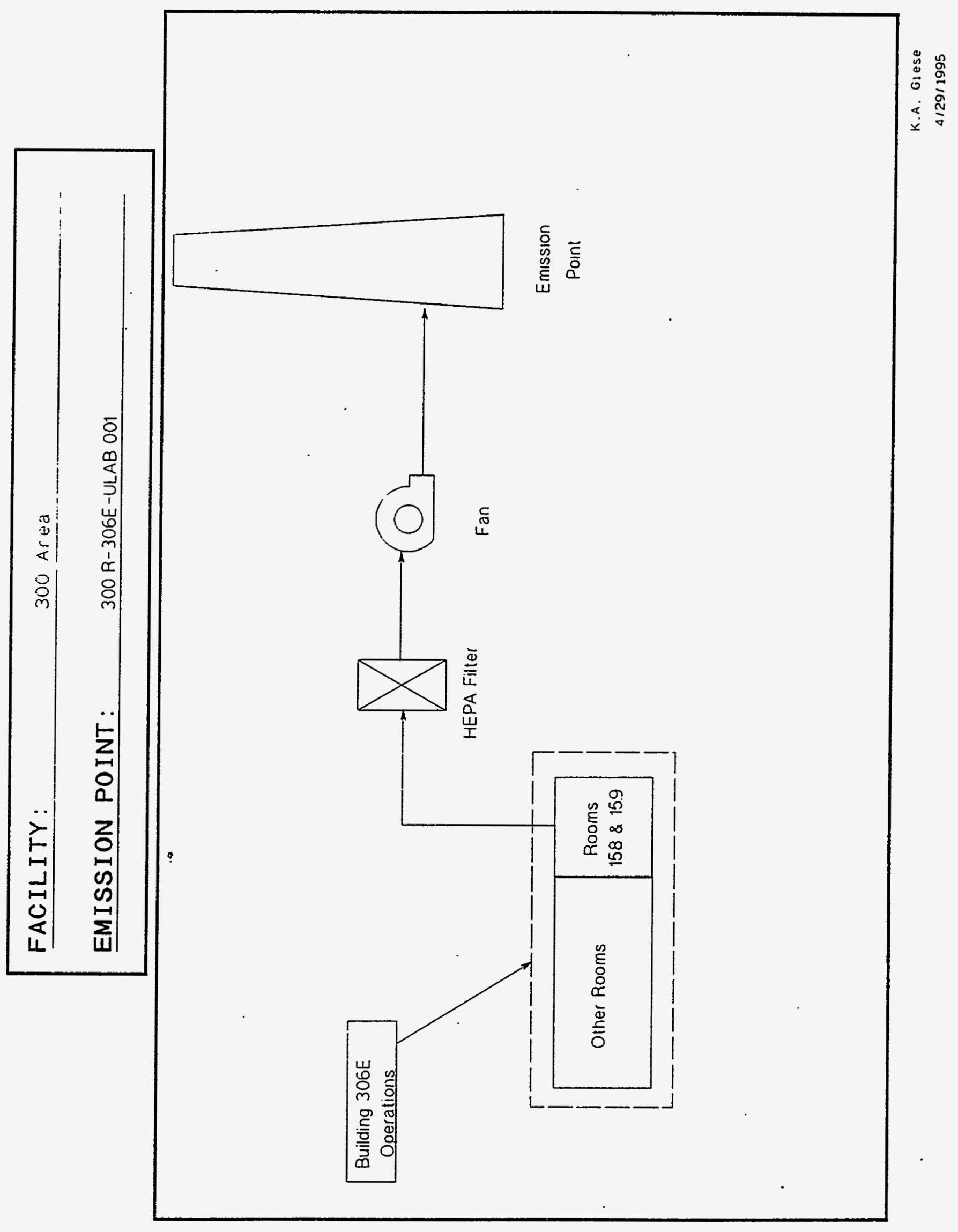



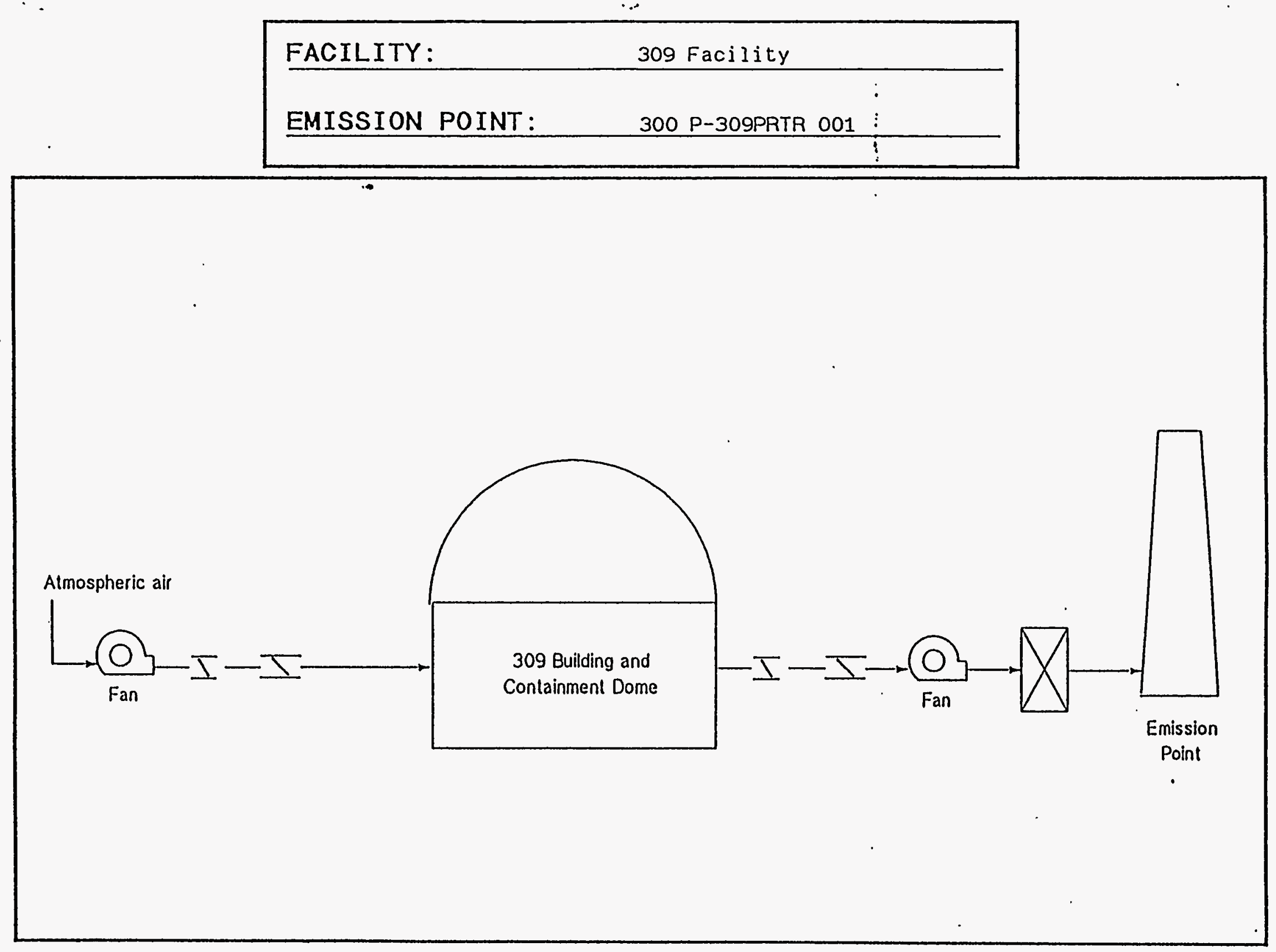


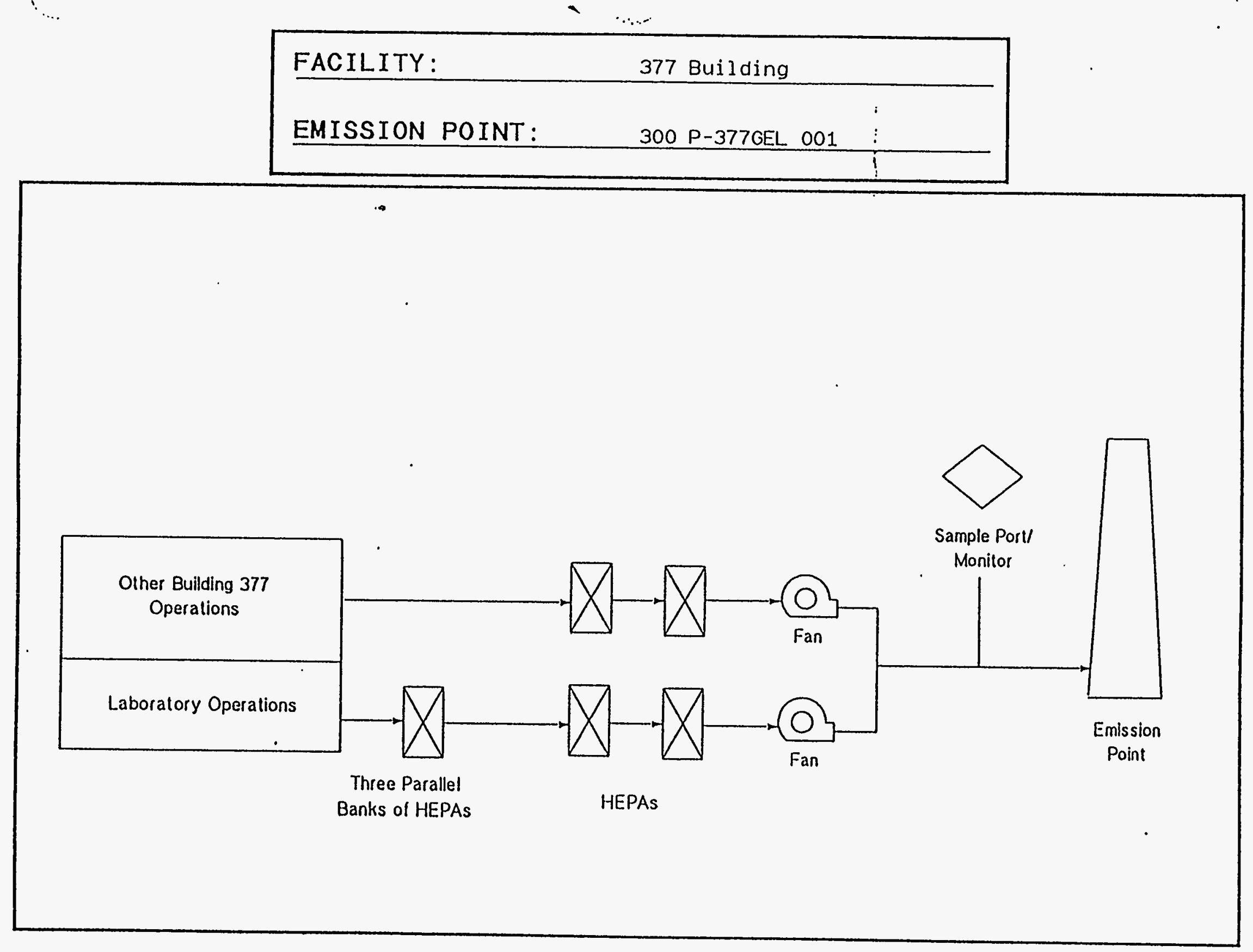




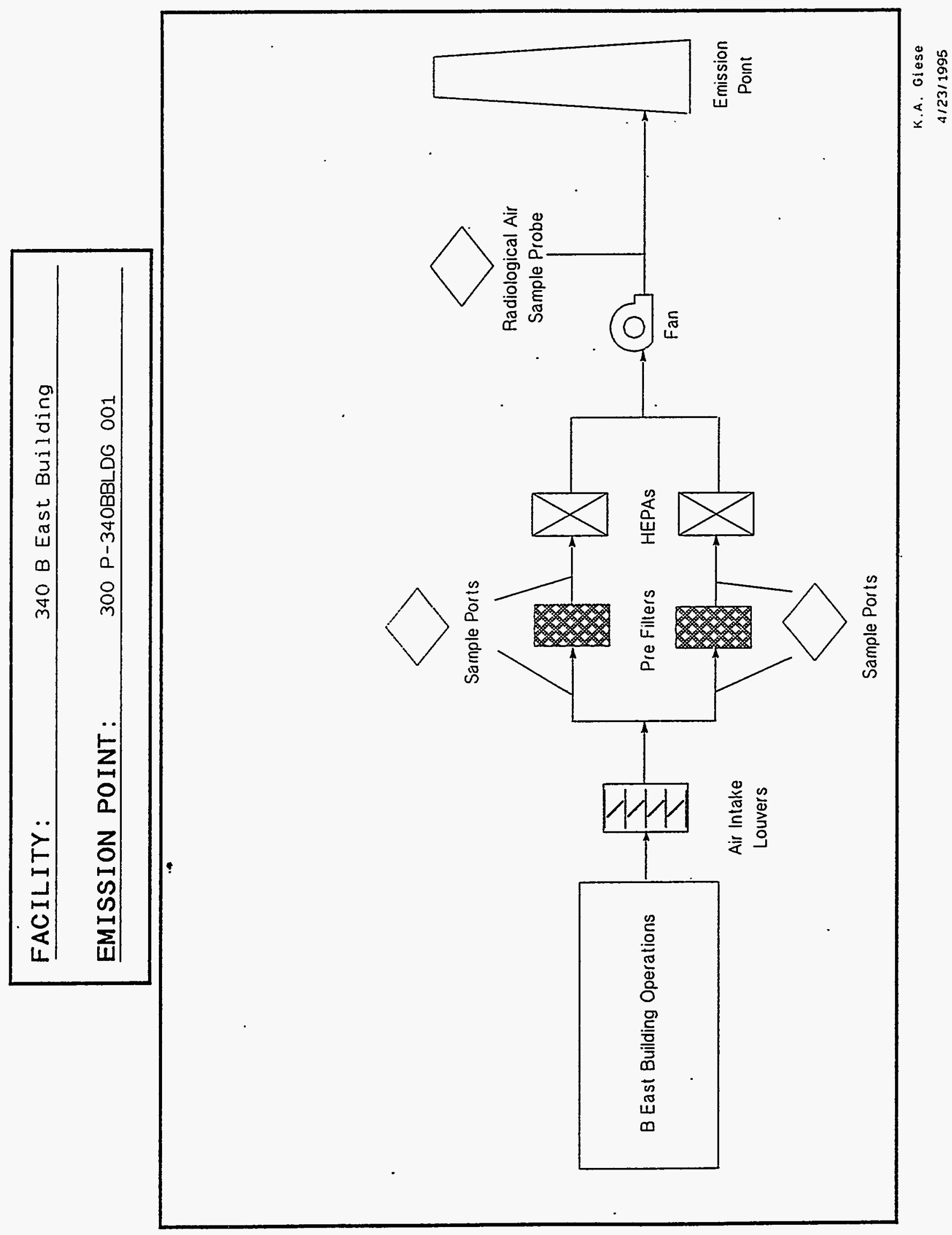




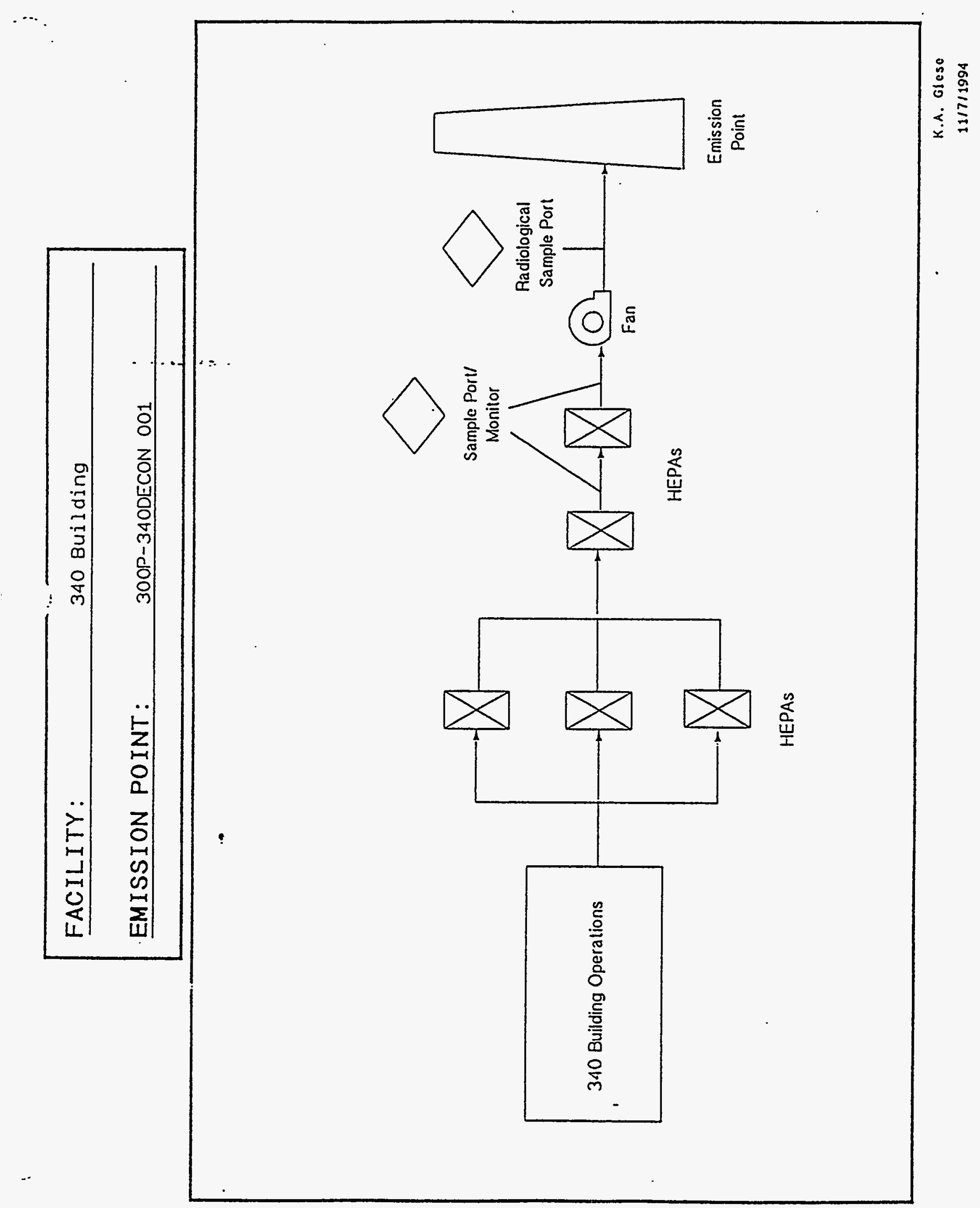




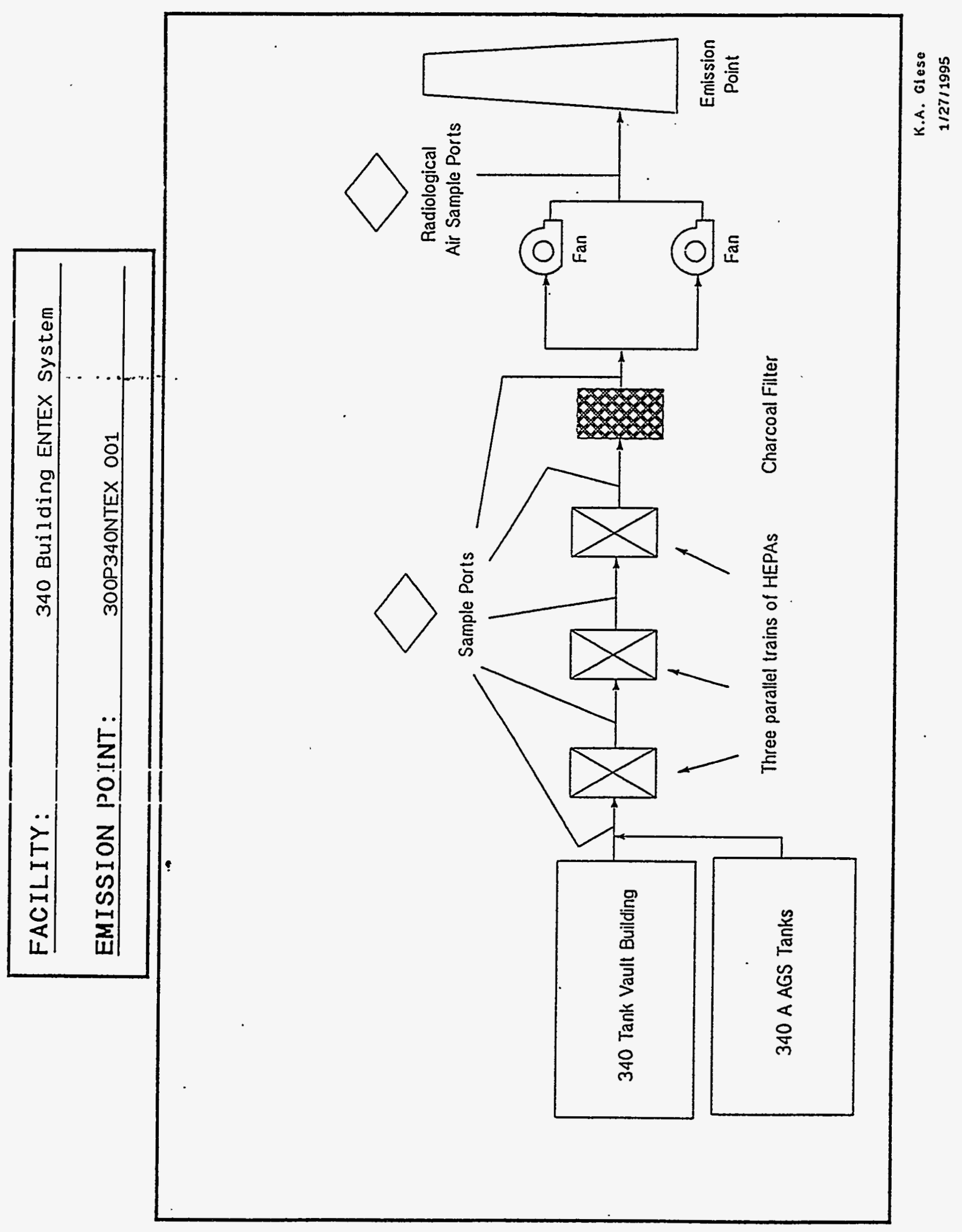


FACILITY: $\quad 300$ Area Treated Effluent Disposal Facility

EMISSION POINT: 300 Z-TEDF 001

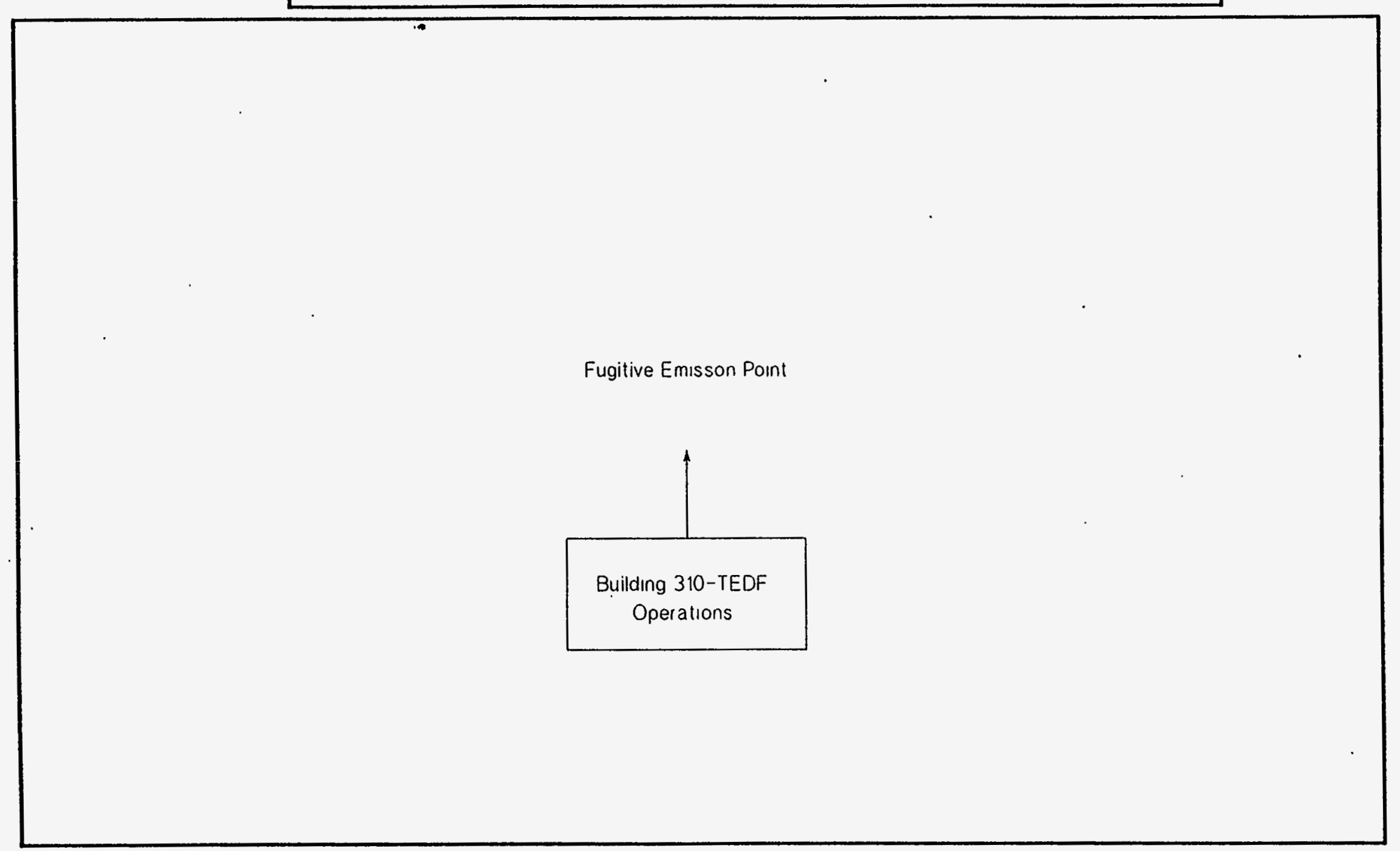




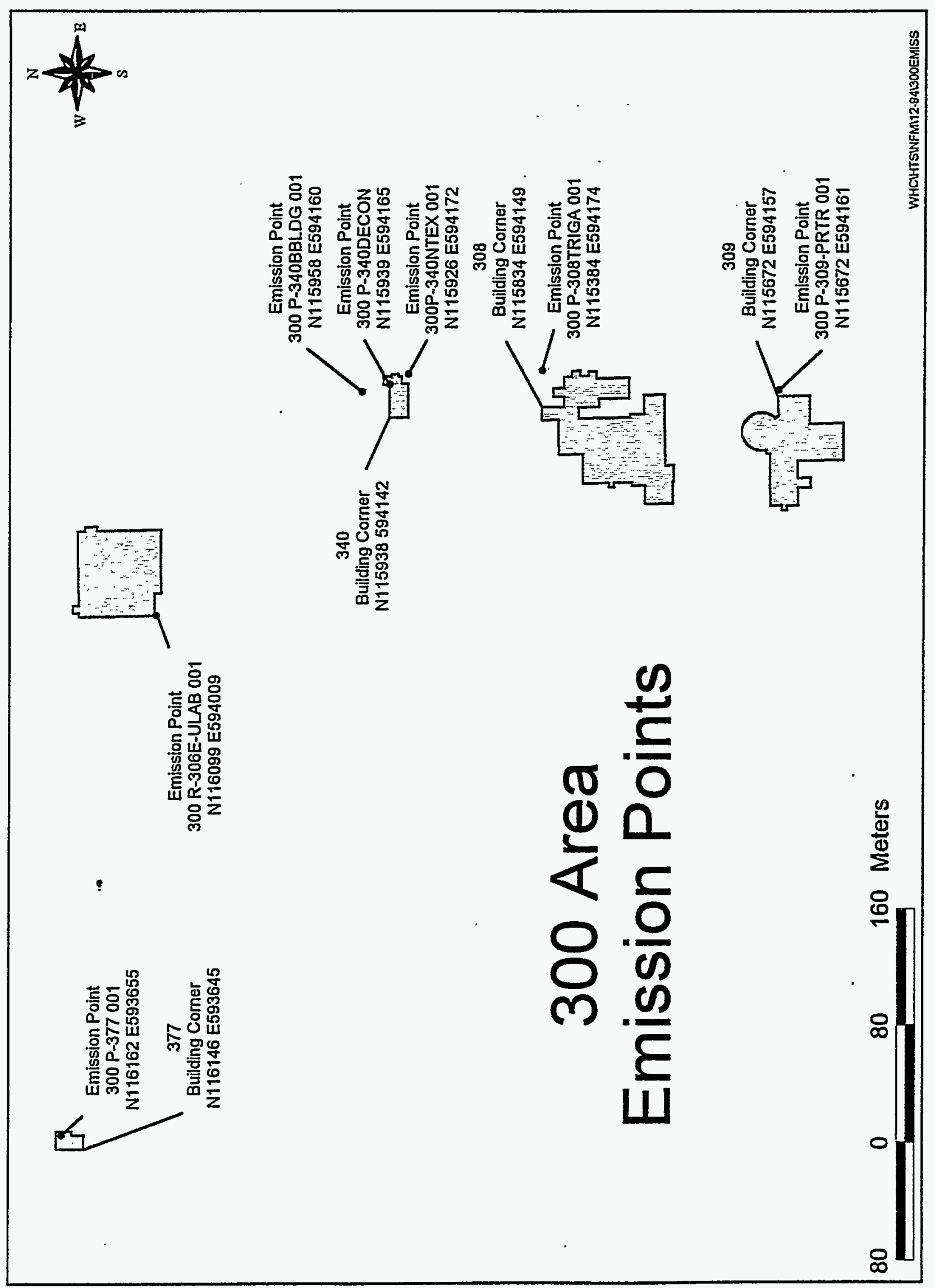




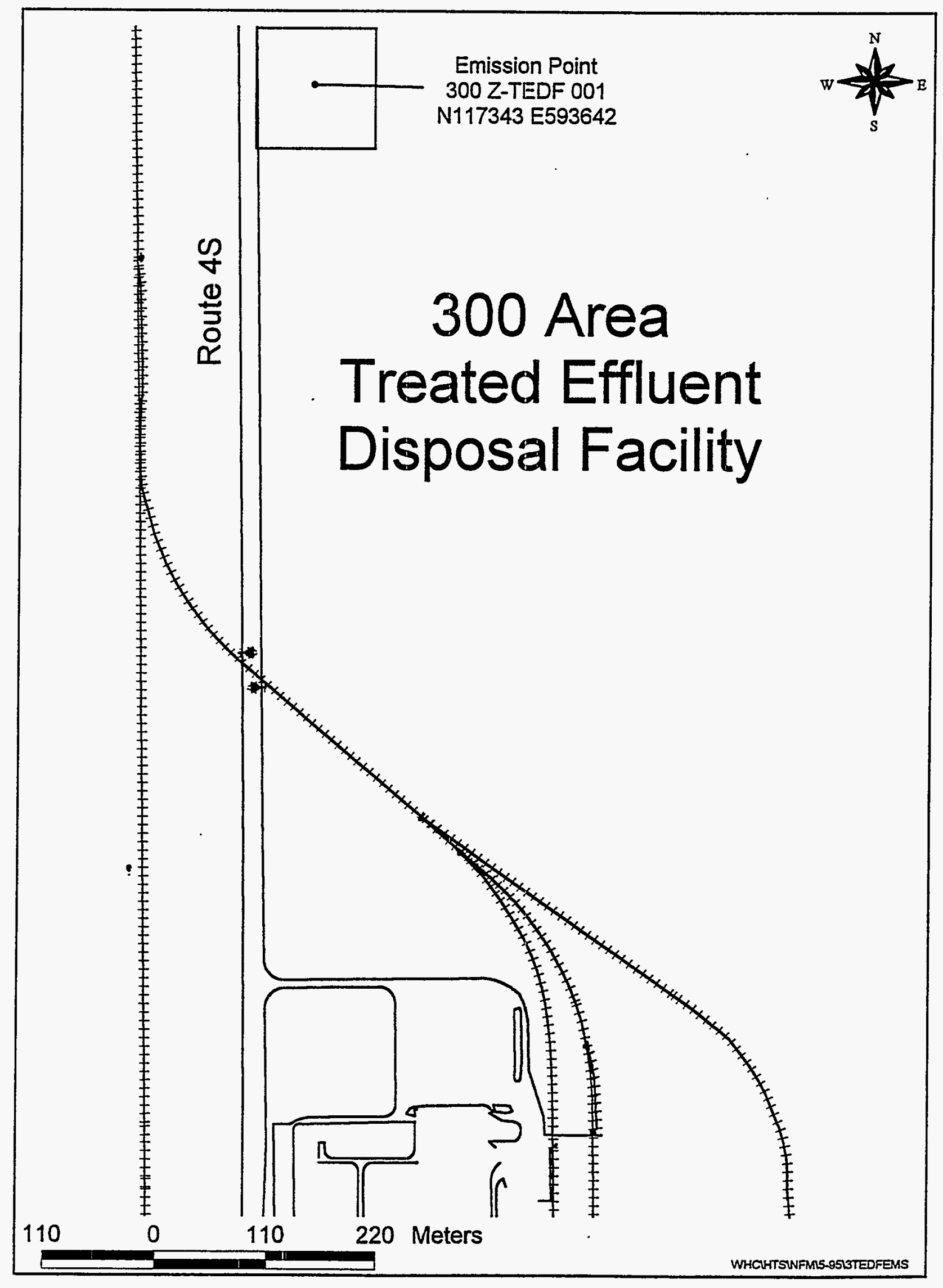




\section{APPENDIX F-2}

384 POWERHOUSE

\section{Chapter I. General Information}

\section{I-B.1. Facility Description}

The 384 Powerhouse is located in the 300 Area. The 384 Powerhouse has six boilers capable of operating. Three of the boilers are coal-fired, and the other three boilers are fired with fuel oil. All of the boilers are used to exclusively generate steam. Steam generated is used in nearby process facilities for heating and process operations. The total rated capacity of the six boilers is 360,000 pounds per hour of steam.

The three coal-fired boilers burn bituminous coal. Each of these boilers is rated for 40,000 pounds per hour of 250 pounds per square inch gauge steam. All three coal-fired boilers did not operate in 1994, nor have the boilers operated since 1989.
1st quarter
0 pounds per hour
2nd quarter
0 pounds per hour
3rd quarter
0 pounds per hour
4th quarter
0 pounds per hour

The three oil-fired boilers burn number six fuel oil. The three boilers are rated for 100,000 , 80,000 , and 60,000 pounds per hour of 300 pounds per square inch gauge steam, respectively. The oil-fired boilers have been in continuous service since 1988. The 1994 average combined steam production rate for these three boilers was:

1st quarter

2nd quarter

3rd quarter

4th quarter
51,031 pounds per hour

15,002 pounds per hour

12,630 pounds per hour

28,738 pounds per hour

\section{I-B.2. Products, Raw Materials, Fuels, Processes}

- Principal products - The only product is 250 to 350 pounds per square inch gauge steam for heating and process operations.

- Process information - Treated boiler feed water is prepared at the powerhouse. Sanitary water is dechlorinate using sodium sulfite before entering sodium zeolite softeners ion exchange units to remove presence of calcium and magnesium salts. Feed water chemistry control is performed using $<4 \%$ potassium hydroxide to prevent scaling and sedimentation. Sodium sulfite is used to scavenge oxygen. Amine is added to each boiler steam outlet line for condensate corrosion control. 
- Raw materials used by processes - There are no raw materials used at the 384 Powerhouse.

- Fuels used by processes - Three of the boilers use bituminous coal; three of the boilers use number six fuel oil.

\section{I-C.1. Emission Points, Controls, Stacks}

The emission point and stack information is found on pages 1 and 2 of the AEI report output. The control information is found on page 7 of the AEI report output.

\section{I-C.2. Facility Process Flow Diagrams}

Attached.

I-C.3. Site Map

Attached.

\section{Chapter II. Regulatory Requirements}

\section{II-A.1. Description of Discharge and Emission Points}

The emission point and stack information is found on pages 1 and 2 of the AEI report output. 
AIR EMISSIONS INVENTORY PERMIT APPLICATION REPORT (UP460)

FACILITY NAME: 384 Facilities and structures

\section{Facility Building List}

\begin{tabular}{|c|c|c|c|c|c|c|c|}
\hline Bldg Id & North & East & Bidg Desc & Custodian(Contractor) & Cust Phone & DOE POC & DOE Phone \\
\hline 384 & 116029 & 593931 & POWER HOUSE BUILDING & CAMP. SAMUEL L. JR. & $376-4019$ & DAVIO G. MURILLO & $373-9179$ \\
\hline
\end{tabular}

\section{Emission Point Listing}

\begin{tabular}{|c|c|c|c|c|c|c|c|c|}
\hline Em Point & Ep Type & North & East & Srce Nbr & Scc Code & Srce Type & & \\
\hline 300 F-384 001 & POINT & 116028 & 593882 & & $\begin{array}{l}10200204 \\
10200204 \\
10200204 \\
10200204\end{array}$ & $\begin{array}{l}\text { EXTERNAL FUEL COMBUSTION } \\
\text { EXTERNAL FUEL COMBUSTION } \\
\text { EXTERNAL FUEL COMBUSTION } \\
\text { SOURCE EMISSIONS ROLL-UP }\end{array}$ & & \\
\hline Stack Configuration & & Diameter $(\mathrm{ft})$ & Length(ft) & idth(ft) & $\operatorname{Area}(s q \mathrm{ft})$ & Exit Veloc(ft/sec) & Ht Abv Grnd(ft) & Ht Abv Struc(f \\
\hline VERTICAL WITHOUT WEA & THER CAP & 7 & & & 38.48 & 337 & 150 & \\
\hline
\end{tabular}

Em Point Ep Type North East Srce Nbr Scc Code

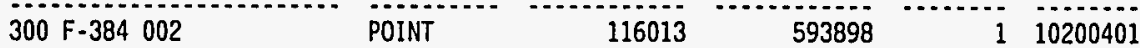
Srce Type Stack Configuration EXTERNAL FUEL COMBUSTION VERTICAL WITH WEATHER CAP Diameter(ft) Length(ft) Width(ft) Area(sq ft) Exit Temp(F) Exit Veloc(ft/sec) Ht Abv Grnd(ft) Ht Abv Struc(ft)

\begin{tabular}{|c|c|}
\hline Em Point & Ер Туре \\
\hline $300 \mathrm{~F}-384005$ & POINT \\
\hline
\end{tabular}

$\begin{array}{rrrr}\text { North } & \text { East Srce } \mathrm{Nbr} \text { Scc Code } \\ 116028 & 593868 & 1 & 10200401\end{array}$

Srce Type VERTICAL WITHOUT WEATHER CAP Diameter(ft) Length(ft) Width(ft) Area(sq ft) Exit Temp(F) Exit Veloc(ft/sec) Ht Abv Grnd(ft) Ht Abv Struc(ft) Eoint 5
5.25 500 Ep Type 300 F -384006

Stack Configuration POINT VERTICAL WITH WEATHER CAP<smiles>[10BH2][14CH3]</smiles>
$300 \mathrm{~J}-384003$ Ep Type FUGITIVE

$\begin{array}{rrr}\text { North } & \text { East Srce Nbr Scc Code } \\ 116013 & 593926 & -\ldots . .\end{array}$

Srce Type

Diameter(ft) Length(ft) Width(ft) Area(sq ft) Exit Temp(F) Exit Veloc(ft/sec) Ht Abv Grnd(ft) Ht Abv Struc(ft)

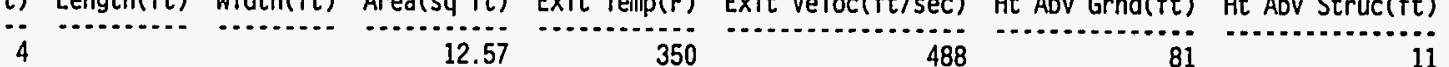

Code Srce Type

Stack Configuration Diameter(ft) Length(ft) Width(ft) Area(sq ft) 0 Exit Temp(F) CRUSHING. HANDLING, AND STORAGE 78
0325

Page \#: 1 of 9 Report Run Date: 27-APR-95 
AIR EMISSIONS INVENTORY PERMIT APPLICATION REPORT (UP460)

FACILITY NAME: 384 Facilities and Structures

\section{Emission Point Listing}

\begin{tabular}{|c|c|c|c|c|c|c|c|c|c|}
\hline Em Point & Ep Type & North & East & Srce Nbr & Sec code & Srce Ty & & & \\
\hline $300 \mathrm{~J}-384004$ & FUGITIVE & 115494 & 594271 & 1 & 50300810 & DISPOSA & $\angle$ AND LANDFILL & & \\
\hline Stack Configuration & & Diameter $(\mathrm{ft})$ & Length(ft) & Width(ft) & $\begin{array}{r}\operatorname{Area}(\mathrm{sq} f \mathrm{ft}) \\
0\end{array}$ & $\begin{array}{r}\text { Exit Temp(F) } \\
78\end{array}$ & $\begin{array}{r}\text { Exit Veloc }(\mathrm{ft} / \mathrm{sec}) \\
.0325\end{array}$ & $\begin{array}{r}H t \text { Abv Grnd(ft) } \\
60\end{array}$ & Ht Abv Struc(ft) \\
\hline
\end{tabular}

\section{Source (Process) Listing}

\begin{tabular}{|c|c|c|c|c|c|c|}
\hline Em Point & Srce Nbr & Srce Type & Hrs Day & Days Week & Wks Year & Summary Process Description \\
\hline $300 \mathrm{~F}-384001$ & 1 & EXTERNAL FUEL COMBUSTION & 24 & 7 & 52 & $\begin{array}{l}\text { INTERNATIONAL COAL-FIRED SPREADER STOKER BOILER, DESIGNATED } \\
\text { AS BOILER } \# 3\end{array}$ \\
\hline Em Point & Srce $\mathrm{Nbr}$ & Srce Type & Hrs Day & Days Week & Wks Year & Summary Process Description \\
\hline $300 F-384001$ & 2 & EXTERNAL FUEL COMBUSTION & 24 & 7 & 52 & $\begin{array}{l}\text { INTERNATIONAL COAL-FIRED SPREADER STOKER BOILER, DESIGNATED } \\
\text { AS BOILER \#4 }\end{array}$ \\
\hline Em Point & Srce Nbr & Srce Type & Hrs Day & Days Week & Wks Year & Summary Process Description \\
\hline $300 F-384001$ & 3 & EXTERNAL FUEL COMBUSTION & 24 & 7 & 52 & $\begin{array}{l}\text { INTERNATIONAL COAL-FIRED SPREADER STOKER BOILER, DESIGNATED } \\
\text { AS BOILER \#5 }\end{array}$ \\
\hline Em Point & Srce Nbr & Srce Type & Hrs Day & Days Week & Wks Year & Summary Process Description \\
\hline $300 F-384001$ & 4 & SOURCE EMISSIONS ROLL-UP & 24 & 7 & 52 & TOTAL EMISSIONS FOR EMISSION POINT F-384-001 \\
\hline Em Point & Srce Nbr & .Srce Type & Hrs Day & Days Week & Whs Year & Summary Process Description \\
\hline $300 F-384002$ & 1 & EXTERNAL FUEL COMBUSTION & 24 & 7 & 52 & $\begin{array}{l}\text { RILEY COAL-FIRED BOILER CONVERTED TO NO. } 6 \text { OIL-FIRED BOILER. } \\
\text { FUEL CONSUMPTION FOR 1992: HEAT CONTENT - } 153000 \text { ASH } \\
\text { CONTENT }-12 \text {, SULFUR CONTENT }-1 \text {. } 75 \text {, HOURLY AVERAGE } 40 . \\
\text { HOURLY AVERAGE FOR } 1993=38 \text {. DESIGNATED AS BOILER \#2 }\end{array}$ \\
\hline Em Point & Srce Nbr & Srce Type & Hrs Day & Days Week & Wks Year & Summary Process Description \\
\hline $300 F-384005$ & 1 & EXTERNAL FUEL COMBUSTION & 24 & 7 & 52 & $\begin{array}{l}\text { PACKAGE BOILER FUEL CONSUMPTION: } 1992 \text { HOURLY AVERAGE = } 100 . \\
\text { I993 HOURLY AVERAGE }=125 . \text { DESIGNATED AS BOILER } \# 1 .\end{array}$ \\
\hline Em Point & Srce $\mathrm{Nbr}$ & Srce Type & Hrs Day & Days Week & Wks Year & Summary Process Description \\
\hline $300 F-384006$ & 1 & EXTERNAL FUEL COMBUSTION & 24 & 7 & 52 & $\begin{array}{l}\text { RILEY COAL-FIRED BOILER CONVERTED TO NO } 6 \text { FUEL OIL FUEL } \\
\text { CONSUMPTION: } 1992 \text { HOURLY AVERAGE - } 92 \text {. } 1993 \text { HOURLY AVERAGE - } \\
\text { 110. DESIGNATED AS BOILER \#6 }\end{array}$ \\
\hline
\end{tabular}


AIR EMISSIONS INVENTORY PERMIT APPLICATION REPORT (UP460)

FACILITY NAME: 384 Facilities and 384 tructures

\section{Source (Process) Listing}

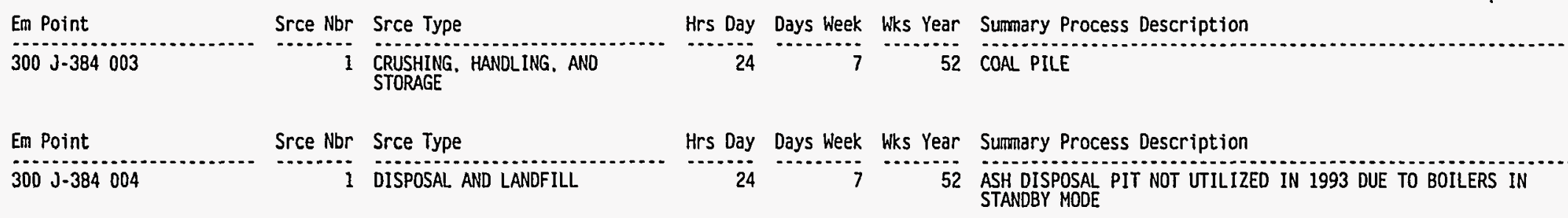

Source Raw Materials Usage

Em Point

Srce Nbr Srce Type

Process Rate Process Uom Material

Fuels Usage by Source Processes

\begin{tabular}{|c|c|c|c|c|c|}
\hline Em Point & Srce Nbr & Srce Type & Qty & Uom & Fuel \\
\hline \multirow[t]{3}{*}{$300 \mathrm{~F} \cdot 384001$} & 1 & EXTERNAL FUEL COMBUSTION & 21000000 & POUND & COAL BITUMINOUS \\
\hline & 2 & EXTERNAL FUEL COMBUSTION & 21000000 & POUND & COAL BITUMINOUS \\
\hline & 3 & EXTERNAL FUEL COMBUSTION & 21000000 & POUND & COAL BITUMINOUS \\
\hline $300 F-384002$ & 1 & EXTERNAL FUEL COMBUSTION & 341875 & GALLONS & NO. 6 HEATING OIL \\
\hline $300 \mathrm{~F}-384005$ & 1 & EXTERNAL FUEL COMBUSTION & 991091 & GALLLONS & NO. 6 HEATING OIL \\
\hline $300 \mathrm{~F}-384006$ & 1 & EXTERNAL FUEL COMBUSTION & 888644 & GALLONS & NO. 6 HEATING OIL \\
\hline
\end{tabular}

Fuels Usage by Control Devices

Em Point

$\mathrm{Ctl} \mathrm{Nbr} \mathrm{Ct} l$ Type

Qty Uom

Fue] 
AIR EMISSIONS INVENTORY PERMIT APPLICATION REPORT (UP460) FACILITY NAME: 384 Facilitíes and Structures

Point Source Criteria Pollutants - Type: Co

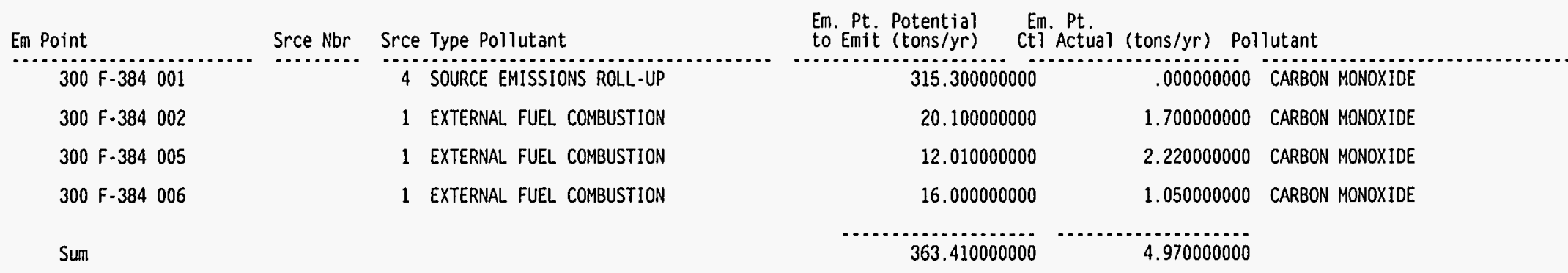

Point Source Criteria Pollutants - Type: LEAD

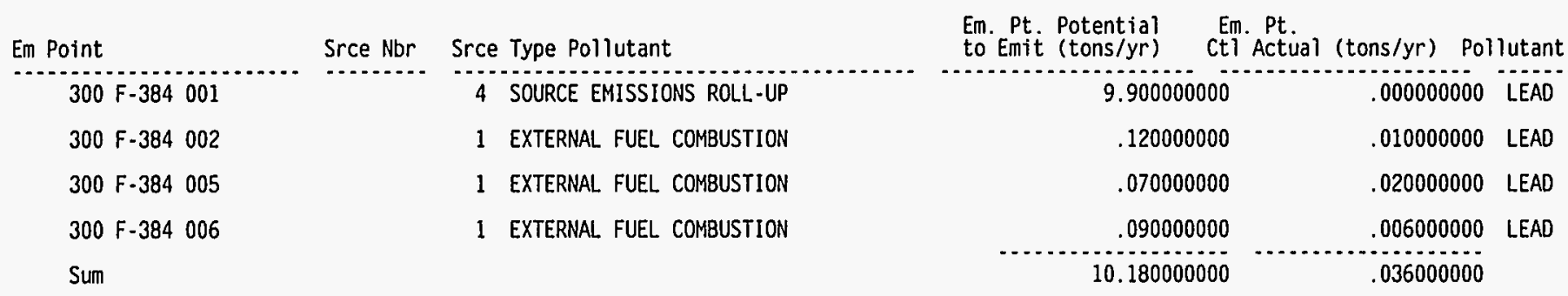

Point Source Criteria Pollutants - Type: NOX

\begin{tabular}{|c|c|c|c|}
\hline Em Point & Srce Nbr & Srce & Type Pollutant \\
\hline 300 F-384 001 & & 4 & SOURCE EMISSIONS ROLL-UP \\
\hline $300 F-384002$ & & 1 & EXTERNAL FUEL COMBUSTION \\
\hline 300 F-384 005 & & 1 & EXTERNAL FUEL COMBUSTION \\
\hline 300 F-384 006 & & 1 & EXTERNAL FUEL COMBUSTION \\
\hline
\end{tabular}

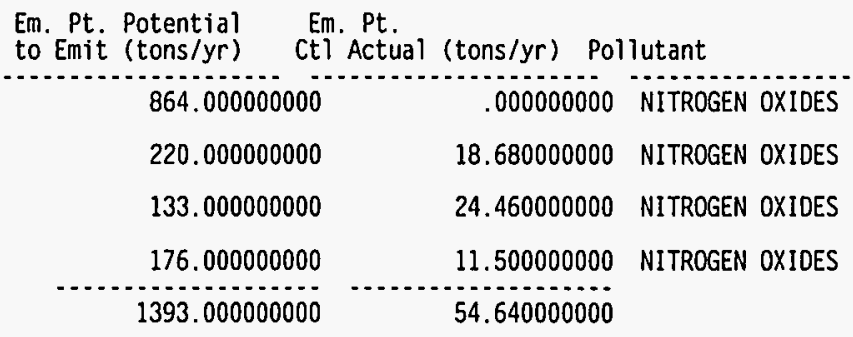

Page \#: 4 of 9 Report Run Date: 27-APR-95 
AIR EMISSIONS INVENTORY PERMIT APPLICATION REPORT (UP460)

FACILITY NAME: 384 Facilities and Structures

Point Source Criteria Pollutants - Type: PM

$\begin{array}{lrl}\text { Em Point } & \text { Srce Nbr } & \text { Srce Type Pollutant } \\ 300 \mathrm{~F}-384001 & 4 & \text { SOURCE EMISSIONS ROLL-UP } \\ 300 \mathrm{~F}-384002 & 1 \text { EXTERNAL FUEL COMBUSTION } \\ 300 \mathrm{~F}-384005 & 1 \text { EXTERNAL FUEL COMBUSTION } \\ 300 \mathrm{~F}-384006 & 1 \text { EXTERNAL FUEL COMBUSTION }\end{array}$

Sum

Point Source Criteria Pollutants - Type: PM10

Em Point
$300 \mathrm{~F}-384001$
Sum

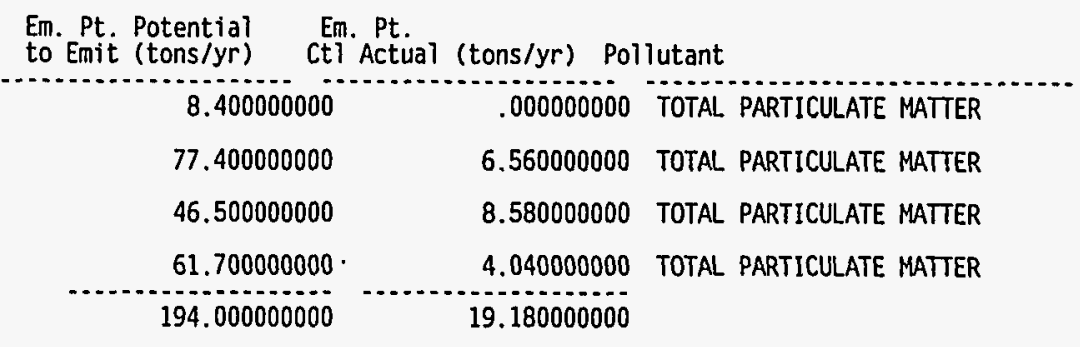

Point Source Criteria Pollutants - Type: Sox

$\begin{array}{ll}\text { Em Point } & \text { Srce Nbr Srce Type Pollutant } \\ 300 \mathrm{~F}-384001 & 4 \text { SOURCE EMISSIONS ROLL-UP } \\ 300 \mathrm{~F}-384002 & 1 \text { EXTERNAL FUEL COMBUSTION } \\ 300 \mathrm{~F}-384005 & 1 \text { EXTERNAL FUEL COMBUSTION } \\ 300 \mathrm{~F}-384006 & 1 \text { EXTERNAL FUEL COMBUSTION }\end{array}$

Sum

Em. Pt. Potential Em. Pt.
to Emit (tons/yr) Ctl Actual (tons/yr) Pollutant

$\begin{array}{cc}1.80000000 & .00000000 \\ 1.800000000 & .000000000\end{array}$

$1.800000000 \quad .000000000$

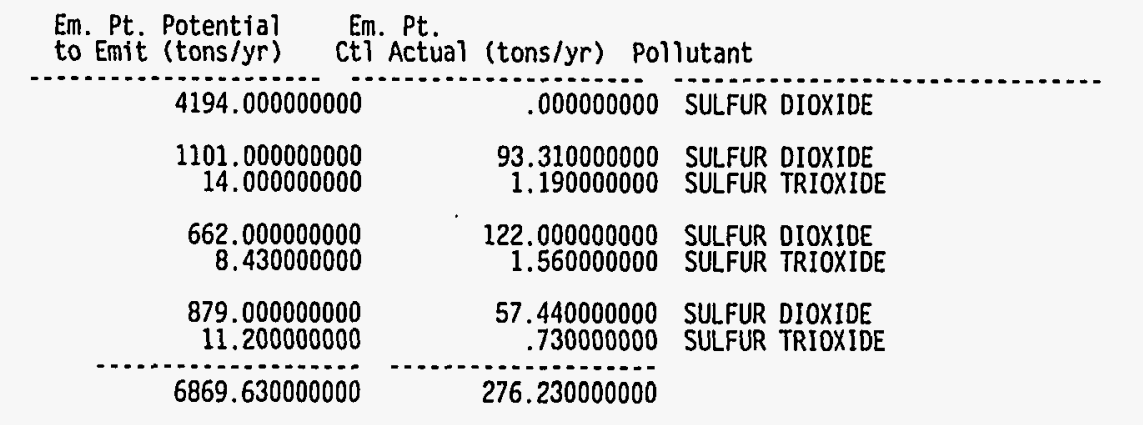


AIR EMISSIONS INVENTORY PERMIT APPLICATION REPORT (UP460)

FACILITY ID : 384
FACILITY NAME: 384 Factlities and Structures

Point Source Criteria Pollutants - Type: VOC

Em Point

300 F -384001

$300 F \cdot 384002$

300 F-384 005

300 F-384 006

Sum

Point Source Emissions - Radiological Pollutants

Em Point

Srce Nbr Srce Type Pollutant

Em. Pt. Potential
to Emit (Ci/yr)

Em. Pt.

Pollutant

Point Source Emissions - HAP Pollutants

Em Point

Srce Nbr Srce Type Pollutant

4 SOURCE EMISSIONS ROLL-UP
300 F -384001 -......
Em. Pt. Potential Em. Pt.
to Emit (tons/yr) Ctl Actual (tons/yr) Pollutant 00000000 FORMALDEHYDE 000000000 NON-METHANE VOLATILE ORGANICS 3. 30000000 020000000 FORMALDEHYDE NON METHANE VOLATILE ORGANICS .250000000 1.170000000
33.600000000 2.850000000 POLYCYCLIC ORGANIC MATTER

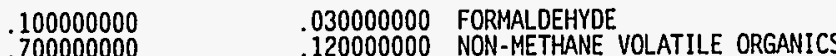
20.70000000

3.740000000 POLYCYCLIC ORGANIC MATTER

200000000

.013000000 FORMALDEHYDE

.200000000 26.900000000

91.520000000 8.693000000
Em. Pt. Potential - Em. Pt.
to Emit (tons/yr) - Ctl Actual (tons/yr) Pollutant

$\begin{array}{rll}10.50000000 & .000000000 & \text { ARSENIC } \\ 1.500000000 & .000000000 & \text { BERYLLIUM } \\ .900000000 & .00000000 & \text { CADMIUM } \\ 30.300000000 & .00000000 & \text { CHROMIUM } \\ 4.20000000 & .00000000 & \text { FORMLLDEHYDE } \\ 9.90000000 & .000000000 & \text { LEAD } \\ 42.000000000 & .000000000 & \text { MANGANESE } \\ .300000000 & .00000000 & \text { MERCURY } \\ 24.90000000 & .00000000 & \text { NICEEL } \\ 3.900000000 & .000000000 & \text { SELENIUM }\end{array}$


AIR EMISSIONS INVENTORY PERMIT APPLICATION REPORT (UP460)

FACILITY NAME: 384 Facilities and Structures

Point Source Emissions - HAP Pollutants

\begin{tabular}{|c|c|c|c|c|c|}
\hline Em Point & Srce $\mathrm{Nbr}$ & Srce Type Pollutant & $\begin{array}{l}\text { Em. Pt. Potential } \\
\text { to Emit (tons/yr) }\end{array}$ & $\begin{array}{l}\text { Em. Pt. } \\
\text { Cti Actual (tons/yr) } P\end{array}$ & Pollutant \\
\hline $300 F-384002$ & & 1 EXTERNAL FUEL COMBUSTION & $\begin{array}{r}.030000000 \\
.070000000 \\
.003000000 \\
.130000000 \\
.080000000 \\
.070000000 \\
.250000000 \\
.120000000 \\
.050000000 \\
.020000000 \\
1.430000000 \\
33.600000000 \\
.020000000\end{array}$ & $\begin{array}{l}.002000000 \\
.006000000 \\
.000200000 \\
.010000000 \\
.007000000 \\
.006000000 \\
.020000000 \\
.010000000 \\
.004000000 \\
.002000000 \\
.120000000 \\
2.850000000 \\
.002000000\end{array}$ & $\begin{array}{ll}0 & \text { ANTIMONY } \\
0 & \text { ARSENIC } \\
0 & \text { BERYLLIUM } \\
0 & \text { CADMIUM } \\
0 & \text { CHROMIUM } \\
0 & \text { COBALT } \\
0 & \text { FORMALDEHYOE } \\
0 & \text { LEAD } \\
0 & \text { MANGANESE } \\
0 & \text { MERCURY } \\
0 & \text { NICKEL } \\
0 & \text { POLYCYCLIC ORGANIC MATTER } \\
0 & \text { SELENIUM }\end{array}$ \\
\hline $300 F-384005$ & & 1 EXTERNAL. FUEL COMBUSTION & $\begin{array}{r}.020000000 \\
.040000000 \\
.002000000 \\
.080000000 \\
.050000000 \\
.040000000 \\
.100000000 \\
.070000000 \\
.030000000 \\
.010000000 \\
.860000000 \\
20.200000000 \\
.010000000\end{array}$ & $\begin{array}{l}.003000000 \\
.010000000 \\
.000300000 \\
.020000000 \\
.010000000 \\
.010000000 \\
.030000000 \\
.020000000 \\
.01000000 \\
.002000000 \\
.200000000 \\
3.740000000 \\
.003000000\end{array}$ & $\begin{array}{ll}0 & \text { ANTIMONY } \\
0 & \text { ARSENIC, } \\
0 & \text { BERYLIUM } \\
0 & \text { CAOMIUM } \\
0 & \text { CHROMIUM } \\
0 & \text { COBALT } \\
0 & \text { FORMALDEHYD } \\
0 & \text { LEAD } \\
0 & \text { MANGANESE } \\
0 & \text { MERCURY } \\
0 & \text { NICKEL } \\
0 & \text { POYYCYCLIC ORGANIC MATTER } \\
0 & \text { SELENIUM }\end{array}$ \\
\hline $300 F-384006$ & & 1 EXTERNAL FUEL COMBUSTION & $\begin{array}{r}.020000000 \\
.060000000 \\
.002000000 \\
.100000000 \\
.060000000 \\
.060000000 \\
.200000000 \\
.090000000 \\
.040000000 \\
.020000000 \\
1.140000000 \\
26.900000000 \\
.020000000\end{array}$ & $\begin{array}{l}.002000000 \\
.00400000 \\
.000100000 \\
.007000000 \\
.004000000 \\
.004000000 \\
.013000000 \\
.00600000 \\
.002000000 \\
.001000000 \\
.075000000 \\
1.760000000 \\
.001000000\end{array}$ & $\begin{array}{l}\text { ANTIMONY } \\
\text { ARSENIC } \\
\text { BERYLIUM } \\
\text { CADMIUM } \\
\text { CHROMIUM } \\
\text { COBALT } \\
\text { FORMALDEHYDE } \\
\text { LEAD } \\
\text { MANGANESE } \\
\text { MERCURY } \\
\text { NICKEL } \\
\text { POLYCYCLIC ORGANIC MATTER } \\
\text { SELENIUM }\end{array}$ \\
\hline Sum & & & 214.497000000 & 8.976600000 & \\
\hline
\end{tabular}

Point Source Emissions - Uncategorized Pollutants

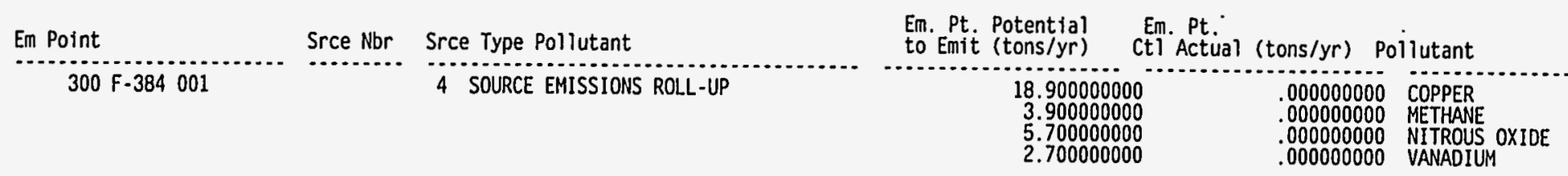

Page \#: 7 of $9 \quad$ Report Run Date: 27-APR-95 
AIR EMISSIONS INVENTORY PERMIT APPLICATION REPORT (UP460) FACILITY NAME: 384 Facilities and structures

Point Source Emissions - Uncategorized Pollutants

\begin{tabular}{|c|c|c|}
\hline Em Point & Srce Nbr & Srce Type Pollutant \\
\hline $300 \mathrm{~F}-384002$ & & 1 EXTERNAL FUEL COMBUSTION \\
\hline $300 F-384005$ & & 1 EXTERNAL FUEL COMBUSTION \\
\hline $300 \mathrm{~F}-384006$ & & 1 EXTERNAL. FUEL COMBUSTION \\
\hline
\end{tabular}

Sum

Em. Pt. Potential Em. Pt.

to Emit (tons/yr) Ct) Actual (tons/yr) Pollutant

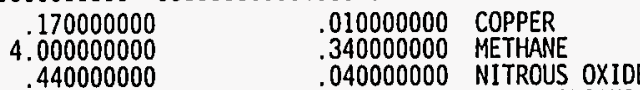

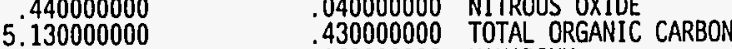

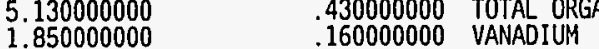

$.100000000 \quad .020000000$ COPPER

$2.410000000 \quad .440000000$ METHANE

$3.080000000 \quad .570000000$ TOTAL ORGANIC CARBON

$1.110000000 \quad .210000000$ VANADIUM

$300 F-384006$

1 EXTERNAL FUEL COMBUSTION

.140000000

.1400000000
3.200000000
.350000000
4.100000000
1.480000000
59.020000000

.010000000 COPPER

.210000000 METHANE

.020000000 NITROUS OXIDE

140000000 VANADIUM

$59.020000000 \quad 2.920000000$

Fugitive Source Criteria Pollutants - Type: PM

\begin{tabular}{|c|c|c|c|}
\hline Em Point & ' & Srce Nbr & Srce Type Pollutant \\
\hline & & & 1 CRUSHING. HANDLING, AND STORAGE \\
\hline
\end{tabular}

Em. Pt. Potential Em. Pt.
to Emit (tons/yr) Ctl Actual (tons/yr) Pollutant

$\begin{array}{ccc}200000000 & .200000000 & \text { TOTAL PARTICULATE MATTER } \\ .200000000 & .200000000\end{array}$

Fugitive Source Emissions - Radiological Pollutants

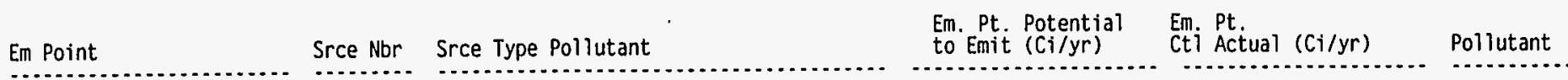

Fugitive Source Emissions - HAP Pollutants

Em Point

Srce Nbr Srce Type Pollutant

Em. Pt. Potential Em. Pt.

(tons/yr) Ctl Actual (tons/yr) pollutant

Sum 
AIR EMISSIONS INVENTORY PERMIT APPLICATION REPORT (UP460)

FACILITY NAME: 384 Facilities and Structures

Fugitive Source Emissions - Uncategorized Pollutants

Em Point Srce Nbr Srce Type Pollutant

Em. Pt. Potential Em. Pt.
to Emit (tons/yr) Ctl Actual (tons/yr) Pollutant

Em Point

Fugitive Source Emissions - Uncạtegorized Pollutants

Em Point

Srce Nbr Srce Type Pollutant

Sum

Insignificant Source Emissions - Radiological Pollutants

Em Point

Srce Nbr Srce Type Pollutant

Em. Pt. Potential

Em. Pt.

Em. Pt. Potential Em. Pt.

to Emit (tons/yr) Ctl Actual (tons/yr) Pollutant

Insignificant Source Emissions - HAP Pollutants

Em Point Srce Nbr Srce Type Pollutant

Sum

Insignificant Source Emissions - Uncategorized Pollutants

Em Point

Srce Nbr Srce Type Pollutant

Em. Pt. Potential Em. Pt.
to Emit (tons/yr) Ctl Actual (tons/yr) Pollutant

Sum

Control Device Listing

Em Point Control Equipment Code

$300 \mathrm{~F}-384001$

016

Em. Pt. Potential Em. Pt.
to Emit (tons/yr) Ctl Actual (tons/yr) Pollutant

..............................................

Page \#: 9 of 9

Report Run Date: 27-APR-95 


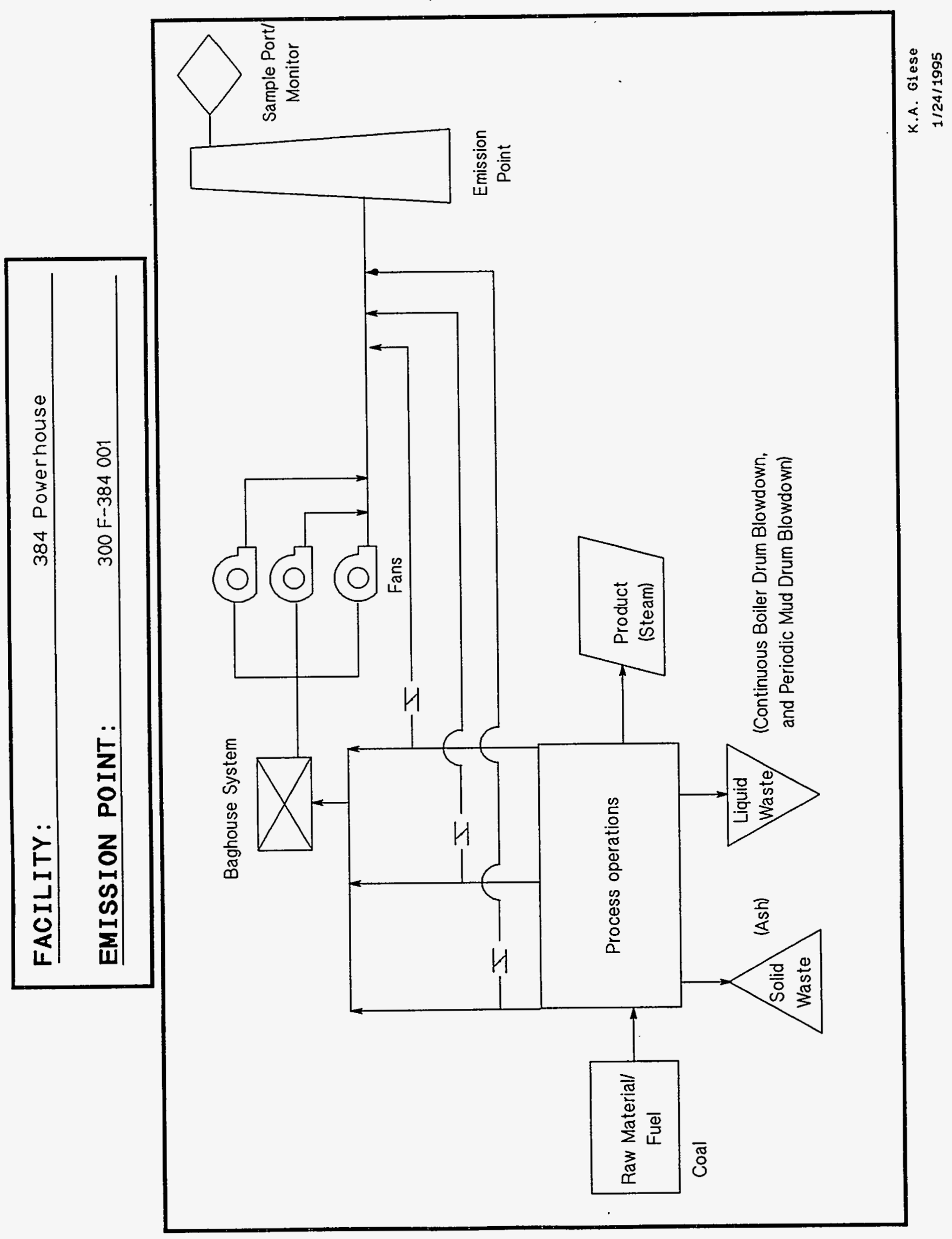




\begin{tabular}{ll}
\hline FACILITY : \\
\hline EMISSION POINT: & 384 Powerhouse \\
\hline
\end{tabular}

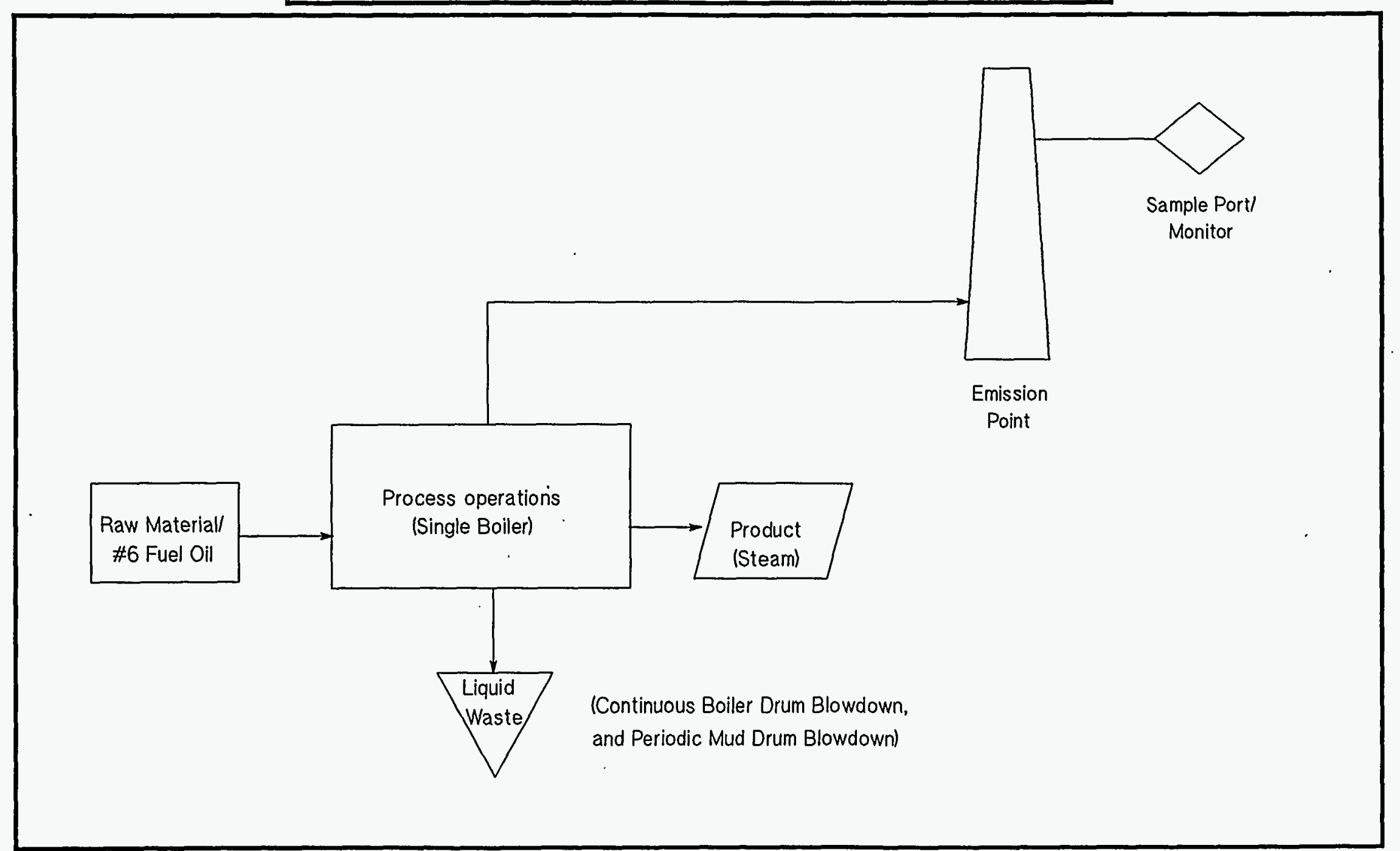




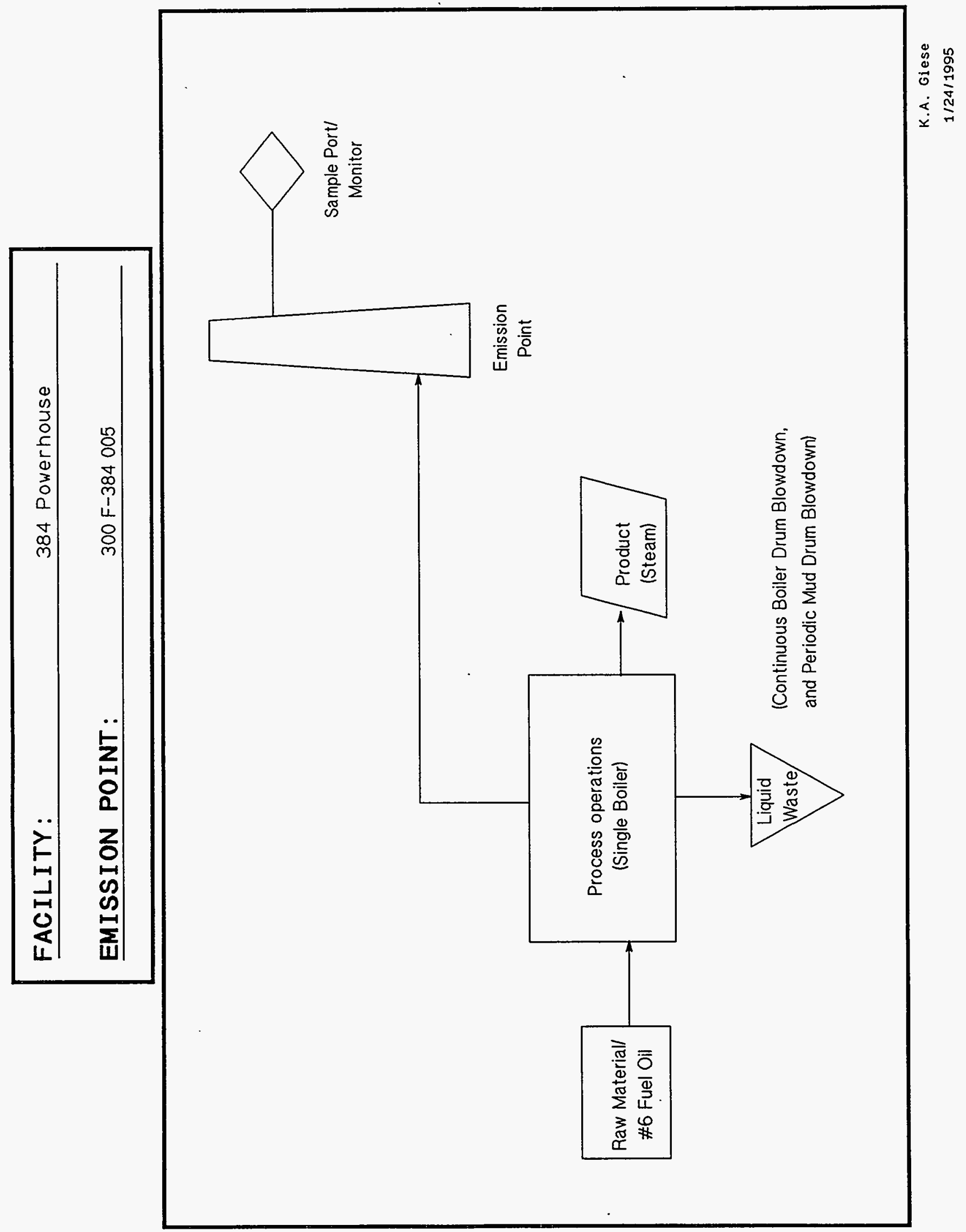




\section{FACILITY:}

EMISSION POINT: $300 F-384006$

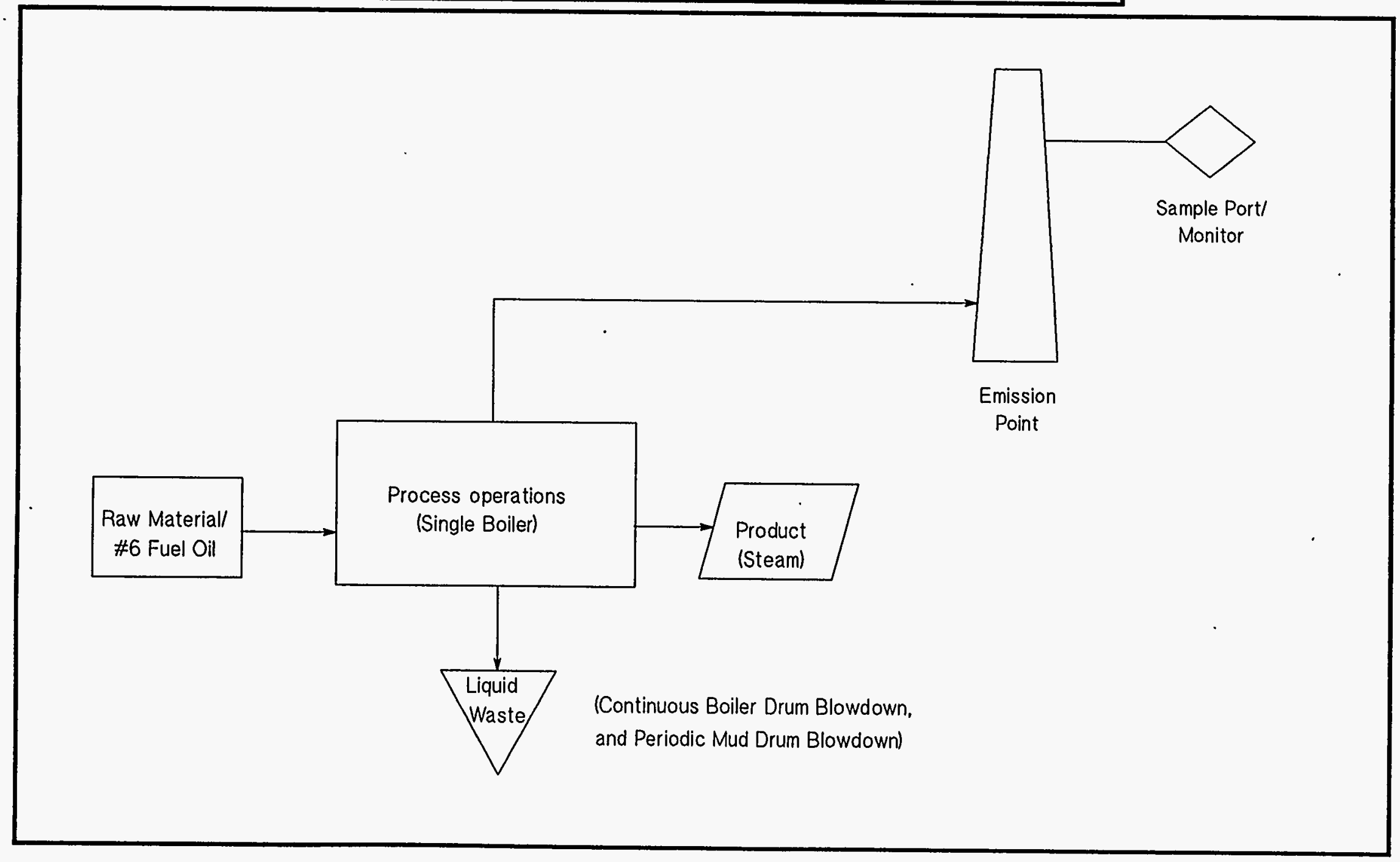




\begin{tabular}{|c|c|}
\hline FACILITY: & 384 Powerhouse \\
\hline EMISSION POINT: & $300 \mathrm{~J}-384003$ \\
\hline
\end{tabular}

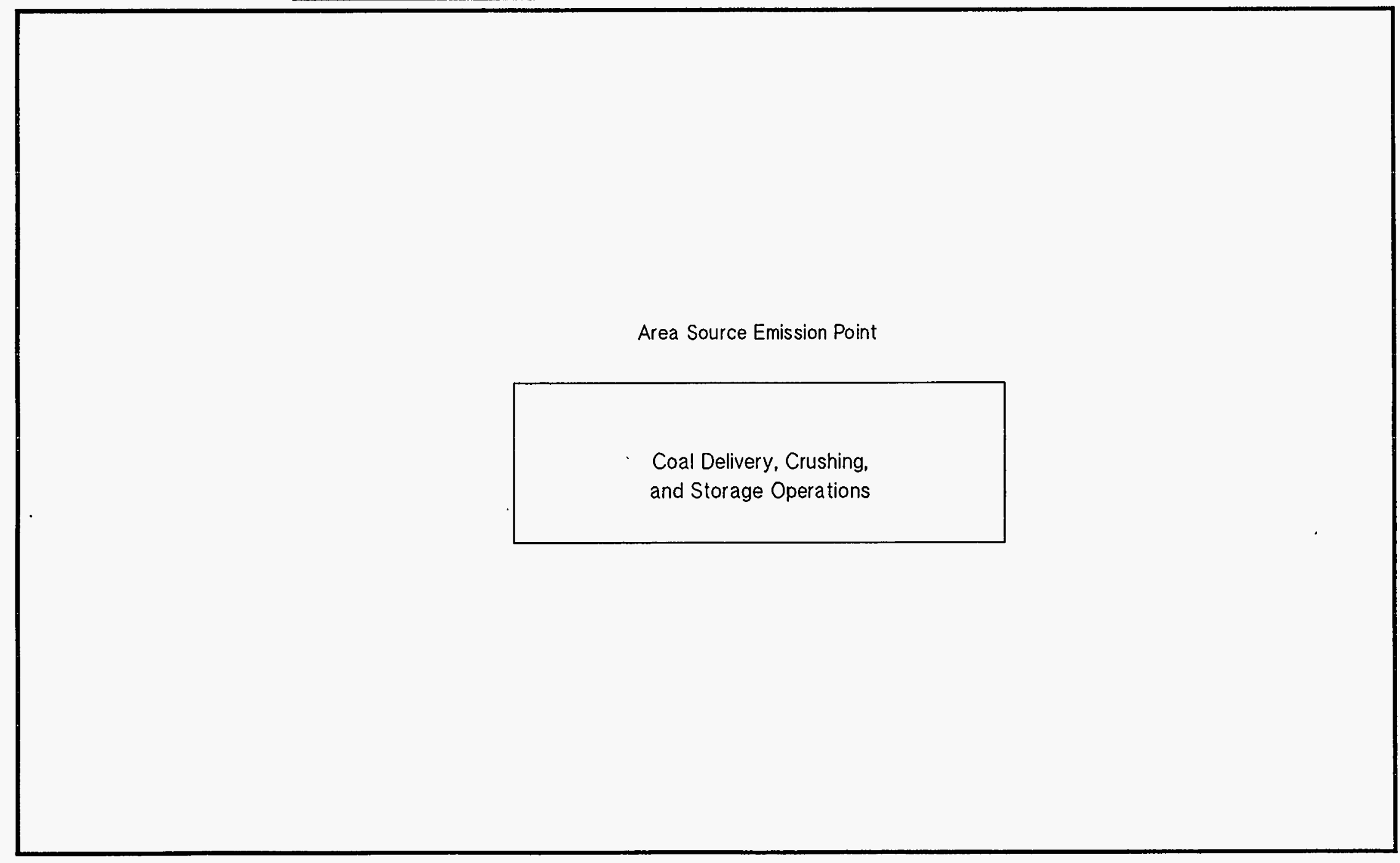




\begin{tabular}{|ll|}
\hline FACILITY: & 384 Powerhouse \\
\hline EMISSION POINT: & $300 \mathrm{~J}-384004$ \\
\hline
\end{tabular}

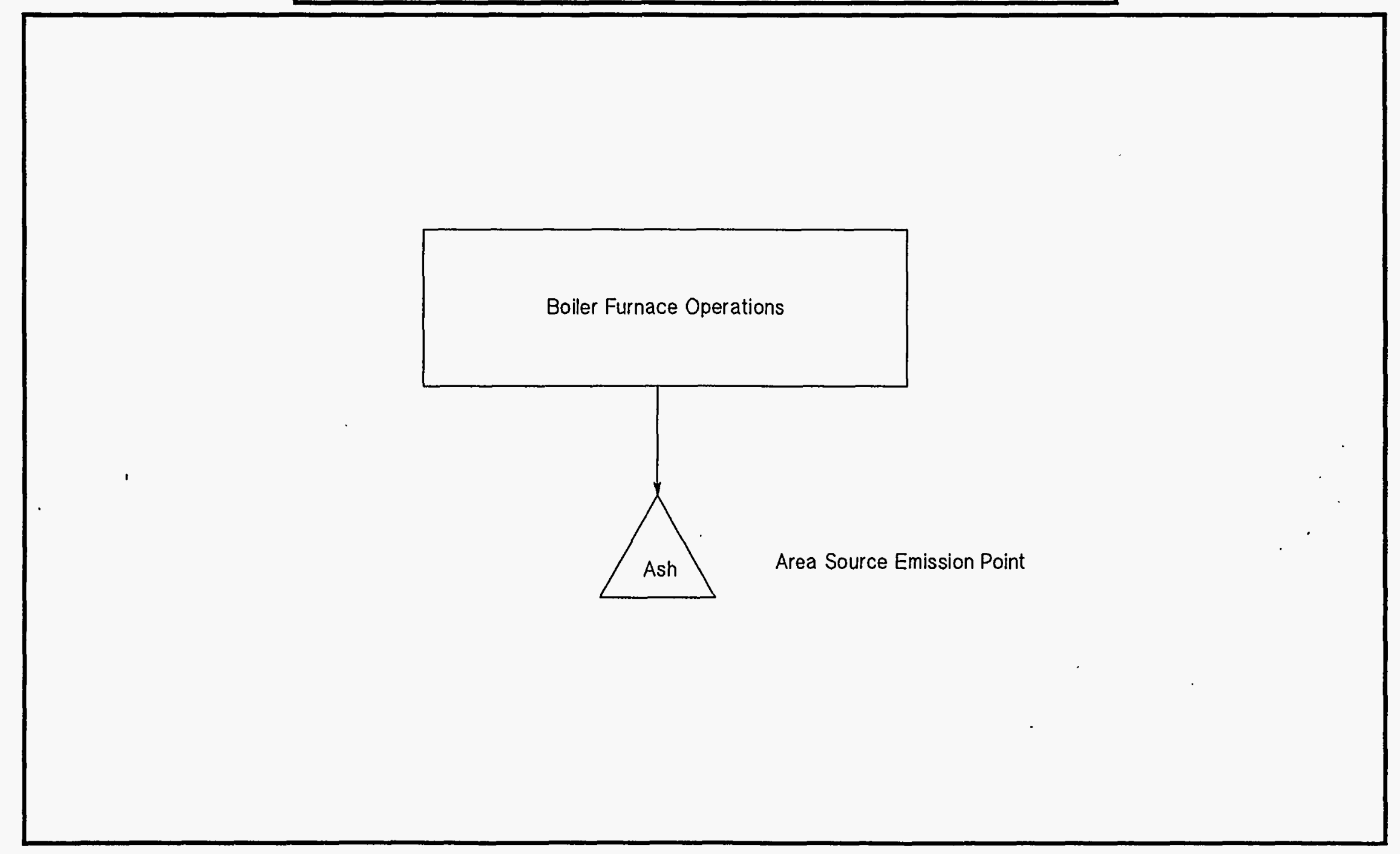




\section{4 \\ Powerhouse Area}

384

Building Corner

N116028 E593902
384

Emission Point 300 F-384 001 N116051 E593906

Emission Point 300 F-384 005

N116028 E593868

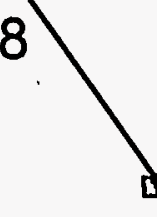
Building Corner N116020 E593931

Emission Point $300 \mathrm{~J}-384003$ N116010 E593933

Emission Point 300 F-384 $002 \quad 300$ F-384 006

N116013 E593898 N116013 E593926 


\section{Area Ash Pits Emission Points}
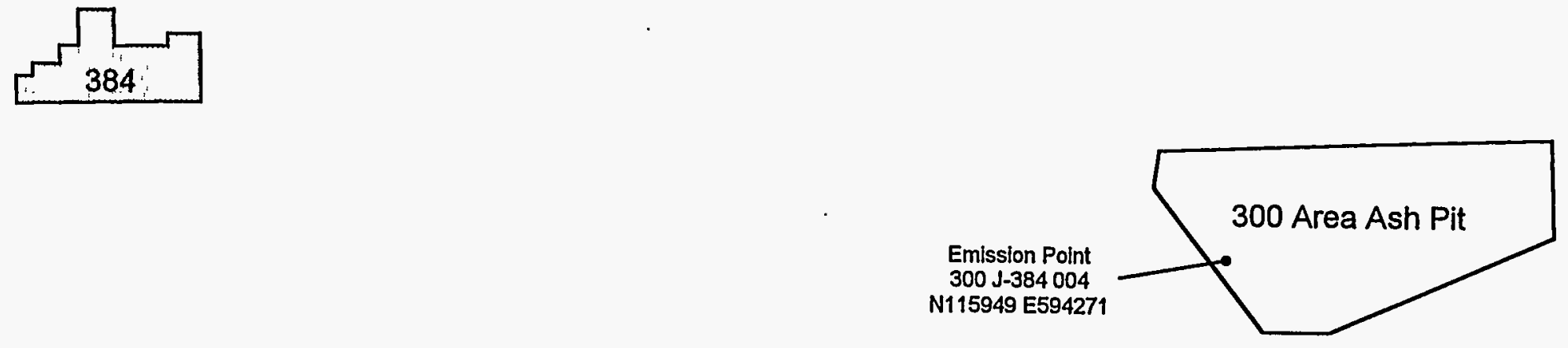


\title{
APPENDIX F-3
}

\section{AREA RESEARCH AND DEVELOPMENT FACILITIES}

\author{
Chapter I. General Information
}

\section{I-B.1.1 324 Facility Description}

The Waste Technology Engineering Laboratory (324 Facility) provides office and laboratory space for Pacific Northwest Laboratory (PNL) scientific and engineering staff conducting multi-disciplinary research in the areas of waste characterization and immobilization, waste remediation and cleanup development, biomass research, spent fuel characterization, tritium development, and cesium chloride encapsulation. The 324 Facility was designed with the necessary safety features to allow radioactive and hazardous material use in support of these activities. The 324 Facility is situated directly east of the 308 Building, and west of the 336 Building.

The 324 Facility consists of four levels (a basement and three floors) and has five distinct operating areas: radioactive hot cells with associated operating galleries and radioactive liquid storage tanks in concrete vaults, radiological engineering development laboratories, SNM storage and assay, nonradiological engineering development laboratories, and administrative offices.

PNL conducts melter testing activities with several small- and pilot-scale melter test systems located in the 324 Building to support the design of the low- and high-level waste vitrification processes for the Hanford Site. This testing also supports future operations, processability, permitting, glass quality/waste form qualification, and safety activities for vitrification of low- and high-level waste.

The supporting subsystems, feed preparation and off-gas treatment, are common to all existing melters; consequently, current testing is limited to the exclusive operation of any one system at a time. The largest pilot-scale melter being tested is a high-temperature melter (HTM) having a melt surface area equal to $80 \%$ of the anticipated scale of a high-level vitrification plant HTM melter. The HTM operations and associated emissions bound all melter operations and are described below.

The HTM process flow is depicted on the process flow diagram for stack EP-324-02-S. Waste simulant is prepared for melter processing by a feed preparation test system and then transferred to the HTM to process the waste into a glass product. Gases produced by the melter are scrubbed by an off-gas system to remove particulate matter and noxious gases. Scrub and condensate solution from the off-gas system are processed in a recycle system and stored for processing in later melter tests or disposal. Melter feed is made-up and concentrated in a feed preparation tank and then transferred to a melter feed tank. The melter feed is transferred from in a feed loop, which allows a portion of the feed to be 
processed by the melter while the rest is recycled back to the feed tank. The HTM vitrifies the waste slurry into glass while the off-gas produced from the process is scrubbed of particulates and noxious gases by a wet scrubber and high efficiency mist eliminator. Condensate from the off-gas is filtered by a solids separator, processed in the recycle treatment system, and stored for reuse in future melter runs or disposal. The melter off-gas is then passed through a high efficiency metal fiber (HEMF) particulate filter and a standard HEPA filter prior to leaving the 324 Building.

The emissions from the melter testing program originate during the formatting process and during melter operation. These include significant emissions of $\mathrm{NO}_{\mathrm{x}}\left(\mathrm{NO}\right.$ and $\mathrm{NO}_{2}$ ) and trace quantities of other materials that are several orders of magnitude below the WAC 173401 thresholds. The actual constituents in the off-gas vary depending on minor changes in raw material composition; however, due to the efficiency of the control technology, emissions other than $\mathrm{NO}_{x}$ are insignificant. The current melter testing program involves two large-scale demonstrations per year, which, based on laboratory data, results in the emission of approximately 10,000 pounds of $\mathrm{NO}_{x}$ per year.

\section{I-B.1.2 325 Facility Description}

The Applied Chemistry Laboratory (325 Facility) houses radiochemistry research, radioanalytical service, radiochemical process development, and mixed waste treatment activities. The laboratories and specialized facilities enable work ranging from that with nonradioactive materials to work with picogram to kilogram quantities of fissionable materials and up to megacurie quantities of other radionuclides. The special facilities include two-shielded hot cell areas that provide for process development or analytical chemistry work with highly radioactive materials and a waste treatment unit for processing mixed, low-level, and transuranic wastes generated by PNL activities.

The 325 Facility is a rectangular-shaped, welded metal frame structure with insulated metal siding erected on reinforced concrete footings, walls, and slabs. Exterior walls are constructed of insulated 1-1/2 inch fiberglass sandwich metal panels. The flat roof is a metal deck with a built-in gravel surface insulated with 1 inch board. Windows are single pane and covered with storm windows.

The building consists of (1) a central portion containing general purpose laboratories modified for low-level radiochemical work by providing special ventilation and work enclosures; (2) a south (front) wing containing office space, a machine and electrical shop, locker rooms, and a lunch room; (3) east and west wings provided with shielded enclosures with remote manipulators for high-exposure radiochemical work; and (4) a filter addition area that provides a final testable HEPA filtration stage for ventilation exhaust air.

The central portion of the building contains laboratories and offices; the laboratories contain hoods and gloveboxes for working safely with radioactive and hazardous materials. Offices also are located on the second floor and on a mezzanine area between the first floor and the 
basement. The basement contains several laboratories, in addition to a portion of the ventilation and waste handling systems. Instrument rooms and the hazardous waste treatment unit have refrigerated air conditioning for temperature and humidity control.

The main heating and ventilation system located on the second floor has four supply fans with heating coils, spray chambers, and filters. Four exhaust fans and a filter room are located in the filter addition annex northwest of the main building. Three fans operate continuously; one is a standby. Pressure barriers for contamination control are achieved by supplying air to offices and corridors, and exhausting it from the laboratories through HEPA filters to a 55-foot-high stack.

Two 50-ton refrigerated air conditioning systems serve special instrumentation rooms located in the northwest corner and north-central portion of the building. Smaller refrigerated air conditioning units serve other special purpose laboratories, as well.

Utility services include steam; hot, cold, process, sanitary, deionized, and distilled water; compressed air; laboratory process vacuum; and contaminated and uncontaminated drains.

\section{I-B.1.3 327 Facility Description}

The Post-Irradiation Laboratory (327 Facility) provides office and laboratory space for PNL scientific and engineering staff conducting multi-disciplinary research in the areas of postirradiated fuels and structural materials. The facility is designed to accommodate the use of radioactive and hazardous materials in the conduct of these activities.

The 327 Facility is a 25,200 square foot single story building with a partial basement and there is 10,000 square feet of laboratory and work areas, 2,100 square feet of offices, and 2,400 square feet of storage areas, along with 10,500 square feet of common areas containing ventilation and auxiliary equipment.

Supply ventilation units are located in the basement, the west end of the canyon in the transfer (truck lock) and storage area, and the northwest storage area of the canyon. The 327 Facility is provided with continuous monitors for air activity, radiation, and criticality. The hot exhaust system takes air from all high-contamination areas such as shielded cells, fume hoods, and dry storage, and filters the air through a roughing filter and two HEPA filters before the effluent air is released.

Stack effluent regulatory monitoring consists of weekly collection of filter samples that are subject to radiological analysis.

\section{I-B.1.4 3720 Facility Description}

The Materials Science Laboratory (3720 Facility) provides office and laboratory space for PNL scientific and engineering staff conducting multi-disciplinary research in the areas of 
materials characterization and testing and waste management. The facility is designed to accommodate using radioactive and hazardous materials to conduct these activities.

The 3720 Facility was built in 1959 and is located in the northern most region of the 300 Area bounded on the north by the 300 Area fence. The 24,412 square foot building is an all-metal frame construction erected on concrete foundations, footings, and floor slab. The building includes 31 offices $(4,188$ square feet), 27 laboratories $(9,302$ square feet), shop space (352 square feet), work and storage space (369 and 308 square feet), respectively, and common space $(9,893$ square feet). The building also contains a 24 foot $\mathrm{x} 109$ foot basement area at the southwest corner.

A one-story concrete block annex (48 feet 2 inch $x 40$ foot 2 inch) was added to the north end of the building in 1980; this addition is, essentially, independent of the rest of the building except for electrical service and air conditioning supply. The annex has its own ventilation exhaust system.

The 3720 Facility is completely air conditioned and fully protected from fire by a wet-pipe sprinkler system. Air conditioning units are located on the southeast side and the north end of the building. Air conditioning chillers are located in the basement. All laboratory hoods containing radioactive materials are double HEPA filtered to minimize atmospheric emissions released through three exhaust stacks. The building is divided into three ventilation regions. The main laboratory building is divided into a north and a south region, each served by an exhaust stack (EP-3720-01-S and EP-3720-03-S, respectively). The third region, the annex laboratory, also has an exhaust stack (EP-3720-02-S).

Stack effluent monitoring consists of a biweekly collection of filters that are analyzed for alpha and beta radioactivity.

\section{I-B.1.5 In Situ Vitrification Operations Description}

In Situ Vitrification (ISV) is a thermal treatment process that converts contaminated soil into a chemically inert and stable glass and crystalline product. PNL uses mobile pilot-scale and large-scale systems for applying this technology at Hanford waste sites. PNL also operates laboratory bench-scale and engineering-scale systems in the 324 Building for ISV research and development purposes.

The potential ISV process emissions vary depending on the radioactive and chemical contaminants present at the waste site selected for treatment. In general, historical performance of the emission control systems has been exceptional, resulting in insignificant emissions of hazardous materials. Future demonstrations of the ISV process will determine potential emissions and notification requirements applicable to the application of this technology on the Hanford Site. 
The general ISV process uses four electrodes which are inserted into the ground to the desired treatment depth. Because soil is not electrically conductive once its moisture is driven off, a conductive mixture of flaked graphite and glass frit is placed among the electrodes to act as a conductive starter path. An electrical current is established in the starter path by applying an electrical potential to the electrodes. The resultant power heats the starter path and surrounding soil up to $2000^{\circ} \mathrm{C}$, well above the typical initial melting temperature of soil $\left(1100^{\circ} \mathrm{C}\right.$ to $\left.1400{ }^{\circ} \mathrm{C}\right)$. The graphite starter path is eventually consumed by oxidation, and the current is transferred to the molten soil, which is now electrically conductive. As the molten zone grows, it incorporates nonvolatile hazardous elements, such as heavy metals, and destroys organic compounds by pyrolysis. The pyrolyzed by-products migrate to the surface of the vitrified zone where they burn in the presence of oxygen. A stainless steel hood placed over the area being vitrified directs the gaseous effluent to an offgas treatment system.

\section{LARGE-SCALE SYSTEM}

Multiple stage off-gas treatment equipment processes the gaseous effluent. This is depicted in the emission point diagram labeled EP-ISV-01-V. The off-gas system has two parallel trains. The off-gas sytem is fitted with a gas cooler, two quench towers, hydrosonic tandem nozzle scrubbers, two heat exchangers, three vane-separated mist eliminators, two scrub solution tanks, two pumps, a condenser, a heater, and high-efficiency particulate air (HEPA) filters. The quenchers remove $90 \%$ of the particulate and semi-volatile radionuclides; the scrubbers are designed to remove $90 \%$ of particulate greater than $0.5 \mu \mathrm{m}$ in diameter and condenses the remaining semi-volatile components. The off-gas system is continuously monitored for radionuclides and chemicals at several locations in the process train. The process effluent stack is monitored continuously by a continuous air monitor unit. In addition, the process off-gas system contains environmental monitors that detect $\mathrm{No}_{x}, \mathrm{So}_{x}$, $\mathrm{CO}$ and $\mathrm{O}_{2}$.

\section{PILOT-SCALE SYSTEM}

The pilot-scale off-gas system is depicted in the emission point diagram labeled EP-ISV-02$V$. The off gas passes through a venturi-ejector scrubber and separator, Hydro-Sonic scrubber, separator, condenser, another separator, heater, two stages of HEPA filtration.

Heat is removed from the off gas by a closed loop cooling system that consists of an air/liquid heat exchanger, a coolant storage tank, and a pump. A 50\% water/ethylene glycol mix is pumped from the storage tank through the shell side of the condenser and the two scrub solution heat exchangers, then through the air/liquid exchanger, where heat is removed from the coolant.

The venturi-ejector scrubber serves both as a quencher and a high energy scrubber. The second scrubber is a two-stage Hydro-Sonic scrubber (tandem nozzle fan drive). The first 
section condenses vapors, removes larger particles, and initiates growth of the finer particles so that they are more easily captured in the second stage. Particulate is captured when the gas is mixed with fine water droplets produced by spraying water into the exhaust of the subsonic nozzle. Large droplets containing the particulate are then removed by a vane separator and drained back into the scrub tank. The unit is designed to remove over $90 \%$ of all particulates greater than $0.5-\mu \mathrm{m}$ dia when operated at a differential pressure of $127 \mathrm{~cm}$ of water. Removal efficiency increases with an increase in pressure differential.

During operation, one filter is used and the other remains as a back-up in case the generating filter becomes loaded. The primary filter can be changed out during operation. The secondstage HEPA filter acts as a back-up if a first-stage filter fails.

\section{I-B.2.1 324 Facility Products, Raw Materials, Fuels, Processes}

The 324 Facility primarily supports the research and development (R\&D) of radioactive and nonradioactive waste vitrification technologies (i.e., ceramic melters, in situ vitrification [ISV], Hanford Waste Vitrification Program [HWVP] design support), spent nuclear fuel studies, biological waste remediation technologies, and waste mixing and transport studies. All of these programs have the potential to release hazardous and/or radioactive material. The potential for discharge would result primarily from (1) conducting research activities using the hazardous material, (2) storing radionuclides and hazardous chemicals, and (3) waste accumulation and storage.

\section{I-B.2.2 325 Facility Products, Raw Materials, Fuels, Processes}

The 325 Facility houses laboratories for organic, inorganic, and radioactive chemistry activities. Also provided are specially shielded, ventilated laboratory space for studies with chemical and mechanical processes that have radiation levels up to $10^{6} \mathrm{R} / \mathrm{h}$. The 325 Facility contains a very comprehensive hot-cell capacity with 4-foot-thick walls and 30-ton and 5-ton bridge cranes to perform process and physical chemistry on high-level samples, such as spent fuels. Shielded analytical cells with 18-inch walls and 3-ton hoists for process chemistry and development also exist. Other processes performed are waste formulation and durability, as well as empirical studies that support the fate of waste. Most of the work performed is analytical chemistry to support environmental restoration and waste management.

\section{I-B.2.3 327 Facility Products, Raw Materials, Fuels, Processes}

The 327 Facility processes that generate solid, liquid, and gaseous effluent are associated with the conduct of testing and metallurgical examinations for supporting sponsors. Examinations and testing performed by staff located include the following:

- gas collection, precision sectioning, metallography, remote machining, alkaline metal reaction, isotope recovery, medical isotope processing 
- specimen/equipment cleaning and decontamination

- irradiated materials preparation and testing

- remote assembly of irradiated components

- radioactive waste management

- shipping and receiving radioactive shipments.

Processes that could potentially result in such discharges include (1) stored radionuclides or chemicals and their usage; (2) liquid waste accumulation and storage; (3) programmatic sample storage; (4) research implementation (e.g., use of gloveboxes and hot cells); and (5) facility operations.

\section{I-B.2.4 3720 Facility Products, Raw Materials, Fuels, Processes}

The 3720 Facility processes that generate solid, liquid, and gaseous effluents are associated with conducting basic and applied research for supporting sponsors. Research performed by staff include the following:

- delineating the fundamental processes and mechanisms that influence the behavior of metals and radionuclides of environmental concern in the subsurface environment

- evaluating waste form stability and the ability of various materials (e.g., grout, glasses, cements) to immobilize contaminants of concern

- preparing and testing/analyzing materials and samples.

Processes also were examined as potential contaminant releases. Processes within the 3720 Facility that could potentially result in such discharges include (1) stored chemicals or radionuclides and their usage; (2) experimental material usage; (3) liquid waste accumulation and storage; (4) programmatic sample storage; (5) research implementation (e.g., use of glove and atmospheric boxes); and 6) facility operation. In the operation area, process evaluation was targeted at the air conditioning system; HEPA filter system; compressed gas storage; and pump systems for condensate return, vacuum and air sampling.

Source terms involving radionuclides are, for the most part (laboratory 202 is the exception), contained within the north portion of the main region of the facility (laboratories 245, 501, $507,506,603$ ) and involve the use in experimental studies of tracer quantities of radionuclides or environmental samples containing low levels of radionuclides. Glove and atmospheric boxes are selectively used to conduct experiments involving radioactive materials, as well as for storing radioactive materials with limited stability. Fume hoods may be used in a similar fashion. The 3720 Facility contains a materials balance area (in 
laboratory 501) for storage of Category 3 SNM isotopes of americium, neptunium, plutonium, and uranium and a laboratory for specialized counting of radioactive samples (laboratory 202). Radioactive liquid and solid wastes are accumulated in satellite storage areas and active inventories maintained.

\section{I-B.2.5 Is Situ Vitrification Products, Raw Materials, Fuels, Processes}

The ISV process generates a solid product which is a vitrified mass of material that is located beneath the surface of the ground. The effluents from the ISV process are associated with conducting basic and applied research for supporting sponsors. The raw materials used in the ISV process are small quantities of carbon (graphite) and silica (glass frit) to aid in the melting process.

\section{I-C.1.1 324 Facility Emission Points, Controls, Stacks}

The 324 Facility ventilation system is designed to ensure safe confinement of airborne radioactive materials under all normal and credible failure modes. The ventilation system is divided into four zones (Zones I, II, II, and IV) using cascading zone pressure levels to maintain proper air flow balance and direction. The system has interlocks, redundancy, failure-to-open control dampers, and back flow prevention dampers to ensure desired zone pressure, and air flow control in the event of partial equipment or power failure.

The Zone I and II exhaust streams are joined downstream of the final (second-stage) filters and exhaust fans, in a common tunnel, and exhaust to the atmosphere via the 150-foot-high stack located outside the northeast corner of the building. Zone I exhausts the high-level radioactive areas such as the radiochemistry and shielded material cells, their accompanying liquid vault tanks, and some gloveboxes and fume hoods. The Zone I exhaust is filtered through at least two testable stages of HEPA filtration and exhausted by three fans (one on standby) out to the atmosphere via the northeast stack.

Zone II exhaust serves potentially contaminated areas in which low-level radioactive materials are handled, such as shielded cell operating galleries, basement laboratories, and the storage vault. All Zone II air is exhausted through at least one testable stage of HEPA filtration. The Zone II system is serviced by two fans running continuously. The Zone II fans discharge to the atmosphere via the northeast stack.

The Zone III exhaust serves normally 'clean' areas such as the Engineering Development Laboratory 102 (EDL-102 is the area north of 101 containing Mods 1-18) and nonradioactive laboratories. All air exhausted from Zone III is filtered through at least one testable stage of HEPA filtration by two continuously-operating fans without any standby fan capability. The Zone III fans discharge directly to the atmosphere through a stack that penetrates the roof. 
Zone IV exhaust serves areas that do not contain any radioactive materials such as offices, change rooms, and administration areas. The Zone IV air is discharged to the atmosphere through various powered or convective roof exhausters or is recirculated, depending on the location.

\section{I-C.1.2 325 Facility Emission Points, Controls, Stacks}

The 325 Facility exhaust system consists of the air circulating first through the uncontrolled corridors, to the controlled corridors, and finally to the laboratories that have at least one HEPA filter at each glovebox and hood. Two restrooms also are in the controlled area that exhaust to the controlled hallway. The air is exhausted through a bank of HEPA filters before being exhausted through the stack. Two restrooms are located in the uncontrolled hallway adjacent to the controlled area and two additional restrooms are in an uncontrolled area that are exhausted via exhaust fans to the atmosphere. The lunchroom, outer conference room, and nonradiation change rooms are exhausted to a nonfiltered exhaust fan.

Airborne emissions from the 325 Facility are primarily via stacks and vents; minor outleakage under upset conditions is possible via doorways and building leaks.

The airborne particulate sample system incorporates a six-nozzle, isokinetic sampling probe assembly positioned in the approximately 86-feet-high by 8-feet in diameter 325 Facility stack (EP-325-01-S). Annual stack volumetric flow rate determinations indicate the stack has a relatively constant flow rate.

The main ventilation air supply units have back draft dampers and do not constitute a viable exhaust point from the 325 Facility. Air intake vents for vaults housing tanks used to store radioactive liquids are located on the east side of the building. These intake vents all have HEPA filters. Several natural gas and steam vents are present on the roof, but are not considered potential release points.

Access doors are separated from potentially contaminated areas by at least one control zone. Hand and shoe counters are located near exit points from control zones to ensure no migration of radionuclide contamination into uncontrolled areas occur.

Airborne radionuclide emission sampling for particulate radionuclides is performed at the stack, using isokinetic sampling systems compliant with National Emissions Standards for Hazardous Air Pollutants (NESHAP) requirements in 40 CFR 61.

The stack, located on the northwest side of the 325 Facility, discharges ventilation exhaust from hot cells, maintenance shops, and canyon areas at an average exhaust rate of 130,000 cubic feet per minute.

I-C.1.3 327 Facility Emission Points, Controls, Stacks 
Almost the entire 327 Facility is in one ventilation zone, within which air balance keeps flow moving from 'clean' to 'dirty' areas and from the atmosphere into the building. Some confinement also is provided by conducting high-inventory operations in hot cells. There are no process offgas systems. All potentially radioactively contaminated air flow passes through at least one stage of HEPA filtration before leaving the building via stack EP-327-01-S, or vent EP-327-02-V.

Airborne radionuclide emission sampling for particulate radionuclides is performed at both EP-327-01-S and EP-327-02-V using isokinetic sampling systems compliant with National Emissions Standards for Hazardous Air Pollutants (NESHAP) requirements in 40 CFR 61.

\section{I-C.1.4 3720 Facility Emission Points, Controls, Stacks}

Airborne emissions from the 3720 Facility are via stacks and vents. Compliance emission sampling is performed at the main stack, at exhaust sample point ESP-3720-01-S. Confirmatory sampling is performed at two locations: the north annex stack, shown as ESP-3720-02-S, and the southeast laboratory stack, shown as ESP-3720-03-S.

Annual stack volumetric flow rate determinations show the main exhaust stack to have a relatively constant flow rate.

The 3720 Facility Stack (EP-3720-01-S) is a 30-foot-high by 3.9-foot-diameter stainless steel cylinder located on the north side of the building. Exhaust from laboratories containing open-faced hoods and glove-boxes discharge via the stack. Building pressures are maintained nearly constant relative to ambient levels by use of control dampers in the intake and exhaust ducts, resulting in relatively constant stack flow rates.

The Southeast Stack (EP-3720-03-S) is a 30-foot-high by 2.5 -foot-diameter steel cylinder stack located on the east side of the building. The stack exhausts offices and laboratories the south portion of the building. Building ventilation systems effectively isolate the south portion of the building from the main and annex laboratory areas.

The Annex Stack (EP-3720-02-S) is a 17-foot-high by 24-inch-diameter steel cylindrical stack located on the north side of the 3720 Facility, adjacent to the annex laboratory addition. Building ventilation systems effectively isolate the annex addition from the remainder of the building. 


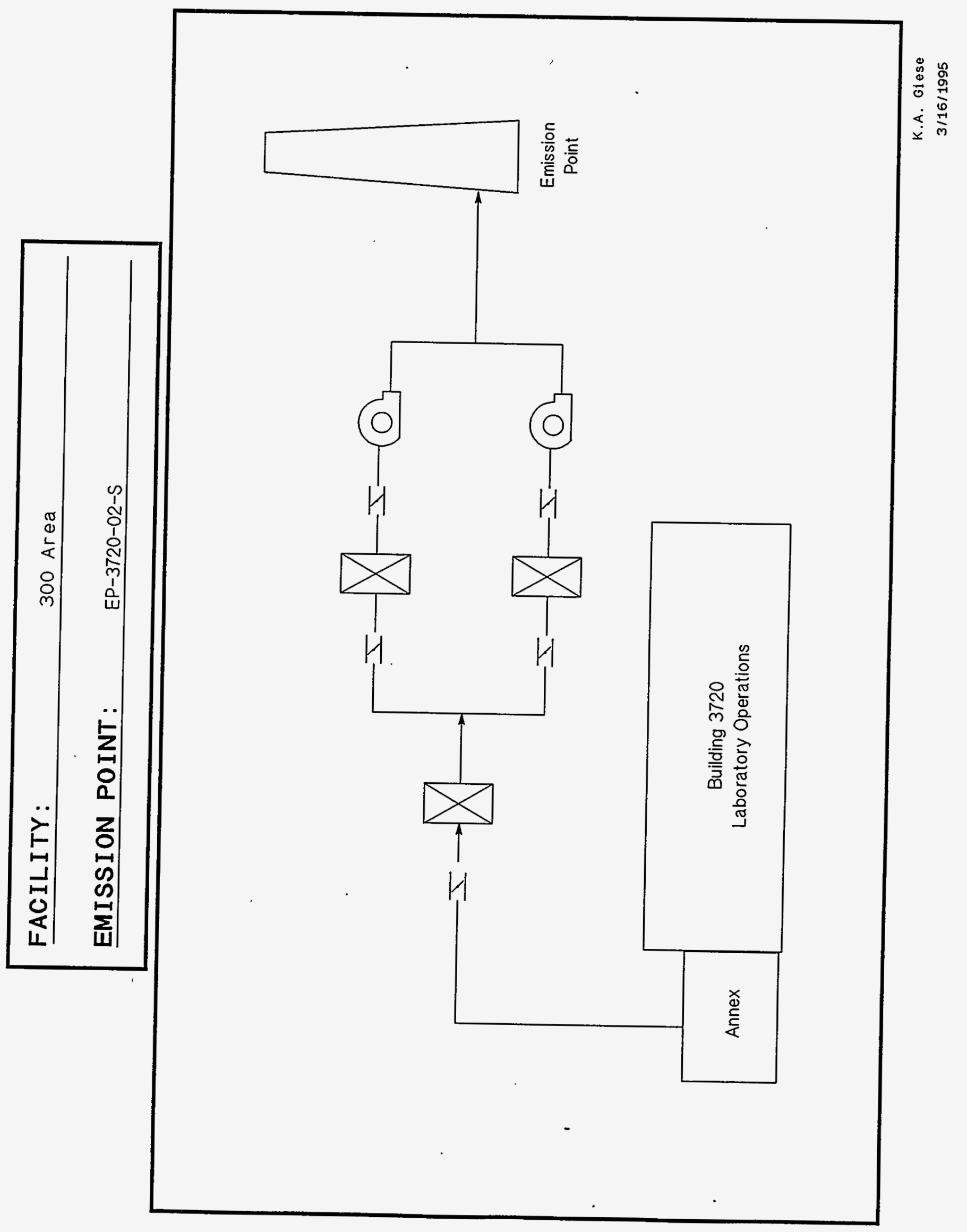




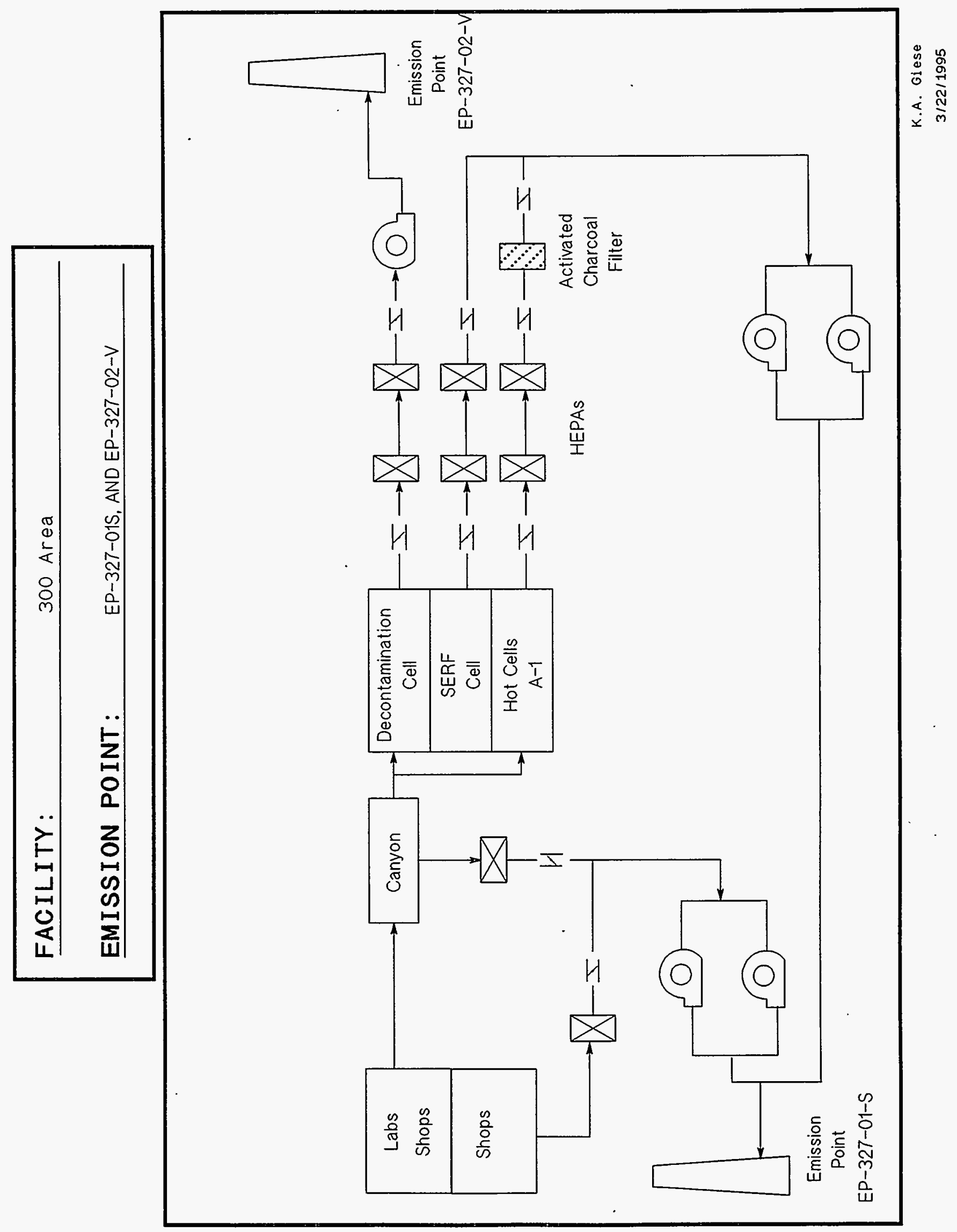




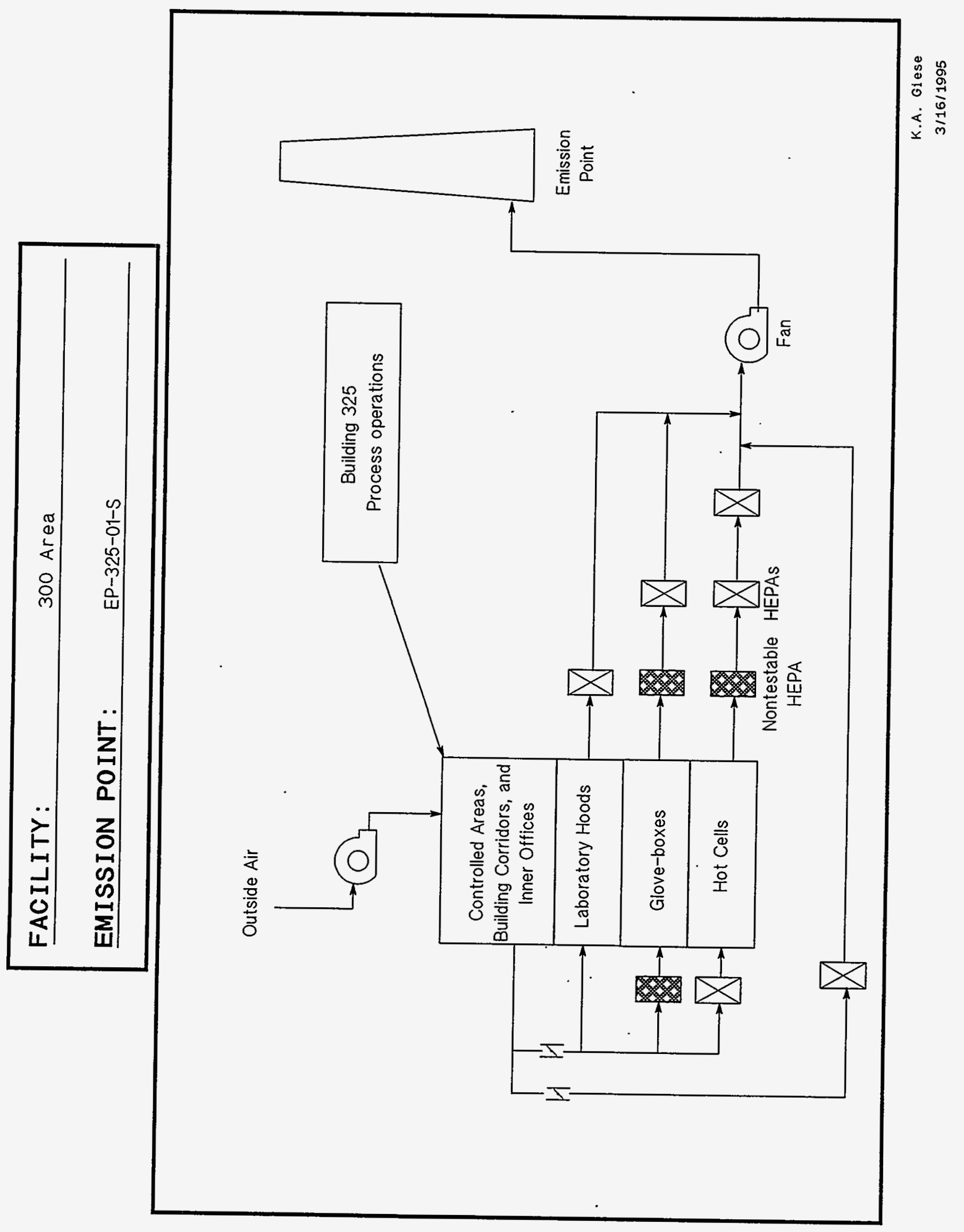




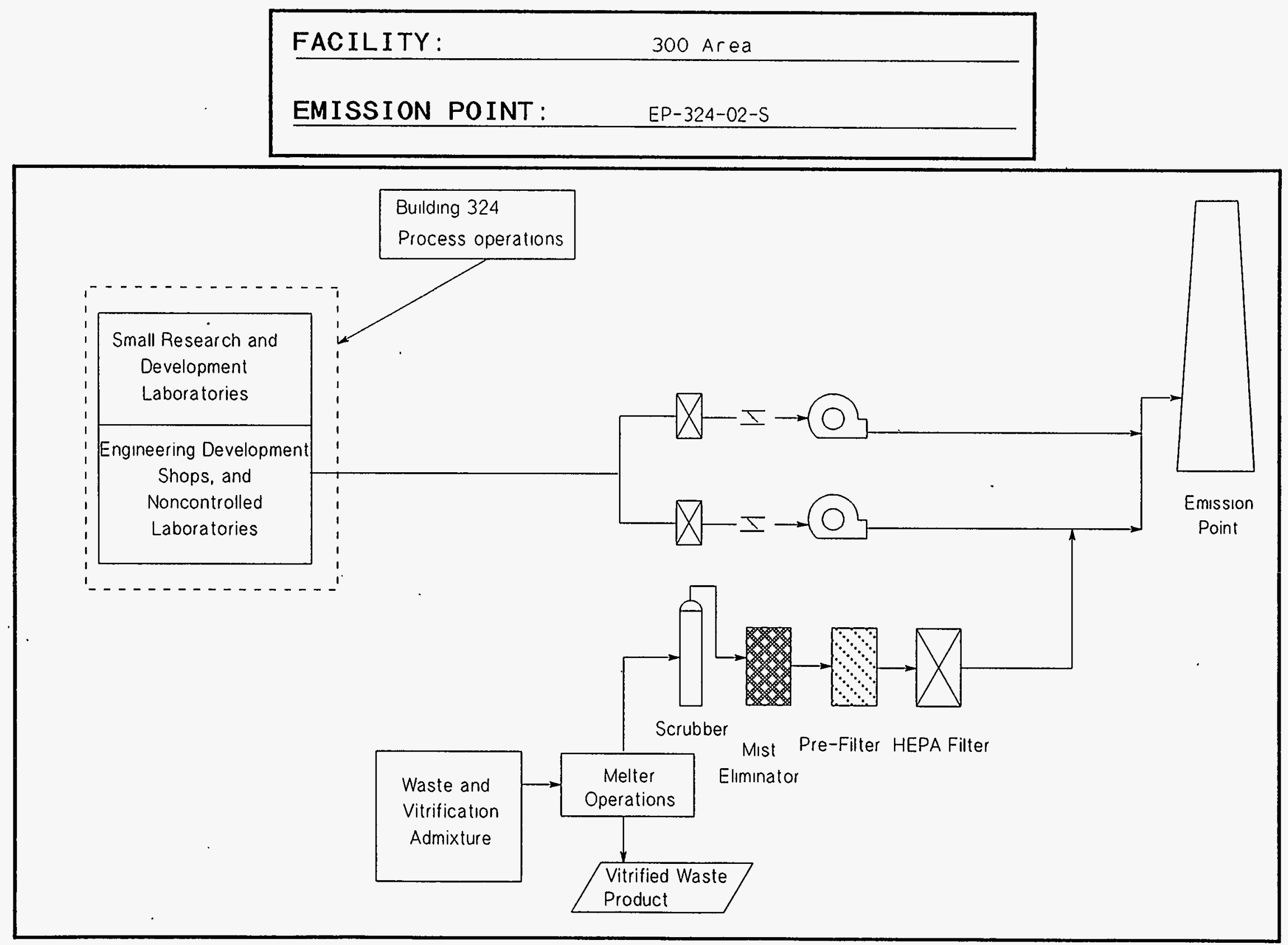




\section{FACILITY:}

300 Area

EMISSION POINT:

EP-324-01-S

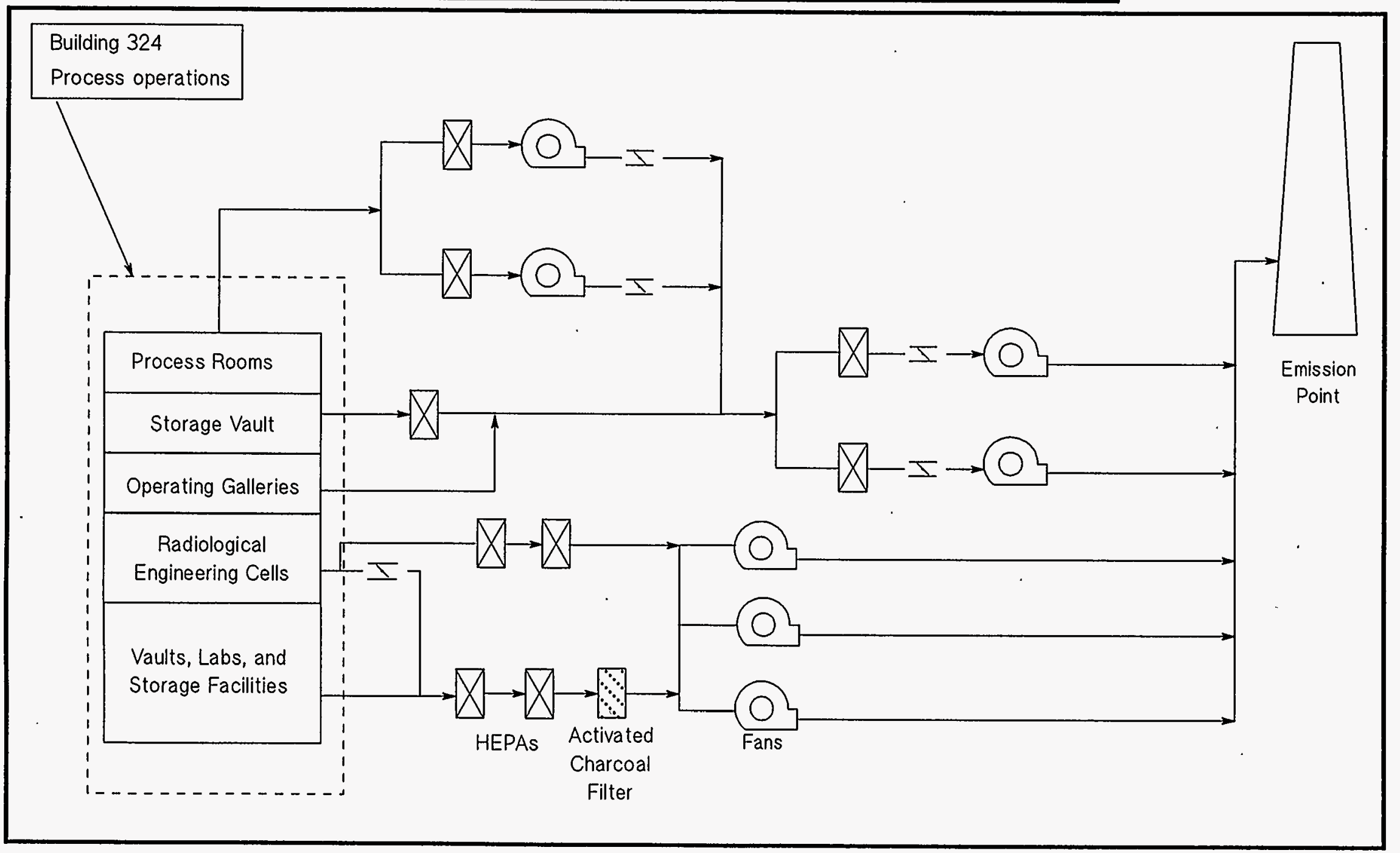




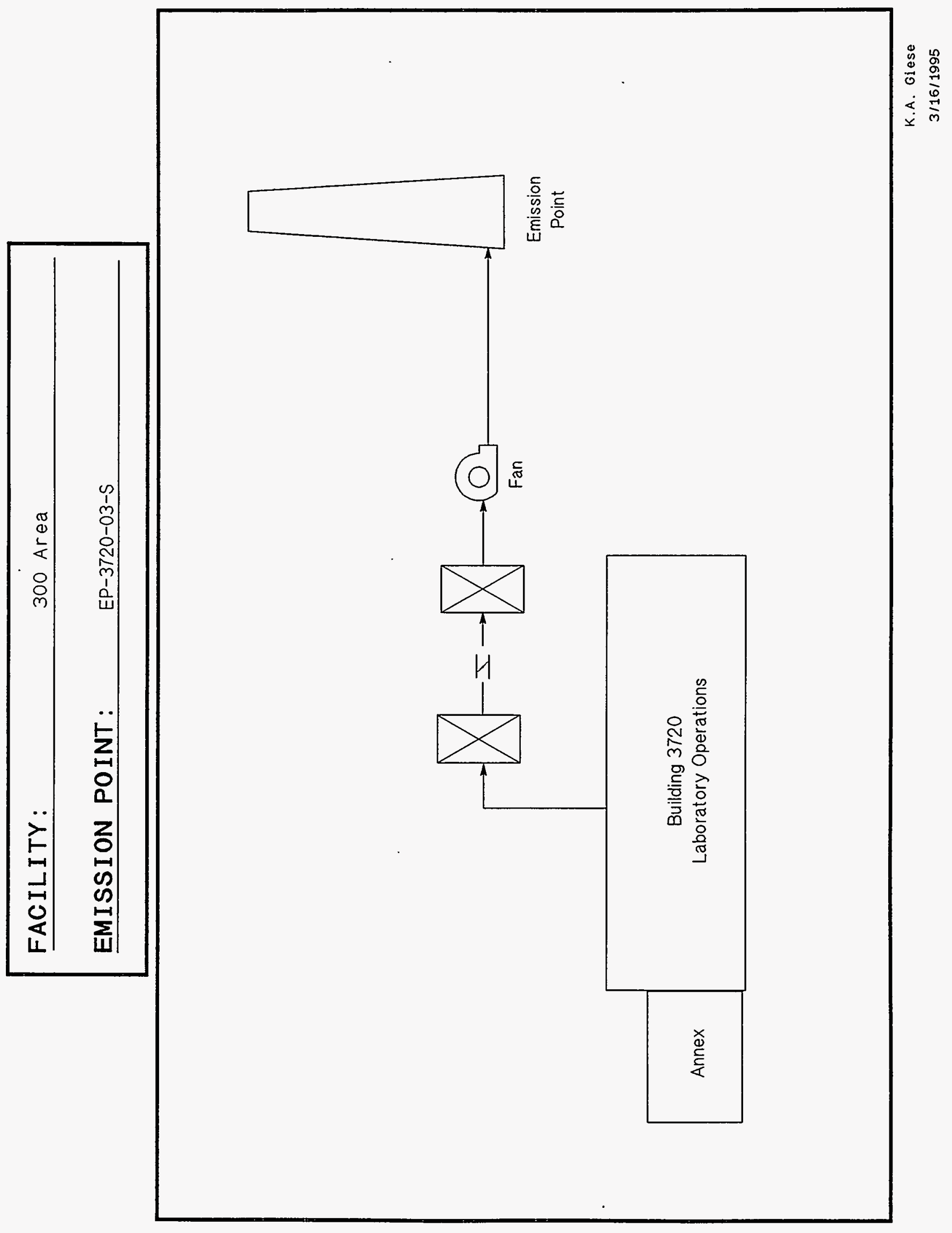




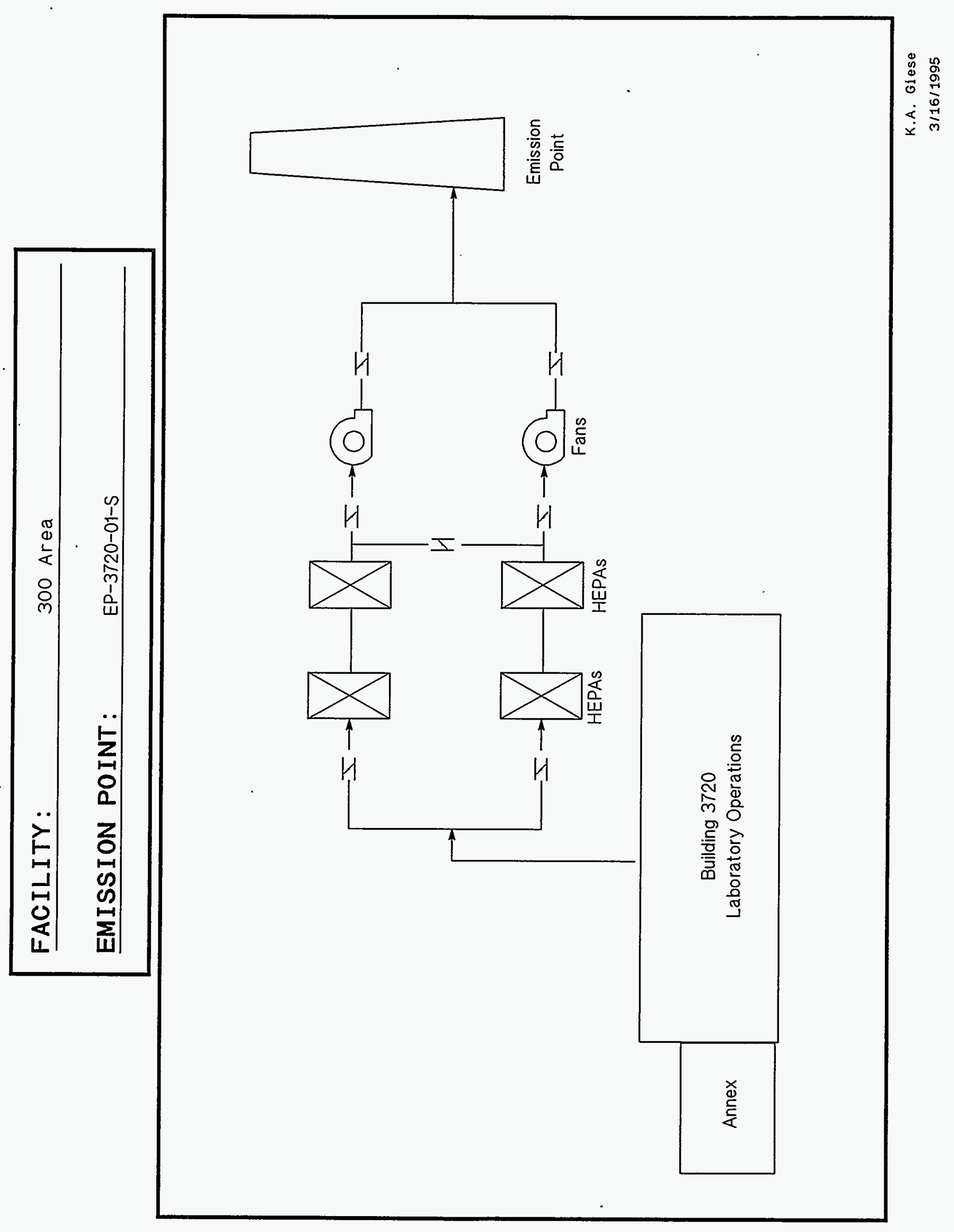




\begin{tabular}{ll} 
FACILITY: & 300 Area \\
\hline EMISSION POINT: & EP-ISV-01-V \\
\hline
\end{tabular}

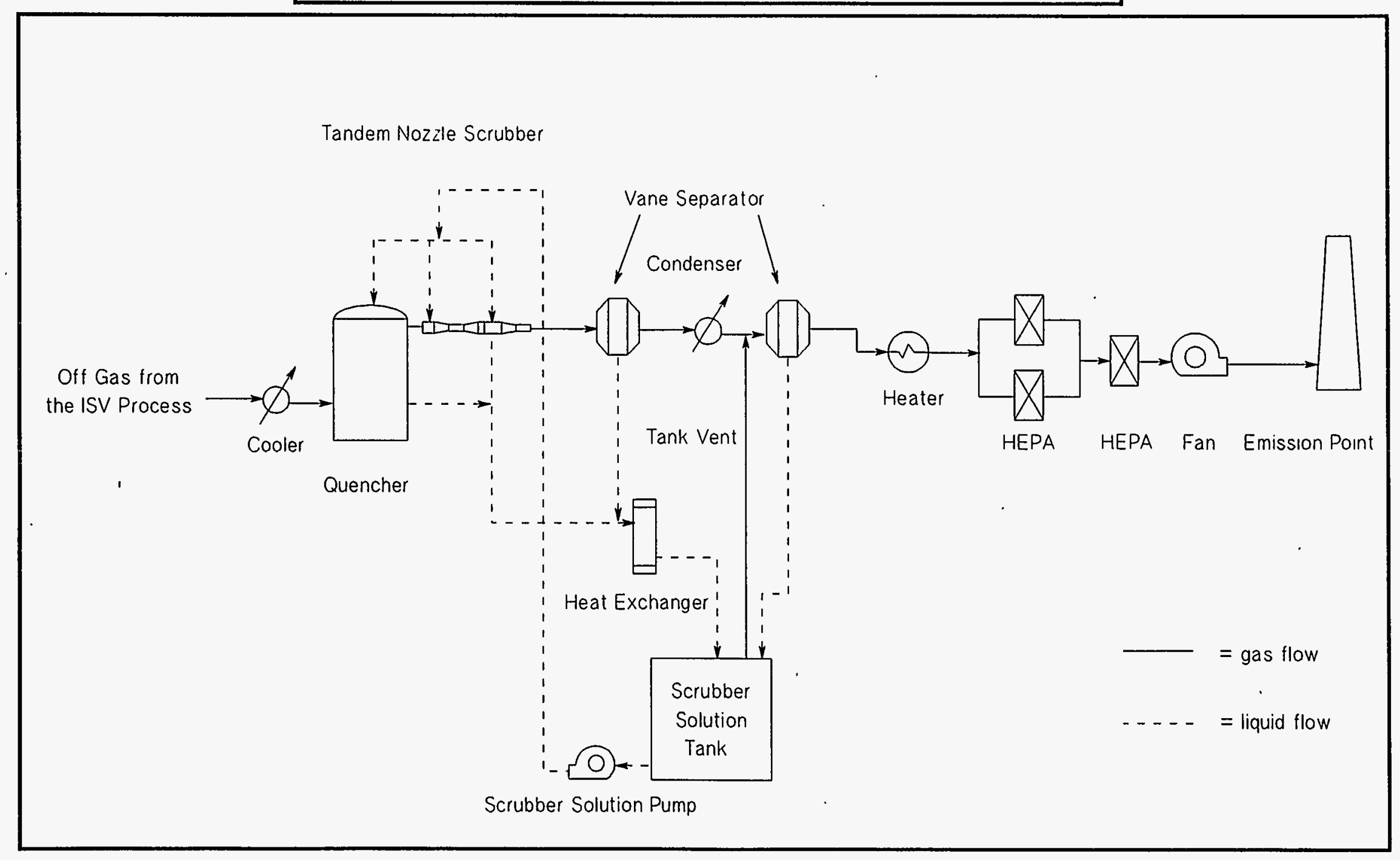




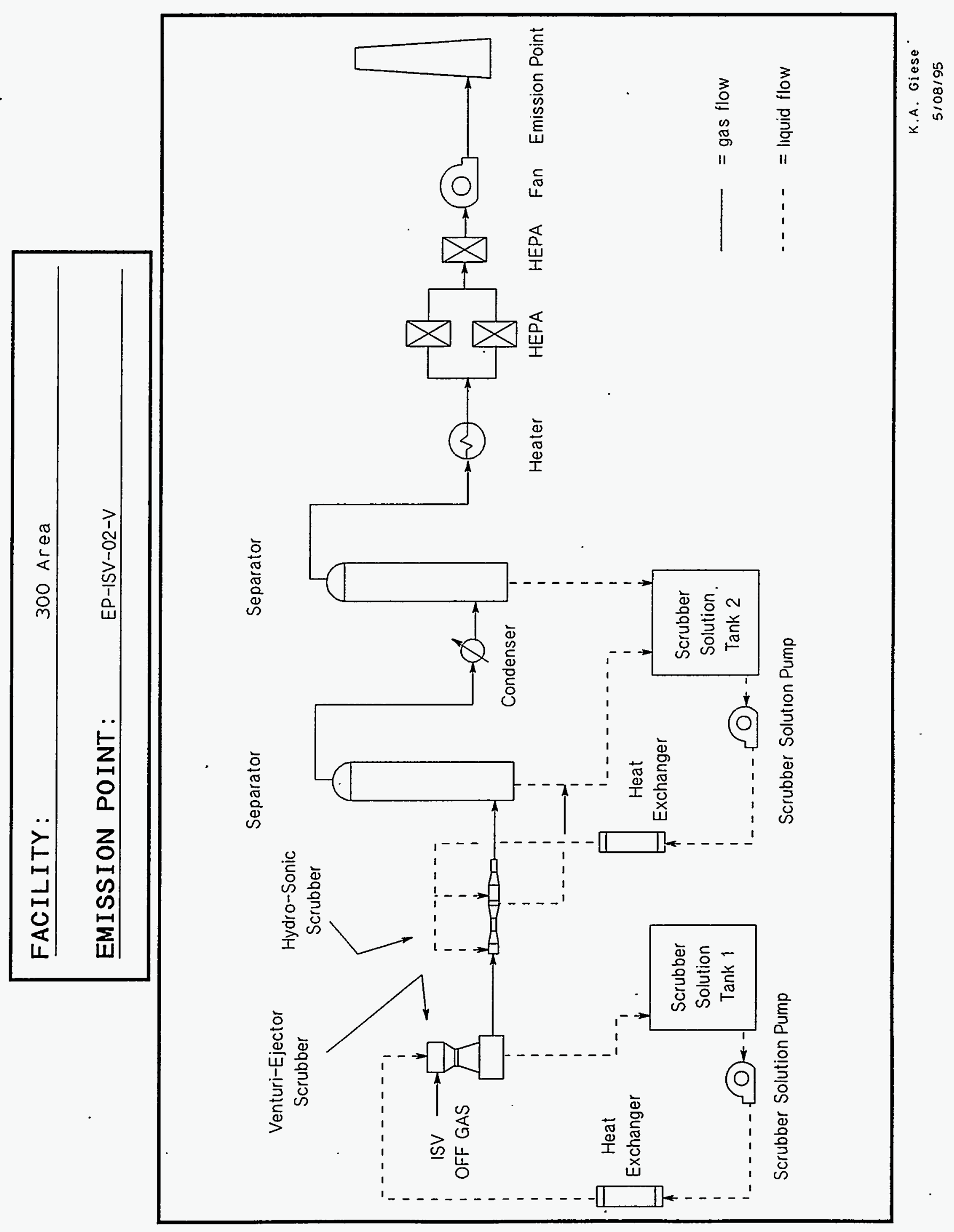




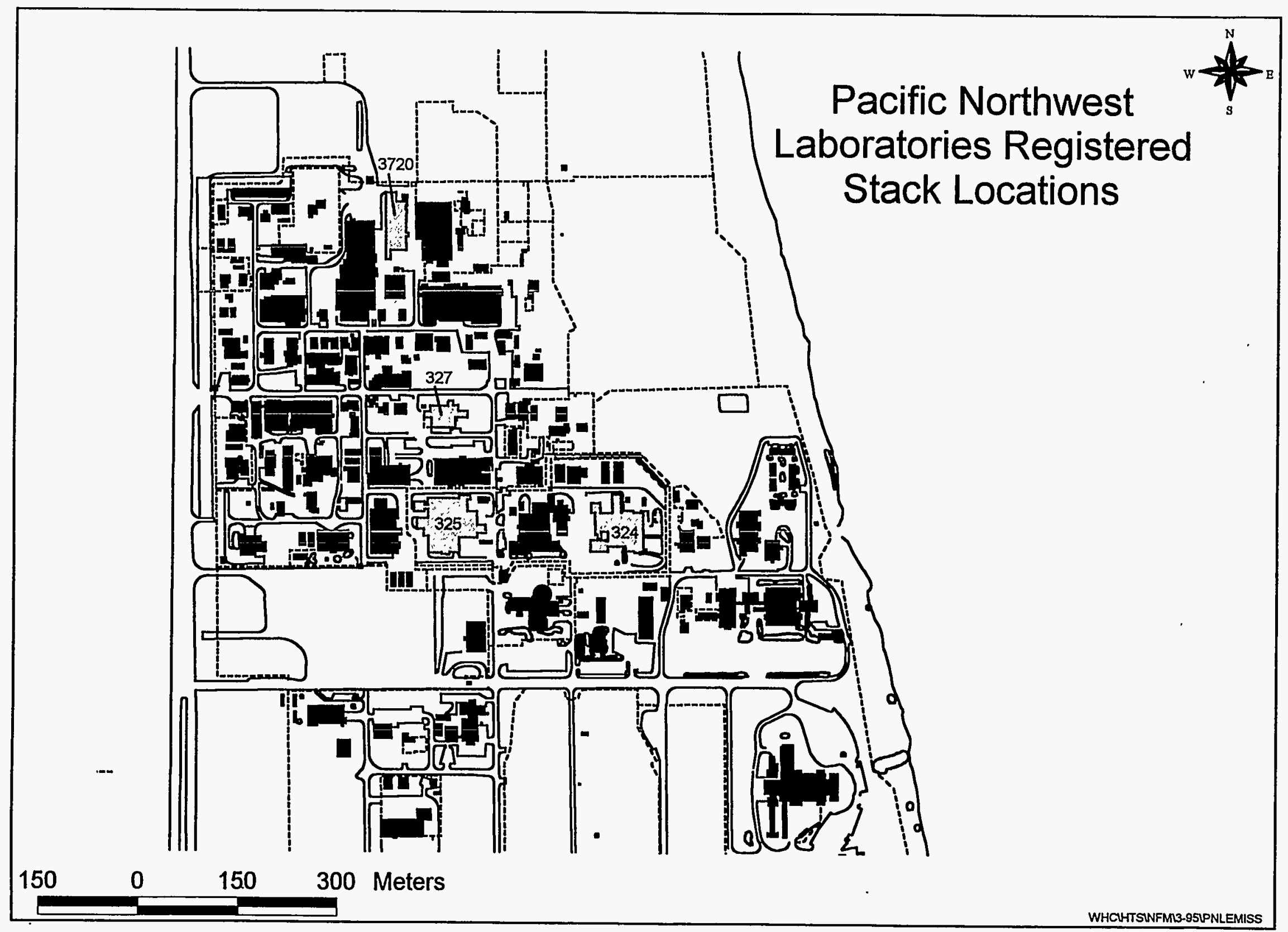




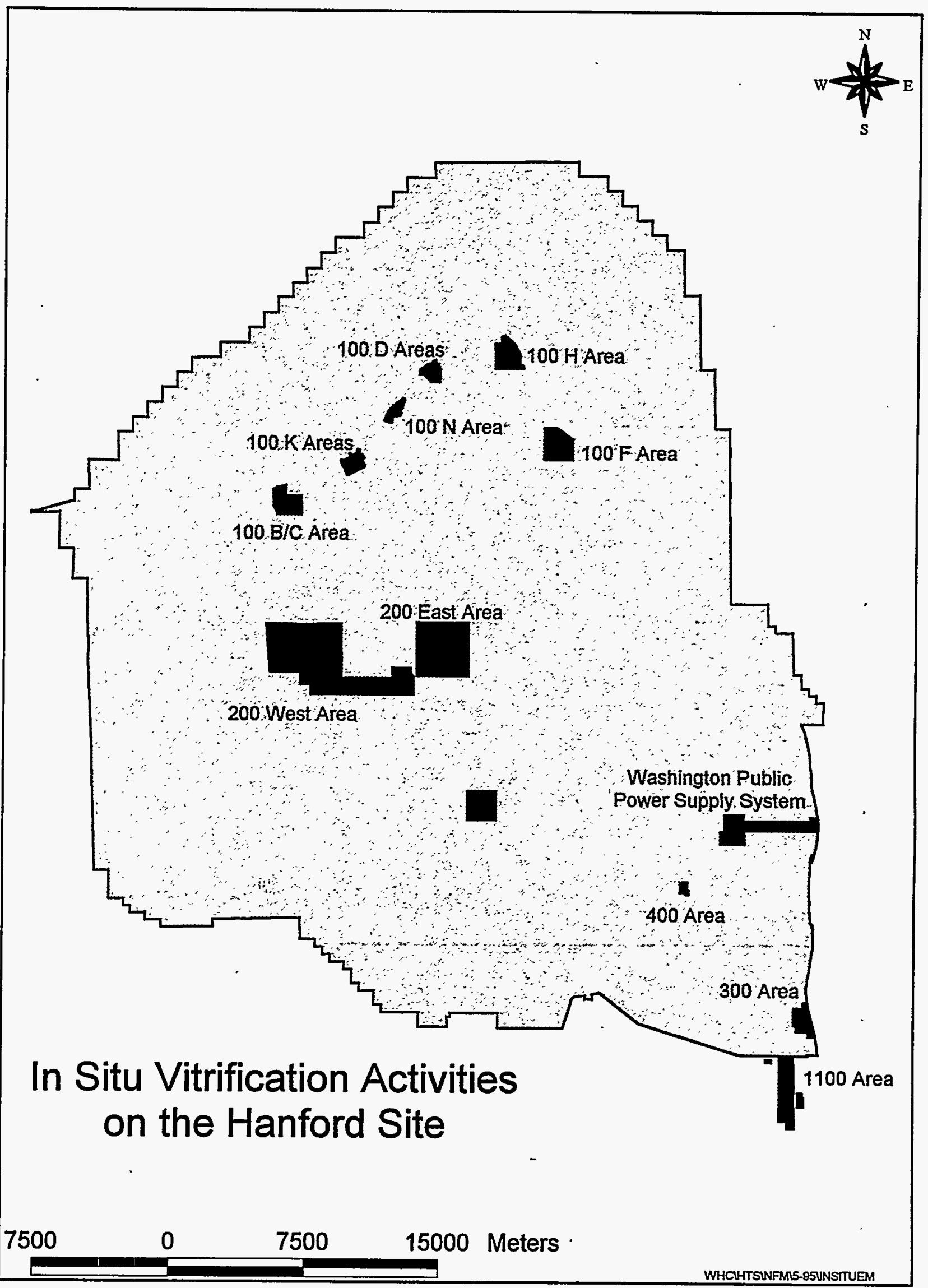




\title{
APPENDIX G
}

\author{
400 AREA
}


Hanford Site Air

Operating Permit Application
DOE/RL-95-07, Rev. 0

05/95

\section{APPENDIX G}

\section{CONTENTS}

G-1 400 AREA

APP G-ii 


\title{
APPENDIX G-1
}

\author{
400 AREA
}

\section{Chapter I. General Information}

\section{I-B.1. Facility Description}

There are two major process areas in the 400 Area, the Fast Flux Test Facility (FFTF) and the Material and Storage Facility (MASF). The FFTF is a 400-megawatt thermal sodiumcooled, fast neutron flux reactor plant designed specifically for irradiation testing of nuclear reactor fuels and materials for liquid metal fast breeder reactors. The operating future of the FFTF has been studied by various political, entrepreneurial, and scientific concerns. On December 15, 1993, the Secretary of the U.S. Department of Energy directed the FFTF be placed in a radiologically and industrially safe shutdown condition.

The MASF is a multipurpose service center that supports the specialized maintenance and storage requirements of the 400 Area facilities. The MASF provides the capability for decontamination, repair, and storage of non-fuel components and hardware for the 400 Area facilities. Work in the MASF includes handling items that are radioactive by neutron activation and items that are contaminated with radioisotopes and/or sodium films. The MASF is operated on a routine basis to meet commitments. Generally, MASF is staffed and operated based on programmatic needs.

\section{I-B.2. Products, Raw Materials, Fuels, Processes}

- Principal products - There are no products produced at the 400 Area.

- Process information - The process operations at the 400 Area are listed as follows.

- The MASF provides the space and equipment for the cleaning, decontamination, storage, and repair of a wide variety of equipment and non-fueled components from the FFTF. This equipment includes reactor grade components, U.S. Department of Transportation-approved shipping casks, sodium-wetted components and contaminated equipment.

- The process operations at the FFTF are centered around placing and maintaining the FFTF in a radiologically and industrially safe shutdown condition. Major processes to achieve this condition are:

- reactor defueling

. unirradiated fuel disposition 
. irradiated fuel washing and dry cask storage.

- sodium system drain and management of sodium residuals

. auxiliary systems shutdown

. bulk sodium conversion to a form suitable for reuse or disposition.

- Raw materials used by processes - There are no raw materials consumed at the 400 Area.

- Fuels used by processes - Process heaters are used to supplement the heat necessary to keep the sodium in the reactor core in a molten state. These heaters burn \#2 fuel oil.

\section{I-C.1. Emission Points, Controls, Stacks}

The emission point and stack information is found on pages 1 and 2 of the AEI report output. The control information is found on page 1 of the AEI report output.

\section{I-C.2. Facility Process Flow Diagram}

Attached.

I-C.3. Site Map

Attached.

\section{Chapter II. Regulatory Requirements}

\section{II-A.1. Description of Discharge and Emission Points}

The emission point and stack information is found on pages 1 and 2 of the AEI report output. 


\section{Facility Building List}

\begin{tabular}{|c|c|c|c|c|c|c|c|}
\hline Bldg Id & North & East & Bldg Desc & Custodian(Contractor) & Cust Phone & DOE POC & DOE Phone \\
\hline $\begin{array}{l}408 A \\
408 B \\
408 C \\
437 \\
\text { 437MN\&ST }\end{array}$ & $\begin{array}{l}123098 \\
123060 \\
123110 \\
123422 \\
123378\end{array}$ & $\begin{array}{l}587662 \\
587582 \\
587548 \\
587584 \\
587536\end{array}$ & $\begin{array}{l}\text { MAIN HEAT DUMP. EAST } \\
\text { MIIN HEAT DUMP. SOUTH } \\
\text { MAIN HEAT DUMP. WEST } \\
\text { MAINTENANCE AND STORAGE FACILITY (MASF) } \\
\text { STACK FOR THE MAINTENANCE AND STORAGE FACILITY } \\
\text { (MASF), BUILDING } 437\end{array}$ & $\begin{array}{l}\text { DAHL. NORMAN R. } \\
\text { DAHL. NORMAN R } \\
\text { DAHL. NORMAN R. } \\
\text { DAHL. NORMAN R. } \\
\text { DAHL. NORMAN R. }\end{array}$ & $\begin{array}{l}376-4788 \\
376-4788 \\
376-4788 \\
376-4788 \\
376-4788\end{array}$ & $\begin{array}{l}\text { KEITH A. BENGUIAT } \\
\text { KEITH A. BENGUIAT } \\
\text { KEITH A. BENGUIAT } \\
\text { KEITH A. BENGUIAT } \\
\text { KEITH A. BENGUIAT }\end{array}$ & $\begin{array}{l}376-8025 \\
376-8025 \\
376-8025 \\
376-8025 \\
376-8025\end{array}$ \\
\hline $\begin{array}{l}4713 B \\
47130 \\
\text { FFTFCBEX }\end{array}$ & $\begin{array}{l}123110 \\
123276 \\
123151\end{array}$ & $\begin{array}{l}587478 \\
587567 \\
587612\end{array}$ & $\begin{array}{l}\text { FFTF MAINTENANE SHOP } \\
\text { INTERIM MAINENANCE AND STORAGE FACILITY } \\
\text { STACK VENTING GASES FROM THE ARGON PROCESSING } \\
\text { SYSTEM, BUILDING } 4621 E\end{array}$ & $\begin{array}{l}\text { DAHL. NORMAN R. } \\
\text { DAHL. NORHAN R. } \\
\text { DAHL, NORMAN R. }\end{array}$ & $\begin{array}{l}376-4788 \\
376-4788 \\
376-4788\end{array}$ & $\begin{array}{l}\text { KEITH A. BENGUIAT } \\
\text { KEITH A. BENGUIAT } \\
\text { KEITH A. BENGUIAT }\end{array}$ & $\begin{array}{l}376-8025 \\
376-8025 \\
376-8025\end{array}$ \\
\hline FFTFHTTR & 123083 & 587614 & $\begin{array}{l}\text { STACK FOR THE HEAT TRANSFER SYSTEM SOUTH, BUILDING } \\
491-S\end{array}$ & DAHL, NORMAN $R$. & $376-4788$ & KEITH A. BENGUIAT & $376-8025$ \\
\hline FFTFRESB & 123156 & 587645 & $\begin{array}{l}\text { LONER REACTOR SERVICE BUILDING STACK. BUILOING } \\
4717\end{array}$ & DAHL. NORMAN R. & $376-4788$ & KEITH A. BENGUIAT & $376-8025$ \\
\hline
\end{tabular}

\section{Emission Point Listing}

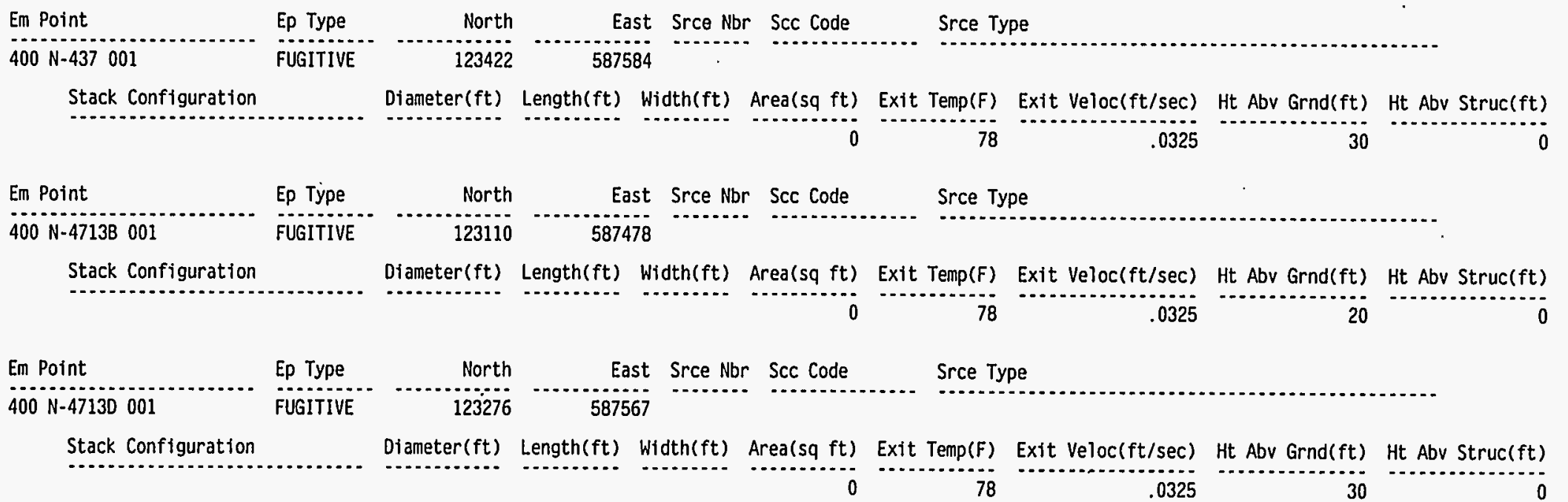




\section{Emission Point Listing}

\begin{tabular}{|c|c|c|c|c|c|c|c|c|}
\hline Em Point & Ep Type & North & East Srce Nbr & $r$ Scc Code & & Srce Type & & \\
\hline $400 \mathrm{P}-408 \mathrm{~A} 001$ & POINT & 123098 & 587662 & $\begin{array}{l}1 \text { not assigne } \\
2 \text { not assigne } \\
3 \text { not assigne } \\
4 \text { not assigne } \\
5 \text { not assigne }\end{array}$ & & $\begin{array}{l}\text { PROCESS AND PRODUCTION } \\
\text { PROCESS AND PRODUCTION } \\
\text { PROCESS AND PRODUTION } \\
\text { PROESS AND PROUCTION } \\
\text { SOURCE EMISSIONS ROLL-UP }\end{array}$ & & \\
\hline Stack Configuration & & Diameter ( $\mathrm{ft}$ ) & Length(ft) Width(ft) & Area(sq ft) & Exit & Exit Veloc(ft/sec) & Ht Abv Grnd(ft) & Ht Abv Struc(ft) \\
\hline VERTICAL WITH WEATHER & CAP & 1 & & .79 & & 32.5 & 17 & -20 \\
\hline Em Point & Ep Type & North & East Srce Nbr & Scc Code & & Srce Type & & \\
\hline $400 \mathrm{P}-408 \mathrm{~B} 001$ & POINT & 123060 & 587582 & $\begin{array}{l}1 \text { not assigne } \\
2 \text { not assigne } \\
3 \text { not assigne } \\
4 \text { not assigne } \\
5 \text { not assigne }\end{array}$ & & $\begin{array}{l}\text { PROCESS AND PRODUCTION } \\
\text { PROCESS AND PRODUCTION } \\
\text { PROCESS AND PRODUTION } \\
\text { PROESS AND PROUUCTION } \\
\text { SOURCE EMISSIONS ROLL-UP }\end{array}$ & & \\
\hline Stack Configuration & & Diameter ( $f t)$ & Length $(f t)$ Width(ft) & Area $(\mathrm{sq} \mathrm{ft})$ & Exit & Temp(F) Exit Veloc(ft/sec) & Ht Abv Grnd(ft) & Ht Abv Struc(ft) \\
\hline VERTICAL WITH WEATHER & CAP & 1 & & .79 & & 32.5 & 17 & -20 \\
\hline Em Point & Ep Type & North & East Srce Nbr & r Scc Code & & Srce Type & & a \\
\hline $400 \mathrm{P}-408 \mathrm{C} 001$ & POINT & 123110 & 587548 & $\begin{array}{ll}1 & \text { not assigne } \\
2 & \text { not assigne } \\
3 & \text { not assigne } \\
4 & \text { not assigne } \\
5 & \text { not assigne }\end{array}$ & & $\begin{array}{l}\text { PROCESS AND PRODUCTION } \\
\text { PROCESS AND PRODUCTION } \\
\text { PROCESS AND PRODUCTION } \\
\text { PROESS AND PROUCTION } \\
\text { SOURCE EMISSIONS ROLL-UP }\end{array}$ & & \\
\hline Stack Configuration & & Diameter ( $f t)$ & Length $(\mathrm{ft})$ & Area $(s q \mathrm{ft})$ & Exit & Exit Veloc $(\mathrm{ft} / \mathrm{sec})$ & Ht Abv Grnd(ft) & Ht Abv Struc(ft) \\
\hline VERTICAL WITH WEATHER & CAP & 1 & & .79 & & 32.5 & 17 & -20 \\
\hline Em Point & Ep Type & North & East Srce Nbr & Scc Code & & Srce Type & & \\
\hline $400 \quad P-437002$ & POINT & 123378 & 587536 & $i$ not assigne & & PROCESS AND PRODUCTION & & \\
\hline Stack Configuration & & Diameter $(\mathrm{ft})$ & Length(ft) Width(ft) & $\operatorname{Area}(s q \mathrm{ft})$ & Exit & Exit Veloc $(\mathrm{ft} / \mathrm{sec})$ & Ht Abv Grnd(ft) & Ht Abv Struc(ft) \\
\hline NONE SPECIFIED & & & .33 & 1.32 & & 18.75 & 37 & 5.5 \\
\hline
\end{tabular}


AIR EMISSIONS INVENTORY PERMIT APPLICATION REPORT (UP460)

FACILITY NAME: 400 AREA Facility and Associated Structures

\section{Emission Point Listing}

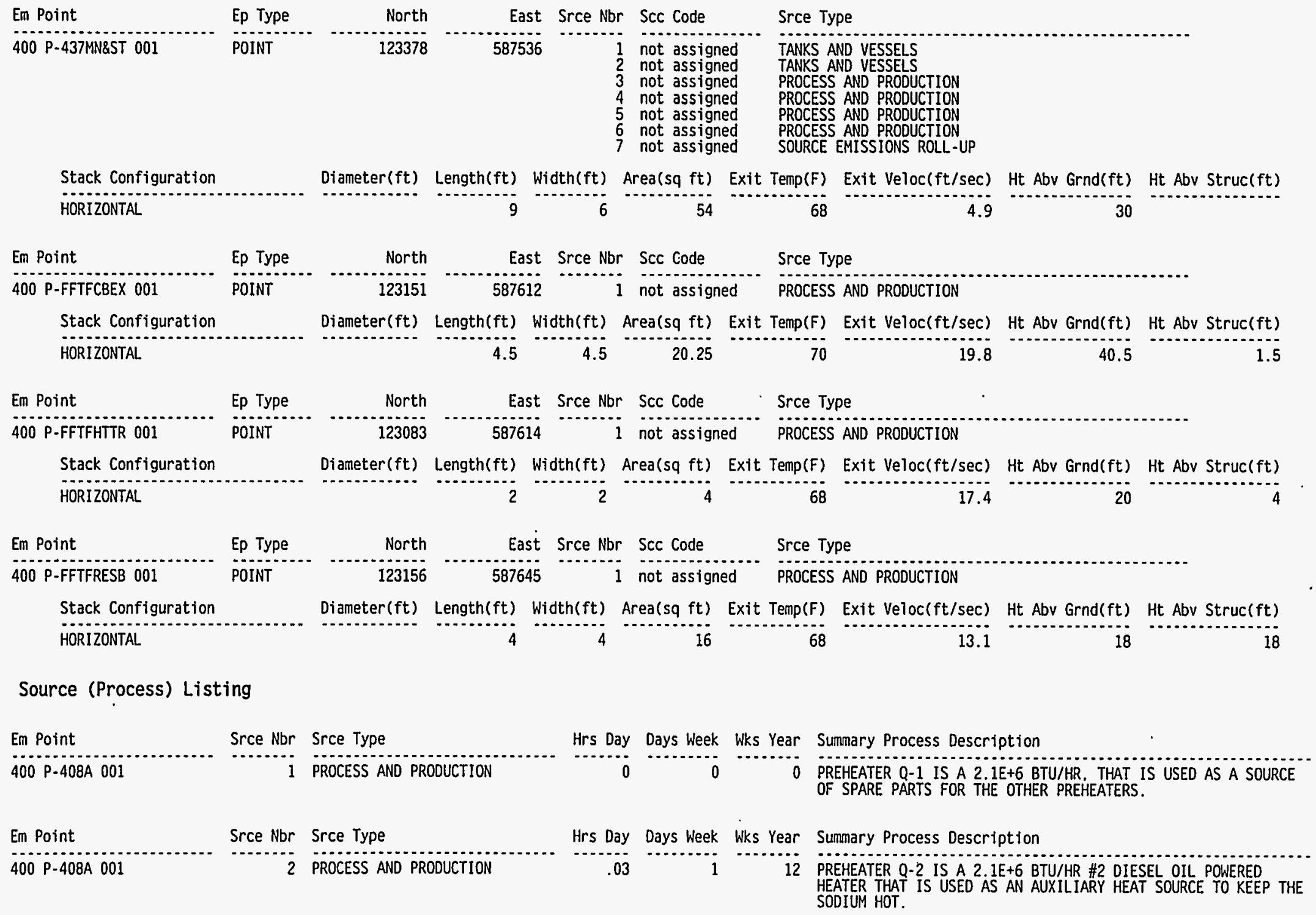

Stack Configuration

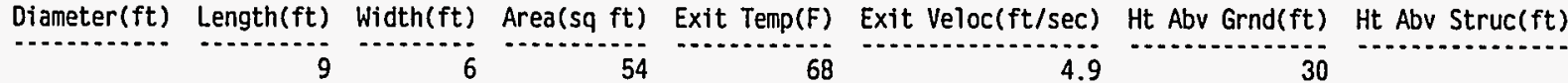
HORIZONTAL

\section{Em Point} Ep Type North East Srce Nbr Scc Code Srce Type POINT

$123151 \quad 587612 \quad 1$ not assigned

PROCESS AND PRODUCTION

Stack Configuration Diameter(ft) Length(ft) Width(ft) Area(sq ft) Exit Temp(F) Exit Veloc(ft/sec) Ht Abv Grnd(ft) Ht Abv Struc(ft) HORIZONTAL

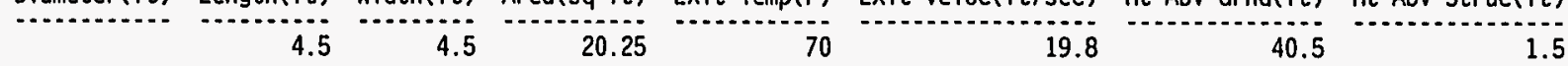

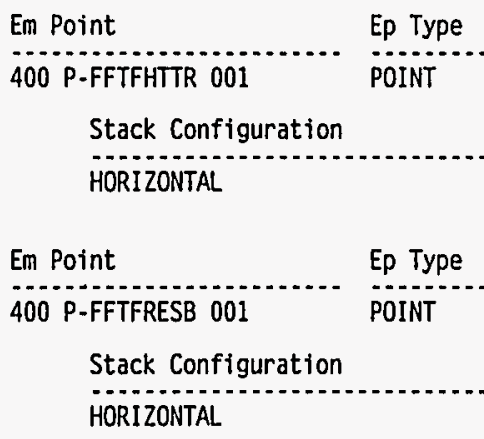

North East Srce Nbr Scc Code Srce Type
$123083 \quad 587614$ i not assigned PROCESS AND PRODUCTION

Diameter(ft) Length(ft) Width(ft) Area(sq ft) Exit Temp(F) Exit Veloc(ft/sec) Ht Abv Grnd(ft) Ht Abv Struc(ft) 2
2

\section{Source (Process) Listing}

\begin{tabular}{|c|c|c|c|c|c|c|}
\hline Em Point & Srce Nbr & Srce Type & Hrs Day & Days Week & Wks Year & Sumnary Process Description \\
\hline 400 P.408A 001 & 1 & PROCESS AND PRODUCTION & 0 & 0 & 0 & $\begin{array}{l}\text { PREHEATER Q-1 IS A } 2.1 E+6 \text { BTU/HR. THAT IS USED AS A SOURCE } \\
\text { OF SPARE PARTS FOR THE OTHER PREHEATERS. }\end{array}$ \\
\hline Em Point & Srce Nbr & Srce Type & Hrs Day & Days Week & Wks Year & Summary Process Description \\
\hline 400 P-408A 001 & 2 & PROCESS AND PRODUCTION & .03 & 1 & 12 & $\begin{array}{l}\text { PREHEATER Q-2 IS A } 2 \text { 1E+6 BTU/HR \#2 DIESEL OIL POWERED } \\
\text { HEATER THAT IS USED AS AN AUXILIARY HEAT SOURCE TO KEEP THE } \\
\text { SODIUM HOT. }\end{array}$ \\
\hline
\end{tabular}


AIR EMISSIONS INVENTORY PERMIT APPLICATION REPORT (UP460)

FACILITY NAME: 400 AREA Facility and Associated Structures

\section{Source (Process) Listing}

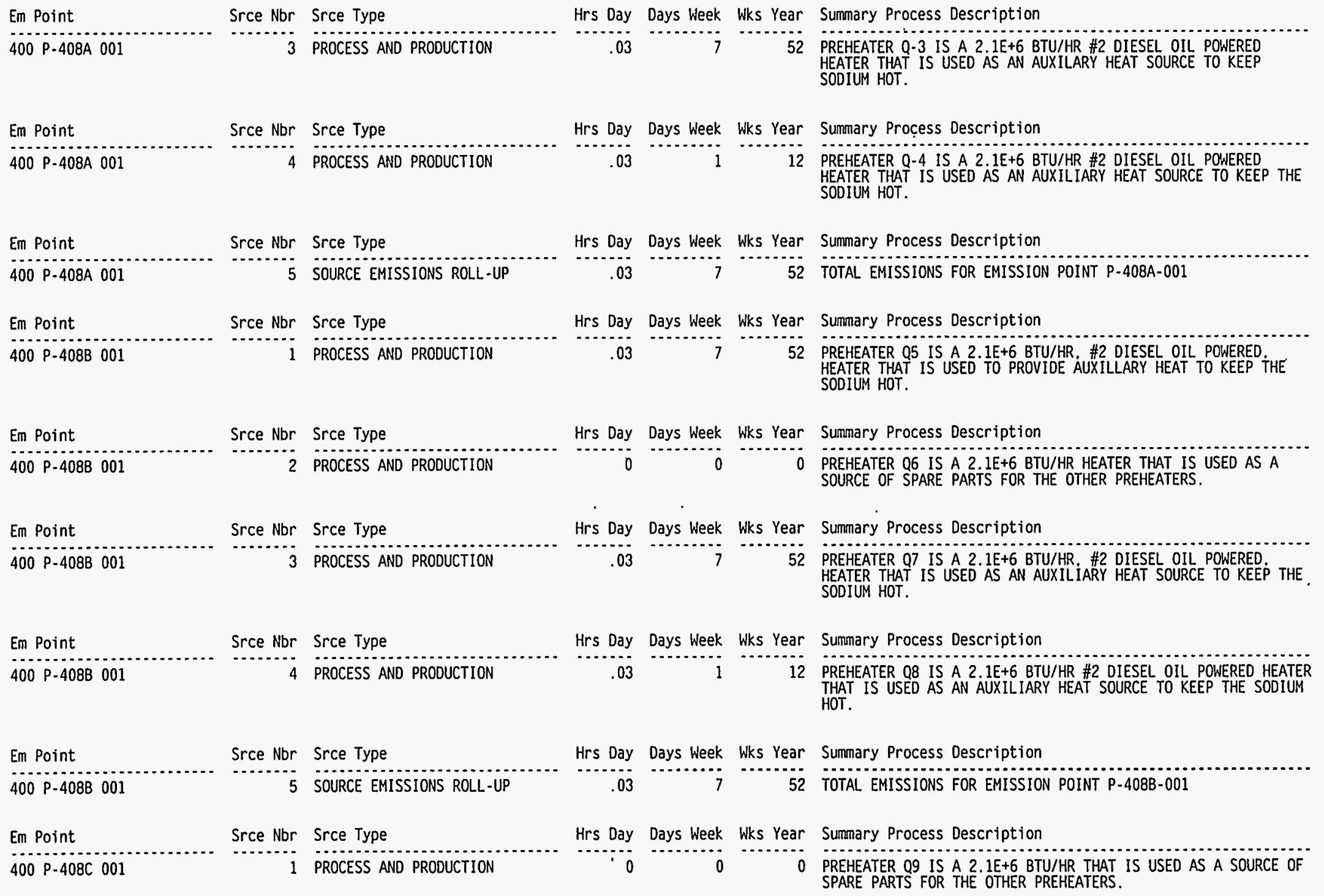




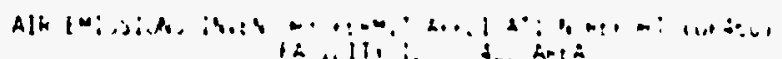

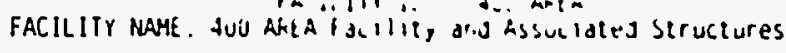

Source (Process) Listing

\begin{tabular}{|c|c|c|c|c|c|c|}
\hline Em Point & Srce $\mathrm{Nbr}$ & Srce Type & Hrs Day & Days Week & Wks Year & Summary Process Description \\
\hline 400 P-408C 001 & 2 & PROCESS AND PRODUCTION & .03 & 7 & 52 & $\begin{array}{l}\text { PREHEATER Q10 IS A } 2.1 \text { E+6 BTU/HR. \#2 DIESEL OIL POWERED, } \\
\text { AUXILLARY HEAT SOURCE THAT IS USED TO KEEP THE SODIUM HOT. }\end{array}$ \\
\hline Em Point & Srce $\mathrm{Nbr}$ & Srce Type & Hrs Day & Days Week & Wks Year & Summary Process Description \\
\hline $400 \quad P-408 C 001$ & 3 & PROCESS AND PRODUCTION & .03 & 7 & 52 & $\begin{array}{l}\text { PREHEATER QI1 IS A } 2.1 E+6 \text { BTU/HR \#2 DIESEL OIL POWERED } \\
\text { HEATER THAT IS USED AS AN AUXILIARY HEAT SOURCE TO KEEP THE } \\
\text { SODIUM HOT. }\end{array}$ \\
\hline $400 \mathrm{P}-408 \mathrm{C} 001$ & 4 & PROCESS AND PRODUCTION & .03 & 1 & 12 & $\begin{array}{l}\text { PREHEATER Q12 IS A } 2 \text {. } 1 E+6 \text { BTU/HR \#2 DIESEEL OIL POWERED } \\
\text { HEATER THAT IS USED AS AN AUXILIARY HEAT SOURCE TO KEEP THE } \\
\text { SODIUM HOT. }\end{array}$ \\
\hline Em Point & Srce Nbr & Srce Type & Hrs Day & Days Week & Wks Year & Summary Process Description \\
\hline $400 \mathrm{P}-408 \mathrm{C} 001$ & $\dddot{5}$ & SOURCE EMISSIONS ROLL-UP & .03 & 7 & 52 & TOTAL EMISSIONS FOR EMISSION POINT P-40BC-00I \\
\hline Em Point & Srce Nbr & Srce Type & Hrs Day & Days Week & Wks Year & Summary Process Description \\
\hline Em Point $\quad$ & Srce Nbr & Srce Type & Hrs Day & Days Week & Wks Year & Summary Process Description \\
\hline 400 P-437MN\&ST 001 & $\overline{1}$ & TANKS AND VESSELS & 24 & 7 & 52 & $\begin{array}{l}\text { RADIATION WASTE STORAGE TANK NO. 1. MONITOR ID NUMBER - RE- } \\
1-62 \text {. }\end{array}$ \\
\hline Em Point & Srce Nbr & Srce Type & Hrs Day & Days Week & Wks Year & Summary Process Description \\
\hline 400 P-437MN\&ST 001 & 2 & TANKS AND VESSELS & 24 & 7 & 52 & RADIATION WASTE STORAGE TANK NO. 2 \\
\hline Em Point & Srce $\mathrm{Nbr}$ & Srce Type & Hrs Day & Days Week & Wks Year & Summary Process Description \\
\hline 400 P-437MN\&ST 001 & 3 & PROCESS AND PRODUCTION & 24 & 7 & 52 & RADIATION WASTE TANK PIT \\
\hline Em Point & Srce Nbr & Srce Type & Hrs Day & Days Week & Wks Year & Summary Process Description \\
\hline 400 P-437MNEST 001 & 4 & PROCESS AND PRODUCTION & 24 & 7 & 52 & DECONTAMINATION ROOMS I AND II (INTERCONNECTED) \\
\hline
\end{tabular}

Page \#: 5 of $10 \quad$ Report Run Date: $29-A P R-95$ 
AIR EMISSIONS INVENTORY PERMIT APPLICATION REPORT (UP460)

FACILITY NAME: 400 AREA Facility and Associated Structures

\section{Source (Process) Listing}

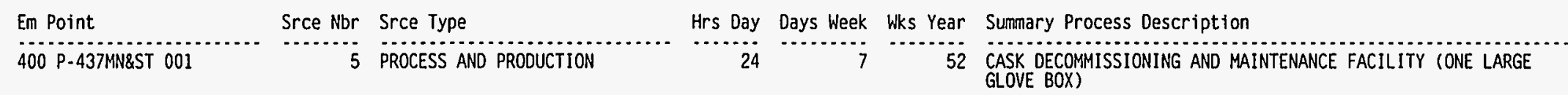

T-3 SHIPPING CASKS ARE WASHED DOWN IN COMF. THE LIQUID WASTE gOES INTO THE RAD WASTE STORAGE TANKS. THERE ARE ONLY 2 CASKS THAT ARE CLEANED YEARLY.

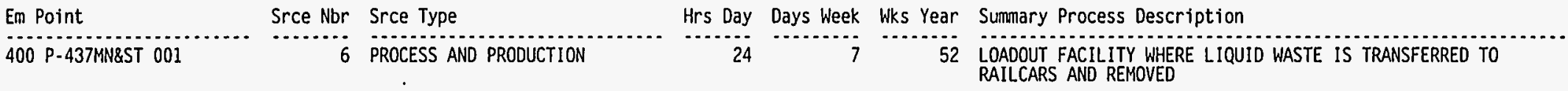

TANK TRANSFERS OCCUR LESS THAN ONE TIME PER YEAR UNLESS THERE WAS AN EXCEPTION OR AN ACCIOENTAL OVER-FILLING. THIS FACILITY IS ALSO KEPT CLEAN. IN

CASE OF A LEAK TRANSFERS ARE IMMEDIATELY STOPPED AND THE SPILL CLEANED.

\begin{tabular}{|c|c|c|c|c|c|c|}
\hline Em Point & Srce $\mathrm{Nbr}$ & Srce Type & Hrs Day & Days Week & Wks Year & Summary Process Description \\
\hline $400 \mathrm{P}-437 \mathrm{MN} \& S T \mathrm{ST} 001$ & 7 & SOURCE EMISSIONS ROLL-UP & 24 & 7 & 52 & TOTAL EMISSIONS FOR EMISSION POINT P-437MN\&ST-001 \\
\hline Em Point & Srce $\mathrm{Nbr}$ & Srce Type & Hrs Day & Days Week & Wks Year & Summary Process Description \\
\hline 400 P-FFTFCBEX 001 & 1 & PROCESS AND PRODUCTION & 24 & 7 & 52 & $\begin{array}{l}\text { EXHAUSTEO ARGON CONTAINS TRACES OF CS- } 137 \text { FROM STOREO SODIUM } \\
\text { COOLANT. SITE MONITOR ID NUMBER C350 CONTAINS 2 EQUIPMENT } \\
\text { PIECES: SERIAL NUMBER RE96010 WHICH MONITORS GROSS BETA. AND } \\
\text { RE9601I WHICH MONITORS NOBLE GASES. }\end{array}$ \\
\hline 400 P-FFTFHTTR 001 & 1 & PROCESS AND PRODUCTION & 24 & 7 & 52 & TRACE CONTAMINATION IN 491-S. MONITOR IO NUMBER - 96061. \\
\hline Em Point & Srce Nbr & Srce Type & Hrs Day & Days Week & Wks Year & Summary Process Description \\
\hline 400 P-FFTFRESB 001 & 1 & PROCESS AND PRODUCTION & 24 & 7 & 52 & TRACE CONTAMINATION \\
\hline
\end{tabular}

Source Raw Materials Usage

Em Point

Srce Nbr Srce Type

Process Rate Process Uom Material

Fuels Usage by Source Processes

Em Point

Srce $\mathrm{Nbr}$ Srce Type

aty Uom

Fuel 
Fuels Usage by Control Devices

Em Point

Ctl Nbr Ctl Type

Qty Uom

Fuel

Point Source Criteria Pollutants - Type: CO

Em Point

400 P-408A 001

- 400 P-408B 001

400 P-408C 001

Sum
Srce Nbr Srce Type Pollutant

5 SOURCE EMISSIONS ROLL-UP

5 SOURCE EMISSIONS ROLL-UP

5 SOURCE EMISSIONS ROLL-UP

Point Source Criteria Pollutants - Type: NOX
Em. Pt. Potential Em. Pt.

to Emit (tons/yr) Ctl Actual (tons/yr) Poilutant

.326170000 $\quad .000447000$ CARBON MONOXIDE

\begin{tabular}{|c|c|c|}
\hline .326170000 & .000447000 & CARBON MONOXIDE \\
\hline .326170000 & .000447000 & CARBON MONOXIDE \\
\hline
\end{tabular}
.001341000

\section{Em Point}

400 P. 408 A 001

400 P. $408 B \quad 001$

400 P-408C 001

Sum
Srce Nbr Srce Type Pollutant

5 SOURCE EMISSIONS ROLL-UP

5 SOURCE EMISSIONS ROLLLUP

5 SOURCE EMISSIONS ROLL-UP

Point Source Criteria Pollutants - Type: PM10

Em. Pt. Potential Em. Pt.
to Emit (tons/yr) Ctl Actual (tons/yr) Pollutant $1.174213000 \quad .001609000$ NITROGEN DIOXIDE (NO2)

$1.174213000 \quad .001609000$ NITROGEN DIOXIDE (NO2)

$1.174213000 \quad$. 001609000 NITROGEN DIOXIDE (NO2)

$3.522639000 \quad .004827000$

Em Point

400 P-408A 001

400 P-408B 001

$400 \quad P-408 C 001$

Sum
Srce Nbr

Srce Type Pollutant

5 SOURCE EMISSIONS ROLL-UP

5 SOURCE EMISSIONS ROLL-UP

5 SOURCE EMISSIONS ROLL-UP

Em. Pt. Potential Em. Pt.

to Emit (tons/yr) Ctl Actual (tons/yr) Polrutant

\begin{tabular}{|c|c|c|}
\hline .163085000 & .000223000 & PARTICULATE MATTER (10 MICRONS OR LESS) \\
\hline .163085000 & .000223000 & PARTICULATE MATTER (10 MICRONS OR LESS) \\
\hline .163085000 & .000223000 & PARTICULATE MATTER (10 MICRONS OR LESS) \\
\hline .489255000 & .000669000 & \\
\hline
\end{tabular}

Page \#: 7 of $10 \quad$ Report Run Date: 29-APR-95 
AIR EMISSIONS INVENTORY PERMIT APPLICATION REPORT (UP460)

FACILITY NAME: 400 AREA Facility and Associated Structures

Point Source Criteria Pollutants - Type: SOX

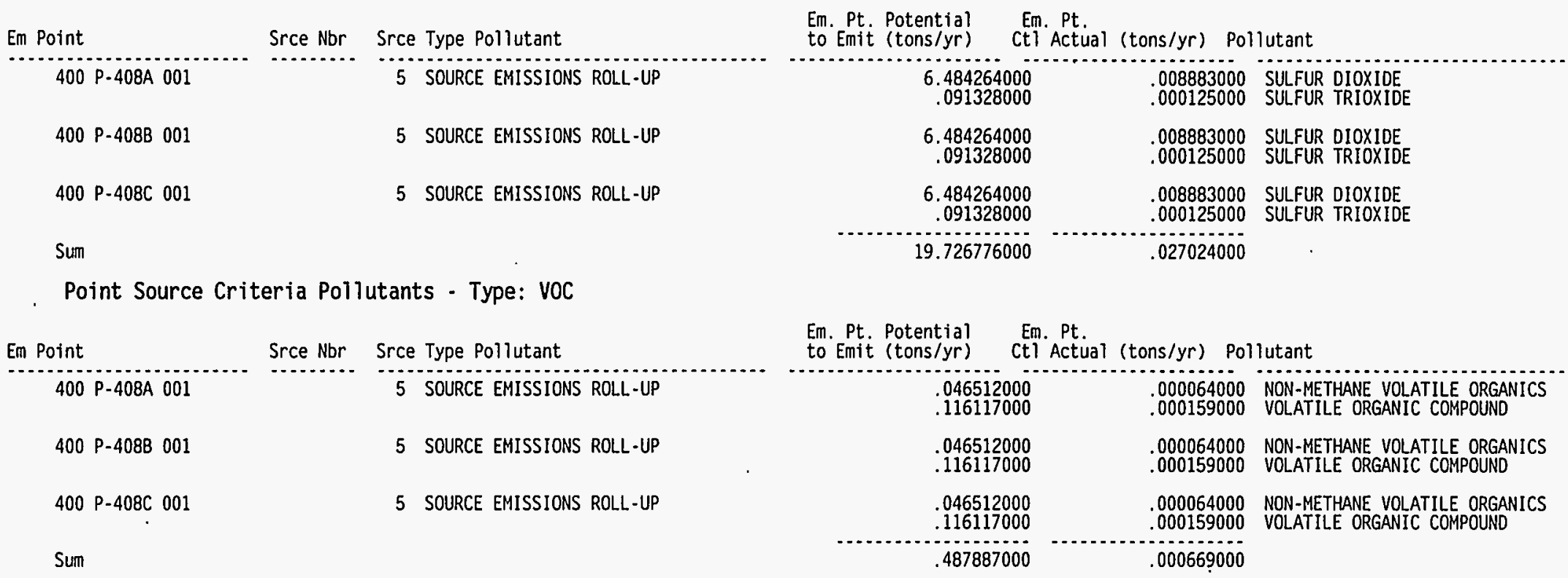

Point Source Emissions - Radiological Pollutants

\begin{tabular}{|c|c|c|c|c|c|}
\hline Em Point & Srce Nbr & Srce Type Pollutant & $\begin{array}{l}\text { Em. Pt. Potential } \\
\text { to Emit }(C i / y r)\end{array}$ & $\begin{array}{l}\text { Em. Pt. } \\
\text { Cti Actual (Ci/yr) }\end{array}$ & Pollutant \\
\hline 400 P-437MN\&ST 001 & $\begin{array}{l}1 \\
2 \\
3 \\
4 \\
5 \\
6\end{array}$ & $\begin{array}{l}\text { TANKS AND VESSELS } \\
\text { TANKS AND VESSELS } \\
\text { PROCESS AND PROUCTION } \\
\text { PROCESS AND PROOUCTION } \\
\text { PROCESS AND PRODUCTION } \\
\text { PROCESS AND PRDDUCTION }\end{array}$ & $\begin{array}{l}\text { Refer to } \\
\text { WHC-SD-EN-RPT-007 } \\
\text { Rev. O Page A-329 }\end{array}$ & $\begin{array}{l}\text { Refer to } \\
\text { DOE/RL }-94-51 \\
\text { Table 2-1 }\end{array}$ & \\
\hline 400 P-FFTFCBEX 001 & $\cdot 1$ & PROCESS AND PRODUCTION & $\begin{array}{l}\text { Refer to } \\
\text { WHC-SD-EN-RPT-007 } \\
\text { Rev. O Page A-326 }\end{array}$ & $\begin{array}{l}\text { Refer to } \\
\text { DOE/RL-94-51 } \\
\text { Table 2-1 }\end{array}$ & \\
\hline 400 P-FFTFHTTR 001 & 1 & PROCESS AND PRODUCTION & $\begin{array}{l}\text { Refer to } \\
\text { WHC-SD-EN-RPT-007 } \\
\text { Rev. O Page A-327 }\end{array}$ & $\begin{array}{l}\text { Refer to } \\
\text { DOE/RL-94-51 } \\
\text { Table 2-1 }\end{array}$ & \\
\hline 400 P-FFTFRESB 001 & 1 & PROCESS AND PRODUCTION & $\begin{array}{l}\text { Refer to } \\
\text { WHC-SD-EN-RPT-007 } \\
\text { Rev. O Page A-328 }\end{array}$ & $\begin{array}{l}\text { Refer to } \\
\text { DOE/RL-94-51 } \\
\text { Table 2-1 }\end{array}$ & \\
\hline
\end{tabular}

Page \#: 8 of $10 \quad$ Report Run Date: 29-APR-95 


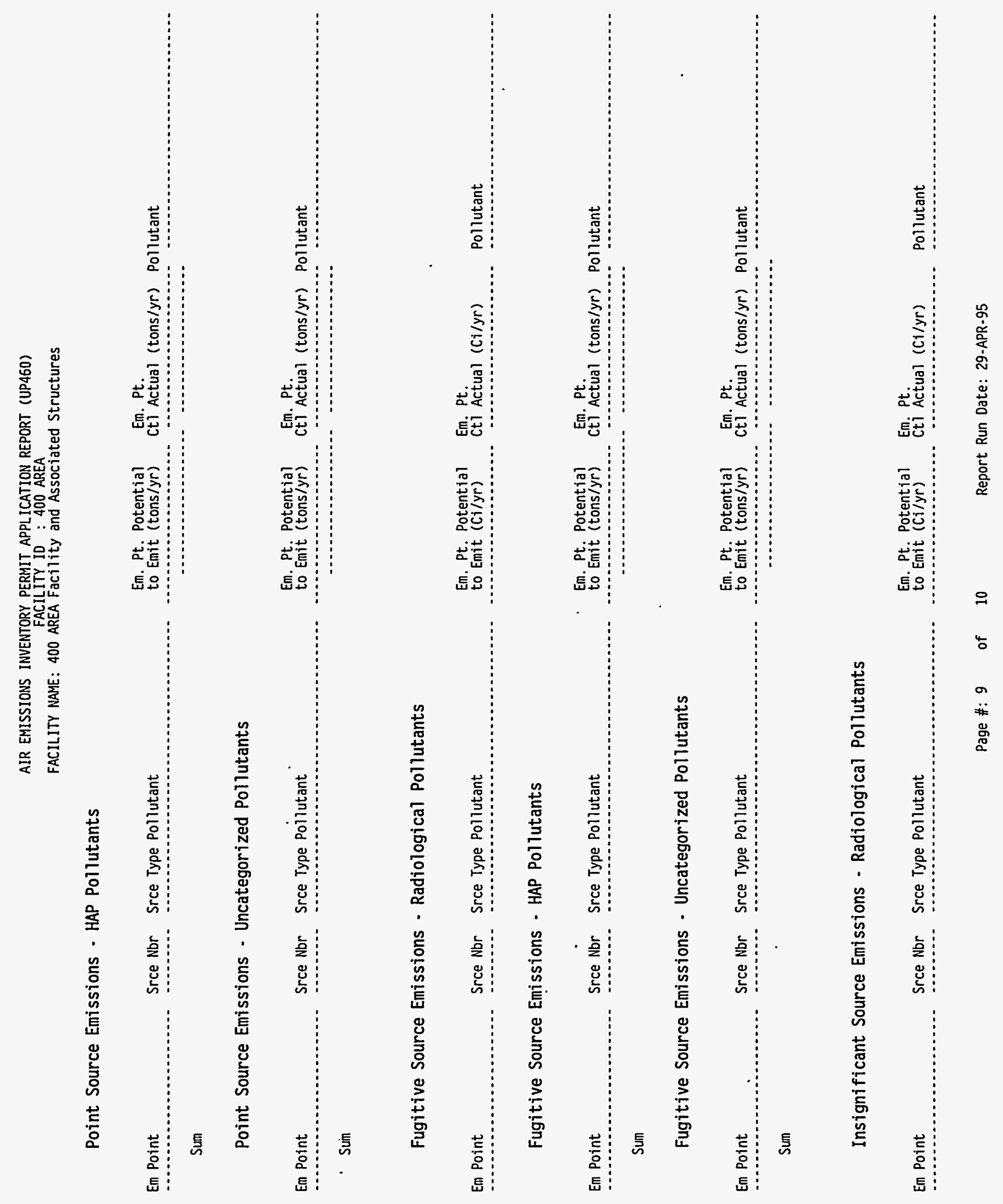


AIR EMISSIONS INVENTORY PERMIT APPLICATION REPORT (UP460)

FACILITY NAME: 400 AREA Facility and Associated Structures

Insignificant Source Emissions - HAP Pollutants

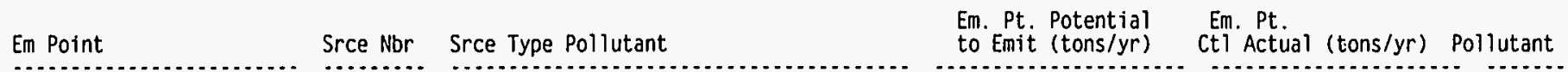

Insignificant Source Emissions - HAP Pollutants

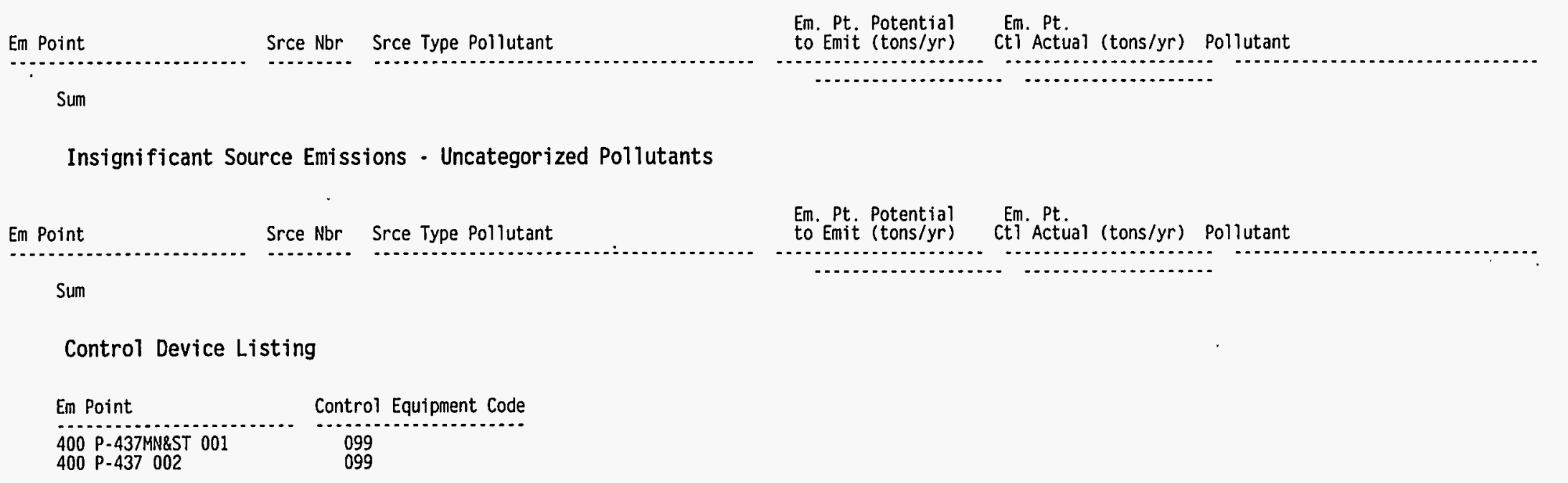




\begin{tabular}{ll}
\hline FACILITY: \\
\hline EMISSION POINT: & 400 Area \\
\hline
\end{tabular}

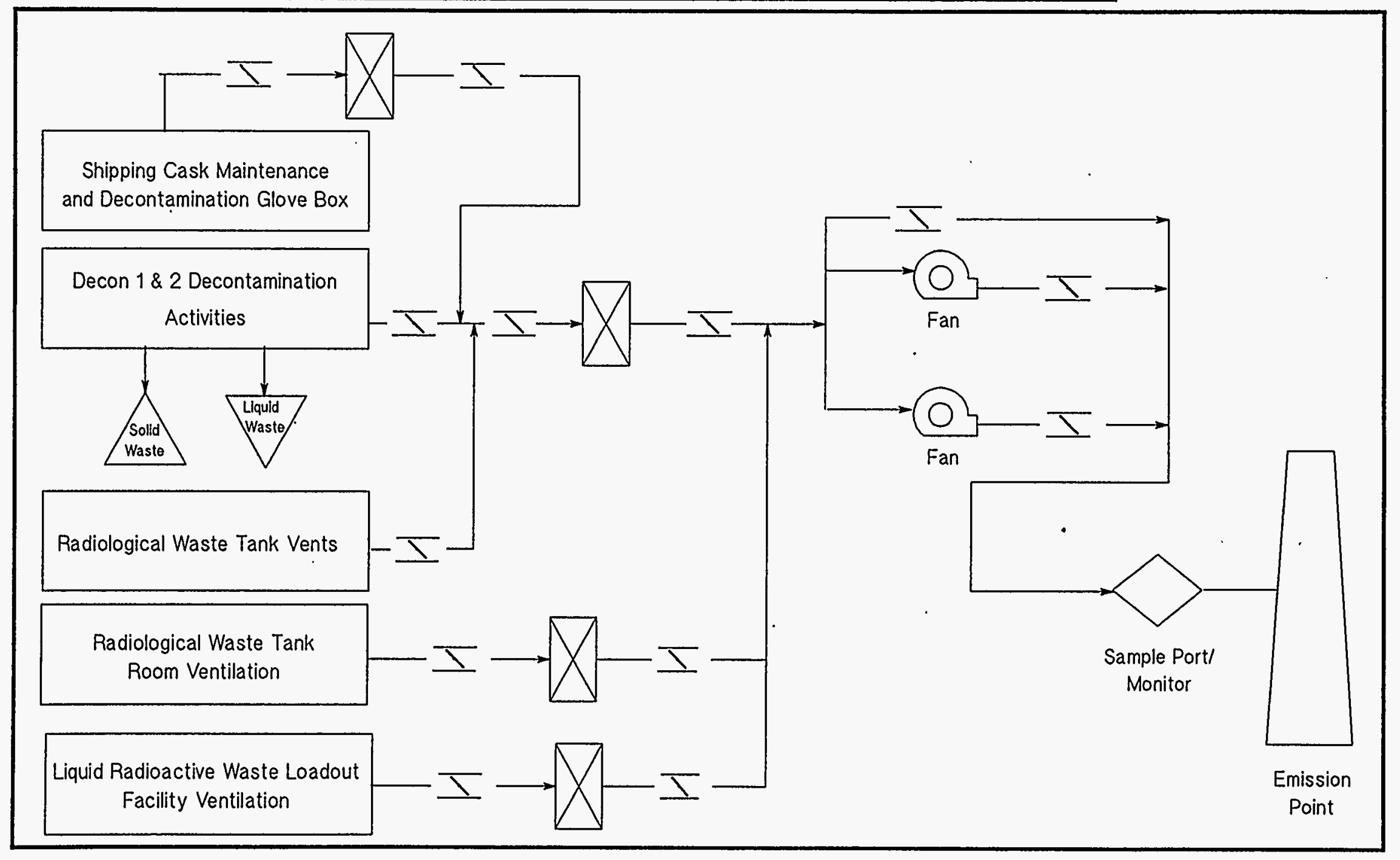




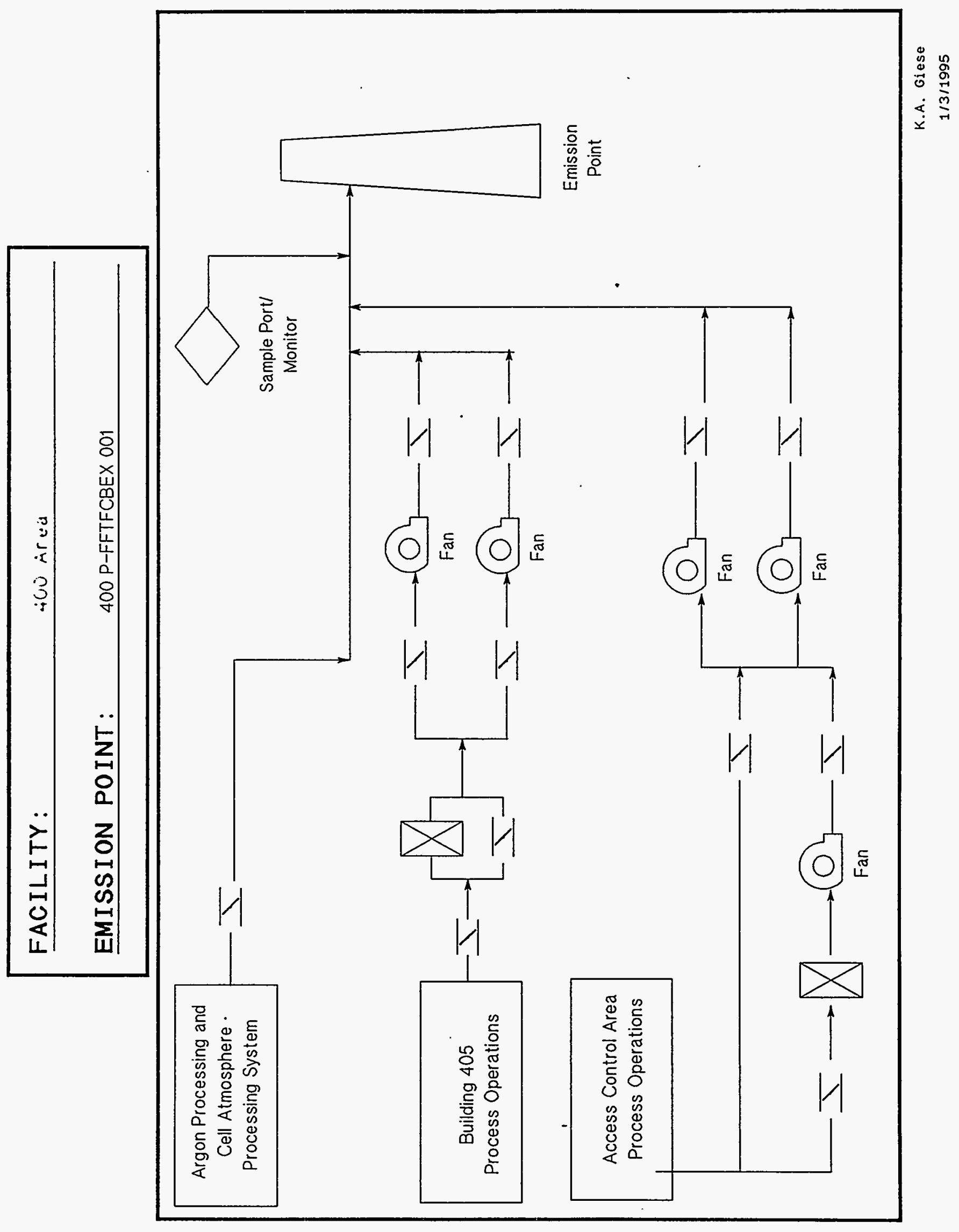




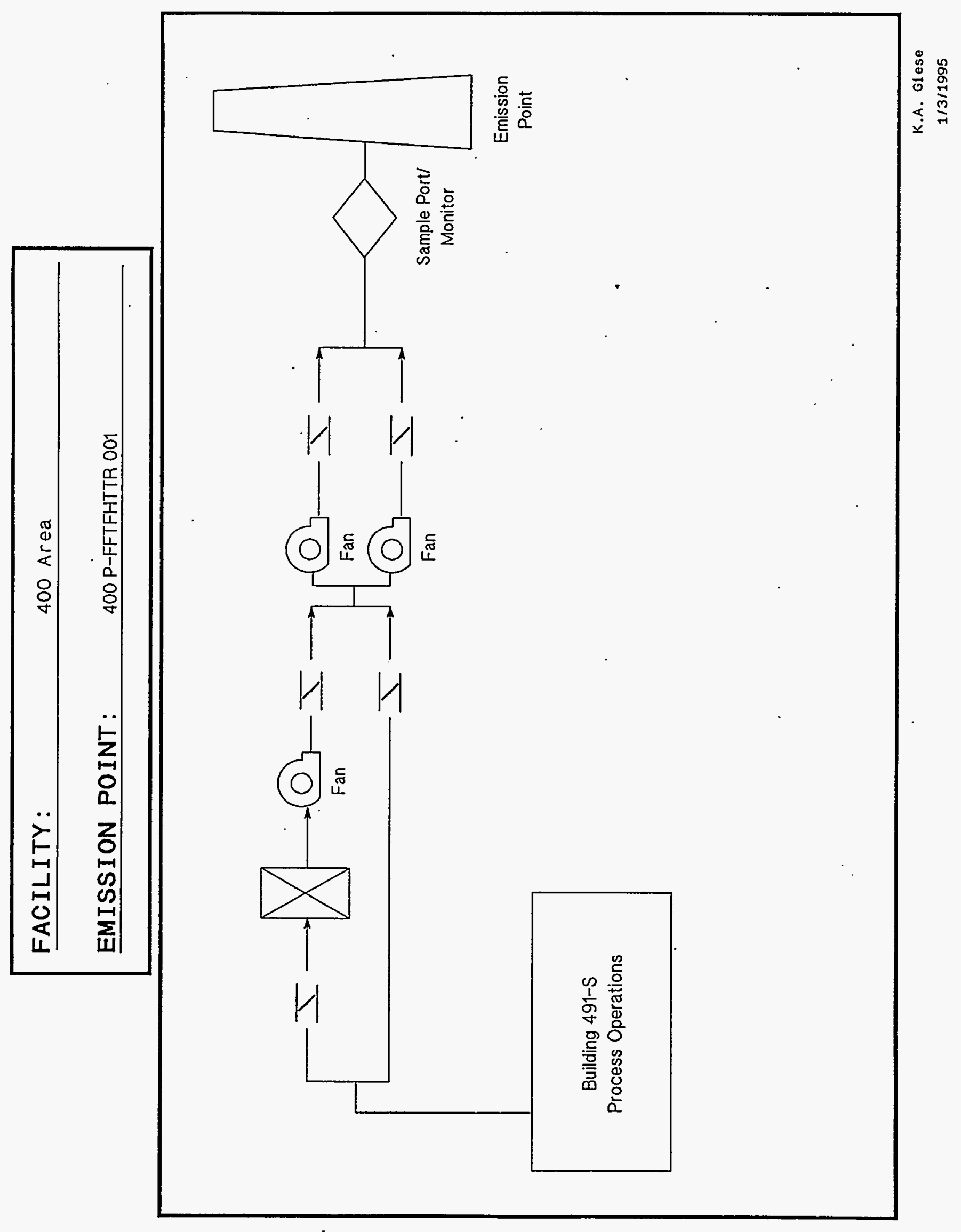




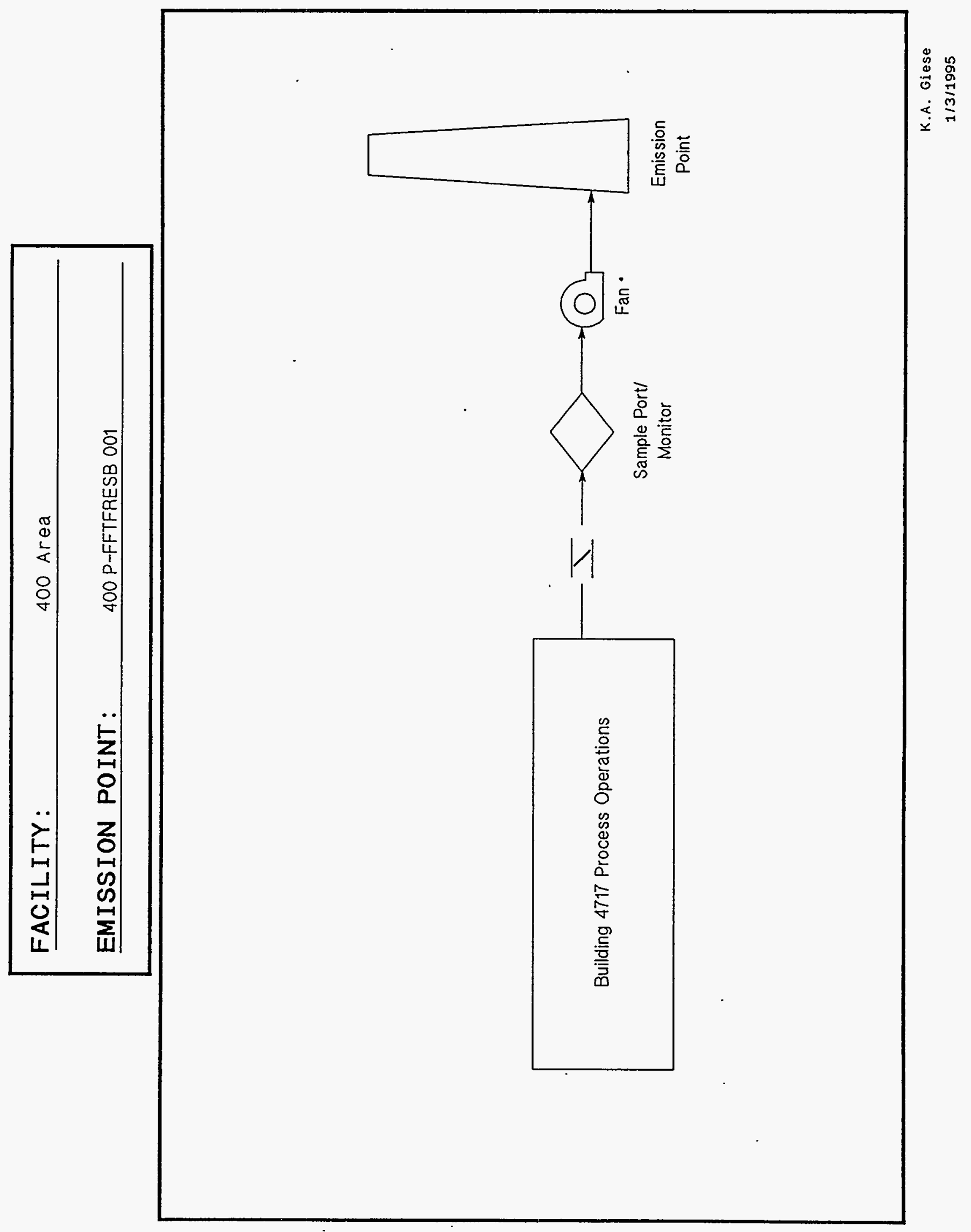




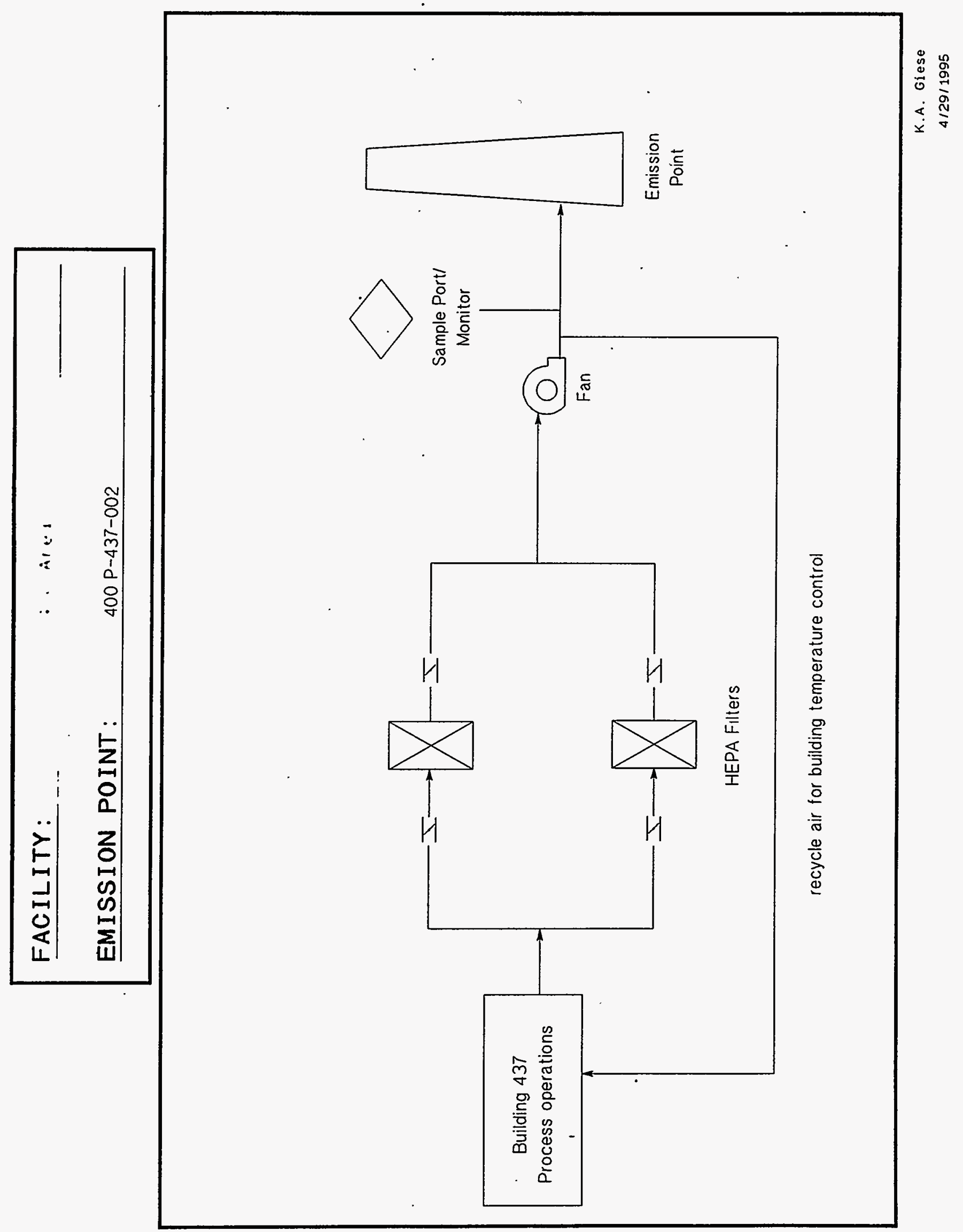




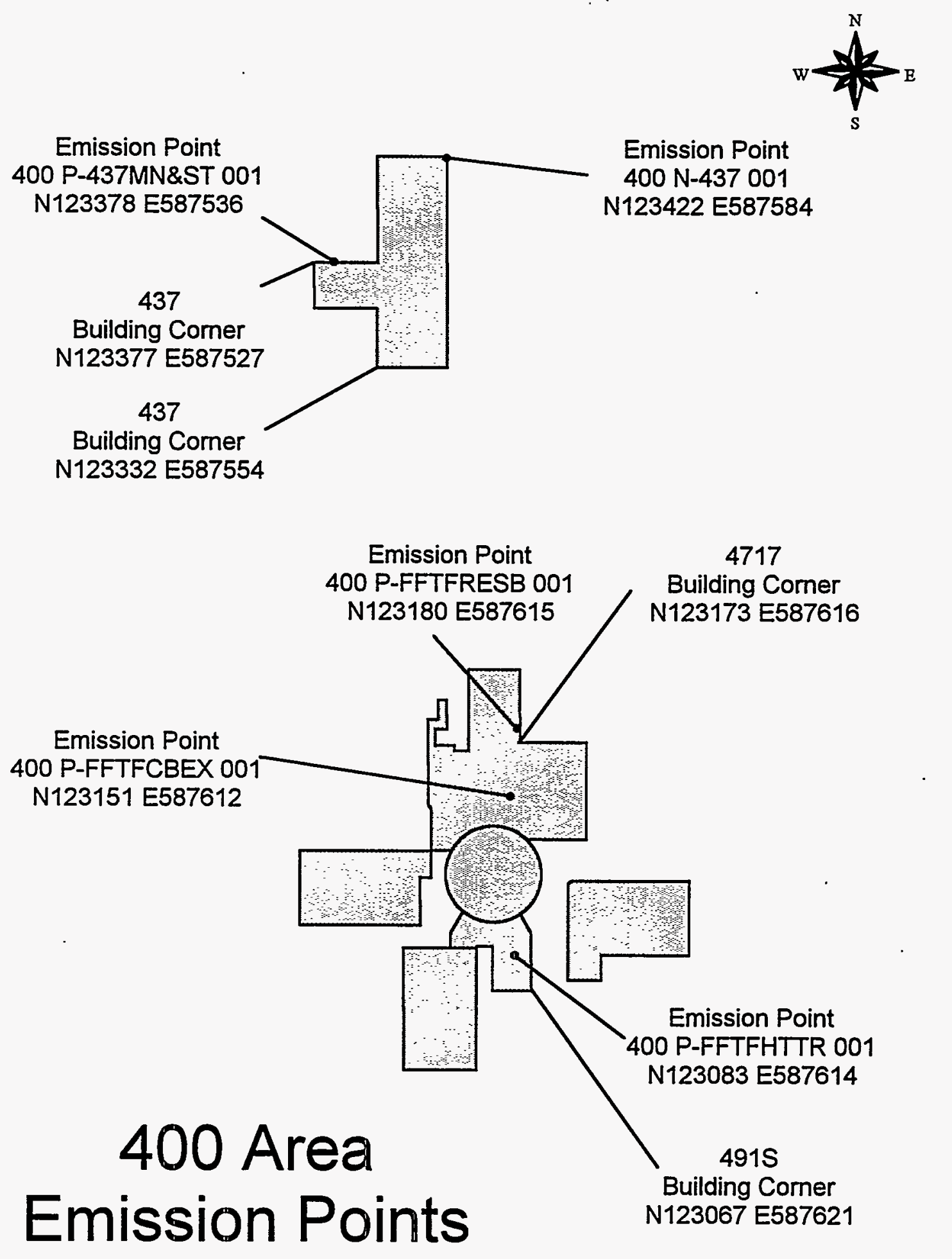


Hanford Site Air

Operating Permit Application
DOE/RL-95-07, Rev. 0

05/95

\title{
APPENDIX H
}

\author{
600 AREA
}




\section{APPENDIX H}

\section{CONTENTS}

H-1 600 AREA TANK FARM VENT STATION

H-2 WASTE SAMPLING AND CHARACTERIZATION FACILITY 


\section{APPENDIX H-1 \\ 600 AREA TANK FARM VENT STATION}

Chapter I. General Information

\section{I-B.1. Facility Description}

The 241-EW-151 DCRT was designed to allow air to enter the cross-site transfer line at the high point in the line. The entry of air behind the transfer breaks the vacuum formed in the line and allows the waste to flow by gravity through the transfer lines with half the waste draining to the 200 West Area and half to the 200 East Area. The vent station tank provides a catch tank for liquids that might enter cross-site transfer vent lines. The primary tank is 4 feet 7 inches in diameter, approximately 6 feet high, has an 800-gallon capacity, and is constructed of stainless steel.

\section{I-B.2. Products, Raw Materials, Fuels, Processes}

- Principle products - There are no products produced.

- Process information - There are no process activities.

- Raw materials used by processes - There are no raw materials used at the 241-EW-151 DCRT.

- Fuels used by processes - There are no fuels used at the 241-EW-151 DCRT.

\section{I-C.1. Emission Points, Controls, Stacks}

The emission point and stack information is found on page 1 of the AEI report output. The control information is found on page 3 of the AEI report output.

\section{I-C.2. Facility Process Flow Diagrams}

Attached.

\section{I-C.3. Site Map}

Attached. 
Hanford Site Air

Operating Permit Application
DOE/RL-95-07, Rev. 0 $05 / 95$

\section{Chapter II. Regulatory Requirements}

\section{II-A.1. Description of Discharge and Emission Points}

The emission point and stack information is found on page 1 of the AEI report output. 


\section{Facility Building List}

\begin{tabular}{|c|c|c|c|c|c|c|c|}
\hline Bldg Id & North & East & Bldg Desc & Point of Contact (POC) & (POC) Phone & DOE POC & DOE Phone \\
\hline 241EW151 & 135304 & 570640 & $\begin{array}{l}\text { DIVERSION BOX VENT STATION, PART OF CROSS-SITE } \\
\text { WASTE TRANSFER SYSTEM }\end{array}$ & CRUMMEL. GARY M. & $373-5175$ & CASEY 0. RUUD & $373-3478$ \\
\hline
\end{tabular}

Emission Point Listing

\begin{tabular}{|c|c|c|c|c|c|c|c|c|c|}
\hline Em Point & Ep Type & North & East & st Srce Nbr & Scc Code & Srce Ty & & & \\
\hline $600 \mathrm{P}-241 E W 151001$ & POINT & 135304 & 57064 & 40 & not assigne & PROCESS & AND PRODUCTION & & \\
\hline Stack Configuration & & Diameter ( $f t$ ) & Length(ft) & Width(ft) & Area (sq ft) & Exit $\operatorname{Temp}(F)$ & Exit Veloc $(\mathrm{ft} / \mathrm{sec})$ & Ht Abv Grnd(ft) & Ht Abv Struc(ft) \\
\hline VERTICAL WITH WEATHE & CAP & .33 & & & .09 & 78 & .0325 & $i$ & \\
\hline
\end{tabular}

\section{Source (Process) Listing}

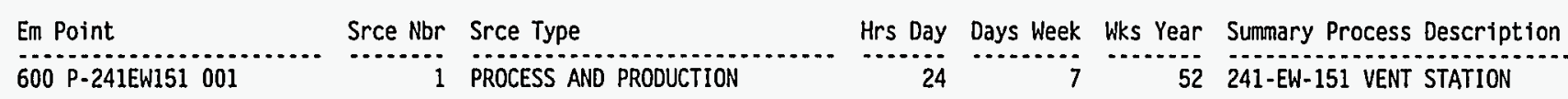

THE 241 -EW-151 DIVERSION BOX IS A CONCRETE UNDERGROUND PIT WITH DIMENSIONS OF $8^{\circ} \times 6^{\circ} \times 3^{\circ} 8^{\prime \prime}$ (LXWXD). THE 241 EW-151 DIVERSION BOX IS THE PRIMARY WASTES TRANSFERRED VIA THE 241 -EW-151 DIVERSION BOX COULD POTENTIALLY INCLUDE ANY 200 AREA WASTE.

\section{Source Raw Materials Usage}

Em Point

Srce Nbr Srce Type

Process Rate Process Uom Material

Fuels Usage by Source Processes

Em Point Srce $\mathrm{Nbr}$ Srce Type

Qty Uom

Fuel

Fuels Usage by Control Devices

Em Point

Ctl Nbr Ctl Type

Qty Uom

Fue? 
AIR EMISSIONS INVENTORY PERMIT APPLICATION REPORT (UP460)

FACILITY NAME: 600 Area Tank Farm and Associated Structures

Point Source Emissions - Radiological Pollutants

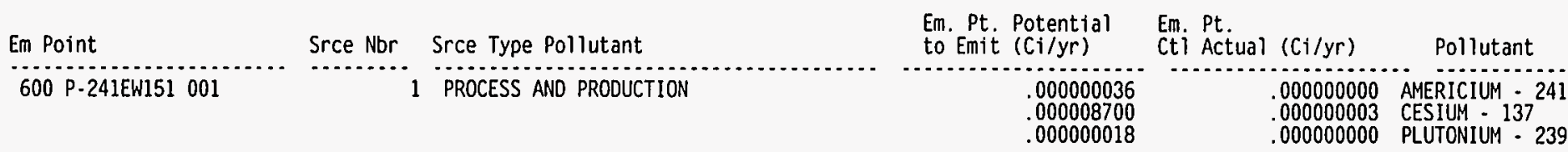

Point Source Emissions - Radiological Pollutants

Em Point $\quad$ Srce Nbr Srce Type Pollutant

600 P-241EW151 $001 \quad 1$ PROCESS AND PRODUCTION

\section{Point Source Emissions - HAP Pollutants}

Em Point

Srce Nbr Srce Type Pollutant

Sum

Point Source Emissions - Uncategorized Pollutants

Em Point Srce Nbr Srce Type Pollutant

Sum

Fugitive Source Emissions - Radiological Pollutants

Em Point

Srce Nbr Srce Type Pollutant

$\begin{array}{ll}\text { Em. Pt. Potential Em. Pt. } & \\ \text { to Emit }(\mathrm{C} i / \mathrm{yr}) & \mathrm{Ct} \text { Actual (Ci/yr) }\end{array}$ Pollutant 
Fugitive Source Emissions - HAP Pollutants

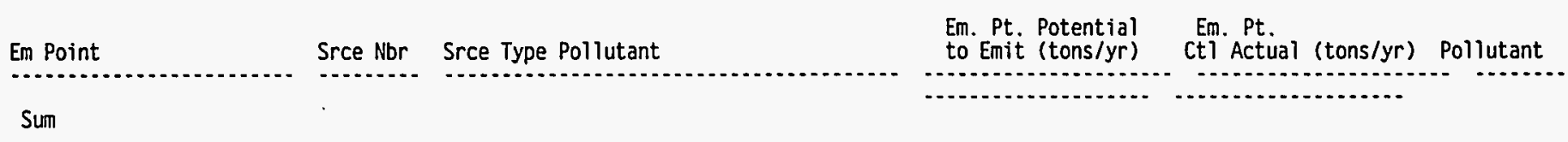

Fugitive Source Emissions - Uncategorized Pollutants

Em Point

Srce Nbr Srce Type Pollutant

Em. Pt. Potential Em. Pt.

(tons/yr) Pollutant

sum

Insignificant Source Emissions - Radiological Pollutants

Em Point

Srce Nbr Srce Type Pollutant

Em. Pt. Potential Em. Pt.

to Emit (Ci/yr) Cti Actual (Ci/yr)

Pollutant

Insignificant Source Emissions - HAP Pollutants

Em Point

Srce Nbr Srce Type Pollutant

Em. Pt. Potential Em. Pt.

(tons/yr) Pollutant

Sum

Insignificant Source Emissions - Uncategorized Pollutants

Em Point

Srce Nbr Srce Type Pollutant

Em. Pt. Potential Em. Pt.
to Emit. (tons/yr) Ctl Actual (tons/yr) Pollutant

Sum

\section{Control Device Listing}

Em Point

Control Equipment Code

600 P-241EW151 001

099 


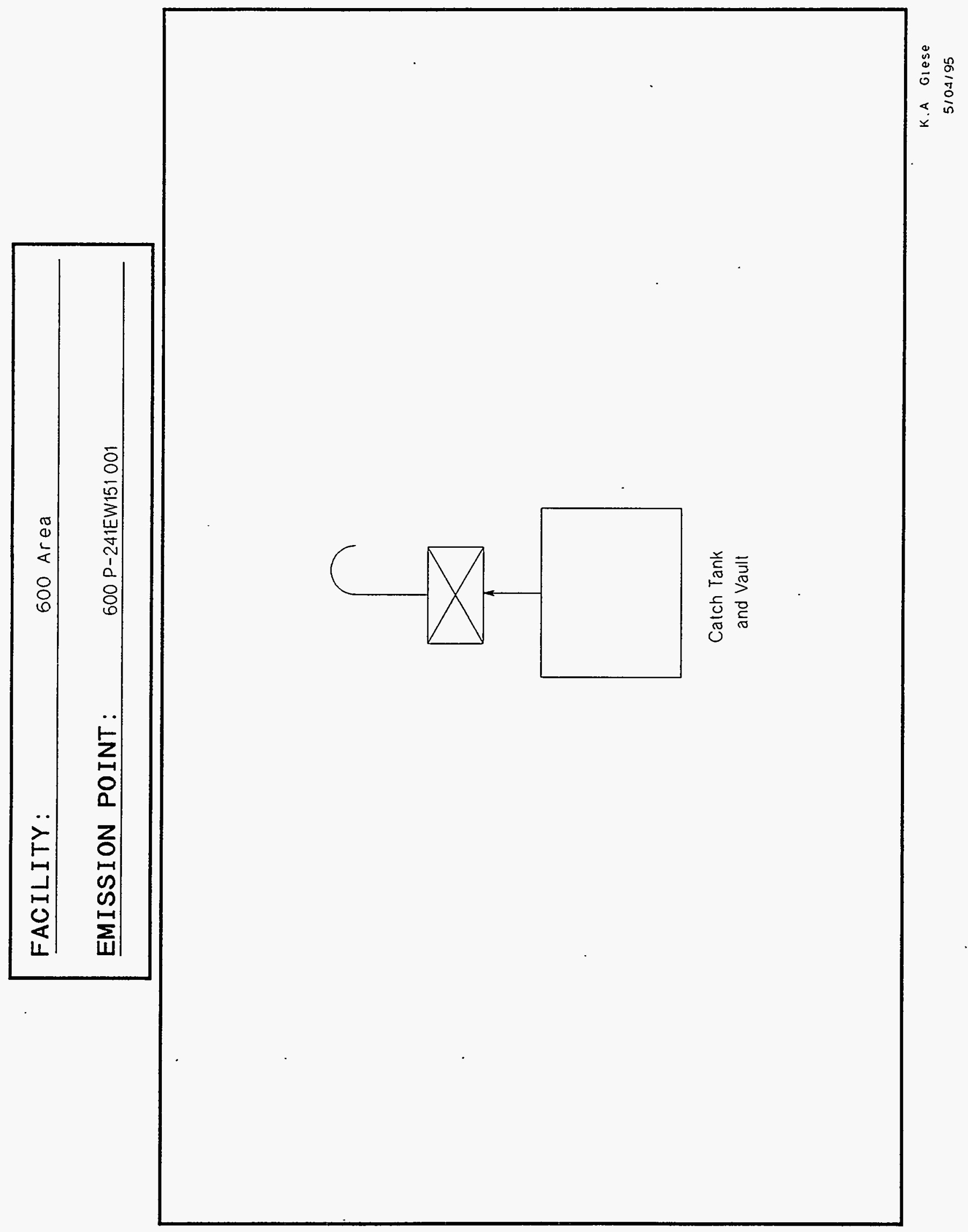




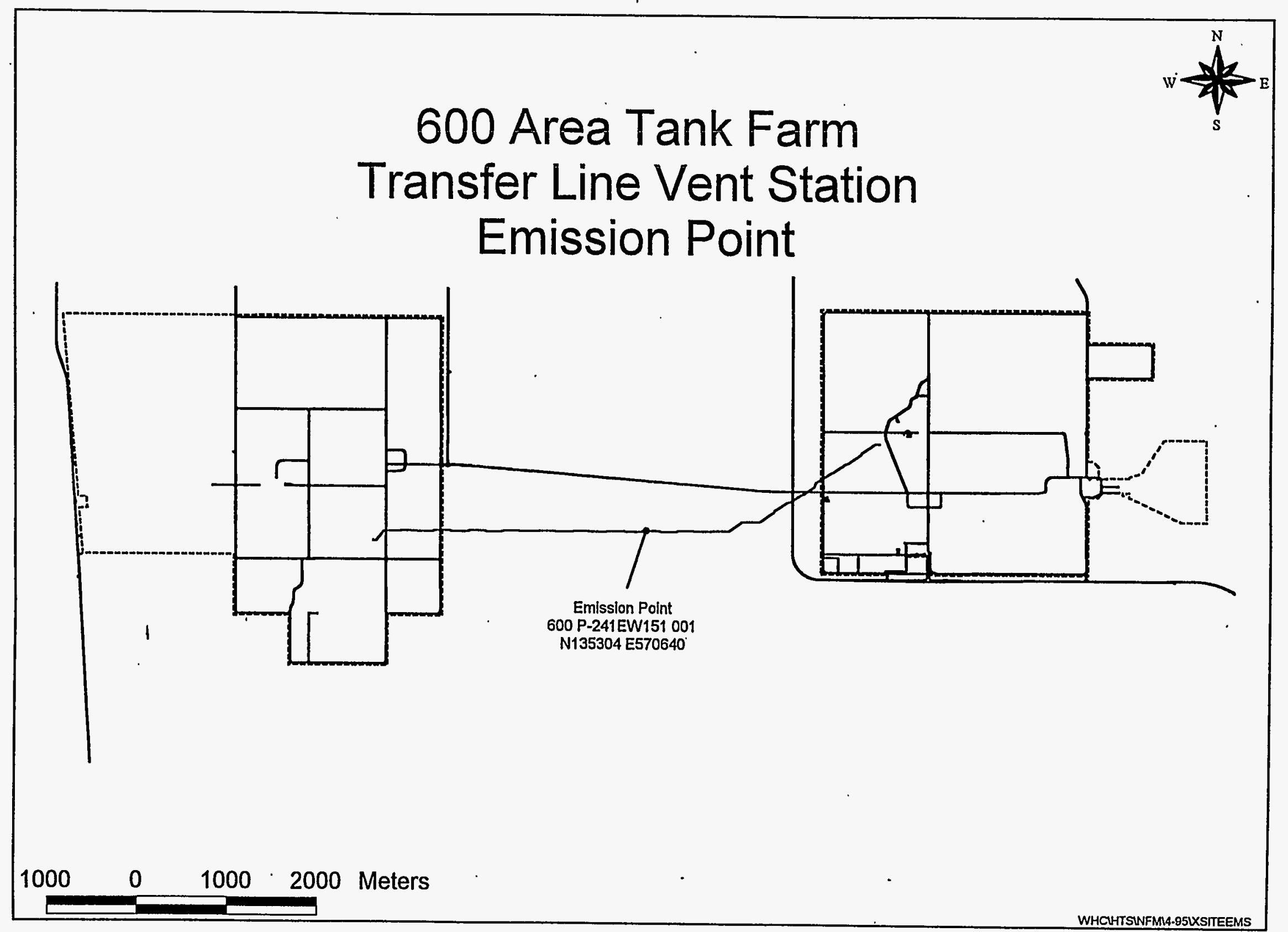




\section{APPENDLX H-2 \\ WASTE SAMPLING AND CHARACTERIZATION FACILITY}

Chapter I. General Information

\section{I-B.1. Facility Description}

The Waste Sampling and Characterization Facility (WSCF) provides for processing of lowlevel and nonradioactive samples. The facility commenced operation in 1994. Current operations are for twenty-four hours per day, 365 days per year.

I-B.2. Products, Raw Materials, Fuels, Processes

- Principle products - There are no products at WSCF

- Process information - There are no process activities at WSCF

- Raw materials used by processes - There are no raw materials used at WSCF

- Fuels used by processes - There are fuels used at WSCF.

\section{I-C.1. Emission Points, Controls, Stacks}

The emission point and stack information is found on page 1 of the AEI report output. The control information is found on page five of the AEI report output.

\section{I-C.2. Facility Process Flow Diagrams}

Attached.

I-C.3. Site Map

Attached.

\section{Chapter II. Regulatory Requirements}

\section{II-A.1. Description of Discharge and Emission Points}

The emission point and stack information is found on page 1 of the AEI report output. 
Hanford Site Air

Operating Permit Application
DOE/RL-95-07, Rev. 0

05/95

This page intentionally left blank.

APP H-2.ii 
AIR EMISSIONS INVENTORY PERMIT APPLICATION REPORT (UP460)
FACILITY ID : 600 LABS

FACILITY NAME: 600 Laboratories and Associated Structures

\section{Facility Building List}

\begin{tabular}{|c|c|c|c|c|c|c|c|}
\hline Bldg Id & North & East & Bldg Desc & Custodian(Contractor) & Cust Phone & DOE POC & DOE Phone \\
\hline 6266 & 136413 & 568856 & WASTE SAMPLING AND CHARACTERIZATION FACILITY & HALL, MICHAEL J. & $373-5719$ & STEVE VEITENHEIMER & $373-9725$ \\
\hline $\begin{array}{l}6266 \mathrm{~A} \\
6269\end{array}$ & $\begin{array}{l}136411 \\
136440\end{array}$ & $\begin{array}{l}568778 \\
568900\end{array}$ & $\begin{array}{l}\text { WSCF) } \\
\text { WSCF BUILDING } \\
\text { WSCF BUILDING }\end{array}$ & $\begin{array}{l}\text { HALL. MICHAEL } \mathrm{J} \text {. } \\
\text { HALL. MICHAEL } \mathrm{j} \text {. }\end{array}$ & $\begin{array}{l}373-5719 \\
373-5719\end{array}$ & $\begin{array}{l}\text { STEVE VEITENHEIMER } \\
\text { STEVE VEITENHEIMER }\end{array}$ & $\begin{array}{l}373-9725 \\
373-9725\end{array}$ \\
\hline
\end{tabular}

Emission Point Listing

\begin{tabular}{|c|c|c|c|c|c|c|c|c|}
\hline Em Point & Ep Type & North & East & Srce Nbr Scc Code & Srce Ty & & & \\
\hline $600 \mathrm{~S}-6266001$ & POINT & 136417 & 568856 & 1 not assigned & ANALYTI & CAL LABORATORY & & \\
\hline Stack Configuration & & Diameter (ft) & Length(ft) & Width(ft) Area(sq ft) & Exit Temp(F) & Exit Veloc $(\mathrm{ft} / \mathrm{sec})$ & Ht Abv Grnd(ft) & Ht Abv Struc(ft) \\
\hline VERTICAL WITHOUT WEATH & THER CAP & 4 & & 12.57 & 78 & 66 & 25 & 8 \\
\hline Em Point & Ep Type & North & East & t Srce Nbr Scc Code & Srce Typ & & & \\
\hline $600 \mathrm{~S}-6266002$ & POINT & 136359 & 568825 & 1 not assigned & ANALYTIC & CAL LABORATORY & & \\
\hline Stack Configuration & & Diameter(ft) & Length(ft) & Width(ft) Area(sq ft) $E$ & Exit Temp(F) & Exit Veloc(ft/sec) & Ht Abv Grnd(ft) & Ht Abv Struc $(\mathrm{ft})$ \\
\hline NONE SPECIFIED & & & $i$ & 3 & 90 & 33.33 & 32.3 & 16.33 \\
\hline Em Point & Ep Type & North & East & t Srce Nbr Scc Code & Srce Ty & & & \\
\hline $600 \mathrm{~S}-6269001$ & POINT & 136443 & 568891 & 1 not assigned & ANALYTIC & CAL LABORATORY & & \\
\hline Stack. Configuration & & Diameter $(f t)$ & Length(ft) & Width $(\mathrm{ft}) \quad \operatorname{Area}(s q \mathrm{ft})$ & Exit Temp(F) & Exit Veloc(ft/sec) & Ht Abv Grnd(ft) & Ht Abv Struc(ft) \\
\hline HORIZONTAL & 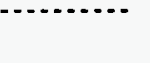 & & .83 & $.83 \quad 0.69$ & 78 & .325 & 10 & 0 \\
\hline Em Point & Ep Type & North & East & t Srce Nbr Scc Code & Srce Ty & & & \\
\hline $600 \mathrm{Z}-6266 \mathrm{~A} 001$ & FUGITIVE & 136411 & 568778 & 1 not assigned & PROCESS & AND PRODUCTION & & \\
\hline Stack Configuration & - & Diameter $(f t)$ & Length $(f t)$ & Width(ft) Area(sq ft) & $\begin{array}{r}\text { Exit } \operatorname{Temp}(F) \\
78\end{array}$ & $\begin{array}{r}\text { Exit } \operatorname{Veloc}(\mathrm{ft} / \mathrm{sec}) \\
.0325\end{array}$ & $\begin{array}{r}H t \text { Abv Grnd(ft) } \\
0\end{array}$ & Ht Abv Struc(ft) \\
\hline Source (Process) Listing & & & & & & & & · \\
\hline Em Point & Srce Nbr & Srce Type & & Mrs Day Days Week & Wks Year & mmary Process Descri & ption & \\
\hline $600 \mathrm{~S}-6266001$ & $i$ & ANALYTICAL LABO & ORATORY & 5 & 52 ANA & $\begin{array}{l}\text { ALYTICAL LABORATORY } \\
\text { AARACTERIZATION }\end{array}$ & FOR WASTE & G AND \\
\hline
\end{tabular}




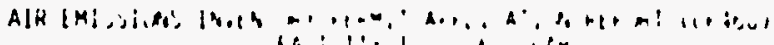

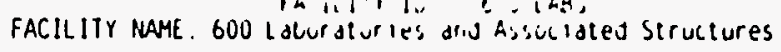

Source (Process) Listing

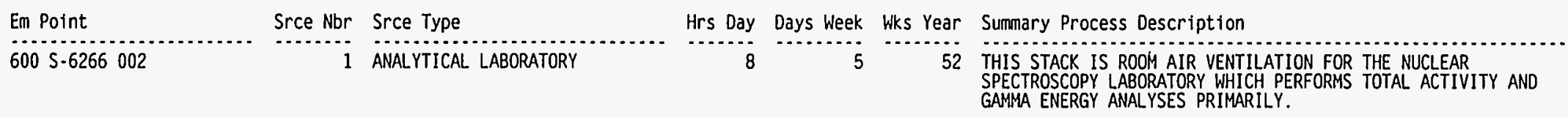

THERE ARE NO FREE CHEMICALS PREPARED OR USED FOR OTHER PURPOSES WITHIN THE ROOM. THE NUCLEAR SPECTROSCOPY LAB IS ALSO USED FOR CQUNTING AIR SAMPLE

PAPERS FROM AROUND THE SITE. THERE IS NO CAM IN THE ROOM OR IN THE STACK.

Em Point

$600 \$-6269001$

Srce Nbr Srce Type

1 ANALYTICAL LABORATORY

Srce Nbr Srce Type

Em Point

$600 Z-6266 A 00$

1 PROCESS AND PRODUCTION
Hrs Day Days Week Wks Year Summary Process Description

52 MOBILE LAB VENT STACK. THE MOBILE SAMPLE LAB CONTAINS A

PROTECTIVE HOOD USED FOR THE CHEMICAL PREPARATION OF SAMPLE BOTILES PRIOR TO TAKING THEM OUT IN THE FIELD. THE HOOD EXITS THROUGH THE WEST SIDE OF THE BUILDING WALL VIA A 10"X10" DUCT.

Hrs Day Days Week Wks Year Summary Process Description

2 TWO CHEMICAL TANKS VENT INTERNALLY THROUGH 2 HEPA FILTERS INTO BUILDING 6266A. THERE ARE NO EXTERNAL VENTS FROM $6266 \mathrm{~A}$.

THE 6266A BUILDING IS A CONTAMINATED LIQUID WASTE RETENTION VAULT. LABORATORY WASTE (AQUEOUS INORGANICS WITH SOME MISCIBLE ALCOHOLS <2\%) IS STORED IN THESE TANKS UNTIL DISPOSAL. THE TANKS HAVE A VENTILATION SYSTEM 2 FLANDERS FILTER HOUSINGS (HEPA) THAT VENT INTO THE OPEN SPACES OF THE BUILDING. THE FILTERS PURPOSE IS THE CAPTURE OF SPURIOUS RADIONUCLIOES
PRESSURE OF 1 , IT IS NOT LIKELY TO OFF GAS IN ANY VOLUME.

\section{Source Raw Materials Usage}

Em Point

Srce Nbr Srce Type

Process Rate Process Uom Materia

Fuels Usage by Source Processes

Em Point

Srce Nbr Srce Type

Qty Uom

Fuel

Fuels Usage by Control Devices

Em Point

Ctl $\mathrm{Nbr}$ Ct] Type

Qty Uom

Fuel

Point Source Criteria Pollutants - Type: H2S04
Report Run Date: 02-MAY-95 
AIR EMISSIONS INVENTORY PERMIT APPLICATION REPORT (UP460)

FACILITY NAME: 600 Laboratories and Associated Structures

Point Source Criteria Pollutants - Type: H2S04

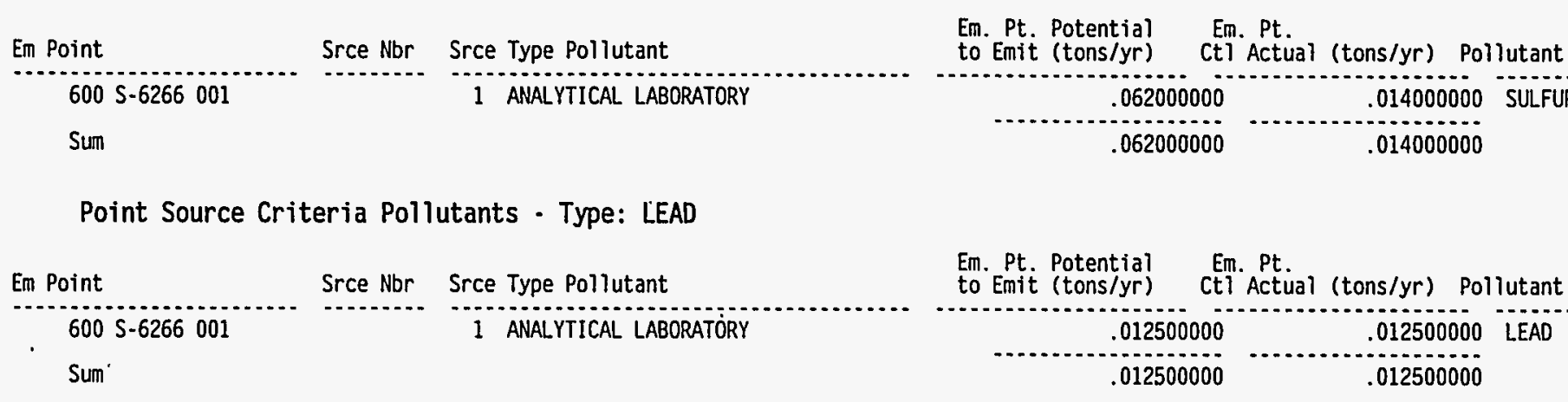

Point Source Criteria Pollutants - Type: VoC

$\begin{array}{lcc}\text { Em Point } & \text { Srce Nbr } & \text { Srce Type Pollutant } \\ 6000 \mathrm{~S}-6266001 & & 1 \text { ANALYTICAL LABORATORY }\end{array}$

Em. Pt. Potential Em. Pt.
to Emit (tons/yr) Ctl Actual (tons/yr) Pollutant

600 S -626600

ANALYTICAL LABORATORY

\begin{tabular}{|c|c|c|}
\hline $\begin{array}{l}.027000000 \\
.000044600 \\
.051000000 \\
.000043500 \\
.000903000 \\
.000048500 \\
.000860000 \\
.550000000 \\
.000048000 \\
.031000000 \\
.004350000 \\
.200000000 \\
.100000000 \\
.000066100 \\
.000055100 \\
.000034700 \\
.000108000 \\
.004200000 \\
.056000000 \\
.017000000\end{array}$ & $\begin{array}{l}.006100000 \\
.000044600 \\
.005790000 \\
.000043500 \\
.000903000 \\
.000048500 \\
.000860000 \\
.130000000 \\
.000048000 \\
.000043000 \\
.004350000 \\
.046000000 \\
.000036400 \\
.000066100 \\
.000055100 \\
.000034700 \\
.000108000 \\
.000960000 \\
.000080700 \\
.000048500\end{array}$ & $\begin{array}{l}2.2 .4-T R I M E T H Y L P E N T A N E \\
2 \text {-HEXANONE } \\
\text { ACETIC ACID } \\
\text { ACETONE } \\
\text { ACETONITRILE } \\
\text { BENZENE } \\
\text { CYCLOHEXANONE } \\
\text { ETHY ALCOHOL } \\
\text { ETHYLBENZENE } \\
\text { ISOPROPYL ALCOHOL } \\
\text { METHYL ALCOHOL } \\
\text { METHYL CHLORIDE } \\
\text { N-HEXANE } \\
\text { NITROBENZENE } \\
\text { NITROSODIMETHYLAMINE } \\
\text { PENTANE } \\
\text { PYRIDINE } \\
\text { TOLUENE } \\
\text { TRICHLOROETHYLENE } \\
\text { XYLENE }\end{array}$ \\
\hline 1.042761500 & .195620100 & \\
\hline
\end{tabular}

Point Source Emissions - Radiological Pollutants

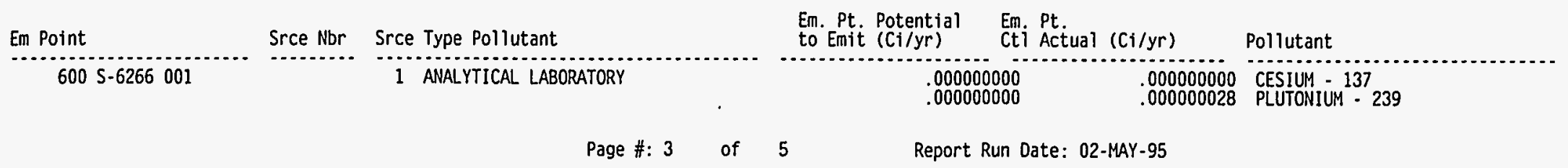


AIR EMISSIONS INVENTORY PERMIT APPLICATION REPORT (UP460)

FACILITY NAME: 600 Laboratories and Associated Structures

Point Source Emissions - HAP Pollutants

\begin{tabular}{|c|c|c|c|c|c|}
\hline Em Point & Srce Nbr & Srce Type Pollutant & $\begin{array}{l}\text { Em. Pt. Potential } \\
\text { to Emit (tons/yr) }\end{array}$ & $\begin{array}{l}\text { Em. Pt. } \\
\text { Ctl Actual (tons/yr) }\end{array}$ & ollutant \\
\hline $600 \mathrm{~S}-6266001$ & & 1 ANALYTICAL LABORATORY & $\begin{array}{l}.027000000 \\
.000903000 \\
.000082700 \\
.000048500 \\
.002040000 \\
.001820000 \\
.000078300 \\
.00215000 \\
.000048000 \\
1.200000000 \\
.012500000 \\
.016000000 \\
.004350000 \\
.200000000 \\
.48000000 \\
.100000000 \\
.001320000 \\
.000066100 \\
.000055100 \\
.001100000 \\
.004200000 \\
.056000000 \\
.017000000\end{array}$ & $\begin{array}{l}.006100000 \\
.000903000 \\
.000082700 \\
.00004800 \\
.002040000 \\
.001820000 \\
.000078300 \\
.002150000 \\
.000048000 \\
.000544000 \\
.012500000 \\
.014900000 \\
.004350000 \\
.046000000 \\
.325000000 \\
.000036400 \\
.001320000 \\
.000066100 \\
.000055100 \\
.001100000 \\
.000960000 \\
.00000700 \\
.000048500\end{array}$ & $\begin{array}{l}2.2 .4-\text { TRIMETHYLPENTANE } \\
\text { ACETONITRILE } \\
\text { ANTIMONY } \\
\text { BENZENE } \\
\text { BERYLLIUM } \\
\text { CADMIUM } \\
\text { CHLORINE } \\
\text { COBALT } \\
\text { ETHYLBENZENE } \\
\text { HYDROCHLORIC ACID (HYOROGEN CHLORIDE) } \\
\text { LEAD } \\
\text { MERCURY } \\
\text { METHYL ALCOHOL } \\
\text { METHYL CHLORIDE } \\
\text { METHYLNE CHLORIDE } \\
\text { N-HEXANE } \\
\text { NICKEL } \\
\text { NITROBENZENE } \\
\text { NITROSODIMETHYLAMINE } \\
\text { SELENIMM } \\
\text { TOLUENE } \\
\text { TRICHLOROETHYLENE } \\
\text { XYLENE }\end{array}$ \\
\hline Sum & & & 2.126761700 & .420231300 & \\
\hline
\end{tabular}

Point Source Emissions - Uncategorized Pollutants

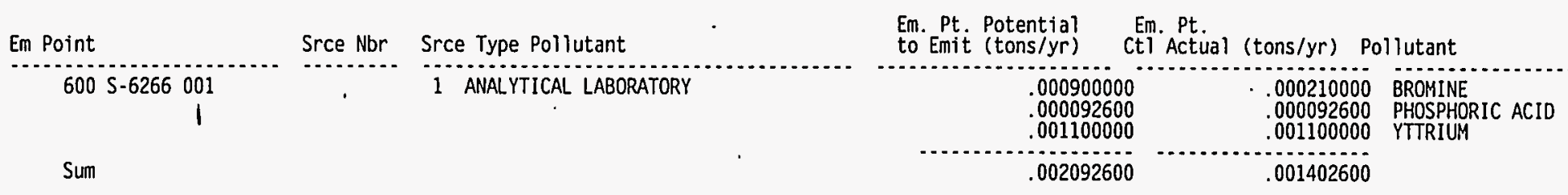

Fugitive Source Emissions - Radiological Pollutants

Em Point

Srce Nbr Srce Type Pollutant

Em. Pt. Potential Em. Pt.

to Emit (Ci/yr) Cti Actual (Ci/yr) Pollutant

Fugitive Source Emissions - HAP Pollutants

\begin{tabular}{|c|c|c|c|c|}
\hline Em Point & Srce Nbr & Srce Type Pollutant & $\begin{array}{l}\text { Em. Pt. Potential } \\
\text { to Emit (tons/yr) }\end{array}$ & $\begin{array}{l}\text { Em. Pt. } \\
\text { Ctl Actual (tons/yr) }\end{array}$ \\
\hline
\end{tabular}

Page \#: 4 of 5 Report Run Date: 02-MAY-95 


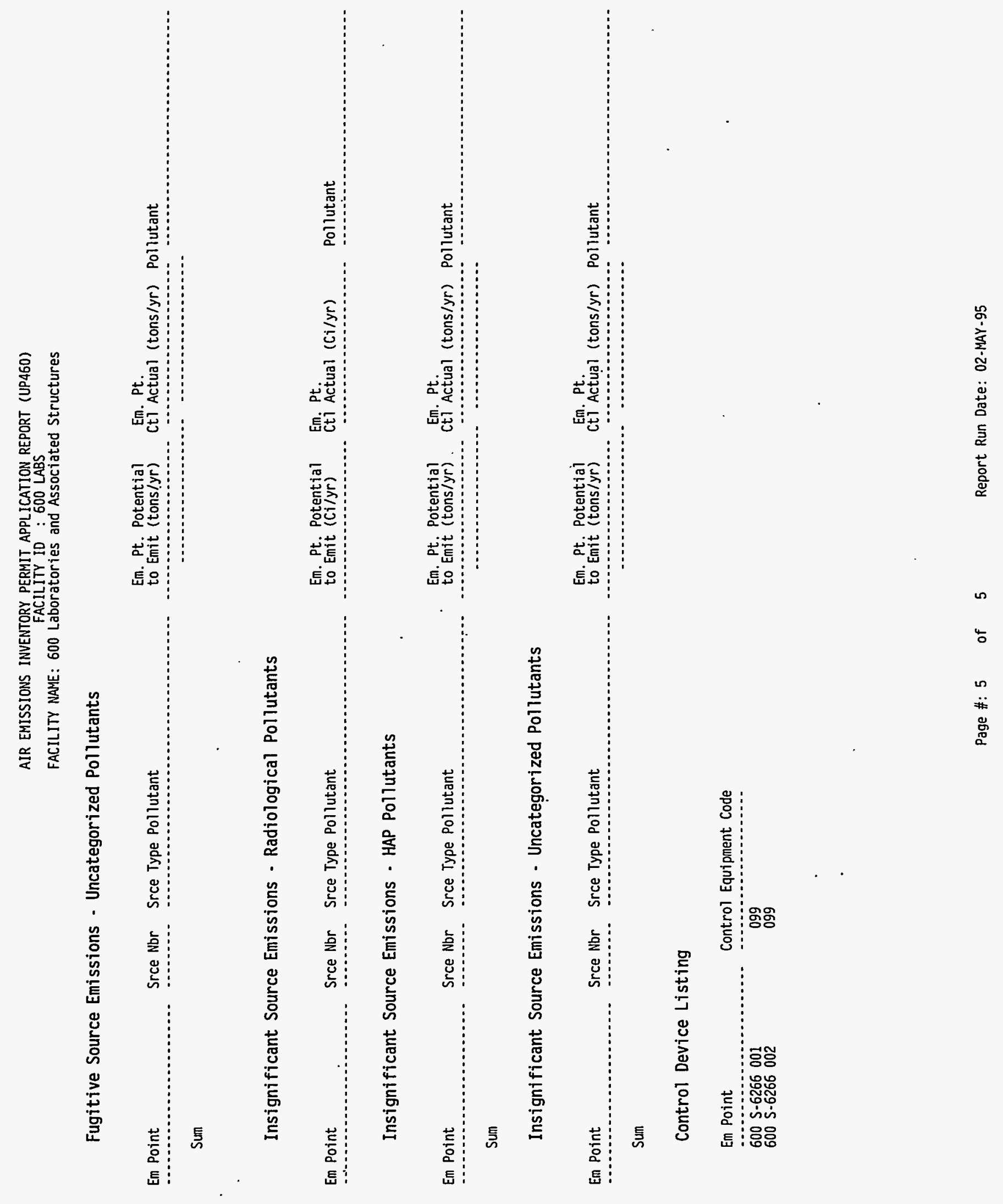




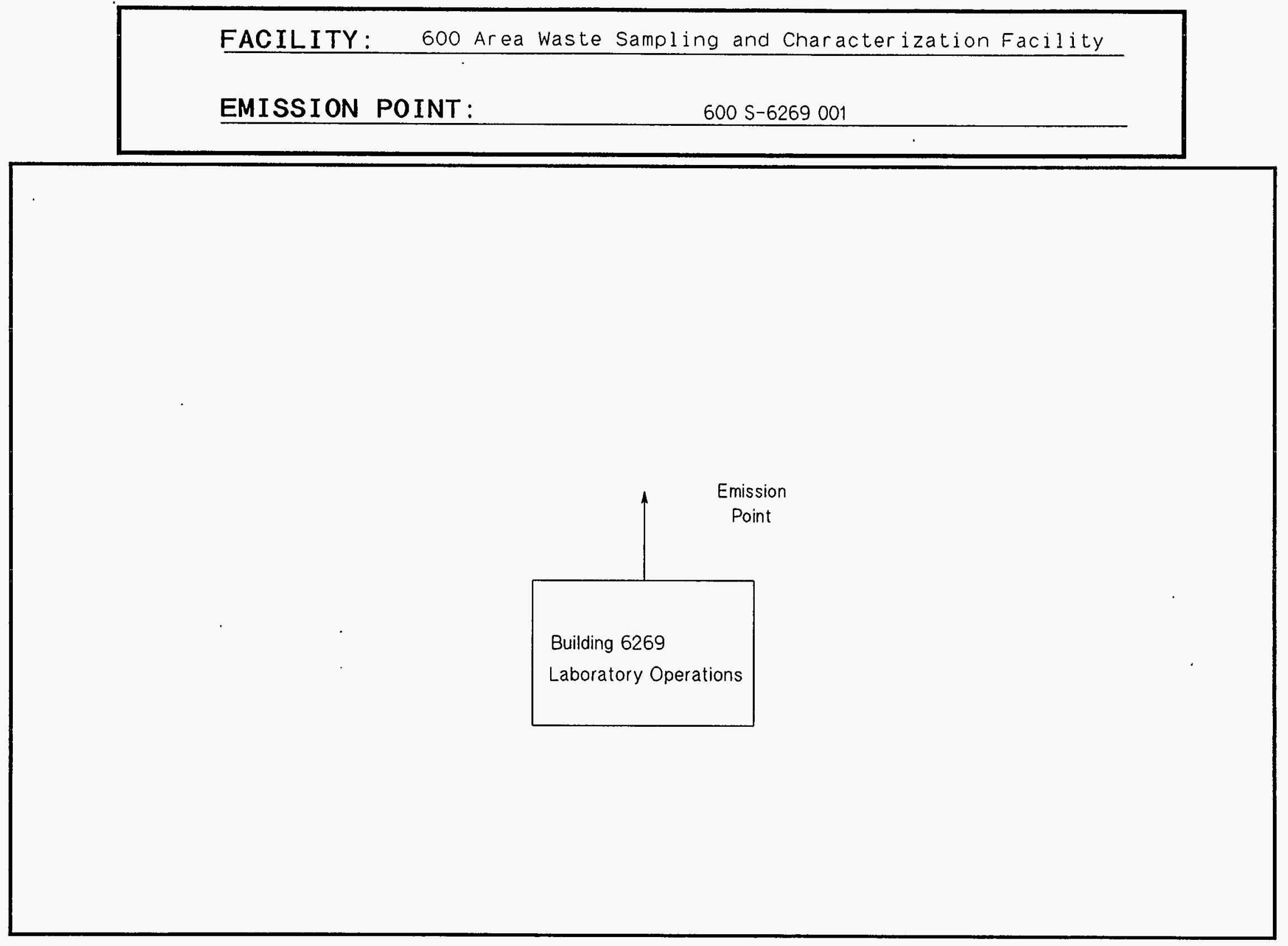


FACILITY: $\quad 600$ Area Waste Sampling and Characterization Facility

EMISSION POINT:

$600 Z-6266 A 001$

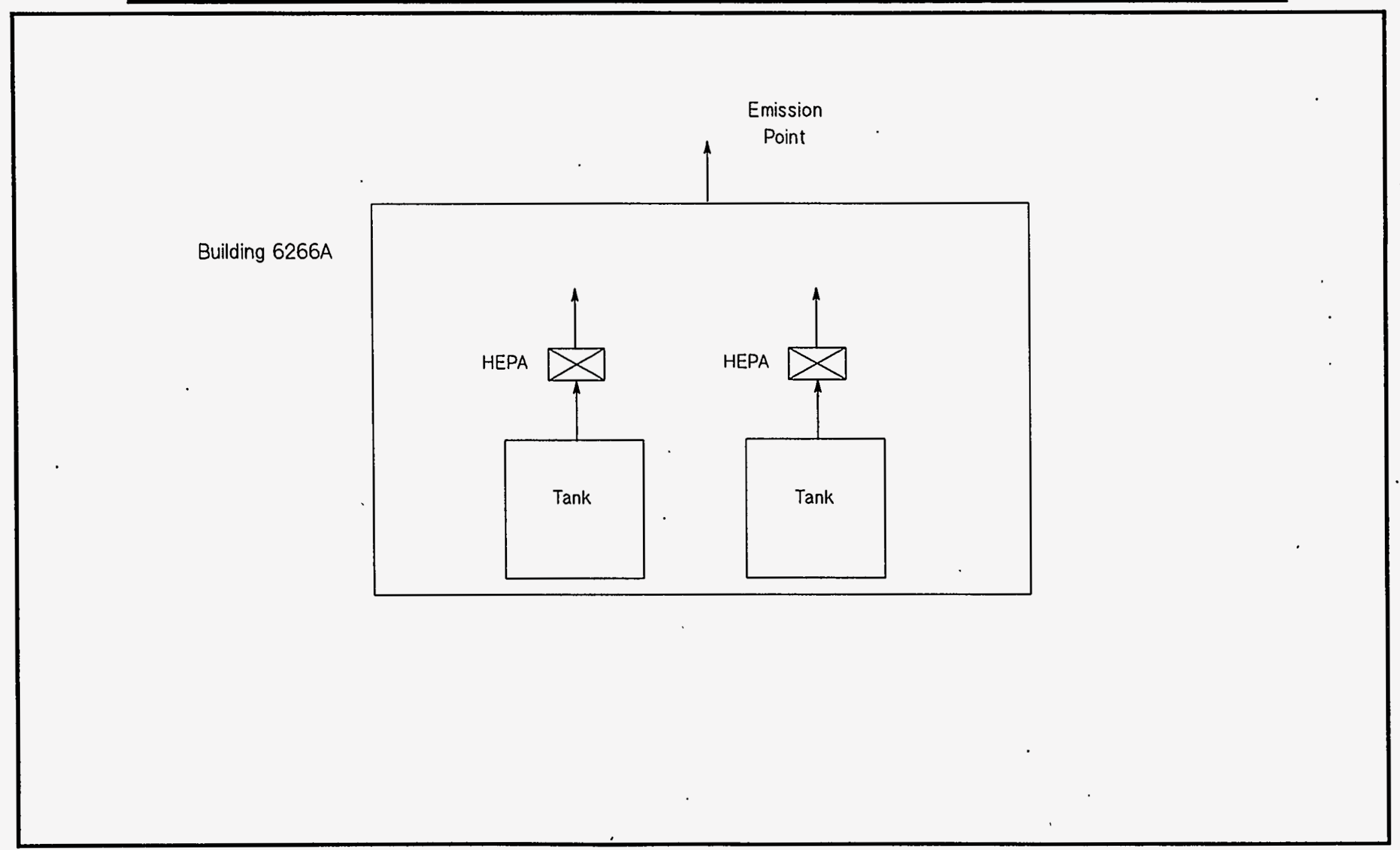

K.A. Glese 


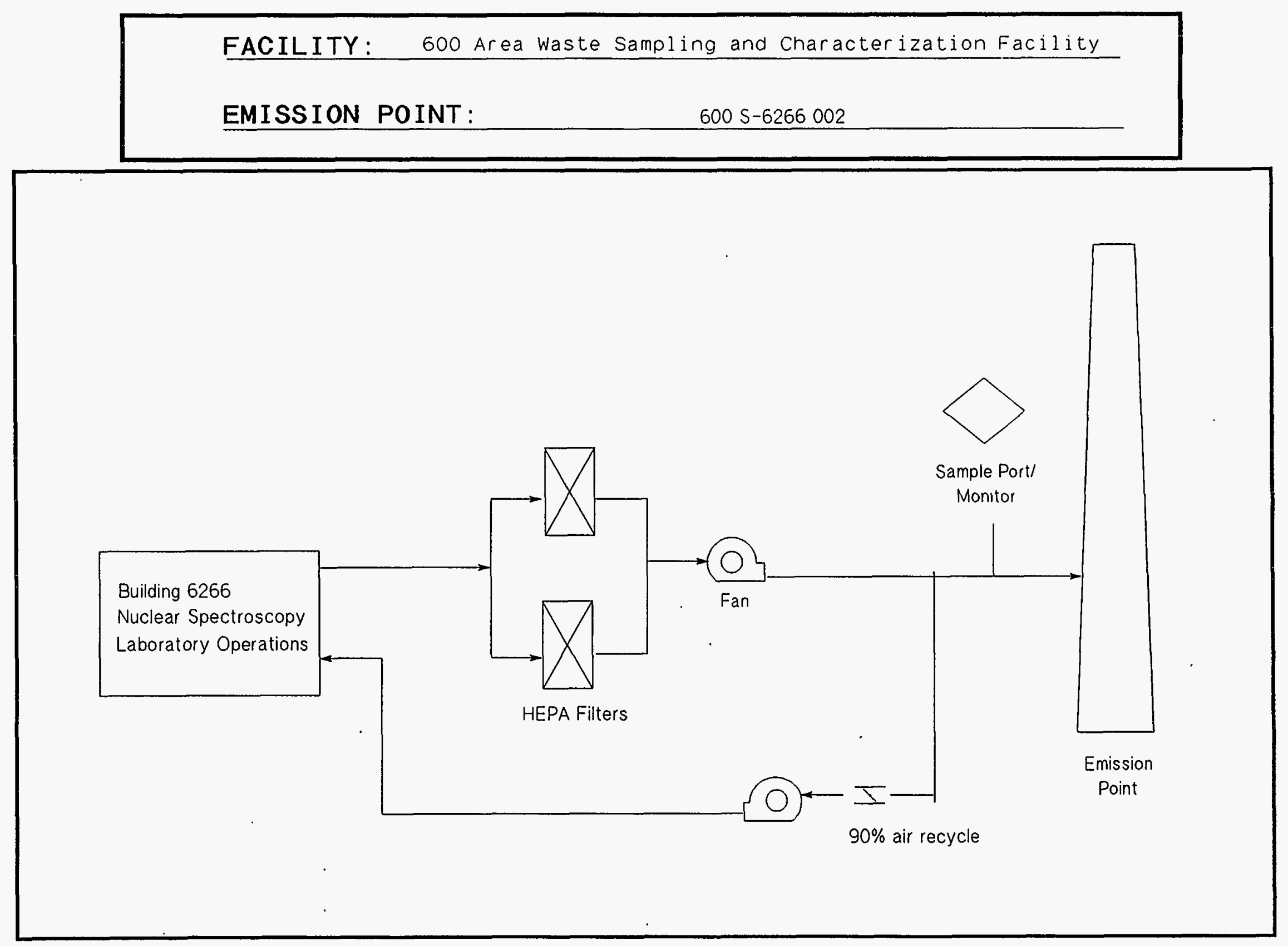

K.A. Giese 
FACILITY: Gill $\therefore$, , , ,

: ..';. : " "., "Lerzation Facility

EMISSION POINT: $600 \mathrm{~S}-6266001$

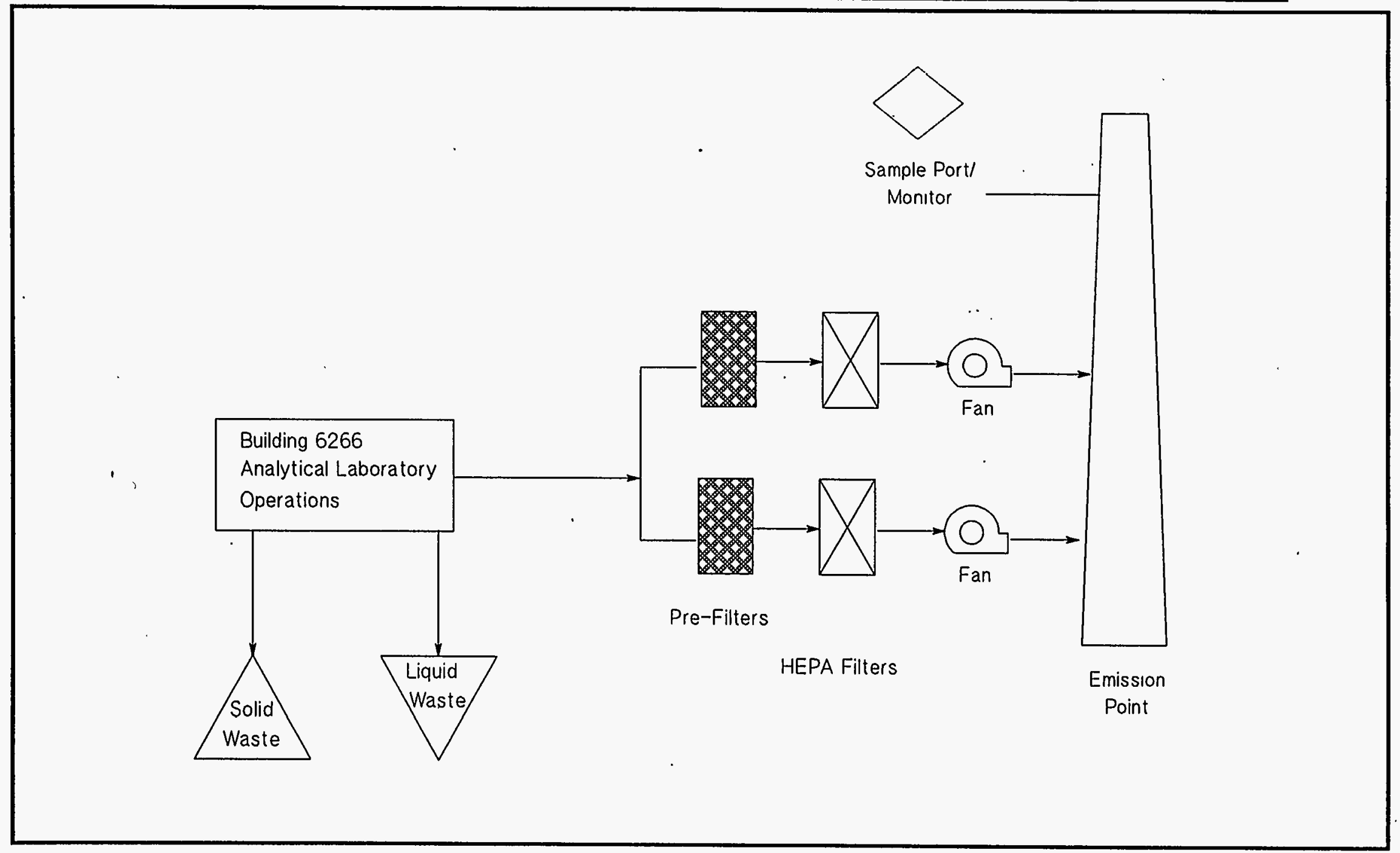




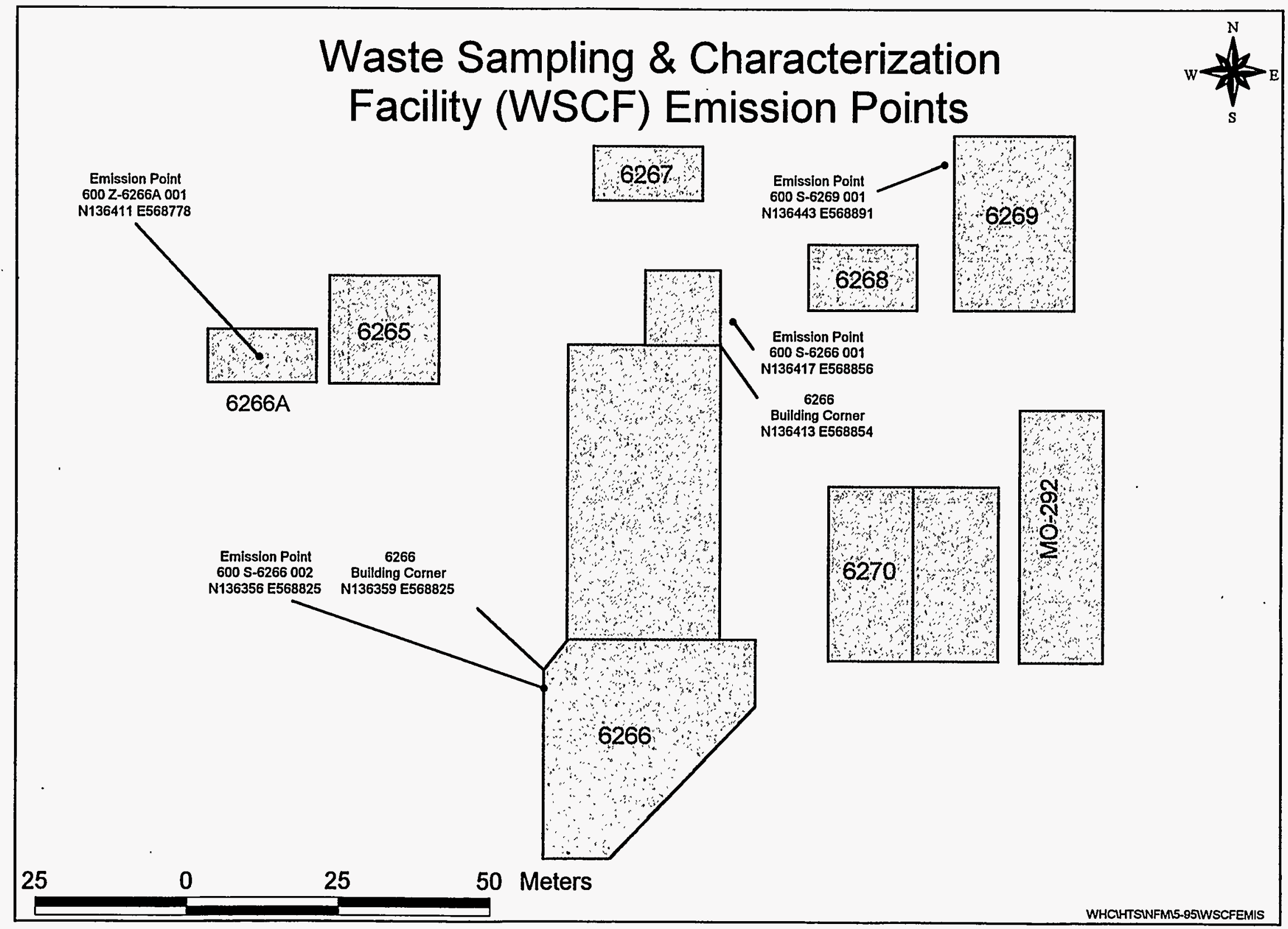


Hanford Site Air

Operating Permit Application
DOE/RL-95-07, Rev. 0

05/95

\section{APPENDIX I}

MAINTENANCE 
Hanford Site Air

Operating Permit Application
DOE/RL-95-07, Rev. 0 05/95

\section{APPENDIX I}

\section{CONTENTS}

\section{I-1 MAINTENANCE}




\section{APPENDIX I-1}

\section{MAINTENANCE}

\section{Chapter I. General Information}

\section{I-B.1. Facility Description}

The maintenance activities span all areas of the Hanford Site. These activities generally are performed in small shops or in open areas. The majority of the maintenance activities are classified as IEUs, and are listed in appendix $\mathrm{K}$. The maintenance activities that are not classified as IEUs are open burning and painting-associated activities.

Open burning is conducted in burn pits in the 200 East Area and the 200 West Area to reduce the volume of tumbleweeds. The burning is conducted for 1 hour per day, 1 day per week, 50 weeks per year. Additionally, tumbleweeds are burned along road sides, fence lines, etc.

Paint activities listed in this appendix are conducted in the 200 East Area, 200 West Area, 300 Area, and the 400 Area. Painting and paint clean-up activities are conducted for 8 hours per day, 5 days per week, 50 weeks per year.

\section{I-B.2. Products, Raw Materials, Fuels, Processes}

- Principle products - There are no products produced.

- Process information - There are no process activities.

- Raw materials used by processes - No raw materials are used as there are no commercial products manufactured on the Hanford Site.

- Fuels used by processes - There are no fuels used for these activities.

\section{I-C.1. Emission Points, Controls, Stacks}

The emission point and stack information is found on pages 1 and 2 of the AEI report output.

\section{I-C.2. Facility Process Flow Diagrams}

Attached. 


\section{Chapter II. Regulatory Requirements}

\section{II-A.1. Description of Discharge and Emission Points}

The emission point and stack information is found on pages 1 and 2 of the AEI report output. 


\section{Facility Building List}

\begin{tabular}{|c|c|}
\hline Bldg Id & North \\
\hline $\begin{array}{l}1167 \\
200 E 001 \\
200 E 002\end{array}$ & $\begin{array}{l}110232 \\
136312 \\
137231\end{array}$ \\
\hline $\begin{array}{l}\text { 200w003 } \\
200 \text { W007 }\end{array}$ & $\begin{array}{l}135893 \\
135473\end{array}$ \\
\hline $\begin{array}{l}3709 \\
4722 C\end{array}$ & $\begin{array}{l}115973 \\
122923\end{array}$ \\
\hline
\end{tabular}

Emission Point Listing

East Bldg Desc

593416 GENERAL STORES COVERED STORAGE BUILDING

573410 PAINT SHOP

$575237200 E$ BURN PIT USEO FOR RUSSIAN THISTLE

567261 (TAMBLEWEED) BURNING.

(PUMBLEED) BURNING.

593685 PAINT SHOP

\begin{tabular}{|c|c|c|c|}
\hline Custodian(Contractor) & Cust Phone & DOE POC & DOE Phone \\
\hline $\begin{array}{l}\text { CARLSON, GARY B. } \\
\text { MCLERRAN, KATHY M. } \\
\text { MARPLE. CANDICE }\end{array}$ & $\begin{array}{l}376-6706 \\
372-1418 \\
373-2202\end{array}$ & $\begin{array}{l}\text { DAVID G. MURILLO } \\
\text { DAVIO G. MURILLO } \\
\text { DAVID G. MURILLO }\end{array}$ & $\begin{array}{l}373-9179 \\
373-9179 \\
373-9179\end{array}$ \\
\hline $\begin{array}{l}\text { MCLERRAN, KATHY M. } \\
\text { MARPLE, CANDICE }\end{array}$ & $\begin{array}{l}372-1418 \\
372-2202\end{array}$ & $\begin{array}{l}\text { DAVID G. MURILLO } \\
\text { DAVID G. MURILLLO }\end{array}$ & $\begin{array}{l}373-9179 \\
373-9179\end{array}$ \\
\hline $\begin{array}{l}\text { POOR, A.D. (DENNIS) } \\
\text { POOR, A.D. (DENNIS) }\end{array}$ & $\begin{array}{l}376-7210 \\
376-7210\end{array}$ & $\begin{array}{l}\text { DAVID G. MURILLO } \\
\text { DAVID G. MURILLO }\end{array}$ & $\begin{array}{l}373-9179 \\
373-9179\end{array}$ \\
\hline
\end{tabular}

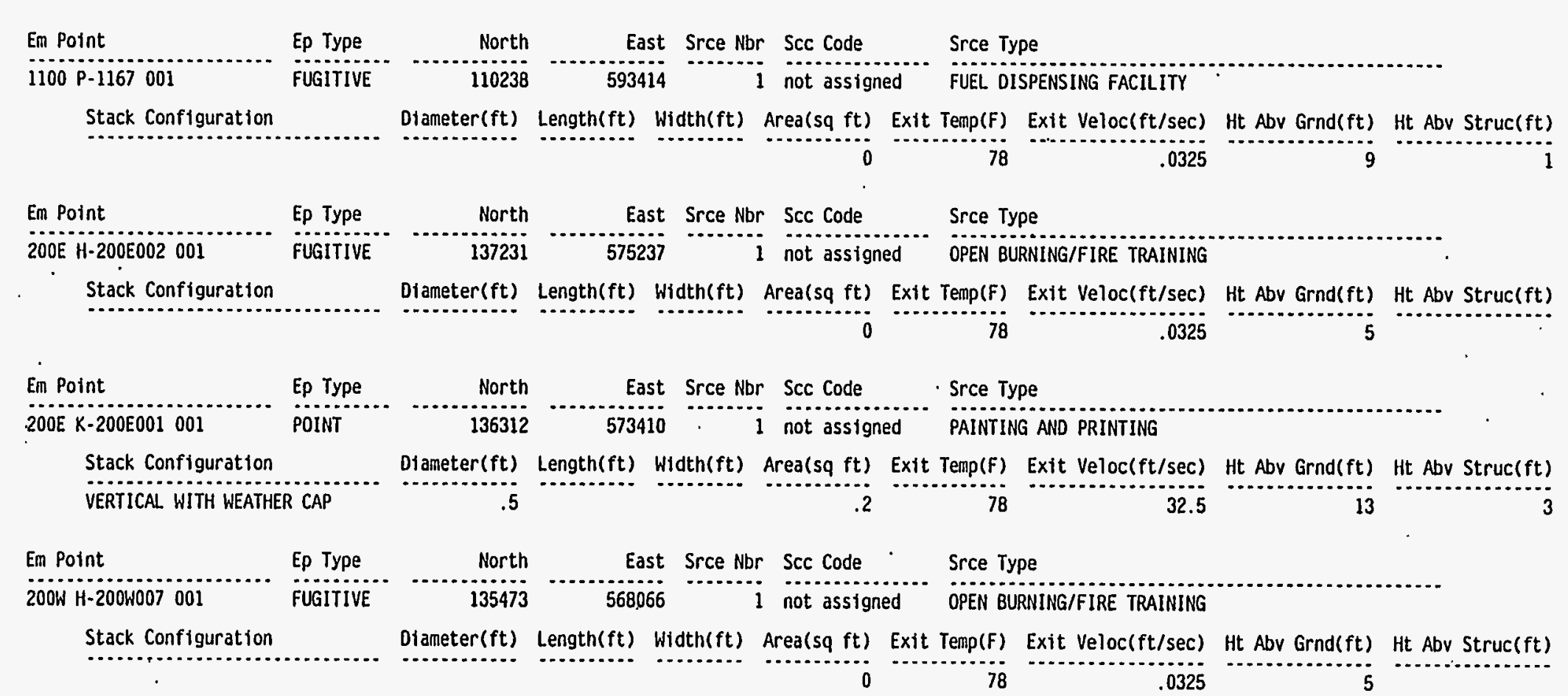

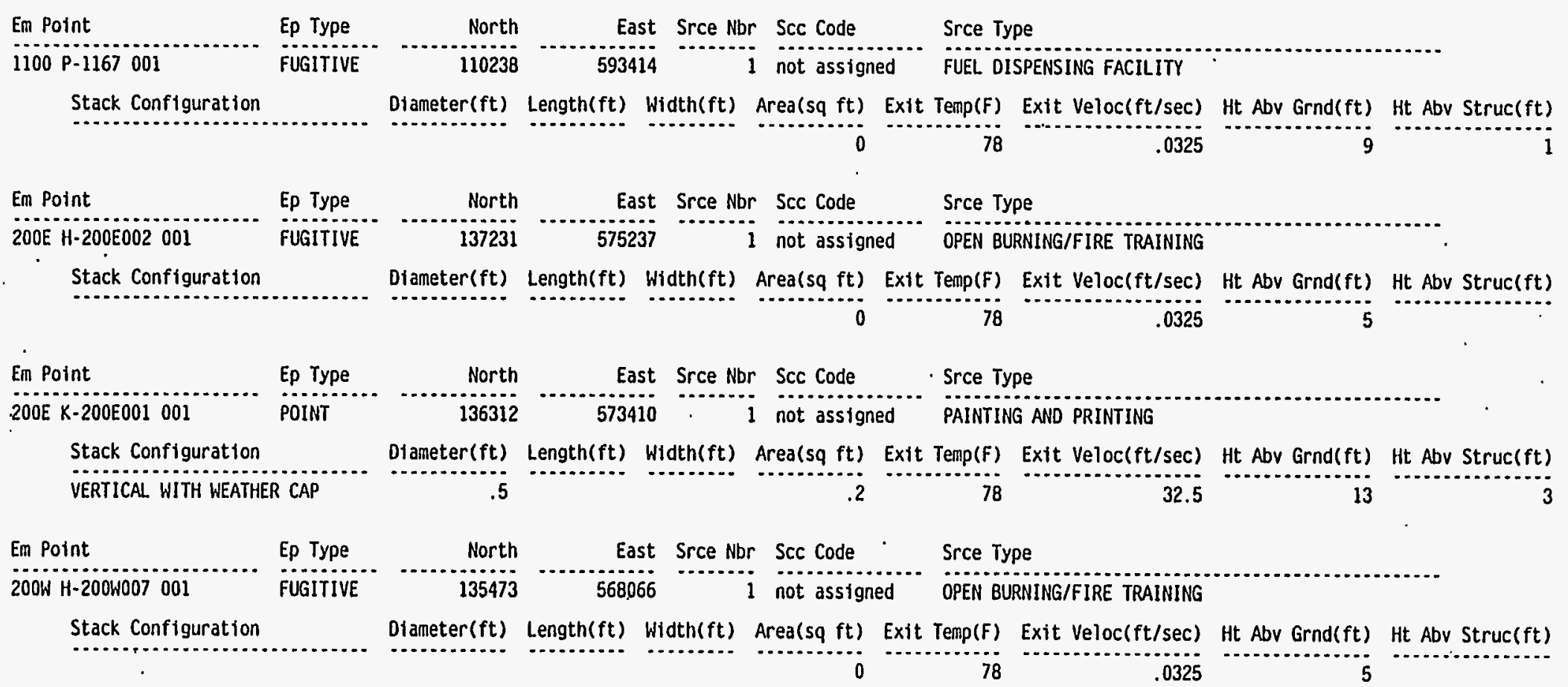

Page \#: 1 of 8 Report Run Date: 10-MAY-95 


\section{Emission Point Listing}

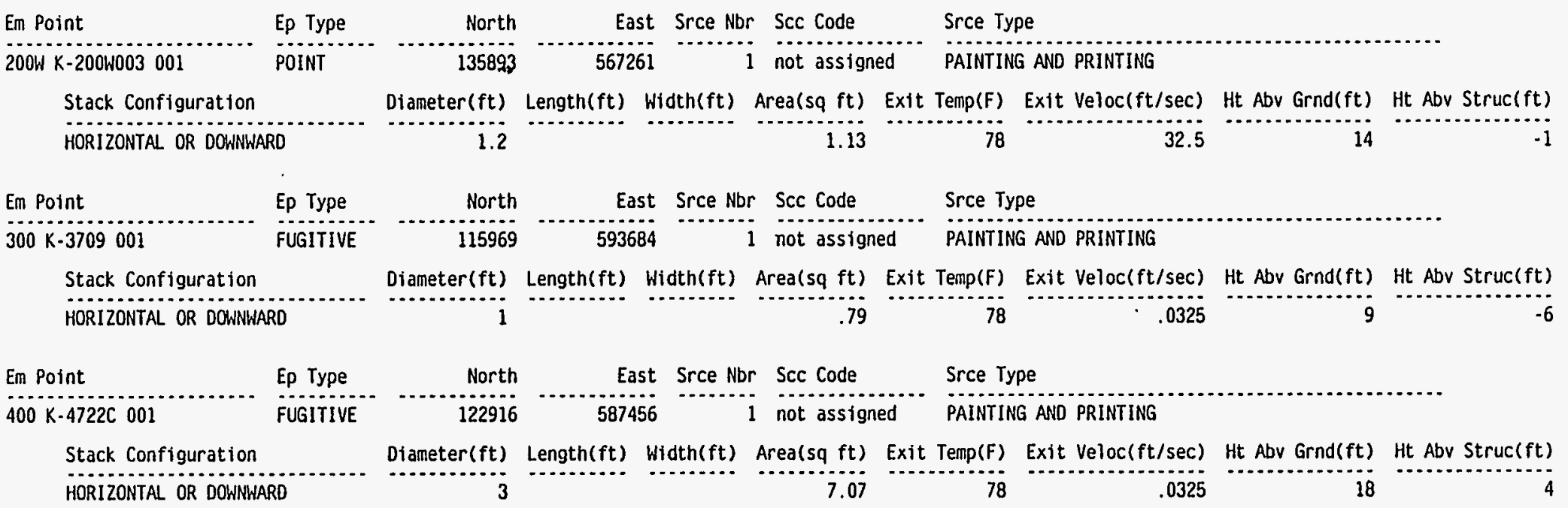

Source (Process) Listing

\begin{tabular}{|c|c|c|c|c|c|c|}
\hline Em Point & Srce Nbr & Srce Type & Hrs Day & Days Week & Wks Year & Summary Process Description \\
\hline $1100 P-1167001$ & 1 & FUEL DISPENSING FACILITY & 8 & 5 & 52 & $\begin{array}{l}\text { LIQUID PROPANE STORAGE TANK AND FILLING DEPOT. } \\
\text { (APPROXIMATELY } 3.000 \text { GALLON CAPACTY) ACTUAL INSTALLED AND } \\
\text { INITIAL OPERATION DATES ARE UNKNOWN (PRE-1980). }\end{array}$ \\
\hline
\end{tabular}

Source (Process) Listing

\begin{tabular}{|c|c|c|c|c|c|c|}
\hline Em Point & Srce $\mathrm{Nbr}$ & Srce Type & Hrs Day & Days Week & Wks Year & Summary Process Description \\
\hline $200 E$ H-200E002 001 & $i$ & OPEN BURNING/FIRE TRAINING & 1 & 1 & 50 & $\begin{array}{l}\text { BURN PIT FOR TUMBLEWEED BURNING. ACTUAL INSTALLED AND } \\
\text { INITIAL OPERATION DATES ARE UNKNOWN (PRE-1990). }\end{array}$ \\
\hline Em Point & Srce $\mathrm{Nbr}$ & Srce Type & Hrs Day & Days Week & Wks Year & Summary Process Description \\
\hline $200 \mathrm{E}$ K-200EO01 O0I & $\ddot{i}$ & PAINTING AND PRINTING & $\ddot{8}$ & $\ddot{5}$ & 50 & PAINT CLEAN-UP ACTIVITIES (1992) \\
\hline
\end{tabular}


AIR EMISSIONS INVENTORY PERMIT APPLICATION REPORT (UP460)

FACILITY NAME: Maintenance Facilities and Assoc. Structures

\section{Source (Process) Listing}

Em Point

Srce Nbr Srce Type

Hrs Day Days Week Wks Year Sumary Process Description

20OW H-200W007 001

1 OPEN BURNING/FIRE TRAINING

50 BURN PIT FOR TUMBLEWEED BURNING. ACTUAL INSTALLED AND

INITIAL OPERATION DATES ARE UNKNOWN (PRE-1990).

TYPE OF MATERIAL BURNED - RUSSIAN THISTLE (TUMBLENEED).

\begin{tabular}{|c|c|c|c|c|c|c|}
\hline Em Point & Srce $\mathrm{Nbr}$ & Srce Type & Hrs Day & Days Week & Wks Year & Summary Process Description \\
\hline $200 W$ K-200W003 001 & 1 & PAINTING AND PRINTING & 8 & 5 & 50 & $\begin{array}{l}\text { PAINT CLEAN-UP ACTIVITIES, ACTUAL INSTALLED AND INITIAL } \\
\text { OPERATION DATES ARE UNKNOWN (PRE-1990). }\end{array}$ \\
\hline Em Point & Srce $\mathrm{Nbr}$ & Srce Type & Hrs Day & Days Week & Wks Year & Summary Process Description \\
\hline $300 \mathrm{~K}-3709001$ & 1 & PAINTING AND PRINTING & 4 & 5 & 50 & VOC EMISSIONS FROM PAINTING OPERATIONS \\
\hline Em Point & Srce $\mathrm{Nbr}$ & Srce Type & Hrs Day & Days Week & Whs Year & Surmary Process Description \\
\hline
\end{tabular}

Source Raw Materials Usage

Em Point

Srce $\mathrm{Nbr}$ Srce Type

Process Rate Process Uom Materlal

Fuels Usage by Source Processes

Em Point Srce Nbr Srce Type

Qty Uom

Fuel

Fuels Usage by Control Devices

Em Point

Ctl Nbr Ctl Type

Qty Uom

Fuel 
AIR EMISSIONS INVENTORY PERMIT APPLICATION REPORT (UP460)

FACILITY NAME: Maintenance Facilities and Assoc. Structures

Point Source Criteria Pollutants - Type: VOC

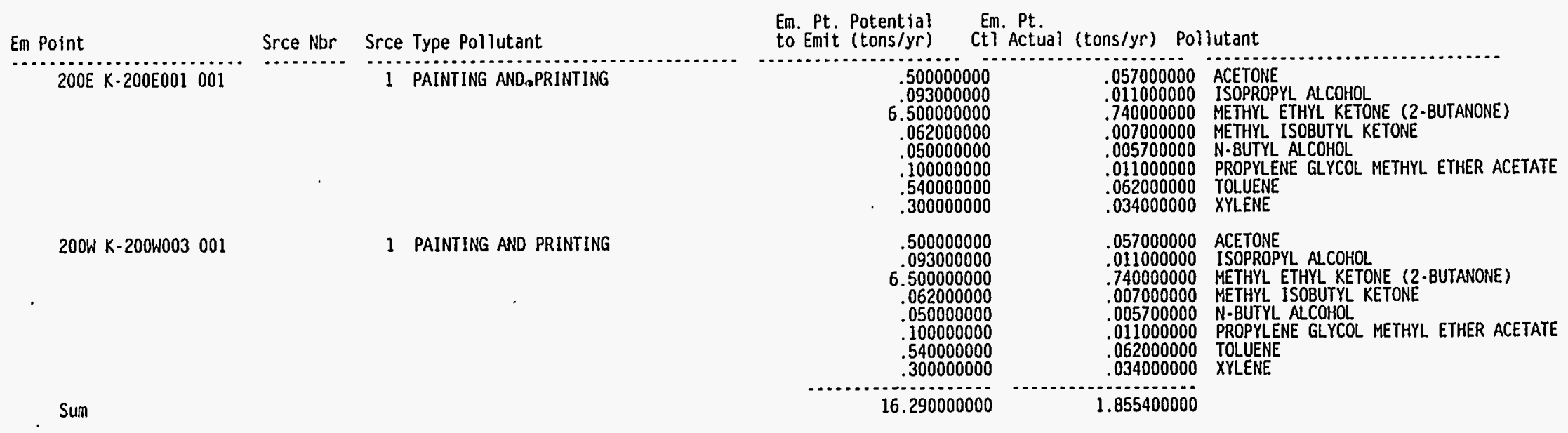

Point Source Emissions - Radiological Pollutants

En Point

Srce Nbr Srce Type Pollutant

Em. Pt. Potential Em. Pt.

to Emit (Ci/yr) Cti Actual (Ci/yr)

Pollutant

Point Source Emissions - HAP Pollutants

\begin{tabular}{|c|c|c|}
\hline Em Point & Srce Nbr & Srce Type Pollutant \\
\hline 200E K-200E001 001 & & 1 PAINTING AND PRINTING \\
\hline
\end{tabular}

Em. Pt. Potential Em. Pt.
to Emit (tons/yr) Ctl Actual (tons/yr) Pollutant

1 PAINTING AND PRINTING

6.500000000
.062000000
.540000000
.300000000

ETHYL KETONE (2-BUTANONE)

000000

.300000000

007000000 METHY ISOBUTYL KETONE

.062000000 TOLUENE

Point Source Emissions - HAP Pollutants

Em Point Srce Nbr Srce Type Pollutant

Em. Pt. Potential Em. Pt.

200W K-200W003 001 PAINTING AND PRINTING

Sum

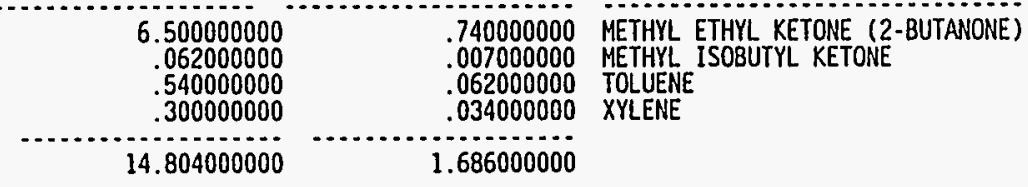

Page \#: 4 of 8 Report Run Date: 10-MAY-95 
AIR EMISSIONS INVENTORY PERHIT APPLICATION REPORT (UP460)

FACILITY NAME: Maintenance Facilities and Assoc. Structures

Point Source Emissions - Uncategorized Pollutants

En Point

200E X-200E001 001

200W K-200W003 001

Sun

Fugitive Source Criteria Pollutants - Type: CO
Em Point

20OW H-200W007 001

Sum

Fugitive Source Criteria Pollutants - Type: PM

1 OPEN BURNING/FIRE TRAINING

1 OPEN BURNING/FIRE TRAINING

Em Point

Srce Nbr Srce Type Pollutant

Sum

Fugitive Source Criteria Pollutants - Type: PM10

Em Point

Srce $\mathrm{Nbr}$

Srce Type Pollutant

1 OPEN BURNING/FIRE TRAINING

200E H-200E002 001

1 OPEN BURNING/FIRE TRAINING

200W H-200W007 001
200E $\mathrm{H}-200 \mathrm{EOO}$ OOI
Em. Pt. Potential Em. Pt.
to Emit (tons/yr) Ctl Actual (tons/yr) Pollutant

$3.200000000 \quad .360000000$ LIGROINE OR VMBP NAPHTHA, OR PETROLEUM NAPHTHA

$3.200000000 \quad .360000000$ LIGROINE OR UM\&P NAPHTHA, OR PETROLEUM

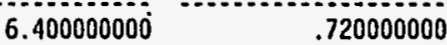

Em. Pt. Potential Em. Pt.
to Emit (tons/yr) Ctl Actual (tons/yr) Pollutant

$20.810000000 \quad 10.410000000$ CARBON MONOXIDE

$20.810000000 \quad 10.410000000$ CARBON MONOXIDE

$41.620000000 \quad 20.820000000$
Em. Pt. Potential Em. Pt.
to Emit (tons/yr) CtI Actual (tons/yr) Pollutant

Sum

Page \#: 5 of 8 Report Run Date: 10-MAY-95 
Fugitive Source Criteria Pollutants - Type: VOC

\begin{tabular}{|c|c|c|c|c|c|c|}
\hline Em Point & Srce $\mathrm{Nbr}$ & Srce & Type Pollutant & $\begin{array}{l}\text { Em. Pt. Potential } \\
\text { to Emit (tons/yr) }\end{array}$ & $\begin{array}{l}\text { Em. Pt. } \\
\text { ti Actual (tons/yr) }\end{array}$ & lutant \\
\hline $1100 p-1167001$ & & 1 & FUEL DISPENSING FACILITY & .930000000 & .930000000 & PROPANE \\
\hline 200E H-200E002 001 & & 1 & OPEN BURNING/FIRE TRAINING & .100000000 & .050000000 & VOLATILE ORGANIC COMPOUND \\
\hline $200 \mathrm{WH}$-200W007 001 & & 1 & OPEN BURNING/FIRE TRAINING & .100000000 & .050000000 & VOLATILE ORGANIC COMPOUND \\
\hline $300 k-3709001$ & & & PAINTING AND PRINTING & $\begin{array}{l}.500000000 \\
.093000000 \\
.750000000 \\
.062000000 \\
.050000000 \\
.100000000 \\
.540000000 \\
.300000000\end{array}$ & $\begin{array}{l}.057000000 \\
.011000000 \\
.086000000 \\
.007000000 \\
.005700000 \\
.011000000 \\
.062000000 \\
.034000000\end{array}$ & $\begin{array}{l}\text { ACETONE } \\
\text { ISOPROPYL ALCOHOL } \\
\text { METHYL ETHYL KETONE (2-BUTANONE) } \\
\text { METHYL ISOBUTYL KETONE } \\
\text { N-BUTYL ALCOHOL } \\
\text { PROPYLENE GLYCOL METHYL ETHER ACETATE } \\
\text { TOLUENE } \\
\text { XYLENE }\end{array}$ \\
\hline $400 \mathrm{~K}-4722 \mathrm{C} 001$ & & & PAINTING AND PRINTING & $\begin{array}{l}.500000000 \\
.093000000 \\
.750000000 \\
.062000000 \\
.050000000 \\
.100000000 \\
.540000000 \\
.300000000\end{array}$ & $\begin{array}{l}.057000000 \\
.011000000 \\
.086000000 \\
.007000000 \\
.005700000 \\
.01100000 \\
.062000000 \\
.034000000\end{array}$ & $\begin{array}{l}\text { ACETONE } \\
\text { ISOPROPYL ALCOHOL } \\
\text { METHYL ETHYL KETONE (2-BUTANONE) } \\
\text { METHYL ISOBUTYL KETONE } \\
\text { N-BUTYL ALCOHOL } \\
\text { PROPYLENE. GLYCOL METHYL. ETHER ACETATE } \\
\text { TOLUENE } \\
\text { XYLENE }\end{array}$ \\
\hline Sum & & & & 5.920000000 & 1.577400000 & \\
\hline
\end{tabular}

Fugitive Source Emissions - Radiological Pollutants

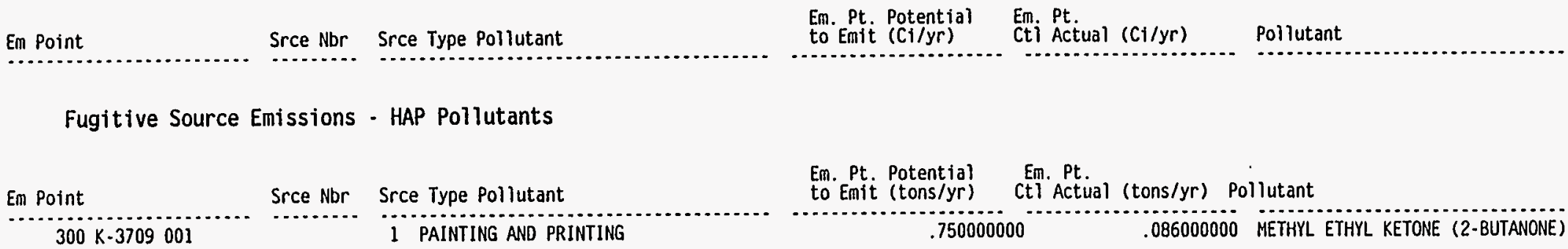




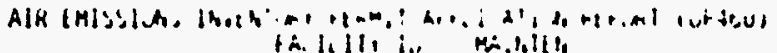

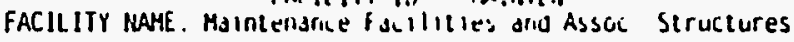

Fugitive Source Emissions - HAP Pollutants

$\begin{array}{lll}\text { Em Point } & \text { Srce Nbr } & \text { Srce Type Pollutant } \\ 300 \mathrm{~K}-3709001 & & 1 \text { PAINTING AND. QRINTING }\end{array}$
$400 \quad K-4722 C 001$
1 PAINTING AND PRINTING

Sum

Em. Pt. Potential Em. Pt.

to Emit (tons/yr) Ct] Actual (tons/yr) Pollutant

\begin{tabular}{ccc}
.062000000 & .007000000 & METHYL ISOBUTYL KETONE \\
.540000000 & .062000000 & TOLUENE \\
.300000000 & .034000000 & XYLENE \\
& & \\
.750000000 & .086000000 & METHYL ETHYL KETONE (2-BUTANONE) \\
.052000000 & .007000000 & METHY ISOBUTYL KETONE \\
.540000000 & .062000000 & TOLUENE \\
.300000000 & .034000000 & XYLENE \\
\hline 3.304000000 & .378000000 &
\end{tabular}

Fugitive Source Emissions - Uncategorized Pollutants.

\begin{tabular}{|c|c|c|}
\hline Em Point & Srce Nbr & Srce Type Pollutant \\
\hline $300 \mathrm{~K}-3709001$ & & 1 PAINTING AND PRINTING \\
\hline $400 \mathrm{~K}-4722 \mathrm{C} 001$ & & 1 PAINTING AND PRINTING \\
\hline
\end{tabular}

Sum

Insignificant Source Criteria Pollutants - Type: PM

Em Point

Srce Nbr Srce Type Pollutant

Em. Pt. Potential Em. Pt.

Em. Pt. Potential Em. Pt.
to Emit (tons/yr) Ctl Actual (tons/yr) Pollutant

\begin{tabular}{|c|c|c|}
\hline .097000000 & .011000000 & $\begin{array}{l}\text { LIGROINE OR VM\&P NAPHTHA. OR PETROLEUM } \\
\text { NAPHTHA }\end{array}$ \\
\hline .097000000 & .011000000 & $\begin{array}{l}\text { LIGROINE OR VM\&P NAPHTHA. OR PETROLEUM } \\
\text { NAPHTHA }\end{array}$ \\
\hline .194000000 & .0220 & \\
\hline
\end{tabular}

(tons/yr) Pollutant

Insignificant Source Emissions - Radiological Pollutants

Em Point

Srce Nbr Srce Type Pollutant

$\begin{array}{ll}\text { Em. Pt. Potentral Em. Pt. } & \\ \text { to Emit (Ci/yr) } & \text { Cti Actual (Ci/yr) Pollutant }\end{array}$

Insignificant Source Emissions - HAP Pollutants

Em Point

Srce $\mathrm{Nbr}$ Srce Type Pollutant

Em. Pt. Potential Em. Pt.

Ctl Actual (tons/yr) Pollutant

Sum

Page \#: 7 of 8 Report Run Date: 10-MAY-95 
AIR EMISSIONS INVENTORY PERMIT APPLICATION REPORT (UP460)

FACILITY NAME: Maintenance Facilities and Assoc. Structures

Insignificant Source Emissions - Uncategorized Pollutants

Em Point $\quad$ Srce Nbr Srce Type Pollutant

Em. Pt. Potential Em. Pt.
to Emit (tons/yr) Ctl Actual (tons/yr) Pollutant

Sum

Control Device Listing

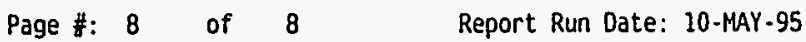



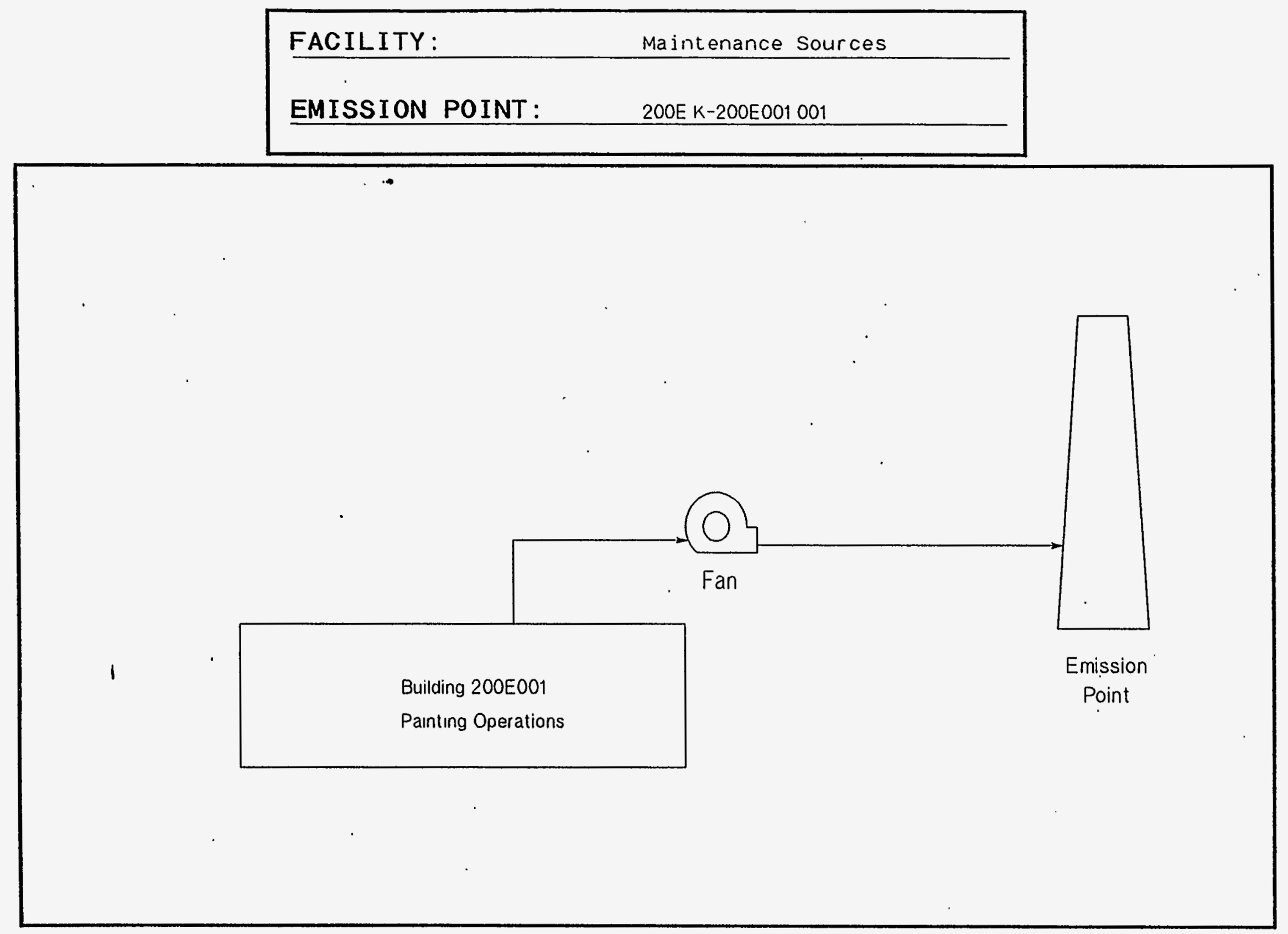


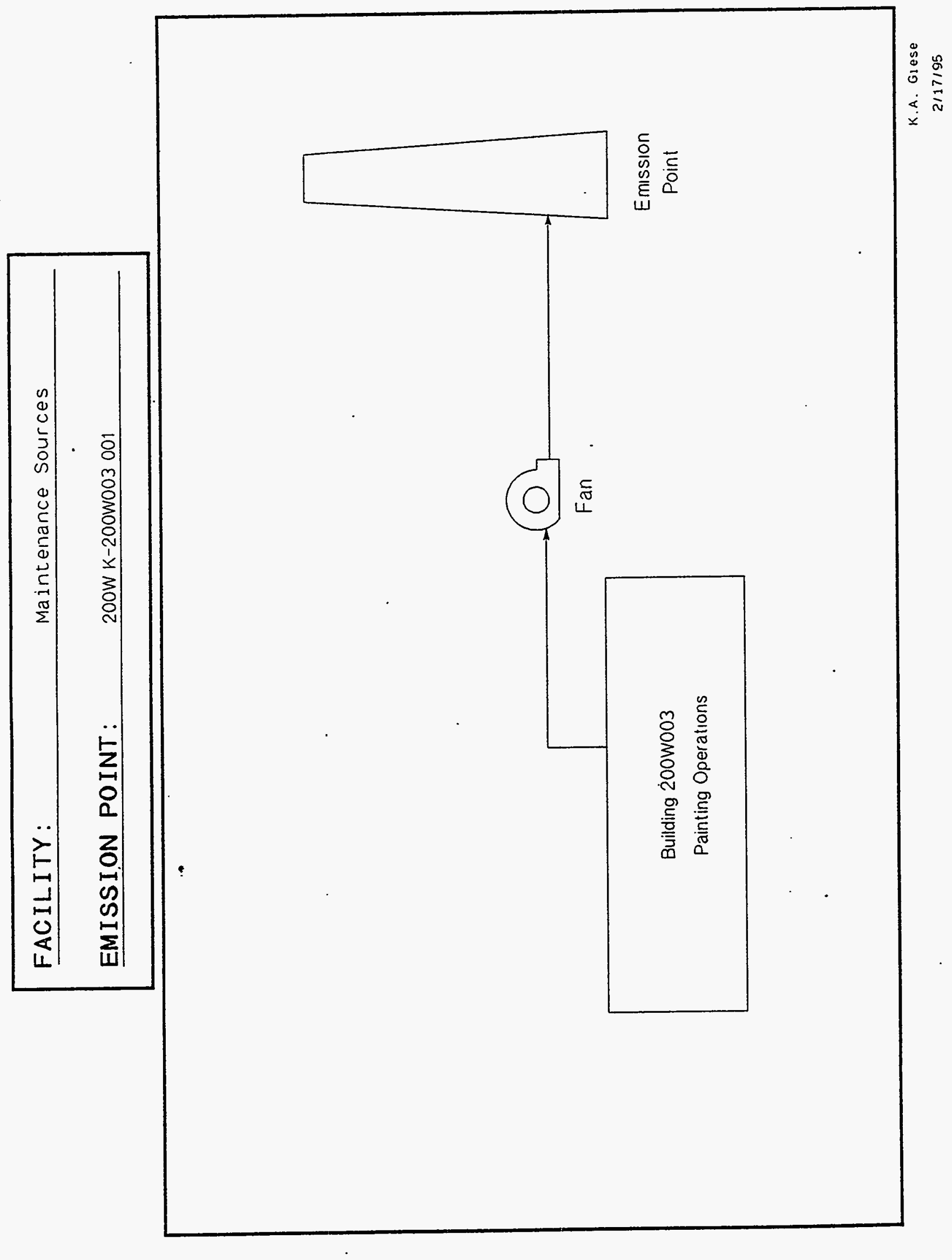




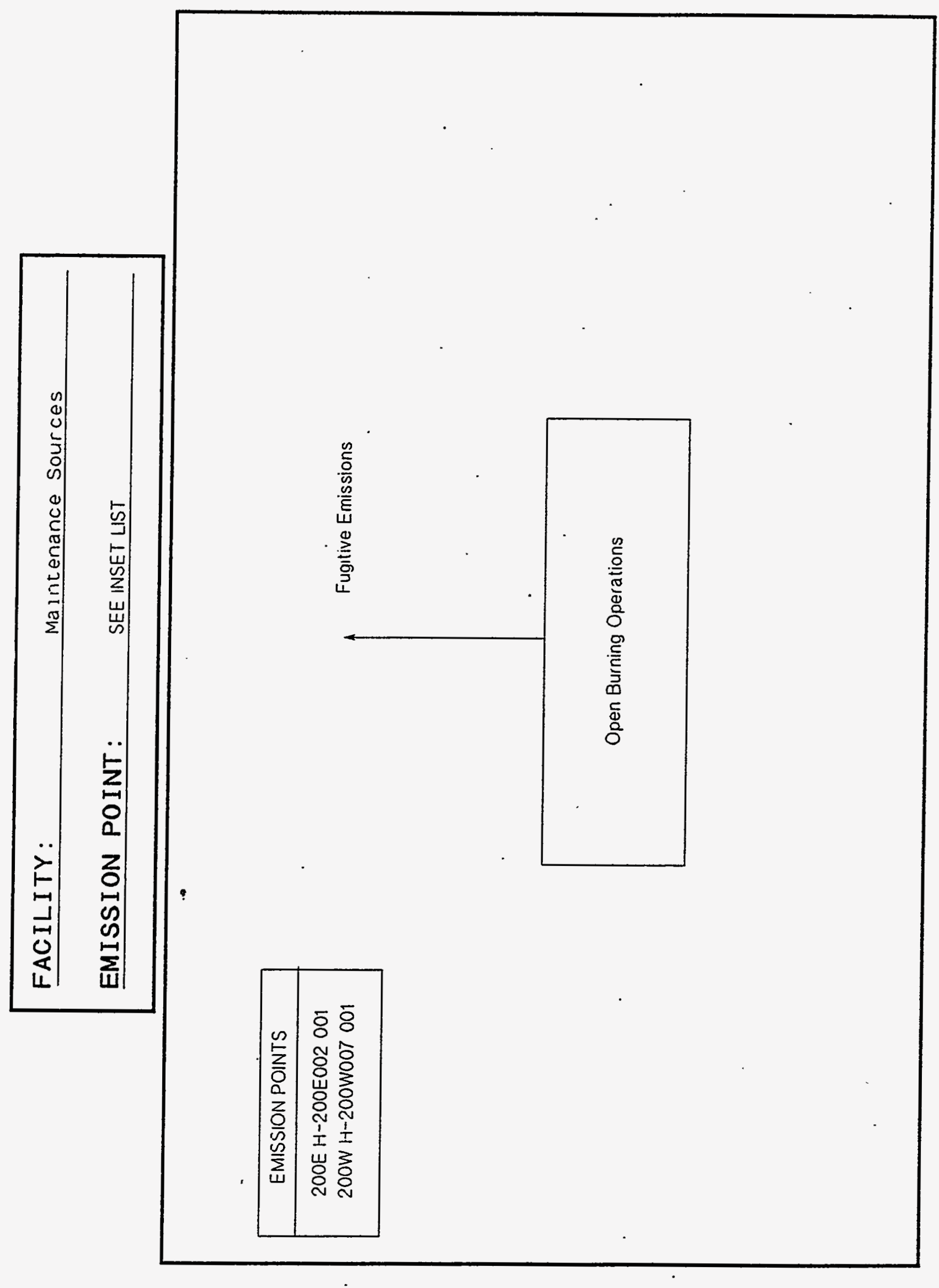




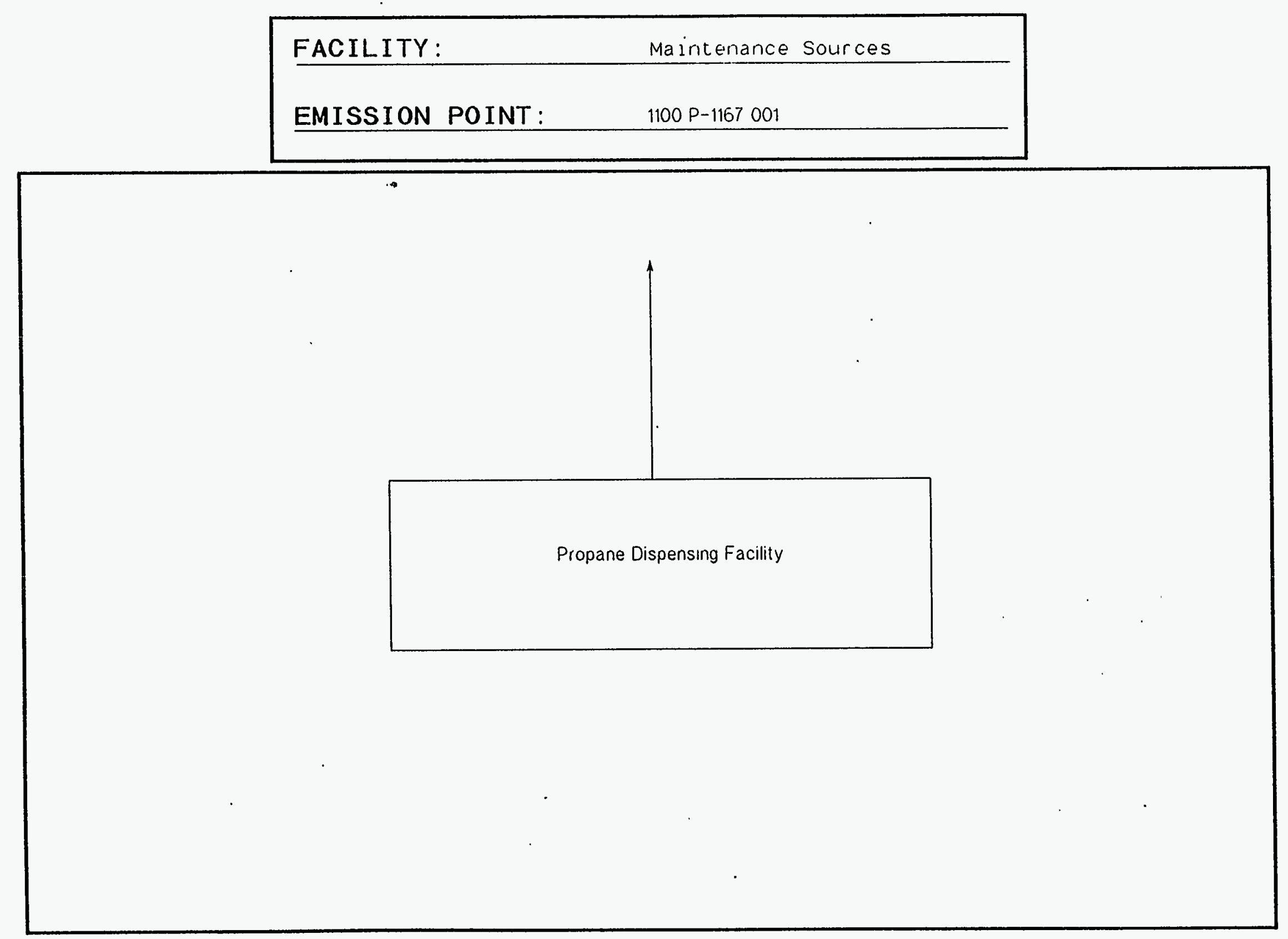

A. A. Giese

$5 / 10 / 95$ 
FACILITY:

EMISSION POINT:

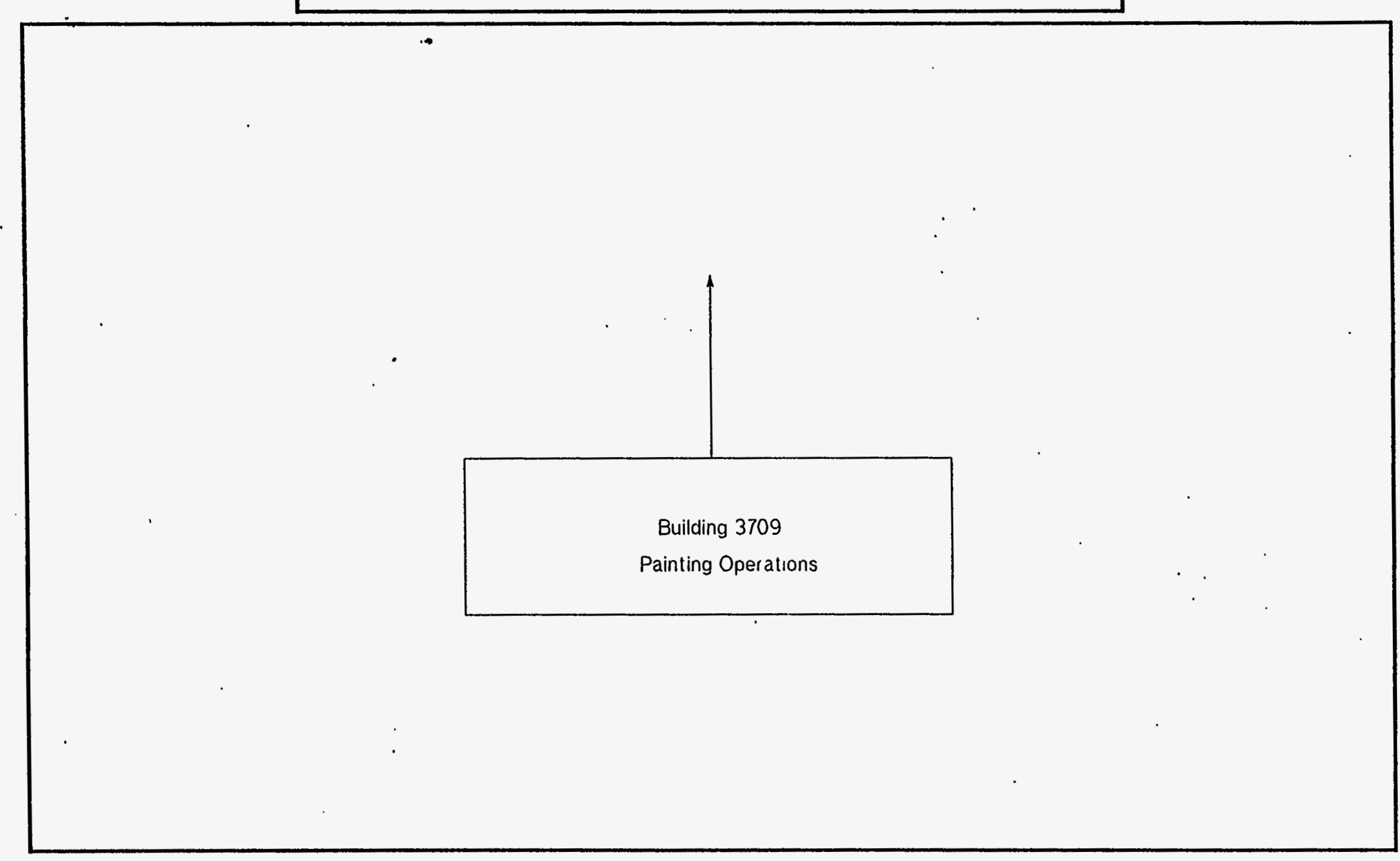




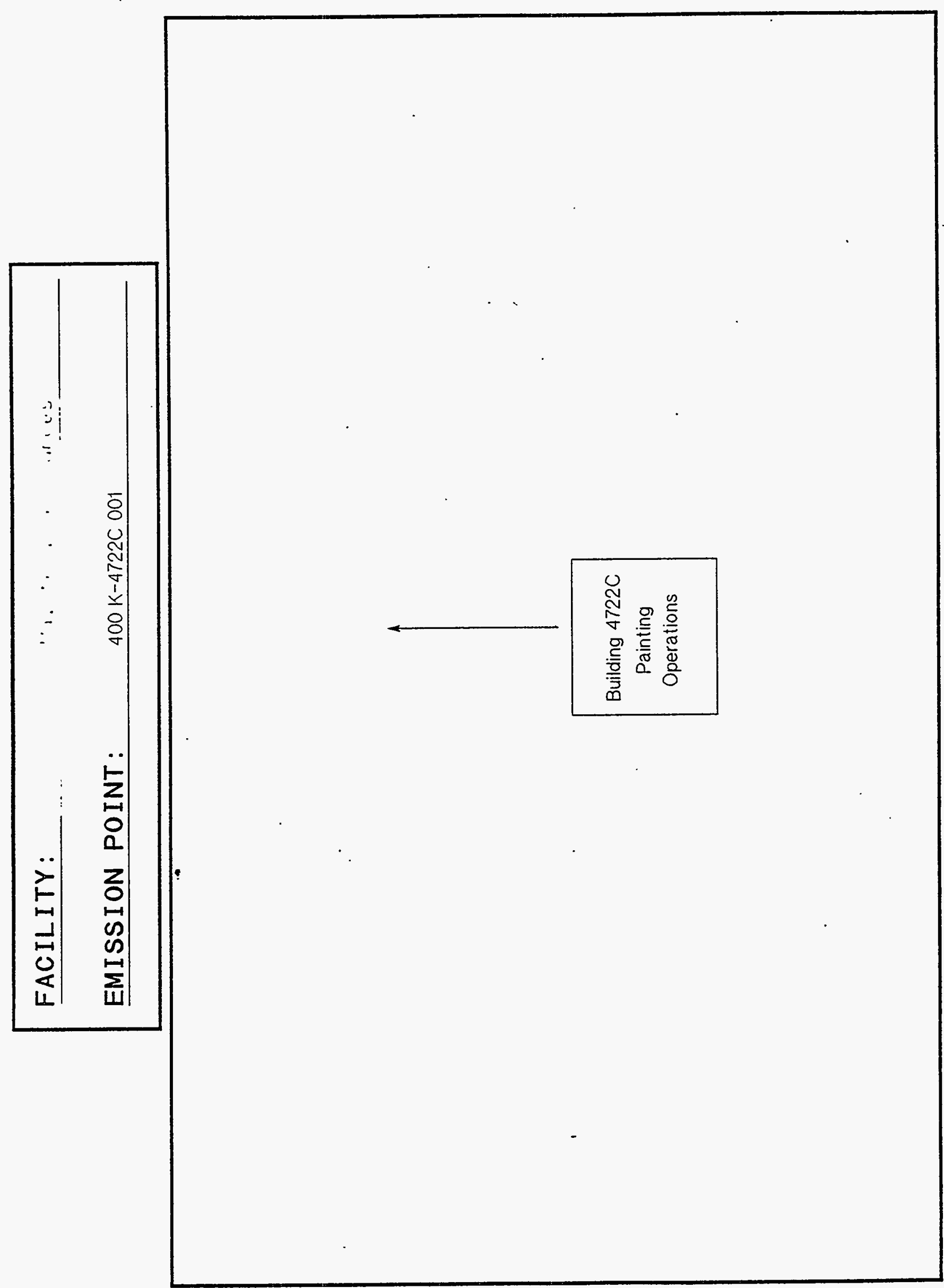




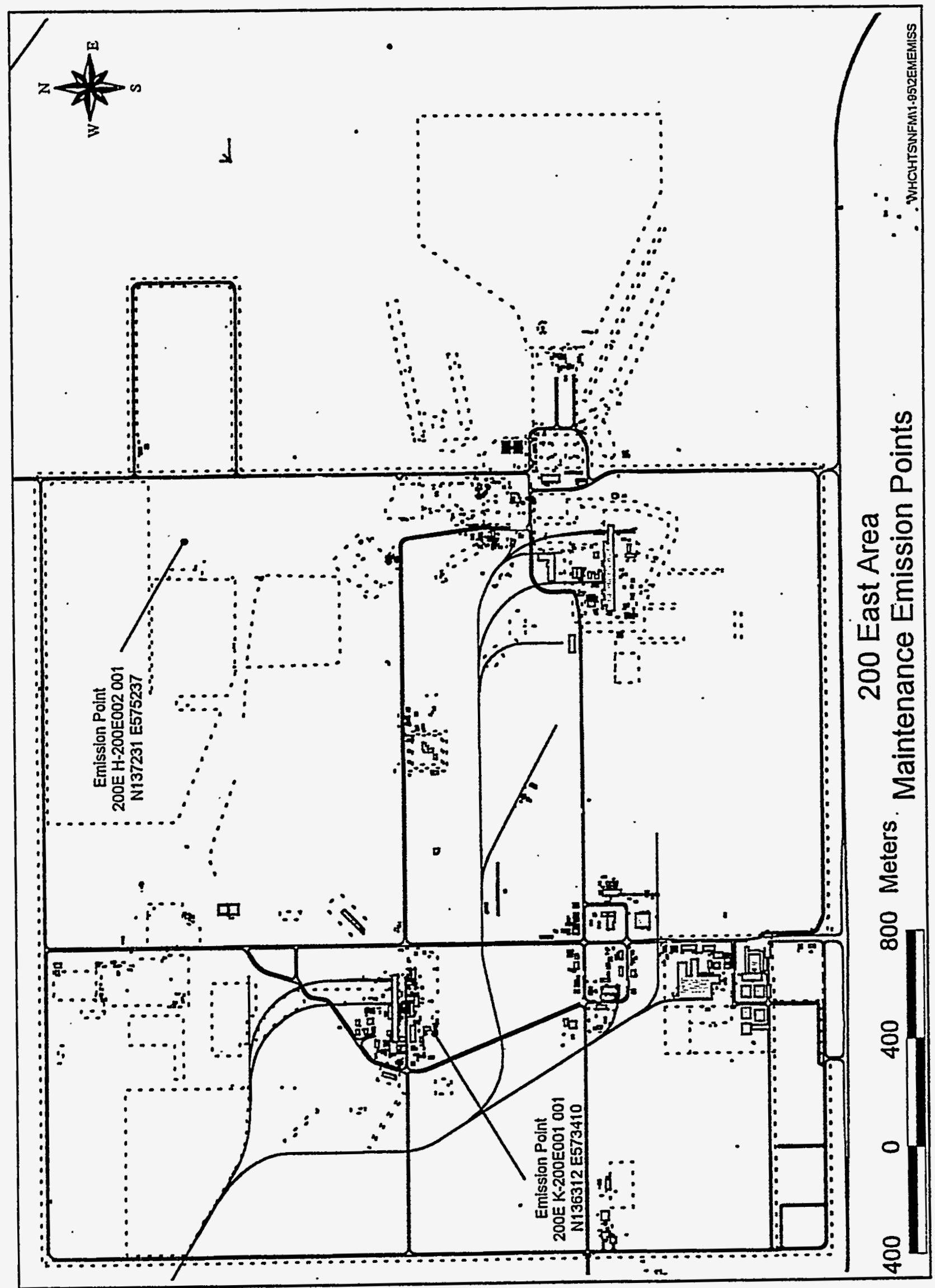




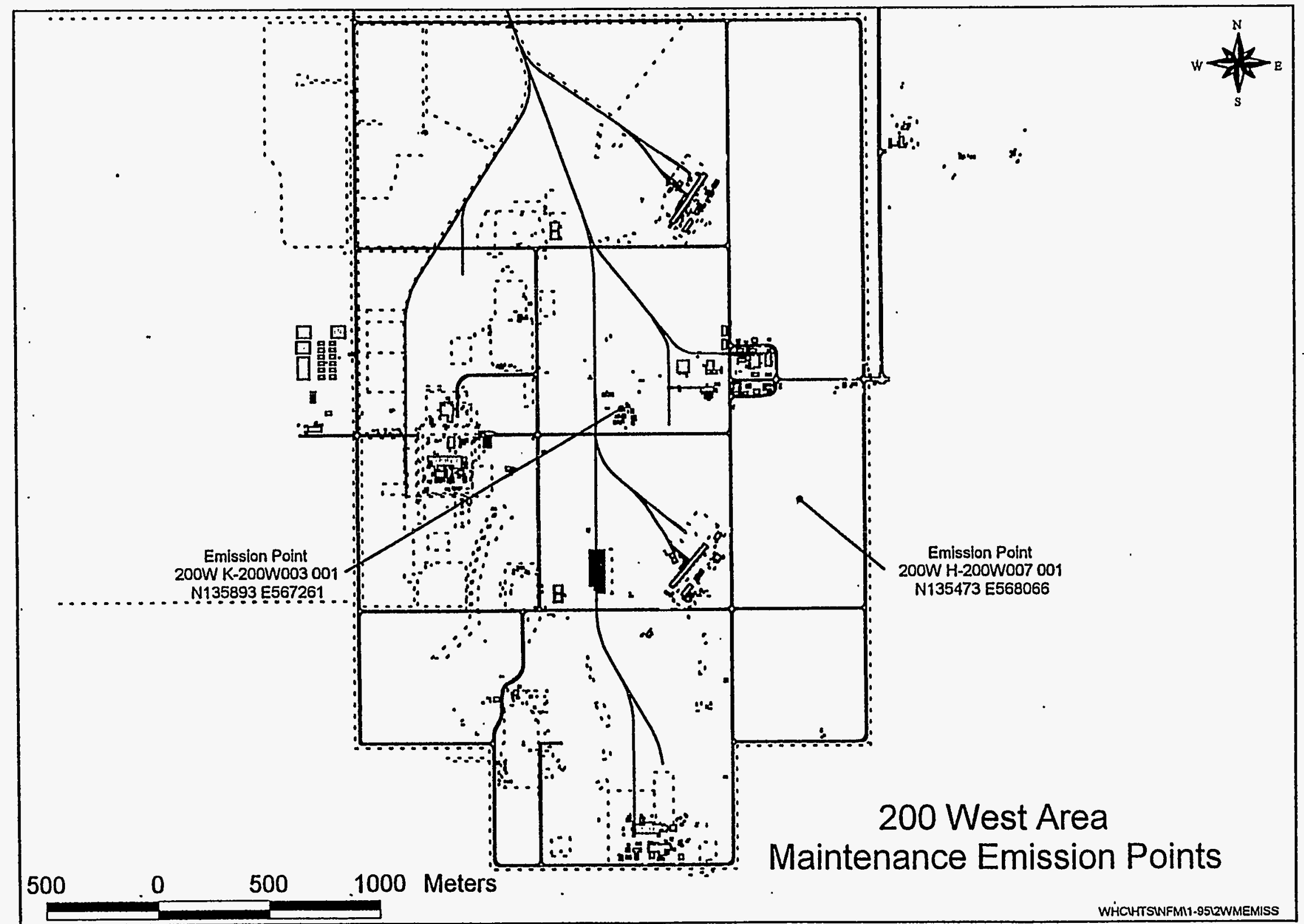




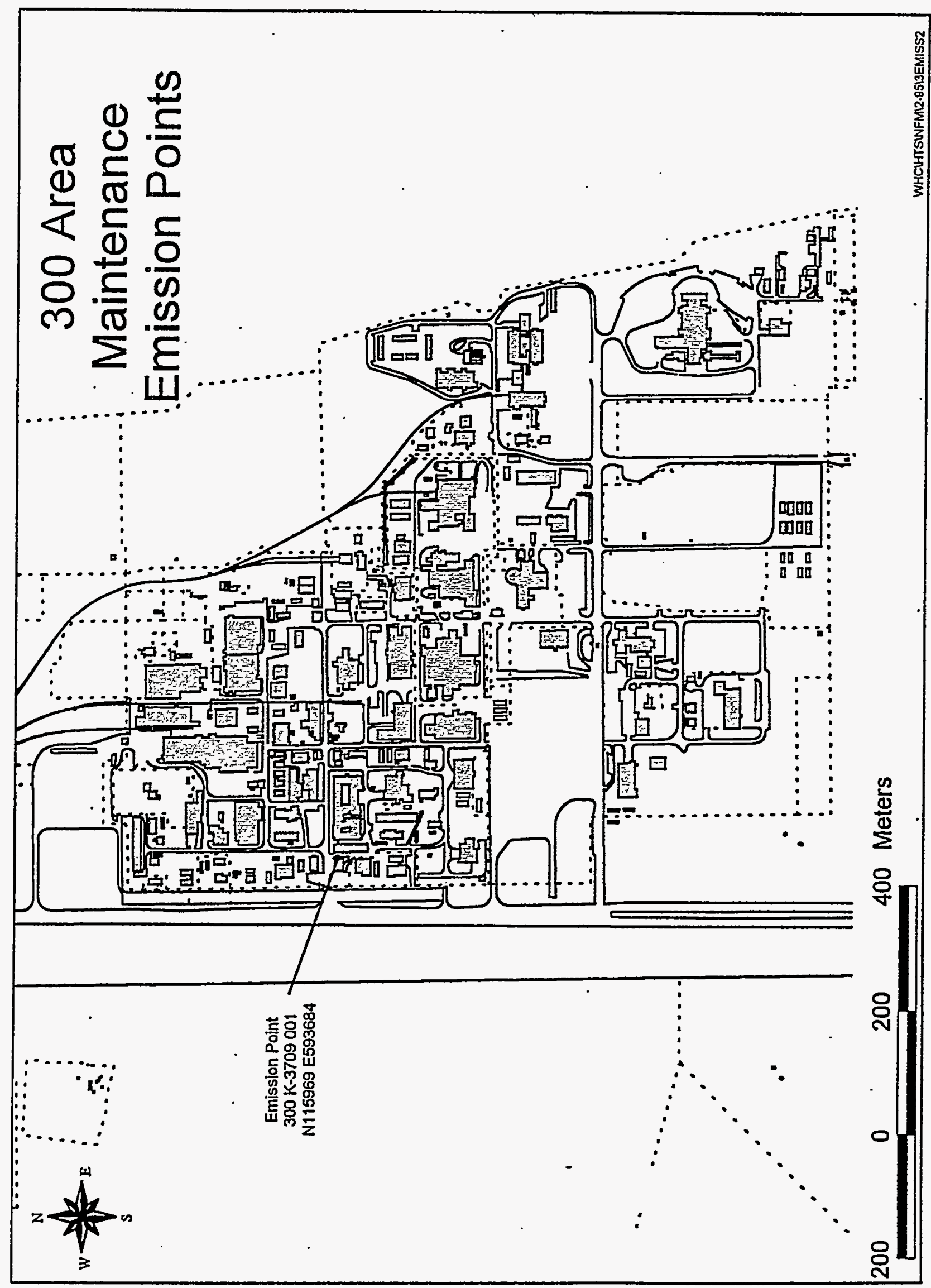




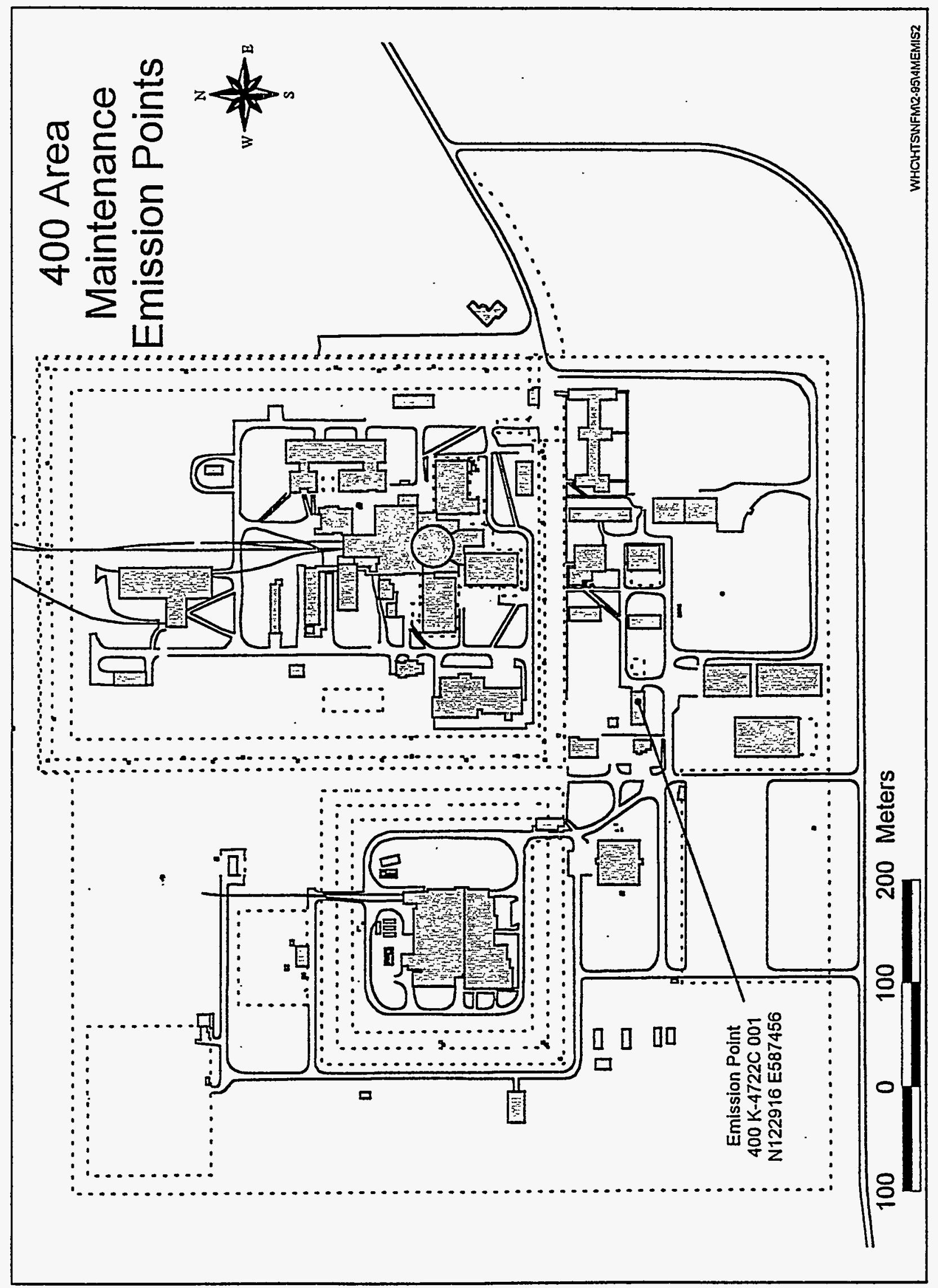




\section{APPENDIX J}

MISCELLANEOUS ITEMS 
Hanford Site Air

Operating Permit Application
DOE/RL-95-07, Rev. 0

05/95

\section{APPENDIXX J}

\section{CONTENTS}

\section{J-1 ROTARY MODE CORE SAMPLING OPERATIONS}




\title{
APPENDIX J-1 \\ ROTARY MODE CORE SAMPLING OPERATIONS
}

\author{
Chapter I. General Information
}

\section{I-B.1. Facility Description}

The rotary mode core sampling system initially was operated on November 17, 1994, and provided an additional capability to sample wastes in single-shell tanks (SSTs) and doubleshell tanks (DSTs). This system was designed to sample soft sludge wastes, and hard saltcake wastes.

For sludge sampling, it usually is not necessary. for the core sampling drill to rotate. The sludge is usually soft enough to allow sample collection by applying pressure to the drill . string. Under these conditions, the sampling method is termed "push mode". During push mode, the exhauster system does not operate.

For sampling of hard saltcake wastes, it might be necessary to drill to obtain the core sample. Under these conditions, the sampling method is termed "rotary mode". During rotary mode operations, a nitrogen purge gas is vented through the core sampling bit for bit cooling and sample integrity. For tanks that have an installed exhauster system, the purge nitrogen is effectively exhausted, and negative pressure is maintained in the tank atmosphere. For passively ventilated tanks, there is a need to use the exhauster system to prevent over pressuring the tank. Use of an exhauster has the potential to increase particulate solid and vapor space gas emissions as it provides a safe pathway to vent the purge gas.

The charter of the rotary mode core sampling operations is to sample all SSTs and DSTs that cannot otherwise be sampled by less labor intensive means. These are approximately 80 SSTs and 30 DSTs that will be sampled. All of these tanks are located in the 200 East and 200 West Areas.

A rotary mode core sampler typically is operated up to 16 hours per day, 5 days per week, 50 weeks per year. When the optional portable exhauster is used, the exhauster runs 24 hours per day until the entire core sample is complete.

\section{I-B.2. Products, Raw Materials, Fuels, Processes}

- Principle products - The product is a complete core sample consisting of 19-inch segments.

- Process information - Tank vapor space sampling and analysis is performed to determine the appropriate level of monitoring, and if subsequent vapor sampling 
will be performed. Push mode is the preferred sampling mode. Once it is determined that push mode operation can proceed no further, the core sampling truck is prepared for operation in the rotary mode. Once a complete core has been obtained, the sampling truck is moved to another riser or another location using the same riser to allow a second core to be obtained. On completion of the second core, the system is disconnected and moved to the next tank.

- Raw materials used by processes - There are no raw materials used on the rotary mode core sampler.

- Fuels used by processes - Diesel fuel is used for a portable generator unit to power the portable exhauster if needed during sampling operations.

\section{I-C.1. Emission Points, Controls, Stacks}

The emission point and stack information is found on page 1 of the AEI report output. The control information is found on page 4 of the AEI report output.

\section{I-C.2. Facility Process Flow Diagrams}

Attached.

\section{I-C.3. Site Map}

Attached.

\section{Chapter II. Regulatory Requirements}

\section{II-A.1. Description of Discharge and Emission Points}

The emission point and stack information is found on page 1 of the AEI report output. 


\section{Facility Building List}

\begin{tabular}{|c|c|c|c|c|c|c|c|}
\hline Bldg Id & North & East & Bldg Desc & Custodian(Contractor) & Cust Phone & DOE POC & DOE Phone \\
\hline CORE & 136054 & 570410 & ROTARY MOOE CORE-SAMPLING SYSTEM & ERMOLD, LEONARO F. & $373-1978$ & CASEY 0 . RUUD & $373-3478$ \\
\hline
\end{tabular}

\section{Emission Point Listing}

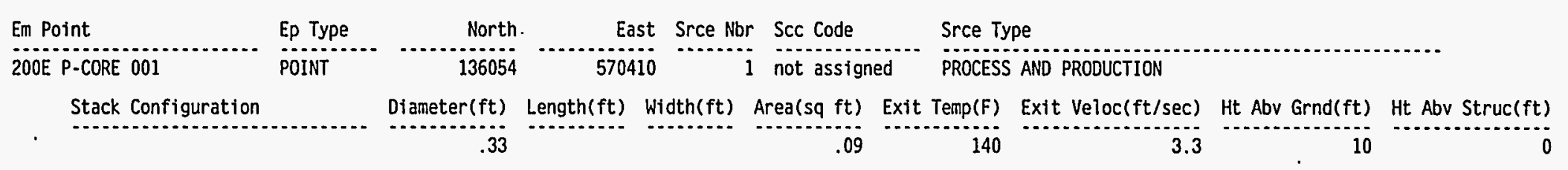

\section{Source (Process) Listing}

\begin{tabular}{lrrr} 
Em Point & Srce Nbr Srce Type & Hrs Day Days Week Wks Year Summary Process Description \\
\hline $200 E$ P-CORE 001 & 1 PROCESS AND PRODUCTION & 16 & 5
\end{tabular}

After the exhaust system and rotary mode core sample system are connected to the tank to be sampled, and all required preoperational tests are complete, rotary mode core-sampling will progress. (Pre-operational tests include sampling of the vapor space for toxic air pollutants.) An attempt necessary to mape to core-sample the waste using push mode. During push mode, it is not necessary to inject nitrogen gas and therefore it is not bit will start rotating. Whenever the drill bit is rotating. nitrogen gas will be injected into the drill string at approximately 1.4 cu.m/min (50 $\mathrm{cu} . \mathrm{ft} / \mathrm{min}$ ) to a maximum of $2.8 \mathrm{cu} . \mathrm{m} / \mathrm{min}$ ( $100 \mathrm{cu} . \mathrm{ft} / \mathrm{min}$ ). Each $48.3-\mathrm{cm}$ (19-in.) segment requires drilling for 5 to $20 \mathrm{~min}$. While the sampler. is being changed out after. each segment. nitrogen will be injected into the drill string at approximately .03 cu.m/min (1 cu.ft.min). This will maintain the pressure head in the drill string (preventing waste from entering the space just sampled) and allows for pressurization and depressurization of the sample receiver as necessary for sampler cha each day).

Once a complete core has been obtained. the position of the rotary mode core-sampling truck will be changed. The rotary mode core-sampling truck will either be repositioned at the same riser. or moved to a different riser on the same tank, to allow a second core to be obtained. Upon completion of the second core, the exhauster and rotary mode core-sampling systems will be disconnected and moved to the next tank to be sampled. During sample left open.

Source Raw Materials Usage

Em Point Srce $\mathrm{Nbr}$ Srce Type

Process Rate Process Uom Material

Fuels Usage by Source Processes

En Point Srce Nbr Srce Type Qty Uom Fuel 


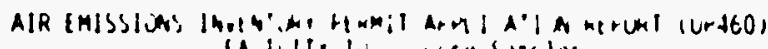

FACILITY RAME Rotary aude Core Sampling unit

Fuels Usage by Control Devices

Em Point

Ctl Nbr Ctl Type

Qty Uom

Fuel

Point Source Criteria Pollutants - Type: H2S

Em Point Srce Nbr Srce Type Pollutant

Em. Pt. Potential Em. Pt.
to Emit (tons/yr) Ctl Actual (tons/yr) Pollutant

200E P-CORE $001 \quad 1$ PROCESS AND PRODUCTION

Sum

010000000

.002000000 HYDROGEN SULFIDE

Point Source Criteria Pollutants - Type: vOC

Em Point

Srce Nbr Srce Type Pollutant

2OOE P-CORE 00

Sum

Point Source Emissions - Radiological Pollutants

Em Point

Srce Nbr Srce Type Pollutant

Em. Pt. Potential

200 P-CORE 001 PROCESS AND PRODUCTION
Em. Pt. Potential Em. Pt.
to Emit (tons/yr) Ct1 Actual (tons/yr) Pollutant

\begin{tabular}{rrr}
.560000000 & .180000000 & ACETONE \\
.003400000 & .000680000 & BENZENE \\
.240000000 & .004600000 & CARBON DISULFIDE \\
.003300000 & .000670000 & CARBON TETRACHLORIDE \\
.150000000 & .019000000 & METHYLAMINE \\
1.800000000 & .460000000 & N-BUTY ALCOHOL \\
.070000000 & .014000000 & N-HEXANE \\
\hline 2.826700000 & .67890000
\end{tabular}

$2.826700000-678950000$

\begin{tabular}{|c|c|c|}
\hline 0 Emit (ci/yr) & $(y r)$ & Pollutant \\
\hline $\begin{array}{r}.002280000 \\
.000001190 \\
.000912000 \\
2.710000000 \\
.00002400 \\
.000005460 \\
.000002890 \\
.000003840 \\
.015700000 \\
.000087300 \\
.001440000 \\
.000336000 \\
.003930000 \\
3.060000000 \\
.001900000 \\
.000037500 \\
.000001930 \\
.000045300\end{array}$ & $\begin{array}{l}.000000001 \\
.000000000 \\
.000912000 \\
.000000677 \\
.000000000 \\
.000000000 \\
.000002890 \\
.000000000 \\
.000000004 \\
.000000000 \\
.00000000 \\
.00000000 \\
.000000001 \\
.000000765 \\
.000000000 \\
.000000000 \\
.000000000 \\
.000000000\end{array}$ & 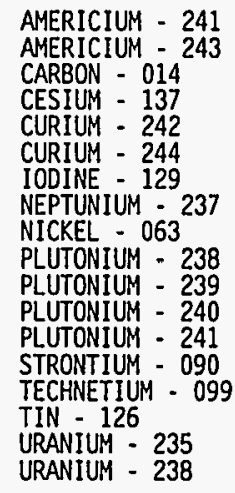 \\
\hline
\end{tabular}

Page \#: 2 of 4 Report Run Date: 09-MAY-95 
AIR EMISSIONS INVENTORY PERMIT APPLICATION REPORT (UP460)

FACILITY NAME: Rotary Mode Core Sampling Unit

Point Source Emissions - HAP Pollutants

En Point

200E P-CORE 001

Sum

Point Source Emissions - Uncategorized Pollutants

\section{Em Point}

200E P-CORE 001

Sum

Fugitive Source Emissions - Radiological Pollutants
Srce Nbr Srce Type Pollutant

1 PROCESS AND PRODUCTION
Em. Pt. Potential Em. Pt.

to Emit (tons/yr) Ct] Actual (tons/yr) Pollutant

$\begin{array}{ccc}.003400000 & .000680000 & \text { BENZENE } \\ .240000000 & .004600000 & \text { CARBON DISULFIDE } \\ .003300000 & .000670000 & \text { CARBON TETRACHLORIDE } \\ .18000000 & .001700000 & \text { HYROGEN CYANIDE } \\ .010000000 & .002000000 & \text { HYOROGEN SULFIDE } \\ .070000000 & .014000000 & \text { N-HEXANE } \\ .506700000 & & .023650000\end{array}$

Em. Pt. Potential Em. Pt.
to Emit (tons/yr) Ctl Actual (tons/yr) Pollutant

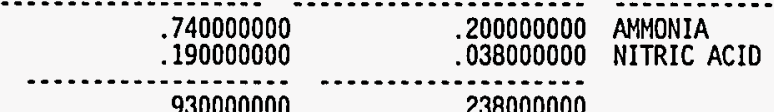

930000000

Em Point

Srce Nbr Srce Type Pollutant

Em. Pt. Potential Em. Pt.
to Emit (Ci/yr)
Cti Actual (Ci/yr) Pollutant

Fugitive Source Emissions - HAP Pollutants

Em Point

Srce Nbr Srce Type Pollutant

Em. Pt. Potential Em. Pt.

to Emit (tons/yr) Ctl Actual (tons/yr) Pollutant

Sum

Fugitive Source Emissions - Uncategorized Pollutants

Em Point

Srce Nbr Srce Type Pollutant

Em. Pt. Potential Em. Pt.
to Emit (tons/yr) Ctl Actual (tons/yr) Pollutant

Sum 

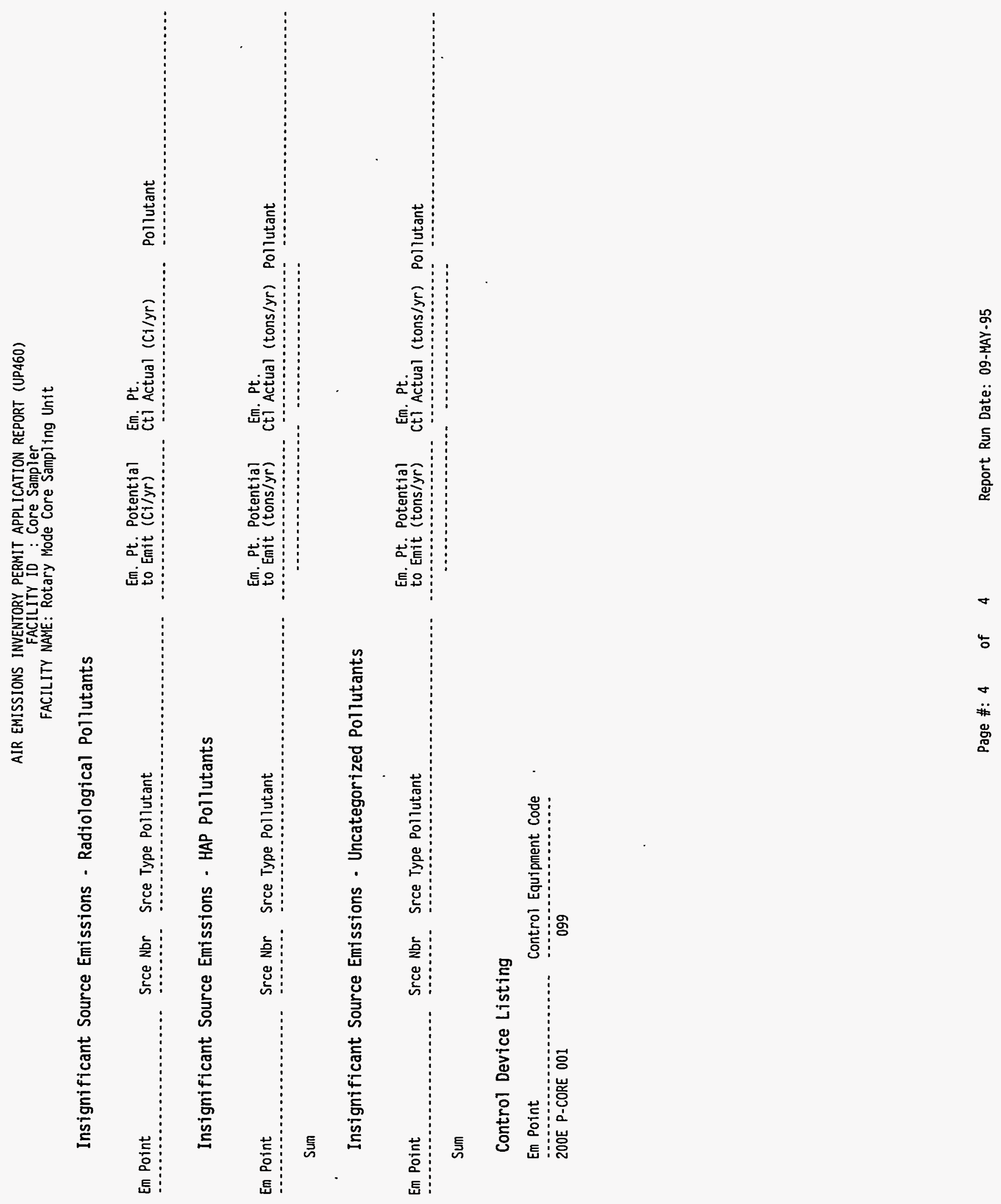


\section{FACILITY:}

EMISSION POINT:

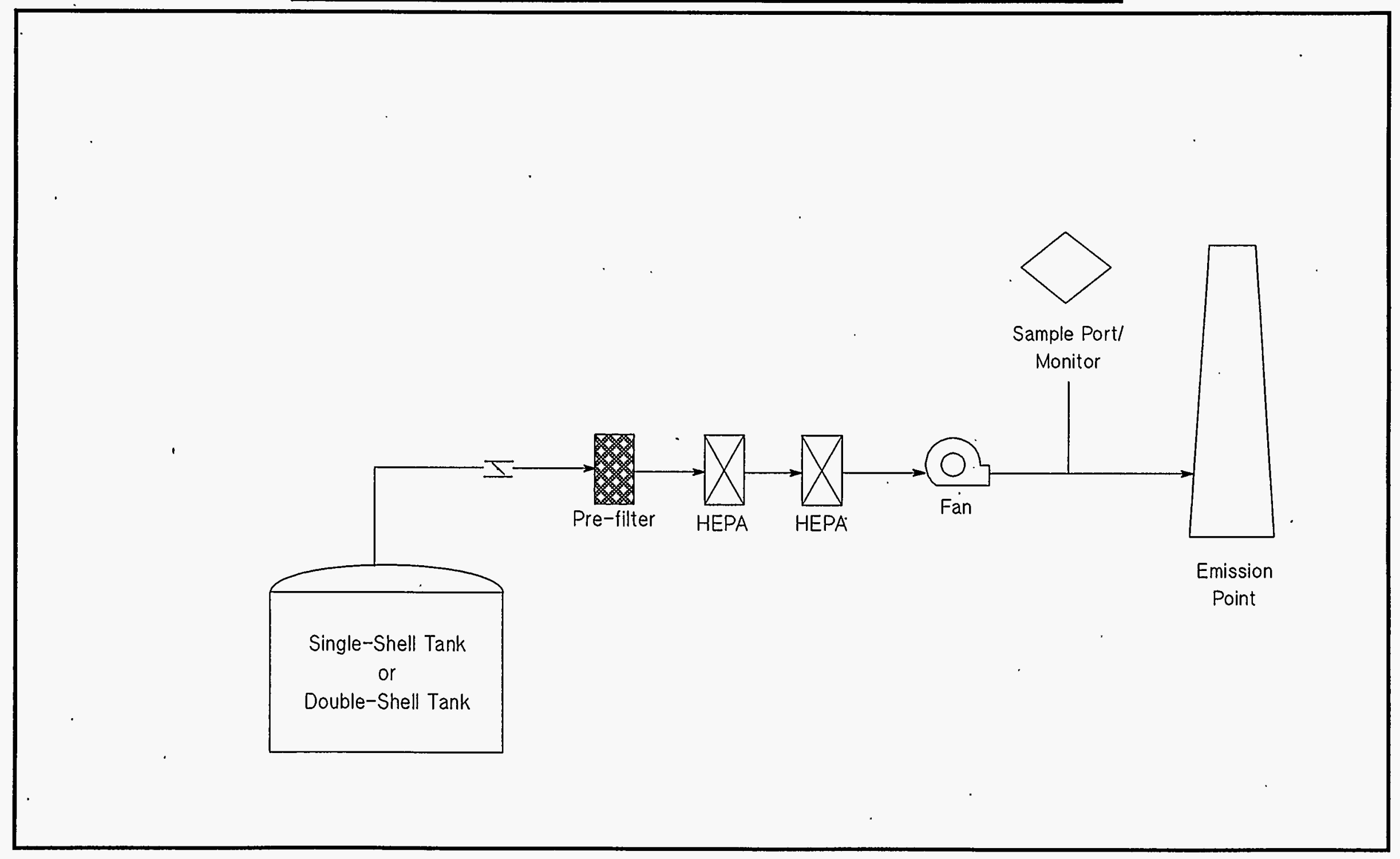




\section{Locations of Rotary Mode Core Sampler Operations Within the Tank Farms on the Hanford Site, Washington}

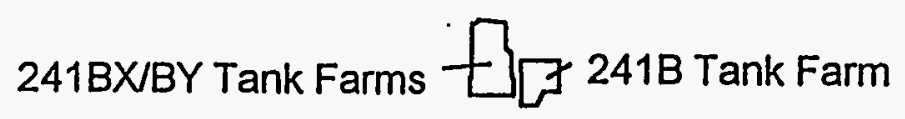

II $241 T$ Tank Farm

?

241TXTY Tank Farms

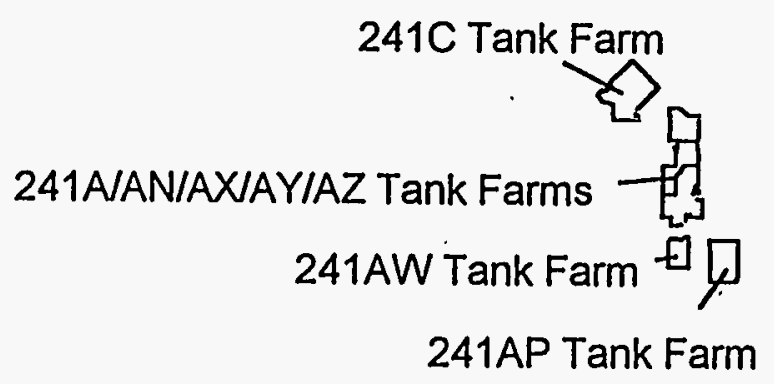

Q $241 \mathrm{U}$ Tank Farm

$[$ 241S/SX/SY Tank Farms

900 
Hanford Site Air

Operating Permit Application
DOE/RL-95-07, Rev. 0

05/95

APPENDIX K

\section{INSIGNIFICANT EMISSIONS UNITS}




\section{APPENDIX K}

\section{INSIGNIFICANT EMISSIONS UNITS}

K.1a. PNL Building List.

K.1b. PNL Building Inventories Compared to Threshold Values.

K.1c. PNL Building Inventories by Room Compared to Threshold Values.

K.2. Insignificant Emission Units Based on Threshold Comparisons [WAC 173-401-530(1)(a)].

K.3. Fugitive Source Insignificant Emission Units [WAC 173-401(1)(d)]. 
Table K.1a. PNL Building List.

\begin{tabular}{|c|c|c|c|c|c|}
\hline $\begin{array}{l}\text { PROPERTY } \\
\text { ID }\end{array}$ & PROPERTY NAME & $\begin{array}{l}\text { PROPERTY } \\
\text { USAGE } \\
\text { PRODOM } \\
\text { CODE }\end{array}$ & LONG DISCRIPTION & $\begin{array}{l}\text { GROSS } \\
\text { SQFT }\end{array}$ & $\begin{array}{c}\text { TOTAL AOP } \\
\text { INVENTORY } \\
\text { CHEMICAL } \\
\text { (TONS) }\end{array}$ \\
\hline $213 \mathrm{~J}$ & STORAGE VAULT & 401 & $\begin{array}{l}\text { PROGRAMMATIC } \\
\text { GENERAL STORAGE }\end{array}$ & 482 & 0 \\
\hline 242B & RADIOACTIVE PAT RES LAB & 401 & $\begin{array}{l}\text { PROGRAMMATIC } \\
\text { GENERAL STORAGE }\end{array}$ & 2713 & $\mathbf{0}$ \\
\hline $242 B L$ & CASK LOADING BUILDING & 401 & $\begin{array}{l}\text { PROGRAMMATIC } \\
\text { GENERAL STORAGE }\end{array}$ & 546 & $\mathbf{0}$ \\
\hline $2718 \mathrm{E}$ & CRITICALMASS STORAGE BUILDING & 412 & $\begin{array}{l}\text { SPECIAL NUCLEAR } \\
\text { STORAGE }\end{array}$ & 1664 & $\mathbf{0}$ \\
\hline $303 C$ & MATERIALS EVALUATION LAB. & 724 & $\begin{array}{l}\text { NUCLEAR PHYSICS } \\
\text { LAB }\end{array}$ & 1296 & 0 \\
\hline $303 \mathrm{~J}$ & MATERIAL STORAGE BUILDING & 401 & $\begin{array}{l}\text { PROGRAMMATIC } \\
\text { GENERAL STORAGE }\end{array}$ & 3652 & 0 \\
\hline $305 \mathrm{~B}$ & HAZARDOUS WASTE STORAGE FAC & 410 & $\begin{array}{l}\text { HAZARDOUS/FLAMMA } \\
\text { BLE STORAGE }\end{array}$ & 9176 & 0 \\
\hline $306 \mathrm{~W}$ & MATERIALS DEVELOPMENT LAB & 751 & MATERIALS LAB & 36531 & 0.3143 \\
\hline 306WTRL2 & OPFICE TRAIILER & 101 & OFFICE & 1569 & $\mathbf{0}$ \\
\hline 306WTRLS & OFFICE TRAILER & 101 & OFFICE & 667 & 0 \\
\hline 306WTRL6 & OFFICE TRAILER & 101 & OFFICE & 1577 & 0 \\
\hline 314 & ENGINEERING DEVEL. LAB & 712 & CALIBRATION LAB & 28192 & 0.1978 \\
\hline $314 B$ & STRESS RUPTURE TEST FAC. & 759 & $\begin{array}{l}\text { OTHER MATERIALS } \\
\text { R\&D TEST BLDG }\end{array}$ & 860 & 0.0168 \\
\hline 318 & RADIOLOGICAL CALIBRATIONS LAB & 704 & CALIBRATION LAB & 32662 & 0.2103 \\
\hline $318 \mathrm{TRL2}$ & OFFICE TRAILER & 101 & OFFICE & 1568 & 0 \\
\hline 318TRL4 & OFFICE TRAIIER & 101 & OFFICE & 3669 & 0 \\
\hline 318TRLS & OFFICE TRAILER & 101 & OFFICE & 1339 & 0 \\
\hline 320 & ANALYTICAL AND NUCLEAR RES LAB & 712 & CALIBRATION LAB & 31437 & 0.296 \\
\hline 320 TRL2 & OFFICE TRAILER & 101 & OFFICE & 667 & 0 \\
\hline 323 & MECHANICAL PROPERTIES LAB & 724 & $\begin{array}{l}\text { NUCLEAR PHYSICS } \\
\text { LAB }\end{array}$ & 4150 & 0 \\
\hline 324 & WASTE TECH ENGINEERING LAB & 781 & $\begin{array}{l}\text { DEMONSTRATION } \\
\text { RESEARCH BLDG }\end{array}$ & 101709 & 0.3052 \\
\hline $324 \mathrm{~A}$ & STACK SAMPLING FACILITY & 694 & OTHER SERVICE BLDG & 144 & $\mathbf{0}$ \\
\hline $324 \mathrm{D}$ & STACK SAMPLING FACILITY & 694 & OTHER SERVICE BLDG & 520 & 0 \\
\hline 325 & APPLIED CHEMISTRY LAB & 712 & CAIIBRATION LAB & 144092 & 0.8567 \\
\hline 325TRLI & ORPICE TRAILER & 101 & OFFICE & 659 & 0 \\
\hline 326 & MATERIALS SCIENCES LAB & 759 & $\begin{array}{l}\text { OTHER MATERIALS } \\
\text { R\&D TEST BLDG }\end{array}$ & 63101 & 0.4535 \\
\hline 326TRL1 & OFFICE TRAILER & 101 & OFFICE & 1556 & $\mathbf{0}$ \\
\hline 326TRL2 & OFFICE TRAIlER & 101 & OFFICE & 1573 & $\mathbf{0}$ \\
\hline 327 & POST IRRADIATION TEST LAB & 759 & $\begin{array}{l}\text { OTHER MATERIALS } \\
\text { R\&D TEST BLDG }\end{array}$ & 26925 & 0.008 \\
\hline 329 & CHEMICAL SCIENCES LAB & 712 & CALIBRATION LAB & 39420 & 0.5824 \\
\hline 329TRL1 & OFFICE TRAILER & 101 & OFFICE & 671 & 0 \\
\hline 329TRL2 & OFFICE TRAIIER & 101 & OFFICE & 671 & 0 \\
\hline 329TRL4 & OPFICE TRAILER & 101 & OFFICE & 1557 & 0 \\
\hline 329TRLS & OFFICE TRAILER & 101 & OFFICE & 1556 & 0 \\
\hline 329TRL6 & OFFICE TRAIIER & 101 & OFFICE & 1562 & 0 \\
\hline
\end{tabular}


Table K.1a. PNL Building List.

\begin{tabular}{|c|c|c|c|c|c|}
\hline $\begin{array}{l}\text { PROPERTY } \\
\text { ID }\end{array}$ & PROPERTY NAME & $\begin{array}{l}\text { PROPERTY } \\
\text { USAGE } \\
\text { PRODOM } \\
\text { CODE }\end{array}$ & LONG DISCRIPTION & $\begin{array}{l}\text { GROSS } \\
\text { SQFT }\end{array}$ & $\begin{array}{c}\text { TOTAL AOP } \\
\text { INVENTORY } \\
\text { CHEMICAI } \\
\text { (TONS) }\end{array}$ \\
\hline 331 & LIFE SCIENCES LAB & 741 & $\begin{array}{l}\text { BIOLOGICAL } \\
\text { RESEARCH LAB }\end{array}$ & 117240 & 1.0763 \\
\hline $331 \mathrm{~A}$ & VIROLOGY LABORATORY & 741 & $\begin{array}{l}\text { BIOLOGICAL } \\
\text { RESEARCH LAB }\end{array}$ & 2800 & 0.1628 \\
\hline 331B & DOG KENNEL & 745 & $\begin{array}{l}\text { ANIMAL RESEARCH } \\
\text { FAC }\end{array}$ & 4225 & 0 \\
\hline $331 \mathrm{C}$ & ANIMAL CARE FAC. STORAGE & 401 & $\begin{array}{l}\text { PROGRAMMATIC } \\
\text { GENERAL STORAGE }\end{array}$ & 5000 & 0.0004 \\
\hline $331 \mathrm{D}$ & BIOMAGNETIC EFFECTS LAB & 749 & OTHER BIO-MED BLDG & 1357 & 0 \\
\hline $331 \mathrm{E}$ & GREENHOUSE & 769 & $\begin{array}{l}\text { OTHER ENVIRON. R\&D } \\
\text { TEST BLDG }\end{array}$ & 1560 & 0 \\
\hline $331 F$ & ANIMAL RESOURCES STORAGE & 401 & $\begin{array}{l}\text { PROGRAMMATIC } \\
\text { GENERAL STORAGE }\end{array}$ & 1120 & 0 \\
\hline $331 G$ & INTERIM TISSUE REPOSITORY & 423 & $\begin{array}{l}\text { TEMPERATURE AND } \\
\text { HUMDITY }\end{array}$ & 1200 & $\mathbf{0}$ \\
\hline $331 \mathrm{H}$ & AEROSOL WIND TUNNEL RES. FAC. & 741 & $\begin{array}{l}\text { BIOLOGICAL } \\
\text { RESEARCH LAB }\end{array}$ & 3557 & 0.1706 \\
\hline $331 \mathrm{~J}$ & INCINERATOR & 450 & SHED STORAGE & 380 & $\mathbf{0}$ \\
\hline 331 TRL4 & STORAGE TRAIlER & 401 & $\begin{array}{l}\text { PROGRAMMATIC } \\
\text { GENERAL STORAGE }\end{array}$ & 516 & 0 \\
\hline 33ITRIS & OFFICE TRAILER & 101 & OFFICE & 1842 & 0 \\
\hline 332 & PACKAGING TEST FACILITY & 801 & OTHER & 400 & 0 \\
\hline 336 & HIGH BAY TEST FACILITY & 781 & $\begin{array}{l}\text { DEMONSTRATION } \\
\text { RESEARCH BLDG }\end{array}$ & 3863 & 0.0023 \\
\hline 337 & TECH MANAGEMENT CENTER & 101 & OFFICE & 70424 & 0.0011 \\
\hline 350 & PLNT OPRNS AND MAINT FAC & 501 & $\begin{array}{l}\text { PRODUCTION/MANUF } \\
\text { AC. BLDG }\end{array}$ & 16320 & 0 \\
\hline $350 \mathrm{~A}$ & PAINT SHOP & 602 & PAINT SHOP & 1400 & 0.0338 \\
\hline $350 \mathrm{~B}$ & WAREHOUSE & 400 & GENERAL STORAGE & 2122 & 0 \\
\hline $350 \mathrm{C}$ & STORAGE BUILDING & 400 & GENERAL STORAGE & 212 & 0 \\
\hline $350 \mathrm{D}$ & OIL STORAGE FACILITY & 410 & $\begin{array}{l}\text { HAZARDOUS/FLAMMA } \\
\text { BLE STORAGE }\end{array}$ & 960 & $\mathbf{0}$ \\
\hline $3614 A$ & RIVER MONITORING STATION & 694 & OTHER SERVICE BLDG & 144 & 0 \\
\hline 3708 & RADIOANALYTICAL LAB & 712 & CALIBRATION LAB & 4027 & 0.0302 \\
\hline $3718 \mathrm{~A}$ & LAB EQUIP. CENTRAL POOL & 411 & $\begin{array}{l}\text { NUCLEAR } \\
\text { CONTAMINATED } \\
\text { STORAGE }\end{array}$ & 6400 & 0 \\
\hline 3718B & LAB EQUIP. CENTRAL POOL & 400 & GENERAL STORAGE & 3200 & 0 \\
\hline $3718 \mathrm{E}$ & STORAGE (324) & 401 & $\begin{array}{l}\text { PROGRAMMATIC } \\
\text { GENERAL STORAGE }\end{array}$ & 3000 & $\mathbf{0}$ \\
\hline $3718 G$ & STORAGE BUILDING & 410 & $\begin{array}{l}\text { HAZARDOUS/FLAMMA } \\
\text { BLE STORAGE }\end{array}$ & 4000 & 1.1705 \\
\hline 37180 & STORAGE BUILDING & 400 & GENERAL STORAGE & 800 & 0 \\
\hline 3720 & ENVIRONMENTAL SCIBNCES LAB & 761 & ENVIRONMENTAL LAB & 29030 & 0.5117 \\
\hline 3723 & SOLVENT/ACID STORAGE & 410 & $\begin{array}{l}\text { HAZARDOUS/FLAMMA } \\
\text { BLE STORAGE }\end{array}$ & 144 & 0.0041 \\
\hline 3730 & GAMMA IRRADIATION FAC & 765 & $\begin{array}{l}\text { RADIATION EFFECTS } \\
\text { LAB }\end{array}$ & 3637 & 0.0042 \\
\hline 3731 & LAB EQUTP. CENTRAL POOL & 400 & GENERAL STORAGE & 3200 & 0 \\
\hline $3731 \mathrm{~A}$ & GRAPHITE MACHINE SHOP & 611 & MACHINE SHOP & 3200 & $\mathbf{0}$ \\
\hline
\end{tabular}


Table K.1a. PNL Building List.

\begin{tabular}{|c|c|c|c|c|c|}
\hline $\begin{array}{l}\text { PROPERTY } \\
\text { ID }\end{array}$ & PROPERTY NAME & $\begin{array}{c}\text { PROPERTY } \\
\text { USAGE } \\
\text { PRODOM } \\
\text { CODE }\end{array}$ & LONG DISCRIPTION & $\begin{array}{l}\text { GROSS } \\
\text { SQFT }\end{array}$ & $\begin{array}{c}\text { TOTAL AOP } \\
\text { INVENTORY } \\
\text { CHEMICAL } \\
\text { (TONS) }\end{array}$ \\
\hline 3745 & RADIOLOGICAL SCIENCES LAB & 792 & $\begin{array}{l}\text { GENERAL LAB } \\
\text { (NUCLEAR) }\end{array}$ & 5152 & 0 \\
\hline $3745 A$ & ELECTRON ACCELERATOR FACILITY & 785 & ACCELERATOR BLDG & 1277 & 0 \\
\hline 3745B & POSTIVE ION ACCELERATOR FAC & 785 & ACCELERATOR BLDG & 6744 & 0.0181 \\
\hline 3745TRL3 & OFFICE TRAIIER & 401 & $\begin{array}{l}\text { PROGRAMMATIC } \\
\text { GENERAL STORAGE }\end{array}$ & 673 & 0 \\
\hline 3746 & IRRADIATION PHYSICS LAB & 721 & PHYSICS LAB & 2203 & 0.00001 \\
\hline $3746 A$ & RADIOLOGICAL PHYSICS LAB & 721 & PHYSICS LAB & 5100 & 0.339 \\
\hline 3760 & TECHNICAL LIBRARY & 290 & LIBRARY & 21700 & 0 \\
\hline 3760TRL1 & OFFICE TRAILER & 101 & OFFICE & 1524 & 0 \\
\hline 3760TRL2 & STORAGE TRAILER & 400 & GENERAL STORAGE & 1573 & $\mathbf{0}$ \\
\hline 3760TRL3 & OFFICE TRAIIER & 101 & OFFICE & 1338 & 0 \\
\hline 3762 & TECHNICAL SECURTTY & 101 & OFFICE & 8030 & 0 \\
\hline 3764 & OFFICE_BUILDING & 101 & OFFICE & 8030 & 0 \\
\hline 3765TRL1 & OFFICE TRAILER & 101 & OFFICE & 1848 & 0 \\
\hline 3765TRL2 & OFFICE TRAILER & 101 & OFFICE & 1848 & 0 \\
\hline 3767 & OFFICE_BUILDING & 101 & OFFICE & 2839 & 0 \\
\hline 614 & MONITORING STATION & 694 & OTHER SERVICE BLDG & 110 & 0 \\
\hline 614BYRL & BYERS LANDING MONITORING STAT & 694 & OTHER SERVICE BLDG & 49 & 0 \\
\hline $622 \mathrm{~A}$ & ELEV ATOR CONTROL BLDG & 761 & ENVIRONMENTAL LAB & 170 & 0 \\
\hline 622B & PILOT BALLOON RELEASE BLDG & 761 & ENVIRONMENTAL LAB & 144 & 0 \\
\hline $622 C$ & STORAGE BUILDING & 400 & GENERAL STORAGE & 1170 & 0 \\
\hline 622D & STORAGE BUILDING & 400 & GENERAL STORAGE & 1013 & 0 \\
\hline $622 \mathrm{~F}$ & PIELD OFFICE & 761 & ENVIRONMENTAL LAB & 3300 & $\mathbf{0}$ \\
\hline 622R & METEOROLOGY LAB & 761 & ENVIRONMENTAL LAB & 8960 & 0.0049 \\
\hline 622TRLI & FIELD OFFICE & 694 & OTHER SERVICE BLDG & 200 & $\mathbf{0}$ \\
\hline 622TRL2 & FIELD OFFICE & 694 & OTHER SERVICE BLDG & 200 & 0 \\
\hline 646 & RADIOECOLOGY FIELD LAB & 761 & ENVIRONMENTAL LAB & 800 & 0 \\
\hline $6652 \mathrm{C}$ & SPACE SCIENCE LABORATORY & 761 & ENVIRONMENTAL LAB & 6359 & 0.0038 \\
\hline 6652CSHED & STORAGE BULLDING & 694 & OTHER SERVICE BLDG & 96 & 0 \\
\hline $6652 \mathrm{D}$ & PUMPHOUSE & 694 & OTHER SERVICE BLDG & 86 & 0 \\
\hline 6652DOME1 & ATMOSPHERIC FACILITY & 729 & OTHER PHYSICS LAB & 463 & $\mathbf{0}$ \\
\hline 6652DOME2 & ATMOSPHERIC FACILITY & 729 & OTHER PHYSICS LAB & 160 & 0 \\
\hline 6652E & LYSIMETER PREPARATION BLDG & 761 & ENVIRONMENTAL LAB & 600 & 0 \\
\hline $6652 \mathrm{G}$ & ALE FIELD STORAGE & 400 & GENERAL STORAGE & 6783 & 0 \\
\hline $6652 \mathrm{H}$ & ALE LABORATORY I & 761 & ENVIRONMENTAL LAB & 3801 & 0 \\
\hline 66521 & ALE HEADQUARTERS & 761 & ENVIRONMENTAL LAB & 4235 & 0 \\
\hline $6652 J$ & ALE LABORATORY II & 761 & ENVIRONMENTAL LAB & 7794 & 0 \\
\hline $6652 \mathrm{~K}$ & PUMPHOUSE & 694 & OTHER SERVICE BLDG & 178 & 0 \\
\hline 6652LP & RATTLESNAKE MTN LOWR PUMPHOUSE & 694 & OTHER SERVICE BLDG & 200 & 0 \\
\hline $6652 \mathrm{M}$ & FALLOUT LABORATORY & 761 & ENVIRONMENTAL LAB & 694 & 0 \\
\hline 66520 & STORAGE BUILDING & 400 & GENERAL STORAGE & 1500 & 0 \\
\hline 6652PH & FIRE PROT PUMPHOUSE & 694 & OTHER SERVICE BLDG & 597 & 0 \\
\hline
\end{tabular}


Table K-1b. PNL Building Inventories Compared to Threshold Values.

\begin{tabular}{|c|c|c|c|c|c|c|c|}
\hline BLDG & CHEMICAL NAME & CASH & FORM & $\begin{array}{l}\text { INVENTORY } \\
\text { (TONS) }\end{array}$ & $\begin{array}{c}\text { 12X } \\
\text { INVENTORY } \\
\text { (TONS) }\end{array}$ & $\begin{array}{l}\text { THRESHOLD } \\
\text { VALUES } \\
\text { (TONS) }\end{array}$ & $\begin{array}{c}12 \mathrm{X} \\
\text { INVENTORY } \\
\text { EXCEEDS } \\
\text { THRESHOLD } \\
\text { VALUE }\end{array}$ \\
\hline $306 \mathrm{~W}$ & 1,1,1-TRICHLOROETHANE & $71-55-6$ & $\bar{L}$ & $2.79 \mathrm{e}-03$ & 0.03348 & 0.5 & $*$ \\
\hline $306 \mathrm{~W}$ & BENZENE & $71-43-2$ & L & $4.60 e-04$ & 0.00552 & 0.5 & * \\
\hline $306 \mathrm{~W}$ & BERYLLIUM & $7440-41-7$ & s & $1.70 e-04$ & 0.00204 & 0.004 & * \\
\hline $306 \mathrm{~W}$ & CHROMIUM & $7440-47-3$ & $\mathrm{~s}$ & $4.50 \mathrm{e}-03$ & 0.054 & 0.5 & t \\
\hline $306 \mathrm{~W}$ & COBALT & $7440-48-4$ & $\mathbf{s}$ & $3.60 e-02$ & 0.432 & 0.1 & EXCEED \\
\hline $306 \mathrm{~W}$ & HEXANE & $110-54-3$ & $\bar{L}$ & $1.38 \mathrm{e}-02$ & 0.16512 & 0.5 & * \\
\hline $306 \mathrm{~W}$ & HYDROFLUORIC ACID & $7664-39-3$ & $\mathbf{L}$ & $1.18 \mathrm{e}-02$ & 0.14136 & 0.5 & - \\
\hline $306 \mathrm{~W}$ & HYDROGEN CHLORIDE & $7647-01-0$ & $\mathbf{L}$ & $2.46 e-02$ & 0.29508 & 0.5 & * \\
\hline $306 \mathrm{~W}$ & LEAD & $7439-92-1$ & $\mathbf{s}$ & $2.50 \mathrm{e}-03$ & 0.03 & 0.005 & EXCEED \\
\hline $306 \mathrm{~W}$ & MANGANESE & $7439-96-5$ & s & $1.10 \mathrm{e}-03$ & 0.0132 & 0.5 & * \\
\hline $306 \mathrm{~W}$ & METHYL ALCOHOL & $67-56-1$ & $\mathbf{L}$ & $1.28 \mathrm{e}-03$ & 0.01536 & 0.5 & + \\
\hline $306 \mathrm{~W}$ & METHYL ETHYL KETONE & $78-93-3$ & $\mathbf{L}$ & $8.40 e-04$ & 0.01008 & 0.5 & 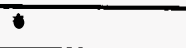 \\
\hline $306 \mathrm{~W}$ & $\begin{array}{l}\text { METHYL ISOBUTYL } \\
\text { KETONE }\end{array}$ & $108-10-1$ & $L$ & $1.67 e-03$ & 0.02004 & 0.1 & * \\
\hline $306 \mathrm{~W}$ & NICKEL & $7440-02-0$ & s & $1.66 \mathrm{e}-01$ & 1.98684 & 0.02 & EXCEED \\
\hline $306 \mathrm{~W}$ & SULFURIC ACID & $7664-93-9$ & $\bar{L}$ & $7.67 e-03$ & 0.09204 & 0.35 & * \\
\hline $306 \mathrm{~W}$ & TOLUENE & $108-88-3$ & L & $7.50 e-03$ & 0.09 & 0.5 & * \\
\hline $306 \mathrm{~W}$ & XYLENE & $1330-20-7$ & $\bar{L}$ & $3.00 \mathrm{e}-02$ & $3.60 \mathrm{e}-01$ & 0.5 & * \\
\hline $306 \mathrm{~W}$ & $\begin{array}{l}\text { VOLATILE ORGANIC } \\
\text { COMPOUNDS }\end{array}$ & & & $2.17 e-03$ & 0.02604 & 2 & * \\
\hline 314 & 1,4-DIOXANE & $123-91-1$ & $\mathrm{I}$ & $1.10 \mathrm{e}-03$ & 0.0132 & 0.5 & * \\
\hline 314 & 2-BUTOXYETHANOL & $111-76-2$ & $\bar{L}$ & $8.48 \mathrm{e}-03$ & 0.10176 & 0.5 & * \\
\hline 314 & ACETONITRILE & $75-05-8$ & $\overline{\mathrm{L}}$ & $1.04 \mathrm{e}-02$ & 0.12456 & 0.5 & * \\
\hline 314 & ANTMONY TRIOXIDE & $1309-64-4$ & s & $2.00 \mathrm{e}-03$ & 0.024 & 0.5 & + \\
\hline 314 & CADMIUM & $7440-43-9$ & $\bar{s}$ & $5.00 \mathrm{e}-04$ & 0.006 & 0.005 & EXCEED \\
\hline 314 & CARBON TETRACHLORIDE & $56-23-5$ & $\mathbf{L}$ & $7.00 \mathrm{e}-03$ & 0.084 & 0.5 & * \\
\hline 314 & CHLOROACETIC ACID & $79-11-8$ & $\mathbf{s}$ & $2.80 \mathrm{e}-04$ & 0.00336 & 0.1 & * \\
\hline 314 & CHLOROFORM & $67-66-3$ & $\mathrm{~L}$ & $6.22 \mathrm{e}-03$ & 0.07464 & 0.45 & * \\
\hline 314 & CHROMIC CHLORIDE & $10025-73-7$ & $\mathbf{s}$ & $5.00 \mathrm{e}-04$ & 0.006 & 0.1 & * \\
\hline 314 & CHROMIUM & $7440-47-3$ & $\bar{s}$ & $2.20 \mathrm{e}-04$ & 0.00264 & 0.5 & * \\
\hline 314 & COBALT & $7440-48-4$ & $s$ & $5.40 \mathrm{e}-04$ & 0.00648 & 0.1 & * \\
\hline 314 & DIMETHYLFORMAMIDE & $68-12-2$ & L & $6.42 \mathrm{e}-03$ & 0.07704 & 0.5 & * \\
\hline 314 & ETHYLENE DICHLORIDE & $107-06-2$ & I & $3.87 e-03$ & 0.04644 & 0.4 & $*$ \\
\hline 314 & FORMALDEHYDE & $50-00-0$ & $\bar{L}$ & $6.00 \mathrm{e}-04$ & 0.0072 & 0.5 & * \\
\hline 314 & HYDROFLUORIC ACID & $7664-39-3$ & $\bar{L}$ & $6.60 \mathrm{e}-04$ & 0.00792 & 0.5 & * \\
\hline 314 & HYDROGEN CHLORIDE & $7647-01-0$ & $\bar{L}$ & $4.70 \mathrm{e}-02$ & 0.56364 & 0.5 & EXCEED \\
\hline 314 & HYDROQUNONE & $123-31-9$ & $\mathbf{s}$ & $1.00 \mathrm{e}-03$ & 0.012 & 0.5 & * \\
\hline 314 & LEAD & $7439-92-1$ & $\mathbf{s}$ & $1.70 \mathrm{e}-02$ & 0.204 & 0.005 & EXCEED \\
\hline 314 & MANGANESE & $7439-96-5$ & $\mathbf{s}$ & $1.00 \mathrm{e}-03$ & 0.012 & 0.5 & * \\
\hline 314 & MERCURIC CHLORIDE & $7487-94-7$ & $\mathbf{s}$ & $2.50 \mathrm{e}-04$ & 0.003 & 0.01 & * \\
\hline 314 & MERCURY & $7439-97-6$ & $\bar{L}$ & $3.30 \mathrm{e}-04$ & 0.00396 & 0.01 & $*$ \\
\hline 314 & METHYL ALCOHOL & $67-56-1$ & L & $9.54 \mathrm{e}-03$ & 0.11448 & 0.5 & * \\
\hline 314 & METHYL IODIDE & $74-88-4$ & $\mathrm{~L}$ & $3.00 \mathrm{e}-04$ & 0.0036 & 0.06 & + \\
\hline 314 & $\begin{array}{l}\text { METHYL ISOBUTYL } \\
\text { KETONE }\end{array}$ & $108-10-1$ & I & $8.80 e-04$ & 0.01056 & 0.1 & * \\
\hline 314 & METHYL METHACRYLATE & $80-62-6$ & $\bar{L}$ & $8.37 e-03$ & 0.10044 & 0.5 & * \\
\hline 314 & NICKEL & $7440-02-0$ & $\mathbf{s}$ & $1.26 \mathrm{e}-03$ & 0.01512 & 0.02 & * \\
\hline 314 & PHENOL & $108-95-2$ & $\bar{s}$ & $1.50 \mathrm{e}-03$ & 0.018 & 0.1 & * \\
\hline
\end{tabular}


Table K-1b. PNL Building Inventories Compared to Threshold Values.

\begin{tabular}{|c|c|c|c|c|c|c|c|}
\hline BLDG & CHEMICAL NAME & CAS\# & FORM & $\begin{array}{l}\text { INVENTORY } \\
\text { (TONS) }\end{array}$ & $\begin{array}{c}12 \mathrm{X} \\
\text { INVENTORY } \\
\text { (TONS) }\end{array}$ & $\begin{array}{l}\text { THRESHOLD } \\
\text { VALUES } \\
\text { (TONS) }\end{array}$ & $\begin{array}{c}12 X \\
\text { INVENTORY } \\
\text { EXCEEDS } \\
\text { THRESHOLD } \\
\text { VALUE }\end{array}$ \\
\hline 314 & $\begin{array}{l}\text { POTASSIUM ANTMONYI } \\
\text { TARTRATE }\end{array}$ & & $\bar{S}$ & $5.00 \mathrm{e}-04$ & 0.006 & 0.5 & \# \\
\hline 314 & POTASSIUM CYANIDE & $151-50-8$ & $\mathrm{~s}$ & $1.18 \mathrm{e}-03$ & 0.01416 & 0.1 & * \\
\hline 314 & QUINONE & $106-51-4$ & s & $1.10 \mathrm{e}-03$ & 0.0132 & 0.5 & * \\
\hline 314 & SODIUM CYANIDE & $143-33-9$ & $\mathrm{~s}$ & $1.30 \mathrm{e}-04$ & 0.00156 & 0.1 & * \\
\hline 314 & SULFURIC ACD & $7664-93-9$ & $\overline{\mathrm{L}}$ & $1.35 \mathrm{e}-02$ & 0.162 & 0.35 & * \\
\hline 314 & TOLUENE & $108-88-3$ & $\overline{\mathrm{L}}$ & $5.00 \mathrm{e}-04$ & 0.006 & 0.5 & * \\
\hline 314 & XYLENE & $1330-20-7$ & $\bar{L}$ & $1.91 \mathrm{e}-03$ & 0.02292 & 0.5 & + \\
\hline$\overline{314}$ & FLUORIDES & & & $3.64 \mathrm{e}-02$ & 0.43728 & 0.15 & EXCEED \\
\hline$\overline{314}$ & $\begin{array}{l}\text { VOLATILE ORGANIC } \\
\text { COMPOUNDS }\end{array}$ & & & $5.34 \mathrm{e}-03$ & 0.06408 & 2 & 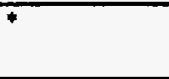 \\
\hline $314 \mathrm{~B}$ & METHYL ETHYL KETONE & $78-93-3$ & L & $1.68 \mathrm{e}-02$ & 0.20136 & 0.5 & $*$ \\
\hline 318 & METHYL ALCOHOL & $67-56-1$ & $\bar{L}$ & $2.02 e-01$ & 2.42832 & 0.5 & EXCEED \\
\hline 318 & TOLUENE & $108-88-3$ & $\bar{I}$ & $7.97 e-03$ & 0.09564 & 0.5 & * \\
\hline 320 & $\begin{array}{l}\text { 2,2,4-TRIMETHYLPENTANE, } \\
\text { HYDROCARBON KIT } 22\end{array}$ & $540-84-1$ & $\mathrm{~L}$ & $4.95 \mathrm{e}-02$ & 0.59448 & 0.5 & EXCEED \\
\hline 320 & 2-ETHOXYETHANOL & $110-80-5$ & $\bar{L}$ & $6.60 \mathrm{e}-04$ & 0.00792 & 0.5 & * \\
\hline 320 & ACETONITRILE & $75-05-8$ & $\bar{L}$ & $1.95 \mathrm{e}-02$ & 0.2334 & 0.5 & * \\
\hline 320 & ANTMONY & $7440-36-0$ & s & $1.50 \mathrm{e}-04$ & 0.0018 & 0.5 & * \\
\hline 320 & ANTIMONY TRIOXIDE & $1309-64-4$ & $\mathbf{s}$ & $5.50 \mathrm{e}-04$ & 0.0066 & 0.5 & * \\
\hline 320 & ARSENIC & $7440-38-2$ & $s$ & $1.65 e-03$ & 0.0198 & 0.002 & EXCEED \\
\hline 320 & BENZENE & $71-43-2$ & $\bar{L}$ & $9.40 \mathrm{e}-04$ & 0.01128 & 0.5 & * \\
\hline 320 & CARBON TETRACHLORIDE & $56-23-5$ & $\bar{I}$ & $6.63 \mathrm{e}-03$ & 0.07956 & 0.5 & + \\
\hline 320 & CHLOROACETIC ACID & $79-11-8$ & $\mathbf{s}$ & $1.50 \mathrm{e}-03$ & 0.018 & 0.1 & * \\
\hline 320 & CHLOROFORM & $67-66-3$ & I & $2.38 \mathrm{e}-03$ & 0.02856 & 0.45 & * \\
\hline 320 & CHROMIC CHLORIDE & $10025-73-7$ & $\mathbf{s}$ & $2.80 \mathrm{e}-04$ & 0.00336 & 0.1 & 7 \\
\hline 320 & CHROMIUM & $7440-47-3$ & $\bar{S}$ & $1.90 \mathrm{e}-04$ & 0.00228 & 0.5 & * \\
\hline 320 & DIBUTYL PHTHALATE & $84-74-2$ & $\mathrm{~s}$ & $5.20 \mathrm{e}-04$ & 0.00624 & 0.5 & * \\
\hline 320 & ETHYLENE DICHLORIDE & $107-06-2$ & $\overline{\mathrm{L}}$ & $2.00 \mathrm{e}-05$ & 0.00024 & 0.4 & * \\
\hline 320 & ETHYLENE GLYCOL & $107-21-1$ & $\bar{L}$ & $1.10 \mathrm{e}-03$ & 0.0132 & $\overline{0.5}$ & 7 \\
\hline$\overline{320}$ & FORMALDEHYDE & $50-00-0$ & $\overline{\mathrm{I}}$ & $6.10 e-04$ & 0.00732 & 0.5 & * \\
\hline 320 & HEXANE & $110-54-3$ & $\bar{L}$ & $5.82 \mathrm{e}-03$ & 0.06984 & 0.5 & * \\
\hline 320 & HYDROFLUORIC ACID & $7664-39-3$ & I & $5.82 e-03$ & 0.06984 & 0.5 & + \\
\hline 320 & HYDROGEN CHLORIDE & $7647-01-0$ & $\bar{L}$ & $3.92 \mathrm{e}-02$ & 0.47076 & 0.5 & * \\
\hline 320 & HYDROQUINONE & $123-31-9$ & $S$ & $1.11 \mathrm{e}-03$ & 0.01332 & 0.5 & $*$ \\
\hline 320 & LEAD & $7439-92-1$ & $\bar{s}$ & $5.00 \mathrm{e}-04$ & 0.006 & 0.005 & EXCEED \\
\hline 320 & MANGANESE & $7439-96-5$ & $\mathbf{s}$ & $5.00 \mathrm{e}-04$ & 0.006 & 0.5 & * \\
\hline 320 & MERCURIC CHLORIDE & $7487-94-7$ & $\mathbf{s}$ & $5.00 \mathrm{e}-04$ & 0.006 & 0.01 & + \\
\hline 320 & $\begin{array}{l}\text { MERCURIC NITRATE, } \\
\text { MONOHYDRATE }\end{array}$ & $10045-94-0$ & $\bar{s}$ & $1.20 \mathrm{e}-04$ & 0.00144 & 0.01 & * \\
\hline 320 & METHYL ALCOHOL & $67-56-1$ & $\bar{L}$ & $6.33 e-02$ & 0.76008 & 0.5 & EXCEED \\
\hline 320 & METHYL IODIDE & $74-88-4$ & $\mathrm{I}$ & $1.10 \mathrm{e}-04$ & 0.00132 & 0.06 & $*$ \\
\hline 320 & METHYL METHACRYLATE & $80-62-6$ & $\mathrm{~L}$ & $5.50 \mathrm{e}-04$ & 0.0066 & 0.5 & * \\
\hline 320 & METHYLENE CHLORIDE & $75-09-2$ & $\bar{L}$ & $5.03 e-02$ & 0.60408 & 0.5 & EXCEED \\
\hline 320 & NAPHTHALENE & $91-20-3$ & $\mathbf{s}$ & $2.80 \mathrm{e}-04$ & 0.00336 & 0.5 & * \\
\hline 320 & NICKEL & $7440-02-0$ & $\mathbf{s}$ & $3.70 e-04$ & 0.00444 & 0.02 & + \\
\hline 320 & PHENOL & $108-95-2$ & s & $5.00 \mathrm{e}-04$ & 0.006 & 0.1 & $*$ \\
\hline 320 & PHOSPHOROUS & $7723-14-0$ & $S$ & $1.10 \mathrm{e}-04$ & 0.00132 & 0.1 & 7 \\
\hline
\end{tabular}


Table K-1b. PNL Building Inventories Compared to Threshold Values.

\begin{tabular}{|c|c|c|c|c|c|c|c|}
\hline BLDG & CHEMICAI NAME & CASH & FORM & $\begin{array}{l}\text { INVENTORY } \\
\text { (TONS) }\end{array}$ & $\begin{array}{c}12 X \\
\text { INVENTORY } \\
\text { (TONS) }\end{array}$ & $\begin{array}{l}\text { THRESHOLD } \\
\text { VALUES } \\
\text { (TONS) }\end{array}$ & $\begin{array}{c}12 \mathrm{X} \\
\text { INVENTORY } \\
\text { EXCEEDS } \\
\text { THRESHOLD } \\
\text { VALUE }\end{array}$ \\
\hline 320 & $\begin{array}{l}\text { POLYCHLORINATED } \\
\text { BIPHENYLS }\end{array}$ & $1336-36-3$ & $\mathbf{s}$ & $1.00 \mathrm{e}-05$ & 0.00012 & 0.0045 & * \\
\hline 320 & POTASSIUM CYANIDE & $151-50-8$ & $\bar{s}$ & $5.50 \mathrm{e}-0.4$ & 0.0066 & 0.1 & * \\
\hline 320 & SELENIUM & $7782-49-2$ & $S$ & $7.40 \mathrm{e}-04$ & 0.00888 & 0.5 & * \\
\hline 320 & SULFURIC ACID & $7664-93-9$ & $\bar{L}$ & $1.44 \mathrm{e}-02$ & 0.17304 & 0.35 & * \\
\hline 320 & TOLUENE & $108-88-3$ & L & $4.46 e-03$ & 0.05352 & 0.5 & * \\
\hline 320 & TRIETHYLAMINE . & $121-44-8$ & $\bar{L}$ & $6.00 \mathrm{e}-05$ & 0.00072 & 0.5 & * \\
\hline 320 & XYLENE & $1330-20-7$ & $\mathbf{L}$ & $3.12 \mathrm{e}-03$ & 0.03744 & 0.5 & * \\
\hline 320 & FLUORIDES & & & $6.75 e-03$ & 0.081 & 0.15 & * \\
\hline 320 & NITRIC OXIDES & & & $2.50 \mathrm{e}-03$ & 0.03 & 2 & * \\
\hline 320 & TOTAL REDUCED SULFUR & & & $1.40 e-04$ & 0.00168 & 0.5 & * \\
\hline 320 & $\begin{array}{l}\text { VOLATILE ORGANIC } \\
\text { COMPOUNDS }\end{array}$ & & & $7.88 \mathrm{e}-03$ & 0.09456 & 2 & * \\
\hline 324 & 1,1,1-TRICHLOROETHANE & $71-55-6$ & $\bar{L}$ & $6.60 \mathrm{e}-04$ & 0.00792 & 0.5 & * \\
\hline 324 & 1,4-DIOXANE & $123-91-1$ & L & $1.32 \mathrm{e}-03$ & 0.01584 & 0.5 & + \\
\hline 324 & 4-AMINOBIPHENYL & $92-67-1$ & S & $2.00 \mathrm{e}-05$ & 0.00024 & 0.5 & * \\
\hline 324 & ANTIMONY & $7440-36-0$ & $\mathrm{~s}$ & $5.50 \mathrm{e}-04$ & 0.0066 & 0.5 & * \\
\hline 324 & ANTIMONY TRIOXIDE & $1309-64-4$ & $\mathbf{s}$ & $1.40 \mathrm{e}-04$ & 0.00168 & 0.5 & + \\
\hline 324 & ARSENIC & $7440-38-2$ & $s$ & $5.50 \mathrm{e}-04$ & 0.0066 & 0.002 & EXCEED \\
\hline 324 & BENZENE & $71-43-2$ & $\mathrm{~L}$ & $3.99 \mathrm{e}-03$ & 0.04788 & 0.5 & * \\
\hline 324 & BERYLLIUM & $7440-41-7$ & S & $8.30 \mathrm{e}-04$ & 0.00996 & 0.004 & EXCEED \\
\hline 324 & CADMIUM & $7440-43-9$ & s & $8.30 \mathrm{e}-04$ & 0.00996 & 0.005 & EXCEED \\
\hline 324 & CARBON MONOXIDE & $630-08-0$ & $\mathbf{G}$ & $1.50 \mathrm{e}-02$ & 0.17964 & 5 & * \\
\hline 324 & CARBON TETRACHLORIDE & $56-23-5$ & $\bar{L}$ & $8.81 e-03$ & 0.10572 & 0.5 & * \\
\hline 324 & CHLORDANE & $57-74-9$ & $s$ & $2.00 \mathrm{e}-05$ & 0.00024 & 0.005 & * \\
\hline 324 & CHLOROFORM & $67-66-3$ & $\bar{I}$ & $1.80 \mathrm{e}-03$ & 0.0216 & 0.45 & + \\
\hline 324 & CHROMIUM & $7440-47-3$ & $\mathbf{s}$ & $2.20 \mathrm{e}-04$ & 0.00264 & 0.5 & $*$ \\
\hline 324 & COBALT & $7440-48-4$ & $\mathbf{s}$ & $8.30 \mathrm{e}-04$ & 0.00996 & 0.1 & * \\
\hline 324 & DIMETHYLFORMAMIDE & $68-12-2$ & L & $2.08 \mathrm{e}-03$ & 0.02496 & 0.5 & * \\
\hline 324 & ETHYLBENZENE & $100-41-4$ & $\bar{L}$ & $9.00 \mathrm{e}-05$ & 0.00108 & 0.5 & + \\
\hline 324 & ETHYLENE DICHLORDE & $107-06-2$ & I & $6.00 \mathrm{e}-05$ & 0.00072 & 0.4 & * \\
\hline 324 & ETHYLENE GLYCOL & $107-21-1$ & $\mathrm{~L}$ & $6.10 e-04$ & 0.00732 & 0.5 & + \\
\hline 324 & FORMALDEHYDE & $50-00-0$ & $\bar{I}$ & $6.10 \mathrm{e}-04$ & 0.00732 & 0.5 & 7 \\
\hline 324 & HEXACHLOROETHANE & $67-72-1$ & $\mathbf{s}$ & $6.60 \mathrm{e}-04$ & 0.00792 & 0.5 & * \\
\hline 324 & HEXANE & $110-54-3$ & $\mathbf{L}$ & $1.21 \mathrm{e}-02$ & 0.14532 & 0.5 & 7 \\
\hline 324 & HYDROFLUORIC ACID & $7664-39-3$ & $\mathrm{~L}$ & $9.20 \mathrm{e}-04$ & 0.01104 & 0.5 & + \\
\hline 324 & HYDROGEN CHLORIDE & $7647-01-0$ & $\bar{L}$ & $5.42 \mathrm{e}-02$ & 0.6504 & 0.5 & EXCEED \\
\hline 324 & HYDROGEN SULFIDE & $7783-06-4$ & $\mathbf{G}$ & $2.40 e-04$ & 0.00288 & 0.5 & + \\
\hline 324 & HYDROQUINONE & $123-31-9$ & $s$ & $5.50 \mathrm{e}-04$ & 0.0066 & 0.5 & + \\
\hline 324 & LEAD & $7439-92-1$ & s & $6.43 e-03$ & 0.07716 & 0.005 & EXCEED \\
\hline 324 & MANGANESE & $7439-96-5$ & $\mathbf{s}$ & $8.30 \mathrm{e}-04$ & 0.00996 & 0.5 & * \\
\hline 324 & MERCURIC CHLORIDE & $7487-94-7$ & $\mathbf{s}$ & $5.50 \mathrm{e}-0.04$ & 0.0066 & 0.01 & * \\
\hline 324 & MERCURY & $7439-97-6$ & $\mathbf{L}$ & $1.19 e-02$ & 0.1428 & 0.01 & EXCEED \\
\hline 324 & METHYL ALCOHOL & $67-56-1$ & $\mathbf{L}$ & $1.13 e-02$ & 0.13608 & 0.5 & $*$ \\
\hline$\overline{324}$ & $\begin{array}{l}\text { METHYL ISOBUTYL } \\
\text { KETONE }\end{array}$ & $108-10-1$ & $\mathbf{L}$ & $8.30 e-04$ & 0.00996 & 0.1 & 7 \\
\hline 324 & METHYLENE CHLORDE & $75-09-2$ & $\mathbf{L}$ & $2.07 e-02$ & 0.24864 & 0.5 & * \\
\hline 324 & NAPHTHALENE & $91-20-3$ & $\mathbf{S}$ & $2.20 \mathrm{e}-04$ & 0.00264 & 0.5 & * \\
\hline
\end{tabular}


Table K-1b. PNL Building Inventories Compared to Threshold Values.

\begin{tabular}{|c|c|c|c|c|c|c|c|}
\hline BLDG & CHEMICAL NAME & CAS\# & FORM & $\begin{array}{l}\text { INVENTORY } \\
\text { (TONS) }\end{array}$ & $\begin{array}{c}12 \mathrm{X} \\
\text { INVENTORY } \\
\text { (TONS) }\end{array}$ & $\begin{array}{l}\text { THRESHOLD } \\
\text { VALUES } \\
\text { (TONS) }\end{array}$ & $\begin{array}{c}12 \mathrm{X} \\
\text { INVENTORY } \\
\text { EXCEEDS } \\
\text { THRESHOLD } \\
\text { VALUE }\end{array}$ \\
\hline 324 & NICKEL & $7440-02-0$ & $\mathbf{S}$ & $5.83 e-03$ & 0.06996 & 0.02 & EXCEED \\
\hline 324 & PERCHLOROETHYLENE & $127-18-4$ & $\mathrm{~L}$ & $9.65 e-03$ & 0.1158 & 0.5 & * \\
\hline 324 & PHENOL & $108-95-2$ & $\bar{S}$ & $9.00 e-05$ & 0.00108 & 0.1 & 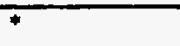 \\
\hline 324 & PHOSPHOROUS & $7723-14-0$ & S & $5.00 \mathrm{e}-04$ & 0.006 & 0.1 & * \\
\hline 324 & SELENIUM & $7782-49-2$ & $\bar{s}$ & $1.10 \mathrm{e}-03$ & 0.0132 & 0.5 & * \\
\hline 324 & SODIUM SELENITE & $10102-18-8$ & S & $3.00 e-05$ & 0.00036 & 0.1 & * \\
\hline 324 & SULFURIC ACID & $7664-93-9$ & $\mathbf{L}$ & $5.98 \mathrm{e}-02$ & 0.71772 & 0.35 & EXCEED \\
\hline 324 & TOLUENE & $108-88-3$ & $\mathbf{L}$ & $3.91 \mathrm{e}-03$ & 0.04692 & 0.5 & * \\
\hline 324 & TRICHLOROETHYLENE & $79-01-6$ & $\bar{L}$ & $1.39 \mathrm{e}-02$ & 0.16632 & 0.5 & + \\
\hline 324 & TRIETHYLAMINE & $121-44-8$ & $\mathbf{L}$ & $1.50 \mathrm{e}-04$ & 0.0018 & 0.5 & * \\
\hline$\overline{324}$ & VINYL CHLORDE & $75-01-4$ & $\bar{L}$ & $5.00 \mathrm{e}-04$ & 0.006 & $\overline{0.1}$ & * \\
\hline 324 & VINYLIDENE CHLORIDE & $75-35-4$ & $\mathbf{L}$ & $6.70 \mathrm{e}-04$ & 0.00804 & 0.2 & * \\
\hline 324 & XYLENE & $1330-20-7$ & $\bar{L}$ & $2.06 \mathrm{e}-02$ & 0.2472 & 0.5 & * \\
\hline 324 & FLUORDES & & & $2.10 \mathrm{e}-03$ & 0.0252 & 0.15 & * \\
\hline 324 & TOTAL REDUCED SULFUR & & & $2.40 \mathrm{e}-04$ & 0.00288 & 0.5 & * \\
\hline 324 & $\begin{array}{l}\text { VOLATILE ORGANIC } \\
\text { COMPOUNDS }\end{array}$ & & & $2.56 e-02$ & 0.30768 & 2 & * \\
\hline 325 & 1,1,1-TRICHLOROETHANE & $71-55-6$ & $\mathrm{~L}$ & $5.58 \mathrm{e}-02$ & 0.67008 & 0.5 & EXCEED \\
\hline 325 & 1,2,4-TRICHLOROBENZENE & $120-82-1$ & $\bar{L}$ & $4.40 \mathrm{e}-04$ & 0.00528 & 0.5 & * \\
\hline 325 & $\begin{array}{l}\text { 2,2,4-TRIMETHYLPENTANE, } \\
\text { HYDROCARBON KIT } 22\end{array}$ & $540-84-1$ & $\bar{L}$ & $1.63 \mathrm{e}-02$ & 0.19608 & 0.5 & * \\
\hline 325 & 2,4,5-TRICHLOROPHENOL & $95-95-4$ & $\mathbf{s}$ & $3.00 e-05$ & 0.00036 & 0.5 & + \\
\hline 325 & 2,4,6-TRICHLOROPHENOL & 88-06-2 & $\mathbf{s}$ & $1.80 \mathrm{e}-04$ & 0.00216 & 0.5 & $*$ \\
\hline 325 & 2,4-DINITROPHENOL & $51-28-5$ & s & $1.10 \mathrm{e}-04$ & 0.00132 & 0.5 & * \\
\hline 325 & 2-BUTOXYETHANOL & $111-76-2$ & $\overline{\mathrm{L}}$ & $1.00 \mathrm{e}-04$ & 0.0012 & 0.5 & $*$ \\
\hline 325 & 2-TOLUIDINE & $95-53-4$ & $\bar{L}$ & $6.60 \mathrm{e}-04$ & 0.00792 & 0.5 & * \\
\hline 325 & 4-AMINOBIPHENYL & $92-67-1$ & $s$ & $5.10 \mathrm{e}-04$ & 0.00612 & 0.5 & 7 \\
\hline 325 & $\begin{array}{l}\text { 4-DMMETHYLAMINOAZOBEN } \\
\text { ZENE }\end{array}$ & $60-11-7$ & $\bar{s}$ & $1.10 \mathrm{e}-04$ & 0.00132 & 0.5 & * \\
\hline 325 & 4-NITROPHENOL & $100-02-7$ & $\bar{s}$ & $3.00 \mathrm{e}-05$ & 0.00036 & 0.5 & * \\
\hline 325 & ACETALDEHYDE & $75-07-0$ & $\bar{L}$ & $1.00 \mathrm{e}-05$ & 0.00012 & 0.5 & * \\
\hline 325 & ACETONITRILE & $75-05-8$ & $\bar{L}$ & $1.54 \mathrm{e}-02$ & 0.18468 & 0.5 & * \\
\hline 325 & ANILINE & $62-53-3$ & I & $1.10 \mathrm{e}-04$ & 0.00132 & 0.5 & * \\
\hline 325 & ANTMONY & $7440-36-0$ & $\mathbf{S}$ & $8.70 \mathrm{e}-04$ & 0.01044 & 0.5 & * \\
\hline 325 & ANTMONY TRIOXIDE & $1309-64-4$ & $\mathbf{s}$ & $3.96 \mathrm{e}-03$ & 0.04752 & 0.5 & * \\
\hline 325 & ARSENIC & $7440-38-2$ & $s$ & $1.37 e-03$ & 0.01644 & 0.002 & EXCEED \\
\hline 325 & BENZENE & $71-43-2$ & $\bar{L}$ & $9.10 \mathrm{e}-04$ & 0.01092 & 0.5 & * \\
\hline 325 & BERYLLIUM & $7440-41-7$ & $\mathbf{s}$ & $1.10 \mathrm{e}-04$ & 0.00132 & 0.004 & * \\
\hline 325 & $\begin{array}{l}\text { BIS(2-ETHYLHEXYL) } \\
\text { PHTHALATE }\end{array}$ & $117-81-7$ & 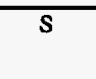 & $5.50 \mathrm{e}-04$ & 0.0066 & 0.5 & 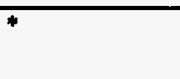 \\
\hline$\overline{325}$ & BROMOFORM & $75-25-2$ & L & $6.37 e-03$ & 0.07644 & 0.5 & 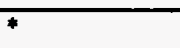 \\
\hline 325 & CADMIUM & $7440-43-9$ & $S$ & $3.24 \mathrm{e}-03$ & 0.03888 & 0.005 & EXCEED \\
\hline 325 & CARBON DISULFIDE & $75-15-0$ & $\mathrm{~L}$ & $2.64 \mathrm{e}-03$ & 0.03168 & 0.5 & * \\
\hline 325 & CARBON TETRACHLORIDE & $56-23-5$ & $\bar{L}$ & $7.74 \mathrm{e}-03$ & 0.09288 & 0.5 & * \\
\hline 325 & CHLOROACETIC ACD & $79-11-8$ & $\mathbf{s}$ & $1.82 \mathrm{e}-03$ & 0.02184 & 0.1 & + \\
\hline 325 & CHIOROBENZENE & $108-90-7$ & $\bar{L}$ & $5.80 \mathrm{e}-04$ & 0.00696 & 0.5 & * \\
\hline 325 & CHLOROFORM & $67-66-3$ & $\mathbf{L}$ & $6.32 \mathrm{e}-03$ & 0.07584 & 0.45 & * \\
\hline 325 & CHROMIC CHLORDE & $10025-73-7$ & $\bar{s}$ & $3.50 \mathrm{e}-03$ & 0.042 & 0.1 & * \\
\hline
\end{tabular}


Table K-1b. PNL Building Inventories Compared to Threshold Values.

\begin{tabular}{|c|c|c|c|c|c|c|c|}
\hline BLDG & CHEMICAL NAME & CAS\# & FORM & $\begin{array}{l}\text { INVENTORY } \\
\text { (TONS) }\end{array}$ & $\begin{array}{c}12 \mathrm{X} \\
\text { INVENTORY } \\
\text { (TONS) }\end{array}$ & $\begin{array}{l}\text { THRESHOLD } \\
\text { VALUES } \\
\text { (TONS) }\end{array}$ & $\begin{array}{c}12 \mathrm{X} \\
\text { INVENTORY } \\
\text { EXCEEDS } \\
\text { THRESHOLD } \\
\text { VALUE }\end{array}$ \\
\hline 325 & CHROMIUM & $7440-47-3$ & $\mathbf{S}$ & $2.20 \mathrm{e}-04$ & 0.00264 & 0.5 & * \\
\hline 325 & COBALT & $7440-48-4$ & $\mathbf{s}$ & $6.30 \mathrm{e}-04$ & 0.00756 & 0.1 & * \\
\hline 325 & DIBUTYL PHTHALATE & $84-74-2$ & $\mathbf{s}$ & $2.80 \mathrm{e}-04$ & 0.00336 & 0.5 & * \\
\hline 325 & DIMETHYLANILINE & $121-69-7$ & $\mathbf{L}$ & $6.20 e-04$ & 0.00744 & 0.5 & * \\
\hline 325 & ETHYLENE DICHLORIDE & $107-06-2$ & $\mathbf{L}$ & $6.01 \mathrm{e}-03$ & 0.07212 & 0.4 & $*$ \\
\hline 325 & ETHYLENE GLYCOL & $107-21-1$ & $\mathbf{L}$ & $1.16 \mathrm{e}-02$ & 0.1386 & 0.5 & * \\
\hline 325 & FORMALDEHYDE & $50-00-0$ & $\mathrm{~L}$ & $2.85 \mathrm{e}-03$ & 0.0342 & 0.5 & $*$ \\
\hline 325 & HEXANE & $110-54-3$ & $\mathrm{~L}$ & $3.96 e-02$ & 0.47556 & 0.5 & $*$ \\
\hline 325 & HYDRAZINE & $302-01-2$ & $\mathrm{~L}$ & $1.66 \mathrm{e}-03$ & 0.01992 & 0.002 & EXCEED \\
\hline 325 & HYDROFLUORIC ACID & 7664-39-3 & $\overline{\mathrm{L}}$ & $2.02 \mathrm{e}-02$ & 0.24288 & 0.5 & $*$ \\
\hline 325 & HYDROGEN CHLORIDE & $7647-01-0$ & $\mathrm{~L}$ & $1.27 e-01$ & 1.5282 & 0.5 & EXCEED \\
\hline 325 & HYDROQUINONE & $123-31-9$ & $s$ & $1.50 \mathrm{e}-03$ & 0.018 & 0.5 & * \\
\hline 325 & LEAD & $7439-92-1$ & $s$ & $7.28 \varepsilon-03$ & 0.08736 & 0.005 & EXCEED \\
\hline 325 & MANGANESE & $7439-96-5$ & $\mathbf{s}$ & $2.05 \mathrm{e}-03$ & 0.0246 & 0.5 & * \\
\hline 325 & MERCURIC CHLORIDE & $7487-94-7$ & $\mathbf{s}$ & $3.80 \mathrm{e}-04$ & 0.00456 & 0.01 & * \\
\hline 325 & MERCURY & 7439-97-6 & $\bar{L}$ & $6.94 e-03$ & 0.08328 & 0.01 & EXCEED \\
\hline 325 & METHYL ALCOHOL & $67-56-1$ & $\bar{L}$ & $9.88 \mathrm{e}-02$ & 1.18512 & 0.5 & EXCEED \\
\hline 325 & METHYL ETHYL KETONE & 78-93-3 & $\mathrm{I}$ & $3.35 \mathrm{e}-03$ & 0.0402 & 0.5 & * \\
\hline 325 & METHYL IODDE & $74-88-4$ & $\bar{L}$ & $6.30 \mathrm{e}-04$ & 0.00756 & 0.06 & 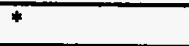 \\
\hline 325 & $\begin{array}{l}\text { METHYL ISOBUTYL } \\
\text { KETONE }\end{array}$ & $108-10-1$ & $\bar{L}$ & $7.74 \mathrm{e}-03$ & 0.09288 & 0.1 & * \\
\hline 325 & METHYLENE CHLORDE & $75-09-2$ & $\mathbf{L}$ & $1.23 e-01$ & 1.47096 & 0.5 & EXCEED \\
\hline 325 & NAPHTHALENE & $91-20-3$ & s & $4.91 \mathrm{e}-03$ & 0.05892 & 0.5 & * \\
\hline 325 & NICKEL & $7440-02-0$ & $s$ & $2.15 \mathrm{e}-03$ & 0.0258 & 0.02 & EXCEED \\
\hline 325 & NITROBENZENE & 98-95-3 & $\mathbf{L}$ & $6.80 \mathrm{e}-04$ & 0.00816 & 0.5 & * \\
\hline $325^{\circ}$ & PENTACHLOROPHENOL & $87-86-5$ & s & $1.10 \mathrm{e}-04$ & 0.00132 & 0.35 & $*$ \\
\hline 325 & PHENOL & $108-95-2$ & S & $2.62 e-03$ & 0.03144 & 0.1 & * \\
\hline 325 & PHOSPHOROUS & $7723-14-0$ & s & $2.80 \mathrm{e}-04$ & 0.00336 & 0.1 & * \\
\hline 325 & $\begin{array}{l}\text { POTASSIUM ANTMONYL } \\
\text { TARTRATE }\end{array}$ & & $\mathbf{s}$ & $5.00 \mathrm{e}-04$ & 0.006 & 0.5 & * \\
\hline 325 & POTASSIUM CYANIDE & $151-50-8$ & $\mathbf{S}$ & $4.10 \mathrm{e}-04$ & 0.00492 & 0.1 & * \\
\hline 325 & QUINOLINE & $91-22-5$ & $s$ & $5.20 \mathrm{e}-04$ & 0.00624 & 0.003 & EXCEED \\
\hline 325 & SELENIUM & $7782-49-2$ & $\mathbf{s}$ & $4.00 e-04$ & 0.0048 & 0.5 & * \\
\hline 325 & SODIUM CYANIDE & $143-33-9$ & $s$ & $1.35 e-03$ & 0.0162 & 0.1 & $*$ \\
\hline 325 & SODIUM SELENATE & $13410-01-0$ & s & $2.20 \mathrm{e}-04$ & 0.00264 & 0.1 & * \\
\hline 325 & SODIUM SELENITE & $10102-18-8$ & $s$ & $1.40 \mathrm{e}-04$ & 0.00168 & 0.1 & * \\
\hline 325 & SULFURIC ACID & $7664-93-9$ & $\mathbf{L}$ & $1.15 e-01$ & 1.3806 & 0.35 & EXCEED \\
\hline 325 & $\begin{array}{l}\text { TITANIUM } \\
\text { TETRACHLORIDE }\end{array}$ & $7550-45-0$ & $\bar{L}$ & $4.48 \mathrm{e}-03$ & 0.05376 & 0.1 & 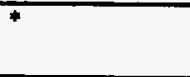 \\
\hline 325 & TOLUENE & $108-88-3$ & $\mathbf{L}$ & $2.27 e-02$ & 0.27192 & 0.5 & $*$ \\
\hline 325 & TRIETHYLAMINE & $121-44-8$ & $\bar{L}$ & $2.90 \mathrm{e}-04$ & 0.00348 & 0.5 & + \\
\hline 325 & XYLENE & $1330-20-7$ & $\mathrm{~L}$ & $6.87 e-03$ & 0.08244 & 0.5 & $*$ \\
\hline 325 & FLUORIDES & & & $6.88 \mathrm{e}-02$ & 0.82512 & 0.15 & EXCEED \\
\hline 325 & NITRIC OXIDES & & & $9.46 e-03$ & 0.11352 & 2 & * \\
\hline 325 & TOTAL REDUCED SULFUR & & & $1.47 \mathrm{e}-03$ & 0.01764 & 0.5 & * \\
\hline 325 & $\begin{array}{l}\text { VOLATIIE ORGANIC } \\
\text { COMPOUNDS }\end{array}$ & & & $2.13 e-02$ & 0.2556 & 2 & * \\
\hline 326 & 1,1,1-TRICHLOROETHANE & $71-55-6$ & L & $8.36 e-03$ & 0.10032 & 0.5 & * \\
\hline
\end{tabular}


Table K-1b. PNL Building Inventories Compared to Threshold Values.

\begin{tabular}{|c|c|c|c|c|c|c|c|}
\hline BLDG & CHEMICAL NAME & $\overline{\text { CASH }}$ & FORM & $\begin{array}{l}\text { INVENTORY } \\
\text { (TONS) }\end{array}$ & $\begin{array}{c}12 \mathrm{X} \\
\text { INVENTORY } \\
\text { (TONS) }\end{array}$ & $\begin{array}{l}\text { THRESHOLD } \\
\text { VALUES } \\
\text { (TONS) }\end{array}$ & $\begin{array}{c}12 \mathrm{X} \\
\text { INVENTORY } \\
\text { EXCEEDS } \\
\text { THRESHOLD } \\
\text { VALUE }\end{array}$ \\
\hline 326 & 1,2,4-TRICHLOROBENZENE & $120-82-1$ & $\overline{\mathrm{L}}$ & $1.00 \mathrm{e}-05$ & 0.00012 & 0.5 & $*$ \\
\hline 326 & $\begin{array}{l}\text { 2,2,4-TRIMETHYLPENTANE, } \\
\text { HYDROCARBON KIT } 22\end{array}$ & $540-84-1$ & $\bar{L}$ & $9.15 e-03$ & 0.1098 & 0.5 & * \\
\hline 326 & 2-METHOXYETHANOL & $109-86-4$ & $\bar{L}$ & $4.25 \mathrm{e}-03$ & 0.051 & 0.5 & * \\
\hline 326 & ACETONITRILE & $75-05-8$ & $\bar{I}$ & $3.12 e-02$ & 0.3738 & 0.5 & * \\
\hline 326 & ACRYLAMIDE & $79-06-1$ & $s$ & $1.32 \mathrm{e}-03$ & 0.01584 & 0.01 & EXCEED \\
\hline 326 & $\overline{\text { ACRYLIC ACID }}$ & $79-10-7$ & $\bar{L}$ & $5.50 \mathrm{e}-04$ & 0.0066 & 0.5 & * \\
\hline 326 & ANILINE & $62-53-3$ & I & $2.80 \mathrm{e}-04$ & 0.00336 & 0.5 & * \\
\hline 326 & ANTIMONY & $7440-36-0$ & $s$ & $1.00 \mathrm{e}-03$ & 0.012 & 0.5 & $*$ \\
\hline 326 & BIPHENYL & $92-52-4$ & $\mathbf{s}$ & $1.10 e-03$ & 0.0132 & 0.5 & * \\
\hline 326 & $\overline{\text { CADMIUM }}$ & $7440-43-9$ & s & $5.00 \mathrm{e}-04$ & 0.006 & 0.005 & EXCEED \\
\hline$\overline{326}$ & CARBON DISULFIDE & $75-15-0$ & $\bar{L}$ & $6.97 e-03$ & 0.08364 & 0.5 & + \\
\hline$\overline{326}$ & CARBON MONOXIDE & $630-08-0$ & $\bar{G}$ & $5.00 \mathrm{e}-04$ & 0.006 & 5 & * \\
\hline 326 & CARBON TETRACHLORIDE & $56-23-5$ & $\bar{L}$ & $2.06 \mathrm{e}-02$ & 0.24768 & 0.5 & * \\
\hline 326 & CHLOROACETIC ACID & $79-11-8$ & s & $1.10 \mathrm{e}-04$ & 0.00132 & 0.1 & * \\
\hline$\overline{326}$ & CHLOROFORM & $67-66-3$ & $\bar{L}$ & $4.60 \mathrm{e}-02$ & 0.55212 & 0.45 & EXCEED \\
\hline 326 & CHROMIUM & $7440-47-3$ & $s$ & $4.69 e-03$ & 0.05628 & 0.5 & * \\
\hline 326 & DMMETHYLANILINE & $121-69-7$ & $\overline{\mathrm{L}}$ & $3.41 \mathrm{e}-03$ & 0.04092 & 0.5 & * \\
\hline 326 & DIMETHYLFORMAMIDE & $68-12-2$ & $\mathrm{~L}$ & $1.82 \mathrm{e}-03$ & 0.02184 & 0.5 & * \\
\hline 326 & ETHYLBENZENE & $100-41-4$ & $\overline{\mathrm{L}}$ & $1.10 \mathrm{e}-03$ & 0.0132 & 0.5 & * \\
\hline 326 & ETHYLENE DICHLORIDE & $107-06-2$ & $\bar{L}$ & $1.38 \mathrm{e}-03$ & 0.01656 & 0.4 & + \\
\hline 326 & ETHYLENE GLYCOL & $107-21-1$ & L & $4.90 \mathrm{e}-03$ & 0.0588 & 0.5 & * \\
\hline 326 & FORMALDEHYDE & $50-00-0$ & I & $6.00 e-04$ & 0.0072 & 0.5 & * \\
\hline 326 & HEXANE & $110-54-3$ & $\bar{L}$ & $1.22 \mathrm{e}-02$ & 0.14664 & 0.5 & * \\
\hline$\overline{326}$ & HYDROFLUORIC ACID & $7664-39-3$ & I & $2.82 \mathrm{e}-02$ & 0.33864 & 0.5 & * \\
\hline 326 & HYDROGEN CHLORWE & $7647-01-0$ & $\bar{L}$ & $3.94 e-02$ & 0.4722 & 0.5 & 7 \\
\hline 326 & HYDROQURNONE & $123-31-9$ & $s$ & $2.32 e-02$ & 0.27816 & 0.5 & * \\
\hline 326 & LEAD & $7439-92-1$ & $s$ & $3.00 e-03$ & 0.036 & 0.005 & EXCEED \\
\hline 326 & MANGANESE & $7439-96-5$ & $\bar{S}$ & $5.20 \mathrm{e}-04$ & 0.00624 & 0.5 & $*$ \\
\hline 326 & MERCURY & $7439-97-6$ & L & $1.10 \mathrm{e}-03$ & 0.0132 & 0.01 & EXCEED \\
\hline 326 & METHYL ALCOHOL & $67-56-1$ & $\mathrm{~L}$ & $3.91 \mathrm{e}-02$ & 0.46884 & 0.5 & † \\
\hline 326 & METHYL ETHYL KETONE & $78-93-3$ & $\bar{L}$ & $4.19 \mathrm{e}-03$ & 0.05028 & 0.5 & * \\
\hline$\overline{326}$ & METHYL IODIDE & $74-88-4$ & I & $1.10 \mathrm{e}-04$ & 0.00132 & 0.06 & * \\
\hline 326 & $\begin{array}{l}\text { METHYL ISOBUTYL } \\
\text { KETONE }\end{array}$ & $108-10-1$ & $\bar{I}$ & $3.34 \mathrm{e}-03$ & 0.04008 & 0.1 & * \\
\hline 326 & METHYL METHACRYLATE & $80-62-6$ & $\bar{L}$ & $7.66 \mathrm{e}-03$ & 0.09192 & 0.5 & + \\
\hline 326 & METHYLENE CHLORIDE & $75-09-2$ & $\overline{\mathbf{L}}$ & $2.63 \mathrm{e}-02$ & 0.31524 & 0.5 & * \\
\hline 326 & NAPHTHALENE & $91-20-3$ & s & $1.39 \mathrm{e}-03$ & 0.01668 & 0.5 & * \\
\hline 326 & NICKEL & $7440-02-0$ & $S$ & $5.17 e-03$ & 0.06204 & 0.02 & EXCEED \\
\hline 326 & NITROBENZENE & $98-95-3$ & $\overline{\mathrm{L}}$ & $6.20 \mathrm{e}-04$ & 0.00744 & 0.5 & + \\
\hline 326 & PHENOL & $108-95-2$ & 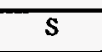 & $3.00 e-05$ & 0.00036 & 0.1 & * \\
\hline 326 & POTASSIUM CYANIDE & $151-50-8$ & $\mathbf{s}$ & $5.00 \mathrm{e}-04$ & 0.006 & 0.1 & * \\
\hline 326 & SODIUM CYANIDE & $143-33-9$ & s & $1.00 \mathrm{e}-03$ & 0.012 & 0.1 & * \\
\hline 326 & SULFURIC ACID & $7664-93-9$ & $\mathbf{L}$ & $2.57 e-02$ & 0.30804 & 0.35 & * \\
\hline 326 & TOLUENE & $108-88-3$ & 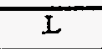 & $3.37 e-02$ & 0.4044 & 0.5 & + \\
\hline 326 & TRIETHYLAMINE & $121-44-8$ & L & $1.59 \mathrm{e}-03$ & 0.01908 & 0.5 & * \\
\hline 326 & URETHANE $99 \%$ & $51-79-6$ & $s$ & $5.30 \mathrm{e}-04$ & 0.00636 & 0.5 & * \\
\hline 326 & XYZENE & $1330-20-7$ & $\bar{L}$ & $1.31 \mathrm{e}-02$ & 0.1566 & 0.5 & * \\
\hline
\end{tabular}


Table K-1b. PNL Building Inventories Compared to Threshold Values.

\begin{tabular}{|c|c|c|c|c|c|c|c|}
\hline BLDG & CHEMICAL NAME & CAS\# & FORM & $\begin{array}{l}\text { INVENTORY } \\
\text { (TONS) } \\
\text {. }\end{array}$ & $\begin{array}{c}12 \mathrm{X} \\
\text { INVENTORY } \\
\text { (TONS) }\end{array}$ & $\begin{array}{l}\text { THRESHOLD } \\
\text { VALUES } \\
\text { (TONS) }\end{array}$ & $\begin{array}{c}12 X \\
\text { INVENTORY } \\
\text { EXCEEDS } \\
\text { THRESHOLD } \\
\text { VALUE }\end{array}$ \\
\hline 326 & FLUORIDES & & & $2.04 \mathrm{e}-02$ & 0.2442 & 0.15 & EXCEED \\
\hline 326 & TOTAL REDUCED SULFUR & & & $3.60 \mathrm{e}-04$ & 0.00432 & 0.5 & * \\
\hline 326 & $\begin{array}{l}\text { VOLATILE ORGANIC } \\
\text { COMPOUNDS }\end{array}$ & & & $1.15 \mathrm{e}-02$ & 0.138 & 2 & * \\
\hline 327 & CARBON TETRACHLORIDE & $56-23-5$ & $\bar{L}$ & $3.31 e-03$ & 0.03972 & 0.5 & * \\
\hline 327 & METHYL ALCOHOL & $67-56-1$ & $\mathbf{L}$ & $2.60 e-04$ & 0.00312 & 0.5 & * \\
\hline 327 & METHYL METHACRYLATE & $80-62-6$ & $\bar{L}$ & $6.20 \mathrm{e}-04$ & 0.00744 & 0.5 & * \\
\hline 327 & SULFURIC ACID & $7664-93-9$ & $\bar{L}$ & $3.84 \mathrm{e}-03$ & 0.04608 & 0.35 & * \\
\hline 329 & 1,1,1-TRICHLOROETHANE & $71-55-6$ & $\mathbf{L}$ & $5.89 \mathrm{e}-03$ & 0.07068 & 0.5 & * \\
\hline 324 & 1,1,2-TRICHLOROETHANE & $79-00-5$ & $\mathbf{L}$ & $1.10 \mathrm{e}-04$ & 0.00132 & 0.5 & * \\
\hline 34 & 1,4-DICHLOROBENZENE & $106-46-7$ & $\mathbf{L}$ & $1.00 \mathrm{e}-05$ & 0.00012 & 0.5 & + \\
\hline 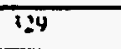 & 1,4-DIOXANE & $123-91-1$ & $\mathbf{L}$ & $3.00 \mathrm{e}-05$ & 0.00036 & 0.5 & $*$ \\
\hline 124 & $\begin{array}{l}\text { 2,2,4-TRIMETHYLPENTANE, } \\
\text { HYDROCARBON KIT } 22\end{array}$ & $540-84-1$ & $\mathbf{L}$ & $2.89 e-03$ & 0.03468 & 0.5 & $*$ \\
\hline 34 & 2,4,6-TRICHLOROPHENOL & $88-06-2$ & $\mathbf{s}$ & $1.00 e-05$ & 0.00012 & 0.5 & + \\
\hline$\because y$ & $\begin{array}{l}\text { 2,4-DICHLOROPHENOXYAC } \\
\text { ETIC ACID }\end{array}$ & 94-75-7 & $\mathbf{L}$ & $1.00 \mathrm{e}-05$ & 0.00012 & 0.5 & * \\
\hline$\because 4$ & 2,4-DINITROTOLUENE & $121-14-2$ & s & $1.10 \mathrm{e}-04$ & 0.00132 & 0.01 & * \\
\hline$\because y$ & $\begin{array}{l}\text { 2,4-TOLUENE } \\
\text { DISOCYANATE }\end{array}$ & $584-84-9$ & 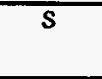 & $2.00 \mathrm{e}-05$ & 0.00024 & 0.05 & * \\
\hline$\because 4$ & 2-ETHOXYETHANOL & $110-80-5$ & $\bar{L}$ & $2.00 e-05$ & 0.00024 & 0.5 & * \\
\hline$\therefore 4$ & 2-NITROPROPANE & $79-46-9$ & $\mathrm{~L}$ & $2.00 \mathrm{e}-05$ & 0.00024 & 0.5 & $\bar{*}$ \\
\hline$\therefore 4$ & 3-METHYLPHENOL & $108-39-4$ & $\mathbf{L}$ & $2.00 \mathrm{e}-05$ & 0.00024 & 0.5 & 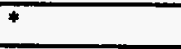 \\
\hline$\because y$ & 4-AMINOBIPHENYI & $92-67-1$ & $s$ & $2.00 \mathrm{e}-05$ & 0.00024 & 0.5 & * \\
\hline$\because 4$ & 4-HYDROXYTOLUENE & $106-44-5$ & $s$ & $1.00 \mathrm{e}-05$ & 0.00012 & 0.5 & * \\
\hline$\because 44$ & 4-NITROBIPHENYL & $92-93-3$ & $\mathbf{s}$ & $1.20 \mathrm{e}-04$ & 0.00144 & 0.5 & * \\
\hline$\therefore 4$ & 4-NITROPHENOL & $100-02-7$ & $s$ & $1.10 \mathrm{e}-04$ & 0.00132 & 0.5 & + \\
\hline 39 & ACETONITRILE & $75-05-8$ & $\bar{L}$ & $5.41 \mathrm{e}-02$ & 0.64944 & 0.5 & EXCEED \\
\hline$\because 4$ & ACETOPHENONE & $98-86-2$ & $\mathrm{~L}$ & $2.00 \mathrm{e}-05$ & 0.00024 & 0.5 & * \\
\hline 329 & ACRYLAMIDE & 79-06-1 & $\mathbf{s}$ & $1.20 \mathrm{e}-04$ & 0.00144 & 0.01 & * \\
\hline 329 & ACRYLIC ACID & $79-10-7$ & $\mathrm{~L}$ & $3.00 \mathrm{e}-05$ & 0.00036 & 0.5 & + \\
\hline 329 & ANILINE & $62-53-3$ & $\bar{L}$ & $5.30 \mathrm{e}-04$ & 0.00636 & 0.5 & $*$ \\
\hline 329 & ANTIMONY & $7440-36-0$ & $s$ & $9.50 \mathrm{e}-04$ & 0.0114 & 0.5 & + \\
\hline 329 & ARSENIC & $7440-38-2$ & $\mathbf{s}$ & $1.06 \mathrm{e}-03$ & 0.01272 & 0.002 & EXCEED \\
\hline 329 & ASBESTOS & $1332-21-4$ & $s$ & $1.30 \mathrm{e}-04$ & 0.00156 & 0.00004 & EXCEED \\
\hline 329 & BENZENE & $71-43-2$ & $\bar{L}$ & $2.04 e-02$ & 0.2442 & 0.5 & * \\
\hline 329 & BENZYL CHLORIDE & $100-44-7$ & $\mathrm{~L}$ & $2.00 \mathrm{e}-05$ & 0.00024 & 0.1 & + \\
\hline 329 & BERYLLIUM & $7440-41-7$ & $s$ & $3.00 \mathrm{e}-04$ & 0.0036 & 0.004 & + \\
\hline 329 & BIPHENYL & $92-52-4$ & $\mathbf{s}$ & $1.11 \mathrm{e}-03$ & 0.01332 & 0.5 & * \\
\hline 329 & CADMIUM & $7440-43-9$ & $s$ & $1.00 \mathrm{e}-05$ & 0.00012 & 0.005 & * \\
\hline 329 & CAPROLACTAM, DUST & $105-60-2$ & $\mathbf{s}$ & $1.00 \mathrm{e}-05$ & 0.00012 & 0.5 & * \\
\hline 329 & CARBON DISULFIDE & $75-15-0$ & $\mathbf{L}$ & $2.20 \mathrm{e}-04$ & 0.00264 & 0.5 & $*$ \\
\hline 329 & CARBON TETRACHLORDE & $56-23-5$ & $\overline{\mathbf{L}}$ & $7.14 e-03$ & 0.08568 & 0.5 & * \\
\hline 329 & CHLOROACETICACID & $79-11-8$ & s & $1.01 \mathrm{e}-03$ & 0.01212 & 0.1 & + \\
\hline 329 & CHLOROFORM & $67-66-3$ & $\bar{L}$ & $4.35 \mathrm{e}-02$ & 0.52236 & 0.45 & EXCEED \\
\hline 329 & CHROMIC CHLORIDE & $10025-73-7$ & $\mathbf{s}$ & $5.00 \mathrm{e}-04$ & 0.006 & 0.1 & * \\
\hline 329 & CHROMIUM & $7440-47-3$ & $\mathbf{s}$ & $5.70 \mathrm{e}-04$ & 0.00684 & 0.5 & * \\
\hline 329 & CHRYSENE & $218-01-9$ & s & $6.00 e-05$ & 0.00072 & 0.005 & * \\
\hline
\end{tabular}


Table K-1b. PNL Building Inventories Compared to Threshold Values.

\begin{tabular}{|c|c|c|c|c|c|c|c|}
\hline BLDG & CHEMICAL NAME & CAS\# & FORM & $\begin{array}{l}\text { INVENTORY } \\
\text { (TONS) }\end{array}$ & $\begin{array}{c}12 \mathrm{X} \\
\text { INVBNTORY } \\
\text { (TONS) }\end{array}$ & $\begin{array}{l}\text { THRESHOLD } \\
\text { VALUES } \\
\text { (TONS) }\end{array}$ & $\begin{array}{c}12 \mathrm{X} \\
\text { INVENTORY } \\
\text { EXCEEDS } \\
\text { THRESHOLD } \\
\text { VALUE }\end{array}$ \\
\hline 329 & COBALT & $7440-48-4$ & $\mathbf{S}$ & $9.50 \mathrm{e}-04$ & 0.0114 & 0.1 & * \\
\hline 329 & CRESOL, O- & $95-48-7$ & $\overline{\mathrm{L}}$ & $1.00 \mathrm{e}-05$ & 0.00012 & 0.5 & * \\
\hline 329 & DIBENZOFURAN & $132-64-9$ & $s$ & $8.30 \mathrm{e}-04$ & 0.00996 & 0.5 & * \\
\hline 329 & DIETHANOLAMINE & $111-42-2$ & $\overline{\mathrm{L}}$ & $1.00 \mathrm{e}-05$ & 0.00012 & 0.5 & * \\
\hline 329 & DIMETHYL SULFATE & $77-78-1$ & $\bar{I}$ & $1.10 \mathrm{e}-04$ & 0.00132 & 0.1 & * \\
\hline 329 & DIMETHYLFORMAMIDE & $68-12-2$ & $\overline{\mathrm{L}}$ & $3.99 \mathrm{e}-03$ & 0.04788 & 0.5 & * \\
\hline 329 & DIMETHYLHYDRAZINE & $57-14-7$ & 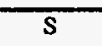 & $1.00 \mathrm{e}-05$ & 0.00012 & 0.004 & + \\
\hline 329 & EPICHLOROHYDRN & $106-89-8$ & $\bar{I}$ & $2.00 \mathrm{e}-05$ & 0.00024 & 0.5 & * \\
\hline 329 & ETHYL SULFATE & $64-67-5$ & $\mathbf{S}$ & $2.00 \mathrm{e}-05$ & 0.00024 & 0.5 & + \\
\hline 329 & HEXACHLOROBENZENE & $118-74-1$ & $\bar{s}$ & $5.60 \mathrm{e}-04$ & 0.00672 & 0.005 & EXCEED \\
\hline 329 & HEXACHLOROBUTADIENE & $87-68-3$ & $\bar{L}$ & $1.00 \mathrm{e}-05$ & 0.00012 & 0.5 & * \\
\hline 329 & HEXACHLOROETHANE & $67-72-1$ & $\bar{s}$ & $1.00 \mathrm{e}-05$ & 0.00012 & 0.5 & $\bar{*}$ \\
\hline 329 & HEXANE & $110-54-3$ & $\bar{L}$ & $2.01 \mathrm{e}-02$ & 0.24072 & 0.5 & * \\
\hline 329 & HYDROFLUORIC ACID & $7664-39-3$ & $\bar{L}$ & $7.85 e-03$ & 0.0942 & 0.5 & + \\
\hline 329 & HYDROGEN CHLORIDE & $7647-01-0$ & $\bar{L}$ & $1.13 e-01$ & 1.36056 & 0.5 & EXCEED \\
\hline 329 & HYDROQUNONE & $123-31-9$ & $\mathbf{s}$ & $5.90 \mathrm{e}-04$ & 0.00708 & 0.5 & + \\
\hline 329 & ISOPHORONE & $78-59-1$ & $\overline{\mathrm{L}}$ & $2.00 \mathrm{e}-05$ & 0.00024 & 0.5 & $\bar{*}$ \\
\hline 329 & LEAD & $7439-92-1$ & $\mathbf{S}$ & $4.18 \mathrm{e}-02$ & 0.50124 & 0.005 & EXCEED \\
\hline 329 & MALEIC ANHYDRIDE & $108-31-6$ & $s$ & $1.00 \mathrm{e}-05$ & 0.00012 & 0.5 & * \\
\hline 329 & MANGANESE & $7439-96-5$ & $\mathbf{s}$ & $1.20 \mathrm{e}-04$ & 0.00144 & 0.5 & * \\
\hline 329 & MERCURIC CHLORIDE & $7487-94-7$ & $\bar{S}$ & $5.00 \mathrm{e}-04$ & 0.006 & 0.01 & * \\
\hline 329 & $\begin{array}{l}\text { MERCURIC NITRATE, } \\
\text { MONOHYDRATE }\end{array}$ & $10045-94-0$ & s & $1.30 \mathrm{e}-04$ & 0.00156 & $\overline{0.01}$ & * \\
\hline 329 & MERCURY & $7439-97-6$ & $\bar{L}$ & $7.46 e-03$ & 0.08952 & 0.01 & EXCEED \\
\hline 329 & METHYL ALCOHOL & $67-56-1$ & $\bar{L}$ & $3.88 \mathrm{e}-02$ & 0.465 & 0.5 & * \\
\hline 329 & METHYL ETHYL KETONE & 78-93-3 & $\bar{L}$ & $2.30 \mathrm{e}-04$ & 0.00276 & 0.5 & * \\
\hline 329 & METHYL IODIDE & $74-88-4$ & $\bar{L}$ & $1.60 \mathrm{e}-03$ & 0.0192 & 0.06 & * \\
\hline 329 & $\begin{array}{l}\text { METHYL ISOBUTYL } \\
\text { KETONE }\end{array}$ & $108-10-1$ & $\overline{\mathrm{L}}$ & $3.00 \mathrm{e}-05$ & 0.00036 & 0.1 & * \\
\hline 329 & METHYL METHACRYLATE & $80-62-6$ & $\bar{L}$ & $3.00 \mathrm{e}-05$ & 0.00036 & 0.5 & * \\
\hline 329 & METHYLENE CHLORIDE & $75-09-2$ & $\bar{L}$ & $5.19 e-02$ & 0.62292 & 0.5 & EXCEED \\
\hline 329 & NAPHTHALENE & $91-20-3$ & $\bar{s}$ & $5.50 \mathrm{e}-04$ & 0.0066 & 0.5 & * \\
\hline 329 & NICKEL & $7440-02-0$ & $\mathbf{s}$ & $3.58 \mathrm{e}-03$ & 0.04296 & 0.02 & EXCEED \\
\hline 329 & NITROBENZENE & $98-95-3$ & $\bar{L}$ & $6.70 \mathrm{e}-04$ & 0.00804 & 0.5 & * \\
\hline 329 & O-ANISIDINE & $90-04-0$ & $\mathbf{S}$ & $2.00 \mathrm{e}-05$ & 0.00024 & 0.5 & * \\
\hline 329 & PERCHLOROETHYLENE & $127-18-4$ & $\bar{L}$ & $2.00 \mathrm{e}-05$ & 0.00024 & 0.5 & * \\
\hline 329 & PHENOL & $108-95-2$ & $\mathbf{S}$ & $1.30 \mathrm{e}-04$ & 0.00156 & $\overline{0.1}$ & * \\
\hline 329 & PHOSPHOROUS & $7723-14-0$ & 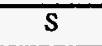 & $8.30 \mathrm{e}-04$ & 0.00996 & 0.1 & * \\
\hline$\overline{329}$ & PHTHALIC ANHYDRIDE & $85-44-9$ & s & $2.00 e-05$ & 0.00024 & 0.5 & * \\
\hline 329 & $\begin{array}{l}\text { POTASSIUM ANTIMONYL } \\
\text { TARTRATE }\end{array}$ & & s & $5.20 \mathrm{e}-04$ & 0.00624 & $\overline{0.5}$ & * \\
\hline 329 & POTASSIUM CYANIDE & $151-50-8$ & $s$ & $1.30 \mathrm{e}-04$ & 0.00156 & 0.1 & * \\
\hline 329 & PROPYLENE DICHLORIDE & $78-87-5$ & s & $1.00 \mathrm{e}-05$ & 0.00012 & 0.5 & $\overline{+}$ \\
\hline 329 & PROPYLENE OXIDE & $75-56-9$ & $\bar{L}$ & $2.00 \mathrm{e}-05$ & 0.00024 & 0.5 & * \\
\hline 329 & SELENIUM & $7782-49-2$ & $\mathbf{s}$ & $8.30 \mathrm{e}-04$ & 0.00996 & 0.5 & + \\
\hline 329 & SODIUM CYANIDE & $143-33-9$ & $\mathbf{s}$ & $1.40 \mathrm{e}-04$ & 0.00168 & 0.1 & 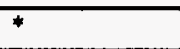 \\
\hline 329 & STYRENE & $100-42-5$ & $\bar{L}$ & $1.10 \mathrm{e}-04$ & 0.00132 & 0.5 & * \\
\hline 329 & STYRENE OXIDE & $96-09-3$ & $\bar{s}$ & $2.00 \mathrm{e}-05$ & 0.00024 & 0.5 & $*$ \\
\hline
\end{tabular}


Table K-1b. PNL Building Inventories Compared to Threshold Values.

\begin{tabular}{|c|c|c|c|c|c|c|c|}
\hline BLDG & CHEMICAL NAME & CAS\# & FORM & $\begin{array}{l}\text { INVENTORY } \\
\text { (TONS) }\end{array}$ & $\begin{array}{c}12 \mathrm{X} \\
\text { INVENTORY } \\
\text { (TONS) }\end{array}$ & $\begin{array}{l}\text { THRESHOLD } \\
\text { VALUES } \\
\text { (TONS) }\end{array}$ & $\begin{array}{c}12 X \\
\text { INVENTORY } \\
\text { EXCEEDS } \\
\text { THRESHOLD } \\
\text { VALUE }\end{array}$ \\
\hline 329 & SULFURIC ACID & 7664-93-9 & $\mathbf{L}$ & $9.63 e-02$ & 1.15536 & 0.35 & EXCEED \\
\hline 329 & TOLUENE & $108-88-3$ & $\mathbf{L}$ & $2.79 \mathrm{e}-02$ & 0.33456 & 0.5 & + \\
\hline 329 & TRICHLOROETHYLENE & $79-01-6$ & $\overline{\mathrm{L}}$ & $8.20 \mathrm{e}-04$ & 0.00984 & 0.5 & 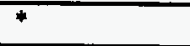 \\
\hline 329 & TRIETHYLAMINE & $121-44-8$ & $\mathbf{L}$ & $1.48 \mathrm{e}-03$ & 0.01776 & 0.5 & * \\
\hline 329 & URETHANE 99\% & $51-79-6$ & $\mathbf{s}$ & $1.00 \mathrm{e}-05$ & 0.00012 & 0.5 & * \\
\hline 329 & VINYL ACETATE & $108-05-4$ & $\mathrm{~L}$ & $2.00 \mathrm{e}-05$ & 0.00024 & 0.5 & * \\
\hline 329 & XỸLENE & $1330-20-7$ & $\bar{L}$ & $1.87 e-03$ & 0.02244 & 0.5 & * \\
\hline 329 & FLUORIDES & & & $9.88 \mathrm{e}-03$ & 0.11856 & 0.15 & * \\
\hline 329 & TOTAL REDUCED SULFUR & & & $8.70 \mathrm{e}-04$ & 0.01044 & 0.5 & $*$ \\
\hline 329 & $\begin{array}{l}\text { VOLATILE ORGANIC } \\
\text { COMPOUNDS }\end{array}$ & & & $3.55 \mathrm{e}-03$ & 0.0426 & 2 & + \\
\hline 331 & 1,1,1-TRICHLOROETHANE & $71-55-6$ & $\mathrm{~L}$ & $4.42 \mathrm{e}-03$ & 0.05304 & 0.5 & + \\
\hline 331 & $\begin{array}{l}\text { 2,2,4-TRIMETHYLPENTANE, } \\
\text { HYDROCARBON KIT } 22\end{array}$ & $540-84-1$ & $\mathrm{~L}$ & $4.19 e-03$ & 0.05028 & 0.5 & \# \\
\hline 331 & 2,4,6-TRICHLOROPHENOL & $88-06-2$ & $\mathbf{S}$ & $1.10 \mathrm{e}-03$ & 0.0132 & 0.5 & * \\
\hline 331 & 2,4-DINITROPHENOL & $51-28-5$ & $s$ & $9.00 \mathrm{e}-05$ & 0.00108 & 0.5 & * \\
\hline 331 & 2-ETHOXYETHANOL & $110-80-5$ & $\mathrm{~L}$ & $5.28 \mathrm{e}-03$ & 0.06336 & 0.5 & + \\
\hline 331 & 2-METHOXYETHANOL & $109-86-4$ & $\mathrm{~L}$ & $9.56 \mathrm{e}-03$ & 0.11472 & 0.5 & $*$ \\
\hline 331 & 3,3'-DIMETHOXYBENZIDINE & $119-90-4$ & s & $3.00 \mathrm{e}-05$ & 0.00036 & 0.5 & $*$ \\
\hline 331 & 4,4'-METHYLENEDLANILINE & $101-77-9$ & $\bar{L}$ & $5.50 \mathrm{e}-04$ & 0.0066 & 0.5 & * \\
\hline 331 & 4-AMNOBIPHENYL & 92-67-1 & $\mathbf{s}$ & $1.00 \mathrm{e}-03$ & 0.012 & 0.5 & $*$ \\
\hline 331 & $\begin{array}{l}\text { 4-DIMETHYLAMINOAZOBEN } \\
\text { ZENE }\end{array}$ & $60-11-7$ & $\mathbf{s}$ & $3.00 \mathrm{e}-05$ & 0.00036 & 0.5 & * \\
\hline 331 & 4-NITROPHENOL & $100-02-7$ & s & $6.00 \mathrm{e}-05$ & 0.00072 & 0.5 & * \\
\hline 331 & ACETAMIDE & $60-35-5$ & s & $3.00 \mathrm{e}-05$ & 0.00036 & 0.5 & * \\
\hline 331 & ACETONITRILE & $75-05-8$ & $\mathbf{L}$ & $2.77 e-01$ & 3.3204 & 0.5 & EXCEED \\
\hline 331 & ACRYLAMIDE & $79-06-1$ & $\mathbf{s}$ & $8.98 \mathrm{e}-03$ & 0.10776 & 0.01 & EXCEED \\
\hline 331 & ANTIMONY & $7440-36-0$ & $s$ & $2.20 \mathrm{e}-04$ & 0.00264 & 0.5 & * \\
\hline 331 & BENZENE & $71-43-2$ & $\bar{L}$ & $1.45 \mathrm{e}-02$ & 0.17388 & 0.5 & + \\
\hline 331 & BENZO(A)PYRENE & $50-32-8$ & $\mathbf{s}$ & $3.00 \mathrm{e}-05$ & 0.00036 & 0.005 & + \\
\hline 331 & BIPHENYL & $92-52-4$ & s & $2.21 \mathrm{e}-03$ & 0.02652 & 0.5 & $\overline{+}$ \\
\hline 331 & CADMIUM & $7440-43-9$ & $\mathbf{s}$ & $1.10 \mathrm{e}-04$ & 0.00132 & 0.005 & * \\
\hline 331 & CARBON DISULFIDE & $75-15-0$ & $\mathbf{L}$ & $3.50 \mathrm{e}-04$ & 0.0042 & 0.5 & * \\
\hline 331 & CARBON TETRACHLORIDE & $56-23-5$ & $\mathrm{~L}$ & $2.08 \mathrm{e}-02$ & 0.25008 & 0.5 & + \\
\hline 331 & CHLOROFORM & $67-66-3$ & $\mathrm{~L}$ & $5.89 \mathrm{e}-02$ & 0.70716 & 0.45 & EXCEED \\
\hline 331 & CRESOL & $1319-77-3$ & $\mathbf{s}$ & $6.00 \mathrm{e}-05$ & 0.00072 & 0.5 & * \\
\hline 331 & DIBENZOFURAN & $132-64-9$ & $s$ & $4.00 \mathrm{e}-05$ & 0.00048 & 0.5 & 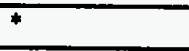 \\
\hline 331 & DIMETHYLFORMAMIDE & $68-12-2$ & L & $8.59 \mathrm{e}-03$ & 0.10308 & 0.5 & * \\
\hline 331 & ETHYLENE GLYCOL & 107-21-1 & $\mathrm{L}$ & $6.58 \mathrm{e}-03$ & 0.07896 & 0.5 & * \\
\hline 331 & ETHYLENE THIOUREA & $96-45-7$ & $\mathrm{~s}$ & $1.10 \mathrm{e}-04$ & 0.00132 & 0.3 & * \\
\hline 331 & FORMALDEHYDE & $50-00-0$ & $\mathrm{~L}$ & $2.75 e-02$ & 0.3294 & 0.5 & * \\
\hline 331 & HEXANE & $110-54-3$ & $\mathbf{L}$ & $2.95 \mathrm{e}-02$ & 0.35412 & 0.5 & + \\
\hline 331 & HYDROFLUORIC ACD & $7664-39-3$ & $\mathbf{L}$ & $5.00 e-04$ & 0.006 & 0.5 & * \\
\hline 331 & HYDROGEN CHLORDE & $7647-01-0$ & $\mathbf{L}$ & $4.67 e-02$ & 0.56016 & 0.5 & EXCEED \\
\hline 331 & HYDROQUINONE & $123-31-9$ & $s$ & $5.50 \mathrm{e}-04$ & 0.0066 & 0.5 & + \\
\hline 331 & LINDANE & $58-89-9$ & s & $3.00 \mathrm{e}-05$ & 0.00036 & 0.005 & 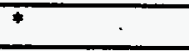 \\
\hline 331 & MERCURIC CHLORIDE & $7487-94-7$ & $\mathbf{s}$ & $1.90 \mathrm{e}-04$ & 0.00228 & 0.01 & * \\
\hline 331 & MERCURY & $7439-97-6$ & $\bar{L}$ & $2.80 \mathrm{e}-04$ & 0.00336 & 0.01 & $*$ \\
\hline
\end{tabular}


Table K-1b. PNL Building Inventories Compared to Threshold Values.

\begin{tabular}{|c|c|c|c|c|c|c|c|}
\hline BLDG & CHEMICAL NAME & CASH & FORM & $\begin{array}{l}\text { INVENTORY } \\
\text { (TONS) }\end{array}$ & $\begin{array}{c}12 \mathrm{X} \\
\text { INVENTORY } \\
\text { (TONS) }\end{array}$ & $\begin{array}{l}\text { THRESHOLD } \\
\text { VALUES } \\
\text { (TONS) }\end{array}$ & $\begin{array}{c}12 X \\
\text { INVENTORY } \\
\text { EXCEEDS } \\
\text { THRESHOLD } \\
\text { VALUE }\end{array}$ \\
\hline 331 & METHYL ALCOHOL & $67-56-1$ & $\mathrm{~L}$ & $1.75 \mathrm{e}-01$ & 2.10504 & 0.5 & EXCEED \\
\hline 331 & $\begin{array}{l}\text { METHYL ISOBUTYL } \\
\text { KETONE }\end{array}$ & $108-10-1$ & $\bar{L}$ & $9.70 e-03$ & 0.1164 & 0.1 & EXCEED \\
\hline 331 & METHYL METHACRYLATE & $80-62-6$ & $\bar{L}$ & $5.00 \mathrm{e}-04$ & 0.006 & 0.5 & * \\
\hline 331 & $\begin{array}{l}\text { METHYL TERT-BUTYI } \\
\text { ETHER }\end{array}$ & $1634-04-4$ & L & $4.90 \mathrm{e}-0.4$ & 0.00588 & $\overline{0.5}$ & * \\
\hline 331 & METHYLENE CHLORIDE & $75-09-2$ & $\bar{L}$ & $5.69 \mathrm{e}-02$ & 0.68292 & 0.5 & EXCEED \\
\hline 331 & NAPHTHALENE & $91-20-3$ & $\mathbf{S}$ & $1.88 \mathrm{e}-03$ & 0.02256 & 0.5 & 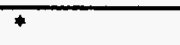 \\
\hline 331 & NICKEL. & $7440-02-0$ & $\mathrm{~s}$ & $1.10 \mathrm{e}-04$ & 0.00132 & 0.02 & * \\
\hline 331 & NITROSODIMETHYLAMINE & $62-75-9$ & $\mathrm{~s}$ & $3.00 \mathrm{e}-05$ & 0.00036 & 0.0005 & * \\
\hline 331 & P-PHENYIENEDIAMINE & $106-50-3$ & $\mathrm{~s}$ & $2.01 \mathrm{e}-03$ & 0.02412 & 0.5 & * \\
\hline 331 & PERCHLOROETHYLENE & $127-18-4$ & $\bar{L}$ & $7.15 \mathrm{e}-03$ & 0.0858 & 0.5 & * \\
\hline 331 & PHENOL & $108-95-2$ & S & $9.26 \mathrm{e}-03$ & 0.11112 & 0.1 & EXCEED \\
\hline 331 & PHOSPHINE & $7803-51-2$ & G & $1.00 \mathrm{e}-05$ & 0.00012 & 0.5 & * \\
\hline 331 & POTASSIUM CYANIDE & $151-50-8$ & $\mathrm{~s}$ & $6.60 e-04$ & 0.00792 & 0.1 & * \\
\hline 331 & PROPYLENE OXIDE & $75-56-9$ & $\bar{L}$ & $1.10 e-03$ & 0.0132 & 0.5 & $\overline{7}$ \\
\hline 331 & QUINONE & $106-51-4$ & $\mathrm{~s}$ & $5.50 e-04$ & 0.0066 & 0.5 & * \\
\hline 331 & SELENIUM & $7782-49-2$ & s & $1.20 \mathrm{e}-04$ & 0.00144 & 0.5 & * \\
\hline 331 & SODIUM CYANIDE & $143-33-9$ & $\mathrm{~s}$ & $5.00 \mathrm{e}-04$ & 0.006 & 0.1 & * \\
\hline 331 & SODIUM SELENATE & $13410-01-0$ & $\mathrm{~s}$ & $1.10 e-03$ & 0.0132 & 0.1 & * \\
\hline 331 & SODIUM SELENITE & $10102-18-8$ & $\mathrm{~s}$ & $1.40 \mathrm{e}-04$ & 0.00168 & $\overline{0.1}$ & * \\
\hline 331 & STYRENE & $100-42-5$ & $\bar{L}$ & $1.10 \mathrm{e}-03$ & 0.0132 & 0.5 & + \\
\hline 331 & SULFURIC ACID & $7664-93-9$ & L & $1.10 \mathrm{e}-01$ & 1.3194 & 0.35 & EXCEED \\
\hline 331 & TOLUENE & $108-88-3$ & $\bar{L}$ & $3.78 \mathrm{e}-02$ & 0.453 & 0.5 & * \\
\hline 331 & TRICHLOROETHYLENE & $79-01-6$ & $\bar{L}$ & $2.42 e-02$ & 0.29004 & 0.5 & + \\
\hline 331 & TRIETHYLAMINE & $121-44-8$ & $\overline{\mathrm{L}}$ & $1.93 \mathrm{e}-03$ & 0.02316 & 0.5 & * \\
\hline 331 & URETHANE 99\% & $51-79-6$ & $\mathrm{~s}$ & $5.10 \mathrm{e}-04$ & 0.00612 & 0.5 & * \\
\hline 331 & XYLENE & $1330-20-7$ & $\bar{L}$ & $4.94 e-02$ & 0.59232 & 0.5 & EXCEED \\
\hline 331 & FLUORIDES & & & $2.41 \mathrm{e}-03$ & 0.02892 & 0.15 & * \\
\hline 331 & TOTAL REDUCED SULFUR & & & $1.12 \mathrm{e}-03$ & 0.01344 & $\overline{0.5}$ & 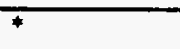 \\
\hline 331 & $\begin{array}{l}\text { VOLATILE ORGANIC } \\
\text { COMPOUNDS }\end{array}$ & & & $5.06 e-02$ & 0.60696 & 2 & * \\
\hline $331 \mathrm{~A}$ & 4-NITROPHENOL & $100-02-7$ & $\mathbf{s}$ & $1.00 \mathrm{e}-05$ & 0.00012 & 0.5 & * \\
\hline $331 \mathrm{~A}$ & $\begin{array}{l}\text { 9,10-DIMETHYLBENZ(A)ANT } \\
\text { HRACENE }\end{array}$ & $57-97-6$ & S & $1.10 \mathrm{e}-04$ & 0.00132 & 0.005 & * \\
\hline $331 \mathrm{~A}$ & ACETONITRILE & $75-05-8$ & $\bar{L}$ & $4.16 e-02$ & 0.4992 & 0.5 & + \\
\hline $331 \mathrm{~A}$ & BENZENE $\cdot$ & $71-43-2$ & $\bar{L}$ & $3.85 e-03$ & 0.0462 & 0.5 & * \\
\hline $331 \mathrm{~A}$ & CARBON MONOXIDE & $630-08-0$ & $\mathbf{G}$ & $1.40 \mathrm{e}-04$ & 0.00168 & 5 & * \\
\hline $331 \mathrm{~A}$ & CARBON TETRACHLORIDE & $56-23-5$ & I & $1.75 \mathrm{e}-03$ & 0.021 & 0.5 & * \\
\hline $331 \mathrm{~A}$ & CHLOROFORM & $67-66-3$ & $\bar{L}$ & $2.04 e-02$ & 0.24468 & 0.45 & * \\
\hline $331 \mathrm{~A}$ & CHROMIUM & $7440-47-3$ & $s$ & $5.20 \mathrm{e}-04$ & 0.00624 & 0.5 & + \\
\hline $331 \mathrm{~A}$ & DIMETHYLFORMAMIDE & $68-12-2$ & $\mathrm{~L}$ & $2.08 \mathrm{e}-03$ & 0.02496 & 0.5 & * \\
\hline$\overline{331 A}$ & FORMALDEHYDE & $50-00-0$ & $\overline{\mathrm{L}}$ & $5.11 \mathrm{e}-03$ & 0.06132 & $\overline{0.5}$ & * \\
\hline$\overline{331 A}$ & HYDROGEN CHLORDE & $7647-01-0$ & I & $1.30 \mathrm{e}-03$ & 0.0156 & 0.5 & * \\
\hline $331 \mathrm{~A}$ & METHYL ALCOHOL & $67-56-1$ & $\overline{\mathrm{L}}$ & $4.03 e-02$ & 0.4836 & 0.5 & 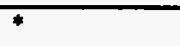 \\
\hline $331 \mathrm{~A}$ & METHYL CHLORDE & $74-87-3$ & $\mathrm{G}$ & $1.10 \mathrm{e}-04$ & 0.00132 & 0.5 & * \\
\hline $331 \mathrm{~A}$ & METHYL ETHY̌L KETONE & $78-93-3$ & $\mathrm{~L}$ & $2.22 \mathrm{e}-03$ & 0.02664 & 0.5 & 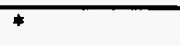 \\
\hline $331 \mathrm{~A}$ & METHYLENE CHLORIDE & $75-09-2$ & $\mathrm{~L}$ & $2.77 e-02$ & 0.33276 & 0.5 & * \\
\hline
\end{tabular}


Table K-1b. PNL Building Inventories Compared to Threshold Values.

\begin{tabular}{|c|c|c|c|c|c|c|c|}
\hline BLDG & CHEMICAL NAME & CAS\# & FORM & $\begin{array}{l}\text { INVENTORY } \\
\text { (TONS) }\end{array}$ & $\begin{array}{c}12 \mathrm{X} \\
\text { INVENTORY } \\
\text { (TONS) }\end{array}$ & $\begin{array}{l}\text { THRESHOLD } \\
\text { VALUES } \\
\text { (TONS) }\end{array}$ & $\begin{array}{c}12 \mathrm{X} \\
\text { INVENTORY } \\
\text { EXCEEDS } \\
\text { THRESHOLD } \\
\text { VALUE }\end{array}$ \\
\hline $331 \mathrm{~A}$ & PHENOL & $108-95-2$ & $s$ & $2.75 e-03$ & 0.033 & 0.1 & + \\
\hline $331 \mathrm{~A}$ & SELENIUM & $7782-49-2$ & $s$ & $1.10 \mathrm{e}-04$ & 0.00132 & 0.5 & * \\
\hline $331 \mathrm{~A}$ & SULFURIC ACID & $7664-93-9$ & $\bar{L}$ & $6.76 \mathrm{e}-03$ & 0.08112 & 0.35 & * \\
\hline $331 A$ & TRIETHYLAMINE & $121-44-8$ & $\mathrm{~L}$ & $6.20 \mathrm{e}-04$ & 0.00744 & 0.5 & + \\
\hline $331 \mathrm{~A}$ & XYLENE & $1330-20-7$ & I & $1.25 e-03$ & 0.015 & 0.5 & $*$ \\
\hline $331 \mathrm{~A}$ & FLUORIDES & & & $1.33 e-03$ & 0.01596 & 0.15 & 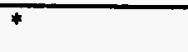 \\
\hline $331 \mathrm{~A}$ & TOTAL REDUCED SULFUR & & & $6.60 \mathrm{e}-04$ & 0.00792 & 0.5 & + \\
\hline $331 \mathrm{~A}$ & $\begin{array}{l}\text { VOLATILE ORGANIC } \\
\text { COMPOUNDS }\end{array}$ & & & $2.09 \mathrm{e}-03$ & 0.02508 & -2 & * \\
\hline $331 \mathrm{C}$ & METHYL ALCOHOL & $67-56-1$ & $\mathbf{L}$ & $4.40 \mathrm{e}-04$ & 0.00528 & 0.5 & $\cdot$ \\
\hline $331 \mathrm{H}$ & 1,1,1-TRICHLOROETHANE & $71-55-6$ & $\mathbf{L}$ & $7.40 e-04$ & 0.00888 & 0.5 & * \\
\hline $331 \mathrm{H}$ & $\begin{array}{l}\text { BIS(2-ETHYLFEXYL) } \\
\text { PHTHALATE }\end{array}$ & $117-81-7$ & $\mathbf{s}$ & $6.60 \mathrm{e}-04$ & 0.00792 & 0.5 & * \\
\hline $331 \mathrm{H}$ & HEXACHLOROETHANE & $67-72-1$ & $S$ & $5.50 \mathrm{e}-04$ & 0.0066 & 0.5 & - \\
\hline $331 \mathrm{H}$ & HYDROGEN CHLORIDE & $7647-01-0$ & $\mathrm{~L}$ & $3.08 \mathrm{e}-03$ & 0.03696 & 0.5 & $\bullet$ \\
\hline $331 \mathrm{H}$ & METHYL ALCOHOL & $67-56-1$ & $\bar{L}$ & $1.64 \mathrm{e}-01$ & 1.9704 & 0.5 & EXCEED \\
\hline $331 \mathrm{H}$ & PHOSPHOROUS & $7723-14-0$ & $\mathbf{s}$ & $3.90 \mathrm{e}-04$ & 0.00468 & 0.1 & * \\
\hline $331 \mathrm{H}$ & $\begin{array}{l}\text { TITANIUM } \\
\text { TETRACHLORIDE }\end{array}$ & $7550-45-0$ & $\overline{\mathrm{L}}$ & $6.60 \mathrm{e}-04$ & 0.00792 & 0.1 & * \\
\hline $331 \mathrm{H}$ & XYLENE & $1330-20-7$ & $\mathrm{~L}$ & $3.10 \mathrm{e}-04$ & 0.00372 & 0.5 & * \\
\hline 336 & CHLORINE & $7782-50-5$ & $\mathbf{G}$ & $1.50 \mathrm{e}-03$ & 0.018 & 0.1 & * \\
\hline 336 & METHYL ALCOHOL & $67-56-1$ & L & $4.10 \mathrm{e}-04$ & 0.00492 & 0.5 & $*$ \\
\hline 336 & TOLUENE & $108-88-3$ & $\mathrm{~L}$ & $3.80 \mathrm{e}-04$ & 0.00456 & 0.5 & * \\
\hline 337 & CARBON MONOXIDE & $630-08-0$ & $\bar{G}$ & $1.40 e-04$ & 0.00168 & 5 & $*$ \\
\hline 337 & HYDROGEN SULFIDE & $7783-06-4$ & $\mathbf{G}$ & $1.70 \mathrm{e}-04$ & 0.00204 & 0.5 & * \\
\hline 337 & TOTAL REDUCED SULFUR & & & $1.70 \mathrm{e}-04$ & 0.00204 & 0.5 & * \\
\hline 337 & SULFUR OXIDES & & & $3.20 \mathrm{e}-04$ & 0.00384 & 2 & 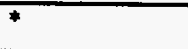 \\
\hline 337 & $\begin{array}{l}\text { VOLATILE ORGANIC } \\
\text { COMPOUNDS }\end{array}$ & & & $2.80 \mathrm{e}-04$ & 0.00336 & 2 & * \\
\hline $350 \mathrm{~A}$ & TOLUENE & $108-88-3$ & $\mathrm{~L}$ & $3.38 \mathrm{e}-02$ & 0.40512 & 0.5 & 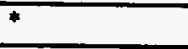 \\
\hline 3708 & ANTMMONY & $7440-36-0$ & $s$ & $1.40 \mathrm{e}-04$ & 0.00168 & 0.5 & * \\
\hline 3708 & ARSENIC & $7440-38-2$ & 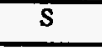 & $1.40 \mathrm{e}-04$ & 0.00168 & 0.002 & * \\
\hline 3708 & BERYLLIUM & $7440-41-7$ & $\mathbf{s}$ & $1.40 \mathrm{e}-04$ & 0.00168 & 0.004 & $*$ \\
\hline 3708 & CADMIUM & $7440-43-9$ & $\mathbf{s}$ & $6.40 \mathrm{e}-04$ & 0.00768 & 0.005 & EXCEED \\
\hline 3708 & CHROMIUM & $7440-47-3$ & $s$ & $1.50 \varepsilon-04$ & 0.0018 & 0.5 & * \\
\hline 3708 & COBALT & $7440-48-4$ & $\mathbf{s}$ & $1.40 \mathrm{e}-04$ & 0.00168 & 0.1 & $*$ \\
\hline 3708 & HYDROFLUORIC ACD & 7664-39-3 & $\mathrm{L}$ & $2.33 \mathrm{e}-03$ & 0.02796 & 0.5 & * \\
\hline 3708 & HYDROGEN CHLORIDE & $7647-01-0$ & $\bar{L}$ & $1.04 \mathrm{e}-02$ & 0.1248 & 0.5 & * \\
\hline 3708 & LEAD & $7439-92-1$ & $\mathbf{s}$ & $4.70 e-04$ & 0.00564 & 0.005 & EXCEED \\
\hline 3708 & MANGANESE & $7439-96-5$ & $\mathrm{~s}$ & $2.00 \mathrm{e}-04$ & 0.0024 & 0.5 & * \\
\hline 3708 & MERCURIC CHLORIDE & $7487-94-7$ & $s$ & $1.40 \mathrm{e}-04$ & 0.00168 & 0.01 & + \\
\hline 3708 & MERCURY & $7439-97-6$ & $\mathbf{L}$ & $1.94 \mathrm{e}-03$ & 0.02328 & 0.01 & EXCEED \\
\hline 3708 & NICKEL & $7440-02-0$ & $\mathrm{~s}$ & $2.70 e-04$ & 0.00324 & 0.02 & 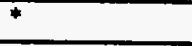 \\
\hline 3708 & PHOSPHOROUS & $7723-14-0$ & s & $1.40 \mathrm{e}-04$ & 0.00168 & 0.1 & - \\
\hline 3708 & SELENIUM & $7782-49-2$ & 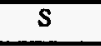 & $1.40 \mathrm{e}-04$ & 0.00168 & 0.5 & * \\
\hline 3708 & SULFURIC ACID & $7664-93-9$ & L & $4.50 \mathrm{e}-03$ & 0.054 & 0.35 & * \\
\hline 3708 & $\begin{array}{l}\text { VOLATILE ORGANIC } \\
\text { COMPOUNDS }\end{array}$ & & & $8.33 e-03$ & 0.09996 & 2 & * \\
\hline
\end{tabular}


Table K-1b. PNL Building Inventories Compared to Threshold Values.

\begin{tabular}{|c|c|c|c|c|c|c|c|}
\hline BLDG & CHEMICAL NAME & $\overline{\text { CASH }}$ & FORM & $\begin{array}{l}\text { INVENTORY } \\
\text { (TONS) }\end{array}$ & $\begin{array}{c}12 \mathrm{X} \\
\text { INVENTORY } \\
\text { (TONS) }\end{array}$ & $\begin{array}{l}\text { THRESHOLD } \\
\text { VALUES } \\
\text { (TONS) }\end{array}$ & $\begin{array}{c}\text { 12X } \\
\text { INVENTORY } \\
\text { EXCEEDS } \\
\text { THRESHOLD } \\
\text { VALUE }\end{array}$ \\
\hline $3718 \mathrm{G}$ & ANIDMONY TRIOXIDE & $1309-64-4$ & $\mathbf{s}$ & $1.00 \mathrm{e}-03$ & 0.012 & 0.5 & * \\
\hline $3718 \mathrm{G}$ & ETHYLENE GLYCOL & $107-21-1$ & $\bar{L}$ & $1.04 e+\infty$ & 12.4242 & 0.5 & EXCEED \\
\hline $3718 \mathrm{G}$ & HEXANE & $110-54-3$ & $\mathrm{~L}$ & $4.36 \mathrm{e}-03$ & 0.05232 & 0.5 & * \\
\hline $3718 \mathrm{G}$ & HYDROFLUORIC ACID & $7664-39-3$ & $\bar{L}$ & $6.90 e-04$ & 0.00828 & 0.5 & $*$ \\
\hline$\overline{3718 G}$ & HYDROGEN CHLORIDE & $7647-01-0$ & $\bar{L}$ & $1.73 e-02$ & 0.20712 & 0.5 & * \\
\hline $3718 G$ & $\begin{array}{l}\text { MERCURIC NITRATE, } \\
\text { MONOHYDRATE }\end{array}$ & $10045-94-0$ & $\mathbf{S}$ & $2.50 \mathrm{e}-04$ & 0.003 & 0.01 & + \\
\hline $3718 \mathrm{G}$ & METHYL ALCOHOL & $67-56-1$ & L & $9.08 e-03$ & 0.10896 & 0.5 & * \\
\hline $3718 \mathrm{G}$ & SULFURIC ACD & $7664-93-9$ & $\bar{I}$ & $1.15 e-02$ & 0.138 & 0.35 & $*$ \\
\hline $3718 G$ & FLUORIDES & • & & $9.10 \mathrm{e}-02$ & 1.092 & 0.15 & EXCEED \\
\hline 3720 & 1,1,1-TRICHLOROETHANE & $71-55-6$ & $\overline{\mathrm{L}}$ & $9.92 \mathrm{e}-03$ & 0.11904 & 0.5 & * \\
\hline 3720 & $\begin{array}{l}\text { 1,1,2,2-TETRACHLOROETHA } \\
\mathrm{NE}\end{array}$ & $79-34-5$ & $\overline{\mathrm{L}}$ & $2.80 \mathrm{e}-04$ & 0.00336 & 0.15 & * \\
\hline 3720 & 1,1,2-TRICHLOROETHANE & $79-00-5$ & $\bar{L}$ & $2.37 e-03$ & 0.02844 & 0.5 & * \\
\hline 3720 & 1,2-DIPHENYLHYDRAZINE & $122-66-7$ & $\mathbf{s}$ & $1.10 \mathrm{e}-04$ & 0.00132 & 0.045 & * \\
\hline 3720 & 1,3-BUTADIENE & $106-99-0$ & $\bar{G}$ & $1.00 \mathrm{e}-03$ & 0.012 & 0.035 & $*$ \\
\hline 3720 & 1,4-DIOXANE & $123-91-1$ & $\bar{L}$ & $1.17 e-02$ & 0.13992 & 0.5 & * \\
\hline 3720 & $\begin{array}{l}\text { 2,2,4TRIMETHYLPENTANE, } \\
\text { HYDROCARBON KIT } 22\end{array}$ & $540-84-1$ & $\bar{L}$ & $5.77 \mathrm{e}-03$ & 0.06924 & 0.5 & * \\
\hline 3720 & 2-ETHOXYETHANOL & $110-80-5$ & $\bar{L}$ & $6.60 \mathrm{e}-04$ & 0.00792 & 0.5 & * \\
\hline 3720 & 2-METHOXYETHANOL & $109-86-4$ & $\bar{L}$ & $1.10 \mathrm{e}-04$ & 0.00132 & 0.5 & * \\
\hline 3720 & 2-TOLUIDINE & $95-53-4$ & $\bar{L}$ & $6.60 \mathrm{e}-04$ & 0.00792 & 0.5 & + \\
\hline 3720 & 3,3'-DIMETHYL BENZIDINE & $119-93-7$ & s & $5.50 \mathrm{e}-04$ & 0.0066 & 0.004 & EXCEED \\
\hline 3720 & 3-CHLOROPROPENE & $107-05-1$ & $\bar{L}$ & $1.10 \mathrm{e}-03$ & 0.0132 & 0.5 & 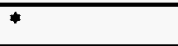 \\
\hline 3720 & 3-METHYLPHENOL & $108-39-4$ & $\mathrm{~L}$ & $2.20 \mathrm{e}-04$ & 0.00264 & 0.5 & * \\
\hline 3720 & 4,4'-BIPHENYLENEDIAMINE & $92-87-5$ & $s$ & $1.30 \mathrm{e}-04$ & 0.00156 & 0.00015 & EXCEED \\
\hline 3720 & 4-AMINOBIPHENYL & $92-67-1$ & $s$ & $2.15 e-03$ & 0.0258 & 0.5 & * \\
\hline 3720 & ACETALDEHYDE & $75-07-0$ & $\bar{L}$ & $9.60 \mathrm{e}-04$ & 0.01152 & 0.5 & * \\
\hline 3720 & ACETAMDE & $60-35-5$ & $s$ & $5.50 \mathrm{e}-04$ & 0.0066 & 0.5 & $*$ \\
\hline 3720 & ACETONITRIIE & $75-05-8$ & $\mathrm{~L}$ & $3.46 \mathrm{e}-03$ & 0.04152 & 0.5 & * \\
\hline 3720 & ACETOPHENONE & $98-86-2$ & $\overline{\mathrm{L}}$ & $1.21 \mathrm{e}-03$ & 0.01452 & 0.5 & * \\
\hline 3720 & ACROLEIN & $107-02-8$ & I & $1.21 \mathrm{e}-03$ & 0.01452 & 0.04 & * \\
\hline 3720 & ACRYLIC ACID & $79-10-7$ & $\bar{L}$ & $3.14 e-03$ & 0.03768 & 0.5 & * \\
\hline 3720 & ANILINE & $62-53-3$ & $\bar{L}$ & $5.30 \mathrm{e}-04$ & 0.00636 & 0.5 & $*$ \\
\hline 3720 & ANTIMONY & $7440-36-0$ & $\mathbf{s}$ & $5.50 \mathrm{e}-04$ & 0.0066 & 0.5 & $*$ \\
\hline 3720 & ANTIMONY TRIOXIDE & $1309-64-4$ & s & $1.17 \mathrm{e}-03$ & 0.01404 & 0.5 & $*$ \\
\hline 3720 & ARSENIC & $7440-38-2$ & $s$ & $2.20 \mathrm{e}-04$ & 0.00264 & 0.002 & EXCEED \\
\hline 3720 & BENZENE & $71-43-2$ & $\bar{L}$ & $2.40 \mathrm{e}-04$ & 0.00288 & 0.5 & * \\
\hline 3720 & BERYLLIUM & $7440-41-7$ & $s$ & $1.10 \mathrm{e}-04$ & 0.00132 & 0.004 & $*$ \\
\hline 3720 & BROMOFORM & $75-25-2$ & $\bar{L}$ & $6.00 \mathrm{e}-05$ & 0.00072 & 0.5 & 7 \\
\hline 3720 & CADMIUM & $7440-43-9$ & s & $1.55 \mathrm{e}-03$ & 0.0186 & 0.005 & EXCEED \\
\hline 3720 & CARBON DISULFIDE & $75-15-0$ & $\mathbf{L}$ & $2.09 e-03$ & 0.02508 & 0.5 & * \\
\hline 3720 & CARBON OXYSULFIDE & $463-58-1$ & G & $1.00 \mathrm{e}-03$ & 0.012 & 0.5 & $*$ \\
\hline 3720 & CARBON TETRACHLORWE & $56-23-5$ & $\bar{L}$ & $7.83 e-03$ & 0.09396 & 0.5 & * \\
\hline 3720 & CATECHOL & $120-80-9$ & $\mathbf{s}$ & $1.10 \mathrm{e}-04$ & 0.00132 & 0.5 & * \\
\hline 3720 & CHLOROBENZENE & $108-90-7$ & $\bar{L}$ & $1.10 \mathrm{e}-04$ & 0.00132 & 0.5 & * \\
\hline 3720 & CHLOROFORM & $67-66-3$ & $\bar{L}$ & $1.40 \mathrm{e}-02$ & 0.16764 & 0.45 & + \\
\hline
\end{tabular}


Table K-1b. PNL Building Inventories Compared to Threshold Values.

\begin{tabular}{|c|c|c|c|c|c|c|c|}
\hline BLDG & CHEMICAL NAME & CASH & FORM & $\begin{array}{l}\text { INVENTORY } \\
\text { (TONS) }\end{array}$ & $\begin{array}{c}12 \mathrm{X} \\
\text { INVENTORY } \\
\text { (TONS) }\end{array}$ & $\begin{array}{l}\text { THRESHOLD } \\
\text { VALUES } \\
\text { (TONS) }\end{array}$ & $\begin{array}{c}12 \mathrm{X} \\
\text { INVENTORY } \\
\text { EXCEEDS } \\
\text { THRESHOLD } \\
\text { VALUE }\end{array}$ \\
\hline 3720 & $\begin{array}{l}\text { CHLOROMETHYL METHYL } \\
\text { ETHER }\end{array}$ & $107-30-2$ & $\bar{L}$ & $5.50 \mathrm{e}-04$ & 0.0066 & 0.1 & $*$ \\
\hline 3720 & CHROMIC CHLORIDE & $10025-73-7$ & $s$ & $5.00 \mathrm{e}-04$ & 0.006 & 0.1 & * \\
\hline 3720 & CHROMIUM & $7440-47-3$ & s & $5.50 \mathrm{e}-04$ & 0.0066 & 0.5 & 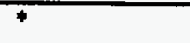 \\
\hline 3720 & CHRYSENE & $218-01-9$ & $\mathbf{s}$ & $6.00 e-05$ & 0.00072 & 0.005 & * \\
\hline 3720 & COBALT & $7440-48-4$ & $\mathbf{s}$ & $2.20 \mathrm{e}-04$ & 0.00264 & 0.1 & * \\
\hline 3720 & COBALT CARBONYL & $10210-68-1$ & $\mathbf{s}$ & $1.70 e-04$ & 0.00204 & 0.1 & * \\
\hline 3720 & CRESOL, O- & $95-48-7$ & $\bar{L}$ & $5.00 \mathrm{e}-04$ & 0.006 & 0.5 & + \\
\hline 3720 & CYSTEINE, N-ACETYL-, L- & $63-25-2$ & $s$ & $1.10 \mathrm{e}-03$ & 0.0132 & 0.5 & * \\
\hline 3720 & DIBUTYL PHTHALATE & $84-74-2$ & $\mathbf{s}$ & $6.61 \mathrm{e}-03$ & 0.07932 & 0.5 & $*$ \\
\hline 3720 & DIETHANOLAMINE & $111-42-2$ & $\mathbf{L}$ & $1.21 \mathrm{e}-03$ & 0.01452 & 0.5 & $*$ \\
\hline 3720 & DIMETHYLFORMAMIDE & $68-12-2$ & $\bar{L}$ & $8.28 e-03$ & 0.09936 & 0.5 & * \\
\hline 3720 & ETHYL ACRYLATE & $140-88-5$ & $\mathbf{L}$ & $2.75 e-03$ & 0.033 & 0.5 & * \\
\hline 3720 & ETHYLBENZENE & $100-41-4$ & $\bar{L}$ & $1.10 \mathrm{e}-03$ & 0.0132 & 0.5 & * \\
\hline 3720 & ETHYLENE DIBROMIDE & $106-93-4$ & $\bar{L}$ & $1.10 \mathrm{e}-04$ & 0.00132 & 0.05 & * \\
\hline 3720 & ETHYLENE DICHLORIDE & $107-06-2$ & $\mathbf{L}$ & $6.90 \mathrm{e}-04$ & 0.00828 & 0.4 & * \\
\hline 3720 & ETHYLENE GLYCOL & $107-21-1$ & $\bar{L}$ & $1.26 \mathrm{e}-02$ & 0.15156 & 0.5 & * \\
\hline 3720 & FORMALDEHYDE & $50-00-0$ & $\bar{L}$ & $8.82 e-03$ & 0.10584 & 0.5 & * \\
\hline 3720 & HEXANE & $110-54-3$ & $\mathrm{I}$ & $6.16 \mathrm{e}-03$ & 0.07392 & 0.5 & † \\
\hline 3720 & HYDROFLUORIC ACID & $7664-39-3$ & $\bar{L}$ & $1.16 \mathrm{e}-02$ & 0.13944 & 0.5 & $*$ \\
\hline 3720 & HYDROGEN CHLORIDE & $7647-01-0$ & $\mathrm{~L}$ & $5.38 \mathrm{e}-02$ & 0.64584 & 0.5 & EXCEED \\
\hline 3720 & HYDROQUINONE & $123-31-9$ & $s$ & $1.65 \mathrm{e}-03$ & 0.0198 & 0.5 & + \\
\hline 3720 & LEAD & $7439-92-1$ & s & $6.01 \mathrm{e}-03$ & 0.07212 & 0.005 & EXCEED \\
\hline 3720 & MALEIC ANHYDRIDE & $108-31-6$ & s & $2.20 \mathrm{e}-03$ & 0.0264 & 0.5 & * \\
\hline 3720 & MANGANESE & $7439-96-5$ & $\mathbf{s}$ & $6.90 \mathrm{e}-04$ & 0.00828 & 0.5 & * \\
\hline 3720 & MERCURIC CHLORIDE & $7487-94-7$ & $s$ & $1.12 e-03$ & 0.01344 & 0.01 & EXCEED \\
\hline 3720 & MERCURY & $7439-97-6$ & $\bar{L}$ & $3.20 \mathrm{e}-02$ & 0.38364 & 0.01 & EXCEED \\
\hline 3720 & METHYL ALCOHOL & $67-56-1$ & $\mathbf{L}$ & $9.55 \mathrm{e}-03$ & 0.1146 & 0.5 & $*$ \\
\hline 3720 & METHYL ETHYL KETONE & 78-93-3 & $\mathbf{L}$ & $4.19 e-03$ & 0.05028 & 0.5 & $*$ \\
\hline 3720 & METHYL IODIDE & $74-88-4$ & $\mathbf{L}$ & $1.70 \mathrm{e}-04$ & 0.00204 & 0.06 & * \\
\hline 3720 & $\begin{array}{l}\text { METHYL ISOBUTYL } \\
\text { KETONE }\end{array}$ & $108-10-1$ & $\bar{L}$ & $2.21 e-03$ & 0.02652 & 0.1 & * \\
\hline 3720 & METHYL METHACRYLATE & $80-62-6$ & $\mathrm{~L}$ & $2.97 e-03$ & 0.03564 & 0.5 & * \\
\hline 3720 & METHYLENE CHLORIDE & $75-09-2$ & $\bar{L}$ & $4.52 \mathrm{e}-02$ & 0.54192 & 0.5 & EXCEED \\
\hline 3720 & NAPHTHALENE & 91-20-3 & $\mathrm{s}$ & $1.14 \mathrm{e}-03$ & 0.01368 & 0.5 & * \\
\hline 3720 & NICKEL & $7440-02-0$ & 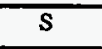 & $1.93 e-03$ & 0.02316 & 0.02 & EXCEED \\
\hline 3720 & PENTACHLOROPHENOL & $87-86-5$ & s & $3.00 \mathrm{e}-05$ & 0.00036 & 0.35 & * \\
\hline 3720 & PERCHLOROETHYLENE & $127-18-4$ & $\bar{L}$ & $5.51 \mathrm{e}-03$ & 0.06612 & 0.5 & * \\
\hline 3720 & PHENOL & $108-95-2$ & $\bar{s}$ & $1.35 e-03$ & 0.0162 & 0.1 & * \\
\hline 3720 & $\begin{array}{l}\text { PHENYLMERCURY } \\
\text { ACETATE }\end{array}$ & $62-38-4$ & $\mathbf{s}$ & $5.00 \mathrm{e}-04$ & 0.006 & 0.01 & * \\
\hline 3720 & PHOSPHOROUS & $7723-14-0$ & s & $1.10 \mathrm{e}-04$ & 0.00132 & 0.1 & * \\
\hline 3720 & PHTHALIC ANHYDRIDE & $85-44-9$ & $s$ & $2.61 \mathrm{e}-03$ & 0.03132 & 0.5 & F \\
\hline 3720 & $\begin{array}{l}\text { POTASSIUM ANTIMONYL } \\
\text { TARTRATE }\end{array}$ & & $s$ & $4.70 e-04$ & 0.00564 & 0.5 & $\bar{*}$ \\
\hline 3720 & POTASSIUM CYANIDE & $151-50-8$ & $\mathrm{~s}$ & $7.00 e-04$ & 0.0084 & 0.1 & $*$ \\
\hline 3720 & PROPIONALDEHYDE & $123-38-6$ & $\mathbf{L}$ & $1.10 \mathrm{e}-04$ & 0.00132 & 0.5 & $*$ \\
\hline 3720 & QUINOLINE & $91-22-5$ & $s$ & $1.04 \mathrm{e}-03$ & 0.01248 & 0.003 & EXCEED \\
\hline 3720 & SELENIUM & $.7782-49-2$ & $\bar{s}$ & $4.80 \mathrm{e}-0.4$ & 0.00576 & 0.5 & * \\
\hline
\end{tabular}


Table K-1b. PNL Building Inventories Compared to Threshold Values.

\begin{tabular}{|c|c|c|c|c|c|c|c|}
\hline BLDG & CHEMICAL NAME & $\overline{C A S} \#$ & FORM & $\begin{array}{l}\text { INVENTORY } \\
\text { (TONS) }\end{array}$ & $\begin{array}{c}12 \mathrm{X} \\
\text { INVENTORY } \\
\text { (TONS) }\end{array}$ & $\begin{array}{l}\text { THRESHOLD } \\
\text { VALUES } \\
\text { (TONS) }\end{array}$ & $\begin{array}{c}12 \mathrm{X} \\
\text { INVENTORY } \\
\text { EXCEEDS } \\
\text { THRESHOLD } \\
\text { VALUE }\end{array}$ \\
\hline 3720 & SODIUM CYANIDE & $143-33-9$ & $\mathbf{s}$ & $6.10 e-04$ & 0.00732 & 0.1 & $*$ \\
\hline 3720 & SODIUM SELENATE & $13410-01-0$ & 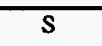 & $2.20 \mathrm{e}-04$ & 0.00264 & 0.1 & 7 \\
\hline 3720 & SODIUM SELENITE & $10102-18-8$ & $\mathrm{~s}$ & $1.10 \mathrm{e}-03$ & 0.0132 & 0.1 & * \\
\hline 3720 & STYRENE & $100-42-5$ & $\bar{L}$ & $1.10 \mathrm{e}-04$ & 0.00132 & 0.5 & + \\
\hline 3720 & SULFURIC ACID & $7664-93-9$ & $\bar{I}$ & $7.32 e-02$ & 0.87864 & 0.35 & EXCEED \\
\hline$\overline{3720}$ & $\begin{array}{l}\text { TITANIUM } \\
\text { TETRACHLORDE }\end{array}$ & $7550-45-0$ & $\overline{\mathrm{L}}$ & $1.30 \mathrm{e}-04$ & 0.00156 & 0.1 & * \\
\hline 3720 & TOLUENE & $108-88-3$ & $\bar{L}$ & $1.30 \mathrm{e}-02$ & 0.15564 & 0.5 & * \\
\hline 3720 & TRICHLOROETHYLENE & $79-01-6$ & $\bar{L}$ & $1.86 \mathrm{e}-03$ & 0.02232 & 0.5 & * \\
\hline 3720 & TRIETHYLAMINE & $121-44-8$ & $\mathbf{L}$ & $3.30 \mathrm{e}-03$ & 0.0396 & 0.5 & + \\
\hline 3721 & VINYL ACETATE & $108-05-4$ & $\bar{L}$ & $1.03 e-03$ & 0.01236 & 0.5 & $*$ \\
\hline$\div-20$ & VINYLIDENE CHLORIDE & $75-35-4$ & $\bar{L}$ & $1.10 \mathrm{e}-04$ & 0.00132 & 0.2 & * \\
\hline$\therefore: 21$ & XYLENE & $1330-20-7$ & $\mathrm{~L}$ & $9.65 e-03$ & 0.1158 & 0.5 & * \\
\hline$:-20$ & FLUORIDES & & & $4.84 \mathrm{e}-02$ & 0.5808 & 0.15 & EXCEED \\
\hline-21 & TOTAL REDUCED SULFUR & & & $3.46 \mathrm{e}-03$ & 0.04152 & 0.5 & * \\
\hline$\therefore$ & $\begin{array}{l}\text { VOLATILE ORGANIC } \\
\text { COMPOUNDS }\end{array}$ & & & $3.75 e-02$ & 0.44976 & 2 & * \\
\hline .93 & HYDROFLUORIC ACID & $7664-39-3$ & $\mathbf{L}$ & $7.80 \mathrm{e}-04$ & 0.00936 & 0.5 & * \\
\hline-23 & METHYL ALCOHOL & $67-56-1$ & $\mathbf{L}$ & $3.30 \mathrm{e}-03$ & 0.0396 & 0.5 & * \\
\hline-91 & 1,1,1-TRICHLOROETHANE & $71-55-6$ & $\mathbf{L}$ & $4.18 e-03$ & 0.05016 & 0.5 & " \\
\hline+46 & 1,1,1-TRICHLOROETHANE & $71-55-6$ & $\overline{\mathbf{L}}$ & $9.76 \mathrm{e}-03$ & 0.11712 & 0.5 & * \\
\hline$\because-75 B$ & CARBON MONOXIDE & $630-08-0$ & G & $5.00 \mathrm{e}-04$ & 0.006 & 5 & \# \\
\hline$\because-95 \mathrm{~B}$ & DIMETHYLANILINE & $121-69-7$ & $\bar{L}$ & $5.00 \mathrm{e}-04$ & 0.006 & 0.5 & + \\
\hline$--9<\mathrm{B}$ & HYDROFLUORIC ACID & $7664-39-3$ & $\bar{L}$ & $1.00 \mathrm{e}-03$ & 0.012 & 0.5 & + \\
\hline$--4 \leq \mathrm{H}$ & HYDROGEN CHLORIDE & $7647-01-0$ & $\bar{L}$ & $5.00 \mathrm{e}-04$ & 0.006 & 0.5 & * \\
\hline$-45 B$ & HYDROGEN SULFIDE & $7783-06-4$ & $\bar{G}$ & $5.00 \mathrm{e}-04$ & 0.006 & 0.5 & * \\
\hline $.95 \mathrm{~B}$ & METHYL ALCOHOL & $67-56-1$ & $\mathbf{L}$ & $8.30 \mathrm{e}-04$ & 0.00996 & 0.5 & * \\
\hline$--45 B$ & TOLUENE & $108-88-3$ & I & $1.49 \mathrm{e}-03$ & 0.01788 & 0.5 & " \\
\hline 17458 & FLUORIDES & & & $2.00 \mathrm{e}-03$ & 0.024 & 0.15 & * \\
\hline $3745 B$ & TOTAL REDUCED SULFUR & & & $5.00 \mathrm{e}-04$ & 0.006 & 0.5 & * \\
\hline $3745 \mathrm{~B}$ & SULFUR OXIDES & & & $5.00 \mathrm{e}-04$ & 0.006 & 2 & * \\
\hline 3746 & $\begin{array}{l}\text { VOLATILE ORGANIC } \\
\text { COMPOUNDS }\end{array}$ & & & $1.00 \mathrm{e}-05$ & 0.00012 & 2 & * \\
\hline $3746 \mathrm{~A}$ & 1,1,1-TRICHLOROETHANE & $71-55-6$ & $\bar{I}$ & $6.97 e-03$ & 0.08364 & 0.5 & * \\
\hline $3746 \mathrm{~A}$ & 1,4-DIOXANE & $123-91-1$ & $\mathrm{~L}$ & $5.28 \mathrm{e}-03$ & 0.06336 & 0.5 & ${ }^{*}$ \\
\hline $3746 \mathrm{~A}$ & CADMIUM & $7440-43-9$ & s & $5.00 \mathrm{e}-04$ & 0.006 & 0.005 & EXCEED \\
\hline$\overline{3746 A}$ & CHLOROFORM & $67-66-3$ & I & $6.57 e-03$ & 0.07884 & 0.45 & + \\
\hline $3746 \mathrm{~A}$ & FORMALDEHYDE & $50-00-0$ & $\bar{I}$ & $5.64 e-03$ & 0.06768 & 0.5 & * \\
\hline $3746 \mathrm{~A}$ & HYDROFLUORIC ACID & $7664-39-3$ & $\mathrm{~L}$ & $6.20 \mathrm{e}-04$ & 0.00744 & 0.5 & $*$ \\
\hline $3746 \mathrm{~A}$ & HYDROGEN CHLORIDE & $7647-01-0$ & $\bar{L}$ & $2.87 e-02$ & 0.34452 & 0.5 & * \\
\hline $3746 \mathrm{~A}$ & METHYL ALCOHOL & $67-56-1$ & $\bar{L}$ & $2.18 \mathrm{e}-01$ & 2.61336 & 0.5 & EXCEED \\
\hline $3746 \mathrm{~A}$ & NAPHTHALENE & $91-20-3$ & $\mathbf{s}$ & $1.10 \mathrm{e}-03$ & 0.0132 & 0.5 & * \\
\hline $3746 \mathrm{~A}$ & PHENOL & $108-95-2$ & $s$ & $3.85 \mathrm{e}-03$ & 0.0462 & 0.1 & * \\
\hline $3746 \mathrm{~A}$ & SULFURIC ACD & $7664-93-9$ & $\mathrm{I}$ & $2.47 e-03$ & 0.02964 & 0.35 & $*$ \\
\hline $3746 \mathrm{~A}$ & TRIETHYLAMINE & $121-44-8$ & $\mathrm{~L}$ & $8.00 \mathrm{e}-05$ & 0.00096 & 0.5 & $*$ \\
\hline $3746 \mathrm{~A}$ & XYLENE & $1330-20-7$ & $\bar{L}$ & $5.28 \mathrm{e}-02$ & 0.63348 & 0.5 & EXCEED \\
\hline $3746 \mathrm{~A}$ & FLUORDES & & & $5.26 \mathrm{e}-03$ & 0.06312 & 0.15 & * \\
\hline
\end{tabular}


Table K-1b. PNL Building Inventories Compared to Threshold Values.

\begin{tabular}{|c|c|c|c|c|c|c|c|}
\hline BLDG & CHEMICAL NAME & CASH & FORM & $\begin{array}{l}\text { INVENTORY } \\
\text { (TONS) }\end{array}$ & $\begin{array}{c}12 \mathrm{X} \\
\text { INVENTORY } \\
\text { (TONS) }\end{array}$ & $\begin{array}{l}\text { THRESHOLD } \\
\text { VALUES } \\
\text { (TONS) }\end{array}$ & $\begin{array}{c}12 X \\
\text { INVENTORY } \\
\text { EXCEEDS } \\
\text { THRESHOLD } \\
\text { VALUE }\end{array}$ \\
\hline $3746 \mathrm{~A}$ & TOTAL REDUCED SULFUR & & & $1.10 \mathrm{e}-04$ & 0.00132 & 0.5 & 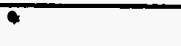 \\
\hline $3746 A$ & $\begin{array}{l}\text { VOLATILE ORGANIC } \\
\text { COMPOUNDS }\end{array}$ & & & $1.31 \mathrm{e}-03$ & 0.01572 & 2 & 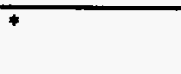 \\
\hline $622 \mathrm{R}$ & METHYL ALCOHOL & $67-56-1$ & $\bar{L}$ & $2.89 e-03$ & 0.03468 & 0.5 & 7 \\
\hline $622 R$ & SULFURIC ACD & $7664-93-9$ & $\bar{L}$ & $2.03 e-03$ & 0.02436 & 0.35 & * \\
\hline $6652 \mathrm{C}$ & XYLENE & $1330-20-7$ & $\bar{L}$ & $3.75 \mathrm{e}-03$ & 0.045 & 0.5 & 7 \\
\hline
\end{tabular}


Table K-1c. PNL Building Inventories by Room Compared to Threshold Values.

\begin{tabular}{|c|c|c|c|c|c|c|c|c|c|}
\hline Bldg. & Room & Pollutants & $\begin{array}{c}\text { CAS } \\
\text { Number }\end{array}$ & Form & $\begin{array}{l}\text { Inventory } \\
\text { (tons/yr) }\end{array}$ & $\begin{array}{c}12 \mathrm{X} \\
\text { Inventory } \\
\text { (tons/yr) }\end{array}$ & $\begin{array}{l}\text { Threshold } \\
\text { Values } \\
\text { (tons/yr) }\end{array}$ & $\begin{array}{l}\text { Release } \\
\text { Fraction }\end{array}$ & $\begin{array}{c}12 X \\
\text { Inventory } \\
\text { Exceeds } \\
\text { Threshold } \\
\text { Value }\end{array}$ \\
\hline $306 \mathrm{~W}$ & 160 & COBALT & $7440-48-4$ & S & 0.0325 & 0.39 & 0.1 & 0.000001 & * \\
\hline $306 \mathrm{~W}$ & 164 & COBALT & $7440-48-4$ & $s$ & 0.0035 & 0.042 & 0.1 & 0.000001 & $*$ \\
\hline $306 \mathrm{~W}$ & 150 & LEAD & $7439-92-1$ & $\mathbf{s}$ & 0.0025 & 0.03 & 0.005 & 0.000001 & * \\
\hline $306 \mathrm{~W}$ & 150 & NICKEL & $7440-02-0$ & $\mathbf{S}$ & 0.00208 & 0.02496 & 0.02 & 0.000001 & * \\
\hline $306 \mathrm{~W}$ & 160 & NICKEL & $7440-02-0$ & $S$ & 0.16085 & 1.9302 & 0.02 & 0.000001 & * \\
\hline $306 \mathrm{~W}$ & 164 & NICKEL & $7440-02-0$ & $s$ & 0.00263 & 0.03156 & 0.02 & 0.000001 & * \\
\hline 314 & 57 & CADMIUM & $7440-43-9$ & $s$ & 0.0005 & 0.006 & 0.005 & 0.000001 & * \\
\hline 314 & 42 & FLUORIDES & & $\bar{L}$ & 0.01094 & 0.13128 & 0.15 & 0.001 & * \\
\hline 314 & 57 & FLUORIDES & & $\mathbf{L}$ & 0.0255 & 0.306 & 0.15 & 0.001 & $*$ \\
\hline 314 & 42 & HYDROGEN CHLORIDE & $7647-01-0$ & L & 0.02404 & 0.28848 & 0.5 & 0.001 & + \\
\hline 314 & 57 & HYDROGEN CHLORIDE & $7647-01-0$ & $\bar{L}$ & 0.02292 & 0.27504 & 0.5 & 0.001 & * \\
\hline 314 & 42 & LEAD & $7439-92-1$ & $\mathbf{s}$ & 0.0135 & 0.162 & 0.005 & 0.000001 & * \\
\hline 314 & 57 & LEAD & $7439-92-1$ & $S$ & 0.0035 & 0.042 & 0.005 & 0.000001 & * \\
\hline 318 & 134 & METHYL ALCOHOL & $67-56-1$ & $\mathbf{L}$ & 0.04187 & 0.50244 & 0.5 & 0.001 & * \\
\hline 318 & 147 & METHYL ALCOHOL & $67-56-1$ & $\mathbf{L}$ & 0.04885 & 0.5862 & 0.5 & 0.001 & * \\
\hline 318 & 149 & METHYL ALCOHOL & $67-56-1$ & $\mathbf{L}$ & 0.11165 & 1.3398 & 0.5 & 0.001 & * \\
\hline 320 & 185 & ARSENIC & $7440-38-2$ & $\mathbf{s}$ & 0.00165 & 0.0198 & 0.002 & 0.000001 & * \\
\hline 320 & 6666 & LEAD & $7439-92-1$ & $\mathbf{s}$ & 0.0005 & 0.006 & 0.005 & 0.000001 & * \\
\hline 320 & 5 & METHYL ALCOHOL & $67-56-1$ & $\bar{L}$ & 0.00087 & 0.01044 & 0.5 & 0.001 & + \\
\hline 320 & 7 & METHYL ALCOHOL & $67-56-1$ & $\mathbf{L}$ & 0.00218 & 0.02616 & 0.5 & 0.001 & $*$ \\
\hline 320 & 65 & METHYL ALCOHOL & $67-56-1$ & $\mathbf{L}$ & 0.00523 & 0.06276 & 0.5 & 0.001 & * \\
\hline 320 & 6666 & METHYL ALCOHOL & $67-56-1$ & $\bar{L}$ & 0.03325 & 0.399 & 0.5 & 0.001 & 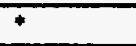 \\
\hline 320 & 138 & METHYLENE CHLORIDE & $75-09-2$ & $\mathbf{L}$ & 0.0321 & 0.3852 & 0.5 & 0.001 & * \\
\hline 320 & 6666 & METHYLENE CHLORIDE & $75-09-2$ & $\bar{L}$ & 0.01824 & 0.21888 & 0.5 & 0.001 & * \\
\hline 324 & 208 & ARSENIC & $7440-38-2$ & $\mathbf{s}$ & 0.00055 & 0.0066 & 0.002 & 0.000001 & + \\
\hline 324 & 208 & BERYLIIUM & $7440-41-7$ & s. & 0.00083 & 0.00996 & 0.004 & 0.000001 & * \\
\hline 324 & 208 & CADMIUM & $7440-43-9$ & $s$ & 0.00083 & 0.00996 & 0.005 & 0.000001 & * \\
\hline 324 & 22 & HYDROGEN CHLORIDE & $7647-01-0$ & $\bar{L}$ & 0.0093 & 0.1116 & 0.5 & 0.001 & $*$ \\
\hline 324 & 207 & HYDROGEN CHLORDE & $7647-01-0$ & L & 0.04029 & 0.48348 & 0.5 & 0.001 & * \\
\hline 324 & 208 & HYDROGEN CHLORIDE & $7647-01-0$ & $\mathrm{~L}$ & 0.00461 & 0.05532 & 0.5 & 0.001 & $*$ \\
\hline 324 & 208 & LEAD & $7439-92-1$ & $s$ & 0.00478 & 0.05736 & 0.005 & 0.000001 & * \\
\hline 324 & 210 & $\overline{\text { LEAD }}$ & $7439-92-1$ & $s$ & 0.00165 & 0.0198 & 0.005 & 0.000001 & * \\
\hline 324 & 22 & MERCURY & $7439-97-6$ & $\mathrm{~L}$ & 0.00439 & 0.05268 & 0.01 & 0.001 & $*$ \\
\hline 324 & 208 & MERCURY & $7439-97-6$ & $\mathrm{~L}$ & 0.00751 & 0.09012 & 0.01 & 0.001 & $*$ \\
\hline 324 & 208 & NICKEL & $7440-02-0$ & s & 0.00333 & 0.03996 & 0.02 & 0.000001 & * \\
\hline 324 & 210 & NICKEL & $7440-02-0$ & s & 0.0025 & 0.03 & 0.02 & 0.000001 & * \\
\hline 324 & & SULFURIC ACID & $7664-93-9$ & $\bar{L}$ & 0.00203 & 0.02436 & 0.35 & 0.001 & * \\
\hline 324 & & SULFURIC ACID & $7664-93-9$ & $\bar{L}$ & 0.00551 & 0.06612 & 0.35 & 0.001 & * \\
\hline 324 & 22 & SULFURIC ACID & 7664-93-9 & $\mathrm{L}$ & 0.0233 & 0.2796 & 0.35 & 0.001 & * \\
\hline 324 & 115 & SULFURIC ACID & $7664-93-9$ & $\mathrm{~L}$ & 0.00344 & 0.04128 & 0.35 & 0.001 & * \\
\hline 324 & 207 & SULPURIC ACID & $7664-93-9$ & $\mathrm{~L}$ & 0.02533 & 0.30396 & 0.35 & 0.001 & + \\
\hline 324 & 208 & SULFURIC ACID & $7664-93-9$ & $\mathrm{~L}$ & 0.0002 & 0.0024 & 0.35 & 0.001 & $\div$ \\
\hline 325 & 300 & 1,1,1-TRICHLOROETHANE & $71-55-6$ & $\bar{L}$ & 0.0007 & 0.0084 & 0.5 & 0.001 & * \\
\hline 325 & 426 & 1,1,1-TRICHLOROETHANE & $71-55-6$ & $\mathbf{L}$ & 0.0007 & 0.0084 & 0.5 & 0.001 & * \\
\hline 325 & 517 & 1,1,1-TRICHLOROETHANE & $71-55-6$ & L & 0.00139 & 0.01668 & 0.5 & 0.001 & $*$ \\
\hline 325 & 700 & 1,1,1-TRICHLOROETHANE & $71-55-6$ & $\bar{L}$ & 0.05305 & 0.6366 & 0.5 & 0.001 & 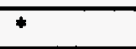 \\
\hline 325 & 300 & ARSENIC & $7440-38-2$ & $\mathbf{s}$ & 0.00074 & 0.00888 & 0.002 & 0.000001 & $*$ \\
\hline 325 & 517 & ARSENIC & $7440-38-2$ & $s$ & 0.00063 & 0.00756 & 0.002 & 0.000001 & * \\
\hline 325 & 300 & CADMIUM & $7440-43-9$ & $\mathbf{S}$ & 0.00124 & 0.01488 & 0.005 & 0.000001 & * \\
\hline
\end{tabular}


Table K-1c. PNL Building Inventories by Room Compared to Threshold Values.

\begin{tabular}{|c|c|c|c|c|c|c|c|c|c|}
\hline Bldg. & Room & Pollutants & $\begin{array}{c}\text { CAS } \\
\text { Number }\end{array}$ & Form & $\begin{array}{l}\text { Inventory } \\
\text { (tons/yr) }\end{array}$ & $\begin{array}{c}12 \mathrm{X} \\
\text { Inventory } \\
\text { (Lons/yr) }\end{array}$ & $\begin{array}{l}\text { Threshold } \\
\text { Values } \\
\text { (tons/yr) }\end{array}$ & $\begin{array}{l}\text { Release } \\
\text { Fraction }\end{array}$ & $\begin{array}{c}12 \mathrm{X} \\
\text { Inventory } \\
\text { Exceeds } \\
\text { Threshold } \\
\text { Value }\end{array}$ \\
\hline 325 & 501 & CADMIUM & $7440-43-9$ & $s$ & 0.0005 & 0.006 & 0.005 & 0.000001 & * \\
\hline 325 & 507 & CADMIUM & $7440-43-9$ & $s$ & 0.0005 & 0.006 & 0.005 & 0.000001 & $*$ \\
\hline 325 & 517 & CADMIUM & $7440-43-9$ & $s$ & 0.001 & 0.012 & 0.005 & 0.000001 & $\div$ \\
\hline 325 & 56 & FLUORIDES & & $\mathrm{L}$ & 0.0015 & 0.018 & 0.15 & 0.001 & * \\
\hline 325 & 95 & FLUORIDES & & I & 0.00314 & 0.03768 & 0.15 & 0.001 & + \\
\hline 325 & 200 & FLUORIDES & & L & 0.00028 & 0.00336 & 0.15 & 0.001 & + \\
\hline 325 & 300 & FLUORIDES & & L & 0.00776 & 0.09312 & 0.15 & 0.001 & * \\
\hline 325 & 305 & FLUORDES & & $\mathrm{L}$ & 0.0035 & 0.042 & 0.15 & 0.001 & * \\
\hline 325 & 316 & FLUORIDES & & $\mathrm{L}$ & 0.00165 & 0.0198 & 0.15 & 0.001 & 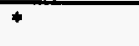 \\
\hline 325 & 400 & FLUORIDES & & $\mathrm{I}$ & 0.00072 & 0.00864 & 0.15 & 0.001 & * \\
\hline 325 & 410 & FLUORIDES & & L & 0.00641 & 0.07692 & 0.15 & 0.001 & * \\
\hline 325 & 419 & FLUORIDES & & $\mathrm{L}$ & 0.00055 & 0.0066 & 0.15 & 0.001 & * \\
\hline 325 & 427 & FLUORIDES & & L & 0.00006 & 0.00072 & 0.15 & 0.001 & * \\
\hline 325 & 501 & FLUORIDES & & L & 0.00955 & 0.1146 & 0.15 & 0.001 & * \\
\hline 325 & 507 & FLUORIDES & & $\mathrm{L}$ & 0.00951 & 0.11412 & 0.15 & 0.001 & $*$ \\
\hline 325 & 510 & FLUORIDES & & $\mathrm{L}$ & 0.0015 & 0.018 & 0.15 & 0.001 & $*$ \\
\hline 325 & 511 & FLUORIDES & & $\mathbf{L}$ & 0.00859 & 0.10308 & 0.15 & 0.001 & * \\
\hline 325 & 514 & FLUORIDES & & L & 0.00055 & 0.0066 & 0.15 & 0.001 & $*$ \\
\hline 325 & 516 & FLUORIDES & & $\mathbf{L}$ & 0.002 & 0.024 & 0.15 & 0.001 & * \\
\hline 325 & 517 & FLUORIDES & & $\mathbf{L}$ & 0.0077 & 0.0924 & 0.15 & 0.001 & + \\
\hline 325 & 524 & FLUORIDES & & L & 0.0033 & 0.0396 & 0.15 & 0.001 & $*$ \\
\hline 325 & 300 & HYDRAZRE & $302-01-2$ & $L$ & 0.00166 & 0.01992 & 0.002 & 0.001 & + \\
\hline 325 & 48 & HYDROGEN CHLORIDE & $7647-01-0$ & $\mathrm{~L}$ & 0.00195 & 0.0234 & 0.5 & 0.001 & * \\
\hline 325 & 56 & HYDROGEN CHLORIDE & $7647-01-0$ & $\mathrm{~L}$ & 0.0039 & 0.0468 & 0.5 & 0.001 & $*$ \\
\hline 325 & 95 & HYDROGEN CHLORIDE & $7647-01-0$ & $\mathrm{~L}$ & 0.00123 & 0.01476 & 0.5 & 0.001 & $*$ \\
\hline 325 & 200 & HYDROGEN CHLORIDE & $7647-01-0$ & $\mathrm{~L}$ & 0.03144 & 0.37728 & 0.5 & 0.001 & * \\
\hline 325 & 300 & HYDROGEN CHLORIDE & $7647-01-0$ & $\bar{L}$ & 0.00791 & 0.09492 & 0.5 & 0.001 & * \\
\hline 325 & 306 & HYDROGEN CHLORIDE & $7647-01-0$ & $\mathrm{~L}$ & 0.00065 & 0.0078 & 0.5 & 0.001 & 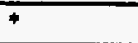 \\
\hline 325 & 309 & HYDROGEN CHLORDE & $7647-01-0$ & $\mathrm{~L}$ & 0.0026 & 0.0312 & 0.5 & 0.001 & * \\
\hline 325 & 312 & HYDROGEN CHLORDE & $7647-01-0$ & $\mathbf{L}$ & 0.00065 & 0.0078 & 0.5 & 0.001 & $*$ \\
\hline 325 & 317 & HYDROGEN CHLORDE & $7647-01-0$ & $\mathrm{I}$ & 0.00065 & 0.0078 & 0.5 & 0.001 & $*$ \\
\hline 325 & 330 & HYDROGEN CHLORIDE & $7647-01-0$ & $\mathrm{~L}$ & 0.00065 & 0.0078 & 0.5 & 0.001 & + \\
\hline 325 & 405 & HYDROGEN CHLORIDE & $7647-01-0$ & $\mathrm{~L}$ & 0.00338 & 0.04056 & 0.5 & 0.001 & + \\
\hline 325 & 410 & HYDROGEN CHLORIDE & $7647-01-0$ & $\mathrm{~L}$ & 0.0077 & 0.0924 & 0.5 & 0.001 & * \\
\hline 325 & 419 & HYDROGEN CHLORIDE & $7647-01-0$ & $\mathrm{~L}$ & 0.00976 & 0.11712 & 0.5 & 0.001 & + \\
\hline 325 & 422 & HYDROGEN CHLORIDE & $7647-01-0$ & $\mathrm{~L}$ & 0.0013 & 0.0156 & 0.5 & 0.001 & * \\
\hline 325 & 426 & HYDROGEN CHLORIDE & $7647-01-0$ & $\mathrm{~L}$ & 0.024 & 0.288 & 0.5 & 0.001 & $*$ \\
\hline 325 & 500 & HYDROGEN CHLORDE & $7647-01-0$ & $\mathrm{~L}$ & 0.0026 & 0.0312 & 0.5 & 0.001 & + \\
\hline 325 & 501 & HYDROGEN CHLORIDE & $7647-01-0$ & $\mathrm{~L}$ & 0.003 & 0.036 & 0.5 & 0.001 & + \\
\hline 325 & 505 & HYDROGEN CHLORIDE & $7647-01-0$ & $\mathrm{~L}$ & 0.00455 & 0.0546 & 0.5 & 0.001 & \# \\
\hline 325 & 507 & HYDROGEN CHLORIDE & $7647-01-0$ & $\mathrm{~L}$ & 0.0039 & 0.0468 & 0.5 & 0.001 & $*$ \\
\hline 325 & 510 & HYDROGEN CHLORDE & $7647-01-0$ & $\mathrm{~L}$ & 0.0026 & 0.0312 & 0.5 & 0.001 & + \\
\hline 325 & 516 & HYDROGEN CHLORIDE & $7647-01-0$ & $\mathrm{~L}$ & 0.00329 & 0.03948 & 0.5 & 0.001 & $*$ \\
\hline 325 & 517 & HYDROGEN CHLORIDE & $7647-01-0$ & $L$ & 0.0025 & 0.03 & 0.5 & 0.001 & * \\
\hline 325 & 525 & HYDROGEN CHLORIDE & $7647-01-0$ & $\bar{L}$ & 0.00715 & 0.0858 & 0.5 & 0.001 & * \\
\hline 325 & 300 & LEAD & $7439-92-1$ & $s$ & 0.00578 & 0.06936 & 0.005 & 0.000001 & * \\
\hline 325 & 510 & LEAD & $7439-92-1$ & $\mathbf{s}$ & 0.001 & 0.012 & 0.005 & 0.000001 & * \\
\hline
\end{tabular}


Table K-1c. PNL Building Inventories by Room Compared to Threshold Values.

\begin{tabular}{|c|c|c|c|c|c|c|c|c|c|}
\hline Bldg. & Room & Pollutants & $\begin{array}{c}\text { CAS } \\
\text { Number }\end{array}$ & Form & $\begin{array}{l}\text { Inventory } \\
\text { (tons/yr) }\end{array}$ & $\begin{array}{c}12 \mathrm{X} \\
\text { Inventory } \\
\text { (tons/yr) }\end{array}$ & $\begin{array}{l}\text { Threshold } \\
\text { Values } \\
\text { (tons/yr) }\end{array}$ & $\begin{array}{l}\text { Release } \\
\text { Fraction }\end{array}$ & $\begin{array}{c}12 X \\
\text { Inventory } \\
\text { Exceeds } \\
\text { Threshold } \\
\text { Value }\end{array}$ \\
\hline 325 & 517 & LEAD & $7439-92-1$ & $\mathbf{s}$ & 0.0005 & 0.006 & 0.005 & 0.000001 & $\bullet$ \\
\hline 325 & 300 & MERCURY & $7439-97-6$ & $\bar{L}$ & 0.00149 & 0.01788 & 0.01 & 0.001 & $\bullet$ \\
\hline 325 & 410 & MERCURY & $7439-97-6$ & L & 0.0025 & 0.03 & 0.01 & 0.001 & $*$ \\
\hline 325 & 419 & MERCURY & $7439-97-6$ & $\mathrm{~L}$ & 0.00045 & 0.0054 & 0.01 & 0.001 & * \\
\hline 325 & 510 & MERCURY & $7439-97-6$ & I & 0.0025 & 0.03 & 0.01 & 0.001 & + \\
\hline 325 & & METHYL ALCOHOL & $67-56-1$ & $\bar{I}$ & 0.0157 & 0.1884 & 0.5 & 0.001 & * \\
\hline 325 & 200 & METHYL ALCOHOL & $67-56-1$ & $\mathrm{~L}$ & 0.00248 & 0.02976 & 0.5 & 0.001 & $*$ \\
\hline 325 & 300 & METHYL ALCOHOL & $67-56-1$ & $\mathrm{~L}$ & 0.00259 & 0.03108 & 0.5 & 0.001 & * \\
\hline 325 & 306 & METHYL ALCOHOL & $67-56-1$ & $\mathrm{~L}$ & 0.00349 & 0.04188 & 0.5 & 0.001 & * \\
\hline 325 & 308 & METHYL ALCOHOL & $67-56-1$ & I & 0.00698 & 0.08376 & 0.5 & 0.001 & + \\
\hline 325 & 312 & METHYL ALCOHOL & $67-56-1$ & L & 0.00087 & 0.01044 & 0.5 & 0.001 & * \\
\hline 325 & 327 & METHYL ALCOHOL & $67-56-1$ & $\mathrm{~L}$ & 0.03489 & 0.41868 & 0.5 & 0.001 & * \\
\hline 325 & 405 & METHYL ALCOHOL & $67-56-1$ & $\mathbf{L}$ & 0.00041 & 0.00492 & 0.5 & 0.001 & * \\
\hline 325 & 410 & METHYL ALCOHOL & $67-56-1$ & $\mathrm{~L}$ & 0.00041 & 0.00492 & 0.5 & 0.001 & * \\
\hline 325 & 416 & METHYL ALCOHOL & $67-56-1$ & $\bar{L}$ & 0.00041 & 0.00492 & 0.5 & 0.001 & * \\
\hline 325 & 426 & METHYL ALCOHOL & $67-56-1$ & I & 0.0048 & 0.0576 & 0.5 & 0.001 & $*$ \\
\hline 325 & 517 & METHYL ALCOHOL & $67-56-1$ & $\mathrm{I}$ & 0.00436 & 0.05232 & 0.5 & 0.001 & 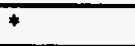 \\
\hline 325 & 524 & METHYL ALCOHOL & $67-56-1$ & I & 0.00044 & 0.00528 & 0.5 & 0.001 & * \\
\hline 325 & 700 & METHYL ALCOHOL & $67-56-1$ & $\mathrm{~L}$ & 0.01744 & 0.20928 & 0.5 & 0.001 & * \\
\hline 325 & 702 & METHYL ALCOHOL & $67-56-1$ & I & 0.00349 & 0.04188 & 0.5 & 0.001 & * \\
\hline 325 & 306 & METHYLENE CHLORIDE & $75-09-2$ & $\bar{L}$ & 0.00584 & 0.07008 & 0.5 & 0.001 & * \\
\hline 325 & 308 & METHYLENE CHLORIDE & $75-09-2$ & $\mathrm{~L}$ & 0.07004 & 0.84048 & 0.5 & 0.001 & * \\
\hline 325 & 700 & METHYLENE CHLORIDE & $75-09-2$ & $\mathrm{~L}$ & 0.01167 & 0.14004 & 0.5 & 0.001 & $*$ \\
\hline 325 & 702 & METHYLENE CHLORDE & $75-09-2$ & $\bar{L}$ & 0.03502 & 0.42024 & 0.5 & 0.001 & * \\
\hline 325 & 300 & NICKEL & $7440-02-0$ & $\mathbf{s}$ & 0.0011 & 0.0132 & 0.02 & 0.000001 & * \\
\hline 325 & 501 & NICKEL & $7440-02-0$ & $s$ & 0.00012 & 0.00144 & 0.02 & 0.000001 & + \\
\hline 325 & 505 & NICKEL & $7440-02-0$ & $\mathrm{~s}$ & 0.00061 & 0.00732 & 0.02 & 0.000001 & $*$ \\
\hline 325 & 507 & NICKEL & $7440-02-0$ & $\mathbf{s}$ & 0.00003 & 0.00036 & 0.02 & 0.000001 & * \\
\hline 325 & 517 & NICKEL & $7440-02-0$ & $s$ & 0.00028 & 0.00336 & 0.02 & 0.000001 & * \\
\hline 325 & 300 & QUINOLINE & $91-22-5$ & $s$ & 0.00052 & 0.00624 & 0.003 & 0.000001 & * \\
\hline 325 & 48 & SULFURIC ACID & $7664-93-9$ & I & 0.00101 & 0.01212 & 0.35 & 0.001 & * \\
\hline 325 & 56 & SULFURIC ACID & $7664-93-9$ & $\mathrm{~L}$ & 0.01013 & 0.12156 & 0.35 & 0.001 & + \\
\hline 325 & 200 & SULPURIC ACID & $7664-93-9$ & $\bar{L}$ & 0.01824 & 0.21888 & 0.35 & 0.001 & + \\
\hline 325 & 300 & SULFURIC ACID & $7664-93-9$ & L & 0.00551 & 0.06612 & 0.35 & 0.001 & $*$ \\
\hline 325 & 305 & SULFURIC ACID & 7664-93-9 & $\mathrm{L}$ & 0.0045 & 0.054 & 0.35 & 0.001 & * \\
\hline 325 & 306 & SULFURIC ACID & $7664-93-9$ & $\overline{\mathrm{L}}$ & 0.004 & 0.048 & 0.35 & 0.001 & $*$ \\
\hline 325 & 309 & SULFURIC ACID & $7664-93-9$ & $\bar{L}$ & 0.0045 & 0.054 & 0.35 & 0.001 & * \\
\hline 325 & 316 & SULFURIC ACID & $7664-93-9$ & $\bar{L}$ & 0.0045 & 0.054 & 0.35 & 0.001 & * \\
\hline 325 & 400 & SULFURIC ACID & $7664-93-9$ & $\mathbf{L}$ & 0.00101 & 0.01212 & 0.35 & 0.001 & * \\
\hline 325 & 406 & SULPURIC ACID & $7664-93-9$ & $\mathbf{L}$ & 0.00101 & 0.01212 & 0.35 & 0.001 & * \\
\hline 325 & 416 & SULFURIC ACID & $7664-93-9$ & $\bar{L}$ & 0.00479 & 0.05748 & 0.35 & 0.001 & * \\
\hline 325 & 419 & SULFURIC ACID & $7664-93-9$ & $\bar{L}$ & 0.00912 & 0.10944 & 0.35 & 0.001 & 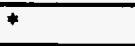 \\
\hline 325 & 426 & SULFURIC ACPD & 7664-93-9 & $\bar{L}$ & 0.009 & 0.108 & 0.35 & 0.001 & * \\
\hline 325 & 500 & SULFURIC ACD & $7664-93-9$ & $\bar{L}$ & 0.0045 & 0.054 & 0.35 & 0.001 & + \\
\hline 325 & 501 & SULFURIC ACID & $7664-93-9$ & $\bar{L}$ & 0.0045 & 0.054 & 0.35 & 0.001 & * \\
\hline 325 & 510 & SULFURIC ACID & $7664-93-9$ & $\bar{L}$ & 0.01621 & 0.19452 & 0.35 & 0.001 & * \\
\hline 325 & 516 & SULPURIC ACID & $7664-93-9$ & L & 0.0035 & 0.042 & 0.35 & 0.001 & 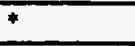 \\
\hline 325 & 517 & SULFURIC ACID & $7664-93-9$ & L & 0.0045 & 0.054 & 0.35 & 0.001 & * \\
\hline 325 & 528 & SULFURIC ACID & $7664-93-9$ & L & 0.0045 & 0.054 & 0.35 & 0.001 & + \\
\hline
\end{tabular}


Table K-1c. PNL Building Inventories by Room Compared to Threshold Values.

\begin{tabular}{|c|c|c|c|c|c|c|c|c|c|}
\hline Bldg. & Room & Pollutants & $\begin{array}{c}\text { CAS } \\
\text { Number }\end{array}$ & Form & $\begin{array}{l}\text { Inventory } \\
\text { (Lons/yr) }\end{array}$ & $\begin{array}{c}12 \mathrm{X} \\
\text { Inventory } \\
\text { (tons/yr) }\end{array}$ & $\begin{array}{l}\text { Threshold } \\
\text { Values } \\
\text { (Lons/yr) }\end{array}$ & $\begin{array}{l}\text { Release } \\
\text { Fraction }\end{array}$ & $\begin{array}{c}12 \mathrm{X} \\
\text { Inventory } \\
\text { Exceeds } \\
\text { Threshold } \\
\text { Value }\end{array}$ \\
\hline 326 & & ACRYLAMIDE & $79-06-1$ & 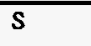 & 0.00011 & 0.00132 & 0.01 & 0.000001 & * \\
\hline 326 & & ACRYLAMIDE & $79-06-1$ & $\bar{s}$ & 0.00121 & 0.01452 & 0.01 & 0.000001 & * \\
\hline 326 & & CADMIUM & $7440-43-9$ & $\bar{s}$ & 0.0005 & 0.006 & 0.005 & 0.000001 & + \\
\hline 326 & & CHLOROFORM & $67-66-3$ & $\overline{\mathrm{L}}$ & 0.00657 & 0.07884 & 0.45 & 0.001 & * \\
\hline 326 & & CHLOROFORM & $67-66-3$ & $\mathrm{~L}$ & 0.01315 & 0.1578 & 0.45 & 0.001 & * \\
\hline 326 & & CHLOROFORM & $67-66-3$ & $\mathrm{~L}$ & 0.02629 & 0.31548 & 0.45 & 0.001 & * \\
\hline 326 & & FLUORIDES & & $I$ & 0.00011 & 0.00132 & 0.15 & 0.001 & * \\
\hline 326 & & FLUORIDES & & $\bar{L}$ & 0.00024 & 0.00288 & 0.15 & 0.001 & * \\
\hline 326 & & FLUORIDES & & $I$ & 0.0005 & 0.006 & 0.15 & 0.001 & * \\
\hline 326 & $26-A$ & FLUORIDES & & $\mathrm{G}$ & 0.0195 & 0.234 & 0.15 & 1 & EXCEEDS \\
\hline 326 & & LEAD & $7439-92-1$ & $\bar{s}$ & 0.001 & 0.012 & 0.005 & 0.000001 & * \\
\hline 326 & & LEAD & $7439-92-1$ & $\bar{s}$ & 0.002 & 0.024 & 0.005 & 0.000001 & $F$ \\
\hline 326 & & MERCURY & $7439-97-6$ & $\bar{L}$ & 0.0011 & 0.0132 & 0.01 & 0.001 & * \\
\hline 326 & & NICKEL & $7440-02-0$ & $S$ & 0.0005 & 0.006 & 0.02 & 0.000001 & * \\
\hline 326 & & NICKEL & $7440-02-0$ & $s$ & 0.00417 & 0.05004 & 0.02 & 0.000001 & * \\
\hline 329 & & ACETONITRILE & $75-05-8$ & $\bar{L}$ & 0.00346 & 0.04152 & 0.5 & 0.001 & * \\
\hline 329 & & ACETONITRILE & $75-05-8$ & $\bar{L}$ & 0.00655 & 0.0786 & 0.5 & 0.001 & $*$ \\
\hline 329 & & ACETONITRILE & $75-05-8$ & $\bar{L}$ & 0.00659 & 0.07908 & 0.5 & 0.001 & 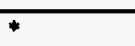 \\
\hline 329 & & ACETONITRILE & $75-05-8$ & $\bar{L}$ & 0.03752 & 0.45024 & 0.5 & 0.001 & 4 \\
\hline 329 & & ARSENIC & $7440-38-2$ & $S$ & 0.00051 & 0.00612 & 0.002 & 0.000001 & * \\
\hline 329 & & ARSENIC & $7440-38-2$ & S & 0.00055 & 0.0066 & 0.002 & 0.000001 & * \\
\hline 329 & 121 & ASBESTOS & $1332-21-4$ & $\mathrm{~s}$ & 0.00013 & 0.00156 & 0.00004 & 0.000001 & * \\
\hline 329 & & CHLOROFORM & $67-66-3$ & $\bar{I}$ & 0.04353 & 0.52236 & 0.45 & 0.001 & * \\
\hline 329 & & HEXACHLOROBENZENE & $118-74-1$ & s & 0.00055 & 0.0066 & 0.005 & 0.000001 & * \\
\hline 329 & & HYDROGEN CHLORIDE & $7647-0110$ & $\bar{L}$ & 0.003 & 0.036 & 0.5 & 0.001 & + \\
\hline 329 & & HYDROGEN CHLORWE & $7647-01-0$ & $\bar{L}$ & 0.0035 & 0.042 & 0.5 & 0.001 & $*$ \\
\hline 329 & & HYDROGEN CHLORIDE & $7647-01-0$ & $\bar{L}$ & 0.006 & 0.072 & 0.5 & 0.001 & * \\
\hline 329 & & HYDROGEN CHLORIDE & $7647-01-0$ & $\bar{L}$ & 0.0078 & 0.0936 & 0.5 & 0.001 & * \\
\hline 329 & & HYDROGEN CHLORIDE & $7647-01-0$ & $\bar{L}$ & 0.008 & 0.096 & 0.5 & 0.001 & $*$ \\
\hline 329 & & HYDROGEN CHLORIDE & $7647-01-0$ & $\overline{\mathrm{L}}$ & 0.00964 & 0.11568 & 0.5 & 0.001 & * \\
\hline 329 & & HYDROGEN CHLORIDE & $7647-01-0$ & L & 0.06425 & 0.771 & 0.5 & 0.001 & * \\
\hline 329 & 121 & HYDROGEN CHLORIDE & $7647-01-0$ & $\bar{I}$ & 0.0077 & 0.0924 & 0.5 & 0.001 & * \\
\hline 329 & & LEAD & $7439-92-1$ & $\mathbf{s}$ & 0.00055 & 0.0066 & 0.005 & 0.000001 & * \\
\hline 329 & & LEAD & $7439-92-1$ & $\mathbf{S}$ & 0.0011 & 0.0132 & 0.005 & 0.000001 & * \\
\hline 329 & & LEAD & $7439-92-1$ & $S$ & 0.00512 & 0.06144 & 0.005 & 0.000001 & * \\
\hline 329 & & LEAD & $7439-92-1$ & $\mathbf{s}$ & 0.035 & 0.42 & 0.005 & 0.000001 & + \\
\hline 329 & & MERCURY & $7439-97-6$ & $\bar{L}$ & 0.00746 & 0.08952 & 0.01 & 0.001 & $*$ \\
\hline 329 & & METHYLENE CHLORDE & $75-09-2$ & L & 0.00552 & 0.06624 & 0.5 & 0.001 & $*$ \\
\hline 329 & & METHYLENE CHLORIDE & $75-09-2$ & $\mathrm{~L}$ & 0.02303 & 0.27636 & 0.5 & 0.001 & * \\
\hline 329 & & METHYLENE CHLORIDE & $75-09-2$ & L & 0.02335 & 0.2802 & 0.5 & 0.001 & * \\
\hline 329 & & NICKEL & $7440-02-0$ & 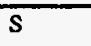 & 0.00002 & 0.00024 & 0.02 & 0.000001 & * \\
\hline 329 & & NICKEL & $7440-02-0$ & $\bar{s}$ & 0.00057 & 0.00684 & 0.02 & 0.000001 & * \\
\hline 329 & & NICKEL & $7440-02-0$ & $S$ & 0.003 & 0.036 & 0.02 & 0.000001 & * \\
\hline
\end{tabular}


Table K-1c. PNL Building Inventories by Room Compared to Threshold Values.

\begin{tabular}{|c|c|c|c|c|c|c|c|c|c|}
\hline Bldg. & Room & Pollutants & $\begin{array}{c}\text { CAS } \\
\text { Number }\end{array}$ & Form & $\begin{array}{l}\text { Inventory } \\
\text { (tons/yr) }\end{array}$ & $\begin{array}{c}12 X \\
\text { Inventory } \\
\text { (tons/yr) }\end{array}$ & $\begin{array}{l}\text { Threshold } \\
\text { Values } \\
\text { (tons/yr) }\end{array}$ & $\begin{array}{l}\text { Release } \\
\text { Fraction }\end{array}$ & $\begin{array}{c}12 \mathrm{X} \\
\text { Inventory } \\
\text { Exceeds } \\
\text { Threshold } \\
\text { Value }\end{array}$ \\
\hline 329 & & SULFURIC ACID & $7664-93-9$ & $\mathbf{L}$ & 0.00101 & 0.01212 & 0.35 & 0.001 & * \\
\hline 329 & & SULFURIC ACID & $7664-93-9$ & L & 0.0045 & 0.054 & 0.35 & 0.001 & $*$ \\
\hline 329 & & SULFURIC ACID & $7664-93-9$ & $\mathbf{L}$ & 0.00891 & 0.10692 & 0.35 & 0.001 & $*$ \\
\hline 329 & & SULFURIC ACID & $7664-93-9$ & L & 0.009 & 0.108 & 0.35 & 0.001 & $*$ \\
\hline 329 & & SULFURIC ACID & $7664-93-9$ & $\bar{L}$ & 0.0135 & 0.162 & 0.35 & 0.001 & * \\
\hline 329 & & SULFURIC ACID & $7664-93-9$ & $\mathbf{L}$ & 0.05936 & 0.71232 & 0.35 & 0.001 & $*$ \\
\hline 331 & & ACETONITRLE & $75-05-8$ & $\mathbf{L}$ & 0.00346 & 0.04152 & 0.5 & 0.001 & $*$ \\
\hline 331 & & ACETONITRILE & $75-05-8$ & L & 0.00865 & 0.1038 & 0.5 & 0.001 & * \\
\hline 331 & & ACETONITRILE & $75-05-8$ & $\mathbf{L}$ & 0.03115 & 0.3738 & 0.5 & 0.001 & $*$ \\
\hline 331 & 102 & ACETONITRILE & $75-05-8$ & $\bar{L}$ & 0.02077 & 0.24924 & 0.5 & 0.001 & $*$ \\
\hline 331 & 116 & ACETONITRILE & $75-05-8$ & $\mathbf{L}$ & 0.00346 & 0.04152 & 0.5 & 0.001 & $*$ \\
\hline 331 & 164 & ACETONITRILE & $75-05-8$ & $\mathbf{L}$ & 0.01384 & 0.16608 & 0.5 & 0.001 & * \\
\hline $3 ! 1$ & 304 & ACETONITRIIE & $75-05-8$ & $\mathrm{~L}$ & 0.03469 & 0.41628 & 0.5 & 0.001 & * \\
\hline 3.3 & 305 & ACETONITRILE & $75-05-8$ & $\mathrm{~L}$ & 0.00692 & 0.08304 & 0.5 & 0.001 & $*$ \\
\hline 81 & 308 & ACETONITRILE & $75-05-8$ & $\mathrm{~L}$ & 0.13481 & 1.61772 & 0.5 & 0.001 & $*$ \\
\hline$\therefore 1$ & 312 & ACETONITRILE & $75-05-8$ & $\mathrm{~L}$ & 0.00013 & 0.00156 & 0.5 & 0.001 & $*$ \\
\hline .11 & 350 & ACETONTRRILE & $75-05-8$ & $L$ & 0.00029 & 0.00348 & 0.5 & 0.001 & $*$ \\
\hline .1 & 354 & ACETONITRILE & $75-05-8$ & $\mathrm{~L}$ & 0.00263 & 0.03156 & 0.5 & 0.001 & $*$ \\
\hline .11 & 6666 & ACETONITRILE & $75-05-8$ & $I$ & 0.01242 & 0.14904 & 0.5 & 0.001 & $*$ \\
\hline .1 & 304 & ACRYLAMIDE & $79-06-1$ & $\mathbf{s}$ & 0.00402 & 0.04824 & 0.01 & 0.000001 & $*$ \\
\hline$\because 1$ & 305 & ACRYLAMIDE & $79-06-1$ & $s$ & 0.00165 & 0.0198 & 0.01 & 0.000001 & * \\
\hline$\because 1$ & 316 & ACRYLAMIDE & $79-06-1$ & $s$ & 0.00055 & 0.0066 & 0.01 & 0.000001 & $*$ \\
\hline .11 & 354 & ACRYLAMIDE & $79-06-1$ & $s$ & 0.00165 & 0.0198 & 0.01 & 0.000001 & $*$ \\
\hline .11 & 357 & ACRYLAMIDE & $79-06-1$ & $s$ & 0.0011 & 0.0132 & 0.01 & 0.000001 & + \\
\hline 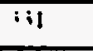 & & CHLOROFORM & $67-66-3$ & $\mathrm{~L}$ & 0.00078 & 0.00936 & 0.45 & 0.001 & * \\
\hline .31 & 116 & CHLOROFORM & $67-66-3$ & $\mathrm{~L}$ & 0.00164 & 0.01968 & 0.45 & 0.001 & $*$ \\
\hline 331 & 175 & CHLOROFORM & $67-66-3$ & $\mathrm{~L}$ & 0.00078 & 0.00936 & 0.45 & 0.001 & + \\
\hline 831 & 304 & CHLOROFORM & 67-66-3 & $\mathrm{L}$ & 0.00986 & 0.11832 & 0.45 & 0.001 & + \\
\hline 331 & 308 & CHLOROFORM & $67-66-3$ & L & 0.01392 & 0.16704 & 0.45 & 0.001 & $*$ \\
\hline 331 & 309 & CHLOROFORM & $67-66-3$ & $I$ & 0.00074 & 0.00888 & 0.45 & 0.001 & * \\
\hline 331 & 312 & CHLOROFORM & $67-66-3$ & L & 0.00164 & 0.01968 & 0.45 & 0.001 & $*$ \\
\hline 331 & 350 & CHLOROFORM & $67-66-3$ & L & 0.00041 & 0.00492 & 0.45 & 0.001 & 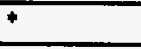 \\
\hline 331 & 354 & CHLOROFORM & $67-66-3$ & $\mathrm{~L}$ & 0.00657 & 0.07884 & 0.45 & 0.001 & * \\
\hline 331 & 359 & CHLOROFORM & $67-66-3$ & $\mathrm{~L}$ & 0.01865 & 0.2238 & 0.45 & 0.001 & * \\
\hline 331 & 365 & CHLOROFORM & $67-66-3$ & $L$ & 0.00082 & 0.00984 & 0.45 & 0.001 & $*$ \\
\hline 331 & 6666 & CHLOROFORM & $67-66-3$ & $\mathrm{~L}$ & 0.00311 & 0.03732 & 0.45 & 0.001 & $*$ \\
\hline 331 & & HYDROGEN CHLORIDE & $7647-01-0$ & L & 0.00599 & 0.07188 & 0.5 & 0.001 & * \\
\hline 331 & 102 & HYDROGEN CHLORIDE & $7647-01-0$ & $\mathrm{~L}$ & 0.003 & 0.036 & 0.5 & 0.001 & * \\
\hline 331 & 146 & HYDROGEN CHLORIDE & $7647-01-0$ & $\mathbf{L}$ & 0.00065 & 0.0078 & 0.5 & 0.001 & $*$ \\
\hline 331 & 158 & HYDROGEN CHLORIDE & $7647-01-0$ & $\mathbf{L}$ & 0.0026 & 0.0312 & 0.5 & 0.001 & $*$ \\
\hline 331 & 164 & HYDROGEN CHLORIDE & $7647-01-0$ & $\mathbf{L}$ & 0.0013 & 0.0156 & 0.5 & 0.001 & $*$ \\
\hline 331 & 175 & HYDROGEN CHLORIDE & $7647-01-0$ & $\mathbf{L}$ & 0.003 & 0.036 & 0.5 & 0.001 & * \\
\hline 331 & 304 & HYDROGEN CHLORIDE & $7647-01-0$ & $\mathbf{L}$ & 0.0026 & 0.0312 & 0.5 & 0.001 & * \\
\hline 331 & 308 & HYDROGEN CHLORIDE & $7647-01-0$ & $\mathbf{L}$ & 0.0039 & 0.0468 & 0.5 & 0.001 & 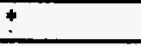 \\
\hline 331 & 312 & HYDROGEN CHLORIDE & $7647-01-0$ & $\mathbf{L}$ & 0.003 & 0.036 & 0.5 & 0.001 & $*$ \\
\hline 331 & 357 & HYDROGEN CHLORIDE & $7647-01-0$ & $\mathbf{L}$ & 0.0011 & 0.0132 & 0.5 & Q.001 & * \\
\hline 331 & 359 & HYDROGEN CHLORIDE & $7647-01-0$ & $\mathbf{L}$ & 0.00039 & 0.00468 & 0.5 & 0.001 & * \\
\hline 331 & 365 & HYDROGEN CHLORIDE & $7647-01-0$ & $\mathbf{L}$ & 0.00195 & 0.0234 & 0.5 & 0.001 & $*$ \\
\hline
\end{tabular}


Table K-Ic. PNL Building Inventories by Room Compared to Threshold Values.

\begin{tabular}{|c|c|c|c|c|c|c|c|c|c|}
\hline Bidg. & Room & Pollutants & $\begin{array}{c}\text { CAS } \\
\text { Number }\end{array}$ & Form & $\begin{array}{l}\text { Inventory } \\
\text { (tons/yr) }\end{array}$ & $\begin{array}{c}12 \mathrm{X} \\
\text { Inventory } \\
\text { (tons/yr) }\end{array}$ & $\begin{array}{l}\text { Threshold } \\
\text { Values } \\
\text { (tons/yr) }\end{array}$ & $\begin{array}{l}\text { Release } \\
\text { Fraction }\end{array}$ & $\begin{array}{c}12 \mathrm{X} \\
\text { Inventory } \\
\text { Exceeds } \\
\text { Threshold } \\
\text { Value }\end{array}$ \\
\hline 331 & 6666 & HYDROGEN CHLORIDE & $7647-01-0$ & $\mathrm{~L}$ & 0.0172 & 0.2064 & 0.5 & 0.001 & 4 \\
\hline 331 & & METHYL ALCOHOL & $67-56-1$ & $\mathrm{~L}$ & 0.00349 & 0.04188 & 0.5 & 0.001 & * \\
\hline 331 & & METHYL ALCOHOL & $67-56-1$ & L & 0.01321 & 0.15852 & 0.5 & 0.001 & * \\
\hline 331 & & METHYL ALCOHOL & $67-56-1$ & $\bar{I}$ & 0.01822 & 0.21864 & 0.5 & 0.001 & * \\
\hline 331 & 102 & METHYL ALCOHOL & $67-56-1$ & L & 0.0109 & 0.1308 & 0.5 & 0.001 & * \\
\hline 331 & 103 & METHYL ALCOHOL & $67-56-1$ & $\overline{\mathrm{L}}$ & 0.00349 & 0.04188 & 0.5 & 0.001 & * \\
\hline 331 & 112 & METHYL ALCOHOL & $67-56-1$ & $\bar{L}$ & 0.00523 & 0.06276 & 0.5 & 0.001 & + \\
\hline 331 & 116 & METHYL ALCOHOL & $67-56-1$ & $\mathrm{~L}$ & 0.01396 & 0.16752 & 0.5 & 0.001 & $*$ \\
\hline 331 & 164 & METHYL ALCOHOL & $67-56-1$ & $\bar{L}$ & 0.01396 & 0.16752 & 0.5 & 0.001 & * \\
\hline 331 & 304 & METHYL ALCOHOL & $67-56-1$ & $\mathrm{~L}$ & 0.01483 & 0.17796 & 0.5 & 0.001 & $*$ \\
\hline 331 & 305 & METHYL ALCOHOL & $67-56-1$ & $\bar{I}$ & 0.00044 & 0.00528 & 0.5 & 0.001 & $*$ \\
\hline 331 & 308 & METHYL ALCOHOL & $67-56-1$ & L & 0.02442 & 0.29304 & 0.5 & 0.001 & $*$ \\
\hline 331 & 309 & METHYL ALCOHOL & $67-56-1$ & I & 0.00523 & 0.06276 & 0.5 & 0.001 & $*$ \\
\hline 331 & 312 & METHYL ALCOHOL & $67-56-1$ & $\bar{L}$ & 0.00044 & 0.00528 & 0.5 & 0.001 & * \\
\hline 331 & 317 & METHYL ALCOHOL & $67-56-1$ & $\bar{L}$ & 0.02093 & 0.25116 & 0.5 & 0.001 & $*$ \\
\hline 331 & 357 & METHYL ALCOHOL & $67-56-1$ & $\bar{L}$ & 0.0075 & 0.09 & 0.5 & 0.001 & * \\
\hline 331 & 359 & METHYL ALCOHOL & $67-56-1$ & $\mathrm{~L}$ & 0.00041 & 0.00492 & 0.5 & 0.001 & * \\
\hline 331 & 365 & METHYL ALCOHOL & $67-56-1$ & $\mathrm{~L}$ & 0.00262 & 0.03144 & 0.5 & 0.001 & 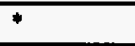 \\
\hline 331 & 6666 & METHYL ALCOHOL & $67-56-1$ & $\mathrm{~L}$ & 0.01614 & 0.19368 & 0.5 & 0.001 & + \\
\hline 331 & & $\begin{array}{l}\text { METHYL ISOBUTYL } \\
\text { KETONE }\end{array}$ & $108-10-1$ & $\mathrm{I}$ & 0.00706 & 0.08472 & 0.1 & 0.001 & + \\
\hline 331 & 6666 & $\begin{array}{l}\text { METHYL ISOBUTYL } \\
\text { KETONE }\end{array}$ & $108-10-1$ & $\mathrm{~L}$ & 0.00265 & 0.0318 & 0.1 & 0.001 & * \\
\hline 331 & & METHYLENE CHLORDE & $75-09-2$ & L & 0.00146 & 0.01752 & 0.5 & 0.001 & * \\
\hline 331 & & METHYLENE CHLORIDE & $75-09-2$ & $\overline{\mathrm{L}}$ & 0.00584 & 0.07008 & 0.5 & 0.001 & + \\
\hline 331 & 304 & METHYLENE CHLORIDE & $75-09-2$ & $\mathrm{~L}$ & 0.02043 & 0.24516 & 0.5 & 0.001 & 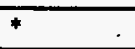 \\
\hline 331 & 308 & METHYLENE CHLORIDE & $75-09-2$ & $\mathrm{~L}$ & 0.02335 & 0.2802 & 0.5 & 0.001 & * \\
\hline 331 & 357 & METHYLENE CHLORDE & $75-09-2$ & $\mathrm{~L}$ & 0.00584 & 0.07008 & 0.5 & 0.001 & $*$ \\
\hline 331 & & PHENOL & $108-95-2$ & $\bar{s}$ & 0.00055 & 0.0066 & 0.1 & 0.000001 & + \\
\hline 331 & 5 & PHENOL & $108-95-2$ & $s$ & 0.0022 & 0.0264 & 0.1 & 0.000001 & + \\
\hline 331 & 305 & PHENOL & $108-95-2$ & $s$ & 0.00143 & 0.01716 & 0.1 & 0.000001 & $*$ \\
\hline 331 & 308 & PHENOL & 108-95-2 & $s$ & 0.00176 & 0.02112 & 0.1 & 0.000001 & * \\
\hline 331 & 312 & PHENOL & $108-95-2$ & $\mathbf{s}$ & 0.0022 & 0.0264 & 0.1 & 0.000001 & * \\
\hline 331 & 316 & PHENOL & $108-95-2$ & $s$ & 0.00011 & 0.00132 & 0.1 & 0.000001 & 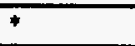 \\
\hline 331 & 350 & PHENOL & $108-95-2$ & $s$ & 0.00028 & 0.00336 & 0.1 & 0.000001 & * \\
\hline 331 & 354 & PHENOL & $108-95-2$ & $s$ & 0.00022 & 0.00264 & 0.1 & 0.000001 & * \\
\hline 331 & 357 & PHENOL & $108-95-2$ & $s$ & 0.0005 & 0.006 & 0.1 & 0.000001 & * \\
\hline 331 & & SULFURIC ACID & $7664-93-9$ & $\bar{L}$ & 0.0225 & 0.27 & 0.35 & 0.001 & * \\
\hline 331 & 102 & SULFURIC ACID & $7664-93-9$ & L & 0.01261 & 0.15132 & 0.35 & 0.001 & * \\
\hline 331 & 116 & SULFURIC ACID & $7664-93-9$ & L & 0.00405 & 0.0486 & 0.35 & 0.001 & * \\
\hline 331 & 158 & SULFURIC ACD & $7664-93-9$ & $\mathbf{L}$ & 0.00904 & 0.10848 & 0.35 & 0.001 & " \\
\hline 331 & 164 & SULFURIC ACID & $7664-93-9$ & $\mathrm{~L}$ & 0.0045 & 0.054 & 0.35 & 0.001 & * \\
\hline 331 & 304 & SULFURIC ACID & $7664-93.9$ & $\mathrm{~L}$ & 0.02432 & 0.29184 & 0.35 & 0.001 & * \\
\hline 331 & 308 & SULFURIC ACID & 7664-93-9 & $\mathbf{L}$ & 0.00619 & 0.07428 & 0.35 & 0.001 & * \\
\hline 331 & 357 & SULFURIC ACID & $7664-93-9$ & L & 0.00041 & 0.00492 & 0.35 & 0.001 & * \\
\hline 331 & 365 & SULFURIC ACID & $7664-93-9$ & $\mathrm{~L}$ & 0.00608 & 0.07296 & 0.35 & 0.001 & * \\
\hline 331 & 6666 & SULFURIC ACID & $7664-93-9$ & L & 0.02026 & 0.24312 & 0.35 & 0.001 & 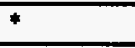 \\
\hline 331 & & XYLENE & $1330-20-7$ & L & 0.00062 & 0.00744 & 0.5 & 0.001 & * \\
\hline 331 & 9 & XYLENE & $1330-20-7$ & $L$ & 0.00062 & 0.00744 & 0.5 & 0.001 & * \\
\hline
\end{tabular}


Table K-1c. PNL Building Inventories by Room Compared to Threshold Values.

\begin{tabular}{|c|c|c|c|c|c|c|c|c|c|}
\hline Bldg. & Room & Pollutants & $\begin{array}{c}\text { CAS } \\
\text { Number }\end{array}$ & Form & $\begin{array}{l}\text { Inventory } \\
\text { (tons/yr) }\end{array}$ & $\begin{array}{c}12 \mathrm{X} \\
\text { Inventory } \\
\text { (tons/yr) }\end{array}$ & $\begin{array}{l}\text { Threshold } \\
\text { Values } \\
\text { (tons/yr) }\end{array}$ & $\begin{array}{l}\text { Release } \\
\text { Fraction }\end{array}$ & $\begin{array}{c}12 \mathrm{X} \\
\text { Inventory } \\
\text { Exceeds } \\
\text { Threshold } \\
\text { Value }\end{array}$ \\
\hline 331 & 114 & XYLENE & $1330-20-7$ & L & 0.00499 & 0.05988 & 0.5 & 0.001 & *. \\
\hline 331 & 151 & XYLENE & $1330-20-7$ & $\mathrm{~L}$ & 0.00187 & 0.02244 & 0.5 & 0.001 & * \\
\hline 331 & 302 & XYLENE & $1330-20-7$ & $\mathbf{L}$ & 0.00062 & 0.00744 & 0.5 & 0.001 & * \\
\hline 331 & 309 & XYLENE & $1330-20-7$ & 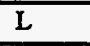 & 0.00066 & 0.00792 & 0.5 & 0.001 & + \\
\hline 331 & 356 & XYLENE & $1330-20-7$ & $L$ & 0.03996 & 0.47952 & 0.5 & 0.001 & $*$ \\
\hline $331 \mathrm{H}$ & 100 & METHYL ALCOHOL & $67-56-1$ & $\mathrm{~L}$ & 0.03214 & 0.38568 & 0.5 & 0.001 & $*$ \\
\hline $331 \mathrm{H}$ & 103 & METHYL ALCOHOL & $67-56-1$ & $\mathbf{L}$ & 0.13206 & 1.58472 & 0.5 & 0.001 & 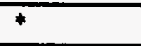 \\
\hline 3708 & 111 & CADMIUM & $7440-43-9$ & $s$ & 0.00014 & 0.00168 & 0.005 & 0.000001 & * \\
\hline 3708 & 116 & CADMIUM & $7440-43-9$ & $\mathrm{~s}$ & 0.0005 & 0.006 & 0.005 & 0.000001 & $*$ \\
\hline 3708 & 111 & LEAD & $7439-92-1$ & $\mathbf{s}$ & 0.00014 & 0.00168 & 0.005 & 0.000001 & $*$ \\
\hline 3708 & 116 & LEAD & $7439-92-1$ & $s$ & 0.00033 & 0.00396 & 0.005 & 0.000001 & $*$ \\
\hline 3708 & 111 & MERCURY & $7439-97-6$ & L & 0.00194 & 0.02328 & 0.01 & 0.001 & * \\
\hline $3718 \mathrm{G}$ & 1 & ETHYLENE GLYCOL & $107-21-1$ & $\mathrm{~L}$ & 1.03535 & 12.4242 & 0.5 & 0.001 & * \\
\hline $3718 \mathrm{G}$ & 1 & FLUORIDES & & $\bar{L}$ & 0.091 & 1.092 & 0.15 & 0.001 & * \\
\hline 3720 & 512 & $\begin{array}{l}\text { 3,3'-DIMETHYL } \\
\text { BENZIDINE }\end{array}$ & $119-93-7$ & S & 0.00055 & 0.0066 & 0.004 & 0.000001 & * \\
\hline 3720 & 508 & $\begin{array}{l}\text { 4,4'-BIPHENYLENEDIAMI } \\
\text { NE }\end{array}$ & $92-87-5$ & $\mathbf{s}$ & 0.00013 & 0.00156 & 0.00015 & 0.000001 & * \\
\hline 3720 & 210 & ARSENIC & $7440-38-2$ & $s$ & 0.00022 & 0.00264 & 0.002 & 0.000001 & 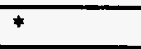 \\
\hline 3720 & 210 & CADMIUM & $7440-43-9$ & $s$ & 0.00011 & 0.00132 & 0.005 & 0.000001 & $*$ \\
\hline 3720 & 233 & CADMIUM & $7440-43-9$ & $s$ & 0.00055 & 0.0066 & 0.005 & 0.000001 & 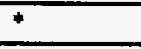 \\
\hline 3720 & 237 & CADMIUM & $7440-43-9$ & $s$ & 0.00011 & 0.00132 & 0.005 & 0.000001 & $*$ \\
\hline 3720 & 508 & CADMIUM & $7440-43-9$ & $s$ & 0.0005 & 0.006 & 0.005 & 0.000001 & * \\
\hline 3720 & 602 & CADMIUM & $7440-43-9$ & $s$ & 0.00028 & 0.00336 & 0.005 & 0.000001 & * \\
\hline 3720 & 2 & FLUORIDES & & I & 0.00483 & 0.05796 & 0.15 & 0.001 & * \\
\hline 3720 & 10 & FLUORIDES & & $\bar{L}$ & 0.0005 & 0.006 & 0.15 & 0.001 & * \\
\hline 3720 & 105 & FLUORIDES & & $\bar{L}$ & 0.00283 & 0.03396 & 0.15 & 0.001 & * \\
\hline 3720 & 206 & FLUORIDES & & $\bar{L}$ & 0.00077 & 0.00924 & 0.15 & 0.001 & * \\
\hline 3720 & 210 & FLUORIDES & & $\bar{L}$ & 0.00151 & 0.01812 & 0.15 & 0.001 & * \\
\hline 3720 & 237 & FLUORIDES & & L & 0.00055 & 0.0066 & 0.15 & 0.001 & * \\
\hline 3720 & 508 & FLUORIDES & & L & 0.01029 & 0.12348 & 0.15 & 0.001 & * \\
\hline 3720 & 512 & FLUORIDES & & $\bar{L}$ & 0.00036 & 0.00432 & 0.15 & 0.001 & * \\
\hline 3720 & 603 & FLUORIDES & & $\bar{L}$ & 0.0005 & 0.006 & 0.15 & 0.001 & * \\
\hline 3720 & 6666 & FLUORIDES & & $\bar{I}$ & 0.02627 & 0.31524 & 0.15 & 0.001 & * \\
\hline 3720 & 2 & HYDROGEN CHLORIDE & $7647-01-0$ & $\mathrm{~L}$ & 0.0026 & 0.0312 & 0.5 & 0.001 & * \\
\hline 3720 & 3 & HYDROGEN CHLORDE & $7647-01-0$ & $\mathrm{~L}$ & 0.0013 & 0.0156 & 0.5 & 0.001 & * \\
\hline 3720 & 7 & HYDROGEN CHLORIDE & $7647-01-0$ & $\mathrm{~L}$ & 0.0033 & 0.0396 & 0.5 & 0.001 & $*$ \\
\hline 3720 & 11 & HYDROGEN CHLORWE & 7647-01-0 & $\mathrm{L}$ & 0.0052 & 0.0624 & 0.5 & 0.001 & $*$ \\
\hline 3720 & 12 & HYDROGEN CHLORIDE & $7647-01-0$ & $I$ & 0.0052 & 0.0624 & 0.5 & 0.001 & + \\
\hline 3720 & 109 & HYDROGEN CHLORIDE & $7647-01-0$ & $\mathrm{~L}$ & 0.00665 & 0.0798 & 0.5 & 0.001 & * \\
\hline 3720 & 201 & HYDROGEN CHLORDE & $7647-01-0$ & L & 0.00588 & 0.07056 & 0.5 & 0.001 & * \\
\hline 3720 & 210 & HYDROGEN CHLORIDE & $7647-01-0$ & 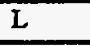 & 0.0052 & 0.0624 & 0.5 & 0.001 & 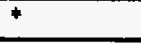 \\
\hline 3720 & 237 & HYDROGEN CHLORIDE & $7647-01-0$ & $\bar{L}$ & 0.0172 & 0.2064 & 0.5 & 0.001 & * \\
\hline 3720 & 322 & HYDROGEN CHLORIDE & $7647-01-0$ & I & 0.0013 & 0.0156 & 0.5 & 0.001 & $*$ \\
\hline 3720 & 7 & LEAD & $7439-92-1$ & $\mathbf{s}$ & 0.00052 & 0.00624 & 0.005 & 0.000001 & $*$ \\
\hline 3720 & 105 & LEAD & $7439-92-1$ & $\mathbf{s}$ & 0.00078 & 0.00936 & 0.005 & 0.000001 & + \\
\hline 3720 & 210 & LEAD & $7439-92-1$ & $\mathbf{s}$ & 0.00017 & 0.00204 & 0.005 & 0.000001 & $*$ \\
\hline 3720 & 233 & LEAD & $7439-92-1$ & $\mathbf{s}$ & 0.00052 & 0.00624 & 0.005 & 0.000001 & $*$ \\
\hline
\end{tabular}


Table K-1c. PNL Building Inventories by Room Compared to Threshold Values.

\begin{tabular}{|c|c|c|c|c|c|c|c|c|c|}
\hline Bldg. & Room & Pollutants & $\begin{array}{c}\text { CAS } \\
\text { Number }\end{array}$ & Form & $\begin{array}{l}\text { Inventory } \\
\text { (tons/yr) }\end{array}$ & $\begin{array}{c}12 \mathrm{X} \\
\text { Inventory } \\
\text { (tons/yr) }\end{array}$ & $\begin{array}{l}\text { Threshold } \\
\text { Values } \\
\text { (tons/yr) }\end{array}$ & $\begin{array}{l}\text { Release } \\
\text { Fraction }\end{array}$ & $\begin{array}{c}12 X \\
\text { Inventory } \\
\text { Exceeds } \\
\text { Threshold } \\
\text { Value }\end{array}$ \\
\hline 3720 & 508 & LEAD & $7439-92-1$ & $s$ & 0.0035 & 0.042 & 0.005 & 0.000001 & * \\
\hline 3720 & 602 & LEAD & $7439-92-1$ & $\mathbf{s}$ & 0.00053 & 0.00636 & 0.005 & 0.000001 & * \\
\hline 3720 & 237 & MERCURIC CHLORIDE & $7487-94-7$ & $\mathbf{s}$ & 0.00011 & 0.00132 & 0.01 & 0.000001 & $*$ \\
\hline 3720 & 508 & MERCURIC CHLORIDE & $7487-94-7$ & $s$ & 0.00101 & 0.01212 & 0.01 & 0.000001 & $*$ \\
\hline 3720 & 210 & MERCURY & $7439-97-6$ & $\mathrm{~L}$ & 0.00597 & 0.07164 & 0.01 & 0.001 & + \\
\hline 3720 & 508 & MERCURY & $7439-97-6$ & I & 0.026 & 0.312 & 0.01 & 0.001 & + \\
\hline 3720 & 302 & METHYLENE CHLORIDE & $75-09-2$ & I & 0.02335 & 0.2802 & 0.5 & 0.001 & * \\
\hline 3720 & 512 & METHYLENE CHLORDE & $75-09-2$ & $\bar{L}$ & 0.02043 & 0.24516 & 0.5 & 0.001 & * \\
\hline 3720 & 602 & METHYLENE CHLORIDE & $75-09-2$ & $\mathrm{~L}$ & 0.00069 & 0.00828 & 0.5 & 0.001 & * \\
\hline 3720 & 603 & METHYLENE CHLORME & $75-09-2$ & $\bar{L}$ & 0.00069 & 0.00828 & 0.5 & 0.001 & * \\
\hline 3720 & 210 & NICKEL & $7440-02-0$ & $\mathrm{~s}$ & 0.00011 & 0.00132 & 0.02 & 0.000001 & $*$ \\
\hline 3720 & 237 & NICKEL & $7440-02-0$ & $\mathbf{s}$ & 0.00011 & 0.00132 & 0.02 & 0.000001 & $*$ \\
\hline 3720 & 508 & NICKEL & $7440-02-0$ & 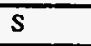 & 0.00165 & 0.0198 & 0.02 & 0.000001 & 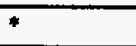 \\
\hline 3720 & 602 & NICKEL & $7440-02-0$ & s & 0.00006 & 0.00072 & 0.02 & 0.000001 & 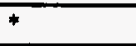 \\
\hline 3720 & 512 & QUINOLINE & $91-22-5$ & s & 0.00104 & 0.01248 & 0.003 & 0.000001 & * \\
\hline 3720 & 2 & SULFURIC ACID & $7664-93-9$ & $\mathrm{~L}$ & 0.02124 & 0.25488 & 0.35 & 0.001 & * \\
\hline 3720 & 7 & SULFURIC ACID & $7664-93-9$ & $\bar{L}$ & 0.01419 & 0.17028 & 0.35 & 0.001 & $*$ \\
\hline 3720 & 109 & SULFURIC ACID & $7664-93-9$ & $\mathbf{L}$ & 0.0075 & 0.09 & 0.35 & 0.001 & $*$ \\
\hline 3720 & 201 & SULFURIC ACID & $7664-93-9$ & $\mathbf{L}$ & 0.00111 & 0.01332 & 0.35 & 0.001 & $*$ \\
\hline 3720 & 206 & SULFURIC ACID & $7664-93-9$ & $\mathbf{L}$ & 0.0015 & 0.018 & 0.35 & 0.001 & $*$ \\
\hline 3720 & 237 & SULFURIC ACID & $7664-93-9$ & $\mathbf{L}$ & 0.009 & 0.108 & 0.35 & 0.001 & $*$ \\
\hline 3720 & 501 & SULFURIC ACID & $7664-93-9$ & L & 0.00405 & 0.0486 & 0.35 & 0.001 & + \\
\hline 3720 & 603 & SULFURIC ACID & $7664-93-9$ & $\mathbf{L}$ & 0.0045 & 0.054 & 0.35 & 0.001 & * \\
\hline 3720 & 604 & SULFURIC ACD & $7664-93-9$ & L & 0.01013 & 0.12156 & 0.35 & 0.001 & $*$ \\
\hline $3746 \mathrm{~A}$ & & CADMIUM & $7440-43-9$ & $\mathbf{s}$ & 0.0005 & 0.006 & 0.005 & 0.000001 & * \\
\hline $3746 \mathrm{~A}$ & & METHYL ALCOHOL & $67-56-1$ & L. & 0.01875 & 0.225 & 0.5 & 0.001 & * \\
\hline $3746 \mathrm{~A}$ & & METHYL ALCOHOL & $67-56-1$ & $\mathrm{~L}$ & 0.18158 & 2.17896 & 0.5 & 0.001 & * \\
\hline $3746 \mathrm{~A}$ & 13 & METHYL ALCOHOL & $67-56-1$ & L & 0.01396 & 0.16752 & 0.5 & 0.001 & * \\
\hline $3746 \mathrm{~A}$ & 16 & METHYL ALCOHOL & $67-56-1$ & $\mathrm{~L}$ & 0.00349 & 0.04188 & 0.5 & 0.001 & + \\
\hline $3746 \mathrm{~A}$ & 16 & XYLENE & $1330-20-7$ & L & 0.05279 & 0.63348 & 0.5 & 0.001 & + \\
\hline
\end{tabular}




\section{Table K.2. Insignificant Emission Units Based on Threshold Comparisons [WAC 173-401-530(1)(a)]}

\begin{tabular}{|c|c|c|c|c|}
\hline Emission Point & Pollutants & CAS Number & $\begin{array}{l}\text { Emission } \\
\text { Threshold } \\
\text { (tons/yr) }\end{array}$ & $\begin{array}{c}\text { Actual } \\
\text { Emissions } \\
\text { (tons/yr) }\end{array}$ \\
\hline 100K N-1706KEL 001 & Hexane, n-hexane & $110-54-3$ & $5.00 \mathrm{E}-01$ & $7.51 \mathrm{E}-03$ \\
\hline $100 \mathrm{~K} \mathrm{~N}-1717 \mathrm{~K} 001$ & 1,1,1-Trichloroethylene & $71-55-6$ & $5.0 \mathrm{E}-01$ & $3.2 \mathrm{E}-01$ \\
\hline $100 \mathrm{~K} \mathrm{~N}-1717 \mathrm{~K} 001$ & 1,2-Butylene oxide & $106-88-7$ & $5.0 \mathrm{E}-01$ & $1.7 \mathrm{E}-02$ \\
\hline $100 \mathrm{~K} \mathrm{~N}-1717 \mathrm{~K} 001$ & Butyl alcohol, tert & $75-65-0$ & $5.0 \mathrm{E}-01$ & $1.7 \mathrm{E}-02$ \\
\hline $100 \mathrm{~K} \mathrm{~N}-1717 \mathrm{~K} 001$ & Dimethoxymethane & $109-87-5$ & $1.7 \mathrm{E}-02$ & \\
\hline $100 \mathrm{~K} \mathrm{~N}-1717 \mathrm{~K} 001$ & Isopropyl alcohol & $67-63-0$ & $5.0 \mathrm{E}-01$ & $1.7 \mathrm{E}-02$ \\
\hline $100 \mathrm{~K} \mathrm{~N}-1717 \mathrm{~K} 001$ & Methyl alcohol & $67-56-1$ & $5.0 \mathrm{E}-01$ & $2.7 \mathrm{E}-04$ \\
\hline $100 \mathrm{~K} \mathrm{~N}-1717 \mathrm{~K} 001$ & Methylene chloride & $75-9-2$ & $5.0 \mathrm{E}-01$ & $1.2 \mathrm{E}-02$ \\
\hline $100 \mathrm{~N}$ M-105N 001 & $\mathrm{PM}<10$ & & $7.5 \mathrm{E}-01$ & $1.0 \mathrm{E}-02$ \\
\hline $100 \mathrm{~N} \mathrm{~N}-1516 \mathrm{~N} 017$ & $\mathrm{PM}<10$ & & $7.5 \mathrm{E}-01$ & $5.2 \mathrm{E}-01$ \\
\hline 200E P-241A101 001 & 1,3-Butadiene & $106-99-0$ & $3.50 \mathrm{E}-02$ & $1.91 \mathrm{E}-06$ \\
\hline 200E P-241A101 001 & 2-Hexanone & $591-78-6$ & $5.00 \mathrm{E}-01$ & 3.47E-05 \\
\hline 200E P-241A101 001 & 2-Pentanone & $107-87-9$ & & $5.51 \mathrm{E}-05$ \\
\hline 200E P-241A101 001 & Acetone & $67-64-1$ & $5.00 \mathrm{E}-01$ & $6.62 \mathrm{E}-04$ \\
\hline 200E P-241A101 001 & Acetonitrile & $75-5-8$ & $5.00 \mathrm{E}-01$ & $3.19 \mathrm{E}-04$ \\
\hline 200E P-241A101 001 & Ammonia & $7664-41-7$ & $5.00 \mathrm{E}-01$ & $3.04 \mathrm{E}-03$ \\
\hline 200E P-241A101 001 & Benzene & $71-43-2$ & $5.00 \mathrm{E}-01$ & $1.51 \mathrm{E}-05$ \\
\hline 200E P-241A101 001 & Carbon monoxide & $630-8-0$ & $5.00 \mathrm{E}+00$ & $4.39 \mathrm{E}-04$ \\
\hline 200 E P-241A101 001 & Heptane & $142-82-5$ & $5.00 \mathrm{E}-01$ & $3.89 \mathrm{E}-05$ \\
\hline 200E P-241A101 001 & Hexane, $\mathrm{n}$-hexane & $110-54-3$ & $5.00 \mathrm{E}-01$ & $4.05 \mathrm{E}-05$ \\
\hline 200E P-241A101 001 & $\begin{array}{l}\text { Methyl N-amyl ketone, 2- } \\
\text { heptanone }\end{array}$ & $110-43-0$ & $5.00 \mathrm{E}-01$ & $3.76 \mathrm{E}-05$ \\
\hline 200E P-241A101 001 & Nitrogen Oxide $\{\mathrm{NO}\}$ & $10102-43-9$ & $2.00 \mathrm{E}+00$ & $2.64 \mathrm{E}-05$ \\
\hline 200E P-241A101 001 & Nonane & $111-84-2$ & $5.00 \mathrm{E}-01$ & $2.11 E-05$ \\
\hline 200E P-241A101 001 & Octane & $111-65-9$ & $5.00 \mathrm{E}-01$ & $2.21 E-05$ \\
\hline 200E P-241A101 001 & $\begin{array}{l}\text { Phosphoric acid, tributyl } \\
\text { ester }\end{array}$ & $126-73-8$ & & $7.95 \mathrm{E}-05$ \\
\hline
\end{tabular}


Table K.2. Insignificant Emission Units Based on Threshold Comparisons [WAC 173-401-530(1)(a)]

\begin{tabular}{|c|c|c|c|c|}
\hline Emission Point & Pollutants & CAS Number & $\begin{array}{l}\text { Emission } \\
\text { Threshold } \\
\text { (tons/yr) }\end{array}$ & $\begin{array}{c}\text { Actual } \\
\text { Emissions } \\
\text { (tons/yr) }\end{array}$ \\
\hline 200E P-241A101 001 & Toluene & $108-88-3$ & $5.00 \mathrm{E}-01$ & $3.08 \mathrm{E}-06$ \\
\hline $200 \mathrm{E}$ P-241A102 001 & 1,3-Butadiene & $106-99-0$ & $3.50 \mathrm{E}-02$ & $4.13 \mathrm{E}-06$ \\
\hline 200E P-241A102 001 & 2-Hexanone & $591-78-6$ & $5.00 \mathrm{E}-01$ & $7.51 \mathrm{E}-05$ \\
\hline 200E P-241A102001 & 2-Pentanone & $107-87-9$ & & $1.19 \mathrm{E}-04$ \\
\hline 200E P-241A102 001 & Acetone & $67-64-1$ & $5.00 \mathrm{E}-01$ & $1.43 \mathrm{E}-03$ \\
\hline 200E P-241A102001 & Acetonitrile & $75-5-8$ & $5.00 \mathrm{E}-01$ & $6.89 \mathrm{E}-04$ \\
\hline 200E P-241A102 001 & Ammonia & $7664-41-7$ & $5.00 \mathrm{E}-01$ & $6.57 \mathrm{E}-03$ \\
\hline 200E P-241A102001 & Benzene & $71-43-2$ & $5.00 \mathrm{E}-01$ & $3.28 \mathrm{E}-05$ \\
\hline 200E P-241A102 001 & Carbon monoxide & $630-8-0$ & $5.00 \mathrm{E}+00$ & $9.50 \mathrm{E}-04$ \\
\hline 200E P-241A102 001 & Heptane & $142-82-5$ & $5.00 \mathrm{E}-01$ & $8.40 \mathrm{E}-05$ \\
\hline 200E P-241A102001 & Hexane, n-hexane & $110-54-3$ & $5.00 \mathrm{E}-01$ & $8.76 \mathrm{E}-05$ \\
\hline $200 \mathrm{E}$ P-241A102 001 & $\begin{array}{l}\text { Methyl N-amyl ketone, 2- } \\
\text { heptanone }\end{array}$ & $110-43-0$ & $5.00 \mathrm{E}-01$ & $8.13 \mathrm{E}-05$ \\
\hline 200E P-241A102001 & Nitrogen Oxide $\{$ NO $\}$ & $10102-43-9$ & $2.00 \mathrm{E}+00$ & $5.72 E-05$ \\
\hline 200E P-241A102 001 & Nonane & $111-84-2$ & $5.00 \mathrm{E}-01$ & $4.57 \mathrm{E}-05$ \\
\hline 200E P-241A102 001 & Octane & $111-65-9$ & $5.00 \mathrm{E}-01$ & $4.79 \mathrm{E}-05$ \\
\hline $200 \mathrm{E}$ P-241A102 001 & $\begin{array}{l}\text { Phosphoric acid, tributyl } \\
\text { ester }\end{array}$ & $126-73-8$ & & $1.72 \mathrm{E}-04$ \\
\hline 200E P-241A102001 & Toluene & $108-88-3$ & $5.00 \mathrm{E}-01$ & $6.67 \mathrm{E}-06$ \\
\hline 200E P-241A103001 & 1,3-Butadiene & $106-99-0$ & $3.50 \mathrm{E}-02$ & $4.13 E-06$ \\
\hline 200E P-241A103 001 & 2-Hexanone & $591-78-6$ & $5.00 \mathrm{E}-01$ & $7.51 \mathrm{E}-05$ \\
\hline 200E P-241A103001 & 2-Pentanone & $107-87-9$ & & $1.19 \mathrm{E}-04$ \\
\hline 200E P-241A103 001 & Acetone & $67-64-1$ & $5.00 E-01$ & $1.43 \mathrm{E}-03$ \\
\hline 200E P-241A103 001 & Acetonitrile & $75-5-8$ & $5.00 \mathrm{E}-01$ & $6.89 \mathrm{E}-04$ \\
\hline 200E P-241A103 001 & Ammonia & $7664-41-7$ & $5.00 \mathrm{E}-01$ & $6.57 \mathrm{E}-03$ \\
\hline 200E P-241A103 001 & Benzene & $71-43-2$ & $5.00 \mathrm{E}-01$ & $3.28 \mathrm{E}-05$ \\
\hline 200E P-241A103 001 & Carbon monoxide & $630-8-0$ & $5.00 \mathrm{E}+00$ & $9.50 \mathrm{E}-04$ \\
\hline 200E P-241A103 001 & Heptane & $142-82-5$ & $5.00 \mathrm{E}-01$ & $8.40 \mathrm{E}-05$ \\
\hline 200E P-241A103001 & Hexane, n-hexane & $110-54-3$ & $5.00 \mathrm{E}-01$ & $8.76 \mathrm{E}-05$ \\
\hline
\end{tabular}


Table K.2. Insignificant Emission Units Based on Threshold Comparisons [WAC 173-401-530(1)(a)]

\begin{tabular}{|c|c|c|c|c|}
\hline Emission Point & Pollutants & CAS Number & $\begin{array}{l}\text { Emission } \\
\text { Threshold } \\
\text { (tons/yr) }\end{array}$ & $\begin{array}{c}\text { Actual } \\
\text { Emissions } \\
\text { (tons/yr) }\end{array}$ \\
\hline 200E P-241A103001 & $\begin{array}{l}\text { Methyl N-amyl ketone, 2- } \\
\text { heptanone }\end{array}$ & $110-43-0$ & $5.00 \mathrm{E}-01$ & $8.13 E-05$ \\
\hline 200E P-241A103001 & Nitrogen Oxide $\{\mathrm{NO}\}$ & $10102-43-9$ & $2.00 E+00$ & $5.72 \mathrm{E}-05$ \\
\hline 200E P-241A103 001 & Nonane & $111-84-2$ & $5.00 \mathrm{E}-01$ & $4.57 \mathrm{E}-05$ \\
\hline 200E P-241A103001 & Octane & $111-65-9$ & $5.00 \mathrm{E}-01$ & $4.79 \mathrm{E}-05$ \\
\hline 200E P-241A103 001 & $\begin{array}{l}\text { Phosphoric acid, tributyl } \\
\text { ester }\end{array}$ & $126-73-8$ & & $1.72 E-04$ \\
\hline 200E P-241A103001 & Toluene & $108-88-3$ & $5.00 \mathrm{E}-01$ & $6.67 \mathrm{E}-06$ \\
\hline 200E P-241A104001 & 1,3-Butadiene & $106-99-0$ & $3.50 \mathrm{E}-02$ & 1.91E-06 \\
\hline 200E P-241A104001 & 2-Hexanone & $591-78-6$ & $5.00 \mathrm{E}-01$ & $3.47 \mathrm{E}-05$ \\
\hline 200E P-241A104 001 & 2-Pentanone & $107-87-9$ & & $5.51 \mathrm{E}-05$ \\
\hline 200E P-241A104001 & Acetone & $67-64-1$ & $5.00 \mathrm{E}-01$ & $6.62 \mathrm{E}-04$ \\
\hline 200E P-241A104 001 & Acetonitrile & $75-5-8$ & $5.00 \mathrm{E}-01$ & $3.19 \mathrm{E}-04$ \\
\hline 200E P-241A104 001 & Ammonia & $7664-41-7$ & $5.00 \mathrm{E}-01$ & $3.04 \mathrm{E}-03$ \\
\hline 200E P-241A104001 & Benzene & $71-43-2$ & $5.00 \mathrm{E}-01$ & $1.51 E-05$ \\
\hline 200E P-241A104001 & Carbon monoxide & $630-8-0$ & $5.00 \mathrm{E}+00$ & $4.39 \mathrm{E}-04$ \\
\hline 200E P-241A104 001 & Heptane & $142-82-5$ & $5.00 \mathrm{E}-01$ & $3.89 \mathrm{E}-05$ \\
\hline 200E P-241A104001 & Hexane, n-hexane & $110-54-3$ & $5.00 \mathrm{E}-01$ & $4.05 \mathrm{E}-05$ \\
\hline 200E P-241A104 001 & $\begin{array}{l}\text { Methyl N-amyl ketone, 2- } \\
\text { heptanone }\end{array}$ & $110-43-0$ & $5.00 \mathrm{E}-01$ & $3.76 \mathrm{E}-05$ \\
\hline 200E P-241A104 001 & Nitrogen Oxide $\{\mathrm{NO}\}$ & $10102-43-9$ & $2.00 \mathrm{E}+00$ & $2.64 \mathrm{E}-05$ \\
\hline 200E P-241A104 001 & Nonane & $111-84-2$ & $5.00 \mathrm{E}-01$ & $2.11 E-05$ \\
\hline 200E P-241A104 001 & Octane & $111-65-9$ & $5.00 E-01$ & $2.21 \mathrm{E}-05$ \\
\hline 200E P-241A104001 & $\begin{array}{l}\text { Phosphoric acid, tributyl } \\
\text { ester }\end{array}$ & $126-73-8$ & & $7.95 \mathrm{E}-05$ \\
\hline 200E P-241A104001 & Toluene & $108-88-3$ & $5.00 \mathrm{E}-01$ & $3.08 \mathrm{E}-06$ \\
\hline 200E P-241A106 001 & 1,3-Butadiene & $106-99-0$ & $3.50 \mathrm{E}-02$ & $4.13 E-06$ \\
\hline 200E P-241A106 001 & 2-Hexanone & $591-78-6$ & $5.00 \mathrm{E}-01$ & $7.51 \mathrm{E}-05$ \\
\hline 200E P-241A106 001 & 2-Pentanone & $107-87-9$ & & $1.19 \mathrm{E}-04$ \\
\hline 200E P-241A106 001 & Acetone & $67-64-1$ & $5.00 \mathrm{E}-01$ & $1.43 \mathrm{E}-03$ \\
\hline
\end{tabular}




\section{Table K.2. Insignificant Emission Units Based on Threshold Comparisons} [WAC 173-401-530(1)(a)]

\begin{tabular}{|c|c|c|c|c|}
\hline Emission Point & Pollutants & CAS Number & $\begin{array}{l}\text { Emission } \\
\text { Threshold } \\
\text { (tons/yr) }\end{array}$ & $\begin{array}{c}\text { Actual } \\
\text { Emissions } \\
\text { (tons/yr) }\end{array}$ \\
\hline 200E P-241A106 001 & Acetonitrile & $75-5-8$ & $5.00 \mathrm{E}-01$ & $6.89 \mathrm{E}-04$ \\
\hline 200E P-241A106 001 & Ammonia & $7664-41-7$ & $5.00 \mathrm{E}-01$ & $6.57 \mathrm{E}-03$ \\
\hline 200E P-241A106 001 & Benzene & $71-43-2$ & $5.00 \mathrm{E}-01$ & $3.28 \mathrm{E}-05$ \\
\hline 200E P-241A106 001 & Carbon monoxide & $630-8-0$ & $5.00 \mathrm{E}+00$ & $9.50 \mathrm{E}-04$ \\
\hline 200E P-241A106 001 & Heptane & $142-82-5$ & $5.00 \mathrm{E}-01$ & $8.40 \mathrm{E}-05$ \\
\hline 200E P-241A106 001 & Hexane, n-hexane & $110-54-3$ & $5.00 \mathrm{E}-01$ & $8.76 \mathrm{E}-05$ \\
\hline 200E P-241A106 001 & $\begin{array}{l}\text { Methyl N-amyl ketone, 2- } \\
\text { heptanone }\end{array}$ & $110-43-0$ & $5.00 \mathrm{E}-01$ & $8.13 \mathrm{E}-05$ \\
\hline 200E P-241A106 001 & Nitrogen Oxide $\{\mathrm{NO}\}$ & $10102-43-9$ & $2.00 \mathrm{E}+00$ & $5.72 \mathrm{E}-05$ \\
\hline 200E P-241A106 001 & Nonane & $111-84-2$ & $5.00 \mathrm{E}-01$ & $4.57 \mathrm{E}-05$ \\
\hline 200 E P-241A106 001 & Octane & $111-65-9$ & $5.00 \mathrm{E}-01$ & $4.79 \mathrm{E}-05$ \\
\hline 200E P-241A106 001 & $\begin{array}{l}\text { Phosphoric acid, tributyl } \\
\text { ester }\end{array}$ & $126-73-8$ & & $1.72 \mathrm{E}-04$ \\
\hline $200 \mathrm{E}$ P-241A106 001 & Toluene & $108-88-3$ & $5.00 \mathrm{E}-01$ & $6.67 \mathrm{E}-06$ \\
\hline 200E P-241AX101001 & 1,3-Butadiene & $106-99-0$ & $3.50 \mathrm{E}-02$ & $4.13 E-06$ \\
\hline 200E P-241AX101 001 & 2-Hexanone & $591-78-6$ & $5.00 \mathrm{E}-01$ & $7.51 \mathrm{E}-05$ \\
\hline 200E P-241AX101 001 & 2-Pentanone & & $107-87-9$ & $1.19 \mathrm{E}-04$ \\
\hline 200E P-241AX101 001 & Acetone & $67-64-1$ & $5.00 \mathrm{E}-01$ & $1.43 \mathrm{E}-03$ \\
\hline 200E P-241AX101001 & Acetonitrile & $75-5-8$ & $5.00 \mathrm{E}-01$ & $6.89 \mathrm{E}-04$ \\
\hline 200E P-241AX101 001 & Ammonia & $7664-41-7$ & $5.00 \mathrm{E}-01$ & $6.57 \mathrm{E}-03$ \\
\hline 200E P-241AX101 001 & Benzene & $71-43-2$ & $5.00 \mathrm{E}-01$ & $3.28 \mathrm{E}-05$ \\
\hline 200E P-241AX101 001 & Carbon monoxide & $630-8-0$ & $5.00 \mathrm{E}+00$ & $9.50 \mathrm{E}-04$ \\
\hline 200E P-241AX101 001 & Heptane & $142-82-5$ & $5.00 \mathrm{E}-01$ & $8.40 \mathrm{E}-05$ \\
\hline 200E P-241AX101 001 & Hexane, n-hexane & $110-54-3$ & $5.00 \mathrm{E}-01$ & $8.76 \mathrm{E}-05$ \\
\hline 200E P-241AX101 001 & $\begin{array}{l}\text { Methyl N-amyl ketone, 2- } \\
\text { heptanone }\end{array}$ & $110-43-0$ & $5.00 \mathrm{E}-01$ & $8.13 E-05$ \\
\hline 200E P-241AX101001 & Nitrogen Oxide $\{\mathrm{NO}\}$ & $10102-43-9$ & $2.00 E+00$ & $5.72 \mathrm{E}-05$ \\
\hline 200E P-241AX101 001 & Nonane & $111-84-2$ & $5.00 \mathrm{E}-01$ & $4.57 \mathrm{E}-05$ \\
\hline 200E P-241AX101 001 & Octane & $111-65-9$ & $5.00 \mathrm{E}-01$ & $4.79 \mathrm{E}-05$ \\
\hline 200E P-241AX101 001 & $\begin{array}{l}\text { Phosphoric acid, tributyl } \\
\text { ester }\end{array}$ & $126-73-8$ & & $1.72 \mathrm{E}-04$ \\
\hline
\end{tabular}


Table K.2. Insignificant Emission Units Based on Threshold Comparisons [WAC 173-401-530(1)(a)]

\begin{tabular}{|c|c|c|c|c|}
\hline Emission Point & Pollutants & CAS Number & $\begin{array}{c}\text { Emission } \\
\text { Threshold } \\
\text { (tons/yr) }\end{array}$ & $\begin{array}{c}\text { Actual } \\
\text { Emissions } \\
\text { (tons/yr) }\end{array}$ \\
\hline 200E P-241AX101 001 & Toluene & $108-88-3$ & $5.00 \mathrm{E}-01$ & $6.67 \mathrm{E}-06$ \\
\hline 200E P-241AX102001 & 1,3-Butadiene & $106-99-0$ & $3.50 \mathrm{E}-02$ & $1.91 \mathrm{E}-06$ \\
\hline 200E P-241AX102 001 & 2-Hexanone & $591-78-6$ & $5.00 \mathrm{E}-01$ & $3.47 E-05$ \\
\hline 200E P-241AX102 001 & 2-Pentanone & $107-87-9$ & & $1.51 \mathrm{E}-05$ \\
\hline 200E P-241AX102001 & Acetone & $67-64-1$ & $5.00 \mathrm{E}-01$ & $6.62 \mathrm{E}-04$ \\
\hline 200E P-241AX102001 & Acetonitrile & $75-5-8$ & $5.00 \mathrm{E}-01$ & $3.19 \mathrm{E}-04$ \\
\hline 200E P-241AX102 001 & Ammonia & $7664-41-7$ & $5.00 \mathrm{E}-01$ & $3.04 E-03$ \\
\hline $200 \mathrm{E}$ P-241AX102 001 & Benzene & $71-43-2$ & $5.00 \mathrm{E}-01$ & $1.51 \mathrm{E}-05$ \\
\hline 200E P-241AX102 001 & Carbon monoxide & $630-8-0$ & $5.00 \mathrm{E}+00$ & $4.39 \mathrm{E}-04$ \\
\hline 200E P-241AX102 001 & Heptane & $142-82-5$ & $5.00 \mathrm{E}-01$ & $3.89 \mathrm{E}-05$ \\
\hline 200E P-241AX102 001 & Hexane, n-hexane & $110-54-3$ & $5.00 \mathrm{E}-01$ & $4.05 \mathrm{E}-05$ \\
\hline 200E P-241AX102 001 & $\begin{array}{l}\text { Methyl N-amyl ketone, 2- } \\
\text { heptanone }\end{array}$ & $110-43-0$ & $5.00 \mathrm{E}-01$ & $3.76 \mathrm{E}-05$ \\
\hline 200E P-241AX102 001 & Nitrogen Oxide $\{\mathrm{NO}\}$ & $10102-43-9$ & $2.00 \mathrm{E}+00$ & $2.64 \mathrm{E}-05$ \\
\hline 200E P-241AX102 001 & Nonane & $111-84-2$ & $5.00 \mathrm{E}-01$ & $2.11 \mathrm{E}-05$ \\
\hline 200E P-241AX102 001 & Octane & $111-65-9$ & $5.00 \mathrm{E}-01$ & $2.21 \mathrm{E}-05$ \\
\hline 200E P-241AX102 001 & $\begin{array}{l}\text { Phosphoric acid, tributyl } \\
\text { ester }\end{array}$ & $126-73-8$ & & $7.95 \mathrm{E}-05$ \\
\hline 200E P-241AX102 001 & Toluene & $108-88-3$ & $5.00 \mathrm{E}-01$ & $3.08 \mathrm{E}-06$ \\
\hline 200E P-241AX103001 & 1,3-Butadiene & $106-99-0$ & $3.50 \mathrm{E}-02$ & $4.13 \mathrm{E}-06$ \\
\hline 200E P-241AX103001 & 2-Hexanone & $591-78-6$ & $5.00 \mathrm{E}-01$ & $7.51 \mathrm{E}-05$ \\
\hline $200 \mathrm{E}$ P-241AX103 001 & 2-Pentanone & $107-87-9$ & & $1.19 \mathrm{E}-04$ \\
\hline $200 \mathrm{E}$ P-241AX103001 & Acetone & $67-64-1$ & $5.00 \mathrm{E}-01$ & $1.43 \mathrm{E}-03$ \\
\hline $200 \mathrm{E}$ P-241AX103 001 & Acetonitrile & $75-5-8$ & $5.00 \mathrm{E}-01$ & $6.89 \mathrm{E}-04$ \\
\hline $200 \mathrm{E}$ P-241AX103 001 & Ammonia & $7664-41-7$ & $5.00 \mathrm{E}-01$ & $6.57 \mathrm{E}-03$ \\
\hline 200E P-241AX103 001 & Benzene & $71-43-2$ & $5.00 \mathrm{E}-01$ & $3.28 \mathrm{E}-05$ \\
\hline $200 \mathrm{E}$ P-241AX103001 & Carbon monoxide & $630-8-0$ & $5.00 \mathrm{E}+00$ & $9.05 \mathrm{E}-04$ \\
\hline 200E P-241AX103001 & Heptane & $142-82-5$ & $5.00 \mathrm{E}-01$ & $8.40 \mathrm{E}-05$ \\
\hline $200 \mathrm{E}$ P-241AX103 001 & Hexane, n-hexane & $110-54-3$ & $5.00 \mathrm{E}-01$ & $8.76 \mathrm{E}-05$ \\
\hline
\end{tabular}




\section{Table K.2. Insignificant Emission Units Based on Threshold Comparisons [WAC 173-401-530(1)(a)]}

\begin{tabular}{|c|c|c|c|c|}
\hline Emission Point & Pollutants & CAS Number & $\begin{array}{l}\text { Emission } \\
\text { Threshold } \\
\text { (tons/yr) }\end{array}$ & $\begin{array}{c}\text { Actual } \\
\text { Emissions } \\
\text { (tons/yr) }\end{array}$ \\
\hline 200E P-241AX103001 & $\begin{array}{l}\text { Methyl N-amyl ketone, 2- } \\
\text { heptanone }\end{array}$ & $110-43-0$ & $5.00 \mathrm{E}-01$ & $8.13 E-05$ \\
\hline 200E P-241AX103 001 & Nitrogen Oxide $\{\mathrm{NO}\}$ & $10102-43-9$ & $2.00 \mathrm{E}+00$ & $5.72 \mathrm{E}-05$ \\
\hline 200E P-241AX103 001 & Nonane & $111-84-2$ & $5.00 \mathrm{E}-01$ & 4.5̣7E-05 \\
\hline 200E P-241AX103 001 & Octane & $111-65-9$ & $5.00 \mathrm{E}-01$ & 4.79E-05 \\
\hline 200E P-241AX103001 & $\begin{array}{l}\text { Phosphoric acid, tributyl } \\
\text { ester }\end{array}$ & $126-73-8$ & & $1.72 \mathrm{E}-04$ \\
\hline 200E P-241AX103 001 & Toluene & $108-88-3$ & $5.00 \mathrm{E}-01$ & $6.67 \mathrm{E}-06$ \\
\hline 200E P-241AX104001 & 1,3-Butadiene & $106-99-0$ & $3.50 \mathrm{E}-02$ & $1.19 \mathrm{E}-06$ \\
\hline 200E P-241AX104001 & 2-Hexanone & $591-78-6$ & $5.00 \mathrm{E}-01$ & $3.47 \mathrm{E}-05$ \\
\hline 200E P-241AX104001 & 2-Pentanone & $107-87-9$ & & $5.51 \mathrm{E}-05$ \\
\hline 200E P-241AX104001 & Acetone & $67-64-1$ & $5.00 \mathrm{E}-01$ & $6.62 \mathrm{E}-04$ \\
\hline 200E P-241AX104001 & Acetonitrile & $75-5-8$ & $5.00 \mathrm{E}-01$ & $3.19 \mathrm{E}-04$ \\
\hline 200E P-241AX104001 & Ammonia & $7664-41-7$ & $5.00 \mathrm{E}-01$ & $3.04 \mathrm{E}-03$ \\
\hline 200E P-241AX104001 & Benzene & $71-43-2$ & $5.00 \mathrm{E}-01$ & $1.51 \mathrm{E}-05$ \\
\hline 200E P-241AX104 001 & Carbon monoxide & $630-8-0$ & $5.00 \mathrm{E}+00$ & $4.39 \mathrm{E}-04$ \\
\hline 200E P-241AX104001 & Heptane & $142-82-5$ & $5.00 \mathrm{E}-01$ & $3.89 \mathrm{E}-05$ \\
\hline 200E P-241AX104 001 & Hexane, n-hexane & $110-54-3$ & $5.00 \mathrm{E}-01$ & $4.05 \mathrm{E}-05$ \\
\hline 200E P-241AX104001 & $\begin{array}{l}\text { Methyl N-amyl ketone, 2- } \\
\text { heptanone }\end{array}$ & $110-43-0$ & $5.00 \mathrm{E}-01$ & $3.76 \mathrm{E}-05$ \\
\hline 200E P-241AX104001 & Nitrogen Oxide $\{\mathrm{NO}\}$ & $10102-43-9$ & $2.00 \mathrm{E}+00$ & $2.64 \mathrm{E}-05$ \\
\hline 200E P-241AX104 001 & Nonane & $111-84-2$ & $5.00 \mathrm{E}-01$ & $2.11 E-05$ \\
\hline 200E P-241AX104 001 & Octane & $111-65-9$ & $5.00 \mathrm{E}-01$ & $2.21 \mathrm{E}-05$ \\
\hline 200E P-241AX104001 & $\begin{array}{l}\text { Phosphoric acid, tributyl } \\
\text { ester }\end{array}$ & $126-73-8$ & & $7.95 \mathrm{E}-05$ \\
\hline 200E P-241AX104001 & Toluene & $108-88-3$ & $5.00 \mathrm{E}-01$ & $3.08 \mathrm{E}-06$ \\
\hline 200E P-241B101 001 & 1,3-Butadiene & $106-99-0$ & $3.50 \mathrm{E}-02$ & $4.13 \mathrm{E}-06$ \\
\hline 200E P-241B101 001 & 2-Hexanone & $591-78-6$ & $5.00 \mathrm{E}-01$ & $7.51 \mathrm{E}-05$ \\
\hline 200E P-241B101 001 & 2-Pentanone & $107-87-9$ & & $1.19 \mathrm{E}-04$ \\
\hline 200E P-241B101 001 & Acetone & $67-64-1$ & $5.00 \mathrm{E}-01$ & $1.43 E-03$ \\
\hline
\end{tabular}


Table K.2. Insignificant Emission Units Based on Threshold Comparisons [WAC 173-401-530(1)(a)]

\begin{tabular}{|c|c|c|c|c|}
\hline Emission Point & Pollutants & CAS Number & $\begin{array}{l}\text { Emission } \\
\text { Threshold } \\
\text { (tons/yr) }\end{array}$ & $\begin{array}{c}\text { Actual } \\
\text { Emissions } \\
\text { (tons/yr) }\end{array}$ \\
\hline 200E P-241B101 001 & Acetonitrile & $75-5-8$ & $5.00 \mathrm{E}-01$ & $6.89 \mathrm{E}-04$ \\
\hline 200E P-241B101 001 & Ammonia & $7664-41-7$ & $5.00 \mathrm{E}-01$ & $6.57 \mathrm{E}-03$ \\
\hline 200E P-241B101 001 & Benzene & $71-43-2$ & $5.00 \mathrm{E}-01$ & $3.28 \mathrm{E}-05$ \\
\hline 200E P-241B101 001 & Carbon monoxide & $630-8-0$ & $5.00 \mathrm{E}+00$ & $9.50 \mathrm{E}-04$ \\
\hline 200E P-241B101 001 & Heptane & $142-82-5$ & $5.00 \mathrm{E}-01$ & $8.40 \mathrm{E}-05$ \\
\hline 200E P-241B101 001 & Hexane, n-hexane & $110-54-3$ & $5.00 \mathrm{E}-01$ & $8.76 \mathrm{E}-05$ \\
\hline 200E P-241B101 001 & $\begin{array}{l}\text { Methyl N-amyl ketone, 2- } \\
\text { heptanone }\end{array}$ & $110-43-0$ & $5.00 \mathrm{E}-01$ & $8.13 \mathrm{E}-05$ \\
\hline 200E P-241B101 001 & Nitrogen Oxide $\{\mathrm{NO}\}$ & $10102-43-9$ & $2.00 \mathrm{E}+00$ & $5.72 \mathrm{E}-05$ \\
\hline 200E P-241B101 001 & Nonane & $111-84-2$ & $5.00 \mathrm{E}-01$ & $4.57 \mathrm{E}-05$ \\
\hline 200E P-241B101 001 & Octane & $111-65-9$ & $5.00 \mathrm{E}-01$ & $4.79 \mathrm{E}-05$ \\
\hline 200E P-241B101 001 & $\begin{array}{l}\text { Phosphoric acid, tributyl } \\
\text { ester }\end{array}$ & $126-73-8$ & & $1.72 \mathrm{E}-04$ \\
\hline 200E P-241B101 001 & Toluene & $108-88-3$ & $5.00 \mathrm{E}-01$ & $6.67 \mathrm{E}-06$ \\
\hline 200E P-241B102001 & 1,3-Butadiene & $106-99-0$ & $3.50 \mathrm{E}-02$ & $4.13 \mathrm{E}-06$ \\
\hline 200E P-241B102001 & 2-Hexanone & $591-78-6$ & $5.00 \mathrm{E}-01$ & $7.51 \mathrm{E}-05$ \\
\hline 200E P-241B102 001 & 2-Pentanone & $107-87-9$ & & $1.19 \mathrm{E}-04$ \\
\hline 200E P-241B102 001 & Acetone & $67-64-1$ & $5.00 \mathrm{E}-01$ & $1.43 \mathrm{E}-03$ \\
\hline 200E P-241B102 001 & Acetonitrile & $75-5-8$ & $5.00 \mathrm{E}-01$ & $6.89 \mathrm{E}-04$ \\
\hline 200E P-241B102 001 & Ammonia & $7664-41-7$ & $5.00 \mathrm{E}-01$ & $6.57 \mathrm{E}-03$ \\
\hline 200E P-241B102 001 & Benzene & $71-43-2$ & $5.00 \mathrm{E}-01$ & $3.28 \mathrm{E}-05$ \\
\hline 200E P-241B102 001 & Carbon monoxide & $630-8-0$ & $5.00 \mathrm{E}+00$ & $9.50 \mathrm{E}-04$ \\
\hline 200E P-241B102 001 & Heptane & $142-82-5$ & $5.00 \mathrm{E}-01$ & $8.40 \mathrm{E}-05$ \\
\hline 200E P-241B102001 & Hexane, n-hexane & $110-54-3$ & $5.00 \mathrm{E}-01$ & $8.76 \mathrm{E}-05$ \\
\hline 200E P-241B102001 & $\begin{array}{l}\text { Methyl N-amyl ketone, 2- } \\
\text { heptanone }\end{array}$ & $110-43-0$ & $5.00 \mathrm{E}-01$ & $8.13 \mathrm{E}-05$ \\
\hline 200E P-241B102 001 & Nitrogen Oxide $\{\mathrm{NO}\}$ & $10102-43-9$ & $2.00 \mathrm{E}+00$ & $5.72 \mathrm{E}-05$ \\
\hline $200 \mathrm{E}$ P-241B102 001 & Nonane & $111-84-2$ & $5.00 \mathrm{E}-01$ & $4.57 \mathrm{E}-05$ \\
\hline 200E P-241B102 001 & Octane & $111-65-9$ & $5.00 \mathrm{E}-01$ & $4.79 \mathrm{E}-05$ \\
\hline 200E P-241B102001 & $\begin{array}{l}\text { Phosphoric acid, tributyl } \\
\text { ester }\end{array}$ & $126-73-8$ & & $1.72 \mathrm{E}-04$ \\
\hline
\end{tabular}


Table K.2. Insignificant Emission Units Based on Threshold Comparisons [WAC 173-401-530(1)(a)]

\begin{tabular}{|c|c|c|c|c|}
\hline Emission Point & Pollutants & CAS Number & $\begin{array}{c}\text { Emission } \\
\text { Threshold } \\
\text { (tons/yr) }\end{array}$ & $\begin{array}{c}\text { Actual } \\
\text { Emissions } \\
\text { (tons/yr) }\end{array}$ \\
\hline 200E P-241B102 001 & Toluene & $108-88-3$ & $5.00 \mathrm{E}-01$ & $6.67 \mathrm{E}-06$ \\
\hline 200E P-241B103 001 & 1,3-Butadiene & $106-99-0$ & $3.50 \mathrm{E}-02$ & $4.13 E-06$ \\
\hline 200E P-241B103 001 & 2-Hexanone & $591-78-6$ & $5.00 \mathrm{E}-01$ & $7.51 \mathrm{E}-05$ \\
\hline 200E P-241B103001 & 2-Pentanone & $107-87-9$ & & $1.19 \mathrm{E}-04$ \\
\hline 200E P-241B103001 & Acetone & 67-64-1 & $5.00 \mathrm{E}-01$ & $1.43 \mathrm{E}-03$ \\
\hline 200E P-241B103 001 & Acetonitrile & $75-5-8$ & $5.00 \mathrm{E}-01$ & $6.89 \mathrm{E}-04$ \\
\hline 200E P-241B103001 & Ammonia & $7664-41-7$ & $5.00 \mathrm{E}-01$ & $6.57 \mathrm{E}-03$ \\
\hline 200E P-241B103001 & Benzene & $71-43-2$ & $5.00 \mathrm{E}-01$ & $3.28 \mathrm{E}-05$ \\
\hline 200E P-241B103001 & Carbon monoxide & $630-8-0$ & $5.00 \mathrm{E}+00$ & $9.50 \mathrm{E}-04$ \\
\hline 200E P-241B103 001 & Heptane & $142-82-5$ & $5.00 \mathrm{E}-01$ & $8.40 \mathrm{E}-05$ \\
\hline 200E P-241B103 001 & Hexane, n-hexane & $110-54-3$ & $5.00 \mathrm{E}-01$ & $8.76 \mathrm{E}-05$ \\
\hline 200E P-241B103001 & $\begin{array}{l}\text { Methyl N-amyl ketone, 2- } \\
\text { heptanone }\end{array}$ & $110-43-0$ & $5.00 \mathrm{E}-01$ & $8.13 \mathrm{E}-05$ \\
\hline 200E P-241B103 001 & Nitrogen Oxide $\{\mathrm{NO}\}$ & $10102-43-9$ & $2.00 \mathrm{E}+00$ & $5.72 \mathrm{E}-05$ \\
\hline 200E P-241B103 001 & Nonane & $111-84-2$ & $5.00 \mathrm{E}-01$ & $4.57 \mathrm{E}-05$ \\
\hline 200E P-241B103 001 & Octane & $111-65-9$ & $5.00 \mathrm{E}-01$ & $4.79 \mathrm{E}-05$ \\
\hline 200E P-241B103 001 & $\begin{array}{l}\text { Phosphoric acid, tributyl } \\
\text { ester }\end{array}$ & $126-73-8$ & & $1.72 \mathrm{E}-04$ \\
\hline 200E P-241B103 001 & Toluene & $108-88-3$ & $5.00 \mathrm{E}-01$ & $6.67 \mathrm{E}-06$ \\
\hline 200E P-241B104 001 & 1,3-Butadiene & $106-99-0$ & $3.50 \mathrm{E}-02$ & $1.91 E-06$ \\
\hline 200E P-241B104 001 & 2-Hexanone & $591-78-6$ & $5.00 \mathrm{E}-01$ & $3.47 \mathrm{E}-05$ \\
\hline 200E P-241B104 001 & 2-Pentanone & $107-87-9$ & & $5.51 \mathrm{E}-05$ \\
\hline 200E P-241B104 001 & Acetone & $67-64-1$ & $5.00 \mathrm{E}-01$ & $6.62 \mathrm{E}-04$ \\
\hline 200E P-241B104 001 & Acetonitrile & $75-5-8$ & $5.00 \mathrm{E}-01$ & $3.19 \mathrm{E}-04$ \\
\hline 200E P.241B104 001 & Ammonia & $7664-41-7$ & $5.00 \mathrm{E}-01$ & $3.04 \mathrm{E}-03$ \\
\hline 200E P-241B104 001 & Benzene & $71-43-2$ & $5.00 \mathrm{E}-01$ & $1.51 \mathrm{E}-05$ \\
\hline 200E P-241B104 001 & Carbon monoxide & $630-8-0$ & $5.00 \mathrm{E}+00$ & $4.39 \mathrm{E}-04$ \\
\hline 200E P-241B104 001 & Heptane & $142-82-5$ & $5.00 \mathrm{E}-01$ & $3.89 \mathrm{E}-05$ \\
\hline 200E P-241B104 001 & Hexane, n-hexane & $110-54-3$ & $5.00 \mathrm{E}-01$ & $4.05 E-05$ \\
\hline
\end{tabular}




\section{Table K.2. Insignificant Emission Units Based on Threshold Comparisons [WAC 173-401-530(1)(a)]}

\begin{tabular}{|c|c|c|c|c|}
\hline Emission Point & Pollutants & CAS Number & $\begin{array}{l}\text { Emission } \\
\text { Threshold } \\
\text { (tons/yr) }\end{array}$ & $\begin{array}{c}\text { Actual } \\
\text { Emissions } \\
\text { (tons/yr) }\end{array}$ \\
\hline 200E P-241B104 001 & $\begin{array}{l}\text { Methyl N-amyl ketone, 2- } \\
\text { heptanone }\end{array}$ & $110-43-0$ & $5.00 \mathrm{E}-01$ & $3.76 \mathrm{E}-05$ \\
\hline 200E P-241B104 001 & Nitrogen Oxide $\{\mathrm{NO}\}$ & $10102-43-9$ & $2.00 \mathrm{E}+00$ & $2.64 \mathrm{E}-05$ \\
\hline 200E P-241B104001 & Nonane & $111-84-2$ & $5.00 \mathrm{E}-01$ & $2.11 \mathrm{E}-05$ \\
\hline 200E P-241B104001 & Octane & $111-65-9$ & $5.00 \mathrm{E}-01$ & $2.21 \mathrm{E}-05$ \\
\hline 200E P-241B104001 & $\begin{array}{l}\text { Phosphoric acid, tributyl } \\
\text { ester }\end{array}$ & $126-73-8$ & & 7.95E-05 \\
\hline 200E P-241B104001 & Toluene & $108-88-3$ & $5.00 \mathrm{E}-01$ & $3.08 \mathrm{E}-06$ \\
\hline $200 \mathrm{E}$ P-241B105 001 & 1,3-Butadiene & $106-99-0$ & $3.50 \mathrm{E}-02$ & $1.91 \mathrm{E}-06$ \\
\hline 200E P-241B105 001 & 2-Hexanone & $591-78-6$ & $5.00 \mathrm{E}-01$ & $3.47 \mathrm{E}-05$ \\
\hline 200E P-241B105 001 & 2-Pentanone & $107-87-9$ & & $5.51 \mathrm{E}-05$ \\
\hline 200E P-241B105 001 & Acetone & $67-64-1$ & $5.00 \mathrm{E}-01$ & $6.62 \mathrm{E}-04$ \\
\hline 200E P-241B105 001 & Acetonitrile & $75-5-8$ & $5.00 E-01$ & $3.19 \mathrm{E}-04$ \\
\hline 200E P-241B105 001 & Ammonia & $7664-41-7$ & $5.00 \mathrm{E}-01$ & $3.04 \mathrm{E}-03$ \\
\hline 200E P-241B105 001 & Benzene & $71-43-2$ & $5.00 \mathrm{E}-01$ & $1.51 \mathrm{E}-05$ \\
\hline 200E P-241B105 001 & Carbon monoxide & $630-8-0$ & $5.00 \mathrm{E}+00$ & $4.39 \mathrm{E}-04$ \\
\hline 200E P-241B105 001 & Heptane & $142-82-5$ & $5.00 \mathrm{E}-01$ & $3.89 \mathrm{E}-05$ \\
\hline 200E P-241B105 001 & Hexane, n-hexane & $110-54-3$ & $5.00 \mathrm{E}-01$ & $4.05 \mathrm{E}-05$ \\
\hline 200E P-241B105 001 & $\begin{array}{l}\text { Methyl N-amyl ketone, 2- } \\
\text { heptanone }\end{array}$ & $110-43-0$ & $5.00 \mathrm{E}-01$ & $3.76 \mathrm{E}-05$ \\
\hline 200E P-241B105 001 & Nitrogen Oxide $\{$ NO $\}$ & $10102-43-9$ & $2.00 \mathrm{E}+00$ & $2.64 \mathrm{E}-05$ \\
\hline 200E P-241B105 001 & Nonane & $111-84-2$ & $5.00 \mathrm{E}-01$ & $2.11 \mathrm{E}-05$ \\
\hline 200E P-241B105 001 & Octane & $111-65-9$ & $5.00 \mathrm{E}-01$ & $2.21 \mathrm{E}-05$ \\
\hline 200E P-241B105 001 & $\begin{array}{l}\text { Phosphoric acid, tributyl } \\
\text { ester }\end{array}$ & $126-73-8$ & & $7.95 \mathrm{E}-05$ \\
\hline 200E P-241B105 001 & Toluene & $108-88-3$ & $5.00 \mathrm{E}-01$ & $3.08 \mathrm{E}-06$ \\
\hline 200E P-241B106001 & 1,3-Butadiene & $106-99-0$ & $3.50 \mathrm{E}-02$ & $4.13 \mathrm{E}-06$ \\
\hline 200E P-241B106 001 & 2-Hexanone & $591-78-6$ & $5.00 \mathrm{E}-01$ & $7.51 \mathrm{E}-05$ \\
\hline 200E P-241B106 001 & 2-Pentanone & $107-87-9$ & & $1.19 \mathrm{E}-04$ \\
\hline 200E P-241B106 001 & Acetone & 67-64-1 & $5.00 \mathrm{E}-01$ & $1.43 \mathrm{E}-03$ \\
\hline
\end{tabular}


Table K.2. Insignificant Emission Units Based on Threshold Comparisons [WAC 173-401-530(1)(a)]

\begin{tabular}{|c|c|c|c|c|}
\hline Emission Point & Pollutants & CAS Number & $\begin{array}{l}\text { Emission } \\
\text { Threshold } \\
\text { (tons/yr) }\end{array}$ & $\begin{array}{c}\text { Actual } \\
\text { Emissions } \\
\text { (tons/yr) }\end{array}$ \\
\hline 200E P-241B106 001 & Acetonitrile & $75-5-8$ & $5.00 \mathrm{E}-01$ & $6.89 \mathrm{E}-04$ \\
\hline $200 \mathrm{E}$ P-241B106 001 & Ammonia & $7664-41-7$ & $5.00 \mathrm{E}-01$ & $6.57 \mathrm{E}-03$ \\
\hline 200E P-241B106 001 & Benzene & $71-43-2$ & $5.00 \mathrm{E}-01$ & $3.28 \mathrm{E}-05$ \\
\hline 200E P-241B106 001 & Carbon monoxide & $630-8-0$ & $5.00 \mathrm{E}+00$ & $9.50 \mathrm{E}-04$ \\
\hline 200E P-241B106 001 & Heptane & $142-82-5$ & $5.00 \mathrm{E}-01$ & $8.40 \mathrm{E}-05$ \\
\hline 200E P-241B106 001 & Hexane, n-hexane & $110-54-3$ & $5.00 \mathrm{E}-01$ & $8.76 \mathrm{E}-05$ \\
\hline 200E P-241B106 001 & $\begin{array}{l}\text { Methyl N-amyl ketone, 2- } \\
\text { heptanone }\end{array}$ & $110-43-0$ & $5.00 \mathrm{E}-01$ & $8.13 \mathrm{E}-05$ \\
\hline 200E P-241B106 001 & Nitrogen Oxide $\{$ NO $\}$ & $10102-43-9$ & $2.00 \mathrm{E}+00$ & $5.72 \mathrm{E}-05$ \\
\hline 200E P-241B106 001 & Nonane & $111-84-2$ & $5.00 \mathrm{E}-01$ & $4.57 \mathrm{E}-05$ \\
\hline 200E P-241B106 001 & Octane & $111-65-9$ & $5.00 \mathrm{E}-01$ & $4.79 \mathrm{E}-05$ \\
\hline 200E P-241B106 001 & $\begin{array}{l}\text { Phosphoric acid, tributyl } \\
\text { ester }\end{array}$ & $126-73-8$ & & $1.72 \mathrm{E}-04$ \\
\hline 200E P-241B106 001 & Toluene & $108-88-3$ & $5.00 \mathrm{E}-01$ & $6.67 \mathrm{E}-06$ \\
\hline 200E P-241B107 001 & 1,3-Butadiene & $106-99-0$ & $3.50 \mathrm{E}-02$ & $1.91 \mathrm{E}-06$ \\
\hline 200E P-241B107 001 & 2-Hexanone & $591-78-6$ & $5.00 \mathrm{E}-01$ & $3.47 \mathrm{E}-05$ \\
\hline 200E P-241B107 001 & 2-Pentanone & $107-87-9$ & & $5.51 \mathrm{E}-05$ \\
\hline 200E P-241B107 001 & Acetone & $67-64-1$ & $5.00 \mathrm{E}-01$ & $6.62 \mathrm{E}-04$ \\
\hline 200E P-241B107 001 & Acetonitrile & $75-5-8$ & $5.00 \mathrm{E}-01$ & $3.19 \mathrm{E}-04$ \\
\hline 200E P-241B107 001 & Ammonia & $7664-41-7$ & $5.00 \mathrm{E}-01$ & $3.04 \mathrm{E}-03$ \\
\hline 200E P-241B107 001 & Benzene & $71-43-2$ & $5.00 \mathrm{E}-01$ & $1.51 \mathrm{E}-05$ \\
\hline 200E P-241B107 001 & Carbon monoxide & $630-8-0$ & $5.00 \mathrm{E}+00$ & $4.39 \mathrm{E}-04$ \\
\hline 200E P-241B107 001 & Heptane & $142-82-5$ & $5.00 \mathrm{E}-01$ & $3.89 \mathrm{E}-05$ \\
\hline 200E P-241B107 001 & Hexane, n-hexane & $110-54-3$ & $5.00 \mathrm{E}-01$ & $4.05 \mathrm{E}-05$ \\
\hline 200E P-241B107 001 & $\begin{array}{l}\text { Methyl N-amyl ketone, 2- } \\
\text { heptanone }\end{array}$ & $110-43-0$ & $5.00 \mathrm{E}-01$ & $3.76 \mathrm{E}-05$ \\
\hline 200E P-241B107 001 & Nitrogen Oxide $\{\mathrm{NO}\}$ & $10102-43-9$ & $2.00 \mathrm{E}+00$ & $2.64 \mathrm{E}-05$ \\
\hline 200E P-241B107 001 & Nonane & $111-84-2$ & $5.00 \mathrm{E}-01$ & $2.11 E-05$ \\
\hline 200E P-241B107 001 & Octane & $111-65-9$ & $5.00 \mathrm{E}-01$ & $2.21 \mathrm{E}-05$ \\
\hline 200E P-241B107 001 & $\begin{array}{l}\text { Phosphoric acid, tributyl } \\
\text { ester }\end{array}$ & $126-73-8$ & & $7.95 \mathrm{E}-05$ \\
\hline
\end{tabular}


Table K.2. Insignificant Emission Units Based on Threshold Comparisons [WAC 173-401-530(1)(a)]

\begin{tabular}{|c|c|c|c|c|}
\hline Emission Point & Pollutants & CAS Number & $\begin{array}{l}\text { Emission } \\
\text { Threshold } \\
\text { (tons/yr) }\end{array}$ & $\begin{array}{c}\text { Actual } \\
\text { Emissions } \\
\text { (tons/yr) }\end{array}$ \\
\hline 200E P-241B107 001 & Toluene & $108-88-3$ & $5.00 \mathrm{E}-01$ & $3.08 \mathrm{E}-06$ \\
\hline 200E P-241B108 001 & 1,3-Butadiene & $106-99-0$ & $3.50 \mathrm{E}-02$ & $4.13 \mathrm{E}-06$ \\
\hline 200E P-241B108 001 & 2-Hexanone & $591-78-6$ & $5.00 \mathrm{E}-01$ & $7.51 \mathrm{E}-05$ \\
\hline 200E P-241B108 001 & 2-Pentanone & $107-87-9$ & & $1.19 \mathrm{E}-04$ \\
\hline 200E P-241B108 001 & Acetone & $67-64-1$ & $5.00 \mathrm{E}-01$ & $1.43 \mathrm{E}-03$ \\
\hline 200E P-241B108 001 & Acetonitrile & $75-5-8$ & $5.00 \mathrm{E}-01$ & $6.89 \mathrm{E}-04$ \\
\hline 200E P-241B108 001 & Ammonia & $7664-41-7$ & $5.00 \mathrm{E}-01$ & $6.57 \mathrm{E}-03$ \\
\hline 200E P-241B108 001 & Benzene & $71-43-2$ & $5.00 \mathrm{E}-01$ & $3.28 \mathrm{E}-05$ \\
\hline 200E P-241B108 001 & Carbon monoxide & $630-8-0$ & $5.00 \mathrm{E}+00$ & $9.50 \mathrm{E}-04$ \\
\hline 200 E P-241B108001 & Heptane & $142-82-5$ & $5.00 \mathrm{E}-01$ & $8.40 \mathrm{E}-05$ \\
\hline 200E P-241B108 001 & Hexane, n-hexane & $110-54-3$ & $5.00 \mathrm{E}-01$ & $8.76 \mathrm{E}-05$ \\
\hline 200E P-241B108001 & $\begin{array}{l}\text { Methyl N-amyl ketone, 2- } \\
\text { heptanone }\end{array}$ & $110-43-0$ & $5.00 \mathrm{E}-01$ & $8.13 \mathrm{E}-05$ \\
\hline 200 E P-241B108 001 & Nitrogen Oxide $\{\mathrm{NO}\}$ & $10102-43-9$ & $2.00 \mathrm{E}+00$ & $5.72 \mathrm{E}-05$ \\
\hline 200E P-241B108 001 & Nonane & $111-84-2$ & $5.00 \mathrm{E}-01$ & $4.57 \mathrm{E}-05$ \\
\hline 200 E P-241B108 001 & Octane & $111-65-9$ & $5.00 \mathrm{E}-01$ & $4.79 \mathrm{E}-05$ \\
\hline 200E P-241B108 001 & $\begin{array}{l}\text { Phosphoric acid, tributyl } \\
\text { ester }\end{array}$ & $126-73-8$ & & $1.72 \mathrm{E}-04$ \\
\hline 200 E P-241B108 001 & Toluene & $108-88-3$ & $5.00 \mathrm{E}-01$ & $6.67 \mathrm{E}-06$ \\
\hline 200E P-241B109 001 & 1,3-Butadiene & $106-99-0$ & $3.50 \mathrm{E}-02$ & $1.91 \mathrm{E}-06$ \\
\hline 200E P-241B109001 & 2-Hexanone & $591-78-6$ & $5.00 \mathrm{E}-01$ & 3.47E-05 \\
\hline 200E P-241B109 001 & 2-Pentanone & $107-87-9$ & & $5.51 \mathrm{E}-05$ \\
\hline 200E P-241B109 001 & Acetone & $67-64-1$ & $5.00 \mathrm{E}-01$ & $6.62 \mathrm{E}-04$ \\
\hline 200E P-241B109 001 & Acetonitrile & $75-5-8$ & $5.00 \mathrm{E}-01$ & $3.19 \mathrm{E}-04$ \\
\hline 200E P-241B109 001 & Ammonia & $7664-41-7$ & $5.00 \mathrm{E}-01$ & $3.04 \mathrm{E}-03$ \\
\hline 200E P-241B109 001 & Benzene & $71-43-2$ & $5.00 \mathrm{E}-01$ & $1.51 \mathrm{E}-05$ \\
\hline 200E P-241B109001 & Carbon monoxide & $630-8-0$ & $5.00 \mathrm{E}+00$ & $4.39 \mathrm{E}-04$ \\
\hline 200E P-241B109 001 & Heptane & $142-82-5$ & $5.00 \mathrm{E}-01$ & $3.89 \mathrm{E}-05$ \\
\hline 200E P-241B109 001 & Hexane, n-hexane & $110-54-3$ & $5.00 \mathrm{E}-01$ & $4.05 \mathrm{E}-05$ \\
\hline
\end{tabular}


Table K.2. Insignificant Emission Units Based on Threshold Comparisons [WAC 173-401-530(1)(a)]

\begin{tabular}{|c|c|c|c|c|}
\hline Emission Point & Pollutants & CAS Number & $\begin{array}{l}\text { Emission } \\
\text { Threshold } \\
\text { (tons/yr) }\end{array}$ & $\begin{array}{c}\text { Actual } \\
\text { Emissions } \\
\text { (tons/yr) }\end{array}$ \\
\hline 200E P-241B109 001 & $\begin{array}{l}\text { Methyl N-amyl ketone, 2- } \\
\text { heptanone }\end{array}$ & $110-43-0$ & $5.00 \mathrm{E}-01$ & $3.76 \mathrm{E}-05$ \\
\hline 200E P-241B109 001 & Nitrogen Oxide $\{\mathrm{NO}\}$ & $10102-43-9$ & $2.00 \mathrm{E}+00$ & $2.64 \mathrm{E}-05$ \\
\hline 200E P-241B109 001 & Nonane & $111-84-2$ & $5.00 \mathrm{E}-01$ & $2.11 E-05$ \\
\hline 200E P-241B109 001 & Octane & $111-65-9$ & $5.00 \mathrm{E}-01$ & $2.21 \mathrm{E}-05$ \\
\hline $200 \mathrm{E}$ P-241B109001 & $\begin{array}{l}\text { Phosphoric acid, tributyl } \\
\text { ester }\end{array}$ & $126-73-8$ & & $7.95 \mathrm{E}-05$ \\
\hline 200E P-241B109 001 & Toluene & $108-88-3$ & $5.00 \mathrm{E}-01$ & $3.08 \mathrm{E}-06$ \\
\hline 200E P-241B110 001 & 1,3-Butadiene & $106-99-0$ & $3.50 \mathrm{E}-02$ & $1.91 \mathrm{E}-06$ \\
\hline 200E P-241B110 001 & 2-Hexanone & $591-78-6$ & $5.00 \mathrm{E}-01$ & $3.47 \mathrm{E}-05$ \\
\hline 200E P-241B110 001 & 2-Pentanone & $107-87-9$ & & $5.51 \mathrm{E}-05$ \\
\hline 200E P-241B110001 & Acetone & $67-64-1$ & $5.00 \mathrm{E}-01$ & $6.62 \mathrm{E}-04$ \\
\hline 200E P-241B110 001 & Acetonitrile & $75-5-8$ & $5.00 \mathrm{E}-01$ & $3.19 \mathrm{E}-04$ \\
\hline 200E P-241B110 001 & Ammonia & $7664-41-7$ & $5.00 \mathrm{E}-01$ & $3.04 \mathrm{E}-03$ \\
\hline 200E P-241B110 001 & Benzene & $71-43-2$ & $5.00 \mathrm{E}-01$ & $1.51 \mathrm{E}-05$ \\
\hline 200E P-241B110 001 & Carbon monoxide & $630-8-0$ & $5.00 \mathrm{E}+00$ & $4.39 \mathrm{E}-04$ \\
\hline 200E P-241B110 001 & Heptane & $142-82-5$ & $5.00 \mathrm{E}-01$ & $3.89 \mathrm{E}-05$ \\
\hline 200E P-241B110 001 & Hexane, n-hexane & $110-54-3$ & $5.00 \mathrm{E}-01$ & $4.05 \mathrm{E}-05$ \\
\hline $200 \mathrm{E}$ P-241B110 001 & $\begin{array}{l}\text { Methyl N-amyl ketone, 2- } \\
\text { heptanone }\end{array}$ & $110-43-0$ & $5.00 \mathrm{E}-01$ & $3.76 \mathrm{E}-05$ \\
\hline 200E P-241B110001 & Nitrogen Oxide $\{\mathrm{NO}\}$ & $10102-43-9$ & $2.00 \mathrm{E}+00$ & $2.64 \mathrm{E}-05$ \\
\hline $200 \mathrm{E}$ P-241B110 001 & Nonane & $111-84-2$ & $5.00 \mathrm{E}-01$ & $2.11 E-05$ \\
\hline 200E P-241B110001 & Octane & $111-65-9$ & $5.00 \mathrm{E}-01$ & $2.21 \mathrm{E}-05$ \\
\hline 200E P-241B110 001 & $\begin{array}{l}\text { Phosphoric acid, tributyl } \\
\text { ester }\end{array}$ & $126-73-8$ & & $7.95 \mathrm{E}-05$ \\
\hline 200E P-241B110 001 & Toluene & $108-88-3$ & $5.00 \mathrm{E}-01$ & $3.08 \mathrm{E}-06$ \\
\hline 200E P-241B111 001 & 1,3-Butadiene & $106-99-0$ & $3.50 \mathrm{E}-02$ & $4.13 \mathrm{E}-06$ \\
\hline 200E P-241B111 001 & 2-Hexanone & $591-78-6$ & $5.00 \mathrm{E}-01$ & $7.51 \mathrm{E}-05$ \\
\hline 200E P-241B111 001 & 2-Pentanone & $107-87-9$ & & $1.19 \mathrm{E}-04$ \\
\hline 200E P-241B111 001 & Acetone & $67-64-1$ & $5.00 \mathrm{E}-01$ & $1.43 \mathrm{E}-03$ \\
\hline
\end{tabular}


Table K.2. Insignificant Emission Units Based on Threshold Comparisons [WAC 173-401-530(1)(a)]

\begin{tabular}{|c|c|c|c|c|}
\hline Emission Point & Pollutants & CAS Number & $\begin{array}{l}\text { Emission } \\
\text { Threshold } \\
\text { (tons/yr) }\end{array}$ & $\begin{array}{c}\text { Actual } \\
\text { Emissions } \\
\text { (tons/yr) }\end{array}$ \\
\hline 200E P-241B111 001 & Acetonitrile & $75-5-8$ & $5.00 \mathrm{E}-01$ & $6.89 \mathrm{E}-04$ \\
\hline 200E P-241B111 001 & Ammonia & $7664-41-7$ & $5.00 \mathrm{E}-01$ & $6.57 \mathrm{E}-03$ \\
\hline 200E P-241B111 001 & Benzene & $71-43-2$ & $5.00 \mathrm{E}-01$ & $3.28 \mathrm{E}-05$ \\
\hline 200E P-241B111 001 & Carbon monoxide & $630-8-0$ & $5.00 \mathrm{E}+00$ & $9.50 \mathrm{E}-04$ \\
\hline 200E P-241B111 001 & Heptane & $142-82-5$ & $5.00 \mathrm{E}-01$ & $8.40 \mathrm{E}-05$ \\
\hline 200E P-241B111 001 & Hexane, n-hexane & $110-54-3$ & $5.00 \mathrm{E}-01$ & $8.76 \mathrm{E}-05$ \\
\hline 200E P-241B111 001 & $\begin{array}{l}\text { Methyl N-amyl ketone, 2- } \\
\text { heptanone }\end{array}$ & $110-43-0$ & $5.00 \mathrm{E}-01$ & $8.13 \mathrm{E}-05$ \\
\hline 200E P-241B111 001 & Nitrogen Oxide $\{\mathrm{NO}\}$ & $10102-43-9$ & $2.00 \mathrm{E}+00$ & $5.72 \mathrm{E}-05$ \\
\hline 200E P-241B111 001 & Nonane & $111-84-2$ & $5.00 \mathrm{E}-01$ & $4.57 \mathrm{E}-05$ \\
\hline 200E P-241B111 001 & Octane & $111-65-9$ & $5.00 \mathrm{E}-01$ & $4.79 \mathrm{E}-05$ \\
\hline 200E P-241B111 001 & $\begin{array}{l}\text { Phosphoric acid, tributyl } \\
\text { ester }\end{array}$ & $126-73-8$ & & $1.72 \mathrm{E}-04$ \\
\hline 200E P-241B111 001 & Toluene & $108-88-3$ & $5.00 \mathrm{E}-01$ & $6.67 \mathrm{E}-06$ \\
\hline 200E P-241B112001 & 1,3-Butadiene & $106-99-0$ & $3.50 \mathrm{E}-02$ & $4.13 E-06$ \\
\hline 200E P-241B112 001 & 2-Hexanone & $591-78-6$ & $5.00 \mathrm{E}-01$ & $7.51 E-05$ \\
\hline 200E P-241B112 001 & 2-Pentanone & $107-87-9$ & & $1.19 \mathrm{E}-04$ \\
\hline 200E P-241B112001 & Acetone & $67-64-1$ & $5.00 \mathrm{E}-01$ & $1.43 \mathrm{E}-03$ \\
\hline 200E P-241B112001 & Acetonitrile & $75-5-8$ & $5.00 \mathrm{E}-01$ & $6.89 \mathrm{E}-04$ \\
\hline 200E P-241B112 001 & Ammonia & $7664-41-7$ & $5.00 \mathrm{E}-01$ & $6.57 \mathrm{E}-03$ \\
\hline 200E P-241B112001 & Benzene & $71-43-2$ & $5.00 \mathrm{E}-01$ & $3.28 \mathrm{E}-05$ \\
\hline 200E P-241B112 001 & Carbon monoxide & $630-8-0$ & $5.00 \mathrm{E}+00$ & $9.50 \mathrm{E}-04$ \\
\hline 200E P-241B112001 & Heptane & $142-82-5$ & $5.00 \mathrm{E}-01$ & $8.40 \mathrm{E}-05$ \\
\hline 200E P-241B112001 & Hexane, n-hexane & $110-54-3$ & $5.00 \mathrm{E}-01$ & $8.76 \mathrm{E}-05$ \\
\hline 200E P-241B112001 & $\begin{array}{l}\text { Methyl N-amyl ketone, 2- } \\
\text { heptanone }\end{array}$ & $110-43-0$ & $5.00 \mathrm{E}-01$ & $8.13 \mathrm{E}-05$ \\
\hline 200E P-241B112001 & Nitrogen Oxide $\{\mathrm{NO}\}$ & $10102-43-9$ & $2.00 \mathrm{E}+00$ & $5.72 \mathrm{E}-05$ \\
\hline $200 \mathrm{E}$ P-241B112 001 & Nonane & $111-84-2$ & $5.00 \mathrm{E}-01$ & $4.57 \mathrm{E}-05$ \\
\hline 200E P-241B112 001 & Octane & $111-65-9$ & $5.00 \mathrm{E}-01$ & $4.79 \mathrm{E}-05$ \\
\hline 200E P-241B112 001 & $\begin{array}{l}\text { Phosphoric acid, tributyl } \\
\text { ester }\end{array}$ & $126-73-8$ & & $1.72 \mathrm{E}-04$ \\
\hline
\end{tabular}


Table K.2. Insignificant Emission Units Based on Threshold Comparisons [WAC 173-401-530(1)(a)]

\begin{tabular}{|c|c|c|c|c|}
\hline Emission Point & Pollutants & CAS Number & $\begin{array}{l}\text { Emission } \\
\text { Threshold } \\
\text { (tons/yr) }\end{array}$ & $\begin{array}{c}\text { Actual } \\
\text { Emissions } \\
\text { (tons/yr) }\end{array}$ \\
\hline 200E P-241B112 001 & Toluene & $108-88-3$ & $5.00 \mathrm{E}-01$ & $6.67 \mathrm{E}-06$ \\
\hline 200E P-241B201001 & 1,3-Butadiene & $106-99-0$ & $3.50 \mathrm{E}-02$ & $1.91 \mathrm{E}-06$ \\
\hline 200E P-241B201 001 & 2-Hexanone & $591-78-6$ & $5.00 \mathrm{E}-01$ & $3.47 \mathrm{E}-05$ \\
\hline 200E P-241B201 001 & 2-Pentanone & $107-87-9$ & & $5.51 \mathrm{E}-05$ \\
\hline 200E P-241B201001 & Acetone & $67-64-1$ & $5.00 \mathrm{E}-01$ & $6.62 \mathrm{E}-04$ \\
\hline 200E P-241B201 001 & Acetonitrile & $75-5-8$ & $5.00 \mathrm{E}-01$ & $3.19 \mathrm{E}-04$ \\
\hline 200E P-241B201 001 & Ammonia & $7664-41-7$ & $5.00 \mathrm{E}-01$ & $3.04 \mathrm{E}-03$ \\
\hline 200E P-241B201 001 & Benzene & $71-43-2$ & $5.00 \mathrm{E}-01$ & $1.51 \mathrm{E}-05$ \\
\hline 200E P-241B201 001 & Carbon monoxide & $630-8-0$ & $5.00 \mathrm{E}+00$ & $4.39 \mathrm{E}-04$ \\
\hline 200E P-241B201001 & Heptane & $142-82-5$ & $5.00 \mathrm{E}-01$ & $3.89 \mathrm{E}-05$ \\
\hline 200E P-241B201001 & Hexane, n-hexane & $110-54-3$ & $5.00 \mathrm{E}-01$ & $4.05 E-05$ \\
\hline 200E P-241B201 001 & $\begin{array}{l}\text { Methyl N-amyl ketone, } 2 \text { - } \\
\text { heptanone }\end{array}$ & $110-43-0$ & $5.00 \mathrm{E}-01$ & $3.76 \mathrm{E}-05$ \\
\hline 200E P-241B201001 & Nitrogen Oxide $\{\mathrm{NO}\}$ & $10102-43-9$ & $2.00 \mathrm{E}+00$ & $2.64 \mathrm{E}-05$ \\
\hline 200E P-241B201 001 & Nonane & $111-84-2$ & $5.00 \mathrm{E}-01$ & $2.11 \mathrm{E}-05$ \\
\hline 200E P-241B201 001 & Octane & $111-65-9$ & $5.00 \mathrm{E}-01$ & $2.21 \mathrm{E}-05$ \\
\hline 200E P-241B201 001 & $\begin{array}{l}\text { Phosphoric acid, tributyl } \\
\text { ester }\end{array}$ & $126-73-8$ & & $7.95 \mathrm{E}-05$ \\
\hline 200E P-241B201001 & Toluene & $108-88-3$ & $5.00 \mathrm{E}-01$ & $3.08 \mathrm{E}-06$ \\
\hline 200E P-241B202001 & 1,3-Butadiene & $106-99-0$ & $3.50 \mathrm{E}-02$ & $1.91 E-06$ \\
\hline 200E P-241B202001 & 2-Hexanone & $591-78-6$ & $5.00 \mathrm{E}-01$ & $3.47 \mathrm{E}-05$ \\
\hline 200E P-241B202001 & 2-Pentanone & $107-87-9$ & & $5.51 \mathrm{E}-05$ \\
\hline 200E P-241B202 001 & Acetone & $67-64-1$ & $5.00 \mathrm{E}-01$ & $6.62 E-04$ \\
\hline 200E P-241B202001 & Acetonitrile & $75-5-8$ & $5.00 \mathrm{E}-01$ & $3.19 E-04$ \\
\hline 200E P-241B202001 & Ammonia & $7664-41-7$ & $5.00 \mathrm{E}-01$ & $3.04 \mathrm{E}-03$ \\
\hline 200E P-241B202001 & Benzene & $71-43-2$ & $5.00 \mathrm{E}-01$ & $1.51 \mathrm{E}-05$ \\
\hline 200E P-241B202001 & Carbon monoxide & $630-8-0$ & $5.00 \mathrm{E}+00$ & $4.39 \mathrm{E}-04$ \\
\hline 200E P-241B202 001 & Heptane & $142-82-5$ & $5.00 \mathrm{E}-01$ & $3.89 \mathrm{E}-05$ \\
\hline 200E P-241B202001 & Hexane, n-hexane & $110-54-3$ & $5.00 \mathrm{E}-01$ & $4.05 \mathrm{E}-05$ \\
\hline
\end{tabular}


Table K.2. Insignificant Emission Units Based on Threshold Comparisons [WAC 173-401-530(1)(a)]

\begin{tabular}{|c|c|c|c|c|}
\hline Emission Point & Pollutants & CAS Number & $\begin{array}{c}\text { Emission } \\
\text { Threshold } \\
\text { (tons/yr) }\end{array}$ & $\begin{array}{c}\text { Actual } \\
\text { Emissions } \\
\text { (tons/yr) }\end{array}$ \\
\hline 200E P-241B202001 & $\begin{array}{l}\text { Methyl N-amyl ketone, 2- } \\
\text { heptanone }\end{array}$ & $110-43-0$ & $5.00 \mathrm{E}-01$ & $3.76 \mathrm{E}-05$ \\
\hline 200E P-241B202 001 & Nitrogen Oxide $\{\mathrm{NO}\}$ & $10102-43-9$ & $2.00 \mathrm{E}+00$ & $2.64 \mathrm{E}-0 \mathrm{~S}$ \\
\hline 200E P-241B202001 & Nonane & $111-84-2$ & $5.00 \mathrm{E}-01$ & $2.11 \mathrm{E}-05$ \\
\hline 200E P-241B202 001 & Octane & $111-65-9$ & $5.00 \mathrm{E}-01$ & $2.21 \mathrm{E}-05$ \\
\hline 200E P-241B202 001 & $\begin{array}{l}\text { Phosphoric acid, tributyl } \\
\text { ester }\end{array}$ & $126-73-8$ & & $7.95 \mathrm{E}-05$ \\
\hline 200E P-241B202 001 & Toluene & $108-88-3$ & $5.00 \mathrm{E}-01$ & $3.08 \mathrm{E}-06$ \\
\hline 200E P-241B203 001 & 1,3-Butadiene & $106-99-0$ & $3.50 \mathrm{E}-02$ & $1.91 \mathrm{E}-06$ \\
\hline 200E P-241B203 001 & 2-Hexanone & $591-78-6$ & $5.00 \mathrm{E}-01$ & $3.47 E-05$ \\
\hline 200E P-241B203 001 & 2-Pentanone & $107-87-9$ & & $5.51 \mathrm{E}-05$ \\
\hline 200E P-241B203 001 & Acetone & $67-64-1$ & $5.00 \mathrm{E}-01$ & $6.62 \mathrm{E}-04$ \\
\hline 200E P-241B203 001 & Acetonitrile & $75-5-8$ & $5.00 \mathrm{E}-01$ & $3.19 \mathrm{E}-04$ \\
\hline 200E P-241B203 001 & Ammonia & $7664-41-7$ & $5.00 \mathrm{E}-01$ & $3.04 \mathrm{E}-03$ \\
\hline 200E P-241B203 001 & Benzene & $71-43-2$ & $5.00 \mathrm{E}-01$ & $1.51 E-05$ \\
\hline 200E P-241B203 001 & Carbon monoxide & $630-8-0$ & $5.00 \mathrm{E}+00$ & $4.39 \mathrm{E}-04$ \\
\hline 200E P-241B203 001 & Heptane & $142-82-5$ & $5.00 \mathrm{E}-01$ & $3.89 \mathrm{E}-05$ \\
\hline 200E P-241B203 001 & Hexane, n-hexane & $110-54-3$ & $5.00 \mathrm{E}-01$ & $4.05 \mathrm{E}-05$ \\
\hline 200E P-241B203 001 & $\begin{array}{l}\text { Methyl N-amyl ketone, 2- } \\
\text { heptanone }\end{array}$ & $110-43-0$ & $5.00 \mathrm{E}-01$ & $3.76 E-05$ \\
\hline 200E P-241B203 001 & Nitrogen Oxide $\{\mathrm{NO}\}$ & $10102-43-9$ & $2.00 \mathrm{E}+00$ & $2.64 E-05$ \\
\hline 200E P-241B203 001 & Nonane & $111-84-2$ & $5.00 \mathrm{E}-01$ & $2.11 E-05$ \\
\hline 200E P-241B203 001 & Octane & $111-65-9$ & $5.00 \mathrm{E}-01$ & $2.21 \mathrm{E}-05$ \\
\hline 200E P-241B203 001 & $\begin{array}{l}\text { Phosphoric acid, tributyl } \\
\text { ester }\end{array}$ & $126-73-8$ & & $7.95 \mathrm{E}-05$ \\
\hline 200E P-241B203 001 & Toluene & $108-88-3$ & $5.00 \mathrm{E}-01$ & $3.08 \mathrm{E}-06$ \\
\hline 200E P-241B204001 & 1,3-Butadiene & $106-99-0$ & $3.50 \mathrm{E}-02$ & $1.91 \mathrm{E}-06$ \\
\hline 200E P-241B204 001 & 2-Hexanone & $591-78-6$ & $5.00 \mathrm{E}-01$ & $3.47 \mathrm{E}-05$ \\
\hline 200E P-241B204 001 & 2-Pentanone & $107-87-9$ & & $5.51 \mathrm{E}-05$ \\
\hline 200E P-241B204 001 & Acetone & $67-64-1$ & $5.00 \mathrm{E}-01$ & $6.62 E-04$ \\
\hline
\end{tabular}


Table K.2. Insignificant Emission Units Based on Threshold Comparisons [WAC 173-401-530(1)(a)]

\begin{tabular}{|c|c|c|c|c|}
\hline Emission Point & Pollutants & CAS Number & $\begin{array}{c}\text { Emission } \\
\text { Threshold } \\
\text { (tons/yr) }\end{array}$ & $\begin{array}{c}\text { Actual } \\
\text { Emissions } \\
\text { (tons/yr) }\end{array}$ \\
\hline 200E P-241B204 001 & Acetonitrile & $75-5-8$ & $5.00 \mathrm{E}-01$ & $3.19 \mathrm{E}-04$ \\
\hline 200E P-241B204001 & Ammonia & $7664-41-7$ & $5.00 \mathrm{E}-01$ & $3.04 \mathrm{E}-03$ \\
\hline 200E P-241B204 001 & Benzene & $71-43-2$ & $5.00 \mathrm{E}-01$ & $1.51 \mathrm{E}-05$ \\
\hline 200E P-241B204001 & Carbon monoxide & $630-8-0$ & $5.00 \mathrm{E}+00$ & $4.39 \mathrm{E}-04$ \\
\hline 200E P-241B204001 & Heptane & $142-82-5$ & $5.00 \mathrm{E}-01$ & $3.89 \mathrm{E}-05$ \\
\hline 200E P-241B204001 & Hexane, n-hexane & $110-54-3$ & $5.00 \mathrm{E}-01$ & $4.05 \mathrm{E}-05$ \\
\hline 200E P-241B204001 & $\begin{array}{l}\text { Methyl N-amyl ketone, 2- } \\
\text { heptanone }\end{array}$ & $110-43-0$ & $5.00 \mathrm{E}-01$ & $3.76 \mathrm{E}-05$ \\
\hline 200E P-241B204001 & Nitrogen Oxide $\{\mathrm{NO}\}$ & $10102-43-9$ & $2.00 \mathrm{E}+00$ & $2.64 \mathrm{E}-05$ \\
\hline 200E P-241B204 001 & Nonane & $111-84-2$ & $5.00 \mathrm{E}-01$ & $2.11 E-05$ \\
\hline 200E P-241B204 001 & Octane & $111-65-9$ & $5.00 \mathrm{E}-01$ & $2.21 E-05$ \\
\hline 200E P-241B204001 & $\begin{array}{l}\text { Phosphoric acid, tributyl } \\
\text { ester }\end{array}$ & $126-73-8$ & & $7.95 E-05$ \\
\hline 200E P-241B204001 & Toluene & $108-88-3$ & $5.00 \mathrm{E}-01$ & $3.08 \mathrm{E}-06$ \\
\hline 200E P-241BX101 001 & 1,3-Butadiene & $106-99-0$ & $3.50 \mathrm{E}-02$ & $1.91 \mathrm{E}-06$ \\
\hline 200E P-241BX101 001 & 2-Hexanone & $591-78-6$ & $5.00 \mathrm{E}-01$ & $3.47 \mathrm{E}-05$ \\
\hline 200E P-241BX101001 & 2-Pentanone & $107-87-9$ & & $5.51 E-05$ \\
\hline 200E P-241BX101001 & Acetone & $67-64-1$ & $5.00 \mathrm{E}-01$ & $6.62 E-04$ \\
\hline 200E P-241BX101 001 & Acetonitrile & $75-5-8$ & $5.00 \mathrm{E}-01$ & $3.19 \mathrm{E}-04$ \\
\hline 200E P-241BX101 001 & Ammonia & $7664-41-7$ & $5.00 \mathrm{E}-01$ & $3.04 \mathrm{E}-03$ \\
\hline 200E P-241BX101 001 & Benzene & $71-43-2$ & $5.00 \mathrm{E}-01$ & $1.51 \mathrm{E}-05$ \\
\hline 200E P-241BX101 001 & Carbon monoxide & $630-8-0$ & $5.00 E+00$ & $4.39 \mathrm{E}-04$ \\
\hline 200E P-241BX101 001 & Heptane & $142-82-5$ & $5.00 \mathrm{E}-01$ & $3.89 \mathrm{E}-05$ \\
\hline 200E P-241BX101 001 & Hexane, n-hexane & $110-54-3$ & $5.00 \mathrm{E}-01$ & $4.05 \mathrm{E}-05$ \\
\hline 200E P-241BX101 001 & $\begin{array}{l}\text { Methyl N-amyl ketone, 2- } \\
\text { heptanone }\end{array}$ & $110-43-0$ & $5.00 \mathrm{E}-01$ & $3.76 E-05$ \\
\hline 200E P-241BX101 001 & Nitrogen Oxide $\{\mathrm{NO}\}$ & $10102-43-9$ & $2.00 \mathrm{E}+00$ & $2.64 \mathrm{E}-05$ \\
\hline 200E P-241BX101 001 & Nonane & $111-84-2$ & $5.00 \mathrm{E}-01$ & $2.11 E-05$ \\
\hline 200E P-241BX101 001 & Octane & $111-65-9$ & $5.00 \mathrm{E}-01$ & $2.21 E-05$ \\
\hline 200E P-241BX101 001 & $\begin{array}{l}\text { Phosphoric acid, tributyl } \\
\text { ester }\end{array}$ & $126-73-8$ & & $7.95 \mathrm{E}-05$ \\
\hline
\end{tabular}


Table K.2. Insignificant Emission Units Based on Threshold Comparisons [WAC 173-401-530(1)(a)]

\begin{tabular}{|c|c|c|c|c|}
\hline Emission Point & Pollutants & CAS Number & $\begin{array}{l}\text { Emission } \\
\text { Threshold } \\
\text { (tons/yr) }\end{array}$ & $\begin{array}{c}\text { Actual } \\
\text { Emissions } \\
\text { (tons/yr) }\end{array}$ \\
\hline 200E P-241BX101001 & Toluene & $108-88-3$ & $5.00 \mathrm{E}-01$ & $3.08 \mathrm{E}-06$ \\
\hline 200E P-241BX102 001 & 1,3-Butadiene & $106-99-0$ & $3.50 \mathrm{E}-02$ & $1.91 \mathrm{E}-06$ \\
\hline 200E P-241BX102 001 & 2-Hexanone & $591-78-6$ & $5.00 \mathrm{E}-01$ & 3.47E-05 \\
\hline 200E P-241BX102 001 & 2-Pentanone & $107-87-9$ & & $5.51 \mathrm{E}-05$ \\
\hline 200E P-241BX102001 & Acetone & $67-64-1$ & $5.00 \mathrm{E}-01$ & $6.62 E-04$ \\
\hline 200E P-241BX102 001 & Acetonitrile & $75-5-8$ & $5.00 \mathrm{E}-01$ & $3.19 \mathrm{E}-04$ \\
\hline 200E P-241BX102 001 & Ammonia & $7664-41-7$ & $5.00 \mathrm{E}-01$ & 3.04E-03 \\
\hline 200E P-241BX102 001 & Benzene & $71-43-2$ & $5.00 \mathrm{E}-01$ & $1.51 \mathrm{E}-05$ \\
\hline 200E P-241BX102 001 & Carbon monoxide & $630-8-0$ & $5.00 \mathrm{E}+00$ & 4.39E-04 \\
\hline 200E P-241BX102 001 & Heptane & $142-82-5$ & $5.00 \mathrm{E}-01$ & $3.89 \mathrm{E}-05$ \\
\hline 200E P-241BX102 001 & Hexane, n-hexane & $110-54-3$ & $5.00 \mathrm{E}-01$ & 4.05E-05 \\
\hline 200E P-241BX102 001 & $\begin{array}{l}\text { Methyl N-amyl ketone, 2- } \\
\text { heptanone }\end{array}$ & $110-43-0$ & $5.00 \mathrm{E}-01$ & $3.76 \mathrm{E}-05$ \\
\hline 200E P-241BX102 001 & Nitrogen Oxide $\{$ NO $\}$ & $10102-43-9$ & $2.00 \mathrm{E}+00$ & $2.64 \mathrm{E}-05$ \\
\hline 200E P-241BX102 001 & Nonane & $111-84-2$ & $5.00 \mathrm{E}-01$ & $2.11 \mathrm{E}-05$ \\
\hline 200E P-241BX102 001 & Octane & $111-65-9$ & $5.00 \mathrm{E}-01$ & $2.21 \mathrm{E}-05$ \\
\hline 200E P-241BX102 001 & $\begin{array}{l}\text { Phosphoric acid, tributyl } \\
\text { ester }\end{array}$ & $126-73-8$ & & $7.95 \mathrm{E}-05$ \\
\hline 200E P-241BX102 001 & Toluene & $108-88-3$ & $5.00 \mathrm{E}-01$ & $3.08 \mathrm{E}-06$ \\
\hline 200E P-241BX103001 & 1,3-Butadiene & $106-99-0$ & $3.50 \mathrm{E}-02$ & $4.13 \mathrm{E}-06$ \\
\hline 200E P-241BX103001 & 2-Hexanone & $591-78-6$ & $5.00 \mathrm{E}-01$ & $7.51 \mathrm{E}-05$ \\
\hline 200E P-241BX103001 & 2-Pentanone & $107-87-9$ & & $1.19 \mathrm{E}-04$ \\
\hline 200E P-241BX103001 & Acetone & $67-64-1$ & $5.00 \mathrm{E}-01$ & $1.43 \mathrm{E}-03$ \\
\hline 200E P-241BX103001 & Acetonitrile & $75-5-8$ & $5.00 \mathrm{E}-01$ & $6.89 \mathrm{E}-04$ \\
\hline 200E P-241BX103001 & Ammonia & $7664-41-7$ & $5.00 \mathrm{E}-01$ & $6.57 \mathrm{E}-03$ \\
\hline 200E P-241BX103001 & Benzene & $71-43-2$ & $5.00 \mathrm{E}-01$ & $3.28 \mathrm{E}-05$ \\
\hline 200E P-241BX103001 & Carbon monoxide & $630-8-0$ & $5.00 \mathrm{E}+00$ & $9.50 \mathrm{E}-04$ \\
\hline 200E P-241BX103 001 & Heptane & $142-82-5$ & $5.00 \mathrm{E}-01$ & $8.40 \mathrm{E}-05$ \\
\hline 200E P-241BX103 001 & Hexane, n-hexane & $110-54-3$ & $5.00 \mathrm{E}-01$ & $8.76 \mathrm{E}-05$ \\
\hline
\end{tabular}


Table K.2. Insignificant Emission Units Based on Threshold Comparisons [WAC 173-401-530(1)(a)]

\begin{tabular}{|c|c|c|c|c|}
\hline Emission Point & Pollutants & CAS Number & $\begin{array}{l}\text { Emission } \\
\text { Threshold } \\
\text { (tons/yr) }\end{array}$ & $\begin{array}{c}\text { Actual } \\
\text { Emissions } \\
\text { (tons/yr) }\end{array}$ \\
\hline 200E P-241BX103001 & $\begin{array}{l}\text { Methyl N-amyl ketone, 2- } \\
\text { heptanone }\end{array}$ & $110-43-0$ & $5.00 \mathrm{E}-01$ & $8.13 E-05$ \\
\hline 200E P-241BX103001 & Nitrogen Oxide $\{\mathrm{NO}\}$ & $10102-43-9$ & $2.00 \mathrm{E}+00$ & $5.72 \mathrm{E}-05$ \\
\hline 200E P-241BX103 001 & Nonane & $111-84-2$ & $5.00 \mathrm{E}-01$ & $4.57 \mathrm{E}-05$ \\
\hline 200E P-241BX103001 & Octane & $111-65-9$ & $5.00 \mathrm{E}-01$ & $4.79 E-05$ \\
\hline 200E P-241BX103 001 & $\begin{array}{l}\text { Phosphoric acid, tributyl } \\
\text { ester }\end{array}$ & $126-73-8$ & & $1.72 \mathrm{E}-04$ \\
\hline 200E P-241BX103 001 & Toluene & $108-88-3$ & $5.00 \mathrm{E}-01$ & $6.67 \mathrm{E}-06$ \\
\hline 200E P-241BX104001 & 1,3-Butadiene & $106-99-0$ & $3.50 \mathrm{E}-02$ & $4.13 E-06$ \\
\hline $200 \mathrm{E}$ P-241BX104 001 & 2-Hexanone & $591-78-6$ & $5.00 \mathrm{E}-01$ & $7.51 \mathrm{E}-05$ \\
\hline 200E P-241BX104001 & 2-Pentanone & $107-87-9$ & & $1.19 \mathrm{E}-04$ \\
\hline 200E P-241BX104 001 & Acetone & $67-64-1$ & $5.00 \mathrm{E}-01$ & $1.43 \mathrm{E}-03$ \\
\hline 200E P-241BX104 001 & Acetonitrile & $75-5-8$ & $5.00 \mathrm{E}-01$ & $6.89 \mathrm{E}-04$ \\
\hline 200 E P-241BX104001 & Ammonia & $7664-41-7$ & $5.00 \mathrm{E}-01$ & $6.57 \mathrm{E}-03$ \\
\hline $200 \mathrm{E}$ P-241BX104 001 & Benzene & $71-43-2$ & $5.00 \mathrm{E}-01$ & $3.28 \mathrm{E}-05$ \\
\hline $200 \mathrm{E}$ P-241BX104 001 & Carbon monoxide & $630-8-0$ & $5.00 \mathrm{E}+00$ & $9.50 \mathrm{E}-04$ \\
\hline $200 \mathrm{E}$ P-241BX104 001 & Heptane & $142-82-5$ & $5.00 \mathrm{E}-01$ & $8.40 \mathrm{E}-05$ \\
\hline 200E P-241BX104 001 & Hexane, n-hexane & $110-54-3$ & $5.00 \mathrm{E}-01$ & $8.76 \mathrm{E}-05$ \\
\hline 200E P-241BX104001 & $\begin{array}{l}\text { Methyl N-amyl ketone, 2- } \\
\text { heptanone }\end{array}$ & $110-43-0$ & $5.00 \mathrm{E}-01$ & $8.13 E-05$ \\
\hline 200E P-241BX104001 & Nitrogen Oxide $\{\mathrm{NO}\}$ & $10102-43-9$ & $2.00 \mathrm{E}+00$ & $5.72 E-05$ \\
\hline 200E P-241BX104001 & Nonane & $111-84-2$ & $5.00 \mathrm{E}-01$ & $4.57 \mathrm{E}-05$ \\
\hline 200E P-241BX104 001 & Octane & $111-65-9$ & $5.00 \mathrm{E}-01$ & 4.79E-05 \\
\hline 200E P-241BX104001 & $\begin{array}{l}\text { Phosphoric acid, tributyl } \\
\text { ester }\end{array}$ & $126-73-8$ & & $1.72 \mathrm{E}-04$ \\
\hline 200E P-241BX104 001 & Toluene & $108-88-3$ & $5.00 \mathrm{E}-01$ & $6.67 \mathrm{E}-06$ \\
\hline 200E P-241BX105001 & 1,3-Butadiene & $106-99-0$ & $3.50 \mathrm{E}-02$ & $4.13 E-06$ \\
\hline 200E P-241BX105 001 & 2-Hexanone & $591-78-6$ & $5.00 \mathrm{E}-01$ & $7.51 \mathrm{E}-05$ \\
\hline 200E P-241BX105 001 & 2-Pentanone & $107-87-9$ & & $1.19 \mathrm{E}-04$ \\
\hline 200E P-241BX105001 & Acetone & $67-64-1$ & $5.00 \mathrm{E}-01$ & $1.43 \mathrm{E}-03$ \\
\hline
\end{tabular}


Table K.2. Insignificant Emission Units Based on Threshold Comparisons [WAC 173-401-530(1)(a)]

\begin{tabular}{|c|c|c|c|c|}
\hline Emission Point & Pollutants & CAS Number & $\begin{array}{c}\text { Emission } \\
\text { Threshold } \\
\text { (tons/yr) }\end{array}$ & $\begin{array}{c}\text { Actual } \\
\text { Emissions } \\
\text { (tons/yr) }\end{array}$ \\
\hline 200E P-241BX105 001 & Acetonitrile & $75-5-8$ & $5.00 \mathrm{E}-01$ & $6.89 \mathrm{E}-04$ \\
\hline 200E P-241BX105 001 & Ammonia & $7664-41-7$ & $5.00 \mathrm{E}-01$ & $6.57 \mathrm{E}-03$ \\
\hline 200E P-241BX105 001 & Benzene & $71-43-2$ & $5.00 \mathrm{E}-01$ & $3.28 \mathrm{E}-05$ \\
\hline 200E P-241BX105001 & Carbon monoxide & $630-8-0$ & $5.00 \mathrm{E}+00$ & $9.50 \mathrm{E}-04$ \\
\hline 200E P-241BX105 001 & Heptane & $142-82-5$ & $5.00 \mathrm{E}-01$ & $8.40 \mathrm{E}-05$ \\
\hline 200E P-241BX105001 & Hexane, n-hexane & $110-54-3$ & $5.00 \mathrm{E}-01$ & $8.76 \mathrm{E}-05$ \\
\hline 200E P-241BX105 001 & $\begin{array}{l}\text { Methyl N-amyl ketone, 2- } \\
\text { heptanone }\end{array}$ & $110-43-0$ & $5.00 \mathrm{E}-01$ & $8.13 E-05$ \\
\hline 200E P-241BX105 001 & Nitrogen Oxide $\{\mathrm{NO}\}$ & $10102-43-9$ & $2.00 \mathrm{E}+00$ & $5.72 \mathrm{E}-05$ \\
\hline $200 \mathrm{E}$ P-241BX105 001 & Nonane & $111-84-2$ & $5.00 \mathrm{E}-01$ & $4.57 \mathrm{E}-05$ \\
\hline 200E P-241BX105 001 & Octane & $111-65-9$ & $5.00 \mathrm{E}-01$ & $4.79 \mathrm{E}-05$ \\
\hline 200E P-241BX105001 & $\begin{array}{l}\text { Phosphoric acid, tributyl } \\
\text { ester }\end{array}$ & $126-73-8$ & & $1.72 \mathrm{E}-04$ \\
\hline 200E P-241BX105 001 & Toluene & $108-88-3$ & $5.00 \mathrm{E}-01$ & $6.67 \mathrm{E}-06$ \\
\hline 200E P-241BX106 001 & 1,3-Butadiene & $106-99-0$ & $3.50 \mathrm{E}-02$ & $1.91 E-06$ \\
\hline 200E P-241BX106 001 & 2-Hexanone & $591-78-6$ & $5.00 \mathrm{E}-01$ & 3.47E-05 \\
\hline 200E P-241BX106 001 & 2-Pentanone & $107-87-9$ & & $5.51 \mathrm{E}-05$ \\
\hline 200E P-241BX106 001 & Acetone & $67-64-1$ & $5.00 \mathrm{E}-01$ & $6.62 \mathrm{E}-04$ \\
\hline 200E P-241BX106 001 & Acetonitrile & $75-5-8$ & $5.00 \mathrm{E}-01$ & $3.19 \mathrm{E}-04$ \\
\hline 200E P-241BX106 001 & Ammonia & $7664-41-7$ & $5.00 \mathrm{E}-01$ & $3.04 \mathrm{E}-03$ \\
\hline 200E P-241BX106 001 & Benzene & $71-43-2$ & $5.00 \mathrm{E}-01$ & $1.51 \mathrm{E}-05$ \\
\hline 200E P-241BX106 001 & Carbon monoxide & $630-8-0$ & $5.00 \mathrm{E}+00$ & $4.39 \mathrm{E}-04$ \\
\hline 200E P-241BX106 001 & Heptane & $142-82-5$ & $5.00 \mathrm{E}-01$ & $3.89 \mathrm{E}-05$ \\
\hline 200E P-241BX106 001 & Hexane, n-hexane & $110-54-3$ & $5.00 \mathrm{E}-01$ & $4.05 \mathrm{E}-05$ \\
\hline 200E P-241BX106 001 & $\begin{array}{l}\text { Methyl N-amyl ketone, 2- } \\
\text { heptanone }\end{array}$ & $110-43-0$ & $5.00 \mathrm{E}-01$ & $3.76 \mathrm{E}-05$ \\
\hline 200E P-241BX106 001 & Nitrogen Oxide $\{\mathrm{NO}\}$ & $10102-43-9$ & $2.00 \mathrm{E}+00$ & $2.64 \mathrm{E}-05$ \\
\hline 200E P-241BX106 001 & Nonane & $111-84-2$ & $5.00 \mathrm{E}-01$ & $2.11 \mathrm{E}-05$ \\
\hline 200E P-241BX106 001 & Octane & $111-65-9$ & $5.00 \mathrm{E}-01$ & $2.21 \mathrm{E}-05$ \\
\hline 200E P-241BX106 001 & $\begin{array}{l}\text { Phosphoric acid, tributyl } \\
\text { ester }\end{array}$ & $126-73-8$ & & $7.95 \mathrm{E}-05$ \\
\hline
\end{tabular}




\section{Table K.2. Insignificant Emission Units Based on Threshold Comparisons} [WAC 173-401-530(1)(a)]

\begin{tabular}{|c|c|c|c|c|}
\hline Emission Point & Pollutants & CAS Number & $\begin{array}{l}\text { Emission } \\
\text { Threshold } \\
\text { (tons/yr) }\end{array}$ & $\begin{array}{c}\text { Actual } \\
\text { Emissions } \\
\text { (tons/yr) }\end{array}$ \\
\hline 200E P-241BX106 001 & Toluene & $108-88-3$ & $5.00 \mathrm{E}-01$ & $3.08 \mathrm{E}-06$ \\
\hline 200E P-241BX107001 & 1,3-Butadiene & $106-99-0$ & $3.50 \mathrm{E}-02$ & $4.13 E-06$ \\
\hline 200E P-241BX107 001 & 2-Hexanone & $591-78-6$ & $5.00 \mathrm{E}-01$ & $7.51 \mathrm{E}-05$ \\
\hline 200E P-241BX107 001 & 2-Pentanone & $107-87-9$ & & $1.19 \mathrm{E}-04$ \\
\hline 200E P-241BX107001 & Acetone & $67-64-1$ & $5.00 \mathrm{E}-01$ & $1.43 E-03$ \\
\hline 200E P-241BX107 001 & Acetonitrile & $75-5-8$ & $5.00 \mathrm{E}-01$ & $6.89 \mathrm{E}-04$ \\
\hline 200E P-241BX107 001 & Ammonia & $7664-41-7$ & $5.00 \mathrm{E}-01$ & $6.57 \mathrm{E}-03$ \\
\hline 200E P-241BX107 001 & Benzene & $71-43-2$ & $5.00 \mathrm{E}-01$ & $3.28 \mathrm{E}-05$ \\
\hline 200E P-241BX107 001 & Carbon monoxide & $630-8-0$ & $5.00 \mathrm{E}+00$ & $9.50 \mathrm{E}-04$ \\
\hline 200E P-241BX107 001 & Heptane & $142-82-5$ & $5.00 \mathrm{E}-01$ & $8.40 \mathrm{E}-05$ \\
\hline 200E P-241BX107 001 & Hexane, n-hexane & $110-54-3$ & $5.00 \mathrm{E}-01$ & $8.76 \mathrm{E}-05$ \\
\hline 200E P-241BX107 001 & $\begin{array}{l}\text { Methyl N-amyl ketone, 2- } \\
\text { heptanone }\end{array}$ & $110-43-0$ & $5.00 \mathrm{E}-01$ & $8.13 \mathrm{E}-05$ \\
\hline 200E P-241BX107 001 & Nitrogen Oxide $\{\mathrm{NO}\}$ & $10102-43-9$ & $2.00 \mathrm{E}+00$ & $5.72 \mathrm{E}-05$ \\
\hline 200E P-241BX107 001 & Nonane & $111-84-2$ & $5.00 \mathrm{E}-01$ & $4.57 \mathrm{E}-05$ \\
\hline 200E P-241BX107 001 & Octane & $111-65-9$ & $5.00 \mathrm{E}-01$ & $4.79 \mathrm{E}-05$ \\
\hline 200E P-241BX107 001 & $\begin{array}{l}\text { Phosphoric acid, tributyl } \\
\text { ester }\end{array}$ & $126-73-8$ & & $1.72 \mathrm{E}-04$ \\
\hline 200E P-241BX107 001 & Toluene & $108-88-3$ & $5.00 \mathrm{E}-01$ & $6.67 \mathrm{E}-06$ \\
\hline 200E P-241BX108 001 & 1,3-Butadiene & $106-99-0$ & $3.50 \mathrm{E}-02$ & $1.91 \mathrm{E}-06$ \\
\hline 200E P-241BX108 001 & 2-Hexanone & $591-78-6$ & $5.00 \mathrm{E}-01$ & $3.47 \mathrm{E}-05$ \\
\hline 200E P-241BX108 001 & 2-Pentanone & $107-87-9$ & & $5.51 \mathrm{E}-05$ \\
\hline 200E P-241BX108 001 & Acetone & $67-64-1$ & $5.00 \mathrm{E}-01$ & $6.62 \mathrm{E}-04$ \\
\hline 200E P-241BX108 001 & Acetonitrile & $75-5-8$ & $5.00 \mathrm{E}-01$ & $3.19 \mathrm{E}-04$ \\
\hline 200E P-241BX108001 & Ammonia & $7664-41-7$ & $5.00 \mathrm{E}-01$ & $3.04 \mathrm{E}-03$ \\
\hline 200E P-241BX108001 & Benzene & $71-43-2$ & $5.00 \mathrm{E}-01$ & $1.51 \mathrm{E}-05$ \\
\hline 200E P-241BX108 001 & Carbon monoxide & $630-8-0$ & $5.00 \mathrm{E}+00$ & $4.39 \mathrm{E}-04$ \\
\hline $200 \mathrm{E}$ P-241BX108 001 & Heptane & $142-82-5$ & $5.00 \mathrm{E}-01$ & $3.89 \mathrm{E}-05$ \\
\hline 200E P-241BX108 001 & Hexane, n-hexane & $110-54-3$ & $5.00 \mathrm{E}-01$ & $4.05 \mathrm{E}-05$ \\
\hline
\end{tabular}


Table K.2. Insignificant Emission Units Based on Threshold Comparisons [WAC 173-401-530(1)(a)]

\begin{tabular}{|c|c|c|c|c|}
\hline Emission Point & Pollutants & CAS Number & $\begin{array}{l}\text { Emission } \\
\text { Threshold } \\
\text { (tons/yr) }\end{array}$ & $\begin{array}{c}\text { Actual } \\
\text { Emissions } \\
\text { (tons/yr) }\end{array}$ \\
\hline 200E P-241BX108 001 & $\begin{array}{l}\text { Methyl N-amyl ketone, 2- } \\
\text { heptanone }\end{array}$ & $110-43-0$ & $5.00 \mathrm{E}-01$ & $3.76 \mathrm{E}-05$ \\
\hline 200E P-241BX108 001 & Nitrogen Oxide $\{\mathrm{NO}\}$ & $10102-43-9$ & $2.00 \mathrm{E}+00$ & $2.64 \mathrm{E}-05$ \\
\hline 200E P-241BX108 001 & Nonane & $111-84-2$ & $5.00 \mathrm{E}-01$ & $2.11 \mathrm{E}-05$ \\
\hline 200E P-241BX108 001 & Octane & $111-65-9$ & $5.00 \mathrm{E}-01$ & $2.21 \mathrm{E}-05$ \\
\hline 200E P-241BX108001 & $\begin{array}{l}\text { Phosphoric acid, tributyl } \\
\text { ester }\end{array}$ & $126-73-8$ & & $7.95 \mathrm{E}-05$ \\
\hline 200E P-241BX108001 & Toluene & $108-88-3$ & $5.00 \mathrm{E}-01$ & $3.08 \mathrm{E}-06$ \\
\hline 200E P-241BX109001 & 1,3-Butadiene & $106-99-0$ & $3.50 \mathrm{E}-02$ & $4.13 \mathrm{E}-06$ \\
\hline 200E P-241BX109 001 & 2-Hexanone & $591-78-6$ & $5.00 \mathrm{E}-01$ & $7.51 \mathrm{E}-05$ \\
\hline 200E P-241BX109001 & 2-Pentanone & $107-87-9$ & & 1.19E-04 \\
\hline 200E P-241BX109001 & Acetone & $67-64-1$ & $5.00 \mathrm{E}-01$ & $1.43 \mathrm{E}-03$ \\
\hline 200E P-241BX109001 & Acetonitrile & $75-5-8$ & $5.00 \mathrm{E}-01$ & $6.89 \mathrm{E}-04$ \\
\hline 200E P-241BX109001 & Ammonia & $7664-41-7$ & $5.00 \mathrm{E}-01$ & $6.57 \mathrm{E}-03$ \\
\hline 200E P-241BX109001 & Benzene & $71-43-2$ & $5.00 \mathrm{E}-01$ & $3.28 \mathrm{E}-05$ \\
\hline 200E P-241BX109001 & Carbon monoxide & $630-8-0$ & $5.00 \mathrm{E}+00$ & $9.50 \mathrm{E}-04$ \\
\hline 200E P-241BX109 001 & Heptane & $142-82-5$ & $5.00 \mathrm{E}-01$ & $8.40 \mathrm{E}-05$ \\
\hline 200E P-241BX109001 & Hexane, n-hexane & $110-54-3$ & $5.00 \mathrm{E}-01$ & $8.76 \mathrm{E}-05$ \\
\hline 200E P-241BX109001 & $\begin{array}{l}\text { Methyl N-amyl ketone, 2- } \\
\text { heptanone }\end{array}$ & $110-43-0$ & $5.00 \mathrm{E}-01$ & $8.13 E-05$ \\
\hline 200E P-241BX109001 & Nitrogen Oxide $\{\mathrm{NO}\}$ & $10102-43-9$ & $2.00 \mathrm{E}+00$ & $5.72 \mathrm{E}-05$ \\
\hline 200E P-241BX109001 & Nonane & $111-84-2$ & $5.00 \mathrm{E}-01$ & $4.57 \mathrm{E}-05$ \\
\hline 200E P-241BX109001 & Octane & $111-65-9$ & $5.00 \mathrm{E}-01$ & $4.79 \mathrm{E}-05$ \\
\hline 200E P-241BX109001 & $\begin{array}{l}\text { Phosphoric acid, tributyl } \\
\text { ester }\end{array}$ & $126-73-8$ & & $1.72 \mathrm{E}-04$ \\
\hline 200E P-241BX109001 & Toluene & $108-88-3$ & $5.00 \mathrm{E}-01$ & $6.67 \mathrm{E}-06$ \\
\hline 200E P-241BX110 001 & 1,3-Butadiene & $106-99-0$ & $3.50 \mathrm{E}-02$ & $1.91 \mathrm{E}-06$ \\
\hline 200E P-241BX110001 & 2-Hexanone & $591-78-6$ & $5.00 \mathrm{E}-01$ & $3.47 \mathrm{E}-05$ \\
\hline 200E P-241BX110 001 & 2-Pentanone & $107-87-9$ & & $5.51 \mathrm{E}-05$ \\
\hline 200E P-241BX110 001 & Acetone & $67-64-1$ & $5.00 \mathrm{E}-01$ & $6.62 \mathrm{E}-04$ \\
\hline
\end{tabular}


Table K.2. Insignificant Emission Units Based on Threshold Comparisons [WAC 173-401-530(1)(a)]

\begin{tabular}{|c|c|c|c|c|}
\hline Emission Point & Pollutants & CAS Number & $\begin{array}{l}\text { Emission } \\
\text { Threshold } \\
\text { (tons/yr) }\end{array}$ & $\begin{array}{c}\text { Actual } \\
\text { Emissions } \\
\text { (tons/yr) }\end{array}$ \\
\hline 200E P-241BX110 001 & Acetonitrile & $75-5-8$ & $5.00 \mathrm{E}-01$ & $3.19 \mathrm{E}-04$ \\
\hline 200E P-241BX110 001 & Ammonia & $7664-41-7$ & $5.00 \mathrm{E}-01$ & $3.04 \mathrm{E}-03$ \\
\hline 200E P-241BX110 001 & Benzene & $71-43-2$ & $5.00 \mathrm{E}-01$ & $1.51 \mathrm{E}-05$ \\
\hline 200E P-241BX110 001 & Carbon monoxide & $630-8-0$ & $5.00 \mathrm{E}+00$ & $4.39 \mathrm{E}-04$ \\
\hline 200E P-241BX110 001 & Heptane & $142-82-5$ & $5.00 \mathrm{E}-01$ & $3.89 \mathrm{E}-05$ \\
\hline 200E P-241BX110 001 & Hexane, n-hexane & $110-54-3$ & $5.00 \mathrm{E}-01$ & $4.05 E-05$ \\
\hline 200E P-241BX110 001 & $\begin{array}{l}\text { Methyl N-amyl ketone, 2- } \\
\text { heptanone }\end{array}$ & $110-43-0$ & $5.00 E-01$ & $3.76 \mathrm{E}-05$ \\
\hline 200E P-241BX110 001 & Nitrogen Oxide $\{\mathrm{NO}\}$ & $10102-43-9$ & $2.00 \mathrm{E}+00$ & $2.64 \mathrm{E}-05$ \\
\hline 200E P-241BX110 001 & Nonane & $111-84-2$ & $5.00 \mathrm{E}-01$ & $2.11 \mathrm{E}-05$ \\
\hline 200E P-241BX110 001 & Octane & $111-65-9$ & $5.00 \mathrm{E}-01$ & $2.21 E-05$ \\
\hline 200E P-241BX110 001 & $\begin{array}{l}\text { Phosphoric acid, tributyl } \\
\text { ester }\end{array}$ & $126-73-8$ & & $7.95 \mathrm{E}-05$ \\
\hline 200E P-241BX110 001 & Toluene & $108-88-3$ & $5.00 \mathrm{E}-01$ & $3.08 \mathrm{E}-06$ \\
\hline 200E P-241BX111001 & 1,3-Butadiene & $106-99-0$ & $3.50 \mathrm{E}-02$ & $1.91 \mathrm{E}-06$ \\
\hline 200E P-241BX111 001 & 2-Hexanone & $591-78-6$ & $5.00 \mathrm{E}-01$ & 3.47E-05 \\
\hline 200E P-241BX111 001 & 2-Pentanone & $107-87-9$ & & $5.51 \mathrm{E}-05$ \\
\hline 200E P-241BX111 001 & Acetone & $67-64-1$ & $5.00 \mathrm{E}-01$ & $6.62 \mathrm{E}-04$ \\
\hline 200E P-241BX111 001 & Acetonitrile & $75-5-8$ & $5.00 \mathrm{E}-01$ & $3.19 \mathrm{E}-04$ \\
\hline 200E P-241BX111 001 & Ammonia & $7664-41-7$ & $5.00 \mathrm{E}-01$ & $3.04 \mathrm{E}-03$ \\
\hline 200E P-241BX111 001 & Benzene & $71-43-2$ & $5.00 \mathrm{E}-01$ & $1.51 \mathrm{E}-05$ \\
\hline 200E P-241BX111 001 & Carbon monoxide & $630-8-0$ & $5.00 \mathrm{E}+00$ & $4.39 \mathrm{E}-04$ \\
\hline 200E P-241BX111001 & Heptane & $142-82-5$ & $5.00 \mathrm{E}-01$ & $3.89 \mathrm{E}-05$ \\
\hline 200E P-241BX111001 & Hexane, n-hexane & $110-54-3$ & $5.00 \mathrm{E}-01$ & $4.05 \mathrm{E}-05$ \\
\hline 200E P-241BX111001 & $\begin{array}{l}\text { Methyl N-amyl ketone, 2- } \\
\text { heptanone }\end{array}$ & $110-43-0$ & $5.00 \mathrm{E}-01$ & $3.76 \mathrm{E}-05$ \\
\hline 200E P-241BX111 001 & Nitrogen Oxide $\{\mathrm{NO}\}$ & $10102-43-9$ & $2.00 \mathrm{E}+00$ & $2.64 \mathrm{E}-05$ \\
\hline 200E P-241BX111 001 & Nonane & $111-84-2$ & $5.00 \mathrm{E}-01$ & $2.11 \mathrm{E}-05$ \\
\hline 200E P-241BX111 001 & Octane & $111-65-9$ & $5.00 \mathrm{E}-01$ & $2.21 \mathrm{E}-05$ \\
\hline 200E P-241BX111 001 & $\begin{array}{l}\text { Phosphoric acid, tributyl } \\
\text { ester }\end{array}$ & $126-73-8$ & & $7.95 \mathrm{E}-05$ \\
\hline
\end{tabular}


Table K.2. Insignificant Emission Units Based on Threshold Comparisons [WAC 173-401-530(1)(a)]

\begin{tabular}{|c|c|c|c|c|}
\hline Emission Point & Pollutants & CAS Number & $\begin{array}{l}\text { Emission } \\
\text { Threshold } \\
\text { (tons/yr) }\end{array}$ & $\begin{array}{c}\text { Actual } \\
\text { Emissions } \\
\text { (tons/yr) }\end{array}$ \\
\hline 200E P-241BX111001 & Toluene & $108-88-3$ & $5.00 \mathrm{E}-01$ & $3.08 \mathrm{E}-06$ \\
\hline 200E P-241BX112 001 & 1,3-Butadiene & $106-99-0$ & $3.50 \mathrm{E}-02$ & $4.13 \mathrm{E}-06$ \\
\hline 200E P-241BX112 001 & 2-Hexanone & $591-78-6$ & $5.00 \mathrm{E}-01$ & $7.51 \mathrm{E}-05$ \\
\hline 200E P-241BX112 001 & 2-Pentanone & $107-87-9$ & & $1.19 \mathrm{E}-04$ \\
\hline 200E P-241BX112 001 & Acetone & $67-64-1$ & $5.00 \mathrm{E}-01$ & $1.43 \mathrm{E}-03$ \\
\hline 200E P-241BX112 001 & Acetonitrile & $75-5-8$ & $5.00 \mathrm{E}-01$ & $6.89 \mathrm{E}-04$ \\
\hline 200E P-241BX112001 & Ammonia & $7664-41-7$ & $5.00 \mathrm{E}-01$ & $6.57 \mathrm{E}-03$ \\
\hline 200E P-241BX112 001 & Benzene & $71-43-2$ & $5.00 \mathrm{E}-01$ & $3.28 E-05$ \\
\hline 200E P-241BX112 001 & Carbon monoxide & $630-8-0$ & $5.00 \mathrm{E}+00$ & $9.50 \mathrm{E}-04$ \\
\hline 200E P-241BX112 001 & Heptane & $142-82-5$ & $5.00 \mathrm{E}-01$ & $8.40 \mathrm{E}-05$ \\
\hline 200E P-241BX112 001 & Hexane, $n$-hexane & $110-54-3$ & $5.00 \mathrm{E}-01$ & $8.76 \mathrm{E}-05$ \\
\hline 200E P-241BX112001 & $\begin{array}{l}\text { Methyl N-amyl ketone, 2- } \\
\text { heptanone }\end{array}$ & $110-43-0$ & $5.00 \mathrm{E}-01$ & $8.13 E-05$ \\
\hline 200E P-241BX112001 & Nitrogen Oxide $\{$ NO $\}$ & $10102-43-9$ & $2.00 \mathrm{E}+00$ & $5.72 \mathrm{E}-05$ \\
\hline 200E P-241BX112001 & Nonane & $111-84-2$ & $5.00 \mathrm{E}-01$ & $4.57 \mathrm{E}-05$ \\
\hline 200E P-241BX112001 & Octane & $111-65-9$ & $5.00 \mathrm{E}-01$ & $4.79 \mathrm{E}-05$ \\
\hline 200E P-241BX112 001 & $\begin{array}{l}\text { Phosphoric acid, tributyl } \\
\text { ester }\end{array}$ & $126-73-8$ & & $1.72 \mathrm{E}-04$ \\
\hline 200E P-241BX112 001 & Toluene & $108-88-3$ & $5.00 \mathrm{E}-01$ & $6.67 \mathrm{E}-06$ \\
\hline 200E P-241BY101 001 & 1,3-Butadiene & $106-99-0$ & $3.50 \mathrm{E}-02$ & $1.91 \mathrm{E}-06$ \\
\hline 200E P-241BY101 001 & 2-Hexanone & $591-78-6$ & $5.00 \mathrm{E}-01$ & $3.47 \mathrm{E}-05$ \\
\hline 200E P-241BY101 001 & 2-Pentanone & $107-87-9$ & & $5.51 \mathrm{E}-05$ \\
\hline 200E P-241BY101 001 & Acetone & $67-64-1$ & $5.00 \mathrm{E}-01$ & $6.62 \mathrm{E}-04$ \\
\hline 200E P-241BY101 001 & Acetonitrile & $75-5-8$ & $5.00 \mathrm{E}-01$ & $3.19 \mathrm{E}-04$ \\
\hline 200E P-241BY101 001 & Ammonia & $7664-41-7$ & $5.00 \mathrm{E}-01$ & $3.04 \mathrm{E}-03$ \\
\hline 200E P-241BY101 001 & Benzene & $71-43-2$ & $5.00 \mathrm{E}-01$ & $1.51 \mathrm{E}-05$ \\
\hline 200E P-241BY101 001 & Carbon monoxide & $630-8-0$ & $5.00 \mathrm{E}+00$ & $4.39 \mathrm{E}-04$ \\
\hline 200E P-241BY101 001 & Heptane & $142-82-5$ & $5.00 \mathrm{E}-01$ & $3.89 \mathrm{E}-05$ \\
\hline 200E P-241BY101001 & Hexane, n-hexane & $110-54-3$ & $5.00 \mathrm{E}-01$ & $4.05 \mathrm{E}-05$ \\
\hline
\end{tabular}




\section{Table K.2. Insignificant Emission Units Based on Threshold Comparisons} [WAC 173-401-530(1)(a)]

\begin{tabular}{|c|c|c|c|c|}
\hline Emission Point & Pollutants & CAS Number & $\begin{array}{l}\text { Emission } \\
\text { Threshold } \\
\text { (tons/yr) }\end{array}$ & $\begin{array}{c}\text { Actual } \\
\text { Emissions } \\
\text { (tons/yr) }\end{array}$ \\
\hline 200E P-241BY101 001 & $\begin{array}{l}\text { Methyl N-amyl ketone, 2- } \\
\text { heptanone }\end{array}$ & $110-43-0$ & $5.00 \mathrm{E}-01$ & $3.76 \mathrm{E}-05$ \\
\hline 200E P-241BY101 001 & Nitrogen Oxide $\{\mathrm{NO}\}$ & $10102-43-9$ & $2.00 \mathrm{E}+00$ & 2.64E-05 \\
\hline 200E P-241BY101 001 & Nonane & $111-84-2$ & $5.00 \mathrm{E}-01$ & $2.11 \mathrm{E}-05$ \\
\hline 200E P-241BY101 001 & Octane & $111-65-9$ & $5.00 \mathrm{E}-01$ & $2.21 \mathrm{E}-05$ \\
\hline 200E P-241BY101 001 & $\begin{array}{l}\text { Phosphoric acid, tributyl } \\
\text { ester }\end{array}$ & $126-73-8$ & & $7.95 \mathrm{E}-05$ \\
\hline 200E P-241BY101 001 & Toluene & $108-88-3$ & $5.00 \mathrm{E}-01$ & $3.08 \mathrm{E}-06$ \\
\hline 200E P-241BY102 001 & 1,3-Butadiene & $106-99-0$ & $3.50 \mathrm{E}-02$ & $1.91 \mathrm{E}-06$ \\
\hline 200E P-241BY102 001 & 2-Hexanone & $591-78-6$ & $5.00 \mathrm{E}-01$ & $3.47 \mathrm{E}-05$ \\
\hline 200E P-241BY102 001 & 2-Pentanone & $107-87-9$ & & $5.51 \mathrm{E}-05$ \\
\hline 200E P-241BY102 001 & Acetone & $67-64-1$ & $5.00 \mathrm{E}-01$ & $6.62 \mathrm{E}-04$ \\
\hline 200E P-241BY102 001 & Acetonitrile & $75-5-8$ & $5.00 \mathrm{E}-01$ & $3.19 \mathrm{E}-04$ \\
\hline 200E P-241BY102 001 & Ammonia & $7664-41-7$ & $5.00 \mathrm{E}-01$ & $3.04 \mathrm{E}-03$ \\
\hline 200E P-241BY102 001 & Benzene & $71-43-2$ & $5.00 \mathrm{E}-01$ & $1.51 E-05$ \\
\hline 200E P-241BY102 001 & Carbon monoxide & $630-8-0$ & $5.00 \mathrm{E}+00$ & 4.39E-04 \\
\hline 200E P-241BY102 001 & Heptane & $142-82-5$ & $5.00 \mathrm{E}-01$ & $3.89 \mathrm{E}-05$ \\
\hline 200E P-241BY102 001 & Hexane, n-hexane & $110-54-3$ & $5.00 \mathrm{E}-01$ & $4.05 \mathrm{E}-05$ \\
\hline 200E P-241BY102 001 & $\begin{array}{l}\text { Methyl N-amyl ketone, 2- } \\
\text { heptanone }\end{array}$ & $110-43-0$ & $5.00 \mathrm{E}-01$ & $3.76 \mathrm{E}-05$ \\
\hline 200E P-241BY102 001 & Nitrogen Oxide $\{\mathrm{NO}\}$ & $10102-43-9$ & $2.00 \mathrm{E}+00$ & $2.64 \mathrm{E}-05$ \\
\hline 200E P-241BY102 001 & Nonane & $111-84-2$ & $5.00 \mathrm{E}-01$ & $2.11 \mathrm{E}-05$ \\
\hline 200E P-241BY102 001 & Octane & $111-65-9$ & $5.00 \mathrm{E}-01$ & $2.21 \mathrm{E}-05$ \\
\hline 200E P-241BY102 001 & $\begin{array}{l}\text { Phosphoric acid, tributyl } \\
\text { ester }\end{array}$ & $126-73-8$ & & $7.95 \mathrm{E}-05$ \\
\hline 200E P-241BY102 001 & Toluene & $108-88-3$ & $5.00 \mathrm{E}-01$ & $3.08 \mathrm{E}-06$ \\
\hline 200E P-241BY103 001 & 1,3-Butadiene & $106-99-0$ & $3.50 \mathrm{E}-02$ & $1.91 \mathrm{E}-06$ \\
\hline 200E P-241BY103 001 & 2-Hexanone & $591-78-6$ & $5.00 \mathrm{E}-01$ & $3.47 \mathrm{E}-05$ \\
\hline 200E P-241BY103 001 & 2-Pentanone & $107-87-9$ & & $5.51 E-05$ \\
\hline 200E P-241BY103 001 & Acetone & $67-64-1$ & $5.00 \mathrm{E}-01$ & $6.62 \mathrm{E}-04$ \\
\hline
\end{tabular}


Table K.2. Insignificant Emission Units Based on Threshold Comparisons [WAC 173-401-530(1)(a)]

\begin{tabular}{|c|c|c|c|c|}
\hline Emission Point & Pollutants & CAS Number & $\begin{array}{l}\text { Emission } \\
\text { Threshold } \\
\text { (tons/yr) }\end{array}$ & $\begin{array}{c}\text { Actual } \\
\text { Emissions } \\
\text { (tons/yr) }\end{array}$ \\
\hline 200E P-241BY103001 & Acetonitrile & $75-5-8$ & $5.00 \mathrm{E}-01$ & $3.19 \mathrm{E}-04$ \\
\hline 200E P-241BY103 001 & Ammonia & $7664-41-7$ & $5.00 \mathrm{E}-01$ & $3.04 \mathrm{E}-03$ \\
\hline 200E P-241BY103 001 & Benzene & $71-43-2$ & $5.00 \mathrm{E}-01$ & $1.51 \mathrm{E}-05$ \\
\hline 200E P-241BY103001 & Carbon monoxide & $630-8-0$ & $5.00 \mathrm{E}+00$ & $4.39 \mathrm{E}-04$ \\
\hline 200E P-241BY103001 & Heptane & $142-82-5$ & $5.00 \mathrm{E}-01$ & $3.89 E-05$ \\
\hline 200E P-241BY103 001 & Hexane, $n$-hexane & $110-54-3$ & $5.00 \mathrm{E}-01$ & $4.05 \mathrm{E}-05$ \\
\hline 200E P-241BY103 001 & $\begin{array}{l}\text { Methyl N-amyl ketone, 2- } \\
\text { heptanone }\end{array}$ & $110-43-0$ & $5.00 \mathrm{E}-01$ & $3.76 \mathrm{E}-05$ \\
\hline 200E P-241BY103001 & Nitrogen Oxide $\{\mathrm{NO}\}$ & $10102-43-9$ & $2.00 \mathrm{E}+00$ & $2.64 \mathrm{E}-05$ \\
\hline 200E P-241BY103 001 & Nonane & $111-84-2$ & $5.00 \mathrm{E}-01$ & $2.11 \mathrm{E}-05$ \\
\hline $200 \mathrm{E}$ P-241BY103 001 & Octane & $111-65-9$ & $5.00 \mathrm{E}-01$ & $2.21 \mathrm{E}-05$ \\
\hline 300E P-241BY103001 & $\begin{array}{l}\text { Phosphoric acid, tributyl } \\
\text { ester }\end{array}$ & $126-73-8$ & & $7.95 \mathrm{E}-05$ \\
\hline 200E P-241BY103 001 & Toluene & $108-88-3$ & $5.00 \mathrm{E}-01$ & $3.08 \mathrm{E}-06$ \\
\hline S(X)E P-241BY104001 & 2-Hexanone & $591-78-6$ & $5.00 \mathrm{E}-01$ & $3.83 \mathrm{E}-06$ \\
\hline 200E P-241BY104 001 & 2-Pentanone & $107-87-9$ & & $8.10 \mathrm{E}-06$ \\
\hline 200E P-241BY104001 & Acetone & $67-64-1$ & $5.00 \mathrm{E}-01$ & $4.10 \mathrm{E}-05$ \\
\hline 200E P-241BY104001 & Acetonitrile & $75-5-8$ & $5.00 \mathrm{E}-01$ & $7.97 \mathrm{E}-06$ \\
\hline 200E P-241BY104 001 & Ammonia & $7664-41-7$ & $5.00 \mathrm{E}-01$ & $2.48 \mathrm{E}-03$ \\
\hline 200E P-241BY104001 & Benzene & $71-43-2$ & $5.00 \mathrm{E}-01$ & $1.93 E-06$ \\
\hline 200E P-241BY104 001 & Heptane & $142-82-5$ & $5.00 \mathrm{E}-01$ & $7.07 \mathrm{E}-06$ \\
\hline 200E P-241BY104 001 & Hexane, n-hexane & $110-54-3$ & $5.00 \mathrm{E}-01$ & $1.62 \mathrm{E}-05$ \\
\hline 200E P-241BY104001 & $\begin{array}{l}\text { Methyl N-amyl ketone, 2- } \\
\text { heptanone }\end{array}$ & $110-43-0$ & $5.00 \mathrm{E}-01$ & $3.22 \mathrm{E}-06$ \\
\hline 200E P-241BY104001 & Methylene chloride & $75-9-2$ & $5.00 \mathrm{E}-01$ & $9.00 \mathrm{E}-08$ \\
\hline 200E P-241BY104001 & Nitrogen Oxide $\{$ NO $\}$ & $10102-43-9$ & $2.00 \mathrm{E}+00$ & $5.29 \mathrm{E}-06$ \\
\hline 200E P-241BY104001 & Nonane & $111-84-2$ & $5.00 \mathrm{E}-01$ & $2.11 \mathrm{E}-06$ \\
\hline 200E P-241BY104001 & Octane & $111-65-9$ & $5.00 \mathrm{E}-01$ & $3.96 \mathrm{E}-06$ \\
\hline 200E P-241BY104001 & $\begin{array}{l}\text { Phosphoric acid, tributyl } \\
\text { ester }\end{array}$ & $126-73-8$ & & $1.28 \mathrm{E}-07$ \\
\hline 200E P-241BY104001 & Toluene & $108-88-3$ & $5.00 \mathrm{E}-01$ & $2.22 \mathrm{E}-06$ \\
\hline
\end{tabular}


Table K.2. Insignificant Emission Units Based on Threshold Comparisons [WAC 173-401-530(1)(a)]

\begin{tabular}{|c|c|c|c|c|}
\hline Emission Point & Pollutants & CAS Number & $\begin{array}{l}\text { Emission } \\
\text { Threshold } \\
\text { (tons/yr) }\end{array}$ & $\begin{array}{c}\text { Actual } \\
\text { Emissions } \\
\text { (tons/yr) }\end{array}$ \\
\hline 200E P-241BY105 001 & 2-Hexanone & $591-78-6$ & $5.00 \mathrm{E}-01$ & $3.18 \mathrm{E}-07$ \\
\hline 200E P-241BY105 001 & 2-Pentanone & $107-87-9$ & & $6.07 \mathrm{E}-07$ \\
\hline 200E P-241BY105 001 & Acetone & $67-64-1$ & $5.00 \mathrm{E}-01$ & $8.54 \mathrm{E}-06$ \\
\hline 200E P-241BY105 001 & Acetonitrile & $75-5-8$ & $5.00 \mathrm{E}-01$ & $1.96 \mathrm{E}-06$ \\
\hline 200E P-241BY105 001 & Ammonia & $7664-41-7$ & $5.00 \mathrm{E}-01$ & $4.30 \mathrm{E}-04$ \\
\hline 200E P-241BY105 001 & Benzene & $71-43-2$ & $5.00 \mathrm{E}-01$ & $3.17 \mathrm{E}-07$ \\
\hline 200E P-241BY105 001 & Carbon monoxide & $630-8-0$ & $5.00 \mathrm{E}+00$ & $8.23 \mathrm{E}-06$ \\
\hline 200E P-241BY105 001 & Heptane & $142-82-5$ & $5.00 \mathrm{E}-01$ & $2.30 \mathrm{E}-06$ \\
\hline 200E P-241BY105 001 & Hexane, n-hexane & $110-54-3$ & $5.00 \mathrm{E}-01$ & $4.36 \mathrm{E}-06$ \\
\hline 200E P-241BY105 001 & $\begin{array}{l}\text { Methyl N-amyl ketone, 2- } \\
\text { heptanone }\end{array}$ & $110-43-0$ & $5.00 \mathrm{E}-01$ & $2.95 \mathrm{E}-07$ \\
\hline 200E P-241BY105 001 & Methylene chloride & $75-9-2$ & $5.00 \mathrm{E}-01$ & $2.59 \mathrm{E}-07$ \\
\hline 200E P-241BY105 001 & Nitrogen Oxide $\{\mathrm{NO}\}$ & $10102-43-9$ & $2.00 \mathrm{E}+00$ & $1.41 \mathrm{E}-06$ \\
\hline 200E P-241BY105001 & Nonane & $111-84-2$ & $5.00 \mathrm{E}-01$ & $4.52 \mathrm{E}-07$ \\
\hline 200E P-241BY105 001 & Octane & $111-65-9$ & $5.00 \mathrm{E}-01$ & $1.01 \mathrm{E}-06$ \\
\hline 200E P-241BY105 001 & $\begin{array}{l}\text { Phosphoric acid, tributyl } \\
\text { ester }\end{array}$ & $126-73-8$ & & $2.70 \mathrm{E}-08$ \\
\hline 200E P-241BY105 001 & Toluene & $108-88-3$ & $5.00 \mathrm{E}-01$ & $4.65 \mathrm{E}-07$ \\
\hline 200E P-241BY106001 & 2-Hexanone & $591-78-6$ & $5.00 \mathrm{E}-01$ & $2.47 \mathrm{E}-07$ \\
\hline 200E P-241BY106 001 & 2-Pentanone & $107-87-9$ & & 5.57E-07 \\
\hline 200E P-241BY106 001 & Acetone & $67-64-1$ & $5.00 \mathrm{E}-01$ & $1.26 \mathrm{E}-05$ \\
\hline 200E P-241BY106 001 & Acetonitrile & $75-5-8$ & $5.00 \mathrm{E}-01$ & $2.90 \mathrm{E}-06$ \\
\hline 200E P-241BY106 001 & Ammonia & $7664-41-7$ & $5.00 \mathrm{E}-01$ & $7.39 \mathrm{E}-04$ \\
\hline 200E P-241BY106 001 & Benzene & $71-43-2$ & $5.00 \mathrm{E}-01$ & $3.35 \mathrm{E}-07$ \\
\hline 200E P-241BY106 001 & Heptane & $142-82-5$ & $5.00 \mathrm{E}-01$ & $1.83 \mathrm{E}-06$ \\
\hline 200E P-241BY106 001 & Hexane, n-hexane & $110-54-3$ & $5.00 \mathrm{E}-01$ & $4.31 \mathrm{E}-06$ \\
\hline 200E P-241BY106 001 & $\begin{array}{l}\text { Methyl N-amyl ketone, 2- } \\
\text { heptanone }\end{array}$ & $110-43-0$ & $5.00 \mathrm{E}-01$ & $2.15 \mathrm{E}-07$ \\
\hline 200E P-241BY106 001 & Methylene chloride & $75-9-2$ & $5.00 \mathrm{E}-01$ & $2.30 \mathrm{E}-08$ \\
\hline 200E P-241BY106 001 & Nitrogen Oxide $\{\mathrm{NO}\}$ & $10102-43-9$ & $2.00 \mathrm{E}+00$ & $1.76 \mathrm{E}-06$ \\
\hline
\end{tabular}


Hanford Site Air

Table K.2. Insignificant Emission Units Based on Threshold Comparisons [WAC 173-401-530(1)(a)]

\begin{tabular}{|c|c|c|c|c|}
\hline Emission Point & Pollutants & CAS Number & $\begin{array}{l}\text { Emission } \\
\text { Threshold } \\
\text { (tons/yr) }\end{array}$ & $\begin{array}{c}\text { Actual } \\
\text { Emissions } \\
\text { (tons/yr) }\end{array}$ \\
\hline 200E P-241BY106 001 & Nonane & $111-84-2$ & $5.00 \mathrm{E}-01$ & $3.47 \mathrm{E}-07$ \\
\hline 200E P-241BY106 001 & Octane & $111-65-9$ & $5.00 \mathrm{E}-01$ & $8.05 \mathrm{E}-07$ \\
\hline 200E P-241BY106 001 & $\begin{array}{l}\text { Phosphoric acid, tributyl } \\
\text { ester }\end{array}$ & $126-73-8$ & & $7.00 \mathrm{E}-09$ \\
\hline 200E P-241BY106 001 & Toluene & $108-88-3$ & $5.00 \mathrm{E}-01$ & $4.49 \mathrm{E}-07$ \\
\hline 200E P-241BY107 001 & 1,3-Butadiene & $106-99-0$ & $3.50 \mathrm{E}-02$ & $1.91 \mathrm{E}-06$ \\
\hline 200E P-241BY107 001 & 2-Hexanone & $591-78-6$ & $5.00 \mathrm{E}-01$ & $3.47 \mathrm{E}-05$ \\
\hline 200E P-241BY107 001 & 2-Pentanone & $107-87-9$ & & $5.51 \mathrm{E}-05$ \\
\hline 200E P-241BY107 001 & Acetone & $67-64-1$ & $5.00 \mathrm{E}-01$ & $6.62 \mathrm{E}-04$ \\
\hline 200E P-241BY107001 & Acetonitrile & $75-5-8$ & $5.00 \mathrm{E}-01$ & $3.19 \mathrm{E}-04$ \\
\hline 200E P-241BY107 001 & Ammonia & $7664-41-7$ & $5.00 \mathrm{E}-01$ & $3.04 \mathrm{E}-03$ \\
\hline 200E P-241BY107001 & Benzene & $71-43-2$ & $5.00 \mathrm{E}-01$ & $1.51 \mathrm{E}-05$ \\
\hline 200E P-241BY107001 & Carbon monoxide & $630-8-0$ & $5.00 \mathrm{E}+00$ & $4.39 \mathrm{E}-04$ \\
\hline 200E P-241BY107 001 & Heptane & $142-82-5$ & $5.00 \mathrm{E}-01$ & $3.89 \mathrm{E}-05$ \\
\hline 200E P-241BY107001 & Hexane, $n$-hexane & $110-54-3$ & $5.00 \mathrm{E}-01$ & $4.05 \mathrm{E}-05$ \\
\hline 200E P-241BY107001 & $\begin{array}{l}\text { Methyl N-amyl ketone, 2- } \\
\text { heptanone }\end{array}$ & $110-43-0$ & $5.00 \mathrm{E}-01$ & $3.76 \mathrm{E}-05$ \\
\hline 200E P-241BY107 001 & Nitrogen Oxide $\{\mathrm{NO}\}$ & $10102-43-9$ & $2.00 \mathrm{E}+00$ & $2.64 \mathrm{E}-05$ \\
\hline 200E P-241BY107 001 & Nonane & $111-84-2$ & $5.00 \mathrm{E}-01$ & 2.11E-05 \\
\hline 200E P-241BY107001 & Octane & $111-65-9$ & $5.00 \mathrm{E}-01$ & $2.21 \mathrm{E}-05$ \\
\hline 200E P-241BY107 001 & $\begin{array}{l}\text { Phosphoric acid, tributyl } \\
\text { ester }\end{array}$ & $126-73-8$ & & $7.95 \mathrm{E}-05$ \\
\hline 200E P-241BY107 001 & Toluene & $108-88-3$ & $5.00 \mathrm{E}-01$ & $3.08 \mathrm{E}-06$ \\
\hline 200E P-241BY108 001 & 1,3-Butadiene & $106-99-0$ & $3.50 \mathrm{E}-02$ & $1.91 \mathrm{E}-06$ \\
\hline 200E P-241BY108 001 & 2-Hexanone & $591-78-6$ & $5.00 \mathrm{E}-01$ & $3.47 \mathrm{E}-05$ \\
\hline 200E P-241BY108001 & 2-Pentanone & $107-87-9$ & & $5.51 E-05$ \\
\hline 200E P-241BY108001 & Acetone & $67-64-1$ & $5.00 \mathrm{E}-01$ & $6.62 \mathrm{E}-04$ \\
\hline 200E P-241BY108001 & Acetonitrile & $75-5-8$ & $5.00 \mathrm{E}-01$ & $3.19 \mathrm{E}-04$ \\
\hline 200E P-241BY108 001 & Ammonia & $7664-41-7$ - & $5.00 \mathrm{E}-01$ & $3.04 \mathrm{E}-03$ \\
\hline 200E P-241BY108 001 & Benzene & $71-43-2$ & $5.00 \mathrm{E}-01$ & $1.51 \mathrm{E}-05$ \\
\hline
\end{tabular}


Table K.2. Insignificant Emission Units Based on Threshold Comparisons [WAC 173-401-530(1)(a)]

\begin{tabular}{|c|c|c|c|c|}
\hline Emission Point & Pollutants & CAS Number & $\begin{array}{l}\text { Emission } \\
\text { Threshold } \\
\text { (tons/yr) }\end{array}$ & $\begin{array}{c}\text { Actual } \\
\text { Emissions } \\
\text { (tons/yr) }\end{array}$ \\
\hline 200E P-241BY108 001 & Carbon monoxide & $630-8-0$ & $5.00 \mathrm{E}+00$ & $4.39 \mathrm{E}-04$ \\
\hline 200E P-241BY108 001 & Heptane & $142-82-5$ & $5.00 \mathrm{E}-01$ & $3.89 \mathrm{E}-05$ \\
\hline 200E P-241BY108 001 & Hexane, n-hexane & $110-54-3$ & $5.00 \mathrm{E}-01$ & $4.05 \mathrm{E}-05$ \\
\hline 200E P-241BY108 001 & $\begin{array}{l}\text { Methyl N-amyl ketone, 2- } \\
\text { heptanone }\end{array}$ & $110-43-0$ & $5.00 \mathrm{E}-01$ & $3.76 \mathrm{E}-05$ \\
\hline 200E P-241BY108 001 & Nitrogen Oxide $\{\mathrm{NO}\}$ & $10102-43-9$ & $2.00 \mathrm{E}+00$ & $2.64 \mathrm{E}-05$ \\
\hline 200E P-241BY108 001 & Nonane & $111-84-2$ & $5.00 \mathrm{E}-01$ & $2.11 \mathrm{E}-05$ \\
\hline 200E P-241BY108 001 & Octane & $111-65-9$ & $5.00 \mathrm{E}-01$ & $2.21 \mathrm{E}-05$ \\
\hline 200E P-241BY108 001 & $\begin{array}{l}\text { Phosphoric acid, tributyl } \\
\text { ester }\end{array}$ & $126-73-8$ & & $7.95 \mathrm{E}-05$ \\
\hline 200E P-241BY108 001 & Toluene & $108-88-3$ & $5.00 \mathrm{E}-01$ & $3.08 \mathrm{E}-06$ \\
\hline 200E P-241BY109 001 & 1,3-Butadiene & $106-99-0$ & $3.50 \mathrm{E}-02$ & $4.13 \mathrm{E}-06$ \\
\hline 200E P-241BY109 001 & 2-Hexanone & $591-78-6$ & $5.00 \mathrm{E}-01$ & $7.51 E-05$ \\
\hline 200E P-241BY109 001 & 2-Pentanone & $107-87-9$ & & $1.19 \mathrm{E}-04$ \\
\hline 200E P-241BY109 001 & Acetone & $67-64-1$ & $5.00 \mathrm{E}-01$ & $1.43 \mathrm{E}-03$ \\
\hline 200E P-241BY109 001 & Acetonitrile & $75-5-8$ & $5.00 \mathrm{E}-01$ & $6.89 \mathrm{E}-04$ \\
\hline 200E P-241BY109 001 & Ammonia & $7664-41-7$ & $5.00 \mathrm{E}-01$ & $6.57 \mathrm{E}-03$ \\
\hline 200E P-241BY109 001 & Benzene & $71-43-2$ & $5.00 \mathrm{E}-01$ & $3.28 \mathrm{E}-05$ \\
\hline 200E P-241BY109 001 & Carbon monoxide & $630-8-0$ & $5.00 \mathrm{E}+00$ & $9.50 \mathrm{E}-04$ \\
\hline 200E P-241BY109 001 & Heptane & $142-82-5$ & $5.00 \mathrm{E}-01$ & $8.40 \mathrm{E}-05$ \\
\hline 200E P-241BY109 001 & Hexane, n-hexane & $110-54-3$ & $5.00 \mathrm{E}-01$ & $8.76 \mathrm{E}-05$ \\
\hline 200E P-241BY109 001 & $\begin{array}{l}\text { Methyl N-amyl ketone, 2- } \\
\text { heptanone }\end{array}$ & $110-43-0$ & $5.00 \mathrm{E}-01$ & $8.13 \mathrm{E}-05$ \\
\hline 200E P-241BY109 001 & Nitrogen Oxide $\{\mathrm{NO}\}$ & $10102-43-9$ & $2.00 \mathrm{E}+00$ & $5.72 \mathrm{E}-05$ \\
\hline 200E P-241BY109 001 & Nonane & $111-84-2$ & $5.00 \mathrm{E}-01$ & $4.57 \mathrm{E}-05$ \\
\hline 200E P-241BY109 001 & Octane & $111-65-9$ & $5.00 \mathrm{E}-01$ & $4.79 \mathrm{E}-05$ \\
\hline 200E P-241BY109 001 & $\begin{array}{l}\text { Phosphoric acid, tributyl } \\
\text { ester }\end{array}$ & $126-73-8$ & & $1.72 \mathrm{E}-04$ \\
\hline 200E P-241BY109 001 & Toluene & $108-88-3$ & $5.00 \mathrm{E}-01$ & $6.67 \mathrm{E}-06$ \\
\hline 200E P-241BY110 001 & 1,3-Butadiene & $106-99-0$ & $3.50 \mathrm{E}-02$ & $1.91 \mathrm{E}-06$ \\
\hline
\end{tabular}




\section{Table K.2. Insignificant Emission Units Based on Threshold Comparisons} [WAC 173-401-530(1)(a)]

\begin{tabular}{|c|c|c|c|c|}
\hline Emission Point & Pollutants & CAS Number & $\begin{array}{c}\text { Emission } \\
\text { Threshold } \\
\text { (tons/yr) }\end{array}$ & $\begin{array}{c}\text { Actual } \\
\text { Emissions } \\
\text { (tons/yr) }\end{array}$ \\
\hline 200E P-241BY110 001 & 2-Hexanone & $591-78-6$ & $5.00 \mathrm{E}-01$ & $3.47 \mathrm{E}-05$ \\
\hline 200E P-241BY110001 & 2-Pentanone & $107-87-9$ & & $5.51 \mathrm{E}-05$ \\
\hline 200E P-241BY110 001 & Acetone & $67-64-1$ & $5.00 \mathrm{E}-01$ & $6.62 \mathrm{E}-04$ \\
\hline 200E P-241BY110 001 & Acetonitrile & $75-5-8$ & $5.00 \mathrm{E}-01$ & $3.19 E-04$ \\
\hline 200E P-241BY110001 & Ammonia & $7664-41-7$ & $5.00 \mathrm{E}-01$ & $3.04 \mathrm{E}-03$ \\
\hline 200E P-241BY110 001 & Benzene & $71-43-2$ & $5.00 \mathrm{E}-01$ & $1.51 \mathrm{E}-05$ \\
\hline 200E P-241BY110 001 & Carbon monoxide & $630-8-0$ & $5.00 \mathrm{E}+00$ & $4.39 \mathrm{E}-04$ \\
\hline 200E P-241BY110 001 & Heptane & $142-82-5$ & $5.00 \mathrm{E}-01$ & $3.89 \mathrm{E}-05$ \\
\hline 200E P-241BY110 001 & Hexane, n-hexane & $110-54-3$ & $5.00 \mathrm{E}-01$ & $4.05 \mathrm{E}-05$ \\
\hline 200E P-241BY110001 & $\begin{array}{l}\text { Methyl N-amyl ketone, 2- } \\
\text { heptanone }\end{array}$ & $110-43-0$ & $5.00 \mathrm{E}-01$ & $3.76 \mathrm{E}-05$ \\
\hline 200E P-241BY110 001 & Nitrogen Oxide $\{\mathrm{NO}\}$ & $10102-43-9$ & $2.00 \mathrm{E}+00$ & $2.64 \mathrm{E}-05$ \\
\hline 200E P-241BY110 001 & Nonane & $111-84-2$ & $5.00 \mathrm{E}-01$ & $2.11 \mathrm{E}-05$ \\
\hline 200E P-241BY110 001 & Octane & $111-65-9$ & $5.00 \mathrm{E}-01$ & $2.21 \mathrm{E}-05$ \\
\hline 200E P-241BY110 001 & $\begin{array}{l}\text { Phosphoric acid, tributyl } \\
\text { ester }\end{array}$ & $126-73-8$ & & $7.95 \mathrm{E}-05$ \\
\hline 200E P-241BY110 001 & Toluene & $108-88-3$ & $5.00 \mathrm{E}-01$ & $3.08 \mathrm{E}-06$ \\
\hline 200E P-241BY111 001 & 1,3-Butadiene & 106-99=0 & $3.50 \mathrm{E}-02$ & $1.91 \mathrm{E}-06$ \\
\hline 200E P-241BY111001 & 2-Hexanone & $591-78-6$ & $5.00 E-01$ & $3.47 \mathrm{E}-05$ \\
\hline 200E P-241BY111001 & 2-Pentanone & $107-87-9$ & & $5.51 \mathrm{E}-05$ \\
\hline 200E P-241BY111 001 & Acetone & $67-64-1$ & $5.00 \mathrm{E}-01$ & $6.62 \mathrm{E}-04$ \\
\hline 200E P-241BY111001 & Acetonitrile & $75-5-8$ & $5.00 \mathrm{E}-01$ & $3.19 \mathrm{E}-04$ \\
\hline 200E P-241BY111 001 & Ammonia & $7664-41-7$ & $5.00 E-01$ & $3.04 \mathrm{E}-03$ \\
\hline 200E P-241BY111 001 & Benzene & $71-43-2$ & $5.00 \mathrm{E}-01$ & $1.51 \mathrm{E}-05$ \\
\hline 200E P-241BY111 001 & Carbon monoxide & $630-8-0$ & $5.00 \mathrm{E}+00$ & $4.39 \mathrm{E}-04$ \\
\hline 200E P-241BY111 001 & Heptane & $142-82-5$ & $5.00 \mathrm{E}-01$ & $3.89 \mathrm{E}-05$ \\
\hline 200E P-241BY111001 & Hexane, n-hexane & $110-54-3$ & $5.00 \mathrm{E}-01$ & $4.05 \mathrm{E}-05$ \\
\hline 200E P-241BY111 001 & $\begin{array}{l}\text { Methyl N-amyl ketone, 2- } \\
\text { heptanone }\end{array}$ & $110-43-0$ & $5.00 \mathrm{E}-01$ & $3.76 \mathrm{E}-05$ \\
\hline 200E P-241BY111 001 & Nitrogen Oxide $\{\mathrm{NO}\}$ & $10102-43-9$ & $2.00 \mathrm{E}+00$ & $2.64 \mathrm{E}-05$ \\
\hline 200E P-241BY111 001 & Nonane & $111-84-2$ & $5.00 \mathrm{E}-01$ & $2.11 E-05$ \\
\hline
\end{tabular}


Table K.2. Insignificant Emission Units Based on Threshold Comparisons [WAC 173-401-530(1)(a)]

\begin{tabular}{|c|c|c|c|c|}
\hline Emission Point & Pollutants & CAS Number & $\begin{array}{l}\text { Emission } \\
\text { Threshold } \\
\text { (tons/yr) }\end{array}$ & $\begin{array}{c}\text { Actual } \\
\text { Emissions } \\
\text { (tons/yr) }\end{array}$ \\
\hline 200E P-241BY111 001 & Octane & $111-65-9$ & $5.00 \mathrm{E}-01$ & $2.21 \mathrm{E}-05$ \\
\hline 200E P-241BY111 001 & $\begin{array}{l}\text { Phosphoric acid, tributyl } \\
\text { ester }\end{array}$ & $126-73-8$ & & 7.95E-05 \\
\hline 200E P-241BY111 001 & Toluene & $108-88-3$ & $5.00 \mathrm{E}-01$ & $3.08 \mathrm{E}-06$ \\
\hline 200E P-241BY112001 & 1,3-Butadiene & $106-99-0$ & $3.50 \mathrm{E}-02$ & $1.91 \mathrm{E}-06$ \\
\hline 200E P-241BY112 001 & 2-Hexanone & $591-78-6$ & $5.00 \mathrm{E}-01$ & 3.47E-05 \\
\hline 200E P-241BY112001 & 2-Pentanone & $107-87-9$ & & $5.51 \mathrm{E}-05$ \\
\hline 200E P-241BY112001 & Acetone & $67-64-1$ & $5.00 \mathrm{E}-01$ & $6.62 \mathrm{E}-04$ \\
\hline 200E P-241BY112 001 & Acetonitrile & $75-5-8$ & $5.00 \mathrm{E}-01$ & $3.19 \mathrm{E}-04$ \\
\hline 200E P-241BY112 001 & Ammonia & $7664-41-7$ & $5.00 \mathrm{E}-01$ & $3.04 \mathrm{E}-03$ \\
\hline 200E P-241BY112001 & Benzene & $71-43-2$ & $5.00 \mathrm{E}-01$ & $1.51 \mathrm{E}-05$ \\
\hline 200E P-241BY112001 & Carbon monoxide & $630-8-0$ & $5.00 \mathrm{E}+00$ & 4.39E-04 \\
\hline 200E P-241BY112001 & Heptane & $142-82-5$ & $5.00 \mathrm{E}-01$ & $3.89 \mathrm{E}-05$ \\
\hline 200E P-241BY112 001 & Hexane, n-hexane & $110-54-3$ & $5.00 \mathrm{E}-01$ & $4.05 \mathrm{E}-05$ \\
\hline 200E P-241BY112001 & $\begin{array}{l}\text { Methyl N-amyl ketone, 2- } \\
\text { heptanone }\end{array}$ & $110-43-0$ & $5.00 \mathrm{E}-01$ & $3.76 \mathrm{E}-05$ \\
\hline 200E P-241BY112 001 & Nitrogen Oxide $\{\mathrm{NO}\}$ & $10102-43-9$ & $2.00 \mathrm{E}+00$ & $2.64 \mathrm{E}-05$ \\
\hline 200E P-241BY112 001 & Nonane & $111-84-2$ & $5.00 \mathrm{E}-01$ & $2.11 \mathrm{E}-05$ \\
\hline 200E P-241BY112001 & Octane & $111-65-9$ & $5.00 \mathrm{E}-01$ & $2.21 E-05$ \\
\hline 200E P-241BY112001 & $\begin{array}{l}\text { Phosphoric acid, tributyl } \\
\text { ester }\end{array}$ & $126-73-8$ & & $7.95 \mathrm{E}-05$ \\
\hline 200E P-241BY112001 & Toluene & $108-88-3$ & $5.00 \mathrm{E}-01$ & $3.08 \mathrm{E}-06$ \\
\hline 200E P-241C101 001 & 1,3-Butadiene & $106-99-0$ & $3.50 \mathrm{E}-02$ & 1.91E-06 \\
\hline 200E P-241C101 001 & 2-Hexanone & $591-78-6$ & $5.00 \mathrm{E}-01$ & $3.47 \mathrm{E}-05$ \\
\hline 200E P-241C101 001 & 2-Pentanone & $107-87-9$ & & $5.51 \mathrm{E}-05$ \\
\hline 200E P-241C101 001 & Acetone & $67-64-1$ & $5.00 \mathrm{E}-01$ & $6.62 \mathrm{E}-04$ \\
\hline 200E P-241C101 001 & Acetonitrile & $75-5-8$ & $5.00 \mathrm{E}-01$ & $3.19 \mathrm{E}-04$ \\
\hline 200E P-241C101 001 & Ammonia & $7664-41-7$ & $5.00 \mathrm{E}-01$ & $3.04 \mathrm{E}-03$ \\
\hline 200E P-241C101 001 & Benzene & $71-43-2$ & $5.00 \mathrm{E}-01$ & $1.51 \mathrm{E}-05$ \\
\hline 200E P-241C101 001 & Carbon monoxide & $630-8-0$ & $5.00 \mathrm{E}+00$ & $4.39 E-04$ \\
\hline
\end{tabular}


Table K.2. Insignificant Emission Units Based on Threshold Comparisons [WAC 173-401-530(1)(a)]

\begin{tabular}{|c|c|c|c|c|}
\hline Emission Point & Pollutants & CAS Number & $\begin{array}{c}\text { Emission } \\
\text { Threshold } \\
\text { (tons/yr) }\end{array}$ & $\begin{array}{c}\text { Actual } \\
\text { Emissions } \\
\text { (tons/yr) }\end{array}$ \\
\hline 200E P-241C101 001 & Heptane & $142-82-5$ & $5.00 \mathrm{E}-01$ & $3.89 \mathrm{E}-05$ \\
\hline 200E P-241C101 001 & Hexane, r-hexane & $110-54-3$ & $5.00 \mathrm{E}-01$ & $4.05 \mathrm{E}-05$ \\
\hline 200E P-241C101 001 & $\begin{array}{l}\text { Methyl N-amyl ketone, 2- } \\
\text { heptanone }\end{array}$ & $110-43-0$ & $5.00 \mathrm{E}-01$ & $3.76 \mathrm{E}-05$ \\
\hline 200E P-241C101 001 & Nitrogen Oxide $\{\mathrm{NO}\}$ & $10102-43-9$ & $2.00 \mathrm{E}+00$ & $2.64 \mathrm{E}-05$ \\
\hline 200E P-241C101 001 & Nonane & $111-84-2$ & $5.00 \mathrm{E}-01$ & $2.11 \mathrm{E}-05$ \\
\hline 200E P-241C101 001 & Octane & $111-65-9$ & $5.00 \mathrm{E}-01$ & $2.21 \mathrm{E}-05$ \\
\hline 200E P-241C101 001 & $\begin{array}{l}\text { Phosphoric acid, tributyl } \\
\text { ester }\end{array}$ & $126-73-8$ & & $7.95 \mathrm{E}-05$ \\
\hline 200E P-241C101 001 & Toluene & $108-88-3$ & $5.00 \mathrm{E}-01$ & $3.08 E-06$ \\
\hline 200E P-241C102 001 & 1,3-Butadiene & $106-99-0$ & $3.50 \mathrm{E}-02$ & $4.13 \mathrm{E}-06$ \\
\hline 200 E P-241C102 001 & 2-Hexanone & $591-78-6$ & $5.00 \mathrm{E}-01$ & $7.51 \mathrm{E}-05$ \\
\hline 200 E P-241C102 001 & 2-Pentanone & $107-87-9$ & & $1.19 \mathrm{E}-04$ \\
\hline 200E P-241C102001 & Acetone & $67-64-1$ & $5.00 \mathrm{E}-01$ & $1.43 \mathrm{E}-03$ \\
\hline 200E P-241C102 001 & Acetonitrile & $75-5-8$ & $5.00 \mathrm{E}-01$ & $6.89 \mathrm{E}-04$ \\
\hline 200E P-241C102 001 & Ammonia & $7664-41-7$ & $5.00 \mathrm{E}-01$ & $6.57 \mathrm{E}-03$ \\
\hline 200E P-241C102001 & Benzene & $71-43-2$ & $5.00 \mathrm{E}-01$ & $3.28 \mathrm{E}-05$ \\
\hline 200E P-241C102 001 & Carbon monoxide & $630-8-0$ & $5.00 \mathrm{E}+00$ & $9.50 \mathrm{E}-04$ \\
\hline 200E P-241C102 001 & Heptane & $142-82-5$ & $5.00 \mathrm{E}-01$ & $8.40 \mathrm{E}-05$ \\
\hline 200E P-241C102 001 & Hexane, n-hexane & $110-54-3$ & $5.00 \mathrm{E}-01$ & $8.76 \mathrm{E}-05$ \\
\hline 200E P-241C102 001 & $\begin{array}{l}\text { Methyl N-amyl ketone, 2- } \\
\text { heptanone }\end{array}$ & $110-43-0$ & $5.00 \mathrm{E}-01$ & $8.13 E-05$ \\
\hline 200E P-241C102 001 & Nitrogen Oxide $\{\mathrm{NO}\}$ & $10102-43-9$ & $2.00 \mathrm{E}+00$ & $5.72 \mathrm{E}-05$ \\
\hline 200E P-241C102 001 & Nonane & $111-84-2$ & $5.00 \mathrm{E}-01$ & $4.57 \mathrm{E}-05$ \\
\hline 200E P-241C102 001 & Octane & $111-65-9$ & $5.00 \mathrm{E}-01$ & $4.79 \mathrm{E}-05$ \\
\hline 200E P-241C102 001 & $\begin{array}{l}\text { Phosphoric acid, tributyl } \\
\text { ester }\end{array}$ & $126-73-8$ & & 1.72E-04 \\
\hline 200E P-241C102 001 & Toluene & $108-88-3$ & $5.00 \mathrm{E}-01$ & $6.67 \mathrm{E}-06$ \\
\hline 200E P-241C103 001 & 1,3-Butadiene & $106-99-0$ & $3.50 \mathrm{E}-02$ & $4.13 \mathrm{E}-06$ \\
\hline 200E P-241C103 001 & 2-Hexanone & $591-78-6$ & $5.00 \mathrm{E}-01$ & $7.51 \mathrm{E}-05$ \\
\hline
\end{tabular}


Table K.2. Insignificant Emission Units Based on Threshold Comparisons [WAC 173-401-530(1)(a)]

\begin{tabular}{|c|c|c|c|c|}
\hline Emission Point & Pollutants & CAS Number & $\begin{array}{l}\text { Emission } \\
\text { Threshold } \\
\text { (tons/yr) }\end{array}$ & $\begin{array}{c}\text { Actual } \\
\text { Emissions } \\
\text { (tons/yr) }\end{array}$ \\
\hline 200E P-241C103 001 & 2-Pentanone & $107-87-9$ & & $1.19 \mathrm{E}-04$ \\
\hline 200E P-241C103001 & Acetone & $67-64-1$ & $5.00 \mathrm{E}-01$ & $1.43 \mathrm{E}-03$ \\
\hline 200E P-241C103 001 & Acetonitrile & $75-5-8$ & $5.00 \mathrm{E}-01$ & $6.89 \mathrm{E}-04$ \\
\hline 200E P-241C103001 & Ammonia & $7664-41-7$ & $5.00 \mathrm{E}-01$ & $6.57 \mathrm{E}-03$ \\
\hline 200E P-241C103 001 & Benzene & $71-43-2$ & $5.00 \mathrm{E}-01$ & $3.28 \mathrm{E}-05$ \\
\hline 200E P-241C103 001 & Carbon monoxide & $630-8-0$ & $5.00 \mathrm{E}+00$ & $9.50 \mathrm{E}-04$ \\
\hline 200E P-241C103001 & Heptane & $142-82-5$ & $5.00 \mathrm{E}-01$ & $8.40 \mathrm{E}-05$ \\
\hline 200E P-241C103001 & Hexane, n-hexane & $110-54-3$ & $5.00 \mathrm{E}-01$ & $8.76 \mathrm{E}-05$ \\
\hline 200 E P-241C103 001 & $\begin{array}{l}\text { Methyl N-amyl ketone, 2- } \\
\text { heptanone }\end{array}$ & $110-43-0$ & $5.00 \mathrm{E}-01$ & $8.13 E-05$ \\
\hline 200E P-241C103001 & Nitrogen Oxide $\{\mathrm{NO}\}$ & $10102-43-9$ & $2.00 \mathrm{E}+00$ & $5.72 \mathrm{E}-05$ \\
\hline 200E P-241C103001 & Nonane & $111-84-2$ & $5.00 \mathrm{E}-01$ & $4.57 \mathrm{E}-05$ \\
\hline 200 E P-241C103 001 & Octane & $111-65-9$ & $5.00 \mathrm{E}-01$ & 4.79E-05 \\
\hline 200E P-241C103001 & $\begin{array}{l}\text { Phosphoric acid, tributyl } \\
\text { ester }\end{array}$ & $126-73-8$ & & $1.72 \mathrm{E}-04$ \\
\hline 200 E P-241C103001 & Toluene & $108-88-3$ & $5.00 \mathrm{E}-01$ & $6.67 \mathrm{E}-06$ \\
\hline 200 E P-241C104 001 & 1,3-Butadiene & $106-99-0$ & $3.50 \mathrm{E}-02$ & $4.13 E-06$ \\
\hline 200E P-241C104001 & 2-Hexanone & $591-78-6$ & $5.00 \mathrm{E}-01$ & $7.51 \mathrm{E}-05$ \\
\hline 200E P-241C104001 & 2-Pentanone & $107-87-9$ & & $1.19 \mathrm{E}-04$ \\
\hline 200E P-241C104 001 & Acetone & $67-64-1$ & $5.00 \mathrm{E}-01$ & $1.43 \mathrm{E}-03$ \\
\hline 200E P-241C104001 & Acetonitrile & $75-5-8$ & $5.00 \mathrm{E}-01$ & $6.89 \mathrm{E}-04$ \\
\hline 200E P-241C104 001 & Ammonia & $7664-41-7$ & $5.00 E-01$ & $6.57 E-03$ \\
\hline 200E P-241C104 001 & Benzene & $71-43-2$ & $5.00 \mathrm{E}-01$ & $3.28 \mathrm{E}-05$ \\
\hline 200E P-241C104 001 & Carbon monoxide & $630-8-0$ & $5.00 \mathrm{E}+00$ & $7.12 \mathrm{E}-05$ \\
\hline 200E P-241C104001 & Heptane & $142-82-5$ & $5.00 \mathrm{E}-01$ & $8.40 \mathrm{E}-05$ \\
\hline 200E P-241C104 001 & Hexane, n-hexane & $110-54-3$ & $5.00 \mathrm{E}-01$ & $8.76 \mathrm{E}-05$ \\
\hline 200E P-241C104 001 & $\begin{array}{l}\text { Methyl N-amyl ketone, 2- } \\
\text { heptanone }\end{array}$ & $110-43-0$ & $5.00 \mathrm{E}-01$ & $8.13 E-05$ \\
\hline 200E P-241C104 001 & Nitrogen Oxide $\{\mathrm{NO}\}$ & $10102-43-9$ & $2.00 \mathrm{E}+00$ & $5.72 \mathrm{E}-05$ \\
\hline 200E P-241C104001 & Nonane & $111-84-2$ & $5.00 \mathrm{E}-01$ & $4.57 \mathrm{E}-05$ \\
\hline 200 E P-241C104 001 & Octane & $111-65-9$ & $5.00 \mathrm{E}-01$ & $4.79 \mathrm{E}-05$ \\
\hline
\end{tabular}


Table K.2. Insignificant Emission Units Based on Threshold Comparisons [WAC 173-401-530(1)(a)]

\begin{tabular}{|c|c|c|c|c|}
\hline Emission Point & Pollutants & CAS Number & $\begin{array}{l}\text { Emission } \\
\text { Threshold } \\
\text { (tons/yr) }\end{array}$ & $\begin{array}{c}\text { Actual } \\
\text { Emissions } \\
\text { (tons/yr) }\end{array}$ \\
\hline 200E P-241C104001 & $\begin{array}{l}\text { Phosphoric acid, tributyl } \\
\text { ester }\end{array}$ & $126-73-8$ & & $1.72 \mathrm{E}-04$ \\
\hline 200E P-241C104001 & Toluene & $108-88-3$ & $5.00 \mathrm{E}-01$ & $6.67 \mathrm{E}-06$ \\
\hline 200E P-241C107 001 & 1,3-Butadiene & $106-99-0$ & $3.50 \mathrm{E}-02$ & $4.13 \mathrm{E}-06$ \\
\hline 200E P-241C107 001 & 2-Hexanone & $591-78-6$ & $5.00 \mathrm{E}-01$ & $7.51 \mathrm{E}-05$ \\
\hline 200E P-241C107 001 & 2-Pentanone & $107-87-9$ & & $1.19 \mathrm{E}-04$ \\
\hline 200E P-241C107 001 & Acetone & $67-64-1$ & $5.00 \mathrm{E}-01$ & $1.43 \mathrm{E}-03$ \\
\hline 200E P-241C107 001 & Acetonitrile & $75-5-8$ & $5.00 \mathrm{E}-01$ & $6.89 \mathrm{E}-04$ \\
\hline 200E P-241C107001 & Ammonia & $7664-41-7$ & $5.00 \mathrm{E}-01$ & $6.57 \mathrm{E}-03$ \\
\hline 200 E P-241C107 001 & Benzene & $71-43-2$ & $5.00 \mathrm{E}-01$ & $3.28 \mathrm{E}-05$ \\
\hline 200E P-241C107001 & Carbon monoxide & $630-8-0$ & $5.00 \mathrm{E}+00$ & $9.50 \mathrm{E}-04$ \\
\hline 200E P-241C107 001 & Heptane & $142-82-5$ & $5.00 \mathrm{E}-01$ & $8.40 \mathrm{E}-05$ \\
\hline $200 \mathrm{E}$ P-241C107 001 & Hexane, n-hexane & $110-54-3$ & $5.00 \mathrm{E}-01$ & $8.76 \mathrm{E}-05$ \\
\hline 200E P-241C107 001 & $\begin{array}{l}\text { Methyl N-amyl ketone, 2- } \\
\text { heptanone }\end{array}$ & $110-43-0$ & 5.00E-01 & $8.13 \mathrm{E}-05$ \\
\hline 200E P-241C107001 & Nitrogen Oxide $\{\mathrm{NO}\}$ & $10102-43-9$ & $2.00 \mathrm{E}+00$ & $5.72 \mathrm{E}-05$ \\
\hline 200E P-241C107001 & Nonane & $111-84-2$ & $5.00 \mathrm{E}-01$ & $4.57 \mathrm{E}-05$ \\
\hline 200E P-241C107 001 & Octane & $111-65-9$ & $5.00 \mathrm{E}-01$ & $4.79 \mathrm{E}-05$ \\
\hline 200E P-241C107 001 & $\begin{array}{l}\text { Phosphoric acid, tributyl } \\
\text { ester }\end{array}$ & $126-73-8$ & & $1.72 \mathrm{E}-04$ \\
\hline 200E P-241C107 001 & Toluene & $108-88-3$ & $5.00 \mathrm{E}-01$ & $6.67 \mathrm{E}-06$ \\
\hline $200 \mathrm{E}$ P-241C108 001 & Ammonia & $7664-41-7$ & $5.00 \mathrm{E}-01$ & $3.04 \mathrm{E}-03$ \\
\hline 200E P-241C108 001 & Nitrogen Oxide $\{$ NO $\}$ & $10102-43-9$ & $2.00 \mathrm{E}+00$ & $2.64 \mathrm{E}-05$ \\
\hline 200E P-241C109 001 & 1,3-Butadiene & $106-99-0$ & $3.50 \mathrm{E}-02$ & $1.91 \mathrm{E}-06$ \\
\hline 200E P-241C109001 & 2-Hexanone & $591-78-6$ & $5.00 E-01$ & $3.47 \mathrm{E}-05$ \\
\hline 200E P-241C109001 & 2-Pentanone & $107-87-9$ & & $5.51 \mathrm{E}-05$ \\
\hline 200E P-241C109 001 & Acetone & $67-64-1$ & $5.00 \mathrm{E}-01$ & $6.62 \mathrm{E}-04$ \\
\hline 200E P-241C109 001 & Acetonitrile & $75-5-8$ & $5.00 \mathrm{E}-01$ & $3.19 \mathrm{E}-04$ \\
\hline 200E P-241C109 001 & Ammonia & $7664-41-7$ & $5.00 \mathrm{E}-01$ & $3.04 \mathrm{E}-03$ \\
\hline
\end{tabular}


Table K.2. Insignificant Emission Units Based on Threshold Comparisons [WAC 173-401-530(1)(a)]

\begin{tabular}{|c|c|c|c|c|}
\hline Emission Point & Pollutants & CAS Number & $\begin{array}{l}\text { Emission } \\
\text { Threshold } \\
\text { (tons/yr) }\end{array}$ & $\begin{array}{c}\text { Actual } \\
\text { Emissions } \\
\text { (tons/yr) }\end{array}$ \\
\hline 200E P-241C109 001 & Benzene & $71-43-2$ & $5.00 \mathrm{E}-01$ & $1.51 \mathrm{E}-05$ \\
\hline 200E P-241C109 001 & Carbon monoxide & $630-8-0$ & $5.00 \mathrm{E}+00$ & $4.39 \mathrm{E}-04$ \\
\hline 200E P-241C109 001 & Heptane & $142-82-5$ & $5.00 \mathrm{E}-01$ & $3.89 \mathrm{E}-05$ \\
\hline 200E P-241C109 001 & Hexane, n-hexane & $110-54-3$ & $5.00 \mathrm{E}-01$ & $4.05 E-05$ \\
\hline 200E P-241C109 001 & $\begin{array}{l}\text { Methyl N-amyl ketone, 2- } \\
\text { heptanone }\end{array}$ & $110-43-0$ & $5.00 \mathrm{E}-01$ & $3.76 \mathrm{E}-05$ \\
\hline 200E P-241C109 001 & Nitrogen Oxide $\{\mathrm{NO}\}$ & $10102-43-9$ & $2.00 \mathrm{E}+00$ & $2.64 \mathrm{E}-05$ \\
\hline 200E P-241C109 001 & Nonane & $111-84-2$ & $5.00 \mathrm{E}-01$ & $2.11 E-05$ \\
\hline 200E P-241C109 001 & Octane & $111-65-9$ & $5.00 \mathrm{E}-01$ & $2.21 \mathrm{E}-05$ \\
\hline 200E P-241C109 001 & $\begin{array}{l}\text { Phosphoric acid, tributyl } \\
\text { ester }\end{array}$ & $126-73-8$ & & 7.95E-05 \\
\hline 200E P-241C109001 & Toluene & $108-88-3$ & $5.00 \mathrm{E}-01$ & $3.08 \mathrm{E}-06$ \\
\hline $200 \mathrm{E}$ P-241C110 001 & 1,3-Butadiene & $106-99-0$ & $3.50 \mathrm{E}-02$ & $1.91 \mathrm{E}-06$ \\
\hline 200E P-241C110 001 & 2-Hexanone & $591-78-6$ & $5.00 \mathrm{E}-01$ & $3.47 \mathrm{E}-05$ \\
\hline 200E P-241C110 001 & 2-Pentanone & $107-87-9$ & & $5.51 \mathrm{E}-05$ \\
\hline $200 \mathrm{E}$ P-241C110 001 & Acetone & $67-64-1$ & $5.00 \mathrm{E}-01$ & $6.62 \mathrm{E}-04$ \\
\hline $200 \mathrm{E}$ P-241C110 001 & Acetonitrile & $75-5-8$ & $5.00 \mathrm{E}-01$ & $3.19 \mathrm{E}-04$ \\
\hline 200E P-241C110 001 & Ammonia & $7664-41-7$ & $5.00 \mathrm{E}-01$ & 3.04E-03 \\
\hline 200E P-241C110 001 & Benzene & $71-43-2$ & $5.00 \mathrm{E}-01$ & $1.51 \mathrm{E}-05$ \\
\hline $200 \mathrm{E}$ P-241C110 001 & Carbon monoxide & $630-8-0$ & $5.00 \mathrm{E}+00$ & $4.39 \mathrm{E}-04$ \\
\hline 200E P-241C110 001 & Heptane & $142-82-5$ & $5.00 \mathrm{E}-01$ & 3.89E-05 \\
\hline $200 \mathrm{E} P-241 \mathrm{C} 110001$ & Hexane, n-hexane & $110-54-3$ & $5.00 \mathrm{E}-01$ & $4.05 \mathrm{E}-05$ \\
\hline $200 \mathrm{E}$ P-241C110 001 & $\begin{array}{l}\text { Methyl N-amyl ketone, 2- } \\
\text { heptanone }\end{array}$ & $110-43-0$ & $5.00 \mathrm{E}-01$ & $3.76 \mathrm{E}-05$ \\
\hline 200E P-241C110 001 & Nitrogen Oxide $\{\mathrm{NO}\}$ & $10102-43-9$ & $2.00 \mathrm{E}+00$ & $2.64 \mathrm{E}-05$ \\
\hline $200 \mathrm{E}$ P-241C110 001 & Nonane & $111-84-2$ & $5.00 \mathrm{E}-01$ & $2.11 E-05$ \\
\hline 200E P-241C110 001 & Octane & $111-65-9$ & $5.00 \mathrm{E}-01$ & $2.21 E-05$ \\
\hline 200E P-241C110 001 & $\begin{array}{l}\text { Phosphoric acid, tributyl } \\
\text { ester }\end{array}$ & $126-73-8$ & & $7.95 \mathrm{E}-05$ \\
\hline 200 E P-241C110 001 & Toluene & $108-88-3$ & $5.00 \mathrm{E}-01$ & $3.08 \mathrm{E}-06$ \\
\hline
\end{tabular}


Table K.2. Insignificant Emission Units Based on Threshold Comparisons [WAC 173-401-530(1)(a)]

\begin{tabular}{|c|c|c|c|c|}
\hline Emission Point & Pollutants & CAS Number & $\begin{array}{l}\text { Emission } \\
\text { Threshold } \\
\text { (tons/yr) }\end{array}$ & $\begin{array}{c}\text { Actual } \\
\text { Emissions } \\
\text { (tons/yr) }\end{array}$ \\
\hline $200 \mathrm{E}$ P-241C111 001 & Ammonia & $7664-41-7$ & $5.00 \mathrm{E}-01$ & $3.04 \mathrm{E}-03$ \\
\hline $200 \mathrm{E}$ P-241C111 001 & Nitrogen Oxide $\{\mathrm{NO}\}$ & $10102-43-9$ & $2.00 \mathrm{E}+00$ & $2.64 \mathrm{E}-05$ \\
\hline 200E P-241C112 001 & 1,3-Butadiene & $106-99-0$ & $3.50 \mathrm{E}-02$ & $1.91 \mathrm{E}-06$ \\
\hline 200E P-241C112001 & 2-Hexanone & $591-78-6$ & $5.00 \mathrm{E}-01$ & $3.47 \mathrm{E}-05$ \\
\hline 200E P-241C112 001 & 2-Pentanone & $107-87-9$ & & 5.51E-05 \\
\hline 200 E P-241C112 001 & Acetone & $67-64-1$ & $5.00 \mathrm{E}-01$ & $6.62 \mathrm{E}-04$ \\
\hline 200E P-241C112001 & Acetonitrile & $75-5-8$ & $5.00 \mathrm{E}-01$ & $3.19 \mathrm{E}-04$ \\
\hline 200E P-241C112 001 & Ammonia & $7664-41-7$ & $5.00 \mathrm{E}-01$ & $3.04 \mathrm{E}-03$ \\
\hline 200E P-241C112 001 & Benzene & $71-43-2$ & $5.00 \mathrm{E}-01$ & $1.51 E-05$ \\
\hline 200E P-241C112001 & Carbon monoxide & $630-8-0$ & $5.00 \mathrm{E}+00$ & $4.39 \mathrm{E}-04$ \\
\hline 200E P-241C112 001 & Heptane & $142-82-5$ & $5.00 \mathrm{E}-01$ & $3.89 \mathrm{E}-05$ \\
\hline 200E P-241C112 001 & Hexane, n-hexane & $110-54-3$ & $5.00 \mathrm{E}-01$ & $4.05 \mathrm{E}-05$ \\
\hline $200 \mathrm{E}$ P-241C112 001 & $\begin{array}{l}\text { Methyl N-amyl ketone, 2- } \\
\text { heptanone }\end{array}$ & $110-43-0$ & $5.00 \mathrm{E}-01$ & $3.76 \mathrm{E}-05$ \\
\hline 200E P-241C112001 & Nitrogen Oxide $\{\mathrm{NO}\}$ & $10102-43-9$ & $2.00 \mathrm{E}+00$ & $2.64 \mathrm{E}-05$ \\
\hline 200E P-241C112 001 & Nonane & $111-84-2$ & $5.00 \mathrm{E}-01$ & $2.11 \mathrm{E}-05$ \\
\hline 200E P-241C112 001 & Octane & $111-65-9$ & $5.00 \mathrm{E}-01$ & $2.21 \mathrm{E}-05$ \\
\hline 200E P-241C112 001 & $\begin{array}{l}\text { Phosphoric acid, tributyl } \\
\text { ester }\end{array}$ & $126-73-8$ & & $7.95 \mathrm{E}-05$ \\
\hline 200E P-241C112 001 & Toluene & $108-88-3$ & $5.00 \mathrm{E}-01$ & $3.08 \mathrm{E}-06$ \\
\hline 200E P-241C201001 & 1,3-Butadiene & $106-99-0$ & $3.50 \mathrm{E}-02$ & $1.91 E-06$ \\
\hline 200E P-241C201 001 & 2-Hexanone & $591-78-6$ & $5.00 \mathrm{E}-01$ & $3.47 \mathrm{E}-05$ \\
\hline 200E P-241C201 001 & 2-Pentanone & $107-87-9$ & & $5.51 \mathrm{E}-05$ \\
\hline 200E P-241C201001 & Acetone & $67-64-1$ & $5.00 \mathrm{E}-01$ & $6.62 \mathrm{E}-04$ \\
\hline 200E P-241C201 001 & Acetonitrile & $75-5-8$ & $5.00 \mathrm{E}-01$ & $3.19 \mathrm{E}-04$ \\
\hline 200E P-241C201001 & Ammonia & $7664-41-7$ & $5.00 \mathrm{E}-01$ & $3.04 \mathrm{E}-03$ \\
\hline 200E P-241C201001 & Benzene & $71-43-2$ & $5.00 \mathrm{E}-01$ & $1.51 \mathrm{E}-05$ \\
\hline 200E P-241C201001 & Carbon monoxide & $630-8-0$ & $5.00 \mathrm{E}+00$ & $4.39 \mathrm{E}-04$ \\
\hline 200E P-241C201001 & Heptane & $142-82-5$ & $5.00 \mathrm{E}-01$ & $3.89 \mathrm{E}-05$ \\
\hline
\end{tabular}




\section{Table K.2. Insignificant Emission Units Based on Threshold Comparisons [WAC 173-401-530(1)(a)]}

\begin{tabular}{|c|c|c|c|c|}
\hline Emission Point & Pollutants & CAS Number & $\begin{array}{c}\text { Emission } \\
\text { Threshold } \\
\text { (tons/yr) }\end{array}$ & $\begin{array}{c}\text { Actual } \\
\text { Emissions } \\
\text { (tons/yr) }\end{array}$ \\
\hline 200E P-241C201001 & Hexane, n-hexane & $110-54-3$ & $5.00 \mathrm{E}-01$ & $4.05 \mathrm{E}-05$ \\
\hline 200E P-241C201 001 & $\begin{array}{l}\text { Methyl N-amyl ketone, 2- } \\
\text { heptanone }\end{array}$ & $110-43-0$ & $5.00 \mathrm{E}-01$ & $3.76 \mathrm{E}-05$ \\
\hline 200E P-241C201 001 & Nitrogen Oxide $\{\mathrm{NO}\}$ & $10102-43-9$ & $2.00 \mathrm{E}+00$ & $2.64 \mathrm{E}-05$ \\
\hline 200E P-241C201 001 & Nonane & $111-84-2$ & $5.00 \mathrm{E}-01$ & $2.11 E-05$ \\
\hline 200E P-241C201001 & Octane & $111-65-9$ & $5.00 \mathrm{E}-01$ & $2.21 \mathrm{E}-05$ \\
\hline 200E P-241C201001 & $\begin{array}{l}\text { Phosphoric acid, tributyl } \\
\text { ester }\end{array}$ & $126-73-8$ & & $7.95 \mathrm{E}-05$ \\
\hline 200E P-241C201 001 & Toluene & $108-88-3$ & $5.00 \mathrm{E}-01$ & $3.08 \mathrm{E}-06$ \\
\hline 200E P-241C202 001 & 1,3-Butadiene & $106-99-0$ & $3.50 \mathrm{E}-02$ & $1.91 \mathrm{E}-06$ \\
\hline 200E P-241C202 001 & 2-Hexanone & $591-78-6$ & $5.00 \mathrm{E}-01$ & $3.47 \mathrm{E}-05$ \\
\hline 200E P-241C202 001 & 2-Pentanone & $107-87-9$ & & $5.51 \mathrm{E}-05$ \\
\hline 200E P-241C202 001 & Acetone & $67-64-1$ & $5.00 \mathrm{E}-01$ & $6.62 \mathrm{E}-04$ \\
\hline 200E P-241C202 001 & Acetonitrile & $75-5-8$ & $5.00 \mathrm{E}-01$ & $3.19 \mathrm{E}-04$ \\
\hline 200E P-241C202 001 & Ammonia & $7664-41-7$ & $5.00 \mathrm{E}-01$ & $3.04 \mathrm{E}-03$ \\
\hline 200E P-241C202 001 & Benzene & $71-43-2$ & $5.00 \mathrm{E}-01$ & $1.51 \mathrm{E}-05$ \\
\hline 200E P-241C202 001 & Carbon monoxide & $630-8-0$ & $5.00 \mathrm{E}+00$ & $4.39 \mathrm{E}-04$ \\
\hline $200 \mathrm{E}$ P-241C202 001 & Heptane & $142-82-5$ & $5.00 \mathrm{E}-01$ & $3.89 \mathrm{E}-05$ \\
\hline 200E P-241C202 001 & Hexane, n-hexane & $110-54-3$ & $5.00 \mathrm{E}-01$ & $4.05 E-05$ \\
\hline 200E P-241C202 001 & $\begin{array}{l}\text { Methyl N-amyl ketone, 2- } \\
\text { heptanone }\end{array}$ & $110-43-0$ & $5.00 \mathrm{E}-01$ & $3.76 \mathrm{E}-05$ \\
\hline 200E P-241C202 001 & Nitrogen Oxide $\{\mathrm{NO}\}$ & $10102-43-9$ & $2.00 \mathrm{E}+00$ & $2.64 \mathrm{E}-05$ \\
\hline 200E P-241C202 001 & Nonane & $111-84-2$ & $5.00 \mathrm{E}-01$ & $2.11 \mathrm{E}-05$ \\
\hline 200E P-241C202 001 & Octane & $111-65-9$ & $5.00 \mathrm{E}-01$ & $2.21 E-05$ \\
\hline 200E P-241C202 001 & $\begin{array}{l}\text { Phosphoric acid, tributyl } \\
\text { ester }\end{array}$ & $126-73-8$ & & $7.95 \mathrm{E}-05$ \\
\hline 200E P-241C202 001 & Toluene & $108-88-3$ & $5.00 \mathrm{E}-01$ & $3.08 \mathrm{E}-06$ \\
\hline 200E P-241C203001 & 1,3-Butadiene & $106-99-0$ & $3.50 \mathrm{E}-02$ & $1.91 \mathrm{E}-06$ \\
\hline 200E P-241C203001 & 2-Hexanone & $591-78-6$ & $5.00 \mathrm{E}-01$ & $3.47 \mathrm{E}-05$ \\
\hline 200E P-241C203001 & 2-Pentanone & $107-87-9$ & & $5.51 \mathrm{E}-05$ \\
\hline
\end{tabular}


Table K.2. Insignificant Emission Units Based on Threshold Comparisons [WAC 173-401-530(1)(a)]

\begin{tabular}{|c|c|c|c|c|}
\hline Emission Point & Pollutants & CAS Number & $\begin{array}{l}\text { Emission } \\
\text { Threshold } \\
\text { (tons/yr) }\end{array}$ & $\begin{array}{c}\text { Actual } \\
\text { Emissions } \\
\text { (tons/yr) }\end{array}$ \\
\hline 200E P-241C203001 & Acetone & $67-64-1$ & $5.00 \mathrm{E}-01$ & $6.62 \mathrm{E}-04$ \\
\hline 200E P-241C203 001 & Acetonitrile & $75-5-8$ & $5.00 \mathrm{E}-01$ & $3.19 \mathrm{E}-04$ \\
\hline 200E P-241C203 001 & Ammonia & $7664-41-7$ & $5.00 \mathrm{E}-01$ & $3.04 \mathrm{E}-03$ \\
\hline 200E P-241C203 001 & Benzene & $71-43-2$ & $5.00 \mathrm{E}-01$ & $1.51 \mathrm{E}-05$ \\
\hline 200E P-241C203001 & Carbon monoxide & $630-8-0$ & $5.00 \mathrm{E}+00$ & $4.39 \mathrm{E}-04$ \\
\hline 200E P-241C203 001 & Heptane & $142-82-5$ & $5.00 \mathrm{E}-01$ & $3.89 \mathrm{E}-05$ \\
\hline 200E P-241C203 001 & Hexane, n-hexane & $110-54-3$ & $5.00 \mathrm{E}-01$ & $4.05 E-05$ \\
\hline 200E P-241C203 001 & $\begin{array}{l}\text { Methyl N-amyl ketone, 2- } \\
\text { heptanone. }\end{array}$ & $110-43-0$ & $5.00 \mathrm{E}-01$ & $3.76 \mathrm{E}-05$ \\
\hline 200E P-241C203 001 & Nitrogen Oxide $\{\mathrm{NO}\}$ & $10102-43-9$ & $2.00 E+00$ & $2.64 \mathrm{E}-05$ \\
\hline 200E P-241C203 001 & Nonane & $111-84-2$ & $5.00 \mathrm{E}-01$ & $2.11 \mathrm{E}-05$ \\
\hline 200E P-241C203001 & Octane & $111-65-9$ & $5.00 \mathrm{E}-01$ & $2.21 \mathrm{E}-05$ \\
\hline 200E P-241C203 001 & $\begin{array}{l}\text { Phosphoric acid, tributyl } \\
\text { ester }\end{array}$ & $126-73-8$ & & $7.95 \mathrm{E}-05$ \\
\hline 200E P-241C203 001 & Toluene & $108-88-3$ & $5.00 \mathrm{E}-01$ & $3.08 \mathrm{E}-06$ \\
\hline 200E P-241C204 001 & 1,3-Butadiene & $106-99-0$ & $3.50 \mathrm{E}-02$ & 1.91E-06 \\
\hline 200E P-241C204 001 & 2-Hexanone & $591-78-6$ & $5.00 \mathrm{E}-01$ & $3.47 \mathrm{E}-05$ \\
\hline $200 E$ P-241C204 001 & 2-Pentanone & $107-87-9$ & & 5.51E-05 \\
\hline 200E P-241C204001 & Acetone & $67-64-1$ & $5.00 \mathrm{E}-01$ & $6.62 \mathrm{E}-04$ \\
\hline 200E P-241C204 001 & Acetonitrile & $75-5-8$ & $5.00 \mathrm{E}-01$ & 3.19E-04 \\
\hline 200E P-241C204001 & Ammonia & $7664-41-7$ & $5.00 \mathrm{E}-01$ & $3.04 \mathrm{E}-03$ \\
\hline 200E P-241C204 001 & Benzene & $71-43-2$ & $5.00 \mathrm{E}-01$ & $1.51 \mathrm{E}-05$ \\
\hline 200E P-241C204001 & Carbon monoxide & $630-8-0$ & $5.00 \mathrm{E}+00$ & 4.39E-04 \\
\hline 200E P-241C204 001 & Heptane & $142-82-5$ & $5.00 \mathrm{E}-01$ & $3.89 \mathrm{E}-05$ \\
\hline 200E P-241C204001 & Hexane, n-hexane & $110-54-3$ & $5.00 \mathrm{E}-01$ & $4.05 \mathrm{E}-05$ \\
\hline $200 \mathrm{E}$ P-241C204001 & $\begin{array}{l}\text { Methyl N-amyl ketone, 2- } \\
\text { heptanone }\end{array}$ & $110-43-0$ & $5.00 \mathrm{E}-01$ & $3.76 \mathrm{E}-05$ \\
\hline 200E P-241C204 001 & Nitrogen Oxide $\{\mathrm{NO}\}$ & $10102-43-9$ & $2.00 \mathrm{E}+00$ & $2.64 \mathrm{E}-05$ \\
\hline 200 E P-241C204 001 & Nonane & $111-84-2$ & $5.00 \mathrm{E}-01$ & $2.11 \mathrm{E}-05$ \\
\hline 200E P-241C204 001 & Octane & $111-65-9$ & $5.00 \mathrm{E}-01$ & $2.21 \mathrm{E}-05$ \\
\hline
\end{tabular}




\section{Table K.2. Insignificant Emission Units Based on Threshold Comparisons} [WAC 173-401-530(1)(a)]

\begin{tabular}{|c|c|c|c|c|}
\hline Emission Point & Pollutants & CAS Number & $\begin{array}{l}\text { Emission } \\
\text { Threshold } \\
\text { (tons/yr) }\end{array}$ & $\begin{array}{c}\text { Actual } \\
\text { Emissions } \\
\text { (tons/yr) }\end{array}$ \\
\hline 200E P-241C204 001 & $\begin{array}{l}\text { Phosphoric acid, tributyl } \\
\text { ester }\end{array}$ & $126-73-8$ & & $7.95 \mathrm{E}-05$ \\
\hline 200E P-241C204 001 & Toluene & $108-88-3$ & $5.00 \mathrm{E}-01$ & $3.08 \mathrm{E}-06$ \\
\hline 200E P-242A 001 & 2-Hexanone & $591-78-6$ & $5.00 \mathrm{E}-01$ & $9.00 \mathrm{E}-08$ \\
\hline 200E P-242A 001 & Acetone & $67-64-1$ & $5.00 \mathrm{E}-01$ & $2.50 \mathrm{E}-05$ \\
\hline 200E P-242A 001 & Ammonia & $7664-41-7$ & $5.00 \mathrm{E}-01$ & $2.35 E-05$ \\
\hline 200E P-242A 001 & Methyl isobutyl ketone & $108-10-1$ & $5.00 \mathrm{E}-01$ & $1.70 \mathrm{E}-06$ \\
\hline 200E P-242A 001 & Methyl isobutyl ketone & $108-10-1$ & $5.00 \mathrm{E}-01$ & $1.70 \mathrm{E}-06$ \\
\hline 200E P-242A 001 & Methyl isobutyl ketone & $108-10-1$ & $5.00 \mathrm{E}-01$ & $1.70 \mathrm{E}-06$ \\
\hline 200E P-242A 001 & Methyl isobutyl ketone & $108-10-1$ & $5.00 \mathrm{E}-01$ & $1.70 \mathrm{E}-06$ \\
\hline 200E P-242A 001 & Methyl isobutyl ketone & $108-10-1$ & $5.00 \mathrm{E}-01$ & $1.70 \mathrm{E}-06$ \\
\hline 200E P-242A 001 & N-butyl alcohol & $71-36-3$ & $5.00 \mathrm{E}-01$ & $1.88 \mathrm{E}-04$ \\
\hline 200E P-242A 002 & 2-Hexanone & $591-78-6$ & $5.00 \mathrm{E}-01$ & $2.87 \mathrm{E}-05$ \\
\hline 200E P-242A 002 & Acetone & $67-64-1$ & $5.00 \mathrm{E}-01$ & $7.95 \mathrm{E}-03$ \\
\hline $200 \mathrm{E}$ P-242A 002 & Ammonia & $7664-41-7$ & $5.00 \mathrm{E}-01$ & $7.49 \mathrm{E}-03$ \\
\hline $200 \mathrm{E}$ P-242A 002 & Methyl isobutyl ketone & $108-10-1$ & $5.00 \mathrm{E}-01$ & $5.42 \mathrm{E}-04$ \\
\hline 200E P-242A 002 & Methyl isobutyl ketone & $108-10-1$ & $5.00 \mathrm{E}-01$ & $5.42 \mathrm{E}-04$ \\
\hline $200 \mathrm{E}$ P-242A 002 & Methyl isobutyl ketone & $108-10-1$ & $5.00 \mathrm{E}-01$ & $5.42 \mathrm{E}-04$ \\
\hline $200 \mathrm{E}$ P-242A 002 & Methyl isobutyl ketone & $108-10-1$ & $5.00 \mathrm{E}-01$ & $5.42 \mathrm{E}-04$ \\
\hline 200E P-242A 002 & Methyl isobutyl ketone & $108-10-1$ & $5.00 \mathrm{E}-01$ & 5.42E-04 \\
\hline 200E P-242A 002 & N-butyl alcohol & $71-36-3$ & $5.00 \mathrm{E}-01$ & $5.98 \mathrm{E}-02$ \\
\hline 200E P-296A 001 & 1,3-Butadiene & $106-99-0$ & $3.50 \mathrm{E}-02$ & $4.13 \mathrm{E}-06$ \\
\hline 200E P-296A 001 & 2-Hexanone & $591-78-6$ & $5.00 \mathrm{E}-01$ & 7.51E-05 \\
\hline 200E P-296A 001 & 2-Pentanone & $107-87-9$ & & $1.19 \mathrm{E}-04$ \\
\hline 200E P-296A 001 & Acetone & $67-64-1$ & $5.00 \mathrm{E}-01$ & $1.43 E-03$ \\
\hline 200E P-296A 001 & Acetonitrile & $75-5-8$ & $5.00 \mathrm{E}-01$ & $6.89 \mathrm{E}-04$ \\
\hline 200E P-296A 001 & Ammonia & $7664-41-7$ & $5.00 \mathrm{E}-01$ & $3.21 \mathrm{E}-01$ \\
\hline 200E P-296A 001 & Benzene & $71-43-2$ & $5.00 \mathrm{E}-01$ & $3.28 \mathrm{E}-05$ \\
\hline
\end{tabular}


Hanford Site Air

Table K.2. Insignificant Emission Units Based on Threshold Comparisons [WAC 173-401-530(1)(a)]

\begin{tabular}{|c|c|c|c|c|}
\hline Emission Point & Pollutants & CAS Number & $\begin{array}{l}\text { Emission } \\
\text { Threshold } \\
\text { (tons/yr) }\end{array}$ & $\begin{array}{c}\text { Actual } \\
\text { Emissions } \\
\text { (tons/yr) }\end{array}$ \\
\hline 200E P-296A 001 & Carbon monoxide & $630-8-0$ & $5.00 \mathrm{E}+00$ & $1.72 \mathrm{E}-02$ \\
\hline 200E P-296A 001 & Heptane & $142-82-5$ & $5.00 \mathrm{E}-01$ & $8.40 \mathrm{E}-05$ \\
\hline 200E P-296A 001 & Hexane, n-hexane & $110-54-3$ & $5.00 \mathrm{E}-01$ & $8.76 \mathrm{E}-05$ \\
\hline 200E P-296A 001 & $\begin{array}{l}\text { Methyl N-amyl ketone, 2- } \\
\text { heptanone }\end{array}$ & $-110-43-0$ & $5.00 \mathrm{E}-01$ & $8.13 E-05$ \\
\hline 200E P-296A 001 & Nitrogen Oxide $\{\mathrm{NO}\}$ & $10102-43-9$ & $2.00 \mathrm{E}+00$ & $5.72 \mathrm{E}-05$ \\
\hline 200E P-296A 001 & Nonane & $111-84-2$ & $5.00 \mathrm{E}-01$ & $4.57 \mathrm{E}-05$ \\
\hline 200E P-296A 001 & Octane & $111-65-9$ & $5.00 \mathrm{E}-01$ & $4.79 E-05$ \\
\hline 200E P-296A 001 & $\begin{array}{l}\text { Phosphoric acid, tributyl } \\
\text { ester }\end{array}$ & $126-73-8$ & & $1.72 \mathrm{E}-04$ \\
\hline 200E P-296A 001 & Toluene & $108-88-3$ & $5.00 \mathrm{E}-01$ & $6.67 \mathrm{E}-06$ \\
\hline 200E P-296AP 001 & 1,3-Butadiene & $106-99-0$ & $3.50 \mathrm{E}-02$ & $3.30 \mathrm{E}-05$ \\
\hline 200 E Р-296AР 001 & 2-Hexanone & $591-78-6$ & $5.00 \mathrm{E}-01$ & $6.01 E-04$ \\
\hline ¿OOE P-296AP 001 & 2-Pentanone & $107-87-9$ & & $9.54 \mathrm{E}-04$ \\
\hline 2(X0E P-296AP 001 & Acetone & $67-64-1$ & $5.00 \mathrm{E}-01$ & $1.15 \mathrm{E}-02$ \\
\hline 200E Р-296АР 001 & Acetonitrile & $75-5-8$ & $5.00 \mathrm{E}-01$ & $5.52 \mathrm{E}-03$ \\
\hline 200E P-296AP 001 & Ammonia & $7664-41-7$ & $5.00 \mathrm{E}-01$ & $3.52 \mathrm{E}-01$ \\
\hline 200E Р-296AP 001 & Benzene & $71-43-2$ & $5.00 \mathrm{E}-01$ & $2.62 \mathrm{E}-04$ \\
\hline 200Е Р-296АР 001 & Carbon monoxide & $630-8-0$ & $5.00 \mathrm{E}+00$ & $1.38 \mathrm{E}-01$ \\
\hline 200Е Р-296АР 001 & Heptane & $142-82-5$ & $5.00 \mathrm{E}-01$ & $6.72 \mathrm{E}-04$ \\
\hline 200E P-296AP 001 & Hexane, n-hexane & $110-54-3$ & $5.00 \mathrm{E}-01$ & $7.01 \mathrm{E}-04$ \\
\hline 200E P-296AP 001 & $\begin{array}{l}\text { Methyl N-amyl ketone, 2- } \\
\text { heptanone }\end{array}$ & $110-43-0$ & $5.00 \mathrm{E}-01$ & $6.50 \mathrm{E}-04$ \\
\hline 200E P-296AP 001 & Nitrogen Oxide $\{\mathrm{NO}\}$ & $10102-43-9$ & $2.00 \mathrm{E}+00$ & $4.58 \mathrm{E}-04$ \\
\hline 200E P-296AP 001 & Nonane & $111-84-2$ & $5.00 \mathrm{E}-01$ & $3.65 \mathrm{E}-04$ \\
\hline 200E P-296AP 001 & Octane & $111-65-9$ & $5.00 \mathrm{E}-01$ & $3.83 \mathrm{E}-04$ \\
\hline 200E P-296AP 001 & $\begin{array}{l}\text { Phosphoric acid, tributyl } \\
\text { ester }\end{array}$ & $126-73-8$ & & $1.38 \mathrm{E}-03$ \\
\hline 200E P-296AP 001 & Toluene & $108-88-3$ & $5.00 \mathrm{E}-01$ & $5.34 \mathrm{E}-05$ \\
\hline 200E P-LERF 001 & Ammonia & $7664-41-7$ & $5.00 \mathrm{E}-01$ & $1.10 \mathrm{E}-01$ \\
\hline
\end{tabular}


Table K.2. Insignificant Emission Units Based on Threshold Comparisons [WAC 173-401-530(1)(a)]

\begin{tabular}{|c|c|c|c|c|}
\hline Emission Point & Pollutants & CAS Number & $\begin{array}{l}\text { Emission } \\
\text { Threshold } \\
\text { (tons/yr) }\end{array}$ & $\begin{array}{c}\text { Actual } \\
\text { Emissions } \\
\text { (tons/yr) }\end{array}$ \\
\hline 200E P-LERF 001 & Isopropyl alcohol & $67-63-0$ & $5: 00 \mathrm{E}-01$ & $1.20 \mathrm{E}-05$ \\
\hline 200E P-LERF 001 & N-butyl alcohol & $71-36-3$ & $5.00 \mathrm{E}-01$ & $3.00 \mathrm{E}-05$ \\
\hline 200E P-LERF 001 & Nitrosodimethylamine & $62-75-9$ & $5.00 \mathrm{E}-04$ & $0.00 E+00$ \\
\hline 200E L-2703E 001 & Acetone & $67-64-1$ & $5.00 \mathrm{E}-01$ & $9.70 \mathrm{E}-04$ \\
\hline 200E L-2703E 001 & Chloroform & $67-66-3$ & $5.00 \mathrm{E}-01$ & $5.50 \mathrm{E}-04$ \\
\hline 200E L-2703E 001 & Hydrochloric acid & $7647-1-0$ & $5.00 \mathrm{E}-01$ & $2.90 \mathrm{E}-03$ \\
\hline 200E L-2703E 001 & Methylene chloride & $75-9-2$ & $5.00 \mathrm{E}-01$ & $1.50 \mathrm{E}-04$ \\
\hline 200E L-2703E 001 & Methyl isobutyl ketone & $108-10-1$ & $5.00 \mathrm{E}-01$ & $4.00 \mathrm{E}-05$ \\
\hline $200 \mathrm{E}$ L-2703E 001 & $\mathrm{~N}$-amyl acetate & $628-63-7$ & $5.00 \mathrm{E}-01$ & $1.00 \mathrm{E}-04$ \\
\hline 200 E L-2703E 001 & Nitric acid & $7697-37-2$ & $5.00 \mathrm{E}-01$ & $1.75 \mathrm{E}-02$ \\
\hline 200E L-2703E 001 & Phosphoric acid & $7664-38-2$ & $5.00 \mathrm{E}-01$ & $4.00 \mathrm{E}-03$ \\
\hline 200E L-2703E 001 & Potassium hydroxide & $1310-58-3$ & $5.00 \mathrm{E}-01$ & $3.70 \mathrm{E}-04$ \\
\hline 200E L-2703E 001 & Sodium hydroxide & $1310-73-2$ & $5.00 \mathrm{E}-01$ & $7.04 \mathrm{E}-03$ \\
\hline 200E L-2703E 001 & Sodium metabisulfite & $7681-57-4$ & $5.00 \mathrm{E}-01$ & $1.38 \mathrm{E}-03$ \\
\hline $200 \mathrm{E}$ L-2703E 001 & Sulfuric acid & $7664-93-9$ & $5.00 \mathrm{E}-01$ & $1.80 \mathrm{E}-02$ \\
\hline $200 \mathrm{E}$ L-2703E 001 & Xylene & $1330-20-7$ & $5.00 \mathrm{E}-01$ & $1.43 \mathrm{E}-03$ \\
\hline $200 \mathrm{~W} P \quad 241 S 101$ & 2-Hexanone & $591-78-6$ & $5.00 \mathrm{E}-01$ & $7.51 \mathrm{E}-05$ \\
\hline $200 W$ P $241 S 101$ & 2-Pentanone & $107-87-9$ & & $1.19 \mathrm{E}-04$ \\
\hline 200W P $241 S 101$ & Acetone & 67-64-1 & $5.00 \mathrm{E}-01$ & $1.43 \mathrm{E}-03$ \\
\hline $200 W$ P $241 S 101$ & Acetonitrile & $75-5-8$ & $5.00 \mathrm{E}-01$ & $6.89 \mathrm{E}-04$ \\
\hline 200W P $241 S 101$ & Ammonia & $7664-41-7$ & $5.00 \mathrm{E}-01$ & $6.57 \mathrm{E}-03$ \\
\hline 200W P $241 S 101$ & Benzene & $71-43-2$ & $5.00 \mathrm{E}-01$ & $3.28 \mathrm{E}-05$ \\
\hline 200W P $241 S 101$ & Carbon monoxide & $630-8-0$ & $5.00 \mathrm{E}+00$ & $9.50 \mathrm{E}-04$ \\
\hline 200W P $241 S 101$ & Heptane & $142-82-5$ & $5.00 \mathrm{E}-01$ & $8.40 \mathrm{E}-05$ \\
\hline $200 W$ P $241 S 101$ & Hexane, n-hexane & $110-54-3$ & $5.00 \mathrm{E}-01$ & $8.76 \mathrm{E}-05$ \\
\hline $200 \mathrm{~W} P \quad 241 S 101$ & $\begin{array}{l}\text { Methyl N-amyl ketone, } 2 \text { - } \\
\text { heptanone }\end{array}$ & $110-43-0$ & $5.00 \mathrm{E}-01$ & $8.13 \mathrm{E}-05$ \\
\hline 200W P 241S101 & Nitrogen Oxide $\{\mathrm{NO}\}$ & $10102-43-9$ & $2.00 \mathrm{E}+00$ & $5.72 E-05$ \\
\hline $200 W$ P $241 S 101$ & Nonane & $111-84-2$ & $5.00 \mathrm{E}-01$ & $4.57 \mathrm{E}-05$ \\
\hline
\end{tabular}


Table K.2. Insignificant Emission Units Based on Threshold Comparisons [WAC 173-401-530(1)(a)]

\begin{tabular}{|c|c|c|c|c|}
\hline Emission Point & Pollutants & CAS Number & $\begin{array}{l}\text { Emission } \\
\text { Threshold } \\
\text { (tons/yr) }\end{array}$ & $\begin{array}{c}\text { Actual } \\
\text { Emissions } \\
\text { (tons/yr) }\end{array}$ \\
\hline $200 W$ P $241 S 101$ & Octane & $111-65-9$ & $5.00 \mathrm{E}-01$ & 4.79E-05 \\
\hline $200 W$ P $241 S 101$ & $\begin{array}{l}\text { Phosphoric acid, tributyl } \\
\text { ester }\end{array}$ & $126-73-8$ & & $1.72 \mathrm{E}-04$ \\
\hline $200 W$ P $241 S 101$ & Toluene & $108-88-3$ & $5.00 \mathrm{E}-01$ & $6.67 \mathrm{E}-06$ \\
\hline $200 W$ P $241 S 102$ & 1,3-Butadiene & $106-99-0$ & $3.50 \mathrm{E}-02$ & $4.13 E-06$ \\
\hline 200W P $241 S 102$ & 2-Hexanone & $591-78-6$ & $5.00 \mathrm{E}-01$ & $7.51 \mathrm{E}-05$ \\
\hline $200 W$ P $241 S 102$ & 2-Pentanone & $107-87-9$ & & $1.19 \mathrm{E}-04$ \\
\hline $200 W$ P $241 S 102$ & Acetone & $67-64-1$ & $5.00 \mathrm{E}-01$ & $1.43 \mathrm{E}-03$ \\
\hline 200W P 241S102 & Acetonitrile & $75-5-8$ & $5.00 \mathrm{E}-01$ & $6.89 \mathrm{E}-04$ \\
\hline 200W P $241 S 102$ & Ammonia & $7664-41-7$ & $5.00 E-01$ & $6.57 \mathrm{E}-03$ \\
\hline $200 W$ P $241 S 102$ & Benzene & $71-43-2$ & $5.00 \mathrm{E}-01$ & $3.28 \mathrm{E}-05$ \\
\hline 200W P $241 S 102$ & Carbon monoxide & $630-8-0$ & $5.00 \mathrm{E}+00$ & $9.50 \mathrm{E}-04$ \\
\hline 200W P $241 S 102$ & Heptane & $142-82-5$ & $5.00 \mathrm{E}-01$ & $8.40 \mathrm{E}-05$ \\
\hline $200 W$ P $241 S 102$ & Hexane, n-hexane & $110-54-3$ & $5.00 \mathrm{E}-01$ & $8.76 \mathrm{E}-05$ \\
\hline $200 W$ P $241 S 102$ & $\begin{array}{l}\text { Methyl N-amyl ketone, 2- } \\
\text { heptanone }\end{array}$ & $110-43-0$ & $5.00 \mathrm{E}-01$ & $8.13 \mathrm{E}-05$ \\
\hline $200 W$ P $241 S 102$ & Nitrogen Oxide $\{\mathrm{NO}\}$ & $10102-43-9$ & $2.00 \mathrm{E}+00$ & 5.72E-05 \\
\hline 200W P $241 S 102$ & Nonane & $111-84-2$ & $5.00 \mathrm{E}-01$ & $4.57 \mathrm{E}-05$ \\
\hline $200 W$ P $241 S 102$ & Octane & $111-65-9$ & $5.00 \mathrm{E}-01$ & $4.79 \mathrm{E}-05$ \\
\hline $200 W$ P $241 S 102$ & $\begin{array}{l}\text { Phosphoric acid, tributyl } \\
\text { ester }\end{array}$ & $126-73-8$ & & $1.72 \mathrm{E}-04$ \\
\hline $200 W$ P $241 S 102$ & Toluene & $108-88-3$ & $5.00 \mathrm{E}-01$ & $6.67 \mathrm{E}-06$ \\
\hline $200 \mathrm{~W} P \quad 241 \mathrm{~S} 103$ & 1,3-Butadiene & $106-99-0$ & $3.50 \mathrm{E}-02$ & $4.13 \mathrm{E}-06$ \\
\hline $200 W$ P $241 S 103$ & 2-Hexanone & $591-78-6$ & $5.00 \mathrm{E}-01$ & $7.51 \mathrm{E}-05$ \\
\hline 200W P 241S103 & 2-Pentanone & $107-87-9$ & & $1.19 \mathrm{E}-04$ \\
\hline 200W P 241S103 & Acetone & $67-64-1$ & $5.00 \mathrm{E}-01$ & $1.43 \mathrm{E}-03$ \\
\hline $200 W$ P $241 S 103$ & Acetonitrile & $75-5-8$ & $5.00 \mathrm{E}-01$ & $6.89 \mathrm{E}-04$ \\
\hline 200W P 241S103 & Ammonia & $7664-41-7$ & $5.00 \mathrm{E}-01$ & $6.57 \mathrm{E}-03$ \\
\hline 200W P $241 S 103$ & Benzene & $71-43-2 \quad-$ & $5.00 \mathrm{E}-01$ & $3.28 \mathrm{E}-05$ \\
\hline $200 W$ P $241 S 103$ & Carbon monoxide & $630-8-0$ & $5.00 \mathrm{E}+00$ & $9.50 \mathrm{E}-04$ \\
\hline
\end{tabular}


Table K.2. Insignificant Emission Units Based on Threshold Comparisons [WAC 173-401-530(1)(a)]

\begin{tabular}{|c|c|c|c|c|}
\hline Emission Point & Pollutants & CAS Number & $\begin{array}{l}\text { Emission } \\
\text { Threshold } \\
\text { (tons/yr) }\end{array}$ & $\begin{array}{c}\text { Actual } \\
\text { Emissions } \\
\text { (tons/yr) }\end{array}$ \\
\hline $200 W$ P $241 S 103$ & Heptane & $142-82-5$ & $5.00 \mathrm{E}-01$ & $8.40 \mathrm{E}-05$ \\
\hline $200 W$ P $241 S 103$ & Hexane, n-hexane & $110-54-3$ & $5.00 \mathrm{E}-01$ & $8.76 \mathrm{E}-05$ \\
\hline 200W P 241S103 & $\begin{array}{l}\text { Methyl N-amyl ketone, 2- } \\
\text { heptanone }\end{array}$ & $110-43-0$ & $5.00 \mathrm{E}-01$ & $8.13 E-05$ \\
\hline $200 W$ P $241 S 103$ & Nitrogen Oxide $\{\mathrm{NO}\}$ & $10102-43-9$ & $2.00 \mathrm{E}+00$ & $5.72 \mathrm{E}-05$ \\
\hline 200W P $241 S 103$ & Nonane & $111-84-2$ & $5.00 \mathrm{E}-01$ & $4.57 \mathrm{E}-05$ \\
\hline 200W P $241 S 103$ & Octane & $111-65-9$ & $5.00 \mathrm{E}-01$ & $4.79 \mathrm{E}-05$ \\
\hline 200W P $241 S 103$ & $\begin{array}{l}\text { Phosphoric acid, tributyl } \\
\text { ester }\end{array}$ & $126-73-8$ & & $1.72 \mathrm{E}-04$ \\
\hline $200 W$ P $241 S 103$ & Toluene & $108-88-3$ & $5.00 \mathrm{E}-01$ & $6.67 \mathrm{E}-06$ \\
\hline 200W P 241S104 & 1,3-Butadiene & $106-99-0$ & $3.50 \mathrm{E}-02$ & $4.13 \mathrm{E}-06$ \\
\hline 200W P $241 S 104$ & 2-Hexanone & $591-78-6$ & $5.00 \mathrm{E}-01$ & $7.51 \mathrm{E}-05$ \\
\hline 200W P 241S104 & 2-Pentanone & $107-87-9$ & & $1.19 \mathrm{E}-04$ \\
\hline 200W P $241 S 104$ & Acetone & $67-64-1$ & $5.00 \mathrm{E}-01$ & $1.43 \mathrm{E}-03$ \\
\hline $200 W$ P $241 S 104$ & Acetonitrile & $.75-5-8$ & $5.00 \mathrm{E}-01$ & $6.89 \mathrm{E}-04$ \\
\hline 200W P $241 S 104$ & Ammonia & $7664-41-7$ & $5.00 \mathrm{E}-01$ & $6.57 \mathrm{E}-03$ \\
\hline 200W P $241 S 104$ & Benzene & $71-43-2$ & $5.00 \mathrm{E}-01$ & $3.28 \mathrm{E}-05$ \\
\hline 200W P 241S104 & Carbon monoxide & $630-8-0$ & $5.00 \mathrm{E}+00$ & $9.50 \mathrm{E}-04$ \\
\hline 200W P $241 S 104$ & Heptane & $142-82-5$ & $5.00 \mathrm{E}-01$ & $8.40 \mathrm{E}-05$ \\
\hline 200W P $241 S 104$ & Hexane, n-hexane & $110-54-3$ & $5.00 \mathrm{E}-01$ & $8.76 \mathrm{E}-05$ \\
\hline 200W P 241S104 & $\begin{array}{l}\text { Methyl N-amyl ketone, 2- } \\
\text { heptanone }\end{array}$ & $110-43-0$ & $5.00 \mathrm{E}-01$ & $8.13 \mathrm{E}-05$ \\
\hline 200W P $241 S 104$ & Nitrogen Oxide $\{\mathrm{NO}\}$ & $10102-43-9$ & $2.00 \mathrm{E}+00$ & $5.72 \mathrm{E}-05$ \\
\hline 200W P $241 S 104$ & Nonane & $111-84-2$ & $5.00 \mathrm{E}-01$ & $4.57 \mathrm{E}-05$ \\
\hline 200W P $241 S 104$ & Octane & $111-65-9$ & $5.00 \mathrm{E}-01$ & $4.79 \mathrm{E}-05$ \\
\hline $200 W$ P $241 S 104$ & $\begin{array}{l}\text { Phosphoric acid, tributyl } \\
\text { ester }\end{array}$ & $126-73-8$ & & $1.72 \mathrm{E}-04$ \\
\hline $200 W$ P $241 S 104$ & Toluene & $108-88-3$ & $5.00 \mathrm{E}-01$ & $6.67 \mathrm{E}-06$ \\
\hline $200 W$ P 241S105 & 1,3-Butadiene & $106-99-0$ & $3.50 \mathrm{E}-02$ & $4.13 \mathrm{E}-06$ \\
\hline 200W P $241 S 105$ & 2-Hexanone & $591-78-6$ & $5.00 \mathrm{E}-01$ & $7.51 \mathrm{E}-05$ \\
\hline
\end{tabular}


Table K.2. Insignificant Emission Units Based on Threshold Comparisons [WAC 173-401-530(1)(a)]

\begin{tabular}{|c|c|c|c|c|}
\hline Emission Point & Pollutants & CAS Number & $\begin{array}{l}\text { Emission } \\
\text { Threshold } \\
\text { (tons/yr) }\end{array}$ & $\begin{array}{c}\text { Actual } \\
\text { Emissions } \\
\text { (tons/yr) }\end{array}$ \\
\hline 200W P 241S105 & 2-Pentanone & $107-87-9$ & & $1.19 \mathrm{E}-04$ \\
\hline 200W P 241S105 & Acetone & $67-64-1$ & $5.00 \mathrm{E}-01$ & $1.43 \mathrm{E}-03$ \\
\hline 200W P $241 S 105$ & Acetonitrile & $75-5-8$ & $5.00 \mathrm{E}-01$ & $6.89 E-04$ \\
\hline 200W P $241 S 105$ & Ammonia & $7664-41-7$ & $5.00 \mathrm{E}-01$ & $6.57 \mathrm{E}-03$ \\
\hline 200W P 241S105 & Benzene & $71-43-2$ & $5.00 \mathrm{E}-01$ & $3.28 \mathrm{E}-05$ \\
\hline 200W P 241S105 & Carbon monoxide & $630-8-0$ & $5.00 \mathrm{E}+00$ & $9.50 \mathrm{E}-04$ \\
\hline 200W P $241 S 105$ & Heptane & $142-82-5$ & $5.00 \mathrm{E}-01$ & $8.40 \mathrm{E}-05$ \\
\hline $200 W$ P $241 S 105$ & Hexane, n-hexane & $110-54-3$ & $5.00 \mathrm{E}-01$ & $8.76 \mathrm{E}-05$ \\
\hline 200W P 241S105 & $\begin{array}{l}\text { Methyl N-amyl ketone, 2- } \\
\text { heptanone }\end{array}$ & $110-43-0$ & $5.00 \mathrm{E}-01$ & $8.13 E-05$ \\
\hline 200W P 241S105 & Nitrogen Oxide $\{\mathrm{NO}\}$ & $10102-43-9$ & $2.00 \mathrm{E}+00$ & $5.72 E-05$ \\
\hline 200W P 241S105 & Nonane & $111-84-2$ & $5.00 \mathrm{E}-01$ & $4.57 \mathrm{E}-05$ \\
\hline 200W P 241S105 & Octane & $111-65-9$ & $5.00 \mathrm{E}-01$ & 4.79E-05 \\
\hline 200W P 241S105 & $\begin{array}{l}\text { Phosphoric acid, tributyl } \\
\text { ester }\end{array}$ & $126-73-8$ & & $1.72 \mathrm{E}-04$ \\
\hline 200W P $241 S 105$ & Toluene & $108-88-3$ & $5.00 \mathrm{E}-01$ & $6.67 \mathrm{E}-06$ \\
\hline 200W P $241 S 106$ & 1,3-Butadiene & $106-99-0$ & $3.50 \mathrm{E}-02$ & $4.13 \mathrm{E}-06$ \\
\hline 200W P $241 \mathrm{~S} 106$ & 2-Hexanone & $591-78-6$ & $5.00 \mathrm{E}-01$ & $7.51 \mathrm{E}-05$ \\
\hline 200W P $241 S 106$ & 2-Pentanone & $107-87-9$ & & $1.19 \mathrm{E}-04$ \\
\hline 200W P $241 S 106$ & Acetone & $67-64-1$ & $5.00 \mathrm{E}-01$ & $1.43 \mathrm{E}-03$ \\
\hline 200W P 241S106 & Acetonitrile & $75-5-8$ & $5.00 \mathrm{E}-01$ & $6.89 \mathrm{E}-04$ \\
\hline $200 W$ P $241 S 106$ & Ammonia & $7664-41-7$ & $5.00 \mathrm{E}-01$ & $6.57 \mathrm{E}-03$ \\
\hline $200 W$ P $241 S 106$ & Benzene & $71-43-2$ & $5.00 \mathrm{E}-01$ & $3.28 \mathrm{E}-05$ \\
\hline 200W P 241S106 & Carbon monoxide & $630-8-0$ & $5.00 \mathrm{E}+00$ & $9.50 \mathrm{E}-04$ \\
\hline 200W P 241S106 & Heptane & $142-82-5$ & $5.00 \mathrm{E}-01$ & $8.40 \mathrm{E}-05$ \\
\hline 200W P 241S106 & Hexane, n-hexane & $110-54-3$ & $5.00 \mathrm{E}-01$ & $8.76 \mathrm{E}-05$ \\
\hline 200W P 241S106 & $\begin{array}{l}\text { Methyl N-amyl ketone, 2- } \\
\text { heptanone }\end{array}$ & $110-43-0$ & $5.00 \mathrm{E}-01$ & $8.13 \mathrm{E}-05$ \\
\hline $200 W$ P $241 S 106$ & Nitrogen Oxide $\{\mathrm{NO}\}$ & $10102-43-9$ & $2.00 E+00$ & $5.72 \mathrm{E}-05$ \\
\hline 200W P 241S106 & Nonane & $111-84-2$ & $5.00 \mathrm{E}-01$ & $4.57 \mathrm{E}-05$ \\
\hline 200W P $241 S 106$ & Octane & $111-65-9$ & $5.00 \mathrm{E}-01$ & $4.79 \mathrm{E}-05$ \\
\hline
\end{tabular}


Table K.2. Insignificant Emission Units Based on Threshold Comparisons [WAC 173-401-530(1)(a)]

\begin{tabular}{|c|c|c|c|c|}
\hline Emission Point & Pollutants & CAS Number & $\begin{array}{l}\text { Emission } \\
\text { Threshold } \\
\text { (tons/yr) }\end{array}$ & $\begin{array}{c}\text { Actual } \\
\text { Emissions } \\
\text { (tons/yr) }\end{array}$ \\
\hline 200W P $241 S 106$ & $\begin{array}{l}\text { Phosphoric acid, tributyl } \\
\text { ester }\end{array}$ & $126-73-8$ & & $1.72 \mathrm{E}-04$ \\
\hline 200W P 241S106 & Toluene & $108-88-3$ & $5.00 \mathrm{E}-01$ & $6.67 \mathrm{E}-06$ \\
\hline 200W P 241S107 & 1,3-Butadiene & $106-99-0$ & $3.50 \mathrm{E}-02$ & $4.13 \mathrm{E}-06$ \\
\hline 200W P $241 S 107$ & 2-Hexanone & $591-78-6$ & $5.00 \mathrm{E}-01$ & $7.51 \mathrm{E}-05$ \\
\hline 200W P $241 S 107$ & 2-Pentanone & $107-87-9$ & & $1.19 \mathrm{E}-04$ \\
\hline 200W P $241 S 107$ & Acetone & $67-64-1$ & $5.00 \mathrm{E}-01$ & $1.43 \mathrm{E}-03$ \\
\hline $200 \mathrm{~W} P \quad 241 S 107$ & Acetonitrile & $75-5-8$ & $5.00 \mathrm{E}-01$ & $6.89 \mathrm{E}-04$ \\
\hline $200 W$ P $241 S 107$ & Ammonia & $7664-41-7$ & $5.00 \mathrm{E}-01$ & $6.57 \mathrm{E}-03$ \\
\hline 200W P $241 S 107$ & Benzene & $71-43-2$ & $5.00 \mathrm{E}-01$ & $3.28 \mathrm{E}-05$ \\
\hline $200 \mathrm{~W} P \quad 241 S 107$ & Carbon monoxide & $630-8-0$ & $5.00 \mathrm{E}+00$ & $9.50 \mathrm{E}-04$ \\
\hline 200W P $241 S 107$ & Heptane & $142-82-5$ & $5.00 \mathrm{E}-01$ & $8.40 \mathrm{E}-05$ \\
\hline $200 W$ P $241 S 107$ & Hexane, n-hexane & $110-54-3$ & $5.00 \mathrm{E}-01$ & $8.76 \mathrm{E}-05$ \\
\hline 200W P $241 S 107$ & $\begin{array}{l}\text { Methyl N-amyl ketone, 2- } \\
\text { heptanone }\end{array}$ & $110-43-0$ & $5.00 \mathrm{E}-01$ & $8.13 \mathrm{E}-05$ \\
\hline $200 W$ P $241 S 107$ & Nitrogen Oxide $\{\mathrm{NO}\}$ & $10102-43-9$ & $2.00 \mathrm{E}+00$ & $5.72 \mathrm{E}-05$ \\
\hline 200W P 241S107 & Nonane & $111-84-2$ & $5.00 \mathrm{E}-01$ & 4.57E-05 \\
\hline $200 W$ P $241 S 107$ & Octane & $111-65-9$ & $5.00 \mathrm{E}-01$ & 4.79E-05 \\
\hline 200W P 241S107 & $\begin{array}{l}\text { Phosphoric acid, tributyl } \\
\text { ester }\end{array}$ & $126-73-8$ & & $1.72 \mathrm{E}-04$ \\
\hline 200W P $241 S 107$ & Toluene & $108-88-3$ & $5.00 \mathrm{E}-01$ & $6.67 \mathrm{E}-06$ \\
\hline 200W P 241S108 & 1,3-Butadiene & $106-99-0$ & $3.50 \mathrm{E}-02$ & $4.13 \mathrm{E}-06$ \\
\hline 200W P 241S108 & 2-Hexanone & $591-78-6$ & $5.00 \mathrm{E}-01$ & $7.51 \mathrm{E}-05$ \\
\hline 200W P 241S108 & 2-Pentanone & $107-87-9$ & & $1.19 \mathrm{E}-04$ \\
\hline 200W P $241 S 108$ & Acetone & $67-64-1$ & $5.00 \mathrm{E}-01$ & $1.43 \mathrm{E}-03$ \\
\hline 200W P 241S108 & Acetonitrile & $75-5-8$ & $5.00 \mathrm{E}-01$ & $6.89 \mathrm{E}-04$ \\
\hline 200W P 241S108 & Ammonia & $7664-41-7$ & $5.00 \mathrm{E}-01$ & $6.57 \mathrm{E}-03$ \\
\hline 200W P 241S108 & Benzene & $71-43-2$ & $5.00 \mathrm{E}-01$ & $3.28 \mathrm{E}-05$ \\
\hline 200W P $241 S 108$ & Carbon monoxide & $630-8-0$ & $5.00 \mathrm{E}+00$ & $9.50 \mathrm{E}-04$ \\
\hline 200W P 241S108 & Heptane & $142-82-5$ & $5.00 \mathrm{E}-01$ & $8.40 \mathrm{E}-05$ \\
\hline
\end{tabular}


Table K.2. Insignificant Emission Units Based on Threshold Comparisons [WAC 173-401-530(1)(a)]

\begin{tabular}{|c|c|c|c|c|}
\hline Emission Point & Pollutants & CAS Number & $\begin{array}{l}\text { Emission } \\
\text { Threshold } \\
\text { (tons/yr) }\end{array}$ & $\begin{array}{c}\text { Actual } \\
\text { Emissions } \\
\text { (tons/yr) }\end{array}$ \\
\hline 200W P $241 S 108$ & Hexane, $n$-hexane & $110-54-3$ & $5.00 \mathrm{E}-01$ & $8.76 \mathrm{E}-05$ \\
\hline 200W P $241 S 108$ & $\begin{array}{l}\text { Methyl N-amyl ketone, 2- } \\
\text { heptanone }\end{array}$ & $110-43-0$ & $5.00 \mathrm{E}-01$ & $8.13 E-05$ \\
\hline $200 W$ P $241 S 108$ & Nitrogen Oxide $\{\mathrm{NO}\}$ & $10102-43-9$ & $2.00 \mathrm{E}+00$ & $5.72 \mathrm{E}-05$ \\
\hline $\begin{array}{ll}200 W \text { P } & 241 S 108\end{array}$ & Nonane & $111-84-2$ & $5.00 \mathrm{E}-01$ & $4.57 \mathrm{E}-05$ \\
\hline 200W P $241 S 108$ & Octane & $111-65-9$ & $5.00 \mathrm{E}-01$ & 4.79E-05 \\
\hline 200W P 241S108 & $\begin{array}{l}\text { Phosphoric acid, tributyl } \\
\text { ester }\end{array}$ & $126-73-8$ & & 1.72E-04 \\
\hline 200W P $241 S 108$ & Toluene & $108-88-3$ & $5.00 \mathrm{E}-01$ & $6.67 \mathrm{E}-06$ \\
\hline $200 W$ P $241 S 109$ & 1,3-Butadiene & $106-99-0$ & $3.50 \mathrm{E}-02$ & $4.13 \mathrm{E}-06$ \\
\hline 200W P 241S109 & 2-Hexanone & $591-78-6$ & $5.00 \mathrm{E}-01$ & $7.51 \mathrm{E}-05$ \\
\hline 200W P $241 S 109$ & 2-Pentanone & $107-87-9$ & & $1.19 \mathrm{E}-04$ \\
\hline 200W P 241S109 & Acetone & $67-64-1$ & $5.00 \mathrm{E}-01$ & $1.43 \mathrm{E}-03$ \\
\hline 200W P 241S109 & Acetonitrile & $75-5-8$ & $5.00 \mathrm{E}-01$ & $6.89 \mathrm{E}-04$ \\
\hline 200W P $241 S 109$ & Ammonia & $7664-41-7$ & $5.00 \mathrm{E}-01$ & $6.57 \mathrm{E}-03$ \\
\hline 200W P 241S109 & Benzene & $71-43-2$ & $5.00 \mathrm{E}-01$ & $3.28 \mathrm{E}-05$ \\
\hline 200W P $241 S 109$ & Carbon monoxide & $630-8-0$ & $5.00 \mathrm{E}+00$ & $9.50 \mathrm{E}-04$ \\
\hline 200W P $241 S 109$ & Heptane & $142-82-5$ & $5.00 \mathrm{E}-01$ & $8.40 \mathrm{E}-05$ \\
\hline $200 W$ P $241 S 109$ & Hexane, n-hexane & $110-54-3$ & $5.00 \mathrm{E}-01$ & $8.76 \mathrm{E}-05$ \\
\hline 200W P 241S109 & $\begin{array}{l}\text { Methyl N-amyl ketone, 2- } \\
\text { heptanone }\end{array}$ & $110-43-0$ & $5.00 \mathrm{E}-01$ & $8.13 \mathrm{E}-05$ \\
\hline $200 W$ P $241 \$ 109$ & Nitrogen Oxide $\{\mathrm{NO}\}$ & $10102-43-9$ & $2.00 \mathrm{E}+00$ & $5.72 \mathrm{E}-05$ \\
\hline 200W P $241 S 109$ & Nonane & $111-84-2$ & $5.00 \mathrm{E}-01$ & $4.57 \mathrm{E}-05$ \\
\hline 200W P $241 S 109$ & Octane & $111-65-9$ & $5.00 \mathrm{E}-01$ & $4.79 \mathrm{E}-05$ \\
\hline 200W P $241 S 109$ & $\begin{array}{l}\text { Phosphoric acid, tributyl } \\
\text { ester }\end{array}$ & $126-73-8$ & & $1.72 E-04$ \\
\hline 200W P $241 S 109$ & Toluene & $108-88-3$ & $5.00 \mathrm{E}-01$ & $6.67 \mathrm{E}-06$ \\
\hline $200 W$ P $241 S 110$ & 1,3-Butadiene & $106-99-0$ & $3.50 \mathrm{E}-02$ & $4.13 \mathrm{E}-06$ \\
\hline 200W P $241 S 110$ & 2-Hexanone & $591-78-6$ & $5.00 \mathrm{E}-01$ & $7.51 \mathrm{E}-05$ \\
\hline 200W P $241 S 110$ & 2-Pentanone & $107-87-9$ & & $1.19 \mathrm{E}-04$ \\
\hline
\end{tabular}


Table K.2. Insignificant Emission Units Based on Threshold Comparisons [WAC 173-401-530(1)(a)]

\begin{tabular}{|c|c|c|c|c|}
\hline Emission Point & Pollutants & CAS Number & $\begin{array}{l}\text { Emission } \\
\text { Threshold } \\
\text { (tons/yr) }\end{array}$ & $\begin{array}{c}\text { Actual } \\
\text { Emissions } \\
\text { (tons/yr) }\end{array}$ \\
\hline 200 W P $241 S 110$ & Acetone & $67-64-1$ & $5.00 \mathrm{E}-01$ & $1.43 \mathrm{E}-03$ \\
\hline 200W P $241 S 110$ & Acetonitrile & $75-5-8$ & $5.00 \mathrm{E}-01$ & $6.89 \mathrm{E}-04$ \\
\hline 200W P $241 S 110$ & Ammonia & $7664-41-7$ & $5.00 \mathrm{E}-01$ & $6.57 \mathrm{E}-03$ \\
\hline $200 W$ P $241 S 110$ & Benzene & $71-43-2$ & $5.00 \mathrm{E}-01$ & $3.28 \mathrm{E}-05$ \\
\hline $200 W$ P $241 S 110$ & Carbon monoxide & $630-8-0$ & $5.00 \mathrm{E}+00$ & $9.50 \mathrm{E}-04$ \\
\hline $200 W \mathrm{P} \quad 241 \mathrm{~S} 110$ & Heptane & $142-82-5$ & $5.00 \mathrm{E}-01$ & $8.40 \mathrm{E}-05$ \\
\hline $200 W$ P $241 S 110$ & Hexane, n-hexane & $110-54-3$ & $5.00 \mathrm{E}-01$ & $8.76 \mathrm{E}-05$ \\
\hline $200 W$ P $241 S 110$ & $\begin{array}{l}\text { Methyl N-amyl ketone, 2- } \\
\text { heptanone }\end{array}$ & $110-43-0$ & $5.00 \mathrm{E}-01$ & $8.13 \mathrm{E}-05$ \\
\hline $200 W$ P $241 S 110$ & Nitrogen Oxide $\{\mathrm{NO}\}$ & $10102-43-9$ & $2.00 \mathrm{E}+00$ & $5.72 \mathrm{E}-05$ \\
\hline $200 W$ P $241 S 110$ & Nonane & $111-84-2$ & $5.00 \mathrm{E}-01$ & $4.57 \mathrm{E}-05$ \\
\hline $200 W$ P $241 S 110$ & Octane & $111-65-9$ & $5.00 \mathrm{E}-01$ & 4.79E-05 \\
\hline $200 \mathrm{~W} P \quad 241 S 110$ & $\begin{array}{l}\text { Phosphoric acid, tributyl } \\
\text { ester }\end{array}$ & $126-73-8$ & & $1.72 \mathrm{E}-04$ \\
\hline $200 W$ P $241 S 110$ & Toluene & $108-88-3$ & $5.00 \mathrm{E}-01$ & $6.67 \mathrm{E}-06$ \\
\hline $200 W$ P $241 S 111$ & 1,3-Butadiene & $106-99-0$ & $3.50 \mathrm{E}-02$ & $4.13 \mathrm{E}-06$ \\
\hline 200 W P $241 S 111$ & 2-Hexanone & $591-78-6$ & $5.00 \mathrm{E}-01$ & $7.51 \mathrm{E}-05$ \\
\hline 200W P $241 S 111$ & 2-Pentanone & $107-87-9$ & & $1.19 \mathrm{E}-04$ \\
\hline 200W P 241S111 & Acetone & $67-64-1$ & $5.00 \mathrm{E}-01$ & $1.43 \mathrm{E}-03$ \\
\hline $200 W$ P $241 S 111$ & Acetonitrile & $75-5-8$ & $5.00 \mathrm{E}-01$ & $6.89 \mathrm{E}-04$ \\
\hline 200W P 241S111 & Ammonia & $7664-41-7$ & $5.00 \mathrm{E}-01$ & $6.57 \mathrm{E}-03$ \\
\hline $200 \mathrm{~W} P \quad 241 S 111$ & Benzene & $71-43-2$ & $5.00 \mathrm{E}-01$ & $3.28 \mathrm{E}-05$ \\
\hline 200W P 241S111 & Carbon monoxide & $630-8-0$ & $5.00 \mathrm{E}+00$ & $9.50 \mathrm{E}-04$ \\
\hline 200W P $241 S 111$ & Heptane & $142-82-5$ & $5.00 \mathrm{E}-01$ & $8.40 \mathrm{E}-05$ \\
\hline 200W P 241S111 & Hexane, n-hexane & $110-54-3$ & $5.00 \mathrm{E}-01$ & $8.76 \mathrm{E}-05$ \\
\hline 200W P 241S111 & $\begin{array}{l}\text { Methyl N-amyl ketone, 2- } \\
\text { heptanone }\end{array}$ & $110-43-0$ & $5.00 \mathrm{E}-01$ & $8.13 E-05$ \\
\hline 200W P 241S111 & Nitrogen Oxide $\{\mathrm{NO}\}$ & $10102-43-9$ & $2.00 \mathrm{E}+00$ & $5.72 \mathrm{E}-05$ \\
\hline 200W P $241 S 111$ & Nonane & $111-84-2$ & $5.00 \mathrm{E}-01$ & $4.57 \mathrm{E}-05$ \\
\hline $200 W$ P $241 S 111$ & Octane & $111-65-9$ & $5.00 \mathrm{E}-01$ & $4.79 E-05$ \\
\hline
\end{tabular}


Table K.2. Insignificant Emission Units Based on Threshold Comparisons [WAC 173-401-530(1)(a)]

\begin{tabular}{|c|c|c|c|c|}
\hline Emission Point & Pollutants & CAS Number & $\begin{array}{l}\text { Emission } \\
\text { Threshold } \\
\text { (tons/yr) }\end{array}$ & $\begin{array}{c}\text { Actual } \\
\text { Emissions } \\
\text { (tons/yr) }\end{array}$ \\
\hline 200W P 241S111 & $\begin{array}{l}\text { Phosphoric acid, tributyl } \\
\text { ester }\end{array}$ & $126-73-8$ & & $1.72 E-04$ \\
\hline 200W P 241S111 & Toluene & $108-88-3$ & $5.00 \mathrm{E}-01$ & $6.67 \mathrm{E}-06$ \\
\hline 200W P $241 S 112$ & 1,3-Butadiene & $106-99-0$ & $3.50 \mathrm{E}-02$ & $4.13 E-06$ \\
\hline 200W P $241 S 112$ & 2-Hexanone & $591-78-6$ & $5.00 \mathrm{E}-01$ & $7.51 E-05$ \\
\hline 200W P 241S112 & 2-Pentanone & $107-87-9$ & & 1.19E-04 \\
\hline 200W P $241 S 112$ & Acetone & $67-64-1$ & $5.00 \mathrm{E}-01$ & $1.43 \mathrm{E}-03$ \\
\hline 200W P $241 S 112$ & Acetonitrile & $75-5-8$ & $5.00 \mathrm{E}-01$ & $6.89 \mathrm{E}-04$ \\
\hline 200W P $241 S 112$ & Ammonia & $7664-41-7$ & $5.00 \mathrm{E}-01$ & $6.57 \mathrm{E}-03$ \\
\hline 200W P $241 S 112$ & Benzene & $71-43-2$ & $5.00 \mathrm{E}-01$ & $3.28 \mathrm{E}-05$ \\
\hline 200W P $241 S 112$ & Carbon monoxide & $630-8-0$ & $5.00 \mathrm{E}+00$ & $9.50 \mathrm{E}-04$ \\
\hline 200W P 241S112 & Heptane & $142-82-5$ & $5.00 \mathrm{E}-01$ & $8.40 \mathrm{E}-05$ \\
\hline 200W P 241S112 & Hexane, n-hexane & $110-54-3$ & $5.00 \mathrm{E}-01$ & $8.76 E-05$ \\
\hline 200W P $241 S 112$ & $\begin{array}{l}\text { Methyl N-amyl ketone, 2- } \\
\text { heptanone }\end{array}$ & $110-43-0$ & $5.00 \mathrm{E}-01$ & $8.13 E-05$ \\
\hline $200 W$ P $241 S 112$ & Nitrogen Oxide $\{\mathrm{NO}\}$ & $10102-43-9$ & $2.00 \mathrm{E}+00$ & $5.72 E-05$ \\
\hline 200W P 241S112 & Nonane & $111-84-2$ & $5.00 \mathrm{E}-01$ & $4.57 \mathrm{E}-0.5$ \\
\hline 200W P 241S112 & Octane & $111-65-9$ & $5.00 \mathrm{E}-01$ & $4.79 \mathrm{E}-05$ \\
\hline $200 W P \quad 241 S 112$ & $\begin{array}{l}\text { Phosphoric acid, tributyl } \\
\text { ester }\end{array}$ & $126-73-8$ & & $1.72 \mathrm{E}-04$ \\
\hline 200W P $241 S 112$ & Toluene & $108-88-3$ & $5.00 \mathrm{E}-01$ & $6.67 \mathrm{E}-06$ \\
\hline 200W P 241SX113 & 1,3-Butadiene & $106-99-0$ & $3.50 \mathrm{E}-02$ & $1.91 \mathrm{E}-06$ \\
\hline 200W P 241SX113 & 2-Hexanone & $591-78-6$ & $5.00 \mathrm{E}-01$ & $3.47 \mathrm{E}-05$ \\
\hline 200W P 241SX113 & 2-Pentanone & $107-87-9$ & & 5.51E-05 \\
\hline 200W P 241SX113 & Acetone & $67-64-1$ & $5.00 \mathrm{E}-01$ & $6.62 E-04$ \\
\hline 200W P $241 S X 113$ & Acetonitrile & $75-5-8$ & $5.00 \mathrm{E}-01$ & $3.19 \mathrm{E}-04$ \\
\hline $200 W$ P $241 S X 113$ & Ammonia & $7664-41-7$ & $5.00 \mathrm{E}-01$ & $3.04 \mathrm{E}-03$ \\
\hline 200W P 241SX113 & Benzene & $71-43-2$ & $5.00 \mathrm{E}-01$ & $1.51 \mathrm{E}-05$ \\
\hline $200 W$ P $241 S X 113$ & Carbon monoxide & $630-8-0$ & $5.00 \mathrm{E}+00$ & 4.39E-04 \\
\hline 200W P $241 S X 113$ & Heptane & $142-82-5$ & $5.00 \mathrm{E}-01$ & $3.89 \mathrm{E}-05$ \\
\hline
\end{tabular}


Table K.2. Insignificant Emission Units Based on Threshold Comparisons [WAC 173-401-530(1)(a)]

\begin{tabular}{|c|c|c|c|c|}
\hline Emission Point & Pollutants & CAS Number & $\begin{array}{l}\text { Emission } \\
\text { Threshold } \\
\text { (tons/yr) }\end{array}$ & $\begin{array}{c}\text { Actual } \\
\text { Emissions } \\
\text { (tons/yr) }\end{array}$ \\
\hline 200W P $241 S X 113$ & Hexane, n-hexane & $110-54-3$ & $5.00 \mathrm{E}-01$ & $4.05 \mathrm{E}-05$ \\
\hline 200W P $241 S X 113$ & $\begin{array}{l}\text { Methyl N-amyl ketone, 2- } \\
\text { heptanone }\end{array}$ & $110-43-0$ & $5.00 \mathrm{E}-01$ & $3.76 \mathrm{E}-05$ \\
\hline 200W P $241 S X 113$ & Nitrogen Oxide $\{\mathrm{NO}\}$ & $10102-43-9$ & $2.00 \mathrm{E}+00$ & $2.64 \mathrm{E}-05$ \\
\hline 200W P $241 S X 113$ & Nonane & $111-84-2$ & $5.00 \mathrm{E}-01$ & $2.11 \mathrm{E}-05$ \\
\hline 200W P $241 S X 113$ & Octane & $111-65-9$ & $5.00 \mathrm{E}-01$ & $2.21 \mathrm{E}-05$ \\
\hline 200W P $241 S X 113$ & $\begin{array}{l}\text { Phosphoric acid, tributyl } \\
\text { ester }\end{array}$ & $126-73-8$ & & $7.95 \mathrm{E}-05$ \\
\hline 200W P $241 S X 113$ & Toluene & $108-88-3$ & $5.00 \mathrm{E}-01$ & $3.08 \mathrm{E}-06$ \\
\hline 200W P $241 S X 115$ & 1,3-Butadiene & $106-99-0$ & $3.50 \mathrm{E}-02$ & $1.91 \mathrm{E}-06$ \\
\hline $200 W$ P $241 S X 115$ & 2-Hexanone & $591-78-6$ & $5.00 \mathrm{E}-01$ & $3.47 \mathrm{E}-05$ \\
\hline $200 W$ P $\quad 241 S X 115$ & 2-Pentanone & $107-87-9$ & & $5.51 \mathrm{E}-05$ \\
\hline 200W P $241 S X 115$ & Acetone & $67-64-1$ & $5.00 \mathrm{E}-01$ & $6.62 \mathrm{E}-04$ \\
\hline 200W P $241 S X 115$ & Acetonitrile & $75-5-8$ & $5.00 \mathrm{E}-01$ & $3.19 \mathrm{E}-04$ \\
\hline $200 W$ P $\quad 241 S X 115$ & Ammonia & $7664-41-7$ & $5.00 \mathrm{E}-01$ & $3.04 \mathrm{E}-03$ \\
\hline $200 W$ P $\quad 241 S X 115$ & Benzene & $71-43-2$ & $5.00 \mathrm{E}-01$ & $1.51 \mathrm{E}-05$ \\
\hline 200W P $241 S X 115$ & Carbon monoxide & $630-8-0$ & $5.00 \mathrm{E}+00$ & $4.39 \mathrm{E}-04$ \\
\hline 200W P $241 S X 115$ & Heptane & $142-82-5$ & $5.00 \mathrm{E}-01$ & 3.89E-05 \\
\hline 200W P $241 S X 115$ & Hexane, n-hexane & $110-54-3$ & $5.00 \mathrm{E}-01$ & $4.05 \mathrm{E}-05$ \\
\hline 200W P $241 S X 115$ & $\begin{array}{l}\text { Methyl N-amyl ketone, 2- } \\
\text { heptanone }\end{array}$ & $110-43-0$ & $5.00 \mathrm{E}-01$ & $3.76 \mathrm{E}-05$ \\
\hline 200W P 241SX115 & Nitrogen Oxide $\{$ NO $\}$ & $10102-43-9$ & $2.00 \mathrm{E}+00$ & $2.64 \mathrm{E}-05$ \\
\hline 200W P $241 S X 115$ & Nonane & $111-84-2$ & $5.00 \mathrm{E}-01$ & $2.11 E-05$ \\
\hline 200W P 241SX115 & Octane & $111-65-9$ & $5.00 \mathrm{E}-01$ & $2.21 \mathrm{E}-05$ \\
\hline 200W P $241 S X 115$ & $\begin{array}{l}\text { Phosphoric acid, tributyl } \\
\text { ester }\end{array}$ & $126-73-8$ & & $7.95 \mathrm{E}-05$ \\
\hline 200W P $241 S X 115$ & Toluene & $108-88-3$ & $5.00 \mathrm{E}-01$ & $3.08 \mathrm{E}-06$ \\
\hline 200W P 241T101 & 1,3-Butadiene & $106-99-0$ & $3.50 \mathrm{E}-02$ & $4.13 \mathrm{E}-06$ \\
\hline 200W P $241 \mathrm{~T} 101$ & 2-Hexanone & $591-78-6$ & $5.00 \mathrm{E}-01$ & $7.51 \mathrm{E}-05$ \\
\hline $200 W$ P $241 T 101$ & 2-Pentanone & $107-87-9$ & & $1.19 \mathrm{E}-04$ \\
\hline
\end{tabular}


Table K.2. Insignificant Emission Units Based on Threshold Comparisons [WAC 173-401-530(1)(a)]

\begin{tabular}{|c|c|c|c|c|}
\hline Emission Point & Pollutants & CAS Number & $\begin{array}{c}\text { Emission } \\
\text { Threshold } \\
\text { (tons/yr) }\end{array}$ & $\begin{array}{c}\text { Actual } \\
\text { Emissions } \\
\text { (tons/yr) }\end{array}$ \\
\hline 200W P $241 \mathrm{~T} 101$ & Acetone & $67-64-1$ & $5.00 \mathrm{E}-01$ & $1.43 \mathrm{E}-03$ \\
\hline 200W P $241 T 101$ & Acetonitrile & $75-5-8$ & $5.00 \mathrm{E}-01$ & $6.89 \mathrm{E}-04$ \\
\hline 200W P $241 T 101$ & Ammonia & $7664-41-7$ & $5.00 \mathrm{E}-01$ & $6.57 \mathrm{E}-03$ \\
\hline 200W P $241 \mathrm{~T} 101$ & Benzene & $71-43-2$ & $5.00 \mathrm{E}-01$ & $3.28 \mathrm{E}-05$ \\
\hline 200W P $241 \mathrm{~T} 101$ & Carbon monoxide & $630-8-0$ & $5.00 \mathrm{E}+00$ & $9.50 \mathrm{E}-04$ \\
\hline 200W P 241T101 & Heptane & $142-82-5$ & $5.00 \mathrm{E}-01$ & $8.40 \mathrm{E}-05$ \\
\hline $\begin{array}{ll}\text { 200W P } & \text { 241T101 }\end{array}$ & Hexane, n-hexane & $110-54-3$ & $5.00 \mathrm{E}-01$ & $8.76 \mathrm{E}-05$ \\
\hline 200W P 241T101 & $\begin{array}{l}\text { Methyl N-amyl ketone, 2- } \\
\text { heptanone }\end{array}$ & $110-43-0$ & $5.00 \mathrm{E}-01$ & $8.13 \mathrm{E}-05$ \\
\hline 200W P $241 \mathrm{~T} 101$ & Nitrogen Oxide $\{\mathrm{NO}\}$ & $10102-43-9$ & $2.00 \mathrm{E}+00$ & $5.72 \mathrm{E}-05$ \\
\hline $\begin{array}{ll}200 W \\
\end{array}$ & Nonane & $111-84-2$ & $5.00 \mathrm{E}-01$ & $4.57 \mathrm{E}-05$ \\
\hline $\begin{array}{ll}200 W & P \\
241 T 101\end{array}$ & Octane & $111-65-9$ & $5.00 \mathrm{E}-01$ & $4.79 \mathrm{E}-05$ \\
\hline 200W P $241 \mathrm{~T} 101$ & $\begin{array}{l}\text { Phosphoric acid, tributyl } \\
\text { ester }\end{array}$ & $126-73-8$ & & $1.72 \mathrm{E}-04$ \\
\hline 200W P 241T101 & Toluene & $108-88-3$ & $5.00 \mathrm{E}-01$ & $6.67 \mathrm{E}-06$ \\
\hline 200W P $241 \mathrm{~T} 102$ & 1,3-Butadiene & $106-99-0$ & $3.50 \mathrm{E}-02$ & $4.13 \mathrm{E}-06$ \\
\hline 200W P $241 \mathrm{~T} 102$ & 2-Hexanone & $591-78-6$ & $5.00 \mathrm{E}-01$ & $7.51 \mathrm{E}-05$ \\
\hline 200W P 241T102 & 2-Pentanone & $107-87-9$ & & $1.19 \mathrm{E}-04$ \\
\hline 200W P 241T102 & Acetone & $67-64-1$ & $5.00 \mathrm{E}-01$ & $1.43 \mathrm{E}-03$ \\
\hline 200W P 241T102 & Acetonitrile & $75-5-8$ & $5.00 \mathrm{E}-01$ & $6.89 \mathrm{E}-04$ \\
\hline $\begin{array}{ll}200 W ~ P & 241 T 102\end{array}$ & Ammonia & $7664-41-7$ & $5.00 \mathrm{E}-01$ & $6.57 \mathrm{E}-03$ \\
\hline $\begin{array}{ll}200 W & P \\
241 T 102\end{array}$ & Benzene & $71-43-2$ & $5.00 \mathrm{E}-01$ & $3.28 \mathrm{E}-05$ \\
\hline $\begin{array}{ll}\text { 200W P } & \text { 241T102 }\end{array}$ & Carbon monoxide & $630-8-0$ & $5.00 \mathrm{E}+00$ & $9.50 \mathrm{E}-04$ \\
\hline 200W P 241T102 & Heptane & $142-82-5$ & $5.00 \mathrm{E}-01$ & $8.40 \mathrm{E}-05$ \\
\hline 200W P $241 \mathrm{~T} 102$ & Hexane, n-hexane & $110-54-3$ & $5.00 \mathrm{E}-01$ & 8.76E-05 \\
\hline 200W P $241 \mathrm{~T} 102$ & $\begin{array}{l}\text { Methyl N-amyl ketone, 2- } \\
\text { heptanone }\end{array}$ & $110-43-0$ & $5.00 \mathrm{E}-01$ & $8.13 E-05$ \\
\hline $\begin{array}{ll}\text { 200W P } & 241 \mathrm{~T} 102\end{array}$ & Nitrogen Oxide $\{\mathrm{NO}\}$ & $10102-43-9$ & $2.00 \mathrm{E}+00$ & $5.72 \mathrm{E}-05$ \\
\hline 200W P 241T102 & Nonane & $111-84-2$ & $5.00 \mathrm{E}-01$ & $4.57 \mathrm{E}-05$ \\
\hline 200W P 241T102 & Octane & $111-65-9$ & $5.00 \mathrm{E}-01$ & $4.79 \mathrm{E}-05$ \\
\hline
\end{tabular}




\section{Table K.2. Insignificant Emission Units Based on Threshold Comparisons [WAC 173-401-530(1)(a)]}

\begin{tabular}{|c|c|c|c|c|}
\hline Emission Point & Pollutants & CAS Number & $\begin{array}{l}\text { Emission } \\
\text { Threshold } \\
\text { (tons/yr) }\end{array}$ & $\begin{array}{c}\text { Actual } \\
\text { Emissions } \\
\text { (tons/yr) }\end{array}$ \\
\hline $200 \mathrm{~W} P \quad 241 \mathrm{~T} 102$ & $\begin{array}{l}\text { Phosphoric acid, tributyl } \\
\text { ester }\end{array}$ & $126-73-8$ & & $1.72 \mathrm{E}-04$ \\
\hline $200 \mathrm{~W} P \quad 241 \mathrm{~T} 102$ & Toluene & $108-88-3$ & $5.00 \mathrm{E}-01$ & $6.67 E-06$ \\
\hline $200 \mathrm{~W} P \quad 241 \mathrm{~T} 103$ & 1,3-Butadiene & $106-99-0$ & $3.50 \mathrm{E}-02$ & $4.13 \mathrm{E}-06$ \\
\hline 200W P 241T103 & 2-Hexanone & $591-78-6$ & $5.00 \mathrm{E}-01$ & $7.51 \mathrm{E}-05$ \\
\hline 200W P $241 T 103$ & 2-Pentanone & $107-87-9$ & & $1.19 \mathrm{E}-04$ \\
\hline 200W P $241 T 103$ & Acetone & $67-64-1$ & $5.00 \mathrm{E}-01$ & $1.43 \mathrm{E}-03$ \\
\hline $200 W$ P $241 T 103$ & Acetonitrile & $75-5-8$ & $5.00 \mathrm{E}-01$ & $6.89 \mathrm{E}-04$ \\
\hline $200 \mathrm{~W} P \quad 241 \mathrm{~T} 103$ & Ammonia & $7664-41-7$ & $5.00 \mathrm{E}-01$ & $6.57 \mathrm{E}-03$ \\
\hline 200W P $241 \mathrm{~T} 103$ & Benzene & $71-43-2$ & $5.00 \mathrm{E}-01$ & $3.28 \mathrm{E}-05$ \\
\hline 200W P $241 \mathrm{~T} 103$ & Carbon monoxide & $630-8-0$ & $5.00 \mathrm{E}+00$ & $9.50 \mathrm{E}-04$ \\
\hline 200W P $241 \mathrm{~T} 103$ & Heptane & $142-82-5$ & $5.00 \mathrm{E}-01$ & $8.40 \mathrm{E}-05$ \\
\hline $200 \mathrm{~W} P \quad 241 \mathrm{~T} 103$ & Hexane, n-hexane & $110-54-3$ & $5.00 \mathrm{E}-01$ & $8.76 \mathrm{E}-05$ \\
\hline 200W P $241 \mathrm{~T} 103$ & $\begin{array}{l}\text { Methyl N-amyl ketone, 2- } \\
\text { heptanone }\end{array}$ & $110-43-0$ & $5.00 \mathrm{E}-01$ & $8.13 \mathrm{E}-05$ \\
\hline 200W P $241 \mathrm{~T} 103$ & Nitrogen Oxide $\{\mathrm{NO}\}$ & $10102-43-9$ & $2.00 \mathrm{E}+00$ & $5.72 \mathrm{E}-05$ \\
\hline $200 \mathrm{~W} P \quad 241 \mathrm{~T} 103$ & Nonane & $111-84-2$ & $5.00 \mathrm{E}-01$ & $4.57 \mathrm{E}-05$ \\
\hline $200 W$ P $241 T 103$ & Octane & $111-65-9$ & $5.00 \mathrm{E}-01$ & $4.79 \mathrm{E}-05$ \\
\hline 200W P $241 T 103$ & $\begin{array}{l}\text { Phosphoric acid, tributyl } \\
\text { ester }\end{array}$ & $126-73-8$ & & $1.72 \mathrm{E}-04$ \\
\hline 200W P $241 T 103$ & Toluene & $108-88-3$ & $5.00 \mathrm{E}-01$ & $6.67 \mathrm{E}-06$ \\
\hline $200 W$ P $241 T 104$ & 1,3-Butadiene & $106-99-0$ & $3.50 \mathrm{E}-02$ & $1.91 \mathrm{E}-06$ \\
\hline $200 W$ P $241 T 104$ & 2-Hexanone & $591-78-6$ & $5.00 \mathrm{E}-01$ & $3.47 \mathrm{E}-05$ \\
\hline $200 W$ P $241 T 104$ & 2-Pentanone & $107-87-9$ & & $5.51 \mathrm{E}-05$ \\
\hline $200 \mathrm{~W} P \quad 241 \mathrm{~T} 104$ & Acetone & $67-64-1$ & $5.00 \mathrm{E}-01$ & $6.62 \mathrm{E}-04$ \\
\hline 200W P 241T104 & Acetonitrile & $75-5-8$ & $5.00 \mathrm{E}-01$ & $3.19 \mathrm{E}-04$ \\
\hline $200 W$ P $241 T 104$ & Ammonia & $7664-41-7$ & $5.00 \mathrm{E}-01$ & $3.04 \mathrm{E}-03$ \\
\hline 200W P $241 T 104$ & Benzene & $71-43-2$ & $5.00 \mathrm{E}-01$ & $1.51 \mathrm{E}-05$ \\
\hline $200 W$ P $241 T 104$ & Carbon monoxide & $630-8-0$ & $5.00 \mathrm{E}+00$ & $4.39 \mathrm{E}-04$ \\
\hline 200W P $241 \mathrm{~T} 104$ & Heptane & $142-82-5$ & $5.00 \mathrm{E}-01$ & $3.89 \mathrm{E}-05$ \\
\hline
\end{tabular}


Table K.2. Insignificant Emission Units Based on Threshold Comparisons [WAC 173-401-530(1)(a)]

\begin{tabular}{|c|c|c|c|c|}
\hline Emission Point & Pollutants & CAS Number & $\begin{array}{c}\text { Emission } \\
\text { Threshold } \\
\text { (tons/yr) }\end{array}$ & $\begin{array}{c}\text { Actual } \\
\text { Emissions } \\
\text { (tons/yr) }\end{array}$ \\
\hline 200W P 241T104 & Hexane, n-hexane & $110-54-3$ & $5.00 \mathrm{E}-01$ & $4.05 E-05$ \\
\hline 200W P 241T104 & $\begin{array}{l}\text { Methyl N-amyl ketone, 2- } \\
\text { heptanone }\end{array}$ & $110-43-0$ & $5.00 \mathrm{E}-01$ & $3.76 \mathrm{E}-05$ \\
\hline 200W P 241T104 & Nitrogen Oxide $\{\mathrm{NO}\}$ & $10102-43-9$ & $2.00 \mathrm{E}+00$ & $2.64 \mathrm{E}-05$ \\
\hline 200W P 241T104 & Nonane & $111-84-2$ & $5.00 \mathrm{E}-01$ & $2.11 E-05$ \\
\hline 200W P $241 T 104$ & Octane & $111-65-9$ & $5.00 \mathrm{E}-01$ & $2.21 \mathrm{E}-05$ \\
\hline $200 \mathrm{~W} P \quad 241 \mathrm{~T} 104$ & $\begin{array}{l}\text { Phosphoric acid, tributyl } \\
\text { ester }\end{array}$ & $126-73-8$ & & $7.95 \mathrm{E}-05$ \\
\hline 200W P 241T104 & Toluene & $108-88-3$ & $5.00 \mathrm{E}-01$ & $3.08 \mathrm{E}-06$ \\
\hline 200W P 241T105 & 1,3-Butadiene & $106-99-0$ & $3.50 \mathrm{E}-02$ & $4.13 \mathrm{E}-06$ \\
\hline 200W P $241 \mathrm{~T} 105$ & 2-Hexanone & $591-78-6$ & $5.00 \mathrm{E}-01$ & $7.51 \mathrm{E}-05$ \\
\hline 200W P 241T105 & 2-Pentanone & $107-87-9$ & & $1.19 \mathrm{E}-04$ \\
\hline 200W P 241T105 & Acetone & $67-64-1$ & $5.00 \mathrm{E}-01$ & $1.43 E-03$ \\
\hline 200W P 241T105 & Acetonitrile & $75-5-8$ & $5.00 \mathrm{E}-01$ & $6.89 \mathrm{E}-04$ \\
\hline 200W P $241 T 105$ & Ammonia & $7664-41-7$ & $5.00 \mathrm{E}-01$ & $6.57 \mathrm{E}-03$ \\
\hline 200W P 241T105 & Benzene & $71-43-2$ & $5.00 \mathrm{E}-01$ & $3.28 \mathrm{E}-05$ \\
\hline 200W P 241T105 & Carbon monoxide & $630-8-0$ & $5.00 \mathrm{E}+00$ & $9.50 \mathrm{E}-04$ \\
\hline 200W P 241T105 & Heptane & $142-82-5$ & $5.00 \mathrm{E}-01$ & $8.40 \mathrm{E}-05$ \\
\hline 200W P $241 T 105$ & Hexane, n-hexane & $110-54-3$ & $5.00 \mathrm{E}-01$ & $8.76 \mathrm{E}-05$ \\
\hline 200W P 241T105 & $\begin{array}{l}\text { Methyl N-amyl ketone, 2- } \\
\text { heptanone }\end{array}$ & $110-43-0$ & $5.00 \mathrm{E}-01$ & $8.13 E-05$ \\
\hline 200W P 241T105 & Nitrogen Oxide $\{\mathrm{NO}\}$ & $10102-43-9$ & $2.00 \mathrm{E}+00$ & $5.72 \mathrm{E}-05$ \\
\hline 200W P 241T105 & Nonane & $111-84-2$ & $5.00 \mathrm{E}-01$ & $4.57 \mathrm{E}-05$ \\
\hline 200W P 241T105 & Octane & $111-65-9$ & $5.00 \mathrm{E}-01$ & $4.79 \mathrm{E}-05$ \\
\hline 200W P 241T105 & $\begin{array}{l}\text { Phosphoric acid, tributyl } \\
\text { ester }\end{array}$ & $126-73-8$ & & $1.72 \mathrm{E}-04$ \\
\hline 200W P $241 \mathrm{~T} 105$ & Toluene. & $108-88-3$ & $5.00 \mathrm{E}-01$ & $6.67 \mathrm{E}-06$ \\
\hline $200 \mathrm{~W} P \quad 241 \mathrm{~T} 106$ & 1,3-Butadiene & $106-99-0$ & $3.50 \mathrm{E}-02$ & $4.13 \mathrm{E}-06$ \\
\hline 200W P $241 \mathrm{~T} 106$ & 2-Hexanone & $591-78-6$ & $5.00 \mathrm{E}-01$ & $7.51 \mathrm{E}-05$ \\
\hline 200W P 241T106 & 2-Pentanone & $107-87-9$ & & $1.19 E-04$ \\
\hline
\end{tabular}


Table K.2. Insignificant Emission Units Based on Threshold Comparisons [WAC 173-401-530(1)(a)]

\begin{tabular}{|c|c|c|c|c|}
\hline Emission Point & Pollutants & CAS Number & $\begin{array}{l}\text { Emission } \\
\text { Threshold } \\
\text { (tons/yr) }\end{array}$ & $\begin{array}{c}\text { Actual } \\
\text { Emissions } \\
\text { (tons/yr) }\end{array}$ \\
\hline $200 \mathrm{~W} P \quad 241 \mathrm{~T} 106$ & Acetone & $67-64-1$ & $5.00 \mathrm{E}-01$ & $1.43 \mathrm{E}-03$ \\
\hline 200W P $241 \mathrm{~T} 106$ & Acetonitrile & $75-5-8$ & $5.00 \mathrm{E}-01$ & $6.89 \mathrm{E}-04$ \\
\hline 200W P $241 T 106$ & Ammonia & $7664-41-7$ & $5.00 \mathrm{E}-01$ & $6.57 \mathrm{E}-03$ \\
\hline 200W P $241 T 106$ & Benzene & $71-43-2$ & $5.00 \mathrm{E}-01$ & $3.28 \mathrm{E}-05$ \\
\hline 200W P 241T106 & Carbon monoxide & $630-8-0$ & $5.00 \mathrm{E}+00$ & $9.50 \mathrm{E}-04$ \\
\hline $200 \mathrm{~W} P \quad 241 \mathrm{~T} 106$ & Heptane & $142-82-5$ & $5.00 \mathrm{E}-01$ & $8.40 \mathrm{E}-05$ \\
\hline $200 \mathrm{~W} P \quad 241 \mathrm{~T} 106$ & Hexane, n-hexane & $110-54-3$ & $5.00 \mathrm{E}-01$ & $8.76 \mathrm{E}-05$ \\
\hline 200W P 241T106 & $\begin{array}{l}\text { Methyl N-amyl ketone, 2- } \\
\text { heptanone }\end{array}$ & $110-43-0$ & $5.00 \mathrm{E}-01$ & $8.13 E-05$ \\
\hline 200W P 241T106 & Nitrogen Oxide $\{\mathrm{NO}\}$ & $10102-43-9$ & $2.00 \mathrm{E}+00$ & $5.72 \mathrm{E}-05$ \\
\hline 200W P $241 \mathrm{~T} 106$ & Nonane & $111-84-2$ & $5.00 \mathrm{E}-01$ & $4.57 \mathrm{E}-05$ \\
\hline 200W P 241T106 & Octane & $111-65-9$ & $5.00 \mathrm{E}-01$ & $4.79 \mathrm{E}-05$ \\
\hline 200W P 241T106 & $\begin{array}{l}\text { Phosphoric acid, tributyl } \\
\text { ester }\end{array}$ & $126-73-8$ & & $1.72 \mathrm{E}-04$ \\
\hline $200 W$ P $241 T 106$ & Toluene & $108-88-3$ & $5.00 \mathrm{E}-01$ & $6.67 E-06$ \\
\hline $200 \mathrm{~W} P \quad 241 \mathrm{~T} 107$ & 1,3-Butadiene & $106-99-0$ & $3.50 \mathrm{E}-02$ & $4.13 \mathrm{E}-06$ \\
\hline 200W P $241 \mathrm{~T} 107$ & 2-Hexanone & $591-78-6$ & $5.00 \mathrm{E}-01$ & 7.51E-05 \\
\hline 200W P $241 T 107$ & 2-Pentanone & $107-87-9$ & & $1.19 \mathrm{E}-04$ \\
\hline 200W P 241T107 & Acetone & $67-64-1$ & $5.00 \mathrm{E}-01$ & $1.43 E-03$ \\
\hline $200 W$ P $241 T 107$ & Acetonitrile & $75-5-8$ & $5.00 \mathrm{E}-01$ & $6.89 \mathrm{E}-04$ \\
\hline 200W P $241 T 107$ & Ammonia & $7664-41-7$ & $5.00 \mathrm{E}-01$ & $6.57 \mathrm{E}-03$ \\
\hline $200 \mathrm{~W} P \quad 241 \mathrm{~T} 107$ & Benzene & $71-43-2$ & $5.00 \mathrm{E}-01$ & $3.28 \mathrm{E}-05$ \\
\hline 200W P 241T107 & Carbon monoxide & $630-8-0$ & $5.00 \mathrm{E}+00$ & $9.50 \mathrm{E}-04$ \\
\hline 200W P 241T107 & Heptane & $142-82-5$ & $5.00 \mathrm{E}-01$ & $8.40 \mathrm{E}-05$ \\
\hline 200W P 241T107 & Hexane, n-hexane & $110-54-3$ & $5.00 \mathrm{E}-01$ & $8.76 \mathrm{E}-05$ \\
\hline 200W P 241T107 & $\begin{array}{l}\text { Methyl N-amyl ketone, 2- } \\
\text { heptanone }\end{array}$ & $110-43-0$ & $5.00 \mathrm{E}-01$ & $8.13 E-05$ \\
\hline 200W P $241 T 107$ & Nitrogen Oxide $\{\mathrm{NO}\}$ & $10102-43-9$ & $2.00 \mathrm{E}+00$ & $5.72 \mathrm{E}-05$ \\
\hline 200W P $241 T 107$ & Nonane & $111-84-2$ & $5.00 \mathrm{E}-01$ & $4.57 \mathrm{E}-05$ \\
\hline 200W P $241 T 107$ & Octane & $111-65-9$ & $5.00 \mathrm{E}-01$ & 4.79E-05 \\
\hline
\end{tabular}


Table K.2. Insignificant Emission Units Based on Threshold Comparisons [WAC 173-401-530(1)(a)]

\begin{tabular}{|c|c|c|c|c|}
\hline Emission Point & Pollutants & CAS Number & $\begin{array}{l}\text { Emission } \\
\text { Threshold } \\
\text { (tons/yr) }\end{array}$ & $\begin{array}{c}\text { Actual } \\
\text { Emissions } \\
\text { (tons/yr) }\end{array}$ \\
\hline 200W P $241 \mathrm{~T} 107$ & $\begin{array}{l}\text { Phosphoric acid, tributyl } \\
\text { ester }\end{array}$ & $126-73-8$ & & $1.72 \mathrm{E}-04$ \\
\hline 200W P $241 T 107$ & Toluene & $108-88-3$ & $5.00 \mathrm{E}-01$ & $6.67 E-06$ \\
\hline $200 \mathrm{~W} P \quad 241 \mathrm{~T} 108$ & 1,3-Butadiene & $106-99-0$ & $3.50 \mathrm{E}-02$ & $1.91 \mathrm{E}-06$ \\
\hline 200W P $241 \mathrm{~T} 108$ & 2-Hexanone & $591-78-6$ & $5.00 \mathrm{E}-01$ & $3.47 \mathrm{E}-05$ \\
\hline $200 \mathrm{~W} P \quad 241 \mathrm{~T} 108$ & 2-Pentanone & $107-87-9$ & & $5.51 \mathrm{E}-05$ \\
\hline 200W P $241 \mathrm{~T} 108$ & Acetone & $67-64-1$ & $5.00 \mathrm{E}-01$ & $6.62 \mathrm{E}-04$ \\
\hline $200 \mathrm{~W}$ P $241 \mathrm{~T} 108$ & Acetonitrile & $75-5-8$ & $5.00 \mathrm{E}-01$ & $3.19 \mathrm{E}-04$ \\
\hline $200 \mathrm{~W} P \quad 241 \mathrm{~T} 108$ & Ammonia & $7664-41-7$ & $5.00 \mathrm{E}-01$ & $3.04 \mathrm{E}-03$ \\
\hline 200W P $241 \mathrm{~T} 108$ & Benzene & $71-43-2$ & $5.00 \mathrm{E}-01$ & $1.51 \mathrm{E}-05$ \\
\hline $200 \mathrm{~W} P \quad 241 \mathrm{~T} 108$ & Carbon monoxide & $630-8-0$ & $5.00 \mathrm{E}+00$ & $4.39 \mathrm{E}-04$ \\
\hline 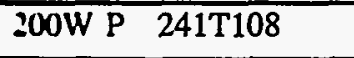 & Heptane & $142-82-5$ & $5.00 \mathrm{E}-01$ & $3.89 \mathrm{E}-05$ \\
\hline $200 W$ P $241 T 108$ & Hexane, n-hexane & $110-54-3$ & $5.00 \mathrm{E}-01$ & $4.05 \mathrm{E}-05$ \\
\hline $\begin{array}{ll}200 W & P \\
241 T 108\end{array}$ & $\begin{array}{l}\text { Methyl N-amyl ketone, 2- } \\
\text { heptanone }\end{array}$ & $110-43-0$ & $5.00 \mathrm{E}-01$ & $3.76 \mathrm{E}-05$ \\
\hline 200W P $241 \mathrm{~T} 108$ & Nitrogen Oxide $\{\mathrm{NO}\}$ & $10102-43-9$ & $2.00 \mathrm{E}+00$ & $2.64 \mathrm{E}-05$ \\
\hline $200 \mathrm{~W} P \quad 241 \mathrm{~T} 108$ & Nonane & $111-84-2$ & $5.00 \mathrm{E}-01$ & 2.11E-05 \\
\hline $\begin{array}{ll}200 W & P \\
241 T 108\end{array}$ & Octane & $111-65-9$ & $5.00 \mathrm{E}-01$ & $2.21 \mathrm{E}-05$ \\
\hline 200W P $241 \mathrm{~T} 108$ & $\begin{array}{l}\text { Phosphoric acid, tributyl } \\
\text { ester }\end{array}$ & $126-73-8$ & & $7.95 \mathrm{E}-05$ \\
\hline 200W P $241 \mathrm{~T} 108$ & Toluene & $108-88-3$ & $5.00 \mathrm{E}-01$ & $3.08 \mathrm{E}-06$ \\
\hline 200W P $241 T 109$ & 1,3-Butadiene & $106-99-0$ & $3.50 \mathrm{E}-02$ & $4.13 E-06$ \\
\hline 200W P $241 \mathrm{~T} 109$ & 2-Hexanone & $591-78-6$ & $5.00 \mathrm{E}-01$ & $7.51 \mathrm{E}-05$ \\
\hline 200W P $241 \mathrm{~T} 109$ & 2-Pentanone & $107-87-9$ & & $1.19 \mathrm{E}-04$ \\
\hline 200W P $241 \mathrm{~T} 109$ & Acetone & $67-64-1$ & $5.00 \mathrm{E}-01$ & $1.43 \mathrm{E}-03$ \\
\hline 200W P $241 T 109$ & Acetonitrile & $75-5-8$ & $5.00 \mathrm{E}-01$ & $6.89 \mathrm{E}-04$ \\
\hline 200W P $241 T 109$ & Ammonia & $7664-41-7$ & $5.00 \mathrm{E}-01$ & $6.57 \mathrm{E}-03$ \\
\hline 200W P $241 T 109$ & Benzene & $71-43-2$ & $5.00 \mathrm{E}-01$ & $3.28 \mathrm{E}-05$ \\
\hline 200W P $241 \mathrm{~T} 109$ & Carbon monoxide & $630-8-0$ & $5.00 \mathrm{E}+00$ & $9.50 \mathrm{E}-04$ \\
\hline 200W P $241 T 109$ & Heptane & $142-82-5$ & $5.00 \mathrm{E}-01$ & $8.40 \mathrm{E}-05$ \\
\hline
\end{tabular}


Table K.2. Insignificant Emission Units Based on Threshold Comparisons [WAC 173-401-530(1)(a)]

\begin{tabular}{|c|c|c|c|c|}
\hline Emission Point & Pollutants & CAS Number & $\begin{array}{l}\text { Emission } \\
\text { Threshold } \\
\text { (tons/yr) }\end{array}$ & $\begin{array}{c}\text { Actual } \\
\text { Emissions } \\
\text { (tons/yr) }\end{array}$ \\
\hline 200W P 241T109 & Hexane, n-hexane & $110-54-3$ & $5.00 \mathrm{E}-01$ & $8.76 \mathrm{E}-05$ \\
\hline 200W P 241T109 & $\begin{array}{l}\text { Methyl N-amyl ketone, 2- } \\
\text { heptanone }\end{array}$ & $110-43-0$ & $5.00 \mathrm{E}-01$ & $8.13 E-05$ \\
\hline 200W P 241T109 & Nitrogen Oxide $\{\mathrm{NO}\}$ & $10102-43-9$ & $2.00 \mathrm{E}+00$ & $5.72 \mathrm{E}-05$ \\
\hline $200 \mathrm{~W} P \quad 241 \mathrm{~T} 109$ & Nonane & $111-84-2$ & $5.00 \mathrm{E}-01$ & $4.57 \mathrm{E}-05$ \\
\hline 200W P 241 T109 & Octane & $111-65-9$ & $5.00 \mathrm{E}-01$ & $4.79 \mathrm{E}-05$ \\
\hline 200W P $241 T 109$ & $\begin{array}{l}\text { Phosphoric acid, tributyl } \\
\text { ester }\end{array}$ & $126-73-8$ & & $1.72 \mathrm{E}-04$ \\
\hline $200 \mathrm{~W}$ P $241 \mathrm{~T} 109$ & Toluene & $108-88-3$ & $5.00 \mathrm{E}-01$ & $6.67 \mathrm{E}-06$ \\
\hline $200 \mathrm{~W} \mathrm{P} \quad 241 \mathrm{~T} 110$ & 1,3-Butadiene & $106-99-0$ & $3.50 \mathrm{E}-02$ & $4.13 \mathrm{E}-06$ \\
\hline 200W P $241 \mathrm{~T} 110$ & 2-Hexanone & $591-78-6$ & $5.00 \mathrm{E}-01$ & $7.51 \mathrm{E}-05$ \\
\hline 200W P $241 \mathrm{~T} 110$ & 2-Pentanone & $107-87-9$ & & $1.19 \mathrm{E}-04$ \\
\hline $\begin{array}{ll}200 W \text { P } & 241 T 110\end{array}$ & Acetone & $67-64-1$ & $5.00 \mathrm{E}-01$ & $1.43 \mathrm{E}-03$ \\
\hline $\begin{array}{ll}\text { 200W P } & 241 T 110\end{array}$ & Acetonitrile & $75-5-8$ & $5.00 \mathrm{E}-01$ & $6.89 E-04$ \\
\hline 200W P $241 T 110$ & Ammonia & $7664-41-7$ & $5.00 \mathrm{E}-01$ & $6.57 \mathrm{E}-03$ \\
\hline 200W P $241 \mathrm{~T} 110$ & Benzene & $71-43-2$ & $5.00 \mathrm{E}-01$ & $3.28 \mathrm{E}-05$ \\
\hline 200W P $241 \mathrm{~T} 110$ & Carbon monoxide & $630-8-0$ & $5.00 \mathrm{E}+00$ & $9.50 \mathrm{E}-04$ \\
\hline $200 \mathrm{~W} P \quad 241 \mathrm{~T} 110$ & Heptane & $142-82-5$ & $5.00 \mathrm{E}-01$ & $8.40 \mathrm{E}-05$ \\
\hline 200W P $241 \mathrm{~T} 110$ & Hexane, n-hexane & $110-54-3$ & $5.00 \mathrm{E}-01$ & $8.76 \mathrm{E}-05$ \\
\hline $200 \mathrm{~W} P \quad 241 \mathrm{~T} 110$ & $\begin{array}{l}\text { Methyl N-amyl ketone, 2- } \\
\text { heptanone }\end{array}$ & $110-43-0$ & $5.00 \mathrm{E}-01$ & $8.13 E-05$ \\
\hline $200 \mathrm{~W} P \quad 241 \mathrm{~T} 110$ & Nitrogen Oxide $\{\mathrm{NO}\}$ & $10102-43-9$ & $2.00 \mathrm{E}+00$ & $5.72 \mathrm{E}-05$ \\
\hline $200 \mathrm{~W} P \quad 241 \mathrm{~T} 110$ & Nonane & $111-84-2$ & $5.00 \mathrm{E}-01$ & $4.57 \mathrm{E}-05$ \\
\hline $200 W$ P 241 T110 & Octane & $111-65-9$ & $5.00 \mathrm{E}-01$ & $4.79 \mathrm{E}-05$ \\
\hline $200 \mathrm{~W} P \quad 241 \mathrm{~T} 110$ & $\begin{array}{l}\text { Phosphoric acid, tributyl } \\
\text { ester }\end{array}$ & $126-73-8$ & & $1.72 \mathrm{E}-04$ \\
\hline $200 \mathrm{~W} P \quad 241 \mathrm{~T} 110$ & Toluene & $108-88-3$ & $5.00 \mathrm{E}-01$ & $6.67 \mathrm{E}-06$ \\
\hline 200W P 241T111 & 1,3-Butadiene & $106-99-0$ & $3.50 \mathrm{E}-02$ & $4.13 \mathrm{E}-06$ \\
\hline 200W P $241 T 111$ & 2-Hexanone & $591-78-6$ & $5.00 \mathrm{E}-01$ & $7.51 \mathrm{E}-05$ \\
\hline $200 W$ P $241 T 111$ & 2-Pentanone & $107-87-9$ & & $1.19 \mathrm{E}-04$ \\
\hline
\end{tabular}


Table K.2. Insignificant Emission Units Based on Threshold Comparisons [WAC 173-401-530(1)(a)]

\begin{tabular}{|c|c|c|c|c|}
\hline Emission Point & Pollutants & CAS Number & $\begin{array}{l}\text { Emission } \\
\text { Threshold } \\
\text { (tons/yr) }\end{array}$ & $\begin{array}{c}\text { Actual } \\
\text { Emissions } \\
\text { (tons } / y r \text { ) }\end{array}$ \\
\hline 200W P 241T111 & Acetone & $67-64-1$ & $5.00 \mathrm{E}-01$ & $1.43 \mathrm{E}-03$ \\
\hline $200 \mathrm{~W} P \quad 241 \mathrm{~T} 111$ & Acetonitrile & $75-5-8$ & $5.00 \mathrm{E}-01$ & $6.89 \mathrm{E}-04$ \\
\hline $200 \mathrm{~W} P \quad 241 \mathrm{~T} 111$ & Ammonia & $7664-41-7$ & $5.00 \mathrm{E}-01$ & $6.57 \mathrm{E}-03$ \\
\hline 200W P 241T111 & Benzene & $71-43-2$ & $5.00 \mathrm{E}-01$ & $3.28 \mathrm{E}-05$ \\
\hline 200W P 241T111 & Carbon monoxide & $630-8-0$ & $5.00 E+00$ & $9.50 \mathrm{E}-04$ \\
\hline 200W P $241 T 111$ & Heptane & $142-82-5$ & $5.00 \mathrm{E}-01$ & $8.40 \mathrm{E}-05$ \\
\hline 200W P $241 T 111$ & Hexane, n-hexane & $110-54-3$ & $5.00 \mathrm{E}-01$ & $8.76 \mathrm{E}-05$ \\
\hline 200W P 241T111 & $\begin{array}{l}\text { Methyl N-amyl ketone, 2- } \\
\text { heptanone }\end{array}$ & $110-43-0$ & $5.00 \mathrm{E}-01$ & $8.13 \mathrm{E}-05$ \\
\hline 200W P 241T111 & Nitrogen Oxide $\{\mathrm{NO}\}$ & $10102-43-9$ & $2.00 \mathrm{E}+00$ & $5.72 \mathrm{E}-05$ \\
\hline $200 \mathrm{~W} P \quad 241 \mathrm{~T} 111$ & Nonane & $111-84-2$ & $5.00 \mathrm{E}-01$ & $4.57 \mathrm{E}-05$ \\
\hline 200W P 241T111 & Octane & $111-65-9$ & $5.00 \mathrm{E}-01$ & 4.79E-05 \\
\hline 200W P $241 T 111$ & $\begin{array}{l}\text { Phosphoric acid, tributyl } \\
\text { ester }\end{array}$ & $126-73-8$ & & $1.72 \mathrm{E}-04$ \\
\hline 200W P 241T111 & Toluene & $108-88-3$ & $5.00 \mathrm{E}-01$ & $6.67 \mathrm{E}-06$ \\
\hline 200W P $241 T 112$ & 1,3-Butadiene & $106-99-0$ & $3.50 \mathrm{E}-02$ & $4.13 \mathrm{E}-06$ \\
\hline 200W P $241 \mathrm{~T} 112$ & 2-Hexanone & $591-78-6$ & $5.00 \mathrm{E}-01$ & $7.51 \mathrm{E}-05$ \\
\hline 200W P $241 T 112$ & 2-Pentanone & $107-87-9$ & & $1.19 \mathrm{E}-04$ \\
\hline 200W P $241 \mathrm{~T} 112$ & Acetone & $67-64-1$ & $5.00 \mathrm{E}-01$ & $1.43 E-03$ \\
\hline 200W P $241 \mathrm{~T} 112$ & Acetonitrile & $75-5-8$ & $5.00 \mathrm{E}-01$ & $6.89 \mathrm{E}-04$ \\
\hline 200W P $241 \mathrm{~T} 112$ & Ammonia & $7664-41-7$ & $5.00 \mathrm{E}-01$ & $6.57 \mathrm{E}-03$ \\
\hline 200W P $241 T 112$ & Benzene & $71-43-2$ & $5.00 \mathrm{E}-01$ & $3.28 \mathrm{E}-05$ \\
\hline $200 W$ P $241 T 112$ & Carbon monoxide & $630-8-0$ & $5.00 \mathrm{E}+00$ & $9.50 \mathrm{E}-04$ \\
\hline 200W P $241 \mathrm{~T} 112$ & Heptane & $142-82-5$ & $5.00 \mathrm{E}-01$ & $8.40 \mathrm{E}-05$ \\
\hline 200W P $241 \mathrm{~T} 112$ & Hexane, n-hexane & $110-54-3$ & $5.00 \mathrm{E}-01$ & $8.76 \mathrm{E}-05$ \\
\hline $200 \mathrm{~W} P \quad 241 \mathrm{~T} 112$ & $\begin{array}{l}\text { Methyl N-amyl ketone, 2- } \\
\text { heptanone }\end{array}$ & $110-43-0$ & $5.00 \mathrm{E}-01$ & $8.13 E-05$ \\
\hline $200 \mathrm{~W} P \quad 241 \mathrm{~T} 112$ & Nitrogen Oxide $\{\mathrm{NO}\}$ & $10102-43-9$ & $2.00 \mathrm{E}+00$ & 5.72E-05 \\
\hline 200W P $241 \mathrm{~T} 112$ & Nonane & $111-84-2$ & $5.00 \mathrm{E}-01$ & $4.57 \mathrm{E}-05$ \\
\hline 200W P $241 \mathrm{~T} 112$ & Octane & $111-65-9$ & $5.00 \mathrm{E}-01$ & 4.79E-05 \\
\hline
\end{tabular}




\section{Table K.2. Insignificant Emission Units Based on Threshold Comparisons [WAC 173-401-530(1)(a)]}

\begin{tabular}{|c|c|c|c|c|}
\hline Emission Point & Pollutants & CAS Number & $\begin{array}{c}\text { Emission } \\
\text { Threshold } \\
\text { (tons/yr) }\end{array}$ & $\begin{array}{c}\text { Actual } \\
\text { Emissions } \\
\text { (tons/yr) }\end{array}$ \\
\hline $200 W$ P $241 \mathrm{~T} 112$ & $\begin{array}{l}\text { Phosphoric acid, tributyl } \\
\text { ester }\end{array}$ & $126-73-8$ & & $1.72 \mathrm{E}-04$ \\
\hline $200 \mathrm{~W} P \quad 241 \mathrm{~T} 112$ & Toluene & $108-88-3$ & $5.00 \mathrm{E}-01$ & $6.67 \mathrm{E}-06$ \\
\hline $200 \mathrm{~W} P \quad 241 \mathrm{~T} 201$ & 1,3-Butadiene & $106-99-0$ & $3.50 \mathrm{E}-02$ & $1.91 \mathrm{E}-06$ \\
\hline 200W P $241 T 201$ & 2-Hexanone & $591-78-6$ & $5.00 \mathrm{E}-01$ & $3.47 \mathrm{E}-05$ \\
\hline 200W P 241T201 & 2-Pentanone & $107-87-9$ & & $5.51 \mathrm{E}-05$ \\
\hline 200W P 241T201 & Acetone & $67-64-1$ & $5.00 \mathrm{E}-01$ & $6.62 \mathrm{E}-04$ \\
\hline $200 \mathrm{~W} P \quad 241 \mathrm{~T} 201$ & Acetonitrile & $75-5-8$ & $5.00 \mathrm{E}-01$ & $3.19 \mathrm{E}-04$ \\
\hline 200W P $241 T 201$ & Ammonia & $7664-41-7$ & $5.00 \mathrm{E}-01$ & $3.04 \mathrm{E}-03$ \\
\hline 200W P $241 T 201$ & Benzene & $71-43-2$ & $5.00 \mathrm{E}-01$ & $1.51 \mathrm{E}-05$ \\
\hline 200W P $241 T 201$ & Carbon monoxide & $630-8-0$ & $5.00 \mathrm{E}+00$ & $4.39 E-04$ \\
\hline 200W P 241T201 & Heptane & $142-82-5$ & $5.00 \mathrm{E}-01$ & $3.89 \mathrm{E}-05$ \\
\hline $200 W$ P $241 T 201$ & Hexane, n-hexane & $110-54-3$ & $5.00 \mathrm{E}-01$ & $4.05 \mathrm{E}-05$ \\
\hline 200W P $241 T 201$ & $\begin{array}{l}\text { Methyl N-amyl ketone, 2- } \\
\text { heptanone }\end{array}$ & $110-43-0$ & $5.00 \mathrm{E}-01$ & $3.76 \mathrm{E}-05$ \\
\hline 200W P 241T201 & Nitrogen Oxide $\{\mathrm{NO}\}$ & $10102-43-9$ & $2.00 \mathrm{E}+00$ & $2.64 \mathrm{E}-05$ \\
\hline 200W P 241T201 & Nonane & $111-84-2$ & $5.00 \mathrm{E}-01$ & $2.11 \mathrm{E}-05$ \\
\hline $200 W$ P $241 T 201$ & Octane & $111-65-9$ & $5.00 \mathrm{E}-01$ & $2.21 \mathrm{E}-05$ \\
\hline 200W P $241 T 201$ & $\begin{array}{l}\text { Phosphoric acid, tributyl } \\
\text { ester }\end{array}$ & $126-73-8$ & & $7.95 \mathrm{E}-05$ \\
\hline $200 W$ P $241 T 201$ & Toluene & $108-88-3$ & $5.00 \mathrm{E}-01$ & $3.08 \mathrm{E}-06$ \\
\hline 200W P $241 \mathrm{~T} 202$ & 1,3-Butadiene & $106-99-0$ & $3.50 \mathrm{E}-02$ & $1.91 \mathrm{E}-06$ \\
\hline 200W P $241 T 202$ & 2-Hexanone & $591-78-6$ & $5.00 \mathrm{E}-01$ & $3.47 \mathrm{E}-05$ \\
\hline 200W P $241 T 202$ & 2-Pentanone & $107-87-9$ & & $5.51 \mathrm{E}-05$ \\
\hline $200 W$ P $241 T 202$ & Acetone & $67-64-1$ & $5.00 \mathrm{E}-01$ & $6.62 \mathrm{E}-04$ \\
\hline $200 \mathrm{~W} P \quad 241 \mathrm{~T} 202$ & Acetonitrile & $75-5-8$ & $5.00 \mathrm{E}-01$ & $3.19 \mathrm{E}-04$ \\
\hline 200W P $241 T 202$ & Ammonia & $7664-41-7$ & $5.00 \mathrm{E}-01$ & $3.04 \mathrm{E}-03$ \\
\hline 200W P 241T202 & Benzene & $71-43-2$ & $5.00 \mathrm{E}-01$ & $1.51 \mathrm{E}-05$ \\
\hline 200W P $241 T 202$ & Carbon monoxide & $630-8-0$ & $5.00 \mathrm{E}+00$ & $4.39 \mathrm{E}-04$ \\
\hline $200 \mathrm{~W} P \quad 241 \mathrm{~T} 202$ & Heptane & $142-82-5$ & $5.00 \mathrm{E}-01$ & $3.89 \mathrm{E}-05$ \\
\hline
\end{tabular}


Table K.2. Insignificant Emission Units Based on Threshold Comparisons [WAC 173-401-530(1)(a)]

\begin{tabular}{|c|c|c|c|c|}
\hline Emission Point & Pollutants & CAS Number & $\begin{array}{l}\text { Emission } \\
\text { Threshold } \\
\text { (tons/yr) }\end{array}$ & $\begin{array}{c}\text { Actual } \\
\text { Emissions } \\
\text { (tons/yr) }\end{array}$ \\
\hline 200W P $241 T 202$ & Hexane, n-hexane & $110-54-3$ & $5.00 \mathrm{E}-01$ & $4.05 E-05$ \\
\hline $200 W$ P $241 T 202$ & $\begin{array}{l}\text { Methyl N-amyl ketone, 2- } \\
\text { heptanone }\end{array}$ & $110-43-0$ & $5.00 \mathrm{E}-01$ & $3.76 \mathrm{E}-05$ \\
\hline $200 W$ P $241 T 202$ & Nitrogen Oxide $\{\mathrm{NO}\}$ & $10102-43-9$ & $2.00 \mathrm{E}+00$ & $2.64 \mathrm{E}-05$ \\
\hline 200W P $241 T 202$ & Nonane & $111-84-2$ & $5.00 \mathrm{E}-01$ & $2.11 E-05$ \\
\hline 200W P $241 T 202$ & Octane & $111-65-9$ & $5.00 \mathrm{E}-01$ & $2.21 \mathrm{E}-05$ \\
\hline $200 \mathrm{~W} P \quad 241 \mathrm{~T} 202$ & $\begin{array}{l}\text { Phosphoric acid, tributyl } \\
\text { ester }\end{array}$ & $126-73-8$ & & $7.95 \mathrm{E}-05$ \\
\hline $200 \mathrm{~W} P \quad 241 \mathrm{~T} 202$ & Toluene & $108-88-3$ & $5.00 \mathrm{E}-01$ & $3.08 \mathrm{E}-06$ \\
\hline 200W P 241T203 & 1,3-Butadiene & $106-99-0$ & $3.50 \mathrm{E}-02$ & $1.91 \mathrm{E}-06$ \\
\hline 200W P $241 T 203$ & 2-Hexanone & $591-78-6$ & $5.00 \mathrm{E}-01$ & $3.47 \mathrm{E}-05$ \\
\hline $200 W$ P $241 T 203$ & 2-Pentanone & $107-87-9$ & & $5.51 \mathrm{E}-05$ \\
\hline $200 W$ P $241 T 203$ & Acetone & $67-64-1$ & $5.00 \mathrm{E}-01$ & $6.62 \mathrm{E}-04$ \\
\hline $200 W$ P $241 T 203$ & Acetonitrile & $75-5-8$ & $5.00 \mathrm{E}-01$ & $3.19 \mathrm{E}-04$ \\
\hline 200W P $241 T 203$ & Ammonia & $7664-41-7$ & $5.00 E-01$ & $3.04 \mathrm{E}-03$ \\
\hline 200W P $241 \mathrm{~T} 203$ & Benzene & $71-43-2$ & $5.00 \mathrm{E}-01$ & $1.51 \mathrm{E}-05$ \\
\hline 200W P $241 \mathrm{~T} 203$ & Carbon monoxide & $630-8-0$ & $5.00 \mathrm{E}+00$ & $4.39 \mathrm{E}-04$ \\
\hline 200W P $241 T 203$ & Heptane & $142-82-5$ & $5.00 \mathrm{E}-01$ & $3.89 \mathrm{E}-05$ \\
\hline 200W P $241 T 203$ & Hexane, $\mathrm{n}$-hexane & $110-54-3$ & $5.00 \mathrm{E}-01$ & $4.05 \mathrm{E}-05$ \\
\hline $200 \mathrm{~W} P \quad 241 \mathrm{~T} 203$ & $\begin{array}{l}\text { Methyl N-amyl ketone, 2- } \\
\text { heptanone }\end{array}$ & $110-43-0$ & $5.00 \mathrm{E}-01$ & $3.76 \mathrm{E}-05$ \\
\hline 200W P $241 T 203$ & Nitrogen Oxide $\{\mathrm{NO}\}$ & $10102-43-9$ & $2.00 \mathrm{E}+00$ & $2.64 \mathrm{E}-05$ \\
\hline 200W P $241 T 203$ & Nonane & $111-84-2$ & $5.00 \mathrm{E}-01$ & $2.11 \mathrm{E}-05$ \\
\hline 200W P $241 T 203$ & Octane & $111-65-9$ & $5.00 \mathrm{E}-01$ & $2.21 \mathrm{E}-05$ \\
\hline 200W P $241 T 203$ & $\begin{array}{l}\text { Phosphoric acid, tributyl } \\
\text { ester }\end{array}$ & $126-73-8$ & & $7.95 \mathrm{E}-05$ \\
\hline 200W P $241 T 203$ & Toluene & $108-88-3$ & $5.00 \mathrm{E}-01$ & $3.08 \mathrm{E}-06$ \\
\hline 200W P 241T204 & 1,3-Butadiene & $106-99-0$ & $3.50 \mathrm{E}-02$ & $1.91 \mathrm{E}-06$ \\
\hline 200W P $241 T 204$ & 2-Hexanone & $591-78-6$ & $5.00 \mathrm{E}-01$ & $3.47 \mathrm{E}-05$ \\
\hline 200W P 241T204 & 2-Pentanone & $107-87-9$ & & $5.51 \mathrm{E}-05$ \\
\hline
\end{tabular}


Hanford Site Air

DOE/RL-95-07, Rev. 0

Operating Permit Application $05 / \mathrm{s}$

Table K.2. Insignificant Emission Units Based on Threshold Comparisons [WAC 173-401-530(1)(a)]

\begin{tabular}{|c|c|c|c|c|}
\hline Emission Point & Pollutants & CAS Number & $\begin{array}{l}\text { Emission } \\
\text { Threshold } \\
\text { (tons/yr) }\end{array}$ & $\begin{array}{c}\text { Actual } \\
\text { Emissions } \\
\text { (tons/yr) }\end{array}$ \\
\hline $200 W$ P $241 T 204$ & Acetone & $67-64-1$ & $5.00 \mathrm{E}-01$ & $6.62 \mathrm{E}-04$ \\
\hline 200W P $241 T 204$ & Acetonitrile & $75-5-8$ & $5.00 \mathrm{E}-01$ & $3.19 \mathrm{E}-04$ \\
\hline 200W P $241 T 204$ & Ammonia & $7664-41-7$ & $5.00 \mathrm{E}-01$ & $3.04 \mathrm{E}-03$ \\
\hline $200 \mathrm{~W} P \quad 241 T 204$ & Benzene & $71-43-2$ & $5.00 \mathrm{E}-01$ & $1.51 \mathrm{E}-05$ \\
\hline $200 W$ P $241 T 204$ & Carbon monoxide & $630-8-0$ & $5.00 \mathrm{E}+00$ & $4.39 \mathrm{E}-04$ \\
\hline 200W P 241T204 & Heptane & $142-82-5$ & $5.00 \mathrm{E}-01$ & $3.89 \mathrm{E}-05$ \\
\hline $200 W$ P $241 T 204$ & Hexane, n-hexane & $110-54-3$ & $5.00 \mathrm{E}-01$ & $4.05 \mathrm{E}-05$ \\
\hline 200W P $241 T 204$ & $\begin{array}{l}\text { Methyl N-amyl ketone, 2- } \\
\text { heptanone }\end{array}$ & $110-43-0$ & $5.00 \mathrm{E}-01$ & $3.76 \mathrm{E}-05$ \\
\hline 200W P $241 T 204$ & Nitrogen Oxide $\{\mathrm{NO}\}$ & $10102-43-9$ & $2.00 \mathrm{E}+00$ & $2.64 \mathrm{E}-05$ \\
\hline 200W P $241 T 204$ & Nonane & $111-84-2$ & $5.00 \mathrm{E}-01$ & $2.11 E-05$ \\
\hline 200W P 241T204 & Octane & $111-65-9$ & $5.00 \mathrm{E}-01$ & $2.21 \mathrm{E}-05$ \\
\hline $\begin{array}{ll}200 W \text { P } & 241 T 204\end{array}$ & $\begin{array}{l}\text { Phosphoric acid, tributyl } \\
\text { ester }\end{array}$ & $126-73-8$ & & $7.95 \mathrm{E}-05$ \\
\hline $\begin{array}{ll}200 W ~ P ~ 241 T 204\end{array}$ & Toluene & $108-88-3$ & $5.00 \mathrm{E}-01$ & $3.08 \mathrm{E}-06$ \\
\hline 200W P $241 T X 101$ & 1,3-Butadiene & $106-99-0$ & $3.50 \mathrm{E}-02$ & $4.13 \mathrm{E}-06$ \\
\hline 200W P $241 T X 101$ & 2-Hexanone & $591-78-6$ & $5.00 \mathrm{E}-01$ & $7.51 \mathrm{E}-05$ \\
\hline 200W P $241 T X 101$ & 2-Pentanone & $107-87-9$ & & $1.19 \mathrm{E}-04$ \\
\hline 200W P $\quad 241 \mathrm{TX101}$ & Acetone & $67-64-1$ & $5.00 \mathrm{E}-01$ & $1.43 \mathrm{E}-03$ \\
\hline 200W P $241 T X 101$ & Acetonitrile & $75-5-8$ & $5.00 \mathrm{E}-01$ & $6.89 \mathrm{E}-04$ \\
\hline 200W P $241 \mathrm{TX101}$ & Ammonia & $7664-41-7$ & $5.00 \mathrm{E}-01$ & $6.57 \mathrm{E}-03$ \\
\hline 200W P 241TX101 & Benzene & $71-43-2$ & $5.00 \mathrm{E}-01$ & $3.28 \mathrm{E}-05$ \\
\hline $200 W$ P $241 T X 101$ & Carbon monoxide & $630-8-0$ & $5.00 \mathrm{E}+00$ & $9.50 \mathrm{E}-04$ \\
\hline $200 \mathrm{~W} P \quad 241 \mathrm{TX} 101$ & Heptane & $142-82-5$ & $5.00 \mathrm{E}-01$ & $8.40 \mathrm{E}-05$ \\
\hline 200W P $241 T X 101$ & Hexane, n-hexane & $110-54-3$ & $5.00 \mathrm{E}-01$ & $8.76 \mathrm{E}-05$ \\
\hline 200W P $241 T X 101$ & $\begin{array}{l}\text { Methyl N-amyl ketone, 2- } \\
\text { heptanone }\end{array}$ & $110-43-0$ & $5.00 \mathrm{E}-01$ & $8.13 \mathrm{E}-05$ \\
\hline 200W P $241 T X 101$ & Nitrogen Oxide $\{\mathrm{NO}\}$ & $10102-43-9$ & $2.00 \mathrm{E}+00$ & $5.72 \mathrm{E}-05$ \\
\hline 200W P $241 \mathrm{TX101}$ & Nonane & $111-84-2$ & $5.00 \mathrm{E}-01$ & $4.57 \mathrm{E}-05$ \\
\hline 200W P $241 T X 101$ & Octane & $111-65-9$ & $5.00 \mathrm{E}-01$ & $4.79 \mathrm{E}-05$ \\
\hline
\end{tabular}




\section{Table K.2. Insignificant Emission Units Based on Threshold Comparisons} [WAC 173-401-530(1)(a)]

\begin{tabular}{|c|c|c|c|c|}
\hline Emission Point & Pollutants & CAS Number & $\begin{array}{l}\text { Emission } \\
\text { Threshold } \\
\text { (tons/yr) }\end{array}$ & $\begin{array}{c}\text { Actual } \\
\text { Emissions } \\
\text { (tons/yr) }\end{array}$ \\
\hline 200W P $241 T X 101$ & $\begin{array}{l}\text { Phosphoric acid, tributyl } \\
\text { ester }\end{array}$ & $126-73-8$ & & $1.72 \mathrm{E}-04$ \\
\hline 200W P $241 T X 101$ & Toluene & $108-88-3$ & $5.00 \mathrm{E}-01$ & $6.67 \mathrm{E}-06$ \\
\hline 200W P $241 \mathrm{TX102}$ & 1,3-Butadiene & $106-99-0$ & $3.50 \mathrm{E}-02$ & $1.91 \mathrm{E}-06$ \\
\hline 200W P $241 T X 102$ & 2-Hexanone & $591-78-6$ & $5.00 \mathrm{E}-01$ & $3.47 E-05$ \\
\hline 200W P $241 \mathrm{TX102}$ & 2-Pentanone & $107-87-9$ & & $5.51 E-05$ \\
\hline 200W P $241 T X 102$ & Acetone & $67-64-1$ & $5.00 \mathrm{E}-01$ & $6.62 \mathrm{E}-04$ \\
\hline $200 \mathrm{~W} P \quad 241 \mathrm{TX102}$ & Acetonitrile & $75-5-8$ & $5.00 \mathrm{E}-01$ & 3.19E-04 \\
\hline $200 \mathrm{~W} P \quad 241 \mathrm{TX} 102$ & Ammonia & $7664-41-7$ & $5.00 \mathrm{E}-01$ & $3.04 \mathrm{E}-03$ \\
\hline $200 \mathrm{~W}$ P $241 \mathrm{TX102}$ & Benzene & $71-43-2$ & $5.00 \mathrm{E}-01$ & $1.51 \mathrm{E}-05$ \\
\hline $.200 \mathrm{~W} P \quad 241 \mathrm{TX} 102$ & Carbon monoxide & $630-8-0$ & $5.00 \mathrm{E}+00$ & $4.39 \mathrm{E}-04$ \\
\hline 200W P $241 \mathrm{TX} 102$ & Heptane & $142-82-5$ & $5.00 \mathrm{E}-01$ & $3.89 \mathrm{E}-05$ \\
\hline 200W P $241 \mathrm{TX102}$ & Hexane, n-hexane & $110-54-3$ & $5.00 \mathrm{E}-01$ & $4.05 \mathrm{E}-05$ \\
\hline $200 \mathrm{~W} P \quad 241 \mathrm{TX} 102$ & $\begin{array}{l}\text { Methyl N-amyl ketone, 2- } \\
\text { heptanone }\end{array}$ & $110-43-0$ & $5.00 \mathrm{E}-01$ & $3.76 \mathrm{E}-05$ \\
\hline 200W P $241 \mathrm{TX102}$ & Nitrogen Oxide $\{N O\}$ & $10102-43-9$ & $2.00 \mathrm{E}+00$ & $2.64 \mathrm{E}-05$ \\
\hline 200W P $241 \mathrm{TX} 102$ & Nonane & $111-84-2$ & $5.00 \mathrm{E}-01$ & $2.11 \mathrm{E}-05$ \\
\hline $200 \mathrm{~W} P \quad 241 \mathrm{TX102}$ & Octane & $111-65-9$ & $5.00 \mathrm{E}-01$ & $2.21 \mathrm{E}-05$ \\
\hline 200W P $241 \mathrm{TX} 102$ & $\begin{array}{l}\text { Phosphoric acid, tributyl } \\
\text { ester }\end{array}$ & $126-73-8$ & & $7.95 \mathrm{E}-05$ \\
\hline 200W P 241TX102 & Toluene & $108-88-3$ & $5.00 \mathrm{E}-01$ & $3.08 \mathrm{E}-06$ \\
\hline $200 \mathrm{~W} P \quad 241 \mathrm{TX103}$ & 1,3-Butadiene & $106-99-0$ & $3.50 \mathrm{E}-02$ & $4.13 \mathrm{E}-06$ \\
\hline $200 \mathrm{~W} P \quad 241 \mathrm{TX103}$ & 2-Hexanone & $591-78-6$ & $5.00 \mathrm{E}-01$ & $7.51 E-05$ \\
\hline 200W P $241 \mathrm{TX103}$ & 2-Pentanone & & $107-87-9$ & 1.19E-04 \\
\hline $200 \mathrm{~W}$ P $241 \mathrm{TX103}$ & Acetone & $67-64-1$ & $5.00 \mathrm{E}-01$ & $1.43 \mathrm{E}-03$ \\
\hline $200 \mathrm{~W} P \quad 241 \mathrm{TX} 103$ & Acetonitrile & $75-5-8$ & $5.00 \mathrm{E}-01$ & $6.89 \mathrm{E}-04$ \\
\hline $200 \mathrm{~W} P \quad 241 \mathrm{TX103}$ & Ammonia & $7664-41-7$ & $5.00 \mathrm{E}-01$ & $6.57 E-03$ \\
\hline $200 \mathrm{~W} P \quad 241 \mathrm{TX} 103$ & Benzene & $71-43-2$ & $5.00 \mathrm{E}-01$ & $3.28 \mathrm{E}-05$ \\
\hline $\begin{array}{ll}200 W \\
\end{array}$ & Carbon monoxide & $630-8-0$ & $5.00 \mathrm{E}+00$ & $9.50 \mathrm{E}-04$ \\
\hline $200 \mathrm{~W} P \quad 241 \mathrm{TX103}$ & Heptane & $142-82-5$ & $5.00 \mathrm{E}-01$ & $8.40 E-05$ \\
\hline
\end{tabular}


Table K.2. Insignificant Emission Units Based on Threshold Comparisons [WAC 173-401-530(1)(a)]

\begin{tabular}{|c|c|c|c|c|}
\hline Emission Point & Pollutants & CAS Number & $\begin{array}{l}\text { Emission } \\
\text { Threshold } \\
\text { (tons/yr) }\end{array}$ & $\begin{array}{c}\text { Actual } \\
\text { Emissions } \\
\text { (tons/yr) }\end{array}$ \\
\hline $200 \mathrm{~W}$ P $241 \mathrm{TX} 103$ & Hexane, n-hexane & $110-54-3$ & $5.00 \mathrm{E}-01$ & $8.76 \mathrm{E}-05$ \\
\hline 200W P $241 \mathrm{TX103}$ & $\begin{array}{l}\text { Methyl N-amyl ketone, 2- } \\
\text { heptanone }\end{array}$ & $110-43-0$ & $5.00 \mathrm{E}-01$ & $8.13 E-05$ \\
\hline 200W P 241 TX103 & Nitrogen Oxide $\{\mathrm{NO}\}$ & $10102-43-9$ & $2.00 \mathrm{E}+00$ & $5.73 E-05$ \\
\hline 200W P $241 T X 103$ & Nonane & $111-84-2$ & $5.00 \mathrm{E}-01$ & $4.57 E-05$ \\
\hline 200W P 241TX103 & Octane & $111-65-9$ & $5.00 \mathrm{E}-01$ & $4.79 \mathrm{E}-05$ \\
\hline 200W P $241 T X 103$ & $\begin{array}{l}\text { Phosphoric acid, tributyl } \\
\text { ester }\end{array}$ & $126-73-8$ & & $1.72 \mathrm{E}-04$ \\
\hline $200 \mathrm{~W}$ P $\quad 241 \mathrm{TX} 103$ & Toluene & $108-88-3$ & $5.00 \mathrm{E}-01$ & 6.67E-06 \\
\hline 200W P 241 TX104 & 1,3-Butadiene & $106-99-0$ & $3.50 \mathrm{E}-02$ & $4.13 \mathrm{E}-06$ \\
\hline 200W P $241 T X 104$ & 2-Hexanone & $591-78-6$ & $5.00 \mathrm{E}-01$ & $7.51 \mathrm{E}-05$ \\
\hline 200W P $241 \mathrm{TX104}$ & 2-Pentanone & $107-87-9$ & & $1.19 \mathrm{E}-04$ \\
\hline $200 \mathrm{~W}$ P $241 \mathrm{TX104}$ & Acetone & $67-64-1$ & $5.00 \mathrm{E}-01$ & $1.43 \mathrm{E}-03$ \\
\hline 200W P $241 \mathrm{TX104}$ & Acetonitrile & $75-5-8$ & $5.00 \mathrm{E}-01$ & $6.89 E-04$ \\
\hline 200W P $241 T X 104$ & Ammonia & $7664-41-7$ & $5.00 \mathrm{E}-01$ & $6.57 \mathrm{E}-03$ \\
\hline 200W P $241 T X 104$ & Benzene & $71-43-2$ & $5.00 \mathrm{E}-01$ & $3.28 \mathrm{E}-05$ \\
\hline $200 W$ P $241 T X 104$ & Carbon monoxide & $630-8-0$ & $5.00 \mathrm{E}+00$ & $9.50 \mathrm{E}-04$ \\
\hline $200 W$ P $241 T X 104$ & Heptane & $142-82-5$ & $5.00 \mathrm{E}-01$ & $8.40 \mathrm{E}-05$ \\
\hline 200W P $241 \mathrm{TX104}$ & Hexane, n-hexane & $110-54-3$ & $5.00 \mathrm{E}-01$ & $8.76 \mathrm{E}-05$ \\
\hline 200W P $241 \mathrm{TX104}$ & $\begin{array}{l}\text { Methyl N-amyl ketone, 2- } \\
\text { heptanone }\end{array}$ & $110-43-0$ & $5.00 \mathrm{E}-01$ & $8.13 \mathrm{E}-05$ \\
\hline 200W P $241 \mathrm{TX104}$ & Nitrogen Oxide $\{\mathrm{NO}\}$ & $10102-43-9$ & $2.00 \mathrm{E}+00$ & $5.72 \mathrm{E}-05$ \\
\hline 200W P $241 \mathrm{TX104}$ & Nonane & $111-84-2$ & $5.00 \mathrm{E}-01$ & $4.57 \mathrm{E}-05$ \\
\hline 200W P 241TX104 & Octane & $111-65-9$ & $5.00 \mathrm{E}-01$ & $4.79 \mathrm{E}-05$ \\
\hline $200 \mathrm{~W} \mathrm{P} \quad 241 \mathrm{TX} 104$ & $\begin{array}{l}\text { Phosphoric acid, tributyl } \\
\text { ester }\end{array}$ & $126-73-8$ & & $1.72 \mathrm{E}-04$ \\
\hline $200 W$ P $241 \mathrm{TX104}$ & Toluene & $108-88-3$ & $5.00 \mathrm{E}-01$ & $6.67 \mathrm{E}-06$ \\
\hline 200W P 241TX105 & 1,3-Butadiene & $106-99-0$ & $3.50 \mathrm{E}-02$ & $1.91 \mathrm{E}-06$ \\
\hline 200W P $241 \mathrm{TX105}$ & 2-Hexanone & $591-78-6$ & $5.00 \mathrm{E}-01$ & $3.47 \mathrm{E}-05$ \\
\hline 200W P $241 T X 105$ & 2-Pentanone & $107-87-9$ & & $5.51 \mathrm{E}-05$ \\
\hline
\end{tabular}


Table K.2. Insignificant Emission Units Based on Threshold Comparisons [WAC 173-401-530(1)(a)]

\begin{tabular}{|c|c|c|c|c|}
\hline Emission Point & Pollutants & CAS Number & $\begin{array}{l}\text { Emission } \\
\text { Threshold } \\
\text { (tons/yr) }\end{array}$ & $\begin{array}{c}\text { Actual } \\
\text { Emissions } \\
\text { (tons/yr) }\end{array}$ \\
\hline 200W P $241 T X 105$ & Acetone & $67-64-1$ & $5.00 \mathrm{E}-01$ & $6.62 \mathrm{E}-04$ \\
\hline 200W P $241 \mathrm{TX105}$ & Acetonitrile & $75-5-8$ & $5.00 \mathrm{E}-01$ & $3.19 \mathrm{E}-04$ \\
\hline 200W P 241TX105 & Ammonia & $7664-41-7$ & $5.00 \mathrm{E}-01$ & $3.04 \mathrm{E}-03$ \\
\hline 200W P 241 TX105 & Benzene & $71-43-2$ & $5.00 \mathrm{E}-01$ & $1.51 \mathrm{E}-05$ \\
\hline 200W P 241TX105 & Carbon monoxide & $630-8-0$ & $5.00 \mathrm{E}+00$ & $4.39 \mathrm{E}-04$ \\
\hline 200W P $241 \mathrm{TX105}$ & Heptane & $142-82-5$ & $5.00 \mathrm{E}-01$ & $3.89 \mathrm{E}-05$ \\
\hline 200W P 241TX105 & Hexane, n-hexane & $110-54-3$ & $5.00 \mathrm{E}-01$ & $4.05 \mathrm{E}-05$ \\
\hline 200W P $241 \mathrm{TX105}$ & $\begin{array}{l}\text { Methyl N-amyl ketone, 2- } \\
\text { heptanone }\end{array}$ & $110-43-0$ & $5.00 \mathrm{E}-01$ & $3.76 \mathrm{E}-05$ \\
\hline 200W P 241TX105 & Nitrogen Oxide $\{\mathrm{NO}\}$ & $10102-43-9$ & $2.00 \mathrm{E}+00$ & $2.64 \mathrm{E}-05$ \\
\hline 200W P 241TX105 & Nonane & $111-84-2$ & $5.00 \mathrm{E}-01$ & $2.11 \mathrm{E}-05$ \\
\hline 200W P $241 T X 105$ & Octane & $111-65-9$ & $5.00 \mathrm{E}-01$ & $2.21 \mathrm{E}-05$ \\
\hline 200W P 241TX105 & $\begin{array}{l}\text { Phosphoric acid, tributyl } \\
\text { ester }\end{array}$ & $126-73-8$ & & $7.95 \mathrm{E}-05$ \\
\hline $200 \mathrm{~W} P \quad 241 \mathrm{TX} 105$ & Toluene & $108-88-3$ & $5.00 \mathrm{E}-01$ & $3.08 \mathrm{E}-06$ \\
\hline 200W P $241 \mathrm{TX106}$ & 1,3-Butadiene & $106-99-0$ & $3.50 \mathrm{E}-02$ & $1.91 \mathrm{E}-06$ \\
\hline 200W P 241TX106 & 2-Hexanone & $591-78-6$ & $5.00 \mathrm{E}-01$ & $3.47 \mathrm{E}-05$ \\
\hline 200W P $241 T X 106$ & 2-Pentanone & $107-87-9$ & & $5.51 E-05$ \\
\hline 200W P 241 TX106 & Acetone & $67-64-1$ & $5.00 \mathrm{E}-01$ & $6.62 \mathrm{E}-04$ \\
\hline 200W P $241 \mathrm{TX106}$ & Acetonitrile & $75-5-8$ & $5.00 \mathrm{E}-01$ & $3.19 \mathrm{E}-04$ \\
\hline 200W P $241 T X 106$ & Ammonia & $7664-41-7$ & $5.00 \mathrm{E}-01$ & $3.04 \mathrm{E}-03$ \\
\hline 200W P $241 T X 106$ & Benzene & $71-43-2$ & $5.00 \mathrm{E}-01$ & $1.51 \mathrm{E}-05$ \\
\hline 200W P $241 \mathrm{TX106}$ & Carbon monoxide & $630-8-0$ & $5.00 \mathrm{E}+00$ & $4.39 \mathrm{E}-04$ \\
\hline 200W P $241 \mathrm{TX106}$ & Heptane & $142-82-5$ & $5.00 \mathrm{E}-01$ & $3.89 \mathrm{E}-05$ \\
\hline 200W P $241 \mathrm{TX106}$ & Hexane, n-hexane & $110-54-3$ & $5.00 \mathrm{E}-01$ & $4.05 \mathrm{E}-05$ \\
\hline 200W P 241TX106 & $\begin{array}{l}\text { Methyl N-amyl ketone, 2- } \\
\text { heptanone }\end{array}$ & $110-43-0$ & $5.00 \mathrm{E}-01$ & $3.76 \mathrm{E}-05$ \\
\hline 200W P 241TX106 & Nitrogen Oxide $\{\mathrm{NO}\}$ & $10102-43-9$ & $2.00 \mathrm{E}+00$ & $2.64 \mathrm{E}-05$ \\
\hline 200W P $241 T X 106$ & Nonane & $111-84-2$ & $5.00 \mathrm{E}-01$ & $2.11 \mathrm{E}-05$ \\
\hline 200W P 241TX106 & Octane & $111-65-9$ & $5.00 \mathrm{E}-01$ & $2.21 \mathrm{E}-05$ \\
\hline
\end{tabular}


Table K.2. Insignificant Emission Units Based on Threshold Comparisons [WAC 173-401-530(1)(a)]

\begin{tabular}{|c|c|c|c|c|}
\hline Emission Point & Pollutants & CAS Number & $\begin{array}{l}\text { Emission } \\
\text { Threshold } \\
\text { (tons/yr) }\end{array}$ & $\begin{array}{c}\text { Actual } \\
\text { Emissions } \\
\text { (tons/yr) }\end{array}$ \\
\hline $200 \mathrm{~W}$ P $241 \mathrm{TX} 106$ & $\begin{array}{l}\text { Phosphoric acid, tributyl } \\
\text { ester }\end{array}$ & $126-73-8$ & & $7.95 \mathrm{E}-05$ \\
\hline $241 \mathrm{TX} 106$ & Toluene & $108-88-3$ & $5.00 \mathrm{E}-01$ & $3.08 \mathrm{E}-06$ \\
\hline $200 \mathrm{~W} P \quad 241 \mathrm{TX} 107$ & 1,3-Butadiene & $106-99-0$ & $3.50 \mathrm{E}-02$ & $4.13 \mathrm{E}-06$ \\
\hline 200W P 241TX107 & 2-Hexanone & $591-78-6$ & $5.00 \mathrm{E}-01$ & $7.51 \mathrm{E}-05$ \\
\hline 200W P $241 \mathrm{TX107}$ & 2-Pentanone & $107-87-9$ & & $1.19 E-04$ \\
\hline 200W P $241 \mathrm{TX} 107$ & Acetone & $67-64-1$ & $5.00 \mathrm{E}-01$ & $1.43 E-03$ \\
\hline 200W P $241 \mathrm{TX} 107$ & Acetonitrile & $75-5-8$ & $5.00 \mathrm{E}-01$ & $6.89 E-04$ \\
\hline 200W P $241 \mathrm{TX} 107$ & Ammonia & $7664-41-7$ & $5.00 \mathrm{E}-01$ & $6.57 \mathrm{E}-03$ \\
\hline $200 \mathrm{~W} P \quad 241 \mathrm{TX} 107$ & Benzene & $71-43-2$ & $5.00 \mathrm{E}-01$ & $3.28 \mathrm{E}-05$ \\
\hline 200W P $241 \mathrm{TX} 107$ & Carbon monoxide & $630-8-0$ & $5.00 \mathrm{E}+00$ & $9.50 \mathrm{E}-04$ \\
\hline 200W P 241TX107 & Heptane & $142-82-5$ & $5.00 \mathrm{E}-01$ & $8.40 \mathrm{E}-05$ \\
\hline 200W P $241 T X 107$ & Hexane, n-hexane & $110-54-3$ & $5.00 \mathrm{E}-01$ & $8.76 E-05$ \\
\hline 200W P $241 \mathrm{TX107}$ & $\begin{array}{l}\text { Methyl N-amyl ketone, 2- } \\
\text { heptanone }\end{array}$ & $110-43-0$ & $5.00 \mathrm{E}-01$ & $8.13 E-05$ \\
\hline 200W P 241TX107 & Nitrogen Oxide $\{N O\}$ & $10102-43-9$ & $2.00 \mathrm{E}+00$ & $5.72 \mathrm{E}-05$ \\
\hline 200W P $241 \mathrm{TX} 107$ & Nonane & $111-84-2$ & $5.00 \mathrm{E}-01$ & $4.57 \mathrm{E}-05$ \\
\hline 200W P $241 \mathrm{TX107}$ & Octane & $111-65-9$ & $5.00 \mathrm{E}-01$ & $4.79 \mathrm{E}-05$ \\
\hline 200W P $241 \mathrm{TX107}$ & $\begin{array}{l}\text { Phosphoric acid, tributyl } \\
\text { ester }\end{array}$ & $126-73-8$ & & $1.72 \mathrm{E}-04$ \\
\hline 200W P 241TX107 & Toluene & $108-88-3$ & $5.00 \mathrm{E}-01$ & $6.67 E-06$ \\
\hline 200W P $241 T X 108$ & 1,3-Butadiene & $106-99-0$ & $3.50 \mathrm{E}-02$ & $4.13 \mathrm{E}-06$ \\
\hline 200W P $241 T X 108$ & 2-Hexanone & $591-78-6$ & $5.00 \mathrm{E}-01$ & $7.51 E-05$ \\
\hline 200W P 241TX108 & 2-Pentanone & $107-87-9$ & & $1.19 \mathrm{E}-04$ \\
\hline 200W P 241TX108 & Acetone & $67-64-1$ & $5.00 \mathrm{E}-01$ & $1.43 \mathrm{E}-03$ \\
\hline 200W P 241TX108 & Acetonitrile & $75-5-8$ & $5.00 \mathrm{E}-01$ & $6.89 \mathrm{E}-04$ \\
\hline 200W P $241 T X 108$ & Ammonia & $7664-41-7$ & $5.00 \mathrm{E}-01$ & 6.57E-03 \\
\hline 200W P $241 \mathrm{TX108}$ & Benzene & $71-43-2$ & $5.00 \mathrm{E}-01$ & $3.28 \mathrm{E}-05$ \\
\hline 200W P $241 \mathrm{TX108}$ & Carbon monoxide & $630-8-0$ & $5.00 \mathrm{E}+00$ & $9.50 \mathrm{E}-04$ \\
\hline 200W P $241 \mathrm{TX108}$ & Heptane & $142-82-5$ & $5.00 \mathrm{E}-01$ & $8.40 \mathrm{E}-05$ \\
\hline
\end{tabular}


Table K.2. Insignificant Emission Units Based on Threshold Comparisons [WAC 173-401-530(1)(a)]

\begin{tabular}{|c|c|c|c|c|}
\hline Emission Point & Pollutants & CAS Number & $\begin{array}{l}\text { Emission } \\
\text { Threshold } \\
\text { (tons/yr) }\end{array}$ & $\begin{array}{c}\text { Actual } \\
\text { Emissions } \\
\text { (tons/yr) }\end{array}$ \\
\hline 200W P $241 T X 108$ & Hexane, n-hexane & $110-54-3$ & $5.00 \mathrm{E}-01$ & $8.76 \mathrm{E}-05$ \\
\hline 200W P $241 T X 108$ & $\begin{array}{l}\text { Methyl N-amyl ketone, 2- } \\
\text { heptanone }\end{array}$ & $110-43-0$ & $5.00 \mathrm{E}-01$ & $8.13 E-05$ \\
\hline 200W P 241 TX108 & Nitrogen Oxide $\{\mathrm{NO}\}$ & $10102-43-9$ & $2.00 \mathrm{E}+00$ & $5.72 \mathrm{E}-05$ \\
\hline 200W P $241 T X 108$ & Nonane & $111-84-2$ & $5.00 \mathrm{E}-01$ & $4.57 \mathrm{E}-05$ \\
\hline 200W P 241 TX108 & Octane & $111-65-9$ & $5.00 \mathrm{E}-01$ & $4.79 \mathrm{E}-05$ \\
\hline 200W P $241 \mathrm{TX108}$ & $\begin{array}{l}\text { Phosphoric acid, tributyl } \\
\text { ester }\end{array}$ & $126-73-8$ & & $1.72 \mathrm{E}-04$ \\
\hline 200W P $241 T X 108$ & Toluene & $108-88-3$ & $5.00 \mathrm{E}-01$ & $6.67 \mathrm{E}-06$ \\
\hline 200W P $241 T X 109$ & 1,3-Butadiene & $106-99-0$ & $3.50 \mathrm{E}-02$ & $4.13 E-06$ \\
\hline 200W P $241 T X 109$ & 2-Hexanone & $591-78-6$ & $5.00 \mathrm{E}-01$ & $7.51 \mathrm{E}-05$ \\
\hline 200W P $241 T X 109$ & 2-Pentanone & $107-87-9$ & & $1.19 \mathrm{E}-04$ \\
\hline 200W P $241 T X 109$ & Acetone & $67-64-1$ & $5.00 \mathrm{E}-01$ & $1.43 \mathrm{E}-03$ \\
\hline $200 W$ P $241 T X 109$ & Acetonitrile & $75-5-8$ & $5.00 \mathrm{E}-01$ & $6.89 \mathrm{E}-04$ \\
\hline 200W P $241 T X 109$ & Ammonia & $7664-41-7$ & $5.00 \mathrm{E}-01$ & $6.57 \mathrm{E}-03$ \\
\hline 200W P $241 T X 109$ & Benzene & $71-43-2$ & $5.00 \mathrm{E}-01$ & $3.28 \mathrm{E}-05$ \\
\hline 200W P $241 T X 109$ & Carbon monoxide & $630-8-0$ & $5.00 \mathrm{E}+00$ & $9.50 \mathrm{E}-04$ \\
\hline 200W P $241 T X 109$ & Heptane & $142-82-5$ & $5.00 \mathrm{E}-01$ & $8.40 \mathrm{E}-05$ \\
\hline 200W P $241 T X 109$ & Hexane, n-hexane & $110-54-3$ & $5.00 \mathrm{E}-01$ & $8.76 \mathrm{E}-05$ \\
\hline $200 W$ P $241 T X 109$ & $\begin{array}{l}\text { Methyl N-amyl ketone, 2- } \\
\text { heptanone }\end{array}$ & $110-43-0$ & $5.00 \mathrm{E}-01$ & $8.13 E-05$ \\
\hline 200W P $241 T X 109$ & Nitrogen Oxide $\{\mathrm{NO}\}$ & $10102-43-9$ & $2.00 \mathrm{E}+00$ & 5.72E-05 \\
\hline $200 \mathrm{~W} P \quad$ 241TX109 & Nonane & $111-84-2$ & $5.00 \mathrm{E}-01$ & 4.57E-05 \\
\hline 200W P $241 T X 109$ & Octane & $111-65-9$ & $5.00 \mathrm{E}-01$ & $4.79 \mathrm{E}-05$ \\
\hline 200W P $241 T X 109$ & $\begin{array}{l}\text { Phosphoric acid, tributyl } \\
\text { ester }\end{array}$ & $126-73-8$ & & $1.72 \mathrm{E}-04$ \\
\hline 200W P $241 T X 109$ & Toluene & $108-88-3$ & $5.00 \mathrm{E}-01$ & $6.67 \mathrm{E}-06$ \\
\hline 200W P $241 T X 110$ & 1,3-Butadiene & $106-99-0$ & $3.50 \mathrm{E}-02$ & $1.91 \mathrm{E}-06$ \\
\hline $200 W$ P $241 T X 110$ & 2-Hexanone & $591-78-6$ & $5.00 \mathrm{E}-01$ & $3.47 \mathrm{E}-05$ \\
\hline $200 \mathrm{~W} P \quad 241 \mathrm{TX} 110$ & 2-Pentanone & $107-87-9$ & & $5.51 E-05$ \\
\hline
\end{tabular}


Table K.2. Insignificant Emission Units Based on Threshold Comparisons [WAC 173-401-530(1)(a)]

\begin{tabular}{|c|c|c|c|c|}
\hline Emission Point & Pollutants & CAS Number & $\begin{array}{l}\text { Emission } \\
\text { Threshold } \\
\text { (tons/yr) }\end{array}$ & $\begin{array}{c}\text { Actual } \\
\text { Emissions } \\
\text { (tons/yr) }\end{array}$ \\
\hline $200 \mathrm{~W}$ P $241 \mathrm{TX} 110$ & Acetone & $67-64-1$ & $5.00 \mathrm{E}-01$ & $6.62 \mathrm{E}-04$ \\
\hline $200 \mathrm{~W}$ P $241 \mathrm{TX} 110$ & Acetonitrile & $75-5-8$ & $5.00 \mathrm{E}-01$ & $3.19 \mathrm{E}-04$ \\
\hline 200W P $241 \mathrm{TX} 110$ & Ammonia & $7664-41-7$ & $5.00 \mathrm{E}-01$ & $3.04 \mathrm{E}-03$ \\
\hline 200W P $241 \mathrm{TX} 110$ & Benzene & $71-43-2$ & $5.00 \mathrm{E}-01$ & $1.51 \mathrm{E}-05$ \\
\hline 200W P $241 \mathrm{TX} 110$ & Carbon monoxide & $630-8-0$ & $5.00 \mathrm{E}+00$ & $4.39 \mathrm{E}-04$ \\
\hline $200 \mathrm{~W} P \quad 241 \mathrm{TX} 110$ & Heptane & $142-82-5$ & $5.00 \mathrm{E}-01$ & $3.89 \mathrm{E}-05$ \\
\hline 200W P $241 \mathrm{TX} 110$ & Hexane, n-hexane & $110-54-3$ & $5.00 \mathrm{E}-01$ & $4.05 \mathrm{E}-05$ \\
\hline $200 \mathrm{~W} P \quad 241 \mathrm{TX} 110$ & $\begin{array}{l}\text { Methyl N-amyl ketone, 2- } \\
\text { heptanone }\end{array}$ & $110-43-0$ & $5.00 \mathrm{E}-01$ & $3.76 \mathrm{E}-05$ \\
\hline $200 \mathrm{~W} P \quad 241 \mathrm{TX} 110$ & Nitrogen Oxide $\{\mathrm{NO}\}$ & $10102-43-9$ & $2.00 \mathrm{E}+00$ & $2.64 \mathrm{E}-05$ \\
\hline 200W P $241 \mathrm{TX} 110$ & Nonane & $111-84-2$ & $5.00 \mathrm{E}-01$ & $2.11 \mathrm{E}-05$ \\
\hline 200W P $241 \mathrm{TX} 110$ & Octane & $111-65-9$ & $5.00 \mathrm{E}-01$ & $2.21 \mathrm{E}-05$ \\
\hline $200 \mathrm{~W} P \quad 241 \mathrm{TX} 110$ & $\begin{array}{l}\text { Phosphoric acid, tributyl } \\
\text { ester }\end{array}$ & $126-73-8$ & & $7.95 \mathrm{E}-05$ \\
\hline 200W P $241 \mathrm{TX} 110$ & Toluene & $108-88-3$ & $5.00 \mathrm{E}-01$ & $3.08 \mathrm{E}-06$ \\
\hline 200W P 241TX111 & 1,3-Butadiene & $106-99-0$ & $3.50 \mathrm{E}-02$ & $1.91 \mathrm{E}-06$ \\
\hline 200W P $241 \mathrm{TX} 111$ & 2-Hexanone & $591-78-6$ & $5.00 \mathrm{E}-01$ & 3.47E-05 \\
\hline 200W P $241 T X 111$ & 2-Pentanone & $107-87-9$ & & 5.51E-05 \\
\hline 200W P 241TX111 & Acetone & $67-64-1$ & $5.00 \mathrm{E}-01$ & $6.62 \mathrm{E}-04$ \\
\hline 200W P $241 \mathrm{TX} 111$ & Acetonitrile & $75-5-8$ & $5.00 \mathrm{E}-01$ & $3.19 \mathrm{E}-04$ \\
\hline 200W P $241 \mathrm{TX} 111$ & Ammonia & $7664-41-7$ & $5.00 \mathrm{E}-01$ & $3.04 \mathrm{E}-03$ \\
\hline 200W P $241 \mathrm{TX} 111$ & Benzene & $71-43-2$ & $5.00 \mathrm{E}-01$ & $1.51 \mathrm{E}-05$ \\
\hline $200 \mathrm{~W} P \quad 241 \mathrm{TX} 111$ & Carbon monoxide & $630-8-0$ & $5.00 \mathrm{E}+00$ & 4.39E-04 \\
\hline 200W P 241TX111 & Heptane & $142-82-5$ & $5.00 \mathrm{E}-01$ & $3.89 \mathrm{E}-05$ \\
\hline 200W P 241TX111 & Hexane, $n$-hexane & $110-54-3$ & $5.00 \mathrm{E}-01$ & $4.05 \mathrm{E}-05$ \\
\hline 200W P 241TX111 & $\begin{array}{l}\text { Methyl N-amyl ketone, 2- } \\
\text { heptanone }\end{array}$ & $110-43-0$ & $5.00 \mathrm{E}-01$ & $3.76 \mathrm{E}-05$ \\
\hline $241 \mathrm{TX} 111$ & Nitrogen Oxide $\{\mathrm{NO}\}$ & $10102-43-9$ & $2.00 \mathrm{E}+00$ & $2.64 \mathrm{E}-05$ \\
\hline 200W P 241TX111 & Nonane & $111-84-2$ & $5.00 \mathrm{E}-01$ & $2.11 \mathrm{E}-05$ \\
\hline 200W P $241 \mathrm{TX} 111$ & Octane & $111-65-9$ & $5.00 \mathrm{E}-01$ & $2.21 \mathrm{E}-05$ \\
\hline
\end{tabular}


Table K.2. Insignificant Emission Units Based on Threshold Comparisons [WAC 173-401-530(1)(a)]

\begin{tabular}{|c|c|c|c|c|}
\hline Emission Point & Pollutants & CAS Number & $\begin{array}{l}\text { Emission } \\
\text { Threshold } \\
\text { (tons/yr) }\end{array}$ & $\begin{array}{c}\text { Actual } \\
\text { Emissions } \\
\text { (tons/yr) }\end{array}$ \\
\hline 200W P 241TX111 & $\begin{array}{l}\text { Phosphoric acid, tributyl } \\
\text { ester }\end{array}$ & $126-73-8$ & & $7.95 \mathrm{E}-05$ \\
\hline $200 \mathrm{~W} P \quad 241 \mathrm{TX} 111$ & Toluene & $108-88-3$ & $5.00 \mathrm{E}-01$ & $3.08 \mathrm{E}-06$ \\
\hline 200W P $241 \mathrm{TX} 112$ & 1,3-Butadiene & $106-99-0$ & $3.50 \mathrm{E}-02$ & $1.91 \mathrm{E}-06$ \\
\hline 200W P 241TX112 & 2-Hexanone & $591-78-6$ & $5.00 \mathrm{E}-01$ & $3.47 \mathrm{E}-05$ \\
\hline 200W P 241TX112 & 2-Pentanone & $107-87-9$ & & $5.51 \mathrm{E}-05$ \\
\hline 200W P $241 \mathrm{TX} 112$ & Acetone & $67-64-1$ & $5.00 \mathrm{E}-01$ & $6.62 \mathrm{E}-04$ \\
\hline 200W P $241 \mathrm{TX} 112$ & Acetonitrile & $75-5-8$ & $5.00 \mathrm{E}-01$ & $3.19 \mathrm{E}-04$ \\
\hline 200W P 241TX112 & Ammonia & $7664-41-7$ & $5.00 \mathrm{E}-01$ & $3.04 \mathrm{E}-03$ \\
\hline 200W P 241TX112 & Benzene & $71-43-2$ & $5.00 \mathrm{E}-01$ & $1.51 \mathrm{E}-05$ \\
\hline 200W P 241TX112 & Carbon monoxide & $630-8-0$ & $5.00 \mathrm{E}+00$ & 4.39E-04 \\
\hline 200W P $241 T X 112$ & Heptane & $142-82-5$ & $5.00 \mathrm{E}-01$ & $3.89 \mathrm{E}-05$ \\
\hline 200W P $241 T X 112$ & Hexane, n-hexane & $110-54-3$ & $5.00 \mathrm{E}-01$ & $4.05 \mathrm{E}-05$ \\
\hline 200W P $241 T X 112$ & $\begin{array}{l}\text { Methyl N-amyl ketone, 2- } \\
\text { heptanone }\end{array}$ & $110-43-0$ & $5.00 \mathrm{E}-01$ & $3.76 \mathrm{E}-05$ \\
\hline 200W P 241TX112 & Nitrogen Oxide $\{\mathrm{NO}\}$ & $10102-43-9$ & $2.00 \mathrm{E}+00$ & $2.64 \mathrm{E}-05$ \\
\hline 200W P 241TX112 & Nonane & $111-84-2$ & $5.00 \mathrm{E}-01$ & $2.11 \mathrm{E}-05$ \\
\hline 200W P $241 \mathrm{TX112}$ & Octane & $111-65-9$ & $5.00 \mathrm{E}-01$ & $2.21 \mathrm{E}-05$ \\
\hline 200W P $241 T X 112$ & $\begin{array}{l}\text { Phosphoric acid, tributyl } \\
\text { ester }\end{array}$ & $126-73-8$ & & $7.95 \mathrm{E}-05$ \\
\hline 200W P 241TX112 & Toluene & $108-88-3$ & $5.00 \mathrm{E}-01$ & $3.08 \mathrm{E}-06$ \\
\hline 200W P $241 T X 113$ & 1,3-Butadiene & $106-99-0$ & $3.50 \mathrm{E}-02$ & $1.91 \mathrm{E}-06$ \\
\hline 200W P 241TX113 & 2-Hexanone & $591-78-6$ & $5.00 \mathrm{E}-01$ & $3.47 \mathrm{E}-05$ \\
\hline 200W P $241 T X 113$ & 2-Pentanone & $107-87-9$ & & $5.51 \mathrm{E}-05$ \\
\hline 200W P $241 \mathrm{TX} 113$ & Acetone & $67-64-1$ & $5.00 \mathrm{E}-01$ & $6.62 E-04$ \\
\hline 200W P 241TX113 & Acetonitrile & $75-5-8$ & $5.00 \mathrm{E}-01$ & $3.19 \mathrm{E}-04$ \\
\hline 200W P $241 \mathrm{TX} 113$ & Ammonia & $7664-41-7$ & $5.00 \mathrm{E}-01$ & $3.04 \mathrm{E}-03$ \\
\hline 200W P $241 T X 113$ & Benzene & $71-43-2$ & $5.00 \mathrm{E}-01$ & $1.51 \mathrm{E}-05$ \\
\hline 200W P $241 T X 113$ & Carbon monoxide & $630-8-0$ & $5.00 \mathrm{E}+00$ & $4.39 \mathrm{E}-04$ \\
\hline 200W P 241TX113 & Heptane & $142-82-5$ & $5.00 \mathrm{E}-01$ & $3.89 \mathrm{E}-05$ \\
\hline
\end{tabular}


Table K.2. Insignificant Emission Units Based on Threshold Comparisons [WAC 173-401-530(1)(a)]

\begin{tabular}{|c|c|c|c|c|}
\hline Emission Point & Pollutants & CAS Number & $\begin{array}{l}\text { Emission } \\
\text { Threshold } \\
\text { (tons/yr) }\end{array}$ & $\begin{array}{c}\text { Actual } \\
\text { Emissions } \\
\text { (tons/yr) }\end{array}$ \\
\hline $200 \mathrm{~W} P \quad 241 \mathrm{TX} 113$ & Hexane, n-hexane & $110-54-3$ & $5.00 \mathrm{E}-01$ & $4.05 \mathrm{E}-05$ \\
\hline 200W P $241 T X 113$ & $\begin{array}{l}\text { Methyl N-amyl ketone, 2- } \\
\text { heptanone }\end{array}$ & $110-43-0$ & $5.00 \mathrm{E}-01$ & $3.76 \mathrm{E}-05$ \\
\hline 200W P $241 \mathrm{TX113}$ & Nitrogen Oxide $\{\mathrm{NO}\}$ & $10102-43-9$ & $2.00 \mathrm{E}+00$ & $2.64 \mathrm{E}-05$ \\
\hline 200W P $241 \mathrm{TX} 113$ & Nonane & $111-84-2$ & $5.00 \mathrm{E}-01$ & $2.11 \mathrm{E}-05$ \\
\hline 200W P $241 \mathrm{TX} 113$ & Octane & $111-65-9$ & $5.00 \mathrm{E}-01$ & $2.21 \mathrm{E}-05$ \\
\hline 200W P $241 \mathrm{TX} 113$ & $\begin{array}{l}\text { Phosphoric acid, tributyl } \\
\text { ester }\end{array}$ & $126-73-8$ & & 7.95E-05 \\
\hline 200W P $241 T X 113$ & Toluene & $108-88-3$ & $5.00 \mathrm{E}-01$ & $3.08 \mathrm{E}-06$ \\
\hline 200W P $241 T X 114$ & 1,3-Butadiene & $106-99-0$ & $3.50 \mathrm{E}-02$ & $1.91 \mathrm{E}-06$ \\
\hline 200W P $241 \mathrm{TX114}$ & 2-Hexanone & $591-78-6$ & $5.00 \mathrm{E}-01$ & $3.47 \mathrm{E}-05$ \\
\hline 200W P $241 T X 114$ & 2-Pentanone & $107-87-9$ & & $5.51 \mathrm{E}-05$ \\
\hline 200W P $241 T X 114$ & Acetone & $67-64-1$ & $5.00 \mathrm{E}-01$ & $6.62 E-04$ \\
\hline $\begin{array}{ll}200 W \\
& 241 T X 114\end{array}$ & Acetonitrile & $75-5-8$ & $5.00 \mathrm{E}-01$ & $3.19 \mathrm{E}-04$ \\
\hline 200W P $241 T X 114$ & Ammonia & $7664-41-7$ & $5.00 \mathrm{E}-01$ & $3.04 \mathrm{E}-03$ \\
\hline $\begin{array}{ll}200 W \text { P } & 241 T X 114\end{array}$ & Benzene & $71-43-2$ & $5.00 \mathrm{E}-01$ & $1.51 \mathrm{E}-05$ \\
\hline $200 W$ P $241 T X 114$ & Carbon monoxide & $630-8-0$ & $5.00 \mathrm{E}+00$ & $4.39 \mathrm{E}-04$ \\
\hline 200 W P $241 T X 114$ & Heptane & $142-82-5$ & $5.00 \mathrm{E}-01$ & $3.89 \mathrm{E}-05$ \\
\hline 200W P $241 \mathrm{TX114}$ & Hexane, n-hexane & $110-54-3$ & $5.00 \mathrm{E}-01$ & $4.05 \mathrm{E}-05$ \\
\hline 200W P $241 T X 114$ & $\begin{array}{l}\text { Methyl N-amyl ketone, 2- } \\
\text { heptanone }\end{array}$ & $110-43-0$ & $5.00 \mathrm{E}-01$ & $3.76 \mathrm{E}-05$ \\
\hline $200 W$ P $241 T X 114$ & Nitrogen Oxide $\{\mathrm{NO}\}$ & $10102-43-9$ & $2.00 \mathrm{E}+00$ & $2.64 \mathrm{E}-05$ \\
\hline 200W P $241 \mathrm{TX114}$ & Nonane & $111-84-2$ & $5.00 \mathrm{E}-01$ & 2.11E-05 \\
\hline 200W P $241 \mathrm{TX114}$ & Octane & $111-65-9$ & $5.00 \mathrm{E}-01$ & $2.21 \mathrm{E}-05$ \\
\hline 200W P $241 T X 114$ & $\begin{array}{l}\text { Phosphoric acid, tributyl } \\
\text { ester }\end{array}$ & $126-73-8$ & & $7.95 \mathrm{E}-05$ \\
\hline 200W P $241 T X 114$ & Toluene & $108-88-3$ & $5.00 \mathrm{E}-01$ & $3.08 \mathrm{E}-06$ \\
\hline 200W P 241 TX115 & 1,3-Butadiene & $106-99-0$ & $3.50 \mathrm{E}-02$ & $1.91 \mathrm{E}-06$ \\
\hline 200W P 241 TX115 & 2-Hexanone & $591-78-6$ & $5.00 \mathrm{E}-01$ & $3.47 \mathrm{E}-05$ \\
\hline $200 \mathrm{~W} P \quad 241 \mathrm{TX} 115$ & 2-Pentanone & $107-87-9$ & & $5.51 \mathrm{E}-05$ \\
\hline
\end{tabular}


Table K.2. Insignificant Emission Units Based on Threshold Comparisons

[WAC 173-401-530(1)(a)]

\begin{tabular}{|c|c|c|c|c|}
\hline Emission Point & Pollutants & CAS Number & $\begin{array}{c}\text { Emission } \\
\text { Threshold } \\
\text { (tons/yr) }\end{array}$ & $\begin{array}{c}\text { Actual } \\
\text { Emissions } \\
\text { (tons/yr) }\end{array}$ \\
\hline 200W P $241 T X 115$ & Acetone & $67-64-1$ & $5.00 \mathrm{E}-01$ & $6.62 \mathrm{E}-04$ \\
\hline 200W P $241 T X 115$ & Acetonitrile & $75-5-8$ & $5.00 E-01$ & $3.19 \mathrm{E}-04$ \\
\hline 200W P $241 T X 115$ & Ammonia & $7664-41-7$ & $5.00 \mathrm{E}-01$ & $3.04 \mathrm{E}-03$ \\
\hline 200W P $241 T X 115$ & Benzene & $71-43-2$ & $5.00 \mathrm{E}-01$ & $1.51 \mathrm{E}-05$ \\
\hline 200W P $241 T X 115$ & Carbon monoxide & $630-8-0$ & $5.00 \mathrm{E}+00$ & $4.39 \mathrm{E}-04$ \\
\hline 200W P $241 \mathrm{TX115}$ & Heptane & $142-82-5$ & $5.00 \mathrm{E}-01$ & $3.89 \mathrm{E}-05$ \\
\hline 200W P $241 T X 115$ & Hexane, n-hexane & $110-54-3$ & $5.00 \mathrm{E}-01$ & $4.05 \mathrm{E}-05$ \\
\hline 200W P $241 T X 115$ & $\begin{array}{l}\text { Methyl N-amyl ketone, 2- } \\
\text { heptanone }\end{array}$ & $110-43-0$ & $5.00 \mathrm{E}-01$ & $3.76 \mathrm{E}-05$ \\
\hline $200 W$ P $241 \mathrm{TX} 115$ & Nitrogen Oxide $\{\mathrm{NO}\}$ & $10102-43-9$ & $2.00 \mathrm{E}+00$ & $2.64 \mathrm{E}-05$ \\
\hline 200W P $241 \mathrm{TX115}$ & Nonane & $111-84-2$ & $5.00 \mathrm{E}-01$ & $2.11 \mathrm{E}-05$ \\
\hline $200 \mathrm{~W} P \quad 241 \mathrm{TX} 115$ & Octane & $111-65-9$ & $5.00 \mathrm{E}-01$ & $2.21 \mathrm{E}-05$ \\
\hline 200W P $241 \mathrm{TX115}$ & $\begin{array}{l}\text { Phosphoric acid, tributyl } \\
\text { ester }\end{array}$ & $126-73-8$ & & $7.95 \mathrm{E}-05$ \\
\hline $200 \mathrm{~W} P \quad 241 \mathrm{TX} 115$ & Toluene & $108-88-3$ & $5.00 \mathrm{E}-01$ & $3.08 \mathrm{E}-06$ \\
\hline 200W P $241 \mathrm{TX116}$ & 1,3-Butadiene & $106-99-0$ & $3.50 \mathrm{E}-02$ & $1.91 \mathrm{E}-06$ \\
\hline $200 W$ P $241 T X 116$ & 2-Hexanone & $591-78-6$ & $5.00 \mathrm{E}-01$ & $3.47 \mathrm{E}-05$ \\
\hline 200W P $241 \mathrm{TX116}$ & 2-Pentanone & $107-87-9$ & & $5.51 \mathrm{E}-05$ \\
\hline 200W P $241 \mathrm{TX116}$ & Acetone & $67-64-1$ & $5.00 \mathrm{E}-01$ & $6.62 \mathrm{E}-04$ \\
\hline $200 \mathrm{~W} P \quad 241 \mathrm{TX} 116$ & Acetonitrile & $75-5-8$ & $5.00 \mathrm{E}-01$ & $3.19 \mathrm{E}-04$ \\
\hline $200 \mathrm{~W} P \quad 241 \mathrm{TX116}$ & Ammonia & $7664-41-7$ & $5.00 \mathrm{E}-01$ & $3.04 \mathrm{E}-03$ \\
\hline 200W P $241 T X 116$ & Benzene & $71-43-2$ & $5.00 \mathrm{E}-01$ & $1.51 \mathrm{E}-05$ \\
\hline 200W P $241 \mathrm{TX116}$ & Carbon monoxide & $630-8-0$ & $5.00 \mathrm{E}+00$ & $4.39 \mathrm{E}-04$ \\
\hline 200W P $241 \mathrm{TX116}$ & Heptane & $142-82-5$ & $5.00 \mathrm{E}-01$ & $3.89 \mathrm{E}-05$ \\
\hline 200W P $241 T X 116$ & Hexane, n-hexane & $110-54-3$ & $5.00 \mathrm{E}-01$ & $4.05 \mathrm{E}-05$ \\
\hline 200W P $241 \mathrm{TX} 116$ & $\begin{array}{l}\text { Methyl N-amyl ketone, 2- } \\
\text { heptanone }\end{array}$ & $110-43-0$ & $5.00 \mathrm{E}-01$ & $3.76 \mathrm{E}-05$ \\
\hline 200W P $241 T X 116$ & Nitrogen Oxide $\{\mathrm{NO}\}$ & $10102-43-9$ & $2.00 \mathrm{E}+00$ & $2.64 \mathrm{E}-05$ \\
\hline 200W P $241 T X 116$ & Nonane & $111-84-2$ & $5.00 \mathrm{E}-01$ & $2.11 \mathrm{E}-05$ \\
\hline $200 W$ P $241 T X 116$ & Octane & $111-65-9$ & $5.00 \mathrm{E}-01$ & $2.21 \mathrm{E}-05$ \\
\hline
\end{tabular}


Table K.2. Insignificant Emission Units Based on Threshold Comparisons [WAC 173-401-530(1)(a)]

\begin{tabular}{|c|c|c|c|c|}
\hline Emission Point & Pollutants & CAS Number & $\begin{array}{c}\text { Emission } \\
\text { Threshold } \\
\text { (tons/yr) }\end{array}$ & $\begin{array}{c}\text { Actual } \\
\text { Emissions } \\
\text { (tons/yr) }\end{array}$ \\
\hline $200 W$ P $241 T \times 116$ & $\begin{array}{l}\text { Phosphoric acid, tributyl } \\
\text { ester }\end{array}$ & $126-73-8$ & & $7.95 \mathrm{E}-05$ \\
\hline 200W P 241TX116 & Toluene & $108-88-3$ & $5.00 \mathrm{E}-01$ & $3.08 \mathrm{E}-06$ \\
\hline 200W P $241 \mathrm{TX117}$ & 1,3-Butadiene & $106-99-0$ & $3.50 \mathrm{E}-02$ & $1.91 \mathrm{E}-06$ \\
\hline 200W P $241 T X 117$ & 2-Hexanone & $591-78-6$ & $5.00 \mathrm{E}-01$ & $3.47 \mathrm{E}-05$ \\
\hline 200W P 241TX117 & 2-Pentanone & $107-87-9$ & & $5.51 \mathrm{E}-05$ \\
\hline 200W P 241TX117 & Acetone & $67-64-1$ & $5.00 \mathrm{E}-01$ & $6.62 E-04$ \\
\hline 200W P $241 T X 117$ & Acetonitrile & $75-5-8$ & $5.00 \mathrm{E}-01$ & $3.19 \mathrm{E}-04$ \\
\hline 200W P $241 \mathrm{TX} 117$ & Ammonia & $7664-41-7$ & $5.00 \mathrm{E}-01$ & $3.04 \mathrm{E}-03$ \\
\hline 200W P $241 \mathrm{TX} 117$ & Benzene & $71-43-2$ & 5.00E-01 & $1.51 \mathrm{E}-05$ \\
\hline 200W P $241 T X 117$ & Carbon monoxide & $630-8-0$ & $5.00 \mathrm{E}+00$ & 4.39E-04 \\
\hline 200W P $241 T X 117$ & Heptane & $142-82-5$ & $5.00 \mathrm{E}-01$ & $3.89 \mathrm{E}-05$ \\
\hline 200W P $241 \mathrm{TX} 117$ & Hexane, n-hexane & $110-54-3$ & $5.00 \mathrm{E}-01$ & $4.05 \mathrm{E}-05$ \\
\hline 200W P 241TX117 & $\begin{array}{l}\text { Methyl N-amyl ketone, 2- } \\
\text { heptanone }\end{array}$ & $110-43-0$ & $5.00 \mathrm{E}-01$ & $3.76 \mathrm{E}-05$ \\
\hline 200W P 241TX117 & Nitrogen Oxide $\{\mathrm{NO}\}$ & $10102-43-9$ & $2.00 \mathrm{E}+00$ & $2.64 \mathrm{E}-05$ \\
\hline 200W P $241 T X 117$ & Nonane & $111-84-2$ & $5.00 \mathrm{E}-01$ & $2.11 \mathrm{E}-05$ \\
\hline 200W P 241TX117 & Octane & $111-65-9$ & $5.00 \mathrm{E}-01$ & $2.21 \mathrm{E}-05$ \\
\hline 200W P $241 T X 117$ & $\begin{array}{l}\text { Phosphoric acid, tributyl } \\
\text { ester }\end{array}$ & $126-73-8$ & & $7.95 \mathrm{E}-05$ \\
\hline 200W P $241 \mathrm{TX} 117$ & Toluene & $108-88-3$ & $5.00 \mathrm{E}-01$ & $3.08 \mathrm{E}-06$ \\
\hline 200W P $241 T X 118$ & Acetonitrile & $75-5-8$ & $5.00 \mathrm{E}-01$ & $7.31 \mathrm{E}-07$ \\
\hline 200W P 241 TX118 & Ammonia & $7664-41-7$ & $5.00 \mathrm{E}-01$ & $6.57 \mathrm{E}-03$ \\
\hline 200W P $241 \mathrm{TX} 118$ & Benzene & $71-43-2$ & $5.00 \mathrm{E}-01$ & $3.97 \mathrm{E}-07$ \\
\hline 200W P $241 \mathrm{TX} 118$ & Carbon monoxide & $630-8-0$ & $5.00 \mathrm{E}+00$ & $1.03 E-04$ \\
\hline 200W P 241 TX118 & Carbon Tetrachloride & $56-23-5$ & $5.00 \mathrm{E}-01$ & $3.91 \mathrm{E}-06$ \\
\hline 200W P $241 \mathrm{TX} 118$ & Ethyl butyl ketone & $106-35-4$ & $5.00 \mathrm{E}-01$ & $1.31 E-05$ \\
\hline 200W P $241 T X 118$ & Methyl chloride & $74-87-3$ & $5.00 \mathrm{E}-01$ & $5.78 \mathrm{E}-07$ \\
\hline 200W P $241 \mathrm{TX} 118$ & Nitrogen Oxide $\{\mathrm{NO}\}$ & $10102-43-9$ & $2.00 \mathrm{E}+00$ & $5.72 \mathrm{E}-05$ \\
\hline 200W P $241 T X 118$ & Tetrahydrofuran & $109-99-9$ & $5.00 \mathrm{E}-01$ & $1.01 E-06$ \\
\hline
\end{tabular}


Table K.2. Insignificant Emission Units Based on Threshold Comparisons [WAC 173-401-530(1)(a)]

\begin{tabular}{|c|c|c|c|c|}
\hline Emission Point & Pollutants & CAS Number & $\begin{array}{l}\text { Emission } \\
\text { Threshold } \\
\text { (tons/yr) }\end{array}$ & $\begin{array}{c}\text { Actual } \\
\text { Emissions } \\
\text { (tons/yr) }\end{array}$ \\
\hline 200W P $241 \mathrm{TX118}$ & Toluene & $108-88-3$ & $5.00 \mathrm{E}-01$ & $1.17 \mathrm{E}-06$ \\
\hline 200W P $241 T X 118$ & Trichlorofluoromethane & $75-69-4$ & $5.00 \mathrm{E}-01$ & $4.89 \mathrm{E}-05$ \\
\hline 200W P 241 TY101 & 1,3-Butadiene & $106-99-0$ & $3.50 \mathrm{E}-02$ & $4.13 \mathrm{E}-06$ \\
\hline 200W P $241 T Y 101$ & 2-Hexanone & $591-78-6$ & $5.00 \mathrm{E}-01$ & $7.51 \mathrm{E}-05$ \\
\hline 200W P $241 T Y 101$ & 2-Pentanone & $107-87-9$ & & $1.19 \mathrm{E}-04$ \\
\hline 200W P $241 T Y 101$ & Acetone & $67-64-1$ & $5.00 \mathrm{E}-01$ & $1.43 \mathrm{E}-03$ \\
\hline 200W P $241 T Y 101$ & Acetonitrile & $75-5-8$ & $5.00 \mathrm{E}-01$ & $6.89 \mathrm{E}-04$ \\
\hline 200W P $241 T Y 101$ & Ammonia & $7664-41-7$ & $5.00 \mathrm{E}-01$ & $6.57 \mathrm{E}-03$ \\
\hline 200W P $241 T Y 101$ & Benzene & $71-43-2$ & $5.00 \mathrm{E}-01$ & $3.28 \mathrm{E}-05$ \\
\hline 200W P $241 T Y 101$ & Carbon monoxide & $630-8-0$ & $5.00 \mathrm{E}+00$ & $9.50 \mathrm{E}-04$ \\
\hline 200W P $241 T Y 101$ & Heptane & $142-82-5$ & $5.00 \mathrm{E}-01$ & $8.40 \mathrm{E}-05$ \\
\hline 200W P $241 T Y 101$ & Hexane, n-hexane & $110-54-3$ & $5.00 \mathrm{E}-01$ & $8.76 \mathrm{E}-05$ \\
\hline 200W P 241 TY101 & $\begin{array}{l}\text { Methyl N-amyl ketone, 2- } \\
\text { heptanone }\end{array}$ & $110-43-0$ & $5.00 \mathrm{E}-01$ & $8.13 \mathrm{E}-05$ \\
\hline 200W P $241 T Y 101$ & Nitrogen Oxide $\{\mathrm{NO}\}$ & $10102-43-9$ & $2.00 \mathrm{E}+00$ & $5.72 \mathrm{E}-05$ \\
\hline 200W P 241TY101 & Nonane & $111-84-2$ & $5.00 \mathrm{E}-01$ & $4.57 \mathrm{E}-05$ \\
\hline 200W P $241 T Y 101$ & Octane & $111-65-9$ & $5.00 \mathrm{E}-01$ & $4.79 \mathrm{E}-05$ \\
\hline 200W P 241TY101 & $\begin{array}{l}\text { Phosphoric acid, tributyl } \\
\text { ester }\end{array}$ & $126-73-8$ & & $1.72 \mathrm{E}-04$ \\
\hline 200W P $241 T Y 101$ & Toluene & $108-88-3$ & $5.00 \mathrm{E}-01$ & $6.67 \mathrm{E}-06$ \\
\hline 200W P 241 TY102 & 1,3-Butadiene & $106-99-0$ & $3.50 \mathrm{E}-02$ & $4.13 \mathrm{E}-06$ \\
\hline 200W P 241TY102 & 2-Hexanone & $591-78-6$ & $5.00 \mathrm{E}-01$ & $7.51 \mathrm{E}-05$ \\
\hline 200W P 241TY102 & 2-Pentanone & $107-87-9$ & & $1.19 \mathrm{E}-04$ \\
\hline 200W P 241TY102 & Acetone & $67-64-1$ & $5.00 \mathrm{E}-01$ & $1.43 \mathrm{E}-03$ \\
\hline 200W P 241 TY102 & Acetonitrile & $75-5-8$ & $5.00 \mathrm{E}-01$ & $6.89 \mathrm{E}-04$ \\
\hline 200W P 241TY102 & Ammonia & $7664-41-7^{\circ}$ & $5.00 \mathrm{E}-01$ & $6.57 \mathrm{E}-03$ \\
\hline 200W P 241TY102 & Benzene & $71-43-2$ & $5.00 \mathrm{E}-01$ & $3.28 \mathrm{E}-05$ \\
\hline $200 \mathrm{~W}$ P $241 \mathrm{TY} 102$ & Carbon monoxide & $630-8-0$ & $5.00 \mathrm{E}+00$ & $9.50 \mathrm{E}-04$ \\
\hline 200W P 241TY102 & Heptane & $142-82-5 \quad-$ & $5.00 \mathrm{E}-01$ & $8.40 \mathrm{E}-05$ \\
\hline
\end{tabular}


Table K:2. Insignificant Emission Units Based on Threshold Comparisons [WAC 173-401-530(1)(a)]

\begin{tabular}{|c|c|c|c|c|}
\hline Emission Point & Pollutants & CAS Number & $\begin{array}{l}\text { Emission } \\
\text { Threshold } \\
\text { (tons/yr) }\end{array}$ & $\begin{array}{c}\text { Actual } \\
\text { Emissions } \\
\text { (tons/yr) }\end{array}$ \\
\hline $200 \mathrm{~W} P \quad 241 \mathrm{TY} 102$ & Hexane, n-hexane & $110-54-3$ & $5.00 \mathrm{E}-01$ & $8.76 \mathrm{E}-05$ \\
\hline 200W P 241 TY102 & $\begin{array}{l}\text { Methyl N-amyl ketone, 2- } \\
\text { heptanone }\end{array}$ & $110-43-0$ & $5.00 \mathrm{E}-01$ & $8.13 E-05$ \\
\hline $200 W$ P 241 TY102 & Nitrogen Oxide $\{\mathrm{NO}\}$ & $10102-43-9$ & $2.00 \mathrm{E}+00$ & $5.72 \mathrm{E}-05$ \\
\hline 200W P 241TY102 & Nonane & $111-84-2$ & $5.00 \mathrm{E}-01$ & $4.57 \mathrm{E}-05$ \\
\hline 200W P 241TY102 & Octane & $111-65-9$ & $5.00 \mathrm{E}-01$ & $4.79 \mathrm{E}-05$ \\
\hline 200W P 241 TY102 & $\begin{array}{l}\text { Phosphoric acid, tributyl } \\
\text { ester }\end{array}$ & $126-73-8$ & & 1.72E-04 \\
\hline 200W P 241 TY102 & Toluene & $108-88-3$ & $5.00 \mathrm{E}-01$ & $6.67 \mathrm{E}-06$ \\
\hline 200W P $241 T Y 103$ & 1,3-Butadiene & $106-99-0$ & $3.50 \mathrm{E}-02$ & $4.13 \mathrm{E}-06$ \\
\hline 200W P 241TY103 & 2-Hexanone & $591-78-6$ & $5.00 \mathrm{E}-01$ & $7.51 \mathrm{E}-05$ \\
\hline 200W P 241TY103 & 2-Pentanone & $107-87-9$ & & $1.19 \mathrm{E}-04$ \\
\hline 200W P 241 TY103 & Acetone & $67-64-1$ & $5.00 \mathrm{E}-01$ & $1.43 \mathrm{E}-03$ \\
\hline 200W P 241TY103 & Acetonitrile & $75-5-8$ & $5.00 \mathrm{E}-01$ & $6.89 \mathrm{E}-04$ \\
\hline 200W P $241 T Y 103$ & Ammonia & $7664-41-7$ & $5.00 \mathrm{E}-01$ & $6.57 \mathrm{E}-03$ \\
\hline 200W P 241TY103 & Benzene & $71-43-2$ & $5.00 \mathrm{E}-01$ & $3.28 \mathrm{E}-05$ \\
\hline 200W P 241TY103 & Carbon monoxide & $630-8-0$ & $5.00 \mathrm{E}+00$ & $9.50 \mathrm{E}-04$ \\
\hline 200W P 241 TY103 & Heptane & $142-82-5$ & $5.00 \mathrm{E}-01$ & $8.40 \mathrm{E}-05$ \\
\hline 200W P 241TY103 & Hexane, n-hexane & $110-54-3$ & $5.00 \mathrm{E}-01$ & $8.76 \mathrm{E}-05$ \\
\hline 200W P 241 TY103 & $\begin{array}{l}\text { Methyl N-amyl ketone, 2- } \\
\text { heptanone }\end{array}$ & $110-43-0$ & $5.00 \mathrm{E}-01$ & $8.13 \mathrm{E}-05$ \\
\hline 200W P 241TY103 & Nitrogen Oxide $\{\mathrm{NO}\}$ & $10102-43-9$ & $2.00 \mathrm{E}+00$ & $5.72 \mathrm{E}-05$ \\
\hline 200W P $241 \mathrm{TY} 103$ & Nonane & $111-84-2$ & $5.00 \mathrm{E}-01$ & $4.57 \mathrm{E}-05$ \\
\hline 200W P $241 T Y 103$ & Octane & $111-65-9$ & $5.00 \mathrm{E}-01$ & $4.79 \mathrm{E}-05$ \\
\hline 200W P 241 TY103 & $\begin{array}{l}\text { Phosphoric acid, tributyl } \\
\text { ester }\end{array}$ & $126-73-8$ & & $1.72 \mathrm{E}-04$ \\
\hline 200W P $241 \mathrm{TY} 103$ & Toluene & $108-88-3$ & $5.00 \mathrm{E}-01$ & $6.67 \mathrm{E}-06$ \\
\hline 200W P 241TY104 & 1,3-Butadiene & $106-99-0$ & $3.50 \mathrm{E}-02$ & $4.13 \mathrm{E}-06$ \\
\hline 200W P $241 T Y 104$ & 2-Hexanone & $591-78-6$ & $5.00 \mathrm{E}-01$ & $7.51 \mathrm{E}-05$ \\
\hline 200W P 241 TY104 & 2-Pentanone & $107-87-9$ & & $1.19 \mathrm{E}-04$ \\
\hline
\end{tabular}


Table K.2. Insignificant Emission Units Based on Threshold Comparisons [WAC 173-401-530(1)(a)]

\begin{tabular}{|c|c|c|c|c|}
\hline Emission Point & Pollutants & CAS Number & $\begin{array}{l}\text { Emission } \\
\text { Threshold } \\
\text { (tons/yr) }\end{array}$ & $\begin{array}{c}\text { Actual } \\
\text { Emissions } \\
\text { (tons/yr) }\end{array}$ \\
\hline 200W P 241TY104 & Acetone & $67-64-1$ & $5.00 \mathrm{E}-01$ & $1.43 \mathrm{E}-03$ \\
\hline 200W P $241 T Y 104$ & Acetonitrile & $75-5-8$ & $5.00 \mathrm{E}-01$ & $6.89 \mathrm{E}-04$ \\
\hline 200W P 241TY104 & Ammonia & $7664-41-7$ & $5.00 \mathrm{E}-01$ & $6.57 \mathrm{E}-03$ \\
\hline 200W P 241TY104 & Benzene & $71-43-2$ & $5.00 \mathrm{E}-01$ & $3.28 \mathrm{E}-05$ \\
\hline 200W P 241TY104 & Carbon monoxide & $630-8-0$ & $5.00 \mathrm{E}+00$ & $9.50 \mathrm{E}-04$ \\
\hline 200W P $241 T Y 104$ & Heptane & $142-82-5$ & $5.00 \mathrm{E}-01$ & $8.40 \mathrm{E}-05$ \\
\hline 200W P 241TY104 & Hexane, n-hexane & $110-54-3$ & $5.00 \mathrm{E}-01$ & $8.76 \mathrm{E}-05$ \\
\hline 200W P 241 TY104 & $\begin{array}{l}\text { Methyl N-amyl ketone, 2- } \\
\text { heptanone }\end{array}$ & $110-43-0$ & $5.00 \mathrm{E}-01$ & $8.13 E-05$ \\
\hline 200W P $241 T Y 104$ & Nitrogen Oxide $\{\mathrm{NO}\}$ & $10102-43-9$ & $2.00 \mathrm{E}+00$ & $5.72 \mathrm{E}-05$ \\
\hline 200W P 241TY104 & Nonane & $111-84-2$ & $5.00 \mathrm{E}-01$ & $4.57 \mathrm{E}-05$ \\
\hline 200W P $241 T Y 104$ & Octane & $111-65-9$ & $5.00 \mathrm{E}-01$ & $4.79 \mathrm{E}-05$ \\
\hline 200W P $241 T Y 104$ & $\begin{array}{l}\text { Phosphoric acid, tributyl } \\
\text { ester }\end{array}$ & $126-73-8$ & & $1.72 \mathrm{E}-04$ \\
\hline 200W P 241TY104 & Toluene & $108-88-3$ & $5.00 \mathrm{E}-01$ & $6.67 \mathrm{E}-06$ \\
\hline 200W P 241TY105 & 1,3-Butadiene & $106-99-0$ & $3.50 \mathrm{E}-02$ & $1.91 \mathrm{E}-06$ \\
\hline 200W P 241TY105 & 2-Hexanone & $591-78-6$ & $5.00 \mathrm{E}-01$ & $3.47 \mathrm{E}-05$ \\
\hline 200W P $241 T Y 105$ & 2-Pentanone & $107-87-9$ & & $5.51 \mathrm{E}-05$ \\
\hline 200W P 241TY105 & Acetone & $67-64-1$ & $5.00 \mathrm{E}-01$ & $6.62 \mathrm{E}-04$ \\
\hline 200W P $241 T Y 105$ & Acetonitrile & $75-5-8$ & $5.00 \mathrm{E}-01$ & $3.19 \mathrm{E}-04$ \\
\hline 200W P $241 T Y 105$ & Ammonia & $7664-41-7$ & $5.00 \mathrm{E}-01$ & $3.04 \mathrm{E}-03$ \\
\hline 200W P $241 T Y 105$ & Benzene & $71-43-2$ & $5.00 \mathrm{E}-01$ & $1.51 \mathrm{E}-05$ \\
\hline 200W P $241 T Y 105$ & Carbon monoxide & $630-8-0$ & $5.00 \mathrm{E}+00$ & $4.39 \mathrm{E}-04$ \\
\hline 200W P 241TY105 & Heptane & $142-82-5$ & $5.00 \mathrm{E}-01$ & $3.89 \mathrm{E}-05$ \\
\hline 200W P 241TY105 & Hexane, n-hexane & $110-54-3$ & $5.00 \mathrm{E}-01$ & $4.05 \mathrm{E}-05$ \\
\hline 200W P 241TY105 & $\begin{array}{l}\text { Methyl N-amyl ketone, 2- } \\
\text { heptanone }\end{array}$ & $110-43-0$ & $5.00 \mathrm{E}-01$ & $3.76 \mathrm{E}-05$ \\
\hline 200W P 241TY105 & Nitrogen Oxide $\{\mathrm{NO}\}$ & $10102-43-9$ & $2.00 \mathrm{E}+00$ & $2.64 \mathrm{E}-05$ \\
\hline 200W P 241TY105 & Nonane & $111-84-2$ & $5.00 \mathrm{E}-01$ & $2.11 \mathrm{E}-05$ \\
\hline 200W P 241TY105 & Octane & $111-65-9$ & $5.00 \mathrm{E}-01$ & $2.21 \mathrm{E}-05$ \\
\hline
\end{tabular}


Table K.2. Insignificant Emission Units Based on Threshold Comparisons [WAC 173-401-530(1)(a)]

\begin{tabular}{|c|c|c|c|c|}
\hline Emission Point & Pollutants & CAS Number & $\begin{array}{l}\text { Emission } \\
\text { Threshold } \\
\text { (tons/yr) }\end{array}$ & $\begin{array}{c}\text { Actual } \\
\text { Emissions } \\
\text { (tons/yr) }\end{array}$ \\
\hline 200W P 241 TY105 & $\begin{array}{l}\text { Phosphoric acid, tributyl } \\
\text { ester }\end{array}$ & $126-73-8$ & & $7.95 \mathrm{E}-05$ \\
\hline 200W P $241 T Y 105$ & Toluene & $108-88-3$ & $5.00 \mathrm{E}-01$ & $3.08 \mathrm{E}-06$ \\
\hline 200W P 241 TY106 & 1,3-Butadiene & $106-99-0$ & $3.50 \mathrm{E}-02$ & $1.91 \mathrm{E}-06$ \\
\hline 200W P 241 TY106 & 2-Hexanone & $591-78-6$ & $5.00 \mathrm{E}-01$ & $3.47 \mathrm{E}-05$ \\
\hline 200W P 241 TY106 & 2-Pentanone & $107-87-9$ & & 5.51E-05 \\
\hline 200W P 241 TY106 & Acetone & 67-64-1 & $5.00 \mathrm{E}-01$ & $6.62 \mathrm{E}-04$ \\
\hline $200 W$ P $241 T Y 106$ & Acetonitrile & $75-5-8$ & $5.00 \mathrm{E}-01$ & $3.19 \mathrm{E}-04$ \\
\hline $200 W$ P 241 TY106 & Ammonia & $7664-41-7$ & $5.00 \mathrm{E}-01$ & $3.04 \mathrm{E}-03$ \\
\hline 200W P 241 TY106 & Benzene & $71-43-2$ & $5.00 \mathrm{E}-01$ & $1.51 \mathrm{E}-05$ \\
\hline $200 W$ P 241 TY106 & Carbon monoxide & $630-8-0$ & $5.00 \mathrm{E}+00$ & $4.39 \mathrm{E}-04$ \\
\hline 200W P 241 TY106 & Heptane & $142-82-5$ & $5.00 \mathrm{E}-01$ & $3.89 \mathrm{E}-05$ \\
\hline 200W P 241 TY106 & Hexane, n-hexane & $110-54-3$ & $5.00 \mathrm{E}-01$ & $4.05 \mathrm{E}-05$ \\
\hline 200W P $241 T Y 106$ & $\begin{array}{l}\text { Methyl N-amyl ketone, 2- } \\
\text { heptanone }\end{array}$ & $110-43-0$ & $5.00 \mathrm{E}-01$ & $3.76 \mathrm{E}-05$ \\
\hline 200W P 241 TY106 & Nitrogen Oxide $\{\mathrm{NO}\}$ & $10102-43-9$ & $2.00 \mathrm{E}+00$ & $2.64 \mathrm{E}-05$ \\
\hline 200W P $241 T Y 106$ & Nonane & $111-84-2$ & $5.00 \mathrm{E}-01$ & $2.11 E-05$ \\
\hline 200W P 241 TY106 & Octane & $111-65-9$ & $5.00 \mathrm{E}-01$ & $2.21 \mathrm{E}-05$ \\
\hline $200 W$ P 241 TY106 & $\begin{array}{l}\text { Phosphoric acid, tributyl } \\
\text { ester }\end{array}$ & $126-73-8$ & & $7.95 \mathrm{E}-05$ \\
\hline 200W P 241 TY106 & Toluene & $108-88-3$ & $5.00 \mathrm{E}-01$ & $3.08 \mathrm{E}-06$ \\
\hline $200 \mathrm{~W} P \quad 241 \mathrm{U} 101$ & 1,3-Butadiene & $106-99-0$ & $3.50 \mathrm{E}-02$ & $1.91 \mathrm{E}-06$ \\
\hline 200W P $241 \mathrm{U101}$ & 2-Hexanone & $591-78-6$ & $5.00 \mathrm{E}-01$ & $3.47 \mathrm{E}-05$ \\
\hline $200 W$ P 241 U101 & 2-Pentanone & $107-87-9$ & & $5.51 \mathrm{E}-05$ \\
\hline $200 \mathrm{~W} P \quad 241 \mathrm{U} 101$ & Acetone & $67-64-1$ & $5.00 \mathrm{E}-01$ & $6.62 \mathrm{E}-04$ \\
\hline 200W P 241U101 & Acetonitrile & $75-5-8$ & $5.00 \mathrm{E}-01$ & $3.19 \mathrm{E}-04$ \\
\hline 200W P $241 \mathrm{U} 101$ & Ammonia & $7664-41-7$ & $5.00 \mathrm{E}-01$ & $3.04 \mathrm{E}-03$ \\
\hline 200W P 241U101 & Benzene & $71-43-2$ & $5.00 \mathrm{E}-01$ & $1.51 \mathrm{E}-05$ \\
\hline $200 \mathrm{~W} P \quad 241 \mathrm{U} 101$ & Carbon monoxide & $630-8-0$ & $5.00 \mathrm{E}+00$ & $4.39 \mathrm{E}-04$ \\
\hline $200 W$ P $241 U 101$ & Heptane & $142-82-5$ & $5.00 \mathrm{E}-01$ & $3.89 \mathrm{E}-05$ \\
\hline
\end{tabular}


Table K.2. Insignificant Emission Units Based on Threshold Comparisons [WAC 173-401-530(1)(a)]

\begin{tabular}{|c|c|c|c|c|}
\hline Emission Point & Pollutants & CAS Number & $\begin{array}{l}\text { Emission } \\
\text { Threshold } \\
\text { (tons/yr) }\end{array}$ & $\begin{array}{c}\text { Actual } \\
\text { Emissions } \\
\text { (tons/yr) }\end{array}$ \\
\hline 200W P 241U101 & Hexane, n-hexane & $110-54-3$ & $5.00 \mathrm{E}-01$ & $4.05 \mathrm{E}-05$ \\
\hline 200W P $241 U 101$ & $\begin{array}{l}\text { Methyl N-amyl ketone, 2- } \\
\text { heptanone }\end{array}$ & $110-43-0$ & $5.00 \mathrm{E}-01$ & $3.76 \mathrm{E}-05$ \\
\hline 200W P $241 U 101$ & Nitrogen Oxide $\{\mathrm{NO}\}$ & $10102-43-9$ & $2.00 \mathrm{E}+00$ & $2.64 \mathrm{E}-05$ \\
\hline $200 \mathrm{~W} \cdot \mathrm{P} \quad 241 \mathrm{U} 101$ & Nonane & $111-84-2$ & $5.00 \mathrm{E}-01$ & $2.11 \mathrm{E}-05$ \\
\hline 200W P $241 \mathrm{U} 101$ & Octane & $111-65-9$ & $5.00 \mathrm{E}-01$ & $2.21 \mathrm{E}-05$ \\
\hline 200W P 241U101 & $\begin{array}{l}\text { Phosphoric acid, tributyl } \\
\text { ester }\end{array}$ & $126-73-8$ & & $7.95 \mathrm{E}-05$ \\
\hline $200 W$ P $241 U 101$ & Toluene & $108-88-3$ & $5.00 \mathrm{E}-01$ & $3.08 \mathrm{E}-06$ \\
\hline 200W P $241 \mathrm{U} 102$ & 1,3-Butadiene & $106-99-0$ & $3.50 E-02$ & 4.13E-06 \\
\hline $200 \mathrm{~W} P \quad 241 \mathrm{U} 102$ & 2-Hexanone & $591-78-6$ & $5.00 \mathrm{E}-01$ & $7.51 \mathrm{E}-05$ \\
\hline 200W P $241 U 102$ & 2-Pentanone & $107-87-9$ & & $1.19 \mathrm{E}-04$ \\
\hline 200W P $241 U 102$ & Acetone & $67-64-1$ & $5.00 \mathrm{E}-01$ & $1.43 \mathrm{E}-03$ \\
\hline 200W P 241U102 & Acetonitrile & $75-5-8$ & $5.00 \mathrm{E}-01$ & $6.89 \mathrm{E}-04$ \\
\hline $200 W$ P $241 U 102$ & Ammonia & $7664-41-7$ & $5.00 \mathrm{E}-01$ & $6.57 \mathrm{E}-03$ \\
\hline 200W P 241U102 & Benzene & $71-43-2$ & $5.00 \mathrm{E}-01$ & $3.28 \mathrm{E}-05$ \\
\hline 200W P $241 U 102$ & Carbon monoxide & $630-8-0$ & $5.00 \mathrm{E}+00$ & $9.50 \mathrm{E}-04$ \\
\hline 200W P $241 \mathrm{U} 102$ & Heptane & $142-82-5$ & $5.00 \mathrm{E}-01$ & $8.40 \mathrm{E}-05$ \\
\hline 200W P $241 U 102$ & Hexane, n-hexane & $110-54-3$ & $5.00 \mathrm{E}-01$ & $8.76 \mathrm{E}-05$ \\
\hline $200 W$ P $241 \mathrm{U} 102$ & $\begin{array}{l}\text { Methyl N-amyl ketone, 2- } \\
\text { heptanone }\end{array}$ & $110-43-0$ & $5.00 \mathrm{E}-01$ & $8.13 \mathrm{E}-05$ \\
\hline 200W P 241U102 & Nitrogen Oxide $\{\mathrm{NO}\}$ & $10102-43-9$ & $2.00 \mathrm{E}+00$ & $5.72 \mathrm{E}-05$ \\
\hline 200W P 241U102 & Nonane & $111-84-2$ & $5.00 \mathrm{E}-01$ & $4.57 \mathrm{E}-05$ \\
\hline 200W P 241U102 & Octane & $111-65-9$ & $5.00 \mathrm{E}-01$ & $4.79 \mathrm{E}-05$ \\
\hline $200 W$ P $241 \mathrm{U} 102$ & $\begin{array}{l}\text { Phosphoric acid, tributyl } \\
\text { ester }\end{array}$ & $126-73-8$ & & $1.72 \mathrm{E}-04$ \\
\hline 200W P 241 U102 & Toluene & $108-88-3$ & $5.00 \mathrm{E}-01$ & $6.67 \mathrm{E}-06$ \\
\hline 200W P 241U103 & 1,3-Butadiene & $106-99-0$ & $3.50 \mathrm{E}-02$ & $4.13 E-06$ \\
\hline $200 W$ P $241 \mathrm{U} 103$ & 2-Hexanone & $591-78-6$ & $5.00 \mathrm{E}-01$ & $7.51 \mathrm{E}-05$ \\
\hline $200 \mathrm{~W} P \quad 241 \mathrm{U} 103$ & 2-Pentanone & $107-87-9$ & & $1.19 \mathrm{E}-04$ \\
\hline
\end{tabular}


Table K.2. Insignificant Emission Units Based on Threshold Comparisons [WAC 173-401-530(1)(a)]

\begin{tabular}{|c|c|c|c|c|}
\hline Emission Point & Pollutants & CAS Number & $\begin{array}{c}\text { Emission } \\
\text { Threshold } \\
\text { (tons/yr) }\end{array}$ & $\begin{array}{c}\text { Actual } \\
\text { Emissions } \\
\text { (tons/yr) }\end{array}$ \\
\hline 200W P 241U103 & Acetone & $67-64-1$ & $5.00 \mathrm{E}-01$ & $1.43 \mathrm{E}-03$ \\
\hline $200 W$ P $241 U 103$ & Acetonitrile & $75-5-8$ & $5.00 \mathrm{E}-01$ & $6.89 \mathrm{E}-04$ \\
\hline 200W P 241U103 & Ammonia & $7664-41-7$ & $5.00 \mathrm{E}-01$ & $6.57 \mathrm{E}-03$ \\
\hline $\begin{array}{ll}200 W \text { P } & 241 \text { U103 }\end{array}$ & Benzene & $71-43-2$ & $5.00 \mathrm{E}-01$ & $3.28 \mathrm{E}-05$ \\
\hline 200W P 241U103 & Carbon monoxide & $630-8-0$ & $5.00 \mathrm{E}+00$ & $9.50 \mathrm{E}-04$ \\
\hline 200W P 241U103 & Heptane & $142-82-5$ & $5.00 \mathrm{E}-01$ & $8.40 \mathrm{E}-05$ \\
\hline 200 W P 241 U103 & Hexane, n-hexane & $110-54-3$ & $5.00 \mathrm{E}-01$ & $8.76 \mathrm{E}-05$ \\
\hline $200 W$ P $241 U 103$ & $\begin{array}{l}\text { Methyl N-amyl ketone, 2- } \\
\text { heptanone }\end{array}$ & $110-43-0$ & $5.00 \mathrm{E}-01$ & $8.13 E-05$ \\
\hline 200W P 241U103 & Nitrogen Oxide $\{\mathrm{NO}\}$ & $10102-43-9$ & $2.00 \mathrm{E}+00$ & 5.72E-05 \\
\hline 200W P $241 \mathrm{U} 103$ & Nonane & $111-84-2$ & $5.00 \mathrm{E}-01$ & $4.57 \mathrm{E}-05$ \\
\hline 200W P $241 \mathrm{U} 103$ & Octane & $111-65-9$ & $5.00 \mathrm{E}-01$ & 4.79E-05 \\
\hline 200W P 241U103 & $\begin{array}{l}\text { Phosphoric acid, tributyl } \\
\text { ester }\end{array}$ & $126-73-8$ & & $1.72 \mathrm{E}-04$ \\
\hline 200W P 241U103 & Toluene & $108-88-3$ & $5.00 \mathrm{E}-01$ & $6.67 \mathrm{E}-06$ \\
\hline $200 W$ P 241 U104 & 1,3-Butadiene & $106-99-0$ & $3.50 \mathrm{E}-02$ & $1.91 \mathrm{E}-06$ \\
\hline $200 W$ P $241 U 104$ & 2-Hexanone & $591-78-6$ & $5.00 \mathrm{E}-01$ & 3.47E-05 \\
\hline 200W P 241U104 & 2-Pentanone & $107-87-9$ & & $5.51 \mathrm{E}-05$ \\
\hline 200W P 241U104 & Acetone & $67-64-1$ & $5.00 \mathrm{E}-01$ & $6.62 \mathrm{E}-04$ \\
\hline 200W P 241U104 & Acetonitrile & $75-5-8$ & $5.00 \mathrm{E}-01$ & $3.19 \mathrm{E}-04$ \\
\hline $200 W$ P $241 \mathrm{U} 104$ & Ammonia & $7664-41-7$ & $5.00 \mathrm{E}-01$ & $3.04 \mathrm{E}-03$ \\
\hline $200 W$ P $241 U 104$ & Benzene & $71-43-2$ & $5.00 \mathrm{E}-01$ & $1.51 E-05$ \\
\hline 200W P $241 U 104$ & Carbon monoxide & $630-8-0$ & $5.00 \mathrm{E}+00$ & $4.39 E-04$ \\
\hline $200 W$ P $241 U 104$ & Heptane & $142-82-5$ & $5.00 \mathrm{E}-01$ & $3.89 \mathrm{E}-05$ \\
\hline 200 W P $241 U 104$ & Hexane, n-hexane & $110-54-3$ & $5.00 \mathrm{E}-01$ & $4.05 E-05$ \\
\hline $200 W$ P $241 U 104$ & $\begin{array}{l}\text { Methyl N-amyl ketone, 2- } \\
\text { heptanone }\end{array}$ & $110-43-0$ & $5.00 \mathrm{E}-01$ & $3.76 \mathrm{E}-05$ \\
\hline $200 \mathrm{~W} P \quad 241 \mathrm{U} 104$ & Nitrogen Oxide $\{\mathrm{NO}\}$ & $10102-43-9$ & $2.00 \mathrm{E}+00$ & $2.64 \mathrm{E}-05$ \\
\hline 200W P $241 U 104$ & Nonane & $111-84-2$ & $5.00 \mathrm{E}-01$ & $2.11 \mathrm{E}-05$ \\
\hline 200W P 241U104 & Octane & $111-65-9$ & $5.00 \mathrm{E}-01$ & $2.21 \mathrm{E}-05$ \\
\hline
\end{tabular}


Table K.2. Insignificant Emission Units Based on Threshold Comparisons [WAC 173-401-530(1)(a)]

\begin{tabular}{|c|c|c|c|c|}
\hline Emission Point & Pollutants & CAS Number & $\begin{array}{l}\text { Emission } \\
\text { Threshold } \\
\text { (tons/yr) }\end{array}$ & $\begin{array}{c}\text { Actual } \\
\text { Emissions } \\
\text { (tons/yr) }\end{array}$ \\
\hline 200W P $241 U 104$ & $\begin{array}{l}\text { Phosphoric acid, tributyl } \\
\text { ester }\end{array}$ & $126-73-8$ & & $7.95 \mathrm{E}-05$ \\
\hline 200W P $241 \mathrm{U} 104$ & Toluene & $108-88-3$ & 5.00E-01 & $3.08 \mathrm{E}-06$ \\
\hline 200W P $241 \mathrm{U} 105$ & 1,3-Butadiene & $106-99-0$ & $3.50 \mathrm{E}-02$ & $4.13 E-06$ \\
\hline 200W P $241 \mathrm{U} 105$ & 2-Hexanone & $591-78-6$ & $5.00 \mathrm{E}-01$ & $7.51 \mathrm{E}-05$ \\
\hline 200W P 241U105 & 2-Pentanone & $107-87-9$ & & $1.19 \mathrm{E}-04$ \\
\hline 200W P 241U105 & Acetone & $67-64-1$ & $5.00 \mathrm{E}-01$ & $1.43 \mathrm{E}-03$ \\
\hline 200W P 241U105 & Acetonitrile & $75-5-8$ & $5.00 \mathrm{E}-01$ & $6.89 \mathrm{E}-04$ \\
\hline 200W P 241U105 & Ammonia & $7664-41-7$ & $5.00 \mathrm{E}-01$ & $6.57 \mathrm{E}-03$ \\
\hline 200W P $241 \mathrm{U105}$ & Benzene & $71-43-2$ & $5.00 \mathrm{E}-01$ & $3.28 \mathrm{E}-05$ \\
\hline $200 W$ P $241 U 105$ & Carbon monoxide & $630-8-0$ & $5.00 \mathrm{E}+00$ & $9.50 \mathrm{E}-04$ \\
\hline 200W P $241 \mathrm{U} 105$ & Heptane & $142-82-5$ & $5.00 \mathrm{E}-01$ & $8.40 \mathrm{E}-05$ \\
\hline 200W P $241 \mathrm{U} 105$ & Hexane, n-hexane & $110-54-3$ & $5.00 \mathrm{E}-01$ & $8.76 \mathrm{E}-05$ \\
\hline 200W P $241 \mathrm{U} 105$ & $\begin{array}{l}\text { Methyl N-amyl ketone, 2- } \\
\text { heptanone }\end{array}$ & $110-43-0$ & $5.00 \mathrm{E}-01$ & $8.13 E-05$ \\
\hline $200 W \mathrm{P} \quad 241 \mathrm{U105}$ & Nitrogen Oxide $\{N O\}$ & $10102-43-9$ & $2.00 \mathrm{E}+00$ & $5.72 \mathrm{E}-05$ \\
\hline 200W P $241 \mathrm{U} 105$ & Nonane & $111-84-2$ & $5.00 \mathrm{E}-01$ & $4.57 \mathrm{E}-05$ \\
\hline 200W P $241 \mathrm{U} 105$ & Octane & $111-65-9$ & $5.00 \mathrm{E}-01$ & $4.79 E-05$ \\
\hline 200W P 241U105 & $\begin{array}{l}\text { Phosphoric acid, tributyl } \\
\text { ester }\end{array}$ & $126-73-8$ & & $1.72 \mathrm{E}-04$ \\
\hline 200W P $241 \mathrm{U} 105$ & Toluene & $108-88-3$ & $5.00 \mathrm{E}-01$ & $6.67 \mathrm{E}-06$ \\
\hline $200 W$ P $241 \mathrm{U} 106$ & 1,3-Butadiene & $106-99-0$ & $3.50 \mathrm{E}-02$ & $4.13 E-06$ \\
\hline $200 W P \quad 241 U 106$ & 2-Hexanone & $591-78-6$ & $5.00 \mathrm{E}-01$ & $7.51 \mathrm{E}-05$ \\
\hline $200 W$ P $241 \mathrm{U} 106$ & 2-Pentanone & $107-87-9$ & & $1.19 \mathrm{E}-04$ \\
\hline $200 W$ P $241 \mathrm{U} 106$ & Acetone & $67-64-1$ & $5.00 \mathrm{E}-01$ & $1.43 E-03$ \\
\hline $200 W$ P $241 U 106$ & Acetonitrile & $75-5-8$ & $5.00 \mathrm{E}-01$ & $6.89 \mathrm{E}-04$ \\
\hline $200 W \mathrm{P} \quad 241 \mathrm{U} 106$ & Ammonia & $7664-41-7$ & $5.00 \mathrm{E}-01$ & $6.57 \mathrm{E}-03$ \\
\hline $200 W$ P $241 U 106$ & Benzene & $71-43-2$ & $5.00 \mathrm{E}-01$ & $3.28 \mathrm{E}-05$ \\
\hline 200W P 241U106 & Carbon monoxide & $630-8-0$ & $5.00 \mathrm{E}+00$ & $9.50 \mathrm{E}-04$ \\
\hline 200W P $241 \mathrm{U} 106$ & Heptane & $142-82-5$ & $5.00 \mathrm{E}-01$ & $8.40 \mathrm{E}-05$ \\
\hline
\end{tabular}


Table K.2. Insignificant Emission Units Based on Threshold Comparisons [WAC 173-401-530(1)(a)]

\begin{tabular}{|c|c|c|c|c|}
\hline Emission Point & Pollutants & CAS Number & $\begin{array}{l}\text { Emission } \\
\text { Threshold } \\
\text { (tons/yr) }\end{array}$ & $\begin{array}{c}\text { Actual } \\
\text { Emissions } \\
\text { (tons/yr) }\end{array}$ \\
\hline $200 \mathrm{~W} P \quad 241 \mathrm{U} 106$ & Hexane, n-hexane & $110-54-3$ & $5.00 \mathrm{E}-01$ & $8.76 \mathrm{E}-05$ \\
\hline 200W P 241U106 & $\begin{array}{l}\text { Methyl N-amyl ketone, 2- } \\
\text { heptanone }\end{array}$ & $110-43-0$ & $5.00 \mathrm{E}-01$ & $8.13 E-05$ \\
\hline $200 \mathrm{~W} P \quad 241 \mathrm{U} 106$ & Nitrogen Oxide $\{\mathrm{NO}\}$ & $10102-43-9$ & $2.00 \mathrm{E}+00$ & $5.72 \mathrm{E}-05$ \\
\hline $200 W$ P $241 U 106$ & Nonane & $111-84-2$ & $5.00 \mathrm{E}-01$ & $4.57 \mathrm{E}-05$ \\
\hline $200 W$ P 241 U106 & Octane & $111-65-9$ & $5.00 \mathrm{E}-01$ & $4.79 \mathrm{E}-05$ \\
\hline $200 \mathrm{~W} P \quad 241 \mathrm{U} 106$ & $\begin{array}{l}\text { Phosphoric acid, tributyl } \\
\text { ester }\end{array}$ & $126-73-8$ & & $1.72 \mathrm{E}-04$ \\
\hline 200W P 241U106 & Toluene & $108-88-3$ & $5.00 \mathrm{E}-01$ & $6.67 \mathrm{E}-06$ \\
\hline 200W P $241 \mathrm{U} 107$ & 1,3-Butadiene & $106-99-0$ & $3.50 \mathrm{E}-02$ & $4.13 \mathrm{E}-06$ \\
\hline 200W P $241 U 107$ & 2-Hexanone & $591-78-6$ & $5.00 \mathrm{E}-01$ & $7.51 \mathrm{E}-05$ \\
\hline 200W P $241 \mathrm{U} 107$ & 2-Pentanone & $107-87-9$ & & $1.19 \mathrm{E}-04$ \\
\hline 200W P $241 \mathrm{U} 107$ & Acetone & $67-64-1$ & $5.00 \mathrm{E}-01$ & $1.43 E-03$ \\
\hline $\begin{array}{ll}200 W \\
P & 241 U 107\end{array}$ & Acetonitrile & $75-5-8$ & $5.00 \mathrm{E}-01$ & $6.89 \mathrm{E}-04$ \\
\hline 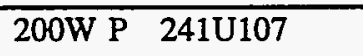 & Ammonia & $7664-41-7$ & $5.00 \mathrm{E}-01$ & $6.57 \mathrm{E}-03$ \\
\hline $200 W$ P $241 U 107$ & Benzene & $71-43-2$ & $5.00 \mathrm{E}-01$ & $3.28 \mathrm{E}-05$ \\
\hline $200 W$ P 241 U107 & Carbon monoxide & $630-8-0$ & $5.00 \mathrm{E}+00$ & $9.50 \mathrm{E}-04$ \\
\hline 200W P $241 U 107$ & Heptane & $142-82-5$ & $5.00 \mathrm{E}-01$ & $8.40 \mathrm{E}-05$ \\
\hline $200 W$ P $241 U 107$ & Hexane, n-hexane & $110-54-3$ & $5.00 \mathrm{E}-01$ & $8.76 \mathrm{E}-05$ \\
\hline 200W P $241 \mathrm{U} 107$ & $\begin{array}{l}\text { Methyl N-amyl ketone, 2- } \\
\text { heptanone }\end{array}$ & $110-43-0$ & $5.00 \mathrm{E}-01$ & $8.13 E-05$ \\
\hline 200W P $241 \mathrm{U} 107$ & Nitrogen Oxide $\{\mathrm{NO}\}$ & $10102-43-9$ & $2.00 \mathrm{E}+00$ & 5.72E-05 \\
\hline 200W P 241U107 & Nonane & $111-84-2$ & $5.00 \mathrm{E}-01$ & $4.57 \mathrm{E}-05$ \\
\hline 200W P $241 \mathrm{U} 107$ & Octane & $111-65-9$ & $5.00 \mathrm{E}-01$ & $4.79 E-05$ \\
\hline 200W P 241U107 & $\begin{array}{l}\text { Phosphoric acid, tributyl } \\
\text { ester }\end{array}$ & $126-73-8$ & & $1.72 \mathrm{E}-04$ \\
\hline 200W P $241 \mathrm{U} 108$ & 1,3-Butadiene & $106-99-0$ & $3.50 \mathrm{E}-02$ & $4.13 \mathrm{E}-06$ \\
\hline 200W P 241 U108 & 2-Hexanone & $591-78-6$ & $5.00 \mathrm{E}-01$ & $7.51 \mathrm{E}-05$ \\
\hline 200W P $241 U 108$ & 2-Pentanone & $107-87-9$ & & $1.19 \mathrm{E}-04$ \\
\hline 200W P $241 \mathrm{U} 108$ & Acetone & $67-64-1$ & $5.00 \mathrm{E}-01$ & $1.43 \mathrm{E}-03$ \\
\hline
\end{tabular}


Table K.2. Insignificant Emission Units.Based on Threshold Comparisons [WAC 173-401-530(1)(a)]

\begin{tabular}{|c|c|c|c|c|}
\hline Emission Point & Pollutants & CAS Number & $\begin{array}{l}\text { Emission } \\
\text { Threshold } \\
\text { (tons/yr) }\end{array}$ & $\begin{array}{c}\text { Actual } \\
\text { Emissions } \\
\text { (tons/yr) }\end{array}$ \\
\hline 200W P 241 U108 & Acetonitrile & $75-5-8$ & $5.00 \mathrm{E}-01$ & $6.89 \mathrm{E}-04$ \\
\hline 200W P 241U108 & Ammonia & $7664-41-7$ & $5.00 \mathrm{E}-01$ & $6.57 \mathrm{E}-03$ \\
\hline 200W P 241 U108 & Benzene & $71-43-2$ & $5.00 \mathrm{E}-01$ & $3.28 \mathrm{E}-05$ \\
\hline 200W P 241U108 & Carbon monoxide & $630-8-0$ & $5.00 \mathrm{E}+00$ & $9.50 \mathrm{E}-04$ \\
\hline 200W P 241U108 & Heptane & $142-82-5$ & $5.00 \mathrm{E}-01$ & $8.40 \mathrm{E}-05$ \\
\hline 200W P $241 U 108$ & Hexane, n-hexane & $110-54-3$ & $5.00 \mathrm{E}-01$ & $8.76 \mathrm{E}-05$ \\
\hline 200W P $241 \mathrm{U} 108$ & $\begin{array}{l}\text { Methyl N-amyl ketone, 2- } \\
\text { heptanone }\end{array}$ & $110-43-0$ & $5.00 \mathrm{E}-01$ & $8.13 \mathrm{E}-05$ \\
\hline 200W P $241 \mathrm{U} 108$ & Nitrogen Oxide $\{\mathrm{NO}\}$ & $10102-43-9$ & $2.00 \mathrm{E}+00$ & $5.72 \mathrm{E}-05$ \\
\hline 200W P $241 \mathrm{U} 108$ & Nonane & $111-84-2$ & $5.00 \mathrm{E}-01$ & $4.57 \mathrm{E}-05$ \\
\hline 200W P $241 \mathrm{U} 108$ & Octane & $111-65-9$ & $5.00 \mathrm{E}-01$ & $4.79 \mathrm{E}-05$ \\
\hline 200W P 241 U108 & $\begin{array}{l}\text { Phosphoric acid, tributyl } \\
\text { ester }\end{array}$ & $126-73-8$ & & $1.72 \mathrm{E}-04$ \\
\hline 200W P 241U108 & Toluene & $108-88-3$ & $5.00 \mathrm{E}-01$ & $6.67 E-06$ \\
\hline 200W P $241 U 109$ & 1,3-Butadiene & $106-99-0$ & $3.50 \mathrm{E}-02$ & 4.13E-06 \\
\hline 200W P $241 \mathrm{U} 109$ & 2-Hexanone- & $591-78-6$ & $5.00 \mathrm{E}-01$ & $7.51 \mathrm{E}-05$ \\
\hline 200W P $241 U 109$ & 2-Pentanone & $107-87-9$ & & $1.19 \mathrm{E}-04$ \\
\hline 200W P $241 \mathrm{U} 109$ & Acetone & $67-64-1$ & $5.00 \mathrm{E}-01$ & $1.43 \mathrm{E}-03$ \\
\hline 200W P $241 \mathrm{U} 109$ & Acetonitrile & $75-5-8$ & $5.00 \mathrm{E}-01$ & $6.89 \mathrm{E}-04$ \\
\hline $\begin{array}{ll}200 W & P \\
241 U 109\end{array}$ & Ammonia & $7664-41-7$ & $5.00 \mathrm{E}-01$ & $6.57 \mathrm{E}-03$ \\
\hline 200W P 241U109 & Benzene & $71-43-2$ & $5.00 \mathrm{E}-01$ & $3.28 \mathrm{E}-05$ \\
\hline 200W P $241 U 109$ & Carbon monoxide & $630-8-0$ & $5.00 \mathrm{E}+00$ & $9.50 \mathrm{E}-04$ \\
\hline 200W P $241 \mathrm{U} 109$ & Heptane & $142-82-5$ & $5.00 \mathrm{E}-01$ & $8.40 \mathrm{E}-05$ \\
\hline $200 W$ P 2410109 & Hexane, n-hexane & $110-54-3$ & $5.00 \mathrm{E}-01$ & $8.76 \mathrm{E}-05$ \\
\hline $200 W$ P $241 U 109$ & $\begin{array}{l}\text { Methyl N-amyl ketone, 2- } \\
\text { heptanone }\end{array}$ & $110-43-0$ & $5.00 \mathrm{E}-01$ & $8.13 \mathrm{E}-05$ \\
\hline $200 W$ P $241 \mathrm{U} 109$ & Nitrogen Oxide $\{\mathrm{NO}\}$ & $10102-43-9$ & $2.00 \mathrm{E}+00$ & $5.72 \mathrm{E}-05$ \\
\hline 200W P $241 \mathrm{U} 109$ & Nonane & $111-84-2$ & $5.00 \mathrm{E}-01$ & $4.57 \mathrm{E}-05$ \\
\hline 200W P $241 U 109$ & Octane & $111-65-9$ & $5.00 \mathrm{E}-01$ & 4.79E-05 \\
\hline 200W P $241 U 109$ & $\begin{array}{l}\text { Phosphoric acid, tributyl } \\
\text { ester }\end{array}$ & $126-73-8$ & & $1.72 \mathrm{E}-04$ \\
\hline
\end{tabular}




\section{Table K.2. Insignificant Emission Units Based on Threshold Comparisons} [WAC 173-401-530(1)(a)]

\begin{tabular}{|c|c|c|c|c|}
\hline Emission Point & Pollutants & CAS Number & $\begin{array}{c}\text { Emission } \\
\text { Threshold } \\
\text { (tons/yr) }\end{array}$ & $\begin{array}{c}\text { Actual } \\
\text { Emissions } \\
\text { (tons/yr) }\end{array}$ \\
\hline 200W P $241 \mathrm{U} 109$ & Toluene & $108-88-3$ & $5.00 \mathrm{E}-01$ & $6.67 \mathrm{E}-06$ \\
\hline 200W P $241 \mathrm{U} 110$ & 1,3-Butadiene & $106-99-0$ & $3.50 \mathrm{E}-02$ & $4.13 \mathrm{E}-06$ \\
\hline $200 W$ P $241 U 110$ & 2-Hexanone & $591-78-6$ & $5.00 \mathrm{E}-01$ & $7.51 \mathrm{E}-05$ \\
\hline 200W P $241 \mathrm{U} 110$ & 2-Pentanone & $107-87-9$ & & $1.19 \mathrm{E}-04$ \\
\hline 200W P $241 \mathrm{U} 110$ & Acetone & $67-64-1$ & $5.00 \mathrm{E}-01$ & $1.43 \mathrm{E}-03$ \\
\hline $200 W$ P $241 U 110$ & Acetonitrile & $75-5-8$ & $5.00 \mathrm{E}-01$ & $6.89 \mathrm{E}-04$ \\
\hline 200W P $241 \mathrm{U} 110$ & Ammonia & $7664-41-7$ & $5.00 \mathrm{E}-01$ & $6.57 \mathrm{E}-03$ \\
\hline $200 W$ P $241 \mathrm{U} 110$ & Benzene & $71-43-2$ & $5.00 \mathrm{E}-01$ & $3.28 \mathrm{E}-05$ \\
\hline $200 W$ P $241 U 110$ & Carbon monoxide & $630-8-0$ & $5.00 \mathrm{E}+00$ & 9.50E-04 \\
\hline $200 W$ P $241 U 110$ & Heptane & $142-82-5$ & $5.00 \mathrm{E}-01$ & $8.40 \mathrm{E}-05$ \\
\hline 200W P $241 \mathrm{U} 110$ & Hexane, n-hexane & $110-54-3$ & $5.00 \mathrm{E}-01$ & $8.76 \mathrm{E}-05$ \\
\hline $200 W$ P $241 \mathrm{U} 110$ & $\begin{array}{l}\text { Methyl N-amyl ketone, 2- } \\
\text { heptanone }\end{array}$ & $110-43-0$ & $5.00 \mathrm{E}-01$ & $8.13 E-05$ \\
\hline $200 \mathrm{~W} P \quad 241 \mathrm{U} 110$ & Nitrogen Oxide $\{\mathrm{NO}\}$ & $10102-43-9$ & $2.00 E+00$ & $5.72 \mathrm{E}-05$ \\
\hline 200W P $241 U 110$ & Nonane & $111-84-2$ & $5.00 \mathrm{E}-01$ & 4.57E-05 \\
\hline 200W P $241 U 110$ & Octane & $111-65-9$ & $5.00 \mathrm{E}-01$ & 4.79E-05 \\
\hline 200W P $241 \mathrm{U} 110$ & $\begin{array}{l}\text { Phosphoric acid, tributyl } \\
\text { ester }\end{array}$ & $126-73-8$ & & $1.72 \mathrm{E}-04$ \\
\hline $200 W$ P $241 \mathrm{U} 110$ & Toluene & $108-88-3$ & $5.00 \mathrm{E}-01$ & $6.67 \mathrm{E}-06$ \\
\hline $200 W$ P $241 \mathrm{U} 111$ & 1,3-Butadiene & $106-99-0$ & $3.50 \mathrm{E}-02$ & $4.13 E-06$ \\
\hline 200W P 241U111 & 2-Hexanone & $591-78-6$ & $5.00 \mathrm{E}-01$ & $7.51 \mathrm{E}-05$ \\
\hline 200W P $241 \mathrm{U} 111$ & 2-Pentanone & $107-87-9$ & & $1.19 \mathrm{E}-04$ \\
\hline 200W P $241 \mathrm{U} 111$ & Acetone & $67-64-1$ & $5.00 \mathrm{E}-01$ & $1.43 \mathrm{E}-03$ \\
\hline $200 W$ P $241 \mathrm{U} 111$ & Acetonitrile & $75-5-8$ & $5.00 \mathrm{E}-01$ & $6.89 \mathrm{E}-04$ \\
\hline 200W P 241U111 & Ammonia & $7664-41-7$ & $5.00 \mathrm{E}-01$ & $6.57 \mathrm{E}-03$ \\
\hline 200W P $241 \mathrm{U} 111$ & Benzene & $71-43-2$ & $5.00 \mathrm{E}-01$ & $3.28 E-05$ \\
\hline 200W P $241 \mathrm{U} 111$ & Carbon monoxide & $630-8-0$ & $5.00 \mathrm{E}+00$ & $9.50 \mathrm{E}-04$ \\
\hline 200W P $241 \mathrm{U} 111$ & Heptane & $142-82-5$ & $5.00 \mathrm{E}-01$ & 8.40E-05 \\
\hline 200W P $241 \mathrm{U} 111$ & Hexane, n-hexane & $110-54-3$ & $5.00 \mathrm{E}-01$ & $8.76 \mathrm{E}-05$ \\
\hline
\end{tabular}


Table K.2. Insignificant Emission Units Based on Threshold Comparisons [WAC 173-401-530(1)(a)]

\begin{tabular}{|c|c|c|c|c|}
\hline Emission Point & Pollutants & CAS Number & $\begin{array}{l}\text { Emission } \\
\text { Threshold } \\
\text { (tons/yr) }\end{array}$ & $\begin{array}{c}\text { Actual } \\
\text { Emissions } \\
\text { (tons/yr) }\end{array}$ \\
\hline $200 W$ P $241 U 111$ & $\begin{array}{l}\text { Methyl N-amyl ketone, 2- } \\
\text { heptanone }\end{array}$ & $110-43-0$ & $5.00 \mathrm{E}-01$ & $8.13 \mathrm{E}-05$ \\
\hline 200W P 241U111 & Nitrogen Oxide $\{\mathrm{NO}\}$ & $10102-43-9$ & $2.00 \mathrm{E}+00$ & $5.72 \mathrm{E}-05$ \\
\hline 200W P $241 \mathrm{U} 111$ & Nonane & $111-84-2$ & $5.00 \mathrm{E}-01$ & $4.57 \mathrm{E}-05$ \\
\hline $200 \mathrm{~W} P \quad 241 \mathrm{U} 111$ & Octane & $111-65-9$ & $5.00 \mathrm{E}-01$ & $4.79 \mathrm{E}-05$ \\
\hline 200W P 241U111 & $\begin{array}{l}\text { Phosphoric acid, tributyl } \\
\text { ester }\end{array}$ & $126-73-8$ & & $1.72 \mathrm{E}-04$ \\
\hline 200W P $241 U 111$ & Toluene & $108-88-3$ & $5.00 \mathrm{E}-01$ & 6.67E-06 \\
\hline $\begin{array}{ll}200 W \text { P } & 241 U 112\end{array}$ & 1,3-Butadiene & $106-99-0$ & $3.50 \mathrm{E}-02$ & $1.91 \mathrm{E}-06$ \\
\hline $200 W$ P $241 \mathrm{U} 112$ & 2-Hexanone & $591-78-6$ & $5.00 \mathrm{E}-01$ & $3.47 \mathrm{E}-05$ \\
\hline 200W P $241 \mathrm{U} 112$ & 2-Pentanone & $107-87-9$ & & $5.51 \mathrm{E}-05$ \\
\hline $200 \mathrm{~W} P \quad 241 \mathrm{U} 112$ & Acetone & $67-64-1$ & $5.00 \mathrm{E}-01$ & $6.62 \mathrm{E}-04$ \\
\hline 200W P 241U112 & Acetonitrile & $75-5-8$ & $5.00 \mathrm{E}-01$ & $3.19 \mathrm{E}-04$ \\
\hline 200W P 241U112 & Ammonia & $7664-41-7$ & $5.00 \mathrm{E}-01$ & $3.04 \mathrm{E}-03$ \\
\hline 200W P $241 \mathrm{U} 112$ & Benzene & $71-43-2$ & $5.00 \mathrm{E}-01$ & $1.51 \mathrm{E}-05$ \\
\hline 200W P 241U112 & Carbon monoxide & $630-8-0$ & $5.00 \mathrm{E}+00$ & $4.39 \mathrm{E}-04$ \\
\hline 200W P 241U112 & Heptane & $142-82-5$ & $5.00 \mathrm{E}-01$ & $3.89 \mathrm{E}-05$ \\
\hline $200 W$ P $241 \mathrm{U} 112$ & Hexane, n-hexane & $110-54-3$ & $5.00 \mathrm{E}-01$ & $4.05 E-05$ \\
\hline $200 \mathrm{~W} P \quad 241 \mathrm{U} 112$ & $\begin{array}{l}\text { Methyl N-amyl ketone, 2- } \\
\text { heptanone }\end{array}$ & $110-43-0$ & $5.00 \mathrm{E}-01$ & $3.76 \mathrm{E}-05$ \\
\hline 200W P $241 \mathrm{U} 112$ & Nitrogen Oxide $\{\mathrm{NO}\}$ & $10102-43-9$ & $2.00 \mathrm{E}+00$ & $2.64 \mathrm{E}-05$ \\
\hline $200 W$ P $\quad 241 U 112$ & Nonane & $111-84-2$ & $5.00 \mathrm{E}-01$ & $2.11 E-05$ \\
\hline $200 \mathrm{~W} P \quad 241 \mathrm{U} 112$ & Octane & $111-65-9$ & $5.00 \mathrm{E}-01$ & $2.21 \mathrm{E}-05$ \\
\hline $200 W$ P $241 U 112$ & $\begin{array}{l}\text { Phosphoric acid, tributyl } \\
\text { ester }\end{array}$ & $126-73-8$ & & $7.95 \mathrm{E}-05$ \\
\hline $200 W$ P $241 U 112$ & Toluene & $108-88-3$ & $5.00 \mathrm{E}-01$ & $3.08 \mathrm{E}-06$ \\
\hline 200W P $241 U 201$ & 1,3-Butadiene & $106-99-0$ & $3.50 \mathrm{E}-02$ & $1.91 \mathrm{E}-06$ \\
\hline 200W P $241 U 201$ & 2-Hexanone & $591-78-6$ & $5.00 \mathrm{E}-01$ & $3.47 \mathrm{E}-05$ \\
\hline $200 \mathrm{~W} P \quad$ 241U201 & 2-Pentanone & $107-87-9$ & & $5.51 \mathrm{E}-05$ \\
\hline 200W P 241U201 & Acetone & $67-64-1$ & $5.00 \mathrm{E}-01$ & $6.62 E-04$ \\
\hline
\end{tabular}


Table K.2. Insignificant Emission Units Based on Threshold Comparisons [WAC 173-401-530(1)(a)]

\begin{tabular}{|c|c|c|c|c|}
\hline Emission Point & Pollutants & CAS Number & $\begin{array}{l}\text { Emission } \\
\text { Threshold } \\
\text { (tons/yr) }\end{array}$ & $\begin{array}{c}\text { Actual } \\
\text { Emissions } \\
\text { (tons/yr) }\end{array}$ \\
\hline 200W P 241U201 & $\overline{\text { Acetonitrile }}$ & $75-5-8$ & $5.00 \mathrm{E}-01$ & $3.19 \mathrm{E}-04$ \\
\hline $200 W$ P $241 U 201$ & Ammonia & $7664-41-7$ & $5.00 \mathrm{E}-01$ & $3.04 \mathrm{E}-03$ \\
\hline $200 W$ P $\quad 241 U 201$ & Benzene & $71-43-2$ & $5.00 \mathrm{E}-01$ & $1.51 \mathrm{E}-05$ \\
\hline 200W P 241U201 & Carbon monoxide & $630-8-0$ & $5.00 \mathrm{E}+00$ & $4.39 \mathrm{E}-04$ \\
\hline 200W P $241 U 201$ & Heptane & $142-82-5$ & $5.00 \mathrm{E}-01$ & $3.89 \mathrm{E}-05$ \\
\hline $200 W$ P $\quad 241 U 201$ & Hexane, n-hexane & $110-54-3$ & $5.00 \mathrm{E}-01$ & $4.05 \mathrm{E}-05$ \\
\hline $200 W$ P $241 \mathrm{U} 201$ & $\begin{array}{l}\text { Methyl N-amyl ketone, 2- } \\
\text { heptanone }\end{array}$ & $110-43-0$ & $5.00 \mathrm{E}-01$ & $3.76 \mathrm{E}-05$ \\
\hline 200W P 241U201 & Nitrogen Oxide $\{\mathrm{NO}\}$ & $10102-43-9$ & $2.00 \mathrm{E}+00$ & $2.64 \mathrm{E}-05$ \\
\hline 200W P $241 U 201$ & Nonane & $111-84-2$ & $5.00 \mathrm{E}-01$ & $2.11 E-05$ \\
\hline 200W P $241 U 201$ & Octane & $111-65-9$ & $5.00 \mathrm{E}-01$ & $2.21 \mathrm{E}-05$ \\
\hline $200 W$ P 241 U201 & $\begin{array}{l}\text { Phosphoric acid, tributyl } \\
\text { ester }\end{array}$ & $126-73-8$ & & $7.95 \mathrm{E}-05$ \\
\hline 200W P 241 U201 & Toluene & $108-88-3$ & $5.00 \mathrm{E}-01$ & $3.08 \mathrm{E}-06$ \\
\hline 200W P 241 U202 & 1,3-Butadiene & $106-99-0$ & $3.50 \mathrm{E}-02$ & $1.91 \mathrm{E}-06$ \\
\hline 200W P 241 U202 & 2-Hexanone & $591-78-6$ & $5.00 \mathrm{E}-01$ & $3.47 \mathrm{E}-05$ \\
\hline $200 W$ P $241 U 202$ & 2-Pentanone & $107-87-9$ & & $5.51 \mathrm{E}-05$ \\
\hline 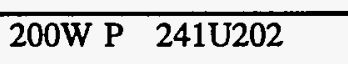 & Acetone & $67-64-1$ & $5.00 \mathrm{E}-01$ & $6.62 \mathrm{E}-04$ \\
\hline 200W P 241 U202 & Acetonitrile & $75-5-8$ & $5.00 \mathrm{E}-01$ & $3.19 \mathrm{E}-04$ \\
\hline $\begin{array}{ll}200 W & P \\
241 U 202\end{array}$ & Ammonia & $7664-41-7$ & $5.00 \mathrm{E}-01$ & $3.04 \mathrm{E}-03$ \\
\hline $\begin{array}{ll}200 W & P \\
241 U 202\end{array}$ & Benzene & $71-43-2$ & $5.00 \mathrm{E}-01$ & $1.51 \mathrm{E}-05$ \\
\hline 200W P $241 \mathrm{U} 202$ & Carbon monoxide & $630-8-0$ & $5.00 \mathrm{E}+00$ & $4.39 \mathrm{E}-04$ \\
\hline $200 W$ P 241 U202 & Heptane & $142-82-5$ & $5.00 \mathrm{E}-01$ & $3.89 \mathrm{E}-05$ \\
\hline 200W P 241U202 & Hexane, n-hexane & $110-54-3$ & $5.00 \mathrm{E}-01$ & $4.05 \mathrm{E}-05$ \\
\hline $200 W$ P $241 U 202$ & $\begin{array}{l}\text { Methyl N-amyl ketone, 2- } \\
\text { heptanone }\end{array}$ & $110-43-0$ & $5.00 \mathrm{E}-01$ & $3.76 \mathrm{E}-05$ \\
\hline $200 W$ P $241 U 202$ & Nitrogen Oxide $\{\mathrm{NO}\}$ & $10102-43-9$ & $2.00 \mathrm{E}+00$ & $2.64 \mathrm{E}-05$ \\
\hline $200 \mathrm{~W} P \quad 241 \mathrm{U} 202$ & Nonane & $111-84-2$ & $5.00 \mathrm{E}-01$ & $2.11 \mathrm{E}-05$ \\
\hline $200 \mathrm{~W} P \quad 241 \mathrm{U} 202$ & Octane & $111-65-9$ & $5.00 \mathrm{E}-01$ & $2.21 \mathrm{E}-05$ \\
\hline $200 W$ P $241 U 202$ & $\begin{array}{l}\text { Phosphoric acid, tributyl } \\
\text { ester }\end{array}$ & $126-73-8$ & & $7.95 \mathrm{E}-05$ \\
\hline
\end{tabular}


Table K.2. Insignificant Emission Units Based on Threshold Comparisons [WAC 173-401-530(1)(a)]

\begin{tabular}{|c|c|c|c|c|}
\hline Emission Point & Pollutants & CAS Number & $\begin{array}{l}\text { Emission } \\
\text { Threshold } \\
\text { (tons/yr) }\end{array}$ & $\begin{array}{c}\text { Actual } \\
\text { Emissions } \\
\text { (tons/yr) }\end{array}$ \\
\hline 200W P 241U202 & Toluene & $108-88-3$ & $5.00 \mathrm{E}-01$ & $3.08 \mathrm{E}-06$ \\
\hline 200W P 241U203 & 1,3-Butadiene & $106-99-0$ & $3.50 \mathrm{E}-02$ & $1.91 \mathrm{E}-06$ \\
\hline 200W P 241U203 & 2-Hexanone & $591-78-6$ & $5.00 \mathrm{E}-01$ & $3.47 \mathrm{E}-05$ \\
\hline 200W P 241U203 & 2-Pentanone & $107-87-9$ & & $5.51 \mathrm{E}-05$ \\
\hline 200W P 241U203 & Acetone & $67-64-1$ & $5.00 \mathrm{E}-01$ & $6.62 \mathrm{E}-04$ \\
\hline $200 \mathrm{~W} P \quad 241 \mathrm{U} 203$ & Acetonitrile & $75-5-8$ & $5.00 \mathrm{E}-01$ & $3.19 \mathrm{E}-04$ \\
\hline 200W P 241U203 & Ammonia & $7664-41-7$ & $5.00 \mathrm{E}-01$ & $3.04 \mathrm{E}-03$ \\
\hline 200W P 241U203 & Benzene & $71-43-2$ & $5.00 \mathrm{E}-01$ & $1.51 \mathrm{E}-05$ \\
\hline 200W P 241U203 & Carbon monoxide & $630-8-0$ & $5.00 \mathrm{E}+00$ & $4.39 \mathrm{E}-04$ \\
\hline $200 W$ P $241 U 203$ & Heptane & $142-82-5$ & $5.00 \mathrm{E}-01$ & $3.89 \mathrm{E}-05$ \\
\hline 200W P 241U203 & Hexane, n-hexane & $110-54-3$ & $5.00 \mathrm{E}-01$ & 4.05E-05 \\
\hline $200 \mathrm{~W} P \quad 241 \mathrm{U} 203$ & $\begin{array}{l}\text { Methyl N-amyl ketone, 2- } \\
\text { heptanone }\end{array}$ & $110-43-0$ & $5.00 \mathrm{E}-01$ & $3.76 \mathrm{E}-05$ \\
\hline $200 \mathrm{~W} P \quad 241 \mathrm{U} 203$ & Nitrogen Oxide $\{\mathrm{NO}\}$ & $10102-43-9$ & $2.00 \mathrm{E}+00$ & $2.64 \mathrm{E}-05$ \\
\hline 200W P 241U203 & Nonane & $111-84-2$ & $5.00 \mathrm{E}-01$ & $2.11 \mathrm{E}-05$ \\
\hline $200 W$ P $241 U 203$ & Octane & $111-65-9$ & $5.00 \mathrm{E}-01$ & $2.21 \mathrm{E}-05$ \\
\hline $200 \mathrm{~W} P \quad 241 \mathrm{U} 203$ & $\begin{array}{l}\text { Phosphoric acid, tributyl } \\
\text { ester }\end{array}$ & $126-73-8$ & & 7.95E-05 \\
\hline $200 W$ P $241 U 203$ & Toluene & $108-88-3$ & $5.00 \mathrm{E}-01$ & $3.08 \mathrm{E}-06$ \\
\hline 200W P 241U204 & 1,3-Butadiene & $106-99-0$ & $3.50 \mathrm{E}-02$ & $1.91 \mathrm{E}-06$ \\
\hline 200W P 241U204 & 2-Hexanone & $591-78-6$ & $5.00 \mathrm{E}-01$ & $3.47 \mathrm{E}-05$ \\
\hline 200W P 241U204 & 2-Pentanone & $107-87-9$ & & $5.51 \mathrm{E}-05$ \\
\hline 200W P 241U204 & Acetone & $67-64-1$ & $5.00 \mathrm{E}-01$ & $6.62 E-04$ \\
\hline 200W P 241U204 & Acetonitrile & $75-5-8$ & $5.00 \mathrm{E}-01$ & $3.19 E-04$ \\
\hline 200W P 241U204 & Ammonia & $7664-41-7$ & $5.00 \mathrm{E}-01$ & $3.04 \mathrm{E}-03$ \\
\hline 200W P 241U204 & Benzene & $71-43-2$ & $5.00 \mathrm{E}-01$ & $1.51 \mathrm{E}-05$ \\
\hline $200 W$ P $241 U 204$ & Carbon monoxide & $630-8-0$ & $5.00 \mathrm{E}+00$ & 4.39E-04 \\
\hline 200W P $241 U 204$ & Heptane & $142-82-5$ & $5.00 \mathrm{E}-01$ & $3.89 \mathrm{E}-05$ \\
\hline 200W P 241U204 & Hexane, n-hexane & $110-54-3$ & $5.00 \mathrm{E}-01$ & $4.05 \mathrm{E}-05$ \\
\hline
\end{tabular}


Table K.2. Insignificant Emission Units Based on Threshold Comparisons [WAC 173-401-530(1)(a)]

\begin{tabular}{|c|c|c|c|c|}
\hline Emission Point & Pollutants & CAS Number & $\begin{array}{c}\text { Emission } \\
\text { Threshold } \\
\text { (tons/yr) }\end{array}$ & $\begin{array}{c}\text { Actual } \\
\text { Emissions } \\
\text { (tons/yr) }\end{array}$ \\
\hline 200W P $241 U 204$ & $\begin{array}{l}\text { Methyl N-amyl ketone, 2- } \\
\text { heptanone }\end{array}$ & $110-43-0$ & $5.00 \mathrm{E}-01$ & $3.76 \mathrm{E}-05$ \\
\hline 200W P 241U204 & Nitrogen Oxide $\{\mathrm{NO}\}$ & $10102-43-9$ & $2.00 \mathrm{E}+00$ & $2.64 \mathrm{E}-05$ \\
\hline 200W P 241U204 & Nonane & $111-84-2$ & $5.00 \mathrm{E}-01$ & $2.11 \mathrm{E}-05$ \\
\hline 200W P $241 U 204$ & Octane & $111-65-9$ & $5.00 \mathrm{E}-01$ & $2.21 \mathrm{E}-05$ \\
\hline 200W P 241U204 & $\begin{array}{l}\text { Phosphoric acid, tributyl } \\
\text { ester }\end{array}$ & $126-73-8$ & & $7.95 \mathrm{E}-05$ \\
\hline 200W P 241U204 & Toluene & $108-88-3$ & $5.00 \mathrm{E}-01$ & $3.08 \mathrm{E}-06$ \\
\hline $200 \mathrm{~W}$ P-241S101 & 1,3-Butadiene & $106-99-0$ & $3.50 \mathrm{E}-02$ & $4.13 \mathrm{E}-06$ \\
\hline 200E P-296AW 001 & 1, 3-Butadiene & $106-99-0$ & $3.50 \mathrm{E}-02$ & $2.48 \mathrm{E}-05$ \\
\hline 200E P-296AW 001 & 2-Hexanone & $591-78-6$ & $5.00 \mathrm{E}-01$ & $4.5 \mathrm{E}-04$ \\
\hline 200E P-296AW 001 & 2-Pentanone & $107-87-9$ & $7.16 \mathrm{E}-04$ & \\
\hline 200E P-296AW 001 & Acetone & $67-64-1$ & $8.59 \mathrm{E}-03$ & $5.00 \mathrm{E}-01$ \\
\hline 200E P-296AW 001 & Acetonitrile & $75-5-8$ & $4.14 \mathrm{E}-03$ & $5.00 \mathrm{E}-01$ \\
\hline 200E P-296AW 001 & Ammonia & $7664-41-7$ & $2.82 \mathrm{E}-01$ & $5.00 \mathrm{E}-01$ \\
\hline 200E P-296AW 001 & Benzene & $71-43-2$ & $1.97 \mathrm{E}-04$ & $5.00 \mathrm{E}-01$ \\
\hline 200E P-296AW 001 & Carbon monoxide & $630-8-0$ & $1.03 \mathrm{E}-01$ & $5.00 \mathrm{E}+00$ \\
\hline 200E P-296AW 001 & Heptane & $142-82-5$ & $5.04 \mathrm{E}-04$ & $5.00 \mathrm{E}-01$ \\
\hline 200E P-296AW 001 & Hexane, n-hexane & $110-54-3$ & $5.26 \mathrm{E}-04$ & $5.00 \mathrm{E}-01$ \\
\hline 200E P-296AW 001 & $\begin{array}{l}\text { Methyl N-amyl ketone, 2- } \\
\text { heptanone }\end{array}$ & $110-43-0$ & $4.88 \mathrm{E}-04$ & $5.00 \mathrm{E}-01$ \\
\hline 200E P-296AW 001 & Nitrogen oxide (NO) & $10102-43-9$ & $3.43 \mathrm{E}-04$ & $2.00 E+00$ \\
\hline 200E P-296AW 001 & Nonane & $111-84-2$ & $2.74 \mathrm{E}-04$ & $5.00-01$ \\
\hline 200E P-296AW 001 & Octane & $111-65-9$ & $2.87 \mathrm{E}-04$ & $5.00 \mathrm{E}-01$ \\
\hline 200E P-296AW 001 & $\begin{array}{l}\text { Phosphoric acid, tributyl } \\
\text { ester }\end{array}$ & $126-73-8$ & $1.03 E-03$ & \\
\hline 200E P-296AW 001 & Toluene & $108-88-3$ & $4.00 \mathrm{E}-05$ & $5.00 \mathrm{E}-01$ \\
\hline 200W S-222SA 001 & Acetone & $67-64-1$ & $5.00 \mathrm{E}-01$ & $5.90 \mathrm{E}-03$ \\
\hline 200W S-222SA 001 & Ammonium hydroxide & $1336-21-6$ & & $6.90 \mathrm{E}-02$ \\
\hline
\end{tabular}


Table K.2. Insignificant Emission Units Based on Threshold Comparisons [WAC 173-401-530(1)(a)]

\begin{tabular}{|c|c|c|c|c|}
\hline Emission Point & Pollutants & CAS Number & $\begin{array}{c}\text { Emission } \\
\text { Threshold } \\
\text { (tons/yr) }\end{array}$ & $\begin{array}{c}\text { Actual } \\
\text { Emissions } \\
\text { (tons/yr) }\end{array}$ \\
\hline 200W S-222SA 001 & Bis(2-ethylhexyl)phosphate & $298-7-7$ & & $2.20 \mathrm{E}-03$ \\
\hline 200W S-222SA 001 & $\begin{array}{l}\text { Hydrochloric acid } \\
\text { (Hydrogen chloride) }\end{array}$ & $7647-1-0$ & $5.00 \mathrm{E}-01$ & $2.10 \mathrm{E}-02$ \\
\hline 200W S-222SA 001 & Isopropyl Alcohol & $67-63-0$ & $5.00 \mathrm{E}-01$ & $1.70 \mathrm{E}-03$ \\
\hline 200W S-222SA 001 & Kerosene & - & & $1.40 \mathrm{E}-02$ \\
\hline 200W S-222SA 001 & Methyl alcohol & $67-56-1$ & $5.00 \mathrm{E}-01$ & $6.30 \mathrm{E}-02$ \\
\hline 200W S-222SA 001 & Methyl isobutyl ketone & $108-10-1$ & $5.00 \mathrm{E}-01$ & $1.80 \mathrm{E}-03$ \\
\hline 200W S-222SA 001 & Methylene chloride & $75-9-2$ & $5.00 \mathrm{E}-01$ & $2.10 \mathrm{E}-02$ \\
\hline 200W S-222SA 001 & N-butyl alcohol & $71-36-3$ & $5.00 \mathrm{E}-01$ & $8.90 \mathrm{E}-04$ \\
\hline 200W S-222SA 001 & Nitrobenzene & $98-95-3$ & $5.00 \mathrm{E}-01$ & $5.30 \mathrm{E}-03$ \\
\hline 200W S-222SA 001 & Petroleum benzin & $8030-30-6$ & & $3.50 \mathrm{E}-03$ \\
\hline 200W S-222SA 001 & $\begin{array}{l}\text { Phosphoric acid, tributyl } \\
\text { ester }\end{array}$ & $126-73-8$ & & $2.30 \mathrm{E}-02$ \\
\hline 200W S-222SA 001 & Pyridine & $110-86-1$ & $5.00 \mathrm{E}-01$ & $2.20 \mathrm{E}-03$ \\
\hline 200W S-222SA 001 & Sulfuric acid & $7664-93-9$ & $5.00 \mathrm{E}-01$ & $5.40 \mathrm{E}-02$ \\
\hline $200 W$ S-222SA: 001 & $\begin{array}{l}\text { Tetrapropylammonium } \\
\text { hydroxide }\end{array}$ & $4499-86-9$ & & $3.90 \mathrm{E}-03$ \\
\hline $200 \mathrm{~W}$ S-222SA 001 & Toluene & $108-88-3$ & $5.00 \mathrm{E}-01$ & $9.60 \mathrm{E}-04$ \\
\hline $200 \mathrm{~W}$ S-222SA 001 & Xylene & $1330-20-7$ & $5.00 \mathrm{E}-01$ & $3.80 \mathrm{E}-03$ \\
\hline $200 \mathrm{~W}$ S-296S021 001 & 2,2,4-Trimethylpentane & $540-84-1$ & $5.00 \mathrm{E}-01$ & $6.10 \mathrm{E}-03$ \\
\hline $200 W$ S-296S021001 & Acetic acid & $64-19-7$ & $5.00 \mathrm{E}-01$ & $1.20 \mathrm{E}-02$ \\
\hline $200 W$ S-296S021 001 & Bromine & $7726-95-6$ & $5.00 \mathrm{E}-01$ & $2.10 \mathrm{E}-04$ \\
\hline $200 \mathrm{~W}$ S-296S021 001 & Ethyl alcohol & $64-17-5$ & $5.00 \mathrm{E}-01$ & $1.30 \mathrm{E}-01$ \\
\hline $200 \mathrm{~W}$ S-296S021001 & Hexane, $\mathbf{n}$-hexane & $110-54-3$ & $5.00 \mathrm{E}-01$ & $2.30 \mathrm{E}-02$ \\
\hline $200 \mathrm{~W}$ S-296S021001 & $\begin{array}{l}\text { Hydrochloric acid } \\
\text { (Hydrogen chloride) }\end{array}$ & $7647-1-0$ & $5.00 \mathrm{E}-01$ & $3.33 \mathrm{E}-02$ \\
\hline $200 \mathrm{~W}$ S-296S021 001 & Isopropyl Alcohol & $67-63-0$ & $5.00 \mathrm{E}-01$ & $7.00 \mathrm{E}-03$ \\
\hline $200 W$ S-296S021 001 & Mercury & $7439-97-6$ & $1.00 \mathrm{E}-02$ & $1.38 \mathrm{E}-08$ \\
\hline $200 \mathrm{~W}$ S-296S021 001 & Methyl chloride & $74-87-3$ & $5.00 \mathrm{E}-01$ & $4.60 \mathrm{E}-02$ \\
\hline $200 \mathrm{~W}$ S-296S021001 & Methylene chloride & $75-9-2$ & $5.00 \mathrm{E}-01$ & $1.10 \mathrm{E}-01$ \\
\hline $200 W$ S-296S021 001 & Sulfuric acid & $7664-93-9$ & $5.00 \mathrm{E}-01$ & $1.40 \mathrm{E}-02$ \\
\hline
\end{tabular}


Table K.2. Insignificant Emission Units Based on Threshold Comparisons . [WAC 173-401-530(1)(a)]

\begin{tabular}{|c|c|c|c|c|}
\hline Emission Point & Pollutants & CAS Number & $\begin{array}{c}\text { Emission } \\
\text { Threshold } \\
\text { (tons/yr) }\end{array}$ & $\begin{array}{c}\text { Actual } \\
\text { Emissions } \\
\text { (tons/yr) }\end{array}$ \\
\hline $200 W$ S-296S021 001 & Toluene & $108-88-3$ & $5.00 \mathrm{E}-01$ & $9.60 \mathrm{E}-04$ \\
\hline $200 \mathrm{~W}$ S-296S021001 & Trichloroethylene & $79-1-6$ & $5.00 \mathrm{E}-01$ & $1.30 \mathrm{E}-02$ \\
\hline 200W S-296S021 001 & Xylene & $1330-20-7$ & $5.00 \mathrm{E}-01$ & $3.80 \mathrm{E}-03$ \\
\hline $200 W$ S-296S023001 & 2,2,4-Trimethylpentane & $540-84-1$ & $5.00 \mathrm{E}-01$ & $3.66 \mathrm{E}-05$ \\
\hline $200 W$ S-296S023001 & Acetic acid & $64-19-7$ & $5.00 \mathrm{E}-01$ & $7.20 \mathrm{E}-05$ \\
\hline $200 \mathrm{~W}$ S-296S023 001 & Bromine & $7726-95-6$ & $5.00 \mathrm{E}-01$ & $1.26 \mathrm{E}-06$ \\
\hline $200 \mathrm{~W}$ S-296S023 001 & Ethyl aclohol & $64-17-5$ & $5.00 \mathrm{E}-01$ & $7.80 \mathrm{E}-04$ \\
\hline 200W S-296S023001 & Hexane, N-hexane & $110-54-3$ & $5.00 \mathrm{E}-01$ & $1.38 \mathrm{E}-04$ \\
\hline $200 \mathrm{~W}$ S-296S023 001 & Hydrochloric acid & $7647-1-0$ & $5.00 \mathrm{E}-01$ & $2.00 \mathrm{E}-04$ \\
\hline 200W S-296S023001 & Isopropyl alcohol & $67-63-0$ & $5.00 \mathrm{E}-01$ & $4.20 \mathrm{E}-05$ \\
\hline $200 \mathrm{~W}$ S-296S023 001 & Mercury & $7439-97-6$ & $1.00 \mathrm{E}-02$ & $8.27 \mathrm{E}-11$ \\
\hline 200W S-296S023001 & Methyl alcohol & $67-56-1$ & $5.00 \mathrm{E}-01$ & $2.76 \mathrm{E}-04$ \\
\hline $200 \mathrm{~W}$ S-296S023001 & Methylene chloride & $75-09-2$ & $5.00 \mathrm{E}-01$ & $6.60 \mathrm{E}-04$ \\
\hline 200W S-296S023001 & Sulfuric acid & $7664-93-9$ & $5.00 \mathrm{E}-01$ & $8.40 \mathrm{E}-05$ \\
\hline 200W S-296S023001 & Toluene & $108-88-3$ & $5.00 \mathrm{E}-01$ & $5.76 \mathrm{E}-06$ \\
\hline $200 \mathrm{~W}$ S-296S023001 & Trichloroethylene & $79-1-6$ & $5.00 \mathrm{E}-01$ & $7.80 \mathrm{E}-05$ \\
\hline $200 \mathrm{~W}$ S-296S023001 & Xylene & $1330-20-7$ & $5.00 \mathrm{E}-01$ & $2.28 \mathrm{E}-05$ \\
\hline $200 W$ P-291Z001 $001^{1}$ & Acetic acid & $64-19-7$ & $5.00 \mathrm{E}-01$ & $5.95 \mathrm{E}-04$ \\
\hline $200 \mathrm{~W}$ P-291Z001 001 & Aluminum nitrate & $13473-90-0$ & & $1.86 \mathrm{E}-04$ \\
\hline $200 \mathrm{~W}$ P-291Z001 001 & Aluminum sulfate & $10043-01-3$ & & $1.65 \mathrm{E}-06$ \\
\hline $200 \mathrm{~W}$ P-291Z001 001 & Anhydrous ammonia & $7664-41-7$ & $5.00 \mathrm{E}-01$ & $3.75 \mathrm{E}-01$ \\
\hline $200 \mathrm{~W}$ P-291Z001 001 & Boric acid & $10043-35-3$ & & $1.09 \mathrm{E}-07$ \\
\hline $200 \mathrm{~W}$ P-291Z001 001 & Calcium nitrate & $10124-37-5$ & & $4.07 \mathrm{E}-06$ \\
\hline $200 \mathrm{~W}$ P-291Z001 001 & Carbon Tetrachloride & $56-23-5$ & $5.00 \mathrm{E}-01$ & $6.78 \mathrm{E}-04$ \\
\hline $200 \mathrm{~W}$ P-291Z001 001 & Ethyl alcohol & $64-17-5$ & $5.00 \mathrm{E}-01$ & $2.18 \mathrm{E}-05$ \\
\hline 200W P-291Z001 001 & Europium nitrate & $10138-01-9$ & & $1.10 \mathrm{E}-04$ \\
\hline $200 \mathrm{~W}$ P-291Z001 001 & Ferrous sulfamate & & & $2.64 \mathrm{E}-05$ \\
\hline
\end{tabular}

'Reported emissions are from the laboratory that are emitted through PFP main stack. 
Table K.2. Insignificant Emission Units Based on Threshold Comparisons [WAC 173-401-530(1)(a)]

\begin{tabular}{|c|c|c|c|c|}
\hline Emission Point & Pollutants & CAS Number & $\begin{array}{c}\text { Emission } \\
\text { Threshold } \\
\text { (tons/yr) }\end{array}$ & $\begin{array}{c}\text { Actual } \\
\text { Emissions } \\
\text { (tons/yr) }\end{array}$ \\
\hline $200 \mathrm{~W}$ P-291Z001 001 & Hydrochloric acid & $7647-01-0$ & $5.00 \mathrm{E}-01$ & $1.16 \mathrm{E}-04$ \\
\hline 200W P-291Z001 001 & Iron II nitrate & & & $8.92 \mathrm{E}-06$ \\
\hline 200W P-291Z001 001 & Magnesium nitrate & $10377-60-3$ & & $1.47 \mathrm{E}-06$ \\
\hline 200W P-291Z001 001 & Naphthylamine sulfonic & $84-86-6$ & & $3.30 \mathrm{E}-06$ \\
\hline 200W P-291Z001 001 & Nitric acid & $7697-37-2$ & $5.00 \mathrm{E}-01$ & $4.50 \mathrm{E}-04$ \\
\hline 200W P-291Z001 001 & Potassium dichromate & $7778-50-9$ & & $2.75 \mathrm{E}-06$ \\
\hline 200W P-291Z001 001 & Potassium persulfate & $7727-21-1$ & & $6.61 \mathrm{E}-03$ \\
\hline 200W P-291Z001 001 & Silver nitrate & $7761-88-8$ & & $1.10 \mathrm{E}-04$ \\
\hline 200W P-291Z001 001 & Silver oxide & $20667-12-3$ & & $5.51 \mathrm{E}-06$ \\
\hline 200W P-291Z001 001 & Sodium fluoride & $7681-49-4$ & & $5.32 \mathrm{E}-05$ \\
\hline 200W P-291Z001 001 & Sodium hydroxide & $1310-73-2$ & $5.00 \mathrm{E}-01$ & $2.64 \mathrm{E}-05$ \\
\hline 200W P-291Z001 001 & Sodium nitrate & $7631-99-4$ & & $2.57 \mathrm{E}-04$ \\
\hline 200W P-291Z001001 & Sodium nitrite & $7632-00-00$ & & $2.77 \mathrm{E}-03$ \\
\hline 200W P-291Z001 001 & Sodium tartrate & $526-94-3$ & & $2.14 \mathrm{E}-04$ \\
\hline 200W P-291Z001 001 & Stannic chloride & $7646-78-8$ & & $2.20 \mathrm{E}-03$ \\
\hline 200W P-291Z001 001 & Sulfanic acid & $121-57-3$ & & $1.32 \mathrm{E}-05$ \\
\hline 200W P-291Z001 001 & Sulfuric acid & 7664-93-9 & $5.00 \mathrm{E}-01$ & $4.28 \mathrm{E}-03$ \\
\hline 200W P-291Z001 001 & Titanium chloride & $7550-45-0$ & $1.00 \mathrm{E}-01$ & 3.30E-03 \\
\hline $200 \mathrm{~W}$ P-291Z001 001 & $\mathrm{ZnCl}_{2}$ & $7646-85-7$ & $5.00 \mathrm{E}-01$ & $1.26 \mathrm{E}-03$ \\
\hline 200E P-296-A05A\&05B 001 & Acetic acid & 64-19-7 & $5.00 \mathrm{E}-01$ & $1.73 \mathrm{E}-03$ \\
\hline 200E P-296-A05A\&05B 001 & Aluminum nitrate & $13473-90-0$ & & $3.22 \mathrm{E}-03$ \\
\hline 200E P-296-A05A\&05B 001 & Ammonia & $7664-41-7$ & $5.00 \mathrm{E}-01$ & $3.75 \mathrm{E}-07$ \\
\hline 200E P-296-A05A\&05B 001 & Ammonium hydroxide & $1336-21-6$ & & $2.32 \mathrm{E}-04$ \\
\hline 200E P-296-A05A\&05B 001 & Ammonium molybdate & $12027-67-7$ & & $2.20 \mathrm{E}-04$ \\
\hline 200E P-296-A05A\&05B 001 & Ammonium nitrate & $6482-52-2$ & & $6.61 \mathrm{E}-05$ \\
\hline 200E P-296-A05A\&05B 001 & Barium chloride & $10361-37-2$ & & $2.29 \mathrm{E}-04$ \\
\hline 200E P-296-A05A\&05B 001 & Boric acid & $10043-35-3$ & & $9.65 \mathrm{E}-06$ \\
\hline 200E P-296-A05A\&05B 001 & Ethyl alcohol & $64-17-5$ & $5.00 \mathrm{E}-01$ & $8.69 \mathrm{E}-03$ \\
\hline 200E P-296-A05A\&05B 001 & Ferric nitrate & $10421-48-4$ & & $3.56 \mathrm{E}-05$ \\
\hline 200E P-296-A05A\&05B 001 & Sodium oxylate & $62-76-0$ & & $6.61 \mathrm{E}-05$ \\
\hline
\end{tabular}


Table K.2. Insignificant Emission Units Based on Threshold Comparisons [WAC 173-401-530(1)(a)]

\begin{tabular}{|c|c|c|c|c|}
\hline Emission Point & Pollutants & CAS Number & $\begin{array}{l}\text { Emission } \\
\text { Threshold } \\
\text { (tons/yr) }\end{array}$ & $\begin{array}{c}\text { Actual } \\
\text { Emissions } \\
\text { (tons/yr) }\end{array}$ \\
\hline 200E P-296-A05A\&05B 001 & Sodium flouride & $7681-49-4$ & & $7.27 \mathrm{E}-05$ \\
\hline 200E P-296-A05A\&05B 001 & Hydrochloric acid & $7647-1-0$ & $5.00 \mathrm{E}-01$ & $1.20 \mathrm{E}-05$ \\
\hline 200E P-296-A05A\&05B 001 & Hydroxylamine HCL & $5470-11-1$ & & $1.54 \mathrm{E}-03$ \\
\hline 200E P-296-A05A\&05B 001 & Nitric acid & $7697-37-2$ & & $6.50 \mathrm{E}-02$ \\
\hline 200E P-296-05A\&05B 001 & $\begin{array}{l}\text { Normal parrafinic } \\
\text { hydrocarbons }\end{array}$ & & & $2.12 \mathrm{E}-02$ \\
\hline 200E P-296-A05A\&05B 001 & Phosphoric acid & $7664-38-2$ & $5.00 \mathrm{E}-01$ & $1.26 \mathrm{E}-01$ \\
\hline 200E P-296-A05A\&05B 001 & Sodium bicarbonate & $144-55-8$ & & $2.22 \mathrm{E}-04$ \\
\hline 200E P-296-A05A\&05B 001 & Sodium carbonate & $497-19-8$ & & $2.62 \mathrm{E}-03$ \\
\hline 200E P-296-A05A\&05B 001 & Sodium hydroxide & $1310-73-2$ & & $6.98 \mathrm{E}-04$ \\
\hline 200E P-296-A05A\&05B 001 & Sodium nitrite & $7632-00-00$ & & $9.40 \mathrm{E}-04$ \\
\hline 200E P-296-A05A\&05B 001 & Sulfamic acid & $5329-14-6$ & & $4.18 \mathrm{E}-03$ \\
\hline 200E P-296-A05A\&05B 001 & Sulfuric acid & $764-93-9$ & $5.00 \mathrm{E}-01$ & $1.71 \mathrm{E}-04$ \\
\hline 200E P-296-A05A\&05B 001 & Sulfanic acid & $121-57-3$ & & $7.93 \mathrm{E}-05$ \\
\hline 200E P-296-A05A\&05B 001 & Tributyl phosphate & $126-73-8$ & & $8.57 \mathrm{E}-03$ \\
\hline 200E P-296-A05A\&05B 001 & Thenoyltriflouroactetane & & & $8.56 \mathrm{E}-04$ \\
\hline 200E P-296-A05A\&05B 001 & Vanadyl sulfate & 27774-13-6 & & $2.48 \mathrm{E}-03$ \\
\hline 200E P-296-A05A\&05B 001 & Xylene & $1330-20-7$ & & $6.83 \mathrm{E}-03$ \\
\hline 200E P-296-A05A\&05B 001 & Zinc chloride & $7646-85-7$ & & $1.20 \mathrm{E}-03$ \\
\hline $1100 \mathrm{G}-1172 \mathrm{~A} 002$ & 2,2,4-Trimethylpentane & $540-84-1$ & $5.00 \mathrm{E}-01$ & $3.70 \mathrm{E}-03$ \\
\hline $1100 \mathrm{G}-1172 \mathrm{~A} 002$ & Benzene & $71-43-2$ & $5.00 \mathrm{E}-01$ & $1.00 \mathrm{E}-02$ \\
\hline $1100 \mathrm{G}-1172 \mathrm{~A} 002$ & Ethyl benzene & $100-41-4$ & $5.00 \mathrm{E}-01$ & $2.80 \mathrm{E}-03$ \\
\hline $1100 \mathrm{G}-1172 \mathrm{~A} 002$ & Hexane, n-hexane & $110-54-3$ & $5.00 \mathrm{E}-01$ & $9.10 \mathrm{E}-03$ \\
\hline 1100 G-1172A 002 & Methyl tert-butyl ether & $1634-4-4$ & $5.00 \mathrm{E}-01$ & $2.90 \mathrm{E}-02$ \\
\hline $1100 \mathrm{G}-1172 \mathrm{~A} 002$ & Toluene & $108-88-3$ & $5.00 \mathrm{E}-01$ & $1.00 \mathrm{E}-02$ \\
\hline $1100 \mathrm{G}-1172 \mathrm{~A} 002$ & Volatile organic compound & - & $2.00 \mathrm{E}+00$ & $9.40 \mathrm{E}-01$ \\
\hline $1100 \mathrm{G}-1172 \mathrm{~A} 002$ & Xylene & $1330-20-7$ & $5.00 \mathrm{E}-01$ & $4.40 \mathrm{E}-03$ \\
\hline $1100 \mathrm{G}-1172 \mathrm{~A} 003$ & 2,2,4-Trimethylpentane & $540-84-1$ & $5.00 \mathrm{E}-01$ & $3.70 \mathrm{E}-03$ \\
\hline $1100 \mathrm{G}-1172 \mathrm{~A} 003$ & Benzene & $71-43-2$ & $5.00 \mathrm{E}-01$ & $1.00 \mathrm{E}-02$ \\
\hline
\end{tabular}


Table K.2. Insignificant Emission Units Based on Threshold Comparisons [WAC 173-401-530(1)(a)]

\begin{tabular}{|c|c|c|c|c|}
\hline Emission Point & Pollutants & CAS -Number & $\begin{array}{l}\text { Emission } \\
\text { Threshold } \\
\text { (tons/yr) }\end{array}$ & $\begin{array}{c}\text { Actual } \\
\text { Emissions } \\
\text { (tons/yr) }\end{array}$ \\
\hline $1100 \mathrm{G}-1172 \mathrm{~A} 003$ & Ethyl benzene & $100-41-4$ & $5.00 \mathrm{E}-01$ & $2.80 \mathrm{E}-03$ \\
\hline $1100 \mathrm{G}-1172 \mathrm{~A} 003$ & Hexane, n-hexane & $110-54-3$ & $5.00 \mathrm{E}-01$ & $9.10 \mathrm{E}-03$ \\
\hline $1100 \mathrm{G}-1172 \mathrm{~A} 003$ & Methyl tert-butyl ether & $1634-4-4$ & $5.00 \mathrm{E}-01$ & $2.90 \mathrm{E}-02$ \\
\hline $1100 \mathrm{G}-1172 \mathrm{~A} 003$ & Toluene & $108-88-3$ & $5.00 \mathrm{E}-01$ & $1.00 \mathrm{E}-02$ \\
\hline $1100 \mathrm{G}-1172 \mathrm{~A} 003$ & Volatile organic compound & - & $2.00 \mathrm{E}+00$ & $9.40 \mathrm{E}-01$ \\
\hline $1100 \mathrm{G}-1172 \mathrm{~A} 003$ & Xylene & $1330-20-7$ & $5.00 \mathrm{E}-01$ & $4.40 \mathrm{E}-03$ \\
\hline $1100 \mathrm{G}-1172 \mathrm{~A} 004$ & Benzene & $71-43-2$ & $5.00 \mathrm{E}-01$ & $2.80 \mathrm{E}-05$ \\
\hline 1100 G-1172A 004 & Hexane, n-hexane & $110-54-3$ & $5.00 \mathrm{E}-01$ & $1.10 \mathrm{E}-04$ \\
\hline $1100 \mathrm{G}-1172 \mathrm{~A} 004$ & Toluene & $108-88-3$ & $5.00 \mathrm{E}-01$ & $1.60 \mathrm{E}-05$ \\
\hline $1100 \mathrm{G}-1172 \mathrm{~A} 004$ & Volatile organic compound & $\therefore$ & $2.00 \mathrm{E}+00$ & $1.00 \mathrm{E}-03$ \\
\hline $1100 \mathrm{G}-1172 \mathrm{~A} 005$ & Benzene & $71-43-2$ & $5.00 \mathrm{E}-01$ & $2.80 \mathrm{E}-05$ \\
\hline $1100 \mathrm{G}-1172 \mathrm{~A} 005$ & Hexane, n-hexane. & $110-54-3$ & $5.00 \mathrm{E}-01$ & $1.10 \mathrm{E}-04$ \\
\hline $1100 \mathrm{G}-1172 \mathrm{~A} 005$ & Toluene & $108-88-3$ & $5.00 \mathrm{E}-01$ & $1.60 \mathrm{E}-05$ \\
\hline $1100 \mathrm{G}-1172 \mathrm{~A} 005$ & Volatile organic compound & - & $2.00 \mathrm{E}+00$ & $1.00 \mathrm{E}-03$ \\
\hline 200E G-2713E 002 & 2,2,4-Trimethylpentane & $540-84-1$ & $5.00 \mathrm{E}-01$ & $3.50 \mathrm{E}-03$ \\
\hline 200E G-2713E 002 & Benzene & $71-43-2$ & $5.00 \mathrm{E}-01$ & $9.50 \mathrm{E}-03$ \\
\hline 200E G-2713E 002 & Ethyl benzene & $100-41-4$ & $5.00 \mathrm{E}-01$ & $2.70 \mathrm{E}-03$ \\
\hline 200E G-2713E 002 & Hexane, $n$-hexane & $-110-54-3$ & $5.00 \mathrm{E}-01$ & $8.60 \mathrm{E}-03$ \\
\hline 200E G-2713E 002 & Methyl tert-butyl ether & $1634-4-4$ & $5.00 \mathrm{E}-01$ & $2.70 \mathrm{E}-02$ \\
\hline 200E G-2713E 002 & Toluene & $108-88-3$ & $5.00 \mathrm{E}-01$ & $9.80 \mathrm{E}-03$ \\
\hline 200E G-2713E 002 & Volatile organic compound & - & $2.00 \mathrm{E}+00$ & $8.90 \mathrm{E}-01$ \\
\hline 200E G-2713E 002 & Xylene & $1330-20-7$ & $5.00 \mathrm{E}-01$ & $4.20 \mathrm{E}-03$ \\
\hline 200E G-2713E 003 & Benzene & $71-43-2$ & $5.00 \mathrm{E}-01$ & $7.30 \mathrm{E}-05$ \\
\hline 200E G-2713E 003 & Hexane, $\mathrm{n}$-hexane & $110-54-3$ & $5.00 \mathrm{E}-01$ & $3.00 \mathrm{E}-04$ \\
\hline 200E G-2713E 003 & Toluene & $108-88-3$ & $5.00 \mathrm{E}-01$ & $4.30 \mathrm{E}-05$ \\
\hline 200E G-2713E 003 & Volatile organic compound & - & $2.00 \mathrm{E}+00$ & $2.60 \mathrm{E}-03$ \\
\hline
\end{tabular}




\section{Table K.2. Insignificant Emission Units Based on Threshold Comparisons} [WAC 173-401-530(1)(a)]

\begin{tabular}{|c|c|c|c|c|}
\hline Emission Point & Pollutants & CAS Number & $\begin{array}{l}\text { Emission } \\
\text { Threshold } \\
\text { (tons/yr) }\end{array}$ & $\begin{array}{c}\text { Actual } \\
\text { Emissions } \\
\text { (tons/yr) }\end{array}$ \\
\hline $200 \mathrm{E}$ K-2715EC 001 & Isopropyl alcohol & $67-63-0$ & $5.00 \mathrm{E}-01$ & $2.30 \mathrm{E}-02$ \\
\hline 200E K-2715EC 001 & $\begin{array}{l}\text { Methyl ethyl ketone (2- } \\
\text { butone) }\end{array}$ & $78-93-3$ & $5.00 \mathrm{E}-01$ & $1.50 \mathrm{E}-02$ \\
\hline $200 \mathrm{E}$ K-2715EC 001 & Methyl isobutyl ketone & $108-10-1$ & $5.00 \mathrm{E}-01$ & $1.50 \mathrm{E}-02$ \\
\hline 200E K-2715EC 001 & Petroleum distillates 3 & $64742-88-7$ & & $2.40 \mathrm{E}-01$ \\
\hline $200 \mathrm{E} \mathrm{K}-2715 \mathrm{EC} 001$ & $\begin{array}{l}\text { Petroleum distillate, } \\
\text { hydrotreated middle }\end{array}$ & $64742-46-7$ & & $5.90 \mathrm{E}-02$ \\
\hline 200E K-2715EC 001 & Toluene & $108-88-3$ & $5.00 \mathrm{E}-01$ & $1.20 \mathrm{E}-01$ \\
\hline 200E K-2715EC 001 & Vm\&p naphtha & $64742-48-9$ & & $1.80 \mathrm{E}-01$ \\
\hline $200 \mathrm{E} N-2101 \mathrm{M} 001$ & Stoddard solvent & $8052-41-3$ & & $8.40 \mathrm{E}-02$ \\
\hline $200 \mathrm{E} \mathrm{N}-2101 \mathrm{M} 002$ & Hydrogen sulfide & $7783-06-4$ & $5.0 \mathrm{E}-01$ & $4.6 \mathrm{E}-09$ \\
\hline $200 \mathrm{E} N-2101 \mathrm{M} 002$ & Sulfur dioxide & $7446-09-5$ & $2.0 \mathrm{E}+0$ & $3.3 \mathrm{E}-09$ \\
\hline $200 \mathrm{E} \mathrm{N}-2101 \mathrm{M} 002$ & Nitrogen dioxide & $10102-44-0$ & $2.0 \mathrm{E}+02$ & $3.0 \mathrm{E}-08$ \\
\hline $200 \mathrm{E}$ N-2101M 002 & Carbon monoxide & $630-08-0$ & $5.0 \mathrm{E}+0$ & $1.5 \mathrm{E}-08$ \\
\hline $200 \mathrm{E} N-2101 \mathrm{M} 002$ & Pentane & $109-66-0$ & $5.0 \mathrm{E}-01$ & $6.4 \mathrm{E}-05$ \\
\hline $200 \mathrm{E}$ N-2101M 002 & Chlorine & $7782-50-5$ & $1.0 \mathrm{E}-01$ & $4.1 \mathrm{E}-08$ \\
\hline $200 \mathrm{E} N-2101 \mathrm{M} 002$ & Methane & $74-82-8$ & $5.0 \mathrm{E}-01$ & $2.1 \mathrm{E}-06$ \\
\hline $200 \mathrm{E} \mathrm{N}-2101 \mathrm{M} 002$ & Isobutylene & $115-11-7$ & & $6.6 \mathrm{E}-07$ \\
\hline $200 \mathrm{E} \mathrm{N}-2101 \mathrm{M} \mathrm{002}$ & Ammonia & $7664-41-7$ & $5.0 \mathrm{E}-01$ & $7.3 \mathrm{E}-08$ \\
\hline $200 \mathrm{E}$ N-2101M 002 & Hydrogen cyanide & $74-90-8$ & $5.0 \mathrm{E}-01$ & $1.1 \mathrm{E}-08$ \\
\hline & & & & \\
\hline 200E N-2242B 001 & $\mathrm{PM}<10 \mu$ & - & $7.50 \mathrm{E}-01$ & $2.50 \mathrm{E}-01$ \\
\hline 200E N-2242B 001 & $\mathrm{PM}>10$ & - & & $6.30 \mathrm{E}-01$ \\
\hline 200E N-2715EC 003 & Total particulate matter & - & & $1.50 \mathrm{E}+00$ \\
\hline 200E N-275E 001 & $\mathrm{PM}<10 \mu$ & - & $7.50 \mathrm{E}-01$ & $4.00 \mathrm{E}-01$ \\
\hline 200E N-275E 001 & PM $>10$ & $-=$ & & $9.90 \mathrm{E}-01$ \\
\hline
\end{tabular}


Hanford Site Air

Operating Permit Application

Table K.2. Insignificant Emission Units Based on Threshold Comparisons [WAC 173-401-530(1)(a)]

\begin{tabular}{|c|c|c|c|c|}
\hline Emission Point & $\begin{array}{c}\text { Pollutants } \\
\text {. }\end{array}$ & CAS Number & $\begin{array}{l}\text { Emission } \\
\text { Threshold } \\
\text { (tons/yr) }\end{array}$ & $\begin{array}{c}\text { Actual } \\
\text { Emissions } \\
\text { (tons/yr) }\end{array}$ \\
\hline 200W M-242AC 001 & $\mathrm{PM}<10 \mu$ & - & $7.50 \mathrm{E}-01$ & $1.40 \mathrm{E}-02$ \\
\hline 200W N-200W001 001 & $\mathrm{PM}<10 \mu$ & -- & 7.50E-01. & $1.00 \mathrm{E}-01$ \\
\hline 200W N-200W001001 & $\mathrm{PM}>10 \mu$ & - & & $2.60 \mathrm{E}-01$ \\
\hline $200 \mathrm{~W} N-277 \mathrm{~W} 002$ & Lead & $7439-92-1$ & $5.00 \mathrm{E}-03$ & $4.80 \mathrm{E}-06$ \\
\hline 200W N-277W 002 & Total particulate matter & - & & $1.90 \mathrm{E}-05$ \\
\hline $300 \mathrm{~N}-328001$ & $\mathrm{PM}<10 \mu$ & - & $7.50 \mathrm{E}-01$ & $1.50 \mathrm{E}-02$ \\
\hline $300 \mathrm{~N}-328001$ & $\mathrm{PM}>10$ & - & & $3.80 \mathrm{E}-02$ \\
\hline $300 \mathrm{~N}-328002$ & Total particulate matter & - & & $3.90 \mathrm{E}-02$ \\
\hline $300 \mathrm{~N}-3713001$ & $\mathrm{PM}<10 \mu$ & - & $7.50 \mathrm{E}-01$ & $4.00 \mathrm{E}-01$ \\
\hline $300 \mathrm{~N}-3713001$ & $\mathrm{PM}>10$ & -- & & $9.90 \mathrm{E}-01$ \\
\hline $300 \mathrm{~N}-3722002$ & $\mathrm{PM}<10 \mu$ & - & $7.50 \mathrm{E}-01$ & $1.10 \mathrm{E}-01$ \\
\hline $300 \mathrm{~N}-3722002$ & $\mathrm{PM}>10$ & - & & $2.90 \mathrm{E}-01$ \\
\hline $400 \mathrm{~N}-4722 \mathrm{~B} 001$ & $\mathrm{PM}<10 \mu$ & - & 7.50E-01 & $2.30 \mathrm{E}-01$ \\
\hline $400 \mathrm{~N}-4722 \mathrm{~B} 001$ & $\mathrm{PM}>10$ & - & & $5.70 \mathrm{E}-01$ \\
\hline $400 \mathrm{~N}-4722 \mathrm{C} 003$ & Total particulate matter & - & & $6.00 \mathrm{E}-03$ \\
\hline $600 \mathrm{~N}-\mathrm{MO} 222001$ & Petroleum distillates 3 & $64742-88-7$ & & $3.10 \mathrm{E}-02$ \\
\hline $600 \mathrm{~N}-\mathrm{MO} 222002$ & Petroleum distillates 3 & $64742-88-7$ & & $3.10 \mathrm{E}-02$ \\
\hline $300 \mathrm{~N}-3717 \mathrm{~B} 001$ & $\begin{array}{l}\text { 1,1,2-Trichloro-1,2,2- } \\
\text { triflouroethane }\end{array}$ & $76-13-1$ & $5.00 \mathrm{E}-01$ & $5.20 \mathrm{E}-02$ \\
\hline
\end{tabular}


Table K.2. Insignificant Emission Units Based on Threshold Comparisons [WAC 173-401-530(1)(a)]

\begin{tabular}{|c|c|c|c|c|}
\hline Emission Point & Pollutants & CAS Number & $\begin{array}{l}\text { Emission } \\
\text { Threshold } \\
\text { (tons/yr) }\end{array}$ & $\begin{array}{c}\text { Actual } \\
\text { Emissions } \\
\text { (tons/yr) }\end{array}$ \\
\hline $300 \mathrm{~N}-3717 \mathrm{~B} 001$ & Ethyl alcohol & $64-17-5$ & $5.00 \mathrm{E}-01$ & $3.30 \mathrm{E}-03$ \\
\hline 600 S-6266-001 & 2-Hexanone & $540-84-1$ & & $4.46 \mathrm{E}-05$ \\
\hline 600 S-6266-001 & Acetic acid & $64-19-7$ & $5.00 \mathrm{E}-01$ & $5.79 \mathrm{E}-03$ \\
\hline 600 S-6266-001 & Acetone & $67-64-1$ & $5.00 \mathrm{E}-01$ & $4.35 \mathrm{E}-05$ \\
\hline 600 S-6266-001 & Acetronile & $75-5-8$ & $5.00 \mathrm{E}-01$ & 9.03E-04 \\
\hline $600 \mathrm{~S}-6266-001$ & Antimony & $7440-36-0$ & & $8.27 \mathrm{E}-05$ \\
\hline $600 \mathrm{~S}-6266-001$ & Benzene & $71-43-2$ & $5.00 \mathrm{E}-01$ & $4.85 \mathrm{E}-05$ \\
\hline 600 S-6266-001 & Beryllium & $7440-41-7$ & $4.00 \mathrm{E}-03$ & $2.04 \mathrm{E}-03$ \\
\hline 600 S-6266-001 & Cadmium & $7440-43-9$ & $5.00 \mathrm{E}-03$ & $1.82 \mathrm{E}-03$ \\
\hline 600 S-6266-001 & Chlorine & $7782-50-5$ & $1.00 \mathrm{E}-01$ & $7.83 \mathrm{E}-05$ \\
\hline 600 S-6266-001 & Cobalt & $7440-48-4$ & & $2.15 \mathrm{E}-03$ \\
\hline 600 S-6266-001 & Cyclohexanone & $110-82-7$ & $5.00 \mathrm{E}-01$ & $8.60 \mathrm{E}-04$ \\
\hline $600 \mathrm{~S}-6266-001$ & Ethyl benzene & $100-41-4$ & $5.00 \mathrm{E}-01$ & $4.80 \mathrm{E}-05$ \\
\hline 600 S-6266-001 & Hexane, n-hexane & $110-54-3$ & $5.00 \mathrm{E}-01$ & $3.64 \mathrm{E}-05$ \\
\hline $600 \mathrm{~S}-6266-001$ & $\begin{array}{l}\text { Hydrochloric acid } \\
\text { (Hydrogen chloride) }\end{array}$ & $7647-1-0$ & $5.00 \mathrm{E}-01$ & $5.44 \mathrm{E}-04$ \\
\hline 600 S-6266-001 & Isopropyl alcohol & $67-63-0$ & $5.00 \mathrm{E}-01$ & $4.30 \mathrm{E}-0.5$ \\
\hline 600 S-6266-001 & Lead & $7439-92-1$ & $5.00 \mathrm{E}-03$ & $1.25 \mathrm{E}-02$ \\
\hline 600 S-6266-001 & Mercury & $7439-97-6$ & $1.00 \mathrm{E}-02$ & $1.49 \mathrm{E}-02$ \\
\hline $600 \mathrm{~S}-6266-001$ & Methyl alcohol & $67-56-1$ & $5.00 \mathrm{E}-01$ & $4.35 \mathrm{E}-03$ \\
\hline $600 \mathrm{~S}-6266-001$ & Methylene chloride & $75-9-2$ & $5.00 \mathrm{E}-01$ & $3.25 \mathrm{E}-01$ \\
\hline $600 \mathrm{~S}-6266-001$ & Nickel & $7440-2-0$ & $2.00 \mathrm{E}-02$ & $1.32 \mathrm{E}-03$ \\
\hline $600 \mathrm{~S}-6266-001$ & Nitrobenzene & $98-95-3$ & $5.00 \mathrm{E}-01$ & $6.61 \mathrm{E}-05$ \\
\hline $600 \mathrm{~S}-6266-001$ & Nitrosodimethylamine & $62-75-9$ & $5.00 \mathrm{E}-04$ & $5.51 \mathrm{E}-05$ \\
\hline 600 S-6266-001 & Pentane & $109-66-0$ & $5.00 \mathrm{E}-01$ & $3.47 \mathrm{E}-05$ \\
\hline 600 S-6266-001 & Phosphoric acid & $7664-38-2$ & $5.00 \mathrm{E}-01$ & $9.26 \mathrm{E}-05$ \\
\hline 600 S-6266-001 & Pyridine & $110-86-1$ & $5.00 \mathrm{E}-01$ & $1.08 \mathrm{E}-04$ \\
\hline 600 S-6266-001 & Selenium & $7782-49-2$ & $5.00 \mathrm{E}-01$ & $1.10 \mathrm{E}-03$ \\
\hline 600 S-6266-001 & Trichloroethylene & $79-1-6$ & $5.00 \mathrm{E}-01$ & $2.76 \mathrm{E}-06$ \\
\hline 600 S-6266-001 & Xylene & $1330-20-7$ & $5.00 \mathrm{E}-01$ & $2.76 \mathrm{E}-06$ \\
\hline
\end{tabular}


Hanford Site Air

Operating Permit Application
DOE/RL-95-07, Rev. 0

$05 / 95$

Table K.2. Insignificant Emission Units Based on Threshold Comparisons [WAC 173-401-530(1)(a)]

\begin{tabular}{|l|l|l|l|c||}
\hline Emission Point & Pollutants & CAS Number & $\begin{array}{c}\text { Emission } \\
\text { Threshold } \\
\text { (tons/yr) }\end{array}$ & $\begin{array}{c}\text { Actual } \\
\text { Emissions } \\
\text { (tons/yr) }\end{array}$ \\
\hline 600 S-6266-001 & Ytrium & $7440-65-5$ & $5.00 \mathrm{E}-01$ & $1.10 \mathrm{E}-03$ \\
\hline & & & & \\
\hline
\end{tabular}


Table K.3. Fugitive Source Insignificant Emission Units [WAC 173-401(1)(d)].

\begin{tabular}{|c|c|c|}
\hline Emission Point & Pollutants & $\begin{array}{c}\text { CAS } \\
\text { Number }\end{array}$ \\
\hline $100 \mathrm{~K} N-1717 \mathrm{~K} 001$ & 1,1,1-Trichloroethane & $71-55-6$ \\
\hline $100 \mathrm{~K}$ N-1717K 001 & 1,2-Butylene Oxide & $106-88-7$ \\
\hline $100 \mathrm{~K}$ N-1717K 001 & Butyl alcohol, tert- & $75-65-0$ \\
\hline $100 \mathrm{~K}$ N-1717K 001 & Dimethoxymethane & $109-87-5$ \\
\hline $100 \mathrm{~K}$ N-1717K 001 & Isopropyl alcohol & $67-63-0$ \\
\hline $100 \mathrm{~K}$ N-1717K 001 & Methyl Alcohol & $67-56-1$ \\
\hline $100 \mathrm{~K}$ N-1717K 001 & Methylene Chloride & $75-9-2$ \\
\hline $100 \mathrm{~N} \mathrm{~K}-1143 \mathrm{~N} 015$ & Methyl ethyl ketone (2-butone) & $78-93-3$ \\
\hline $100 \mathrm{~N} \mathrm{~K}-1143 \mathrm{~N} 015$ & Petroleum Benzin & $8030-30-6$ \\
\hline $100 \mathrm{~N} \mathrm{~K}-1517 \mathrm{~N} 021$ & Methyl ethyl ketone (2-butone) & $78-93-3$ \\
\hline $100 \mathrm{~N} \mathrm{~K}-1517 \mathrm{~N} 021$ & Petroleum Benzin & $8030-30-6$ \\
\hline 100 N M-1515N 020 & $\mathrm{PM}<10 \mu$ & 一 \\
\hline 100 N M-1518N 019 & $\mathrm{PM}<10 \mu$ & - \\
\hline $100 \mathrm{~N} M-1705 \mathrm{~N} 013$ & $\mathrm{PM}<10 \mu$ & - \\
\hline $100 \mathrm{~N} N-1143 \mathrm{~N} 016$ & Methyl ethyl ketone (2-butone) & $78-93-3$ \\
\hline $100 \mathrm{~N} N-1143 \mathrm{~N} 016$ & Petroleum Benzin & $8030-30-6$ \\
\hline $100 \mathrm{~N} N-1143 \mathrm{~N} 016$ & Total particulate matter & - \\
\hline $100 \mathrm{~N} N-1517 \mathrm{~N} 018$ & Total particulate matter & - \\
\hline 200E N-272AW 001 & 2-Butoxy ethanol & $111-76-2$ \\
\hline 200E N-272AW 001 & 2-(2-Butoxyethoxy) ethanol & $112-34-5$ \\
\hline 200E N-272AW 001 & 2-(2-Methoxyethoxy) ethanol & $111-77-3$ \\
\hline 200E N-272AW 001 & 2,2,4Trimethyl 1,3pentadiol & $25265-77-4$ \\
\hline 200E N-272AW 001 & Acetone & $67-64-1$ \\
\hline 200E N-272AW 001 & Butyl Acetate & $123-86-4$ \\
\hline 200E N-272AW 001 & Cyclohexane & $110-82-7$ \\
\hline 200E N-272AW 001 & Cyclohexanone & $108-94-1$ \\
\hline 200E N-272AW 001 & Dibutyl Phthalate & $87-74-2$ \\
\hline 200E N-272AW 001 & Ester alcohol & $25264-77-4$ \\
\hline 200E N-272AW 001 & Ethyl benzene & $100-41-4$ \\
\hline 200E N-272AW 001 & Ethylene glycol & $107-21-1$ \\
\hline 200E N-272AW 001 & Hexane, n-hexane & $110-54-3$ \\
\hline 200E N-272AW 001 & Isobutane & $75-28-5$ \\
\hline 200E N-272AW 001 & Ligroine or vm\&p naphtha, or petroleum & $8032-32-4$ \\
\hline
\end{tabular}


Table K.3. Fugitive Source Insignificant Emission Units [WAC 173-401(1)(d)].

\begin{tabular}{|c|c|c|}
\hline Emission Point & Pollutants & $\begin{array}{c}\text { CAS } \\
\text { Number }\end{array}$ \\
\hline 200E N-272AW 001 & Methyl ether or dimethyl ether & $115-10-6$ \\
\hline 200E N-272AW 001 & Methyl ethyl ketone (2-butone) & $78-93-3$ \\
\hline 200E N-272AW 001 & Methyl isobutyl ketone & $108-10-1$ \\
\hline 200E N-272AW 001 & Methyl N-amyl ketone, 2-heptanone & $110-43-0$ \\
\hline 200E N-272AW 001 & Phosphoric acid & $7664-38-2$ \\
\hline 200E N-272AW 001 & Polyvinyl Chloride Latex & $9002-86-2$ \\
\hline 200E N-272AW 001 & Propane & $74-98-6$ \\
\hline 200E N-272AW 001 & Tetrahydrofuran & $109-99-9$ \\
\hline 200E N-272AW 001 & Toluene & $108-88-3$ \\
\hline 200E N-272AW 001 & Vm\&p naphtha & $64742-48-9$ \\
\hline 200E N-272AW 001 & Xylene & $1330-20-7$ \\
\hline 200W M 272WA & 2-Butoxy ethanol & $111-76-2$ \\
\hline $200 W M \quad 272 W \cdot A$ & 2-(2-Butoxyethoxy) ethanol & $112-34-5$ \\
\hline 200W M 272WA & 2-(2-Methoxyethoxy) ethanol & $111-77-3$ \\
\hline $200 \mathrm{~W} M \quad 272 \mathrm{WA}$ & $2,2,4$ Trimethyl 1,3 pentadiol & $25265-77-4$ \\
\hline $200 \mathrm{~W} M \quad 272 \mathrm{WA}$ & Acetone & $67-64-1$ \\
\hline 200W M 272WA & Butyl Acetate & $123-86-4$ \\
\hline $200 \mathrm{~W}$ M 272WA & Cyclohexane & $110-82-7$ \\
\hline 200W M 272WA & Cyclohexanone & $108-94-1$ \\
\hline 200W M 272WA & Dibutyl Phthalate & $87-74-2$ \\
\hline 200W M 272WA & Ester alcohol & $25264-77-4$ \\
\hline $200 \mathrm{~W} M \quad 272 W A$ & Ethyl benzene & $100-41-4$ \\
\hline $200 \mathrm{~W} M \quad 272 \mathrm{WA}$ & Ethylene glycol & $107-21-1$ \\
\hline $200 \mathrm{~W} M \quad 272 \mathrm{WA}$ & Hexane, n-hexane & $110-54-3$ \\
\hline $200 \mathrm{~W} M \quad 272 \mathrm{WA}$ & Isobutane & $75-28-5$ \\
\hline $200 \mathrm{~W} M \quad 272 \mathrm{WA}$ & Ligroine or vm\&p naphtha, or petroleum & $8032-32-4$ \\
\hline $200 \mathrm{~W}$ M 272WA & Methyl ether or dimethyl ether & $115-10-6$ \\
\hline $200 \mathrm{~W} M \quad 272 \mathrm{WA}$ & Methyl ethyl ketone (2-butone) & $78-93-3$ \\
\hline 200W M 272WA & Methyl isobutyl ketone & $108-10-1$ \\
\hline 200W M 272WA & Methyl N-amyl ketone, 2-heptanone & $110-43-0$ \\
\hline 200W M 272WA & Phosphoric acid & $7664-38-2$ \\
\hline $200 \mathrm{~W} \mathrm{M} \quad 272 \mathrm{WA}$ & Polyvinyl Chloride Latex & $9002-86-2$ \\
\hline 200W M 272WA & Propane & $74-98-6$ \\
\hline $200 \mathrm{~W} M \quad$ 272WA & Tetrahydrofuran & $109-99-9$ \\
\hline $200 \mathrm{~W} \mathrm{M} \mathrm{272WA}$ & Toluene & $108-88-3$ \\
\hline $200 \mathrm{~W} M \quad$ 272WA & Vm\&p naphtha & $64742-48-9$ \\
\hline $200 \mathrm{~W} \mathrm{M} \mathrm{272WA}$ & Xylene & $1330-20-7$ \\
\hline $1100 \mathrm{G}-1172 \mathrm{~A} 001$ & 2,2,4-Trimethylpentane & $540-84-1$ \\
\hline
\end{tabular}


Table K.3. Fugitive Source Insignificant Emission Units [WAC 173-401(1)(d)].

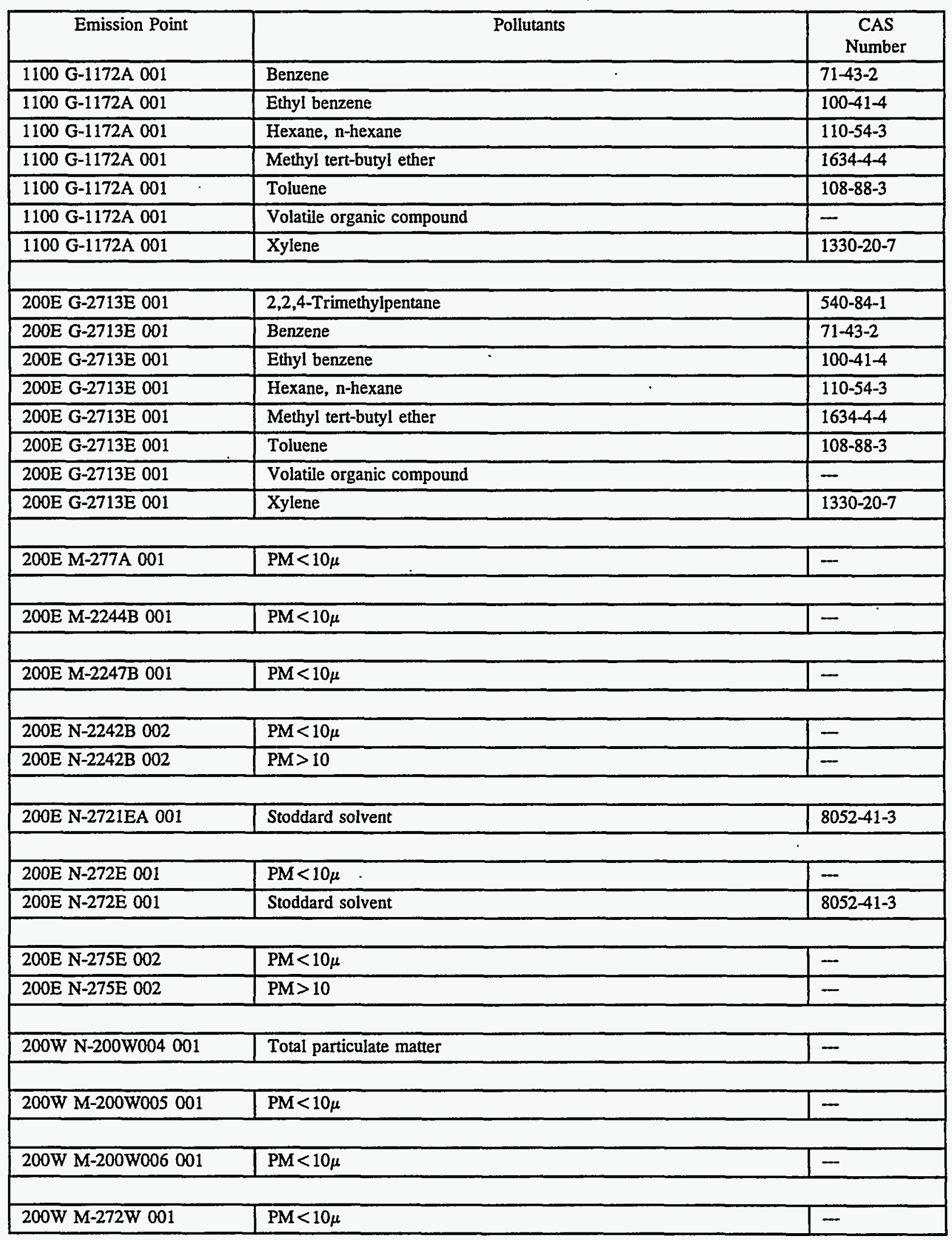


Table K.3. Fugitive Source Insignificant Emission Units [WAC 173-401(1)(d)].

\begin{tabular}{|c|c|c|}
\hline Emission Point & Pollutants & $\begin{array}{c}\text { CAS } \\
\text { Number }\end{array}$ \\
\hline 200W M-277W 001 & $\mathrm{PM}<10 \mu$ & - \\
\hline $200 \mathrm{~W} N-200 \mathrm{~W} 002001$ & $\mathrm{PM}<10 \mu$ & - \\
\hline $200 \mathrm{~W} N-200 \mathrm{~W} 002001$ & $\mathrm{PM}>10$ & - \\
\hline 200W N-275W 001 & $\mathrm{PM}<10 \mu$ & - \\
\hline $200 \mathrm{~W} N-277 \mathrm{~W} 003$ & $\mathrm{PM}<10 \mu$ & - \\
\hline 200W M-2722W 001 & $\mathrm{PM}<10 \mu$ & - \\
\hline 300 M-305A 001 & 1,1,1-Trichloroethane & $71-55-6$ \\
\hline $300 \mathrm{M}-305 \mathrm{~A} 001$ & Isopropyl alcohol & $67-63-0$ \\
\hline $300 \mathrm{M}-305 \mathrm{~A} 001$ & Total particulate matter & - \\
\hline $300 \mathrm{M}-328003$ & $\mathrm{PM} 10 \mu$ & - \\
\hline & & \\
\hline $300 \mathrm{M}-338001$ & Acetone & $67-64-1$ \\
\hline $300 \mathrm{M}-338001$ & Stoddard solvent & $8052-41-3$ \\
\hline $300 \mathrm{~N}-305001$ & Total particulate matter & - \\
\hline 300 N-3713002 & $\mathrm{PM}<10 \mu$ & - \\
\hline $300 \mathrm{~N}-3713002$ & $\mathrm{PM}>10$ & - \\
\hline $300 \mathrm{~N}-3722001$ & $\mathrm{PM}<10 \mu$ & - \\
\hline $300 \mathrm{~N}-3722003$ & $\mathrm{PM}<10 \mu$ & - \\
\hline $300 \mathrm{~N}-3722003$ & $\mathrm{PM}>10$ & - \\
\hline $400 \mathrm{~N}-4704 \mathrm{~N} 001$ & Stoddard solvent & $8052-41-3$ \\
\hline $400 \mathrm{~N}-4704 \mathrm{~N} 001$ & Total particulate matter & $\cdots$ \\
\hline $400 \mathrm{~N}-4722 \mathrm{~B} 002$ & $\mathrm{PM}<10 \mu$ & - \\
\hline $400 \mathrm{~N}-4722 \mathrm{~B} 002$ & $\mathrm{PM}>10$ & - \\
\hline $400 \mathrm{~N}-4722 \mathrm{C} 002$ & Total particulate matter & - \\
\hline $600 \mathrm{M}-6290001$ & $\mathrm{PM}<10 \mu$ & - \\
\hline
\end{tabular}


Table K.3. Fugitive Source Insignificant Emission Units [WAC 173-401(1)(d)].

\begin{tabular}{|c|c|c|}
\hline Emission Point & Pollutants & $\begin{array}{c}\text { CAS } \\
\text { Number }\end{array}$ \\
\hline $600 \mathrm{~N}-661001$ & Hydrogen sulfide & $7783-6-4$ \\
\hline $600 \mathrm{~N}-661001$ & Lead & $7439-92-1$ \\
\hline $600 \mathrm{~N}-\mathrm{MO} 302001$ & Petroleum distillates 3 & $64742-88-7$ \\
\hline
\end{tabular}




\section{DISTRIBUTION}

OFFSITE

R. Jim

Yakima Indian Nation

P.O. Box 151

Toppenish, Washington 98948

Melodie Selby

Nuclear Waste Program

State of Washington Department of Ecology

1315 W. 4th Avenue

Kennewick, Washington 99336

Rick Poeton

Mail Stop At-082

USEPA Region 10

1200 Sixth Avenue

Seattle, Washington 98101

Oliver Wang

Nuclear Waste Program .

State of Washington Department of Ecology

1315 W. 4th Avenue

Kennewick, Washington 99336

Susan May (2)

Radiation Protection Division

State of Washington Department of Health

Air Industrial Park Building 5, LE-13

Olympia, Washington 98504-0095

D.Powaukee

Nez Perce Tribe

P.O. Box 305 Lapwai, Idaho 83540

J. Wilkinson

Confederated Tribes of the Umatilla Indian Nation

P.O. Box 638

Pendleton, Oregon 97801

Distr-1 
Michael Wilson (2)

State of Washington Department of Ecology

300 Desmond Dr. S.E.

Lacey, Washington 98503

ONSITE

MSIN

U.S. Department of Energy,

Richland Field Office (RL)

Paul Krupin (8)

A5-15

Hector Rodriguez (9)

A5- 15

OSTI (2)

L8-07

Reading Room (2)

H2-53

Pacific Northwest Laboratory

Hanford Technical Library

$\mathrm{K} 1-11$

Westinghouse Hanford Company

Barry Curn (61)

H6-25

WHC Central Files

L8-04

ICF/Kaiser Company

Curt Clement

G7-33

Debbie Herman

S2-12

Deanna Klages

S2-51

ERC Team

John Hadley

H4-79 